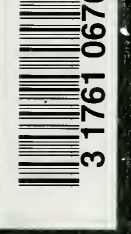




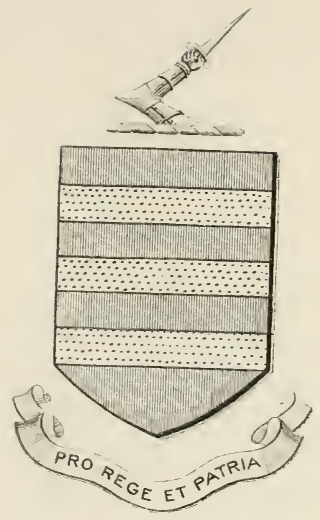

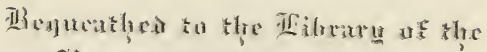

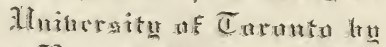

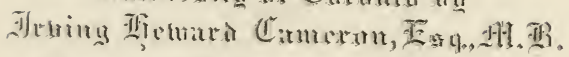

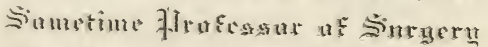

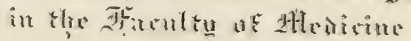



Digitized by the Internet Archive in 2007 with funding from Microsoft Corporation 


TEXT'BOOK OF PHYSIOLOGY. 


$$
\&
$$


MPhy $_{S}$

$$
\text { T' E X T'- B O O K }
$$

OF

\section{PHYSIOLOG Y}

EDITED BY

E. A. (SCHÄFER, LL. I., F.R.S., PROFESSOR OF PHYSIOLOGY, DNIVERSITY OF EDINBURGH.

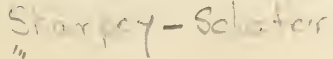

VOLUME SECOND.

EDINBURGH \& LONDON :

YOUNG J. PEN TLANI).

1900 .

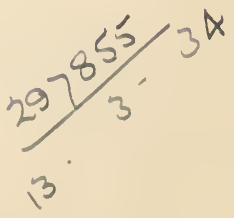


EDINBERGII: PRIXTED FOR YOUXg J. PENTLAND, 11 TEVIOT PLACE, AND 38 WEST SMITHFIELD, LOXDOX, E.C., BY MORRISON AND GIBB LIMITED. October 1900 . 


\title{
CONTENTS OF VOLUME SECOND.
}

\author{
THE MECHANISM OF THE CIRCULATION OF \\ THE BLOOD.
}

BY LEONARD HILL.

Action of Heart-Form of Heart-Valves and Papillary Muscles-Pressure in Cavities-Heart Sounds-Heart Impulse-Work of Heart-Diastolic Filling of Heart-Systolic Output-Action of Nerves-Cardiac Centres and ReflexesDepressor Nerve - Hæmodynamics - Torricelli's Law-Poiseuille's LawGeneral Principles of Circulation-Pressure in Arteries-Velocity of FlowCirculation Time-Effect of Change of Posture on Circulation-The PulseThe Capillary Circulation-Microscopical Examination-Rate of FlowCapillary Pressure-Blood Flow In the Verss-Pressure in Veins-Effect of Iruscular Movements-Rate of Flow-INfudence of Respiration on CirculaTION-VAsomotor Mechasis I-Historical-Methods-Action of Vaso-dilators - Local Tone-Traube-Hering Curves-Vasomotors of Veins-Circulation is Sfecial Parts-Cerebral Cireulation-Pulmonary Cireulation-Blood Flow in Limbs-Renal Circulation-Mesenteric Circulation-Hepatic CirculationSplenic Circulation - Pancreatic Circulation - Coronary Circulation - Vasomotor Reflexes . . . . . . . . . . page 1

\section{THE CONTRACTION OF CARDIAC MUSCLE.}

BY W. H. GASKELL.

The Meaning of the Heart-Beat-The Sequence of the Contractions of the different Parts of the Heart-The Causation of the Beat of the Heart and of the Sequence of the Contractions of the Separate Cavities is the same in Warm-blooded as iu Cold-blooded Animals-The Peculiarities of the Cardiac Muscle by which the Rhythm and Sequence are to be explained-On Fibrillar CoutractionOn the Tonicity of the Cardiac Muscle-The Meaning of the Ganglion Cells in the Heart, and their Relation to the Cardiac Nerves-The Nature of the Action of the Cardiac Nerves: (1) The Inhibitory Nerves, (2) the Augmentor Nerves, (3) the After-Action of the Inhibitory and Augmentor Nerves-The Action of Certain Poisons-On the Nutrition of the Heart . . page 169

\section{ANIMAL MECHANICS.}

BY J. B. HAYCRAFT.

Introductory-The Movements and Positions of the Body-The Mechanism of Joints-The IIuscle as a Machine-The Ceutre of Gravity of the BodyMethods of Recording Movements of the Body-Detailed Deseription of Special Movements 


\title{
THE MUSCULAR AND NERVOUS MECHANISM OF THE PESPIRATORY MOVEAENTS.
}

\author{
BI E. H. STARLING.
}

Jechanical Conditions-Inspiration-Diaphragm-Iutercostal Muscles-Expiration-Accessory Respiratory Novements-Types of Respiration-Movements of the Lungs-Respiratory Rhythm-Pressure in Lungs-Methods of Registering Movements-Nervous Mechanisu-Respiratory Centre - Spinal CentresAutomatism of Centres-Influence of Afferent Nerves-Action of VagusNormal Movements and Effects of Division of Vagi-Effects of Distension and Collapse of Lung-Apnœa-Electrical Stimulation of Vagus-Influence of other Sensory Nerves-Influence of Brain-Asphyxia-Modified Respiratory Movements

\section{THE MUSCULAR AND NERVOUS MECHANISMS OF THE DIGESTIVE TRACT.}

By E. H. STARLING.

Mastication-Sucking-Deglutition-Movements of the Stomach-VomitingYovements of the Intestines-Defrecation

THE MUSCULAR MECHANISMS OF THE URINARY TRACT.

By E. H. STARLING.

Movements of the Ureters-Movenexts of the Bladder-Muscular Tissue of Bladder and Urethra-Mechanism of Closure of Bladder-Intravesical Tension - Nerve Supply of the Bladder-Mechanism of Micturition-Voluntary Control of Micturition .

THE MUSCULAR MECHANISMS OF THE GENERATIVE APPARATUS.

BY E. H. STARLING.

Erection-Nervous Mechanism--Retractor Penis-Parturition-Nervous Mechanism .

page 347

\section{THE MECHANICAL, THERMAL, AND ELECTRICAL PROPERTIES OF STRIPED MUSCLE.}

\section{BY J. BURDON SANDERSON.}

The Mechanical Respoxse-Response to Successive Excitations-Response to Single Stimulus-Influence of Mechanical Conditions on Excitatory ProcessMuscular Tonus-Genesis of Tetanus-Summation of Stimuli-Period of Latency-Rate of Propagation-Conduction of Slow Waves-ExHaustion AND DEATH of MUscle-Fatigue-Causes of Exhaustion-Death of Muscle-Rigor Mortis-Nature of Process concerned in Muscular ContractionProduction of Heat durixg Contraction-Electrical Properties of MUSCLE-In the Unexcited State-Current of Injury-Electromotive Pheno- 
mena of Excited Muscle-Electrical Response to a Single Stimulus-Electrical Response to a Series of Stimuli-Direct Action of Voltaic: Current on Musches-Polarisation of Muscle-Polar Effects during and after Passage of Current-Polar Excitation-Secondary Excitation-Polarising Action of Currents of Brief Duration-CARbiac Muscle-The Mechanical ResponseThe Electrical Response.

\section{NERVE.}

\section{By FRANCIS GOTCH.}

Fundamental Characteristics of Nerve Phexomena-General Review of Nerve Functions-Phenomena of the Excitatory State-Metabolism during Activity --Conductivity of Nerve-Transmission in both Directions-The Excitation of Nerve-Modes of Stimulation-Induced Currents-Undulatory CurrentsCondenser Discharges-Galvanic Currents-Unipolar Excitation-Chemical Excitation-Mechanical Excitation-Conditions determining the Efficiency of Stimuli-Law of Excitation-Influence of Direction of Electrical CurrentsNerve Indefatigability-Limits of Excitation by Repeated Stimuli-THE Circumstances affecting Nerve Excitability and Conductivity-Maintenance of Circulation-Maintenance of Continuity with Nerve Cells-Comparison of Different Nerves and Different Parts of the same Nerve-Alterations of Temperature-Chemical Reagents-Anesthetics-Polarisation ChangesPolar Excitation of Nerve by Electrical Currents-Pflüger's Law of Contraction--Influence of Duration, etc.-Causation of Polar ExcitationClosing and Opening Tetanus-Polar Excitation in Crayfish Nerves-In ManIn Peripheral Śense Organs-Electromotive Changes in Unexcited NerveThe Nerve Current-Its Production-Influence of Various Conditions on its Amount-Electromotive Changes in Excited Nerve-The Negative Variation-Its Discontinuous Character-Electrical Response to One StimulusInfluence of Various Conditions-After-Effects-Electromotive Changes associated with Passage of Currents-Extermal and Internal Polarisation -Electrotonus-Influence of Physiological Changes upon ElectrotonusExcitatory After-Effects--Polarisation and Excitation . . . page 451

\section{THE PHYSIOLOGY OF ELECTRICAL ORGANS.}

\section{By FRANCIS GOTCH.}

General Description-Minute Structure in Raja batis, Gymnotus, Torpedo, and Malapterurus-Functional Activity-The Electrical Changes of Nerve Organ Preparations; Activity Effects-The so-called Current of Rest-The Organ Response-Its Time Relations-Self-Excitation of the Electrical Organs-Effect of Repeated Stimuli-Residual Effect of the Response-Electromotive Force of the Response-Polarisation Phenomena, Secondary Electromotive Effects, Irreciprocal Conductivity-The Reflex Response of the Organ-The Nature of the Activity . . . . . . . . . . . page 561

\section{THE NERVE CELL.}

\section{By E. A. SCHÄFER.}

Classification of Nerve Cclls-Theory of Isolated Units (Neurone Theory)-Structure of Nerve Cells-Changes in Nerve Cells as the result of ExcitationChanges resulting from Section of the Nerve Fibre Process-Conduction in Nerve Cells-Time lost in Transmission from Cull to Cell-Refractory Period . . . . . . page 592 


\title{
THE SYMPATHETIC AND OTHER RELATEI) SYSTEMS
} OF NERVES.

\author{
BY J. N. LANGLEY.
}

Gexeral Accouxt of the Sympathetic Nervous System Sympathetic Nervous Supply of the Head-Of the Skin, except that of the Head and of the AnoGenital Region-Of the External Generative Organs and of the Skin of the Ano-Genital Region-White and Grey Rami ; Some General Features of the Sympathetic Nerve Supply to the Skin-Of the Skeletal Muscles-Of the Thoracic and Abdominal Viscera-Nerve Fibres of the Sympathetic System-Degeneration of Nerve Fibres-Regeneration-Structure of Sympathetic Nerve Cells-Duration of Effects caused by Section of Nerves-Efferent Pre-Ganglionic Fibres in relation to the Anterior and Posterior Roots of the Spinal Nerves Nervous Sistems related to the Srmpathetic Nervous Sistem-Distribution and Aetion of Cranial Autonomic Nerve Fibres-Distribution and Action of Sacral Autonomic Nerve Fibres-The Enteric Nervous System-Possible Autonomic Fibres rumning direct in the Cerebro-Spinal Nerves-Local Nerve Centres other than the Cranial, Sympathetic, and Sacral Ganglia-Questioxs common to the Sympathetic and to Some Other Parts of the Autonomic System-Mode of Action of Inhibitory Fibres-Automatism of Peripheral Ganglia-The Peripheral Ganglia as Centres for Definite Areas-Reflex Action from Autonomic Ganglia-The Number of Nerve Cells on the course of a PreGanglionic Fibre; Commissural Fibres-The Size of Nerve Fibres as a Characteristic of the Autonomic System - Afferent Autonomic Nerve Fibres ; Referred Pain in Visceral Disease-Growth of Pre-Ganglionic Cranial Fibres into Sympathetic Ganglia-General Comparison of the Cranial, Sacral, and Srmpathetic Autonomic Systems . . . . . . . . page 616

\section{THE CEREBRAL CORTEX.}

\author{
Br E. A. SCHÄFER.
}

Gexeral Functions-Results of Removal of whole Cortex-Of one HemisphereResults of Artificial Excitation-Character of Muscular Contractions resulting from Excitation-Other Phenomena resulting from Excitation-Effects on Respiration-On Circulation-On the Pupil-On the Secretions and on the II uscular Tissue of the Viscera-Heat Centres-Epilepsy-Special LocalisaTions-The Motor Areas and Centres-Sensory (?) Functions of Motor AreasResults of Stimulation and Removal of Particular Areas-Associated Movements-The Principal Parts of the Motor Cortex of the Monkey-The Head and Eyes Area-Face Area-Arm Area-Leg Area-Trunk Area-The Motor Centres in the Orang-The Motor Centres in Man-The Sensory Areas of the Cortex-The Visual Area-The Auditory Area-The Olfactory and Gustatory Areas-The Localisation of Tactile Sensibility--Latent Regions of the Cortex - Hypothesis of Association Centres of Flechsig-Aphasia-Connections of Cortical Centres witl one another and with Lower Centres . page 697

\section{THE SPINAL CORD.}

\section{By C. S. SHERRINGTON.}

Reflex Action-The Way In-Recurrent Sensitivity-The Way Out-Relation of Afferent to Efferent Root Cell-Reflex Tonus-Regeneration of Afferent Roots - Functional Anatomy of Mediate Spinal Cell-Systems-The Spinal Grey Matter-Reflex Capacity of Fragments of the Cord-The SPINal AximaLRules of Spread in "Short" Reflexes-In Long Reflexes-Influence of the 
Quality of the Stimulus - Sumnation of Stimuli--Intensity of the StimulusCharacter of the Muscular Response--Influence of the Locus of the Stimulus"Loeal Sign" and Spinal Reflexes-Fatality of Spinal Reflexes-Influence of Condition of the Central Organ itself ; Vaseular Condition-Drugs-Influence of Assoeiated Parts of the Central Organ ; Inhibition and "Bahnung"-Coordinate Character of the Movements-Plasic Variation in Reflex ActivityAutotomy - Spinal "Shock" - Difference between skeletal and Visceral Musculature in regard to Shock-Spinal Visceral Reflexes-Urinary BladderDefrecation-Sexual Spinal Reflexes - Other Visceral Reflexes-Reflexes Affecting the Vascular Musculature-Significance of Spinal Reflexes-SPINoCerebral and Cerebro-Spinal Reactions-Conduction in the Cord-Spinal Traumatic Hyperesthesia-Tonic Functioss of THE Cord-Automatism"Knee-Jerk" and Allied Phenomena-Trophic Fuxctioss of the CordRegeneration of the Spinal Cord

page 783

\title{
THE PALTS OF THE BRAIN BELOW CEREBRAL CORTEX, VIZ. MEDULLA OBLONGATA, PONS, CEREBELLUM, COR- PORA QUADRIGEMINA, AND REGION OF THALAMUS.
}

\author{
BY C. S. SHERRINGTON.
}

The Mrelencephalon or Region of the Medulua Obloxgata-The Root Cell Systems-The Bulbo-spinal Frog-The Bulbo-spinal Mammal-_Centres" in the Bulb-Conduction Paths in the Bulb-The MIetexcephalon or Regiox of the Poxs Varoli axd Cerebellum-Reactions in the Frog-And Rabbit -The Pons - The Root Cell Systems - "Centres" - Conduetion Paths The Cerebellum - Excitation - Lesioxs - Unilateral Ablation - Ablation of the whole Cerebellum - Destruction of the Middle Lobe-Longitudinal Bisection-Ablation in the Bird-Ablation in Lower Vertebrates-Phenomena caused by Section of the Cerebellar Peduncles-Section of Anterior Peduncle-Section of Middle Peduncle - Section of Posterior PeduncleBilateral Section of the Peduncles-Phenomena of Cerebellar Origin in Human Disease-Inferences drawn fron Comparative Anatomy-Functions of the Cerebellum-The Mesexcephalox or Region of the Corpora Quadrigemina -The Root Cell Systems-Excitation of the Corpora Quadrigemina-Reactions of Animals after Äblation of all the Encephalic Regions in front of the Mesencephalon-Lesions of the Corpora Quadrigemina-Decerebrate Rigidity -Conduction Paths through the Mesencephalon-The Diencephalon or Region of the Optic Thalajus-Visual Connections-Excitation-Destructive Lesions

\section{CUTANEOUS SENSATIONS.}

By C. S. SHERRINGTON.

The Sexse of Touch-Toueh-Spots-The Adequate Stimulus-Weber's Law in respect to Tactual Sensations-The Spatial Quality of Touch-Theory of "Localising" Power of Touch-Projection of Touch-Illusions in Tactual Judgments-Reaction Time for Touch-The Sense of Temperature-"Cold" and "Warmth" as Distinct Species of Sense-Distribution of Apparatns for Thermal Sensations in the Skin, etc.-The Adequate Stimulus-Other Stimuli -Paradoxical Sensation of Cold-Some Characters of "Cold" and "Warmth" Sensations-Relation of Thermal Sensation to Pain-Influence of Areal Extent of Stimulus upon Intensity - The Plyysiological "Zero-temperature" and "Adaptation"-The Degree of Projection of Thermal Sensation-The Thermal Sense in relation to Thermotaxis-The Liminal Difference of Intensity of stinulus-Reaction Time-Antagonism of Sensations of "(old" and "Warnth" - Paths of Conduction-Common Swisstion-Cutameous Pains considered in relation to Pain in general-Relation of Pain to Intensity of Reaction-Quality 
of Cutaneous Pain-Scope of the term "Common Sensation"-The Viscera and Common Sensation-The Musculo-articular Apparatus and Common Sensation - Nature of "Physical Pain"-Action of Painful Stimuli-Character of the Motor Reactions provoked-The C'entral Neural Mechanism for Cutaneous Pain-Tickling-Dolorous Reflexes compared with Tactual-_The Spinal Path of Pain-Associated (Referred) Pains-Interference of Pain with other Sensations-The Peripheral Path of Pain-"Pain-Spots" in the Skin-Pain and Nerves of Muscular Sense-Pain and Visceral Nerves-Hunger as a Type of Visceral Sensation-Evolution of Visceral Pain-Cutaneous Pain and Adequate Stimuli-Specific End-organs or Undifferentiated Endings-The Forms of "Excess" of Stimulus-Summation in Production of Pain-"Inertia" of the Neural Apparatus of Pain-The Brain and Physical Pain . . page 920

\section{THE MUSCULAR SENSE.}

\section{By C. S. SHERRINGTON.}

Definition of "Museular Sense"-Views regarding the nature of Muscular SenseCharles Bell's "Sixth Sense"-The Peripheral Apparatus of the Muscular Sense; Histological Data-Nerves of Muscles and Tendons-End-Bulbs and Pacinian Corpuseles-Hypothesis as to the Mode of Excitation of the Peripheral End-Organs of the Muscular Sense-Muscle-Reflexes-The Performances of Muscular Sense-Perception of Posture-Perception of Passive MovementPerception of Active Movement-Objective Effects of Loss of Muscular Sense upon "Willed Movement"-Subconseious Character of Reactions of Muscular Sense-Probable Importance of Tonus for $\mathrm{Co}$-ordination-Apresthesia produces Ataxy of "Willed Movement"-Senso-paralysis-Perception of ResistanceThe Liminal stimulus-The Liminal Difference-" Adaptation"-Interpretation of Results_Fusion of Muscular with other Sensations . . page 1002

\section{VISION.}

\section{By W. H. R. RIVERS.}

The Dioptric Srstem-Accommodation-The Pupil-The Retina and Visual Sensations-Physiological Retinal Processes-Visual Sensations-Adaptation and Induction-The Duration of Stimulus and Sensation-The Brightness of Colour Sensations-Mixture-Vision of Different Regions of the RetinaColour-Blindness, etc. - Binocular Phenomena - Functions of the Retinal Structures-Theories of Colour-Vision-BINocular Vision-Movements of the Eyes-Spatial Perception - page 1026

\section{THE EAR.}

\section{BY JOHN GRAY I'KENDRICK AND ALBERT A. GRAY.}

The Exterxal Ear-The Auricle-The External Auditory Meatus-Movements of the Auricle-The Midple Ear-The Tension of the Membrana Tympani, and its Regulation-Movements of the Membrana Tympani in response to Sound Pressures-The Movements of the Malleus-The Movements of the IncusThe Movements of the Stapes-The Tensor Tympani-The Stapedius-The Movements of the Ossicles as a whole under the Influence of Sound-wavesThe Magnitude of the Movements of the Ossicles-Transmission of Sound to the Labyrinth otherwise than by the Ossicles-Regulation of Atmospheric Pressure in the Tympanum-Auditory Reflexes-Binaural Audition-The Internal Ear-General Mechanism of Internal Ear-The Utricle and Saccule -The Cochlea-Pitch-Beats-Beat Tones-Audibility as affected by Intensity -The Analytic Properties of the Ear-Resonance-Probable Action of the Cochlea-Upper Partial Tones and the Theory of Dissonance-Combination 'Tones-Objections to the Theory of Helmholtz-Other Theories-The Semicircular Canals. 


\title{
ON VOCAL SOUNDS.
}

BI JOHN GRAY M'KENDRICK AND ALBERT A. GRAY.

Voice Production-The Singing Voice-Tone Intensity-Tone Pitch-The Range of the Voice-The Múscular Mechanisms which affect the Vocal Cords-Voice Registers-The Quality of the Voice and Vowel Tones-Theories of Vowel Tones-Wave-forms of Vowel Tones-The Fourierian Analysis of Wave-forms - Phonograms-General Conclusion-The Consonants-Effect of the Cavities in connection with the Larynx and Pharynx upon the Voice-Whispering page 1206

\section{THE SENSE OF TASTE.}

\section{BY J. B. HAYCRAFT.}

The Anatomical Mechanisms concerned in the Production of Taste, and the Regions of Mucous Membrane which are involved-Taste Sensations-The Action of Drugs on Taste-Modality and Quality of Taste-The Relationship between Taste Sensations and the Chemico-Physical Properties of the Stimulus-AfterTastes and Successive and Simultaneous Contrast . . . . page 1237

\section{THE SENSE OF SMELL.}

\author{
BY .J. B. HAYCRAFT.
}

The Anatomical Data-The Passage of Air through the Nose-OlfactometryHyperosmia and Anosmia-The Classification of Odours-The Law of Specific Energy of the Senses-The Relationship between Smell and the ChemicoPhysical Properties of Odorous Bodies-The Physical Condition of the Stimulant-The Sinultaneous Action of Two Odours-The Psychology of Sinell.

page 1246

IXDEX OF SUBJECTS

page 1261

INDEX OF Authors

page 1336 



\section{LIST OF ILLUSTRATIONS.}

FIG.

1. Sections through the frozen thorax of a dog (Haycraft and Paterson) .

2. Diagram showing the resultant pull of the papillary muscles (Ludwig).

3. Multiconvex upper surface of the closed tricuspid valve (Krehl) 8

4. Mitral valve. Insertion of the chordæ tendinea (Krehl) . . 9

5. Diagram of the chordæ tendiner (Krehl) . . . . . . 99

6. Intracardial pressure curves (Chauveau and Marey) . . . 14

7. Fick's straight spring manometer . $\quad$. $\quad . \quad$. $\quad$. 15

8. Goltz and Gaule's combined maximal and minimal manometer, as modified by Hiirthle . . . . . . . . 16

9. Fredericq's sphygmoscope . $\quad$. $\quad$. $\quad . \quad$. $\quad . \quad 17$

10. v. Basch's hollow spring manometer . . . . . 17

11. Hürthle's differential manometer . . . . . . . 17

12. Intraventricular pressure curve (Bayliss and Starling) . $\quad$. $\quad 19$

13. Aortic pressure curve (Bayliss and Starling) . . . . 19

14. Aortic and intraventricular pressure curves (Hürthle) . . . 20

15. $\quad " \quad$ " $\quad$ (Hiirthle) . . . 21

16. Auricular pressure curve (Porter) . . . . . 22

17. Cardiac and aortic pressure curves (Porter) . . . . 24

18. Tracing of the right auricle and carotid pulse (Porter) . . . . . 27

19. Diagram of Hiirthle's experiment . . . . . 30

20. Tracing of pulse and of first and second sounds of the heart (Einthoven and Geluk) . . . . . . . . . . . 31

21. Tracing of sounds of heart from a case of aortic regurgitation (Einthoven and Geluk) . . . . . . . . . . 32

22. Areas of cardiac impulse, when supine and when lying on the left side (Sequeira) . $\quad . \quad . \quad . \quad . \quad . \quad 33$

23. Edgren's cardiograph _ . . . . . . . . 35

24. Impulse curve and sounds of the heart (Edgren) . . . 36

25. High and low aortic pressure curves (Hürthle) . . . . . 3 . 3

26. Simultaneous records of carotid pulse and heart impulse (Edgren) . 38

27. Myocardiograph (Roy and Adami) . . . . . 39

28. Tracing illustrating effect of compression of the thorax (Hill and Barnard) 45

$29 . \quad " \quad, \quad " \quad, \quad$ " $\quad 45$

30. Effect on aortic pressure of artificial ventilation of the lungs (Hill and

Barnard) . . . . . . . . . . . 46

31. Pressure in the air passages and carotid pulse (Mos:o) . . . 47

32. Cardiometer (Roy and Adami) . . . . . . 49 
Flo.

33. Curves illustrating the effect of a rise of aortic pressure upon the pressure in the right and left auricles (Kanders) . . . . . 51

34. Effect of abdominal compression upon curve of aortic pressure . . 51

35 .

36. Cardiographic tracing, illustrating effect on the volume of the heart of alteration in position (Barnard) .

37. Posterior view of medulla ollongata of the eat (Laborde)

38. Reflex inlibition produced by inhalation of ammonia (François-Franck)

39. Dissection of the depressor nerve, rabbit (Ludwig and Cyon)

40. Aortic pressure. Excitation of depressor (Bayliss)

41. Aortic pressure and volume curve of limb (Bayliss)

42. Flow of fluid in cylindrical tubes; piezometer

13. Mode of determining velocity of flow (Nicolls)

44. Flow of fluid in branching tubes.

45. Weber's model, illustrating the principles of the circulation .

46. Distribution of pressure and velocity during diastole and systole of the lieart (Fredericq).

17. Apparatus for testing elasticity of arteries (Marey)

48. Horizontal lines illustrating extension of artery under different pressures (Roy)

49. Effect of injection of saline solution on arterial, portal, and vena cava pressures (Bayliss and Starling) .

50. Poiseuille's hrmodynamometer

51. Hürthle's recording apparatus

52. Hill and Barnard's sphygmometer

53. Ludwig's stromulır, as modified by Tigerstedt

54. Chauveau and Lortet's dromograph

55. Dromograph tracing from carotid artery (Chauveau and Marey)

56. Tachogranı; arm elevated (v. Kries)

57. Sphygmogram; , , (v. Kries)

58. Tachogram; arm dependent (v. Kries)

59. Sphygniogram ; , , (v. Kries)

60. Illustrating effect of change of posture on the circulation

61. Aortic pressure ; effect of posture .

62. " and intracranial pressure ; effect of posture . .

63. " pressure; effect of compression of abdomen, ete. (Hill and Baruard) .

64. Aortic and vena cava pressures ; effect of posture, etc. (Hill and Barnard)

65. Scheme of the circulation (Hill and Barnard)

66. $\quad$ wave passage along elastic tube

67.

68. Section of the forearm passing through the radial suleus (Hill, Barnard, and Sequeira)

69. Superficial view of the radial vessels (Hill, Barnard, and Sequeira) .

70. Pulse tracings, showing effect of venous congestion (Hill, Barnard, and Sequeira) .

71. Pulse tracings, showing effect of venous congestion (Hill, Barnard, and Sequeira) .

72. Tracing, showing effect of occluding the brachial artery and the pulse (Hill, Barnard, and Serueira) .

73. Velocity of pulse wave. Carotid and radial pulse (Edoren)

74. Experiment to show central origin of dicrotic wave (Grasher) . .

75. Tracings illustrating the effect of inllalation of amyl nitrite upon the pulse 
FIG.

76. Curves taken during the escape of the heart from vagal inhibition (v. Frey).

77. Effect of respiration on arterial pressure (Fredericq)

78. Respiratory undulations of aorta and vena cava pressures (Hill and Barnard) .

79. Diagram to illustrate the effect of intrathoracic pressure upon the blood flow through the thorax .

80. Model illustrating the effect of intrathoracic pressure on the circulation (Funke and Latschenberger)

81. Aortic pressure. Effect of artificial respiration (Kowalewsky) .

82. Plethysmograms of lind-limb. Excitation of sciatic nerve (Bowditch and Warren)

83.

$$
\text { , , }
$$$$
\text { , },
$$

84. Traube-Hering curves produced by injection of magnesium sulphate; dog

85. Plethysmographic tracing of arm and record of Cheyne-Stokes respiration ; man

86. Effect of injection of absinthe on blood pressure (Bayliss and Hill)

87. Respiration and cerebral pulse tracings (Mosso) .

88. Cerebral pressure gauge

89. Effect of asphyxia on blood pressure (Bayliss and Hill) .

91. Pulmonary and carotid arterial pressures (Bradford and Dean) .

92. Effect of exciting vasomotor centre on pulmonary and aortic pressures (Bradford and Dean)

93. Pulmonary and carotid arterial pressure ; effect of dividing spinal cord (Bradford and Dean)

94. Pressure curves of pulmonary artery, left auricle, and femoral artery ; effect of excitation of sensory nerves (François-Franck)

95. Limb plethysmograph

96. Record of aortic pressure and the volume of the fore- and hind-limbs (Bradford and Bayliss) .

97. Effect of excitation of a superficial branch of the crural nerve on blood pressure and volume of organs (Hallion and François-Franck).

98. Schäfer's visceral plethysmograph $\quad . \quad+\quad . \quad . \quad 162$

100. Aortic pressure and splenic volume (Schäfer and Moore) _ $\quad 163$

101. Tracing of frog's heart; effect of heating sinus and auricle . . 172

102. Tracing from heart of toad; effect of heating ventricle . . . 173

103. Heart of tortoise . . . . . . . . . . . 173

104. " ", suspended . . . . . . . . 174

105. " " " " to show appearance of preparation when a block is caused by slitting up the auricle . . . . . 181

106,107 . Beat of ventricle of frog, registered by Gaskell's modification of Roy's tonometer. . . . . . . . . . 196

108. Tracing from heart of toad; effect of stimulating intracranial vagus . 204

$109 . \quad, \quad, \quad, \quad, \quad 205$

110-113. Tracing from heart of toad and frog; effects of stimulating ragosympathetic . . . . . . . . . 206

114,115 . Tracings from heart of tortoise; effect of stimulating nerves . $\quad 207$

116. Modification of Roy's tonometer . . . . . . 210

117. Preparation of heart of tortoise to demonstrate the action of the vagus nerve on the quiescent auricles . $\quad . \quad$. $\quad . \quad 212$

118. Curve of demarcation current, caused by heating apex of auricle _ 213 
FIG.

119. Method of expressing the position of a point in reference to two coordinate axes

120. Method of measuring the angular displacement of the arm from a primary vertical position

121. Method of determining the position of a point in reference to three coordinate planes.

122. Showing the position of the centre of gravity of the whole body and of the head, and also the position of the centres of rotation of the chief joints (Braune and Fischer)

123. Method by which the axis of rotation of a ball-and-socket joint is determined

124. Method of finding the position assumed by a point when a limb is rotated

125. Method of compounding axes of rotation .

126. Method of determining the centre of rotation of a joint .

127. Illustrates the fact that a screw is an inclined plane wrapped round the surface of a cylinder

128. To show the geometrical construction of a saddle-shaped joint .

129. To illustrate the formation of an ellipsoidal joint

130. Curves of extension of resting and contracted muscle . . . 24

131.

131. Jerk contractions" tracings from the gastrocnemius muscle of a frog

by the pendulum myograph
133. Jerk contractions; tracings of the movements of the human finger-tip taken with the pendulum myograph

134. Cheese maggots, from an instantaneous photograph . . .

135. Illustrating the position of the common centre of gravity of the hand and lower arm

136. Represents the rotation of a rod around a fulcrum . . .

137. Illustrates Weber's theory of the movements around the ankle-joint .

138. $"$ the method of calculating movements around the anklejoint

139. Represents the method of calculating movements when the forces are oblique to the axes of the body on which they act

140. Illustrates the change in the centre of gravity of the body when the heels are raised

141. To represent the moments around the ankle-joint

142. Illustrates the resolution of moments into their components .

143. Illustrating the resolution of the force of contraction of a muscle into three forces

144. Represents the action of the triceps and brachialis upon the humerus and ulna .

145. Apparatus for determining the centre of gravity of the body . .

146. Posture of the body with the centres of rotations of the principal joints in the same vertical plane

147. To show the position of the centre of gravity in each of the three postures.

148. Centre of gravity and centres of rotation in a soldier at attention .

149. To show the effect of a push in overturning the body, which is viewed as a rigid body resting upon the feet .

150. Shows the area of underpropping and the plumb of the centre of gravity

151. Shoe for indicating when a man's foot comes in contact with the ground (Marey) . 
152. Pedestrian furnished with special shoes and carrying a chronographic apparatus (Marey)

153. Special apparatus for recording the contacts of a horse's feet with the ground (Marey).

154. Chronographic apparatus recording the paces of a horse (Marey)

155. Rhythm curves obtained from a man running (Marey)

156. Chronographic record of the periods of contact of the feet of a man executing various paces (Marey).

157. Chronophotographic method of recording bodily progression (Marey) .

158. Chronophotograph of a man's movements when walking (Marey) .

159. Chronograph of the movements of a man's leg in running (Marey)

160. Illustrates certain mechanical forces operative during progression .

161. To illustrate the surface of the body exposed to the resistance of air in bicycling, in running, and in walking .

162. Illustrates the difference between the centre of gravity of the body in the erect posture and in running

163. Shows the effect of the thrust of the foot against the ground in progression

164. Diagrammatic representation of position of diaphragm during expiration and inspiration

165-167. Diagrams illustrating the action of the intercostal muscles upon the ribs

168. Normal tracing of diaphragm-slips of rabbit: Head's method

169. Diagrammatic section of medulla oblongata below middle of olivary body, to show region of respiratory centre (Bechterew).

170. Effect on respiratory movements of closing trachea at height of inspiratory period (Head)

171. Effect on respiratory movements of closing the trachea during the expiratory pause (Head) .

172. Effects on respiratory movements of dividing first one and then the other vagus (Head)

173. The right vagus has been divided, and the curve shows the effect produced by complete collapse of the left lung (Head)

174. Effects on respiratory movements of positive ventilation with air (Head)

175.

175. " " ", negative

(Head) (Head)

177. Apnœic pause, caused by positive ventilation with hydrogen (Head) .

178. Respiratory tracing showing negative after-effect of inflation of lungs (Head)

179. Tracings of blood pressure and of intratracheal pressure, taken by Einthoven's "differential manometer" .

180. Diminution of respiratory excursions produced by spasm of bronchial muscle (Einthoven)

181-183. Curves obtained during swallowing (Kronecker and Meltzer)

184. Curve obtained by placing a balloon in the oesophagus (Kronecker and Meltzer).

185. Starling's Enterograph $\quad . \quad$. $\quad . \quad$. . .

186. Rhythmical contractions of circular coat of small intestine . . . 328

187. Curve to show effects of exciting peripleral end of right vagus . $\quad 332$

188. Curve showing inhibitory effect on contractions of small intestine $\quad . \quad 333$

188A. Diagram of the nerve supply to the bladder and colon in the cat (Langley and Anderson).

189. The double adductor preparation .

190. Cosine lever, combined with writing lever and tension recorder (Fick). 
FIG.

191. Work-diagram (Fick)

192. Isotonic curve drawn by a double semi-membranosus preparation (Fick)

193. Series of isotonic curves with increasing loads (Fick)

193A. Work-diagram for the same muscle (Fick)

194. Isotonic and isometric curve of the same muscle, represented as springing from the sante base line (Fick)

195. Blix's apparatus for recording isometric and isotonic curves synclironically

195A. Enlarged view of the same apparatus to show the torsion rod from above

196. Series of "after-loading" curves with inereasing load (v. Kries). .

197. v. Kries's apparatus for taking "after-loading" curves and "arrestedcontraction" curves

198. Series of "arrested-contraction" curves (v. Kries)

199. Isotonic curve and arrest curve of the same muscle, as in Fig. 200, cooled to $6^{\circ} \mathrm{C}$. (Schenck)

200. Isotonic curve and arrest curve of a muscle, warmed to $31^{\circ} .5 \mathrm{C}$. (Schenck)

201. Isometric and isotonic curves of the same muscle (Schenck)

202. Schenck's apparatus for taking "jerk eurves"

204. Curves of isotonic and arrested contractions of an unloaded muscle (Kaiser) .

205. (1) Isotonic curve of unloaded muscle, at temperature $14^{\circ} \mathrm{C}$. ; (2) the same, at $25^{\circ} \mathrm{C}$; (3) at $0^{\circ} \mathrm{C} . ;$ (4) at $14^{\circ}$ C., with load on muscle (Kaiser) .

206. Isotonic curves of musele unloaded on the summit (Kaiser) . .

207. " " with same muscle more heavily loaded (Kaiser) .

208. Series of release curves following arrest, with muscle cooled to $0^{\circ} \mathrm{C}$. (Kaiser) .

209. Isotonic and arrest curves of an unloaded muscle. Modified from Kaiser

210. Two isotonic curves of an unloaded muscle (Kaiser)

211. Isotonic curve and release curve, after arrest, of an unloaded muscle (Kaiser)

212. Muscle curve, showing summation of inadequate stimuli (Richet)

213. Method of measuring mechanical latency of muscle . . .

214. Facsimile of the photographic record of the beginning of the contraction of the sartorius

215. Fatigue curve of muscle (Kronecker) . . . . .

216. Muscle curves showing the effect of fatigue (Waller) . .

217. Showing curves of rigor mortis of white and red muscles of rabbit (Bierfreund)

218. Curves of rigor mortis in fresh and exhausted muscle (Nagel) . .

219. Engelmann's apparatus for recording contraction of a violin string on heating .

220. Blix's apparatus for taking the extension curves of muscle with the load either increasing or decreasing (Fick)

221. Two extension curves of the same excited muscle taken continuously with decreasing and increasing loads (modified from Fick) . .

222. Showing the arrangement of the fibres in the gastrocnemius muscle

223. Diagram of method for studying the electrical properties of muscle

224. Non-polarisable electrode .

392

392 
226. Bernstein's differential rheotome.

227. Progress of the wave of excitation, aceording to Bernstein . 416

228. Curves of electrical response to instantaneous stimulation (Matthias) . 417

229. Diagram representing the excitatory variation of muscle . . $\quad 417$

230. Burch's projecting electrometer . . . . . . . . 418

231. Comparison curve for estimating the value of the curves of the eapillary electrometer

232. Diphasic and monophasic curves of the sartorius .

233. To illustrate the point of equipotentiality in the curve of the capillary electrometer

234. To illustrate the excitatory variation of muscle

235. Electrical response to a series of stimulations (du Bois-Reymond) . $\quad 422$

236. Method of leading-off from injured muscle . . . . . . . 423

237. Tetanus curve of injured sartorius . . . . . . . . 424

238. Responses to ten excitations of an uninjured gastrocnemius _ . . 421

239. Response to indirect excitation of a cooled muscle . . . . 425

240. Veratrine curve (sartorius) . . . . . . . . . 425

241. Stryclinine curve of an uninjured gastrocnemius . _ . . . 426

242. Method of showing polarisation of musele

243. Effects on excitability of the passage of a constant current through musele

244. Hering's double myograpl

245. Contractions of sartorius recorded by the double myograph (Biedermann)

246. Contractions of the two ends of the sartorius, as recorded by the double myograph (Biedermann) .

247. Diagram of Frank's apparatus for recording isometric eurve of frog's ventricle.

248. Isometric and isotonic curves of the ventricle of the lieart of the frog (Frank)

249. Excitatory variations of cardiae musele (Einthoven)

250. Einthoveu's electro-cardiogram of man

251. Experiments showing transmission in both directions . . . 456

252. Time relations of induced currents

253. Diagram of rheonome

254-256. Influence of temperature on nerve excitability

257.

270. Facsinile curves of the photographed excursions of a capillary electrometer connected with the frog's sciatic nerve (Gotch and Burch) . $\quad 530$

271. Biphasic response of frog's sciatic nerve (Hermann)

272. Influence of intensity of stimulus on electrical response of nerve (Waller) . 
FIG.

275. The curves show alteration in excitability produced by the vapours of chloroform, ether, and alcohol (Waller) .

276. Effect of faradisation upon the electrical response of nerve (Waller) . 537

277. Excitatory variations in fresh and stale nerves (Waller) . . . 539

278. Influence of $\mathrm{CO}_{2}$ upon the response from "stale" nerve (Waller) . 530

279. To illustrate internal polarisation in a moist conductor . . . . 541

280. Internal polarisation around metallic core of Hermann's model . . 542

281. „ $" \quad$ effects in such a model as Hering's . . . 542

282. Extrapolar polarisation currents on opening polarising current . . 544

283. Spread of current into extrapolar regions . . . . . . . 546

284. Electrotonic effects of constant current . . . . . . . 546

285. Development of electrotonic currents (du Bois-Reymond) . $\quad 548$

286. Helmholtz's experiment on rate of electrotonic propagation . $\quad . \quad 549$

287-289. Influence of excitation upon electrotonic effect . . . 550, 551, 552

290. After-effects of polarising current . . . . . . . 556

291. Electrical organ of skate. Longitudinal section . _ . . . 563

292. $, \quad, \quad, \quad$ Diagrammatic view, to show the structure of a dise

293. Curve of development of excitatory effects in torpedo organ

294. Excitatory effects shown with capillary electrometer . . . . 578

295. Self-excitation of torpedo organ . . . . . . . $\quad . \quad 579$

296. Self-excitation of Malapterurus organ . . . . . . 580

297-299. Experiment with , $\quad$ " . . . . . . . . 580,581

300. Evolution of sensory cell (G. Retzius) . . . . . . 593

301. Nerve cell from the electric lobe of torpedo (M. Schultze) . 595

302. " cells of Hirudo (Apáthy) . . . . . . . . 596

303. Spinal ganglion cell showing intracellular network of fibrils (C. Golgi). $\quad 597$

304. Nerve cell of annelid (Apáthy) . . . . . . 597

305. Diagram of a nerve cell to show the Nissl granules (Cajal) 599

306. Illustrates the "law of axipetal conduction" in motor nerve cells (Cajal) . . . . . . . . . .

307. Illustrates the supposed lines of conduction in a spinal ganglion cell (Cajal) . . . . . . . . .

308. Shows the relative number of the synapses in the visual and auditory organs (G. Retzius)

309. Variations of sympathetic nerve distribution . . . . $\quad$. 630

310. Diagram of course of a grey ramus _ . . . . . $\quad$. 631

311. Relations of nerve roots to lumbar and sacral vertebræ . _ . . 631

312. Diagram to show variation in nerve origin . . . . . $\quad .632$

313. Nerve cell connections of abdominal lymphatic . . . . . 647

314. Left pelvic plexus of cat . . . . . . . . $\quad 666$

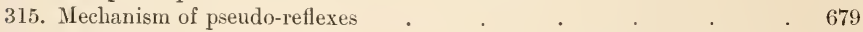

316. Reflexes and pseudo-reflexes . . . . . . 680

317. To illustrate certain connections of pre- and post-ganglionic fibres $\quad .681$

318. Experiment of joining the cut vagus and sympathetic in the neck . 691

319. Method of recording voluntary contraction-curve . . . 707

320. Tracing of a voluntary contraction of the thumb muscles . $\quad 707$

321. Part of a similar tracing, taken on a faster surface and with the movements more amplified . . . . . . . 708

322. Vibrations of the outstretched arm holding a weight (W. Griffiths) . 708

323. Myographic curves from hamstring of monkey (Horsley and Schäfer) . 709

$324 . \quad " \quad$ tracings obtained from stimulation of different parts of the motor path (Horsley and Schäfer) . . . . .

325. Periorl of latent excitation on stimulation of the cortex cerebri, and 
of the subjacent corona radiata respectively (François-Franck and Pitres)

326. Effects upon the volume of the kidney and arterial pressure of exciting the motor area of the cerebral cortex of a dog (François-Franck and Pitres)

327. Myographic curves obtained as the result of strong excitation of the cortex cerebri and of the subjacent corona radiata (François-Franck and Pitres)

328. Curve of the contraction of a muscle during and after cortical excitation, showing the tetanic and epileptoid after-effect (François-Franck and Pitres)

329. Curves showing the effects of cortical excitation productive of epilepsy upon the blood pressure, the pulse rate, and the size of the pupil (François-Franck and Pitres)

330. Brain of monkey, marked out into the so-called "sensory areas" (H. Munk)

331. Diagram showing the chief results obtained by Fritsch and Hitzig (in 1870), on stimulation of the cerebral cortex of the dog .

332. Diagram showing the results obtained by Ferrier on stimulation of the cerebral cortex of the dog

333. Diagram showing the results obtained by Ferrier from stimulation of the cerebral cortex of the cat

334. Diagram showing the results obtained by Ferrier on stimulation of the brain of the rabbit

335. Diagram showing the results obtained by Ferrier on excitation of the cerebral cortex of the monkey

336. Diagrams of the motor zone of the monkey's brain, sliowing the five areas into which it is divisible.

337. Diagrams from Beevor and Horsley, showing effect of stimulation within the hallux area.

338. Diagrams of the lateral and mesial surfaces of the monkey's brain, showing the effects obtained from stimulation of the excitable areas

339. The chief motor areas in the brain of the orang-utan (Beevor and Horsley) .

340. Diagrams indicating the probable localisation of the chief motor and sensory areas of the cerebral cortex of man

341. Diagram of arrangement of apparatus for determining the comparative latency periods of two parts of the cerebral cortex

342. Curves showing the comparative periods of latency for the ocular muscles, on stimulation of the frontal and occipital cortex respectively

343. Brain of a monkey from which one occipital lobe had been entirely removed, but the angular gyrus left intact (Schäfer and Sanger Browu)

344. Brain of a monkey from which both occipital lobes liad beell cut away (Schäfer and Sanger Brown)

345. Diagran of connection between the several parts of the retina and occipital lobes in the dog, according to H. Munk

346. Diagram showing the probable relations between the parts of the retinie and the visual area of the cortex in the monkey, according to Schäfer

347. Corresponding points of the two retine and of the two halves of the visual cortex

348. Brain of a monkey, showing complete removal of the superior tenuporal gyrus on both sides (Schäfer and Sanger Brown) 
FIG.

349. Diagram to show the connections of the olfactory organ with the hippocampal region (Elliot Smith)

Diagram of a frontal section through the brain of a new-born child, to show the course and destination of fibres from the optic thalamus to the cortex (Flechsig)

351. Diagrams to show the supposed division of the cerebral cortex into projection and association areas.--Modified from Flechsig .

353. $"$ to show the manner in which lesions of internuncial fibres may produce aphasia.-Modified from Gowers .

354. Diagram of the arrangement of segmental skin-fields in the hind-limb of the monkey

355. Diagram showing arrangement of the root filaments of two spinal nerves (van Gehuchten) .

356. Diagram of the different movements of the head and fore-limb (spinal cat) caused by stimuli applied to the fore-paw and to the pinna respectively

357. Diagram of irradiation of reflex movement in spinal cat .

358. ", " the predominant uncrossed flexor reflex of the hind-limb inhibiting the crossed extensor reflex

359. Scheme of the arrangenent of the nerves and muscles of chief importance in the knee-jerk

360. Diagrams to show the situation of the chief nerve nuclei in the nedulla oblongata and pons near the floor of the fourth ventricle (Schafer) .

361. Transverse section of the medulla oblongata sonrewhat above the middle of the olivary body (Schïfer)

362. Transverse section of pons Varolii through the origin of the auditory nerve (Schäfer)

363. Posture assumed after removal of the right half of the cerebellum (Thomas) .

364. Foot-prints of a normal dog (Luciani)

365. Footprints of a dog ten days after ablation of the middle lobe of the cerebellum (Luciani)

366. Gait after destruction of the vermis (Thomas)

367. Pigeon after removal of the cerebellum (Dalton) .

368. Map showing the relative distribution of the sensitivity to touch, warmth, and cold in the palm of the hand (Goldscheider) .

369. Map of the skin distributions of the seventh, eighth, ninth, and tenth afferent nerve roots of the frog.

370. Transverse sections of a nerve-twig supplying a portion of the vastus medialis muscle (knee-jerk muscle) of the cat .

371. Diagram of ophthalmometer

372. Method of using ophthalmometer .

373.

374. Images observed in above method.

to determine position of pupil

375. Determination of centre of rotation of eyeball (Donders).

376. Diagram of ellipsoidal curvature of cornea (Landolt)

377. To show imperfect centring of eye (Helmholtz) .

378. Determination of angles $a$ and $\gamma$

379. Spectrum of visual purple and of visual yellow (Kiihne)

380. Bleaching action of spectral colours on visual purple (Kühne)

381-383. Retinal currents of action (Steiner and Kühne)

384. Experiment on simultaneous contrast (Hering)

355. ,,$\quad$ to illustrate colour contrast . 
PIG.

386. Experiment to test Talbot-Plateau law (Helmholtz)

387, 388. ", upon the influence of contrast on flicker sensation (Sherrington)

PAGE

1069

389. Fechner's experiment . . . . . . . . . 1075

390. Experiments on flicker (Haycraft) _ . . . . . 1079

391. Colour triangle . . . . . . . . . . 1081

392. Curve of luminosity in total colour-blindness (König) . . . 1089

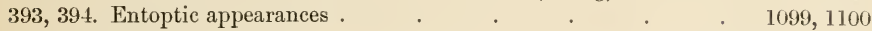

395. Helmholtz's experiment on delicacy of local discrimination in retina . 1101

396. Curves illustrating the Young-Helmholtz theory of colour vision

(König and Dieterici)

397. Curves of sensation in red-green blindness (König and Dieterici)

398. Curve of spectral and fundamental colours

399

$$
\text { , , (Helmholtz) }
$$

(Helmholtz)

1106

1107

1108

1109

400. Experiment on single and double vision (Volkmann)
401. , , , (Wheatstone)

1125

401. " " " "

1126

1127,1128

405, 406. To illustrate Listing's law (Hering) .

- 1130

407. Apparatus for investigating visual localisation (Greeff)

. 1133

408-410. To illustrate geometrical illusions

411. Diagram to explain geometrical illusions (Kundt)

1141,1142

412.

" " , " , (Hering)

1142

1143

1143

414. Auerbach's experiment . . . . . . . . . . 1144

415, 416. To illustrate Einthoven's explanation of colour stereoscopy . . 1145

417. Spiral disc illusion _ . . . . . . . . . . . 1147

418. Posterior wall of the cartilaginous and osseous meatus (Politzer) _ 1151

419. Curvature of tympanic membrane (Helmholtz) . . . . . 1153

420. Illustrates the effect of the curve of the radial fibres of the membrana tympani .

421. Relations of the malleus and incus (Helmholtz) .

1154

422. Ear manometer (Politzer) .

423. Various forms of wave curves

424. Form of wave produced by combining two simple waves. . . 1173

1158

425. Compound wave form according to Max Meyer . . . . . 1193

426. Diagram showing range of registers of human voice _ . . . 1210

427. Aperture of glottis during production of low note or lower register (Bosworth)

428. Aperture of glottis during production of high note of lower register (Bosworth)

429. Aperture of glottis during production of a note in the head register (Bosworth)

430. Pitch of vowels according to Helmholtz . . . . . 1215

431. " " " " König . . . . 1216

432. König's apparatus for illustrating the quality of vowel tones by a manometric flame

433. Flame pictures of the vowels $O U, O$, and $A$ (König) . . . . 1219

434. Diagram showing the first step in analysing wave-forms . . . 1221

435. Diagram showing the amplitude as indicated by the height of ordinates, of the partials of the Swedish $i$, sung at pitch of 293 vibs. (Pipping) .

436. Diagram showing the amplitude in the partials of the German long $a$, sung and analysed by Hermann .

437. Diagram showing the application of Pipping's method . . . 1224

438. Examples of rowel curves obtained by Hermanil. 
FIG.

439. Pitch of the vowels according to Hermann

PAGE

440. Diagram showing Boeke's theory of measurement of depth of curve $\quad$. 1227

441. Examples of vowel curves obtained by Hermann . . . 1228

442. Examples of tracings obtained by M'Kendrick's phonograph recorder $\quad$ • 1229

443. Curves by Boeke . $\quad$. $\quad$. $\quad$. $\quad$. $\quad$. $\quad . \quad 1231$

444. Diagram showing harmonic analyses of the curves in Fig. 443 . . 1231

445. Diagram showing the course of the nerve fibres concerned with taste $\quad 1238$

446,447 . Diagrams showing the position and extent of the olfactory mucous nembrane

1246, 1247

448. Diagram of olfactory path (Cajal) _ . . . . . . . $\quad$. 1247

449. Diagram of Zwaardemaker's olfactometer . . . . . . . 1250 


\section{TEXT-BOOK OF PHYSIOLOGY.}





\title{
TEXT-BOOK OF PHYSIOLOGY.
}

\author{
THE MECHANISM OF THE CIRCULATION OF \\ THE BLOOD.
}

\author{
By Leonard Hill. ${ }^{1}$
}

Contents:-Action of Heart.-Form of Heart, p. 1-Valves and Papillary Muscles, p. 8--Pressure in Cavities, p. 14-Heart Sounds, p. 28-Heart Impulse, p. 33-Work of Heart, p. 40-Diastolic Filling of Heart, p. 45Systolic Output, p. 48-Action of Nerves, p. 54-Cardiac Centres and Reflexes, p. 54-Depressor Nerve, p. 60-Hжmonynamics.-Torricelli's Law, p. 63-Poiseuille's Law, p. 65-General Principles of Cireulation, p. 69-Pressure in Arteries, p. 78-Velocity of Flow, p. 83-Circulation Time, p. 89Effect of Change of Posture on Circulation, p. 91-The Pulse, p. 97-Tне Capillary Circulation.-Mieroseopical Examination, p. 113-Rate of Flow, p. 115-Capillary Pressure, p. 116-Blood-Flow in the VeIns, p. 119Pressure in Veins, p. 120-Effeet of Museular Movements, p. 121-Rate of Flow, p. 122-Influence of Respiration on Circulation, p. 123-Vasomotor Ifechanisu. - Historical, p. 131-Methods, p. 133-Action of Vaso-dilators, p. 136-Local Tone, p. 138-Traube-Hering Curves, p. 139-Vasomotors of Veins, p. 141-Circulation in Special Parts. - Cerebral Cireulation, p. 142-Pulmonary Circulation, p. 149-Blood-Flow in Limbs, p. 156-Renal Cireulation, p. 160 -Mesenteric Cireulation, p. 161-Hepatic Cireulation, p. 162-Splenic Circulation, p. 163 -Pancreatic Cireulation, p. 165-Coronary Cireulation, p. 165Vasomotor Reflexes, p. 166.

\section{ACTION OF THE HEART.}

\section{The Form of the Heart.}

The changes in form of the heart during systole and diastole can be studied in an animal, the heart of which has been exposed by opening the thorax under an anesthetic, artificial respiration being meanwhile maintained. When the heart is watched beating in full vigour and rapidity, it is an impossible task to unravel, by the eye alone, the sequence of events which form the cardiac cycle.

Harvey felt this difficulty, and writes: "When first I gave my mind to vivisections, as a means of discovering the movements and uses of the heart, and sought to discover these from actual inspection, and

I The writer of this article is greatly indebted to Tigerstedt's "Lehrbuch der Physiologie des Kreislaufes," Leipzig, 1893 . To this work the reader is referred for an exhaustive analysis of the literature of the circulation.

VOL. II. - I 
not from the writings of others, I found the task so truly arduous, so full of difficulties, that I was almost tempted to think (with Fracastorius) that the movement of the heart was only to be comprehended by God. For I could neither rightly perceive at first when the systole and when the diastole took place, nor when and where dilatation and contraction occurred, by reason of the rapidity of the movement, which in many animals is accomplished in the twinkling of an eye, coming and going like a flash of lightning; so that the systole presented itself to me now from this point, now from that-the diastole the same; and then everything was reversed, the movements occurring, as it seemed, variously and confusedly together. ${ }^{1}$

"When the heart begins to flag, to move more slowly, and, as it were, to die, the movements then become slower and rarer, the pauses longer, by which it is made much more easy to perceive and unravel what the movements really are, and how they are performed.

"In the pause," Harvey says, "as in death, the heart is soft, flaccid, exhausted-lying, as it were, at rest. In the movement and interval in which this is accomplished, three principal circumstances are to be noted-

" 1 . That the heart is erected, and rises upwards to a point, so that at this time it strikes against the breast, and the pulse is felt externally.

"2. That it is everywhere contracted, but more especially towards the sides, so that it looks narrower, relatively longer, and more drawn together.

" 3 . The heart being grasped in the hand is felt to become harder during its action. Now, this hardness proceeds from tension; precisely as when the forearm is grasped its tendons are perceived to become tense and resilient when the fingers are moved.

" 4. It may further be observed in fishes and the colder blooded animals, such as frogs, serpents, etc., that the heart, when it moves, becomes of a paler colour ; when quiescent, of a deeper red colour." 2

There is also to be noticed in the heart " a certain obscure undulation and lateral inclination in the direction of the axis of the right ventricle, as if twisting itself slightly in performing its work." 3

Analysing the movements of the chambers of the heart, Harvey determined that "first of all the auricle contracts, and in the course of its contraction forces the blood (which it contains in ample quantity as the head of the veins, the storehouse and cistern of the blood) into the ventricle, which, being filled, the heart raises itself straightway, makes all its fibres tense, contracts the ventricles, and performs a beat, by which beat it immediately sends the blood supplied to it by the auricle into the arteries."

Little can be added to Harvey's admirable description. Turning to a nineteenth-century author-Sibson-we find the action of the heart described thus : ${ }^{4}$

"The heart, when in action, presents to the eye the most remarkable contrasts in the size, position, colour, and form of its various cavities and great

\footnotetext{
${ }^{1}$ Harvey, "An Anatomical Dissertation upon the Movements of the Heart, etc.," ch. i., Canterbury, 1894.

${ }^{2}$ Ibid., eh. ii. $\quad$ "Jbid., eh. v.

4 "Merdical Anatomy," London, 1869, p. 73.
} 
vessels. When the ventricles contract they do so with a twisting movement, their walls become rigid and corrugated, their apex and base approximate, the descent of the base being greater than the ascent of the apex, and the arteries and veins on their surface become prominent and tortuous. The auricles become swollen with blood, and seem to push before them the retreating ventricles at the base. The great arteries are distended and lengthened, being apparently drawn downwards at their origin by the contracting ventricles. When, however, the ventricles expand, the aspect of all the parts is reversed. The ventricles enlarge in every direction, but especially towards the base. Their walls are flaccid and smooth; and the arteries and veins on their surface becorne straight and small. The auricles shrink, becoming pale and wrinkled, and are in great part replaced by the ventricles, into which they empty their blood. The great arteries lessen in size, and shorten and ascend at their origin, being apparently raised by the upward enlargement of the ventricles.

"The chief movement observable in the auricles is the sudden retraction of the appendices, which almost instantly become refilled with blood as the ventricles contract. There is a slight twisting movement of the whole heart during systole, both on its longitudinal and on its transverse axis. By its rotation on its transverse axis the apex of the heart is tilted forwards; by its rotation on the long axis, the left ventricle, which, during the diastole, looks backward, is turned forwards. The combination of the two movements gives to the apex a twisting movement from left to right and forwards."

Sibson measured the change of form of the heart by placing a millimetre scale across the organ, and noting the systolic and diastolic measurements of its parts. By Ludwig ${ }^{1}$ the heart of the cat was likewise measured in situ. He observed that in systole the antero-posterior diameter increased, the apex-base diameter shortened, the transverse diameter diminished, while the circumference of the base changed from an ellipse to a circle.

The following measurements are a type of his observations :-

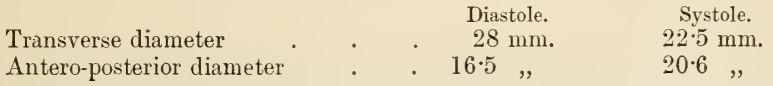

These measurements were taken with the animal in the horizontal position, lying on its back. Now the position of the heart and the pressure of the surrounding organs considerably affect the form of the heart.

During the period of diastole the heart becomes a flaccid bag, and thus passes entirely under the influence of gravity. When an excised frog heart is so placed on a glass plate that the base rests on the plate, the organ is seen to become smaller in systole while the apex rises forward. If the heart be placed with its posterior surface on the plate, the base becomes rounded in systole, and the apex lifts itself up from the plate. Lastly, if the heart be suspended with the apex hanging vertically downwards, then, while the base becomes round, the apex moves nearer to the base during systole.

Since the pressure of the surrounding organs, and especially of the lungs, influences the form of the heart, it is impossible to arrive at any accurate knowledge of the normal variations, so long as the experimental observations are conducted in abnormal conditions with the thorax opened. To obviate this difficulty, Bricke ${ }^{2}$ implanted needles into the

${ }^{1} Z$ tschr. f. rat. Med., 1849, Bd. vii. S. 205.

2 Sitzungsb. d. k. Akad. d. Wissensch., Wien, 1855, Bd. xiv. S. 348 ; "Vorlesungen," 1874 , S. 172 . 
hearts of rabbits throngh the walls of the closed thorax, and watched the movements of the needle heads. The needles acted as levers, the thoracic wall formed the fulcrmm, the movements of the needle heads gave the reverse of the movements of the needle points within the heart. From the result of these olsservations Bricke maintained that all parts of the ventricles move downwards in systole with the exception of the apex, the amount of movement increasing from below upwards. The apex of the heart undergoes less change of position than any other part. Its upward movement is neutralised by the descent of the whole heart. This descent is occasioned by the elastic recoil of the great vessels. ${ }^{1}$ Haycraft ${ }^{2}$ has likewise passed needles through the closed thorax into the mammal's heart. The needles indicated the contraction of the organ, both in the transverse and antero-posterior diameter. They did not show any sign of that twisting of the heart as a whole, which is seen when the chest is opened. Comparing these results with those of Ludwig, it is elear that by opening the thorax, and allowing the lungs to collapse, a material difference is made in the change of form of the heart. This follows from the removal of those structmres which naturally support the heart. Thus Haycraft observed that if an animal be suspended horizontally, with the belly downwards, and the chest be opened, the heart moves downwards towards the stermmm in diastole, and springs towards the vertebral column during systole. While, if the animal be placed on one side, the heart sinks towards that side during diastole, and springs towarls the other side during systole. In diastole the heart always follows the pull of gravity, in systole the direction of the spring of the apex depends upon the fulcrum, or, in other words, upon that part of the heart which is supported by some neighbouring structure. When the animal lies on its back, the fulcrum to the heart is afforded by the fibrous structure of the pericardium. The base of the left ventricle rests securely upon this strong membrane, upon which it is slung as upon a hammock.

In the closed thorax the falling back of the heart in diastole is largely prevented by the resistance of the expanded lungs. Thus, when a dog is placed symmetrically on its back, and the lungs are allowed to collapse, the heart falls backwards and to the left during diastole, while with each systole it springs mpwards and towards the right, and the twisting movement from left to right is observed. If the lungs be now inflated so that the heart is fully supported by the lungs on both sides, then no rotation of the organ as a whole takes place.

Ludwig and Hesse took casts of excised hearts, which had previously been distended with blood at a pressure of $150 \mathrm{~mm}$. $\mathrm{Hg}$, so as to imitate the diastolic form. Other casts were taken of empty hearts in a state of heat contraction produced by the sudden immersion of the organs in a solution of potassium bichromate heated to a temperature of $50^{\circ} \mathrm{C}$. From the measurements of these casts they determined that the heart is diminished in heat systole in all its diameters except the vertical, while the areas of the auriculo-ventricular orifices are diminished by one-half.

As the museulature of the heart is, speaking generally, composed of an inner and an outer longitudinal layer, and a middle circular layer, we must suppose that in heat-rigor the contraction of these layers strikes a balance, so that the vertical diameter does not shorten.

\footnotetext{
1 Halford, "On the Movements and Sounds of the Heart," London, 1861, p. 12.

${ }^{2}$ Journ. Physiol., Cambridge and London, 1891, vol, xii. p. 452.
} 
This method of investigation is open to considerable fallacy. The hearts are excised and unsupported by surrounding structures, and while the diastole is artificially produced by a high injection pressure, the systolic contraction is not normally obtained, for, contrary to the natural systole, it takes place against no resistance. Thus it comes about that the great contraction of the auriculoventricular orifices, found by Ludwig in the rigored heart, is not confirmed by observations on the living heart. Recently Hayeraft and Paterson have cut a series of sections through the frozen thorax of dogs, after the heart has been fixed either in diastole or systole. ${ }^{1}$ From these sections the form of the lieart has been reconstructed. The great difficulty is to obtain the death of the animal with the heart in either the true diastolic or systolic shape. Death nearly always takes place with the heart in diastole, but, according to the distribution of the blood at the moment of death, the heart may be dilated more or less. To obtain death with the heart in systole is a matter of considerable difficulty. Hayeraft and Paterson obtained the best results by the injection of a solution of corrosive sublimate into the jugular vein.

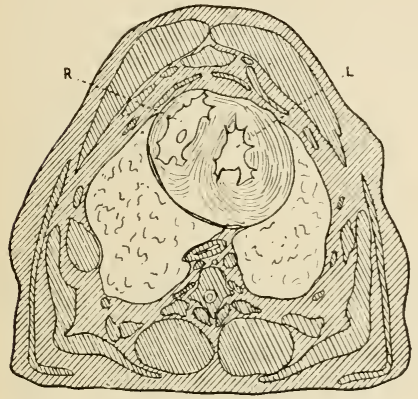

$A$.

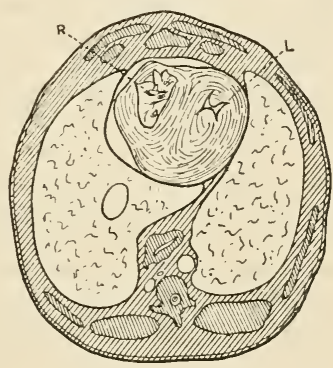

B.

Fig. 1.-Sections through the frozen thorax of a dog. $A$, Heart in diastolic death ; $B$, In systolic death.-Haycraft and Paterson.

In the figure are presented the appearances observed in sections from two dogs, and seen from below. In $A$ the heart is in diastolic death, and in $B$ in systolic death.

In a third figure these authors illustrate the alteration in the position of the heart produced by placing the animal after death on the left side. The heart has fallen over to the left, and has, while compressing the left lung, expanded the right. This illustrates the interaction of the thoracie organs on one another; by merely altering the position of the body, and thus of the lieart, air may be driven out of one lung and sucked into the other.

By taking plaster casts of the interior, after fixing the heart in diastole or systole, Ludwig and Hesse studied the form of the ventricular cavity. As to the accuracy of this methorl, the same reservations must be made as above. In diastole the cavity of the left ventricle forms a sloort and wide tube, while the auriculo-ventricular opening is separated from the aortic orifice by the pendulous anterior flap of the mitral valve. In leat-rigor, a condition of contraction greater no doubt than that produced by any systole during life, the cavity appears as a narrow slit which is star-shaped in the region of the p. 496 .

${ }^{1}$ Hayeraft and Paterson, Journ. Physiol., Cambridge and London, 1896, vol. xix. 
papillary muscles. Above this region there lies a space, which in the ox's heart forms a very irregular cone pointing downwards. ${ }^{1}$ The heart, therefore, never empties itself completely in systole.

The outer muscular layers of the heart take an oblique direction from right to left, the inner layers take an oblique direction from left to right, and the intermediate fibres run more or less transversely. Ludwig has shown that in every piece of the walls of either chamber the fibres on the inner surface take a direction across those on the outer surface, the intermediate fibres taking, in regular order, a corresponding change of direction.

The various fibrous structures of the base are all associated together, forming one common fibrous membrane. This surrounds the aortic, the mitral, and the tricuspid orifices, and, resting upon the base of the ventricles, gives a general insertion to the muscular fibres of both of these cavities, and a general origin to the muscular fibres of both auricles.

In the $\log$ the heart within its pericardium is slung above and below by fibrous cords, which allow considerable displacement of the organ. In man the pericardium is suspended above by the stout cords of the deep cervical fascia; below it is attached to the central tendon of the diaphragm, in such a way as to form a triangular box of considerable strength and rigidity. In this box the heart is slung. Almost all lateral movement of the heart is thereby prevented, and since the central tendon of the diaphragm is a fixed point, ${ }^{2}$ the heart likewise cannot descend. This fixation of the pericardium is a striking characteristic of the human body, and its evolution in man can be traced to the assumption of the erect posture.

By means of a special mechanical contrivance (Fig. 27, p. 39), Roy and Adani ${ }^{3}$ have measured the approximation of any two given points on the exposed heart during systole. They determined that if two lines be drawn parallel either to the intraventricular furrow or the auriculo-ventricular groove, these lines approximate in systole. Therefore the exposed heart shortens both in its longitudinal and transverse diameters.

It is agreed by nearly all observers that the apex does not move up to the base, but the base moves down towards the apex. The latter remains practically a fixed point. The explanation of this fact is partly to be found in the recoil of the heart. When the blood is shot out into the aorta, the heart "kicks back" like a gun on its discharge. The momentum of recoil equals the momentum of output. Thus the heart is jerked downwards with considerable force, and at the dead point there must be a slight pause before it returns. If the veins which feed the heart be ligatured, this recoil fails to take place, and the base does not move towards the apex. ${ }^{4}$

The recoil is probably not the whole explanation of the descent of the base. The aorta and pulmonary artery as they leave the heart form a spiral figure; the blood thrown into them on systole not only extends these arteries but partly undoes the spiral. This may contribute to the descent of the base.

The middle muscular layers of the lieart are called by Krehl ${ }^{5}$ the

\footnotetext{
${ }^{1}$ Sandborg and Worm Müller, Arch.f. d. ges. Physiol., Bonn, 1880, Bd. xxii. S. 424.

${ }^{2}$ Grouiroes, Anat. Anz., Jena, 1897, Bd. xiii. S. 562. The evidence is furnished by Röntgen ray photographs.

3 Practitioner, London, 1890 , vol. i. p. 82.

${ }^{4}$ Guttmann, Virchow's Archiv, 1875, Bd. lxv. S. 540.

${ }^{5}$ Abhandl. d. math. -phys. Cl. d. k. sächs. Gescllsch. d. Wissensch., 1891, Bd. xvii. S. 346.
} 
driving mechanism. These fibres run in circles and almost transrersely round the ventricles, forming, when separated from the rest of the musculature, a cylinder open above and below. The external layer of muscle arises from the auriculo-ventricular groove, runs obliquely down the ventricle, and turns in at the apical whorl; thence the fibres ascend in the internal layer to be inserted once more into the auriculoventricular fibrous septum. These hook-shaped fibres shorten the cavity of the ventricle, while the middle circular layers compress it. The papillary 1nuscles and the colummæ carneæ shorten, approximate, and at length come into close contact, pressed together by the contraction of the circular layer. Thus the many-celled structure of the lower part of the ventricular cavity contracts, driving the blood out of its meshes, and finally disappears, being replaced by solidified and thickened walls.

Harvey noticed this point. He writes: "Not only are the ventricles contracted in virtue of the direction and condensation of the walls, but further, that those fibres or bands, styled nerves by Aristotle, which are so conspicuous in the ventricles of the lower animals, and contain all the straight fibres (the parietes of the heart containing only circular ones) when they contract simultaneously by an admirable adjustment, all the internal surfaces are drawn together as if with cords, and so is the charge of blood expelled witlı force." I

In cousequence of the packing together and thickening of the papillary muscles and fleshy columis during the systole, the extent of the contraction of the muscular walls required to empty the cavity is materially lessened, and their expulsive force is thereby economised. The papillary muscles and columnæ carneæ fill up the whole interior of the ventricle, with the exception of the small conical space situated just behind the septum, and in the direction of the aortic opening. Blood is left in contact with the auriculo-ventricular valves up to the end of systole, and thus their closure is maintained. The contraction of the ventricle proceeds in a twisting manner ; the blood is as it were wrung out of the cavity, and with a current that naturally takes the spiral direction of the spring and arch of the aorta.

In the region of the aortic opeuing, the internal surface is perfectly smooth, and offers a free passage to the exit of the blood. In the right ventricle the conus arteriosus is also free from the presence of musculi pectinati, and a space is left therein which is never completely emptied of blood, although the conus arteriosus is shortened by its longitudinal, and contracted by its transverse fibres. In each systole the diastolic swelling of the conus disappears, and its long axis shortens. In the right ventricle muscular bands cross the cavity, act as stays or "braces," 2 and prevent its over-distension. The musculi papillares and chordre tendinece also take the part of stays in both cavities. The community of the musculature determines the synchrouism of the two ventricles. The researches of Chauveau and Marey ${ }^{3}$ on the heart of the hor'se, and of François-Franck ${ }^{4}$ on a case of ectopia cordis in man have yielder records of this synchronism.

\footnotetext{
${ }^{1}$ Harvey, op. cit., eh. ii.

2 Ibid, , ch. i.

3 Mém. Acad. de méd., Paris, 1863, tome xxvi. p. 302.

" "Trav. du labor. de Marey," 18 7 , tome iii. p. 316.
} 


\section{The Valves of the Heart and the Action of the Papillary Muscles.}

The auriculo-ventricular valves. - If the auricles be removed from the freshly excised heart of a mammal, and the rest of the organ be gently placed under water, these valves immediately float upwards and almost close the auriculo-ventricular orifices. This is not due to the

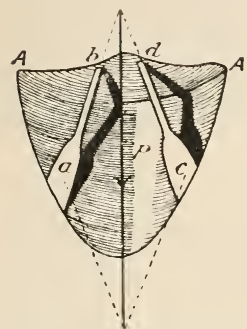

Fig. 2,-Diagram slıowing the resultant pull of the papillary museles.-Ludwig. specific gravity of the valves, for an excised valve placed in water slowly sinks. The closure of the valves is caused by eddies. In its expanded state the thin and light valve offers a large surface to the action of such eddies.

On pouring a stream of water from the height of a few inches through the auriculo-ventricular opening into either ventricle of an excised heart, the valve takes the shape of a fumnel. The point of this funnel projects into the middle of the eavity of the ventricle.

The segments of the valves cannot be driven against the wall of the ventricles by the entry of blood from the auricle. This is rendered impossible by the central position of the papillary muscles, as well as by the shortness of the chordie tendinee. In diastole the papillary muscles are wide apart, and their tips diverge from one another and point towards the base of the ventricle. Consequently the flaps of the valves are drawn by the cords ontwards and downwards, so that the tricuspid orifice is thrown widely open. As the blood flows in from the anricle, the eddies cause the valves to float $u p$ and their edges to approach each other. At the end of the auricular systole the flaps are in such a position that complete closure immediately follows, so soon as the ventricular pressure rises to the slightest degree above the auricular pres-

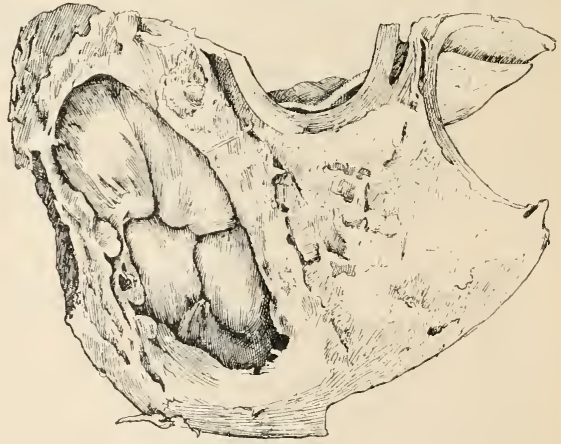

Fig. 3.-Multiconvex upper surface of the closed tricuspicl valve.-Krehl.

sure. Thus, on systole of the ventricles, the thin edges of the valves are already in apposition, and the valves then shut with such rapidity that in all probability no regurgitation takes place.

From the results gained by manometric observations of the auricular pressure, Krehl ${ }^{1}$ concluded that there is no regurgitation into the auricle so long as the conditions of the circulation are normal. 
Fredericq ${ }^{1}$ and Porter, ${ }^{2}$ by more delicate methods of investigation, have obtained distinct signs of a slight rise in auricular pressure, which occurs synchronously with the upstroke of the ventricular pressure curve, but this is probably not cansed by the regurgitation of blood, but by the ballooningout of the auriculo-ventricular valve.

By plugging the pulmonary orifice with wax, and then directing into the ventricle a stream of water from the height of one foot, Basungarten ${ }^{3}$ observed that the auriculoventricular valves closed firmly immediately after the cessation of the stream. This results from the pressure exerted on the fluid in the cavity by the elastic rebound of the muscular walls. The result of such an experiment cannot be

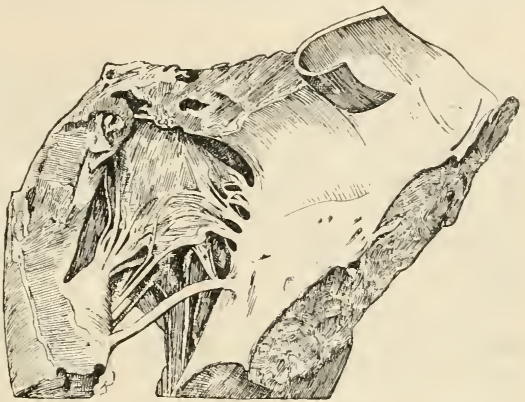

Fis, 4.- Mitral valve. Insertion of the chordæ tendimere.-Krehl. directly applied as an explanation of the closure of the auriculoventricular valves in the living heart, for the filling pressure in the auricle is often extremely slight, and can give origin to but little elastic rebound.

As the blood presses equally on either side of the margins of the

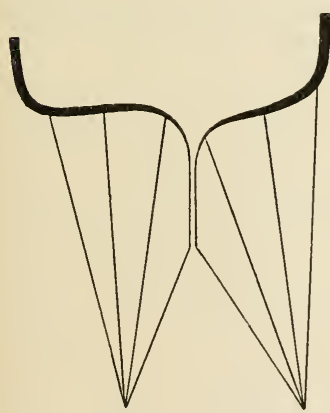

FIG. 5.-Diagram of the chorle tendinere.-Krehl. valves when placed in apposition, these need no great strength, and are in fact exceedingly delicate. The remainder of the valve, on the other hand, bears the full strain of the pressure, and, consequently, is much tougher. The chordæ tendinere are affixed both to the ventricular surfaces and close to the free edges of the valve flaps. Each flap is supplied with cords from two papillary muscles, and these are situated opposite to each other. The resultant pull of the cords is shown by the direction of the arrow in Fig. 2. ${ }^{4}$

By the insertion of a finger into the heart of a living horse, Chauveau and Faivre ${ }^{5}$ determined that an elevation of the auriculo-ventricular valves can be felt to take place before the flaps come absolutely into apposition. When closed, the valvular diaphragm appears slightly ballooned out towards

1 "Trav. du labor. de Liège," 1888, tome ii. 1. 116.

2 Porter, Journ. Physiol., Cambridge and London, 1892, vol. xiii. p. 524. Cf. Gottwalt, Arch. f. d. gcs. Physiol., Bonn, 1881, Bd. xxv. S. 1 ; Riegel, Deutsches Arch. f. klin. Mcd., Leipzigr, 1862, S. 1 and 471.

${ }^{3}$ Arch. f. Anat., Physiol. u. uissensch. Med., Berlin, 1843, S. 463. This experiment was originally performed by Lower.

+ Lndwig, "Lehrbuch der Physiologie," 1861, Bd. ii. S. 81.

${ }^{5}$ Gaz. méd. de Paris, 1856, 1. 410. 
the auricle. Above, its surface is multiconvex (Fig. 3), while below it is multiconcave. Beneath the concave surface a quantity of blood is perceptible, which is not expelled by the ventricular systole. The chordie tendineæ which are inserted on the edges of the flaps are shorter than those attached to the under surface. ${ }^{1}$

Hesse $^{2}$ measured the base of the heart in both the state of diastole and in the systole produced by heat-rigor. In the latter state he found the diameter of the base of the heart was much diminished.

If this diminution takes place in the systole of the living heart, it must not only aid in the closing of the auriculo-ventricular orifice, but must increase the sacculation of the valvular diaphragm. Chauveau ${ }^{3}$ was, however, by the insertion of his fingers in the heart of the horse, not able to detect any very great diminution of the basal diameter. Sibson ${ }^{4}$ has likewise denied any marked diminution of the diameter of the auriculo-ventricular orifice.

The condition of heat-rigor produced by Hesse is very different to that which obtains in the normal systole, and the observations on the living heart must be accepted as of far greater worth.

Many views have been held as to the action of the papillary muscles. Among such are the following :-

1. That they approximate the flaps of the valves and aid in their closure ;

2. That they open the valves, because they are the last to receive the wave of contraction, and therefore continue to shorten after the rest of the ventricle has passed into diastole;

3. That synchronously with the systole of the ventricle they contract, and, in proportion as the longitudinal axis of the ventricular cavity shortens, pull in the slack chordie teudiuer, and thus prevent the valves from being forced too far into the auricles.

An attempt has been made by Roy and Adami to record the time of action of the papillary muscles, by passing a wire hook through the auricle, and this they hooked on to a flap of the mitral valve. The hook they connected to their myocardiograph (see p. 39). The contraction of the ventricular wall was simultaneously recorded. From the records thus obtained, these author's concluded that the contraction of the papillary muscles began later and ended earlier than that of the ventricular wall, and in the proportion of 5 to $8 .^{5}$ The movement of the flap of the valve is rapid at first, and afterwards reaches a maximum somewhat slowly; this maximum is maintained for a brief space, and is followed by a sudden relaxation.

Fenwick and Overend, ${ }^{6}$ Haycraft and Paterson,${ }^{7}$ have directly recorded the contraction of the papillary muscles in the excised heart. In order to effect this, the heart is with the greatest rapidity cut out and laid open, the base of a papillary muscle is then pinned down to the table, while hooks are attached to the apex of the muscle, and to the ventricular wall. These hooks are connected by threads to writing

\footnotetext{
${ }^{1}$ Krehl, Arch.f. Physiol., Leipzig, 1889, s. 290.

2 Arch.f. Anat. u. Entwcklngsgesch., Leipzig, 1880, S. 344.

3 l.oc. cit. 4"Medical Anatomy," 1869, j. 80.

5 Roy and Adami, Practitioner, London, 1890, vol. xliv. pp. 88-94.

6 Brit. Mid. Journ., London, 1891, vol. i. p. 1117.

7 Journ. Physiol., Cambridge and London, 1896, vol. xix. p. 262.
} 
levers. The first two observers fomm that the contraction of the papillary muscles occurred very shortly after that of the ventricular wall; by Haycraft and Paterson the contraction in vigorons hearts was found to be synchronous with that of the ventricular wall, while it subsequently became later when the rhythm of the heart began to fail.

Roy and Adami's method is not free from fallacy. The movements of the hook and lever connected with the valve camnot correspond exactly to the contraction and relaxation of the papillary muscles. For until the pull of these muscles on the auriculo-ventricular valve becomes of such a strength that it surpasses the difference in pressure between the auricle and ventricle, the flaps will not move in either direction. Roy and Adami found that the first effect of the contraction of the ventricle is to push the valvular diaphragm towards the auricle. The reverse movement does not necessarily mark the beginning of the contraction of the papillary muscles, but marks the moment when the pull of these muscles becomes greater than the intraventricular pressure. Likewise, the fall of Roy and Adami's curve does not mark the end of the contraction of the papillary muscles, but the time when the intraventricular pressure has become higher than the tension exerted by those muscles. The variations, then, in Roy and Adami's curves must be referred not only to the contraction of the papillary muscles, but also to the changes in intracardiac pressure.

The most reasonable view to hold is that the papillary muscles contract synchronously with the ventricular wall, for the rate of propagation of the contraction wave in the heart is so fast, reaching 5 metres a second or more, that, for all practical purposes, we may say that the contraction of the whole ventricle is synchronons. Thus, in the rabbit's heart, beating 180 times in a minute, each complete cardiac cycle occupies $\frac{1}{3}$ second. The ventricular systole occupies about $\frac{1}{3}$ of that time, that is $\frac{1}{9}$ second. The length of the ventricle is some $20 \mathrm{~mm}$., the rate of propagation of the contraction wave can be taken as $5000 \mathrm{~mm}$. a second. The whole ventricle would therefore have passed into systole in $\frac{1}{250}$ second, while it continues in systole for $\frac{1}{9}$ second. Considering that the papillary muscles are in direct continuity with the general musculature of the heart, it is difficult to conceive how the wave of contractility could be delayed from reaching these muscles, even for so long as $\frac{1}{20}$ second in the rabbit. The records of the action current of the heart, obtained with the capillary electrometer, suggest no such delay as is found by Roy and Adami.

It has been stated that the mitral valve closes much more perfectly than the tricuspid valve, and that, therefore, considerable regurgitation can take place through the latter when the right heart is over-distended. ${ }^{1}$

The normal heart must be greatly distended before actual regurgitation takes place past any of the valves. The perfect structure of the valves effectually prevents this. A back effect, causing a congestion of the blool in the venous system, can be brought about quite simply and without the aid of systolic regurgitation. The heart fails to empty its cavities at each systole, and thus the diastolic pressure is increased at a time when the valves are naturally open. The blood does not regurgitate during systole, but at each diastole the veins are unable to empty their full quota of blood into the ventricle, for the ventricular cavity is already partially filled. On asphyxiating a curarised animal, regurgitation from the right ventricle is

1 Flint, "Physiology," 1866, p. 201. 
found to take place only in the final stage, when the arterial pressure is falling to zero.

In pathological conditions of hypertrophy and dilatation of the heart, due to a prolonged continuance of obstruction in the pulmonary circulation or in the orifices of the heart, regurgitation takes place, and the great veins may then pulsate equally with the arteries.

The semilunar valves.-It was maintained by Burdach ${ }^{1}$ that the semilunar valves close at the end of systole, in consequence of their own elasticity. This is at once disproved by the fact that these valves do not assume the closed position when the aorta and ventricle are filled with fluid at the normal aortic pressure. By Weber ${ }^{2}$ the closure was attributed simply to the agency of the aortic pressure at the moment when the ventricle passes into diastole. Since by its systole the ventricle never completely empties itself, there would be, if Weber's hypothesis were true, a column of blood extending through the arterial orifice at the termination of systole, and considerable regurgitation would thus take place before the closure was effected.

Now the curves of intraventricular pressure do not give us the slightest evidence of regurgitation. Some other method of closure must therefore exist. Light is thrown upon this problem by the following experiment. ${ }^{3}$ A long glass syringe is filled with water containing a fine powder such as lycopodium spores. The syringe is held in the vertical position, and the fluid column is set in motion by a slight upward push given to the piston. The velocity of movement in the axis of the column is double as great as the velocity of the whole column. Close to the wall of the syringe, owing to viscosity, the movement of the Huid is so slow that the lycopodium spores are seen to be here overtaken by the piston. When this happens, an eddy drags the spores back into the axis, they thence mount rapidly to the top of the column, and there by an eddy once more reach the wall of the syringe. At the moment when the piston ceases to move, the column of fluid is divided into an immer core which runs upward with the axial velocity, and an outer mantle, in which the movement is in the opposite direction. It is this centripetal eddy which produces the closure of the valves in the aorta and pulmonary artery. The sinuses of Valsalva are dilatations in which eddies are bound to be produced, and there can be no doubt that these eddies cause the valves to approximate more and more, and finally close them at the instant when the ventricular pressure ceases to be higher than that in the aorta.

It has been suggested that the closure of the valves is likewise aided by the contraction of the longitudinal bands of muscle fibres which spring from the margins of the aortic or pulmonary orifices. These muscular bands are assumed to contract the orifices into cleft-like openings, bringing the segments of the valves almost into apposition at the end of systole. ${ }^{4}$ The eddies in the sinuses of Valsalva complete the work of these muscular bands.

Moens ${ }^{5}$ explained the closure of the semilunar valves in a different manner.

1 Ber. d. anat. Anstalt zu Königsberg, Leipzig, 1820, Bd. iii. S. 25.

${ }^{2}$ Letter to Hamernjk, Vrtljschr.f. d. praht. Hcilk., Prag, 1848, Bd. xx. S. 106.

${ }^{3}$ Ceradini, "Die Mechanismus der halbmondformigen Klappen," Leipzig, 1872, S. 46 ; Centralbl.f. d. med. Wissensch., Berlin, i8 72 , S. 135.

${ }^{+}$lirehl, Abhandl. d. math.-phys. Cl. d. k. sächs. Gesellsch. d. Wissensch., 1891, Bd. xvii. s'. 348 .

${ }^{5}$ Arch. f. d. ges. Physiol., Bonn, 1879, Bd. xx. S. 524. 
He supposed that the intraventricular negative pressure, which is known to exist, was systolic in time. He believed that the blood, aided by its momentum, was shot out with a velocity which the ventricular wall could not follow. To this fact he ascribed the origin of the negative pressure, which, in its turn, produced the closure of the valves, and that before any regurgitation could take place. This theory is entirely unsupported by the curves of intraventricular pressure.

The position of the semilunar valves during the time of systolic output has been a subject of considerable controversy. An old doctrine of Thebesius was revived by Bricke. ${ }^{1}$ He maintained that the valves swing back against, and close, the orifices of the coronary arteries during the systolic period. Thus, he taught, the heart is only fed with blood during diastole. While, in systole, the want of blood brings the contraction to a close, the re-entry of blood in diastole leads to the expansion of the heart.

The publication of this doctrine led to a fierce discussion, but Briicke defended his position with such brilliancy and wealth of argumentative resource, that for a time his theory was accepted by most of the schools. Meanwhile, sereral anatomists chronicled cases in which the valves were not sufficiently large to cover the coronary orifices, and in these no abnormality of the heart had been observed during life. Chauveau and Rebatel ${ }^{2}$ finally overthrew Bricke's theory by simultaneously measuring the velocity and the pressure in the coronary artery. The former was recorded by means of Chauveau's instrument, the hremodromograph (see "Velocity of Flow," p. 85).

At the beginning of systole the relocity and pressure in the coronary arteries increase; later, the velocity becomes negative, and the pressure rises, or, in other words, the blood hegins to flow in the reverse direction back to the aorta. At this stage the ventricular wall is so strongly contracted that the muscular tension rises above that of the aorta; the coronary arteries and their tributaries are therefore compressed, and the blood driven back into the aorta. In the final stage, when the diastole is commencing, the velocity once more increases.

By Newell Martin and Sedgwick ${ }^{3}$ the pressures in the coronary artery and the aorta were synchronously recorded. They found that the coronary tracing was always a duplicate of that of the aorta.

We may conclude, then, that the coronary arteries are not closed by the valves, but that the cardiac vessels are compressed within the muscle, and emptied back towards the aorta. This is confirmed by experiments of Klug, ${ }^{4}$ who ligatured the base of the heart in the period of systole, and again in the period of diastole. In the systolic heart the vessels within the deeper layers of the musculature were found to be emptied of blood. Injection of the coronary arteries with fluid at the pressure of the aorta having been found to produce a negative pressure in the cavity of the ventricles, we must admit the truth of part of Briicke's hypothesis. Fach systole does partially ent off the heart muscle from its blood supply, and at each diastole the injection of the coronary arteries does aid in the dilatation of the heart.

${ }^{1}$ Sitzungsb. d. k. Akad. d. Wissensch. math.-naturw. Cl., Wien, 1855, Bd. xiv. S. 345; "Der Versehluss der Kranzschlagadem durch die Aortenklappen," Wien, 1855.

2 "Rech. expér. sur la circ. dans les artères coronaires," Thèse, Paris, 1872.

${ }^{3}$ Journ. Physiol., Cambridge and London, 1882, vol. iii. p. 165.

+Centralbl. f. d. med. Wissensch., Berlin, 1876, s. 133. 
But both these conditions are brought about by a method entirely different from that supposed by Briicke. The sinuses of Valsalva have been evolved for the attaimment of two objects. The first of these is the prevention of the semilmnar valves swinging back so as to adhere to the wall of the artery; the second is the provocation of eddies which close the valves at the instant systole ceases. Ceradini ${ }^{1}$ has experimentally determined that, when fluid is suddenly driven from the ventricle into the pulmonary orifice, the valves do not swing back against the wall of the sinuses.

\section{The Changes of Pressure in the Heart during the Cardiac Cycle.}

Method.-Dissatisfied with the differences of opinion arising from the mere personal observation of the sequence of events in the cardiac cycle, Chauvean and Marey ${ }^{2}$ explored the intracardiac pressure. For

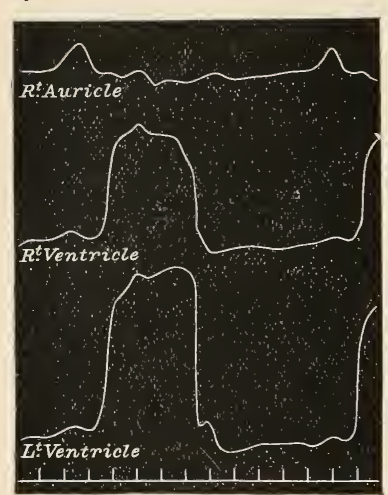

FIG. 6.-Intracardial pressure curves. Chauveau and Marey. this purpose they invented a special instrument, the cardiac sound or sphygmoscope, by which the variations of pressure in one or other chamber of the heart could be recorded.

The sound consisted of a hollow metal tube, of such a bore that it could be easily passed down the jugular vein or carotid artery of the horse. At one end of the tube was a wire framework, over which was drawn a thin rubber bag. This end was passed into a cavity of the heart, and the other end was put in connection with a recording tambour. The rubber bag transmitted the changes of pressure in the heart to the recording tambour, and was at the same time, by means of the framework, prevented from completely collapsing.

In some experiments, Chauveau and Marey employed a byway sound, consisting of a double tube and two bags. When this was passed down the jugular vein, one bag was so situated as to lie in the right auricle, and the other in the right ventricle. Each tube of the byway sound passed to a recording tambour, and the levers connected with each tambour wrote exactly beneath one another, and thus the auricular and ventricular pressures were simultaneously recorded on a revolving drum. These records can be taken while the horse quietly eats at its manger, oblivious of the instrument within its heart.

These and similar instruments are experimentally calibrated against the readings of a mercury manometer. ${ }^{3}$

Many investigators have followed in the steps of Chauveau and Marey. Magini and Rolleston passed hollow trocars directly through the thorax into the cavities of the heart, while Fick, Hürthle, Fredericq, v. Frey, Krehi, and others, have passed cannulas down the jugular

${ }^{1}$ Loc. cit., S. $60 . \quad 2$ Mém. Acad. de méd., Paris, 1863, tome xxvi. p. 272.

3 Marey, "Circulation du sang," Fig. 45, p. 111. 
vein and carotid artery, or directly through the auricular appendix into the heart. These tubes have, in each case, been connected with manometers of special construction, and all more or less adapted to the recording of rapid changes of pressure. None of these methods materially interfere with the circulation. After the introduction of such tubes, the sounds of the heart remain the same as before. ${ }^{1}$ Huirthle has shown that the curve given by the cardiac impulse also remains unaltered. ${ }^{2}$ Even if a semilunar valve be punctured during the passing of a tube down the carotid, no harm results, since the tube fills the hole it has made, the valves remain otherwise competent, and the pressure curves are unaltered.

The truth of the records obtained depends entirely on the perfection of the registering apparatus employed. Chauveau and Marey did not give any proofs of the capabilities of their instrument, and the accuracy of their curves. These authors used air transmission, and thus, no doubt, most rapid variations of pressure could be conducted to the recording tambours. Donders ${ }^{3}$ has since proved that a sensitive Marey tambour gives readings of considerable accuracy, although the lever has a swing of its own, due to the momentum of its mass, which in rapid variations of pressure tends to make the excursions too great.

It is an extremely difficult problem to contrive a manometer which will answer, without much inertia, to extremely quick changes of intraventricular pressure. A mercury manometer is useless, because of the momentum of its mass. Thus this instrument gives a lower reading of intraventricular pressure than of aortic pressure, simply because it has not time to follow the rise of intraventricular pressure. Such a result is, of course, absurd.

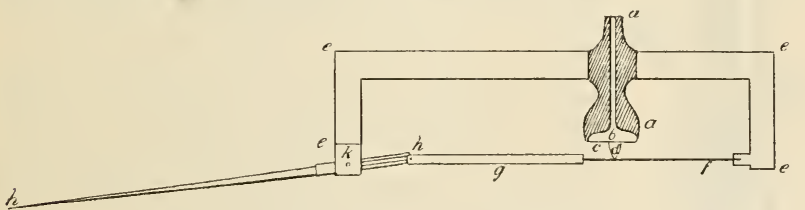

FIG. 7.-Fick's straight spring manometer.

The manometer must, in all its moving parts, be made of the smallest mass, and the object in hand is best attained by the use of some form of elastic counterpoise to resist the moving part of the instrument. Fick's straight spring manometer, in which air is employed as the agent of transmission, satisfies most of the conditions required. ${ }^{4}$

It consists of a tube $a \alpha$, which opens into a very small chamber, the floor of which is formed by a rubber nembrane. In the middle of this membrane is fixed an ivory knob, which presses against a strong steel spring. $f$. The exceedingly small movements of this spring are greatly magnified by the long lever $h$. This instrument has been proved to be accurate by the following experiment.

${ }^{1}$ Chanveau and Marey, loc. cit., p. 301.

2 Arch.f. d. ges. Plyysiol., Bonn, 1891, Bd. xlix. S. 93.

3 Onderzoek. ged. in h. physiol. Lab. Utrecht, Hoogeseh., Tweede Reeks, 1867, vol. i. pp. 11-18.

${ }^{4}$ Fick, Arch. f. d. ges. Physiol., Bonn, 1883, Bd. xxx. S. 597. v. Frey's tonometer is constructed essentially on the same principle, air being used as the medium of transnission. 
By means of a cannula a pressure record is taken in the left ventricle, the cannula is then pulled out into the aorta, and another record taken. The two readings thus obtained closely tally. As the intraventricular pressure rises about $160 \mathrm{~mm} . \mathrm{Hg}$ in one second, this instrument can record a variation of pressure equal to $1600 \mathrm{~mm}$. Hg per second, without either shooting on too far or lagging behind.

Hürthle's ${ }^{1}$ spring manometer consists of a small tambour, covered with a rubber membrane only $5.5 \mathrm{~mm}$. in diameter. A button attached to the membrane works against a steel spring, which acts as a counterpoise. The movement of the spring is magnified and recorded by means of a lever provided with a writing-

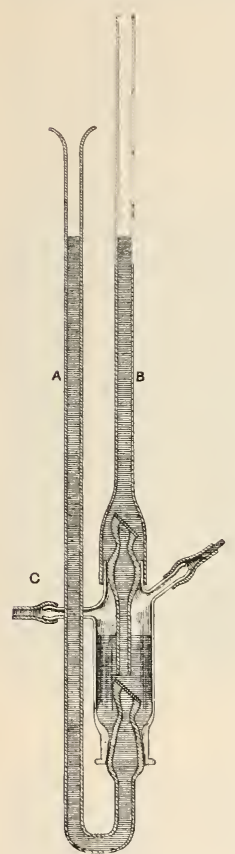

FIG.8,-GoltzandGaule's combined maximal, $B$, and minimal manometer, $\mathbf{A}$, as modified by Hürthle. ${ }^{+}$

mal or minimal instrument.

style. Fluid is used as the transmission agent, and the great principle of the instrument is the rerluction of this fluid to the smallest amount. The use of a practically incompressible fluid, as a medium of transmission, is open to one objection. Once set in motion, the fluid column, extending from the ventricle to the manometer, after the original force has ceased to act upon it, still continues to move. The degree of this inertia is known to be proportional to the mass and the square of velocity of the fluirl. In a properly constructed manometer, the smallest mass of fluid practicable must enter for a given increase of pressure. The practical limit is reached somewhat before this mass becomes so small that its movements are appreciably affected by the friction of the lever, or, above all, by the friction of the writing point against the smoked paper. ${ }^{2}$ The Hürthle tambour is therefore made of the smallest dimensions, and the swing of the instrument is further capable of being damped down by the partial turning of a stopeock, placed between the tambour and the artery, which increases the friction of the fluid, and so diminishes its velocity. This stopeock is turned until a point is experimentally found where the inertia is removed with as little sacrifice as possible of the speed of the instrument in following the changes of pressure. When the stopcock is fully open, and the instrument undamped, the Hürthle manometer is found to record changes of pressure equal to $200 \mathrm{~mm} . \mathrm{Hg}$ in 02 second. This is equivalent to $10,000 \mathrm{~mm}$. $\mathrm{Hg}$ per second.

Goltz and Gaule contrived another and valuable instrument, the maximum and minimum manometer, by which can be recorded the highest or lowest pressure occurring during a lengthened perior of observation in any chamber of the heart. ${ }^{3}$ This is effected by placing valves between the intracardial canmula and the mercury manometers, and then, according to the direction of the valves, the manometer becomes a maxi-

A simple form of valve can be constructed in any laboratory out of a tube formed of thick glass, ground to a slant at one end, and brought to a perfectly smooth surface. The orifice of the tube at this end is $1 \mathrm{~mm}$. in diameter. Over this is affixed a flap of oil-silk, which forms an efficient and

${ }^{1}$ Arch.f. d. ges. Physiol., Bonn, 1888, Bd. xliii. S. 420.

${ }^{2}$ Porter, Journ. Physiol., Cambrilge and London, 1892, vol. xii. p. 518.

${ }^{3}$ Goltz and Gaule, Arrh. f. d. ges. Physiol., Bonn, 1878, Bd. xvii. S. 100.

${ }^{4}$ Hürthle, ibid., Bonn, 1888, Bi. xliii. S. 438; Porter, Journ. Physiol., Cambridge and London, 1892, vol. xiii. p. 516. 
simple valve. An instrument so constructed is shown in Fig. 8 . If $\mathrm{C}$ be placed in connection with the intracardiac cannula, $A$ acts as a ininimal, and $\mathrm{B}$ as a maximal manometer.

Using Hürthle's spring manometer in conjunction with maximal and minimal manometers, we can arrive at the true changes of intracardiac pressure, for the first will give us the time, sign, and velocity, and approximately the amount of the pressure variations, while the last will give us the true maximal and minimal pressures.

The sphygmoscope of Chauveau and Marey, as modified by Fredericq, is

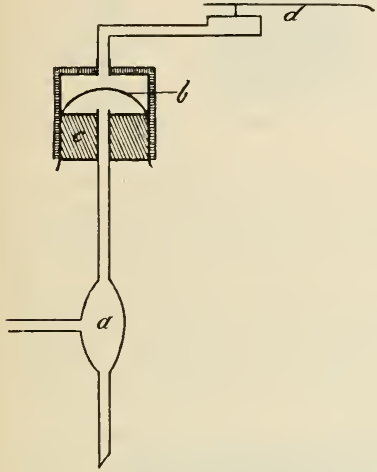

FIG. 9.-Diagram of Fredericq's sphygnoscope. $a$, cannula ; $b$, rubber finger stall ; $c$, cork ; $d$, lever of tambour.

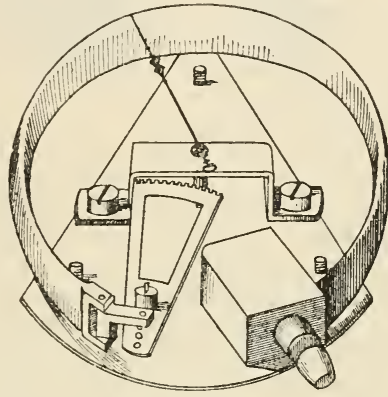

Fig. 10.-Diagram of v. Basch's hollow spring manometer. The index reeords the pressure on a graduated scale. A recording style can be affixed to the end of the hollow C-spring.

probably as perfect an instrument as the Hurthle manometer, and is far simpler in construction. ${ }^{1}$

The end of a rubber finger-stall is drawn over the end of a rubber cork, and a glass tube connected with the intracardiac cannula passes through the rubber cork into a very small air space, which is left beneath the apex of the finger-stall at the top of the cork. The cork is inserted into the end of a glass thistle-shaped funnel, and this is in its turn connected with a delicate Marey tambour. Every part of the instrument is reduced to the smallest

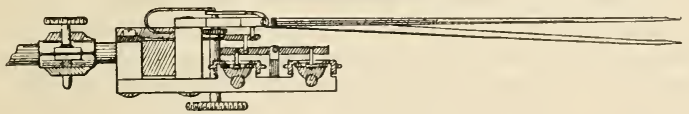

Fig. 11.-Hiirthle's differential manometer.

dimensions, and the rubber tip of the finger-stall acts as an elastic counterpoise to the variations of pressure.

One of the most convenient forms of manometer for accurate work is that of v. Basch, who has constructed a C-spring manometer on the smallest possible scale. The alvantages of this instrument are, that a permanent hollow spring replaces a tambour covered with a perishable rubber membrane,

1 Fredericq, "Manipulations de physiologie," Paris, 1892, p. 174.

VOL. II. -2 
and that the pressure height can be read off directly on a dial, while the pressure curve is simultaneously recorded. ${ }^{1}$

For the determination of the relations of the pressure changes in any two cavities, Hürthle has constructed a differential spring manometer. In this instrument two cannule are brought into connection with tambours placed on either side of the fulcrum of a lever. This lever works against a spring, which sets in motion a writing-style. When the pressures are equal, the style will remain at rest, and will rise or descend according as the pressure in the one rises above or falls below that of the other. ${ }^{2}$

The curve of intraventricular pressure. - The tracings of Chauveau and Marey, Fick, Hürthle, Fredericq, all exhibit a curve which is marked by a very steep ascent, followed by a maintained plateau which either rises or falls slightly, or remains horizontal. On this plateau oceur two or three small waves; thence follows the steep line of descent. ${ }^{3}$

The curves obtained by v. Frey and Krehl, Rolleston, Roy and Adami are of a different type: these show either no plateau, or else a kind of rounded plateau which subsides quickly into the descent.

The differences in the curves depend ahnost entirely on the instruments employed, because all the researches have been carried out on dogs, except those of Chauveau and Marey on the horse. The best instrument, as we have seen, is that with the shortest delay, so long as the inertia of the instrument is not too great.

Contejean has passed a hollow needle into the right ventricle, and allowed the blood to spirt on to a revolving drum covered with white paper; in this way he has obtained a hremautograph of the intraventricular pressure. A plateau is found on such a tracing. ${ }^{4}$

Lastly, Bayliss and Starling have demonstrated the accuracy of the plateau curves by a new method. They connected the intracardiac camnula with a capillary glass tube, which had been partially filled with $\mathrm{MgSO}_{4}$ solution, and had then been sealed at the top. They photographed on a moving sensitized plate the movements of the meniscus of the Huid in the capillary tube. At the same time the movements of a style affixed to a vibrating tuning-fork were also photographed.

Within the rates of pressure variations which occur in the heart, the inertia of the fluid was found to be nil, while the volume of fluid moved for a rise of $100 \mathrm{~mm}$. Hg pressure was only 0335 c.mn. In Huirthle's smallest manometer, this volume for the same rise of pressure was $100 \mathrm{c} . \mathrm{mm}^{5}$. This method is therefore by far the most perfect yet invented for arriving at the true curve and time relations of intracardiac pressure. Unfortunately, it is too inconvenient to employ habitually. By its means, however, we are assured that the Hurthle manometer and the sphygmoscope are instruments of precision. ${ }^{6}$

The curves obtained by Bayliss and Starling are no doubt a true representation of the intraventricular pressure changes, and it is

${ }^{1}$ Centralbl. f. Physiol., Leipzig u. Wien, 1896, Bd. x. S. 330.

${ }^{2}$ Hurthle, Arch. $f$. d. ges. Physiol., Bonn, 1891, Bd. xlix. S. 45.

${ }^{3}$ Chauveau and Marey, Mém. Acad. de méd., P'aris, 1863, tonse xxvi. p. 298.

4 Arch. de physiol. norm. et path., Paris, 1894, p. 821.

5 Arch. f. d. ges. Physiol., Bonn, 1888, Bd. xliii. S. 409

${ }^{6}$ Bayliss and Starling, Internat. Monatschr. f. Anat. u. Physiol., Leipzig, 1894, Bd. xi. S. 426 . 
evident, on comparison, that the original tracings obtained by Chauveau and Marey (Fig. 6) also nearly represent these.

The small waves which are seen on the top of the plateau have been explained in several ways. They have been attributed to the vibrations of the auriculo-ventricular valves. By Marey they were ascribed to

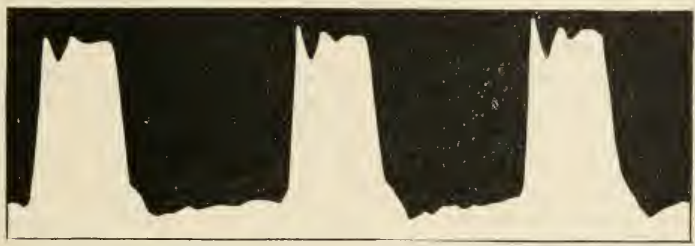

F16. 12.-Intraventricular pressure eurve.-Bayliss and Starling.

waves set up in the large arteries during the systolic output. He supposed that these waves passed back into the ventricle, and were reflected from the ventricular wall and auriculo-ventricular valves. It is highly probable that such vibrations would be produced by the sudden tension of such an elastic system as is formed by the heart and large arteries. Tigerstedt ${ }^{1}$ and Porter ${ }^{2}$ have attributed the waves to the swing of the instruments employed. Fredericq, ${ }^{3} \mathrm{~d}^{\prime}$ Espine, ${ }^{4}$ and Stefani ${ }^{5}$ have ascribed them to three successive muscular contractions of the

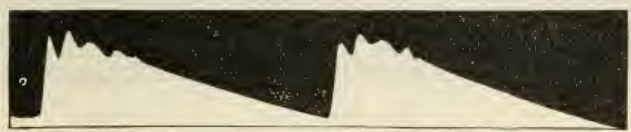

Fig. 13.-Aortie pressure curve.-Bayliss and Starling.

ventricle. This is a far-fetched explanation, and is controverted by the curves of the negative variation of the heart, obtained with the capillary electrometer by Waller, Bayliss and Starling, and others.

The plateau and its wavelets are only obtained when the ventricle is filled with blood, and are not seen on curves of contraction obtained from the empty ventricle. ${ }^{6}$ It is to be concluded that the plateau is the sign of the sustained tension on the blood by the ventricle wall during the systolic output, and that the wavelets are due to vibrations set up in the elastic circulatory system by the sudden systolic increase of tension.

The period of rising tension.-Part of the period of ventricular systole is spent in raising the tension of the blood up to and above that in the aorta.

During this period all the valves in the ventricle remain closed, and the capacity of the ventricular cavity does not vary. The muscle bands

1 "Lehrbuch d. Phys, des Kreislaufes," Leipzig, 1893, S. 101.

2 Journ. Exper. Hed., N. Y., 1896, vol. i. p. 296.

3 "Trav. du labor. Liege," 1888, tome ii. 1. 91.

${ }^{4}$ Pex. de méd., Paris, 1882 , tome vii. p. 17.

5 Mem. Accad. med.-chir. di Ferrara, il 5 Agosto, 1891, p. 69 ; Centralbl. f. Physiol., Leipzig u. Wien, 1893 , s. 79.

"Laulanié, Compt. rend. Soc. de biol , Paris, 1892, p. 557 ; E. Meyer, A rch. de physiol.; norm. et path., Paris, 1892, tome xxvi. Ip. 670-678; Marey, "Cireulation du saug," Paris, 1881, p. 95. 
on the outside of the ventricle assume the shortest position which is possible without change of the cavity, and therefore the external form of the ventricle becomes more spherical in shape. As the tension rises higher, the blood in the coronary vessels becomes more and more expelled from the musculature of the heart. Chauveau and Marey took synchronous curves of the intraventricular and aortic pressures. They found the rise of aortic pressure occurred 1 sec. later than the beginning of the systolic curve. ${ }^{1}$

This, therefore, was the period of rising tension in the horse. The whole ventricular contraction, up to the point of steep descent at the end of the plateau, covered a period of 4 sec.

With improved apparatus Huirthle found the time of rising tension to be considerably shorter; in the dog it was ' 02 to $04 \mathrm{sec}^{2}$

If the aortic pressure be very low this period becomes even shorter. When the aortic pressure was high, Huirthle found no constant relation between the height of the pressure and the duration of the

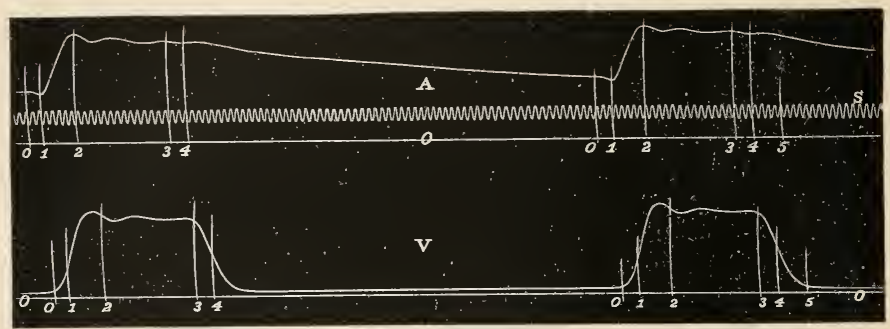

Fig. 14. - A, aortic, and $\mathrm{V}$, intraventricular pressure eurves; $\mathrm{O}$, abscissa ; $\mathrm{S}$, tine trace = $\frac{1}{100}$ sec, ; 0-5, corresponding ordinates. - Hurthle.

period of rising tension. On taking measurements of the difference between the diastolic pressure in the ventricle and the aortic pressure, Hürthle determined that with a pressure difference of 58 to $60 \mathrm{~mm}$. $\mathrm{Hg}$, the period of rising tension was 017 sec., while it was 03 sec. with a pressure difference of $111 \mathrm{~mm}$. $\mathrm{Hg}$. The variations of the period of rising extension are therefore most insignificant, and remain so even when the rhythm of the heart is altered by section of the vagi or excitation of the accelerator nerves. This is equivalent to saying that the heart can meet great demands on its powers, without any appreciable loss of time.

The period of systolic output.-With what smoothness the cardiac mechanism works is strikingly evidenced by the fact that neither the opening nor closing of the valves produces any peak or point on the curves of intracardiac pressure. Similarly, the ventricle fills from the auricle so smoothly, that the auricular systole is scarcely marked on the intraventricular pressure curve.

The point on the intraventricular pressure curve at which the semilunar valves shut, has been a subject of much discussion. The moment when the second sound of the heart is heard has been marked with a signal on tracings of such curves, and this, after

${ }^{1}$ Chauveau and Marey, Mém. Acad. de méd., Paris, 1863, tome xxvi. p. 305.

2 Arch. f. d. ges. Physiol., Bonn, 1891, Bd. xlix. S. 59. 
deduction of the observer's reaction time, has been taken as the point of closure.

The second sound, however, is not produced by the closure itself, but by the tension of the valves which follows the closure, some fraction of a second later. The closure takes place at the moment when the ventricular pressure falls to the slightest degree below the aortic pressure.

By Hürthle, the differential manometer ${ }^{1}$ was employed to settle the moment. A byway cannula was passed into the aorta and ventricle, and the time marked on the intraventricular pressure curve when the aortic pressure surpassed the ventricular pressure. ${ }^{2}$ The aortic and ventricular pressure curves are taken simultaneously with the differential curve by means of $\mathrm{Y}$ tubes. The point found corresponds in time

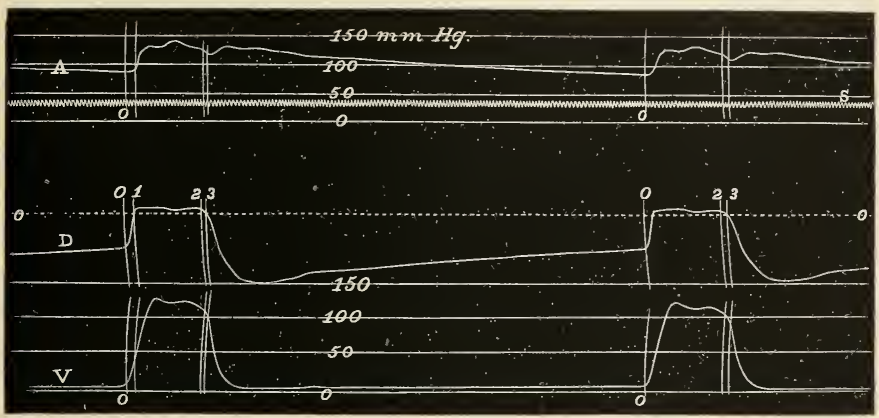

FIG. 15. - A, aortic; D, differential ; V, ventricular pressure. - Hürthle.

with the bottom of the dicrotic notch on the aortic curve, and is at the end of the plateau (Fig. 15, 3). Fredericq, Porter, and Starling and Bayliss agree with this conclusion.

Chauveau and Marey placed the closure much lower down on the descending limb of the curve, at a point where a notch occurs in the descent. ${ }^{3}$ Chauveau has recently attempted to confirm this conclusion by an ingenious experiment. He passed a byway sound down the carotid of the horse, until one ampulla lay in the aorta, and the other in the ventricle. At the scat of the semilunar valves, an electric contact was fixed to the sound; the contact was made by the closure of the valve, and a signal wrote the moment of contact on the tracing. ${ }^{4}$ It is most probable, however, that the contact would be made, not by the closure, but by the tension of the valves, for a certain amount of pressure would probably be required to effect the contact.

The differential manometer also shows the moment when the intraventricular pressure overtops the aortic pressure, and the semilunar valves open (Fig. 15, 1). This moment is found to be a little below the

1 See p. 18 and Fig. 11, p. 17.

2 Arch. f. d. ges. Physiol., Bom, Bd. xlix. S. 54.

3 Mém. Acad. de méd., Paris, 1863, tome xxvi. p. 298.

4 Compt. rend. Acad. d. sc., Paris, tome exviii. 1'). 686, 690. 
middle of the ascending limb of the ventricular curve. The period of systolic output extends from the opening to the closure of the semilunar valves, and that is the period during which the ventricular pressure is higher in the differential tracing (Fig. 15,1 to 3 ). This time of output, according to Hurthle, is from 178 to 195 sec. in the dog, and is, as already stated, but slightly altered either by variations in aortic pressure or in the frequency of the heart.

The maximal pressure in the ventricles.-By means of their graduated cardiac sounds Chauveau and Marey ${ }^{1}$ reckoned the maximal pressure in the right ventricle of the horse to be 24 to $30 \mathrm{~mm}$. $\mathrm{Hg}$; in the left ventricle 95 to $140 \mathrm{~mm}$. Hg. With the maximal manometer, Goltz and Gaule found the pressure in the dog to be-

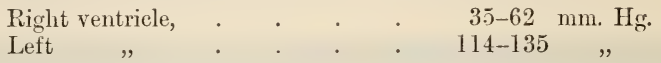

de Jager ${ }^{2}$ employed a maximal manometer, but lessened the resistance by using large camnulie. He measured the maximum in the ventricle, and then, after pulling the cannula out into the aorta, immediately measured the aortic maximum. The results recorded were-

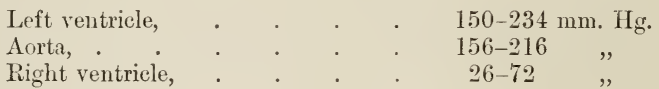

These results were obtained during morphia narcosis, and are perhaps too high. The relation of the right to the left ventricular maximum is therefore from $1: 3$ to $1: 6$. This agrees closely with the relation found between the pulmonary arterial and the aortic pressures. Thus by Beutner ${ }^{3}$ this was found to be $1: 3$ in $\operatorname{dogs}, 1: 5$ in cats, $1: 4 \mathrm{in}$ rabbits, and by Colin, ${ }^{4} 1: 3$ or $1: 4$.

In order that the systole may be efficient, the intraventricular pressure must rise to a higher point than the aortic pressure. In certain conditions of the heart some of the beats may be inefficient, and so produce no sign on the aortic curve.

After considerable loss of blood, the intraventricular pressure at each systole exceeds the aortic pressure by a comparatively greater amount.

The aurieular pressure curve.-The systolic pressure in the auricle

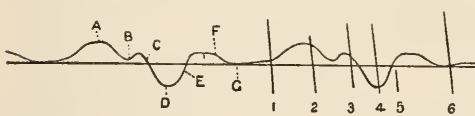
reaches its maximum in 1 sec. in the horse, while the ventricular contraction up to the end of the plateau lasts 4 sec.

Fif: 16.-Auricular pressure curve. - Porter. This short duration of the anricular systole found by Chauveau and Marey has not been confirmed by all observers. ${ }^{5}$

The pressure curve of the auricle has been obtained by the same instruments as were employed for ventricular olservations. The tracings obtained by Chauvean, Marey, and Frederieq with the sphygmoscope, and

${ }^{1}$ Loc. cit., p. $305 . \quad{ }^{2}$ Arch. f. d. ges. Physiol., Bonn, 1S83, Bd. xxx. S. 504.

3 Ztschr. j. rat. Mted., 1852, N. F., Bd. ii. S. 118.

¿ Bull. Acad. de méd., Paris, 1874, p. 398.

5 “Trav. du labor. de Marey," 18i7, tome iii. p. 311. Cf. Samways, "Le Rôle de l'oreilette gauche," Paris, 1896. 
by Porter with the Hürthle manometer, agree fairly closely in the main. outlines.

By means of a large cannula placed in the auricular appendix, and connected with a Hiirthle tambour, Porter ${ }^{1}$ took curves (Fig. 16), in which the following points are to be observed:-

A, The systolic rise of the auricular pressure $=+9 \mathrm{~mm} . \mathrm{Hg}$.

A.B, A first diastolic fall

B.C, A first diastolic rise

C.D, A second diastolic fall

$$
\begin{aligned}
& =+5 \quad, \\
& =-10 \quad, \\
& =+5 \quad, \\
& =+0.5 \quad,
\end{aligned}
$$

E, A second diastolic rise

$\mathrm{F}, \mathrm{A}$ third diastolic fall

G, A period of no pressure.

Upon the second curve given in the figure the following relations are marked :-

1. Beginning of the auricular systole.

2. Beginning of ventricular systole.

3. Opening of semilunar valves.

4. The plateau stage of the ventricular systole.

5. Beginning of ventricular downstroke.

6. Fnd of ventricular downstroke.

A considerahle negative pressure appears in the auricle during the height of the ventricular systole (Fig. 16, D, 4), a negative pressure which must materially aid in the filling of the auricle. This is given as follows:-

$$
\begin{aligned}
& \text { Chauveau and Marey }-2 \text { to }-33 \mathrm{~mm} \text {. } \mathrm{Hg} \text {. } \\
& \text { Goltz and Gaule . }-7 \text { to }-10 \cdot 4, \\
& \text { Porter . . }-10
\end{aligned}
$$

By Chauveau and Marey the phenomenon was explained by the supposition that the descent of the base of the ventricle during systole pulls on the auricular wall, and thus dilates the auricular cavity. This is scarcely probable, as the auricle is free to move as a whole, and would therefore follow the ventricle. v. Frey, Krehl, and Fredericq suggest a different explanation. During the ventricular systole, the ventricles, by the expulsion of blood, become smaller; by their diminution the negative pressure in the thorax is increased, and thus the auricles, large veins, and lungs are dilated. So long, therefore, as the thorax is closed, the heart can act in this way, not only as a force pump, but also as a suction pump. Each systole of the ventricles helps to fill the auricles. Porter has further put forward an ingenions theory to explain the second diastolic fall. He supposes that when the papillary muscles contract they pull down like a diaphragm the auriculo-ventricular valves, which are closed and ballooned out towards the ventricular cavity. Thus the cavity of the auricle is increased. The commencement of the second diastolic fall agrees closely with the beginning of the downward movement of the valves, as determined by Roy and Adami in their research on the papillary muscles (see p. 10 and p. 39).

Probably this, the most important fall in the auricular pressure, is produced, not by one only, but hy both of these factors combined. With an increasing frequency of heart-beat, Porter las found that the

1 Journ. Physiol., Cambridge and London, 1892, vol. xiii. p. 513. 
period of the second diastolic fall, like the period of ventricular systole, remains almost constant. The second diastolic rise, E (Fig. 16),

A

B

$\mathbf{C}$

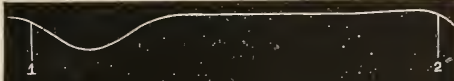

D

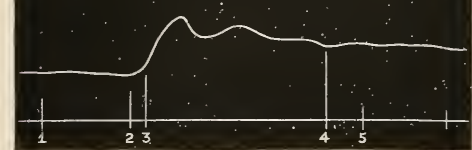

Fis. 17.

Cardiac and aortic pressure curves (Porter) -

$A$, Contraction curve of the left auricular appendix.

$B$, Differential tracing of the auricular and ventricular pressures.

C, Ventricular pressure curve.

D, Aortic pressure curve.

The corresponding times are marked by ordinates, thus-

ORDinate 2. The pressure in the auricle and ventricle is equal. An instant later the pressure is higher in the ventricle, and the auriculoventricular valves close.

3. There is marked here the beginning of the aortic rise, that is to say, the moment when the semilunar valves open.

4. This marks the bottom of the dicrotic noteh. At this point the semilunar valves close.

5. The auricular and ventricular pressures are aqain equal, and immediately after the valves open, as the auricular pressure rises above the ventricular. is no doubt produced by the filling of the auricles from the veins; at the same time, the intraventricular pressure may rise above the tension of the papillary muscles towards the end of the systole, and so again tend to drive the auriculo - ventricular valves out towards the auricular cavity. The third diastolic fall $(\mathrm{F})$ is synchronous with the relaxation of the ventricle, and is due to the flow of blood from the auricle into the ventricle.

The time of the opening and closing of the auriculoventricular valves is obtained by taking tracings of the auricular and ventricular pressures, simultaneously with a differential trace recorded by the differential manometer (see Fig. 17).

The first diastolic rise (BC) seen in Porter's auricular curve (Fig. 16) has not been found by all observers. When this rise is obtained, it must be due cither to a slight regurgitation or to the ballooning out of the auri-

culo-ventricular valves. Owing to the great capacity of the veins, and the absence of elastic tension of the venous wall, this rise is generally not sufficient to produce a venous pulse.

Observations on the maximal pressure in the auricle has yielded very different results-

Chauveau and Marey estimated it as $2.5 \mathrm{~mm}$. Hg in the horse, right auricle.

Goltz and Gaule

Magini

Porter

v. Frey

$\begin{array}{ll}, & 20 \\ , & 20-22 \\ , & 1-2 \\ , & 10\end{array}$

,
, in the dog,

,

,

,
,

,

, 9

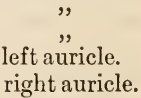

The pressure in the cavities of the heart is undoubtedly subject to great variation. On what conditions these variations depend we have as yet no exact knowledge. There is no reason to doubt the results of the above observers, but further rescarches into the causes of the variations are obviously needed. The fact of the thorax being opened or closed, the efficiency or inefficiency of the vasomotor tone, the 
position of the animal, will all markedly influence the filling pressure of the auricle, and no doubt the maximal auricular pressure depends far more on the filling pressure than on the force of the auricular contraction.

The negative pressure in the heart.-The diastole of the ventricles is apparently performed with considerable energy. It has been maintained, therefore, that the diastole, as well as the systole, results from muscular action. It has been impossible, experimenting upon the frog, to prove the existence of any diastolic force in the excised heart, although Luciani has attempted to do so. ${ }^{1}$

Roy and Tunnicliffe found that the intracardiac pressure in the frog must be 5 to $1 \mathrm{~cm}$. $\mathrm{H}_{2} \mathrm{O}$ above the extracardiac for diastole to take place. ${ }^{2}$ Tunnicliffe placed the frog-heart in a chamber, in which the extracardiac pressure could be varied. By means of a byway cannula the heart was fed with a nutrient fluid, at a pressure which also could be varied." The diastole did not take place unless the pressure in the feeding tube was made positive.

The same conclusion was arrived at by Newell Martin and Donaldson, ${ }^{4}$ with regard to the excised mammalian heart. After placing an inflow tube in the vena cava superior, and an outflow tube in one subclavian artery, Martin ligatured the branches of the arch of the aorta, the thoracic aorta, and the venæe cavie. All connections of the heart with the general circulation were thus removed. The pulmonary circulation and the coronary circulation still continued, for artificial respiration was maintained in the lungs, while a circulation of defibrinated blood was established through the heart at a constant temperature. Under such conditions the heart continued to beat normally for many hours. These authors concluded that if the aspiration of the thorax be removed, the right auricle will not receive blood, unless it be supplied to it under a decided, if small, positive pressure.

On passing a cannula connected with a manometer into one or other ventricle of the dog, Goltz and Gaule obtained evidence of a considerable negative pressure, which appeared in the ventricular cavities at some period of the cardiac cycle. ${ }^{5}$ In one experiment with the thorax open, these authors obtained a minimal pressure of $-23.5 \mathrm{~mm} . \mathrm{Hg}$ in the left ventricle. In another experiment with the thorax closed, $-52 \mathrm{~mm}$. $\mathrm{Hg}$ was recorded. The negativity was only marked when the systole was strong, and the heart emptied well. No evidence could be obtained of a negative pressure during the beat of an excised and empty heart. These results were confirmed by de Jager, ${ }^{6}$ who obtained, with the thorax open, the following minimal pressures:-

$$
\begin{aligned}
& -5 \text { to }-38 \mathrm{~mm} \text {. Hg. in the right ventricle. } \\
& -2,-6,, \text { in the right auricle. }
\end{aligned}
$$

Rolleston ${ }^{7}$ obtained a minimal negative pressure in the left ventricle

${ }^{1}$ Luciani, “Dell' attivita della diastole cardiaca," Bologna, 1871, 1874, and 1876 ; Mosso and Pagliani, "Critica sperimentale della attivita diastolica cardiaca," ete., Torino, 1876 .

${ }^{2}$ Journ. Physiol., Cambridge and London, 1878-79, vol, i. p. 465.

3 Ihid., 1896, vol. xx. p. 50.

${ }^{4}$ Mem. Biol. Lab. Johns Hopkins Univ., Baltimore, 1887, vol. iv. p. 37.

5 Arch.f. d. ges. Physiol., Bonn, 1878, Bd. xvii. s. 108.

6 Ibid., 1883, Bd. xxx. S. 506.

7 Journ. Physiol., Cambridge and London, 1887, vol. viii. p. 250. 
equal to $-20 \mathrm{~mm}$. Hg. Evidence of negative pressure is found at the end of systole in the intraventricular pressure curves taken by the sphygmoscope and by the Huirthle manometer, or by the method employed by Bayliss and Starling.

The origin of the negative pressure has received many explanations. It has been supposed that by shortening the long diameter of the heart certain longitudinal nuscle bands enlarge its cavity, and produce the diastole. ${ }^{1}$ It has also been suggested that if the circular fibres continue to contract after the longitudinal fibres have ceased to shorten the ventricle, these fibres will help to extend and dilate the ventricles. ${ }^{2}$ When the output of blood takes place the aortic orifice is distended, and this distension must be shared by the adjacent portions of the ventricle (conus arteriosus). ${ }^{3}$ Many of the spiral ventricular fibres take origin thence, and it is conceivable that the dilatation of the aorta by the undoing of the spiral of these muscle bands may contribute to the production of diastole. ${ }^{*}$

From the arrangement of the musculature of the heart, it is obvious that a great part of the muscle nust be strongly compressed by the contraction of the remainder. The columnie carnese will, for example, be driven like so many elastic cushions against one another. So soon as contraction ceases this will lead to an elastic rebound of the walls. Again, in liastole, the walls of the heart become like those of a flaceid bag, and under the influence of gravity fall apart. Thus, if an excised heart be placed in a vessel of water, and be rhythmically squeezed, the fluid can be pumped out from the aorta and pulmonary orifices, for, after each compression, the heart, in consequence of the elastic rebound and the force of gravity, relaxes. Goltz and Gaule obtained, after sudden compression, a negative pressure in the empty heart. This must have been entirely brought about by the elastic rebound, following on the removal of the compressing agent.

The flow of blood in the coronary arteries may be another cause of diastole. Chauveau and Rebatel ${ }^{5}$ have proved that the blood is driven back towarls the aorta and out of the coronary system during systole, while the current once nore sets into those vessels at the end of systole. It has been demonstrated by Donders ${ }^{6}$ that a high-pressure injection of these arteries in the excised heart produces a negative pressure in the ventricular cavity, and that this pressure by expanding the wall aids in the causation of diastole. In the same way as the distension of a hollow curved and elastic tube opens ont the curvature, so the injection of the coronary arteries dilates the heart. If this be the true cause of the negative pressure, some relationship should be found between its amount and the height of the aortic pressure.

Moens urged that the negative pressure is systolic, and not diastolic in time, and endeavoured to explain its origin by the following experiment: ${ }^{7}$ -

\footnotetext{
${ }^{1}$ Spring, Hém. Acad. roy. de méd. de Belg., Bruxelles, 1861, tome xxxiii. p. 78.

2 v. Frey, "Die Untersuch. des Pulses," Berlin, 1892, S. 94.

3 Mink, Centralbl. f. Physiol., Leipzig u. Wien, 1890, S. 568.

${ }^{4}$ Gaule, ibid., S. 617.

${ }^{5}$ Rehatel, "Rech. expér. sur la circ. dans les art. coronaires," Thèse de Paris, 1872.

6 "Physiol. des Mensch.," 1859, Bd. ii. S. 42.

7 Arch.f. d. ges. Physiol., Bonn, 1879, Bd. xx. S. 529.
} 
He attached a rubber bag to a tube and filled it with water. By a second tube the bag was put in connection with a minimum manometer. On strongly compressing the bag, and driving the water out, a negative pressure equal to $80 \mathrm{~mm}$. Hg appeared. This is due to the momentum of the fluid, which escapes more rapidly than the walls of the bag can follow. If this be so in the heart, it is clear that the negative pressure should also be fomd in the root of the aorta, for the momentum of the blood would carry it on beyond the ventricle. de Jager ${ }^{1}$ has controverted this explanation by recording an aortic pressure of $130 \mathrm{~mm}$. $\mathrm{Hg}$ occurring at the same instant as a pressure of $-36 \mathrm{~mm} . \mathrm{Hg}$ in the left ventricle. Moreover, in all the curves of intraventricular pressure, the negative pressure occurs after the closure of the semilunar valves.

Porter $^{2}$ has investigated this difficult subject by means of his minimum manometer in combination with a Huirthle manometer. The intraventricular cannula was connected by a T-tube to both these instruments. He found that the negative pressure in the left ventricle is sometimes entirely absent: often slight, occasionally great. With the thorax open, in five out of fifteen experiments no negative pressure was observed. In one out of six experiments with the thorax closed there was no sign of a negative pressure. The minimum varied from 0 to $-58 \mathrm{~mm}$. Hg. Sometimes, when the heart was beating well and powerfully, no negative pressure was apparent; while, on the other hand, a considerable negative pressure was often present in the left ventricle without any synchronous sign of negative pressure in the left auricle. So also in the tracing taken by Frederic of the right auricular pressure, there

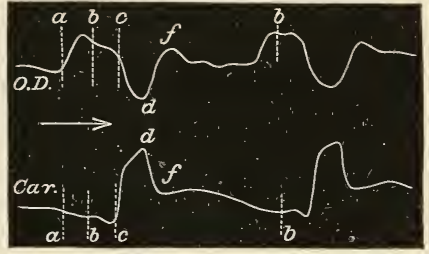

FIG. 18.

$O . D$. right auricle.

Car, carotid pulse.

In the auricular tracing, $a b$ is the auricular sys. tole; $b c$ the thrust outwards of the auriculoventricular valves, or slight regurgitation of ventricular valves, or slight regurgitation of
blood at the commencement of ventricular blood at the commencement of ventricular
systole; cd the negative pressure produced by the contraction of the papillary museles causing the descent of the floor of the auricle, and by the diminution of the volume of the heart in the systole; $d f$ the refilling of the auricle from the veins. The coincident points on the carotid tracing are similarly marked.

At the point $d$, where the downstroke of intraventricular pressure is taking place, the auricular pressure is just beginning to rise.

is no fall below zero synchronous with the period of negative pressure in the right ventricle. ${ }^{3}$

According to Porter, the negative pressure in the ventricle has no appreciable effect on the filling of the heart. He explains this anomaly on the following grounds:-

The ventricular negative pressure may occur so rapidly and last so short a time that it is almost over before the auriculo-ventricular valves can open. The cavity of the closed and relaxing ventricle need become but a few cubic millimetres larger than its contents, in order to produce a great negative pressure, just as the suction of a rery few drops of fluid from the camnula has a great effect on the manometer. If this be so, the entry into the ventricle of the smallest quantity of blood from the auricle, would abolish the nogative pressure. As the auricle stands in open comnection with the reins, so slight is

${ }^{1}$ Arch. f. d. ges. Physiol., Bonn, 1883, Bil. xxx. S. 506.

2 Journ. Physiol., Cambridge and London, 1892, vol, xiii. p. 541.

3 "Trav. du labor. de Liège," 1888, tome ii. p. 116. 
suction of blood would have no perceptible effect on the auricular pressure.

There is some evidence of two phases of negative pressure in the ventricle, one very short in duration, appearing very early in diastole, the other presystolic in time, and lasting longer. This latter has been ascribed by Chauveau and Marey to the aspiration of the thorax, acting on the heart at a time when the tone of the muscle had decreased. The whole subject of the intraventricular negative pressure is still in obseurity ; all that can be said is, that we are certain that a negative pressure usually appears, though we do not know why it is inconstant and variable. We are uncertain whether it has any effect on the filling of the ventricle, and must suppose that its origin is complex, depending, probably, on the elastic rebound of the walls, on the filling of the coronary arteries, and especially on the aspiration of the thorax.

\section{The Heart Sounds.}

The existence of these sounds was not concealed from Harvey. He writes: "When there is the delivery of a quantity of blood from the veins to the arteries a pulse takes place, and can be heard within the chest." 1 To Laennec, ${ }^{2}$ the founder of the art of auscultation, is owing the first accurate description of the character of the sounds and their significance in the diagnosis of diseases of the heart. To the ventricular contraction he ascribed the first sound, and the second sound to the auricular contraction. The second sound he aptly compared to the sound produced by a dog lapping water. Laennec's authority remained unquestioned until Turner ${ }^{3}$ pointed out that the second sound occurred, not at the end but at the beginning of the diastolic pause, and therefore could not derive its origin from the auricular systole. He suggested that the second sound might be caused by the dilatation and suction action of the heart at the end of systole. From then till now the cause of the sounds has been a subject of endless controversy. Dunglison, ${ }^{4}$ in 1856, tabulated the opinions of a large number of authors, showing what an extraordinary amount of discordance has existed upon this matter; nor is there even yet a consensus of opinion on the question. Sandborg, in $1881,{ }^{5}$ collected as many as forty different theories.

That so many theories should have arisen is not surprising, since so many different phenomena attend each contraction of the heart, any one of which may be capable of producing a sound. The mistake has been that each observer, according to his particular theory, has attributed the sounds to one simple factor, and has failed to recognise that the sounds are in reality not pure tones but are compound in origin. Thus the first sound has been ascribed to the following causes:-

1. The impulse of the heart against the parietes of the thorax.

2. The rush of blood through the narrowed orifice of the aorta and pulmonary artery.

3. The contraction of the muscular wall of the ventricles.

1 "De motu Cordis" ch. v.

2 "De l'alscultation médiate," Paris, 1819, tome ii. p. 210.

3 Trans. Med.-Chir. Soc. Edinburgh, 1829, p. 226.

4 "Human Physiology," 1856, vol. i. p. 140.

5 "Résumé des études sur les bruites du cour," Christiania, 1881, p. 6. 
4. The sudden collision of the ventricular contents with the column of blood in the aorta and pulmonary artery.

5 . The sudden tension of the blood caused by a collision of the blood particles in the ventricles.

6. The rub of the heart against the pericardium.

7. The vibration of the chest wall from the sudden rigidity and pressure of the heart.

8. The tension of the auriculo-ventricular valves.

9. The sudden opening and consequent vibration of the semilunar valves.

The first sound.-It was shown by C. J. B. Williams ${ }^{1}$ and a committee of the British Association, that the first sound can be heard in the excised heart, and this, even if the auriculo-ventricular valves be held open with the fingers.

Ludwig and Dogiel ${ }^{2}$ listened to the heart, after tying in succession the venæ cavie, the pulmonary artery and vein, and the aorta. The first sound continued, and seemed to be but slightly altered in character. These experiments entirely overthrow the view that the first sound is of simple valvular origin. The valves ean be held back by hooks or by the finger, and neither the papillary muscles, nor any other suggested mechanism, can throw the valves into tension, yet a systolic sound continues. $^{3}$

Sibson and Broadbent ${ }^{4}$ listened to the exposed heart of the ass and of the dog. They record that the first sound is apparently of equal loudness over the whole ventricle, that it begins and continues with a rumble, and that it ends with an accent or sharp sound, which is coincident with the extreme contraction of the cavity, and with a faint shock felt just then over the ventricle. If the blood be shut off by tying the venæ cavæ, the accent appears to be quite lost and the rumble is materially weakened. The tone of the sound is undoubtedly altered by removing the action of the auricular-ventricular valves, and it has been determined that the sudden throwing into tension of the auriculo-ventricular valves in the dead heart does produce a tone.

The first sound has been by Wintrich ${ }^{5}$ resolved into two tones, one deep and the other high. These are, doubtless, the rumble and the accent described by Sibson. Haycraft ${ }^{6}$ has found that the deeper tone alone persists in the excised heart, while the higher tone can be produced in the dead heart by throwing the auriculo-ventricular valves into tension.

By some authors the first sound has been attributed to the bursting open of the semilunar valves, but any vibration of these valves must be entirely damped down in a fluid which is of almost the same specific gravity as the vaive itself. There is no evidence in favour of the view that these valves are suddenly forced open. On the contrary, it is highly probable that they quietly open at

1 Rep. Brit. Ass. Adv. Sc., London, 1836, p. 269.

2 Ber. d. k. sächs. Gesellsch. d. Wissensch., math.-nat. Cl., Leipzig, 1868, Bd. xx. S. 89.

${ }^{3}$ Halford has denied the occurrence of any sound in the heart after ligation of the venæ eave and pulmonary veins ("The Action and Sounds of the Heart," London, 1860, pp. 25-27).

"Sibson, "Medical Anatomy," 1869, p. 89.

5 Sitzungsb. d. phys.-med. Soc. zu Érlangen, 1875, Bd. vii. S. 51.

${ }^{6}$ Journ. Physiol., Cambridge and Loudon, 1890, vol. xi. p. 486. 
the moment when the intraventricular tension just rises above the aurtic. $^{1}$

Although the impulse of the heart is not the principal cause of the first sound, as was maintained by Magendie, yet it may be an auxiliary and occasional cause. The first sound can be heard when the chest wall is removed, and also when, by filling the pericardium with fluid, the heart

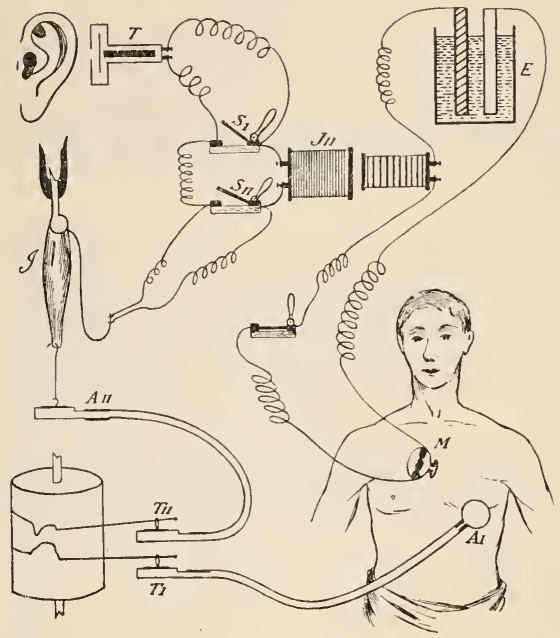
is prevented from striking against the stethoscope. If, however, a board be applied to the exposed heart, the first sound is increased in loudness. In quietude and the supine position the impulse is probably of no account, but under the opposite circumstances the first sound may very considerably increase. According to Chaureau and Faivre, the vibrations causing the sounds of the heart can be felt by a finger placed over the region of the valves. Recently the sounds have been successfully recorded by means of the micro-

FIg. 19.-Diagram of Hürthle's cx periment. $M$, nicrophone; phone. Hürthle in$E$, battery; In, induction coil ; $S^{\prime \prime}, S^{\prime \prime}$, keys; $T$, tele- serted the microphone phone; $g$, frog gastrocuemius; $A^{\prime}$, cardiograph ; $A^{\prime \prime}$, in the primary circuit
myograph ; $T^{\prime \prime}, T^{\prime \prime}$, tambours. of a du Bois Reymond coil, and placed a frog muscle preparation in connection with the secondary circuit. A lever attached to this muscle marked the moment of excitation, when the sound of the heart reached the microphone, and closed the primary circuit. ${ }^{2}$

By Einthoven and Geluk ${ }^{3}$ a similar method was employed, but they registered the effect produced by the sounds on the microphone circuit by means of a capillary electrometer. The movements of the electrometer are photographed on a moving sensitized plate. Holowinski has photographed the sounds by means of the optical telephone invented ly Babinet and Fizeau. ${ }^{4}$

That the sounds are compounded of several tones, is shown by the photographs of Einthoven and Geluk. Each sound gives rise to a succession of vibrations of the mercury meniscus. The first sound was found to show itself earlier when the microphone was applied

${ }^{1}$ R. Quain denies either the valvular or muscular origin of the first sound. He ascribes the sound to the impact of the blood against the resistance in the aortic orifices (Lancet, London, 1897, vol. i. p. 1672 ; Proc. Pivy. Soc. London, 1897, vol. 1xi. p. 331), but there are many reasons for regarding this view as an impossible one.

${ }^{2}$ Arch. f. d. ges. Physiol., Bonn, 1895, Bd. Ix. S. 263.

3 Ibid., 1894 , Bd. lvii. S. 617.

${ }^{4}$ Holowinski, Arch. de physiol. norm. et path., Paris, 1896, tome xxviii. p. 893. 
to the apex, than when it was applied over the region of the arterial orifices.

It is concluded that the first somnd is formed of many component tones, and is caused by the sudden tension and vibration of the cardiac muscle, of the auriculo-ventricular valves, and of the blood, and that the sound may be augmented by the stroke of the heart beating against the parietes of the chest. The vibrations which are seen on the platean of the curve of the intraventricular pressure probably appear as sound vibrations in the curves of the capillary elcetrometer, such as are obtained by the microphonic method of Einthoven.

The second sound.-Carswell and Rouanet ${ }^{1}$ were the first to assert that the second sound of the heart was dependent upon the return shock of the blood against the semilunar valves, when, at the end of systole, the intraventricular pressure falls rapidly below zero and the elastic reaction of the arteries comes into play.

A sound is produced when, in the excised aorta, the semilumar valves are suddenly thrown into tension, and this sound is similar in character to the second sound of the heart. The most satisfactory method of obtaining this result is, after ligaturing all other orifices and placing the

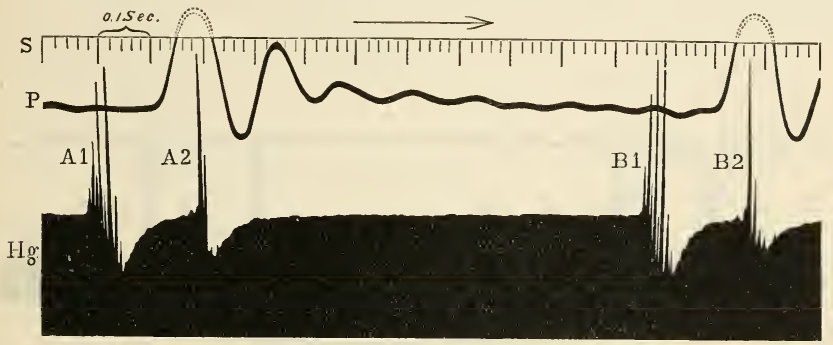

Fit. 20. - Tracing of pulse (P), first $\left(A^{1}, B^{1}\right)$ and second $\left(A^{2}, B^{2}\right)$ sounds of the heart.-Einthoven and Geluk.

aorta and valves in a vessel of water, to connect one coronary artery with a pressure bottle. On suddenly opening the tap interposed between the coronary artery and the pressure bottle, the valves are thrown into tension and a sound is heard. A tube connected with the vessel of water is used as a stethoscope.

To C. J. B. Williams ${ }^{2}$ we owe the first and conclusive experiments on the living animal. He noticed that the second sound was suspended on applying pressure over the region of the orifice of the aorta and pulmonary artery, and also when, by laying open the auricles, the heart became emptied of blood. In both cases the first sound continued. Williams further passed an ordinary dissecting-hook into the pulmonary artery, and caught up and hooked back one of the semilumar flaps. The second sound was then changed in character. He next passed a shoemaker's curved awl into the aorta, and hooked back an aortic Hap. The sound now gave way to a hissing noise. On withdrawing the hook and awl, the second sound of the heart was again heard, and the hissing ceased. For the purpose of these experiments he used asses, supplied

1 "Analyse des bruits du cour," Paris, 1832.

2 Rep. Brit. Ass. Adv. Sc., London, 1836, 1. 269. 
them with artificial respiration, and placed them under curari. The committee of the British Association further record, in their investigations on the causes of the heart sounds, that the second sound disappears when the blood is allowed at each systole to escape-not into the arteries, but through openings made in the ventricles.

The semilunar valves close at that moment when the aortic tension rises the slightest degree higher than the intraventricular pressure; the closure will therefore be effected gently, and will lead to no sound. In the next moment of time the intraventricular pressure rapidly falls below zero, and the valves will be thrown into tension by the back swing of the blood, which is in its turn occasioned by the elastic rebound of the arteries. The question has been raised as to whether it is the vibration of the valves, or the vibration of the column of blood in the arteries, which produces the second sound. Talma ${ }^{1}$ experimented with long glass tubes: to the end of each of these he tied the root of the pulmonary artery with its valves. The tubes after being filled with water were held upright. If, now, the valves were pushed upward with the finger, and then suddenly let go, a sound was heard. If, by varying the height of the Huid columns, the tones in any two tubes were made similar, then, on further varying the height of the column of Huid

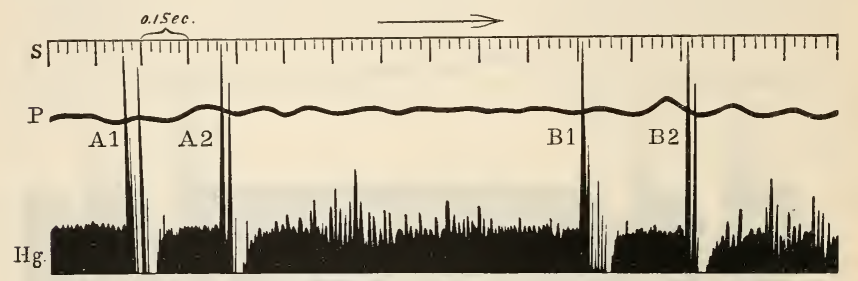

Fig. 21. - Tracing of sounds of heart from a case of aortic regurgitation.-Einthoven and Geluk.

in one tube, the tone was found to vary relatively to the height. On these grounds, Talma attributed the sound to the vibration of the column of fluid. These experiments were repeated by Webster, ${ }^{2}$ who, in place of the pulmonary valves, employed bladders. He found that if the height of the column of blood were kept constant, the tone varied with the thickness of the bladder. Thus the sound consists of tones produced by the vibration of the membrane, and of others dependent on the vibration of the column of fluid.

The photographic method of Einthoven makes apparent the exact time relations of the second sound (see Fig. 20).

It is highly interesting to note the change in the curve of the second sound, resultant on the valves being rendered incompetent. The vibrations are seen to be much more numerous, and to last over a far longer period. In Fig. 21 we see, for the first time recorded by the graphic method, those vibrations which produce a regurgitant murmur. The conclusion reached in regard to the second sound is therefore that it is not simple in origin, but is a sound compounded of two or more tones. These constituent tones arise from vibrations

${ }^{1}$ Arch. f. d. ges. Physiol., Bonn, 1880, Bd. xxiii. S. 275.

2 Journ. Physiol., Cambridge and London, 1882, vol. iii. p. 294. 
of the valves, thrown after closure into sudden tension, from the vibrations of the blood columns in the multitudinous branches of the arteries, and from vibrations of the arterial walls. Although both the first and second sounds are double in origin, yet the constituent somuls are normally so far synchronous as not to be separable by the ear.

Hæmic murmurs.-Experimenting on the flow of fluids through tubes of glass, thin rubber, and intestines and arteries of animals, Nicolls found that even with velocities much greater than that of the blood-flow, it is impossible to produce sounds in a moving liquid, so long as it is free from air, no matter what dilatations or contractions occur in the pipes. Hence he concludes that hæmic murmurs, such as are heard over the pulmonary artery in cases of oligocythæmia, are produced like vocal sounds, by the vibrations of elastic substances. Likewise the sounds of the heart are produced in all eases by membranous vibration, and not by fluid friction, as the velocity is too small to produce audible sounds by this means. ${ }^{1}$

\section{The Cardiac Impulse.}

Synchronously with the systole of the ventricles, the chest wall receives a blow from the heart, at the point where the mass of the ventricle comes in contact with the parietes.

The position of the impulse varies considerably with the position of the body. Although it is generally stated that the cardiac impulse is normally visible in the fifth left intercostal space, 2 in. below the nipple, and $1 \frac{1}{2}$ in. to its stemal side, yet in 67 per cent. of men it is stated to be visible in the fourth intercostal space - that is to say, when the body is in the supine position. $^{2}$ The impulse is felt slightly lower in men than in women, and in the old than in the young. By

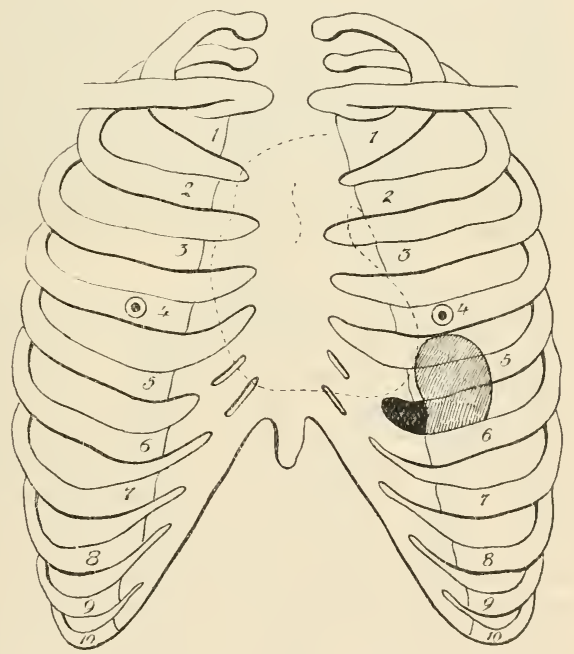

FIs, 22.-Areas of cardiac impulsc, when supine (black), and when lying on the left side (Jightly shaded). - Sequeira. Ransome ${ }^{3}$ it was found that the seat of the impulse varied much more when the body was rolled from one side to the other, than when the body was raised from the horizontal to the vertical position. In rising from the supine to

1 Nicolls, Joum. Physiol., Canıbridge and London, 1896, vol. xx. pl). 413 and 426.

2 Mariannini and Namias, Arch. ital. de biol., Turin, 1883, vol. iv. p. 143.

${ }^{3}$ Journ. Anat, and Physiol., London, 1895, vol. ix. 1'. 137.

VOL. II. -3 
the standing position, the apex beat shifts downwards for about $\frac{1}{4}$ to 1 in., and by the same amount to the left.

When a man rolls over on to his right side, the seat of the impulse moves to the right, and may completely disappear beneath the sternum. It has been observed to shift $3 \frac{3}{4}$ in. from left to right. Similarly, when a man rolls over on to his left side, the impulse is found considerably to the left side of the nipple line (see Fig. 22).

This shifting of the impulse is due to the effect of gravity on the heart within the pericardium. On passing neelles through a certain given spot in the thoracic wall into the heart, with the body placed in the three positions-supine, on the right, and on the left side-the needles are found to pierce the heart at different places. In each position a different part of the heart, owing to the weight of the organ, is brought into close contact with the chest wall. The heart rotates in the pericardium. This membrane is too strong to allow of much lateral displacement of the whole heart.

If the finger be pressed up against the apex, it is shot down by the systole. If the whole heart be grasped by the hand, the fingers sink into the flaccid walls during diastole, but are driven asunder with great force by systole. Wherever the heart is touched it appears to drive the finger outward, and yet the heart diminishes in two if not in all its diameters.

The origin of the impulse has been attributed to many factors-(1) That the curvature of the aorta is straightened out by the blood shot therein. Since the heart is attached to the aorta, the apex is forced to describe an are of a circle and impinge against the chest wall. (2) That the diastolic filling of the left auricle, which lies between the spine and the base of the heart, pushes the heart forward. ${ }^{1}$ Haller experimentally showed that injection of the left auricle caused the heart to "approach the mamma with vivacity." (3) That the systolic out-thrust of the auriculo-ventricular valves raises the pressure in the left auricle, and thus drives the heart forward.

A more reasonable suggestion, and one put forward among others by Harvey, is (4) that the heart erects itself from the collapsed position, which it assumes in diastole. A fulcrum for this movenent is afforded by the base resting on the pericardium (Ludwig). ${ }^{2}$ In consequence of this erection, the heart strikes the chest wall. This explanation is sufficient for the conditions which exist when the body is supine or vertical, but it is scarcely satisfactory when the body is prone, for then the heart is in apposition with the thoracic wall, and the impulse is most diffuse and intense. (5) The recoil of the heart, due to the expulsion of the blood into the aorta, and the straightening out of the spiral figure formed by the aorta and pulmonary artery, have respectively served their turn as an explanation of the cardiac impulse. ${ }^{3}$ These suggestions are at once negatived by the fact that the output of blood from the ventricle occurs later than the commencement of the impulse. (6) During the period of rising tension within the ventricle, the blood presses upon every point of the internal surface with a force equal to that by which it is itsclf compressed. The pressure of the blood reacts thus on the wall, for the heart contracts against the

1 Senae, "Traité de la structure du eœur," Paris, 1749, p. 356.

2 Ludwig, "Lehrbueh d. Phys," Leipzig, 1861, Bd. ii. S. 86.

${ }^{3}$ Skoda, "Abhandl. ueber Pereussion und Auscultation," Wien, 1842, S. 147 ; liornitzer, Sitzungsb. d. k. Akad. d. Wissensch., math.-naturw. Cl., Wien, 1857, Bil. xxiv. S. 120. 
fluid, the exit of which is hindered by the aortic pressure. If the blood be shut off from the heart, then a finger placed on the ventricle is forced out on systole to a far less extent than before. An outward thrust is still perceptible, however, and this must be so, for muscle layers compress other muscle layers. Thus the columne carnere and papillary muscles are forced together. In this case, it is the elastic resilience of the compressed muscle which produces the impulse.

The points to be remembered in regard to the origin of the impulse are these-

1. The impulse is synchronous with the commencement of the period of rising intraventricular tension.

2. The inpulse occurs where the ventricular wall touches the parietes of the chest.

3. By no locomotion of the whole heart can its origin be entirely explained, for it persists after the suppression of the auricular contraction, and of the recoil due to the output.

4. The primary factor is the hardening of the muscular mass, the secondary factor the erection of the ventricles upon the base of the heart.

5. The impulse is felt at a different spot in each position of the body, owing to the influence of gravity acting on the heart.

Cardiographs. - The impulse of the heart can be recorded by various forms of apparatus. In animals a receiving tambour has been inserted between the chest wall and the heart, and placed in connection with a recording tambour. ${ }^{1}$ In obtaining curves from man, the receiving instrument has necessarily to be applied to the external wall of the thorax over the seat of impulse. Probably the best form of cardiograph is that of Edgren, which is a modification of Marey's instrument. ${ }^{2}$

In this instrument the receiving tambour (c) is placed within an outer rigid cham ber (AB), which is fastened to the thorax by elastic bands $(f g)$, which encircle the chest. The pressure of the button (K) of the tambour on the seat of impulse can be varied by a toothed wheel and $\operatorname{rod}(h)$, while small spiral springs

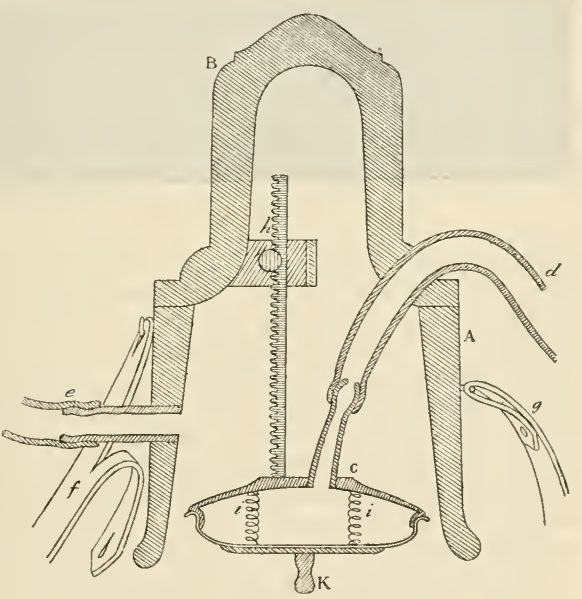

Fig. 23.-Edgren's cardiograph. (i) placed between the roof of the tambour and the rubber membrane give to the latter the requisite amount of tension. The tube $(d)$ passes to the recording instrument, and the tube $(e)$ enables the observer to listen to the sounds of the heart, at the same time that the impulse is recorded. By this apparatus the movements of the chest wall are excluded, for the whole apparatus moves with the thorax. Only the cardiac impulse affects

${ }^{1}$ Chaureau and Marey, Mém. Acad, de méd., Paris, 1863, tome xxvi. p. 284.

${ }^{2}$ Skandin. Arch.f. Physiol., Leilyig, 1889-1891, Bd. i. S. 71. 
the tambour, and the instrument, therefore, yields the same form of curve as does a tambour placed between the heart and the chest wall in an animal.

The curve of the impulse, or cardiogram, taken when the heart is beating normally, is entirely different from a cardiogram taken when the vessels are ligatured and the heart bloodless. ${ }^{1}$

The cardiogram is not, therefore, a true muscle curve, but is a combined curve of pressure and volume. The form of the curve is, moreover, endlessly varied by the application of the receiving tambour to different parts of the heart, and by alteration of the pressure with which the instrument is applied. Thus the instrument is not by itself a perfectly sure or trustworthy guide, and the main physiological interest which it possesses is that, by its means, certain time relations in the cardiac eycle can be decided in man. By simultaneous record of the impulse curve, the time of the sounds of the heart, and the carotid or radial pulse, interesting results can be arrived at, such, for example, as the period of rising intraventricular tension.

The occurrence of the sounds of the heart can be marked below the impulse curve by means of an electric signal worked by the hand of the observer who is charged with the duty of auscultating the heart. The reaction time of a practised experimenter for the making of signals in answer to sounds is known to be from 0.15 to 0.20 seconds. With practice the reaction time for regular periodic excitations is

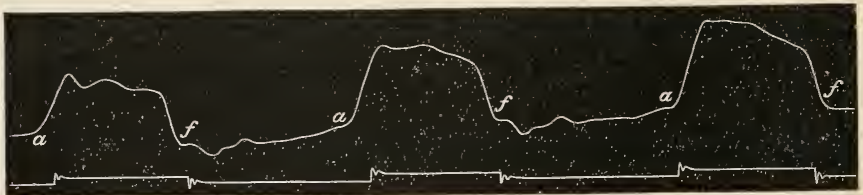

Frg. 24.-Impulse curve and somnds of the heart. - Edgren.

found to become very constant, and the error due to variations in this may only be $0 \cdot 01$ sec. As the constancy of the reaction time depends on the regular periodicity of the excitation, this method of marking the time of the heart sounds can only be employed on healthy hearts beating with a regular rhythm. In such cases the constancy of the period of each pulse varies by no more than 0.03 to 0.04 sec. The total error of observation is therefore equal to about 0.04 sec. With the use of Edgren's apparatus the observer can discard the method of signalling, and by watching the tracing of the impulse can see, at the same time as he listens to the heart, the points on the curve at which the sounds are heard. Since the heart sounds have been successfully recorded by the combined use of the microphone and the capillary electrometer, it will be possible in the future to improve on the above method, and simultaneously to obtain curves of the impulse and the sounds of the heart.

Marey, ${ }^{2}$ Edgren, ${ }^{3}$ and others find that the second sound of the heart corresponds in time with a small nick or elevation at the

1 v. Frey, "Untersuch, des Pulses," S. 112.

2 "La circulation du sang," Paris, 1881, p. 127. Cf. Fredericq, "Trav. du labor. de Liege," 1888 , tome ii. \%. 84 .

${ }^{3}$ Skundin. Arch. f. Ph.ysiol., Leipzig, 1889-91, Bd. i. S. 88. 
bottom of the descending limb of the impulse curve, marked $f$ in Edgren's tracing.

By Hilbert ${ }^{1}$ the second sound is placed earlier-at the beginning of the descending limb of the curve.

The second sound does not mark the exact moment of closure of the semilunar valves, but the time at which these valves are thrown into tension by the back swing of the blood. Syuchronous or differential curves of intraventricular and aortic pressure show that the valves shut earlier than the occurrence of the second sound, and without noise, at the moment when the aortic pressure rises to a point higher than the intraventricular pressure.

Another method of obtaining the moment of closure of the semilunar valves on the impulse curve is as follows:-

The cardiac impulse and carotid or radial pulse curves are sinultaneously recorded. The time relation of thebottoin of the dicrotic notch is then marked upon the impulse curve. The time lost in the

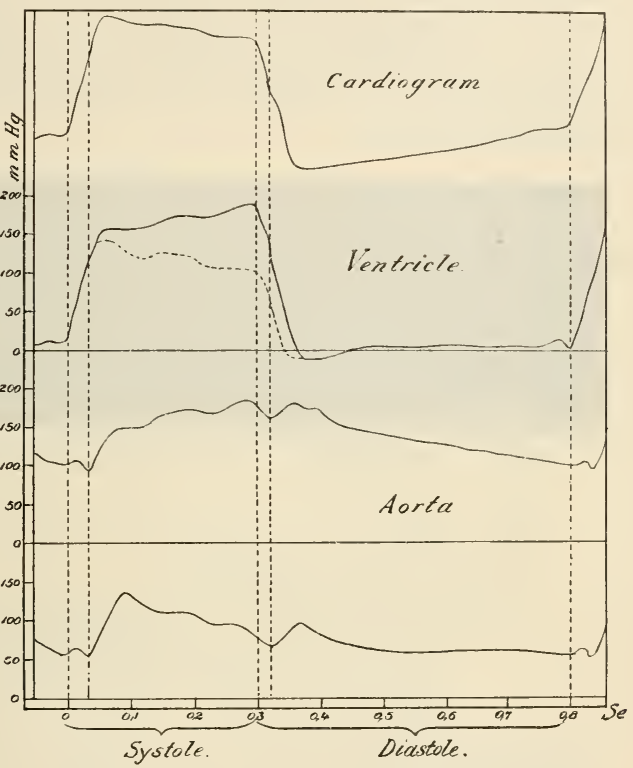

FIG. 25.-Both high and low aortic pressure curves are shown.-Hiirthle.

transmission of the pulse-wave from the heart to the artery where the pulse is recorded must be first deducted. By the observations on intraventricular and aortic pressure, curves it has been abundantly proved that the dicrotic notch occurs immediately on the closure of the valves, and synchronously with the commencement of the downstroke of the intraventricular pressure curve. Fredericq ${ }^{2}$ has thus found that the dicrotic notch corresponds in time with the beginning of the downstroke of the impulse curve, and he believes that the nick, which occurs synchronously with the second sound at the bottom of the descending limb of the cardiogram, is caused by the inflow of blood into the ventricle at the commencement of diastole. The waves on the top of the plateau of the carliogram have no doubt the same

${ }^{1}$ Ztschr. f. Klin. Med., Berlin, 1591, Suppl. Heft, Bd. xix. S. 158. Cf. Bramwell and Murray, Brit. Med. Joum., London, 1888, vol. i. p. 10; Martius, Ztschr. f. Klin. Med., Berlin, 1891, Brl. xix. S. 108.

2 C'entralbl. f. Physiol., Leipzig u. Wien, 1892, Bd. vi. S. 257. 
origin as the waves seen on the plateau of the intraventricular pressure curve.

The moment when the semilumar valves open can be marked on the impulse curve by synchronously recording the carotid pulse, and deducting the time spent in the transmission of the pulse wave. The commencement of the upstroke of the pulse curve after the necessary deduction marks the beginning of the systolic output when the valves open (see Fig. 25). ${ }^{1}$ By Edgren ${ }^{2}$ and Martius ${ }^{3}$ this moment is placed at the top of the ascent of the impulse curve, where the plateau commences. By signalling the position of the first sound on the impulse curve, the moment of closure of the auriculo-ventricular valves is obtained with sufficient accuracy. All these points are marked in the accompanying figure-

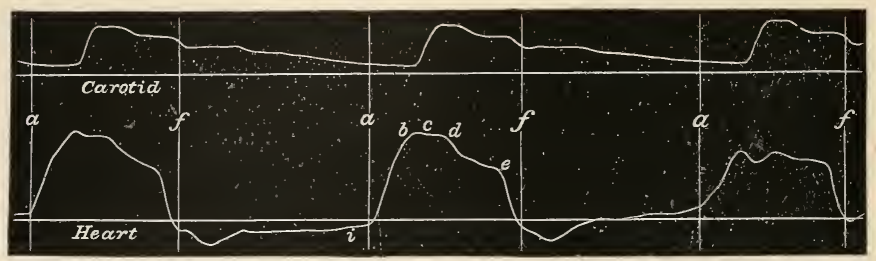

Fig. 26. - Simultaneous records of carotid pulse and heart inıpulse. --Edgren.

$i$, the auricular systole.

$a$, the commencement of ventricular systole, the time of occurrence of the first sound.

$b$, the opening of the semilumar valves.

$i, d$, waves on the top of the plateau.

$e$, the closure of the semilunar valves and commencement of diastole.

$f$, the tension of the semilumar valves and the occurrence of the second sound. From these points the following time periods ean be arrived at in man :$a-b$, The time of rising tension in the ventricle; $a-e$, the period of ventricular contraction; $b-e$, the period of ontput of blood; $a-f$, the time between the commencement of the two heart sounds; $e-a$, the period of diastole of the ventricles.

Beyond these data, it must be remembered that the impulse curve tells nothing of value, for the form of the curve lears no accurate relation to the contraction of the heart.

In a man with a pulse frequency of 70 , Edgren determined the duration of systole to be 0.379 sec., of diastole 0.483 sec. The relation between the duration of the systole and the frequency is shown in the following table:-

Pulse Frequeney.
$74-94$
$63-124$
$55-65$
$55-113$
$47-128$

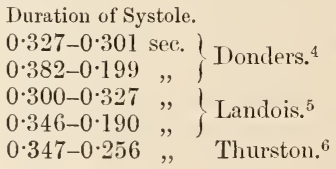

${ }^{1}$ Hiurthle, Arch. f. d. ges. Physiol., Bonn, 1891, Bd. xlix. plate viii. fig. $a$.

2 Skandin. Arch. $f$. Physiol., Leipzig, 1889 , Bd. i. S. 127.

${ }^{3}$ Ztschr. f. klin. Med., Berlin, 1888, Bd, xiii. S. 346.

+ Dublin Journ. Med. Sc., 1868, p. 225.

5 . Die Lelıre vom Arterienpuls," S. 307.

6. Journ. Anat. and Physiol., London, 1876, p. 499 ; cf. on the time relations of the eardiac novements in man, Gibson, Ilid., 1879, yol. xiv. p. 234. 
If the pulse frequency vary in the proportion $100: 270$, the duration of systole varies in the proportion 136:100. In other words, when the frequency increases, the diastolic period of rest shortens far more than the systolic period.

The following observations on animals confirm this conclusion :-

Normal duration, $0.149: 0.373$ see.

, , $0.253: 0.299$, $\begin{array}{ccc}\text { On excitation of vagus, } & \text { Systole. } & \text { Diastole. } \\ \text {, } & 0.158: & 0.635 \mathrm{sec}^{1} \\ & \end{array}$

By Roy and Adami an instrument has been constructed by which the actual contraction of the mammalian heart can be investigated. ${ }^{3}$

In their myocardiograph, one end of the $\operatorname{rod}(a)$, as is shown in the figure, can, by means of gimbals, freely oscillate from $b$. The other end is fastened to a chosen spot on the ventricle. On this rod is fixed a small pulley, over which there passes a silk thread. The one end of this thread is attached by a hook to some other part of the heart, while the other end is connected with a writing lever. By the arrangement shown, the shortening between any two points on the external surface of the heart can be recorded; and, further, by passing the hook through an auricle and affixing it to a flap of an auriculo-ventricular valve, the movements of the valve and the pull of the papillary muscles ean be studied. For the ventricular end of the $\operatorname{rod}(\alpha)$, by which the pulley is carried, remains a fixed point, while the hook attached to the thread is in systole approximated to, in diastole driven from, this point. A myographic curve of the ventricle thus obtained can be compared with intra-

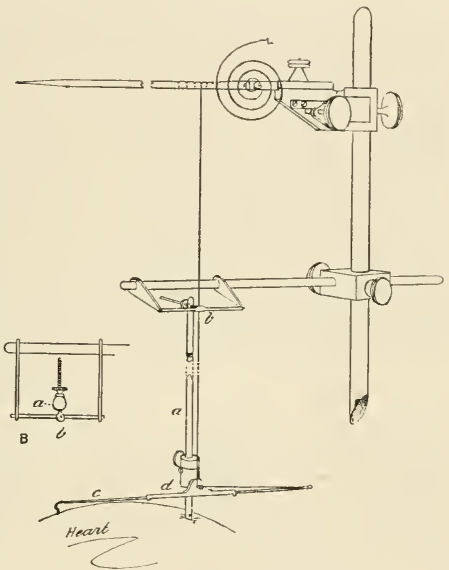
ventricular pressure curves or aortic Fis. 27. - Myocardiograph. a, connecting rod; curves recorded synchronously. ${ }^{4}$

The cessation of the output is placed by these authors at the moment when the ventricle has $b$, frame with guiding hole for thread; $c$, hooked needle moving with heart muscle; $d$, guide for needle ; B, frame viewed from above.-Roy and Adanii.

reached its maximum of contraction, aud according to them the ventricle remains contracted for a certain interval after the cessation of the output. They further consider that the contraction of the papillary muscles pulls down the auriculo-ventricular valves, and, by thus increasing the intraven- tricular tension, causes a temporary arrest in the myocarliographic curve. They find that the apex of the aortic pulse curve corresponds with this moment, and therefore this apex is by Roy and Adami ascribed to the rise of intraventricular tension, produced by the pull of the papillary muscles. The ventricular wall and the papillary muscles remain in contraction for a period which corresponds to the plateau stage in the curve of intraventricular pressure. Reasons have, however, already been given for regarding many of these deductions as incorrect.

${ }^{1}$ v. Frey, "Die Untersuch. des Pulses," Berlin, 1892, S. 86.

2 Baxter, Areh. $f$. Physiol., Leipzig, 1878, s. 132.

${ }^{3}$ Roy and Adani, Phil. 'Trans., London, 1592, vol. clxxxiii. B, 1. $20 \%$.

+ Roy and Adami, Iractitioner, London, 1890, vol. xliv. 1. 414. 


\section{The Pressure and Work of the Heart.}

The average mean arterial tension in the brachial artery of a healthy young man is 11 to $12 \mathrm{cms}$. $\mathrm{Hg}$, and it is probably not much higher in the aortá. If the intraventricular pressure be taken as equal to $13 \mathrm{cms}$. Hg, the pressure exerted by every square centimetre of the inner wall of the left ventricle can then be reckoned thus-

$$
P=13 \text { ems. } \times 13.5 \text { (sp. gr. of } H g \text { ) }=175.5 \text { grms. per sq. cm. }
$$

It is obvious that, other things remaining equal, the greater the superficial area of the inner wall, the greater will be the total stress on the ventricle.

The positive tension of a membrane enclosing a fluid under any given pressure is not a fixed quantity, but, as the radius of curvature at any part of the membrane is greater, so is the tension greater. On account of the larger size of the filled intraventricular cavity, and necessarily greater radii of the curvature of its walls, the distended heart must make a far greater effort at contraction, in order, by means of the tension of its walls, to raise the contained blood to the pressure of that in the aorta. The thinner the heart wall, the fewer the muscle fibres in a given width of its transverse section, the more will each fibre have to exert itself to bring about a given tension. When the exertion of each individual fibre is taken as constant, the fluid pressure per unit area must vary inversely as the cube of the radius of curvature, or, if the pressure be regarded as constant, the exertion. of each fibre must vary as the cube of the radius of curvature. If the cardiac chamber be considered a sphere with a wall of uniform substance and elasticity, ${ }^{1}$ then $P=\frac{2 t e}{r}$ approximately, where $P=$ the blood pressure sustained by each unit of surface of the cardiac wall; $t=$ the muscular tension per unit of section; $e=$ the thickness of the cardiac wall at any time; and $r=$ the radius of the cardiac chamber at the time. Now $e$, when the wall is thin, varies as $\frac{1}{r^{2}}$, therefore $P$ varies as $\frac{1}{r^{3}}$.

For example, if the auricle contract and reduce its diameter by one-half, it would be able to increase the intra-auricular pressure eightfold, supposing the force of contraction of its constituent muscle fibres remained constant. Samways ${ }^{2}$ suggests that an auricle, towards the latter part of its contraction, might thus have the advantage over a full ventricle, and continue in certain cases to force blood therein, even after the systole of the ventricle had begun.

On the other hand, if the auricle or ventricle be suddenly enlarged to twice its diameter; the muscle fibres must be exerted eight times as much as before, to produce the same pressure. It is perhaps on account of this relation between the radius of curvature, and the work of the muscle, that the thickness of the musculature of the ventricle varies more or less directly as the radius of curvature at any point. The columnæe carnee and papillary muscles, by acting as stays, also compensate for the disadvantage attending the distension of the cavity at the beginning of systole. At the end of systole, when the radii of

${ }^{1}$ The application of this formula to the heart ean be only approximately correct, since this condition is only partially fulfilled.

2 "Le role de l'oreillette gauche," Paris, 1896, p. 36. 
curvature of the ventricle have become less, the action of these muscles is no longer needed. ${ }^{1}$

The total stress in a dilated heart will be far greater than in a contracted heart. It is for this reason advantageous that the diastolic pressure of the heart be, by the mechanism of the veins, kept very low and fairly constant. If, during diastole, the heart be too forcibly distended, it may in this distended state be quite unable to contract. This condition is seen in asphyxia and in chloroform poisoning. It can readily be produced by pushing chloroform to excess, and by following this with forcible compression of the abdomen with the hand, so that the abdominal veins empty into the right heart. On relieving the distension by letting blood, the right ventricle will begin to beat once more.

It is found on examination that the heart when dilated does not empty; the residual blood is seen to increase on vagal inhibition, on compression of the aorta, on abdominal compression, or on injection of fluid into the veins. ${ }^{2}$

The force of the heart is increased by flushing the coronary arteries with blood, and improving the nutrition. If a maximal manometer be placed in the right ventricle, and the pulmonary artery be temporarily occluded, the maximal tension exerted by the wall of the ventricular cavity can then be estimated. If, after making such an observation, the aorta be temporarily occluded so as to increase the blood supply of the coronary arteries, and the maximal pressure in the right ventricle be again estimated, it will be found to be higher. Thus a rise of aortic pressure may increase the force of the heart, and this may even more than counterbalance the increase of work demanded. ${ }^{3}$

Tension also seems to increase the power of muscle, and thus a physiological advantage may compensate for a physical disadvantage. The effect of tension is seen in the case of the arteries. The arteries react to tension with a force that is greater than the force that distends them. Similarly, the power of the isometric muscle preparation when contracting against the pull of a steel spring is greater than that of a muscle which is after-weighted, and not in a state of tension at the moment of excitation. Likewise, the apex preparation of a frog's heart can be made to beat by raising the intracardiac tension; the inhibitory power of the vagus can be diminished by raising the tension of the blood within the chambers of the heart; the beat of the suail's heart can often be arrested by merely letting the blood escape from within it.

In the case of the skeletal muscles, Maggiore ${ }^{4}$ has shown that there is a definite weight and rate of contraction essential to the execution of the greatest amount of work in a given time. Either increasing the weight or the rate of contraction lessens the total output of work, and hastens fatigue. With a given light weight, the abductor indicis can be made to contract and raise this weight once a second for two and a half hours without any sign of fatigue. On increasing the rate of contraction, or the weight to be lifted, fatigue rapidly super-

1 Woods, Journ. Anat. and Physiol., London, 1891-2, vol. xxvi. p. 362 ; Samways, Brit. Med. Journ., London, 1897, vol. i. p. 703.

${ }^{2}$ Roy and Adami, Phil. Trans., London, 1892, vol. clxxxiii. B, I. 210.

3 Ibid., p. 269.

${ }^{4}$ Arch. f. Physiol., Leipzig, 1890, s. 191. 
venes. Thus, there is no extraordinary difference between the heart and the skeletal muscle. A skeletal muscle, like the heart, can rhythmically perform work once a second, without fatigue, for a long period of time. The heart, like the skeletal muscle, executes most work with a certain weight and at a certain rate. Increase of either beyond a certain point hastens the fatigue and lessens the power of the heart.

More than 150 years ago, Stephen Hales ${ }^{1}$ attempted to measure the pressure and work of the heart. He injected nielted beeswax into the left ventricle and obtained a cast of the cavity. This cast he covered over with small pieces of paper, pinning the pieces on, in exact apposition to each other. He then removed the pieces of paper, and, placing them once more in apposition on a flat surface, measured the superficial area of the cavity of the ventricle. From this measurement he calculated the output of the ventricle, and, having experimentally obtained the height of the normal arterial pressure, he drew conclusions as to the power and work of the heart. Ingenious as this method is, there are many fallacies. It is impossible to obtain from the dead heart the diastolic capacity of the living heart; moreover, the ventricle never completely empties itself in systole.

The encryy of the circulation.-The energy of the circulation can be reckoned in the following way by the use of hydrodynamic formulie.

The work done by a force is proportional to the force, and to the distance through which it moves its point of application. The energy of a body is its capacity for doing work, measured by the work to which it is equivalent. ${ }^{2}$

1. Let $Q$ be the systolic output of the heart in cubic centimetres, $v$ the mean velocity of the issuing stream, $t$ the time of escape, $A$ the area of the arrtic orifice. Let $A^{\prime}$ be the area of the ascending aorta, $u$ the mean velocity of the blood in it, $t^{\prime}$ the time of a complete cardiac cycle-

Then $A v t=A^{\prime} u t^{\prime}=Q$.

That is to say, the output of the left ventricle can be reckoned, if the mean velocity of the blood-flow in the ascending aorta be found by experiment, and if the area of the ascending aorta and the time of a cardiac cycle be known.

The following example is given by Nicolls. ${ }^{3}$ Assuming the following data, namely, the time of the cardiac cycle, $0.8 \mathrm{sec}$; the time of the systolic output, 0.1 sec.; the diameter of the aortic orifice, $2 r=2.5 \mathrm{cms}$.; of the aorta, $2 r^{\prime}=2 \cdot 8 \mathrm{cms}$; the mean velocity in the aorta, $u=32 \mathrm{cms}$.-

$$
\begin{aligned}
A v t=A^{\prime} u t^{\prime} & =Q \\
\pi r^{2} \times v \times 1 & =\pi\left(r^{\prime}\right)^{2} \times u \times 0 \cdot 8 \\
\pi_{2}^{1}(2 \cdot 5)^{2} \times v \times 1 & =(2 \cdot 8)^{2} \times u \times 0.8 \\
25^{2} v & =28^{2} \times 8 u \\
625 v & =6272 u \\
v & =10 u=320 \mathrm{cms} . \text { per sec. }
\end{aligned}
$$

1 "Statical Essays," 1733. vol. i.

2 Car. Gri. Sec. Uxits.

Dyne.-The force which, acting upon $1 \mathrm{grm}$., produces per sec. velocity of $1 \mathrm{~cm}$. per sec.

Ery.-The work done by a dyne when it has moved its point of application through $1 \mathrm{em}$.

Density.-Grm. jer c.c.

Velocity. - Cns. per sec.

Accelerution. - Cns. per sec. per sec.

Pressure.-Dyne per sq. cm.

3 Journ. Physiol., Cambridge and London, 1896, vol. xx. 
the result arrived at being that the velocity of output is ten times the mean velocity in the aorta. To obtain the output of the left ventricle-

$$
\begin{aligned}
Q & =A v t \\
& =\pi r^{2} \times 320 \times 0 \cdot 1 \\
& =5 \times 320 \times 0.1 \\
& =160 \text { c.c. }{ }^{1}
\end{aligned}
$$

The diameter of the pulmonary orifice being reckoned as $3 \mathrm{cms}$., and the output of the right ventricle being the same as the left, the velocity of output at the pulmonary orifice will be in inverse proportion to its sectional area as compared with that of the aortic orifice.

$(3)^{2}:(2 \cdot 5)^{2}:: 320: 222 \mathrm{cms}$. per sec. (velocity of output at pulmonary orifice).

The kinetic energy of the velocity of output is largely changed into potential energy in the aorta and pulnowary artery. During diastole the potential energy stored up by the distension of the elastic arterial walls becomes again kinetic. It is spent in maintaining the velocity of flow, and in overcoming the viscosity of the blood in the arterioles and capillaries.

To estimate the work of the heart, the mean pressure and relocity in the aorta, and the volume of the systolic output must be obtained.

If $W$ be the work done during systole of the left ventricle in gramme centimetres; $Q$, the volume of the output in cubic centimetres; $M$, the mass of the output in grammes; $P$, the specific gravity of blood $(. \cdot M=P Q) ; V$, the mean velocity in the aorta; $H$, the mean aortic pressure in grammes per centimetre; $g$, the acceleration due to gravity $=981 \mathrm{cms}$. per second;

$$
\text { Then, } \begin{array}{r}
W=Q H+\frac{M V^{2}}{2 g} \\
\therefore W=Q H+\frac{P Q V^{2}}{2 g}
\end{array}
$$

Now, the mean aortic pressure may be taken to be $12 \mathrm{cms}$. of mercury (sp. gr. of mercury=13.5). The volume of the systolic output has been reckoned above as 160 c.c.; but experimental determinations of the output in animals lead to the conclusion that the output in man does not much exceed 100 c.c. (see p. 49). For the purposes of this calculation a mean value of 110 c.c. may be taken as the output. Substituting these data in the above equation, one obtains-

$$
W^{*}=110 \times 12 \times 13.5+\frac{1.05 \times 110 \times 32^{2}}{2 \times 981}=17,880 \text { gramme-centimetres. }
$$

It is obvious that the work spent in maintaining the aortic velocity is almost negligeable (1 : 300 nearly) compared with that spent in overcoming resistance.

If in the case of the right ventricle the mean pressure in the pulmonary artery be taken to be $4 \mathrm{cms}$. of mercury, the work of that ventricle will be one-third of that of the left ventricle.

Thus the total work of each systole of the heart will be-

$$
17,880 \times \frac{4}{3}=23,640 \text { gramme-centimetres, }
$$

and the total work of the heart per diem about 24,000 kilogramme-

1 This is, however, undoubtedly too ligh an estimate (see 1). 49). 
metres (or 1000 kilogramme-metres per hour). ${ }^{1}$ The day's work of the heart is therefore equivalent to $56 \cdot 6$ kilo-calories, or about one-fiftieth of the total amount of heat produced in the body, in addition to which, it must be remembered that there is a constant production of energy in the form of heat in the heart muscle, as in other muscle, as a result of its metabolic changes, and this heat is also taken up by the blood. Thus the heart is an important mechanism for the production as well as for the distribution of heat in the body.

\section{The Diastolic Filling of the Heart.}

In the excised heart no evidence of any suction power has been observed; indeed, the heart will only fill when supplied with blood under a positive pressure. Similarly, when the thorax is opened, the heart cannot fill itself by its own power of suction; this force must be aided by a positive pressure in the thoracic veins.

If the pressure in the venæ cavie be not positive, then any negative pressure which oceurs in the heart eavities leads to a collapse of the thinwalled vence eave, and not to suetion of blood from the veins. In hydraulic engineering the efforts of engineers are directed towards making the water enter without shock. If the negative pressure had any decided action, the blood would enter the heart with considerable shock, and consequent loss of energy. This is contrary to what we should expect in nature. The driving force of the heart is sufficient by itself to complete the circulation and return the blood to the heart, when the action of the respiratory pump is abolished by injection of curari, and the thorax is opened.

Normally, the filling of the heart is largely under the control of the respiratory pump. In the closed thorax the pressure is less than that of the atmosphere by the amount of tension which is required to overcome the pulmonary elasticity, and expand the lungs to the size of the thoracic cavity. In ordinary inspiration this tension is equivalent to $9 \mathrm{~mm}$. $\mathrm{Hg}$; in the position of deepest inspiration it may be $30 \mathrm{~mm}$. $\mathrm{Hg}$. On the one hand, the extrathoracic veins are under a pressure which is nearly that of the capillaries, and is thus somewhat above the atmospheric pressure; on the other hand, the intrathoracic veins and the heart are under a slight but constant negative pressure, which in inspiration may be -9 to $-30 \mathrm{~mm}$. Hg. The venous blood is by these alterations in thoracic pressure aspirated into the heart. If, in sequence to, or synchronously with, the inspiratory movements of the thorax, the abdominal muscles be thrown into contraction, then the abdominal veins are compressed, and the respiratory museles act on the venous circulation, not only as a suction- but also as a force-pump. If the pressure in the thorax be made sufficiently great, the efficiency of the heart as a pump ceases, for the heart is unable to fill. Thus the diastole of the heart can be prevented by raising the intrapericardial pressure.

To carry out this experiment, a pressure bottle filled with oil is connected by a T-piece with an oil manometer, and a tube tied into the pericardial sac. So soon as the pressure of the oil rises to $60-70 \mathrm{~mm}$. oil, the arterial pressure falls by $20-30 \mathrm{~mm}$. $\mathrm{Hg}$, while the vena cava pressure

\footnotetext{
1 Estimates of more than double this amount are frequently given, bnt they have been based nyon a far larger output and a higher aortic pressure than (for reasons subsequently to be given) have here been adopted.
} 
rises to about $60 \mathrm{~mm}$. soda solution. By a pressure of at most 240 $300 \mathrm{~mm}$. oil, the arterial pressure is driven to zero. ${ }^{1}$ By no possible means can the vena cava pressure rise beyond this pressure, and thus the leart is unable to fill. "The quantity of blood thrown into the aorta by each contraction of the left ventricle, should accurately corresyond to that issuing from the arteries into the capillaries during the period which elapses from the commencement of the systole to the end of the following diastole. It is the maintenance of this proportion that is the source of the constant mean tension of the arteries." 2

In the dog either the vena cava superior or the vena cava inferior below the liver may be completely occluded, and yet no change of pressure is indicated by the arterial manometer. On compressing the vena cava inferior between the liver and the heart, on the other hand, there is an immediate and marked descent of the arterial tension. Thus, too, a rabbit, in which the portal vein has been ligatured, perishes within a few minutes, owing to the rapid accumulation of the blood in the portal tributaries. The capacity of these is so great that they are sufficient to

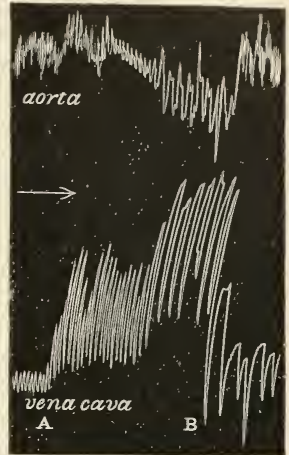

FIG. 23.-Compression of the thorax (A-B). Compensatory effect of powerful inspirations, alternating with forced expiratory efforts (glottis elosed).-Hill and Barnard.

hold all the blood at a low tension. The filling of the heart is thus enormously diminished while the aorta continues to empty itself, by its elastic reaction, into the portal system.

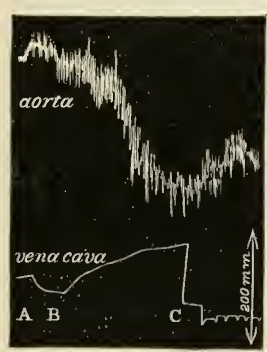

FIG. 29.-A, vertical feetdown position ; $\mathrm{B}-\mathrm{C}$, effect of compressing the thorax.-Hill and Barnard.

Stefani ${ }^{3}$ found that the intrapericardial pressure must, in order to stop the diastole of the heart, be raised to a higher point if the ragi are intact than if the vagi are divided. This need not prove, as Stefani believes, that the vagus actively influences the diastole of the heart muscle. The result can be explained by the alteration in the action of the respiratory pump which follows division of these nerves. By dividing both vagi the rate of respiration is greatly slowed, and thus the action of the respiratory pump, so far as regards the filling of the heart, is lessened.

On compression of the thorax of a $\mathrm{dog}$, the arterial pressure falls towards zero, owing to the increased intrathoracic pressure, while the vena cara pressure rises. The gravily of the effect depends greatly on the rigidity of the thorax and resisting power of the animal. ${ }^{4}$ In man, compression of the chest produces the same result, and Weber thereby induced in himself loss of consciousness and

${ }^{1}$ Cohnheim, "Lectures on General Pathology," New Syd. Soc. Trans., vol. i. 1. 23.

${ }^{2}$ Cohnlieim, ibid., p. 25.

3 "Cardiovolume pressione pericardica e attivita della diastole," $1 / \mathrm{cm}$. Accad. med. chir. di Ferrara, il 5 Agoste 1891.

${ }^{4}$ Hill and Barnard, Journ. Physiol., Cambridge and London, 1597, vol. xxi. 1. 333. 
slight convulsive spasms, owing to the production of acute cerebral anæmia.

In the vertical feet-down posture, when the full hydrostatic effect of gravity opposes the return of blood from the parts below the heart, the efficiency of the respiratory pump becomes of vital importance. Thus the establishment of forcible artificial respiration by raising the intra-

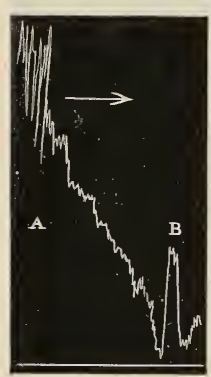

F1G. 30.-A, eflect on aortic pressure of artificial ventilation of the lungs in the vertical feet-down posture; B, compensatory effect of abdo: minal compression. - Hill and Barnard. thoracic pressure, while the animal is in the feetlown position, forces the arterial pressure to zero. In the horizontal posture the fall of arterial pressure is considerable, but not so dangerous. Compression of the abdomen immediately restores the circulation. This compensates for the positive pressure in the thorax, and fills the heart with blood. ${ }^{1}$

When the larynx is blocked by some hard impacted mass of food, too hastily swallowed, death is hastened by the violent expiratory spasms, which raise the intrathoracic pressure, and lead to deficient filling of the heart.

So, in the fatal crushes of panic-stricken crowds, death is produced by compression of the thorax and circulatory failure. The women and children with collapsible chests are the first to die, while the men with rigid chests escape.

The case of Colonel Townsend, as reported by Chcyne, ${ }^{2}$ has always aroused considerable interest. Colonel Tuwnsend was in the last stage of some form of nephritic disease, and life was at its lowest ebb. Cheyne reports the experiment he witnessed together with two other medical men. "The man could die or expire when he pleased, and yet, by an effort or somehow, he could come to life again. He composed himself on his back, and lay in a still posture some time. While I held his right hand, Dr. Baynard laid his hand on his heart, and Mr. Skrine held a clear lookingglass to his mouth. I found his pulse sink gradually, till at last I conld not feel any, by the most exact and nice touch. Dr. Baynard could not feel the least motion in his heart, nor Mr. Skrine the least soil of breath on the bright mirror he held to his mouth; then each of us by turns examined his arm, heart, and breath, but could not by the nicest scrutiny discover the least symptom of life in him. We began to conclude that he had indeed carried the experiment too far, and at last were satisfied he was actually dead, and were just ready to leave him. As we were going away, we observed some motion about the body, and upon examination found his pulse and the motion of his heart gradually returning; he began to breathe gently and speak softly." $\mathrm{He}$ died the same evening.

The explanation of this case, which appeared so full of mystery to Cheyne, is probably to be found in the following facts. The circulation was failing, the blood pressure very low, and the vascular tone probably almost entircly lost; such was evidenced by his thready and feeble pulse. The heartbeat was so feeble, that the vis-a-teryo was insufficient, even in the recumbent posture, to return the blood to the auricle without the aid of respiration. When, therefore, Colonel Townsend held his breath, the circulation ceased, while the empty heart continued to beat, but so fcebly as to be imperceptible. If anæmia of the bulbar centres is slow in origin, spasm is never provoked by the total cessation of the circulation. On asphyxiating a curarised animal, the heart frequently is found to continue to beat for fifteen or even twenty

${ }^{1}$ Hill and Barnard, Journ. Physiol., Cambridge and London, 1897, vol. xxi. p. 333.

2 Cheyne, "English Malady," London, 1733, p. 307. 
minutes, for the supply of oxygen is not exhausted, owing to the absence of asphyxial spasms. Remembering these experimental facts, it is possible to conceive that the heart of Colonel Townsend continued to beat imperceptibly for some time after the cessation of respiration, and that at the end of that time the respiratory centre discharged a group of respirations, which re-established the circulation. The man, in fact, had sunk into the condition of a hibernating animal, where the metabolic processes are at the lowest ebb.

It must be borne in mind that the heart, during its systole, by diminishing its own volume, increases the negative pressure in the thorax. In proportion as the ventricles become smaller, the lungs, the thoracic reins and auricles must become larger. On passing a tube down the jugular vein of a horse or dog, there can be olserved, besides the respiratory oscillations, short undulations, synchronous with the heart-beat. The fall of pressure in these is synchronous with the systole. ${ }^{1}$ On removing the front of the chest wall from a rabbit, without injury to the pleura, a to-and-fro movement of the lung can be seen to take place synchronously with the beat of the heart. Similarly, the chest wall sinks in, outside the area of the cardiac impulse during systole, ${ }^{2}$ and if the breath be held, a negative pressure appears at the same time in the air passages. This last phenomenon can be recorded ${ }^{3}$ by means of a tube placed in one nostril, while the mouth and the other nostril are held closed. The tube is connected with a delicate tambour. The larynx must be kept open during the observation.

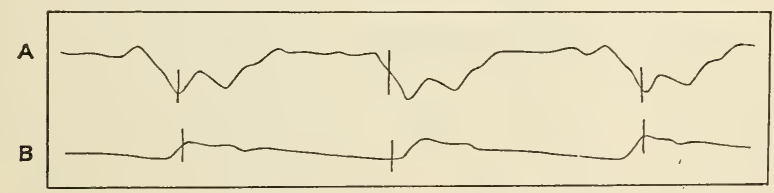

FIG. 31.-A, Pressure in the air passage ; B, carotid pulse.-Mosso.

In Fig. 31, a curve of the carotid pulse is simultaneously shown with that of the pressure in the air passages. During the rise of the former, the latter is seen to fall. The fall is found to begin earlier than the rise of the carotid pulse, because it is dependent on two factors. The first of these is the contraction of the ventricle, which drives ontwards the wall of the thorax; the second factor is the expulsion of the blood from the thoracic cavity. The first factor produces its effect on the nasal tracing earlier than the second, while the rise of pressure in the carotid artery is produced only lyy the latter. Part of the difference in time is due to the fact that the rate of transmission in the air-tubes is far greater than that of the pulse wave along the arteries. If, in the course of this experiment, the larynx be closed, the pressure is found to rise in the nose with each systole, owing to the expansion of the arteries in the mouth, nose, and pharynx.

1 Werlemeyer, "Untersuch. ueber d. Kreislauf des Blutes," Hanover, 1828; Weyrich, "De cordis aspiratione," Dorpat, 1853.

2 Lovén, Nord. med. Ark., Stockholm, 1870, No. 19, vol. ii.

3 Mosso, "Die Diagnostik des Pulses," Leipzig, 1879, S. 42. 


\section{The Systolic Output of the Heart and the Arterial Pressure.}

The determination of the exact output of the heart has been a matter of considerable difficulty. In the calculation of the energy of the circulation, it is shown that the output can be arrived at approximately by hydrodynanic formulæ, if certain data are obtained experimentally. To arrive at one of these data, namely, the velocity of flow in the ascending aorta, has always been a formidable matter.

Volkmann ${ }^{1}$ and Vierorlt ${ }^{2}$ estimated the velocity of the blood-flow in the carotid artery, and, hy comparing the sectional area of this with that of the aorta, reckoned the velocity of flow in the aorta, and thus the output of the heart per second. Now, the flow in sueh a tube as the earotid depends at any moment on the proportionate relation whieh obtains between the resistanees in the earotid vaseular area and in the remainder of the arterial system. It is therefore impossible, from the velocity of flow in the carotid artery, to accurately reckon the velocity of flow in the aorta. It is equally impossible to determine the diastolic capacity of the ventricle in the dead animal-not only because of the fallacies which arise from the inadequacy of any method to imitate the conditions of the living heart, but from the fact that the ventricle does not completely empty itself during systole.

Stolnikow ${ }^{3}$ attempted to measure the output in a different manner. He tied all the aortic branches except the axillary, and the outflow from that artery he drew off into a graduated vessel. From thence the blood was sent back to the heart by way of the exterual jugular vein. Under these conditions, with a low resistance to flow, with the arterial pressure rising to 30 or $40 \mathrm{~mm}$. $\mathrm{Hg}$ only, and with ample filling of the heart in diastole, the systolic output was probably maximal. He found it from .00032 to 0016 of the body weight. Tigerstedt ${ }^{4}$ attacked the problem directly, and, by means of an improved form of stromuhr, measured the How in the aorta of the rabbit. In fourteen experiments, wherein the blood pressure averaged 88.5 to $176 \mathrm{~mm}$. $\mathrm{Hg}$, and the heart frequency 193, the mean outflow was 00027 of the body weight, and the output per sec. 00085 of the body weight. Other observers have availed themselves of indirect methods of arriving at the same resuit. Thus Zuntz ${ }^{5}$ estimated the blood-gases in arterial and venous blood, and the gases of the expired air. He found that a resting horse consumed 2733 c.e. of oxygen per minute, while the arterial blood contained 10:33 per cent. more oxygen than venous blood. It follows from these data that every 100 c.c. of blood which traversed the lungs took up 10.33 per cent. of oxygen; but, as in one minute 2733 c.c. of oxygen was carried away by the blood passing through the lungs, therefore $\frac{100 \times 2733}{10.33}=26,457$ c.c. of blood must have passed in the given time. This, then, was the output per minute of the right ventricle. The output per second was 00122 of the body weight (this was about 360 kilos.). By a similar investigation, the output per

1 “Die Himodynamik," Leipzig, 1850, S. 204.

2 "Die Erscheinungen und Gesetze der Stromgeschwindigkeiten des Blutes," Frankfurt, 1858 , S. 104 .

${ }^{3}$ Arch. f. Physiol., Leipzig, 1886, S. 1.

${ }^{4}$ Skandin. Arch. f. Physiol., Leipzig, 1892-93, Bd. iii. S. 145

5 Deutsche med. Wehnschr., Leipzig, ] 892, s. 109 ; ef. Gréhant and Quinquand, Compt. rend. Soc. de biol., Paris, 1886, p. 159. 
second in a dog was found to be 00157 of the body weight (25 kilos.).

Obtained by such different methods, Zuntz and Tigerstedt's results roughly agree. Thus the output per second was-

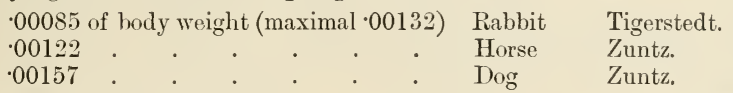

Stewart has recently estimated the output by a new method. ${ }^{1}$ A definite amount of 1.5 per cent. solution of $\mathrm{NaCl}$ is injected into the right auricle through the jugular vein at a uniform rate. From the femoral artery samples of blood are collected (1) just before, (2) during the injection. The quantity of 1.5 per cent. $\mathrm{NaCl}$ solution necessary to add to sample 1 , to make its $\mathrm{NaCl}$ content equal to that of sample 2, is determined. This determination yields the amount by which the injection is diluted with blood in the heart. If the quantity of the injection in a given time be known, and the extent of its dilution, it is obvious that the output of the heart can be calculated for that time. Stewart's results agree closely with those of Zuntz.

From these data the systolic output in man can be calculated to be a little more than 100 c.c. By the use of hydrodynamical formulie the output was reckoned to be 160 c.c. (see p. 43 ), but this, as already stated, is probably much too high.

Such methods of investigation as the above are unsuitable for prolonged researches on the output of the heart under the varying conditions of experiment. An attempt has therefore been made to use the pericardial sac as the chamber of a plethysmograph, and thus obtain records of the changes of cardiac volume. The pericardial wall is not, however, sufficiently rigid for the purpose; moreover, the diastole of the heart is impeded whenever this sac is distended with fluid. The experimental difficulties in the way of inquiry have been recently solved by Roy and Adami's invention of the cardiometer. $^{2}$

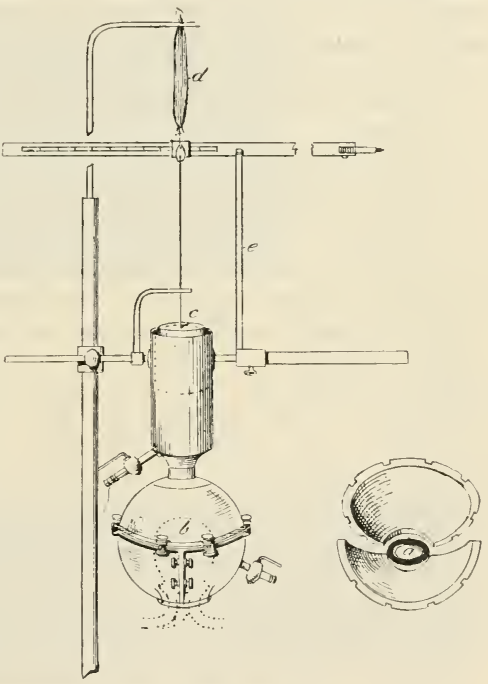

FIG. 32.-Cardiometer.-Roy and Adami.

The cardiometer consists of a metal box, constructed in two jarts, these are uniter by a linge joint. After the thorax has been opened, and the pericarlium slit open, the heart is slipped within the opened cardioneter, and this is then closed. The vessels issue from the heart through an orifice in the cardiometer, and to the elge of this opening the parietal layer of the peri-

1 Journ. Physiol., Cambridge and London, 1897, vol. xxii. p. 159.

2 Phil. Trans., London, 1892, vol. el xxxiii. B, 1). 202.

VOL. II. -4 
cardium is securely fastened. By this means all leakage from the chamber of the instrument is prevented. Finally, the cardiometer is filled with oil, and connected by a tube to a piston recorder. The instrument is experimentally calibrated, so that a certain extent of stroke is known to signify a certain change in volume, and an automatic counter has been devised by Roy and Adami, which records a notch on the tracing at every period when the sum of the upstrokes of the lever corresponds to an output of 100 e.c. of blood from the heart. A simple and adequate form of cardiometer can be made out of a tennis ball. A tube is fixed into the ball and connecterl with a piston recorder. On the opposite side to this tube a hole is cut large enough to admit the heart. The pericardium is tied round the ball, and the junction made air-tight by the application of thick vaselin. ${ }^{1}$

The efficiency of the systole can be estimated in yet another way. This is by the simultaneous record of the left auricular and aortic pressures. If the ventricle fail to empty itself, and the residual blood increase, then both the diastolic volume and pressure rise. While the cardiometer records the increase of volume, a manometer in connection with the left auricle registers the increase in diastolic pressure.

By one or other of these methods, interesting and important results have been obtained. It is known that, under high pressure, the elastic coefficient of the aorta increases; the energy of the systole must correspondingly increase, in order that the output when the pressure is high may be maintained at the same volume as when the pressure is low. From his researches on the output of the heart, Tigerstedt concludes-

1. That within certain limits the systolic output is independent of the resistance.

2. That under favourable conditions a rise of resistance may increase the systolic output.

3. That with increasing peripheral resistance the systolic output as a rule decreases.

4. That as the arterial pressure generally increases in spite of the diminished systolic output, the diminution in output per second must be proportionately smaller than the increase in resistance. ${ }^{2}$

The diminution of the output per second is usually shown most strikingly during the rise of arterial tension which is occasioned by asphyxia. In the asphyxial condition the heart muscle rapidly fails and passes into paralytic dilatation; while the output from the ventricles is opposed, the venous input is enormously assisted by the action of the respiratory spasms.

A large area of arterioles, mainly the splanchnic, is thrown into constriction, and the blood in the first stage is propelled from the constricting vessels into the veins, ${ }^{3}$ while the velocity of flow through the locomotor organs is accelerated owing to a compensatory dilatation of the vessels in these organs, and thus the diastolic filling of the heart is increased. At the same time, under favourable conditions, the heart is accelerated, the systolic output increases, the left auricular pressure falls, and the arterial pressure rises. In the second stage, the vagal centre in the spinal bulb is excited by the high arterial tension, the heart frequency is lessened, and the output diminished. Further, so soon as the arterial pressure reaches a certain point, the heart becomes unequal to the strain of emptying itself against the resistance, the

${ }^{1}$ Hill and Barnard, Brit. Mcl. Journ., London, 1897.

2 Slaandin. Arch. f. Physiol., Leipzig, 1891, Bd. ii. S. 430.

${ }^{3}$ Mall, Arch. $f$. Physiol., Leipzig, 1892, s. 418. 
output then becomes imperfect, the residual blood increases, the left auricular pressure rises, and the blood is congested back upon the venous system.

A greater rise of arterial pressure can be produced by excitation of the splanchnic nerves than by closure of the thoracic aorta with a clip. ${ }^{1}$ This difference in result is to be explained by the following facts:-

1. On closing the aorta with a clip, the driving
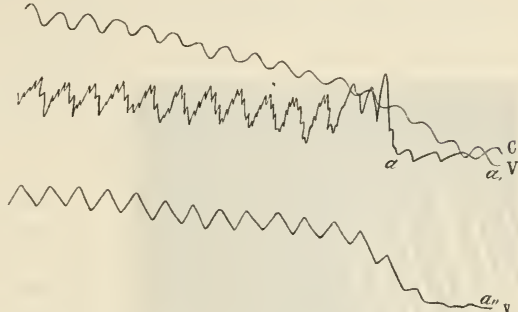

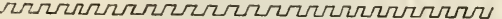

FIG. 33.-Curves illustrating the effect of a rise of aortic pressure $\left(\alpha^{\prime}\right)$ upon the pressure in the right $\left(\alpha^{\prime \prime}\right)$ auricles; to be real from right to left.-Kauders. power of the heart is cut off from the lower parts of the vascular system, the blood stagnates therein, and thus the return of the renous blood to the heart is impeded. This is not so in the case of splanchnic constriction.

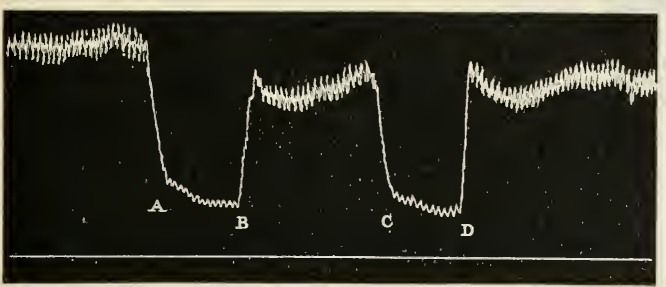

2. The power

FIG. 34.-Aortic pressure. A, vertical feet-down position; B C, is thrown in syseffect of abdominal compression; D, horizontal position.- tole is enormHill. ously diminished.

Whenever the left ventricle fails to maintain its output, the pressure rises in the left auricle. Subsequently, in consequence of the back effect through the lungs, it rises in the right auricle. ${ }^{2}$ Figure 33 illustrates the effect on auricular pressures of a rise in aortic pressure. The points marked $a, a^{\prime}, a^{\prime \prime}$, give the corresponding moments in time on the three curves.

The dependence of

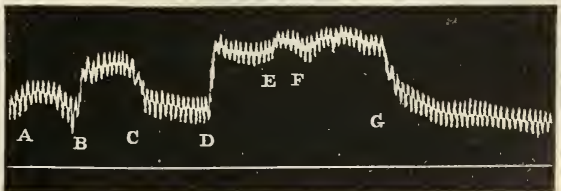
the arterial pressure and Fig. 35.-Aortic pressure. A, horizontal position; B C, the systolic output on the diastolic filling of the abdomen compressed; D, vertical feet-up position ; E F, abdomen compressed; G, horizontal.-Hill. heart is shown by the experimental results obtained on compressing the abdomen or thorax.

1 v. Basch, Ber. d. k. sächs. Gesellsch. d. Wisscnsch. math.-phys. Cl., Leiprig, 1875, S. 383 ; S. Mayer, Sitzungsb. d. k. Akad. d. Wissensch. math.-naturw. Cl., Wien, 1879, Bd. Ixxix. Abth. 3, S. 19.

${ }^{2}$ Kauders, Ztschr. f. Klin. Med., Berlin, 1892, Bd. xxi. S. 68. 
If the thorax be compressed, the pulmonary circulation is obstructed, and the filling of the left heart opposed. If the abdomen be compressed, the filling of the right heart is aided, and the output per second may be doubled or trebled. In the one case the arterial pressure falls, in the

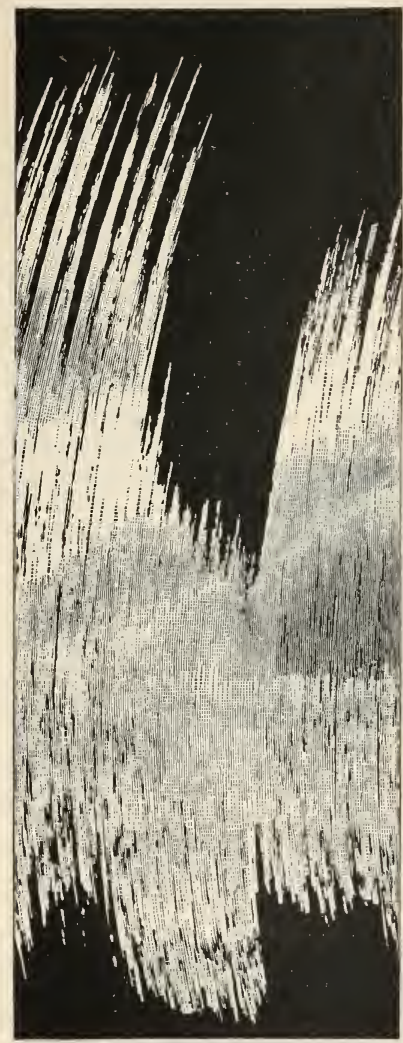

A

FIเ. 36. - Cardiograplic tracing. Effect on the volume of the heart of alteration in position. A, horizontal; B, vertical feet-down; C, horizontal. In the feetdown posture the heart slirinks in volume, and the systolic upstroke is lessened.-Harold Barnard. other case it rises. Compression of the abdomen, however, only increases the systolic output up to a certain limit.

Thus, in Figs. 34,35 , is shown the comparative effect of compression when the animal is placed in the vertical feet-down, horizontal, and vertical feet-up positions. In the feet-down position the rise of arterial tension is great, in the horizontal position it is slight, and in the vertical feet-up position almost nothing. In the last case the heart has already enough and more than enough blood at its disposal. Beyond a certain point the increased filling of the heart works unfavourably; that is, when the arterial pressure has risen to such a height that the ventricle is unable to rise to the strain of maintaining the output. In such conditions a vicious circle is established, for the greater the input the less the output, since it has been shown (p. 40) that if the ventricle be suddenly enlarged to twice its diameter the muscle fibres must be exerted eight times as much as before to produce the same pressure.

Thus, very powerful compression of the abdomen not only dilates the right heart, but opposes the emptying of the left ventricle. And, as Stephen Hales graphically says, "The heart being in this case like a watermill, that is, surcharged with a flood both before and behind, its force must needs abate and grow languid." When the heart muscle is weakened by inhalation of chloroform, or by asphyxia, forcible compression of the abdomen may throw the right ventricle into a condition of paralytic dilatation. ${ }^{1}$

Besides chloroform, alcohol may be mentioned as another drug which, while it renders the systolic output incomplete, increases the diastolic pressure and the dilatation of the heart. If the heart be

${ }^{1}$ Hill, Journ. Physiol., Cambridge and London, 1895, vol. xviii. p. 51 ; Brit. Med. Journ., London, 1897. 
confined in the pericardium, and these drugs be pushed to excess, the heart ceases to have room for diastole when the distension reaches a certain point; cutting the pericardium relieves the heart for a time, but final cessation in diastole takes place eventually. The circulation of calves' blood through dogs' hearts has the same effect. ${ }^{1}$ The diastolic distension of the heart can be immediately relieved by placing the body in the vertical feet-down position; the right heart is thus emptied into the splanchnic area. ${ }^{2}$

The action of the inhibitory and accelerator nerves on the output of the heart is of considerable interest.

The effect of excitation of the peripheral end of the vagus nerve has been recorded by the cardiometer. ${ }^{3}$ The output per second is lessened, the residual blood is increased, the expansion of the heart in diastole is made more evident. The excitation of this nerve weakens or arrests the auricular contractions, and, while it slows the ventricles, the venous pressure rises because the output is lessened. A considerable rise of pressure in the left auricle accompanies the great fall of aortic pressure. ${ }^{4}$

By moderate stimulation of the vagus, the output per second may be diminished 20 to 30 per cent. The ventricles are more expanded both in diastole and in systole, owing to the increase of the diastolic filling pressure. Excitation of the accelerator nerves, on the other hand, increases the output per second by augmenting the force of the heart, and especially that of the auricular contraction. The venous pressure falls in consequence, the arterial pressure rises, and less residual blood remains behind within the ventricle. As a rule, the output per systole is, owing to the increase of diastolic filling, greater when the heart beats slowly, less when the heart is accelerated. The opposite is true for the output per second.

So long as the output per second remains unchanged, the arterial pressure will not vary, other factors remaining constant; but if the ontput decreases the pressure will fall, and if the output increases the pressure will rise. Changes in cardiac frequency, however, affect the mean arterial pressure only when the peripheral resistance is sufficiently great, but not otherwise.

Thus, if the arterioles are in a state of tone and the peripheral resistance is considerable, then acceleration of the heart raises the mean arterial pressure. Such, for example, is markedly the case when both vagi are divided in a morphinised dog. The blood is then pumped into the arteries faster than it can escape through the arterioles. On the other hand, when the peripheral resistance is greatly diminished by section of the spinal cord in the cervical region, then, on dividing both vagi, or exciting the accelerator nerves, only an insisnificant change of mean arterial pressure is to be observed. ${ }^{5}$ In this case the blood escapes with great rapidity into the veins. This is a fact of the utmost importance, for it is evident that unless the arterioles are closed down to a certain extent, the heart cannot, between its contractions, maintain the arteries in a condition of distension.

1 Newell Martin, Phil. Trans., London, 1883, vol. clxxiv. 1) 663.

${ }^{2}$ Hill, Brit. Med. Journ., London, 1897.

3 Roy and Adami, Phil. Trans., London, 1892, vol. clxxxiii. B, p. 215.

+ Kauders, Zischr. f. klin. Med., Berlin, 1892, Bd. xxi. S. 67 .

5 Ilunzel, Arch. $f$. Physiol., Leipzig, 1887, S. 136 ; François-Franck, "Trav. du labor. de Marey," is80, Bd. iv. S. 296. 


\section{The Centres of the Cardiac Nerves and Cardiac Reflexes.}

After placing one electrode within the nostril of a frog, and the other on a cross-section of the spinal cord at the level of the fourth or sixth vertebra, the astounding observation was made by the brothers Weber that tetanic excitation caused standstill of the heart. This experiment led to the discovery of the function of inhibition. By further experiments the Webers localised the seat of the inhibitory power to the spinal bulb, in the region which lies between the optic lobes and the lower end of the calamus scriptorius. ${ }^{1}$ By using the prick of a needle as a method of excitation, Eckhard ${ }^{2}$ more minutely localised the inhibitory centre in the frog to be between the cerebellum and the tip of the calamus scriptorius. In rabbits, after dividing the spinal cord immediately below the medulla oblongata, excitation of the

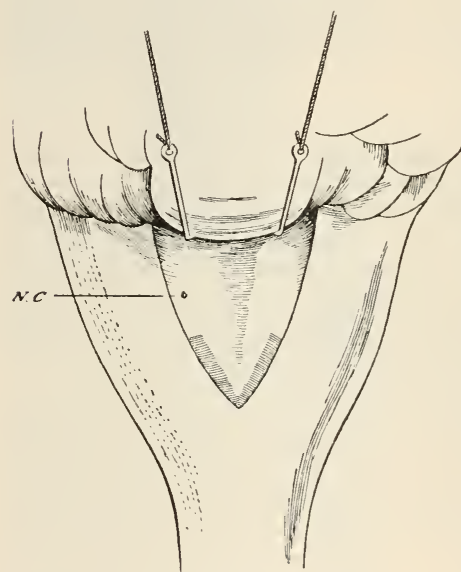

FIG. 37.-Posterior view of medulla oblongata of the cat, showing $N . C$, position of vagus centre ; semi-diagrammatic.-Laborde. fifth nerve reflexly slows the heart. $^{3}$ By the needle method of excitation, Laborde ${ }^{4}$ localised the vagus centre in the cat to a point in the middle of the spinal bulb lying in the lateral part of the floor of the fourth ventricle. At this spot there is situated a mass of grey matter, which forms the accessory nuclei of the hypoglossal, spinal accessory, and glosso-pharyngeal nerves.

The vagal centre can not only be excited by the stimulation of afferent nerves, but it can also be influenced by the direct action of blood on the centre, and from the cerebrum. The frequency of the pulse can be altered by psychical and emotional conditions. In many men the pulse may be slowed by directing the attention to the contemplation of painful subjects. It is constantly accelerated in emotional patients by mere physical examination on the part of a physician. In the case of an individual counting his own pulse, the frequency often becomes diminished.

Tarchanoff observed a medical student who could alter his pulse rate at will. ${ }^{5}$ He found that men with this faculty had generally a remarkable power over their muscles; for example, they could bend the third joint of a finger independently of the other joints, or contract the platysma muscle.

In conditions of hypnotism, the pulse rate has been observed to

1 Ed. Weber, "Handwörterbuch d. Physiol.," 1846 (2), Bel. ii. S. 45.

2 Beitr. z. Anat. u. Physiol. (Eckhard), Giessen, 1878, Pid. viii. S. 187.

3 François-Franck, "Trav. du labor. de Marey," 1876, Bd. ii. p. 255.

4 Arch. de physiol. norm. et path., Paris, 1888, p. 397.

5 Arch. f. d. ges. Physiol., Bonn, 1885, Bd. xxxy. S, 109. Cf, Pease, Boston Ifed, and S, Journ., 1889, vol. exx. p. 525 , 
change markedly under the influence of suggestion. Inquiry for cerebral cardiac centres has been made by the methods of cortical excitation. If, by the use of suitable strengths of current, the excitation of epileptic fits be avoided, François-Franck finds that stimulation of any part of the motor area accelerates the heart when a weak current is employed. On the other hand, a strong current slows the heart. From other parts of the cerebrum the heart can also be influenced. All these cerebral effects are similar to those excited reflexly by stimulation of afferent nerves. The vagus centre maintains over the heart a tonic bridle action, and the question arises as to whether this tonus is kept up reflexly by the constant instreaming of afferent impulses, or whether the centre possesses automatism.

According to Soltmann and others, vagal tone is not to be found in the new-born animal. ${ }^{1}$ On the other hand, pressure on the head of the new-born child slows the pulse, ${ }^{2}$ and thus the centre can be reflexly excited. In support of the hypothesis that the tone depends on afferent impulses, the following experiments may be quoted :-

1. After dividing the cervical spinal cord, the heart is said not to be accelerated by a subsequent division of the vagi.

2. After section of the splanchnic nerves, and thus diminishing the sensory field from which reflexes are excited, the pulse frequency is increased.

Neither of these experiments, however, proves that the tone depends on afferent nerves, for the arterial tension is greatly lessened by both operations. In consequence of any great fall of arterial tension, the heart is accelerated. This acceleration may be so great that the frequency is not increased to any further degree by section of the vagi. There can be no doubt that the arterial pressure is one of the most important agencies by which the vagus centre is constantly influenced, and since it is quite impossible to cut off all afferent impulses from this centre, and at the same time remove the influence of the arterial pressure, the automatism of the centre can neither be proved or disproved. The theoretical assumption of such a power is not required.

After section of both ragi, excitation of the medulla oblongata causes cardiac acceleration. This acceleration takes place after section of the splanchnic nerves, and thus is independent of rasoconstriction and the rise of arterial tension, which normally follows such excitation. A centre for the accelerator nerves is localised in the spinal bulb, and is said to maintain a tonic influence over the heart. Tschirjew, ${ }^{3}$ and also Stricker and Wagner, ${ }^{4}$ have witnessed, after section of the vagi, a decrease of the pulse rate to take place on excision of the inferior cervical and first thoracic ganglia. It is also affirmed that, after section of the accelerator fibres, inhibition by excitation of the vagus can be induced with greater ease than before. The inhibitory effect is likewise obtained more easily after section of the cervical spinal cord. ${ }^{5}$

Since, by the operative procedures of exposing the accelerator nerve,

1 Jahrb. f. Kinderh., 1877 , s. 101.

2 Engström, "Förlossningens inverkan på fostrets respiration," Helsingfors, 1889, S. 245, eited after Tigerstedt.

${ }^{3}$ Arch.f. Physiol., Leipzig, 1877, S. 164.

4 Wien. med. Jahrb., 1878, s. 370 .

${ }^{5}$ Sustschinsky, Untersuch, a. d. physiol, Lab. in Würzburg, 1868, Bd, iii, S. 164, 
or dividing the spinal cord, the action of the respiratory pump is greatly diminished, the above experiments cannot be accepted as convineing proof of the tonic action of the accelerator nerves. The best proof of such tonicity is the fact that Timofeew ${ }^{1}$ has kept animals for some time after section of these nerves, and has observed in them a persistent decrease in pulse frequency. The tonicity of the accelerator centre is normally held in check by the stronger tonicity of the vagus centre.

The influence of the blood pressure on the frequency of the heart has been studied both on the isolated heart and on the heart in situ in the normal conditions of life. The results obtained have been contradictory and confusing, because the effects of the two factors of most importance, namely, the venous filling pressure and the arterial or systolic output pressure, have not been in all the researches carefully and separately analysed. If the inflow or venous pressure be kept constant in the excised heart of the frog or tortoise, in which an artificial circulation has been established, then it is said a variation of the arterial pressure, by increasing the resistance to outflow, produces 110 effect on the frequency. On the other hand, an increase of venous pressure accelerates the heart. ${ }^{2}$ Newell Martin drew the same conclusion from observations made on the excised heart of the dog maintained in functional activity by a circulation of clefibrinated blood at a constant temperature.

When the normal circulation is maintained with the heart in situ, and all the cardiac nerves are divided, then by compression of the abdominal aorta or excitation of the peripheral end of the spinal cord or of the splanchnic nerves, the arterial tension is raised, and acceleration of the heart is caused, and the more so in proportion to the suddenness of the change of pressure. This result has been obtained by several observers, and, since all the nerves have been divided, the acceleration must be provoked by the direct action of the pressure on the heart itself. ${ }^{3}$ By all these methods of raising aortic pressure, not only the filling pressure of the heart, but the temperature of the blood may be changed. The variation in frequency camnot therefore be ascribed with certainty to the rise of arterial tension. Since a high intracardiac pressure has been found by all authors to render vagus inhibition more difficult, we must suppose that either the intracardiac centre of this nerve is hindered from action by the rise of diastolic pressure, or that the part of the cardiac musculature at which the contractions originate is rendered thereby hyper-excitable. ${ }^{4}$ If all the cardiac nerves be intact, a rise of arterial pressure always slows the heart, and a fall accelerates it. This mechanism, first recognised by Marey, ${ }^{5}$ is of great importance, for by its means the constancy of the arterial pressure is maintained. So long as the vagi are intact, the arterial pressure cannot be greatly raised. Whether the centres are normally excited by afferent impulses, or directly by the blood pressure, is not

1 Centralbl. f. Physiol., Leipzig n. Wien, 1889, S. 235.

2 Howell and Wartich, Johns Hopkins Univ. Stud. biol. lab., Baltimore, 1881, vol. ii. Pp. 235-245 ; Tschirjew, Arch.f. Physiol., Leipzig, 187\%, S. 187; J. M. Ludwig and Luchsinger, Arch. f. d. yes. Physiol., Bomn, 1881, Bd. xxv. S. 228.

3 Johanssen, Arch. f. Physiol., Leipzig, 1891, S. 111.

${ }^{4}$ Sustchinsky, Untersuch. a. d. physiol. Lab. in Würluerg, 1868, Bd. iii. S. 165 ; Lndwig and Lllehsinger, Arch.f. d. ges. Physiol, Bonn, 1881, Bd. xxv. S. 235 ; Stewart, "Proc. Phys. Soc." Journ. Physiol., Cambridge and London, 1891, vol. xii. p. xxx. ; Hill and Barnard, ibid., 1897, vol. xxi. p. 338.

5 "La circulation du sang," Paris, 1881, p. 334. 
fully determined. The heart itself possesses afferent nerves, and by means of these it is quite conceivable that it can reflexly control its own beat. The afferent nerves of the heart do not include nerves which excite sensations of touch or pain, at any rate when the organ is in a normal condition. On touching the heart of Viscount Montgomery, who had, through an accident, lost part of the thoracic wall, Harvey was unable to excite any signs of consciousness. General reflex movements can, however, be excited. ${ }^{1}$ Thus François-Franck succeeded in producing reflex inhibition of the respiration by chemical stimulation of the inner wall of the heart. ${ }^{2}$

By excitation of the central ends of the different nerves which are found rumning superficially on the anterior wall of the ventricle, Wooldridge obtained the following results: ${ }^{3}$

1. Slowing of the pulse rate and a rise of arterial pressure.

2. Slowing of the pulse rate, followed by acceleration and a fall of arterial pressure.

On stimulation of the nerves which course over the posterior wall of the ventricle, he observed-

1. Acceleration of the pulse rate.

2. Slowing of the pulse rate and a fall of arterial pressure.

3. A rise of arterial pressure.

4. A fall of arterial pressure.

While stimulation of the central end of one vagus nerve-if the other end be intact-slows the heart, the arterial pressure may rise or fall, according as depressor or pressor fibres are in the ascendancy. The result varies in different individuals. From the right or left vagi, opposite effects on the arterial pressure may be obtained. The afferent nerves examined by Wooldridge run in the vagi. The arterial pressure may reflexly excite the cardiac centres in the bulb by way of these afferent fibres. In the case of the depressor nerve we have an analogous example.

After carrying out a division of the spinal cord in the cervical region, a sudden injection of blood at a high pressure into the peripheral end of the carotid artery provokes slowing of the heart. ${ }^{4}$ Sudden anamia of the bulbar centres has the same effect, whether it be produced by ligation of the cerebral arteries or by compression of the brain. The vagus centre is first excited and then paralysed. Since acute anæmia of the spinal bulb primarily causes a slow beat and a rise of arterial tension, it is curious that the vagus centre should be directly excited to greater activity by a rise of arterial tension, which increases the supply of blood to the spinal bulb. It is conceivable in the above experiment that the sudden expansion of the cerebral arteries by a high-pressure injection momentarily pinches the spinal bulb within the closed cranial cavity, and renders it ancemic. ${ }^{5}$ If this suggestion be accepted, and it be agreed that the vagus centre is in all cases directly excited by anamia, how is the normal interdependence of the arterial tension and cardiac inhibition to be explained? This is a question which cannot be settled, since the afferent nerve of the heart is also the afferent nerve or channel of inhibition.

${ }^{1}$ Goltz, Virchow's Archiv, 1863, Bd. xxvi. S. 5.

2 "Trav. du labor. de Marey," 1880 , tome iv. p. 382.

${ }^{3}$ Arch. f. Physiol., Leipzig, 1883, S. 539.

+Francois-Franck, "Trav. dn labor. de Marey," 187 7 , tome iii. p. 276. p. 135

Hill, "The Physiology and Pathology of the Cerebral Circulation," London, 1896, 
On the whole, there is much to be said in favour of the supposition that the terminations of the afferent nerves on the inner wall of the heart may be stimulated by intracardiac pressure, and thus the controlling centres of the circulation-vagus, vasomotor, and respiratory-excited or depressed. If this be the case, the heart possesses a reflex mechanism for regulating, not only its own rate, but the whole circulation.

By moderate expansion of the lungs the heart is accelerated. ${ }^{1}$ The afferent nerves of this reflex are are the vagi. The frequency of the pulse is increased by singing, coughing, sipping water, reading aloud, and forced breathing. By starvation the excitability of the vagus centre is said to be greatly increased. The normal difference between the pulse frequency in inspiration and expiration becomes then very marked.2 The heart is normally greatly accelerated by severe muscular exertion, but slightly so after division of the vagi, and not at all after also dividing the accelerators. These observations have been marle on the rabbit. ${ }^{3}$

According to François-Franck, ${ }^{4}$ stimulation of the mucous membrane of the lungs and the upper part of the larynx produces slowing of the heart. Similarly, excitation of the nasal branch of the fifth nerve always

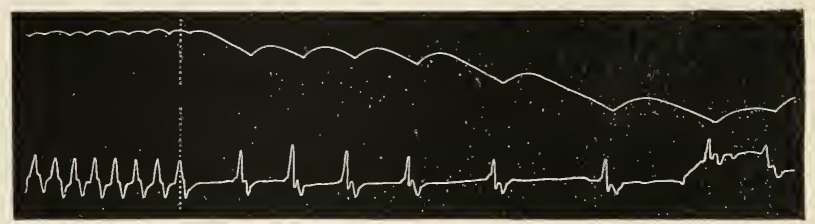

FIG. 38.-Reflex inhibition produced by inhalation of ammonia. The upper line is the trace of arterial pressurc, the lower line that of the pulse. -- Francois-Franck.

diminishes the frequency of the pulse (Fig. 38). ${ }^{5} \quad$ Thus, cardiac inhibition is of usual oceurrence during the primary stage of inhalation of chloroform.

In the frog, inhibition of the heart is produced with the greatest ease by tapping the intestines. ${ }^{6}$ The reflex are for this effect is formed by the abdominal sympathetic, the spinal cord and bulb, and the vagi. ${ }^{7}$

Almost any form of stimulation of the abdominal viscera produces slowing of the heart. Sudden distension of the stomach may be especially mentioned as a means by which this reflex may be evoked. ${ }^{8}$ Excitation of the central end of the sciatic or splanchnic nerves arouses to activity both the vagal and the accelerator centres. The accelerator centre is usually the more strongly excited of the two, so that increased frequency is usually the resultant effect. The excitation of the vagal centre either lasts the longer or else becomes relatively increased by

${ }^{1}$ Hering, Sitzungsh. d. k. Akad. d. Wissensch., math.-naturu. Cl., Wien, 1871, Bd. lxiv. S. 332 ; Sommerbrodt, Ztschr. f. klin. Med., Berlin, 1881, Bd. ii. S. 601.

2 Aduceo, Areh. ital. de biol., Turin, 1894, vol. xxi. p. 419.

${ }^{3}$ H. E. Hering, Centralbl. f. Physiol., Leipzig u. Wien, 1894, Bd. viii. S. 75.

" "Trav. du labor. de Marey," 1880 , tome iv. p. 378 .

"Francois-Franck, "Ibid.,"1876, tome ii. p. 226.

'Goltz, Tirchow's Archir, 1863, Bd. xxvi. S. 10.

7 Bernstein, Arch.f. Physiol., Leipzig, 1864, S. 622.

${ }^{8}$ Mayer and Pribram, S'itzungsh. d. k. Akah. d. Wissensih., math.-naturw. Cl., Wien, 1872, Bd. lxvi. Abth. 3, S. 102. 
the concomitant rise of arterial tension, so that after a time inhibition takes the place of acceleration. In some cases the vagal effect may be the stronger from the first. ${ }^{1}$ The frequency of the heart can similarly be affected by stimulation of every other afferent nerve in the body. The nasal branch of the trigeminus seems to stand alone as the one afferent nerve which always produces slowing and never acceleration of the pulse.

\section{The Depressor Nerve.}

In the rabbit, from the junction of the vagus and superior laryngeal nerves, there arises by two roots a fine nerve, which courses down the neck.

On excitation of the central end of this nerve, Ludwig and C'yon ${ }^{2}$ witnessed a surprising fall of arterial pressure, accompanied by a slight decrease in cardiac frequency. The peripheral end failed to give any response to stimulation. Thus the purely afferent nature of the nerve was established. On repeating the excitation of the central end, after section of both vagi, the arterial pressure fell to an undiminished extent, but the pulse rate, in this case, remained unchanged. After section of all the cardiac nerves, the result was still the same. This nerve was named, by Ludwig and Cyon, the depressor, for it possessed the power of depressing the arterial tension by 30 to 50 per cent. Ludwig and Cyon observed that, on stimulating the depressor, the kidney flushed red with blood, while the fall of arterial tension became insignificant after section of the splanchnic nerves.

In the lower parts of its course the depressor nerve joins the nervous strands which issue from the first thoracic or stellate ganglion; thence, passing behind the aorta, and through the connective tissue which lies between the latter and the pulmonary artery, it reaches the heart. The fibres eventually gain the cardiac wall, and course over the ventricle. At this point they have been stimulated by Wooldridge. ${ }^{3}$ Some depressor fibres are found to rum in the vagi of the rabbit.

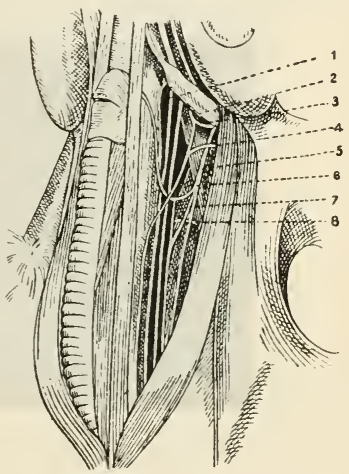

Fig. 39.-Dissection of the depressor nerve, rabbit. 1, sympathetic (the white line next to it is the line of the carotid artery); 2, hypoglossal ; 3 , descending branch of hypoglossal ; 4 , branch to this from cervical plexus; 5 , vagus; 6 , origin of superior larnygeal; 7 and 8, origin of de. pressor from vagus and superior laryngeal.-Ludwig and Cyon.

In differeut mammals, many variations are to be marked in the course of this nerve, but, in all, fibres with a depressor function are to be found. The same arrangement as in the rabbit, is met with in horses, hares, pigs, and hedgehogs. In dogs, and sometimes in cats, the depressor fibres are bound up with the vago-sympathetic trunk, but can often be separated as a distinct strand from the rest of that nerve. No sign of a depressor nerve has been found in any cold-blooded animal,

1 Roy and Allami, Phit. Trans., Louton, 1892, vol. elxxxiii. B, p. 258.

${ }^{2}$ Ber. d. k. süchs. Gesellsch. d. Wissensch. math.-phys. C., Leipzig, 1866, S. 307.

${ }^{3}$ Arch. f. Physiol., Leipzig, 1883, S, 539. 
although it has been diligently sought for by several observer's. In man, the homologous nerve arises from the junction of the vagus and superior laryngeal nerves, but quickly joins again the main trunk of the vagus.

The depressor seems to exert no tonic action on the vasomotor centre. Neither during normal conditions, nor in the state of asphyxia, is any rise of arterial pressure produced by sudden section of both depressors. $^{1}$ The depressor seems incapable of fatigue. It has maintained its full functional effect during a period of excitation lasting seventeen minutes. ${ }^{2}$

If the vagi be cut, excitation of the depressor is often followed by acceleration of the heart. This is the ordinary effect of a fall of blood pressure. If, while one depressor is under excitation, the other be stimulated, a summation of effect is produced.

The activity of the depressor abolishes or very much diminishes Traube-Hering curves, when these are present on the tracing of arterial pressure. At the same time, it lessens the extent of the respiratory undulations. On simultaneously exciting a pressor nerve, such as the anterior crural, together with the depressor, an antagonism is provoked,

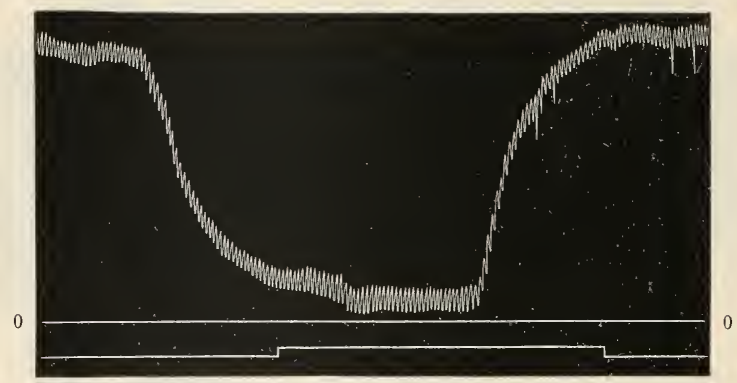

Fig. 40.-Aortic pressure. Excitation of depressor. The drum was stopped in the middle of the curve, and the excitation maintained for seventeen minutes. The line of zero pressure $(0,0)$ slıould be $30 \mathrm{~mm}$. lower than is here shown.-Bayliss.

and the strongest produces its effect. So when the depressor is excited, and asphyxia produced, the pressor effect overcomes and abolishes the depressor influence, while, if the excitation be continued, and respiration be supplied, the depressor effect, after a short period, regains the mastery. ${ }^{3}$ Neither pressor nor depressor effects can be obtained after the injection of strychnine, nor does the depressor act while the arteries are contracted by intravenous injection of suprarenal extract. ${ }^{4}$

Excitation of the depressor increases the resistance in the central nervous system in such a way as to prevent the tonic influence which is maintained by the vaso-constrictor centre. It does not appear, however, to produce anabolism of this centre during the period of rest or inhibition, for the rise of arterial tension produced by asphyxia is no greater after than before stimulation of the depressor.

${ }^{1}$ Konow and Stenbeck, Skandin. Arch. f. Physiol., Leipzig, 1889, Bd. i. S. 424.

2 Bayliss, Journ. Physiol., Cambridge and London, 1893, vol. xiv. p. 314.

3 Ibid., p. 319.

${ }^{4}$ Oliver and Sehäfer, Journ. Physiol., Cambridge and London, 1895, vol. xviii. 
An alternative view is that the depressor excites a hypothetical vaso-dilator centre. There are, however, certain differences between the depressor and other vaso-dilator nerves. The latter are easily exhausted by artificial stimulation, while the former is inexhaustible. On the other hand, according to Bayliss, section of the spinal cord at the level of the second lumbar nerve-root ${ }^{1}$ prevents, when the depressor is excited, any manifestation of dilatation in the lower limbs; and fibres producing vaso-dilatation of the legs have been found, by Bradford and Bayliss, by direct excitation of the third, fourth, fifth, sixth, and seventh lumbar roots. The question is one not yet definitely settled, and no conclusion as to the exact method of action of this nerve can be drawn. Light should be thrown on the subject by experiments on such a gland as the salivary, which possesses a distinct and powerful supply of both vaso-dilators and vaso-constrictor nerves. It should be possible to determine by section of one or other of these nerves the channel by which the depressor acts. The depressor stands alone as a distinct type of nerve. No other nerve in the body can, on excitation

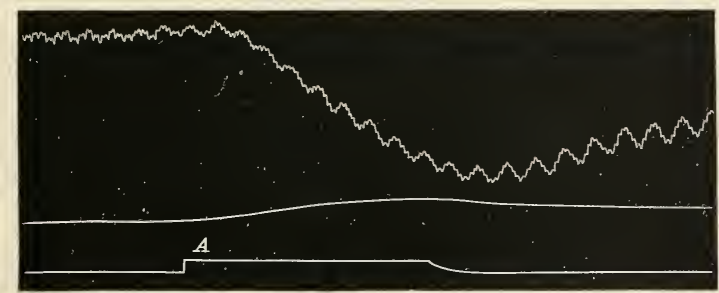

Fig. 41.-Aortic pressure and volume curve of limb. A, Excitation of depressor.-Bayliss.

of the central end, uniformly produce a reflex fall of blood pressure. By all other nerves, either pressor or depressor effects are provoked, and, generally, on prolongiug the excitation, the first effect is followed by the second. The depressor is purely an afferent dilator nerve.

Almost all parts of the vascular system can be thrown into dilatation by the depressor nerve. While compression of the thoracic aorta does not entirely abolish the fall of arterial tension produced by excitation of this nerve, section of the spinal cord in the cervical region does do so. It follows that parts of the body above the abdomen must share in the reflex dilatation.

By Bayliss the question has been minutely studied. He employed the plethysmographic method. The limbs were enclosed in a glass cylinder, the connection being made air-tight by means of an indiarubber collar greased with vaselin; the variations of volume were recorded by means of a tube passing from the cylinder to a delicate tambour. In such experiments, in order to avoid disturbing elcments, due to movements or cardiac inhibition, it is necessary to place the animal under curari, and to divide both vagi. The limbs dilate on exciting the depressor, but the latent period is long. ${ }^{2}$ This is in eonsequence of the primary passive constricting effect produced in the limb by the fall of arterial tension.

1 The vaso-constrictor nerves for the lower limb come off above this level.

${ }^{2}$ Bayliss, Journ. Physiol., Cambridge and London, 1893, vol. xiv. p. 304. 
After clamping the thoracic aorta, or dividing the spinal cord in the dorsal region, excitation of the depressor still causes a fall of arterial pressure. This fall is abolished by section of the cervical sympathetic nerves. The vessels of the head and neek therefore share in the production of the depressor effect, and, on plethysmographing the tongue, this organ is found to dilate. So, too, the thigh muscles, free from skin, and the foot, which is practically all skin and bone, are both found to dilate (Bayliss). Thus we may conclude that the action of the depressor is almost universal, nevertheless the splanchnic area is by far the most important seat of dilatation. It is by means of this afferent nerve that the heart can relieve itself of too great a systolic strain. The splanchnic floodgates can be thrown rapidly open thereby, and the peripheral resistance lowered.

When an animal is placed in the feet-down position during excitation of the depressor, the arterial pressure, owing to splanchnic dilatation, falls to a very marked extent. ${ }^{1}$

\section{HENODYNAMICS.}

The circulation of the blood follows certain definite laws; unfortunately the conditions of flow are so complicated that these laws remain for the most part still undetermined.

A viscous fluid driven by an intermittent pump which circulates through a system of branching elastic tubes of varying capacity; a system of tubes into and out of which passage of fluid takes place either by osmosis, filtration, or secretion ; a fluid which varies in viscosity ; a pump which varies in force, and tubes which have an ever-changing diameter and coefficient of elasticity. It is in the first case helpful to consider the flow of fluids in certain simple models, where the conditions are constant. The application of theoretical deductions thus obtained must be tested by experimental inquiry on the living animal.

Torricelli's law.-If a circular hole be pierced in the bottom of a vessel of water, the outflowing fluid, according to the law deduced by Torricelli, should have a velocity $V=\sqrt{?-g h}$.

Now, the velocity of a free falling body, whell it has fallen through a space $h$, equals $\sqrt{2} g \%$. Therefore the velocity of the fluid escaping through the hole in the bottom of the vessel is equal to that which the fluid would attain if it had fallen free through the height of the column of water. In other words, the velocity of outflow is that velocity which a free falling body, starting from a state of rest at the surface, would gain on reaching the orifice. Given the velocity of outflow, and the sectional area of the orifice, the product of these two is equal in a given time to the volume of the output. The output is equivalent to the cylindrical column of fluid which has fallen through the orifice in the given time. If $A$ is the volume of the output, then $A=\pi r^{2} \sqrt{2 g \%}$.

Experiment, however, yields an actual result which shows that the outflow is less than that theoretically deduced. This is because the motion at the orifice is not steady. Eldies are there set up; and in consequence of their counter-action, the well-known phenomenon of the vena contracta is produced.

Thus, whenever fluid in motion meets a sharp edge, there is a breach of continuity and a consequent loss of energy. This loss can be avoided by imitating the form of the vena contracta and making the orifice bell-shaped. With a bell-shaped orifice the observed velocity is 0.99 times the theoretical value. Such an arrangement exists in the branches of the aorta. Thoma has determined this by injecting molten paraffin at blood pressure into the arterial circulation, and cutting sections at the points of branching. The shape of

${ }^{2}$ Hill and Barnard, Journ. Physiol., Cambridge and London, 1897, vol. xxi. p. 335. 
the curve formed by a plane section through the axis of a main artery and its branches is approximately a parabola, and this is found by experiment to be the best shape. Hence there is no energy lost when blood passes from a main artery into its branch. ${ }^{1}$

If a tube be fixed to the orifice, then the mean velocity of outflow through such a tube leading from the bottom of the vessel is found to be less than through the simple orifice. This is due to the resistance to the flow in the tube, owing to viscosity of the fluid; that is, in a simple case, where the fluid wets the inner wall of the tube. Now, "rigid mathematical calculation can only be applied to the motion of actual fluids, as long as it is not turbulent; when a certain limit of velocity is exceeded the motion is disturbed."

By introdueing a small stream of coloured liquid into transparent tubes, in which water was moving with a velocity nearly equal to that of the blood, Nicolls found the motion not turbulent in tubes of different diameters. We may assume, therefore, that the flow in the bloor vessels is not turbulent.

Flow of fluid in cylindrical tubes.- In a cylindrical tube, the fluid particles, flowing under constant pressure, move parallel with the axis, but with varying velocity. In the axial layer the velocity is at its greatest, at the wall it is practically nil. The material of which the wall is made has no influence on the velocity, the whole friction lies between the particles of fluid. In any two tubes, equally long and witle, it is only the chemical and physical qualities, i.e. the viscosity of the circulating fluid, which influence the relative velocity of flow. Since the threads of fluid which are at the same distance

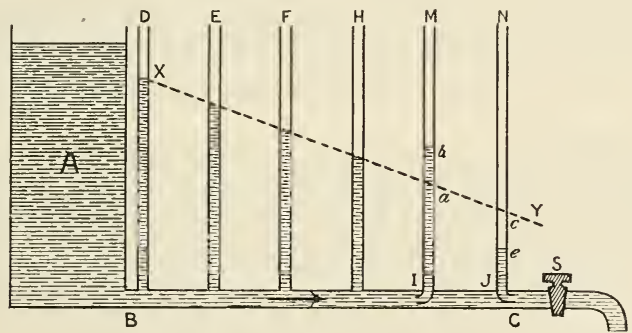

FiG. 42.

from the axis will move at the same velocity, the fluid may be considered as consisting of an infinite number of concentric cylindrical surfaces, which glide over one another, and move the more rapidly, the smaller their radius. The velocity which is reckoned from the outflow per second per sectional area of the tube, yields us the mean velocity of all these cylinders of fluid.

The mean velocity in such tubes as we are considering, where the motion is stearly, is equal to lialf the axial velocity. ${ }^{2}$ - It is the friction between the particles of fluid, or the viscosity, that causes the outflow from a tube to be less in a given time than from a free opening of the same bore. Part of the head of pressure is spent in overeoming this viscosity. The amount of head so lost in overcoming fluid friction is in a uniform tube proportional to the length of tube which has been traversed by the fluid. This may be shown experimentally by means of the apparatus sketched in Fig. 42 .

In the figure, $A$ is a vessel filled with fluid to the level shown; $B C$ is a uniform tube attached horizontally to $\mathrm{A} ; \mathrm{D}, \mathrm{E}, \mathrm{F}, \mathrm{H}, \mathrm{M}, \mathrm{N}$, are vertical tubes (piezometers) to indicate the lateral pressure at different points along

1 Nicolls, Journ. Physiol., Cambridge and London, 1896, vol. xx. p. 416.

${ }^{2}$ v. Kries, Arb. a. d. physiol. Anst. $\approx u$ Leip $\approx i$ ig, 1887, S. 101. 
BC, of which $\mathrm{M}$ and $\mathrm{N}$ are provided with bent nozzles, turned in the case of M to face the direction of flow, in the ease of $\mathrm{N}$ in the direction of flow; $\mathrm{S}$ is a stopeock, by means of which the fluid can be allowed to flow along BC with different velocities.

So long as $\mathrm{S}$ is kept closed, the level of the fluid in all the tubes is the same as that in the vessel (A); but as soon as $\mathrm{S}$ is opened, the level falls in all the tubes, and the fall is greater, the greater the distance along BC. This gradual fall in pressure along $\mathrm{BC}$ is clue to the loss through fluid resistance, and as the amount of this loss is proportional to the length of tube traversed (when the tube is uniform and the flow constant), it follows that the fall in head in the tubes $(\mathrm{D}, \mathrm{E}, \mathrm{F}$, and $\mathrm{H}$ ) will be proportional to the length at which each of these tubes is situate along BC, measured from the opening into the reservoir (A), and will be represented by the line (XY). Since the fluid resistance in a tube varies with the square of the velocity of the fluid, it follows that the loss in head when the velocity is varied will be directly as the square of the velocity. Hence, when the flow is made faster by opening more fully the stopcock (S), the line of fall of pressure becomes more steeply inclined. The tangent of the angle of slope of this line to the horizontal line (BC) is called the "pressure gradient." The pressure gradient is from this evidently equivalent to the fall in pressure per unit length.

Besides this gradual fall, due to fluid resistance along BC, there is a sudden drop in lateral pressure just at the beginning of $\mathrm{BC}$, which is not due to fluid resistance, but to a portion of the pressure due to the head in $\mathrm{A}$ being used to give velocity to the fluid as it enters BC. The amount of head so lost is called the "velocity head." Its value is easily calculated when the velocity is known, for equating potential energy lost to kinetic energy gained, $2 g h=v^{2}$, or $h=\frac{v^{2}}{2 g}$, where $h$ is velocity head, $v$ is velocity, and $g$ accumulation due to gravity.

The dependence of the velocity head on the velocity can be shown by manipulating the tap (S). When the flow is made very slow, the pressure in $D$ is nearly the same as that in A; when the flow is increased, the level falls in $\mathrm{D}$, showing that the initial loss in pressure due to velocity has increased. At the same time, the effect of the increased velocity on the pressure gradient is shown by the increased steepness of the line (XY). That the loss in lateral pressure due to velocity head persists all along the tube (BC) can be shown by the tubes (M and $\mathrm{N}$ ). In M the impact of the fluid against the mouth of the tube raises the level in $\mathrm{M}$ above the height due to the lateral pressure alone at that point by an amount $(a b)$ equal to the velocity head. Similarly, in the tube (N), the suction of the fluid passing the bent end depresses the column in $\mathrm{N}$ by an amount (ce) equal again to the velocity head. The lateral head at any given point is often called the "effective head;" and the loss in head due to fluid resistance up to any point, the "resistance head." It is evident that the sum of velocity head, resistance head, and effective head must equal the total head, or reservoir head.

In the arteries the velocity head is very small, because the velocity of flow is not great; taking the velocity in the aorta as 32 cms. per second, the velocity head, $h=\frac{32^{2}}{2 \times 981}=\frac{1}{2} \mathrm{~cm}$. of blood.

Poiseuille's law.-In tubes wherein the threads of fluid move in parallel lines, Poiseuille has laid down the law that the mean velocity is directly proportional to the sectional area of the tube and the pressure gradient. In other worls, we can find the mean velocity by the product of three factors-sectional area, pressure gradient, and a constant coefficient $(C)$, which depends on the viscosity or physico-chemical nature of the fluid in the conditions of experiment. This coefficient can be defined as that mean 
velocity which a current would have, with a unit pressure gradient in a tube of unit sectional area.

Jacobson ${ }^{1}$ passed water through a tube of constant bore, $437 \mathrm{~mm}$. long and $1.147 \mathrm{~mm}$. in radius, with a mean velocity of $802.54 \mathrm{~mm}$. per second. The pressure gradient was $0.5485 \mathrm{~mm}$., and the cross-section of the tube 4.132 sq. mm. According to Poiseuille's law, then

$$
802.54=C \times 4.132 \times 0.5485 . \cdot \text { the coefficient } C=353.85 \text {. }
$$

Having once found the value of the coefficient, the velocity, if Poiseuille's law be right, can be reckoned in any experiment carried out under the same conditions, where the sectional area and the pressure gradient are known. Jacobson experimentally found that this was so. Let $R=$ the radius of a tube, $V=$ the velocity of any particle at a distance $(A)$ from the centre. Let $l=$ length of tube, $p p^{\prime}$ the pressures at the two ends of the tube, $\mu$ the coefficient of viscosity. Then $V=\frac{p-p^{\prime}}{4 \mu l}\left(R^{2}-A^{2}\right) \therefore$ the velocity varies inversely as the viscosity. The axial velocity $=\frac{p-p^{\prime}}{4 \mu l} R^{2}$. The mean velocity $=\frac{p-p^{\prime}}{4 \mu l} \cdot \frac{R^{2}}{2}$ The quantity of fluid which flows through in a given time $t=\frac{p-p^{\prime}}{4 \mu l} \cdot \frac{\pi R^{4} t}{2}$

The coefficient at one and the same temperature varies for different fluids, and is found to be smaller in proportion to the viscosity of the fluid. Nicolls investigated the viscosity of blood by means of Poiseuille's viscometer. The liquid was allowed to escape from a reservoir through a capillary tube in a horizontal position. The two orifices at the ends were bell-shaped, and at the end where the fluid issued there was a lip to prevent the resistance of surface tension. The viscosity is obtained from the time occupied in emptying the reservoir.

The viscosity of blood was found by Ewald ${ }^{2}$ and by Nicolls to be about five times, by Lewy ${ }^{3}$ three and a half times, that of distilled water. A mixture of blood and water is less viscous than blood. The viscosity of blood diminishes rapidly as the temperature rises. Assuming that this is true in the living body, it follows, if the quality of the blood remains unchanged, that the rise of temperature in fever diminishes the resistance, and consequently the heart has less work to do.

Duncan and Gamgee found that the velocity of flow in glass capillaries of 0.9289 to $1.259 \mathrm{~mm}$. diameter was greater for unclotted than for defibrinated blood. ${ }^{4}$ The viscosity of the blood is increased by addition of $\mathrm{CO}_{2}$, ether and chloral, decreased by laking the blood.

By experiments upon the flow of distilled water in capillary glass tubes, 0.15 to $0.65 \mathrm{~mm}$. in diameter, Poiseuille reached the following conclusions :-

1. That the amount of outflow is proportional to the head of pressure.

2. That the time spent in the outflow of a certain volume of fluid at a constant pressure is, if the diameter be constant, directly proportional to the length of the tube.

3. That with the same heal of pressure, the time spent in the outflow of a certain volume of fluid through equally long tubes is inversely proportional to the fourth power of the diameter. Thus, if $A$ be the volume of outflow per second,

$$
A=\frac{l \cdot r^{4}}{l} H, \text { and } V=\frac{A}{\pi r^{2}}=\frac{l r^{2}}{\pi l} H,
$$

where $r$ is the radius, $l$ the length of the tube, $H$ the pressure head, and $l$;

${ }^{1}$ Arch. f. Anat., Physiol. u. wisscrsch. Med., Leipzig, 1860, S. 85. Quoted from Tigerstedt's "Physiol. des Kreislaufes," S. 312

${ }^{2}$ Arch.f. Physiol., Leipzig, 1877, s. 217.

${ }^{3}$ Arch. f. d. ges. Physiol., Bonn, 1897, Bd. 1xv. S. $447,461$.

${ }^{4}$ Journ. Anat. and Physiol., London, 1871, vol. v. p. 155.

VOL. II. -5 
a coefficient depending on the viscosity and temperature of the fluid, but independent of the structure of the tube wall so long as this is wetted by the fluid; $k$ is known as the transpiration coefficient.

In such a model as is shown in Fig. 42 , the velocity head can be easily determined by an instrument which can be directly applied to the circulation. ${ }^{1}$ The instrument is a glass tube (nqs, Fig. 43).

"From $n$ to $q$ the tube is of a fine bore, and at $n$ there is a small bell-shaped opening. When the tube is plunged into moving liquid, so that the orifice (n) faces the current, as at I (Fig. 42), the fluid will rise to the height Ib, due to velocity and pressure; if $\mathrm{n}$ be turned at right angles to the current, the height will be Ia; and if turned so that $\mathrm{n}$ is with the current as at $J$, the height Je will be less than the pressure head by an amount ce, which is nearly equal to the velocity head. By means of this instrument very accurate results can be obtained, especially for velocity, as, by taking the diflerence of two

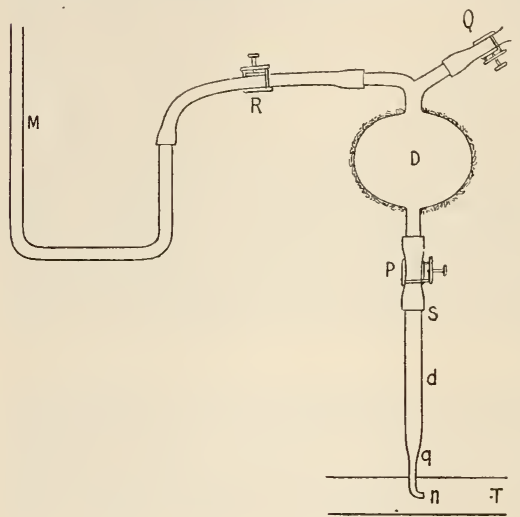

Fig. 43. heights, the effect of capillarity disappears. When the pressure is high, as in an artery, the following modification can be adopted. The end of the tube (s) is connected with a rubber tube $(\mathrm{P})$ provided with a clamp. $\mathrm{P}$ is, in its turn, connected with a glass reservoir (D) containing air ; this is covered thickly with cottonwool, to prevent changes of surrounding temperature affecting the air. The glass reservoir is connected with a mercury manometer (M) and a syringe by tubes $(\mathrm{R}$ and $\mathrm{Q})$ provided with clamps. The tube $(\mathrm{P})$ is clamped, and the pressure is raised in the reservoir to a point which is known to be about that of the arterial tension. The tube.(nq) is then introduced into the artery, and the clamp (Q) is closed and P opened."

The tube (nq), in experiments with this instrument, is filled with a solution of oxalate of potash to prevent clotting. In one of Nicoll's researches, the inferior mesenteric artery was exposed and divided in a dog, and through it the end $(n)$ was introduced into the abdominal aorta. At the beginning of the experiment, the height of the fluid in ns indicated a pressure of $145 \mathrm{~mm}$. $\mathrm{Hg}$. The velocity head was a little over $6 \mathrm{~mm}$. $\mathrm{Hg}$, indicating a mean velocity $=\sqrt{12 g}=346 \mathrm{~mm}$., for $V^{2}=2 g h$. The change of height in ns due to pulsation was $9 \mathrm{~mm}$. Hg.

The flow in a tube of varying diameter.- Since fluid is incompressible, an equal amount must flow in the unit of time through every section of the tube, and thus the velocity in any part of a tube which varies in diameter stands in inverse proportion to the sectional area. In such a tube the pressure gradient is steepest in the narrowest section, for there the velocity and the friction is greatest. In two sections of equal diameter the pressure gradient is the same. Where a wide section follows upon a narrower, the lateral pressure may either sink, remain unaltered, or even rise. How this can be so will appear from the following considerations. At any point of the tube the whole pressure head $(H)$ equals the sum of the velocity head $\left(h^{\prime}\right)$ and the resistance head $\left(h^{2}\right)$.

1 Nicolls, Journ. Physiol., Cambridge and London, 1896, vol. xx. p. 417. 
Now, $H$ decreases uniformly along the tube, and since where the tube widens the velocity becomes less, and $h^{\prime}$ suddenly diminishes, it follows that $h^{2}$ increases, and it is conceivable that $h^{2}$ in the wide section may become higher than $h^{2}$ in the narrow section. In other words, since more of $H$ is spent in maintaining the velocity in the narrow section, the lateral pressure may be lower there than in the succeeding wide section.

The flow in branching tubes.-When a tube branches into a number of smaller tubes, and these collect again into one tube, we have two opposing factors to consider-

1. The increase of sectional area. The velocity is inversely proportional to the sum of the sectional area of the branches.

2. The increase of resistance, due to the great extent of surface contact between the moving fluid and the fluid that wets the walls of the tubes. The resistance is proportional to the surface area, nearly proportional to the square of the velocity, and inversely proportional to the sectional area. The formula used by engineers for what they call skin friction is $R=h: S v^{2}$, where $R=$ resistance ; $k$, a constant ; $S$, surface area ; $v$, velocity.

On passing from $a$ to $b$ (Fig. 44 ), the velocity has decreased, but great resistance has been overcome; the total effect therefore will be a much lower lateral pressure at $b$. On passing from $b$ to $c$ the velocity increases, and a

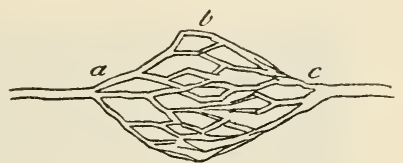

F1G. 44 . further great fall of lateral pressure will have taken place owing to the resistance that has been overcome between $b$ and $c$.

The flow in elastic tubes. - The same laws which have been discussed in connection with rigid tubes obtain in elastic tubes, if the flow of fluid be continuous.

If the inflow be intermittent:-in the case of the rigid tube the whole column of fluid is driven on, step by step, by each stroke of the pump, and the outflow is also intermittent. In an elastic tube, on the other hand, if the resistance to the output be made great, as by constricting the orifice, the elastic wall of the tube is extended by the input of the pump, and the elasticity of the wall comes into play, and continues the output after the stroke of the pump has ceased.

By the heart the blood is rliythmically driven into the arteries; the arterial walls are elastic ; in the small arteries, arterioles, and capillaries, there exists great resistance. All the conditions are present for converting the intermittent flow from the heart into the continuous flow through the capillaries. ${ }^{1}$ If the arteries were rigid tubes, the heart would be required to drive on the whole blood at one and the same time in all the vessels, in place of intermittently forcing a certain small volume of blood into the large arteries. The heart is, on the contrary, saved this large expenditure of work, and after each systole retires into its diastolic rest, leaving the elasticity of the large arteries to continue the circulation. Further, if two tubes be taken, one elastic and the other rigid, and they be chosen so that each delivers the same output when fed by a continuous inflow, then, if the inflow be made intermittent, it is found that the elastic tube now yields the greater output. ${ }^{2}$

Beyond these advantages, due to the intermittent pump and elastic tubes, there is another physiological fact which as yet has received no explanation. It has been found that, if normal saline be artificially circulated by continuous pressure through the vessels of an animal, œdema of the tissue soon arises, and the outflow decreases. If, on the other hand, the inflow be made rhythmic, as in the natural condition, then the ordema is insignificant, and the outflow

'Stephen Hales, "Statical Essays," vol. ii.

${ }^{2}$ Marey, Ann. d. sc. nat. Zool., Paris, 1857, tome viii. p. 330. 
is fourfold as great as in the first case. ${ }^{1}$ The efficacy of an impulsive compared with a steady force is a matter of everyday observation, and we may perhaps attribute this result to the opening of the capillaries by the impulsive action of wave motion. ${ }^{2}$

\section{A Discussion of Some of the General Principles of the Circulation.}

By E. H. Weber ${ }^{3}$ the model represented in Fig. 45 was constructed. This can be conveniently used as the basis for the discussion of some of the main principles of the circulation. In this model, a piece of small intestine, fashioned as a single chamber, represents the heart $(H)$. The valves are formed of short pieces of intestine, which are firmly tied on to pieces of glass

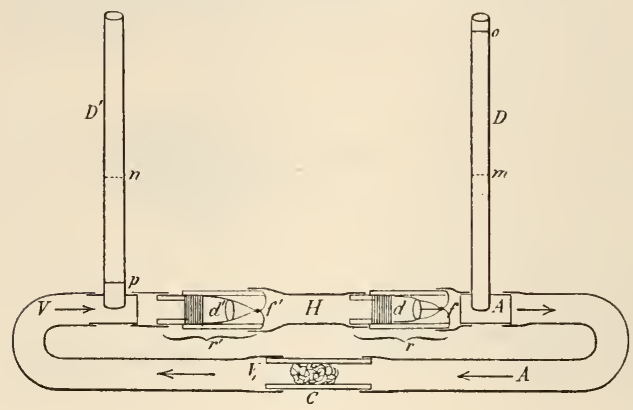

Fig. 45 . tube. These are fixed within and to either end of the heart. The valves are prevented from turning inside out under pressure by short guiding strings, which, fastened as they are to the edges of the valves, take the place of the chordæ tendinex. The artery $(A)$ and the vein $(V)$ are formed of a long loop of intestine, and a

sponge $(C)$ is inserted within this loop to take the place of the arterioles and capillaries. The model is distended with water to an extent represented by $m$ and $n$ in the manometers $D$ and $D^{\prime}$.

Suppose the model be laid upon a horizontal surface, so that the effect of gravity is eliminated, and the conditions made as simple as possible. If by squeezing it with the hand, $H$ be now made to contract, the fluid contained therein is directed by the valves and forced on into $A A$. If $A A$ were a rigid tube, the whole column of fluid would be forced on at one and the same time, and the flow of fluid into $V$ would last only just so long as the contraction of $H$ was maintained. As, however, $A A$ is an extensile elastic tube, the projection of the fluid is successive. The systolic output first finds a place by distending the tube $(A)$, and produces therein a positive wave, which travels with a certain velocity through the system. This velocity is independent of the velocity of flow. If the valves were not competent, and the systole passed into diastole at the moment the blood was expelled, then the expanded tube $(A)$ would drive part of the blood back again into $H$, and a negative wave would arise, which, following the positive wave, would also travel with a certain velocity through the system. In the entire absence of valves, the fluid particles would swing backwards as much as forwards, and in the end would suffer no change of place. As, however, the valves are competent, there is no back swing into $H$, and each systole pushes on the fluid a step further.

If all valves were absent from $H$, the fluid would stream into it from both sides of the system at the moment when the pressure on it was relaxed;

${ }^{1}$ Hamel, Ztschr. f. Biol., Miinchen, 1888, Bd. xxv. S. 474.

2 Nicolls, privately communicated to the author.

${ }^{3}$ Ber. ü. d. Verhandl. der königl. Sächs. Gesellsch. der Wissensch., Leịzig, 1850, S. 186. 
thus two negative waves would appear; one of these would travel through $A$, the other through $V$. As on diastole the valves $(d)$ shut to while the valves $\left(d^{\prime}\right)$ open, the fluid from $V$ alone can stream into $H$, and a negative wave appears in $V$ only. Thus the model is so constructer that, during the periodic systole and diastole, only waves of positive pressure appear in $A$, and waves of negative pressure in $V$. Both waves ail one another, and cause the fluid to move in one and the same direction.

If the system were formed of wide tubes, free from constrictions, each positive wave would travel with so great a velocity, that the whole system would reach the same pressure, and before the next systole occurred a condition of equilibrium would be established. On the other hand, on account of the resistance in the sponge, which represents the arterioles and capillaries, it is otherwise. The internal friction or viscosity of the fluid particles, as they pass through the interstices of the sponge, prevent the fluid from pressing through with anything like the velocity of transmission of the positive wave. Thus the positive wave becomes reflected at the sponge, and runs to and fro in

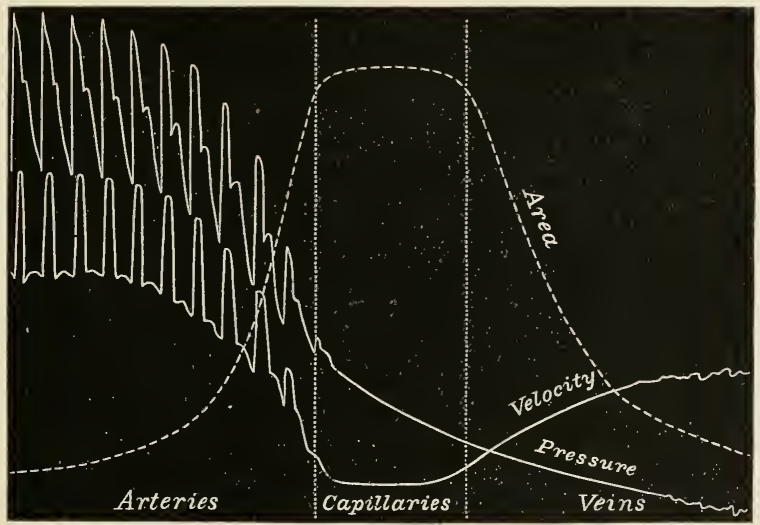

FIG. 46. - Distribution of pressure and velocity during diastole and systole of the heart.-Frederieq.

$A$, until it is finally damped down and lost, so that no pulse reaches $V$. Should, however, the sponge be loose in structure, and possess wide pores, then the pulse night pass through into $r$.

If the periodic systole of $H$ be made to occur with a sufficient frequency, the fluid is piled up in $A$, for at each systole a fresh quantity of fluid is driven into $A$, while, in the same time, a less quantity escapes through the sponge into $V$.

With each systole the pressure mounts in $A$. In $V$, the pressure, on the other hand, may sink, but it cannot sink below zero, in spite of the continued abstraction of fluid, owing to the fact that the flaccid wall of $V$ cannot support any part of the atmospheric pressure. Suppose the pressure in the arterial manometer $(D)$ has risen to $O$, and in the venous manometer has sunk to $p$, and at this point imagine the action of $H$ to cease, the pressure will fall in $D$ and rise in $D^{\prime}$, at first with greater and then with decreasing velocity, until the pressure in the two manometers is once more the same. In other words, the action of $H$ has upset the balance of tension, and this balance is regained by the flow of fluid in the direction of the arrows, at first with 
great, and then with an ever-decreasing velocity, until the velocity reaches zero at the moment when the pressure in $D$ and $D^{\prime}$ becomes the same. It is therefore clear that the flow of fluid is produced by the difference of pressure in $A$ and $V$. If this pressure difference be greater, the velocity of flow is greater; if it be smaller, the velocity is smaller.

So soon as the pressure difference between the artery and vein is established, the fluid will move through the sponge into the vein, not in jets, but with a constant flow. If in the model, $H$ be working with a constant rhythm and energy, and a constant flow be established, it is obvious that any change in the output per second of $H$, or in the peripheral resistance, will upset the constancy of the flow, until a new condition of equilibrium is once more established.

In such a continuous system as Weber's model, a hydrostatic mean pressure is obtained when the heart is at rest. This is the average fluid pressure, as already stated, which pertains to the system, when the capacity of the model is constant, and the fluid is in a state of stasis.

The hydrokinetic mean pressure is the average fluid pressure throughout the system when the fluid is in motion. These means are obtained by measuring the pressure at each unit of length along the tube and dividing by the number of units. Weber maintained that the hydrostatic and hydrokinetic mean pressures are equivalent; that is to say, the pump cannot increase the mean pressure of the system. By so much as it increases the pressure on the arterial side it will decrease it on the venous side. This deduction of Weber's is not admissible, for the arterial tension can be raised, while the tension in the vein caunot fall below zero, for, as already stated, the flaccid wall of the vein is unable to support any part of the atmospheric pressure.

It has also been held that contraction of arterioles, by the influence of vasomotor nerves, not only increases the peripheral resistance, but also diminishes the total capacity of the system, so that we have the same effect produced as if more fluid were poured into the system. Thus, it has been said, an increase in peripheral resistance must always connote a rise in the hydrostatic mean pressure of the system brought about by the diminution of its capacity. According to this supposition, a rise of arterial tension, occasioner by vasomotor activity must be explained by the coincidence of two factors - $(a)$ increased peripheral resistance; $(b)$ diminished capacity of blood vessels. ${ }^{1}$ Now, if the model be not filled to distension, then any diminution of its capacity will not raise the fluid hydrostatic pressure above zero, until the reduction of capacity has reached the point when the walls of the system are thrown into tension. In other words, if 1000 c.c. be introduced within an elastic bag, capable of holding without distension 2000 c.c., the capacity of the bag can be reduced from 2000 c.c. to 1000 c.c. without any alteration of the tension of the wall. On turning to the evidences of experiment, we find that the vascular system is not filled to distension. The proofs for this statement are the following :-

1. Owing to the elastic resilience of the arteries, and the influence of gravity, the blood, after the arrest of the heart, passes into the venous side, and to the most dependent parts of the body. Thus the large arteries are found after death to be empty of blood.

2. It is not possible to distend the entire vascular system of a recently killed animal even by injecting into an artery a volume of fluid equal to that of the blood. Even after the injection of 500 c.c. of water into a dog, the tension in the arteries falls with the utmost rapidity. The fluid flows until it has collected in the most dependent parts of the body. By alternately placing the animal in the horizontal and vertical feet-down postures, the fluid can be

${ }^{1}$ Bayliss and Starling, Journ. Physiol., Cambridge and London, 1894, vol. xvi. p. 165 ; ef. Mall, Arch. f. Physiol., Leipzig, 1892, S. 409. 
pumped from the arteries past the venous valves into the veins, and the arterial tension can be rapidly driven to zero.

3. In a normal living dog, the heart is arrested by stimulation of the vagus. During the standstill of the heart, the animal is alternately placed in the vertical feet-down and horizontal positions. By these means the blood is quickly pumped from the arteries into the veins. In the vertical feet-down position, the pressure in both the aorta and the vena cava superior becomes zero. On then opening the femoral artery, only a few drops of blood escape, and on turning the animal into the vertical feet-up position, air is sucked into the artery.

From the consideration of these experimental facts, it is clear (1) that the system is not filled to distension; (2) that various pressures can occur in different parts of it, when the heart is in arrest; (3) that a reduction in total capacity of the system need not raise the mean pressure of the whole. It is owing to the great capacity of the veins that the vascular system is not filled to distension. Normally, the larger number of veins have a flattened instead of a cylindrical shape, and, owing to this fact, a large amount of fluid can pass into them, before the elasticity of their walls is brought into play. On the other hand, the arteries are fashioned of a highly elastic distensile tubing, the capacity of which is very small when undistended, while the elasticity is so great that considerable tension is required to increase the capacity. The whole of the blood within the body can pass into the roomy reservoirs of the veins, and yet the walls of the veins will scarcely be thrown into tension. It is partly owing to these differences that the blood vacates the arteries, and collects in the veins after death. Brunner ${ }^{1}$ found that, thirty seconds after complete cessation of the heart-beat and respiration, the fall of pressure in the carotid artery was thirty-eight to fifty-seven times as great as the fall in the jugular vein.

So long as the action of the heart and respiratory pump remain constant, neither in the vena cava, nor in the pulmonary artery, is there any rise of pressure during periods of vaso-constriction. ${ }^{2}$ Thus, on excitation of the peripheral end of the spinal cord (divided between the sixth and seventh dorsal nerves), a rise of aortic pressure from 72 to $230 \mathrm{~mm}$. $\mathrm{Hg}$ has been recorded, while the pressure in the pulmonary artery remained at from 20 to 21 mm. Hg. ${ }^{3}$ In such experiments the vena cava or pulmonary pressure does not rise until the heart fails to maintain the systolic output in face of the increased peripheral resistance. Similarly the fall of arterial pressure which takes place on general vaso-dilatation, is not occasioned in any part by the increase in the total capacity of the vascular system. This is further shown by the fact that the vena cava pressure is generally unaltered (it may sometimes either rise or fall slightly) when the spinal cord is divided in the upper dorsal region. The rise of arterial pressure which takes place on constriction of vascular areas, is due to the power of the heart and the increased peripheral resistance. The constriction of the arterioles acts in the same way as the closing of a clip placed on the aorta or on the tube $(A)$ in Weber's model. The closing of the clip scarcely diminishes the capacity of the system, but the pressure rises behind the resistance.

One of the most striking phenomena in the circulation is the comparative independence of the systemic arteries, the pulmonary vessels, and the systemic veins. Large oscillations of pressure in the systemic arteries produce little or even no effect on the blood pressure in the venæ cavæ or pulmonary artery. The vascular system is especially constructed as a discontinuous system, in order that great changes of pressure may be brought about on the arterial side, without any very great alteration of the pressures in the venous or pulmonary

${ }^{3}$ Ztschr. f. rat. Med., 1855, S. 336.

${ }^{2}$ Hill and Barnard, Journ. Physiol., Cambridge and London, 1897, vol. xxi. p. 343.

${ }^{3}$ Bradford and Dean, ibid., 1894, vol. xvi. 1. 34, lig. 1. 
side of the circulation. Thus the venæ cavæ have a constant low head of pressure, from which the heart draws its supply, and the diastolic pressure within the heart is kept constant during the enormous variations of arterial pressure which are brought about by vaso-constriction.

The variations in vena cava pressure under different circumstances can be seen in the following experiments carried out on $\operatorname{logs}:^{1}$

\begin{tabular}{|c|c|c|c|c|c|c|c|c|c|}
\hline \multirow[b]{2}{*}{1.} & \multicolumn{4}{|l|}{. } & & \multicolumn{2}{|c|}{ Femoral Artery: } & \multicolumn{2}{|c|}{ Vena Cava. } \\
\hline & $\begin{array}{l}\text { Normal } \\
\text { On excitation }\end{array}$ & of spla & anchnic n & erve & & $\begin{array}{c}92-94 \\
111-132\end{array}$ & .. $\mathrm{Hg}$ & $\begin{array}{l}5 \cdot 2-6 \cdot 3 \\
6 \cdot 3-6 \cdot 7\end{array}$ & $\begin{array}{l}\text { m. } \mathrm{Hg} \\
,,\end{array}$ \\
\hline 2. & $\begin{array}{l}\text { Normal } \\
\text { On excitation }\end{array}$ & of vag & us : & . & . & $\begin{array}{l}87-90 \\
10\end{array}$ & ,", & $\begin{array}{l}3 \cdot 2-3 \cdot 4 \\
6 \cdot 2\end{array}$ & ," \\
\hline 3. & $\begin{array}{l}\text { Normal } \\
\text { Bled } 150 \text { c.c. }\end{array}$ & $\begin{array}{l}\cdot \quad \cdot \\
\cdot \quad\end{array}$ & $\dot{.}$ & . & . & $\begin{array}{l}74 \\
26\end{array}$ & , & $\begin{array}{l}3 \\
1 \cdot 7\end{array}$ & ", \\
\hline 4. & $\begin{array}{l}\text { Normal } \\
\text { Bled to death }\end{array}$ & . & . & . & 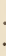 & $\begin{array}{l}98 \\
10\end{array}$ & ,", & $\begin{array}{l}7 \\
6 \cdot 5\end{array}$ & ," \\
\hline 5. & $\begin{array}{l}\text { Normal . } \\
\text { Injection of } 50 \\
\text { Ten minutes } 1\end{array}$ & $\begin{array}{l}00 \text { c.c. } \\
\text { ater. }\end{array}$ & of saline & $\dot{.}$ & & $\begin{array}{l}102 \\
124 \\
122\end{array}$ & $\begin{array}{l}\text {," } \\
, "\end{array}$ & $\begin{array}{r}2 \cdot 5 \\
17 \cdot 3 \\
7 \cdot 7\end{array}$ & ", \\
\hline
\end{tabular}

If at any moment the right heart be unable to empty itself, owing to obstruction in the pulmonary circulation-as, for example, in Valsalva's experiment-- the large veins and the liver form a reservoir for the congested blood. In those animals which remain long under water-such as the seal, otter, and diving birds-large dilatations of the vena cava inferior occur. During the time of impeded respiration accumulation of venous blood takes place in the vena cava without an unusual distension of the right heart. ${ }^{2}$

If the left heart be unable to completely empty itself, and its ontput per second decrease owing to great increase in peripheral resistance, the pulmonary veins and capillaries will then, in the first place, form a reservior for the congested blood; and when the pulmonary obstruction tells back on the right heart, the liver and systemic veins form a second line of defence. It is to be concluded that the venous side forms a low-pressure reservoir for the circulatory system, by which the diastolic pressure of the heart can be maintained constant.

The seat of peripheral resistance lies in the small arteries. That this is so is shown by the enormous fall of arterial pressure which takes place when the splanchnic arterioles are dilated by section of the splanchnic nerves. Normally, the sum of the resistances which, at each moment, oppose the flow from the aorta is maintained at the same value. When one vascular area dilates, another constricts, and thus the arterial pressure is maintained constant. Owing to the vasomotor mechanism the necessary blood quantum is greatly reduced, for, since the blood can be sent to any organ that requires it, all the available space in the vascular system need not be filled. A highpressure main runs to all the organs of the body. By means of the vasomotor nerves the stream can be turned on here or there, and any part flushed with blood, while the supply to the remaining parts is proportionately diminished.

\section{The Elasticity of Arteries.}

The coefficient of elasticity of an excised strip of an artery is not a constant factor, for with the extension it increases. The curve

1 Bayliss and Starling, Journ. Physiol., Cambridge and London, 1894, vol. xvi.

2 Allen Thomson, "Todd's Encyclopiedia," 1836, p. 679. 
obtained by equal increments of tension from such a strip is hyperbolic in form. ${ }^{1}$

The variations in the volume of an artery examined under different degrees of internal tension has been measured by Marey, ${ }^{2}$ Roy, and others. This can be done by the apparatus shown in Fig. 47 .

In healthy arteries, placed under observation immediately after death, the volume by equal increments of internal tension will be found to increase by equal increments up to a certain point. Under continued increase of tension the extensibility decreases, or, in other words, the coefficient of elasticity increases. Thus the elasticity curve of a healthy artery is a true hyperbola. In the rabbit's aorta the maximum extensibil-

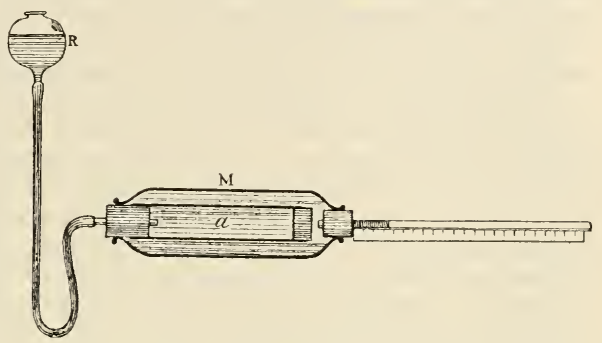

Frg. 47.-R, pressure bottle ; $a$, artery; M, plethysmograph.-Marey.

ity, or the point at which the change to a decreased ratio of extensibility takes place, is reached at abont $70 \mathrm{~mm}$. $\mathrm{Hg}$; in the cat's, at 110 to 120 $\mathrm{mm}$. Hg. In the living dog, by measurements of the diameter, the maximum extensibility of the aorta has been found at a pressure of 75 to $100 \mathrm{~mm}$. $\mathrm{Hg}^{3}$ In many arteries, taken from the post-mortem room, the extensibility is found to be greater at lower increments of tension. This is the case with all wasting diseases; and although, on microscopical examination, no structural change is to be found, yet the more advanced the marasmus preceding death, the lower is the pressure which corresponds to the maximum extensibility of the arteries. With the advance

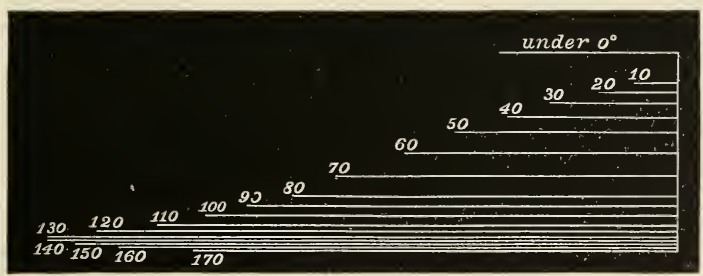

FIG. 48. - The height between the horizontal lines represents extension of artery under different pressures. The pressures are noted at the end of the lines. The difference in length of the lines is merely for the convenience of placing the numerals. - Roy.

of age the arteries become less and less extensile. When the arterial pressure is high, it follows from the above fact that any increase in the

1 Wertheim, Ann. de chim. et phys, , Paris, 1847, tome xxi. p. 385.

2 "Trav. du labor. de Marey," 1880 , tome iv. p. 78 ; Roy, Journ. Physiol., Cambridge and London, 1881, vol. iii. p. 125; 1888, vol. ix. p. 227.

3 Zwaardemaker, Nederl. Tijdschr. v. Geneesk., Ansterdam, Tweede Reeks, 18s8, vol. xxiv. p. 61 . 
output of the heart must increase the blood pressure by a very significant amount. The arteries reach their maximum extensibility at the normal blood tension, 90 to $120 \mathrm{~mm}$. Hg. Beyond this point the arteries become more and more rigid, and the heart must then at each beat push on a longer and still longer column of blood.

The elasticity of an artery is very complete. On removing the tension the volume returns exactly to its previous condition. On raising the internal tension up to the normal blood pressure the cubic volume of an artery very greatly increases, e.g. four to six times.

If the heart-beat be brought to a standstill by vagus inhibition, the arteries almost entirely empty themselves of blood into the veins.

When an artery is dilated by an injection of fluid, it reacts, according to John Hunter, with a force greater than that of the injection pressure. ${ }^{1}$ This resilience is much greater in the living artery than in the dead artery. It is probable that when the heart, on vagus inhibition, ceases to beat, the arteries empty themselves by contraction of their muscular coat. Some arteries exhibit a propulsive wave of contraction, as is seen in the contractile bulbus arteriosus of amphibia and some fishes. The same phenomenon is to be seen in the rhythmic contractility of the arteries of the ear, in the saphenous artery of the rabbit, and in the vessels of the frog's web. ${ }^{2}$

The breaking strain of a healthy artery is very great. In some experiments of Volkmann, the carotid of a goat successfully withstood a pressure of $2250 \mathrm{~mm}$. Hg, i.e. about fourteen times the normal blood pressure. It takes an internal pressure of 3000 to $8500 \mathrm{~mm}$. $\mathrm{Hg}$ to rupture the carotid artery of a dog. In the case of the human carotid the smallest rupturing pressure was found to be $1290 \mathrm{~mm}$. $\mathrm{Hg}$. The larger arteries rupture more easily than the smaller, and thus the aorta breaks asunder at a lower tension than the radial. ${ }^{3}$

According to Nicolls, to attain the greatest strength with a given amount of material, a cylinder should be constructed of layers the elastic coefficient of which gradually increases from within outwards. Now, this is exactly the arrangement of the different layers of the arterial wall; thus the greatest strength is attained by the smallest amount of material consistent with safety. Similar reasoning applies to the walls of a sphere, from which it appears that the main source of weakness in an aneurysm is, that the wall practically is composed of only one coat. ${ }^{4}$ As the sac enlarges the total strain increases as the cube of the radius of curvature.

The influence of the amount of blood in the body on the systolic output and arterial pressure.-The average amount of blood in the body, estimated by Welcker's method, can be taken as equivalent to 6 to 9 per cent. of the body weight in dogs, 5 to 9 per cent. in cats, 4 to 8 per cent. in rabbits. In man it has been estimated as equal to 7 per cent. of the body weight. ${ }^{5}$ On transfusion of fluid, the striking fact becomes apparent, that the arterial tension cannot possibly be raised by such means above that height which the tension can reach when the vessels contain a normal amount of blood. This was so even when

1 "Works of John Hunter," London, 1837, vol. iii. p. 157.

${ }^{2}$ Riegel, Arch. f. d. ges. Physiol., Bonn, 1871, Bd. iv. S. 350.

${ }^{3}$ Stephen Hales, "Statical Essays," vol. ii.; Grélhant and Quinquand, Journ. de l'anat. et physiol., etc., Paris, 1885, tome xxi. 1). 287 .

4 Nicolls, Journ. Physiol., Cambridge and London, 1896, vol. xx. p. 410.

5 Bischoff, Ztschr. f. wissensch. Zool., 1856, Bd. vii. S. 331. 
Worm Müller ${ }^{1}$ transfused fluid to the extent of $12 \cdot 7$ per cent. of the body-weight.

Similarly, bleeding to the extent of 1.88 to 2.82 per cent. of the body-weight causes only an insignificant fall of arterial tension. The velocity of the escape of the blood has a considerable influence on the change of arterial tension produced by bleeding. Sudden anæmia of the bulbar centres excites, while slowly advancing anæmia, without any preliminary stage of excitation, paralyses these centres. Thus it is easier to obtain a fall of tension by slowly tapping a vein, than it is by allowing the same quantity of blood to escape rapidly from an artery. After rapid hæmorrhage the heart is accelerated, the action of the respiratory pump increased, and the arterioles constricted. The blood is taken from the veins, and piled up in the arteries. It has been observed in the frog that the vessels contract and the tissue lymph passes into the circulation after the loss of blood. ${ }^{2}$ At the same time the secretions become diminished.

Transfusion of blood or of saline solution, in the normal animal, at first raises the arterial pressure to about 170 to $180 \mathrm{~mm}$., but the pressure never passes much beyond this limit. If the cervical cord be divided, and the arterial pressure thus lowered previous to the transfusion, the pressure may reach to the same limit, and not beyond. The reason for this is not far to seek. So soon as the arterial tension reaches a certain height, the systolic output of the heart becomes less complete, the residual blood in the left ventricle increases, and the diastolic pressure rises. The diastolic rise of pressure in the left ventricle tells back in turn on the left auricle, the pulmonary circulation, the right heart, and the venous circulation. The transfused fluid thus congests in the capacious reservoirs of the veins and capillaries, especially in the lungs, the liver, and the abrlominal organs. According to Cohnheim, ${ }^{3}$ injections of saline equal to 25 to 30 per cent. of the body-weight, or four times that of the amount of the blood, does not notably increase for any length of time the pressure in the femoral vein. Still larger injections may raise the venous pressure to 18 or $20 \mathrm{~mm}$. Hg. "These experiments show that the vascular system is vastly more capacious than the accommodation of the normal blood quantum demands." On microscopical examination of the circulation, some of the capillaries are always seen to be empty and collapsed, except in times of vaso-dilatation. Likewise the large veins appear on examination normally more or less flattened, not round and distended with blood. By the filling out of these vessels, the capacity of the vascular system is enormously increased. On transfusion of large quantities of saline, the liver becomes swollen, hard, and tense; the lungs and other tissues odematous. Fluid is transuded, and the abdoninal organs becone without exception markedly dropsical. Large quantities of urine collect in the bladder, and the stomach and intestines become distended with fluid. Almost every one of the secretions is increased, and the lymph flow from the thoracic duct may become twenty-five times greater than at the commencement of the experiment. In fact, the leakage of fluid finally becomes as fast as the rate of transfusion, and

1 Ber. d. k. sächs. Gesellsch. d. Wissensch. math.-phys. Cl., 1873, Leipzig, S. 573-664. Cf. Magendie, Compt. rend. Acad. d. sc., Paris, 1838, tome i. 1. 55.

${ }^{2}$ Klemensiewiez, Sitzungsb. d. k. Akad. d. Wisscnsch., Wien, 1887, Bd. 96, Abth. iii. S. 57 .

3 "Lectures on Pathology," New S'yd. Soc. Translation, vol. i. p. 457. 
thus neither the arterial nor the venous pressure can be driven up beyond a certain point; and this point, it is noticeable, is not very far removed from the normal pressure. After the transfusion of large quantities of fluid, both the venous and arterial pressures return rapidly to normal. On injecting a volume of fluid equal to that of the blood, Grosglik $^{1}$ estimated that 69 to 79 per cent. transuded in the first hour. On injecting saline coloured with methyl blue into the circulation, the blue colour appears in five to ten minutes in the stomach and the bladder. Dastre and Loye ${ }^{2}$ found that, on injecting saline at the rate of 1 c.c. per minute for each kilo. of body weight in the dog, the transfusion was compensated by an equally rapid excretion from the kidney.

In the diagram (Fig. 49) is exhibited the effect on the arterial, portal, and vena cava pressures produced by the injection of 500 c.c. of normal saline solution; an amount fully equal to the blood quantum of the dog. It will be seen that five minutes after the injection was finished, the vena cava pressure had risen only $60 \mathrm{~mm}$. $\mathrm{MgSO}_{4}$ solution, or scarcely $5 \mathrm{~mm}$. Hg. In another experiment, 350 e.c. of normal saline were

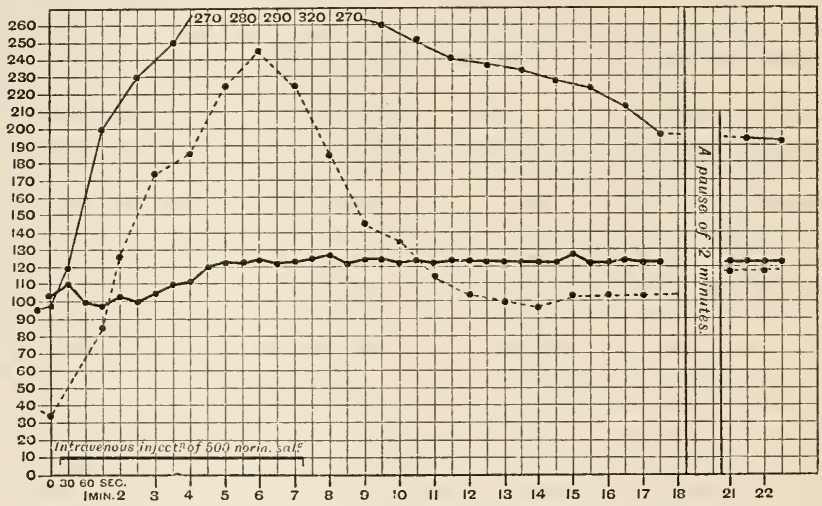

Fig. 49.-Effect of injection of 500 c.c. saline solution on the arterial pressure (black line), portal pressure (fine line), and vena cava pressure (interrupted line). -Bayliss and Starling.

injected during the space of two and a half minutes into a dog. During the injection the arterial pressure rose from 60 to $70 \mathrm{~mm}$. $\mathrm{Hg}$, the portal pressure from 87 to 167 , and the vena cava pressure from 34 to 124 mm. $\mathrm{MgSO}_{4}$ solution. Ten minutes later the pressures had become -arterial $72 \mathrm{~mm}$. Hg, portal $110 \mathrm{~mm}$, and vena cava $50 \mathrm{~mm}$. $\mathrm{MgSO}_{4}$ solution. ${ }^{3}$ After the injection of saline the velocity of the blood stream is very considerably accelerated, not merely during the injection, and simultaneously with the rise of blood pressure, but also lastingly, long after the pressure has again become normal. The eause of this is the lessening of the viscosity of the blood by dilution. The increase of velocity can be observed in the frog's web after injection of 1 c.c. of normal saline into the vena abdominalis, " the light-coloured blood shoots with such rapidity

${ }^{1}$ Arch. de physiol. norm. et path., Paris, 1890, p. 704.

2 Ibid., 1888, 1. $93 ; 1889$, p. 253.

${ }^{3}$ Bayliss and Starling, Journ. Physiol., Cambridge and London, 1894, vol. xvi. p. 128. 
through the vessels, that one is absolutely unable to recognise an individual corpuscle in the capillaries." 1 More than half an hour elapses before the velocity commences to slacken. No such increase of velocity is to be observed on the injection of blood; that is to say, after the injection has ceased and the blood pressure has returned to normal.

After the injection of blood, Worm Muiller found that during the next few hours a very considerable portion of the injected plasma passed out of the vessels. The surplus corpuseles disappeared in the course of a few days.

The functional power of the vascular system is lowered by pronounced plethora. Venesection of a dog, in which artificial plethora has been produced, causes the arterial pressure to fall so rapidly, that it is impossible to obtain even the quantity of blood originally transfused. When the injection of fluid into a vein is maintained for some time, the heart must be strained by the increased diastolic pressure. It is probably owing to cardiac failure that animals sometimes die during continuous injections of large quantities of fluid. The collapse of the heart may sometimes be prevented by the letting of blood. ${ }^{2}$ The heart has not only to do extra work when the diastolic pressure is raised, but the emptying of the coronary veins is impeded, and thus the coronary circulation impaired. The fact that asphyxia produces no rise of arterial pressure in plethoric dogs, with the fact that they are easily killed by hremorrhage, shows that there is general vasomotor paralysis produced by over-distension of the vascular system. The addition of blood to the extent of 150 per cent. of the normal blood quantum is the outside limit beyond which life is directly threatened.

\section{The Pressure in tile Arteries.}

The lateral pressure of the blood in the large arteries was first accurately measured by Stephen Hales. ${ }^{3}$ He writes-

"In December 1733, I caused a mare to be tied down alive on her back; she was fourteen hands high, and about 14 years of age, had a fistula on her withers, was neither very lean, nor yet lusty. Having laid open the left crural artery about 3 in. from her belly, I inserted into it a brass pipe whose bore was one-sixth of an inch in diameter; and to that, by means of another brass pipe, which was fitly adapted to it, I fixed a glass tube of nearly the same diameter, which was $9 \mathrm{ft}$. in length; then untying the ligature on the artery, the blood rose in the tube $8 \mathrm{ft} .3 \mathrm{in}$. perpendicular above the level of the left ventricle of the heart. When it was at its full height it would rise and fall at and after each pulse 2, 3, or $4 \mathrm{in.}$; and sometimes it would fall 12 or 14 in., and have there for a time the same vibrations up and down, at and after each pulse, as it had when it was at its full height, to which it would rise again after forty or fifty pulses."

Hales drew the correct inference from his experiments that "the real force of the blood in the arteries depends on the proportion which the quantity of blood thrown out of the left ventricle in a given time bears to the quantity which can pass through the capillary arteries into the veins at that time."

Simple as these experiments are, they afford us the most striking

${ }^{1}$ Cohnheim, loc. cit., p. 457 .

${ }^{2}$ Johansson and Tigerstedt, Skandin. Arch. f. Physiol., Leipzig, 1889, Bd. i. S. 394.

3 "Statical Essays," vol. ii. 
demonstration of arterial pressure. Nevertheless the method of Hales is open to two objections-first, the inconvenience of a long tube; second, the speedy coagulation of the blood.

By Poiseuille ${ }^{1}$ both these sources of difficulty in the method of experiment were removed, when he substituted for Hales' glass tube a mercurial manometer, and to prevent coagulation filled the tube of connection between the artery and the manometer with a solution of sodium carbonate.

Ludwig ${ }^{2}$ completed the work of Poiseuille by adding to the mercury

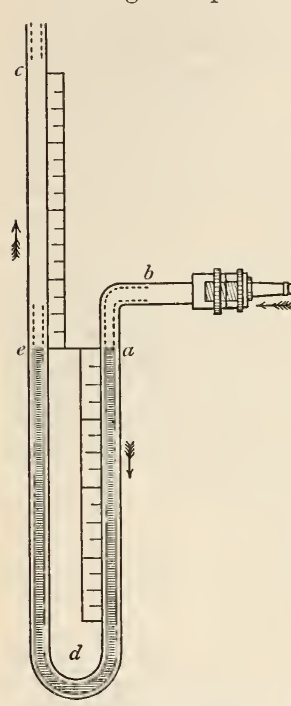

Fig. 50.-Poiseuille's hæmodynamometer. manometer a float carrying a writing style, and by recording thereby on a revolving drum the variations of arterial pressure. To this device of Ludwig (kymographion) the graphic method in physiology owed its origin. ${ }^{3}$

Owing to the inertia of the mercury, the mercurial manometer is not fitted to give a true record of the pressure changes which occur during the cardiac cycle. When the heart is beating slowly, the maximal aortic pressure, when read by this instrument, is too high, and the minimal pressure is too low; with a rapid heart the reverse obtains. Thus, no knowledge as to the true systolic and diastolic variations of pressure can be gained by it.

The mercurial manometer is nevertheless the most convenient and useful instrument for obtaining records of the mean arterial pressure. It is stated by v. Kries ${ }^{4}$ that the manometer should be constructed of tubing $4 \mathrm{~mm}$. in diameter. The error in the mean pressure is then not greater than 3 per cent. With the use of wider or narrower tubes the error becomes greater. If the manometer tube be very considerably constricted in one part of its course, then the instrument, whatever be its diameter, gives the true mean pressure, for the oscillations of the mercury are almost entirely damped down by the constriction. The speed of the instrument is thereby sacrificed, for it takes some time to rise to the mean pressure. The instruments of precision now largely employed for investigations on the systolic and diastolic variations of arterial pressure, are Fredericq's modified form of sphygmoscope and the Fick, Hiirthle, or v. Basch manometers (see intracardiac pressure). Before use these manometers must be graduated against a mercury manometer.

For the purpose of obtaining continuous records of arterial pressure, the writing style of the arterial manometer is brought to bear on a band of paper, covered with lamp-black. By means of clookwork this band can be made to revolve at varying speeds round two drums placed at either end of a long frame. There are also brought to write on the paper an electric signal, by which points of excitation can be marked during the course of an experiment,

1 " Rech. sur la force du cœur aortique," Thèse, Paris, 1828.

2 Arch.f. Anat., Physiol. u. wissensch. Med., 1847, S. 261.

${ }^{3}$ For an improved method of constructing the float of Ludwig's kymograph, see Anderson Stuart, Journ. Physiol., Cambridge and London, 1891, vol. xii. p. 147.

4 Arch. f. Physiol., Leipzig, 1878, S. 430. 
and a time-marker. The band of paper is of sufficient width to enable the experimenter to record, simultaneously with the arterial pressure, the venous pressure, the respiratory movements, the intraventricular pressure, or any other phenomena which require investigation.

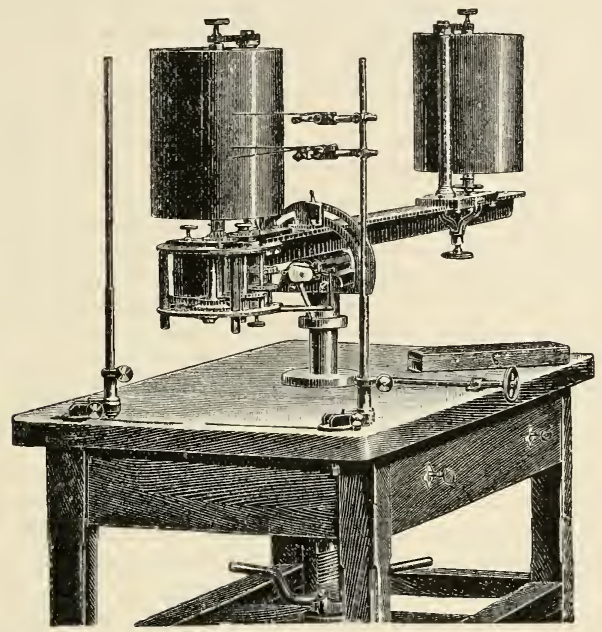

Fig. 51.-Hurthle's recording apparatus.

A convenient form of arrangement of such drums is that of Hering, as modified by Huirthle, which is shown in the figure; the two drums are united by a continuous band of smoked paper.

Several instruments have been contrived for estimating the arterial pressure

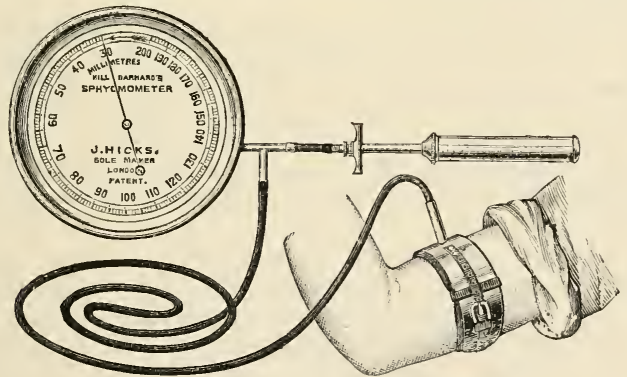

FIG. 52.-Hill and Barnard's sp'hygmometer.

in man. ${ }^{1}$ The one which has been most extensively used is v. Basch's ${ }^{2}$ sphygmomanometer. This consists of a small capsule; the cavity of this is connected with a manometer. The floor of the capsule is formed of a rubber nembrane, and this is pressed upon the radial artery until the pulse vanishes

${ }^{1}$ See George Oliver, "Pulse Gauging," London, 1895.

2 Wien. med. Wchnschr., 1883, S. 673; Ztschr. f. klin. Lled., Berlin, Bd, ii. S. 79 ; Berl. klin. Wchnschr., 1887, S. 181. 
beyond the seat of compression. The reading of the manometer at this point is taken to be equal to that of the arterial pressure. The fallacies in the use of such an instrument are very considerable. It is impossible to avoid compressing the venæ comites radialis, the skin and surrounding tissues, together with the artery, and it is difficult to note the exact moment when the pulse ceases to be perceptible. Experimentally, it has been found that the errors may be as great as 30 to $70 \mathrm{~mm}$. $\mathrm{Hg}$.

Recently a sphygmomanometer has been invented, which possesses considerable accuracy. This instrument consists of a broad armlet, which is strapped round the upper arm. The armlet is formed of a watch spring or stiff leather band, on the inside of which is a bag of thin indiarubber. The rubber bag is connected by a $T$-tube with an air-pump and a spring manometer. The pressure within the bag is raised until the pulsation in the manometer becomes of maximal excursion. At this point the pressure indicated by the manometer is read, and this pressure is the mean arterial pressure. ${ }^{1}$

The instrument has been tested thuns: It was placed round the neck of a dog (excluding the trachea), and simultaneous readings were then taken, both of the pressure in the femoral artery, as indicated by a mercurial manometer, and of the pressure in the carotid arteries, as indicated by the instrument. The maximal excursion of the pulse gave a sharp and accurate index of the mean arterial pressure.

In the case of healthy young men the pressure in the brachial artery is from 110 to $130 \mathrm{~mm}$. $\mathrm{Hg}$. The pressure in a normal healthy individual is maximal in the vertical and minimal in the horizontal position. $^{2}$ A hot bath diminishes, and a cold bath increases, the pressure. Violent exercise raises the tension by $20 \mathrm{~mm}$. $\mathrm{Hg}$; this rise lasts for some fifteen minutes, and is followed by a fall. Alcohol lowers the arterial tension. During chloroform anæsthesia the tension falls 20 to $40 \mathrm{~mm}$. Hg. During deep sleep the pressure may be 20 to $30 \mathrm{~mm}$. Hg lower than in the active waking state. This lowered pressure is not, however, peculiar to the condition of sleep, for just as low a pressure may obtain if the body be kept at rest, and this is so even although the mind be engaged in quiet work.

It must be remembered, in using such an instrument for the purpose of obtaining a series of accurate comparative results, that certain principles of experiment must be complied with. Thus, the subject of the experiment must be placed uniformly in one position, and the arm which is under investigation must be put at a uniform level in relation to the heart. If this be not done, large errors will arise in the readings, owing to variations in the hydrostatic effect of gravity and the efforts of the vasomotor meclianism to compensate for the same.

Faivre has directly measured the arterial pressure in man during the amputation of a limb. His results gave a pressure equal to 110 to $120 \mathrm{~mm}$. $\mathrm{Hg}$. The arterial pressure is considerably higher in warmthan in cold-blooded animals; in the former it is at least three times greater. The pressure is independent of the size of the animal, and thus may be as great in the cat as in the horse. ${ }^{3}$ In the sheep's embryo (in utero) the pressure has been found to be from 43 to $84 \mathrm{~mm}$. Hg. ${ }^{4}$ The total work of the heart, however, is greater in large than

${ }^{1}$ Maximal pulsation was first used as an index of mean tension in an instrument invented by Roy and Adami (Practitioner, London, 1890). Mosso has recently enployed the same index (Arch. ital. de biol., Turin, 1895, vol. xxiii. p. 196).

${ }^{2}$ In eonditions of asthenia the opposite obtains.

3 Volkmann, "Die Hämorlynamik," Leipzig, 1850, S. 177.

${ }^{4}$ Cohnstein and Zuntz, Arch. f. d. ges. Physiol., Bonn, 1884, Bd. xxxiv. S. 215. 
in small animals, because the ventricular output increases with the size of the heart. The work per unit weight of heart muscle in different animals has never been estimated. The mean arterial pressure, when the animal is in the horizontal position, is, within certain limits, nearly the same in arteries of different calibre, and at varying distances from the heart. Poiseuille ${ }^{1}$ found that the mercury in the hæmodynamometer rose to nearly equal heights when the instrument was connected in turn with the iliac, carotid, radial, facial, and other arteries. This is so, on account of the slight amount of resistance in the larger arteries compared to that in the smaller arteries and arterioles.

If the measurements of mean pressure are repeated in different arteries when the animal is placed, not in the horizontal, but in the vertical feet-down posture, then the arterial pressure varies owing to the effect of gravity (see p.90).

Although, in the horizontal posture, the mean pressure in different arteries varies but little, records of the systolic pressure show considerable differences. With his spring manometer Huirtlle has obtained a series of comparative measurements, from which the following table of diastolic and systolic pressures is takell :-

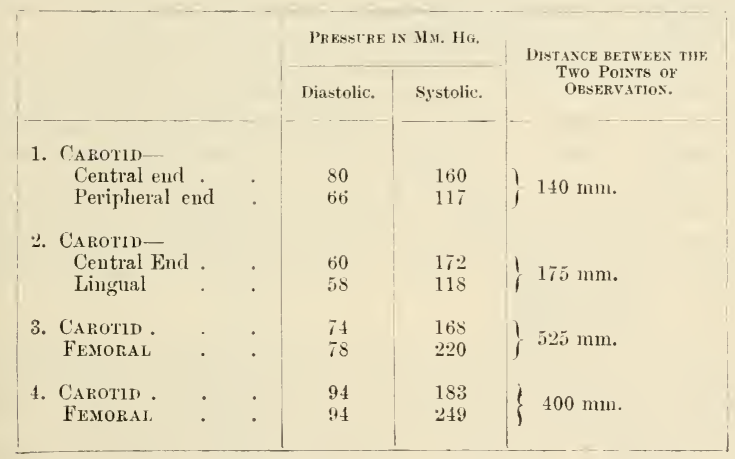

The two most striking results to be observed in this series are, first, the near agreement of the diastolic pressures; and, second, the fact that in the femoral, the systolic pressure is higher than in the carotid artery. This last result has been explained by supposing that the first centripetal positive pressure wave, which is reflected from the periphery, is of such a period that it adds itself in this artery to the primary centrifugal positive wave before the latter has passed away. It is, however, possible that the result is due to instrumental error. The rise of systolic pressure lasts longer in the femoral than in the carotid artery, and may thus be more correctly recorded. The experiments require repetition with the use of a maximal manometer.

The maintenance of a mean arterial pressure of constant height is the object of the circulatory mechanism. On the one hand, we are convinced that this object is attained during life; on the other hand, we know that countless and ceaseless variations are occurring in all parts of the circulatory system. The whole system must therefore be so craftily built and so delicately balanced, that every variation in one part is compensated by a simultaneous

VOL. 11. -6

1 "Reeh. sur la force du eneur aortique," Thèse, Paris, 1828. 
and contrary variation in another part, and thus, throughout the wear and tear of life, the aortic pressure is kept at a constant mean height. Pawlow ${ }^{1}$ possessed a dog so excellently trained that it lay still during any period of observation. In this dog, by means of an insignificant skin cut, Pawlow inserted a cannula into a superficial artery on the inner side of the knee. By this means he was able to measure the arterial pressure on successive days, connecting the cannula with the manometer on each occasion. As a rule, the mean pressure did not vary more than $10 \mathrm{~mm}$. Hg. Neither when the animal was fed on dry food after twelve hours' abstention from water, nor after the imbibing of large quantities of drink, did the pressure vary to a greater extent.

Pawlow observed the following pressures (in millimetres of mercury) in his dog on different days in the course of a month's observation :-

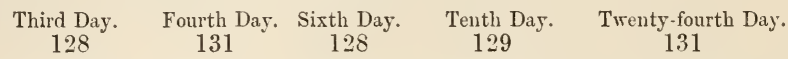

The same constancy has been observed in man by means of the sphygmomanometer.

\section{The Velocity of Blood Flow.}

In order that the circulation may be constantly maintained throughout the vascular system, an equal quantity of blood must on the arerage pass through each section of the system in the same time.

The output per second of the heart, divided by the total sectional area of the rascular system at any point, gives the mean relocity per second at that point. Thus the velocity is inversely proportional to the sectional area; and since the total sectional area of the arterial system increases from the aorta to the capillaries, the mean velocity must steadily decrease towards the periphery. On the other hand, since from the capillaries to the vena cava the total sectional area decreases, the mean velocity in the veins must increase centripetally (see Fig. 46, p. 69).

The velocity and the tension of the blood in the aorta are dependent on the energy of the heart and the resistance in the peripheral vascular system. In the living animal both of these factors are constantly varying, and we can consider the effect of such variations in the following examples:-

1. The energy of the heart is constant: $(a)$ the peripheral resistance increases. The tension in the aorta will become greater and the velocity less. (b) The peripheral resistance decreases. The velocity will become greater and the tension less.

2. The resistance remains constant: $(a)$ the energy of the heart increases. Both the tension and the velocity become greater. (b) The energy of the heart decreases. Both the tension and the velocity become less.

3 . The energy of the heart increases: the resistance at the same time becomes greater, and in proportion to the increase of cardiac energy. The tension rises and the velocity remains constant.

4. The energy of the heart increases: the resistance at the same time decreases, and in proportion to the increase of cardiac energr. The tension remains constant and the velocity becomes greater.

5. The energy of the heart decreases : the resistance increases, and in proportion to the decrease of cardiac energy. The tension remains constant and the velocity becomes less.

6. The energy of the heart decreases: the resistance becomes less, and in

${ }^{1}$ Arch. f. d. ges. Phy/siol., Bonn, 1878, Bd. xvi. S. 266 ; 1879, Bd. xx. S. 213. 
proportiun to the decrease of eardiae energy. The tension falls and the velocity remains constant.

Since under the influenee of the vasomotor mechanism any one section of the arterial system may become constrieted, while at the same moment any other section may become dilated, it is possible that the tension and velocity in the aorta may remain constant; for a balance may be struek between sueh variations when of opposite sign. It follows from the above that (1) the veloeity can vary in an opposite sense in different parts of the vascular system ; (2) the velocity in any partieular artery bears no absolute relation to the pulse frequency or the general arterial tension.

The velocity cannot be measured by simple division of an artery, and estimation of the outflow in a given time, for the peripheral resistance is almost entirely abolished thereby, while the output of the heart is rapidly diminished by the loss of blood. Tolkmann ${ }^{1}$ was the first to more or less accurately measure the velocity of blood-How by introducing a $\mathrm{U}$-shaped glass tube into the course of an artery. This tube was filled with a solution of a neutral salt, and provided with a tap. At a certain moment, the tap was turned and the rate of How of the columm of blood along the tule directly measured. Supposing the sectional area of the tube to be the same as that of the artery, the velocity in the latter is obtained without further correction. But it is practically impossible to select a glass tube which shall have the exact calibre of the artery to he measured, nor is the method suitable for comparative estimations of velocity during the course of an experiment. Among the more recent methods of investigation, Ludwig's stromuhr, ${ }^{2}$ by which the amount of blood passing in a given time may be accur-

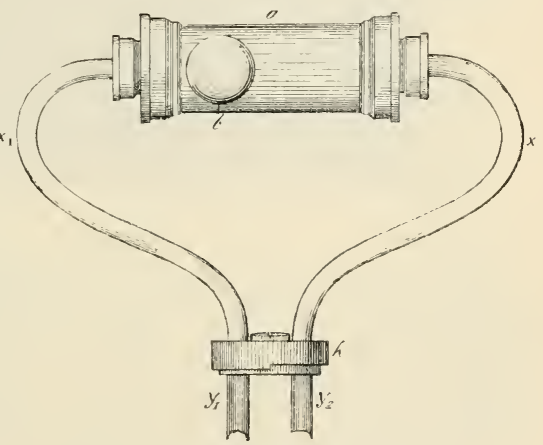

FIG. 53.-Ludwig's stromuhr, as modifier by Tigerstedt. ately measured, or its moditied form, as contrived by Tigerstedt, deserves description. $^{3}$

In using this instrument, the tube $\left(y_{1}\right)$ is placed in connection with the eentral end, and the tube $\left(y_{2}\right)$ in eomneetion with the peripheral end of the cut artery which is under investigation. The whole instrument is washed out with oil to prevent clotting, and filled with defibrinated blood. So soon as the blood is allowed to flow from the artery, the metal ball (b) is driven over by the current till it reaches the end of the eylinder $(a)$. The instrument is then rapidly rotated on the drum $\left(l_{i}\right)$, so that the tube $\left(x_{2}\right)$ comes to lie over the tube $\left(y_{1}\right)$, and the tube $\left(x_{1}\right)$ over the tube $\left(y_{2}\right)$. The metal ball is now once more driven by the current to the opposite end of the eylinder. This procedure is repeated several times, and the number of revolutions during the period of observation is noted. The eapacity of the cylinder (a), minus the volume of the ball (b), multiplied by the number of revolutions, gives the

i “Die Hämodynamik," Leipzig, 1850, S. 185.

2 Dogiel, Ber. d. k. Sächs. Gesellsch. d. Wissensch., Leipzis, 1867, Bd. хх. S. 200.

"Tigerstedt, skandin. Arch.f. Physiol., Leipzig, 1891, Bd. iii. S. 152. 
volume of blood which has passed during the period of observation, and this volume, divided by the time and the sectional area of the artery, gives the mean velocity per second. In using the stromuhr, the mean velocity in an artery is found to vary greatly. This, for the most part, is owing to the variations of resistance in the peripheral arterioles. During the operative procedure the blood-flow must for a time be cut off by clips in order that the tubes of the stromuhr may be introduced within the artery. From the anæmia thus produced there results a temporary paralysis of the tone in the arterioles. Variations of resistance arise when the blood-flow is rc-established and the tone commences to recover. By means of Ludwig's stromuhr, for example, Dogiel ${ }^{1}$ found the velocity to vary from moment to moment thus :-

\begin{tabular}{|c|c|c|c|c|c|c|}
\hline \multicolumn{4}{|c|}{ Time. } & \multicolumn{2}{|c|}{ Jean Velocity. } & \\
\hline $\begin{array}{l}0-15 \text { sec. } \\
15-25, \\
25-44, \\
44-65, \\
65-80,\end{array}$ & $\begin{array}{l}\dot{.} \\
\dot{.} \\
\dot{.}\end{array}$ & $\begin{array}{l}\text {. } \\
\text {. } \\
\text {. }\end{array}$ & $\begin{array}{l}. \\
\dot{.} \\
\text {. } \\
\text {. }\end{array}$ & $\begin{array}{l}489 \\
733 \\
386 \\
349 \\
489\end{array}$ & 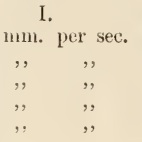 & $\begin{array}{l}\text { Dogr:-Carotid artery. } \\
\text { Diameter, } 2.7 \mathrm{~mm} \text {. }\end{array}$ \\
\hline $\begin{array}{r}0-30 \text { sec. } \\
30-36 \quad,, \\
36-54,, \\
54-70 \\
70-84, \\
84-90, \\
90-98, \\
98-100,\end{array}$ & $\dot{.}$ & $\begin{array}{l}\dot{5} \\
\dot{5} \\
\dot{5} \\
\text {. }\end{array}$ & . & $\begin{array}{r}226 \\
188 \\
125 \\
141 \\
161 \\
188 \\
141 \\
94\end{array}$ & 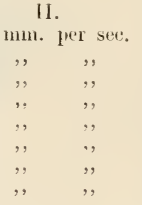 & $\begin{array}{l}\text { Rabbit. Carotid artery. } \\
\text { Diameter, } 1.4 \mathrm{~mm} .\end{array}$ \\
\hline
\end{tabular}

Tigerstedt has by means of the stromuln been able to measure lirectly in the rabbit the velocity of the blood-flow in the root of the aorta, and thus the systolic output of the heart. Other instruments have been invented for obtaining contimuons records of the variations of velocity of How in any given vessel. (hauveau and Lortet, employing the principle of the hydrometric pendulum, constructed an instrument of this kind, named the dromograph. ${ }^{2}$

As is seen in the diagram, this instrument consists of a chamber (B), which communicates with a tube $(\mathrm{A})$, and is closed at the top by a membrane. The

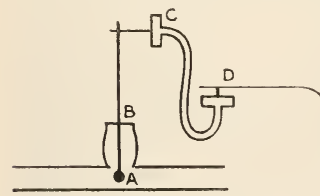

Fig. 54.-Diagram of (hauveau and Lortet's droniograplh. tube $(A)$ is inserted in the course of the artery which is under investigation. In this tube there hangs a small pendulum, which issues through the membrane in the roof of the chamber (B). The membrane affords a fulcrum to the pendulum, and the movements of the latter are brought to bear upon a tambour, which communicates by an indiarubber tube with the recording tambour (I)).

If the mass of the pendulum be small, the accuracy of this instrument is found to be considerable. Before use, the excursion of the dromograph must be calibrated by recording the flow of water at known velocity through it.

In Fig. 55 is shown the synchronous record obtained of the relocity

1 ber. d. k. Süchs. Gescllsch. d. Wisscnsch., Math.-Phys. Cl., Leipzig, 1867, S. 239.

2 Journ. de physiol. de l'homme, Paris, 1860, tome iii. 1. 695; Lortet, "Rech. sur la vitesse dn conrs du sang," Paris, 1867. 
and pressure in the carotid artery of the horse. ${ }^{1}$ It is noticeable that the curve of velocity $\left(\mathrm{V}^{\prime}\right)$ reaches its maximum before the curve of tension. This is so, because, as the arteries become overfilled, the heart cannot maintain the initial velocity of ontput. Chaureau found that the velocity in the carotid artery of the horse reached $520 \mathrm{~mm}$. per second during systole, while at the time of the dicrotic wave the velocity sank to $220 \mathrm{~mm}$. per second, and in diastole to $150 \mathrm{~mm}$. per second. Continuous records of the velocity curve afford us a valuable means of arriving at the volume of blood flowing through the vascular area supplied by the artery in question. Thus the effect on the blood-flow of vasomotorial excitement or functional activity can be investigated.

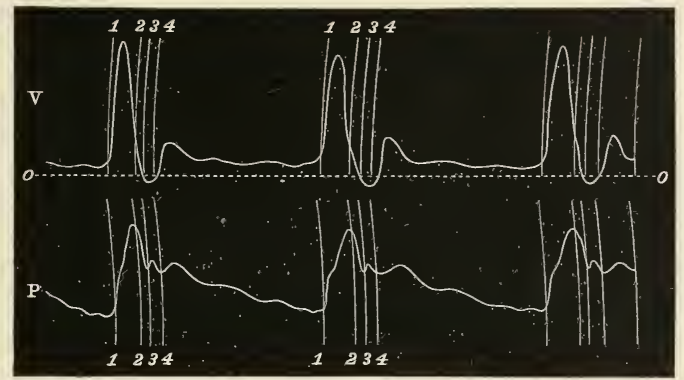

Fic, 55.-Carotid artery. $V$, velocity ; $P$, jressure eurves; $O, O$, abscissa of velocity curve; $1,2,3,4$, simultaneous points on both eurves. - Chauveau and Marey.

By means of the dromograph, ('hauveau and Lortet determined that, during the rise of systolic tension, the blood is driven from the coronary arteries back towarls the aorta. Lortet determined that the velocity of How in the carotid is five or six times greater when a horse is actively masticating than when at rest. In one experiment, while the nornal velocity in the carotid artery during systole was $540 \mathrm{~mm}$. per second, Chauvean noted that after section of the cervical sympathetic, and in consequence of the loss of vascular tone, the velocity became equal to $750 \mathrm{~mm}$. per second. After section of the spinal cord in the upper dorsal region, the velocity in the arterial systen becomes greatly accelerated during systole, and greatly diminished during diastole. Owing to the lowering of the peripleral resistance from the loss of vascular tone, the heart is able to clischarge the blood with the greatest rapidity into the venous system, and in consequence, during diastole, the arterial system is emptied of blood to a great extent. If this condition be pushed to an extreme, and the frequency of the heart be small, the blood hecomes discharged into the veins intermittently.

Comparative records have heen obtained of the velocity of flow in the carotid and facial arteries. At the end of the diastole the velocity is small in the carotid, and relatively great in the facial artery. At the beginning of systole the primary wave of velocity rises rapidly in the carotid, and is proportionately small in the facial artery. The secondary

1 Marey, "Circulatiou du sang," p. $32 \%$. 
increase of velocity which is produced by the licrotic wave, is far nore evident on the carotid than on the facial curve.

These results show how the intermittent energy of the heart is stored up by the elasticity of the large arteries, and expenderl in maintaining a continuous flow throngh the small arteries. In the root of the ascending aorta, the nean velocity during systole, according to the calculations of Nicolls, ${ }^{1}$ is ten times greater than the mean velocity in the abrominal aorta (see pp. 42, 43).

Another method of recording variations in velocity depends on the principle of Pitot's tubes. Suppose that a current is flowing constantly through the uniformly wide tube (BC) in the direction of the arrow (Fig. 42), then the lateral pressure in the piezometer (M) will be considerably ligher than that in $\mathrm{N}$. The pressure head in the piezometer (M) is an expression of the velocity head and the effective head, in the piezometer $(\mathrm{N})$ of the effective head, minus the velocity head.

When an alteration of resistance arises in the outflow, the lateral pressures at points nearer the periphery vary by larger fractions than those at points further up the stream. As the velocity in an artery varies inversely as the peripheral resistance, any change in the velocity may be estimated by noting the changes in the lateral pressures in two such manometers placed in comnection with the same artery at two different points. In Marey's apparatus the two lateral tubes are connected with two tambours. The buttons of these tambours are arranged so as to press on either side of a lever which works upon a third tambour. The pressure in this tambour varies in relation to the proportionate pressures in the other two tambours, and the variations are registered by a recording tambour. ${ }^{2}$

Cybulski contrived an arrangement, by which the two lateral Pitot-tubes are prolonged into two manometric tubes. These, connected together at the top, are filled in the lower part with fluid, in the upper part with air. The meniscus of the fluid in each tube is photographed on to a moving sensitised plate, and thus the difference in the pressures recorderl.

In the following experiments, Cybulski determined the velocity to be-

\begin{tabular}{|c|c|c|c|c|}
\hline & & SrstoLe. & DICRotic WaVE. & Diastole. \\
\hline & & Mm. per second. & IIm. per second. & MIm. per second. \\
\hline 1. Carotid Artery & . & $248-238$ & $248-225$ & $12 \bar{\imath}-156$ \\
\hline Femoral ,. & . & 356 & 300 & $17 \pi$ \\
\hline 2. Carotid ,. & . & 238 & $\ldots$ & 177 \\
\hline,$\quad$ (vagi cut) & . & 440 & $\ldots$ & 425 \\
\hline 3. Femoral . & . & 287 & $\ldots$ & $\ldots$ \\
\hline,$\quad$ (vagi cut) & . & 457 & $\cdots$ & $\ldots$ \\
\hline
\end{tabular}

Owing to the cardiac acceleration, and the consequent increase of the output of the heart, the velocity is seen to be greatly increased by section of the vagi.

From the study of the variations of the velocity of the blood, we may conclude that the following general statements, first formulated by Marey, are true:-

1 Journ. Physiol., Cambridge and London, 1896, vol. xx.

2 Jarey, "Circulation du sang," p. 310. 
If the resistance increases, and the output of the heart remains constant, then the arterial tension rises and the velocity becomes less.

If the output of the heart increases, and the resistance remains constant, then both the tension and the velocity become greater.

All experiments point to the fact that mere records of arterial tension give us no insight into the velocity of flow. The latter depends, not on the amount of the tension, but on the difference in tension between any two parts of the vascular system.

Certain attempts have been made to arrive at a method of recording variations in the relocity of the arterial blood in man. If a volume curve of the human arm is taken by the ordinary plethysmographic method, and the outflow of venous blood is constant, then the variations in rolume of the passive arm can only be produced by the systolic expansion of the arterial system. Thus, when the rolume curve rises the arterial inflow is greater than the venous outflow, and when the curve falls the venous outflow is greater than the arterial inflow. It follows that the more rapid the change of volume, the more rapid must be the variation in blood-flow. If, then, the steepness of the rolunie curve is estimated at different points, an approximate idea of the variations in velocity can be reached, and a velocity curve can be plotted out. The absolute worth of the measurements cannot of course be obtainerl, and since the venous outflow is not constant the results must be considered as only approximative. It is, however, noteworthy that the velocity curve thus obtained bears a striking resemblance to the curves recorded by the dromograph.

v. Kries has contrived an ingenious and delicate method of thus obtaining the relocity curve in man. The arin is placed in a plethysmographic chamber in the usual manner, and

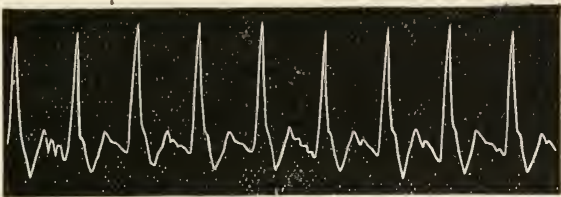

FIG. 56.-Tachogram ; arm elevated.-v. hiries.

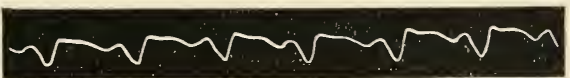

Fig. 57.-Sphyggmogram; arm elevaterl.-v. Kries.

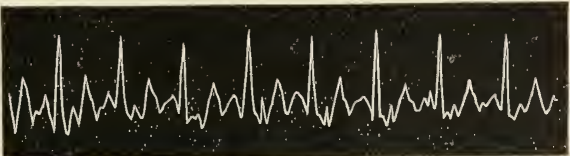

FIG. 58.-Taehogram; arm dependent.-v. Kries.

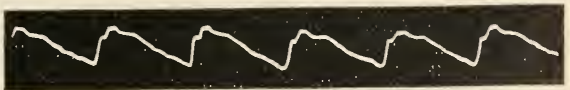

FIG. 59.-Sphygmogram; arm dependent.-v. Kries. this is connected by a tube with a sensitive gas-burner. Every expansion of the arm drives gas out of the plethysmographic chamber, and causes the flame to leap up. The movements of the flame are photographed on a moving sensitised plate.

The records, or tachograms, thus obtained are entirely different from the pressure curves obtained with the sphygmograph (see Figs. 56-59). 
The primary elevation rapidly reaches its maximum, and is followed by a fall which is not found on the arterial tension curves. This fall closely resembles that on the velocity curves obtained with the dromograph.

\section{The Circulation Time.}

Ed. Hering injected a solution of potassium ferrocyanide into one jugular vein of a horse, while from the opposite jugular vein he collected samples of blood at intervals of five seconds. The samples thus obtained were allowed to coagulate, and after expression of the serum the latter was tested with ferric chloride. He found that in 20 to 30 seconds the salt is received by the right heart, propelled through the lungs, received by the left heart, and propelled through the carotid arteries and capillaries, into the jugular vein. ${ }^{1}$ So astounding a result was at first received with incredulity, but Hering's experiments were fully confirmed by Poiseuille and Blake. ${ }^{2}$

After injection of barium nitrate into the jugular vein of a horse, Blake obtained evidence of the presence of this salt in the carotid artery on the opposite side in 15 seconds. I'oisenille ${ }^{3}$ found that on employing as an index weak solutions of ammonimm acetate or potassium nitrate, the circulation time was accelerated, while on the addition of alcohol the period was lengthened. This agreed with results which Poiseuille had obtained in investigations on the flow of water in capillary glass tubes. By Vierordt ${ }^{4}$ the experimental method was improved. He affixed a number of small cups to a dise which was rotated at a uniform and known rate. The blood, subsequently to the moment of injection, was allowed to flow from the vein into these cups. Hermann ${ }^{5}$ replaced the cups by a contimnous sheet of filter-paper, which was divided into sections corresponding to intervals of time. The venous blood flowed in a continuous stream on to the filter-paper as the drum rotated. At the end of the experiment the filter-paper was dried and tested for the presence of the salt. The innocuons ferrocyanide of solim is more suitable than the potassium salt. Vierordt ${ }^{6}$ determined the circulation time in seconds in the following animals to be-

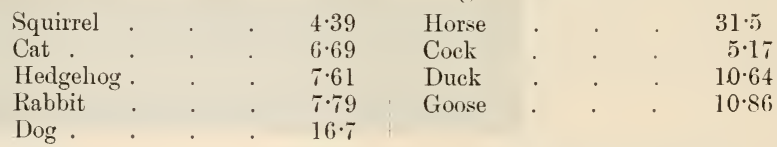

The length of the circuit is fomml to make but little difference. This is so because the time is chiefly spent in the passage of the blood, not through the arteries and veins, but through the capillaries. Thus Vierordt obtained the following mean results in four double determinations :- -

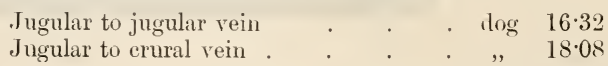

Recently other ingenious methods of determining the circulation time

${ }^{1}$ Ztschr. f. Physiol., 1829, Bd. iii. S. 85 ; 1833, Bd. v. S. 58.

"Edin. Hed. Journ., Oct. 1841, p. 104.

3 Ann. d. sc. mat., Paris, Sér. 2, Zool., 1843, tome xix. p. 20.

4 "Die Erschein. u. Gesetze d. Stromgeschwindigkeiten des Blutes," Frankfurt, 1858. S. 56 .

5 Arch.f. d. ges. Physiol., Bonn, 1884, Brl. xxxiii. S. 169.

\& "Phys. d. Menseh.," 1861, s. 111. 
have been devised by Stewart.' In these methods no collection or testing of the blood is required, and the whole procedure is extremely simple and easy of demonstration.

In his first method he proceeds as follows: The carotid artery is exposed and placed upon a sheet of insulating material; non-polarisable electrodes are then applied to the artery, and the portion of the artery between these is inserted as a resistance in one arm of a Wheatstone's bridge. After the resistances in the bridge have been balanced, and the galvanometer brought to rest, a small quantity of a solution of common salt is injected into the opposite jugular vein. So soon as the salt reaches the carotid artery the resistance of the blowl is altered, the balance of the TWheatstone's bridge is upset, and the galvanometer swings.

The period between the moment of injection and the commencement of swing can be noted with a stop-wateh with great precision.

In a second and still simpler method, Stewart ${ }^{2}$ employs a solution of methylene blue, which he injects into the jugular vein of the rabbit. The carotid artery on the opposite side is exposed, placed upon a sheet of white paper, and illuminater with a strong light. The time is taken with a stopwatch from the moment of injection to the moment when the blue colour is seen to appear in the carotid artery.

In a rabbit weighing 2 kilos., Stewart found the time of the lesser circulation varied from 2 to 3 seconds, and the jugular to jugular time from 5 to 7 seconds. The longest circulation times were found in the kidner, the portal system, and the lower limbs. By section of the rasomotor nerves of any organ the circulation time is lessened in that organ, while it is lengthened by excitation of the vaso-constrictors.

There is yet another means of arriving at the circulation time, and that is by the injection into a vein of a powerful poison. Thus, in the horse, nitrate of potash passes from the jugular vein into the coronary circulation, and arrests the heart in 16 seconds. In the same period strychnine produces its effect on the spinal cord. In the $\log , 12$ secomils is sufficient for the manifestation of the action of these poisons; in the rablit, 4 to 4.5 seconds; and in the fowl, 6 to 6.5 seconds.

The jugular to jugular result obtained by Hering's or. Stewart's method yields, not the circulation time of the whole of the hlood, lint the shortest possible time which any particle of hlood can travel throngh the shortest pathway romd both the lesser and greater circnlation. According to Poiseuille and v. Kries, ${ }^{3}$ the maximal or axial velocity is double the mean velocity. A particle of blood swept along in the axial strean, therefore, travels with far greater speed than another particle which rubs along the wet walls of the ressels. Again, the blood that takes a broad, open jathway, throngh wide or dilated capillaries, will arrive far more speedily at the opposite jugular vein than blood which ereeps through tortuons and constricted capillaries. It is impossible to arrive by this method at the circulation time of the whole blood. Part of the blood may travel from the left to the right ventricle, and from the right to the left ventricle, with great rapidity, and part with extreme slowness. The direct observations of Tigerstedt ${ }^{4}$ on the output of the left ventricle show that the circulation time of the whole blood is at least five times

1 Journ. Physiol., Cambridge and London, 1894, vol. xv. p. 1.

2 "Mantial of Physiology," London, 1896.

${ }^{3}$ Beitr. z. Anat. u. Physiol. als Festgabe C: Ludurig, Leipzig, 1887, s. 109.

"Skandin. Arch. f. Physiol., Leipzig, 1891, Bd. iii. S. 233 ; "Physiol. des Kreislaufes," 1893, s. 467 . 
as long as the period arrived at by the Hering method. It is therefore fallacious to use the circulation times arrived at by Hering's or Stewart's method as a basis for calculating the quantity of blood in the body.

\section{The Effect of Change of Posture on the Circulatios.}

The circulation is so contrived that it remains efficient, not only in the horizontal position, but also when the living animal is ceaselessly shifting the position of his body. The hydrostatic influence of gravity must have had a most important bearing on the evolution of the mechanisms which control the circulation. Especially is this so in the case of an erect animal like man, 5 to $6 \mathrm{ft}$. in stature. In a man $6 \mathrm{ft}$. high the hydrostatic pressure of a column of blood reaching from the vertex to the sole of the foot is equal to $140 \mathrm{~mm} . \mathrm{Hg}$, and from the vertex to the middle of the abdomen about $50 \mathrm{~mm}$. $\mathrm{Hg}$. It is obvious, then, that the influence of gravity demands careful inquiry.

Suppose a closed and rigid tube (AI) filled with water and fixed to a board (Fig. 60). When the board is placed in the horizontal position (I) the pressure
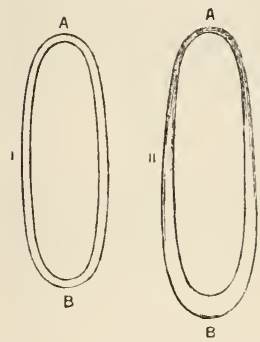

FIG. 60 .

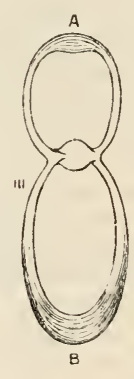
in all parts of the tube (AB) will be the same. If the board be turnerl into the vertical position, then the pressure at 1 will become nil, while the pressure at $B$ will be equal to the height of the column of fluid (AB). The fluid will still equally pervade the tube in all its parts. This must be so, because the fluid is incompressible and the tube is rigid and mnyiclding in structure.

If the rigid tube be now replaced by an elastic tube, and this at the points $A$ and $B$ be made to expand into thin-walled elastic

bags, then the conditions become markedly different (II). On placing such a model in the vertical position, the lower end (B) bags under the pressure of the column of fluid $(\mathrm{AB})$, and while the water flows into $\mathrm{B}, \mathrm{A}$ empties and collapses under the atmospheric pressure.

Suppose a pump be placed in such a model, and that the pump work with perfect uniformity and maintain a constant circulation, if the outflow tubes or arteries be made of small capacity and considerable extensibility and elasticity, and the inflow tubes or veins be valved, and be made of considerable capacity and slight extensibility and elasticity, and if a sponge be inserted as a resistance in the ends ( $A$ and $B$ ), then many of the conditions of the systemic circulation are closely represented in the model. $\mathrm{A}$ is now equivalent to the capillary area of the head, B to the splanchnic area of capillaries (III).

Let the distensibility of the splanchnic capillaries and veins (B) be great, and let the pump be at rest and the model be placed in the vertical position, the fluid will then collect in $\mathrm{B}$, and $\mathrm{A}$ will empty. The increase of the pressure in the splanchnic area by the height of the column (AB) tends to enlarge the capacity of that area while it diminishes the capacity in A. Let the pump be at work, then the circulation through $\mathrm{A}$ will cease if the pump is unable to raise the fluid from $B$, or to lift the fluid to $A$, and drive it through the resistance in $A$. If the model be turned with $B$ at the top, these results will 
be exactly reversed. These hydrostatic influences are compensated for in the normal animal by vital changes in the blood vessels, in the heart, and in the respiratory movements.

In the elastic model the fluid sinks under the influence of gravity if the venous and capillary tubes be made distensible by the pressure of the column of fluid within them. In man the venous column is broken into short segments by valves in the veins of the arms, in the root of the jugular veins, in the femoral and other veins of the lower extremities. The longest column of venous blool unbroken by any valve is that which extends from the intracranial sinuses, down the intravertebral sinuses to the azygos veins, and thence to the end of the inferior vena cava. From the innominate veins down the superior vena cava to the end of the inferior vena cava there also extends an unbroken column of blood. The valves are most numerous in the veins of the extremities, and are also foumd in the portal system, in the veins to the stomach, spleen, and intestine. Some of the portal valves become functionless in adult life. The suspension of the heart by the deep cervical fascia, and constraint of the pericardial sac, prevent the heart from rolling over and kinking the venæ cavæ during changes of posture. The vena cava inferior passes through the central tendon of the diaphragm, at a point which is immobile during the respiratory movements. It is protected in its passage behind the liver from the weight of that organ, for it lies in a tunnel formed by the Spigelian lobe of the liver, which, in its turn, rests upon the vertebral column.

The great and capacious abdominal veins are maintained and supported under the hydrostatic stress of gravity, by means of the expiratory muscles of the abdominal wall. The tone of these muscles, and therefore the capacity of the veins, depends upon the integrity of the respiratory centre. In consequence of the valves, the veins of the limbs are constantly emptied onwards by the contraction of the skeletal muscles, and by changes in posture. If a limb be kept motionless and in the dependent posture, the veins quickly become congested. The amount of this congestion depends on the tore of the skeletal muscles, the arterioles, and the walls of the veins. By such means the distension of the veins and capillaries in the erect posture is largely prevented.

The arterioles are held in a state of tone by the rasomotor centre, and limit the output into the veins. When an animal passes from the horizontal to the vertical feet-down position, the peripheral resistance in the splanchnic area can be increased and the capacity diminisher by the contraction of the arterioles; thus the circulation through the head is maintained. The blood in all cases takes the pathway of least resistance. The great splanchnic area forms the resistance box of the circulation; if it be contracted, the blood takes the pathway through the locomotor organs; if it be dilated, the blood passes into the capacious veins of the abdomen. When the splanchnic area is fully dilated, the whole of the blood can collect within the abdomen, and if the animal be in the vertical feet-down position the influence of gravity will bring about this result. The splanchnic vasomotor paralysis can, howerer, be then compensated for by the action of the respiratory pump. The capacity of the abdominal vessels can be reduced by the compressive action of the respiratory muscles, and while the output from the arterioles is thus held in check, the blood is pressed up the veins, and can be aspirated into the right heart by the action of the thoracic muscles.

To study this question experimentally, cannula are passed down the external jugular vein and carotid artery of the dog, so that the ends of the cannulæ lie respectively in the vena cava superior and the aorta, 
the animal-hoard being constructed to turn round an axis passing through the body at the level of the intravascular openings of the cannula. ${ }^{1}$ Then the effect of posture on the venous and arterial pressures is recorded." On placing the animal in the feet-down posture the arterial $^{3}$ and venous pressures immediately fall. The venous pressure remains down nntil the horizontal posture is once more assumed (Fig. 61). The arterial pressure rapidly rises again to normal, and often

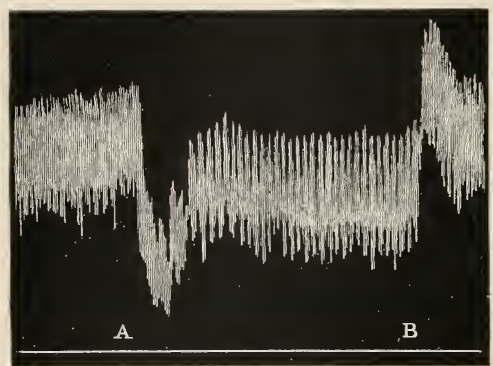

Fig. 61.-Aortic pressire. A, feet-down : B, horizontal posture. - Hill. it may be found to rise above normal. The respiratory undulations are frequently intensified while the animal is in the feet-down postmre.

If the spinal cord be dividerl at the level of the first dorsal vertebra, the influence of the bullar centres on the parts below the section is removed. Alrdominal and intercostal respiration is paralyser, and the breathing lecomes purely diaphragmatic. The tone of the great splanchinic area of arterioles is lost; the tone of the abdominal wall is abolished, and thus the capacity of the abdominal vessels is greatly increaserl. The total effect on the animal,

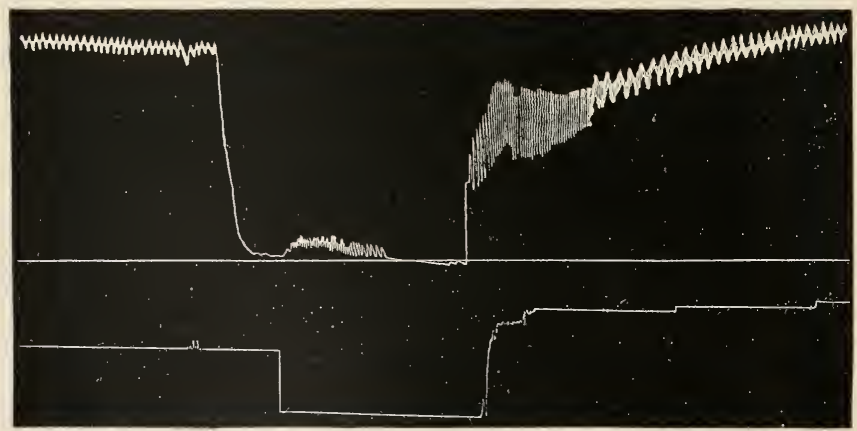

FIc. 62.-Aortic pressure and intraeranial pressure. Spinal cord dividerl at first dorsal vertebra. Effect of feet-down posture.-Hill.

when lying in the horizontal posture, is a considerable fall of arterial pressure, and a marked diminution of the respiratory undulations of pressure.

If the animal be now dropped into the vertical feet-down posture, the arterial pressure falls rapidly, and may reach zero (Fig. 62); the

${ }^{1}$ Any alteration in the level of the extravasenlar column of fluid in the manometer tubing is thus avoided.

"Hill, Journ. Physiol., Cambridge and London, 1895, vol. xviii. p. 15; Hill and Bamard, ibid., 1897, vol, xxi. p. 323 ; Hill, "Cerebral Cireulation," Lonclon, 1896.

${ }_{3}$ This fall had been previously observed by Cyhulski, St. Petrsb. Wed. W' Hinschr., 1878, S. 93 ; and Friedman, Med. Jahrb., Wien, 1882, S. 225. 
circulation is then at an end. This is so, hecause the great abdominal veins bag out runder the hydrostatic pressure; in them the whole of the blood collects, for it can pass rapidly through the dilated arterioles; there is no mechanism left for filling the heart; thus the heart, empty of blood, continues to beat to no purpose.

If the abdominal wall be compressed with the hand, the capacity of the veins and splanchnic area is reduced, the right heart is once more filled with blood, the arterial pressure rises, and the circulation is renewed. On taking off the hand, the heart once more empties, the arterial pressure falls, and the circulation ceases. When the animal is returned to the horizontal position, the influence of gravity is abolished, and the circulation immediately becomes re-established. Such experiments prove that in the normal animal there are two chief compensatory mechanisms by which the hydrostatic effect of gravity is overcome, namely, the vasomotor mechanism of the arterioles and the respiratory muscles. It is necessary to examine these separately, and estimate the relative power of each.

The vasomotor tone of the great splanchnic area can be easily abolished, and without affection of the respiratory mechanism, by section of the splanchnic nerves; that is to say, if these nerves are reached by a lumbar incision, and all interference with the thorax or abdominal wall is avoider.

The effect of section of the splanchnic nerves is shown in Fig. 63. The arterial pressure in the vertical feet-down posture falls very considerably, but nevertheless the circulation remains efficient, on account of the action of the respiratory muscles. A form of respiration is evoked which consists of thoracic inspiratory aspirations, combined

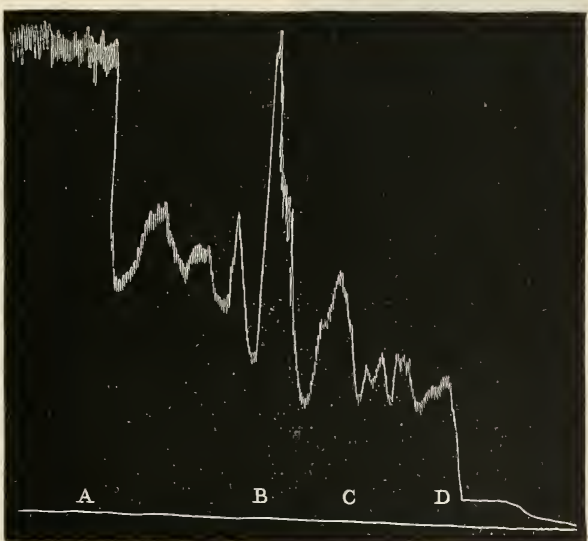

FIG. 63.-Aortic pressure. Morphinised dog.-Hill and Barnard.

A, Vertical feet-down position. Splanchnic nerves divided. B, Effect of compressing abdomen.

$\mathrm{C}$, Abdominal wall divided.

D, Thorax opened.

with powerful expiratory abdominal compressions. Therely the diastolic filling of the heart is maintained, and the velocity of flow thromgh the splanchnic capillaries checked. On dividing the abdominal wall by a crucial incision, the support of the abdominal muscles is withdrawn, the splanchnic vessels dilate, and the pressure falls to a further extent. Finally, on suddenly opening the thorax, the pressme falls to zero, and the circulation ceases. By compression of the abdomen, or by a returm to the horizontal postmre, the circulation can be once more renewed.

This experiment shows that the respiratory museles can compensate for the influence of gravity when the vasomotor mechanisin is paralysed. 
The respiratory mechanism can he thrown out of gear by itself, and without damage to the vasomotor mechanism by the injection of curari. The power of the heart is then sufficient by itself to maintain the circulation in the feet-down position, so long as the capacity of the abdominal vessels is kept under control by the vasomotor nerves.

The effect on the circulation of rendering the intrathoracic pressure positive, is dealt with in the section relating to the diastolic filling of the heart. Here it may be briefly stated that the blood stagnates in the abdomen, and the circulation ceases, whenever, by any means, the thoracic pressure is rendered sufficiently positive to overcome the venous pressure produced by the driving power of the heart. Owing to

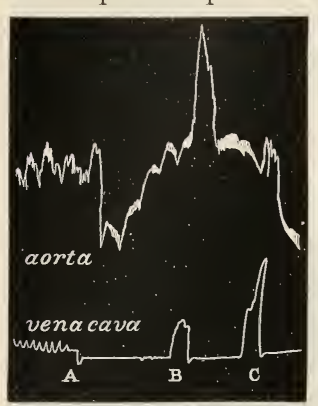

Fig. 64.-Aortic and vena cava pressures. Morphinised and curarised dog. - Hill and Bar. nard.

A, Artificial respiration withdrawn. Vertical feet-down posture.

A-B, Asphyxial rise of aortic pressure. Traube-Hering curves.

$B$ and $\mathrm{C}$, Effect of compression of abdomen. At $\mathrm{C}$ this produced paralyticdilatation of the heart. the influence of gravity, this state of affairs is brought about more easily in the vertical feet-down position than in the horizontal posture. Compensation for the positive intrathoracic pressure is supplied by firm compression of the abdomen, the heart then fills, and the arterial pressure regains its normal level.

The arterial pressure depends on the energy of the heart, and on the peripheral resistance. It is clear that the arterial pressure likewise depends on the hydrostatic effect of gravity. If a normal man be placed in the vertical feet-down position, the pressure in the femoral artery will be higher than the pressure in the common carotid artery, by the height of the column of blood which reaches from the carotid to the femoral artery. In a man $6 \mathrm{ft}$. in stature this difference will amount to about $45 \mathrm{~mm}$. $\mathrm{Hg}$. On the assumption of the vertical feet-up or headdown posture the pressure in the common carotid will rise to a like extent above that of the femoral artery, owing to the influence of gravity. In the feet-up posture there exists no great vascular field like the splanchnic area to expand under the hydrostatic pressure. The face and neck alone become injected, for the brain is confined within the rigid wall of the cranium, and thus the cerebral vessels cannot expand.

In changes of posture, it is apparent, from the experiments related, that the pressure in the carotid artery is maintained at practically a constant height by the tone of the splanchnic arterioles and action of the respiratory muscles. If the splanchnic arterioles are in an efficient state of tone, and if the abdominal veins are supported by the tone of the abdominal wall, then the splanchnic vessels are similar in nature to a model of rigid tubes, and will not dilate under the hydrostatic stress of gravity. The nervous mechanism involved is probably of the simplest nature, for if the arterial pressure suddenly rise or fall at the moment of change in posture, the bulbar centres are thereby either directly or reflexly excited to decreased or increased activity. A sudden fall of arterial tension always provokes acceleration of the heart, amplified respiration, and increased vaso-constriction. A sudden rise of tension, on the other hand, provokes a slow heart, shallow respiration, and vaso-dilatation. 
When the compensatory mechanism is abolished by destruction, exhaustion, or inhibition of the bulbar centres, the circulation fails and becomes inadequate to maintain life in the vertical feet-down posture. The blood passes into the capacious reservoirs of the toneless abdominal veins, the heart empties, and the cerebral circulation ceases. There can be no doubt that the control of this compensatory mechanism is one of the most important and absolutely necessary functions of the group of bulbar centres, a function which must have been evolved to its highest point as the anthropoids slowly assumed the erect posture.

During the course of each day the compensatory mechanism becomes exhausted, and especially is this so after severe muscular exertion. ${ }^{1}$ By sleep the compensatory power is restored. In conditions of neurasthenia, weakness and exhaustion after disease, shock after severe injury or hemorrhage, this power may be almost entirely lost.

By sudden fright in the standing posture the respiration is often arrested, the vasomotor tone inhibited, and syncope induced by the rush of blood from the brain to the abdomen. Recovery from syncope is brought about by the assumption of the horizontal position. When the compensatory mechanism is entirely lost, the circulation is only possible in the recumbent position, and life is at its lowest ebb. Among the anæsthetics in common use, chloroform

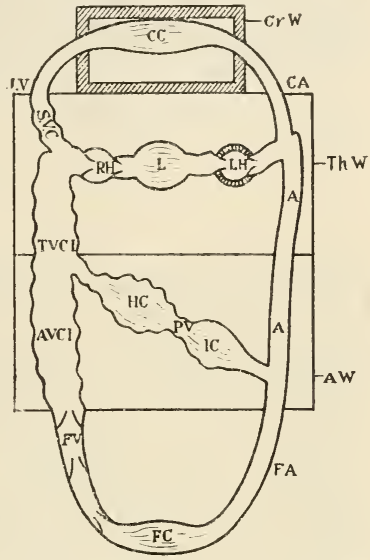

Fif. 65.-Selieme of the circulation.Hill and Barnard.

ThW, thoracic ; AW, abdominal wall; CrW, rigid cranial wall; CC, cerebral capillaries $\mathrm{JV}$, jugular vein ; CA, caroticl artery ; SVC, vena cava superior; TVCI and ATCI, thoracic and abdominal vena cava; $F$. femoral vein; $\mathrm{RH}, \mathrm{LH}$, right and left heart; L, pulmonary capillary area; $A$, aorta ; FA, femoral artery ; FC, femoral capillary area; $\mathrm{IC}, \mathrm{HC}$, intestinal and hepatic capillary areas; $\mathrm{PY}$, portal vein. stands prepotent as a drug which has the power to abolish the compensatory mechanism. Chloroform causes cardiac and vascular dilatation, weakens the respiration, and abolishes the tone of the abdominal muscles."

A useful clinical guide to the condition of the compensatory mechanism in man is afforded by the rate of the pulse on change of posture. If the heart becomes greatly accelerated on rising from the horizontal to the vertical position, the vasomotor tone is deficient.

The alteration in the distribution of the blood by the influence of gravity has been studied on man by Mosso. ${ }^{3}$ He balanced the body on a horizontal board, turning on a transverse axis. The head-end soon becomes the heavier, owing to the increased outflow from the legs in the horizontal posture. If the legs have previously been dilated by standing in a hot bath, this effect becomes more marked. The change in weight may be as much as 260 c.c. of blood. At each inspiration the foot-end becomes heavier on account of the compression of the vena cava inferior by the descent of the diaphragm. The volume of one arm is increased if the other be raised up, and similarly in the case of the legs.

${ }^{1}$ George Oliver, "Pulse Gauging," London, 1895.

${ }^{2}$ Leonard Hill, Brit. Med. Journ., London, 1897, vol. i. P. 957.

3 Arch. ital. de liol., Turin 1884, vol. v. 1. 130. 


\section{The Pulse.}

The most difficult problem connected with the hydrodynamics of the circulation is that of wave motion.

Suppose an extensile elastic tube $(\mathrm{AB})$ to be divided into two parts by the membrane $(0)$ (Fig. 66). ${ }^{1}$ If the membrane he made to move towards $\mathrm{A}$, a positive wave will be propagated along $\mathrm{OA}$, and a negative wave along $\mathrm{OB}$. If, however, the membrane be imperfect, then a wave,

$B$

FIG. 66. partly positive and partly negative, will

A be propagated in both directions. The pulse wave is formed in a similar manner. Suppose O to be the aortic orifice, $\mathrm{B}$ the heart, and the membrane the aortic valve. As blood is forced from $\mathrm{B}$ into $\mathrm{A}$, it develops a positive wave along $\mathrm{OA}$. The fluid is driven into $\mathrm{OA}$, with first, an increasing, and secondly, a diminishing velocity, and finally the force of propulsion comes to rest. If we consider the tube (OA), which is now expanded, to be divided into sections $a, b, c, d, c, f, g, h, i$ (Fig. 67), ${ }^{2}$ it is obvious that, when the ventricular output ceases, pressure will be exerted by the expanded elastic wall in the direction shown by the arrows: that is to say, the fluid particles are propelled towards $i$, retarded and brought to rest towards $a$.

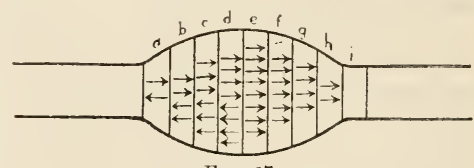

FIG. 67.

Thus section a returns to rest and its original diameter, while section $i$ expands. Likewise the sections $f, y, h$ swell, since more particles of Huid are propelled through the wider sections $d, e$ than can escape in the same time through the narrow sections $f, g, h$. Similarly, the sections $a, b$, $e$ will shrink. Thus the wave of expansion travels from section to section along the tube $(A)$. Since the return of the blood towards $A$, in the direction of the arrows, will close the aortic valve $(O)$, and drive it as a whole towards $\mathrm{B}$, a negative wave will be developed in OA. This wave will likewise be propagated along the tube $(A)$. Un the motion towards $B$ coming to rest, the valve $(O)$ will again move towards $\mathrm{A}$, giving rise to a second positive wave, which in its tur'n will travel down the tube $(A)$. Thus are produced in the arterial system the primary wave of expansion, and the secondary wave of expansion (dicrotic wave).

A wave in an elastic tube to a certain extent produces a translation of the particles of fluid, but the wave must be regarded mainly in the light of a translation of a change of form. The propulsion of each particle of thuid occasions a successive disturbance of the stability of contiguous particles; thus the impact of the blood, which is thrown from the heart into the aorta, travels down the arterial system. So an impact travels from truck to truck, when an engine strikes the end of a lomg railway train. If the train be in motion at the moment

${ }^{1}$ Nicolls, Journ. Physiol., Cambridge and London, 1890, vol. xx. 1. 425.

${ }^{2}$ Weber, Ber. d. k. Süchs. Gesellsch. d. Wissensch., Math.-Nat. Cl., Lei]rig, 1850, Bd. i. s. $164-204$. 
of impact, the rate with which the impact travels from truck to truck will be quite independent of the constant velocity of the train, while the impact will momentarily increase the velocity of each truck and translate it forwards. That a wave must be considered mainly as the propagation of a change of form, is shown by the consideration of the following case. Suppose a long trough to be filled with water, and the water be pumped rhythmically out of it, then the water, with each stroke of the pump, will move towards the exit, but a negative wave at the same time, and in a contrary direction, will travel along the trough. The negative wave will slightly retard the velocity of flow towards the exit, but in itself it is a propagation of a change of form, which is entirely independent of the velocity of the fluid.

From mathematical consideration of a wave in an artery, where the blood and the vessel wall are held to be constants, the motion of the blood is assumed to be irrotational, and, to avoid the complication of gravity, the portion of the artery is supposed to be horizontal, Nicolls draws the following conclusions :-

1. That all particles of blood which lie on any cross-section continue to lie on the same cross-section during the passage of a wave; that is to say, each cross-section moves forward as a whole, and thus each particle, when the wave has passed, is left in advance of its original position.

2. The distance to which each particle is advanced is equal to the total increase in volume, livided by the area of the cross-section. Thus the passage of a positive wave slightly increases the velocity of the blood.

3 . That the square of the velocity of the wave varies inversely as the density of the fluid and the radius of the tube.

4. That the less elastic the ressel the greater the velocity.

5. That the amplitude of the wave varies directly as the radius. In vessels of very small diameter the pulse becomes imperceptible, since, for a given wave length, the artery must have a certain diameter before pulsation be possible.

6. That the effect of the viscosity of the blood on the pulse is too small to be of any importance.

7. That normally the pulse wave is mainly the effect of lateral vibration, but in conditions where the range of pressure is very great, as in aortic regurgitation, longitudinal vibrations, which become visible as capillary pulsations, may occur.

Some of these deductions are in close agreement with the experimental determination of Moens.

The wave of expansion, according to Moens, ${ }^{1}$ travels through such a tube as an artery with a constant velocity, which is expressed by the following formula :-

$$
v=k \sqrt{\frac{g e a}{\mathrm{D} d}}
$$

where $v=$ the velocity per second.

$g=$ the acceleration due to gravity.

$e=$ the elastic coefficient of the wall.

$a=$ the thickness of the wall.

$d=$ the diameter of the tube.

$\mathrm{D}=$ the density of the fluid.

$k=\mathrm{a}$ constant.

It is postulated that the thickness of the wall is small, and the wave leugth

1 "Die Pulscurve," Leyden, 1878, s. 87, ef. Grummach, Arch. f. Physiol., Lcipzig, 1888 , S. 129 ; v. Kries, "Stud. zur Pulslehre," Freiburg, 1892, S. 7.

VOL. II. -7 
very long in comparison with the diameter of the tube. Thus, according to Moens, the velocity of the wave varies inversely as the square root of the diameter of the tube and the density of the fluid, and directly as the square root of the thickness and elastic coefficient of the wall.

As a wave travels down an elastic tube it is altered in form, owing to the viscosity of the fluid. Thus it becomes smaller, less steep, and more drawn out the further it travels from its source.

If water be rhythmically pumped into an elastic tube, a positive wave starts with each input and travels through the tube; and similarly, with each cessation of the input, there starts a negative wave. If the tube be sufficiently long, these waves are entirely spent; but if the tube be short, the waves may be reflected and run to and fro several times. Thus there may arise-

1. The primary positive or input wave;

2. The first centripetal reflected wave;

3. The second centrifugal reflected wave;

4. The second centripetal reflected wave;

and so on until the input wave is entirely spent.

Similarly, on the cessation of input there may arise-

1. The primary negative wave;

2. The first centripetal reflected wave;

3. The first centrifugal reflected wave; and so on until the wave is spent.

If the end of the elastic tube be shut, the wave is completely reflected, and without change of sign. If the end be fully open, the wave is completely reflected with change of sign.

In the case of a wave travelling up and down a tube, one end of which is open and the other shut, the change of sign takes place at the open end only. If one end of a tube be partly open and partly closed, then the sign of the wave when reflected will be partly changed and partly unchanged. Thus reflected waves of opposite sign arise, and these, according to their comparative size, may more or less interfere with one another. It follows that with a certain size of opening the reflected waves will completely interfere, and thus they will disappear.

It has been experimentally determined by $\mathrm{v}$. Kries that if an elastic tube be of sufficient width, and no reflection of the wave takes place, the seat of maximal and minimal velocity in the tube will be found to be the same as the seat of maximal and minimal pressure. Thus, if the pressure be found to increase in an artery, where the velocity decreases, a significant proof of reflection is obtained.

In addition to the waves which travel through the fluid in an elastic tube, the wall of the tube is itself thrown into vibration with each throb of the pump, just as a rubber band vibrates when it is suddenly and momentarily thrown into tension. These vibrations, according to $\mathrm{v}$. Kries, are of such frequency as to be extraordinarily difficult to observe, and they cannot be considered to have any effect on the main features of the pulse curve.

Thus far we have considered the simplest possible conditions, the production of waves in an elastic tube of uniform bore and of uniform structure. In the circulatory system the conditions are, on the other hand, of considerable complexity. The arteries continually change, their walls become more or less elastic, the lumen wider or narrower, and finally 
the arteries end in a multitude of narrow branches. Reflected waves are produced by any change in the conditions of the tube through which the primary waves are passing. An increase of frictional resistance acts in just the same way as a change in the diameter of the lumen or the elastic coefficient of the wall. Thus v. Kries ${ }^{1}$ inserted fibres of cotton-wool in an elastic tube. The sectional area of the tube was 150 sq. mm., while the total sectional area of the cotton-wool fibres was only $3 \mathrm{sq} . \mathrm{mm}$. Reflected waves were produced in this case almost to the same degree as if the tube had been conipletely blocked. Similarly v. Frey, ${ }^{2}$ in experiments with a preparation of the aorta and its branches, found that reflected waves were less easily obtained when the vessels were filled with salt solution, than when filled witlı defibrinated blood. The blood corpuscles increase the resistance in the capillaries, and thus probably lead to the reflection of waves.

In the multitudinously branched system of tubes which the arteries form, any reflected wave, arising in any branch, will propagate itself through all the other branches. We have then, in the arterial system, all the conditions for the origin of primary and of reflected waves, and for the interference of these waves, and it necessarily becomes a matter of the greatest difficulty to determine the origin of the secondary waves wlich contribute to the peculiar form of the pulse curve. To the consideration of the experimental methods of obtaining and analysing the pulse curve, we must now turn, seeing that the theoretical treatment of such a subject, where most of the conditions are as yet $n$ known, is of very slight value.

Terms used in describing the pulse.-The exploration of the pulse by the finger of the physician has led to the origin of many descriptive terms, which are commonly used to define the quality of the beat. Thus, according to the number of beats per minute, the pulse is described as frequent or rare. The size of the excursion of each beat is defined as large or small; the speed with which the excursion is executed, as quick or slow. If the pulse is compressible beneath the finger, it is said to be soft; if incompressible, hard.

A strong pulse is both large and hard. A weak pulse is both small and soft. A contracted pulse is small and hard. In a full pulse largeness rather than harduess is the predominant quality, and in an empty pulse smallness rather than softness.

Variations in the rhythm of the pulse are likewise described by set terms. Thus, when an imperceptible beat occurs in a series of regular beats, the pulse is said to be intermittent, and when one or more small beats occur in a row of regular and larger beats, the pulse is termed intereurrent.

Sphygmography. - Vierordt ${ }^{3}$ first applied the graphic method to the investigation of the pulse, and Marey ${ }^{4}$ in 1860 constructed an instrument, the sphygmograph, which possessed all the essential qualities of accuracy.

A pelotte, attached to a steel spring, rests upon the radial artery, and its movements are communicated by a toothed rod and wheel to a lever. The inertia of the instrument is very small, and since the movements of the spring

\footnotetext{
I v. Kries, "Stud. zur Pulslehre," Freiburg, 1892, S. 24.

2 "Die Untersuch. des Pulses," S. 173.

3 "Die Lehre vom Arterienpuls," Braunschweig, 1855, S. 21.

4 Journ. de physiol. de l'homme, Paris, 1860, tome iii. p. 241.
} 
are also very small, the error due to the momentum of the mass is inappreciable. Buisson ${ }^{1}$ experimentally proved that the instrument is free from any swing of its own.

If a small mirror ${ }^{2}$ be fastened over the radial artery, and a beam of light thrown upon the mirror, the excursion of the pulse can be photographed on a moving sensitised plate. If a small puncture be made in an artery, the jet of blood can be brought to play on a sheet of white paper moved by clockwork, and a hæmautograph can be thus obtained. ${ }^{3} \quad$ Both these methods yield exactly the same type of curve as the Marey sphygmograph. The instrument has been modified in many ways. Dudgeon has constructed a less cumbersome and more convenient form, suitable for the obtaining of clinical records.

These sphygmographs are less suitable for taking continuous records during long periods of observation. For this purpose air transmission is employed, and the pulse trace is obtained by means of a receiving and a recording tambour.

The frequency of the pulse.-If for a space of twenty-four hours a man be kept in bed, perfectly quiet, uniformly covered with clothes, and without food, it will be found that the variations of the pulse rate during the day are very small. Such variations as arise are due to changes in temperature. Much clothing increases the frequency, while exposure to cold lowers it. The variation due to the change of clothing may be as much as ten beats per minute. ${ }^{4}$

The hot room of a Turkish bath greatly increases the pulse rate. In an air-chamber at $65^{\circ} \mathrm{C}$., the heart may attain to a frequency of 160 per minute. In the tropics the pulse of a traveller quickens, while in the arctic regions it becomes slower. Drinking hot water accelerates the pulse, while the imbibing of a large quantity of cold water slows it. Every sensation of warmth, pressure, or discomfort in the stomach accelerates the heart. The taking of cold foods does not alter the rate, but hot meals increase it by as much as ten per minute. The heart lies in apposition to the stomach, warmth increases the rapidity of the heart; and we may therefore assume that the warm food in the stomach directly raises the excitability of the rhythmic mechanism of the heart. The effect of meals on the pulse rate is shown in the following example: ${ }^{5}$

\begin{tabular}{|c|c|c|c|c|c|}
\hline Hovr. & Frequency. & & Hotr. & Frequenct. & \\
\hline $\begin{array}{c}7-8 \text { A.M. } \\
8-8.30,, \\
8.30-9 \quad,, \\
9-10 \quad, \\
10-11 \quad, \\
11-12 \quad, \\
12-1 \quad \text { Р.M. } \\
1-2 \quad, \\
2-3 \quad,\end{array}$ & $\begin{array}{l}69 \\
79 \\
75 \\
77 \\
73 \\
73 \\
67 \\
69 \\
73\end{array}$ & $\begin{array}{c}\text { Breakfast, } \\
8 \mathrm{A.M} .\end{array}$ & $\begin{array}{cc}3-4 & \text { P.M. } \\
4-5 & , \\
5-6 & , " \\
6-7 & , \\
7-8 & , " \\
8-9 & , " \\
9-10 & , " \\
10-11 & , " \\
11-12 & , "\end{array}$ & $\begin{array}{l}74 \\
72 \\
67 \\
68 \\
68 \\
72 \\
67 \\
63 \\
70\end{array}$ & $\begin{array}{l}\text { Supper, } \\
10 \text { P.M. }\end{array}$ \\
\hline
\end{tabular}

1 Marey, "La eirculation du sang," Paris, 1881, p. 217.

${ }^{2}$ Czermak, Sitzungsb. d. k. Akad. d. Wissensch., math.-naturw. Cl., Wien, 1863, Bd. xlvii. Abth. 2, S. 438.

${ }^{3}$ Landois, Arch.f. d. ges. Physiol., Bonn, 1874, Bd. ix. S. 71.

4 Bleuler and Lehmann, Arch. $f$. Hyg., München u. Leipzig, 1s88, Bd. iii. S. 224-231.

' Adapted from Tigerstedt, "Physiol. des Kreislaufes." Leipzig, 1893, S. 27. 
In a condition of fasting and resting the pulse rate decreases slightly during the day, up to 2 P.M., and is then slightly accelerated again.

The seasons have little effect on the pulse rate. Coste, ${ }^{1}$ in a period of five years' examination, found his pulse to beat less frequently in the summer, and more frequently in the winter.

Change of position of the body, whether such change be brought about actively or passively, has a marked effect on the pulse rate. In one hundred men averaging 27 years in age, Guy ${ }^{2}$ found the average rate of pulse to be-

$\begin{array}{llllll}78.9 & . & . & . & . & \text { Standing } \\ 70 \cdot 1 & . & . & . & . & \text { Sitting. } \\ 66.6 & . & . & . & . & \text { Lying. }\end{array}$

After debilitating disease the change of frequency lietween the horizontal and erect postures may reach 30 to 50 beats per minute. ${ }^{3}$ Active movement has a very great effect on the pulse rate. The frequency may, for example, be 100 when slowly walking, and rise to 150 on running as fast as possible. The exertion of climbing steps may raise the pulse 58 per minute, and produce marked dicrotism. ${ }^{4}$ After a series of pendulum movements executed for a period of 30 seconds by the right arm, which was loaded with a weight of $2 \mathrm{lb}$., the pulse rate was noted to rise in one case from 82 to 156 per minute. When active movements have been executed for a short period only, the frequency quickly returns to normal, while if continued long the pulse rate may subsequently remain increased for an hour or more. Sleep lowers the rate of heart-beat slightly in adults, and markedly in children.

The pulse frequency is greater in women than in men, but this difference almost disappears if men and wormen of equal stature are compared.

Large animals always have a slower pulse rate than small animals; thus elephants have a frequency of 25 to 30 , and rabbits 150 ; and tall men have as a rule slower rates than short men. The acceleration in small animals is due to the fact that the surface exposure is greater in these, in proportion to the cubic contents of the body; therefore, as the loss of heat is greater, and the metabolism more rapid, a quicker circulation is required. Individual variations in healthy men may be very great. It is said that Napoleon had a constant pulse rate of 40 . Rates of 20 and 26 have even been recorded. On the other hand, a constant rate of 120 has been noted in a healthy woman. ${ }^{5}$ We can sum up from the above facts that a small size of body, the female sex, muscular movement, hot food, and rise of temperature of body, increase the pulse rate. In the following table is shown the average pulse rate at different ages up to 20 years. The observations were taken in the resting and sitting posture, and not immediately after food. ${ }^{6}$ From 20 years onward the rate remains practically constant; in old age there may be a very slight increase.

1 Naturc, London, May 14, 1891.

2 "Cyelopedia of Anat. and Physiol.," 1852, vol. iv. p. 186.

${ }^{3}$ Grave's "Clinieal Medicine," Nex Syd. Soc., 1848, vol. i. p. 56.

${ }^{4}$ Christ, Deutsches Arch. f. klin. Med., Leipzig, 1894, Bd. liii. S. 102.

5 Milne-Edwards, "Leçons sur la phys. comp.," 1859 , tome iv. p. 89.

${ }^{6}$ Guy, loc. cit., p. 184. 


\begin{tabular}{|c|c|c|}
\hline Age in Years. & Pulse Rate. & \\
\hline $\begin{array}{c}\text { Fctus } \\
0-1 \\
1-2 \\
3-4 \\
5-6 \\
9-10 \\
12-13 \\
16-17 \\
19-20\end{array}$ & 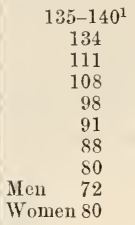 & $\begin{array}{l}\text { At the third month the } \\
\text { infant's pulse may be } \\
\text { faster than at birth, } \\
\text { owing to the increase } \\
\text { of muscular activity. }{ }^{2}\end{array}$ \\
\hline
\end{tabular}

The velocity of the pulse wave.-The fact that the carotid pulse is earlier in time than the radial pulse is determinable ly the fingers of a practised observer. With the help of a clock beating thirds of a second, E. H. Weber ${ }^{3}$ found the difference time between the perception of the pulse in the maxillary and anterior tibial artery to be respectively $\frac{1}{6}$ to $\frac{1}{7}$ second. From this result the velocity of transmission was calculated to be 7.92 to 9.24 metres per second.

Later observers have obtained, by means of tambours, synchronous graphic records from two different arteries. Some selected examples of the results obtained by different workers are given in the following table : ${ }^{4}$

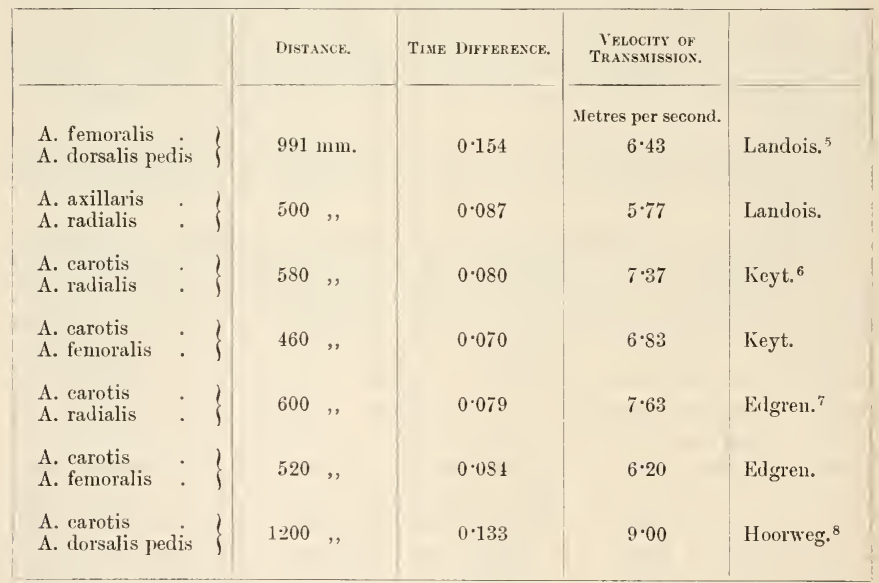

The variations in the rate of transmission depend on the elastic coefficient of the arterial wall. With a rise of arterial pressure the

1 Nägele found in six hundred observations the extremes to be 90 and 180 , the average 135 , "Die geburtshülfliche Auscultation," Mainz, 1838, S. 35.

2 Milne-Edwards, loc. cit., p. 57 .

3 "Annotationes anatomice et plyysiologice," Leipzig, 1834, Bd. i. ; Ber. d. k. Süchs. Gescllsch. d. Wissensch., math.-phys. Cl., Leipzig, 1850, S. 196.

"After Tigerstedt, "Physiol. des Kreislaufes," 1894, S. 385.

5 "Die Lehre vom Arterienpuls," Berlin, 1872, S. 297.

6 "Sphygmography and Cardiography," New York, 1887, p. 41.

${ }^{7}$ Skandin. Arch. $f$. Physiol., Leipzig, 1889, Bd. i. S. 96.

${ }^{8}$ Arch. f. d. gcs. Physiol., Bonn, 1889, Bd. xlvi. S. 132. 
velocity of transmission and the coefficient of elasticity increase. Thus Moens ${ }^{1}$ determined that in the same individual the velocity was greater when the arterial pressure was higher. In Valsalva's experiment the filling of the heart is impeded by the increase of intrathoracic pressure, hence the arterial pressure falls. During this condition, the velocity of the pulse wave has been estimated to be 7 metres per second, while during quiet respiration the velocity rose to $\delta$ metres per second.

Before stimulation of the peripheral end of the vagus, Moens estimated the velocity to be 11.5 metres per second. During the first six beats, after the cessation of cardiac inhibition, the velocity was 4.5 metres, 4.5 metres, 6.0 metres, 7.5 metres, 12 metres, 13.5 metres, respectively, per second. Similarly, when the arterial pressure is lowered by a condition of deep anesthetisation, or by dividing the spinal cord, the velocity is lessened, while it is increased by excitation of the divided spinal cord. ${ }^{2}$

By placing the arm in hot water, the velocity of transmission from the carotid to the radial artery may be lowered by 14 per cent., owing to the local dilatation of the blood vessels. The velocity may be found to be different in the arm and in the leg, owing to the variations in the elastic coefficient of the arteries of these parts-variations which may depend upon the structure of the arterial wall, upon the hydrostatic effect of gravity, or upon the local condition of vascular dilatation. When the arteries are sclerosed, and the rigidity of the wall is increased, as in the degenerative condition which accompanies chronic Bright's disease, the velocity is increased.

The length of the pulse wave is the product of the velocity of transmission of the wave into the time occupied by the wave in passing any given point; which time, in the case of the pulse wave, is about the time of a cardiac cycle, i.e. 0.8 second.

If the velocity of transmission be taken as 6 metres per second, then the length of the wave will be 6 metres $\times 0.8$, i.e. about 5 metres. From this it follows that the pulse wave reaches the periphery long before it has left the aorta.

The characteristic features of the pulse curve. - The radial artery is surrounded by veins. A vena comes lies on either side, whilst a superficial vein (a branch of the median or radial) often lies directly over or just to the side of the artery (Fig. 68, $e$ ).

In Figs. 68, 69, there is represented (drawn to scale) the arrangement of the veins and artery. The plane of the section (Fig. 68) passes through the spot where the radial pulse is usually explored by the finger or the sphygmo. graph. The artery and veins lie in a space between the styloid process of the radius and the tendon of the flexor carpi radialis. This space may be conveniently termed the radial sulcus.

If, after ligation of the upper forearm in a corpse, the radial artery be distended from above, and the venæ comites from below, it appears to the finger as if one broad flat band filled the radial suleus. If the artery be then pulsed by rhythmically compressing the tube connected with the arterial pressure bottle, both artery and veins seem to share in the pulsatile movement.

1 "Die Pulscurve," Leyden, 1878, S. 111.

${ }^{2}$ Grunmach, Arch. f. Physiol., Leipzig, 1879, s. 424. 
To the finger, both the breadth of the pulse and the apparent diameter of the radial artery is increased by distension of the venæ comites. Such being the case, on examining the radial sulcus with the finger, we can speak of-

1. The size or breadth of the band-like swelling, composed of the artery and the veins, which more or less occupy this space.

2. The tension of the band. By this we mean the sense of pressure necessary to obliterate the calibre of the artery, and stop the pulse.

3. The condition of the arterial wall, which can be investigated by flattening the artery with the finger and rolling it upon the bone.

4. The oscillation or wave of expansion.

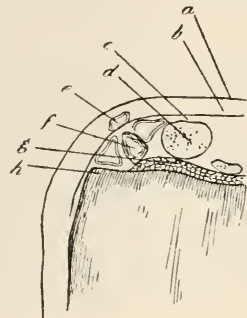

FIG. 68.-Section of forearm passing through the radial sulcus. - Hill, Barnard, and Sequeira.

$a$, Skin.

, Fat.

$c$, Deep fascia.

$d$, Flexor carpi radialis.

$e$, Superficial vein.

$f$, Radial artery and its ven: comites.

$g$, Pronator quadratus.

$h$, Styloid process of radius.

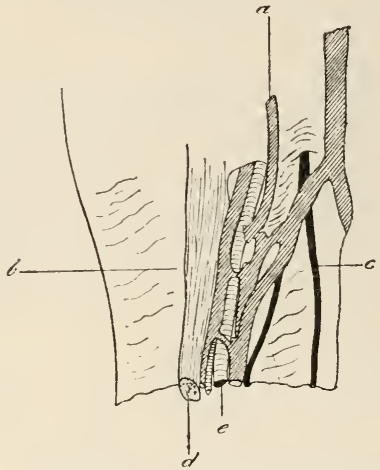

FIG. 69.-Superficial view of the radial vessels.-Hill, Barnard, and Sequeira.

a, Vena comites.

$b, d$, Flexor carpi radialis. $c$, Post. div. radial nerve. $e$, Radial artery.

The wave of expansion admits of description in these particulars -(1) Frequency; (2) regularity;

(3) amplitude of excursion; period of excursion; (5) dicrotism.

A radial sphygmogram is a pressure curve, for the movements of the lever of the sphygmograph are produced by the changes in pressure in the vessels which lie in the radial sulcus. The scale by which the ordinates of the curve should be measured is, however, an unknown quantity. The arterial pressure acts not only on the arterial wall, but also on the surrounding tissues; and since the pad of the instrument rests partly upon the venæ comites, not only the arterial tension, but any turgidity arising from venous congestion, affects the height of the sphygmographic curve. ${ }^{1}$ That this is so, is shown by the effect of compression of the veins of the arm.

In Fig. 70 , tracing 1, the whole of the upper arm at the point (A) was compressed by the hands of the observer; at the point (B) the compression p. 148.

${ }^{1}$ Hill, Barnard, and Sequeira, Journ. Physiol, Cambridge and London, 1897, vol. xxi. 
was withdrawn from the brachial artery, but maintained in the veins. As the veins fill, the abscissa line of the sphygmographic curve shows a gradual rise. This rise is considerable. The excursion of the wave is at the same time diminished. On withdrawing the compression, as the veins empty, the pulse tracing slowly returns to the normal condition.

To the finger, the breadth or fulness of the vessels in the radial sulcus aplears greater during the compression of the veins, and the amplitnde of the excursion is diminished. On compressing the brachial artery, at the moment when the elevation of the tracing produced by compression of the veins is at its maximum, the line of the trace does not fall, because the venous outlets are entirely blocked (Fig. 70, tracing 2).

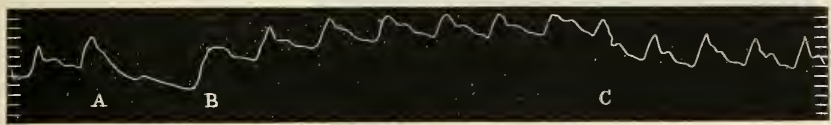

TRACING I
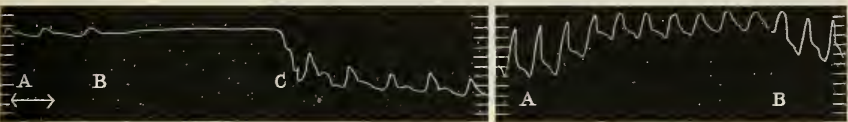

TRACING 2

TRACING 3

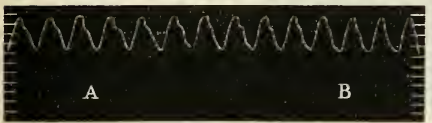

TRACING 4

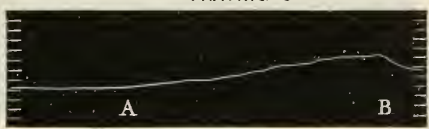

TRACING 5

FIG 70 .

Tracing 1.-A, Compression of the veins of the arm and the hrachial artery; B, compression of the veins of the arm only; $\mathrm{C}$, compression withdrawn.

Tracing 2.-A, Veins of the arm compressed; the double arrow marks the height of the base line before the compression was applied; B, brachial artery occluded ; C, conspression withdrawn from the artery and veins.

Tracing 3.- Sphygmograph resting on the femoral artery and vein of a dog. A, Fensoral vein compressed; $\mathrm{B}$, compression withdrawn.

Tracing 4.-Sphygmograph resting on the femoral artery of the same dog. A, Femoral vein compressed; 1 , compression withdrawn.

Tracing 5.-Splygmograph resting on the femoral vein of the same dog. A, Femoral vein compressed; B, compression withdrawn.-Hill, Barnard, and Sequeira.

In Fig. 70, tracing 3, there is recorded the effect of compressing the femoral vein in the dog, while the pad of the sphygmograph rested upon the femoral artery and vein. In this experiment all the other venous exits were blocked by means of a ligature, which was drawn round the thigh, excluding the femoral vessels. As the vein is compressed it can be seen to gradually distend, until in diameter it more than equals the artery. When entirely occluded, the tension within the vein rises to that of the artery. By the distension of the vein the pad of the sphygmograph is elevated above the artery; thus the line of the tracing rises, while the excursion of each pulse is considerably diminished. Owing to the elevation of the pad of the instrument by the vein above the artery, the bottom of each curve is cut off. That the alteration of the tracing is due to the rise of pressure within the vein, is shown by the next two figures. In Fig. 70, tracing 4, the femoral vein was compressed, while the pad of the sphygmograph rested on the femoral artery only, and in Fig. 70, traciug 5, the experiment was repeated while the instrument rested on the femoral vein only. 
Valsalva's experiment-forced expiration with the mouth and nose shutproduces the same effect on the pulse as compression of the veins of the arm. By the rise of intra-thoracic and abdominal pressure, the outlet of the veins of the limbs and head and neck is blocked, and the pressure in the venæ comites radiales rises to the mean arterial tension. Thus the pad of the

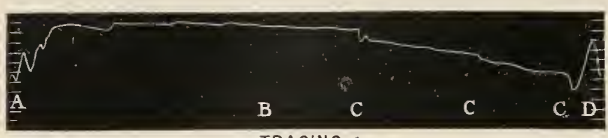

TRACING 1

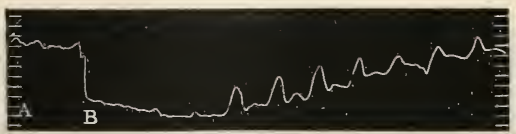

TRACING 2

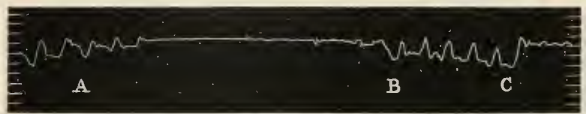

TRACING 3

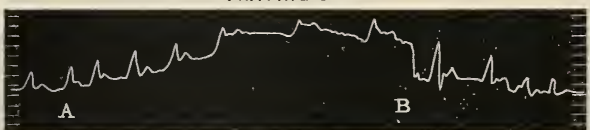

TRACING 4

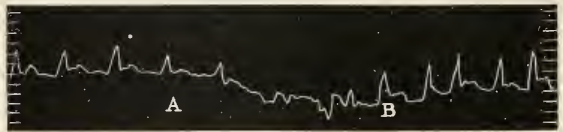

TRACING 5

FIG. 71.

Tracing 1.-Valsalva's experiment. A, Forced expiration ; ished. Owing to these B, brachial artery oceluded ; C C C, inspirations ; D, con- effects, the arterial tenpression of brachial artery withdrawn.

Tracing 2.-Valsalva's experiment. A, Forced expiration of sion rises to a slight severe intensity ; B, cessation of expiratory effort followed extent. In the next by inspiration.

Tracing 3.-Sphygmograph resting on the femoral artery and vein of a dog. $A$, Expiratory spasm; $B$, cessation of expiratory spasm; C, conpression of the femoral vein. Tracing 4.-Passive compression of the thorax, while the
breath is lield. A, Compression applied ; B, compression withdrawn.

Tracing 5.-Passive compression of the thorax while the breath is allowed to cscape. A, Compression applied; B, compression withdrawn. - Hill, Barnard, and Sequeira. to the mean arterial tension. The arterial pressure may then begin to gradually fall, but before any considerable fall has taken place the expiratory effort ceases from exlaustion of the subject of the experiment, and a deep gasping inspiration is taken. During this inspiration the whole of the blood delivered by the right heart is swallowed up in the roomy reservoirs of the expanded lungs; thus several beats of the left ventricle become abortive (Fig. 71, tracing 2). The radial pulse is then felt with the finger to stop, the face blanches, and the subject turns faint owing to acute cerebral anæmia. 
At the moment of the deep inspiration, not only are the peripheral veins emptied into the thorax, but the blood in the peripheral arteries is also sucked back into the thoracic aorta. Thus the tension in the radial vessels falls below zero; in fact, the systemic circulation entirely ceases until the left ventricle once more becones filled with blood from the pulmonary veins. These experiments are performed with far greater effect by a man with a compressible than with a rigid thorax.

The sphygmogram shows variations synchronous with the respiratory movements. Normally these are slight, but they become marked whenever the respiration is either deep or partially obstructed. During deep inspiration the line of the tracing falls, whilst the excursion of the primary wave decreases, and the dicrotic wave becomes more evident. The vence comites radiales are here emptied, and the expanded lungs swallow up the blood so that the arterial tension falls slightly. When expiration follows, the line of the tracing rises, and the excursion of each pulse is increased. During a deep inspiration the vessels in the radial sulcus appear to the finger of less breadth and lower tension. In obstructed breathing the pulse beats may, during inspiration, almost disappear, for the air cannot then enter freely, and thus the force of the inspiratory movement is thrown largely on to the intra-thoracic vessels and the heart.

Passive compression of the thorax produces the same effect on the pulse as Valsalva's experiment, if the breath be held (Fig. 71, tracing 4). If, on the other hand, the breath be allowed to escape as the thorax is compressed, the line of the tracing falls, and the pulse beats become abortive (Fig. 71, tracing 5). In this latter condition the outlet of the peripheral veins is not blocked, while the beat of the heart is mechanically opposed by the compression.

So soon as the fact is once grasped that the veins which surround the radial artery bear an important share in the production of the phenomena of the pulse, almost all the difficulties which have hitherto surrounded the explanation of sphygmographic tracings under these conditions disappear. In place of these, an absolute agreement between the results obtained on animals by manometric observations, and those recorded on man with the sphygmograph, becomes apparent.

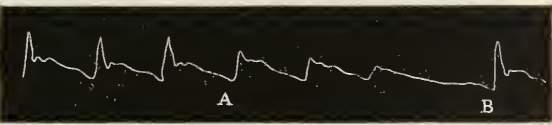

TRACING 1

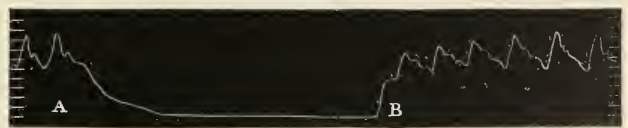

TRACING 2

In all patholog- FIG. 72.-Two examples of the effect of occluding the brachial ical conditions of artery. A, Artery compressed; B, compression withdrawn.obstruction of the Hill, Barnard, and Sequeira.

circulation, arising from pulmonary or cardiac disease, wherein by back pressure the general venous tension is raised, and the arterial tension lowered, the veins which surround the artery must have an important share in giving to the pulse the quality of breadth or fulness which is so commonly combined with that of low tension. When the quality of fulness is estimated to be present between the pulse beats, this does not betoken a high arterial tension, but rather an engorgement of the venous system. 
By recording with the sphygmograph the effect of occlusion of the lrachial artery, we possess a method of investigating the condition of the circulatory system. If the fall of the line of the curve is steep and rapid on sudden compression of the artery, a high arterial tension, a low venous pressure, and a rapid escape of the venous blood is indicated (Fig. 72 , tracing 2); if the fall is shallow and slow, the opposite conditions exist, for then the indication is that the arterial and venous pressures are more approximate to each other, and that the escape of the venous blood is olstructed ${ }^{1}$ (Fig. 72, tracing 1).

Analysis of pulse curve.-Each pulse curve begins with an ascending limb of considerable steepness, which corresponds with the positive wave produced by the systolic ontput of the heart. As a rule, the ascent forms an umbroken line until the apex of the curve is reached, and the descent commences. The descending limb is marked by several minor waves, of which one, the dicrotic, is present in all forms of pulse curve.

The minor waves on the descending limb of the curve are termed katacrotic, and if such waves appear on the ascending limb these are termed anacrotic. ${ }^{2}$
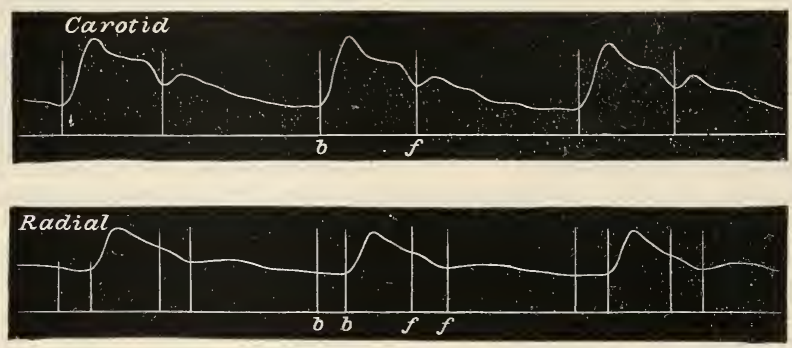

FIG. 73.-Velocity of pulse wave. Carotid and radial pulse.-After Edgren.

The difference in time between $b$ and $b={ }^{\circ} 0786 \mathrm{sec}$. The distance between radial and earotid $=$ about $60 \mathrm{~cm}$.

$\therefore$ velocity of transmission $=$ about $7 \mathrm{~m}$. per sec.

The dicrotic wave.-There has been much dispute over the origin of the secondary waves, and the matter cannot yet be cousidered definitely settled. It camnot be doubted that the dicrotic wave is a positive centrifugal wave, for, if synchronous tracings be taken from two arteries, the dicrotic wave, like the primary wave, appears earlier in time in the artery which is nearer the heart. Although the dicrotic wave runs centrifugally, it does not follow that it is entirely central in origin. Several authors have maintained that the dicrotic wave travels with less velocity than the primary wave down the arteries of the lower limb, while it travels with approximately the same velocity down the arteries of the upper limb. ${ }^{3}$

${ }^{1}$ In taking such observations the sphygmograj,h must be arranged so as in each case to give the maximal pulsation. The wrist also shonld be placed on the same level as the heart.

2 Landois, "Die Lehre vom Arterienpuls," S. 108.

3 v. Kries, Arch.f. Physiol., Leipzig, 1887, S. 275; Edgren, Shandin. Arch. f. Physiol., Leipzig, 18s9, Bd. i. S. 104 ; for the contrary conclusion, see Hurthle, Arch. f. d. ges. Physiol., Bonn, 1890, Bd. xlvii. S. 28. 
Such results as these have been obtained:-

\begin{tabular}{|ll|l|l|l|}
\hline & & \multicolumn{2}{|c|}{ VeLocirr. } \\
\cline { 3 - 4 } & & \multicolumn{2}{|c|}{ Primary Wave. } & Dicrotic Wave. \\
\hline Upper extremity &. &. & $7 \cdot 32$ metres per sec. & $7 \cdot 32$ metres per sec. \\
Lower extremity &. & $6.59 \quad$, & $5 \cdot 24 \quad,$, \\
\hline
\end{tabular}

If this be so, it is argued, the dicrotic wave must either be modified, or produced, by waves reflected from the periphery. On the other hand, this difference in velocity time may be due simply to differences in arterial pressure, which may exist at the period of the dicrotic wave. The greater the tension of the arterial wall, the greater is the velocity. No doubt reflected waves may be produced in such a system of branching tubes as the arteries form. Such reflected waves must arise at different distances from the heart, spread into every branch of the system, and modify, not only each other, but the primary waves by interference.

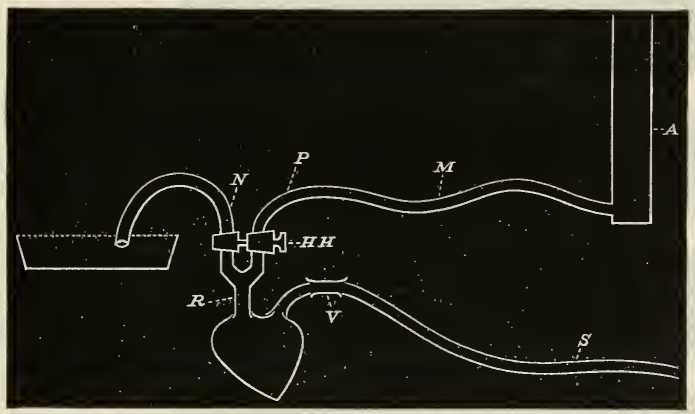

FIG. 74.-Experiment to show central origin of dierotic wave.-Grashey.

v. Kries bled an animal to death, placed a spring manometer in connection with the femoral artery, and a bag filled with fluid in connection with the aorta. ${ }^{i}$ This bag was suddenly compressed, and retained in the compressed position. Thus a primary positive centrifugal wave was propagated through the arterial system. This gave rise to secondary waves, which v. Kries attributed to reflection. On the other hand, it must be borne in mind that these waves were obtained under the most favourable conditions, and it is very doubtful whether such waves would be visible when produced under the conditions of the ordinary pulse curve. Moreover, the waves may have been occasioned rather by oscillations of the wall of the aorta, than by reflections from the periphery.

In order to demonstrate the central origin of the dicrotic wave, Grashey ${ }^{2}$

${ }^{1}$ v. Kries, "Studien zur Pulslehre," S. 62, ef. v. Frey, "Die Untersuch. des Pulses," S, 164 .

2 "Die Wellenbewegung elasticher Röhren," Leipzig, 1881, S. 165. 
employed the arrangement shown in Fig. 74. Into the left auriculo-ventricular opening was fastened a tube $(R)$. This tube ended in two branches $(P$ and $N)$. The branch $(P)$ was connected with a pressure bottle $(A)$, and the branch $(N)$ formed an exit tube. A tap was placed in these branches, so that $N$ was opened when $P$ was closed, and $P$ was opened when $N$ was closed. Into the aorta was fixed a long elastic tube $(V)$. Water was allowed to flow from the pressure bottle through the heart and the aorta. If the tap was now suddenly turned so as to shut $P$ and open $N$, the pressure sank to zero in the ventricle, and a positive wave appeared in the aortic tube, which was produced by the closure and vibration of the semilunar valves, and not by reflection from the periphery.

Hoorweg ${ }^{1}$ employed a circulatory model in which a current through a Rumkorff coil was completed by the closure of the semilunar valves. The coil sparked on to the smoked paper, and knocked away the soot at the point where the lever style recorded the pulse wave on the drum. The closure of the valves was thus found to take place exactly at the foot of the ascent of the dicrotic wave.

Hoorweg found that the dicrotic wave appeared at the same interval of time, after the commencement of the primary wave, in
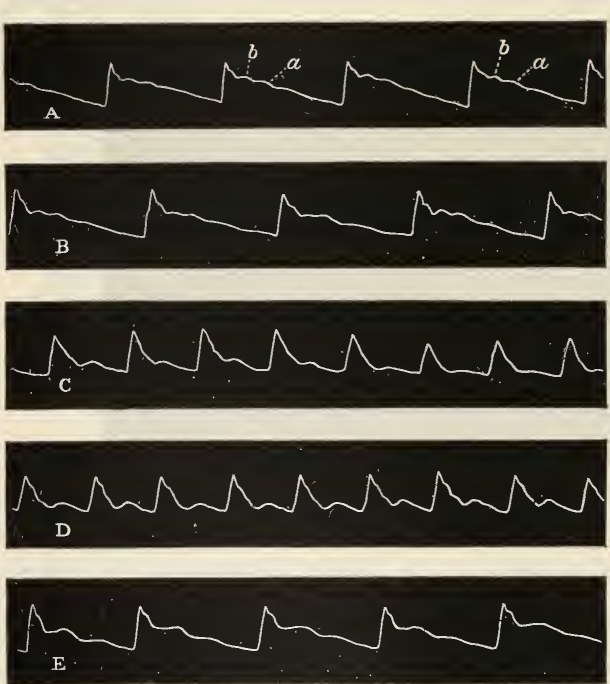

Fig. 75. - Effect of inhalation of amyl nitrite. A, Normal trace; C, maximal effect of the drug. a dwarf as in a man of tall stature. If the dicrotic wave were of peripheral and reflected origin, it should assuredly have occurred earlier in the dwarf, for in the dwarf the length of artery to be travelled by the reflected wave would be much smaller.

Under the influence of inhalation of amyl nitrite, the dicrotic wave may, it is stated, almost disappear during the maximal effect of the drug (Fig. 75). Those who seek to explain the origin of this wave by the production of reflected waves suggest that this is so, because the arterioles are so dilated by the drug that little reflection can take place. ${ }^{2}$ The evidence in favour of this statement is, however, unsatisfactory, and, holding to the central origin of the dicrotic wave, the phenomenon can equally well be explained by the fact that the heart force is diminished while the peripheral resistance is lowered, and therefore the back swing of the blood against the

1 Arch. f. d. ges. Physiol., Bonn, 1890, Bd. xlvi. S. 143.

${ }^{2} \mathrm{v}$. Kries, loc. cit. 
semilunar valves is greatly enfeebled. On a review of the whole evidence, the central origin of the dicrotic wave must be taken as an assured fact.

Reflected waves, however, if synchronous in time, may possibly modify the dicrotic wave, and, according to the sign of these waves, the height of the dicrotic wave may be diminished or increased in different arteries; the time relations of the dicrotic wave to the primary wave may also be altered thereby.

The question of interference between reflected waves and the primary pulse waves is inextricably complicated, owing to the fact that it is necessary to take into account not only the centripetal reflected waves which arise in the peripheral field of an artery, but also the reflected waves which arise in other arterial fields, and which, after being again centrally reflected, course centrifugally down the observed artery.

That the pulse wave is affected by purely local conditions, is shown by the following facts:-

1. The primary and dicrotic waves of the radial pulse are greatly diminished by occlusion of the veins of the arm, by the dependent position of the limb, or by plunging the arm into cold water.

2. They are greatly increased when the arm is elevated above the head, or plunged into hot water. ${ }^{1}$

When the general and not the local conditions of the circulation are varied, the dicrotic wave is found to be most marked in the following conditions :-

1. When the arterioles are dilated, the arterial tension low, and the systole of the heart vigorous; for example, after a hot bath, or in the first stage of asthenic fever.

2. When the systolic output of the left ventricle is, from any cause, diminished; for example, on taking a deep inspiration, in the second stage of a forcible expiratory effort, and after severe hæmorrhage.

The dicrotic wave is most marked in youth when the arteries are elastic. In any individual it is increased at times of exhaustion, and diminished in times of vigour. The amplitude of the dicrotic wave, so long as the local conditions are not varied, can be taken as a sign of low arterial tension.

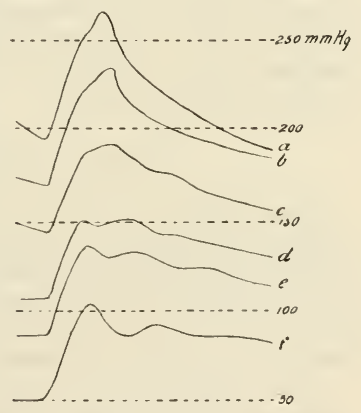

The predicrotic wave, which is sometimes observed preceding the dicrotic wave, is, according to v. Kries, due to reflection for a comparison of the pressure and velocity curves shows that, at the point of occurrence of this wave, the pressure and velocity are most divergent. If the predicrotic wave become very marked, it may rise to a higher elevation than the primary wave. The pulse curve is then marked by an anacrotic wave. The curve is anacrotic in type when the

${ }^{1}$ Hill, Barnard, and Sequeira, Journ. Physiol., Cambridge and London, 1897, vol. xxi. 
arterial pressure is very high, katacrotic when the pressure is low. The change of form, as the arterial tension rises, is seen very well in the six curves taken with a spring manometer during the escape of the heart from vagal inhibition (Fig. 76). By compression of the abdominal aorta, the carotid pulse can easily be made to exhibit an anacrotic wave.

To sum up, then-1. The form of the pulse curve in any artery is produced by (1) the aortic centrifugal primary waves; (2) the centripetal waves reflected from the peripheral field of the observed artery; (3) the waves reflected from other arteries. These waves modify each other by interference. The sphygmograms from different arteries show certain main and constant features, which in each artery are modified by peculiar local characteristics. For example, in femoral tracings, the primary and dicrotic waves are much more prolonged than in radial curves.

2. Since the form of each pulse curve depends not only upon the systole of the heart, but also on the peripheral resistance, the tone of the arteries, the turgescence of the venæe comites, and the amplitude of the reflected waves, it is obvious that no sure deductions can be drawn from the amplitude of the oscillation, either as to the power of the heart or the height of the arterial tension.

Finally, it must be remembered that the sphygmograph can never be applied twice to the same artery in exactly the same way, and with the same amount of pressure. This vitiates the instrument for comparative work. The best rule to follow is to always so adjust the instrument as to obtain the maximal excursion that can be possibly obtained in any given case.

\section{THE CAPILLARY CIRCULATION.}

The blood is brought into intimate relation with the tissues by diffusing through the endothelial wall of the capillaries, and this wall is of the greatest tenuity; thereby takes place that exchange of material which maintains the combustion of the body and the fire of life. The whole aim of the circulation is attained, so long as the arterialized blood is driven with a constant velocity through the capillaries.

The capillaries vary in size in different organs. In the brain the length has been estimated to be $0.709 \mathrm{~mm}$. (pons), and $0.42 \mathrm{~mm}$. (optic thalamus), in the mucosa of the stomach $0.6 \mathrm{~mm}$., and in the liver 0.5 to $1.1 \mathrm{~mm} .^{1}$ The diameter of the capillaries varies from 007 to 013 $\mathrm{mm}$.

Malpighi ${ }^{2}$ (1661) first observed under the microscope the capillary eirculation. He examined the lung, the mesentery, and the bladder of the frog. It has since been seen in many other transparent or translucent parts of animals.

The microscopical examination of the circulation.-By using a low power it is possible to simultaneously examine arteries, capillaries, and veins in the same field. The first thing which strikes the observer is the different direction of the stream in the arteries and in

${ }^{1}$ Lewy, Arch.f. d. ges. Physiol., Bonn, 1896, S. 461.

2 "De pulmonibus epistola," ii., Opera omnia, London, 1686. 
the veins. On account of the reticular arrangement of the capillaries, the direction of the stream through them is by no means constant; for a period there may be a complete cessation of the How in a capillary channel, or the direction of the current may even be reversed for a longer or shorter time. The flow through the arteries is by far the most rapid. In the veins also the stream is so rapid that it is difficult to catch the contour of the corpuscles. The stream is slower in the small veins, and in the capillaries the movement is, as a rule, so tardy that the individual corpuscles can be followed without any difficulty. The inconstancy of the capillary stream is generally apparent. If a group of capillaries be kept for some time under observation, the blood is occasionally seen to hurry suddenly through a number of these with increased rapidity ; this continues for a while, and then the stream becomes again slower and slower, till after an interval it reassumes the usual quiet rate of flow which has been maintained without interruption in the neighbouring capillaries. These variations depend on alterations in the lumen of the afferent arteries. ${ }^{1}$

The arterial stream is pulsatile, and each systole may be recognised even in very small arteries by the rhythmical acceleration and retardation of the blood stream. Such a rhythmical movement is absent from the capillaries and veins in a normal condition; the stream is a continuous one in both. In the arteries the mass of red corpuscles does not completely occupy the lumen, but moves along the axis of the stream. To the outside there lies a clear layer of plasma, in which here and there a stray white corpuscle rolls along. In the veins there is also a similar peripheral plasmatic layer, in which the white corpuscles roll slowly along, sticking now and again to the wall of the vessels in their course. In the smallest capillaries the plasma layer cannot be distinguished, the red corpuscles march in single file, and often become distorted and bent as they pass. These capillaries are invisible if by any chance the file of corpuscles ceases to pass through them. Thus in the course of an observation capillaries may be seen to appear and vanish from view. In the angles of the capillary network, red corpuscles may sometimes be seen to stick and hang in the balance, bent round the angle, half in one branch and half in another, until finally swept on into the rush and hurry of the stream.

The white corpuscles progress with a slow rolling motion in the plasmatic layer. The axial stream travels with the greater velocity, and thus the side of the leucocyte which at any moment lies nearer the axis is driven on with the greater speed; hence the rolling movement. The white corpuscles travel in the peripheral layer, the red in the axial layer, for the latter are the heavier. It is not, as has been supposed, that the white are lighter and the red corpuscles heavier than the plasma; both forms are of a higher density than the plasma.

Schklarewsky ${ }^{2}$ found that, when he circulated particles of graphite and carmine through glass capillaries, the carmine travelled in the peripheral layer. When he substituted resin in the place of graphite, the carmine travelled in the axis. When he circulated pus corpuscles and milk globules, the pus corpuscles occupied the axial stream.

The capillary circulation no longer remains pulseless, if the resistance

${ }^{1}$ Cohnheim, "Lectures on General Pathology," New Syd. Soc. Translation, 1889, vol. i. pp. $152,153$.

${ }^{2}$ Arch. f. d. ges. Physiol., Bonn, 1868, Bu. i. S. 603.

vOL. II. -8 
in the arterioles be lowered to a certain point. Bernard stimulated the chorda tympani nerve, and found that the blood issued in pulses from the veins of the submaxillary gland. By plunging the hand in very hot water, the pulse may be seen to reach even the turgid veins on the back of the hand. In cases of aortic insufficiency, a capillary pulse is readily obtained in any area of congestion which is produced by scratching the skin.

The effect of vaso-dilatation can be observed under the microscope with the greatest ease. On brushing the tongue of a curarised frog, an appearance of intense redness shows that arterial congestion has set in. In the words of Cohnheim, a really magnificent spectacle now presents itself. All the vessels, arteries, capillaries, and veins are wide and strongly distended with blood; innumerable capillaries are perceptible at a glance, where previously a few redcoloured threads were toilsomely sought for; and in all these vessels, small and large, the blood rushes on with the greatest rapidity-so rapidly that even in the capillaries the eye in vain strives to catch the outline of a single corpuscle.

By the application of a piece of ice to the tongue of the frog, vasodilatation can be converted into constriction. The arteries become narrow, the tongue pale. The eye has difficulty in finding any except the larger vessels; few capillaries appear to contain blood; and where a considerable quantity of blood is still present, as in the arteries and veins, the flow is tardy, and even in the arteries the individual corpuscles can now generally be recognised.

In a warm-blooded animal the results of exposure to an irritant are much more rapidly established ; after exposure of the rabbit's ear to water at $56^{\circ} \mathrm{C}$., the bloorl is altogether unable to penetrate the arteries. A change has taken place in the relations between the blood and the vessel wall as regards friction and adhesiveness, and thus complete stasis of the circulation arises. If the change be less intense the porous nature of the vessel is affected, and a quantitative and qualitative change in the transudation from the capillaries ensues. The rabbit's ear, with exception of the central artery and vein, may be entirely separated from the body. After thus dividing all the vasomotor nerves, vascular dilatation will be greatly increased by rubbing the ear, and all the phenomena of inflammation occur in just the same way after the application of an irritant or as a result of ischæmia. We have here to deal, not with a nervous mechanism, but with a molecular change of the vessel wall. The circulation through the capillaries is possible only so long as the vessel wall is in the normal physico-chemical condition which characterises the living state.

In spite of considerable variations of internal tension, the capillaries show only slight variations in width. After amputation of the hind-limb of a frog, the capillaries in the swimming-web scarcely become reduced in size.

Rate of flow.-Hales ${ }^{1}$ reckoned the velocity of a blood corpuscle in the capillaries of the frog's muscles to be 0.1 inch in 9 seconds $(0.28 \mathrm{~mm}$. per second). The method most conveniently used is to employ an ocular micrometer, and to follow the course of a corpuscle during a period of time given by a clock beating one-fifth seconds. The velocity has been thus found by various observers to be $0 \cdot 25$ to $0.57 \mathrm{~mm}$. per second in cold-blooded animals.

Vierordt ${ }^{2}$ reckoned the velocity in the retinal capillaries by the entoptic method. He projected the shadow of the corpuscles on an

I "Statical Essays," 1733, vol. ii. p. 63.

2 "Dic Erscheinungen und Gesetze der Stromgeschwindigkeit des Blutes," Frankfurt, 1858 , S. $41,111$. 
illuminated ground-glass screen, held 11 to $16 \mathrm{cms}$. from the eye, and on this screen he reckoned the progression of a corpuscle in a given time. He found that a blood corpuscle could be thus followed over a space of 20 to $30 \mathrm{~mm}$. If $A$ represents the distance of the screen from the anterior nodal point, and $B$ the distance of the retina from the posterior nodal point of the eye, and $C$ the distance travelled by the corpuscle on the screen, then $\frac{B C}{A}=x$, the real distance which is covered by the corpuscle in the capillary.

The mean velocity was thus found to be $0.75 \mathrm{~mm}$. per second. The red corpuscles travel in the axial parts of the stream, and as the mean velocity is less than the axial velocity, the true mean velocity of flow is less than the above; it may be taken to be about $0.5 \mathrm{~mm}$. Since the velocity at any point in a system of tubes stands in inverse proportion to the sectional area, the relationship of the total sectional area of the capillaries, at any one time patent, to that of the aorta can be reckoned. ${ }^{1}$ Thus, if the mean velocity be taken as $320 \mathrm{~mm}$. per second in the aorta and $0.5 \mathrm{~mm}$. per second in the capillaries, the relation is $1: 640$. In man the sectional area of the aorta is $4 \cdot 4 \mathrm{sq}$. cms. The total sectional area of the capillaries would thus be equal to about $2800 \mathrm{sq}$. cms. This result is, of course, only roughly approximative.

The capillary blood pressure. - The pressure was first obtained by v. Kries. ${ }^{2}$ He placed a glass plate, 2.5 to $5 \mathrm{sq} . \mathrm{mm}$. in size, in a suitable place on the skin, such as the last joint of the finger. There depended from this glass plate a small scale pan. On this, weights were placed until the pressure was reached at which the skin was blanched and the capillaries compressed.

In using this method, the reading is taken when the change of colonr in the skin just begins to appear, that is, when the superficial capillaries are obliterated. Thus the error due to the elasticity of the epidermis is avoided as far as possible. It is presupposed that the elasticity of the capillary wall is negligeable, and that the blood pressure, in all the area of capillaries under observation, is equally great.

If a weight of $g$ grammes be placed on a surface of $f$ square millimetres, then the formula $h=\frac{g}{f}$ gives the hydrostatic pressure in $m m \cdot \mathrm{H}_{2} \mathrm{O}$ which is required to obliterate the capillaries. Thus, suppose the square surface of the glass plate be $4 \mathrm{sq}$. mm., and the weight be $1 \mathrm{grm}$., then an area of $4 \mathrm{sq}$. mml carries 1 grm. $=1$ c.c. $\mathrm{H}_{2} \mathrm{O}=1000$ cub. mm. The height of the column of water supported by one sq. mm. is therefore $\frac{1000}{4}=250 \mathrm{~mm}$. $\mathrm{H}_{2} \mathrm{O}$.

0.25 grms, is the smallest difference in weight which can produce the index of colour change. To obtain the index with a smaller glass plate, a heavier weight is needed than with a larger plate. The method is therefore very rough and inaccurate.

Roy and Graham Brown ${ }^{3}$ employed a method of directly determining how great a hydrostatic pressure is necessary to obliterate the capillaries while under microscopic observation. The web or mesentery of a frog

1 Vierordt, Arch. f. physiol. Heilk., Stuttgart, 1848, S. 184.

${ }^{2}$ Bcr. d. k. Sächs. Gesellsch. d. Wissensch., math.-phys. Cl., Leipzig, 1875, S. 148.

${ }^{3}$ Journ. Physiol., Cambridge and London, 1879, vol. ii. p. 328. 
is laid on a glass plate, and compressed by a thin and transparent membrane, which forms the base of a glass capsule. The latter is filled with water, and connected with a pressure bottle and manometer.

They found that a pressure of 100 to $150 \mathrm{~mm}$. $\mathrm{H}_{2} \mathrm{O}$ is sufficient to stop the circulation in the capillaries and veins of the frog's web; to expel the blood from the arteriole 200 to $350 \mathrm{~mm}$. is necessary. From time to time the pressure varies 20 to $30 \mathrm{~mm} . \mathrm{H}_{2} \mathrm{O}$. On producing splanchnic paralysis, by striking the abdomen, the pressure sank to zero, and then rose again in the veins to 70 or $100 \mathrm{~mm}$., owing to venous congestion. Temporary ancmia of the web caused dilatation of the vessels, and this produced in its turn a higher capillary pressure.

v. Kries found that, in the sitting posture, the position of the arm influenced the capillary pressure in the fingers very markedly. Thus the pressure varied with the distance from the vertex of the head in the following way :-

$\begin{array}{cc}\mathrm{Mm} . \mathrm{H}_{2} \mathrm{O} . & \text { Distance of finger below vertex. } \\ 328 & 0 \mathrm{~mm} . \\ 397 & 205, \\ 513 & 490 ", \\ 738 & 840 ",\end{array}$

The pressure is seen not to increase by the full hydrostatic effect, and v. Kries failed to explain this fact. It is probably due to a compensation for the effect of gravity, which is brought about by the vasomotor mechanism. In the ear, v. Kries found the mean capillary pressure to be $20 \mathrm{~mm}$. $\mathrm{Hg}$; in the gums of the rabbit $33 \mathrm{~mm} . \mathrm{Hg}$; or one-third of the aortic pressure.

If the finger be ligatured, so as to block the venous exits, the capillary pressure rises to the statical arterial tension; after loosening the ligature the capillary pressure remains for some minutes at a high level, owing to the paralytic dilatation of the arterioles produced by the venous blood. ${ }^{1}$

In the brain, the capillary venous pressure can be estimated by finding the tension which is just sufficient to balance that which the brain exerts against the skull (see p. 143). This pressure in the horizontal position of the animal is usually about $10 \mathrm{~mm}$. $\mathrm{Hg}$. The capillary pressure varies widely with changes in the general venous and arterial pressures, and with the position of the animal. Thus, in the brain, the pressure may fall below zero in the vertical feet-down position, and rise to almost $50 \mathrm{~mm}$. $\mathrm{Hg}$ during the height of strychnine convulsions.

The capillary pressure stands in closer relationship to the venous pressure than to the arterial pressure. Between an artery and its capillaries lies the unknown and varying resistance of the arterioles; between the capillaries and vein there is no such resistance. It is most fallacious to argue that a fall of arterial pressure necessarily produces a fall of pressure in all the capillary areas of the body. In the want of a good method of direct determination of the capillary pressure, we can only judge of changes which take place under varying experimental conditions in that pressurc, by recording the tension in both the afferent and the efferent vessel. Thus, for example, in dealing with the capillary pressure in the liver, the tension both in the portal vein and vena cava

${ }^{1}$ Natanson, Arch.f. d. ges. Physiol., Bonn, 1886, Bd. xxxix. S. 386. 
inferior must be estimated. In the brain, similarly, the pressure in the carotid artery and in the venous sinuses must be taken.

If in any area the resistance in the arterioles does not vary, then if both arterial and venous pressures rise together, or if one pressure rise while the other remains constant, we may say with certainty that the capillary pressure is increased. If both pressures fall, or if one falls while the other remains constant, the capillary pressure is diminished. A rise of arterial pressure, with a fall of venous pressure, or a fall of arterial with a rise of venous pressure, renders the determination of the capillary pressure a matter of considerable difficulty. ${ }^{1}$ This difficulty is intensified when the arterioles in the area under investigation also vary in calibre.

Variations in capillary pressure are given in the following experimental conditions ${ }^{2}$ (the animals are placed in the horizontal posture) :-

Experiment 1.-Section of Spinal Cord at First Dorsal Vertebra.

Intestinal. Fall.
Hepatic.

Slight rise or fall.
Cerebral. Fall.

In this case the capacity of the venous system is greatly iucreased, and the peripheral resistance in the arterioles greatly lowered. The pressure in the intestinal and cerebral capillaries falls in consequence of the fall of arterial pressure. The hepatic capillary pressure varies but slightly, for the vena cava pressure remains almost unaltered.

\section{Experiment 2. - Arrest of the Heart by Excitation of the Vagus. \\ Intestinal. Fall. \\ Hepatic. Rise. \\ Cerebral. Fall.}

The intestinal and cerebral capillary tensions fall in sequence to the enor mous fall of arterial pressure. The hepatic capillary pressure rises in sequence to the congestion of blood in the venous system.

\section{EXPERIMENT 3.-Asphyxia (Animal curarised).}

\section{Intestinal.}

Preliminary rise, then fall.
Hepatic.
Rise.
Cerebral.

Rise.

During asphyxia the arterial pressure rises, and finally falls; the venous pressure rises when the heart fails. The portal pressure rises at first, owing to the forward expression of blood by the constricting splanchnic arterioles, then falls in consequence of the diminished input. The hepatic pressure rises, in sequence to the venous rise, owing to the back effect from the failure of the heart to maintain the systolic output. The cerebral pressure rises greatly, owing both to the arterial and venous rise of tension.

\section{Experiment 4.--Severe Bleeding.}

$\begin{array}{ccc}\text { Intestinal. } & \text { Hepatic. } & \text { Cerebral. } \\ \text { Fall. } & \text { Fall. } & \text { Fall. }\end{array}$

Here, there is a general fall of venous and arterial pressure.

${ }^{1}$ Bayliss and Starling, Journ. Physiol., Cambridge and Loudon, 1894, vol. xvi. p. 160.

2 Ibid., 1894, vol. xvi. p. 190; Hill and Payliss, lbid., 1895, vol. xviii. p. 334; Hill, "Cerebral Cireulation," London, 1896. 
Experiment 5.-Injection of a Large Quantity of Normal Saline.
Intestinal.
Rise.
Hepatic.
Rise.
Cerebral.
Rise.

Here, there is a general rise of venous and arterial pressure.

Experiment 6. - Obstruction of the Thoracic Aorta.

$\begin{array}{ccc}\text { Intestinal. } & \text { Hepatic. } & \text { Cerebral. } \\ \text { Fall. } & \text { Rise. } & \text { Rise. }\end{array}$

The intestinal pressure falls in consequence of the diminished input, the cerebral tension rises owing to the increased pressure in the cerebral arteries. The hepatic pressure rises by a back effect, for the heart, in the face of the increased resistance, is unable to maintain the systolic output.

Experiment 7.-Obstruction of the Inferior Vena Cava above the Liver.
Intestinal.
Doubtful.
Hepatic.
Rise.
Cerebral.
Fall.

The blood congests in the abdominal veins, therefore the hepatic pressure rises, the arterial pressure falls enomonsly, and thus the cerebral tension decreases. On the one side of the intestinal capillaries the arterial pressure falls, on the other side the venous pressure rises greatly; which effect will have the most influence is doubtful.

liy many authors it has been suggested that osmotic force, capillary attraction, and the ceaseless removal of particles from the blood to supply the materials of the various secretions, may be factors in the maintenance of the capillary circulation. ${ }^{1}$ Hitherto this theory has defied experimental proof. We may rest assured that if the action of the heart cease, no other force operates sufficiently to cause a continued and progressivemotion of the blood.

\section{THE CONDITIONS OF BLOOD-FLOW IN THE VEINS.}

The most striking difference between the structure of an artery and its venæ comites is a decrease of elastic and muscular tissue in the veins, together with an increase of white connective tissue. Accompanying these changes, there is, on the side of the vein, a general and considerable decrease in the thickness of the wall, and increase in the capacity. In consequence of these structural differences, the maximal extensibility of the veins, as measured by their increase in cubic volume, is found to occur at very low pressures. At higher pressures the extensibility rapidly decreases. The veins are tubes with flaccid walls, which fall together when empty, and under very slight pressure expand to their full capacity. Beyond this point, the walls, on account of the quantity of connective tissue entering into their structure, can extend but little. Thus a rise of pressure from 0 to about $40 \mathrm{~mm}$. $\mathrm{Hg}$ doubles the cubic content of a vein, and enlarges it to its utmost capacity. It follows that the great variations of volume which are seen to occur in the veins are occasioned less by changes of pressure than by changes in the volume of blood passing into the veins." In this respect an artery shows

1 Alison, "Outlines of Physiology," 3rd edition, 1839, P. 63 ; Cappie, "The Intracranial Circulation," Edinburgh, 1890.

${ }^{2}$ Roy, Journ. Physiol., Cambridge and London, 1881, vol. iii. 1. 136. 
a marked contrast to a vein, for by a rise of internal tension from 0 to $200 \mathrm{~mm}$. Hg the cubic content increases three and a half to seven times, and therefore the arterial capacity entirely depends on the magnitude of the internal tension.

The resistance to a breaking strain on the part of the veins is very great. It requires a higher pressmre to rupture a vein than the corresponding artery. ${ }^{1}$ The rupturing strain is found to be some $3 \cdot 7$ to $9 \cdot 2$ atmospheres. ${ }^{2}$

If, by external compression, or by a forced expiratory effort, the various outlets be blocked, the tension may rise in the vein to the full statical pressure in the artery. For this reason the veins must be strong enough to bear any such increased strain. There is, however, another need for strength of veins, and that is, that they may be able to bear the strain which may arise from external violence. The superficial reins are endowed with more muscular and elastic tissue than those more deeply situated; while those veins which run in the muscles and in the bones, and are thus protected from violence and supported by firm structure, possess no muscular elements. When exposed, a superficial vein contracts on mechanical stimulation and on cooling, while it may be made to dilate by applying warmth. On rough mechanical stimulation, the external jugular vein is often seen to shrink up so as completely to obliterate the lumen, and some minutes may elapse before the blood again finds a pathway through the constricted portion.

Pressure in the venous system.-The venous pressure is very low. In investigations on the sulject, all conditions which affect the How must be borne in mind. The manometer tube must not be inserted into a vein in the direction of the periphery, but must be placed in a branch forming a T-junction with some larger vein. It is necessary to destroy any valve which may lie at the seat of junction. The side pressure in the larger vein is then obtained. Thus, when a cannula is pushed down the jugular vein, the lateral pressure of the vena cava superior is obtained. Hales ${ }^{3}$ estimated the venous pressure in a limb to be 6 in. $\mathrm{H}_{2} \mathrm{O}$. The mean pressure in the vence cavæ within the thorax is negative. ${ }^{4}$ Weyrich, by means of a $\mathrm{Hg}$ manometer, estimated the negative pressure in the right auricle to be -2 to $-3 \mathrm{~mm}$. $\mathrm{Hg}$ on each cardiac oscillation, and -5 to $-8 \mathrm{~mm}$. $\mathrm{Hg}$ on each respiratory undulation. ${ }^{5}$ Several observers have recorded the venous pulse thus produced, but the reading of the complicated curves so far obtained is as yet too undetermined for discussion to be profitable. ${ }^{6}$ The venous pressure is recorded by manometric readings, taken at stated intervals of time, from which curves can afterwards be plotted out. The use of either a delicate tambour, or piston-recorder, which has been previously calibrated against a manometer, will give equally satisfactory

1 Wintringliam, "Exper. Inquiry on Some Parts of Animal Strueture," London, 1740, p. 100.

${ }^{2}$ Gréhant and Quinquaud, Journ. de l'anat. et physiol., etc., Paris, 1885, tome xxi. p. 296.

3 "Statical Essays," London, 1735, vol. i. p. 42.

+ Mogk, Ztschr.f.rat. Med., 1845, Bd. iii. S. 33 ; Volkmann, "Hämodynamik," Leipzig, 1850, S. 356.

5 "De cordis aspiratione," Dorpat, 1853.

${ }^{6}$ See Frederieq, Trav. du labor. de Liege, 1890, tome iii. p. 85 ; and Francois-Franck, Arch. de physiol. norm. et path., Paris, 1889, 1. 85 ; Riegel, Deutsches Arch. f. klin. Med., Leipzig, 1882, Bd. xxxi. S. 1, 471. 
results. The manometer employed for venous work is filled with a semi-saturated solution of sodium or magnesium sulphate.

Jacobson ${ }^{1}$ found in the sheep, during normal quiet respiration, the following venous pressures:-

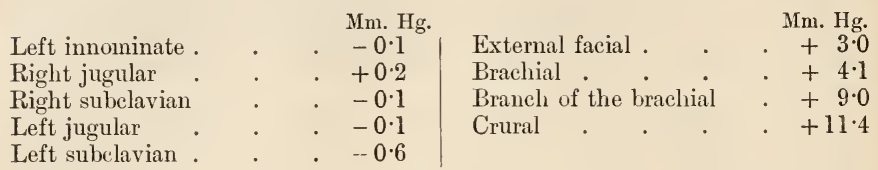

The pressure is lowest nearest the heart and thorax, and becomes ligher in proportion as the vein is placed more peripherally. The negativity in the central veins is due to the suction of the thoracic cavity produced by the elastic pull of the lungs. Owing to this negative pressure, when a large vein is opened in the neighbourhood of the thorax, air can be sucked into the circulation. Air that has thus obtained an entry has been observed to pass right through the pulmonary circulation, and to enter the arteries. The danger of air entering during operations has been over-rated. A large amount of air can be slowly injected into a vein withont killing an animal.2

A rapid injection, such as would be caused by blowing air into the venous cannula, kills by causing frothing in the heart, embolism in the lungs, and perhaps embolism in the coronary arteries. The danger of embolism from the entry of air is much greater in a small than in a large animal, for the smaller the heart the less the amount of air required to hinder its action by frothing. ${ }^{3}$

Effect of muscular movements.-Muscular movement is a most important agency which aids the venous flow. On account of the valves the blood can only be expressed from those veins which are compressed by muscular contraction, in one direction, viz. towards the heart. Continued tetanic spasm stops the flow, but clonic or repeated movements, by the alternate filling and expression of the veins, materially favours the flow. The pressure in the vena cava may be considerably raised by general tetanic spasm of the whole body, as is seen after injection of strychnine or essential oil of absinthe; and this is so, in spite of a general arterial constriction which diminishes the inflow into the veins. The rise of venous pressure in such a condition of tetanus is of mixed origin. It is due, first, to compression of the veins by the spasm of the muscles; and, secondly, to general vaso-constriction of arteries and portal veins. Thus, while the capacity of the venous system is diminished, the output of the heart is, owing to the high arterial pressure, lessened, and the blood is congested into the veins.

Pressure of the body against external objects influences the venous flow in the same manner as muscular contraction. Sitting or lying in one posture retards the venous circulation, while, on the other hand, exercise and constant change of posture not only accelerate the circulation on the arterial side, but also aid the flow in the veins.

By rhythmic compression of the thigh muscles the blood can be discharged in jets from the femoral vein of a dog, and the flow of blood greatly increased. The rhythmic contractions of the spleen and the

1 Arch. f. Anat., Physiol. u. wisscnsch. Med., 1867, S. 226.

${ }^{2}$ Laborde and Muron, Compt. rend. Soc. de biol., Paris, 1873, tome lvii. pp. 84, 131 ; Jurgensen, Deutsches Arch. f. Klin. Med., Leipzig, 1882, Bd. xxxi. S. 453.

${ }^{3}$ Hill and Barnard, Journ. Physiol., Cambridge and London, 1897, vol. xxi. p. 335. 
peristalsis of the intestines greatly promote the portal venous flow. Respiration not only aspirates and compresses the venous blood into the right heart, but promotes the flow into the left heart from the pulmonary veins.

It was determined by Braune ${ }^{1}$ that, if the thigh be rolled outwards and backwards, the pressure in the femoral vein under Poupart's ligament falls 5 to $10 \mathrm{~mm}$. $\mathrm{H}_{2} \mathrm{O}$ below zero, and becomes positive again when the leg is placed in the opposite position. The bones, the muscles, and the fascia, together with the vein, make a suction and force pump, and thus, by this mechanism, walking can aid the flow in the femoral vein. Extension of the veins in their long diameter has been found to increase their capacity, and thus aspiration may be produced therein by alternate extension and flexion of the body or limbs.

When the arterial supply to any part of the body is entirely cut off, the pressure in the vein which drains the part in question sinks to the pressure of the great vein into which it opens. When, on the other hand, all the venous exits from any part are ligatured, then the pressure rises, peripherally to the seat of ligature, to the arterial pressure.

If the arteries conveying blood to the part are few and narrow, a few veins are sufficient to secure an adequate escape of blood; conversely, where the arterial supply is great, a moderate obstacle to the venous flow causes marked stasis and transudation. For example, on ligaturing in the rabbit all the large veins of both ears, and dividing the cervical sympathetic nerve on one side, the ear on this side will, within a few hours, display a considerable swelling, while the other will remain unchanged. If, on occlusion of the central end of the femoral vein, the pressure therein is equal to 80 to $100 \mathrm{~mm}$. $\mathrm{MgSO}_{4}$ solution, it will be found, after division of the sciatic nerve, to rise to 280 $\mathrm{mm} . \mathrm{MgSO}_{4}$ solution in a few minutes. ${ }^{2}$

Rate of flow in the veins.-Turning to the question of the velocity of the venous flow, it is obvious that the average input of the veins per second must equal the average output per second, in order that the circulation may continue. If the veins that enter the heart were of the same sectional area as the arteries that leave it, then the velocity would be the same in these veins as in the arteries. When the venæ cavæ are filled with blood, their total sectional area is found to be considerably greater than that of the aorta. But, as normally these veins are never filled to their full capacity, it is probable that the velocity of flow in them is approximately equal to that in the aorta. The velocity in the carotid artery and jugular vein, or in the umbilical artery and vein of the sheep's embryo, has been measured with Ludwig's stromuhr, and has been found to be almost the same. ${ }^{3}$

That the progression of the blood in the veins is primarily maintained by the driving force of the heart, was shown by Magendie. He divided the whole of the thigh, except the femoral artery and vein, from the body. Pressure on the artery immediately stopped the flow in the vein. The velocity, on account of the centripetal diminution of the capacity of the venous system, must increase from point to point, while the pressure must steadily decrease. The oscillations of pressure and velocity are not conducted from the arteries into the veins, because the pulse waves are reflected from the resistance in the arterioles and

${ }^{1}$ Ber. d. k. Sächs. Gesellsch. d. Wissensch., math. phys. Cl., Leipzig, 1870, S. 261.

2 Cohnheim, "Lectures on Pathology," New Syd. Soc. Translation, vol. i. p. 159.

${ }^{3}$ Cyon and Steinman, Mélanges biol. Acad. imp. d. sc. de St. Péterrbourg, 1871, vol. viii. p. 53 ; Zuntz and Cohnstein, Arch. f. d. ges. Physiol., Bonn, 1884, Bd. xxxiv, S. 224. 
capillaries, and lost in the arterial system. In conditions of local vasodilatation, where the peripheral resistance is greatly lessened, the pulse may travel through the capillaries into the veins. The steady increase of velocity and decrease in pressure are masked in the venous system by other agencies, besides the systole of the heart, which affect the venous circulation. The discussion of these will be found in the sections on the influences of gravity and respiration on the circulation.

\section{Influence of Respiration on the Pulmonari and Systemic Circulation.}

In his classical researches on blood pressure, Stephen Hales ${ }^{1}$ records the following observation:- "When the blood has subsided a little in the tubes which were fixed to the arteries of these $\operatorname{dogs}$, it would, as in the horse's, rise on a sudden considerably on deep sighing, as also on pressing the dogs' bellies hard with the hand, the blood would immediately rise about six inches, and subside as much on taking off the hand; and it was the same on several repetitions." Farly in this century the inspiratory suction power of the thorax was urged by Carson $^{2}$ and Barry, ${ }^{3}$ as a mechanism for forwarding the progression of the blood in the veins. Carson estimated the elasticity of the expanded lung of the sheep as equal to the tension of a column of water seven inches high. Barry observes that if one end of a bent tube be introduced down the jugular vein, and the other end rest in a vessel containing water, then the water within the tube can be seen to rise during each inspiration, and sink during each expiration. Although by this experiment Barry proved that a negative pressure is established in the vente cave during inspiration, yet the importance of the suction power of the thorax was not generally admitted. In opposition to such a doctrine, Davies ${ }^{4}$ declared that on compressing the vena cava inferior in the abdomen, the upper portion of the vein is not emptied by the aspiration of the thorax. On the other hand, on obliterating the vena cava just below the auricles, after first squeezing the blood within it onward into the heart, the vein immediately fills up again from below. Thus, he argued, the driving force of the left heart is by itself sufficient to return the blood to the right heart, and complete the circulation. In experiments with the hemalynamometer, Poiseuille and Magendie ${ }^{5}$ noticed an elevation of arterial pressure during the period of expiration, and so soon as Ludwig, ${ }^{6}$ by the completion of the kymograph, succeeded in taking traciugs of the arterial pressure, he observed that variations in arterial tension constantly occurred synchronously with the movements of respiration.

Accepting the explanation of these undulations which at first sight appeared obvious, Ludwig taught that the expiratory rise of intrathoracic pressure aids the systolic output of the heart, propels the blood onward into the arteries, and raises the arterial tension. The researches of Donders, on the changes of intrathoracic pressure during the phases of

${ }^{1}$ Hales, "Statical Essays," London, 1733, vol. ii. p. 33.

2 "Inquiry into the Causes of the Motion of the Blood," Liverpool, 1815.

3 " Exper. Researches on Influence of Atmospheric Pressure upon Progression of Blood in the Veins," London, 1826.

+ Lancet, London, vol. xi. p. 606.

5 Journ. de physiol. expér., Paris, tome i. p. 132.

6 Arch. f. Anat., Physiol. u. wissensch. Med., 1847, S. 242. 
respiration, for a time lent support to the doctrine of Ludwig, but this had to be abandoned so soon as the time relations of the respiratory movements and the undulations of arterial pressure were definitely settled.

The synchronous records of respiration and arterial tension obtained by Ludwig and Einbrodt, ${ }^{1}$ showed that the rise of arterial pressure takes place mainly in the inspiratory and not in the expiratory phase.

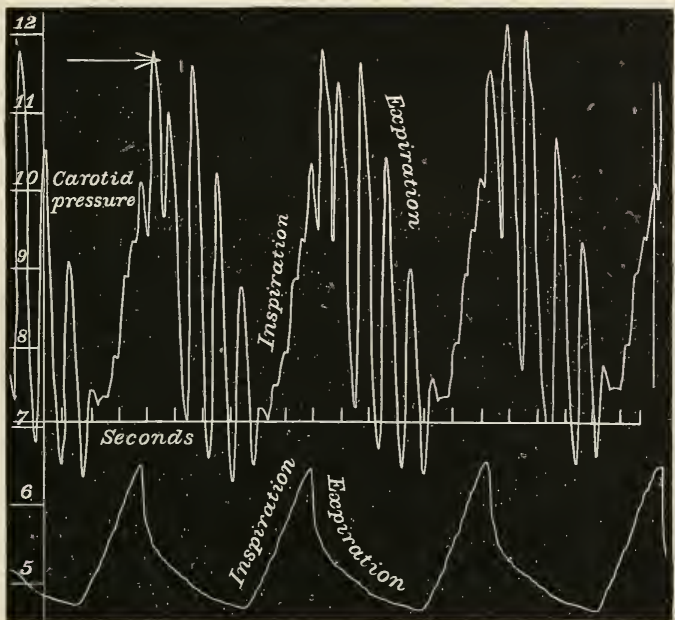

Fig. 77.--Effect of respiration on arterial pressure. The numbers on the left denote centimetres of nercury pressure.-Fredericq.

The respiratory undulations of arterial tension have been recorded, not only in mammals, but also in birds.

By Einbrodt three different types of the respiratory effect are distinguished:-

1. The respiration is quick and shallow. No marked effect either on the freqnency of the heart or the blood pressure is seen.

2. The respiration is deep, the inspiratory movement is executed more rapidly than the expiratory, and the periodicity is such that several heart-beats occur during each complete act of respiration. The arterial pressure falls at the beginning of inspiration, rises luring inspiration until expiration has commenced, and falls during expiration. The heart-beat increases in frequency during inspiration.

3. The respiration is deep and slow, and the heart-beat is frequent; these conditions are established by vagotomy. The changes of arterial tension are the same as in 2 , but increased in amplitude.

In order to explain the origin of the respiratory waves of arterial tension, it is necessary to grasp the conditions which pertain to the thoracic cavity. The heart and the large blood vessels which enter and leave the heart lie in a closed and air-tight chamber, the thoracic cavity. The remainder of this space is occupied by the lumgs. The thorax is formed of a bony and muscular wall, which possesses rigidity sufficient to

${ }^{1}$ Sitzungsb. d. k. Akad. d. Wissensch., math.-naturw. Cl., Wien, 1860, Bd. xl. s. 361. 
withstand the pressure of the atmosphere. If the thoracic cavity be freely opened, it appears to be far larger than its contents, for, at the moment of opening, the lungs collapse. In the fotus, on the other hand, the opened thorax is exactly filled by its contents. When the new-born child takes its first respiration the thoracic walls assume a new position, and the cavity then becomes permanently enlarged. The explanation of this is not quite clear. It has been suggested that the expiratory mechanism is over-stretched by the first inspiration, and is not endowed with sufficient elasticity to pull the chest back into its old position. ${ }^{1}$

In consequence of the enlargement of the thorax, the intrathoracic pressure becomes negative at the first breath of the child, and the lungs are expanded to fill the partial vacuum thus formed. The expanding force is the pressure of the atmosphere acting down the trachea. On one side of the pulmonary air-cell is the full pressure of the atmosphere; on the other side, the sub-atmospheric pressure within the thoracic

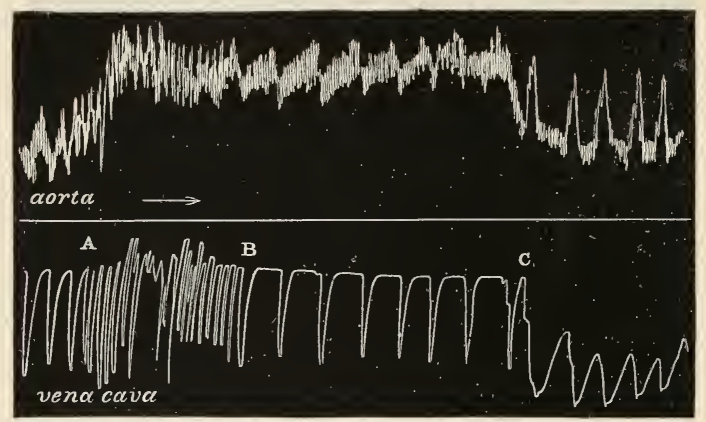

Fig. 78. - Respiratory undulations of aortic and vena cava pressures. Effect of vagotomy and the vertical feet-down position. A-B, vagi divided; C, feet-down position. The downstroke of the vena eava trace signifies inspiration.-Hill and Barnard.

cavity. The lungs are elastic bags; their structure is permeated by elastic tissue; in virtue of this elasticity, the lungs, when expanded, oppose a force contrary in sign to that of the atmospheric pressure. In consequence of this elasticity, the lungs collapse when the thorax is freely opened. In the natural state the pressure within the thorax is at any moment less than that of the atmosphere by that amount of the atmospheric tension which is required to overcome the elasticity of the lungs and to distend these organs to the size of the thoracic cavity. The intrathoracic pressure is measured by the insertion of a tube through an intercostal space into the cavity of the chest. This tube is connected with a manometer.

By a series of such determinations Donders ${ }^{2}$ arrived at the following values of the intrathoracic negative pressure or elastic pull of the lungs in man :-

In the position of ordinary quiet inspiration $-9 \mathrm{~mm}$. $\mathrm{Hg}$.

$$
\begin{array}{lll}
" \quad \text { expiration } & -7 \cdot 5 \text {. } \\
, \quad & -30 \text { to }-40 .
\end{array}
$$

${ }^{1}$ J. Bernstein, Arch. f. d. ges. Physiol., Bonn, 1878, Bd, xvii. S. 617.

"Ztschr. f. rat. Med., 1853, Bd, iii. S. 287. 
In smaller animals, such as the dog and rabbit, the negative pressure within the pericardium is equal to -3 to $-5 \mathrm{~mm}$. $\mathrm{Hg}$, rising in dyspnoea to -9 nim. $\mathrm{Hg}^{1}$

Within the lungs the tension of the air varies but slightly during the phases of quiet respiration. In inspiration the pressure of the intratracheal air equals $-0.1 \mathrm{~mm}$. $\mathrm{Hg}$; in expiration $+0.13 \mathrm{~mm}$. $\mathrm{Hg}$. In certain conditions, however, the intra-pulmonary pressure may vary enormously. Thus in Valsalva's experiment, when, after a deep inspiration, the mouth and nose are shut and a forcible expiratory movement is made, the intra-pulmonary pressure may rise to $100 \mathrm{~mm}$. $\mathrm{Hg}$. On the other hand, in Miller's experiment, when, after a deep expiration, a forced inspiratory movement is executed with the mouth and nose shut, the negative pressure within the thorax and lungs may sink as much below zero. Thus it seems clear that, while in quiet inspiration the extrathoracic vessels lie under the full pressure of the atmosphere, the intrathoracic vessels are exposed to a pressure of $-9 \mathrm{~mm}$. Hg. Likewise the pressure on the pulmonary capillaries = $-0 \cdot 1$, while the pressure on the pulmonary arteries and veins within the thoracic cavity $=-9 \mathrm{~mm}$. Hg. In Valsalva's experiment both the intrathoracic and intra-pulmonary pressure will increase, but the latter will always remain higher than the former by the amount of elastic tension exerted by the lungs. In Miiller's experiment both pressures will diminish, but the intra-pulmonary pressure will again remain higher than the intrathoracic pressure by the amount of elastic tension exerted by the lungs.

The changes in intrathoracic pressure naturally affect the calibre of the thin-walled inelastic veins and auricles far more than the elastic and thick-walled ventricles and arteries. The veins and auricles are flaceid bags, which can be easily distended up to their full capacity, while to expand the arteries and the ventricles a considerable amount of force is required. A negative pressure of $9 \mathrm{~mm}$. $\mathrm{Hg}$ has but an insignificant effect on the capacity of the aorta, wherein the tension is equal to $120 \mathrm{~mm}$. Hg, or more. The total effect of the negative pressure within the thorax is to increase, during inspiration, the diastolic filling of the heart, while the systolic output is not materially hindered.

Experimental facts.-1. Poiseuille ${ }^{2}$ discovered that the circulation of a certain volume of blood through the pulmonary vascular system occupied a longer time when the lungs were blown out than when they were collapsed.

2. On microscopical examination of the pulmonary circulation in the frog, it can be seen that the bloodflow is less rapid the more the lungs are distended.

3 . If the thorax be opened in a inammal, and artificial respiration

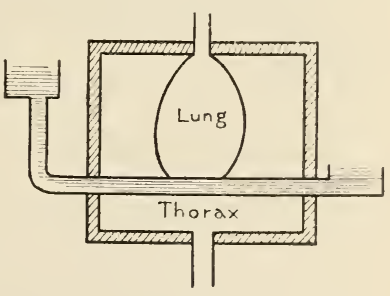

Fig. 79. supplied, the aortic pressure mounts when the lungs collapse, while it falls when the lungs are expanded.

4. If the lungs be excised and placed within an artificial thorax, and

\footnotetext{
${ }^{1}$ Adamkiewicz and Jacobson, Centralbl. $f$. d. med. Wissensch., Wien, 1873, S. 483.

${ }^{2}$ Compt. rend. Acad. d. sc., Paris, 1852, tome xli. p. 1072.
} 
the natural expansion of the lungs be imitated by the aspiration of this chamber, it is then found that a How of blood through the pulmonary vessels is quickened by expansion and lessened by collapse. Experiments 3 and $t$ thus yield exactly contrary results. Now, on examining the diagram (Fig. 79), it becomes obvious that the elastic tube which passes through the thorax and along the base of the lung would be

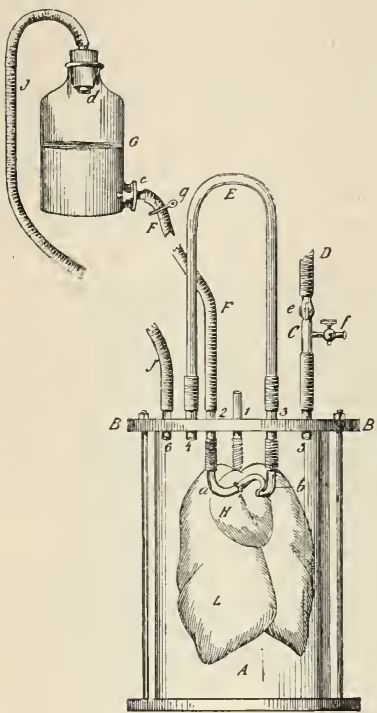

FIG. 80.-Model illustrating the effect of intrathoracic pressure on the circulation.-Funke and Latschen. berger. compressed by a rise of pressure in the lung. On the other hand, if the thorax were aspirated, this elastic tube would expand. ${ }^{1}$

It must be noted, however, that the conditions in the thorax are not exactly imitated in this model, for the head of pressure, or pump, should also lie within the chamber.

Funke and Latschenberger have more exactly imitated the natural conditions in the following way (Fig. 80). ${ }^{2}$ The lungs and heart are placed in an artificial thorax; the pulmonary artery is fed with defibrinated blood (tube 2) from a pressure-bottle, which is so arranged as to come under the influence of the intrathoracic pressure (tube 6). The outflow from the left auricle into the thoracic chamber is measured (tubes 3 and 4). Under these conditions an expansion of the lungs brought about by thoracic aspiration (tube 5) favours the flow, while an expansion produced by blowing down the trachea (tube 1) lessens the pulmonary circulation.

There are four chief factors which influence the pulmonary circulation :-

1. The positive pressure in the force-pump, the right ventricle.

2. The negative pressure in the suck-pump, the left auricle.

3. The pressure of the alveolar air exerted on the pulmonary capillaries.

4. The calibre of the pulmonary capillaries dependent upon the expansion of the lung.

On artificial expansion of the lungs, the thorax being opened, the third and fourth factors are increased, but the former is greater in effect than the latter, and the circulation is lessened. By natural inspiration the third factor is decreased, the second and fourth increased; the circulation is therefore augmenterl.

In artificial respiration, when the lungs are blown out, the pulmonary vessels are compressed by the rise of intra-pulmonary pressure. At first the blood within them is expelled, and subsequently the How of blood through the lungs is diminished. When the lungs collapse the

1 Quincke and Pfeiffer, Arch. f. Physiol., Leipzig, 1871, S. 90 ; de Jäger, Arch.f. d. ges. Physiol., Honn, 1879, Bd. xx. S. 426.

2 Arch.f. d. ges. Physiol., Bonn, 1878, Bd. xvii. S. 547. 
pulmonary vessels dilate. At first the blood collects in the dilated vessels; subsequently the blood-flow is increased. If the thorax be closed, the intrathoracic pressure is increased when the lungs are expanded, and decreased when the lungs are collapsed. The diastolic filling of the heart is diminished by expansion, thus the pressure in the pulmonary artery falls. It follows from the above that, with a slow rate of artificial respiration, the maximal pressure is obtained in the aorta at the commencement of expansion, the minimal at the beginning of collapse. If the rate of artificial respiration he rapid, the first effect only has time to appear in either case; that is to say, the expulsion of the blood from the pulmonary capillaries in expansion, and the retention of the same in collapse. In consequence, the pressure rises in inspiration and falls in expiration. ${ }^{1}$

In natural respiration the results are diametrically opposite. During inspiration, the intrathoracic pressure falls, the intra-pulmonary pressure exerted on the pulmonary capillaries becomes less, the sectional area of these capillaries increases, the diastolic filling of the heart is favoured. The blood at first fills up the enlarged pulmonary ressels,

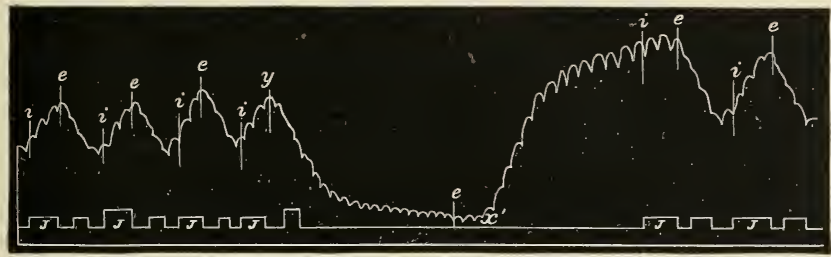

FIG. 81.-Aortic pressure. Artificial respiration. $y-\ell$, effect of clamping trachea, the lungs being expanded; $e, x^{\prime}, i$, effect of removing clamp.-Kowalewsky.

and subsequently flows in increased volume. Thus the pulmonary arterial pressure falls, ${ }^{2}$ while the aortic tension rises after a preliminary fall. During expiration the intra-pulmonary pressure increases, the sectional area of the pulmonary vessels decreases, the intrathoracic pressure rises, and the diastolic filling of the heart is lessened. The blood is at first expelled from the pulmonary vessels, and subsequently flows in diminished volume. Thus the pulmonary arterial pressure rises, while the aortic tension falls after a preliminary rise. These typical results of natural breathing can be observed in the most marked form when the respiration is slow and at the same time deep; for example, after section of the vagi. In rapid breathing only the first phase has time to appear in either case; that is to say, the retention of the blood in the pulmonary capillaries in inspiration, and the expulsion of the same in expiration.

In addition to the influence of the thoracic aspiration, the diastolic filling of the heart is aided by the inspiratory descent of the diaphragm, and expiratory contraction of the abdominal inuscles, both of which compress the abdominal contents and drive the blood into the heart. Respiration acts both as a suction and a force pump.

After section of the spinal cord at the first dorsal vertebra, in

1 Kowalewsky, Arch. $f$. Physiol., Leipzig, 1877, S. 419 et seq.

${ }^{2}$ Talma, Arch. f. d. ges. Physiol., Bonn, 1882, Bd. xxix. S. 332. 
consequence of the paralysis of the thoracic and the abdominal wall, the respiratory undulations on the arterial tracing are greatly reduced.

According to Marey, the rise of arterial pressure in man is inspiratory when the breathing is diaphragmatic, and expiratory when the breathing is thoracic in type. In the rabbit, an animal which breathes rapidly, the respiratory waves of aortic pressure can be reversed in time by turning the animal alternately on its back and on to its belly.

Heger and Spehl ${ }^{1}$ split the sternum without opening the pleural cavities, and passed a ligature round the aorta and pulmonary arteries. In one animal, they tightened this ligature in the inspiratory phase, and in a second animal in the period of expiration. The lungs were in each case excised, and the amount of blood therein estimated. They found that in the phase of natural inspiration, $\frac{1}{12}$ to $\frac{1}{13}$ of the whole blood in the body was contained in the lungs; in the phase of expiration, $\frac{1}{15}$ to $\frac{1}{18}$. On the other hand, when the lungs were artificially blown out, only $\frac{1}{60}$ of the whole blood was found therein. The amount of blood found in the lungs in the state of deepest expiration may be called the residual blood. The difference between this amount, and that found in ordinary expiration, will then be known as the supplemental blood, while the increase of the volume of blood in ordinary inspiration is called the tidal blood. Lastly, the difference between the volume of blood in ordinary inspiration, and the deepest inspiration, may be named the complemental blood. Thus, the changes in blood volume ean be brought under the same nomenclature as the changes in the volume of intra-pulmonary air. In natural breathiny, the air and the blood volume change together, and in the same way.

The effect of raising the intrathoracic pressure can be studied in animals by placing the tracheal tube in connection with a chamber in which the pressure of the air can be suddenly raised or lowered. On maintaining the intra-pulmonary pressure at a certain positive height, the arterial pressure falls to zero, and the blood congests in the veins. Conversely, on lowering the intra-pulmonary pressure considerably, the arterial tension is increased. ${ }^{2}$ These effects are similar to, but more pronounced than, those produced by expansion and collapse of the lungs in ordinary artificial respiration.

The breathing of compressed or rarefied air has been applied as a therapeutic agent by means of pneumatic cabinets. ${ }^{3}$ It is found that-

1. If the expiratory air be conducted by a tracheal tube into compressed air, while the inspiratory air be drawn from rarefied air, then both the normal expiratory and inspiratory effects are heightened, and the respiratory waves on the arterial trace are magnified. ${ }^{4}$

2. If, on the other hand, the inspiratory air be drawn from compressed air, while the expiratory air be conducted into rarefied air, then the normal effects are opposed, and the respiratory waves of arterial tension are lessened. By the inspiration of highly compressed air through a tracheal tube the circulation is rendered difficult, owing to the rise of intra-pulmonary pressure. In consequence, an asphyxial rise of arterial tension may take place, arising from the lack of oxygenation of the bulbar centres.

3. If the lungs be supplied with air at the normal atmospheric pressure through a tracheal tube, and the body be exposed to a lessened pressure in the pneumatic cabinet, then the arterial pressure falls very considerably. Thus an extrathoracic pressure of $-27 \mathrm{~mm}$. $\mathrm{Hg}$ produced a fall of arterial pressure equal to $120 \mathrm{~mm}$. $\mathrm{Hg}$. In this condition, the intra-pulmonary pressure is

${ }^{1}$ Spehl, "De la répartition du sang, etc.," Bruxelles, 1883.

2 Einbrodt, loc. cit.

3 Waldenburg, Berl. klin. Wchnschr., 1873, S. 465.

+Zuntz, Arch. f. d. ges. Physiol., Boun, 1878, Bd. xvii. S. 374. 
raised above the pressure exerted on the extrathoracic vascular system, the blood congests in the veins, and the heart fails to fill.

In the case of the balloonist or the diver, both the intra-pulmonary and extrathoracic pressures fall or rise together, and thus the circulatory mechanism remains unatfected.

The frequency of the heart is increased by inspiration, and as this change is brought about by a lessening of the vagal tone, it fails to appear when the vagi are cut. How the vagus centre is thus influenced is not clear. Moderate artificial expansion of the lung increases the frequency of the heart. Fredericq finds the change in frequency still occurs synchronously with the respirations when the thorax is widely open, the lungs collapsed, and the respiration ineffectual. ${ }^{1}$ The vasomotor centre is also said to be influenced rhythmically and directly by the respiration. To show this, Fredericq opened the thorax and belly of an animal, divided both vagi and phrenic nerves, and stopped the artificial respiration. The aortic pressure then rose with each expiratory and fell with each inspiratory spasm. This experiment is not conclusive, for although the suction action of the thorax and the compressing action of the abdominal muscles were to a great extent abolished, yet the general asphyxial spasms of the inuscles still promoted the venous flow and the diastolic filling of the heart. The three centres-the respiratory, the cardio-inhibitory, and the vasomotor-lie closely bound up together in the medulla oblongata, and it is therefore conceivable, although not proved, that the other two may be directly influenced by irradiation of the rhythnic discharge from the respiratory centre. The exciting agent is certainly not the chemical nature of the blood, for the alteration of frequency takes place when the thorax is opened and the respiration is totally ineffectual. The inspiratory acceleration may be due to local mechanical excitation of the heart, since, during vagal inhibition, respiratory gasps or compressions of the abdomen excite beats of the heart.

From the preceding discussion the following conclusions can be tabulated ${ }^{2}$ as to the effect of respiration on the circulation :-

Conditions of Heart and Blood Flow, favoured or the reverse.

1. Aspiration of blood to right heart

2. Diastole of heart

3. Systole of heart .

4. Sectional area of pulnonary vascular system

5. Flow of blood through the lungs

6. Retention of blood in pulmonary vascular system

7. Pressure on abdominal contentsBy abdominal muscles.

8. Frequency of heart By descent of diaphragn

The total effect on the aortic tension in slow-breathing animals is maximal at the commencement of expiration, minimal at the beginning of inspiration.

1 Arch. de biol., Gand, 1882, tome iii. p. 86.

${ }^{2}$ After Tigerstedt, "Phys. des Kreislaufes," S. 460.

VOL. II. -9 
In animals with quick respiration, such as cats, rabbits, and guinea-pigs, the total effect is maximal at the height of expiration, minimal at the depth of inspiration.

\section{The Vasomotor Mechanism.}

Historical.-As early as 1733 the notion that the small arteries change their calibre was put forward by Stephen Hales. ${ }^{1}$ Among the many ingenious experiments he devised is the following:-Tying a brass tube into the aorta of a dog, and employing a head of pressure equal to the normal aortic tension, he injected water and measured the outflow, per minute, from the divided vessels of the intestine. He found that while cold water diminished, hot water increased the outflow. Further, from investigations on the action of such drugs as alcohol and infusion of cinchona bark, he concluded that while one set of agents, by constricting the vessels, lessens the outflow, another set widens the vessels and increases the outflow.

On scratching the femoral or the carotid artery with the point of a knife, the local constriction of these vessels ean be evoked. Likewise by chemical irritation, or the application of the galvanic current, the peripheral arteries of the frog can be thrown into contraction. Observations of this kind were recorded by several experimentalists towards the end of the eighteenth and the beginning of the nineteentl centuries.

Hunter observed that the carotid artery of the dog gradually contracts if it be exposed to the air, while Parry obtained the same result on loosely tying a ligature round an artery. Mayo ${ }^{2}$ records that the diameter of an artery is markedly increased by rubbing it for the space of half a minute between the finger and thumb.

Some time after this it was recorded, and more than once, that congestion of the head and neck follows after division of the cervical sympathetic nerve, or excision of the superior cervical ganglion. ${ }^{3}$

By Krimer ${ }^{4}$ the interesting observation was made, that the application of salt to a frog's web does not, when the nerves of the part are injured, produce its usual effect of dilatation of the capillaries.

By E. H. Weber ${ }^{5}$ the direct proposition was made, for the first time, that pallor and blushing could be explained, if it were once taken for granted that the calibre of the peripheral arteries could under nervous influence become smaller or larger.

The position in which the question of the contractility of the arteries stood in 1836 is illustrated by the following observations of Allen Thomson :-

"It may be remarked that the coldness and impaired nourishment common in palsied limbs, the known increase or diminution of the various secretions from mental emotions, and direct or sympathetic affections of the nerves belonging to the glands or other secreting organs, the phenomena of blushing, erection, inflammation, and the like, are all very direct and satisfactory proofs that the small vessels and the capillary circulation may be influenced by affections of the nerves." 6

Physiological ideas drawn from experiments thus forestalled anatomical discovery, for the muscular coat of the arteries remained undiscovered until

1 "Statical Essays," 1733, vol. ii.

2 Mayo, "Outlines of Human Physiology," London, 1833, p. 67.

3 See Milne-Edwards, "Leçons sur la Physiologie," Paris, 1859, tome iv. p. 207.

4 " Physiol. Untersuch.," Leipzig, 1820.

5 Hildebrandt's "Handbuch der Anatomie," 4th edition, 1831, pt. iii. S. 75.

6 Todd's "Cyclopædia of Anat. and Physiology," 1836, vol. i. pp. 672, 680. 
1840. In this year Henle ${ }^{1}$ established the existence of a plentiful supply of involuntary muscle cells in the middle coat of the small arteries, and thus laid the anatomical foundation of the doctrine that while the general circulation of the blood depends upon the heart, its distribution to any one part depends on the calibre of the arteries supplying that part. The final link in the chain of anatomical evidence was not wanting, for many observers had traced filaments of nerves along the course of the arteries as far as their ultimate ramifications in the tissues. To these nerves Stilling ${ }^{2}$ gave the name "vasomotor," ascribing to them the function of maintaining the tone and ordering the contractions of the eapillaries, arteries, and veins.

The exact discovery of the vasomotor mechanism, a discovery fraught with the utmost importance to the advance of physiological and pathological knowledge, dates from Claude Bernard's observations on the cervical synpathetic nerve of the rabbit, published in 1851. ${ }^{3}$ He found, after dividing this nerve, or excising the superior cervical ganglion, a noticeable increase in the vascularity and the temperature of the ear on the same side as the lesion.

In such case the temperature may be raised some $4^{\circ}$ to $6^{\circ} \mathrm{C}$. above that of the opposite ear. Bernard was at first inclined to believe that the sympathetic nerve, apart from its influence on the blood supply, exerted control over the production of heat. ${ }^{4}$

In 1852, Brown-Séquard crowned Bernard's discovery by the publication of this observation: "If galvanism is applied to the superior portion of the sympathetic, after it has been cut in the neck, the vessels of the face and of the ear after a certain time begin to contract; their contraction increases slowly, but at last it is evident that they resume their normal condition, if they are not even smaller. Then the temperature and the sensibility diminish in the face and the ear, and they become in the palsied side the same as in the sound side. When the galvanic current ceases to act, the vessels begin to dilate again, and all the phenomena discovered by Dr. Bernard reappear." 5

Unwitting of the fact that Brown-Séquard had forestalled him, Bernard $^{6}$ himself discovered that excitation of the cervical sympathetic nerve produced constriction of the blood vessels of the ear. The same conclusion was arrived at independently by Waller. ${ }^{7}$

The amount of dilatation in the ear which follows section of the cervical sympathetic nerve is a sign of the strength of vascular tone. It must be borne in mind that dilatation is not always produced thereby, seeing that the tone of any particular vascular area is constantly varying. Thus, if the rabbit be placed in a warm atmosphere, division of the cervical sympathetic will induce but little change in the vascularity or temperature of the ear ; while, if the atmosphere be cold, the temperature of the ear may rise after the operation $5^{\circ} \mathrm{C}$. or even $10^{\circ} \mathrm{C}$.

Schiff made a further discovery of great importance. After dividing one cervical sympathetic nerve, he found, on exciting the animal, or taking it for a run in the warmth of the sun, the ear on the uninjured side becaine slightly the warmer, and the vascularity of the ear more pro-

1 “Allgemeine Anatomie," Leipzig, 1841, S. 525, 690.

2 "Physiol. Untersuch, ueber die Spinalirritation," 1840, S. 163, 275.

${ }^{3}$ Compt. rend. Soc. de biol., Paris, 1851, p. 163.

${ }^{4}$ Compt. rend. Acad. d. sc., Paris, 1852, tome xxxiv. P1. 472-475.

5 Med. Exam., Phila., Aug. 1852, p. 490.

${ }^{6}$ Compt. rend. Soc. de biol., Paris, 1852, p. 169.

${ }^{7}$ Compt. rend. Acad. d. sc., Paris, 1853, tome xxxvi. p. 378. 
nounced. So soon, however, as the animal became quiet these conditions were once more reversed. Schiff explained this result by supposing the existence of vaso-dilator nerves. ${ }^{1}$

The existence of vaso-dilator nerves, thus foreshadowed by Schiff, was conclusively proved by Bernard. ${ }^{2}$ Exposing the submaxillary gland of a $\mathrm{dog}$, he opened one of the efferent veins, and observed the outflow of blood. On dividing the cervical sympathetic nerve, the blood flowed in increased volume and became more arterial in colour. On exciting the same nerve, the outflow entirely ceased, and the gland became pale in colour. He next excited the chorda tympani nerve, the gland blushed red, and almost immediately bright arterial blood gushed out from the vein. The vascular dilatation was in this case so great that the blood, with each pulsation of the artery, flowed from the vein in jets.

By this fundamental experiment, Bernard proved that there exist two kinds of vasomotor nerves. The one, the vaso-constrictors, which exert a tonic action, for on section of these the gland blushes; the other, the vaso-dilators, which exert no tonic action, but can, when excited, throw the vessels into the widest dilatation.

Evidence that the vaso-constrictor nerves have their origin in the spinal cord was afforded by Schiff, who found that vascular dilatation and a rise of temperature in various parts of the body followed destruction of certain parts of the cord.

Budge, ${ }^{3}$ by unilateral extirpation of the cord between the last cervical and third thoracic nerve, obtained the same vaso-dilatation as is seen after section of the cervical sympathetic. Waller ${ }^{4}$ constricted the vessels of the ear by excitation of the cervical cord. The vasomotor fibres issue by the anterior roots. The first observation of this fact (on the frog) is attributed to Pfluiger. ${ }^{5}$

These experiments of Bermard, Brown-Séquard, Waller, and Schiff have led to a vast amount of inquiry into the physiological action and anatomical distribution of vasomotor nerves.

The methods which have been employed are these :-

1. Direct observation of the colour of any part, or of the amount of blood which flows from a wound.

This method has been used with advantage in the case of the rabbit's ear, the submaxillary gland, the mucous membrane of the mouth. Microseopical observation and measurement of the calibre of the arterioles has been employed in investigations of the frog's web, mesentery, mylohyoid muscle.

2. Thermometric observations of the temperature of any organ. This is best carried out by means of thermo-electric junctions.

3. Estimation of the output of blood per minute from the efferent vein of an organ.

4. Records of the velocity of blood flow in the artery supplying an organ. This ean be effected either by using Chauveau's dromograph or Cybulski's photohæmatochometer.

5. Estimation of the changes in volume of an organ, such as the spleen, the kidney, the brain, the lung, the intestines, or the limbs. This is effected by means of the plethysmograph. The organ is placed in an air-tight box, and this in its turn is connected with a recording tambour.

1 Berner Schriften, 1856, p. 69 ; "Untersuch. neber Zuckerbildung in der Leber," Wuirzburg, 1859, S. 153-156.

2 Journ. de la physiol. de l'homme, Paris, 1858, tome i. pp. 237, 649.

${ }^{3}$ Compt. rend. Acad. d. sc., Paris, 1853, tome xxxvi. p. 378.

${ }^{4}$ Ibid., p. 381.

${ }_{5}$ Allg. med. Centr.-Ztg., Berlin, 1855. 
6. Observation of the lateral pressure in the peripheral end of an artery, that is to say, at a point peripheral to the origin of anastomotic branches, which are sufficient to maintain the circulation through the organ. Hiirthle employed this method in a research on the brain. He recorded the pressure in the peripheral end of the internal carotid artery. Dastre and Norat similarly observed the lateral pressure in the peripheral end of the femoral artery, below the origin of the profunda femoris.

7. Simultaneous records of the lateral pressure in the afferent artery and the efferent vein of any organ. This method has been used in researches on the portal system, the brain, and the lungs.

8. Estimation of the capillary pressure. The intra-cranial and intra-ocular pressures have been studied by this method.

The greatest accuracy and scientific acumen is required in the execution of researches on the more delicate vasomotor mechanisms. It must constantly be borne in mind that changes in the vascularity of an organ may depend on central as well as peripheral alterations in the circulatory system. Thus, for example, during the rise of arterial pressure which occurs during asphyxia, the kidneys decrease in volume, and the limbs increase; the vessels of the rabbit's ear may either contract or dilate; the vessels of the intestines contract; in either the cerebral or the pulmonary veins the pressure rises, while the pressure in the portal vein may either rise or fall. From the mere observation of the circulatory changes in any of these organs, we cannot tell whether the above effects are passive or active. The question of peripheral change is complicated in each case by the passive effects produced by general changes. Owing to this fact, it is usually necessary to record the aortic and vena cava pressures simultaneously with the observations of the organ which are made by one or other of the above methods.

If the general venous and arterial pressures remain constant, while the circulation in the organ varies, then unequivocal evidence is obtained of the existence of a vasomotor mechanism in that organ. Such is the case also if the circulation in the organ vary in the opposite sense to the changes which take place simultaneously in the general circulation.

In many cases it is necessary to abolish, as far as possible, all passive effects produced by changes in the general circulation.

Thus, to exclude the effect of the respiratory pump, the animal can be curarised and a constant artificial respiration supplied. In the case of the brain or fore-limbs, the circulation, by ligature of the thoracic aorta and vena cava inferior, can be limited to these parts; thus all changes occurring in the splanchnic circulation can be eliminated. The same result can be arrived at by section of the spinal cord above the origin of the splanchnic vasomotor nerves. The splanchnic vasomotor mechanism is by far the most powerful in the body, and in investigations on the limbs, lungs, or brain, it is absolutely necessary to eliminate its action in order to obtain unequivocal evidence for or against a vasomotor supply to these parts. Attempts have been made to avoid all these difficulties by the establishment of an artificial circulation through the particular organ under investigation. Vasomotor tone is, however, so easily abolished by alterations in the temperature and quality of the blood, that this method has as yet neither proved trustworthy nor of any particular value.

To distinguish the existence of vaso-constrictor and vaso-dilator fibres, running in one common nerve trunk, special means must be 
employed. The fact that both kinds of nerves exist in the common nerve trunks was first demonstrated by Goltz. ${ }^{1}$

1. If vaso-constrictors and dilators are stimulated simultaneously, the constrictor influence at first overpowers the dilator. The dilator effect, however, appears as an after-result, for the vaso-dilator fibres are the less easily exhausted. This result can be observed in the salivary gland, while the chorda tympani and cervical sympathetic nerves are excited at the same time. ${ }^{2}$ That the vaso-constrictors and dilators are antagonistic in action, is shown by an experiment of v. Anrep and Cybulski, ${ }^{3}$ who recorded the variations in volume of the tongue by the plethysmograph method, and determined that the constriction of the tongue, induced by stimulation of the hypoglossal nerve, could be put aside by an excitation of the lingual nerve. Similarly, the vaso-dilation induced by excitation of the lingual could be put aside by stimulation of the hypoglossal nerve. The constrictor influence is usually by far the strongest. Thus Bayliss ${ }^{4}$ found, on asphyxiating an animal during excitation of the depressor nerve, that the arterial pressure rose, and the

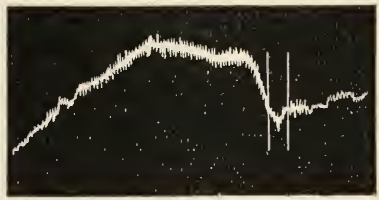

A

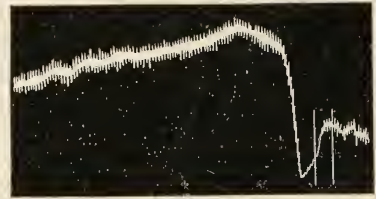

$B$

Fig. 82.-Plethysmograms of hind-limb. Exeitations of seiatic nerve. $A$, One per second. $B$, Four per seeond. The tracings in this and the next figure are to be read from right to left.--Bowditeh and Warren.

pressor completely overcame the depressor effect. On renewing the respirations, the depressor nerve again exerted its power, and the arterial tension fell once more below the mean.

2 . If the leg be cooled, excitation of the sciatic nerve evokes vasodilatation, while if the leg be warmed it produces vaso-constriction. ${ }^{5}$ Similarly, if the rabbit's ear be warm, asphyxia provokes constriction; if cold, dilatation.

3. After separation of the vasomotor nerves from the central nervous systcm, the vaso-constrictor fibres degenerate much more rapidly than the vaso-dilator fibres; the vaso-dilator fibres maintaining their excitability for some six to ten days after division, while the vasoconstrictor became inexcitable on the third or fourth day. ${ }^{6}$

4. While the vaso-dilator fibres are excited by single induction shocks repeated at intervals of not more than 5 per sec. (Fig. 82), the constrictor fibres are aroused to action most easily by a rapid tetanising current (Fig. 83). ${ }^{7}$

5 . If the tetanising current be strong, primary vaso-constriction

1 Arch.f. d. ges. Physiol., Bonn, 1874, Bd. ix. S. 174-197.

${ }^{2}$ v. Frey, Arb. a. d. physiol. Anst. zu Leipzig, 1876, S. 89-107.

3 Jehresb. ü. d. Fortschr. d. Anat. u. Physiol., Leipzig, 1884, Bd. ii. S. 52.

+ Journ. Physiol., Cambridge and London, 1893, vol. xiv. p. 319.

${ }^{5}$ Lépine, Compt. rend. Soc. de biol., Paris, 1876, p. 26.

${ }^{6}$ Dziedziul, Jahresb. ï. d. Fortschr. d. Anat. u. Physiol., Leipzig, 1880, Bd. ii. S. 68.

7 Bowditch and Warren, Journ. Physiol., Cambridge and London, 1886, vol. vii. p. 32 ; Bradford, Ibid., 1889, vol. x. p. 390 . 
always appears; if the current be very weak, primary vaso-dilatation usually oceurs.

6. On cooling the sciatic nerve to $3^{\circ} \mathrm{C}$., the vaso-constrictor fibres are paralysed before the dilator. ${ }^{1}$

For the vaso-dilator nerves, the latent period (between excitation and result) is usually longer than it is for the vaso-constrictor fibres. In the case of the hind-limb, the vaso-dilator latent period is about 3.5 seconds, the vaso-constrictor 1.5 seconds. The latent period of the nervi erigentes is recorded as being 3.4 to 4.0 seconds. $^{2}$ Likewise, the maximal dilator effect is reached much more slowly, and dies away much less rapidly than the maximal constrictor effect.

To sum up, then, the vaso-dilator fibres are more easily excited by an infrequent rate and weak form of stimulus. The vaso-constrictors

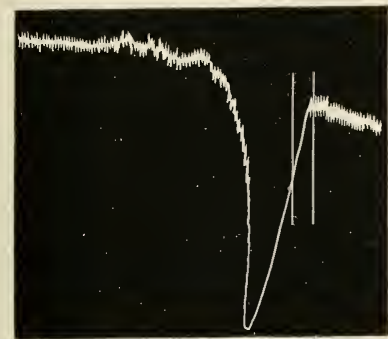

A

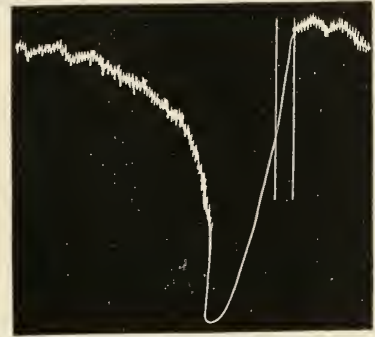

B

FIG. 83.-Plethysmograms of hind-limb. Excitations of sciatic nerve. $A$, Sixteen per second. $B$, Sixty-four per second.-Bowditeh and Warren.

degenerate more rapidly after division, and are paralysed more easily by cold. The vaso-dilator effect appears after a longer latent period, while it reaches its maximum and disappears more slowly.

Hypotheses on the action of vaso-dilator nerves.-It is easy to imagine that, excited by the influence of the vaso-constrictor nerves, the muscular coats of the arterioles should constrict the calibre of these vessels. It is a more difficult task to explain by what method the vasodilator nerves work their effect.

The hypothesis that first suggests itself is that the vaso-dilator inhibit the tonic action of the vaso-constrictor fibres. Before accepting this hypothesis, we are confronted with the difficulty that the vessels of any organ are dilated to a greater degree by excitation of the vasodilators than they are by paralysis of the vaso-constrictors. In spite of the destruction of the vaso-constrictor nerves, the vessels, after a certain period, recover their tone. That the tone is not even for a time abolished completely, is shown by the fact that a local dilatation can be produced by certain chemical irritants. Thus it is clear that some peripheral inechanism exists, a mechanism which, in its turn, can be paralysed by the local application of such a drug as chloroform.

' Howell, Budgett, and Leonard, Journ. Physiol., Cambridge and London, 1894, vol. vi. p. 306 .

${ }^{2}$ Bowditch and Warren, loc. cit., p. 440 ; v. Anrep and Cybulski, Juhresb. ü. d. Fortschr. d. Anat. u. Physiol., Leipzig, 1884, S. 53. 
It seems highly probable that the vascular muscles are maintained in a state of tone by the tension of the blood within the vessels. This would be in close analogy with the fact that the heart is excited to contract by a rise of internal tension. Accepting this hypothesis, we may then suppose that the vaso-constrictor nerves increase the excitability of the vascular muscle to the stimulus of blood tension, while the vaso-dilator nerves diminish the excitability. The vaso-dilator fibres would thus be brought into analogy with the cardio-inhibitory nerves. These lessen the irritability of the heart towards the intracardiac tension, ${ }^{1}$ and relax the cardiac muscle.

On this hypothesis, section of the vaso-constrictor nerves abolishes that tonic condition of exalted excitability to internal tension which is maintained by the vaso-motor centre. It does not, however, entirely destroy the excitability of the vascular muscle to tension, and with time the tone is fully restored. On the other hand, the vaso-dilator influence completely relaxes the muscle by altogether inhibiting its excitability to tension. It is conceivable that the quality as well as the tension of the blood may be the exciting cause of vascular tone. An increase in the alkalinity of the blood favours the development of tone. Certain glands, such as the suprarenals, appear to secrete a material into the blood which produces vascular contraction.

Vasomotor centres.-Ludwig and Dittmar were the first to localise a vasomotor centre. By excitation of the central end of the sciatic nerve, after the great brain had been separated from the mednlla oblongata, they obtained a reflex rise of arterial pressure. This centre was minutely localised by Ludwig and Owsjannikow. While a section of the brain carried out below the corpora quadrigemina did not prodnce any noteworthy change in the arterial pressure, they found, on slicing away the spinal bulb by successive cuts, that the pressure fell with each cut, until a maximal depressor effect was reached. By such experiments the centre was located in the rabbit to a spot 3 to $4 \mathrm{~mm}$. in length, commencing 1 to $2 \mathrm{~mm}$. below the corpora quadrigenina, and ending $4 \mathrm{~mm}$. above the tip of the calamus scriptorius. ${ }^{2}$ The centre is bilateral, and in the area which it covers there is situated a group of nerve cells known as the antero-lateral nucleus of Clarke. ${ }^{3}$

The bulbar vasomotor centre is constantly in action, maintaining the tone of the small arteries.

Thus Bernard noticed, after section of the cervical cord, that the arterial pressure fell markedly. By excitation of the peripheral end of the cervical cord, v. Bezold ${ }^{4}$ drove the pressure up to no less than seven times the height it reached after section of the cord. Ludwig and Thiry ${ }^{5}$ slowed that the same result can be obtained after section of all the cardiac nerves; and, moreover, that while tying of the aorta below the renal arteries causes but a slight rise of tension, ligation of the thoracic aorta causes a very great rise. These experiments indicate the overwhelming importance of the splanchnic vaso-constrictor nerves.

The vasomotor centre is not only excited reflexly, but responds to every change in the circulation through the spinal bulb. A rise of

${ }^{1}$ Hill and Barnard, Joum. Physiol., Cambridge and London, 1897, p. 338.

2 Ber. d. k. Sächs. Gesellsch. d. Wissensch., math.-phys. Cl., Leipzig, 1871, S. 135.

${ }^{3}$ Dittmar, Ibid., 1873 , S. 460.

4 " Untersuch. neber d. Innervation des Herzens," 1863, Abth. ii. S. 221.

${ }^{5}$ Sitzungsb. d. k. Akad. d. Wissensch., math.-naturw. Cl., Wien, 1864, Bd. xlix. Abth. 2, S. 421. 
pressure in the cerebral arteries provokes a fall of aortic tension; conversely, a fall of pressure in the cerebral arteries provokes a rise. In other words, cerebral anæmia, however produced, excites the centre, and increases vascular tone, while cerebral hyperamia decreases vascular tone.

Besides the bulbar centre, there are other subsidiary centres in the spinal cord. (The word centre is here used to signify nothing more than a portion of the central nervous system, wherein afferent impulses are changed into efferent impulses.) The existence of these spinal centres was first established by Goltz. After destruction of the whole brain of a frog, the vascular tone is not abolished completely. It is abolished after destruction of the spinal cord. ${ }^{1}$

In mammals the same statement holds true. If, after section of the spinal cord in the lower dorsal region, a $\log$ be maintained alive, reflex vascular dilatation causing erection of the penis can be evoked, and, while the vessels of the lower limbs are at first dilated, they return with the lapse of time to a state of tone. The temperature of the lower limbs, at first much raised, returns gradually to the same as that of the fore-limbs. On destruction of the lumbar cord, the temperature in the hind-limbs once more rises, for the vessels again dilate. ${ }^{2}$ After section of the spinal cord in the mid-dorsal region, evidence of reflex vasomotor effects can be obtained. Thus, on sensory excitation of one leg, constriction of the blood vessels in the other limb has been oloserved. ${ }^{3}$

An asphyxial rise of arterial pressure is sometimes witnessed after section of the spinal cord. This rise is not obtained if the cord be destroyed. ${ }^{4}$

Other observations showing the existence of subsidiary vasomotor mechanisms in the spinal cord are (1) that, after division of the spinal cord in the upper cervical region, section of the splanchnic nerves causes a further depression of arterial tension, and (2) excitation of the central end of one sciatic nerve causes a considerable rise of tension.

A certain amount of evidence as to the path of the vasomotor fibres down the spinal cord has been brought forward. By studying the results of hemisection of the cord, it has been determined that these fibres for the most part follow a direct but partly a crossed pathway. If the cord be completely divided in the cervical region, and be hemisected in the mid-dorsal region, then, on faradising the cervical cord, both kidneys shrink in volume, but the kidney on the side of the hemisection contracts less than that on the other side. ${ }^{5}$

After section of the posterior columns and grey matter of the cord, Dittmar ${ }^{6}$ obtained the usual pressor effect which follows excitation of the central end of the sciatic. We must conclude, then, that the tracts pass in the antero-lateral columns.

Local vasomotor tone.-Even after complete separation of the vascular areas from the central nervous system, recovery of tone in the vessels eventually takes place. Thus, when Goltz and Ewald removed the lower part of the spinal cord of a dog for the length of $80 \mathrm{~mm}$. or

1 Virchow's Archiv, 1864, Bd. xxix. S. 11.

${ }^{2}$ Goltz, Arch. f. d. ges. Physiol., Bonn, 1874, Bd. viii. S. 82.

3 Vulpian, "Leçons sur l'appareil vaso-motenr," Paris, 1875. tome i. p. 292.

${ }^{4}$ Kowalewsky and Adamik, Centralbl. f. d. med. Wissensch., Berlin, 1868, S. 582 ; Konow and Stenbeck, Skandin. Areh, f. Physiol., Leipzig, 1889, Bd. i. S. 407.

5 Nicolaides, Areh. f. Physiol., Leipzig, 1892, S. 29.

${ }^{6}$ Dittmar, Ber. d. k. Säehs. Gesellsch. d. Wissensch., math.-phys. Cl., Leipzig, 1873, S. 455. 
more, the hind-limbs, which were at first hotter and more Hushed with blood, became eventually as cool or cooler than the fore-limbs. In pathological cases, the same phenomenon is constantly observed to follow on section of all the nerves.

The tone of the great splanchnic area is completely restored some eight days after section of all the splanchnic nerves.

It has been suggested that the sympathetic ganglia are centres of reflex vasomotor action. An experiment quoted in favour of this view is the following:- If the first thoracic ganglion be entirely separated from connection with the spinal cord, and the ganglionic end of one branch of the annulus Vieussenii be stimulated, the vessels of the head and neck contract. This and similar phenomena are perhaps not true reflexes. The effect may be produced by the stimulus running up the efferent fibres and thence down the collaterals of the same fibres. These collaterals pass into several branches of the ganglionic system. ${ }^{1}$

To show the tonic influence of the peripheral ganglion cells, Dastre and Morat bring forward this experiment. On section of the cervical sympathetic nerve below the superior cervical ganglion, searcely any vaso-dilatation is to be seen in the mueous membrane of the mouth, but on destruction of the ganglion the dilatation becomes unmistakable. $^{2}$

In certain places rhythmic contractions and dilatations of arterics can be observel, movements which are apparently independent of the central nervous system, or of any change taking place in the general vascular system. ${ }^{3}$ This phenomenon can be witnessed in the central artery of the ear or saphena artery of the rabbit (especially when the animal is excited), and in the web or mesenteric arteries of the frog. The contractions or dilatations may not affect the whole length of an artery, but be localised to one or other part. Certain drugs, such as chloroform and amyl nitrite, when applied locally, produce dilatation of the blood vessels. These drugs act on the peripheral mechanism, for if injected into a carotid artery they primarily excite the vasoconstrictor centre; when injected into a jugular vein they produce, on the contrary, primary vaso-dilatation. According to Stefani, dyspnoeic blood locally causes dilatation of the blood vessels, centrally it provokes vaso-constriction. ${ }^{4}$

An extract of the medulla of the suprarenal gland exerts a most powerful local vaso-constrictor effect. If one drop of a 1 per cent. extract of the dried organ in normal saline be placed upon the mesentery of a frog, the arterioles are constricted for thirty to sixty minutes, and to such a degree that the onward flow of blood in the eapillaries may entirely cease, and be replaced by a back-eddy from the veins. The same result is obtained after destruction of the spinal cord. Similarly, after the conjunctiva of the rabbit's eye has been congested by the application of acetic acid, the vessels may be again constricted by a drop of suprarenal extract. ${ }^{5}$

Traube-Hering curves.-In addition to the ordinary respiratory

1 Langley and Anderson, Journ. Physiol., Cambridge and London, 1894, vol. xvi.

"Dastre and Morat, "Recherches expér. sur le système nerv. vaso-moteur," p. 326.

${ }^{3}$ Sehiff, Arch. $f$. physiol. Heilk., Stuttgart, 1854, Bd. xiii. S. 525 ; Riegel, Arch. $f . d$. yes. Physiol., Bonn, Bd. iv. S. 350 ; Klemensiewicz, Sitzungsb. d. k. Akad. d. Wisscnsch., math.-naturw. Cl., Wien, 1887, Bd. xevi. Abth. 3, S. 71.

${ }^{4}$ Stefani, Arch. ital. de biol., Turin, 1894, vol, xxi. pp. 245-248.

${ }^{5}$ George Oliver, Proc. Physiol. Soc., March 1897. 
oscillations, rhythmic variations of tension frequently appear in the tracings of arterial pressure.

These variations are known as Traube-Hering curves. ${ }^{3}$ They can be evoked with the greatest ease by the injection of a little magnesium sulphate solution into the circulation of the dog (Fic. 84). During the asphyxial rise of arterial pressure in the curarised dog, these curves are of constant occurrence, and also after injection of a large dose of

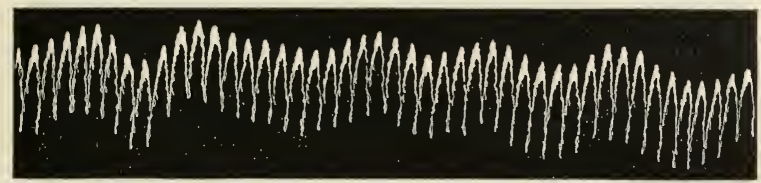

Fıd. 84. - Traube-Hering curves produced by injection of magnesium sulphate ; dog.-Hill.

morphia. In conditions of anxmia of the bulbar centres, prodnced either by tying the cerebral arteries or compression of the brain, or after injection of chloroform into the cerebral arteries, Traube curves frequently become apparent. Very often these oscillations of arterial pressure are accompanied by a periodicity of the respiration, which is known as Cheyne-Stokes breathing. It would appear as if each oscillatory rise of arterial pressure excited the respiratory centre, and evoked a group of respirations. ${ }^{2}$

These oscillations of arterial pressure are produced by periodic constriction and dilatation of vascular areas. On recording the volume of the kidney, this organ is found to contract when the arterial tension rises. ${ }^{3}$

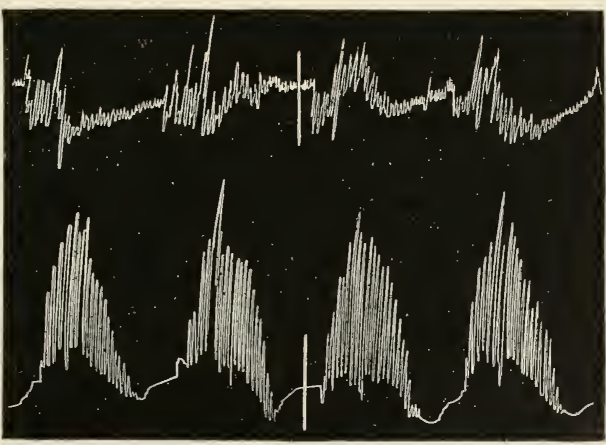

They are usually to be ascribed to a periodical excitement

FIG. 85.-Plethysmographic tracing of arm and record of Cheyne-Stokes respiration; manl.-Hill.

of the vasomotor centre. A deficient circulation through the bulb excites the centre, the arterial pressure in consequence rises, the rise of pressure flushes the bulbar centres with more blood, the centres then cease to be excited, the pressure falls again, and the cycle of events is repeated. Traube curves may in some cases be peripheral, and not central, in origin.

By the injection of essential oil of absinthe in a curarised animal, the vasomotor centre can be excited to the discharge of a succession of powerful

1 Traube, Centralbl. $f$. $d$. med. Wissensch., Berlin, 1865, S. 882 ; Hering, Sitzungsb. $d$. k. Akad. d. Wissensch., Wien, 1869, Bd. lx. S. 839 .

2 Hill, "Phys. and Path. Cerebral Circulation," London, 1896, p. 130 et seq.

${ }^{3}$ Roy, Journ. Physiol., Cambridge and London, 1882, vol, iii. p. 217 et scq. 
spasms, by each of which the arterial pressure is driven up to a great height. ${ }^{1}$ This phenomenon suggests a clonic fit of the centre, the clonus of involuntary muscle naturally taking place at a far slower rate than that of skeletal muscles.

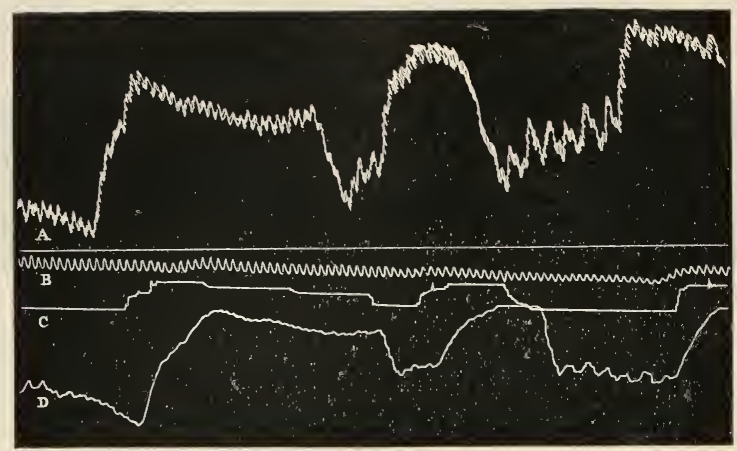

FIG. 86. - Injeetion of ausinthe. Curarised dog. A, aortic pressure ; B, vena eava pressure ; C, intracranial pressure; D, eerebral venous pressure. Bayliss and Hill.

The vasomotor mechanism of the veins.-The portal venous system. - If the thoracic aorta be clamped, the pressure in the carotid artery rises; if the splanchnic nerves be then excited, the pressure in the carotid artery is raised to a still higher level. This further rise is to be attributed to the increased diastolic filling of the heart, which in its turn is caused by the expulsion of the blood from the portal system, an expulsion which is brought about by constriction of the splanchuic and hepatic vessels. After ligature of the portal vein, the same result is obtained, owing to constriction of the hepatic vessels. ${ }^{2}$

Bayliss and Starling localise the outflow of vaso-constrictor nerves to the portal system in from the third to the eleventh anterior thoracic roots. By excitation of the eighth to the eleventh roots, they obtained, first, a rise of pressure in the portal vein, due to constriction of the mesenteric arteries, forcing blood on into the portal vein; secondly, a fall due to the diminished flow of blood through the mesenteric arteries; and, lastly, a rise due to constriction in the hepatic branches of the portal vein. ${ }^{3}$

The portal venous system is peculiar in its structural arrangement, and generally bears a close analogy to an arterial vascular area. As to the innervation of the other veins we know very little. Thompson, ${ }^{4}$ after tying the crural artery and on excitation of the peripheral end of the divided sciatic nerve, has witnessed contraction of the superficial veins of the rabbit's leg. He has also witnessed the same in abdominal veins, on stimulation of the peripheral end of the divided cervical spinal cord. The contraction is usually in the form of band-like constrictions, between which the veins appear full of blood. Such contractions appear

\footnotetext{
${ }^{1}$ Bayliss and Hill, Journ. Physiol., Cambridge and London, 1895, vol. xviii. 1. 350.

2 Mall, Arch. f. Phy/siol., Leipzig, 1892, S. 409.

3 Journ. Physiol., Cambridge and London, 1894-5, vol. xvii. p. 120, ef. Cavazzani and Manea, Arch. ital. de biol., Turin, 1895, vol. xxiv. pp. 33, 295.

${ }^{4}$ Arch. f. Physiol., Leipzig, 1893, S. 102.
} 
on mere exposure of the veins. The skeletal muscles, and especially the abdominal respiratory muscles, act as a tonic mechanism to the veins. Thus the abdominal wall is of the utmost importance, in so far as it tonically maintains the calibre of the great abdominal veins, and can at any moment compress these vessels or allow them to expand. ${ }^{1}$

\section{CIRCULATION IN SPECIAL PARTS.}

\section{The Circulation in the Head.}

Vasomotors of face and mouth. - The vaso-constrictor fibres for the head issue by the upper thoracic anterior roots ( 1 to 5 in the $\log$ ), and pass into the cervical sympathetic nerve. ${ }^{2}$

The vaso-dilator supply to the face and mouth has been investigated by Dastre and Morat. ${ }^{3}$ These fibres issue also from the upper thoracic roots, and pass up the cervical sympathetic nerve to the Gasserian ganglion, and thence to the fifth nerve. Some of the dilator fibres issue directly from the cranial origin of the fifth nerve, for stimulation of this nerve between the pons and the Gasserian ganglion causes Hushing of the face. Excitation of the lingual and glosso-pharyngeal nerves dilates the vessels of the tongue, while stimulation of the hypoglossal nerve causes them to contract. These constrictor fibres come from the superior cervical ganglion.

The circulation in the mucous membrane of the mouth has been examined by direct observation, while the volume of the tongue can be investigated with a suitable plethysmograph. ${ }^{4}$ By observing the effects of section or excitation of the cervical and thoracic sympathetic nerve, during ophthalmoscopic examination of the eye in the curarised rabbit, both vaso-constrictor and vaso-dilator nerves have been found to pass to this organ. ${ }^{5}$

Vasomotors of salivary glands.-The vaso-constrictor fibres to the submaxillary gland issue from the first and second, or the second and third, thoracic anterior roots, and pass up the cervical sympathetic nerve. The cell stations for these fibres lie in the superior cervical ganglion. ${ }^{6}$

The vaso-dilator fibres to the submaxillary gland pass by way of the chorda tympani from the facial nerve, and have their cell stations in contiguity with the gland. The vaso-dilator fibres to the parotid gland issue from the glosso-pharyngeal nerve, and pass to the gland by way of the auriculo-temporal nerve (see vol. i. p. 482). The circulation in these glands has been studied by placing a cannula in an efferent vein and observing the outflow, or by exposing the gland and directly inspecting its colour.

Cerebral circulation.-Observations on the movements of the brain are to be found recorded in the earliest medical writings. Whenever the brain is exposed by defects in the wall of the cranium, the brain can be seen to pulsate. This phenomenon can be demonstrated with

${ }^{1}$ Hill and Barnard, Journ. Fhysiol., Cambridge and London, 1897, vol. xxi. p. 323.

2 Claude Bernard, Journ. de la physiol. de l'homme, Paris, 1862, tome v. p. 413 ; Dastre and Morat, "Recherches sur le syst. nerv. vaso-moteur," Paris, 1884, p. 325 ; Langley, Phil. Trans., London, 1892, vol. clxxxiii. p. 85.

3 Loc. cit., p. 115 et seq.

${ }^{4}$ Piotrowski, Centralbl. $f$. Physiol., Leipzig u. Wien, 1887, s. 454.

${ }^{5}$ Morat and Doyan, Arch. de physiol. norm. et path., Paris, 1892, 1. 60, ef. v. Schulten, Arch.f. Ophth., Leipzig, 1884 (4), Bd. xxx. S. 65.

${ }^{6}$ Langley, Phil. Trans., London, 1892, vol. elxxxiii. p. 97 ; Joum. Physiol., Cambridge and London, 1890, vol. xi. 1. 123. 
the greatest ease, by applying a sensitive recording tambour to the fontanel of a newborn child. ${ }^{1}$

Galen and his followers thought that by the diastole of the brain, the $\pi v \varepsilon \tilde{u}, \mu \alpha$, the spirit of life, was sucked in through the cribriform plate, and mingled with the vital spirit, which ascended by the arteries from the heart. It was supposed that the dura mater pulsated, and by driving onward the animal spirits, produced movements of the muscles, and stimulated the soul. De la Mure, Haller, ${ }^{2}$ and Lorry, ${ }^{3}$ in the eighteenth century, by finally establishing the cardiac and respiratory

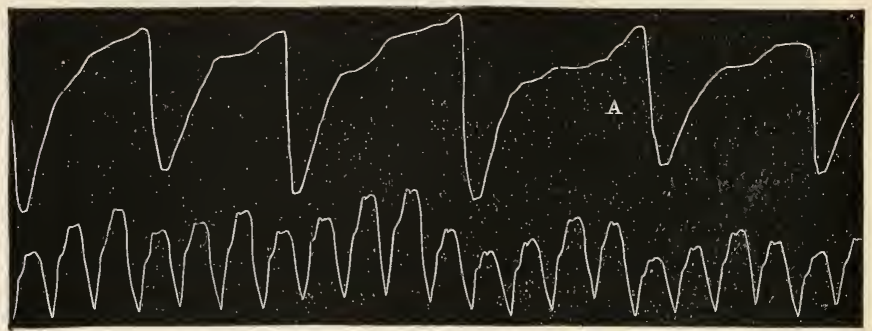

F1G. 87.--Respiration (A) and cerebral pulse tracing. Downstroke=inspiration.-Mosso. origin of the cerebral pulsation, proved that the dura possessed no independent power of movement in itself. They showed that the collapse of the chest wall pressed upon the thoracic veins, and drove back the venous blood, thus causing the expiratory expansion of the brain.

The movements of the brain in the closed cranium can be demonstrated in the following way:-

The skull is trephined, the trephine hole wormed with a mechanic's tap,

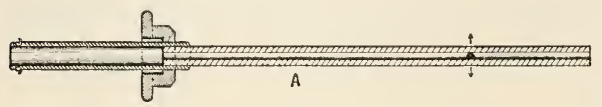
the dura mater carefully divided without causing any hæmorrhage, and a piece of steel tubing (B) screwed into the hole.

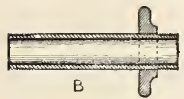
Over the end of a piece of brass tubing $(\mathrm{A})$, of smaller diameter than the steel tube, a piece FiG. 88.-Cerebral pressure gange.-Hill. of thin indiarubber membrane is tied. This second tube is passed into the steel tube, and the two are screwed together by means of a male and female screw. ${ }^{4}$ By means of this mechanism the indiarubber membrane comes into exact apposition with the brain. Attached to the end of the brass tubing is a piece of glass tubing of a fine bore. This is connected with a T-piece, one branch of which leads to a pressure bottle, and another to a mercury manometer. The whole apparatus is completely filled with water, and a bubble of air, to act as an index, is introduced within the fine-bored glass tubing. Before the apparatus is screwed in, the normal no-pressure position of the air index, when the tube is held

${ }^{1}$ Leyden, Virchow's Archiv, 1866, Bd. xxxvii. S. 519 ; Salathé, “Trav. du labor. de Marey," 1876, tome ii. p. 345; Mosso, "Ueber den Kreislauf des Blutes im menschliehen Gehirn," Leipzig, 1881.

2 Haller, "Elem. Physiol.," lib. x. vol. vii. p. 301.

${ }^{3}$ Lorry, Mém. de math. et phys. Acad. roy. d. sc., Paris, 1760, vol. iii. p. 277.

" Hill, "Cerebral Circulation," p. 9. 
horizontally and the membrane is flat, is marked on the glass tube. On screwing in the apparatus, the air index, which is at first forced out, is again, by raising the pressure bottle, brought to the normal position. That is to say, the brain, which is slightly bulged outwards by the intracranial pressure, is forced into that flattened position, which it naturally must assume against the closed cranial wall. The elevation of the manometer, consequent on this procedure, gives the normal intracranial tension. The pressure of the cranial contents against the skull wall is exactly balanced, and the skull becomes once more a closed cavity. The air index in the "cerebral pressure gauge" exhibits cardiac and respiratory undulations of intracranial pressure.

The brain pulsates in the direction where the resistance is least. Thus, when the cranium is trephined, and the dura opcned, the pulse can scarcely be seen in the occipito-atlantal membrane, for the trephine hole has become the seat of least resistance. Similarly, when the trephine hole is closed by a glass window, the occipito-atlantal membrane becomes the seat of least resistance, and the pulsation appears there.

The diastole and systole of the brain are made possible in the unyielding box of the cranium in two ways-

1. By the ebb and flow of the cerebro-spinal fluid. The occipito-atlantal and other vertebral ligaments extend in cerebral diastole, and allow the fluid to escape from the cranial cavity, while in systole, through the elasticity of these ligaments coming into play, it is driven back.

2. By expression of the blood in the cerebral veins another mechanism is provided. The cerebral venous pressure can be studied in the following way :In the dog, the superior longitudinal sinus, with its tributaries, opens into a large venous cavity within the occipital protuberance. This bony cavity is the torcular Herophili. The transverse sinuses lie within the osseous part of the tentorium cerebelli, and lead out of this cavity. Part of the blood finds its exit by the post-glenoid foramen, and reaches the external jugular vein, but a large portion passes into the large sinuses which run down the vertebral canal. It is quite simple to drill a hole into the torcular, and then immediately to screw into the hole a brass tube filled with magnesium sulphate solution. This tube is connected with a manometer containing $\mathrm{MgSO}_{4}$ solution.

The manometer can be directly observed, or, if connected with a delicate tambour, its movements can be recorded. In this way a continuous record of cerebral venous pressure can be obtained with little dissection and considerable ease. The manometer exhibits all the cardiac and respiratory pulsations, the latter in a marked way.

The brain is an organ which pulsates with every stroke of the heart, and with every respiratory movement, and, owing to its peculiar conditions, enclosed as it is in the unyielding cranium, the cardiac pulse is not entirely spent in distending the arterioles and capillaries, but is transmitted to the venous sinuses. The brain is lifted up by the stroke of the arteries at its base, and is thrown against the pial veins and the venous sinuses. By rise of pressure in the thoracic veins in expiration, the blood is dammed back into the sinuses, and the brain must then expand more, at the expense of the cerebro-spinal fluid. The contrary occurs in inspiration; the veins are emptied by the suction action of the thorax, the brain collapses, and the cerebro-spinal fluid re-enters the cranial cavity. Normally, the brain is more affected by the expiratory rise of general venous pressure; this is in contrast with such an organ as the kidney, which follows the inspiratory rise of general arterial pressure. If the inspiratory arterial rise of cerebral capillary tension becomes in any way increased, so as to be greater than the expiratory venous rise, then the respiratory curve of the brain is reversed. The 
cerebral pulsation is least when the intracranial tension is normal. In the living animal, the chief contents of the cranial cavity, beside the brain substance, is blood, and not cerebro-spinal fluid. The living brain, with its circulating blood, almost entirely fills the cranium, and the fluid that moistens its surfaces is little more in amount than the synovial fluid in a joint. The cerebral subarachnoid space is chiefly a potential rather than an actual space. The rate of transudation of the cerebro-spinal fluid seems to depend directly on the difference between the cerebral venous and the subarachnoid pressure.

In 1783, Alexander Monro the younger ${ }^{1}$ put forward the view that the quantity of blood within the cranium is almost invariable. "For, being enclosed in a case of bone," he writes, "the blood must be continually flowing out of the veins, that room may be given to the blood which is entering by the arteries. For, as the substance of the brain, like that of the other solids of our body, is nearly incompressible, the quantity of blood within the head must be the same, or nearly the same, at all times, whether in health or disease, in life, or after death, those cases only excepted in which water or other matter is effused or secreted from the blood vessels; for, in these, a quantity of blood equal in bulk to the effused matter will be pressed out of the cranium."

Monro's conjecture is confirmed by experiment. If a glass plate be screwed into a trephine hole, on compressing the innominate and subclavian arteries, the pial vessels can be seen to become less in size. The brain, however, does not collapse or retreat from the glass window. If, on the other hand, the glass window be faultily placed, and allow leakage into the cranial cavity, air passes within, and the brain collapses under atmospheric pressure. This experiment proves that the brain in the closed cranium can by no means completely empty itself of blood, even though the arterial pressure should fall to zero.

The brain can only empty itself of blood in so far as the cerebrospinal fluid-and this is small in amount-can take the place of the blood. If an animal be placed in the vertical feet-down position, and the skull be trephined, then, on opening the dura mater, the brain, which before was in close apposition with that membrane, can be seen collapsing, as it is emptied of blood by atmospheric pressure.

It is evident that in all physiological conditions the blood content of the brain can vary suddenly only to a slight degree by the ebb and flow of the cerebro-spinal fluid, and that Monro's doctrine is, to all intents and purposes, true. No sure evidence of the condition of the cerebral circulation can be drawn from examination of the brain after death, for in many different ways the relative volume of blood and of serous fluid within the cranium may be altered by post-mortem changes.

The following methods have been employed in investigations on the cerebral circulation :-

1. Direct observation of the pia mater.

2. A record of the volume of the brain.

3. A measurement of intracranial pressure.

4. Measurement of the blood pressure in the cerebral vessels.

5. Measurement of the velocity of blood flow in the cerebral vessels.

1 "Observations on the Structure and Functions of the Nervous System," Edin., 1783. Cf. Abercrombie, Edin. Med. and Surg. Journ., 1818, vol. xiv. p. 553 ; "Pathological and Practical Researches on Diseases of the Brain," etc., 1828. 
The essential point which is required in all experiments on the cerebral circulation is, that the cranial cavity should be a closed cavity, as in the normal condition.

Changes in intracranial tension cannot be registered by screwing an ordinary manometer ${ }^{1}$ into the skull, because, with any increase of tension, the brain floats up against the trephine hole, and acts towards it as a valve, so that no more fluid can escape into the manometer. The cranio-vertebral cavity cannot be put in comnection with a tambour, or used as an oncometer, ${ }^{2}$ because the brain on its expansion closes up, not only a trephine hole in the roof of the cranium, but the foramen magnum in the base. Moreover, the cerebro-spinal fluid leaks from the cavity.

Of considerably greater value, as a method, is the record of the outflow of blood, per second, from the lateral sinuses. ${ }^{3}$ Huirthle and Cavazzani have recorded the arterial pressure in the circle of Willis ${ }^{4}$ by placing a cannula in the peripheral end of the carotid artery, after tying all but the cerebral branch.

Cybulski, by means of his instrument, the photohæmatochometer, has investigated the rate of flow in the internal carotid artery under varying conditions of experiment. ${ }^{5}$

Roy and Sherrington, ${ }^{6}$ by an ingenious plethysmographic method, recorder the variations of the vertical diameter of the brain witl the cranium open. The conditions here are not normal. In the closed cranium, large alterations of blood volume are impossible. In the open cranium, with the atmospheric pressure brought to bear on the cerebral capillaries, the physical conditions of the cerebral circulation are altered.

Hill and Bayliss ${ }^{7}$ have simultaneously recorded the aortic pressure, the vena cava pressure, the intracranial pressure, and the cerebral venous pressure; the cranium being, as in the normal condition, a closed cavity. The intracranial pressure is measured by means of the brain-pressure gauge, while the cerebral venous pressure is obtained by placing a cannula in the torcular Herophili. The pressure of the cerebro-spinal fluid can be measured by trephining the atlas, opening the dura mater, screwing a tube into the trephine hole, and connecting this tube with a water manometer.

By these means, the following principles of the cerebral circulation have been determined.

When the aortic pressure rises, the expansion of cerebral volume can take place only to a certain limited amount, for, as soon as all the cerebro-spinal fluid has been driven out from the cranium, the brain is everywhere in contact with the rigid wall of the skull. Any further expansion of the arteries can only take place by an equivalent compression of veins, for the semifluid brain matter is incompressible. The reservoirs of blood in the veins will therefore be so far constricted, until the cerebral venous pressure rises to the pressure of the brain against these veins. Thus, as the arterial tension rises, the whole

${ }^{1}$ Salathé, "Trav, du labor. de Marey," 1876, tome ii. p. 362.

${ }^{2}$ Falkenheim and Naunyn, Arch. f. exper. Path. u. Pharmakol., Leipzig, 1887, Bd. xxii. S. 261; Dean, Journ. Path, and Bacteriol., Edin. and London, 1892, vol. i. p. 26 ; Knoll, Sitzungsb. $l$. k. Akad. $d$. Wissensch., math.-naturw. Cl., Wien, 1886 (3), Bd. xciii. S. 227 .

${ }^{3}$ Gärtner and Wagner, Wien. med. Wchnschr., 1897, S. 602, 639.

${ }^{4}$ Hürthle, Arch. $f$. $d$. ges. Physiol., Bonn, 1889, Bd. xliv. S. 582; Cavazzani, Arch. ital. de biol., Turin, vol. xvi. p. 23 ; vol. xix. p. 24 ; Centrulbl. $f$. Physiol., Leipzig u. Wien, 1896.

${ }^{5}$ Cybulski, "O ucisku mózgu," Krakow, 1891 ; Centralbl. f. Physiol., Leipzig u. Wien, 1890 , S. 834.

${ }^{6}$ Roy and Sherrington, Journ. Physiol., Cambridge and London, 1890, vol. xi. p. 85. p. 40.

Bayliss and Hill, ibid., 1895, vol. xviii. p. 334; and Hill, "Cerebral Circulation," 
circulatory systen of the brain will assimilate itself more and more to a scheme of rigid tubes. The velocity of the blood flow will be increased, and the relative distribution of the blood in the arteries, capillaries, and veins will be changed. A rise of arterial pressure does not by any means, as has been supposed, ${ }^{1}$ produce an anæmia of the brain, through compression of the cerebral capillaries and reins, but rather it causes an increased velocity of blood flow.

The intracranial pressure is, in all physiological conditions, the same as the cerebral capillary and venous pressure. The intracranial tension is, in fact, that tension which remains after the force of the heart has been expended in driving the blood through the cerebral arterioles. ${ }^{2}$ It is therefore an ever-varying quantity.

In nommal conditions, with the animal in the horizontal position, the intracranial pressure in the dog is generally equal to about $100 \mathrm{~mm}$. $\mathrm{H}_{2} \mathrm{O}$.

There is no compensatory mechanism by which the brain matter can be protected from great changes of circulatory pressure. In the spasms of strychnine poisoning, the intracranial tension may rise to 40 or $50 \mathrm{~mm}$. $\mathrm{Hg}$. This is due, not only to the rise of arterial tension, but to the enormous diminution of capacity of the venous system and rise of vena cava pressure produced by general muscular spasm. On the other hand, the writer has measured the intracranial tension in a certain patient when standing upright, and found it to be slightly below zero. This patient had been trephined for symptoms of lunacy. Men in the spasms of strychnine poisoning are conscious, and the above patient was also conscious. It is clear, therefore, that the functions of the brain can continue at circulatory pressures varying from zero to $50 \mathrm{~mm}$. Hg.

No evidence has been found of the existence of cerebral vasomotor nerves, either by means of stimulation of the vasomotor centre, or of the central end of the spinal cord, after division in the upper dorsal region; or by stimulation of the stellate ganglion. That is to say, the whole of the cranial nerve supply, and the whole of the sympathetic supply to the carotid and vertebral arteries, have been stimulated, and the result has been negative. ${ }^{3}$

Nor is there any anatomical evidence of the existence of a local vasomotor mechanism. Gulland has entirely failed, by every known histological method, to demonstrate vasomotor nerves in the vessels of the pia mater. ${ }^{4}$

In every experimental condition the cerebral circulation passively follows the changes in the general arterial and venous pressures. The intracranial or cerebral venous pressure varies directly and absolutely with vena cava pressure, but only proportionately with aortic pressure. If both alter, the cerebral venous or intracranial pressure is affected by both, and an alteration of pressure of equal amount, and in opposite directions in both, will affect the intracranial or cerebral venous pressure from the venous side to a far greater degree than from the arterial side, because it is on the arterial side that the peripheral resistance lies. If the torcular Herophili be opened in the freshly-killed animal, and the

\footnotetext{
${ }^{1}$ Grashey, Festschr. f. L. A. Buchner, München, 1892, S. 61 ; Geigel, "Die Mechanik der Blutversorgung des Gehirns," Stuttgart, 1890; Virchow's Archiv, 1890-91, Bd. cxix. S. 93 ; cxxi. S. 432 ; exxiii. S. 27 ; exxv. S. 92.

${ }^{2}$ Lewy, ibid., 1890, Bd. cxxii. S. 146.

${ }^{3}$ Hill, "Cerebral Circulation," p. 42.

* Journ. Physiol., Cambridge and London, 1895, vol. xviii. p. 361.
} 
abdominal veins be compressed, venous blood can be driven out of the torcular in a continuous stream. If in the living animal the torcular venous pressure and the vena cava pressure he simultaneously recorded in manometers placed side hy side, and if at the same time the abdomen be compressed, then the pressure rises in both by equal increments, when the arterial pressure remains constant, and by greater increments in the torcular if the arterial pressure rises also. Between the vena cava and the torcular there lies no appreciable resistance, between the aorta and the cerebral capillaries or veins there lies the unknown resistance of the arterioles. Thus, the variations of arterial pressure, read in millimetres of mercury, only produce variations in cerebral venous pressure which can be read in millimetres of water.

Every change in the position of an animal, owing to the influence of gravity on the vascular system, affects the cerebral circulation. Every

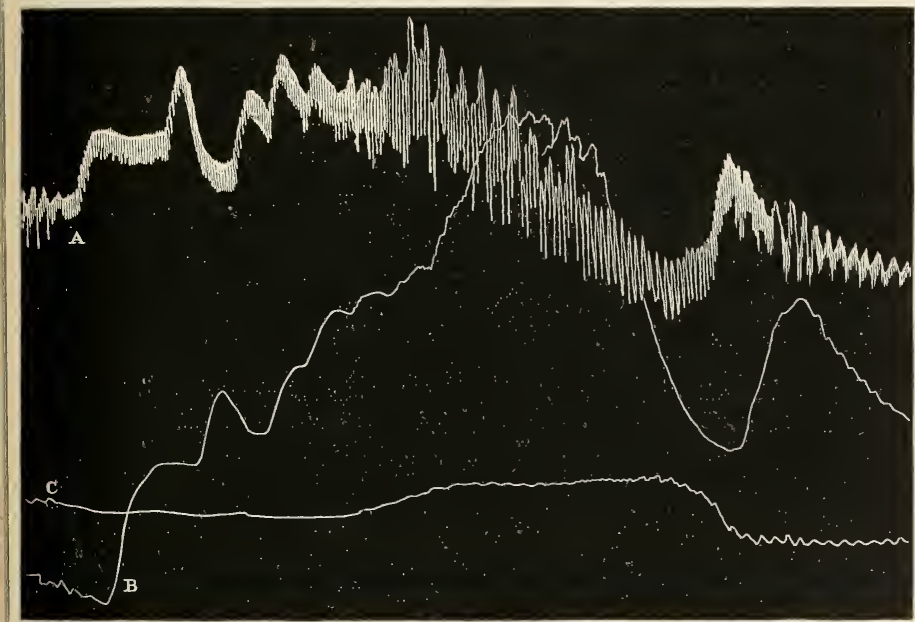

12

3

4

Fig. 89.-Effect of asphyxia. Curarised dog. A, aortic pressure ; B, cerebral venous pressure ; $\mathrm{C}$, vena cava pressure. 1 , fall of $\mathrm{C}$ and $\mathrm{B}$ due to cessation of artificial respiration ; 2, asphyxial rise of $A$ and $B ; 3$, rise of $C$ and $B$ and fall of $A$; 4 , reestablishment of artificial respiration. Note.-The tambour used for recording B was more delicate than that used for recording C.-Bayliss and Hill.

variation in respiration and every muscular movement is followed by passive changes in the circulation within the brain. Compression of the jugular veins or of the abdomen causes a marked rise in cerebral venous pressure. The movements of the muscles of the neck by pressing on the jugular vein are sufficient to affect the cerebral circulation.

Every stimulus that enters the organism and affects the general vasomotor centre produces a passive effect on the cerebral circulation. It is by means of the great splanchnic area that the blood supply to the brain is controlled. An anremia of the spinal bulb excites the vasomotor centre, the splanchnic vessels contract, the blood pressure 
rises, and more blood is driven through the brain. At the same time, the respiratory centre is excited, and by the increased action of the respiratory pump more blood is driven to the right heart, and thence to the brain. We have in the vasomotor centre a protective mechanism, by which blood can be drawn at need from the abdomen and supplied to the brain. At the moment that excitation from the outside world demands cerebral response, the splanchnic area contracts, and more blood is driven through the brain. The brain has no direct vasomotor mechanism, but its blood supply can be controlled indirectly by the vasomotor centre acting on the splanchnic area. The rasomotor centre is part of the central nervous system, and feels the same needs and is stimulated by the same centripetal impulses as affect the rest of that system, and thus it maintains a supply of blood to the central nervous system which corresponds to its functional activity.

The arterial smpply to the lirain is in the lower animals so superabundant, that, in the dog,

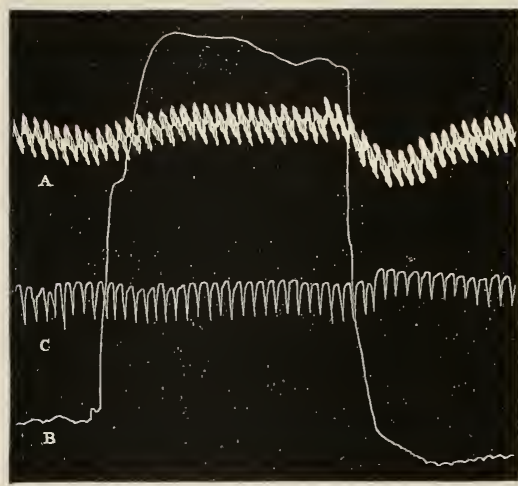

F16. 90.--Effect of drawing a ligature gently roumd the neck, after placing a cannula in the trachea. $A$, aortic pressure; $B$, cerebral venous pressure ; C, vena eava pressure.-Bayliss and Hill. four of the arteries - two common carotids and two vertebrals - which supply the brain can be tied in the course of ten minutes, and yet the animal either at once, or after a temporary period of idiocy and paralysis, completely recovers. In the monkey, on the other hand, ligation in one operation of one vertebral artery, in addition to the two carotids, produces either death in twentyfour hours or softening of the great brain, accompanied ly idiocy and paralysis of movement and sensation. The efficiency of the anastomosis through the circle of Willis varies in different individuals. Sudden compression of one common carotid artery in some inen produces epileptic spasm, and ligation of this artery has been followed in some by more or less temporary paralysis on the opposite side of the body; in others the effect is nil.

\section{The Pulmonary Circulation.}

The first physiologist who succeeded in making observations on the pressure in the pulmonary artery was Beutner. ${ }^{1}$ The more important recent investigations on this subject are those of Bradford and Dean, ${ }^{2}$ and François-Franck. ${ }^{3}$ By Bradford and Dean, dogs were placed under the influence of morphia and curari, and artificial respiration was established. The artery to the lower lobe of the left lung was reached by

${ }^{1}$ Ztschr. f. rat. Med., 1852, Bi. ii. S. 97 .

2 Proc. Roy. Soc. London, 1889, vol. xlv. p. 362 ; Journ. Physiol., Cambridge and London, 1894, vol. xvi. p. 34 .

${ }^{3}$ Arch, de physiol, norm. et path., Paris, 1896, pp. 178, 193. 
resection of two or three ribs in the back, and a cannula was inserted therein. Both the carotid and pulmonary arterial pressures were recorded synchronously with mercurial manometers.

Chauveau and Faivre passed a trocar connected with a manometer directly into the pulmonary artery of the horse, through an intercostal space, and without opening the thorax. This method, since the conditions are absolutely normal, is far preferable, but it is only possible to carry it out in large animals, such as the horse.

The following determinations of the pulmonary arterial pressure, with the chest opened, have been recorded:-

\begin{tabular}{|c|c|c|c|c|c|c|}
\hline \multicolumn{3}{|c|}{ Name. } & Aniınal. & \multicolumn{2}{|c|}{ Hean Pressure. } & $\begin{array}{l}\text { Proportionate Relation } \\
\text { to Aortic Pressure. }\end{array}$ \\
\hline Bentner & . & . & Rablit & \multicolumn{2}{|c|}{$12 \cdot 07 \mathrm{~mm} .1 \mathrm{gs.}$} & $1-4 \cdot 2$ \\
\hline , & . & . & C'at & $17 \cdot 6$ & , & $1-5 \cdot 3$ \\
\hline ", & . & . & Dogg & $29 \cdot 6$ & , & $1-3 \cdot 1$ \\
\hline Knoll ${ }^{1}$. & . & . & Rabbit & $12 \cdot 2$ & , & $1-6 \cdot 8$ \\
\hline Bradford & nd Dean & . & Dog & $16-20$ & , & $1-5$ \\
\hline
\end{tabular}

There can be no doubt that these figures are lower than normal, owing to the severity of the operative procedure, and to the necessity of upening the thoracic cavity.

By passing a camnula into the right ventricle, de Jager found the intraventricular pressure to be equal to 27 to $59 \mathrm{~mm}$. $\mathrm{Hg}$. By Chauveau and Faivre's method, which is free from the objections pertaining to the other procedures, the relation of pulmonary to aortic pressure was foumd to be as 1 to 3 . If the aortic pressure in man be equal to 100 to $120 \mathrm{~mm}$. $\mathrm{Hg}$, the pulmonary arterial tension is probably equal to 30 to $40 \mathrm{~mm}$. $\mathrm{Hg}$.

In some experiments, conducted under conditions of artificial respiration with the thorax open, Lichtheim ${ }^{2}$ found that the greater number of the hranches of the pulmonary artery could be ligatured without lessening the input into the left heart, or lowering the aortic pressure. After tying the left pulmonary artery, and driving melted paraffin by way of the jugular vein into the branches of the right pulmonary artery, Lichtheim even occluded as much as three-fourths of the channels of communication between the right and left hearts, and yet obtained no fall of aortic pressure. This result was not due to any compensatory constriction of the systemic arterioles.

Astounding as these experiments seem, yet their results are probably entirely to be explained as due to the effect of artificial respiration. To begin with, the capacity of the circulatory system of the lungs must be very great in comparison with that of any other organ, for in a given time as much blood must stream through the lungs as through the rest of the body. Secondly, the high tension of the air artificially blown into the lungs diminishes the capacity of the pulmonary vessels. So long, therefore, as the vascular system is not reduced by ligature beyond that amount which is rendered impervious by the artificial respiration, the aortic pressure will not vary. The conclusion thus reached is that only about one-third of

${ }^{1}$ Sitzungsh. d. k. Akad. d. Wissensch., math.-naturw. Cl., Wien, 1888, Bd. xcvii. Abth. 3, S. 212 .

2 "Die Störungen des Lungenkreislaufes," Berlin, 1876, S. 20. 
the circulatory pathway through the lungs is open during vigorous artificial respiration.

In confirmation of this view, Landgraf's observations on the rabbit may be quoted. ${ }^{1}$ He split the sternum in the middle line without opening the pleural cavities or interfering with natural respiration. In this condition he found that ligature of one pulmonary artery lowered the aortic pressure, markedly lessened the filling of the left lieart and dilated the right heart. The experiments of Lichtheim, and also those of Landgraf, prove how markedly different the pulmonary circulation is in the states of artificial and natural breathing.

Apart from the effect of respiration, the pulmonary circulation may be influenced passively by the following agencies:-

1. By changes in the rate and force of the heart-beat, the circulatory system otherwise remaining constant.

2. By congestion in the left auricle, a "back effect," consequent on increased resistance to the aortic outflow.

3. By a fall of pressure in the left auricle, due to diminished resistance in the aortic outflow.

4. By a "forward effect," or increased input into the right heart, such as is produced by the surlden constriction of a large area of arteries emptying the blood into the veins, or by compression of the abdomen.

5 . By a diminished input into the right heart, such as is produced by loss of vascular tone.

6. $\mathrm{Br}$ the influence of gravity and the effect of position, producing an increased or diminished imput into the right heart.

It is important to consider in further detail how, by a "back effect," a rise of aortic pressure may congest the pulmonary circulation.

The left ventricle has to vary its power of contraction in accordance with alterations of arterial pressure which are far greater than ever fall to the lot of the right ventricle. The left ventricle is thus found to become exhausted, while the right still continnes unaffected. Thus on inducing asphyxia, after dividing the ragi, the left heart begins, when the aortic pressure reaches its maximum, to give weak and irregular beats, while the right heart continues for a time to contract strongly and regularly.

On stimulating the cervical spinal cord, Waller ${ }^{2}$ found that the left ventricle could not empty itself against the high aortic pressure consequent on the general condition of raso-constriction. The left auricle thus became congested, and even ceased to show any signs of contraction. Meanwhile the right heart continued to beat normally, and while the pressure in the left auricle rose to 25 or $30 \mathrm{~mm}$. $\mathrm{Hg}$, the pressure in the right auricle was only $5 \mathrm{~mm}$. Hg. The lungs act to the left heart as a blood reservoir, as the liver and large veins stand in the same relation to the right heart. Thus the "back effect" on the right heart is small during a short period of increased resistance to the aortic outflow. By the combined effect of stimulation of the spinal cord, ligature of the aorta, and compression of the abdomen, the pressure in the right auricle has been raised to $22 \mathrm{~mm}$. $\mathrm{Hg}$. In this case a "back effect" is combined with a "forward effect," and the input into the right heart is increased while the output is resisted. Finally, when the output of the right heart also becomes incomplete, and the reservoirs of the lungs are filled, the blood congests in the liver and large veins.

Bradford and Dean observed that compression of the thoracic aorta,

1 Ztschr. f. klin. Med., Berlin, 1892, Bd. xx. S. 181.

${ }^{2}$ Arch.f. Physiol., Leipzig, 1878, S. 525. 
if maintained for only a short period, produced no "back effect" on the pulmonary artery. When, in one experiment, the period of compression was increased, the pressure rose in the pulmonary artery from 24 to 30 $\mathrm{mm}$. $\mathrm{Hg}$, while the aortic pressure rose from 90 to $135 \mathrm{~mm}$. $\mathrm{Hg}$. On excitation of the peripheral end of the great splanchnic nerve, the pulmonary arterial tension at first rises rery slightly, and at the same time there occurs a great rise of aortic pressmre. By continuing the excitation the blood congests in the left heart, and the pressure in the pulmonary vessels then rises.

The devising of experimental means by which purely active changes can be evoked in the pulmonary circulation, and thus the proof of a vasomotor control established, has been extremely difficult; so that some physiologists have gone so far as to maintain that the vascrlar system of the lungs is not under the direct influence of the central nervous system. The difficulty arises from the susceptibility of the pulmonary circulation to passive changes such as we have discussed, and which arise secondarily to active alterations in the conditions of the

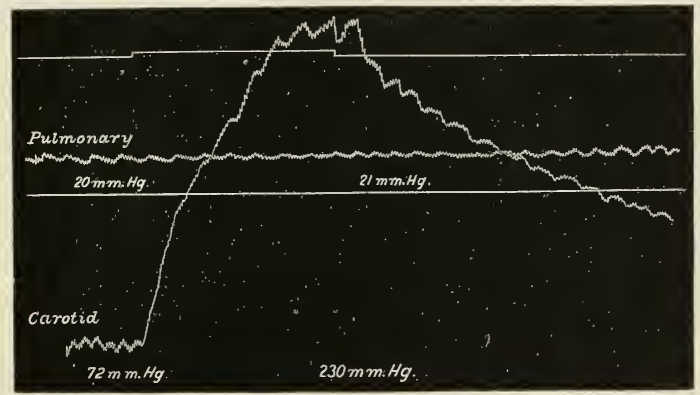

FIG. 91.-Pulmonary and carotid arterial pressmres. Curarised dog. Vagi divided. Cord divided at level of seventh dorsal root. Effect of excitation of peripheral end of cort.-Bradford and Dean.

systemic circulation. It is necessary in the experimental investigation of this question to provoke vasomotor changes in the pulmonary circulation by the excitation of some portion of the nervons system, while the diastolic filling of the right heart and the systolic output of the left heart remain unaltered by any synchronous variation in the systemic circulation. These postulates have not been carried out in most of the experiments which have been made on the pulmonary vasomotors. ${ }^{1}$

The most exhaustive research on this difficult subject has been carried out by Bradford and Dean. ${ }^{2}$ Their evidence in favour of a vasomotor mechanism appears to be conclusive, and is based upon the following experiments :-

1 Brown-Séquard, Lancet, London, 1872 ; Compt. rend. Soc: de biol., Paris, 1872, 1. 180 ; Badaud, "Ueber den Einfluss des Hirns auf den Blutdruck in dem Lungen-arterie," Würburg, 1874; Couvreur, Compt. rend. Soc. de biol., Paris, 1889, p. 731; Bokai, Jahresb. ü. d. Fortschr. d. A nat. u. Physiol., Leipzig, 1880, Bd. ii. S. 71; Henriques, Skandin. Arch. f. Physiol., Leipzig, 1892, Bd. iv. S. 229 ; Waller, Arch. $f$. Physiol., Leipzig, 1878, S. 525 ; Lichtheim, "Störungen des Lungenkreislaufes," Berlin, 1876; Openchowski, Arch. $f . d$. ges. Physiol., Bonn, 1882, Bd. xxvii. S. 233.

"Bradford and Dean, Journ. Physiol., Cambridge and London, 1894, vol. xvi. p. 34. 
1. On excitation of the central end of the divided vagus in a curarised dog, the pressure in the pulmonary vein fell, in the pulmonary artery rose, in the aorta fell. These results, brought about by reflex excitation of the vasomotor centre, seem to point conclusively to constriction in the pulmonary arterioles.

2. After section of both vagi in a curarised dog, excitation of the peripheral end of the spinal cord, divided at the level of the seventh dorsal root, produced a rise of aortic pressure equal to $160 \mathrm{~mm}$. Hg. During the period of stimulation, the pulmonary arterial pressure rose $1 \mathrm{~mm}$. $\mathrm{Hg}$ (Fig. 91). On the other hand, excitation of the vasomotor centre immediately afterwards produced a rise of $57 \mathrm{~mm}$. $\mathrm{Hg}$ in the aorta, and $6 \mathrm{~mm} . \mathrm{Hg}$ in the pulmonary artery (Fig. 92).

The rise of pressure by $6 \mathrm{~mm}$. Hg, in the latter case, cannot well be explained by a passive back effect, because, just before, a risc of aortic: pressure thrice as great had only raised the pressure in the pulmonary artery $2 \mathrm{~mm}$. Hg. Sinee the vagi were divided this experinental result cannot be explained by change in the rate of the heart.

Carrying the investigation a step further, these authors divided the

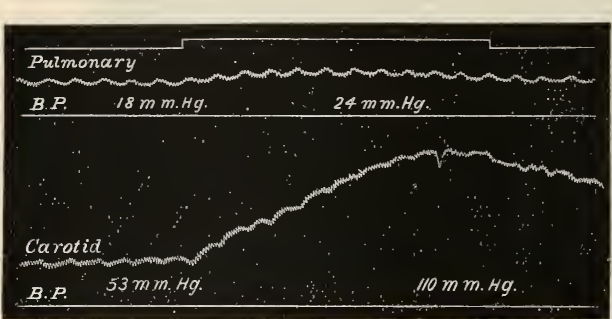
upper thoracic nerve roots, and stimulated the peripheral ends. Accepting the anatomical rescarches of Gaskell, it is from this part of the spinal cord that we should expect to find the outtlow of the pulmonary vasoconstrictor nerves.

FIG. 92.-Continuation of experiment shown in Fig. 91. Effect Excitation of the of excitation of vasomotor centre, - Bradford and Dean. third thoracic root accelerated the heart, raised the tension in the pulmonary artery at the same time, and frequently caused a slight fall in aortic pressure. Here the aortic fall is neither produced by loss of cardiac power, nor ly dilatation of any vascular area, and must be ascribed to the diminished input, consequent on contraction of the pulnonary arterioles.

On excitation of the succeeling roots, the proportionate change in the aorta and pulmonary artery were found to be as follows:-

Fourth thoracic (1) Aurta, . + +10 mm. Puhmonary artery, . + +4 mm.

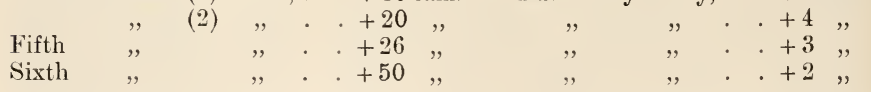

These results show conclusively that the pulmonary rise is not produced passively. On passing down from root to root, the excitation produces less effect on the pulmonary vascular system, and more on the systemic systcm, for the splanchnic fibres become stimulated towards the middle thoracic region.

3. On producing asphyxia in the curarised animal, Bradford and Dean observed the following:-(1) A great rise in aortic tension, followed 
by a more gradual fall; (2) a considerable rise in the pulmonary artery, synchronous with the commencement of the aortic rise but overlasting the latter, and followed by a gradual fall; (3) a great rise in the pulmowary venous pressure synchronous with the aortic fall.

After complete division of the spinal cord at the level of the sixth or seventh dorsal root, asphyxia produced the same rise in the pulmonary

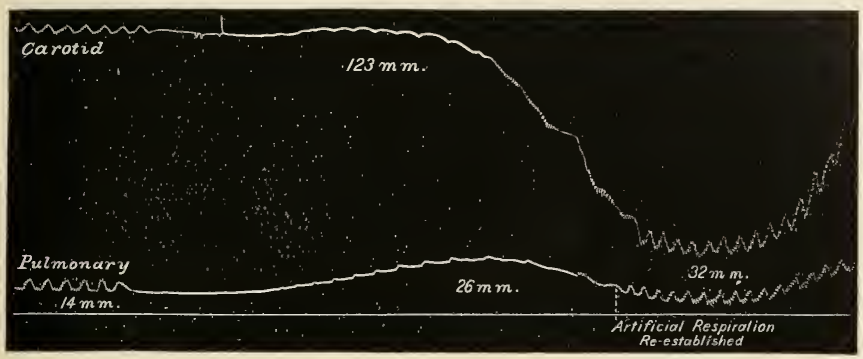

FlG. 93. - Pulmonary and carotid arterial pressures. Curarised dog. Spinal cord divided at level of sixth dorsal root. Effect of asphyxia.-Bradford and Dean.

arterial pressure, and this was now accompanied by but a slight rise in aortic pressure (Fig. 93).

Thus in one case the aortic pressure rose from 117 to $123 \mathrm{~mm}$. $\mathrm{Hg}$, while the tension in the pulmonary artery rose from 14 to $26 \mathrm{~mm}$. $\mathrm{Hg}$. The rise of pulmonary venous pressure in the last stage of asphyxia is due to failure of the left heart, and a consequent decrease in the systolic output. In all these experiments the animals were curarised as

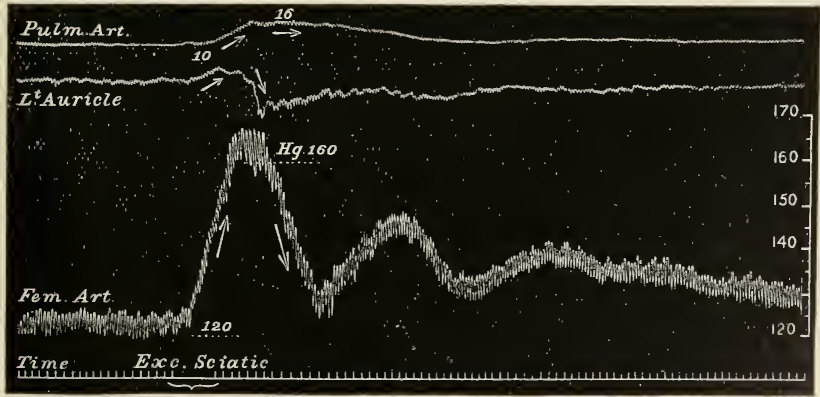

Fig. 94.-Pressure curves of pulnonary artery, left auricle, and femoral artery. Effect of excitation of central end of crural nerve.-François-Franck.

well as anæsthetised, in order to eliminate all changes in the circulation which might arise from muscular movements. Turning to the effects of reflex excitation of the pulmonary vasomotor mechanism, Bradford and Dean noted that stimulation of the central end of any posterior root in the upper thoracic region caused a rise both in aortic and pulmonary arterial pressure, but the rise was proportionately greater in the latter. 
On excitation of the central end of an intercostal nerve, there occurred a rise of aortic pressure followed by a fall, or a fall was obtained from the first. The pressure in the pulmonary artery fell also, but not proportionately. It is thus evident that the pulmonary circulation can be affected to a certain extent by sensory stimulation of the chest wall. In confirmation of Bradford and Dean's work, François-Franck ${ }^{1}$ obtained, by excitation of the central end of the anterior crural nerve, a rise of pressure in the pulmonary artery and in the aorta, accompanied by a fall of pressure in the left auricle. On stimulation of the central end of the splanchnic nerve, he recorded a maintained rise of tension in the pulmonary artery, while the aortic pressure varied, owing to the reflex excitation of the dilator as well as the constrictor fibres which govern the splanchnic vascular system.

François-Franck recorded the volume of one lobe of the lung by the plethysmographic method. A paradoxical result is obtained on plethysmography of the lung, since the organ does not diminish, but swells on contraction of the arterioles. This is owing to the distension of the large and extensile pulmonary arteries, in consequence of the increased resistance to the outflow, and the continuance of the input from the right heart. Excitation of the peripheral end of the vagus in the atropinised animal was found by Bradford and Dean to have no effect on the pressure in the pulmonary artery, and they were unable to find any evidence of pulmonary raso-dilator fibres in this or any other nerve.

On stimulating the depressor nerve, both the aortic and the pulmonary arterial pressures fall together.

Among the points which become manifest in these experiments the following are of especial interest :-

1. The lesser circulation is independent of the systemic circulation, within certain limits. Increased resistance to aortic flow does not immediately produce a back effect in the pulmonary vascular system, but only when the aortic tension has been maintained at a high level for a considerable period, and when the ventricle becomes fatigued. The left heart then fails to keep up the systolic output. Even when this happens, the lungs act as reservoirs for the blood, and the pressure does not materially rise in the right heart till the back effect has continued for some time. Finally, the output of the right heart is affected, and the blood congests in the venous system. To grasp how back effects are produced in the circulatory system is of the utmost importance for the proper understanding of the pathology of the circulation.

2, The pulmonary vascular system is undoubtedly innervated from the upper thoracic nerve roots, and thus comes under the influence of the central vasomotor mechanism. From the results of the experiments which have been considered, it might be concluded that the vasomotor effects are very small. In the light, however, of Lichtheim and Landgraf's researches on the ligature of the pulmonary arteries, this conclusion may be wrong. If ligature of threefourths of the arterial channels produces no effect when the thorax is opened and artificial respiration is maintained, we cannot expect that a constriction of the arterioles should produce a very great result in Bradford and Dean's experiments, since these authors worked with the thorax open, and with the aid of artificial respiration. In the normal animal, the vasomotor phenomena may be found to be somewhat more important, just as Landgraf discovered that a great fall of aortic pressure and a rise of tension in the right heart is produced when he ligatured one pulmonary artery without opening the pleural cavity.

${ }^{1}$ A reh. de physiol. norm, et path., Paris, 1896, p. 184. 


\section{The Circulation through the Limbs.}

By far the greater quantity of the blood, 50 to 70 per cent., lies within the roomy reservoirs of the abdominal and thoracic organs. Thence, by means of the vasomotor mechanism, the blood is distributed at need to the locomotor organs.

In the organs of locomotion the quantity of blood during rest has been roughly estimated to be 36 per cent., and during activity 66 per cent. of the whole blood quautum. ${ }^{1}$

Spehl reckoned the proportionate weight of each organ, washed free of blood, to the weight of the whole body washed free of blood. He likewise estimated the proportionate weight of the blood found in each organ to the weight of the whole blood in the body. Dividing the proportionate weight of the bloodless organ by the proportionate weight of the blood within it, he obtained the blood capacity of the organ. If the blood were equally distributed throughout the parts of the body, the blood capacity should equal 1. In the lungs he found the blood capacity to be 11 to 13 in inspiration, 9 to 12 in expiration. The blood capacity of the muscles was 0.327 to $0.34: 3$ during rest, and 0.520 to 0.664 during maximal activity. ${ }^{2}$

Chauveau and Kaufmamn estimated the amount of blood flowing in one minute through the levator labii superioris of the horse to be five times as great during activity as during rest of the muscles. ${ }^{3}$

The blood flow from the deep femoral vein of the dog during an epileptic fit (excited by essential oil of absinthe) is three to five times as great as during rest. ${ }^{4}$ The clonic contractions alternately suck the blood into the vessels of the muscles, and then compress the blood on into the veins.

Similarly, massage greatly increases the flow of blood through the muscles. Massage of a considerable muscular area produces a fall of general arterial pressure, in consequence of the derivation of blood into the dilated muscular vessels. This fall may amount to one-fifth of the initial pressure. ${ }^{5}$

Gaskell ${ }^{6}$ observed the circulation in the mylo-hyoid muscle of the frog directly, and measured the diameter of the ressels with a micrometer. On stimulating the trigeminus, the vessels dilated after a latent period of 5 to 6 seconds. Venous congestion occurred only at the commencement of tetanus, owing to the pressure of the muscle fibres. During the rest of the period of excitation the blood flow was increased.

In the curarised animal the outflow from the muscular veins of the lower limb is increased on excitation of the motor nerves. Vaso-dilator fibres must run, therefore, in these nerves. On the other hand, Gaskell obtained evidence of a diminished outflow on stimulation of the peripheral end of the abdominal sympathetic in the curarised dog. We must therefore admit the existence of vaso-constrictor fibres. They are, however, but poorly developed in the muscles.

At times of great muscular effort, a man closes his glottis, and main-

${ }^{1}$ Ranke, "Die Blutvertheilung und der Thatigkeitswechsel der Organe," Leipzig, 1871, S. 69,87 .

'Spehl, "De la répartition du sang circulant daus l'écononie," Thèse, Bruxelles, 1883.

"Kaufmann, Arch. de physiol. norm. et peth., Paris, 1892, p. 283.

${ }^{4}$ Hill and Nabarro, Journ. Physiol., Cambridge and London, 1895, vol. xviii. p. 218.

5 Brunton and Tunnicliffe, ibid., 1894-5, vol. xvii. p. 362 ; Kleen, Skandin. Avh.f. Physiol., Leipzig, 1889, Bu. i. S. 247.

${ }_{6}^{6}$ Journ. Physiol., Cambrilge and London, vol. i. p. 262. 
tains his tholax and abdomen in the position of forced expiration. By this means the intrathoracic pressure is raised, the outlet from the veins blocked, and the muscles are distended with blood. The pressure rises in the capillaries and veins of the muscles almost to arterial pressure.

The control of the blood supply to the limbs has been studied by the thermometric and plethysmographic methods. The temperature in

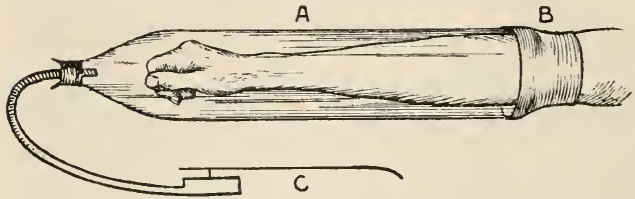

Fis. 95.-Limb plethysmograph. $\Lambda$, rlass chamber filled with air ; B, broad indiarubber band; C, Marey tambour. The junction between the limh and the plethysmograph is rendered air-tight by the free application of thick vaseline beneath the rubber-band (B) nerve. The vasomotor fibres have been traced from this nerve to the lower thoracie roots. They pass down the sympathetic chain in the lower lumbar region. ${ }^{2}$ On excitation of these vasomotor nerves, the greatest change of temperature is found in the feet. This is so, because the pad of an animal's foot is free froll a furr coat, and thus formis one of the nore important places for the regulation of the temperature of the body."

By Bradford and liayliss the vasomotor nerves of the limbs have been minutely studierl, with the aid of the plethysmograph. According to these authors, the vaso-

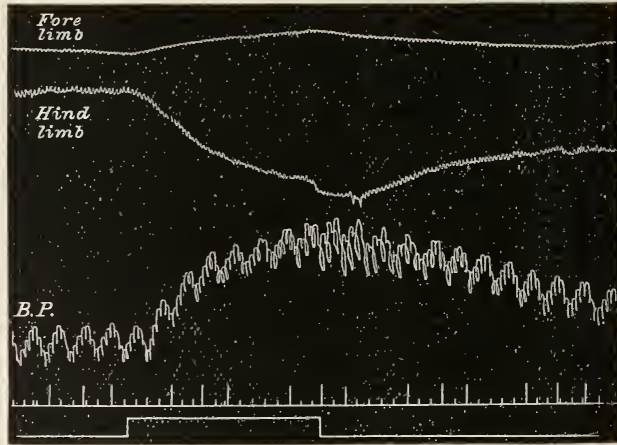
constrictor fibres lik. 96.--Record of aortic pressure and the volume of the fore which supply the and hind limbs. Curarised dog. Effeet of excitation of the fore-liub lhirteenth dorsal root. Passive dilatation of the fore-limb, the anterior roots of from the third to the eleventh thoracic nerves. The hind-limb is supplied by fibres from the eleventh thoracic to the third lumbar nerve. By each root the volume of the whole limb is affected.

${ }^{1}$ Sehiff, "Untersnehungen, ete.," Frankfurt-am-Maine, 1855, S. 176; Bernard, Compt. rend. Aead. d. sc., Paris, 1862, tome lv. p. 305; Cyon, Ber. d. k. Säehs. Gesellsch. d. Wissenseh., Leipzig, 1868, S. 73.

${ }^{2}$ Bernard, Compt. rend. Acud. d. se., Paris, 1862, tome lv. p. 232 ; Brown-Séquard, Comp. rend. Acad. d. sc., Paris, 1854 , tome xxxviii. p. 76 ; Ostronmoff, Areh. f. d. ges. Physiol., Bonn, 1876, Bd. xii. S. 261.

${ }^{3}$ Lewaschew, Arch.f. d. yes. Physiol., Bonn, 1882, Brl. xxviii. s. 397 scq.

${ }^{4}$ Journ. Physiol., Cambridge and Lonilon, 1894, vol. xvi. p. 10. 
The chief outflow of constrictor fibres occurs from the sixth dorsal to the first lumbar nerve. Thus the limbs and the abdominal viscera get their supply approximately from the same part of the spinal cord. The vasomotor mechanism of the limbs is not powerfully developed.

By stimulation of any sensory nerve the vessels of the limbs can be constricted. Usually, however, the limbs expand owing to the passive

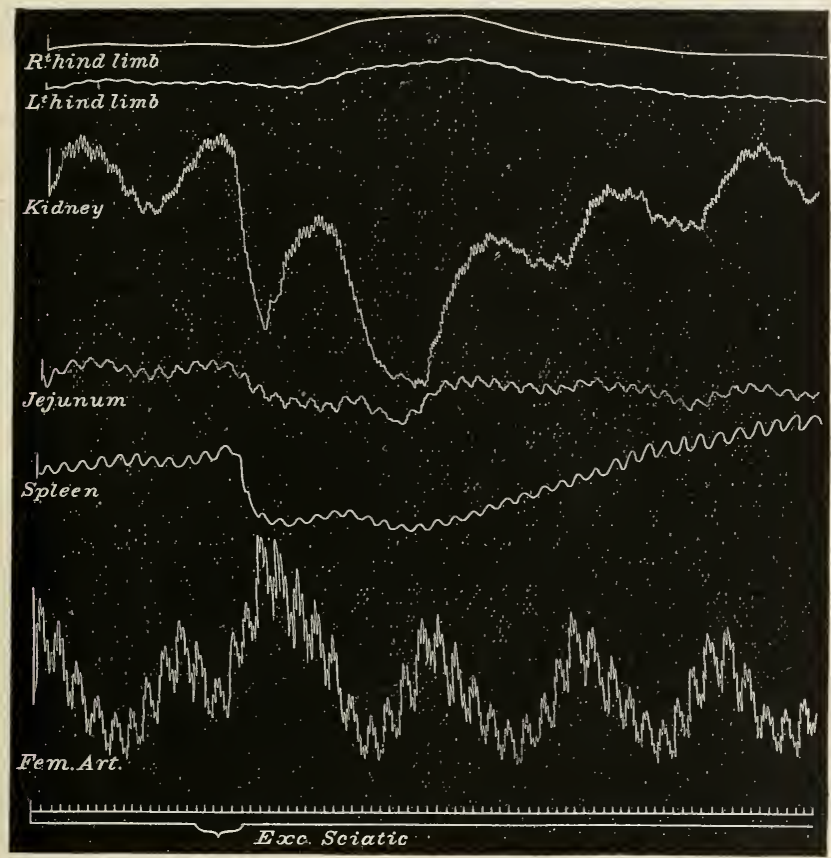

FIs. 97.-Effect of excitation of a superficial branch of the crural nerve on the arterial pressure, and on the volume of the spleen, jejunum, kidney, and feet.-Hallion and Franeois-Franck.

dilatation produced by the rise of aortic pressure which follows reflex constriction of the splanchnic vessels. The reflex constriction of the limbs can be obtained after section of the splanchnic nerves.

Langley's ${ }^{1}$ observations on the vaso-constrictor fibres of the feet agree closely with those of Payliss and Bradford. He further finds that the sympathetic cell stations for the fibres of the upper limb are in the stellate ganglion; of the hind-limb, in the sixth and seventh lumbar and first sacral ganglia. The vasomotor fibres to the trunk follow the same distribution as the pilomotor fibres.

The general changes which take place on exciting the central end of an afferent nerve are seen exceedingly well in Fig. 97 . In this there is shown a simultaneous record of-

1 Journ. Physiol., Cambridge and London, 1891, vol. xvi. p. 375; 1894-5, vol, xvii. p. 307. 
1. The pressure in the femoral artery.

2. The volume of the spleen.

3 . The volume of the jejunum.

4. The volume of the kidney.

5. The volume of the left hind-limb.

6. The volume of the right hind-limb.

On exciting the central end of the sciatic nerve, the arterial pressure rose, the spleen, jejunum, and kidney contracted. The feet ${ }^{1}$ expanded (perhaps passively by the rise of arterial pressure). The arterial pressure then fell somewhat (owing to cutaneous vaso-dilatation and pulmonary vaso-constriction). ${ }^{2}$

The volume of the limbs depends both upon the arterial and the rena cara pressures. If the arterial pressure remain constant, and the vena cava pressure rise, as on performing a Valsalva experiment, then the volume of the limb increases greatly. Here the venous tension rises towards the mean arterial tension, for the outlet of the reins is blocked by the rise of intrathoracic pressure. If the vena cava pressure rise while the arterial pressure fall, the two effects may halance each other, and the volume of the limb remain constant.

The tracing of the limb volume shows all the respiratory and cardiac oscillations. The limb may expand more, either with expiration or with inspiration, according as the expiratory rise of vena cava pressure, $\mathrm{or}^{\circ}$ the inspiratory rise of arterial pressure, has the greater effect upon the capillary pressure.

During Traube-Hering oscillations of arterial pressure the volume of a limb follows the rise and fall of aortic pressure.

It has been said that an antagonism exists between the vasomotor mechanisms of the splanchnic and locomotor organs. Thus, while during asphyxia the splanchnic vascular area contracts, the vessels of the skin and muscles dilate. ${ }^{3}$ The dilatation of the latter, however, is in all probability not occasioned by active dilatation, but is due to the over-mastering power of the splanchnic constrictors. By the rise of aortic tension the vessels in the remaining parts of the body are passively lilated, and the blood flow is thus increased through the skin and muscles. That this is so is suggested by the fact that after the circulation has, by ligature of the thoracic aorta and vena cava inferior, been limited to the fore-part of the body, either asphyxia or excitation of the vasomotor centre produces a slight rise of arterial tension, owing to the constriction of the vascular areas of the face and fore-limbs. Previous to the double ligature the same excitation produces splanchnic contraction, a great rise of aortic tension, and dilatation in the face and fore-limbs. Bayliss ${ }^{4}$ has obtained plethysmographic evidence of contraction of the leg during asphyxia.

By driving normal saline through the vessels of a recently dead animal, and measuring the outflow, Stefani ${ }^{5}$ determined that a given head of pressure dilates the vessels of the skin and muscles to a far greater extent than the splanchnic vessels. The cerebral vessels enclosed in the rigid cranium dilate

${ }^{1} \mathrm{By}$ means of the foot the eirculation in the skin is studied.

" Hallion and François-Franck, Arch. de physiol. norm. et path., Paris, 1896, p. 502.

${ }^{3}$ Heidenhain, Arch. f. d. ges. Physiol., Boun, 1870, Bd. iii. S. 78 ; 1872, Bd. v. S. 504 ; Dastre and Morat, "Recherches exp. sur le syst. nerv. vaso-moteur," p. 287 ; Hallion and Francois-Franck, loc. cit.

${ }^{4}$ Journ. Physiol., Cambridge and London, 1893, vol. xiv. p. 307.

${ }^{5}$ Arch. ital. de biol., Turin, 1894, vol. xx. pp. 91-109. 
least. It is noteworthy that the dilated pulmonary vessels recover their original calibre very slowly.

Whether the dilatation of the locomotor organs be active or passive is of no particular importance. The fact remains that the splanchnic area forms the resistance box of the circulation, and when the splanchnic: vessels are contracted, the blood is driven with increased velocity through the locomotor organs, and is determined from the deep to the superficial parts of the body.

\section{The Renal Circulation.}

The circulation in the kidney is studied with great ease by the plethysmographic method. Cohnheim and Roy ${ }^{1}$ constructed a metal oncometer for this purpose. A box similar to that used by Schäfer and Moore in their investigations upon the spleen (see Fig. $99)$, can be moulded without difficulty out of guttapercha. ${ }^{2}$ The kidney having beell exposed by a lumbar incision, is drawn ont of the wound and placed in the box. The pedicle of the kidney passes out through a groove in one side of the chamber. The box is closed by a glass cover, and this is made air-tight by a free application of thick vaseline. The alterations in the volume of the kidney are recorded by means of a tambour. The tracing of renal volume follows exactly the curve of arterial pressure, and exhibits both the cardiac and respiratory oseillations.

By the use of Roy's oncometer, Bradford $^{3}$ localised the renal vasomotor fibres to the anterior roots of from the sixth to the thirteenth thoracic nerves. Most of the renal vasomotor fibres are found in the eleventh, twelfth, and thirteenth nerves.

By reflex excitation, it is more common to obtain contraction than expansion of the kidney, but expansion is frequently witnessed when the central

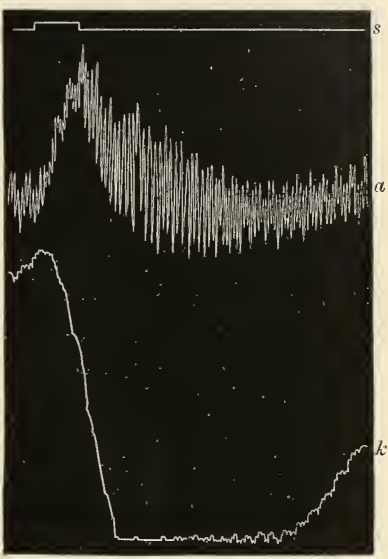

A

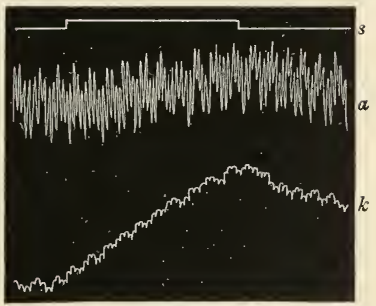

B

Fig, 98.-Aortic pressure and renal volume. Exeitation of thirteenth dorsal root. $A$, fast rate of excitation contracts; $B$, slow rate dilates the kidney ; $a$, arterial pressure ; $h$, kidney volume; $s$, signal.-Bradford. end of the eleventh, twelfth, and thirteenth posterior thoracic roots are stimulated. Vaso-dilator fibres in the anterior roots are evidenced by employing a slow rate of excitation (one per second). Rapid excitation of the splanchnic nerve causes marked contraction of the kidney of its own

1 Virchow's Archiv, Berlin, 1883, Bd. xcii. S. 424.

${ }^{2}$ Halliburton and Mott, "Proc. Physiol. Soc.," Journ. Physiol., Cambridge and London, 1897, vol. xxi. p. xviii.

3 Journ. Physiol., Cambridge and London, 1889, vol. x. p. 358. See also Preobrasehensky, Hermann's Jahresb., 1892, S. 74. 
side, and sometimes contraction of the kidney of the opposite side as well (Cohnheim and Roy).

Landergren and Tigerstedt ${ }^{1}$ have studied the circulation in the kidney by estimating the velocity of blood flow in the renal artery. They employed for this purpose Tigerstedt's stromuhr. After section of the renal nerves, they found that injections of normal saline, of 2 to 3 per cent. nitrate of soda solution and of caffein greatly increased the velocity in the renal artery. Hence the diuretic action of these drugs. If urea be injected, it produces local dilatation of the kidney, while it excites the vasomotor centre and causes general vaso-constriction. ${ }^{2}$

\section{The Circulation in the Generative Organs.}

Eckhard ${ }^{3}$ showed that excitation of the first and second sacral nerves in the dog produces erection of the penis (nervi erigentes), and Goltz ${ }^{4}$ determined the existence of a centre in the lumbar region of the cord, by means of which erection could be reflexly excited. In the rabbit, Gaskell ${ }^{5}$ found the vaso-dilators to the penis in the second and thirr anterior sacral roots. The same fact has been determined for the monkey and the cat. ${ }^{6}$ The vaso-constrictor fibres issue from the third, fourth, and fifth lumbar anterior roots.

The changes in volume of the penis have further been recorded by the plethysmographic method, ${ }^{7}$ and by measurement of the rate of How of blood from the veins of the organ. The outflow from the vena pudenda communis is increased as much as eight times on excitation of the nervi erigentes. ${ }^{8}$

The internal generative organs are supplied with vaso-constrictor nerves from the lumbar anterior roots.

All the vaso-constrictor fibres to the generative organs pass through cells stationed in the inferior mesenteric and sacral ganglia of the sympathetic. The vaso-dilator nerves, as elsewhere, pass through cell stations in scattered ganglia, situated in or near the organs they supply.

\section{The Mesenteric Circulation.}

The vaso-constrictor nerves to the intestines are supplied by the splanchnic nerves. The course of the fibres is interrupted by nervecells placed in the semilunar ganglia. Painting these ganglia with nicotine abolishes the usual effect which follows excitation of the splanchnic nerves. ${ }^{9}$

Hallion and François-Franck have investigated the origin of the intestinal vasomotor nerves by the plethysmographic method. ${ }^{10}$

The animal is placed by these authors belly downwards, the abdominal

${ }^{1}$ Skandin. Arch. f. Physiol., Leipzig, Bd. iv. S. 241.

2 A. Cavazzani, Arch, ital. de biol., Turìn, vol. xviii. p. 158.

3 Beitr. z. Auat. u. Physiol. (Eckhard), Giessen, 1863, Bd. iii. S. 125.

4 Arch. f. d. ges. Physiol., Bonn, 1874, Bd. ix. S. 174.

5 "Proe. Phys. Soc.," Journ. Physiol., Cambridge and London, 1887, vol. viii. p. iv.

6 Sherrington, ibid., 1892, vol. xiii. p. 686 ; Langley and Anderson, ibid., 1895, vol. xix. p. 119.

7 Francois-Franck, Arch. de physiol. norm. ct path., Paris, 1895, tome xxvii. pp. 122 and 138; Piotrowski, Arch. f. d. ges. Physiol., Bonn, Bd. lv. S. 240.

8 Eckhard, loc. cit., S. 153.

y Langley and Dickinson, Proc. Roy. Soc. London, 1889, vol. xlvi. p. 423 ; Langley, Journ. Physiol., Cambridge and London, 1896, vol. xx. p. 223.

10 Arch. de physiol. norm. et path., Paris, 1896, p. 488. 
wall is freely opened, and a loop of intestine ligatured at either end, and, separated from the remaining guts, is allowed to hang down into a glass vessel partly filled with warm saline. At the orifice of the glass vessel the mesentery is surrounded by a broad band of thin indiarubber, which is kept sufficiently tight by means of a clip. By this means the chamber is rendered air-tight, and yet the mesenteric vessels are not over-compressed. From the top of the chamber a tube passes to a sensitive tambour. Bunch has also worked at the innervation of the intestinal vessels, using Schäfer's visceral plethysmograph (Fig. 99). ${ }^{1}$

Hallion and Franeois-Franck ${ }^{2}$ applied plethysmographs to other abdominal organs, and took simultaneous tracings of the volume of the kirlney, intestine, and spleen, together with the arterial blood pressure (see Fig. 97).

They found that excitation of the central end of the sciatic nerve produces reflex vaso-constriction in the small intestine, the spleen, the liver, and the kidney, and vaso-dilatation of the colon, and that excitation of the peripheral end of the vagus in the atropinised animal (i.c. cardio-inhibitory fibres paralysed) produces marked vaso-dilatation in the intestines. ${ }^{3}$

The vaso-constrictor fibres issue from the fifth thoracic nerve downwards. The upper roots supply the duodenum and jejunum, the lower roots the ileum and colon. On exciting the eleventh, twelfth, and thirteenth thoracic, and first and second lumbar roots, marked vasodilatation follows, after a fugitive preliminary constriction. This vasodilatation is accompanied by a fall of arterial pressure. The usual effect on exciting the splanchnic nerves is a great rise of arterial pressure, followed by a fall. This double effect is due to the presence of both vaso-constrictors and dilators in these nerves. The vaso-constrictors are exhausted before the vaso-dilators.

\section{The Hepatic Circulation.}

By simultaneous records of aortic and portal pressures, Bayliss and Starling ${ }^{4}$ determined that the constrictor fibres to the branches of the portal vein issue by the anterior roots from the third to the eleventh thoracic nerves. Cavazzani and Manca ${ }^{5}$ attempted to investigate the innervation of the hepatic blood vessels by artificially circulating warm normal saline solution through the organ, and measuring the output from the vena cava inferior. They ascribe both constrictor and dilator fibres to the splanchnic nerves, and dilator fibres to the vagus.

François-Franck and Hallion have investigated the hepatic circulation by the plethysmographic method. On excitation of the central end of the seiatic nerve, the liver vessels are constricted. The pressure in the portal vein rises slightly, in spite of the concomitant contraction of the mesenteric vessels. On exciting the central end of the vagus, either contraction or dilatation follow. By stimulation of the thoracic sympathetic cord, blood was actively expelled from the liver. If the hepatic nerves were divided, this stimulation of the sympathetic, by causing contraction of the spleen, produced dilatation of the liver. Nicotine, when injected into the hepatic circulation, contracted the

1 "Proc. Phys. Soc.," Journ. Physiol., Cambridge and London, March 1898.

2 Arch. de physiol. norm. et path., Paris, 1896, p. 502.

${ }^{3}$ Bunch was unable to obtain an effect upon the intestiual vessels on stimulating the peripheral vagus after atropine.

4 Journ. Physiol., Cambridge and London, 1894, vol. xvii. p. 120.

${ }^{5}$ Arch. ital. de biol., Turin, 1895 , vol. xxiv. pp. $33,295$.

VOI. II. - I I 
hepatic vessels, and raised the pressure in both the portal vein and the hepatic artery. ${ }^{1}$

The liver is exceedingly vascular, and forms an enormous reservoir for the venous blood at periods when the diastolic filling of the right heart is impeded; for instance, during an intense muscular effort, or a prolonged dive under water. The portal circulation is aided considerably by the action of the respiratory pump. The abdominal wall compresses the blood, while the thorax sucks the blood from the liver. The circulation through the liver is thus greatly accelerated by muscular exercise.

\section{The Splenic Circulation.}

The spleen must be looked upon as a sponge full of blood, the trabeculæ of the spongework and the capsule being formed of muscular tissue. Roy ${ }^{2}$ found the spleen to be normally subject to regular alternations of volume (systole and diastole). These succeed one another in the case of the $\operatorname{dog}$ and cat, without intermission, at regular intervals of about a minute. In consequence of this power of contractility, the circulation through the spleen is somewhat modified, and the spleen may itself act as a pump to the portal system.

The method employed for investigating the splenic circulation is that of the plethysmograph. The first records were obtained by Roy, with the help of a special hinged metal box or oncometer, in which the spleen was enclosed.

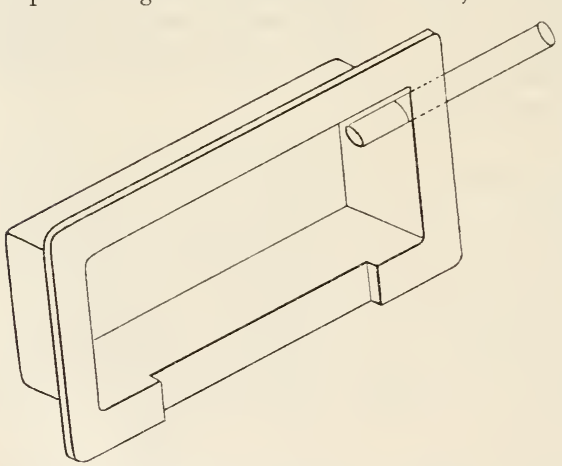

Fig. 99.-Schäfer's visceral plethysmograph.

The oncometer was, in its turn, connected with a piston recorder, termed the oncograph.

A suitable box for the spleen, or for any other separable viseus, ean be made of guttapercha. ${ }^{3}$ It should have flattened edges, on which a flat plate of glass accurately fits.

The viseus is exposed by a free abdominal incision, and placed within the plethysmograph, the blood supply to the organ passing over one side of the box, which is made lower than the others. The glass cover is maintained in position with the help of indiarubber bands, and the box is rendered air-tight by a free application of thick vaseline. Any gap at the side of the vessels is plugged with cotton-wool soaked in vaseline. A tube leads from the plethysmograph, and this is connected by an indiarubber tube with a recording tambour or a piston recorder. The whole apparatus is filled with air.

Fig. 100 shows a typical spleen tracing. Two splenic contraction waves are visible, and upon these are seen the respiratory and cardiac undulations of arterial pressure.

\footnotetext{
1 Arch. de physiol. norm. et path., Paris, 1897, pp. 434, 448.

${ }^{2}$ Roy, Journ. Physiol., Cambridge and London, 1880-2, vol. iii. p. 203.

3 Schäfer and Moore, Journ. Physiol., Cambridge and London, vol. xx. p. 5.
} 
By the use of the above instrument Schäfer and Moore found-

1. That the spleen volume is extremely responsive to all fluctuations in the general blood pressure, and not independent of these, as had been supposed by Roy.

2. That when the nerves going to the organ are intact, other fluctuations in the volume of the spleen may occur, which exactly correspond to Traube-Hering curves of arterial pressure. On the other hand, these fluctuations of volume may not correspond, and the arterial curve may be rising while the splenic is falling. In the first case, the variations in splenic volume are purely passive, and follow the rise and fall of arterial pressure. In the second case, the spleenarterioles, like those of the kidney, share in the general rhythm of contractility, contracting when the arterioles contract, and expanding when the arterioles generally dilate.

3. That the specific rhythmic variations in splenic volume are almost entirely abolished by chloroform.

4. That rhythmic contraction and dilatation occur in the spleen when excised and fed by an artificial circulation of defibrinated blood.

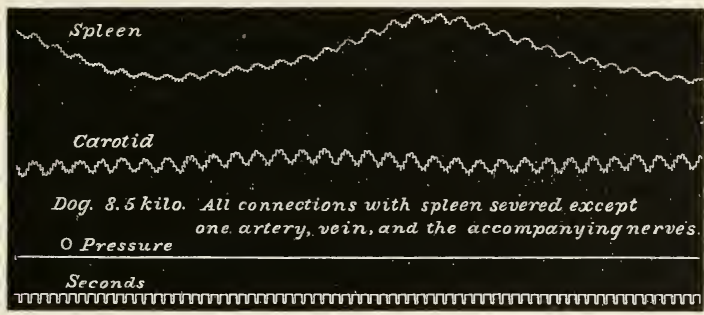

Fig. 100.-Aortic pressure and splenic volume. ${ }^{1}$ - Schafer and Moore.

These movements may therefore be carried out quite independently of the central nervous system.

5. That certain conditions, such as diminution of oxygen in the blood, produce not only a general contraction of the organ, but also a subsequent increase in the extent of its rhythmic movement. Extract of suprarenal gland produces an extreme condition of contraction.

6. That certain drugs, such as curari, or a watery extract of dried brain, greatly increase the contractions of the spleen. Anæmia of the organ acts in the same way, whether produced by compression of the artery, or otherwise.

The innervation of the spleen was first investigated by Bulgak, ${ }^{2}$ and has recently been worked out more fully by Schäfer and Moore. Bulgak observed the spleen directly, and described the nerves in the gastro-splenic omentum as of two kinds, afferent and efferent. Stimulation of the peripheral end of the efferent fibres caused marked contraction of the spleen. The organ became pale in colour, its edges rounded, and its surface crinkled. Excitation of the central end of the afferent nerves produced well-marked reflex contraction of the organ. Bulgak

1 The line of zero blood-pressure should be placed some millimetres lower.

'Bulgak, Virchow's Archiv, 187\%, Bd. lxix. S. 181. 
traced the efferent fibres to the semilunar ganglion and to the greater splanchnic nerve of the left side only. Thence he traced them to the anterior roots of the thoracic nerves on the left side from the third to the tenth. Schäfer and Moore have shown, however, that contraction of the spleen is obtained on stimulating either splanchnic nerve. ${ }^{1}$ They determined the outflow of splenic fibres to arise from the anterior roots of both sides, from the third to the fourteenth post-cervical nerves. The largest effect was obtained by excitation of the sixth, seventh, and eighth roots. Each left root produced a relatively greater contraction than the corresponding right one. They were unable to confirm Bulgak's observations as to the afferent fibres of the organ.

In one case dilatation of the spleen was obtained by weak excitation of the splanchnic nerve, and constriction by strong excitation, and hence they conclude that this nerve contains both constrictor and dilator fibres.

They further found, in opposition to Roy, that excitation of the peripheral end of the thoracic or of the atropinised cervical vagus produces no effect on the spleen. There appeared to be no means of separating the vasomotor effects on the splenic vessels from the contractility of the muscular tissue of the spleen itself. Apparently the whole organ acts in the same manner as the muscular wall of the arterioles, and thus the organ controls its own blood supply.

\section{The Pancreatic Circulation.}

After tying some of the branches of the arteria pancreatico-duodenalis, François-Franck and Hallion ${ }^{2}$ placed the pancreas in a plethysmograph of suitable shape. They obtained evidence of vaso-constricton on excitation of the splanchnic nerves on either side, and traced the origin of the constrictor fibres to the anterior roots from the fifth thoracic to the first lumbar nerves. Expansion of the pancreas seemed to follow excitation of the peripheral end of the vagus. Reflex constriction of the organ was produced by stimulation of any sensory nerve, or of the cerebral cortex; reflex dilatation, by excitation of the central end of the vagus. During the first stage of an asphyxial rise of blood pressure the pancreas is contracted in volume.

\section{The Coronary Circulation.}

In the dog, the left coronary artery supplies the left ventricle and intraventricular septum. From thence arise the ramus descendens and the ramus circumflexus. The ramus descendens passes down the intraventricular groove, and gives off the septal branch; the ramus circumflexus passes to the left along the auriculo-ventricular groove. The right coronary artery supplies the right ventricle and auricle; it is much smaller than either branch of the left coronary artery. The branches of these arteries are physiologically, but not anatomically, terminal. The resistance in the narrow communicating branches is too great for an efficient circulation to be maintained through them. Thus, closure of any one of them is followed by sudden anæmia and infarction of the capillary areas which they supply. That the part of the heart wall supplied by the infarcted artery degenerates, has experimentally been determined in the dog.

${ }^{1}$ Loc. cit.; cf. Schiff, “ Leçons sur la physiol. de la digestion,” Paris, 1867, tome ii. p. 451.

${ }^{2}$ Compt. rend. Soc. de biol., Paris, 1896, p. 561. 
In 1698 , Chirac $^{1}$ tied one of the coronary arteries in the $\mathrm{dog}$, and found that the heart soon ceased to beat. Jenner established a pathological connection between angina pectoris and the reduction of the coronary circulation attending calcification of these arteries.

"The death of John Hunter in a paroxysm of angina, and the discovery that his coronary arteries were indeed calcareous, as Jenner had predicted, was a great stimulus to the clinical and pathological study of these vessels." Erichsen ${ }^{2}$ tied the coronary arteries in the $\mathrm{dog}$, and found the ventricles ceased to beat in a few minutes. The musculature of the ventricles speedily passes into inco-ordinate fibrillar contractions (delirium cordis). Ligaturing one of the large branches only is frequently sufficient to cause arrest, ${ }^{3}$ especially when the heart is lowered in vigour by cooling and exposure, or when the circulation is feeble. Arrest is produced in over 60 per cent. of the cases when the ramus circumflexus is ligatured alone. Ligature of either the right coronary artery or the ramus descendens far less frequently produces a fatal result. As the heart begins to fail after the ligation has been effected, the intraventricular and the aortic pressures steadily fall, while the diastolic pressure in the left ventricle and auricle rises, the ventricular beat becomes irregular, and the systolic output greatly lessened.

The heart after arrest, and even after it has passed into delirium cordis, can often be revived by re-establishing the coronary circulation. The failure of the heart is due to the animia produced. ${ }^{4}$ This is so, because (1) the severity of effect depends on the size of the artery; (2) the arrest can be produced by the injection of lycopodium spores ${ }^{5}$ into the coronary circulation, and without any mechanical interference with the heart; (3) the arteries may be exposed and prepared for ligation, and no arrest follows until the ligatures are drawn tight. ${ }^{6}$

Closure of all the coronary veins produces fibrillar contraction of the heart in the rabbit. This does not occur in the dog, owing to the fact that the blood can still find an exit into the cavities of the heart through the venæ Thebesii.

On establishing an artificial circulation through the left coronary artery of the isolated heart of a cat, Porter ${ }^{7}$ found that strong and regular contractions were maintained by a circulation of $4 \mathrm{~cm}$. of blood per minute. Probably about 10 to $20 \mathrm{cms}$. of blood per minute must pass through the coronary circulation to maintain the heart of a moderate-sized dog in efficiency. While the force of the ventricular contraction is immediately increased by a greater flow of blood through the coronary arteries, the frequency of the heart is largely independent of the blood supply. ${ }^{8}$

1 "De motu Cordis," Monspelii, 1698, 1. 121.

2 Lond. Hosp. Gaz., 1842, vol. ii. 561-564.

${ }^{3}$ Cohnheim and Rechberg, Virchow's Archiv, 1881, Bd. 1xxxv. S. 503 ; MacWilliam, Journ. Physiol., Cambridge and London, 1887, vol. viii. p. 296; Bethelheim, Ztschr. f. klin. Med., Berlin, 1892, Bd. xx. S. 436 ; Porter, Arch. f. d. ges. Physiol., Bomn, 1894, Bd. lv. S. 336; Journ. Physiol., Cambridge and London, 1894, vol. xv. p. 121.

4 Fenoglio and Drogoul, Arch. ital. de biol., Turin, vol. ix. p. 49.

5 Sée, Bochefontaine, and Roussy, Compt. rend. Acad. d. sc., Paris, 1881, p. 86.

${ }^{6}$ Porter, Centralbl. $f$. Physiol., Leipzig u. Wien, 1896, S. 57.

7 Porter, loc. cit.; cf. Bohr and Henriques, Bull. Acad. d. sc. de Danemark, 1893 ; Magrath and Kennedy, Journ. Exper. Med., New York, 1897, 1. 13.

${ }^{8}$ Magrath and Kennedy, loc. cit. 
The circulation in the heart is peculiar, because, during each systole, the tension of the ventricular muscle rises above the aortic tension. The blood in the coronary arterioles and arteries is thus driven on into the veins, and back towards the aorta, and the cardiac vessels empty during systole and fill again during diastole. Porter's observations on the veins of the heart, made with minimum manometers, give no support to the view that the heart acts on the coronary circulation to any extent as a suction pump, although efficiently as a force pump. ${ }^{1} \quad$ A shortening of the diastolic period lessens the nutritive supply to the heart. Similarly, diastolic distension of the left heart by "back pressure" lessens the coronary flow. These are facts of great pathological importance.

Newell Martin ${ }^{2}$ observed that the coronary arteries dilated on excitation of the peripheral end of the vagus nerve, and during asphyxia. In the latter case, the dilatation occurred before the rise of aortic pressure. These effects Martin ascribed to the influence of coronary vasomotor nerves. Poy and Adami ${ }^{3}$ also obtained some evidence of the existence of vaso-dilators passing to the heart by the vagus and vaso-constrictors by the sympathetic.

\section{VASOMOTOR REFLEXES.}

The arterial pressure is affected reflexly by stimulation of any sensory nerve in the body. Sudden pallor of the face resulting from severe pain, blushing from emotional causes, erection of the cavernous tissue of the genitals from sexual excitement, are examples of common observation. Experimental stimulation of any sensory nerve in the body can produce vaso-constriction; it may, in certain conditions, produce vaso-dilatation.

It seems probable that vasomotor reflexes can be excited from excitation of sensory nerves in the walls of the vessels themselves. ${ }^{4}$

In regard to local reflex vasomotor changes, the following observations of Marey are of considerable interest. If the point of a pencil be drawn across the forearm, a white line due to the expression of the blood immediately results. This line rapidly disappears, but after an interval of half a minute reappears once more, and then lasts for some minutes. The white line, on its second appearance, irradiates into neighbouring parts. If the pencil point be drawn down the arm with violence, the primary white line is replaced by a red line. Around this there appears secondarily a white area of some extent. The irradiation of the white area into neighbouring parts, and its slow appearance, suggest that the phenomenon is of reflex origin. ${ }^{5}$

Reflex vasomotor effects generally appear in the vascular area from which the sensory excitation arises. Thus, on stimulating the tongue, the submaxillary gland flushes. ${ }^{6}$ On exciting the posterior auricular nerve in the rabbit, the vessels of the ear either first contract and then

${ }^{1}$ Porter, Rep. Brit. Ass. Adv. Sc., London, 1897.

2 Trans. Med.-Chir. Fac. of Maryland, 1891.

3 Phil. Trans., London, 1892, vol. elxxxii. B.

4 Latschenberger and Deahna, Arch.f. d. ges. Physiol., Bonn, 1876, Bd. xii. S. 178; Zuntz, ibid., 1878, Bd. xvii. S. 404 ; Heger, Beitr. z. Physiol. C. Ludwig z. s. Geburtst. gew., Leipzig, 1887, S. 193.

5 Ann. d. sc. nat., Paris, 1858, Zool., Sér. 4, tome ix. p. 68.

6 Bernard, Journ. de la physiol. de l'homme, Paris, 1858, tome i. p. 656. 
dilate, or primarily dilate. ${ }^{1}$ On stimulation of the central ends of the posterior roots of the eleventh to the thirteenth thoracic nerves, the kidney expands. ${ }^{2}$ Excitation of the entrance to the glottis flushes the inylo-hyoid muscle of the frog. ${ }^{3}$

The sensory excitation of one side of the body frequently evokes vasomotor changes on both sides. Thus a drop of mustard, introduced into the lymph sac of the frog's leg, causes congestion of the leg on the same side, and contraction of the vessels on the opposite side. ${ }^{4}$ Compression of the median artery of the rabbit's ear with the finger-nail causes dilatation of that ear and contraction of the opposite ear. Stimulation of the central end of the posterior auricular or sciatic nerve on one side, may cause vasomotor changes in both ears or both hind-limbs. Excitation of the mucous membrane of the nose may cause vascular dilatation all over the face, but more on the same side as the stimulus. ${ }^{5}$

It is a simple experiment to verify the fact that the vessels of the one arm contract, if the other be placed in hot water. Similarly, by electrical excitation of the arm, vasomotor changes may be evoked in the opposite arm. ${ }^{6}$ The latent time between the excitation and the reflex results usually occupies several seconds. The place and the kind of stimulus influence the reflexes obtained. For example, greater changes of general arterial pressure are evoked by excitation of the posterior roots of the thoracic nerves than by stimulation of the peripheral nerves. ${ }^{7} \quad$ The afferent fibres from the viscera produce the most marked vasomotor reflexes. ${ }^{8}$

In lightly curarised rabbits, a rise of arterial pressure can be evoked by blowing on or stroking the skin, while more violent stimulation by chemical irritants or heat scarcely produces any effect.

Although the usual result of stimulating a sensory nerve is a reflex rise of arterial pressure, a fall may occur. There is one afferent nerve, and one only, which constantly produces this result, and that is the depressor nerve (see p. 59). Efforts have been directed towards finding depressor fibres in the common nerve-trunks. General reflex dilator effects are often obtained on excitation of a sensory nerve, when the vasomotor tone is weakened after prolonged experiment, ${ }^{9}$ or during administration of chloral and chloroform.

Hunt ${ }^{10}$ has succeeded in demonstrating afferent depressor fibres in the central end of the sciatic nerve by the following methods:-

1. On regeneration of the nerve after section, the depressor fibres are the first to recover their function. Thus, a rise of arterial tension results from stimulation of the central end of a normal sciatic, a fall of tension from excitation of the regenerating sciatic. 2. Excitation with a feeble strength of current is more likely to produce a fall, while a strong current produces a rise of pressure. 3. After cooling or long exposure of the sciatic nerve, depressor effects may be evoked.

Hunt classifies the afferent nerves in reference to their reflex vasomotor

${ }^{1}$ Lovén, Ber. d. k. Sächs. Gescllsch. d. Wissensch., math.-phys. Cl., Leipzig, 1866, S. 92.

2 Bradford, Journ. Physiol., Cambridge and London, 1889, vol. x. p. 396.

${ }^{3}$ Gaskell, Journ. Anat. and Physiol., London, 1877, vol. xi. 1. 742.

${ }^{+}$Ranvier, Compt. rend. Acad. d. sc., Paris, tome cxiv. p. 629.

${ }^{5}$ François-Franck, Arch. de physiol. norm. et path., Paris, 1889, p. 550.

6 Maragliano and Lissano, Arch. ital. de biol., Turin, 1889, vol. xi. p. 246.

7 Bradford, Journ. Physiol., Cambridge and London, 1889, vol. x. p. 400.

${ }_{9}$ Heidenhain and Gritzner, Arch.f. d. ges. Physiol., Bonm, 1878, Bd. xvi. S. 54.

${ }^{9}$ Cyon, ibid., 1874, Bd. viii. S. 334.

${ }_{10}$ Journ. Physiol., Cambridge and London, 1895, vol. xviii. p. 381. 
powers as follows:-1. Nerves which always produce a fall of arterial tension (e.g. the depressor). 2. Nerves which sometimes produce a fall, sometimes a rise (e.g. the vagus). 3. Nerves which usually occasion a fall (e.g. the glossopharyngeal). 4. Nerves from which primary depressor effects can only be obtained by special means (e.g. the sciatic, anterior crural, uhnar, median, and intercostal).

He states that the power of the depressor nerve, which acts nainly on the splanchnic area, is not abolished by curari. On the other hand, the depressor power of other afferent nerves, which act mainly on the vascular areas of the skin and muscles, is abolished by curari. ${ }^{1}$

${ }^{1}$ It has been stater on p. 146 that Gulland has failed to demonstrate vasomotor nerves in the vessels of the pia mater. Since then, however, various observers, including Gulland himself, have been able to show the usual perivascular nerve-plexuses upon the cerebral vessels, Brit. Med. Journ., London, 1898, vol. ii. p. 781. The existence of cerebral vasomotor nerves cannot therefore be denied, and we must assume that the experimental evidence remains as yet inconclusive, owing to the extreme difficulty of separating passive changes, brought about by the slightest alterations in the general circulation, from active vasomotor effects. - Note added in September 1898. 


\title{
THE CONTRACTION OF CARDIAC MUSCLE.
}

\author{
By W. H. Gaskell.
}

Contents:-The Meaning of the Heart-Beat, p. 169-The Seqnence of the Contractions of the different parts of the Heart, p. 180-The Causation of the Beat of the Heart and of the Sequence of the Contractions of the Separate Cavities is the same in Warm-blooded as in Cold-blooded Animals, p. 186The Peculiarities of the Cardiac Muscle by which the Rhythm and sequence are to be explained, p. 188-On Fibrillar Contraction, p. 192-On the Tonicity of the Cardiac Muscle, p. 194-The Meaning of the Ganglion Cells in the IIeart, and their Relation to the Cardiac Nerves, p. 197-The Nature of the Action of the Cardiac Nerves - (1) The Inhibitory Nerves, 1. 203; (2) The Augmentor Nerves, p. 216; (3) The After-action of the Inhibitory and Augmentor Nerves, p. 219-The Action of Certain Poisons, 221-On the Nutrition of the Heart, p. 224.

\section{The Meaning of tile Heart-Beat.}

Is the early days of physiology, e.g. in the time of Haller, the beat of the heart was supposed to be caused by the direct action of the blood as it flowed through the cavities of the heart. It was soon, however, apparent that the heart of such an animal as the frog could beat out of the body when there was no blood flowing through it; the heart-beat, consequently, was elearly not due to the movement of the blood, but to some peculiarity of the heart-tissue itself; it was therefore said that the tissue of the heart possessed in itself automatic rhythmical power. Then came the discovery of Remak, ${ }^{1}$ that groups of ganglion cells were present in the sinus venosus of the frog's heart, with the subsequent discovery of Bidder ${ }^{2}$ of the two large masses of ganglion cells at the junction of auricles and ventricle, and of Ludwig ${ }^{3}$ of the ganglion cells in the interauricular septum. At this period the automatic rhythm of respiration was ascribed to the action of groups of nerve cells in the respiratory centre, so that it was natural, indeed inevitable, that a similar mechanism should be assumed to explain the automatic cardiac rhythm. Hence arose the conception that Remak's ganglia in the sinus venosus acted the part of an automatic rhythmical centre, sending out impulses to the cardiac muscular tissue at regular intervals, and so caused the beat of the heart.

This view received apparently its confirmation and its proof by the well-known experiments of Stamius, ${ }^{4}$ which, according to Riosenthal, ${ }^{5}$ were to be explained on the same principles as he had already laid down for the respiratory centre.

${ }^{1}$ Arch.f. Anat., Physiol. u, wissensch. Med., 1848, S. 189.

2 Bidder and Rosenberger, ibid., 1852, S. 172.

3 Ibid., 1848, S. 139.

4 Ibid., 1852 , S. 85.

5 "Bemerkungen neber die Thätigkeit der automat. Nerveneentra insbesondere ueber die Athembewegungen," Erlangen, 1875. 
In both cases Rosenthal ascribed the rhythmical activity to the automatic action of groups of easily excitable nerve cells. In the case of respiration, these cells were those governing the movements of the ordinary muscles of inspiration ; in the case of the heart, the cells in the sinus venosus. In both cases, additional, subsidiary, less excitable centres were assumed to exist, which required a stronger stimulus to make their presence manifest; such centres were, in the case of respiration, the centres connected with the extraordinary muscles of respiration, which were thrown into activity by increasing venosity of the blood ; and in the case of the heart, Bidder's ganglia in the auriculo-ventricular groove, which, though normally inactive, were eapable of being aroused by a sufficiently strong stimulus. The first Stannius ligature, or eut, at the junction of sinus and auricles, caused standstill, according to this view, because it removed the automatic motor centre or Remak's ganglia; the second Stamiuss ligature, in the auriculo-ventricular groove, caused the ventricle to beat again, because it supplied the extra stimulus necessary to set in action the less excitable motor cells of Bidder's ganglia.

Such, then, was the conception of the heart-beat. A motor centre was supposed to exist in the sinus, which was usually active, thus resembling the ordinary centre for respiration, and which sent out discrete impulses to the muscular tissue of the auricles and ventricle. This centre was supposed to be supplemented by a less excitable motor centre between auricle and ventricle, which was normally inactive, but available, like the extraordinary centres of respiration, for employment upon an emergency.

This view soon began to receive modification, when, in consequence of the experiments of Eckhard ${ }^{1}$ and Foster and Dew Smith, ${ }^{2}$ it was proved that the ganglion-free apex of the frog's heart was thrown into rhythmical activity by the passage of a constant current through it, and also that this same apex would beat rhythmically when supplied with a nutrient fluid under pressure, as proved by the experiments of Bowditch $^{3}$ and Merunowicz ${ }^{4}$ in Ludwig's laboratory.

These experiments were proof positive that, under certain conditions, the muscular tissue of the heart would beat rhythmically without the presence of motor nerve centres. It was however argued, that such rhythmical contractions had nothing to do with the normal beating of the heart, but were due to the foreign stimulus of the electrical current on the one hand, and of the chemical ingredients of the nutritive fluid on the other; and Bernstein ${ }^{5}$ showed, by clamping the ventricle between the base and apex, that if the apex were cut off from the base, then, although kept still supplied with blood from the contractions of the base, it yet remained motionless, and never contracted of itself. In other words, the stimulus of its own blood was not sufficient to arouse rhythmical contractions in the apex of the frog's ventricle. Bernstein, however, did not realise the importance of another factor in the causation of rhythm in the isolated apex, namely, the pressure in its cavity; and at Foster's suggestion I was able to show, by clamping the aorta, and so increasing the pressure in the cavity of the ventricle, that the apex of the frog's heart, when isolated after Bernstein's method,

\footnotetext{
'Beitr. z. Anat. u. Physiol. (Eckhard), Giessen, 1858, Bd. i.

2 Journ. Anat. and Physiol., London, 1876, vol, x.

${ }^{3}$ Ber. d. k. Sachs. Gesellsch. d. Wissensch., Leipzig, 1871, S. 682.

${ }^{4}$ Ibid., 1875, S. 254.

${ }^{5}$ Centralbl. f. d. med. Wissensch., Berlin, 1876, s. 385.
} 
would beat rhythmically, provided that there was sufficient tension of its walls. ${ }^{1}$ The importance of the tension of the walls in the production of rhythmical contractions of the apex of the heart was also, about the same time, insisted on by J. M. Ludwig and Luchsinger, ${ }^{2}$ who showed that the rate of rhythm was closely associated with the pressure within the cavity.

From these experiments the conclusion followed, that the muscular tissue of the heart was of such a character, that a sufficiently powerful constant stimulus, whether electrical, chemical, or mechanical, did not produce a constant tonic contraction, but was manifested as a rhythmical series of contractions.

The net result of these facts upon the minds of the upholders of the motor ganglia theory was to cause them to put forward the proposition, that the impulses sent out by the centre in the sinus were not discontinuous but continuous, and that the cardiac muscle responded to this continuous stimulation by a rhythmical contraction.

Pari passu with the question of the meaning of the cardiac rhythm goes the question of the sequence of the contractions of the different parts of the heart, and here again we see the ganglion cells conspicuously pressed into service. The original view held by Haller, and others of his time, was that a peristaltic wave of contraction passed over the heart from the great veins to the aorta. This conception of a peristaltic muscular wave was given up largely, because it was noticed by Schiff and others that the ventricle of the dying heart frequently beats at a different rate from that of the auricle; it was therefore asserted that such a phenomenon could not be due to a peristaltic wave, but showed that a co-ordinating mechanism of a presumably nervous nature had got out of gear, and therefore the ventricle no longer responded in due sequence to the auricle.

This co-ordinating mechanism was held to be situated in the cells of Bidder's ganglia, in consequence mainly of the experiments of Eckhard ${ }^{3}$ and Marchand, ${ }^{4}$ which appeared to indicate that the sequence of the contraction of ventricle upon that of auricle depended upon the integrity of these ganglia; also, that the time which elapsed before the ventricle contracted upon stimulation of a point in the auricle was too long for the passage of a wave of muscular contraction; this greater length of time must therefore be explained by a delay caused by the passage of the impulse through a special nerve centrein other words, through Bidder's ganglia.

So far, then, the conception formed was:--Motor ganglia in the sinus send out a continuous impulse along nerve fibres to auricle and ventricle ; such impulse produces a discontinuous rhythnical result, owing to the nature of the muscular tissue; the sequence of the contraction of the ventricle upon that of the auricle is due to a delay in the impulse, owing to its passage through the ganglia at the auriculo-rentricular junction.

Such was the condition of things when I commenced my investigations in 1881, and it seemed to me that the question whether continuous

1 "On the Tonicity of the Heart and Blood Vessels," Jou 'n. Physiol., Cambridge and London, 1880-82, vol. iii. p. 51.

2 Med.-chir. Centralbl., Wien, 1879, S. 404 ; Arch. f. d. ges. Physiol., Bonn, 1880, Bd. xxv. S. 211.

${ }^{3}$ Bcitr. z. Anat. u. Physiol. (Eckhard), Giessen, Bd. vii. S. 191.

+ Arch. f. d. gcs. Physiol., Bonn, 1878, Bd. xvii. S. 137. 
or discontinuous impulses passed from the sinus to auricle and ventricle, could be easily answered by increasing the excitability of ventricle or amricle respectively. A more excitable ventricle must respond to a constant stimulus with contractions at a more rapid rate than a less excitable one; while, on the other hand, the rate would remain unaltered if the impulses from the sinus were discontinuous, and the excitability of the ventricle only were altered.

For this purpose I made use of a method of registering the heart contractions which up to that time had not been used, and has sinec proved of great value; this method is the suspension method. The contraction of the auricle and ventricle are registered separately by means of two levers, which are attached by means of threads to the apex of the ventricle and auricle respectively ; the one lever is pulled downwards against an elastic spring, and the other upwards. The heart is held fast by means of a serew-clamp in the

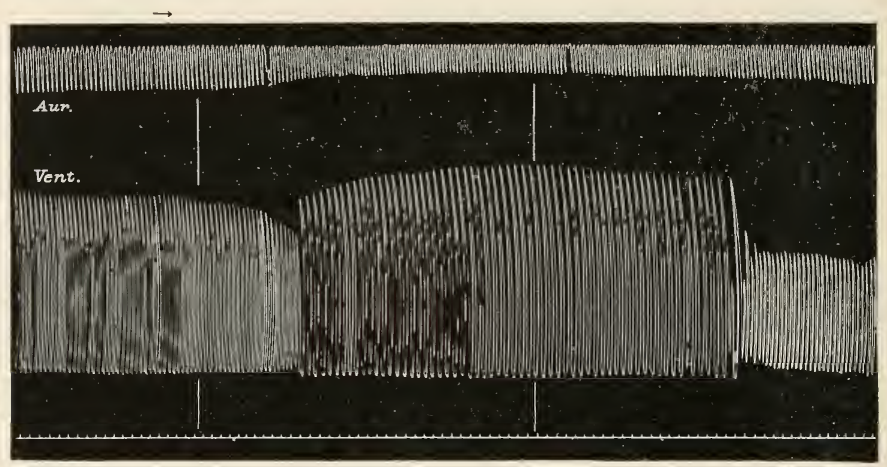

$a$

Fig. 101.-Frog's heart. Suspension method. Clamp in auriculo-ventricular groove. Auricles and sinus above clamp, ventricle below. Coil round auricles and sinus; heated between the vertical lines. The ventricular contractions at first keep pace with the quickening auricular, then are unable to do so, and at $a$ the ventricle contracts to every second of the auricular contractions.

aurieulo-ventricular groove. This clamp is provided with a fine serew, which can easily be adjusted so as to hold the heart firmly, without injuring the tissue. In this way the contractions of auricle and ventricle can be registered separately.

The excitability of a tissue can be increased by raising its temperature up to a certain limit. In my experiments I made use of a coil of thin wire, which was heated by the passage of a galvanic eurrent through it. This eoil was placed so as to surround either the auricles and sinus or the ventricle, so that in this way the auricular or ventricular side of the heart could be heater separately. Curves so obtained are given in Figs. 101 and 102, taken from my paper in the Philosophical Transactions, ${ }^{1}$ and it is seen that, whereas heating the auricles and sinus eauses a great inerease in the rapility of the beats both of auricles and ventricle, no alteration of rate of rhythm in the ventricle is produced by heating the ventricle alone, but the force of its contraction is altered.

1 "On the Rhythm of the Heart of the Frog and the Nature of the Action of the Vagus Nerve," Phil. Trans., London, 1882, p. 993, Figs. 1 and 2, Pl. lxvii. 
From this experiment I concluded, on the assumption that the motor ganglia in the sinus are the cause of the heart-beat, that they send out discrete and not continuous stimuli to the muscular tissue of the various cardiac chambers. The next question that arose in my mind was, Is it really clear that the sinus ganglia do originate nerve impulses to the cardiac muscle? or is it not that, whereas all parts of the

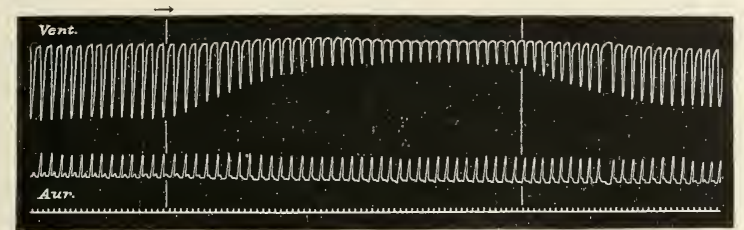

Fig. 102. - Heart of toad. Suspension method. Clamp in auriculo-ventricular groove. Ventricle above clamp, auricle and sinus below. Coil round ventricle heated between the vertical lines. The ventricular contractions diminish in force during the heating of the ventricle, but no alteration whatever oceurs in the rate of beat.

muscular tissue of the heart possess the power of rhythmical contraction to a greater or less degree, some parts possess that power to such an extent that their contractions are automatic?

To answer this question it appeared to me very necessary to investigate other hearts than that of the frog, for in the frog the nerves and ganglion cells between the sinus and ventricle are situated in the septum of the auricles, and are therefore not easily exposed for the purposes of experiment. On the other hand, in the case of the tortoise, the septum between the auricles is free from nerve trunks and nerve cells, and all the nerves and cells lie superficially in the flat base which connects the two auricles, and continues on from the sinus to the ventricle. ${ }^{1}$

This flattened part or junction-wall between the two auricles is represented in Fig. 103, $J$. On each side of it is seen the reticulated tissue of the auricle

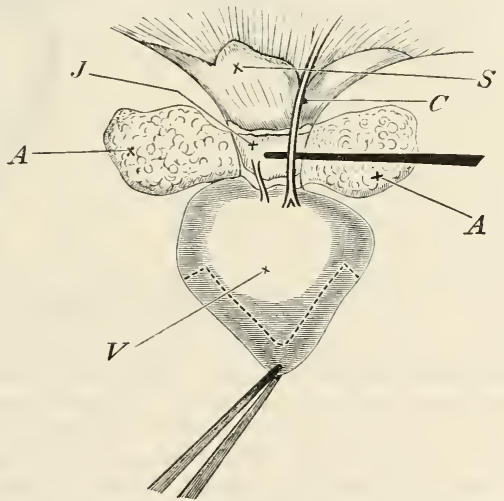

FIG. 103.-Heart of tortoise. Deseription of letters in text. proper $(A)$, and the manner in which the junction-wall connects the sinus $(S)$ and ventricle $(V)$ is also shown. This basal auricular wall exists also in fishes (described by MacWilliam ${ }^{2}$ as sinus-extension), in crocodiles, and in Chelonia generally, and in all cases carries the nerve trunks and coronary veins wherever

\footnotetext{
${ }^{1}$ Gaskell, "On the Innervation of the Heart, with especial reference to the Heart of the Tortoise," Journ. Physiol., Cambridge and London, 1883, vol. iv. 1. 43.

2 "On the Structure and Rhythm of the Heart of Fishes, with espeeial reference to the Heart of the Eel," Journ. Physiol., Cambridge and London, 1885, vol. vi. p. 192.
} 
a coronary system is present. A well-defined coronary system exists for the ventricle of the tortoise, and the coronary veins empty into the sinus venosus. In the land-tortoise (Testudo greca) it very frequently happens that one of these coronary veins runs free from ventricle to sinus, so that, as is shown in Fig. 103, a seeker can be slipped under it without injury to any part of the heart; it also usually happens that one of the nerve trunks which pass from the sinus to the auriculo-ventricular junction, accompanies this free coronary vein, so that in this animal we have a free intracardiac nerve between sinus and ventricle, whose functions can be examined without injury to the rest of the heart. This nerve, which experiment shows to be connected with the vagus, I have called the coronary nerve. In Fig. 104 the appearance of the heart when suspended is shown, and the position of the coronary nerve, $C$. In the tortoise, as in all other animals, the cardiac ganglion cells are found in connection with the main trunks of the intracardiac vagus nerves. As described in my paper (p. 62), the largest accumulations of them are found at the bifureation of the larger vagus nerve trunks in the sinus, in the junction-wall or sinus-

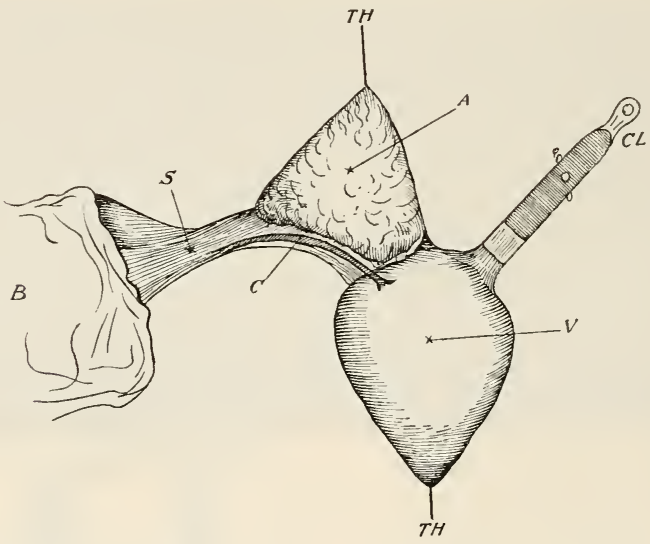

FIG. 104.-Heart of tortoise as suspended. $\quad B$, body of tortoise ; $T H$, thread to lever ; $C L$, clamp holding aorta.

extension between the two auricles, and in the termination of this wall in the auriculo-ventricular ring. In all these places the ganglion cells are also found on the smaller branches of the nerves which ramify over the sinus, and form a rich plexus in the junction-wall between the two auricles, and at the junction between auricles and ventricle. The nerves, with their accompanying ganglion cells, are distributed around the whole junction of the sinus and the auricles, and from this ring, as well as from both sides of the junctionwall between the two auricles, nerve fibres with ganglia are plainly seen passing into the auricular tissue; as the nerves pass further into the tissue the ganglia become more and more scarce, and soon disappear. If, then, we call the junction-wall between the two auricles the flattened part, and the rest of the auricles the bulged part of the aurieles, we find that the larger nerve trunks and ganglia of the auricles are found only in those parts of the bulged portion which are in the immediate vicinity of this flattened part. Again, at the junction of the auricles and ventricle the nerve trunks passing from the junction-wall of the two auricles form a rich plexus, containing large 
groups of ganglion cells along the line where this junction-wall passes into the auriculo-ventricular ring-along, that is, the side of the ring furthest removed from the aorta, which may be called the basal part of the ring. Thence a plexus of nerve fibres, also containing ganglia, though much more sparingly than the basal portion, passes round the auriculo-ventricular ring. From this ganglionic nervous ring, fibres ramify over both the ventricle and the auricles, in each case accompanied by ganglion cells for a short distance.

We see, then, that the ganglion cells in the sinus and sino-auricular junction correspond to Remak's ganglia in the frog. The cells in the junction-wall between the two auricles correspond to those on the inter-auricular nerves in the septum between the auricles of the frog, and the cells of the auriculoventricular ring correspond to Bidder's ganglia in the frog.

In the tortoise, as in the frog, the Stannius experiment succeeds; we see here, however, very plainly, what had been already observed by Stannius ${ }^{1}$ in the frog, that the standstill after removal of the sinus is not permanent; the auricle and ventricle commence after a time to beat again, at first slowly, then gradually quicker and quicker, until at last a good steady rate of rhythm is observed. This rhythm of development is characteristic. If the cut is anywhere between sinus and ventricle, the same thing may be observed; the difference being that the primary standstill is longer the nearer to the ventricle the cut is made; and the rate of rhythm finally obtained is liable to be less the nearer to the ventricle. In all cases the first effect of the section is to cause one or more contractions followed by standstill.

When the cut is made close against the ventricle in the auriculoventricular groove, i.e. corresponds to the second Stamius ligature, the ventricle beats rapidly for a tine, gradually slowing more and more, until at last it stands still; then after a time it begins to beat again, at first very slowly, steadily gaining in rate until it has reached a steady uniformity.

We see, in fact, that the ligature or section in the auriculo-ventricular groove produces two different rhythmical phenomena in the ventricle; on the one hand, a series of rhythmical contractions take place immediately upon the section or ligature, which are characterised by the peculiarity that the rate of the contractions is quickest at the commencement, and then gradually becomes slower and slower, until a standstill occurs; this may be called a rhythm of excitation. On the other hand, in contradistinction to this rhythm of excitation, a subsequent more permanent series of rhythmical contractions, which may be called the rhythm of development, occurs under favourable conditions in the ventricle, after the rhythm of excitation is over.

This rhythm of development, like the similar rhythm in the auricle after the first Stannius ligature, or section, is characterised by its slow rate at the commencement, and its gradual development to a steady rate, the rapidity of which depends upon the rhythmical modulus of the tissue and the conditions of nutrition, temperature, etc. If, then, we can remove this rhythm of excitation, the effect of the ligature at the auriculo-ventricular groove would be no different from that of the ligature at the junction of sinus and auricles, or across any part of the auricles. This can be done in the frog by means of a clamp with a fine screw adjustment, for it is then possible, by very slowly tightening the clamp in the auriculo-ventricular groove, to bring the ventricle to a

${ }^{1}$ Stannius, Arch.f. Anat., Physiol. u. wissensch. Med., 1852, S. 81. 
standstill without provoking any more rhythm of excitation than an occasional beat or two upon each separate turn of the screw, just as may be seen when the ligature or the clamp is applied to any part between the sinus and the ventricle.

I conclude, then, that the phenomena following upon section are of the same kind wherever that section is situated; the part in connection with the sinus continues its regular beat; the part separated from it remains still for a variable time, and then, according to its inherent rhythmical power, develops a rhythmical beat of its own, the rate of that rhythm when fully developed, and the length of time that the standstill lasts, being correlated with the rhythmicity of the tissue. Further, the same experiment can be applied to every part of the heart, for I have seen the same kind of standstill, followed by a rhythm of development, take place when a strip of muscle, taken from the apex of the ventricle or the auricle, is suspended in the same way as the whole ventricle or auricle. ${ }^{1}$ It is true that the development of this spontaneous rhythm is assisted by the occasional stimulation of the strip by means of a weak tetanising current, and the regular testing of the condition of the strip by means of single induction shocks; but it is not true that such rhythm is dependent upon such artificial stimuli, and owes its existence to polarisation phenomena in the strip, as suggested by Langendorff, ${ }^{2}$ for, as pointed out in my paper, ${ }^{3}$ I have seen control strips develop a spontaneous rhythm, when they have simply been suspended, and have not received a single electrical stimulus of any kind: the only difference between the two strips being, that the one which had been taught to beat commenced its spontaneous contractions quicker than the control.

In addition, as mentioned in the same paper, ${ }^{4}$ the strip from the ventricle, when once spontaneously beating, will continue to beat for as long as thirty hours; since then I have once observed a strip from the apex of the ventricle, suspended in a moist chamber, beat for as long a time as a hundred hours. Clearly, then, this rhythm cannot be explained by any artificial electrical action. It is, I think, highly probable that the tension of suspension plays a distinct part in its causation, just as the tension of distension in the case of the apex of the frog's ventricle; though, that it is not entirely dependent on the stimulus of tension, is seen by the fact that the strip or even parts of the strip will continue beating rhythmically in a watch glass, after the spontaneous rhythmical beat has once become well established. The main condition necessary for the establishment of this rhythm is, it appears to me, some unknown factor in the nutrition of the tortoise, for since the publication of that paper I have attempted to demonstrate to my class the spontaneous beat of the strip whenever I have managed to obtain a tortoise, and have been much amnoyed to find that again and again I have failed to obtain any sign of rhythmical activity in the strip, and, even more than that, I have sometimes failed to obtain any spontaneous activity in the isolater entire ventricle. During the years 1882 and 1883 , when my tortoise experiments were in constant progress, I had a large supply of animals, carefully tended, fat and healthy, in the very best condition, and I never failed once in obtaining the results described in my paper.

\footnotetext{
${ }^{1}$ Op. cit., pp. 51-56.

2 "Rhythmik und Automatik des Frosch-herzens," Leipzig, S. 57.

3 Op. cit., 1. 54.

${ }^{4}$ Ibid., p. 53.
} 
The evidence, then, points absolutely to the conclusion that the muscular tissue of the heart possesses the power of rhythmicity, i.e. answers to a sufficient constant stimulus with a series of contractions, and that certain parts are more rhythmical than others, so that in them the rhythmical contraction takes place automatically, without the necessity of any continuous external stimulus.

Upon what does this difference of rhythmicity depend? It is acknowledged on all sides that such difference is real, and it has been largely supposed that the difference is due to the presence or absence of ganglion cells, because ganglion cells are found in the sinus, in the auriculoventricular junction, and between sinus and ventricle-in those parts, in fact, where the spontaneous rhythm is most easily observed ; also, because a rhythmical action is supposed to necessitate a central nervous system, and finally because there is apparently nothing else to which to attribute this difference of rhythmical power.

But is there really nothing else peculiar to those parts where rhythm is easiest?

If we consider its mode of development, we find that in all vertebrates the heart arises from two longitudinal veins, or venous sinuses, which coalesce to form the sub-intestinal vein and heart; the simple tube so formed, with its circular muscular coat, becomes twisted on itself, and parts of it develop bulgings, the commencement of the auricles and ventricles, in consequence of which the simple circular arrangement of muscle fibres becomes the complex arrangement which is ultimately found. With this special growth of the parts of the tube by which the heart cavities are formed, is correlated a more rapid contraction of the specialised parts of the muscular walls, so as to ensure a more rapid emptying of the separate cavities, and thereby make the heart more efficient as a force-pump. The development of this nearer approach to striated muscle is made at the expense of the original rhythmical power, so that finally the muscular tissue of the heart becomes differentiated into muscle of varying rhythmical power, according to the amount of deviation from the original embryonic rhythmical muscle.

Those parts which remained least altered, both in the circular arrangenent of their fibres and in their physiological status, are naturally the large veins, sinus, and junction of sinus and auricles; then the circularly arranged fibres of the auriculo-ventricular groove, i.e. the compressed remains of that portion of the original tube which, known as the canalis auricularis, separated the auricular from the ventricular bulgings ; and, lastly, the conus arteriosus, which represents the final part of the tube beyond the ventricular bulging. In addition, there is in the tortoise a basal portion of the tube leading from sinus to ventricle, from which the bulged part of the auricles springs; this may be looked upon as a portion of the original tube, which has been only very slightly differentiated.

In fact, as can be readily understood, if the auricular and ventricular bulgings are special modifications of the muscular tissue, for the purpose of forming rapidly contracting roomy chambers-in other words, for the purpose of forming a heart rather than a rhythmically contractile tube -then the very parts which were left ummodified would naturally continue to carry the main intracardiac nerves with their accompanying ganglion cells; so that in the sinus, in the auricles, and in the auriculo-

VOL. II. - I 2 
ventricular ring, the ganglionic structures and the less modified muscular tissue would be coterminous.

If, then, the rhythmical power depends upon the presence of more embryonic muscle, we ought to find those parts which we suppose to contain such muscle especially rhythmical, quite independently of the presence or absence of ganglion cells in them. We ought, in fact, to find, according to our theory, that the bulbus arteriosus contracts rhythmically with great ease, either spontaneously, or with such slight constant stimulus as is implied by a steady distension of its walls; we ought to find that the muscular auriculo-ventricular junction will respond to a stimulus with a series of contractions, and not with a single contraction, as in the case of the auricular or ventricular muscles proper-in this way, explaining incidentally the phenomenon of the second Stannius ligature; we ought to find that in the large veins, even in those parts which are outside the region of ganglion cells, spontaneous rhythmical contractions take place upon isolation.

With respect to the bulbus, the experiments of Engelmann ${ }^{1}$ in 1882 have shown most conclusively how easily this part, when isolated, will beat rhythmically; and the observations of Munk, ${ }^{2}$ Löwit, ${ }^{3}$ and others, with respect to the presence of ganglion cells in the bulbus, have failed to convince Engelmann that ganglion cells are really present there. What Engelmamn has observed for the bulbus arteriosus in the frog can be still better seen in the conus arteriosus of the skate, where there is a long stretch of circularly arranged muscular tissue, the merest touch of any part of which is sufficient to start a regular rhythmical series of contractions.

Passing now to the remains of the canalis auricularis, the auriculoventricular ring of muscle, we see that the second Stannius ligature, or cut, not only stimulates this ring of tissue, but also the two Bidder's ganglia as well, so that the rhythmical series of contractions which results may be due to the stimulus affecting the special rhythmical tissue of the ring, or directly stimulating the nerve cells of Bidder's ganglia. The supporters of the motor ganglia theory of the heart's beat have always attributed this rhythm to a stimulation of Pidder's ganglia, and the latest supporter of this theory, viz. Kaiser, ${ }^{4}$ asserts definitely that, if Bidder's ganglia are removed, then ligature or section of the auriculo-ventricular junction produces no special effect; that, in fact, each stimulus applied now produces only a single contraction, just as in the case of the auricular or ventricular muscle, and not, as is always the case before the removal of the ganglia, a series of contractions. It is impossible to imagine a stronger piece of evidence that the rhythm is dependent on motor ganglia than this - a particular part of the heart always responds with a series of contractions to a single stimulus, but upon removal of the ganglion cells gives only a single contraction to a single stimulus. If this experiment is true, then most assuredly the motor ganglia theory is put on a basis such as it never had before, and, however improbable it may appear, must be taken into serious consideration.

Kaiser's argument is-Any stimulus applied here always gives a series of contractions, not a single contraction; this is due to the stimulus exciting a nerve

${ }^{1}$ Arch. f. d. ges. Physiol., Bonn, 1882, Bd. xxix. S. 425.

2 Arch. f. Physiol., Leipzig, 1878, S. 569.

+Ztschr.f. Biol, München, 1894, Bd. xxx. s. 302.

3 lbid., 1S81, S. 399. 
centre in the shape of Bidder's ganglia, because when that centre is removed then only a single contraction takes place upon a single stimulus. It follows, therefore, that if Bidder's ganglia and the muscular tissue of the auriculoventricular ring be exposed, a stimulus to either ganglion ought to give a series of contractions, while a stimulus to the auriculo-ventricular muscular ring ought to give only a single contraction.

It is possible to test this question in the following manner:- Slit up one auricle and the ventricle, and pin back so as to expose the septum; the two vagi nerves on the septum, with their terminations in Bidder's ganglia, are then most easily seen, as well as the muscular ring between auricles and ventricle, and the whole field can be explored under a lens with the point of a needle before and after removal of the septum and Bidder's ganglia. Now, eut away the sinus, the whole preparation remains quiescent; touch the outlying auricular or ventricular tissue with the needle point, each time a single contraction takes place, and no more; tonch either of Bidder's ganglia, not a single contraction takes place; pierce it again and again, and pull it ahout, no sign of contraction. Touch the auriculo-ventricular ring of muscle with the slightest stimulus, immediately a series of rhythmical contractions occurs. It is most striking to see, after removal of the septum, how every portion of auricular and ventricular tissue can be explored up to the very eilge of the ring, without obtaining more than a single contraction, while immediately the needle touches the muscular ring a series of rapid contractions results. Conversely, the two Bidder's ganglia can be ent away, and yet stimulation of the ring will proluce a rhythmical result. In this latter experiment, it is of course difficult to say whether all the cells have been removed; and it is this fact, that the certainty of having removed all the cells of the ganglia earries with it the probability of nearly complete destruction of the auriculo-ventricular ring, which renders Kaiser's experiment of doubtful value. The exploration of the uninjurerl tissues by the fine point of the needle is much more valuable, and appears to me conclusive against his view. Unfortunately, the experiment, simple as it appears to be, is not always successful, because it is not always possible to olstain standstill by the removal of the sinus when the heart has been cut open. I performed the experiment successfully many times cluring the winter months, but have hitherto failed to obtain standstill during the spring months. Further investigation of this crucial experiment is alvisable.

Finally, Engelmamn, ${ }^{1}$ in a recent paper, has shown that parts of the large veins which continue to beat normally and spontaneously after separation, which in fact are part of the normal rhythmical tissue from which the heart-beat starts, are outside the region of ganglion cells, and that, therefore, the spontaneous heart-beat starts from a nonganglionic region of muscular tissue.

It still seems then, to me, that the explanation which I gave in 1883 , of the reason why different parts of the heart differ in rhythmical power-an explanation dependent upon morphological differences of the muscular tissne and not upon the presence or absence of ganglion cellsis right. Nothing that has since been written has tended to make me alter that opinion. On the contrary, Engelmann ${ }^{2}$ and W. His, junior, ${ }^{3}$ in their recent papers, have both come to the conclusion that the varying rhythmicity of different parts of the heart is dne to exactly the same morphological arrangement of the muscular tissue as I had already pointed out.

${ }^{1}$ Arch. f. d. ges. Physiol., Bomn, 1896, Bd. lxv. S. 109.

2 Ibid., 1897, Bd. lxv. S. 535.

${ }^{3}$ Arb. a. d. med. Klin, zu Leiprig, 1893, Bd. i. S. 14-49. 
We may then sum up this section by saying that the beat of the heart of cold-blooded vertebrates depends upon the rhythmical power of the muscular tissue of the large veins and sinus being greater than the rhythmical power of the other parts of the heart, and that in all cases the greater or less rhythmicity of any part of the heart depends upon the nature of the muscular fibre of which that part is composed, and not upon the presence or absence of ganglion cells.

\section{ON the SEQUexce of the Contractions of the Different Parts of the Heart.}

In the same way precisely as we have been driven to the conclusion that the beat of the heart depends upon the inherent rhythmical power of its muscular tissue, and that some parts beat more easily than others, because their rhythmical power is greater, so also are we driven to the conclusion that the sequence of the contractions of the different parts of the heart is due to a peristaltic wave of contraction which starts from the most automatically rhythmical tissue, and travels over the rest of the heart with varying speed, quickest over the tissue which has become modified so as to approach more nearly in its properties to ordinary striated muscle, namely, the reticulated bulged portion of the auricle and ventricle, more slowly over those parts which retain a more embryonic character, namely, the auriculo-ventricular muscular ring, and the bulbus or conus arteriosus. Starting with a tube of equal character throughout, we see how, by the modification of portions of that tissue in the two regions of the auricle and ventricle into a more rapidly contracting, more rapidly conducting, less rhythmical tissue, we obtain an efficient heart, which shall not merely pass the blood on, but, by the forcible and nearly simultaneous contraction of all parts of its auricular and ventricular walls respectively, keep up in a most efficient manner a high blood pressure in the arterial system, while at the same time, owing to the absence of modification of the tissue between the auricles and ventricles, i.e. the canalis auricularis, whereby it still retains its original more rhythmical but slower conducting power, the useful purposeful pause between the auricular and ventricular contractions is brought about.

The experimental evidence which I brought forward in $1883,{ }^{1}$ shows clearly that such an explanation is the true one, and that therefore the sequence of the contractions of the different parts of the heart is not dependent upon the presence of ganglion cells any more than the heartbeat itself.

The fundamental experiment, and it is one of very great importance for all questions connected with the heart, is an experiment of the same character as that originally performed by Romanes ${ }^{2}$ on the muscular tissue of the Medusce; an experiment, namely, of cutting the muscular tissue of the heart, so as to leave only a small bridge of tissue over which the contraction must pass, and then studying the block to the passage of that contraction caused by reducing this bridge more and more.

If the heart of the tortoise be suspended, as in Fig. 104, and one auricle be cut away, then a section through the basal part of the auricles into the auricular tissue proper will divide all the nerves with their ganglion

1 Journ. Physiol., Cambridge and London, 1883, vol. iv. pp. 61-81.

2 Phit. Trans., London, 1875, vol. elxvi. p. 269. 
cells which pass from the sinus to the auriculo-ventricular junction, so that, as seen in Fig. 105, if the cut be carried far enough, the auricle is divided into two parts, $A s$, a part connected with the sinus, and $A v$, a part comnected with the ventricle, and the only connection still left between sinus and ventricle is by means of a narrow bridge of tissue at the apex of the auricle which connects $A s$ and $A v$. Conversely, owing to the presence of the free coronary nerve between the sinus and auriculo-ventricular junction, it is possible to cut through the whole of the auricle and leave only the coronary nerve as connection between sinus and ventricle.

These two experiments show conclusively that the sequence of ventricular upon auricular contraction is not dependent upon the integrity of the nerve trunks which pass from the sinus to the auriculoventricular groove. For, in the first case, the sequence is never affected until the bridge of tissue at the apex of the auricle has become very small : and, in the second case, when the coronary nerve only is left, the rhythm of the beats of the ventricle and that part of the auricle still in connection with it never bears any relationship to the rhythm of the sinus, and that part of the auricle still left in connection with the sinus.

From these experiments it is Fig. 105. - Heart of tortoise, suspended as in Fig. 104, to show seen that the vell- appearance of preparation when a block is caused by slitting tricle contracts in

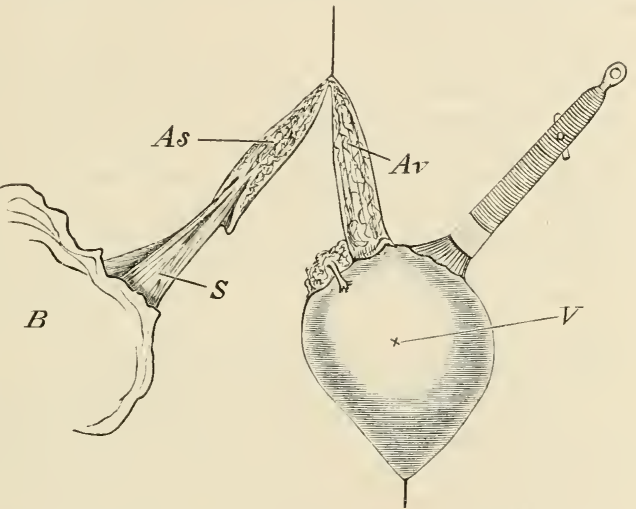
up the auricle.

sequence to the auricle, only when a wave of contraction is able to pass along $A s$ and $A v$.

I was able further to show that if the piece of tissue between $A s$ and $A v$ is reduced sufficiently, then a block to the passage of the contraction from $A s$ to $A v$ takes place of such a character, that, if the block is very slight, a distinct pause occurs at this point when the contraction wave which passes from the sinus along $A s$ reaches it before it passes along $A v$, a pause distinctly comparable to and resembling the pause which takes place at the auriculo-ventricular junction between the contraction of $A v$ and the ventricle. If the block is more severe, then, instead of every contraction passing the blocking point, only every second contraction is able to pass, and therefore a contraction of the ventricle follows upon only every second auricular contraction. With a still more severe block it is possible to see only every third or every fourth contraction able to pass, and therefore to see the ventricle beating to every third or fourth contraction of the auricle. This latter rhythm cammot be obtained for any length of time, but it is 
easy to obtain a partial block, so that every second contraction passes, which may last a long while unaltered. Finally, if the cut is more severe still, then the block becomes absolute, and no contractions are able to pass; consequently $A v$ and the ventricle remain absolutely still until they commence to beat with their own independent rhythm. Even when the block is apparently absolute, as long as a very small bridge of intact tissue remains, it is as a rule possible by vagus stimulation and other methods to overcome the block to a greater or less extent, with the result that as soon as a contraction passes, the quiescent ventricle immediately responds with a contraction.

A precisely similar experiment can be performed on the frog by means of the screw-clamp in the auriculo-ventricular groove. ${ }^{1}$ According to the tightness of the clamp, the ventricle can be made to beat synchronously with the auricles, to respond to every second contraction of the auricles, to respond to every third, fourth, or other contraction, or to remain quiescent.

In fact, we see that we can artificially reproduce, by means of blocking to a greater or less extent the passage of the contraction wave from one portion of auricular muscle to the other, all the phenomena which are characteristic of the normal blocking at the auriculo-ventricular junction between the contractions of the auricle and ventricle. The muscular tissue of all parts of the heart can be blocked in this wayventricle or auricle, it is all the same; by means of the clamp the apcx of the ventricle can be made to respond to only every sccond contraction of the base, just as the whole ventricle to every second contraction of the auricle; it matters not the least whether nerve trumks and ganglion cells are present, all that is necessary is a continuity of muscular substance along which a contraction can travel.

Clearly, then, the natural pause between the contractions of auricle and ventricle, and the sequence of ventricular upon auricular beat, can be explained without the intervention of any special nervous mechanism, if the contraction of the heart signifies the passage of a contraction wave from one end of the heart to the other, over muscular tissue of varying power of conductivity. If part of the museular tissue has been modified, so as to contract with greater rapidity, the contraction wave will necessarily pass more quickly over that part than over the more primitive, less modified tissue of other parts; in other words, the contraction wave will pass more rapidly over the reticulated tissue of auricle and ventricle than over the maltered canalis auricularis, i.e. auriculo-ventricular muscular ring, or the conus artcriosus.

Precisely as in the question of the rhythm, so also of the sequence, the more rhythmical tissue of the auriculo-ventricular ring, and the conus arteriosus, conducts the contraction wave more slowly than the less rhythmical, more rapidly contracting tissue of the ventricle and auricle proper.

In strict accordance with this explanation of the meaning of the sequence, are the observations of $\mathrm{Fano}^{2}$ on the rate of the contraction wave in the heart of the embryo. He found that the rate of travel of the wave in the heart of a chick on the third day of incubation, at $38^{\circ} \mathrm{C}$., varied from 11.5 to $3.6 \mathrm{~mm}$. per second, very much less than the rate observed by Engelmann, ${ }^{5}$ and by

1 Phil. Trans., London, 1882, pp. 999 and 1031.

2 Areh. per le sc. med., Torino, 1890 , vol. xiv.

${ }^{5}$ Arch. f. d. ges. Physiol., Bonn, Bd. xi. S. 480. 
Marchand, ${ }^{1}$ in the museular tissue of the ventricle of the frog, which they estimated as greater than $20 \mathrm{~mm}$. per second.

If it be necessary still further to prove that the sequence of the ventricular upon the aurieular contraction has nothing to do with the presence of ganglion eells at the auriculo-ventrieular junction, it may be mentioned that the removal of the aurieulo-ventricular ring in the tortoise bit by bit shows that those parts of the ring which contain ganglion cells in largest quantity, namely, the parts where the large nerves enter the ring, are not so efficient in maintaining the sequence of the eontractions as those parts near the aorta where ganglion cells are comparatively scanty. It is also evident that the experiments which have been relied upon as strongly suggesting a co-ordinating centre for the due sequence of ventricular and auricular contractions, can be explained on the theory of blocking of a muscular wave of contraction. The three prineipal arguments used are-

1. Schiff's observation, that in the dying leart the ventricle beats more slowly than the auricle, and therefore the eontraction is not of the nature of a peristaltic wave. The phenomena of the apparent independence of the beats of the various heart cavities during the process of dying are all beautiful illustrations of a gradually increasing block, first at the auriculo-ventricular junction, and then at the sino-auricular.

2. Ecklard's ${ }^{2}$ observation that section of the septum together with its nerves in the frog causes the ventricle to beat independently of the auriele. In this ease the slower beat of the ventricle observed hy Eckhard was clearly not independent of the auricular beat, for he himself says that both auricular and ventricular contractions were stopped by stimulation of the vagus nerve, although the nerves of the septum were cut; plain proof that the ventricular rhythm in his experiments was not independent of the auricular, and that in cutting through the septum he had damaged the anriculo-ventricular muscular ring, and so caused a partial block.

3. Marchand's ${ }^{3}$ observation, that the time taken before the ventricle contracts when a stimulus is applied to the auricle is too long to be accounted for by the travel of a wave of contraction along muscular tissue; the delay he accounted for as due to processes going on in Bidder's ganglia, and to prove this he removed Bidder's ganglia, and then found the sequence gone. The first part of Narchand's proof is based on the erroneous assumption, that the rate of travel in the muscular tissue from auricle to ventricle is the same as that shown by Engelmann's observations in the ventricular muscle, whereas we have seen that the low conducting power of the auriculo-ventricular muscular ring is quite sutticient to explain Marchand's figures. As to his second argument, it is very difficult to remove Bidder's ganglia without seriously damaging the aurieulo-ventricular muscular ring, especially if, as in Narchand's ease, the attention is not particularly directed towards this ring. I have, however, succeeded in doing so, and found, ${ }^{4}$ as indeed did Bidder himself, no alteration of sequence whatever as the result of such removal. I conclucle, therefore, that Marehand, as well as Eckliard, caused a hlock by damage to the auriculo-ventricular ring when he removed the two ganglia.

This, then, is the explanation of the sequence of the contractions. A rhythmical series of contractions start from the sinus, traverse the heart as peristaltic waves, and come out at the bulbus arteriosus; and with such an explanation one thing clearly follows-such contractions must pass over the heart, whether they start at one end or the

1 Arch. f. d. ges. Physiol., Bonn, 1878, Bd. xvii. S. 137.

${ }^{2}$ Beitr. z. Anat. u. Physiol. (Eckhard), Giessen, Bd. vii. S. 191.

${ }^{3}$ Arch.f. d. gcs. Physiol., Bomn, 1878, Bd. xvii. s. 137.

4 Journ. Physiol., Cambridge and London, 1883, vol. iv. 1. 75. 
other; we must, in fact, be able to obtain a reversal of beats, as in the heart of the Ascidian. The ease with which a reversal of the sequence of the beats of the heart can be observed is well exemplified in the hearts of the skate ${ }^{1}$ and tortoise, and in both cases it is clearly seen that the only factor requisite is to start a rhythm with a rate quicker than that of the natural sinus rhythm; that, as might be expected, the heart-beat starts from the place where the rate of the spontaneous contractions is quickest. If the heart of a large skate is taken out of the body, and the auricle slit up so as to make a long strip of tissue, then it is very easy to see the peristaltic wave travel along this strip to the ventricle, and reappear after the quick, apparently simultaneous contraction of the ventricle as a peristaltic wave slowly traversing the long inuscular conus arteriosus. If, then, the conus arteriasus be stimulated by any pointed instrument, it is excited to a rhythm of excitation, the rate of which at first may be quicker than that of the sinus rate. Immediately the peristaltic wave is reversed, and all the beats can be seen travelling in the reverse direction along the elongated auricular strip, until, with the subsidence of the stimulation, the rate of the conus beat becomes just less than that of the sinus beat, when forthwith all the contractions pass along in the normal direction. The same thing can be seen in the tortoise ${ }^{2}$ by starting a spontaneous beat in the ventricle and auriculo-ventricular junction with a rhythm quicker than that of the sinus. This can be obtained as follows: The auricle is slit up, not sufficiently to cause any blocking, but so as to allow every contraction to travel easily, and a cannula is placed in the aorta in connection with a pressure bottle filled with a blood solution. The pressure of the fluid closes the aortic valves, and forces blood into the coronary system, which is developed to a certain extent in the tortoise. This blood drops out of the cut coronary veins, and does not reach the sinus. In this way the rhythmical tissue of the auriculo-ventricular ring is brought nearer the condition of spontaneous rhythmical activity, but still the sinus holds its own. Now cool down the sinus, which, owing to the conditions of the experiment, can easily be done without affecting the ventricle, the sinus beat gets slower and slower, and at last every contraction suddenly passes in the reverse direction, first $V$, then $A v$, then $A s$, and finally sinus. If now the sinus be cut away, there is no Stannius standstill, but the ventricle and auricle continue to beat with the same rate of rhythm as before removal of the sinus.

We come, then, to the conclusion that the beat of the heart starts from that part which is most rhythmical, i.e. which beats spontaneously at the quickest rate, and travels as a wave of contraction over the rest of the heart at rates of speed which vary in different parts according to the nature of the muscular tissue.

So far I have not attempted to decide the question whether the sequence in question is due to the actual passage of a wave of contraction over muscular fibres, or whether, owing to the presence of a nerve plexus coincident with the muscular structures, or to the nervous nature of the muscular substance itself, it is possible to conceive of a stimulation wave travelling along the neuromuscular tissue without an accompanying contraction. Granted, in fact, that the nerve trunks and nerve cells are not concerned in the sequence, is it still possible that the ventricle may contract, in response to a contraction of the sinus, without an intermediate contraction of the auricle?

${ }^{1}$ (iaskell, op. cit., 1. 78.

${ }^{2}$ op. cit., 1). 88. 
Such is asserted to be capable of experimental proof, and is the strongest argument used against the sequence being simply due to the passage of a wave of contraction. Two methods have been especially prominently brought forward by Engelmanu. ${ }^{1}$

1. Biedermann ${ }^{2}$ has shown that ordinary striated muscle can be completely deprived of all power of shortening its fibres, without any loss of its excitability to electrical stimulation, or its power of conduction, by soaking it in ordinary water, so that the part of the muscle so treated behaves to the rest of the muscle like a motor nerve. Engehmann has made trial of this same method in the case of the heart, and asserts that if the auricles and sinus of the isolated heart are dipped under water, while the ventricle is left free and its contractions are registered, then, after a time, the auricles lose all muscular character, and behave as though they were motor nerves to the ventricle; each stimulus of the auricle producing a contraction of the ventricle without the slightest trace on the ventricular curve of any antecedent auricular contraction.

2. Engelmann has sometimes succeeded in reducing the contractions of the auricles to invisibility, while the contractions of the ventricle still continue, by means of a reflex stimulation of the vagus nerve on the heart, in consequence of stimulation of the wall of the stomach. This implies, according to him, that the normal impulse from the sinus can reach the ventricular muscle, and cause it to contract without any necessary intermediate contraction of the auricular muscle.

To my mind it is very doubtful whether either of these experiments are conclusive, for the simple reason that it is very difficult to be quite certain that no contraction passes along any part of the auricle.

The difficulty of being certain that no part of the heart is contracting, when it is apparently absolutely still, was brought home to me very strongly when I was making the experiments recorded on p. 217, for the purpose of seeing whether the accelerator (augmentor) nerve was able to make a quiescent heart beat again; as mentioned there, I was deceived again and again; in each case the heart was apparently absolutely motionless, stimulation of the nerve made it beat vigorously. Any graphic method, such as that used by Engelmanu, would most certainly have led to the conclusion that the augmentor nerve was able to set up rhythmic contractions in the non-beating heart, and yet such a conclusion would have been wrong, for in every case examination of the sinus with a powerful lens showed that the heart had not really ceased to beat, but only that the beats, which were perfectly regular, were confined to infinitesimal movements of the muscular tissue of the sinus. So also with respect to the vagus nerve, I have shown in my paper in the Philosophical Transactions (Fig. 12, $a$ and $b$ ) that the vagus is able upon stimulation to reduce the contractions of the auricle to invisibility, as far as registration on a blackened surface goes, and yet, from the very comparison of $12 b$ with $12 a$, I feel sure that in both cases the auricle was contracting.

Again, MacWilliam ${ }^{3}$ has pointed out that a contraction wave may travel from the sinus to the ventricle in the case of such a heart as that of the tortoise or eel in two different ways-(1) Over the auricular tissue proper, i.e. the reticulated portion; (2) over the flattened basal part of the two auricles or sinus extension. If then, as is possible, the septum between the auricles in the frog's heart corresponds to the sinus extension of the tortoise's heart, a contraction might reach the ventricle through the muscular tissue of the septum, while the auricles proper remained absolutely quiescent.

Engelmann, too, has demonstrated most conchusively that the rate of travel of the wave of stimulation over the heart is strong evidence that such wave

${ }^{1}$ Arch. f. d. ycs. Physiol., Bomn, 1894, Bd. lvi. s. 199.

2 Sitzunysb. d. k. Akad. d. Wissensch., Wien, 1888, Bd. xevii. s. 101.

${ }^{3}$ Journ. Physiol., Cambridge and London, 1885, vol. vi. p. 192. 
takes place in muscular rather than in nervous tissue, so that when he considers that his experiments prove that the ventricle may contract after the sinus without an intermediate contraction of the auricles, he still considers at the same time that the stimulus to contraction of the ventricular muscle reaches the ventricle by way of the muscular tissue of the auricle, although the molecular change in the auricular muscle is not sufficient to cause a change of form in it.

Such a conclusion is greatly strengthened by Fletcher's ${ }^{1}$ recent observation that water rigor in striated muscle does not cause a discharge of $\mathrm{CO}_{2}$ similar to leat rigor, and by Biedermann's observation that the electromotive phenomena of water-clotted muscle are not at all comparable with those of true rigor, since the difference of potential between a normal and water-clotted part of the muscle is insignificant. Botl these observations point to the conclusion. that the action of distilled water upon the muscle is pliysical and not chenical ; that therefore the wave of contraction would travel along the water-clotted auricle in Engelmann's experiment without showing any alteration of form, just as it would along an auricle which was mechanically prevented from altering its form.

Considering, then, the difficulty of decilling whether in any given ease a contraction does or does not take place, we may sum up this spetion by saying that in all cases the sequence of the contractions of the different parts of the heart is most probably due to the passage of a wave of contraction along muscular tissue, even though under certain conditions this contraction may be so small as not to be visible by ordinary methods of investigation.

The causation of the beat of the heart and of the sequence of the contractions of the separate cavities is the same in warm-blooded as in cold-blooded animals. - A considerable amount of very difficult and laborious experimental investigation has been carried out by various observers upon the mammalian heart on the assumption that it is not safe to draw the conclusion that what is known to be true of the coldblooded heart will necessarily be true of the warm-blooded; and it has even been argued again and again, that although it may be proved up to the hilt that the beat of the frog's heart is due to the rhythmical properties of its muscular tissue, yet the beat of the dog's heart may be totally different, and may be due to the presence of special nerve centres.

This view of a fundamental difference between the innervation of the hearts of the cold- and warm-blooded animals has received especial support from the supposed impossibility in the case of warm-blooded animals, of attributing the sequence of ventricular upon auricular beat to the passage of a peristaltic wave over museular tissue at the auriculoventricular junction. The explanation of the sequence of beats in the mammal must, it is said, be due to special nervous arrangements, because the muscular tissue of the auricles is absolutely discomnected from that of the ventricles. As might have been anticipated, all recent work on the mammalian heart tends more and more strongly to prove that there is no fundamental difference between it and the cold-blooded heart, any difference there may be being a difference in degree, not in kind.

Thus the observations of Kent, ${ }^{2}$ and of Krehl and Romberg, ${ }^{3}$ have shown that there is muscular continuity between the auricles and ventricles quite sufficient to account for the passage of a contraction

1 Joum. Plyysiol., Cambridge and London, 1897, vol. xxiii. p. 85.

2 Ibid., 1892, vol. xiv, p. 223.

3 Aveh. f. exper. Path. u. Pharmakol., Leipzig, 1892, Bd. xxx. S. 71. 
wave, just as in the hearts of the frog, tortoise, ${ }^{1}$ eel, ${ }^{2}$ etc. Further, MacWilliam ${ }^{3}$ has shown that in the mammalian, just as in the coldblooded heart, a reversal of the sequence takes place when the rentricle is made to beat at a quicker rate than the great veins and auricles; so also does a block take place at the auriculo-ventricular junction, of precisely the same character as in the cold-blooded, so that the ventricle responds to every second contraction of the auricle, ete. Another difference of a fundamental character was said to exist by Waller and Reid, ${ }^{4}$ who concluded, from the measurement of the time relations of the electrical variation at two points on the ventricle, that the apex of the ventricle in the beat of the warm-blooded heart contracts before the base, while in the beat of the cold-blooded the base contracts before the apex. Bayliss and Starling, ${ }^{5}$ however, have shown that this conclusion is erroneous, and that the contraction travels over the mammalian ventricle from base to apex, just as in the hearts of the frog and other cold-blooded animals.

With respect to the rlythmical power of the different parts of the heart, we find the same results as in the cold-blooded, the main difference being that the mammalian ventricle, when isolated, appears to possess greater rhythmical power than that of cold-blooded animals. Thus the observations of Wooldridge, ${ }^{6}$ of Tigerstedt, ${ }^{7}$ by means of the atriotome, and of MacWilliam, ${ }^{8}$ all show that the ventricle when separated from the auricles beats immediately with its own rhythm, without any marked preliminary standstill. The mammalian heart, therefore, is said not to correspond to that of the cold-blooded in this important respect, that the Stannius standstill does not exist. In these experiments, it is important to remember one thing, namely, that the circulation of blood in the ventricle is retained, in Wooldridge's experiment, by the removal of the écraseur; in Tigerstedt's, by the application of the atriotome, while in the experiments upon cold-blooded hearts, the ligature or section between simus and ventricles removes all blood supply from the ventricle. In the case of the tortoise, I have to all intents and purposes seen the same result as described for the manmalian heart, by means of the simple expedient of supplying the ventricle with blood through its coronary system (as already mentioned). Then, just as in the mammalian heart, the Stannius standstill is either cut short, or does not occur, and the ventricle commences imnediately upon isolation to beat with its own rhythm. In this respect, then, the mammalian ventricle does not fundamentally differ from that of the cold-blooded animal.

Quite recently, still further proofs that the same explanation of the cause of the heart-beat applies to the heart of the warm-blooded as to that of the cold-blooded animal, has been given by Porter, ${ }^{9}$ for he has shown, in the first place, that the isolated strip from the rentricle of the mammalian heart resembles that from the leart of the tortoise in its

1 Gaskell, Journ. Physiol., Cambridge and London, 1883, vol. iv. 1) 70.

2 MacWilliam, ibil., 1885, vol. vi. p. 195.

3 lbid., 1888, vol. ix. 1) 185.

+ Phil. Trans., London, 1887, 1. 215.

${ }^{5}$ Proc. Roy. Soc. London, 1891, vol. iv. 1. 211 ; and Internat. Monatseler. $f$. Anat. u. Physiol., Leipzig, 1892, Bd. ix.

${ }^{6}$ Arch. f. Physiol., Leipzig, 1883.

7 Ibid., 1884.

8 Op. cit., p. 177 .

9 "On the Cause of the Heart-beat," Journ. Exper. Med., New Iork, 1897, vol. ii. 1. 391. 
inherent rhythmical power; and, in the second place, Pratt, ${ }^{1}$ working with Porter, has shown that the isolated ventricle of the cat can be tied on to a perfusion cannula and fed with blood at the temperature of the body through the foramina Thebesii, and then, just as in the case of the frog's ventricle, it will beat regularly and well for many hours. The strip from the mammalian ventricle, even when taken from the apex of the ventricle, will beat regularly at its own rate of rhythm, apart from the beating of the rest of the heart, provided that care be taken to keep intact the circulation of blood through the coronary vessels supplying the strip.

Owing to this inherent rhythmical power of the mammalian ventricle, when supplied with blood through the coronary system, it seems, according to the general consent of all experimenters on the mammalian heart, that it is much more easily possible to obtain standstill of the auricles with the ventricle still beating than in the case of the cold-blooded animal. This is said to be of special frequent occurrence on stimulation of the vagus. MacWilliam, ${ }^{2}$ Roy and Adami, ${ }^{3}$ Kreh] and Romberg, ${ }^{4}$ and others, have all noticed cases where vagus stimulation has caused cessation of auricular beats, while the ventricle has continued to beat, though with altered rhythm. It is said, in fact, that stimulation of the vagus has brought to light the independent ventricular rhythmical beat, which could not manifest itself previously owing to the greater rapidity of the veno-auricular beat. MacWilliam here gives the same warning as I have already given, as to the difficulty of being sure that the standstill of the auricles is absolnte; and he expressly mentions cases where his curves show complete cessation of auricular contractions, although upon close inspection he convinced himself that the auricles were still beating very feebly. The fact, however, that an independent ventricular rhythm can be unmasked by a vagus stimulation, which causes standstill of the auricles, is shown most clearly by an example given by me in my paper on the tortoise heart (Fig. 8, PLATE IV.), where an independent ventricular rhythm, with a reversed sequence of auriculo-ventricular contraction, was unmasked at every stimulation of the vagus which caused sino-auricular standstill. The development of this ventricular rhythm could be seen, by means of a series of stimulations of the vagus nerve, to take place in the same way as its development in an isolated ventricle; and its complete independence of the auricles and sinus was seen at the end of the experiment by simply cutting away the auricles; the ventricle then immediately went on beating at the rate it had been shown to have attained at the last stimulation of the vagus. In the case of the mammalian heart, this last decisive proof of the unmasked ventricular rhythm being truly independent, is unfortunately wanting.

The peculiarities of the cardiac muscle by which the rhythm and sequence may be explained.-As is well known, the cardiac muscle, though striated, differs in respect to stimulation from the ordinary striated skeletal muscle in various important points, of which the principal are :-

\footnotetext{
1 " The Nutrition of the Heart through the Vessels of Thebesius and the Coronary Veins," Am. Journ. Physiol., 1898, vol. i. p. 86.

"Journ. Physiol., Cambridge and London, 1888, vol. ix. pp. 167, 34.

3 Thil. Trans., London, 1892.

+ op. cit., 1892.
} 
1. The strength of the contraction does not vary with the strength of the stimulus. The heart's motto, as Ranvier and Kronecker and Meltzer put it, is, "All or none" ; either it will not contract at all, or it will contract to the fullest extent possible at the time, whether the stimulus be weak or strong.

2. The cardiac muscle cannot be tetanised.

3. The cardiac muscle possesses a long refractory period.

These three facts of stimulation of cardiac muscle are due almost certainly to one common cause, and to that same cause is due in all probability the rhythmical power of the cardiac muscle and the peculiarities of the conduction which are manifested in the due sequence of the contraction of the various chambers.

At the present time it is impossible to give any explanation of the difference between striated skeletal and cardiac muscle, which will account for the fact that the extent of contraction in the former varies with the strength of the stimulus, while in the latter the extent of the contraction is independent of the strength of the stimulus. It is possible to imagine that, in the former case, only a portion of the muscle is thrown into contraction with a weak stimulus, while in the latter the whole of the muscle is affected; or it is possible to imagine that the greater slowness of the metabolic processes, both anabolic and katabolic, which take place in cardiac muscle, as evidenced by the greater length of the refractory period, combined with a difference in the stability of the contractile material when formed, may account for the fact that minimal stimuli are at the same time maximal. Certainly, as far as the other two peculiarities of cardiac muscle are concerned, the absence of tetanus and the length of the refractory period, they are to be explained as a necessary consequence of a slow rate of repair after a contraction. The impossibility of tetanising the cardiac muscle is a necessary corollary of the fact that the tissue is inexcitable to the strongest stimulus for some time after the commencemeut of a contraction.

In all striated muscles this refractory period is said to exist; in all striated muscles, therefore, a time must elapse after a contraction before the tissue is ready for a new contraction; the only difference then is, that this time is longer in cardiac muscle than in skeletal. During this inexcitable period, it is natural to suppose that a process of repair is going on in the tissue, so as to bring it back to the condition it was in before the contraction took place; if this is so, we may express the difference between cardiac and skeletal muscle, by saying that the process of repair after a contraction takes place more slowly in the former than in the latter.

It is then possible, it seems to me, to find for all three phenomenathe length of the refractory period, the absence of tetanus, and the absence of any relation between strength of contraction and strength of stimulus-one common hypothetical cause, namely, the metabolic processes, which accompany and are caused by a contraction, take place in the cardiac muscle much more slowly than in ordinary striated muscle.

The length of the period of inexcitability after an effective stimulus explains why a continuously acting stimulus, such as the constant current, or the pressure of suitable fluids within the cavity of the ventricle, etc., or the tension of the suspended muscular strip, causes a series of contractions instead of one continuous contraction; and if we 
choose to use the term "inner stimuli" to express the causation of the internal changes which are going on in the automatic rhythmical tissue of a normally nourished heart, then we can explain the normal rhythm in the same way as the artificially produced rhythm, as a necessary consequence of a constantly acting internal stimulus upon a muscular tissue in which the refractory period is exceedingly long.

By the refractory period was originally meant by Budge and Marey, the time during which the tissue was inexcitable to any stimulus of any strength. This period of complete inexcitability lasts during the time of systole; afterwards, during the diastole and pause, the excitability recovers itself, not suddenly but gradually, so that, according to Engehmann's ${ }^{1}$ latest researches, the power of response to very weak stimuli is not regained until after the completion of the diastole. By the refractory period, therefore, we mean at the present day a sudilen complete loss of excitability, caused by the contraction, with a gradual slow recovery of excitability after the contraction is over. It follows from Engelmann's measurements that the inner stimuli upon which the normal rhythmical beat depends must be extremely weak.

What is true of the rhythmical tissue in the sinus and large veins on which the beat depends, is true also of the less rhythmical tissue over which the contraction wave passes, such as the reticulated tissue of auricle and ventricle; here, too, each contraction is followed by a slow recovery of conductivity, and the question whether the next contraction wave, which reaches any particular spot, will be able to cause a further travelling of the wave or not, depends upon the extent to which the muscular tissue there has been able to recover its conductivity. Anything, therefore, which diminishes the conductivity of any part of the tissue, will tend to cause a block in the contraction wave at that part; and the question whether any block will be manifested or not, will depend upon the rate of the heart-beat in relation to the extent of the diminution of conductivity in the damaged part. A very excellent example of the dependence of a blocking upon the rate of beat can be shown in the following manner :-

Slit up the amricle of the tortoise so that every eontraction is just able to pass the blocking point, then remove the sinus and apply electroles, arranged so as to give single induction shocks at regular intervals to the part of $A s$ near the sinus. Owing to the removal of the sinus, the auricle and ventricle remain still, and now by means of the electrodes on $A s$, we can produce a regular artificial rhythm, the rate of which can be varied at will; we can, for instance, send through single shocks every ten seconds, with the result that every contraction of $A s$ causes a contraction of $A v$, and therefore of $V$. We may then sec that single induction shocks sent in to $A s$ every five seconds will cause a regular series of contractions in $A s$, but only every second contraction can pass the blocking point and cause a contraction of $A v$, and therefore of $V$. We see, in fact, a longer time after a contraction is required for the recovery of the conductivity of the tissue at the blocking point than of that of the rest of the auricle.

This experiment illustrates many important facts concerning the heart : it shows that that part of the heart which possesses the quickest rhythm must of necessity be the leader; under normal conditions, therefore, the great veins and sinus. It shows also, that in the beating ${ }^{1}$ Arch. f. d. yes. Physiol., Bonn, 1894, Bil. lix. S, 309. 
heart the wave of contraction must travel always in one direction, for all the parts immediately in the rear of the part which is contracting are blocked (being in the inexcitable phase), so that no backward wave is possible.

The experiments of Bowditch, ${ }^{1}$ Kronecker, ${ }^{2}$ and v. Basch ${ }^{3}$ have shown that a series of single induction shocks, sent in to the muscle of the frog's ventricle, are able to produce an infallible response to each shock only when they are sent in at a slow rate-two seconds being the limit-and that if they are sent in at a quicker rate than this, then a contraction occurs only with every second stimulus. In fact, whether the contraction be due to the travelling of a contraction wave, or to a direct external stimulus, it will not take place unless sufficient time be allowed for the tissue to recover its conductivity or excitability respectively after a previous contraction.

From these experiments of Kronecker and v. Basch, combined with my experiments upon the blocking of contraction waves, we see that very similar laws apply to the recovery of excitability and the recovery of conductivity after a contraction, and it makes no difference at any spot whether that contraction is the natural contraction due to the normal beating of the heart, or an artificial contraction induced by an interpolated stimulus between the natural contractions; just as such stimulus cannot cause a contraction in the ventricle unless it be applied a sufficient time after the commencement of the natural contraction, so also the normal contraction will be blocked if it reaches the ventricle before the tissue has had time to sufficiently recover its conductivity after the commencement of the interpolated or forced contraction. For this reason it is self-evident that an interpolated contraction of the ventricle in the case of a normally beating heart may be followed by a longer pause than usual, because the normal contraction following the beat of the auricle, which ought to take place during that pause, cannot take place, as it arrives luring the inexcitable phase of the interpolated contraction. This lengthened pause has been called the "compensatory pause," and attempts have been made to look upon it as an inhibitory phenomenon. It is, however, so clearly due to a blocking of the natural contraction wave by the interpolated contraction, owing to the existence of the refractory period, that it is not necessary to discuss it further.

In comnection with the observations of Kronecker ${ }^{4}$ and v. Basch, ${ }^{5}$ upon the effects of sending in single induction shocks to the cardiac muscle at definite intervals, are observations of v. Basch upon the effect of sending in single induction shocks, the strength of each one of which is insufficient to cause a contraction. He finds that such stimuli will produce a rhythmical series of contractions, the rate of which is less than the rate at which these insufficient stimuli are sent in. The explanation appears to be ${ }^{6}$ that a stimulus which is insufticient to cause a contraction increases the excitability of the muscle, and so enables a second or third stimulus of the same strength to be powerful enough to cause a contraction. This observation of $\mathrm{v}$. Basch is in accordance with

${ }^{1}$ Ber. d. k. Sächs. Gesellsch. d. Wissensch., Leipzig, 1871.

2 "Das eharaet. Merkmal d. Herzmuskelbeweg.," Beitr. z. Anat. u. Physiol. als Fcstgabc C. Ludwig, Leipzig, 1874.

${ }^{3}$ Sitzungsb. d. k. Akad. d. Wissenseh., Wien, Bd. 1xxix.

+0 p. cit. 5 Op. cit.

${ }^{6}$ v. Basch, Arch.f. Physiol., Leipzig, 1880, S. 283. 
another phenomenon, observed originally by Bowditch, ${ }^{1}$ namely, the phenomenon of the "Treppe," or staircase phenomenon, which consists in the fact that, when a series of single induction shocks of the same strength are sent in to the muscular tissue of the quiescent frog's ventricle or apex, the contractions are not all of the same size, but the first is the smallest, and they then gradually increase up to a maximum. From this experiment it is concluded that every stimulus which causes a contraction produces in addition a beneficial change in the muscular tissue, so that it is more excitable and able to contract more strongly than before the contraction. How such beneficial effect is brought about, whether, as Hering would say, by the assimilation process which is produced by the dissimilation stimulus being greater than is necessary to produce autonomous equilibrium, or in some other way, such, for instance, as by means of nerve fibres, which are excited simultaneously with the muscular tissue, cannot be determined at present. The question will, however, be considered again in connection with the action of the intracardiac nerves.

\section{On Fibrillar Contractions.}

Another question which arises in connection with the conductivity of cardiac muscle, is the question of the co-ordination of the different parts of the ventricle or auricle. Why is it that the contraction of the ventricle takes place as a whole, and not as a series of separate contractions? In the case of warm-blooded animals, a marked inco-ordination of the different parts of the muscular tissue of the ventricle, known by the name of fibrillar contractions, can be brought about by the application of a strong tetanic current to the surface of the ventricle, or by the ligature of one or more coronary arteries. How are these fibrillar contractions to be explained?

According to the observations of Kronecker and Schmey, ${ }^{2}$ a puncture in a particular spot on the septum between the ventricles always brings about this kind of inco-ordination, and they conclude, therefore, that the ventricle possesses here a co-ordination centre which harmonises the contractions of all the ventricular muscle fibres, and so causes a single contraction of the whole ventricle at each heart-beat, instead of the disorderly disjointed contractions of separate muscle-strands, such as are seen in the condition of fibrillar contraction. Against this view it has been pointed out by MacWilliam, ${ }^{3}$ that nothing of the nature of any nerve centre is to be found at the place indicated; that after the destruction of this supposed centre the ventricle will frequently recover from these inco-ordinated movements, and beat again regularly and well, and that the same fibrillar contractions can be induced in the regularly beating isolated apex of the heart-in tissue, therefore, entirely removed from this supposed co-ordination centre.

With respect to the fibrillar contractions produced by strong tetanic stimulation, they are, it would appear, confined to the hearts of warm-blooded animals. In these animals there is a distinct difference between auricle and ventricle in this respect-when the ventricle muscle is thrown into this condition of inco-ordinated fibrillar contraction, it is said not to recover either by nerve stimulation or other means; when, however, similar movements are

${ }^{1}$ Op. cit. $\quad{ }^{2}$ Sitzungsb. d. k. Akad. d. Wissensch. zu Berlin, 1884, S. 87.

3 Journ. Physiol., Cambridge and London, 1887, vol. viii. p. 296. 
induced in the auricle by strong stimulation, the recovery to a normal coordinated contraction takes place quickly and easily after the stimulus is over. According to Gley, ${ }^{1}$ fibrillar contractions may be induced in the ventricle of a new-born dog upon strong stimulation just as in older dogs, but the ventricular muscle at this stage behaves like the auricular muscle of the adult, and the inco-ordination can pass off easily and give place to regular normal contractions. After the dogs are twenty-nine to thirty-three days old, no such recovery is possible under ordinary conditions. But Porter ${ }^{2}$ has shown that even in adult animals the normal contractions may be renewed, provided the nutrition of the muscular substance be restored by perfusing blood of the same animal, at the temperature of the body, through the muscular substance, either by way of the coronary arteries or by way of the vessels of Thebesius, as in Pratt's experiment (cf. p. 188). In many cases the artificial circulation alone will not transform the condition of fibrillation into that of regular co-ordinated beating; it is, however, always possible to obtain this result if the fibrillar contractions are first caused to cease by the application of cold, and then, when the ventricle is quite motionless, the warmed blood solution is sent through its muscular substance.

Besides electrical stimulation, these fibrillar contractions have been observed as the effect of bromide of potassium in large doses, under conditions of anæmia and other weakening agencies, and exceptionally as the result of the intravenous injection of extract of the suprarenal capsules, ${ }^{3}$ the actual cause in this case being probably excessive contraction of the coronary arterioles. It would appear that the fibrillar contractions, which oceur upon ligature of the coronary arteries, must be ascribed to animia and not to any stimulation of nerve fibres by the ligature, ${ }^{4}$ and it is very instructive to notice the order of sequence of the events which follow ligature of the coronary arteries, according to Sée 5 and Cohnheim. ${ }^{6}$ After the arteries have been tied, the first effect is that the ventricle drops beats, i.e. does not respond to every auricular beat; this partial block between auricle and ventricle is then followed by a complete block, so that the ventricle remains still; this standstill is then followed by fibrillar contractions of the ventricle.

These observations suggest very strongly that the condition of fibrillar contraction is brought about by a blocking in the connections between the branching muscle cells of the ventricle itself; that, in fact, a difference of conductivity takes place in the different portions of the ventricular muscle, owing to anæmia, just as in the tissue of the auriculoventricular ring; in consequence of which contractions travel at different rates, and are blocked at different distances throughout the whole ventricular muscular mass, thus giving the appearance of inco-ordinated contractions, known as fibrillar contractions. That this is the probable explanation of fibrillar contractions follows, it seems to me, from my experiments on the stimulation of a strip of muscle cut from the apex of the tortoise ventricle. In such a strip the very action of the section has of necessity brought about a series of blocking places; in consequence of this, the single indnction shocks applied to one end of the strip at first cause contractions which are limited to the tissue in the immediate neighbour-

1 Compt. rend. Soc. de biol., Paris, 1892.

2 Am. Joum. Physiol., 1898, vol. i. p. 71.

* Oliver and Schäfer, Journ. Physiol., Cambridge and London, 1895, vol. xviii. p. 261.

4 Porter, ibid., 1894, vol. xv. p. 121.

${ }^{5}$ Sée, Bochefontaine, and Roussy, Compt. reut. Soc. de biol., Paris, 1881, p. 86.

"Cohnheim and v. Schulthess Rechberg, Firchoe's Arehir, 1881, Bd. Ixxiv. s. 503. VOI. II. - I 3 
hood of the electrodes; then, as the single stimuli continue, and especially if at intervals a weak tetanising current is sent through the whole strip, we see that the contractions are not all of the same strength; some are stronger, some weaker, although the strength of the induction shocks remains the same. The reason of this variation is clear on inspection: those contractions which are stronger travel further along the strip from the point of stimulation than the shorter contractions; we see, in fact, that every now and then a contraction succeeds in forcing a previously existing blocking place, so that different contractions travel to different degrees along the strip. As time passes, more and more of the blocking places are overcome, until at last a regular series of contractions takes place, every contraction passing rapidly and easily from one end of the strip to the other. When this has occurred, when the conduction power has been fully restored along the whole strip, then is the time when the strip begins to contract rhythmically of its own accord, without the necessity of any regular external stimuli.

\section{On the Tonicity of the Cardac Muscle.}

So far we have considered various properties of the cardiac muscle, such as rhythmicity, excitability, conductivity, and have found that different parts of the heart differ in the extent of their possession of these different properties. It is impossible at present to lay down absolute laws of the relations which exist between these several properties in the different parts of the heart, but still we may with advantage sum up our knowledge by suggesting that the present direction of research seems to point roughly to some such series of laws as the following:-

1. The rhythmicity of the cardiac muscle varies inversely as its conductivity.

2. The rhythmicity of the cardiac muscle varies directly as its excitability.

3. The rate of recovery of the excitability, after a contraction, varies directly as the rate of recovery of the conductivity after a contraction.

In other words, the most quickly contracting parts of the heart are the least rhythmical, and, conversely, a block in the passage of the contraction wave is most likely to occur in the more rhythmical tissue.

The end result of the different modifications of the different parts is not only to form an efficient force-pump, the different chambers of which shall always act in regular order, but also, as Engelmann ${ }^{1}$ has pointed out, to render as harmless as possible to the economy any accidental irregularity in the beat of the pump. For we see from his experiments, that not only does a contraction travel at different rates over the different muscular tissues of the heart, but also the rate of travel in any one tissue depends upon the phase of that tissue at the time. For just as the excitability of the tissue is restored gradually after a contraction, so also is the restoration of the full power of conductivity a gradual one after a contraction. It follows, therefore, that an interpolated wave of contraction, starting at the sinus soon after a normal contraction, will travel to the ventricle over tissue which has not yet regained its full power of conduction, and consequently will take a longer time to reach the ventricle than the previous normal ${ }^{1}$ Arch. f. d. ges. Physiol., Bonn, 1897, Bd. 1xv. S. 153. 
contraction wave. Therefore the time between the normal and interpolated ventricular contractions will be longer than the time between the normal and interpolated contractions of the sinus; in other words, any irregularity in the beat at headquarters will tend to be compensated by the time the ventricle contracts, so that the contractions of the ventricle will occur at much more regular intervals than those of the sinus. Seeing that the main importance to the economy is to ensure a regular ventricular beat, it is clear that this more or less complete obliteration of any irregularity in the rhythm, by means of the varying rates of conduction at different phases of the beat, is a very important factor in maintaining the efficiency of the heart.

All these different properties are possessed, not only by cardiac muscle, but also by all other contractile tissue, so that the differences of the cardiac muscle are differences of degree rather than of kind. In addition to these properties, other muscular tissues are said to possess tonicity, a property which is especially well marked in the muscles of the vascular system; by analogy, the cardiac muscle ought also to possess tone, and it would not be unreasonable to expect that different parts of the heart should vary in the extent of their tonicity, just as they vary in the extent of their rhythmicity, in their power of rapidity of contraction, and their other properties.

The question whether cardiac muscle does or does not possess tone is by no means easy to answer off-hand, owing to the beating of the heart; for it is clear that the extent of relaxation between the contractions depends, not only upon the tone of the muscular tissue, but also upon the rate of beat: with a slower beat there is more time for complete relaxation to take place than with a quicker beat, and it is very doubtful whether, with the normal rate of beat of the cold-blooded heart, complete relaxation does take place between the beats. It is therefore difficult to speak of an alteration of tone in the cardiac muscle, if such apparent alteration is accompanied with the corresponding change in the rate of beat. Still there is evidence which points distinctly to a power of tonicity in the cardiac muscle. This evidence is especially dependent upon the action of various drugs and solutions upon the nature of the cardiac contraction. Such solutions may be divided into two groups: those which tend to produce a tonic contraction of the ventricle or apex of the ventricle, so that a less and less complete relaxation takes place l.etween the beats, and finally the ventricle remains in a condition of systolic standstill; and those which tend to produce an atonic condition of the ventricle or apex, characterised by the greater and greater feebleness of the contractions, until finally the ventricle stands still in the condition of complete relaxation or diastolic standstill. The type of the first group is given by the action of alkaline sodium compounds, and of the second by the action of acid substances, such as lactic acid. ${ }^{1}$

The nature of the action of sodium hydrate in very weak solution (1 part to 20,000 normal saline) is shown in Fig. 106, which represents samples $a, b, c, d, e$, taken at intervals from a continuous curve, ${ }^{2}$ and the corresponding action of lactic acid (1 part to 10,000 normal saline) in Fig. 107. In both cases the time when the solution was sent in is represented by a vertical arrow. We see from the figures that not only is the relaxation after each beat hindered more and more by the alkaline salt, but also the muscle remains at each beat a longer time in the

\footnotetext{
${ }^{1}$ Gaskell, Journ. Physiol., Cambridge and London, 1880-82, vol. iii. p. $53 . \quad{ }^{2}$ Ibid.
} 
condition of complete contraction, till at length it is almost or quite impossible to recognise the separate beats at all.

Precisely similar curves can be obtained by the action of such drugs as antiarine, veratrine, digitalin, which are known to act upon ordinary striated muscle in a similar manner; and it is of great interest for the physiology of the heart, to find that muscarine solutions act in the opposite direction, and produce a diastolic standstill of the same nature as that produced by lactic acid. The opposing effect of the acid and alkaline solutions can be seen in the same preparation, so that all the intermediate steps between a systolic and a diastolic standstill can be brought about by the administration of the alkaline solution, followed by that of the acid solution, and vice versa. Precisely similar curves can be obtained by the alternate administration of digitalin and muscarine solutions, ${ }^{1}$ so that we are, it seems to me, driven to the conclusion that muscarine acts upon the apex of the ventricle, or upon the whole ventricle, in the opposite sense to digitalin, and therefore, if, as we have every reason to believe, digitalin produces its effect by

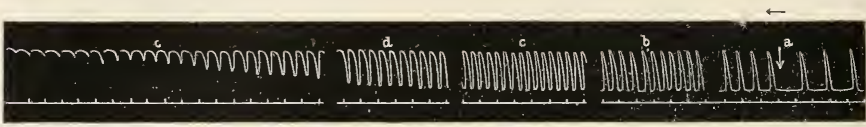

FiG. 105. - Beat of ventricle of frog registered by Gaskell's modification of Roy's tonometer. Perfusion camula in ventricle; blood solution flowing through at constant pressure; at arrow, sodium hydrate solution was sent through instead of blood solution.

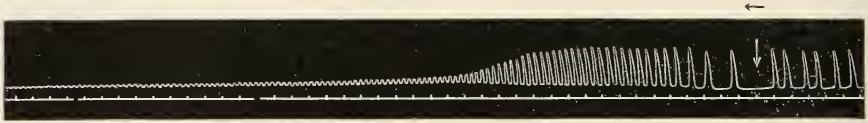

Fig. 107.- Same experiment as Fig. 106. After Fig. 106 had been obtained, blood solution was sent through for seven minutes, and then at arrow the lactic acid solution was sent through instead of the blood solution.

direct action upon the muscle substance, in the direction of bringing about a tonic condition of that muscle, muscarine must also directly act upon the muscle in the opposite direction, and produce its effect by bringing about an atonic condition of that muscle. This action of muscarine will be considered further in a subsequent section. Of such nature is the evidence that the cardiac muscle possesses tonicity as well as rhythmicity.

In addition to the evidence of the action of chemical substances, Fano ${ }^{2}$ has published evidence of another kind of tonic contraction which is more inysterious and more difficult to understand. He finds that a clamp or ligature placed in the auriculo-ventricular groove, will cause in Emys europoea a rhythmical variation of tone in the auricles, of a most marked and striking character. The phenomenon is confined to the auricles, and is not seen in any other animal to the extent described in the ease of Emys. The rate of rhythm is not very regular; the phenomenon, according to Fano, very often does not appear until the beats of the auricle have been registered for a long time. It

1 Gaskell, op. cit., Fig. 106, Pl. III.

2 "Ueber Tonuschwankungen der Atrien des Herzens von Emys europœea," Beitr. z. Physiol. C. Ludwig z. s. 70 Geburtst. gew., Leijzig, 1887. 
is seen in the isolated heart out of the body, and necessitates a clamp in the auriculo-ventricular groove for its production. I have never seen anything of the sort in the auricle of the land-tortoise.

Since the publication of Fano's paper, Botazzi ${ }^{1}$ has observed a somewhat similar phenomenon in the auricles of Rana esculenta and Bufo viridis. He has failed to see it in Testudo greca, Tropidonotus natrix, Lacerta viridis, Anguilla vulgaris, Rana temporaria. In Bufo vulgaris he has observed something of the same kind.

The curves which Fano obtained from the auricle of Emys resemble closely the eurves obtained by v. Frey, ${ }^{2}$ when a muscle is stimulated with rhythmical isochronic stimuli, and at the same time supported to different extents. In consequence of this resemblance, Fano was led to the conclusion that the rhythmical tonic contractions were due to slow rhythmical contractions of some contractile substance, different to that to which the normal or fundamental contractions were due. This different contractile substance may be a different kind of museular tissue, more nearly approaching unstriped, interspersed among the auricular fibres proper, or, according to Botazzi, the phenomenon may be due to the contraction of the sareoplasm as distinet from that of the anisotropic substance of the muscle. This latter explanation seems to me difficult to accept, considering that the phenomenon is confined to the auricles of the hearts of only a few cold-blooded animals; the other explanation seems more possible ; it requires, however, careful histological examination of the auricles of Emys, such as has not yet been done.

\section{The Meaning of the Ganglion Cells in the Heart, and their Rielation to the ('ardiac Nerves.}

So far, I have explained all the phenomena, both of the beat of the heart and the sequence of its contractions, etc., without bringing in ganglion cells at all, and the upholders of special nerve centres in the heart have a perfect right to ask, What then are the ganglion cells in the heart? What function do you attribute to them? That is a question which I am ready to answer, and to answer with confidence, as follows:-The ganglion cells in the heart are part of the great group of ganglion cells which are situated on the course of the smallfibred efferent nerves supplying the viscera. These cells form the outlying vagrant groups of nerve cells which are known by the name of the sympathetic and cerebro-spinal ganglia. In the case of the heart, the ganglion cells are the cells belonging to the small-fibred efferent cardiac fibres of the vagus, just as some of the cells in the ganglion stellatum and in the inferior cervical ganglion are the cells belonging to the small-fibred efferent cardiac fibres of the augmentor nerve. There is no more reason to assign special functions to these cells than to any of the other peripheral efferent nerve cells. They are cells connected only with the inhibitory fibres of the vagus, and as such are simply part and parcel of the mechanism of inhibition, just as the corresponding cells in the ganglion stellatum are simply part and parcel of the augmentor mechanism.

The evidence upon which these statements is based will be best appreciated, if I relate the history of our present knowledge of the relations between the cardiac nerves and these peripheral ganglion cells.

1 Journ. Physiol., Cambridge and London, 1897, vol. xxi. p. 1.

${ }^{2}$ Beitr. z. Physiol. C. Ludwig z. s. 70 Geburtst. geu., Leipzig, 1887, S. 55. 
In 1845 , when the brothers Weber discovered the inhibitory action of the vagus nerve upon the heart, it was known that the heart was also supplied with fibres from the sympathetic system, and E. Weber, ${ }^{1}$ in Wagner's "Handwörterbuch," considered that the two nerves which went to the heart, vagus, and sympathetic, were of opposite function; that the latter were motor nerves, and the former inhibitory. He was unable to obtain any effect from stimulation of the sympathetic fibres themselves, but evidently looked upon those nerves as entering at the aortic end of the heart, while the vagus entered at the venous end; for he says that the sympathetic fibres in the frog are too delicate to stimulate, but by direct stimulation of the aorta he was able to obtain acceleration, which he imagined to indicate that sympathetic fibres passed into the heart in this direction. Continuing this idea, he pointed out the difference between direct stimulation of the bulbus and ventricle on the one hand, and of the vena cava and auricle on the other. In the former case he obtained acceleration and increased systolic contraction, in the latter case standstill in diastole and weaker contractions.

Such then was Weber's conception: the heart was supplied with two nerves, which entered at opposite ends, and their stimulation was opposite in effect both on rate of rhythm and force of contraction. Of these two nerves he and his brother clearly demonstrated that the inhibitory fibres ran in the vagus nerve, but were unable to demonstrate the course and existence of the accelerator fibres either in warm or cold-blooded animals.

Following upon the great discovery of the brothers Weber, physiologists were engaged for many years upon a controversy as to the meaning of this standstill, the majority agreeing with Weber, that the inhibition of the heart is caused by the vagus in the same way as inhibition by nervous action in the central nervous system; that, in fact, Weber's discovery did not imply the discovery of a new class of nerves which acted on the muscular tissue in the opposite lirection to motor nerves, but rather that a motor nerve centre, in the shape of the ganglion cells, existed in the heart, of the same character as the centres in the central nervous system, the action of which the vagus was able to inhibit. The strongest opponent of this view was Schiff, ${ }^{2}$ who objected in toto to the conception of inhibition, and maintained that the vagus was the motor nerve of the heart up to its termination in the muscular tissue, that the beat of the heart depended upon the stimulation of the terminal nerve fibres in the heart itself, and that the so-called inhibition was due to over-stimulation, causing exhaustion of these motor nerve fibres. Schiff laid especial stress on the fact that direct stimulation of the muscular tissue of the ventricle caused a local diastole of that part of the heart, so that it remained relaxed and distended when the rest of the ventricle was contracting; also, this effect was removed by atropine, just like the corresponding effect of vagus stimulation. In other words, Schiff argued that the terminal fibres of the motor vagus nerve in the muscular tissue could be lirectly exhausted by over-stimulation as well as indircetly by stimulation of the vagus trunk, and that therefore this experiment was clear evidence that the vagus did not act by inhibiting any central nervous apparatus in the shape of ganglion cells.

Schiff's view that the vagus was the motor nerve of the heart, which was easily exhausted by over-stimulation, was largely based upon the fact that a weak stimulation of the frog's vagus often produced acceleration when a stroug stimulation produced inhibition. This acceleration upon stimulation of the frog's vagus was noticed by many observers, especially by Wundt and Schelske ${ }^{3}$ in 1859, after strong curari poisoning, but it was not until 1870 that Schmiede-

1 " Handwörterbuch d. Physiol.," 1846 (3), Bd. ii. S. 42-48.

${ }^{2}$ Arch. f. physiol. Heilk., Stuttgart, 1849, Bd. viii.

3 Verhandl. d. naturh.-med. Ver. zu Heidelberg, 1859. 
berg ${ }^{1}$ (and Ludwig) discovered that, after the administration of nicotine, stimulation of the vagus in the frog always produced acceleration, and concluded from their experiments that the vagus of the frog contained accelerator fibres as well as inhibitors. Previously to this, v. Bezold, ${ }^{2}$ in 1862 and 1866 , had shown, by section of the cord in the upper thoracic region and stimulation of the cervical cord, that accelerating fibres left the spinal cord of mammals to reach the heart at the region of the first thoracic ganglion; and the brothers Cyon, ${ }^{3}$ in 1867 , showed that destruction of the ganglion stellatum removed this accelerating effect of stimulation of the cervical cord. Finally, in 1871, Schmiedeberg ${ }^{4}$ and Ludwig traced out the course of the accelerator nerves in the dog, and thus completed the proof of Weber's suggestion, that the sympathetic nerves to the heart produce the opposite effect to the vagus nerves.

This paper of Ludwig and Schmiedeberg definitely settled the anatomical course and position of the accelerator nerves in the mammal, and we see that, whereas the inhibitory nerves were first discovered in the vagus of the frog, and then found to exist in the same nerve in the mammal, the accelerator fibres were first discovered in the mammal, and it was supposed for some time that the accelerator fibres in the vagus of the frog belonged to that nerve, and that therefore the cold-blooded differed from the warm-blooded animal in this important respect; in fact, one finds, even up to the present day, in the literature of the innervation of the heart, continued assertions that what is true for the coldblooded heart is not necessarily true for the warm-blooded, as the differences between them are so great.

In $1881^{5}$ I read a paper before the International Medical Congress, London, the substance of which was afterwards (1882) published more fully in the Philosophical Transactions of the Royal Socicty, in which I showed, among other things, that the frog's vagus on stimulation was able to cause not only acceleration, but also very striking augmentation of the force of the contractions both of the auricle and ventricle. Heidenhain, ${ }^{6}$ in 1882, described the same thing; and further showed that stimulation of the medulla oblongata, with the cord cut just below it, never produced either acceleration or augmentation, but only pure inhibitory effects. From this he concluded that the accelerator fibres in the vagus did not leave the medulla oblongata in the roots of the vagus nerve, but joined the vagus after its exit from the brain.

In order to clear up the question of the accelerators in cold-blooded animals, and to determine definitely their course, I determined to investigate them in the higher cold-blooded animals, and for that purpose obtained, through the kindness of Sir Joseph Fayrer and Dr. Kynsey of Colombo, a number of young crocodiles from Ceylon. I was rewarded by finding a very definite accelerator nerve, which arose from the first ganglion below the cervical region and passed directly to the heart. ${ }^{7}$ Passing to the tortoise, ${ }^{8}$ I found that here also accelerator nerves passed from the ganglion belonging to the first nerve posterior to the brachial

${ }^{1}$ Ber. d. k. Sächs. Gesellsch. d. Wissensch., Leipzig, 1870.

2 "Untersueh. ueber die Innervation des Herzens," 1863; and v. Bezold u. Bever, Untersuch. a. d. physiol. Lab. in Wür zburg, 1867, Bd. ii.

${ }^{3}$ Arch. f. Anat., Physiol. u. wissensch. Med., 1867.

+ Ber. d. k. Sächs. Gesellsch. d. W Wissensch., Leipzig, 1870.

5 Trans. Internat. Med. Congress, London, 1881, p. 254.

${ }^{6}$ Arch. f. d. ges. Plyysiol., Bonn, 1882, Bd. xxvii. S. 388.

7 Journ. Physiol., Cambridge and London, 1884, vol. v. p. 46.

${ }^{8}$ Gaskell and Gadow, ibid., vol. v. p. 362. 
plexus, and that in this animal a regular annulus of Vieussens, strictly corresponding to the arrangement in mammals, existed. I then carefully investigated the sympathetic system of the frog and toad, ${ }^{1}$ feeling sure that they could not prove an exception, and found that from the ganglion on the third nerve, i.e. the first postbrachial nerve, an anmulus of Vieussens passed to the ganglion on the second nerve, i.e. the brachial plexus, and from thence the sympathetic nerve passed to the vagus at the region of the vagus ganglion, some of the fibres turning back with the vagus to go to the heart, while others went on to the Gasserian ganglion of the trigeminal. Here, then, was precisely the same arrangement as in the mammal or tortoise, and the experiment showed that stimulation of any part of the course of these fibres, between the anterior roots of the third nerve and the vagus ganglion, gave pure augmentor and accelerator effects; while, on the other hand, stimulation of the roots of the vagus within the cranium gave pure inhibitory effects.

Thus the proof was obtained that the innervation of the heart is the same both in cold-blooded and warm-blooded animals. In all cases the inhibitory fibres leave the central nervous system in the roots of the vagus, while the augmentor fibres leave in the roots of the nerves immediately succeeding those which form the brachial plexus; and the reason was clearly seen why Weber and others following him were unable to find the accelerators in the frog, for they expected them to leave by the third or fourth ganglion and go direct to the aorta, and so to the heart; they were therefore stimulating the nerve in the wrong direction.

In the course of these investigations I was struck with the histological difference between the two sets of nerves on their way to the heart; the inhibitory nerves of the vagus being medullated, while those of the accelerator were non-medullated. This led to the investigation of the histological nature of the accelerator nerves in the whole of their course, and to the conclusion that both inhibitory and accelerator nerves belonged to the great system of efferent ganglionated nerves; a system characterised by the fineness of their fibres, which are always medullated when they leave the central nervous system, by the presence on each one of these fine medullated fibres, in some part or other of its course, of a ganglion cell, from which fibres of the same physiological character pass to the end-organ. This system includes, not only the cells of the sympathetic system, but all vagrant motor cells, such as are found on the course of the accessory part of the vagus in the ganglion trunci vagi, on the course of the third nerve in the ciliary ganglion, etc. The fibres which pass from these cells are, in the majority of cases, non-medullated. ${ }^{2}$ Seeing, then, that the vagus fibres entered the heart as medullated fibres, and that no medullated fibres are to be found in the rentricle proper or auricles, but only non-medullated, it was immediately evident that the ganglion cells in the heart had the same relation to the medullated inhibitory fibres of the vagus, as the ganglion cells of the ganglion stellatum to the medullated accelerator fibres in the white rami communicantes, from the second, third, and fourth thoracic nerves. In other words, the intrinsic cardiac ganglion cells belong to the same system as the extrinsic cardiac and vasomotor ganglion cells, and there is no justification whatever for ascribing special central nervous system

${ }^{1}$ Gaskell and Gadow, Jonrn. Physiol., Cambridge and London, 1884, vol. v. p. 362.

2 Gaskell, "On the Structure, Distribution, and Function of the Nerves which innervate the Visceral and Vascular System," Joum. Physiol., Cambridge and London, 1885, vol. vii. 
qualifications to the one and not to the other. One possibility remained, llamely, that the ganglion of the inhibitory system, which corresponded to the ganglion stellatum, etc., of the augmentor system, was not to be looked for in the intrinsic ganglia of the heart, but in the ganglion trunci vagi, and that the intrinsic ganglion cells formed a still further ganglionic system upon which both sets of nerve fibres could act. This seemed possible, because, on the one hand, I had shown clearly that this ganglion in the crocodile, which in this animal is situated at the entrance of the thoracic region, is a motor ganglion, from some of the cells of which the terminal motor nerves to the osophagus and stomach arise; and, on the other hand, that the fibres of the short ciliary nerves are medullated, although they are the terminal nerves of those cells in the ciliary ganglion, which are the motor cells of the fine medullated fibres of the third nerve. It seemed, therefore, plausible that the cardiac fibres of the vagus might enter the lieart as fine medullated fibres, even though they were connected with cells in the ganglion trunci vagi. This question received its answer ${ }^{1}$ by means of section of the vagus nerve in the crocodile, in the neck, between therefore the ganglion jugulare and ganglion trunci vagi, and subsequent degeneration of the fibres. I then found that, whereas after periods varying from twenty-five days to three hundred days, stimulation of the peripheral end of the cut nerve in the neck produced neither inhibition nor contraction of the esophagus, stimulation of the nerve peripheral to the ganglion caused as good a contraction of the cesophagus as before, but no sign of any inhibitory effect upon the heart. This experiment conclusively proved that the cardiac tibres of the vagus did not connect with any ganglion cells of the ganglion trunci vagi, and further that the cells on the course of a motor visceral nerve were nutritive to the fibres in the peripheral direction only. I was also able in the dog to trace those fine medullated fibres, among which the inhibitory fibres must have been, from the accessory roots of the vagus, past the ganglion jugulare into the ganglion trunci vagi, without a possibility of the presence of any ganglion cells on that part of their course, so that we may say with certainty there are no efferent cells on the course of the cardiac fibres of the vagus nerve, until we reach the intrinsic ganglia of the heart, such as Remak's and Bidder's ganglia and the other ganglion cells found on the course of the nerve fibres in the heart itself. Finally, Langley's ${ }^{2}$ experiments with nicotine have shown that the action of this drug is to break the connection between the efferent medullated fibre from the central nervous system or pre-ganglionic fibre and its vagrant nerve cell, while it does not impair the action of the processes from the nerve cell to the muscular tissue, i.e. the post-ganglionic fibres; and by a series of such observations, he has come to the conclusion that each efferent visceral nerve possesses upon its course, from the central nervous system to its termination in the peripheral tissue, one and only one nerve cell.

Taking into consideration also the recent investigations of Ramón y Cajal and others, ${ }^{3}$ as to the connection of nerve fibres with nerve cells,

${ }^{1}$ Gaskell, "On the Structure, Distribution, and Function of the Nerves which innervate the Visceral and Vaseular System," Journ. Physiol., Cambridge and Loudon, 1885, vol. vii. 1). 22 .

2 Langley and Dickinson, Joum. Physiol., Cambridge and London, 1890, vol. xi. pp. 265 and 509 ; Langley, ibid., vol. xi. p. 123 ; Langley and Anderson, ibid., vol. xiii., ete.

"See article on the "Nerve Cell" in this volume. 
we may formulate our present knowledge of the meaning of the ganglion cells in the heart as follows:-

Efferent fibres of opposite function leave the central nervous system to supply the viscera; they pass out of the central nervous system as the fine inedullated processes of nerve cells in the central nervous system. These nerve fibres, the pre-ganglionic fibres, do not pass directly into the tissue of the peripheral organ, but each one terminates in connection with an efferent nerve cell, from which a number of fibres, the postganglionic fibres of the same physiological value, pass to the tissue. The efferent nerve cells of the augmentor system are situated in the ganglia of the so-called sympathetic system; the corresponding efferent nerve cells of the inhibitory system are situated in the heart itself.

This simple explanation of the meaning of the cardiac ganglion cells, combined with Langley's discovery that nicotine acts upon the junction between the nerve fibre and its efferent cell, like curari upon the termination of the motor nerve in striated muscle, while it does not affect the termination of the post-ganglionic nerve fibre in the end tissue, immediately gives (as pointed out by Langley and Dickinson ${ }^{1}$ ) a straightforward explanation of Schelske's and Sclnniedeberg's experiment with respect to the action of the vagus nerve in the frog, after curari and nicotine respectively; for it is clear that in stimulating the so-called vagus in this case, they were really stimulating the postganglionic fibres of the augmentor system and the pre-ganglionic fibres of the inhibitory system. The former fibres, therefore, were unaffected by the poison, while the junction between the latter and their efferent ganglion cells was interrupted, consequently acceleration and augmentation alone took place, although direct stimulation of the venous sinus still caused standstill, because, with the electrodes in that position, the post-ganglionic fibres from Remak's ganglia were stimulated. Atropine, on the other hand, prevented the inhibition upon direct stimulation, because, as will be shown later, it paralyses the action of the post-ganglionic fibres upon the muscular tissue.

Again, the evidence that these intrinsic ganglion cells belong to the same system as the extrinsic efferent ganglia, is borne out by the results of embryological investigation, which may be shortly summed up by saying that the observations of Balfour ${ }^{2}$ and Onodi ${ }^{3}$ have led to the conclusion that the sympathetic chain of ganglia is formed as offshoots from the spinal ganglia; and the recent observations of His, ${ }^{4}$ and of His and Romberg, ${ }^{5}$ have shown that the same method of origin applies also to the cells in the heart itself. They are found in connection with the vagus nerves as offshoots from the vagus ganglia, at first outside the forming heart, and afterwards become included within the heart itself. These workers further confirm previous observations, to the effect that the heart has already begun to beat before the ganglion cells have become included in its tissues. Although in most animals the intracardiac ganglion cells belong to the vagus nerve, yet in some, especially in man, according to His, they are chiefly derived from the sympathetic ganglia. If such a difference really exists, then it is possible that in all animals a varying number of fibres belonging to the augmentor nerves may not reach their ganglion cell until they enter the heart itself.

I Journ. Physiol., Cambridge and London, 1890, vol. xi. 1) 277.

2 "Conparative Einbryology," vol. ii. p. 384 .

3 Arch. f. Anat. u. Entwcklngsgesch., Leipzig, 1884, S. 145.

${ }^{4}$ His, jun., Abhandl. d. k. Süchs. Gesellseh. d. Wissensch., No. 1, Bd. xviii.

5 Verhandl. $d$. ix. Cong. f. imnere Med., Wiesbaden, 1890. 
But the upholders of the special cardiac nerve centre theory may argue, "You have given away your case; you acknowledge that the ganglion cells of the heart must be looked upon as precisely of the same character as the ganglia of the sympathetic system; and seeing that there is evidence that these latter do act as an independent central nervous system, so it must also be with the former."

Undoubtedly, the efferent ncrve cells, both in the heart and in other places, are the trophic centres of the fibres which spring from them, and in so far as the nutrition of the muscle fibre and its condition of wellbeing is dependent upon the integrity of its efferent nerves, these nerve cells may be considered as nerve centres governing the muscle; but a central nervous system means more than this, it means essentially a system of nerve cells and fibres connected with both afferent and efferent nerves, by means of which reflex action is able to take place; and it is because there is no evidence in any part of this vagrant ganglionic system of any connection between afferent and efferent nerves constituting a true reflex are, that I for one am unable to attribute the function of a central nervous system to any part of it.

The evidence, as far as it goes at present, seems to point directly to the conclusion that the trophic centre of all the afferent visceral nerves is situated in the stationary ganglia of the posterior roots, and that, therefore, all the nerve cells of the vagrant ganglia are efferent; that, further, as shown by Langley and Anderson, ${ }^{1}$ the so-called reflexes from isolated sympathetic ganglia are not true reflexes, but rather actions along branching efferent nerves. All the conditions necessary for a reflex action of this kind are present in the heart, and in all probability the same explanation as that given by Langley and Anderson for the hypogastric reflex holds good for the reflex which I have described in the heart of the tortoise, ${ }^{2}$ upon stimulation of the ventricle, when the auricles are cut away from the ventricle, so that the coronary nerve is the only communication left between ventricle and sinus. In such an experiment the sinus and auricles are beating regularly, and the auricular contractions are registered, while the ventricle remains quiescent. Stimulation of the coronary nerve near the ventricle, or of the surface of the ventricle in the neighbourhood of the coronary nerve, caused, whenever I tried it, a slowing of the rate of beat and a diminution in the strength of the auricular contractions; caused, in fact, the same effect as a weak vagus stimulation. The strength of stimulus to the ventricle, which produced this effect, was not necessarily enough to cause a contraction in the quiescent ventricle. It is to be remarked that the effect was always in the direction of inhibition, as must necessarily be the case if the ganglion cells of the heart are efferent vagus cells, and if the effect of the stimulation was not due to an escape of current but to an efferent reflex action of the kind suggested.

\section{The Nature of the Actiox of the Cardiac Nelites.}

1. The action of the inhibitory nerves.-As already stated, Weber pointed out that stimulation of the vagus in the frog not ouly slows the heart, but also weakens the contractions. In the controversies that followed, this latter fact was largely lost sight of, until, in Ludwig's laboratory

${ }^{1}$ Journ. Physiol., Cambridge and London, 1894, vol. xvi. 1). 410.

2 llivl., 18s3, vol. iv. p. 92 . 
in 1869 , Coats ${ }^{1}$ pointed out that the contractions of the heart registered by a mercurial manometer were markedly diminished in force upon stimulation of the vagus nerve. These observations were followed by those of Nuël, ${ }^{2}$ who observed that the contractions of the auricle were diminished to a greater extent than those of the ventricle, and were afterwards confirmed both by myself and Heidenhain in 1881 and 1882. This very fact, that a diminution in strength of contraction as well as in rate, taken in connection with the observed fact that the force of the cardias contraction does not vary with the strength of the stimulus, ought of itself to have been sufficient to convince physiologists that the inhibitory nerve acts on the muscle itself, and not by diminishing a stimulus from motor ganglia. So strong, however, is the incubus of a dominant theory, that even up to this day the action of the vagus nerve is spoken of as diminishing impulses to the muscle rather than as affecting the muscle itself.

Quite early in the investigation of the action of the vagus nerve on

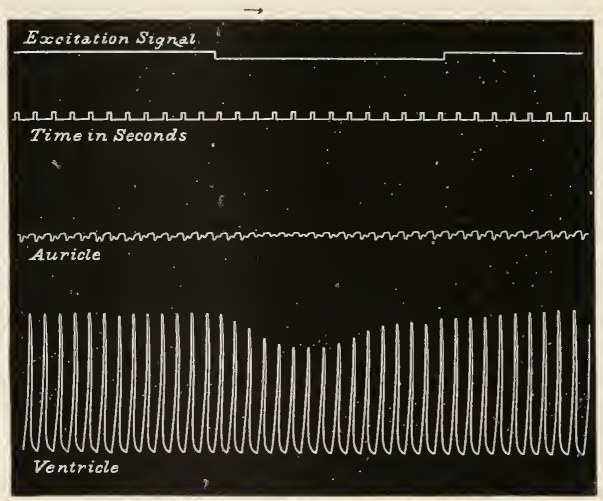

Fig. 108.- Heart of toad. Suspension method. Clamp in auriculo-ventricular junction. Weak stimulation of the intracranial roots of the vagus nerve. the heart, Schiff ${ }^{3}$ pointed out that the muscular tissue, both of auricle and ventricle, in the frog, responded less readily to a stimulus during standstill, or even would not respond at all, although the stimulus was a strong one. This was confirmed by Eckhard, ${ }^{4}$ and is now universally allowed, and is evidence that the vagus nerve diminishes the excitability of the muscular tissue.

Next came the work of myself and Heidenhain, which showed the necessity of separating the inhibitory from the accelerator fibres in the frog's vago-accelerator nerve, in order to obtain the pure effects of inhibitory action. The contrast between the stimulation of the ragus in the frog or toad, as ordinarily given, and that of its two components, the intracranial vagus on the one hand and the sympathetic on the other, is given in Figs. 108, 109, 110, 111, 112, 11:3; and in my paper in the Plitosophical Transactions, a number of tracings show how variable is the extent of the inhibitory effect when the augmentor fibres are stimulated simultaneously. In my experiments upon the effect of stimulation of the intracranial vagus, I have been struck with three phenomena, ${ }^{5}$ which

${ }_{1} \mathrm{Bcr}$. d. k. Sächs. Gesellsch. d. Wissensch., Leipzig, 1869, S. 360.

2 Arch.f. d. ges. Physiol., Bonn, 1874, Bd. ix. S. 83.

3 "Modus der Herzbewegung," Areh. $f$. physiol. Heilk., Stuttgart, 1850.

4 "Erregung des durch Vagusreizung zum Stillstand gebrachten Herzen," Bcitr. * Anat. u. Physiol. (Eckhard), Giessen, 1883, Bel. x. S. 23 ,

${ }_{5}$ Trans. Internat. Med. Cong., Copenhagen, 1884, Section of Physiol., p. 29. 
are in marked contrast with what is seen when the vagus is stimulated in the ordinary mamner. In the first place, I have been able to obtain absolute standstill with a strength of current immensely weaker than what is required to obtain any effect when the vago-augmentor nerve is stimulated. In one case the strength of current was as weak as is just necessary to cause contraction of a striated muscle upon stimulation of its motor nerve, the secondary coil being at $35 \mathrm{cms}$. from the primary with one Daniell cell; at the distance of $12 \mathrm{cms}$. from the primary it was just perceptible to the tongue. In the second place, it is astonishing how long the standstill sometimes lasts after the stimulation is over. Thus one of my tracings shows that a stimulation of the intracranial vagus, lasting thirty-eight seconds, caused the suspended heart to remain absolutely still for 290 seconds, i.e. for 252 seconds after the end of the stimulation. In the third place, it is possible to keep the heart absolutely still by means of continued stimulation for a very much longer time than is possible when the vagus is stimulated in the ordinary manner. Thus, as I mentioned at Copenhagen, I have kept the heart absolutely quiescent for as long a time as twenty-eight minutes by continued stimulation of the intracranial vagus with a weak stimulus (sec. coil between 12 and 11 cms. from primary).

In order to investigate more fully the action of the inhibitory fibres alone, I turned my attention to the land-tortoise (Testudo graca) an animal which not only has the advantage that its inhibitory nerve is separate from its accel-

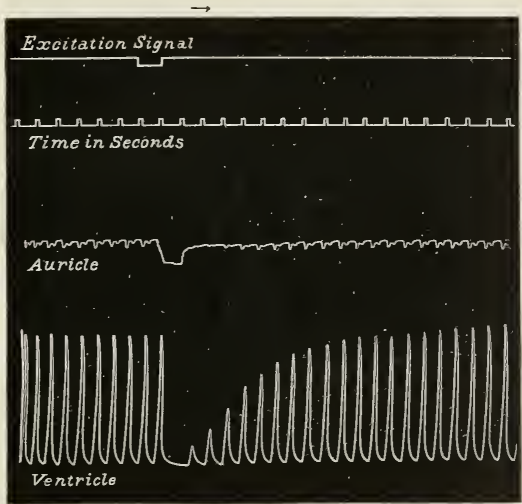

Fig. 109.-Heart of toad. Suspension method. Clamp in aurieulo-ventricular groove. Short stimulation of the intracranial vagus roots with current of moderate strength. The change of level in the auricular tracing during the stimulation is due to the movement of the laryngeal and trapezius muscles. erator, but also possesses, as already described, a free coronary nerve, the fibres of which belong to the vagus system. It is possible to cut this nerve and stimulate it without in any way damaging the heart, and so an insight can be obtained into the action of the inter-auricular nerves in an intact heart. The presence of this free nerve, between the sinus and auriculo-rentricular junction, has proved invaluable in increasing our knowledge of the action of the inhibitory nerves. Fig. 114 shows the effect of stimulation of the isolated coronary nerve, the sinus end having been cut and ligatured. If Fig. 114 be compared with Fig. 115, which shows the effect of stimulation of the right vagus in the neck, we see that the difference between the two curves consists simply in this, that the coronary nerve diminishes the strength of the contractions of the auricle without affecting the rate of the beat, while the vagus diminishes both the rate and the strength of the auricular contractions. In this instance the slowing of the rate of 


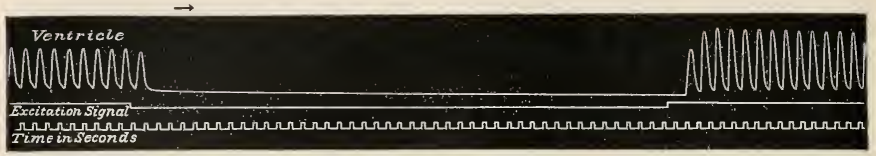

FIG. 110,-Heart of toad. Suspension method. Ventricle only registered. Long stimulation of the intracranial vagus roots with moderately strong current.

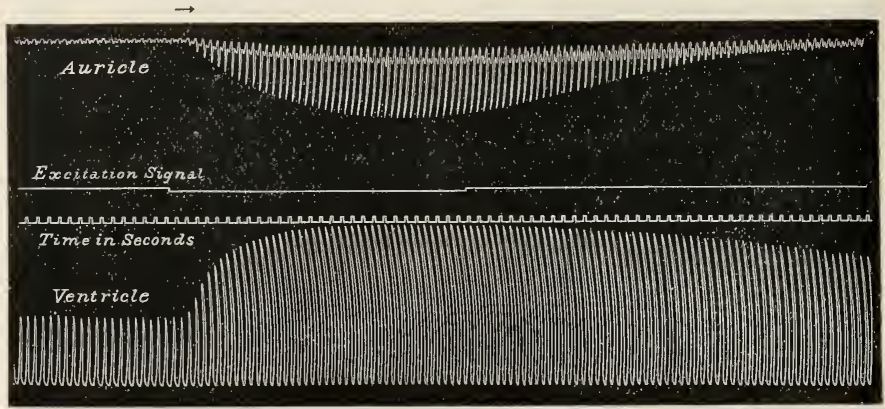

Fig. 111.-Heart of toad. Suspension method. Clamp in auriculo-ventricular groove. Stimulation of augmentor (sympathetic) nerve before it joins the vagus.

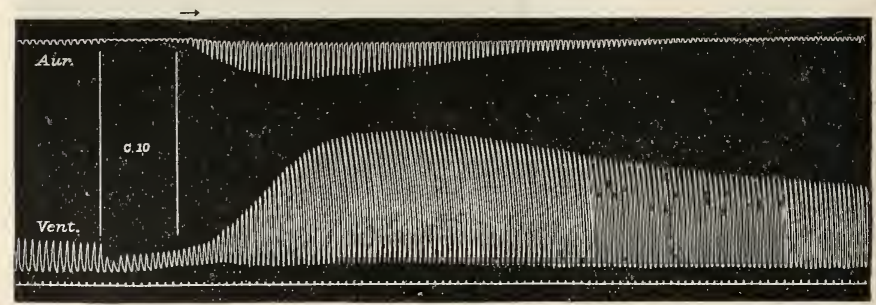

FIG. 112,-Heart of toad. Suspension method. Clamp in auriculo-ventricular groove. Stimulation of vagus (i.e. vago-sympathetic) nerve in neck.

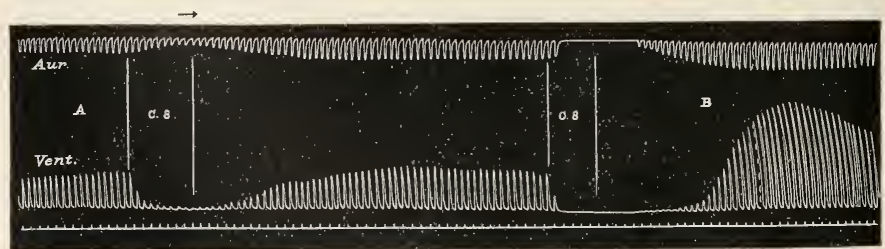

Fig. 113. - Heart of frog. Suspension method. Clamp in auriculo-ventricular groove. Stimulation of the vagus in the neck (i.e. the combined vagus and sympathetic nerve), showing the effect of the simultaneous stimulation of the two nerves. 
the auricular, and therefore also of the ventricular, contractions is not due to a slowing of the rate of the heart-beat, for, as is seen, the sinus continues beating at the same rate as before; but is due to a partial blocking at the sino-auricular junction, caused by the stimulation of the vagus nerve. In the majority of instances the vagus of the tortoise

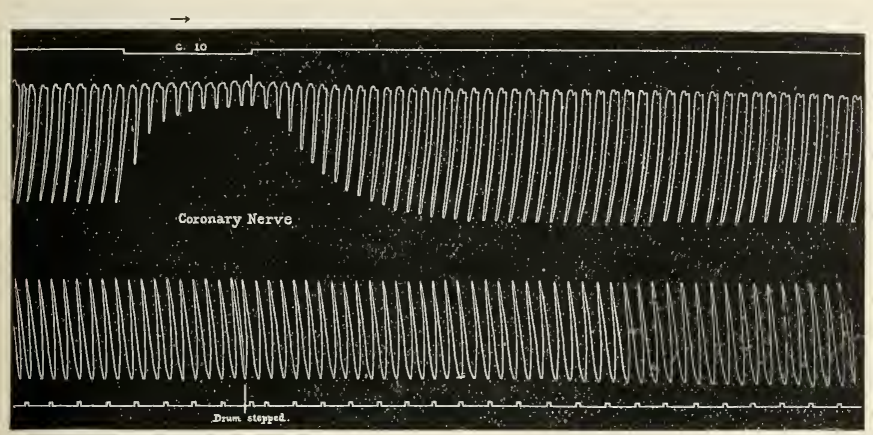

Fig. 114. - Heart of tortoise. Suspension method. Stimulation of the peripheral end of the cut coronary nerve.

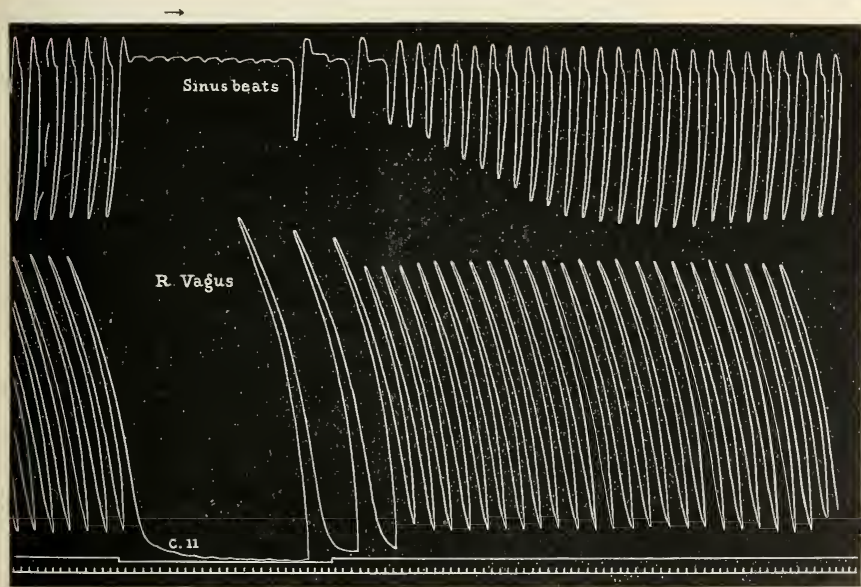

FIG. 115.-Heart of tortoise. Suspension method. Stimulation of right vagus nerve in the neek.

(especially the right vagus) causes a slowing or stoppage of the whole heart-beat, just as in other animals. It is further noticeable in these two curves that when, as in Fig. 114, the rate of the auricular contractions is unaltered, the contractions of the ventricle remain absolutely unaffected, while in Fig. 115 the contractions of the ventricle, instead of being diminished by the stimulation of the nerve, are increased in strict accordance with the amount of slowing of the auricular contractions. 
Such curves are to be explained as follows:-

If the muscular fibres are initiating a rhythm, then the effect of stimulating the fibres of the vagus which supply them is to diminish both rate and contraction force, as is the case in the sinus muscle; if the muscle fibres are not initiating a rhythm, but only responding to a regular series of contraction waves from the sinus, then the stimulation of the fibres of the ragus which supply these muscle fibres cannot influence rate, but only the force of their contraction, as is seen in the case of the auricle when the coronary nerve is stimulated.

This experiment proves that the nerve fibres of the vagus which diminish the force of the auricular contractions act in the same manner after they have passed the ganglia in the sinus as before. Still it does not prove that the post-ganglionic fibres which pass directly into the auricular muscle act in the same way as the pre-ganglionic fibres, for at the junction of auricle and ventricle groups of nerve cells exist with which the fibres of the coronary nerve or some of them-for many, if not all of them, are medullated-do certainly have comnection ; and it is possible to argue that these cells are part and parcel of an inhibitory centre, just as much as those in the sinus. It is, however, easy to show that the post-ganglionic nerve fibres in the muscle itself still produce the same effect upon the auricular contractions as the pre-ganglionic fibres.

A strip of muscle is taken from the apex of the auricle, suspended and attached by means of a thread at one extremity to a lever, so as to register its contractions. Single induction shocks at regular intervals are sent in to the muscle at the fixed end; and by means of very thin wires, fixed at the two extremities of the muscle, which do not interfere with the free movements of the muscle strip, a weak interrupted current can be sent through the whole length of the strip. When the strip is first suspended, each contraction is able to travel only a little way, owing to the number of blocking places caused by the section.

These blocking places are gradually removed, their removal being assisterl by the application of a weak interrupted current at intervals, until at last every single induction shock produces a strong contraction which travels quickly and uniformly from one eml of the strip to the other. When this result has been obtained, the strip will often begin to beat spontaneously. If now a weak interrupted current, too weak to cause any contraction, is sent through the strip, whether the contractions are spontaneous or not, these contractions are diminished in size, or even inhibited altogether, just as if the vagus nerve had been stimulated. Further, atropine applied to the strip removes this effect of the interrupted current, just as it removes the action of the vagus nerve. Finally, if the strip be clamped in the middle, and the single induction shocks applied to the lower half only, while the weak tetanising current is applied to the upper half, it is possible to clamp so tightly that only the lower half will respond to the single induction shocks, and yet these contractions will be diminished in size by the stimulus of the weak interrupted current applied to the upper half only. Clamp tighter, and then the weak interrupted current produces no effect upon the contractions of the lower half. In other words, the upper part of the strip behaves like a nerve to the lower half of the strip.

These experiments are, it appears to me, very strong evidence that the post-ganglionic inhibitory fibres in the auricular muscle itself act in the same way as the pre-ganglionic, and that such fibres are stimulated 
simultaneously with the muscle, whenever the muscle is directly stimulated. The interpretation, in fact, which Schiff gave to the local diastole, caused by direct stimulation of the frog's ventricle, is confirmed and elucidated by these experiments on the behaviour of the strip of tortoise auricle to direct stimulation.

One of the most striking primary depressing effects of stimulation of the vagus nerve is the depression of conductivity. In the intact heart such depression takes place at the two natural blocking points, namely, the sino-auricular and auriculo-ventricular junctions; in my experiments with the suspended heart I have frequently obtained upon stimulation of the vagus, especially in the case of the crocodile, tortoise, and snake, an absolute standstill of both auricle and ventricle, although the sinus has continued to beat regularly. In these cases it was perfectly clear upon inspection that the contractions of the sinus stopped absolutely at the sino-auricular junction, and did not pass into the auricle. Fig. 115 affords an example of this phenomenon.

So also in the case of the suspended heart of the frog, with the clamp at the auriculo-ventricular junction, I have seen instances where stimulation of the vagus nerve has caused the ventricle during the stimulation to respond to only every second auricular contraction. In such cases, owing to the simultaneous stimulation of the accelerator nerve fibres, the rate of rhythm was not slowed, and in consequence the diminution in conductivity was able to be manifested.

This depression of conductivity at the auriculo-ventricular junction, as the result of stimuli of inhibitory fibres, gives a simple explanation of Eckhard's experiment, ${ }^{1}$ that stimulation of the cut septal nerves in the frog causes the ventricle to stand still, although both auricle and sinus continue beating.

Perhaps the clearest evidence of the primary influence of the vagus nerve upon the conductivity of the auricular muscle is given by stimulation of the nerve when the auricle of the tortoise is slit up so as to cause a partial block in the passage of the contraction wave over the two parts of the auricle as in Fig. 105. In this case for a long time the contractions of $A v$ and $V$ follow only upon every second contraction of $A s$, and the result of stimulation of the inhibitory fibres supplying the auricle is to make the block complete, so that during the stimulation no contractions pass from $A s$ to $A v$, and the ventricle therefore remains still. Naturally this experiment succeeds only when stimulation of the vagus does not cause a standstill of the whole heart, and is therefore most easily seen upon stimulation of the coronary nerve, rather than of the vagus nerve itself.

It is clear from what has been said, that apparent standstill of the heart, i.e. of the auricle and ventricle, may be produced by stimulation of the vagus in three distinct ways-

1. By the depression of rate in the simus.

2. By the depression of conductivity at the sino-auricular junction, so that the sinus continues beating, but the auricle and ventricle remain still.

3. By the depression of the force of the contraction to invisibility, so that, although the contractions pass from the sinus into the auricle, these latter are so diminished in force as to be not apparent.

Such a depression of the force of the contractions of both auricle

${ }^{1}$ Beitr. z. Anat. u. Physiol. (Eckhard.), Giessen, 1876, Bri. vii.

VOL II. - I 4 
and ventricle has only been observed by me in the heart of the toad or frog, where, in consequence of the combination of the accelerator nerves with the vagus, the diminution of contraction force has been accompanied with acceleration of rate.

In addition to the depression of rhythmical power, of excitability, of contraction force and of conductivity, the vagus produces as a primary effect a depression of tonicity, so that the muscular tissue relaxes when the nerve is stimulated.

Ever since the action of the vagus has been studied by physiologists, the flaccid condition of the heart distended with blood, which is seen during standstill, has attracted their attention, and has been, again and again, attributed to an active relaxation of the cardiac muscle due to the stimulation of the inhibitory nerve. The difficulty has always been to prove that such relaxation does in reality take place, owing to the difficulty already mentioned

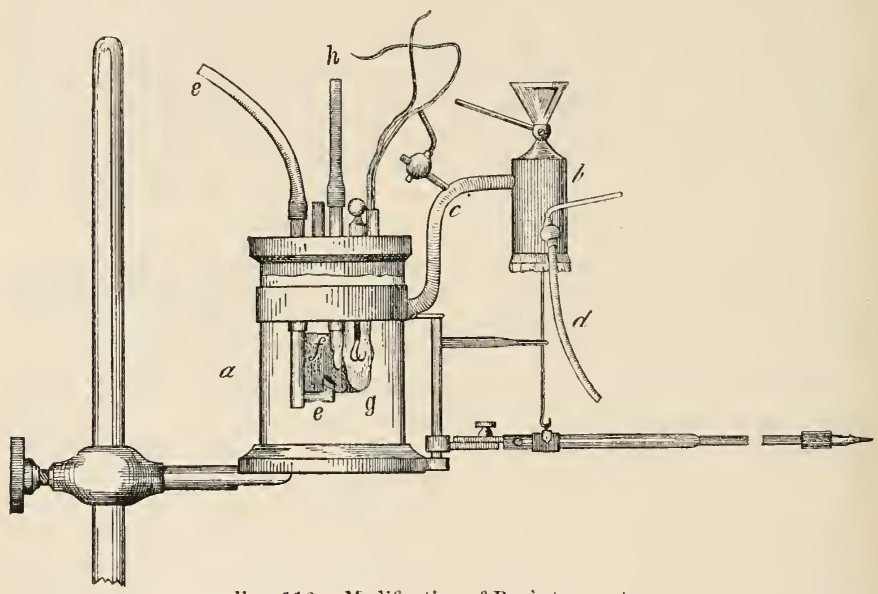

FIg. 116.-Modification of Roy's tonometer.

of proving that the cardiac muscle possesses tone in addition to its rhythmical contraction. The extent of relaxation between the contractions depends on the rapidity of rate of beat; any influence, therefore, such as the action of the vagus nerve, which slows rate, must increase the relaxation between the contractions without necessarily altering the tone of the muscle. In order, then, to be sure that the nerve does alter the tonicity of the muscle, the rate of beat must be unaltered or even quickened at the same time that the relaxation of the muscle is observed. Also it is clear that the extent of relaxation can be more easily observed by the measurement of the volume of the ventricle or auricle, than by any linear measurement, such as is given by the method of suspension. In order to estimate this relaxation, I have made use of a modified Roy's tonometer, ${ }^{1}$ as seen in the accompanying Fig. $116 .^{2}$

It consists essentially of two parts-a glass chamber $a$, which contains the heart and its nerve, and a recording chamber $b$, which contains oil, and is

${ }^{1}$ Roy, Journ. Physiol., Cambridge and London, 1878, vol. i.

2 Cf. Phit. Trans., London, op. cit., p. 1019. 
fitted with a piston and membrane as in Roy's tonometer. The chamber $a$ is hermetically sealed with a vulcanite stopper, which holds a cannula $e$, which can be fitted into the rena rava inferior, a small glass cannula $h$, which is fixed into one of the aortic trunks, a cork slab $f$, on which the heart is fixed, and a glass tube /, containing the platinum electrodes, which is closed, except for two small holes which are blown exactly opposite each other in the middle of this tube. Through these holes the vagus nerve can be drawn by a thread attached to its extremity, and by pinning the threar on to the cork slab $\dot{f}$, the nerve will always remain in contact with the electrodes. The fluid within the chamber $a$ c innot penetrate within this glass tube, and therefore the nerve lies on the electroiles surrounded with moist air, although the chamber $a$ is filled with salt solution or other fluid. The lower extremity of the reservoir $b$ is on the same level as the top of the chamber $a$, so that $b$ can be filled with oil without any fear of contamination from the salt solution or other fluid in $a . \quad A$ vulcanite plug is provided in one of the holes in the vulcanite stopper, for the purpose of removing all air from the chamber $a$. The tap and indiarubber tube $d$ is for the purpose of allowing oil to pass out of $b$, and so, by means of the negative pressure thus caused, of raising the piston until the lever is in the horizontal position. In tying the superior vena cava care must be taken not to injure the vagus nerve.

By means of this apparatus the lieart can be kept beating for days, if necessary, and the vagus stimulated. The fluid which flows through the heart may be varied at will, and the effect of the vagus tested with different fluids. The heart may be placed in a fluid of a composition different to that which is flowing through its cavities. The whole heart may be used, or only the ventricle or apex.

The curves so obtained are difficult to interpret, owing to the fact that the volume measured at any moment is the volume, not of the ventricle or auricle alone, but of the whole heart; so that if, as seems the case in the normal beat of the frog's heart, there is no moment of time when both auricles and ventricle are simultaneously fully relaxed, it necessarily follows that an action like that of the vagus, which causes a simultaneous cessation of their contractions, must cause a marked increase in the volume registered by the apparatus. As mentioned in my paper, I have endeavoured to elininate the action of the auricles by raising the pressure within them to a height sufficient to prevent their contraction, and therefore to estimate the change of volume caused by the stimulation of the vagus nerve in a heart consisting of quiescent auricles and a contracting ventricle. Also, owing to the presence of the accelerator nerves in the frog's vagus, I have been able to obtain curves in which an increase of volume has been distinctly shown without any slowing of rate of beat, or any indication of auricnlar contractions. From these curves I conclude that the vagus is able to diminish the tonicity of the frog's ventricle and a fortiori of the frog's auricle.

So far I have described the primary effects of the vagus nerve upon the beating heart, and shown that such effects are produced by the direct action of the nerve upon the active muscular tissue. It is therefore of great interest to show the nature of this action upon the cardiac muscle when that muscle is at rest. Such an experiment is possible in the case of the tortoise, owing to the presence of the coronary nerve, for by means of that nerve the vagus in the neck still remains in commumication with the auricle and ventricle when the sinus is cut away without injuring the coronary nerve. In such a preparation-equivalent, 
to the first Stannius ligature-the auriele and rentricle at first remain absolutely quiescent naturally, and then hegin to contract with their own independent rhythm. It is then found that the contractions of the auricle are diminished in force when the vagus nerre in the neck is stimulated, showing that the vagus in the neck can affect the auricular muscle, through the corourary nerve.

This experiment shows that the ragus produces an effect upon the muscle of the auricle, which is manifested as a diminution of the force of its contractions, as soon as the auricle begins to beat. It follows clearly that this action of the nerve on the muscle must still exist during the period of quiescence, before the spontaneous contractions begin, although the stimulation of the nerve at this time produces no visible change of form.

This invisible aetion of the nerve on the muscle can be rendered

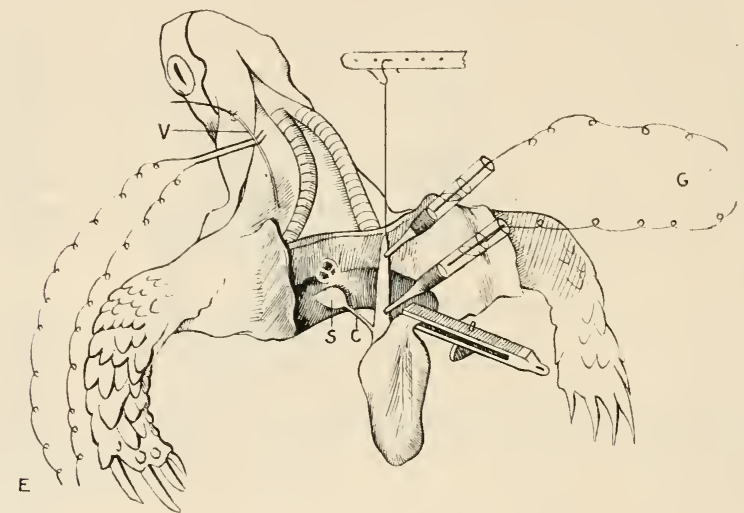

F16. 117.- Method of preparation of the heart of the tortoise in order to demonstrate the action of the vagus nerve on the quiescent aurieles. $\mathrm{S}$, sinus and part of auricle; $\mathrm{C}$, the coronary nerve and vein; $\mathrm{V}$, the right vagus nerve: $\mathrm{E}$, electrodes connected with interrupted current ; $\mathrm{G}$, non-polarisable electrodes connected with the galvanometer.

visible by means of the galvanometer, and it is then seen that the action of the inhibitory nerve is of the opposite character to that of a motor nerve, in that it produces a positive and not a negative variation of the demarcation current. ${ }^{1}$

The experiment is carrierl out in the following way :

The heart is ent through between the sinus and the auricles so as to leave the coronary nerve intact, and the apex of one auricle and that of the ventricle are attached to the levers in the usual way. Fig. 117 shows the preparation. The apex of the auricle is heated, so as to cause a strong demarcation current, and non-polarisable electrodes are placed on the heated apex and on the uninjured auricular tissue near its base. The electrodes are attached to the galvanometer, and the demarcation current is registered every five seconds by an observer stationed at the galvanometer, who is therefore not necessarily aware

1 Gaskell, Beitr. z. Physiol. C'. Ludurig a. s. 70 Geburtst. geu., Leipzig, 1887, S. 114 ; also Joum. Physiol., Cambridge and London, 1887, vol. viii. p. 104. 
when the nerve is stimulated. The curve so obtained is given in Fig. 118, and shows clearly the fact of the positive electrieal variation in eonsequener of vagus stimulation, and also that such electrical change takes place so slowly that its contrse can easily be registered by an observer who is noting the position of the galvanometer needle only every five seconds. Such a rate of change is remarkably different from the rapid norement in the opposite direction, which ocenrs when a contraction takes place. This action of the vagus is abolished by atropine, or hy ligaturing the coronary nerve tightly, so as to destroy its plyysiological continuity with the vagus in the neck.

Such, then, are the primary effects of the stimulation of the ragus nerve: depression of rate, depression of excitability, depression of con-

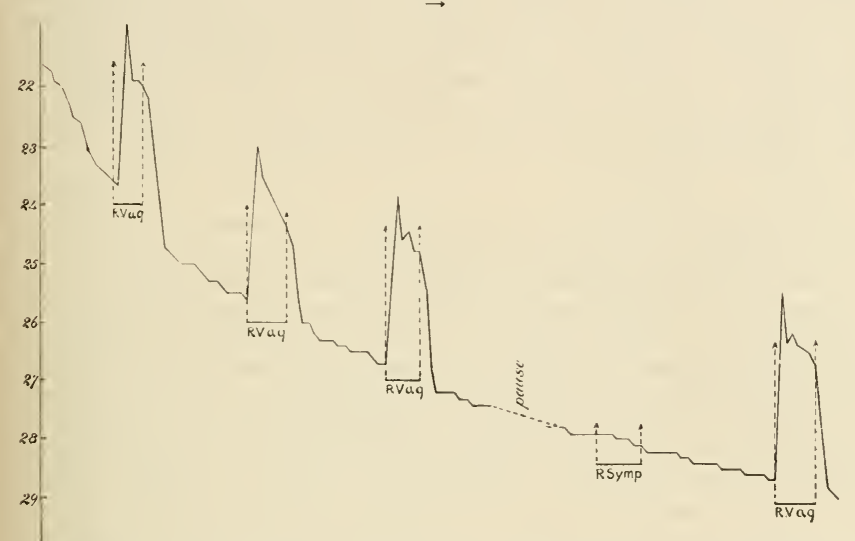

FIG 118. - Curve of demarcation current caused by heating apex of auricle. Divisions of ordinate represent divisions of the galvanometer scale in centimetres. Fine divisions of abscisse represent intervals of five seconds. As is seen, the demareation current diminishes at first rapidly, then more slowly, during the course of the experiment. At intervals, as represented by the arrows, the right vagus or right augmentor nerves were stimulated.

traction force, depression of conductivity, depression of tonicity, and increased positivity of the muscular substance.

We may therefore conclude that the nerve acts in the same way on all the attributes of auricular muscle, that its terminal fibrils in the muscle act in the same way as its main fibres in the neck, and that atropine paralyses these terminal fibrils; and that, therefore, as far at all events as the auricle is concerned, any explanation of inhibition must be consistent with a direct action of an inhibitory nerve on muscle.

The evidence shows, further, that in all cold-blooded animals, without exception, the vagus acts powerfully on the auricle. When, however, we consider its action upon the ventricle, we come immediately face to face with one of the most puzzling and most important facts in the innervation of the heart of cold-blooded vertebrates, namely, that as far as is at present known the vagus has no effect whatever upon the ventricle of any cold-blooded vertebrate except the Amphibia. In my experiments ${ }^{1}$

${ }^{1}$ Journ. Physiol., Cambridge and London, 1587, vol. viii. PI. X1I. Figs. 1 and 2, $a$, 
on the tortoise, I could not find any dimimution in the force of the contraction of the ventricle, snch as is well known to oceur in the frog and toad (cf. Figs. 114 and 115 and Figs. 108 and 109), but, on the contrary, have always found that the ventricular contractions are unaltered in strength if the rate of heat is unaltered (Fig. 114), or increased in strength according to the amomnt of slowing of the rate of beat caused by the stimulation of the nerve (Fig. 115); and MacWilliam ${ }^{1}$ says that during the standstill dhe to vagus stimulation there is no diminition of excitability in its ventricle, as certainly occurs in the case of the frog. The same indifference of the ventricle to ragus stimulation was seen in the case of the eel by MacWilliam, of the crocodile and snake by me, of the terrapin and toad-fish by Wesley Mills: ${ }^{2}$ in other words, in all the cases of Teleostei, Chelonia, and Reptilia which have been observed, the vagus does not in any way affect the ventricle. On the other hand, the vagus dnes aflect the ventricle in the same way as the auricle-though to a less degree-in the frog and toad, in the newt according to MacWilliam, and in Menobranchus according to Wesley Mills, ${ }^{3}$ all instances which are included in Amphibia.

Further, in absolntely strict accordance with this absence of effect upon stimnlation of the nerve in the neck, is the alsence of inhibitory effect 1 pon direct stimulation of the muscle.

Thus, as already mentioned, direct stimulation of the ventricle of the frog produces a local liastole which may last for some time, and is removable by atropine-produces, in fact, on the frog's ventricle an inhibitory effect which exactly corresponds with what I have describer as the effect of direct stimulation of the suspended strip of the auricle of the tortoise. With, however, the suspended strip of the ventricle of the tortoise matters are very different. Here, just as in the case of the antricle, a series of blockings to the passage of the contraction are first got rid of, so that finally each of the single induction shocks causes a contraction which passes freely from one end of the strip to the other, but at no time does the passage of a weak interrupted current diminish the force of these contractions; even if the strip is beating spontaneously there is no evidence of any inhibition when the interrnpted current is sent through.

A precisely similar negative result upon direct stimulation of the ventricle has heen observed by me in the crocodile, and by MacWilliam in the eel.

Another somewhat mexpected coincidence is bronght out by the comparison of ventricnlar muscle whose contractions are diminished by vagus stimulation, and ventricular muscle whose contractions are not so diminished: namely, that the staircase phenomenon obtains only in the former case and not in the latter.

This phenomenon of the staircase (Treppe) was discovered, as has been already mentioned, by Bowditch ${ }^{4}$ in the apex preparation of the frog's ventricle, and consists in this, that in the quiescent apex, through which a series of efficient single induction stimuli are sent, the first contraction is not the strongest, but the contractions increase in staircase fashion, until a certain maximnm is reacherl at which the con-

1 Journ. Physiol., Cambrilge and London, 1585, vol. vi. 1) 224.

2 Ibid., vol, vi. p. 246 ; anil vol. vii. 1. 81 .

3 1hid., 1886, vol. vii. p. 81.

+ Op. cit. 
tractions remain steady for some time. MacWilliam ${ }^{1}$ has been unable to confirm this observation of Bowditch when the ventricle of the eel or tortoise is used instead of that of the frog. In these cases he says there is no sign of any ascending staircase, the first contraction is as large as any of the others. I have seen the same thing in the strip of the tortoise ventricle; here it is true that the single induction shocks, when first sent into the strip, cause very small contractions, and gradually and more or less steadily the contractions increase in size, until they attain their maximum; this phenomenon, however, is clearly due to the gradual removal of the blocking points in the strip. When the strip has recovered, so that every contraction passes freely from end to end, then I have found that the first contraction after a pause, whether long or short, is as large as any of the subsequent contractions. It is very suggestive in this connection, that Bowditch ${ }^{2}$ found that atropine removed this staircase effect in the frog's ventricle. The atropinised frog's ventricle, in fact, behaves in this respect, as in so many others, similarly to the non-atropinised ventricle of the tortoise, and this suggests that the phenomenon of the Treppe is due to the direct stimulation of inlibitory fibres in the frog's ventricle.

The investigation of the primary action of the vagus on the heart of cold-blooded animals brings out clearly two facts of great importance-

1. The inhibitory action is a direct one of nerve fibre upon muscle fibre, and affects all the properties of the muscle fibre in the same wayrhythmicity, excitability, conductivity, contraction-force, and tonicity.

2. The cold-blooded vertebrates are divisible into two sets, namely, those of the amplibian type, in which the ventricular muscle is affected in the same way as the auricular, but to a less degree, and those of the same type as Teleostei, Chelonia, and Reptilia, in which the inhibitory action of the vagus nerve appears to be confined to the auricles; at all events, no action is apparent on the ventricles.

This extraordinary and unexpected difference in the behaviour of the vagus nerve in these two groups leads at once to the idea that we are dealing here with a deep-seated morphological difference, and that the study of the physiology of the cardiac nerves may give very important help in the classification of the vertebrata; thus, if the birds have arisen from reptiles and the mammals from amphibia, we might expect to find that the vagus nerve does not affect the ventricle in the bird's heart, but does act upon the mammalian ventricle in the same way as upon that of the frog or toad. As far as the latter question is concerned, the experimental researches of MacWilliam ${ }^{3}$ point to the conclusion that the vagus does diminish the force of the ventricular as well as the auricular contractions in the various mammalia investigated (cat, dog, rabbit, rat, hedgehog, guinea-pig), and indeed that the nerve acts upon rhythm, excitability, contraction-force, etc., of the different parts of the heart, precisely in the same way as in the case of the amphibian; so that, as far as the heart of the mammal is concerned, the physiologist would agree that it is closely related to the amphibian. With respect to the heart of the bird, I have been unable to find any evidence ; ${ }^{4}$ I cannot find that anyone has ever observed the effect of

1 Journ. Physiol., Cambridge and London, 1885, vol. vi. p. 212.2 op. cit.

3 "Inhibition in the Mammalian Heart,"Joum. Physiol., Cambridge and London, 1888, vol. ix. p. 345 .

${ }^{4}$ Knoll, who has recently published a paper on the action of the vagus nerve in warmblooded animals (Arch. f. d. ges. Phy iol., Bonn, Bd. xlvii. S. 595), descril es some experiments on the hearts of pigeons as well as those on mammals. In this paper he points out that stimulation of the vagus nerve does diminish the strength of the contractions of the 
the vagus upon the force of the ventricular contractions of the avian heart; it would undoubtedly be a very interesting subject of research. So also, in the case of Elasmobranchii, I cannot find anything definite; it does not appear to be known whether they behave like Amphibia or Teleosts. It would also be interesting to investigate the lung-breathing fishes, Protopterus, etc., which I should expect to behave like Amphibia.

It is perfectly conceivable, as Roy ${ }^{1}$ has pointed out, that the strength of the auricular contractions is the most important factor in the beat of the heart, and that, therefore, it is not so necessary that the force of the ventricular contractions should be regulated to the same extent as the auricular; but still it is difficult to believe that in the evolution of the heart one part of its muscular structure was supplied with nerves, and not the other; we must, it seems to me, imagine that in the protovertebrate, the whole of the muscular tissue of the longitudinal venous sinuses was supplied with the inhibitory fibres, and that, as the different groups evolved themselves with the formation of auricles and ventricles, the inhibitory fibres still supplied all parts of the heart. It is possible that the difference in the behaviour of the ventricles in the two groups is due to a difference in the evolution of the striated muscular tissue of the ventricle, and that it still remains to be found out what change the inhibitory fibres do produce in the ventricular muscle of such an animal as the tortoise. It has certainly seemed to me difficult to believe in the absence of any diminution of tone in the muscular tissue of the ventricle upon stimulation of the vagus, when one sees the enormous increase in the volume of the whole heart when the nerve is stimulated. Still, on the other hand, it is not easy to understand how a nerve can produce a muscular relaxation without any accompanying diminution in the force of the contractions. We must wait for further investigations of the action of the cardiac nerves on the tortoise ventricle before it is possible to decide this question.

2. The action of the augmentor nerves.-The primary action of the augmentor nerves is in every respect exactly the opposite of that of the inhibitory. The evidence both in cold-blooded and warm-blooded animals shows that the rate of rhythm is increased, that the force of the contractions is increased, and that the conductivity is increased. Here, just as in the case of the inhibitory nerve, it is difficult to make any positive statement as to any increase in tonic contraction, apart, of course, from the diminished relaxation that of necessity accompanies an increased rate of contraction in a normally beating heart. Of these different phenomena I have already illustrated the alteration of the rate and force of the contractions in the case of the toad or frog, and it may be noticed that the augmentor nerve takes a very much longer time than the inhibitory nerve to reach its maximum effect, whether that effect consists of an alteration of rate or of contraction force; it is partly for this reason that, in the conjoint stimulation of the two nerves, the action of the inhibitory nerve is always most apparent at first, and that

ventricle in the mammalian heart, thongh to a less extent than those of the auricle. When a small dose of atropine is given, then it frequently occurs that the contractions of the ventricle are slightly larger dming stimulation of the nerve, provided that such stimulation has eansel some slowing of rate. He finds, therefore, that the mammalian ventricle behaves like the amphibian, but the atropinised ventricle behaves like the chelunian. In the ease of the pigeon he stater exmessly, and illustrates his statement by tracings (Fig 1, Taf. II., and Figs. 1 and 8, Taf. IV.), that the ventricle of its heart reacts nornally withont atropine to vagus stimulation in the same manner as the atropinised mammalian ventricle, i.e. its contractions are not diminished but inereased in size if the rate is dininished : that, in fact, the avian ventricle behaves to vagus stimulation like the chelnnian and reptiliaı and nut like the amphibian ventricle.

1 Journ. Physiol., Cambridge and London, 1878, vol. i. p. 452. 
of the augmentor most conspicuous later. I have chiefly studied the influence of the augmentor nerve upon conduction in the heart of the frog and toad, by means of the clamp in the auriculo-ventricular groove. When the clamp is tightened so that a partial block is caused, and the ventricle is responding to every second or more contraction of the auricle, then stimulation of the augmentor nerve forces the block, so that every contraction passes, even although the rate is increased by the stimulation. The same action upon the passage of a contraction wave over a block, as the result of stimulation of the augmentor nerve, has been described by MacWilliam for the eel, and by Bayliss and Starling ${ }^{1}$ for the mammalian heart.

The position of the augmentor nerves seems clear. They belong, as already mentioned, to the same great group of visceral nerves as the vaso-constrictors; they increase the rhythmical power of that part of the heart which is initiating the rhythm; they increase the contraction force and conductivity of the cardiac muscle. They may distinctly be considered as the cardio-motor nerves, the difference between them and other motor nerves being due to the fact that they are motor to a rhythmical instead of a quiescent muscle. The question then arises, Can such a notor nerve cause a quiescent heart to beat? At first sight it would appear that the answer to this question must be in the affirmative, for as long ago as 1860 , Schelske ${ }^{2}$ asserted that the vagus, i.e. the conjoint vagus and augmentor nerves, could make the frog's heart beat again when it was reduced to standstill by raising the temperature. I myself have found that if the heart of the frog or toad be left till next day, it is often apparently quite motionless, and then stimulation of the conjoint vagus and augmentor nerves will make it beat again strongly. I found, as might be expected, first, that this effect was due to the augmentor fibres, and, secondly, that in reality the heart was not still. A powerful lens showed in every case the existence of exceedingly minute but regular contractions of the sinus and great veins, so that although the auricles and ventricle were absolutely motionless, the sinus contracted regularly. The standstill, in fact, was due to a block between sinus and auricles, just as in the ordinary case of a dying heart a block is first caused between auricles and rentricle, and then between auricles and sinus. The case, then, was not a case of motor action upon a quiescent heart, but a very striking example of the power of the augmentor nerve in removing a block to the passage of the contraction wave. Similarly, with regard to Schelske's experiment, the blocking power of heat upon the passage of the contraction from auricle to ventricle, and the removal of that block by stimulation of the augmentor fibres of the vagus nerve, is shown in my Philosophical Transactions paper, Fig. 17, Pl. LXIX. On the other hand, Löwit ${ }^{3}$ showed, in 1882, that when the heart of the frog had been brought to standstill by the application of muscarine, stimulation of the vagus nerve was able to cause such a quiescent heart to beat strongly. I myself ${ }^{4}$ have repeated his experiment, and found that here too it is the augmentor nerve which causes this effect, and apparently without any sign of beating in the sinus. I found, however, that a further dose of

1 Journ. Physiol., Cambridge and London, 1892, vol, xiii. p. 407.

2 "Ueber die Veränderungen der Erregbarkeit durch die Wärme," Heidelberg, 1860, S. 20.

s Arch.f. d. ges. Physiol., Bonn, 1882, Bd. xxviii. S. 333 .

4 "On the Action of Muscarine upon the Heart, ete.," Journ. Physiol., Cambridge and London, 1887, vol. viii. p. 404 . 
muscarine prevented any such action of the nerve, and am therefore inclined to believe that in this case also the motor effect cannot be produced, unless some portion of the heart (in this case perhaps the large veins) is still beating.

Is it then possible to demonstrate any action of the augmentor nerve upon the quiescent muscular tissue of the heart? The evidence appears clearly to show that the augmentor fibres in the frog and toad supply the muscular fibres of all parts of the heart-sinus, auricles, and ventricle-and eause such ehanges in that tissue that the rhythmical tissue of the sinus beats more quickly and more strongly, and the contractions of the auricle and ventricle increase in force, while yet they are unable to produce any visible effect in any part of the heart which is quiescent. Can we by any means isolate the ventricle, and yet leave the augmentor nerves to the ventricle intact, so as to test their action on the quiescent ventricle? This can be done either by causing a block between sinus and auricles, by means of the application of muscarine to the sinus, or by clamping between auricles and ventricle. In both cases the ventricle remains still, and in both cases stimulation of the augmentor nerve is able to force the block, and thus produce a series of ventricular contractions. These contractions are increased in force, showing that the augmentor fibres to the ventricle are intact. Increase the muscarine very carefully, or slightly tighten the clamp, and it is then seen that the block cannot be forced, and so the ventricle still remains quiescent when the nerve is stimulated, and no visible effect is produced. However, upon testing this quiescent muscle by means of the galvanometer in the same way as already described in the case of the vagus nerve, a distinct variation in the muscle current was seen upon each stimulation of the nerve, of the opposite character to that seen upon vagus stimulation in the case of the tortoise auricle. ${ }^{1}$ Give still more muscarine, or clamp still tighter, and then stimulation of the nerve is absolutely without effect, showing that the previous effect was due to a true nervous action.

This experiment shows that the augmentor nerve causes an electrical change in the muscular tissue, of the opposite kind to that caused by the inhibitory nerve, and it is worthy of note that the process to which this increase of negativity is due is like the corresponding positive change, a slow slight one, very different indeed from the rapid excessive process which initiates a contraction. The contrast between this slow motor effect of the augmentor nerve and the rapid movement of the galvanometer needle in the same direction when a contraction takes place, is most striking. The nature of the electrical change in the case of both nerves seems to me to point to the conclusion that the change which occurs in the quiescent muscle, upon stimulation of the inhibitory nerve, is of the nature of a relaxation or diminution of tone, while the change which occurs upon stimulation of the augmentor nerve is of the nature of an increase of tone. In neither case have I succeeded in actually measuring any difference in length of the muscle, coincidently with the electrical change, so that the alteration of tone must be slight.

It seems to me that an alteration of toue in the two directions, due to an activity emanating from the ganglion cells of the two sets of nerves, is in accordance with the known facts of the function of the efferent vagrant ganglia, and the nerves emanating from them in other cases, e.g. the vasomotor and viscero-motor ganglia and nerves.

The augmentor nerves, like the inhibitory, appear to affect the different parts of the heart to a different degree in different animals, and apparently

${ }^{1}$ Journ. Physiol., Cambridge and London, 1887, vol. viii. p. 404, Pl. XIII. 
the difference of effect is in the same direction as in the case of the inhibitory fibres. Thus we see in all animals the rhythmical tibres of the large veins and sinus are stirred up to increased rate of beat, and the fibres of the auricles are made to contract more vigorously, while in the amphibians the ventricular muscle is also most markedly influenced by the augmentor fibres which supply it. On the other hand, I am not at all clear whether any augmentor effect can be proved on the ventricle of the crocodile or tortoise; certainly, as far as my curves show, the effect is insignificant in comparison with the increase in the force of the auricular contractions, and I have grave doubt: whether there is any true effect at all produced, whether in fact the ventricle of Chelonia and Reptilia is not as indifferent to stimulation of augmentor fibres as to stimulation of inhibitory fibres. In the case of these nerves, too, the mammalian heart behaves like the amphibian, according to the observations of Roy and Adami, ${ }^{1}$ and of Bayliss and Starling. ${ }^{2}$ The comparative physiology of the vertebrate heart requires further investigation, and would, I believe, yield results of very great value to the morphologist as well as to the physiologist.

3. The after-action of the inhibitory and augmentor nerves.From what has been said, we may conclude that the muscular tissue of the heart is supplied by two nerves of opposite action, the one of which, speaking broadly, canses a process of relaxation, or an atonic condition, and the other a tonic condition in the tissue. In order to try to understand the nature of these processes, we must investigate, not only the primary effects of stimulation of the nerves, but also their after-effects. Thus we consider that the action of an ordinary motor nerve upon muscle can be classed as katabolic, i.e. the metabolism caused is destructive in its character, not only because of the evidence given during the contraction, bnt also because of the subsequent exhaustion which follows a series of contractions.

What, then, are the after-effects of stimulation of the inhibitory and augmentor nerves respectively, and how far do they throw light on the meaning of inhibition?

In endeavouring to formulate any view of cardiac inhibition, there are, it seems to me, three possible methods of surveying the problem.

The first is the view which has been and is still so largely held, namely, that the inhibitory process takes place outside the muscular tissue, and can be explained either by Rosenthal's resistance theory, or some theory of interference of vibrations, similar to what is well known in the case of light. This theory is absolutely untenable, in view of the fact that the nerve acts directly on the muscle and diminishes its contractions. Of the other two possibilities, we see that such substances as lactic acid and carbonic acid cause a weakening of the contractions, and final stoppage in diastole, so that it is not impossible to imagine that changes detrimental in character to the activity of the tissue might produce the symptoms of inhibition. On the other hand, it is conceivable that a sudden increase in the anabolic process would manifest itself for the time being as a diminution of the katabolic process, and so bring about the diminisher contraction and other inhibitory effects, although at the same time the net result would be an increase in that process.

According to the first view, inhibition, as far as the muscle is concerned, means cessation of extermal stimuli. According to the second, it means a process of exhaustion; according to the third, a beneficial process, the aftereffects of which must be the opposite of exhaustion.

${ }^{1}$ (op. cit., p. 244.

Op. cit., p. 413. 
The most marked after-effect of the stimulation of the inhibitory nerve is its effect upon the conductivity of the tissue. When a block has bcen caused by a partial section of the auricle in the tortoise, so that only every sccond contraction passes the block, it is very common to see, as an after-effect of vagus stimulation, that every contraction passes, and with frequent vagus stimulation I have found that more and more tissue must be cut away, in order to keep the contractions blocked. Similarly, in the frog's heart, the clamp in the auriculo-ventricular groove requires to be screwed up tighter and tighter after a series of stimulations of the intracranial vagus, in order to cause a partial block. ITe see, in fact, just the reverse of what occurs in the dying heart, and also the reverse of what is seen after stimulation of augmentor fibres; for in the latter case I have noticed that after a series of stimulations of the auginentor nerve in the frog or toad, a partial block at the auriculo-ventricular junction, so that the ventricle responds to every second auricular contraction, is very apt to be produced, although with the clamp in the same position it responded to every contraction before the commencement of the series of stimulations. So, too, with respect to the force of the contractions, a series of stimulations of the augmentor nerve distinctly exhaust the muscular tissue of auricle and ventricle, so that it contracts more weakly than it would have done in the same time if no nerve had been stimulated. On the other hand, a series of stimulations of the intracranial vagus in the frog or toad keeps the heart up to a condition of vigorous contraction, which affords a very striking contrast to the condition of exhaustion of the heart which has been acted on by the augmentor nerve. So, too, after repeated stimulations of the augmentor nerve, not only does the heart beat weakly, but also these weak contractions cannot be augmented on stimulation of the nerve to any very great extent; under these circumstances I have frequently seen a decided improvement in the augmentor action, in consequence of the interpolation of two or three intracranial vagus stimulations. In fact, the evidence as to contraction-force and conductivity is distinctly to the effect that in each case the after-effect is in the opposite dircetion to the primary effect.

The after-effect upon rhythm is not so clear. My own experience is that, if I have been stimulating the augmentor nerve alone for a long time at intervals, the net result has been a quick rate of beat throughout the whole time; and if I have been stimulating in the same way the intracranial vagus alone, then, throughout, the heart has been beating slowly. I do not mean to assert that there is never any evidence of an increase of rate after each vagus stimulation, but simply that the exhaustion of the rhythmical tissue of the sinus, which is presumed to follow after repeated stimulations of the augmentor nerve, does not manifest itself in a marked slowing of the beat, any more than the presumed beneficial effect of a series of vagus stimulations manifests itself by a marked quickening of the beat. I do not feel clear how far it is possible to argue with respect to rate in the same way as with respect to force of contractions; an exhausted muscle must necessarily contract more weakly, but does it necessarily follow that an exhausted rhythmical muscle beats at a slower rate of rhythm?

The conclusion to which I came in $1881^{1}$ was that the vagus nerve was the anabolic nerve of the heart, and that inhibition or relaxa-

${ }^{1}$ Phil. Trans., London, vol. elxxiii. p. 1029. 
tion of museular tissue was a sign of anabolism in that tissue, just as contraction of the tissue is a sign of katabolism in that tissue. Subsequently, I have put forward the proposition that all muscular tissues are probably supplied with anabolic and katabolic nerves, the one causing relaxation of the tissue and diminution of its contractions, the other causing contraction of the tissue.

Such double nerve supply has been proved for very many muscular tissues, both unstriped and striped. Thus the muscles of the vascular system are supplied by vaso-dilator as well as vaso-constrictor nerves; the unstriped muscles of the bladder and other visceral muscles are supplied with two opposite kinds of nerves; among these the recent observations of Langley and Anderson ${ }^{1}$ have shown a specially good example in the case of the retractor penis, supplied as it is with motor nerves from the lumbar region and inhibitory nerves from the sacral region; the adductor muscles of Anodon are supplied with two nerves of opposite function, according to Pawlow ${ }^{2}$ and Heidenhain. And the most suggestive case of all, in connection with the question of the action of antagonistic striated muscles in vertebrates, - the adductor and abductor muscles of the claw of the crayfish are, according to Biedermann's researches, supplied with two nerves, of which the one is motor and the other inhibitory.

In close connection with this question of the meaning of relaxation of tone, when brought about by the action of an inhibitory nerve, is the meaning of the relaxation phenomenon, brought about in a tonically contracted muscle by the passage of a constant current through it. Such relaxation has been shown by Biedermann to occur in the snail's ventricle, when the ventricle has been thrown into a state of tonic contraction by too great a pressure within its cavity, in the tonically contracted muscle of Anodon, in the veratrinised sartorius, and has led him to the conclusion that such relaxation of muscle upon the passage of a constant current is evidence of assimilation.

This is, however, not the place to discuss the nature of the action of the constant current upon muscular tissue; it is sufficient to point out that the view of the action of the inhibitory nerves of the heart which I put forward in 1881, and which was subsequently in 1882 restated by Löwit ${ }^{3}$ in the terms of Hering's theory, is a view which is not opposed by the observations of Bielermann and others upon the phenomena of relaxation of muscle, brought about by the direct action of an electrical current.

It may, I think, fairly be said that this view, that the action of the vagus nerve is to promote the anabolic or assimilatory processes in the cardiac muscle, is steadily gaining ground; while the alternative view, that a special inhibitory nervous mechanism is situated in the heart, which causes inhibition by interference with motor impulses, is becoming more and more discredited. Such a riew has been supposed to receive special support from the study of the action of various heart poisons, especially curari, nicotine, atropine, and muscarine.

With respect to these poisons, there is no evidence that they possess any selective power over one set of nerve fibres and nerve cells ratler than another; the question is rather, do they act on the junction of the pre-ganglionic nerve fibre and the ganglion cell, or on the junction of the post-ganglionic nerve fibre and the muscle, or on the muscle itself?

The Action of Certain Poisons.-Nicotine, as already mentioned, acts in all probability on the junction of the pre-ganglionic fibre with the nerve cell.

1 Jow'n. Physiol., Cambridge and London, 1895, rol. xix. p. 71.

^ Arch.f. d. ges. Physiol., Bomn, 1885, Bd. xxxvii. S. 6.

3 Ibid., Bil. xxix. S. 503. 
The action of curari is upon the motor end-plate of the ordinary striated muscle, and takes the place of atropine in cases where the muscle becomes striated in character; thus in the bird's pupil, where the fibres are striped, curari not atropine causes dilatation. In the œsophagus of various mammals where the muscle is partly striped, partly unstriped, atropine prevents the action of the nerve on the unstriped, curari on the striped muscle; and, curiously enough, in the hearts of the snail, octopus, etc., according to Ransom's ${ }^{1}$ observations, where the muscle is not only markedly striped, but also varies in strength of contraction according to the strength of stimulus, and can be tetanised, the action of the inhibitory nerve is not affected by atropine, but is put out of play by curari. In all these cases the curari in all probability acts on the endings of the nerve in the muscle. In the case of the heart of the frog and toad, and doubtless of other vertebrate hearts, the curari acts like nicotine on the junction of the pre-ganglionic fibre with the nerve cell, before it acts upon the junction of the post-ganglionic fibre with the muscle.

Atropine prevents the action of the motor nerves on the unstriped muscle of the œsophagus, of the iris, of the secretory nerves of the gland cells, whether sweat glands or salivary, of the inhibitory nerves of the lieart of vertebrates, and from its local action it is clear that it paralyses the post-ganglionic fibres as well as the pre-ganglionic. In addition, it appears to act upon the muscle fibre itself.

The removal by atropine of Bowditch's "staircase" phenomenon, of the "local diastole" of Schiff, of the inhibitory action of the interrupted current on the strip from the tortoise auricle, are all to be explainer by the action of the drug on the terminations of the post-ganglionic fibres in the muscle. On the other hand, Ransom ${ }^{2}$ has shown that, in the case both of the cephalopod and of the snail, atropine must be classed with drugs, such as digitalin, antiarine, etc., which produce an increased tonic contraction of the cardiac muscle. Romanes ${ }^{3}$ has shown the same thing with respect to the muscular tissue of the Medusce, and I myself, in my investigations on the action of various drugs on the apex of the frog's ventricle ${ }^{4}$ came to the conclusion that atropine acts in a much less degree but in the same direction as those drugs which increasc the muscular contraction, and diminish relaxation.

The direct action of atropine upon the muscle-substance is rendered probable by its antagonising action to drugs which weaken muscular contraction by their direct action upon muscle.

This is especially seen in the case of such poisons as muscarine, which is apparently a muscle-poison, not a stimulant to inhibitory fibres or inhilitory mechanisms.

We see from the evidence brought forward by Luchsinger and his pupils, ${ }^{5}$ that a series of substances, such as potash salts, and the whole group of substances containing trimethyl-ammonium, to which muscarine belongs, all act in the same way upon the muscular tissue of the heart, namely, in the direction of weakeuing the contractions, and ultimately bringing the inuscle into the condition of diastolic standstill; all behave, in fact, in the same manner as lactic acid, according to my observations. In opposition to this series of substances, we find another series which

1 Journ. Physiol., Cambridge and London, 1885, vol. v. p. 261.

2 Op. cit., pp. 301, 331 .

3 Phil. Trans., London, vol. clxvii. p. 736.

4 Joum. Physiol., Cambridge and London, 1880, vol. iii. p. 14.

5 Luchsinger and Am. Glause, "Zur Kenntniss der Hemmungsmechanismen des Herzens," Inang. Diss., Bern, 1884; Olga Sokolow, "Physiol. u. toxikolog. Studien am Herzen," Inaug. Diss., Bern, 1881. 
act in the same way as sodium salts, namely, increase the strength and leugth of the contractions, and ultimately bring the apex or ventricle into a condition of systolic standstill. This series iucludes such poisons as antiarine, digitalin, veratrine, and, to a less degree, atropine.

Further, my own experiments have shown that, just as the alkaline sodium salt and the lactic acid solution can antagonise each other ou the apex muscle, so also muscarine and digitalin ${ }^{1}$ can antagonise each other: and Luchsinger and his pupil Amalie Glause have shown that all the members of the muscarine group behave in the same way to atropine. The evidence, then, is strong that both atropine and muscarine act directly upon the muscular tissue of the heart in opposite directions. What the precise nature of these opposite actions is, whether the poisons form loose chemical combinations with the contractile tissue, as originally suggested by Langley and afterwards by Ringer and myself, or whether they act in some other way, is at present unknown.

The weakening effect of muscarine upon the muscular tissue is shown, not only by the diminution of the strength of the contractions and the relaxation of the tissue, but also by the diminution of the conductivity.

I know no more striking experiment to slow the opposing action of muscarine and atropine, than is afforded by a strip from the auricle of the tortoise, which has been taught to respond vigorously and well to single induction shocks sent through a pair of electrodes applied to one end of the strip. With each induction shock a strong contraction occurs through the whole strip. Now, let a little muscarine solution drop over the strip; immediately the contraction is seen to consist of two parts-first, that of the small piece of muscle between the electrodes, and then, quickly following, the rest of the muscle; a little later, the pause between the contraction of the two parts of the muscle becomes very marked, and a partial block often occurs, so that only every second contraction of the piece between the electrodes is followed by a contraction of the rest of the strip, that contraction itself being weaker; finally, the block is complete, and the only part which contracts is the small piece between the electrodes. Now, apply a drop of atropine solution over the strip; like magic each contraction passes the block, and in a remarkably short time the whole strip contracts to every induction shock as strongly and rapidly as before. We see, in fact, in the strip a miniature representation of the action of the two poisons on the whole heart.

Again, the presence of the coronary nerve in the tortoise enables the question, whether muscarine applied to the sinus stimulates inhibitory mechanisms there, to be tested by means of the electrical changes which occur in the isolated quiescent auricular muscle, when the sinus and therefore the fibres of the coronary nerve are stimulated. I find ${ }^{2}$ that, whereas a crystal of salt applied to the sinus will produce the same electrical variation as stimulation of the vagus nerve, muscarine applied to the siuns produces 110 effect whatever; there is not the slightest indication of any stimulating action.

It has heen further argued by Kobert ${ }^{3}$ that, as a dose of muscariue, which will easily cause standstill when applied to the sinus, will not arrest the contractions of the isolated ventricle, therefore it acts

\footnotetext{
1 Gaskell, Journ. Physiol., Cambridge and London, 1880, vol. iii. p. 12, PI. III.

2 Ibid.. 1887, rol. viii. p. 404.

${ }^{3}$ Arch. f. exper. Puth. u. Pharmakol., Leipzig, 1885, Bd. xx. S. 92.
} 
in a special manner on the sinus. In accordance with this, he finds, and Pickering ${ }^{1}$ confirms his observations, that muscarine is ineffective on the hearts of embryos when they first begin to beat. In other words, muscarine is asserted to be ineffective unless certain nerve cells, which are supposed to constitute an inhibitory centre, are present. Curiously enough, Pickering finds that under the same circumstances pilocarpine, which in other respects acts like muscarine, is effective at a time when muscarine is unable to produce standstill. It seems to me that we are dealing here only with a question of degree, and not with a question of fundamental difference of action. Some tissues are more resistant than others, and their resistance in the embryo differs from that in the adult.

Finally, Ransom's ${ }^{2}$ experiments upon the heart of Cephalopods give very conclusive evidence upon this question, for he has shown, in the first place, that atropine does not paralyse the inhibitory nerve, though curari does; in the second place, that muscarine will produce standstill in this heart as in others, and yet that atropine will antagonise the action of muscarine. Clearly in this case the antagonistic action of atropine to muscarine cannot be explained by the supposition that muscarine stimulates inhibitory fibres.

Vulpian also asserts that muscarine will produce standstill in the snail's heart, and atropine removes that standstill.

We may, it seems to me, sum up the action of these poisons on the vertebrate heart in physiological amounts thus:-

Nicotine and curari break the connection of the pre-ganglionic fibre with the nerve cell.

Atropine acts on the muscular tissue to a slight extent tonically, breaks the connection between the post-ganglionic fibre and the muscle, and also the connection between the pre-ganglionic fibre and the nerve cell.

Muscarine, etc., act on the muscular tissue atonically.

Digitaline, etc., act on the muscular tissue tonically.

\section{The Nutrition of the Heart.}

The beating of the isolated ventricle or apex is dependent, not only on the pressure of the fluid in its cavity, but also upon the nature of that fluid, and a large number of observations have been made for the purpose of finding out what are the constituents which a fluid must contain in order to maintain good contractions of the cardiac muscle. The earlier experiments largely emanated from Ludwig's laboratory, under the guidance especially of Kronecker, and were started by Bowditch's ${ }^{3}$ discovery that delphine was able to make the isolated apex of the heart beat more or less rhythmically. This paper was followed in 1872 by that of Luciani, ${ }^{4}$ who showed not only that the frog's ventricle was able to beat when supplied with serum, but also that its contractions were not always regular like those of the intact heart, but during a portion of the time of action of the serum occurred in distinct groups, with pauses of varying lengths between the groups. Two distinct questions thereupon arose-(1) What are the constituents of serum which enable the ventricle to continue beating? and (2) what is the meaning of this periodicity?

1 Journ. Physiol., Cambridge and London, vol. xiv. p. 449.

$\because 0$. cit.

+ Ber. d. k. Särhs. Gesellsch. d. M־issmseh. Leipzig, 1873, ‥ 11. 
With respect to the first question, the object of all investigators has been to remove the blood out of the ventricle or apex-preparation by means of a normal saline solution, and then when, in consequence of the washing out with the salt solution, the ventricle has ceased to beat, or is beating very weakly, to send in the fluid to be tested, and see whether the heart recovers its contractions. By the experiments of Merunowicz ${ }^{1}$ in 1874 , it was shown that the apex of the heart, when reduced to standstill by washing out with normal saline solution, could be made to beat again by sending through it a solution of the ashes of blood or serum ; and among the different constituents in such ashes Merunowicz supposed that sodium carbonate was the most important, and that a solution of sodium carbonate and sodium chloride alone was able to resuscitate the motionless apex. Gaule, ${ }^{2}$ following on Merunowicz and using Kronecker's perfusion cannula and the whole heart, proved that sodium hydrate was more efficient than sodium carbonate, and that when the heart no longer responded to the alkaline solution it could again be brought to renewed activity by sending through it for a second time the alkaline solution which had already passed through.

He attributed its efficiency over the simple alkaline salt solution to albuminous substances taken up from the heart; he came to the conclusion that amongst such substances peptones were especially efficient when added to the alkaline fluid. This conclusion of Gaule's was tested, and not confirmed, by Martius ${ }^{3}$ in Kronecker's laboratory. He found peptones absolutely useless, after the heart had been thoroughly washed out by an alkaline fluid; and came to the conclusion that the whole value of the alkaline solution was to remove the acid products of metabolism; and he and Kronecker came to the conclusion that the perfused fluid must contain serum albumin, and, further, that any fluid which maintained the cardiac contractions must contain this substance.

In a series of papers commencing in $1882,{ }^{4}$ Ringer showed that a solution, containing sodium chloride, potassium chloride, and calcium chloride alone, would act as an efficient circulating fluid for the frogventricle, provided the salts in question were present in certain proportions. He found (as had been done by Merunowicz) that sodium chloride alone soon brings the heart to a standstill in diastole, but he further showed that the addition of a lime salt causes the beat to be prolonged by delaying the diastole, and eventually produces stillstand in systole. ${ }^{5}$ The potassium salt, on the other hand, when added to this mixture, antagonises the effect of the lime salt, and produces a normal beat. The solutions must be prepared with distilled water, since nearly all natural water contains an appreciable quantity of lime in solution.

The circulating fluid which Ringer eventually adopted is made as follows:-

A 0.6 per cent. solution of sodium chloride is saturated with calcium phosphate, and to each 100 c.c. of the mixture, 3 c.c. of a 1 per cent. solution of potassium chloride is added.

Howell and Cooke ${ }^{6}$ lave lately shown that, if the heart is washed out with saline solution and Martius' fluid to exhaustion, and then supplied with Ringer's fluid, not only will it beat again, but they have seen such beating

${ }^{1}$ Ber. d. k. süchs. Gesellsch. d. Wissensch., 1875, p. 252.

${ }^{2}$ Arch. f. Physiol., Leipzig, 1878, S. 291.

+ Joun. Physiol., Cambridge and London, vols. iii., iv., and vi.

Ibid., vol. iv. pl. 29, 222.

6 Ibid., 1893 , vol. xiv. p. 198.

VOL. II. - I 5

Ibid., 1882, S. 548. 
last for thirty hours. According to Kronecker, this ought to be proof that Ringer's fluid contains serum alhumin, especially in view of the statement of Martius, that from one and a half to two hours is sufficient to thoroughly exhaust the heart.

The great point of Ringer's work was the establishment of the importance of calcium salts for the contraction of the muscular tissue, and the recognition that a definite supply of solium and potassium must also be contained in the circulating fluid; in fact, that the ideal circulating fluid must contain a variety of salts in right proportion. It does not at all follow that the fluid recommended by Ringer, although unqnestionably effective, yet contains the salts in the absolutely best proportion. In fact, Howell and Cooke ${ }^{1}$ removed the proteids from serum, and then found that the remaining solntion of the salts in the serum was more efficacious than Ringer's solution. On the other hand, the proteids of the serum, after removal of the salts, were ineflective.

In view of this, the conchusions of White, ${ }^{2}$ working with Kronecker, who, finding that serum would restore a heart after it had ceased contracting with perfusions of Ringer's fluid, supposed this action to be due to the proteid of the serum, are evidently not justified.

Another series of experiments hy Heffter, ${ }^{3}$ Albanese, ${ }^{4}$ and Ohrn, ${ }^{5}$ have led them to the conclusion, that a good circulating fluid must not only contain the right proportion of salts, but also must possess the same physical properties as blood with respect to riscosity, osmotic coefficient, etc. For this purpose they added gum arahic to Martius' solution, and assert that such a solution is as efficacious as serum in keeping up the contractions of the heart.

Howell and Cooke, ${ }^{6}$ and later, Locke, ${ }^{7}$ have pointed out that gmm arabic is a combination of arabin with calcium and potassium salts, so that, in this ease also, the salts, rather than the physical character of the fluid, are in all probability the important factors.

To sum up the evidence, the artificial circulating flnid which is most efficient in keeping the heart beating must contain the right proportion of sodium chloride, of a calcium salt, and of a potassium salt. The experiments so far do not prove that any special proteid is necessary in this mixture, and give no definite answer to the question whether the heart contracts at the expense of its own substance, or contracts only as long as a suitable proteid diet (according to Kronecker, serum albmmin) is constantly supplied to it. Considering, however, the length of time it can be washed out with a fluid like Ringer's, and yet continue beating, it seems very unlikely that any sermm albumin or other proteid can be still left in the capillary spaces, and therefore the probability is that the heart can continue to contract at the expense of its own substance upon an absolutely inorganic supply.

Luciani's groups. - The second question raised by Lnciani's experiments is the question of the meaning of the secondary or periodic rhythm, observed by him when serum was sent through the ventricle. Subsequent experiments, especially by Langendorff, 8 have endeavoured to determine the conditions necessary for the appearance of such groups as Luciani deseribed; and the general opinion of physiologists at the present time is that the grouping does not appear unless a condition of asphyxia has heen induced in the cardiac tissue. According to Langeudorff, it is possible to ohtain this periodic rhythmical contraction, not only in the ventricle, but also in the whole heart, by keeping a curarised frog under water for a sufficient length of time, so that, according to his observations, as well as those of Luciani and Rossbach on the isolated ventricle, this method of beating in groups, instead of with a normal

1 Op. cit. $\quad 2$ Journ. Physiol., Cambridge and London, 1896, vol. xix. p. 344.

3 Arch. f. exper. Path. u. Pharmakol., Leipzig, 1891, Bd. xxix. S. 41.

+ Ihid., 1893, Bd. xxxii. S. 297.

5 Ibid., 1894, Bd. xxxiv. S. 29.

6 op. cit., p. 216.

7 Journ. Physiol., Cambridge and London, 1895, vol. xviii. p. 332.

8 op. cit. 
regular rhythm, is due to some alteration in the rhythmical organ itself. In all these experiments the contractions of the ventricle alone are registererl, the auricular contractions being only registered oceasionally by the accident of their appearance on the ventricular curve. It is therefore not elear to my mind how far these groups of ventricular beats may be due to the passage of a series of contractions into the ventricle over a blocking-place, somewhere between the starting-place of the rlythm and the ventrieular tissue.

Certainly I have occasionally obtained, by means of tightening the clamp in the anriculo-ventricular groove, a series of ventricular contractions which for a time were arranged in groups just like Luciani's groups, and Fano mentions the same thing as the eflect of clamping. The separate ventricular contractions in these groups usually corresponded to every second contraction of the auriele, thongh occasionally a few contractions were able to respond to every aurieular contraction, so giving curves resembling some of those figured in Langendorff's paper. In fact, luring the group the conductivity of the tissue at the blocking point altered from that of a complete block to that of a partial block. Again, one of the most striking phenomena observed and recorded by me in my paper in the Philosoplical Transactions, was the manner in which the vago-sympathetic nerve was able to cause a group of ventricular contractions, when, by means of heating or clamping, a complete block had been formed between auricle and ventricle. This group of contractions was most elearly dependent on the auricular contractions, being either all synchronous with those of the auriele, or mostly synchronous, with a few at the beginning or end of the group synchronous with cvery second auricular contraction. When we remember also that it is very difficult, without most careful investigation, to be sure that the sinus is not beating regularly when the heart is apparently still, that Luciani and Rossbach tied on to the cannula, not the ventricle alone, but the whole heart, and that it is astonishing how tightly it is necessary to serew up a clamp (or in their case to tie a ligature) between auricle and ventricle, so as to cause a block sufficiently complete to prevent the effect of the stimulation of the vago-sympathetic nerve, it is, I think, more probable than not that the so-called Lueiani's groups, or periodic beating, owe their origin to a partial blocking of the contraction wave into the ventricle, and not to a peculiar periolicity of rhythm. Such an explanation is in eomplete harmony with the view that a condition of asphyxia is most favourable for the production of these groups, for such a condition is certain to produce a greater or less amount of blocking. 


\title{
A N I M A L M ECHA N I CS. ${ }^{1}$
}

\author{
By J. B. Haycraft.
}

Contents :- Introductory, p. 228 -The Movements and Positions of the Body, p. 228-The Mechanism of Joints, p. 233-The Muscle as a Machine, p. 241The Centre of Gravity of the Body, p. 257-Nethods of Recording Novements of the Body, p. 264-Detailed Description of Special Movements, p. 267.

Introductory.-Animal mechanics is for the most part merely a special branch of ordinary mechanics, and the mechanical engineer would at once recognise that many of the problems to be solved are already discussed in textbooks of mechanics. Thus, the properties of the shoulder-joint are already known as soon as we rccognise that it is a joint of three degrees of freedom, and of the nature of a ball-and-socket joint. But although we may understand the mechanical principles of the shoulder-joint, the movements which take place there are excessively difficult to follow. In the first place, the joint is in the living subject embedded in flesh, and we cannot be certain of its position in space; in the second place, we find in practice that a pure movement at the joint almost never oceurs, but that it is accompanied by translations of the whole joint, due to movements of the shoulder-girdle. The study of the movements which actually take place at the joint is therefore a mechanical problem of the greatest complexity, and one which it is at present impossible to study with appropriate exactiturle. This is typical of the movements of the limbs in general. There are other problems which a previous training in mechanics will not enable us to follow. For these we require a training in physiology, a study of the properties of muscles, and a knowledge of the action of the nervous system, the initiator and controller of our bodily movements. Our knowledge of the facts relative to muscular activity bearing upon locomotion is largely due to the investigations of the brothers Weber of Helmholtz, and A. Fick. The application of the study of mechanics to the human body is due chiefly to the brothers Weber, H. Meyer, and Marey, and recently, to the work of Braune and Fischer.

In attempting to epitomise these results we shall procecd synthetically, studying first the mechanical properties of the bones, joints, and muscles, and finally the whole body in its upright position and during progression.

\section{The Movements and Positions of the Body, Described by reference to Co-ordinate Planes or Axes.}

In their description of the position of parts of the body, anatomists have for long been accustomed to speak of these in their relationship with certain planes and axes. Thus, the liver is on the right-hand side of

\footnotetext{
${ }^{1}$ Professor Hayeraft having been prevented by illness from seeing this artiele through the press, the proofs were submitted to Dr. René du Bois-Reymond, who has carefully revised them, and has supplied several additional paragraphs, which have been incorporated in the article.
} 
the mesial plane of the body; a rotation of the head is described as taking place in a horizontal plane. By the use of co-ordinate planes and axes - a method extensively employed in mechanics-it is possible to express in exact terms and without ambiguity the position of any portion of the body in space, and to define its position relative to any other part. By this means we can exactly measure the extent and direction of a movement, say that which occurs at a joint, and determine what new position the limb has assumed as a result of that movement.

This method has been of late years so extensively used by those who are attempting to obtain more exact data in connection with animal mechanics, that it is necessary to master its principle at the outset, in order to understand the results at which they have arrived.

Let us, in the first case, confine our attention to space of two dimensions, and study the methods for determining the position of points which lie, or which change their position, in one plane. There are two methods, known as the method of rectangular, and the method of polar co-ordinates.

In the first of these we describe the position of any given point, by reference to two lines at right angles to each other. If, for example, we want to define the positions of a series of points in the upper limb, and to investigate their changes in position during the movement of that

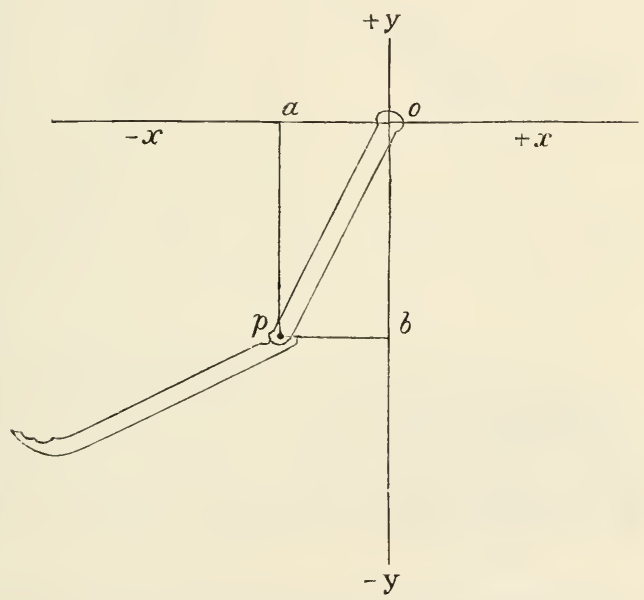

FIG. 119.--This figure represents the method of expressing the position of a point $p$ - the centre of rotation of the elbowjoint-in reference to two co-ordinate axes ( $x x$ and $y y)$.

limb in a given

plane, we choose two convenient reference lines. As the plumb-line is a definite direction in space, and rearlily determined by a thread and plummet, we take as one of our reference lines the plumb-line $(+y-y)$ falling through the centre of rotation of the shoulder-joint. The other reference line must intersect this at right angles, and in the plane in which displacements are to be studied; this second line $(+x-x)$ may itself pass through the shoulder-joint (Fig. 119), or, in many cases, may more conveniently form the ground-line.

If we draw the lines $p a, p b$ at right angles to the reference lines, the position of the point $p$ is defined without ambiguity, by saying that its ordinate is $-p a$, and its abseissa is $-p b$, which lengths we may express in centimetres. 
In the methor of polar eo-ordinates we require to find the angle bop, whieh is usually denoted by $\theta$. This is regarded as plus, if it be measured round the point $o$ from a reference line in a direction the reverse of the movement of the hands of a watch, and minus if in the same direction. The radius veetor op, denoted usually by $r$, must also be known; it is always taken as positive. ${ }^{1}$

If we simply require to determine an angular displacement of a limb, we proeeed as follows. A eertain position of the limb, say the vertieal, as in

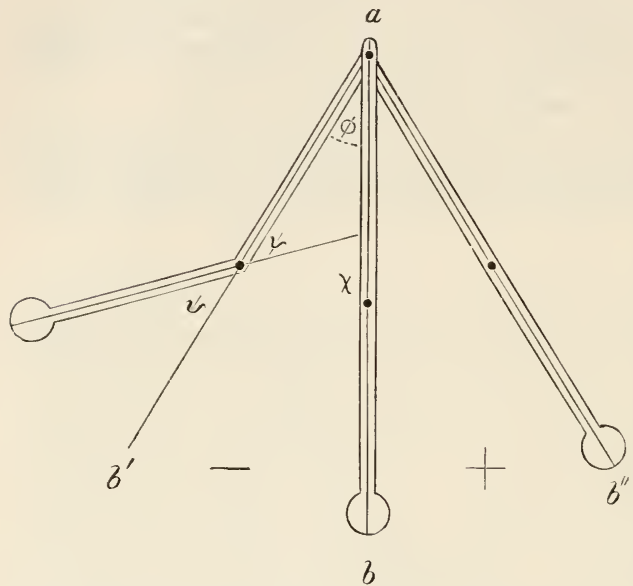

FIG. 120.-This figure indicates the method of measuring the angular displacement of the arm from a primary vertical josition $(a b)$. forearm upon the arm. One may readily, however, pass from one method of expressing the position to the other, for the angle $\psi=\psi$ and the angles $\psi, \phi$, and $\chi$ are simply related to one another; for

$$
\chi=\psi+\phi
$$

If the angle $\phi$ be a rotation forwards, say of $30^{\circ}$, we may eall this a negative rotation of $30^{\circ}$, and we must in that ease term an extension at the shoulder baekwards to $a b^{\prime \prime}$ as a rotation of $+30^{\circ}$.

So far we have limited our attention to the description of the positions or changes of position of points in space for two dimensions, but the shoulder-joint can move on more than one axis, and in more than one plane. In fact, to denote most of the changes of position of the limbs which are brought about by muscular contraction, and to denote completely the position in space occupied by various parts of the body, we have to deal with a problem in tridimensional space, and must refer these positions to three co-ordinate planes at right angles to each other. In Fig. 121 these three planes are represented. The horizontal plane or floor is that through which the lines $y y$ and $x x$ pass. One vertical plane is that through which the lines $y y$ and $z z$ pass; the other vertical plane

${ }^{1}$ It is quite immaterial which direction is considered as plus and which as minus, so long as this is once settled and adhered to. 
is that through which pass the lines $z z$ and $x x$. The lines in the figure are each the lines of intersection of two planes; the point $O$ is the point of intersection of the three planes. If now we wish to define the position of the point $p$ in reference to these three co-ordinate planes, we can drop a line vertical to the horizontal plane, cutting it at $a$. $p a$ is the distance of the point $p$ from the horizontal plane $x x$ and $y y$.

If, now, we draw a line $a b$ perpendicular to the vertical plane az and $y y$, this will give the distance of $p$ from that plane. The distance bo gives finally the distance of $p$ from the second vertical plane $z z$ and $x x$. It is necessary further to notice the signs of $p a$, $a b$, and ob, which, in the case under consileration, are all positive. Were $p$ helow the floor, $p a$ would be negative; for the movement of $p$ around $O$ would be in the direction of the hands of a watch. The method of describing the position of the body in reference to three co-ordinate planes has been greatly used by those who have studied the animal mechanisms, and by no one more elaborately than by Braune and Fischer.

In their investigation into the centre of gravity of the human boly, they start with an erect position, in which the chief joints of the borly, and the centres of gravity of the trunk and limbs, are all in one frontal plane. In Fig. 122, $B$, simplified from their original figure, this plane is vertical to the paper, and runs through the line $z z$. In Fig. 122, $A$, the plane is seen "from the front," and is mapped out in equal squares, giving distances along the line

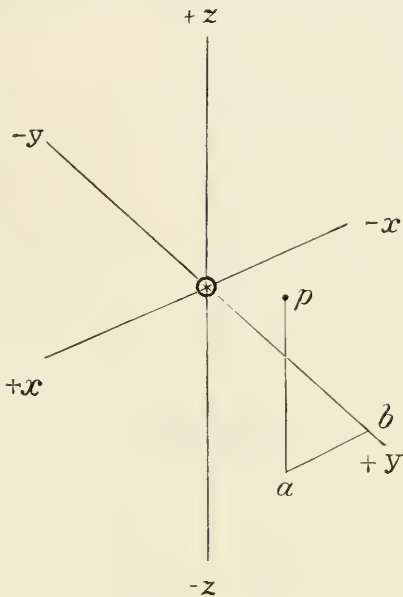

FIG. 121.-Shows the method of determining the position of the point $(p)$ in reference to three co-ordinate planes intersecting in the point $\mathrm{O}$. $y y$ from the centre of the plane $z z$, these being reckoned as negative to the right-hand side, and positive to the left-hand side. Along this plane can also be measured distances from the ground plane in a vertical direction.

Cutting this plane through the line $z z$, and rumning in the mesial plane of the borly, is the sagittal plane, scen from the left in Fig. 122, B. This is also divider into equal squares; and distances forwards from the axis $z z$ of intersection of the two planes are counted as positive, while distances backwarts are counterl as negative, both passing in the direction $r x$ of the figure.

Co-ordinates on $\mathrm{X}, \mathrm{Y}$, and Z Planes.

\begin{tabular}{|c|c|c|c|c|c|}
\hline Joint. & & & $x$. & $r$. & $Z$. \\
\hline Atlanto-oceipital & . & . & 0 & 0 & 152 \\
\hline Right hip & . & . & 0 & $+8 \cdot 5$ & 87 \\
\hline
\end{tabular}

In deseribing the position of any point of the borly, say the atlintoaceipital joint, this will, by construction, be in the frontal plane, and therefore its deviation in the direction will be 0 . As it is also in the mesial 
plane, its deviation along the $y y$ direction will also be nothing. It will be some distance, however, from the floor in a vertical direction; in the case of Braune and Fischer's subject, $152 \mathrm{cms}$. Again, let us take the centre of

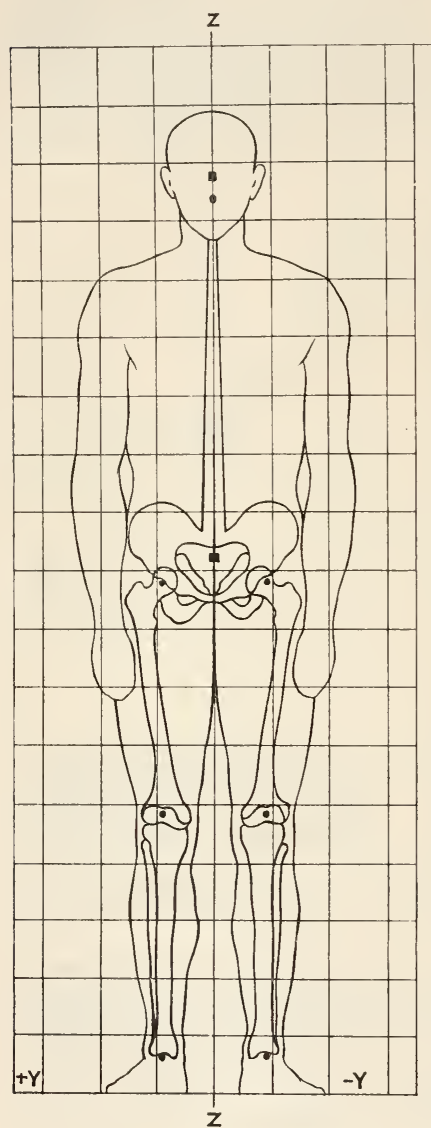

$A$.

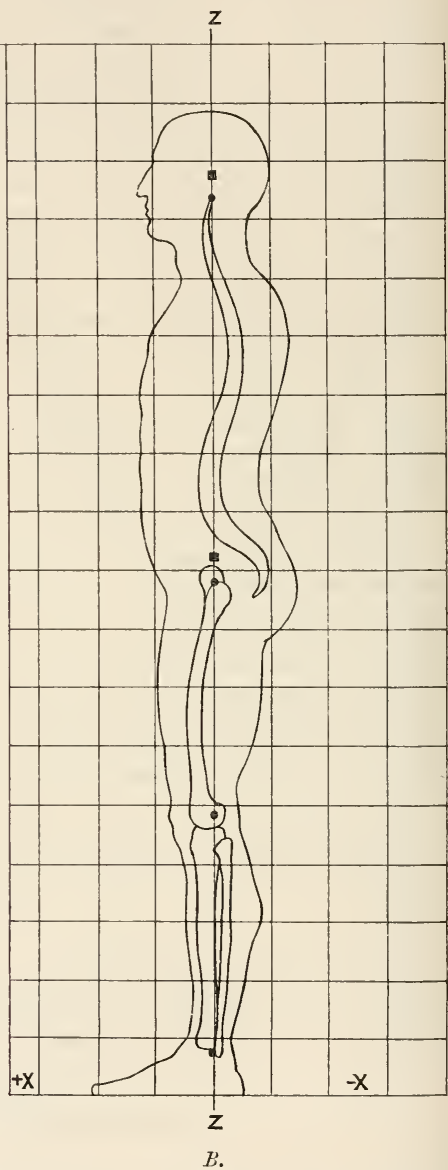

FIG. 122.- Showing the position of the centre of gravity of the whole body and of the head $(-)$, and also the position of the centres of rotation of the chief joints $(\bullet)$ in reference to three co-ordinate planes. - Braune and Fischer.

rotation of the right hip. By construction this also will fall in the frontal plane, and its deviation in the $x x$ direction will be nothing. It is not, however, in the mesial plane, lying in fact to its right. In the subject under discussion it is +8.5 c.c. on the $y y$ direction. Its distance from the ground in a vertical direction is $87 \mathrm{ems}$. 
The above being the relation of certain given parts of the body to the co-ordinate planes in the position or pose selected for study, any movement of the body will displace these points, which will again take up new positions in relation to these planes. Thus, on bending forwards, the right hip will move out of the frontal plane, and move in the $x x$ direction, say to +12 , and will occupy a lower position in the vertical direction, say $80 \mathrm{cms}$. from the ground.

When the whole body is translated forwards, as in walking or running, these planes must also be conceived of as being translated, one point of the body, at any rate, preserving its position in one of the co-ordinate planes.

If it be desired merely to describe a movement of a limb, the method of polar co-ordinates described at the head of p. 230 is more generally employed. If the movement take place in a plane, the position of this plane is noted with reference to three co-ordinate planes,-one sagittal, the other frontal, and the third horizontal,-all intersecting at the centre of rotation. The plane in which displacement occurs may also be defined by giving the direction of the axis of rotation (which is perpendicular to that plane) with regard to the three co-ordinate planes. Iovements not in a plane are either described by resolving them into several movements in different planes, or by compounding the different axes of rotation in the way described on p. 236.

In addition to the movements hitherto spoken of, in which there is displacement of a limb, a different kind of movement can take place in some joints, e.g. the hip and shoulder-joint. The limb may remain practically stationary in space while revolving to a certain extent upon its own longitudinal axis. In this case the axis of rotation coincides with the longitudinal axis of the limb. There is no angular displacement of the limb itself, but the amount of longitudinal rotation can be measured by the angular displacement of an indicator-rod fixed to the limb perpendicularly to its axis. In the case of the lower limb, the tip of the toes will serve as such an indicator, pointing forward in the ordinary position, and nearly lateral in extreme outward longitudinal rotation of the leg.

Any actual movement not confined to a plane, as for instance waving the arm in a circle, or any movement in which rotation upon the longitudinal axis is combined with the angular displacement, cannot be accurately described by any definite number of rotations round fixed axes. Such movements are said to take place on shifting or instantaneous axes.

\section{The Mechanism of Joints.}

From a mechanical standpoint we may, with A. Fick, divide joints into two classes. In the first class we may place joints, the bones of which have one fixed position of equilibrium, to which they return as soon as any displacing force is withdrawn. To this class belong the synchondroses, such as the vertebra, united by white fibro-cartilage, and the amphiarthroses, such as the joints between the tarsal and carpal bones. The attachments of the bones to each other is here so firm that but little movement is possible, and that only by a displacement or deformation of the tissue of the joint, which thus suffers a strain, against which it reacts when released, and resumes its original condition of equilibrium. 
In the second class are placed those joints which more especially concern us, such as the hinge and ball-and-socket joints. Here, the bones forming the joint may be placed in a great number of positions, in any one of which they can remain during muscular relaxation.

Joints belonging to the second class are formed by bones whose extremities are covered by a layer of articular cartilage, and they are enclosed in capsules and accessory ligaments of white fibrous tissue, sufficiently lax to permit of a more or less free play of action. Synovial fluid lubricates the joint and diminishes the friction between the articular ends. The friction between two surfaces is proportional to the pressures of these two surfaces-unless that pressure be very great. The friction is independent of the extent of the surfaces which touch one another. It is greatly influenced by the lubricating material, being, in the case of the joints, greatly diminished by the synovial fluid; and it is independent of the velocity of the movement. In the joints the coefficient of friction ${ }^{1}(\mu)$ is very small, and while movement between the surfaces is occurring, the coefficient of kinetic friction $(K)$ is still less, so that practically it may always be regarded as nil.

The area of contact at articular surfaces. - It is generally believed that the articular surfaces are in close contact with each other, but it would appear that this is only the case when considerable pressure has been applied. König ${ }^{2}$ was the first to show that the articular surfaces in a joint like the hip are not in contact in the dead subject; for in frozen sections, where the positions of parts are unaltered, a layer of frozen synovia is always found between them. König further showed that if the head of the femur be firmly wired to the acetabulum, the contact produced takes place over but a small area of the articular surfaces. If the pressure applied by König at all represented the pressures to which the joint is normally subject under the influence of muscular contraction and of the body weight, the joint would not conform in its movements to a true ball-and-socket joint, where there is extensive contact over two geometrically adapted surfaces. Its surfaces would, in consequence of this, slip or roll over each other.

Braune and Fischer ${ }^{3}$ doubt whether this pressure applied by König was sufficient, and they found much more extensive contact when the articular surfaces were pressed together by the help of screws. The action of the pressure is to mould the elastic cartilaginous surfaces upon each other, and to make them conform to pure geometrical figures. It is to be regretted that these experiments are but of a qualitative nature, the pressures applied not having been measured.

The forces which resist displacements of the articular surfaces. - The articular surfaces are bound together by the capsular and other ligaments of the joint, but these ligaments are only put upon the stretch when the surfaces are forcibly separated from each other or when the joint is bent. A certain separation of the surfaces can take place, therefore, without the intervention of these ligaments. This separation is, however, hindered by other forces which we have now to study. The muscles passing over a joint, and attached to the bones on either

${ }^{1}$ If a body press upon a plane surface with a pressure $P$, and a force $F$ is required to move it along that surface, the coefficient of friction (generally written $\mu$ ) is $\frac{F}{P}$.

2 Deutsche Ztschr. f. Chir., Leipzig, 1873, S. 256.

3 " Die Bewegungen des Kniegulenks," Leipzig, 1891. 
side of it, exert more or less continuous pull upon the bones, jamming the articular surfaces together. This contraction has been estimated at about 20 kilos. in the case of the hip-joint, even during the resting condition of the limb, but will be a very considerable power when the muscles are voluntarily or reflexly contracted.

Edward Weber drew attention to the action of atmospheric pressure in keeping the articular surfaces in contact. He assumed that the joints, having closed cavities, cannot be enlarged, as they would be if the articular surfaces were separated, without the action of a force greater than that of the pressure of the atmosphere. In the case of a large joint like the hip, the atmospheric pressure would be such that a weight of about 20 kilos. would be capable of overcoming it, and of pulling the surfaces of the joint apart and rendering the joint-ligaments tense. These ligaments would, owing to their inextensible nature, be able to resist a weight of over 400 kilos. before complete rupture could take place. Weber divided all the muscles surrounding the hip-joint, leaving, however, the capsule of the joint intact. The weight of the limb was not sufficient to draw the articular surfaces apart. On opening the joint cavity, by boring a hole through the acetabulum, the head at once fell downwards. It is, however, doubtful whether the result of this experiment may be applied to any other than the hip-joint. A strain of 500 grms. put upon a metacarpo-phalangeal joint during muscular relaxation causes the articular surfaces to come apart, the space being filled by the surrounding substance closing in. For the living subject the articular cavity of the hip-joint may perhaps likewise be considered as being in partial communication with the outer pressure.

Ball-and-socket joints.-A spherical surface is produced by the rotation of a circle round any one of its diameters. If such a surface be received into a cup of the same, or of a slightly longer, radius of curvature, these two surfaces may be caused to move in relationship to each other. Putting on one side any "translation" of the cup-and-ball in space, and considering the cup as fixed-for example, when the arm or leg is raised from the shoulder or hip-we find that the centre of the spherical mass will remain fixed in space, and movements of the ball will take place around axes passing through the fixed point. This point is therefore termed the centre of rotation, and the axes are termed the axes of rotation. If, on the other hand, the ball be fixed in space, the socket may move upon it, as when the thigh or arm are fixed, and the trunk moves upon one of these fixed limbs. Here we have a centre and axis of rotation for the cup, but these do not lie within the substance of the cup. We must consider them as a point and line in space, the space occupied or partly occupied liy the ball itself. In the body we may have simultaneous rotations of the ball and of the socket, as in many movements of the arm, where not only does the humerus rotate, but the shoulder-blade and glenoid cavity change their positions too. Confining our attention to the rotation of the ball, and remembering that the centre of rotation is a point fixed in space, any movement of the ball is equivalent to a rotation around a certain axis passing through the centre of rotation.

If the displacement of any two points in the splhere be known, neither of which is a point lying in the centre of rotation, nor in a line with it and 
the other point, then we can determine not only the axis upon which the rotation took place, but also the position assumed by any other point resultant from the same displacement. In Fig. 123 let the points $a$ and $b$ be points upon the surface of a sphere with a large radius, and the points $a^{\prime}$ and $b^{\prime}$ the position which they oceupy subsequent to a given movement.

Join $a$ and $a^{\prime}, b$ and $b^{\prime}$ by ares of a great circle, and bisect each of these

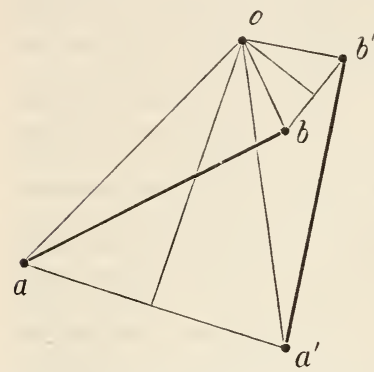

Fig, 123.-Figure to show a method by which the axis of rotation of a ball-and-socket joint is determined, when the line $a b$ is carried into the position of the line $a^{\prime} b^{\prime}$. ares. From the point of bisection draw lines perpendicular to $a a^{\prime}$ and $b b^{\prime}$, meeting at the point $o$. The point $o$ will have remained stationary during the rotation, and so will $C$, the centre of rotation, as well as every point on the line joining them. The line passing from the centre through $o$ will therefore be the axis of rotation required. For join $o a, o b$, and $o a^{\prime}$ and $o b^{\prime}$, then the triangles oab and $o^{\prime} a^{\prime} l^{\prime}$ are equal and similar, hence the displacement of $o$ is zero. The angle of rotation will be coa'.

To find the position assumed by any other point $p$, during the displacement of the points $a b$ to $a^{\prime} b^{\prime}$, perform the following construction (see Fig. 124).

Join $p a$ and $p b$ and $a b$ to make the triangle pab. Join $a^{\prime} b^{\prime}$, and upon $a^{\prime} b^{\prime}$, the final position assumed by the line $a b$, erect a triangle $a^{\prime} p^{\prime} b^{\prime}$ equal and similar to $a b p$. The point $p^{\prime}$ will be the position assumel by $p$ at the end of the rotation.

Compounding axes of rotation.-If rotation occurs around two axes, one after the other, the sane result may be obtained by a single rotation around a definite single axis. 'To find this single axis, atopt the following coustruction. In order that the line of the axis may be made to represent the direction of rotation, select that half of each axis which, if it rums through the length of the body of an inaginary olsserver, with his feet upon the centre of rotation, will place him in such a position that the direction of rotation will correspond to the direction of rotation of the hands

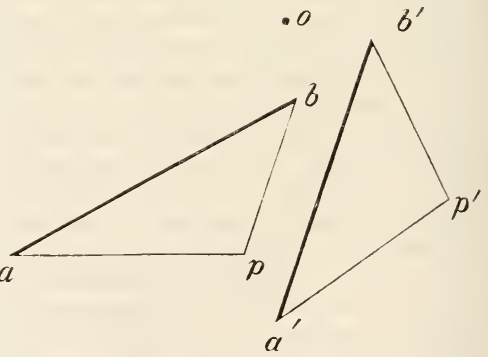

FIG. 124.- Shows the method of finding the position assumed by the point $p$, when the line $a b$ is rotated into the position $a^{\prime} b^{\prime}$. of a watch.

Let the points $\mathrm{O}$ and $\mathrm{O}^{\prime}$ represent the points at which these axes cut the surface of the sphere; join $O$ and $O^{\prime}$. If the rotation at $O$ be equal to $\theta$, measure off the angle $\mathrm{O}^{\prime} \mathrm{O} x$ equal to $\frac{\theta}{2}$ and in a direction opposite to the rotation around $O$. If the rotation around $\mathrm{O}^{\prime}$ be equal to $\theta^{\prime}$, measure off the angle OO'r equal to $\frac{\theta^{\prime}}{2}$ and passing in the same direction. The position of the 
point of intersection $x$ will be unaffected by the double rotation, the centre of rotation will be at the same spot, and the line joining the centre of rotation with $x$, will he what is termed the resultant axis of rotation. For the rotation $(\theta)$ around the point $O$ would carry the point $x$ to the point $x^{\prime}$, and the subsequent rotation around $\mathrm{O}^{\prime}$ would bring it to the position $x$ from which it starterl.

If the order of rotation be reversed, occurring first around $\mathrm{O}^{\prime}$ and then

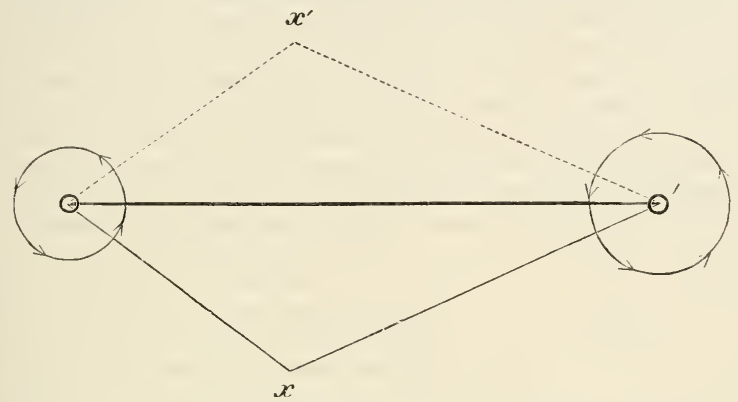

FIG. 125.-To illustrate the method of compounding axes of rotation.

around $\mathrm{O}$, the resultant axis of rotation would pass through the point $x^{\prime}$. Upon the other sile of $\mathrm{OO}^{\prime}$ draw the angles $\mathrm{O}^{\prime} \mathrm{O} x^{\prime}$ equal to $\mathrm{O}^{\prime} \mathrm{O} x$, and $\mathrm{OO}^{\prime} x^{\prime}$ equal to $\mathrm{OO}^{\prime} x$. The two triangles will be equal in every respect.

By constructions similar to those detailed above, the movements of any given solid in space can be exactly determined, provided the movements of three points (not in a straight line) of the solid be known. This axiom was applied by Braune and Fischer in their study of the movements at the knee. Since accuracy of observation depends on the distance traversed by the three points, rods of some length were fixed to the foot of the subject. The excursions of the tips of these rods, recorded by the photographic method described on p. 266, supplied the data for determining the movements of the knee-joint with accuracy. For most purposes simpler methods are sufficient.

In the case of the sloulder, as an example, we proceed as follows:-Attach to the outer surface of the ar'm, so as to rum

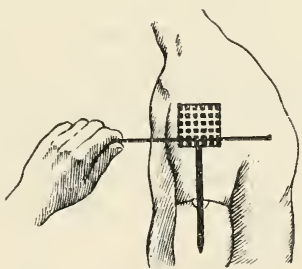

Fig. 126. - Shows the anthor's method of determining the centre of rotation of a joint. During the experiment the arn of the subject is allowed to swing backwards and forwards like a penduhum.

vertically and parallel with the axis of the limb, a light wooden rod bearing a small board 2 in. square, and marked out in equal squares. The board should centre somewhere over the spot at which the centre is supposed to lie. The arm is now allowed to swing pendulum-wise from side to side.

The observer now places himself with his eyes on a level with the joint, and holds a straight rod-a pencil-so that it is parallel with one of the horizontal lines on the board of equal squares (Fig. 126). 
As the limb swings, the horizontal straight line on the board will be found to oscillate about a definite point in the line of the pencil, provided this line rums through the centre, and this point will therefore mark the position of the centre. If the horizontal line lies ahove or below the centre, it will, while the arm swings, alternately intersect the line of the pencil in two points. A line drawn vertically from a point bisecting the line joining these two points will pass through the centre, and this line may be drawn upon the skin. The observer should now hold his penci! parallel with a vertical line, and determine in the same way the position of the centre of the joint in an axis which is horizontal. The point of intersection of the two lines will mark the position of the centre of the joint. It is advisable in such an experiment to support the pencil, not by the hand, but by some kind of stand. In the case of a joint like the shoulder, the shoulder girdle should be steadied by an assistant, and the observer himself should move the arm backwards and forwards.

Special ball-and-socket joints. - In the shoulder-joint considerable movement can oceur. In its ordinary position the humerus can rotate through an angle of $90^{\circ}$ round its longitudinal axis. Round a sagittal axis the humerus can rotate through $120^{\circ}$, round a frontal axis through an angle of $170^{\circ}$. By an added movement of the whole shoulder the humerus may be moved through an angle of $170^{\circ}$ round a sagittal axis, and through an angle of $190^{\circ}$ round a frontal axis. With the combined movement of pronation and supination of the bones of the lower arm, the hand in all can be rotated through an angle of $280^{\circ}$.

In the case of the hip-joint we have a smaller range of movement, due in the first place to the capsular structure of the joint. This powerful and very inextensible structure consists of a capsule, strengthened by accessory ligaments, formed many of them by fascial sheaths of the surrounding museles which are attached to it. The most important of these is the ileo-femoral ligament, which hinders eversion and too great extension of the limb. The actual movements of the limbat this joint are likewise hampered by the thick muscles of the hip, either by their passive resistance to pressure or by their extensibility. The femur can rotate through an angle of $45^{\circ}$ round an axis rumning in the length of the limb. Around a sagittal axis rotation ean take place through an angle of $80^{\circ}$, and round a frontal axis it can rotate through an angle of $140^{\circ}$.

The lower limb is not so movable upon the trunk as is the upper one, not only on account of the more limited movement of the hip, as compared with the shoulder-joint, but also because the acetabular surface occupies a fixed position on the trunk, whereas the glenoid cavity is itself movable.

Hinge-joints. - In these cases one articular surface forms a portion of a cylinder, and we may conceive it as having been generated by the rotation of a straight line around a central axis. This articular cylinder is received into another concave surface of sinilar shape. The surfaces are not always true cylinders, in fact more often grooved, or of a conical shape. In hinge-joints the capsular ligaments are characterised by greater strength at the sides, and are here termed the lateral ligaments of the joint. Rotation can oceur around a single axis, every portion of the surface of the joint describing in its movements a portion of a circle. In these joints the axis is rarely at right angles with the long axis of the bones concerned; thus the trochlear surface and axis 
of rotation of the ulna is set olliquely to the axis of the humerus, salient outwards at $105^{\circ}$, so that the forearm, when extended, forms an angle with the upper arm re-entrant outwards. ${ }^{1}$

Many joints which we may, on superficial observation, regard as hinge-joints are really screw-joints; and we may now discuss the character of the surface of a screw, and the movements which such a surface can make. In moving the nut upon the surface of a screw, or, more simply still, in moving a small hody up the thread of a serew, the movement of any particle can be resolved into rotation, and what is termed translation. The body or any portion of the surface of the nut will rotate around the cylindrical axis of the screw, and at the same time it will move in a direction parallel with the axis of this cylinder.

A serew is really an inclined plane rolled upon the surface of a cylinder, the hypotenuse forming a spiral or helix (see Fig. 127). The "pitch" of the screw is the vertical distance between two coils of the spiral, and the inclination of the screw is the angle bac.

The motion of the trochlea in the sigmoid cavity is that of a serew moving in a nut, the inclination of the screw is $8^{\circ}$, and in the $140^{\circ}$ through which the elbow can pass the translation amounts to $2.5 \mathrm{~mm}$. In most cases the right elbow is a right- and the left a left-handed screw. ${ }^{2}$

The serew character of a joint like the ellow may be demonstrated in the following manner. Clamp the humerus in such a position that the axis of rotation of the ellow-joint, whose ligamentons attachments are undisturbed, is vertical, the forearm being extended. Then stretch a thread horizontally between two retort stands, and
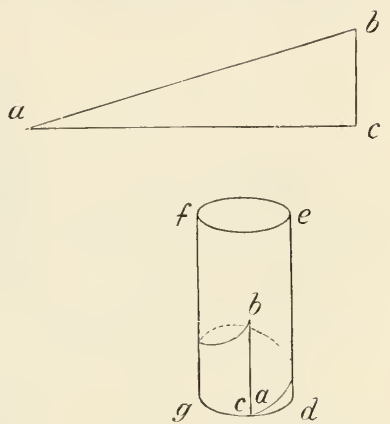

FIG. 127.-This figure illustrates the faet that a sercw is an inclined plane wrapped around the surface of a eylinder. get the eve in such a position that a marked spot on the coronoid process of the ulna is on a level with the thread. When the limb is flexed, the markerl point moves round the axis of rotation, and is also displaced upwards or downwards over the thread towards the radial side of the limb.

The elbow-joint, as anatomically defined, contains two physiologically distinct articulations. The bones of the forearm together form a hingejoint with the humerus, but the radius is also attached to the ulna by a similar joint, which allows it to rotate on its axis, its perfectly rounded hear remaining in unchanged relation to the articular surface of the humerus. It may be remarked that in the radio-ulnar articulation the axis of rotation coincides with the longitudinal axis of the bone. In this respect the joint between radius and ulna, as well as that between atlas and ilens epistrophei, represent a particular type of joint, rather to be compared to the pintles of a rudder than to the common form of hinge.

Padius and ulna being united at the wrist in nearly the same way,

1 Macalister, "A Text-Book of Human Anatomy," 1. 141.
"Hultkrantr, "Das Ellenbogengelenk," Jena, 1892, S. 24. 
the distal end of the radius can be swung around the comparatively immovable ulna. The head of the radius remaining stationary while the lower end moves in a circle round the distal end of the ulna, the shaft of the radius moves on the surface of a cone, of which the axis passes through the centre of the capituhum radii above and through the styloid process of the ulna below. This is the mechanism of the movements termed pronation and supination.

The movements at the knee-joint were carefully studied by the Webers. ${ }^{1}$ According to these observations, movements oceur in a sagittal plane (flexion and extension), through an angle of about $150^{\circ}$. The axis of rotation is, however, not a fixed axis, bnt shifts during the acts of extension and flexion. The articular surface of the tibia is almost horizontal, and upon it the condyles of the femur roll like the wheels of a carriage, rolling forwards during extension, and rolling back-

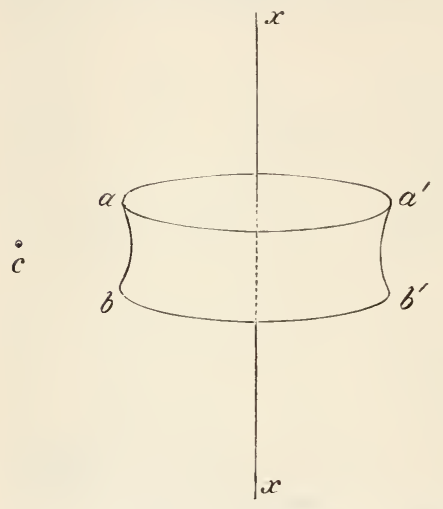

Fig. 128.-To show the geometrical construction of a saddle-shaped joint. wards during flexion. But this rolling is not a simple rolling in one plane, but it is combined with a rotation upon a vertical axis ; the condyles moving in relation to the smrface of the tibia, like the fore-wheels of a carriage as it turns a corner move on -c the ground.

c Mever, ${ }^{2}$ like the Webers, in addition to that rotation which is bound up, as it

were, with the act of llexion, recognises some rotation upon a vertical axis, which can be produced in the bent but not in the extended limb. Sappey $^{3}$ described slight lateral movements of the joint.

Braune and Fischer, ${ }^{4}$ who have investigated in the living subject the movements of the knee in their relation to co-ordinate planes, agree in the main with the conchusions of Weber. Their most important additions appear to be the following. In respect of the rotation which accompanies and is bound up with the act of flexion, they find that, commencing with the extended position, and ending with that of complete flexion, there is at first, for about $20^{\circ}$, a rotation inwards. This amounts in all to $6^{\circ}$. Then follows a slighter rotation outwards, but as this lasts longer than the inward rotation that preceded it, it finally reaches $6^{\circ}$ in the opposite direction, one effect counterbalancing the other.

Saddle joints and ellipsoidal joints. - We may conceive the saddleshaped surface as generated in the following manner (Fig. 128).

Take the are $a b$ of a small circle, the centre of which is at $c$, and rotate it upon the axis $x x$, and it will produce the figure $a b b^{\prime} a^{\prime}$.

1 "Gehwerkzeuge," s. 170.

2 "Statik und Mechanik des menschlichen Knochengeruistes," Leipzig, 1873, S. 357.

" "Auatonie descriptive." \& "Die Bewegungen des Kniegelenks," Leipzig, 1891. 
The sides of this figure will possess a double curvature like a saddle, a curvature of a small circle in vertical, a curvature of a large circle in horizontal, direction. A saddle-shaped surface can glide on its counterpart to a certain extent around either of two axes (in the figure around an axis passing through $c c$ or $x x$ ). Thus the surfaces may be brought to a multitude of new positions, by first rotating on one and then on the other axis. In this respect the saddle-joint lies between the hinge and ball-and-socket joint. Its freedom of movement is principally due to the absence of lateral ligaments. The trapeziometacarpal joint is an example of a saddle-joint.

We may conceive an ellipsoidal joint as formed in the following manner. Take the arc $a b$ of a circle whose centre is at $c$, and allow the arc to revolve around an axis $x x$ on the same side of the arc as its centre, and the figure $\left(a b b^{\prime} a^{\prime}\right)$ will be formed. At the sides of this figure the surface resembles an ellipsoid, and will, like the saddle-joint, allow of rotation in two axes, namely, an axis passing through $c$ and the axis $x x$. The first carpal joint is ellipsoidal.

R. du Bois Reymond, ${ }^{1}$ says, "as a matter of practical experience, a certain movement of rotation is possible because the surfaces do not geometrically correspond, the stronger convexity not fitting the weaker concavity. A saddle-joint or ellipsoidal joint with rigid and perfectly adapted surfaces would not be capable of movement at all."

\section{The Muscle as a Machine.}

The phenomena of muscular contraction are fully discussed in the portion of this work directed to the special physiology of muscle. We may, however, select for discussion a few of the points which have to be borne in mind, when studying the part

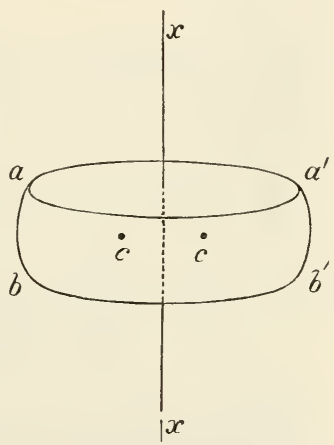

FIg. 129. - To illustrate the formation of an ellipsoidal joint.

these structures play in producing the movements of the trunk and limbs. When an unexcited muscle is stretched by a force, such as the weight of a limb, or other load, it elongates until a point is reached at which there is equilibrium. At this point the stress applied is equalled by the stress in the muscle acting in the contrary direction. While with most inorganic matter the elongation or distortion is proportional to the distorting force (ut tensio sicut vis), in the case of the muscle it follows the formula $y^{2}=a x^{2}+b x$, where $y$ is the elongation, $x$ the weight, and $a$ and $b$ are constants. ${ }^{2}$ The curve representing the elongation of a muscle when loaded with increasing weights is given in Fig. 130, and it will be seen that the muscle becomes less and less extensible as the weight increases. It is on this account that muscles are able to play an important rôle in the maintenance of equilibrium, quite apart from their action in shortening. They play the part of ligaments and prevent too free a movement of the body in undesirable directions. Thus, over-extension of the knee is in part prevented by

${ }^{1}$ Arch.f. Physiol., Leipzig, 1895.

2 Wertheim, Aun. de chim., Paris, tomes xii. and xxi.

VOL. 11. - I 6 
the over-extension of the muscles of the back of the thigh thereby induced, and the weight of the head which plumbs in front of the atlanto-occipital joint is partly supported by the mechanically stretched muscles at the back of the neck.

An ordinary stretched muscle is, from a histological point of view, a complex of muscular fibres and connective tissue, consisting for the most part of the inextensible white variety. It is on this account that they are able to resist over-extension, and it will be found that those muscles which are frequently called upon to do this, which have in fact to perform the part both of ligaments and muscles, have powerful tendinous prolongations passing through them. In some vertebrates this is very especially the case; for instance, in the horse, where the flexor metatarsi muscle is really a combination of a muscle and a check ligament. In this muscle there is a long tendon, which arises from the

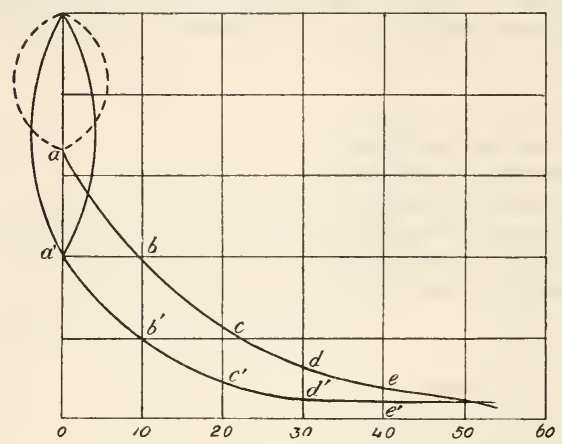

FrG. 130.-Two curves are here represented; the upper one $(a b c d e)$ is the extension of a tetanised muscle by $10,20,30$, ete., grms. The lower curve $\left(a^{\prime} b^{\prime} c^{\prime} d^{\prime} e^{\prime}\right)$ represents the extension by the same weights of a muscle at rest. The two curves are seen to cross towards their lowest points, when the muscles are extended by weights a little over 50 grms.

force of its muscular contraction. anterior and inferior portion of the femur, and passes over the stifle (knee) down the front of the tibia and over the hock (ankle), and is inserted into the metatarsal bone. As this teudon passes over two joints, the knee and the ankle, when one of these is bent the other one is bent too, in purely a mechanical manner. This round inextensible cord receives the attachment of a strong band of muscular fibres springing from the tibia itself, and is directly pulled upon by the

When a muscle with parallel fibres contracts and lifts a load, each muscular fibre bears its fraction of the load, so that if the load be $w$ and the number of fibres $n$, each fibre raises a load of $\frac{w}{n}$. It follows that the load which can be lifted will vary with the number of fibres, and therefore with the sectional area of the gross muscle. When the fibres are not parallel but obliquely set, as in the gastrocnemius, we have a greatly extended transverse area of muscular fibres, which act therefore very powerfully, though, on account of their short length, they can exercise their pull through but a comparatively short distance.

If a weight be attached to a muscle, and if that muscle be stimulated to contract, it will exert a pull or force upon the weight, the mass of which will then be acted upon in opposite directions by two forces, that of gravity and the muscular force. The absolute museular force may be measured, after Weber, by so adjusting the mass of the load, that the muscle on passing into activity neither lifts the load nor is itself 
elongated. In this case the force of the muscle exactly balances gravity, and may be measured by it. We may say that the force of a particular muscle per square centimetre of its cross-section is a weight of 3 kilos. If we wish to express this in units of force, or dynes, we must multiply the grammes or units of the mass, namely 3000 , by 981 , the acceleration due to gravity.

\section{Force $=$ mass $\times$ acceleration.}

In this case force $=3000 \times 981=2,943,000$ dynes.

The dynamometer may be used with advantage to gauge absolute muscular force. It is a spring in some form or another, whose distortions under the action of gravity have been previously ascertained, as by suspending weights from it and recording its elongations. If the spring be now pulled upon by a muscle, and elongated an inch, an elongation previously produced by gravity acting upon a miass of 3 kilos. suspended from it, we conclude that the force of the muscle is equal to 3 kilos., or 2,94:3,000 dynes.

The absolute muscular force is not the same for the muscles of all animals. Thus Rosenthal ${ }^{1}$ found that per square centimetre of cross section the gastrocnemii of a frog can just raise 3 kilos., while in warm-blooded animals the same sectional area will raise about twice as much. ${ }^{2}$ It is probahle that the muscular force is not the same for all museles of the body, and it is subject to physiological and pathological variation in one and the same nusele. Exercise is said to increase it, and the greater power of the right arm over the left is sometimes instanced as an example of this increase. It is, however, to be borne in mind that the girth of an exercised muscle, e.g. that of the right arm, increases, so that in this case it is not clear that per unit area the muscular force is increased. Disuse and degenerative ehanges greatly diminish the museular force, and fatigue operates in a similar direction.

It had long been known that many of the lower animals, insects for instance, are, for their size, remarkably strong. ${ }^{3}$ Thus, while a horse can barely drag its own weight, many inseets can drag a weight over sixty times their body-weight. But the comparison between the force of the pull of different animals, when this is expressed in terms of the body-weight, yields no data by which we may compare their absolute muscular forces. The reason of this is, that whereas in ascending a scale of size the body-weight increases as the cube, the cross-section of the museles, on which the absolute force depends, increases only as the square. If, then, the absolute musele force be the same throughout the whole animal kingdom, the larger animal would be able to lift or drag a smaller proportion of his body-weight than the smaller one; indeed, the insect who ean drag sixty times its own body-weight may have museles which are, per cross-section, weaker than those of a horse which cannot drag its body-weight.

When a muscle is stretched to or beyond its full physiological length, ${ }^{4}$ it is able to exert more power in raising a weight; its absolute force is greater. As it shortens, its force becomes less and less. ${ }^{5}$ This is clear from Fig. 130, in which is represented the curve of extension of a contracted inuscle, the abscissæ being weights applied, and the

${ }^{1}$ Compt. rend. Acad. d. sc., Paris, 1867, tome lxiv. 1. 1143.

2 Haughton, "Prineiples of Animal Mechanisu," 2ud edition, London, 1873 ; Knorz,

Diss., Marburg, 1865 ; Henke, Ztschr. f. rat. Mcd., 1865, Bd. xxiv. S. 247.

${ }^{3}$ Plateau, Bull. Acad. roy. d. sc. de Bely., Brux., 1865, p. 732.

${ }^{4}$ The full physiological length is the greatest length it ever assumes during life.

${ }^{5}$ Schwann, Miuller's "Handbuch d. Phys.," 1837, Bd. ii. S. 59. 
ordinates the shortening which follows upon the application of a uniform stimulus. Let us consider the position of the muscle at the three points $a, b$, and $c$. At $a$ the muscle is loaded with 10 grms., and it is raised to a considerable height; the muscle is short. Its strain balances the stress of the 10 grms., and more than 10 grms. would elongate it. At $b$ it is loaded with 20 grms., and at $c$ with 30 grms. In each case there is equilibrium between the muscular force and that of gravity, and we notice that this muscular force is smaller when the muscle is shorter.

A simple method of demonstrating this fact is the following. Stand upright in front of a table, bend one knee backwards so that the toes touch the ground. Load the foot as by the weight of a child sitting in front of the heel. If the child is of the right weight, you can just lift it by trying to bend the knee still more. Now, bend the body forward on the table so as to flex the thigh upon the trunk, and the child can be raised with ease. In the latter case the flexor muscles of the knee are nearly at their full physiological length, and their absolute force is greater.

The amount of shortening during contraction.-When the muscle contracts under the action of a given stimulus, it will shorten and raise a weight to a height that will bear a certain ratio to its length. Two similar muscles fastened tandem-wise to a weight will, on contracting, raise it to twice that height. This distance will bear the same ratio to their combined lengths, and with the same results these two muscles might be replaced by one muscle of the length of the two together; and we may say, therefore, that the shortening of a muscle is proportional to its length. This accounts for the fact that the extensive movements of the fingers are not brought about by muscles situated in the hand, but are produced by longer muscles lying in the forearm.

By the same muscle, and with the same stimulus or effort, the lift diminishes as the load increases.

In Fig. 130 the abscissæ represent the weights attached, and the ordinates, lengths of muscles. The lower curve represents a muscle at rest when loaded with $0,10,20$, etc., grms., and the upper curve represents the positions assumed by the same point of the tetanised muscle when loaded in a similar manner. It will be observed that the lift ( $\left.a a^{\prime \prime}\right)$ of the unloaded muscle is greater than when the muscle is loaded with 10 grms. $\left(b b^{\prime}\right)$, and so on. Weber believed that the curves ultimately cross, as represented in the figure. According to this observer, on loading the muscle, with say 50 grms., and then stimulating it, it lengthens instead of shortening. This is denied by more recent observers, such as Fick. ${ }^{1}$

While Weber described a muscle as shortening 70 per cent. of its length, when unweighted, more recent observers incline to put the shortening at 20 to 30 per cent. of its length.

Shortening of individual muscles.-In order to investigate upon the skeleton the possible shortening of an individual muscle, during a movement of a limb, the following plan may be adopted. A hook is driven into the exact place of insertion of the muscle to be investigated. A staple is driven into the position of origin of the same muscle. A piece of twine attached to the hook is passed through the staple and tied to a weight, by means of which it is kept taut. The limb is placed I "Untersuch. ueber MLuskelarbeit," Basel, 1867. 
in such a position that the weight is pulled up towards the staple as far as it will go. The length of the thread from hook to staple gives the length of the muscle at its fullest physiological extension. The weight is then allowed to pull the hook towards the staple, and the distance in this case gives the length of the muscle in complete physiological contraction. The difference between these two lengths is equal to the shortening which takes place during contraction.

The terms full physiological extension and full physiological contraction have been used to indicate that the measurements relate to the extensions and contractions which can actually take place in the living body. An excised muscle can of course be extended beyond the point to which it is stretched, when it is physiologically extended within the body. In the same way, an excised muscle can contract to a shorter length than it ever reaches during life. A muscle in situ is in fact always stretched to a certain degree; thus when we flex the shoulder, rotate it inwards, bend the elbow, and supinate the hand, the points of attachments and origin of the biceps muscle are as close together as they will come. The muscle can, however, in this position be made to contract by an effort of the will, and this contraction can be felt by the other hand.

Weber ${ }^{1}$ was the first to investigate the amount of shortening which takes place during the contraction of the individual muscles of the body.

The following table gives some of the measurements of $\mathrm{E}^{\text {. Fick }}{ }^{2}$ :-

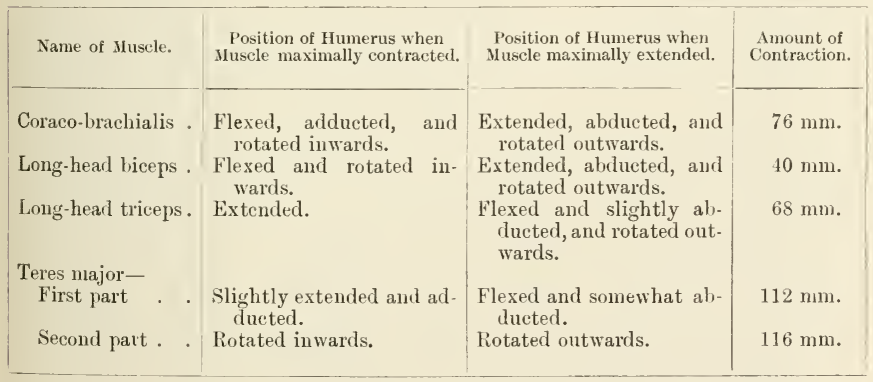

Work done by a contracting muscle.-When a force acts upon a body and moves it, that force is said to perform work, which work is measured by the product of the force into the space traversed in the direction of the force-

$$
W=F \times S .
$$

When a muscle contracts, its force is capable of lifting a body against gravity, and, as has already been shown, the muscular force is measured as against that of gravity itself. If a muscle contracts and lifts a unit of mass, 1 grm., the force employed in dynes is

$$
1 \times 981 \text { (the acceleration due to gravity). }
$$

If the gramme be lifted through unit of space (1 cm.), the work

${ }^{1}$ Ber. d. k. Sächs. Gesellsch. d. Wissensch., Math.-Phys. Cl., 1851.

${ }^{2}$ E. Fiek, Verhandl. d. phys.-med. Gesellsch, in Würburg, N. F., Bd. xi. S. 349. 
done $=F^{\prime}(1 \times 981) \times S(1)=981$ ergs, or units of work, in absolute measure.

The pull of gravity on the unit of mass is often taken to represent the unit of force, and in these gravitation units-

$$
W=M \times S .
$$

Thus, if $10 \mathrm{lb}$. be lifted $10 \mathrm{ft}$., we have the work of 100 foot-lbs. If 10 grms. be lifted $10 \mathrm{cms}$, we have $100 \mathrm{grm}$.-cms. of work.

While the force exerted by a muscle depends upon its cross-section and not upon its length, the work done will be proportioned not only to the cross-section but also to the length, inasmuch as the space through

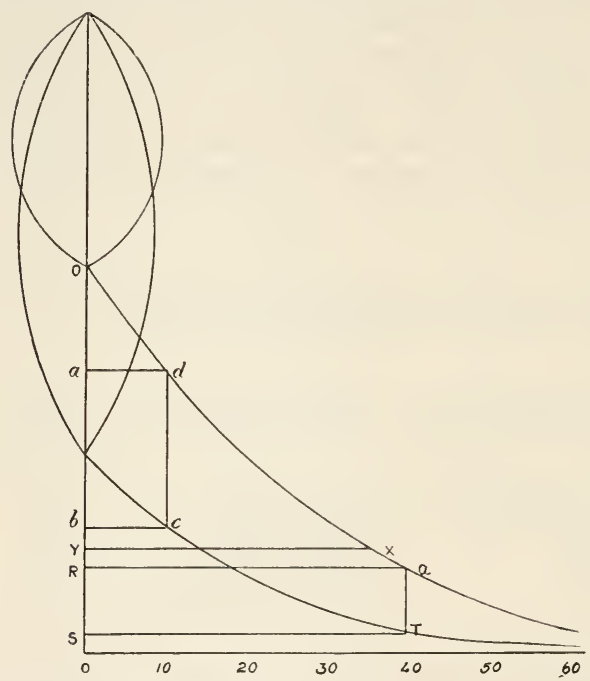

FIG. 131.- Represents the curves of a resting and eontracting musele, when loaded with $0,10,20$, etc. grms. On loading a musele with 10 grms. and stimulating it, the weight will be raised from $c$ to $d$, and the work done will be represented by the area $a b c d$. The areas QRST and XYRQ arc other areas representing work done (see text). which the force acts is one of the determinants of the work done, and the work done will therefore bear a proportion to the whole mass of the muscle. Many of the muscles of the body, such as the long head of the biceps, pass over two joints ; and one advantage of its length is the fact that the work done by such a muscle is greater than can be accomplished by a shorter one of the same girth. The mass which a given musele has to lift modifies in an important manner the amount of work which it will perform. As the muscle is gradually loaded, the distance through which the loads are raised diminishes, as we have already seen; but the product of this diminished space and the increased weight becomes greater and greater. After a time a maximum is reached, and after this the work done falls gradually to zero, at which point $S=O$.

We may represent the work done by an area. Thus, if in Fig. 131 the muscle lifts a weight of $10 \mathrm{grms}$. through $10 \mathrm{~mm}$, the muscle performs 100 grin.-mm. of work, which is represented by the area $a b c d$.

The work may be increased, as Fick has shown, ${ }^{1}$ by any mechanical device which diminishes the load while a muscle is contracting. It will be understood by consulting Fig. 131, that, on loading the muscle with 40 grms., it

1 Hermann's "Handbueb," Bd. i. S. 241. 
will contract on stimulation, and lift the load from $T$ to $Q$. In so doing it will perform work represented by the area QRST. If, lowever, where the muscle has contracted to $\mathrm{Q}$, a fraction of the load be removed, it will lift the rest and perform more work. If, for instance, $5 \mathrm{grms}$. be removed, it will lift the remaining $35 \mathrm{grms}$. to $\mathrm{X}$, and do an adlitional amount of work represented by the area XYRQ. If this be continued, it will finally contract to $\mathrm{O}$, and perform its maximum work represented by the area OSTQ. This area may be measured and compared with the parallelogram abcd either by using a planimeter, or by cutting out corresponding areas in a piece of cardboard and weighing them, or by plotting out on squared paper. Many muscles in the body encounter, during the course of their contraction, less and less opposing force, and approximate in some degree to the ideal case rejresented above. The reason for this will be understood when the movements around the joints are studied, for then it will be seen that, according to the position of the limbs, the force of muscular contraction is very frequently opposed to a diminishing resistance (see p. 253).

From the definition of the tern "work," as given above, a peculiar difficulty arises in discussing animal mechanies. It would seem from it that, when a muscle remains contracted and supporting a weight, it does no mechanical work while this is going on. "Work" being the product of a force into a space, there can be 110 "work" done when no space is traversed. But the muscle after a time becomes exhausted, and it is found to contain products of chemical action. This shows conclusively that work is aetually being done, though it does not appear as external work, and cannot be gauged by the ordinary mechanical standard.

The rapidity of muscular contractions. - When a muscle contracts it does so with a certain rapidity, shortening for a certain distance in a certain interval of time. A survey of animal movements shows us that these differ greatly in their velocity, from the slow movements of the muscular walls of the stomach to the rapid movements of the insect's wing. We find that there are, in nature at least, three ways by which increased velocity of movement may be obtained. In the first case, the muscular fibres themselves acquire the faculty of moving more quickly and imparting a greater velocity to the hard tissue to which they are attached. This change is associated with a marked structural alterationthey become striped. Thus the muscular tissue of molluses in general is unstriped; but certain species, together with more active contraction, have acquired striped muscular tissue.

In the second case, the muscle is attached nearer to the fixed point of a lever on which it acts. Thus, while the biceps, attached to the radius near the elbow-joint, moves its point of attachment with hut a small velocity, the hand at some distance from the ellow moves comparatively rapidly through space.

But there is a third method, by which a greatly increased velocity may be obtained, a method almost entirely overlooked by physiological investigators, although it has not escaped the observation of A. Fick. Helmholtz ${ }^{1}$ has shown that the full force of muscular contraction takes some time to develop. The muscle, as it were, gets up steam, so that a movement is made, not at full steam pressure, but begins as soon as the absolute muscular force just overbalances the resistance. It follows from this that if the point of attachment of the muscle be fixed in space, for, say, a second after the muscle has begun to contract (but not to shorten), and if it be then let go, the muscle will have developed a

${ }^{1}$ Arch. f. Anat., Physiol. u. wiscensch. Med., 1850, S. 276; and 1852, S. 199. 
greater force, and the attachment of the muscle will be pulled at a much greater velocity.

In Fig. 132 are two tracings from the gastrocnemius muscle of a frog. The muscle was weighted with 5 grms., and, where a tetanising

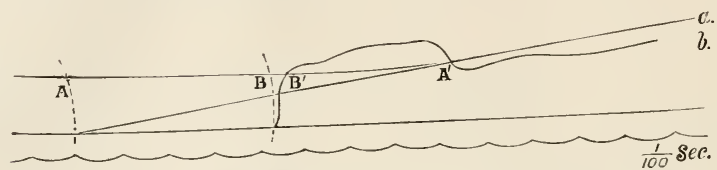

Fig. 132.-Reduced tracings taken from the frog's gastrocnemius musele by the pendulum myograph. In $a$ a tetanic current was suddenly passed through the muscle. In $b$ the muscle was prevented from contracting until the current had operated for a short time. The npright broken lines mark the position of the path of the lever on a stationary plate. In $a$ the point of the lever is raised to $A^{\prime}$ in about $\frac{8}{10 \sigma}$ of a second, giving a velocity of about 6 in. per sccond. In $b$ the point of the lever was raised to the same height $\left(D^{\prime}\right)$ while the plate travelled the distance $B B^{\prime}$, or in abont $\frac{1}{200}$ of a second, giving a velocity of about 100 in. per second.

current was passed through it, it raised the point of the lever from the gromnd line up to $A^{\prime}$, corresponding to a shortening of the muscle of about $4 \mathrm{~cm}$. This took place while the plate was travelling from

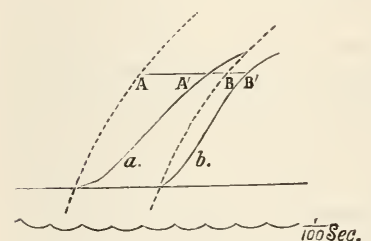

Fig. 133.--Reduced tracings taken of the movements of the human fingertip with the pendulum myograph. Curve $a$ represents the path of a sudden voluntary flexure. Curve $b$, the path described by the same finger, fixed during the first part of contraction, and then suddenly released. The broken lines sloping upwards are the paths of the finger with a stationary plate. In $\alpha$ the finger is raised to $\mathrm{A}^{\prime}$ (about an inch) during the time that the plate has travelled from $\mathrm{A}$ to $\mathrm{A}^{\prime}$, or in abont $\frac{3}{100}$ of a second; its mean velocity is therefore about 33 in. per sccond. In $b \quad a$ movement the same height has oceurred while the plate has been travelling from $\mathrm{B}$ to $\mathrm{B}^{\prime}$, or in about $\frac{1}{150}$ second. Its velocity is therefore about 150 in. per second, or about five times that of $\alpha$.

In Fig. 133 a tracing is made by a style attached to the finger. During the movement of the plate the finger is volmntarily bent as quickly as possible. The style moved from the ground line to $\mathrm{A}^{\prime}$ during the time the plate travelled from $A$ to $A^{\prime}$ - in about $\frac{1}{40}$ second. The second curve represents the path of second, giving a velocity of about $5 \mathrm{cms}$. per second. In the case of tracing $b$, the lever was held for a short time after the tetanising current had passed through the muscle, and then it was suddenly released. It will be noted that the point of the lever attached to it has moved upwards to the same distance, namely, to $\mathrm{B}^{\prime}$, hut that it has done so while the recording surface travelled from $\mathrm{B}$ to $\mathrm{B}^{\prime}$, which event occupied $\frac{1}{200}$ of a second only, giving a velocity of about 80 cms. per second, a velocity sixteen times as great as in the first case.

We have all found out empirically the fact that the above experiment illustrates, and when we wish to impart great velocity, we check the movement during the initial stages of the muscular contraction. Thus, when we wish to project a pellet of paper with considerable velocity, we place it on the tip of the thumb, press the nail of the middle finger into the soft skin of the thumb and behind the pellet; then we forcibly extend the middle finger, but do not at once release it. A to $A^{\prime}$, a period of about $\frac{8}{100}$ of a 
contraction of the finger, supported during the initial stage of the contraction, and then suddenly releasedsnapped, in fact. The same movement is made while the plate travels from $\mathrm{B}$ to $\mathrm{B}^{\prime}$ in about one-fourth of the time.

Amongst many lower animals this "hold and let go" method of increasing velocity eharacterises their most important movements. The elater (click - beetle, Springlirifer), when he falls upon his back is unahle to right limself in the manner usual with insects, on account of the shortness of his legs. He hends his prothorax backwards, and hooks a spine into a depression of his mesothorax. He then contracts his extensor muscles, sudilenly lets go, and springs many inclies into the air.
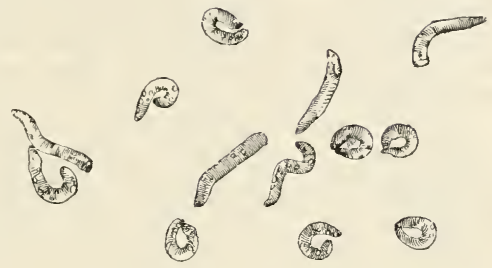

Fig. 134...-Drawn from an instantaneous photograph of cheese maggots. Some are curling round to fix the tail to the head. The upper one has already done this, is straining to extend itself, and is just abont to mnfix itself and jump into the air.

The cheese maggot bends its body round and inserts a hook attached to

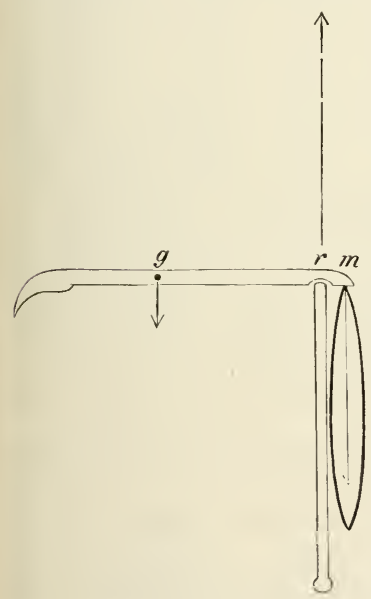

Fig. 135.-The letter $g$ marks the position of the common centre of gravity of the hand and lower arm, the letter $r$ the position at which the lower is supported by the upper arm, and $m$ the position at which the triceps musele is attached. The arrows represent the forces acting at these positions. of application from the point considered (in this case $r$ ). If the distance $g r$ be five times as great as the
distance $r m$, and if the force $w$ be $5 \mathrm{lb}$, and the force $m 25 \mathrm{lb}$., its tail into a grooved plate, like a file, near its head. It then contracts its straightening muscles, the hook is released suddenly from the file, and the maggot is projected with great velocity many times its own length. (Fig. 134).

Moments.-Let us suppose that the upper arm and trunk are fixed in space, the upper arm being raised, and that the lower arm and hand are fixed together so as to form a single rigicl hody free to move at the elbow-joint upon the upper arm (Fig. 135). The centre of gravity of the lower arm and hand (the weight of which is $w$ ) is at the point $g$, and the line of action of gravity will be in the direction of the downwardly pointing arrow. The triceps will exert a force pulling in the direction of the arrow descending from $m$. The point $r$ is a point through which passes the axis of rotation of the lower upon the upper arm.

If the two forces $m$ and $w$ are balancing each other, and if we then shift the force $w$ nearer to the hand, it will overeome the force $m$, and the limb will sink. Shift it towards $r$, and it will itself he overcome by $m$, and the limb will rise. This "valıe" of a force is termerl the "moment" of the force, and is measured by the product of the force, and by the distance of its line 
the moments of the two forces are equal, and there is rest or equilibrium.

$$
g r(5 \text { units of length }) \times 5=m r(1 \text { unit of length }) \times 25 .
$$

Thus the smaller force acting on the longer arm balances the greater force acting on the shorter one. From its situation near the joint the force of the muscular contraction of the triceps needs to be very great, in comparison with the force of gravity pulling down the limb. This muscle may be taken as typical of those of the body generally, and when we push, or pull, or lift, the force of muscular exertion is often many times as great as the force against which it is opposed.

If in Fig. 136 the horizontal line grm represent the rigid lower arm, we may represent the forces acting upon it by straight lines, which can represent these forces, not only in the direction in which they act, but, as in the figure, their lengths may be made to correspond with the magnitudes of the forces. The consileration of these balanced forces is one of statics, but when equilibrium is disturbed by the moments on one or the other side being increased or diminished, a movement of rotation around the point $r$ will occur, and we enter the region of kinetics. If we suppose that

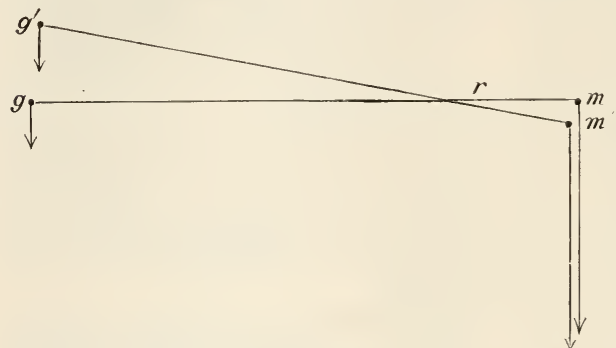

FiG. 136.-Represents the rotation of a rorl $\mathrm{gm}$ to the position $g^{\prime} m^{\prime}$ around a fulcrum at $r$. the force at $m$ (the contraction of the triceps) be increased, rotation of the limb will take place, and after a certain interval of time it will have assumed the position $g^{\prime} r m^{\prime}$. In the performance of this movement $g$ will have travelled five times the distance that $m$ has travelled, and in an opposite direction, so that if the force of

contraction of the triceps at $m$ has to he just a little more than five times as great as the force of gravity acting at $\%$, the point $g$ will be lifted five times as far as the point $m$ will be lowered. What is lost in power is therefore gained in amount of movement, so that an extensive motion of the hand occurs when the muscles acting at the elbow-joint shorten to but a slight extent.

When we take into account the element of time, we learn that the velocity of the point $g$ will be five times as great as that of the point $m$. Movements of $g$ to $g^{\prime}$ and $m$ to $m^{\prime}$ take place in the same interval of time, and $V=\frac{s}{t}$. The aforesaid muscles may, on account of the distance of the hand from the elbow-joint, canse the hand, as in the act of throwing a ball, to move with considerable velocity.

Although the hand carrying a weight may be moved through a great space and with a great velocity, there is no more work done by the arm than is put into it by the muscle. A lever or other machine does not make work; it only performs work in such ways as may be desired and arranged for. This is affirmed in general principles by the law of the conservation of energy, and may readily be shown from Fig. 136.

The muscular contraction of the triceps lowers the point $m$ to $m^{\prime}$, with a force of $25 \mathrm{lh}$. The work is therefore $25 \times \mathrm{mm}^{\prime}$. Although, as the result of this contraction, the point $y$ is raised to $y^{\prime}$ through a space five times 
as great, the protuct (=the work done) remains the same, for the force applied at $g$ is only one-fifth of that at $m$.

Action and reaction.-Returning once more to Fig. 136, it will be evident that unless the point $r$ be fixed in space, the weight of the limb acting at $g$ and the force of the contracting triceps will carry the upper arm bodily downwards. This they tend actually to do with a force which acts upon the upturned articular surface of the humerus. 'This action produces (Newton's third law) an equal reaction in the opposite direction, which reaction, if equilibrium be established, must exactly counterbalance the two forces acting at $g$ and at $m$.

In the case of most muscular actions we have to consider three forces-that of gravity, that of muscular force, and that of the reaction at a joint. It is by investigating the movements around a joint that we can solve many problems of movement; thus, given the force of gravity and its moment, and given the distance of the point of application of the muscular force from the centre of rotation of the joint, we call calculate the muscular force necessary to counterbalance that of gravity acting upon the limb, or any weight supported by the limb.

Levers.-Although the term has not yet been used, it is evident that the bones fall under the definition of a lever. This is defined as a rigid bar, with a fixed point around which it can rotate, which is acted upon by two or morc forces, and the reaction of the fixed point called a fulcrum.

The raising of the body by the action of the calf muscles.-In his well-known article in Wagner's Handwörterbuch, Bả. iii. S. 88 ,

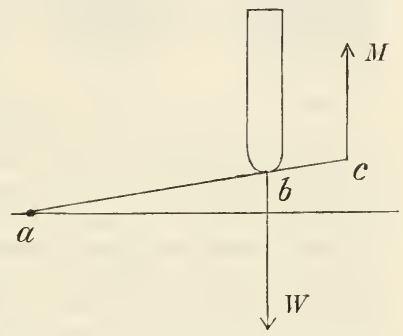

Fig. 137.-To illustrate Weber's theory of the movements around the anklejoint. a marks the centre of rotation of the ball of the great toe; $b$ that of the ankle-joint; and $c$ the point of attachment of the calf muscles.

entitled "Muskelbewegung," Edward Weber describes the foot as a lever of the second order. When we stand upon the ground, and then raise the body on the toes, the fulcrum, according to Weber, is sitnated at the ball of the foot, the weight of the body falls vertically on the position of the ankle-joint, and the muscular pull is at the point of insertion of the tendo Achillis in the hecl. Weber therefore supposed the forces and movements to be related as follows:-If $M=$ the muscular pull and $W$ the weight of the body (see Fig. 137), $M \times a e=$ $W \times b a$, overlooking the fact that the shortening of the calf muscle is the real measure for the movement of the point of insertion, whatever space that point may travel through at the same time from other causes.

Knor ${ }^{1}$ and Henke ${ }^{2}$ rectified this error and gave the true formula, $M \times b c=W \times b a$.

Richard Ewald ${ }^{3}$ explains the matter in the following way:- If a man were to stand on his head and support on his toes another

1 “Ein Beitrag zur Bestimmung der absoluten Muskelkraft," Marburg, 1865.

2 Ztschr.f. rat. Mcd., Leipzig u. Heidelberg, 1865, Bd. xxiv.

3 Arch.f. d. ges. Physiol., Bonn, 1894, Bd. lix. S. 251 ; and 1896, Bd. lxiv. S. 53. 
nan of his own weight, then his ankle-joints would be fulcra fixed in space, and would be situated in the middle points of the levers; the feet would be levers of the first order, or two-armed lever's. If now he were to stand on his own feet, and raise himself on his own toes,

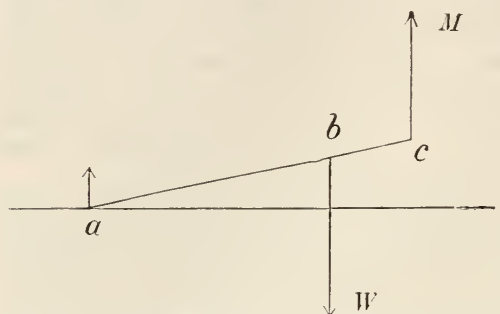

FIG. 138.-Diagram to illustrate the method of calculating movements around the ankle-joint. Letters as before.

ever, werely one of words and definitions.

Fischer ${ }^{2}$ has given the subject as a general problem an exact and comprehensive study. It is evident that the same reasoning will apply to many different movements, as for instance raising the body by extending the arms on parallel bars.

Forces acting obliquely upon a bone. -So far, we have studied forces such as those of gravity or muscular contraction, acting in a direction at right angles to the bone, and in calculating their moments we have measured the distance along the bone from their point of action to the fixed point. We cannot do this if they act obliquely, the moments being in this case calculated from the nearest distance from the fixed point to the line of direction of the force. This nearest distance will be that of a straight line passing from the fixed point, and falling vertically upon the line of direction of the forces. Thus, in Fig. 139, gravity $\times g r$ will give the moments on one side of $r$, and muscular action $\times r m$ will give the moments upon the other side. The moments of the muscle thus varying with position, it is evident that when trying to overcome a constant resistance a flexor muscle exerts least traction when the limb is fully extended, but that the moment will increase as flexion takes place. If the moment of the resisting force does not, as is the case with gravity, augment in the same proportion, it will therefore become less, compared to the moment of the muscular force, and the muscle will do its work under the most favourable conditions, i.e.

${ }_{1}$ Arch. f. d. gcs. Physiol., Bonn, Bd. 1xii.

2 Arch.f. Anat. u. Entwcklngsgcsch., Leipzig, 1895, S. 101. 
diminishing resistance. It must be borne in mind that the direction of traction may be greatly modified by the tuberosities over which the tendon may pass before insertion. A calculation of the moments at the ankle-joint will serve to illustrate the important principle alluded to above. The calculation is only true for the position at which the calculation is made, and the reason is that the moments vary with every change in position.

Knorz ${ }^{1}$ and Henke ${ }^{2}$ pointed out that before the heel is raised the body is instinctively thrown forwards, so that the centre of gravity falls over the base of the toes on which the body rests, before the heel actually leaves the ground. As Bedart $^{3}$ pointed out, it is on this account that when we stand with our toes touching a vertical wall it is impossible to rise on the toes (Fig. 140). When in this position we lean forwards against the wall, the centre of gravity plumbs over a point midway between the ankle and the base of the toes, the wall prevents the experimenter from leaning forward enough to throw the centre plumb over

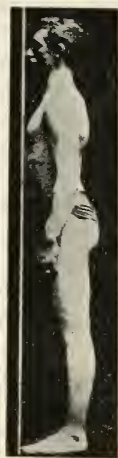

$a$

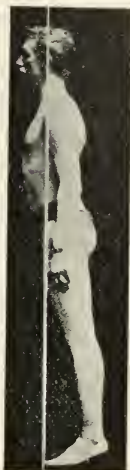

b

Fis. 140.- In $a$ the tip of the toes, the front of the ehest, and the ehin are in the same vertical plane. In $b$ the individual rises on his toes, and in so doing the body moves forwards into the position indicated. Were a wall to oceupy the position of the vertical line, this action would be impossible.

the toes. He has therefore no support for his body and camnot raise it.

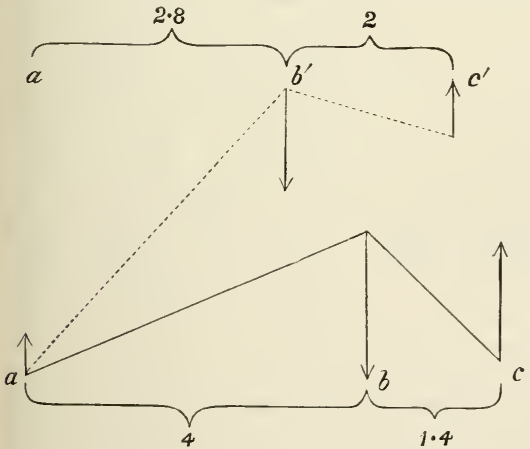

FIf. 141.-Diagram to rejresent the moments around the ankle-joint $(b)$ resting on the ground, and when it is raised to the position $b^{\prime}$. $a$ marks the centre of the ball of the great toe, and $c$ and $c^{\prime}$ the point of insertion of the calf muscle.
Thus, when we raise ourselves higher and higher on the toes, the centre of gravity shifts slightly forwards towards the tips of the toes. One can assure oneself of this fact by standing in front of the door, with the toes about $2 \mathrm{in}$. from the door. If one can just raise the heel, say half an inch, and keep it in that position without falling backwards, it will be found impossible to raise the body to its full height. This can, however, readily be done on standing back, say half an inch. Still more inportant is the fact that

1 "Ein Beitrag zur Bestimmung der absoluten Muskelkraft," Marburg, 1865.

${ }^{2}$ Ztschr. f. rat. Med., Leipzig u. Heidelberg, 1865, Bd. xiv.

${ }^{3}$ Compt. rend. Soc. de biol., Paris, 1892. 
when we raise the body on the toes, the heel end of the lever becomes relatively longer as compared with the toe end, so that the muscular pull required becomes less and less. Fig. 141 illustrates this in the case of a boy's foot. With the foot upon the ground the distance $a b$ is to $b c$ as 4 to $1 \cdot 4$. On raising the heel somewhat more than 2 in.- - see dotted line-the relations are altered, $a b^{\prime}$ being to $b^{\prime} c^{\prime}$ as 2.8 to 2 . It is true that it is very tiring to stand with the body raised high on the toes, but it is probable that this is due to the fact that the calf muscles are shortened, and their pull in consequence greatly diminished.

The resolution of moments into their components.--The moment of a force is, as appears from the construction given, smaller than the force itself. The reason is that a part of the force spends itself by exerting pressure on the joint. Thus in a muscle acting obliquely on a bone there are two component forces to be considered, one acting in the direction of the bone and producing pressure on the joint, the other acting perpendicularly to the first, and producing rotation. The lines

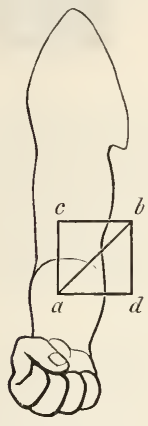

Fig, 142 . representing the component forces form one half of what is termed the parallelogram of forces.

So far we have studied the action of a muscle lying in the plane of movement of the bone upon which it acts. If we are dealing with a joint under constraint, which can, like the elbow-joint, move in one plane only, we have to consider the action of muscles like the pronator radii teres, which are oblique to that plane. If, as in Fig. 142, we represent in force and direction the action of the muscle by the straight line $a b$, we can resolve this force by the parallelogram of forces into two, one of which ac lies in the plane in which movement can actually take place, and the other at right angles to it. The length of ac will represent the force of the muscle acting so as to produce the movement. The moments of flexion will be the force in this direction, multiplied by the perpendicular distance between the point of rotation and the line of action of the force. The rest of the force will be spent in pressure and lateral strain on the joint.

When we are dealing with the action of a muscle upon an unconstrained joint, like that of the shoulder, the direction of the resulting movement will be determined by the direction of the muscle itself. If we take that plane which yasses through the centre of rotation of the joint, the point of insertion, and the point of origin of the muscle (or the line of action of the muscle), then its contraction will cause a rotation around an axis normal to that plane, and passing through the centre of rotation. We may further, by means of three co-ordinate planes, determine the position of these points and the position of the centre of rotation, and then calculate by trigonometrical methods the position of this axis, and the new positions assumed by the limb, resultant upon the muscular contraction.

It is customary to describe the action of muscles in relationship to three co-ordinate planes. Thus, a portion of the deltoid is described as abducting the humerus, slightly flexing it, and rotating it inwards. In other words, abduction may be taken as a plus rotation on the frontal plane, and about a horizontal sagittal axis; flexion, a plus rotation along a sagittal plane about 
a frontal and horizontal axis; and rotation inwardly, as a plus movement in a horizontal plane and about a vertical axis. If we represent the muscle by a line, the length of which represents in force and direction the action of the muscle, we may resolve these into forces acting in three directions by the ordinary parallelepipedon of forces. Thus, in Fig. 143, if $a$ represents the insertion, and $b$ the origin of the muscle, and the line $a b$ represents the action of the muscle in force and direction, we can resolve $a b$ into forces acting along axes intersecting at the point $a$. The lines $a d$, ae, and $a c$ will represent the three components in three planes, at right angles to each other.

In practice we may proceed as follows:-

The bone from which the muscle arises is fixed in space by a clamp. The limb is then brought into the position at which the action of the muscle is to be studied. A cord is then attached to the bone at the point of insertion of the muscle, and the cord passes through an eye at the point of its origin, and to the end of this is attached a weight to keep it taut. By this means the distance from the point of insertion to the point of origin of the muscle may be measured along the cord, which can be graduated for that purpose. If the muscle is a pure flexor, when the limb is flexed it will be found that the points are at their nearest possible approximation. If it be mainly an adductor, and a flexor to but a slight extent, then movement in the direction $x$ (flexion) will but slightly approximate the points ; while movement through the same angle in the direction $y$ (adluction) will produce a relatively greater approximation. The components are, therefore, found by moving the bone through a small angle, say $10^{\circ}$, in each of the coordinate planes, and reckoning the shortening of the

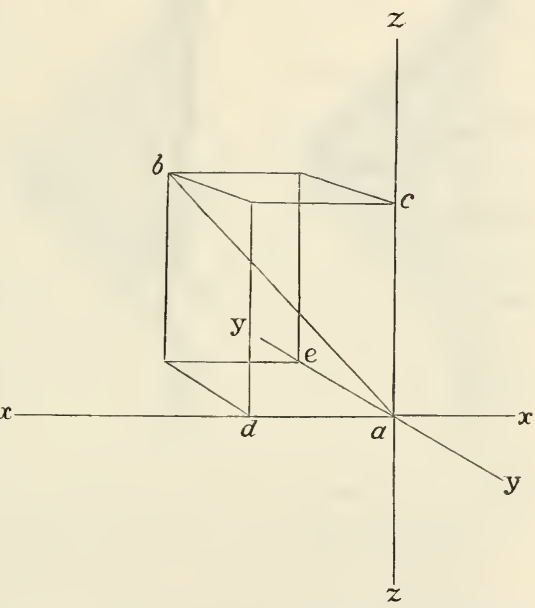

FIG. 143.-The resolution of the force of contraction of a nuscle, represented by the line $a b$, into three forces acting in three co-ordinate planes, namely, into $a c$ along $z z$, ad along $x x$, and ae along $y y$. cord which occurs in each case.

The double action of a muscle at both insertion and origin.So far we have, for the sake of simplicity, considered the point of origin of a inuscle as being fixed in space and not free to move. The latter is sometimes the case, as when the upper arm is fixed by muscles from the shoulder, and then the forearm is moved upon it. But, in the great inajority of cases, both the points of origin and of insertion are inore or less free, and inove as a result of the muscular pull applied to them. If a muscle passes directly between its point of origin and insertion, and if it contracts with a force $F$, this acts equally upon the point of origin and of insertion. Even if the muscle by its tendon passes round a corner, the extensors of the thigh over the knee, or the long head of the biceps over the shoulder-joint, the same still holds good, 
for a force is transmitted without diminution by a perfectly flexible cord passing over a perfectly smooth surface, and the tendon may, for all practical purposes, be considered as perfectly flexible, and the surface over which it passes as perfectly smooth.

A muscle like the brachialis, which passes from the anterior surface of the humerus, and is inserted into the coronoid process of the ulna, not only exerts a force upon the ulna, but exerts an equal and opposite force upon the humerus from which it springs. If the humerus be free to move at the shoulder-joint, this force may cause a rotation of the humerus as well as a rotation of the ulna at the elbow-joint. Fischer, ${ }^{1}$ who has investigated this subject with great care, studied chiefly the actions of the brachialis muscle and the triceps. He made a mechanical model of the arm, corresponding to it not only in gross

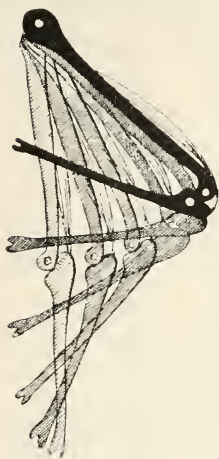

A

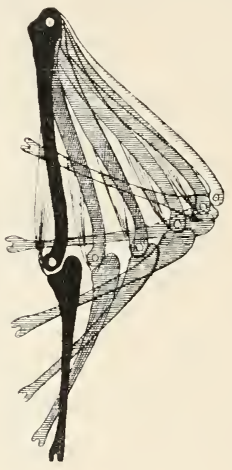

B

Fig. 144. - $A$ represents the action of the triceps upon the humerus and ulna. As the ulna is extended the hmmerus flexes. $B$ represents the action of the brachialis which flexes the ulna, and at the same time extends the humerus. In both $A$ and $D$ the black figures represent the bones before the muscles have contracted. - After Fischer. When the band represented the triceps it extended the elbow, and at the same time flexed forward the humerus (Fig. 144).

Stimulation of these muscles in the living body by faradic currents gave similar results.

It appears, therefore, that a muscle which only passes over one joint does yet effect movements on neighbouring joints, and this as a rule in an opposite direction; thus a flexion at the elbow causes extension at the shoulder, and extension at the elbow causes flexion at the shouller.

Muscles which pass over two joints.-While certain muscles, such as those which move the jaws-the deltoid, the brachialis anticus, etc.-pass over but one joint in their course from origin to insertion, many muscles, especially those of the limbs, pass over two joints, or even more. Thus the long head of the biceps passes over both the shoulder-

\footnotetext{
'Fischer, "Beitr. zu einer Muskeldynamik," Leipzig, 1895, S. 56-197.
} 
and elbow-joints; the hamstring muscles inserted into the bones of the lower leg arise from the pelvis and pass over both the hip- and knee-joints. There are certain advantages gained from this construction of the boly, one of these being that, as the amount of shortening of a muscle is in proportion to its length, a greater amount of shortening is obtained from the long muscles under consideration. As Fick ${ }^{1}$ has pointed out, we are able by this arrangement to move two joints by means of one muscle, and thus there is an economy in the amount of muscular substance required. Thus, in order to perform the same anount of work in extending the hip-and bending the knee-joint by muscles passing over one joint alone, it would be necessary to lave two sets of nuuscles, each set equal to the combined nasses of the hamstring muscles. Not only, then, is the amount of movement of a limb considerably increased, but the amount of work which can be performed, say by bending the knee, is very greatly increased by this arrangement.

\section{The Centre of Gravity of the Body.}

Johames Alphonsus Borellus ${ }^{2}$ and the brothers Wilhelm and Eduard Weber, ${ }^{3}$ taking for granted that the centre of gravity lies in the middle line of the body, determined its distance from the sole of the foot, but failed to determine its position in the antero-posterior plane. They placed a man flat on his back upon a board, and balanced the board see-saw wise upon a wedge. The centre is sitnated roughly between the pubes and buttocks, and is farther from the sole of the foot than from the crown of the head. If the whole height of a man be taken as 100 , the distance of the centre of gravity from the ground will, in the erect position, be 57 per cent. of the total height. This result was verified by Harless ${ }^{4}$ and Meyer, ${ }^{5}$ whose nleasurements vary within the limits of 3 per cent. This is, however, to be expected, for no two individuals possess the same build-a man with muscular armis and shoulders will have his centre of gravity relatively high, another man with strong legs and sloping shoulders will have his centre of gravity relatively low.

Meyer further determined the position of the centre of gravity in the antero-posterior plane by the following method. A man standing at "attention" bent forwards so that the weight of the body fell upon the front of the feet; a line plumb to this point passed through his centre of gravity. He then extended his limbs at the ankle-joint until he nearly fell backwards over his heels; the line plumb to his heels passed through the centre of gravity, which lay in the point of intersection of these two lines. Meyer found the centre to lie in the body or canal of the second sacral vertebra.

Recently Braune and Fischer ${ }^{6}$ have ascertained the position of the centre of gravity. They proceeded as follows:-A "subject" placed flat on the back upon a board was frozen in this position. The frozen

1 "Handbuch der Physiol.," Bd. i. Th. 2, S. 284.

2 "De Motu Animalium," Lugduni Batavorum, 1679.

3 "Mechanik der menschlichen Gehwerkzeuge," Göttingen, 1836.

+ "Die statischen Momente der menschlichen Gliedmaasen," Königl. Bayr. Akad. d. Wissensch., 1860.

5 "Statik und Mechanik des menschlichen Knochengeriistes," Leipzig, 1873.

${ }^{6}$ Abhandl. d. math.-phys. Cl. d. k. süchs. Gesellsch. $l$. Wisscnsch., Bd. xv. VOL. II. - I 7 
body was then pierced by steel "spits," placed in three axes at right angles to each other. From these spits the body was in turn suspended, and inasmuch as the centre of gravity must in each case be in the plane vertical to the axis of suspension, it could be readily fixed by finding the point of intersection on these three planes. Having found the centre of gravity for the whole body, the head and limbs were removed from the trunk, and their centres of gravity determined by the same method.

In a typical subject, to which special reference is made, the centre of gravity for the whole body was $4.5 \mathrm{cms}$. above the axis joining the centres of the heads of the two femora, and on a level with the upper border of the third sacral vertebra. The centre of gravity of the head was plumb above the atlanto-occipital joint, and those of the limbs almost exactly in the line joining the neighbouring joints; thus, the centre of gravity of the arm and thigh lay in the lines joining the centres of the heads of the humerus and femur with the middle point of the axis of the elbow- and ankle-joint. The centre of gravity of the torso lay in the line joining the atlanto-occipital joint to the centre of the line joining the centres of the humeral heads.

The centres of gravity of the different portions of the body lying in the lines joining the principal joints, it was possible to arrange a skeleton of a subject similar in build to the frozen subject, so that the axes of all the joints were in the same frontal plane. In this case, were the skeleton clothed with flesh, the centres of gravity would fall in this plane. The skeleton was photographed from the front and profile, and the centres of gravity, the positions of which were calculated from the observations made upon the frozen subject, were indicated, together with the joints on two coordinate planes, one in a frontal and the other in a sagittal direction. These planes were mapped out in equal squares of $1 \mathrm{~mm}$., and the position of any point, say the centre of gravity of a limb, could be indicated as a plus or minus distance from the line of intersection of these planes in both a frontal and in a sagittal direction, together with a distance along the line of intersection. Thus the centre of gravity of the whole body falling in the frontal plane would give the deviation $O$ along the sagittal plane; those for the head and trunk lying in the lines of intersection would show deviation $O$ for both the frontal and sagittal plane, while the centres for the arms and legs, lying as they do to the side, would give a deviation in the frontal plane of $\pm x \mathrm{cms}$ (see Fig. 122).

The next step taken by Braune and Fischer was to utilise the services of a sollier of nearly the same proportions as the cadaver used. The positions of his joint-axes were indicated on the surface of the body, and he stood in such a manner that these joint-axes plumbed one over the other; and, by inference from the dead sulject, his centre of gravity and those of his limbs lay in the same frontal plane. This was termed the normal position (Normalstellung). A profile photograph was then obtained, on which was projected a millimetre network of equal squares, from which plus and minus deviations from the line of intersection of the frontal and sagittal planes could be measured on the sagittal plane. When the soldier assumed the military position (German), or a position of ease, the joints and centres of gravity occupied new positions, moving forwards or backwards, downwards or upwards, on the sagittal plane. Photographs were taken of the soldier in these new positions; and the new positions of his centres of gravity of limbs, trunk, head, etc., measured on the co-ordinate planes. From these data the position of the centre of gravity of the whole body was calculated, by the 
method of finding the resultant of two or more parallel forces due to the action of gravity on the centres of the limbs.

One cannot read the work of these authors without the greatest admiration for the eare with which they have tried to settle an important problem, but it is very doubtful if the mathematical calculations they have so rigorously used can be applied with advantage to the case in point. To apply data supplied from a corpse stretched upon its back, and subject before freezing to the distortions of gravity acting in the front to back direction, to a somewhat similar living man standing upright and subject to the distortion of gravity acting in a vertical direction, is not a basis upon which we can lope, with further work, however careful and exact, to obtain accurate results.

As will presently be shown, the determination of the position of the centre of gravity is of importance in determining the position of stable and unstable equilibrium. It is here a matter of chief interest to find out whether the centre plumbs in front or behind the hip- and kneeand ankle-joints; to determine, in fact, its position in the sagittal plane. This can easily be determined by an apparatus, contrived by Dr. W. Sheen and myself, which is so simple and easily manipulated that it can be used by the physician as a clinical instrument. It consists of a box, on which the patient stands upon both feet (Fig. 145). The board
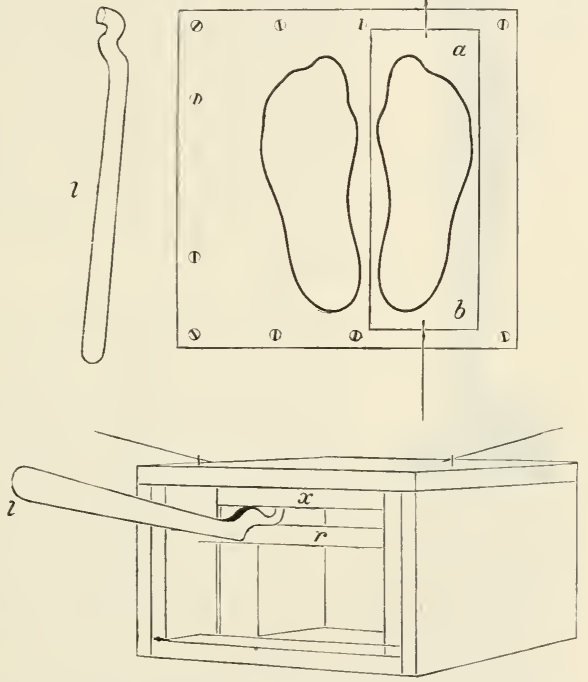

Fic. 145.-Apparatus for determining the centre of gravity. Above, the upper view of the box is shown. $a b$ is the board on which one foot rests, and which is tilted by the lever $l$. Below is shown an oblique view of the same. $\quad r$ is the rod on which the lever $l$ rests, and $x$ the runner carrying the board $a b$. The indices at either end of the board $a b$ show which end is raised.

$a b$, on which one foot rests, is provided below with a steel runner like that of a skate. The runner is supported at each end in a slot of metal. The oblique front view of the apparatus is seen in the lower part of Fig. 145. Below the runner is a strong steel rod, and by introducing between the rod and the runner the bent lever, the rumner can be prised up at any point in its length. If the patient be standing with his centre of gravity falling over the middle of the foot, and if we prise up the runner in front, the front part will be raised, the runner resting on the lever and the back slot. On prising the rummer behind the ankle, the back part will be raised, for the centre is in front. On prising the runner nearer and nearer the plumb-line through the centre 
of gravity alternately behind and in front of it, we shall gradually reach the plumb-line and carry the rumner balanced on the lever. It is quite easy to determine this to within $\cdot 3 \mathrm{~cm}$. By arranging a plumbline to fall through this point, as in Fig. 146, we can determine by photography the relation between the centre of gravity and the centres of rotation of the joints previously determined.

Braune and Fischer, as we have seen, give as the Normal-stellung that erect posture in which all the axes of rotation of the joints lie in the same frontal plane. In this position the centre of gravity of the body is found by them to fall in the same plane. This we

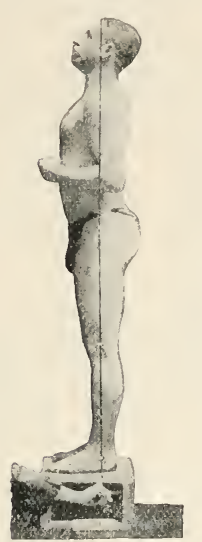

FIG. 146.-The posture is arranged that the dots indicating the centres of rotation of the principal joints fall in the same vertical plane, which is indicated by the vertical line. The posture is a very constrained one, and the centre of gravity of the borly falls in this individual slightly in front of that plane.

the hip come into play. The head was believed by Weber to be exactly balanced on the atlas in its normal position. Thus the whole body would be sustained in the erect position without intervention of muscles, provided it were poised in equilibrium on the ankle-joints.

Now, according to the brothers Weber, the centre of gravity plumbs behind the hip, in front of the knee, and above the ankle-joint, when the body is standing in the position of greatest ease. For this reason muscular effort is only required to establish and retain the position described. Meyer agrees in the main with the Webers, except that he states that the centre plumbs in front of the ankle-joint, thus calling upon the extended calf muscles to contribute towards the support of the body. Meyer, moreover, places the centre of gravity of the head 
a little in front of the atlanto-occipital joint, so that the head is largely supported without muscular exertion by the stretching of the muscles and ligaments at the back of the neck. These views were still further modified by Henke. ${ }^{1}$

The fact that the thigh may be rotated considerably farther backwards from its position in standing, shows that the backward rotation of the pelvis is not at its maximum, the ligaments therefore are not taut. Braune and Fischer place the centre of rotation of the knee before the line between hip and ankle-joint, and thus prove the necessity of muscular exertion to maintain extension of the knee. The fact adduced as counter-evidence, namely, that when the knees are straight the extensor muscles of the thigh are perfectly flaccid, as tested by the patella, which can be moved from side to side without resistance, is not a valid argument, as extension of the knee in the upright

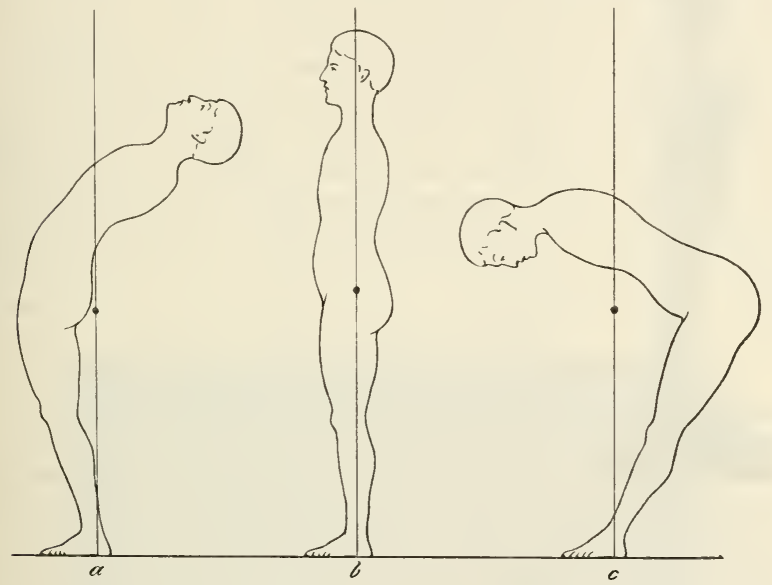

Fig 147. - The position of the eentre of gravity is indieated by a dot in each of the postures $a, b$, and $c$. In $a$ and $c$ it lies outside the body altogether.

position ean doubtless be enforced by the muscles at the back of the leg. The increase of metabolism during standing upright, and its variations with varying positions, offer proof of considerable muscular exertion.

According to Braune and Fischer, who corroborate the results obtained by Parow, ${ }^{1}$ the centre of gravity plumbs in the line joining the hip-joints-in the Normal-stellung, and but very slightly behind it in the bequeme-haltung, and very slightly in front of it in the position of attention.

These somewhat conflicting views are no doubt due to the difficulty in defining what is the erect position. As a matter of fact, there are many erect postures more or less comfortable, and the centre of gravity may be made to plumb either in front of, above, or behind either the hip, the knee, or the ankle. The erect position of the brothers ITeber

1 "Anatomie u. Meehanik der Gelenke," Leipzig u. Heidelherg, 1863, S. 215.

${ }^{2}$ 'irchow's Archie, Bd. $\mathrm{xxxi}$. 
probably did not correspond to that described by Meyer and termed by him militärisch, and this again certainly does not correspond with the bequeme-haltung of Braune and Fischer.

In Fig. 147 three figures of a man are representerl $(a)$ bending backwards, (b) standing erect, and $(c)$ bending forwards. In $a$ and $c$ the centre of gravity actually falls outside the body, and it will readily be understood how the slightest movement in $b$, either forwards or backwards, will shift the position of the centre in relation to the joints.

The following points may be readily ascertained by the aid of the apparatus for ascertaining the position of the centre of gravity mentioned on p. 259 . Where the centre plumbs over the ankle-joint, or lies behind it, the body is held uncomfortably erect, and there is a feeling that it would easily be caused

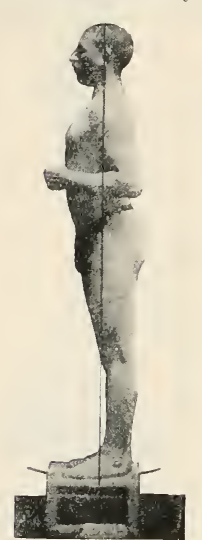

FıG. 148. - $\Lambda$ soldier in the erect posture. 'The black vertical line plumbs with centre of gravity. The centres of rotation of ankle, knee, and lip fall behind this plumbline. to fall backwards. In the most comfortable position the centre plumbs in front of the ankle, but when it is caused to fall over, or nearly over, the ball of the great toe, as in the position of attention, there is a feeling of strain, and the posture cannot be maintained long without great discomfort. On this account soldiers are not kept long at attention by officers of experience; indeed, it is very doubtfil whether this position should be retained at all, for it is questionable whether a smart appearance, and the gaining of half a second in commencing to march, compensate for the waste of considerable muscular activity.

There is no erect posture of the body which can be long maintained without exhaustion. ${ }^{1}$ If we are called upon to stand for some length of time, we instinctively change our posture from time to timc, throwing the strain first on one set of muscles and ligaments and then upon another. The position of the centre of gravity changes with any deformation of the figure. Thus, a protuberant belly, either caused hy pregnancy or by excessive development of fat, eauses the centre to move forwards, so that the obese or pregnant person has to hohl his or her head and shoulders well back, in order that it may plumb within the area of ground covered by the feet. $A$ burden on the back, on the contrary, causes the centre common to the burden and the individual carrying it, to move backwards, so that the bearer has to bend forwards in order that the centre nay fall phmb with the feet.

Viewing the body as a rigid body, we shall find that its stability will depend upon its height and the area of its underpropping (see Fig. 149). If we imagine a force (push) acting through the centre of gravity in the direction of the line $a c$, it will, if great enough, cause the body to fall over the toes at the point $f$. In so doing, it will overcome the force of gravity acting downwards at the distance fil.

The question is really one of the moment around the point $j$. At the instant the overturning is just about to commence, the moments around $f$ will

${ }^{1}$ In the horse, the weight of the animal is largely suplorted by what are termed "check liganents," found both in the fore- and hind-legs. The animal ean on this account remain standing for a consillerable time without fatigue, and can sleep in the standing position (F. S'nith). 
be equal. The moments are found by drawing the shortest line $f^{\prime}$ l from $f$ to the direction of the force $a c$, and the line $f d$ to the direction along

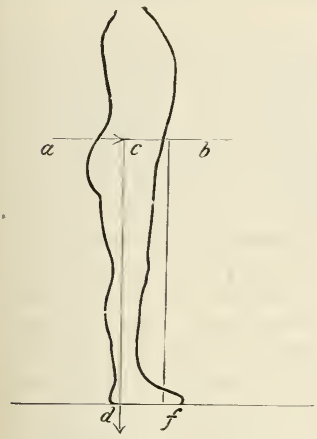

FIG. 149. - To sliow the effeet of a push acting in the direction of the arrow ac in overturning the body, which is viewed as a rigid body resting on the feet.

which gravity acts. Then $b f \times$ push $=f d \times$ weight of body.

If the push be greater than $\frac{f d}{b f} \times$ weight of body, then the body will be pushed over.

It also follows that the longer $f d$ is, the greater must be the push in order that overturning may take place; indeed, we may say that the larger the area of underpropping the more stable is the body. In the erect position at attention the area of underpropping is as 1 in Fig. 150, and coincides with the area covered by the feet, together with the area between them. Under ordinary eircumstances we stand with the feet somewhat apart as in 2, when the area of underpropping is increased. When we wish to resist a push from the sides, we stand as in 3 ; and when, as in fencing or boxing, we wish to resist a push from the front, we stand as in 4.

Strictly speaking, the body not being a rigid mass, there can be no question of stable or even unstable equilibrium. In fact a living subject is constantly in motion, even when trying hard to stand "motionless." Vierordt ${ }^{1}$ by a simple recording method obtained tracings of the swaying movements of persons standing upright. Leitersdorffer ${ }^{2}$ proved by this

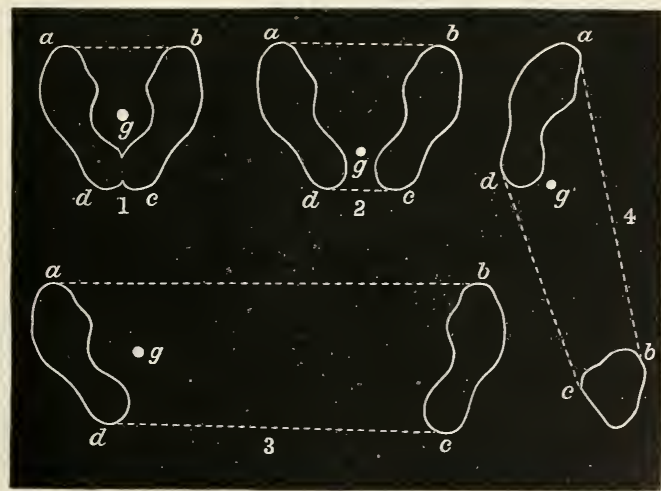

Fig. 150. - Shows the area of underpropping and the plumb of the centre of gravity $(y)$.- (1) At attention. (2) In an erect and easy attitude. (3) When resisting a force applied from the ieft. (4) When resisting a force applied from the front, as in boxing.

method the influence of the area of underpropping and the difference between trained and untrained subjects.

1 "Physiol, des Menschen," Tübingen, 1862, S. 365.

2 "Das militärische Training," Stuttgart, 1894, S. 42. 


\section{Methods of Recording the Movements of Walking and Running.}

The Webers ${ }^{1}$ studied the actions of walking and running, on a track

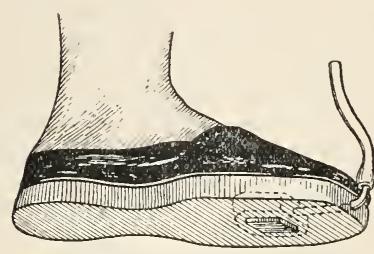

F1G. 151.-Shoe for indicating when a man's foot comes in contact with the ground; a transmitting tubc effects a communication between the air-chamber and the chronographic tambour.-After Marey. of measured length. By means of a watch they could determine the period of any given pace; for, knowing the space traversed, the time taken to traverse it, and the number of paces indicated by the foot-prints, the mean duration and length of a step could easily be calculated. Further, they were able by this means to determine the relationship between the length of the step and its duration, and arrived at conclusions which will presently be alluded to.

By observation they established the fact that, in walking, the trunk and centre of gravity sink to a lower level than obtains in the erect position, and they found, moreover, that this sinking is greater the longer the stride.

Chronographic methods.-M. Marey, who has rendered such signal service to the physiologist by inventing useful methods for rccording the movements of the heart, the blood vessels, and the muscles of the body, has also been the pioneer in inventing chronographic methods for recorling such movements of the whole body as those of walking, rumning, swimming, etc. ${ }^{2}$ These methods do not vield very full information, but they give useful data as the exact times during which the several limbs remain in eontact with the ground.

The apparatus for recording the "paces" of a man is as follows:- $A$ pair of shocs are constructed (Fig. 151), with thick indiarubber soles, within each of which is a hollow cavity, communicating by a long tube with a Marey's writing tambour. The two tambours arc arranged to rccord in vertical serics upon a revolving drum, which is carricd in the hand (Fig. 152). When a foot touches the ground, a wave of pressure travels to the corresponding writing-lever, which is

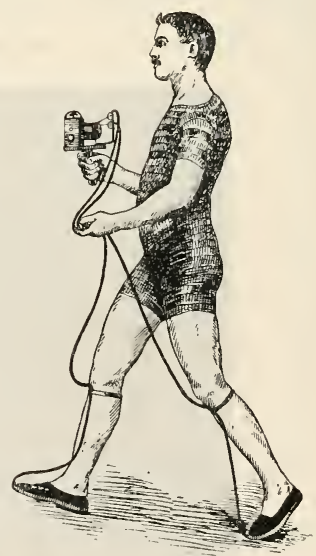

FIG. 152.-Pedestrian furnished with special shoes and earry. ing a chronographic apparatus.-After Marey. caused to trace an ascending curve upon the cylinder.

The paces of the horsc have naturally called forth much observation, and

1 Wilhelm and Eduard W cber, "Mechanik der menschlichen Gehwerkzeuge," Göttingen, 1836. Recently republished in vol. iv. of Wilhclm Weber's "Gesammelte Werke."

${ }^{2}$ Ann. d. sc. nat., Zoologie, Paris, 1872. His work recently cpitomised in "Le muvement," English transl. by Pritehard, 1895. 
for their study a similar piece of apparatus has been used by Marey (Fin. 153). Indiarubber bags, stuffed with hair, are fixed in the hollow of the hoof, communicating, by means of a long indiarubber tube, each with a writing-lever. The ricler (Fig. 154) carries a revolving drum, with the four writinglevers arranged in vertical series. The tracings obtained from a man running are shown in Fig. 155. The continuous curve is that of the right foot, while the discontinuous curve is that of the left. The upper curve represents the oscillations of the centre of gravity. In both eases, descent of the lever is due to pressure of the foot upon the ground. Ascent is due to the sole of the foot leaving the gromd. Marey usually rejresents his results directly obtained by means of the above curves in the notation seen on the lower part of this figure and in figure 156. The methor of its derivation from the curves will readily be understood by reference to figure 155 .

Vierordt modified Marey's method, using electrical recorders instead of air recorders. In other experiments, tiny outflow pipes from a reservoir of coloured fluid, carried on the shoulders of the runner or walker, were attached at various points, such as the hip, kwee, shoulder, etc. From these outflow pipes a continuous flow spurted upon a piece of

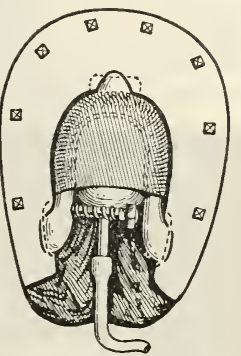

Fig. 153.--Special apparatus for recording the contacts of a horse's feet with the ground; a transmitting tube effects a communication between the air-chamber and the chronographic t a mbour. $-\Lambda$ fter Marey. paper, fixed vertically by the side of the person under observation, and thus recorded the novements which were taking place.

Chronophotographic method-This method is a modern one, dating from the year 1878, when Janssen took a series of photographs of the

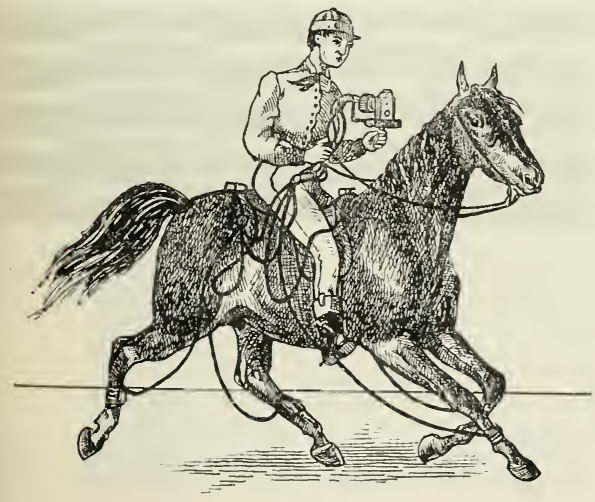

F1G. 154.-The chronographic apparatus for recording the paces of a horse.-After Narey. planet Venus while it was traversing the sun's disc, and suggesterl a similar methorl for the investigation of animal locomotion. It has since been used with remarkable success by Muybidge, Marey, Anschutz, Braune, and Fischer. Muybrilge, in stulying the movements of the lorse, causerl that animal to run in front of a white screen along a track across which were stretched a series of wires, each one of which formed part of the circuit of an electro-magnet, which closed the shutter of a photographic camera. When the horse broke the wires, one by one, a shutter was released each time, and the horse was photographed. ${ }^{1}$

1 "The Horse in Motion, as shown by Instantaneous Photography," London, 1882. See also Londe, "La photo. méd.," Paris, 1893. 
Marey, using but one camera, provided with a rotating series of diapliragms, obtained a series of exposures - ten a second, each lasting $\frac{1}{500}$ secondupon his single plate. In photographing a man running, he caused him to pass in front of a black background, consisting of an enormous box, blackened on the inside, and opening towards the camera, and protected from the direct influence of the sum's rays. He thus obtained a series of photographs, say at intervals of $\frac{1}{10}$ second. When, however, he dealt with slow

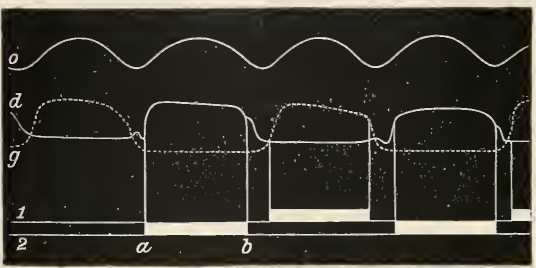

Fig. 155.-Marey's curves obtained from a man running; 0 , oscillations of the centre of gravity; $d$, eurve described by pressure of right foot; $y$, the same of left foot.

parts during each phase of even a slow motion.

A second method consisted in using a sensitive plate, or preferably a roll of sensitised gelatin, which travelled at a known rate, and was at intervals, say, of $\frac{1}{12}$ of a second, brought to a standstill. At rach moment, during which the plate became stationary, the shutter of the camera was automatically opened and a photograph taken. In order to keep the camera pointed at the moving object-a bird, for instance-the camera was reduced as much as possible in size, sighted like a gun, and moved after the bird by the observer.

W. Braune and Fischer ${ }^{1}$ used a moditication of Marey's method. The person under observation was clothed in black, and upon important parts of the head and limbs a series of eleven Geissler's tubes were attached. They were filled with nitrogen, and con-

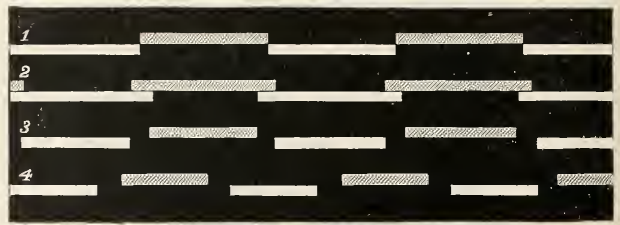

Fis. 156.--Chronographic record of the periods of contact of the feet of a man executing varions paces. The contact of the right foot is represented by a white band, that of the left foot by a shaded band.-After Marey.

1. Represents walking on the level ground.

2. Represents walking upstairs.

3. Represents running.

4. Represents running at a greater speed.

nected with the secondary coil of a Rulnmkorff's machine. In the primary coil was placed an interrupter, which caused a current to pass through the tubes at regular intervals of $\frac{1}{25}$ of a second. The experinent was conducted in a dark room, and the periodic flashes of the Geissler's tubes were photographed. Nitrogen was placed within the tubes, because its light is especially rich in chemically active rays. The most important advance made by these observers was the exact record of their results in relationship to co-ordinate planes. The observed person's movements were recorded by cameras placed 
on either side, with their optical axis perpendicular to the plane of his path, and at the same time his movements were photographed from the front or from behind. By this means, and by the hely' of networks of equal squares, these observers were able to record photographically the movements of any part - say the shoulder-in all three dimensions of space, a result not hitherto satisfactorily achieved.

\section{Detaileid Description of Movements.}

Walking.--On level ground one foot leaves the ground as the other reaches it. On walking uphill or in carrying a weight the advancing foot does not leave the ground until its companion has become firmly planted (Fig. 156, ${ }^{1}$ and ${ }^{2}$ ).

In studying the details of the movement we may consult Fig. 158.

This is obtained chronophotographically from a man clothed in black, with white stripes in the axis of the limbs

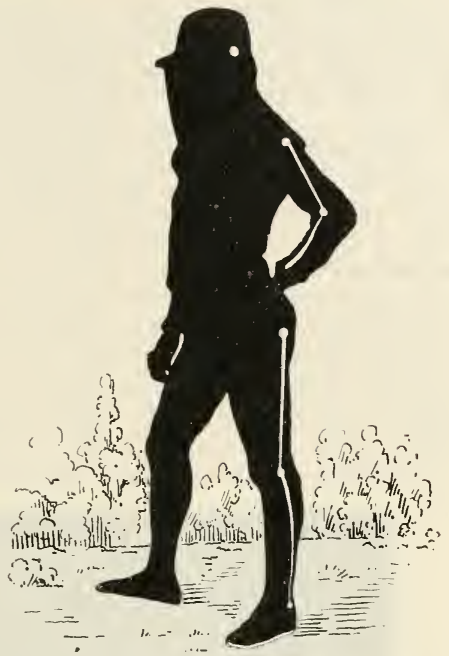

Fig. 157.-Chronophotographic method of re. cording bodily progression. The axes of rotation of the joints and axes of the limbs are represented in white on a dark back. ground. These are photographed during progression.-After Marey.

(see above). It agrees remarkably with the results more recently

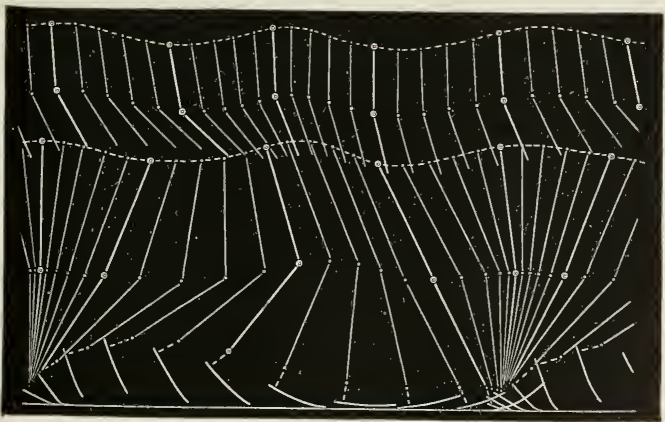

FIG. 158.-Chronophotograph of a man's movements when walking. Rearlfom left to right.-After Marey.

obtained by Braune and Fischer, ${ }^{1}$ but differs somewhat from the older observations obtained by less exact methods.

Examining the figure representing the movement of one side of ${ }^{1} \mathrm{O}_{1}$. cit., Leipzig, 1895 , plate $x$. 
the body, we notice that the trunk oscillates upwards and downwards on a vertical plane. A crest occurs about the middle of the period during which the limb rests upon the ground. There is another crest which occurs while the leg is off the ground, and this coincides with the middle of the period during which the other leg-not represented in the figure-is in contact with the ground.

If we study the position of the limb just before it leaves the ground, it will be noted that the limb is fully extended and forms the hypotenuse of a right-angled triangle, whose vertical side falls from the hipjoint to the ground. The trunk is now falling, and in order that the leg may be brought forward free of the ground, it is bent by muscular exertion and reaches its maximum of bending. It then extends until the heel reaches the ground and the limb is once more straight. While the foot remains upon the ground, and while the hip is still advancing, the knee is once more bent, but to a slighter extent. The limbs are straight, therefore, at the moment when they reach and leave the ground; they are bent at all other times.

Running.-In the case of running, the body remains suspended for a

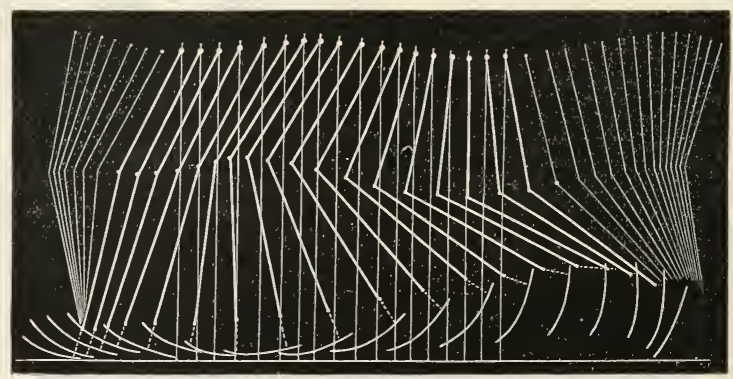

FIG. 159. - Chronograph of the movements of a man's leg in running. Read from right to left. - After Marey.

moment in the air (see Fig. $156,{ }^{3}$ and ${ }^{4}$ ), that is to say, one limb leaves the ground a little before the other leg reaches it. On exanining Marey's chronophotographic figure (Fig. 159) representing a running man, it will be noticed that although the limbs are never quite extended, as in walking, yet the greatest extension is at the time the foot reaches, and then again at the time it leaves, the ground.

In running, the length of the stride may be greater than in walking, and the frequency of stride may also be greater. On this account it is often stated that a man can run three times as fast as he can wall. This is undoubtedly an error, as the rumning and walking "records" conclusively show. While the record for the mile rum is 4 minutes 18 seconds, the record for the mile walked is 6 minutes 36 seconds $^{1}$. Even if we calculate for running on the running pace for 100 yds. (10 seconds), this gives for the mile run 2 minutes 56 seconils.

Relationship between duration and length of stride.-According to the brothers Welser, ${ }^{2}$ there is in walking a relationship between the ${ }^{1}$ Ansateur records up to 1895 . 2 "Meehanik der Gehwerkzeuge." 
duration of a stride ${ }^{1}$ and its length, the longer strides being taken in the shorter periods of time.

The Webers also investigated this relationship in the case of a running man, and found that the duration of the stride suffered but slight variations, and that in consequence differences of velocity are due chiefly to differences in the length of stride.

Marey investigated this matter by somewhat improved methods, and found that, as the number of steps was increased in walking from 40 to 75 , the length of the stride was increased, too, but that in still quicker walking the length diminished very considerably.

In the face of this conflicting evidence, it is difficult to believe in any necessary relationship between the length of a stride and its duration. Indeed, without any scientific appliances at all, we can convince ourselves that the step can voluntarily be lengthened and shortened at will within very wide limits, without changing the duration of the pace. It is very questionable whether we can accept either the Webers' or Marey's results as true, except for the particular experiments which they carried out. For instance, in Marey's experiments, the subjects of experiment were required to walk in time with the sound of a bell struck at regular intervals. Knowing the distance traversed, and the time which this took, the mean length of the stride could be calculated. The men-they were soldiers-when they had to walk to a quicker time would instinctively lengthen the pace, the object of quick time being to get over ground, and this they were able to do up to a certain limit. We may indeed walk or run in many fashions, and even the Webers recognised that the time relationship between length and duration of pace was not the same in "Sprunglauf" as in "Eillauf." Nevertheless, there is probably for each individual a walking and running pace of certain duration involving the least expenditure of energy. The step is quicker in short than in tall persons, and the reason for this will be apparent on perusal of the next section.

Pendulum movements of the limb.-The Webers ${ }^{2}$ taught that, in walking or running, the forward movement of a limb, from the moment at which it is lifted from the ground to the moment it reaches it again, is a pendulum-swing, due not to the action of muscles but of gravity itself. This view is generally held to be true, and some writers such as A. Fick ${ }^{3}$ consider that the swings of a hanging thigh are merely damped by the surrounding muscles, which alter the amplitude but not the period of the swing: they would deny the aid of muscles altogether.

The evidence in favour of this theory is as follows:- The lower limb hanging lax and allowed to swing has a period of 0.692 seconds. In walking or running, half a swing is executed by each limb during the period of a single pace, and a whole swing during the period of two paces. This is true especially in the case of rapid rumning, when the feet touch the ground and remain at rest for a very short period of time. Now two such paces are accomplished in 0.664 seconds, rather less, in fact, than the time taken by the swing of a lax leg. This somewhat shorter duration is accounted for, by those who advocate the pendulum theory, by the fact that in running the leg is flexed, and that on this account the period of its swing would be diminished. Again, it is maintained by the followers of the Webers that by the pendulum theory alone can the fact be explained that the longer the stride the shorter is the period

\footnotetext{
${ }^{1}$ The length of a stride is the distance, say, between the point of the toe of one foot as it leaves the ground, and the point of the other foot when it, in its turn, leaves the ground.

2 "Mechanik der Gehwerkzeuge."

${ }^{3}$ Herman's "Handbuch," Bil. ii. Th. 1, S. 330, 331.
} 
taken. Moreover, it is a well known and undoubted fact that people with short legs walk in quicker time than those with long ones, when they assume a natural pace, as free as possible from muscular exertion.

The investigations of Marey just quoted have, however, shown that there is no necessary relationship between the length and the duration of the stride.

Mechanical forces operative during progression.-In walking and running there are certain forces which have to be so equilibrated that the pace is neither increased nor diminished, and that the body retains its elevated position from the ground. There is, in the first place, the weight of the whole mass of the body tending to seek its lowest level. Secondly, there is the resistance of the air, which will vary with the velocity of progression, and the extent of surface presented by the body, etc.; and, thirdly, the reaction of the ground upon which progression is performed. As against these forces we have the muscular force of the body which tends to straighten the legs, raise the

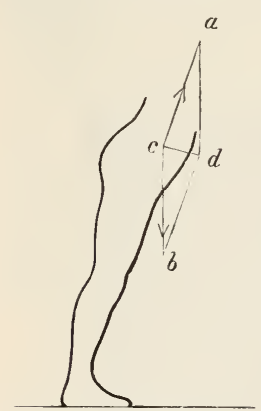

Fis. 160. - The upward thrust of the leg is represented by the arrow $c a$. From $c$, the centre of gravity, the line $c b$ represents the force of gravity. By completing the parallelogram $c a d b$, we obtain the line $c d$ which represents the displacement of $c$ that will actually take place.

when the body is bent, a , and probably moves rhythmically to some slight extent as the limbs swing. These movements also we must discard as being relatively unimportant, and we must view the centre of gravity as fixed in the body, and in the median plane just above and somewhat in front of the middle of the line joining the centre of rotation of the hip-joints. The chief movements in space of this centre-which we may fix by the movements in space of the hip-joint-are a movement forwards and a movement up and down. ${ }^{1}$ Marey's chronophotographic figure (Fig. 158) shows that the hip and shoulder, and therefore the centre of gravity, as well as moving forwards, are displaced upwards and downwards several centimetres during each stride. The moment at which the leg

${ }^{1}$ During walking the mean position of the centre is nearer the ground than it is in the erect position. It is still lower during the act of rumning. 
on the left-hand side of the figure leaves the ground, the push of the leg would be in the direction of the arrow ca (Fig. 160). If the line ca represents the push of the leg in amount and direction, and the line $c b$ the force of gravity, by completing the parallelogram we obtain the actual displacement of the centre $c$, which would take place under the influence of these two forces. The push of the leg under discussion and gravity combined would cause the body to be projected forwards and downwards, but for the fact that at this very time the other leg touches the ground in the extended position, and prevents this downward displacement of the body. The momentum of the body, therefore, carries it forward, and it swings forward on the second leg, which is like the travelling radius describing the are of a circle. Thus the centre of gravity is raised upon the extended leg, and then falls, until raised again, when the first foot next reaches the ground.

The resistance of the air is not a very considerable force, but its rate of increase is in proportion to the square of the rate of progression.
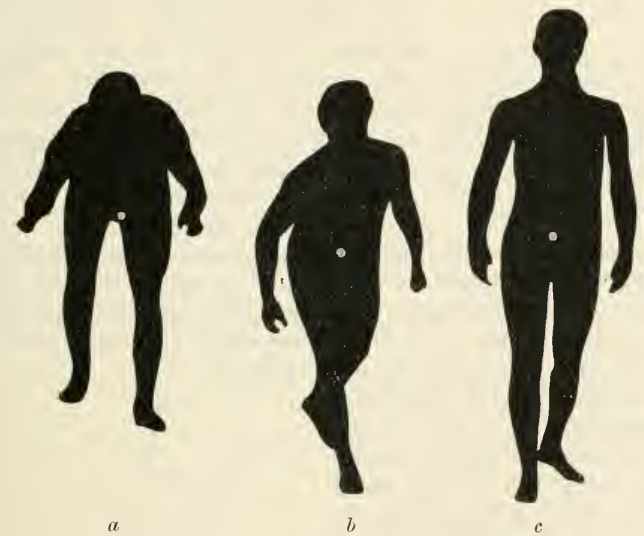

FI6. 161.-To illustrate the surface of body exposed to the resistance of air $(a)$ in bicycling, $(b)$ in running, (c) in walking. The centroid is indicated by a white spot.

This resistance is met by the changes in the posture of the body which invariably accompany progression. During the act of rapid walking or running the body is bent forward, so that a diminished surface is presented in the direction of movement. In bicycling, the racer also bends down upon his handles, presenting a diminished surface in the direction of resistance. Fig. 161 is constructed from three photographs taken at equal distances from the same individual bicycling $(a)$, running $(b)$, and walking $(c)$, the areas being respectively $100,7:$, and 72.1

Not only does the act of bending forward diminish the surface

1 The areas were calculated by projecting the photographs upon pieces of cardboard, outlining the figures, cutting them out and weighing them. The centre of the surface (centroid) was found in the usual way, by suspending each of them from two different points and finding the point of intersection of the plumb-lines of these points. 
exposed to the resistance of the air, but it is a necessary action, which must occur if equilibrium is to be established without the aid of excessive muscular exertion. This will be readily understood on reference to Fig. 162.

In the erect position the centre of gravity of the body is approximately above the centre of rotation of the hip, and it is steadied upon
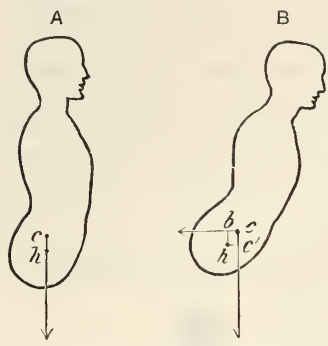

Frg. 162.--In A, the crect position, the centre of gravity $(c)$ plumbs above hip $h$. B represents the body of a man rumning, the resistance of the wind is represented by the line $c b$, and (c) the centre of gravity plumbs in front of the hip-joint $(h)$. the hip by the aid of muscular effort. Were the body to progress forwards in the erect position, and encounter the resistance of the air, it would be toppled backwards, unless for muscular adjustments. In point of fact, we instinctively lean forwards, so that the centre of gravity is well in front of the hip-joint, as in B (Fig. 162). If, as in the figure, we consider the centre of the vertical surface exposed to the resistance of the air (the centroid) as corresponding with the centre of gravity, we may consider the line $b c$ as repressing in force and direction the resistance of the wind. Its moments around $h$, the centre of rotation of the hip, will be $b c$ multiplied by the shortest distance between $h$ and the direction of $b c$, namely, $h b$. Now the centre of gravity must, in order that equilibrium

be established, move forwards, until its moments are equal, until the force of gravity multiplied by $h c^{\prime}$ is equal to the resistance of the air multiplied by $h b$. In more rapid progression, resistance of the air increases as the square, and, in consequence, the movement forward of the centre of gravity must be much greater.

The reaction of the ground upon which progression is male is the last of the external forces acting upon the body which we need discuss. Were the ground perfectly smooth, a condition which is not reached by the smoothest ice, we should be unable to progress, for a perfectly smooth surface has a reaction in the vertical direction only, and the feet would at once slip backwards. On smooth ice progression is difficult, because of the large amount of slipping which takes place, but on an ordinary floor there is sufficient roughness to give a grip to the foot.

When the foot is about to leave the ground, pushing the body for-

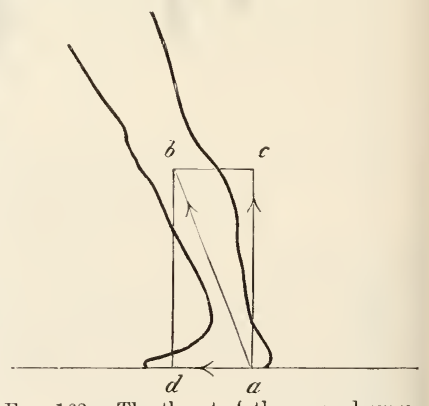

Fic. 163.-The thrust of the ground represented by the arrow $a b$ may be resolved into a vertical component $a c$ and a horizontal component ad. This latter is the force which will prevent the leg from slipping backwards. wards, the thrust or reaction of the ground will be in the direction of the arrow (Fig. 163, ab). If $a b$ represents this reaction in force and direction, we may resolve it by the parallelogram of forces into a vertical force $(a c)$ and a horizontal force $(a d)$. The vertical force is that which 
balances the vertical weight of the body through the leg, and the horizontal force $(\mathrm{ad})$ balances the backward slipping of the foot which would otherwise occur.

The forces which we have now considered may be termed external: and in contradistinction to these are the internal forces of the body, the friction occurring at the joints, the tension of ligaments, tendons, and over-stretched muscles; and, over and above all these, the force of muscular contraction. In all forms of progression muscular contraction is that which opposes the external forces which act upon the body. 


\title{
THE MUSCULAR AND NERVOUS MECHANISU OF THE RESPIRATORY MOVEMENTS.
}

\author{
By E. H. Starling.
}

Contents.-Dechavical Conditions, p. 274--Inspiration, p. 275-1)iaphragm, p. 276-Intercostal Nuscles, p. 277-Expiratiou, p. 279-Accessory Respiratory Movements, p. 279-Types of Respiration, p. 280-Movements of the Lungs, 1. 280-Respiratory Rhythm, p. 281-Pressure in Lungs, p. 281-Methods of Registering Movements, p. 282-Nervous Mechaniss, p. 283-Respiratory ('entre, p. 284 Spinal Ceutres, p. 286-Automatism of Centres, p. 288Influence of Afferent Nerves, p. 292-Action of Vagus, p. 292- Normal Movements and Effects of Division of Vagi, p. 296-Effects of Disteusion and Collapse of Lung, p. 298-A puœa, p. 298-Electrical Stimulation of Yagus, p. 301 -Iuthuence of other Sensory Nerves, p. 304--Influence of Brain, p. 305Asphyxia, p. 306-Modified Respiratory ILovements, p. 308.

\section{The Mechanical Condtions of Respiration.}

SINCE the lungs are contained in a elosed cavity, their onter surface being in direct contact with the inner wall of the thorax, it is evident that any changes in the eapacity of the thorax must lead to corresponding changes in the volume of the lung. In inspiration, the cavity of the thorax is enlarger in all directions, in consequence of the action of certain muscles. The lnngs must therefore expand to take up the increased space in the thorax, and air is drawn into them. The existence of the pleural cavity insures the uniform expansion of the lung, even although some parts of the thoracic wall move more freely than others. The inspiratory enlargement of the lungs not only acts against gravity in raising the ribs, but also stores mp potential energy in conseqnence of a stretching of the rib cartilages and of the elastic lungs. As soon, therefore, as the muscles relax, these forces-gravity and elastic reaction of thoracic wall and pulmonary tissue-come into play, bringing the thorax back to its original size, and thens causing an expulsion of air from the lungs-an expiration. On the other hand, the cavity of the thorax can be diminished below that corresponding to its position of rest by contraction of certain expiratory muscles. In this case, relaxation of the muscles would be followed by a return to the normal size of the thorax, and therefore by an inspiration. It is evident, therefore, that respiration might be carried ont in one of two ways-by an active inspiratory effort, followed by a passive respiration due to elastic reaction; or an active expiratory effort, followed by an elastic inspiratory reaction. We find that in all mammalia the former mode of breathing is the normal one, inspiration being due to the contraction of muscles, whereas expiration is largely passive. 
In order that the lungs may follow accurately the changes in capacity of the bony cage in which they are enclosed, it is necessary that they should be highly elastic organs; and we know that the greater part of their framework is made up of a meshwork of elastic fibres. In consequence of this elasticity, the distension of the lungs will require the expenditure of a certain amount of energy, which, as soon as the distending force is removed, will become active and cause collapse of the lungs. In the new-borll child, before it has breathed, the lungs are collapsed and airless, and their tendency to contract further is nil. As soon as the first breath has been taken, the eavity of the thorax becomes permanently larger than the volume of the contracted lung, so that, dnring the whole of life, the lung is in a condition of distension, its collapse being prevented by the resistance of the thoracic wall. This tendency of the lungs to collapse can be shown by piercing the thoracic wall of an animal. As soon as this oceurs, the pressures on the outer and inner walls of the lung vesicles become identical; the lung collapses, and air rushes in through the hole in the pleura to take the place formerly occupied by the distended lung.

When this experiment is performed on the dead body. the expulsion of air from the lungs is never complete, owing to the fact that the smaller bronchioles collapse and prevent the total emptying of the alveoli by the elastic contraction of the alveolar wall. The elastic reaction of the wall is however not complete until the alveoli are empty. This only happens when in the living animal the inspiratory force or pull of the thoracic wall is prevented from acting on a portion of the lung, in consequence of the accumulation of fluid in the pleural cavity or obstruction of a bronchus; in this event the part of the lung that is thrown out of action becomes airless. The air that could not be forced through the bronchioles has been absorbed by the blood circulating through the pulmonary capillaries.

Since the lungs are in a continual state of tension, they must exert a pull on the thoracic wall and also on all the other structures contained in the thoracic cavity. Hence there is a negative pressure in the pleural cavity. This negative pressure is increased when the rolume of the lungs is enlarged, as in a condition of inspiration, and diminished during expiration, but with normal lungs it could only be abolished by a shrinking of the thoracic walls until they enclose a cavity no larger than the atelectatic lung. This negative pressure naturally affects all the other organs enclosed in the thoracic cavity, and therefore exercises an important influence on the flow of blood into and from the heart, as well as on the lymph flow (cf. vol. i. p. 300).

Inspiration.-The inspiratory increase in the thoracic cavity which takes place in all three diameters, is effected by two processes,the elevation of the ribs and the descent of the diaphragm. The articulation of the ribs to the spinal column behind and to the sternum in front allows of a donble novement of these bones. Thus, when they are raised, they revolve about an axis passing through the heads of two corresponding ribs, so that the front part of the ril is raised, the angle between rib and costal cartilage straightened out, and the sternum pushed forwards and mpwards. At the same time, a slight rotation of the rib occurs, and its outer surface, which in a condition of rest was directed somewhat downwards, is thus caused to look outwards. Moreover, since the transverse diameter of the thorax increases from 
the first to the ninth rilss, an elevation of the ribs, by bringing, say, the ninth rib into the position previously occupied by the eighth rib, must cause an increase in the transverse diameter of the thorax at this point. This increase in transverse diameter would be attended by a corresponding shortening of the vertical diameter of the thorax, but this shortening is more than comnteracted by the fixation of the lower ribs and the descent of the diaphragm. In the relaxed condition, the diaphragm, in consequence of the pressure of the abdominal viscera and elastic reaction of the abdominal muscles, is pressed upwards into the thorax, presenting a convex surface to this cavity. Moreover, the circumferential parts of the diaphragm come into close apposition with the lower portion of the thoracic wall, the two layers of parietal pleura being in contact. When the muscular fibres of the diaphragm contract, two events occur, first, a lowering of the diaphragm; and,

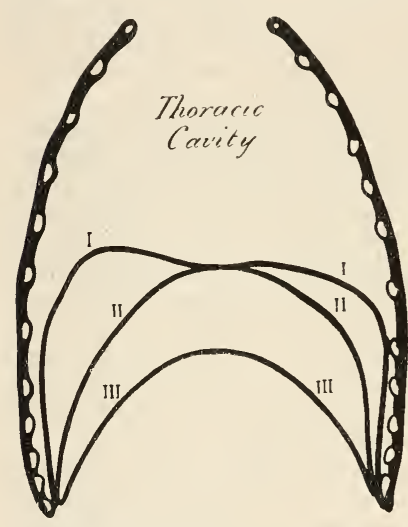

Fig. 164-Diagrammatic representation of position of diaphragn during expiration (I), normal inspiration (II), and forced inspiration (III). second, a flattening of its circumference, which is thus drawn away from the chest wall, the space left being filled by the descent of the lung. Of these two results of the diaphragmatic contraction, the secoud is by far the most important in ordinary respiration. It may be shown that, under these conditions, the central tendon of the diaphragm is practically motionless. As soon, however, as the respiration becomes laboured, there is an actual movement downwards of the central tendon.

The immobility of the central tendon in nornual respiration and its descent in laboured inspiration ean be shown in the dog by passing a sound provicled with a rubber eapsule on the end down through the jugular vein into the inferior vena cava, and then distending the capsule until it is held by the walls of the vessel.

It will be seen that the projecting end of the sound only moves with respiration when the animal is rendered dyspnoeic in any manner. The difference in the changes between the slape in the diaphragmatic floor of the thorax in normal and laboured respiration is shown diagrammatically in Fig. 164.

In consequence of the attachments of the diaphragm to the six lower ribs, contraction of the diaphragm will tend to pull the ends of the upper three of these downwards and inwards, of the lower three upwards and inwards. Under normal circumstances, this tendency is counteracted by the simultaneous expansion of the rib-cage effected by the inspiratory muscles, and the fixation of the lower ribs by the serratus posticus inferior, quadratus lumborum, and the lower portions of the sacro-lumbalis. In cases of paralysis of the muscles which receive their innervation from the cord below the origin of the phrenic nerves, and much more frequently in children where the ribs are wanting in rigidity, the contraction of the diaphragn may cause a well-marked depression, rumning downwards and outwards on each side in front of the chest, corre- 
sponding to the costal origins of this muscle. This groove is frequently seen in rickety children, where the softness of the bones has bcen con1binerl with respiratory troubles.

The contraction of the diaplragm may also cause a widening of the lower part of the thorax, since in its descent it presses upon the abdominal viscera, so distending the abdomen and pushing out the lower ribs.

The elevation of the ribs in respiration is carried out by means of the contraction of a number of muscles, of which the external intercostals are usually regarded as the most important. Other muscles involved in the act are the scalenus anticus, the serratus posticus superior, and the levatores costarum. Whereas these muscles raise the upper ribs towards the spinal column and fix it in this elevated position, the external intercostals raise all the ribs with the exception of the first, each intercostal acting from its attachment to the rib ahove as a fixed point.

The functions of the external and internal intercostal muscles have been the subject of considerable discussion ever since Hamberger ${ }^{1}$ put forward his views concerning these muscles, and the question cannot even yet be regarded as finally decided.

In discussing the action of the intercostal muscles, we must distinguish three portions-

1. The external intercostal muscles, which run from one rib to the next rib below in an oblique direction, forwards and downwards. They extend from the levatores costarum behind to the rib cartilages in front.

2. The internal intercostal muscles, which extend from the angles of the ribs behind to the rib eartilages in front. The fibres are directed downwards and backwards, and are somewhat shorter and less oblique than those of the external intercostals.

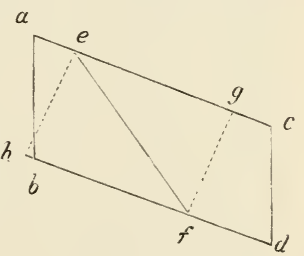

Fif. 165 .

3. The musculi intercartilaginei, which are generally regarded by anatomists as part of the internal intercostals, their tibres being directed, like those of the latter, downwards and outwards.

With regard to the actions of the external muscles, most physio$\operatorname{logists}^{2}$ have accepted the view put forward by Hamberger, that they are inspiratory in function, and that their action is to raise the ribs. Hamberger founded his theory on the following geometrical considerations. If in the Fig. $165 a c$ and $b d$ represent two ribs, which can rotate in a vertical direction about two points $a$ and $b$, and $c$ l. represent the sternum, then abed represents a parallelogram, of which only one side $a b$ is fixed. The line of corresponds in direction to a fibre of the external intercostal muscles. The force with which this fibre tends to move the two ribs may be represented by the lines he and gf. Of these he represents the force acting at the point $e$, and tending $t_{1}$ depress the rib $a c$, while $f g$ represents the force acting at $f$, and tending to raise the rib brl. Since the component acting in an upward

1 "De Respirationis mechanismo" (quoted from Rosenthal, Hermann's "Handbuch," Bl. iv. S. 189).

"Hutchinson, Todd's "Cyclopedia," London, 1852, vol. iv. 1. 1043 ; Yolkmann, Zlschr. f. Anat. u. Entweklngs., Leipzig, Bd. ii. S. 159 (quoted from Rosenthal); Newell Martin, Journ. Physiol., Canihridge and London, 1879, vol. ii. 1. 24. 
direction works on a longer lever arm than the force tending to depress the rib, the total result of the contraction of the muscle must be to raise the lower rib and with it the upper rib.

Hamberger showed, moreover, that an elevation of the ribs must be associated with a widening of the thorax. Thus, in Fig. 166, it is evident

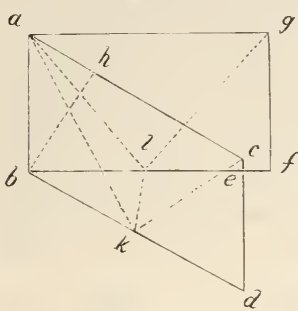

Fis, 166.

$g$ that the distance from $b$ to the vertical line $g f$ is greater than the distance from the point $b$ to the vertical line $c d$, i.e., that in the inspiratory condition, the distance between sternmm and spine, that is to say, the antero-posterior $f$ diameter of the thorax, is increased. Again, since $b h$ is smaller than $b a$, the elevation of the ribs is attended with a widening of the intercostal spaces. It is also obvious that $a l$ is shorter than $a k$; in other words, the contracted condition of the muscular fibres corresponds with a raised condition of the ribs.

Analogous reasoning applied to the internal intercostal muscles shows that these, so far as they lie between the bony ribs, must be expiratory in function and tend to depress the ribs. The intercartilaginous portions, on the other hand, although they continue the general direction of the internal intercostal muscles, yet hear a different relation to their attachments, i.e. the cartilages, since these are directed upwards and inwards. As will he seen from Fig. 167, the action of these filnes on the rib cartilages appears to resemble the action of the external intercostals on the bony ribs, namely, to raise the cartilages and to flatten out the angle between them and the bony ribs. They must, therefore, it is 8 argued, be inspiratory in function.

The views of Hamberger as to the action of the external intercostal muscles have been confirmed by $l^{\prime}$ physiological experiment. Newell Fis, 167.-ac, he, two ribs with their earMartin and Hartwell ${ }^{1}$ recorded the contraction of a segment of the internal intercostal muscles after they har been separated from the rest of the body by dividing the ribs and intereostal spaces on each side of the muscle, leaving only the intercostal artery and the nerve supply to the internal intercostal intact. They found that these muscles acted alternately with the diaphragm, and were therefore expiratory. On the other hand, the segment of the muscles between the costal cartilages acted synchronously with the rest of the internal intercostals, and were therefore also expiratory. Since this result with regard to the intercartilaginous muscles is difficult to understand from a mechanical point of view, it has been put to the test of a further experimental investigation by Masoin

\footnotetext{
1 Journ. Physiol., Cambridge and London, 1879, vol. ii. p. 24.
} 
and R. du Bois-Reymond, ${ }^{1}$ who, while accepting Martin and Hartwell's view with regard to the intercostals between the bones, show conclusively that the intercartilaginous muscles contract synchronously with the diaphragm, and are therefore, as Hamberger thought, inspiratory. The method they employed was practically identical with that devised by Martin. In normal respiration, the contraction of these muscles was not noticeable; it only became evident in laboured respiration. ${ }^{2}$ After apnoea has been produced, the intercartilaginous muscles begin to contract later than the diaphragm. Division of the phrenic nerve has no influence on their contractions.

Expiration.-The diminution of the thoracic cavity which gives rise to expiration is under normal conditions not associated with the active contraction of any muscles. As the inspiratory muscles relax, the lung, in virtue of its elasticity, tends to contract and drag the chest wall with it. Moreover, the ribs which have been raised against gravity and the elastic resistance of the rib cartilages, return to their previous condition, while the slack diaphragm is pushed up into the chest by the retraction of the abdominal walls pressing upon the abdominal viscera.

Laboured respiration.-In forced inspiration, such as occurs when there is any obstruction to the entrance of air into the lungs, or if the aeration of the blood be prevented in any way, in addition to a more powerful action of the inuscles already mentioned, the aid of other muscles is called in. In order to secure an inspiratory action of these accessory umscles, a fixed attachment must be provided by the elevation and support of the head, shoulder, and arm. The chief of these accessory muscles are the sterno-mastoid, the pectoralis minor, the lower part of the pectoralis major, and the lower slips of the serratus magnus.

The chief muscles concerned in forced expiration are those forming the abdominal wall. These press on the contents of the abionen and pull down the sternum and ribs, thus diminishing the cavity of the thorax in all directions. They are assisted in the depression of the lower ribs by the serratus posticus inferior and portions of the sacrolumbalis. In the strong expiratory convulsions of asphyxia, an enormous number of muscles come into play, and every muscle which can depress the ribs or press on the abdominal viscera, or afford a fixed point for muscles having these actions, is forcibly contracted.

Accessory respiratory movements.-Besides the movements of the thorax which bring about changes in the capacity of this cavity, and so serve to draw air into, or expel air from, the lungs, certain other movements are connected with the respiratory act, known as accessory or concomitant respiratory movements. These have no influence on the size of the thoracic cavity, but serve simply to facilitate the entrance and exit of air to and from the lungs. Many of them are wanting in normal respiration, and are only seen when the breathing becomes laboured from any cause.

1. Movements of the larynx.-Even in quiet respiration there is a movement of the larynx up and down, the upward movement corresponding to expiration. The depression of the larynx during inspiration is due partly to contraction of the sterno-hyoid and sterno-thyroid muscles,

${ }^{1}$ Aich. f. Physiol., Leipzig, 1896, S. 85.

"Weidenfelr, Sitzungsb. d. k. Akail. d. Wissensch., Wien, 1894 (3), Bd. ciii., had personally come to the conclusion, from his experiments, that the intercostals take no part in the respiratory movements. 
partly to the drawing down of the lungs and trachea, in consequence of the deepening of the thoracic cavity. The elevation during expiration is due chiefly to the recovery of the lungs, partly to the contraction of the thyro-hyoid muscles.

2. Movements of the vocal cords.-During quiet breathing the rima glottidis is moderately open, and shows but slight movements. If, however, deep inspirations be made, the glottis is widely dilated with each inspiration, by means of contraction of the posterior crico-arytenoid muscles. Even in quiet hreathing, however, the muscles of the larynx must be in a certain state of activity, since in total paralysis of all the muscles the position of the glottis differs from that in the normal individual. In the former case the vocal cords are placed rather obliquely, so that their upper surface looks outwards and their lower inwards. The effect of paralysis is to make the rima into a valve-shaped opening, which may close by the apposition of the two cords if any sudden inspiratory effort be made. There will, however, be no impediment to expiration.

3. Movements of alce nasi.-During each inspiration the nostril is dilated by the action of the dilatator naris, contracting again during expiration. This contraction is chiefly due to the elastic reaction of the nasal cartilages, but may be assisted by the action of the compressor naris (n. nasalis). The movements of the alæ nasi are more evident in children than in adults, and become especially well marked in the former when there is any obstruction to the entry of air into the lungs.

Other accessory muscles of respiration are those which fix the head, shoulder, spinal column, etc., and which only come into play during laboured respiration.

Different types of respiratory movements. - The amount to which each diameter of the thorax is altered with respiration differs, not only according to the depth of the respiratory movements, but also with the sex. ${ }^{1}$

Thus in man, ordinary quiet respiration is effected almost entirely by contraction of the diaphragm, so that the chief external sign is a pushing out of the abdominal wall and the lower ribs. In woman, on the other hand, inspiration is mainly carried out by means of the thorax, so that the ablomen recedes with inspiration. ${ }^{2}$ In laboured respiration these differences almost disappear, the breathing being mainly thoracic in both cases, and the abdomen receles with each inspiration. Mosso ${ }^{3}$ has shown that during sleep the respiration in the male sex is also chiefly thoracic.

Movements of the lungs. - Since the lungs in inspiration expand all over, while the increase in the thoracic cavity is more marked in certain diameters (especially the vertical), it follows that there must be a movement of the lung with regard to the thoracic wall as it expands. This movement of the lungs occurs in two directions from two fixed points, being in the vertical direction from the apex towards the base of the chest, and from the attachment of the lungs to the bronchi towards the front and sides of the chest, and is rendered possible, as already mentioned, by the existence of the pleural cavity.

1 Cf. Hutchinson, loc. cit.

2 It is stated that the difference is mainly produced by the habit of wearing stays, and that in women who have never adopted this habit, the ordinary respiration is abdominal (Wilherforce Smith, Brit. Hed. Jomrn., London, 1890, vol. ii. p. 843).

"Arch.f. Physiol., Leipzig, 1878, s. 441. 
Hence, as the chest expands from the expiratory to the inspiratory position, the lower border of the lungs moves from the level of the sixth or seventh rib to that of the eleventh. There is, moreover, a movement of the anterior margins of the lungs forward, consequent on the lateral expansion of these organs. On this account the area of cardiac dulness is markedly decreased, or even abolished, during a deep inspiration.

On applying the ear to the chest of a healthy individual, a delicate rustling sound is heard with each inspiration. This sound is known as the "vesicular murmur," and is supposed to be due to the entry of air into the alreoli. Expiration is either noiseless or is accompanied by a much fainter murmur of the same character, which only lasts during the first third of the expiration. If we auscultate over the trachea or bronchi, a sound is heard which is quite distinct in character from the vesicular murmur, and is known as "bronchial breathing." It is a loud blowing nurmur, resembling the sound produced by approximating the root of the tongue to the soft palate and expiring, as in pronouncing the Greek letter \%. It differs from the vesicular murmur, moreover, in that the expiratory part is as loud as or louder than the inspiratory. It is probably generated in the larynx, since complete division of the trachea below the glottis abolishes the sound.

Respiratory rhythm.-In normal conditions each inspiration is followed immediately by an expiration, which is succeeded by a slight pause before the next inspiratory movement takes place. The inspiration is generally shorter than the expiration, the time occupied by the two phases being in the proportion 10 to 12. The pause between two respirations lasts about one-third to one-fifth of the total time between the beginning of one inspiration and the next.

Both the frequency of respiration and the amount of air respired vary within wide linits in different individuals, and under various conditions; these are fully discussed in the article on the "Chemistry of Respiration" in Vol. I.

Pressure in the lungs and pleural cavities.-We have already seen that, even in conditions of expiration, the lumgs are still in a stretched condition, and therefore exert a pull on the thoracic walls, until they are allowed to collapse by puncture of the wall. The amount of this elastic force has been measured by connecting a mercurial manometer with the trachea in the dead body, and then puncturing the thorax. Measured in this way it amounts to between 2 and $5 \mathrm{~mm}$. Hg. According to Donders, ${ }^{1}$ however, this is less than the actual pressure in the living body, owing to a loss of elasticity by the lungs. Donders calculated the elastic pull of the lungs in the expiratory condition as about $7.5 \mathrm{~mm}$. $\mathrm{Hg}$. After an ordinary inspiration this is increased to $9 \mathrm{~mm}$, and after the deepest possible inspiration to about $30 \mathrm{~mm}$. It is evident that these figures also represent the negative pressure in the thoracic cavity under the same conditions.

During ordinary respiratory movements there are slight changes of pressure in the alveoli and tracheal air. In inspiration the pressure in the trachea is from -2 to $-3 \mathrm{~mm}$. $\mathrm{Hg}$, in expiration about $+2 \mathrm{~mm}$. $\mathrm{Hg}$. By connecting the closed trachea or air passages with a manometer, we may measure the total possible force exerted by the respiratory muscles in drawing air in or expelling air from the lungs. These

${ }^{1}$ Ztschr. f. rat. Meal., 1853, N. F., Bi. iii. (quoted from Rosenthal, loc. cit.). 
pressures amount to from -30 to $-74 \mathrm{~mm}$. $\mathrm{Hg}$ in inspiration, and to from about +62 to $+100 \mathrm{~mm}$. $\mathrm{Hg}$ in expiration (Donders).

Methods of registering the respiratory movements.-The methods of registration can be diviled into four classes-

1. In the first class the expansion and contraction of the thorax are recorded. To this end Marey devised his meumograph, which consists of a cylinder with elastic ends, the interior of which is connected with a recording tambour. A strap or cord attached to the membrane, closing the two ends of the cylinder, is passed round the chest. As the thorax expands, the membrane at each end of the cylinder is drawn out. The rarefaction of the air in the cylinder thus produced is transmitted to the tambour and causes a fall of the lever.

Burdon Sanderson's stethometer consists of a rigid rectangular framework, formed of two bars joined by a cross piece. The distance between the bars can be adjusted to fit the antero-posterior diameter of the subject under investigation. The free ends of the bars are provided, one with an ivory button which rests on the spine of a vertebra, the other with a tambour, the knob of which presses against the sternum. An increase in the antero-posterior diameter of the chest presses on the button of the tambour, and the inovement thus produced is transmitted by a rubber tube to another recording tambour.

2. Another method is to record in animals the movements of the column of air which enters and leaves the lungs at each respiration. One method of doing this is to attach a T-piece to the tracheal cannula, one limb of the $\mathrm{T}$ being left open, while the other is attached to a large vessel of air, which is also connected with a Marey's tambour. When it is desired to record the movements the free end of the T-piece is clamped, so that the animal now breathes in and out of the receiver, and the consequent changes of pressure in this vessel are transmitted to the Marey's tambour and recorded. This method suffers from the drawback that the air in the receiver soon becomes vitiated, so that the breathing is altered, and it is necessary to interrupt the observation in order to renew the air.

Gad ${ }^{1}$ has used a modification of this method in nearly all his researches. This observer's pneumatograph, which is substituted for the Marey's tambour employed in the method just described, is a light open box of mica, which is counterpoised so as to rest face downwards in a vessel of water. To the bottom of the box is attached a lever, the movements of which can be recorded on a blackened surface. The interior of the box is connecterl with a large air chamber into which the animal breathes. Each inspiration causes a sucking of air out of the box into the air chamber, so that the pneumatograph sinks in the water, whereas each expiration will increase the volume of air, and so cause a rise of the pneumatograph with its lever.

3. Since each respiratory movement causes a change in the intrathoracic pressure, it is evident that a record of these variations may be used as evidence of the respiratory movements. These variations may be studied by introducing a cannula connecter with a manometer or tambour into the pleural cavity, or by introducing an elastic sound connected with a registering tambour through the mouth into the esophagus. The latter methorl has been used especially by Rosenthal. ${ }^{2}$

4. Since the diaphragm is in the higher animals the most important inspiratory muscle, and in rabbits is practically the only active uuscle in quiet respiration, it is obviously of advantage to record its movements directly. Iany methods for recording the contractions of the diaphragm have been derised.

1 Awh.f. Physiol., Leipzig, 1879, s. 181.

2 Hermann's "Handbuch," Bd. iv. Th. 2, S. 163-286. 
In one method an elastic bag is inserted through an opening in the abdominal wall, between liver and diaphragm. The elastic bag is connected by a tube with a recording tambour. Each descent of the diaphragm in inspiration presses on the bag, and the increased pressure transmitted to the recording tambour can be recorded as a respiratory movement.

Kronecker's phrenograph ${ }^{1}$ consists of a lever, one broad spoon-shaped end of which is inserted between the liver and diaphragm, while the other end is connected by threads running on pulleys to a writing lever. Rosenthal's phrenograph differs from the preceding in that the movement is transmitted by air to a tambour instead of by means of threads.

5. All these methods, however, suffer from the defect that, although they directly or indirectly give a fairly correct record of the movements of the respiratory apparatus, they are unable to discriminate between passive and active movements. Thus, if the lungs be artificially distended, there will he a descent of the diaphragm and a movement of the phrenograph lever, just as if the inspiration were brought about by an active contraction of the muscle. In dealing with the innervation of respiration, we shall see that it is very important to be able to study the effect of artificial inflation or collapse of the lungs on the activity of the respiratory muscles; and such information can be only very imperfectly afforded by any

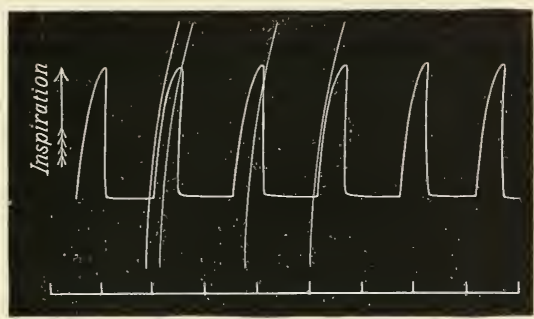

Fic. 168, - Normal tracing of diaphragm-slips of rabbit.-(Head's method.) of the graphic methods already lescribed. The method which best meets these difficulties is that devised by Head. ${ }^{2}$ This observer has shown that in the rabbit the anterior part of the diaphragm, which is connected to the ensiform cartilage, is so disposed that it may be isolated as two parallel slips from the rest of the muscle without injury to the blood- or nerve-snpply. These slips may be clamper at the tendinous end, and the contractions recorded by a lever attached by a thread to the other end, which is cut free from the cartilage. It is then found that the slips contract synchronously with the rest of the diaphragm, and their contractions (Fig. 168) may be taken as a sample of the contraction of the whole diaphragm, while they are bnt little affecterl by any passive movements which may be imparted to the thoracic wall. The results arrived at by this method will be described later on.

\section{The Nervous Mechanisi of Respiration.}

We have seen that each act of inspiration involves the contraction of a number of muscles, which are added to whenever the need for greater respiratory interchanges occurs. Expiration also, although normally effected by the passive retraction of the stretched lungs and costal wall, yet, with the smallest additional call on the respiratory apparatus, involves the co-ordinated actions of a number of other inuscles. These co-ordinated rhythmical contractions must be initiated in the central nervous system, and we have in this section to discuss p. 9 .

${ }^{1}$ v. Marckwall, "The Movements of Respiration," translated by Haig, London, 1888,

¿ Journ. Physiol., Cambrilge and London, 18s9, vol. x. [1]. 1 and 279. 
the seat and mode of the activities of the central nervous system which determine the respiratory movements.

The respiratory muscles receive their nerve supply from various parts of the cerebro-spinal axis. Thus the muscles moving the alæ nasi are innervated from the seventh nerve; the museles opening the glottis, from the accessory part of the vagus; the diaphragm, from the cervical cord by the fourth and fifth cervical nerves; and the intercostal muscles, from the whole of the dorsal cord. From what we know of the anatomy of the cord, we may affirm almost certainly that the motor fibres going to the inspiratory muscles are derived from cells in the grey matter of the anterior cornua of the cord, or from the corresponding part of the grey matter in the medulla oblongata. All these cells represent, therefore, centres for the innervation of the inspiratory muscles, and any contraction of these muscles can only be brought about during life through the intermediation of these cells. Under normal conditions, the motor cells of the cord are excited to activity by the impression on them of nervous impulses derived from sensory fibres which have entered the cord by the sensory roots, or from fibres originating in cells situated in the higher parts of the nervous system, e.g. pyramidal tract fibres.

The normal performance of the respiratory movements is dependent, then, upon a rhythmic and co-ordinate activity of a number of cells extended over a considerable length of the medulla oblongata and spinal cord. The question we have to discuss is the manner in which the activities of these cells are roused and co-ordinated. Are they so constituted that their normal metabolism leads to rhythmic automatic discharges, or is their activity excited by changes in the blood passing to them or by afferent impulses arriving from the periphery? Is the co-ordination of their activities dependent on intercentral communications between the motor cells at various levels of the cord, or are they harmonised in obedience to impulses proceeding to them from higher parts of the central nervous system? In the latter case, can we by experiment localise any part of the nervous system which carries out this function of origination and co-ordination, and so plays the part of a respiratory centre?

The first attempts to answer these questions by systematic experiments were made by Legallois. ${ }^{1}$ His investigations were amplified and confirmed by Flourens. ${ }^{2}$ These experiments were as follows:-If the spinal cord be divided on a level with the seventh cervical nerve, respiration continues, but is carried out solely by means of diaphragmatic contractions, the chest walls remaining motionless or being moved passively by the contractions of the diaphragm. If the spinal cord be divided at the third cervical nerve (i.e. above the origin of the phrenic nerves), the respiratory movements of the thorax either cease entirely, or are confined to spasmodic elevations of its upper part, induced by contractions of the sterno-mastoid and trapezius muscles. The animal therefore dies of asphyxia if artificial respiration be not resorted to. Respiratory movements, although not effectual in aerating the lungs, are still to be observed in the laryngeal muscles, and in the nuuscles of the face. These experiments seem to indicate that the spinal centres

1 " (Euvres completes," Paris, 1824, tome i.

2 "Recherches expérimentales sur les propritités et fonctions du système nerveux," 2me edition, Paris, 1842 ; Compt. rend. Acul. d. sc., Paris, 1858, tome xlvii. 1. 803. 
of the respiratory nerves are unable, of their own initiative, to carry out rhythmic respiratory movements, but are subsidiary to the action of a co-ordinating centre situated somewhere above the lower end of the medulla oblongata.

If, on the other hand, a section be made through the upper part of the medulla oblongata, separating this and the spinal cord from the higher parts of the brain, respiration is absolutely unaffected. It is evident, therefore, that the respiratory centre, or the part of the central nervous system which originates and co-ordinates the respiratory movements, is situated in the medulla oblongata, somewhere between its lower part and the strix acusticæ. A number of attempts have been made to localise the situation of the respiratory centre more exactly. From the time of Flourens it has been recognised to be bilateral, as is proved by the fact that bisection of the medulla oblongata by an anteroposterior incision in the middle line does not alter in any way the rhythm of respiration. According to Flourens ${ }^{1}$ the respiratory centre is represented by a circumscribed spot just below the apex of the calamus scriptorius, of the size of a pin's head. This spot was designated by its discoverer the noud vital, and according to him, its destruction causes total cessation on the side on which it was destroyed, or, if the lesion be bilateral, an instant and permanent cessation of the respiratory

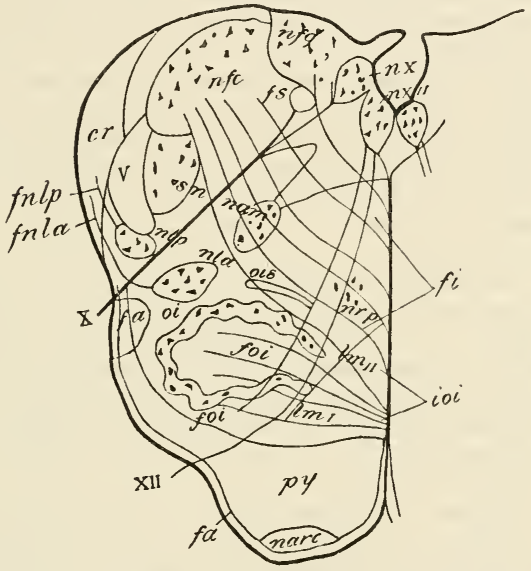

Fig. 169.-Diagrammatic section of medulla oblongata below middle of olivary boly, to show region of respiratory centre. $\omega_{x}$, nucleus of tenth nerve; $X$, root of tenth nerve; mxi, nucleus of twelfth nerve; $f s$, funiculus solitarius ; $f t$, arcuate fibres ; nam, nucleus ambiguus ; $c r$, restiform body ; $p y$, pyramidal tract ; $V$, ascendiug root of fifth nerve; $u l j$, nucleus lateralis; $n f c$, nucleus of funiculus cuneatus ; $u f y$, nucleus of funiculus gracilis.-After Beehterew.

movements on both sides, and consequently the death of the animal.

In 1873 , careful experiments were carried out by Gierke ${ }^{2}$ in Heidenhain's laboratory, in order, if possible, to determine the cells or groups of cells contained in Flourens' centre. Gierke was unable to localise the respiratory centre in any special group of cells, and found that the grey matter lying in the situation of Flourens' centre might be destroyed to a considerable extent, without causing a cessation of the respiratory noveinents. The only part of the medulla oblongata which, according to him, seemed to be essential for the carrying out of these movements, was a longitudinal bundle of white filores on each side of the median line. This bundle, which was termed the "respiratory bundle" by Krause, and is generally known as the funiculus solitarius, is situateil 
outside and below the nucleus of the pneumogastric and glossopharyngeal nerves. It is surrounded by gelatinous grey matter with many small nerve cells. "Its fibres appear to lose themselves among the cells of the enclosing grey matter, and this and the bundle gradually disappear when traced towards the spinal cord. Traced upwards, they pass out with the bundles of nerve-roots which go to form the vagus and glosso-pharyngeal." 1

Mislawsky, ${ }^{2}$ who experimented on cats, concluded that the respiratory centre was to be found, not in Gierke's bundle, but in two cell groups lying in the formatio reticular is on each side of the median raphe internal to the roots of the hypoglossal nuclei. The latest attempts to localise the centre have been made by Gad and Marinesco. ${ }^{3}$ In order to destroy the medulla oblongata bit by bit, these observers used minute hot glass beads, the destructive action of which could be accurately localised. In this way they clain to have burnt away the whole of the medulla oblongata corresponding to the situations laid down by Flourens, Gierke, and Mislawsky for the respiratory centre, without altering the respiratory movements. They themselves locate the centre in the cells of the formatio reticularis, and consider that it oceupies an extensive area in this formation on each side of the middle line; it certainly cannot he restricted to a part the size of a pin's head, as was supposed by Flourens.

Although the evidence in favour of the localisation of the respiratory centre in the medulla oblongata is apparently so convincing, certain observers have denied altogether the adequacy of the experiments adduced in its support. Thus Brown-Séquard, ${ }^{4}$ in 1860, showed that, if young animals were kept alive by artificial respiration for some time after the division of the cord below the medulla oblongata, on ceasing the artificial respiration co-ordinated movements of thorax and diaphragm might still for a time be observed. He considered therefore that the centre of respiration was not confined to a circumscribed area in the medulla oblongata, but extended throughout the spinal cord. The effects on respiration which follow section of the cord below the medulla oblongata, he ascribed to inhibition of these spinal centres consequent on the lesion acting as a stimulus, and compared the result to the phenomena accompanying shock. Twenty years later this idea was warmly taken up by Langendorff, ${ }^{5}$ who carried out a number of experiments similar to those of Brown-Séquard, in order to prove the independent automatisn of the spinal centres. Wertheimer ${ }^{6}$ also pointed out that if an adult animal (dog) were kept alive by means of artificial respiration for some hours after section of the cervical cord, rhythmic respiratory movements of the thorax take place as soon as the artificial respiration is discontinued; he states that in one case a dog survived the cessation of artificial respiration about three-quarters of an hour, respiration all this time being carried out by the spinal centres. These experiments have been repeated by a number of other observers, who, while confirming to a certain extent the previous results, were unable to attribute to them the same importance. As I have already pointed out, the cells from

\footnotetext{
${ }^{1}$ Schäfer in "Quain's Anatouy," vol. iii. jart 1, 1. 55.

${ }^{2}$ Centralbl. f. d. med. IVissensch., Berlin, 1885, s. 465.

3 Aich.f. Physiol., Leipzig, 1893, S. 175.

4 Journ. de la physiol. de l'homme, Paris, 1860, tome ii. p. 153.

5 Arch. f. Physiol., Leipzig, 1880, S. 518 ; ibid., 1887, S. 237 ; ibid., 1888, S. 283.

${ }^{6}$ Journ. de l'anat. ct physiol., etc., Paris, 1886, tome xxvi. p. 458.
} 
which the nerve fibres to the respiratory muscles arise can, like the motor cells of other parts of the cord, be affected by impulses reaching them along various paths. We may conclude that their normal activity in respiration is determined by impulses reaching them from the medulla oblongata. But they can also be affected along other tracts derived ultimately from the posterior roots, either at the same or at a higher or lower level of the cord. We know that the spinal cord, separated from the upper parts of the central nervous system, under the influence of asphyxia or strychnine, gives rise to spasmodic motor discharges, which have a tendency to take on a more or less rhythmic character. These rhythmic discharges affect the limbs as well as the muscles of the trunk, and it is evident that, if we were tracing the movements of the thorax or diaphragm, they might resemble the effect produced by normal respiratory movements. But, strictly speaking, we camnot locate respiratory centres in the cord, since this property of rhythmic discharge, in consequence of asphyxia or strychnine, is shared hy all the motor cells or centres of the cord, and is not limited to those supplying the respiratory muscles. As a matter of fact the pseudorespiratory movements observed under these conditions are extremely inadequate for the renewal of air in the lungs, and one often obtains simultaneous spasms of inspiratory and expiratory muscles. So long, therefore, as an animal is warm-blooded, they are of very little use for the maintenance of life. In Wertheimer's experiments, in consequence of the prolonged ventilation of the lungs, the animal had become practically cold-blooded, and under these circumstances it is not wonderful that it should have survived cessation of artificial respiration for fortyfive minutes. In an anæsthetized and curarised animal, after a long experiment in which the animal has had time to get cold, the heart may go on beating for half an hour or more after artificial respiration has been discontinued.

Moreover, Porter ${ }^{1}$ has shown that such a lesion as dividing the medulla oblongata below the respiratory centre does not produce an appreciable amount of shock, i.e., does not affect injuriously the subordinated activities of these spinal respiratory centres. Thus, if the bulb be hemisected below the respiratory centre, the respiratory movements of the diaphragm at once cease on that side. This result, according to Brown-Séquard or Langendorff; would be due to shock or inhibition of the diaphragm centre in the cord. If now the phrenic nerve be divided on the other side, the diaphragm on the side of the hemisection at once commences to contract, the impulses coming from the other side of the medulla oblongata having crossed from one side of the spinal cord to the other by means of commissural fibres situated at the level of origin of the phrenic nerves. This immediate result shows that the diaphragmatic centre in the cord was previously inactive, not in consequence of inhibition due to the hemisection, but because the impulses from the respiratory centre in the medulla oblongata on the same side had been cut off by the hemisection.

We may conclude, therefore, that for the normal performance and co-ordination of the respiratory movements, the integrity of certain parts of the medulla oblongata situated on each side of the median line is necessary, and we are therefore justified in giving to these parts the name of respiratory centre.

\footnotetext{
${ }^{1}$ Journ. Physiol., Cambridge and Jondon, 1895, vol. xvii. p. 455.
} 
Since each inspiration is followed by an expiration, which, though passive during quiet breathing, may and frequently does involve the co-ordinated activity of a number of muscles, we might, from a purely physiological point of view, divide the respiratory centre into two parts, one always active and presiding over inspiration, the other only active when a special call is made on the respiratory mechanism and presiding over expiration. Nevertheless we have no experimental evidence of any anatomical separation between these two centres. Localised stimulation applied to the medulla oblongata may call forth inspiration or expiration according to the condition of the centre and the phase of respiration at which the stimulus is applied; inspiration, however, predominates in most cases over expiration. No point of the respiratory centre has been found as to which we can say with certainty that we shall obtain only cxpiration or only inspiration as the result of the stimulus. We do not know, therefore, whether some groups of cells originate or transmit inspiratory and others expiratory impulses, or whether both sets of muscles are innervated from the same cell-groups.

We have now to inquire into the nature of the activity by virtue of which this centre is able to excite rhythmic co-ordinated movements of the respiratory muscles. This inquiry involves two distinct questions-

1. How is the activity of the centre aroused? Is it aroused by stimuli arriving at the centre from the periphery along afferent nerves, or by changes occurring in the cells of the centre itsclf, the results of their normal metabolism, or by changes in the fluids-lymph and blood -surrounding the cells?

2. What is the cause of the natural rhythm of this activity?

In deciding the first point, i.e. whether the activity is automatic or reflex, we must attempt to study the behaviour of the centre when separated as much as possible from the influence of all afferent impulses. Rach, ${ }^{1}$ who was the first to make this attempt, came to the conclusion that the respiratory centre separated from all aflerent impulses was unable to originate any movements. His experiment consisted in dividing the cord below the fifth cervical nerve, and the medulla oblongata above the strix acusticæ, and then cutting both vagi and all the posterior roots of the upper cervical nerves. Subsequent repetition of Rach's experiment by Rosenthal and Marckwald ${ }^{2}$ failed to confirm these results. We may consider here shortly the effects on the respiratory movements of the various operative procedures employed in this experiment.

Section of the lower part of the cervical cord in the rabbit leaves the diaphragmatic movements practically unaltered, since in this animal the chief part of inspiration is carried out by the diaphragm. The section through the upper part of the medulla oblongata, separating the respiratory centre from the higher parts of the brain, is equally without effect on the depth and rhythm of the respiratory movements. A great change is observed, however, if the vagi are subsequently divided under these conditions. The first effect is a long inspiratory spasm of the diaphragm, which may last as long as a minute. Its relaxation then follows, to be succeeded again by another inspiratory spasm many times exceeding in duration the ordinary respiratory contraction of the

1 “'Inaug. Diss.," Königsberg, 1863.

2 "The Movements of Respiration," translated by Haig, London, 1888. 
diaphragm. The animal thus continues to breathe by a series of prolonged inspiratory spasms. The centre continues to discharge rhythmically, but its activity is not adequate to the normal aeration of the blood, and the spasms get gradually slower and slower, until the animal finally dies of asphyxia. According to Rosenthal, these imperfect respiratory movements are not altered further by dividing all the posterior roots of the upper cervical nerves, and he concludes, therefore, that the isolated respiratory centre is capable of an automatic rhythmic activity apart from the influence of afferent impulses.

It must be remembered, in attempting to draw conclusions from this experiment, that it is necessarily an imperfect one, since afferent impulses must be ascending to the centre in consequence of stimulation of the cut ends of the nerves as they lie in the wound, and the animal camnot be kept alive sufficiently long for the irritating effects of the section to pass off. These impulses are constant and not rhythmic in character, so that the only certain conclusion to be drawn from the experiment is that the centre is able to convert constant into rhythmic discharges. We do not know certainly whether the centre separated from all afferent impulses would discharge at all. H. E. Hering, ${ }^{1}$ who has investigated the activity of the spinal cord in the frog after division of all the posterior roots, has concluded that in this case the motor centres are unable to discharge, even under the influence of strychnine, when removed from all peripheral stimuli, and it is possible that, were we able to perform a similar experiment on the respiratory centre of mammals, we should meet with like results.

If we conclude with Rosenthal that the activity of the centre is automatic, we must seek to find out what are, apart from afferent impulses, the local changes in or around the nerve cells by which this activity is evoked. Among the environments of the cell the most important factor in evoking this activity is the condition of the blood eirculating through the blood vessels of the part. It has long been known that the activity of the centre is intimately dependent on the condition of the blood, and is especially excited by increased venosity of the blood and allayed by improved arterialisation. Every hindrance to the free renewal of the blood-gases in the lungs calls forth increased respiratory movements, which, if the obstacle be not removed, may involve almost every muscle in the body; this condition is known as dyspnoa. On the other hand, free renewal of the gases in the blood leads to a condition in which the respiratory movements are rendered shallower or even abolished. This condition, which is known as apnoa, is, as we shall see later, most readily produced by reflexes from the lungs, but may also, in the absence of these impulses, be brought about by free aeration of the blood (see p. 301).

Ever since dyspnøa has been the subject of scientific investigation, the question has been much discussed whether the excitant is to be sought in the increased amount of carbon dioxide, or in the diminished quantity of oxygen in the blood. Opinions at first were divided on this point; J. Muiller ${ }^{2}$ and, in his earlier work, Posenthal, ${ }^{3}$ considering the deficiency of oxygen as the stimulus, while Traube ${ }^{4}$ and Thiry ${ }^{5}$ ascribed

1 Arch. f. d. ges. Physiol., Bonn, 1893, Bd. liv. S. 614.

2 Sitzungsb. d. k. Akad. d. Wissensch., Wien, Bd. xxxiij. S. 99 (quoted from Rosenthal).

3 Loc. cit. ("Die Athembewegungen, etc.," Berlin, 1862).

+ Ges. Beitr. z. Pat'l. u. Physiol., Berlin, Bd. i.

${ }^{5}$ Rec. de tiav. Soc. med. allem. de Paris, 1865 (quoted from Rosenthal). VOL 1I. - I 9 
the activity of the centre to the larger quantity of carbon dioxide. It was later on shown by Pfluger and Dohmen ${ }^{1}$ that either of these two moments - increased carbon dioxide or diminished oxygen-might serve as a respiratory stimulus. These authors controlled their results by analyses of the blood.

Where dyspnoea followed inhalation of hydrogen or nitrogen, they found that the blood only differed from normal blood in containing less oxygen. On the other hand, when the animals were made to breathe mixtures of carbon dioxide and oxygen, the blood contained a normal amount of oxygen, but an excess of carbon dioxide. In both these cases dyspnœa was produced, although in the one instance deficiency of oxygen, and in the other excess of carbon dioxide, were the only deviations from the normal. It is in the first stages alone that the results of these two experiments are similar. If the inhalation of the abnormal gaseous mixtures be continued, it is found that deprivation of oxygen leads to ever-increasing dyspnœea, and finally general convulsions, the animal dying of asphyxia. When, on the other hand, the animal breathes a mixture of carbon dioxide and oxygen, the respiration, which is deepened and slightly quickened at first, undergoes very little further alteration. The animal can continue to breathe the mixture for a considerable time without apparent ill effects; then respirations gradually become slower, the animal passes into a deep sleep, and finally dies without convulsions, as under the influence of a narcotic poison.

Bernstein ${ }^{2}$ attempted to show that carbon dioxide dyspnœa differed from that produced by absence of oxygen, in the fact that carbon dioxide chiefly augmented expiration, while absence of oxygen increased inspiration. More careful experiments by $\mathrm{Gad}^{3}$ have prover that this is not the case, but that in each instance the dyspnoea is mainly inspiratory.

Some physiologists have found a difficulty in conceiving how the absence of a substance (oxygen) could act as a stimulus. It has therefore been suggested that the exciting agent was probably represented by such "reducing substances" as have been described by Schmidt ${ }^{4}$ and Pflüger ${ }^{5}$ as occurring in the blood of asphyxiated animals.

According to the theory of Pfliger, metabolic changes are constantly occurring in living cells, including nerve cells, leading to the formation of molecules, or groups of molecules, with a high affinity for oxygen. It is these intracellular reducing substances which combine in the first instance with the oxygen leaving the vessels, or are able to tear away the oxygen from such substances as methylene blue, as in Ehrlich's experiments. $^{6}$ It is possible that these bodies, when they meet with insufficient oxygen for their saturation, act as excitants on the nerre cells; so that the normal activity of these cells is maintained by a condition of auto-stimulation, the stimulus being supplied by their own products of metabolism. On the other hand, when oxygen is supplied in excess, the aftinities of the reducing substances are satisfied. No

1 Untersuch a. d. physiol. Lab. zu Bonn. 1875, S. 83.

${ }^{2}$ Arch.f. Physiol, Leipzig., 1882, S. 313.

3 1bid., 1886, S. 388 (Verhandl. d. Bert. pllys. Gesellsch.), confirmed by Max Rosenthal, ibid., 18S6, Suppl., S. 248.

${ }^{4}$ Arb. a. d. pihysiol. Anst. zu Leipzig, 1867.

${ }_{5}$ Arch. f. d. ges. Plyysiol., Bonn, 1868, Bd. i. S. 61.

6 "Das Sauerstoftbedurfniss des Organismus," Berlin, 1885. 
excitation, therefore, takes place, and a condition of apnoea (apnoca vera) is produced.

Whatever explanation we choose to adopt of the exact mode in which the excitation is brought about, there can be no doubt that the absence of oxygen, or rather the need of the centre for oxygen, is a very considerable factor in determining its activity. We have hitherto only considered the cases in which this need was increased or diminished by altering the amount of oxygen supplied to the centre. But we may also alter it by increasing or diminishing the metabolic activity of the centre itself, by heating or cooling the medulla oblongata. Thus Fick ${ }^{1}$ and Goldstein ${ }^{2}$ surrounded the exposed carotid arteries of a dog with a vessel with hollow walls, between which hot or cold water could be passed, so changing the temperature of the blood flowing to the medulla oblongata. They found that in each case the respiratory rhythm underwent marked alterations. If the blood is warmed above its normal temperature, so increasing the rapidity of the metabolic changes in the centre, the respirations become much quicker and shallower (tachypnoca, Gad); whereas, when the metabolic changes are depressed by lowering the temperature of the blood, the movements become much slower. The latter result may also be produced, as Frederica ${ }^{3}$ pointed out, by direct application of pieces of ice to the exposed medulla oblongata,-a further proof of the fact that the respiratory impulses are originated there, and not in the cord, since we cannot imagine that the application of cold should act as an excitant of inhibitory impulses.

There are thus two kinds of stimuli which may act on the respiratory centre in the medulla oblongata, and provoke its activity. The stimulus derived from the blood is, under normal conditions of respiration, constant. The stimulus derived from the afferent nerves is subject to rhythmical variation, and, as we shall see later, this variation is of considerable importance for the maintenance of the normal respiratory rhythm. The centre can, however, go on discharging rhythmically in the absence of any rhythmic afferent stimulation. We have therefore to discuss how the centre is able to respond to a constant stimulus with a rhythmic discharge.

The simplest explanation of this process, if explanation it can be called, is to say that it is a property of the nerve cells as of the muscle cells of the heart to respond to a constant stimulus by a series of rhythmic discharges. Many physiologists, especially Pfliiger, have attempted to form a conception of the intra-molecular processes which lead to this transformation. Pfluiger ${ }^{4}$ considers that the molecular processes in a living cell may be divided into two sets-those which tend to produce a discharge of energy, and those which tend to prevent a discharge. In more modern terms, we might speak of these two processes as katabolic or dissimilative, and as anabolic or assimilative. In a condition of rest, these processes balance one another exactly. The effect of applying a stimulus to the cell is to increase the metabolic processes. In order that these excitatory processes may prevail against the inhibitory, it is necessary that they should attain a certain superiority above the latter. As soon as this condition is reached, the excitatory processes break

${ }^{1}$ Arch. f. d. ges. Physiol., Bonn, 1872, Bd. v. S. 38.

2 Verhandl. d. phys.-med. Gesellsch. in Würzburg, 1872, N. F., Bd. ii.

3 Arch. f. Physiol., Leipzig, 1883, Suppl., S. 51.

4 "Untersuch. neber d. Physiol. des Elektrotomis," Berlin, 1879. 
through the resistance of the inhibitory, and an explosive discharge is the result. After the discharge the inhibitory processes are in the ascendant, and the stimulus from without has to continue acting for a further length of time before the excitation can gather fresh strength to break again through the resistance.

This conception, in default of a better, has played a great part in the speculations of physiologists on the nature of inhibition and the production of rhythmic activity. It suffers, however, as was pointed out by Gad, from the drawback that the molecular processes have to be considered as endowed with inertia. Otherwise, as soon as the excitatory were in excess of the inhibitory processes, there would be a leaking discharge, and a constant stimulus would cause a constant activity. We shall have occasion to discuss further applications of this theory later on when dealing with the nature of the action of the vagus on the respiratory centre. I have already mentioned the opinion held by some physiologists, that the respiratory centre, if separated altogether from afferent stimuli, would be unable to originate any respiratory movements. How, under this hypothesis, could we explain the action of oxygen-hunger on the respiratory centre? It is evident that the results of deprivation of oxygen might be equally well explained by assuming that its action was similar to that of strychnine, only differing from this drug in its more pronounced specific effect on the medullary centres. Like strychuine, asphyxia may cause convulsions of spinal origin after separation of the spinal cord from the medulla oblongata. Whether we consider that the activity of the respiratory centre is inaugurated by afferent stimuli, or by the molecular life of the cells themselves, it is evident that a lowering of the resistance to the passage of the impulses from the cells in all directions will lead first to an increase in the respiratory movements, as in dyspnoea, and later on to the general convulsions of asphyxia.

We may conclude that respiration is dependent on the integrity of a part of the central nervous system situated in the medulla oblongata, and which is therefore termed the respiratory centre. This centre is so arranged that it can respond to constant stimulation by intermittent rhythmic discharges. Under normal conditions this constant stimulation may be afforded by-

1. The sum of afferent inpulses arriving at the centre.

2. The venosity of the circulating blood.

3. The normal metabolic changes in the nerve cells. These two latter factors may probably be classed together as the oxygen-hunger of the cells.

The influence of afferent nerves on respiration. - I have already mentioned the fact that, on cutting off the centre as far as possible from all afferent impulses, the respiratory movements, though rhythmic, differ widely from the normal. They consist of a series of spasms, recurring at ever-increasing intervals, which are inadequate for the maintenance of life. This fact shows that, for the normal performance of the respiratory functions, the arrival of afferent impulses at the centre is necessary, and we have now to consider the paths of these afferent impulses and the nature of the influence they exert on the centre.

Action of the vagus on respiration.-Of all the sensory nerves in the body, the vagi are the only ones whose section produces a marked and permanent change in the respiratory rhythm. These, therefore, have 
been looked upon, and rightly, as the most important for the carrying out of normal respiratory movements, since they must convey, during the whole of life, impulses which affect and regulate these movements. We have, therefore, to discuss the nature of the influence exerted by the vagi on the respiratory centre and the mode in which these impulses are initiated.

As in all other cases, where we wish to study the functions of a nerve trunk or nerve centre, our experiments can be divided into two main classes: Those in which the normal function is abolished by section or extirpation, and those in which this function is initiated or exaggerated by artificial stimulation.

The earlier experiments of this nature were inconclusive, conflicting results having been obtained by different observers. It is to Rosenthal $^{1}$ that we owe the first accurate investigation of the subject, with a clearly defined hypothesis of the normal working of the vagus and respiratory centre founded on experiment. This observer showed in the first place that the constant effect of section of both vagi was to make the respiratory movements deeper and less frequent. The amount of air taken in during a given time was, however, unaltered, the increase in depth of the respirations exactly counteracting the slower rhythm. Before Rosenthal's time, the effect of electrical excitation of the central end of one vagus was found to produce sometimes expiration, sometimes inspiration. Rosenthal showed that the trunk of the vagus contains two definite sets of fibres. Excitation of one set produces reflex contraction of the inspiratory muscles, especially of the diaphragm, while stimulation of the second set produces relaxation of the diaphragm, and might therefore be looked upon as inhibitory of inspiration. The first set of fibres is, according to Rosenthal, distributed solely to the lungs, so that inspiration or a tetanic spasm of the diaphragm is an invariable sequence of excitation of the nerve in the lower part of the neck. If the exciting current be weak, the result may be not inspiratory spasm, but simply a quickening of the respiratory rhythm to that which obtained before the section of the vagi. On the other hand, a purely expiratory effect is produced by stimulating the central end of the superior laryngeal nerve, and Rosenthal ascribed the occasional expiratory effects, observed by his predecessors on stimulation of the vagus in the lower part of the neck, to an escape of current to the superior laryngeal nerves. As a result of his investigations, Rosenthal founded a theory of the normal working of the respiratory centre in the medulla oblongata, based to some extent on a hypothesis conceived about twelve years earlier by Ludwig and $\mathrm{Hoffa}^{2}$ to account for the action of the vagus on the heart. These observers, on recording the contractions of the mammalian heart by a lever resting on the surface of the exposed ventricle, found that, during stimulation of the vagus, the heart beats, though less frequent, appeared much more powerful, and they concluded that the total amount of work performed in any given time by the heart muscle was the same under all circumstances, the only result of the vagus stimulation being to increase the resistance to the motor discharge, so that the tendeney to discharge had to rise to a

1 "Die Athembewegungen, u. ihre Beziehung zum Nervus vagus," Berlin, 1865. See also the section on the respiratory movements by the same author in Hermann's "Handbuch," Bd. iv. (2) S. 165.

${ }^{2} Z$ tschr. f. rat. Med., 1850, Bd. ix. S. 129. 
greater extent before it could be effective. Although this hypothesis was shown to be untenable by Coats, ${ }^{1}$ working under Ludwig's guidance, Rosenthal made use of a very similar conception to explain the activity of the respiratory centre. I have already mentioned that (according to Rosenthal) the cause of the respiratory rhythm is to be sought in the existence of a resistance to the passage of impulses from the centre to the inspiratory muscles. The centre itself is kept in a condition of constant stimulation, in consequence of the unsaturated condition of the blood circulating through it. So long as the normal conditions for the conveying of oxgyen to the blood are maintained, the activity-and total work done by the centre-could be regarded as constant, an increase of the resistance to discharge serving only to make each discharge more forcible, but the interval between consecutive discharges longer. In the normal animal this resistance is diminished by tonic influences ascending both vagi from the lungs. If both vagi be cut, these inHuences are abolished, with the result of an increased resistance to discharge of the centre. We thus get the familiar picture of slow deep inspirations following section of both vagi. Stimulation of the central end of the vagus fibres coming from the lungs diminishes this resistance, so that the respiration may be quickened, or, if the resistance be entirely

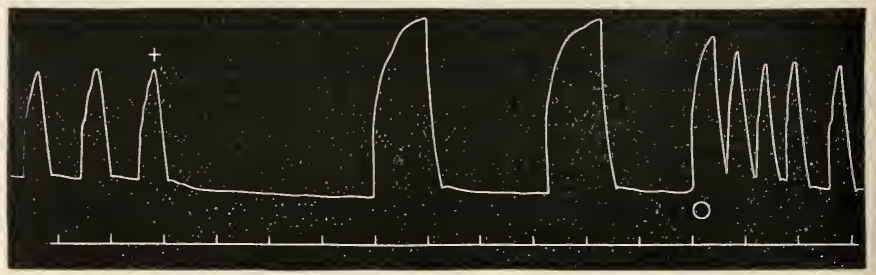

To be read from left to right

FIG. 170.-Effect of elosing trachea at height of inspiratory period. The trachea was suddenly closed at + and again opened at o.-Head.

abolished, there may be a continuous flow of energy from the respiratory centre to the inspiratory muscles, so producing a tetanic spasm of the diaphragm. Stimulation of the superior laryngeal has the reverse effect. It increases the resistance to discharge, so that inspiration is abolished. If stimulation be prolonged, the store of energy in the respiratory centre rises to such an extent that, being unable to burst through the normal resistance to inspiratory impulses, it escapes through the resistance between it and the expiratory muscles, thus producing an active expiration.

A complete revolution in our idea of the relationship of the vagus to the respiratory movements was effected by the experiments of Hering and Breuer ${ }^{2}$ in 1868. These observers considered that, in order to arrive at some idea of the normal functions of the vagus, it was important to employ a stimulus for these nerves resembling as far as possible the normal stimulus. Proceeding on the assumption that the normal stimulus for the vagus was to be sought in the alternate distension and contraction of the lungs, they attempted to exaggerate these influences by closing the trachea at the end sometimes of an expiratory, sometimes of an inspiratory movement. They found that, if the trachea

1 -Avb. a. d. physiol. Anst. zu Leipzig, 1869.

2 Sitzungsb. d. k. Akad. d. Wissensch., Wien, 1868, Bd. lviii. S. 909. 
be closed at the end of an inspiration, the result is an immediate relaxation of the diaphragm, and the expiration thus produced is enormously prolonged (Fig. 170). In those cases where tonic contraction of the diaphragin was present during the expiratory period, the muscle continues to relax, and its fibres finally reach a condition of complete elongation. After a varying interval, the pause is broken by an inspiratory contraction considerably stronger than the normal inspirations. The elongation which follows is again complete and a second long pause follows, which is, however, shorter than the one which preceded it, though longer than the normal pauses. If the tracheal cannula be now opened, the respiratory movements become very rapid.

If, on the other hand, the trachea is closed during the expiratory pause, the breathing suffers the converse change. The pause, during which the trachea is closed, lasts the usual time, and is followed by an inspiratory contraction of the normal rapidity. But when the inspiration has reached the normal strength, instead of ceasing abruptly, it goes on increasing until the curve reaches a point far above the crests of the normal inspiratory contractions (Fig. 171). Thus closure of the trachea in the expiratory phase of respiration causes an increase in the strength and duration of the inspiratory contractions.

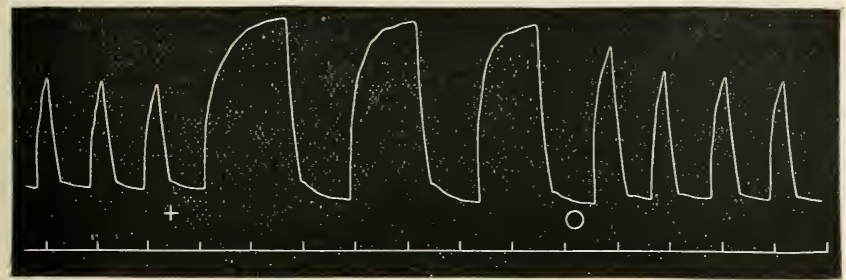

FIG. 171.-Effect on respiratory movements of closing the trachea during the expiratory pause. The trachea was closed at + and opened at o.-Head.

Still more marked results were produced by inflating the lungs, or by allowing them to collapse entirely by making an opening in the thoracic wall. The effect of blowing up the lungs was invariably to cut short an inspiratory movement which might be proceeding, and to cause a long expiratory pause. On allowing the lungs to collapse, a strong inspiratory spasm of the diaphragm was produced, which lasted in the rabbit from eight to ten seconds, giving place to gradually increasing oscillations of the diaphragm, and finally, with the onset of dyspncea, to complete relaxations of the diaphragm between the spasmodic inspirations.

Here we have evidently the mechanism for an automatic regulation (Selbststeuerung) of the respiration, each expansion of the lungs in inspiration tending to stop the inspiratory movement which caused it, and so bring on the following expiration. The collapse of the lungs during expiration would, on the other hand, send inspiratory impulses up the vagi, which would excite again the activity of the inspiratory centres. We see at once that the influence of the vagi must therefore be to quicken the respiratory movements, and, by cutting them short, to make them shallower, so that the results of cutting both vagi, by withdrawing these quickening impulses, must be a slowing and deepening of the respirations. 
Subsequent investigations, while confirming the account given by Hering and Breuer of their experiments, have introduced certain modifications into their interpretation of them. It is curious to note that, whereas Rosenthal regarded the lung fibres of the vagus as purely inspiratory in function, the researches since the publication of Hering and Breuer's paper have tended more and more to exalt the expiratory functions of these fibres, some authors going so far as to deny the existence in the vagus of any fibres whose function itnormally is to promote inspiration.

Of these investigations, the most important are those carried out by H. Head ${ }^{1}$ in Hering's laboratory, since they not only embrace a number of experiments of the most varied kind on the functions of the vagi, but they also represent the only series of researches in which the method of experiment was free from objection and really adapted to the object in view. In order to record the respiratory movements, all other investigators have used the depression of the diaphragm, the elevation of the ribs, or the movements of the air in the lungs. These methods are obviously inapplicable when we wish to study the normal mode of stimulation, i.e. distension or collapse of the lungs, since these events cause a passive movement of the recording lever which it is difficult to dissociate from the active movement caused by the contraction of the inspiratory muscles. In Head's method, as has been already described, the activity of the respiratory centre is gauged by recording the contractions of an isolated slip of the diaphragm, which is practically unaffected by passive movements of the chest wall. In the succeeding account the results of Head's experiments will be mainly followed.

1. The normal respiratory movements.-Each inspiratory contraction of the diaphragm, as sampled by the isolated slip, begins rapidly, but soon becomes slower, though the lever is still rising when the contraction suddenly ceases. The first part of the expiratory relaxation is extremely rapid, but the fall of the lever generally gets slower towards the end of the relaxation. The writing point then traces a horizontal line, representing the complete elongation of the muscular slip (Fig. 168). In the rabbit the expiration is in most cases entirely passive. Where an active expiratory movement takes place, it begins gradually towards the end of the expiratory pause, and increases until it is suddenly broken in upon by the rapid inspiratory contraction of the diaphragm, which is accompanied by a complete relaxation of the expiratory muscles. Very often, when the breathing is rapid, the respiratory pause may be entirely wanting, and in such cases it is often found that the lever does not quite descend to the base line between each inspiratory contraction, so that each contraction starts, as it were, from the summit of a tonic contraction of the diaphragm. The existence of this tonus may be easily shown by dividing the phrenic nerves, when the lever at once sinks to the base line of total relaxation.

2. Effects of division of the vagi.--Division of the vagi has been performed by every physiologist who has investigated the immervation of respiration, but with varying results. The typical effect, as described by Rosenthal, I have already described. In many cases, however, the issue may be quite different. The breathing may become irregular, and accompanied with restlessness of the animal, while in other cases division may be followed by long expiratory pauses. Now, in all instances where the nerves are divided by section or ligature and

${ }^{1}$ Journ. Physiol., Cambridge and London, 1889, vol. x. pp. 1-70, and pp. 279-290. 
allowed to fall back into the wound, the effects of division of the conducting tracts may be complicated by the occurrence of excitation in consequence of closure of the demarcation current at the surface of the cut nerve. In order, therefore, to observe the effects of pure division, it is better to use the method adopted by Gad, ${ }^{1}$ of freezing the nerve. Gad has shown that, when the conductivity of an afferent nerve is abolished in this way, no effective stimulation, so far as can be judged from the reflexes, is produced, although, if a notor nerve be treated in the same way, fibrillar twitchings of the muscle supplied by the nerve are observed. In addition to this method, Heal made use of another devised by Biedermann, for extinguishing the conductivity of the nerve. The vagus is placed in a tube of indiarubber, so constructed that ether vapour can be passed over it without affecting the atmosphere which the animal breathes. In a few seconds the nerve is completely ancesthetised and incapable of conducting impulses. On removing the ether vapour, by blowing air through the tube, the nerve completely recovers its conducting power, and this process can be repeated several times without any apparent injury to the nerve.

Since the results of dividing one vagus in this manner are very transitory, and resemble in a smaller degree those due to division of both nerves, we may

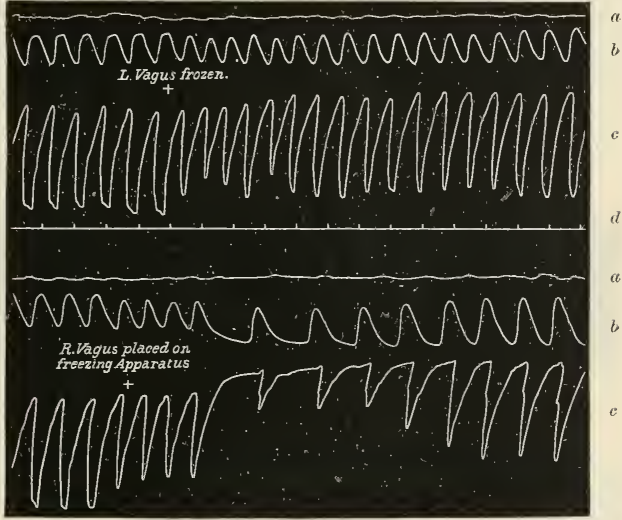

Fig. 172.-Eflects on respiratory movemeuts of dividing (freezing) first one, and then the other vagus. $\alpha$, $\alpha$, control eurve, showing jassive movements of the chest wall; $b, b$, curve taken by a tambour, showiug changes in pressure in a bottle of air connecterl with the trachea; $c$, $c$, myogram of diaphragmatic slip; $d$, time-marking in seconds.-Head.

pass at once to the effect of the latter proceeding. It is found invariably that removal of the influence which the vagi nomally exert on the breathing leads to an increase in the force and duration of the inspiratory contractions, the first contraction which follows the division far exceeding in extent both the previous and the subsequent contractions. Division of the vagi affects the duration rather than the height of each contraction of the diaphragn. The expiratory relaxations are as a rule incomplete, so that the base line of the curve is raised, i.e. the diaphragm is in a state of tonic contraction (Fig. 172). In course of time the inspiratory tonus gradually diminishes and the expiratory pause is more pronounced, so that finally the breathing assumes the type which has already been described as typical after section of both vagi. If the animal be deeply narcotiser? or in a bad condition, or if there be any dyspnoa, the prolonged 
initial contraction and the subsequent tonic contraction of the diaphragm are absent; the respirations become at once deeper and less frequent, each inspiration being followed by an expiratory pause which may be accompanied with active contraction of the expiratory muscles.

3. Effects produced by increasing the duration or intensity of the normal action of the vagi. - The results of increasing the duration of the normal vagal stimulation by closing the trachea at the end of inspiration or of expiration, as observed by Hering and Breuer, have been already described. The effects of increasing the extent of the stimulation by artificial inflation or collapse of the lungs were also studied by these observers. Since, however, certain new facts were brought to light by Head's experiments, some further mention of them is necessary.

If the lungs be gently inflated, the first result is an immediate cessation of the rhythmic inspiratory contractions, so that a pause is produced which may last fifteen to twenty seconds, but is always, sooner or later, broken by an inspiratory contraction. If any tonic contraction of the diaphragm were previously present, the lever, after its first rapid fall to the ordinary expiratory position, continues to sink slowly until, if the pause last long enough, the muscles attain complete relaxation.

If the inflation is carried out too violently, an inspiration may

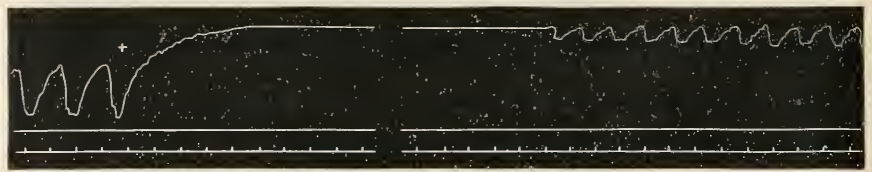

FIG. 173.--The right vagus has been divided, and the curve shows the effect produced by complete collapse of the left lung. The left pleural cavity was opened at + , and the resulting tonic contraction of the diaphragm lasted twenty-five seconds (only the beginning and end of the panse are shown).-Head.

precede the expiratory pause, due probably to the stimulation of nerves in the body wall,--since it may be produced even after division of the vagi.

I have already mentioned that collapse of the lungs produces a prolonged contraction of the diaphragm. Since the further course of the experiment is complicated by the onset of dyspnoea, we may only perforate one pleura. In this case, since the resulting inspiratory dilatation of the sound lung tends to inhibit the inspiratory movement which produces it, the vagus nerve must be divided previously on the sound side. If, as is usually the case, each lung is supplied only by the vagus nerve of its own side, the result of the unilateral collapse is to cause a quick contraction of the diaphragm, which may continue in this condition for over twenty seconds. The first incomplete expiratory elongations then appear, but it is many miutes before the respiratory movements in any way approach the normal type. Finally, the breathing assumes a form different from the normal in the strength of the inspirations and the presence of a certain amount of inspiratory tone, very similar to that resulting from division of both vagi (Fig. 173).

A similar result (inspiratory spasm and increased inspiratory tone) may be produced by momentary suction applied to the tracheal tube.

Mechanical apnæa.--The increased inspiratory activity following 
collapse of the lung or a momentary suction of air out of the air tubes, as well as the diminished activity caused by inflation or by closing the trachea at the end of inspiration, outlast the events which give rise to then by a considerable time. Thus, a monentary suction will give rise to an increased tone of the diaphragin which lasts for four or five seconds, or during the five or six respirations following the suction. These after-effects are designated by Head the "positive after-action."

Now this observer, in confirmation of observations by Gad, has

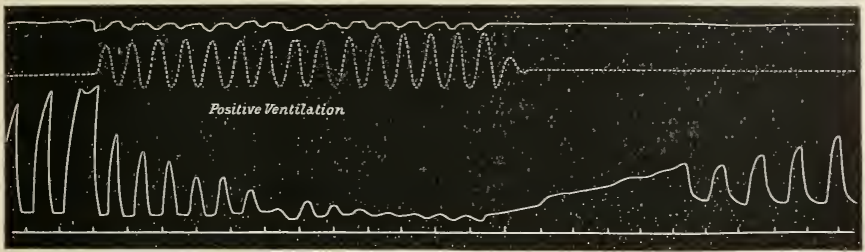

Fig. 174.-Etfects of positive ventilation with air on respiratory movements. (The upper line shows the passive movements of the sternum, the niddle line the strokes of the pump, and the lower line the respiratory movements of the slip of diaphragm.) - Head.

shown that, by repeating the stimulation at sufficiently short intervals, we may have a summation of the effects (the positive after-action), so that finally there is a standstill of respiration in a condition of expiration, or of continuous inspiration, or in a position halfway between the two. In order to carry out the series of rhythmic inflations or suctions, Head employed a double-action pump derised by Hering. When the whole pump is in action the first stroke pumps a certain quantity of air or gas into the lungs, and the next stroke remores exactly the same quantity. By connecting one-half only of the pump with the trachea, it is possible to produce only periodic inflations (positive rentilation), while by using only the other half the ventilation consists simply of periodic suctions (negative ventilation), the lungs in each case tending to return to the previous condition between the strokes of the puinp, in virtue of the elasticity of the chest wall.

When positive ventilation is employed (Fig. 173), every

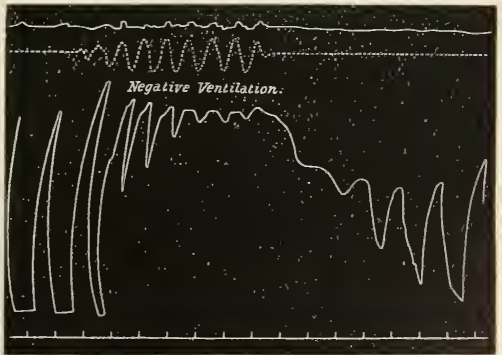

Fig. 175.--Effeets of negative ventilation on respiratory movenients.-Head. inflation not only produces an expiratory pause luring the time that it lasts, but this depressing influence on the inspiratory activity of the centre continues even after the linngs have returned to their normal volume. Hence the subsequent contractions suffer from the inlibition, and the inspiratory movements get smaller and smaller with every stroke of the pump, until they entirely disappear, the pause continuing for a time even after the ventilation is stopped; this conclition is expiratory apncea. 
On using negative ventilation the respiration is modified in a very different manner. Each suction causes a strong inspiratory contraction, which ceases as soon as the suction stops, but leaves behind it a condition of raised inspiratory activity, evidenced by the imperfect relaxation of the diaphragm. As the suctions are repeated, the augmentor effects
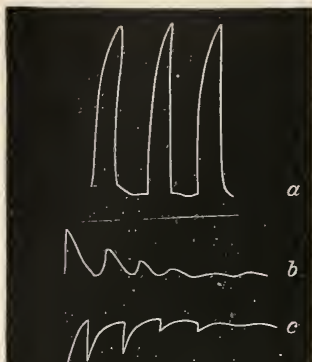

$\int$

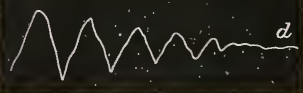

Frg. 176.-Diagrammatic representation of effects of different kinds of ventilation. $a$, normal breathing; $b$, apncea with positive ventilation; $c$, apncea with negative ventilation ; $d$, apucea with compound ventilation. Head.

A cogent objection to Rosenthal's interpretation is to be found in the fact, that the arterial blood is normally so nearly saturated with oxygen that increased ventilation can cause very little increase in the amount of oxygen carried by the blood. Moreover, it has been shown that towards the end of an apnœic pause, before the respiratory movements have recommenced, the blood becomes of a venous hue on both

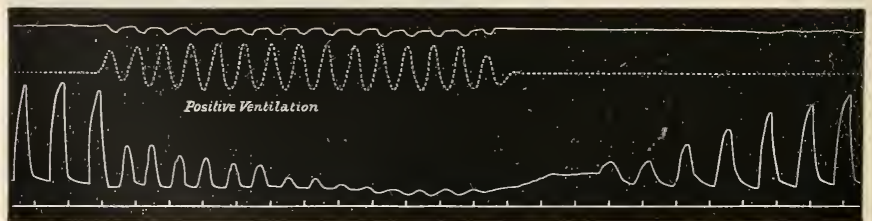

FIG. 177.-A [nocic pause, caused by positive ventilation with hydrogen.-Head.

sides of the heart. The oxygenation of the blood is in fact less than in a normal animal. The most striking proof, however, that the apnoea is determined solely by recurring stimulation of the vagus fibres, is afforded by the fact that a distinct apnœic pause can be produced by positive or compound ventilation with some inert gas, such as hydrogen or nitrogen, which is powerless to support life (see Fig. 177).

It night be thought from these experiments that it would be 
impossible to produce apnœa after section of both vagi, and Knoll stated that such was the case. As a matter of fact, however, although difficult shortly after section of the vagi, it is not impossible to produce an apnœic pause by ventilation with air. The ease with which this effect is produced increases with the length of time that the vagi have been divider.

How is the production of apnoea under these conditions to be explained? Head ascribes its production to the fact that, after section of the vagi, the aeration of the blood is not efficiently carried out, and the blood gradually gets more and more venous. In consequence of its lowered nutrition, the respiratory centre becomes adapted to this dyspnoic condition of the blood. If active ventilation be now carried out, the blood, being normally aerated, is over-oxygenated with reference to the condition of the centre, so that in this case the causation of the apnoea is the same as that imagined by Rosenthal for the apnoea in animals with intact vagi.

As might be expected, it is impossible, after division of the vagi, to cause apnoea by ventilation with hydrogen or nitrogen. The fact that it is possible, even after section of the vagi, to produce an apnoic pause by means of artificial respiration, was first established by Miescher, ${ }^{1}$ who therefore distinguished apnoe vera or chemical apnoea, due to (relative) over-oxygenation of the centre, from apnoa vagi or mechanical apnoea, due to the inhibitory effects of repeated distension of the lungs.

Where does the summation of the stimuli which cause the apnœic pause take place? Gad, who was the first to explain the apnoea as due to the summation of certain stimuli, believed that the summation takes place in the peripheral end-organs of the vagi. Head has shown, however, that if the vagi be divided at the begimning of the apnœic pause, this pause, instead of being cut off, is much more pronounced than under normal conditions, showing conclusively that the summation of stimuli must take place in the centre itself rather than in the vagal terminations.

Effects of artificial stimulation of the central end of the divided vagus nerve.-Piosenthal pointed out that electrical stimulation (by induction currents) of the central end of the vagus in the neck causes an increase in the inspiratory rhythm, and with stronger currents an actual tetanus of the inspiratory muscles. He ascribed all expiratory effects to the escape of current into the superior laryngeal nerves. Since then it has been shown by numerous observers that the constant effect of the weakest current which can cause any change at all in the respiratory movements is inhibitory. The pauses between the inspiratory contractions become longer than usual, and the inspirations are generally less powerful. Exactly the same effect is produced when the divided nerve is stimulated by its own demarcation current, or when a constant current is passed through it in an ascending direction (Langendorff and Oldag). ${ }^{2}$ The statement of these observers, that on opening the current, or on closing the current in a descending direction, an inspiratory effect is produced, has been contested by later authors, e.g. Lewandowsky. ${ }^{3}$ With the ordinary induction currents this primary expiratory effect is difficult to obtain with certainty, since a very slight

1 Arch.f. Physiol., Leipzic, 1885, S. 355.

2 Arch.f.d.ges. Physiol., Bonn, Bd. Iviii. S. 201 ; cf. also Bonttan, ibid., Bd. 1xi. S. 39.

${ }^{3}$ Arch.f. Physiol., Leipzig, 1896, s. 195. 
increase in the strength of the current produces the inspiratory effects described by Rosenthal. The inspiratory tetanus in the latter case is brought about chiefly by a decrease in the completeness of the expiratory relaxation of the diaphragm, which finally enters into a state of tonic contraction. A further increase in the strength of the exciting current leads to active expiratory contractions (possibly due to escape of current to the superior laryngeal nerves?).

Negative after-action.-Head has described an important aftereffect of changes in the volume of the lung on the respiratory centre, under the term "negative after-action." The nature of this effect may be best explained by two or three illustrations.

If the lungs be inflated for a short time, the return of these organs to their normal volume is often followed by a slow inspiratory contraction of great duration but of comparatively small strength, and the elongation after the next two or three inspirations is incomplete. Here the positive effect of the inflation is an abolition of inspiratory tone. The negative after-effect is increased inspiratory tone.

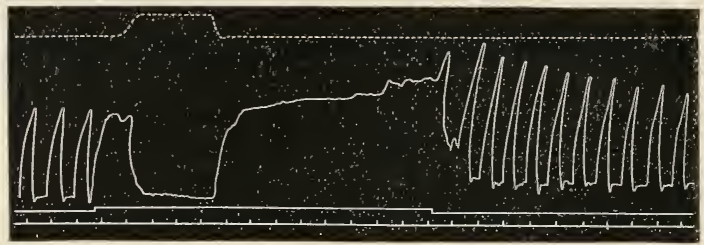

FiG. 178. - Respiratory tracing showing negative after-effect. The left vagus was stimulated electrically with currents just insufficient to cause Rosenthal's 'tetanus.' The period of stimulation is marked by the rise of the line above the seconds time-tracing. During this time both lungs were inflated for a period marked by the rise of the tracheal manometer curve (dotted line). This inflation caused instant elongation of the inspiratory muscles. As soon as the lungs were allowed to return to their normal volume, the mnscles again contracted nuder the influence of the vagal stimulation, and fell into a condition of complete tonic contraction.-Hear.

During the normal breathing of a rabbit, with partial pneumothorax on one side, the diaphragm is in a condition of slight tonic contraction. If we wait until the tone has disappeared and then inflate the lungs, we notice, on the resumption of the movements after the arrest immediately following the intiation, that the tone has reappeared. It disappears again after a little time, but can be again recalled by another inflation.

If the central end of the divided vagus on one side be stimulated by means of an interrupted current, it is possible so to graduate the strength of the current that it is just insufficient to cause a complete 'tetanus' of the diaphragm. If the lungs be now inflated, the diaphragm at once elongates. As soon, howerer, as the lungs return to the normal volume, the diaphragm contracts powerfully and completely; the stimulus which was previously ineffectual being now able to evoke a complete tetanus, since it is reinforced by the negative after-action due to the infiation (Fig. 178).

What light do these olservations throw on the functions of the ragus in normal respiration?

We have seen that the immediate effect of section of both vagi is to 
cause the appearance of an inspiratory tone and to lengthen the individual inspiratory contractions. This effect is still more marked if the division of the vagi be carried out after the medulla oblongata has been separated from the upper parts of the brain by a section through the striæ acusticæ. In this latter case the inspiration which follows division of the second vagus may last from forty-five seconds to one and a-half minutes. At the end of this time there is a quick complete relaxation of the diaphragm, followed by a slow expiratory contraction of the abdominal muscles. When this active expiration has reached a considerable height, the abdominal muscles suddenly relax, and the inspiratory muscles contract violently, producing a second prolonged inspiratory spasm. Gad ${ }^{1}$ pointed out that these effects indicate that the impulses previously ascending the vagi were inhibitory in character, rather than inspiratory, as was supposed by Rosenthal. Whereas Hering and Breuer had imagined that the vagus is traversed alternately by expiratory and inspiratory impulses, Gad concluded that the only impulses travelling up the vagi are expiratory or inhibitory in nature. These impulses are excited by the condition of distension of the lung, and are therefore present even at the end of a normal expiration, being only abolished by total collapse of the lung.

It is evident that we can explain the results of distension or collapse of the lungs just as well on this hypothesis as on that of Hering and Breuer. When the lungs are forcibly distended, the normal inhibitory influence of the vagus is increased, and an expiratory pause is the result. Sudden collapse of the lungs causes a diminution in the normal inhibitory stimuli, and therefore increased inspiratory activity.

Head, though agreeing with Gad in thinking that the collapse of the lung in normal quiet expiration acts only by a removal of inhibitory stimuli, does not accept his view that the only impulses travelling up the vagi are inhibitory. If this were the case, sudden collapse of both lungs should be exactiy equivalent to section of both vagi; whereas Head, using a more perfect method of investigation than Gad, has shown that the results of collapse are far more pronounced than those of simple section of the vagi. Moreover, it is difficult to understand the summation of effects produced by negative ventilation on the assumption that each suction merely causes a diminution of previously existing stimuli.

Assuming that the inhibitory impulses which ascend the vagi are the more important under normal conditions of respiration, how can we explain the quickening effect of these stimuli? In endeavouring to answer this question, it must be remembered that every stimulus, whether produced by inflation or collapse, is followed by a positive and a negative after-reaction. After the division of the vagi, the breathing finally acquires the type characterised by long powerful inspirations, separated by complete expiratory pauses. If now we could suddenly reinstate the vagi, it is evident that in the very next inspiration, before the movement could be completed, the dilatation of the lungs would send an inhibitory impulse up to the centre, checking the inspiratory movement and causing a subsequent pause (positive after-action). As soon as this positive after-action was sufficiently diminished, a second

$$
{ }^{1} \text { Aich. f. Physiol., Leiprig, 1380, S. 1; ibid., 1881, S. } 53 \text { s. }
$$


inspiration wonld occur, which would be again cnt short before it had reached its full height. Not only, however, will the height of the contractions be diminished by this automatic governing arrangement, but each inhibitory impulse ascending the vagi will have a negative after-action, tending to increase the vitality or inspiratory activity of the centre. The effect of this increased inspiratory activity will be to diminish the pauses between the different inspirations, so that finally we get a condition in which the breathing remains rhythmic, but the inspiratory contractions are smaller and more frequent than before the reinstatement of the vagi. Moreover, the centre is in a condition of increased vitality, and able to respond with much greater promptitude to any inspiratory stimulus. The vagi thus act like the governor of a steam-engine in economising the labour expended, and in preventing the centre from wearing itself out by excessive exertion. When these nerves have been divided, the centre finally settles down into a condition of "spendthrift activity," regulated only by the impulses from the centres in the higher parts of the brain, and we have already seen that, even with their aid, the aeration of the blood, the object of the respiratory mechanism, becomes more and more ineffectually carried out.

Of the other views with regard to the nature of vagus action, I need only mention those of S. J. Meltzer. ${ }^{1}$ This author, after showing that in all animals excitation by means of strong currents of the central end of the divided vagus produces expiration, followed after the cessation of the stimulus by a strong inspiratory movement, concludes that the vagus contains two classes of fibres,- the inspiratory and the inhibitory or expiratory fibres. These differ in the time relations of their response to stimuli, resembling in this particular the accelerator and inhibitory cardiac nerves. When they are both stimulated together, the primary effect is therefore expiration, the more lasting effect inspiration. He imagines that the inspiratory expansion of the lungs excites both sets of fibres simultaneously. During the excitation, the inhibitory effects predominate over the inspiratory, so that the first result is a stoppage of inspiration with relaxation of the inspiratory muscles. The expiratory collapse which follows removes the stimulus, and therefore the more lasting after-effect of the excitation, namely, that due to the inspiratory fibres, can make itself felt, and we get a new inspiration.

The influence of other afferent nerves on respiration.-Although there are hardly any afferent nerves in the body, stimulation of which may not cause some change in the respiratory rhythm, yet in no case can we regard their influence on respiration as equivalent to that of the vagus, since their division is without effect on the respiratory rhythm.

Superior laryngeal nerve.-As was first shown by Rosenthal, stimulation of the central end of this nerve has a nearly pure expiratory effect. Stimulation with a weak current causes an instant cessation of inspiration and an expiratory pause. Stronger stimulation may evoke strong contractions of the expiratory muscles. Besides this effect on the respiratory rliythm, excitation of the superior laryngeal also affects the swallowing centre in the medulla, and so gives rise to elevation of the larynx, followed by peristaltic contractions of the osophagus. As was pointed out by Steiner ${ }^{2}$ and by Meltzer, ${ }^{3}$ the first phase of each act of deglutition is accompanied by a short twitch of the diaphragm. A tracing of the diaphragm during stimulation of this nerve shows

${ }^{1}$ Arch. f. physiol., Leipzig, 1892, s. 340.

3 Thid., 1883, S. 209.

${ }^{2}$ Ibid., 1883, S. 57. 
therefore a series of small elevations rising from the base line corresponding to the condition of relaxation.

Inferior laryngeal nerve.-Excitation of this nerve is frequently attended by inhibition of iuspiration, with sonetimes active expiratory movements.

Glosso-pharyngeal nerve._-Kronecker and Meltzer, ${ }^{1}$ in studying the mechanism of deglutition, found that the small inspiration mentioned above, which accompanies the first phase of this act, is followed by a period during which respiration is entirely inhibited. This result they ascribed to an irradiation from the centre of deglutition to the respiratory centre. Marckwald ${ }^{2}$ has shown, however, that the pause is originated reflexly by means of the glosso-pharyngeal nerve. If this nerve be cut and its central end stimulated, after a latent period of half to one second, an absolute stoppage of respiration takes place. This stoppage differs from that produced by any other nerve, in that the muscles remain in the condition in which they were when the stimulation began to act, whether during inspiration, expiration, or midway between the two conditions. This cessation of respiration lasts for about the time occupied by the two or three preceding respirations. Respiration then recommences, even though stimulation be continued, with an inspiration which starts from the position at which the diaphragm was arrested at the beginning of stimulation.

Other sensory nerves. - Gentle stimulation of most sensory nerves causes quickening and increase in the inspiratory movements; with a stimulation strong enough to evoke signs of pain, the animal becomes restless, and the breathing is mainly of the active expiratory type. The application of gentle stimuli to the stomach or abdominal viscera, or to the splanchnic nerves, is, however, invariably expiratory, and displays itself either as an increase in the expiratory pauses, or more often as a strong contraction of the expiratory muscles.

A pure expiratory reflex may also be brought about by gentle stimulation of the nasal mucous membrane of the rabbit, as by application of chloroform vapour. A similar expiratory pause is caused in many animals by dipping the nose into water, or even by plunging the lower half of the body into water (Tauchreflex). The temperature of the water is of no influence on the results of the experiment. Fredericq ${ }^{3}$ has shown that a specially long expiratory pause may be produced in a diving animal, such as the duck, by allowing a stream of water to flow on its beak. The teleological importance of these reflex cessations of respiration, which have been classed together by Miescher-Rusch ${ }^{4}$ as apnoea spuria, is obvious.

Influence of the higher parts of the brain on the respiratory centre.-We have seen that a complete section of the bulb above the respiratory centre is without influence on the respiratory rate, the centre with the help of the vagi being quite sufficient to carry out the normal respiratory rhythn. If, however, the vagi have been previously divided, the severance of the paths from the higher parts of the brain has an enormous influence. The previous rhythmic respiratory movements are practically abolished, and give place to a series of prolonged inspiratory spasms, which are separated by long pauses during which

1 Arch.f. Physiol., Leipzig, 1888, Suppl.

2 "Movements of Respiration," 1888, ". 88.

: Arch. f. Physiol., Leipzig, 1893, Suppl., S. 65.

${ }^{4}$ Ibid., 1885 , S. 355.

VOL. II. -20 
the inspiratory museles are completely relaxed. Towards the end of the pause, active contraction of the expiratory muscles takes place. These spasms are inadequate for the proper aeration of the blood. As the blood gets more venous, the spasms become less and less frequent, and the animal, at the end of an hour or so, dies of asphyxia. We must conclude from these results that, in default of the normal vagus stimulation, the activity of the respiratory centre can be regulated and controlled to a large extent by vicarious influences from the higher parts of the brain. In searching for the origin of these impulses, we naturally think first of the afferent nerves which enter the cerebro-spinal centres above the medulla oblongata, especially since we know that respiration may be altered in an inspiratory or expiratory direction by excitation of the olfactory, optic, auditory, and fifth nerves. A total division of these nerves would be probably impracticable. Loewy ${ }^{1}$ has shown that bilateral section of the fifth nerves at their exit from the pons does not alter the respiratory rhythm after division of both vagi. Attempts have therefore been made to localise the origin of these impulsesfirst, by carrying out the extirpation of the brain in successive stages from above downwards; and, secondly, by observing the effects of stimulation of certain parts of the higher portions of the brain.

By the first method it has been determined that, after division of both vagi, no further alteration takes place in the breathing until the section has bcen carried through the level of the anterior corpora quarlrigemina.

By the second method, certain localised areas of the brain have been found to give, on excitation, definite changes in the respiratory rhythm. Thus mechanical, thermal, or electrical stimulation of the inner wall of the optic thalamus on each side (Budge, Christiani) causes either a standstill of the diaphragm in an inspiratory condition, or increases the rhythm and depth of the inspiratory movements. On the other hand, an expiratory centre has been described by Christiani ${ }^{2}$ in the substance of the anterior corpora quadrigemina close to the aqueductus Sylvii, and an inspiratory centre by Martin and Booker ${ }^{3}$ midway between the anterior and posterior corpora quadrigemina. It would seem that a section has to pass through or behind these two last-named centres, in order to produce the effects already described of cutting off the higher centres from the medulla oblongata after division of the vagi.

Spencer ${ }^{4}$ obtained slowing and arrest of respiration by faradic stimulation of the cortex just outside the olfactory lobe in front of the point where the tract joins the temporo-sphenoidal lobe, and increased action on stimulating a spot on the convex surface of the cortex within the motor area. Excitation of the junction of the olfactory bulb and tract, as well as of the uncinate gyrus, produced super-inspiratory clonus, while super-inspiratory tomus was obtained on stimulating the descending motor tract.

The experimental facts at our command are not adequate at present to permit of our arriving at any definite conclusions as to the nature or mode of origin of the vicarious impulses which reach the medulla oblongata from these centres. We have, however, no reason to suppose

' Arch. f. Physiol., Leipzig, 1893, S. 185.

${ }^{2}$ Monatsb. d. k. Akad. d. Wissensch. zu Berlin, 1881, S. 213.

3 Journ. Physiol., Cambridge and London, 1879, vol. i. p. 370.

4 Phil. Trans., London, 1891, vol. el xxxii. p. 201. 
that they are active during normal respiration, for which the integrity of the medullary centre and vagi is all that is necessary.

Asphyxia.-This condition is brought about by all circumstances which hinder the normal respiratory changes of the blood passing to the medullary centres. It may therefore be caused by any agency which prevents the respiratory movements as well as by obstructing the trachea, or by opening both pleural cavities, so that the respiratory movements no longer dilate the lungs. Asphyxia may be brought about by cutting off the blood supply to the medulla oblongata, either by ligature of all the vessels going to the brain, as in the Kussmaul-Tenner experiments, ${ }^{1}$ or by producing universal anæmia by a large hæmorrhage. So far as the respiratory movements are concerned, the phenomena of asphyxia may be divided into three stages.

In the first stage, the respiratory movements are increased in rhythm and anplitude. This stage is sometimes known as hyperpnoa. If any tonic contraction of the diaphragm had been previously present, this disappears at the onset of dyspnœa, the relaxation of the diaphragm at the end of each inspiration being complete.

In the second stage (dyspnca) the expiration becomes active, and gradually the expiratory movements become increased out of all proportion to the inspiratory, so that finally respiration is made up of a series of prolonged expiratory convulsions, separated by short inspirations. In these forcible expirations nearly every muscle of the body may be involved.

At the end of the second minute after ligature of the trachea (if this mode of producing asphyxia has been adopted), the expiratory convulsions cease almost suddenly, and give way to slow deep inspirations. With each inspiratory spasm, the animal stretches itself out, and opens its mouth widely, as if gasping for breath. The whole stage is one of exhaustion; the pupils are widely dilated, and the animal is perfectly insensitive, being unaffected by the strongest sensory stimulation. The pauses between each inspiration become longer and longer and each inspiration shallower, till, at the end of four or five minutes, "with extended limbs and straightened trunk, with the head thrown back, the mouth widely open, the face drawn and the nostrils dilated, the last breath is taken." 2

The effects of deprivation of oxygen upon the circulation are different in the different stages of asphyxia, and naturally vary according to the mode by which asphyxia is produced. If by occlusion of the trachea, the variations in intrathoracic pressure which are brought about by the dyspnœic movements of the chest are enormous, and produce corresponding effects upon the arterial and venous pressures. In addition to this, and whatever be the method by which asphyxia is caused, the venous blood circulating in the medulla oblongata stimulates both the vasomotor and the cardio-inhibitory centres, so that the arterioles are contracted and the heart-beat slowed. The effect of the vaso-constriction is, however, greater than that of cardio-inhibition, and the blood pressure markedly rises. There are also usually produced very well-marked Traube-Hering curves, indicating a rhythmic action of the vasomotor centre. These changes go on during the first and second stages of asphyxia, but after this the heart muscle, over-strained by the raised peripheral resistance and inadequately supplied with

1 "On Epileptiform Convulsions from Hæmorrhage," by Kussnaul and Temner, Ncw Syd. Soc. Translation, London, 1859.

2 M. Foster, "Text-book of Physiology," 6th edition, p. 640. 
oxygen, begins to fail. Each beat becomes more and more feeble, the quantity of residual blood left in the cavities at the end of systole increasing at each beat, until finally the heart is enormously dilated, and each contraction is marked only by a feeble flutter of the cardiac walls. In consequence of this heart failure, the pressure sinks steadily until circulation is at an end and the pressure is the same all over the vascular system (about $10 \mathrm{~mm} . \mathrm{Hg}$ ). A vigorous animal, with wellmarked vasomotor reaction, will die of heart failure, before any relaxation of the vessels has taken place. This is shown by the fact that if artificial respiration be suddenly employed, when the blood pressure is approaching its lowest point, the access of oxygen benefits the heart earlier than the vasomotor centre, and the pressure may rise to a point even higher than that reached during the primary asphyxial rise.

Failure of the circulation, however, gradually impairs the activity of the medullary centres, as is shown by the gradual decline in arterial and venous pressures after all circulation has ceased (in consequence of increased capacity of vascular system).

At the end of asphyxia, therefore, all cavities of the heart are distended with blood, the distension being most pronounced in the cavities with thin walls, i.e. auricles and right ventricle. The difference thus occasioned between right and left ventricles is generally increased after rigor mortis has set in, and it is supposed that the post-mortem contraction of the left ventricle expels the contents of that cavity; so that it may be found almost empty unless examined immediately after death.

\section{Modified Respiratory Movements.}

Coughing.-This consists in the first place of a deep inspiration, followed by closure of the glottis. This is succeeded by a sudden powerful expiratory effort, with total relaxation of the inspiratory muscles. The air is forced suddenly through the resistance offered by the glottis, and the blast of air thus produced serves to expel through the upper air-passages and mouth any foreign body lodged in the larynx. Coughing is usually excited by stimulation of the terminations of the superior laryngeal nerve in the mucous membrane of the larynx. But it may also be due to stimulation of certain of the vagus endings in the lungs, or of the nerves supplied to the pleura.

Sneezing is very allied in its general characters to coughing. In each case an inspiration is followed by a powerful expiratory effort. In sneezing, the resistance to the outburst of air seems to be afforded in the first place by the approximation of the dorsum of the tongue to the soft palate, so that the first part of the expired air passes through the nasal passages. The greater part of the air is, however, expelled through the mouth. Sneezing is usually excited reflexly by stimulation of the nasal branch of the fifth nerve; when it is produced by sudden exposure to a bright light, the afferent impulses probably travel up the optic nerve.

In laughing and crying, a deep inspiration is followed by a series of short spasmodic expirations. The glottis is freely open during the whole time, and the vocal cords being tense, are thrown into vibration, so occasioning the characteristic sounds of the two actions. The chief difference between the two actions is in the accompanying facial expressions. 
Yawning consists of a deep inspiration accompanied by a wide opening of the mouth and of the glottis, often attended by movements of the arms.

Cheyne-Stokes respiration. - A special modification of the respiratory rhythm, known under the above title, occurs in certain pathological cases. In this form the activity of the respiratory centre becomes periodic. Thus the respirations gradually decrease in amplitude until they cease altogether. After a pause of greater or less duration, a feeble inspiration ensues, to be succeeded by a somewhat stronger one, and the inspirations gradually return to, or somewhat above, their normal height, to then undergo a diminution as before. Mosso' has shown that, even in the normal state, a certain periodicity during respiration may be observed during sleep. Well-marked CheyneStokes breathing has often been observed in cases where there was increased cerebral pressure or deficient blood supply to the brain. Langendorff $^{2}$ discovered that a similar condition might be produced in frogs by ligature of the aorta, or by cutting out the heart, or by poisoning with muscarin. In the latter case, the phenomenon could not be abolished by subsequent administration of atropin. In animals a periodic respiration has sometimes been observed when a section between the brain and the medulla oblongata has been carried out too low, so as to affect the respiratory centre injuriously. Although we can assume with a high degree of probability that the cause of the Cheyne-Stokes phenomenon is to be sought in a morbid condition of the respiratory centre, we are not yet in a position to form a definite conclusion as to the exact changes involved in this condition.

\section{THE INNERVATION AND ACTION OF THE BRONCHIAL MUSCLE FIBRES. ${ }^{3}$}

A contraction of the smooth muscle fibres which surround the large and smaller air tubes must have several effects, namely-(1) A constriction of the bronchi or bronchioles. (2) A diminution of the air space of the lungs, and therefore, in the absence of compensatory changes in blood contents, of the volume of the whole lung. (3) An increased resistance to the passage of the air into and out of the alveoli.

From the time when Longet, ${ }^{4}$ from direct observation of the bronchioles, concluded that the vagus nerve carried motor fibres to the bronchial muscle, the subject has been investigaterl by a number of physiologists. Although in some cases purely negative results were obtained, the accuracy of Longet's statement must be accepted as beyond doubt. Among those who obtained evidence of bronchial constriction by various methods, more or less imperfect, may be mentioned Schiff, ${ }^{5}$ Paul Bert, ${ }^{6}$ Gerlach, ${ }^{7}$ MacGillavry, ${ }^{8}$ Roy and Brown, ${ }^{9}$

1 Aich. f. Physiol., Leipzig, 1886, Suppl., S. 37.

2 llid., 1880, S. 241 ; 1881 , S. 33.

3 This chapter is based on the very full accounts of the subject given by Einthoven, "Ueber d. Wirkung d. Bronchialmuskehn, ete.," Arch. $f$. d. ges. Physiol., Bonn, 1892, Bd. li. S. 367 ; and by Th. Beer, "Ueber d. Einfluss d. peripheren Vagusreizung auf d. Lunge," Arch.f. Physiol., Leipzig, 1892, Suppl., S. 101.

${ }^{4}$ Compt. rend. Acad. d. sc., Paris, tome xv. p. 500.

5 Areh. f. $d$. ges. Physiol., Bonn, Bd. iv. S. 226.

6 "Leçons sur la physiol. comp. de respiration," Paris, 1870, p. 375.

7 Arch. f. d. ges. Physiol., Bonn, Bd. xiii. S. 491.

${ }^{8}$ Arch. neérl. d. sc. exactes, ete., 1877, S. 445.

${ }^{9}$ Journ. Physiol., Cambridge and London, 1885, vol. vi. 
Sandmann, ${ }^{1}$ F. Franck, ${ }^{2}$ Riegel and Edinger, ${ }^{3}$ Einthoven and Th. Beer. Einthoven made use of two methods, both based on the increased resistance to the passage of air through the bronchioles which would be occasioned by contraction of the bronchial muscles. In the first method, artificial respiration was carried out, a constant volume of air being blown in and sucked out at each respiration. Any diminution in the ealibre of the bronchioles must increase the resistance to the incoming current, and so cause a rise of pressure in the tracheal tube. Instead of recording the variations of pressure throughout the whole of each stroke of the pump, an arrangement was made use of, by means of which the mercurial manometer was connected with the trachea only for a brief space of time, during one part of the inspiratory phase. The lever recording the excursions of the mercury in the manometer recorded therefore an almost unbroken line during the experiment. Any increased

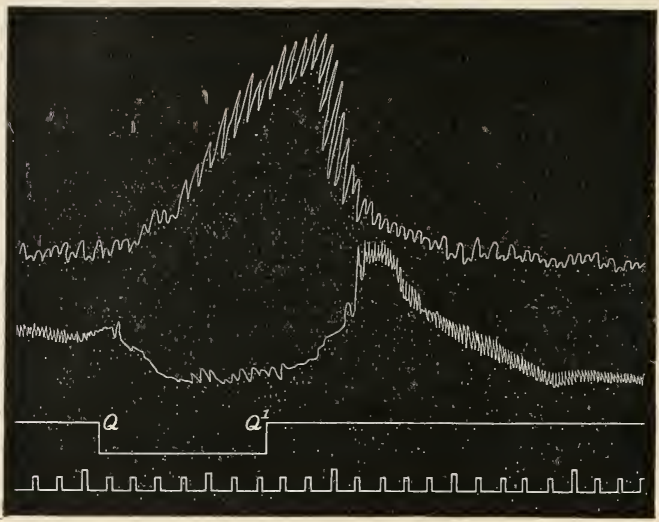

F1G. 179. - Tracings of blood pressure (middle eurve) and of intratraeheal pressure (upper curve), taken by Einthoven's "differential manometer." Between Q \& $Q^{\prime}$ the peripheral end of one vagus was stimulated. Time marking $=$ seconds. resistance to the current of air raised the pressure during the whole inspiration, and therefore during the moment at which the manometer was put into connection with the tracheal tube. A rise of the lever was thus produced, which continued with each stroke of the pump, until the level of the mercury corresponded with the true pressure obtaining at the moment of connection, during constric-

tion of the bronchioles. Einthoven calls this arrangement a "differential manometer," since its principle is the same as that of Bernstein's differential rheotome.

In the second method, artificial respiration at a constant pressure is made use of. Any change in the bronchioles will in this case affect the volume of air entering the lungs at each stroke of the pump, and can be measured by recording the passive respiratory movements of the chest wall or diaphragm. In both cases the animal must be curarised, so as to abolish spontaneous respiratory movements.

The influence of stimulation of the peripheral end of the vagus.-If in a curarised animal the peripheral end of either vagus be stimulated, the effect is invariably a constriction of the bronchioles. This is rendered evident by a rise of the manometer lever, if Einthoven's first method of observation be adopted (Fig. 179); or by a diminution of the respiratory movements of the thoracic wall, if the second method be

${ }^{1}$ Arch. f. Physiol., Leipzig, 1890, S. 252.

${ }^{2}$ Arch. de physiol. norm. et path., Paris, 1889, tome i. p. 538.

${ }^{3}$ Ztschr. f. klin. Med., Berlin, Bd. v. S. 413. 
employed (Fig. 180); the effect is increased within limits, by increasing the duration of the stimnlus. The latent period which elapses between the beginning of the excitation and the commencement of the bronchomotor effect, lies between 1.0 and 1.5 seconds.

It might be thought that this bronchial spasm was directly caused by the fall of blood pressure, due to the action of the vagus on the heart. It is not possible to exclude this explanation by administration of atropin, since this drug paralyses completely the bronchomotor fibres of the vagus. Einthoven has shown, however, that there is no proportionality between the effect on the heart and the amount of bronchial constriction, and that, in fact, a well-marked and typical bronchial constriction may be brought about by vagal stimulation, even after the death of the animal and consequent cessation of the blood flow.

Tonic action of the vagus. - In the dog there is, as a rule, very slight evidence of a tonic influence of the vagus on the bronchial muscle. Section of both vagi either does not alter the respiratory curve (Einthoven's method), or lowers it by 2 to $10 \mathrm{~mm}$. water. It is, however, in all cases, easy to bring about a vagus tonus by allowing the animal to inhale air containing an excess ( 3 or 4 per cent.) of $\mathrm{CO}_{2}$. As the $\mathrm{CO}_{2}$ tension in the blood rises, the bronchi constrict slowly. This constriction is at once cut short by dividing both vagi. It is not due to commencing asphyxia (oxygen hunger), since it is not produced by allowing the animal to inhale pure nitrogen.

Chauveau ${ }^{1}$ states that in the horse the vesicular murmur disappears after section of the vagi. This fact would tend to point to a marked tonic action of the vagi on the bronchial muscles in this

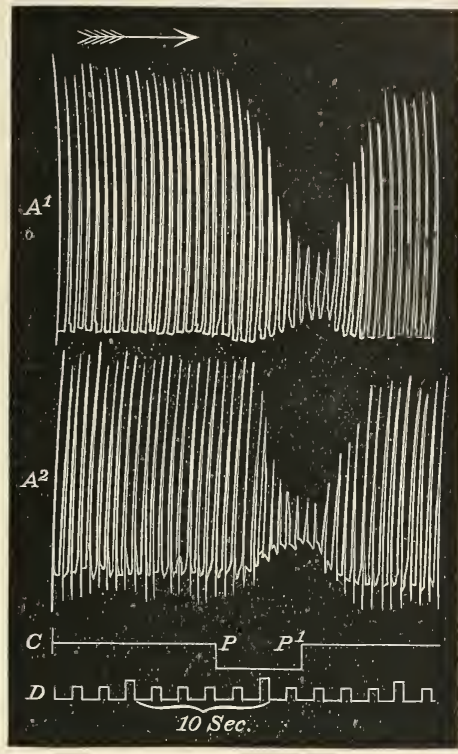

FIf. 180.-Diminution of respiratory exeursions produced by spasm of bronchial musele (artificial respiration at constant pressure). $A^{\prime}=$ thoracic wall, $\mathrm{A}^{2}=$ diaphragm. Vagus stimulated between $\mathrm{P}$ and $\mathrm{P}^{\prime}$.- Einthoven. animal.

When present the vagus tonus may present slight slow rhythmical oseillations, and may sometimes be inhibited by stimulation of the central end of a sensory nerve such as the sciatic. In one casc, Einthoven observed a reflex broncho-constriction on stimulating the central end of the sciatic in a curarised dog. There seems to be no satisfactory evidence for assuming the existence of broncho-dilator fibres in the vagus.

The effects of bronchial constriction; Asthma.-Under the influenec of vagal stimulation or of $\mathrm{CO}_{2}$, the pressure necessary to drive the normal amount of air into the lungs way be raised in the $\log$ from 125 to $300 \mathrm{~mm} . \mathrm{H}_{2} \mathrm{O}$.

We should therefore expect that, in cases where bronchial constriction is present, there would be difficulty both in inspiration and expiration. There

1 Quoted by Einthoven, loc. cit., S. 373. 
is, however, a difference in the mechanical conditions of the bronchi during the two phases of a respiratory movement. Normally the elastic structure of the lungs is drawing upon the bronchial wall, tending to maintain it patent, and so opposing the action of the bronchial muscle. During inspiration this expanding force is increased, so that in the presence of bronchial constriction the access of air is rendered the easier, the more powerful the contraction of the inspiratory muscles. In expiration all parts of the lung collapse, drawing with them the chest wall ; the pull of the lung tissue on the bronchial wall is lessened, but is still present. If, however, the expiratory muscles contract vigorously, the intrapleural pressure becomes positive, and the pull of the lung tissue on the bronchial walls is changed into a pressure tending to obliterate their lumen, and so impede the outflow of air.

It is evident, therefore, that in the presence of a spasmodic contraction of the bronchial muscles the inspiration will be forcible and rapid, but all contractions of muscles must be avoided, so far as possible, during expiration, which must be left to the elastic reaction of the lungs, and becomes slow and prolonged. Moreover, it will be of advantage to keep the lung as nearly as possible in the inspiratory position, so as to reinforce the elastic forces which dilate the bronchioles and aid expiration. We thus get the typical breathing which occurs in man in eases of spasm of the bronchial muscles, known as asthma nervosum. This type of breathing is often described as being marked by expiratory dyspnoea. This description is, however, erroneous. The muscles which in these cases are contracted to their uttermost, are the inspiratory muscles; the expiratory muscles, such as the abdominal, will be found to be quite flaccid even during expiration. 


\title{
THE MUSCULAR AND NERVOUS MECHANISMS OF THE DIGESTIVE TRAC'T.
}

\author{
Bi E. H. Starling.
}

Contents :-Mastication, p. 313-Sucking, p. 314-Deglutition, p. 314-Movements of the Stomach, p. 321-Vomiting, p. 324-Movements of the Intestines, p. 326-Defreation, p. 335.

\section{Mastication.}

Is order to prepare the solid food-stuffs for the action of the digestive juices in the alimentary canal, they are reduced to a fine state of subdivision in the mouth by the process of mastication. In this act the incisors divide the lump of food into smaller portions, which are then ground up between the molar teeth. The necessary movements for these processes are carried out by the muscles acting on the lower jaw, the temporo-maxillary articulation allowing of elevation and depression of the lower jaw, as well as forward, backward, and side-to-side movements. This dental triturating apparatus would be of no avail without some means of keeping it constantly supplied with fresh particles of food. The renewal of the food between the teeth is carried out by movements of the tongue and cheeks. Bilateral paralysis, sensory or motor, of the tongue, renders mastication almost impossible. Paralysis of the cheek (buccinator) on one side causes the food to collect and form a pouch outside the molar teeth. In such cases, the patient assists mastication by emptying the pouch with his finger.

With regard to the muscles involved in the various movements by which mastication is performed, the lower jaw is raised by the action of the masseter, temporal, and internal pterygoid muscles. Depression of the lower jaw is largely passive, but is also aided by the digastric and, to a slighter extent, by the mylohyoid and geniohyoid muscles. The grinding movements of the jaw-sidewards, forwards, and backwardsare carried out by contractions of the external pterygoid muscles of the two sides in varying degree.

Of the muscles affecting the tongue, the genioglossus and transverse fibres move this organ forwards, while the hyoglossi, palato- and styloglossi cause retraction. Movement from one side to the other is effected by contraction of the longitudinal muscular fibres. Contraction of the hyoglossi alone will make the upper surface of the tongue eonvex from side to side; contraction of the genioglossi will make it concave.

The act of mastication is a voluntary one, but, like most other coordinated muscular actions, is dependent for its guidance on sense impressions. The sensory nerve involved is the fiftl. The efferent 
impulses travel by the motor root of the fifth cranial nerve to the elevators of the jaw and the mylohyoid, along the seventh nerve to the digastric and muscles of the lips and cheek, and along the hypoglossal nerve to the muscles of the tongue.

\section{Sucking.}

During the first year of life, before the development of the teeth, the child is dependent for its sustenance on liquid food, which it obtains under normal circumstances from the breast of the mother. In this act the lips enclose the nipple, adherence being assisted by the existence of a special membranous projection of the front part of the gums of both jaws. During suction, the respiration goes on through the nose without check. Normally the dorsum of the tongue lies against the hard palate, but, according to Donders, ${ }^{1}$ at the back part it is separated from the soft palate by a small space. Owing to the weight of the jaw, there is a negative pressure in this space of $2-4 \mathrm{~mm}$. Whether such a space is present under ordinary conditions or not, it is certain that the jaw is maintained in its position, not by muscular effort, but by the pressure of the air; so that, if a tube from a manometer be passed between the tongue and the palate, the manometer shows a slight negative pressure corresponding to the weight of the jaw. If, therefore, the nipple of the breast or feeding-bottle be passed between the tongue and palate, there will be a tendency for the fluid to flow into this space. During active sucking the space is enlarged and the negative pressure increased by means of movements of the tongue, this organ being retracted and also hollowed out on its upper surface by means of the genioglossi and palatoglossi.

\section{Deglutition.}

Muscular mechanisms.-Since the time of Magendie, physiologists have been accustomed to divide the act of swallowing into three stages.

In the first stage, the bolus of food is carried past the anterior pillars of the fauces; the second stage comprises the period during which the food is conveyed through the pharynx, past the openings of the nasal cavities and larynx; and during the third stage it is passed through the lower part of the pharynx and down the osophagus. Since, however, there is no pause between these various stages, but the muscular movements involved follow one another continuously, it is better to consider the act of deglutition as one process.

The food, when sufficiently masticated, is collected as a bolus on the dorsum of the tongue by the movements of this organ. A pause then occurs in the process of mastication, a slight movement of the diaphragm usually takes place ("respiration of swallowing"), and a sudden elevation of the tongue occurs, which travels from the apex to the base, so squeezing the bolus backwards past the isthmus faucium. At this moment the bolus has arrived at a part of the pharynx which is common to the functions of respiration and deglutition. It has therefore to be conveyed quickly past the openings of the air passages into the pharynx,

1 Arch. f. d. ges. Physiol. Bonn, 1875, Bd. x. S. 91. 
and means must be taken to prevent any of the food escaping into the air passages. We have therefore to consider the means whereby(1) The bolus is propelled downwards into the grasp of the constrictors; (2) the nasal passages are closed; (3) the larynx is guarded.

1. The chief factor in the propulsion of the bolus through the back of the pharynx is the contraction of the mylohyoid muscle, which presses the tongue against the palate and pushes it backwards. In this action it may be aided by the contraction of the styloglossus and palatoglossus muscles, which pull the base of the tongue suddenly backwards. These muscles, moreover, especially the palatoglossi, serve to close the isthmus faucium, thus preventing any return of the food towards the mouth.

2. The shutting off of the naso-pharyngeal cavity is a somewhat complicated act. As soon as, or even before, the bolus touches the soft palate, this structure is raised and put on the stretch by a simultaneous contraction of the levator palati and palato-pharyngeus muscles on each side. At the same time the contraction of the last-named museles, which form the posterior pillars of the fauces, approximates and straightens the edges of these two structures, and raises to a certain extent the upper part of the pharynx. The soft palate, which has thus been raised, comes in contact with an elevation on the posterior wall of the pharynx, an elevation due to the contraction of fibres of the superior constrictor of the pharynx. The uvula, which is also raised slightly by the azygos uvulæ, hes between and fills up the opening between the straightened posterior pillars of the fauces. The soft palate is thus formed into a tense sloping roof, which guides the bolus down the pharynx.

3. More important still is the shutting off of the lower air-passages from the pharynx. The contraction of the mylohyoid muscles, which accompanies the first act of deglutition, can be seen to be followed almost immediately (at an interval of 0.07 seconds) by an elevation of the larynx. Observations on animals and on men in whom the parts involved have been extensively exposed by operation, have shown that this elevation of the larynx is accompanied by a closure of the glottis as well as of the superior opening of the larynx. That this latter is important is shown by the fact that the nucous membrane of the vestibule of the larynx is extremely irritable, so that any particle of food falling on it will cause a spasmodic cough. It has generally been supposed to be carried out by a movement of the dorsum of the tongue backwards, effected by the stylo- and palato-glossal muscles. In consequence of this movement, combined with contraction of the muscular fibres in the aryteno-epiglottidean folds, the expanded portion of the epiglottis has been supposed to be folded like a lid over the opening of the larynx, thus furnishing a sloping floor for the passage of the food. But observations by Anderson Stuart ${ }^{1}$ have shown that this bending over of the epiglottis probably does not occur, that the epiglottis remains erect, and that the food passes not over its upper (anterior) but over its lower (posterior) surface; the anterior resting against the root of the tongue. Magendie and Schiff had already pointed out that extirpation of the epiglottis caused very little disturbance to an animal, which could swallow both solids and fluids as well after the operation as before.

The laryngeal opening is bounded in front by the epiglottis, behind by the tips of the arytenoid cartilages, and at the sides by the aryteno-

$$
{ }^{1} \text { Proc. Roy. Soc. Loment, 1891, vol. 1. 1. } 323 .
$$


epiglottidean folds, in which are contained the two small cartilages of Santorini and Wrisberg. During normal respiration this cavity is widely open, and the arytenoids lie against the posterior wall of the pharynx. When deglutition takes place, the arytenoid cartilages leave the posterior wall of the pharynx, are rotated and moved inwards and forwards, with their internal faces in contact. In consequence of this movement of the hinder boundaries of the laryngeal opening forwards, this opening assumes the form of a triradiate fissure, the vertical limb being short, while the transverse limb is rounded, owing to the pulling inwards of the margins of the epiglottis. Round the central point of the triradiate fissure three prominences are to be observed, namely, the cushion of the epiglottis, and on each side of this the cartilages of Wrisberg. Thus, according to Stuart, closure of the larynx is effected chiefly by the pulling of the posterior boundaries of its orifice forwards, and not, as often described, by the movement of its anterior boundary, the epiglottis, backwards.

The muscles which are actively involved in this closure are the external thyro-arytenoid and arytenoid, and the ary-epiglottidean and the lateral crico-arytenoid muscles. The external thyro-arytenoid muscle, which ruus from the thyroid cartilage in front to the outer surface of the arytenoid cartilage behind, pulls the latter forcibly forwards and rotates it inwards. It is therefore, with the arytenoideus muscle, the chief factor in the closure of the larynx. The function of the ary-epiglottidean muscles is to make tense the folds of mucous membrane which bound the entrance on each side. The lateral cricoarytenoids aid the thyro-arytenoids by pulling the bases of the arytenoid cartilages forwards and rotating them inwards.

By the action of these muscles, both the true and false vocal cords are approximated. This closure of the glottis is not, however, essential for the carrying out of deglutition. Longet showed that swallowing was performed without passage of food into the windpipe, even if the vocal cords were held apart by passing a pair of forceps up between them from an opening in the trachea.

The approximation of the posterior to the anterior boundary of the laryngeal opening is only rendered possible by the elevation of the whole larynx under the hyoid bone, which is chiefly effected by the thyro-hyoid muscles. Hence the act of deglutition is rendered impossible by fixation of the larynx.

Thus the openings into the posterior nares and into the larynx being both closed, and the entrance from the pharynx into the œsoplagus widened by the pulling forward of the arytenoid cartilages and the contraction of the palato- and stylo-pharyngeus muscles, the bolus is shot rapidly past these openings into the region of the middle and inferior constrictors of the pharynx. If it is large, it is seized by these muscles and thrust down into the œsophagus by the successive contraction of their fibres from above downwards. With a smaller bolus, however, or when liquids are swallowed, the movement of the back part of the tongue is sufficient to propel the substance past the constrictors and through the oesophagus to its lower end, or even through the cardiac orifice into the stomach, as shown by Kronecker and Meltzer. ${ }^{1}$ In such cases, the rapid passage of the bolus is followed by a contraction of the constrictors and by a peristaltic contraction of the œsophagus, which ${ }^{1}$ Arch. f. Physiol., Leipzig, 1880, S. 446 ; and 1883, Suppl., S. 328. 
serve merely to drive any particles of food that may be still adherent to the walls of the tube, down into the stomach (Figs. 181-184).

This view, which was first put forward by Kronecker and Falck, ${ }^{1}$ is supported by the fact that in cases where corrosive Huids have been swallowed, the œsophagus has been attacked only at three points, where it is narrowed, and not along its whole length, as would be the case if the Huid were propelled gradually down the tube by the peristaltic contraction of its walls.

The anthors showed, further,

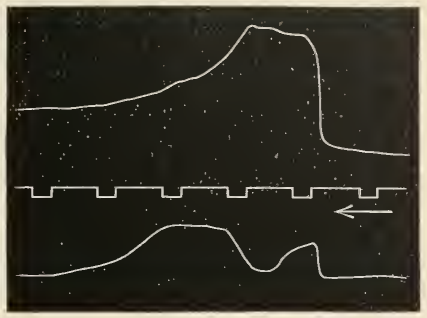

FIG. 181. that the act of $\mathrm{s}$ w a $110 \mathrm{w}$ in $\mathrm{g}$ causes an elevation of pressure of 20 cms. water in the œsophagus, and regard this elevation of pressure, due (chiefly) to the contraction of the mylohyoid muscles, as the active force in

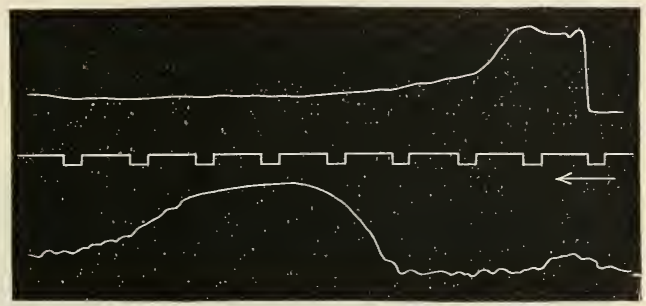

FIG. 182. the onward propulsion of the food.

The movement of the bolus through the fauces and phar y $\mathrm{x}$ is carried out entirely by striated muscles. In the œsophagus the bolus, if too large to be shot through the lax tube, is carried down more slowly by the peri-

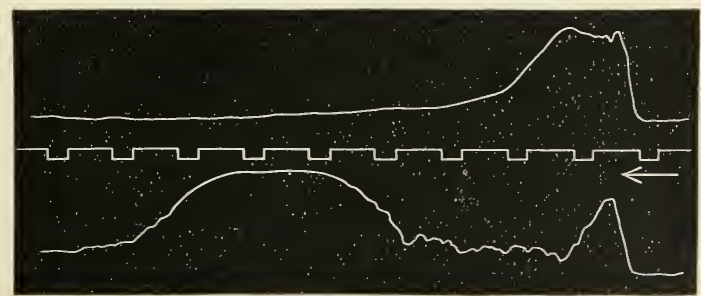

FIG. 183.

Figs. 181-183. - Curves obtained during swallowing by placing two rubber balloons, one (the upper curve) in the pharynx, the other (the lower curve) in the osophagus. In Fig. 181 the second balloon was 4 cms. ; in Fig. 182, 12 cms. ; and in Fig. 183, $16 \mathrm{cms}$. from the upper end of the esophagus. In each curve it will be noticed that the excursion of the upper lever is followed immediately by an excursion of the lower lever (due to passage of the swallowed fluid and the consequent rise of pressure), and then, after an interval of time varying with the distance of the second from the first balloon, by another rise of the esophagcal lever due to the contraction of the wall of that tube, i.e. to the advancing peristaltic waves,-Kronecker and Meltzer.

staltic contraction of its walls. These walls, in the cervical part of the osophagus, are composed of striated muscle. In the upper

${ }^{1}$ Arch. f. Physiol., Leipzig, 1880, S. 296, 446. 
part of the thorax, striated and unstriated muscular fibres are associated together, while the lower part consists entirely of unstriated muscle. Corresponding to these differences in structure, Kronecker and Meltzer have found differences in the duration and rapidity of propagation of the contraction wave in each part. Thus, in the first section (cervical) of the œsophagus, which is about $6 \mathrm{cms}$. long, the contraction wave lasts 2.0 to 2.5 seconds. From the beginning of this contraction to the commencement of the contraction of the second part, an interval of 1.8 seconds elapses. The contraction of the second part, which is about $10 \mathrm{cms}$. long, lasts 6 to 7 seconds. The contraction of the third part, the length of which these observers could not determine accurately, lasts about 9 to 10 seconds, and commences 3 seconds after that of the second part. Each of these parts seems to contract almost simultaneously throughout its whole length. Kronecker and Meltzer ${ }^{1}$ consider that in all probability the contraction travels from one end of the section to the other, but that the rapidity of propagation of the wave was too rapid to be accurately determined by the means at their disposal.

When a second act of deglutition succeeds the first within a sufficiently short interval, the nervous changes which originate the
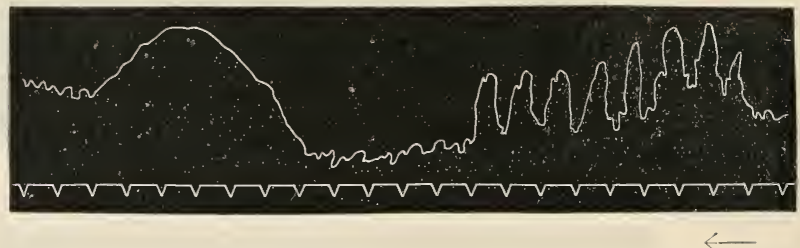

Fig. 15t. - Curve obtained by placing a balloon in the asophagus 12 cms. from its upper end. In this case it is seen that a series of swallowing movements is only followed by one contraction, showing that the osophaseal walls are inhibited during the passage of the swallowed fluid.-Kronceker and Meltzer.

pharyngeal movements of the second act, or rather the second act itself, causes a reflex inhibition of the œsophagus-contraction, which would otherwise follow the first act. The osophagus thus remains in a lax condition, and allows of the free rapid shooting of the food downwards until the movements of deglutition have come to an end, when the peristaltic contraction of the osophagus oceurs and sweeps all remaining adherent particles of food into the stomach.

Under normal circumstances the circular fibres of the lower end of the cesophagus, which form a ring round the cardiac orifice of the stomach, are in a state of tonic contraction, in order to prevent the regurgitation of food from the stomach. When one mouthful of food or liquid is swallowed, it may be either squirted directly through the opening into the stomach, or it may remain at the lower end of the œsophagus until the following peristaltic wave forces it through the orifice. When several acts of deglutition succeed one another, the cardiac sphincter seems to share in the inhibition of the osophageal walls, so that it offers no resistance to the direct propulsion of food from the mouth into the stomach.

1 Arch.f. Physiol., Leipzig, 1883, Suppl., S. 328. 
The following table is intended to show the time relations of the contractions of the chief muscles engaged in deglutition, as determined by Kronecker and Meltzer, and by Marckwald:-

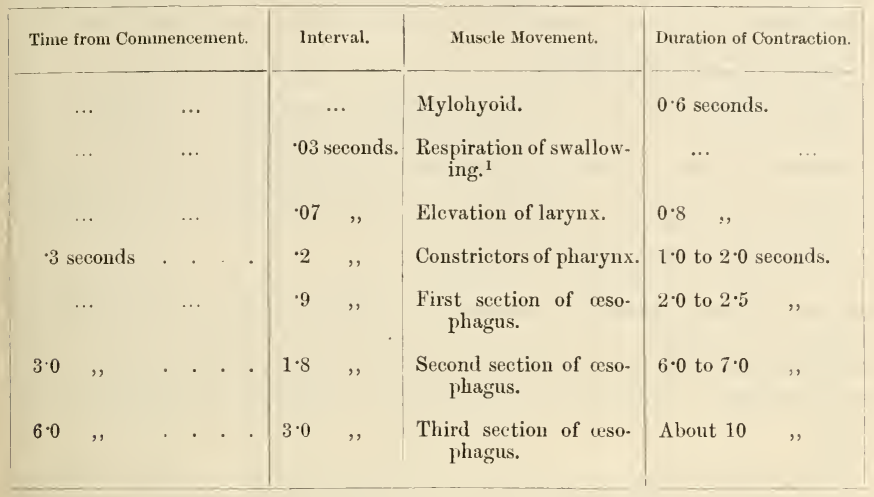

${ }^{1}$ See 1. 314 .

Thus a bolus of food, if carried down entirely by the peristaltic contractions of the gullet, reaches the stomach six seconds after leaving the mouth. As a rule, on auscultating over the lower end of the nesophagus, two sounds are heard to follow one swallowing action. The first sound is coincident with the passage of the bolus, and occurs immediately after this has left the mouth. A second sound is then heard about six seconds later, as the peristaltic contraction reaches the cardiac orifice and forces through it food particles and bubbles of air which had adhered to the walls of the tube during the passage of the bolus.

The process of deglutition has been lately studied by Cannon and Moser ${ }^{1}$ in unanæsthetised animals, by the use of the Röntgen rays, using liquid or semiliquid food, to which the opaque subnitrate of bismuth had been added. For a solid bolus bismuth was administered in gelatin capsules. These observers find that the rapid injection or squirting of the food, by the action of the mylohyoids, only occurs in man and the horse, and then only in the case of liquid food. With seniliquid or solid food the greater part of the act is carried out by the peristaltic contractions of the œsophagus in all the animals investigated (fowl, eat, dog, horse, and man). Thus in the dog the total time for the descent of a bolus is from four to five seconds. The food is always propelled rapidly in the upper œsophagus and moves more slowly below. This rapid movement is frequently continued further with liquid food. No distinct pause was observed when the movement of the bolus changed from the rapid to the slower rate.

The nervous mechanism of deglutition. - Although swallowing can be started by the will, and is therefore to that extent voluntary, as a whole it is a reflex act, and cannot take place unless sone stimulus be applied to the fauces. When we swallow voluntarily we supply the necessary initial stimulus, either by touching the fauces with the tongue

\footnotetext{
${ }^{1}$ Am. Jouru. Physiol., Boston, 1898, vol. i. 1. 435.
} 
itself, or by forcing a certain amount of saliva into the fauces. The afferent impulses for this reflex act travel by branches of the second division of the fifth, and by the glosso-pharyngeal nerve and the pharyngeal branches of the superior laryngeal nerve. By excitation of the latter nerve, it is possible to evoke either a single act or a whole series of acts of deglutition.

The centre of deglutition lies in the medulla oblongata, in the neighbourhood of the nucleus of the vagus nerve. According to Marckwald, ${ }^{1}$ it can be destroyed without injuring the adjacent respiratory centre. After such destruction, stimulation of the superior laryngeal nerve causes total relaxation of the diaphragm, unaccompanied by the small twitches (respirations of swallowing) which accompany or precede the normal acts of deglutition.

The efferent impulses from the centre pass by the hypoglossal nerve to the muscles of the tongue, by the fifth to the nylohyoid muscle, by the glosso-pharyngeal, the pharyngeal branches of the vagus, the fifth, and the spinal accessory nerves, to the muscles of the fances and pharynx. The closure of the larynx is effected by impulses which travel through the superior and inferior laryngeal branches of the vagus.

The distribution of nerves to the nophagus varies in different animals. In man the upper part of the œsophagus receives branches from the recurrent laryngeals, the lower part from the pulmonary and œsophageal plexuses of the vagus. In the rabbit and also in the guinea-pig some of the fibres to the upper portion of the œsophagus run in a distinct nerve, which rises from the trunk of the vagus and passes upward to its destination. ${ }^{2}$ In the horse and dog the innervation of the lower segment of the osophagus resembles that in man. Most of the fibres to the upper segment are derived, however, from a small branch of the pharyngeal nerve, which travels alongside of the œsophagus down into the chest, sending numerous branches into the muscular wall on its way.

The effects of section of the osophageal nerves vary in the different segments of this tube. Whereas the upper part becomes permanently relaxed, the lower segment, including the cardiac sphincter, enters into a condition of tonic contraction, which may last several days. These results suggest that, although the contraction of the wesophagus resembles in many particulars the autocthonous, peristaltic contractions of other muscular tubes, such as the intestine and ureter, it will probably differ from these in being dependent on nervous influences from outside. As a matter of fact, Mosso ${ }^{3}$ has shown that the propagation of contraction down the œsophagus is a reflex act, and requires the integrity of the nervous connections of the œesophagus with the centre of deglutition. If the œsophagus be ligatured or cut across, waves of contraction, originated from the pharynx, pass across the lesion from one separated end to the other, as before. On the other hand, section of the nerves going to the osophagus abolishes its peristaltic contractions. The peristaltic contraction of the osophagus, therefore, like that of the pharynx, is a reflex started from the stimula-

1 "The Movements of Respiration," trans. by Haig, London, 1888, p. 134.

2 Steiner, Verlandl. d. naturh.-med. Ver. $\approx u$ Heidelberg, 1879 , N. F., Bd. ii. S. 183 (quoted by S. Mayer, Hermann's "Haudbuch," Bd. v. S. 424).

${ }^{3}$ Untersuch. $\approx$ Naturl. d. Nensch. $u$. d. Thiere, 1876, Bd. xi. S. 331. 
tion of the palate and fauces. It is interesting to note that it can only be inaugurated in this way, and that stimulation applied to the mucous surface of the csophageal tube is without effect and produces no contraction.

There is an important interdependence between the functions of respiration and deglutition. The small inspiratory movement depends on intra-bulbar connections between the two centres. ${ }^{1}$ More important, however, is the inhibition of respiration which occurs during swallowing. Without this inhibition, food particles might be drawn by the ingoing current of air into the bronchi and lungs, and would there set up inflammation. This inhibition of respiration is a reflex act, and is carried out by the intermediation of the glosso-pharyngeal nerve. As has been already mentioned, in dealing with the respiratory movements, ${ }^{2}$ excitation of the central end of the glosso-pharyngeal nerve at once causes a cessation of respiration, in whatever phase it may happen to be. This cessation lasts for five or six seconds, i.e. a sufficient length of time for a whole series of acts of deglutition. Respiration then recommences, and the inhibition cannot be prolonged by continuing the stimulation of the glosso-pharyngeal nerve.

\section{Movemfnts of the Stomach.}

The movements of the stomach have a twofold purpose. In the first place, they serve to churn up the ingesta with the gastric juice, and, in the second place, to propel the semi-digested chyme onwards into the duodenum and intestines.

The muscular coat of the stomach consists of three layers-an outer longitudinal layer, continuous with the longitudinal fibres of the œsophagus; a middle or circular; and an inner incomplete layer of oblique fibres, passing from the left of the cardiac orifice, and merging at the pyloric end in the middle or circular coat.

The pyloric part of the stomach is divided by a special thickening of the circular fibres, the "transverse band" 3 or "sphincter antri pylorici," ${ }^{4}$ into two parts, the antrum, between the band and the pylorus, and the pre-antral portion or middle region of the stomach.

If the stomach be examined in a fasting animal, care being taken to avoid stimulation by cooling or drying on exposure to the air, it will be found to be in a state of rest in slight tonic contraction. Owing to this contraction, the cavity of the stomach is small, the walls are fallen together, and the mucous membrane, which cannot retract with the muscular coat, is thrown into rugie. When food is taken, the walls of the stomach relax in proportion to the distension of its cavity, each act of swallowing being, inoreover, accompanied by a special dilatation of the cardiac orifice, carried out mainly by the radiating longitudinal fibres in the outer muscular coat. Shortly after the taking of food irregular contractions of the gastric wall begin, and these become more active as digestion advances. According to Beaumont's observations on Alexis St. Martin, ${ }^{5}$ these movements are of such a character as to pro-

1 Marekwald, loc. cit.

2 Vide p. 305.

${ }^{3}$ William Beaumont, "Experiments and Observations on the Gastric Juice and the Physiology of Digestion," edited by William Combe, Edinburgh, 1838, p. 107. S. 7 .

${ }^{4}$ Hofmeister and Schiitz, Arch. f. exper. Puth. $u$. Pharmakol., Leipzig, 1586, Bel. xx.

5 Beaumont, op. cit.

VOL. II. -2 I 
duce a movement of the gastric contents, from the cardia along the greater curvature to the pylorus, and then back agair along the lesser curvature. These movements, together with subsidiary currents which are set up, effect a thorough mixing of food and gastric juice. After a time, which depends on the nature of the food taken, the stomach movements acquire more of the characters of a peristaltic contraction. We may observe a deep groove, produced by the contraction of the circular muscles of the stomach, midway between pylorus and cardia (the "transverse band"). This groove or constriction travels down towards the pylorus, so tending to heap up the food, and to press it against the pyloric opening. Finally, on the arrival of one of these waves at the pylorus, a partial relaxation of this opening is brought about, partly by inhibition of the circular sphincter pylori, partly by contraction of the longitudinal muscular fibres. In consequence, the fluid portion of the food in contact with the opening is pressed through this opening into the duodenum. At the beginning of digestion only fluids can pass from stomach into duodenum. As digestion advances, the propulsive contractions of the stomach, especially at its pyloric end, become more and more marked, and the relaxation of the pyloric sphincter more complete, so that, towards the end of digestion, undigested pieces of food can be forced through, and the stomach may empty itself entirely.

The description of the stomach movements given by Hofmeister and Schuitz, is very similar to that of Beaumont. These observers studied the movements of the excised stomach, placed in a warm moist chamber. They describe a typical movement as consisting of two phases. At first a constriction of the circular fibres starts near the cardia and passes towards the pylorus, gradually increasing in strength until it reaches the region in front of the antrum ("preantral constriction"). The transverse band now contracts highly, while the preantral constriction is relaxing, shutting off the antrum from the rest of the stomach, and this is followed by a general contraction of the muscles of the antrum.

Cannon, ${ }^{1}$ who has investigated the movements by observing the stomach after administration of bismuth, by means of the Röntgen rays, has shown that the movements observed by Beaumont and the two last-mentioned physiologists were hardly physiological, and were produced by the abnormal character of the irritant (thermometer tube in Beaumont's case), or other abnormal conditions of the stomach.

According to Cannon (whose results confirm those of Rossbach, ${ }^{2}$ obtained by direct observation of the exposed viscus in dogs), movements of the stomach commence a few minutes after taking food, and consist of slight constrictions, which appear near the middle of the stomach, and course slowly towards the pyloric end. As digestion goes on, the antrum elongates, and the contractions grow stronger, but do not entirely divide the cavity until the stomach is nearly empty. A wave takes about 30 seconds (in the cat) to move from the middle of the stomach to the pylorus. The waves recur regularly at intervals of 10 seconds, so that when one wave is beginning several others are running in order before it, causing the stomach to be bulged out between rings of constriction. The fundus during this time acts as a reservoir for the food, and merely squeezes its contents gradually into the pyloric part. Since no churning movements take place in the fundus, salivary digestion can go on in this region for a considerable time, before it is stopped by the acid gastric juice.

1 Am. Journ. Physiol., Boston, 1898, vol. i. p. 359.

${ }^{2}$ Deutsches Arch.f. Klin. Med, Leipzig, 1890, Bd. xlvi. S. 296. 
The pylorus does not open at the approach of every wave, but only at irregular intervals. The arrival of a hard morsel inhibits the opening of the sphincter, and thus interferes with the passage of the already digested food. Thus the motor functions of the stomach, which result in the mixing, trituration, and expulsion of the food, are carried out almost entirely by the pyloric half of the stomach.

von Mering ${ }^{1}$ has shown that when fluids alone are taken by the mouth, their first discharge into the duodenum may occur within ten minutes after the time at which they reach the stomach, and the stomach may completely empty itself within twenty to twenty-five minutes after the taking of fluid. This observer also pointed out that a state of repletion of the small intestines reflexly slows the evacuation of the stomach. The same effect may be produced by psychical excitement.

With regard to the causation of the gastric movements we know very little. The stomach can be separated from all its nervous connections, and indeed cut out of the body, without abolishing the contractions. These, therefore, must have their origin in the walls of the stomach itself. A number of small ganglia are to be found in the walls of this organ, and have been regarded as local centres for the production and co-ordination of the contractions. ${ }^{2}$ It is more probable, however, that here, as in the heart, the contraction wave is originated and propagated in the muscular tissue itself. That the stimulus to contraction is not in the main afforded by the distension of the stomach, is shown by the fact that the movements become more vigorous as digestion proceeds, and as the stomach empties more of its contents into the duodenum. It has been thought, therefore, that the chief exciting cause of the movements is the acidity of the gastric contents, which increases steadily with the progress of digestion.

Although contractions of the stomach can be produced locally, this local reaction is aided by reflex impulses carried out through the intermediation of the central nervous system. The stomach is supplied with nerves from two sources, namely, from the vagi, and from the dorsal region of the spinal cord through the sympathetic chain and splanchnics. The exact nature of the impulses carried by these nerves cannot yet be regarded as finally settled. All observers, however, agree in describing the vagus as a motor nerve for the stomach. According to S. Mayer, ${ }^{3}$ the results of exciting the peripheral end of the vagus in the neck may be one of three-(1) A contraction of the cardiac end, which gradually advances towards the pylorus; (2) the groove present in the region of the transverse band deepens, and similar constrictions appear in other parts of the stomach; (3) the whole muscular wall enters into a state of tonic contraction, which comes on slowly and lasts a considerable time.

More or less similar results have been obtained by Longet, ${ }^{4}$ Goltz, ${ }^{5}$ v. Braam Houckgeest, ${ }^{6}$ Openchowski, ${ }^{7}$ Morat, ${ }^{8}$ and others. According to v. Braam Houckgeest and Morat, the motor effects are obtained more

1 Verhandl. d. xii Cong. $f$. innere Med., Wiesbaden, 1893.

2 Cf. Openchowski, Arch. f. Physiol., Leipzig, 1889, S. 549.

${ }^{3}$ Hermann's "Handbuch," Bd. v. (2), S. 431.

4 "Traité de physiologie," 3rd ed., tome i. p. 148 (quoted by S. Mayer).

${ }^{5}$ Arch. f. d. ges. Physiol., Bonn, 1872, Bd. vi. S. 616.

6 Ibid., 1872, Bd. vi. S. 266.

7 Arch. f. Physiol., Leipzig, 1889, S. 549.

${ }^{8}$ Arch. de physiol. norm. et path., Paris, (5), tome v. p. 142. 
certainly and promptly if the animal be in state of digestion, or if the splanchnic nerves be previously divided.

Langley ${ }^{1}$ has recently shown that the vagus conveys both motor and inhibitory impulses to the stomach. If curari be given, stimulation of the vagus causes, as a rule, contraction of the osophagus and cardiac end of the stomach. After administration of atropin, stimulation of the vagus causes relaxation of the cardiac end, followed, on cessation of the stimulus, by a strong contraction. At the pyloric opening, the general result of vagal stimulation is contraction, though occasionally dilatation of the orifice may be observed.

According to Schiff, motor fibres also reach the stomach from the sympathetic chain, by way of the splanchnics. Morat also observed one case in which the rhythmical contractions of the stomach (and intestine) were augmented on stimulation of the splanchnics. As a rule, however, excitation of these nerves caused dininution of the tonus, as well as of the rhythmic cortractions of the stomach.

The movements of the stomach, however excited, may be inhibited reflexly by stimulation of the central end of the vagus, or indeed of any sensory nerve. Cannon noticed that any mental disturbance (anger, pain) of the cats under investigation, at once checked contractions of the stomach, which had been induced by feeding the animal.

According to Head, ${ }^{2}$ the sensory fibres to the stomach in man probably run in the sixth, seventh, eighth, and ninth dorsal nerve-roots, the cardiac end being especially associated with the sixth and seventh, and the pyloric end with the ninth roots.

Vomiting.-Under normal circumstances, the contractions of the stomach wall are directed solely to the mixing of the food with the gastric juice, and to the furtherance of the passage of chyme into the duodenum. If, however, irritating substances have been taken or the stomach over-distended, it is important that this organ should have some means of ridding itself of its contents, and this is mainly accomplished by the contraction of the abdominal muscles and diaphragm pressing against it. Vomiting is usually excited by the direct contact of irritants with the gastric mucous membrane, but it may be also caused by reflex irritation from other parts of the body, and especially of the alimentary tract, as is the case with the vomiting caused by acute intestinal obstruction. It may also occur in the colic produced by the passage of a renal or biliary calculus, or from inflammation of the peritoneum or any of the abdominal viscera. Besides this reflex causation, vomiting may be brought about directly by events occurring in the central nervous system. Thus it may be excited by emotions or smells, and is a very frequent symptom of diseases of the brain, such as tumour and inflammation. Sea-sickness, although complex in origin, is probably chiefly determined by reflex stimulation originating in the semicircular canals.

Vomiting is generally preceded by a feeling of nausea, which is accompanied by copious salivation. The saliva which is swallowed carries down with it a quantity of air, which distends the stornach and may assist in the subsequent dilatation of the cardiac orifice. Retching movements then occur. These consist essentially in deep inspiratory efforts, attended with closure of the glottis. Since no air can be drawn

1 Journ. Physiol., Cambridge and London, 1898, vol. xxiii. p. 407.

2 Brain, London, 1893, vol. xvi. p. 66. 
into the lungs, the contraction of the diaphragm drags in the lower ribs and presses on the stomach, and at the same time air is drawn into the pharynx and into the osophagus. After a few ineffectual efforts of this description, a sudden, violent expiratory contraction of the abdominal muscles takes place, accompanied by a contraction of the diaphragm. The stomach is thus compressed between the abdominal muscles and diaphragm. Immediately before this effort the cardiac orifice of the stomach is brought close up to the opening in the diaphragm by a contraction of the longitudinal fibres at the lower end of the oesophagus, and is dilated by the contraction of these fibres and the relaxation of its sphincter. Thus a way is opened for the escape of the contents of the stomach, and these are pressed out by the contraction of the abdominal muscles, and to some extent by that of the muscular wall of the stomach itself, and are ejected forcibly up the osophagus and out through the mouth. At the same time the soft palate is raised, and the posterior pillars of the fances approximated, so as to shut off, though in many cases ineffectually, the passage through the nose. A strong expiratory effort is made immediately after the passage of the gastric contents, in order to prevent any remnants of the vomit passing in to the larynx.

If the movements of the stomach be observed, either by opening the abdomen (Openchowski), or by means of the Röntgen rays (Camnon) after the administration of apomorphine, the first change noticed is an extreme relaxation of the cardiac end. Then a deep constriction starts about $3 \mathrm{cms}$. below the cardia, and, growing in strength, moves towards the pylorus. On reaching the transverse band, this contracts tightly, and a wave of contraction passes over the antrum. This is followed by ten or twelve other waves, and then the firm contraction at the beginning of the antrum divides the stomach into two parts, one tightly contracted, and the other, the fundus, relaxed and flaccid. A sudden contraction of the diaphragm and abdominal muscles, accompanied by the opening of the cardiac orifice, now forces the gastric contents into and through the œesophagus.

During vomiting there is generally a discharge of bile from the gall bladder into the duodenum. A little of this may be pressed through the closed pyloric orifice, so that, after the emptying of the stomach, the later efforts of vomiting may bring up a little bile-stained mucus.

It was long a subject of discussion whether the contraction of the stomach or that of the abdominal wall was the more essential factor in the act of vomiting. Magendie ${ }^{1}$ thought he had decided the question when he pointed out that vomiting might be produced by the injection of tartar emetic into the blood, even after the stomach had been replaced by a bladder. It was shown subsequently, however, that this was only true if the bladder were tied into the lower end of the oesophagus. If the cardiac orifice be left intact, and merely the greater part of the stomach replaced by the bladder, vomiting cannot be brought about. ${ }^{2}$ This fact proves that there are two necessary factors in vomiting, nainely, active dilatation of the cardiac orifice and compression of the stomach. So far as the latter event is concerned, it is probable that Magendie was correct, since, in a curarised animal, it is impossible to

1 "Mémoire sur le vomissement," Paris, 1813. Cited by S. Mayer.

${ }^{2}$ Tanbini Omodei, Ann. univ. di med. e chir., Milano, 1824, (S. Mayer); Schiff, Untersuch. z. Naturl. d. Mensch. u. d. Thiere, 1867, Bd. x. S. 353. 
excite vomiting, though, after paralysis of the abdominal muscles alone, the expulsion of the gastric contents can be still brought about by the agency of the diaphragm. The intrinsic contraction of the stomach itself, though unable to empty this organ, as in vomiting, can cause the escape of gas or of small quantities of food or of fluid, as occurs in pyrosis, or water-brash.

Since vomiting involves the co-ordinated activity of a number of muscles, voluntary as well as involuntary, it is evident that the proper carrying out of this process must depend on the integrity of the central nervous system. Vomiting is generally said to occur through the agency of a vomiting centre in the medulla oblongata. This has been thought to be closely related in situation to the respiratory centre; ${ }^{1}$ and it is probable that, like the latter, it is intimately related to the central connections of the afferent and efferent fibres of the vagus.

Vomiting can be excited reflexly in many ways. The most usual stimulus is one affecting the stomach. Both in this case, and in all other cases when the exciting cause is some affection of the abdominal viscera, the afferent impulses are probably carried by the vagi. Vomiting can also be excited reflexly through the olfactory, the fifth and glosso-pharyngeal, or the auditory nerve, or directly from the higher parts of the brain. The efferent channels of the act are:-The vagus, to stomach, cardiac sphincter, osophagus, larynx; through chorda tympani, and probably tympanic branch of glosso-pharyngeal to salivary glands; through phrenics and various spinal nerves to respiratory muscles.

Emetics, or substances which excite vomiting, may act either reflexly from the stomach (e.g. mustard and water), or directly on the centre (apomorphin), or in both ways, as in the case of tartar emetic.

\section{Movements OF THE INTESTINES.}

After the chyme has arrived in the duodenum, it has to be carried slowly from one end of the intestine to the other, in order that the digestive juices poured into different parts of the gut may have time to complete their action on the food-stuffs, and that all assimilable constituents may be absorbed. Thus only the indigestible portions of food, together with a certain amount of epithelial débris and mucus, arrive at the lower end, to be extruded in the act of defrecation.

The investigation of the mechanism of intestinal movements is beset with many difficulties, so that we still find a considerable divergence among various authors of repute, even as to the character of the movements which are the subject of investigation.

The following are the chief factors which contribute to this divergence of results, and which must be taken into account in working on the intestinal movements, namely, the varying reactions of the intestines in different animals, the disturbing action of drugs and anæstheties, but, above all, the fact that each segment of the intestines is subject to augmentor and inhibitory influences, partly originating in other portions of the gut and transmitted along the walls of the intestine, partly

${ }^{1}$ Grimm, Arch. f. d. ges. Physiol., Bonn, 1871, Bd. iv. S. 205. The identity has been contested by Harnack on pharmacological grounds; Arch.f. exper. Path. u. Pharmakol., Leipzig, 1874, Bd. ii. S. 154. 
originating in the intestine or elsewhere and transmitted reflexly through the central nervous system.

As is well known, the muscular wall of the small intestine consists of two layers of unstriated muscular fibres separated by a thin layer of connective tissue, in which is embedded a rich plexus of ganglion cells and non-medullated nerve fibres, known as Auerbach's plexus. Fine fibres from this plexus form networks round the individual fibres of the two layers. These layers are separated from the mucous membrane by the submucosa, a tough, fibrous membrane which, according to Mall, ${ }^{1}$ determines the limit of distension of the intestine. The two muscular coats are so closely bound together, that it is difficult, by inspection, to deternine whether any given change of form of the intestine is caused by a relaxation of one coat or by a contraction of the other coat. It is possible, however, to record the contractions of the two coats independently; when it is found that they nearly always contract simultaneously, the function of the longitudinal coat being to aid the contraction of the circular, and not to assist in the relaxation of the intestine.

The contraction of the longitudinal or circular coats may be conveniently registered by means of the enterograph (Fig. 185).

In a brass plate $a a$, two steel needles, $b$ and $c$, are fixed in a siot; $b$ can be shifted nearer to or farther from $c$ by loosening its fixing screw ; $c$ is

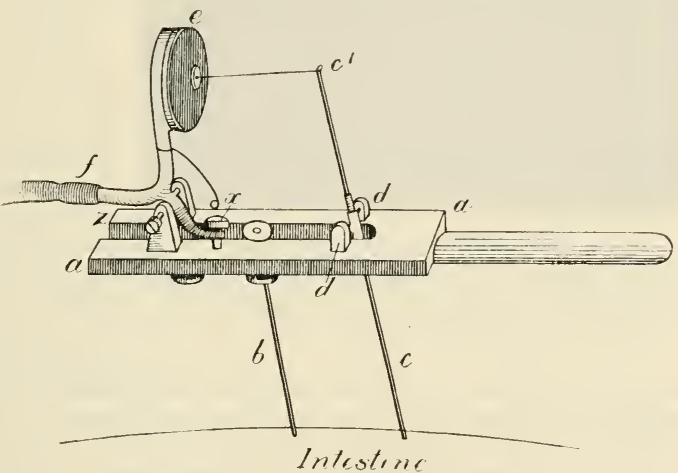

FIG. 185. prolonged through the slot, and revolves as a lever round the axis $d d$. The upper end $c^{\prime}$ is fastened by a thread to the disc on the rubber of the tambour $e$, which communicates by a tube $f$, with a piston recorder. The lower ends of $b$ and $c$ are pierced with holes. Through these holes pass fine threads, which are carried by a needle through the outer layers of the intestinal wall, and fastened. The muscle fibres running from $b$ to $c$ can only contract by pulling $c$ towards $b$. This causes a movement of the upper end of $c$ in the opposite direction, and a consequent pull on the membrane of the tambour, which is registered by the piston recorder. The distance of $e$ from $c^{\prime}$ and the tension on the muscle fibres between $b$ and $c$ can be regulated by means of the screw $x$, or roughly by moving the tambour in the slot $z$.

The contractions of the circular coat alone can be registered by introducing an indiarubber bulla, which is slightly distended with air or water, in to the interior of the intestine through an opening in the free border, and connecting

${ }^{1}$ Johus Hopkins Hosp. Rep., Baltimore, 1896, vol. i. p. 37. 
the bulla with a recording tambour. Those of the longitudinal coat alone can be registered by an ingenious contrivance devised by New, ${ }^{1}$ consisting of two glass tubes sliding the one within the other, so arranged that contractions of the longitudinal coat diminish the common cavity and raise the lever of a recording tambour, while contractions of the circular coat are unable to affect it.

Nature of the intestinal movements. - In order to avoid the disturbing effects of drying and cooling on the intestines, it is best to open the abdomen under a bath of warm normal saline (von Braam Houckgeest). ${ }^{2}$ The appearance of the intestines will vary according to the state of the animal. If it has not received food for two or three days, the intestines will be seen to be anæmic, motionless, and in a state of tonic contraction. After food, however, the intestines are hyperæmic, relaxed (i.e. thicker and shorter), and the various loops present the swaying movements which were described by Ludwig ${ }^{3}$ as Pendelberegungen. Very much the same appearance will be observed also in

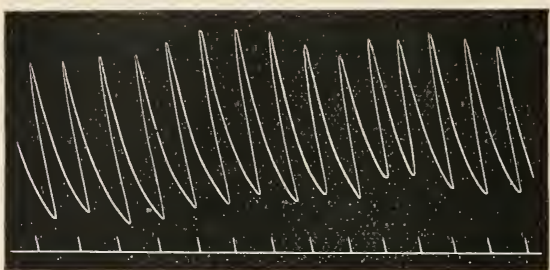

FIG. 186. - Rhythmical contractions of circular coat of small intestine. A rubber balloon distended with air was placed in lumen of gut, and connected with a piston recorder. Time marking $=$ six-second intervals. Contraction causes upward movement of lever. fasting animals, if both splanchnic nerves have been previously divided (Fig. 186).

1. The swaying movements have been generally regarded as occasioned by contractions of the longitudinal muscles, since the translatory movements of the loops are more apparent than the changes in their calibre. If, however, we record the movements of the intestine by connecting its interior to a piston recorder, or by inserting a small balloon, which is connected with a tambour, into the lumen of the gut, we find in most cases that the wall is undergoing a series of rhythmical contractions, repeated regularly ten to thirteen times in the minute, so that each individual contraction lasts from $5 \frac{1}{2}$ to 6 seconds. This intestinal rhythm is remarkably independent of external conditions, although the amplitude of the contractions may be angmented or diminished. Thus, distension of the intestine or of a rubber capsule in its interior causes increased height of contraction, or may evoke contractions, if these were previously absent. The contractions are better marked in a fed animal, or when the intestines are hyperæmic. They may be diminished or abolished by shutting off the arterial blood supply to the intestines. They are propagated rapidly down the intestine (2 to $5 \mathrm{cms}$. per second), but may originate at any point of the gut, so that an exact determination of their rate of propagation is very difficult. They are as well marked in isolated loops as in those which retain their functional continuity with the rest of the intestine. Both coats of the intestine act together in these contractions. In the normal intestine these waves

1 "Proc. Physiol. Soc.," March 18, 1899, Journ. Physiol., Cambridge and London, vol. xxiv.

${ }^{2}$ Arch. f. d. ges. Physiol., Bonn, 1872, Bd. vi. S. 266.

3 "Lehrbuch d. Physiologie," Aufl. 2, Bd. ii. S. 615. 
nearly always travel from above downwards. After paralysis of the local nervous mechanism (Auerbach's plexus) by nicotin or cocain, the waves are still present in undiminished force, but now travel indifferently in either direction. They therefore originate in the muscle fibres, and probably are transmitted from one muscle cell to the next, i.c. the contractions are myogcnic and myodromic.

The post-mortem "vermicular" contraction described by Engelmann in the rabbit is probably merely an exaggerated wave of the type just described, since it may be excited by local stimulation, and travels equally well in either direction at a rate of 3 to 4 cms. per second.

Mall, however, would place this form of contraction in a class by itself, and distinguishes, therefore, three types of contraction, namely, pendular or rhythmic, vermicular, and peristaltic.

2. True physiological peristalsis is a slow process. According to Cash, ${ }^{1}$ it takes in the cat about an hour and a half to travel from one end of the intestine to the other. On observing the intestines in an animal, after division of the splanchnic nerves, such waves of constriction may be seen passing slowly down the gut, or, if absent, may be excited by mechanical stimulation. They may be studied by introducing a bolus of wax, or cotton-wool and vaseline, into the intestine at some point. Almost immediately it will be noticed that the segment of intestine just above the bolus enters into a strong tonic contraction. At the same time the intestine for a considerable length below the bolus is inhibited and relaxed, as may be shown by recording the contractions of either muscular coat by means of the enterograph. In consequence of this contraction above and relaxation below, the bolus moves onwards down the intestine, followed up and driven by the advancings ring of constriction. If the intestine be less irritable, the introduction of the bolus may cause merely an augmentation of the rhythmic contractions of the segment above, and the inhibition below may be only slightly marked. In this case the movement of the bolus still occurs, but is incomparably slower than under the conditions just described. It may be quickened if we increase the stimulus due to the presence of the bolus by pinching the gut at this point, each pinch being followed by a temporary inhibition of the gut below, and a corresponding acceleration of the movement of the bolus. The progression of the bolus down the intestine depends, therefore, on two events, which are excited by the mere presence of the bolus, namely, augmentation of contraction above, and inhibition of contraction below, the bolus. This double effect is the characteristic response of the intestine to local stimulation. If we record the contractions at any point of the gut, it will be found that a pinch applied from 1 inch to 12 inches above the recorded spot gives an instant inhibition of the rhythmic contractions; whereas a pinch applied from $\frac{1}{2}$ inch to 3 inches below produces, after a varying latent period, an augmentation of the contractions.

It is evident that the true peristaltic contraction is a co-ordinated reflex, carried out by the local nervous centres in the wall of the gut. By means of the injection of nicotin or the local application of cocain, we can paralyse the nerve centres, leaving the muscle fibres intact. WV then find that, although the rhythmic contractions are even better

\footnotetext{
${ }^{1}$ Proc. Roy. Soc. London, vols. xl. and xli.
} 
marked than before, running as waves up or down the gut with a rapidity of 2-4 cms. per second, they are totally unaffected by the application of a strong stimulus to the intestine, either above or below the segment which is under observation. Since the double effect of local stimulation, augmentation above and inhibition below, is wanting, the power of the intestine to move its contents in any definite direction is also gone. A bolus placed in any part of the paralysed loop remains absolutely motionless.

Nothnage ${ }^{1}$ pointed out that the application of a crystal of sodium chloride to the outer surface of the intestine causes in the rabbit and cat a ring of constriction, not at the stimulated point, but immediately above it. He rightly refers this effect to the stimulation of Auerbach's plexus, and contrasts it with that produced by potassium salts, which excite the muscle fibres directly, and therefore cause a ring of constriction at the stimulated spot. This observer also noticed the inhibition in front of an advancing wave of constriction, which we have shown to be of so great importance for the normal progress of the intestinal contents.

The fact that the true peristalsis, dependent on the activity of the local nerve centres, is only propagated in one direction, is well illustrated by an experiment carried out by Mall. If a seginent of intestine be separated from the rest, and then resected in its former position, the functions of the bowel are carried out in a perfectly normal fashion. If, however, the resected loop be turned round, and connected with the cut ends of the remaining intestine, so that its previously lower end is comnected with the upper part of the intestine, and its upper end is directed towards the rectum, the animal dies after a time of intestinal obstruction, due to the accumulation of indigestible material in the gut above the resected loop. These experiments show that the propulsive movements of any part of the intestine always occur in one direction.

We have, therefore, in the normal animal to take into account only two kinds of movement. The effect of the pendulum movement is to thoroughly mix the contents of the intestine and bring them in intimate contact with the mucous membrane of the wall. If an animal be fed with pills containing bismuth subnitrate, and observed under the Röntgen rays, it will be seen that the opaque pills move continually to and fro in the lumen of the intestine, their actual progress towards the colon being very small in comparison with the distance traversed (Griitzner). ${ }^{2}$ If the contents of the bowel are fluid, the mixing effects due to the pendulum movements may predominate to such an extent over the progression due to peristalsis, that foreign substances introduced with large enemata into the rectum may travel up past the ileo-cæcal valve into the small intestine, or in rare cases may even enter the stoniach. Such a phenomenon has often been regarded as a proof of the existence of anti-peristalsis in the normal gut, but is probably sufficiently accounted for by the factors I have already mentioned.

Conditions of intestinal activity-Circulation.-The activity of the intestines may be modified or called into play by a number of local conditions. Chief among these are to be reckoned changes in the circulation. According to some observers, local anæmia, induced by

1 “Physiologie u. Pathologie des Darmes," Berlin, 1884.

${ }^{2}$ Arch.f. d. ges. Physiol., Bonn, 1898, Bd. Ixxi. S. 492. 
obstruction of the aorta, causes or strengthens the intestinal contractions (Schiff). ${ }^{1}$ Nasse, ${ }^{2}$ Mayer and v. Basch ${ }^{3}$ state that obstruction of the aorta arrests the movements for a time, but they return afterwards with increased vigour. Most observers (Betz, ${ }^{4}$ v. Braam Houckgeest, ${ }^{5}$ Mall), however, agree that ancmia inhibits all the movements of the intestines, a view which I can confirm. If the rhythmical contractions of the small intestines in the dog be recorded by the insertion of a small balloon communicating with a piston recorder, obstruction of the aorta in the chest causes an almost immediate cessation of the movements, which lasts as long as the obstruction is continued (up to 15 minutes). During this time the lever sinks slowly, i.e., there is a gradual diminution of intestinal tonus. If, now, the blood be let in, the intestines contract immediately, once or twice, then pause, and then recommence their rhythmic movements, the contractions often increasing in force for a couple of minutes. This recommencement is associated with a considerable diminution in the diastolic volume of the intestines, i.e. increased tonus. Mall points out that ligation of the superior mesenteric artery causes strong contractions. In this case the determining cause appears to be, not the ischæmia, but the hamorrhagic infarction which is produced by the ligation, so that the ultimate effect resembles in some extent that produced by venous obstruction.

It is probable that the varying statements on this subject depend on the frequent coincidence of anæmia and local asphyxia. The excitatory effect of the circulation of venous blood through the intestines has been frequently pointed out (Engelmann, v. Basch, etc.). In general asphyxia, one may indeed observe a preliminary inhibition of intestinal movements, but this is due to the splanchnic excitation, and consequent anæmia. I must confess, however, that I have often missed altogether an excitatory effect of asphyxia on the intestinal movements, and have only observed it in rabbits.

Influence of the nervous system on intestinal movements.The intestines receive a rich nerve supply from the spinal cord, by way of the sympathetic chain, great and small splanchnics (from first three lumbar ganglia), and solar and mesenteric plexuses. They also receive fibres from the vagus through the communicating branches from the anterior and posterior gastric plexuses to the solar plexus.

Vagus.-A number of authors have described the vagus as the motor nerves of the intestines. Thus stimulation of the vagus in the neck has been said to evoke active contractions of both stomach and intestines (Budge, ${ }^{6}$ Ludwig and Kupffer, ${ }^{7}$ Engelmann ${ }^{8}$ ). According to v. Braam Houckgeest, ${ }^{9}$ the vagus has no direct action on the intestines, the contractions being simply propagated from the stomach. Mayer ${ }^{10}$ states, however, that he has oliserved movements of the small and large intestines on stimulation of the vagus, even after ligation of the duodenum. There is no doubt that, in a number of cases, stimulation

1 "Lehrbuch d. Physiologie," 1858-59, S. 105.

2 "Beitr. z. Physiol. d. Darmbewegung," Leipzig, 1866.

${ }^{3}$ Sitzungsh. d. k. Akad. d. Wissensch., Wien, 1870, Bd. 1xii.

+ Virchow's Archin, Bd. i. ${ }^{5}$ Arch. f. d. ges. Physiol., Bonn, 1872, Bd. vi. S. 266.

6 "Lehrbuch d. Physiologie," 1887, S. 225.

7 Ztschr.f. rat. Med., 1858 , Bd. ii. S. 357.

8 Arch. f. d. ges. Physiol., Bonn, 1871, Bd. iv.

${ }^{10}$ Hermann's "Handbuch," Bd. v. (2).

${ }^{9}$ Ibid., Bd. vi. 
of the vagus has no apparent effect on the intestines. Bunch ${ }^{1}$ only observed an effect (inhibition) once in a large number of experiments, and hence denied that the vagus is to be regarded as a motor nerve. Jacobi ${ }^{2}$ states that in the rabbit (after a few days' starvation) stimulation of the vagus is without effect, unless the splanchnics or the fibres which run from the suprarenals to the solar plexus be divided, and hence regards these fibres and the vagi as mutually antagonistic. In rabbits he describes the intestinal fibres as conveyed chiefly in one vagus, either right or left, while the other sends fibres chiefly to the stomach. These discordant results are due to the influence of various disturbing factors, among which may be mentioned the influence of anæsthetics and the exposure and handling of the intestines, with the circulatory changes thereby induced. More important than these, however, are the inhibitory influences to which every part of the intestine is subject, either originating in the higher parts of the gut, and travelling down the

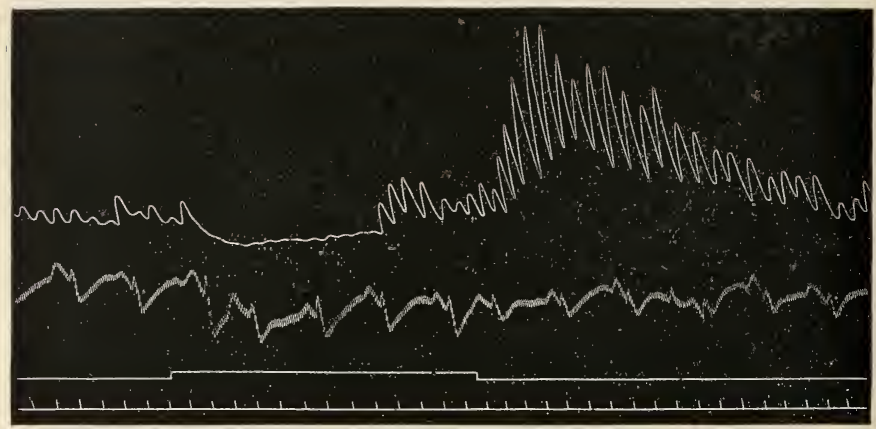

Fit. 187.-Curves obtained by balloon method, to show effects of exciting peripheral end of right vagus. Upper curve $=$ intestinal contractions, sceond curve $=$ artcrial blood pressure, third curve $=$ excitation marker, lowest curve $=$ time marking in six-second intervals. During the period shown on the excitation marker, the vagns was excited with strong induced currents (tentl excitation).

intestinal wall, or started by any sensory stimulation of the gut itself, and transmitted reflexly through the spinal cord. It is necessary, therefore, to cut out such influences as much as possible, by dividing both splanchnics and by avoiding any lesion of the intestine above the observed point, such as a ligature, which might set up descending inhibitory impulses. If these precautions are observed, stimulation of the vagus in the neck, after paralysis of the cardio-inhibitory fibres by means of atropin, will always produce an effect upon the intestinal movements; the effect, which may be little marked at first, but increases with each succeeding stimulation, is twofold-(1) An inhibition with very short latent period (less than one second), leading to the dropping of one or two beats, and (2) an augmentation of the rhythmic contractions, which gradually develops after the lapse of 10 to 30 seconds, and lasts for some length of time after the cessation of the stimulus. The

\footnotetext{
1 Journ. Physiol., Cambridge and London, 1897, vol. xxii. p. 357. (This contains an excellent summary of previous work on intestinal innervation, with full references.)

${ }_{2}$ Arch. f. exper. Path. $u$. Pharmakol., Leipzig, 1892, Bd. xxix. S. 171.
} 
vagus effect may come on simultaneously at all points of the small intestine, and is not abolished by ligaturing the second part of the duodenum or the upper part of the jejunum. The vagus fibres must therefore reach the intestine at all points, and do not run down from the stomach or duodenum between the two coats. The effects are better marked in those portions of the intestine which are already excited by the presence of a bolus or of a balloon for the purpose of recording the movements.

We must conclude, therefore, that the vagus contains two sets of fibres to the muscular coat of the intestine-one set inhibitory, with a very small latent period; the other set augmentor or motor, with a very long latent period. The action of the latter can be easily prevented by the presence of inhibitory influences acting on the intestine at any point.

Sympathetic.-The main sympathetic supply reaches the intestines by means of the splanchnic nerves. Pflüger ${ }^{1}$ was the first to show

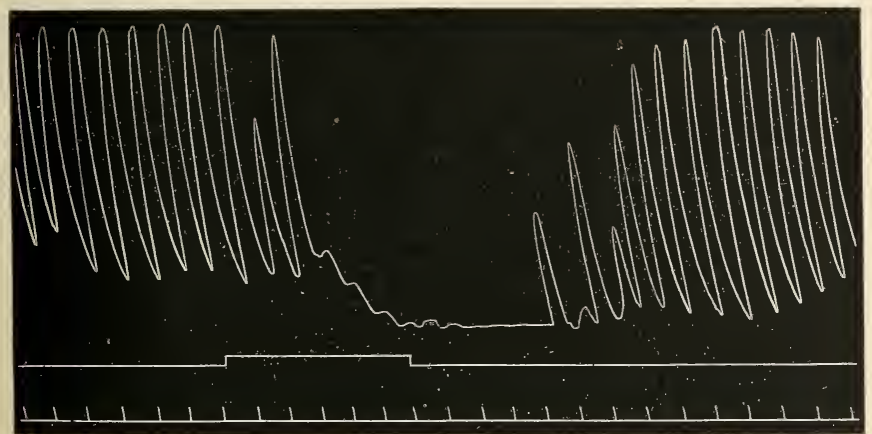

FIG. 188. - Curve showing inhibitory effect on contractions of small intestine, of stimulat. ing peripheral end of right splanchnic nerve. The middle line shows the duration of stimulation. Time marking $=$ six-second intervals. Balloon method.

that the stimulation of these nerves imhibits the intestinal movements. This observation has been confirmed by almost every subsequent observer, though there has been considerable discussion as to the exact mode in which the inhibitory action is brought about. It has been already pointed out that anæmia of the intestines checks the movements, and S. Mayer and v. Basch ${ }^{2}$ ascribed the inhibitory action of the splanchnics to their vaso-constrictor influence on the intestines. According to $\mathrm{v}$. Braam Houckgeest, this view is negatived by the following experiment. The intestines of a rabbit are exposed to the air until they become reddened by vasomotor paralysis. Stimulation of the splanchnics now has no effect on the blood vessels. The movements of the intestines, however, are inhibited, as in a normal animal. Jacobi states that the intestinal inhibitory fibres of the splanchnics take a

1 "Ueber d. Hemmungsnervensystem f. d. peristaltisehen Bewegungen d. Gedärme," Berlin, 1857.

${ }^{2}$ Sitzungsb. d. k. Alkel. d. Wissensch., math.-naturm. Cl., Wien, 1870, Bd. lxii., Abtl. 2, S. 811. 
different course from the vasomotor fibres, and that section of the nerves running from the suprarenals to the solar plexus annuls the inhibitory action of the splanchnics without affecting their vasoconstrictor effect.

Besides these inhibitory effects, various observers have recorded a motor effect on stimulating the splanchnics (Schiff, Ludwig and Kupffer, Bechterew and Mislawsky, Bunch). The last-named observer has given graphic records of intestinal movements, in which stimulation of the splanchnics caused in some animals augmentation, in others inhibition, of intestinal tone, and in some cases primary augmentation followed by inhibition; and concludes that nerve fibres of opposed function run in the splanchnics, and that the result obtained is due to the preponderating influence of one or other kind in the particular animal employed. I have myself never obtained any other effect than inhibition on excitation of these nerves.

In 1885 , Ehrmann ${ }^{1}$ put forward the view, that the intestines conformed to v. Basch's idea of "crossed innervation," and that the splanchnics were motor for the longitudinal but inhibitory for the circular coat. Courtade and Guyon, ${ }^{2}$ on the other hand, found that the splanchnics produced contraction of the circular and inhibition of the longitudinal coat. Their method of recording the movements of the two coats does not appear to offer any criterion for distinguishing active from passive movements of the intestinal wall. These authors find that when the intestines are in an abnormal condition, Ehrmann's results may be obtained, and conclude, therefore, that the splanchnics contain motor and inhibitory fibres for both layers of muscle.

Observations by Bayliss and myself ${ }^{3}$ lend no support to the theory of crossed innervation in either of its versions. Recording the contractions of each coat by means of two enterographs, placed at right angles to one another, we have found that both coats contract synchronously, and that the contractions of both are inhibited on stimulating the peripheral end of either splanchnic nerve. So far as concerns the vagus, although the initial inhibitory effect is produced equally on both coats, the subsequent augmentation is confined almost entirely to the circular coat.

Bechterew and Mislawsky ${ }^{4}$ and Bunch have investigated the spinal nerve-roots which contribute fibres through the sympathetic to the intestines. According to Bunch, the nerve fibres pass to the splanchnic from the anterior roots, from the sixth thoracic to the second, third, fourth, or fifth, lumbar nerves, and have one cell station on their course (in the ganglia of the solar plexus).

Central and reflex influences.-According to Bechterew and Mislawsky, stimulation of the sigmoid convolution in dogs, or of the posterior corpora quadrigemina, influences the intestinal contractions; these being inhibited or augmented according to the exact localisation of the stimulus. Hence they locate "centres for intestinal movements" in these parts of the brain. Somewhat similar results were obtained by Bochefontaine, ${ }^{5}$ and by Pal and Bergrün. ${ }^{6}$

It is probable that the intestinal movements, like those of the stomach, may be inhibited or reinforced under the influence of

${ }^{1}$ Med. Jahrb., Wien, 1885. ${ }^{2}$ Arch. de physiol. norm. et path., Paris, 1897, tome ix.

3 Journ. Physiol., Cambridge and London, 1899, vol. xxiv.

4 Arch.f. Physiol., Leipzig, 1889, Suppl. S. 254.

5 Arch. de physiol. norm. et path., Paris, 1876, tome iii., p. 164.

" Med. Jahrb., Wien, 1888, Bd. viii. (quoted by Mislawsky). 
emotions. They are certainly checked reflexly by painful stimulation of sensory nerves, especially if the stimulus affect the abdominal viscera. If the splanchnic nerves be intact, the application of an interrupted current to any point in the intestines causes a ring of constriction at the excited spot, with a simultaneous reflex inhibition of the spontaneous contractions, and tonus of all other parts of the small intestine. This inhibitory effect is almost abolished by section of both larger splanchnic nerves.

The innervation of the alimentary canal in the frog.-It has been stated by Steinach and Wiener, ${ }^{1}$ that the alimentary canal of the frog receives its motor innervation chiefly from the posterior roots of the spinal nerves. The following table shows the distribution of these nerves to the various parts of the canal, according to these observers :-

Esophagus . . Vagus, third and fourth posterior roots.
Stomach.
Anterior half of small in- ", fourth and fifth (and sixth) posterior roots.
testine .
Posterior half of smail in- ", fifth and sixth
testine. . . " , sixth and seventh
Rectum . . . ,

Stimulation of the anterior roots of all these nerves (with the exception of the seventh and eightlı) had no effect on the intestines. Somewhat similar results had already been obtained by Waters, ${ }^{2}$ without, however, distinguishing between the anterior and posterior roots. The following represents the results obtained by this observer:-

$\begin{aligned} & \text { Fourth spinal nerve } \\ & \text { Fifth , } \\ & \text { Sixth }\end{aligned}$
$\begin{aligned} & \text { Seventh , } \\ & \text { Eighth , }\end{aligned}$
$\begin{gathered}\text { Feeble" contractions of intestines ; vaso-constric- } \\ \text { tion in the upper part of intestines. } \\ \text { Vaso-constriction in lower two-thirds of in- } \\ \text { testine; occasional peristalsis. }\end{gathered}$

A repetition of these experiments by Horton Smith ${ }^{3}$ under Langley's directions failed to confirm them. Horton Smith regards the vagus as the sole motor nerve for the œesophagus, stomach, small intestine, and upper part of the large intestine, while the lower part of the large intestine and the rectum are supplied by the anterior roots of the ninth and tenth spinal nerves. The matter cannot be regarded as definitely settled.

\section{DEFACATION.}

As the products of digestion pass down the colon they lose their fluid consistence, in consequence of the absorption of water. The mass thus formed is carried down by the peristaltic contractions of the colon towards, and tends to accumulate in, the sigmoid flexure. That it is not driven down at once into the rectum is due largely to the fact that the contractions which start at the ileo-crecal valve become less powerful on their way down the intestine, and do not as a rule affect the rectum. In healthy subjects, except just before defrcation, the rectum is empty, and the presence of fæces in this part of the bowel at once gives rise to discomfort, and to a desire to defæcate.

\footnotetext{
${ }^{1}$ Arch. f. d. ges. Physiol., Bonn, 1895, Bd. lx.

2 Journ. Physiol., Cambridge and London, 1885, vol. vi.

3 lbid., 1897, vol. xxi. p. 101.
} 
When the freces have reached the rectum, the passing is still kept in check by the tonic contraction of the two sphincters of the anus, the internal sphincter composed of involuntary and the external sphincter of voluntary muscle. When the contents of the rectum are fluid, or when they are forced against the sphincters by strong peristaltic contractions of the lower bowel, the action of the sphincters may be voluntarily reinforced by active contraction of the levator ani muscles, which raise and compress the lower segment of the rectum or anal canal.

Defæcation is normally started by a voluntary act, although it may take place involuntarily, as is shown by the fact that it may occur in a dog whose spinal cord has been divided in the mid-dorsal region (Goltz).

The steps of normal defæcation are as follows:-The glottis being closed, a forcible expiratory contraction of the abdominal muscles takes place. The perineal muscles being relaxed at the same time, the lower part of the rectum is straightened, and a portion of the contents of the sigmoid flexure is forced down into the lower part of the rectum. The presence of a foreign body in this part of the rectum irritates the mucous membrane and excites reflexly the rest of the act. Strong peristaltic contractions take place along the whole of the descending colon, sigmoid flexure, and rectum, while both sphincters are relaxed, thus forcing out the contents of the bowel. The last section of the rectum at the close of the act is emptied by a forcible contraction of the levator ani and the other perineal muscles.

The carrying out of this reflex act is dependent on the integrity of a certain part of the lumbar spinal cord. If this centre be destroyed, the tonic contraction of the sphincter muscles disappears. Budge ${ }^{1}$ found that stimulation of the spinal cord in the rabbit, at the level of the fourth lumbar vertebra, caused contraction of the rectum. He considered this portion of the cord to be a centre for the rectum, bladder, and vasa deferentia, and named it therefore the genito-spinal centre. His view that motor fibres left this centre to run to the rectum by the sacral nerves was confirmed later by Masius. ${ }^{2}$ Nasse ${ }^{3}$ showed that, in addition to this supply from the sacral nerves, the rectum receives fibres from the lumbar nerves through the sympathetic chain and mesenteric plexus.

The nerve supply to the pelvic viscera has been recently the subject of careful investigation by Langley and Anderson. ${ }^{*}$ These authors come to the following conclusions with regard to the descending colon, rectum, and anus.

These parts of the alimentary canal receive fibres from two sources: an upper supply in the rabbit from the second, third, fourth, and fifth lumbar nerves; in the cat from the second, third, and fourth lumbar; and a lower supply from third and fourth sacral nerves in the rabbit, with some fibres either from the second sacral or first coccygeal nerve; and in the cat from the second and third sacral nerves, and sometimes also from the first sacral.

\footnotetext{
${ }^{1}$ Virchow's Archiv, 1858, Bd. xv. S. 115 ; Ztschr.f. rat. Med., Leipzig u. Heidelberg, 1864, Bd. xxi.

${ }^{2}$ Bull. Acad. roy. d. sc. de Bcly., Brux., 1867, tome xxiv. p. 312 ; and 1868, tome xxv. p. 491 .

3 "Beitr. z. Physiol. der Darmbewegung," Leipzig, 1866 (quoted by Langley and Anderson).

${ }^{4}$ Journ. Physiol., Cambridge and London, 1895, vol. xviii. p. 67; 1895, vol. xix. p. 71 ; and 1896 , vol. xx. p. 371 .
} 
The upper set of fibres pass into the lumbar sympathetic chain, and thence in the mesentery to the inferior mesenteric ganglia, and so by the colonic and hypogastric nerves to the rectum. They are connected with nerve cells chielly in the inferior mesenteric ganglia, and supply the descending colon, rectum, and internal sphincter. A few fibres pass along the sympathetic chain to the sacral ganglia, and thence by the grey rami of the ganglia to the sacral nerves; in these they follow the somatic branches of the sacral nerves, and end chiefly in the unstriated muscles of the skin round the anus.

The lower set of fibres run in the pelvic nerves, or nervi erigentes. They pass into the hypogastric plexus, and have ganglion cells either as they pass the side of the rectum, or more peripherally.

The object or use of this double nerve supply to the rectum is difficult to ascertain. It is natural to imagine that these two sets of nerve fibres should be antagonistic to one another, as is the case with other organs, such as the heart, which are supplied with nerves from two sources. Fellner, working in v. Basch's laboratory, has indeed come to the conclusion that the theory of "crossed innervation," put forward by the latter, also applies to the rectum. According to this theory, when a muscular tube containing circular and longitudinal fibres in its walls, is supplied with two sets of nerve fibres, one set of nerves will be inhibitory for the longitudinal and motor for the circular muscles, while the other nerves will be motor for the longitudinal and inhibitory for the circular muscles. Fellner, ${ }^{1}$ as the result of experiments on the dog, concluded that the sacral nerves are motor for the longitudinal muscles of the rectum, including the recto-coccygeal muscle, and inhibitory for the circular muscles, whereas the fibres which run from the hypogastric nerves cause contraction of the circular fibres and relaxation of the longitudinal. We have already seen that this theory is inadmissible for the intestine, and Langley and Anderson, while not denying the possible accuracy of Fellner's experiments, so far as the rectum of the dog is concerned, have shown that they do not apply to other animals. In rabbits and cats they find that the fibres from the lumbar nerves may cause either inhibition or contraction of either muscular coat. The usual result was inhibition of both coats of the rectum, which might be preceded or followed by a slight contraction. On the internal sphincter the effects were again variable, though here also inhibition predominated.

Excitation of the sacral nerves produced much more marked results than did that of the hypogastric nerves. In most cases a strong contraction of the recto-coccygeal muscle and of the longitudinal fibres of the rectum was accompanied by marked relaxation and dilatation of the internal sphincter. Strong peristaltic contractions were also set up in the lower part of the bowel, so that stimulation of these nerves gave rise to defæcation. Here, therefore, both kinds of fibres were caused to contract in the upper part of the rectum. In a few cases the relaxation of the internal sphincter was replaced or succeeded by a contraction of this muscle.

The voluntary external sphincter and levator ani are also supplied from the sacral nerves; in man, from the fourth sacral.

1 Med. Jahrl., Wien, 1883, S. 571, and Arch. f. d. ges. Physiol., Bonn, 1893, Bd. Ivi. S. 542. The tracings given in the latter paper do not, however, by any means support Felluer's contentions.

VOL. II. -22 


\title{
MUSCULAR MECHANISHS OF THE URINARY TRACT.
}

\author{
By Ernest H. Starling.
}

Contents :-Movements of the Ureters, p. 338-Movements of the Bladder, p. 339-Muscular Tissue of Bladder and Urethra, p. 339-Mechanism of Closure of Bladder, p. 340-Intravesical Tension, p. 341-Nerve Supply of the Bladder, p. 342-Mechanism of Micturition, p. 344-Voluntary Control of Micturition, p. 345 .

\section{Movements OF THE URETERS.}

THE urine, which is secreted continuously, though at varying rates, passes to the bladder through the ureters, partly in consequence of the pressure at which it is secreted, partly in consequence of peristaltic contractions of these tubes.

These contractions begin at the upper end of the ureters, and travel down to the bladder, but do not affect the musculature of the latter. Although, by artificial stimulation, contraction waves may be started at any point in the ureter, from which they will then travel upwards as well as downwards, under normal conditions the contractions always take place from above downwards, no spontaneous antiperistaltic waves having ever been observed. The contraction waves, according to Engelmann, ${ }^{1}$ travel at about 20 to $30 \mathrm{~mm}$. in the second, and are repeated at the rate of about three in the minute.

If the ureter is divided about its middle, the upper half still continues to present rhythmic waves of peristalsis. The lower half often begins to contract on its own account, but its rhythm now no longer coincides with that of the upper half.

The contractions seem to be initiated by the muscular tissue of the walls of the ureter. Although (in man) the ureter receives fibres from the mesenteric, spermatic, and hypogastric plexuses, and contains a few ganglion cells, the middle third of the ureter is, according to Engelmann, free from nervous elements, and yet contracts rhythmically when isolated from the other segments. According to Dogiel, ${ }^{2}$ Maier, ${ }^{3}$ and Protopopow, ${ }^{4}$ however, ganghion cells may be found in all parts of the ureter, although more abundant at the two ends. The contractions are altered to a certain extent by distension of the ureter, yet they are largely independent of the urimary flow, and occur almost as frequently in animals which have been deprived of water, as when the urinary flow is excessive in consequence of administration of large amounts of fluid.

Contractions of the ureter can be excited by stimulation of the

1 Arch. f. d. gcs. Physiol., Bonn, 1869, Bd. ii.

${ }^{2}$ Arch. f. mikr. Anat., Bonn, Bd. xv. S. 64.

${ }^{3}$ Virchow's Archiv, 1881, Bd. Ixxxii. S. 49.

+ Arch. f. l. ges. Physiol., Bonn, 1897, Bd. Ixvi. S. 1. 
splanchnic nerve of the same side. They are said to cease permanently shortly after ligature of the upper end of the ureter, in consequence of the severance of the nerve fibres. They may occur, however, in the isolated ureter. That this tube is well supplied with sensory fibres is evidenced by the severe pain of renal colic, which occurs on the impaction of a calculus in the ureter.

The reflux of urine from the bladder into the ureters is prevented by the oblique manner in which these enter the bladder, a sort of valvular opening being thus formed.

\section{Movements of the Bladder.}

The urine, which is constantly trickling down the ureters, accumulates in the bladder, whence it is expelled at intervals by the contraction of the muscular wall of this viscus. The act of micturition is in the young child purely reflex and dependent on the tension in the bladder. With advancing age, however, the individual acquires more or less voluntary control over the reflex act. It will be convenient to first consider the purely reflex act of micturition.

Muscular tissue of bladder and urethra.-The muscular wall of the bladder is generally described as being composed of three layers-an outer longitudinal, a middle circular, and an inner longitudinal or oblique coat. The division between these coats is, however, by no means well marked, and, as Griffiths has shown, ${ }^{1}$ bundles which are longitudinal may become circular or oblique, and bundles of different coats may be continuous with one another. The longitudinal fibres are best marked in the middle of the anterior and posterior surfaces of the bladder. These coats are practically continuous in the female with a similar series of coats forming the muscular wall of the urethra. In the male, according to Griffiths, the bundles are gradually lost in the connective tissuc of the neck of the bladder, and are not inserted into any special part, such as the capsule of the prostate of the gland. On the lateral surfaces of the bladder, the oblique and transverse fibres are most apparent. The bundles of muscular tissue on the two sides of the bladder are continuous. The longitudinal fibres of the bladder, with or without the transverse fibres, are often described as the detrusor urino.

The apparent necessity for some muscle which by its contraction would close the vesico-urethral orifice, has led many anatomists to ascribe an internal sphincter muscle, formed by a thickening of the circular coats of the bladder at its junction with the urethra. An account of this internal sphincter was given by Charles Bell, ${ }^{2}$ and has been repeated in other works on anatomy. Careful dissection, however, fails to show any thickening of muscle around the commencement of the urethra, sufficient to constitute a sphincter. The absence of such a sphineter has been especially emphasised by Griffiths, and can be easily verified by dissection. There are, however, round the urethra, collections of unstriated and striated muscle, which by their contraction close this canal, and check the urinary flow. Thus the first part of the urethra is surrounded by a muscular coat, consisting of inner longitudinal fibres and an outer thicker circular layer, continuous with the inner and middle layer of the bladder. There is also a sheet of striped muscle, which surrounds the greater part of the urethra, and in the male extends from the level of the entrance of the vasa deferentia into the urethra, to within a couple of inches of the urinary meatus. In man, the first part of this muscle is represented by a few trans-

1 Journ. Anat, and Physiol., London, 1894, vol. xxv. p. 540.

2 "Anatomy of the Human Body," 6th edition, 1826, vol. iii. p. 390. 
verse fibres on the ventral surface of the lower half of the prostate gland, and is here known as the external splincter of Henle. Round the membranous part of the urethra the sheet is termed the constrictor urethræ, and round the penile portion forms the accelerator urina. In the dog we find a similar collection of striated muscle fibres round the small portion of the urethra which intervenes between the prostatic and membranous parts. This is designated Wilson's muscle.

Mechanism of closure of the bladder.-The mechanism of closure of the bladder has been the subject of much discussion. The retention of urine has been variously ascribed to the elastic resistance of the tissues at the neck of the bladder, or to the contraction of the internal sphincter or of the external sphincter (striated muscle) of Henle. Rosenthal's ${ }^{1}$ view, that the closure was due to the elasticity of the sphincter, which had to be forcibly overcome by the contraction of the detrusor in micturition, was discredited by the statement of Heidenhain and Colberg, ${ }^{2}$ that the bladder is able to hold fluid at a much greater pressure in the living than in the dead subject. These authors found that the intravesical pressure necessary to overcome the resistance of the sphincter amounted to between 21 and $33 \mathrm{cms}$. of water in the living rabbit, and to between 2.5 and $7.5 \mathrm{cms}$. of water in the dead rabbit. In the dog, the bladder in the dead animal is able to hold fluid at a pressure of 18 to $20 \mathrm{cms}$. of water, whereas in the living animal the pressure may reach $100 \mathrm{cms}$. before the contents of the bladder begin to trickle through the urethra. If the lumbar spinal cord be destroyed, the difference in favour of the living animal is abolished, showing that it depends on the existence of a tonic contraction under the influence of this part of the cord. Griffiths has pointed out that there is no such striking difference between the pressure necessary to open the urethra and force out urine in living and dead animals. In the latter case, however, if the urethra has once been forced open by raising the pressure in the bladder, it remains more or less patent, so that on subsequent occasions a flow of urine is obtained at a low pressure. This author finds that the resistance to the escape of urine, which in the male dog varies from 16 to 20 in. of water, remains the same after division of the pelvic nerves, of the hypogastric nerves, or even of the spinal cord in the dorso-lumbar region.

There can be no doubt that the greater part of the resistance to the outflow of urine is occasioned at the urethro-vesical orifice, only a small part being offered by the urethra itself. Thus, in surgical operations, the urethra may be incised up to the neck of the bladder without any escape of urine, and in the cat ${ }^{3}$ the urethra between the neck of the bladder and the prostate gland may be divided completely without any of the urine escaping. Reyfisch has excised the prostate in dogs without producing any incontinence. Moreover, if the intravesical pressure necessary to cause a flow of urine be determined, by connecting the interior of the bladder through a tube in the ureter with a reservoir of fluid (a catheter having previously been placed in the urethra), it is found that this pressure remains the same, whatever may be the situation of the catheter. It is not until the catheter is actually pushed into the

1 " De tono cum musculorum, tum eo imprimis, qui sphinctorum tonus vocatur," Diss., Königsberg, 1857.

2 Arch. f. Anat., Physiol. u. wissensch. Med., Berlin, 1858, S. 437.

${ }^{3}$ Griffiths, loc. cit. 
bladder that there is any fall of the pressure at which urine escapes. These facts show that the resistance to the outflow of urine is situated at the neck of the bladder, and must be due either to a tonic contraction of circular fibres at this point, or to the elastic retraction of this orifice, aided, perhaps, by the apposition of the mucous surfaces along the whole length of the urethra, and the tendency of the weight of the contained urine in the relaxed bladder to press the two walls of the urethra together. Griffiths concludes that this resistance is in the main physical, from the fact that it remains the same after death (on the first occasion tried) as during life. ${ }^{1}$

Intravesical tension.-In the empty bladder the wall of this organ is contracted, so that the cavity is obliterated. The pressure in the empty bladder can be regarded as practically zero. As urine is gradually secreted, the tension in the bladder rises. Since, however, the wall of the bladder is normally in a condition of greater or less contraction, it follows that the rise in internal pressure of the bladder is not proportional to the increased amount of fluid in its cavity. If the tonus of the bladder wall is well marked, the intravesical pressure may be raised to a considerable extent by the injection of only a small amount of fluid. On the other hand, the bladder is able to accommodate large quantities of fluid without any corresponding rise in pressure, in consequence of a relaxation of the tonic contraction of its muscular wall. The amount of fluid which the bladder is able to accommodate depends largely on the rate at which the fluid enters the bladder. Thus, in man, the state of tension of the bladder wall which is associated with a desire to micturate, equals about $15 \mathrm{cms}$. water. This state of tension is attained by the injection of about 150 c.c. of fluid, although, when the bladder is allowed to fill naturally with urine secreted slowly by the kidneys, an intravesical pressure of $15 \mathrm{cms}$. corresponds to about 250 c.c. of urine. ${ }^{2}$ When, on account of a continued accession of urine, the tension reaches a certain height, slow rhythmical contractions of the bladder wall begin, weak at first, but growing gradually stronger, with increasing distension. Thus in the bladder, as in most other hollow viscera, the effect of tension on the sheet of unstriated muscle forming the wall of the organ is twofold.

1. A relaxation (not a stretching), accommodating the capacity of the viscus to the incoming fluid.

2. An excitatory effect, bringing about rhythmic contractions of the wall, the extent and strength of the contractions being directly proportional to the intravisceral tension (i.e. to the stretching of the muscle fibres).

Both these effects seem to be independent of the nervous system, and nay be observed in most hollow viscera after separation from all nervous connections with the body, and must therefore be dependent on local nervous mechanisms or on fundamental properties of the muscle fibres themselves.

Each rhythmic contraction of the bladder lasts on an average from

1 This experiment of Griffiths does not put a muscular tonic action absolutely out of court. The retractor penis muscle in the dog retains its irritability and contractility for two or three days after the death of the animal, and would, when the body had cooled down, be in a strongly contracted condition. It seems possible, therefore, that the unstriated muscle at the neck of the bladder might similarly be in a state of tonic contraction one or even two days after death.

2 Genouville, Arch. de physiol. norm. et path., Paris, 1894, tome vi. 
40 to 60 seconds, that is to say, the bladder rhythm is similar to the spleen rhythm, first described by Roy. ${ }^{1}$

Since the mere accumulation of urine in the bladder increases the intravesical tension and brings about rhythmic contractions, increasing in force with growing distension, it would appear theoretically possible that at a given distension a contraction might raise the pressure high enough to overcome the resistance at the neck of the bladder, and cause expulsion of the urine. According to v. Zeissl, this actually does occur. This observer has found that micturition may be accomplished normally in a dog after section of all the nerves to the bladder. ${ }^{2}$ As a rule, however, this evacuation will be only partial, the escape of urine being checked as soon as the intravesical pressure falls below a certain point. Thus, so-called incontinence of urine, which is met with in various affections of the spinal cord, is invariably an overflow of urine from a full bladder.

It is evident, then, that for the normal carrying out of micturition we require one or both of the following events:-

1. A strong contraction of the muscular wall, continued until the bladder is emptied.

2. A relaxation of the "sphincter" at the neck of the bladder.

For either or both of these events to occur, the local mechanisms of the bladder must be reinforced by impulses from the central nervous system.

Nerve-supply of the bladder.-We have now to inquire into the nature and paths of the impulses which reach the bladder. The walls of this organ are supplied with nerve fibres, which rum to the neck of the bladder from the hypogastric plexus. This plexus consists of nerve fibres and small collections of ganglion cells lying in the loose connective tissue on both sides of the middle part of the rectum. Some ganglion cells are also found in the course of the fibres in the walls of the bladder itself. The hypogastric plexus receives its fibres from two sources-from the sacral nerves, and from the inferior mesenteric ganglion. ${ }^{3}$ The branches from the sacral nerves which run to the plexus are two or, more rarely, three in number, and are derived in the cat from the second and third sacral nerves. They are known as the nervi erigentes or pelvic nerve (Langley), and run straight to the plexus without passing through the sympathetic chain. The fibres from the inferior mesenteric ganglion to the plexus run in the hypogastric nerves, two grey nerve-trunks which pass downwards on each side of the rectum to take part in the formation of the plexus near the neck of the bladder. The inferior mesenteric ganglion consists of four small ganglia connected by fine fibres, lying round the origin of the inferior mesenteric artery. This ganglion receives three nerves from the lumbar sympathetic chain, and sends branches down along the inferior mesenteric artery, and two fine branches upwards to the superior mesenteric ganglion, and so to the solar plexus. The fibres which reach the inferior mesenteric ganglion from the sympathetic chain are derived from the third, fourth, and fifth lumbar nerve-roots

1 A tracing of these contractions is given by Sherrington, Journ. Physiol., Cambridge and London, 1892, vol. xiii. pl. xxii. fig. 10.

2 Wien. klin. Wchnschr., 1896.

3 Nawrocki and Scabitschewsky, Arch. f. d. ges. Physiol., Bonn, 1897, Bd. xlviii. S. 335 . 
(in the cat). They are comnected, as Langley and Anderson ${ }^{1}$ have shown by means of the nicotin method, with cells in the inferior mesenteric ganglion, and make no connection with the cells in the ganglia of the lumbar sympathetic chain. In the monkey, according to Sherrington, ${ }^{2}$ these nerves are derived from the second, third, and fourth lumbar in most cases, rarely from the third, fourth, and fifth.

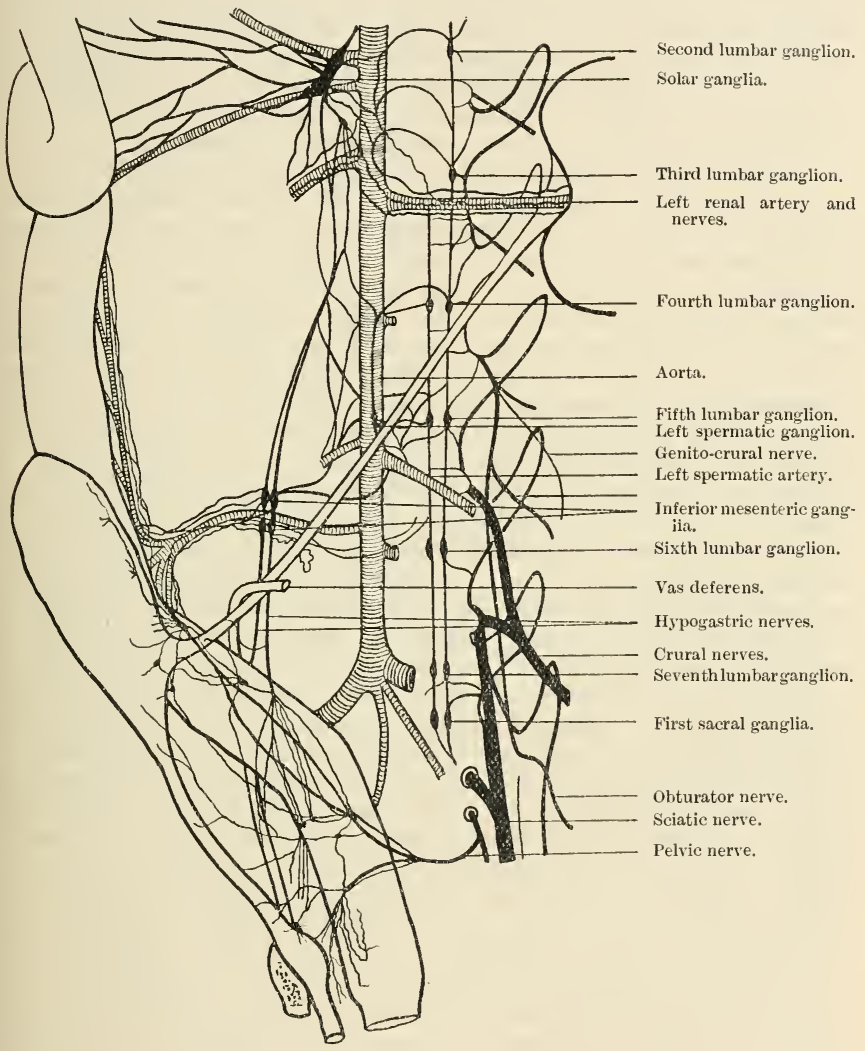

FIG. 188A.-Diagram of the nerve supply to the bladder and colon in the cat (Langley and Anderson, Journ. Physiol., Cambridge and London, 1896, vol. xx. p. 372.

With regard to the functions of these two sets of nerves, almost all observers agree that they convey motor impulses to the bladder. On exciting the lumbar nerve-roots or the mesenteric branches to the ganglion, a weak bilateral contraction of the bladder is obtained-a

1 Journ. Physiol., Cambridge and London, 1895, vol. xix. p. 71 ; 1896, vol. xix. p. 372 ; 1896, vol, xx. p. 372.

${ }^{2}$ lbid., 1892 , vol. xiii. p. 678. 
contraction which is unable to overcome the resistance of the sphincter and to give rise to micturition. The bilateral character of the contraction is due to the fact that certain of the fibres cross in the inferior mesenteric ganglion, so that the lumbar nerves of one side send fibres to both sides of the bladder. Stimulation of one hypogastric nerve may produce a contraction which is more or less unilateral. Stimulation of the sacral nerve-roots or of the nervi erigentes gives rise to a strong contraction of the bladder, which is unilateral if the nerves of only one side be stimulated, and which generally causes an expulsion of urine, i.e. an active micturition. Stimulation of the branches of the hypogastric plexus causes contraction of the bladder wall, limited to the area of distribution of the excited filaments (Griffiths).

Under some circumstances, it seems that stimulation of the upper set of nerve fibres may cause a relaxation of the bladder, ${ }^{1}$ or may hasten the process of relaxation, after a contraction has been excited by stimulation of the pelvic nerves. According to von Zeissl, ${ }^{2}$ the hypogastric nerves are the physiological antagonists of the sacral nerves. If fluid be forced into the bladder by means of a cannula in one ureter, at such a pressure that it is just able to overcome the resistance of the sphincter and trickle through the urethra, stimulation of the hypogastric nerves will at once stop the flow by reinforcing the contraction of the sphincter. On the other hand, if the fundus of the bladder be opened and tied round a glass tube connected with a pressure-bottle, and the latter be so arranged that the fluid is just unable to overcome the resistance of the sphincter, then stimulation of the nervi erigentes will cause a relaxation of the sphincter, and a flow of fluid through the urethra. In this case, the fundus being opened, any contraction of the muscular wall of the bladder is ineffectual to raise the pressure in the bladder, so that the result can only be ascribed to the relaxation of the sphincter. The accumulation of urine in the bladder would therefore be effected through the intermediation of the upper set of fibres, while the evacuation would be brought about by stimulation of the sacral fibres. Langley objects to these experiments, that the results obtained by von Zeissl on stimulating the hypogastric nerve might be due to a longitudinal contraction of the basal portion of the bladder drawing the urethra forwards, whereas a strong contraction, such as that produced by stimulating the nervus erigens, even if it involved the urethra, would tend to widen the urethral orifice, and so give the apparent effect of relaxation of the sphincter. I am of opinion that von Zeissl's results are due to a fallacious method of experiment. I have been unable to confirm him as to the inhibitory influence of the pelvic nerve on the "sphincter vesicæ." If his experiments be repeated, it will be found that even when the fundus is connected by a fairly wide tube with a reservoir, the sudden contraction of the bladder wall, on stimulation of the pelvic nerve, causes a considerable rise of pressure in the bladder. The expulsion of urine is caused by this rise of pressure, and not by any relaxation of the sphincter. If a tube one inch in diameter be tied into the fundus, and fluid poured into the tube until it just drops from the urethra, stimulation of the pelvic nerve does not alter the rate of

1 Griffiths, Journ. Anat. and Hhysiol., London, 1894, vol. xxv. p. 540 ; and 1895, vol. xxvi. pp. 60 and 225 .

${ }^{2}$ Arch. f. d. ges. Physiol., Bonn, 1893, Bd. liii. S. 560. 
flow, the free escape from the bladder through the wide tube having prevented any rise of intravesical tension. Von Zeissl performed his work in von Basch's laboratory, and looks upon his experiments as confirmatory of the theory of crossed innervation already put forward by von Basch. He regards the hypogastric nerve as motor for the circular, and inhibitory for the longitudinal (detrusor) fibres of the bladder, the nervi erigentes having the reverse effect. Courtade and Guyon ${ }^{1}$ also conclude that the pelvic nerve is motor for the longitudinal, the hypogastric for the circular fibres. I have already mentioned, however, that there is, according to Griffiths, no demarcation to be made out between circular and longitudinal or oblique fibres, all being apparently continuous with one another.

The nerve centre which presides over the tonus and contraction of the bladder is situated in the lumbar spinal cord. If this centre and its connections be intact, micturition may be carried out normally, even after section of the cord in the dorsal region. The centre can be excited reflexly by stimulation of almost any sensory nerve, such as the sciatic or the fifth nerve. ${ }^{2}$ In many cases where, in consequence of obstruction to the passage of impulses from the higher parts of the central nervous system, micturition is delayed, this act may be excited by the application of cold or hot sponges to the perineum, and it is well known that almost any irritation of the pelvic organs in children may give rise to reflex involuntary micturition.

The mechanism of micturition.-It is evident that the reflex act of micturition is originated by afferent impulses ascending to the centre in the lumbo-sacral cord, and started either by the increasing tension of the bladder wall (most probably), or by stimulation of the urethra. According to Goltz, ${ }^{3}$ Kuss, ${ }^{4}$ and Finger, ${ }^{5}$ with increasing tension the rhythmic contractions increase in force until one of them is sufficient to overcome the resistance of the sphincter and to drive a little urine into the urethra. The stimulus of this urine in the urethra starts the whole reflex chain of events leading to micturition. The sphincter, by the intermediation of the spinal centre, is inbibited. At the same time, the longitudinal and circular fibres of the bladder wall contract, thus emptying the bladder. The evacuation may be aided by an associated contraction of the abdominal muscles. At the end of the act, the last drops are expelled from the urethra by rhythmical contractions of the perineal muscles, especially the accelerator urinæ and levator ani. There is no doubt that in this act the contraction of the 'detrusor' (i.e. of the whole bladder wall) is the most important factor in bringing about the expulsion of the urine. Without the co-operation of the bladder wall, the strongest contraction of the abdominal muscles is inadequate to empty the bladder. It is much more uncertain what part, if any, in the process, is played by the structures at the neck of the bladder. Nearly all authorities lay a great stress on the importance of a dilatation of the sphincter, either by the direct action of the detrusor (longitudinal) fibres (Kohlrausch, ${ }^{6}$ and Henle), or by an inhibition of the

${ }^{1}$ Arch. de physiol. norm. et path., Paris, 1896 (5), tome viii. p. 622.

${ }^{2}$ Bert, ibid., 1869, tome iii. p. 650 ; S. Mayer, Hermann's "Handbuch," Bd. v. (2), S. 462 .

${ }^{3}$ Arch.f. d. ges. Physiol., Bonn, 1874, Bd. viii. s. 477 .

+ "Lehrbuch d. Physiologie."

${ }^{5}$ Allg. Wien. med. Ztg., 1893.

6 "Zur Anat. u. Physiol. der Beckenorgane," Leipzig, 1854, S. 14. 
circular fibres at the neck of the bladder. The main events in reflex micturition may be summarised as follows :-

1. Accumulation of urine. 2. Slow rise of intravesical pressure (to $15 \mathrm{cms}$. water). 3. Rhythmical contractions of bladder, increasing in force with increasing tension. 4. Afferent impulses ascending from bladder to lumbo-sacral cord. 5. Reflex discharge of impulses by hypogastric and pelvic nerves, causing contraction of the whole bladder wall, and a rise of intravesical tension to 20 or $30 \mathrm{cms}$. water. 6. The resistance at the neck of the bladder is overcome, the beginning of the urethra is straightened out, and the adhesion of the walls of the empty tube overcome. 7. Discharge of urine and emptying of bladder. 8. Contraction of the unstriated and striated muscles surrounding the urethra and emptying this canal.

At 5 there will be in nearly all cases a reinforcing contraction of the abdominal muscles.

Voluntary control of micturition.-In the adult, the processes of retention and evacuation of urine are modified and controlled by voluntary effort. The normal action of the sphincter mechanism may be aided by the contraction of the perineal muscles, which keep the urethra closed. The reflex process of micturition may be set in motion by the contraction of the abdominal muscles, by which the pressure in the bladder is increased and the resistance to the passage of urine into the urethra overcome. It is possible, too, that the individual has a certain extent of voluntary power over the unstriated muscles of the bladder, and that the contraction of the muscular wall may be augmented by inipulses proceeding from the cortex to the micturition centre in the bladder cord. In this case, the initiation of the act would be analogous to that in which micturition is caused by stimulation of some sensory nerve, since in both cases the centre in the lumbar cord must first be influenced by the stimulus.

This view is favoured by the fact that contraction of the detrusor urinæ can be evoked by stimulation of the crura cerebri, or of the medulla oblongata or anterior columns of the cord (Budge, ${ }^{1}$ Mosso ${ }^{2}$ ), and that this effect may be observed even after exclusion of contraction of the abdominal muscles by opening the abdomen or by the administration of curari. Bechterew and Mislawsky ${ }^{3}$ obtained contraction of the bladder on stimulating the sigmoid gyrus in dogs.

The series of striated muscles which surround the urethra are under the control of the will, so that micturition can be stopped at any time by their forcible contraction. According to some authors, however, there is direct voluntary control over the unstriated fibres at the neck of the bladder, the so-called "interual sphincter," which, as I have already mentioned, does not exist as a distinct muscle. According to Reyfisch, ${ }^{4}$ the "internal sphincter" may be inhibited or set into contraction directly by voluntary impulses.

Thus, if a catheter be pushed up the human urethra until its opening lies in the prostatic part of the urethra just outside the bladder, no urine flows, unless micturition be inaugurated voluntarily by the person experimented on. When micturition is proceeding, the flow can be instantly checked at the will of the person experimented on. Reyfisch considers that the voluntary muscles surrounding the urethra are in this case powerless to stop the stream, since the closure of the canal is prevented by the presence of the rigid catheter. $\mathrm{He}$ concludes, then, that the stoppage can only be accounted for by a voluntary contraction of the internal sphincter. It seems to me, however, possible that a lifting up of the base of the bladder and the prostate by the anterior fibres of the levator ani might be responsible for the sudden stoppage in the urinary flow observed in Reyfisch's experiments.

${ }^{1}$ Arch. f. d. ges. Physiol., Bonn, 1869, Bd. ii. S. 511 ; Virchow's Archiv, 1865, Bd. xv. S. 115 ; Ztschr. f. rat. Med., 1864, Bd. xix. S. 434.

${ }^{2}$ Arch. ital. de biol., Turin, 1882.

${ }^{3}$ Neurol. Centralbl., Leipzig, Bd. vii.

+ Virchow's Archiv, 1897, Bd. el. S. 111. 


\title{
THE MUSCULAR MECHANISMS OF THE GENERATIVE APPARATUS.
}

\author{
By Ernest H. Starling.
}

Contents :-Erection, p. 347-Nervous Mechanism, p. 348-Retractor Penis, p. 349 -Parturition, p. 349-Nervous Mechanism, p. 351.

THE act of impregnation consists in the introduction of spermatozoa into the female genital tract, where they may come in contact with and fertilise the ovum, which has been discharged from the ovary by a bursting of the Graafian follicle. We have therefore to describe the changes in the generative organs in both sexes, which accompany this act, and the means by which these changes are brought about.

Erection.--The mechanism by which the erection of the penis is brought about is twofold. The most important factor, as was shown by Eckhard ${ }^{1}$ and Loven, ${ }^{2}$ is an active dilatation of the vessels of the penis, especially of the medium and smaller sized arteries. This is shown by the fact that, if the penis be cut across, venous blood merely trickles away from the cut surface, whereas, if erection be excited, the flow of blood from the cut surface is increased eight to ten times, and the blood becomes bright arterial in colour. That this vaso-dilatation is the most important factor in erection, is shown by the fact that it is possible to excite erection in an animal, in whom the second factor has been abolished by paralysing the muscles by the injection of curare.

The second factor is the contraction of the ischio-cavernosus or erector penis muscle, certain fibres of which pass over the dorsal vein of the penis, and compress this vessel when they contract. Although this mechanism undoubtedly assists in the production of erection, yet it is unable by itself to cause erection, since ligature of the veins coming from the penis is unable to do so.

Before or at the beginning of coitus, analogous erectile changes occur in the female organs, leading to erection of the clitoris and of the other erectile structures of the vulva. The friction between the glans and the wall of the vagina causes a reflex discharge of motor impulses in both female and male. In the male, the muscle in the walls of the vasa deferentia and vesiculæ seminales enters into peristaltic contractions, thus forcing the contents of these receptacles into the urethra. The semen is pressed along the urethra by rhythmical contractions of the bulbo- and ischio-cavernosi muscles, which empty this canal from behind forwards. At the same time it seems probable that movements take place coincidently in the uterus, consisting in a change in the

\footnotetext{
${ }^{1}$ Beitr. z. Anat. u. Physiol. (Eekharl), Giessen, 1863, Bd. iii. S. 125.

${ }^{2}$ Arb. a. d. physiol. Anst. zu Leipzig, 1866.
} 
position of this organ, so that its axis more nearly coincides with that of the vagina, and, according to observations on animals, in an active antiperistaltic contraction of uterus and Fallopian tubes, by which the semen becomes forwarded towards the ovaries. Some such movement as this seems necessary to explain the fact that the fertilisation of the ovum takes place, as a rule, in the Fallopian tubes, and may take place on the surface of the ovaries or in the abdominal cavity.

Nervous meehanism.-Although, in both sexes, coitus is attended by a high degree of psychical excitement, yet it is essentially a reflex act, and can be carried out when all impulses from the higher centres are cut off by section of the cord in the dorsal region. The centre presiding over the act is situated in the lumbar spinal cord; and we have now to consider the paths, afferent and efferent, which are concerned in the various phases of the act.

The first accurate information on these points was furnished by the discovery by Eckhard ${ }^{1}$ of the function of the nervi erigentes. Since this time, the nerves to the generative organs have been the subject of investigation by Gaskell, ${ }^{2}$ Morat, ${ }^{3}$ Sherrington ${ }^{4}$ and Langley and Anderson. ${ }^{5}$ I shall follow the very full account given by the two lastnamed observers.

The external generative organs, like the bladder, are supplied from two sets of nerve fibres-from the lumbar nerves through the sympathetic, and from the sacral nerves. The fibres from the lumbar nerves arise in the cat from the second, third, and fourth, or the third, fourth, and fifth lumbar nerve-roots, and in the dog from the thirteenth thoracic, and the first to the fourth lumbar roots. They run in the white rami communicantes to the sympathetic chain, whence they may take two paths.

(a) The great majority of the fibres run down the sympathetic chain to the sacral ganglia, whence fibres are given off in the grey rami communicantes to the sacral nerves; their further course is by the pudic nerves, none running in the nervi erigentes.

(b) A few fibres go by the hypogastric nerves to the pelvic plexus.

Excitation of these fibres causes strong contraction of the arteries of the penis, and of the unstriated muscles of the tunica dartos of the scrotum. In animals which possess a retractor penis muscle, excitation of the lumbar nerves causes strong contraction of the muscle.

The fibres from the sacral nerve can be divided into two classesvisceral and somatic. The visceral branches run in the pelvic nerves, or nervi erigentes. Stimulation of these fibres produces active dilatation of the arteries of the penis or vulva, and also inhibition of the unstriated muscle or body of the penis, the retractor muscle of the penis, when present, and of the vulva muscles. The somatic branches supply motor nerves to the ischio- and bulbo-cavernosi, as well as the constrictor urethre. In the female, they supply the analogous muscles, namely, the erector clitoridis (ischio-cavernosus), and the sphincter vaginie (bulbo-cavernosus). Both these sets of fibres are therefore involved in the erection of the generative organs which accompanies coitus.

1 Beitr. z. Anat. u. Physiol. (Eckhard), Giessen, 1866.

${ }^{2}$ Journ. Physiol., Cambridge and London, 1886, vol. vii. 1) 1.

3 Arch. de physiol. norm. et path., Paris, 1890, tome ii.

4 Journ. Physiol., Cambridge and London, 1892, vol. xiii. p. 675.

5 lbid., 1895, vol. xix. p. 85 . 
The internal organs, i.e. the uterus and vagina in the female, and vasa deferentia, seninal vesicles, and uterus masculinus in the male, differ from the external organs in receiving no efferent nerve fibres from the sacral nerves, as has been pointed out by Langley and Anderson. They are supplied with fibres which pass out through the anterior roots of the third, fourth, and fifth lumbar nerves (in the rabbit and cat), and run through the sympathetic to the inferior mesenteric ganglia, whence they proceed by the hypogastric nerves. On stimulating these fibres, two effects are produced on the uterus and vagina, namely, a contraction of the small arteries, leading to pallor of the organs, and a strong contraction of the muscular coats. In the vagina, the contraction can usually be seen to start from one end and spread to the other. The whole then remains for a time in a state of powerful tonic contraction, which affects both longitudinal as well as circular muscles. In the male, stimulation of these nerves excites strong contraction of the whole musculature of the vasa deferentia and seminal vesicles, which may be strong enough to cause emission of semen from the penis. Here again both sets of muscular fibres are involved. These effects on the uterus and seminal vesicles are not abolished by injection of atropine.

The course of the sensory fibres from the generative organs to the lumbo-sacral cord has not yet been fully made out, but it seems probable that it corresponds to the course taken by the efferent fibres.

An accessory genital muscle, the retractor penis, which is found in the dog, cat, horse, donkey, hedgehog (not in the rabbit or man), presents considerable physiological interest. It was first described by Eckhard as the Afterruthenband, and consists of a thin band of longitudinally arranged unstriated muscle (15 to $20 \mathrm{cms}$. long in a spaniel weighing about $15 \mathrm{kilos}$.), ${ }^{1}$ which is inserted at the attachment of the prepuce, and is continued backwards in a sheath of connective tissue to the bulb, when it divides into two slips which pass on either side of the anus. A few striated fibres are found in the back part of this muscle, derived from the external sphincter of the anus and the bulbo-cavernosus muscles.

This muscle is extremely sensitive to changes of temperature, and is at the same time very tenacious of life. Thus it may be cut out of the body and kept in serum or blood, in a cool place, for two days. At the end of this time it will, on warming, relax, and enter into spontaneous rhythmic contractions. At about $40^{\circ} \mathrm{C}$. the muscle is quite flaccid. On cooling slightly (to $35^{\circ}$ ) it will shorten, and at the same time may enter into slow rhythmic contractions. If cooled to $15^{\circ} \mathrm{C}$. the muscle will contract to about a quarter of its previous length. The same shortening may be produced on exciting the muscle with strong interrupted currents.

The muscle is innervated from two sources, the two nerves having antagonistic actions. The motor fibres to the muscle are derived from the lumbar sympathetic (i.e. the upper set of nerve roots), and run to the muscle in the internal pudic nerve. The pelvic nerves, on the other hand, carry inhibitory impulses to the muscle, thus enabling the concomitant vascular dilatation to take effect in producing erection of the penis.

Parturition.-During pregnancy, marked changes take place in all the generative organs, the most important and striking being those affecting the uterus. This organ undergoes an enormous hypertrophy involving all its tissues, so that, whereas in a virgin the uterus is about

\footnotetext{
${ }^{1}$ Langley and Anderson, loc. cit., 1. 88.
} 
$6.5 \mathrm{cms}$. long, at the end of pregnancy it is $35 \mathrm{cms}$. in length by 24 cms. in width, and is twenty-four times as heavy as the unimpregnated uterus. This growth is mainly due to the hypertrophy of the muscular tissues, the larger muscular cells being increased seven- to eleven-fold in length, and two- to five-fold in breadth. Moreover, a number of fresh muscle cells are developed, apparently from cells which have the character of embryonic muscle cells. At the same time there is a marked growth in the mucous membrane which forms the decidua, and in all the connective tissue structures of the uterus, including blood vessels and lymphatics.

Along with the growth of the ovum, the uterus increases in size and becomes more distended, its irritability becoming greater, so that it is easily excited to contract. The stimulus may be supplied from adjacent abdominal organs, from the brain-as by emotions, or by direct excitation of the internal surface of the uterus, in consequence of movements of the fœetus. In many cases no antecedent stimulus can be discovered, and the automatic contraction of the uterus seems to be analogous to that which occurs in the distended bladder. These contractions ordinarily give rise to no sensations, and are only felt when they are augmented in consequence of reflex stimulation. During the greater part of pregnancy they have little or no effect on the contents of the uterus. During the last weeks or days of pregnancy, however, these contractions, which have now become more marked, have a distinct physiological effect. Not only do they, by pressing on the foetus, cause it, in most instances, to assume a suitable position for its subsequent expulsion, but, affecting the whole body of the uterus, including the longitudinal muscular fibres surrounding its neck, they assist the general enlargement of the organ in dilating the internal os, so that the upper part of the cervix is obliterated and drawn up into the body of the uterus some little time before labour has commenced.

With these changes in the uterus are associated changes in the round ligaments and in the vagina and vulva. The muscular fibres of the round ligaments become much hypertrophied and lengthened, and these structures can therefore aid appreciably the uterine contractions in the subsequent expulsion of the foetus. The vaginal walls become thickened and of looser texture, so as to afford less resistance to distension during the passage of the foetal head.

Considerable discussion has taken place as to the cause for the onset of the processes comprised under the heading of labour or parturition, at a nearly constant period of two hundred and seventy-two days after conception. Most of the explanations which have been suggested, such as the great irritability of the uterus at the termination of pregnancy, the loosening of the fotal membranes, the return of the menstrual congestion after tell months, thrombosis of the placental sinuses, simply replace one question by another. According to Spiegelberg, the phenomena accompanying the birth of twins, which are often born at a considerable interval from each other, the onset of contractions of the uterus at the right time in normal as well as in extrauterine fœtation, the fact that the extra-uterine foetus dies when it has become mature, all go to show that the reason why labour occurs at a definite time must be sought for in fœetal rather than in uterine changes. This author suggests that some substances which had previously been used up by the fotus gradually accumulate in the maternal 
blood as the fœtus becomes mature, and provoke, by their direct action on the uterus or spinal cord, the uterine contractions which give rise to labour.

Actual parturition is in man generally divided into two stages. In the first stage, the contractions are confined to the uterus, and chiefly act in dilating the os uteri. In this dilatation two factors are involved, namely, the active dilatation brought about by the contraction of the longitudinal muscular fibres which form the chief constituent of the lower part of the uterine wall; and, in the second place, a passive dilatation by the pressure of the foetal bag of membranes, which is filled with amniotic fluid, and forced down as a fluid wedge into the os by the contractions of the uterine fundus.

The uterine contractions are essentially rhythmical, being feeble at first, and increasing gradually in intensity to a maximum which endures a certain time, and then gradually subsides. The frequency and duration of the contractions increase as labour advances.

As soon as the os is fully dilated and the foetal head has entered the pelvis, the contractions change in character, being much more prolonged and frequent, and attended by more or less voluntary contractions of the abdominal muscles. This action of the abdominal muscles is associated with fixation of the diaphragin and closure of the glottis, so that pressure is brought to bear on the whole contents of the abdomen, including the uterus. No expelling force can be ascribed to the vagina, since it is too greatly stretched by the advancing fotus.

In this way the foetus is gradually thrust through the pelvic canal, dilating the soft parts which impede its progress, and is finally expelled through the vulva, the head being born first. The membranes generally rupture towards the end of the first stage of parturition.

A third stage of labour is generally described. This consists in a renewal of uterine contractions about twenty to thirty minutes after the birth of the child, and results in the expulsion of the placenta and fœtal membranes.

Nervous mechanism.-We possess little experimental knowledge of the nervous mechanism of parturition. The most important observation on this point is the already quoted experiment by Goltz, ${ }^{1}$ in which this physiologist observed the normal performance of menstruation (heat), impregnation, and parturition in a bitch whose spinal cord had been completely divided in the dorsal region during the previous year. On the other hand, destruction of the lumbar cord completely abolishes the normal uterine contractions of parturition, so that this act must be regarded as essentially reflex, presided over by a controlling 'centre' in the grey matter of the lumbar cord.

The activity of the centre can be inhibited or augmented by impulses arriving at it from the peripheral parts of the body, as by the stimulation of sensory nerves, or from the brain, as under the influence of emotions. The nerve paths from the centre to the uterus have been already described in the previous section.

${ }^{1}$ Arch. f. $d$. ges. Physiol., Bonn, 1874, Bd, ix. S. 552. 


\title{
THE MECHANICAL, THERMAL, AND ELECTRICAL PROPERTIES OF STRIPED MUSCLE.
}

\author{
By J. Burdon Sanderson.
}

Contents.-The Mechanical Response-Response to Successive Excitations, p. 353-Response to Single Stimulus, p. 358-Influence of Mechanical Conditions on Excitatory Process, p. 363-Muscular Tonus, p. 377-Genesis of Tetanus, p. 378-Summation of Stimuli, p. 380-Period of Latency, p. 381-Rate of Propagation, p. 383-Conduction of Slow Waves, p. 384-ExHAUstion AND DEATH of Muscle-Fatigue, p. 385-Causes of Exhaustion, p. 389-Death of Muscle, p. 390-Rigor Mortis, p. 390-Nature of Process concerned in Muscular Contraction, p. 393 - Production of Heat during Contraction, p. 397 Electrical Properties of Muscle-In the Unexcited State, p. 407-Current of Injury, p. 411-Electromotive Phenomena of Excited Muscle, p. 413Electrical Response to a Single Stimulus, p. 414-Electrical Response to a Series of Stimuli, p. 422-Direct Actiox of Voltaic Current on MusclesPolarisation of Muscle, p. 426-Polar Effects during and after Passage of Current, p. 429-Polar Excitation, p. 431-Secondary Excitation, p. 436-Polarising Action of Currents of Brief Duration, p. 438-CARDiac MUscLE-The Mechanical Response, 1. 439-The Electrical Response, p. 443.

THE function of a muscle is to contribute to the accomplishment of bodily movements, or to the performance of external work, by changing its form in obedience to influences received by it from the central nervous system.

These influences are comprised under the general term "stimuli." The characteristic of a stimulus is that it evokes the specific function of an excitable organ; and the characteristic of an excitable organ is that its function is a response to stimulation. Muscle is the type of such an organ. According to the mode of observation employed, the phenomena of muscular function which present themselves to the observer are chemical, mechanical, thermal, or electrical. The present article comprises the last three of these four classes. ${ }^{1}$

\section{I.-The Mechanical Response.}

In the examination of the mechanical changes which follow excitation, we may begin either. with the mechanical effect of a single momentary excitation, or by comparing the mechanical properties of a muscle when in a state of excitation with those which it possesses when at rest. For two reasons the latter course is preferable. One reason is that the natural action of a muscle consists in its passing from the unexcited into the excited state, under the influence of a stimulus which acts con-

${ }^{1}$ The chemical ehanges which aecompany the activity of muscle are dealt with in vol. $i$. The phenomena of museular contraction which require the aid of the mieroscope for their observation are described in works on histology, and are therefore omitted here. 
tinuously; the other, that the response to a single stimulation consists of two processes or stages, in one of which it enters into the state of activity, in the other returns to that of rest. The interference of these two states involves complications which do not present themselves in a muscle which is continuously excited.

Mechanical response to a succession of instantaneous stimuliTetanus.-A state of excitation comparable to voluntary contraction can be produced for the purpose of investigation, by subjecting a muscle to the action of a succession of instantaneous stimuli of sufficient frequency. The response to excitation of this kind is technically called tetanus. ${ }^{1}$ In using this tern, it should be carefully borne in mind that there are other forms of continuous contraction which are not evoked by repeated stimuli; and it is by no means certain that voluntary contraction is not of the number.

The methods of producing tetanus will be discussed more fully in a subsequent section. For our present purpose it is sufficient to say that the tetanic state (the state of excitatory shortening) can be produced either directly or indirectly, i.e. by subjecting either the muscle itself (direct method) or its nerve (indirect method) to a succession of stimuli. For the purpose now in view it is essential that the stimulations should be not only frequent, but of uniform efficiency; and to insure this, they must be of such strength as to make the muscle contract to the utmost.

The characteristic phenomena of tetanus can be best observed in a muscle which is excited directly by induction currents in alternately opposite directions, of which the frequency is from 30 to 100 per second. It is assumed in what follows that this method is employed.

For experiments relating to the mechanical response to stimulation, the muscle preparation which has been used by preference is that of the pair of internal thigh muscles of the frog-the gracitis and semimembranosus. These are easily recognised by the tendinous inscription with which each is provided. They take their origin from the ischio-pubic symphysis and run nearly parallel to each other, to be inserted into the inner aspect of the head of the tibiafibula. The preparation is made by cutting through the two heads on each side and removing with each the two muscles in question, together with the ischio-pubic ridge from which they spring. The advantage of this preparation (hereafter referred to as the "double adductor preparation") is that each of the muscles consists of fibres all of which run in the same direction. It is a further advantage that when prepared as above described the two groups act for all practical purposes as one muscle, the length of which, if they are used lengthwise, may be, in a good-sized frog, as much as 3 in. (Fig. 189).

The phenomena which present themselves when a muscle is completely and continuously excited, differ according to the mechanical conditions under which it is placed. If before and during excitation its opposite attachments are so fixed that they cannot be brought nearer together by the effort of the muscle to contract, the excitation is said to occur under isometric conditions. If, on the other hand, one end of the muscle is left free, so that it can shorten on excitation, and in

${ }^{1}$ The fact that continuous contraction can be produced by repeated stimulation was known to Volta (1792). The word "tetanise" was introduced into physiology by Matteucei (1838). "Tetanus" is now defined in inost text-books as the effect of rapidly repeated stimulation (see Hermann's "Handbuch," Bd. i. S. 41). du Bois Reymond, who first used this method for investigating muscular contraction, did not limit the meaning of the word to the result of this kind of stimulation.

VOL. 11. -23 
so doing lift a weight which is attached to it, the excitation is said to take place under isotonic conditions.

In the former case the form of the muscle remains nearly the same as it was before it was stimulated, although, owing to a certain amount

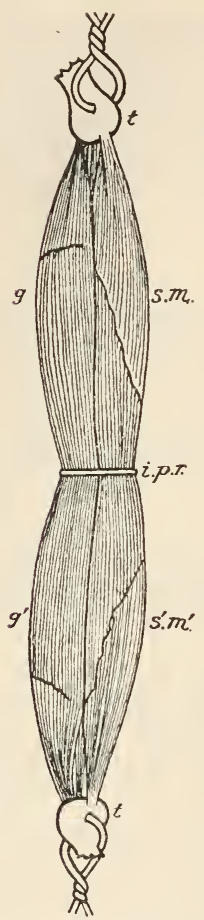

FIG. 189. - The double adductor preparation. sin, $s^{\prime} m^{\prime}$, semimembranosus ; $g g^{\prime}$, gracilis of right and left legs respectively of a Rana esculcnta; ipr, ischiopubic ridge ; $t, t$, head of tibia-fibula of right and left side respectively. of internal displacement, consequent on unequal contraction of different parts, it is not precisely the same. ${ }^{1}$ The muscle which was before relaxed now becomes tense. It thus acquires properties resembling those of a spiral steel spring of which the ends are attached to two fixed points, at a distance from each other which considerably exceeds the length of the spring when left to itself.

According to the simplest theory of muscular contraction, the work done by a muscle in passing from the relaxed to the contracted state, and thereby lifting a weight, is equal to the work done by the same weight on the same muscle in extending it from its length when so shortened to its length when relaxed. Experiment, however, shows that it is only under exceptional conditions that this equality is realised. It is on these conditions, therefore, that the efficiency of muscular action depends. For, just as a stretched spring cannot do more external work when released than was required to stretch it, but may do less, so a loaded muscle, when excited, lifts its load with an efficiency which may equal but cannot exceed that which would be exercised on the excited muscle by the load in extending it to the same length. The relation between the two quantities, i.e. between the amount of work done on the excited muscle in extending it, and the amount of external work which can be done by the same muscle in lifting a weight or overcoming a resistance, has been investigated experimentally under a variety of conditions by Fick, who embodied the results in a small book on the physiology of muscle some seventeen years ago. ${ }^{2}$ As it is of great importance that the subject should be treated experimentally, I propose to begin the discussion of it by giving an account of a form of experiment in which the amount of actual work done is equal to that estimated.

The amount of work which must be done on an excited muscle in order to stretch it to its "natural" length when not excited, is, as in the case of a spiral spring or an indiarubber band, the product obtained by multiplying half the increment of length by the weight which is required to extend it. With regard to muscle, this statement is not absolutely exact; for when an excited muscle is stretched, it does not

\footnotetext{
${ }^{1}$ See Blix, Skandin. Arch. f. Physiol., Leipzig, Bd. vi. S. 245.

2 Fick, "Mechanische Arbeit und Wärme-entwickelung bei der Muskelthätigkeit," Leipzig, 1882.
} 
lengthen in exact accordance with Hooke's law, i.e. the successive increments of length which are produced by equal increments of load are not perfectly equal. In other words, it is not perfectly elastic. It is, however, so nearly elastic that the statement above given is sufficiently correct.

In all experiments the external work actually done is represented by the lifting of a weight, and estimated in gramme-millimetres by multiplying the weight by the height to which it is lifted; but the amount so measured varies according to the mechanical conditions under which the effort is made. In order to obtain the most favourable result, these must be such as to enable the muscle, throughout the period during which it is being excited, to act to the best advantage. Now, in a muscle excited under isotonic conditions, the stress rapidly diminishes as the muscle shortens. If, for example, it shortens from its natural length when unexcited to its natural length in tetanus, the tension diminishes pari passu with the length, from a considerable amount to zero. If the load is nearly as much as it can lift at the beginning of the effort, there will be a slight initial shortening followed by a long period during which at first no work is being done by it, but afterwards "negative" work is being done on it. If the load is very small, the muscle will at once nearly complete its contraction, but will thereby do little work; for, though the lift is great, the load is too small to yield a considerable product. If the original loar

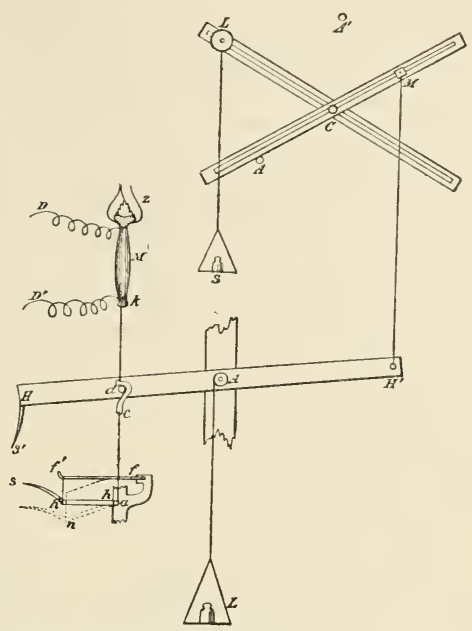

FIG. 190.-Cosine lever, combined with writing lever and tension recorder. $H \boldsymbol{H}^{\prime}$, steel writing lever, $32 \mathrm{ems}$. long, rotating on the axle $A$, which bears two pulleys, of 4 and $8 \mathrm{~mm}$. diameter respectively, from one of which a scale-pan $L$ is suspended. $M{ }^{\prime}$, the musele preparation, fixed at $z$; its free end, $k$, being attached by a fine wire to one arno of the writing lever at a distance half-way between the axis and the writing point $s^{\prime}$, i.e. $8 \mathrm{cms}$. from $A$. $D D^{\prime}$, wires from the secondary coil of an inductorium. To the extremity of the other arm of the writing lever, at $H^{\prime}, i . e$. $16 \mathrm{cms}$. from $A$, one bar of the cosine lever is connected by a long thread, the whole length of which (about $60 \mathrm{cms}$.) is not shown in the figure. $M I$, the movable block on this bar to which the thread is attached. $C$, axle of the cosine lever, fixed to a stand, so that the two brass eross-bars shall be in the same vertical plane as the lever, $H H^{\prime} . \quad L$, sliding weight of 200 grms. on the second eross-bar. $S$, seale-pan for additional weights. ' $A A^{\prime}$ ', stops on the stand for preventing the weight $L$ from acting on the muscle before and after the experiment respectively. $h h^{\prime}$, baniboo tension-recording lever, with its fixed axle $a$ bearing a small pulley, only $1 \mathrm{~mm}$. in diameter, round which is strung a pliable but inextensible thread, ending in a brass hook $c$, by means of which it can be attached to a pin $d$ on the length-recording lever $H H^{\prime}$, at the same point at which the free end of the muscle is attached, and readily detached. $f f^{\prime}$, steel spring fixed at $f$, its other end, $f^{\prime}$, being connected with the lever $h h^{\prime}$ by a stiff wire. $s$, writing point of lever $h h^{\prime}$. $n a$, position that $h h^{\prime}$ would assume on exeitation of the musele.-After Fiek.

is reduced to half, the result of the first part of the effort will be nuch 
larger than in either of the two other cases; but during the succeeding part nothing further will be accomplished. There would, however, be no such fruitless period if the load were diminished throughout at the same rate as the length of the muscle; for in that case the muscle would at every moment have as much work as it could manage.

Acting on this principle, Fick has contrived an instrument (Fig. 190) which may be called a cosine lever. When this is interposed between the muscle and the weight it has to lift, the former is enabled to work under conditions which approximate to those required.

It consists of two bars which can be screwed together at any angle. They rotate on the axle $C$. From the left upper arm a weight can be hung at any distance from $C$. On the right upper arm at $M$, the muscle acts. The rotation of the lever is checked at $A$ and $A^{\prime}$. The cross must be so arranged that, during the period of shortening of the muscle, the rotatory moment of the weight shall diminish from a maximum-that of the initial tension-to zero; and $C M$ must be of such length that the muscle in contracting with a minimal load shall rotate the bar $M 60^{\circ}$, in which case the distance through which $M$ descends must be equal to $C M I$. For this purpose, it is convenient that the levers should be set at an angle of $60^{\circ}$ to each other.

The mode of experimenting is as follows:-After being tetanised, the muscle is allowed to shorten, and in so doing to act on the shorter end of the lever $H H^{\prime}$, the long arm being attached by a long cord to the cosine lever at $M$. In an experiment, of which the data are given by Fick, a weight of 200 grms., $L$, was attached to the lever at $90 \mathrm{~mm}$. distance from $C$, while the muscle, which had been previously found to shorten from 50 to $24 \mathrm{~mm}$., acted on the other lever at $52 \mathrm{~mm}$. distance from the same axis. Obviously, the rotatory moment of $L$ on the axis $C$ is proportional to the cosine of the angle of inclination of the bar $L C$. The completely tetanised muscle had been found to exercise at $d$ an initial traction of 800 grms., consequently at $M$ of $400 \mathrm{grms}$., for $A H^{\prime}$ is twice as long as $A d$. Its rotatory moment was therefore $52 \times 400 \times \cos 30^{\circ}=20,800 \cos 30^{\circ}$. As the rotation of $C$ produced by the contraction of the muscle amounted to $60^{\circ}, M$ was pulled down $52 \mathrm{~mm}$., and $L$ raised $45 \mathrm{~mm}$., so that the amount of work done was $9000 \mathrm{grm} . \mathrm{mm}^{1}{ }^{1}$

The amount of work which, according to calculation, would have been required to stretch the same excited muscle from its natural or equilibrium length in tetanus, to its equilibrium length when at rest, was $9200 \mathrm{grm} . \mathrm{mm}$., an amount which so nearly corresponds with that of the external work it was found to be able to do, as to afford no sufficient reason for doubting that, under the conditions in question, the whole of the available mechanical energy of the muscle was brought into play. By a slight increase of the distance $L C$, the correspondence might have been made closer.

Other experiments show that it is not possible to make a muscle do the amount of external work which corresponds to the negative work done on it in extending it from its excited to its unexcited length, except under such conditions as have been stated, and that the more nearly these are approached the larger is the proportion of work actually done to what theoretically might be done. The muscle acts advantageously, because, during the whole period of its action, the work it is called upon to do is always as much as it can master, and diminishes as it becomes less fit for it; or, to use the language in which

${ }^{1}$ From $30^{\circ}$ to $0^{\circ}$ the cosines decrease nearly at the same rate as the angles. Between $60^{\circ}$ and $30^{\circ}$ they obviously decrease more slowly. This, however, does not appear to interfere with the result. It is possible to give a simpler form to the cosine lever. I prefer, however, not to modify Fick's figure, 
the principle is formulated by Fick, "If a muscle is completely tetanised, and thereby overcomes an external force which at each moment nearly balances its tension, the mechanical effect, i.e. the negative work overcome, is exactly equal to the positive work as calculated from the extension curve." 1

The utmost amount of external work which can theoretically be done by a tetanised muscle during the period of effort is, in accordance with the principle which has just been enunciated, obtained by multiplying the load lifted by half the lift. It may be represented by a triangular area (Fig. 191), hereafter to be referred to as the work-diagram. If $a c$ be the equilibrium-length of the muscle when tetanised, $a b$ its equilibrium-length when relaxed, and $b g$ represent the amount of the load required to stretch the excited muscle from the length $a c$ to the length $a b$, it is obvious that the triangle $c b g$ represents the product in question, i.e., on the one hand the smallest amount of negative work required to extend the tetanised muscle to its unexcited length, and, on the other, the maximum amount of positive external work which it is capable of accomplishing under the most favourable circumstances.

The normal length of a muscle in the body, when said to be at rest, is not that of equilibrium, but of extension, i.e., its length at the moment of beginning any effort exceeds that which it would possess if it were perfectly free to assume its natural form. This fact is advantageous to the organism in a way that the diagram helps to explain. In constructing it, it was assumed that, in order to extend the excited muscle to the length $a b$, a weight of 700 grms. was required. If, before excitation, an extending force of say 250 grms. had been applied to it, so that it would have assumed the length $a d$, it would require, as the diagram shows, in

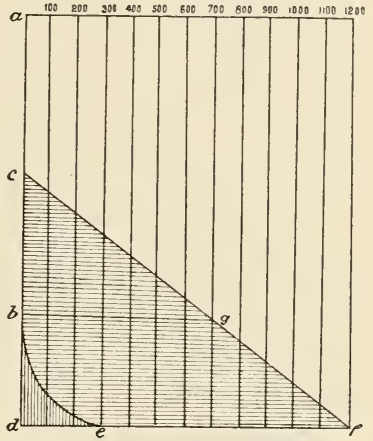

FIG. 191.- Work-diagram. $\alpha b$, length of unexcited muscle; and $b c$, its extension curve. $a c$, length of musele when excited; and $c f$, its extension curve. $c d f$, the whole work capable of being done by an excited muscle in shortening from a length $\alpha d$, to its proper length ac. The vertically shaded area represents the work done on the muscle in bringing it to the length ad before excitation; the horizontally shaded area the "useful effect." - After Fick. order to stretch it to the same length in the excited state, the weight of 1200 grms. It would then be able, under perfectly favourable conditions, to do, when released from extension, an amount of work represented by the much larger area $c d f$. As the diagram also shows, by far the greater part of this work would be accomplished by the proper energy of the muscle at the expense of material contained in itself. But the remainder, indicated by the vertically shaded area $b d c$, would be assignable to the negative work which must have been done on the muscle before excitation, in order to bring it to the length $a d$. The curved line $b c$ is, according to Fick, the "extension curve" of unexcited muscle, a curve of which the ordinates represent the successive lengths of such a muscle when extended by a load increasing 
by equal increments from 0 to 250 grms. The concavity of this curve is always upwards; had it heen rectilinear, the area would have been larger, but still inconsiderable as compared with the whole area $c d f$, so that we arrive at this conclusion, namely, that the stretching of a muscle beforc it is excited increases its efficiency in a degree out of all proportion to the amount of extrinsic work required for the purpose, the reason being that the amount of positive work done depends not on the very inconsiderable force actually used to extend the muscle, but on the negative work which would have been done had it been necessary to extend the cxcited muscle to the same length.

Response to a single instantaneous stimulus.-The twitch.-The mechanical change which takes place in a muscle in response to an instantaneous stimulation, is in all respects more complicated than that which is determined by continuous excitation, for whereas in studying the latter we have to compare a more or less persistent state which lasts as long as its cause, with the still more permanent condition of the muscle when inactive, we have in the former a process consisting of a succession of states, to the whole of which the instantaneous exciting cause is antecedent, and of which the duration and time-relations are entirely dependent on the physiological endowments of the muscle.

The knowledge which we possess of this process is gained by observing it and recording its effects under the two conditions already referred to-that in which the muscle is allowed to shorten without hindrance (isotonic contraction), and that in which no diminution of its length is allowed to take place. In the latter (isomctric contraction) the force with which it strives to shorten may be recorded with the aid of suitable methods.

The graphic record of the response to a single excitation under isotonic conditions.-For recording the diminution of length which a

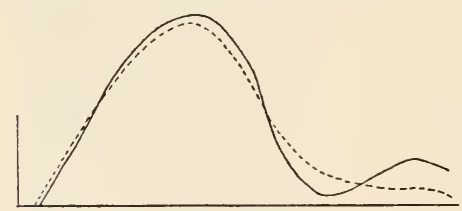

FIG. 192.-Isotomic curve drawn by a double semi-nembranosus preparation attached to a lever similar to $H H$ of Fig. 190 . The dotted line shows the curve described when the lever is made as light as possible.-Fick. muscle undergoes when instantaneously excited, Fick employed the lever of the form shown in Fig. 190, $H H^{\prime}$. The weight to be lifted hangs by its cord from the sicle of the axial pulley, the radius of which is $4 \mathrm{~mm}$. The muscle acts on the lever at a distance of $80 \mathrm{~mm}$., so that if the weight is $200 \mathrm{grms}$. the tension of the muscle when at rest is only 10 grms. If the rate of horizontal movement of the recording surface is about 10 in. per second, the curve at ordinary temperatures has the form shown in Fig. 192. Even when the lightest possible writing lever is used, its movement is somewhat affected by its inertia; so that the real form of the curve is approximately that represented by the dotted line.

It is a familiar fact, that when, in a succession of experiments on the same muscle, the strength of the instantaneous stimulus, which at the beginning is very inconsiderable, is increased step by step, there is at first no response, that the muscle then shortens scarcely perceptibly, but that, when this point has been gained, a very slight further increase of the strength of the stimulus evokes a complete or, as it is technically called, a maximal contraction. This term is used to signify 
that no further augmentation of effect can be gained by the same method.

The height to which the free end of the muscle is raised when contracting under isotonic conditions (which may be conveniently designated the lift), obviously depends on the load. If, as before, we measure the load in grammes and the lift in $\mathrm{mm}$., and multiply the one by the other, we have the work done during the shortening of the muscle.

To investigate the relation of load to lift, we cause the muscle to inscribe a succession of curves, the load being increased by an equal amount after each inscription, in such a way that the corresponding points in all the curves are in the same vertical line, as showl in Fig. 193. As the attached (upper) end of the
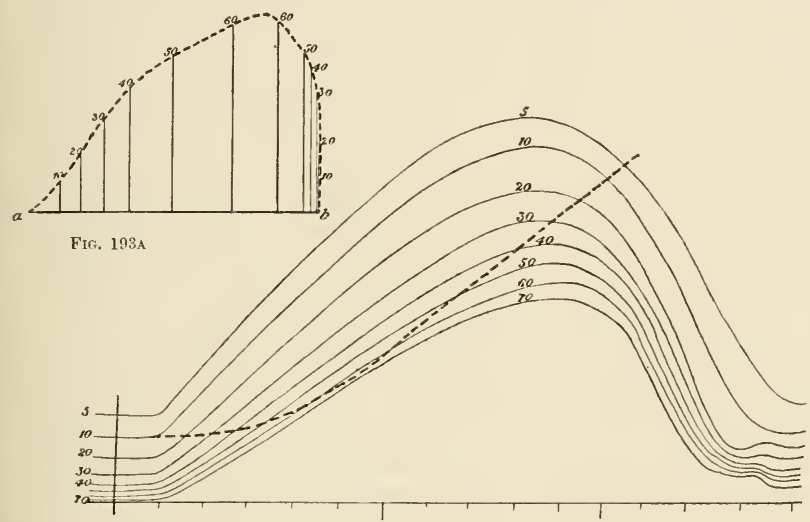

Fig. 193.

Fig. 193.- Series of isotonic curves with increasing load. The number to each curve indicates the weight on the muscle in granmes. The broken line represents the first part of an inertia curve, taken with the same muscle with a load of 10 grms. See p. 360.-After Fick.

Fic. 193A.-Work-diagram (to be read from right to left) for the same muscle as it would be if the tension were a fraction of the length of the muscle at any particular moment after the beginning of the contraction. Diminution of length is expressed by the distance from $b$, in the base line $b a$, to the foot of the vertical line, of which the lieight expresses the corresponding tension. Both figures liave been reduced in the same proportion. In the original, $1 \mathrm{grm}$. of tension was represented by $0.5 \mathrm{~mm}$.-After Fick. See Note 1.

muscle is in the same position throughout, the writing lever attached to the free end takes a lower position in the successive curves, the difference between the base lines of the curves expressing the increase of length which the additional load has produced. In the series of isotonic contractions of which the curves are given in the figure, the loads and lifts were respectively as follows : ${ }^{2}$

$\begin{array}{lrrrrrrrrr}\text { Loads (in grms.) } & \text {. } & 5 & 10 & 20 & 30 & 40 & 50 & 60 & 70 \\ \text { Lifts (in mm.) . } & . & 22 \cdot 5 & 23 \cdot 5 & 22 \cdot 0 & 20 \cdot 5 & 19 \cdot 0 & 18 \cdot 5 & 17 \cdot 5 & 16 \cdot 5 \\ \text { Products (grm.-nm.) } & . & 117 \cdot 5 & 235 & 440 & 615 & 760 & 925 & 1050 & 1155\end{array}$

1 The reader will understand this diagram (Fig. 193A) more easily if he imagines it placed on Fig. 193 with the base line vertical, and $b$ on the beginning of the broken line.

${ }^{2}$ Fick, op. cit., S. 112. 
In attempting to form a conception of the change which a muscle undergoes in response to a single excitation, in its transition from the unexcited to the contracted state, we derive assistance from the provisional hypothesis, that it is capable of assuming two forms-a longer and a shorter-and that in each of them it is in elastic cquilibrium. By this expression is meant that the internal elastic forces which are in operation in the structure of the organ are in each position so balanced, that if the organ is put out of shape by an external force, its equilibrium length is restored from the moment that this force ceases to act. In the case of a muscle arranged to contract under isotonic conditions in response to an instantaneous stimulus, we determine approximately the first of these positions, $(\alpha)$ when, without exciting the muscle, we employ an extremely light load; and the second $(b)$ when, under similar mechanical conditions, we stimulate maximally; the excitatory change of form is a transition from $a$ to $b$. The change, as it is observed by the graphic method, is found to occupy some time (in the muscles of the frog at ordinary temperatures about $\frac{8}{100}$ of a second), but what is recorded is the result of an infinite number of constituent changes, which do not occur simultaneously in all parts. The transition from the unexcited to the excited state must be supposed to last in each part for a less time than the whole period of shortening of the muscle; so that, between its accomplishment and the attainment by the muscle of the new form, we may think of it as striving towards a new state of elastic equilibrium. ${ }^{1}$

If we could consider the state of elastic equilibrium of a momentarily excited muscle, designated $b$, to be constant under the same physiological conditions, it would follow that the tension of a muscle at any moment during its period of shortening would always depend on its relative length, provided that the time lapsed since excitation remained the same, i.e., that if in a muscle actually contracting in response to an instantaneous stimulus, the tension were measured at a given moment (say 4, 5, 6 or 7-hundredths of a second after excitation), it would always be the same in a muscle of the same length, by whatever means it had been brought to this length. Unless it can be shown that this is so, the provisional assumption set forth above must be accepted, with the reservation, not that a muscle in contracting is actuated by other than elastic forces, but that these forces or some of them are not constant.

The question whether or not a muscle of a certain length, at a certain temperature and at a certain time after excitation, will always, so long as it is in a normal condition, have the same tension, presents itself in relation to the following experiment: ${ }^{2}$ -

A series of isotonic curves having been drawn under the conditions described in the preceding paragraph, an additional curve is drawn with a load of 10 grms., with this difference, that to the light lever $H H^{\prime}$ (Fig. 190), previously used, a heavy equilibrated oscillating bar is attached, so that the one cannot move without the other. For this purpose a wooden bar is used, which rotates in the same vertical plane as $H H^{\prime}$. It carries at either end a sliding weight of lead. The two weights, which are equal, can be so adjusted that the centre of gravity of lever and weight is in the axis. The writing point follows the course indicated by the broken line in Fig. 193, showing that the

1 Weber, Wagner's “"Handwörterbuch," Bd. iii. Abth. 2, S. 110.

Fick, op. cit., S. 120. 
free end of the muscle, instead of at once ascending rapidly, as in the curve previously drawn with the light lever from the same starting point, rises very slowly at first, afterwards, $i$.e. at a period of about $\frac{4}{100}$ of a second later, as rapidly as when unhampered by having to set in motion an inert equilibrated mass. It will be noticed that this curve, which may be called an "inertia curve," crosses each of the previous ones twice. Each point of intersection indicates that the muscle has on two occasions been of the same length, at the same time after excitation. On each of the first occasions the tension of the muscle is known, for each occurred in the course of an isotonic curve of known tension. If the hypothesis above stated is correct, the tension on the two occasions must in each case have been the same. Consequently if, on the base line $a b$ (Fig. 193A), we set off the lifts, i.e. the heights of the free end of the muscle corresponding to the intersections, and draw from the points thus marked ordinates, each representing the tension as indicated by the isotonic curve at the points of intersection, then a line joining the free ends of these ordinates (broken line in the figure) exhibits the relation of tension to length during the period that the lever was acted on by the contracting muscle. The space included between the broken line and the base line expresses, in gramme-millimetres, the work which would be done on the lever and oscillating bar during the same period, if the tension were a function of the length. The bar is made to do external work (in lifting a weight) which can be measured. ${ }^{1}$ The amount of this work, i.e. of the actual work done by the muscle when acting on the lever, can therefore be compared with the amount estimated from the diagram. In numerous experiments, made by Professor Fick, it was found that the actual work done exceeds-in some instances considerably-the amount estimated. It therefore appears that a muscle when resisted in the way above described, acquires a tension during its period of effort, which is greater than it possesses under what seem to be precisely the same physiological conditions, and is extended to the same length by a weight which it is lifting without any further resistance. In other words, the effort made by a muscle at any given moment after an instantaneous excitation, does not depend exclusively on its length, but is also affected by the mechanical conditions under which it shortens. The question whether this "reaction" of a muscle to resistance is physiological or physical will be considered later.

Considering that a muscle in the discharge of its normal function is always resisted, it is of more immediate importance to inquire how it deals with resistance, than to investigate, as we have done in the preceding pages, its behaviour when allowed to contract so as to lift a weight attached to its free end. At a very early period in the progress of experimental physiology, Schwann, one of the founders of the science, enunciated the fundamental principle that, on the whole, under the same physiological conditions, the tension of a contracting muscle varies with its length. For this purpose he used a very simple method, now called the method of "after-loading." He attached the upwardly directed tendon of the gastrocnemius of the frog to one end of a balance, of which the other end supported a weight. The beam at the muscle end was prevented from rising by a stop, so that the muscle, so long as it was relaxed, was not stretched by the weight. The experiment consisted in exciting the muscle, and observing what weight was just insufficient to hold the beam against the stop-in other words, what

1 This is effected by hanging the weight on a pulley on the axis of the equilibrated bar, in such a way that the weight begins to be lifted at the moment that the shortening of the muscle is completed. The height to which the weight is carried is recorded graphically. From the record, the work done on the equilibrated bar can be estimated. 
weight the muscle could just lift under the mechanical conditions present. This having been ascertained, the stop was lowered so that the weight began to act a little later. The weight, which was now in excess, having been adjusted as before, the stop was then again lowered and the observation was repeated with a similar result, and so on. It was thus found that the longer after excitation the muscle encountered the resistance of the weight (or, in other words, the more it had already contracted), the less able was it to overcome that resistance. ${ }^{1}$

The aim of Schwann's experiment was limited to the investigation of the relation between the length to which an excited muscle is extended, and the force which, when so extended, it is able to exert in contraction. Helmholtz ${ }^{2}$ subsequently applied the method of afterloading in an investigation which differed from that of Schwann in this respect, that instead of determining the tensions ("forces") corresponding to a series of lengths, he made the length constant, and observed the

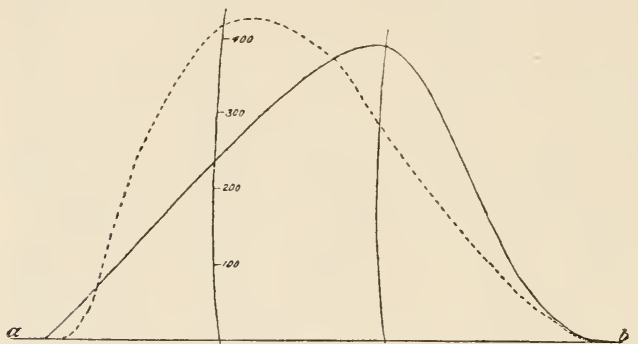

FIG. 194.-Isotonic (continuous line) and isometric (dotted line) curve of the same musele, represented as springing from the same base line. The two ascending lines designate the fourth and eighth-hundredth of a second after excitation. On one of them the tension in grammes for the isometric curve are indicated. The isotonic curve is drawn with the muscle loaded with 30 grms.-After Fick (modified from Fig. 28, loc. cit., p. 132).

which are identical with those of the isometric musele curve (see Fig. 194) as now obtained by a single observation. The most important outcome of the facts which have so far presented themselves, in relation to the isometric curve, is that which was shadowed forth in the preceding paragraph, namely, that the tension of a resisted muscle, especially during the first part of its period of effort, is much greater in proportion to its length than that of the same muscle when it attains the same length in the course of an isotonic contraction; and consequently that, although in muscle, whether in the relaxed or contracted state, we have sufficient evidence that the relation between tension and length is that which characterises elastic bodies, it cannot be asserted, even with the proviso that the comparison is made at the same stage in the excitatory process, that, the length being known, the tension can be estimated from it. As Professor Fick expresses it, a muscle is always

${ }^{1}$ Schwamn's experiment was first deseribed in Müller's "Physiologie," 1837, Bd. ii. S. 59-61.

${ }^{2}$ Arch.f. Anat., Physiol. u. wissensch. Med., Leipzig, 1850, S. 303. 
an elastic structure, but not always the same elastic structure, even when observed under the same physiological conditions. ${ }^{1}$

Influence of mechanical conditions on the course of the excitatory process.-In the preceding paragraph it has been shown that resistance exercises a very material influence on the character of the mechanical response to instantaneous stimulation. Although the question how this influence is exercised has during the last ten years engaged the attention of some of the most skilful experimenters, it has not yet been completely elucidated. What strikes us as remarkable is that the order of the phenomena which characterise the excitatory process in muscle, so long as the muscle contracts freely, gives place to a new order when the muscle is prevented from shortening. Call we so define the fundamental change which is undergone in a muscle when it is excited, as to be able to deduce therefrom an explanation sufficient to account, on physical principles, for both orders; or must we have recourse to the hypothesis of a secondary excitatory action of a physiological nature, caused by resistance, in which the primary change evoked by the stimulus excites in its turn secondary changes, which add their influence to the change which evoked them? Each of these views has at the present time strenuous supporters. I propose in the following pages to give an account of the experimental data which appear to be of most value as a basis for the discussion of this question.

Instead of using the term "resistance," it is convenient to refer to the condition which is produced by resistance to contraction, of an extended elastic body. This condition is commonly designated "tension." We shall afterwards find it convenient to designate as contractile stress that particular form of tension which expresses the effort made by a muscle in response to excitation, to overcome a resistance-whether that of a load which it is able to lift, or of a spring which it is able to bend or stretch. Whether we use the isotonic or the isometric method, we measure contractile stress in grammes.

Although it has been long recognised that the character of the mechanical response to an instantaneous stimulation depeuds not only on the strength of the stimulus, but also on the resistance to be overcome, it was not until seventeen years ago that J. v. Kries (now Professor at Freiburg), when working in Ludwig's laboratory, showed experimentally ${ }^{2}$ that mechanical conditions affecting the process at any stage in its progress, may alter its character. Referring to this action in a more recent paper, ${ }^{3}$ he appears to admit the possibility of its being due to a kind of secondary stimulation-the muscle being re-excited by the very effort which it makes in contracting or striving to contract. A similar view has been advanced by other writers in connection with a doctrine which has since become the subject of much controversy - the doctrine that the excitatory process is made up of two antagonistic component processes, one of which is associated with shortening, the other with relaxation, and that, inasmuch as the contractile component manifestly prevails at the beginning, that of relaxation towards the end, we may associate the two periods of a contraction with these two processes respectively. As it

\footnotetext{
1 Fick, op. cit., S. 138.

2 Arch. f. Physiol., Leipzig, 1880, S. 370.

${ }^{3}$ Ibid., 1892, S. 3.
} 
appeared to Professor Fick necessary that the whole subject of the mechanical conditions which affect muscular activity, especially with reference to their influence on the terminal stage, should be worked out, his pupil and assistant, Dr. Schenck, ${ }^{1}$ has with infinite perseverance subjected these conditions to experimental investigation. Regarding resistance to shortening as the most important of them, he aims at showing how the tension so produced acts directly on the two processes which he supposes to be concerned in the twitch- "the one which causes shortening, and the one which effectuates relaxation" 2_-the most characteristic effect of tension being, according to him, that it hastens the supervention of the "second process." Schenck regards the two processes as complementary to each other, and consequently holds that any alteration of the intensity or time-relations of either constituent process must affect the character of the whole. Whilst this view was being set forth at Würzburg, under Fick's immediate influence, Professor Gad of Berlin, and his pupil Kohnstamm, had been led by Fick's previous suggestions to speculate in a very similar direction. There are, according to them, ${ }^{3}$ two processes by which energy is transformed in the muscular response to stimulation. Both are heat-producing, but the first, which is associated with contraction, more so than the second, which is associated with relaxation. Both are favoured more or less by increase of temperature, as well as by increased intensity of stimulus, but the first only is favoured by increase of tension. The difference between the two theories lies in the fact that Gad does not recognise what appears to be Schenck's fundamental notion, namely, that whenever a muscular contraction is resisted, the action of the primary instantaneous stimulus is supplemented or reinforced by a second stimulation, having its source in the tension which the resistance produces. Both authors show that the course (order of phenomena) of the excitatory process can be modified during its progress by external conditions, and think that these modifications can be best understood by supposing that the process itself is dual, i.e. that it is made up of two constituent processes, more or less antagonistic to each other, and that the effect of resistance in modifying the course of the excitatory process depends on its influence on the two constituent processes.

The sketch I have given of these speculations, although scanty, may serve the purpose of facilitating the understanding of the very remarkable influence of tension on the excitatory process, of which the experiments made during the last half-dozen years by v. Kries, Schenck, Gad, Kaiser, and others, give evidence. The examination of these observatious will afford us the opportunity for such theoretical comments as may appear necessary.

Methods and instruments.-In Helmholtz's original myographic method, the changes recorded were partly of length, partly of tension. Marey was the first to show that, if it is desired to register changes of length correctly, very light writing-levers must be used, so as to avoid the errors arising from inertia; and it was he who taught us how to obtain myograms, the forms of which approach that of the true isotonic curve. Then, twenty years ago, Fick, by discovering and showing how to apply the isometric method, opened a new line of inquiry. His first tension-writer (represented in Fig. 190, p. 355) was

\footnotetext{
${ }^{1}$ Arch. f. d. ges. Physiol., Bonn, 1891, Bd. 1. S. 166 ; 1892, Bd. lii. S. 117.

${ }^{2}$ Sehenck, ibid., Bd. 1v. S. 626.

${ }^{3} \mathrm{Gad}$, Arch. f. Physiol., Leipzig, 1893, S. 170.
} 
the parent of many other contrivances for the same purpose. It consists of a light lever $h h^{\prime}$, round the axis of which, $a$, runs a pliable but inextensible thread with a hook at its free end, $c$, which is attached to the lever $H H^{\prime}$ previously described, at the same point $d$ at which the muscle is attached; connected with the lever $h h^{\prime}$ by a stiff wire is a rigid bar, $f f^{\prime}$, fixed at $f$.

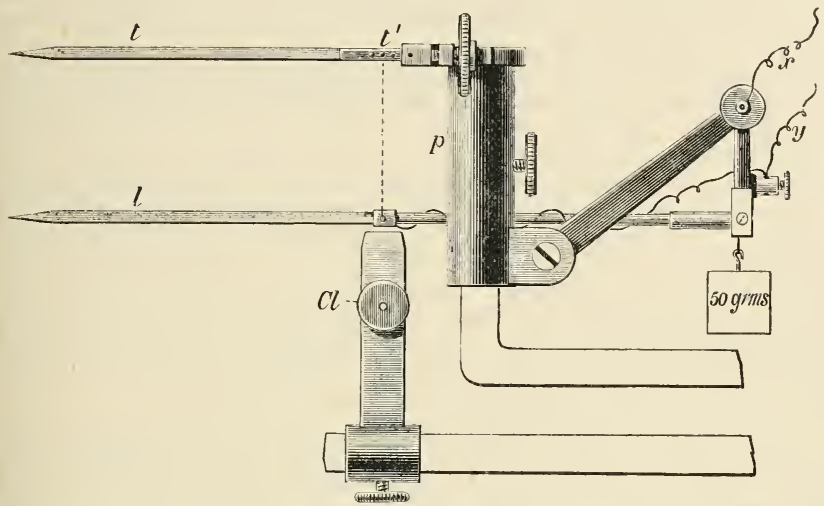

Fig. 195.-Blix's apparatus for recording isometric and isotonic curves synchronically. $p$, the support ; $t t^{\prime}$, isometric lever ; $l$, isotonic lever ; $x$ and $y$, wires from the secondary coil of an inductorium. The muscle occupies the position indicated by the broken line. For taking an isometric curve, the lever $l$ and the attachment of the muscle to it, are made immovable by means of the clamp, $C l$. When the clamp is open, $l$ records an isotonic curve. Its movement is resisted by a weight, the thread supporting which is wound round the axle. A serew serves to adjust the isometric lever in the way shown in Fig. 195A.

When the muscle is excited it strives to rotate the axis $a$, but is opposed in this effort by the steel spring $f f^{\prime}$. The tension it acquires is recorded graphically by a writer $s$. If during the existence of this tension the hook $c$ is unhitehed from its pin $d$, the excited muscle at once shortens to the length which it possesses when left to itself. This shortening can also be recorded by a writer $s^{\prime}$, on the lever $H H^{\prime}$.

Fick's first purpose was to register the changes of tension in a muscle striving to contract, but entirely prevented from doing so. Subsequently, the method was extended to the case in which a muscle contracts against resistance, and instruments were contrived by which tension

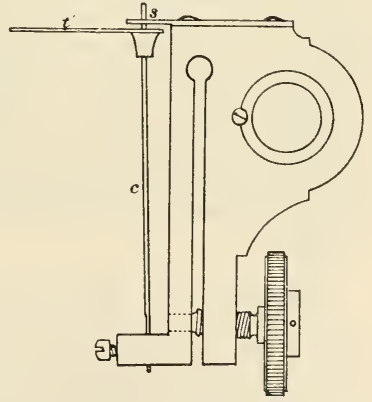

FIg. 195A.-Enlarged view of the same apparatus, to show the torsion rod from above.

taneously. Although Fick's original myotonograph gave curves which exhibit to a considerable extent the deformation which arises from friction and inertia, the records obtained by the more perfect tension-writer of his pupils Schönlein and Schenck are not open to this objection. ${ }^{1}$

${ }^{1}$ Schenck, Arch.f. d. ges. Physiol., Bonn, 1892, Bd. lii. S. 108. 
A tension-writer which has the further advantage of greater simplicity is that of Professor Blix ${ }^{1}$ (see Fig. 195 and 195A). The muscle acts by torsion on a cylindrical rod of steel $e, 5 \mathrm{~mm}$. long, and nearly $1 \mathrm{~mm}$. thick. This is fixed horizontally at one end ; the other is free, but is supported at $s$. From a point near the free end a metal bar $t^{\prime}$ springs, of which the tension-recording arm $t$ is a continuation. At known distances the bar is perforated for the reception of a steel hook, to which the muscle is attached, the usual distance being $3 \mathrm{~mm}$. from the axis. The resistance which the steel rod offers to. torsion is such that a weight of 10 to 20 grms. depresses the writing end only $1 \mathrm{~mm}$. The arm is $9 \cdot 6 \mathrm{cms}$. long, and together with the steel rod weighs only $0.05 \mathrm{grm}$. The figure shows the way in which the tension-writer is combined with the shortening-writer, so as to register the isometric and isotonic curves synchronically, i.e. the curves occupying corresponding positions on the recording surface of the cylinder. The shortening-writer $l$ is a strip of bamboo, of which the free end is $18 \mathrm{cms}$. from its axis. It is perforated transversely by a needle about $8 \mathrm{cms}$. from the axis ; to this needle the lower end of the muscle is attached. This instrument is not only very free from the errors due to inertia and friction, but has the advantage that in employing it for drawing an isometric curve alone, the length of the muscle diminishes extremely little as the tension increases. A tension of $100 \mathrm{grms}$. can be attained with a diminution of length of no more than $0.06 \mathrm{~mm}$.

It is desirable for all myographic purposes that the rate of rotation of the recording cylinder should be constant and equable, that the position of the lever at the moment of excitation should be invariable, and that the vibrator which marks the time should start simultaneously with the record. In the instrument designed by Blix all these ends are secured. The rotation of the cylinder is urged by a spring, but is restrained by a detent, on the withdrawal of which the cylinder flies round. In doing so, it liberates a reed which writes its vibrations on the blackened surface, and at a later moment opens the primary circuit of the induction coil. The heavy cylinder, which before this happens has ceased to be acted on by the spring, continues its rotation at a nearly uniform rate, until, after the curves have been drawn, it is arrested by a mechanism which need not be here described. Before the next observation, it must of course be brought back to its original position and adjustment. Although the apparatus above described may admit of further improvement, it answers for the present the requirements of the investigator. It enables him to obtain a true graphic representation of each of the mechanical changes which a muscle undergoes in consequence of excitation by an instantaneous stimulus; and, by registering tension and length synchronically, to determine both their quantitative relation to each other at any moment, and the relation existing at any moment between their rates of change.

Experiments in which the mechanical conditions are varied during the response.- In v. Kries' first series of experiments relating to this subject, the conditions were such that, although when in the unexcited state the muscle is of its natural length and has no tension, it cannot contract without encountering resistance, which, however, it overcomes as soon as it has gathered sufficient strength. The moment at which this happens is the longer delayed the heavier the weight. In this case the excitatory process begins isometrically and proceeds isotonically. The figure (Fig. 196) shows what happens.

Curve 1 in both series is drawn by the unloaded muscle, Curves 2, 3, 4, and 5 by the same muscle, when loaded successively with increasing weights. All ${ }^{1}$ Skandin. Arch. f. Physiol., Leipzig, 1892, Bd. iii. S. 295. 
the curves culminate about the same time, but the inclination of the rise, i.e. the rate of shortening, diminishes as the load increases. The changes of tension are shown in the curves of the upper series. In each case the horizontal part of the tracing indicates that, during the time to which it corresponds, the muscle is working isotonically. Curve 5 of this series is identical with Helmholtz's " energy curve" (see p. 362). In this case it is evident that, as the load increases the rate of increase of length lessens, while the tension remains the same.

Fig. 197 shows

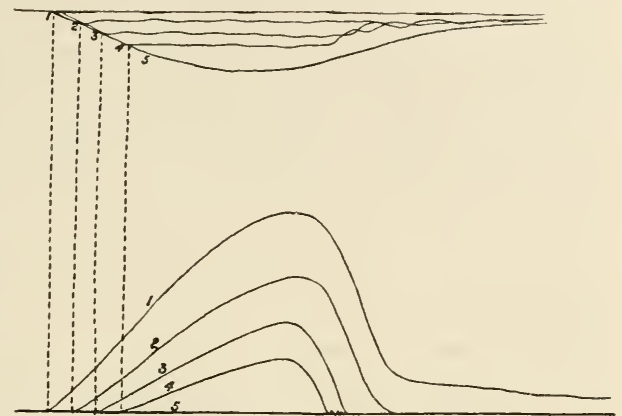

Fig. 196.-Series of "after-loading" curves, with increasing load.-After v. Kries.

the simple mechanism with the aid of which these curves are recorded. Before the muscle begins to shorten, the descent of the load is arrested by the stop So, the height of which is so adjusted that until the moment of excitation there shall be no tension. The stop $S u$ in this case is not used. When the muscle relaxes, and the prolongation of the lever again strikes So, the curve again becomes isometric.

In another series of experiments the same contrivance was employed to produce sudden increase of tension at a later perior during the response, the stop $S u$ being now brought into play, the stop So not being used. The contractions recorded are of the kind called by v. Kries, Anschlagszuckungen (arrested contractions). The load is increased as before, after each

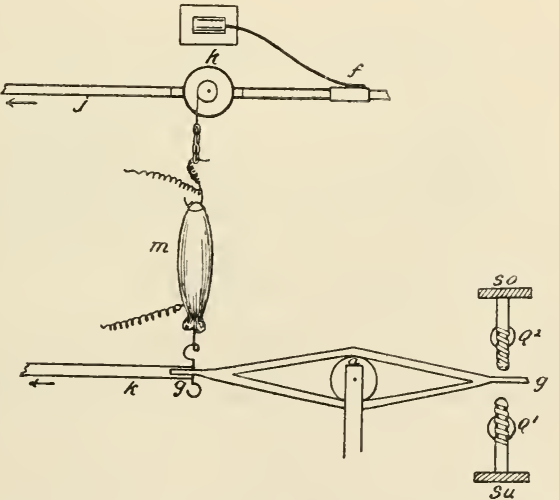

FIG. 197.-v. Kries' apparatus for taking "after-loading" curves and "arrested-contraction" curves. $j$, isometric lever with its axis at $k ; g g$, isotonic lever with its axis at $a, 30 \mathrm{~mm}$. to the right of $g$; So and $S u$, serews held by two horizontal arms of a support, $Q^{2}$ and $Q ; m$, muscle (Fick's double adductor preparation, with the two groups of museles used abreast). observation, and consequently each successive eurve begins at a lower level. In order that the length of the muscle may be the same at the moment of arrest in each case, the stop $S u$ must remain throughout at the same height. It is therefore encountered by the prolongation of the shortening lever later each time, and 
the interval of time between the moment of encounter and that of culmination diminishes. In this case each response begins isotonically, and proceeds isometrically, as seen in Fig. 198. ${ }^{1}$ When the lever, on the relaxation of the muscle, leaves the stop, the curve again follows an isotonic course.

To make clear the bearing of these empirical data, some further explanation is necessary. The reader must first understand that the purpose of both the methods above described is to investigate at any moment in the response, the two functional activities (Leistungsfühigkieiten) of a muscle-the Fähigkeit Verkïrzung zu leisten, and the Fühigkieit Spannung zu erzeugen. These are represented by the slant of the isometric and isotonic curves respectively, at the moment that the lever parts from So or encounters $S u$. To indicate these times the vertical broken lines have been added to von Kries' figure. The important point in the curve of after-loading is that whereas the isotonic curve rises with an initial inclination which diminishes as the load increases, the isometric has nearly the same initial inclination throughout. This means that "the ability of the muscle to generate tension" is unchanged, while its "ability to produce shortening" diminishes. The relation between these he calls "the apparent extensibility" of the muscle. In the experiment given in Fig. 198, the load which is at first minimal is increased after each observation ;

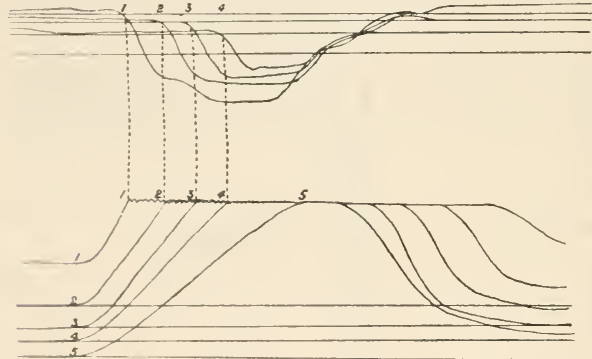

Fig. 198, - Series of "arrested contraction" curves, with increasing load, the length of the muscle being the same at the moment of arrest in each ease.-After v. Kries. but as it is not supported, the lift begins at a lower and lower level. At the moment of arrest, which of course occurs later and later, the length of the muscle is always the same. Its rate of shortening diminishes as in the previous case, but its rate of increase of tension is not constant as before, but diminishes with the rate of shortening; so

that the "apparent extensibility" remains nearly the same. The explanation given by $v$. Kries is as follows:-In a muscle, notwithstanding that length and tension remain the same, the relation between the two functional capacities dliffers according to the "way in which the portion of the response which precedes the transition" has occurred (see p. 369). $\mathrm{He}$ supposes that what happens at the beginning of the contraction influences the subsiduent state of the muscle by its influence in determining the mumber of fibres which participate in the process, and surmises that the effect of initial tension (starlie Spannung im Moment der Reizung) is to augment the number of acting fibres, and thereby to bring about a condition in which the rate of change of tension corresponds to that of change of form. The datil seem to me to admit of a simpler explanation. The contractile stress of the nulscular fibres at the moment of transition is in each case truly indiented ly the rate of augmentation of tension-that is, by the initial inclination of the isometric curve, and is accordingly greater in the first saries than at the corresponding period in the second. But the reason why this is so, is thut the measurenents relating to the first series are made at an 
earlier stage in the response than those relating to the second; and, consequently, that the diminution of the rate of augmentation of tension in that series means nothing more than that the contractile stress of the muscle has already passed its maximum.

v. Kries also made experiments in which the load (and therefore the initial length of the muscle) was constant, but in which its length at the time the stop was applied, varied. A method founded on the same principle was adopted by Schenck in investigating the influence of tension on the timerelations of the excitatory process. His experiment is as follows :-A muscle is made to contract isotonically with a small load. It draws the curve indicated by the dotted line in the figure (Figs. 199 and 200). Immediately

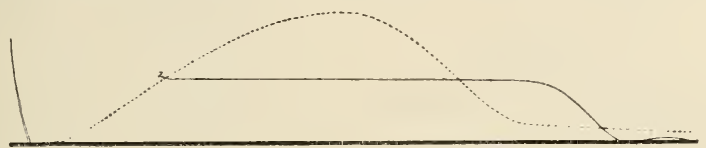

FIg. 199.--Isotonic curve (dotted line) and arrest curve (continuous line) of the same musele as in Fig. 200, cooled to $6^{\circ} \mathrm{C}$.

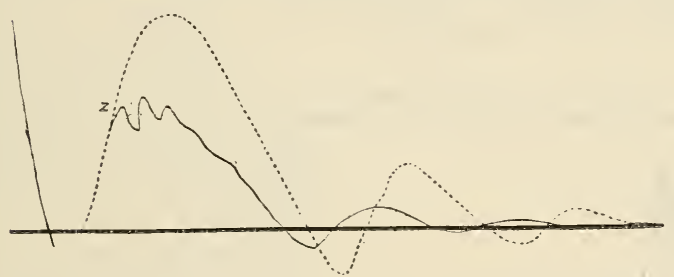

FIG. 200.-Isotonic curve (dotted line) and arrest curve (continuous line) of a muscle warmed to $31^{\circ} 5 \mathrm{C}$. (double adductor preparation, the two groups of muscles arranged as shown in Fig. 189), the arrest taking place when the point of the writing-lever was at z.-After Schenck.

afterwards a second curve is drawn, with the stop so placed as to arrest the shortening of the muscle half-way. He found that if the observation is made at a low temperature, e.g. $6^{\circ} \mathrm{C}$. (Fig. 199), the period of contraction is prolonged; if at a high temperature, e.g. $31^{\circ} .5$ C. (Fig. 200), it is abbreviated, as compared with an unarrested isotonic contraction at the same temperature. At an intermediate temperature the fall of the arrested curve coincides with that of the isotonic curve. ${ }^{1}$

Schenck explains these apparently inconsistent results by assuming that tension has a twofold action-that it "strengthens and protracts the process of contraction," and that at the same time it " hastens relaxation." "In the warm muscle, the second of these actions predominates; in the cold, the first." 2 He also found that the first effect is the more pronounced the less the muscle is loaded, and connects this with the fact, to be referred to in a later section, that the result of increasing the load in isotonic contractions is to increase the production of heat. It is to be noted that Schenck applies the arrest when the muscle is half-way between its natural unexcited length and that

${ }^{1}$ Schenek, Arch. f. d. ges. Physiol., Bonı, 1894, Bd. Iv. S. 629.

schenck, ibid., 1894, Bd. lvii. S. 606 .

VOL. II. - 24 
which it eventually attains, and that this position is different in relation to the former in a cooled muscle from what it is in one that has been warmed. It will be seen later, that in this observation he is comparing very different stages in the progress of a muscle from the unexcited to the excited condition.

Similar considerations led Schenck to investigate the effect of different kinds of augmentation of tension, having regard especially to its amount,

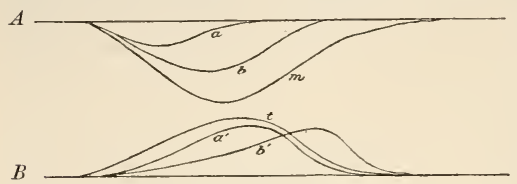

FIG. 201.- $m$, Normal isometric curve; $t$, normal isotonic curve of the same muscle ; $a a^{\prime}$, inertia curves of tension and shortening respectively, the heavy lever deseribed on P. 360 being used but not weighted; $b b^{\prime}$, similar eurves with the lever weighted on either side of the axis with 500 grms.-After Schenck.

muscle is delayed by the inertia of the bar before the commeneement of shortening, or later, so long as the increase of tension ceases during the first
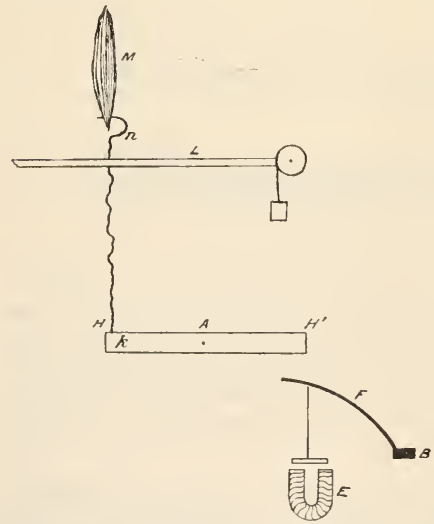

Fig. 202.--Schenck's appraratus for taking "jerk curves." $L$, isotonic lever : $H H^{\prime}$ light wooden lever, working on the axle $A$, with one arm attached to lower end of muscle and isotonic lever by the thread $x k ; F$, spring fixed at $B$, which can be held down by the elcetro-magnet $E$., and which, when let go, strikes $H^{\prime}$.-After Schenck. in this way) coincided in its decline
with the isotonic curve. The later the jerk took place, the sooner the relaxation of the muscle occurred (Fig. 203, B). The contrast between the happens when the bar is unloaded, the fall of the curve of shortening $a$, is either in front of, or almost coincides with, that of the isotonic curve $t$. If, however, the moment of inertia of the bar is augmented by weighting its ends, so that the augmentation of tension lasts much longer (curve $b$ ), the maximum of contraction is reached later, and the fall of the curve is beyond what it would have been in the absence of the bar.

$\mathrm{He}$ subsequently experimented with more sudden and transient augmentations of tension by jerking the writing-lever downwards during an isotonic contraction. ${ }^{1}$ By an ingenious contrivance, shown in Fig. 202, this could be done at any desired period, and it was found that if done at the very beginning of an isotonic contraction, the jerk scarcely affected the time of relaxation (Fig. 203, A); for the jerk curve (as I propose to call curves produced in this way) coincided in its decline rapidity of development, and duration. $\mathrm{He}$ experimented first with the equilibrated oscillating bar, coupled with the isotonic lever, in a similar way to that used by Fick (p.359), thus obtaining Schleuderauclingen, the curves of which (as shown in Fig. 193, or in Fig. 201, $B$ ) are what I have already called "inertia curves." By taking the accompanying tension-curves, he shows (Fig. 201, A) that whether the contraction of the half of the period of contraction, as 'Schenck, Arch.f. d. yes. Physiol., Bomn, 1895, Bd. lxi. s. 77 . 
last set of experiments and the preceding inertia experiment needs scarcely to be indicated. When the jerk method was used, the rise of tension was abrupt and forcible. In the contractions with the equilibrated bar, it rose and subsided gradually.

Schenck also made experiments with arrested contractions, for the purpose of comparing the effects of the arrest on loaded and unloaded muscles, ${ }^{1}$ and found that similar differences present themselves. If the contraction of a lightly loaded muscle is arrested when already half accomplished, it gives way so promptly that the writer at once leaves the stop in the same way as in Fig. 200. The more the load is increased, the less the relaxation is hastened, until eventually the writer is held against the stop during the whole period of isotonic shortening, or even longer. In one case the tension is augmented at the moment of arrest, from a very small amount to one which is fifty or a hundred times as great; in the other, from an amount already large to another a little larger. ${ }^{2}$ As has already been stated, cold has a similar effect to load, so that the same muscle which at $20^{\circ} \mathrm{C}$. promptly relaxes during the period of arrest, holds the writer against the stop during the whole

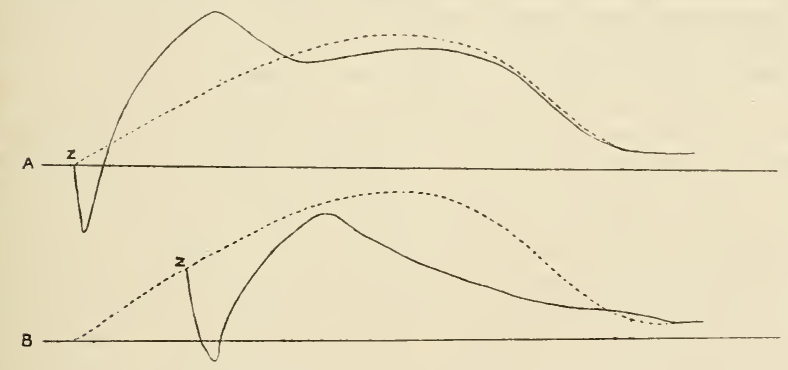

FIG. 203.--Isotonic (dotted line) and jerk eurves (continuous line); $z$, position of point of writing-lever when jerk took place.-After Schenck.

period of isometric contraction, when the temperature approaches $0^{\circ} \mathrm{C}$. (Fig. 199).

Schenck sums up his conclusions as to the influence of augmentation of tension on the isotonic contraction of a lightly loaded muscle as follows : ${ }^{3}$-Sudden and extensive alterations of tension hasten relaxation; changes of longer duration, which develop slowly and are of small amount, tend to increase contraction and to defer relaxation. Of the two effects, the former follows the change of tension immediately, the other often not until a little later.

It can scarcely be questioned that this statement is in accordance with the experimental data which have been given; but when we pass from the observed facts to their explanation, we find ourselves in considerable perplexity. Schenck finds the link which connects the hastening of relaxation (verfrühte Erschlaffung), with the conditions which produce it, in the paradox of inhibitory excitation. He imagines

${ }^{1}$ Sehenck, Arch. f. d. ges. Physiol., 1894, Bi. Ivii. S. 608.

2 It is here again to be noted that the length of the muscle at the time that the arrest was applied in these experiments was not the same.

${ }^{3}$ Sehenck, Arch.f. d. ges. Physiol., Bonn, Bd. 1xi. S. 85, 103. 
it possible for a muscular fibre to be stimulated into relaxationwakened into sleep. After again stating that the more abrupt and transitory the change of tension, the more marked is its relaxing action, he continues (p. 104): “A contracted muscle is, according to Pfliger's theory, a labile group of atoms." These he supposes to form a chain, of which the "loosely joined links" are more easily sundered by a "short forcible jerk than by slow persistent tension"; the latter, on the other hand, is more effectual in evoking contraction, for the very reason that it is itself of relatively slow development.

The case which seems chiefly to require such a theory is that of the jerk relaxation. Schenck himself tells us that in this experiment the muscle receives an Erschütterung-a shatter-a process by which we know experimentally that heat is liberated. It is difficult to accept a doctrine which identifies this with inhibition, and much more easy to suppose that the influence of jerk or shatter is simply to put a stop to the whole process, by changing the mode in which energy is liberated, substituting the production of heat for that of work, and to regard both the changes which follow excitation-relaxation no less than contraction-as parts of the same process. We may then inquire whether or not it is necessary to call in the aid of an auxiliary theory of secondary excitation. ${ }^{1}$

The "simple experiment" which led Schenck to the discovery of the remarkable fact, that a muscle which is arrested in the course of an isotonic contraction, if lightly loaded, promptly relaxes, rather suggests questions than answers them. It has been subjected to criticism by other experimenters, particularly by Kaiser, ${ }^{2}$ who has shown that both at high and low temperatures, the question whether the muscle relaxes or not inmediately after the arrest, depends on the height of the stop, i.e. on the length of the muscle at the time the stop is applied as compared with its natural length when unloaded and unexcited. On this observation he has founded what may be called a new method of observing the phenomena of isotonic contraction and relaxation, which promises to be very fruitful. Starting from Weber's conception of the process, according to which the excited contractile molecular structure, at the moment of excitation, takes the place of the unexcited, and is replaced by it at the end of the period of excitation (see p. 360), he begins by seeking for a criterion by which the state of excitation may be characterised in a muscle which is allowed to change its form in response to an instantaneous stimulus without mechanical interference. The character by which an excited muscle can be most easily distinguished from an unexcited one is its length as recorded in the isotonic curve. He therefore takes as the object of observation, a muscle (gastrocnemius) arranged for writing its curve isotonically with the lightest possible load.

The upper end of the muscle is fixed during the whole course of each series of observations; consequently the horizontal line which corresponds to the natural length (Fig. 205, 7 ) of the muscle when unexcited and unloaded is also constant. When in the progress of the observation the muscle is loaded,

\footnotetext{
1 The paper on this subject already referred to (Arch. $f$. d. ges. Physiol., Bonn, Bd. Iv. S. 629) is entitled "Ein einfaeher Versuch zur Demonstration des Einflusses der Spannung, u.s.w."

2 "Zur Analysc der Zuckungsenrve des quergestreiften Muskels," Ztschr. f. Biol., Minchen, 1896, Bd. xxii. S. 157-178; "Untersuch. neber die Natur der bei der Contraetion des quergestreiften Muskels wirksame Kräifte," S. 361.
} 
care is taken to avoid the slightest change of position of the fixed end, so that the graphic record of each length, due to extension by the load, may be absolutely reliable.

The first step is to determine and record graphically in the unweighted muscle its "natural" length when excited by a maximal stimulus. If we call this length $\lambda$ we may designate the horizontal line corresponding to it by the same letter. The position of that line is determined as follows: - An ordinary isotonic curve having been drawn with an "unloaded" muscle, a series of arrest curves are taken from the same starting-point, as slown in the figure (Fig. 204), the stop being slightly raised after each excitation. At first the lever hesitates at the stop for several hundredths of

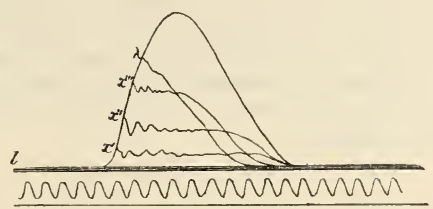

Fic. 204.-Curves of isotonic and arrested contractions of an unloaded muscle, with maximal stimulation at ordinary temperature. $x^{\prime}, x^{\prime \prime}, x^{\prime \prime \prime}$, and $\lambda$, points at which the lever was arrested in successive curves. - After Kaiser. a second; but, as the succeeding curves show, the period of hesitation gets less and less, until finally the lever falls immediately. The same thing happens, whatever the temperature of the muscle; and if the experiment is repeated with the same unweighted muscle, at temperatures, say, of $0^{\circ}, 14^{\circ}$, and $30^{\circ} \mathrm{C}$., it is found that

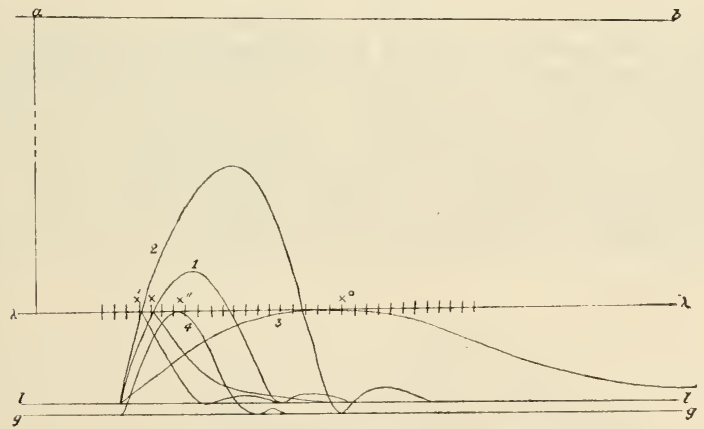

F1G. 205.

(1) Isotonic curve of unloaded muscle at temperature $14^{\circ} \mathrm{C}$. ; (2) the same, at $25^{\circ} \mathrm{C}$. ; (3) at $0^{\circ} \mathrm{C}$. ; (4) at $14^{\circ} \mathrm{C}$, with load on muscle. $a b$ indicates level of fixed end of muscle. $l$ indicates level of free end of muscle in its first equilibrium position (i.e. when mloaded and at rest); $\lambda$ denotes the length of muscle in its second equilibrium position (i.e. that which it tends to assune when unloaled, in response to a single naximal stimulation); $g$, line representing length of muscle when so loaded that the summit of the curve is at $\lambda ; x, x^{\prime}$, point at which the lever falls at once on arrest, in curves drawn with ruuscle at $14^{\circ}$ and $25^{\circ} \mathrm{C}$. respectively; $x^{\circ}$ and $x^{\prime \prime}$, summit of eurves drawn with muscle at $0^{\circ} \mathrm{C}$. and when loaded respectively. The marks on the $\lambda$ line indicate hundredths of a second. $-\Lambda$ fter Kiaiser.

the height at which there is no lesitation of the lever is the same for each, however much the height of the summit of the curve, and its duration may vary. A horizontal line at this height is therefore the one required. It is represented in the diagram (Fig. 205), in which its relation to the summit of 
the curve, under varying physical conditions, is shown. As regards temperature, this relation may be stated generally thus:-At $0^{\circ} \mathrm{C}$. the summit of the curve does not rise above $\lambda$, and only rises to this level slowly, whereas above $0^{\circ} \mathrm{C}$. the height of the summit increases with the temperature, as does also the rapidity with which it attains this height. Kaiser therefore concludes that there is a certain limit in the response of a muscle to a single instantaneous excitation, at which the contractile stress which causes shortening ceases to act; and he believes that any further diminution of length is due to the velocity imparted to the process of shortening by this stress, and varies accordingly.

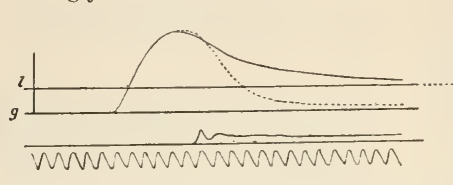

FIG. 206.-Isotonic curves of muscle so loaded that its summit is in the second equilibrium position, in the one case (continuous line) unloaded on the summit. $l$, line indicating equilibrium length of nusele at rest when unloaded; $g$, the length when loaded.-After Kaiser.

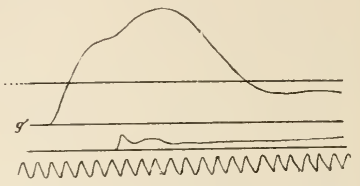

Fia. 207.-Isotonic eurve with the same musele more heavily loaded to begin with, and unloaded on the summit. $g^{\prime}$, line indicating length of muscle when loaded. - After Kaiser.

To complete the evidence that this limit is that of the excitatory equilibrium position of the muscle (i.e. that it is $\lambda$ ), Kaiser seeks to demonstrate that it is constant under other varying physical conditions besides temperature. He has given satisfactory proof that this is so in the case of load, which, after temperature, is the physical condition of most importance in determining the "form" of an isotonic contraction. His method consisted in unloading differently weighted muscles at different points on their course, whereby he found that it is only when the muscle is unloaded at the moment that it is at the length $\lambda$, as ascertained from the isotonic curve of an unweighted

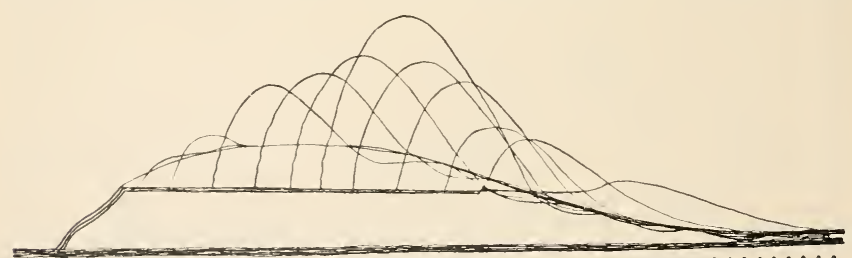

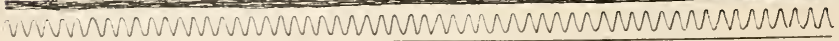

FIc. 208.- Series of release curves following arrest, with muscle cooled to $0^{\circ} \mathrm{C}$.

muscle, that the free end of the muscle returns at once to its original unexcited level, $l$. Two of Kaiser's curves showing this are given in Figs. 206 and 207. In the first experiment (Fig. 206) the muscle was so loaded that the summit of the curve was at the level of $\lambda$; it was relieved of its load at the moment of culmination of the curve, and it is seen that it relaxes at once to its natural length, l-apparently indicating that there was no more available contractile stress at the moment when unloading took place. In the experiment to which Fig. 207 refers, the weight on the muscle was heavier, so that the summit of the curve was below the level of $\lambda$. It was again relieved of its load at the moment of the culmination of the curve, and the occurrence of 
a second twitch indicated that contractile stress was then still present. The series of twelve curves, reproduced in Fig. 208, were obtained by a similar method, with the exception that before each release of the lever it was arrested at a level about halfway between $l$ and $\lambda$, and that the muscle had been cooled to a temperature of $0^{\circ} \mathrm{C}$.

These curves show that, although in each muscular element the transition from the unexcited to the excited state may be instantaneous, a certain time is required in order that the contractile stress of the whole may attain its maximum. This time Kaiser refers to as that of the Entwickelung der Verkürzungskraft. Thus, although a low temperature does not affect the position of $\lambda$, and accordingly does not increase the contractile stress, it may yet enable the muscle to raise a weight to a greater height by postponing the moment at which the contractile stress ceases to act.

It having been found that the second equilibrium length of the muscle is independent of the physical conditions under which it may be placed, the next step is to demonstrate its relation to the physiological conditions. Now, the means by which function is evoked in a muscle is excitation. We may regard a muscle when "maximally" stimulated (and not fatigued) as in a definite physiological condition. The condition is altered when a supramaximal response is evoked, as may be done by applying two instantaneous stimuli, both of which are maximal, within a period of one- or two-hundredths of a second, or a submaximal one, by applying a stimulus weaker than that required to produce a maximal contraction. Comparing the curves so obtained with a normal maximal curve of the same muscle, Kaiser found that in the case of supramaximal stimulation (Fig. 209), although the summit of the curve is not much higher, there is a hesitation of the writing-lever when it is arrested at a height which corresponded to $\lambda$ in a maximal contraction, indicating

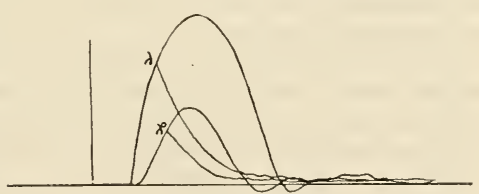

FIG. 210.--Two isotonic curves of an unloaded muscle, the one with maximal and the other with submaximal stimulation. $\lambda$ and $\lambda^{\circ}$ the positions representing the second equilibrium length in the two eases. - Two figures com. hined, after Kaiser.

that the second equilibrium position of a muscle excited by a summation of stimuli, is at a higher level, say at $\lambda^{2}$. Conversely, when the lever is arrested at different points in the course of a submaximal response (Fig. 210), its sudden fall occurs when it is at a considerably lower level than that of $\lambda$ in the curve of maximal stimulation, indicating that the second equilibrium position of a muscle, submaximally stimulated, is at a lower level, say at $\lambda$. It thus appears that the equilibrium length of an excited muscle varies with the degree of action of the exciting cause, 
so that $\lambda$ is the expression of the capacity of the muscle to respond to a maximal instantaneous stimulation, and we are justified in designating the contractile stress which determines the change from the first to the second equilibrium position as "physiological," although it acts as if it were "elastic." Kaiser gives the name of Verkiirzungskraft to this contractile stress.

The preceding summary will be sufficient to give the reader an idea of the scope of Kaiser's research, a main purpose of which was to elucidate the relation between the length of a muscle, when allowed to shorten freely in response to a single maximal stimulation, and the isotonic curve of such a contraction. The experimental data obtained, lead him to reject the theory that the single contraction is a dual process. Following out Weber's original idea, he thinks of the "forces" operative in the process as elastic, and accounts for all that happens by supposing that shortening expresses transition from the "natural" unexcited condition to the "natural" excited condition ; and that lengthening is a process which is simply the reverse of the other. The first is the direct response to excitation, the second a necessary consequence of the first. The proof that the stage of relaxation is a mere

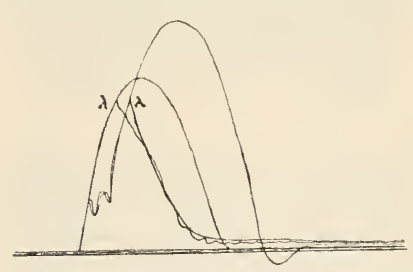

FIG. 211.-Isotonic curve and release curve, after arrest, of an unloaded muscle. -After Kaiser. consequence of that of contraction, he finds in the fact that temperature, which has so marked an influence on the first part- that in which the muscle is actively contracting-has no effect on the second, for the curve drawn by the same muscle in relaxing is always precisely the same, provided that it is unloaded and that the muscle at the starting-point of relaxation is of its natural length when excited, i.e., that its unattached end has to accomplish, in transition from the excited to the unexcited state, the distance above designated as $l-\lambda_{0}{ }^{1}$

Before concluding the consideration of the equilibrium length of excited muscle, it may be well once more to refer to the difference between the maximum degree of contraction which is evoked by a single instantaneous stimulation, and that more intense state of excitation which exists in tetanus.

When a lightly loaded muscle is excited by maximal instantaneous excitations which succeed one another with sufficient frequency (100 per second), the contraction being recorded isotonically, the lever rapidly

\footnotetext{
${ }^{1}$ To the reader who consults Kaiser's papers it may be of use to note that he employs the terms erster and sweiter Fusspunkt to designate the position of the muattached end of the unextended muscle in the unexcited and excited states respectively. I have in the text made no reference to Kaiser's theorctical explanation of the isotonic curve. He attributes the descent of the lever at the moment indicated by $\lambda$ in Fig. 204, not to the rebound of the lever against the stop, bit to a downwards impulse of the muscle, and connects this with the theory that so nuch of the ascent of the lever as is above $\lambda$ is due, not to the action of the lever, but to the action on it of the muscle, which, after attaining its equilibrium length $\lambda$, shortens, by virtne of its previous motion, to a length less than its cquilibrium length. Actual experiment docs not favour this conchusion, for Schenck has shown that if the lever is supported at the height of $\lambda$, before the muscle is excited, it remains motionless (Arch.f. d. ges. Physiol., Bonn, Bd. lxv. S. 325). This bcing the case, it is clear that, so far as concerns the part of the isotonic cmrve which is above $\lambda$, the lever is not actuated by the muscle.
} 
rises to its maximal height, after which it remains for a time at the same level-indicating that the muscle is fully tetanised. In this state it is free from stress of any kind, so that its actual length and equilibrium length, which may be designated $\Lambda$, are the same. Although we have no experimental data which enable us to state what the relation of $\lambda$ to $\Lambda$ is, there is reason to believe that the former length exceeds the latter by about three-quarters. In a muscle excited in the manner above described, the tetanic state is produced not so much by the superposition of effects (as in Helmholtz's well-known experiments), as by the summation of stimuli; for each excitation occurs before the contraction evoked by its predecessor has begun. Consequently, in the transition of a muscle from the imperfect state of excitation to which $\lambda$ relates, to that of complete tetanus, there may be any number of stages of contraction, each characterised by an equilibrium length intermediate between $\lambda$ and $\Lambda$. In this connection it will be remembered that in the response to two maximal stimuli, following each other at a very short interval, the equilibrium length $\lambda^{2}$ takes a higher position in the curve than in that which follows a single excitation of the same strength (Fig. 209).

Muscular tonus.-Throughout the preceding discussions relating to the mechanical properties of muscle, it has been found convenient to regard the length of an unexcited muscle as constant, and to designate it by the letter $l$. There are, however, many instances which show that a muscle may, although at rest and under conditions which cannot be regarded as abnormal, be of a different length at different times. States of relaxation, in which muscles are longer than they are ordinarily, are not recognised; so that we need not consider them. It is only necessary to designate those conditions in which the fibres are permanently short. All such states come under the term Tonus.

The lengthening which the muscles of the higher animals undergo, in consequence of destruction of the motor centres by which they are innervated, may be taken as evidence that in the natural state their fibres are not perfectly at rest. This state, to which the term "skeletal tone " 1 is applied, is essentially one of continuous indirect excitation; for it manifestly depends on the influence of the nervous system. There are, however, conditions of tone in the production and maintenance of which the central nervous system appears to take no part. Of these the most instructive examples are (1) the tone of the claw muscles of the crayfish; and (2) that of the heart of Helix.

The first of these has been most recently investigated by Piotrowsky, who has shown that lengthening of the abductor muscle can be produced as easily by direct as by indirect stimulation, provided that the muscle when stimulated is in a tonic state. The phenomenon can, however, be only observed in winter, and then only when the temperature is about $8^{\circ} \mathrm{C}$. or lower. At higher temperatures the muscle contracts normally when stimulated, so that, according to the degree of tonus present, you may have contraction, relaxation, or a mixed result.

The heart of the snail affords quite as striking an example of the same relation between excitatory relaxation and tonus. The mode of observing it is as follows:- A narrow glass tube is drawn out at one end, and shaped into a cannula, to which the ventricle is tied. The

\footnotetext{
${ }^{1}$ Foster's "Physiology," ith edition, part 3, p. 996.
} 
cavity of the ventricle and the tube are filled with salt solution, which, when the tube is fixed in an upright position, stands in it at the height of a few centimetres. The heart thereupon begins to beat, but eventually stops in systole. By a suitable arrangement, a voltaic current is led through the ventricle from base to apex, or the reverse. On closing the current the ventricle relaxes at the anode, and the wave of relaxation is propagated towards the cathode. If the closure is of short duration, the ventricle returns, when the current is opened, to its previous state of tonus, and the observation may be repeated. Here again it is seen that the effect of the current is to annul an existing state of excitation, rather than to bring into existence a new state.

The fact that, in these instances, the elements of muscular structure lengthen when subjected to mechanical or electrical disturbance, is difficult to understand, chiefly because it appear's to be against the purpose to which those elements are adapted in the organism, i.e., against their function. If, therefore, stimulation is the equivalent of Auslösung-the discharge of function-we cannot designate as stimulation an action by which activity is quelled. A living organism cannot be waked into inactivity.

Yet the fact remains that the agents which we are in the habit of regarding as stimuli may, by their direct action, quell function. Since Ptliger discovered that by the voltaic current we can at will modify the vital condition of a motor nerve in either of the two possible directions, -augmentation and diminution,-we have recognised in nerve and in other excitable structures, an up and a down state. The essence of what we know about these states is, first, that the down state is a concomitant of activity, the up state is similarly associated with rest; and, secondly, that the latter is the antecedent condition of restitution or repair, the former of capacity for function (Functionsfähigkeit). Hence the two states constitute one cyclical process, in which each state is alike the consequence and cause of the other. But no sooner have we expressed this intimate relation, than again the essential difference between the two presents itself-the contrast between functional activity and repose. The contrast consists mainly in this, that while in the former the thermogenetic and electrical changes are sudden, easily recognised, and are so conspicuous that they constitute the most characteristic phenomena of animal life, those of assimilation or restitution are so little obvious that their existence is rather a matter of inference than of observation.

Summation of a series of mechanical responses-Genesis of tetanus.-Tetanus has already been defined as the response to a succession of instantaneous stimuli. The ty pical form of this response (complete tetanus) is obtained when the frequency of the excitations is such that the intervals between them are each equal to the time lequired for the muscle, excited by a single instantaneous stimulus under isometric conditions, to attain its greatest tension. A muscle, so tetanised, has an equilibrium length $\Lambda$, which, as we have already seen, is considerably less than $\lambda$, the length which it strives to attain (and may overshoot) in response to a single instantaneous excitation; in other words, the distance $l-\Lambda$ is considerably greater than $l-\lambda$. When an "unloaded" muscle, i.e. one with a load say of $0.5 \mathrm{grm}$., is tetanised, its actual length and its equilibrium length $(\Lambda)$ are identical. The same unloaded muscle shortens nearly to the same extent when excited by an instantaneous 
stimulus, but, as we have seen, the summit of the isotonic curve does not usually correspond to the equilibrium length $\lambda$. The question why a muscle shortens to a less equilibrium length when it is tetanised, than when it is maximally excited by a single stimulus, will be better understood when we have had under consideration the order of the changes which occur in response to a single stimulation. It will be seen that the first of these changes is oxidation, of which the excitatory electrical change is the sign and probably indicates the duration, and that as soon as it has attained its full development it is at once converted into mechanical tension (contractile stress). We shall also see that, although the link which connects the chemical change with the mechanical change is an unknown one, there is good reason for believing that in whatever way mechanical potential energy may arise out of chemical energy, no measurable time is lost in the conversion. Its visible effect, on the other hand, i.e. the change of form, is a process which requires time, and consequently overlasts its efficient cause. At the moment that the change of form is beginning, the electro-chemical change has already culminated. The mechanical effect subsides the moment it has reached the degree called maximal, its efficient cause being no longer operative. If, however, the excitation is repeated before this subsidence begins, the effect of the second excitation is added to that of the first, so that the curve may, as Helmholtz's well-known experiment shows, attain nearly double the height of the single isotonic curve. If a series of such excitations follow one another at equal time-intervals, their effects are so summed that the muscle shortens to a definite length, the difference between which and $\Lambda$ depends on the load. So that, when maximal excitations are used, it is the summation of the effects of successive excitatory changes, which causes the muscle to shorten to an equilibrium length less than that attained by it in response to a single such excitation. With reference to this summation, Fick's measurements (to be referred to later), determining that in such a succession of excitations the chemical changes as judged by their thermal effects are more intense in the first than in the subsequent responses, must be borne in mind in connection with the fact that, in the summation of effects, the increments due to the second and immediately following excitations are also much larger than those due to succeeding ones.

That the rise of the tetanus curve is due to something more than summation of stimuli, i.e. in large measure to the summation of effects, seems to be indicated by the fact that the electrical change, by which the chemical one can best be made apparent, shows no sign of summation. The moment a muscle is tetanised, the so-called "action current" has its full strength. Certain observations made by v. Frey, ${ }^{1}$ relating to "supported" muscles, may have a similar bearing. He alleges that if the load is so supported that the muscle does not begin to lift it in response to a single excitation, until the muscle has attained a length but little in excess of its length when tetanised, it raises the load to the same height as that to which it would be raised in tetanus; the reason being that the amount of shortening to be accomplished being less, the limited time during which the muscular effort is at or near its maximum, has become

${ }^{1}$ v. Frey, "Versuche zur Auflösung der tetanischen Nuskeleurve," Beitr. z. Physiol. Carl Ludwig, z. s. 70 Geburtst., Leipzig, 1886. 
sufficient for the completion of the contraction. ${ }^{1}$ The question, however, whether, in the rise of tetanus, we can distinguish between the summation of excitations and the summation of their effects, requires further experimental investigation.

Summation of inadequate stimuli.-It was long ago observed by Helmholtz that when an instantaneous stimulus, which in itself is incapable of evoking a maximal response, is followed during the period of latency by a second of the same strength, a maximal response may be obtained. If the time-interval between the two stimuli is sufficiently short (less than $\frac{1}{500}$ second), there may, as we have already seen, be an increase of effect, even when the stimuli are maximal (see Fig. 209). Again, a succession of stimuli of sufficient frequency may be effectual, although each stimulus when alone is so weak as to produce no response. An instance of this is given in Fig. 212, which

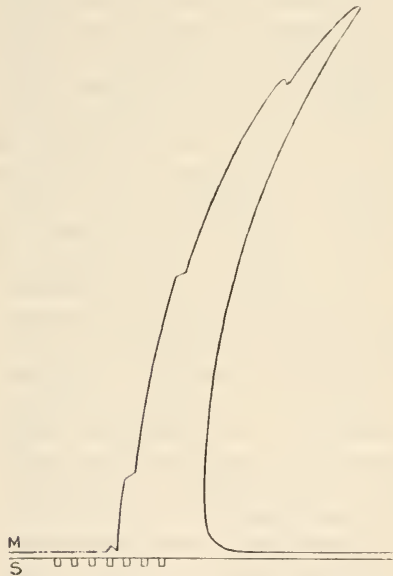

Fir. 212.-Muscle curve, showing the effect of a succession of stimuli, each one of which alone was inadequate to produce contraction.--Richet. represents a tracing made by Richet ${ }^{2}$ with the claw-muscle of the crayfish. Seven stimuli, each inadequate in itself to produce an effect, were sent through the muscle in succession. The first three gave rise to no response, the fourth to a very slight one, while the fifth, sixth, and seventh evoked contractions each stronger than that of its predecessor.

Such summation of submaximal stimuli can be much more easily observed in slowly contracting skeletal muscles, such as the claw-muscle of cray-fish, than in quickly contracting ones. The cardiac muscle of the frog, when stimulated by induction currents which singly are of insufficient strength to produce an effect, responds when these are repeated with sufficient frequency, the response being slower in occurring the less the frequency. Thus Engelmann found that in the bulb of the aorta ${ }^{3}$ a frequency of fifty inadequate stimulations per second produced a response after a delay of one second, a frequency of four per second after two and a half seconds. In smooth muscle even strong single induction shocks are often inadequate to produce a response, at the same time that tetanisation with comparatively weak stimuli may be effectual. ${ }^{4}$

We must distinguish between instances such as those above referred to,

${ }^{1}$ From Kaiser's observations, we have learned that at the moment of culmination of the clcctro-chemical response to a maximal instantaneous stimulus (Case 1), a muscle, even when after-loadel, tends to assume the length $\lambda$, whereas, under the conditions of $v$. Frey's experiment (Case 2), it shortens to its eventual length in tetanus $\Lambda$. There is in reality no contradiction. In Case 2 the contraction eulminates immediately after it begins, i.e. at a time when, as Bernstcin has lately shown, the process by which ehemical energy is transformed into muscular foree is at its maximum. In Case 1 that process is already declining at the moment that its meehanical effect is at maximum. This may be expressed by saying that it is for want of time that the mechanical effeet of a single stimulus falls short of that of repeated excitation. See Kiaiser's eriticism of v. Frey's interpretation of his observation, loc. cit., p. 367.

" "Physiol. des muscles et des nerfs," Paris, 1882.

3 Arch. f. d. ges. Physiol., Bonn, 1852, Bd. xxix. S. 453.

"Engelmann, ibid., 1870, Bd. iii. S. 280. 
in which two submaximal stimuli acting practically simultaneously, produce the effect of a single maximal one, from those in which the first of two excitations following one another with an interval between them prepares for its successor, as in the so-called "staircase" phenomenon, first described by Bowditch in the heart. ${ }^{1}$ When, after having cut off the apex of the ventricle of the frog's heart, single induction eurrents are led through it at intervals of from five to ten seconds, by electrodes at a very short distance from one another applied to its surface, the heights of the curves show that the second beat is stronger than the first, the third than the second, and so on, the increments diminishing progressively until the heights of the curves become uniform. In 1875 it was observed by Tiegel ${ }^{2}$ that a similar augmentation takes place in skeletal museles. It is best seen when a curarised gastroenemins, in which the circulation is carefully maintained, is excited for a long time at regular intervals, in the same way as in Kronecker's experiments on exhaustion. In this case the augmentation of effect when the stimulation is maximal, may go on for some three hundred excitations, after which the heights of the successive curves begin to diminish. When stimuli which are not quite maximal are used, the increase is maintained longer, and was observed by Tiegel to be accompanied by a remarkable degree of vascular congestion in the muscle.

Period of latency of the mechanical response.-About half a century ago Helmholtz discovered that a considerable interval of time intervenes between the excitation of a muscle by an instantaneous stimulus, and the beginning of the response. He designated this interval the period of latent stimulation, and from his measurements of its duration-employing methods which have served all subsequent observers as models-concluded that it was about one-hundredth of a second. Although Helmholtz clearly indicated that the delay was influenced by the strength of the stimulus and by the excitability of the muscle, his estimate was regarded for many years in physiology as if it had the authority of a physical law, which could be applied without reserve, not only to the whole organ, but to each of its constituent structural elements. It was not until 1879 that $\mathrm{Gad}^{3}$ showed that about half of this delay was due to mechanical conditions which had been previously overlooked; for even when the load was very inconsiderable, he found that there was loss of time from inertia, which could be much diminished by interposing an elastic band between the weight and the lever. He further observed that when a parallel-fibred muscle is excited by an induction current led through its lower half, the contraction is not only delayed, but may even be preceded by a slight elongation. This he rightly attributed to the extension of the unexcited part, which takes place at the moment the excited part begins to shorten; and he pointed out that the yielding either of muscular parts not excited or of non-muscular parts is a second cause of delay.

A few years later similar conclusions were arrived at by Tigerstedt, ${ }^{4}$ both as regards the true duration of the delay, and the reason why, when observed in the ordinary way, it appears to be so long. His method of measurement consisted in recording the time after excitation at which a very delicately adjusted contact is broken by the contraction. He found that the gastrocnemius, when excited directly by an ascending over-maximal break induction

1 Arb. a. d. physiol. Anst. zu Leipziy, Bd. i. S. 156.

2 Ibid., 1875 , S. 37 . S. 250 .

: "Ueber das Latenzstadium des Muskelelementes," Arch. f. Physiol., Leipzico, 1879,

4Arch. f. Physiol., Leipzig, 1895, S. 111. 
current, and loaded with 4 grms., shortens sufficiently to break the contact $4 \cdot 3$ thousandths of a second after excitation. Under other conditions the delay was somewhat greater. Ten years later the present writer was able to show,

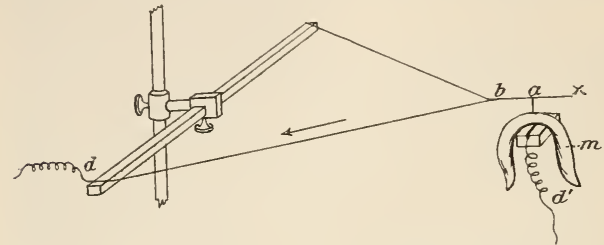

FIG, 213, - The induction current flows through the nuscle $m$, from $d^{\prime}$ to $d$, the cathode being at $a$. The square rod is of vulcanite, the wircs are of steel. by the photographic method, 1 that the period is shorter by nearly another thousandth of a second. The expansion in thickness of the muscular fibres of the sartorius was registered photographically with the aid of the apparatus for pliotographic measurements, to be

described further on in its application to the capillary electrometer (p. 418). The spring indicator shown in Fig. 213 was applied to the muscle, the movement photographed being that of its point $x$. In the plotograph (Fig. 214)

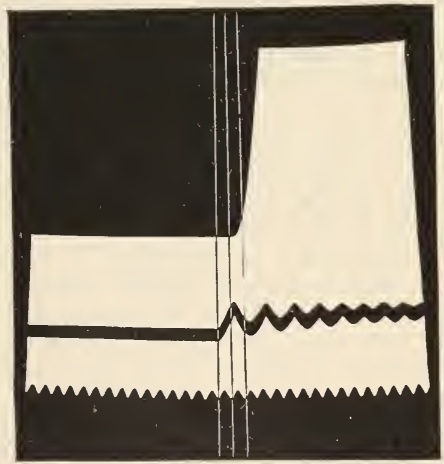

FIG. 214.-Faesimile of the photographic record of the beginning of the contraction of the sartorius. The white space is limited by two polar ordinates, which converge to a centre half a metre distant. In the toothed line each tooth corresponds to of sec. Above it, the signal line indicates the instant of break of the primary circuit of the induction coil (first of the three vertical lines). It is scen that the time which intervenes between the break excitation and the response is about one and a half times the duration of a tooth, i.e. $10^{3} 00 \mathrm{sec}$.

shortest he was able to observe. He, however, gives no reason for declining to accept the shorter estimate.

The writer of this article has shown that, as measured photographically,

${ }^{1}$ Burlon Sanderson, Journ. Physiol., Cambridge and London, vol. xviii. p. 146.

2 Arch. f. d. ges. Physiol., Bonn, 1897, Bd. lxvii. S. 207. dredth of a second apart, the distance between them corresponding to the vibration period of a fork of 500 double vibrations per second. The opening of the key by which the primary circuit of the inductorium is broken, is at the same time photographed on the plate, so that no doubt can exist, either as to the time after which the induction current must have reached the muscle, or as to the time before which the change of form must have begun. The photograph therefore affords incontrovertible evidence that a muscular fibre begins to contract under ordinary conditions not later than 0.0035 sec. after excitation.

The same question has been recently discussed by Bernstein. ${ }^{2}$ $\mathrm{He}$ gives the data of elaborate experiments made by him in 1890 , in which, as in those just referred to, the lateral expansion of a muscle was registered photographically. He concludes that the latency period is not less than 0.004 second, that being the
e, however, gives no reason for

the converging lines are one five-hun- 
the minimum delay which intervenes between instantaneous excitation of the nerve and the first observable change of form is about 7 -thousandths of a second, and that in direct excitation this is diminished to about $3 \frac{1}{2}$-thousandths. The well-known observations of Bernstein ${ }^{1}$ on the period of latency, which were published in 1882, and made by the ordinary myographic method, gave of course a much longer delay in both cases; but the difference in this respect between direct and indirect stimulation was as nearly as possible the same, namely, 0.0032 sec. The inference drawn from the observation of this difference was that in indirect stimulation about $\frac{1}{300}$ sec. is lost in transmission through the end-organs of the nerve. Doubt was thrown on the significance of Bernstein's experimental data by Hoisholt ${ }^{2}$ in 1885, who concluded that inasmuch as when an induction current is led through a muscle the muscle fibres are excited partly by its direct action on them, partly by its action on intramuscular nerves, the greater promptitude of the response might be attributed to the summation of the two actions. The question has lately been reinvestigated by Boruttau, ${ }^{3}$ who has shown that, provided that strong ("overmaximal ") currents are used, the abbreviation of the delay in direct stimulation is always in accordance with Bernstein's statement; he found, however, that when the current strength is slightly diminished, the period of latency acquires the duration which it possesses when the muscle is stimulated through its nerve.

Rate of propagation of the mechanical response.-When a curarised parallel-fibred muscle is subjected to the direct action of an instantaneous stimulus at any spot in its length, in such a way that all the fibres are affected by it, a thickening occurs at the excited spot; this at once divides into two parts, which separate from one another, and are propagated in opposite directions. Each of these advancing thickenings is called a contraction wave. In ordinary skeletal muscle of the frog, it travels, provided that the preparation is fresh and vigorous, at a rate which approaches 3 metres per second, and persists at each point in its course for about $\frac{1}{10}$ of a second, so that if the muscle were sufficiently long, it would be observed that at the moment the thickening ceases at one point it begins about $30 \mathrm{cms}$. further on in its course. In other words, the contraction wave has a length of about $\frac{3}{10}$ of a metre.

The contraction wave is a phenomenon intimately connected with two others; with the excitation wave on the one hand, and with the duration of the twitch, or response to instantaneous stimulation, on the other. By the term excitation wave is meant the initial change in the excitable mechanism contained in every contractile element which, in its progress, precedes the change of form, and is the harbinger of its advance. We shall learn subsequently how it can be observed and its rate of propagation determined. The duration of the twitch is the time which intervenes between the moment at which any particular part of a fibre or bundle of fibres departs from, and that at which it returns to, its normal form. It can therefore be better determined by measuring the change in thickness than the change in length of the muscle.

The first measurements of the rate of progress of the contraction

1 "Die Erregungszeit der Nervenendorgane in den Muskeln," Arch.f. Physiol., Leipzig, 1882 , S. 329.

2 Journ. Physiol., Cambridge and London, vol, vi. p. 1.

3 "Zur Frage der spec. Erregungszeit der motor. Nervenendigungen," Arch. $f$. Physiol., Leipzig, 1892, S. 454. 
wave itself led to erroneous results. Bezold ${ }^{1}$ (1861) measured the time-interval between the excitation and the beginning of response, when the muscle, clamped in the middle in such a way as to prevent the change of form, but not the excitation, from passing, was excited at one end and observed at the other. Aeby $^{2}(1860)$ caused a muscle excited at one end, to record simultaneously, on the same rapidly moving surface, its thickening at two points as far removed from one another as possible; two curves being thus obtained, of which the one drawn by the lever furthest removed from the exciting electrodes was a certain distance behind the other in time. Both of these modes of determining the rate of propagation gave results which fell short of the truth. Ten years later, Bernstein ${ }^{3}$ applied the more correct method of comparing the moments at which successive waves, travelling in the same direction from different points, reached a particular spot at a known distance from each of them. Bernstein used muscles of which the fibres are interrupted by tendinous insertions. Hermann, ${ }^{4}$ criticising this, made similar experiments with the sartorius, using for measurement Pouillet's instead of the graphic method, and obtained a mean result of $2 \cdot 7$ metres per second as the rate of propagation therein. Notwithstanding the disadvantages just referred to, Bernstein's estimate, although it closely approximated to that of Hermann, was a little higher, so that we may accept the statement usually made, that the rate in frog muscles is about 3 metres per second, as correct. In mammalian muscle the rate is, as a rule, much greater than this; the elaborate investigations of Rollett ${ }^{5}$ show that, although it is not more than 3.4 metres in the red muscles of the rabbit, the rate of propagation may be as much as 11 metres per second in the white. The duration of the twitch is correspondingly longer in the red muscle than in the pale, the observations of Kronecker and Stirling ${ }^{6}$ having shown that it is three times as long in the one as in the other. Cash ${ }^{7}$ has demonstrated that similar, though less considerable, differences in duration present themselves in the different muscles of the frog, a single twitch of the gastrocnemius, c.g., lasting 0.12 sec., while that of the hyoglossus may last as long as $0.3 \mathrm{sec}$.

Conduction of slow waves in transversely striated muscle.-On this subject very little is definitely known, for the existence of slow waves has only been established as regards muscles which are under abnormal conditions. They are most readily observed in insect muscles. Rollett ${ }^{8}$ has shown that in the living larva of Corethra they occur side by side with the quick contractions or twitches, and may alternate with them or be interrupted by them. They are therefore independent phenomena; and whereas the twitch seems to be dependent on influences emanating from the nervous system, predominating when the animal is most vigorous, the slow contractions seem to be idiomuscular, i.e., to emanate from the muscle itself; for they only begin to appear as the animal loses its vivacity and as the control exercised by the nervous system is withdrawn. They persist even when death

1 "Untersuch. ueber die elect. Erregungen von Muskeln und Nerven," 1861, s. 156.

2 Arch. f. Physiol., Leipzig, 1860, S. 253 ; and "Untersuch. neber die Fortpflanzungsgeschw. der Reizung im quergest. Muskelfasern," Braunschweig, 1863. S. 79 .

3 "Untersueh. ueher den Erregungsvorgang im Nerven- und Muskel-systeme," 1871,

4 Arch. f. d. ges. Physiol., Bonn, 1874, Bd. x. S. 48.

- Ibid., Bd. lii. S. 224.

6 Arch. f. Physiol., Leipzig, 1878, s. 1.

7 Ibid., 1880.

${ }^{8}$ Loc. cit. 
is approaching, and after the quick contractions have already ceased. Rollett also observed them in strips of excised beetle muscle, and estimated the rate of propagation of the wave to be about $0.116 \mathrm{~mm}$. per second. He found it impossible to measure the rate of propagation of the twitch in insects, on account of its extreme quickness, which must considerably exceed that of the quickest muscles of vertebrates. The length of the slow wave he found to be very inconsiderable, being only about $0.097 \mathrm{~mm}$. i.e., involving from twelve to twenty-nine transverse striations of the fibre.

In those vertebrates in which they have been observed, ${ }^{1}$ slowly progressing waves are only called into existence by mechanical stimuli applied directly to the muscle when it is in some peculiar, but as yet undefined, condition, which is not that of diminished excitability. The local muscular swellings first described by Schiff, ${ }^{2}$ which may be produced in much the same way, also under abnormal but equally indefinable conditions, may represent arrested slow waves which are prevented by loss of excitability and conductivity of the muscle from being propagated. They will be again referred to in the next section.

\section{Exhaustion and Death of Muscle.}

Exhaustion (fatigue).-By the word exhaustion, as applied to muscle, is meant a state consequent on previous exertion, in which the maximal effect of stimulation, whether instantaneous or tetanic, as measured either by increase of tension or diminution of length, is less than normal. The notion suggested by the word, namely, that the chemical material which is the source of mechanical energy set free in contraction has been during the antecedent period of exertion used up more rapidly than it has bcen replaced, is substantially true. Exhaustion may also be defined as a condition in which the normal relation of tension to length is altered. An excited muscle requires, when exhausted, a less weight to extend it to any given length than it did before; the quotient $\frac{\text { tension }}{\text { length }}$ is therefore diminished at the same time that the value of $\Lambda$-i.e. the equilibrium length of the muscle in the excited state-is increased. This may be also expressed by saying that the extensibility of excited muscle is increased by exhaustion. As is implied by the lengthening of $\Lambda$, the same stimulus which before was adequate to raise a weight to a certain height becomes inadequate in muscular exhaustion. To obtain the same result as before-that is, to shorten $\Lambda$ to its original length, and to restore the relation of tension to length to its previous value-the strength of the stimulus must be increased.

In the muscles of the frog two changes relating to its power of contracting, present themselves for examination, namely-(1) The gradual lengthening of a tetanised muscle, which immediately follows the attainment of its maximum degree of shortening; and (2) the much slower diminution of the height to which a weight is lifted, when a muscle is successively excited at time-intervals of such length that the effect of each excitation passes off before that of the succeeding one begins. The first of these effects was described by Ed. Weber fifty years ago, and was afterwards recorded graphically by Wundt, who found that the descending limb of the curve of a prolonged tetanus is concave upwards, indicating that the muscle lengthens quickly at first, less and less so afterwards. The first investigation of the fatigue produced by subjecting a loaded

I See Biedermann, “Elektrophysiologie," S. $129 \mathrm{ff}$.

2 "Lehrbuch d. Physiologie," S. 26.

VOL. II. -25 
muscle to a succession of instantaneous stimuli of unvarying strength and frequency, was made by Volkmann, ${ }^{1}$ but it was not until ten years later that the elaborate research of Kronecker in Ludwig's laboratory afforded the basis for the more exact knowledge of the subject which we now possess. ${ }^{2}$

The muscle used was the triceps femoris of the frog. It remained in situ, so that the circulation could be maintained, and worked under isotonic conditions, either loaded or after-loaded. The load varied in different series of experiments, but never exceeded 50 grms. The nerve was excited by opening induction currents, of which the direction was reversed between each excitation and the succeeding one, regularity being insured by the employment of automatic arrangements throughout. When the method of after-loading was used, it was established that, under the conditions above described (equal stimuli of equal frequency), the successive diminutions of the excitatory shortening of the muscle were exactly equal; so that, when the contractions were recorded on a slowly moving surface, the tops of the vertical marks which represented the successive twitches were in a straight line (p. 204). The inclination or slope of this line depends on the frequency of the excitations, so that if at any time during the decline an interval of longer duration occurs, the difference in height between the record of the preceding and of the succeeding twitch is correspondingly diminished. The graphic record therefore shows that in after-loaded muscles the decline is equable throughout, the height to which the weight is raised being diminished by equal amounts, until the muscle no longer shortens to a length less than its equilibrium length $l$; for although it continues to contract after this point has been reached, the weight is not lifted, and consequently the contractions are not recorded. In the graphic records of loaded muscle with the weight unsupported, the contractions are recorded until they cease. In this case it is observed that towards the close of the observation the curve of decline becomes more and more concave upwards, approaching the base line (the line which corresponds to the length of the muscle when unexcited, but extended by the weight) asymptotically. It is further found that the load may be varied in different experiments, up to a certain limit, without changing the rate of fatigue, i.e., that the height to which at any moment during the decline the muscle can lift a given load, depends not on the weight which it has up to that moment lifted, and consequently not on the work it has done, but exclusively on the number of times that it has been maximally excited. This result was subsequently confirmed by Kronecker and Gotch ${ }^{3}$ as regards exhaustion by tetanus.

Kronecker found that the triceps would go on contracting more than two thousand times when excited every two minutes. When he diminished the interval to a quarter of a minute, the height of the contractions declined more rapidly. This appeared to be also the case when, with a frequency of six per second, the contractions became eventually blended; whence it appears that there is no essential difference between the exhaustion produced by tetanus (as seen in the decline of the tetanus curve) and that which results from less frequently repeated stimulations.

1 Arch. f. Anat., Physiol. u. wissensch. Med., Leipzig, 1860.

2 "Ueber die Ermuidung und Erholung der quergestreiften Muskeln," Arb. a. $d$. physiol. Anst. zu Leip $\approx i q, 1871$, S. 177.

${ }^{3}$ Arch. f. Physiol., Leipzig, 1880, S. 438. 
If exhaustion is regarded as meaning diminution of the height to which a muscle is able to lift a given weight, it is evident that the assumption frequently made cannot be accepted, namely, that so long as the time occupied in doing the work remains the same, exhaustion increases with the sum of the work which has produced it; in other words, that it may be expressed as the product obtained by multiplying the number of efforts by the work done in each. If we represent the successive lifts in Kronecker's series by the vertical lines enclosed in the triangular space $A B C$ (Fig. 215), it is obvious that the sum of the work done at the moment that any contraction (e.g. at $D$ ) takes place is represented by the area $A B D E$, and that this area increases very rapidly during the first part of the period represented by the base of the triangle, very slowly towards the end. A muscle, for example, which contracts and lifts a weight a hundred times at equal intervals, is only half exhausted when it has made fifty efforts, though it has done threequarters of the work.

Further evidence that the rate of exhaustion does not depend on the amount of work previously done, may be obtained by comparing the graphic records of two similar muscles after-loaded with different weights. In these the lines of decline begin at a level which is the higher the less the weight. They are both straight lines, and their inclination is the same, so that if they were written one above the other on the same recording surface, they would be parallel. As however the difference between the heights to which a weight is

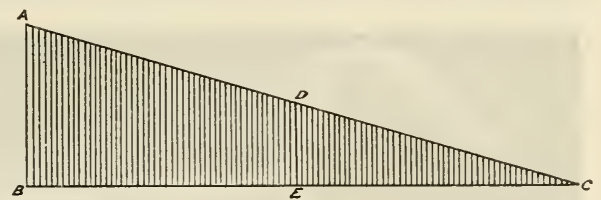

FIG. 215 . raised in two successive contractions in each series is always the same so long as the interval is unchanged and the excitation is maximal, it follows that when a muscle is heavily after-loaded, the contractions disappear from the record earlier than when the after-load is less. The earlier disappearance does not mean that the muscle is sooner exhausted, but only that its actual length when excited and extended by the weight, becomes greater than $l$ a longer time before it is exhausted; for, as has been already seen, when the after-loading method is used, no contraction, in which the muscle fails to shorten to this length, is recorded.

The application of these results to the study of muscular exhaustion in man is attended with considerable difficulty. Professor Mosso has lately invented an instrument (ergograph) by which graphic records of fatigue, resembling those of Kronecker, may be obtained in human muscle. The muscle of which the contractions are recorded is the flexor of the fingers, the nerve is the median, the movement of a weight lifted by the middle finger being inscribed on a suitable recording surface. The weight is hung upon the finger, and supported during the intervals between successive excitations, so that the nuscle is "after-loaded." In general the line of decline is nearly straight, but, as might be expected, it is more variable than in Kronecker's experiments. Although it is difficult to say whether the numerous observations made by this method have materially contributed to the understanding of the nature of exhaustion, they have proved to be of great value as a means of determining the influence of psychical, pharmacological, and other conditions on the process. Another 
instrument for investigating muscular fatigue in man has been employed by Dr. Waller; the essential difference between the two methods being, that in the one the muscle contracts isotonically, in the other isometrically.

The exhausting effect of continuous voluntary contraction was investigated by Donders, in man, with special reference to the case in which a weight is sustained by the hand with the forearm supine and horizontal. The method is analogous to that previously described. A cord is attached to the weight, by the severing of which the forearm is liberated. Instantly the hand rises, hastening to assume a position which corresponds to the equilibrium length of the acting muscle, the biceps. This length can be calculated with approximate accuracy from the angular measurement of the movement executed by the forearm in assuming its new position. The experiment affords direct proof that the effect of muscular fatigue is, as has been already explained, to increase the equilibrium length of the excited muscle; whence it follows that the longer the effort lasts and the oftener it is repeated, the stronger is the influence which must be exercised on the muscle by the central nervous system. The sensation of fatigue which by a conscious effort we strive to overcome, in keeping up the weight as long as possible, is probably a fairly true index of the gradual intensification of the excitatory process in the motor cell and motor nerve.

Effect of exhaustion on the duration of the response to single excitations. - The observation was originally made by Helmholtz, that when a muscle

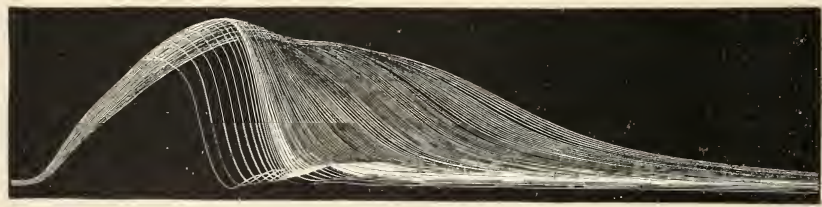

FIG. 216.-Muscle curves showing the effect of fatigue.-After Waller.

is excited repeatedly by an instantaneous stimulus, the time occupied by the response increases at the same time that the height of the curve diminishes. This was subsequently shown in a striking way by Wundt, who so arranged a muscle that each time it relaxed after excitation, it re-established an exciting contact which, in contracting, it immediately broke. The diminishing frequency of the contractions thus automatically produced, showed that each lasts a little longer than its predecessor. The character of the change of form of the isotonic curve which is thus produced, is well seen in Fig. 216, which represents a succession of such curves with their starting-points coincident. Their heights at first increase, then diminish as the spans of the curves become greater. It has been stated that the increase of duration is due to failure in the process of relaxation, but of this such curves can afford no evidence. All that they show is that the state of excitation and the contractile stress by which it expresses itself, both increase and diminish more slowly.

To understand the prolongation of the mechanical response in a tired muscle, we have only to consider that the response itself is a "wave of contraction" which is normally propagated at a certain rate; and if we assume that the wave length remains the same, and that the propagation rate is diminished, the duration of the effect at any point over which the wave passes must increase proportionally to that diminution. 
It must, however, be remembered that the diminution of the rate of propagation in fatigue has not been proved experimentally.

Efficient causes of exhaustion.--It was shown long ago by Ranke ${ }^{1}$ that the definition of exhaustion which we have characterised as in the main correct-that which regards it as a state in which restitution lags behind waste-is not wholly adequate. Without denying that mere disturbance of balance of nutrition in the muscle substance may produce the effects of fatigue, he showed that in the frog these may be got rid of by the circulation through the muscle of normal salt solution, i.e. of a liquid which has no nutritive properties; and that a state which cannot be distinguished from fatigue can be produced in fresh muscles, by the injection of aqueous extract of the fatigued muscles of another animal.

Mosso $^{2}$ has shown much more recently, that even the introduction of the blood of a fatigued animal (e.g. a dog) into the circulation of a normal one will give rise in the latter to all the symptoms of fatigue (respiratory, cardiac, etc.). It would appear, therefore, that some toxic substance is produced in the muscles by tetanising them. Ranke at first inferred from what was known at the time as to the production of lactic acid in protracted muscular exertion, that the acid was the true cause of exhaustion; and he was thus led to make the well-known experiments by which it was proved that the introduction into the circulation of normal physiological salt solution, containing a very small percentage of this acid, produces exhaustion, and that the muscles regain their natural vigour when the lactic acid is withdrawn, and the circulating fluid rendered alkaline by a trace of sodium carbonate. He soon, however, began to surmise that the fatiguing action belonged to lactic in common with other acid bodies, and this led to the discovery that, even when no free acid was present, a reaction due to the monophosphates of the alkali metals was sufficient to produce the result. It was subsequently found that the presence of lactates had no effect in a neutral solution, so that exhaustion can only be attributed to lactic acid, in so far as the production of this body is the immediate cause of the acid reaction of fatigued muscle. The facts just stated prove no more than that acid reaction is one of the conditions of exhaustion. It is not probable that it is its only cause; for it has been shown by Abelous as well as by Mosso, that the alkaline serum of a fatigued animal also produces fatigue. It cannot, therefore, be doubted that among the products of muscular activity there exists a substance endowed with the property of rendering muscle less competent, although we have as yet no sufficient knowledge either of the nature or the mode of action of this substance.

The remarkable fact insisted on by Waller, ${ }^{3}$ that in the ordinary process of exhaustion of muscle, the indirect excitability disappears while the direct excitability persists, has been also shown to obtain by Abelous ${ }^{4}$ with fresh muscle (frog) when supplied with blood derived from fatigued muscle. His method consisted in severing one sciatic, and then throwing the whole muscular system with the exception

1 "Grundzüge der Physiologie," S. 632.

2 Trans. Internat. Med. Cong., Berlin, 1890.

3 "Physiology," 2nd edition, p. 380.

${ }^{4}$ Abelous and Langlois, Arch. de physiol. norm. et path., Paris, 1893, tome v. pp. 437-448; and 1894 , tome vi. p. 432 . 
of the paralysed limb, into tetanus by faradising the spinal cord, the circulation remaining intact. In this way the paralysed muscles, although inactive, become fatigued. The process of fatigue is tested at regular intervals by direct and indirect stimulation, and it is found that the indirect excitability disappears first. Abelous therefore concludes that, as nerve is inexhaustible, the end-organs are more directly affected by the "fatigue substance" than the muscular fibres themselves.

Death of muscle.-When muscle is immersed in boiling water it at once shortens and thickens, losing its elasticity and becoming opaque. In muscle so treated the proteids coagulate, and all functional chemical changes are brought suddenly to an end. This method is therefore frequently used for the purpose of ascertaining the chenical condition of a muscle at any desired moment, so far as relates to those constituents which are known not to be aflected by boiling temperature. If the temperature of a muscle is raised gradually, the change of form occurs in stages. According to the recent observations of Brodie, a first shortening occurs at $32^{\circ} \mathrm{C}$., ceasing at about $40^{\circ} \mathrm{C}$.; a second hegins at $46^{\circ} \mathrm{C}$. and ceases at $50^{\circ} \mathrm{C}$; ; a third at $56^{\circ} \mathrm{C}$., ending at $60^{\circ} \mathrm{C}$; ; and at $63^{\circ} \mathrm{C}$. there is a final shrinking which ceases at $75^{\circ} \mathrm{C}$. As these temperatures closely correspond with the temperatures of coagulation of the several proteid bodies contained in musele, ${ }^{1}$ the observations afford better evidence than had before been given of the connection of coagulation with the changes produced in a muscle by warming it. But although coagulation may serve to account both for the loss of elasticity and the opacity produced by heat, it does not sufficiently explain its shortening; nor does there seem to be any reason why, even when the process is very gradual, the successive diminutions of length should not be in part attributed to contractility. The shortening may be a response to the stimulating action of the temperature, and the opacity may be due to coagulation.

The production of heat rigor is a more simple process than the similar change which occurs spontaneously at a certain time after death -true rigor mortis, or, as it is called by German writers, Zeitstarre. The same question, however, presents itself as regards the immediate cause in the two cases, for in rigor mortis we have, on the one hand, shortening which may be compared to the effect of excitation; on the other, opacity and loss of elasticity, which may be attributed to solidification of previously fluid constituents of the muscular substance. We will first inquire what grounds there are for supposing that muscle in the process of dying, passes into a state analogous to that produced by excitation.

The notion that it does so is an old one. Nysten (1811) compared rigor with vital contraction, and spoke of it as the last effort of dying muscle. This vitalistic ilea, after holding its ground for more than a generation, was displaced by the theory usually attributed to Brücke, that the process is solely one of coagulation. It may be questioned whether Brücke took so exclusive a view as these words imply; but, in any case, the physiologists of thirty years ago were justified by experimental results, in regarding the solidification of myosin as the most important consequence of the cessation of vital activity. Külne had shown ${ }^{2}$ that a muscle cau be kept in a living state, i.e. prevented

\footnotetext{
I See Halliburton's artiele in vol. i. of the present book, and Ternon's paper in the Journal of Physioloyy, 1899.
}

2 "Lehrbuch der physiologisehen Chenie," 1866, S. 274, 285. 
from going into rigor, by subjecting it immediately after removal to a temperature approaching $0^{\circ} \mathrm{C}$, and that a liquid squeezed out of it by pressure when in this state, remains fluid so long as this temperature is maintained, but that myosin separates from it as soon as the temperature rises ; and, further, that although a certain quantity of liquid can be squeezed out of muscle which not having been kept cold had become rigid, this is incapable of spontaneous coagulation, inasmuch as it contains no myosin. It thus appeared as if the characteristic proteid of muscle were affected by low temperature in the same way as the nuuscular tissue itself, and this seemed to afford a cogent proof that in the properties of niyosin the explanation of rigor was to be found. There were, however, always those who held to the old view of Nysten, that in muscle the process of death is continuous with that of life. Brown-Séquard indicated as the result of numerous observations, partly clinical, partly experimental, that rigor occurs under conditions which can have nothing to do with coagulation, that it occurs in muscles which are still "living," that it associates itself with excitatory states, and that it can be warded off by passive movement, recurring when this is discontinued. Schiff ${ }^{1}$ also found it to be closely related to certain forms of contraction, particularly that localised form to which he applied the term "idiomuscular."

The true relation of the spasmodic to the other phenomena of rigor was not ascertained with accuracy until in 1881 the inquiry was taken up by Hermann and worked out by his pupils. By them it was shown, both in the frog and in mammals, that rigor was retarded in the muscles of a limb by severing its nerves, or ly hemisection of the spinal cord, the operation being in either case performed immediately after death, so as to avoid the possibility of interference with the previous state of the circulation or nutrition of the muscles. It thus appeared that the nervous system continues to exercise a certain influence on the muscles after death, and that interference with the innervation of a muscle is unfavourable to the development of rigor. The following experiment of Nagel $^{2}$ makes it probable that this statement may be extended to the peripheral parts of motor nerves:-

A frog, in which one hind-limb has been protected by ligature, is curarised ; the spinal cord is destroyed, and both sciatics are immediately divided. It is then found that rigor is retarded on the curarised side as compared with the other side, a result which shows that, even when separated from the central nervous system, the peripheral parts of the motor nerves exercise an influence in initiating the post-mortem contraction of the muscles to which they are distributed.

From the observations of Bierfreund, ${ }^{3}$ it appears that muscles which contract differently also behave differently with regard to rigor. Thus, in the red muscles of the rabbit the post-mortem contraction is so much retarded as compared with that of the pale muscles, that the contraction of the latter may have ceased at the time that that of the other is only half over (Fig. 217).

Other differences in the behaviour of muscles in rigor, depending upon individual peculiarities of the muscles themselves, have been observed by Langendorff and Gerlach. ${ }^{4}$ When the hind-limbs of a frog are freely suspended in normal salt solution, the attitudes which they assume indicate that the flexors pass into rigor before the extensors. Nagel has confirmed this observation as regards toads and certain specimens of $R$. temporaria which had been kept through the winter. In other frogs he found that both sets of muscles went into rigor simultaneously.

I 1858, In "Beitr. 7. Physiol.," 1894, Bd. ii. S. 9, 18.

${ }^{2}$ Arch. f. d. ges. Physiol., Bonn, Bd. lviii. S. 279.

3 Ibid., 1888 , Bd. xliii. S. 195.

${ }^{4}$ Ibid., Bd. Iviii. S. 279-308. 
As regards the cessation of rigor, Bierfreund showed that it was neither due, as Brïcke supposed, to the development of acid reaction, nor, as other observers have thought, to the commencement of putrefactive cliange, but that the time of its occurrence is rather dependent on individual peculiarities of the muscle used. That it is not due to sepsis, is proved by the fact that it occurs in muscles perfectly sterilised by the injection of salt solution containing car-

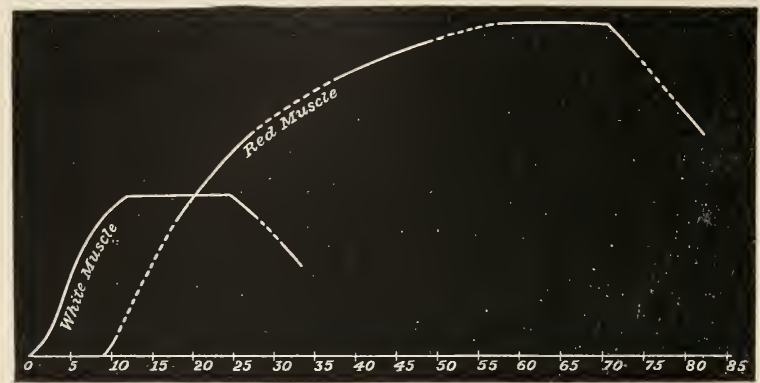

Fig. 217. - Showing curves of rigor mortis of white (gastrocnemius) and red (soleus) muscles of rabbit. Rigor occurs eight hours after death in the former, fifteen hours in the latter. The red muscle also shortens more than the pale. On comparing these curves with the ordinary isotonic curves of the same muscles, their heights are found to be in similar proportion to each other.-After Bierfreund.

bolic acid. This, lowever, does not exclude the possibility that a putrefactive change, if present, might arrest rigor; and Nagel ${ }^{1}$ has mentioned instances in which the early occurrence of putrefaction was associated with the absence of rigor. Nagel, however, agrees with Bierfreund in not regarding putrefaction as the ordinary cause of cessation of rigor, for he has also found many cases of rigor persisting in muscles which have undergone a good deal of putre-

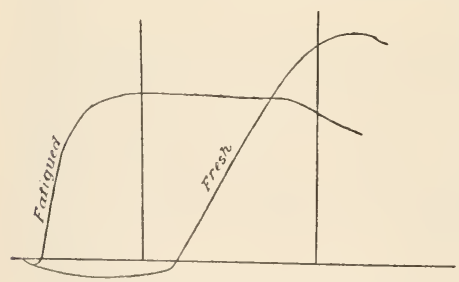

F16. 218.-Curves of rigor mortis in fresh and exhausted nuscle.-After Nagel. factive change, and some in which it has appeared, although no putrefactive change could be perceived.

The well-known fact that a inuscle exhausted by previous tetanisation, or by the spasms of strychnine, passes more quickly into rigor than a normal muscle, is well illustrated by an experiment of Nagel, of which Fig. 218 gives the result. The shortening of a gastrocnemius, which had been repeatedly tetanised, was compared with the unfatigued gastrocnemius in another frog. Rigor supervened sixteen hours later in the one than in the other.

It has already been mentioned that Schiff, at an early period, connected the incipient stage of rigor with localised or "idliomuscular" contractions. He imagined that it might be initiated by the direct action on the still excitable muscular substance, of some product coming into existence either after death or when death was approaching. The

\footnotetext{
${ }^{1}$ Loc. cit., 1894, Bd. lv. S. 481.
} 
following phenomena, many of which have been indicated by Schiff or his pupils, tend to support this view : ${ }^{1-}$ (1) Whenever rigor comes on very gradually, the muscles continue for some time to respond to appropriate stimuli (interruption of a battery current led through the muscle, or tapping of the surface). If the stimulated parts are afterwards compared with other parts, they are found to be rigid and unexcitable. This can be observed both in the frog and in mammals. (2) If a muscle in the transition state above referred to, is observed with the aid of the electrometer, the meniscus is seen to oscillate in such a way as to indicate the existence of irregular action currents. In some muscles, when going into rigor, irregular contractions are olservable. This is chiefly seen in mammalian muscles (Brown-Séquard), although it is also noticeable in some amphibians, e.g. in toads and, to a less extent, in Rana temporaria. In $R$. csculenta the passing into rigor is almost always a continuous process. (3) Schiff finds it impossible to determine when the initial shortening of muscles deprived of their supply of arterial blood by ligature, as in Stenson's experiment, ceases to be contraction and becomes rigor. The distinction between them is so gradual, that the muscles can at first be brought back to their normal state by restoring the circulation; later, this becomes more and more difficult. (4) In a frog poisoned by strychnine after one sciatic nerve has been divided, the spasm of strychnine in the poisoned limb passes so immediately into that of rigor, that it is difficult to distinguish them. Here Schiff attributes the initial contraction to the chemical stimulus (lactic acid?) brought into existence by the previous spasm. For, according to his pupil, C. Schipiloff, the first effect of the circulation of salt solution containing $0 \cdot 1$ per cent. of sarcolactic acid, is to produce spasm. The muscles, however, retain their translucency, and can be brought back to the normal by substituting alkaline for acid salt solution. If this is not done, the spasm passes into rigor.

The general result of the preceding considerations relating to the nature of rigor mortis is-(1) That rigor is a mixed process, of which the first stage is spasmodic, the second coagulatory; (2) that although the spasmodic phenomena for the most part precede the coagulatory, the two may present themselves simultaneously; (3) that although there is no sufficient evidence that the presence of free lactic acid is the cause of the dying contraction of muscle, acid reaction is its earliest concomitant; (4) that post-mortem spasm is of similar nature to those continuous contractions which, although they occur during life, are characterised by $(a)$ extreme slowness or even absence of propagation, $(b)$ slowness of development, and $(c)$ by the fact that they are relatively more readily evoked by stimuli of a certain duration than by instantaneous stimuli.

\section{Nature of the Process by which Chemical Energy is Transformed into Mechanical Energy in Muscular Contraction.}

The mechanical potential energy which suddenly comes into existence when a muscle is excited, whether this is allowed to manifest itself in lifting a weight, or is compelled to appear exclusively as tension, has its ultimate source in the chemical potential energy of certain constituents 1 "Beitr. z. Physiol.," 1894, S. 97-124. 
of muscular structure. The mechanical effects, although the consequence rather than the concomitant of the chemical change, follow it instantaneously and invariably. What further happens depends upon mechanical conditions, in relation to which three cases may be distinguished. If (Case 1) the muscle is prevented from shortening, heat is produced in quantity equal to the heat value of the material disintegrated. If (Case 2 ) the muscle is allowed to do external work, e.g. in lifting a weight, the energy so used is transferred to the weight, and lost; but if (Case 3) the weight after being lifted is allowed to fall, that energy is returned to the muscle at the moment of its relaxation, and manifests itself by producing in it an amount of heat which is the equivalent of the potential energy immediately before transferred to the weight. Cousequently, the eventual result in Cases 1 and 3 is of the same kind. In both, the first mechanical result is the appearance of tension; in Case 1, potential mechanical energy is transformed directly into heat; in Case 3, it is not transformed until a certain proportion of it has undergone translation and retranslation.

The chemical change which, as has been stated above, precedes all others in the response of a muscle to stimulation, is an oxidation. This does not necessarily mean that the chemical energy which is concerned in the combination of oxygen atoms with those of carbon and hydrogen, is directly transformed into the mechanical energy which manifests itself in muscular effort. It is prima facie quite as reasonable to suppose that muscle in doing work may act after the manner of a thermodynamic inachine, such as a steam-engine; i.e., that it may first produce heat by oxidation, and then use that heat in the doing of work. If this is so, we have to suppose that between the initial stage of chemical change and the manifestation of potential mechanical energy in the form of tension, there is an intermediate stage or phase in which the whole of the energy concerned in the process becomes kinetic, as heat; in other words, that between chemical attraction aud mechanical tension, there is a thermogenetic link in the chain of events, so that the immediate agent in the production of the mechanical response is heat, not chemical attraction.

The doctrine set forth above was first enunciated in 1845 by $\mathrm{J}$. R. Mayer, ${ }^{1}$ in the treatise which was the foundation of all that we know as to the relation of heat and work in the living organism. It was not until 1882 that the objections to it were clearly stated by Fick ${ }^{2}$ in the book already so often referred to, and, more at length, in his "Medical Physics." These are as follows: In every thermodynamic machine, i.e. in every machine in which work is produced as in the steam-engine at the expense of heat, the process by which this is effected consists in the translation of heat from a body of higher temperature $A$, to a body of lower temperature $B$, under such conditions that a certain part of the heat so translated is converted into work. The utmost quantity of work so producible can be determined with absolute certainty, provided that the quantity of heat transnitted, the temperature of $A$ and $B$, and that of the medium of transmission, are known, and that the process is a "cychical" one, i.e., that the final state of the medium of transmission is

1 "Die organische Bewegung in ihrem Zusammenhang mit dem Stoffwechsel," Heilbronn, 1845 . S. 206 .

"Mechanische Arbeit., u.s.w.," S. 154, 158; "Medizinische Physik," 1885, Aufl. 3, 
the same as the initial. The nature of this relation is set forth in the second law of thermodynamics. It is such that, unless a very high estimate be taken of the difference of temperature which in muscular contraction can exist between $A$ and $B$, i.e. between the source of heat and its environment, the quantity of work resulting from the heat transmitted would be a small fraction only of the quantity actually produced by the combustion of an amount of material of corresponding heat value. Thus, if muscle could be made the medium of a thermodynamic process, i.e., could be made to do work at the expense of heat transmitted through it from outside, the quantity of heat transformed into work would bear so small a proportion to the quantity transmitted, that no comparison could be made between the hypothetical experiment and what actually happens in contracting muscle.

Such a hypothetical process as is described in the following lines, is suggested by Fick:-

A muscle is extended by a weight, at a temperature of $20^{\circ} \mathrm{C}$., and then warmed to $30^{\circ} \mathrm{C}$. It shortens as soon as it is allowed to do so, and lifts the weight. It is then made to do further work, by gradually unloading it at this temperature until the extending weight $=0$. The muscle is next cooled to $20^{\circ} \mathrm{C}$., so that it reassumes its unextended length at that temperature. Work is then done upon it by extending it gradually, until the extending weight is of the original amount.

The muscle is again warmed to $30^{\circ}$, and so the cycle recommences. There is therefore in each repetition of the process a gain of external mechanical work, the amount of which is in accordance with the second thermodynamic law.

This law may be expressed as follows:-In every thermodynamic cyclical process, the heat value of the work $Q$, which can be produced by the translation of a given quantity of heat $Q_{1}$, from a warmer to a colder body, depends (1) on the absolute temperature of the medium $T$, i.e., the temperature at which the process takes place, and $(2)$ on the difference of temperature between $A$ and $B\left(T_{1}-T_{2}\right){ }^{1}$ Consequently, unless it is possible to suppose the existence of very large differences of temperature in the environment of the acting parts of muscular substance, the doing of work in muscular contraction cannot be regarded as a thermodynamic process.

The reader will find in Engelmann's Croonian Lecture, ${ }^{2}$ his solution of this problem. He contends that a rise of temperature of the whole substance of the contracting muscle, amounting to $\frac{1}{1000}{ }^{\circ} \mathrm{C}$., would involve a very high temperature of the chemically active particles, the number of which he estimates to be exceedingly small. The objection that the high temperature required is incompatible with life, he meets on the same ground, namely, the extremely small proportion to the whole of the part in which this high temperature exists. In the same lecture, experiments were adduced which seemed to give support to a thermodynamic theory of muscular contraction. Of these the most important related to the work which can be got out of either soaked fiddlestrings or caoutchouc, by warming these substances. The method is as follows:-

1 The formula by which this is expressed is-

$$
\frac{Q}{Q_{1}}=T\left(\frac{T_{1}-T_{2}}{T_{2} T_{1}}\right)
$$

If the difference between the value of $T$ and that of $T_{1}$ or $T_{2}$ is relatively small, the right

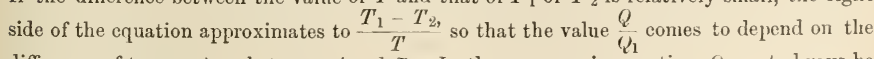
difference of temperature between $A$ and $B$. In the case now in question, $Q$ must always be extremely small as compared with $Q_{1}$.

${ }^{2}$ Proc. Roy. Soc. London, vol. lvii. pp. 411-433. 
A bit of Estring $2 \mathrm{in.} \mathrm{long} \mathrm{is} \mathrm{so} \mathrm{arranged} \mathrm{that} \mathrm{its} \mathrm{shortening} \mathrm{may} \mathrm{be} \mathrm{recorded}$ myographically (Fig. 219). It is extended by a load of 50 grms. and surrounded by a coil of thin platinum wire in connection with the two poles of a fairly strong battery, so that it can be promptly heated or brought back again to the temperature of the water in which it is immersed, by closing or opening the current which flows through the wire. Before making the experiment, the water is warmed gradually to a temperature above that at which the experiment is to be made, namely, to about $50^{\circ} \mathrm{C}$., in consequence of which the string is permanently shortened to a certain length. Under these conditions momentary closure of the current is followed by a rapid rise of the writinglever, which describes a curve resembling a myogram.

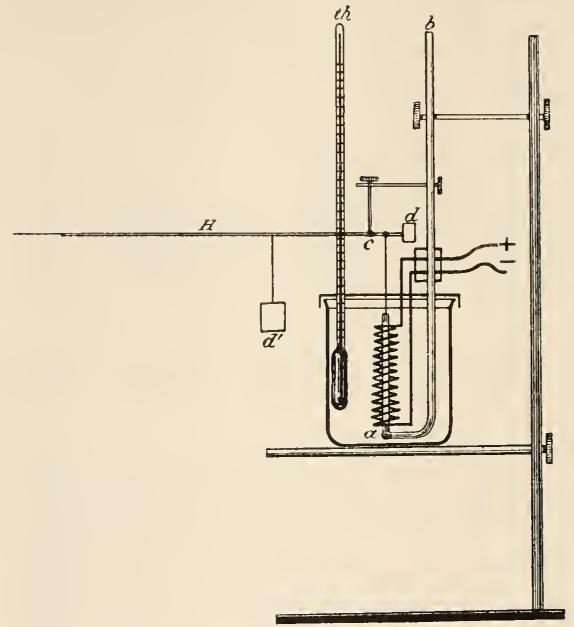

FIG. 219.-Engelmanu's apparatus for recording contractions of a violin string on heating. $b a$, support, to the one end of which $(a)$ the previously swollen violin string is attached. $H$, writing-lever, supported at $c$, to which the other end of the string is attached. $d$ and $d^{\prime}$, weights for altering tension. + and -, the two ends of the platinum wire coiled round the string leading to the two poles of a battery. th, thermometer with its bulb in the same bath in which the string is immersed.

human muscles of the same transverse sections." 2

By soaking and heating the cord before the experiment, it has been made to assume a constant condition, i.e., one in which the observation can be repeated any number of times. We have therefore before us a process in which work, comparable to that of muscular contraction, is done by a "cyclical" process at the expense of heat; a tension of 300 grms. being, for example, developed in a string $30 \mathrm{~mm}$. long and only 1 square $\mathrm{mm}$. in transverse section, by raising the temperature $75^{\circ}$ C. 1 If the same experiment be made with stretched indiarubber instead of violin string, the effect is even greater; if heated by only about $20^{\circ} \mathrm{C}$. it shortens in the direction in which it has been extended, and can be made thereby to do an amount of work "sixty times as great as the maximum afforded by

The three substances - catgut, stretched caoutchouc, and muscle - all resemble one another in being doubly refractive or containing doubly refractive elements. The property of contracting when heated is one which, according to Engelmann, is met with in doubly refractive bodies, and varies with the degree in which they are so. ${ }^{3}$ It is for this reason that stretched indiarubber, which is made up entirely of doubly refractive substance, can do more work than either a muscle or catgut string when similarly heated. A further resemblance that he finds between musele and other such substances is, that within

1 Engelmann, "Sur l'origine de la force musculaire," Arch. néerl. d. sc. exactes, vol. xxvii. p. 24. See also Engelmann, "Ueber einige gegen meine Ansicht vom Ursprung der Muskelkraft erhobene Bedenkeu," Arch.f. d. ges. Physiol., Bonn, Bd. liv. S. 637.

${ }^{2}$ Croonian Lecture, p. 426. 3 Ibid., p. 419. 
certain limits the shortening power increases with the load to be lifted. ${ }^{1}$ This he attributes also to the fact that extension in all these substances increases the property of double refraction. He concludes, therefore, that the cause of the development of contractile stress in living muscle is to be sought for in the warming of its doubly refractive particles. Heat sufficient for this purpose he thinks can be produced by the transformation of the chemical potential energy of the constituents of muscle, which follows excitation. Having regard to the "infinitesimally small number of particles concerned in a contraction," he can see no reason why the difference of temperature between the heat-producing and the work-producing particles should not be sufficient to account for the existence of such a relation between the amounts of work heat and of translated heat as would be required by the second thermodynamic law. He does not maintain that his catgut and indiarubber models represent all that happens in living muscle, but points out that they present phenomena which are among the most important of those of muscular contraction.

From the preceding considerations it will be seen that, in regarding the origin of muscular force as a thermodynamic process, Engelmann recognises that the development of high temperature at the foci of origin of mechanical energy in muscle is a necessary link in the process. Before we accept Fick's conclusion that no such link is wanted, let us remember that the view usually entertained by physiologists, that at the moment of excitation the attraction of carbon and oxygen atoms is directly converted into axial tension, involves difficulties of which the character can be best judged by reading the pages in which Fick himself sets forth what he regards as the simplest molecular mechanism by which the transformation of chemical into mechanical energy can be effected. ${ }^{2}$ The question how the former is converted into the latter is rather physical than physiological. The physiological chemist may proceed with his inquiries as to the sources of chemical energy, and the experimental physiologist pursue the lines of investigation which will be sketched in the next section, without waiting for the decision of the question whether muscular contraction is or is not a thermodynamic process. It may be surmised that, whenever that question is answered, it will be by following the indications given by Engelmann in his Croonian Lecture, namely, by the investigation of an analogous process in a non-living structure.

\section{The Production of Heat in Muscular Contraction.}

The reasons which compel the physiologist, in studying the mechanical phenomena of muscular contraction, to have recourse, in the first instance, to the muscles of the frog, have even greater force when his purpose is to investigate the changes of temperature which are associated with these phenomena. For here the quantities to be measured are very small, and the experiments require an even greater degree of precision than those which relate to the mechanical effects of stimulation. This can only be secured by repeating the observations a sufficient number of times under constant conditions. In the muscles of a homœothermic animal, such constancy is scarcely attainable; for the necessity of maintaining the circulation introduces sources of error which it is hard to guard against.

\footnotetext{
1 Croonian Lecture, p. 422.

2 Fick, Arch. f. d. ges. Physiol., Bonn, 1893, Bd. liii. S. 611.
} 
It was one of the great discoveries of Helmholtz, that when a muscle is tetanised its temperature increases. The method of measuring this increase, and of determining its relation to the other phenomena of the excitatory state, was subsequently elaborated by Heidenhain. ${ }^{1}$

Methods.-It need scarcely be mentioned here that both of these great observers used the thermo-electric method. Heidenhain employed a small thermopile specially made for the purpose, of fifteen bismuth and antimony couples, of which one set of junctions was covered by the muscle to be investigated (the gastrocnemius of the frog), while a dead muscle was similarly applied to the junctions on the opposite side of the thermopile; and this, when the method is carried out in accordance with his directions, is found to be abundantly sensitive for its purpose. But this very sensitiveness brings the observer face to face with great technical difficulties; for it is found that even if two inactive muscles are compared with each other by applying them severally to the opposite sides of the thermopile, it is extremely difficult, even when the instrument is kept in a "moist chamber" and protected from changes of temperature in the environment, to bring the needle to a fixed position. As no observations can be made until the accidental disturbances which prevent this have been got rid of, much time is unavoidably lost. Nor is this the only practical objection to the method, for it has further to be borne in mind in dealing with the result, that what is measured is not the temperature of the muscle itself, but that of the junctions, because, from the form of the thermopile, these cannot be brought into such proximity to the muscle that the two temperatures can be considered the same. These considerations induced Fick, ${ }^{2}$ more than twenty years ago, to give to the thermopile such a form that, although the instrument is rendered less sensitive, it affords more reliable indications of the changes which occur in the muscle. Fick's thermopile consists of very slender strips of iron and German silver. The end of each strip is filed away on one side, and is soldered to its fellow in such a way that the surface of junction of the two ends does not extend more than half a millimetre. As their united ends are as thin as paper, their mass is so inconsiderable that they assume the temperature of the muscular substance by which they are surrounded, without altering it. The end of this thermopile resembles a comb of six teeth, each of which is a junction; its form renders it possible to introduce it between the fibres of a muscle with very little injury ; it is, however, more advantageous to avoid all risk of this kind by introducing it between the two adductor groups of muscles (Fig. 189) placed abreast of each other. These, when properly arranged and excited simultaneously, apply themselves by their opposed internal surfaces to the junctions as perfectly as if its knife-like edge were plunged into the muscular substance. Whatever form of thermopile is used, it must be graduated. The data for graduation are-(1) The deflection obtained with a larger element of the same material as that used in making the thermopile, with a known resistance in circuit and a known (considerable) difference of temperature between the junctions; and (2) the resistance of the thermopile circuit, as actually used for measurement.

Relation between thermogenetic and inogenetic processes.Before proceeding to the discussion of the influence of physiological and other conditions on the production of heat in a contracting muscle, it will be useful to set forth the general results of experiments as to the

1 “Mechanische Leistung, Wärmeentwickelung n. Stoffumsatz bei der Muskelthätigkeit," Leipzig, 1864, S. 63.

22 "Ueber die Wärmeentwickelung bei der Muskelzuckung," Arch. f. d. ges. Physiol., Bonn, 1877, Bd. xiv. S. 31. 
relation between the thermogenetic and inogenetic processes. In all experiments in which it is desired to obtain quantitative results, the muscle to be investigated is regarded as if it were a calorimeter. Having placed it under such conditions as to prevent the transference of heat to or from the surrounding media during the period of observation, the amount of heat produced during any thermogenetic process is estimated by multiplying the increase of temperature by the weight of the muscle, and the product by the specific heat of muscular substance. Measurements of this kind are regarded as reliable, because it is found that when the experiment is so made that the whole of the heat which disappears in doing work can be measured, its relation to the quantity of work done is in accordance with the first thermodynamic law.

To prove this eonformity, the amount of work done is measured in a series of instantaneous excitations, following one another with such frequency as just to allow the muscle to relax between each contraction and its successor. This is done by a contrivance called a "work-collector," consisting of a wheel around the horizontal axle of which a cord supporting a weight is wound. Each time the muscle contracts, it works on the periphery of the wheel so as to rotate it and thereby lift the weight. As the wheel is prevented from moving back by a catch, the weight is raised a little by each contraction, but never descends. The total lifting work done in the series can thus be measured. The heat produced during the series is measured at the same time. If $H$ denotes the heat value of the material oxidised, and $W h$ the heat equivalent of the work done, $H-W h$ denotes the result of this measurement. To ascertain the value of $H$ the collector is dispensed with, and a second experiment is made, in which the heat produced in the muscle is measured in an exactly similar series of excitations, with the weight hung on to the muscle itself. In this case the weight falls between each contraction and its successor, so that the heat lost during contraction in doing external work is returned to the muscle during relaxation. Consequently the amount of heat produced during this series exceeds that measured in the first experiment by $W$, i.e., the equivalent of the work done. If it were possible to make this experiment with perfect exactitude, this difference, measured in microcalories, would be to the work done, measured in gramme-millimetres, as 1 to 425 .

From experiments of this kind we learn what was the relation between the quantity of material oxidised and the work done in any particular case investigated. But no general inference can be drawn from it as to the normal relation of material expended to work produced in muscle, unless it is known that the conditions under which the muscle worked were favourable, i.e., that they were such that the amount of material used yielded the largest attainable result in work. What these conditions are will be shown in the following paragraphs. For the present purpose it is sufficient to say that for every muscle there is a certain amount of load which cannot be either increased or diminished without disadvantage, as regards the relation between the work done and the material expended. If we seek to increase the work done by increasing the load, we soon find that the effect of doing so is more than made up for by the diminution of the lift, and similarly we cannot increase the lift without finding the effect more than counterbalanced by the diminution of the load.

For the gastrocnemius of the frog such a load is about 200 grms. When this is employed, the relation obtained between the heat-value of the work done and that of the material used is about 1 to 4 . In man 
it is estimated from recent experimental data, that the heat produced during a period of active muscular exertion in the whole body is about five times as great as the heat-equivalent of the external work done; so that the work-heat is to the heat-equivalent of the total chemical oxidation of the body as 1 to 6 . Assuming this estimate to be correct, about three-fifths of the total heat set free in the body during exercise, must be produced in the muscles (i.c. the quotient $H-W h$ Wh is, in muscle 3 , in the whole body 5), provided always that the proportion above given, 1 to 4 , holds for mammalian muscle. ${ }^{1}$

Recurring to the first and third of the three cases referred to at the beginning of the last section, p. 394, we find that whether, as in Case 1, the muscle is prevented from shortening when stimulated, or, as in Case 3, is allowed to lift a weight which falls when the effort ceases, the whole of the chemical work done is represented by its equivalent in heat, and indicated by the rise of temperature consequent on the excitation. When a muscle is subjected to instantaneous stimulation, of which the intensity exceeds the maximum, no corresponding increase of the response is observed, and hence it night be inferred that the effect of such a stimulation is to "explode" the whole of the oxidisable material which the muscle at the time has at its disposal. The most important result of Heidenhain's researches was the proof which they afforded that this is not the case, ${ }^{2}$ and that, although we are quite right in regarding the muscular response as a process of discharge, the amount of chemical potential energy liberated by excitation varies, even when the strength of the stimulus remains the same, according to the circumstances under which the discharge takes place. Of these circumstances resistance is the most influential. This is plainly shown when the thermal responses, in a series of excitations in which the muscle is allowed to lift and let fall different weights-say, 30, 60, and 90 grns.-are compared with each other. It is found that the quantity of heat produced is not the same in the three experiments, as we should expect it to be if it were dependent on the amount of available energy in store, but increases with the quantity of work done in each lift of the weight. It does not, however, increase in the same proportion with the work done, for Heidenhain found in all his experiments ${ }^{3}$ that in maximal stimulation the ratio of heat produced to work done diminishes as the quantity of work done increases. In other words, the muscle works more economically when moderately loaded than when lifting a very small weight.

It was further found that when a muscle is called upon to make repeated efforts in lifting and letting fall the same weight, the exhaustion thereby produced tells much more rapidly on thermogenesis than on working power. The exhausted muscle does less work, but does it more economically. In like manner it appeared that when a muscle is "after-loaded," so that, contracting at first without obstacle it encounters the resistance subsequently, the effect of a given load on heat production is not so great as it would be if the muscle were loaded in the ordinary way. From these data Heidenhain concluded, that in

1 See Gad and Heyman's "Lehrbuch," S. 504.

2 Heidenhain, loc. cit., S. 85.

${ }^{4}$ Heidenhain, loc. cit., S. 103.

${ }^{3}$ Heidenhain, loc. cit., S. 88 . 
general the chemical work done by a muscle, as indicated by the heat produced in contracting in response to an instantaneous stimulus, is determined by the resistance offered, up to a certain limit. If this limit is exceeded, the thermal effect cannot be increased, and may even be diminished by increasing the load.

Relation of thermogenetic effect to strength of stimulus.-The question whether and to what extent the chemical, i.e. the thermal, response is dependent on the strength of the stimulus, was investigated many years ago in Heidenhain's laboratory. ${ }^{1}$ For this purpose the increase of temperature produced in lifting and letting fall a weight, was measured in a series of excitations with "inadequate" stimuli of graduated strengths. The difficulty of making such measurement is considerable, for the strength of a maximal excitation differs so little from that of an excitation which is just sufficient to evoke a measurable response, that the range of excitation values which can be compared experimentally is very limited. On the whole, it was found that in isotonic contractions the thermal effect varies with the stimulus, in the same way that the work varies; but that, if the load remains the same, the increase of heat production which results from increasing the stimulus is more than proportional to the greater lift. The meaning of this fact will be considered later.

The experimental results which have been already referred to might all be understood as consistent with the notion that the one condition which is influential in determining the amount of the thermal response to a single maximal stimulation, is tension or strain. It was, however, recognised by Fick many years ago that the evidence in favour of so simple a relation between tension and thermogenesis was insufficient. It was therefore necessary to devise an experimental method by which the question could be elucidated. If the thermal response depended solely on tension, it was obvious that its amount must be greater under isometric conditions than under any other. This could be tested by comparing two cases, ${ }^{2}$ in one of which $(a)$ the muscle was under isometric conditions during the whole of the mechanical response, while in the other $(b)$ it was released at the moment of maximum tension and allowed to lift a weight of $20 \mathrm{grms}$. The result showed that, notwithstanding that in $b$ the tension was at once reduced at the moment of release, the thermogenesis was greater than in $a$. The numbers, each of which represents a deflection of the galvanometer, were as follows:-
(b) Wo work done Weight lifted and let fall
25
$\left.29\right|^{22}|28| 2$
$22|29|^{22}|28|^{\text {etc. }}$

The numbers relate to successive excitations.

By this experiment it was shown for the first time that in inuscular action the sum of the thermogenetic values of tension and work is greater than the maximum thermogenetic value of tension by itself. In our study of continuous contraction we shall see to what important results this leads.

Production of heat in tetanus.-It does not necessarily follow from the facts which have been stated, that persistent contraction,

\footnotetext{
${ }^{1}$ J. Nawalichin, "Myothermische Untersuchungen," Arch. f. a. ges. Physiol., Bonn, Bd. xiv. S. 293.

${ }^{2}$ Fick, "Myothermische Fragen, u.s.w.," Verhandl. d. phys.-med. Gesellsch. zu Würzburg, 1884, N. F., Bd. xviii. S. 12, Sep. Abd.
}

VOL. I1. -26 
whether evoked by repeated or continuous excitation, should be a heat producing process. If a muscle to which a weight is attached contracts persistently, it does no external work, whatever the load may be. And if we are to regard the state of contraction as nothing more than one in which the natural length of each fibre (i.e. the length it possesses when unexcited) is diminished, there is no more reason why we should expect that in the state of extension the excited muscle should produce heat, than that an elastic band, or an unexcited muscle, under the same conditions, should do so. We know that it actually does produce heat when contracted, although no external work is done, and therefore conclude that the difference between the excited and unexcited muscle does not consist exclusively in the difference of the relation of length to tension. To explain the active thermogenesis of a muscle which holds up a load persistently at the same height, it must possess some property which does not belong to a stretched elastic cord.

This consideration reminds us of the suggestion that every muscular contraction consists essentially of two processes, of which the second is mechanically a reversal of the first; and that when a muscle is subjected to frequently repeated excitation (tetanus), so as to alternate between states of increasing and diminishing tension, these are accompanied, or rather ushered in, by corresponding chemical actions. In the first stage it has been suggested that, as the immediate result of stimulation, some body (e.g. lactic acid) comes into existence ${ }^{1}$ and occasions an increase of tension, which in the second is annulled by the oxidation of its efficient cause. Both processes are in the chemical sense disintegrative, i.e., consist in the transformation of bodies of greater heat-value into others of less ; in other words, both are thermogenetic.

Even as regards tetanus produced by repeated stimulations, it is difficult to see how this explanation facilitates the understanding of the result; for if we suppose the frequency of the excitations to be no more than 100 per second, it is difficult to imagine that the muscular element can pass through the two stages of the chemical process above referred to, in the interval between two successive excitations. The difficulty is still greater in applying this explanation to prolonged contraction of central origin, in respect of which we have no evidence that the excitation by which it is evoked is discontinuous. It therefore seems that we must seek for some other solution of the fact that all continuous muscular effort, whether effectual or not, is accompanied by the production of heat, in quantity which in the main depends on the intensity of the effort. Just as, in comparing the thermal effect of single excitations which are not adequate to produce a maximal contraction, it was found that this effect depends on the efficacy of the excitation as indicated by the lift, so in tetanus, as long as the frequency of the excitations is insufficient to evoke a maximal tetanic contraction, the heat produced by a tetanus of given duration increases with the height to which the load is lifted. Thus the thermogenetic effect, no less than the mechanical effect, depends on the frequency of the excitations. But from the moment that the contraction becomes maximal, frequency ceases to have any influence; so that, in a series of similar experiments, in each of which a muscle is completely tetanised but with stimuli of different frequency, the quantity of heat produced in the same time is always the same. It is also found, by comparing equal periods of a tetanus brought about by a series of maximal stimuli of constant frequency, that more than twice as much heat is produced in the first period, i.e., that in which the muscle shortens, than in any succeeding period. Provided that

${ }^{1}$ Gad, "Zur Theorie der Erregungsvorgänge im Muskel," Arch. f. Physiol., Leipzig, 1893, S. 166. 
the excitations are strong enough and frequent enough to produce a full thermal effect, they may be altered in both these respects without interfering with the result. When the frequency of the stimuli is not only insufficient to produce a maximal tetanus, but fails to evoke a continuous contraction at all, as is the case in a series of maximal excitations, following one another at intervals of such length as to allow the muscle time to relax between each contraction and its successor, more heat is produced than in a tetanus, whether complete or incomplete, of the same duration.

It may be noted that Fick ${ }^{1}$ summarises these results by the statements(1) That in tetanus the quantity of heat which is assignable to each excitation varies inversely with the frequency, and (2) that in a series of responses to instantaneous excitations, the thermogenetic effect of each excitation far exceeds that which is attributable to each member of a series of stimulations of such frequency as to evoke complete tetanus. ${ }^{2}$

For the reasons stated on p. 394, oxidation must be considered as the first in order of time of the processes which constitute the response of a muscle to excitation. In the excitation of a muscular element, the direct action of the stimulus is to induce in it chemical changes, whereby either heat is produced or work is done. But we have seen that not only the proportion between the inogenetic and thermogenetic changes varies according to the mechanical conditions, but that the total amount of chemical work done is also dependent on them. We are thus led, with Fick, to adopt the general principle that the " chemical process is in part determined by the external conditions under which the muscle is placed when it is excited." How far this influence extends is one of the most important problems in muscle physiology; for in all discussions of the relation between the useful result obtained, and the quantity of material expended in its production, it is this problem that we have to encounter.

In voluntary movement we may well suppose that when the central nervous system sends its mandate to a inuscular element to contract, it does so without definite information (if I may so express myself) as to the amount of material which it will be necessary to use or waste in fulfilling that mandate, and that if the demand first made is found to be inadequate, as, e.g., in consequence of unanticipated resistance, the supply will be proportionately increased. There is, in short, no difficulty in supposing that during the performance of a voluntary act, expenditure is continuously adapted to requirement. In skilled movements, such as those of drawing, writing, or playing on an instrument, it is difficult to see how such adaptation could be dispensed with. We might without difficulty found upon this consideration the hypothesis that in general the adaptation of supply to demand is a function of the nervous system. Inasmuch, however, as in muscles rendered nerveless by curare, this adaptation is as remarkable (see p. 361), though not so perfect, as in muscles working under the guidance of nerve cells, no such general statement can be made.

We have already seen (p. 357) that if a muscle sustaining a weight which it is just able to lift, is tetanised for a limited period, during which its load is gradually diminished in such a way that throughout the period of excitation the lifting power of the muscle is only just in excess of the resistance it has to overcome, it will do more work than under any ordinary conditions, for throughout its effort it is doing its utmost. It eventually attains its maxinum degree of shortening, at

$$
1 \text { " Mech. Arbeit," S. } 216 .
$$

${ }^{2}$ Loc. cit., S. 213. 
which moment it no longer has any load to lift. If the same muscle is tetanised for the same period, sustaining a weight which, at first approximately 0 , is gradually increased to the amount of the initial weight in the previous case, it will then (after first promptly shortening in response to the excitation) be stretched to the length which it had before, when charged as it is now with a load it was only just able to lift.

In the two cases the excitation is the same; the contrast between them consists in the mechanical conditions under which the muscle responds. In the first case it does its utmost amount of work; in the second case it not only does no work, but is worked upon by the descending weight which stretches it. In both, the temperature of the muscle is raised, but in the former this represents only a part of the chemical change (oxidation) which takes place; the remainder becomes external in work. In the latter the warming of the muscular substance represents more than the heat production due to oxidation, for a considerable part of it is due to the action of an external cause, the extension of the muscle by the weight.

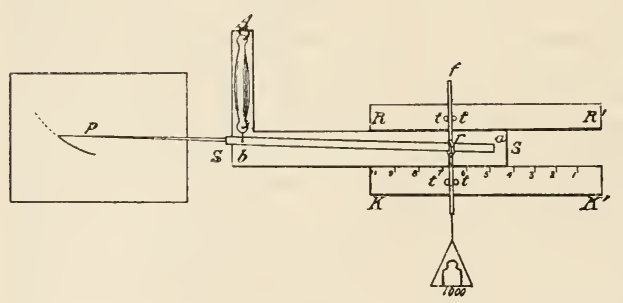

FiG. 220.-Blix's apparatus for taking the extension curves of muscle with the load either increasing or decreasing.After Fick.

The results of the experimental comparison of the two cases liave been given by Fick in one of his recent researches. ${ }^{1}$ The method will be readily understood with the aid of the diagram (Fig. 220), which represents Blix's ingenious contrivance for obtaining the extension curve of a muscle. The slider $S S$ can be freely moved backwards and forwards on the rails $R R^{\prime}, K K^{\prime}$, between which it works. At one end of it there is a support $A$, from which the muscle is suspended. The other end of the muscle is attached to the myographic lever at $b$. The axle $a$ on which this lever rotates is carried by the slider. The load is sustained by a vertical rod $f$, which rests on the lever at $r$, and moves freely up and down between the pins $t t$ and $t t^{\prime}$; consequently the distance between the point at which the load is sustained by the lever, and the axle $a$ varies according to the position of $S S$. The plate on which the lever writes is fixed. The curved lines inscribed on it are such as would be drawn by an up-and-down movement of $p$, if the index of the slider were brought in succession to the points $1,2,3$, etc., on the scale and back again. It will be seen at once that if the muscle is unexcited, $p$ will describe its extension curve on the plate, whenever the slider is moved along its whole course in either direction. If the load is 1000 grms., and the maximum distance between $r$ and $a$ one-half of the distance between $a$ and $b$, each division of the scale (i.e. one-tenth of this distance) will correspond to $50 \mathrm{grms}$. of tension. If during the time (say 2 seconds) that the slider accomplishes its backward and forward journeys, the muscle is tetanised, it draws in like manner the extension curve of tetanised muscle. If contracted muscular fibre were to behave in

1 Fick, "Ncue Beiträge zur Kenntniss von der Wärmeentwickelung im Muskel," Arch. f. d. ges. Physiol., Bonn, 1892, Bd. li. S. 541. 
exactly the same way as an elastic corl, the curve drawn when the slider was moving from 0 to 10 would be identical with that drawn during the return movement from 10 to 0 . The curves (Fig. 221) show that it is not so. With diminishing load the writer follows the line $a b$; with increasing load, the line $b^{\prime} a^{\prime}$, or rather $b a^{\prime}$; nor does it make any difference (with the exception indicated in the description of the figure) which of the two curves is drawn first. Now, as was explained in p. 357, every extension curve serves as a "work diagram." The area included between the line $b a$, the vertical line $b e$, and the horizontal line $e a$, represents the amount of positive work done by the muscle in contracting when carrying a diminishing load. Similarly, the area limited by the lines $b a^{\prime}, b e, e a^{\prime}$, expresses on the same scale the negative work done by the weight on the muscle in the act of extension. As indicated by the figure, this area is always larger than the other, exceeding it by the area enclosed between $a a^{\prime}$ and the two curves drawn by the muscle in extension and in shortening respectively. Consequently, in order to get at the true amount of heat attributable to the chemical change which has taken place in the muscle during the period of observation, the amount of heat corresponding to the excess of the negative over the positive area must be deducted from the total quantity indicated by the increase of temperature.

As has been already stated, it makes no difference as regards the amount of work done, whether the load first increases then diminishes, or first diminishes then increases; but, as regards the total production of heat, the experiments yielded a very remarkable though not unexpected result, for it was found that the heat-effect is nearly twice as great when the muscle is allowed to do extermal work during the first half of the period of excitation, than in the contrary case. The meaning of this fact will be better understood if we first refer to the results of preliminary experiments, in which the heat production was measured of a muscle tetanised for equal times, e.g., 1.9 seconds, in one case $(A)$ with diminishing, in another $(B)$ with increasing, load. In $A$ the total amount of heat produced, as indicated by the rise of temperature, was 123.4 microcalories, and the heat equivalent of the positive work done during the period of shortening was $18 \cdot 3$ microcalories.

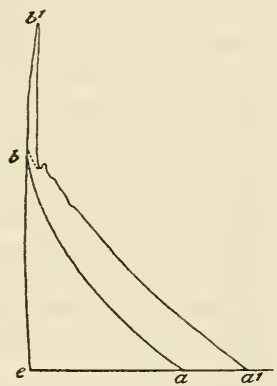

FIG. 221. - Two extension curves of the same excited musele taken continuously with deereasing $(a b)$ and increasing $\left(b a^{\prime}\right)$ loads. The figure is modified from the original (Fick, loc. cit., Fig. 6, p. 550), by the addition of the dotted line which indicates the course the writer must have actually followed in the case described. When the order of the experiment is reversed, so that the inerease of load precedes the decrease, the tracing follows the continuorts line $e b^{\prime} a^{\prime} a b$. The part of the tracing above $b$ is no doubt chiefly due to the inertia of the lever. Hence the total heat product of the oxidation which took place during the same period, was $131 \cdot 7(=h+v h)$. In $B$ the total thermogenesis was 118.5 microcalories, and the heat equivalent of the negative work done $32 \cdot 3$. The heat value of the material oxidised was therefore $86.2(h-w h)$. The chemical process was thus more active when the work done was positive than when it was negative, in the proportion of $\frac{1.53}{1}$. In the form of experiment $(C)$ in which the thermogenetic effects of two excitations of equal duration are compared, in one of which ( $a$ after $b$, i.e. $b a$ ) a diminishing load is preceded, in the other ( $b$ after $a$, i.e. $a b)$ is followed, by an increasing one, there was a very considerable difference between $b a$ and $a b$. The measurements in microcalories were when $b$ followed $a, 137$, when $b$ preceled $a, 92$. As in each case the amount of heat produced by stretching, namely 19 micro- 
calories, i.e., the amount of heat corresponding to the excess of the negative over the positive work, must be deducted, we have, as the actual amount of heat produced by oxidation during the period in question-for $a b, 118$ microcalories ; for $b a, 63$ microcalories.

The question on which these experimental results throw light, is that of the conditions which dctermine the cxpenditurc of oxidisable material in a muscle whon in the excited statc. With reference to this question, they may be set forth as follows:-

From the data of experiments $A$ and $B$, it is seen that during a brief period of excitation (two seconds) the expenditure of the excited muscle is greatest when the resistance opposed to its shortening is such as it can overcome, so as to do its utmost amount of external work ; and that it is least when the resistance is considerably greater than it can overcome, i.e., when the opposing force of the load is sufficient to lengthen it to its utmost. Between these two contrasting conditions comes that in which the muscle neither prevails nor yields-that in which it is excited isometrically. Here the heat produced must be less than in contraction against resistance, greater than when the effort to contract is overpowered by an antagonistic force-or, putting it otherwise, to hold on against an opposing force involves expenditure, of which the amount is proportional to the effort made; but, provided that the effort remains the same, to hold on against an unyielding resistance is less expensive than to overcome an opposing force, more expensive than to be overcome by it.

From experiment $C$ it appears that if an excited muscle is allowed to shorten, and thereby to do work under the conditions which we know to be most favourable, i.e., in lifting a diminishing load, the expenditure of material which accompanies this effort is affected in a remarkable way by antecedent mechanical conditions; if (case $a b$ ) it is excited when it is at its natural length, and at the same moment its shortening is opposed by a decreasing load, of which the initial amount is the greatest that it can lift, it expends much more material than if (case $b a$ ), before beginning its effort, it is stretched from its natural length when excited $(\lambda)$ to its natural length when relaxed $(l)$. The contrast between the two cases can only be explained by supposing that the negative work, which in case $b a$ has been done on the muscle towards the end of the previous period of extension, leaves behind it mechanical potential energy which, in the succeeding act of shortening, takes the place of part of the mechanical energy which in case $a b$ was derived from the transformation of chemical energy. The theory of this transformation can of course not be given, so long as the problem of the origin of muscular force remains unsolved.

The practical importance of both results lies in their bearing on the conditions of exhaustion-meaning thereby that kind of exhaustion which depends on the using up of oxidisable material. Experience teaches that different kinds of muscular effort differ from each other in a remarkable way in their relative aptness to induce fatigue, and that this does not depend on the muscular tension with which the effort is accompanied. Going up hill is exhausting because external work is done against resistance. Descending is not exhausting, although the muscles are as frequently in action, and as tense when in action as in ascending. The reason why there is so little fatigue is that each muscular effort is overcome by a superior opposing force-the weight of 
the body. The muscles work with relatively little expenditure of thermogenetic material, because, for the most part, each effort is preceded and prepared for by previous extension.

Looking at this subject in a more general way, and seeing that even curarised muscles have a certain power of regulating their own expenditure in accordance with the work they are required to do, the experimental data which we have had before us indicate that this regulation is such as must unquestionably tend to the advantage of the organism.

\section{The Electrical Properties of Muscle.}

In all excitable organs differences of potential present themselves between different parts of the surface, which cannot be attributed to accidental or external, physical or chemical conditions. These have a definite relation to the structure of the organ, to its general state of functional activity, and to the relative state of functional activity of its parts; so that when the whole organ or any part of it passes from the state of rest (relative inactivity) to that of activity, or the reverse, this change is indicated by a corresponding change in the distribution of electrical tension (i.e. of differences of potential) at its surface. These changes of tension can be investigated with the aid of suitable instruments. They derive their importance from their correlation with the physiological changes which they accompany. This correlation can be determined by the comparison of concomitant variations of different orders, i.e., of variations of functional activity on the one hand, with variations of distribution of electrical tension on the other. If the parts of the surface of an organ between which differences of potential exist, are so connected with a galvanoscope that they are included in its circuit, they are of course indicated in the instrument as currents. As some form of galvanoscope is generally used as the means of investigation, the variations or states so observed are ordinarily spoken of as "currents." Those currents which indicate the normal distribution of tension on the surface of muscle are called currents of rest, or "muscle currents." Those which appear as concomitants of transition from relative rest to functional activity, are called "action currents." With reference to all of them, it has to be remembered that what is observed and measured is not what occurs in the organ under investigation, but in the galvanoscope. For although currents actually exist in the organ itself, we learn nothing by our observation, either as to their direction or relative strength. All that is revealed to us, when we compare the two parts of the surface by applying to them the terminals of our galvanoscopic circuit, is the measurable difference of potential between the two parts.

Electromotive phenomena of muscle in the unexcited state.Inasmuch as the electrical changes which accompany increase, diminution, or cessation of functional activity, can only be spoken of in their relation to the normal state from which the structure has departed, and to which it tends to return, it is obviously desirable that the study of that state-the state of relative rest-should precede that of activity or disturbance.

It is important in the employment of muscles for the investigation of electromotive phenomena, to distinguish between those which are 
parallel-fibred and others. In some parallel-fibred muscles the fibres are as long as the whole muscle. Each fibre is provided with one or (in the frog) more nerve-endings, and these are disposed either near the middle, or on either side of it; none occur at the ends. Consequently, when the muscle is excited through the nerve, the excitatory influence reaches the middle of the muscle first. The tendons extend so short a distance into the muscle, that muscular fibres underlie the surface everywhere. Of such a muscle the sartorius is a type. If this muscle is prepared with due care, its surface is found to be nearly equipotential, i.e., no appreciable current is indicated by a galvanometer of which the terminal electrodes are applied to its surface, whatever the situation of the contacts. In some parallel-fibred muscles, as in the adductor internus or gracilis and the semi-membranosus, the fibres are interrupted by tendinous "inscriptions" (see Fig. 189), the presence of which, as will be seen subsequently, modifies the electromotive properties of the
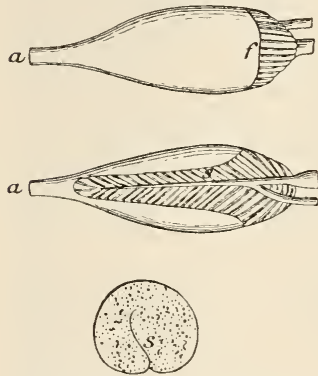

FIG. 222. - Diagram showing the arrangement of the fibres in the gastrocnemius musele. The letter $f$ is on the dorsal surface, close to the edge of the tendinous expansion ; $s$, on the ventral surface, marks the edge of the dorsoventral tendinous plate. In the third figure this plate is seen in section. gh resistance ( 8000 to 20,000 ohms) is used. The galvanometer is provided with a shunt, by means of which $\frac{1}{10}, \frac{1}{100}$, or $\frac{1}{1000}$ respectively, of any current may be led through the coils. The circuit may be arranged according to some such plan as is shown in Fig. 223. From the principal terminals of the shunt, $n$ and $s$, the wires lead to the north and south terminals of the galvanometer. When the plug between $n$ and $s$ is withdrawn, the galvanometer is in the circuit, but with a plug in the position marked $\frac{1}{9}$ in the figure, only one-tenth of the current in the circuit flows through the galvanometer. When the keys $K, K^{1}$, and $K^{4}$ are plugged, the circuit is completed through the plugs. When the plug of $K$ is withdrawn, it is completed through the preparation $p r d$, the wire from $d$ leading to the north terminal of the galvanometer, the wire from $m$ to the south terminal. When the key $K^{1}$ is unplugged, so much of the wire $B C$ as intervenes between $C$ and the sliding contact is included in the circuit. The wire stretched between the two blocks $C$ and $B$ is a rheochord through which, when the key $K^{3}$ is plugged, the current of a battery flows. $B C$ is a metre long, and is 
provided with a millimetre scale, by means of which the distance of the sliding contact from the block $C$ can be measured. Between the block $B$ and a block $A$ is a resistance coil. The resistance of the wire $B C$ having been determined, that of the coil must be so adjusted that the former is to the latter as 1 to 13.56 , for a reason which will appear immediately.

The wire which connects the battery with $C$ passes through a rheostat. The terminals of a Latimer Clark's standard battery can be connected with $C$ and $A$, by closing a key. Between the key and $A$ is a galvanoscope $g$, by which it can be observed whether any current is flowing between $A$ and $C$ or not. The branch of the Daniell battery which flows through $g$ is opposed to the current of the standard cell. Before making the experiment, it is reduced with the aid of the rheostat, until $g$ indicates no current. Whell this is the case, the difference of potential between $C$ and $A$ is 1.456 volt, i.e., the electromotive force of the standard cell. Consequently, as the resistance of $C B$ is to that of the coil as 1 to 13.56 , the difference of potential between $C$ and $B$ is to that between $B$ and $A$ as 1 to 13.56 ; and hence to that between

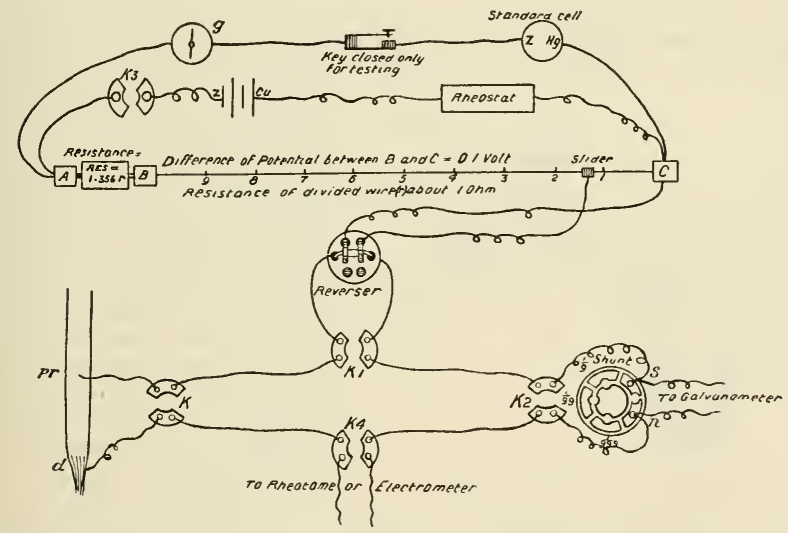

FIG. 223.

$C$ and $A$ as 1 to 14.56 , or 0.1 to $1 \cdot 456$. That between $C$ and $A$ being 1.456 volt, the difference between the two ends of the metre wire will be 0.1 volt, and each centimetre of it will correspond to a difference of $\frac{1}{1000}$ volt. ${ }^{1}$

The arrangement just described (the rheochord in connection with the "balanced" cell) is called a "Compensator." The use of an iustrument of this kind, for measuring physiological differences of potential, dates from 1861, when du Bois-Reymond described the two forms of compensator which are used in Germany, the "long" and the "round." Of these, and the method of using them, a description will be found in Hermann's "Muskel-physik." The arrangement described above has the advantage, as compared with these, that so long as the standard cell for which the scale is graduated is used, no calculation is required.

The ends of the wires at $p r$ and $d$ are connected with the surface of the muscle by non-polarisable electrodes. Of such electrodes a suitable form

1 The form of instrument mentioned above was described by the writer in 1873 , in the "Practical Exercises" for the Laboratory of University College, London. 
is represented in Fig. 224. A glass U-tube $u$, containing saturated solution of zine sulphate, is supported by a brass block $b$ and holder which slides on a horizontal vuleanite rod $r$. The block also supports an L-shaped zine wire $L$, of which the arm dips into the solution in the U-tube. It is also provided with a terminal, for attaching the wire by which the electrode is connected with the circuit. The arm of each zine rod is flattened by hammering, at the end which dips into the zine solution, so as to take the form of the blade of an oar, and its surface is earefully smoothed, and then amalgamated. The straight tube $T$ (too narrow in the figure) is of such width that it fits loosely into the other end of the U-tube; it is prevented from slipping in by the flange $f$. It is charged with paste, made by kneading kaolin with the zinc solution. To prepare the electrodes for use, a lump of kaolin paste $k$, made with 0.6 per cent. solution of sodium chloride, is stuck on the summit of the straight tube, a groove being made across the top of this lump with an ivory knife, in which the end of a "cable" of cotton wick thoroughly soaked in physiological salt solution is placed. By this means the clay is brought into connection with the surface of the muscle to be examined.

Before establishing this connection, it is necessary to test the electrodes by making contact between the two lumps. If the galvanometer is sufficiently sensitive, some current will generally be indicated, the electromotive force

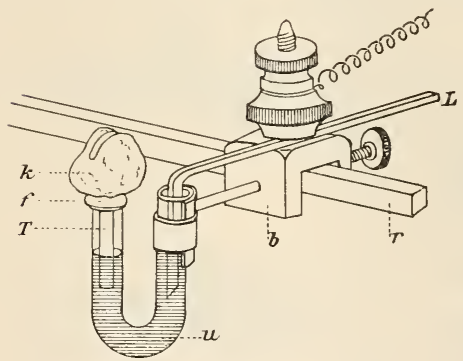

Fí. 224.--Non-polarisableelectrode. and direction of which can be readily ascertained with the aid of the compensator. If it is considerable (electromotive force exceeding $\frac{1}{10000}$ volt), the electrodes must be remade. To avoid mistakes of direction, it is desirable to label the electrodes $N$ and $S$ respectively -it being understood that the wire of the $N$ electrode runs to the north terminal of the galvanometer, and accordingly that a current flowing therefrom is indicated by a movement of the spot of light southwards.

In exploring a muscle in the resting state galvanometrically, the differences of potential between different parts of its surface are measured in hundredths of the divided metre of the compensator, i.e. in millivolts. In such observations the "muscle current" is balanced by a current of opposite direction from the compensator, by working the slider until the galvanometer is at zero.

When the capillary electrometer is substituted for the galvanometer, the arrangement of the circuit is the same. But, with the aid of a scale by which the excursion of the mercurial meniscus from its normal or zero position is measured, the observer is enabled to determine approximately the difference of potential between the two contacts, provided that the value of the graduation in decimals of a volt is known.

In the gastrocnemius, as has been indicated above, however carefully it is prepared, a considerable difference of potential usually presents itself between the tendon end and the most positive part (Fig. 224, f) of the natural surface, notwithstanding that Hermann found that under conditions which absolutely exclude sources of injury this difference is inappreciable. The difference, when present, diminishes as the electrode on the tendon is brought step by step nearer to the other. In uninjured parallel-fibred muscles, on the other hand, the differences 
of potential between different parts are, as has been said, scarcely measurable, for which reason these are best adapted for the study of the natural electromotive phenomena of muscle, and their modifications.

"Current" of injury.-When the tendinous expansion of the tendo Achillis is injured by heat or otherwise, a very much larger difference of potential comes into existence between it and the proximal contact at $f$. Heat injury may be effected either by dipping the end of the muscle into salt solution at any temperature above $55^{\circ} \mathrm{C}$., or by applying a camel-hair pencil, which has been dipped in hot salt solution, to the surface of the muscle at the tendon end. Whichever plan is used, the ends of the fibres are made to shorten and swell. In the gastrocnemius the difference may amount to more than $\frac{4}{100}$ of a volt. When the sartorius is similarly treated, the difference seldom exceeds $\frac{2}{100}$. A muscle injured at one end in the way above described is referred to by physiological writers as having a "thermal section." As this expression is founded on du Bois-Reymond's "doctrine of the muscle cylinder," it will now be convenient to discuss this subject.

It may be assumed that the essential physiological properties of a muscle are possessed by each of its constituent fibres. If it were possible to divide a muscle fibre into parts by cross-sections without injuring it, each part might be expected to exhibit the endowments of the whole muscle. Proceeding from this consideration, the theory in question further supposes that each such cylindrical part of a fibre is built up of structural elements which have the same properties; these in their turn consist of electromotive molecules, to the endowments of which those of the whole organ are attributable. It is obvious that cylinders cut from single fibres could not be subjected to direct investigation. The experimental basis for the theory of electromotive molecules had therefore to be sought for in the data to be obtained by the examination of a truncated, cylindrical, and parallel-fibred muscle. A cylinder so prepared possesses a natural and two cut surfaces. These are called by du Bois-Reymond respectively "longitudinal" and "transverse," designations which are open to the objection that they conceal the all-important fact that each transverse sectional surface is that of a wound. At the centres of the opposite cut surfaces there are found to be spots which are mutually equipotential, but relatively negative to all other parts; and on the natural surface there is an equatorial zone which divides the cylinder into two, and is positive to all other parts. If the other parts of the surface are explored galvanometrically, by placing one electrode on the equator, the other first on the centre of an end surface, and then successively on spots nearer and nearer to the equator, it is found that the difference of potential in each successive observation is less than in the preceding one. The exact relation between these differences has been minutely investigated both theoretically and empirically by du Bois-Reymond, but has little physiological interest. The essential point is that the distribution of tension on either side of the equator is symmetrical. If the cylinder is cut from a muscle of irregular construction like the gastrocnemius, no such symmetry is observed; for the central spot of the cut surface which faces the tendon is negative to the corresponding spot in the surface which looks in the opposite direction. The reason of this must now be considered.

The muscle rhombus.-Long ago, du Bois-Reymond discovered that 
if, in preparing the muscle cylinder from a parallel-fibred muscle, the fibres are cut obliquely, so that a section parallel to the long axes of the elliptical cut surfaces is rhombic, the sharp and blunt edges of such a rhombus are not equipotential, but the sharp edge is relatively negative to the other, for a reason which the diagram (Fig. 225) will help to explain. In an obliquely cut sectional surface, the fibres are imbricated. In each fibre, $a$ is negative to $a^{\prime}, b$ to $b^{\prime}$, and so on; for, as we have already seen, a more injured part is negative to a less injured. The difference of potential $(\Delta)$ between them is the same in all. $b$ is also negative to $a^{\prime}$, and $c$ to $b^{\prime}$, and so on ; but this difference is smaller than $\Delta$ by a certain amount $(\delta)$, which is consequently the difference of potential between $a$ and $b, b$ and $c$, and so on. And if the cut surface is of the width of a hundred fibres, there will be a difference of potential of a hundred times the amount $\delta$ between the two borders. In other words, the ends of the fibres in an obliquely cut surface act like voltaic elements in series; whereas in a transverse surface they act as if in multiple arc. These considerations help us to understand the fact that the tendon end of the intact gastrocnemius is ordinarily strongly negative to the middle. If the tendinous expansion were uot there, the relation of the exposed ends of the fibres would be precisely that which has just been described in an obliquely cut surface. Any part further fron $f$ would be negative to any nearer spot.

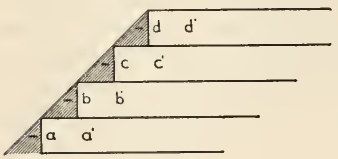

FIG. 225.

Before proceeding to the study of the excitatory response, it will be convenient to inquire, in a preliminary way, whether the facts we have had before us relating to the electromotive properties of unexcited muscles-particularly those relating to the equipotentiality of certain muscles when sufficiently carefully prepared, and to the readiness with which the slightest damage renders any part of the surface, more especially in the neighbourhood of the tendon, negative to the rest-can be comprehended under any general statement.

In any excitable organ two kinds of functional difference may exist between parts. They may either have permancntly differing functional endowments, e.g. nerved and nerveless parts of the same muscles, or they may be at the moment of observation in different states of fitness for the discharge of function, this being due to the circumstance that they are in different stages of actual discharge of function or of exhaustion therefrom; or dependent upon injury, difference of temperature, or other exterual conditions which affect function. It may be generally stated as regards excitable organs, such as muscle and nerve, that in comparing parts which are similarly endowed, those which are functionally in a more capable state are positive to those which are functionally less capable. No such "law" applies to organs which do not possess the property of responding to stimulation. It appears to me impossible to suppose that this fundamental difference between the one group of organs and the other-those organs, namely, in which functional activity has no concomitant electrical phenonena, and those, on the other haud, in which this occurs without fail-should manifest itself independently of the existence in the one set of organs of a special mechanism which in the other set is absent. Nor can it be doubted 
that this mechanism is molecular, i.e., that the electromotive function of muscular substance or of nerve substance is dependent on their respective molecular structures. Although, therefore, du Bois-Reymond's molecular theory, in the form given to it by its author, does not appear to aid in the understanding of the relation between the mechanical, chemical, and electrical processes which are concerned in the response of a muscular element to stimulation, the recent development by Bernstein of the chemical aspect of the theory appears to be of greater value.

Du Bois-Reymond's "molecules" were electromotive only. They were of prismatic form, and were supposed to be arranged in series end to end, their ends or poles being negative to their equators. Bernstein's molecules are also prisms arranged in the same way, but their fundamental attribute is chemical, that is, their poles are able to attach to themselves electro-negative groups of atoms (oxidisable material), while their sides attach oxygen. This change, which may be designated polarisation, is the first effect of excitation. It is the immediate antecedent of the explosive oxidation of which the transformation of chemical into mechanical energy is the consequence. Any molecule which is the seat of this change is able to communicate a similar state to the molecules on either side, of which the poles face its poles; and so we have the propagation in two directions of the "excitatory wave."

The chemical processes above described necessarily involve mechanical and thermal consequences. At the moment of explosive oxidation, mechanical stress takes the place of the chemical tension which is thereby relieved. This mechanical stress is itself transformed into heat and external work, in proportions which are dependent on the conditions set forth in the preceding section. As regards the order in which these changes occur, Bernstein's scheme is in accordance with views which have been already stated. Chemical explosion comes first with its concomitant electrical manifestations, then follow the thermal and mechanical phenomena of muscular contraction.

Such is Bernstein's theory. It explains nothing, but it is of use as enabling us to think of excitation, propagation, oxidation, electrical change, mechanical stress, change of form, production of heat, as all parts or manifestations of one and the same process.

The simple statement already made, that in muscle and nerve, relative capability of function (i.e. fitness for its performance) is indicated by relative electrical positivity (that is to say, that of two parts of identical function, in structural continuity with each other, the one which is at the time most capable of performing that function is positive to the other), springs out of experimental results independently of any theory. In accordance with this statement, it will be shown in the next paragraph that action is always attender by disappearance or diminution of the capability of acting; a part, in becoming active, becomes at the same time relatively negative to other parts.

Electromotive phenomena of muscle when excited.-The two kinds of mechanical change which were studied in the first chapterthe single response which follows an instantaneous stimulation and the persistent one which is evoked by continuous stimulation-have their counterparts in the electrical phenomena which we have now to examine. The continuous electrical change which corresponds to tetanus has been long known. It was recognised by Matteucci, and investigated by du Bois-Reymond, who bestowed on it the designation Negative Variation, 
in which the word "negative" is used in its algebraic, not in its electrical sense. The transitory change which corresponds to the single contraction is of very short duration, and is propagated in such a way that by the time it reaches the end of a muscle furthest from the seat of excitation, it may have already ceased in the neighbourhood of the excited part. This being so, it is not difficult to understand that the response to instantaneous stimulation remained unrecognised for many years after the electromotive properties of muscle in the resting state and the "negative variation" (in the original sense of du BoisReymond) were well known.

Whereas the "negative variation" is persistent and not propagated, the response to instantaneous stimulation is transitory and spreads at a rate of niany feet per second. In both cases the change manifests itself by relative electrical negativity of the affected part of the surface. In discussing these changes, it is necessary to bear in mind the caution already given, that the phenomena observed are those of the instruments we employ rather than those of the organs we explore. If we talk of

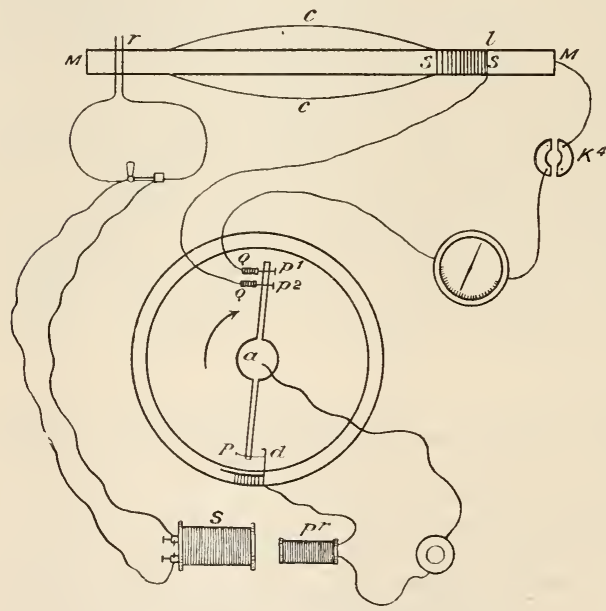

FIc. 226. - Bernstein's differential rheotome. "action currents," we refer to currents as they really exist in the galvanometric circuit; if we say that a certain spot is "negative," we mean that there is a difference of potential between the surface of contact to which we refer, and another contact surface to which the other electrode is applied-which difference we measure either by the compensator or the electrometer.

(a) The electrical response to an instantaneous

stimulus-Its time-relations. - It was known from an experiment made by Helmholtz, which will be referred to further on (p. 421), that the electrical response to an instantaneous excitation is of very short duration, and that it precedes the change of form. But it was not until twenty years later that Bernstein devised a method for investigating its time-relations, and particularly its rate of propagation, and was further enabled to distinguish the differences between the phenomena of the electrical response, as they present themselves in injured and in uninjured muscles respectively. The description of the instrument, which he called a Differential Rheotome, is now to be found in most textbooks. It will be sufficient, therefore, to refer to the original diagram, which is reproduced in Fig. $226 .^{1}$

1 From Bernstein's "Lehrbuch der Physiologie," S. 354. 
A bar of non-conducting material revolves on a vertical axis $a, 5$ to 10 times per second; $p^{1}$ and $p^{2}$ are isolated metal points which dip into the mercury pools, $Q Q$; when both of them touch the mercury, the galvanometer circuit is closed; this happens every time the bar revolves. The opposite end of the bar carries a pin $p$ which touches the wire $d$ and so closes the primary circuit just before $p^{1}$ and $p^{2}$ dip into the mercury. The time-interval between the two contacts - at $d$ and $Q$-can be determined by reference to the scale, which is divided into hundredths of a revolution. At the same moment that $p$ touches $d$, the muscle is excited at $r$. As long as the interval between the two contacts remains the same, each repetition of the stimulus as the bar goes on revolving, produces the same electrical effect. Consequently the similar effects rapidly following one another may be taken as one, which has been so magnified by repetition as to be measurable.

To make an observation, the instrument is brought into circuit by closing the key, $K^{4}$ (Fig. 226), the binding screws of which are connected with $Q$ and $Q$. The contacts of $p^{1}$ and $p^{2}$ with $Q Q$ may be so adjusted, that, supposing the rate of revolution of the bar to be 10 per second, the duration of closure of the galvanometer circuit shall be $\frac{2}{100}$ of the period of revolution, i.e. 0.002 second. The rheotome is set in motion, and the key in the circuit of the secondary coil, $S$, opened. If any deflection is observable, it is compensated, and the key again closed. The contact $p d$ is then so adjusted that $d$ is struck by $p$, say 0.01 second before the galvanometer circuit is closed at $Q Q$; then, the rheotome being still in motion, the same key is opened, for as short time as is necessary to obtain a full effect, and the deflection noted. The time-interval between the contacts is then reduced from 0.01 second to, say, 0.008 second, and the observation repeated, and so on with further reduction of the time-interval by the same amount each time until there is no longer any deflection, a result which will be obtained when the interval has been reduced to 0.002 . This having been accomplished, the first observation (time-interval 0.01 second) is repeated, and a second series made in which the time-interval is lengthened between each two observations, in the same way as that in which it was before shortened. In this way a series of numbers, each representing a galvanometric deflection, is obtained. The corresponding time-intervals between excitation and closure of the galvanometric circuit vary from 0.002 second to 0.020 second. If the circuit is arranged as in Fig. 223, the first set of deflections will probably be southwards, the second set northwards. It is important to note that between each observation and its successor, the muscle current (pre-existing difference of potential) must be carefully compensated, for a muscle cannot be subjected to repeated excitations without its electrical condition being altered.

The result of such a series of observations is stated by Bernstein as follows:-When a muscle is excited at $r$ at a given moment, by an induction shock, a certain time elapses before the excitatory variation begins at $l$ (i.e. at the proximal leading-off electrode). Here the effect culminates very rapidly, and disappears so promptly, that it has already ceased by the time that the "wave of contraction" has reached the same point. The shading represents the "part of the fibre," the elements of which participate in the process of negative change at the moment that $l$ is reached. This process lasts in each "element," according to Bernstein's observations, about $\frac{1}{2} \frac{1}{50}$ to $\frac{1}{300}$ second. It advances along the muscular fibre at a rate of 3 or $4 \mathrm{cms}$. in $\frac{1}{10}$ second. He therefore designated it the Reizwelle or Excitation-wave. What he supposes actually to happen in an uninjured muscle is illustrated by the diagram (Fig. 227). Each excitation wave that starts from $r v$ reaches first $l^{1}$ then $l^{2}$. When it is at $l^{1}$ a current exists in the direction of the arrow $\rightarrow$, when it is at $l^{2}$ there is a current in the opposite direction. In such a muscle 
the variation, i.e. the galvanometric effect, as observed with the aid of the rheotome, is diphasic. During the exceedingly short period that the wave is under the electrode $l^{1}$, a current from $l^{2}$ to $l^{1}$ passes through the galvanometer. During the similar period that it is under the electrode $l^{2}$, a current flows in the opposite direction. If we accept Bernstein's estimate of the rate of propagation and of the duration of the wave, and assume the electrodes to be over $2 \mathrm{cms}$. apart, there will be an interval of time between the two periods during which there will be no current either way. It will be seen further on that the excitatory change lasts longer than Bernstein supposed.

In a muscle truncated at one end, with the electrode $l^{2}$ on the injured part, the case is different. The variation has one phase only. The wave, as in the other case, makes its way along the fibres, but on its arrival at the injured part it is suppressed, so that its presence there is without galvanometric effect. Consequently, in the injured muscle, from the moment that the excitatory change has ceased at the electrode $l^{1}$ the variation is at an end. Hence the whole monophasic variation of the injured muscle represents the first phase of the diphasic of the uninjured.

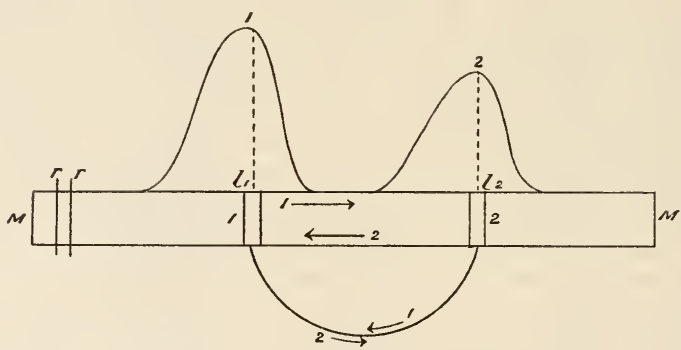

FIG. 227.-Progress of the wave of excitation, according to Bernstein.

From Bernstein's description of the single excitatory variation, founded on his observations with the rheotome, we may pass to Hermann's more recent (1893) account of the same phenomenon, based on a different method of using the same instrument. This method is of such importance that it must be shortly described.

From the description of the rheotome given above, it will be seen that during each observation of the series, i.e. during each period of excitation of the muscle, the time-interval between excitation and the beginning of the "closing time" remains unaltered. For the better understanding of Hermann's method, let it be supposed that the excitation is continued for a period of two seconds, that this period is divided into ten equal parts, and that at the end of each part the interval between excitation and closure of the galvanometer circuit is increased by $\frac{2}{1000}$ second. It is obvious that during each successive part or period of $\frac{1}{5}$ second, the difference of potential between the leading-off electrodes represents that which actually exists during the corresponding periol of $\frac{1}{5} 00$ second, and that if the ten measurements were arranged on an abscissal axis, the ordinates representing relative negativity of the proximal electrode being directed upwards, those representing relative positivity of the same electrode downwards, and the ends of the ordinates joined, we should have a graphic representation of the 
variation. The observations extending over a period of two seconds, divided into ten parts, would represent an actual period of $\frac{2}{100}$ second, made up of successive periods of $\frac{2}{1000}$ second. It is this method that Hermann has followed, with the important modification that instead of increasing the time-interval periodically, he increases it continuously and equably during the whole period. His period of excitation is also relatively longer, each hundredth of a second in the actual variation being represented by $3 \cdot 47$ second. ${ }^{1}$ The process thus prolonged can be followed by a galvanometer with a light aperiodic magnet, so as to give a true graphic record of the variations.

Observations were made both with uninjured muscles, and with muscles of which the surface of contact at the tendon end had been injured by lunar caustic. By bringing together the two curves $a$ and $b$ resulting from these observations, so that they lie with corresponding points over one another on the same axis, and drawing a third curve $c$, any ordinate of which when summed algebraically with the corresponding ordinate of $a$ would give the corresponding ordinate of $b$, the combination reproduced in Fig. 228 was obtained, in which $a$ represents the curve of variation at the proximal electrode (the curve of injury), $b$ the diphasic curve of the uninjured muscle, and $c$ the (deduced) curve of variation at the distal electrode. The figure shows that the relative duration of the instrumental effect of the first phase of the diphasic variation is

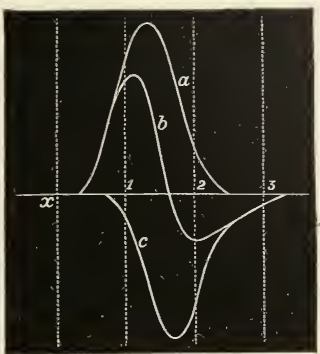

FIG. 228.-Curves of electrical response to instantaneous stimulation(gastrocnemius). Contacts at $17 \mathrm{~mm}$. distance on dorsal surface and tendon; $x$, moment of cxcitation. The vertical lines indicate hundredths of scconds after $x$. $a$, curve of response of injured muscle ; $b$, diphasic curve of uninjured muscle; $c$, derived curve of variation at distal electrode.-From Matthias. considerably less than that of the monophasic variation. This, of course, does not imply that the actual process which takes place at the proximal electrode in the muscle itself is different in the two cases.

In Fig. $229 \mathrm{I}$ have endeavoured to show the time-relations of the same two processes according to Bernstein's estimate of the duration of each, represented on the same scale as Hermann's curves, with the contacts at the same distance from one another. The reader will see at once that the difference between them consists in this, that the duration of each phase is according to Bernstein much shorter, and that, although the maxima of the phases are about equal distance from one another in the two cases, there is, according to Bernstein's conception, an interval between them during which the two contacts are equipotential, so that the curve is as it would appear if the galvanometer were acted upon by the summits of the waves only.

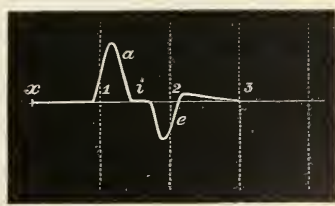

FIG. 229, - Diagram (by the writer, not after Bernstein) representing the same phenomenon as it would be if the duration of each variation werc $\frac{1}{300}$ sec. $i$, interval between first and second phases, $a$ and $e$.

We may now pass to the further knowledge which we are able to obtain by means of the capillary electrometer. But in order to make the experimental data on which that knowledge is founded intelligible, it will be necessary to state shortly the principles by which observers

1 Matthias, "Ueber graphische Darstellung der Actionströme des Muskels." Arch. f. d. ges. Physiol., Bonn, 1893, Bd. liii. S. 72 .

VOL. II. -27 
must be guided in interpreting them. The data in question are, as most readers now know, curves obtained by projecting the image of the mercury column of an electrometer (Fig. 230) on to a photographic plate, which moves at an equable rate behind a slit. The instrument takes the place of the galvanometer and shunt, in the circuit represented in Fig. $223,{ }^{1}$ the electrode on the injured end of the muscle, marked $d$ in that figure, being supposed in the following paragraphs to be connected

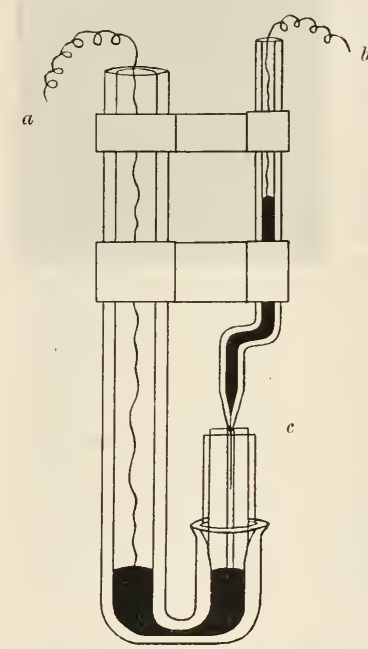

Fig. 230.-Burch's projecting electrometer. The shorter limb of the wide tube contains a piece of thick walled tube $c$, which is ground in like a stopper. The front of this tube is ground away so that its bore is laid open from end to end, forming a trough wide enough to contain the capillary. A thin cover glass closes the trough in front, which is kept full of liquid by the surface tension of the acid. For further description see "The Capillary Electrometer," London, 1896. with the capillary, and that on the muscular surface, marked $p r$, with the sulphuric acid; the current of a muscle injured at one end and placed as shown in the figure, would therefore be indicated by a movement of the meniscus from the point of the capillary. The shadow of a vibrating prong of a tuning-fork falls on the same plate at the same time, so that the time-relations of the movements of the meniscus of the mercurial column can be measured; and, by an automatic arrangement, the moment at which the circuit of the primary coil of the inductorium which serves to excite the muscle preparation, is opened, is also shadowed on the plate. The discovery which furnished the key to the interpretation of the curves so obtained, as indicating the nature and time-relations of the almost instantaneous electromotive changes we have now under consideration, was made by Mr. G. Burch, in the course of experiments carried out in the Oxford Physiological Laboratory, the results of which were communicated to the Royal Society in $1892 .^{2}$ He showed that the difference of potential at the moment corresponding to any point in such a curve of electrical variation, can be readily estimated from quantities which may be determined by the examination of the curve itself at that point. These are-(1) The distance through which the meniscus has moved from its zcro position, and (2) the inclination of the curve representing the velocity of the mercury, at the particular moment. Each of these quantities has its value
in positive or negative difference of potential. To estimate their values, another curve must be obtained by photographing the excursion when a known difference of potential (say of 0.01 volt), derived from the compensator, is allowed to act on the electrometer, so as to determine a movement of the mercury towards the point of the

${ }^{1}$ If it is desired to use the galvanometer at the same time, the electrometer may be connected with $\mathrm{K}^{4}$.

${ }^{2}$ Burch, Phil. Trans., London, vol. clxxxiii. p. 81. 
capillary tube. This photograph of what may be called a "normal" or "comparison curve" having been taken, the reverser of the compensator is turned over, and a second photograph of the same kind is obtained with the same electromotive force acting in the opposite direction. The first of these curves is reproduced in Fig. 231. Its character is such that, at the moment corresponding to any point whatever in its course, the difference of potential acting on the electrometer is to the total such difference (in this case 0.01 volt) as the distance from that point to the asymptote $\alpha$ is to the distance of this line from the starting point of the curve. From the latter distance, the true value in volts of the distance (1) referred to above, can be readily calculated. If, for example, the difference in question be $10 \mathrm{~mm}$., and the corresponding difference of potential 0.01 volt, the value of each millimetreis then known to be one millivolt. To find (2), i.e. the value of the angle of inclination at any point in the variation curve, the point in the comparison curve at which the inclination is identical must be sought. The distance in $\mathrm{mm}$. of this point in the comparison curve from the asymptote then gives the corresponding difference of potential in

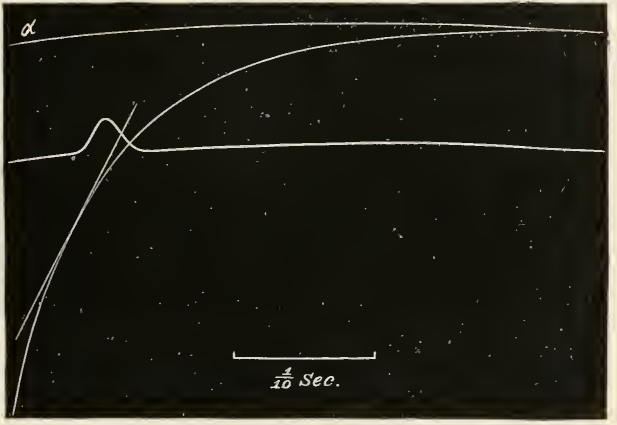

Fig. 231.-Comparison curve. It is of such a eharacter that any two ordinates at an angular distance of one degree from each other are, as measured from the asymptote $a$, in the proportion of $1: 1 \cdot 3$. The straight line touching the curve is drawn as nearly as possible parallel to the steepest part of the ascent of the diphasic curve, and is, where it touches the comparison curve, $23 \mathrm{~mm}$. from the asymptote. If it be assumed that 0.01 volt corresponds to $10 \mathrm{~mm}$., the greatest acting difference of potential during the first phase is 23 millivolts. Adding to this estimate the difference due to the previous rise of the mereury up to the point of contact, we have 25 millivolts, the height above the base line being about $2 \mathrm{~mm}$. The fact that the curve is inscribed as if on the surface of a dise, must be noted. To obtain strictly correct results, the corresponding points in the two eurves should be at the same distance from the centre of the disc on which they are inscribed. If they are not so, a correction is necessary. Thus, in the case figured, the inclination of the experimental curve must be increased about 1 per cent.

millivolts. For reasons which need not be here entered upon, the comparison curves must be taken under the same conditions as the variation curve to be referred to them, and the points compared in the two curves must be at the same distance from the centre of rotation of the disc.

The photographic record of a diphasic response is shown in Fig. 232; the time of excitation, i.e. the monent at which the primary circuit is broken, being indicated by the radial line. The ascending part of the curve correspouds in the main to the first phase-the descending to the second. ${ }^{1}$ The essential points to be determined by the investigation of

${ }^{1}$ Elaborate analyses of several curves kindly made for me by Mr. Burch by his method, have been published elsewhere (see Journ. Physiol., Cambridge and London, 1895, vol. xviii. 1. 130). 
such curves of an uninjured parallel-fibred muscle are-(1) The rate of propagation of the excitatory wave; (2) the time after excitation at which the two contacts become equipotential; and (3) the moments at which the first and second phases respectively attain their maxima, and the differences of potential which exist at these moments. The propagation-rate is determined by the comparison of the curves of two observations in which the distance between the seat of excitation and the contact nearest to it is different (the distance between the leadingoff electrodes remaining the same). The time-interval between the moment of excitation and the moment corresponding to the summit

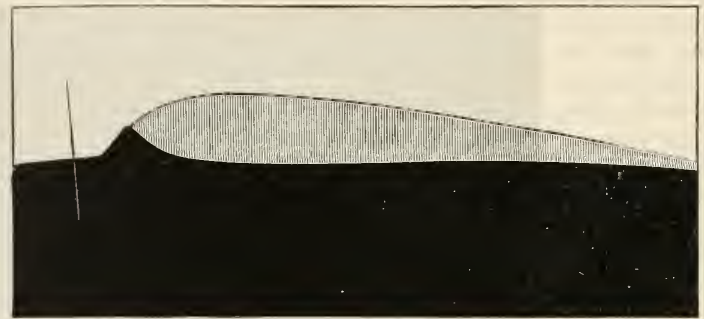

FIG. 232.-Copies of the diphasic (black) and monophasic (grey) eurves of the sartorius, as they are seen when the two photographic records are placed one over the other, so that the beginnings coincide. Up to the beginning of the second phase the curves are identical. Rate of movement of the plate as in Fig. 231.

of the curve in each case can thus be estimated and compared. The moment of equipotentiality is that at which the positive difference of potential, indicated by the downward inclination of the curve, becomes equal to the negative, corresponding to the upward displacement of the meniscus. This event is indicated in the diagram, Fig. 233, by the vertical line, and coincides with the arrival of the excitatory wave at the midpoint between the contacts. The rate of propagation and the moment of equipotentiality having thus been determined, the times of culmination of each of the two phases of the excitatory wave can be readily deduced from them. Thus, if the propagation-rate

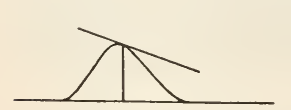

FIG. 233. be $2000 \mathrm{~mm}$. per second, and the distance between the electrodes $20 \mathrm{~mm}$., it is obvious that the excitatory change must arrive at the proximal electrode 0.005 seconds before the moment of equipotentiality, at the distal electrode 0.005 second after that event. It may be seen from the photographic curve reproduced in Fig. 232 that the culmination of the first phase coincides with the middle of the ascending limb of the curve, that of the second phase with the beginning of the descending limb. The photographic record of a monophasic response, as obtained from the same sartorius muscle when one end has been devitalised by immersion in normal salt solution at the temperature of over $60^{\circ} \mathrm{C}$., is shown in Fig. 232 , by the shaded area. It will be observed that it differs from that yielded by the uninjured muscle chiefly by the absence of the second phase. In both cases the arrangement of the leading-off contacts was the same, the proximal contact being near the 
middle of the muscle, the distal near its tibial end, which in the one case was devitalised, but not in the other. It is seen that the ascending limb is identical in the two curves, but that, from the point at which the diphasic curve approaches its culmination, the "injury curve" diverges from it, continuing to ascend. The inclination, however, rapidly diminishes until, from $\frac{3}{100}$ to $\frac{5}{100}$ of a second after excitation, the line becomes first horizontal and then begins slowly to decline. Shortly after the beginning of the decline, the two leading-off contacts become equipotential. The meniscus, however, does not reach its zero position until later. During the period which corresponds to the second phase in the uninjured muscle, the difference of potential in the injured muscle, which at the moment corresponding to the divergence of the two curves is still strongly negative, diminishes at first rapidly, then very gradually, until it finally disappears.

The estimate usually given of the duration of the monophasic variation is mainly founded on a beautiful experiment of Helmholtz's, in which lie measured the latency-period in the same muscle when excited under different conditions. In one case the nerve was excited directly by an induction shock, and the muscle through it; in the other, the nerve of the same muscle was excited by the monophasic variation of another muscle, of which the nerve was excited directly. In the first case the period of latency was shorter than in the other by about $\frac{1}{20} \overline{0}$ second. He therefore concluded that the rheoscopic limb receives the excitation to which it responds, $\frac{5}{100}$ of a second later than it would have received it had its nerve been directly excited-or, as he then supposed, at the middle of the period of latent stimulation of the exciting muscle. Our photographic records tell us that at that moment the column of the electrometer is shot up by the sudden rise of tension at the proximal electrode; and it can scarcely be doubted that it is by this change that, in Helmholtz's experiment, the nerve of the recording muscle is excited.

At the moments of culmination of the diphasic and monophasic variations respectively, the difference of potential is expressed in much larger proportion

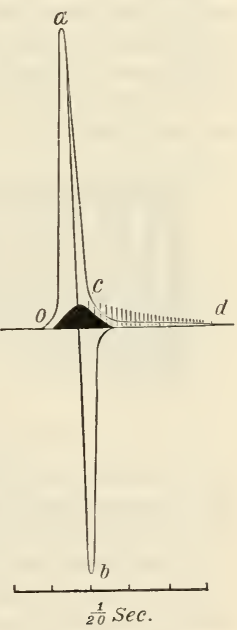

FIG. 234. by the inclination of the curve at the point corresponding to that moment, than by the distance of that point from the zero line. Thus, in the examples published in $1895,{ }^{1}$ the maximum "negativity" was found to be from $\frac{f_{0}}{100}$ to $\frac{8}{100}$ of a volt, of which amount only $\frac{3}{1000}$ or $\frac{4}{1000}$ were due to the height of the meniscus above its zero position. Fig. 234, which must be understood to be nothing more than a diagram, serves to illustrate this. The black area is that of the diphasic variation, the black and shaded together make up that of the monophasic. The line o abd is that of the "tension curve" of the diphasic, the line o acd that of the monophasic. The difference of potential between the contacts at the monent of culmination of the first phase is two or three times as great as that of the "negative variation," as estimated by du Bois-Reymond. This important fact was proved some years ago by a method of investigation employed by myself in association

\footnotetext{
'Burdon Sanderson, "The Electrical Response to Stimulation of Muscle," Journ. Physiol., Cambridge and London, 1895, vol. xviii. p. 117.
} 
with Professor Gotch. ${ }^{1}$ The sartorius muscle is connected with the galvanometer circuit, which is arranged as in Fig. 223. The nerve is excited by a rheotome, the galvanometer wires of which go to $\mathrm{K}^{4}$. This also closes the galvanometer circuit automatically at any desired time after excitation. The "closing time" of the rheotome is very short-usually $\frac{1}{500}$ second, it having been found that the greatest (negative) deflection is obtained when the closure begins about 0.006 second after excitation. The slider of the compensator is so adjusted that the derived current is in the opposite direction, and of just sufficient strength to annul the deflection when the excitation is repeated. In the gastrocnemius the mean difference of potential in five series of observations was found to be 0.084 volt.

(b) The electrical response to a series of stimulations.--The nature of the change which accompanies tetanus, when observed in an injured muscle, with leading-off contacts on the natural and cross sections respectively, was investigated by du Bois-Reymond some fifty years ago. ${ }^{2}$ He interpreted the imperfect data which the methods then available afforded, with wonderful sagacity-drawing therefrom inferences which were surprisingly near the truth. It was more than twenty years later $^{3}$ that, with the aid of the much better instruments which his own ingenuity and the progress of science had placed in his hands,

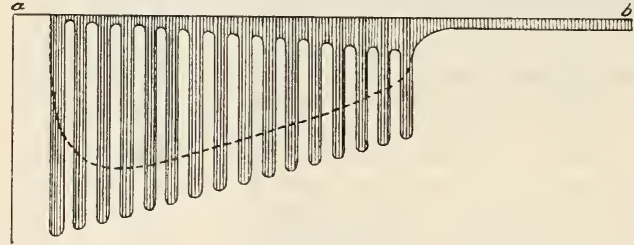

FIG. 235.-After du Bois-Reymond. he published an account of the phenomena to which he applied the term "negative variation." His general view of what happens in tetanus can be best understood by referring to

the curves by which he represents it, as reproduced in Fig. 235. The broken line is the curve of what he calls the Gesammtschwankung, the integral curve of the "variation." The successive effects of which it is supposed to be made up are represented by the teeth of the "ctenoid curve," the relation of which to the integral curve is such that, if as many equidistant ordinates were drawn to the latter as there are teeth, the space enclosed between the horizontal line $a b$, the curve, and any two neighbouring ordinates, would represent the integral of the differences of potential existing between the contacts during the corresponding period. Each tooth, therefore, represents the response to a single instantaneous excitation. The figure further denotes that tetanisation is followed by an "after-effect" - a slight diminution of the previous difference of potential.

The "negative variation" of uninjured muscle was also investigated by du Bois-Reymond in the same exhaustive way, particularly that of the gastrocnemius, and he represented his conception of it graphically. He found that, even when there was previously no difference of potential between the contacts, the proximal contact became negative during

1 See "Proc. Physiol. Soc.," Journ. Physiol., Cambridge and London, 1891, vol. xii. p. 43. 2 du Bois-Reymond, "Untersuch. ueber thierische Eiectricitat," 1894, Bd. ii. S. 91, Fig. 89 .

${ }^{3}$ du Bois-Reymond, "Gesammelte Abhandlungen," Bd. ii. S. 559. 
tetanic excitation. The change, however, was less in amount than in injured muscle, and irregular in development, and moreover lasted longer after the excitation ceased than when the distal electrode was on an injured surface. As has already been pointed out, du Bois-Reymond regarded the "negative variation" as a diminutional effect, using the word negative in its algebraic sense. In order to explain its presence when there was no muscle current, he had recourse to the auxiliary theory, that the "natural cross-section," i.e. the end surface of every muscle, presents peculiarities of molecular structure, which he designated by the term "parelectronomy." He was thus able to account in the most ingenious way for the irregularities he observed, as resulting from the interference of parelectronomic effects having their seat in the ends of the fibres, with the normal ones.

As has been already stated, the molecular theory of du Bois-Reymond is not now accepted by physiologists. The other theory, however, with which it is associated, namely, that which attributes the negative variation, no less of injured than of uninjured muscle, to the summation of individual electrical responses evoked by instantaneous stimuli, has not as yet, so far as I know, been questioned.

In the case of injured muscles with the leading-off contacts, as in Fig. 236, A, the mollophasic character of the single response appears to be adequate to explain the course of the tetanic variation; but as regards uninjured muscles it is at first sight difficult to see why the summed effect of a series of diphasic variations should take the direction of the first phase, as was found to be the case in the gastrocnemius by du BoisReymond. Many years ago (1868), Her-

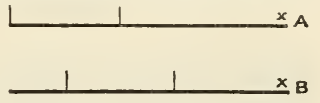

FIG. 236. $X$ X denotes the seat of excitation; the upright lines show the positions of the leading-off contacts. mann suggested that when it occurs, it is due to the fact that every excitatory wave becomes weaker as it progresses. Soon afterwards, Bernstein, as the result of his rheotome experiments, was led to believe this surmise to be correct, and Hermann, later, in repeating an old experiment, showed that in a sartorius with the electrodes arranged as in Fig. 236, B, there is usually a "decremental" tetanic effect-the proximal contact becoming negative to the distal. ${ }^{1}$ The total difference of potential in the tetanic response of an uninjured muscle was explained by him, on the supposition that each excitatory wave becomes weaker as it progresses, the second phase of each diphasic effect being weaker than the first. The complete absence of negative variation in tetanus of the human forearm indicates that in perfectly normal muscles there is, as Hermann says, no decrement. ${ }^{2}$

The tetanic variation, or, as Hermann calls it, the "tetanic actioncurrent," can be observed in different ways. When a galvanometer of high resistance is used, the needle being so damped as to be nearly aperiodic, only $\frac{1}{100}$ part of the current passing through the bobbins, the spot assumes a position which corresponds to the integral difference of potential, the value of which can be easily determined from the deflection with the aid of a compensator. As the time required for the

\footnotetext{
${ }^{1}$ Hermann's "Handbuch," Bd. i. S. 214 ; du Bois-Reymond, "Gesammelte Abhandlungen," Bd. ii. S. 560.

"Im ganz normalen Muskel ist kein Decrement der Erregungswelle vorhanden," loc. cit., S. 224 .
} 
needle to come to rest depends on instrumental, not on physiological, conditions, it has no special interest.

When the capillary electrometer is used, a toothed curve is obtained (uninjured sartorius), which brings to mind the ideal representation of the "negative variation" in tetanus given by du Bois-Reymond. In the photographic curve shown in Fig. 237, obtained from the injured sartorius, it is easy to distinguish the general or integral form on which the humps are inscribed, each corresponding to the description given of a single monophasic variation (Fig. 232). If the frequency of excitation be about 30 per second, the starting-point of each individual curve of the series will coincide with the very beginning of the decline of the

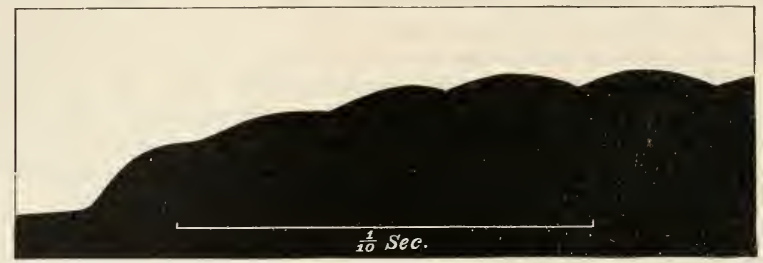

Fig. 237.-Tetanus curve of injured sartorius.

preceding one. The form of the integral curve is such as to show that the electrical change which takes place at the beginning of a tetanus is a sudden one. This is proved by the following experiment:-

The apparatus is so arranged that immediately the advancing edge of the plate passes behind the slit, the tetanising induction currents begin to act. The excursion in the electrometer is photographed, and a comparison curve is then made in the way already described, by introducing at a given moment a known difference of potential with the same muscle in circuit. The general form of this comparison curve is the same as that produced by excitation, with the exception that the comparison curve has of course no inequalities. What we learn from this correspondence is that in each case the difference of potential attains its eventual amount at once.

If an uninjured muscle, with the contacts arranged in a similar way, is observed, it may happen that the meniscus remains nearly at the

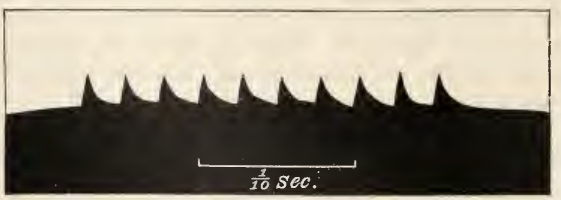

Fig. 238.-Responses to ten excitations of an uninjured gastrocnemius. Frequeney about 40 per second. same height throughout the period of tetanic excitation. In this case the integral curve is nearly parallel to the zero line. The spikes which represent the successive excitations project upwards from the curve, each having the form of a normal diphasic response to a single instantaneous stimulation (Fig. 238).

The writer ${ }^{1}$ has shown that other forms of continuous contraction, evoked either by a single instantaneous stimulation, or otherwise, are accompanied by electrical changes which, while they resemble in general

$$
1 \text { Loc. cit., p. } 142 .
$$


that of tetanus produced by repeated stimulation, differ from this in certain important particulars. Among these forms of continuous contraction are-(1) That which is evoked when a muscle is excited by the passage of a constant current through its nerve; (2) that caused by a single instantaneous stimulation when a muscle has been poisoned by an extremely small dose of veratrine; and (3) the reflex spasm of strychnine.

To observe Case 1, it is best to use the muscle of a frog which has been kept at a temperature approaching $0^{\circ} \mathrm{C}$. In such a preparation ${ }^{1}$ it is easy to

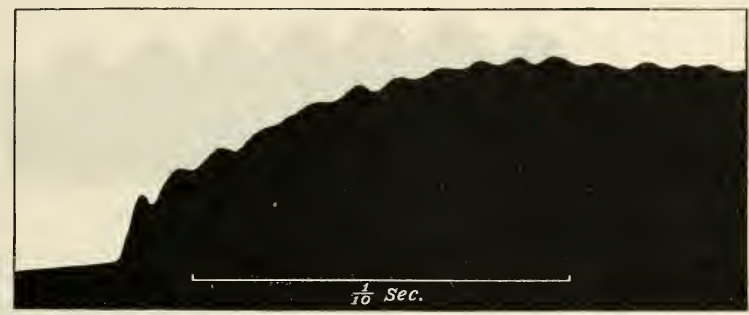

Fig. 239.-Response to indirect excitation of a cooled muscle, injured at the tendon end by plunging into hot salt solution, on closure of a constant current (gastrocnemius).

bring about a sufficiently persistent contraction, even with currents of moderate strength. The form of the curve is seen in Fig. 239. It gives the same evidence as before, that the muscle passes promptly into the excited state. The curve exhibits undulations which occur with surprising regularity, the significance of which cannot as yet be stated. A muscle which has been

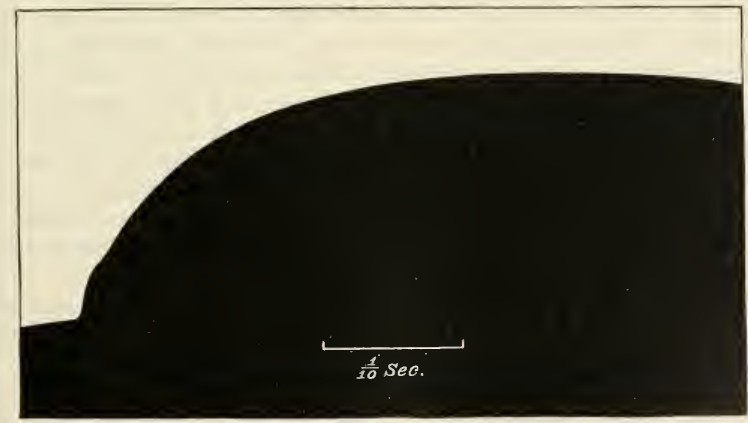

FIG. 240--Response to direct excitation of a veratrinised sartorius devitalised at the tibial end.

thoroughly veratrinised (Case 2), furnishes similar curves in response either to direct or indirect stimulation, which, however, do not exhibit any undulations (Fig. 240). For the observation of the strychnine spasm (Case 3), a frog must be used which has received by injection into the lymph sac, an extremely small dose of the drug. After exposing the muscle to be observed, the leading-off electrodes are applied with as little interference with

${ }^{1}$ Hering, "Biological Memoirs," Oxford, vol. i. p. 136. 
the circulation as possible. An observation may be made when a condition has been reached in which an induction current led through the skin of the flank evokes a prolonged contraction instead of an instantaneous twitch. Here, again, the integral curve obtained resembles the tetanic curve of injury ; the proximal electrode becomes, on the whole, negative to the distal. This general movement of the meniscus is interrupted by alternating upward and downward movements (Fig. 241). The ascents are produced by spasms, each of

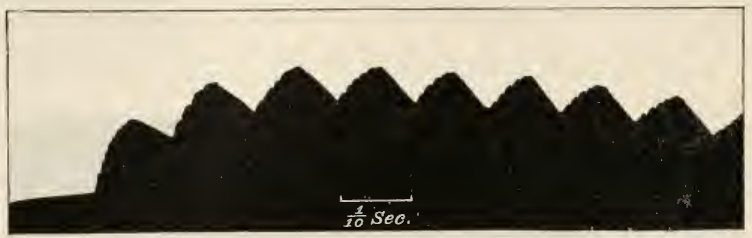

FIG. 241.-Response to a reflex excitation-of an uninjured gastrocnemius-(strychnine).

which lasts $1 \frac{4}{00}$ or $\frac{5}{100}$ of a second. The descents correspond to the intervals between these spasms, and continue longer, the two together having a duration of from $\frac{8}{100}$ to $\frac{12}{100}$ of a second. It is noticeable that undulations are observed in each ascent, similar to those shown in Fig. 239. The larger oscillations of the meniscus were long ago described by Lovén, who regarded each as a response to a single instantaneous stimulus emanating from the spinal cord. ${ }^{1}$

\section{Phenomena which are produced by the Direct Action of Voltaic Currents on Muscles.}

Polarisation of muscle.-Experimental data.-In 1883 it was shown by du Bois-Reymond that, when a battery current is led for a very short period from end to end through a living muscle by means of terminal electrodes, the muscle being so connected by the same electrodes with a galvanometer that its circuit can be closed through the muscle immediately after the opening of the battery circuit, currents present themselves of which the direction varies according to the conditions of the experiment. These were called by du BoisReymond "secondary." They are also termed "polarisation" currents, and the current which produces them is termed the "polarising" current. If this current is of sufficient duration, the direction of the secondary current is opposed to that of the polarising current. If it is strong and of short duration, its direction is the same as that of the polarising current. Both of these phenomena were described by $\mathrm{du}$ Bois-Reymond, under the term secondary electromotive phenomena.

The simplest way of demonstrating the two phenomena is indicated in the diagram (Fig. 242), which shows an ordinary Pohl's reverser made of hard paraffin without cross-wires, so arranged as to enable the observer to direct the current of the battery $(B)$ by the hand through the muscle. When the bridge is turned over, the muscle is in the circuit of the galvanometer. For investigating the phenomena, however, an automatic key or rheotome ${ }^{2}$ must be

${ }^{1}$ Lovén, Centralbl. $f$. d. med. Wissensch., Berlin, 1881, No. 7. On the subject of the preceding section further details will be found in two papers published by the writer since this article was in typc. See Journ. Physiol., Cambridge and London, November 1898, and Proc. Roy. Soc. London, 1899 (Croonian Lecture).

${ }^{2}$ Such an instrument is described in the Phil. Trans., London, 1888, p. 421. 
used. This consists of a revolving bar, which carries platinum contacts so arranged that the battery circuit is closed at each revolution, and immediately afterwards the galvanometer circuit is closed. The duration of the closure can be varied at will.

The opposed secondary current resembles so closely the so-called negative polarisation current of electrolytes, investigated many years ago by Peltier, that there need be no hesitation in regarding it as a physical phenomenon, and applying to it the term polarisation, in the sense in which it is used by physicists. The effect in question shows itself immediately after the polarising circuit is opened, and is very transitory. The most important facts relating to it are-(1) That it is not confined to the anode or cathode, but can be shown to exist throughout the region through which the polarising current has flowed; (2) that it subsides very rapidly; and (3) that its amount is in obvious relation to the strength and duration of the polarising current. In all these respects the results obtained in experiments on muscle (e.g. on the sartorius) correspond with those observed when a conductor of similar form, consisting of porous material soaked with liquid containing electrolytes, is investigated in a similar way (Peltier's polarisation). Another point of importance, as showing that it is dependent on the structural and chemical properties of muscle, not on its functional activity, is that although absent in muscles of which the structure has

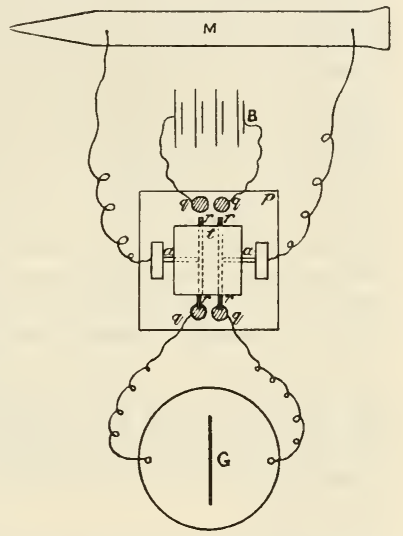

FIG. 242. $-p$ is a paraffin block with mercury pools at $q q q q$; $t$ is also a paraffin block, and tilts on its bearings at $a a$. When $r r$ on the battery side dip into $q q$, the inuscle is in circuit with the battery. In the diagram it is represented in circuit with the galvanometer.

been altered by subjecting them to high temperature, or otherwise, the negative after-effects can be nearly as well observed in an exhausted muscle which fails to respond to stimulation, as in a fresh muscle.

The secondary current, of which the direction is the same as that of the polarising current, is of a different nature. Its characteristics are-(1) That it chiefly manifests itself in the neighbourhood of the anode, i.e., that the effect is best observed when the galvanometer contacts are placed, one at the anode, the other at a short distance from it; (2) that in this case the effect is increased by shortening the duration and increasing the intensity of the polarising current ${ }^{1}$; (3) that it fails to appear unless the muscle experimented on is fresh and vigorous. The phenomenon appears to be dependent on the functional disturbance which the muscular structure undergoes in consequence of the passage of the current. By du Bois-Reymond it was called polarisa-

${ }^{1}$ Four to six Daniells, for from a half to a quarter of a second, may be used for the sartorius. 
tion, because it associates itself so closely with ordinary polarisation, that it is often difficult to distinguish the one effect from the other; positive; because it is in the same direction as the led-through current. The significance of these characteristics becomes at once plain from the moment that it is understood that this phenomenon is of the nature of excitation.

After the opening of a current flowing through a muscle, the presence of the effect just described indicates that the region of the anode, that is, the seat of disturbance, is itself relatively negative. If the experimental current is strong, this effect is so immediate and powerful that the polarisation effect proper is overpowered by it. If it is weaker, a diphasic movement of the needle is observed, in which the transitory true polarisation effect is followed by the other. If in a succession of experiments the strength of the "polarising" or external current is gradually increased, the brief duration remaining the same, the effects which are at first opposed in direction to the external current become diphasic (the movement due to polarisation being followed by that due to the anodic disturbance), and eventually have ouly the direction of the external current; and this gradual change is best seen when one galvanometer contact is at the anode. Perhaps the best proof of the excitatory nature of the phenomenon is obtained when, the muscle having been injured by heat so as to produce a "thermal section" at its tibial end, one electrode is placed on the injured part, the other on the natural surface at the pelvic end. In this case, if the anode of the polarising current is on the injured surface, the effect is scarcely perceptible, whereas with the polarising current reversed (anode on the uninjured pelvic end) a descending after-current is observed.

These experiments justify the application to the phenomena in question, in preference to du Bois-Reymond's original designation (positive polarisation), of the term "anodic after-effect" used by other writers, as denoting the localisation of the effect at the anode and the time of its occurrence, without suggesting any theory of its nature. Considering that relative negativity of a part is a sign of the excitatory state, and that in nerve the anode is the seat of the excitatory effects which take place at the opening of a current led along it, it is not unreasonable to regard the effect as excitatory. The reader will find that some difference of statement exists between leading physiologists as to the degree in which the anodic after-effect is limited to the anode. According to some observers, the whole of the anelectrotonic region becomes relatively negative.

The phenomena which have just been described are those which are best known. The admission, however, that the anodic after-effect is the result of a polar change, dependent on the cessation of the inflow of the current, carries with it the suggestion that the cessation of the outflow at the cathode may have a corresponding after-effect, that is, one in which the cathodic region becomes relatively positive. Phenomena which are in accordance with this surmise have been observed by Biedermann in the sartorius; but in order to make them appreciable, one end of the muscle must be steeped in salt solution containing veratrine. The part thus treated can be thrown by a single instantaneous excitation into a state of continuous spasm of sufficient duration to make it possible to examine its properties experinentally. 
If a voltaic current is led through such a muscle, with the cathode on the exeited and veratrinised end, no effect is produced so long as the current is closed, but on opening it the anelectrotonic state manifests itself by relative positivity of the anodic part.

In the adductor muscle of Anodonta, which is normally in a state of tonus, Biedermann has shown that the cathodic relaxation which follows the opening of a polarising current is accompanied by a corresponding electrical state (relative positivity), but this observation does not bear on the question whether or not, in the absence of tomus (produced either naturally, by veratrine, or by other chemical agencies), an inhibitory after-effect, marked by relative positivity and muscular relaxation, can be observed at the cathode in normal skeletal muscle. ${ }^{1}$ On this point the only information we possess is derived from certain observations of Hering to which reference is made by Biedermann.

In all experiments on polarisation care must be taken to select muscles which are free from tendinous inscriptions, for, as Hering conclusively showed, these exhibit the physiological properties of cathodes and anodes. At each inscription the current leaves the muscular substance to enter tendon, and again leaves tendon to enter muscle. An after-effect in the same direction may consequently manifest itself even when both galvanometer electrodes are at a distance from the cathode and anode, provided that an inscription occurs in the region from which the effect is led off.

In nerve the phenomena of polarisation can be studied more advantageously than in muscle, and are of greater physiological importance. The reader will therefore find it useful to refer to the Article on Nerve, in connection with the preceding paragraph.

Polar effects on excitability during and after the passage of a voltaic current.-If a curarised sartorius be so arranged that during the flow of a battery current from end to end, an induction current is led through the same electrodes in the same direction, it is observed that the response to the induction eurrent is much augmented. Thus an induction current of which the strength is such as to produce a minimal contraction when the voltaic current is open, at once evokes a maximal response if this current has previously been closed, - a fact which led v. Bezold, who first observed it, to the erroneous supposition that a general increase of excitability of the muscle is produced by the flow of a voltaic current through it. ${ }^{2}$

The arrangement of this old experiment will be best understood from the description of the diagram (Fig. 243), with the addition that the metallic electrodes originally used should be replaced by non-polarisable ones. It affords evidence that during the passage of a current, excitability is augmented at the anode, diminished at the cathode, but gives no indication of the state of the region between the poles.

As in the case of nerve, so also in that of muscle, the observation of interpolar excitability by means of electrical excitation is attended with great difficulty, consequent on the interference of the testing current with the

1 With reference to the apparently similar case, in which a polarising current is lerl through a sartorius with a devitalised tibial end, see Locke's paper, Arch. $f$. d. ges. Physiol., Bonn, Bd. liv. S. 520.

2 "Biological Memoirs," Oxford, 1887, "On the Phenomena of Inhibition, ete., and on Positive Cathodic Polarisation," p. 331. See also "Elektrophysiologie," S. 386 ; Translation, vol. i. p. 454 . 
polarising one. For, in the experiment just referred to, the current of each circuit must, if both are closed at the same time, flow into the other, in proportion dependent on its resistance. The same thing would happen if the testing electrodes were separated from the others, to whatever part of the

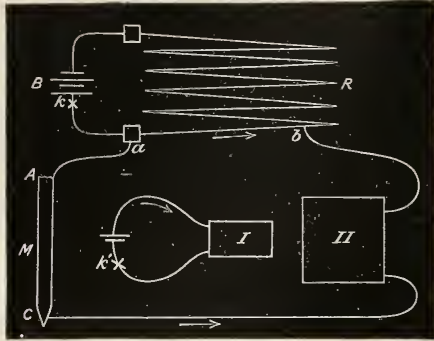

Fis. 243. $-I$ and $I I$ the primary and secondary coils of the Inductorium. $R$, rheochord through which the eurrent of $B$ flows in the direction of the arrow. A current derived therefrom flows from $a$ to $b$ through the muscle $M$ and the secondary coil. The current through the primary coil can be closed by the key $k^{\prime}$. At the moment that this takes place a closing induction shock flows through the circuit $a M b$ in the same direction as the battery current. muscle they were applied. Consequently, the existence of these derivation currents would modify the excitability of the parts explored, and thus vitiate the results of the comparisons. Biedermann ${ }^{1}$ seeks to avoid this difficulty by introducing into the exciting circuit a resistance so great, that the derivation currents in question are too inconsiderable to have any effect. By this method he has arrived at the conclusion, that in the curarised sartorius, so long as the testing electrodes are applied outside of the polar regions, no alteration of excitability can be detected during the flow of the polarising current.

In experiments of this kind, the current should be so weak that its closure scarcely evokes an excitation. So long as this is the case, the effect continues without alteration. By increasing its intensity the effect is at first increased, but beyond a certain degree of intensity, which is soon reached, it begins to diminish. ${ }^{2}$ The effect is strongest during the fifteen or twenty seconds immediately following closure, and disappears if the circuit remains closed. A few seconds later the response to the induction shock may be so weakened as to suggest that the increased excitability which at first presents itself has produced a sort of fatigue. The previous state of increased response, Biedermann speaks of as a "latent state of excitation." 3 The changes of excitability at the anode during the passage of a voltaic current have also been investigated by him, and have yielded results which appear to be in harmony with those above stated.

We have seen that in curarised muscles the cathodic increase of excitability which shows itself immediately after the closure of a voltaic current, diminishes in a few seconds, and is eventually replaced by an opposite state. If a current of sufficient strength to evoke a strong response at closure is broken, and after a very short interval of time again closed, the second closure is often without effect. But on closing the current in the opposite direction the response is as strong as before. This phenomenon is analogous to that which in nerve has been long known as that of "voltaic alternatives." The failure of the second closure is evidently another manifestation of the state resembling fatigue already referred to. It can, however, be shown that a muscle of which the excitability has been diminished by real

1 “Elektrophysiologie," 1895, S. 238 ; Translation, vol. i. p. 281.

2 Loc. cit., S. 242 ; Translation, vol. i. pp. 284-286.

${ }^{3}$ Loc. cit., S. 244. 
fatigue, regains it at the cathode the moment a voltaic current is led through it; whence it is evident that the cathodic after-effect cannot be of the nature of exhaustion. Another reason for this conclusion is that the decrease of the excitability at the cathode after opening, has its counterpart in increase at the anode. The preceding facts are comprised in the general statement that, during closure of the voltaic current, each of the polar states first brought into existence (the cathodic augmentation and the anodic diminution) gives way to the contrary state, and the relation between these two effects can be best understood by saying that the normal state of muscle is intermediate between two opposite states, which contrast with each other both as regards the ability of the tissue to contract and the readiness with which it responds to stimulation. This being so, we may expect that the loss of the second of these qualities at a pole of the voltaic current would also be attended with loss of conductivity. That this is so can be easily shown experimentally, for if a curarised sartorius is so arranged that a current is led to the middle of the muscle from its pelvic end, and if on its cessation the tibial end is excited by an induction current, no response takes place at the pelvic end, the wave of contraction being stopped halfway by the cathode of the voltaic current. If the current is strong enough, the block persists for some time after opening, but after the passage of the same current in the reverse direction the status quo is restored. What the cathode has done the anode has annulled, so that the wave of contraction passes freely once more from end to end. ${ }^{1}$

Polar excitation of muscle by the voltaic current.-Localisation of the excitatory effects at the poles.-When a voltaic current is led for a short time through a curarised muscle in the direction of the fibres, it contracts when the current is closed. This closing contraction starts at the cathode.

The first experimental indication that the closing excitation is localised at the cathode, was given by Schiff about half a century ago. ${ }^{2}$ He found that in a muscle in which the mechanical response to electrical stimulation is no longer propagated, in consequence of poisons or cessation of circulation, a very slight "idiomuscular hump" is formed at the cathode when a battery current is closed through it, which does not spread. Not many years later, v. Bezold ${ }^{3}$ showed that the localisation thus indicated had no necessary connection with the absence of propagation, or the peculiar conditions of Schiff's experiment, but could be demonstrated on fresh eurarised muscle. His method was founded on the consideration that, inasmuch as the period of latency in curarised muscle increases with the distance from the seat of excitation of the part where the change of form is being observed, this distance can be computed from the loss of time. A sartorius was laid in a shallow groove cut in a cork plate, and kept in its place by two wires wlich crossed it at a distance of half a centimetre from each other. These served as electrodes for the battery current. The tendon end of the muscle was attached to a writing lever, and the muscle so arranged that the latent period could be measured graphically. This was found to be longer at closure when the current was directed from the tendon, than when it was in the opposite direction. This difference in the period of latency in the two cases evidently indicated that the starting-point of the excitation wave must in either case have been the cathode.

1 Biedermann, loc. cit., S. 252 ; Translation, vol. i. p. 296.

2 Schiff, see "Beitr. z. Physiol.," Bd. ii. S. 13, 14.

3 "Untersuch. ueber die electrische Erregung der Nerven und Muskeln," Leipzig, 1861 , S. $239-244$. 
The prolonged contraction.-The important distinction between the persistent contraction which is scarcely, if at all, propagated, and the twitch or wave of contraction, was further illustrated by Engelmann, ${ }^{1}$ by a method of preparing the sartorius which has since proved to be of great value in experimental work. The middle of the muscle is grasped by a holder tight enough to prevent it from slipping when the tibial end is made to lift a very light writing lever attached to the tendon, but not tight enough to interfere with the propagation of excitation. The battery electrodes are placed on opposite sides of the holder, and the current is closed first from, then towards the tendon. In the former case the curve has the character of the single twitch, in the latter there is in addition a prolonged contraction.

The specific character of the prolonged contraction, i.e. its claim to be regarded as a phenomenon by itself, and not merely as an after-effect or sequel to the primary response or twitch, is indicated, not only by its persistence and the fact that its tendency to spread is inappreciable, but also by the analogy of the conditions under which it manifests itself with those which favour the production of Schiff's "idiomuscular contraction." The resemblance is sufficient to justify the surmise that they are closely related phenomena.

Some of the experimental facts by which earlier observers proved the localisation of the make-excitations can be very satisfactorily demonstrated

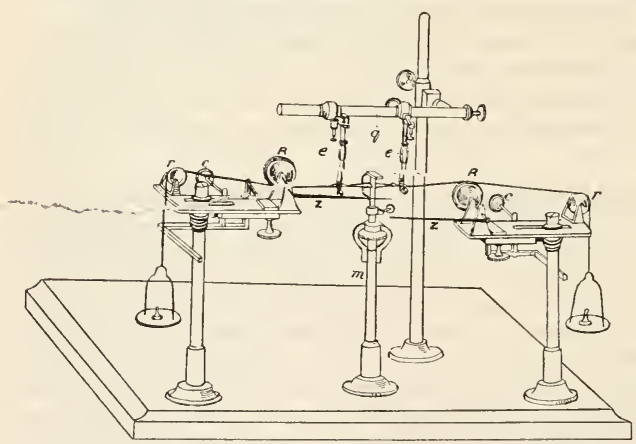

FIG. 244.-Hering's double myograph. The sartorius muscle is held in the middle between two pards of oil-clay (underneath $q$ ), so that each half ean be cxtended horizontally independently of the other. Non-polarisable electrodes $e e$ are applied to the bony attachments of the muscle. These are snpported on the vulcanite rod $q$ in such a way that they follow the movements of the ends of the muscle, so that when it is excited through them it can contract freely. Each end is attached to a weight by a thread which passes over the pulleys $R, r$. $R R$ are provided with pointers $z z$, which record their movements.-After Hering. the sartorius. In the instance given, the current was descending, i.e. direeted towards the tibial tendon; the contraction of the tibial (cathodic) half began 0.034 second after excitation, that of the pelvic (anodic) half 0.045 second.

${ }^{1}$ Engelmann, "Beitr. z. allg. Muskel- und Nerven-physiologie," Arch.f. d. ges. Physiol., Bonn, Bd. iii. S. $316-318$. 
Comparing these time-intervals with the corresponding distances of the cathodes, we have a rate of propagation of from 1 to 2 metres per second. The double myograph can also be used for comparing the character of the two contractions, both myograms being recorded for this purpose on a slowly moving surface. They show (Fig. 246) that the closure of a descending current evokes twitches of about equal vigour in both halves of the muscle, but in the tibial (cathodic) half, the contraction is prolonged until the current is broken, the reason being that, whereas the twitch is rapidly propagated from the tibial to the pelvic end as a wave of contraction, a prolonged contraction occurs in the tibial half which shows but slight, if any, tendency to spread. For the same reason, when the direc-

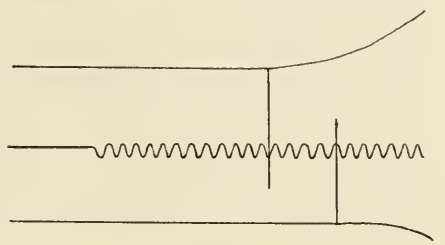

FIG. 245.-After Biedermann. tion of the current is reversed, both halves twitch, but the pelvic end, which before relaxed instantly to its natural length, is now cathodic and behaves accordingly.

If the muscle is injured at one end (by dipping it into hot salt solution), these phenomena are considerably modified. If the cathode is placed on the devitalised part, there is scarcely any response on closing; ${ }^{1}$ both halves twitch slightly. If the injured end is anodic, the responses on closure are the same as they would have been had there been no injury.

One of the facts stated above as to the polar effects of the voltaic current may be confirmed by direct inspection with the aid of a lens of

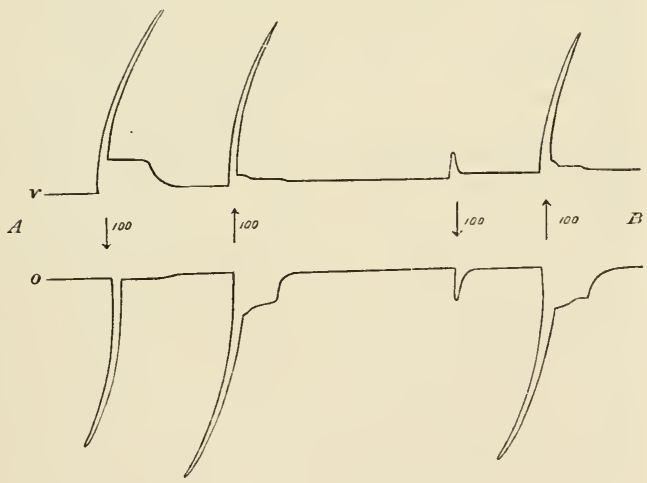

FIG. 246.-Contractions of the two ends of the sartorius, as recorded by the double myograph. $\quad v$ is the reeord of the tibial half, $o$ of the pelvic half of the muscle. Note that the deseending arrows indieate an ascending current. The numbers indicate that the strength of the eurrent was the same throughout. - After Biedermann.

the surface through which the current enters or escapes. It can thus be seen that if a current is led from end to end of a muscle, the cathodic end swells during its passage. Other details may be made out by a method devised by Biedermann-that of streaking the surface with a paste of sepia, rubbed up with 0.6 per cent. salt solution. If the streaks cross the fibres and are equidistant, it is easy to see that at the cathode the streaks are drawn together from each side, and that this effect is a

1 This faet was discovered at the sane time (1880) by Engelmann and Biedermann. See Engelmann, Arch. f. d. ges. Physiol., Bonn, Bd. xxvi. S. 97; and Biedermann, Sitzungsb. d. k. Akad. d. Wissensch., Wien. Bd. lxxx. Abtl. 3, S. 367.

VOL. II. -28 
protracted one which does not spread. A change of an opposite nature has been described at the anode; a slight depression presents itself at the surface of contact, on either side of which the streaks sweep in curves.

The conditions under which the cathodic persistent contraction presents itself have not as yet been sufficiently studied. The utmost that can be said on the subject is, that the appearance of this phenomenon is favoured by any cause which tends to diminish the promptitude with which the muscular elements directly excited respond to the stimulus, as well as the readiness with which the excitatory state is taken up from them by neighbouring elements. The best known of the conditions in question is that of cold. In the sartorius of a frog which has been kept at a temperature a little above that of freezing, as Hermann has shown, the cathodic continuous contraction is very readily produced.

The anodic closing contraction.-It occasionally happens, when a current of considerable strength is led through the sartorius from the tibial to the pelvic end, that a contraction appears at the anode simultaneously with that at the cathode. When the pelvic end is injured, so as to annul the cathodic effect, this contraction is more obvious, and has been recorded graphically by Biedermann. By careful observation with the lens, Biedermanu has satisfied himself that the seat of contraction is a part of the muscle between the anode and the middle, and that in fact the fibres at the anode itself are relaxed. A similar appearance presents itself when the current is led in by the anode only, the cathode being at a distance. Biedermann no doubt rightly attributes this phenomenon to the passing of threads of current from one muscular fibre to another. It derives special interest from its connection with a much more striking effect of very strong currents on the sartorius-the galvanic wave. By this is meant that when a very strong current is led through the sartorius, visible waves of contraction follow each other in the direction of the current with a peculiar surging motion. This only happens when the muscle is in a good state and its fibres are relaxed so as to be more or less wavy. Professor Hermann, who has studied the phenomenon minutely, has no doubt that this, like the anodic closing contraction just referred to, is due to want of conformity between the threads of current and the muscular fibres, and that although each wave starts from near the anode, its origin at any point in a muscular fibre is in reality cathodic, for it is determiner by the passing out of the fibre of a thread of current.

Influence of currents led through muscular fibres transierscly.-If, as appears from the facts we have had before us, the actions of the two poles are antagonistic to each other, so that the exciting effect of the cathode is "inhibited" by the quelling effect of the anode, we should expect to find that neither of these effects could be produced on any muscular element, unless the direction of the current either coincided with that of the axis of the element or crossed it obliquely. For, if it crossed it transversely, the action of the two poles would neutralise one another. In other words, we should expect that if the current threads were parallel to each other, and the fibres of the muscle were also parallel, a current led across a muscle would have no effect whatever. The difficulty of testing this anticipation by experiment is so great, that there has been considerable difference of statement with reference to it. 
The method by which the question has now been settled is as follows: ${ }^{1}$ -

An oblong trough is divided by upright partitions of plaster of Paris into three chambers, of which the middle one is square, the two end ones being narrow but of the same length as one side of the square. The middle chamber contains physiological salt solution, each of the end ones saturated solution of zinc sulphate. Into these last are plunged amalgamated zinc plates, in which the battery circuit ends. The current flows in nearly parallel threads through the salt solution from one porous partition to the other. The muscle is prepared with its bony attachments intact, and supported independently of the trough, and is so held that it is immersed in the salt solution with its fibres horizontal. As the trough can be freely rotated, without moving the muscle, the threads of current may be made to cross the fibres at any desired angle.

It can thus be proved that the excitatory response to closure of the current is strongest when the direction of the fibres is the same as that of the current; that it is much weaker when there is a difference between them of $45^{\circ}$, and is annulled when they cross at right angles. In muscles with injured ends no effect is produced unless the strength of the current is excessive; whence it may be inferred that the ends of muscular fibres are much more susceptible to this kind of stimulation than the other parts. This fact is analogous to that referred to above - the refusal of an injured muscle to respond to cathodic excitation, when the cathode is on the injured part.

From the preceding facts we have learned that the exciting effects of the current on curarised muscle are chiefly of two kinds, those which are evoked by its sudden closure, and those which are dependent on its continuance.

The first are of the nature of the excitatory responses which (p. 414) have been attributed to instantaneous stimulation. These are (1) the excitation wave with its concomitant electrical change, and (2) the wave of contraction. The intimate relation between these is shown by the fact that each is propagated in the direction of the fibres at the same velocity, and that both affect each muscular element for a strictly limited period of exceedingly brief duration. The dependence of rapidly propagated effects (Reizwellen and Contractionswellen) on the suddenness with which the change which produces them is accomplished, has been studied with great exactness in nerve. With reference to it, du BoisReymond enunciated many years ago his well-known "Law of Excitation,"2 according to which the excitatory efficiency at any moment of a current led along a nerve depends on "the rate of change of the current density" (i.e., of the relation between current strength and sectional area). There are no experimental data which would justify the extension of du Bois-Reymond's law to the case of curarised muscle. It is, however, so evident that in muscle as in nerve, rapidly propagated excitatory effects are exclusively produced by sudden changes of current strength, that we may fairly assume the relation in muscle between rate of change and these characteristic effects, to be analogous to that which is expressed as regards nerve in du Bois-Reymond's law. This being admitted, there is little difficulty in understanding why excitatory effects of the kind in

${ }^{1}$ Leicher, "Ueber den Einfluss des Durchströmungswinkels, etc.," Untersuch. a. d. physiol. Inst. d. Univ. Halle, 1888, S. 1.

2 "Untersuchungen," 1848 , Bd. i. S. 258. 
question are chiefly polar; for it is at the anode and cathode that the changes of current density are most abrupt. This consideration, however, affords us no explanation of the other fact of which we have such convincing proofs, namely, that in muscle as in nerve, excitation waves start from the cathode at the moment of closure, from the anode at the moment of opening of the current. As regards nerve, the fact just stated has been expressed by Pflüger in the form of a law as well known as that of du Bois-Reymond, according to which it is an essential condition of the efficiency of a polar change, not only that it should be abrupt, but that it should be towards cathelectrotonus or from anelectrotonus-neaning by these terms the condition or state which exists at the cathode or anode respectively-in other words, from the normal to the cathodic state, or from the anodic to the normal. It is obvious that the changes in the opposite direction, namely, from normal to anodic at the anode on closure, and from cathodic to normal on opening, although they are no less abrupt, have no exciting effect-a fact which will be more advantageously dealt with in connection with the discussion of the corresponding phenomena in nerve.

As regards the excitatory effects of the second kind, those which are dependent on the duration of the current, nothing further need be added. Attention has, however, not been drawn to the remarkable difference which presents itself in this respect between nerve and muscle. Physiologists are so little inclined to admit that a nerve is continuously excited by the flow along it of a current, that the doctrine of du Bois-Reymond, that so long as a current is of constant strength it has no exciting effect, has the authority of a "law"; whereas in muscle the contrary has been familiar to us ever since "Wundt's curves" have been copied into every text-book. At first sight the contrast appears very striking. More careful consideration leads us, however, to surmise that the difference may be more phenomenal than essential. In muscle, contraction is the sign of the cathodic state; in nerve, increased excitability. In the former it is transitory, soon giving way to "exhaustion." In nerve, which is incapable of fatigue, it is persistent. These differences seem, however, to afford no ground for doubting that the underlying process is the same in the two cases.

Secondary muscular and neuro-muscular electrical excitation.-More than half a century ago, du Bois-Reymond ${ }^{1}$ proved by experiments, in which conductors of different kinds were interposed between the exciting muscle and the excited nerve of the physiological rheoscope, that the change in the former which evokes the response of the latter, is electrical. In the ordinary form of the experiment it is accordingly found that the secondary neuro-muscular response is best observed when the parts bridged over by the nerve are, either in the unexcited or in the excited state, at considerably different potentials. Experience, however, shows that if both preparations are in good condition, the rheoscopic limb reacts even when the position of the nerve is apparently very unfavourable-as, e.g., when it is applied to the sartorius in such a way as to cross the fibres transversely. ${ }^{2}$ The best results are, as might be expected, obtained when winter frogs are used, which have been kept at a temperature approaching that of freezing. In this case, if the primary muscle is rhythmically excited

1 Loc. cit., Bd. ii. S. 87 .

2 Biedermann, "Elektrophysiologie," S. 355. 
through its nerve at intervals, say of $\frac{1}{5}$ second, the secondary twitches are exact counterparts of the primary; and if the frequency is sufficiently increased, tetanic contractions are obtained which cannot be distinguished from primary ones. When the same experiment is made after the primary muscle has become exhausted, it often happens that the secondary muscle responds to tetanus of the primary by a single initial twitch, in which case the normal result may be expected to reappear if the muscle is allowed to rest.

It is not by any means essential that the primary excitation should be indirect. A curarised sartorius, whether excited by a single induction current, or tetanised in the same way, evokes corresponding neuromuscular responses; but this effect, namely, the secondary twitch in response to direct excitation of the primary muscle, is best obtained when a battery current is led for a few millimetres along the fibres of the uninjured muscle to a terminal cathode. ${ }^{1}$

The secondary neuro-muscular response has long been recognised as a means of distinguishing different kinds of continuous contraction. Its value for this purpose can be best judged of by referring to the instances in which it fails :-

1. No continuous contraction of central origin, whether cerebral or spinal, has as yet been observed to evoke a secondary neuro-muscular response of the same character as the primary, with the striking exception that the reflex muscular contractions of frogs poisoned by strychnine induce in the physiological rheoscope neuro-muscular contractions which have a strong superficial resemblance to tetanus. When they are recorded graphically, they are seen to consist of a series of twitches, following each other with a frequency of about eight or ten per second, each of which corresponds to the beginning of one of the series of spasms which constitute the primary response. ${ }^{2}$

2. Secondary neuro-muscular spasm is, as a rule, not produced by any of the prolonged contractions which are evoked by continuous stimulation, whether direct or indirect. As examples may be mentioned the closing continuous contraction by indirect excitation (Ritter's tetanus); ${ }^{3}$ the spasm consequent on partial drying of a nerve; or that produced by the action of common salt thereon. ${ }^{4}$ There is, however, an exception to this general statement of such importance that it must be specially considered.

Some years ago, the fact that a muscle can be excited directly by its own current, or by that of a beating heart of the tortoise, suggested to Kühne the inquiry whether an excited muscle could be made to communicate its state of excitation directly to another muscle at rest, if the two were brought into relation with each other in a suitable manner. The result of this investigation was the discovery that, although the mere contact of an excited muscle with an unexcited produces no effect whatever in the latter, it is different if contact is accompanied by pressure, and particularly that if the ends of two sartorius muscles are squeezed together in such a degree as not seriously to injure the compressed parts, the two muscles so "welded" together respond to the excitation of either as if they were structurally united.

${ }^{1}$ Biedermann, loc, cit.

2 Kühne, Untersuch. a. d. physiol. Inst. d. Univ. Heidelberg, 1880, Bd. iii. S. 59.

3 Friedrich, Sitzungsb. d. k. Akad. d. Wissensch.. Wien, 1875, Bd. Ixxii. Abth. 3, S. 413 .

+ Kühne, S. 61. 
But this union is not the only, or even the most important way in which the physiological action of compression manifests itself. A muscle of which one end is squeezed, passes in consequence into such a state of abnormal excitability, that a stimulus which would ordinarily evoke a single twitch is followed by a persistent tetanus-like spasm. So long as this spasm lasts, the muscle acts on the physiological rheoscope with the greatest energy, producing secondary contractions which, when they are recorded graphically, seem to consist of an arhythmical series of unequal twitches. Kühne explains this effect as produced by a kind of electrical storm in the compressed muscle, and attributes to the intensity of the electromotive changes of which it is the seat so long as the spasm lasts, the extraordinary energy with which it acts on the rheoscopic limb. ${ }^{1}$

A state of altered excitability similar to that which is produced by compression, manifests itself in muscles which have been subjected to partial dessication, e.g. in the limbs of frogs, which, after having been stripped, are left exposed. In limbs so prepared, slight mechanical irritation produces spasm which spreads not only to the whole of the muscle irritated, but to its neighbours. Biedermann has also found that if two muscles, of which the surfaces have been allowed to dry, are applied without pressure to each other, the spasm of the muscle primarily excited is faithfully imitated by the other; and, further, that muscles so acted on have the same power of exciting secondary neuromuscular spasm in the rheoscopic limb as compressed muscles. The analogy thus indicated between the two effects has led both Biedermann and Kuihne to believe that the abnomal excitability is attributable in the two cases to the same cause-privation of water. In the one case water is squeezed out of the compressed part, which thereby becomes in the excited muscle a focus from which irregular waves of excitation emanate; in the other, the surface fibres which have lost water by evaporation play the same part.

Voltaic currents of brief duration - Induction currents. When a current is closed for a very small fraction of a second, e.g. $\frac{1}{100}$, through a curarised muscle, those characters of the response which are dependent on its duration disappear. The effect produced is that of a single instantaneous excitation, the seat of which is at the cathode. An excitation wave, with its accompanying contraction wave, starts from the cathode in either direction. If the current is of sufficient strength, a closure of 0.005 seconds evokes a maximal response. If the closure is shortened, the height of the resulting twitch diminishes with the duration. This can be best shown by a method devised by Fick many years ago. The instrument ${ }^{2}$ used for the purpose is such that a brass tongue, springing from a revolving horizontal bar, rubs against an insulated triangular brass plate in its revolution, the brass plate and the tongue being in circuit. The time of contact can be varied (1) by varying the rate of rotation, and (2) by adjusting the plate so that the tongue rubs over a greater or less extent of its surface. Experiments made on Fick's plan show that even when the closure is so short that the anodic excitation is indistinguishable, the excitatory process may yet be of a measurable duration.

1 Loc. cit., Bd. xxvi. S. 222.

2 A full description of the instrument will be found in Fick's." Medizinische Physik," 1866 , S. 425 . 
All that has been said on the mode of action of battery currents of short closure applies to induction currents. The induction current which is produced in the secondary circuit of a du Bois-Reymond's inductorium at closure of the primary circuit, evokes an excitation which has its origin exclusively at the cathode. It has been long known that nerveless muscles react relatively nore readily to closure of the voltaic current than to the induction current. This is in accordance with the observation, that in muscles of which the motor nerves have been severed for a sufficient time to produce complete degeneration, evell strong induction shocks evoke no contraction, notwithstanding that they respond readily to battery currents of moderate strength.

In connection with these facts, it may be noted that in unstriped muscles a relation presents itself between duration of closure and response, similar to that in Fick's experiment. The two cases are, however, scarcely comparable. In striped muscle we are concerned with thousandths of a second, in smooth muscle with durations of several seconds; and that the difference is not one of degree only, but of kind, is probable, for, as we have already seen, there is in striped muscle a second kind of effect introduced when currents of long duration are used. In Engelmamn's observations on the ureter of the rabbit, it was found that even with very strong currents a closure of a quarter of a second was required to evoke a response; and that, if the strength of the current was diminished, it was necessary to close it for a still longer time. Similarly, Biedermann could not obtain a full contraction of the adductor of Anodonta with a shorter closure than one of four seconds. Shorter closures evoked responses, but of much smaller amount.

\section{CARDIAC MUSCLE.}

In the preceding account of the physiological properties of striped muscular fibre, only occasional reference has been made to that form of it which exists in the heart.

The physiological properties of cardiac muscular tissue may be discussed in the same way as those of skeletal muscle. It responds similarly to an instantaneous stimulus, and can be excited to contraction either directly or indirectly. Further, the response (of the ventricle) to indirect stimulation (i.e. of the auricle) cannot be distinguished from the beat evoked by the natural stimulus, arising at and propagated from the venous orifices. The phenomena of the beat may therefore be studied in the same order as those of a single muscular contraction. As, however, we possess but little information as to the chemical and thermal changes which in the heart are associated with activity, the present subject need only be discussed here under the following headings :-

1. The mechanical phenomena of the response;

2. The electrical phenomena of the response; and

3. The physiological differences between cardiac excitability and that of ordinary muscle.

\section{The Mechanical Response.}

For reasons similar to those previously given in relation to skeletal muscle, the systole of the ventricle of the frog's heart may be taken to represent the normal action of cardiac muscle. The most striking 
phenomenon of the systole of this organ is the apparent diminution of its volume. As in the case of skeletal muscle, this diminution is only apparent. Each of the individual muscular fibres of which the organ is made up, occupies approximately the same space in systole as it did in diastole; it is its shape that has altered. All the fibres have widened and shortened, and, in consequence, the blood which previously occupied the central cavity and the innumerable channels which, in the frog's ventricle, extend therefrom almost to the surface, has been squeezed out. If the expulsion of the blood takes place freely, each fibre assumes its new form with very inconsiderable production of tension. If it is so resisted that no blood can escape, a state of effort comes into existence, in which each fibre sooner or later participates. During the very brief period occupied by the spread of the contraction in the cardiac tissue (about $\frac{1}{20}$ second), there may be a change of form due to the action of the

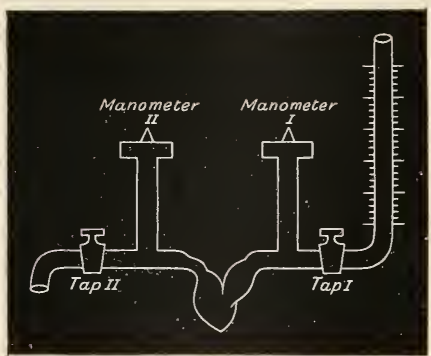

Fig. 247.-Diagram of Frank's apparatus for recording isometric curve of frog's ventricle with different initial impletions. Tap $\mathrm{I}$. is closed and the heart allowed to empty itself. Then Tap II. is closed and tracings are taken with Manometer II. with no impletion, and then with gradually inereasing impletion-a known quantity of circulating fluid being let in from the graduated tube on the right between each two successive observations.-Frank. excited fibres on those which are as yet relaxed, but it may be considered certain that all eventually occupy the same space as they did in diastole. In the case just described the effort is isometric. It corresponds to the isometric response of skeletal muscle. If the resistance is due to a constant lateral pressure exercised by the blood against the wall of the ventricle, which the ventricle is able to overcome, the organ contracts under isotonic conditions.

The isotonic contraction of the ventricle is best observed by a plethysmograph, ${ }^{1}$ the changes of volume being measured by a pistonrecorder, or by Marey's tambour, ${ }^{2}$ or they may be recorded photographically. The curve so obtained corresponds, as has been shown by Otto Frank ${ }^{3}$ in a series of important researches, to the ordinary isotonic myogram, and is modified by mechanical conditions, in ways already studied in relation to skeletal muscle.

The form of the isometric curve of the ventricle is shown in Fig. 248, $\alpha$. It is obtained by arranging the heart as shown in the diagram (Fig. 247). As in ordinary muscle, the height of the curve, i.e. the maximum pressure attained during the response, varies with the length of the muscular fibres when relaxed, i.e. with the previous fulness of the ventricle. In a good-sized ventricle, if the initial content is very small, this maximum is correspondingly inconsiderable, but when the initial capacity is increased, say to a third of a cubic centimetre, the maximum may attain to over $60 \mathrm{~mm}$. Beyond some such point as this, Frank

${ }^{1}$ Sehäfer, Journ. Physiol., Cambridge and London, vol. v. p. $130 . \quad 2$ Ibid. p. 127.

${ }^{3}$ Frank, "Zur Dynamik des Herzmuskels," Ztschr.f. Biol., 1895, Bd. xxxii. S. 370, and "Die Wirkung von Digitalis auf das Herz,"Sitzungsb. d. Gesellsch. f. Morphol. u. Physiol. in München, Heft 2, S. 30. 
found that no further increase of intraventricular pressure could be obtained, so that there appeared to be a certain initial impletion which was most advantageous, and which could neither be exceeded nor reduced without loss of effect. In skeletal muscle we measure the "absolute force" by estimating the relation between the sectional area of the contracting fibres and the weight which the muscle can just lift. The same mode of estimation cannot be applied to the heart, because the area of the cardiac fibres concerned in systole cannot be measured.

It is probable that the product of the number and the mean sectional areas of the fibres in the ventricle may be considered as proportional to its size, so that we may express the absolute force of the ventricle correctly by the maximum pressure which it is able to produce when contracting against resistance. This can be easily got at by recording the isometric curve in the way above indicated, in a series of experiments in which the initial impletion of the ventricle is increased after each observation, until the point is reached at which the maximum pressure in question is attained. The observations can be made so quickly that the results can be verified by repetition, without risk of exhausting the organ (l.e. p. 386).

The effect of after-loading.-It is not within the scope of this article to deal with the mechanical relations of the circulation. It must suffice to indicate the application of elementary principles derived from the investigation of ordinary striped muscle, to the ventricle doing its normal work. As we have already seen, the action of the ventricle when contracting automatically does not differ in any respect from its response to stimulation of the auricle by an induction shock. Both are comparable to the response of a muscle when made to contract under corresponding mechanical conditions. In the method of after-loading a skeletal muscle, which is illustrated in Fig. 198, p. 368, we have seen that the mechanical response to a single induction shock is divisible into three periods, during the first and third of which the muscle is under isometric conditions, while during the intervening period it does its work isotonically. The normal action of the ventricle is, as Frank shows, in a similar manner divisible into three stages-those of distension, expulsion, and relaxation, the first being separated from the second by the opening of the aortic valve, the second from the third by its closure. During the first period, contractile stress rapidly increases; during the third, it more gradually diminishes. During the intervening stage, that of expulsion, the relation between change of tension and change of volume differs according to the lateral pressure, this being determined by the resistance in front and the initial change. The effect of increasing the resistance in front, in the case of the ventricle, corresponds to that of increasing the load in the case of muscle. Empirical evidence of this correspondence was oltained by Frank by comparing the curves of intraventricular pressure in a series of experiments, in which the resistance in front was successively increased by equal increments. In skeletal muscle the effects of increasing the after-load are to defer the beginning of the isotonic period, to shorten its duration, to diminish the velocity of the upward movement of the free end of the muscle, and consequently to diminish the lift. In the ventricle, the corresponding results of increasing the resistance in front are to defer the beginning of the period of expulsion, i.e. the opening of the aortic valve, to shorten the period betwecn opening and closure of the valve, and to diminish the rate of flow through the aortic 
orifice, and consequently to diminish the systolic discharge. Stated numerically, the result of such experiments on the frog's ventricle is that when the resistance increases from a pressure of, in round numbers, $10 \mathrm{~mm}$. to $40 \mathrm{~mm}$. of mercury, the flow decreases from 0.6 c.c. per second to 0.2 c.c. per second, and the period of expulsion diminishes from 0.56 second to 0.51 second, the systolic discharge consequently diminishing from 0.33 c.c. to 0.08 c.c. As throughout the series of experiments on which this statement is founded the initial impletion of the ventricle was the same, it is obvious that the quantity of fluid which remains when the valve closes, i.e. when the flow through the aortic orifice ceases, increases with the diminution of the systolic discharge.

In the experiments just referred to, the element of elasticity is, for the sake of simplicity, excluded. It is easy to modify the apparatus used so as to include it. The effect of doing this is, that the attainment of the maximum of intraventricular pressure is postponed, and that, in connection therewith, the notch produced by the back-flow from the aorta at the moment that the valve closes is accentuated.

The preceding considerations may be summarised as follows:-(1) The isometric curve of muscle is represented by the pressure curve of the systole
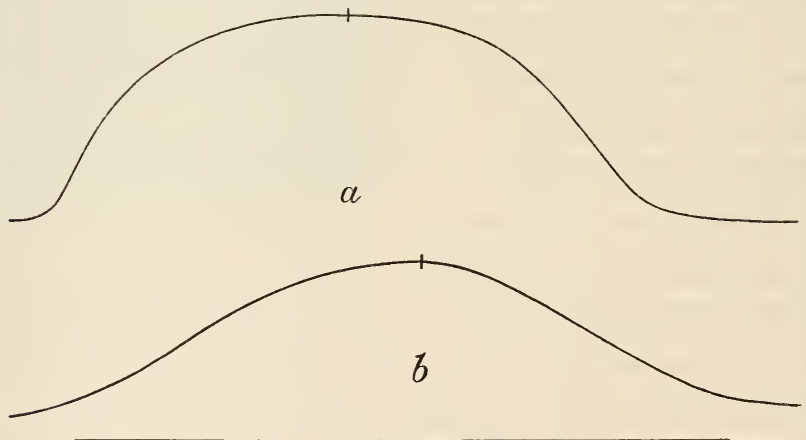

Two seconds.

FIG. 248.-Isometric and isotonic curves of the ventricle of the leart of the frog (given to the writer by Dr. O. Frank). The rate of movement of the recording surface is indicated by the horizontal line. $a$, The isometric eurve is the record of a membrane manometer (Frank's) in connection with the cavity of the ventricle, of which the impletion was constant. $b$, The isotonic curve is the record of a Marey's capsule in connection with a plethysmographic chamber containing the heart. In the cavity of the ventricle the lateral pressure was constant, and amounted to $10 \mathrm{~cm}$. of water.

of the charged ventricle with arrested discharge. (2) The isotonic curve of muscle is represented by the volume curve of the ventricle; the load, by the resistance in front as measured by the aortic pressure. (3) As in muscle the isometric curve culminates before the isotonic, so in the ventricle the isometric pressure curve culminates sooner than the isotonic volume curve (Fig. 248). The pressure curves and volume curves recorded in a ventricle working under conditions which exist in the circulation, resemble in general the after-loading curves of muscle. If the arterial pressure during the discharge is constant, the resemblance is complete. The want of this constancy in the circulation is dependent on conditions which lie outside our present inquiry. 
Certain points in respect of which cardiac and skeletal muscle seem to differ, will be referred to in the concluding paragraph of this section. In the meantime we are justified by the analogies indicated above relating to the mechanical phenomena of the response, in stating that, for their elucidation, cardiac muscle is quite as available as skeletal. There are, indeed, several reasons why the former ean be referred to with greater advantage; the long duration of the ventricular systole, and the facility with which the organ can be preserved in a normal state after removal from the animal, being conditions extremely favourable to exact observation. If, for example, the theory that is now entertained by many physiologists, that the character of the second part of a muscular contraction is affected by the stimulating influence of resistance or other conditions present during the first part, we should expect to be able to observe this more readily in the deliberate systole of the heart than in the almost instantaneous twitch of a muscle. So far as I am aware, no suggestion of the kind has been offered.

\section{Rate of Propagation.}

It was discovered by Engelmann in $1875,{ }^{1}$ that the change by which each muscular element of the ventricle passes from the unexcited state into that of excitation is propagated from element to element with equal facility in every direction. This he showed to be the case by the following experiment:--The ventricle of a freshly-killed frog is separated into a number of small pieces, united to one another by bridges of muscular substance not more than a millimetre wide, and is left for half an hour or so in a moist chamber, in order that the living tissue may recover from the immediate effects of the injury. It is then found that a contraction wave proceeds with equal facility in any direction, from any portion which has been stimulated. In a ventricle so injured, the rate of conduction was not more than $20 \mathrm{~mm}$. per second; but Engelmann recognised that in uninjured tissue the rate must be much greater. By recently published experiments, he has now shown that, when the circulation is going on under normal conditions, it may be more than $150 \mathrm{~mm}$. per second in the auricle, and rather less than this in the ventricle. ${ }^{2}$

In other experiments with the "suspended" heart, in which the organ was removed from the body, the rate was nuch less. By removing it, however, Engelmann was able to show that cardiac muscle deprived of its power of visibly contracting by immersing it in 0.2 per cent. $\mathrm{NaCl}$, still retained its power of propagating the excitatory state.

\section{The Electrical Phenomena of the Response.}

Historical.-In 1856, Kölliker and H. Miiller ${ }^{3}$ made the discovery that the "negative variation" can be observed in the beating heart. They applied the nerve of a rheoscopic limb to the pulsating ventricle, and found that whether the nerve was laid across the ventricle, or in the direction of its length, the muscle of the preparation contracted a "scarcely preceptible time before the systole." In certain cases a second feebler twitch was observed in the beginning of the liastole. This they considered to be dependent on a "positive variation" of the

\footnotetext{
${ }^{1}$ Engelmann, Arch. f. d. ges. Physiol., Bonn, Bd. xi. S. 465.

2 Ibid., 1894, Bd. lvi. S. 149.

3 Verhandl. d. phys.-med. Gescllsch, in Wiirzbury, Bd. vi. S. 528.
} 
muscle current of the ventricle, coincident with relaxation. Subsequently (1872), Donders ${ }^{1}$ confirmed these observations, and, by skilful application of the graphic method, determined the time relations of the phenomena. The subject was next taken up by Engelmann ${ }^{2}$ in 1873 , who then established that, when at rest, "the surface of the uninjured heart exhibits no difference of electric tension" (i.e. is equipotential), and by means of the galvanometer and rheotome, investigated the duration and time-relations of the excitatory change. He found that, in general, when the ventricle was excited at a point near to one of the leading-off electrodes and remote from the other, "the nearer of the two became on excitation first negative, then positive to the more remote." As the result was the same whether the contacts were at base and apex, or on opposite sides of the ventricle, it was concluded that every, excited part becomes momentarily "negativelectromotorisch wirksam," and that this negativity "is propagated from the seat of excitation in all directions" 3 (loc. cit., p. 73). Such a diphasic effect was termed by Engelmann a Doppelschwankung. When the negativity of the nearer contact culminated at 0.16 second after excitation, it was succeeded 0.14 second later by relative positivity (positive Schwankung), i.e. negativity of the further contact, and had completely subsided half a second after excitation. Considering that each phase was of very short duration, and that the wave of excitation may reach the second electrode before it has culminated at the first, he concluded that the whole period occupied by the electrical disturbance is not more than $\frac{4}{10}$ of a second.

In $1879 \mathrm{I}$ made, in conjunction with $\mathrm{Mr}$. Page, ${ }^{4}$ an extensive series of experiments, in which the electromotive phenomena of the response were investigated with the galvanometer and rheotome, and also with the electrometer, the photographic method being used for recording (see p. 418). For both series of observations the preparation chiefly used was the "Stannius heart," i.e. one brought into a state of atonic quiescence by a ligature applied between the sinus and the auricle.

The rheotome observations showed that in such a heart, at $15^{\circ} \mathrm{C}$., the variation evoked by excitation of the auricle consists of two phases, separated from one another by a period during which the surface of the ventricle is equipotential. The first lasts about 0.05 second, the equipotential period 1.3 second. The duration of the second phase exceeds that of the first, but its difference of potential is relatively very inconsiderable. The duration of the ventricular systole was recorded graphically at the same time, and found to be about $1 \cdot 1$ second, so that it did not last quite as long as the interval between the two phases. There was no difficulty in explaining the first phase of the variation in accordance with the principles already laid down by Engelmann; but the existence of a second phase, separated from the other by a long period of equipotentiality, was a new fact which neither he nor anyone else excepting Kölliker and Müller had observed, for Engelmann applied the term Doppelschwankung exclusively to the initial period of about a third of a second, during which (in the normal beat) the base becomes first negative, then positive, to the apex. It seemed probable from the

\footnotetext{
${ }^{1}$ Donders, Onderszoch. ged. in h. physiol. Lab. d. Utrecht. Hoogesch., 1872, Bd. i. S. 256.

2 Engelmann, ibid., Bd. iii. S. 806.

3 Arch.f. d. ges. Physiol., Bonn, 1874, Bd. xvii. S. 68.

${ }^{4}$ Journ. Physiol., Cambridge and London, vol. ii. p. 384.
} 
incidence of the terminal phase almost immediately after the end of the systole, that it marked the duration of the electrical change which is a concomitant of functional activity. To ascertain whether this relation was a constant one, a comparison was made of the relative durations of the systole and of the interval in question at different temperatures, an arrangement being adopted by which the rheotome observations could be repeated at temperatures varying from $12^{\circ}$ to $20^{\circ} \mathrm{C}$. It was found, as was anticipated, that the duration of the interval of equipotentiality always a little exceeded that of the systole.

The interpretation applied to these observations eighteen years ago has proved to be correct. As was then indicated, the fact of the existence of a relatively long period of equipotentiality (over 2 seconds in a cooled heart) does not necessarily imply that both contacts are in a state of electrical inactivity, but may mean that the electromotive forces at work at the one electrode happen to balance precisely those that are at work at the other. ${ }^{1}$

Latent period.-For the purpose of determining the earliest moment at which an electrical change can be observed, experiments were made in which the exciting electrodes were placed one on either side of the proximal leading-off electrode. With this mode of stimulation, the first effect was not observed until more than 0.02 sec. after excitation. The period of closure was always 0.03 second. When it terminated 0.02 sec. after excitation, there was no effect, showing that up to that moment there was no response.

The injured ventricle.-Further proof of the long duration of the excitatory electrical change was obtained by a similar mode of observation, the only difference being that the surface under one of the leadingoff electrodes was devitalised. It was already known that in the heart, as in other striped muscle, an injured surface is negative to a sound one, but it was by no means clearly understood that the relative negativity was due, not to any abnormal activity at the injured part, but to unbalanced activity of the living tissue at the opposite electrode. As had already been shown by Engelmann, the resting difference of potential in question (i.e. the relative positivity of the uninjured surface) immediately after the injury is very considerable, amounting to $\frac{2}{100}$ of a Daniell, or more; but it soon subsides. In a heart recently injured at the apex, during the whole period of the systole the sound surface at the base becomes relatively negative to the devitalised part at the apex; so that the previous difference suffers a diminution to about lialf its initial amount.

The meaning of the prolonged negativity of the uninjured surface during systole is not difficult to understand,- that surface, in common with all other uninjured parts of the excited ventricle, being in a state of functional activity, in which the injured part alone does not participate. Its duration, like that of the systole and of the equipotential period, varies with temperature. Inasmuch as the duration of this "negative variation" can be easily measured, it affords the readiest means of proving the important fact, that the excitatory electrical change lasts about the same time as the systole.

Effect of local application of heat on the variation.-Local inequalities of temperature may be produced in two ways, namely, either by placing the heart upon a support of which one part is warmer

$$
{ }^{1} \text { Loc. cit., p. } 398 .
$$


than the other, or by bringing near to the surface which it is desired to warm, a minute coil of platinum wire, heated to any required amount by leading a battery current through it. By the first method it may be shown that a very slight difference of temperature between the apex and base of the ventricle is sufficient to alter the character of the variation. If, with the proximal contact on the former and the distal on the latter, the temperature of the apex is slightly raised, the effect shows itself in the strengthening of the terminal phase, and shortening of the isoelectric interval. The second method has the advantage that it enables us to localise the action of warmth with more exactitude, and to prove that, whether the contacts are on the opposite sides of the ventricle, or at opposite ends, the electrical response of the unwarmed contact to stimulation is always greater than when both contacts are at the same temperature. It is further found that, in the absence of excitation, a warmed surface becomes slightly positive to an unwarmed, the amount of difference of potential, however, not exceeding $\frac{1}{1000}$ of a volt, and soon giving way to an effect in the opposite direction.

The excitatory variation as observed by the capillary electrometer.-Two years later, the rheotome results above described

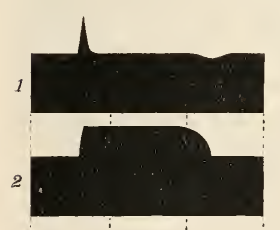
were verified and extended by this method, which was then new. ${ }^{1}$ A large number of photographic records were taken, relating to the ventricle of the heart of the frog and of the tortoise, in which the time-relations of the excitatory variation were fully demonstrated. The character of the results can be best understood by the following series of tracings (Fig. 249), copied from photographs made in August 1882:-

No. 1 represents the normal variation of the

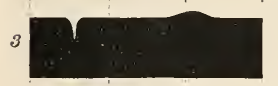
ventricle of a Stannius preparation excited from the auricle, with the proxinal leading-off

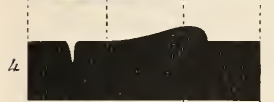
electrode at the base, the distal on the apex. Period of latency, 0.45 sec.; interval between first and second phase, 1.4 sec.

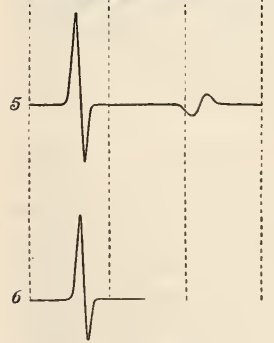

Fig. 249.

No. 2. The same with the apex devitalised by heat.

No. 3. Uninjured ventricle, leading-off electrodes as before; excitation at the apex close to what was before the distal contact.

No. 4. The same; the now proximal contact warmed by a platinum coil placed at a distance of $3 \mathrm{~mm}$. from the surface.

No. 5 shows the actual differences of potential during the two phases of the variation represented in No. 1, as deduced from more recent photographs obtained with a more sensitive instrument. The first phase corresponds, both as regards differences of potential and timerelations, to Engelmann's curve, published in $1878,{ }^{2}$ representing the mean result of thirty-three observations of the variation in the

${ }^{1}$ Sanderson \& Page, Journ. Physiol., Cambridge and London, vol. iv. p. 327.

2 Engelmann, Arch. f. d. ges. Physiol., Bonn, Bd. xvii. S. 68. 
uninjured ventricle, with the leading-off electrodes at a similar distance apart. According to Engelmann's curve (Fig. 1 in his paper), the relation between the differences observed and the time elapsed after excitation was as follows:-

$\begin{array}{cccc}\text { Up to } & 6 & \text { hundredths of a second } \\ \text { At } & 7 & , & , \\ " & 16 & , & " \\ \text { " } & 26 & , & , \\ " & 30 & , & , \\ , & 56 & , & ,\end{array}$
No deflection.
Beginning of variation.
Negative maximum.
Equipotentiality.
Positive maximum.
End of variation.

No. 6 shows the appearance that such a curve would represent if reduced to the same scale as No. 5.

The rate of propagation of the electrical response, which is the sign of the excitatory state, admits of being determined by two methods. The first is founded on the comparison of two measurements, i.e., of the time after excitation at which the first indication of electrical changes are appreciable at the proximal contact, and of the distance of that contact from the seat of excitation. This method was used by Engelmann, and led in his hands to the conclusion that the rate of propagation is less than $50 \mathrm{~mm}$. per second. We were, however, able to show in 1882, that correct observations cannot be obtained by a single comparison of the distance between the seat of excitation and any point in its progress, with the time at which its arrival at that point is indicated, because neither the exact starting-point of the excitation wave, nor the moment at which it starts, can be determined with certainty. This being so, the only satisfactory way of measuring the rate of transmission by this method is to compare the results of two observations, in each of which the wave of excitation starts from the same point, but in the one, the proximal electrode rests on a spot nearer to the starting-point than in the other. The relation between the difference of time at which each of the two points is attained and their distance from each other, accurately represents the rate of propagation. An equally correct method on the same principle is to compare two observations in which, the position of the proximal electrode being unchanged, the exciting electrodes are at varying distances from it. It was thus determined that at $12^{\circ} \mathrm{C}$. the rate of propagation might be as much as $125 \mathrm{~mm}$. per second, but only so long as the ventricle was perfectly fresh (cf. p. 443).

The second method, which consists in measuring the time-interval between the beginning and culmination of the first phase, yields similar results, but it has not been much used. There is, however, no reason to doubt that it is equally reliable.

Electromotive phenomena of the mammalian heart.-In 1892, Bayliss and Starling ${ }^{1}$ investigated the electrical phenomena of the normal beat in the mammalian heart with maintenance of circulation. Their experiments showed that in the ventricle of the dog, when the leading-off electrodes to the electrometer are applied to the base and apex respectively, the excursions of the mercurial column are such as to indicate that "the variation is always diphasic, . . . negativity of the base preceding that at the apex," whether the pericardium be opened or not. They found that difference of temperature and differences of physiological state between the two contacts produce modifications

${ }^{1}$ Internat. Monatschr. f. Anat. u. Physiol., Leipzig, 1892, Bd. ix. Heft 7. 
of the normal variation, which correspond with those described by myself and $\mathrm{Mr}$. Page in the ventricle of the frog. There is, however, one important difference between their results and ours, namely, that in general they observed no interval between the diphasic variation which constitutes Engelmann's Doppelschwankung, and the later movement which marks the end of the contraction. The absence of this interval Bayliss and Starling attribute to the short duration of the beat. Here it must be mentioned that one of these observers (Bayliss, loc. cit., p. 13) has made observations on the heart of the frog and the tortoise, the photographic records of which show that, in his preparations, Engelmann's second phase (see Fig. 249,6) was apparently much stronger than in ours. This relatively unimportant discrepancy cannot be explained without further investigation.

The fact that the electromotive changes which accompany the

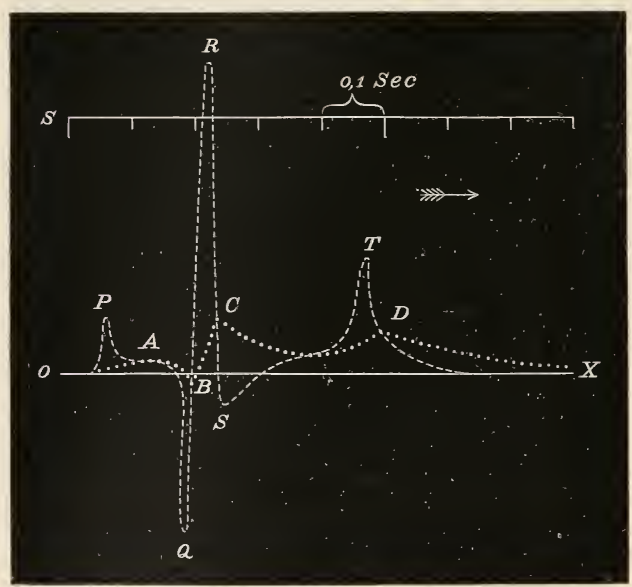

FIG. 250.-Einthoven's Electrocardiogram of man. action of the heart can be observed in man was discovered by Waller some ten years ago. The effect observed varies according to the mode of leading off. This may consist either in placing one electrode on the chest at the seat of impulse, and the other on the right hand, or the one oll the right hand and the other on the left. The former of these methods has been employed by Bayliss and Starling, the latter by Einthoven. The form of the photographic record ${ }^{1}$ indicates that the variation begins with a strongly marked change, in which the base becomes first negative then positive to the apex, followed by a more prolonged movement in the former direction. In Einthoven's experiments the contacts were on the right and left hands respectively. The character of the photographic record is shown in Fig. 250 ( $A B C D X$ ). From it the curve (OPQRSTX) is deduced by a process of analysis, which is essentially the same as that of Burch, and leads to similar results. It may therefore be compared with curve 5 of Fig. 249. Einthoven declines to go further in the physiological interpretation of this curve, than to point out that $R$, which corresponds to the negative phase of Engelmann's Doppclschwankung, denotes the beginuing of the ventricular systole, and that its end is indicated by $T$ (or $D$ ).

${ }^{1}$ Einthoven "Ueher die Form des menschlichen Electroeardiograms," Arch. f. d. ges. Physiol., Bonn, Bd. lx. S. 101. 


\section{Physiological Peculiarities of Cardiac Muscle as COMPARED WiTH SKeletal.}

There are two respects in which the excitatory properties of cardiac muscle appear to differ from those of skeletal, namely-(1) The existence of periodic variations of excitability; (2) the characteristic relation of response to stimulus known as the "all or none" principle, according to which the vigour of the response evoked by a stimulus adequate to produce an effect, is independent of the strength of the stimulus.

1. Periodic variations of excitability.-There can be no doubt that any part of the heart tends to respond rhythmically to a stimulus which acts continuously. The continuous stimulus may be mechanical, e.g., an increase of intracardiac pressure, or it may be electrical. A notable instance of the effect of the former may be seen in the snail's heart, which, when quite quiescent, can be made to beat rhythmically by distending it. The same thing happens in nearly as striking a way in a motionless apex preparation of the ventricle of the heart of the frog, when similarly treated. The rhythmical effect of a continuous electrical stimulus, such as that of a succession of alternating induction currents following one another with very great frequency, can be well seen in a strip of muscle cut from the border of the ventricle of the heart of the tortoise. It is, I think, usual to explain such instances of the apparent transformation of a constant excitatory action into a recurrent one, by saying that cardiac fibres can only respond rhythmically - that they are essentially incapable of tetanus, etc. According to a similar mode of explanation, this rhythmicality is attributed to the liability of the heart to be "refractory" for a certain period after each excitation. Neither of these modes of expression brings us, however, any nearer to an understanding of the phenomenon we have under consideration; they are merely different ways of stating it. There are so many non-physiological examples (such as, e.g., the dropping of water from a narrow aperture under a constant pressure) of the conversion of a continuous action into a rhythmical one, that we need not be surprised at the same thing happening in muscular tissue. The difficulty lies rather in the fact that in skeletal muscle no such conversion appears to take place, than that it occurs in cardiac muscle. Before, however, we admit that it does not occur in the former, we must inquire what form it would assume in such muscle, if it could be observed. In so far as the response to a single stimulation is strictly limited in duration, varying with the muscle eniployed and with temperature, skeletal muscle has also its period. What the period is may be estimated either from the mechanical or from the electrical response to an instantaneous stimulation, but much more easily from the latter than from the former. The monophasic response lasts less than one-fiftieth of a second; so that a "refractory period," if such there be, should, by analogy with the heart, have a similar duration. In the heart the corresponding monophasic variation, at a temperature of $12^{\circ} \mathrm{C}$., lasts about two seconds, so that where cardiac muscle responds once a second, we should expect, under the same conditions, skeletal muscle to respond a hundred times a second. It has not yet been proved, experimentally, that a continuously excited muscle does thrill mechanically at any such rate, but there are indications, not yet investigated, of an electrical thrill of this order of frequency produced by continuous stimulation.

VOL. II. -29 
2. The "all or none" principle.-The second respect in which cardiac muscle differs physiologically from skeletal muscle, namely, its property of responding fully to any stimulus to which it responds at all, whatever the strength of the stimulus, is much easier to explain, inasmuch as it may be accounted for by the structural peculiarities of cardiac muscle. In curarised skeletal muscle there is no communication of the excitatory state from element to element. In the ventricle, such communication is essential to the production of a single contraction or beat. In order that it may happen, the excitatory change produced at the seat of excitation must have a certain intensity, for which a certain strength of stimulus is necessary.

The same "law" with regard to the propagation of the excitatory process prevails, for a similar reason, in the excitable tissues of plants as, e.g., in the leaf of Dionca, where also a stimulus, whether mechanical or electrical, is either fully adequate or else inadequate. If a stimulus applied to one lobe is adequate to evoke a response in the opposite lobe, the latter responds in the same way, whatever be the strength of the stimulus. 


\section{NERVE.}

\section{By Francis Gotch.}

Contents :-Fundamextal Characteristics of Nerve Phexomena.-General Review of Nerve Functions, p. 451-Phenomena of the Excitatory State, p. 453 -Metabolism during Aetivity, p. 453-Conductivity of Nerve, p. 455-Transmission in both Directions, p. 456-The Excitation of Nerve.-Modes of Stimulation, p. 459-Induced Currents, p. 459-Undulatory Currents, p. 461Condenser Discharges, p. 461-Galvanic Currents, p. 462-Unipolar Excitation, p. 463-Chemical Excitation, p. 464-Mechanical Excitation, p. 467--Conditions determining the Efficiency of Stimuli, p. 468-Law of Excitation, p. 469 - Influence of Direction of Electrical Currents, p. 472-Nerve Indefatigability, p. 473-Limits of Excitation by Repeated Stimuli, p. 474-Tне CIRcumstances affecting Nerve Excitability and Conductivity.-Maintenance of Circulation, p. 477-Maintenance of Continuity with Nerve-Cells, p. 478Comparison of Different Nerves and Different Parts of same Nerve, p. 480Alterations of Temperature, p. 484-Chemical Reagents, p. 487-Anasthetics, p. 491-Polarisation Changes, p. 493-Polar Excitation of Nerve by ElecTrical Currents. - Pflüger's Law of Contraction, p. 503-Influence of Duration, etc., p. 505-Causation of Polar Excitation, p. 507-Closing and Opening Tetanus, p. 510-Polar Excitation in Crayfish Nerves, p. 512-In Man, p. 515 -In Peripheral Sense Organs, p. 516-Electromotive Chaxges in Unexcited Nerve.-The Nerve Current, p. 518-Its Production, p. 519-Influence of Various Conditions on its Amount, p. 520-Electro-Motive Changes iN Excited Nerve.-The Negative Variation, p. 524-Its Discontinuous Character, p. 527-Electrical Response to One Stinulus, p. 529-Influence of Various Conditions, p. 533-After-Effects, p. 537-Electromotive Changes associated with Passage of Currents. - External and Internal Polarisation, p. 540-Electrotonus, p. 546-Influence of Physiological Changes upon Electrotonus, p. 550-Excitatory After-Effects, p. 556-Polarisation and Excitation, p. 557 .

\section{The Fundanental Characteristics of Nerve Phenomena.}

General review of nerve functions. - The physiological phenomena. exhibited by nerve may be considered under three different aspects, indicated by the terms "excitability," "conductivity," and the "state of excitation."

Excitability is pre-eminently the attribute of nervous tissue; hence the phenomena of nerve comprised under this expression represent more definitely than those of other excitable structures the conditions which determine its possession, and the circumstances which modify its manifestation. A tissue is said to be excitable if it responds by the development of specific changes to the local action of external change in its immediate environment. The altered condition thus produced is termed "the excitatory state," whilst the external agent which has evoked this is termed the "stimulus" or " exciting cause." 
The excitatory state may be successively assumed by other portions of the excited tissue, and is thus propagated from the point of stimulation to more remote structures; the changes evoked in such structures are the objective sign of the possession by the stimulated nerve of the property of "conductivity."

The special structure of nerve fibres is the undoubted basis for the high development in these of this attribute of "conductivity," the natural and fundamental function of a living nerve, inasmuch as propagation is the purpose which the structure is especially framed to subserve.

It is evidently closely associated with that of excitability, but although the relationship is an intimate one, the experimental investigations of recent years seem to show that the two attributes, "conductivity" and "excitability," must be regarded as more or less distinct, since conditions may modify the manifestations of the one without affecting those of the other.

There is no doubt not only that an axis cylinder transmits in both directions the excitatory state evoked by the stimulus, but that it transmits this state along all its branches.

The isolation of the excitatory nerve change just referred to is an expression for the familiar fact that, when a particular group of nerve fibres is excited, the excitatory state thus evoked is propagated along the excited fibres and their loranches, and along these only. The definite character of the experimental results observed in the excitation of efferent nerves, whether medullated or non-medullated, is due to this definite restriction of the path along which the propagation of the change occurs. This localisation, although so familiar as to be generally accepted without criticism, is by no means easily explained.

It has been suggested that the sheaths, and in particular the medullated sheath, may play an important part in securing adequate insulation, but although it is not improbable that some such rôle may be attributed to them, the fact of the insulation being present where such sheaths fail (in non-medullated nerves) forbids the assumption that their presence is essential for its attainment. It must be regarded as the property of the axis cylinder itself, and the sheaths, if they play any such part, can only afford additional security.

Although it appears that axis cylinder continuity is a sufficient guarantee that the change, which is successively assumed by its various portions, shall be localised in the nerve fibre in which it started, it is evident that where such continuity ceases the isolation of the propagation also comes to an end, and that conduction will now become diffuse. This is the case at the distal and central terminal branches. In the case of the distal terminations, if these are motor, the specific character of the ending, and its restriction to special muscle fibres, renders the subsequent events still precise in their locality; but, as regards the central terminations, spread must take place, and the subsequent propagation appears to be determined very largely by the extent of such spread and the proximity of other terminal branches. Thus the excitatory state must be assumed to leave its axis cylinder path where this ends, and, crossing an intermediate region, to enter another path, preferably that in more immediate contiguity. This passage from one 
fibre to another is associated with very special characteristics, which will be referred to later in considering the excitatory changes present in nerve-endings. It is mentioned here, in order to guard against the inference that the functional isolation now under consideration is all absolute one; it is evident that it is only relative isolation; wherever the axis cylinder path exists, the propagation is restricted to it, when it ceases a passage can be forced through other routes.

Phenomena of the excitatory state.-The excitatory state evoked by a stimulus manifests itself in nerve fibre by electromotive changes, and, as far as our present knowledge goes, by these only. This state may spread to successive portions of the nerve beyond the region subjected to the action of the stimulus, the spread being accompanied by similar electromotive changes. All theories as to the nature of the hidden events which take place in the living tissue, and constitute this excitatory state, must embrace these demonstrable facts.

It is further demonstrable that any alteration in the nerve, evoked by the operation of an external agent, is succeeded on the removal of the producing cause by a reversed state. Just as, in a muscle, relaxation succeeds contraction, and the whole cycle constitutes the response, so, in a nerve, the excitatory state is in fact, as in conception, composed of changes in one direction, succeeded after a very brief interval by others of opposite character.

The conception of such an excitable living tissue as nerve, implies that of a molecular state which is in stable equilibrium; this equilibrium can be readily upset by an external agency, the stimulus, but the term "stable" expresses the fact that a change in any direction must be succeeded by one of opposite character, this being the return of the living structure to its previous state. Thus the electrical manifestation of the excitatory state is one whose duration depends upon the time during which the external agent is able to upset and retain in a new poise the living equilibrium, and if this is extremely brief, then the recoil of the tissue causes such manifestation to be itself of very short duration. Whether our conception of the actual physico-chemical conditions of this living equilibrium, whose disturbance is excitation, be mechanical (du Bois-Reymond, Pflüger), chemical (Hering, Biedermann), electrochemical (Bernstein, Hermann, Boruttau), the above statement is equally true. No departure from the normal in consequence of excitation takes place without being succeeded by the opposite condition. Throughout the whole of the phenomena of nerve this consideration must be always kept in view. Such well-known experimental investigations as those of the polar changes in excitability are frained for its direct demonstration, and there is little likelihood in these of its being forgotten, but in others, in which its determination is not the direct object of inquiry, it is apt to be passed over. It is for this reason that this fundamental conception is introduced at this early stage in the consideration of nerve phenomena.

Metabolism during nerve activity.-During the activity of a muscle, changes of four different kinds are revealed-mechanical, electrical, chemical, and thermal. In nerve the electrical changes are readily demonstrable, but the chemical ones, upon which these are presumably based, are extremely slight, whilst any thermal effects, in consequence of such chemical processes, have not hitherto been ascer- 
tained. The negative character of the evidence of metabolism is an important circumstance in connection with the rationale of nerve phenomena, and such positive data as exist support the conclusion that nerre metabolism must be very small in amount.

Waller considers the close agreement between the effect upon the functional changes of external $\mathrm{CO}_{2}$, and of previous nerve activity, to be an indication that $\mathrm{CO}_{2}$ is produced during the active state. ${ }^{1}$ Funke found that nerve fibres had an acid reaction after prolonged activity, such as that caused by strychnia, although alkaline under normal conditions: ${ }^{2}$ the statement has, however, been contradicted by other observers. ${ }^{3}$ It is agreed that destruction by heating to $50^{\circ} \mathrm{C}$., mechanical pressure, etc., causes the nerve to have this acid reaction. The electrical organ of fish is a nervous structure, being a nerve-ending of a most extensive kind. In the resting state it is faintly alkaline in the uninjured living fish, but after excision it has been observed to be neutral or faintly acid, and it becomes acid when heated to $50^{\circ} \mathrm{C} .{ }^{4} \mathrm{By}$ means of intravital injection of acid fuchsine, a dye which is blanched by the normal alkalinity of the tissues, it has been found that the two organs of a strychninised torpedo differ in appearance, if the nerves of one had been divided before the injection. The active-nerved organ was seen to be red, whilst the paralysed one was blanched. ${ }^{5}$ This method was found by Dreser to give marked results in muscle; it gave no definite result in nerve. It appears, therefore, that the evidence of acidity during nerve activity is scanty and uncertain. Finally, the blood supply of nerve is too limited to admit of any pronounced metabolism, and nerve fibres appear to be incapable of fatigue in consequence of their activity.

There is the same want of evidence of any thermal change during nerve activity. Such change was described by Schiff, ${ }^{6}$ but the most delicate methods had failed to reveal its development in the hands of other observers. ${ }^{\top}$ A careful investigation by Rolleston, with the exceedingly sensitive method of Callander, confirmed the absence of any appreciable thermal effect. ${ }^{8}$ On the other hand, post mortem changes, such as are produced by chemical reagents or heat, always cause an appreciable development of heat. A development of heat during the activity of the cerebral hemispheres has been observed by Mosso, ${ }^{9}$ whose extensive investigations along this line have been taken as proving the localisation of such thermal changes in the central nerve substance. The validity of this conclusion has, however, been disputed; moreover, the results are opposed by the observation that the production of $\mathrm{CO}_{2}$ during cerebral activity is very slightly, if at all, augmented. ${ }^{10}$ From these data it is evident that metabolism during activity is either

1 Waller, "Croonian Lecture," Phil. Trans., London, 1896.

2 Arch. f. Anat., Physiol. u. wissensch. Ired., 1859, S. 835 ; Centralbl. f. d. med. Wissensch., Berlin, 1869.

${ }^{3}$ Heidenhain, Centralbl. f. d. med. Wissensch., Berlin, 1868.

+ Boll, Arch. f. Physiol., Leipzig, 1873, S. 99; Weyl, ibid., 1883, Suppl., S. 109 ; Marcuse, "Inaug. Diss.," Breslau, 1891.

${ }^{5}$ Röhmann, Arch. f. Physiol., Leipzig, 1893, S. 423.

${ }^{6}$ Arch. f. d. ges. Physiol., Bonn, 1871, Bd. iv. S. 230.

${ }^{7}$ Helmholtz, Arch. f. Anat., Physiol. u. wissensch. Med., 1848, S. 158 ; Heidenhain, Stud. d. physiol. Inst. zu Breslau, 1868, Bd. iv. S. 250.

${ }^{8}$ Journ. Physiol., Cambridge and London, 1890, vol. xi. p. 208.

9 Ifosso, Proc. Poy. Soc. London, 1892, p. 83; "Die Temp. des Gehirns," Leipzig, 1894.

${ }^{10}$ Hill and Naharro, Journ. Physiol., Cambridge and London, 1895, vol. xviii. p. 227. 
extremely small, or is such that any chemical dissociation is rapidly compensated for by subsequent association.

The conductivity of nerve - Rate of transmission. - The propagation of the excitatory state along the nerve fibre, from the seat of excitation, is probably the most fundamental fact in nerve physiology. The experimental basis of the fact is the series of observations made by Helmholtz; these showed that, when the sciatic nerve of the frog was excited at its central end, the period between such excitation and the response evoked in the attached muscle was perceptibly longer than in the case of excitation at the muscular end of the nerve. The period of delay was increased in the former case by 001 sec. when, with the nerve at $15^{\circ} \mathrm{C}$., a distance of $27 \mathrm{~mm}$. lay between the central and peripheral seats of excitation. In order to ascertain the interval of time between the excitation and the muscular response, Helmholtz employed the galvanometric method of Pouillet. ${ }^{1}$ The movement of a suitable key caused an induced current to traverse and excite the nerve, whilst it closed, at the same instant, a galvanic current of known intensity through a circuit comprising a galvanometer. The contraction of the muscle evoked by the arrival of the excitatory state was utilised to break a contact in this galvanometer circuit, and thus the duration of the galvanic current comprised the period between the nerve excitation and the lift of the contact through the muscle response. As the extent of the deflection of the needle was, in the instrument used, proportional to the time of current flow, the difference between this time with a central and a peripheral stimulation could be readily ascertained.

Similar experiments were carried out by Helmholtz on man, the muscles in the ball of the thumb being used as the index, and the nerve excited at the root of the neck and bend of the elbow respectively.

The experiments have been repeated under many varying conditions, the movement of the muscular response being recorded by any one of the methods employed for the accurate determination of the period of delay in the response of muscle to indirect or direct excitation.

The transmission is undoubtedly due to each successive portion of the continuous nerve fibre being thrown into the state of excitation, in consequence of the production of this state in the neighbouring portion. This view is confirmed by the determination of the rate of propagation of the excitatory state itself, as evidenced by its accompanying electromotive changes. These are transmitted in the case of the sciatic nerve of the frog at a rate which is similar to that deduced from the timerelations of the muscle response. ${ }^{2}$

The propagation rate ( 27 metres per second) is slow compared with that of sound, light, etc., but is much faster than exists in the case of muscle. It differs in different classes of nerves, and may be altered by a large number of agencies, which will be considered in the succeeding sections.

The alteration is one not merely of the rate but also of the intensity of transmitted effect. Thus, on passing through a locally cooled region, the transmission is both delayed in time and diminished as to intensity : the resultant muscular response thus occurs later, and may be smaller than it otherwise would be.

${ }^{1}$ Helmholtz, Arch.f. Anat., Physiol. u. wissensch. Med., 1850 and 1852.

2 Bernstein, "Untersuch. u. d. Erregungsvorgang in Nerven u. Muskeln," 1891. 
Transmission in both directions.-Another aspect of this fundamental fact of propagation is that known as transmission in both directions. If a nerve fibre is stimulated in the course of its continuity, the excitatory state is transmitted along both its peripheral and its central portious. The property possessed by the nerve is thus one of conductivity in both directions.

It is clear that it is not easy to secure proofs of such doubly directed propagation, since the index for either the efferent or the afferent nerve is afforded by the response of a structure (muscle or central nervous system) situated at one end only. In order to secure a double terminus, each capable of responding on the arrival of the excitatory state in precisely the same way, the division of efferent axis cylinders in the neighbourhood of their terminations has been taken advantage of. In the sartorius muscle of the frog a suitable nerve

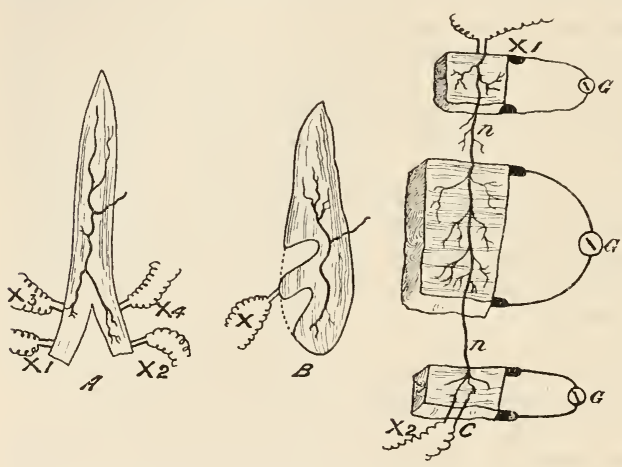

FIG. 251. - $A$, Sartorius split at iliac end ; excitation at $\mathrm{x}_{1}$ or $\mathrm{x}_{2}$ evokes contraction of excited half only; excitation at $x_{3}$ or $\mathrm{x}_{+}$evokes contraction in both halves. $B$, Gracilis with peninsula of muscle cut, so that the continuity of its fibres is interrupted. Excitation of nerve in muscle peninsula $\left(x_{1}\right)$ evokes contraction in all parts of the muscle. $C$, Three portions of Malapterurus electrical organ, connected by single nerve fibre. Excitation at either $\mathrm{x}_{1}$ or $\mathrm{x}_{2}$ evokes an electrical response in all threc portions. evokes a response on both sides. The gracilis muscle may be so cut that different regions shall have independent muscle fibres, but retain a nerve supply from the divisions of the same axis cylinders (Fig. 251). A similar double response of its separated regions is evoked when any one part with its contained nerve fibres is stimulated. ${ }^{1}$ The electrical organ of Malapterurus is a notable instance of an enormous nerve supply attained by the divisions of a single axis cylinder. It can be detached from the fish, and the nerve with its single axis cylinder can be divided where it courses upon the inner surface of the organ, and excited at either its central or its peripheral end. Further, any single branch may be cut off from its peripheral connections, and similarly excited. The response of the electrical organ is a very distinct one, and is evoked in every part, whether a peripheral twig or the main

${ }^{1}$ Kiihne, Ztschr. f. Biol., Bd. xxii. S. 305. 
stem of the nerve fibre is excited. ${ }^{1}$ The only possible interpretation of these phenomena is the transmission of the excitatory state up the efferent nerve fibre to the main stem, and along every one of its branches; if these lead to a terminal organ which responds to the arrival of the state, such response is evoked.

A question of some interest does not appear as yet to have been investigated. Is the rate of such propagation the same whether it is opposed to that of the normal functional activity, or is similarly directed to this? In other words, is there any evidence of irreciprocal conductivity? The writer has attempted to obtain information on this point by utilising the comparatively long single axis cylinder of the electrical nerve of Malapterurus. The difficulties are largely due to the slight differences in time measurement which are involved. As far as the series of experiments throws light upon the subject, it appears that the rate of transmission in the preparations used was not materially affected by the direction of such transmission; at any rate, it may be safely said that the average rate was the same, whether the propagation ascended or descended this efferent nerve (Fig. 251, $C$, excitation at $x_{1}$ and $x_{2}$ respectively).

A further proof of this conductivity in both directions is that afforded by the electrical changes accompanying the development of the excitatory state in the nerve. This, in the case of efferent nerves, is shown by the division of an anterior root of the sciatic plexus in an animal such as the cat, and the connection of the root with an appropriate galvanometer. Excitation of the trunk of the sciatic evokes both a response in the muscles of the limb and electrical changes in the anterior root, due to the propagation of the excitatory state upwards towards the central nervous system. A remarkable feature of this propagation up efferent nerves is its absolute extinction on its arrival at the central nervous system, there being no trace of any excitatory electrical change in the spinal cord. ${ }^{2}$ Similar methods may be applied to afferent nerves; here it is sufficient to expose and excite a posterior root, and determine the existence of excitatory electrical changes in the central end of the cut sciatic. These are produced not only by the stimulation of the posterior root, but by excitation of the spinal cord after all the anterior roots have been cut, due to the circumstance that the posterior root fibres are directly prolonged by branches into the posterior columns of the cord.

Various expedients were formerly resorted to in order to demonstrate the possibility of reversed propagation along afferent nerves. Among these is that of dividing the hypoglossal and lingual nerves in the dog, and then suturing the peripheral portion of the former and the central portion of the latter. It was thought that the fact of the contraction of the tongue muscles, which could after some weeks be evoked by the excitation of the lingual, showed transmission along the afferent fibres of this nerve, which had become united to the efferent ones of the hypoglossal. Such experiments, and those of Paul Bert, which involved a transplantation of the tail of the rat, by grafting its cut tip in a fresh situation on the skin, are all futile, since they were carried out before the phenomena of nerve degeneration, and nerve regeneration had been adequately appreciated. There is no evidence of such direct union of nerve fibres as that demanded by these experiments, nor, if

${ }^{1}$ Babuchin, Arch.f. Physiol., Leipzig, 1877, S. 66.

${ }^{2}$ Goteh and Horsley, Phil. Trans., London, 1891, vol. clxxxii. B., p. 485. 
this highly improbable premise were granted, would the deduction as to transmission in the lingual be sound, since this is known to possess efferent fibres derived from the chorda tympani. Langley ${ }^{1}$ has recently recorded experiments on the rabbit, which seem to show that the central end of the cervical vagus can establish physiological connection with the cells of the superior ganglion of the cervical sympathetic, if the cut cephalic ends of these two nerves are brought into contiguity and left for from seventy-three to 123 days.

The conductivity of any nerve fibre is influenced by a large number of conditions ; these are especially changes in its enviromment, and will be considered in connection with alterations in excitability due to the same causes. One influence only will be referred to now, namely, that due to the intensity of the excitatory state. It was observed by Helmholtz, in his experiments on the rate of propagation along the nerves of the arm in man, that the excitatory state evoked in nerve by an intense stimulus was propagated more rapidly than that caused by a weaker one. This result has been confirmed in the motor nerve of the frog by Vintschgau, ${ }^{2}$ and in the non-medullated nerves of Anodon by A. Fick. ${ }^{3}$

It is, however, questionable whether this result is true for all forms of stimulation, mechanical as well as electrical; the employment of the latter as the means of starting the excitatory state is open to the criticism that, when intense, it is difficult to localise it. Since the precise localisation of the starting point is essential for experiments upon the rate of propagation, any uncertainty as to its being strictly limited must render the results dubions. The apparent decrease in the period between nerve excitation and muscle response, when the nerve is stimulated with stronger induced currents, may, in many cases, be due to the spread of such currents along the nerve, and the consequent stimulation of portions which lie between the electrodes and the responding muscles.

The essential facts of nerve transmission may be summed up as dependent upon the successive excitation of the various contiguous portions of the nerve fibre. Two conditions must be assumed to determine the realisation of this succession, the potency of the exciting cause, and the degree of susceptibility of the tissue which has to be aroused. Our present knowledge leads us to regard the exciting cause as an agent able to produce a particular molecular rearrangement in the nerve; this constitutes the state of excitation, and is accompanied by localised electrical changes as an ascertained physical concomitant. It has been suggested that these local electrical changes are themselves the agents concerned in the stimulation of the quiescent portions of nerve in immediate proximity, and that this process is carried on in succession down to the nerve-endings, where it forms the releasing agent for the muscular response. ${ }^{4}$ It is certain that electrolytic processes play a nost important part in the whole process, and that the intensity of any one local excitatory effect determines, other things being equal, that of the effect aroused in the neighbouring portion. The view is supported by other considerations, which will be alluded to later.

1 Proc. Roy. Soc. London, 1898, vol. lxii. p. 331 ; "Proc. Physiol. Soc.," in Jouru. Physiol., Cambridge and London, 1899, vol. xxiv.

${ }_{2}$ Arch.f. d. ges. Physiol., Bonn, Bd. xxx. and $\mathrm{xl}$.

3 "Vergleich. Physiol. der irritabl. Substanzen," 1863.

"Kiihme, "Causation of Vital Movement," Croonian Lecture, 1888, Proc. Roy. Soc. London. 
Attention may now be drawn to the second condition for propagation, the susceptibility of the unexcited neighbouring portion. The possession of this susceptibility, when the stimulating agent is an external change, is the attribute of excitability; when the stimulating agent is the excitatory state itself in a neighbouring portion, it is expressed as conductivity. It is evident that the two are phases of one fundamental characteristic, but they are certainly more or less independent phases of this. The evidence for such separation will be furnished in detail in the sections dealing with the influence of various conditions upon excitability and conductivity.

\section{The Excitation of Nerve.}

Modes of stimulation.-The term "excitability" is conveniently limited to the possession by living nerve of a capability of responding by a definite change to the action of an external agent, and every external agency which is capable of evoking such a response is an efficient or adequate stimulus. Such "excitability" may be measured in terms of the intensity of the minimal efficient stimulus, if the exciting agent or stimulus is itself a measurable one. The direct index of a nerve response is the electrical change, which is the sole physical alteration at present ascertained in active nerve, but an indirect index is afforded by the propagation of the excitatory change from the seat of the local stimulus to such a peripheral organ as a muscle, a contraction of which is evoked on the arrival of the transmitted nerve effect. It is evident that this indirect index is complicated by being the response of another structure, and that all conclusions deduced from experimental investigations in which this method is used, can only be made after full allowance for this. In the great majority of the investigations upon nerve this muscular index has been employed, and the results are thus, for the most part, confined to motor nerves. The electrical response will be treated in the concluding chapters, so that in the present and immediately succeeding sections the subject matter will be largely limited to the evidence afforded by what may be termed the indirect index. In the case of afferent nerves, a similar indirect index is the reaction of the central nervous system as indicated either by reflex effects in peripheral organs, or by the development of those changes which are transmuted in the crucible of consciousness into sensation. A large variety of external agencies can act as local stimuli for nerve fibres; they may be grouped as electrical, mechanical, chemical, and thermal.

Of these the electrical stimulus is that principally employed on account of the ease with which it can be applied to a definite region of the nerve, the facilities its employment offers for graduating the intensity of the stimulus, and the comparatively small amount of injury which it causes in the tissue subjected to its action.

Electrical stimulation.-(1) Make and break induced currents.The forms of electrical stimulus most frequently made use of are the induced currents occurring in the secondary coil on the commencement or the cessation of a current flow through the primary coil of an induction machine.

They are susceptible of very accurate graduation as to intensity by altering the distance between the secondary and primary coils, and, in 
consequence of comparatively large electromotive force, brief duration, and rapidity of development, they form the most effective of all nerve stimuli.

Their employnent necessitates the adoption of certain precautions due to their character and method of production. These currents are largely modified by the presence of the extra current in the primary coil, and therefore by the nature of this coil ; if, as in most induction apparatus, this contains a core, then such modification is very pronounced. Under any circumstances the "make" induced effect is slower in its development and subsidence than the "break" (Fig. 252, A). This is due to the variation in the counteracting influence of the extra current, ${ }^{1}$ which, on elosing the primary circuit, is of some intensity, but on opening is comparatively feeble, since, in the latter case, the circuit being opened, it must traverse the gap as a spark. The time-relations of the break induced current are thus dependent upon the velocity with which the key in the primary circuit is opened. It will be seen that rapidity of development is an inportant factor in determining the stimulating intensity of a current; hence, in the case of the break induced current, its excitation value is dependent upon the rate at which the circuit of the primary coil is opened.

For many experiments it is necessary that the time-relations of each one of
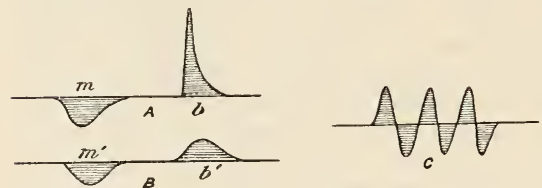

Fig. 252.- $A$, Time relations of make $(m)$ and break $(b)$ induced eurrents (ordinates = current intensity. $B$, Time relations of induced currents obtained by Helmholtz side wire method. $C$, Time relations of magneto-induced currents obtained by rapid rotation machine.

a series of stimulating induced currents should be absolutely uniform; to insure this, a key must be used, such that the primary circuit shall be always opened at a constant velocity. If all other conditions are constant, then the more quickly the primary circuit is opened, the more rapid will be the development of the break induced current, and the more powerful its stimulating effect. A large number of different forms of apparatus of varying degrees of efficiency have been employed to effect this end; a spring which on its release separates two surfaces, or a traveller driven by either a weight or some constant force, which opens at a uniform velocity an appropriate key.

It is less easy to secure a constant closure, hence the break is to be preferred to the make induced current.

A useful and sufficiently uniform stimulus is that produced by a closure of the primary circuit for a definite but very short period, such as can be effected by the striking of a steel needle as it passes over and lightly touches a stretched wire ; this is made use of in many forms of rheotome, and, when produced by a rotator travelling at a constant and sufficiently rapid velocity, causes a series of uniform induced currents in the secondary circuit.

The production of such a rapid series of uniform induced currents is ordinarily effected by the use of the spring hammer of the inductorium, which causes make and break induced currents recurring at regular intervals. The objection that the fact of the break being different in time of development to the make effect, must, when both rapidly recur, cause an overpowering series of currents in one direction, is got rid of by the interposition of a side wire, which is so disposed that the interrupter opens and closes a short circuit of the primary coil. Under these circumstances the

\footnotetext{
1 See Rosenthal, "Electricitätslehre f. prakt. Aerzte."
} 
extra current is approximately the same, whether the current in the primary coil is augmented or decreased (Fig. 252, B); thus a series of induced currents, recurring at rapid intervals, can be produced, alternate in direction and of approximately equal intensity. Various devices have been employed in order to insure mechanical accuracy in the closing and opening of the contacts effected by the oscillations of the spring or fork; of these the most effectual is Kronecker's capillary contact, in which the spring causes a platinum wire to dip in and out of mercury, whilst a stream of water removes any electrolytic products formed at the point of contact. A rotator with two strikers and two batteries, coupled up by opposite poles, produces very good results, and obviates the necessity for the introduction of the side wire.

(2) Undulatory induced currents.-For precise stimulation with a rapid series of alternating currents, probably the most effectual method is the employment of a magneto-electric machine. A convenient form consists of two sets of small coils of wire, fixed in a frame a short distance above one another, so as to allow of the rapid passage of a magnet or series of magnets between them. One set of coils is connected with a battery, the other with the exciting circuit, or with the primary coil of an induction machine. The disc with the magnets is rotated by means of a motor at a rapid rate, and a series of precisely similar alternating currents are thus induced in the apparatus. If these are allowed to traverse the primary coil of an induction machine, this acts as a transformer, and a series of currents are produced in the secondary, the varying intensity of which would, if represented graphically, assume the form of a symmetrically undulating curve (Fig. $252, C$ ), and may, by appropriate choice of coils, be made strictly sinusoidal in character. ${ }^{1}$

The production of single or slowly recurring currents of similar physical character to those just described, can be obtained by allowing a magnet to fall with constant velocity through the centre of a coil. ${ }^{2}$ The elaborate instrument used by Mareš for the purpose may be replaced by the following simple form.

A primary coil containing an internal core of the usual character is fixed upon a pendulum and is connected with a battery $;^{3}$ a secondary coil is fixed immediately below the stationary position of the pendulum and its coil. Each swing of the pendulum produces an effect in the stationary secondary coil, which can be made of such character as to have a current intensity augmenting and diminishing in a perfectly regular way; the effect of several swings is thus to produce a series of alternating currents, each of which is precisely similar as regards its time-relations. By varying the shape of the magnetised core, the time of development may be altered and the variations made sinusoidal in character. The uniform character of the time-relations of these effects insures uniformity in the physical characters of the stimulus.

(3) Condenser discharges.-Theoretically, the condenser discharge is in almost all respects an ideal form of electrical stimulus.

Its practical employment necessitates several precautions: two condenser plates, on being connected with an electromotive source, are charged up to the PD of this source; on disconnecting, the charge remains; but on connecting, through a second circuit, including a nerve, the charge rapidly equalises itself, and a current thus flows through the nerve. The rate at which the equalisation occurs depends upon

1 Burch, Journ. Physiol., Cambridge and London, 1897, vol. xxi. p. 231.

2 Mareš, Proc. Internat. Cong., Liége, 1892 ; Centralbl. $f$. Physiol., Leipzig u. Wien, 1892, Bd. vi. S. 408 .

${ }^{3}$ Gotch and Macdonald, Journ. Physiol., Cambridge and London, 1596, vol. xx. p. 217. 
the resistance of the circuit and the capacity of the plates, being slower as either of these increases. The character of the discharge is thus expressed by a fall from the full potential to nothing, which fall varies in the time necessary for its completion. As condenser plates are liable to allow leakage between the opening of the charging and the closure of the discharging circuit, a useful method is to place the condenser plates in direct connection, through the nerve, with the electromotive source, and use one circuit only; on short circuiting the electromotive source the discharge of the plates occurs through the nerve.

The elaborate investigations of d'Arsonval, Hoorweg, Cybulski, and Waller ${ }^{1}$ show that in this method of nerve excitation, the physical constants of the stimulus are capable of being accurately determined, and thus the stimulus energy in any one instance can be expressed in terms strictly comparable with that in another.

(4) The galvanic current.-The peculiar features of the excitation of nerve by galvanic currents will be considered in detail later. As a mode of stimulation, its employment has the disadvantage that the duration of the current causes electro-chemical changes in the tissue of a more pronounced character than those produced by the former methods.

These changes may, however, be reduced to a small amount by employing currents of very short duration, such as are produced by a rapidly rotating striker which effects a closure by lightly striking a stretched wire; and if, by a further arrangement, a second similar closure is also effected, two short galvanic currents opposite in direction may be produced, thus reducing the possibility of prolonged electrolytic changes to a minimum. By means of a rheochord the intensity of the current, and thus the stimulus, may be graduated with great precision, and a mode of stimulation can be thus obtained which has several points in its favour. The duration and character of the currents used for stimulation are constant, and can be determined with great precision, whilst the electromotive force employed-is also readily ascertained.

(5) Other modes of electrical excitation.-The passage of any electrical current of sufficient intensity may act as a stimulus to a medullated motor nerve, especially if the time of its development is sufficiently short; thus the discharge between the knobs of a frictional machine will excite the nerve of a frog's muscle nerve preparation, when this is placed in air in the neighbourhood of either pole. ${ }^{2}$ An anomaly, however, appears to exist in the case of electrical oscillations of very great frequency, such as those to which attention was drawn by Hertz. The Hertz waves, even when adequate to cause an incandescent lamp to glow, will only stimulate an exposed nerve at the moment of their onset, whilst, during their continuance, they appear to cause a paralysis of conductivity. ${ }^{3}$

The currents produced by Bell's telephone have been made use of for stimulation; a striking instance is their employment by Hürthle, in order to record the time-relation of the sounds of the heart, the currents thus caused being adequate to excite an exposed nerve. ${ }^{4}$

${ }^{1}$ Cybulski, Arch. f. d. ges. Physiol., Bonn, 1894, Bd. lvi.; Hoorweg, ibid., 1893, Bd. liii.; ibid., 1895, Bd. lvii. ; Waller, Proc. Roy. Soc. London, 1899.

${ }^{2}$ Loeb, Arch. f. d. ges. Physiol., Bonn, ] 897, Bd. lxvii. S. 483 ; Centralbl. f. Physiol., Leipzig u. Wien, 1897, Bd. xi. S. 13.

${ }^{3}$ D'Arsonval, Compt. rend. Soc. de biol., Paris, 1893 ; ibid., tome cxvi. p. 630.

${ }^{4}$ Huirthle, Proc. Intcrnat. Cong., Liége, 1892; Centralbl. f. Physiol., Leipzig u. Wien, 1892, Bd. vi. S. 398. 
Finally, the vital electromotive changes present during the functional activity of electrical organs, voluntary muscle, cardiac muscle, and even nerve itself, may under appropriate conditions be employed as nerve stimuli. ${ }^{1}$

(6) Unipolar excitation.-The employment of electrical currents for stimulation is, unless special precautions are observed, liable to lead to erroneous conclusions, through difficulty in localising the seat of their application. This is especially the case when the electromotive source is one of high potential, and one fruitful source of error is the existence of what is termed unipolar excitation. This phenomenon is readily demonstrated when induction currents are employed. If the sciatic nerve of the frog is connected with one terminal only of the secondary coil, the opening or closing of the primary circuit may still evoke a muscular response. The most careful insulation of the preparation is ineffectual to eliminate the exciting effect, but it disappears if the terminal connected with the nerve is in addition adequately connected with the earth by means of water-pipes, or the like. If the intensity of current employed is just inadequate to evoke the response, it becomes adequate when either the unconnected terminal of the coil, or any part of the insulated preparation, is touched with the finger: it is even possible to cause an excitation by approaching the nerve with the moist finger.

The cause of the phenomenon is the unequal surface distribution of electrical potential through the whole conducting mass, including the preparation. $^{2}$ With high potential, such as that of the induced current, any alteration in surface distribution is necessarily accompanied by the passage of considerable currents during the period of alteration; the rapidity with which this takes place is very considerable, owing to the quick development of the electromotive force, and thus increases the exciting value. It is possible to obtain such variations in surface distribution by suspending an insulated nerve muscle preparation in air, in the neighbourhood of Leyden jars connected with the poles of a suitable frictional machine; every time the distribution is altered by a discharge between the poles, a response occurs, owing to a similar variation of distribution in the preparation, and such response is augmented if the preparation is touched by the finger.

The use of electromotive changes of high potential is thus always liable to produce unipolar effects; it is for this reason that in the case of induced currents, especially break induced currents, the presence of a closing and opening key directly interposed in the secondary exciting circuit is inadmissible, and a short circuiting key, with effective metal contacts, must be employed.

Unipolar effects are a fruitful source of error, as changes will be constantly produced by the existence of currents which, being inadequate to evoke muscular effects, are disregarded; in all investigations upon the electromotive phenomena of nerve, the possibility of their presence must be always considered, and as far as possible guarded against. ${ }^{3}$ The employment of unipolar excitation offers some special advantages, and in the hands of Charpentier and others has yielded results which are obtained with difficulty, if at all, by other modes of stimulation. ${ }^{4}$

${ }^{1}$ Hering, "Biological Memoirs," Oxford, 1889 ; Sitzungsb. d. k. Akad. d. Wissensch., Wien, 1882, Bd. lxxxv.

${ }^{2}$ E. du Bois-Reymond, "Untersuch. ii. thier. Elektricität."

${ }^{3}$ Hering, Sitzungsb. d. k. Akad. d. Wissensch., Wien, 1884, Bd. lxxxix. Abth. 3.

${ }^{4}$ Charpentier, Compt. rend. Soc. de biol., Paris, 1894; Arch. de physiol. norm. ct path., Paris, 1894, ete. 
Chemical modes of stimulation. - Many chemical substances when applied locally to nerve act as stimuli; the exciting effects are irregular in character, and appear to be due partly to the withdrawal of water from the nerve, and partly to the specific effect of the chemical substance upon the molecular state of the nerve.

Although the subject will be referred to again in the section which deals with the influence of chemical substances upon nerve excitability and conductivity, it is advisable at this stage to enumerate those forms which appear to be most effective, and the node of their employment.

(1) The withdrawal of water by drying.-The effect of drying a motor nerve is to cause first increased susceptibility to excitation by other modes of stimulation, then an irregular excitation which shows itself in the attached muscles by flickering irregular muscular tetanus, and finally loss of both excitability and conductivity. The effect increases with the length of the nerve thus dried, and when the drying is very rapid the final stage is reached so quickly that no responses are obtained. ${ }^{1}$ Different nerves and different portions of the same nerve vary considerably as regards their susceptibility to stimulation by this agency. Thus in the case of the sciatic nerve of the frog, the lower end is far more readily excited than the upper, probably owing to the larger amount of connective tissue surrounding the upper fibres, which protects the essential structure. A large number of chemical agents act by withdrawing water, glycerine and sodium chloride being familiar instances.

(2) Neutral salts. - Almost all the neutral'salts cause nerve excitation, whether they are applied by allowing a nerve to lie for some minutes in a bath containing an appropriate solution of the salt, or whether the salt is applied locally to a portion of the nerve. The exciting value varies directly with the degree of concentration, until a limit is reached at which the nerve functions are suspended, if not destroyed, by the rapid action of the strong solution. The investigation has been largely carried out on motor nerves, particularly the sciatic nerve of the frog, the muscular response being taken as the index of effective excitation, which resembles in its general characters that produced by drying.

Sodium chloride is the form which has been most frequently employed, and the salt tetanus evoked by its application has been studied by Eckhard, Kölliker, and others. An extended inquiry into the exciting value of different salts has been made by Grïtzner. ${ }^{2}$ In order to obtain solutions of different salts which should admit of accurate comparison, the solutions used were not of equal percentage, but equimolecular. Thus, in comparing $\mathrm{NaF}, \mathrm{NaCl}, \mathrm{NaBr}$ and $\mathrm{NaI}$ (the varying molecular weights of which are respectively $41 \cdot 9,58 \cdot 3,102 \cdot 7,149 \cdot 4)$, equimolecular solutions are those containing corresponding proportions by weight of the salts, namely, $4.2 \mathrm{NaF}, 5 \cdot 8 \mathrm{NaCl}, 10.2 \mathrm{NaBr}$, 14.9 NaI. The effectiveness of these solutions for stimulating the sciatic nerve of the frog was found to be in the following order: $\mathrm{NaF}$, $\mathrm{NaI}, \mathrm{NaBr}, \mathrm{NaCl}$. The action of $\mathrm{NaF}$ is very intense; if this is excepted, the remaining salts act more strongly the higher their molecular weight, and even with equal percentages the same relationship holds good. It therefore appears that the withdrawal of water is not

${ }^{1}$ Harless, Ztschr. f. rat. Med., 1859, Bd. vii.

${ }^{2}$ Grützner, Arch.f. d. ges. Physiol., Bonn, 1893, Bd. liii. s. 83. 
the sole factor which determines the exciting value of the salt, but that this must be largely dependent upon the specific action of the chemical compound upon the nerve. This is confirmed by the results of such substances as $\mathrm{KI}, \mathrm{KBr}$, and $\mathrm{KCl}$, which excite motor nerves hardly at all.

The exciting value of equimolecular solutions of various neutral salts, when applied to motor nerves, may be contrasted with one another as follows:-

$$
\begin{aligned}
\mathrm{NaF}>\mathrm{NaI} & >\mathrm{NaBr}>\mathrm{NaCl} \\
\mathrm{C} \cong \mathrm{Cl} & >\mathrm{RubCl}>\mathrm{KCl} \\
\mathrm{BaCl} & >\mathrm{SrCl}>\mathrm{CaCl}
\end{aligned}
$$

The stimulation of afferent nerves by such salts presents some remarkable features. In the first place, such stimulation, as evidenced by reflex responses, has been found to be so capricious and uncertain as to lead many investigators to state that sensory nerves are more or less refractory to such excitation. Grützner ingeniously utilised the wellknown smarting sensation which follows the application of saline solutions to cuts upon the fingers. The production of this sensation is undoubted evidence that the sensory nerves are stimulated; on comparing various salts in equimolecular solution, he obtained with sodium salts results of which the following is a typical example:-

With $\mathrm{NaI}$ (14.9 per cent.) the sensation was evoked in five seconds, with $\mathrm{NaBr}(10.2$ per cent.) in ten seconds, with $\mathrm{NaCl}(5.8$ per cent.) in fifty seconds. This order is the same as that above referred to as prevailing with motor nerves. But he found, further, that whereas potassium salts have little exciting value for motor nerves, they are extraordinarily effective for sensory nerves. Solutions of all the potassium salts produced the smarting sensation when tested by the skin-cut method, and this when in a state of concentration which, with equimolecular solutions of sodium salts, was far too weak to be effectual. Similar results are obtained by experiments on the coujunctiva, whilst, in the frog, salts of potassium evoke reflexes, when applied to the skin, much more readily than those of sodium. Moreover, the form of potassium salt which is most effective is the chloride, while the chloride of sodium is the least effective.

The following table shows the comparative exciting efficiency of various neutral salts when applied to afferent nerves:-

$$
\begin{aligned}
& \mathrm{KCl}>\mathrm{KBr}>\mathrm{KI} \\
& \mathrm{NaI}>\mathrm{NaBr}>\mathrm{NaCl} \\
& \mathrm{CaCl}_{2}>\mathrm{RubCl}>\mathrm{KCl}
\end{aligned}
$$

The extraordinary contrast between the marked exciting value of $\mathrm{KCl}$ when applied to afferent nerves, and its inadequacy as a stimulating agent to motor nerve, is shown further by the following experiment. The sciatic plexus of a narcotised frog is exposed and divided on both sides. On applying potassium chloride to the central end of one plexus and to the peripheral end of the other, reflex movements of the flanks, etc., are evoked through the former, but no movement of the lower limb is produced by the latter application. On the other hand, a similar experiment with sodium chloride causes movements of the muscles whose nerves are directly excited, followed after some little interval by reflex effects in the flanks and other muscles.

VOL. II. -30 
These differences in the apparent excitability of afferent and efferent nerves to chemical stimulation may be dependent upon the difference between the responding end-organ present in the two cases. On this hypothesis, even though all the nerve fibres are excited, the indication of such excitation in the case of a motor nerve, i.e. the resultant muscular response, is one which is readily evoked by the transmitted excitatory states produced by sodium salts, but not by those started by potassium salts; on the other hand, in the case of afferent nerves, the excitatory state aroused by the potassium salt is of a character to evoke central (reflex) activity, and this in a more marked degree than is the case with that due to sodium salts. Any explanation must include those phenomena of isolated nerve, in which an electrical reponse is the index of activity. These are referred to in the section dealing with the electromotive phenomena of nerve.

(3) Stimulation by bases.-Considerable discrepancy exists as to the stimulating effects upon motor nerves of such alkalies as $\mathrm{NaHO}$, KHO, and ammonia. According to the majority of observers, the first two will excite a motor nerve, even when weak, e.g. 0.1 per cent. ${ }^{1}$ (On the other hand, Grützner found that KHO rarely if ever excites the motor nerve.) As regards ammonia, the great majority of observers are agreed that it does not excite such nerves. In all cases, these alkalies, if at all concentrated, rapidly destroy all functional activity, - a circumstance which may account for the discrepancies in the observations.

As regards afferent nerves, Gritzner found that all three alkalies excited when tested by the skin-cut method, and that their comparative exciting efficiency was as follows, Ammonia, $\mathrm{KHO}, \mathrm{NaHO}$, thus showing again the marked contrast between the central system response and that of the peripheral muscle to chemical excitation of the centripetal and centrifugal nerves respectively.

(4) Stimulation by aeids.-Acids excite both motor and sensory nerves. ${ }^{1}$ As regards inorganic acids, their comparative exciting value is in strict accordance with their chemical avidity. Thus $\mathrm{HNO}_{3}$ and $\mathrm{HCl}$ excite in weaker solution than $\left.\mathrm{H}_{2} \mathrm{SO}\right)_{4}$, whilst $\mathrm{H}_{3} \mathrm{PO}_{4}$ excites very feebly. ${ }^{2}$ The organic acids excite only when concentrated, and some of them, such as oxalic acid, impair vitality without excitation.

(5) Stimulation by salts of the heavy metals. - The majority of metallic salts destroy nerve vitality without excitation. Mercuric nitrate, zine chloride, zinc sulphate, and chloride of iron are exceptions. These excite, when solntions are used of a concentration which causes a comparatively slow injury. The first is by far the most potent, and the others differ as regards exciting efficiency in the order above given. ${ }^{1}$

(6) Stimulation by organie substanees.-Glycerin, if concentrated, excites notor nerves, probably through the withdrawal of water; and, as in the case of drying, the lower end of the frog's sciatic is far more readily stimulated than the upper end. Urea and sugar, if sufficiently concentrated, excite in a similar way; the former is remarkable inasmuch as it does not produce the eventual loss of vitality which is the common result of the prolonged action of other exciting chemical sub-

${ }^{1}$ Eckhard, Ztschr. f. rat. Med., 1851 (2), Bd. i. ; Kiihne, Arch.f. Anat., Physiol. ". wissensch. Med., 1859.

f fruitzner, loc. cit. 
stances. Hence the permanent impaiment of tissue vitality is not a necessary termination to prolonged chemical excitation. ${ }^{1}$

Mechanical stimulation.-Various modes of mechanical excitation have been devised. The simplest is that of a rapid compression, such as is caused by dividing the nerve trunk. In order to secure the repetition of the stimulus, du Bois-Reymond ${ }^{2}$ employed a small toothed wheel which compressed successive portions of the nerve, and thus always affected a new region. Heidenhain used a hammer, which struck the nerve where it lay upon a vulcanite block, whilst by means of a small cylinder a fresh portion was brought under the vulcanite anvil in the interval between the ascent and descent of the hammer. ${ }^{3} \mathrm{~A}$ similar "tetanomotor" was employed by Wundt; it was materially modified by Tigerstedt, the hammer being made very light, and the force of the blow rendered capable of adjustment by altering the position of a small load, placed between the fulcrum of the hammer and its head. ${ }^{4}$ With this apparatus carefully adjusted, it is possible to excite once every few seconds upon the same spot many times in succession. Langendorf employed an entirely different method; the cut end of the sciatic nerve of the frog was ligatured and attached to the free end of a tuning-fork, which was then adjusted so as to lightly pull the nerve. On setting the tuning-fork in vibration, the nerve was alternately stretched and relaxed with each vibration and thus stimulated. ${ }^{5}$ The conditions which determine effective mechanical stimulation have been investigated by Uexküll, ${ }^{6}$ who states that this is best achieved by an arrangement in which the hammer is rigid but the counterresistance extremely yielding. This condition is fulfilled when the nerve is rapidly shaken against an elastic pad by a vibrating ivory bar. The apparatus devised by Uexkiill, and termed by him the nerve shaker, consists of a thin ivory lever, moving on a central fulcrum, and held in position by an adjustable spring. By means of a rotating toothed wheel one end of this lever is lightly struck in rapid succession, and the whole thus set in vibration. The nerve either rests upon a yielding pad under the further end of the lever, or is twisted round the lever point. The vibration of the lever is communicated to the nerve, and the shatter thus produced excites it. Each shatter of the lever can be registered by means of a small contact, which opens and closes an electrical circuit. Uexkiill found that, with this apparatus, the nerve could be excited many times at the same spot, and that thus the injurious effects caused by striking with the hammer could be eliminated. The shatter appears to excite the motor nerve in the same way as the induced current, at least in so far as the time relations of the resulting muscular contraction are concerned.

A distinct mode of stimulation is that caused by suddenly lifting a compressing weight; it appears doubtful whether this is in reality a form of mechanical excitation, since it is possible that the release of the compression may cause the nerve to regain its condition of moisture, which during the squeeze has undoubtedly been diminished; the demarcation nerve current due to the injury of the squeeze is thus suddenly

${ }^{1}$ Buehner, Ztschr.f. Biol., 1876, Bd. xii.

2 E. du liois-Reymond, "Untersuchungen," 1849, Bd. ii.

${ }^{3}$ Heidenhain, Untersuch. z. Naturl. d. Menseh. u. d. Thiere, 1858, Bel. iv.

+ Tigerstedt, Britr. ₹. Physiol. Carl Luduig z. s. 70 Goburtst., Leiprig.

${ }^{5}$ Langendorff, Centralbl. f. d. med. Wissenscli., Berlin, 1882, S. 114.

${ }^{6}$ Uexkiill, Ztschr.f. Biol., 1895, Bd. xxxi. and xxxii. 
increased in intensity as in Hering's experiment, and this may excite the nerve.

According to Uexküll, all modes of mechanical stimulation are effective only in proportion as they shatter the nerve, and there is good ground for the belief that the withdrawal of water caused by drying, and by many chemical stimuli, act in a similar manner.

The method of mechanical stimulation eliminates all error due to the spread of the exciting agency to other portions of tissue, such as occur's when electrical modes are employed. The methods of Uexkiill, or modifications of these, are likely to be of great service in the future, as their employment is not accompanied by nerve injury.

The conditions which determine the efficiency of a stimulus.The excitation of a nerve by an external stimulus is favoured by two entirely independent sets of conditions,-one of these, it need hardly be said, is physiological, and is expressed by the term maintenance of high excitability; the circumstances which play upon this physiological state will be detailed in a succeeding section. The other conditions are incident to the exciting cause, and involve the consideration of the special characters which the external change must possess in order to render it an effective stimulus.

1. The intensity of the physical change.-The most obvious of these characters is that of the force of the exciting agency, in other words, the intensity of the stimulus, although it is a familiar fact that there are minimal and maximal limits to its potency. Thus, an electrical current of definite character, if very weak, is inadequate; but when gradually increased in intensity, without any alteration in its time relations, it will, at some given point, become sufficiently potent to stimulate.

It is, however, by no means a simple matter to determine for any one agent the point when its intensity has sufficiently developed to arouse a response in a nerve. What is the indication that such a response has been arouserl? In the case of a motor nerve, the contraction of the attached muscle shows that it has been aroused sufficiently to produce an excitatory change of such extent that, when transmitted through the nerve-endings, it evokes the muscle response. But it is certain that the nerve itself must be affected by external agents which are inadequate to cause an indirect muscular contraction. The only physical indication of the excitatory change is the electrical response of the nerve.

In ascertaining, therefore, the relation between the intensity and the exciting value of any stimulating agent, the electrical response is the most reliable index of effective excitation.

Such comparison has been made by Waller, who recorded the effect produced when the strength of the exciting agent was gradually increased. ${ }^{1}$ The record consisted of a series of galvanometric excursions, photographed on a slowly moving surface, each being the instrumental summation of a number of electrical responses of a nerve, evoked by a definite number of electrical stimuli (induction currents). The stimulation was repeated at regular intervals of one minute, and was gradually increased in intensity. The electrical nerve changes were contrasted with the records of the muscle lift due to the nerve excitation by the

${ }^{1}$ Brain, London, 1895, vol. xviii. p. 209. 
same series of stimuli. The character of the two sets of results is shown in the following table :-

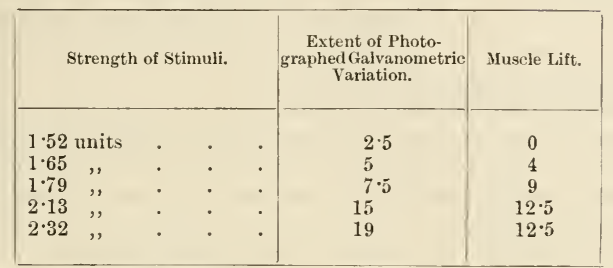

It will be noticed that the nerve change increases in proportion as the intensity of the stimulus is augmented, and that this increase still occurs when the muscular response, having reached its maximum limit, is unable to indicate the augmentation. The relationship of electrical response to nerve stimulus will be again referred to in the section dealing with the electromotive phenomena.

2. The time relations of the change (the law of excitation). These have been especially studied in connection with electrical modes of stimulation, and their consideration may be appropriately prefaced by a statement of the results arrived at by du Bois-Reymond, the consideration of which caused him to formulate the two propositions which constitute his "law of excitation." 1

(a) It is not the absolute intensity of a current at any given moment which constitutes its effectiveness for the excitation of a motor nerve, but the variation in such intensity from one moment to another.

(b) The excitation by current variation with any given quantity is the more pronounced the more rapidly such variation is effected, i.e., the greater the amount of such variation in a unit of time. In other words, the potency of an electrical current for the excitation of an excitable tissue such as nerve, is not so much a function of I as of $\frac{d I}{d T}$ where I represents the intensity and $T$ the time.

There are here two statements, - the first indicates that it is the increase or decrease, the onset or cessation, of the flow of a current, and not its maintenance, which determines its exciting efficiency ; the second, that in proportion as the onset or cessation is more rapidly effected, the exciting efficiency is increased.

The foundation for the first statement is a series of well-known and uncontested facts; they may all be sufficiently illustrated by one example, that of the exciting value of a galvanic current, when this is led through the sciatic nerve of the frog. The muscle to which such nerve is attached, responds by a single twitch synchronous with the closure, and by a second twitch synchronous with the opening, but during the maintenance of the closure it remains quiescent.

Although this and other facts of a similar nature appear to prove the validity of the first part of du Bois-Reymond's law, there are many considerations which forbid its acceptance as a general law of excitation.

As already indicated, the muscular response, when present, is an ${ }^{1}$ E. du Bois-Reymond, "Untersuchungen," 1849, Bd. i. 
undoubted index of nerve excitation, but its absence cannot be taken as convineing proof of the failure of such nerve excitation. It is conceivable that the nerve equilibrium, although upset more potently at the moment of onset and cessation, may be similarly disturbed during and after the flow of a current, and indeed we have experimental proofs (electrotonus) that such is the case.

This prolonged disturbance is not termed in ordinary physiological parlance an excitation simply because the attached musele does not respond on its being propagated to the motorial nerve-endings. The electromotive changes, which are the index of the production of the true nervous disturbance, are however present, and the disturbance may under favourable conditions be so pronounced that a muscular response is evoked, thus definitely proving its existence. It is well known that in the case of cooled nerves, or those which have been treated by chemical reagents $(\mathrm{NaCl}$, ete.), either the closing or opening muscular twitch may be succeeded by a prolonged tetanic contraction.

In the case of other nerves, such as those of the adductor and abductor muscles of the crayfish claw, prolonged excitatory effects, evoking relaxation or augmentation of the muscular tonic contraction, occur during and following the closure of the current, whilst voluntary muscle responds by a continuous contraction to the passage of a galvanic current through its substance. The sciatic nerve of the cooled frog may be persistently stimulated by the demarcation current caused by its section, the excitation evoling a continuous muscular contraction. ${ }^{1}$ It has been shown that stimulation of the afferent fibres in the vagus nerve of the mammal is produced during the whole time of the passage of such a current, as evidenced by the respiratory movements." The glandular response to excitation of the glosso-pharyngeal nerve is similarly evoked, not merely at the onset, but still more effectually during the flow of a galvanic current through this nerve. $^{3}$ Finally, it was shown by König ${ }^{4}$ that the duration of a galvanic current was an important factor in determining its efficiency as a nerve stimulus; this is strikingly the case in involuntary muscle, but it is easily demonstrable in cooled nerve, that for any given intensity there is a minimal period of closure below which the stimulating effect is decreased. Since the rise of the current intensity is practically instantaneous, this fact can only be interpreted as meaning a less efficient excitation, in consequence of the shortness of the period of How.

As regards nerve, therefore, the first statennent in the law of excitation requires limitation. Nerve being of all structures that in which the properties of excitability are most pronounced, is differentiated, so that it is more susceptible to variations in current intensity than to the maintenance of such intensity; but the excitatory condition, though produced at the onset and the cessation of the current, can be also evoked, though in less degree, both during and after the current flow.

The second portion of du Bois-Reymond's statement is, as regards the notor nerves of the frog and the mammal, of far more general

${ }^{1}$ Hering, Sitzungsb. d. k. Akad. d. Wissensch., Wien, 1882, Bd. lxxxv. Abth. 3, S. 237 ; also, "Biological Memoirs," Oxford, 1887 , p. 136.

${ }^{2}$ Langendorff and Oldag, Arch.f. d. ges. Physiol., Bonn, 1894, Bd. lix.

${ }^{3}$ Biedermann, ibid., 1893, Brl. liv.

${ }^{4}$ König, Sitzungsb. d. k. Alkad. d. Wissensch., Wien, 1870, Abth. 2. 
application. With any given intensity the stimulating effect of the onset or cessation of a galvanic current is largely determined by the time relations, i.e., by the rapidity with which either of these is effected. This is readily demonstrated by a rheonome, an apparatus designed so as to constitute a varying rheochord. ${ }^{1}$

Fleischl's rheonome consists of a narrow circular canal, filled with saturated solution of zinc sulphate; a battery is connected with two fixed points in this canal at opposite ends of one diameter, whilst two revolving contacts dipping into the canal are connected with the derived or exciting circuit, and thus with the nerve (Fig. 253). At each complete revolution the difference of potential in the derived circuit is augmented and decreased in alternate directions with a rapidity dependent upon the rate of revolution, and since the apparatus provides for equal revolutions in equal times the variation is linear. With a very slow rate of revolution the current increase and decrease can be effected without causing a nerve excitation which shall be adequate to evoke

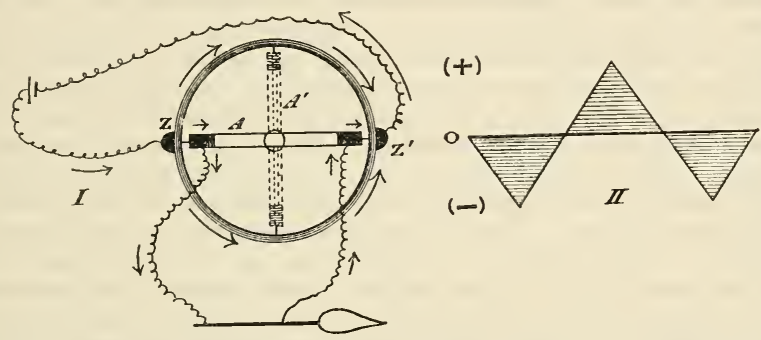

Fig. 253.-I, Diagram of rheonome. Circular trough containing saturated solution of $\mathrm{ZnSO}_{4}$, connected by zines at $\mathrm{ZZ}^{\prime}$ with battery circuit ; the revolving $\operatorname{arm}(A)$ dips by its end contacts into the trough; when it reaches the position $\left(A^{\prime}\right)$ the two points of contact in the trough are equipotential. $I I$, Diagrammatic representation of the variation in current, through the wires attached to the ends of the revolving arm, when this is slowly rotated.

a muscular contraction; as the rate increases the stimulus becomes effectual, evoking minimal and subsequently maximal contractions when a certain rapidity of development has been attained. The conditions are such that the maximum of current intensity is the same at whatever rate the revolving contacts move, hence the rapidity of development $\left(\frac{d I}{d T}\right)$ is the effectual factor in letermining the exciting efficiency.

The difference between the make and the break induced currents is largely due to varying rapidity of development; the break induced current, reaching its maximum more rapidly than the make, is more effectual.

The muscular nerves of the frog and of most mammals are differentiated so as to be extraordinarily susceptible to rapid variations of current intensity; the excitation of such a nerve by the static spread from the neighbourhood of the poles of a frictional machine or by telephone currents are illustrations of such susceptibility.

But, in spite of this, du Bois-Reymond's statement camnot be accepted as a fundamental law of excitation for excitable tissues

${ }^{1}$ Fleischl, Sitzungsb. d. k. Alud. d. Wissensch., Wien, 1877, Bd. Ixxvi. Abth. 3, S. 3 ; v. Kries, Arch. f. Physiol., Leipzig, 1884. 
generally. In many such tissues, involuntary muscle for instance, there is a limit beyond which increased rapidity of current change ceases to augment its excitatory value, and there are instances of such results even in nerve. Thus the motor nerves of the toad are more effectually excited by the make than by the break induced current, ${ }^{1}$ and even the motor nerve of the frog, when locally cooled, will respond to slowly developed better than to very rapidly developed variations in current intensity. ${ }^{2}$ Finally, the increased effectiveness for cooled nerve of condenser discharges, when with constant electromotive force the condenser capacity is augmented, shows that the duration of such discharge is a potent factor. ${ }^{3}$

All that can be said is that, as a rule, effectiveness of stimulation in the case of electrical currents is increased in proportion as the rate of variation in current flow is accelerated.

3. The influence of the direction of current upon its exciting value.-The above considerations relate to the intensity and time relationship of a current, a third important factor is its direction. It will be seen in a subsequent section that, owing to a blend of excitability and conductivity changes, the muscular response is affected to a very considerable degree by such alterations in direction as are implied by the terms "ascending" and "descending." There is, however, one feature in regard to direction which must be referred to at this stage, since it brings forward an important point in reference to the electrical stimulation of nerves. This is the great decrease in exciting value, when the current flow is so directed as to be in a plane which cuts the nerve fibres at right angles to their length. The attainment of such direction is by no means easy; it can, however, be approximately achieved by stretching the immersed nerve in a bath of physiological saline, as in Bernstein's trough experiment with the sartorius muscle. ${ }^{4}$ The stretched nerve is arranged so as to be strictly parallel to the facing sides of the trough which form the contacts through which the current is led into the liquid. Under these conditions the nerve appears to be almost inexcitable. In this connection the electrical resistance of nerve must be taken into account; this resistance for any given bulk of the tissue is five times as great in the transverse as it is in the longitudinal direction; but the increased resistance, although large, is insufficient to account for the failure in exciting efficiency. The most plausible explanation of the failure is the close proximity of the changes caused at the poles, which in this case are on either side of each single nerve fibre.

It may be remarked, in conclusion, that although the various factors which determine the exciting value of an external agent have been ascertained in the case of electrical currents, in their fundamental aspects they are true of other stimuli. Thus, mechanical pressure is most effectual at the moment of onset; and the exciting value of such onset is increased in proportion as this is more rapidly effected. By an ingenious arrangement, it can be shown that mechanical press-

1 Schott, Arch. f. d. ges. Physiol., Bonn, 1891, Bd. xlviii. S. 354.

2 v. Kries, Arch. f. Physiol., Leipzig, 1884; Gotch and Macdonald, Journ. Physiol., Cambridge and London, 1896, vol. xx. 1. 247.

${ }^{3}$ Hoorweg, Arch.f. d. ges. Physiol., Bonn, 1892, Bd. lii. S. 87 ; ibid., Bd. liii. S. 587 ; Waller, Proc. Roy. Soc. London, 1899.

${ }^{4}$ Albrecht and Mayer, Arch. f. $d$. ges. Physiol., Bonn, 1880, Bd. xxi.; see also Leicher, Untersuch. a. d. physiol. Inst. d. Univ. Hallc, 1888, S. 1, where previous literature is given. 
ure may be wholly inadequate to evoke an indirect muscular response when slowly applied to a motor nerve, whilst the same pressure, rapidly produced, is fully adequate. A good illustration of the increased exciting value of mechanical change when this is rapid is that afforded by the use of the nerve shaker; the nerve may be thus adequately stimulated by very small, but rapidly effected, changes.

In regard to chemical stimuli, such comparisons are hardly possible, but in so far as these act by the withdrawal of water (glycerin, $\mathrm{NaCl}$, etc.), the rapidity of such withdrawal is obviously increased by the strength of the chemical substance used. Provided that the nerve is not injured by the agent, the exciting value of such substances as those mentioned is in proportion to their degree of concentration. Finally, in regard to thermal agencies, it is only sudden and extensive change which causes these to have any exciting value at all.

The effect of the repetition of the stimulus.-The indefatigability of nerve.-To what extent can a stimulus be repeated and still remain efficient? The investigation of this question opens up a strictly physiological line of inquiry; for the efficiency can only fail on repetition, in consequence of the inability of the living tissue to respond to the constantly recurring physical change.

The subject has been investigated in several ways, which may be broadly divided into two groups. In the first, the nerve is excited with an efficient stimulus recurring at comparatively long intervals, and of uniform character and intensity. In the second, the nerve is excited by a series of stimuli which recur at great rapidity. Does nerve show signs of fatigue when subjected to excitations of the first kind ? Does it show inability to respond when subjected to those of the second?

In the case of a motor nerve, the index of excitation afforded by the indirectly evoked muscle response is itself susceptible of fatigue; the gradual failure in this contraction, when the nerve is excited by a series of stimuli recurring every few seconds, is thus no indication of a failure in the nerve. It is possible to avoid this source of error by eliminating the muscle. This can be done in the mammal by the use of curare. $A$ sufficient dose is given to paralyse the motor nerve-endings, and artificial respiration is employed until, after some hours, the effects of the drug disappear, in consequence of its elimination. ${ }^{1}$ The exposed nerve is stimulated at intervals during the whole period, and, as the curare paralysis wears off, it is seen by the muscular contractions which now appear, that although the nerve has been subjected to this prolonged excitation, it still responds to the action of the stimulus.

Bernstein prevented the muscular contraction in the frog by blocking the transmission from the seat of nerve excitation to the muscle, this being effected by leading a galvanic current through a portion of the nerve. The nerve was then excited for a prolonged period above the block; on opening the galvanic current, the muscular contraction showed that the nerve was still being adequately excited. ${ }^{2}$

This method has been improved by using synchronous short periods for closing both the excitation and the blocking currents; by alternate reversal of the latter, any injurious effect due to their prolonged closure is largely got rid of. ${ }^{3}$ It is found that after such excitation

${ }^{1}$ Bowditeh, Arch.f. Anat., Physiol. u. wissensch. Med., 1870.

2 Berustein, Arch.f. l. ges. Physiol., Boun, 1877, Bd. xv.

${ }^{3}$ Wedenskii, Centralbl. f. d. med. Wissensch., Berlin, 1884. 
has continued for six hours, with the sciatic nerve of the frog the nerve still responds to the stimulation, for, on the removal of the block, the muscular contraction reappears in its initial intensity.

The propagation has been blocked by the local application of ether vapour to a portion of nerve intervening between the excited region and the muscle; on removing the ether, the excitation of the nerve still caused the muscle to respond after twelve hours. ${ }^{1}$ The secretory nerveendings of the chorda tympani can be paralysed by atropin, and the continuons excitation of these fibres is seen to become effective as the atropin wears off. ${ }^{2}$

The electrical changes in the nerve have been taken as an index by Waller. In this case the indication of each group of electrical responses was obtained by photographing the galvanometric variation. The nerve was submitted to a short series of rapid excitations of similar intensity which recurred at regular intervals of one minute, and the resulting effects showed no evidence of diminution through fatigue. ${ }^{3}$

Nerve fibres are thus remarkable in being so differentiated as to be unaffected by repeated activity, and they offer a striking contrast in this respect to other parts of the nerrous system. Thus the nerve junctions in the central nervous system and the neuromuscular connections are readily fatigued, the latter more readily than muscle itself; so that if a muscle is indirectly excited, until it can no longer respond, it will be foumd that it is still capable of contracting to direct stimulation.

In these cases we may assume either that the terminal nerve fibres differ from the fibres of the main trunk, or that the fatigue is localised in the field of conjunction which lies on the further side of these terminal fibres, whether in muscle, gland, or central grey matter. In this connection the fatigability of the isolated electrical organ may be referred to, the activity of which is in all probability that of the terminal nerve fibres. ${ }^{4}$

The limits of efficient stimulation by rapidly repeated stimuli. -The second method of inquiry, although apparently of a different character, involves to some extent the same issues. Can a nerve be excited by a series of stimuli, when these recur at very rapid intervals, and if so, at what point is a limit reached for such excitation? The muscular response is in this case an adequate index of nerve excitation, and the simplest method of obtaining information is one in which two electrical excitations of the nerve occur in rapid succession. Under these circumstances, the two muscular responses must obviously be fused, and when the second excitation occurs, at a very brief interval after the first, this summated muscular effect may be so slight as to be with difficulty distinguished from that of the single twitch. Those cases in which such a difference can be detected furnish undoubted evidence that the nerve has been adequately excited by the two stimuli. Various methods have been employed for obtaining two excitatory states in rapid succession. The nerve may be simultaneously excited at two points, one near, the other remote from the muscle. In this case, with a distance of $15 \mathrm{~mm}$. between the seats of excitation, the difference in the length of the nerve along which the

1 Masehek, Sitznngsb. d. k. Akad. d. Wissensch., Wien, 188i, Bd. xev. Abth. 3.

${ }^{2}$ Lambert, Compt. rend. Soc. re biol., Paris, 1894.

3 Waller, Croonian Lecture, Phil. Trans., London, 1896.

${ }^{4}$ Goteh and Burch, ibid., 1896, vol. elxxxvii. B, p. 347. 
excitatory states are propagated to the muscle is such that at $15^{\circ} \mathrm{C}$ the one will reach the motorial nerve endings only 0005 sec. in advance of the other. The results are complicated ly the conditions of the experiment, since the part of the nerve subjected to the electrical stimulation is changed as regards both conductivity and excitability, these changes being dependent upon the direction and the intensity of the exciting currents. In the most favourable conditions, it has been found that the effect of the two excitations is to evoke a contraction which is larger than that evoked by either excitation alone. This may mean that two excitatory states recurring at $\frac{1}{2000}$ sec. interval can be propagated along the nerve without blending; in other words, the upset of nerve equilibrium by the first stimulus does not interfere with the production of a fresh disturbance by a second one at this interval. ${ }^{1}$

Another method is that of exciting the nerve at the same point by two successive electrical stimuli. The alterations as regards both conductivity and excitability are even more pronounced uuder these conditions, but in spite of these it has been found possible, by the use of minimal stimuli, to evoke, with two excitations $\frac{1}{1000}$ sec. apart, a response which is larger than that produced by either single excitation. ${ }^{2}$ Pecent experiments carried out under the direction of the present writer have, however, limited the extent of this capacity of the nerve to respond to a second stimulus. ${ }^{3} \quad$ These show that a summated muscle contraction camnot be evoked by double excitation of a nerve at $3^{\circ} \mathrm{C}$, unless the stimuli are $\frac{1}{200}$ sec. apart, whereas at $15^{\circ}$ there is evidence of summation with stimuli at $\frac{1}{1000}$ sec. to $\frac{2}{10^{2} 00}$ sec. apart.

A large number of rapidly recurring excitations of the same point of the nerve have also been used. The difficulty in this case is the uniform production of the rapid series of electrical currents. By the use of a small dynamo, it is possible to obtain a series of sinusoidal current variations, at a rate of 1800 to 2000 per second. With this rate the nerve at $15^{\circ} \mathrm{C}$ can be excited, and the muscular response is a prolonged tetanic contraction. ${ }^{4}$ It was observed by Bernstein that with a rapid series of make and break induction shocks, the resultant muscular response, when these recurred at 500 per second, was not a tetanic but a single initial contraction. ${ }^{5}$ It was therefore surmised that only the first excitatory state was adequately produced. The facts just mentioned are opposed to any generalisation from these experiments. The failure of the tetanic response undoubtedly implies that at the temperature employed a relatively smaller nerve disturbance is produced by the successive excitations, as compared with that evoked by the first of the series, but it does not mean that at intervals of $\frac{1}{500}$ sec. uerve excitation is impossible. ${ }^{6}$ This is shown by experiments in which telephone currents, augmented by an appropriate microplone, are used for purposes of excitation. With these, Roth found that it was possible to excite the nerve so as to obtain a tetanic muscular response, with excitations recurring at from $\frac{1}{1000}$ sec. to $\frac{1}{5000}$ sec. interval. He observed, however, that as the rate increased the intensity of the stimuli

I Werigo, Arch. f. d. ges. Physiol., Bonn, Bd. xxxvi.

${ }^{2}$ Sewall, Journ. Physiol., Cambridge and Londou, 1880, vol. ii. ; 1881, vol. iii.

${ }^{3}$ Boycott, ibid., 1899, vol. xxiv.

- Langdon and Schenck, Cincin. Lancet-Climic, 1896.

5 Bernstein, "Ueber d. Erreg. Nerven- u. Muskelsysteme," 1871.

6 Engelmann, Arch.f. d. ges. Physiol., Bomn, 1871, Bd. iv., who also found that failure of excitability occurred in nerve stinulated by rapidly recurring eurrents. 
had to be augmented, and that if such augmentation was not made, the muscular response was an initial twitch. ${ }^{1}$ Kronecker used magnetoinduced currents, produced by the vibration of a steel core, within the primary coil of a specially constructed induction apparatus. The production of a note indicating 20,000 vibrations per second was effectual for the excitation of the nerve, the muscular response being at first a tetanus, but on repetition it became an initial twitch, and subsequently failed. ${ }^{2}$ In this connection attention may be drawn to the fact that the Hertz oscillations, which are of a different order, being far in excess of any of these rates, only excite when very intense, and then generally evoke initial twitch. ${ }^{3}$

It thus appears that the nerve at $12^{\circ} \mathrm{C}$ is capable of being excited by stimuli recurring at very rapid intervals, but that the susceptibility to excitation is greatest at the commencement of such stimulation, and diminishes with the repetition. In explanation of this, Bernstein and others have laid stress on the possibility of functional interference due to the overlapping of the successive nerve responses, which thus become fused; it is, however, probable that no such fusion occurs. The duration of the excitatory disturbance in nerve is suggested by that of the electrical nerve response. This has been variously estimated in frog's nerve as lasting from $\frac{1}{1500}$ sec. to $\frac{1}{100}$ sec., and undoubtedly the duration is greatly increased at low temperatures. An overlapping is thus possible, particularly at low temperature, but the real question is how far the response to a second stimulus is prohibited under these conditions, by the fact that the response to a previous stimulus is still in progress. In other words, is there an inexcitable or " refractory" period during activity? Wedenskii determined the electrical excitatory changes by connecting a telephone with four sciatic nerves, placed side by side, and exciting with rapid stimulation at two points. The telephone note caused by the excitatory changes was always the same pitch, whether the excitation was double or single,- the only perceptible difference being that in the former case the character and intensity of the note varied at rhythmical intervals, due to alterations in nerve excitability. ${ }^{4}$

The graphic records of the electrical responses, evoked in nerve by two successive stimuli, and obtained by the capillary electrometer method, appear to show that a second response cannot be evoked if the change evoked by its predecessor is still in progress. The exact interval necessary for the second stimulus to be effective thus obviously depends upon the duration of the first change, and this is largely determined by temperature..$^{5}$

Finally, it must be borne in mind that the experiments just referred to deal almost exclusively with the medullated motor nerves of the frog and mammal. At present there is no reliable information as to the possession of the same attributes by the non-medullated nerves or the afferent medullated nerves, as the determination by means of either the muscular index or the electrical nerve response presents great technical difficulties. On general grounds, it may be assumed as

\footnotetext{
${ }^{1}$ Roth, Arch. f. d. ges. Physiol., Bonn, 1888, Bd. xlii. S. 91.

"Kronecker: "Die Genesis des Tetanus," Arch. f. Anat., Physiol. u. wissensch. Med., 1868.

${ }^{3}$ Lodge and Gotch, Rep. Brit. Ass. Adv. Sc., London, 1894, p. 818.

${ }^{4}$ Wedenskii, Compt. rend. Soc, de biol., Paris, 1893, tome cxvii. p. 4.

${ }^{5}$ Gotch and Burch, "Electrical Response of Nerve to Two Successive Stimuli," Journ. Physiol., Canıbridge and London, 1899, vol. xxiv. p. 410.
} 
probable that nerves differ as to the possession of relative indefatigability and susceptibility to stimulation by rapidly recurring excitations. The rapid failure of non-medullated nerves, when removed from their connections, appears to indicate that they do not possess such properties to the same extent as medullated motor nerves.

\section{Circumstances affecting Nerve Excitability and Conductivity.}

Maintenance of the circulation.-It is common knowledge that the blood supply is essential for the maintenance in their normal condition of the living structures of higher animals, and that the failure of vital activity in consequence of its cessation is one which is more rapidly produced in warm- than in cold-blooded animals. Thus the blood can be replaced in the frog by water containing suitable proportions of $\mathrm{NaCl}$, $\mathrm{KCl}, \mathrm{CaCO}_{3}$, etc., and the animal kept alive in an apparently normal state for many days. A similar variation, as regards dependence upon blood supply, is observed in the different structures of any one animal, and in the different parts of the nervous system. It is well known that the central portions of this system are the first to fail in consequence of the cessation of the circulation, and that the cerebral cortex is more rapidly impaired than the bulbo-spinal system, whilst excised nerve trunks retain their excitability for a very considerable time. Such retention implies the possession by the fibres of nerve trunks of properties which enable them to resist the gradual invasion of those changes that bring about their death. This resistance depends very largely upon the general condition of the animal previous to the time at which the nerve is excised. Thus frogs kept in captivity during the summer become anæmic, and their nerves more rapidly fail on exposure than those of winter frogs.

It is to be expected, in view of the facts as to indefatigability, etc., brought forward in the preceding chapter, that nerve fibres should offer a very considerable resistance to functional failure, in cousequence of the arrest of the circulation, their metabolism being such as to require but little assistance from extraneous sources.

If injurious effects of other kinds, such as loss of water through drying, temperature changes, etc., are avoided, the medullated fibres of a nerve trunk maintain their excitability for many hours, and, in the case of the frog, for days after the circulation has ceased.

Ritter-Valli law.-In the medullated fibres of the frog's sciatic, the failure is one which is gradual in its development, and is more rapidly produced in the central than the distal portions of the nerve. This is enunciated in the Ritter-Valli law, which states that the susceptibility of the nervous system to excitation, as indicated by the muscle response, fails first in the central portions, then in the peripheral nerve trunks, and, last, in the terminal arborisations within the muscle. ${ }^{1}$

The experimental proof of these statements is, as regards a nerve trunk, complicated by the alterations produced by nerve-section; this always produces a very considerable rise in excitability, due to the electromotive changes caused in the neighbourhood of the section. These changes are such that a persistent current (demarcation current) flows through the nerve fibres from the injured to the uninjured portions

${ }^{1}$ Vide Budge, "Lehrbuch. d. Physiologie," Leipzig, 1862, S. 665. 
in the immediate neighbourhood; as this spreads along the fibres, in accordance with the known distribution of currents in moist conductors, the nerve in the vicinity of the section is most subjected, and the further portions less subjected to its influence. Wherever it leaves the fibres, these are placed into a cathodic state of heightened excitability. The method, therefore, of preparing a nerve with its attached muscle, freeing it from all other connections and placing it in a moist chamber, involves both the alterations due to arrest of the circulation and those due to the section of the nerve at its central end, together with other similar changes wherever a branch from the main trunk has been cut. In order to diminish the extent of these injury effects, the sciatic nerve of the chloralised frog has been used. The removal of the sensorium, the exposure of the heart, the bleeding of the animal, and the slight dissection for the adequate exposure of the sciatic nerve in a central and a peripheral region, are the only operative procedures employed. In such a preparation the changes in excitability are ascertained by determining the intensity of a single given localised stimulus, which is adequate to evoke a minimal muscular twitch,i.c. the minimal exciting value of a local stimulus. Some form of electrical stimulus has been generally used in order that the physical factors may remain unaltered, and the particular one chiefly employed has been the break induced current. The minimal exciting value of such a stimulus for the more central regions is at first attained with slightly lower intensity than for the peripheral; subsequently, the conditions are gradually altered, until finally the central end demands a more intense stimulus than the peripheral one. Two sets of factors may contribute to the production of this final condition. The excitability alone may change, or the conductivity, or both functions; the phenomena would be equally present, whether failure in conductivity or in excitability existed in different degrees throughout the nerve. The separation between the properties of conductivity and excitability clearly admits of these two possible interpretations of the experimental facts.

Indeed, it has been suggested that the initial apparent increase in excitability observed in the neighbourhood of a nerve section is not due merely to the circumstance that the excitatory state at this point is more easily produced, but also to the development of an avalanche effect, occurring during the propagation of the state along the nerve to the muscle, the excitatory state gathering volume as it progresses. ${ }^{1}$ There is, however, little confirmatory evidence of such an increment. It is certainly not present with all stimuli, for on using chemical stimulation the peripheral part of the nerve appears to be more excitable than the central, ${ }^{2}$ whilst the experiments of Tigerstedt with mechanical excitation show that in an uninjured nerve all parts are at first equally excitable. ${ }^{3}$ Even in the case of induced currents, experiments made under the direction of the writer by Dear show that, with nerves kept in appropriate saline solutions, the excitability after eight hours gradually fails in all parts of the nerve equally,--thus contradicting the Ritter-Valli law.

Maintenance of continuity with nerve cells.-Every nerve fibre, whether medullated or non-medullated, is the specialised process of

1 Pflüger, "Unter'sueh. ueber Electrotonus," Perlin, 1859, S. 140.

2 (triitzner, Arch.f. d. ges. Physiol., Bomn, 1893, Bd. liii. S. 83.

3 Tigerstedt, "Studien u. mechanisch. Nervenreizung," 1880. 
some nerve cell. The severance of the fibre cuts off the distal portion from such cellular connection. If this is carried out in the manmal, with no appreciable disturbance of the circulation, and the animal kept alive, changes manifest themselves in the distal portion (Wallerian law of degeneration). The substance of the sheath in medullated nerve is altered, so that this, instead of being a continuous mantle, is changed to a series of discontinuous swollen masses; the axis cylinder is broken up, and the primitive sheath nuclei are multiplied. Finally, the whole is invaded by wandering cells, the fragments of axis cylinder and inedullary sheath disappear, and the nerve fibre is replaced by a strand of connective tissue. All these changes are gradually developed, commencing within a few days of the section, and being completed after some weeks or months. The initial change may commence within forty-eight hours of the section in the mammal, and soon presents itself throughout the whole length of the distal portion of the fibre. The maintenance of the normal condition of a nerve fibre, both structurally and functionally, is thus dependent upon the preservation of functional continuity with the cell of which it is a process, and there is reason to believe that the nutritive influence exerted by the cell is associated more especially with its nucleus. A manumalian nerve with unimpaired circulation, if severed from its cell comnection, soon loses its fundamental physiological properties. In the case of the sciatic of the rabbit, stimulation below the point of severance evokes little or no response in the muscles to which it is distributed. This functional failure may appear forty-eight hours after the division of the nerve; it pervades the whole distal portion, and is evidently associated with loss of conductivity dependent upon the breaches in axis cylinder continuity, produced by degenerative changes.

Galvanometric observations made by the writer appear to show that, after three or four dlays, the rabbit's sciatic still gives indications of a localised electrical response, although no transmitted one can be detected.

The time of onset of the failure of function in consequence of degeneration differs very greatly, not only in different mammals, but in different nerves of the same animal. Thus Arloing found that with corresponding motor nerves of the $\log$ and monkey, the section in the former caused total loss of functional conductivity in four days, whereas in the latter this failure was not established until a longer period. The time of onset of the failure differs in the sciatic, median, facial, and vagus of the same monkey. The different nerve fibres of any one nerve trunk vary in this respect; the well-known experiment as to vaso-constrictor and vaso-dilator fibres in the sciatic of the frog is an illustration of this, the latter maintaining their excitability longer than the former. The different fibres of the divided vagus nerve of the monkey lose their excitability at different times, the cardio-inhibitory fibres in eight days, whilst the motor fibres for the larynx and nesophagus may be still excitable after thirteen days. ${ }^{1}$

The regeneration of medullated nerve appears, from the work of Ramier, which has been recently confirmed by Ströebe, ${ }^{2}$ to be largely due to the growth of new medullated fibres from the central encls of

${ }^{1}$ Arloing, Arch. de physiol. norm. et path., Paris, 1896, p. 75; see also Langley and Anderson, Journ. Physiol., Cambridge and London, 1896, vol. xix. p. 381.

2 Briti. $\approx$ path. Anut. u. $\approx$ ally. Puth., Jena, 1893, Bil. xiii. s. 160. 
the normal nerves. These invade the degenerated tracts, which offer especially favourable media for their growth, and serve as guides to the appropriate terminal structures. The suture of a divided mammalian nerve, such as the sciatic of the rabbit, is followed by an outgrowth of afferent fibres, whose excitation is capable of evoking reflex effects in eight days; the establishment of motor connections of the efferent fibres with the muscles is slower, movement not being possible until about forty days. The actual time of recovery must differ both in different animals and nerves.

$\mathrm{Erb}^{1}$ states that after local compression of the nerve, the restoration of movement through spinal cord activity takes place at a date when the nerve peripheral to the injury is still inexcitable. This result the writer has failed to confirm; if true, it is an instance of the recovery of nerve conductivity for specific impulses, when excitability to external stimulation is still unrestored, and furnishes another instance of the separation of these two aspects of nerve activity.

Excitability and conductivity in different nerves, and in different parts of the same nerve.-The comparative excitability of different nerves may be ascertained by allowing the same stimulating electrical current to traverse simultaneously different nerves; or the bundles of different nerve fibres, which are bound up by epineurium into one nerve trunk. A well-known observation is that first made by Ritter, and confirmed by Rollet, ${ }^{2}$ as to the minimal intensity of nerve stimulus adequate to arouse responses in the groups of muscles supplied respectively by the peroneal and tibial branches of the sciatic nerve of the frog. If the whole trunk is excited, the first-named group of museles contract with an intensity of electrical stimulus which is inadequate to evoke a response in the second group; hence the neuromuscular apparatus of the flexors and abiuctors is more excitable than that of the extensors and adductors, and on etherisation a stage may be reached when exeitation of the sciatic causes contraction of flexors only. It does not necessarily follow from these and other analogous experiments, that the nerves of the flexors possess greater excitability, since the whole neuromuscular comnection is involved in the production of the effect. Thus Albanese has shown that a similar result is obtained after simultaneous local impairment of function in the peripheral neuro-muscular mechanism of both flexors and extensors. ${ }^{3}$ The difference between flexor and extensor response obtains for the rabbit as for the frog and toad, and for niechanical and chemical as well as electrical stimuli.* Differences have been also observed in the neuromuscular mechanism of the larynx, the adductors responding to a weaker stimulus of the laryngeal branch of the vagus than that necessary to evoke a response in the abductors; and as regards the normal response to central nervous discharge, the abductors fail first through the local action of ether, cold, etc. ${ }^{5}$

A striking example of the varying excitability of the different nervefibres composing a nerve is furnished by the remarkable response of the crayfish muscles. The excitation of the nerve, with stimulus of

\footnotetext{
${ }^{1}$ Deutsches Arch. f. Klin. Med., Leipzig, 1869, Bd. v.

2 Sitzungsb. d. k. Akad. d. Wissensch., Wien, 1876, Bd. vi. Abth. 3.

"Arch. f. exper. Path. u. Pharmakol, Leipzig, 1894, Bd. xxxiv.

+ Arch. f. d. ges. Physiol., Bonn, 1868, Bd. i.

${ }^{5}$ Horsley and Semon, Brit. Med. Journ., London, 1886, vol. ii.
} 
minimal intensity, evokes a contraction of the muscle opening the claw; with stronger stimuli the normal tonicity of this is actually diminished, whilst the muscle closing the claw is thrown into action. The results appear to show that there are two sets of nerve fibres, augmentor and inhibitory, for each muscle; hence the degree of excitability of these four sets of neuromuscular mechanisms is different for each set.

It is well known that the vago-sympathetic of the frog contains two sets of nerve fibres, the excitation of which causes in the one case inhibition and diminution, in the other augmentation and acceleration of the cardiac contraction. The former are stimulated by electrical currents which are too weak to excite the latter; on the other hand, the inhibitory fibres lose their excitability more rapidly than the augmentor. ${ }^{1}$

The difference in the excitability of different nerves of the same animal, of which the above are illustrations, might be exemplified by many other instances; probably no two nerves are equally excitable. It is not, therefore, surprising that great differences exist in the nerves of widely different animals. One of the most striking instances of this is furnished by the electrical nerves supplying the electrical organs of fishes (Torpedo, Malapterurus, Gymnotus, etc.). These all possess a comparatively low degree of excitability. E. du Bois-Reymond claims for the whole nervous system of these fish, a low excitability, which confers upon them relative immunity to excitation by their own electrical shocks. Although the existence of such lessened excitability is questioned by Schönlein, ${ }^{2}$ there is, in the opinion of the writer, little doubt as to its existence in Malapterurus. In this fish the nerve call only be adequately excited by an intensity of current out of all proportion to that requisite for the excitation of the frog's sciatic, the ratio being at least 100 to 1 ; whilst in neither Torpedo nor Malapterurus is the nerve at all readily excited by chemical agencies. ${ }^{3}$

A strict comparison of the excitability of different nerves is undoubtedly more or less vitiated by inequalities in the electrical resistance, by variations in the position and character of the sheathing investments, and still more by the unknown extent of alterations produced by exposure and dissection. Hence variations in excitability exist even with different regions of any single nerve, such as the frog's sciatic. Any dissection must inflict local injuries, and thus cause local heightened excitability. For this reason the upper two-thirds of the frog's sciatic, for some little time after dissection, is more excitable to electrical stimulation than the lower third, since more branches must be divided in exposing the upper part. ${ }^{4}$ The character of the electrical stimulus appears also to be a factor which has to be taken into account. Thus Fleischl found the excitability different for ascending and descending exciting currents, the former evoking a muscle response more easily in the distal than in the central portions, the latter the reverse. ${ }^{5}$ It has been already mentioned, that the

1 See the article on the "Heart," by Gaskell, in this volume.

2 Ztschr. f. Biol., 1898, Bd. xxxi.

${ }^{3}$ Schönlein, ibid., Bd. xxi. S. 461 ; Gotclı and Burch, Phil. Trans., London, 1896, vol, clxxxvii. $B$, p. 370 .

${ }^{4}$ Fleischl; Heidenhain, Stud. d. physiol. Inst. zu Breslau, Leipzig, 1861.

${ }^{5}$ Fleischl, Sitzungsb. d. $k$. Akad. d. Wissenseh., Wien, 1880, Bd. lxxiv. Abth. 3. See also Weiss, Areh.f. d. ges. Physiol., Bonn, 1898, Bd. lxxii. S. 15.

VOL. 11. - 3 I 
sciatic nerve in the living animal, with the circulation and central connections undisturbed, is equally excitable throughout its whole length to mechanical stimuli. ${ }^{1}$ On the other hand, chemical stimuli (glycerin, $\mathrm{NaCl}$, etc.), are far more effective with the peripheral than with the central portions of such a nerve. The results thus appear to be conflicting as regards comparative excitability of different regions of the same nerve, but undoubtedly some portions are less tolerant of the injurious effects of exposure than others; it is not improbable that unequal changes of this kind are the chief factors in determining the different excitability of the various nerves already referred to.

The conductivity of different portions of any nerve does not vary in so striking a manner as the excitability. There are, however, very great differences in the rate of transmission of structurally different nerves. Thus in the non-medullated nerves of the involuntary muscles, the rate of transmission was estimated by Chauveau as at most 8 metres per second; nearly four times as slow as that of medullated nerves to voluntary muscles. In the non-medullated nerves of Octopus a rate of 4 metres per second, in those of the Anodon a far slower rate of $1 \mathrm{~cm}$. per second was found; whilst in the nerves to the mantle of Eledone, Uexküll found a rate of only one-half to $1 \mathrm{~mm}$. per second. ${ }^{2}$ In the medullated fibres of the electrical nerve of Torpedo, the rate is from 8 to 20 metres per second, ${ }^{3}$ whilst in the single medullated nerve fibre of the electrical organ of the Malapterurus, it is as rapid as that of the frog's sciatic, 28 metres per second. ${ }^{*}$

Attempts have been made to estimate the rate in afferent nerves, by utilising the reflex movement as an index of the amount of the transmitted excitatory state at the central nervous system. The results are undoubtedly ambiguous, through the uncertainty in the time relations of the reflex response, and when, as in experiments on man, the response is a voluntary one, involving the "personal time" of the individual, they become still more dubious. The only reliable data are rheotomic observations of the electrical response, and the rate of transmission in the afferent nerves of the frog appears with these methods to resemble closely that in efferent nerves. An inquiry of very great interest is that of the alteration in rate which occurs in the fine terminal arborisations of the nerves. Whether central or peripheral, these endings are such that the excitatory state has finally to leave the continuous axis cylinder and pass into other excitable tissues. It is known that an appreciable delay occurs under these circumstances; the central delay for afferent endings in the spinal cord is at least .006 sec., and may be considerably more; the peripheral delay in the case of a voluntary muscle has been already alluded to in the section dealing with muscle, it amounts to from 003 sec. to 005 sec. $^{5}$ The electrical organ of Malapterurus furnishes an admirable object for the further investigation for this delay, as in this organ the nerve fibres split up, making a rich network in each electrical plate. The response of the organ producing an electrical shock is one which is

1 Tigerstedt, "Studien u. meehanisch. Nerveureizung," 1880.

2 Ztschr. f. Biol., Bd. xxx.

3 Jolyet, Ann. d. sc. nat., Bordeaux, Sér. 2, tome ii.; Mem. Soc. d. sc. phys. et nat. de Bordeaux, 1883, tome v.

${ }^{4}$ Gotch, Phil. Trans., London, 1887, B, p. 510 ; Gotch and Burch, ibid., 1896, B, p. 347.

${ }^{5}$ Bernstein, Arch.f. Physiol., Leipzig, 1882, S. 329. 
readily evoked by excitation, either of the nerve trunk or of the whole organ and its contained nerves. But whereas the muscular response is retarded by 003 sec. to .005 sec., with indirect as compared with direct modes of stimulation, no such difference is found in the electrical organ of either Malapterurus or Torpedo, provided the nerve trunk is excited close to its point of entry into the organ. In both cases a minimal delay of 003 sec. to 005 sec. occurs. ${ }^{1}$ It would appear, therefore, that the electrical response is not that of another organ apart from the nerves, but is the excitatory change in the nerveendings. If this is so, then the delay of 003 sec. to .005 sec. must be regarded as due to retarded transmission in the nerve arborisations. It is not improbable that a portion of the so-called excitatory time (.003 sec. to 005 sec.) of the motorial nerve-endings of muscle is in reality the time consumed by the slower rate of transmission along these fine nerve twigs, which thus resemble the non-medullated nerves in their retarded rate of propagation.

As regards the central terminations, we have no means at present of ascertaining how the extra delay is to be apportioned between the fine nerve-endings, the unknown field of conjunction, and the branches of the efferent cell through which it is discharged into the issuing efferent fibres. It is, however, logical to assume that in each one of these three situations the difference of structure involves a corresponding difference in the rate of propagation, and that central delay is the sum of the retardation in all these situations.

The transmission time through the substance of a nerve cell is commonly assumed to differ from that along a nerve fibre; but evidence of such difference cannot be said to be at all conclusive. For its ascertainment the preparation must be one in which the cell is bipolar, with processes on both sides of considerable length. Exner estimated the rate of propagation of the electrical nerve response along the sciatic nerve of the frog, through the posterior root ganglion into the posterior root, and found that the interposition of the ganglion did not appreciably affect the time relations of such excitatory electrical changes. $^{2}$ In opposition to this result is that of Wundt, ${ }^{3}$ who found the reflex response to be delayed when the ganglion formed part of the conducting afferent tract. Excitation on the proximal and distal side of the ganglion stellatum in Eledone moschatus is associated with an alteration in the muscular responses. ${ }^{4}$ Finally, Gad showed that the interposition of the vagus ganglion caused a very considerable retardation of from $\cdot 03$ sec. to $\cdot 04$ sec. in the respiratory centre inhibition, which is produced by excitation of the vagus trunk..$^{5}$ It is not impossible that the change of the excitatory state in its passage through the ganglion cell may be of a complex kind, and that it may be so altered in character as to be a weaker afferent stimulus on its arrival at the central endings. Such a supposition would of itself account for the extra delay in the central response to impulses which have traversed the ganglion, irrespective of any additional retardation; hence the p. 347 .

${ }^{1}$ Gotch, Phil. Trans., London, 1887, B, p. 510 ; Goteh and Burch, ibid., 1896. B,

2 Exner, Arch.f. Physiol., Leipzig, 1877, S. 567. Confirmed by Moore and Reynolds,

"Proc. Phys. Soc.," March 1898, Journ. Physiol., Cambridge and London.

${ }^{3}$ Wundt, "Untersuch. z. Mechanik der Nerven u. Nerven Centrum," Bd. ii. S. 45.

${ }^{4}$ Uexkuill, Ztschr.f. Biol., Bd. xxx. S. 317.

${ }^{5} \mathrm{Gad}$, Arch. f. Physiol., Leipzig, 1889, S. 199. 
results, although of great importance as indicating specific change in consequence of the interposition of the ganglion, cannot be taken as clear evidence that this change is brought about by decreased rapidity of transmission through the nerve cell. The delay, which is so marked a feature in the central nervous system, is obviously one involving nerveendings and interposed breaches of continuity as well as nerve cells. ${ }^{1}$

Influence of temperature upon nerve excitability and conductivity. - It has long been known that within certain limits the temperature of a nerve may be altered without causing any permanent impairment of its functions. The lower limit does not appear to be reached, even by a fall to $0^{\circ} \mathrm{C}$.; the higher limit differs slightly in different animals, but is reached in the case of the frog by a temperature of $40^{\circ} \mathrm{C}$., and in most mamnials by one of $50^{\circ} \mathrm{C}$.

In a remarkable work published in 1824, W. F. Edwards ${ }^{2}$ dealt with the influence of many physical agents upon life, and described a number of interesting experiments as to the effects upon frogs, fish, etc., of prolonged immersion in the Seine when at different temperatures. The results showed that the low temperature of winter was far more favourable to life than the higher temperature of summer. The differences between winter and summer frogs are not improbably associated with the effect of rise of temperature, which augments the general tissue metabolism. It is well known that the nerves of summer frogs are less tolerant of exposure, but in addition the nerve functional activity as a whole is lowered. Thus the rate of propagation in the freshly prepared sciatic nerves of $R$. esculenta may be only 14.5 metres per sec. in summer, at $15^{\circ} \mathrm{C}$., i.e., half the rate obtained in winter specimens. ${ }^{3}$ These differences are caused by temperature affecting the whole organism. As regards the direct action of changes of temperature upon nerve trunks, permanent impairment is obviously caused by temperature alterations which exceed certain limits $\left(45^{\circ} \mathrm{C}\right.$.), and temporary diminution or even abolition of function is produced by smaller variations within the limits, $0^{\circ} \mathrm{C}$. to $40^{\circ} \mathrm{C}$. One function, that of conductivity, appears to be very generally lowered by fall and augmented by rise of temperature up to $35^{\circ} \mathrm{C}$.

It is otherwise with the property of excitability. It was long held that this was similarly augmented by a rise of temperature; but in opposition to this view was the well-known fact that the nerves of cooled frogs were far more excitable than those of frogs kept at the normal temperature.

In order to ascertain changes of excitability, it is necessary to eliminate as far as possible changes in the conductivity which may otherwise lead to erroneous conclusions as to excitability ; this can be approximately affected by localising the change of temperature to a small portion of the nerve, and ascertaining the extent to which the minimal exciting intensity is changed in this region only. A further fruitful source of error is present when electrical currents are used as the exciting stimulus; this is due to the very marked alteration in the electrical resistance of the moist conductor which is produced by a change of temperature. Warming a moist electrolyte diminishes, cooling increases the resistance. A simple proof of this is afforded by the following experiment. An exciting induced current is allowed to traverse in succession similar portions of nerve in each of two muscle-nerve preparations

1 See on this subject Schäfer's article "Nerve Cell" in this volume, p. 592.

2 "De l'influence des agents physiques sur la vie," Paris, 1824.

${ }^{3}$ Popielski, Centralbl.f. Physiol., Leipzig u. Wien, 1896, No. 9, Bd. x. 
( $A$ and $B)$, placed in the same circuit. A small glass tube through which water at different temperatures can flow is placed under the traversed region of $A$, and the intensity of the current is adjusted so that with both $A$ and $B$ at the normal temperature of $15^{\circ} \mathrm{C}$, it is just adequate to excite and evoke in $B$ a minimal contraction. On now warming the nerve of $A$ a few degrees the contraction of $B$ becomes maximal, on cooling it the contraction fails. Since the nerve of $B$ is unaltered during the experiment, the apparent physiological alteration in its excitability is in reality due to physical increase and decrease in the current intensity through the change in the electrical resistance of $A{ }^{1}$

The experiments of Hirschberg, Efron, Howells, ${ }^{2}$ etc., were made by stimulating with induction currents without providing against sources of error; hence the conclusion arrived at by them and others that nerve excitability is augmented by rise of temperature up to $35^{\circ} \mathrm{C}$. cannot be legitimately deduced from their observations. The introduction of a sufficiently large external resistance, as in Fig. 254, renders any change of resistance too small to cause any marked alteration in current intensity. By using these and other precautions, it has been ascertained that nerve excitability is raised by local cooling for the following forms of external stimulus : galvanic currents, exciting either at closure or at opening; condenser discharges of large capacity; sinusoidal magneto-induction currents; mechanical and chemical stimuli. On the other hand, the excitability is definitely lowered by local cooling from $35^{\circ}$ C. to $2^{\circ} \mathrm{C}$., for the break induced currents (see Fig. 255) or discharges from condensers of small capacity. ${ }^{3}$ Galvanic and sinusoidal currents of very short duration, 002 sec. or under, appear to excite

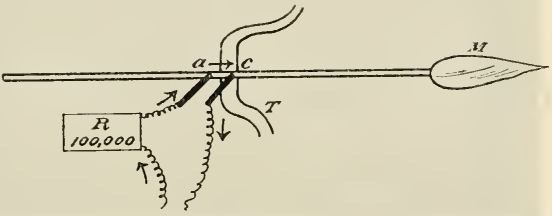

FIG. 254. $-T=$ Tempcrature tube under nerve. $R=$ large resistance placed in exciting circuit, the nerve contacts of which are at $a$ and $c$. The stimulating current is descending, and thus its closure excites at $C$.

rather better when the tissue is locally warmed from $2^{\circ} \mathrm{C}$. to $35^{\circ}$ C., but this augmentation is not so marked as in the case of the break induced current. It thus appears that whilst change of temperature alters the susceptibility of the nerve to excitation, the character of the alteration is one which is not the same for all methods of excitation. The results are the same for the motor nerves of the frog, rabbit, and cat, and for the afferent nerves. In the case of mechanical stimulation the augmentation of the excitability through cold is in harmony with the observation of Steinach, that in cooled frogs a single mechanical excitation can evoke a prolonged electrical response in the nerve. ${ }^{4}$

These facts can only be interpreted as indicating what various observations suggest, that break induced currents excite by the production of changes which are not of the same kind as those caused by

${ }^{1}$ Gotch and Macdonald, Journ. Physiol., Cambridge aurl Loudon, 1896, vol. xx. p. 247.

${ }^{2}$ Arch. f. d. ges. Physiol., Bonn, 1886, Bd. xxxix. S. 75 ; ibid., 1885, Bd. xxxvi. S. 488 ; Journ. Physiol., Cambridge and London, 1894, vol. xvi. p. 298.

${ }^{3}$ Gotch and Macdonald, loc. cit.; Waller, "Proc. Physiol. Soc.," Journ. Physiol., Cambridge and London, Feb. 1899, vol. xxiv.

${ }^{4}$ Steinach, Arch.f. d. ges. Physiol., Bonn, 1894, Bd. lviii. S. 490. 
more prolonged galvanic currents; the warmed nerve being in a state of greater molecular mobility, is best excited by energy which is rapidly applied for the purpose. ${ }^{1}$

They also demonstrate that there is a difference between the susceptibility to respond to the action of an external agent (excitability), and the susceptibility to respond to the internal agent, that is, the excitatory state in the neighbouring portion of the nerve; this latter is the basis of nerve transmission (conductivity).

The propagation of the excitatory state is altered, as already stated, by changes of temperature, as regards both its rate and the magnitude of the transmitted excitatory state.

The alteration was observed by Helmholtz, who found that, in the case of the motor medullated nerves of the frog, the rate of transmission was lessened by cold and increased by heat up to $25^{\circ} \mathrm{C}^{2}$ In every class of nerve, and indeed in every excitable tissue through which an excitatory state is propagated by its successive establishment in

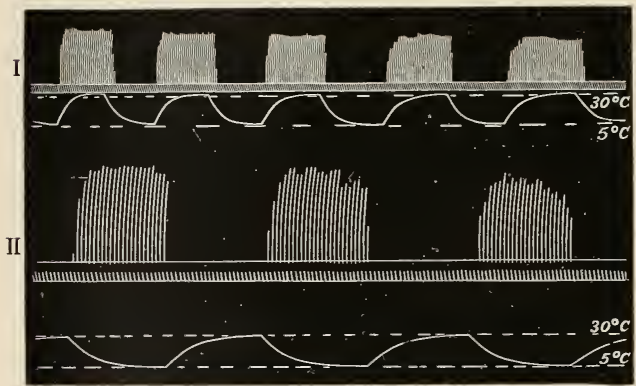

FIG. 255.-1, Response of gastrocnemius muscle to nerve excitation every 2 seconds by a break induced eurrent. Temperature of nerve at seat of excitation varied from $5^{\circ}$ to $30^{\circ}$, as indicated by recording thermometer, the rise of the line corresponding to rise of temperature. The stimulus becomes inadequate when the temperature falls. II, Nerve excited every 5 seconds by closure of a descending galvanic eurrent. The stimulus becomes inadequate as the temperature rises. different portions, the same alteration in the transnission of the excitatory change is produced by variations of temperature. On cooling a portion of a nerve, not only is the rate lessened, but the excitatory state diminishes in amount as it traverses the cooled region, and on emerging from this it continues with the same diminished intensity, so that it evokes a smaller muscular response than it would otherwise do.

The employment of a series of uniform electrical stimulations recurring at regular intervals, and of minimal or nearly minimal exciting intensity, demonstrates this with great clearness. On cooling to $5^{\circ} \mathrm{C}$. the muscular response is diminished or fails, on warming to $35^{\circ}$ the response increases and becomes maximal (Fig. 256, $A$, left portion of record).

A striking discrepancy is sometimes obtained by the use of special modes of stimulation. Thus, if a short galvanic current or a condenser discharge, arranged to ascend the nerve, is used for the excitation of a portion of the frog's sciatic remote from the muscle, and the peripheral portion of the nerve is cooled $30 \mathrm{~mm}$. below the seat of excitation,

1 See Waller, "On the Characteristic of Nerve," Journ. Physiol., Cambridge and London, vol. xxiv., loc. cit., and Proc. Roy. Soc. London, 1899.

${ }^{2}$ Helmholtz, loc. cit. 
it often happens that the muscular response is increased in intensity when the propagation has traversed such a cooled region of the nerve, and yet the rate of propagation is retarded (Fig. 256, $B$ ). ${ }^{1}$

The cause of this anomaly may possibly be found in the simultaneous propagation of the condition produced at the proximal electrode of the ascending exciting current, the condition known as anelectrotonus. This is far more rapidly propagated than the excitatory state, and it appears to be less readily produced in the cold than in the warm. As it is a state of the nerve opposed to the change which constitutes an excitation, any local circumstances which diminish its extent will ceteris paribus augment that of the excitatory state by removing its inhibitory influence. The marked difference between excitation by descending and ascending currents under these circumstances favours this explanation of the anomaly.

The result opens up a most suggestive line of thought, since it implies that propagation is not necessarily the simple transmission of an excitatory change, but that it may be a complex disturbance, one element of which is derived from a specific alteration produced at the pole of a stimulating current. The transmitted effect thus may bear a specific character, determined by the nature of the external stimulating agent which evoked it. We are thus confronted by the possibility that an excitatory state, constituting by its
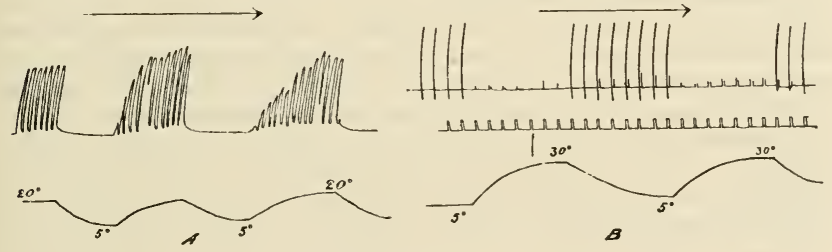

FIG. 256.

transmission what is familiarly termed a nervous impulse, may be different in kind when it is evoked by a different form of stimulus. It is scarcely necessary to dwell upon the importance of such variability in the character of the transmitted nerve effect due to variations in the character of the exciting cause. If true, its application to the excitatory phenomena of the sense organs would modify the doctrine of Johannes Miiller, which assumes the peripheral sense organ to have been elaborated so as to be able, in response to specific stimuli, to start excitatory states, which are then propagated along nerve fibres having distinct terminal connections. Instead of this, it would be possible on physiological grounds to assume that excitatory states, evoked by different specific stimuli of the sense organ, might be transmitted along the same nerve fibre, and yet be so different as to cause in each instance a specific central response. ${ }^{2}$

Influence of chemical substances on excitability and conductivity.-The use of chemical substances for excitation has already been alluded to, and the response evoked by such means has been described as a series of muscular twitches, passing into a flickering tetanus and culminating in a prolonged continuous contraction.

If the kaolin paste forming the point of contact of a non-polarisable electrode is the medium for the salt, and arrangements are made such

${ }^{1}$ Gotch, Journ. Roy. Inst., London, 1892.

${ }^{2}$ See also Rollett, Arch. f. d. ges. Physiol., Bonn, 1899, Bd. lxxiv. S. 163. 
that this is the cathode of an exciting galvanic or induced current, then, in the case of strong $\mathrm{NaCl}$, the gradual alteration in excitability which precedes the appearance of these twitches may be readily observed. It will be found that an intensity of current, just inadequate to excite immediately after the electrode is placed in position, is in a very few minutes adequate, and that the passage of either the galvanic current or the induced current now evokes a maximal contraction. With a little longer time for the operation of the salt, the closure of the galvanic current evokes a closing continuous muscular contraction. The increased excitability thus renders the closing cathodic excitation fully adequate. In a similar way, if the anodic electrode is the salt contact, the opening of a current is rendered effectual, and an opening continuous contraction is evoked in the muscle, with an intensity of current which was inadequate under normal conditions to excite the nerve sufficiently to evoke even a twitch. The salt, $\mathrm{NaCl}$, has thus raised the local excitability. The continuous contraction is due to the salt throwing the nerve into a state of continuous feeble excitation, but at first it stimulates it so slightly that the resultant transmitted nerve effect is unable to evoke the muscular response. The cathodic closure or anodic opening, with its rise of excitability, is sufficient to render this "latent excitation" adequate.

The most plausible view of the mode in which the nerve excitability is augmented by the salt is the following. The molecular equilibrium of the nerve is upset by the salt in the same manner as it is by any stimulating agent. The disturbance, however, is not in the first instance of sufficient magnitude to constitute an evident excitation, but it gradually becomes more pronounced, and may finally attain the requisite magnitude. In the earlier stages the equilibrium of the molecular state being upset, and thus rendered less stable, the action of any additional external disturbing agent readily causes the necessary magnitude of disturbance which constitutes an excitation. We have here an illustration of a fundamental characteristic of nerve. All stimulating agents, when too weak to be able to evoke an adequate excitation, operate upon the nerve so as to disturb it in a direction which, if more intense, would constitute an excitation, and they thus render it easier for an external stimulus to evoke the true excitatory effect, that is to say, they increase local excitability.

In the case of $\mathrm{NaCl}$ it is most probable that the modus operandi largely consists in the withdrawal of water from the nerve fibre, and that glycerin acts in a similar way. The withdrawal of water which takes place in drying, produces the same rise of excitability, passing on into a continuous excitation. It is difficult to localise its operation with the precision which the use of $\mathrm{NaCl}$ permits, but the careful experiments of Harless, Grïnhagen, and others show that a nerve is more excitable to such stimuli as galvanic currents, when it is gradually dried. ${ }^{1}$ The demonstration of this is carried out by experiments similar in character to that described with $\mathrm{NaCl}$, but the drying of the nerve is effected far more slowly than the salt effect, even when, with a dry surrounding of high temperature, evaporation is favoured. The results of both $\mathrm{NaCl}$ and drying are most clearly brought forward by Biedermann in his Elektrophysiologie. ${ }^{2}$

${ }^{1}$ Harless, Ztschr. f. rat. Med. (3), Bd. vii. ; Grinhagen, ibid., Bd. xvi.

${ }^{2}$ Biedermann, "Elektrophysiologie," 1895, S. 582. 
A further point in connection with both these agencies is significant. Having first increased excitability, and then actually excited, they generally end by permanently impairing functional activity. This final extinction occurs rather suddenly, the excitability being first gradually diminished, and then rapidly disappearing, whilst conductivity is not notably affected for some little time afterwards. We have here another link in the chain of evidence which supports the distinction between these two forms of nerve activity.

Although there is but little doubt as to the general nature of the modus operandi of $\mathrm{NaCl}$ in augmenting nerve excitability, it is possible that other neutral salts may affect nerve by causing more specific chemical change than an alteration in the water content of the tissne, since this view, first put forward by Eckhard in regard to nerve, and extended by Kunkel to the action of ${ }^{1}$ neutral salts on muscle, does not explain the peculiar effect of certain salts.

Thus, in the case of $\mathrm{KNO}_{3}$, though the effect described by Ranke is an initial increase with subsequent impairment of excitability, the latter phase is really dependent upon the nature of the stimulus. ${ }^{2}$ Biedermann found that if the sciatic nerve of the frog is treated with a 1 per cent. solution of this salt, its excitability is lowered for most stimulating agencies. The passage of induced currents, or the closure of a galvanic current, evokes a far less pronounced muscular response than that produced by the same intensity of stimulating current before the treatment. On the other hand, the opening of the current now causes an excitation producing an opening muscular twitch, with a current intensity which, in the normal condition of the nerve, was quite inadequate to evoke an opening response. ${ }^{3}$

By placing the nerve in physiological $\mathrm{NaCl}(0.6$ per cent.) for a few minutes, the conditions are again changed, and the nerve excitability restored to its normal condition. This remarkable opening effect will be alluded to again in dealing with the phenomena of polar excitation. It is introduced here to show that chemical agencies which lower excitability for one form of stimulation may raise it for another.

The comparative effects of various chemical substances upon nerve excitability has formed the subject of prolonged investigations carried out by Gritzner. It has been already pointed out in connection with nerve excitation that such comparison can only be made when equimolecular solutions of the various salts are employed. The results of most interest as regards motor nerves are those relating to the action of neutral salts-their acid moieties, and their basic moieties.

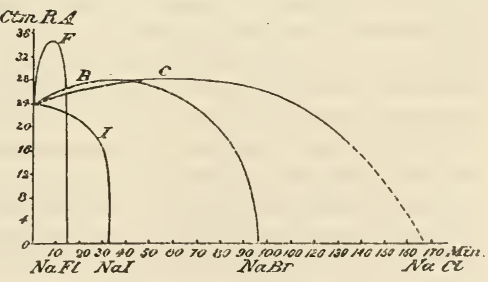

F1G. 257.

Influence of neutral salts.-The alterations in local excitability of motor nerves through the action of neutral sodium salts is best expressed by the diagram (Fig. 257), in which the abscissa indicate the

${ }^{1}$ Eckhard, Ztschr.f. rat. Med., 1851 (2), Bd. i.; Kunkel, Arch. f. d. ges. Physiol., Bonn, 1855, Bd. xxxvi. S. 353 .

2 "Lebenshedingungen der Nerven," 1868.

${ }^{3}$ Biedermann, "Elektrophysiologie," 1895, S. 593. 
time after the application of the salt, the ordinates the minimal exciting intensity of an induced current applied to the upper end of the nerve.

It is seen that sodium fluoride, $2 \cdot 1$ per cent., produces a very rapid rise in excitability, which soon subsides; and that sodium bromide $5 \cdot 14$ per cent., and chloride 2.92 per cent., each causes a rise which subsides comparatively slowly, whilst sodium iodide, $7 \cdot 14$ per cent., causes a fall. The middle and lower ends of the nerve are not so rapidly affected, and the rise when produced lasts longer; moreover, in these situations, all four salts cause a rise. Unlike sodium salts, all potassium salts lower the excitability for such stimuli as induced currents-potassium iodide more than potassium bromide, and potassium chloride least. Injurious effects are produced by any salt of sufficient concentration. These may amount to irreparable loss of excitability, or to an abeyance of this, from which the nerve may be recovered by immersion in isotonic indifferent liquids, blood serum or physiological saline solution. This loss of excitability is produced by some salts more rapidly than by others, the effect, as far as the bases are concerned, being in the following order:-K, Rub, Cœes, $\mathrm{Ca}, \mathrm{Sr}, \mathrm{Ba}, \mathrm{Na}$. As regards the acid moieties of the neutral salts, the injurious action of iodine is more rapid than that of chlorine. It thus appears that initial rise of excitability to stimulation by induced eurrents is caused by appropriate solutions of sodium salts, and initial fall by potassium salts. In the section dealing with chemical stimulation, remarkable facts were referred to which showed that sensory nerves are more readily excited by potassium than by sodium salts, and it appears that the same difference is true as regards excitability, though the evidence on this point is more ambiguous.

The effect of salts mpon the propagation of the excitatory state has not been so thoroughly investigated, owing to technical difficulties; the points of chief interest are those which show that conductivity is not affected to the same degree as excitability. It does not appear that an excitatory state is ever augmented during its propagation through a portion treated with $\mathrm{NaCl}$; on the other hand, a gradual diminution in the intensity of the transmitted effect is often observed, and after prolonged action of the chemical reagent all propagation may cease. The final injurious influences upon conductivity, due to different salts, appears to be produced in the same order as those previously referred to in connection with excitability; in motor nerves potassium salts are more powerful in effecting them than sodium salts, and iodides than chlorides. All these chemical agencies, whether neutral salts, acids, alkalies, or other compounds, which, by their continued action, lower and finally abolish nerve excitability, will, if their action is further prolonged, lower and abolish conductivity; but, in most instances, excitability begins to fail some time before conductivity is appreciably affected. Thus the susceptibility to internal stimulation, which lies at the basis of nerve propagation, remains practically unaffected when, by the constant operation of a chemical substance, the susceptibility to stimulation by an external agent has partially or even wholly disappeared. A remarkable exception is that in which the sciatic nerve is treated in the middle of its course by such an agent as $\mathrm{NaI} 6.1$ per cent. It can retain its diminished excitability to the induced current at this point when excitation of the central portion is quite ineffectual to evoke the muscular response; here conductivity appears to have 
failed completely, whilst excitability is still present, though diminished. ${ }^{1}$

The influence of anæsthetics.-It is the peculiar property of this class of chemical substances (ether, chloroform, carbonic acid, etc.) that they can diminish the functional attributes of living nerve fibres without causing any permanent impairment, and can do this when reaching the tissues through the blood, by inhalation, etc. The degree to which they affect the two main attributes of nerve fibres, excitability and conductivity, has been comparatively recently investigated in isolated nerve, and the results obtained by different observers are in some instances conflicting. The method for determining their direct action upon motor nerves is that first employed by Grünhagen in determining the effects of $\mathrm{CO}_{2}$ gas. $^{2}$ This consisted in enclosing the middle portion of the sciatic nerve of a muscle-nerve preparation of the frog in a small chamber through which the gas could be passed (Fig. 258). The nerve could be excited either in the chamber or on the central side, and it was found that the passage of $\mathrm{CO}_{2}$ gas caused the excitation by the induced current at $\mathrm{X}_{2}$ within the chamber to be ineffectual, whilst similar excitation of the central end at $\mathrm{X}_{1}$ still evoked the muscular response. Similar experiments by Luchsinger, ${ }^{3}$ in which the vapours of ether, chloroform, alcohol, and ammonia, as well as $\mathrm{CO}_{2}$, were employed, showed results which varied with the duration of the action of the vapour or gas. In the early stages the nerve in the chamber could no longer be excited so as to evoke a muscle response by an intensity of stimu-

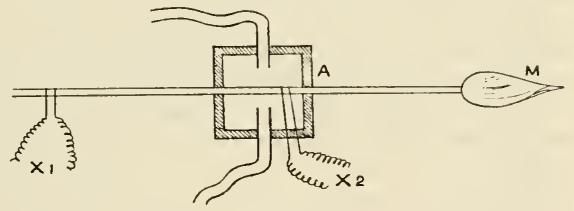

FIG. 258. lus which aroused it before the passage of the vapour, whilst excitation on the central side was unaffected; a subsequent stage was reached, in which even the strongest excitation of the central portion failed to evoke the response, whilst such strong excitation of the nerve in the chamber was still effective. This stage could be reached by $\mathrm{CO}_{2}$ after fifteen to twenty minutes. Hirschberg repeated these experiments with $\mathrm{CO}_{2}$ gas, but did not obtain the failure to the strong central excitation.

It is evident that the experiment is one of considerable importance in determining the extent to which excitability and conductivity are independent. The whole sulject has been investigated with great care by Piotrowski. ${ }^{4}$ His observations were made along the same lines as the preceding, but were extended to include the action of several different modes of excitation, whilst the alterations of the nerve functions through the anæsthetic were determined in a variety of ways, including the variation in the extent of the muscle response evoked by an unaltered intensity of nerve stimulus, the variation in the intensity of the minimal adequate nerve stimulus, and the alteration in the rate of propagation of the nerve excitatory state.

1 See Griitzner, Arch. f. d. ges. Physiol., Bonn, 1893, Bd. liii. S. 113.

2 Ibid., Bd. vi.

3 Ibid., Bd. xxiv.

+ Arch. f. Physiol., Leipzig, 1893, S. 205-290. 
The results were the same, whether the nerve stimulus was the induced current, the galvanic current, the condenser discharge, or mechanical compression; in all cases $\mathrm{CO}_{2}$ or $\mathrm{CO}$ caused a marked diminution of the nerve excitability in the chamber, but had no such obvious effect upon the conductivity of this portion of nerve; on the cessation of the gas the excitability was rapidly restored to its normal value. On the other hand, alcohol vapour caused an initial increase in the local excitability and to a less degree in conductivity; this soon changed, the conductivity becoming rapidly impaired before the local excitability diminished; on the cessation of the alcohol vapour the normal conductivity and excitability were reinstated, but the latter returned quickly, the former much more slowly. A stage in the alcohol treatment was always obtained in which the excitation of the central end of the nerve was quite ineffectual, whilst that of the altered nerve in the chamber was increased in effectiveness. This result cannot be due simply to the fact that propagation occurs along a considerable length of the affected nerve when the excitation is central, but along a much smaller length when this occurs in the gas chamber. It is not due to the spread of the vapour or gas beyoud the chamber to the central end, since simultaneous observation of the electrical change at this end shows that the central stimulus is not influenced as to its exciting value by the play of the gas or vapour on the more peripheral portion of the nerve. It must therefore be concluded that $\mathrm{CO}_{2}$ and $\mathrm{CO}$ are agents which influence local excitability more than conductivity, whilst alcohol influences conductivity more than local excitability. The distinction between the effects of $\mathrm{CO}_{2}$ and alcohol as regards conductivity is further demonstrated by determining the rate of propagation from the central excitation to the motorial nerveendings. $\mathrm{CO}_{2}$ and $\mathrm{CO}$ have but little influence upon this, whereas alcohol vapour causes a retardation from 28 to 4 metres per second.

Ether and chloroform act by diminishing both local excitability and conductivity; the former, however, is dinimished much earlier and in a more pronounced manner than the latter. Thus, in one of the writer's experiments, ether vapour for thirty seconds produced a fall of local excitability such that the stimulus (break induction current) became quite inadequate, whilst conductivity, as tested by a similar central stimulus, was unimpaired. When the vapour was allowed to act for two minutes, the excitability was very much diminished, but still longer exposure was necessary to produce impairment of conductivity. On the cessation of the ether, the central excitation became again adequate in two minutes, showing the return of conductivity, whilst the local excitability did not reappear until fifteen minutes had elapsed, and even then was not restored completely for another ten minutes. Chloroform is far more toxic than ether; it produces the same effects, but is apt to permanently injure the nerve.

To the above experiments with ether, obtained under the direction of the present writer, may be added some with $\mathrm{CO}_{2}$. These show that it is always possible to abolish conductivity by this gas, but that excitability is invariably affected first, and the subsequent impairment of conductivity occurs only when the action of $\mathrm{CO}_{2}$ is prolonged. Just as with ether, recovery takes place rapidly as regards conductivity, and far more slowly as regards excitability. Similar experiments with nitrous oxide showed no anæsthesia as affecting either nerve function; the local excitability seemed to be always slightly augmented by the 
action of the gas, possibly through drying. The results may be compared with those obtained by Waller in isolated nerve, in which the electrical response is taken as the index of nerve excitation; these are referred to in detail in the section dealing with the electromotive phenomena.

Influence of electrical currents upon nerve excitability and conductivity-Polarisation changes.-The passage of electrical currents through a nerve causes profound changes in both its excitability and its conductivity. Since the nerve is a moist conductor, such passage produces electrolytic changes, which are most pronounced at the poles, i.e. the point of entry (anode) and point of exit (cathode). They spread in diminishing amount from these situations along the nerve fibres. These polarisation changes are presumably based upon the formation of electrolytic products or ions ; they manifest themselves during closure by the establishment of an increased electrical resistance and extrapolar currents, on opening by the establishment of counter electromotive changes or polarisation currents, which in the intrapolar region are of opposite sign to the original or polarising current. The formation of ions, and thus the polarisation, may be distinguished broadly according to its locality as external and internal. When a current is led through a nerve by means of metallic electrodes, an extensive electrolytic change occurs at the actual points of metallic contact. The changes may be so pronounced as to produce on the opening of the polarising current a counter polarisation current of sufficient intensity to excite the nerve. Hence, if by means of metallic electrodes a galvanic current is led through the sciatic nerve of the frog, then, on the battery being disconnected, the closure and opening of the connections attached to the electrodes is accompanied by an excitation indicated by a muscular response.

Such external polarisation must mask the effect of any similar change in the nerve fibres themselves, and must be eliminated or reduced to very small proportions in all experiments in which electrical currents are used for nerve stimulation. It is reduced by employing platinum electrodes and currents of very short duration, and by arranging that, when a rapid series of these are used, they shall be alternate in direction, and as far as possible of similar duration and intensity. The make and break induced currents obtained by Helmholtz's modification (the side wire of the induction coil), are employed in order to attain this end. It is still further reduced by employing non-polarisable electrodes.

The most common form of such electrodes is that in which the metallic wire terminates in a zinc rod dipping into saturated sulphate of zinc, and this is connected to the nerve by means of an intervening moist conductor of physiological salt solution. Kaolin forms a convenient non-polarisable medium for the retention of the liquids, as it can be made into a paste and moulded to any desired shape. The actual contacts may be such paste, fine brushes, threads, or filter paper, steeped with the salt solution, or plastered by paste similarly steeped. Silver wires coated with a fine layer of fused silver chloride, and then covered with filter paper steeped in physiological salt solution, have been employed by D'Arsonval for similar purposes. Although advantageous in many respects, these electrodes offer a very large resistance, and are difficult to make as free from polarisation as the other form.

When a current is led through a nerve by non-polarisable electrodes, 
although the external polarisation is comparatively insignificant, there is every indication of the formation of electrolytic ions within the nerve; this must be termed internal polarisation. It is dependent upon the structural and physiological properties of living nerve, and is especially marked in the case of medullated nerve fibres. It can be produced in any structure consisting of a better conducting continuous core, and a worse conducting moist envelope, such as the model devised by Hermann, in which a platinum wire lies within a tube of sulphate of zine or saline solution. On closing the current, the internal polarisation changes are most intense at the poles (anode and cathode), but spread into the adjoining intrapolar and extrapolar regions.

These polarisation products are themselves electromotive, so that the nerve is in a peculiar condition termed by its discoverer, du BoisReymond, electrotonus. ${ }^{1}$ It would be unprofitable at this stage to enter in any letail into these electrical effects, the foregoing reference being intended merely to introduce the excitability changes which are produced at the same time as these alterations in electrical state, and which have been therefore termed the electrotonic alterations in excitability.

Polar changes in excitability.-Pflüger's ${ }^{2}$ work on this subject culminated in a series of general conclusions, deduced from a study of nerve, so careful and exhaustive, that the results have stood the test of time as few other physiological data have done. Subsequent work has extended but not materially modified the original statements, which may be briefly summed up as follows:-

1. Under the influence of a constant current flowing through a nerve, there is an increase in the nerve excitability, at and near the negative pole (cathode), a decrease at and near the positive pole (anode).

2. On the cessation of the current these changes are reversed, the cathode being the seat of a fall, and the anode that of a rise in excitability.

3. The alterations in excitability are most intense at the poles, but spread, diminishing with the distance into the intrapolar and extrapolar regions.

4. At some point in the intrapolar region the boundary between the two polar extensions is reached; this point is therefore unaffected, and is termed the indifference point.

5. The excitability changes are true for all forms of stimulation, electrical, mechanical, or chemical, and for both efferent and afferent nerves.

The experimental proof of the first two propositions is readily effected in a muscle-nerve preparation, by arranging so that a uniform stimulus of minimal exciting intensity is applied to the nerve just beyond the pole of the polarising current which is nearest to the muscle. If this pole be made the anode, the stimulus becomes, during closure, ineffectual; if the cathode, it becomes of maximal exciting intensity, and the alteration persists during the whole time of the closure of the current. A similar method demonstrates the anodic rise and the cathodic fall of excitability immediately after closure. The pronounced character of the alterations at the poles themselves can be ascertained by using such an arrangement as that employed by Grühagen, in which a uniform exciting stimulus, the break or the make induced

1 E. du Bois-Reymond, "Untersuchungen," loc, cit.

2 "Electrotonus," Berlin, 1859. 
current, is allowed to traverse the nerve by the same electrodes as those employed for the galvanic current, and is so directed as always to excite at the pole nearest the muscle.

The changes in an afferent nerve are demonstrable by similar experiments on the brainless frog, the more central pole and its neighbourhood being the seat of the uniform exciting stimulus, and the reflex response the index of the extent of the excitation thus produced.

The investigation of the changes in the intrapolar region is more difficult, since the employment of electrical modes of stimulation is impracticable, owing to the formation, at the exciting contacts, of a subsidiary intrapolar anode and cathode. By the use of mechanical or chemical modes of excitation, the intrapolar anodic and cathodic changes can be ascertained, and the position of the indifferent point determined. ${ }^{1}$

The degree of anelectrotonic fall and catelectrotonic rise of excitability during closure, together with the extent of the spread of the two states, varies with the intensity and duration of the polarising current, and with the length of the nerve traversed by the current (i.e. the distance between the poles).

The influence of varying current intensity is shown diagrammatically in the left-hand portion of Fig. 259 ; the curves below the base line indicate anodic
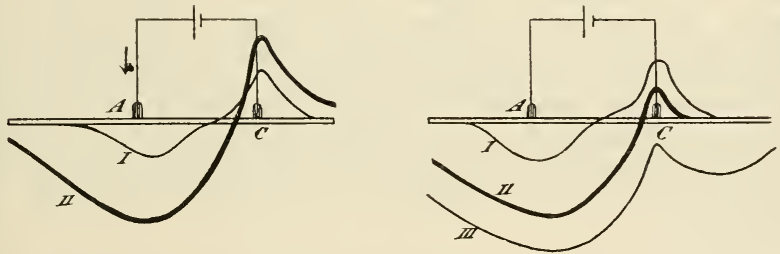

FIG. 259.

fall, those above cathodic rise, of excitability, with $I$. weak, and $I I$. strong currents respectively. It will be observed that the anodic fall tends to increase more than the cathodic rise, so that the indifferent point gradually gets closer to the cathode. The righthand portion of the diagram (Fig. 259) shows the influence of varying duration; here again the anodic change tends to spread at the expense of the eathodic. With currents of considerable intensity and prolonged duration, as in $I I I$, the cathodic change may be completely swamped by the spread of the anodic into both extrapolar regions. The influence of increasing the length of traversed nerve is easily

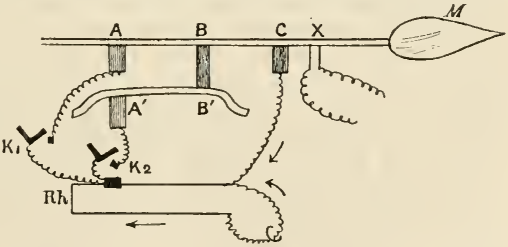

FIG. 260.-The closure of $K_{1}$ allows a descending current to traverse the length of nerve from A to C. By opening $\mathrm{K}_{1}$ and closing $\mathrm{K}_{2}$ the current traverses the two lengths of nerve $\left(A^{\prime} B^{\prime}\right.$ and $\left.B C\right)$. The length $\mathrm{AC}=\mathrm{A}^{\prime} \mathrm{B}^{\prime}+\mathrm{BC}$.

demonstrated by passing the current through a long length of nerve, AC (Fig. 260), and testing the extrapolar excitability by induced currents at $\mathrm{X}$. This is compared with that produced when the same current traverses two

1 Tigerstedt, Mitth. v. physiol. Lab. d. Carolin, med. chir. Inst. in Stockholm, 1882, Bd. i. 
portions of nerve together, equal in length to $\mathrm{AC}$. Of these portions, one $\left(A^{\prime} B^{\prime}\right)$ is an isolated portion to make up the necessary length, the other $(B C)$ is half the original nerve. The total resistance being the same in both cases, the current intensity is unaltered, but the anodic fall and cathodic rise at and near $\mathrm{C}$ is found to be far less rnarked in the second case.

The time relations of the effects.-(a) Polar.-Grünhagen interposed an induction coil in the polarising circuit, and excited with the make induction current caused by closing the polarising circuit; the nerve was thus stimulated the moment that the current flow cominenced. Such an experiment establishes the fact that both the cathodic rise and anodic fall commence at the poles with no appreciable delay on the closure of the current. The electromotive effects of the polarisation changes have also been detected in the immediate neighbourhood of the poles, at an interval of 001 sec. after the closure of a very brief intense current, and are therefore in all probability instantaneous.

It may be pointed out that the absence of any delay is in harmony with the instantaneous character of the nerve electrical response to an exciting current. ${ }^{1}$

(b) Extrapolar.-In regard to the development of the excitability changes in the extrapolar regions, there is considerable difference of view. The electromotive effects (electrotonic currents) have been detected by Hermann, Boruttau, etc., in the extrapolar regions of models and of nerve, very soon after their production at the poles, and hence the rate of their propagation has been considered as extremely rapid, 500 metres per second. On the other hand, Bernstein's investigation with the differential rheotome showed comparatively slow propagation of these electrotonic changes, 6 to 13 metres per second; the probable reasons for this discrepancy will be given in connection with electrotonus.

The recent work of Asher appears to indicate that the anelectrotonic fall spreads at a rate closely identical with that of the so-called nerve impulse. Kiihne had shown by ingenious experiments that the motorial nerve-endings could be placed in the state of anelectrotonic decreased excitability. Thus a curarised sartorius is most excitable at the point of entry of its nerve, but this portion loses its predominant excitability when a galvanic current is led through the nerve, so that the anode is near the muscular termination. Further, glycerin excites the sartorius only through its nerves; it fails to do so if the muscle, with its entering nerves, is the anode of a galvanic current. Starting with such possibility of throwing the motorial nerve-endings into the anelectrotonic state, Asher arranged that a polarising current should be passed through the nerve, so that the anode should lie a known distance from the nerve termination. The muscle was excited directly by an induced current, and the polarising current closed either at the same instant, or at a definite interval before the excitation. There was no diminution in the response, unless the polarising current was closed $\cdot 0007$ sec. before the excitation; and on measuring the distance from the anode to the muscle, a propagation rate for the anelectrotonic decrease was deduced which corresponded with that of the nerve excitatory state, 30 metres per 1 sec. $^{2}$ The experiment led to the further interesting discovery that if the muscular response had commenced, the establishment of anelectrotonus in the nerve-endings had no effect upon the contraction. But grave doubt

${ }^{1}$ Bernstein, "Untersuch. ü. Nerven-u. Muskelsysteme."

2 Asher, Ztschr.f. Biol., 1895, Bd. xxxii. 
is thrown upon the accuracy of Asher's conclusions by recent work done in Hermann's laboratory. ${ }^{1}$ (See also footnote.)

Although the extrapolar development of the excitability alteration and of the electrotonic effects may not at present be considered as contemporaneous, the time relations of the development of these two states at the actual poles are closely similar. This parallelism between the formation of the electrotonic currents and the production of the excitability changes suggests a very close connection between the two classes of effects. It is probable that the electrotonic effects are due to what may be termed two different reactions in the nerve fibre, one the formation of ions, an electrolytic process rendered possible by the physical conditions presented by the living nerve structure, the other the establishment of prolonged excitatory changes with their accompanying electromotive phenomena. The further consideration of this may with advantage be postponed to the section dealing with the electromotive phenomena of nerve, but it may be pointed out that this second reaction is to a great extent the electrical indication of the alteration in excitability, which forms the proper subject of this section. It is not, therefore, surprising to find that the time relations of the development, in close proximity to the poles of excitability and of electrotonic changes, are to a large extent identical.

The two changes, excitability and electrotonic, both commence at the moment of closure, and they are both developed in increasing amount during the first period of such closure. Investigation with the rheotome shows that the anodic fall of excitability, and the anelectrotonic change, gradually increase at the anode during the continued flow of the current, and then slightly decrease; on the other hand, the cathodic rise and catelectrotonic change are both developed quickly, the development culminates much sooner than that of the anodic states, and may subside whilst the latter is still going on, if the closure is sufficiently long.

It is for this reason among others that duration of closure, apart from the intensity of the current, has such an appreciable effect upon the production and spread of excitability changes; the longer the duration, the more will the anodic fall overpower the subsiding cathodic rise, the indifference point moving at first nearer the cathode, until finally the anodic state may spread into the cathodic extrapolar region. For similar reasons a very short closure produces a cathodic rise of excitability, which is more obvious than the anodic fall, and thus a series of alternating induced currents, recurring at rapid intervals, will excite sufficiently to evoke a flickering muscle response, when one induced current of similar intensity and character is inadequate.

The excitability changes on the cessation of the current flow are to be regarded as due to a rebound of the tissue equilibrium, when released

${ }^{1}$ Hermann and Weiss, Arch. f. d. ges. Physiol., Bonn, 1898, Bd. Ixx. S. 237-296. The writer of this article agrees with the position taken up by Hermann, namely, that botl the excitability and the electrotonic modifications commence almost instantaneously in all parts of the extrapolar regions. The fact that such modifications develop the more slowly and less extensively in the extrapolar region, as the region investigated is removed from the pole, accounts for many of the contradictory results hitherto obtained. A certain degrce of change must occur before evidence, whether of decreased excitability or electrotonic effect, can be obtained; this degree can be attained instantaneously at the pole, but it may not be detected in the extrapolar region, although a feeble modification may have begun instantaneously even in this part. Such would be pre-eminently the case with the anodic effects, which have been those chiefly investigated.

VOL. II. -32 
from the new poise caused by the current. The anodic rise and cathodic fall both commence at the poles, on the moment of cessation with no appreciable delay; they probably extend from their polar seats of origin into extrapolar regions, although this has not been satisfactorily proved. The anodic polar rise is the most obvious; this is probably due to the fact that such opening effects are more demonstrable after long closures, and with these the anodic condition is always accentuated. Hence, during such a closure, the anodic fall is one which has to a great extent swamped the cathodic rise in consequence of its greater development, so that, on opening, the resulting anodic "rebound" is very appreciable, and a rise of excitability ensues, which increases to a maximum and then declines.

On the other hand, the circumstances which favour the cathodic rebound are, cocteris paribus, those which favour the production of the preceding cathodic rise; since, however, this rapidly attains its maximum and then subsides even during closure, the physiological condition which constitutes the rebound may be said to have begun even before the cessation of the current flow; hence, when such cessation occurs, the rebound constituting a cathodic fall of excitability is not a prominent feature. It is, however, demonstrable with closures of short duration, particularly by the use of mechanical excitation in the neighbourhood of the poles; this method has been employed by Tigerstedt with great success in the determination of the four polar excitability changes. ${ }^{1}$

The whole cycle of change due to the passage of a current may be summed up as follows:-

\section{Closure.}

Anodic fall of excitability (i.e. greater stability of molecular equilibrium), established instantaneously, increasing slowly to a maximum, and slowly declining.

Cathodic rise of excitability (i.e. less stable molecular equilibrium), established instantaneously, increasing rapidly to a maximum, and subsiding quickly.

\section{Cessation.}

Anodic rise of excitability (rebound to lessened stability), established instantaneously, increasing, and subsiding slowly. ${ }^{2}$

Cathodic fall of excitability (rebound to greater stability), established instantaneously and subsiding rapidly.

It has been suggested that the causation of the changes on cessation is to be found in the reversed or polarisation current, which is present in the nerve, and which is unmasked when the polarising current ceases; this, if present, would turn the former anode into a cathode, and vice versa. The changes on cessation would thus be, both as to causation and character, simply those due to the closure of a reversed polarisation current, which is unmasked by the cessation of the polarising one. This simple explanation has been applied, as it was logically compelled to be, to the explanation of the opening excitation as well as the excitability changes. ${ }^{3}$ There are, however,

Tigerstedt, loc. cit.

2 From experiments on the excitability produced on the nerves of man, Waller has concluded that the anodic fall produced by a closure may last for ${ }^{\circ} 03 \mathrm{sec}$. after the current has been opened, Arch. de physiol. norm. et path., Paris, 1882, 1. 383.

3 Tigerstedt, Mitth. v. physiol. Lab. d. Carolin. med.-chir. Inst. in Stockholm, "Biological Memoirs," Oxford, 1887, vol. i. p. 49. 
many considerations which render it impossible to regard this as the sole cause. In the first place, it has been already indicated that the excitability after effects on cessation do not correspond in their time relations with those during closure; the anodic after effect is prolonged, unlike the cathodic closing effect. Secondly, the opening anodic excitation may be proluced by nerely diminishing the intensity of the polarising current to such a point that the reduced value of this is still in excess of the polarisation current, so that the latter does not come into play. Thirdly, as will be seen in the section dealing with the electromotive effects produced by currents, the anodic electrical response may be so pronounced and prolonged, on the cessation of the current, as to overpower the actual polarisation after effect, through its being of opposite sign, in which case no such reversal of poles can occur. Finally, in non-medullated nerves, such as those of the crayfish, etc., the amount of polarisation is relatively small, but the excitability changes both on closure and opening are extremely pronounced. There can be no question that the electrolytic phenomena, in so far as these are displayed by polarisation, profoundly modify the nerve conditions, but several facts render it difficult to assume that the polar changes of excitability are entirely dependent upon their presence. In many instances the two effects appear to be concomitant tissue reactions, but even in these cases the excitability changes, although intimately associated with the formation of ions, are presumably based upon a more subtle chemical rearrangement. As regards the nature of such rearrangement, the utmost that can be said is, that it presents itself in two phases, one of augmented, the other of lessened molecular stability. If an agent, such as a current, enforces by its continued passage the predominance of either phase, its cessation is followed by a rebound towards that of the other.

The alterations of excitability, due to the passage of electrical currents, may be caused by muscle or nerve demarcation currents. The latter is developed in the neighbourhood of a cut section or injury, the tissue here becoming galvanometrically negative to its surroundings; a current thus flows along the nerve fibres from the cut section, it then leaves the fibres to return by the juices of the tissue to the seat of its causation. The neighbourhood of a cut section is thus thrown into the condition of catelectrotonic rise of excitability, and in this way the increased excitability due to injury is explained.

Conductivity changes.-Nerve conductivity is also altered by the passage of electrical currents; this shows itself as an alteration, both in the magnitude of the transmitted excitatory change, and in the rate of propagation. The simplest demonstration of the former is that afforded by the following experiment. If the central part of the frog's sciatic be excited by a stimulus of minimal intensity, the resulting muscular response fails or is much diminished when a galvanic current is closed in either direction through the peripheral portion of the nerve. A more or less effective block for transmitted excitatory states is thus shown to be produced on and during closure at the anode; i.e., the nerve in this region is rendered less responsive to the internal stimulus which is the basis of the transmission. The effect lasts for a little time after the cessation of the current, especially if this has been moderately intense and of short duration, but this after block is now localised at the cathode. 
The closing anodic and opening cathodic blocks are evidenced in a different form of experiment, namely, that which affords evidence for the well-known third stage of Pflüger's law of contraction. Here the passage of an ascending current of considerable intensity excites, on closure, at the upper or central pole (the cathode), the transmission from this has to traverse the lower pole, the anode, and the size of the transmitted effect may in consequence be so much reduced as to be inadequate to evoke a muscular response. In a similar way the feeble muscular response evoked on opening a strong descending current is due to the inability of the opening excitation which occurs at the upper anodic contact, to force the after block present at the cathode. Both experiments show that the interference with conductivity must be established at the instant of closure and opening respectively. The terms block and after-block thus imply a condition of the tissue at the anode on closure, at the cathode on opening, in which conductivity is impaired. The degree of impairment, i.e. the extent of the block, depends primarily upon the intensity of the polarising current causing the change, but it also depends upon the duration of the closure; for although the anodic blocking commences at the instant of closure, it resembles the anodic fall in excitability in being augmented during the continued flow of the current. Thus a current of very short duration does not impair anodic conductivity to the same extent as one of long duration, and it may often happen that the stimulation of that portion of a nerve, central to the polarising current, may be at first adequate to force the block and evoke the muscle response, but may cease to be adequate as the polarising current continues to flow, rendering the block far more complete. An experiment illustrating this is the following. A series of galvanic currents of sufficient but uniform intensity are closed at regular intervals in the ascending direction through the sciatic nerve of the frog. The duration of closure is gradually increased from a minimal time of about 001 sec. or less. As the duration of each current is increased, the muscular response, which at first is not present, commences and increases in magnitude; it then diminishes and can utterly fail; finally, it reappears again, and may reattain its initial maximal size. ${ }^{1}$ The failure, termed by Fick, the gap, is obviously due to an effective block at the lower anodic pole, and the resultant interference with the propagation of the excitatory effect evoked on closure at the upper cathodic pole. This block is only rendered adequate when a certain duration of current flow has been reached; beyond this point the return of the contraction is due to the opening excitation at the anodic lower pole. Increased duration thus acts like increased intensity in augmenting the anodic block, and the variation of either of these factors when the other is kept uniform can produce similar results. Thus the passage of a series of very brief galvanic currents of constant duration, but variable intensity, causes the same series of phenomena; the muscular responses appear when a certain intensity is reached, increase in size, and then diminish until they fail, and finally they reappear again and attain a definite maximum. The same results can even be obtained by the use of ascending induced currents, and their employment furnishes another proof of the truth of the statement, that the anodic interference, although produced at the xlviii.

${ }^{1}$ Fick, Sitzungsb. d. k. Akad. d. Wissensch., Wien, 1862-63, Abth. 3, Bde. xlvi., xlvii., 
instant of closure, is increased with the duration of the current which causes it, for Tigerstedt found that the more prolonged make induced currents gave the results far more easily than the more rapidly developed currents caused by the complete break of the primary coil circuit. ${ }^{1}$

The above are experimental demonstrations in motor nerve of the effect of the anodic block, or cathodic after-block, upon the intensity of the excitatory change which has to be propagated through the altered region.

These alterations in conductivity, like those in excitability, have been shown to be valid for excitatory states evoked by all varieties of local stimuli, electrical, mechanical, and chemical, as well as for those due to discharge from the central nervous system. They are present in afferent as well as efferent nerves, and can be demonstrated in these by using the reflex response as the index of the propagation.

A further question upon which there is at present but little information is, whether transmission through the cathodic region during closure, or through the anodic on opening, augments the propagated excitatory state. As a rule the blocking effects more than counterbalance any such augmentation.

In order to reduce these, the most effective method is that in which tripolar leads are used for the galvanic currents. The arrangement allows of three poles, for instance, an anode $B$, with a cathode on each side, at $A$ and $C$, as in Fig. 261. During closure the excitation of a break induced current at $X$, above the

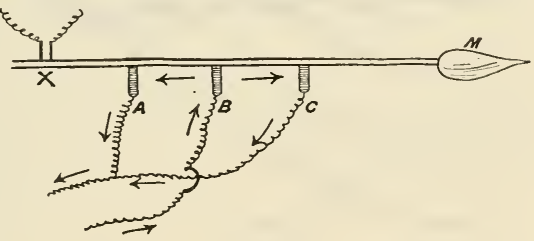

FiG. 261. centripolar cathode $A$, evokes a far more pronounced muscle response than can be obtained by the reversed arrangement, with the cathode at $B$ and the anodes at $A$ and $C$. In both cases the same length of nerve is subjected to current flow; hence it may be inferred that the excitatory change in the first tripolar arrangement gathers force during its transmission through the second cathodic region. ${ }^{2} \mathrm{~A}$ similar but less marked augmentation has been observed by the present writer, when, with the cathode in the middle and two anodes, one on each side, the nerve is excited at $X$ immediately after the galvanic current is opened. In both instances the closure must be of short duration, and the current intensity comparatively low.

In addition to these changes in the magnitude of the transmitted effect, the polar conditions undoubtedly affect the rate of propagation.

The simplest proof of such alteration in rate is furnished by the experiments of von Bezold, whose work forms a valuable extension of Ptlüger's. ${ }^{3}$ The method was utilised by Rutherford, ${ }^{4}$ and has been employed by the present writer. In its simplest form it consists in the

1 Tigerstedt, Mitth. v. physiol. Lab. d. Carolin. med.-chir. Inst. in Stockholm, Bd. iii.; Tiegel, Arch.f. d. ges. Plyysiol., Bonn, Bd. xiii.

2 See Danilewsky, Centralbl. $f$. Physiol., Leipzig u. Wien, 1595, Bd. ix. S. 398.

${ }^{3}$ v. Bezold, "Elektr. Erreg. Nerven u. Muskeln," Leipzig, 1861.

${ }^{4}$ Rutherford, Journ. Anat. and Physiol., London, 1868, vol. ii. p. 87. 
repetition of the classical transmission experiment of Helmholtz, with the addition of a polarising current on the central side of both exciting contacts (Fig. 262).

During the passage at $A$ of an ascending galvanic current (of at

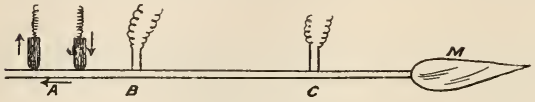

FIG. 262 . most 0.5 Daniell), the maximal muscular responses evoked by excitation, with adequate induced current at the proximal and distal points

$B$ and $C$, are separated by a much larger interval than is normally the ease; if the current is descending, the interval is less than the normal. von Bezold found that both currents increased the difference, but Rutherford found that the descending current diminished it, and the

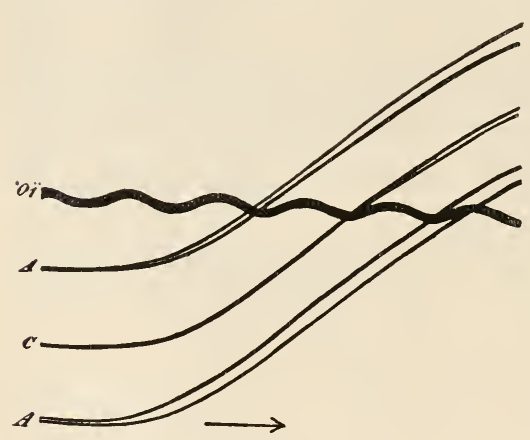

FIG. 263. present writer can fully confirm this observation. The curves in Fig. 263 are instances of such cathodic acceleration and anodic retardation; $C$ being cathodic, $A$ anodic, and the uppermost curves normal.

It will be observed that by this method the exciting stimuli are applied to the extrapolar region. If the whole polar region is interposed between the exciting electrode and the muscle, the conditions are far more complex. I have been unable to find evidence of any marked alteration in propagation rate, unless the polarising current is intense or of prolonged duration, in which case it is always retarded. The presence of two polar regions, a cathodic accelerating and an anodic retarding, causes the one change to counterbalance the other; when, however, the conditions are such that the anodic effect is intense and the cathodic diminished, then retardation is observed. von Bezold's experiments involved the use of currents of considerable intensity and duration, hence the retardation found by him in all cases.

Even the opening changes can be seen to influence the rate of propagation. I have found that the opening cathodic retards more than the opening anodic, provided that the current has been of small intensity, short duration, and that excitation by induced currents occurs within one second of the opening.

Another proof of such polar effect on the rate of propagation is furnished by the increased duration of the delay of the indirect muscular response to polar excitation of its nerve by the galvanic current.

It is evident that the only cases of marked increase in delay are those in which the cathodic closing and anodic opening excitation are situated, so that the intrapolar region lies between them and the muscle, and the galvanic current is of some intensity. It appears to the writer that the chief factor in the production of the more marked delay is the slower propagation which 
occurs in a nerve, the total functional capacity of which has fallen through the experimental procedure; such a fall, particularly in the intrapolar region, is produced in consequence of the passage of currents of considerable intensity, and is manifested by delayed transmission lasting for some little time after the current has ceased. Localised changes of a less pronounced type, at the anode on closure and at the cathode on opening, undoubtedly cause slight retardation, and account for those cases where the extra delay is small.

Duration of Periods of Delay in Muscle Response to Excitation of its Nerve by Galvanic Current. ${ }^{1}$

\begin{tabular}{|c|c|c|c|c|c|c|}
\hline \multicolumn{4}{|c|}{ Intensity of Current. } & $\begin{array}{l}\text { Direction of } \\
\text { Current. }\end{array}$ & $\begin{array}{l}\text { Moment of } \\
\text { Excitation. }\end{array}$ & Character of Delay. \\
\hline $\begin{array}{l}\text { Moderate } \\
\text { Strong . } \\
\text { Very strong } \\
\text { Moderate } \\
\text { Strong. } \\
\text { Moderate } \\
\text { Strong. } \\
\text { Moderate } \\
\text { Strong. }\end{array}$ & $\dot{\bullet} \cdot \vec{\cdot}$ & $\begin{array}{l}\dot{\cdot} \\
\dot{\cdot} \\
\dot{\cdot} \\
\dot{\cdot} \\
\dot{\cdot} \\
\dot{\cdot}\end{array}$ & $\dot{.}$ & $\begin{array}{l}\text { Ascending. } \\
\text {,", } \\
\text { Descending. } \\
\text {,, } \\
\text { Ascending. } \\
\text {,", } \\
\text { Descending. }\end{array}$ & $\begin{array}{c}\text { Closure. } \\
\text {," } \\
\text {," } \\
\text { Opening. } \\
\text {,", } \\
, " \\
\text {," }\end{array}$ & $\begin{array}{l}\text { Slight increase in delay. } \\
\text { No perceptible difference. } \\
\text { Bfarked delay. } \\
\text { Slight increase in delay. } \\
\text { No difference. } \\
\text { Slight increase in delay. } \\
\text { No difference. } \\
\text { Evident increase in delay. } \\
\text { Marked increase in delcy. }\end{array}$ \\
\hline
\end{tabular}

\section{Polar Excitation of Nerve by Electrical Currents.}

Pfluiger's law of contraction.-The passage of a galvanic current through a motor nerve of sufficient duration to allow the muscular response evoked at closure to be distinct from that at opening, causes effects which vary in accordance with the intensity and direction of the current; they may be tabulated as regards their production into stages as follows:-

Pflüger's Law of Contraction.

\begin{tabular}{|c|c|c|c|c|c|c|}
\hline & & \multirow{2}{*}{$\begin{array}{l}\text { CURRENT } \\
\text { INTENSITY. }\end{array}$} & \multicolumn{2}{|c|}{ Ascending DiRECTION. } & \multicolumn{2}{|c|}{ DESCENDING DIRECTION. } \\
\hline & & & Closure. & Opening. & Closure. & Opening. \\
\hline First Stage & . & Weak. & $\begin{array}{c}\text { Minimal } \\
\text { twiteh. }\end{array}$ & $\ldots$ & $\begin{array}{c}\text { Minimal } \\
\text { twitch. }\end{array}$ & $\cdots$ \\
\hline Second Stage & - & Moderate. & $\begin{array}{c}\text { Maximal } \\
\text { twitch. }\end{array}$ & $\begin{array}{c}\text { Minimal } \\
\text { twitch. }\end{array}$ & $\begin{array}{c}\text { Maximal } \\
\text { twitch. }\end{array}$ & $\begin{array}{c}\text { Minimal } \\
\text { twiteh. }\end{array}$ \\
\hline Third Stage & . & $\{$ Stronger. & $\begin{array}{r}\text { Minimal } \\
\text { twiteh. }\end{array}$ & $\begin{array}{c}\text { Maximal } \\
\text { twitch. }\end{array}$ & $\begin{array}{r}\text { Maximal } \\
\text { twitch. }\end{array}$ & $\begin{array}{c}\text { Minimal } \\
\text { twitch. }\end{array}$ \\
\hline & & (Very Strong. & $\cdots$ & $\begin{array}{r}\text { Maximal } \\
\text { twitch. }\end{array}$ & $\begin{array}{r}\text { Naximal } \\
\text { twitch. }\end{array}$ & $\cdots$ \\
\hline
\end{tabular}

The muscular effects produced in the first two stages are dependent upon the circumstance that, at closure, the nerve excitation takes place at the cathode, and is associated with the sudden development of the state of lessened molecular stability; at the moment of cessation it takes place at the anode, and is associated with the sudden rebound

1 The delay in the response to nerve excitation by the elosing or opening of the galvanic current is in each case compared with that present when the nerve is excited by an induced current. 
from the state of increased stability. Of these the first is the most effectual; it is therefore produced by weaker currents than those necessary to evoke the opening effect (Pflüger).

The effects in the third stage are due to such excitation, modified by the polar interferences with conductivity described in the preceding chapter. The nerve is locally excited, both at the cathode on closure and at the anode on cessation, the stimulation being more intense than in the preceding stages; but in the case of a motor nerve the excitatory state started by the cathodic excitation of an ascending current has to traverse the anodic region, and is greatly diminished in the passage, so that it evokes either a minimal or no nuscular response.

Similarly, the descending current excites at both poles, but that started at the anode on its cessation has to traverse a region in which catelectrotonus is disappearing, and its propagation is interfered with in the same way, but complete block is more difficult to attain.

In afferent nerves the reflex response is used as the index, but the same laws hold good, although modified in the third stage. Since the effective propagation is now towards the central nervous system, it follows that in the third stage it is the descending current which fails to evoke the closing, and the ascending one which fails to evolie the opening reflex effect. ${ }^{1}$

That the excitation on closure does actually occur at the cathode, and that on opening at the anode, may be directly demonstrated in nerve by experiments of the following kind. If a portion of a motor nerve be locally raised in excitability by such an agent as fall of temperature, then the closure of a very weak galvanic current causes a local excitation adequate to evoke a maximal muscular effect, provided that the cathode coincides with the altered region; on the other hand, when the anode coincides with this region, then, with an intensity of current too weak to adequately excite at closure, an opening response may be obtained. By locally raising the temperature, and thus diminishing the susceptibility to stimulation, the reverse effects can be produced. Finally, by locally raising the temperature at one pole and lowering it at the other, the passage of a current of appropriate intensity in either direction will excite only at the pole which is situated upon the cooled region; if this is the cathode the excitation occurs on closure, if the anode it occurs on opening. ${ }^{2}$

Any agent which causes a local alteration in excitability may be utilised for demonstrating the polar site of the excitation; temperature is convenient as it produces no permanent effect, but the local application of $\mathrm{NaCl}$ may be used for increasing local excitability, and gives the same results; the closure being effective when the salt is on the cathodic region, the opening when on the anodic. Ammonia may be used for abolishing local excitability, and the failure of cathodic closing or anodic opening excitation is then observed when either of these poles corresponds with the impaired region. By similar experiments it can be shown that the intrapolar region is not the seat of this sudden stimulation on closure and opening; it is limited to the neighbourhood of the poles. As the nerve loses its excitability by any of the previously described agencies, arrest of circulation, etc., the exciting effects of the stronger currents alter from the third to the second and finally to the first of Pflüger's three stages. ${ }^{3}$

1 Pflüger, loc. cit.

${ }^{2}$ Gotch and Maedonald, Journ. Physiol., Cambridge and London, 1896, vol. xx. p. 257.

${ }^{3}$ Rosenthal and v. Bezold, Arch.f. Anct., Physiol. u. wissensch. Med., 1859. 
Apart from such variations in the general excitability of the nerve, the effectiveness of these polar excitatory phenomena is dependent upon the intensity of the polar change itself. This is mainly determined by four factors: intensity of current, direction of current, length of nerve subjected to the influence of the current, duration of current.

These have all been briefly referred to in the section dealing with modes of excitation. It has been already stated that a current loses its exciting value if the direction of flow is across the nerve fibres; this is not due to the increased resistance, nor to the failure of polarisation, since polarisation, owing to the multiple anodes and cathodes (each nerve fibre traversed having its own set), is more prominent than when an equal length of nerve is subjected to a longitudinally directed current flow. It must therefore be due to the interference of the two nerve disturbances, the cathodic lessened stability and the anodic augmented stability of the nerve equilibrium so balancing each other on the current closure that excitation (i.e. sudden lessened stability) becomes impracticable.

The local separation of these two states is thus an important factor in determining efficient polar excitation. It is for this reason that a nerve is excited more effectually the greater the length which is subjected to the longitudinal flow. In order to ascertain the fact, it is essential to eliminate the effect of increased electrical resistance, and the consequent diminution in current intensity produced by the greater length of nerve through which the current is led. The elimination may be approximately effected by using a very large external resistance, in comparison with which the alteration in nerve resistance becomes a negligible quantity. In this way du Bois-Reymond showed that the extrapolar electromotive changes associated with electrotonus are augmented by increase in the length of nerve between the poles. ${ }^{1}$

Marcuse placed the nerve in a trough of physiological $\mathrm{NaCl}$ solution, arranged so that a current could be led through it; alteration in the length of nerve dipping in the trough now makes no appreciable difference in resistance. ${ }^{2}$ He found that the minimal exciting current was of less intensity in proportion as the length of nerve traversed was increased; beyond a certain limit, the favourable influence of polar separation became gradually diminished, and disappeared. The result was the same whether the direction of current flow was ascending or descending. The simple method described in connection with excitability (see Fig. 260) also affords a ready means of ascertaining the same fact. A more remarkable influence is that afforded by multipolar contacts, first suggested by Setschenow, ${ }^{3}$ and employed in a modified form by Danilewsky. ${ }^{4}$

The arrangement for such eontacts consists in placing upon the nerve three electrodes $(A, B, C)$, at equal distances as in Fig. 264 ; these are so connected with the electromotive source that the current flow has its point of exit at the middle electrode, and its points of entry at the peripheral ones; by reversing the current direction, the points of exit are peripheral and central to the point of entry. A special side attachment with another nerve permits of

I E. du Bois-Reymond, "Untersuchungen," (1), Bd. ii. S. 337.

2 Verhandl. d. phys.-med. Gesellsch. in W Wurzburg, 1877, Bd. x.; also Tschiriew, Arch. f. Physiol., Leipzig, 1877.

${ }^{3}$ Centralbl. $f$. d. med. Wissensch., Berlin, 1895.

${ }^{4}$ Centralbl. $f$. Physiol., Leipzig u. Wien, 1895, Bd. ix. 
the exclusion of the centripolar electrode $(A)$, without interfering with the resistance or current intensity ; in the figure the opening of $K_{1}$ and $K_{3}$, and the closure of $K_{2}$, must produce the familiar bipolar arrangement of contacts $B, C$.

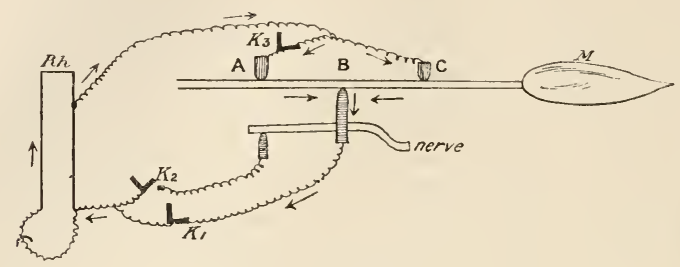

FIG. 264 .

Danilewsky found that all stages of Pflüger's law were demonstrable with the tripolar as with the ordinary bipolar arrangement, but, with the tripolar arrangement, excitation both at closure and at opening was effectually produced by an intensity of current which was inadequate with bipolar contacts. This difference was obtained whether the direction of the currents was through the myopolar and centripolar regions of nerve to the middle point, or the reverse. It thus appears that the doubling of the intrapolar region favours excitation by electrical currents, even when this doubling is one which involves the presence of two symmetrically altered regions meeting at a middle point.

Both the length of nerve submitted to the action of the current and the number of poles are thus clearly shown to be important factors in determining the efficiency of excitation by electrical currents. The importance of the duplication of poles is further demonstrated by experiments in which alternating induced currents are used for excitation of a motor nerve. With the tripolar arrangement a muscular response can be evoked with induced currents which are quite inadequate to cause the response with the ordinary bipolar contacts.

The effect of the duration of the current upon polar excitation has been incidentally mentioned several times. The rapid development of catelectrotonus, and the slower development of anelectrotonus, causes currents of moderate strength, but of short duration, to excite only on closure, i.e. at the cathode. This is the case with condenser discharges, break induced currents, and short galvanic currents. In the case of the latter, a limit can be reached even for this cathodic excitation of medullated nerves, the minimum exciting duration at $15^{\circ} \mathrm{C}$. being $\cdot 0015$ sec. $;^{1}$ it appears to be longer in nerves cooled to $0^{\circ} \mathrm{C}$., amounting then to 02 sec., ${ }^{2}$ and is also longer in nerves the excitability of which has been lowered by excision, etc. ${ }^{3}$ With non-medullated nerves (Anodon) the limit and consequent failure is reached with still longer duration. ${ }^{4}$

The experimental proof of the main fact, that induced currents, unless intense, excite at the cathode only, is readily effected by the use of local alterations of temperature in the manner previously indicated, for these currents excite better when the seat of excitation is

${ }^{1}$ Fick, "Untersuch. u. elektr. Nervenerreguug," 1864.

2. König, Sitzungsb. d. k. Akad. d. Wissensch., Wien, 1870, Bd. 1xii. Abth. 2.

${ }^{3}$ Neumann, Arch. f. Anat., Physiol. u. wissensch. Med., 1864, S. 554.

4 Biedermann, "Elektrophysiologie," 1895, S. 546. 
locally warmed. It can be easily demonstrated that an excitation of minimal exciting intensity becomes more effectual when the cathode of the induced current is on the region whose excitability to the stimulating agent has been thus locally augmented, but remains unaltered when the anode is on this region.

It has, however, been already pointed out that it is possible to obtain evidence of anodic alterations in both excitability and conductivity with rapid induced currents of sufficient intensity, and by similar means it is possible to demonstrate the existence of opening anodic excitation. The experiments previously referred to, in which, through a series of ascending induced currents of gradually increasing intensity, a series of muscular responses are evoked, which attain a maximum, then fail, and finally reappear, afford an illustration of this. The responses occurring after the failure are characterised by two peculiarities: the earlier ones have a prolonged period of delay, the characteristic of those evoked by weak opening anodic excitation, and the later ones are often larger than those of a single twitch. This is most probably due to a summation of effect in the muscle through two effective nerve stimulations occurring in succession, on closure at cathode and on opening at anode. The anodic block is insufficient to resist the intense state transmitted from the cathode, whilst the response to the anodic opening excitation, since it occurs later, is sufficiently retarded to produce with the former a summated muscular effect. In similar experiments with descending currents of short duration, there is no such failure, but the muscular response with gradually increasing intensity becomes augmented. The absence of failure is in strict accordance with Pflüger's law of contraction, since in this case the cathodic seat of the closing excitation is nearest the muscle; the sudden development of the augmented response implies a summated effect, due to the propagation of two successive nerve impulses, the first evoked on closure at the cathode being followed on the cessation of the brief current by one evoked at the anode. ${ }^{1}$

That both poles can be the seats of an effective nerve excitation by intense induced currents, is confirmed by experiments in which the anodic pole is placed on the nerve near the muscle, and the cathodic near the central end. If the cathode only were the exciting point, then the period of delay between excitation and muscular response must be longer when the current is ascending than when it is descending, since the cathodic seat of excitation is in the former case distal, in the latter proximal to the muscle. If, however, a double excitation can occur at both poles, there should be, with adequate current intensity, but little perceptible difference in the time relations dependent upon change of direction. The latter appears, according to Mareš, to be the case. $^{2}$

Pfliiger's law of contraction in its first and second stages, and to some extent in its third stage, thus holds gool for currents of short duration, though the anodic results are only obtained with currents of very considerable intensity.

The causation of the closing and opening excitation.-As stated by P'fligger, the closing excitation is associated with the sudden establishment of catelectrotonus, the opening one with the sudden disappearance of anelectrotonus.

1 Fick, loc. cit.

2Sitzungsb. d. k. böhm. Gcscllsch. d. Wissensch., Prag, 1891. 
There is little doubt that this statement expresses the general conditions underlying polar excitation of medullated nerves; instances, however, occur, due to the interference of unsuspected currents in the nerve, which have led some writers to attempt to associate all excitation with catelectrotonus only.

Influence of the demarcation eurrent. - The opening contraction is generally obtained only on the cessation of currents of some intensity; it can, however, be obtained by much weaker currents, under the following conditions ${ }^{1}$ :-

If the nerve is divided from the spinal cord, and the cathode placed near the cross section, the opening excitation is produced with very weak ascending currents. As these increase in intensity, it fails to reappear again with currents of moderate strength. The earlier opening excitation of the ascending current may be due to the unmasking of the descending demarcation current. There are, however, other currents which will reinforce this. The galvanic current is succeeded by polarisation after effects; these polarisation currents are such as to make the anode into a cathode; they are unmasked by the cessation of the

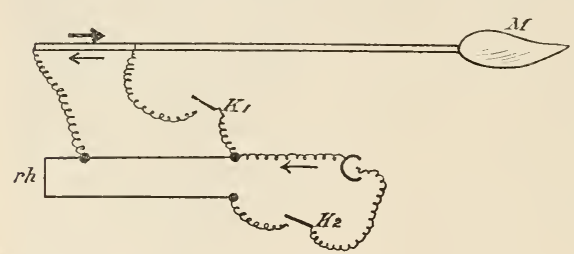

FIG. 265. $-K_{1}=$ key in derived eireuit of rheochord $(r h)$; $K_{2}=$ key in battery eirenit of rheochord. The darker arrow indieates the nerve demareation eurrent, the thin arrow indicates the battery derivation. current flow, and may be adequate to evoke an excitation, especially if they coincide in direction with the feeble demarcation current. The opening excitation, if caused in this way, is in reality based not upon the disappearance of anelectrotonus, but, like the closing one, on the production of catelectrotonus, through feeble polarisation currents in the opposite direction to the original current summing with that of the nerve demarcation change.

That the demarcation current when it exists in some intensity, as in freshly injured nerve, can profoundly modify the results which are expressed in Pfluger's law, is shown, further, by Hering's experiments. ${ }^{2}$ In these a nerve was used with a fresh cut section, and it was observed that the minimal intensity of the battery current adequate for excitation varied with the position $K_{1}$ and $K_{2}$ of the key which effects the closure and opening (see Fig. 265). If this is in the electrode circuit of the rheochord, at $K_{1}$, its closure makes a circuit not only for the derivation of the battery current, but in addition for that of any nerve current present. If it is at $K_{2}$, in the battery circuit of the rheochord, it makes a circuit for the voltaic current only, whilst the nerve current is continually flowing through the derived part of the rheochord circuit. It thus depends upon the respective directions of the battery current and nerve current what the precise variation will be as regards closing and opening excitation.

If the rheochord derivation is about equal to the nerve current, and

${ }^{1}$ Griitzner, Arch. f. d. ges. Physiol., Bonn, Bd. xxvii. S. 1882. S. 237 .

${ }^{2}$ Hering, Sitwungsb. d. k. Akad. d. Wissensch., Wien, 1882, Bd. lxxxv. Abth. 3, 
the battery circuit key, $K_{2}$, is kept closed, then the closure of $K_{1}$ simply completes the circuit for two equal and opposite derivations, one from the rheochord, the other from the nerve; it has therefore no exciting effect either on closure or on opening. With the electrode key, $K_{1}$, closed, and $K_{2}$ open, a circuit for the nerve derivation is completed; although the closure of $K_{2}$ may not evoke contraction, its opening evokes one. The closure under these conditions brings about, in reality, the sudden cessation of the nerve current through the oppositely directed battery current, and the opening causes the reinstatement of the nerve current in its full intensity plus polarisation currents.

With a descending current since this is in the same direction as the nerve current, both the closure and the opening of $K_{1}$ evoke effects far more readily than the closure of $K_{2}$.

Influence of polarisation currents.-A second means for increasing the exciting efficiency of the opening of a current is closely related to the above. It is efficacious in any region of the nerve, and in uninjured nerves (i.e. connected with the spinal cord) as well as in those with a cross section. It is best illustrated by an experimental description. A nerve having been prepared, is excited by the closure of a current too weak to excite on its cessation; an intense current is now used, which excites both at closure and cessation; and it is then found that, following this, the former weak current now excites both at closure and at opening. The polarisation after-current is here increased in its intensity by sunming with that caused by its predecessors; and on opening it is unmasked, and, being suddenly established, excites at the anode, which is now its eathode.

The above facts show that there are circumstances under which an apparent opening excitation is in reality the closure of an oppositely directed current; hence the excitation in these instances is, as in the closing one, based upon the sudden development of catelectrotonus, which, owing to the altered direction of the after effects, occurs at what was the anode of the polarising current.

This view is further supported by the fact that such opening excitations, with their resultant muscular contractions, have the same time relations as the closing ones, the period of delay being identical in the two cases; whilst Tigerstedt found that such opening excitation is favoured by all agents which increase the actual amount of the polarisation current, as estimated by appropriate galvanometric methods. ${ }^{1}$

It thus appears that, since the sudden establishment of catelectrotonus produces a closing single excitation, the same sudden establishment of catelectrotonus through polarisation currents, etc., if it occurs on opening at the anode, must produce an opening excitation. There is, however, ample proof that this is not the only factor concerned in the opening excitation. The matter has been already partly referred to; further evidence will now be adduced to show that we are still bound to employ Pflüger's expression, and assume that the disappearance of the state of anelectrotonus determines an excitation as well as the appearance of that of catelectrotonus.

Excitation in consequence of the disappearance of anelectrotonus is to be regarded as a rebound of the tissue from a greater state of stability (inexcitability) towards one of molecular instability. Biedermann has brought forward demonstrative evidence that the excitation

${ }^{1}$ Tigerstedt, "Biological Memoirs," Oxford, 1887, vol. i. pp. 49-74. 
thus produced may possess special characters of which the most prominent is that of its duration. The evidence is founded upon the phenomena known as Ritter's tetanus, in which excitation is prolonged both during closure and after opening, but the conditions which favour the closing do not necessarily favour the opening effects.

Opening and closing tetanus.-The passage of strong currents through a motor nerve may cause not merely the third stage of Pflüger's law, but a prolonged excitation indicated by a continuous contraction of the muscle. This starts in the case of the ascending current at the moment of cessation, the phenomenon being known as Ritter's opening tetanus. In the case of the descending current it may occur during closure as a closing tetanus. In both cases the term "continuous contraction" expresses the condition far more precisely than the term "tetanus," since there is no proof of repeated excitation.

The opening tetanus with ascending currents is due to a state of prolonged excitation at the anode. This is shown by the following facts. If the effect is present, the severance of the nerve in the intrapolar region above the anodic pole does not stop the continuous contraction. It ceases if the current is again closed through the intact nerve, so as to make the anode the seat not of opening but of closing changes. The effect, if present, is increased if a current is closed in the reverse direction, thus making what was formerly the anode into a cathode. A continuous opening contraction can be obtained after the prolonged action of weaker currents, especially if the direction has been reversed several times, and the circuit opened when the current is ascending. This opening contraction is undoubtedly associated with the production of extensive anelectrotonus, which, owing to its spread, takes some time to disappear, and prolonged excitatory electromotive changes are now found in the nerve in the region of the anode.

Both the closing and the opening prolonged effects can be obtained with comparatively weak currents, if the region of the exciting pole is the seat of a continuous excitation too weak to evoke a muscular response; such a state is produced either by local drying, or by the local application of concentrated $\mathrm{NaCl}$ solution. If the whole nerve is treated with $\mathrm{NaCl}$, then, since it is throughout in this condition of slight inadequate excitation, the closure of a comparatively weak descending current, or the opening of such an ascending one, now evokes a prolonged contraction in the muscle.

In these instances, of which Fig. 266 is an example, the current effect acts by producing changes in excitability rather than true excitation phenomena; continuous excitation is produced by the chemical or mechanical stimuli; it is made effectual on closure at the cathode, and on opening at the anode. 1 In other words, a "latent" (i.e. inadequate) nerve excitation is rendered adequate by the increased excitability which accompanies the closure at the cathode and follows the opening at the anode. That this condition may produce the results was shown by Grïnhagen." A nerve is traversed near its central end by an inadequate series of rapidly recurring induced currents; the passage of a current may now cause either a closing or an opening tetanus resembling in its time relations and form that just described. There is thus no evidence, so far, of any prolonged excitation by the

${ }^{1}$ Eugelmann, Arch. f. d. ges. Physiol., Boun, 1870, Bd. iii. S. 403.

2 Ibid., 1871, Bd. iv. S. 548. 
galvanic current, but only of the familiar alterations in excitability; and as regards the closing tetanus, it appears that in the medullated nerve of the frog the phenomenon is generally of this nature.

It is otherwise with the facts to be now detailed as to the opening contraction, in which it will be noted that, although the alterations in excitability are in all cases present, the effects camnot be accounted for by "latent" excitation.

It was shown by Monmsen ${ }^{1}$ that weak alcoholic solutions of $\mathrm{NaCl}$ (1 per cent.) slightly increase the excitability of motor nerves which have been immersed in it. If such a nerve is subjected, on removal from the solution, to stimulation by weak ascending galvanic currents at periodic intervals, it is found that the closure evokes a large single contraction, and that in a few minutes the opening of the current evokes not merely the single twitch, but following this a prolonged contraction.

That the nerve itself is not in this instance continuously stimulated by the solution, is shown by the fact that no spontaneous contraction is evoked by its action alone; the excitability increases to a certain point,

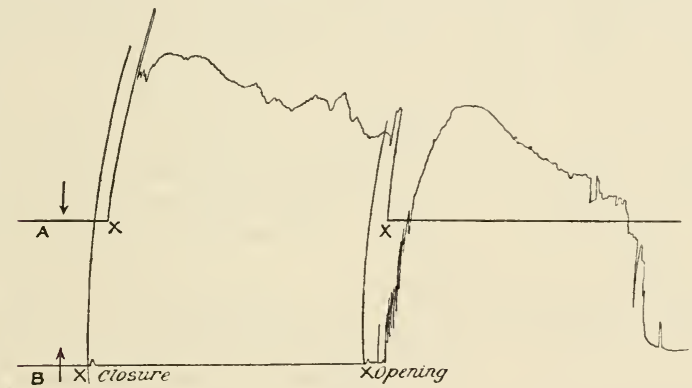

Fig, 266.

and then remains in this condition. There is little doubt that the part played by the current in causing this continuous opening contraction is not the mere production of a prolonged local rise of excitability, but a true prolonged opening excitation, which is effectual because of the greater general excitability of the nerve.

The muscular response to the opening excitation shows two peculiarities: it is delayed in its appearance so as to occur at a distinct interval after the opening twitch, and it is dependent as to size and form upon the duration of the current closure.

In the last respect it differs from the opening tetanus, which has been previously described as produced in a nerve which is continuously but inadequately excited by drying or by concentrated $\mathrm{NaCl}$. In these cases, when the opening of a weak current evokes the continuous effect, it is impossible to separate its start from that of the opening twitch. In the nerve treated with the alcoholic solution, the prolonged opening effect is produced only on the cessation of moderately strong currents, and varies directly with the length of the previous closure. ${ }^{2}$

1 Virchow's Archiv, Bd. Ixxxiii. S. 261.

${ }^{2}$ Biedermann, "Elektrophysiologie," 1895, S. 587. 
A necessary condition for its production is the increased excitability of the nerve. Other chemical agents may be employed for increasing nerve excitability. In cooled nerves it sometimes happens that the opening of an ascending current evokes the single twitch, followed by a prolonged contraction, having the same characters as that seen in the above case.

The opening of the current may thus produce two excitation phenomena,--one sudden and rapidly developed, indicated by the initial opening twitch; the other prolonged and more slowly developed, indicated by the continuous contraction. These cannot be identical as to their causation, since it is possible to place the nerve under conditions which favour the production of the one, although they disfavour that of the other. General increase in excitability, such as that produced by immersion in 1 per cent. $\mathrm{NaCl}$ (alcoholic solution), favours the production of both effects; but a general decrease in excitability can be caused by appropriate reagents, which will disfavour the second whilst favouring the first. The most striking of these reagents are 10 per cent. solution of $\mathrm{NaCl}$ and 1 per cent. solution of $\mathrm{KNO}_{3}{ }^{1}$ If the nerve is immersed in the latter solution, the following facts are observed. Immediately after its removal a single twitch is evoked in the muscle by the closure of an ascending current, and on opening an initial single twitch is evoked which may, under appropriate conditions, be succeeded by a prolonged muscular response. As the experiment is repeated, the closing excitation response becomes feebler, showing gradual decrease in excitability; the opening initial single twitch becomes larger, whilst the prolonged opening effect becomes small and finally disappears. By placing the nerve in physiological salt solution, all these phenomena are replaced by those obtained in a normal nerve; they may be again produced by fresh immersion in the $\mathrm{KNO}_{3}$ solution.

Hence it appears that the two opening effects differ as regards the condition of nerve most suitable for their manifestation. The single excitation evoking the twitch is favoured by some conditions which cause general lowering of excitability; the prolonged response is favoured by general rise of excitability. The probable causation of the initial opening effect is the polarisation after effect, aided by the demarcation nerve current, if this is of sufficient intensity and appropriate direction; the causation of the second continuous response is a prolonged molecular change, coincident with the gradual disappearance of anelectrotonus, in other words, a rebound of the nerve equilibrium from an enforced state of greater stability into one of instability.

As already stated, the closing tetanus in the frog appears to be an effect in which a continuous "latent" excitation becomes adequate at the cathode, through the local rise in excitability during closure. There are, however, clear indications that in some nerves there are prolonged excitation phenomena, even at closure.

The most striking instances of such closing effects are furnished by the results of the elaborate experiments made by Biedermann upon the non-medullated nerves of the muscles which open and close the claw of the crayfish; these experiments also afford demonstrative evidence of the validity of the above conclusions as to the character of the opening excitation. ${ }^{2}$

1 Biedermann, "Elektrophysiologie," 1895, S. 590-594.

2 Biedermann, Sitzungsb. d. k. Akad. d. Wissensch., Wien, 1888, Bd. xcvii. Abth. 3, S. 49. 
Polar excitation of the nerves of the crayfish.-The response to the excitation of the nerves supplying the claw is remarkable, inasmuch as a weak adequate stimulus, such as the faradic current, evokes augmentation of the tonic contraction of the abductor or opening muscle, at the same time that it causes relaxation of that of the adductor or closing muscle; on the other hand, a more intense stimulus of a similar kind causes an augmentation in the adductor contraction, accompanied by the relaxation of the abductor.

To account for this, we must assume the existence of four sets of nerves differing in the degree of their excitability, and in their susceptibility to respond to different external stimuli; the four sets comprise an augmentor and inhibitory set for each muscle.

The polar excitation of such a nerve trunk by galvanic currents is thus extraordinarily complicated, since to the inhibitory influences of the poles themselves must be added those produced by the polar excitation of the inhibitory nerves.

The simplest experiments are those made upon a preparation in which one muscle is dissected free of its insertion, and employed when it has lost its tonicity. In such a preparation all the three stages of Pfliuger's law can be demonstrated, but there is this difference between
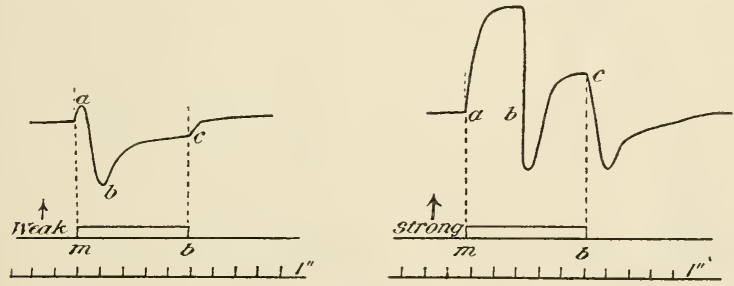

FIG. 267.

the results and those observed in the nerve-muscle preparation of the frog, the muscular contractions evoked at opening and closing are in all cases prolonged. ${ }^{1}$ If, on the other hand, the muscle retains its tonicity, then the passage of a current in either direction evokes more complex effects. In the case of the adductor muscle (Fig. 267) the results are as follows:-

When the nerve is excited by weak currents, three changes are seen- $a$, an initial contraction on closure ( $m$ in Fig. 267), or an augmentation of tonicity, due to the excitation of the augmentor fibres; $b$, a persistent relaxation succeeding $a$, slowly subsiding during the closure, due to the inhibition of the effect; $c$, the return of the muscle to its normal state of tonicity on opening the current.

With more intense currents these effects are profoundly altered (see Fig. 267, right-hand figure). (a) The initial augmentation is fused with a persistent one, due to a prolonged excitation of the augmentor overpowering that of the inhibitory fibres; $(b)$ this may be succeeded during closure by relaxation, and this again by contraction, so that a conflict between the response due to persistent excitation of augmentor and inhibitory nerve fibres occurs, in this conflict the augmentor effect has the advantage; $(c)$ on opening, there is a relaxation of the muscle

1 Biedermann, "Elektrophysiologie;" 1895, s. 602-604.

VOL. II. -33 
due to a weak opening excitation of the inhibitory fibres, as in $1, b$.

The results show that, as far as the closing excitation is concerned, this is of a twofold character, one set of nerve fibres being excited strongly on the instant of closure, whilst the other set is excited by the persistence of such closure, and that by altering the intensity of the current either set may be excited during the whole time that the circuit is closed.

Experiments with the abductor muscle prepared in a similar way give, as might be anticipated, different results; if the muscle still possesses tonicity, then the following facts are observed when the nerve is excited by weak and strong currents respectively (Fig. 268) :-

Wcak currents (abductor musclc). - Closure - (a) An initial relaxation due to excitation of the inhibitory fibres; (b) a prolonged persistent augmentation of tonicity (i.e. contraction) succeeding (a), lasting during closure, and due to the excitation of the augmentor fibres. Opening-(c) The return of the muscle to its normal state, this being fused with a more prolonged relaxation, which slowly passes away,
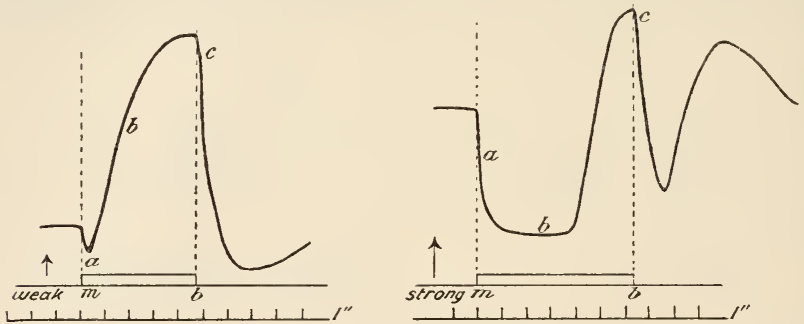

Fig. 268.

due to the weaker opening excitation of the inhibitory fibres, evoking a repetition of $(\alpha)$.

With stronger currents (Fig. 268, right-hand figure) the effects are changed- $(a)$ The initial relaxation on closure becomes fused with a more prolonged effect of the same kind; $(b)$ a conflict occurs during closure between the prolonged relaxation now evoked and a succeeding augmentation, due to simultaneous prolonged excitation of both sets of fibres, in which conflict the relaxation has the advantage for some time, but is finally overpowered; $(c)$ on opening, an initial relaxation occurs succeeded by a prolonged augmentation, due to the overpowering effect of excitation of the augmentor fibres.

These results show that both at closure and at opening a twofold excitation occurs, those at the opening of a strong current being similar in character to such closure effects as are evoked by a weak one.

The interpretation here given rests on the supposition that the effects are all due to the excitation of augmentor and inhibitory nerve fibres supplying each muscle. It might, however, be urged that the second prolonged effect is in each case an actual polar inhibition; thus the closure effects in the abductor muscle might be excitation of the inhibitory set of fibres at the cathode, succeeded by overpowering inhibition of such excitation at the other pole, anode. This would 
obviously cause an initial relaxation, succeeded by a return to tonicity, i.e. an augmentation. A further experiment makes it clear that such bipolar interference effects are not the causative factors. The abductor muscle free from tonus cannot relax in response to the excitation of its inhibitory nerve fibres, and any subsequent tonicity cannot therefore be àe to anodic interference with such cathodic excitation of inhibitory fibres. Such a muscle shows no alteration at the instant the current is made, but the tonicity still develops during the closure. It must therefore be due to prolonged excitation of the augmentor nerves, the muscular effect of which is retarded in its onset. This retardation is undoubtedly due to the impairment of conductivity, since it becomes more pronounced as the stimulating current is increased in intensity.

The nerves to the muscles of the crayfish claw, owing to their different degrees of excitability, and to the distinctive characters of the muscular response evoked by their excitation, thus furnish an admirable object for demonstrating the possibility of polar excitation being a prolonged state.

The results do much to confirm the truth of Biedermann's views as to the twofold character of polar excitation in the medullated nerves of voluntary muscle. ${ }^{1}$ These are pre-eminently susceptible under normal circumstances to the onset or cessation of current flow, but they can be so altered as to respond, in addition, to the prolonged polar change following current flow. The nerves of some other structures respond normally to both the initial and the prolonged changes. E. du Bois-Reymond's law of excitation, which places rapidity of variation in current intensity as the effective determinant of exciting efficiency, applies only to the production of the initial excitations, and is not therefore of general application. The efferent nerve appears to be differentiated, so that its susceptibility to the form of stimulus shall be related to the activity of the peripheral responding mechanism; if a continuous response is that most appropriate for the discharge of its functions, the nerve is susceptible to the continuous form of excitation.

It is in consequence of such varying susceptibility that certain nerves are incapable of electrical stimulation by currents of short duration, unless these are rapidly repeated. Thus the vasomotor nerves, whose stimulation in the cervical sympathetic causes constriction of the vessels in the rabbit's ear, are not easily excited by a single induced current, though readily excited by a series of such currents; the secretory nerves, such as the glosso-pharyngeal, the excitation of which causes secretory and corresponding electromotive changes in the frog's tongue, are readily stimulated by the closure and opening of galvanic currents of some duration, but are only slightly excited, if at all, by induced currents. ${ }^{2}$

Polar excitation in man.- The polar excitation of a nerve is complicated when this is surrounded by a mass of conducting tissue. In all the experiments previously referred to, care is taken to insure that the nerve between and on each side of the poles is free; but when, as in the cxcitation of nerves in man, one electrode is applied to the skin over the course of a nerve, it is clear that, though this may represent truly

\footnotetext{
${ }^{1}$ Biedermann, "Elektrophysiologie," 1895, S. 596, 597.

${ }^{2}$ Biedermann, Arch.f. d. ges. Physiol., Bonn, 1893, Bd. liv. S. 241.
} 
either an anode or a cathode, the polar region thus constituted is surrounded on all sides by what may be termed a peripolar region of opposite sign. The conditions are such that in the polar region of the actual electrode the current density will be most marked, whilst in the peripolar region it will be far less. With gradually increasing intensities of current, the muscular response to stimulation over the entry of the nerve must vary in consequence of these conditions. This is shown by the results given in the following table: ${ }^{1}$

\begin{tabular}{|c|c|c|c|c|c|}
\hline $\begin{array}{l}\text { Currrent } \\
\text { Intensity. }\end{array}$ & & $\begin{array}{l}\text { Character of } \\
\text { Surface Pole. }\end{array}$ & $\begin{array}{l}\text { Condition } \\
\text { of Circuit. }\end{array}$ & Effect observed. & Explanation of Result. \\
\hline $\begin{array}{c}\text { Weak } \\
\text {,, } \\
\text { ", } \\
\text { Moderate } \\
\text {," } \\
\text {," } \\
\text { Strong }\end{array}$ & . & $\begin{array}{l}\text { Cathode. } \\
\text { Anode. } \\
\text { Cathode. } \\
\text { Anode. } \\
\text { Cathode. } \\
\text { Anode. } \\
\text { Cathode. } \\
\text { Anode. } \\
\text { Cathode. } \\
\text { Anode. } \\
\text { Cathode. } \\
\text { Anode. }\end{array}$ & $\begin{array}{l}\text { Closure. } \\
\text {,", } \\
\text { Opening. } \\
\text { Closure. } \\
\text { Opening. } \\
\text {,", } \\
\text { Closure. } \\
\text { Opening. } \\
\text {,", }\end{array}$ & $\begin{array}{l}\text { Minimal response. } \\
\text { Nil. } \\
\text { Nil. } \\
\text { Nil. } \\
\text { Maximal response. } \\
\text { Minimal response. } \\
\text { Nil. } \\
\text { Minimal response. } \\
\text { Prolonged re- } \\
\text { sponse. } \\
\text { Maximal response. } \\
\text { Minimal response. } \\
\text { Maximal response. }\end{array}$ & $\begin{array}{l}\text { Polar cathodic excitation. } \\
\qquad \ldots \\
\ldots \\
\ldots \\
\text { Polar cathodic excitation. } \\
\text { Peripolar cathodic excitation. } \\
\text { Polar anodic excitation. } \\
\text { Polar cathodic excitation. } \\
\text { Peripolar cathodic excitation. } \\
\text { Peripolar anodic excitation. } \\
\text { Polar anodic excitation. }\end{array}$ \\
\hline
\end{tabular}

A portion of the peripolar region is obviously on the myopolar side of the polar, and thus may cause conductivity changes when the current is sufficiently intense.

This and the great difference between the denser polar and diffuse peripolar regions readily explains the occurrence of the responses, which are therefore really in accordance with Pfliger's law as determined in isolated nerve. Two interesting features come into special prominence by the use of this method upon man. First, the prolonged response on the closure of strong currents with cathode upon surface; secondly, the marked increase in the period of delay in the muscular response to the opening polar anodic excitation. This was found by Waller to amount to 0.03 sec. It appears that the delay is associated with impairment in conductivity from which the nerve does not recover until $0.03 \mathrm{sec}$. after the current has ceased to flow. ${ }^{2}$ Although, therefore, the excitation occurs at the moment of opening, the impairment of conductivity persists and delays the propagation of the excitatory state.

In these respects the results confirm those obtained on isolated nerve, though in man they are modified by the diffuse peripolar and intense polar condition previously referred to.

Polar excitation of peripheral sensory organs. - Taste organs.-If a current traverses the tongue in man, an acid taste is localised at the contact when the anode is on the tongue, an alkaline or salt taste when the cathode is placed there. The sensations last during the whole time of closure, are reversed on the cessation of the current, and can be obtained with unpolarisable electrodes. ${ }^{3}$ The susceptibility of the taste organs to such excitation is extremely delicate. The acid anodic

1 Waller and de Watteville, Phil. Trans., London, 1882 ; Proc. Roy. Soc. London, 1882.

2 Waller, Arch. de physiol. norm. et path., Paris, 1882, p. 383.

${ }^{3}$ Rosenthal, Arch. $f$. Anat., Physiol. u. wissensch. Med., 1860; Biol. Centralbl., Bd. vi. S. 120 . 
effect is appreciated when non-polarisable electrodes are used with a current intensity of only $\frac{1}{156}$ milliampere. ${ }^{1}$

The effects may be due to the excitation of specific taste organs, or to the presence of electrolytic products. It is undoubtedly due to the stimulation of the peripheral organ at the pole, and not to excitation of the larger branches of the gustatory nerves, since it is much diminished by the local application to the surface of cocain, which dulls all taste sensations. That it is not the mere effect of acid and alkali, due to electrolytic decomposition, is shown by the circumstance that the sensations are present when no evidence of such chemical products can be found. ${ }^{2}$

The change in the sensation on the cessation of the current may be caused by the reversed polarisation current coming into play, but in any case it is of the nature of an opening excitatory change. The production of greater instability (dissimilation process) in a peripheral sense organ constitutes an excitation of a specific kind, and the resultant excitatory state, when propagated to the higher centres, is thus the physiological basis of a specific sensation. If the production of greater stability (assimilation process) is also an excitation, it must be of fundamentally different character. Both the propagated nerve states and the result in consciousness will thus differ from those associated with greater instability. The two states are linked together, since the production of either is followed by a rebound into the other condition. This is the view held by Hering in reference to visual contrast, and the taste effects are so nearly parallel, as far as the antagonism of effect with after-effect is concerned, as to fall in with his conceptions.

The visual organs.-If the poles of a galvanic current of sufficient intensity are placed one on the forehead and one on the neck, both the closure and the opening cause an effect which appears to consciousness as the sensation produced by a flash of light. More intense currents produce effects not only at but during closure. These differ with the direction of the current, and are best obtained when one electrode is placed over the shut eyelid.

There is considerable disagreement as to the character of the sensation experienced under these conditions, but the majority of observers concur in the following particulars. ${ }^{3}$ When the anode is on the eyelid, the Hash at closure has the appearance of a violet field with dark centre. This is succeeded by field illumination during the maintenance of the current flow, which slowly subsides, and often appears of a violet or blue hue. On opening the current, there is a bright blue centre with an outside field of yellowish green, or, according to Helmholtz, orange.

With the cathode on the eyelid, the effects are more or less reversed. At the instant of closure the centre is bright and blue, the outside field dark. This persists, but with diminishing intensity, during closure, and, on cessation of the current, is succeeded by opposite effects-the centre being greenish yellow and the external field blue. Hence the centre appears of a hue in contrast with its surrounding, and is, according to Helmholtz, associated as to its peripheral origin with the entry of the optic nerve or blind spot. On this supposition it represents a retinal area which is incapable of appropriate stimulation by the current.

The results as to the visual characters of the field appear to show

${ }^{1}$ Laserstein, Arch. f. d. ges. Physiol., Bonn, Bd. xlix. 8. 519.

" v. Vintschgau, see Hermann, "Handbuch," Bd. iii. (2), S. 186.

${ }^{3}$ Helmholtz, "Physiol. Optik," 2te Aufl. S. 243 et seq. 
that during the flow of a current directed through the retina from the layer of nerve cells to that of the rods and cones (anode on the eyelid), the retinal excitation is such as to give the consciousness of bright colour, the flow in the opposite direction is such as to give the consciousness of a dark contrast colour. In such instances the visual characters of the field must be taken as those associated with the retinal excitation, the visual characters of the centre being due to contrast.

These results, although showing discrepancies in the hands of different observers, may be taken as indicating, first, the existence of both initial and prolonged closing and opening excitation effects; and, secondly, the existence of polar excitatory results of opposite kind, but so linked that the production of the one is succeeded by that of the other. They are thus in conformity with the views as to the nature of polar excitation of nerve, which have been set forth in the preceding pages.

\section{Electromotive Cimanges in Unexcited Nerve.}

General characters of the nerve current. - It was discovered in 1845 by du Bois-Reymond that persistent electromotive changes might be detected in all nerves. ${ }^{1}$ The main features of such changes in an excised nerve may be summed up as follows:-

1. Two symmetrical points on the longitudinal surface of a nerve are equipotential.

2. If one point is nearer a cross-section than the other, a difference of potential is observed, the former being galvanometrically negative to the latter.

3. The maximum difference of potential is observed when one point is the cross section itself and the other the longitudinal surface, the tissue at the section being now markedly negative to that of the surface.

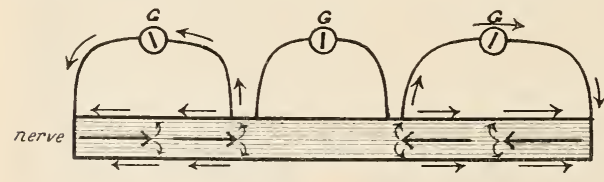

FIG. 269.

The nerve fibres of an excised nerve are thus traversed by axial currents, directed from the cross section along the fibres; and since the nerve is a moist conductor, a closed

circuit for the spread of such currents is afforded by the adjacent nerve fibres and by the moist surroundings. The electrical distribution and direction of the currents are indicated in the annexed diagram (Fig. 269), and since these are present in nerves which may be regarded, at any rate for the present, as unexcited, they may be conveniently described as currents of rest.

The amount of the difference of potential varies with the degree of approximation of the terminal contact to the cross section; hence the source of the electromotive change is undoubtedly to be found in the difference between the molecular state of the nerve at any point of its length, and that in the immediate vicinity of the cross section; the critical point is localised in the zone of demarcation between the 1 du Bois-Reymond, "Untersuchungen," Bd. ii. (1), s. 289. 
normal tissue and the altered portion of the actual cross section, the resting nerve current has been therefore termed the demarcation current.

Methods of producing so-called resting or demarcation nerve currents.-Cross section.-Every cross section of a nerve brings into prominence the above-mentioned difference. It is, however, somewhat difficult in the case of a small nerve to adjust the contacts, so that one shall be effectively connected with the cross section; a more certain method for attaining the same result is furnished by "thermal section." This is accomplished by dipping the end of an excised nerve for a brief space into physiological saline at $60^{\circ} \mathrm{C}$., so that several $\mathrm{mm}$. of the nerve may be suddenly destroyed; the boundary zone between such a destroyed portion and the normal tissue is practically the same as that present on a cross section, whilst the destroyed end now serves as an indifferent moist conductor to connect the whole of the cross section with the necessary contact.

Loealised injury.-Instead of the end, any intermediate portion of the nerve may be similarly injured by local heat, compression, etc.; its boundary zones on each side are demarcation areas between the normal and the injured fibres, and the injured part is thus galvanometrically negative to either portion of the normal longitudinal surface. Partial localised injuries of this kind involving superficial fibres of a nerve trunk may be readily produced by nerve exposure, with the result that, owing to the formation of demarcation zones, the electromotive changes just referred to are now observed.

Temperature.-Localised alterations of the temperature of nerve fibres are accompanied by electromotive changes, even when the temperature change is such as to cause no persistent injury. Thus, with two symmetrical contacts showing isoelectrical conditions, localised warmth to $30^{\circ} \mathrm{C}$., in the immediate neighbourhood of one causes the tissue at this point to become galvanometrically positive to that of the cooler region; localised cooling produces the opposite condition.

The methods of ascertaining the characters of the nerve current.-The experimental demonstration of resting nerve currents in the tissue under any of the preceding conditions is one in which the recording instrument may be a high resistance galvanometer, a capillary electrometer, or such a physiological galvanoscope as is afforded by a muscle-nerve preparation. The special precautions attending such demonstration cannot be entered upon here, but some features presented by nerve in common with other moist conductors must be briefly referred to. These are related to the use of the compensator, the restriction of all such investigations to the examination of derivation effects, and the electrical resistance of the nerves themselves.

The compensator is a graduated rheochord of definite resistance, so constructed that it allows the experimental circuit to comprise a known electromotive force, the amount of which can be varied at will. It is thus possible to exactly counterbalance any current in the instrumental circuit due to electromotive changes at the tissue contacts, by means of one of precisely equal amount but opposite sign. The amount of the antagonistic electromotive force which has thus to be employed is a measure of the amount of that present under the tissue contacts. This latter is, in reality, that produced when an elcctromotive source is surrounded by a moist conductor, and contacts are placed upon two points of the surface of the latter; such a condition, for instance, as is present when two small pieces of dissimilar metals are embedded within a cylinder of kaolin soaked in 0.6 per cent. $\mathrm{NaCl}$. It is obvious that the whole of this moist conductor is now traversed by derivation currents, and 
that different points of its surface will show corresponding differences of electrical potential. When a current is obtained in the experimental circuit between any two points of the surface, and is counterbalanced by a suitable external electromotive source, the latter is not a measure of the electromotive change in the interior of the investigated source, but only of the surface differences. Supposing, therefore, that the surface itself is altered, and its electrical resistance changed by increase of moisture, then the current compensation is no longer adequate, since alterations in electrical distribution occur in the new moist envelope. The estimation of the amount of the electromotive force in resting nerve is in like manner that of the amount of surface distribution, and the real value of the change present at the electromotive source is not determined. Other things being equal, the surface change varies directly with that of the interior; but the surface change may be but a fraction of the amount of the electromotive difference present within the nerve fibres. Since the nerve is a moist conductor, its electrical resistance is increased by cooling and diminished by warming. The resistance is very large; in the case of the sciatic nerve of the frog it is about 100,000 ohms for each longitudinal centimetre of nerve; it is also greater to currents flowing across the nerve fibres than to those flowing in the direction of their axis, being five times as great in the former case. The resistance is best determined by balancing the nerve resistance against a variable one, after the method of Kohlrausch. In this a series of alternating induced eurrents traverse a Wheatstone bridge and a portion of nerve; a telephone suitably connected indicates by its silence when the balance is complete.

The influence of various conditions upon the amount and duration of the resting currents.-The amount of the electromotive difference between the surface and cross section of a nerve, as indicated by the amount of the surface derivation, varies in accordance with the structural character and physiological condition of the tissue.

(a) The character of the nerve.-Any mixed nerve, such as the sciatic, will show an electromotive difference between surface and cross section. The amount of this difference does not appear to be entirely dependent upon cross sectional area, although the surface distribution must be largely influenced by this. Thus both the slender nerve of the frog and the larger one of the dog are capable of giving a high difference (.02 D), whilst the large nerve of the horse and the smaller one of the monkey give low differences $(\cdot 008 \mathrm{D}){ }^{1} \quad$ This is also the case in such a nerve as the large second electrical nerve of Torpedo, which, with 24,000 fibres, gives only $007 \mathrm{D}^{2}{ }^{2}$ The amount is thus related to the source of the nerve, i.e. the animal from which it is taken, and is probably an expression of the varying susceptibility of the nerve to the particular molecular change which produces the demarcation effect. This is itself dependent upon the extent to which the uninjured surface of the nerve retains its vital properties and still possesses the particular molecular state which characterises the living tissue. All circumstances which impair functional capacity diminish the magnitude of the effect.

The differences just referred to may thus be related to variations in the functional attributes of the nerves of the various animals mentioned. But, in addition to this, the alnount of the resting electrical difference is associated with structural peculiarities of the nerve fibres. Thus non-medullated nerves give far larger differences of potential than medullated ones. The fine nerves in such inverte-

${ }^{1}$ See Biedermann, “Elektrophysiologie,” 1895, S. 638.

${ }^{2}$ du Bois-Reymond, Sitzungsb. d. k. Preuss. Akad. d. Wissensci., Berlin, 1883. 
brates as the lobster give $048 \mathrm{D},{ }^{1}$ in Anodon and in Eledone $026 \mathrm{D}^{2}$ In vertebrates, the olfactory of the pike gives $.02 \mathrm{D}$, whereas medullated nerves from this fish of the same cross sectional area gave only $01 \mathrm{D}^{3}$ A still greater contrast is that between the sciatic nerve and the spinal cord; the latter gives a very large cross sectional difference of potential, $046 \mathrm{D}$ in the cat, and $.029 \mathrm{D}$ in the monkey. These latter differences are not due to the larger cross sectional area, since, on comparing the cord of a small monkey with a sciatic nerve from a large animal of the same species, the cord difference was found to be three times that of the nerve. ${ }^{4}$

Finally, in many nerves, the difference varies with the locality of the cross section. A striking illustration of this is afforded by comparing the lumbar posterior roots with the whole sciatic nerve. In the cat the seventh lumbar root gave $026 \mathrm{D}$, whereas the whole sciatic nerve gave only 018 D. ${ }^{4}$ It appears, further, that in many purely afferent nerves a more central cross section is more effective than a more peripheral one, so that, on connecting the two cross sections by appropriate contacts, the more central is galvanometrically negative to the more peripheral, and a similar difference is found between two points on the longitudinal surface, when equidistant from their respective cross sections. ${ }^{5}$ On the other hand, in some purely efferent nerves, this relationship is reversed. Thus, in the efferent electrical nerves of Torpedo, the increased negativity of a peripheral cross section over a more central one amounts to $002 \mathrm{D}^{6}$

It is not improbable that these results are, to some extent, associated with the varying susceptibility of the nerve fibres to injury through external agencies owing to differences in the sheaths, etc., of the different regions.

If the phenomena in question are present, the one in all afferent, the other in all efferent fibres, then, in a mixed nerve, the results must vary in proportion as the afferent fibres are more or less nunerous than the efferent ones. As a matter of fact, such a mixed nerve as the sciatic gives results which vary with the position of the cross section.

The large electromotive effect obtained with non-medullated nerves shows that the medullary sheath plays little or no part in the production of the difference. If the sources of the change are localised in the axis cylinders, the absence of such an additional moist envelope would serve to bring the experimental contacts into far closer proximity with the electromotive source, and the large difference would be explicable.

It is probable, however, that although the absence of the sheath must be a prominent factor in producing larger effects, it is the electromotive characters of the axis cylinders themselves which are the most potent factor. Hence we are led to infer that different axis cylinders vary as regards the amount of the electromotive change, which can be brought into prominence by their cross section.

\section{(b) Temperature.-In any nerve which shows the resting cross}

${ }^{1}$ Fredericq, Arch. f. Physiol., Leipzig, 1880, s. 68, 71.

${ }^{2}$ Biedermann, Sitzungsb. d. k. Akal. d. Wissensch., Wien (3), Bd. xeiii. ; Fuchs, ibid., 1894, Abth. 3.

${ }^{3}$ Kühne, Untersuch. a. d. physiol. Inst. d. Univ. Heiulelberg, Bd. iii. S. 149.

4 Gotch and Horsley, Phil. Trans., London, 1891, vol. elxxxii. B, p. 267 et seq.

s Schiff, "Lehrbuch d. Muskel- u. Nerven-Physiol.," 1859; Mendelssolin, Arch. f. Physiol., Leipzig, 1885, S. 381.

${ }^{6}$ du Bois-Reymond, loc. cit. ; "Living Torpedoes in Berlin," "Biological Memoirs," Oxford, 1887 , p. 464 . 
sectional difference, local warming in the neighbourhood of the cross section up to $35^{\circ} \mathrm{C}$. increases, whilst local cooling diminishes the amount of the electrical derivation. It is not clear how far such increase and diminution is dependent upon the alteration in the resistance of the moist conductor, with its consequent variation in electrical distribution. There are, however, other considerations in connection with excitatory electromotive changes which afford a basis for the belief that temperature also acts upon the source of the electromotive change in such a way that warmth increases the susceptibility of the axis cylinders to the alterations produced in the neighbourhood of the cross section.

(c) Connection with nerve eells.-The functional and structural alterations which follow the severance of their cellular connection cause very marked changes in the nerve fibres as regards the production of the resting electromotive differences. As might be assumed, the onset of the degenerative processes is associated with diminished demarcation difference. It is interesting to note that such difference, although diminished in amount, can be observed in degenerated mammalian nerve after the functional attribute of conductivity has disappeared. The interruption of continuity, which is the cause of the latter, occur's at intervals along the whole course of the fibres, but histological examination in this earlier stage of the process shows that any cross section may comprise fragments of axis cylinders with their medullary sheaths. The existence of the cross sectional difference of potential shows that even in their altered condition such fragments are still susceptible of the change which is the basis of the resting difference.

The electromotive source under these circumstances is presumably localised in the fragments of swollen axis cylinders which happen to be included in the area of the cross section, for, when complete degeneration has been attained, no cross sectional difference is found. Thus, if a portion of a nervous column be completely degenerated, the normal amount of the resting difference of such a tissue is very much diminished. Examples of this are the following : the difference in the normal spinal cord of the cat on the lower dorsal region was found to average $.03 \mathrm{D}$; after section of all the lumbar posterior roots three months previously, the difference was only $012 \mathrm{D}$; after hemisection above the investigated area three months previously, it was $018 \mathrm{D}$, and $02 \mathrm{D}$ after degeneration through section of one posterior column below the investigated area. ${ }^{1}$ In these cases the lower values are chiefly due to the substitution of some nerve fibres by a layer of indifferently conducting tissue, thus increasing the moist envelope; but it is possible that the remaining fibres, although apparently normal in structure, may have lost their susceptibility to the production of the electromotive effect, and that in addition to the increased connective tissue envelope, there is a diminution in the amount of the electromotive change produced in the apparently sound fibres.

(d) Arrest of the cireulation.-The arrest of the circulation, or the removal of the nerve from the body, is followed by changes in the amount of the difference, the consideration of which involves the question as to the duration of the change in freshly excised nerves.

The prominent feature exhibited by such nerves is a steady decrease in the amount of the resting difference, this being much more marked in warm-blooded than in cold-blooded animals. In the case of the sciatic nerve of the frog, the difference may in two to four hours sink

${ }^{1}$ Gotch and Horsley, Phil. Trans., London, 1891, vol. clxxxii. B, p. 267 et seq. 
to half the amount present in the freshly excised nerve, and in twelve hours to a comparatively small amount. This is undoubtedly due to the passing away of the specific change in the neighbourhood of the cross section, since it may be restored to nearly its full amount by a new cross section. ${ }^{1}$ The gradual decline is more rapid in summer than in winter frogs; though here also, after the difference has subsided, it can be renewed by a fresh cross section.

The decline and the susceptibility to renewal by fresh cross section are phenomena common to medullated nerves, the spinal cord, and nonmedullated nerves. There is thus little ground for the belief either that the decline is due to the gradual physiological failure of the nerve fibres from the point of cross section as far as the nearest node, or that the renewal with a fresh section is due to parts being involved above such nodes. Both the decline and the renewal are rather to be regarded as indicating that the sudden installation of the difference is a functional change, localised to the neighbourhood of the cross section; the duration of this change is determined by the functional capacity of the uninjured nerve and the extent of the alterations produced by the injury.

It often happens that the difference in a freshly excised nerve increases in amount for a short time after its preparation; this might be due to loss of liquid from the nerve through evaporation, thus increasing the resistance, and so altering the distribution of the derivation surface currents, since moistening the nerve always causes the reverse effect.

It is probable, however, that in most instances both the functional capacity of the uninjured tissue and the extent of the reaction of the altered tissue increases for a certain time, since such augmentation is a familiar phase of excitation phenomena in the case of both nerve and other excitable tissues.

An interesting fact connected with the circulation and the maintenance of resting electromotive phenomena is demonstrable on mammalian nerve. The sciatic nerve, or the exposed spinal cord, of the cat or monkey is ligatured and divided, and the end having been freed from the neighbouring structures, is connected with a galvanometer. In this condition a cross sectional difference is obtained which declines very slowly, provided that the few centimetres of exposed nerve are in structural and physiological connection with the deeper undisturbed portions of the same tissue. On the death of the animal, or the complete excision of the tissue, the difference commences to decline with considerable rapidity. It thus appears that the maintenance of a connection with functionally active portions of the tissue, prolongs the duration of the cross sectional difference in the exposed nerve of the mammal. ${ }^{2}$

Finally, although the resting difference of an excised nerve declines, even in the case of the frog's sciatic, to little or nothing in twenty-four hours, it may be reproduced by fresh cross section several days after excision, provided that the nerve is kept from drying, by placing it in 0.6 per cent. $\mathrm{NaCl}$, containing traces of $\mathrm{Ca}$ salts; and the same is true of the excised mammalian nerve. The amount of the resting differences in such stale mammalian nerves is notably less than those of fresh ones, as might be expected from the impairment which has taken place in their functional attributes.

(e) The influence of previous excitation.-The gradual decline in the difference which occurs in an excised nerve is greatly modified by

I Engelmann, Arch. f. d. ges. Physiol., Bonn, 1877, Bd. xv. S. 138.

"Gotch and Horsley, Phit. Trans., London, loc. cit. 
previous excitation. These modifications will be referred to in detail in connection with the electromotive phenomena of excited nerve. It will be sufficient, at this stage, to mention that if a nerve is subjected to short periods of excitation occurring at regular intervals, and the amount of the resting difference is noted between these intervals, the difference declines more rapidly than it does if the nerve is left unstimulated. Similarly, if a nerve is subjected to prolonged rapid stimulation, the difference after such excitation is generally found to be more diminished in amount than can be accounted for by the general decline. On the other hand, it occasionally happens that with the fresh nerves of cooled or winter frogs, ${ }^{1}$ and with nerves excised and left in a bath of physiological saline, ${ }^{2}$ the after effect of excitation is to cause an increase in the difference. If the diminution is considered as, to a large extent, due to an impairment in the functional capacity of the uninjured surface, then the augmentation is explicable, in cooled frogs, by the greater functional capacity, which is a prominent feature of such nerves.

The whole matter may be complicated by the presence of prolonged excitatory electromotive changes, and the possibility that these are similar or opposed to the difference; if present, they must sum algebraically with the persistent resting difference, thus the decline may, in some cases, be accelerated, in others arrested or converted into temporary rise.

It is clear that there is a most intimate connection between the electromotive changes in resting and in excited nerve. Such a relationship is implied in all theories which have been put forward to explain the electromotive phenomena of nerve, whether the molecular theories of du Bois-Reymond and of Bernstein, or the alteration theory of Hermann, and its modification by Hering. The further consideration of the so-called after-effects of excitation may be thus profitably deferred until the actual excitatory changes have themselves been discussed.

\section{Excitatory Electromotive Changes of Nerve.}

The excitatory "negative variation."-The discovery of the resting nerve current by du Bois-Reymond was immediately succeeded by that of electromotive changes evoked by excitation. These show themselves when an excised nerve is connected with a high resistance galvanometer by longitudinal and cross sectional contacts, and the distal portion remote from these contacts is stimulated by a rapid series of excitations. If the nerve is the sciatic of the frog, and the excitation consists of a series of alternating induced currents, a fall in the resting difference is observed, which, on the cessation of the stimulation, passes away more or less completely. Such a decline was termed by du BoisReymond ${ }^{3}$ the excitatory "negative variation," the term "negative" being used in its algebraic sense. The decline was rightly considered by its discoverer as due to electromotive changes set up in the tissues which respond to the excitation; these are such that the portion under the surface contact becomes less positive to the cross section than it was in the unexcited state, and currents, termed "action currents," thus flow through the nerve fibres, from the portion under surface contact

${ }^{1}$ Head, Arch.f. d. ges. Physiol., Bonn, 1887, Bd. xl. S. 207.

3 Waller, Phil. Trans., London, $1897, B$, p. 47.

3 E. du Bois-Reymond, "Untersuchungen," loc. cit. 
to that under the contact on the cross section. The same result is obtained if the distal portion of the nerve is excited by chemical means, ${ }^{1}$ or by a series of mechanical stimuli. ${ }^{2}$ Changes of a similar kind also occur in an efferent nerve left attached to the intact spinal cord whenever a prolonged reflex discharge from the central nervous system is evoked. The efferent medullated nerves of other animals, including those of mammalia, give corresponding effects. In the monkey, cat, rabbit, etc., an excitatory negative variation may be observed in the central end of the divided sciatic, when the nerve, the spinal cord, or the cerebral cortex is roused by either electrical, mechanical, or chemical stimulation. ${ }^{3}$

The negative variation may be observed equally well in afferent nerves; thus, on dividing a lumbar posterior root in the mammal, and connecting the portion peripheral to the section with a galvanometer circuit by means of contacts on its surface and cross section, the negative diminution of the resting nerve change is observed when distal portions of the sciatic nerve are stimulated by induced currents. So, too, the cross sectional difference in the lower fragment of the divided spinal cord shows a marked diminution when either a posterior root or the sciatic nerve is excited, and since, in the latter case, such diminution is abolished by section of all the posterior roots of the nerve, it is clearly due to the excitation of afferent nerves only.

Just as the negative variation in an efferent nerve can be obtained in consequence of the reflex discharge from the central nervous system, so, too, a similar variation has been obtained in some afferent nerves by the natural stimulus of the peripheral nerve-ending. In the frog, du Bois-Reymond observed the effect in the upper portion of a divided sciatic when the skin was acted on by acids, etc. ${ }^{4}$ S. Fuchs has observed the variation in the peripheral end of the trigeminus of the Torpedo divided near its cranial origin, when the skin containing Lorenzini's ampullæ and Savi's vesicles was pressed. ${ }^{5}$ Finally, Beck observed electromotive changes in the occipital lobe of the rabbit when the retina was stimulated by light, ${ }^{6}$ and similar effects have been found to occur in other portions of the cerebral cortex in response to excitation of other afferent nerves. ${ }^{7}$

There can therefore be little doubt that the negative variation is the electrical expression of excitatory changes in the nerves. Further proofs of this are furnished by the following characteristics of the electrical change. It is diminished or abolished by such agents as ether, which lower the functional attributes of excitability and conductivity. It is present during the period of excitation, and disappears when, on cessation of the stimulus, the other indirect signs of functional activity, such as the muscle responses, subside. It is best produced by such stimuli as are found pre-eminently adapted by their character to evoke the functional activity of the nerve, as judged by

\footnotetext{
${ }^{1}$ Grützner, Arch. f. d. ges. Physiol., Bonn, 1878, Bd. xvii.; 1881, Bd. xxv.; Kühne, Unsersuch. a. d. physiol. Inst. d. Univ. Heidelberg, 1881, Bd. iii.

${ }^{2}$ Hering, "Lotos," Prag, 1888, Bd. ix.; Steinach, Arch.f. d. ges. Physiol., Bonn, Bd. vi. S. 516 .

${ }^{3}$ Gotch and Horsley, Plit. Trans., London, 1891, vol. elxxxii. $B$.

${ }^{4}$ E. du Bois-Reymond, "Untersuchungen," Bd. ii. S. 473, loc. cit.

${ }^{5}$ Fuchs, Areh.f. d. ges. Physiol., Bonn, 1895, Bd. lix. S. 468.

${ }^{6}$ Beck, Centralbl. f. Physiol., Leipzig u. Wien, 1890, Bd. iv. S. 16.

${ }^{7}$ Caton, Brit. Med. Assoc. Meeting, London, 1875 ; Fleisch. v. Marlow, Centralbl. $f$. Physiol., Leipzig u. Wien, 1890, Bd. iv. S. 18 ; Danilewsky, ibid., 1891, Bd. v. S. 1.
} 
the response of the end organs with which this is associated. Thus the non-medullated nerves which innervate the muscles of Anodon are excited by the longer galvanic currents far more effectively than by the rapid induced currents, as evidenced by the motor responses, and in these the excitatory electrical change is observed with the former method of stimulation rather than the latter. ${ }^{1}$

The discovery of these excitatory electrical effects formed a notable advance in nerve physiology, since their presence affords the only positive data derived from nerve itself as to the characteristics of the alteration which the tissue undergoes when it passes from a condition of rest into one of activity.

It must not, however, be assumed that the electrical change is the whole excitatory alteration; nor indeed, in the present state of our knowledge, are we justified in the less sweeping assumption that it is a complete or absolutely faithful record of such alteration. It has already been pointed out that the arrest of the circulation and the excision of an efferent nerve are followed by profound changes in excitability and conductivity, as estimated by the response of the muscle to which it is distributed. When, in consequence of this arrest, muscular responses have ceased to be evoked by indirect excitation, the nerve will still give the resting difference on cross section; but a more remarkable feature is that, as long as structural continuity is unimpaired, such a nerve may also give the excitatory negative variation. Thus Hermann, Fredericq, and others ${ }^{2}$ found the excitatory electrical change to be present for many hours in the excised sciatic of the rabbit, as indeed is also and more notably the case in the same nerve of the cat. ${ }^{3}$ Steinach ${ }^{4}$ noticed that the frog's sciatic gave the excitatory effect when, having been dried, it was soaked again in 0.6 per cent. $\mathrm{NaCl}$, and Boruttau ${ }^{5}$ observed electrical changes on mechanical stimulation of the same nerve eight days after its excision, and in the vago-sympathetic of the dog two days after its removal from the body. It must, however, be remembered that the muscular response to indirect excitation requires the functional activity of the nerve-endings. It is therefore not improbable that this manifestation should completely fail, and yet the nerve fibres themselves maintain, to some extent, their functional attributes. Still the facts above mentioned warrant the caution that, although the electrical change is a concomitant of the real excitatory process, the two are not identical, and the former may be perceptible when other evidence of the existence of the latter fails. ${ }^{6}$

In order to accentuate the difference between the vera causa and its excitatory electrical concomitant, the latter will in these pages be now referred to as the excitatory electrical response of the nerve.

${ }^{1}$ Biedermann, Sitzungsb. d. k. Akad. d. Wissensch., Wien (3), Bd. xciii.

2 Hermann, "Handbuch," 1879 , Bd. ii. S. 120 ; Fredericq, Arch. $f$. Physiol., Leipzig, 1880 , S. 65 .

${ }^{3}$ Gotch and Horsley, Phil. Trans., London, loc. cit.

+ Steinach, Arch. f. d. ges. Physiol., Bonn, Bd. Iv.

5 Boruttan, ibid., 1894, Bd. lviii. S. 29.

6 Quite recently Goteh and Burch have obtained evidence of the dissociation of the electrical response from the excitatory process. In the sciatic nerve of the frog it is rossible with two stimuli in rapid succession to obtain only one elcetrical response ncar the seat of excitation which has been cooled, whilst two such responses occur in a more peripheral warmer region._- "Proc. Physiol. Soc.,"Journ. Physiol., Cambridge and London, 1899, vol. xxiii. ; 1899, vol. xxiv. 
Before proceeding to discuss in detail the characters of this electrical response, one other aspect of the negative variation must be alluded to, namely, its relation to the cross sectional difference. The negative variation is dependent not merely upon effective stimulation, but upon the condition of the nerve contacts, and is thus related to the resting nerve current. The larger the demarcation difference, the larger, cateris paribus, will be the negative variation. Most of the conditions alluded to in the previous section as affecting the amount of the resting difference, influence in similar manner that of the negative variation. Thus the spinal cord and those non-medullated nerves which give large demarcation currents give with appropriate stimulation large excitatory effects.

At this stage it will be convenient to anticipate a portion of the subject matter of the succeeding pages, and state in general terms what is known as to those electromotive changes in an excited nerve which produce the familiar negative variation. When the excitation producing this galvanometric deflection is a rapid series of stimuli, each of these evokes an excitatory state of brief duration, with its accompanying electromotive change. A corresponding series of such states and changes will thus be evoked at the seat of stimulation, and be propagated in strict succession along the nerve. The arrival of each member of the series in the neighbourhood of the contact on the longitudinal surface is contemporaneous with a brief electromotive change at this point, such that the tissue there becomes for a short period galvanometrically negative to the unexcited portions. It is the sum of such a series which affects the galvanometer, and if, on the subsequent arrival of the several transmitted states at the cross section, similar changes were produced, it will be obvious that the tissue here would also exhibit the same condition, when compared with any unexcited portions. Under these circumstances the recording instrument would be acted upon by a series of brief currents of alternate direction, one from cross section to surface being in an extremely brief period (less than 001 sec.), followed by one from surface to cross section. It is scarcely necessary to point out that under such conditions there would be no deflection of the galvanometer needle. The considerable deflection which occurs when one contact is upon the surface and the other on the cross section, thus shows that the electromotive change on the arrival of the excitatory state under the former contact is far more intense than that occurring subsequently in the neighbourhood of the cross section, and thus the change resulting in galvanometric negativity of the surface contact becomes prominent. With repeated stimuli the deflection is, in reality, the sum of a series of such electromotive changes under the surface contact, corresponding to the series of stimuli by which they are evoked. Hence the relation of the negative variation to the cross sectional difference must be to a large extent dependent upon the circumstance that, under these conditions, one contact alone is upon tissue which gives the full excitatory response. When both contacts are so arranged on the surface that there is little or no difference, it is obvious that there must be little or no galvanometric iudication of negative variation.

The discontinuous character of the electromotive change evoked in nerve by a series of excitations.-Non-medullated nerves are remarkable, inasmuch as the closure or opening of a single galvanic current may cause an obvious galvanometric indication of a diminution 
in their resting difference; ${ }^{1}$ but in medullated nerves a succession of stimuli must be employed. The proofs that the negative variation produced by such successive stimuli is not a uniform continuous electromotive change, but a series of the same rhythm as the excitation, are similar in character to those which exist in the case of muscle, and differ merely in detail.

Observations with rheotome.-If the differential rheotome referred to in connection with muscle is employed, it is found that when the rate of revolution is 20 per second, a large negative variation occurs if the galvanometric contacts of the instrument are so arranged as to comprise a time of from one to ten thousandths of a second after each excitation; whilst with contacts arranged in any part of the remaining twentieth of a second, which intervenes between the successive excitations, there is no such effect. ${ }^{2}$

Observations with the telephone.-If a delicate Siemen's telephone is arranged so as to be in metallic contact with the surface and cross section of a large excised nerve or group of nerves, and the nerve be excited near its further end by a rapid series of induced currents (100 per second), a note similar to that of the vibrating exciter is heard. That this is due to the production and transmission of a series of true electrical responses, and not to the physical spread of the exciting induced currents, is proved by observing that it may be augmented or diminished by placing the excited region in the condition of catelectrotonus and anelectrotonus respectively, that it may be blocked by ether, and other agents which impair nerve conductivity, and that it is abolished by destroying the functional continuity between the excited and observed regions. ${ }^{3}$

Observations with the capillary electrometer. - An instrument combining great sensitiveness and rapidity of movement is highly magnified, and the image of the mercurial meniscus thrown upon a photographic plate which travels at about $50 \mathrm{cms}$. in $1 \mathrm{sec}$. When the instrument is connected with the excised sciatic nerve of the frog, a movement of the level of the meniscus is recorded on the plate in response to each single stimulus of the nerve trunk, whether electrical or mechanical. When the nerve is excited by a series of stimuli of 100 per second or less, a movement is shown on the record corresponding to each member of the series of exoitations. ${ }^{4}$

Obscrvations with the physiological rheoscopc.-The phenomena of secondary tetanus can be obtained when two excitable nerves are in juxtaposition. ${ }^{5}$ Such excitation of one nerve by the excitatory electrical response in an adjoining one was first demonstrated by Hering as follows. In the cooled frog the sciatic branches are prepared near the knee, ligatured and divided below this, and then dissected up a short way. On now exposing and dividing the upper sciatic plexus, the excitation of the lower sciatic branches evokes a tetanic contraction in the adductor muscles. This contraction is not due to an electrotonic escape, such as that which causes the paradoxical contraction of du Bois-Reymond. This is proved by the circumstance that it is evoked with a pro-

${ }^{1}$ Biedermann, Sitzungsb. d. k. Akad. d. Wissensch., Wien, loc. cit.

${ }^{2}$ Bernstein, "Untersuch. ueher Nerv. u. Musk.," Heidelberg, 1891.

3 Wedenski, Compt. rend. Acad. d. sc., Paris, 1893, tome cxvii. p. 4.

+ Goteh and Bureh, Proc. Roy. Soc. London, 1898, vol. lxiii. p. 300.

5 Hering, Sitzungsb. d. k. Akad. d. Wissensch., Wien, 1882, Bd. lxxxv. Abth. 3, S. 237. See also "Biological Mermoirs," Oxford, 1889. 
portionately weaker stimulus the nearer the excitation is to the ligatured end of the knee branches, and thus to the more excitable end of these nerves. Indeed, the response fails if the stimulus is shifted nearer the muscles, and away from this ligatured cross section. It thus fails if the stimulated nerve is not sufficiently excitable, and it fails also if the excitability of the sciatic plexus is not raised by previous cross scction. The group of nerves in the plexus comprises the upper extremities of the fibres which are being stimulated near the knee, and also others which, lying in the plexus bundle, emerge lower down to supply the adductor muscles. Although the two sets of fibres are physiologically and structurally independent, excitation of the latter is produced by the electrical responses occurring in the former. The seat of this excitation is the combined nerve trunk of the plexus, the excitability of whose fibres has been raised by its division, so that the electrical response in the knee fibres, on arriving at the plexus, becomes adequate to arouse other fibres lying in immediate proximity. The fact that the rapid stimulation evokes the secondary tetanus, can only be explained on the supposition that a series of electromotive disturbances are produced and propagated up the primary nerve bundle, and that each member of this rhythmical series is adequate to arouse the fibres of the secondary nerve bundle.

The negative variation caused by repeated stimulation thus comprises a series of electromotive disturbances, each member of the series being transmitted along the nerve, and evoked by a single member of the series of stimuli. The further consideration of the excitatory change resolves itself into that of the electrical response evoked by a single stimulus.

The electrical response of nerve to a single stimulus.-For the reasons already referred to, the simplest mode of investigating this is one in which the electromotive difference between the surface and cross section of a nerve is obtained, and any variation in consequence of excitation observed. The variation is of such brief duration that galvanometric indications of its presence can only be obtained by summing up a number of such variations by means of the repeating rheotome. The capillary electrometer, if constructed so that the surface of the mercury moves with very great rapidity, is, however, able to follow the brief electromotive change. In order to observe the movement, the surface of the mercurial meniseus is projected upon a travelling sensitive plate, so that a quick movement of the magnified image may be recorded, it being impossible to follow the rapid oscillation by the eye. Under these conditions, an oscillation, indicating a rapid electromotive change, has been recorded by the capillary when the nerve is excited by a single stimulus.

The character of the recorded excursion is always such as to show a rapidly developed change, in which the contact nearest the seat of the stimulus becomes relatively negative to that further off. The subsidence of this relative negativity of the tissue under the proximal contact is greatly influenced by the condition of the nerve, but when the distal contact is one involving a cross section of the nerve, it is always somewhat prolonged. This is indicated in Fig. 270 , in which the upward movement indicates negativity of the proximal contact. As the moment of the stimulus is indicated on the plate, the time relations of the electrical response to the single

VOL. Il. -34 
stimulus may be readily calculated. Outlines of such records are shown in Fig. 270, and the results confirm those obtained by means of the repeating rheotome. ${ }^{1}$

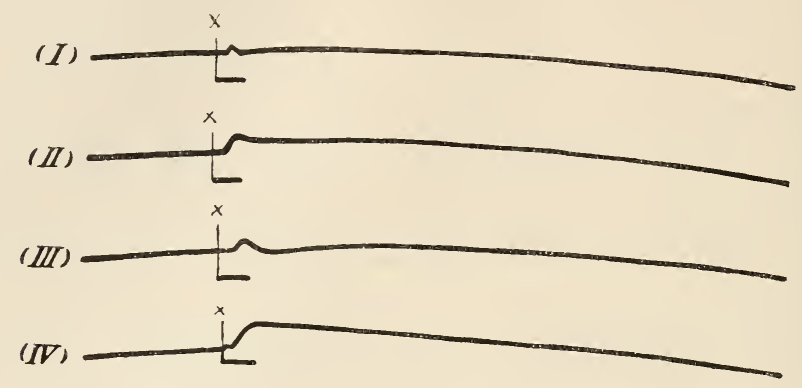

Frg. 270.-Facsimile curves of the photographed excursions of a capillary electrometer connected with the frog's sciatic nerve. Excitation, $30 \mathrm{~mm}$. from proximal capillary contact by a single induced current at moment marked $x$. The short dark horizontal line indicates 01 sec. (I), Uninjured fresh nerve; (II), fresh nerve with distal contact on warmed area; $(I I I)$, excised nerve kept twenty-four hours in 0.6 per ecnt. $\mathrm{NaCl}$ solution; $(I V)$, excised nerve with distal contact on a recent eross-section.-Gotch and Burch.

(a) Propagation time.-The interval of time between the stimulus and the appearance of the change under the surface contact is strictly proportional to the distance between the seat of excitation and this contact. Hence, if two seats of excitation are so situated that one is twice as far as the other from the surface contact, the interval in question is in the former case twice as long. In the frog's sciatic the interval is such that at a temperature of $15^{\circ} \mathrm{C}$. it is 001 sec. for $30 \mathrm{~mm}$. of nerve. The electrical response is thus transmitted at a rate of 30 metres per second, i.c. the same rate as the excitatory process, when estimated by the indirect muscle response, according to the method devised by Helmholtz. Further, since the time at which the effect appears is strictly proportional to the distance of the surface contact from the seat of the excitation, it is obvious that the loss of time must be due to transmission and to this only. In other words, there is no delay except that necessitated by uniform propagation at the above rate; there is, therefore, no period of delay or latent time in the electrical response of nerve. ${ }^{2}$ The propagation time of the electrical response varies in any given nerve, when subjected to changed conditions of temperature, etc.; it also varies in different parts of a nerve, in different nerves, and in the nerves of different animals. Thus, in the non-medullated nerves of Eledone moschatus the rate of such propagation has been found, in the winter, to be 1 metre per second; in the summer Boruttau ${ }^{3}$ found a rate of from $3 \frac{1}{2}$ to $5 \frac{1}{2}$ metres per second, both in Elcdone and in Octopus. ${ }^{4}$

${ }^{1}$ Goteh and Burch, Proc. Roy. Soc. London, 1898, vol. lxiii. p. 300 ; Goteh and Horsley, ibid., vol. xlv.

2 Bernstein, "Erregungsvorgang im Nerven- u. Muskelsysteme," 1871.

${ }^{3}$ Fuchs, Sitzungsb. d. k. Akad. d. Wissensch., Wien, Bd. eiii. Abth. 3, S. 209.

+ Boruttau, Arch. f. d. ges. Physiol., Bonn, 1897, Bd. lxvi. S. 285. 
(b) The duration of the response.-The electrical change (negativity) under the surface contact reaches its maximum in the frog's sciatic very rapidly, and then subsides; the duration of the whole effect, at $15^{\circ} \mathrm{C}$., was found by Bernstein to be as little as 0007 sec. It is probable that this time is too short; Hermann estimated it as .005 sec. at $5^{\circ} \mathrm{C}$., and Head as 02 sec. $^{1}$ These results, obtained with the repeating rheotome, may be compared with those calculated from the records of the capillary electrometer excursions. From these it appears that at $15^{\circ} \mathrm{C}$. the change under the surface contact reaches its maximum in 001 sec. after its first appearance, and subsides in 01 sec. to a small amount, which, however, is followed by a second increase of low electromotive force, lasting several hundredths of a second, this being the so-called after effect. The duration is much prolonged by low temperature (.003 sec. at $5^{\circ} \mathrm{C}$.), and diminished by rise up to $30^{\circ} \mathrm{C}$. It is probably of different value in different nerves. In non-medullated nerves, such as that of the cephalopod, it has been estimated by Fuchs ${ }^{2}$ as varying between .01 sec. and 008 sec., a result which has been in the main confirmed by Boruttau, though in his observations, carried out upon summer animals, the duration was found to be a little shorter.

(c) The electromotive forcc of the response.-This cannot be accurately determined by the rheotome method; the only reliable estimation is that afforded by analysing the curve of the capillary electrometer variation, as photographed upon a rapidly moving sensitive plate. It is roughly determined by comparing the total deflection obtained in rheotome observations with that produced by a known electromotive force, the currents of which are led through the repeating rheotome contacts. In this way Hermann showed that the excitatory response may in all probability involve an electromotive force, equal to or surpassing that of the cross sectional difference. In some instances in the frog's sciatic, it was nearly twice as great. The analysis of electrometer records appears to show that the maximal electromotive force of the initial change may in the frog's sciatic, at $5^{\circ} \mathrm{C}$., amount to .03 volt, whilst that of the more prolonged after effect is only $\frac{1}{10}$ of this, i.e. 003 volt. $^{3}$

(d) The character of the rcsponse with two surface contacts.-The rapidity with which the excitatory electrical change is propagated from one part to another of a nerve, renders it difficult to determine the character of the change with two surface contacts. In this case, first the proximal and then the distal contact will be the seat of the same electromotive alteration. The proximal electrode thus becomes negative to the distal, until the change in the latter has developed sufficiently, when it becomes positive to this. Since, however, the distal change in nerve would occur only 001 sec. after the proximal, if $30 \mathrm{~mm}$. of nerve intervened between the two contacts, the experimental demonstration of this diphasic effect is only rendered possible when the rate of propagation is very much slowed. By taking a bundle of six sciatic nerves from the frog and cooling these to $5^{\circ} \mathrm{C}$., Hermann has been able to demoustrate the diphasic effects with the rheotome. The proximal surface coutact was found to become first negative and then positive to the distal oue. As the negative effect under the proximal contact has not subsided when that under the distal contact has commenced, the total result is still

\footnotetext{
${ }^{1}$ Hermann, Arch. f. d. ges. Physiol., Bonn, Bd. xxiv. S. 246 ; Head, ibid., 1887, Bd. xi. S. 207.

2 Loc. cit.

${ }^{3}$ Goteh and Burch, Proc. Poy. Soc. London, 1898.
} 
the algebraic sum of two electromotive changes, and the isolated components must be inferred from the time relations of the whole effect.

The curves $a, b, c$, and $d, e, f$, marked by thin lines in Fig. 271, represent the proximal and distal change respectively. They would produce

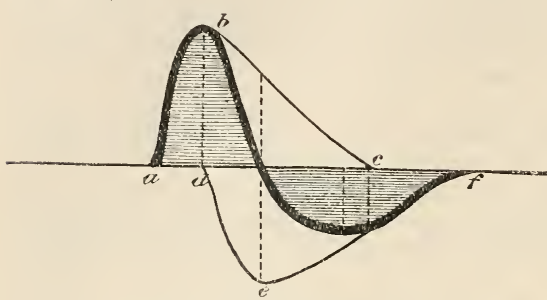

Fig, 271.-After Hermann. by their combination the observed diphasic effects indicated by the strongly marked lines enclosing the shaded area. In the diagram the abscissa represent time, and the ordinates the extent of galvanometric deflection when the circuit was closed at difierent moments after excitation.

It is noteworthy that in all Hermann's results the distal electromotive change and the proximal one are slightly different; these differences are such that the total area of the two curves, $a, b, c$, and $d, e, f$, are the same, but the forms of the two are not absolutely identical. ${ }^{1}$

In the non-medullated nerves of Octopus and Elcdone, the slower rate of transmission has enabled Boruttau to observe similar diphasic effects with the rheotome method of galvanometric observation. The

1 The analysis of such electrometer curves as those outlined in Fig. 270, I, IV, 111 , gives the following results :-

\begin{tabular}{|c|c|c|c|}
\hline $\begin{array}{c}\text { Time after Excitation } \\
\text { of Nerve } 30 \mathrm{Mm} \text {. } \\
\text { from Proximal } \\
\text { Contact. }\end{array}$ & $\begin{array}{c}\text { I. } \\
\text { Temp., } 5^{\circ} \text { C. Uninjured } \\
\text { Nerve. Electrometer } \\
\text { Contacts } 10 \mathrm{~mm} \text {. apart } \\
\text { and both on } \\
\text { uninjured Surfaces. }\end{array}$ & $\begin{array}{c}\text { IV. } \\
\text { Temp., } 5^{\circ} \mathrm{C} \text {. Nerve with } \\
\text { Cross Section. Proximal } \\
\text { Contact on uninjured } \\
\text { Surface + to } \\
\text { Distal on Cross Section. }\end{array}$ & $\begin{array}{c}\text { III. } \\
\text { Temp., } 5^{\circ} \mathrm{C} \text {. Nerve Excised } \\
\text { and kept Twenty- } \\
\text { four Hours in } 0.6 \text { per } \\
\text { cent. NaCl. Proximal } \\
\text { contact on Surface, } \\
\text { Distal on end. }\end{array}$ \\
\hline $\begin{array}{l}\text { See. } \\
0.0020 \\
0.0025 \\
0.0030 \\
0.0035 \\
0.0040 \\
0.0045 \\
0.0050 \\
0.0055 \\
0.0060 \\
0.0065 \\
0.007 \\
0.008 \\
0.009 \\
0.010 \\
0.011 \\
0.012 \\
0.013 \\
0.014 \\
0.016 \\
0.018 \\
0.020 \\
0.030 \\
0.040 \\
0.050 \\
0.060\end{array}$ & $\begin{array}{c}\text { Voltage E.M.F. } \\
\quad \text { Start } \\
-0.0218 \\
-0.0218 \\
-0.0218 \\
-0.0002 \\
+0.0145 \\
+0.0128 \\
+0.0113 \\
+0.0101 \\
+0.0099 \\
+0.0054 \\
+0.0007 \\
+0.0004 \\
+0.0000 \\
\ldots \\
\ldots \\
\ldots \\
\ldots \\
\ldots \\
\ldots \\
\ldots \\
\ldots\end{array}$ & $\begin{array}{l}\text { Voltage E.M.F. } \\
\begin{array}{l}\text { Start } \\
-0.0262 \\
-0.0176 \\
-0.0144 \\
-0.0127 \\
-0.0103 \\
-0.0081 \\
-0.0054 \\
-0.0029 \\
-0.0004 \\
+0.0015 \\
+0.0016 \\
+0.0020 \\
+0.0023 \\
+0.0023 \\
+0.0023 \\
+0.0026 \\
+0.0029 \\
-0.0002 \\
-0.0019 \\
-0.0023 \\
-0.0019 \\
-0.0013 \\
-0.0006 \\
0.0000\end{array}\end{array}$ & $\begin{array}{c}\text { Voltage E.M.F. } \\
\text { Nil } \\
, " \\
, \\
, \\
\text { start } \\
-0.0093 \\
-0.0145 \\
-0.0178 \\
-0.0093 \\
-0.0002 \\
+0.0041 \\
+0.0050 \\
+0.0113 \\
+0.0116 \\
+0.0024 \\
+0.0011 \\
+0.0010 \\
+0.0007 \\
+0.0003 \\
0.0000 \\
\ldots \\
\ldots\end{array}$ \\
\hline
\end{tabular}


duration of each phase is altered by various nerve conditions, and particularly by low temperature, which prolongs the time relations, and thus increases the effect. The diphasic character of the effect, when the tissue under each contact is susceptible of undergoing the change, is beautifully shown in capillary records. ${ }^{1}$ With two surface contacts on an uninjured nerve, the record is of the character shown in Fig. 270, curve $I$, the rapid upward displacement being succeeded by one of opposite direction. If the sciatic nerve is excised and left for over twelve hours in $0.6 \mathrm{NaCl}$, the subsidence of the injury at the cross section places the whole nerve in the same physiological state, and a diphasic effect may now be obtained wherever the two contacts are placed on the tissue. Propagation being slowed, the effect is more evident than in the fresh nerve. An example is given in Fig. 270, curve III. On warming locally the tissues at the distal contact, the record resembles that of curve $I I$, the distal tissue being now incapable of responding to its full extent, whilst on making a cross section the records are those of $I V .^{2}$

The influence of various conditions upon the negative variation.-The intensity of the stimulus.-The electromotive change increases in magnitude with the increasing intensity of stimulus. The character of the increase is well shown in the photographic records of the galvanometric deflections due to a brief series of successive excitatory changes. ${ }^{3}$ With one contact on the surface, and the

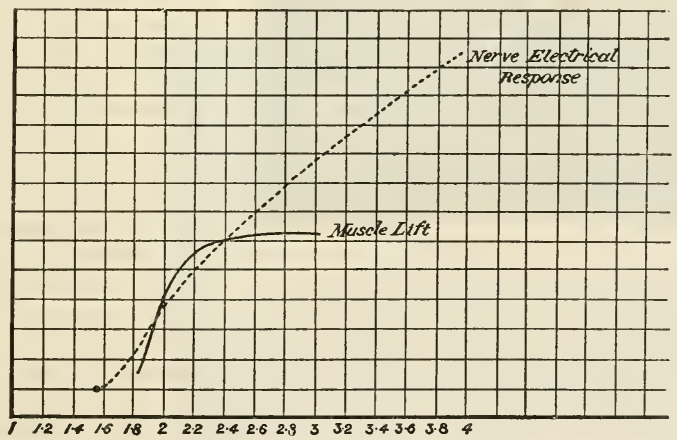

FiG. 272.-Abseissæ indicate increasing intensity of stinuulus. Ordinates indicate magnitude of response.-After Waller.

other on the cross section of the nerve, a short series of about ten induced currents, occurring at a rate of fifcy per second, is allowed to excite the excised nerve every fifteen minutes. The intensity of this stimulus is regularly increased, and the resultant deflections are recorded on a slowly moving photographic plate. Within limits the excitatory negative variations, as estimated by the extent of the galvanometric deflections, are augmented in strict proportion to the increase of the stimulus intensity.

\footnotetext{
${ }^{1}$ Gotch and Burch, Proc. Roy. Soc. Londom, 1898.

2 See analysis on previous page (footnote).

3 Waller, Brain, London, 1895, vol. xviii. p. 210 ; Croonian Lecture, Phil. Trans., London, 1896.
} 
If the results are portrayed diagrammatically in a curve whose abscisse represent units of intensity of stimulns, whilst the ordinates represent those of galvanometric deflection, it will be seen that the curve has three portions (Fig. 272). The first portion, when the stimulating intensity is inadequate to evoke muscular response, is slightly convex to the abscissæ, i.e., the effect increases with increasing increments in the exciting agent. This is succeeded by a portion which is an ascending straight line, the effect increasing in strict proportion to increase in the stimulus. The final portion is slightly concave to the abscissa, the effect increasing less and less in proportion to the augmentation of the stimulus. ${ }^{1}$

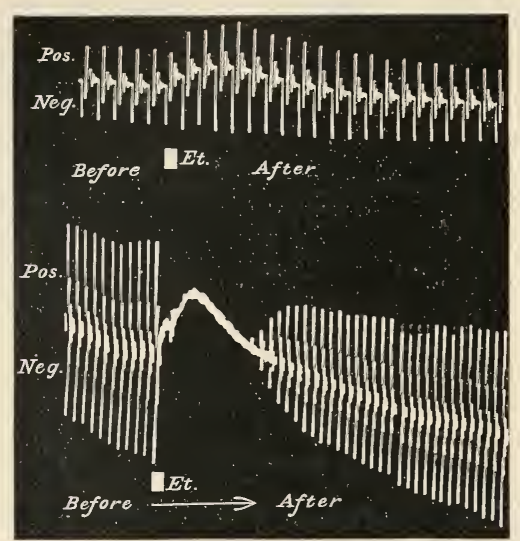

Temperature.-On warming the nerve up to $35^{\circ}$ or $40^{\circ}$ C., the duration of electrical response is much diminished; on the other hand, it becomes prolonged in nerves cooled to $5^{\circ} \mathrm{C}$. The low temperature acts in two ways, it retards propagation, and it causes a slow rate of development of the response in any one portion of nerve. The total result is thus a marked increase in the negative variation. It is not clear whether there is an alteration in the electromotive force of the response apart from this change in its time relations. The influence of ands-

FIG. 273.-Effect of ether unon the electrical response of nerve. The upper tracing shows the increased response after slight etherisation during period marked Et.; the lower tracing shows temporary abolition after more profound etherisation during period marked $E t$. The series reads from left to right. In this and all similar figures the vertical lines are the photographed deflections of a galvanometer needle.

thetic gases and drugs.These have been investigated by Waller in much the same way as in the experiments just described. Negative variations are evoked by a series of induced currents, short periods of excitation being alternated with longer periods of repose. The nerve with both its galvanometric and exciting contacts lies in a small chamber, through which the gas is passed, and any alteration in the responses is indicated by a change in the photographic record of the galvanometric deflections. ${ }^{2}$

Ether, when in very small amount, causes an augmentation in the effects; when a little more pronounced, a primary augmentation is succeeded by diminution and possibly by abolition; when still stronger, the primary augmentation is not seen, and there is complete abolition. All these effects soon pass away if the ether is removed, and even in cases where complete abolition has been produced, the deflections return to at least their original value on the removal of the vapour (Fig. 273).

Chloroform, when very weak, causes primary augmentation, but

${ }^{1}$ Waller, Brain, London, 1895, vol. xviii. p. $210 . \quad{ }^{2}$ Ibid., vol. xix. p. 43. 
this is succeeded by gradual diminution, which is prone to pass into complete abolition with very gradual recovery. These subsequent effects come on even when attempts have been made at quite an early stage to remove the vapour from the chamber. A stronger dose of chloroform abolishes the variations, and on removal of the vapour the electrical effect may never reappear, but even when it does it is liable to be permanently diminished in amount. Alcohol vapour causes an augmentation, which persists far longer than in the case of weak ether, and $a$ fortiori than in that of weak chloroform (Fig. 275). The augmentation is seen when the vapour is comparatively strong, and depressant effects are only observed as the result of prolonged action. A large number of other gases have been investigated in the same way, the details of which cannot be entered into here. There is, however, one gas the action of which

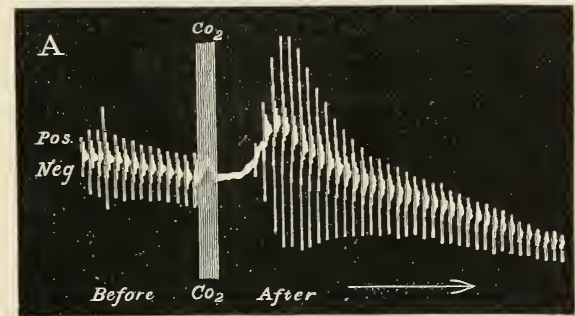
is of great physiological importance, namely, carbon dioxide.

Carbon dioxide. - The effects of the passage of $\mathrm{CO}_{2}$ gas through the chamber containing the nerve are as follows. A slight amount causes, during the passage, a primary augmentation followed by a diminution. These efferts are succeeded by a marked secondary increase in the negative variations, which continues for

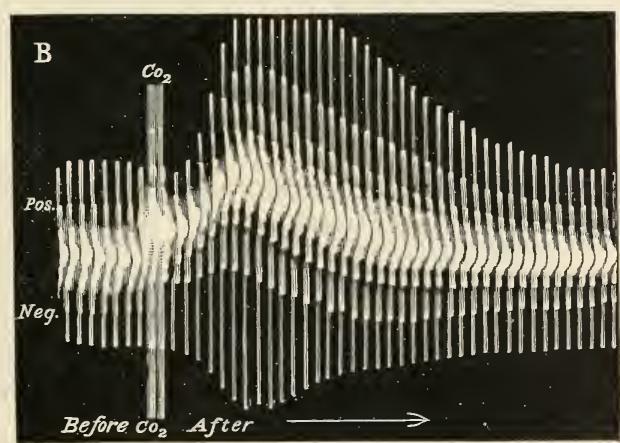

FIG. 274. - The upper record shows the full effect of "much" earbon dioxide on the negative variation. Temporary abolition followed by secondary angmentation. The lower record shows the effect of little $\mathrm{CO}_{2}$ producing secondary augmentation of negative variation. - Waller.

some little time

after the cessation of the gas, and then gradually subsides. The effects are well displayed in Fig. 274. If the $\mathrm{CO}_{2}$ is strong, then the primary augmentation is not observed, and the diminution passes into complete abolition of the electrical effects. The abolition lasts for some little time after the cessation of the $\mathrm{CO}_{2}$, and is then succeeded by most marked secondary augmentation.

It will be observed that all the agents above referred to-ether, chloroform, alcohol, and $\mathrm{CO}_{2}$ - exert both augmentor and depressant effects, possibly related to increased and decreased nerve excitability, 
but obviously due to an increased and decreased susceptibility of the nerve to the production of negative variations. The secondary angmenting effect of $\mathrm{CO}_{2}$ is so pronounced, that when this gas is combined with chloroform it tends to neutralise the depressing effect produced by that vapour.

Influence of solutions of certain salts.-The investigation of this influence has been carried out by Waller in the following way. The excised sciatic of the frog is soaked in 0.6 per cent. $\mathrm{NaCl}$, and the negative variations produced by short periods of faradisation is recorded by the photographic method previously described. 'The normal saline is now replaced by solutions containing definite percentages of some other salt.

Neutral salts.-The immersion of the nerve in decimolecular solutions of these salts profoundly modifies the amount of the electrical changes. Potassium salts are markedly depressant; calciun and strontium salts, on the other hand, augment the variations; sodium

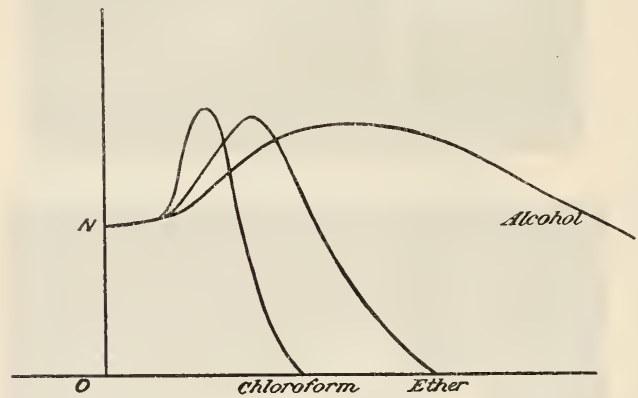

FIG. 275. $-N=$ level of normal excitability. $O=$ level of zero excitability. The curves show alteration in excitability, produced by continuance of the three vapours indicated. Ordinates indicate amount of negative variation. Abscissie indicate time after exposure to vapour.-After Waller. salts are slightly depressant, but the depressing action is in no way comparable with that of potassium. 'The nature of the acid moiety modifies the result; thus potassium bromide is rather more depressant than the chloride.

Salts of various alkaloids. - Most alkaloids appear to exercise a depressing action upon the negative variation. Aconitin and veratrin in 1 per cent. solution rapidly abolish the effect; curarin, digitalin, and morphin diminish its amount, whilst strychnin causes an augmentation.

The method of soaking the nerve in solutions of the salts produces an alteration in electrical resistance, which need not invalidate the conclusions derived from the photographic records of the responses, since any change due to this cause can be ascertained from the records of excursions caused by a known electromotive force before and after the alteration.

Influence of previous aetivity.-A short snccession of stimuli may be repeated over and over again in the case of the frog's sciatic nerve, without altering perceptibly the extent of the negative variations thus evoked. At the commencement of such a series, comprising short periods of excitation with longer intervals of repose, the effect increases in amount, then it attains a maximum and continues undiminished. The negative variation in a nerve trunk thus shows no sign of fatigue in consequence of the repetition of the external stimulus. This indefatigability has been already alluded to as a characteristic of the excita- 
tory process in nerve fibres ; it is evidently an equally striking feature of the electrical response.

The initial stage of augmentation is undoubtedly related with the conditions produced in the first few responses, and is thus increased if such changes occur on a more extensive scale.

It will be remembered that nerve excitability, as ganged by the indirect muscular response, is raised by previous excitation; the negative variations show a similar augmentation.

If the nerve is subjected to a prolonged series of rapid alternating induced currents, during the regular and periodic excitation by the shorter series of such currents, then, on the cessation of the prolonged stimulation, the negative variations evoked by the periodic series become augmented in amount (Fig. 276). This augmentation closely resembles that produced

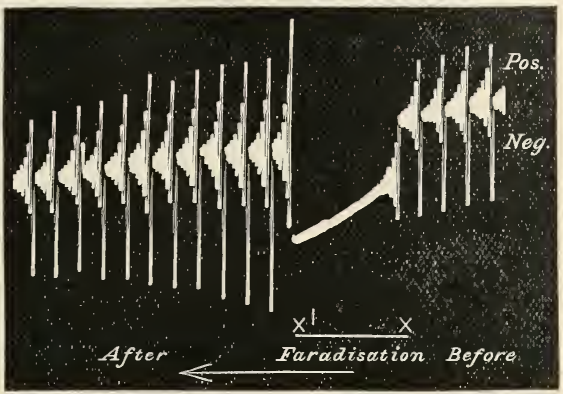

FIG. 276.-Effect of faradisation upon the electrical response evoked by each of a series of stimulations, repeated at considerable intervals. The faradic currents stimulated nerve during period $\mathrm{x} \mathrm{x}^{1}$, the additional electrical responses thus evoked are fused, and the members of the series are thus masked; on the cessation of the faradisation, the subsequent members of the series are augmented. The series of events is to be read from right to left.-After Waller.

in nerve through subjection to a $\mathrm{CO}_{2}$ environment. It has been, therefore, supposed by Waller, that the augmentation following prolonged activity is due to the $\mathrm{CO}_{2}$ set free in the nerve in consequence of its activity. There is, undoubtedly, a close correspondence between the two classes of effect, those of external $\mathrm{CO}_{2}$ and of internal prolonged activity, which will be made more evident by the facts to be next detailed. The absence of any thermal effects during nerve activity renders it, however, somewhat difficult to accept this explanation of the effect. ${ }^{1}$

Negative and positive after effects.-It has been already pointed out that the excitatory negative variation observed in a nerve, when connected by surface and cross sectional contacts with a galvanometric circuit, may exhibit itself as a prolonged change in the resting demarcation current. This is generally of such a character as to cause its diminution, but the presence of effects in the other direction may be detected under certain conditions. The diminution of the difference is termed a negative after effect, the augmentation a positive one, these terms being here employed to indicate a prolonged decrement or increment of the denarcation difference.

It appears from the capillary records previonsly referred to, that any circumstance causing an impairment in the physiological condition of the tissue under the distal contact (i.e. that farthest from the seat of the excitation) produces a negative after effect; as this follows the pro-

1 Waller, Croonian Lecture, Phil. Trans., London, 1896. 
nounced negativity, which is the evidence of the electrical response, it blends with the main effect, and may be regarded as the normal wake of the single response. With uninjured contacts, this wake presumably occurs as the successor of both the change under the proximal and that under the distal contact, and the algebraic sum of the two practically reduces it to nothing. If, however, the distal tissue is rendered less capable of responding (injury, local heat, etc.), the unbalanced effect under the proximal electrode becomes evident. The negative after effect seen under these conditions is thus the tail or wake of the explosion, and occurs under normal conditions as a constant concomitant of this. On the other haud, diminished capacity of the tissue under the proximal contact must allow the wake of the change under an uninjured distal contact to appear. It must show itself as positivity of the proximal contact, and thus give rise to a positive after effect. There are, however, other conditions capable of developing after effects of this type. Thus in the nerves of winter or cooled frogs, the negative variation, as evidenced by the galvanometer, is often succeeded by augmentation of the resting difference. Head, who first drew attention to the phenomenon, considered that the essential condition which favoured its appearance was vital power or vigour. In summer frogs it gives place to the customary demarcation decrement.

Another set of conditions which favours the production of positive after effects has been observed by Waller. He found that the excised sciatic nerve of the frog, if kept for some time in physiological salt solution, responds to electrical stimulation in quite a different manner to that of the freshly excised nerve. ${ }^{1}$

In such "stale" nerves, the following electromotive phenomena are observed. After immersion for a few hours, the variation evoked by a short period of rapid stimulation is often of a biphasic character, the deflections indicating surface negativity, followed by positivity. These effects are succeeded by the usual demarcation decrement, i.e. decline in the resting difference.

If the nerve has been kept for a still longer time in the bath, the positive phase of the variation becomes still more prominent. The excitatory variation now becomes one in which a positive phase may precede the negative, and may finally be such as to indicate positive phases only.

Photographic reproductions of such positive deflections are given in Fig. 277.

The frequent repetition of the excitation causes a gradual diminution in these positive effects, and thus the most modified condition, in which the variation is purely positive, may become transformed into one showing a positive succeeded by a negative, or into one showing a negative followed by a positive.

There are thus four distinct stages as regards the character of the excitatory galvanometric effects-

First stage.-Each set, with effect - (ummodified nerve).

Second stage.-Each set, first - then + (slightly modified nerve).

Third stage.-Each set, first + then - (more modified nerve).

Fourth stage.-Each set, + only (profoundly modified nerve).

${ }^{1}$ Waller, Croonian Lecture, Phil. Trans., London, 1896. 
The essential factor in the production of the modification is the duration of the immersion of the nerve in 0.6 per cent. $\mathrm{NaCl}$.

The modified nerve giving positive variations can be rendered less susceptible to such production by two agents, prolonged activity and $\mathrm{CO}_{2}$ gas, both of which agents augment the negative variation of the freshly excised nerve. These agencies thus decrease the positive

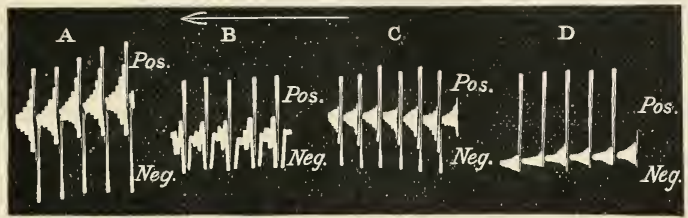

FIG. 277.-Excitatory variations in fresh and stale nerves. A, Excitatory variation in fresh nerve, large negative (down) effect followed by oscillations and negative after effect; $\mathrm{B}$ and C, excitatory variation in early stage of stale nerve, small negative (dowil) effect, followed by conspicuous positive (up) effect; in $\mathrm{C}$, there is a positive after effect; $\mathrm{D}$, excitatory variations in later stages of stale nerve, the excitatory variation is now positive (up) only. The tracings in the above series are to be read from right to left.--After Waller.

and augment the negative one. If prolonged excitation of a nerve in the fourth stage is interposed among the members of the series of short groups of excitations, then the extensive deflection thus produced is seen to be first positive, rapidly passing into negative. On the cessation of the prolonged excitation, each excitation group erokes an effect of the second stage, being first negative and then positive; this gradually changes into the third, and finally may again reach the fourth stage, i.e. + only (Fig. 278).

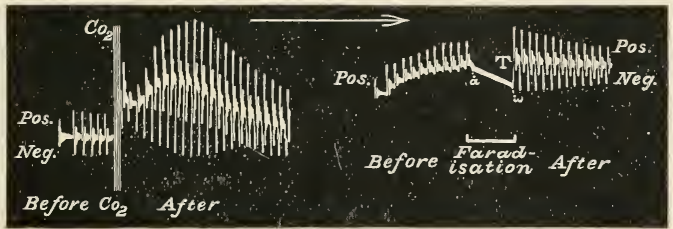

Fig. 278. - The portion upon the left hand of the figure shows the influence of external $\mathrm{CO}_{2}$ upon the variation obtained from modified or stale nerve. Before the $\mathrm{CO}_{2}$ the variations are positive (1p), followed by negative (down); after $\mathrm{CO}_{2}$ they are large negative (down), followed by positive (up). The right hand portion shows the influence of prolonged faradisation upon such variation. The faradisation occurs from $a$ to $w$, the variation before is positive (up); after it is negative (down), followed by positive (up).-After Waller.

A nerve in a less modified stage, similarly treated, may give a large negative effect only during the prolonged excitation; on the cessation of this, each group of the responses resembles that of the fresh nerve, being negative only; this gradually gives way to - + and finally to +- effects. 
Finally the positive after effect or demarcation increment seems, in many cases, to be similarly changed into the more usual negative after effect, i.e. demarcation decrement.

Precisely similar changes are caused by $\mathrm{CO}_{2}$ gas, the most modified effects being transformed into less modified, and these latter into those of the freshly excised nerve. Examples of both these sets of conditions are given in Fig. 278.

It is thus clear that a close similarity exists between the action of previous activity and that of $\mathrm{CO}_{2}$ on the electromotive phenomena of stale or modified nerves, and this is the foundation for Waller's view, that the changes following previous activity are caused by the development of $\mathrm{CO}_{2}$ in the nervous substance, in consequence of the metabolism accompanying such activity.

From these experiments Waller infers that the electrical responses of nerve are in reality the algebraic sum of changes of opposite sign. In the freshly excised nerve those indicating negativity (i.e. diminution of the resting (ifference) are far the larger, in excised nerves kept for some hours those indicating negativity have declined, and positivity may be so pronounced as to swamp the feeble negative changes. The action of prolonged excitation and of $\mathrm{CO}_{2}$ gas in sweeping away the positive effects, may be due either to their diminishing the molecular change which is the basis of these, or more probably to their increasing the change which underlies the negative effects. If the galvanometric effects are to be regarded as a faithful indication of the excitatory processes, then their twofold character must be interpreted as meaning that, in nerve, two excitatory changes of opposite sign are transmitted along an excited nerve.

It must be confessed that the capillary electrometer records previously described do not support this view, since, whatever the character of the after effect, one change only occurs in kept nerves as the initial effect, i.e. relative negativity. The results obtained by Waller may be explained on the view that the positive effects are all effects due to the development of relative negativity of the tissue under the distal contact. The method used by him, unless extended to comprise rheotome observations, is incapable of giving precise information as to the seat of the changes producing the positive effects. This is of essential importance in connection with his conceptions of the excitatory processes, and until we have evidence that the positive change occurs under the proximal contact, the views of Waller, based upon the supposition that it does occur there, cannot be regarded as well founded.

\section{Electromotive Changes in Nerve associated with the Passage of Electrical Currents.}

Polarisation effects-Electrolysis-External and internal polarisation.-The passage of a galvanic current through a moist conductor is attended by well-known electrolytic phenomena, which subside more or less rapidly on its cessation. The phenomena in question are due to the production of electrolytic changes attaining their maximal development at the points where the current enters and leaves the moist conductor, i.e. at the metallic contacts of the battery circuit with this. The electrolytic changes produced by strong currents are such that bubbles of oxygen emerge at the anode, bubbles of hydrogen at the cathode; with feebler currents no obvious evolution of gas takes place, and the products of electrolysis, or electrolytic " ions," are less evident. Evidence of the formation of ions in these instances is obtained, when the moist conductor contains neutral salts, by acidity of the liquid at the anode and alkalinity at the cathode, and an appropriate 
dye will change colour in accordance with the reaction. Methylene blue, such as is used for intravital injection, which blanches on reduction and becomes of a more pronounced tinge on oxidation, is a very suitable dye for the purpose; it is observed to blanch at the cathode and become more intense in colour at the anode. A far more delicate proof is that afforded by the fact that the products are themselves electromotive. Thus, if a "polarising" current is passed through a moist conductor by means of metallic contacts, and these are disconnected from the battery and connected with a galvanometer, the anode is now found to be strongly positive to the cathode (Fig. 279). The electromotive changes thus caused are such that a current flows along the moist conductor from the cathode of the battery circuit to the anode; it is thus opposed to the "polarising" current, and is termed the polarisation current. It is sometimes further defined as the "negative" polarisation current in consequence of its direction; the term is a useless refinement, since in all cases the anodic ions of polarisation are galvanometrically positive to the cathodic, and therefore electro-negative to those at the cathode of the moist conductor. The anodic ions being acid are for simplicity denoted in the figures thus, $\mathrm{O}-$; the cathodic being basic thus, $\mathrm{H}+$.

Internal polarisation.-Since a nerve is a moist conductor, the passage of an electrical current through it, by means of metallic contacts on its surface, must be accompanied by similar changes. The main seat of these, under such conditions, is at the surface junctions of the metals with the liquid of the nerve, hence the polarisation (i.e. the electrolytic change) is said to be external.

When non-polarisable electrodes are used, such external polarisation is

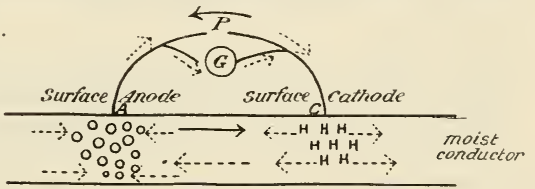

FIG. 279.-The polarising current $(P)$ has been led through the moist conductor in the direction of the black arrow by means of metallic contacts from $A$ to $C$. On opening the eireuit and connecting it with the galvanometer $(G)$, polarisation currents are found directed as shown by the dotted arrows.

reduced to a minimum, but nerve fibres are still polarisable. This is especially the case in medullated nerves, in which there is galvanometric proof of "negative" polarisation, following the passage of a galvanic current. The nerve substance itself is thus polarisable.

Effects very similar in character to those of nerve can be obtained when a current is passed through any schematic arrangement, comprising a good conducting core, surrounded by a moist envelope.

Hermann's model consists of a glass tube, with side openings. The tube contains a thin core of platinum wire, forming its central axis. Both the central and the side tubes are filled with saturated solution of $\mathrm{ZnSO}_{4}$, and into the latter dip amalgamated zinc rods, which serve for the polarising and the galvanometric contacts.

An effectual and simple model consists of a fine copper wire, well covered with cotton. The cotton is soaked in 0.6 per cent. $\mathrm{NaCl}$, and the whole plastered with kaolin similarly moistened. It is used in a moist chamber to diminish evaporation, and the contacts are made by non-polarisable electrodes of the usual type.

Hering's model is without a metal core. It consists of a tubular stem of hollow grass, which is soaked in water, and is filled with strong solution of $\mathrm{NaCl}$ just before use. The concentrated $\mathrm{NaCl}$ in the 
interior furnishes the good conducting core, the less concentrated liquid in the wall is the moist envelope. All these models give polarisation effects, although external polarisation is eliminated; hence these effects must be due to the surface junction existing between the moist envelope and the internal core. ${ }^{1}$ The core is a better conductor than the surrounding envelope, so that the passage of the polarising current is followed by the formation of ions around the platinum wire in Hermann's model, and both around and within the moist core of Hering's model.

Polarisation in Hermann's model.-This is presumed to be of the following character. At the anodic contact the current entering

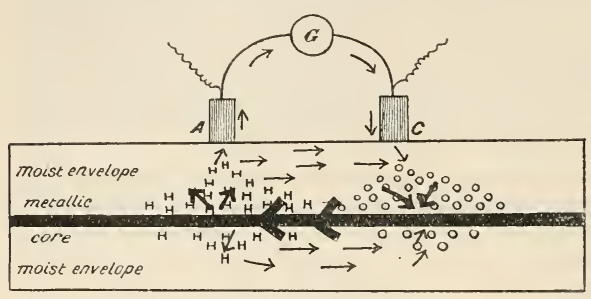

FIG. 280.-Internal polarisation around metallic core of Hermann's model. The polarising current has been led through the model from $A$ to $C$. The polarisation after effects are indicated by the ions and lines of current flow.
Hermann's model will traverse the envelope, and leave this liquid to enter the betterconducting core. There is, therefore, at this point an internal cathode (Fig. 280) for the liquid envelope immediately around the core, and cathodic ions are thus developed in this region. A similar internal anode for the envelope occurs at the point where the current leaves the core to enter the envelope (Fig. 281). At both these places, in the neighbourhood of the better conducting core, electrolytic polarisation will be at its maximum, and may be termed, from its position, "interual." The ions of such internal polarisation under the surface anode $(A)$, being,

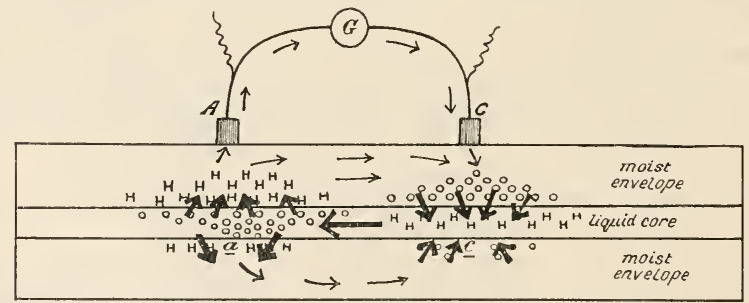

FIG. 281.-Internal polarisation effects around and within liquid core of such a model as Hering's. The polarising current has traversed the complex moist conductor from $A$ to $C$.

as far as the moist envelope is concerned, cathodic, will be $\boldsymbol{H}$, i.e. electro-positive to other portions of the moist conducting envelope. Those under the surface cathode $(C)$ will, for similar reasons, be 0 , i.c. electro-negative. A current will thus pass through the moist envelope from $A$ to $C$, outside the core, and back in this from the portion of core under the eathode to that under the anode as shown in

${ }^{1}$ Hermann, Arch. f. d. ges. Physiol., Bonn, Bd. vi. S. 319 ; Bd. xxxiii. S. 103. 
Fig. 280. If a galvanometer is connected with the surface at or near the polarising contacts, a portion of the surface derivation will pass through the instrument from the anodic to the cathodic contact. Hence the internal polarisation around a central metallic core, lying in a moist envelope, will be evidenced by the fact that on the cessation of the polarising current, the anodic contact is found to be galvanometrically positive to the cathodic.

Polarisation in Hering's model. - In this model both the envelope and the core are liquid, hence the surface junction is the seat of dissimilar polarisation, and ions are formed of one kind in the envelope and of another in the core. ${ }^{1}$ The change in the envelope is precisely similar to that just described as occurring in the metallic core model, i.e. $\mathrm{H}$ ions are found under the surface anode $(A)$, where the polarising current leaves the envelope to enter the core, and 0 ions under the surface cathode $(C)$, where the current leaves the core to enter the envelope. These envelope changes must produce a current, Howing from $A$ to $C$ in the liquid envelope, and from $c$ to $a$ in the core.

But, in addition to these, the core is itself the seat of ions. Under the surface anode at $a$, the polarising current enters this new liquid, producing 0 ions; under the surface cathode at $c$, it leaves this, producing $\mathrm{H}$ ions. A current thus passes from $c$ to $a$ within the core, which is the seat of these changes. It leaves this core at $a$, and passes in the liquid envelope from $A$ to $C$. It thus augments that produced by the envelope polarisation (see Fig. 281). If the polarising circuit is opened, and a galvanometer connected with the surface at or near the polarising contacts, then a derivation of the current in the moist envelope passes through the galvanometer from the anode to the cathode. The former is therefore galvanometrically positive to the latter.

Polarisation in nerve.-Analogous effects to those just described are observed in nerve. They are far more pronounced in medullated than in non-medullated nerve. It is therefore concluded that the sources are similar to those present in the above models, i.e. a twofold polarisation occurs within the nerve fibres at the surface junction of the liquids of the fibre sheaths with those of the axis cylinders.

On the cessation of a current through a nerve, the anode is found to be galvanometrically positive to the cathode, and it may be thus inferred that the portion of the fibres beneath the surface anode contain basic $\mathrm{H}$ compounds in the envelope, and acid o compounds in the axis cylinder, whilst a reversed condition is presumably present under the surface cathode.

Extrapolar polarisation currents.-(a) After effects.-The passage of a current through either the model or the nerve, is followed not only by intrapolar polarisation currents, but by extrapolar effects. The character of these is due to the circumstance that the part immediately under the surface contact is the seat of maximum electrolytic change. Thus, on the cessation of the polarising current, the portion of envelope under the anode will be galvanometrically positive to all other parts of the whole envelope. A current will therefore flow from this, not only to the intrapolar, but to the extrapolar portions of the envelope. In the anodic extrapolar region, this will enter and return along the core from more remote points to those near the anode. Hence, when

${ }^{1}$ Hering, "Lotos," Prag, 1888, Bd. ix. 
two parts of the extrapolar anodic region are connected with a galvanometer, a derivation of the surface effect will pass through it from the contact nearer the anode to the more distant one, i.e., the proximal anodic extrapolar contact will be galvanometrically positive to the distal one, and a current will thus pass in the core from the latter to the former. Similarly, the proximal cathodic extrapolar contact is galvanometrically negative to a distal one. If the direction of the polarising current in the axis cylinder or interual core has been such as to be represented thus $\longrightarrow$, then, on its cessation, the intrapolar polarisation effect in this will be $\longleftarrow$; the anodic extrapolar effect will be $\longrightarrow$, and the cathodic $\longrightarrow$, as shown in Fig. 282, I.

As a matter of fact, the above results are complicated when both anode and cathode are present by bipolar electrolytic changes, anodic and cathodic, occurring in close proximity to one another. The effect of the changes in the neighbourhood of any one pole, "idiopolar," can be thus disturbed by the spread of charges from the other pole. In order to simplify the matter, a model may be made so as to be unipolar, one contact only being in connection with the moist envelope, and the

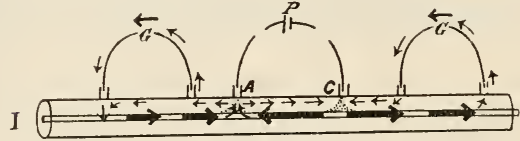

II

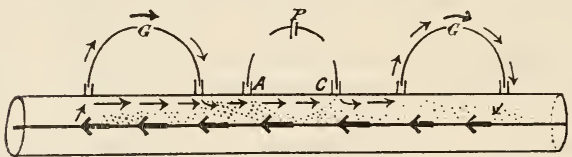

FIG. 282.-I. Extrapolar polarisation currents on opening a polarising current led through model, with thin envelope from $A$ to $C$ (idiopolar effects). II. Extrapolar polarisation currents in model, with large envelope and tine core (bipolar effects). other being attached to the projecting end of the central metallic core.

Under these conditions the idiopolar effects only are-seen, and are always those described; i.e., on the cessation of the polarising current, currents flow through the core towards the previous anode and away from the cathode. With bipolar contacts, the bipolar polarisation effect may swamp the idiopolar. In the intrapolar region the bipolar polarisation is such that the anodic region of the envelope is galvanometrically positive to the cathodic; but if the polarisation has spread along the moist envelope into extrapolar regions, then such region on the anodic side will be also galvanometrically positive to the cathodic, or to all points nearer this cathodic portion. A current will thus flow in the envelope from a distal point of the anodic extrapolar region towards a point nearer the cathodic one, returning through the core the opposite way. Hence, if the direction of the polarisation current in the core is, as before, represented by $\longrightarrow$, although the intrapolar polarisation after effect is as before $\longleftarrow$, the anodic extrapolar effect will be $\longleftarrow$. A similar state of affairs may exist in the cathodic extrapolar region, giving a cathodic extrapolar current directed $\longleftarrow$. It will be observed that these bipolar effects are in the opposite direction to the idiopolar effects just described (Fig. 282, II).

By the use of very thin envelopes, the resistance offered by the liquid envelope to bipolar polarisation effects is greatly increased, 
whilst polarisation spread is also altered; hence the bipolar effect is so lessened that the idiopolar one comes into prominence as in the unipolar arrangenent, and a turning-point can be found by suitable adjustment of envelope and core. ${ }^{1}$ In nerve this thin sheathing envelope exists, so that the extrapolar changes are chiefly those of the idiopolar type. The following table shows the direction of the after effects in various cases of polarisation :-

\section{Polarisation After Effects. ${ }^{2}$}

\begin{tabular}{|c|c|c|c|}
\hline & $\begin{array}{l}\text { Model. } \\
\text { Thick Envelope. }\end{array}$ & $\begin{array}{l}\text { Model. } \\
\text { Thin Envelope. }\end{array}$ & Nerve. \\
\hline Polarising current. . & $>\longrightarrow$ & $>>$ & $>\longrightarrow$ \\
\hline $\left.\begin{array}{c}\text { Intrapolar polarisation } \\
\text { current } \\
. .\end{array}\right\}$ & $\leftarrow$ & $\leftarrow$ & $\leftarrow$ \\
\hline $\left.\begin{array}{l}\text { Anodic extrapolar } \\
\text { polarisation current }\end{array}\right\}$ & & $\longrightarrow$ & $\{\stackrel{\longleftarrow}{\longrightarrow}$ \\
\hline $\left.\begin{array}{l}\text { Cathodic extrapolar } \\
\text { polarisation current }\end{array}\right\}$ & $\leftarrow$ & $\longrightarrow$ & $\{\longleftarrow$ \\
\hline
\end{tabular}

(b) Effects during current flow.-All the polarisation currents just referred to are observed on the cessation of the polarising current. They are therefore after effects, but the electrolytic changes, which they represent, commence the moment that the polarising current begins to flow. In the intrapolar region this is evidenced by a diminution in the polarising current; in the extrapolar regions it is shown by the establishment of extrapolar currents during closure. The moment a current begins to flow through any of the models previously referred to, extrapolar currents are observed; the anodic, directed through the core towards the anodic contact; the cathodic, through the core from the cathodic contact; such currents pass through the envelope in the reverse direction in each case. It will be thus evident that during the passage of a current or a series of currents through such a polarisable moist conductor as a medullated nerve, similar changes must be set up outside the line of current flow, so that every point nearer the anode becomes galvanometrically positive to one more remote, and every point nearer the cathode negative to one more remote. If the characters of these changes are compared with those of the excitatory electrical response, it will be noted that since in the latter the transmitted excitatory effect is one in which the point nearer the seat of excitation is negative to one more remote, the character of the excitatory change resembles the polarisation effects produced during closure in the cathodic region. Now, since both polarisation effects, the anodic and the cathodic, extend during closure into the extrapolar regions, electromotive changes of this type might be successively assumed by more remote parts, and possibly be propagated, like those of the excitatory electrical response. Hence the electro-

${ }^{1}$ Hermann, Arch. f. d. ges. Physiol., Bonn, Bd. xxxiii. S. 103 et scq. ; see also "Biological Memoirs," Oxford, 1887, for translation of Hermann's paper, p1. 312-319.

${ }^{2}$ Arrows indicate direction in core or axis cylinder.

VOL. $11,--35$ 
motive effects produced in nerve by an electrical current or a series of currents may consist of the algebraic sum of (1) the excitatory electrical response, (2) the similarly directed cathodic extrapolar effects, and (3) the oppositely directed anodic effects. The similarity existing between the excitatory electrical response and the cathodic extrapolar change has led Boruttau to frame the hypothesis that the excitatory response may itself be regarded as an electrolytic change of the kind produced at the cathode of a polarising current: ${ }^{1}$ the hypothesis will be referred to again later. With regard to the causation of these currents, it is evident that internal polarisation (i.e. formation of

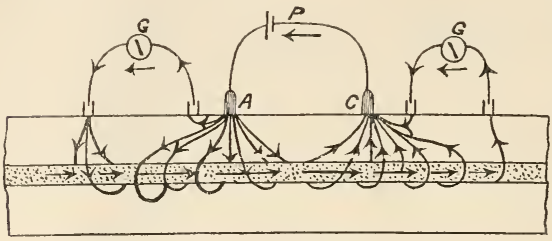

FIG. 283.-Spread of current into extrapolar regions during the flow of a polarising current through the model from $A$ to $C$. electrolytic products around and within the conducting core) must produce extrapolar currents during the passage of the polarising eurrent. The polarisation operates in two waysfirst, by setting up new electromotive sources around and within the internal core, in the manner already described: and, secondly, by bringing into play, through the formation of ions, an increased resistance to the flow of the polarising current through the envelope into the core. This increased resistance causes the lines of current flow to spread more and more into the extrapolar region, in order to enter and leave the core respectively. ${ }^{2}$ The spread is indicated in Fig. 283 ; it obviously will produce extrapolar surface currents of the same direction as those previously referred to.

In nerve such changes observed during closure in the extrapolar regions constitute the familiar phenomena of electrotonus discovered by du Bois-Reymond in 1843.

The electromotive changes of electrotonus.-On passing a galvanic current through a portion of medullated nerve, the extrapolar

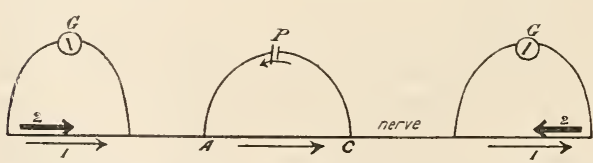

FIG. 284.-The thin arrows (1) represent the clcctrotonic currents present during the flow of a polarising current through a nerve from $A$ to $C$; the dark arrows (2) represent the direction of the demarcation currents. regions show an alteration of electrical state; the new condition on the side of the anode is termed anclectrotonus, on that of the cathode catelectrotonus. As in the polarisable model, these changes are such that a current flows through the nerve towards thus galvanometrically positive to a more remote one, whilst each part nearer the cathode is galvanometrically negative to a more remote one.

If the nerve is an exciserl one with a cross section at each end, then, as shown in Fig. 284, the anelectrotonic change, being of the same

${ }^{1}$ Boruttau, Arch. f. d. ges. Physiol., Bonn, 1894, Bd. 1viii. S. 29.

2 Grinhagen, ibid., 1873, Bd. viii. S. 419. 
sign as the demarcation current, increases this, whilst the catelectrotonic current, being opposed, diminishes it.

The amount of the eleetrotonie effeets.-The amount of these electrotonic currents varies under the following conditions:-

(a) As the intensity of the polarising current is increased the electrotonic effects are angmented; a maximum can be reached, but this may be as much as twenty times the nerve demarcation current.

(b) The effects vary directly with the length of nerve subjected to the polarising current, provided that the alteration of electrical resistance due to the longer nerve is allowed for or eliminated. In other words, the electrotonic effects increase in proportion as the poles are separated.

(e) The condition of the nerve affects the production of electrotonus. Excised nerves continue to show the changes as long as the structural continuity is maintained, but in diminishing amount. Degenerative changes, which interfere with such continuity, diminish the electrotonic effects.

(d) The polarisability of the nerve. All circumstances which diminish the polarisation constant of the nerve, and thus decrease the susceptibility to electrolytic change, diminish the electrotonic currents. One circumstance of special interest in this respect is the change produced by a rapid series of excitations; the results of such stimulation appear to show that excited nerve is less polarisable, and therefore less susceptible to the production of electrotonic effects.

(e) The effects diminish in amount in proportion as the extrapolar region under investigation is removed from the neighbourhood of the polarising current.

The above conditions affect the changes, not only in medullated nerve, but also in the artificial model; according to Boruttau, even the excitatory changes of a nerve can be reproduced on the model if one end is subjected to a series of alternating induced currents, such as are used to excite the end of the nerve.

One of the most important features of the electrotonic currents is that of their time relations. These present two aspects for investigation, the rate of development at the seat of the change, the pole, and the time of appearance at various distances outside the pole, i.e. the rate of extrapolar propagation.

The polar development of eleetrotonic effeets.-This has been investigated by Hermann, Bernstein, and others, by means of the rheotome, which is arranged so that at each revolution both the polarising circuit and extrapolar galvanometer circuit are closed for a short period of definite duration; the time interval between the two closures is, as in all experiments of this nature, susceptible of alteration.

The experiment shows that both in nerve and in the artificial model, extrapolar electrotonus can be appreciated in the neighbourhood of either pole within 001 second after the polarising current has commenced to flow. ${ }^{1}$ At the actual poles the electrolytic dissociation probably commences at the moment of closure. ${ }^{2}$

It will be remembered that in nerve the polar changes in excitability also begin at the moment of closure. ${ }^{3}$ P'fliger has shown that

${ }^{1}$ Bernstein, Untersuch. a. d. physiol. Inst. d. Univ. Halle, 1888, S. 75 ; Naturwiss. Rundsch., 1887, S. 9.

Hermann, Arch.f. d. ges. Physiol., Bonn, 1888, Bd. 1xii. s. 1 to 83.

${ }^{3}$ Grimhagen, lor, cit. 
the anodic lowering of excitability and the anelectrotonic current appear simultaneously, ${ }^{1}$ and there is good ground for the belief that the further time relations of these two sets of changes are identical.

The anelectrotonic current, like the anodic fall in excitability, attains its maximum slowly, and subsides but little during closure; the catelectrotonic attains its maximum rapidly, and may subside whilst the former change is still increasing. The time relations of the development of the electrotonic currents in the immediate neighbourhood of the poles were expressed diagrammatically by du Bois-Reymond, as shown in the curves, Fig. 285, where the abscissæ represent time after closure has begum, and the ordinates the intensity of the respective electrotonic currents. $^{2}$ The curves are constructed from a series of successive observations, in each of which the galvanometer was connected with the extrapolar region for a definite period after the closure of the polarising current.

The propagation of the clectrotonic cffects.-The electromotive changes of electrotonus are undoubtedly present in the extrapolar regions, and there appears to be a delay between the moment of closure and

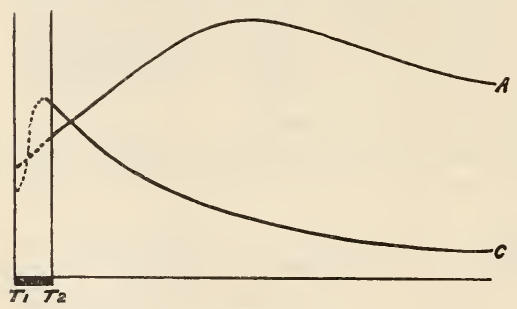

FIG. 285. $-A=$ anelectrotonic ; $C=$ catelectrotonic effect. $T_{1}$, commencement of closure of polarising circuit; $T_{2}$, commencement of observed extrapolar effect. between 6 and $12 \mathrm{~mm}$. per second. ${ }^{3} \quad$ 'These results are rendered inconclusive by the circumstance that the electromotive change at some distance from the pole is much diminished, and thus any comparison between a distant and a near point must be made under dissimilar conditions. The later commencement in the distant region may be chiefly an instrumental lag due to the smallness of the change. ${ }^{4}$ A further complication may be the presence of the excitatory negative variation.

The rate of propagation of the electrotonic change has been investigated also by the response of a nerve muscle preparation used as a galvanoscope. If one of the two branches of the sciatic nerve of the frog is dissected out and divided near its muscular termination, then, on passing a galvanic current through this portion, the other branch is excited, the excitation being indicated by a response in the supplied muscles. This "paradoxical contraction" 5 is due to the electrotonic

${ }^{1}$ Pflitger, loc. cit.

2 E. du Bois-Reymond, Aich. f. Anat., Physiol. u. wissensch. Med., 1867, S. 446 ; also "Gesammelte Abhandl.," Bd. ii. S. 545 .

${ }^{3}$ Bernstein, Arch. f. Physiol., Leipzig, 1886, S. 197 ; also Tschiriew, ibid., 1879,

${ }^{4}$ Hermann, Arch. f. d. ges. Physiol., Bonn, 1880, Bd. xxi. S. 423.

5 E. du Bois-Reymond, "Untersuchungen," 1849 (1), Bd. ii. S. 528. 
current in the polarised branch spreading to the region where the two branches are in close proximity, at which point it traverses and excites the second nerve. The phenomenon was utilised by Helmholtz for estimating the rate of the electrotonic propagation from the seat of the galvanic current to the point of apposition of the nerve branches ( $a b$ in Fig. 286). He found that the period of delay of the secondary muscle was identical with that obtained by electrical excitation at a corresponding distance ( $b c$ in Fig. 286) along the secondary nerve itself, and inferred that the electrotonic change was propagated at about the same rate as the true excitatory process. ${ }^{1}$ Hermann has pointed out that the only conclusion to be drawn from this experiment is that the electrotonic disturbance is developed to the same extent in both branches of the nerve. ${ }^{2}$

The polar alterations in excitability have been utilised as an index, and on the assumption that these are identical as regards origin and time relations with the electromotive changes of electrotonus, the determination of the rate of transmission of the former might throw light on the propagation of the latter. The results have already been referred to in connection with polar changes excitability; it will be remembered that they exhibit considerable discrepancies. Thus, according to Griinhagen, excitability alterations occur in all extrapolar regions almost instantaneously $^{3}$; experiments in Hermann's laboratory show that the anelectrotonic decrease in excitability is propagated at least as fast as 165 metres per second, ${ }^{4}$ whilst Asher

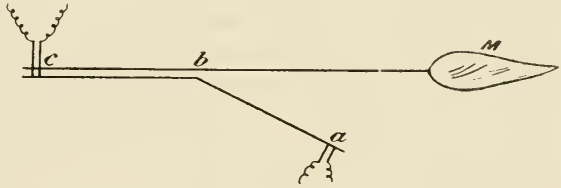

FIG. 286. found a rate of 30 metres per second $;^{5}$ finally, Wundt found that the cathodic increase was transmitted at the same rate as the excitatory state, whilst the anodic decrease was transmitted at 1 metre per second. ${ }^{6}$ In regard to these discrepancies, the most that can be said is that both states are developed at the poles instantaneously, whilst the diminution of the effects in portions far removed from the poles renders all the slower times open to suspicion.

In Hermann's model the rate of propagation of the electrical changes has been estimated by rheotome observations as at least 60 metres per second. ${ }^{7}$ Boruttau, working with polarising currents of short duration and alternate direction, found a rate of cathodic spread in the same model of over 100 metres per second. ${ }^{8}$ In association with Burch, the present writer found a rate of over 100 metres per second for both anodic and cathodic states in a model of similar type, the method used being that of the photographic record of the capillary electrometer excursion produced by an extrapolar effect. Quite recently

${ }^{1}$ Helmholtz, Monatsbl. Akad. d. Wissensch., Berlin, 1854, S. 329.

2 Hermann, "Handbuch der Physiologie," 1879, Bd. ii. S. 162.

3 Grünhagen, Arch. f. d. ges. Physiol., Bonn, 1871, Bd. iv. S. 547.

${ }^{4}$ Baranowsky, ibid., 1880 , Bd. xxi. S. 449 ; Hermann, ibid., 1880, Bd. xxi. S. 443.

5 Asher, Ztschr. f. Biol., 1895, Bd. xxxii. S. 473.

6 Wundt, "Untersuch. a. d. Nerven.," 1871 , Abth. 1.

${ }^{7}$ Herniann and Samways, Arch.f. d. ges. Physiol., Bonn, 1885, Bd. xxxv. S. 1.

${ }^{8}$ Boruttau, ibid., 1894, Bd. Iviii. S. 1 . 
Hermann has extended his inquiry to nerve, and has obtained results which lead to the conclusion that the rate of propagation is so rapid as to imply that electrotonus is instantaneously produced in all parts of the extrapolar regions; the only differences between distal and proximal parts being in the amount, not in the time relations of the change. ${ }^{1}$

The influence of physiological alterations upon electrotonic currents. - The great similarity between the cathodic electrotonic currents and the currents of action has already been alluded to. The striking experiments of Boruttau with the inodel bring this similarity prominently forward. He found that when induced currents alternating in direction were passed through a portion of the model, the resulting extrapolar effects on both sides were those of catelectrotonus, i.e., negativity of the part nearest the polarising contacts. This result was not changed when the polarising currents were reversed, and was present when, by means of sinusoidal induced eurrents, the alternating polarising flow was equal in the two directions. ${ }^{2}$ It admitted of only one explanation: both the anodic and cathodic electromotive disturbances are present in both extrapolar regions of the model, but, owing to their different intensities of development, the algebraic sum of the two opposing effects is one which shows that catelectrotonus overpowers anelectrotonus. It must be remembered that the essential conditions for the production of the result are the short duration of each individual polarising current, the alternation of these currents, and their frequent repetition. An effect can thus be produced in an artificial model closely resembling the negative variation of nerve. In view of this similarity, it is a matter of great theoretical importance to ascertain the extent to which the physiological conditions of a nerve affect polarisation and electrotonus.

Influenee of exeitation upon electrotonus, and of eleetrotonus upon the eleetrical response.-An excited nerve shows a diminution of the

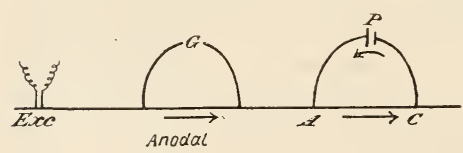
electrotonic currents, and if these are the extrapolar phenomena of polarisation, then during excitation polarisation is apparently diminished. ${ }^{3} \quad$ The diminution has been deduced from the following experiment:

The sciatic nerve (frog) is placed in connection with three

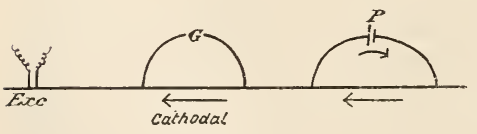
independent circuits, the middle set of contacts being that of the galvanometric circuit, whilst the other two are those of the FIG. 287. polarising and exciting circuits respectively. As the galvanometric contacts are both upon the longitudinal surface of the nerve, the resting difference and the negative variation due to excitation will be extremely small. The polarising circuit is now closed, so as to obtain an anodic extrapolar effect in the region of the galvanometer contacts. The anodic electrotonic current is thus present in

${ }^{1}$ Hermann and Weiss, Arch.f. d. ges. Physiol., Bonn, 1898, Bd. 1xxi. S. 237-295.

2 Boruttau, ibid., 1895 , Bd. lix. S. 47.

${ }^{3}$ Bernstein, Arch. f. Anat., Physiol. u. wissenseh. Med., 1866, S. 614. 
the galvanometric circuit (Fig. 287). If the nerve is excited under these conditions, a perceptible diminution in this anodic, extrapolar current is produced. The reversal of the polarising current calses a cathodic extrapolar effect, opposed in direction to the previous anodic one. On exciting, this is also diminished. The excitatory diminution has been explained on the assumption that during excitation the susceptibility of the nerve to polarisation is decreased, and thus there will be a corresponding diminution in the anount of the electrotonic currents.

The same decrease in the electrotonic currents during excitation may be demonstrated when the galvanometer contacts include the nerve cross section (Fig. 288).
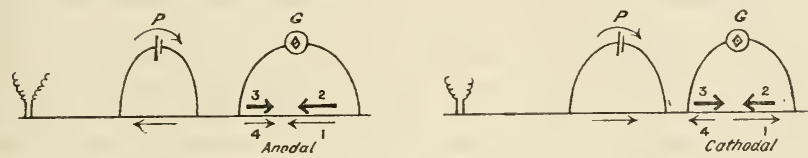

FIG. 288.

In this case the direction of the demarcation current in the nerve may be represented by the arrow 2 of Fig. 288, and the excitatory negative variation by the arrow 3 . If the galvanometric contacts lie in the anodic extrapolar region, an electrotonic current during closure represented in the figure by the arrow 1, is produced. This sums with the resting demarcation current, and on excitation a much larger negative variation occurs, since both resting and anelectrotonic currents are diminished, represented by arrows 3 and 4 . If, on the other hand, the contacts lie in the cathodic extrapolar region, the catelectrotonic current diminishes that of the resting difference. On excitation, a very small excitatory negative variation occurs, as both the demarcation and the catelectrotonic currents diminish, and, being opposed, their diminutions ( 3 and 4 in the figure) are counter to one another.

What is the rationale of the apparent excitatory decrease in the polarisation? The excitatory electrical responses, starting from the seat of stimulation, are propagated through the polarised region; they will cause the apparent decrease in polarisation if they should become more pronounced as they approach the anode, less pronounced as they approach the cathode. The result of such alterations would be that the anodic portion of the nerve would tend to become galvanometrically negative to the cathodic portion, and axial currents would thus tend to flow through the nerve, directed from the anorle to the cathode, i.e. counter to polarisation. There is evidence, by rheotomic observations, that such an alteration in the electrical response is actually present near the poles, and that the polarisation currents, being counter to this, are thus decreased. ${ }^{1}$ But, further, the electrical response, when propagated through the extrapolar regions, is profoumlly affected. Every point nearer the anode will, owing to the electrolytic change present in its neighbourhood, be the seat of a more intense electrical response than one further off (Fig. 289). The whole anodic extrapolar region will thus become the seat of brief excitatory currents, lirected so that the

${ }^{1}$ Hermann, "Handbuch," 1879, "Allg. Nervenphysiologie," Bd. ii. Th. 1, S. 166-167 ; Fleischl von Mlarxow, Sitzungsb. d. k. Akad. d. Wisscnsch., Wien, 1878, Bd. Ixxvii. Abth. 3. 
point proximal to the pole is galvanometrically negative to the more distal one, these being opposed in direction to the anelectrotonic current.

A similar line of argument applies to the cathodic extrapolar region. In this case the excitatory electrical change gains in galvanometric value as it travels from the cathode, and thus brief currents traverse the nerve from the more distal to the proximal contacts, i.e. in the opposite direction to the cathodic electrotonic current (see Fig. 289). By means of rheotome observations, Hermann has conclusively demonstrated the existence of these changes in the propagated electrical response of cooled nerves. ${ }^{1}$ Experiments with the capillary electrometer have confirmed and extended this demonstration. It appears that the single electrical diphasic response of an uninjured nerve, having the character shown in Fig. 270 (I. or $I I I$.), is completely altered when the proximal contact, i.e. that nearest the seat of excitation, is rendered persistently positive to the distal one by extrapolar electrotonus. ${ }^{2}$ The record under these circumstances is like that produced when the distal contact lies on a warmed or injured region (Fig. 270, II., IV.). If the polarisation is of opposite sign, the descending limb of the electrometer curve becomes more extensive as compared with the

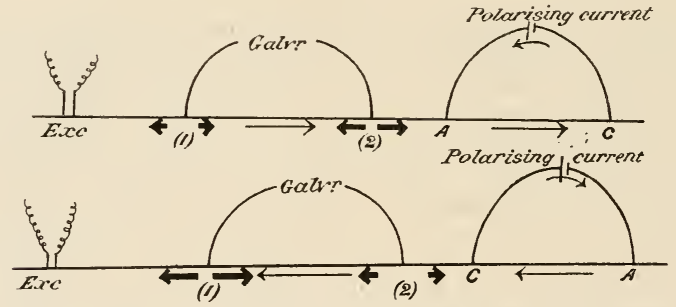

Frg. 289.-The upper portion represents the anodic extrapolar effects. The excitatory electrical change is more pronounced under the contact (2), nearer the anode than under the distal contact (1). The lower portion represents the cathodic effect, the electrical change due to excitation being more pronounced at (1) than at (2).

ascending one. Hence relative positivity is associated with greater functional capacity of the tissue, so that, when this is aroused, the state of excitation is of large amount; relative negativity is associated with diminished functional capacity.

Influence of ancsthetics. - The natural tendeney to regard the changes of electrotonus as states of electrolytic polarisation has been strongly resented by some investigators, and particularly by Biedermann, who differentiates between the "physical" (electrolytic) and the "physiological" factors concerned in the production of the phenomena. ${ }^{3}$ The theoretical value of the distinction is most certainly open to question, since the electrolytic phenomena displayed by a living nerve are admitted by all to be dependent upon its molecular condition, and as this is one thing during the living and another during the dead state, they must be logically regarded as physiological. In this sense, the

${ }^{1}$ Hermann, Arch. f. d. ges. Physiol., Bonn, 1872, Bd. vi. S. 359 ; 1873 , Bd. vii. S. 349 ; Bd. x. S. $215 ; 1875$, Bd. xii. S. 157 .

2 Goteh and Burch, Proc. Roy. Soc. London, 1898, vol. lxiii. p. 300.

${ }^{3}$ Biedermann, "Elektrophysiologie," S. 694. 
only physical phenomena are those due to current escape. The distinction has, however, some practical value, since it brings into prominence other aspects of electrotonic phenomena, which are at any rate not so obviously associated with electrolysis as the preceding. The first of these is that due to the comparison of the extrapolar effects in normal and in etherised nerve respectively.

It has been already stated that the cathodic and anodic electrotonic effects differ in their time relations. In a normal nerve, with extrapolar leads at $1 \mathrm{~cm}$. distance from those of the polarising current, it is notable that the anodic electrotonic effect of a prolonged closure (several seconds) is far larger than the cathodic one, as judged by the galvanometric deflection. ${ }^{1}$ Thus in one of Biedermann's experiments the anodic effect gave a deflection of 46 , the cathodic only 25 ; on increasing the intensity of the polarising currents, the anodic gave 96 , the cathodic 60 . The nerve was now etherised; it gave no excitatory electrical response, and the deflections were, anodic 24, cathodic 24, with the first intensity of polarising current; anodic 68, cathodic 66, with the second one. The inequality between the anodic and cathodic electrotonic effects thus disappear in etherised nerve; but it will be observed that both effects are still present although excitability and conductivity are in abeyance. The explanation given by Biedermann is that in the etherised nerve the condition of electrotonus during closure is a true expression of the polar electrolytic changes, but that in normal nerve, owing to physiological response, a further concomitant effect occurs. This is especially prominent on the anodic side, and spreads from the pole in diminishing amount through the extrapolar region; since it produces electromotive changes of similar sign to those of the anodic electrolytic change, the total anodic extrapolar effect is thus augmented. In the etherised nerve the production of this physiological response is in abeyance, hence the total anodic effect is diminished, and is now nearly equal to the cathodic one. In support of this, he states that etherised nerve shows little or 110 electrotonic effect at some distance from the polarised region, whilst the normal nerve shows at the same distance the anelectrotonic current due to the physiological response. The anodic electrotonic changes observed during closure in a normal sciatic nerve, within $1 \mathrm{~cm}$. of the polarising current, are thus regarded as the sum of electrolytic spread, and of a physiological response of the nerve at the anode, rendering all parts at or near this galvanometrically positive to those more remote; this response subsides when the polarising current is broken. The basis of the response is a reaction corresponding with an assimilation process in Hering's sense; it is present in diminishing amount through the anodic extrapolar region, and corresponds with greater functional capacity, i.e. galvanometric positivity, of the tissue.

Further support for this view is afforded by other facts to be referred to; it must, however, be noted that Waller has found that both the cathodic and the anodic electrotonic effects and their excitatory diminution are influenced by all those agencies which affect the electrical respouse of nerve. Thus ether and $\mathrm{CO}_{2}$ may abolish both electrotonic effects, the abolition being succeeded by secondary augmentation. When the gases are weak, an augmenting effect immediately

${ }^{1}$ Biedermann, "Elektrophysiologie," s. 696 ; Sitzungsb. d. k. Akad. d. Wissensch., Wien, 1888, Bd. xcvii. Abth. 3, S. 84 . 
succeeds an initial slight diminution. ${ }^{1}$ It is thus extremely doubtful whether the observations of Biedermann can be taken to indicate a separation between "physical" and "physiological" electromotive factors; if susceptibility to change through the action of ether is to be taken as a crucial test, then we must regard the whole effect as physiological.

The character of the nerve.-The non-mednllated nerves of Anodon, Eledone, and Octopus, are readily excited by the passage of a galvanic current, and an electrical excitatory response is propagated at each closure of such a current along the nerve. The electrical response evidently differs in its time relations from that present in medullated nerve, since a single closing excitation causes an obvious galvanometric deflection in a distant portion of the nerve; it is thus far more prolonged than in the case of medullated nerve. It is, however, of the same general character, the proximal surface contact becomes galvanometrically negative to the more distant contact on the cross section.

This initial excitatory effect on elosing a polarising current has the same sign whatever the direction of the current, but the subsequent electrical changes differ in accordance as the extrapolar region is anodic or cathodic. On the anodic side it is quickly succeeded and overpowered by an electrical change of opposite sign, the anodic extrapolar electrotonic current; on the cathodic side it is, according to Biedermann, maltered, being neither augmented nor diminished. ${ }^{2}$ If the galvanometer contacts are both placed on the surface, so that the resting difference and the excitatory electrical response produce but very slight galvanometric effects, then the appearance of the anelectrotonic change and the absence of the catelectrotonic one become very conspicuous. As the galvanometric contacts approach the polarising ones, the anelectrotonic change increases in intensity, although such increase is small when compared with the similar augmentation in medullated nerve. The amount of the effect also increases with that of the polarising current, but a maximum is soon reached, and further increase of polarising intensity does not cause any perceptible augmentation. The structure of non-medullated nerves is not favourable for electrolytic spread, and this factor is therefore reduced to comparatively small proportions. Biedermann thus concludes that the physiological reaction of the tissue at the anode is the chief if not the sole source of the anodic electrotonic current in such nerves. The change spreads with diminishing intensity into the anodic extrapolar region.

These facts have not been confirmed by Boruttau. In the nonmedullated nerves of large Cephalopoda, Octopus and Eledone moschatus, Boruttau observed both anelectrotonic and catelectrotonic extrapolar currents. Thus in Octopus the anelectrotonic effect gave a galvanometric deflection of 64 , the catelectrotonic one of 49 , and, on increasing the intensity of the polarising current, the effects were anelectrotonic 95 , catelectrotonic 70 , when $18 \mathrm{~mm}$. of nerve intervened between polarising and galvanometric contacts. ${ }^{3}$ Further, both effects showed the excitatory

${ }^{1}$ Waller, "Proc. Physiol. Soc.," Joum. Physiol., Cambridge and London, 1896, vol. xix.

Pielermann, "Elektrophysiologie," 1895, S. 685; Sitzungsb. d. k. Akad. d. IVissensch., Wien, Bd. xeiii. Abth. 3.

${ }^{3}$ Bortuttan, Arch. f. d. ges. Physiol., Bonn, 1897, Bd. lxvi. S. 285. 
diminution when the nerve was stimulated. Boruttau thus concludes that these non-medullated nerves show electrotonic changes of precisely the same character as those present in medullated nerves, and that such difference as exists is merely a quantitative one, due to the diminished susceptibility to electrolytic polarisation, consequent upon the small extent of the nerve sheath.

Influence of temperature.-The sciatic nerves of large cooled frogs, if kept at a low temperature during the experiments, show results which Biedermann considers to be analogous to those of the Anodon non-medullated nerve. If the galvanometer electrodes are connected at a distance of $3 \mathrm{cms}$. from the polarising, and contacts made with the portion of cooled nerve left in its natural connection with the gastrocnemius muscle, then the resting difference and excitatory electrical response are both so small as not to affect the galvanometer. Under these circumstances an anelectrotonic extrapolar effect is noticed, whilst the catelectrotonic effect is very small. In one of Biedermann's experiments, the former amounted to 6 , the latter being absent. ${ }^{1}$ On now approaching the galvanometric contacts to $17 \mathrm{~mm}$. from the polarising ones, the anodic effect was 20 , the cathodic 2. At $11 \mathrm{~mm}$. the anodic was 34 , the cathodic 13. The nerves of the cooled frog are thus in a state which accentuates the normal discrepancy between the amounts of the anodic and cathodic electrotonic effects. This accentuation is regarded by Biedermanu as due to increased susceptibility of the nerve to the physiological reaction at the anode. It appears, however, that the influence of temperature is a complicated one. Waller has observed that both electrotonic currents fail when the temperature of the whole sciatic nerve is raised to $40^{\circ} \mathrm{C}$. or lowered to $0^{\circ} \mathrm{C}$, returning when the temperature is brought back to $15^{\circ} \mathrm{C}^{2} \quad$ He observed, also, that after prolonged exposure to these temperatures, electrotonic effects are not produced at ordinary temperatures, whilst after a moderate exposure to $40^{\circ} \mathrm{C}$. the effects at $15^{\circ} \mathrm{C}$. are perceptibly altered, the cathodic being increased and the anodic diminished so much that the latter may becoine less than the former.

It is therefore doubtful how far the experimental data brought forward by Biedermann justify the arbitrary division of electrotonic phenomena into two classes. The particular terms entployed to describe these two classes, "physical" and "physiological," the present writer is unable to accept. Such a distinction in no way facilitates the physiological conception of the changes in a living nerve. For it is clear that the physiological changes which Biedermann desires to accentuate must have a physical basis as their causative factor. As Boruttau has most pertinently remarked, some physical change must be assumed even by those who take Biedermann's view, and this is presumed to be some chemical rearrangement of a synthetic type at the anode, which is obviously produced by the passage of the current. ${ }^{3}$ The only known chemical rearrangement at the anode is electrolytic in character. Whether, therefore, electrotonic phenomena have an electro-chemical basis only, or involve, as Biedermann insists, an electro-chemical basis, plus a further "unknown" chemical change, the releasing causative factor is physical,aul

${ }^{1}$ Biedermann, "Elektrophysiologie," S. 691.

2 Waller, "Proc. Physiol. Soc.," Journ. Physiol., Cambridge and London, vol. xx.

${ }^{3}$ Boruttau, Arch.f. d. ges. Physiol., Bonn, 1896, Bd. lxiii. S. 145, 158. 
on either view the resultant phenomena in a living nerve are physiological. Probably the qualifying expression "unknown" is the characteristic which Biedermann desires to accentuate. Those who maintain that the resultant phenomena are in themselves the electrical expression of electrolytic change, are in his opinion open to the criticism that the "vital" character of nerve change is ignored; but surely electrolytic polarisation in nerve must depend upon the chemical and mechanical conditions of the structure, and it is the peculiarity of the living state that these conditions are eminently favourable for its production. Thus the whole group of phenomena remain physiological, and even the use of the term "physical" for the current escape along the nerve is only justified in so far as such escape is independent of electrolytic changes produced in the tissue, in consequence of its living molecular state.

Excitatory after effects-Secondary electromotive phenomena. -The electromotive changes which follow the passage of galvanic currents through tissues have been termed "secondary electromotive phenomena." These after effects, like those in muscle, are partly the more obvious polarisation changes previously described, and are partly localised excitatory effects produced in the tissue by such changes.
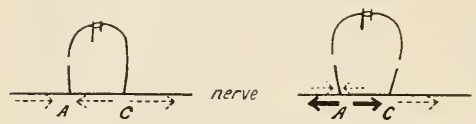

Frg. 290.-In the left-hand portion of the figure the ordinary polarisation after effects, intra. polar and extrapolar, are shown; the flow of the polarising current through the nerve has been from $A$ to $C$, and has just ceased; the right-hand portion shows the anodic excitatory after effects, the direction of the eurrents thus produced being indicated by the dark arrows; the polarisation currents are indicated by the dotted lines.

The discovery by du BoisReymond that the cessation of the passage of a galvanic current of sufficient intensity and of short duration was accompanied by so-called "positive polarisation," has been already alluded to in the case of muscle. ${ }^{1}$ The same phenomenon is readily produced in nerve. The causation is in both instances of the same character. It is a prolonged opening excitation localised at the anode, and of such character that the nerve at this point is galvanometrically negative. Hence currents flow along the nerve from more distant points to the anode, and these may be so intense as to overpower the proper polarisation effects. The results of this anodic excitatory after effect upon current distribution are shown in Fig. 290.

The proof that the effect is due to a localised anodic effect is due to Hermann. ${ }^{2}$ With a long nerve, and thus a considerable separation of the two polar contacts, galvanometric investigation of the anodic extrapolar region shows that excitatory effects are present, when the polarising current ceases, in sufficient amount to overpower the anodic polarisation change, whilst in the cathodic extrapolar region the polarisation current alone manifests itself. Any localised injury of the anodic region abolishes or largely diminishes the effect. The anodic opening effect is thus explained on the supposition that this region, on opening a current, is the basis of a prolonged localised

I E. du Bois-Reymond, Sitzungsb. k. Preuss Akad. d. Wissensch., Berlin, 1888, S. 404 .

2 Hermann, Arch. f. d. ges. Physiol.. Bonn, 1884, Pd. xxxiii. S. 103. 
excitation; this prolonged opening response, evoked at the anode, causes anodic negativity such as must occur in consequence of any prolonged excitation. Such an excitation can undoubtedly occur in a muscle, where it is evidenced by an initial propagated twitch and a prolonged localised contraction, the latter being produced on opening intense currents. Both of these opening contractions fail if the anodic tissue is injured.

In nerve, no evidence of a similar prolonged localised excitation at the anode, when a current is opened, had up to the discovery of the phenomena now referred to, been found. Opening tetanus is a proof that a series of propagated excitatory states may be produced, but a localised change without propagation can only be indicated by a localised electrical effect. If the anodic opening after effect is such an indication, the phenomena of nerve excitation are brought into strict accord with those of muscle. In both tissues the excitatory anodic after effect fails, or is markedly diminished if the anodic contact is upon a thermal section, ${ }^{1}$ and is decreased in amount if the tissue is in a state of excitation. ${ }^{2}$

A point raised by the presence of this anodic change has reference to the causation of such opening contractions as are obtained in the second stage of Pfliuger's law of contraction.

The discovery of the opening excitatory effect at the anode, renders it difficult to accept the theory advanced by Grutzner and by Tigerstedt as to the causation of opening excitation. ${ }^{3}$ According to this theory, the polarisation current, which comes into full play when a current is opened, is the presumed cause of the anodic excitation, for this is now the cathode of the polarisation counter after effect; hence all excitation is on this view reduced to the production of cathodic changes. It is evident that a prolonged excitation on breaking a current intense enough to cause an anodic excitatory after effect cannot be due to such counter polarisation current, since this is itself overpowered and replaced by an electrical change of opposed sign. Under these circumstances, the anode does not become the cathode of a counter current.

Polarisation and excitation.-It follows from the whole of the facts previously detailed, that the comnection between the electrolytic action of a current and its exciting efficiency is, in the case of nerve, a very intimate one. Indeed, the two appear to be so blended that the determination of the conditions which influence polarisation, as well as the character of the polarisation changes, appear to many to constitute the surest road by which the physiologist may reach his goal and acquire more exact conceptions of the excitatory state.

It is therefore a matter of great interest to consider, in conclusion, how far the phenomena of nerve justify the conclusion that the relationship between the electrolytic action and the exciting efficiency of electrical currents is so indissoluble, that the possession of the latter involves, as a necessity, the production of the former, and vice versî.

In the first place, what evidence is there that an exciting current

${ }^{1}$ Hermamn, Arch. f. d. ges. Physiol., Bomn, Bd. xxxiii. S. 103.

2 Stewart, Journ. Physiol., Cambridge and London, 1888, vol. ix. 1), 26 and 199.

${ }^{3}$ Gritzner, Arch.f. d. ges. Physiol., Bom, 1883, Bd. xxxii. S. 357. 
is one that produces electrolysis? The only absolutely reliable evidence of excitation is the production of an undoubted response in an attached muscle or other recipient organ; the only reliable evidence of electrolysis is the presence of the electromotive state which we term polarisation. That either of these may exist apart from such evidence is by no means improbable; but the present inquiry may be advantageously limited to the indications above mentioned. With this limitation, it will be seen that there is little difficulty in the assumption that, when a current is able to excite, it must have produced polarisation; on the other hand, there are serious objections to the second assumption, namely, that if a current produces polarisation of sufficient intensity, such production must, in an excitable structure, lead to excitation.

It is remarkable what a large number of conditions influence both exciting efficiency and the production of polarisation in a similar manner. The intensity of the current, its duration, the length of the intrapolar region, are all factors the variation of which influence both effects in the same way; and most conditions which increase the rapidity of polarisation development are, catcris paribus, those which increase exciting value. Finally, the nerves most susceptible to polarisation effects (c.g. medullated nerves of the frog) are those most readily stimulated by electrical currents.

Against the second assumption is the well-known fact, that currents directed across the fibres of either nerves or muscle are unable to excite, yet, owing to the inultiple anodes and cathodes between the poles (there being a pair for each fibre), the polarisation is extremely pronounced. The seat of electrolytic change in nerve must be at the surface junction of each medullated axis cylinder with its envelope, and the counter after effect is a clear indication, both of its existence and of the separation of the two groups of products. Polarisation may thus be produced in large amount, and yet all evidence of excitation may completely fail. A still greater difficulty is the behaviour of nerve when subjected to local change of temperature; local cold increases nerve susceptibility to excitation by galvanic currents and condenser discharges; it lecreases such susceptibility for make or break induced currents. Local cooling undoubtedly modifies polarisation, but it is difficult to frame any hypothetical link between excitation and polarisation which may account, on the one hand, for the increased exciting efficiency of prolonged condenser discharges as the temperature falls, and, on the other, for the increased exciting efficiency of rapid condenser discharges as the temperature rises. Finally, the fact that excitation can itself produce electromotive changes of sufficient magnitude to overpower all evidence of polarisation, as in the anodic opening after effect, appears to indicate that the two conditions cannot be connected so intimately that the one is an essential concomitant of the other.

Some of these difficulties have been recognised by Bernstein, who, anxious to bring excitation and polarisation into complete accord, framed an electro-chemical hypothesis to explain the most conspicuous of the difficulties. ${ }^{1}$ He supposes that the internal conductor is in reality a discontinuous one, and that electrolytic change takes place both on the surface and in the intervals between the portions of such an interrupted core. Surface polarisation alone does not constitute excitation; it is

${ }^{1}$ Bernstein, Untersuch. a. d. physiol. Inst. d. Univ. Halle, 1888 (1); "Lehrbuch d. Physiol.," 1894,1 p. 359, 450. 
the intermolecular dissociation of oxygen which is the vera causa. The hypothesis has been obviously framed to account for the exciting efficiency of longitudinally-directed currents and the inadequacy of cross-directed ones, and is by far the most satisfactory of all attempts to give a picture of the molecular basis of an excitable tissue which shall be in harmony with our modern knowledge. In spite of this, it appears to the present writer to be inadequate, since it explains neither the varying phenomena produced by temperature, nor the still more subtle differences which justify the conclusion that the phenomena of nerve conduction and of nerve excitation are to a great extent independent of each other. The theory would probably need considerable modification in order to embrace these. It has, however, the great merit of accentuating the crux of the present position, namely, that there is no definite standpoint except that which links excitation with polarisation. The latest development of this view has been made by Boruttau. He regards the electrical response of nerve as in reality a catelectrotonic effect; this necessitates the assumption that polarisation and excitation are different aspects of the same state, since catelectrotonus must be understood as the presence of those electrolytic changes which are produced at the cathode of a polarising current. ${ }^{1}$ Hermann ${ }^{2}$ has recently drawn attention to the circumstance that in 1872 he framed a conception of the excitatory state in muscle and nerve, based upon the following lines:-(1) Such tissue is excited by the establishment of an adequate cathodic and the disappearance of an adequate anodic polarisation (i.e., electrolytic change of a certain type); (2) the excited tissue becomes at the seat of its excitation relatively negative to those unexcited portions which are in the neighbourhood; (3) cathodic polarisation is thus produced in the latter districts, and on this attaining an adequate intensity, the state of excitation occurs in such districts ; (4) this state of excitation must itself similarly influence the neighbouring unexcited parts, and be thus successively assumed by such parts (i.e., it is propagated). The fundamental distinction between the state of excitation and cathodic polarisation is that, whereas the former may be propagated, the latter is not, but is estab. lished simultaneously throughout the tissue in a diminishing quantity. A certain intensity of cathodic change is necessary for the production of the state of excitation, and this is only reached in the immediate neighbourhood of the changed tissue; the electrotonic decrement which, in accordance with the laws of electrical distribution, is present in every polarisable moist conductor, thus becomes an important factor in determining the production of a localised state of excitation.

In regard to the whole subject, it must be remembered that the chemical changes which undoubtedly underlie vital activity must, like all others of this nature, be resolved into the interaction of electrolytes. Electrolysis, such as gives rise to the electromotive phenomena of polarisation, is but one result of such interaction, and it is unquestionable that electrolytes may be present although no electrical indications of their presence have been observed. Although, therefore, in our present state of knowledge, the comnection between exciting efficiency

${ }^{1}$ Boruttau, Arch. f. d. ges. Physiol., Bonn, 1894, Bi. lviii. S. 51 ; ibid., 1896. Bd. Ixiii. S. 154 .

2 lbid., 1897, Bd. lxvii. S. 257 ; ibid., 1898, Bd. 1xxi. S. 277-294. 
and such polarisation as we can recognise, may not appear to be so close as to make the two identical, there is sufficient evidence that the relationship is an extremely intimate one. As knowledge increases, the ties of this relationship appear stronger, and a special interest is thus given to the opinion expressed fifty years ago by du BoisReymond, that electrical excitation is nothing more than a stage in the process of electrolysis. ${ }^{1}$

${ }^{1}$ E. du Bois-Reymond, "Untersuchungen," Bd. ii. S. 387. 


\title{
THE PHYSIOLOGY OF ELECTRICAL ORGANS.
}

\author{
By Francis Gotch.
}

Contents :-General Description, p. 561-Minute Structure in R. batis, Gymnotus, Torpedo, and Malapterurus, p. 563-Functional Activity, 1.568-The Electrical Changes of Nerve-Organ Preparations; Activity Effects, p. 571-The so-called Current of Rest, p. 571-The Organ Response, p. 572-Its Time Relations, 1. 575-Self-Excitation of the Electrical Organs, p. 579-Effect of Repeated Stimuli, p. 582-Residual Effect of the Response, p. 583-Electromotive Force of the Response, p. 583 -Polarisation Phenomena, Secondary Electromotive Effects, Irreciprocal Conductivity, p. 584-The Reflex Response of the Organs, p. 588-The Nature of the Activity, p. 590.

The possession by certain fishes of a peculiar power, which is now known to be that of giving electric shocks, is such a remarkable phenomenon, that it attracted the special attention of early naturalists, and is referred to in ancient literature. The earliest record is probably the hieroglyphic representation of the Malapterurus electricus in the Egyptian tomb of $\mathrm{Ti}$; this fish, belonging to the Silurida, is still found in the Nile, and in the West African rivers. The numbing force exerted by the electric ray of the Mediterranean caused this still more formidable fish to be termed vapжn by the Greeks, and torpedo by the Romans. The powers of the electric eel, or Gymnotus, found in the branches of the River Orinoco, have always been feared by the inhabitants of this portion of South America; it is in many respects the most remarkable of all electrical fish, since it attains a large size, 8 to $10 \mathrm{ft}$. in length, and its electric power is thus very great. The shock of all these fish may be sufficient to kill small animals and stun large ones, but although the numbing effects on man were well known, their causation remained a mystery until Dr. Walsh, in 1773, discovered that the power of the Torpedo was due to the development of intense electrical currents, through the functional activity of special organs situated one on each side in the lateral mass of the body of the fish. ${ }^{1}$ The general structure of the organs in Torpedo was described by Lorenzini in 1678, and a detailed account was given by John Hunter, who also described the organ of Gymnotus. ${ }^{2}$ Organs of small size have been found in certain species of skate and in the Mormyrus, the latter a fresh-water fish found in the rivers of North Africa (Nile, etc.). These organs were termed "pseudo-electric," because, though resembling in general structure those of Torpedo and Gymnotus, the electric shocks produced by their activity were insufficient to attract notice by their effects upon

1 Walsl, Phil. Trans., London, 1773, 11p. 461-477.

2 Lorenzini, "Osservazioni intorno alle Torpedini," Firenze, 1678; J. Hunter, "Works," London, 1835, vol. iv.

VOL. II. -36 
man. The term "pseudo-electric" has now been abandoned, and is obviously misleading, since the structures in question, though diminutive and less highly differentiated than those of Torpedo, are fundamentally the same as these, both as regards structure and functional activity.

All electric organs possess a characteristic structure which determines the peculiar features of their functional activity, and they all have an unmistakable appearance when examined in the fresh state. This is well displayed in the recently killed Torpedo, when the ventral or dorsal skin is removed. Two large masses now come into view placed between the gill slits and the margin of the fish. Each has a concave inner border, and consists of a semi-transparent material, of the consistency of jelly, in which may be seen connective tissue septa and numerous nerve branches. The septa are so arranged as to divide the mass into hexagonal columns, the ends of which lie immediately beneath the dorsal and ventral skin, so that the skin surface of the whole organ presents an appearance resembling the tops of basaltic columns. On making a transverse section of the fish, the organs are seen to extend from the ventral to the dorsal surface, and to contain a number of closely packed columns arranged side by side; as these run from the ventral to the dorsal skin, some of them are displayed in the section, which has cut them in the direction of their length.

In Gymnotus, the dissection of the fish shows four such organs, two dorsal and two ventral, the former being the larger. These present a similar transparent appearance in the fresh state, and consist of columns of jelly-like material separated and bounded by connective tissue septa. The organs extend from the neighbourhood of the cephalic to that of the caudal end of the fish, and the columns run for variable distances in the same direction. A section at right angles to the spinal canal thus reveals organs and columns in cross-section.

In Mormyrus there are four slender organs situated between the muscles, with their long diameter parallel to the spinal column, thus resembling in general disposition the arrangement present in Gymnotus.

The organs found in the skate (Raia batis) are situated one on each side of the caudal portion of the spinal column. Each is a slender body tapering at its two ends, and exhibits in the fresh state the characteristic transparent material enclosed within connective tissue septa. A cross-section of the tail at right angles to the spinal column shows that each organ consists of columns which lie closely packed with their long axes parallel to the spinal column; they are thus displayed in such a section as translucent masses, bounded by hexagonal walls.

Finally, in Malapterurus, the organ attains a high degree of differentiation. Unlike those just described, it is situated within the skin, which is correspondingly thickened. The organ is imperfectly divided into two halves by a connective tissue septum upon the ventral surface, and enwraps the whole body except the fins and a small portion of the cephalic end. It has not been developed at the expense of any of the muscles used in locomotion, and hence the rapid movements which characterise these fish are in striking contrast with the sluggish motions of the Torpedo and Cymnotus. The jelly-like organ is bounded externally by the surface layers of the skin, and internally by a strong layer of connective tissue, which is loosely connected with the subjacent muscles. On making sections through the organ, the characteristic 
translucent material is seen to be divided up by connective tissue septa, but there is no obvious arrangement of columns.

Minute structure.-The most important features of the minute structure of the organs just described are those of the columnar constituents.

Every column bounded by connective tissue walls, is itself subdivided at regular intervals by connective tissue cross septa, which are fixed at their peripheral edges to the boundary walls; the column thus consists of a pile of superimposed compartments. Further, each of these compartments contains a special protoplasmic mass in which efferent nerves end, whilst the remaining portion of the space offered by the compartment is filled by a transparent material. The protoplasmic mass, with its supporting fibres and nerve terminations, constitutes what is termed the electric plate or disc, and is well supplied with blood vessels.

The structure of the plates differs as regards minute detail in the various groups of fish just mentioned, although all present general features of resemblance. It is best understood by an examination of the least complicated organ, that of the skate.

In the organ of the skate, the columns of which it is composed are divided into the compartments previously referred to ; along the connective tissue walls of these compartments run the blood vessels and nerves which enter to be distributed in the plate (see Fig. 291).

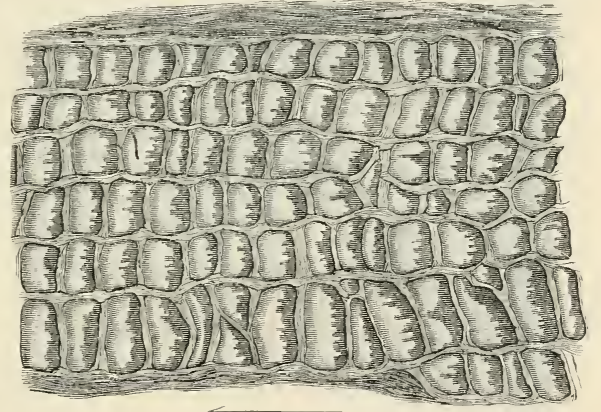

Fig. 291.-Electrical organ of skate. Longitudinal section of upper part of organ, as seen under a low power. The arrow indicates the direction of the shock.

The plate or dise is slung across the compartment, being attached by its peripheral edges to the lateral walls, and in contact by its free surfaces with the semi-fluid albuminous material which fills the renainder of the compartment, as shown in Fig. 292. ${ }^{1}$ The cephalic border receives a dense network of nerve fibres, the dichotomous divisions of which enter along this surface. Beneath this the nerve branches appear to end in groups of twigs, with terminal knobs lying in a multinucleated layer of granular protoplasm. Immediately beneath this in the protoplasmic layer is a remarkable band of wavy fibres; these are all parallel, and run across the plate between the two lateral attachments, in such a position as to form the most central portion of the disc. These unbranched fibres are quite distinct from one another, and are rery prominent in preparations which have been fixed in osmic acid. This

${ }^{1}$ For structure of organ of skate, see Stark, Proc. Roy. Soc. Edin., 1844-45, vol. ii. ; Sanderson and Gotch, Journ. Physiol., Cambridge and London, 1888, vol. ix.; Ewart, Phil. Trans., London, 1888, vol. clxxix.; 1892, vol. clxxxiii.; Engelmann, Arch. f. d. ges. Physiol., Bown, 1894, Bd. lvii.; G. Retrius, Biol. Untersuch., Stockholm, 1898, Bd. viii. S. 83 . 
meandering layer is probably the vestige of the cross striations of those voluntary muscle fibres which have been transformed into the plates, since in the embryonic stage of $R$. batis and the adult stage of $R$. circularis, the layer can be identified with the materials forming the cross striæ of a slightly modified muscle fibre, and the meandering layer of the organ in adult $R$. batis still possesses those optical properties which characterise striated muscle. The protoplasmic layer on the caudal border of each plate or disc contains many nuclei, and is thrown upon its free surface into a number of projecting processes, upon which extended surface blood capillaries ramify. Every column consists of a series of such compartments, each containing a plate of essentially the

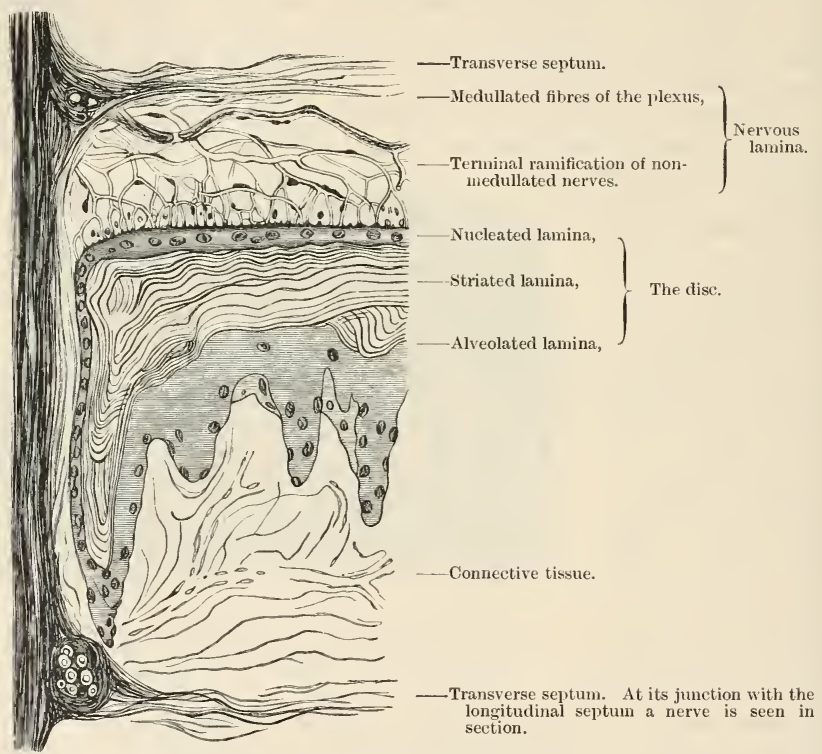

FIG. 292.-Electrical organ of skate. Diagrammatic view to show the structure of a disc.

same structure. As the fish grows in size, the organs also increase, but the number of compartments and plates is fixed at an early stage of growth, and thus the organ of large specimens affords the most favourable specimens for the examination of the plates, since the plates are now some distance apart. The nerves which supply the columns and plates arise from the caudal portion of the spinal cord, and appear to come from special nerve cells situated on the dorsal side of those which give rise to the nerve fibres innervating the muscles. An exception to the structure just described occurs in the embryonic condition of $R$. batis and the adult condition of certain species, e.g. R. cireularis. Here the compartments composing the columns are irregular, since each contains a modified muscle fibre forming a cross striated multinucleated mass. 
This has a flask-like shape, the cephalic extremity being wide and cupshaped, the caudal long and tapering. The former is seen to be connected with numerous branches of nerve fibres, and corresponds to the nervous layer of the plate just described.

The columns which compose the four organs of Gymnotus resemble those of the skate in having compartments, each enclosing a protoplasmic mass which constitutes the electric plate or disc. This presents the same different layers, though with certain modifications. The nerve fibres enter the caudal aspect of each disc, and appear to end in granular protoplasm containing very conspicuous nuclei; this caudal surface is thrown into conspicuous folds with secondary villous projections, all of which are richly supplied with nerve fibres. The meandering layer is not conspicuous, being represented by a few fine parallel lines (Pacini), running through the interior of the whole protoplasmic mass, as in the skate organ; finally, the surface of the cephalic portion is thrown into very prominent papillae, upon which blood vessels ramify. It is evident that the great difference between this organ and that of the skate is the position of the plate layers with regard to the body extremities, the nerved side being caudal in Gymnotus and cephalic in the skate.

A noteworthy feature is the striking difference which exists between different portions of the whole organ of Gymnotus. The columns near the tail run somewhat obliquely from the ventral towards the dorsal aspect, are yellowish red in colour, and are subdivided into compartments of very considerable size, in which the protoplasmic plate occupies but a small part of the available space, the clear albuminous substance filling the remainder of the compartment. These columns were supposed by Sachs to be less highly developed; his view is rendered plausible by the microscopic characters of the contained discs, the cephalic surface of each such disc being distinguished by having a large number of long tapering processes, in which may be detected traces of transverse striæ with doubly refracting properties, analogous to those of muscle fibres. All the columns receive nerves from the spinal cord; these emerge from the whole length of the cord, and are the axis cylinder processes of special large cells, the electrical nerve cells. They form a vesicular column on each side, and are seen in any transverse section of the cord to be situated in the grey matter, dorsal and somewhat lateral to the central canal. ${ }^{1}$

The organs of Mormyrus are in many respects analogous to those of Gymnotus, although, owing to the small number of discs and their imperfect development, the total electromotive force of the columns is small. The compartments contain discs athwart the line of the columns, which latter is parallel to the spinal axis. The nerves enter the caudal side of each disc and are derived from special spinal cells like those of Gymnotus. A noteworthy feature is that some fibres decussate and supply the half of the organ which is upon opposite side. The nerve cells also are remarkable since their processes join by short anastomoses. ${ }^{2}$

There are several different varieties of Torpedo, all possessing two electrical organs symmetrically placed on each side of the borly. The

\footnotetext{
${ }^{1}$ For structure of organ in Gymnotus, see Sachs, "Untersuch. an Zitteraal," Leipzig, 1881 ; Pacini, "Sulla struttura intima dell' organo elettrico," Firenze, 1852 ; Schultz, Arch. f. Anat., Physiol. $u$. wissensch. Med., 1858, 1862 ; Fritsch, "die elektrischen Fische," Leipzig, 1887.

${ }_{2}$ Fritsch, Sitzungsb. d. k. prenss. Akcul. d. Wissensch., Berlin, 1891, Bd. xliv.
} 
varieties differ, not only as regards adult size, shape, and skin markings, but also in the number of columns which compose each of the electrical organs. Thus in Torpedo occidentalis there are on an average 1069 columns, in 7 . californica 895 , in T. marmorata 450 to $500 .^{1}$

In all varieties the columns are extremely distinct and consist of a series of superimposed compartments, but these are more closely approximated than in the case of the skate or Gymnotus organ, and the electric discs which they contain are proportionately thimner. Since the columns extend from the ventral to the dorsal surface of the organ, and the dises are slung athwart the columns, the surfaces of the dises are ventral and dorsal respectively. The nerves enter to be distributed upon the ventral surface, which presents an extraordinarily beautiful example of the dichotomous division of nerve fibres. This is readily demonstrated in the organ of a young torpedo, after fixation in warm osmic acid. If a single plate is stripped off, and spread out with its ventral surface uppermost, microscopic examination shows innumerable nerve twigs, and it may be seen that the twigs of any one branch do not join those of any other. The nuclei of the primitive sheath of the nerve fibres are conspicuous objects in such a preparation, as also those of the granular protoplasmic mass in which the fibres are situated. In cross-sections of the disc the deeper portions of this multinucleated protoplasmic layer exhibit the ultimate endings of the axis cylinder branches, which, according to Ogneff, are groups of nerve twigs with slight bulbous enlargements, each twig being free, i.e. not connected with its neighbours. A faint fibrillation running across the dise parallel to the surface probably represents the meandering layer of the skate organ, reduced in the torpedo to very small proportions. The dorsal surface of the plate is not thrown into folds as in the organs just referred to.

The nerves which supply each organ spring from prominent elevations, situated upon the dorsal aspect of the medulla. These elevations, one on each side, constitute the electrical lobes of the medulla, and contain a number of large multipolar nerve cells, each of which has one axis cylinder process continuous with an electrical nerve fibre. The total number of such nerve cells (and thus of electrical nerve fibres) in the case of $T$. marmorata is 58,000 on each side, the fibres being massed for distribution into five nerves, of which the middle are the largest. Each nerve fibre, on entering the connective tissue septa, between the columns of the organ, divides, giving off eighteen branches; this multiple division of the axis cylinder presents an appearance likened by Wagner to a bouquet. Each of the eighteen branches enters the column at one angle of eighteen successive discs, and as there are six such sets of branches coming from six nerve fibres, and entering the angles of each hexagonal disc, it follows that the nerve cells supply three times their number of dises. ${ }^{2}$ The direct enumeration of the discs bears out this numerical relationship, there being about 180,000 dises in an organ of T. marmorata, i.e. 450 columns, with an average of 400 discs in each column. ${ }^{3}$

${ }^{1}$ Fritsch, Arch. f. Physiol., Leipzig, 1886, S. 362.

${ }^{2}$ Fritseh, Sitzungsb. d. k. preuss. Aliad. d. IVissensch., Berlin, 1889, Bd. lii.

${ }^{3}$ For strueture of organ in Torpedo, there is an extensive literature comprising among others: Wagner, "Ueber den Bau des elektr. Org. im Zitterrochen," Göttingen, 1847 ; A. Ewald, "Torpedo," Heidelberg, 1880; Schultze, Arch. f. Anat., Physiol. u. wissensch. Med., 1862; Fritsch, "Die elektrisehen Fische," Leipzig, 1887, 1890; Ballowitz, Aich. f. mikr. Anat., Bonn, 1893, Bd. xlii.; Ogneff, Arch.f. Physiol., Leipzig, 1897. 
As regards Malapterurus, it has been already pointed out that there is no obvious columnar arrangement in the organ, but that the connective tissue septa divide it into a very large number of irregularly shaped compartments. ${ }^{1}$ Cross-sections made in different planes show that these compartments are more or less lozenge shaped, the acute angle of the lozenge being towards the surface and deep layers of the skin respectively, whilst the obtuse angles are cephalic and caudal in position. These lozenge shaped compartments are dovetailed into their neighbours, and thus practically constitute a large number of columns parallel with the spinal axis. The compartments may be seen with a magnifying lens in a fish $30 \mathrm{cms}$. in length; they are filled with the usual transparent jelly-like substance, and contain discs slung athwart them. The protoplasmic layer of each disc lies near the walls which form the caudal boundary of each compartment, and the whole organ contains a very large number of such layers, arranged in series from head to tail one behind the other. The nerve fibres enter the compartments at the lateral acute angles, and, rumning along close to the wall, pass finally to a central stalk projecting from the caudal surface of each protoplasmic disc. Each disc is shaped like a peltate leaf with the central stalk projecting from its caudal surface; the cephalic surface is smooth and has a slight depression opposite the emergence of the stalk from the other caudal surface. The nerve fibre which enters the stalk was formerly supposed to be traceable through this on to the cephalic surface, thus piercing the plate by means of the stalk. Sections show that under the cephalic surface the protoplasm has a punctiform appearance, and this was thought to indicate the section, either real or optical, of the finest divisions of axis cylinder twigs. The observations of Ballowitz do not confirm this view, for the nerve fibre ends in the base of the stalk in free branches with terminal knobs. The actual disc thus contains no nerves at all. The nerve which supplies the organ is probably unique as regards its structure. It consists on each side of one axis cylinder, the process of one gigantic nerve cell, situated in the grey matter, lateral and somewhat dorsal to the central canal, at a level between the exit of the first and second spinal nerve roots. The two cells, one on each side of the cord, were as much as $\frac{1}{5} \mathrm{~mm}$. in cross-section, in a specimen of the fish $25 \mathrm{cms}$. long. They are further characterised by conspicuous nuclei, fibrillæ, and cell processes. The processes are extremely numerous, and those of one cell often join another, but it appears that they do not join the processes either of the other electrical nerve cell or of any neighbouring nerve cells. The cell itself is traversed by special blood vessels, an arrangement which secures an adequate blood supply. The single axis cylinder process of each cell, large in its origin, soon dwindles, and coursing along the inner surface of the organ, accompanied by blood ressels, gives off at regular intervals branches for the supply of the organ. It is surrounded by an extensive connective tissue sheath, consisting of numerous laminæ, prolongations of which extend along the subdivisions as far as the angles of the organ compartments. The number of the organ compartments is very large, there being little short of two millions in each lateral half of the entire organ. Since these are all

\footnotetext{
${ }^{1}$ For structure of organ in Malapterurus, see Bilharz, "Elektr. Org. d. Zitterwels," Leipzig, 1857 ; Fritseh, loc. cit.; Ballowitz, Anat. Anz., Jena, 1898, Bd. xv. S. 85 ; ibid., "Das elektrische organ des Afrikanischen 'itterwelses," Jena, 1899.
} 
innervated by the branches of one axis cylinder, and each compartmental branch itself divides, forming fine terminations in the protoplasmic lining of the compartmental wall, it will be evident that the Malapterurus organ affords an instance of the peripheral distribution of a single nerve cell process upon a far more extensive scale than can be traced in any other known structure. It has been estimated that the chief compartmental branches entering the organ have a cross-sectional area 350,000 times that of the axis cylinder process of the nerve cell.

The foregoing brief description of the structural features of electrical organs is by no means an exhaustive one. It is merely intended to portray the most striking of such features, and the reader is referred to the excellent account given by Biedermann in his " Electro-physiology" for other details. ${ }^{1}$ The space allotted to the subject adinits of but a restricted reference to the chief points in connection with it, and the same is true in regard to the functional activity of the organ. This can only be dealt with in the present article so as to touch upon its most prominent characteristics, but attention will be drawn to such recent discoveries as appear to have a general interest in connection with nerve muscle phenomena.

\section{The Functional Activity of the Organ.}

The peculiar features of the structure of an electrical organ, as described in the preceding paragraphs, are the existence of distinct protoplasmic expansions or dises, their situation in separate compartments arranged in series one behind the other, and the presence on each dise of an innervated surface. Such an arrangement is eminently adapted for the functional purposes of an electric organ. For if the functional activity of any one disc is dependent upon the nerveendings it contains, and displays itself by electromotive changes such that electrical currents traverse the disc from one surface to the other, then, since the liquid contents of the compartment furnish a conducting medium of low resistance for such currents, these must traverse the whole series of superimposed discs. When, therefore, similar electromotive changes are simultaneously established in all the plates of a column, the resultant effects must be summed, and a large total electromotive change is thus produced. The principle upon which each organ is constructed resembles in this respect that of the voltaic pile with columns of superimposed pairs of elements arranged in series; in such a pile the ends of the columns will be the points of maximal potential difference which, with an extensive pile of discs, may be very considerable.

As regards the direction of the electrical currents, which are present during functional activity, the simplest experiments show that this is definite and unvarying for any organ, and that the line of flow is through the dises from one surface to the other. Pacini first drew attention to a supposed relationship between such direction and the minute structure of the protoplasmic disc. In the organs then accurately known, the surface of the disc which receives the entering nerves is so altered during the excitatory state, that it becomes galvanometrically negative to the opposite one, and thus the currents, which during activity traverse

\footnotetext{
1 Biedermann, "Electro-Physiology," translated by F. A. Welby, London, 1898, vol. ii. p. $357-407$.
} 
the substance of the discs, flow through these from the nerve entry layer to the opposite side. However, Pacini's generalisation obviously breaks down since it is opposed to the condition of affairs in Malapterurus. ${ }^{1}$ This will be rendered clear by the following table:-

\begin{tabular}{|c|c|c|c|}
\hline Fish. & & Position of Nervous Layer of Dise. & $\begin{array}{l}\text { Direction of the Current of the } \\
\text { Organ Response through the } \\
\text { Body of the Fish. }\end{array}$ \\
\hline Skate . & . & Head aspeet of each dise. & Head to tail. \\
\hline Gymnotus & - & Tail aspect of each dise. & Tail to head. \\
\hline Mulapterums & . & $\begin{array}{l}\text { Tail aspect of each dise. It was sup- } \\
\text { posed to pieree this and become } \\
\text { cephalic, but it really ends in the } \\
\text { caudal stalk. }\end{array}$ & Head to tail. \\
\hline Torpedo . & . & Ventral aspect of each disc. & Ventral to dorsal surface. \\
\hline Mormyrus & - & Tail aspect of each disc. & Tail to head. \\
\hline
\end{tabular}

These and several other general characteristics of the activity of electrical organs may be readily ascertained, by experiments upon living specimens of fish when the specimen is placed in a suitable experimental tank. In the case of the sluggish torpedo, the fish is allowed to rest with the ventral surface upon a zine plate, forming the floor of the tank, whilst a second zine plate is brought into contact with the dorsal surface; if both metallic contacts are connected by means of wires with a suitable galvanometer, a forcible movement of the needle occurs whenever the fish is aroused by mechanical means. The active Malapterurus may be caught in an appropriate net, into which two sets of wires have been woven, so that one shall be in contact with the cephalic and the other with the caudal extremity. Finally, in the case of the Gymnotus, it is sufficient to immerse two zine plates or rods at each end of a long narrow tank, along the floor of which the Gymnotus lies extended.

In this way it has been ascertained that, when the fish is aroused, intense currents flow through its body, causing strong derivations to pass through the surrounding water and along the wires connected with the metallic contacts.

If the ends of the wires are grasped by the hands, shocks are felt; if connected with a telephone, sounds are heard; if connected with a coil of wire surrounding a soft iron core, this is magnetised (hence an electro-magnetic signal is effected); and if connected with liquid capable of electrolysis, the products of chemical dissociation occur. It is difficult to obtain from even a large torpedo any indication of an electrical spark, for even when the external wires are separated by only $005 \mathrm{~mm}$., no such effect was observed by du Bois-Reymond; but a small spark has been seen on separating the external wires from contact with each

1 The ending of the nerve in the eaudal stalk of the dise of Malapterurus organ forbids at present any generalisation from structure to function. The nerved part becomes in Malapterurus galvanometrically positive to the unnerved, since the organ shock is from head to tail; it is thus the opposite of the condition in the other electrical fishes. A further difficulty is raised by the localisation of the nerve-endings to the tip of the stalk, the large expanded dise containing no nervous twigs. 
other just as the organ discharges. The explanation of this is related to the character and situation of the organ in the fish. This is itself a moist conductor, surrounded by other moist tissues, which afford a perpetual short circuit for any current flow which is caused by electromotive changes in the organ dises. It is impossible, therefore, to completely open the circuit, for the separation of the external wires merely opens one of the derivation circuits. ${ }^{1}$

The short circuit afforded by the body and surroundings of the fish inclined the early physicists to dispute the possibility of an external derivation being adequate for the production of the shock-effect on man; but Cavendish demonstrated that shocks similar to those given by Torpedo could be easily obtained from a model containing an electromotive source, when immersed, like the fish-organ, in moist surroundings. ${ }^{2}$

It is obvious that the intense electrical currents produced by the simultaneous activity of the dises in Gymnotus, Malapterume, or Torpedo traverse the columns, and that the lines of current-flow must comprise the moist-conducting tissues of the body of the fish. These tissues are not only such excitable tissues as muscle and nerve, but the central nervous system itself. In the case of the Gymnotus, the head forms, as it were, one terminal of the system of powerful batteries constituted by the functional activity of the very extensive organs, yet although an extremely inteuse current must traverse the brain of this creature, the vitality of the tissue is not impaired. The excitable structures of such electrical fish as Gymnotus, Torpedo, and Malapterurus thus appear to have become relatively immune to the passage of intense electrical currents.

The simultaneous development of electromotive changes in all the dises causes each column to be transformed into a series of batteries, coupled up in series for intensity, and when, as in the organ of Gymnotus and Malapterurus, the series of discs is a very extensive one, the arrangement is one eminently adapted for a circuit comprising a large external resistance. Such a resistance is offered by the fresh water in which these fishes live. On the other hand, groups of shorter columns, stacked side by side, afford an arrangement like that produced by coupling up batteries in parallel series, and adapted for a circuit which comprises a low external resistance. This is more or less the case in the organ of Torpedo, in which the columns are comparatively short, but so arranged that from 400 to 1000 are placed side by side in each organ; in this connection, du Bois-Reymond pointed out that the electrical resistance of sea water is one million times less than that of fresh water. ${ }^{3}$

As to the releasing cause of the activity of an electrical organ in the entire fish, it is undoubtedly a central nervous discharge, the excitatory state reaching the organ through the electrical nerves; but, like that of muscle, the activity can be evoked either reflexly in the entire fish, or in separated masses of the organ by excitation of the entering nerves.

A mass of electrical organ, when removed with its attached nerves from a recently killed fish, thus resembles a muscle nerve preparation, inasmuch as it affords opportunities for the study of the response of a

${ }^{1}$ du Bois-Reymond, Sitzungsb. d. k. pieuss. Akad., d. Wissensch., Berlin, 1883, 1885.

2 Cavendish, Phit. Trans., London, 1776, vol. lxvi. pt. 1.

3 du Bois-Reymond, lor, cit. 
definite portion of tissue when excited by means of an efferent nerve. The characteristics of muscle activity have been ascertained by noting and recording the changes occurring in isolated nerve-muscle preparations; so also those of the electrical organ are best determined by investigating the response in isolated nerve-organ preparations. The changes which such a preparation undergoes when its nerve is excited, being entirely electrical in character, have to be studied and recorded by appropriate instruments. Such records must form the basis of our knowledge as to the functional activity of the organ when wielded by the living fish, and the study of the nerve-organ response thus has the same importance as that of the contractile process in a muscle.

The electrical changes of nerve-organ preparations; activity effects.-It has already been stated that, when a portion of the electrical organ with its nerves is cut away from a recently killed Torpeclo, Malapterurus, or skate, such a portion responds to excitation of its nerves by the development of the intense electromotive change, for the production of which the tissue is differentiated. In the case of Torpedo, the excised organ may be further separated by incisions running from the dorsal to the ventral surface, and a block thus made containing in its interior a number of entire and uninjured columns. If the nerve entering this mass be carefully dissected out, so that its columnar attachments remain intact, a suitable nerve organ preparation is obtained for the investigation of the response. The ends of the strip may be left covered by their portions of dorsal and ventral skin respectively, and brought into connection with a galvanometer, capillary electrometer, telephone, or rheoscopic muscle-nerve preparation.

In an organ preparation, connected with one of these instruments, it is seen that each single stimulus of the nerve evokes an intense electrical response of short duration, the electromotive change being such that a current flows through the organ columns from the ventral to the dorsal skin.

The response is evoked with each excitation of the nerve by an electrical stimulus, whether an induced or galvanic current, provided this is of sufficient intensity; it is also evoked with great ease by even slight mechanical disturbance of the nerve, but is not readily caused by those chemical agents ( $\mathrm{NaCl}$, etc.) which so easily excite muscular nerves, ${ }^{1}$ although it can be produced by ammonia.

The so-called current of rest.-When connected with a galvanometer, the piece of Torpedo organ constituting the preparation generally shows a resting electromotive difference, the dorsal surface being galvanometrically positive to the ventral. A persistent current under these circumstances must be flowing through the excised organ from its ventral to its dorsal surface, i.e., in the same direction as that due to the excitatory response; it was termed by du Bois-Peymond the organ current. The theoretical importance attached by du Bois-Reymond to the existence of such resting electromotive changes, led him to make an extended series of observations on their character. ${ }^{2}$ It appears, however, that the fundamental fact of the existence of this resting difference in uninjured resting organ is problematical.

In an entire Torpedo, when quiescent, the difference between the

${ }^{1}$ Schönlein, Ztschr. f. Biol., München, Br. xxxi. S. 461 ; also Goteh aml Burch, Phil. Trans., London, 1896, vol. elxxxvii. B, p. 370.

2 du Bois-Reymonl, loc. cit. 
ventral and dorsal skin is extremely small, and only appears as the after-effect of any prolonged organ activity. In the excised organ there is always a difference, but this is dependent upon the extent of the mutilation involved in its excision. The amount is always greatest immediately after the necessary incisions have been made, and then dwindles until in half-an-hour it is comparatively small. It may, however, be renewed by a fresh mutilation, especially that produced by dipping the organ preparation for a brief period in $0.6 \mathrm{NaCl}$ at $80^{\circ} \mathrm{C}$.; such immersion causes death of the surface tissue, whilst the internal columns remain uninjured. ${ }^{1}$

A piece of organ treated in this way shows a large difference, the dorsal end being galvanometrically positive to the ventral. The sign of the electromotive change is independent of the seat of the injury; it is indifferent whether the sides, dorsal end, or ventral end is the part affected; the resultant effect is always that the dorsal end becomes galvanometrically positive to the ventral. The extent of the change, on the other hand, is definitely related to the amount and position of the injury; it is greatest when the injury involves all the sides of the columns, i.e. all the entering nerves; it is small when the ends alone are injured, since under these conditions the mutilation spreads but a short distance along the sides of the subjacent columns. An effective mutilation is thus one which involves local injury and hence excitation of the nerves which enter at the sides of the columns, whilst the resultant electromotive change is always similar, both as regards sign and character, to that of functional activity. It thus seems not improbable that the resting organ current of du Bois-Reymond should be regarded as an instance of that prolonged residual effect which will be described as succeeding every intense organ response, and if so, then no organ current apart from that due to activity has been observed.

This view is confirmed by experiments upon the organ of the skate, where the excitatory response is one in which the caudal end of the organ columns becomes galvanometrically positive to the cephalic end. Here, too, the organ current may be produced by injury, and, when thus caused, is always similar both in direction and character to the residual effect of the response.

In the organ of Malapterurus, very little residual effect is observed to follow functional activity, and the response appears to be never succeeded by that prolonged residuum which is so characteristic a feature in Torpedo; it is most suggestive that in this fish the resting organ current is either absent or very small, even when excised strips through the entire organ are examined. ${ }^{2}$

Conditions affecting the magnitude of the excitatory response of the organ.-The functional response of a nerve organ preparation can be evoked by such a stimulus as the passage of a sufficiently intense electrical current, whether this traverse the nerve or the isolated organ. For the present the terminology employed in muscle may be used to denote this distinction, the first being termed a response to indirect, the second that to direct electrical stimulation. Whichever method is employed, the magnitude of the response, as determined by that of either the galvanometric deflection or the excursion of the capillary electrometer, varies under a variety of conditions as follows :-

\footnotetext{
${ }^{1}$ Goteh, Phil. Trans., London, 1887, vol. elxxviii. B.

2 Goteh and Burch, ibid., 1896, vol, clxxxvii. B.
} 
(a) The number of struetural elements.-This is best illustrated in the case of the torpedo organ preparation. The response is the summed effect of changes of like sign, occurring simultaneously in each of a superimposed series of electrical discs; the number of such discs thrown into simultaneous activity must therefore be a potent factor in determining the magnitude of the total effect. A simple experimental proof is the comparison of the galvanometric effects produced when a portion of the columns and the whole of the columns respectively are traversed by the same exciting current. The exciting current which is thus allowed to traverse the tissue and excite the organ directly, is prevented, by the use of an appropriate rheotome, from entering the galvanometer circuit, which is coupled up with the preparation about 005 secs. after the exciting induced current has been passed through the organ strip. If the portion of organ traversed by the exciting current is small, then the response is small; as it increases in length, so as to include greater lengths of column, the response shows a proportionate increase in intensity.

A further consideration is suggested by this simple experiment. The response is an effect, localised in each transverse disc or plate with its innumerable nerve-endings. There can be no propagation of the excitatory change from one plate to another, for the simple reason that each plate is structurally complete in itself, and is not connected by its differentiated protoplasm with its neighbours. Simultaneous activity is ensured by simultaneous excitation of the nerves to all the plates of the series, and the response of the organ thus differs from that of either a muscle or nerve fibre, since in these the change at the point first aroused is successively assumed by neighbouring portions of the protoplasmic continuum, which have not been the seat of the initial excitatory disturbance.

(b) The size of the structural elements.-The intensity of the response increases as the plates increase in size; and since the size is greater the larger the fish, it is always more pronounced in fully grown than it is in young fishes. The increase is too pronounced to be accounted for by the diminution in the electrical resistance offered by the larger organs, for such diminution is small compared with the greatly augmented intensity of the organ response. Further, the capillary electrometer shows a similar increase, hence it is due to the greater capacity for electromotive change possessed by each plate of the adult organ.

(c) The intensity of the stimulus.-As in all excitable tissues, so in the electrical organ preparation, a stimulus must be of a certain intensity before it can evoke an appreciable response. When this intensity is reached, the response can be further increased in magnitude by augmenting the stimulation. The minimal exciting intensity of electrical induced currents is much greater than that adequate to evoke a response in a muscle preparation of the frog, whether this is aroused by indirect or direct stimulation. In the case of the Malapterurus organ, the ratio between the amounts of such a minimal stimulus as the break-induced current applied, on the one hand to the nerve of the organ, and on the other to the nerve of the gastrocuemius of the frog, may be as much as 100 to 1 .

It has been already pointed out that the threshold of exciting efficiency is so high in the case of chemical stimuli, that such reagents as $\mathrm{NaCl}$, etc., evoke no response in the organ preparation, even when 
applied to the exposed nerve. On the other hand, the organ readily responds to mechanical stimulation of the nerve, even when this is the mere vibration obtained by Uexküll's nerve-shaker.

The alteration in the magnitude of the response of the organ to the stimulation of its nerve by induced currents, resembles that of a motor nerve with its nerve-endings rather than that of a muscle. Thus the organ response increases with the augmentation of the exciting current, through a much larger range than does that of a muscle, whilst the resemblance to nerve-endings is shown by the circumstance that the organ response is diminished by previous activity. It is true that an isolated piece of organ, if kept cool and moist, can be excited several times before there is any very marked diminution in the size of the response; but such diminution does occur, and has been studied by Schönlein, in torpedo strips repeatedly stimulated by the repeating rheotome; hence fatigue can be produced, and the organ thus resembles nerve-endings as regards fatigability. ${ }^{1}$ It should, however, be remarked that the discharge of the entire organ through the central nervous system becomes far more rapidly weakened, unless ample periods of repose alternate with those of activity; this is due to central fatigue.

(d) Influcnee of arrest of circulation.-In close connection with the question of fatigability is the extent of modification which portions of tissues show when kept in the cold and in appropriate moist surroundings for many hours. Such portions may respond even after thirty-six hours to exciting stimuli, but are far more susceptible to fatigue and are evidently in a condition of lowered excitability. This is shown by the small extent to which the phenomena of self-excitation, to be referred to later on, are now observed. The altered condition of the tissue is further shown in Malapterurus preparations, by the circumstance that careful analysis of the electrometer record shows the response to be slower in development than is that of the freshly excised organ. In both Torpedo and Malaptcrurus, portions of kept organ sometimes exhibit a further phenomenon. A rapid series of responses affecting the recording instruments may suddenly be observed in the absence of any obvious stimulus. This often appears to usher in a gradual diminution and final extinction of excitability; and since the organ is refractory to chemical, but very susceptible to mechanical, stimulation, it is probably due to the nerve-shatter caused by drying, and resembles the familiar muscle tetanus, which is so readily produced by the drying of portions of a motor nerve.

Influence of such drugs as affect muscles and glands.-The comparative indifference of the organ to such drugs as curari and atropin is a remarkable and suggestive fact. Torpedoes can be curarised by placing them in salt water containing the alkaloid, until they are quite immovable, yet, when touched, they give violent shocks, showing that the functional activity of the organ is still at the beck and call of the nervous system. ${ }^{2}$ The organ of Malapterurus with its nerve, can be soaked in 1 per cent. curari solution, without undergoing any appreciable alteration in its susceptibility to respond to nerve excitation. ${ }^{3}$

It has been recently found that when large doses of curari (15 c.c. of

1 Schönlein, Ztschr. f. Biol., München, Bd. xxxi.; Bd. xxxiii. S. 408.

2 Moreau, Ann. d. sc. nat., Paris, 1862, tome xviii.; Gotch, "Proc. Physiol. Soc.," 1888, p. 6, Journ. Physiol., Cambridge and London, vol. ix.

${ }^{3}$ Goteh and Burch, loc. cit. 
4 per cent. solution) are injected into the blood stream of Torpedo, there is a final diminution in the organ response to both indirect and direct excitation. ${ }^{1}$ In this connection it is interesting to note that Waller has observed a diminution in the nerve electrical response to follow the action of large doses of curari. It thus appears that, unlike muscle, the nerve organ mechanism is one in which there are no connecting links between nerve-endings and organ to be affected by curari, and that no distinction between direct and indirect excitability can be brought out in the case of the electrical organ by the use of this drug. ${ }^{2}$

The same is true of solutions of atropin, which paralyse many of the neuro-muscular and neuro-glandular mechanisms. Portions of Malapterurus immersed in such solutions are as readily excited, and give as intense responses, as they did before the immersion.

Experiments made by the author show that when portions of Malapterums organ are submitted to chloroform and ether, the responses fail; in the case of ether, recovery may follow the removal of the vapour.

It will be clear from this brief description of the influence of various conditions upon the excised organ, that the response presents far more striking analogies to that of efferent nerves and nerve-endings than to that of muscle, although the plates of which the organ is composed appear to have been developed by the transformation of muscle. With reference to such transformation, it should be remembered that the essential elements are nervous and that these are the processes of central cells, which do not correspond in position with those innervating the muscles. The difficulty thus offered to those who would view the organ as a transformed muscle, has not so far received the attention it merits. ${ }^{3}$

The time relations of the organ response.-The most interesting features of the response are undoubtedly those brought to light by the study of its time relations. In the first place, the response is so intense, even in isolated portions of tissue, that its commencement can be ascertained with more precision than that of the excitatory electromotive change in other forms of excitable tissue. In the second place, the analogy between the pronounced organ response and the comparatively feeble nerve excitatory change makes the precise determination of the former most suggestive in regard to the characters of the latter.

The time relations may be most easily determined by photographing the excursions of a known and accurately calibrated capillary electrometer, but, owing to the difficulties in getting fish, in suitable condition, to laboratories containing photographic appliances for this purpose, this has at present been done in the case of one organ only, that of the Malapterurus.

The time relations of the response have been determined in the case of the organs of Torpedo and of Raia by means of rheotome methods, the galvanometer, a special telephone, or the nerve muscle preparation of the frog, being used as the recording instrument. A convenient rheotome is that of the spring myograph, or pendulum, which at each passage opens three keys. The opening of the first key $\left(\mathrm{K}_{1}\right)$ causes an exciting break-induced current; that of the second $\left(K_{2}\right)$ opens a short circuit,

\footnotetext{
${ }^{1}$ Sehönlein, Ztschr. f. Biol., Mümchen, Bd. xxxi.

2 Gotch and Burch, loc. cit.

3 Sec Fritsch, "Die elektrischen Fische," Leipzig, 1887, 1890.
} 
and allows any electrical effect in the organ to traverse the galvanemeter; that of the third $\left(\mathrm{K}_{3}\right)$ breaks the galvanometer circuit, and hence the instrument is only influenced by electromotive changes occurring between the opening of $K_{2}$ and of $K_{3}$. By suitable arrangements, the time between $K_{1}$ and $K_{2}$ may be varied, whilst that between $K_{2}$ and $K_{3}$ remains always of some fixed short duration, such as 001 sec. or less.

By moving $K_{2}$ and $K_{3}$ towards or away from $K_{1}$ before each passage of the traveller, and noting the galvanometric deflections produced by such a passage with its consequent excitation, data are obtained from which the total development of the response may be inferred. The repeating rheotome has also been used in the Naples laboratory, by Schönlein, for the same purpose.

The first and most obvious fact is the existence of a period of delay between the excitation and the commencement of the response. Every preparation shows such a period of delay, the so-called latent period. If the preparation is excited by the stimulation of its nerve, then the delay is in the case of Torpedo perceptibly increased, in proportion as the seat of the stimulus is removed from the nerve termination in the organ. The increase has been shown to be accounted for by the propagation rate along the nerve of the excitatory state thus aroused, which, in the case of the electrical nerves of Torpedo, may be as little as 9 metres per second at $5^{\circ} \mathrm{C}$, although at $20^{\circ} \mathrm{C}$. it is 30 metres per second. At $15^{\circ}$ C. a rate of from 15 to 20 metres per second has been observed. ${ }^{1}$

In Malapterurus preparations, on the other hand, change in the delay, due to the seat of the nerve excitation, is far less marked, and the propagation rate in the electrical nerve of this organ is, at $12^{\circ} \mathrm{C}$., at least as fast as that in the motor nerve, i.e. 40 metres per second. But even when the nerve is excited close to the organ, a distinct delay occurs, and a fact of great significance is the remarkable circumstance that, when the exciting current is allowed to traverse the organ itself, the duration of the period of delay is not perceptibly different. ${ }^{2}$ It will be remembered that in frog's muscle a very appreciable diminution, amounting to 0.003 second, at a temperature of $15^{\circ} \mathrm{C}$., occurs in the delay to direct, as compared with that to indirect, excitation.

The absence of any such diminution in the case of the electrical organ is in favour of the view that the organ response is that of a structure which cannot be separated from the nerve-endings. These are excited whether the stimulus is applied to the entering trunk of the nerve, or to the nerve fibres contained in the dises. It follows from this that the organ owes its susceptibility to excitation entirely to the nerves which it contains, and that, apart from these, its substance is inexcitable. A further inference may be hazarded. If the plates, apart from the nerveendings, are inexcitable, then the seat of the excitatory change, that is the electrical response, being localised in the nerve-endings of the plate, the function of the protoplasmic substance remains at present unknown. An additional confirmation of the view that the discs apart from the nerves are inexcitable, is furmished by the observations of Garten on the result of nerve section. Torpedoes, the electrical nerves of which had been previously divided on one side, showed, nineteen days after section, complete inexcitability of the organ of this side to both

${ }^{1}$ Schönlein, loc cit.; Goteh, Phil. Trans., London, 1887, vol. clxxviii. B.

2 Gotch and Burch, loc. cit. 
indirect and direct excitation. ${ }^{1}$ A suggestive fact in connection with the delay which occurs in the response of the organ to the passage of an exciting current through its substance, is the minimal duration of the period. This, in Torpedo, Malapterurus, and skate, is 003 sec., a time identical with the nerve-ending time in motor nerves, as ascertained by Bernstein. The delay is prolonged by lowering the temperature, the minimal limit of $003 \mathrm{sec}$. at $30^{\circ} \mathrm{C}$. being in all the above organs increased, until at $5^{\circ} \mathrm{C}$. it may amount to 01 sec. Here, again, the alteration is one which affects almost equally the delay to the so-called "direct," as that due to indirect, stimulation. From the above remarks, it will be clear that the writer considers the term "direct," as applied to the stimulation of a portion of organ by the passage through it of an electrical current, to be inappropriate; the passage of a current through the substance of the organ acts by exciting the contained nerves.

When the response begins, it develops in Torpedo organ with great rapidity, reaching its maximum in $0.003 \mathrm{sec}$. under favourable conditions. $^{2}$ The rate of development is accelerated by warmth, and slowed by cold. This is seen by the curves given in Fig. 293, which are plotted from a number of rheotome observations obtained in the organ of Torpedo by myself. In Malapterurus the development may be completed in less than 0.002 sec. at $30^{\circ} \mathrm{C}$. The magnitude of the change in this organ is increased by cold, and diminished by heat (see Fig. 294).

The subsidence of the electromotive change of the response is slower than the development, occupying 0.005 sec. at $15^{\circ} \mathrm{C}$., and $0.01 \mathrm{sec}$. at $5^{\circ} \mathrm{C}$. In the final stages it is generally much prolonged, each response being thus accompanied by an after-effect, having the same sign as the response itself.

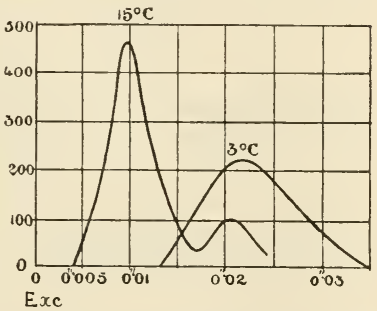

Frg. 293.-The ordinates indicate the galvanometric deflections when the galvanometer was connected with a strip of torpedo organ for 0.002 sec. at various intervals after the excitation of the strip, which occurred at 0 .

It is for this reason that, after a rapid series of responses, a more or less persistent electromotive change presents itself, having the same sign as that of the response, the so-called organ current of du Bois-Reymond. Attention may here be drawn to the fact that the subsidence is not caused by any counter-effect, due to changes occurring in more remote parts. In both muscle and nerve, with contacts on uninjured tissue, the change under the contact proximal to the seat of excitation having been propagated to the distal region, is counteracted by a second change of like sign under this distal portion. The total effect under any one contact in these tissues is thus diphasic, i.e. the response is one in which the initial change rapidly subsides through the development of one of opposite sign counteracting it. In the electrical organ there is no such counter-effect, because there is no protoplasmic continuum along which the excitatory change can be propagated, and

${ }^{1}$ Garten, Centralbl. $f$. Physiol., Leipzig u. Wien, 1879, Bd. xiii. S. 1.

2 Schönlein has found that the development of the change in the organ of vigorous Torpedoes may take place in less than 0.002 sec. (Ztschr. $f$. Biol., Minchen, Bd. xxxiii. S. 408).

VOL. II. -37 
the change is thus strictly monophasic. This is well shown by the character of the single excursion of the mercurial meniscus of the capillary electrometer, produced by a single response of the excised organ of Malapterurus, when in a low state of excitability. An examination of this excursion, as photographed (see Fig. 294, A), shows that, after the application of the stimulus at the time $(x)$, a period of delay occurs
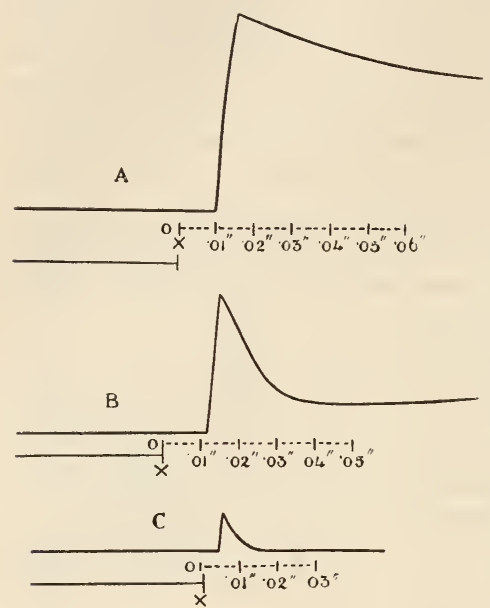

FIG. 294.-Outlines of records obtained by photographing the eapillary electrometer. A, Mat apterurus organ strip of low excitability; electrometer connected with caudal and cephalic ends of strip, with short circuiting shunt of 80 chms. Temperature of strip $=5^{\circ} \mathrm{C}$. The nerve was excited by a single induced current at the moment marked $x$. The single monophasic response commences at $0.01 \mathrm{sec}$. and is fully developed by $0.02 \mathrm{sec}$. $\mathrm{B}$. Same strip as that used in A, but connected with a pair of condenser plates; the electrometer was connected with another set of plates at $3 \mathrm{~mm}$. distance. The change in the organ commences at 0.01 sec. after the excitation of the nerve producing a condenser charge; it ceases at $0.016 \mathrm{sec}$., producing a discharge of opposite sign. Temperature of organ strip, $5^{\circ}$ C. Condenser 537 C.G.S. units. C, Same as B, but temperature of strip $30^{\circ} \mathrm{C}$. Time of commencement of response after excitation of nerve, 0.006 see. ; cessationlof response indicated by discharge of opposite sign at $0.008 \mathrm{sec}$. succeeded by a rapid rise, due to the development of the change; the subsidence is far more gradual, and shows no evidence of any counter electromotive effect bringing the meniscus back to the initial position, such movement as exists being largely the discharge of the charged capillary through the short circuiting shunt. The character of the excursion may be contrasted with that of $B$ in the same figure (Fig. 294), where a counter-effect exists. This was obtained in the following way. The preparation was connected with one pair of condenser - plates, a second pair of plates at $3 \mathrm{~mm}$. distance being connected with the capillary electrometer. With this arrangement, both the development and the cessation of a monophasic change should produce an electromotive disturbance in the condenser plates, the former causing a change of one sign, the latter one of the opposite sign. If a monophasic change occurs in the organ preparation, it should produce a diphasic effect in the condensers connected with the capillary. The photographed records of the electrometer excursion, outlined in B, Fig. 294, is clearly of this diphasic character, the sudden rise being succeeded by a sudden fall, which brings back the level of the meniscus. The records $\mathrm{A}$ and $\mathrm{B}$ are both those due to responses of the organ at a low temperature; in C, Fig. 294, the record obtained from the same organ at $30^{\circ} \mathrm{C}$. shows that the response culminates in $\frac{2}{1000}$ sec. after its commencement. ${ }^{1}$ 
Self-excitation of the electrical organ.-In the excised Malapterurus organ it is but seldom that the response to a single stimulus is the single monophasic change indicated by such electrometer excursions as are outlined in Fig. 294. It generally happens that the subsidence of such an effect is succeeded by the development of a second one, this by a third one, and so on. This tendency of the organ to respond to a single excitation by a rhythmical series of changes, although apparently most marked in Malapterurus, appears to be a property of all electrical organs. In the Torpedo it is demonstrable by a sufficiently wide range of rheotome readings, when the galvanometer is used as the recording instrument. In the portion of organ investigated a series of electromotive changes are now found, occurring one after another at definite intervals, which at $5^{\circ} \mathrm{C}$. are $\frac{1}{100}$ sec. (see Fig. 295). A more striking demonstration of the same characteristic is afforded by the photographic records of the capillary electrometer, when connected with the excised Malapterurus organ. A series of from ten to twenty monophasic changes is now seen to be evoked by the single stimulus. In consequence of the slow subsidence of the meniscus, the successive fresh developments are superimposed one upon another, but the rhythm may be made plainer by converting each monophasic change into diphasic condenser discharges, through the interposition of the condenser plates alluded to in the preceding paragraph. Two illustrations, with the organ at different

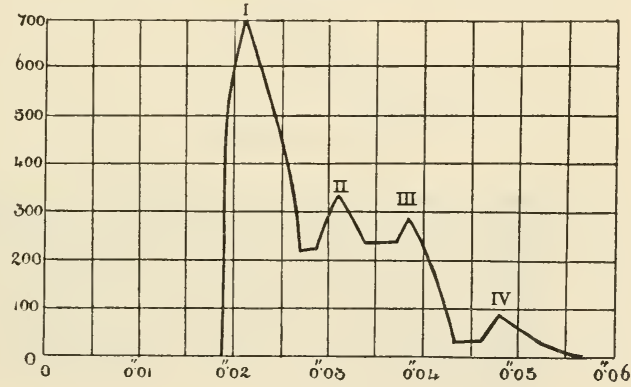

Fig. 295. temperatures, are given in Fig. 296. The extent of this " peripheral organ rhythm" varies with the temperature, the state of excitability of the organ, and the intensity of the individual responses which compose it. ${ }^{1}$

The multiple response is evoked by a single stimulus of either the nerve trunk or the organ strip. This discovery, made by me in 1886 , was confirmed by Schönlein, working with the repeating rheotome, who termed the phenomenon, Selbsttetanisirung. The essential factor concerned in its production is undoubtedly the passage through the tissue of the intense current due to the response; this excites the tissue again, and the same effect is repeated. The following facts, among others, prove that the phenomena are due to such auto-excitation. ${ }^{2}$ If two strips of Malapterurus organ, A and B, are arranged as in Fig. 297, and the nerve of one, the primary strip, excited by a single stimulus, then the current due to the activity of this primary strip A will traverse the secondary strip B. By placing the secondary strip B the opposite way round to $A$, it is easy to see whether it responds to the current produced by the functional activity of $\mathrm{A}$, for if $\mathrm{B}$ responds, the electrical effect of its

\footnotetext{
${ }^{1}$ Gotch, loc. cit.; Gotch and Burch, loc. cit.; Schönlein, loc. cit.

2 Gotch and Burch, loc. eit.
} 

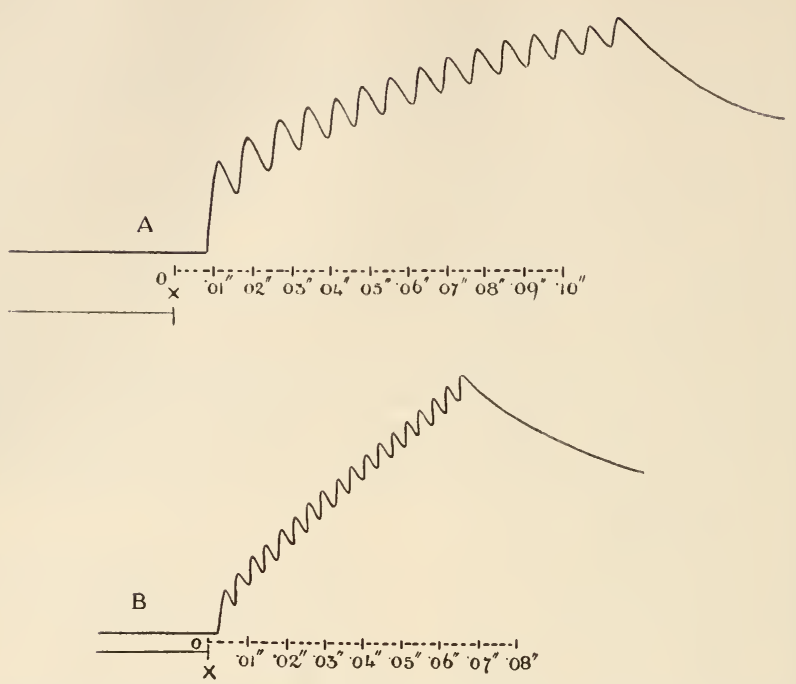

FIG. 296.-Outlines of excursions of capillary electrometer records when the capil. lary is connected to one pair of condenser-plates, and another pair, at $3 \mathrm{~mm}$. distance from these, is connected with the two ends of a strip of Malapterumes organ. In $\mathrm{A}$ the organ was excited by stimulating the nerve with a single induced current at the moment marked $x$. The temperature of the strip was $15^{\circ} \mathrm{C}$. The secondary responses occur at an average interval of $0.0076 \mathrm{sec}$; initial period of delay, 0.0080 sec. In $B$ the organ strip was excited directly by an induced current (heterodromous) ; the temperature of strip was $25^{\circ} \mathrm{C}$.; initial period of delay, 0.0035 sec.; average interval between secondary responses, $0 \cdot 0045 \mathrm{sec}$.

response passing from the head to the tail end of the strip must be of opposite sign to that of A. When the two strips, A and B, are successively connected with a capillary electrometer, records of their

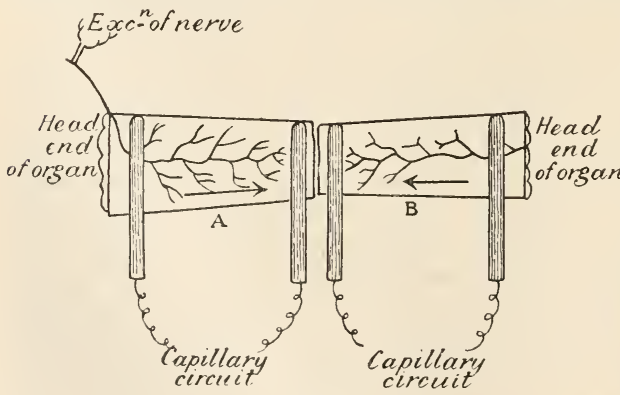

FIG. 297. responses are obtained, of which the curves $\mathrm{A}$ and $\mathrm{B}$ in Fig. 298 are an illustration. It is obvious that the lower curve is the response of $B$, due to the excitatory change of A traversing its substance. The response of the secondary preparation $\mathrm{B}$, differs from that of the primary one A, in having an extra period of delay. It is clear, there- 
fore, that the electrical current of an active strip is fully capable of exciting another strip, but it can also excite the nerves of its own plates. This is demonstrated by section of the single nerve which lies upon the under surface of a Malapterurus strip, so that one end only is left with its nerve fibres in connection with the nerve outside the organ, as in Fig. 299. On exciting the nerve of the preparation, and connecting successively the two halves, proximal and distal respectively, with the electro meter, responses are obtained in both, but the record of the distal one differs from that of the proximal in having an extra period of delay.
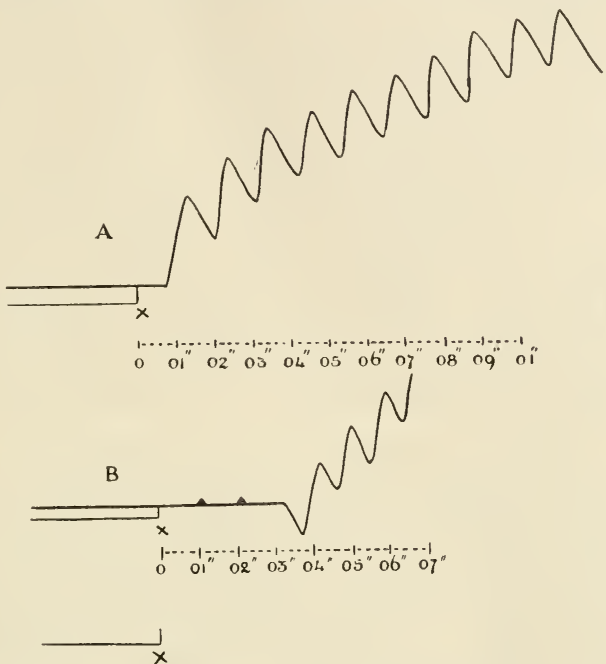

Fig. 298.-A, Outline of electrometer excursion obtained from primary strip of Malapterurus organ in response to a single excitation of its nerve. $\mathrm{T}=15^{\circ} \mathrm{C}$. First response occurs $0^{\circ} 008 \mathrm{sec}$. after excitation. B, Outline of excursion obtained from a secondary strip, through which current produced by $\mathrm{A}$ has been led. The secondary strip, having been arranged the opposite way round, will show, when excited by the current of $\mathrm{A}$, a response of opposite direction to that of $\mathrm{A}$. First response 0.03 scc. after excitation of $\mathrm{A}$ at moment $\mathrm{x}$. The derivations of two of the responses of $A$ are seen in front of the response of $B$ at 0.01 sec. and $0.021 \mathrm{sec}$. respectively.

Thus, in one instance, the two periods of delay were $\cdot 01$ sec. and $\cdot 04$ sec. respectively.

The successive responses in Malapterurus follow one another with the utmost regularity, the rapidity of the succession being influenced by temperature. Thus at $30^{\circ} \mathrm{C}$., a rate of succession of 300 per sec. was recorded; whilst at $5^{\circ} \mathrm{C}$. it sank to 100 . The state of excitability of the tissue has a great effect upon the production of the phenomenon. A freshly prepared organ strip and the organ in the living fish always

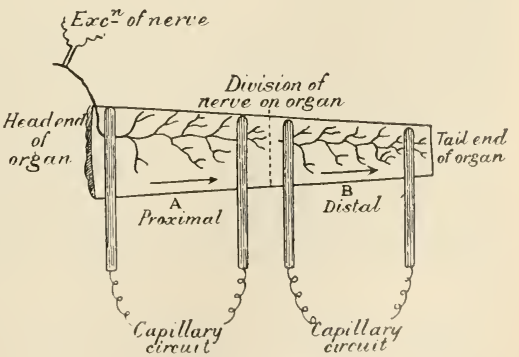

FIG. 299. show the multiple effect, but it is possible by keeping exciscd strips in 
0.6 per cent. $\mathrm{NaCl}$ to obtain a lowered state of excitability, in which the initial response alone presents itself. Even then any circumstance raising the excitability may develop the normal multiple effect, such, for instance, as section of the nerve near the organ. Schönlein has observed an interesting instance of the abolition of the multiple response, when the excitability is sufficiently lowered; this occurred in Torpedo on exciting the nerve trunk by an ascending galvanic current, the lowered excitability being in this case that of the anodic extrapolar region of the nerve extending to the nerve-endings in the organ discs.

The members of the whole multiple response become feebler as they are successively evoked, and when the intensity of one sinks below the threshold of exciting efficiency, the reduplication of the effect ceases. The duration or number of members is thus very variable, being increased by great intensity of initial effect and high organ excitability. In the living Malapterurus such a rhythmical response may last 0.5 sec., and comprise 150 individual members, but as a rule the multiple effect only lasts 0.2 sec., and seldom has more than fifteen members.

Similar auto-excitation has been observed in motor nerve, when rendered highly excitable through cold, ${ }^{1}$ and in the sartorius muscle when locally squeezed $;^{2}$ in these instances, the causation of the phenomenon is of the same character, the electrical change of the active tissue exciting it to renewed activity, but in no structure is the production of secondary, tertiary, etc., effects so marked as in the electrical organ. In Malapterurus this is so pre-eminently the case, that the efficiency of the organ, as a weapon for protection and attack, is thus enormously increased.

The effect of repetition of the stimulus.-It does not appear possible by rapid repetition of the stimuli to evoke responses which shall be superimposed upon their predecessors. Probably the tissue change, which is the basis of the response, cannot be reproduced until the electromotive effect, which is its objective sign, has more or less subsided. The presence of the change would be then associated with a period of diminished excitability. It appears probable from recent researches by Gotch and Burch, that nerve shows a similar incapacity for the production of a second electromotive change whilst that of a precursor is still in progress. ${ }^{3}$ The potency of the organ discharge, when wielded by the fish, is thus not increased, like that of a muscle, by such summation of mechanical effect as exists in the fused tetanic contraction. It is modified by the magnitude of the initial effect, which is itself dependent upon that of the exciting stimulus, by the extent of the auto-excitation which causes a repetition of the effect, and by the number of the successive central discharges which reach the organ.

The gradual diminution in the magnitude of the response, when the organ is excited many times at such intervals (say five times per sec.), as to insure its excitation, has been already alluded to. In Torpedo preparations, Schönlein ${ }^{4}$ found that a rheotome experiment, involving at least 500 excitations, could be repeated about ten times when the tissue

${ }^{1}$ Hering, Sitzungsb. d. k. Akad. d. Wissensch., Wien, 1882 (3), Bd. lxxxv. S. 237.

2 Kühne, Ztschr. f. Biol., München, N. F., Bd. viii. S. 203.

3 "Proc. Physiol. Soc.," January 1899, Journ. Physiol., Cambridge and London, vol. xxiii.

${ }^{4}$ Schönlein, Ztschr.f. Biol., München, Bd. xxxiii. S. 408. 
was fresh, although only two or three times when it had been kept for over twenty-four hours.

The residual effect of the response.-In Torpedo the electromotive changes, due to the excitatory explosion, are followed by others of the same sign; these may be termed residual effects, and are not improbably strictly analogous to the negative after-effect observed in both muscle and nerve. In the organ of Malapterurus they are of small amount, and it is of interest to note that, in this fish, the so-called resting current of du Bois-Reymond is also inconsiderable. In the organ of Torpedo and in that of the skate, such residual effects are far more marked. ${ }^{1}$ The simplest experiment suffices to show the change. A strip of organ with its attached nerve is connected with a galvanometric circuit, and any existing difference of potential is carefully compensated. The galvanometer circuit is now disconnected, and the organ excited several times by stimulating its nerve. On again connecting the galvanometer, although the excitatory explosions have had ample time to develop and subside, the dorsal end of the Torpedo columns is found to be galvanometrically positive to the ventral. This wake or residuum of the excitatory disturbance slowly subsides; it resembles the initial response in sign, but is unlike it in other respects, since the change is of comparatively low electromotive force, and subsides slowly. It is of interest, not merely because it accounts for the so-called resting difference, but also because its presence shows that the molecular disturbance, which is the basis of the pronounced shock, is such that the tissue does not completely regain its poise for some considerable time. It must be remembered that, since the residual effect is present in every disc, the change observed in a preparation is the sum of such effects in all the dises constituting the columns. In any one disc it is thus of very small amount, but is magnified into a distinct effect by the pile arrangement of the tissue.

The electromotive force of the response.-Various methods have been employed for this interesting determination. These have been chiefly concerned with the electromotive force of the discharge which is obtained from an uninjured electrical fish, but it is probable that the intermittent character of the effect in such cases only allows of an approximate determination. In Torpedo the telephone results of Schönlein gave an electromotive force of 30 volts, or over; ${ }^{2}$ in Malapterurus the total electromotive force must certainly be at least six times as great as this. With the excised organ the electromotive force of a single response may be ascertained within certain limits, either by balancing the effect, when a known rheotomic fraction of the response is permitted to traverse a galvanometric circuit, or by analysing the photographed curves of the excursion of the capillary electrometer produced by such a response. If the strip of organ stimulated is subsequently prepared for microscopic purposes, the number of dises placed in series in the strip can easily be counted, and thus the electromotive force of the change in any one disc can be calculated, on the supposition that all such discs are the seat of similar changes. In this way it has been found that in the comparatively feeble organ of the skate the change in each disc amounts to about 0.03 volt, in Torpedo to about 0.04 volt, in the powerful organ of the Malapterurus it may reach as mucl as

${ }^{1}$ du Bois-Reymond, loc. cit.; Goteh, loc. cit.

a Sehönlein, Ztschr. f. Biol., Mümehen, Bd. xxxiii. S. 408. 
0.045 volt. ${ }^{1}$ In regard to the physiological effects produced by the electrical discharge of an entire fish, it must be remembered that the small quantity, the rapid character of the development, and the sudden cessation, causes the physiological effects of the high potential upon the tissues of other animals to be of a different order to those produced by a similar voltage of longer duration; and since the seat of electrical production, the discs, is set in the moist tissues of the organ, only a derivation of the currents produced by the electromotive changes will pass through such external conductors as are supplied by the tissues of any other animals which chance to be in the neighbourhood.

Polarisation phenomena in electrical organs.-The peculiar structure of the electrical organ must undoubtedly complicate the production of such polarisation effects as are demonstrable in nerve, during and after the passage of electrical currents. The main features of polar changes in nerve are those associated with alterations in the excitability and in the electromotive conditions of the tissue respectively. Evidence of similar effects is not wanting in the case of electrical organs, but this evidence can be only rendered intelligible after a brief consideration of the polarisable media presented by such a series of compartments as exist in these structures. Each compartment has a protoplasmic disc, with a dense array of nerves, both being polarisable structures; it is bounded on both sides by a more liquid mass, the electrical conductivity of which is on a par with that of 0.6 per cent. $\mathrm{NaCl}$ solution. ${ }^{2}$ The disc thus offers to the passage of currents along the column length a thin central plate of higher resistance, and when non-polarisable electrodes are used for the passage of a current from an external source through the length of a column, the polarisation interfaces will obviously be situated on each surface of each disc. In a column, therefore, there will be, as far as the discs and the contained nerves are concerned, multiple anodes and cathodes, an anode and a cathode being present in each disc. The polar changes in excitability, if present, will thus resemble those produced when a current is directed more or less transversely through a series of nerve fibres lying side by side. We know that the more the direction of a current is transverse in these latter structures, the less becomes its exciting efficiency, and the less, therefore, any evidence of polar interference with such excitability, in consequence of reversal of the direction of the traversing current. We should not, therefore, expect, on a priori grounds, any pronounced change in the response of an electrical organ, dependent upon altered direction of a polarising current, when this is allowed to traverse the plates in the direction of the columns. On the other hand, if the polarising current is applied to the trunk of the electrical nerve, then the nerve divisions in the plate will be the seat of extrapolar changes, and the response of the organ should be affected in much the same way as is that of a muscle under similar conditions.

${ }^{1}$ Recent observations by Goteh and Burch, made in 1899, have enabled an aecurate calculation of the electromotive force of the effect obtained from a strip of JIalapterurus organ $15 \mathrm{~mm}$. long. The electromotive force of the strip reached a maximum of 26 volts, giving for the whole fish a maximum of 200 volts, and for each disc a maximum of 0.044 volt, since the strip contained 500 dises in series (Proc. Roy. Soc. London, 1899).

2 Recent experiments on Malapterurus organ, made at Oxford, show that the resistance for currents directed longitudinally through the columns is four times as great as that offered to transverse currents. The greater resistance in the former easc is an indication of that of the discs. The resistance to transverse currents was found to be the same as that of $0^{\circ} 6$ per cent, NaCl. (Proc. Roy. Soc. London, 1899). 
In the case of polarising currents which traverse the dises, the most appropriate nomenclature for indicating their direction is that first employed by du Bois-Reymond, who termed all currents traversing the discs in the same direction as those produced by the functional organ response, homodromous, whilst those of opposite direction were described as heterodromous. ${ }^{1}$

In Torpedo organ it is found that homodromous currents excite a response, on the whole more effectually than heterodromous ones; with the former the trunks of the nerve fibres entering the dises, to be distributed on its ventral aspect, are evidently in the region of the anodic polarisation, whilst the terminations of such nerves in the disc would lie in regions of cathodic polarisation. ${ }^{2}$ The reverse will be the case with heterodromous currents, the nerve-endings being now anodic rather than cathodic. Any increased exciting efficiency of hornodromous currents may be thus reasonably supposed to be related to cathodic polar changes in the nerve-endings contained in these. It appears, however, from recent experiments by Schönlein, that there is considerable variation as regards exciting efficiency, and it is extremely doubtful if any definite augmentation of excitability can be produced by homodromous currents.

In Malapterurus organ the present writer has always observed that the heterodromous current excites better than the homodromous. It must be remembered that the discs in this organ are peculiar, since the nerve fibres end in the candal stalk and do not ramify in the expansion of the disc. The current due to functional activity is directed through the discs from the cephalic to the caudal aspect; this would therefore be the direction of homodromous exciting currents. On the passage of such homodromous currents, the nerve-endings would, owing to their position, be in an anodic region, whilst, with heterodromous currents, the nerve-ending layer would, for similar reasons, be within the cathodic region. We should therefore expect, provided these regions are the seat of polar changes in excitability during the flow of a current, that the heterodromous would be more effectual for evoking the response than the homodromous. Such results as are definite both in Malapterurus and Torpedo are thus consistent with the view that polar changes of excitability may occur in the nerve-endings of electrical organs.

This is confirmed by observations upon the exciting efficiency of currents applied to the nerve trunk outside the organ. It has been found by Schönlein that an ascending galvanic current (directed up the electrical nerve) evokes a response in Torpedo of much less marked character than one directed down the nerve. ${ }^{3}$ In the former case, the peripheral nerve branches are in the region extrapolar to the anode (anelectrotonic), in the latter catelectrotonic, and the nerveendings undoubtedly share in the extrapolar state. The augmented response to the descending current, and the diminished response to the ascending one, appear to indicate that the excitability of the nerve-endings in the supplied discs is angmented in the one case, diminished in the other. The result might be due to polar changes in the nerve outside the organ, interfering with the adequate propagation of the excitatory state along the nerve trunk; but such interference is insufficient to account for the difference in the organ responses, for the following reason. The response to the nerve excitation by the descend-

\footnotetext{
${ }^{1}$ du Bois-Reymond, loc. cit.

${ }^{2}$ Goteh, loc. cit.

${ }^{3}$ Schönlein, loc. cit.
} 
ing voltaic current is not merely augmented but shows self-excitation, whilst that due to excitation by the ascending current is a single small effect. Since self-excitation occurs when the excitability of the nerveendings is high, it appears most probable that we have in this experiment a demonstration of the establishment of extrapolar changes in excitability along the nerve trunk to its ending in the organ.

The second great feature of the changes caused in nerve by the passage of currents is the production of secondary electromotive effects. The simplest experiment upon this subject is the familiar fact that the passage of a galvanic current is succeeded in polarisable media by the establishment of counter-electromotive changes. In strips of organ this is readily demonstrated, provided the galvanic current used be not intense enough to excite. If, however, it should excite the strip, then the intense current due to the response must obviously sum algebraically with any polarisation after-effect. For this reason, homodromous polarising currents, though followed by opposite, i.e. negative, polarisation effects when weak, are, when stronger, followed by a marked positive or similarly directed change, that of the response itself. On the other hand, heterodromous currents, which when weak are followed by the counter-current of negative polarisation, are, when stronger, succeeded by counter-effects of very great intensity, since the excitatory change, being of like sign with that of polarisation, now sums directly with this.

The presence of the response appears to have been overlooked by $\mathrm{du}$ Bois-Reymond, who considered that two sorts of polarisation effects, negative and positive, could be produced in the electrical organ. ${ }^{1}$ There seems little doubt that when experiments are so arranged as to exclude the strong excitatory effect which immediately follows the cessation of a strong current, but little evidence of such doubly directed polarisation exists.

Excitatory and Polarisation After-Effects in Torpedo Organ.

\begin{tabular}{|c|c|c|c|c|}
\hline \multicolumn{3}{|c|}{ Polarising Currext. } & \multirow{2}{*}{$\begin{array}{l}\text { GALTANOMETRIC } \\
\text { AFTER-EFFECT } \\
\text { (TIOT SHUNT). }\end{array}$} & \multirow{2}{*}{$\begin{array}{c}\text { Causation } \\
\text { of } \\
\text { EFFECT. }\end{array}$} \\
\hline Intensity. & Duration. & Direction. & & \\
\hline Seven Groves. & $\cdot 005^{\prime}$ & Homodromous $\uparrow$ & $\begin{array}{l}\text { Homodromous } \\
\uparrow 50 \text { scale }\end{array}$ & $\begin{array}{l}\text { Response } \uparrow \text { over- } \\
\text { powering nega- } \\
\text { tive polarisa- } \\
\text { tion } \downarrow\end{array}$ \\
\hline ", & $1^{\prime \prime}$ & , & $\begin{array}{l}\text { Heterodromous } \\
\downarrow 60 \text { scale }\end{array}$ & $\begin{array}{l}\text { Polarisation } \downarrow \\
\text { overpowering } \\
\text { response } \uparrow\end{array}$ \\
\hline
\end{tabular}

The particular positive after-effect, or positive polarisation, was admitted by du Bois-Reymond to occur, as a secondary phenomenon, only in the case of homodromous polarising currents. This in itself suggests that the change is due to an excitation, since the response is always in one direction, however aroused. It will be remembered that a similar positive polarisation, declared by the same authority to exist in muscle and nerve, has been shown to be due to local polar

${ }^{1}$ du Bois-Reymond, Arch. f. Physiol., Leipzig, 1885, S. 86 ; 1887, S. 51 ; Sitzungsh. d. k. Akad. d. Wissensch., Berlin, 1888, Bd. xxï. S. 531. 
excitation. The present writer, working with strips of Malapterurus organ, in 1894, convinced himself that if the strip was narcotised or otherwise altered, so that the excitatory response failed, no homodromous after-effect occurred on the cessation of the homodromous polarising current. Indeed, even in perfectly excitable strips of Torpedo organ, it is possible to do away with the homodromous after-effect by very simple means. For if the polarising current is not too strong (maximum, 7 Groves), although a short closure of .005 sec. to $\cdot 01$ sec. is succeeded by an homodromous after-effect, due to the excitatory response overpowering negative polarisation effects, yet, on lengthening the period of closure to 1 sec., the negative polarisation is sufficiently increased to swamp the excitatory change, and the usual heterodromous after-effect appears. This will be made clear by the above example.

The failure to recognise excitation phenomena involved du BoisReymond in a further unwarrantable statement, namely, that the electrical conductivity of an organ strip was very much less for homodromous than for heterodromous currents. The evidence for such irreciprocal resistance is quite as unconvincing. Many of the observations were made with induced currents, which were allowed to traverse both the organ and an appropriately arranged galvanometer. If this current was homodromous, the galvanometric deflection was large; if heterodromous, small; but rheotome observations have shown that, under these circumstances, the former large galvanometric effect was due to the summed effect of the homodromous induced current and the homodromous excitatory response, whilst the small effect was the algebraic sum of the dissimilar heterodromous induced current with the homodromous response. In regard to the observations with voltaic currents of long duration, such interference of the excitatory effect is not so obvious. The subject has been reinvestigated by Schönlein, who used Kirchhoff"s well-known methods for estimating electrical resistance and Kohlrausch's bridge. ${ }^{1} \mathrm{He}$ found that when a current of from 20 Daniells or more was led through the excised organ for 30 secs. in either direction, the tissue lost its excitability, and did not respond to ordinary excitation. Accompanying this failure is a diminution of the living electrical resistance. When such currents were led through the tissue for only 0.1 sec., the tissue showed most irregular results. At one time the homodromous, at another the heterodromous, appeared to have the advantage. There is thus no evidence of such marked irreciprocal resistance as du Bois-Reymond imagined to exist, an irreciprocity so great as only to be accounted for, in his opinion, by assuming an electromotive force of over 30 volts, due to a particular arrangement of those hypothetical molecules with which his name is identified.

The refutation of positive polarisation and of marked irreciprocal conductivity may seem a matter of small moment, but as their existence was considered by du Bois-Reymond as almost a demonstratio ad oculos of the existence of those special electromotive molecules which form the basis of his theory, it is really of some little importance. It cannot be said that the great electro-physiologist contributed much towards the elucidation of the functional activity of electrical organs, and it was the error of ignoring the true excitatory response which appears to have barred his progress. The phenomena of electrical organs may seem somewhat inexplicable, on any present conception as to the source of

\footnotetext{
${ }^{1} Z$ tschr.f. Biol., München, Bd. xxxiii. S. 408.
} 
vital electromotive changes, but they appear to the author to be far more in harmony with the alteration theory than with any other; and if, as experiments seems to show, the change is that of nerve-endings magnified by the peculiar symmetry of their arrangements, this theory can embrace them as harmoniously as it does those of nerve itself.

The reflex response of the organ.-The nerve fibres which supply the electrical organs are in all cases the axis cylinder branches of special nerve cells. In Gymnotus and Mormyrus, these cells form a vesicular column on each side of the spinal cord, but in Torpedo they are massed into special lobes of the medulla oblongata. In the case of Malapterumus, there being only one nerve fibre for each lateral organ, there is only one giant nerve cell in each half of the cord; this is situated between the exit of the first and second spinal nerves. In all these fish the organ can be made to respond reflexly to special sensory stimulation, the most efficient being electrical or mechanical excitation of the skin. Mechanical pressure appears to be a particularly efficacious means of evolving the reflex. Thus in the skate, a reflex response of the organs in the tail is readily evoked by rubbing the rough spines upon the dorsal surface. Torpedo and Malapterurus discharge when the surface is pressed. The telephone forms a convenient mode of ascertaining the presence of the response, the fish being placed in a suitable tank and connected by means of either plates or an appropriate net with the necessary metallic leads. The reflex responses are enormously increased by the action of strychnia, the organ like the muscles being thrown into a series of prolonged intermittent phases of functional activity; they are diminished by narcotisation and by prolonged cold.

In the case of the active Malapterurus, discharges of the organ often occur when it is swimming about, and the same appears to be true of Torpedo. The Gymnotus, kept in a tank, will discharge when small live fish are placed in the water. The discharge stuns a certain number of the fish, and the sluggish eel is thus able to devour them at his leisure.

These natural discharges present two points of interest. In the first place, the discharge is a succession of organ-effects; thus the electromagnetic signal vibrates, and a singing sound is heard in the telephone. In the second place, both the rate of succession and the intensity of the organ responses are extremely variable. The writer recorded with an electro-magnetic signal a torpedo discharge of 110 successive electrical changes in the second. At Naples, working with the recording telephone, Schönlein found a maximum of 187 in the second. This rapid succession cannot be kept up for any length of time, the longest period of rapid discharges observed at Naples was only 0.896 sec., but in this time ninetytwo separate responses occurred. ${ }^{1}$ It appears also that the responses of both organs are evoked simultaneously, so that the numbers just given must indicate the rate of discharge of each of the organs.

A special interest attaches to the study of the reflex organ response, in consequence of the light which it may throw upon the working of the central nervous system. For if, as seems probable, the organ response is due to the functional activity of nerve-endings, then the reflex discharge should give a tolerably faithful picture of the character and rate of the outflow from those nerve cells with which the nerve-endings are structurally continuons.

${ }^{1}$ Schönlein, loc, cit. 
In the case of both Torpedo and Gymnotus, there is apparent evidence that a succession of excitatory states leaves the electrical nerve cells at a rate which may be at least 100 per second. It would be rash to assume that this rate of outflow occurred from each central electrical nerve cell, for in both these fish there are a large number of such cells. But in the case of Torpedo the nerve cells are all definitely localised in a portion of the medulla oblongata, and thus form as a whole the efferent cells of one nerve centre ; the whole centre may possibly discharge at this rate. It is, however, not clear in either Torpedo or Gymnotus what share the self-excitation of the organ takes in the production of the rapid succession of responses. Until the organ discharge has been recorded by means of such an instrument as the capillary electrometer, the responses evoked by fresh central nervous impulses cannot be separated from those of the organ rhythm. At present, therefore, any such rapid rate of central outflow of nervous impulses is not proved, and is on a priori grounds improbable.

The most interesting case is undoubtedly that of the Malapterurus, for, as each organ is innervated by the terminal branches of one axis cylinder, the character of the reflex response in this fish must indicate that of the efferent output of the single nerve cell from which the axis cylinder springs. The reflex response of the organ of Malapterurus has been studied in the living specimen by the aid of the capillary electrometer, and the following characters have been thus determined. ${ }^{1}$ When the skin of the fish is excited, either mechanically or electrically, a reflex response is evoked after a comparatively long period of delay. This delay may be as little as 0.023 sec., or as much as $0.5 \mathrm{sec}$. The smaller number, after deduction of the transmission time along the main nerve-paths, and of the delay in the organ itself, leaves a remainder of 0.008 sec. for the central time. This duration, 0.008 sec., is thus the time occupied by the transmission of the excitatory state through the central are.

As regards the character of the response itself, a difficulty arises, due to the capacity for self-excitation previously described; from the absolute regularity of the effects produced during this peripheral organ activity, it is, however, easy to distinguish, in the photographic records of electrometer excursions, between the results of such auto-excitation and the arrival at the organ of a new excitatory state along the efferent nerves.

This distinction is further facilitated by the circumstance that, in Malapterurus, whereas the separate responses due to auto-excitation occur at rates of from 100 to 300 per second, those due to a fresh central outflow are of a very different order. The shortest interval between two separate responses of a reflex character yet observed was $\frac{7}{12}$ sec. $(0.085)$, and the average interval was as much as $\frac{1}{4}$ sec. $(0.25)$. Even this slow rate of central discharge could not be maintained, the maxinum number in any series being only five, and the average number two to three. If the reflex organ response, when evoked by impulses reaching it through its efferent nerves, gives a true picture of the central discharge, then in the Malapterurus a second excitatory state cannot emerge from the single cell at an interval of much less than $\frac{1}{10}$ second, whilst the cell itself, after two or three such displays of activity, is fatigued. The same susceptibility to central fatigue occurs in both Gymnotus and Torpedo, and hence it is not improbable that the so-called immunity of

\footnotetext{
1 Goteh and Burch, loc, cit.
} 
electrical fish to their own discharges is only relative. It seems far more likely that the discharges, if forcible or prolonged, exercise deleterious effects upon both central and peripheral parts of the nervous system. In confirmation of this is the observation of Schönlein and others, that after repeated reflex discharges of a violent character, the excised organ is in a far lower state of excitability than it is when repeatedly excited by stimulation of its nerves. ${ }^{1}$

\section{The Nature of the Activity of Electrical Organs.}

A few remarks may be made, in concluding the present article, with reference to the question as to how far the activity of electrical organs is to be regarded as based upon changes which are sui generis, rather than upon tlose present in other excitable structures such as muscle and nerve. The great electromotive force of the change is, at first sight, only to be accounted for on the supposition that the organ is the seat of special processes unlike those present in muscle or nerve; it has, however, been insisted upon in this article, that the extent of this electromotive force is due to the disposition of the elements in series, and that in each element of the series no such enormous development occurs. The columnar structure of the electrical organ thus modifies rather than determines the characteristics of the activity. The separate elements constituting the essential structural features of the organ, have in the case of Torpedo, Mormyrus, Raia, and Gymnotus been developed from neuro-muscular elements, and whereas the nervous part persists, the muscular part has been transformed. It is thus not unreasonable to consider, in the first place, how far the activity of the organ can be viewed as in its essence of the same nature as that which produces the electromotive changes accompanying muscular activity. Although there is no disparity between the electromotive force of the change in an organ element and that of such a muscle as the sartorius, there are very serious objections to any causal connection between organ and muscular electrical effects. In Malapterurus the morphological evidence tends to show that glandular elements have been utilised in the evolution of the organ, not muscular ones. Further, the multinucleated protoplasm which constitutes the greater part of the dise of Raia and the whole of the Malapterurus dise is homologous neither with muscle nor with gland, but with the protoplasmic sole of the nerve end-plate. The whole question, therefore, is narrowed down to the two following possibilities:-(1) Is the disc the effective part of the organ element? If so, then the change, though analogous with that presumably present in nerve end-plates, is in reality something sui generis, for no electrical effect has been observed in such end-plates. (2) Is the disc merely a means for reinforcing electrical changes occurring in the nerves themselves? If so, then the effective part of the organ-elements is nervous, and the change is based upon the same processes as are present in nerve when roused to activity. (1) The view that the disc is an excitable structure responding to nervous impulses which reach it through its nerves, is contradicted by all experimental observations. Drugs, such as curari and atropin, appear to have no specific paralysing effect upon the organ, unless such doses are employed that the nerves themselves are affected. There is no evidence that the dises can be excited apart from their contained nerves; all agents which annul the excitability of the electrical nerves annul at the same time the response of the organ to electrical currents which traverse its substance. One of the most suggestive facts bearing on this point is the complete inexcitability of an organ when deprived of its nerves through nerve degeneration in consequence of previous section. Direct excitation of the organ is direct only in appearance, not in reality; there is always nerve excitation, whether the 
excited nerves lie outside or inside the organ columns. In one organ, that of Malapterurus, the expanded disc has no nerves in its substance, these all ending in the slender stalk which projects from the disc ; and the disc expansion itself affords no structural differences to account for corresponding electromotive differences between its surfaces.

(2) The only excitable structures in the organ are thus the nerves and their fine terminations, and since these are known to be the seat of electrical changes during activity, it seems more reasonable to presume that the organ change is in reality closely related to the production of molecular disturbances in its contained nerves. Apart from the consideration that the nerves are the only structures present which we know to be capable of developing such electrical changes, there are other circumstances which favour this view. The time relations of the organ shock and those of nerve closely correspond; it is true that the organ shows a period of delay, but this can be accounted for by transmission time either along nerve trunks or fine nerve-endings. There are in fact only two discrepancies of moment; the organ can be fatigued by activity, and the electromotive force of the change in each organ element is greater than that present during nerve activity. With regard to organ fatigue, it may be pointed out that this occurs even in nerve as far as the finer terminations are concerned, and as regards the electromotive force discrepancy it has been recently shown that the sciatic nerve of the frog can develop a change of more than .03 volt in response to a single stimulus. ${ }^{1}$ The electromotive force of each organ element varies between .03 and .05 volt; the discrepancy is thus not so great as to offer any insuperable objection to the view that the organ effect is essentially that of active nerve. It is not fanciful to imagine that the disc itself may play somewhat the same secondary part in regard to the primary and essential nervous disturbance, as a resonator can play in the case of sound, or an electrophorus in the case of the electrified body which charges it. ${ }^{2}$

But whether the protoplasmic expansion of the disc plays such a reinforeing rôle or not, the clear fact remains that the essential primary disturbance which constitutes the organ shock is a nervous one; without nerves, the dises, having nothing to reinforce, would be impotent and absolutely inexcitable. The primary cause of the organ shock is thus in all probability a change identical in nature with that producing the electromotive phenomena of nerve, and if so, then it will be interesting to see how far the various theoretical explanations of these phenomena can be extended so as to comprise those of the electrical organ. One such hypothesis can undoubtedly be so extended-the alteration theory, as given either by Hermann or by Hering. It is far more difficult to extend any molecular theory in this direction, or indeed any view which involves the pre-existence of electromotive elements. Electrical organs show no resting electrical effects; all the effects are connected with excitation and its concomitant alteration. This may be produced by the sudden stimulus of electrical currents or the prolonged one of a persistent localised injury of nerve trunks. In all cases the alteration in the nerve is accompanied by the rapid development of electrical changes, whilst the structural disposition of the organ is such that the changes present in each group of nerve terminations can, by summing with those in the neighbouring groups, become conspicuous.

1 Gotch and Burch, Proc. Roy. Soc. London, 1898, vol. lxiii. p. 300.

2 Compare Kühne's views on the causative value of electrical changes in motor nerveendings for the production of indirect muscular contraction. The above interpretation of the phenomena of the electrical organ undoubtedly supports Kuihne's suggestion that "a nerve throws a muscle into contraction by means of its eurrents of action." Kühne, Croonian Lecture, "Ueber die Entstehung der vitalen Bewegung," Proc. Roy. Soc. London, 1888, vol. xliv. pp. 429-446. 


\title{
THE NERVE CELL.
}

\author{
By E. A. SCHÄFER.
}

Contents.-Classification of Nerve Cells, p. 592-Theory of Isolated Units (Neurone Theory), p. 593-Structure of Nerve Cells, p. 594-Changes in Nerve Cells as the result of Excitation, p. 599-Changes resulting from Section of the Nerve Fibre Process, p. 600-Conduction in Nerve Cells, p. 605-Time lost in Transmission from Cell to Cell, p. 608-Refractory Period, p. 614.

NERVE CELLS may be broadly classified into-(1) afferent cells, which receive impressions at the periphery, and convert such impressions into impulses which pass towards the central nervous system; (2) efferent cells, which send out nervous impressions towards the periphery; (3) intermediary cells, which receive impressions from afferent cells and transmit them directly or indirectly to efferent cells; (4) distributing cells, which occur near the periphery, and, receiving impulses from efferent cells, distribute them to involuntary muscles and secreting organs. The cells classed under (1) and (2) are also known as root cells. The greater number of the nerve cells of the brain and cord belong to the intermediate class; they serve the purposes of association and co-ordination, and afford a physical basis for psychical phenomena; the cells classed under (4) belong to the so-called sympathetic system.

Originally the afferent or sensory root-cell is situated at the periphery, in some part of the integument, and is connected with the central nervous system by a process (nerve-root fibre) which ramifies amongst the nerve-cells of the central organ. This primitive condition is found in the earthworm, and it is also persistent in the olfactory cells of all vertebrates. In the process of evolution the peripheral cell sinks below the integument, leaving a process at the surface. Ultimately the body of the cell is found close to the central nervous system (in the spinal ganglion), and the peripheral process is found to have developed into the peripheral sensory nerve (Fig. 300). The typical efferent root cell is the cell of the anterior horn of the spinal cord, which sends out a nerve fibre directly to one or more muscle fibres or gland cells.

Every nerve fibre is a process of a nerve cell. As was first made clear by the researches of Deiters, ${ }^{1}$ most nerve cells have, besides the nerve fibre or axis cylinder process (neuron, ${ }^{2}$ axon), an uncertain number of other processes (dendrons), ${ }^{3}$ which differ from the nerve fibre process

1 "Untersuch. u. Gehirn. u. Rüickenmark," Braunschweig, 1865.

${ }^{2}$ From $\nu \in v \rho o \nu$, a nerve. This term was suggested by me (Brain, London, 1893, vol. xvi. p. 134) to denote that the process in question is a nerve fibre, and I regret that only one or two prominent neurologists have employed it in this sense. The terms neuraxon, neurite, are also used by authors to express the same idea.

3 Dendrites of some authors. 
in certain details of structure, and also in the fact that at once on leaving the cell body they begin to branch dendritically, and the resulting branches terminate in arborisations in the neighbouring grey matter. The dendrons of some nerve cells are beset in their whole course with short and minute processes which have been termed "thorns" or "gemmulæ."1 Many nerve cells possess no dendrons; this is the case with the typical cells of the spinal ganglia, and with those of the corresponding ganglia of the cranial nerves, but it is doubtful whether any nerve cell possesses only dendrons, and is destitute of a nerve fibre process. ${ }^{2}$ On the other hand, a nerve cell may possess more than one; thus the bipolar cells of the spinal ganglia of osseous fishes and the cells of the
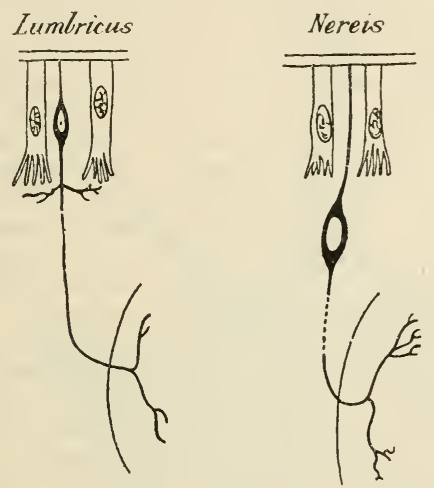

Tertelrata

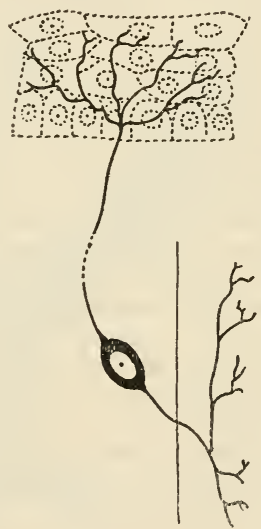

Fig. 300.-Diagram to show the primitive condition of the afferent nerve cell, and the manner in which it has become altered in the process of evolution of the nervous system.-G. Retzius.

ganglion cochleæ possess two, issuing from opposite ends of the cell (bineural cells); and cells have been described by Ramón y Cajal in the molecular layer of the cortex cerebri which have three or more (polyneural cells).

Cajal ${ }^{3}$ distinguishes nerve cells into those with a long axis cylinder process (type 1 of Golgi), and those with a short axis cylinder process (type 2 of Golgi), but it is doubtful if these types represent any physiological distinction, as supposed by Golgi, or if, indeed, they are morphologically distinct from one another.

Theory of isolated units. Neurone theory of Waldeyer.-Each nerve cell, inclusive of the nucleated body and all its processes, irrespect-

${ }_{1}^{1}$ See on these structures, A. Hill, Brain, London, 1897, vol. xx. p. 131.

2 The "amacrin" cells described by Ramón y Cajal in the retina, may be an exception to this rule. See Quain's "Anatomy," vol. iii. pt. 3.

${ }^{3}$ Rev. de cien. méd. de Bareelona, 1891.

VOL. II. $-3^{8}$ 
ive of their number, length, complexity, character, and position, forms a distinct unit, which is believed, as the result of the employment of the chromate of silver methods introduced by Golgi, to have no structural continuity with any other unit. This "theory of isolated units," which is often spoken of as the "neurone ${ }^{1}$ theory," from the name "Neuron" having been applied by Waldeyer ${ }^{2}$ to the nerve cell as above defined (including all its processes), is not conclusively proved, nor is it accepted by some prominent histologists, including Golgi himself. But it is supported by the anatomical observations which have just been referred to, and is found to fit in with many known physiological facts, and to lend itself better than the "continuity theory" of nervous elements, which it has in large measure supplanted, to the explanation of phenomena which were previously obscure; on this account it has been widely adopted by neurologists, many of whom, following the example of Waldeyer, habitually speak of nerve cells as neurones, although the term is quite synonymous with "nerve cell"; nor has the newer name any advantage either on the score of brevity or of etymology over the older and more general expression. Strong reasons, moreover, as will presently appear, have lately been urged against the acceptance of the theory which is associated with the newer name, so that it would seem wiser to retain the term "nerve cell." The practice of restricting the use of "nerve cell" to the body of the cell ("perikaryon" of some writers) should for obvious reasons be dropped.

Structure of nerve cells.-The structure of nerve cells has of late years been the subject of numerous researches. ${ }^{3}$ The general result of these may be shortly given as follows:-The nucleus of the nerve cell is characterised by its large size, and the fact that its chromatin is in relatively small amount, except in the nucleolus, which is large. ${ }^{4}$ The protoplasm of the cell body resembles that of other cells in exhibiting a reticular or sponge-like structure (spongioplasm), with a clear or finely granular substance (hyaloplasm) in its meshes. ${ }^{5}$ But there are two points in which it is peculiar. One consists in the occurrence of fibrils which run through the protoplasm. Such fibrils were observed by Max Schultze, ${ }^{6}$ who found them in both fresh and osmic preparations of nerve cells (Fig. 301), and described them as passing into and out of the cell by its processes, and as having the same finely varicose character as the fibrils which he had also found in the axis cylinder and its terminations. Of late years there has been shown a tendency to doubt the existence of such fibrils, as structures distinct from the general reticulum of the protoplasm. For it is obvious that the elongation of the meshes of a reticulum in definite directions might simulate fibrils, as indeed is probably the case in many elongated cells, such as columnar epithelium, and smooth muscle. This view of the matter was taken

1 Often erroneously written "neuron." Gr. $\nu \epsilon v \rho \omega ́ \nu$.

2 Deutsche med. Wchnschr., Leipzig, 1891.

3 A concise account of these researches, with vcry full bibliography, is given by W. Ford Robertson in Brain, London, 1899, vol. xxii. pp. 203-327.

${ }_{4}$ The structure of the nucleus is dealt with by Levi in various papers in the Riv. di patol. nerv., Firenze, for 1896,1897 , and 1898 .

${ }^{5}$ Like other cells, it contains a centrosome (v. Lenhossék, Arch. f. mikr. Anat., Bonn, 1895 , Bd. xlv. S. 345). The protoplasm (interfibrillar substanee) of the axis cylinder is somewhat different chemically-as shown by its reaction to certain stains and to osmic acid-from the protoplasm of the cell-body and dendrons (Mönckeberg and Bethe, ibid., 1899, Bd. xliv. S. 135).

"Stricker's "Histology," vol. i. Syd. Soc. Translation. 
originally by Frommann, ${ }^{1}$ and adopted by several recent writers, such as Rámon y Cajal, ${ }^{2}$ v. Lenhossék ${ }^{3}$ v. Gehuchten, ${ }^{4}$ Held ${ }^{5}$ and Marinesco. ${ }^{6}$

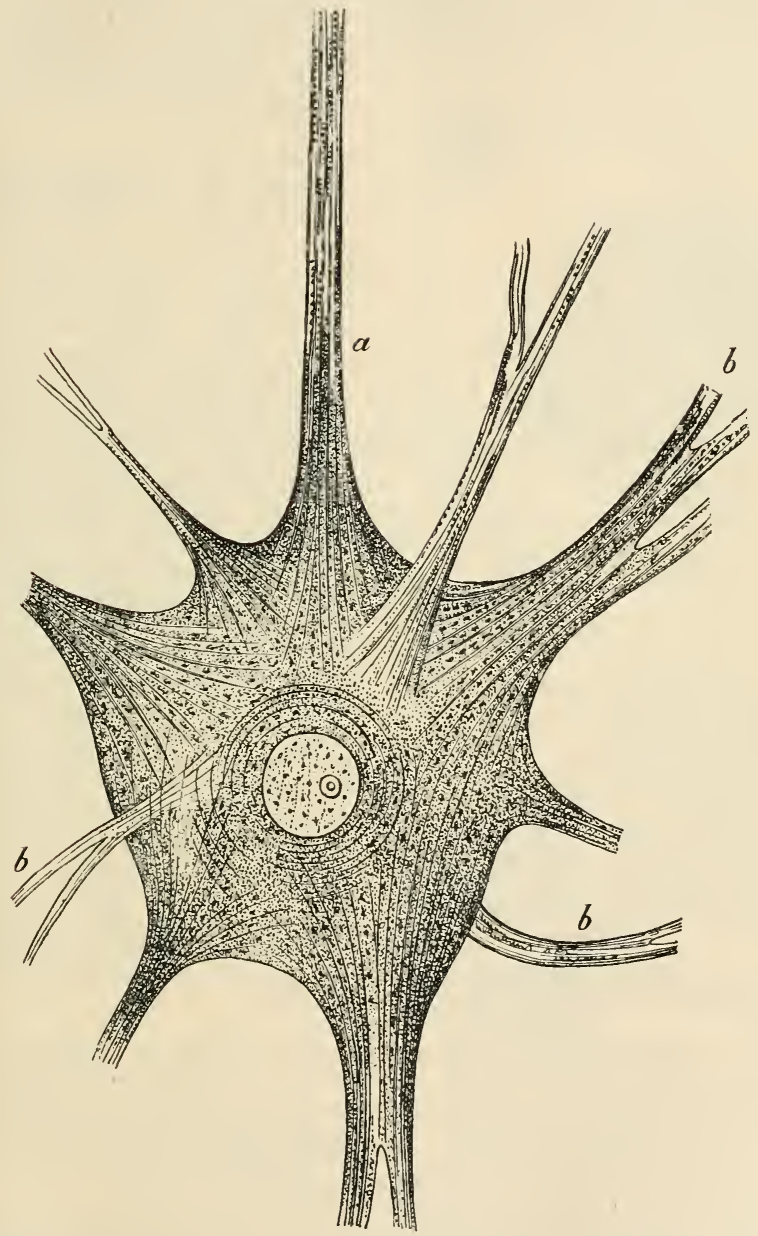

FIG. 301.-Nerve cell from the electric lobe of Torpedo. $\quad a$, axis eylinder ; $b, b$, dendrons. - M. Scliultze.

${ }^{1}$ Jenaische Ztschr. f. Naturw., Jena, 1875 ; Ztschr. f. Psychol. u. Physiol. d. Sinnesorg, Hamburg u. Leipzig, 1890, Bd. xlvi. S. 498. ${ }^{2}$ Rev. trim. de histol., 1896. 3 " Der feinere Pau des Nervensystems, etc.," Berlin, 1895; Arch. f. Psychiat., Berliu, 1897, Bd. xxix. + "Le système nerveux," 1897.

${ }^{5}$ Arch. f. Anat. u. Entweklngsgesch., Leipzig, 1897.

- Presse méd., Paris, 1897. 
Others, such as Flemming, ${ }^{1}$ Lugaro, ${ }^{2}$ Dogiel, ${ }^{3}$ Levi, ${ }^{4}$ Cox, ${ }^{5}$ Mann, ${ }^{6}$ and Bethe, ${ }^{7}$ have, on the other hand, insisted upon the existence of fibrils, distinct from the reticulum, running through the protoplasm from and into the several processes, and this view is now generally held, and has

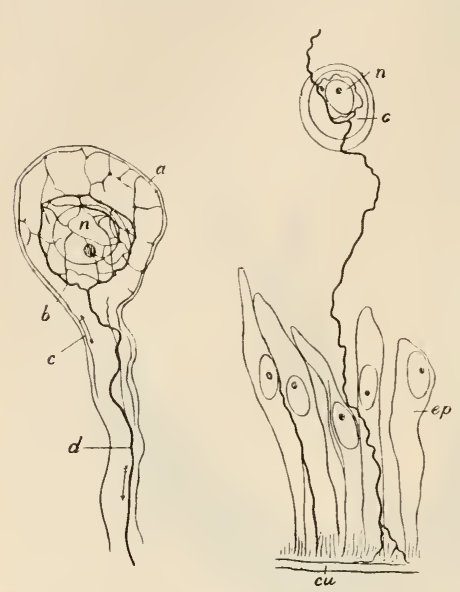

A.

been adopted even by those who until quite lately were of the opposite opinion.

While it is easy, by certain methods and in certain cells, to show a fibrillation both in the cell body and in its processes, with other cells it is more difficult on account of the complexity of course of the fibrils within them. The axis cylinder of a nerve is an especially favourable object for the observation of nerve fibrils, and they become naturally dissociated at the termination of nerves, where in some cases, as in the cornea, the axis cylinders break up into pencils of fibrils. It must be noted that these terminal fibrils are almost invariably branched, and sometimes even form a network; which appears to indicate that there may be lateral communications between them, and that they may not be always entirely distinct even throughout their course in the nerve fibre. ${ }^{8}$ Such lateral communications are sometimes apparent in the fibrillations of the dendrons, as well as in those of the cell body, and although less obvious in the course of the axis cylinder process, they may not be altogether absent in this. Nor are there any physiomotor nerve cell ; $a$, network of newrofibrils in cell body near surface; $b$, network near nucleus, $n$; $c$, afferent neurofibrils ; $d$, efferent neuro-fibril. $B$, Sensory nerve cell, bipolar; $a$, body of cell ; $n$, nucleus. A neuro-fibril is seen passing from its ramification between the epidermis eells, ep, close to the cuticle, $c u$, to form a network within the nerve cell, before passing on to the central nervous system.-Apathy.

logical facts which would lead to the belief that nervous impulses are conducted within the nervous units by isolated fibrillæ.

The question of the existence or non-existence of fibrillæ may be now regarded as having been settled by the discovery by Apáthy ${ }^{9}$ of definite fibrils, entirely different from any spongioplastic reticulum, in the nerve cells of annelids. The fibrils form a network with com-

${ }^{1}$ Beilr. z. Anat. u. Embryol. als Festgabe J. Henle, Bonn, 1882; Arch. f. mikr. Anat., Bonn, 1895, Bd. xlvi. S. 379 ; Arch. f. Psychiat., Berlin, 1897, Bd. xxix.

${ }_{2}^{2}$ Riv. di patol. nerv., Firenze, 1896.

3 Arch. f. mikr. Anat., Bonn, 1893, Bd. xli. S. 62 ; Anat. Anz., Jena, 1897 ; Monthly Internat. Journ. Anat. and Physiol., 1897.

${ }^{4}$ Riv. di patol. nerv., Firenze, 1897.

5 Monthly Internat. Journ. Anat. and Physiol., 1898, vol. xv. p. 209.

6 Journ. Anat. and Physiol., London, 1894, vol. xxix.; and Verhandl. d. anat. Gesellsch., Jena, 1898 , Bd. xii. S. 39.

7 Verhandl. d. anat. Gesellsch., Jena, 1898, Bd. xii. S. 39 ; Morphol. Arb., Jena, 1898, Bd. viii. S. 95 .

8 Cf. G. Retzius, Biol. Fören. Förhandl. Verhandl. d. biol. Ver. in Stockholm, 1898, S. 83.

${ }^{9}$ Mitth. a. d. zool. Station zu Neapel, Leipzig, 1897, Bd. xii. 
paratively open meshes in most parts of the cell, but sometimes more closely arranged immediately around the nucleus. The cell network is continuous with fibrils in the cell processes (Fig. 304).

Apáthy ${ }^{1}$ has shown that cells which are apparently unipolar (unineural), may show, when appropriately stained, a central fibril in the centre of the nerve fibre process, which is traceable from a network of fibrils encircling the nucleus, and two or more fibrils situated peripherally in the process and connected with another network, intercommunicating with the first, and situated at the periphery of the cell (Fig. 302 A). He suggests that the central fibril is efferent, the others afferent in function.

A fibril network of a somewhat similar character has also been demonstrated by Golgi 2 in various cells of the central nervous system and in the cells of the spinal ganglia of mammals, so that the existence of such a network is probably to be looked upon as of general occurrence. Apáthy's observations in annelids go, however, further than this, for he has been able to show that the fibrillæ which he describes are not only continuous throughout the whole of any one nerve cell and its

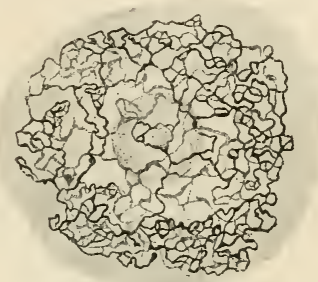

FIG. 303.-Spinal ganglion cell, showing intracellular network of fibrils.-C. Golgi. processes, but exhibit continuity from cell to cell, and form a network in the intercellular substance, or grey matter, of the nerve centres. If this should prove to be the case in vertebrates, as Nissl inclines to

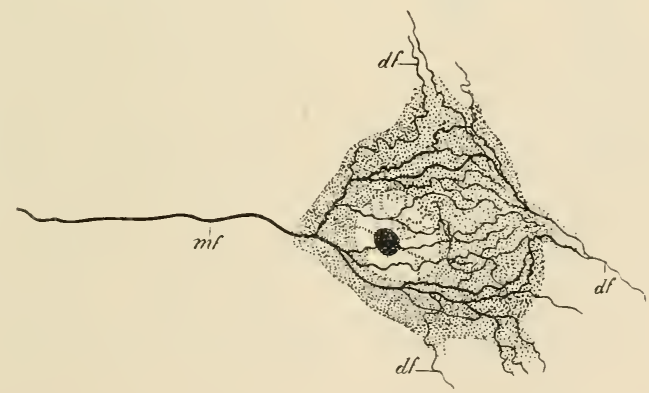

F1G. 304.-Nerve cell of annelid, showing neuro-fibruls witlin the cellbody. $d f$, fibriis of dendrons; $m f$, fibril of axon. The two sets of fibrils form a network within the cell.

believe, ${ }^{3}$ our conception of the nervous system must undergo important modification. At any rate, it appears clear that, at least in annelids, the neurone theory will not hold good, although the structure and

1 Loc. cit.

2 Boll. l. Soc. med.-chir. di Pavia, Milano, 1898, Fase. $1^{\circ}$, and ibid., 15 Luglio 1898. Cf. Donaggio, Riv. sper. di freniat., Reggio-Emilia, 1896, p. 265 ; Nartinotti, Ann. di freniat., Torino, 1897 ; E. Holmgren, Anat. Anz., Jena, 1899, Bd. xvi. S. 161.

${ }^{3}$ Nissl, Mü̈nchen. Med. Wchnschr., 1898, Bd. xlv. S. 988, Cf. also Bethe, Arch. f. mikr. Anat., Bown, Bd. 1, and li. 
arrangement of their nervous system was at one time regarded as affording one of the surest bases for its foundation; and we must find some other reason than want of continuity of the fibrillæ for the explanation of the fact that, in preparations made by the chromate methods of Golgi, the nerve cells always appear distinct from one another and never actually united. But if we hold with Leydig (see below, note 4 ) that the fibrille are not the true conducting material, but merely supporting structures, and that the conducting function is carried on by the perifibrillar substance, which, it is possible to suppose, does not share the continuity of the fibrillæ, we can, whilst accepting the conclusions of Apathy regarding the continuity of the nerve fibrils, still hold the neurone theory, so far as concerns the perifibrillar substance. According to Mönckeberg and Bethe, ${ }^{1}$ the perifibrillar substance is discontinuous at the nodes of Ranvier.

There is some reason to believe that nerve fibrils are tubular. The fact that they are prone after death and under the influence of reagents to become varicose, is an indication of such a structure. Mönckeberg and Bethe ${ }^{2}$ account for the varicosities by supposing that the interfibrillar substance may form droplets around the fibrils. But in sections of nerve fibres, the axis cylinders of which have not undergone any marked shrinking in the process of preparation, the fibrils, when examined or photographed with a very high magnifying power, have a distinctly tubular appearance. ${ }^{3}$

It is generally assumed, the existence of fibrils in the nerve element being presupposed, that these are the parts of the element along which the conduction of nerve impulses actually takes place. ${ }^{4}$ If this is so, we can conceive that the nervous impulses might pass along such minute tubes, in the form of a chemical change propagated from molecule to molecule, or as a physical change in the form of waves, started either by external mechanical means (tactile nerves, auditory nerves), or by contraction of the body of a nerve cell (motor nerves (?), olfactory nerves (?), visual nerves). The fact that nerve fibres are practically unfatiguable is in favour of a purely physical explanation of their peculiar manifestation of activity ; moreover, no chemical change and no development of heat ${ }^{5}$ can be shown to occur in them as a result of their excitation. This is not the case with the body of the nerve cell, for the grey matter of the nerve centres does tend to become acid and also warmer ${ }^{6}$ after excitation. The fatigue of nerve centres is in fact a matter of every-day experience, and it has also been shown by Mosso $^{7}$ that such fatigue, produced by mental strain, is accompanied by the development of fatigue products, which cause, through the circulation, fatigue effects on the voluntary muscles. It must, however, be conceded that both the unfatiguability and the absence of demonstrable chemical change in nerve fibre does not necessarily prove that no chemical changes are occurring; for such changes may be so slight as to be entirely beyond our means of observation, owing to the very small

${ }^{1}$ Loc. cit. According to Engelmann, this discontinuity at the nodes of Ranvier involves the nerve fibrils of the axis cylinder (Arch.f. d. ges. Physiol., Bonn, 1880, Bd. xxi.).

2 Loc, cit.

3 See, for example, Fig. 116 in my "Essentials of Histology," London, 1898. For the recent literature of the structure of nerve cells, see further' Flemming, "Morphologie der Zelle" in "Ergebnisse der Anatomie u. Entwickl.," Wiesbaden, 1898, Bd. vii. ; Goldscheider and Flatau, "Norm. u. path. Anat. d. Nervenz. u.s.w.," Berlin, 1898 ; Nissl, "Nerven u. graue Substanz," Mïnchen. med. Wehnschr., 1898, Nos. 31, 32, 33.

"Leydig loolds that it is more probably the interfibrillar substance which is actually, the conducting part of the nerve fibre. "Der reizleitende Theil des Nervengewebes," Arch. f. Anat. u. Entwcklngsgesch., Leipzig, 1897, S. 431.

${ }^{5}$ H. D. Rolleston, Journ. Physiol., Cambridge and London, 1890, vol. xi. p. 208.

${ }^{6}$ Mosso, Croonian Lecture, Plitl. Trans., London, 1892, vol. clxxxiii. B. p. 299.

7 Trav. du lab. dc physiol., Turin, 1890. 
amount of true conducting matter within the nerves, and there may besides be a rapid process of reparation going on at the same time. There is, indeed, insufficient basis upon which we can at present found a probable theory as to the mode of origination and conduction of nervous impulses, or as to the exact nature of these impulses.

Another point of peculiarity displayed by the protoplasm of many nerve cells, is the existence within it of peculiar angular granules having a special affinity for basic aniline dyes, such as methylene-blue and toluidin-blue. The demonstration of these granules and the evidence of their importance in the physiology of the nerve cell we owe to Nissl, ${ }^{1}$ and they are usually called after him the "Nissl bodies": they are also known as "chromatophil" or "tigroid" granules. They occur scattered in variable amount in all parts of the cell body and in its dendrons, but they are absent from the axis cylinder process and also from the part of the cell body to which this process is immediately attached. Their disposition within the cell body varies. In some cells they are chiefly disposed in the neighbourhood of the nucleus, whereas in others they are uniformly distributed or are more numerous at the periphery, but whether this implies differences in function or in the functional condition of the cells, is uncertain.

The most important physiological fact regarding these granules is that, under certain conditions of either normal or abnormal activity of the nerve cell, they undergo a change which has been termed by Marinesco chromatolysis, and which appears to consist in a gradual disintegra-

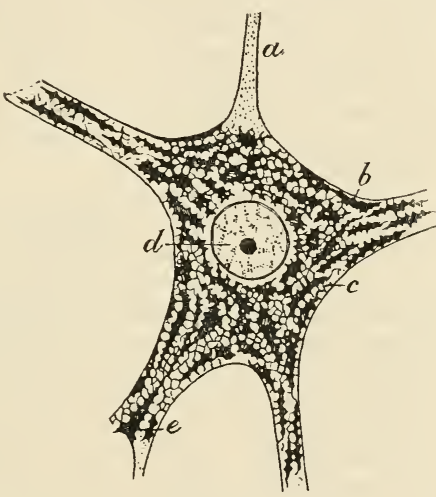

Fig. 305.-Diagram of a nerve cell to show the Nissl granules. $-a$, axis cylinder process ; $b$, Nissl bodies; $c$, achromatic substance between them ; $d$, nucleus ; $e$, dendron, with Nissl body at division.--Ramón y Cajal.

tion of the substance of the granules, and its diffusion in the juices of the protoplasm: this change is at first accompanied by a swelling of the cell, together with a displacement of the nucleus to one side; subsequently the cell may diminish in size, with disappearance complete or partial of the chromatophil substance.

The structural changes in the nerve cell which result from its excitation, whether this be direct excitation of a ganglion, or indirect by means of a nerve passing to it, have been studied by Hodge, ${ }^{2}$ Vas, ${ }^{3}$ Mann, ${ }^{4}$ and others. Hodge has also studied the changes which result from natural excitation of nerve cells in the motor ganglia of insects, and in the cerebellum, cerebrum, and spinal cord of birds, both before and after a period of prolonged activity; and observations of a similar

${ }^{1}$ Ally. Ztschr. f. Psychiat., etc., Berlin, 1892, Bd. xlviii. S. 197; see also Neurol. Ccntralbl., Leipzig, 1894, Bd. xiii. S. 676.

${ }_{2}^{2}$ Am. Jonrn. Psychol., 1887 to 1890 ; Joum. Morphol., Boston, 1892, vol. vii. 1. 95 ; 1894, vol. ix. p. 449 ; Journ. Physiol., Cambridge and London, 1894, vol. xvii. p. 129.

${ }^{3}$ Arch. f. mikr. Anut., Bonn, 1892, Bd. xl. S. 375 .

${ }^{4}$ Journ. Anat. and Physiol., London, 1894, vol. xxix. 1' 100. 
character have been made by Mann upon the motor nerve cells of the brain and spinal cord, and the nerve cells concerned in visual perceptions (in the retina and the occipital lobe). Hodge did not examine the condition of the Nissl bodies, but notes that active nerve cells, as compared with those at rest, show a diminution of volume and a lessened power of reducing osmic acid, the nucleus also becoming shrunken and showing a tendency to stain more deeply. $\operatorname{Vas}^{1}$ and Lambert ${ }^{2}$ described the stimulated nerve cell as becoming clear in the centre and chromophil towards the periphery, and Pugnat ${ }^{3}$ found that, with more prolonged stimulation, the Nissl bodies disappear entirely. Mann described the cell body and nucleus as becoming at first somewhat enlarged as the result of activity, but with fatigue there succeeds diminution in bulk of both nucleus and protoplasm; he also noted a diminution in the chromatic substance of the cell protoplasm. Nissl ${ }^{4}$ himself, in repeating these experiments, was unable to obtain such marked results. ${ }^{5}$ Nevertheless, it appears clear that the effect of activity is to induce certain chemical changes within the body of the nerve cell, its functional activity being attended with a diminution in the anount of chromatic substance in the cell protoplasm.

Much more definite effects are obtained as the result of section of the nerve fibre process. The first study of these effects we also owe to Nissl; ${ }^{\boldsymbol{b}}$ his observations have been confirmed and extended by many other's, and especially by Lugaro, ${ }^{7}$ Marinesco, ${ }^{8}$ and v. Gehuchten. ${ }^{9}$ Their results may be briefly summarised as follows:- When a motor nerve is cut, the cells from which its axis cylinders are derived begin, within from twenty-four to forty-eight hours after the lesion, to show indications of chromatolysis. The granules of Nissl become less defined, and the neighbouring portions of the protoplasm diffusely coloured. Concomitantly with, or soon after this, the cell body becomes swollen and the nucleus is often displaced to one side. This process goes on for about fifteen days, at the end of which time most of the cells of origin of the nerve are converted into rounded bodies, staining nearly uniformly with methylene-blue and showing no Nissl granules or only small ones, and these confined to the periphery of the cell. The change begins apparently at the place where the axis cylinder leaves the cell, and invades first the perinuclear portion of the protoplasm, and then its successive layers, the peripheral being last affected. It also extends gradually into the dendrons. After fifteen to twenty-four days the process of chromatolysis is at an end, and a process of reparation begins; this proceeds very slowly, so that it may take three months to be completed. At the end of that time the cells have for the most part resumed their original appearance, even although the cut nerve may not have undergone regeneration.

Loc. eit.

${ }^{2}$ Compt. rend. Soc. de biol., Paris, 1893.

${ }^{3}$ Compt. rend. Acad. d. sc., Paris, 1897, tome exxv. p. 736.

${ }^{4}$ Ally. Ztschr. f. Psychiat., etc., Berlin, 1897, Bd. liv.

${ }^{5}$ Nor did Eve, working with the rabbit's sympathetic. Journ. Physiol., Cambridge and London, 1896, vol. xx. p. 334.

${ }^{6}$ Ally. Ztschr.f. Psychiat., etc., Berlin, 1892, Bd. xlviii. S. 197 ; Centralbl. f. Nervenh. и. Psychiat., Coblenz u. Leipzig, 1894, S. 337.

7 Riv. di patol. nerv., Firenze, 1896 ; Sperimentale, Firenze, 1895.

8 Presse méd., Paris, 27 Janvier 1897 ; and Rev. gén. d. sc. mures et appliq., Paris, 30 Mai 1897 ; also in Arch. f. Physiol., Leipzig, 1899, S. 89.

${ }^{9}$ Cellule, Lierre et Louvain, 1897 , tome xiii. 
The corresponding changes in sensory nerve cells after section of the peripheral nerve, have been studied by Lugaro, ${ }^{1} \mathrm{R}$. Fleming, ${ }^{2} \mathrm{Cox},{ }^{3}$ and others; partial atrophy and chromatolysis in the spiual ganglion cells having been noted by them. ${ }^{4}$ Lugaro failed to find any definite changes in the posterior ganglion cells on cutting the root between the ganglion and the spinal cord, or at most a very slight amount of chromatolysis. This is in accordance with the observation that in tabes, with complete atrophy of the fibres of the posterior columns of the cord, the spinal ganglion cells may be intact. van Gehuchten, ${ }^{5}$ with Nélis, has pursued similar investigations upon the effects on the cells of the ganglion of the vagus, of cutting that nerve peripherally to the ganglion. These observers find that changes, quite comparable to those above described for motor nerves, proceed in the cells of this ganglion, but that the process of disintegration is not replaced by a process of reparation, so that at the end of three months the cells have almost all disappeared. The complete disintegration of some motor cells, as a result of division of motor nerves, has also been affirmed by Nissl. I have found a similar change to take place with the cells of Clarke's column of the spinal cord, after section nearer the bulb of the afferent (cerebellar) tracts. The cells on the side of the section undergo chromatolysis, atrophy, and ultimately almost disappear. ${ }^{6}$

In all cases the nerve fibres cut off from the cell body undergo disintegration and disappear in a few days. In the case of the medullated nerves, their section also involves a breaking up and gradual removal of the substance of the medullary sheath, accompanied by multiplication of the nuclei which are embedded in this sheath. This is the wellknown Wallerian degeneration of nerve fibres, ${ }^{7}$ and the first signs of it appear about twenty-four hours after section of the nerve, in the commencing fragmentation of the medullary sheath. This in two or three days is broken up into segments of variable size, and these, as the degeneration progresses, become gradually resolved into drops and droplets of myelin. Simultaneously with or immediately before these alterations in the myelin sheath, a change in the chenical constitution of the fibrils of the axis cylinder appears, as shown by an alteration in their capacity for staining, ${ }^{8}$ and it is about this time that the peripheral part of the nerve ceases to be excitable by electrical stimulation, although for the first few hours its excitability was increased. The fibrils next become wavy and varicose, and soon break up into isolated portions (granules), which break up still further, so that they are soon indistinguishable from the perifibrillar substance. Similar changes occur for a certain distance along the central part of the cut nerve. The degenerated axis cylinder may show complete

${ }^{1}$ Op. cit. ; Riv. di patol. nerv., Firenze, 1896, vol. i.

${ }^{2}$ Edin. Med. Journ., 1897, N.S., vol. i. p. 174; and Brain, I.ondon, 1897, vol. xx. 1. 56.

${ }^{3}$ Jonthly Internat. Journ. Anat. and Plyysiol., 1898, vol. xv. p. 211.

${ }^{4} \mathrm{Cox}$ (loc. cit.) finds that the changes occur in some cells carly, in others considerably later, and points out the possibility of there being two kinds of cells in the ganglia, as Dogiel (Amut. Anz., Jena, Bil. xii.) had also supposed on other grounds.

${ }_{5} O_{p}$. cit. xxiv.

6" "Proe. Physiol. Soe.," March 18, 1899, Journ. Physiol., Cambridge and London, vol.

7 Augustus Wallcr, "Experiments on the Section of the Glosso-Pharyngcal and Hypoglossal Nerves, and Observations on the Alterations produced in the Structure of their Primitive Fibres," Phit. Trans., London, 1850, pt. 2, p. 423 ; "Nouvelle métliode pour l'étude du système nerveux, etc.," Compt. rend. Acad. d. sc., Paris, 1851, tome xxxiii. p. 606.

${ }^{8}$ Moinckeberg and Bethe, loc. cit. 
breaches of continuity, ${ }^{1}$ or may remain continuous. According to Mönckeberg and Bethe, the changes occur first near the lesion, and gradually extend towards the periphery, the rate of progress varying in different fibres, e.g., proceeding more rapidly in sensory than in motor fibres. $^{2}$ Eventually, almost all the myelin, as well as the broken-down axis cylinder, disappears, and the nerve is only represented by its connective tissue sheaths and the tubes of neurolemma, which are occupied by a granular multinucleated protoplasm. Within these tubes fine fibres may become developed, ${ }^{3}$ but whether these are of a nervous nature cannot be stated; they cannot function as nerves, for they are not in continuity with any other nerves or nerve cells. Regeneration only occurs by an outgrowth from the central end of the cut nerve fibres. Each of these appears ultimately to throw out several root-like sprouts, which, unless they become entangled in cicatricial tissue, tend to grow down along the sheaths of the degenerated peripheral nerve fibres, and thus eventually to find their way to the periphery. Probably only one of the sprouts ultimately carries on the functions of the fibre, and the others disappear. The new fibres become surrounded early by a thin medullary sheath, which gradually becomes thicker, and a neurolemma also ultimately becomes developed around them. Under the most favourable circumstances, the new nerve fibres take from one to two months to become sufficiently regenerated to perform their function, and cases in which a rapid recovery of function has been reported to succeed nerve section, must be explicable either by a duplicate source of innervation (this may especially be the case with sensory nerves) or by the section having been incomplete. If the observation of v. Gehuchten and Nélis upon the disappearance of the sensory nerve cells of the vagal ganglion, after section of their peripheral fibres, is correct, and is a phenomenon of general occurrence, it is difficult to see how the sensory fibres in a cut nerve ever become regenerated. Restoration of function in such cases may perhaps often be explained by an ingrowth of sensory nerve fibres from adjacent areas of distribution. Other observers have, however, not hitherto confirmed these statements so far as the spinal root ganglia are concerned; indeed, the cells of these ganglia are stated to show rather less change after section of their nerves than do the anterior horn cells of the spinal cord.

No clear evidence has been obtained in mammals of regeneration of nerve fibres within the nerve centres, after artificially produced lesions. ${ }^{*}$ A priori, one would expect such regeneration to occur, and certain observations seem to indicate a commencement of regeneration, ${ }^{5}$ but it is never completed. Probably the cicatricial tissue offers an insuperable obstacle to the growth of the sprouting fibres. Some regeneration of severed posterior root-fibres may occur, ${ }^{6}$ but it does not appear to extend into the posterior column of the spinal cord. Sherrington ${ }^{7}$ found in the central stump fine medullated fibres, resembling those

${ }^{1}$ Ranvier, "Traité technique"; Beer, Jahrb. f. Psychiat., 1s93, Bd. xi.

2 Mönckeberg and Bethe, loc. cit.

3 R. Kennedy, Phil. Trans., London, 1897, vol. clxxxviii. B. p. 257.

${ }_{4}$ Sherrington, Journ. Physiol., Cambridge and London, 1893, vol. xiv. p. 271.

${ }^{5}$ Eichhorst and Naunyn, Arch. f. expcr. Path. u. Pharmakol., Leipzig, 1874, Bd. ii. S. 377 .

"Kahler, Prag. med. Wchnschr., 1884, S. 301 ; Chipault, "Études de chir. médullaire," Paris, 1893.

7 Journ. Physiol., Cambridge and London, 1894, vol. xvii. p. 218. 
of regenerating nerve, even after the posterior root ganglion liad been removed.

It is a remarkable but unexplained fact, that the regenerating fibres which are growing along the old nerve sheaths find their way eventually to the same structures (muscles, skin, blood vessels) that they were distributed to before section and degeneration had occurred. In this connection, Langley's observations upon the regeneration of the cut cervical sympathetic of the cat ${ }^{1}$ may be noticed. These show that the nerve fibres in that nerve, which are of very different functional distribution, some carrying impulses to the dilator pupillæ, others to blood vessels, others to hair muscles, others to secretory cells, nevertheless, after section and regeneration, are found to effect connection with the same cells in the superior cervical ganglion as before; e.g., the fibres in the first thoracic anterior root still give, on stimulation after regeneration, dilatation of the pupil and movement of the membrana nictitans; those in the third thoracic root still mainly produce contraction of ear vessels, and so on. Even more striking are the experiments of the same observer upon the results of joining the central cut end of the vagus to the peripheral cut end of the cervical sympathetic. These show that the vagus fibres, when regenerating under these circumstances, make connections with the cells of the superior cervical ganglion of the sympathetic, so that all the effects of stimulating the cervical sympathetic can now be produced through the vagus.

It is not difficult to understand why the part of the nerve fibre which is cut off from the nucleated body of the parent cell, should undergo degeneration, for this is a phenomenon which is exhibited by all cells; the nucleus being essential for the maintenance of nutrition of all parts of the cell. But it is much more difficult to comprehend the occurrence, under these circumstances, of the changes which have been described under the name of chromatolysis, and the degeneration which follows those changes in the case of certain cells. Actual degeneration does not extend along the axis cylinder backwards for any great distance ; the greater part of the central end of the nerve fibre remains to all appearance unaltered. Nor can the chromatolysis be alone due to arrest of function of the nerve cells, since, although this might be alleged for the sensory cells, which no longer receive the usual nervous impulses from the periphery, the same cannot be admitted for the motor cells, which must receive impulses by reflex and voluntary paths as before. Perhaps the explanation is to be found in the fact that, in the first instance, the section of the nerve and the chemical and electrical changes thereby set up, and, secondly, the inflammatory and cicatricial changes in the wound, maintain a constant excitation of the cut ends of the nerve fibres, and thus keep the cells in continual activity. The changes produced (chromatolysis and swelling of the cells) are, in fact, changes which accompany activity, and they can be also brought about by the production of local inflammation, and by the application of a chemical stimulus (salt) to nerve-endings, as well as by the section of the nerve. This would not suffice to explain the difference which has been described by v. Gehuchten between the ultimate behaviour of motor and sensory nerve cells, the former of which undergo, after about fifteen days, a gradual process of reparation; whereas the latter, at least those of the vagus ganglion, fail to become repaired, but, on the contrary, are eventually completely degenerated. This may, however, well be due to

${ }^{1}$ Journ. Physiol., Cambridge and London, 1892, vol. xiii.; and 1895, vol. xviii. p. 280. See also this volume, article "Sympathetic," p. 691. 
the prolonged cessation of function of the sensory cells, since, after the irritation produced by the severance of the nerve and cicatrisation of the wound had passed off, they would be receiving no nervous impulses, and would then tend to obey the general law, that atrophy results from loss of function. The motor cells, on the other hand, would still be receiving reflex and possibly also voluntary impulses, and their activity would thus be maintained until the cut nerve fibres were regenerated. In the event of these failing to become regenerated-as in the case of an amputated limb-the motor nerve cells also become atrophied. ${ }^{1}$

Various other conditions besides those above noticed may determine chromatolysis in nerve cells, e.g., certain drugs, such as acetate of lead, ${ }^{2}$ arsenic, ${ }^{3}$ bromine, antipyrin, cocain, ${ }^{4}$ strychnin $;^{3}$ intoxicants such as alcohol in large doses, ${ }^{3,5}$ and malonic nitrite ${ }^{6}$ (which can be antagonised by hyposulphite of soda); poisoning by disease-toxins, e.g., the toxins of rabies and tetanus ${ }^{3}$ (which can be antagonised by the corresponding antitoxins) $;^{7}$ acute local anæmia (as by compressing the aorta), ${ }^{8}$ and a rise of body temperature experimentally induced. ${ }^{9}$

Whether the nucleated cell body plays any part in the starting of nerve impulses, or in their modification, or has any other function than that of regulating the nutrition of the nerve cell and its processes, is a matter not easy to decide. Bethe (cited on p. 597, note 3 ) found (in the crab) that the cell bodies are not essential to the performance of reflex actions. In the case of the fibres of the posterior roots of mammals and most vertebrates, there is no necessity for the nervous impulses to go through the nerve cell at all, nor is their passage appreciably delayed in traversing the ganglion. ${ }^{10}$ It would, however, be of interest to

1 Warrington (Journ. Physiol., Cambridge and London, 1898, vol. xxiii. p. 112) has found chromatolysis to occur a certain time after section of posterior roots in the motor cells of the anterior horn of the same side (chicfly of the postero-lateral group), and in a few cells on the other side of the cord. He ascribes this result to the loss of afferent impulses which normally impinge upon those cells. After section of an anterior root, nearly all the cells of the corresponding segment of the cord show some sort of change of the same kind.

${ }^{2}$ Schaffer, Abstract in Ncurol. Ccntralbl., Leipzig, 1894, No. 24. Numerons references on this subject will be found in Warrington's paper, above quoted.

${ }^{3}$ Nissl, Allg. Ztschr. f. Psychiat., ctc., Berlin, Bd. xlviii., l., and liv.; Centralbl. f. Nervcnh. u. Psychiat., Coblenz u. Leipzig, 1896, S. 544. Cf. also Lugaro, Riv. di patol. ncrv., Firenze, 1897.

4 Pandi, Abstract in Neurol. Centralbl., Leipzig, 1894, No. 24.

${ }^{5}$ Dehio, Centralbl. f. Nervenh. $u$. Psychiat., Coblenz u. Leipzig, 1895, Bd. vi. S. 113 ; C. C. Stewart, Journ. Expcr. Mcd., N. Y., 1896, vol. i. No. 4 ; Marineseo, Presse méd., Paris, 1897, No. 8; and Rev. gén. d. sc. purcs et appliq., Paris, Mai 30, 1897.

${ }^{6}$ Goldscheider and Flatau, "Pathologie der Nervenzelle," Fortschr. d. Mcd., Berlin, 1897.

${ }^{7}$ Goldscheider and Flatau, loc. cit.; Chantemesse and Marinesco, Prcssc méd., Paris, 1898. For many other references on the effects of drugs, etc., on the nerve cell, see Barker, "The Nervous System," 1899, chap. xxv.

${ }^{8}$ Sarbó, Neurol. Centralbl., Leipzig, 1895, No. 15, S. 664 ; Marinesco, loc. cit. ; Gilbert Ballet and Dutil, Arch, de neurol., Paris, 1897, tome iv. p. 430.

${ }^{9}$ Goldscheider and Flatau, loc. cit.; see also Turner, Brain, London, 1899, vol. xxii. p. 105.

10 This statement, due originally to Exner (Arch. f. Physiol. Leipzig, 1897, S. 567) has been confirmed as the result of a series of experiments upon the posterior roots of the frog, which were performed at my request by B. Moore and H. W. Reynolds " Internat. Cong. Physiol.," Aug. 1898, Journ. Physiol., Cambridge and I tondon, 1898, vol. xxiii. Suppl., 1. 56). They were carcful to insure that the nervous impulses took the same path through the cord to produce the excitation of the muscle, which was to serve as the signal, botl when the root was stimulated between the cord and the ganglion, and when the nerve was stimulated beyond the ganglion, by cutting all the root bundles of the nerve employed except one. A contrary result, namcly, a delay of some 0.02 second in traversing the ganglion, which was got by Wundt, was probably due to the fact that, on account of the method which he employed, the nervous impulses in the two cases did not take exactly 
ascertain whether there is any delay in cases where the nerve impulse is compelled to pass through the body of the nerve cell, as it must do, for example, in bipolar cells; this is a point which has yet to be determined.

The only chemical change which has been noticed in the bodies of nerve cells, as the result of activity, is a change in the direction of acidity, due apparently to the formation of lactic acid, as in muscle. That the fatigue products of the nerve centres and of muscles are similar, is shown, first, by the fact that the muscles may give evident signs of fatigue, merely as the result of prolonged nervous (mental) activity $;^{1}$ and, secondly, by the evident fact that muscular fatigue is detrimental to nervous (intellectual) activity in general, and not only to the particular parts of the central nervous system which come into action during the muscular effort. In nerve fibres the only change which is apparent as the result of excitation is the change of electric potential which accompanies the passage of the nervous impulse. It might be expected that when the nerve centres are set in activity, the cells in which the impulses are started would also exhibit changes of potential. But, probable as this is, the demonstration of the fact is beset with serious difficulties; and, in spite of attempts which have from time to time been made to overcome these difficulties, our knowledge on this subject has remained in a very fragmentary and indecisive condition. ${ }^{2}$

The question whether the "sense of effort," which is closely associated with the "sense of fatigue," is due to changes originating in the nerve cells, or to sensory impressions passing to the nerve centres from the contracting muscles, has been much debated. ${ }^{3}$ The probability is, that its origin is from both sources, and that the effects are accentuated, with the supervention of fatigue, by the accumulation of waste products of both nervous and muscular metabolism in the circulatory fluid.

Conduction within and between nerve cells.-It was conjectured by Golgi that the dendrons of a nerve cell were not for the conduction of nerve impulses, but might merely serve the purpose of rootlets, and thus aid to obtain pabulum for the nutrition of the cell. This conjecture has, however, been abandoned, since it has been discovered that in many cases nervous impulses, in passing along a nerve cell, must of necessity traverse one or more dendrons as well as the axon. Granting, however, that both dendrons and axons serve as nerve conductors, the question suggests itself, Is there any specific difference in the manner or direction by which the two kinds of processes conduct impulses?

van Gehuchten ${ }^{4}$ and Ramón y Cajal $^{5}$ have attempted to lay down, as a general law of conduction in the nerve cell, the proposition that all nervous impulses within the cell are axipetal, that is, that they all pass

the same path through the cord to produce the reflex movement which was used as a measure of the relative time occupied.

1 Mosso, "Die Ermiidung"; Arch. f. Physiol., Leipzig, 1890, S. 129.

2 See on this subject R. Caton, Rep. Brit. Ass. Adv. Sc., London, 1875 ; Beck, Centralbl. f. Physiol., Leipzig u. Wien, 1890, Bd. iv.; Fleischl von Marxow, ibid.; Danilewsky, ibid., 1891, Bd. v.; Beck and Cybulski, ibid., 1892, Bd. vi.

${ }^{3}$ For discussion of this and cognate points in neural physiology, see Waller, "On the Sense of Effort," Brain, London, 1891, vol. xiv. p. $179 ;$ and "On the Functional Attributes of the Cerebral Cortex," ibid., 1892, vol. xv. p. 329. Cf. also Bastian, "On the Neural Process underlying Attention and Volition," ibid., 1892, p. 1.

4 "Système nerveux," 1897.

${ }^{5}$ Rev. trim. de histol., 1897. 
towards the attachment of the axis cylinder and leave the cell by that process. In other words, that the direction of conduction in the dendrons is centripetal, in the axon centrifugal; this has been termed the "law of axipetal conduction." This is included in the more general statement by W. James, ${ }^{1}$ that nerve impulses take always a particular direction in the nervous system, and never naturally the reverse direction ("law of forward direction"): that there is, in short, a kind of valved action which permits passage of impulses in one direction only.

The law of axipetal conduction is no doubt true for many cells, but it will not hold good for the typical sensory cell. This, in its simplest and original form, is a bipolar cell with two

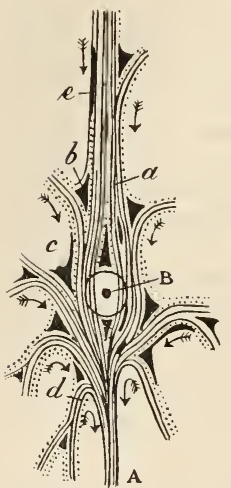

Fig. 306.-Diagram to il. lustrate the "law of axipetal conduction" in motor nerve cells. A, axis cylinder process. $\mathrm{B}$, nucleus ; $a$, fibrils of cell body ; $b, c, e$, Nissl bodies; $d$, fibrils of dendron. The arrows indicate the direction of nerve impulses.-Cajal.

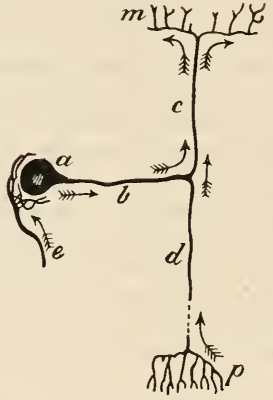

FIG. 307.-Diagram to illustrate supposed lines of conduction in a spinal ganglion cell. $-a$, body of cell; $b$, its axis cylinder process; $c$, fibre of posterior root; $d$, peripheral fibre; $e$, termination of axis cylinder of another cell (? sympathetic) ; $m$, ramifications of nerve fibre in spinal cord ; $p$, ramifications at sensory surface-Cajal. axons and no dendrons, and the nervous impulses produced at the periphery pass towards the cell by one axon, and away from it by the other. The contention that the peripheral process, although possessing all the characters of an axon, must be considered a dendron, because it conducts centrifugally, merely begs the question.

Although nothing whatever has been ascertained regarding the nature of the change which is set up in the nerve element, and which travels along it as what is called the nervous impulse, it is clear, from an examination of the electrical change which accompanies its passage along a nerve, that it passes in the form of a wave, rising at any point of its course to a certain intensity, and gradually falling from this crest to a point at or even slightly below the normal. It would further appear, from observations conducted by the same method upon the nerve centres, that if the body of the cell is stimulated by a prolonged or intense stimulus, the nervous impulses which are discharged tend, even if the stimulus be uninterrupted, to become discontinuous; in other words, to exhibit a rhythm of a more or less regular character. Such rhythm can be also investigated by the effect it produces upon the muscles which are connected with the nerve elements in activity. Tracings of muscular contraction, or of the changes in electrical potential which accompany the contraction, exhibit a curve with waves corre1 "Principles of Psychology," 1890, vol. ii. 
sponding to the rhythm at which the nervous impulses have passed along. By both these methods it appears that such nerve cells as those of the motor region of the cerebral cortex, and the motor cells of the anterior horn of the spinal cord, tend, whether stimulated either directly or indirectly (e.g., reflexly), to respond by discharging nervous impulses at the rate of about ten or twelve per second, unless the stimulation be at a less rate than this, in which case the response is at the same rate as the stimulation. Under some circumstances, however, the rate of rhythmic discharge may be more rapid than ten or twelve per second, or it may be as slow as five per second, the muscular response in the latter case being of a clonic character. What it is which determines this difference in different cases and under different conditions, is not at present known; in discussing the manner in which the nervous impulses pass from one cell to another, we shall have occasion again to refer to it.

We have so far confined our attention almost entirely to the changes which occur within the limits of a single nerve cell; we have next to inquire how these changes may be propagated from one nerve cell to another.

The introduction of the method of Golgi, and especially its employment by Cajal, dealt a severe blow to the then prevalent hypothesis, that the propagation of nervous impulses from cell to cell took place through a continuity of their dendrons, which were thought to be united in a network which everywhere pervaded the grey matter (J. Gerlach). At the same time, it exhibited an explanation by which this hypothesis could be replaced, since it showed that although there may be no continuity of processes of nerve cells, there undoubtedly is close contiguity or even actual contact. This is sometimes effected by the embracing of the body of one cell by the ramifications of the axon of another, ${ }^{1}$ sometimes by the interlocking of the dendrons of one cell with the dendrons or with the ramified axon of another, or even of more than one other cell. It is convenient to have a short term by which to speak of the union of one nerve cell with another by this close contiguity of processes with cell body or of processes with processes, presumably without actual continuity of substance. Foster ${ }^{2}$ has employed the term "synapse" 3 to denote such conjunction, an expression which may provisionally be adopted.

It has been conjectured that the extent of contact of the adjacent nerve cells at the synapses may vary from time to time, such variations being brought about by a contraction or expansion of the ramified processes by which the contact is effected, analogous to the amœboid movements of protoplasm in general; and, further, that the effects of drugs in diminishing or increasing the resistance of the nerve centres to the passage of nervous impulses might be produced in

${ }^{1}$ W. A. Turner and W. Hunter (Brain, London, 1899, vol. xxii. p. 123) have described a network covering the cell body of nerve cells in the grey matter of the central nervous system, which they regard as the terminal apparatus of the axis cylinder processes of other nerve cells. A basket-like ending, extending over both cell body and dendrons, was described by Semi Meyer (Ber. d. k. sächs. Gescllsch. d. Wissensch., math.-phys. Cl., Leipzig, 1897, S. 475), who came to the sanie conclusion; Martinotti, Ann. di freniat., Torino, 1897, p. 253 ; and Golgi, Boll. d. Soc. med.-chir. di Pavia, Milano, April 19, 1898. Cf. also Held, who describes a concrescence of the axon of one nerve cell with the body or dendron of another cell, Arch. $f$. Anat. $u$. Entwcklugsgesch., Leipzig, 1891, 1893, and 1897.

2 "Text-book of Physiology," 1897, vol. iii. p. 929.

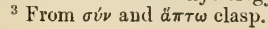


a similar way. ${ }^{1}$ This conjecture opens up a wide field of speculation, since it is possible to extend it so as to embrace the explanation of many physiological and psychical conditions. ${ }^{2}$ Such speculations, however, unless they have been tested experimentally, do not lie within the scope of this work. No one has been able to see any amceboid movement in the nerve cells of vertebrates, and the only direct evidence of such movement in nerve cells, that we have any knowledge of, has been furnished by Wiedersheim, ${ }^{3}$ who observed slow changes of form to occur in ganglion cells of the central nervous system of a minute crustacean. It is true that various observers have described in Golgi preparations, appearances in the processes of nerve cells after excitation, or after the action of anæsthetics and other drugs, which they interpret as indicating a withdrawal or retraction of the finer processes. But it cannot yet be accepted as proved that the changes which are described are invariable concomitants of alterations in the functional condition of the cells, still less that they are the cause of the functional conditions. ${ }^{4}$

Every nervous path is formed of a chain of nerve cells. The chain of the simplest reflex process may be conceived as composed of only two cells, the sensory and the motor, or afferent and efferent, rootcells, and in such a chain there would be only one place of adjunction, where the central process of the sensory cell comes in contact with the dendrons or the cell body of the motor cell. On the other hand, a path which includes any of the higher nerve centres or any complex nerve processes, must have a chain of several cells, with a synapse at the place of contact between each two links in the chain. There is reason to believe that the additional delay ("lost time"), which is characteristic of the passage of nervous impulses through the nerve centres, is due to a block at each synapse; that, in fact, the nervous impulses are momentarily arrested at these places of contact of the nerve-cells with one another. And it is not improbable that the relative number of these blocks will furnish a key to the differences which are found to obtain in the reaction time for different reflexes and psychical processes. ${ }^{5}$ The differences of reaction time are too great to be accounted for simply by the fact that the nervous impulses are sent along paths of different

${ }^{1}$ It appears certain that nicotin, in blocking the passage of nervous impulses along a nerve path, acts upon the synapses (Langley).

2 The production of both natural and hypnotic sleep has been supposed to be due to retraction of processes of the nerve cell, leading to a partial separation of the synapses between some cells (Lépine, Compt. r'cnd. Soc. dc biol., Paris, 1895; Duval, ibid., 1895 ; and "L'amoboidisme du système nerveux," Rev. scient., Paris, 1898); Rabl-Rnckhard, Neurol. Centralbl. Leipzig, 1890, Bd. ix. S. 199). Lingaro (Riv. di patol. nerv., Firenze, 1898), on the other hand, considers that the condition of sleep is concomitant with a general diffusion or protraction of nerve cell processes, and the state of attention with retraction of some of the processes and gemmules, so that the paths of conduction for nerve impulses are thereby narrowed, and the impnlse waves become intensified.

3 Anat. Anz., Jena, 1890, Bd. v. S. 673.

4 See on this subject v. Kölliker, Sitzungsb. d. phys.-med. Gesellsch. zu Würzburg, 1895 ; Ramón y Cajal, Arch. f. Anat. $u$. Entwcklngsgesch., Leipzig, 1895 ; Rénant, Presse méd., Paris, 1895, p. 297 ; Monti, Arch. ital. de biol., Turin, tome xxiv. ; Demoor, "De la plasticité morpholog. des nemrones," Arch. de biol., Gand, 1896, tome xiv. ; Stefanowska, Compt. rend. Soc. de biol., Paris, 13th Nov. 1897; Manouélian, ibid., and Année Psychologique, 1898 ; Heger, Bull. Acad. roy. de méd. de Belg., Bruxelles, 1898, tome ix. p. 831 ; Pupin, "Le neurone," Thèse, Paris, 1898 ; Deyber, Thèse, Paris, 1898 ; M. Duval, Compt. rend. Soc. de biol., Paris, 1895 ; Rev. d. sc. méd., Paris, 1898, 12 Mars ; Soukhanoff; Cellule, Lierre et Louvain, 1898, tome xiv. ; Havet, ibid., 1899, tome xvi.

5 The relative strength of the blocks in different individuals may also be the physical cause of the individual differences in reaction time for the same stimulus. As is well known, these differences gave rise amongst astronomers to the term "personal equation" being applied to the difference in noting the time of an observation which is found to obtain, with a considerable degree of constancy, between any two observers. 
length in the various cases; it is more than probable that they pass through an increasing number of nerve units (i.e. nerve cells), according to the increased complexity of the psychical process involved. And this, according to the theory of isolated units (neurone theory), would mean the passage across as many synapses, which are probably the parts of the nerve chain where relative blocks occur.

If we assume (and the assumption is not a very improbable one) that in the same individual, under the same conditions, the lost time of block at each synapse is about the same, we can arrive at a general idea of the relative number of synapses which are passed; in other words, the number of nerve cells in a chain called into action in various psychical processes.

A careful study of the differences in reaction time, and of other examples of "lost time," in the passage through nerve centres, may help us to form a conception of the amount of delay which each synapse is responsible for. We may therefore advantageously consider some of the facts which have been accumulated upon this point.

The reduced reflex time which occurs in the lower part of the frog's spinal cord, when a reflex contraction of the homonymous gastrocnemius is produced by stimulation of a posterior root, has been determined by Wundt, ${ }^{1}$ who found the minimal amount to be only 0.008 second. ${ }^{2}$ For a crossed reflex the delay over and above this was only about 0.004 second, and the same for a reflex movement of the other limb on the side stimulated. If we assume that for the crossed reflex only one additional nerve cell is interpolated, we obtain 0.004 second as the lost time of the synapse; whereas, if two additional cells are assumed to be interpolated, the lost time at each synapse would be only 0.002 second. Since the reflex has a lost time of 0.008 second, at least two, and perhaps four, synapses are involved in the path of the most simple reflex through the nerve centre.

The reduced reflex time ${ }^{3}$ of the closure of the eyelid on excitation of the skin of the lid, was found by Exner to be as much as 0.047 second. $^{4}$

It would appear probable, therefore, that the time occupied by any simple reflex act is far too long to be produced merely by the passage of the nervous impulses across a single set of synapses (e.g. between the sensory and the motor cells), but we must assume a far greater complexity of arrangement, and the intermediation of several intercalated cells.

The great difference between the time taken in the eyelid reflex and that produced by stimulation of a posterior root, may be due to the fact that there is a choice of paths for afferent impulses; that under ordinary circumstances of natural excitation of the nerve-endings in the skin, the nervous impulses take a course tending to produce co-ordinate movements and involving a chain of several nerve cells, but that in the abnormal conditions of direct and more severe excitation of the nerve fibres, the impulses take the shortest possible course, and call into play a less complex nervous mechanism.

It was found by Exner ${ }^{5}$ that the total lost time between the excitation of

1 "Untersuch. z. Mechanik d. Nerven u. Nervencentren," Stuttgart, 1876, Abth. 2.

${ }^{2}$ Gotch found the reduced reflex for the electrical discharge in Malapterurus to be also 0.008 second (see this volume, p. 589).

3 1.e. the time obtaincd after leducting from the total time of the response-(1) That occupied in the passage of the sensory and motor nervous impulses from and to the eyelicl; and (2) that occupied by the perior of latent stimulation of the orbicularis palpebrarum.

4 Arch. f. d. ges. Physiol., Bomm, 1874, Bd. viii. S. 526.

5 "Exper. Untersuch., ete.," Arch.f. d. ges. Physiol., Bonn, Bd. viii. s. 532.

VOL. II. -39 
the frog's cerebral hemisphere and the response of the leg muscles is 0.05 second. ${ }^{1}$ Langendorff and Krawsoff ${ }^{2}$ further found a difference of about 0.02 second between the lost time obtained on stimulating the cerebrum and that obtained on stimulating the cut surface of the medulla oblongata. This, therefore, represents in the frog the lost time of the cerebral cortex itself. Almost the same figure $(0.015$ second to 0.02 second) was obtained for the cortex of the dog by François-Franck and Pitres, ${ }^{3}$ Bubnoff and Heidenhain, ${ }^{4}$ and by Novi and Grandis. ${ }^{5}$ Wilson ${ }^{6}$ gives a closely corresponding result for the latency of the optic lobes of the frog.

The time of response (reaction time) by a voluntary movement (usually of the hand) to a stimulus applied to one of the sense organs, varies, as has been said, with different individuals and in the same individual according to his physiological condition at the time. There
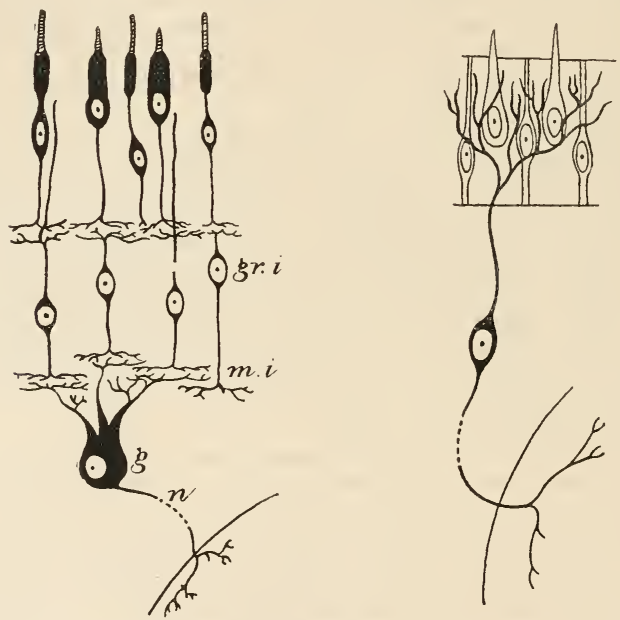

Fig. 308. -Diagram to show the relative number of the synapses in the visual and auditory organs.-G. Retzius.

is, further, a notable difference between the reaction times for different sense organs stimulated in the natural way. This is shown by the following figures from Richet, ${ }^{7}$ which represent the mean results arrived at by various authors :-

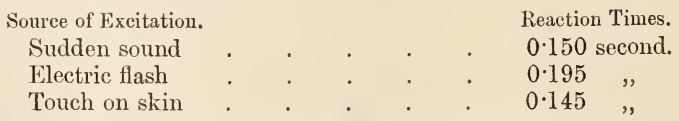

${ }^{1}$ Similar results have been got in the dog by Franck and Pitres (François-Franck, "Lecons," 1887) and others, and in the monkey by myself (Internat. Monatschr. f. Anat. u. Physiol., Leipzig, 1888, Bd. v.).

2 Arch. f. Pliysiol., Leipzig, 1879, S. 90.

3 Trav. du lab. de Murey, 1878-9, p. 413.

${ }^{4}$ Arch. f. d. ges. Physiol., Bonn, 1881, Bd. xxvi.

5 Riv. sper. di freniat., Reggio-Emilia, 1888, tome xiii.

6 Journ. Physiol., Cambridge and London, 1890, vol. xi. p. 504.

7 "Dictionnaire de physiologie," Paris, tome iii. p. 19. 
Even with the same sense differences are observed, for it has been noted that the response to an electrical stimulus of the skin of the forehead may demand a longer time than an electrical stimulus of the hand; ${ }^{1}$ hence, probably, the difference of acuteness of perception of the different terminal organs influences the result.

In the case of the eye and ear, the length of path being approximately the same, the reaction times might have been expected to be equal, whereas this is far from being the case. It is a significant fact that there are a larger number of synapses in the nerve chain along which nervous impulses, started by the falling of light upon the retina, pass to reach the sensorium, than is the case with the auditory path, for in the retina alone there are at least two synapses, namely, between the cone-fibres and the inner granules, and between the inner granules and the ganglion cells, whereas in the cochlea there are no synapses, these being first met with in the part of the auditory path which lies in the medulla oblongata (Fig. 308).

Another explanation which has been put forward regarding the length of the optical reaction time, depends upon the hypothesis that the peripheral excitation of the nerve fibres is of the nature of a chemical change, as contrasted with the mechanical excitation of anditory and tactile nerve fibres. This, if true, may account for a part of the delay, but can hardly be responsible for the whole, since it is not likely to be longer (nor even so long) in the case of the retinal elements as in muscle, where it is certainly not more than 0.0025 second (see article on "Muscle" in this volume, p. 169).

The reaction time is dependent upon many conditions, and is therefore extremely variable. The following are the most important sources of variation :-

1. The temperament of the individual.-Contrary to what might perhaps have been expected, individuals of a phlegmatic temperament have, as a rule, a shorter reaction time than those of a mercurial temperament-probably because they are less liable to have their attention distracted from the act which they are performing.

2. Age.-In old age the reaction time becomes slow.

3. Practice and attention.-These tend up to a certain point to diminish the time.

4. Fatigue, on the other hand, tends to increase it.

5. Intensity of stimulus. - The reaction time is shortened by increasing the intensity up to a certain point ; this shortening may be very marked.

6. Season.-The reaction time is shorter in winter than in summer.

7. Drugs.-Alcohol and morphia increase, coffee diminishes it. It is further affected by various other drugs. It also varies in disease.

The total reaction time is of course compounded of several factors. There is-(1) The time of propagation of the stimulus to the terminal nerve apparatus (which may be practically disregarded as far as most sensations are concerned); (2) the latency (inertia) of the terminal or recipient apparatus; (3) the time occupied by the passage of the nerve impulses to and within the lower centres; (4) the transmission from these to the higher centres (sensory and association centres) and that occupied by changes taking place in the nerve cells here; $(5)$ the transmission to and within the motor cerebral centres; $(6)$ the passage of the volitional impulses herein generated to the nerve cells of the spinal cord; (7) the generation of fresh nerve impulses in these cells ; (8) their passage

${ }^{1}$ Kries and Auerbach, Arch. f. Physiol., Leipzig, 1877 ; Hall and Kries, ibid., 1879, Suppl., S. 1; Vintsehgau, Arch.f. d. ges. Physiol., Bomn, 1880, Bd. xxii. s. 87. 
down the motor nerves to the voluntary muscle employed for giving the signal; and, lastly, (9) there is the period of latency of this muscle.

Attempts have been made to estimate the reaction time in the higher centres, or, in other words, the time taken by the processes (perception, apperception, and volition) going on in the nerve cells of the cerebral cortex, by making allowance for each and all the other processes, and subtracting the sum from the gross reaction time. The resultant would be the time occupied by a psychical act of the simplest kind, and has been termed "reduced reaction time"; but, considering that many of the above factors are not ascertainable with any degree of accuracy, it is obvious that the mode of its estimation renders the number obtained ( 0.08 second is given by Exner for a special case) of very little value. A better result might be got by ascertaining the time occupied in performing a movement as a simple reflex, and subtracting this from the gross reaction time performed as a voluntary act in response to a stimulus; it would still be necessary, however, to make an allowance for the time of transmission between the lower and higher level centres. Nevertheless, for want of anything more accurate, we may provisionally accept 0.08 second as the time taken up by a voluntary reaction, i.e. for the block occurring in transmission between the nerve cells of the brain.

If the psychical process be rendered somewhat more complex than is needed for the performance of a simple voluntary reaction, by the presentation of a dilemma,- - e.g., as when the person experimented upon is required to react only when the skin is stimulated at one of two places, or when one of two sounds is heard or pictures seen,- the reaction time is found to be lengthened by from 0.03 to 0.14 second. ${ }^{1}$ Or if there is not only the simple dilemma, but also a choice between two modes of response to the signal, it may become lengthened by as much as 0.184 second, according to Wundt (but other observers have not obtained so marked an increase). ${ }^{2}$ Or there may be a choice not between two but between several modes of stimulation; and this, according to the complexity of the psychical process involved, still further lengthens the reaction time. ${ }^{3}$ But the differences observed with different individuals become much more marked as the complexity of the psychical process is increased.

A modification of the ordinary reaction time experiment was employed by Donders, to determine the extra time involved in deciding the path which a voluntary impulse shall take, the person experimented on being stimulated upon one foot, and being called upon to react with the hand of the same side, or to react to a vowel sound by repeating the same sound. The extra time involved over and above the ordinary reaction time for the simple psychieal proeess involved was found in such a case to be about 0.036 second; this has sometimes been termed the "volitional" time. ${ }^{4}$ The introduetion of the simplest possible arithmetical process, such as the response to a number

1 Donders, Arch.f. Anat., Physiol. u. wissensch. Med., 1868 ; Wundt, "Elements of Physiological Psyehology," vol. ii. Sometimes termed "discrimination time."

${ }_{2}^{2}$ E.g. Cattell, Mind, London, $18 s 6$, vol, xi.

3 The literature of this subject is given at some length by Richet, "Dietionnaire de physiologie," tome ii. 1'l. 34 to 36.

${ }^{4}$ Cattell found that, although the reaction time is shortened by an increase in the intensity of the stimulus, the volitional time is not affeeted (Brain, London, 1885, vol. viii. p. 512). 
not by the same number but by a certain small multiple of it, has been found to produce a further delay of about 0.075 second, and so on.

Another question which may be here briefly considered, is, whether the nervous impulses are actually passed on from one cell to the other at the synapses, or whether new impulses are set up in the second cell, as a result of a stimulation received from the first one. The only facts which we have to guide us here are furnished by observations of the following character:-One of the limb muscles of an animal (dog, monkey) is connected with a myograph, and the part of the cerebral cortex being first found, excitation of which produces contraction of the muscle, the grey matter of that part of the cortex is removed, and the electrodes are placed on the subjacent corona radiata. If now the fibres of this, containing of course the fibres of the pyramidal tract derived from the cells which have been removed, are stimulated by a rapidly interrupted faradic current (50 per second), the muscle curve shows an incomplete tetanus, with waves of about 10 or 12 per second. ${ }^{1}$ Now, since nervous impulses are being generated in the fibres of the pyramidal tract at the rate of 50 per second, if they passed unaltered to the cells from which the nerves to the muscle employed take origin, the muscle should respond by a complete tetanus, as it would do if the motor nerve itself were stimulated at this rate; it is therefore clear that a new rate of rhythm has been started in the motor cells of the anterior horn, and it is probable that fresh nervous impulses are set up within them. ${ }^{2}$ In such a case as this, we may conceive the stimulus which provokes the nerve impulses in the second nerve cell to be the change of electrical potential (action-current) which accompanies the nervous impulses in the fibres artificially stimulated. But whatever the nature of the stimulus, if we assume that for each nerve cell there is, as with other protoplasmic structures, a period of latent excitation, the block which occurs at each synapse would thereby be accounted for.

It would appear that, while the synaptic mechanism produces only a delay in the passage of nerve impulses which are travelling in the direction which they normally take, it forms a complete block to passage of impulses in the reverse direction ("valve" action). Thus when, after the posterior roots are cut, the mixed nerve is stimulated, no effect whatever is produced upon the spinal cord. This is in marked contrast to the effects got when the anterior roots are cut and the posterior left intact; for stimulation of the mixed nerve under these conditions produces, of course, very pronounced reflex effects. When, under the same conditions (anterior roots cut, posterior intact), the spinal cord is stimulated and the mixed nerve led off to the galvanometer, a decided effect is obtained. ${ }^{3}$ In the latter case, the course of the nerve impulses is of course the reverse of normal, but since there is no synapse between the peripheral sensory nerves and the fibres which pass up the posterior

${ }^{1}$ Horsley and Schäfer, Journ. Physiol., Cambridge and Loudon, vol. vii.

2 The same thing is scen when a movement is produced reflexly by excitation of a sensory nerve or of the skin. The excitation may be very rapid, or it may be continnous, but it is responded to by the museles with a relatively slow rhythm of eontraction. This rhythm must in all probability be set up in the motor nerve cell, unless we are to assume that it is due to a tendeney of the muscle itself to contraet with this rhythm. It does not do so if its nerve be directly stimulated at a rapirl rate, althongh, if very excitahle, it may respond to a continnous electrical stimulation with a prolonged contraction which simulates an irregular tetanus.

${ }^{3}$ Gotch and Horsley, Phil. Truns., London, 1891, vol. clxxxii. B, p. 267. 
column of the cord, no block is met with, and the nerve impulses can pass freely in either direction.

Refractory period of nerve cells.-When two successive stimulations are applied to the same sensory nerve cell, nerve fibre, or nerve termination, they are only appreciated as two, if a certain small interval of time has elapsed between their application; in other words, the nerve substance only responds to a second stimulus after a certain interval has elapsed since the removal of the first. This is a general phenomenon of living substance, and is quite similar in character to the refractory period of cardiac and of skeletal muscle. The difference is found to be by no means the same for different terminal organs, being very small (0.002 second) in the case of the auditory organ (noise of two successive sparks), much longer $(0.017$ second) in the case of the eye (image of two successive sparks falling on the same spot of the retina), and still longer $(0.0277)$ in the case of the sense of touch (two successive touches of the skin of the finger). The relative amount of this so-called "minimal difference" in these cases is in all probability merely a measure of the relative inertia of the different kinds of terminal apparatus. This difference of inertia is probably also in a measure responsible for the fact that if stimuli be applied simultaneously to two different sense organs, as when a sound is emitted at the same instant that a spark is rendered visible, or at the same moment that the skin is excited, the two sensations are not appreciated simultaneously; in order that they should be so, the one must operate a certain time sooner than the other. The difference is known as the "differential time" between two senses. In this case, the difference may be partly due to a difference in the time taken by the nerve impulses in traversing the nerve paths, and this, again, is probably mainly due to a difference in the number of synapses which have to be passed in the two cases.

The following measurements are taken from Exner $:^{1}$

Smallest possible interval between two stimuli to produce two distinct sensations.

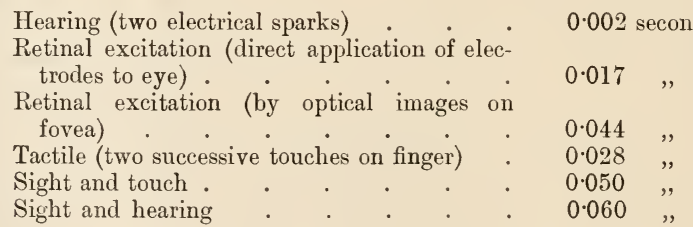

Richet ${ }^{2}$ has attempted directly to determine the refractory period of the nerve cell of the motor cerebral cortex in the anrsthetised dog. He found that, if stimuli succeed one another at a rate of 10 per second,

${ }^{1}$ Hermann's " Handbuch," Bd. ii. S. 262, where further details regarding the differential time, and also the reaction time, will be found. See also Exner, "Exper. Untersuch. $d$. einfachsten psychischen Processe," Arch. f. d. ges. Physiol., Bonn, Bd. vii. and xi.; Wundt, "Neuere Leistungen auf d. Gebiete der physiol. Psychologie," Vrtljschr. $f$. Psychiat., Neuwied, 1867, Bd. i.; Donders, "Schnelligkeit psychischer Processe," Arch. f. Anat., Physiol., u. wissensch. Med., 1868; Kries and Auerbach, Arch. f. Physiol., Leipzig, 1877 ; Richet, "Dictionnaire de physiologie," loc. cit., 1899.

2 Rep. Brit. Ass. Adv. Sc., London, 1897. Lecture to British Association in Nature, Oct. 26,1899 . 
there is no longer a (muscular) response to each excitation, as with lower rates; and he concludes that the refractory period of the cerebral cell is 0.1 second. This is in conformity with the experiments of Horsley and myself, already alluded to (p. 613), who found the rate of response to cortical excitation in the monkey to be only about 12 per second, even when the excitation rate was very much more rapid, and with the conclusions regarding the rate of volitional impulses arrived at independently by v. Kries ${ }^{1}$ and myself ${ }^{2}$ (about 10 per second).

It would further appear that it is not possible to perform a succession of voluntary movements such as is involved in the movements of the fingers in playing a musical instrument, or the movements of the mouth in speaking, at a greater rate than this, and Richet found from experiments upon himself that he was unable to "think" words at a greater rate than about 10 to 12 syllables per second. Thus the rapidity of neural processes has a physiological limit imposed upon it by virtue of the refractory period of the nerve cell.

1 Arch. f. Physiol., Leipzig, 1886.

2 Journ. Physiol., Cambridge and London, 1886, vol. vii. p. 114. Cf. Canney and Tunstall, "Proe. Physiol. Soe.," March 1885, Journ. Plıysiol., Cambrilge and London, 1885, vol. vi. 


\title{
THE SYMPATHETIC AND OTHER RELATED SYSTEMS OF NERVES.
}

\author{
By J. N. Langley.
}

Contents. ${ }^{1}$ - Generai Account of the Sympathetic Nervous System, p. 617 Sympathetic Nervous Supply of the Head, p. 617-Of the Skin, except that of the Head and of the Ano-Genital Region, p. 625-Of the External Generative Organs and of the Skin of the Ano-Genital Region, p. 635-White and Grey Rami ; Some General Features of the Sympathetic Nerve Supply to the Skin, p. 637-Of the Skeletal Museles, p. 639-Of the Thoracic and Abdominal Viscera, p. 642-Nerve Fibres of the Sympathetic System, p. 648-Degeneration of Nerve Fibres, p. 652-Regeneration, p. 652-Structure of Sympathetic Nerve Cells, p. 655-Duration of Effects caused by Section of Nerves, p. 655Efferent Pre-Ganglionic Fibres in relation to the Anterior and Posterior Roots of the Spinal Nerves, p. 658-Nervous Systems RELATED to THE SyMpatheTrC Nervous Srstey, p. 659-Distribution and Action of Cranial Autonomic Nerve Fibres, p. 660-Distribution and Action of Sacral Autonomic Nerve Fibres, p. 666-The Enteric Nervous System, p. 668-Possible Autonomic Fibres running direct in the Cerebro-Spinal Nerves, p. 668-Local Nerve Centres other than the Cranial, Sympathetic, and Sacral Ganglia, p. 671Questions common to the Srmpathetic and to Some Other Part of the Autonomic Sister, p. 672-Mode of Action of Inhibitory Fibres, p. 672Automatism of Peripheral Ganglia, p. 676-The Peripheral Ganglia as Centres for Definite Areas, p. 678-Reflex Action from Autonomic Ganglia, p. 678The Number of Nerve Cells on the course of a Pre-Ganglionic Fibre; Commissural Fibres, p. 682-The Size of Nerve Fibres as a Characteristic of the Autonomic System, p. 685-Afferent Autonomic Nerve Fibres; Referred Pain in Visceral Disease, p. 686-Growth of Pre-Ganglionic Cranial Fibres into Sympathetic Ganglia, p. 691-General Comparison of the Cranial, Sacral, and Sympathetic Autonomic Systems, p. 692.

In this account I propose to treat in somewhat general terms of the course of the sympathetic nerve fibres from the spinal cord to the peripheral ganglia, of the distribution and nature of the fibres given off by the ganglia, and to compare broadly the sympathetic fibres with similar fibres given off by the cranial and by the sacral nerves. Since sympathetic fibres run to all, or nearly all, parts of the body, the sections of this text-book which treat of the several organs and tissues have already given some historical account of many of the facts relating to the connections and functions of the sympathetic fibres; and in consequence, in bringing the facts together, as is done in the following pages, it will, in many cases, be unnecessary to enter into detail either as to the facts themselves or as to the original authorities.

The relation of the sympathetic to the central nervous system, the relation of its several ganglia to one another, and the function of its ganglia, have been subjects of controversy from the beginning of the

${ }^{1}$ A brief account of the papers relating to the sympathetic system which have appeared since August 1898, the date of the completion of this article, save for verbal corrections, is given in an Appendix, p. 695. 
century. A discussion of the various views which have been held, even of those which have been held in recent times, would take more space than it is desirable to occupy here. I shall, then, in the main restrict myself to stating the views which seem to me to rest on the most satisfactory experimental basis.

\section{GENERAL ACCOUNT OF THE SYMPATHETIC NERVOUS SYSTEM.}

Vertebral and pre-vertebral ganglia.-The ganglia of the sympathetic system are divided into vertebral or lateral, and pre-vertebral or collateral. The vertebral ganglia are the ganglia of the sympathetic chain or trunk, and I shall include in this also the superior cervical ganglia. The pre-vertebral ganglia are the ganglia of the solar plexus (solar ganglion, epigastric ganglion), the inferior mesenteric ganglia, and some small ganglia peripherally of the main masses. I shall include in this class the inferior cervical ganglion.

The characteristic of the vertebral ganglia is, that they send nerve fibres to the cerebro-spinal nerves. The superior cervical ganglion sends fibres in most mammals to the first three cervical nerves-in man to the first three or four-as well as to the fifth, and to some other cranial nerves. And each ganglion of the sympathetic chain sends fibres to one or more of the spinal nerves. The pre-vertebral ganglia do not, so far as is known, send nerve fibres to spinal nerves; they send their fibres to the thoracic, abdominal, and pelvic viscera. It is true that the inferior cervical ganglion is described in man and in some other mammals as sending strands to the lower cervical nerves; but, as there is at present no experimental evidence that the inferior cervical ganglion exercises any sympathetic action in the region supplied by these nerves, I have provisionally included it in the class of pre-vertebral ganglia.

We will consider successively the sympathetic nervous supply of the head, of the skin, of the trunk and limbs, of the skeletal muscles, and of the thoracic and abdominal viscera.

\section{Sympathetic Nerves to the Head.}

Effects produced by stimulating the cervical sympathetic.Stimulation of the cervical sympathetic produces on the side stimulated the following effects:-

1. Dilatation of the pupil.-This is the result of contraction of the dilatator pupillæ; the contractile substance probably consists, not of ordinary unstriated muscle, but of the processes of the cells covering the posterior surface. The dilatation is more marked in the cat and dog than in the rabbit.

2. Retraction of the nietitating membrane; the movement of the nictitating membrane is stronger in the cat and dog than in the rabbitin man and ape the nictitating membrane is rudimentary. Separation of the eyelids, the movement being greater in the lower than in the upper eyelids. Projection of the eye and direction of its axis straight forwards.

3. Contraetion of the blood vessels of the shin and mucous membrane of the head, and of the salivary and other glands.-The rapidity and extent of contraction varies in different parts of the head. It is usually very great in the salivary glands and in the ear ; it is especially obvious in the ear of the rabbit. It is marked in the conjunctiva, the iris, and in most 
animals in the skin and adjoining mucous membrane of the nose and lips, and in the mucous membrane of the hard palate; it is less marked in the gums and mucous membrane of the cheeks, and slight only in the tongue.

4. Dilatation of vessels in the bucco-facial region of the dog. - This was first shown by Dastre and Morat. ${ }^{1}$ They found that strong and moderately strong stimulation of the cervical sympathetic produced intense flushing in the mucous membrane of the lips, gums, cheeks, hard palate, nose, and in the neighbouring parts of the skin. With weak currents the first effect produced is contraction of the blood vessels. In other animals, primary contraction appears always to be produced, whatever the strength of the current. Thus the occurrence of vaso-dilator fibres -at any rate in sufficient number to overcome the vaso-constrictor fibres-is, so far as we know, peculiar to the dog. In this animal they are probably of use in regulating the temperature of the body.

5 . Secretion ${ }^{2}$ from the salivary glands, the lachrymal glands, the glands of the mucous membrane of the mouth, nose, and pharynx, and from the sweat glands of the skin where these occur.

6. Erection of hairs ${ }^{3}$ in animals which have erectile hairs. In the cat, erection of hairs is obtained in a triangular area between the eye and ear, the base of the triangle lying in front of the ear; in the occipital region, and occasionally in the whole dorsal region of the head; and in the skin of the back of the neck supplied by the third cervical nerve. In the ape it is obtained on the forehead, on the anterior half or more of the scalp, on the temple and cheek.

In the hedgehog the cervical sympathetic causes erection of quills in the head and upper part of the neck. ${ }^{4}$ The cervical sympathetic causes in the dog slight contraction of the angle of the nostril, and in the ape slight retraction of the pinna of the ear.

All of the above actions are well established. Others have been described, about which opinion is conflicting, and the evidence is not satisfactory. Such ${ }^{5}$ are contraction and dilatation of the vessels of the retina, inhibition of the ciliary muscle and the sphincter pupillæ, and, notably, contraction and dilatation of the arteries of the brain.

Some early observations on the cervical sympathetic.--Petit, ${ }^{6}$ in 1727 , described in the dog certain effects of section of the vagus in the neck, and

${ }_{1}$ Dastre and Morat, Compt. rend. Acad. d. sc., Paris, 1880, tome xci. pp. 393, 441 ; cf. also Bochefontaine and Vulpian, Compt. rend. Soc. de biol., Paris, 1880, p. 319 ; Dastre and Morat, "Système nerveux vaso-moteur," Paris, 1884, p. 102 ; Morat, Arch. de physiol. norm. et path., Paris, 1898, p. 204; Langley and Dickinson, Proe. Roy. Soc. London, 1890, vol. xlvii. p. 380 .

2 See articles on "Salivary Glands and on Secretion by the Skin," Vol. I. p. 475.

${ }^{3}$ Langley and Shcrrington, Journ. Physiol., Cambridge and London, 1891, vol. xii. p. 278 ; Langley, ibid., 1893 , vol. xv. p. 176 . Cf. also H. Miiller, Würzb. naturw. Ztschr., 1861 , Bd. ii. S. 54 ; Sehiff, Imparziale, Firenze, Anno x.

4 Langley, "Proc. Physiol. Soc.," 1893, vol. i. ; Journ. Physiol., Cambridge and London, vol, xiv. For the effect of the cervical sympathetic on the wattles, the feathers, and the eye in the fowl and turkey, ef. Schiff (1862 and 1867), Gesam. Beitr., Lausanne, 1894, Bd. i. S. 183 ; Jegorow, Arch.f. d. ges. Physiol., Bonn, 1887, Bd. xli. S. 326 ; Areh. f. Physiol., Leipzig, 1890, Suppl, S. 33.

${ }^{5}$ Cf. Jegorow, Arch. f. Physiol., Leipzig, 1886, S. 174 ; Morat and Doyon, Arch. de physiol. norm. et path., Paris, 1892, p. 60 ; ibid., 1891, p. 507 ; Jessop, Proc. Roy. Soc. London, 1886, vol. xl. p. 478; Langley and Anderson, Joum. Physiol., Cambridge and London, 1892, vol. xiii. p. 583. For innervation of the vessels of the brain, ef. this Text-Book, article on "Circulation."

${ }^{6}$ Hist. de l'Acad. Roy. des Se., 1727. Cf. also Dupuy, Jonm. de méd., chir. et pharmacol., Bruxelles, 1816, tome xxxvii.; Reid, "Physiological, Anatomical, and Pathological Researches," 1848 . 
of the "nerf intercostal" (the cervical sympathetic). As the result of section of the latter nerve, he found decrease in the size of the pupil, projection of the "membrane particulière du grand coin de l'oil" (the nictitating membrane), reddening of the conjunctiva, an increase in the fluid bathing the eye, and a decrease in the convexity of the cornea.

Biffi, ${ }^{1}$ in 1846 , noticed that stimulation of the cervical sympathetic caused dilatation of the pupil.

Bernard, ${ }^{2}$ in 1852 , observed that section of the cervical sympatletic led to the dilatation of the vessels of the ear, and increase of temperature of the skin of the head on the side of the cut nerve. This was soon followed by observations of the effect of stimulating the cervical sympathetic. Bernard,"3 BrownSéquard, ${ }^{4}$ and Waller ${ }^{5}$ all found contraction of the blood vessels of the ear, and decrease of temperature. The two latter observers attributed the contraction of the vessels to the stimulation of vasomotor fibres, and the temperature changes to variations in the amount of blood; whilst Bernard was inclined to attribute the variations in temperature, in part at least, to special calorific and frigorific nerves.

Tonic action; effect of section of cervical sympathetic.-The sympathetic nervous system exercises a tonic action on certain of the structures to which it runs, but not on all of them. In the cervical sympathetic, the vaso-constrictor fibres, the pupillo-dilator fibres, and the constrictor fibres to the unstriated muscle of the eye, are in a state of constant action, only broken by occasional inhibition occurring in the central nervous system; whilst the secretory fibres, the pilo-motor fibres in the cat, ${ }^{6}$ and the vaso-dilator fibres to the bucco-facial region of the dog, are normally at rest, broken only by an occasional excitation. Section of the cervical sympathetic will, then, only cause paralytic effects in those tissues which are in a state of tonic contraction. It causes a dilatation of the blood vessels, and an accompanying rise of temperature in the ear and the skin of the head. The vascular effects in other parts are less pronounced; they are generally marked in the conjunctiva, but slight or absent in the mucous membrane of the mouth. The hyperæmia of the lachrymal and salivary glands leads to a greater secretion than normal, whenever secretion is in any way set up. And, similarly, the hyperæmia of the nasal mucous membrane may lead to frequent sneezing. Section of the cervical sympathetic causes also a considerable narrowing of the palpebral aperture and projection of the nictitating membrane, and some retraction of the globe of the eye.

The duration of the several paralytic effects we shall consider later (p. 655).

Origin of fibres of the cervical sympathetic.-The origin of the fibres of the cervical sympathetic from the spinal cord, by which is meant the spinal nerves which convey fibres from the spinal cord to the sympathetic, can be determined by stimulating the several spinal nerves in the vertebral canal, after removal of the corresponding portion of the spinal cord, and observing which of them cause the effects also caused by stimulating the cervical sympathetic. The effects being noted, the

1 Serafino Biffi, "Milanese Inaug. Diss.," Pavia, 1846. (Quoted.)

2 Compt. rend. Acad. d. sc., Paris, 1852, tome xxxiv. p. 472.

3 Compt. rend. Soc. de biol., Paris, October and November 1852.

+ Phila. Med. Exam., 1853.

5 Compt. rend. Acad. d. sc., Paris, 1853, tome xxxvi. p. 378.

${ }^{6}$ In the ape, after section of the cervical sympathetic, the hairs of the hear on the ontside are somewhat less erect than on the intact side (Sherrington). 
cervical sympathetic is cut, and the stimulation repeated, in order to make certain that the effects were due to impulses passing by this nerve. It is thus found that the different classes of tibres have different origins; they differ also, though not very greatly, in different animals.

The pupillo-dilator fibres arise from the first three thoracic nerves. In the cat and dog the third thoracic has much less effect than the other two. On the other hand, in the rabbit, and apparently also in the ape, the third thoracic may have a greater effect than the first, though never so great as the second thoracic nerve.

The nerve fibres for the nictitating membrane, the eyelids, and the muscles of the orbit, arise from a greater number of nerves than those for the pupil, and their origin is on the whole more posterior. They arise in the cat and dog from the first five thoracic nerves; but the fifth thoracic has a very slight effect, and the fourth less than the first three. Of the first three, the second and third are more effective than the first.

The vaso-constrictor and vaso-dilator fibres are again, as a whole, a little more posterior in origin than the fibres supplying the nictitating membrane. They arise from the second to the fifth thoracic nerves inclusive, and occasionally a few from the first; the maximum effect is obtained from the third, or from the third and fourth. In the rabbit the origin of the vasomotor fibres to the ear is more extensive; some pass by the sixth and seventh, and occasionally a few by the eighth thoracic. It is not, however, certain whether these fibres of lower origin run to the cervical sympathetic, or make their way by the ramus vertebralis of the ganglion stellatum (cf. p. 624).

The submaxillary gland of the dog receives fibres also from the second to the fifth nerves inclusive, but the maximum effect is obtained from the second thoracic.

Lastly, we have the pilo-motor nerves. Those in the cat are of much lower origin than the others. They arise from the fourth to the seventh thoracic nerves inclusive, and the maximum effect is obtained from the fifth and sixth. ${ }^{1}$

It will facilitate a general view of these facts to put those which are best established in a tabular form:-

\section{Effects of Stimulation of Nerve Roots, indicating Origin of Filres of the} Cervical Sympathetic in the Cat.

\begin{tabular}{|lll|c|c|c|c|}
\hline \multicolumn{2}{|c|}{ Spinal Nerve. } & & $\begin{array}{c}\text { Dilatation of } \\
\text { Pupil. }\end{array}$ & $\begin{array}{c}\text { Retraction of } \\
\text { Nictitating } \\
\text { Membrane, etc. }\end{array}$ & $\begin{array}{c}\text { Vessels of } \\
\text { Ear and } \\
\text { Conjunctiva. }\end{array}$ & $\begin{array}{c}\text { Hair of Face } \\
\text { and Back of } \\
\text { Head. }\end{array}$ \\
\hline First thoracie &. & . & Good. & Moderate. & None or \\
slight. \\
Second
\end{tabular}

${ }^{1}$ In the ape, Sherrington found the pilo-motor fibres for the head to issue by the second to the fifth thoracic nerves. 
The origin of the other fibres of the cervical sympathetic are omitted, as they have not been studied in sufficient detail. "Good," "moderate," and "slight" in the table indicate the relative effects produced by the several spinal nerves.

It must be remarked that there is some evidence ${ }^{1}$ that the vasomotor fibres for the iris have the same origin as the fibres for the dilatator pupillæ, and thus a different origin from that of the vasomotor fibres for the conjunctiva and ear, but not many experiments have been made on the point.

The earliest observations on the origin from the spinal cord of the cervical sympathetic fibres were made by Budge and Waller in 1851, with regard to the dilator fibres for the pupil. They found dilatation of the pupil in the rabbit, on stimulating the second thoracic nerve. The results obtained by various observers are conflicting. The account I have given above is based upon my own experiments; in many respects it agrees with that given by some one or other of the earliest observers; thus the account of the origin of the fibres for the dilatator pupillæ is essentially the same as that given by Bernard. It may be mentioned that a considerable number of observers state that fibres run to the cervical sympathetic from the lower cervical nerves. I append below a list of papers for reference. ${ }^{2}$

Nerve cell connection of the nerve fibres.-. The nerve fibres which run from the spinal cord to the cervical sympathetic and to the superior cervical ganglion all end in connection with the nerve cells of this ganglion, ${ }^{3}$ for if the ganglion in the cat or rabbit be brushed over with 0.5 to 1 per cent. nicotin, in amount insufficient to appreciably affect the conductivity of nerve fibres, stimulation of the cervical sympathetic will have no effect.

The absence of effects must be due to a loss of conductivity somewhere in the ganglion; and in accordance with what we know generally of the action of alkaloids, as well as in accordance with the results of recent histological inquiry, we may fairly conclude that the break of conductivity occurs in the terminations of the sympathetic fibres around the cells of the superior cervical ganglion. In other words, we may say that the absence of effect on stimulating the cervical sympathetic is due to the fact that all its fibres form nerve-endings in the ganglion, and that

${ }^{1}$ Langley and Anderson, Journ. Physiol., Cambridge and London, 1892, vol. xiii. p. 577.

2 Pupillo-Dilator Fibres. - Budge and Waller, Compt. rend. Acad. d. sc., Paris, 1851, tome xxxiii. p. 370 ; Budge, ibid., 1852 , tome xxxv. p. 255 ; Arch. f. physiol. Heilh., Tübingen, 1852, S. 773 ; "Ueber d. Bewegung der Iris," Braunselweig, 1855, S. 111 ; Cl. Bernard, Journ. de la physiol. de l'homme, Paris, 1862, tome v. p. 383; Salkowski, 2tsehr. f. rat. IIcd., 1867, Bd. xxix. S. 167; François-Franck, Trav. du Lab. de Narcy, 1880, tome iv. 1. 26 ; Ferrier, Proc. Roy. Soc. London, 1883, vol. xxxv. p. 229 ; Nawrocki u. Przybylski, Arch.f. d. ges. Physiol., Bonn, 1891, Bd. i. S. 262 ; Langley, Phil. Trans., London, 1892, vol. elxxxiii. p. 85 ; Journ. Physiol., Cambridge and London, 1897, vol. xxii. p. 217 ; Sherrington, ibid., 1892, vol. xiii. p. 702.

Vasomotor Fibres,-Budge, Compt. rend. Acad. d. sc., Paris, 1853, tome xxxvi. p. 377 ; Cl. Bernard, ibid., 1862, tome lv. p. 381 ; Salkowski, Ztschr. f. rat. Med., 1867, Bd. xxix. S. 167; Dastre and Morat, Compt. rend. Arcul. d. sc., Paris, 12th Feb. 1883 ; "Systime nerveux vaso-noteur," Paris, 1884, 1. 132; Langley, Phil. Trans., London, 1892, vol. elxxxiii. p. 85 ; Joum. Physiol., Cambridge and London, 1897, vol. xxii. 1. 217 .

Pilo-Motor Fibres.-Langley and sherrington, Journ. Physiol., Cambridge and London, 1891, vol. xii. p. 166 ; Langley, ilid., 1893, vol. xv. p. 213 ; 1897, vol. xxii. p. 217.

Secretory Fibres.-Langley, Phil. Trans., London, 1892, vol. clxxxiii. 1) 104.

${ }^{3}$ Langley and Diekinson, Proc. Roy. Soc. London, 1889, vol. xlvi. p. 424 ; ibid., 1890, vol. xlvii. p. 379 ; Langley, Joum. Physiol., Cambridge and London, vol. xi. p. 146. 
these endings are paralysed by nicotin, just'as nicotin in rather larger doses paralyses motor nerve-endings in skeletal muscle.

Occasionally, the cervical sympathetic has a slight effect on the nictitating membrane and eyelid, after application of nicotin to the superior cervical ganglion. In such cases it is found that there are groups of nerve cells in the trunk of the nerve at or centrally of the point stimulated. In fact a few of the nerve cells of the superior cervical ganglion occasionally occur in the trunk of the nerve.

The fibres of the cervical sympathetic, as we have seen, pass through one or more of the upper thoracic sympathetic ganglia, and the inferior cervical ganglion, but the cautious application of nicotin to all of these ganglia does not prevent any one of the cervical sympathetic actions from being obtained by stimulation of the spinal nerve. It follows from this result that no appreciable number of any kind of nerve fibre present in the cervical sympathetic has a relay station in any of the upper thoracic ganglia, or in the inferior cervical ganglion; and one may fairly conclude-and the conclusion is supported by other facts-that all the fibres which pass to the cervical sympathetic from the spinal cord reach the nerve without having nerve cells on their course.

A further question is, whether the nerve fibres given off by the superior cervical ganglion run direct to the various peripheral structures, or end in connection with the cells of some other ganglia. In order to determine this, nicotin is injected into a blood vessel. The injection paralyses the cervical sympathetic, just as application of nicotin to the superior cervical ganglion paralyses it, and with the same occasional restriction we have mentioned above. But, on stimulating the several branches given off by the ganglion, all the normal effects can be produced, and to a maximal degree; that is, the nerve-endings of the fibres given off by the ganglionic nerve cells are not paralysed, and in consequence we conclude that they do not run to other sympathetic nerve cells, but proceed straight to the tissue.

Conclusions similar to these deduced from the action of nicotin may be legitimately deduced from the results of degenerative section of the cervical sympathetic and of the branches of the superior cervical ganglion. ${ }^{1}$ Waller and Budge found that on section of the sympathetic in the neck, the part in connection with the superior cervical ganglion showed the microscopical signs of degeneration, but that the part still in connection with the spinal cord and the part beyond the ganglion remained unaltered. They found also that stimulation of the part attached to the superior cervical ganglion no longer caused dilatation of the pupil, but stimulation of the ganglion itself promptly caused dilatation. The microscopical observations have been confirmed, and the experimental results extended to other classes of nerve fibres. Waller observed, further, that on degenerative ${ }^{2}$ section of the internal carotid branches of the ganglia, degenerated fibres could be traced to the

${ }^{1}$ Cf. Papers by Waller and by Waller and Budge in Compt. rend. Acad. d. sc., Paris, 1851-1853 ; Ztschr. $f$. wissensch. Zool., Leipzig, 1851, Bd. iii. S. 347 ; Waller, "Nonvelle methode anat., ete.," Bomn, 1852 ; Budge, Areh. f. physiol. Heilk., Stuttgart, 1852, S. 773 ; Langley, Journ. Physiol., Cambridge and London, 1885, vol. vi. p. 87; 1896, vol. xx. p. 70 ; 1897, vol. xxii. p. 228 ; Bradford, ibid., 1888, vol. ix. 1. 302 ; Langley and Sherrington, ibid., 1891, vol. xii. p. 278.

"I use the term "degenerative section" for the cases in which a nerve is cut, but the animal is not killed until degeneration is sufficiently advanced to stop the passage of nervous impulses. 
ophthalmic branch of the fifth nerve, and that stimulation of this nerve-normally producing dilatation of the pupil-no longer had an effect.

That the degeneration, after section of the internal carotid branches, spreads to the periphery, is shown by stimulating the sclerotic before and after the degenerative section. In the former case, there is a double effect-local contraction of the radial muscle leading to local enlargement of the pupil, and local contraction of the circular muscle of the iris; in the latter case, the radial contraction is lacking, the circular takes place as before.

General plan of arrangement.-The general idea of the sympathetic supply to the head, which we gather from the foregoing, is as follows: - The upper part of the thoracic spinal cord sends out fibres by the anterior roots of the spinal nerves of this region. The fibres make no halt at the ganglia until they reach the superior cervical ganglion. This is a relay station for the sympathetic nerve supply of the whole of the head; in it all the nerve fibres form nerve-endings-synapses as they are called by Foster' probably, indeed, as we shall see later, each nerve fibre branches and so forms several nerve-endings, each in connection with a nerve cell. Each nerve cell sends off a nerve fibre, which runs to the periphery, where it branches and supplies a group of unstriated muscle or gland cells. On the course of a nervous impulse from the spinal cord to the periphery, there are, then, two nerve cells, one with cell body in the spinal cord, the other with cell body in the local sympathetic ganglion. The nerve fibre of the first nerve cell we may call a pre-ganglionic fibre, that of the second a postganglionic fibre.

In introducing the terms pre-ganglionic and post-ganglionic, ${ }^{1} \mathrm{I}$ mentioned also the terms pre-cellular and post-cellular. I rejected the latter, since they are less distinctive. A pre-ganglionic fibre is post-cellular in relation to the nerve cell from which it arises. Kölliker, ${ }^{2}$ however, prefers to use either pre-cellular and post-cellular, or "Motorische Eingeweide-Fasern, I. und II. Ordnung."

Course of post-ganglionic fibres.-A further point that concerns us is the course taken by the post-ganglionic fibres of the superior cervical ganglion on their way to the periphery. Our information with regard to this is not very full or well assured. The majority of the fibres which have been traced, pass to the fifth cranial nerve, and are distributed with its sensory fibres. ${ }^{3}$ Thus the pupillo-dilator fibres and other fibres to the eye pass to the ophthalmic branch of the fifth, and the long ciliary nerves; the fibres-whether constrictor or dilator-to the blood vessels of the lips and mucous membrane of the mouth and nose, pass by the superior and inferior maxillary branch of the fifth. Broadly

1 Journ. Physiol., Cambridge and London, 1893, vol. xv. p. 178.

2 "Handbuch d. Gewebelehre," Bd. ii. S. 855.

${ }^{3}$ Waller and Budge, Compt. rend. Acad. d. sc., Paris, 1851, tome xxxiii. p. 418 (pupillodilator fibres); Wegner, Arch. f. Ophth., Leipzig, 1866, Bd. xii. S. 16 (vaso-constrictor fibres to iris); Francois-Franck, Trav. de Lab. de Marey, 1880. (According to FrançoisFranck, the vaso-constrictor fibres to the iris rum in the interual carotid plexus and not in the cervico-Gasserian strand.) Jegorow, Arch. f. Physiol., Leipzig, 1886, S. 149; Luehsinger, Hermanu's "Handbnch," 1883, Bd. v. Th. 1, S. 421 et seq. (sweat fibres); Dastre and Morat, "Systeme vaso-moteur," Paris, 1884, 1) 116 (vaso-dilator); Morat, Arch. dc physiol. norm. et path., Paris, 1889 , p. 196. I have found that the pilo-motor nerves for the face area accompany the fifth nerve. 
speaking, we may say that all parts of the skin and mucous membrane, which receive their sole sensory supply from the fifth nerve, receive their sympathetic supply also by way of the fifth nerve. Sympathetic fibres pass to the posterior part of the tongue, the pharynx, and the larynx, by way of the glosso-pharyngeal nerve, the pharyngeal and superior laryngeal branches of the vagus. The tongue receives fibres by way of the hypoglossal, but they are few, for the superior cervical ganglion has no great influence on the vessels of the tongue.

The ear ${ }^{1}$ receives sympathetic fibres by more than one route, and there are considerable differences in different animals. In the cat, the grey rami, running from the superior cervical ganglion to the first three cervical nerves, contain fibres which accompany the cutaneous branches of these nerves, and thus run to the ear, the occipital region of the head, and the upper part of the neck. The anterior branches of the superior cervical ganglion also contain vasomotor fibres for the ear, but by what course they reach their destination is not known. In the rabbit most of the fibres for the ear are contained in the strand which runs from the superior cervical ganglion to the external carotid, and they follow the branch of the artery which runs to the ear. These fibres, on stimulation, cause contraction of the whole of the central artery, but the effect is sometimes slight at the tip of the ear. As in the cat, some fibres run in the anterior strands of the ganglia, chiefly to the basal third of the ear. It may be mentioned that in the rabbit vasomotor fibres commonly run to the ear from the ganglion stellatum ${ }^{2}$ by way of the ramus vertebralis and third cervical nerve; these, on stimulation, cause contraction, chiefly at the tip of the ear, ${ }^{3}$ though the contraction spreads a variable distance towards the base. In the dog most of the vasomotor fibres for the ear run by the anterior branches of the superior cervical ganglion, their further course being unknown, and some pass by the branch to the external carotid artery.

The sympathetic fibres to the large salivary glands, pass by the strand which runs to the external carotid, and reach the glands by way of their arteries.

Three bundles of post-ganglionic fibres from the superior cervical ganglion remain for mention. There is that which accompanies the internal carotid; it would naturally be supposed to convey vasomotor fibres to the vessels of the brain, but this view, as we have said, is contested. And there are two bundles, one passing to the third and the other to the sixth cranial nerve, these might be supposed to convey vasomotor fibres to the vessels of the eye muscles, but so far experiments have not shown that they do so.

The general scheme of the distribution of the post-ganglionic fibres of the superior cervical ganglion appears then to be, that those which run to skin and mucous membrane accompany the cranial and cervical sensory fibres running to the skin and mucous membrane, and that those which run to deep visceral structures-the salivary glands-accompany the arteries. There is, however, in the rabbit the striking exception, that vasomotor fibres run to the ear by the artery. And this, together

\footnotetext{
${ }^{1}$ Morat, Arch. de physiol. norm. et path., Paris, 1891, p. 87; Langley, "Proe. Physiol. Soe.," p. ii., Journ. Physiol., Cambridge and London, 1893, vol. xiv.

2 Fleteher, Journ. Physiol., Cambridge and London, 1898, vol. xxii. p. 259.

${ }^{3}$ The vasomotor aetion of the great aurieular nerve was first deseribed by Sehiff, and its loeal aetion by Lovèn, Arb. $a . d$. physiol. Arst. zu Leipzig, 1866. An anatomieal aeeount of the nervous supply of the ear of the rabbit is given in this paper.
} 
with the inadequacy of detailed experiment on the course taken by the post-ganglionic fibres, makes it obvious that the scheme just memtioned is only to be regarded as approximate and provisional.

\section{Sympathetic Fibres to the Skin, except that of the Heav and of the Ano-Genital Region.}

Effects produced by stimulation.-The actions set going in the skin by the sympathetic nerves, naturally vary in different parts and in different animals, in accordance with the kind of structures which have developed in them. Thus, in rabbits, the only action produced in the skin of the trunk and limbs is a vasomotor one. Sweat glands and erector muscles for the hairs are absent. The effects which can be produced in the skin by stimulation of some part or other of the sympathetic chain are as follows, omitting the effects produced on the skin of the genital region, which will be given in the account of the external generative organs :-

1. Contraction of the arteries. - This can be brought about in all parts of the skin, but apparently more easily in some parts than in others. Thus, with equal nervous stimulation, it is said that there is greater contraction in the vessels of the foot than in the vessels of the thigh. The sympathetic fibres have not been shown to cause directly any change in the capillaries. They have been found to cause contraction in the veins of the wing of the bat, ${ }^{1}$ and in the superficial veins of the leg of the cat and rabbit. ${ }^{2}$ The former have rhythmic contractions and dilatations, like those which occur in the arteries of the ear of the rabbit; the rhythm depends upon impulses passing from the spinal cord.

2. Secretion from sweat glands.-Observations on the distribution of secretory fibres to the sweat glands of the skin, other than that of the head, have only been made upon the cat and dog; and in the cat and dog the glands only occur on the pads of the feet.

3. Erection of hairs.-This is also a local occurrence. In the cat it occurs in the dorsal strip of skin which is innervated by the posterior cutaneous branches of the spinal nerves, and in the tail. In the dog and ape the hairs are affected in a similar dorsal strip. In the hedgehog the region in which quills are moved is co-extensive with the region in which quills are found, so that here the sympathetic causes movement in the region of the lateral cutaneous as well as in that of the posterior cutaneous skin branches.

The "goose skin" which occurs in man is a similar phenomenon, and is no doubt also produced by sympathetic nerve fibres.

We may note here that the action of the sympathetic chain is unilateral on the trunk; it is unilateral also on the limbs, except for an occasional very trifling effect on the opposite hind-limb, but it is bilateral on the tail and on the external generative organs, though generally stronger on the same side. The bilateral action is connected with the fact that the vertebral ganglia from the second sacral, and sometimes from the first sacral downwards, are connected together on the two sides, and it is these ganglia, as we shall see, which send fibres to the tail and to the external generative organs.

${ }^{1}$ Cf. Wharton Jones, Arch. de physiol. norm. et path., Paris, 1849 ; Schiff, Arch. f. d. ges. Physiol., Bonn, 1881, Bd. xxvi. S. 456.

"Bancroft, Am. Journ. Physiol., Boston, 1898, vol. i. p. 477. VOL. II. -40 
4. Flushing of the skin, or a rise of temperature in it, are at times and in certain circumstances produced by stimulation of the sympathetic; and it is generally believed that the changes are due to the presence of vaso-dilator nerves. ${ }^{1}$ The evidence, on the whole, is in favour of the presence of such nerve fibres, but it is, I think, premature to regard the question as settled.

Some of the statements made do not harmonise with well-established facts. Thus it has been said that weak tetanising currents stimulate vaso-dilator more than vaso-constrictor fibres, although, as we have already mentioned, the cervical sympathetic in the dog, when stimulated with weak currents, produces contraction, and when stimulated with strong currents, produces dilatation of the vessels of the bucco-facial region.

The strongest evidence for the presence of vaso-dilator fibres in the sciatic is that afforded by the series of well-known experiments initiated by Goltz, ${ }^{2}$ on the effect of stimulating the sciatic nerve some two to four days after cutting it. The thermometric method, the plethysmographic method, and direct observation have given similar results, namely, that the vaso-constrictor action becomes less as time goes on, and that in the last day or two, before irritability completely disappears, the vascular dilatation is out of proportion to the preliminary contraction, or occurs without any contraction at all.

It is not an unfair deduction that the vascular dilatation is due to the presence of vaso-dilator fibres, which become prepotent some days after section of the nerve, in consequence of their preserving their conductivity rather longer than the vaso-constrictor fibres do. But, on the other hand, it has not been shown by experiments in typical dilator and constrictor fibres, that the latter degenerate more slowly than the former, nor is it inconceivable that the changes which take place in the nerve and the tissue after section of the nerve should be of a nature enabling stimulation of constrictor fibres to cause the described degree of dilatation.

According to Dziediul, ${ }^{3}$ the vaso-dilator fibres of the submaxillary gland (chorda tympani) take eleven days to lose their irritability, and those of the nervus erigens nine days. These observations are, I think, untrustworthy. I have found the vaso-dilator fibres of the chorda tympani of the cat to be completely without effect in five days. Anderson and myself ${ }^{4}$ found that the sacral nerves seven days after section had no effect of any kind. ${ }^{5}$

There is some evidence also for the presence of vaso-dilator fibres in the sympathetic supply to the skin, in the effects produced by stimulating fresh and normal nerves. Primary dilatation is comparatively rarely obtained with any strength or form of stimulation, but not uncommonly the after-dilatation seems out of proportion to the primary contraction. ${ }^{6}$ When a part only of sympathetic supply to a given region is stimulated, the dilatation may occur during the stimula-

\footnotetext{
${ }^{1}$ For reference to papers, in addition to those mentioned in this section, ef. article on "Circulation" in this volume.

${ }^{2}$ Arch. f. d. ges. Physiol., Bonn, 1874, Bd. ix. S. 174. Of later papers, ef. especially Ostroumoff, ibid., 1876, Bd. xii. S. 219; and Bowditch and Warren, Journ. Physiol., Cambridge and London, 1886 , vol. xii. p. 416 .

${ }^{3}$ Jahresb. ü. d. Fortschr. d. Anat. u. Physiol., Leipzig, 1880, pt. 2, S. 68.

4 Journ. Physiol., Cambridge and London, 1896, vol. xix. p. 372.

${ }^{5}$ For a further criticism of the evidence relating to vaso-dilator fibres of the limbs, and especially of the muscles, see p. 640 .

${ }^{\circ} \mathrm{Cf}$. Dastre and Morat, Compt. rend. Acal. c. sc., Paris, 1878, tome lxxxvii. p. 880.
} 
tion; indeed, if the vessels are already contracted, there may be no observable primary contraction, but dilatation only. And when, as first shown by Ostroumoff, slowly repeated shocks-about 1 in 2 seconds to 1 in 5 seconds-are used as the stimulus, there may be primary dilatation. The most obvious conclusion from these results is, that the dilatation of the vessels is due to the presence of the vaso-dilator fibres; but it may perhaps be doubted whether the evidence is conclusive that in these cases the stimulation does not lower the irritability and conductivity of the peripheral vasomotor nerve fibres, and thus prevent tonic vasomotor impulses from coming into play. Lastly, we must mention a line of evidence first put forward by Schiff, ${ }^{1}$ and adopted by Ostroumoff and others. This consists in showing that the dilatation of the vessels of a given region of the skin which can be produced by impulses from the central nervous system-for example, by excitation of afferent fibres-is greater than that produced by section of the vaso-constrictor and all other nerves to the region. Unfortunately for the conclusiveness of these observations, the reflex dilatation has not-in most cases, at any rate-been compared with the maximal dilatation caused by section, but with the dilatation existing after the vessels have recovered some degree of tone.

Course of post-ganglionic fibres.-The sympathetic fibres which run to the skin leave the sympathetic chain by the grey rami, they run to the spinal nerves, and follow the cutaneous branches to the skin. ${ }^{2}$ It has been generally believed that some sympathetic fibres make their way to the periphery along the sheaths of the arteries. It is easily shown that this is not the case with the pilo-motor nerves, for if the dorsal cutaneous nerves are cut, the sympathetic is no longer able to cause movement of the hairs. Similarly, after section of the sciatic, the sympathetic is no longer able to cause secretion from the sweat glands of the foot. There is some difference of opinion with regard to vasomotor fibres. For it has been found by some observers that, after section of the sciatic, changes take place in the calibre of the vessels of the foot, which can only be due to impulses coming from the central nervous system. But in these cases the crural nerve was left out of account. And it has been shown that sometimes, at any rate, the crural nerve contains vasomotor fibres for the foot. ${ }^{3}$ In mammals, then, there is no evidence that sympathetic fibres pass to the skin of the trunks or limbs by way of the arteries, and, taking all the facts into account, we may conclude that they all pass by way of the spinal nerves. In the frog, experiments have been made upon the vasomotor nerves to the hind-limb; the general result of which is the same as in mammals, although the statement that the fibres accompany the arteries has been more positively put forward.

In this connection there is one point to keep in mind-the sympathetic sends some fibres direct to the aorta, and these appear to spread some way down the large arteries. It is not unlikely that these nerve

1 Schriften d. Berner Naturf. Gesellsch., 1856, S. 69.

${ }^{2}$ Langley, Journ. Physiol., Cambridge and London, 1891, vol. xii. p. 368 (sweat fibres); ibid., p. 375 (vasomotor fibres); ibill., 1893, vol. xv. p. 199 (pilo-motor fibres); ibid., 1894 , vol. xvii. p. 296 (secretory and vasomotor fibres). Cf. these papers also for origin and nerve-cell connection of the sympathetic fibres to the skin.

${ }^{3}$ Lewaschew, Arch. f. d. grs. Physiol., Bonn, 1882, Bd. xxviii. S. 431 ; Dastre and Morat, "Système nerveux vaso-moteur," Paris, 1884, p. 262 ; Spallitta and Consiglio, Arch. ital. de biol., Turin, 1897, tomo xxviii. p. 257 . 
fibres can cause some contraction in the large arteries. In this way a modification of blood supply to the skin, and to the muscles, may take place, without any direct effect upon the peripheral vessels.

Distribution of grey rami.-Broadly speaking, the successive spinal nerves to the neck and the trunk are found anatomically to supply successive bands of skin encircling the deeper lying tissues. These rings are broken where the nerves pass to the fore- and hind-limbs; there the several nerves cease to run separately, but join to form the brachial and the lumbo-sacral plexus. One or more of the nerves in the middle of the series of nerves forming the plexus send no cutaneous fibres to the trunk, but run entirely to the limb. Since each spinal nerve has its grey ramus, and since, as we have seen, the grey ramus contains sympathetic fibres running to the periphery with the spinal nerve, the probability is great, that the distribution of the fibres of the successive grey rami is the same in its general plan as that of the cutaneous branches of the successive spinal nerves.

The question is easily tested as regards pilo-motor fibres, by stimulating the successive grey rami in the cat, and observing the areas of skin in which erection of hairs occur. In this way, it is found ${ }^{1}$ that the area of skin innervated by successive effective grey rami are successive, overlapping by a millimetre or two only, and that the areas correspond to the anatomical areas of the successive spinal nerves as nearly as could be expected from the lack of accuracy of the anatomical method. A similar result is obtained by observing the contraction of the small arteries to the skin, although the result is less constant and precise, on account of the variability on the course of the arteries.

I have said that the grey rami which are effective supply successive strips of skin with pilo-motor nerves. There are one or more grey rami about the middle of each of the series which form the brachial and lumbo-sacral plexuses, which have no pilo-motor nerves. These rami run to nerves which have no dorsal cutaneous branches. In the cat these nerves are the eighth cervical, often with either the seventh cervical or the first thoracic in the region of the brachial plexus; and the sixth lumbar, often with either the fifth lumbar or the seventh lumbar in the region of the lumbo-sacral plexus. When the sixth and seventh lumbar nerves liave no dorsal cutaneous branches, the sixth and seventh lumbar grey rami have no pilo-motor nerve fibres, and send no fibres to the skin over the vertebræ, the dorsal cutaneous branch of the first sacral nerve follows immediately on that of the fifth lumbar, the first sacral grey ramus causes erection of hairs in a strip of skin immediately posterior to that affected by the fifth lumbar grey ramus.

From these and other experiments, it is clear, as regards the skin of the neck and trunk, that the grey ramus running from the sympathetic to a spinal nerve may be regarded as having broadly the same skin distribution as the cutaneous branches of the nerve to which it runs. These branches contain sensory fibres of the skin. Sherrington ${ }^{2}$ has shown that the successive spinal nerves which send sensory fibres to the skin of the neck and trunk supply successive areas, but that these areas overlap considerably, so that in general each area includes a half of the area above, and a half of the area below. On this plan it will be noticed each portion of the skin sends afferent fibres to two nerve roots. As we have said, the efferent fibres of the grey rami do not overlap to this

${ }^{1}$ Langley, Journ. Physiol., Cambridge and London, 1893, vol. xv. p. 199.

${ }^{2}$ Phil. Trans., London, 1893, vol. clxxxiv. p. 641. 
extent; they encroach very little on their neighbour's territory. We must conclude, then, that although the efferent sympathetic fibres accompany the sensory spinal fibres to the skin, they spread out less in the skin than do the sensory fibres. Thus the efferent sympathetic nerve region lies in the afferent spinal nerve region.

Turning now to the distribution of the grey rami which run to the limbs, we have no pilo-motor fibres to serve as indicators, and although the vasomotor fibres afford some aid, we have for accurate observations on distribution to rely upon the sweat glands in the pads of the cat's foot. $^{1}$ As a rule, four grey rami send secretory nerves to the foot of the cat, namely, the sixth lumbar to the second sacral; the second sacral grey ramus occasionally has no effect. In a typical case the sixth lumbar grey ramus causes secretion in the inner part of the foot, the second sacral grey ramus causes secretion on the outer part of the foot, the other two cause copious secretion on the whole of the foot, but the seventh lumbar most on the inner part, and the first sacral most on the outer part. Here, then, each part of the skin receives fibres from three grey rami. Some parts, in some cases, receive fibres from four grey rami. Türck ${ }^{2}$ and Sherrington ${ }^{3}$ have shown that the afferent fibres of the nerves which go to make up the sciatic, overlap very largely. The overlapping described by them is, however, hardly so great as that which occurs in the secretory fibres of the several grey rami. In the limbs, then, unlike the trunk, the sympathetic efferent areas of the grey rami appear to overlap rather more than do the afferent skin fibres of the corresponding spinal nerves:

Origin of grey rami from the ganglia.-Having thus determined the course taken by sympathetic fibres, on passing from the sympathetic chain to the skin, our next step is to determine from what nerve cells the nerve fibres arise. This can be done by the use of nicotin, in the manner already given in treating of the cervical sympathetic. The result of such experiments is, that the great majority of the fibres of a grey ramus, in some cases all of them, arise from the corresponding vertebral sympathetic ganglion, and run to the periphery without having further sympathetic nerve cells on their course. The few fibres which do not arise from the corresponding ganglion arise from the ganglion next above or below, and in the case of the lumbar grey rami from the ganglion next above; in the case of the thoracic and sacral ganglia there appears to be some variation, but the details have not been fully worked out.

Broadly speaking, then, the vertebral sympathetic ganglia are segmental, and each supplies sympathetic fibres to its own spinal nerve.

There is a divergence from this rule, though functionally not a very important one; the white rami not infrequently receive a few fibres from the sympathetic ganglia. Where the white rami run posteriorly before joining the sympathetic chain, as they do in the cat, from the tenth or eleventh thoracic to the fourth or fifth lumbar, a white ramus may receive fibres from the nerve cells of the ganglion of the nerve immediately posterior to it (ef. Fig. 309).

${ }_{1 .}^{1}$ Langley, Joum. Physiol., Cambridge and London, 1891, vol. xii. 1. 368; 1894, vol. xvii. p. 299.

${ }_{2}$ Sitzungsb. d. k. Akad. d. Wissensch., Wien, 1856, Bd. xxi. S. 586 ; Denkschriften d. k. Akad. d. Wissensch., Math.-Nat. Cl., Wien, 1869, Bd. xxix. S. 299.

'Phil. Trans., London, 1893, vol. elxxxiv. 1' 641 . 
A complication occurs, in that the ganglia may unite with one another and form a compound ganglion. A compound ganglion gives off a grey ramus to more than one nerve. In some places compound ganglia are constant in occurrence, in others they are occasional. The constant compound ganglia are the ganglion stellatum which sends grey rami to the cervical nerves from about the third downwards, and to the

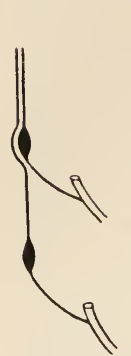

A

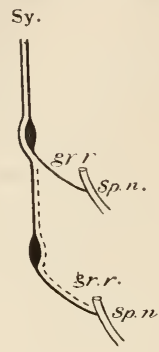

B

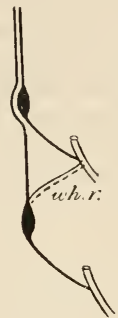

C

FIG. 309.-The variations we have mentioned are represented in the diagrams A, B, and C. In A each ganglion sends its nerve fibres to the corresponding spinal nerve. In $\mathrm{B}$ the upper ganglion sends the great majority of its nerve fibres to its own nerve, and a few (represented by the dotted line) to the nerve below in the grey ramus of that nerve. In $\mathrm{C}$ each ganglion sends the majority of its fibres to its own nerve, but the lower sends a few (represented by the dotted line) to the nerve above in the white ramus of that nerve.

Lastly, we have to determine the connection which exists between the spinal cord and the several ganglia, or rather we have to determine with which ganglia the efferent fibres in the roots of each spinal nerve are connected. The fundamental line of experiment is to stimulate each spinal nerve near its exit from the spinal cord, and to observe the area of the skin in which sympathetic effects occur. Since we know, within narrow limits, the area of the skin innervated by each ganglion, we can tell within narrow limits which ganglia are in connection with each spinal nerve-supposing always that the effects are brought about by means of the ganglia.

The spinal nerves which, on stimulation, give rise to sympathetic effects in the skin in the regions we are here considering, are, in the cat, the fourth thoracic (occasionally the third) to the third or fourth lumbar inclusive. The region, it will be remembered, is that of the whole body, except the head and the ano-genital region.

The distribution of the several nerves has been determined by observation on the pilo-motor nerves to the neck, back, and tail in the cat; and on the secretory and vasomotor nerves to the feet and limbs. Some cunfirmatory observations have been made in the vasomotor nerves to the back and trunk. As we shall see, there are some differences in the distribution of the different classes of nerve fibres, although the differences are inconsiderable.

The pilo-motor nerves present especial advantages in dealing with this question, because the movement of hairs is readily observed. And 
this allows the area of skin actually supplied with pilo-motor fibres by each grey ramus to be determined without difficulty, after the area supplied by each spinal nerve has been determined. Moreover, the region of skin having erectile hairs receives its nerves from the posterior primary division of the spinal nerves, and this, with the fibres of the grey ramus accompanying it, leaves the anterior primary division close to the posterior root ganglia. Thus, by pulling the spinal nerve a little into the vertebral canal, or by using a strong current-so that it spreads

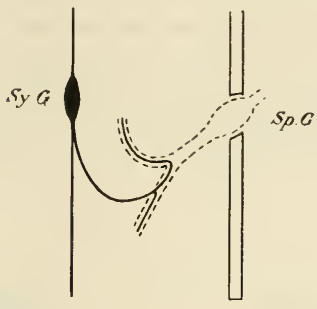

A

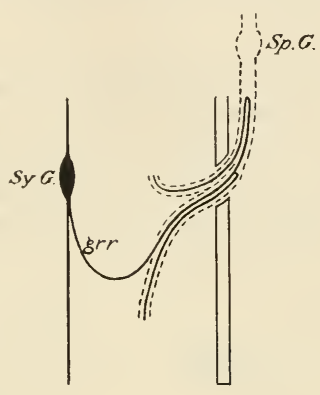

B

FIG. 310.-Diagram of course of a grey ranus. A, in a thoracic spinal nerve. $B$, in a sacral spinal nerve; $S y$. G., sympathetic ganglion; $S p . G$., spinal ganglion. In A the branch of the grey ramus to the posterior primary division of the nerve is outside the vertebral canal, but can be stimulated if the nerve is pulled a short distance into the canal. In B the grey ramus follows the nerve a short distance into the vertebral canal.

down the nerve-the pilo-motor fibres of each grey ramus can be stimulated without opening the abdominal cavity. This will be obvious from the diagram given (Fig. 310).

Before taking the connections of the individual nerves, we must mention a cornmon feature presented by their sympathetic fibres. We have seen that none of the nerve fibres, which pass from the spinal cord to

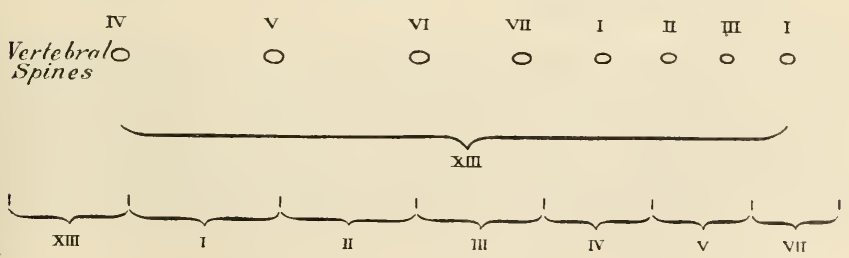

Fig. 311.

the head by way of the cervical sympathetic, run direct to the periphery, but that they all form nerve-endings (synapses) around sympathetic ganglion cells. The same law holds for the nerve fibres ruming from the spinal cord by way of the sympathetic to the skin of the trunk and limbs. They all form nerve-endings in one or other of the pre-vertebral ganglia. This is shown by experiments with nicotin. 
We may now return for a moment to the stimulation of the spinal nerves in the vertebral canal. I have said that in certain ways the pilo-motor nerve fibres of the grey ramus of the nerve can be stimulated. But these are post-ganglionic fibres, and they are not paralysed by nicotin. So that, after observing the area of the skin in which the hairs move on stimulation of each spinal nerve, we have only to inject nicotin into the blood, and to stimulate the nerves a little past the posterior root ganglia, or to stimulate it with a strong current, to observe also the area supplied by each grey ramus.

Thus, to take an example from an experiment. The thirteenth thoracic nerve roots caused the hairs to become erect from a spot a little below the third lumbar vertebra (fourth spine) to a little below the first coccygeal vertebra (cf. long bracket XIII. in Fig. 311). In the middle part the hairs were strongly erected; in the ends, especially the lower end, they moved slightly only. Then nicotin was injected. After this, stimulation of the thirteenth thoracic nerve had no effect, unless the nerve was stimulated peripherally of the ganglion or with strong currents, in which case a small patch of hairs just above the

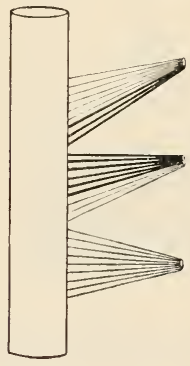

A

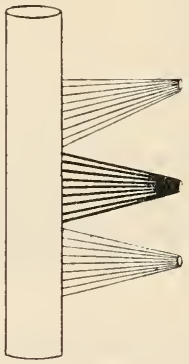

M

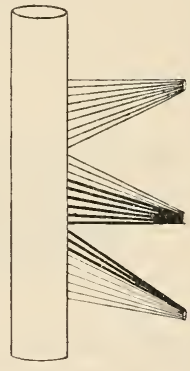

$\mathrm{P}$

F1G. 312,-Diagram to show variation in nerve-origin. $\mathrm{M}=$ median arrangement. The upper nerve roots of $\mathrm{M}$ pass into the nerve above in the anterior arrangement $(A)$, and the lower nerve roots of $M$ pass into the nerve below in the posterior arrangement $(\mathrm{P})$.

previous limit moved sharply and strongly; and on stimulating similarly each of the following nerves, small successive patches were obtained, as indicated in the lower line of brackets in the figure. In this case the pre-ganglionic fibres of the thirteenth thoracic sent fibres to all the lumbar sympathetic ganglia, sending most to the second, third, fourth, and fifth ganglia. It will be noticed that no area is given for the sixth lumbar grey ramus; as we have said above, this runs entirely to the limb.

On investigation in this fashion, it becomes clear that there are individual variations. In part these can be referred to a difference in the functional activity of the tissues in different individuals; in some, for example, the contractile muscular tissue of the hairs is much better developed than in others, and thus spinal nerves which produce a weak erection of hairs in the one, may have no observable effect in the other. In part, however, they are due to structural variations, which are correlated with a number of other structural differences. The roots of 
the spinal nerves leave the grey matter in a continuous band. Adjacent portions of this continuous band are gathered up into bundles and form the spinal nerves. In this gathering up into bundles there is a certain amount of individual variation. The fibres, which in one individual are gathered up so as to make the lowermost fibres of a given bundle, may, in another individual, be taken to form the uppermost fibres of the bundle below. The variation rarely involves more than two-thirds of the fibres of a nerve.. It may be represented diagrammatically as in Fig. 312, A, M, P.

The three arrangements may be spoken of as anterior (A), median $(\mathrm{M})$, and posterior $(\mathrm{P})$ arrangement of nerves. This relation of roots to nerves affects a whole series of nerves simultaneously, and to very nearly the same relative extent. Thus, if one lumbar nerve is anterior in arrangement, all the lumbar and sacral nerves will also be anterior in arrangement. On this basis it follows that the relative size of the strands which the several spinal nerves send to the trunks issuing from the lumbo-sacral plexus ${ }^{1}$ will be different with each arrangement, and thus the anatomical characters of the lumbo-sacral plexus form a ready means of determining whether the arrangement of nerves is anterior, middle, or posterior. In consequence, instead of saying that in a given individual the arrangement of nerves is anterior, we may say, simply, that the plexus is anterior. The brachial nerves appear to vary much less frequently than the lumbo-sacral nerves.

The variation in the two sets of nerves is not necessarily in the same direction; the brachial plexus may be anterior, and the lumbo-sacral plexus posterior, but the statistics are insufficient to show how frequently this is the case.

The importance of this, for the subject we are considering, is that, in order to state at all accurately the several sympathetic ganglia to which each lumbar nerve sends nerve fibres in any one species of animal, it is imperative to know whether the arrangement of nerves is anterior, middle, or posterior. At the same time, it must be remembered that these variations are very numerous, and that the grouping of them into three forms is purely arbitrary.

The connections of the spinal nerves in relation to these variations have been only worked out in detail in the cat. I shall take the connections with an anterior arrangement of nerves as a basis for description; other forms I shall not deal with in detail; a few illustrations will be sufficient to show the general trend of the variations.

It need hardly be said that the spinal nerves in one species have only approximate homologues in the spinal nerves of another species. But as the structural outlines not only of species but of all mammals are the same, information as to the connections of the sympathetic fibres known for one animal, can, with proper allowance for anatomical peculiarities, be applied to any other.

${ }^{1}$ Cf. Langley, Journ. Physiol., Cambridge and London, 1891, vol. xii. p. 347 ; 1893 , vol. xv. p. 210 ; 1894, vol. xvii. p. 296 ; Langley and Anderson, ibid., 1896, vol. xx. 1. 392 ; Sherrington, ibid., 1892, vol. xiii. 1. 639 . In this paper references will be foumd to earlier observations bearing on the subject. Sherrington divides the lumbo-sacral plexus into two classes, pre-fixed and post-fixed. Eisler, "Der Plexus lumbo-sacralis des Menschen," Diss., Halle, 1892; cf. also Thane, Quain's "Anatomy," vol. iii. pt. 2, 1. 313. 
Connections of the Spinal Nerves with the Vertebral Ganglia, so far as these supply the Skin, except that of the Head and of the Ano-genital Region.

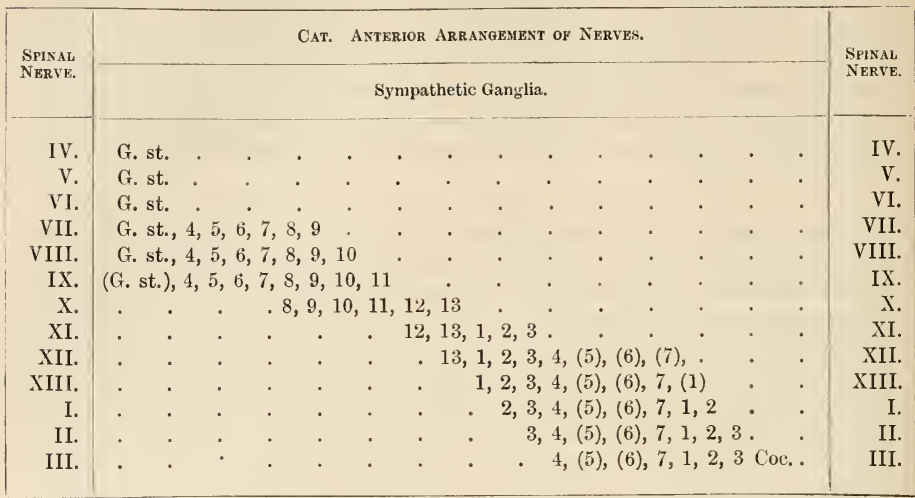

In this table a number enclosed in brackets indicates that the connection of the spinal nerve with this ganglion has been determined by vasomotor fibres to the limb, or by secretory fibres to the foot. We have already seen (cf. 628) that the fifth and sixth lumbar sympathetic ganglia do not-when the plexus is anterior-send off pilo-motor fibres; nor do the seventh and eighth cervical, but these ganglia are merged in the compound ganglion stellatum.

As we have said, the connections of the several spinal nerves have been chiefly determined for pilo-motor fibres; we may make a brief statement with regard to the other kinds of nerve fibres.

1. The connection of the vasomotor fibres with the dorsal cutaneous branches of the intercostal and lumbar arteries, has not, in the experiments I have made, presented any constant difference from that of the pilo-motor nerves, though sometimes the connection was a little more extensive.

2. The secretory and vasomotor fibres for the hind-foot of the cat arise from the twelfth thoracic to the third lumbar inclusive. The ganglia which give off secretory fibres are the sixth and seventh lumbar, and the first and second sacral. With one or more of these ganglia, then, the twelfth to the third lumbar nerves must be connected. The exact connections have not been determined, but it appears probable that the twelfth thoracic sends a few fibres secretory and vasomotor to the seventh lumbar ganglia, and that the thirteenth thoracic sends a few such fibres to the first sacral ganglion; and this connection has, in consequence, been inserted in the table. The nerve having the maximum effect on secretion is the first or second lumbar.

The eleventh thoracic nerve occasionally sends a few secretory fibres to the foot, so that in these eases the eleventh thoracic must send fibres either to the fifth or sixth lumbar ganglion; this is not represented in the table.

3. The secretory and vasomotor fibres for the fore-foot arise from the spinal cord by the fourth to the ninth thoracic nerves inclusive, and 
end in the ganglion stellatum, from which the post-ganglionic fibres are given off to the brachial nerves. The origin of these fibres closely resembles that of the pilo-motor fibres (cf. table), with the exception that the ninth thoracic nerve, whilst sending secretory and vasomotor fibres to the ganglion stellatum, does not send pilo-motor fibres to it. The maximum effect is usually obtained with the seventh nerve, the nerves above and below giving progressively less effect.

Occasionally there is slight secretion and pallor in the fore-foot on stimulating the third thoracic, possibly also from the tenth. Bayliss and Bradford found, in the dog, that the tenth and sometimes the eleventh nerve caused vaso-constriction in the fore-limb, and that the eleventh to the third lumbar caused vaso-constriction in the hind-limb.

4. The vasomotor fibres for the tail have very nearly the same origin and connections as the pilo-motor fibres for this region. They appear, however, to be a little more posterior.

General differences accompanying a posterior plexus.-With a posterior arrangement of nerves, the fourth lumbar nerve in the cat sends fibres to the coccygeal ganglion, and, according to the degree of downward shift, to more and more of the ganglia above up to the fifth lumbar. The posterior arrangement also considerably affects the connection of the seventh to the eleventh thoracic nerves. Of these nerves, four always send some fibres up and some down in the sympathetic chain; the remaining one, the eleventh thoracic nerve, may or may not do so. For details with regard to the variations in ganglionic connection of these nerves, the original papers must be referred to. The connection of the other nerves-except, perhaps, the third lumbar-are comparatively little affected by a shifting in their origin.

\section{Sympathetic Nervous Supply of the Skin of the External Generative Organs and of the Ano-Genital Region. ${ }^{1}$}

Effects produced by stimulation.-Stimulation of the lumbar sympathetic chain causes contraction of the unstriated muscles and blood vessels of the skin of the ano-genital region, and of the penis or vagina. The degree of contraction varies considerably in different parts and in different animals. We may mention the effects in the cat, dog, and rabbit in the male. In the female the effects are mutatis mutandis the same as in the male, but most of them are much less marked.

In all three animals the lumbar sympathetic causes strong contraction of the blood vessels, of the unstriated muscles of the body of the penis and of the prepuce, and of the retractor muscle of the penis. The retractor muscle is well developed in the $\mathrm{dog}$, and, as in some other animals, it is a thin band of longitudinally arranged unstriated muscle, inserted at the attachment of the prepuce, and continued backwards in the middle line over the ventral surface of the corpus spongiosum and bulbus urethræ. In the eat and dog there is strong contraction of the tunica dartos of the scrotum: but the blood vessels of the scrotum are not strongly affected. In the rabbit the scrotal contraction is very slight. In the cat and dog there is little movement of the skin of the ano-genital region, apart from that of the scrotum. In the rabbit the skin covering the body of the penis contracts strongly.

${ }^{1}$ For list of papers and more detailed account, ef. Langley and Amerson, Joum. Physiol., Cambridge and London, 1895, vol. xx. p. 85 . 
Course and origin of post-ganglionic fibres.-In the main the sympathetic fibres producing these effects have the same general arrangement as the nerves to the skin-that is, they leave the sympathetic by grey rami, pass to the spinal nerves, and follow the branches of these to the periphery. The branches followed in this case are the pudic nerves, which give rise to the nervus dorsalis penis, the genitoanal nerve or nerves, and the scrotal nerve. It must be noticed that the nervus dorsalis and the genito-anal nerves cause contraction of one or more of the striated intrinsic muscles of the penis.

The fibres of the grey rami which run to the pudic nerve follow, as regards their origin, the general rule of the fibres of the grey rami which run to the skin, i.e. they arise from the nerve cells of the corresponding ganglion. The ganglia in the cat and dog are the three sacral ganglia. But the ganglia in the sacral region often fuse together, so that a grey ramus is less restricted to a corresponding ganglion here than elsewhere; and the first sacral grey ramus not infrequently receives from the seventh lumbar ganglia some fibres for the external generative organs.

The pudic nerves vary in origin, and in correspondence with this there is some variation in the particular sympathetic ganglia, from which the fibres for the external generative organs arise.

Whilst the great majority of the sympathetic fibres for the external generative organs have the same general arrangement as the sympathetic fibres for the skin, a few have a different arrangement, passing by the inferior mesenteric ganglion (cf. p. 646), i.e. by a pre-vertebral ganglion, and reaching their destination by the hypogastric nerve and the pelvic plexus. The origin of these fibres from the spinal cord is the same as that of the fibres which run by way of the vertebral ganglia, and as they are few in number, we need not enter into any detailed account of them.

Origin of pre-ganglionic fibres from spinal nerves. - We have seen, in treating of the sympathetic fibres which supply the skin of the body generally, that the sacral ganglia receive pre-ganglionic fibres from the thirteenth thoracic and the first three lumbar nerves (cf. table, p. 634) in the cat. Since the sacral ganglia send fibres to the external generative organs, we might expect that the spinal nerves which influence the external generative organs, by way of the sympathetic, would be the thirteenth thoracic and the first three lumbar nerves; and we might expect that the second and third lumbar would have a greater influence than the first lumbar, and the first lumbar than the thirteenth thoracic nerve. And this, in fact, is the case. But there is an additional fact, which could not be deduced simply from the connections of the cutaneous fibres of the sacral ganglia, namely, that the fourth lumbar nerve, which has no effect on the hairs of the tail or on the blood vessels or glands of the foot, causes marked contraction of the musculature of the external generative organs. This difference is constant. Thus when, in the cat, the arrangenient of nerves is posterior, and the fourth lumbar nerve sends some fibres to the tail and to the foot, the fibres for the external generative organs are shifted also, and the fifth lumbar nerve will cause retraction of the penis.

It is natural enough that the external generative organs should have a more extensive nervous supply than the sweat glands of the foot, or the hairs of the tail. But the fact that it is so, involves a selective arrangement of pre-ganglionic fibres with particular nerve cells, which 
presents certain difficulties in considering the whole question. We need not, however, dwell on these. It is sufficient at present to point out that a bundle of pre-ganglionic fibres may run to a single vertebral ganglion, and restrict its connections with cells which supply a given region, entirely passing over the cells which supply fibres to other regions.

The external generative organs and a portion of the ano-genital skin receive efferent fibres from the sacral nerves by way of the pelvic nerve (nervus erigens), but these fibres do not belong to the sympathetic system, and will be considered later.

\section{Some General Features of the Sympathetic Nerve Supply} TO THE SKIN.

White and grey rami as paths for fibres from the spinal cord.It appear's from the preceding account that in the cat, the skin of the body receives in all cases sympathetic fibres from the first thoracic to the fourth lumbar nerves inclusive, and from no others, ${ }^{1}$ except in those cases in which the nerves have a more posterior arrangement, when the skin receives some sympathetic fibres from the fifth lumbar nerve also. These nerves are the only ones which, on anatomical and microscopical investigation, are found to have white rami connecting them with the sympathetic chain. Since every nerve is connected with a vertebral ganglion by a grey ramus, the conclusion may fairly be drawn that all the sympathetic nerve fibres which run to the skin from the spinal cord, run to the sympathetic chain, on their way to the skin, by the white rami. And the conclusion is confirmed by the entire absence of sympathetic effect, when the roots of a lower thoracic or upper lumbar nerve ${ }^{2}$ are stimulated after section of its white ramus, its grey ramus remaining intact.

The adoption of the view that the efferent fibres of the sympathetic pass to it by the white rami, is due to Gaskell. ${ }^{3}$ He arrived at his conclusions chiefly from a histological investigation of the white and grey rami and of the nerve roots. $\mathrm{He}$ pointed out that since the nerve roots contained very few non-medullated fibres, and since. the few present might reasonably be supposed to end in the vessels of the spinal cord, it must be concluded that all the fibres running from the cord to the sympathetic are medullated fibres. Further, since medullated fibres are numerous in the white rami, and very scanty in the grey rami, it must be concluded that the white rami form the channel of communication between the spinal cord and the sympathetic.

This line of argument is satisfactory up to a certain point, but it is only conclusive as regards the great majority of fibres. It does not exclude the possibility of some efferent fibres passing by the grey rami. In fact, some grey rami contain a considerable number of medullated fibres (cf. p. 649).

If efferent fibres run to the sympathetic chain by way of the grey rami, they would degenerate on section of the nerve roots. Some experiments 4 have been made on the lines thus suggested. As a rule, section of the nerve roots caused no degeneration of medullated fibres in the grey rami. In sonie

${ }^{1}$ A considerable number of observers have described sympathetic effects as occurring on stimulation of nerves which have grey rami only. As I have been unable to obtain any such effects, I attribute them to experimental errors.

" The uppermost thoracic white and grey rami are too closely associated to be cut separately.

3 Journ. Physiol., Cambridge and London, 1886, vol. vii. p. 1.

4 Langley, ibid., 1896, vol. xx. p. 60. 
cases one or two degenerated fibres were found these could be referred either to an implication of the spinal ganglion in the injury, or to fibres passing to the grey ramus from the white ramus of one of the cut nerves.

Limits of white rami in different mammals.-Of the cat we have already spoken. With regard to the white rami in other animals, our knowledge is less accurate than could be wished; but there is more or less evidence for the following statements. The first thoracic nerve is in all animals the first nerve to give off a white ramus. Relatively, as well as absolutely, there are fewer fibres in the first thoracic white ramus of the rabbit than in that of the cat, dog, monkey, and man. In the dog the last white ramus comes from the third lumbar nerve, with anterior arrangement of nerves, otherwise from the fourth. In the rabbit, the last nerve with a white ramus is the fourth or fifth. In man, it is, so far as is known, the second lumbar; ${ }^{1}$ whether the second lumbar nerve has always a white ramus, and whether the third lumbar nerve has a white ramus when the arrangement of nerves is markedly posterior, has not been sufficiently investigated.

Course of post-ganglionic sympathetic fibres to the skin.On reviewing the course of the sympathetic efferent fibres to the skin, we see that the peripheral fibres all arise from the ganglia of the sympathetic chain. The post-ganglionic fibres, almost without exception, pass by grey rami to the cerebro-spinal nerves, and accompany the cutaneous branches of these nerves. And, further, the fibres of each grey ramus have in the main the same distribution in the skin, as the afferent cerebro-spinal fibres of the nerve it accompanies.

General scheme of the connections of the white rami with the vertebral ganglia.-A consideration of the connections of the several spinal nerves given above, shows that they are arranged with a certain symmetry (cf. especially table, p. 634). Each spinal nerve, from the first thoracic to the fourth lumbar (cat, anterior arrangement), sends fibres to a series of ganglia, varying usually from six to nine, and in no case to a single segmental ganglion. Each spinal nerve sends its rays of fibres a little more anteriorly than the spinal nerve behind it, and a little more posteriorly than the one in front of it. Thus, speaking broadly, the successive spinal nerves give off sympathetic fibres to successive largely overlapping portions of the skin of the body.

Although each spinal nerve sends fibres to a series of ganglia, it does not send an equal number to each of them. In each case it sends more fibres to one or two ganglia than to any others; and from this position of maximum supply there is a regular diminution in the number of fibres sent to the several ganglia of the series. The position of maximum supply is not necessarily, or even usually, in the middle of the series, but it would take us too far to give here an account of the details of distribution.

A compound ganglion behaves in some respects like a series of simple ganglia. We have seen thatt he ganglion stellatum sends grey rami to a series of spinal nerves. The nerve cells giving off these grey rami are connected with the spinal nerves in much the same way as if they formed a series of separate ganglia. Thus, a spinal nerve may be connected with some of the grey rami, and not with the rest, but the former will in all cases

${ }^{1}$ Langley and Latham, Journ. Physiol., Cambridge and London, 1893, vol. xv. p. 242 ; Harman (Joum. Anat. and Physiol., London, 1898, vol. xxxii.) finds that in man the rami of the second lumbar nerve have about two hundred medullated fibres, and that the rami of the third lumbar have about twenty only. 
form a series, as they would if they arose from separate ganglia. So also with the coccygeal ganglion, this sends grey rami to six coccygeal nerves; when the arrangement of nerves is posterior, the pilo-motor connections with the several grey rami may be as follows:-The second lumbar nerve sends a few fibres to the first coccygeal ramus, and none to the rest; the third lumbar nerve sends a considerable number of fibres to the first, second, and third grey rani, and a few to the rest; the fourth lumbar nerve sends fibres to all the grey rami.

A question which naturally arises from the fact that one spinal nerve is connected with a number of ganglia, is whether a single nerve fibre runs to one ganglion only, or whether it divides and runs to some or to all. Since a given spinal nerve does not affect equally all the ganglia of the series to which it runs, it is fairly certain that the constituent nerve fibres in it do not divide into branches equal in number to that of the ganglia, and send one branch to each ganglion. That in some cases the constituent fibres run to comparatively few ganglia, is obvious from a comparison of the innervation with different arrangements of the spinal nerves. In the anterior arrangement, the third lumbar nerve causes strong erection of hairs in the whole of the tail and over the sacrum, i.e. it sends fibres to the fourth lumbar and all the lower ganglia, and the fourth lumbar nerve has no effect at all upon the hairs. As, however, the nerves shift downward, some of the fibres of the third lumbar nerve pass into the fourth; these will naturally be the ones contained in the lowermost nerve roots. Thus a certain number of fibres ordinarily contained in the third lumbar nerve become separated from the rest, and pass into the fourth lumbar nerve. On stimulating these, it is found in some cases that they do not affect the hairs in the whole of the region from the sacrum downwards, but in part of it only ; for example, only in the latter two-thirds of the tail. Thus these pilo-motor fibres send no branches to the ganglia from the fifth lumbar to the third sacral, but end entirely in connection with the coccygeal ganglion. As the coceygeal ganglion is made up of about six single segmental ganglia, the nerve fibres may still divide and give a branch to each. We may conclude, then, that the ganglionic connection of individual nerve fibres in a spinal nerve is usually less extensive than that shown in the table (p. 634) for the several spinal nerves. We shall later consider a method of determining approximately the number of single ganglia with which individual nerve fibres are connected (cf. p. 681).

It will be noticed also that a spinal nerve sending fibres to a ganglion, even when this is a single segmental ganglion, does not necessarily send fibres to all the kinds of nerve cells in the ganglion. Thus the fourth lumbar nerve sends fibres to the sacral ganglia, but these fibres are in connection with the cells which innervate the external generative organs, and not in connection with the cells which innervate the vessels and hair muscles of the root of the tail.

\section{Sympathetic Nerves supplying the Skeletal Muscles.}

We have seen that the grey ramus running to a spinal nerve contains fibres which accompany the cutaneous branches of the nerve, and which supply various structures occurring in the skin in the region to which the cutaneous branches run. We might, then, naturally expect that the grey ramus would also send fibres to the other branches of the spinal nerve, and thus supply vasomotor fibres to the arteries of the skeletal muscles.

We shall consider separately the evidence with regard to vasoconstrictor and vaso-dilator fibres. 
Vaso-constrictor fibres.-According to Grützner and Heidenhain, ${ }^{1}$ the abdominal sympathetic trunk in the dog contains vaso-constrictor fibres for the muscles of the limb. They found that stimulation of the abdominal sympathetic trunk causes a slight fall of temperature in the gastrocnemius, and a fall of blood pressure in the veins carrying blood from the extensor group of muscles of the thigh.

Gaskell ${ }^{2}$ observed directly the blood-flow from the extensor group of muscles of the thigh; he obtained a slight, but only a slight, decrease of blood-flow on stimulating the abdominal sympathetic. The result is not convincing as to the presence of vasomotor fibres, since Gaskell obtained only a transient increase of blood-flow by section of the sympathetic, and no decrease by stimulating the fifth lumbar grey ramus. I have not seen any change of colour in the muscles of the limbs in the cat and rabbit, on stimulation of the abdominal sympathetic, though sometimes there appeared to be a slight constriction of the larger arteries. We may conclude that, if there is a sympathetic vasoconstrictor supply for the muscles of the limbs, it is at any rate not well developed.

The circulation in some of the small muscles of the eye and of the larynx can be examined under the microscope. On stimulation of the cervical sympathetic, I have not found any certain change in the size of the small arteries of the muscle; sometimes there was a slight slowing in the blood-flow in the capillaries, suggesting a contraction of some larger arteries outside the muscle.

Vaso dilator fibres.-The presence of vaso-dilator fibres in the nerves supplying the muscles of the limb has been advocated by Gaskell, ${ }^{3}$ and by Heidenhain with his collaborators. ${ }^{4}$ Gaskell did not deal with the origin of these fibres. Heidenhain considered that they ran to the muscular nerves from the abdominal sympathetic.

We are here only concerned with the question of vaso-dilator fibres of sympathetic origin, but as there is some ground for believing that if vaso-dilator fibres are present in the muscular nerves they arise from the sympathetic, it is advisable to mention, though briefly, the chief experiments which have been brought forward to show that the muscles receive vaso-dilator fibres.

Gaskell observed the rate of blood-flow from the extensor muscles of the thigh of the dog on stimulation of the crural nerve. Normally, this caused great increase of blood-flow, after a preliminary decrease. When curari was given in minimal amount, just sufficient to paralyse the motor nerves to the muscle, an increase in the blood-flow was sometimes obtained on stimulating the crural. When a larger dose of curari was given, the crural became ineffective.

Now, the amount of curari which paralyses the vaso-dilator action of the crural does not paralyse any known vaso-dilator nerve fibres, neither those of the chorda tympani, nor of the nervus erigens, nor of the dog's cervical sympathetic. In other words, the vaso-dilator fibres of muscle,

1 Arch. f. d. ges. Physiol., Bonn, 1878, Bd. xvi. S. 1.

2 Joum. Physiol., Cambridge and London, 1878, vol. i. p. 108.

${ }^{3}$ Arb. a. d. physiol. Anst. zu Leipzig, 1876 (reprinted with slight additions in Journ. Anat. and Physiol., London, 1877, vol. xi. p. 360); Journ. Physiol., Cambridge and London, 1878, vol. i. p. 262.

${ }^{4}$ Grützner and Heidenhain, Arch. f. d. ges. Physiol., Boun, 1878, Bd. xvi. S. 1 ; Heidenhain, Alexander u. Gottstein, ibid., S. 31. 
if they exist, are much more easily paralysed by curari than the other known vaso-dilator fibres. In view of this, it is impossible to avoid the suspicion that the dilatation of the vessels, which was observed when a minimal dose of curari was given, was accompanied by an unnoticed slight contraction of the skeletal muscular fibres.

Heidenhain experimented on the sciatic nerve. He found that when the sciatic nerve was stimulated four days after it had been cut, a rise of temperature was produced in the gastrocnemius. The rise, however, was slow and slight. It was produced on completely curarised animals, and, according to Gaskell, curari paralyses the vaso-dilator nerves to muscle.

It is obvious that the conclusive form of experiment, as regards vasodilator fibres of sympathetic origin, is to observe the effects of stimulating the abdominal sympathetic. Gaskell found a slight decrease of the blood-flow through the muscles. Heidenhain found a slight fall of temperature. Neither found, on direct stimulation, evidence of the presence of vaso-dilator fibres. The absence of vaso-dilator action has been attributed to the antagonistic action of the vaso-constrictor fibres. What we know of the antagonism of vaso-constrictor and vaso-dilatation fibres, makes it permissible to assume that a few vaso-dilator fibres may be present in the abdominal sympathetic, but is directly opposed to the assumption that there are sufficient vaso-dilator fibres to produce the very large increase of blood-flow which occurs during contraction of a muscle. It was pointed out by Gaskell that the increase of blood-flow accompanying contraction, however produced, is comparable to the increased blood-flow produced elsewhere by the chorda tympani and by the nervus erigens. If a few vaso-constrictor fibres are stimulated simultaneously with either of these nerves, there is either vaso-dilatation at once or after a preliminary constriction; if many vaso-constrictor fibres are stimulated with either of these nerves, there is complete vaso-constriction, with or without an after-dilatation. In no case are results obtained similar to those so far obtained by stimulating the abdominal sympathetic, namely, with all strengths of stimulus a slight constriction without after-dilatation.

Further, on Heidenhain's view that both the vascular effects in muscle on stimulating a muscle nerve are due to fibres coming from the abdominal sympathetic, it is inexplicable why the muscle nerve and the sympathetic should produce such very different results.

There is, then, no satisfactory evidence that the sympathetic sends vaso-dilator fibres to the skeletal muscle, although it is possible that it sends a few. As a provisional hypothesis, we may suppose that the increased blood-flow which takes place in muscle, on stimulation of its motor nerve, is brought about by changes of some kind in the muscle fibres.

In the frog, it was shown by Gaskell ${ }^{1}$ that the branch of the fifth nerve which supplies the mylo-hyoid muscle, contains vaso-dilator filores for it. This muscle, however, is more related to the muscles of the tongue-to which in mammals vaso-dilator fibres run-than to the muscles of the limbs, so that the behaviour of the mylo-hyoid does not justify us in drawing any conclusion as to the immervation of the limb muscles, or of the skeletal museles of the body in general. It may be noticed the vaso-dilator fibres of the mylo-hyoid are not paralysed by

${ }^{1}$ Journ. Anat. and Physiol., London, 187\%, vol. xi. p. 720.

VOL. 11. -4 I 
curari. Whether these fibres arise from a cranial nerve, or reach the fifth nerve from the sympathetic, has not been investigated.

\section{Sympathetic Fibres supplying the Thoracic and ABDominal Viscera.}

Thoracic viscera.-The relations of the sympathetic nerves to the heart and lungs have been already noticed in previous articles in this volume, so that I need only mention a few points here.

The accelerator fibres for the heart proceed from a rather more anterior series of the spinal nerves than do the vasomotor fibres for the lungs. The accelerator fibres are contained chiefly in the second, third, and fourth thoracic nerves, some fibres also at times being present in the first and fifth. ${ }^{1}$ The vasomotor fibres for the lungs arise, according to Bradford and Dean, from the third to the seventh thoracic nerves inclusive. ${ }^{2}$

The fibres proceed partly by strands leaving the ganglion stellatum, forming what is sometimes called the nervus accelerans, and partly by strands leaving the inferior cervical ganglion. The nerve cell comnection of the pre-ganglionic fibres has not been worked out, but it is probable that the majority end in connection with the cells of the ganglion stellatum, and the minority in connection with the cells of the inferior cervical ganglion. Assuming that the accelerator fibres do end in the ganglion stellatum, we have the curious fact that, whilst the origins of the nerve fibres supplying the external carotid and the heart are practically the same, the nerve cells with which the nerve fibres are connected have become widely separated, and that the first, second, and usually also the third thoracic nerves are connected with no cells of the ganglion stellatum, except those supplying the thoracic viscera.

Upper abdominal viscera.-The nerve fibres to the upper abdominal viscera run by way of the splanchnic nerves, the solar ganglia and the bundles of nerve fibres accompanying the coliac, the superior mesenteric, and the renal arteries. The splanchnic nerves on each side consist of the main splanchnic, and of two or three smaller nerves. When there are three nerves only, as is generally the case, they are sometimes spoken of as the great, the small, and the smallest splanchnic. Not infrequently the term "splanchnic nerve" is used to include all three nerves.

The solar ganglia, which, it will be remembered, are pre-vertebral ganglia, consist of a group of ganglia joined by nerve strands. These are in close connection with the cœliac, superior mesenteric, and renal arteries. From the ganglia proceed nerve fibres, which accompany and innervate the arteries, and innervate also the organs to which the arteries run. The fibres which accompany the arterial trunks arise in large part, though by no means wholly, from different ganglionic masses, so that we may speak of the cœliac ganglia, the superior mesenteric ganglia, and the renal ganglia, as sending their fibres in the main to their respective arteries. Certain of the renal ganglia are close to the hilum of the kidney, bnt it is convenient to include them in the solar ganglia.

The several abdominal organs, with the exception of the kidney,

${ }^{1}$ Cf. Phit. Trans., London, 1892, vol. clxxxiii. p. 107.

2 Proc. Roy. Soc. London, 1889, vol. xlv. p. 369. 
receive fibres from the splanchnic nerves of both sides. And, so far as is known, the fibres proceeding from the corresponding ganglia of the two sides, for example the two superior mesenteric ganglia, have the same peripheral distribution.

The earliest attempts to determine which spinal nerves innervate a given abdominal viscus was made by Bulgak ${ }^{1}$ (1877). He obtained contraction of the spleen, on stimulating the third to the tenth thoracic nerves on the left side. No further observations of the kind were made until 1889. Bradford ${ }^{2}$ then investigated the origin of the vasomotor fibres of the kidney by Roy's oncometer method. He found that vasomotor nerves run to the kidney in the dog in the fourth thoracic to the fourth lumbar nerves inclusive; the maximum effect was obtained from the eleventh, twelfth, and thirteenth thoracic nerves, a moderate effect from the sixth to the tenth thoracic, a very slight and inconstant effect from the other nerves.

Bechterew and Mislawsky, ${ }^{3}$ also in 1889 , obtained evidence of the presence of inhibitory fibres for the small intestine of the dog, in the sixth thoracic to the first lumbar nerves inclusive, and especially in the ninth, tenth, eleventh, and twelfth thoracic nerves. In one experiment inhibitory effects were obtained by stimulating the nerves above the sixth thoracic.

In the last few years a considerable number of observations have been made.

Bayliss and Starling ${ }^{4}$ (1894) have found that a rise of portal blood pressure is produced in the dog by stimulating the third to the eleventh thoracic nerves inclusive; a considerable effect being produced by the fifth, sixth, seventh, eighth, and ninth; a less effect by the fourth and tenth; and a slight effect only by the third and eleventh. On general grounds, partly anatomical and partly physiological, $\mathrm{I}^{5}$ came to the conclusion (1895) that in the cat (with anterior lumbo-sacral plexus) the sixth thoracic to the second lumbar nerves inclusive send pre-ganglionic fibres to the solar ganglia, and that these ganglia send post-ganglionic fibres to all the abdominal viscera.

Schäfer and Moore ${ }^{6}$ (1896) reinvestigated the inmervation of the spleen in the dog, using the plethysmographic method. They found motor nerves to be present in the third thoracic to the first lumbar inclusive (on both sides). The most marked effect was obtained on stimulating the sixth, seventh, and eighth thoracic nerves; a very distinct contraction on stimulating the fifth, ninth, and tenth nerves; a slight contraction on stimulating the third, fourth, eleventh, twelfth, and thirteenth thoracic and the first lumbar nerve.

${ }^{1}$ Virchow's Archiv, 1877, Bd. lxix. S. 181. For other literature, ef. Schäfer and Moore (op. cit. infra).

${ }^{2}$ Journ. Physiol., Cambridge and London, 1896, vol. x. p. 358. I have seen in the eat prompt and great pallor of the kidney and of the upper part of the rreter on stinulating the first lumbar nerve, and slower, though still great, pallor on stimulating the second lumbar nerve. It appears to me unlikely that the third lumbar nerve, and still more unlikely that the fomth lumbar nerve, send fibres to the kidney in the dog.

3 Arch. de physiol. noin. et path., Paris, 1889, Sulpl., p. 243.

${ }^{4}$ Journ. Physiol., Cambridge and London, 1894, vol. xvii. 1. 120 ; the filres to the lortal vein run in the splanchnic nerves. It had already been shown by Mall (Arch. $f$. Physiol., Leipzig, 1892, S. 409) that the splanchnic coutains vasomotor fibres for the portal vein.

5 "Short Aceount of the Sympathetic System," 1895.

6 Journ. Physiol., Cambridge and London, 1896, vol. xx. 1. 1. 
François-Franck and Hallion ${ }^{1}(1896,1897)$ have investigated in the dog the vasomotor supply of the liver, the pancreas, and the small intestine, and the first part of the large intestine. They found that the vasomotor fibres in each case leave the spinal cord by the sixth thoracic to the second lumbar nerve inclusive. The vessels of the large intestine only occasionally receive fibres from the sixth thoracic; the vessels of the small intestine sometimes receive vasomotor fibres from the fifth, and occasionally from the fourth thoracic. The maximum effect on the intestine appeared to be obtained from the twelfth and thirteenth thoracic nerves. The second lumbar had a slight effect only upon the vessels of the pancreas.

Lastly, Bunch ${ }^{2}$ has experimented on the viscero-motor and visceroinhibitory action of the spinal nerves on the small intestine of the dog and cat. He finds the innervation to extend from the sixth thoracic to the second, third, fourth, or fifth lumbar. The greatest effect was obtained from the eighth thoracic to the first lumbar.

The several observations given above do not enable us to form a simple and consistent scheme of the innervation of abdominal viscera, and we may consider the matter from another point of view.

By dissection, it is easy to see that a large proportion of the splanchnic nerve fibres arise from the white rami communicantes, running in the sympathetic chain for a variable distance. And there is good reason to believe that all the splanchnic fibres are the direct continuation of the fibres of the white rami (cf. p. 646). If this be so, it follows that if any white ramus can be shown not to send fibres to the splanchnic nerves, the spinal nerve from which the white ramus springs, does not send fibres to the solar ganglia.

In the cat and dog the fibres of the great splanchnic nerve can be traced upwards in the sympathetic chain as far as the sixth thoracic ganglion, ${ }^{3}$ and this was one of the facts which led me earlier to place the upper limit of connection of the spinal cord with the solar ganglia at the sixth thoracic nerve. But, on examining the course of the white rami, some fibres of the fifth thoracic ramus can be seen to descend in the sympathetic chaill. As these fibres do not run to the vertebral ganglia (cf. p. 634), we may conclude that they run to the splanchnics. It is possible that a few fibres also descend from the fourth white ramus. But, so far as I have seen, none of the fibres of the third white ramus (cat) run downwards. We may then conclude that the uppermost nerve to send fibres to the solar ganglia is the fourth or fifth thoracic.

The fibres of the lowest splanchnic strand in the cat usually leave the sympathetic chain a little above the third lumbar white ramus. ${ }^{4}$ There is some variation here, according as the arrangement of nerves is anterior or posterior. On tracing the white rami, it can be seen that the second lumbar white ramus constantly sends fibres to the smallest splanchnic, and that the third lumbar white ramus sends a few fibres to it, if the arrangement of nerves is posterior. Probably, in the dog, the

${ }^{1}$ Arch. de physiol. nowm. et puth., Paris, 1896, 1. 478 (intestine) ; ibid., 1896, p. 921 (liver); ibid., 1897 , 1. 661 (pancreas). These autliors stimulated the sympathetic chain or the white rami, and not the spinal nerves in the vertebral canal.

2 Journ. Physiol., Cambridge and London, 1898, vol. xxii. p. 357.

${ }^{3}$ In man, it is said that the splanclmic fibres ean be followed up to the level of the second or third thoracie ganglion.

${ }^{4}$ In man, the smallest splanchnic leaves the sympathetic chain about the level of the twelfth thoracic ganglia. 
lowest nerve sending fibres to the splanchnic is, with an anterior arrangement of nerves, the first lumbar, and with a posterior arrangement of nerves, the second lumbar.

It will be seen that the conclusions thus reached agree very closely with the experimental results of Francois-Franck and Hallion, on the vasomotor supply of the liver, pancreas, and intestine in the dog. The nerves which had a marked effect were the sixth thoracic to the first lumbar; the second lumbar had a slight effect-probably there would have been none if the arrangement of nerves had been anterior; the fifth thoracic had an effect sometimes, and the fourth very rarely.

On the other liand, the third thoracic nerve is stated by Bulgak and by Schäfer and Moore to have an effect on the spleen; the third and fourth lumbar are said by Bradford to cause contraction of the kidney vessels; and the third, fourth, and fifth lumbar nerves are said by Bunch to cause contraction or inhibition of the small intestine. But in all cases the effects were slight, and in none were the nerve fibres producing them shown to pass by the splanchnics. With regard to fibres from the third and fourth lumbar nerves, it is possible that some might pass to the intestine, etc., by way of the inferior mesenteric ganglia, but I think this is very unlikely. It is also to be borne in mind that in the dog, so far as is known, the fifth lumbar nerve never has a white ramus, and that sometimes (anterior plexus) the fourth lumbar nerve also has no white ramus.

We have said that the cœliac artery, the superior mesenteric artery, and the renal artery receive their nerves in large part, but not altogether, from separate ganglia. A comparison of the facts we have learnt with regard to the connections of the vertebral ganglia, does not give us any sure guidance as to the connection of the several pre-vertebral ganglia, but it suggests that a more anterior set of nerves will pass to the cœliac than to the superior mesenteric ganglia, and to the superior mesenteric than to the renal ganglia, and that these sets will overlap considerably. It suggests further that, as regards the connection of the spinal nerves with the several arteries, there will be an additional overlap, due to fibres from one ganglion passing to more than one artery.

It is not worth while to discuss this matter at present, since there are practically no anatomical or histological data, and the experimental data are insufficient. It may, however, be pointed out that the spinal nerves which have the maximum effect on the spleen are the mid-thoracic (Schäfer and Moore), whilst those which have the maximum effect upon the vessels of the intestine (François-Franck and Hallion) and of the kidney (Bradford) are the lower thoracic. It may be expected that further investigation will show that the maximum effect on the intestine is obtained from a more anterior set of nerves than that which gives the maximum effect on the kidney.

Lower abdominal viscera.-The sympathetic nerve strands which supply the lower abdominal and pelvic viscera, are three or four on each side. Since these strands correspond to the splanchnic nerves, we may call them the inferior splanchnic nerves. ${ }^{1}$

The inferior splanchnic nerves run to the inferior mesenteric ganglia. In the cat, rabbit, and many other animals, there are two on each side, one above and one below the inferior mesenteric artery, connected with one another by nerve strands. As the connecting strands are often short, the whole group is often spoken of as a single ganglion, the inferior mesenteric ganglion.

${ }^{1}$ They have also becn called the rami efferentes of, and the spinal branches to, the inferior mesenteric ganglion. 
The inferior mesenteric ganglion gives off several strands - the colonic nerves-which accompany the inferior mesenteric artery. They give off also, on each side, a hypogastric nerve which rums downwards to the pelvic plexus, and ascending branches which run partly to the descending colon and partly to the solar plexus.

Stimulation of the colonic branches causes great pallor, with inlibition and sometimes contraction of the descending colon and rectum ; and, in the cat and dog, slight contraction and pallor of the internal anal sphincter.

The ascending branches have a similar but smaller effect upon the upper part of the descending colon.

The hypogastric nerves cause great pallor with more or less contraction of the Fallopian tubes and uterus, or corresponding organs in the male; weak contraction of the bladder, chiefly in the region around the ureters, but little or no contraction of the blood vessels; pallor with inhibition or contraction of the rectum and end of the descending colon; slight contraction and pallor of the internal anal sphincter with its mucous membrane, and of the external generative organs.

The inferior mesenteric ganglia in the cat receive nerve fibres from the first to the fourth lumbar nerves, and, when the arrangement of nerves is posterior, from the fifth lumbar nerve also; occasionally they receive a few fibres from the lower thoracic nerves. In the dog the origin of the fibres is a little higher, and in the rabbit it is a little lower, than in the cat.

Taking the matter generally, it may be said that each spinal nerve which sends fibres to the inferior mesenteric ganglion, is capable of producing all the effects which we have mentioned above as produced by stimulating the nerves given off by the ganglia.

The intensity of the effects with an anterior arrangement of nerves is marked with the third or fourth lumbar, moderate or slight with the second lumbar, and slight or absent with the first lumbar.

With a posterior arrangement of nerves the fifth lumbar also produces an effect, the effect becoming greater as that of the third lumbar diminishes. ${ }^{1}$

Nerve-cell connection of the sympathetic fibres supplying the abdominal viscera.- It can easily be shown in the rabbit that the very great majority of the fibres running from the spinal cord to the abdominal viscera end in the pre-vertebral ganglia. After injection of a small amount of nicotin in the rabbit, the nerve roots, the splanchnics proper, ${ }^{2}$ and the inferior splanchnics ${ }^{3}$ have either no effect or a mere trace on the blood vessels or abdominal and pelvic viscera; but all the normal effects can be readily obtained by stimulating the fibres given off by the pre-vertebral ganglia. And on degeneration of the anterior roots of a nerve sending fibres to a splanchnic strand, numerous

${ }^{1}$ For the differences in the innervation of the several organs which occur in correspondence with the arrangement of the spinal nerves, for the slight differences which exist in the innervation of the several organs, and for an account of the literature of the subject, see Langley and Anderson, Journ. Physiol., Cambridge and London, 1895, vol. xviii. p. 67 (lower part of intestine); ibid., 1895, vol. xix. p. 71 (bladder); p. 86 (external generative organs); p. 122 (internal generative organs).

${ }^{2}$ Langley and Dickinson, Proc. Roy. Soc. London, 1889, vol. xlvi. p. 424 ; Langley Journ. Physiol., Cambridge and London, 1896, vol. xx. p. 223; Bunch (ibid., 1897, vol. xxii. p. 357) comes to the same result as regards the motor and inhibitory fibres of the small intestine.

${ }^{3}$ Langley and Anderson, ibid., 1895, vol. xix. p. 131. 
degenerated fibres can be traced into the strand. In the cat, after degenerative section of the first five lumbar nerve roots, it was found by Anderson and myself ${ }^{1}$ that the several inferior splanchnic nerves in nearly every case had no effect when stimulated, although the colonic fibres and the hypogastrics caused contraction of arteries and the remainder of their customary actions.

Occasionally, after injection of nicotin in the rabbit, a rise of 1 or $2 \mathrm{~mm}$. of arterial pressure may follow on stimulation of the splanchnics. Theoretically, I see no adequate reason why some nerve cells which send their fibres to the abdominal viscera, should not be situated in the vertebral ganglia, for it is a probable assumption that the pre-vertebral and the vertebral ganglia have separated from a common mass, and in that case it might easily happen that the separation would not be strictly carried out. In the ganglion stellatum the
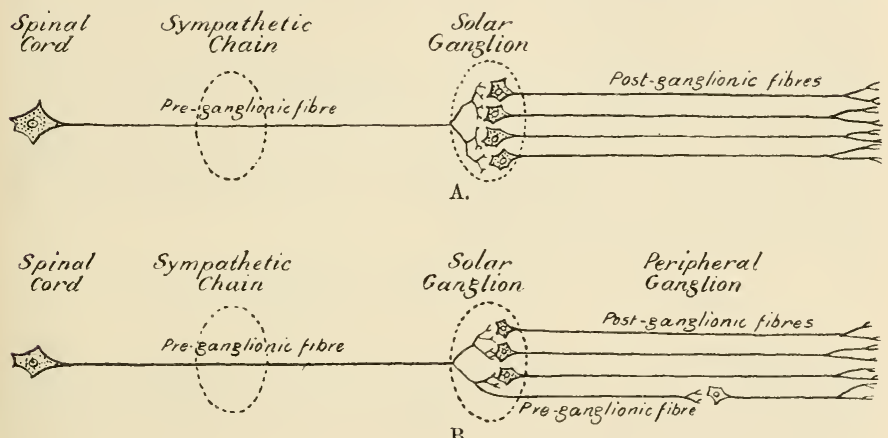

B.

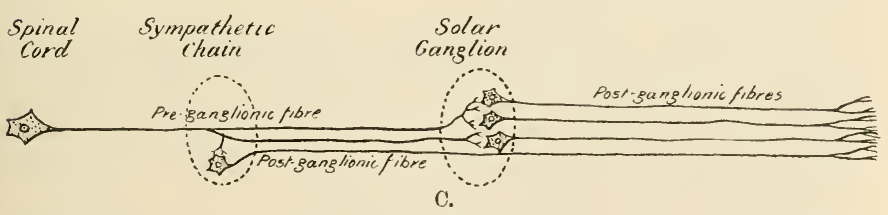

Fig. 313.

separation does not appear to have taken place, or only partially. But if the slight rise of blood pressure mentioned above be taken as showing the occasional presence of a few fibres proceeding from cells in the vertebral ganglia, it is clear that they run to the periphery without having nerve cells in their course, unless we suppose that the connection of sympathetic nerve cells with other nerve cells is not paralysed by nicotin, a supposition on which I shall later have something to say (p. 685).

There is, however, good evidence that some efferent fibres rum through the inferior mesenteric ganglion, pass down the hypogastric nerves, and end in connection with cells of the pelvic plexus. A sufficient proof of this is the degeneration of nerve fibres which takes place in the hypo-

\footnotetext{
1 Journ. Physiol., Cambridge and London, 1894, vol. xvi. p. 432.
} 
gastric nerves after section of the roots of the lumbar nerves. ${ }^{1}$ And this is, no doubt, also the case with the solar ganglion; some fibres traverse it and run to small peripheral groups of ganglion cells, such as those which occur in the pancreas. Whether such fibres send a branch to the main pre-vertebral ganglion, as they traverse it, requires experiments of a different kind to determine (cf. p. 679). We may for the present regard the splanchnic and inferior splanchnic nerve fibres as arranged as in Fig. 313 ; the great majority, as in A; comparatively few, as in B; and possibly, occasionally, a few as in C.

It has long been known that the major splanchnic contains a considerable number of non-medullated fibres, and it is in large part this fact which has led to the unquestioned belief that the ganglia of the sympathetic chain send fibres to the solar ganglia or to the abdominal viscera. It is a priori possible that the non-medullated fibres in the splanchnic nerve should arise from the solar ganglion and run to the ganglia of the sympathetic chain, or to their grey rami. But this possibility I put on one side, since I have not observed any pilo-motor, vasomotor, or other similar effect, on stimulating the central end of the splanchnic nerve after removal of the spinal cord. If, then, the view I have given above be accepted, namely, that very few if any fibres pass from the cells of the vertebral ganglia to the splanchnic nerves, we must take the non-medullated fibres to be pre-ganglionic fibres which have lost their medulla (cf. p. 650).

\section{The Nerve Fibres of the Sympathetic System.}

Distribution of medullated and of non-medullated fibres.-A striking feature of the sympathetic system is the predominance of small medullated fibres in it. This was pointed out by Bidder and Volkmann ${ }^{2}$ in 1842. The great majority of its medullated fibres vary from $2 \mu$ to $3.5 \mu$ in diameter.

Other larger medullated fibres are present to about $12 \mu$. These vary in number in the different strands, and although all sizes, from 3.5 to $12 \mu$, occur, they may, broadly speaking, be divided into two classesmedium-sized fibres about $5 \mu$, and large fibres of about $8 \mu$. The size of small, medium, and large vary somewhat in different animals. Very large medullated fibres, 15 to $20 \mu$, which are common in the roots of the spinal nerves, do not occur in the sympathetic system.

The large fibres of the sympathetic occasionally divide. In this case the branches are of somewhat less diameter than the parent fibre. Most of the large fibres in the cat can be traced to Pacinian bodies.

In the trunk of the sympathetic the large and medium fibres are most numerous in the lower dorsal and upper lumbar regions. Here, too, they are somewhat larger than in the lower lumbar region. Most of them pass outwards in the superior and inferior splanchnics. Below the lowest inferior splanchnic strand, there are few fibres larger than $5 \mu$ in diameter, and they diminish in number in passing downwards. In the cervical sympathetic there are sometimes a few medium and 1). 183

${ }^{1}$ Langley and Anderson, Journ. Physiol., Cambridge and London, 1894, vol. xvii.

2 “Die Selbstständigkeit des sympathisehen Nervensystems," Leipzig, 1842. Numerous observations of the size of the medullated fibres in the different nerves of vertebrates are here given. 
large fibres, and sometimes none; the few which may be present probably arise from the vagus. In the cat, the sympathetic chain-apart from its ganglia-contains few non-medullated fibres. Some of these are fibres we have spoken of above (p. 629) as passing from one ganglion to the ramus of a neighbouring ganglion. In the rabbit, non-medullated fibres are more numerous, and in the sacral region they are the chief constituent, very few medullated fibres being present. These nonmedullated fibres I take to be largely pre-ganglionic fibres which have lost their medulla (cf. p. 650).

The non-medullated fibres make up about one-fifth of the transverse section of the great splanchnic nerve (cf. p. 642). There are fewer in the small splanchnics, still fewer in the inferior splanchnics and in the cervical sympathetic.

In the branches proceeding from the superior cervical ganglion and the pre-vertebral ganglia the non-medullated fibres preponderate. Some have but few medullated fibres, and others have many.

The internal carotid branches of the superior cervical ganglion contain in the cat several hundred small medullated fibres, in the rabbit there are a few only, and usually a few only in the dog. The external carotid branches of the superior cervical ganglion contain only a few medullated fibres. A small nerve filament, consisting chiefly of medium and large fibres, runs to these branches from the vago-glosso-pharyngeal nerve. The cardiac accelerator nerve, which runs from the ganglion stellatum, has a few medullated fibres up to about $5 \mu$ in diameter. The branches from the solar ganglia vary greatly in the number of medullated fibres they contain. The hypogastric nerve ${ }^{1}$ in the cat contains 350 to 900 medullated fibres; of these, one or two only, and sometimes none are large.

In the grey rami the great majority of the nerve fibres are nonmedullated. The limit of size of the medullated fibres is about 1.8 to $10 \mu$ in diameter; they are scattered amongst the non-medullated fibres. The number of medullated fibres in the grey rami varies considerably in different mammals. In the rabbit there are few, in the cat there are many. Thus in the seventh lumbar grey ramus of the cat there may be more than 300 medullated fibres.

The variation in the number of medullated fibres in the grey rami affects almost entirely the small ones, and the most constant form is the medium fibre. Most of the small medullated fibres of the grey rami are post-ganglionic fibres (cf. p. 650). Others are pre-ganglionic fibres on their way to aberrant sympathetic cells. Others, again, medium and large, as well as small, are afferent fibres which run in the white rami to the sympathetic chain from the spinal ganglia, and after a course of varying length leave the chain and accompany the post-ganglionic efferent fibres. When the grey ramus divides, part running centrally and part peripherally, medullated fibres, small and medium, accompany both branches. Both, then, on stimulation, should produce some reflex effect. This, however, so far, has not been obtained. ${ }^{2}$ There is reason to believe that one or two of the medium and large fibres of the grey rami have their trophic centre in the corresponding spinal ganglia, ${ }^{3}$ i.e. that not all the afferent fibres run to the spinal cord by the white rami,

${ }^{1}$ Langley and Anderson, Journ. Plyssiol., Cambridge and London, 1894, vol. xvii. p. $17 \%$.

${ }^{2}$ Langley, Phit. Trans., London, 1892, vol. elxxxiii. p. 120.

${ }^{3}$ Langley, Jourı. Physiol., Cambridge and London, 1896, vol. xx. p. 55. 
but that a few run to it by the grey rami. There is not, however, any definite physiological evidence for the existence of such afferent fibres.

Loss of medulla by pre-ganglionic fibres.-We have already seen reason to believe that some of the pre-ganglionic fibres of the splanchnic nerve lose their medulla on their way to the pre-vertebral ganglia (cf. p. 648). The evidence that pre-ganglionic fibres are not necessarily medullated up to their terminations, is still stronger in the case of the vertebral chain.

By the degeneration and by the nicotin method, it can be shown that in the cat fibres run from the upper lumbar white rami to the sacral and coccygeal ganglia without passing through nerve cells. In the rabbit the nicotin method only has been tried; it gives the same results. We may then conclude that in the rabbit there are preganglionic fibres, stretching from the upper lumbar white rami to the sacral and coccygeal ganglia. But since the sympathetic in the sacral and coccygeal region of the rabbit contains very few medullated fibres, it follows that the pre-ganglionic fibres in this region must be nonmedullated, and as they are medullated in the white rami, they must become non-medullated in passing down the sympathetic chain. In other words, pre-ganglionic fibres may become non-medullated some distance from their termination in the vertebral ganglia.

Post-ganglionic medullated fibres.-Although Remak discovered non-medullated nerve fibres (organic fibres) in 1838,1 their nervous nature was not generally accepted till about 1860 . Thus, most of the early observers who believed that the sympathetic ganglia gave off nerve fibres at all, necessarily believed that they gave off medullated nerve fibres (primitive nerve fibres, tubular fibres). Those observer's, on the other hand, who accepted the nervous nature of Remak's fibres, were inclined to think that these alone were sympathetic fibres, and that all medullated fibres were of cerebro-spinal origin. As time went on, the observations of Bidder and Volkmann ${ }^{2}$ on the origin of small medullated fibres from sympathetic and spinal ganglia seemed more and more irreconcilable with the results of research, and in consequence less and less attention was paid to them. Thus, some writers passed by the question of the medullation of ganglionic nerves, without even considering it, and others took for granted that no further proof was required to show that all fibres given off by sympathetic nerve cells are non-medullated.

The proof brought forward by Bidder and Volkmann, that in the frog small medullated fibres arise from sympathetic ganglia, was fairly conclusive. The essential part of the proof was the fact that many more small medullated fibres run from the sympathetic ganglia to the periphery, than run to the ganglia from the central nervous system. As at that time there was practically no knowledge of the connection of nerve cells with nerve fibres, or of the finer structure of the ganglia, it might have been objected that the increase in the number of fibres peripherally of the ganglia was merely due to a division of nerve fibres in them. But, in the light of our present knowledge of the ganglia, Bidder and Volkmann's proof must be regarded as conclusive. They showed also, and in the same way, that the ciliary ganglion gives off medullated fibres.

${ }^{1}$ Remak, "Observ, anat. et micros. de systematis nervosi structura," Berlin, 1838.

2 "Die Selbstständigkeit des sympathischen Nervensystems," Leipzig, 1842. 
The observations of Waller ${ }^{1}$ on the superior cervical ganglion of mammals showed that this ganglion also gives off medullated nerve fibres. He did not himself make this deduction, presumably because he, like many others at the time, considered the question to have been settled by Bidder and Volkmann. He found that section of the cervical sympathetic caused degeneration of the fibres up to the superior cervical gallglion, but not beyond it, and that section of the internal carotid branches of the ganglion caused degeneration in the peripheral branches; degenerated fibres were followed as far as the ophthalmic nerve.

These experiments I have repeated, ${ }^{2}$ without at the time knowing of Waller's experiments on the internal carotid strands, and with the same results, except that after section of the internal carotid branches a few sound fibres were found in the peripheral end, and a few degenerated fibres in the end attached to the ganglion.

Kölliker in 1844 , in teasing out the fourth thoracic sympathetic ganglion of the cat, found in some cases that one of the processes of the cell could be traced into a medullated fibre. Numerous other similar instances have been recorded in various vertebrates, and in some of the recent observations on sympathetic ganglia by the Golgi and the methylene-blue method, the axons of the cells have been found to become medullated.

By the degeneration method it can also be shown ${ }^{3}$ that the great majority of the small medullated fibres of the grey rami arise from the nerve cells of the corresponding sympathetic ganglion.

A few of the medullated fibres arise occasionally from an adjoining ganglion, and a few are pre-ganglionic fibres, which are on their way to the aberrant nerve cells of the grey rami. The inferior mesenteric ganglion, and the ganglia of the pelvic plexus, send off very few, and possibly at times no non-medullated fibres. ${ }^{4}$ The same appears to be the case with the ganglia on the cranial nerves, ${ }^{5}$ with the exception of the ciliary ganglion, but the observations are not very complete.

So far as investigation has gone, the efferent medullated fibres which arise from nerve cells are all small. ${ }^{6}$

Summing up, then, we find that medullated post-ganglionic fibres are present in mammals, in amphibia, and probably in all vertebrates. 'They are relatively very much more numerous in amphibia than in mammals; in amphibia, indeed, non-medullated fibres are fewer than medullated, and they vary in number in different mammals. Thus, few medullated fibres are given off by the sympathetic ganglia in the rabbit, but a considerable number in the cat.

We should naturally expect to find some difference in function between the medullated and the non-medullated post-ganglionic fibres, but there is not at present any evidence of such difference.

1 "Nouvelle méthode anatonique, etc.," Bonn, 1852.

2 Langley, Journ. Physiol., Cambridge and London, 1896, vol. xx. p. 70.

3 Langley, op cit.

${ }^{4}$ Langley and Anderson, Journ. Physiol., Cambridge and London, 1894, vol. xvii. p. 177.

${ }^{5}$ On the spheno-palatine ganglion in the dog, ef. Prevost, Arch. de jhiysiol. norm. et path., Paris, 1867 , p. 207.

${ }^{6}$ In some cases apparently they are as much as $4 \mu$ in cliameter, that is, in the border line between small and medium. 


\section{Degeneration of Sympathetic Nerve Fibres after Section- Duration of IrRitability.}

The medullated nerve fibres of the sympathetic undergo the same series of changes in degeneration as other medullated fibres. But the changes are in most cases more rapid; since most of the nerves are small, and the medulla, which has to be absorbed, is less in amount. Thus, after section of the sympathetic trunk, all the fatty portion of the medulla may be absorbed in two or three weeks, and the degeneration which has taken place may only be indicated by a few rows or small clumps of granules.

In non-medullated nerves, section produces extremely little histological change, when the nerves are treated with most reagents ${ }^{1}$; but Tuckett ${ }^{2}$ has shown that the core of the non-medullated nerve in about two days ceases in the fresh state to stain with methylene-blue. The conducting core appears to become granular and then to be absorbed, the sheath still remaining.

Broadly speaking, sympathetic nerves lose their irritability in the dog, cat, and rabbit, about the fourth day after section, sometimes a little earlier, sometimes a little later. There is no satisfactory evidence that there is any constant difference in the time after section at which different classes of nerve fibres lose their irritability (cf. above, p. 626). The time is longer in old animals than in young; it varies in different animals, and with the state of nutrition of the animal; it varies also with the size of the nerve cut. In the rabbit, Tuckett found that the branches of the superior cervical ganglion which run to the internal carotid artery, lose their irritability two days after section; but it may be doubted whether early degeneration is characteristic of non-medullated fibres, since the vaso-constrictor fibres of the sciatic of the dog retain their irritability up to about the fourth day.

Waller ${ }^{3}$ was the first to make observations on the duration of irritability of the cervical sympathetic. He found that, at the end of the third day, stimulation of the peripheral (upper) end no longer caused dilatation of the pupil. Budge, ${ }^{4}$ as the result of experiments on rabbits, stated that the irritability of the pupillo-dilator fibres of the sympathetic lasts four to six days, and occasionally even longer.

\section{Regeneration of Sympathetic Fibres.}

Pre-ganglionic fibres.-In the earlier observations on the regeneration of nerve fibres, pre-ganglionic fibres were at times the subject of experiment, but no distinction was made between them and any other nerve fibres. Some attention was directed to observing differences in the time of return of function to nerve fibres of different classes, as motor and sensory, vaso-constrictor and vaso-dilator, but the inherent differences attending regeneration in the pre-ganglionic and post-ganglionic fibres were not even mentioned. Waller ${ }^{5}$ found that, two months after section of the cervical sympathetic, dilatation of the pupil could be obtained by

${ }^{1}$ Langley, and Anderson, Journ. Physiol., Cambridge and London, 1894, vol. xvii. p. 183.

${ }_{2}^{2}$ Ibid., 1896, vol. xix. p. 291

${ }^{3}$ Compt. rend. Acal. d. sc., Paris, 1851.

4 "Ueber die Bewegung der Iris," Braunschweig, 1855.

${ }^{5}$ Compt. rend. Acad. d. sc., Paris, 1853, p. 378. 
stimulating the cut nerve; and in the subsequent observations of Schiff, v. Beke Callenfels, and others, on the duration of the effects of excision of the sympathetic, it was pointed out that a considerable length of nerve must be excised to prevent regeneration. I have found ${ }^{1}$ a return, though not to the full extent, of all the functions of the sympathetic in the cat twenty-four days after section. The return of function probably begins in about a fortnight. Regeneration, then, is fairly rapid in the cervical sympathetic, and hence probably in other pre-ganglionic sympathetic fibres.

We have seen that in the cat seven thoracic nerves send fibres to the superior cervical ganglion, and that each of these produces its own peculiar effects. Hence, no two thoracic nerves send fibres to exactly the same cells in the superior cervical ganglion. Moreover, since the highest of the series of thoracic nerves do not produce any of the effects of the lowest of the series, the highest and the lowest must run to a completely different set of cells in the ganglion.

Now, when the several thoracic nerves in the cat are stimulated after regeneration of the cut cervical sympathetic, ${ }^{2}$ each produces, in the majority of cases, its normal effects.

Thus (usually), the first thoracic nerve causes good dilatation of the pupil, opening of the eye, and retraction of the nictitatiug membrane, but causes little or no contraction of the arteries of the ear, and no effect on the erectile hairs; the third thoracic nerve causes very little dilatation of the pupil, moderate opening of the eye, and retraction of the nictitating membrane, good contraction of the arteries of the ear, but (usually), like the first thoracic nerve, it has no effect upon the erectile hairs; the sixth thoracic nerve produces none of the effects mentioned above, but causes more or less marked movement of the erectile hairs.

It is conceivable that in regeneration the nerve fibres should grow through the superior cervical ganglion and become directly connected with peripheral structures. But since nicotin, both when applied locally to the ganglion and when injected into the blood, has its normal effect, we must conclude that the nerve fibres have formed new terminations in connection with the nerve cells.

We arrive, then, at the remarkable fact that, in most cases, each spinal nerve becomes connected with nerve cells similar to, if not the same as, those with which it was previously connected, and with no others. It is difficult to account for this, except on the supposition that the regenerating fibres are guided to their appropriate nerve cells by some chemiotactic attraction. The guiding influence might either lie in the remains of the old fibres or in the characters of the nervo cells.

The selective connection of nerve fibres with nerve cells occurs also if a regenerated nerve be cut; and the second regeneration takes place as readily as the first.

It may be mentioned that not infrequently, though by no means always, an upper thoracic nerve, after regeneration of the cervical sympathetic, has not quite so strong an action as normally, as if the regeneration were not quite complete. This is probably due to a failure of some of the nerve fibres to make their way through the

1 Journ. Physiol., Cambridge and Loudon, 1897, vol. xxii. p. 228.

${ }^{2}$ Langley, ibid., 1895, vol. xviii. p. 280 ; and 1897, vol. xxii. p. 215. 
cicatricial tissue which forms early between the cut ends of the nerves.

The return of function to the sympathetic takes place before the freshly formed fibres have become medullated. Thus, in the case mentioned above, in which regeneration had occurred twenty-four days after section of the cervical sympathetic, no medullated fibres were found in the peripheral portion.

Seven to eight weeks after section of the nerve, the medullated fibres in the regenerated part of the nerve are still few in number. Even fifteen months after section, the regenerated nerve has fewer medullated and more non-medullated nerves than normal.

Although the fibres from each spinal nerve usually take on during regeneration the functions they possessed before section, they do not always do so. Thus the first and second thoracic nerves may after regeneration cause erection of hairs, and the fourth and fifth thoracic nerves may cause dilatation of the pupil, effects which they do not normally produce. The selective action of the nerve fibres has therefore its limits. And we may conclude that a sympathetic nerve fibre of one class, as pupillo-dilator, is capable of becoming connected with a nerve cell of another class as pilo-motor.

Post-ganglionic fibres. - In the accounts which have been given of observations on the regeneration of the nerves to the limbs, mention is occasionally made of recovery of vasomotor action. Since we have seen reason to believe that the vasomotor fibres in limb-nerves are post-ganglionic, we may conclude that post-ganglionic fibres can regenerate. Extremely few direct observations have, however, been made on the matter. Tuckett ${ }^{1}$ found, in a rabbit, that the anterior strands of the superior cervical ganglion regenerated in 259 days. Stimulation of the cervical sympathetic caused dilatation of the pupil, and treatment of the internal carotid with dilute methylene-blue showed the presence of regenerated non-medullated fibres.

The problem already dealt with in relation to the pre-ganglionic nerves obviously occurs also, in a slightly modified form, in relation to the postganglionic nerves. The fibres from the several cells run to different tissues. When the fibres re-grow, will they end in connection with the tissue with which they were previously connected? I have, as yet, made one experiment only on this point. The anterior branches of the right superior cervical ganglion were cut in a cat; after nineteen weeks, the upper thoracic nerve roots were stimulated on both sides. On the left side, the usual effects were obtained. But on the right side, the first six thoracic nerves, instead of the first three only, caused dilatation of the pupil. A part of the region of the face in which the hairs are normally strongly erected, by stimulation of the fifth and sixth thoracic nerves, was barely affected by stimulation of any nerve. The explanation of this which at once presents itself, is that some of the fibres which normally run to the hairs of the face, had, in the process of regrowth, been carried out of their way, and had formed new nerve-endings in the pupil. If this explanation is correct, it follows that a nerve cell does not necessarily send its axis cylinder process to a particular kind of peripheral tissue; and it suggests that there is no specific difference between the different classes of post-ganglionic fibres, and that the function which each serves is in a way accidental, depending upon the kind of peripheral tissue with which it first comes in contact.

1 Jouru. Physiol., Cambridge and London, 1896, vol. xix. p. 297. 


\section{Some Features of the Structure of Sympathetic Nerve Cells.}

Our knowledge of the minute structure of sympathetic nerve cells has been greatly furthered by the use of the Golgi and methylene-blue methods of staining. ${ }^{1}$ IVe need, however, only mention those points which bear upon the experimental results.

In mammals, the sympathetic nerve cell has a single nerve fibre process and a considerable number of dendrons. In this it resembles most of the nerve-cells of the central nervous system; but in most cases, at any rate, a characteristic appearance is given it by the arrangement of the dendrons.

In amphibia and in some other lower vertebrates, dendrons are not always present. Thus the spiral cells, discovered by Beale and Arnold, have no dendrons, the straight process is the nerve fibre.

In such sympathetic ganglia as have been investigated, fine nerve fibres are found penetrating the ganglion and ending in it, in more or less close relation with the nerve cells. In amphibia, such fibres form the spiral "process" of the pyriform cells, and end in fine branches underneath the cell capsule. In some cases the entering nerve fibres divide and end in connection with more than one nerve cell. In the sympathetic chain of birds and mammals a fibre may be seen giving off a branch to end in a ganglion, and then continuing on its way, presumably to end in another ganglion. We may reasonably take the fibres ending in the ganglion to be the pre-ganglionic fibres, and the fibres given off by the nerve cells to be the post-ganglionic fibres, which we have described on experimental grounds. So far, the histological and the physiological results are plainly in harmony.

\section{The Degree of Permanence of the Effects caused by Section of Sympathetic Nerves.}

Speaking generally, it may be said that on section of all, or of the great majority of sympathetic nerves to a tissue, paralytic effects may be observed in a few seconds. But in anæsthetised animals, at any rate, the degree of paralysis immediately following the section differs widely in different cases. It is probable that when the symptoms develop slowly, there is some condition of stimulation, either of the cut ends of the nerve fibres or of their peripheral terminations, which only slowly dies out. When vaso-constrictor fibres are cut, the extent of the flushing will depend in part upon the arterial blood pressure. And in cases of section of the majority only of the sympathetic fibres to a tissue, the degree of paralysis will, of course, depend upon the number and activity of the uncut fibres.

These effects may be maximal in a few seconds after section of the

${ }^{1}$ Kölliker, Sitzungsb. d. phys.-med. Gesellsch. zu Wiur $b u r g, 1889$; Verhanall. d. naturf. Gesellsch. in Wien, 1892 ; Sitzungsb. d. phys.-med. Gesellsch. zu Würzburg, 1894 ; Wien. klin. Wchusehr., 1894, No. 40-41. Snirnow, Arch. f. mikr. Amat., Boun, 1890, Bd. xxxv. S. 407. Cajal, Arch. f. Anat. u. Entwcklngsgesch., Leipzig, 1893, S. 319: "Atlas d. pathol. Histologie d. Nervensystem," Berlin, 1895, Lief. 4. v. Gehuehten, Cellule, Lonvain, tome viii. 1. 83 ; Anat. Anz., Jena, 1893, Bd. viii. Retzius, Biol. Untersuch., Stockholm, 1892, N.F., Bd. iii. Sala, Arch. ital. de biol., Turin, 1893, tome xviii. p. 439. Lenhossék, "Beitr. z. Histol. des Nervensystems, u.s.w.," Wiesbaden, 1894. A. S. Dogiel, Arch. f. mikr. Anat., Bonn, 1895, Bd. xlvi. S. 305.'Arnstein and Plosehko, Aurt. Anz., Jena, 1897, Bd. xiii. S. 17. Other references will be found in Kiolliker, "Handbuch d. Gewebelehre," 1896, Bd. ii. S. 870, and in Huber, Journ. Comp. Neurol., 1897, vol. vii. 
nerve, or they may only become so in anæsthetised animals after some delay.

In the frog the development of the paralytic symptoms in the eye (and tongue), after section of the sympathetic or extirpation of the superior-cervical ganglion, is usually, if not always, gradual.

In the rabbit there is an early stage in the condition of the pupil, ${ }^{1}$ after section of the post-ganglionic fibres, which has not been noticed in the cat or dog. After about twenty-four hours the pupil begins to dilate, slowly enlarges, and on the third to the fifth day again becomes small and remains smaller than normal. This may be taken to be due to stimuli set up in the fibres during degeneration, though it is curious that this reaction of degeneration should not occur, so far as we know, in the vaso-constrictor fibres of the rabbit, or in any of the fibres in the sympathetic of the eat and dog.

Apart from this temporary escape from the eflect of section, there is, in all animals, as time goes on, some degree of return to the normal condition. This varies in different animals; there is much difference of opinion with regard to it. The recovery is generally held to be greater and more complete in the rabbit than in the cat and dog.

Section of the cervical sympathetic causes a permanent diminution in the size of the pupil. In the cat and dog this is obvious, in the rabbit it is much less; even a week or two only after section, the difference from the normal pupil may be very slight.

Less attention has been paid to the other effects produced on the eye by section of the nerve. In the cat, the narrowing in the palpebral fissure is for several months more or less obvious on the side of the lesion; the nictitating membrane, which for a few days covers about a half of the cornea, is gradually withdrawn until it projects very little more than the normal one. The increase in the secretion poured on the surface of the eye lasts in the dog two or three weeks only.

The nictitating membrane does not maintain a constant position ; a month or more after section, it may at times be almost completely withdrawn, and at times project and cover about a third of the cornea.

As we have said, the great dilatation of the vessels of the ear, which is seen in the first few days after section of the cervical sympathetic, or after excision of the superior cervical ganglion, gradually diminishes, at any rate as a rule.

Not infrequently in the rabbit the vessels of the ear on the side of the lesion return nearly to normal in four or five days. The rapid recovery in these cases we must attribute to the presence of uncut vasomotor fibres, passing to the ear by way of the vertebral ramus of the ganglion stellatum and the great auricular nerve (cf. p. 624).

In the cat and dog the decrease in the flushing is slow, going on for some weeks; and it is doubtful whether the normal tone is ever completely regained. In some cases, indeed, in the rabbit as well as in the cat and dog, it is said that the paralytic effects continue for a very long period - a year and more-without any considerable diminution.

S. 125 . This was first noticed by Budge, "Ueber d. Bewegung. d. Iris," Braunschweig, 1855, 
The principal papers dealing with the duration of the paralytic effects are quoted below. ${ }^{1}$ It will be sufficient here to mention the comparatively few instances in which paralysis of a long duration has been described, either after section of the sympathetic or removal of the superior cervical ganglion. Bernard in one case, in a dog, found that the paralytic effects on the iris, eyelids, and vessels of the ear, were still intense one and a half years after extirpation of the superior cervical ganglion. Schiff observed one case in a dog, in which there was hardly any decrease in intensity in the hyperæmia of the ear for two years after section of the cervical sympathetic. Budge stated that, in rabbits, section of the cervical sympathetic caused a decrease in the size of the pupil, lasting a year after the operation. And Pye-Smith found that the paralytic effects lasted indefinitely in rabbits and cats; in one case, indeed, in a rabbit, after section of the cervical sympathetic and of the spinal auricular nerves, the paralytic effects remained maximal or nearly maximal for more than five years.

Observations have also been made upon the duration of paralytic effects on the vessels of the hind-foot in dogs. Section of the sciatic causes a dilatation of arteries, and a rise of temperature in the foot: according to Goltz, ${ }^{2}$ the temperature begins to fall in four or five days, and becomes in three or four weeks the same as that on the intact side.

The recovery of tone in this case may fairly be referred to vasomotor fibres passing to the foot by the crural nerve, for Lewaschew ${ }^{3}$ found that, as a rule, section of the crural nerve caused a rise of temperature of the foot, and that stimulation of the peripheral end caused a fall of temperature. Spallitta and Consiglio state that on removal of the lumbo-sacral sympathetic chain on one side, the rise of temperature in the foot, though diminishing somewhat, remains considerable for an indefinite period. Their observations, however, do not seem to have extended over a greater period than three months.

It appears certain that unstriated muscle, when severed from the central nervous system, recovers some degree of tone, and it is reasonable to suppose that inherent vitality and responsiveness to stimuli vary in different tissues. Schiff showed that after section of all the nerves to the ear of the rabbit, the arteries still retained their irritability and contractibility, and that irregular automatic contractions and dilatations of the arteries still occurred. There can be little doubt,

${ }^{1}$ Reid, Edin. Med. Journ., 1839 (reprinted in "Physiological, Pathological, and Anatomical Researches," Edinburgh, 1848, p. 291). In this an account of earlier papers will be found. Bernard, "Recherches expérimentales sur le grand sympathetique, ete," Paris, 1845 (sec also "Lecons sur la systeme nerveux," Paris, 1858, tome ii.). Schiff, "Leęons sur la physiol. de la digestion," Turin, 1867, p. 233 et seq. In this Schiff gives a summary of his views and of his experiments from 1854 to 1867. (See "Récueil des mémoires physiologiques," de M. Schiff, Lausanne, 1894, tome i.) Budge, "Ueber die Bewegung des Iris," Braunsehweig, 1855. Van der Beke Callenfels, Ztschr. f. rat. Med., 1855, S. 157. (Full abstracts of the observations made in the immediately prceeding years are given in this paper.) J. Ogle, Med.-Chir. Trans., London, 1858, vol. xli. (observations on man). W. Ogle, ibid., London, 1869, vol. lii. (observations on man and animals). Pye-Smith, Jouri. Physiol., Cambridge and London, 1887, vol. viii. p. 25. Panctl, Centralbl. f. Physiol., Leipzig u. Wien, 1887, s. 272.

${ }^{2}$ Arch. f. d. ges. Physiol., Bonn, 1874, Bd. ix. S. 174. Schiff, however, states that if certain precautions be taken, the foot on the eut side is permanently of a somewhat higher temperature than that of the opposite side.

${ }^{3}$ Ibid., 1882, Bd. xxviii. S. 431. Cf. also Spallitta et Consiglio, Arch. ital. de biol., Turin, 1897, tome xxviii. p. 257. Dastre and Morat "Système nervellx vaso-moteur," Paris, 1884, p. 262) observed pallor of the skin of the toes on stimulating the peripheral end of the crural nerve in dogs.

VOL. II. -42 
then, that the degree of contraction of arteries having no nervous connection with the spinal cord, will to some extent vary with the temperature, the state of nutrition, and so forth.

Whether an area, the vasomotor nerves of which have been cut, is at any moment warmer than the corresponding area of the opposite side of the body, clearly must depend upon a number of independent variables. Since the vessels on the intact side are still under the influence of the central nervous system, they will at times be fully contracted and at times fully dilated. On the cut side the variation is relatively slight. It follows, then, that the tissues on the uncut side will sometimes be warmer and sometimes be colder than on the cut side. And it was shown by Schiff that, after extirpation of the superior cervical ganglion on one side, the ear on the opposite side-ordinarily the cooler-became the warmer when the animal was placed in a heated chamber, when it became hot from exercise, when a feverish condition was induced, and in certain emotional states. On the cut side there was also an increase in temperature, but comparatively a slight one. Similar facts are known with regard to the limbs.

The more or less complete recovery of tone which we have just spoken of might conceivably be due to a peripheral nervous mechanism, and not, as we have suggested, to the inherent nature of the nuuscular tissue. This we will consider in a later section (p. 671).

What has been said above for the eat and dog, with regard to the cervical sympathetic and the superior cervical ganglion, holds also for man. The paralytic effects of section of the cervical sympathetic continue indefinitely, but they diminish in intensity; the recovery is greater in the blood vessels of the ear and face than in the dilator pupillæ. ${ }^{1}$ The presence of sweat glands on the face allows an additional point to be noticed, namely, the permanent absence of secretion of sweat on the side of the lesion. In some cases it has been observed that, during exercise, when the face on the sound side flushes, the face on the side of the lesion becomes paler. This is probably a mechanical effect, the general flushing causing a lowering of blood pressure.

\section{Efferent Pre-Ganglionic Fibres in relation to the Anterior and Posterior Roots of the Spinal Nerves.}

The efferent fibres which pass from the spinal cord to the sympathetic ganglia and to the pelvic nerve ${ }^{2}$ (nervus erigens) form, in mammals at any rate, no exception to the law of Bell and Magendie. They pass outwards in the anterior and not in the posterior roots of the spinal nerves. This is shown both by the absence of effect on stimulating the peripheral ends of the posterior roots, and by the absence of degeneration $^{3}$ in the peripheral ends of the posterior roots when they are cut.

It is true that Stricker and others have found in the $\operatorname{dog}$ a rise of

1 The recovery of tone in the blood vessels appears often to be complete; this suggests either that partial regeneration of the cervical sympathetic has occurred, or that some vasomotor fibres take a path other than that of the cervical sympathetic.

2 Cf. below, p. 666 .

${ }^{3}$ Cf. Sherrington, Journ. Physiol., Cambridge and London, 1897, vol. xxi. 1) 209. References to early work on degeneration in the nerve roots are given in this paper. 
temperature in the limbs, on stimulating the posterior roots of the nerves supplying the limbs. The experiments we consider elsewhere (cf. p. 668), but the observations on degeneration in the posterior roots after section, show that the increase of temperature observed cannot be due to the presence in the posterior roots of efferent fibres arising from the spinal cord.

Budge ${ }^{1}$ found in the rabbit that dilator fibres for the pupil run in the anterior and not in the posterior roots of the first and second thoracic nerves. In the following year he stated $^{2}$ that section of certain anterior roots in the rabbit causes flushing of the ear, whilst section of the corresponding posterior roots has little or no effect. Since that time numerous observations have been made, ef. Dastre and Morat ${ }^{3}$ on vaso-dilator fibres to the buccofacial region ; Gaskell ${ }^{4}$ on erector nerves of the penis; Bradford ${ }^{5}$ on vasomotor nerves to the kidney; Langley ${ }^{6}$ on pilo-motor nerves; Langley and Anderson ${ }^{7}$ on nerve fibres running to the end of the intestine, the bladder, the internal and external generative organs.

In vertebrates other than mammals the matter is less clear. In the chick the axons of nerve cells in the spinal cord have been traced into the posterior roots, but the function of these axons has not been determined. There are no satisfactory degenerative observations on any lower vertebrate. Experimental observations have been chiefly made on the frog, but the conclusions drawn from them are contradictory.

Pflüger ${ }^{8}$ found that the vasomotor fibres to the mesentery and web of the frog run in the anterior and not in the posterior roots of the spinal nerves.

Steinach and Wiener ${ }^{9}$ state that in Rana esculenta electrical and mechanical stimulation of the posterior roots of the second to the ninth spinal nerves causes movements in some part or other of the alimentary canal or in the bladder. They find that the effect on the rectum and bladder is not confined to the posterior roots, but occurs to a varying extent on stimulating the anterior roots. Horton Smith, ${ }^{10}$ however, was unable to confirm these results. He readily obtained contraction of the rectum and bladder on stimulating the anterior roots of certain of the lower spinal nerves, but did not obtain contraction from the posterior roots.

\section{Systems of Nerves Related to the Sympathetic.}

Nomenclature.-The sympathetic system, as we have seen, supplies nerve fibres to certain structures in all parts of the body. In some parts of the body these structures receive nerve fibres from other sources than the sympathetic. It is, in consequence, convenient to have some term to include the whole nervous supply. The words "organic," "vegetative," ganglionic," and "involuntary" have all been used, but they have also been used in senses other than that we require. The term "visceral nervous system " has been employed by Gaskell and others, including myself. The word "visceral," however, is obviously

1 Arch. f. physiol. Heilh., Stuttgart, 1852, S. 773.

${ }^{2}$ Compt. rend. Acad. d. sc., Paris, 1853 , tome xxx. p. 377.

3 Arch. de physiol. norm. et path., Paris, 1882, p. 190.

" "Proc. Physiol. Soc.," 1887, p. iv., Journ. Physiol., Cambridge and London, vol. viii.

5 Ibid., 1889, vol x. p. 363.

'Tbid., 1893, vol. xv. p. 181

I Ibid., 1895, vols. xviii. and xix.

${ }^{8}$ Ztschr. f. rat. Med., 1857, III. Reihe, Bd. i. S. 346.

${ }^{9}$ Arch. f. d. ges. Physiol., Bonn, 1895, Bd. lx. S. 593.

${ }^{10}$ Journ. Physiol., Cambridge and London, 1897, vol. xxi. p. 101. Steinach (Arch. $f$. d. ges. Physiol., Bonn, 1898, Bd. Ixxi. S. 533), after renewed experiments, upholds the conclusion he had previously come to. 
inapplicable to some of the structures brought under it, such as the nerve fibres which run to the skin. I propose, then, following a suggestion of Professor Jebb, to use the word "autonomic," including under that term the contractile cells, unstriated muscle, cardiac muscle, and gland cells of the body, together with the nerve cells and fibres in connection with them.

The autonomic nervous system consists of the sympathetic system, which we have already in large part considered, of the cranial autonomic system, the sacral autonomic system, and the enteric system (the plexuses of Auerbach and Meissner), which we will now pass on to consider. The interstitial cells of Cajal, if they are nervous, also belong to the autonomic system.

\section{Distribution and Action of Cranial Autonomic Nerve Fibres.}

Autonomic nerve fibres leave the brain by way of the third, seventh, ninth, tenth, and eleventh cranial nerves.

Third nerve.-The third cranial nerve sends a branch to the ciliary ganglion, and in this the efferent fibres end. The connection is shown both by the nicotin ${ }^{1}$ and by the degeneration method. ${ }^{2}$ If the third nerve be eut in the skull, numerous degenerated fibres are found running to the ganglion, but no degenerated fibres are found peripherally of it. The post-ganglionic fibres form-with the addition of a few fibres from the fifth nerve-the short ciliary nerves, which give motor fibres to the sphincter pupille and to the ciliary muscle.

Seventh and ninth nerves. - The seventh and the ninth nerves we may to a certain extent consider together, since the autonomic fibres of the seventh are, so far as is known, contained in the intermediate nerve of Wrisberg, and this may be regarded as the continuation anteriorly of the ninth. Moreover, the two together probably supply with autonomic fibres the whole of the mucous membrane of the nose and mouth.

The seventh and the ninth nerves together supply in the dog secreting fibres to the orbital gland, the glands of the lips and cheek, and the salivary glands. The fibres run in the several branches of the fifth nerve supplying the respective glands, for stimulation of these branches causes secretion, and, after section of any one branch, stimulation of the seventh and ninth nerves has no longer an effect in the region to which the cut branch runs.

The seventh and ninth nerves have also been shown to send vasodilator fibres by way of the fifth to the greater part of the region to which they send secretory fibres, namely, to all the salivary glands, to the tongue, to the mucous membrane of the floor of the mouth, and to part of the soft palate. They have not been shown to send vaso-dilator fibres to the mucous membrane of the cheeks and gums. According to Vulpian, indeed, stimulation of the seventh and ninth nerves intracranially does not cause flushing of the lips and cheeks, but since it does cause secretion from glands in this region, and since in other cases the

\footnotetext{
${ }^{1}$ Langley and Anderson, Journ. Physiol., Cambridge and London, 1892, vol. xiii. p. 460 .

2 Jegorow, Arch. slaves de biol., Paris, 1887, tome iii. p. 322. (Jegorow, notwithstanding his results, was inclined to consider the ciliary ganglion as homologous to a posterior root ganglion.) Langley and Anderson, Journ. Physiol., Cambridge and London, 1894, vol. xvii. p. 184.
} 
secretory and vaso-dilator fibres run together, the question can hardly be considered as definitely settled.

The fibres of the seventh nerve, which run by way of the lingual branch of the fifth to the submaxillary and sublingual glands, end in the submaxillary and sublingual ganglia, from which fibres (postganglionic), both vaso-dilator and secretory, are given off. ${ }^{1}$ We might expect, then, to find similar ganglia on the course of the fibres which the seventh and ninth send to the other branches of the fifth nerve. The ganglia which occur in connection with the other branches occur early on the course of the fifth instead of in or near the peripheral organs. The spheno-palatine ganglion is in connection with the superior maxillary nerve, and the otic ganglion in connection with the inferior maxillary nerve. Since nicotin stops the effects of stimulating the seventh and ninth nerves in the tympanic cavity, and does not stop the effects of stimulating the peripheral branches of the fifth nerve, there is some, though by no means conclusive, evidence that the sphenopalatine and otic ganglia are on the course of the autonomic fibres proceeding from the seventh and ninth nerves to the branches of the fifth, other than the lingual. It is to be borne in mind that the fibres of the seventh and ninth nerves which run to the fifth do not, so far as we know, accompany the branches of the fifth which run to the skin, but only those which run to the mucous membrane and glands. We have, then, only ground for considering that the spheno-palatine and otic ganglion send fibres to the branches of the fifth which run to the mucous membrane and to the glands of the head.

On this basis we should conclude that the spheno-palatine ganglion sends vaso-dilator and secretory fibres to the mucous membrane of the nose, soft palate, tonsils, uvula, roof of the mouth, upper lip and gums, and upper part of the pharynx, and that the otic ganglion sends similar fibres to the mucous membrane of the lower lip, cheek, and gums, the parotid and orbital glands; that the ganglia on the course of the lingual send similar fibres to the anterior part of the tongue, the floor of the mouth, the submaxillary and sublingual glands.

In view of the anatomy of the parts, we should expect to find that the spheno-palatine ganglion receives its pre-ganglionic fibres chiefly from the seventh nerve, the otic ganglion chiefly from the ninth nerve, and this is in general accordance with the result of physiological experiment.

The scheme just given with regard to the arrangement of the cranial autonomic fibres has simplicity on its side, and it is not inconsistent with any well-established facts ; it is, however, in large part conjectural, and we must await further experimental evidence for proof or disproof of it.

There still remains to consider the cranial autonomic fibres to that part of the tongue which receives its sensory fibres not by the fifth but by the glosso-pharyngeal nerve. Experiments on this point were made by Vulpian. He found that stimulation of the roots of the glossopharyngeal nerve intracranially caused flushing of the posterior part of the tongue; and he considered that the vaso-dilator fibres ran direct in the glosso-pharyngeal. We have no information as to any nerve cell comnection of these fibres.

Some account of the observations which have been made upon the cranial origin of the secretory and vaso-dilator nerves of the salivary glands

1 See art. "The Salivary Glands," this Text-Book, Vol. I. 1. 475. 
have been given elsewhere. ${ }^{1}$ Prevost ${ }^{2}$ was the first to obtain evidence of vasodilator fibres in the nasal mucous membrane on stimulating the spheno-palatine ganglion. Vulpian ${ }^{3}$ found dilator fibres for the vessels of the anterior part of the tongue in the lingual nerve, and for the posterior part in the glossopharyngeal nerves. He traced the fibres to the seventh and ninth nerves respectively. ${ }^{4}$ Jolyet and Laffont ${ }^{5}$ observed flushing in the nasal and parts of the buccal mucous membrane on stimulating the superior maxillary nerve. For further information, see the papers quoted below in the footnotes.

Fifth nerve.-Whether the roots of the fifth nerve have any autonomic fibres must, I think, be regarded as doubtful. They have been said to contain secretory fibres, constrictor and dilator fibres for the pupil, constrictor and dilator fibres for the blood vessels. The view that the fifth nerve has secretory fibres is now practically abandoned. There is no evidence worth considering here that it contains vaso-constrictor fibres. ${ }^{6}$ Many observers have advocated the view that the fifth nerve sends constrictor fibres of a peculiar kind to the iris of the rabbit. The chief evidence alleged is that, after atropin has been given and the cervical sympathetic cut, stimulation of the first and second cervical segments of the cord, of the bulb, and of the fifth nerve, causes constriction of the pupil. ${ }^{7}$ But in the cat and dog the tifth nerve has apparently no action on the iris.

There is, on the other hand, some evidence for the existence of vasodilator fibres in the roots of the fifth. Stimulation of the fifth nerve in the skull with a strong current will cause in the dog vascular dilatation of the mouth, lips, and part of the adjoining skin, as well as dilatation of the pupil. Part of the effect is certainly due to an escape of current to the sympathetic filaments which join the fifth nerve at or near the Gasserian ganglion. This is shown by making the current weaker when there is no longer an effect on the pupil or on the skin of the face. ${ }^{8}$ Thus a strong current stimulates the sympathetic grey ramus to the fifth in just the same way as we have seen the grey ramus to a spinal nerve can be stimulated (cf. p. 631). The question then is, whether the Hushing of the mucous membrane of the mouth, when it alone is obtained by stimulating the roots of the fifth, is also due to an escape of current or not. According to Laffont, ${ }^{y}$ stimulation of the superior maxillary nerve in the dog causes flushing twenty days after extirpation of the superior cervical ganglion, i.e. at a time when the sympathetic fibres in the fifth have degenerated. There still remains the possibility of an escape of current to the spheno-palatine ganglion or to fibres running to it from the seventh or ninth nerve. If Vulpian's statement, that these nerves have no vaso-dilator fibres for the mucous membrane of the cheek and gums, were confirmed, the balance of evidence would be

\footnotetext{
${ }^{1}$ See art. "The Salivary Glands," this Text-Book, Vol. I. pl. 479-485.

2 Arch. de physiol. norm. et path., Paris, 1868, pp. 7 and 207.

3 Compt. remi. Acad. d. sc., Paris, 1873 , tome lxxvi. p. 622 ; ibill., 1875, tome lxxx, 1. 330 ; uf. also Lépine, Aib. a. d. physiol. Anst. zu Leipzig, 1870.

${ }^{4}$ Compt. rend. Acad. d. sr., Paris, 1885, tome ei. pp. 851, 981, 1037.1448.

5 Jbid., 1879, tome lxxxix. p. 1038.

"An account of observations on the matter will be found in Tigerstenlt's "Physiologie des Kreislaufs," Leipzig, 1893, S. 480, 481.

7 Cf. Eckhard, Centralbl. f. Physiol., Leipzig u. Wien, 1s92, S. 129 ; Grünhagen, ibid., S. 326 ; and S. Mayer's account of the earlier ohservations in Hermann's "Handbuch," Leipzig, 1879, Bd. ii. Th. 1, S. 248-252.

8 Vulpian, Compt. rend. Acad. d. se., Paris, 1885, tome ci. p. 981.

${ }^{9}$ Compt. rend. Soc. de biol., Paris, 1880, p. 297.
} 
decidedly in favour of vaso-dilators in the fifth, but in the meantime we may regard the view as still requiring proof.

Tenth and eleventh nerves.- The autonomic fibres of the tenth and eleventh nerves are in their central origin homologous with the autonomic filbres of the ninth and seventh, which we have considered above. The fibres run in the vagus. The vagus ${ }^{1}$ supplies motor fibres to the cesophagus, stomach, and probably the rest of the gut, as far as the descending colon, and inhibitory fibres to the heart; it sends also motor fibres to the unstriated muscle of the lungs, and, according to recent observations, secretory fibres to the gastric glands and to the pancreas. There is evidence also that the vagus contains inhibitory as well as motor fibres for the gut, but this is not admitted by all observers.

The presence in the vagus of motor fibres for the spleen is denied. ${ }^{2}$ According to Schiff, the vagus contains accelerator fibres for the heart, and Rutherford found (1869) that stimulation of the vagus caused acceleration of the heart after atropin had been injected. Brown-Séquard (1854) and Porter (1896) state that stimulation of the vagus in the lower cervical region causes contraction of the coronary arteries. These effects on the heart and blood vessels may possibly be due to fibres running to the vagus from the superior cervical ganglion.

So far as the experiments have gone, all the autonomic fibres of the vagus arise from the medulla, and none from the cervical spinal cord. The œsophageal fibres arise from the roots of the tenth nerve, the cardiac inhibitory fibres arise from the medullary roots of the eleventh nerve, and, according to Kreidl, from the uppermost of these. ${ }^{3}$ According to Vulpian, ${ }^{4}$ both the tenth and the eleventh nerves give motor fibres to the stomach.

The efferent autonomic nerve fibres of the tenth and eleventh are apparently not connected with either the cells of the jugular ganglion or of the ganglion of the trunk of the vagus.

It was found by Waller ${ }^{5}$ that, when the vagus was eut between the jugular ganglion and the ganglion of the trunk, and time left for degeneration, stimulation of the vagus in the neck had no effect upon the "lungs, larynx, etc." He concluded that the motor fibres of the vagus were not connected with the ganglion trunci vagi.

Gaskell ${ }^{6}$ found in the crocodile and alligator that, after degenerative section of the vagus in the neck, stimulation of the nerve peripherally of a ganglion, which occurs at the entrance of the vagus into the thorax, caused contraction of the lower part of the osophagus and of the stomach, but not inhibition of the heart. As he considered that this ganglion was homologous with the ganglion trunci vagi of mammals, he inferred that the ganglion trunci vagi was on the course of the efferent fibres to the œsophagus and stomach.

Garl and Joseph ${ }^{7}$ repeated Waller's experiment ; they found that the motor fibres for the œesophagus were not connected with the ganglion trunci

${ }^{1}$ For details and references, ef. the sections of this Text-Book dealing with the several organs or tissues.

2 Cf. Schäfer and Moore, Journ. Physiol., Cambrilge and London, 1896, vol. xx. p. 1.

${ }^{3}$ Kreidl, Sitzungsb. d. k. Akud. d. Wissensch., math.-naturw. Cl., Wien, 1897, Bd. cvi. Abth. 3, S. 197. References to earlier observations on the functions of the several roots will be found in this paper.

${ }^{4}$ Compt. rend. Acad. d. sc., Paris, 1885, tome ci. p. 851.

5 Ibid., 1852, tome xxxiv. p. 582.

${ }^{6}$.Jouru. Physiol., Cambridge and London, 1886, vol. vii. p. 20.

7 Arch. f. Physiol., Leiprig, 1889, S. 209. 
vagi. In the cat I have made a similar experiment, and come to the same result as regards the fibres to the œsophagus (lower cervical and thoracic portion), the stomach, the intestines, and heart.

We may conclude that the autonomic fibres of the vagus end in connection with peripheral nerve cells, since in the rabbit a small dose of nicotin prevents any effects being obtained by stimulating the nerve in the neck. It is probable that the nerve fibres for each organ end in small ganglia situated in or near the organ itself. This is shown, as regards the cardiac inhibitory fibres in the mammal and frog, by the continued inhibitory power of muscarin, after nicotin injection; and in the frog, also, by the inhibition obtained by local stimulation after injection of nicotin.

Twelfth nerve.-The peripheral part of the hypoglossal nerve has a slight vaso-constrictor action upon the arteries of the tongue. The vaso-constrictor fibres come in part, at any rate, from the superior cervical ganglion. It has been supposed that some issue with the roots of the hypoglossal nerve, but the evidence is not satisfactory. In some animals the hypoglossal nerve has constantly, in others occasionally, a small gangliated root; the ganglion is apparently homologous with the jugular ganglion, i.e. it belongs to the system of spinal ganglia, and not to that of the autonomic ganglia.

Characters of the cranial autonomic nerve fibres.-The nerve strand running from the third nerve to the ciliary ganglion consists chiefly of small medullated fibres. The fibres proceeding from the ganglion also consist chiefly of small medullated fibres; they are three to four times as numerous as the fibres rumning to the ganglion. These facts were pointed out by Bidder and Volkmann ${ }^{1}$ in 1842.

The chorda tympani, Jacobson's nerve, the superficial petrosals, have very few medium or large fibres.

One or two medium or large fibres usually run to the submaxillary glandsometimes branching as they go-by way of the duct; the chorda tympani, after leaving the lingual, has usually no fibre larger than $3.5 \mu$; in the tympanic cavity it has more medium and large fibres.

The post-ganglionic fibres of the otic, spheno-palatine, and submaxillary ganglion are chiefly, at any rate, non-medullated. The nerve cells on the course of the cranial nerves so far considered have the same general characters as those of the sympathetic system.

The vagus at its exit from the skull contains many large fibres, as well as many small ones. The large fibres pass off chiefly to the pharynx and larynx. The branches of the nerve which run to the œsophagus and to the stomach consist of non-medullated fibres, small medullated fibres, a considerable number of medium, and hardly any large fibres. The branches to the lungs contain more large fibres than those to the oesophagus and stomach.

Bidder and Volkmann ${ }^{2}$ give the proportion of small to large fibres in the branches of the vagus of the cat as follows:-In the superior laryngeal, $2: 1$; in the inferior laryngeal, $1: 8$; in the branches to the lungs, about $2: 1 ;$ in the branches to the osophagus and stomach, hundreds of small, and hardly any large, with much 'Zellgevebe,' i.e. Remak's fibres.

\footnotetext{
1 “Die Selbstständigkeit des sympathischen Nervensystems," Leipzig, 1842.
}

${ }^{2} O_{l}$. cit., S. 62. 
The depressor consists chiefly of medium fibres, the other varieties being also represented.

Gaskell ${ }^{1}$ pointed out that numerous non-medullated fibres appear in the vagus immediately below the ganglion trunci vagi. If the view given above (p. 663) be accepted, namely, that the nerve cells of the ganglion of the trunk are posterior root cells, it follows either that afferent fibres may become non-medullated a long way from their endings, or that the efferent pre-ganglionic fibres do.

In the sympathetic system it can hardly be doubted that preganglionic fibres may become non-medullated a considerable distance centrally of the cells to which they run (cf. above, p. 650), and, in consequence, we may fairly assume that the same may be the case with the similar fibres of the vagus.

Degeneration.-What has been said with regard to the degeneration of sympathetic fibres (p. 652) holds in general for the autonomic fibres of the cranial nerves. It may be noted that, as the vagus is a comparatively large nerve, the absorption of degenerative products, after the nerve has been cut, goes on much more slowly than in the cervical sympathetic. Clumps and granules and granular cells may be conspicuous for two or three months. The irritability of the vagus is lost, as a rule, at any rate, on the fourth to fifth day after section of the nerve.

Schiff ${ }^{2}$ stated that the vagus in the dog lost its inhibitory power on the heart on the fourth or fifth day after section of the nerve. According to the same observer, acceleration of the heart can in most cases be obtained by stimulating the peripheral end of the cut nerve, five, seven, and even fourteen days after the nerve has been cut.

Arloing, ${ }^{3}$ who holds, as did Schiff, that cardiac accelerator fibres are present in the vagus, finds that in the dog the accelerator fibres are irritable on the fifth day, but that the cardiac inhibitory and the motor osophageal tibres have lost their irritability. In the donkey he finds that the accelerator fibres retain their irritability about eight days after section, and that the motor œsophageal fibres may be still effective thirteen days after section. He states also that, in the donkey, the sciatic nerve, eight days after section, may still cause contraction of the blood vessels of the hind-leg.

Regeneration of vagus.-A recovery of function, after section of the vagus, has not been observed so far as regards its autonomic fibres, but not many observations have been made on the matter. We should, of course, expect that the vagus, when cut in the neck, would take a considerable time to regenerate, since the distance from the point of section to the peripheral structures is considerable.

In an experiment on a cat, $\mathrm{I}^{4}$ obtained no effect on stimulating the peripheral end of the vagus twelve months after scetion, although the nerve had largely regenerated, and contained numerous medullated fibres. Tuckett ${ }^{5}$ did not find regeneration of the œsophageal fibres of the vagus in the rabbit 231 days after section of the nerve. According to Vanlair, ${ }^{6}$ the recurrent laryngeal in the dog recovers its functions about eleven months after section of the cervical vagus.

\footnotetext{
1 Journ. Physiol., Cambridge and London, 1886, vol. vii. p. 19.

- Arch. f. d. ges. Physiol., Bonn, 1878, Bd. xviii. S. 172.

: Arch. de physiol. norm. et path., Paris, 1896, p. 75.

+Journ. Physiol., Cambridge and London, 1895, vol. xviii. 1. 283.

s Ibid., 1896, vol. xix. p. 297.

${ }^{6}$ Arch. de physiol. norm. et path., Paris, 1894, 1. 217.
} 


\section{Distribution and Action of Sacral Autonomic Nerve Fibres.}

The sacral autonomic nerve fibres all run to the pelvic nerve, commonly called the nervus erigens.

The origin of the pelvic nerve ${ }^{1}$ varies somewhat in different animals,

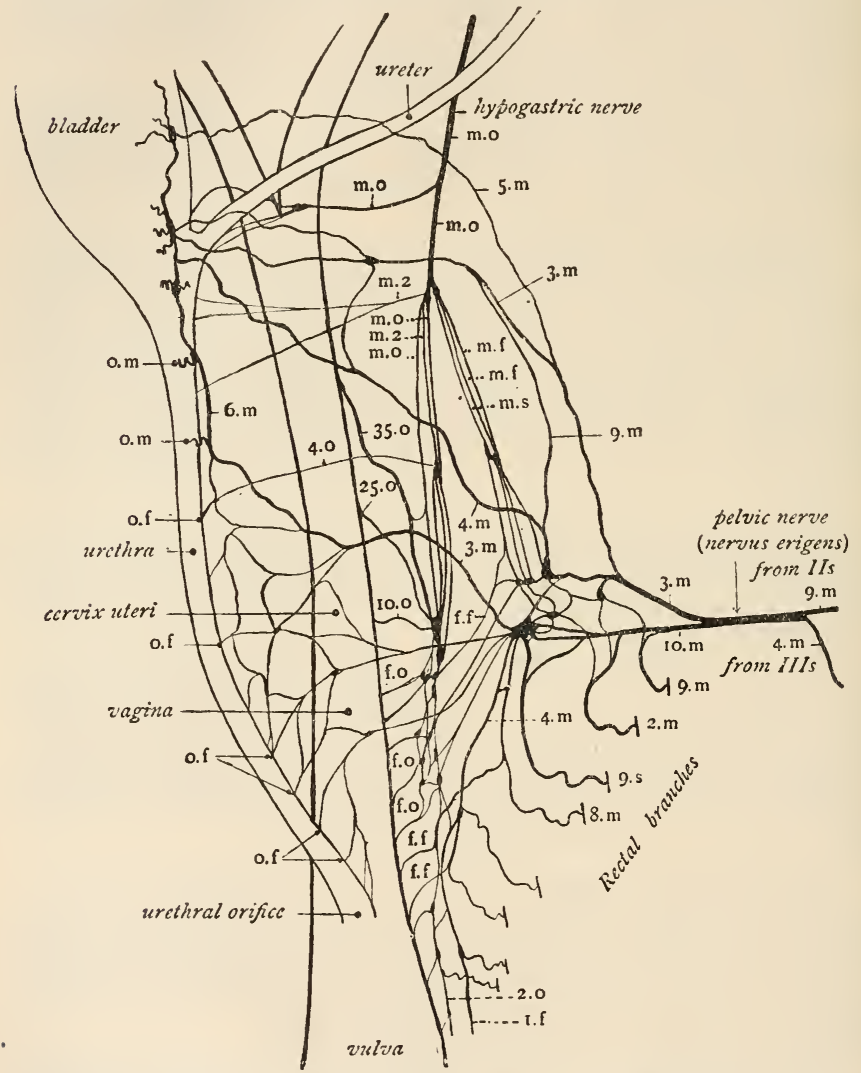

Fig. 314.-Left pelvic plexus of cat. The three sacral nerves and the first coceygeal nerve were cut peripherally of the spinal ganglia, and eleven days allowed for degeneration.

The numbers placed opposite each strand show the number of normal and of degenerated fibres present in it,- the number of normal fibres being placed first. Thus $35^{\circ} 0$ indicates that the strand contained thirty-five normal fibres and not one degenerated; $m$ signifies many fibres (more than twenty-five); $s$ signifies some (ten to twenty-five); and $f$, a few fibres (nime or less). Thus $9 . m$ signifies that nine sound anil more than twenty-five degenerated fibres were present in the strand.

and in each animal varies in correspondence with the origin of the lumbo-sacral nerves (cf. p. 633). In the cat, the second sacral nerve

' Langley and Anderson, Journ. Physiol., Cambridge and London, 1896, vol. xx. 1. 372. 
always sends a considerable number of fibres to the pelvic nerve, and the third generally does; the first sends comparatively few fibres, and none when the arrangement of nerves is posterior. In the rabbit, the pelvic nerve arises chiefly from the third and fourth sacral nerves, the second sacral nerve also sending fibres to it in the anterior and median classes; the first coccygeal nerve sending some fibres to it in the posterior class.

Stimulation of the pelvic nerve ${ }^{1}$ causes strong contraction of the bladder, but has no certain effect on the blood vessels of the organ; it causes contraction, varying in strength, of both coats of the descending colon and rectum, the effect being much more constant, and generally greater, in the rabbit than in the cat and dog; strong contraction of the recto-coccygeal muscle and of other special longitudinal muscles of the rectum; dilatation of the vessels of the mucous membrane of the end of the rectum, and of the external generative organs; inhibition of the proper unstriated muscle of the external generative organs, notably of the retractor penis; inhibition in the rabbit of the internal anal sphincter, and of some unstriated muscle in the skin of the ano-genital region.

It has been commonly said that the pelvic nerve contains notor fibres for the uterus. Anderson and myself ${ }^{2}$ in numerous experiments found the pelvic nerve and the sacral nerves to be entirely ineffective on the uterus. Further, section of the sacral nerves ${ }^{3}$ in the cat or rabbit caused no degeneration in nerve fibres running to the uterus or to the vagina, though it caused considerable degeneration in the strands running to the rectum and bladder, and some in the strands to the urethra and vulva; and we concluded that the sacral nerves do not send nerve fibres to the internal generative organs. It is, however, possible that some of the afferent fibres which run to the vulva may run on past this structure, and it is to be remembered that human anatomists have described filaments as passing from the sacral nerves to the uterus.

The origin from the spinal cord of the nerve fibres to the several viscera supplied by the pelvic nerve, is in correspondence with the anatomical origin of the nerve, stated above, except that the nerve fibres to the external generative organs are rather more anterior than those to the bladder and the end of the intestine.

The pelvic nerve soon breaks up into a plexus of nerve fibres containing numerous small ganglia. In these, the nerve fibres end. Speaking broadly, the ganglia in the neighbourhood of each organ are on the course of the fibres for that organ ${ }^{4}$ (cf. Fig. 314). Thus, in the rabbit, after injection of nicotin, the pelvic nerves have no effect, but on stimulating the peripheral parts of the plexus all the usual effects are obtained.

The pelvic nerve consists chiefly of small inedullated fibres, but both medium and large fibres are present; it has near its origin few or no non-medullated fibres.

In the eat, the pelvic nerve contains ${ }^{5}$ about 3500 medullated fibres. Of these, one to two dozen are $7 \mu$ to $12 \mu$ in diameter; several hundreds, $+\mu$ to

1 Cf. Langley and Anderson, Journ. Physiol., Cambridge and London, 1895, vol. xviii. p. 67 ; vol. xix. p]. $72,85,122$. References to earlier observations are given in these papers.

2 Ibid., 1895, vol. xix. p. 122.

${ }^{3}$ Ibid., 1895, vol. xix. 1. 372 ; vol. xx. 1) 379.

+ Langley and Anderson, ibid., 1895, vol. xix. p. 131.

'Langley and Andersou, ibid., 1895, vol. xix. 1'. $37 \%$. 
$5 \mu$ in diameter. In the rabbit the pelvic nerve has few fibres other than small. The histological characters of the nerve cells in the course of the pelvic nerve are, so far as is known, the same as those of the sympathetic ganglia.

\section{The Enteric Nervous System.}

The plexuses of Auerbach and Meissner are peculiar to the gut; they extend from the beginning of the unstriated portion of the nosophagus to the end of the rectum. They have usually been considered to belong to the sympathetic system, but it appears to me preferable to place them in a class by themselves. We may speak of them as forming the enteric nervous system. The chief reasons for placing the enteric nerve cells in a class by themselves are, first, that they differ in many of their histological characters from the nerve cells of the vertebral and pre-vertebral ganglia; and, secondly, that we do not know whether they are connected with the central nervous system by sympathetic or by cranial and sacral autonomic fibres.

The injection of a certain amount of nicotin into a blood vessel in a rabbit prevents stimulation of the spinal nerves having any effect upon the intestine, so that nicotin paralyses all the pre-ganglionic fibres. The results of local application of nicotin to the inferior mesenteric ganglion gives evidence that the pre-ganglionic fibres for the gut end in the pre-vertebral ganglia. If these impulses pass to the enteric nerve cells from the central nervous system, by way of the sympathetic, they inust pass by the post-ganglionic fibres of the pre-vertebral ganglia. But the injection of nicotin does not prevent stimulation of the postganglionic fibres from producing their usual effects, so that either the post-ganglionic fibres send collaterals only to the enteric cells, or, if they end entirely in synapses with the enteric cells, the synapses are not paralysed by nicotin. In either case, the arrangement is different in the enteric and in the sympathetic (vertebral and pre-vertebral) ganglia. In view of this, it is natural to suppose that the enteric cells are in the course of the vagus fibres in the upper part of the gut, and in the course of the sacral fibres in the lower part of the gut. The matter has not been sufficiently investigated to allow any decisive opinion to be arrived at. The experiments that have been made have been chiefly on the lower part of the gut; the results have tended to show that the sacral fibres end in cells of the sympathetic type, and not in the cells of the enteric plexuses.

\section{Possible Autonomic Fibres running direct in the Cerebro-Spinal Nerves.}

The view that some vasomotor fibres leave the cord by the roots of a spinal nerve, and accompany the branches of the spinal nerve to the periphery, is an old one, but no definite experiments were alleged in support of it before Schiff's account of the innervation of the vessels of the ear and of the vessels of the limbs.

There are one or two general considerations with regard to these fibres which must be borne in mind.

If such fibres exist in the particular cases mentioned, we should expect to find them in every spinal nerve, for there is nothing in the function of the nerves to the ear or limbs which makes it reasonable to suppose that they have 
a special kind of autonomic fibre unrepresented in other spinal nerves. And such fibres, if they exist, must be special forms of nerve fibres, distinct from all others.

We have seen that the fibres running from the central nervous system to the sympathetic, to the cranial and sacral autonomic systems, end in connection with nerve cells. The spinal nerves have no nerve cells of the sympathetic type on their peripheral course, so that, if the alleged direct fibres end at all in cells of the sympathetic type, it must be in the posterior root ganglion. Even in this case they would form a class of nerve fibres distinct from other autonomic nerve fibres. But there is no evidence of the presence of characteristic sympathetic nerve cells in the posterior root ganglia, and if this be so, the fibres must differ widely from other autonomic fibres, for either they are connected with a special form of nerve cell, or they have no nerve cell at all on their course.

We may turn now to the particular cases which have been brought forward. These are-(1) The third cervical nerve (vasomotor fibres); (2) the nerves running to the brachial plexus (vasomotor and secretory fibres); (3) the nerves forming the lumbo-sacral plexus (vasomotor and vaso-dilator secretory fibres).

As we have already said (p. 624), Schiff showed that the great auricular nerve in the rabbit contains vaso-constrictor fibres for the ear, and Lovèn that the vaso-constrictor fibres run chiefly to the distal part of the ear. Schiff considered that these fibres issue from the spinal cord in the roots of the cervical nerves, which give rise to the great auricular. He found ${ }^{1}$ that degeneration of the fibres which run from the superior cervical ganglion did not diminish the vasomotor action of the auricular nerve. This observation, however, does not touch the question, for it has been shown by Fletcher that in the rabbit vasomotor fibres run to the great auricular nerve from the ganglion stellatum. In order, then, to show that the cervical nerve roots in the rabbit have vaso-constrictor fibres, the cervical nerves should be stimulated a week or more after extirpation of both the ganglion stellatum and the superior cervical ganglion. This has not been done in the rabbit, but one case has been placed on record by Morat, ${ }^{2}$ in which the operation was performed. Morat stimulated the great auricular nerve, after allowing time for degeneration, and found, as a result, dilatation of the vessels of the ear. The experiment, however, can hardly be regarded as a conclusive one, since normally the great auricular nerve does not cause vascular dilatation.

I have made a few experiments in the cat and rabbit, and have not found any effect on the vessels of the ear when the cat's upper cervical nerves are stimulated close to the dura mater. Sometimes, when the electrodes were shifted up to the end of the nerve from the vertebral canal, there was contraction of the artery at the tip of the ear, from the second cervical nerve in the cat, and the third in the rabbit.

On the whole, I think the evidence is against the view that vasomotor fibres issue in the roots of upper cervical nerves.

The experiments brought forward to show that direct autonomic fibres occur in the nerves to the limbs, fall into two classes- $(\alpha)$ Those which are said to show that vasomotor fibres pass out in the anterior

1 Schiff, "Leçons sur la physiol. de la digestion," Turin, 1867, Leçon 11.

"Arch. de physiol. norm. et path., Paris, 1891, p. 93. 
roots; and $(b)$ those which are said to show that vaso-dilator fibres pass out in the posterior roots.

Most of those observers who support the presence of one class of fibres, deny the existence of the other.

Schiff was the chief advocate of the view that direct vasomotor fibres are present in the anterior roots. His experiments were solely on the effect of section of the nerves; he did not stimulate them. According to him, ${ }^{1}$ section of the anterior roots going to the sciatic in the dog causes lasting and considerable rise of temperature in the foot and the lower third (about) of the limb. Bernard,2 on the other hand, cut the lower lumbar and sacral nerves in the spinal canal, so that there was complete paralysis and lack of sensation in the limb, and found no increase of temperature of the foot. And this is in agreement with general experience.

Schiff ${ }^{3}$ stated also that section of the anterior roots of the nerves running to the brachial plexus causes rise of temperature in the lower part of the forelimb. This also is opposed to the results obtained by Bernard, by Cyon (1868), and by other observers.

Schiff's view was, that some vasomotor fibres run direct by the spinal nerves, and that others run by way of the sympathetic. If this were the case, stimulation of the anterior roots of the nerves supplying the limbs should cause pallor in the foot. There is little difficulty in making the experiment in a cat or dog with light-coloured feet, curari being given to paralyse the muscles. I do not find that the nerves which supply motor and sensory nerves to the foot have any effect upon the tint of the skin. The result is the more obvious, in that complete pallor and subsequent flushing is produced by stimulating the anterior roots of certain of the upper lumbar nerves (see p. 634), which send no fibres to the foot except by way of the sympathetic. Even if curari is not given, the difference in the action in the two sets of nerves is unmistakable, although naturally some pallor may be caused by the contraction of the muscles of the limb.

A considerable number of observers have arrived at the conclusion, that vaso-dilator fibres pass to the lower limb of the dog by way of the posterior roots of the nerves which supply it with somatic fibres.

The view was first advanced by Stricker. ${ }^{4}$ He found that mechanical irritation of the cut posterior roots of the sixth and seventh lumbar nerves-he spoke of them as the fourth and fifth-caused constantly an increase, and sometimes a very great increase, in the temperature of the foot. He did not obtain constant results if curari or anæsthetics were given, and in consequence found it necessary, as a preliminary to the experiment, to cut the cord in the lower thoracic region, and wait for a week to a fortnight, by which time the vascular dilatation produced by the section had passed off. The experiment could then be made without anæsthetics and without pain. Nor did he obtain satisfactory results by electrical stimulation of the posterior roots, nor when adult dogs were used.

Cossy, ${ }^{5}$ repeating Stricker's experiments, did not obtain constant results, but sometimes found a rise of temperature from stimulating the

1 Compt. rend. Acad. d. sc., Paris, 1862, tome lv. p. 400.

2 Journ. de la physiol. de l'homme, Paris, 1862.

${ }^{3}$ Compt. rend. Acad. d. sc., Paris, 1862, tome lv. p. 425.

${ }^{4}$ Sitzungsb. d. k. Akad. d. Wissensch., Wien, 1876, Bd. lxxiv. Abth 3. ; Med. Jahrb., Wien, 1878 , S. 409 ; cf. also Bonuzzi, ibid., 1885, S. 473.

${ }^{5}$ Arch. de physiol. norm. et path., Paris, 1876, p. 832. 
posterior roots. On the other hand, neither Vulpian ${ }^{1}$ nor Kuhlwetter ${ }^{2}$ found any effect.

Girtner ${ }^{3}$ confirmed Stricker, and insisted on the necessity of the precautions pointed out by Stricker, namely, young dogs, no curari or anæsthetics, stimulation by ligature and not by the interrupted current, previous section of the thoracic cord.

Since none of these precautions are necessary in the case of the known vaso-dilator nerves, it follows, so far, that however the rise of temperature is produced, it is not produced by the action of ordinary vaso-dilator nerves.

The question was taken up by Morat. ${ }^{4}$ He observed the colour of the hairless, unpigmented parts of the foot in the dog and cat. According to him, the only precaution necessary is that the animals should be young. Werziloff, 5 returning to the use of the thermometer and using also the plethysmographic method, again confirmed Stricker's results. He apparently did not find necessary any of the special precautions mentioned by Stricker and Gärtner.

Since in numerous cases in anæsthetised cats I have not found any flushing in the foot, on stimulating the nerves which run to the fore- and hind-limbs, I am inclined to attribute the rise of temperature and flushing, which has been observed by others on stimulating the posterior roots, to some other cause than the stimulation of vaso-dilator fibres in these nerve roots.

One fact which has been overlooked in experiments on this question, is the possibility of an escape of current to the ramus of the nerve. This takes place much more readily in young than in old animals, and it is curious that three out of the four observers who have found positive effects, lay stress on the advantage of experimenting with young animals. It is also more likely to occur the stronger the stimulation, and, according to Morat, strong stimulation is required. On the other hand, we should expect that an escape of current to the grey ramus would take place as readily when the anterior roots are stimulated as when the posterior roots are stimulated. But all observers find slight or no effects with the anterior roots. It may be objected also that an escape of eurrent to the grey ramus would cause a fall of temperature, and not a rise; this is to a certain extent true, but the rise might be due to an after-dilatation of the vessels. There are also other ways in which, in the conditions of the experiments, vascular dilatation might be produced, although there were no efferent vaso-dilator fibres in the posterior roots. It would, however, take up too much space to dwell on the various possibilities. It is sufficient to say that the existence of these fibres has not yet been proved, and that, as the question is one of considerable importance, it is most desirable that further careful experiments should be made.

\section{Local Nerve Centres other than the Cranial, Sympathetic, AND Sacral Ganglia.}

Goltz, ${ }^{6}$ in experiments on dogs, found that section of the sciatic nerve did not cause a permanent rise of temperature in the foot, but that the temperature returned to normal in a week or two. He dis-

\footnotetext{
${ }^{1}$ Arch. de physiol. norm. et path., Paris, 1878, p. 336.

${ }^{2}$ Beitr. z. Anat. u. Physiol. (Eckharl), Giessen, 1885, Bd. xi. S. 25.

3 IVien. klin. Wchnschr., 1889.

+ Arch. de physiol. norm. et path., Paris, 1892, p. 689.

${ }^{5}$ Centralbl. $f$. Physiol., Leipzig u. Wien, 1896, Bd. x. S. 194.

${ }^{6}$ Arch. f. d. ges. Physiol., Bonn, 1875, Bd. xi. S. 92.
} 
cussed the several possible explanations of the recovery of tone, and adopted the view that in the foot and in the body generally the tone of the blood vessels was maintained by the action of local ganglion cells. The progress of histological inquiry has shown that the hypothetical local ganglion cells of the type of sympathetic nerve cells do not exist. And unless we fall back upon "interstitial cells," the view of the maintenance of tone by local nerve cell centres must be abandoned. It is very doubtful whether the "interstitial" cells are nerve cells at all. And, on the whole, the most probable explanation of such recovery of tone as occurs is the inherent contractile power of the plain muscular tissue itself.

It must be noticed that the degree of recovery of tone, after section of all vasomotor fibres, is still a matter in dispute (cf. p. 655), and that possibly the rapid recovery in the tone of the vessels of the foot, after section of the sciatic, is due to vasomotor fibres taking the course of the crural nerve (cf. p. 657).

In the stomach and intestine, the nerve cells of the plexuses of Auerbach and Meissner may well act as the local centres, but nothing definite can be said at present with regard to this.

\section{QUESTIONS COMMON TO THE SYMPATHETIC AND SOME OTHER PARTS OF THE AUTONOMIC SYSTEM OF NERVES.}

We have considered separately the sympathetic, the cranial autonomic, and the sacral autonomic systems, so far as this could be done conveniently, but a separate treatment is inconvenient for certain questions. These we shall consider in the following pages.

\section{Mode of Action of Inhibitory Fibres.}

Claucle Bernard was the first to attempt to explain the action of inhibitory nerves. This he did with regard to the vaso-dilator fibres of the chorda tympani. He considered that the fibres of the chorda tympani inhibit the action of a local vaso-constrictor centre. On this hypothesis the local vaso-constrictor centre is in constant action : sympathetic fibres run to it and increase its action; chorda tympani fibres run to it and decrease its action; thus the nerve cells would receive fibres from two sources. This view of the mechanism of inhibition was advocated by Vulpian, and is supported by Dastre and Morat.

There are many facts which show that this view is untenable. We will consider the two chief instances of vaso-dilator fibres, the chorda tympani and the nervus erigens.

The local nerve centre with which the chorda tympani fibres is connected is the submaxillary ganglion in the hilum of the gland. This is shown by the action of nicotin. On Bernard's hypothesis, stimulation of the fibres proceeding from the local centre should cause contraction of the arteries. But, after the chorda tympani fibres have been paralysed by nicotin, stimulation of the fibres past the ganglion causes dilatation of the arteries of the gland, and does not cause contraction; hence the nerve fibres given off from the local nerve centre are not vaso-constrictor fibres but vaso-dilator fibres. So that the chief assumption of the hypothesis is incorrect. Moreover, when the sympathetic fibres are followed by means of serial sections into the gland, it is seen that they 
accompany the arteries and run past the submaxillary ganglion without entering it. Further, it is easily shown that the sympathetic fibres are connected with nerve cells in the superior cervical ganglion, and that stimulation of the nerve fibres on either side of the ganglion has the same result, namely, contraction of the arteries.

In the case of the nervus erigens, the facts are of the same nature. Nearly all the sympathetic fibres - which are vaso-constrictor-end in connection with nerve cells in the sacral sympathetic ganglia; stinulation on either side of these ganglia causes great contraction of the vessels of the penis-section on either side of these ganglia causes great vascular paralytic dilatation. The fibres given off by the sacral ganglia have no nerve cells on their course to the periphery, for nicotin does not paralyse them. On Bernard's hypothesis, then, the sacral ganglia would be the local centres which are inhibited by the nervus erigens, but in fact the nervus erigens has no connection whatever with these ganglia. If, then, the nervus erigens inhibit local vaso-constrictor ganglia, there must be some such ganglia in addition to the sacral ganglia, and situated more peripherally. All the evidence, however, goes to show that the ganglia in the course of the nervus erigens are vaso-dilator and not vaso-constrictor. Stimulation peripherally of these ganglia causes vaso-dilatation, and nicotin annuls the effect of stimulating centrally of the ganglia, but does not annul the effect of stimulating peripherally of them.

Both for the chorda tympani and for the nervus erigens we have, then, satisfactory evidence that the vaso-dilator fibres run to nerve cells, and that both pre-ganglionic and post-ganglionic fibres produce vasodilator effects; further, that the vaso-constrictor fibres run to nerve cells, and that both pre-ganglionic and post-ganglionic fibres produce vaso-constrictor effects; lastly, that if the two systems communicate, it can only be in the region of the post-ganglionic fibres.

The state of things is essentially the same in the case of the vasodilator fibres of the bucco-facial region of the dog, the inhibitory fibres of the heart, and the inhibitory fibres of the stomach and intestine. And we may conclude for all cases that the inhibition which we are considering is not produced by a lowering of the activity of sympathetic or similar nerve cells.

We must, look, then, for the mechanism of inhibition either in interference of nervous impulses in the peripheral region of the postganglionic fibres, or in a direct action of the inhibitory fibres upon the tissue.

On the one alternative, constant motor impulses would be annulled, and no longer reach the tissue; on the other, the tissue itself would be affected, so that such motor impulses as did reach it would not have their usual effect.

The former is essentially the mode of action suggested by Bernard, but the action takes place in a different part of the nervous apparatus. The peripheral apparatus in which it is conceivable it should take place is either- $(a)$ a nerve cell apparatus, the existence of which we have at present no sufficient reason for believing; or $(b)$ a nervous network, into which both motor and inhibitory fibres run.

But such a network could not of itself keep up a tonic constrictor action, so that, if inhibition is produced after degenerative section of the constrictor fibres, it can only be produced by a direct action on the tissue.

vOL. II. -43 
Now, after extirpation of the superior cervical ganglion in the rabbit, and consequent degeneration of the vaso-constrictor fibres to the submaxillary gland, vaso-dilatation is still produced by stimulation of the chorda tympani. ${ }^{1}$ In this instance, then, the inhibition must be caused by nervous impulses affecting directly the tissue. And we may take it as probable that peripheral inhibition by autonomic nerves is always brought about in this manner.

It must be mentioned that, according to Fletcher, ${ }^{2}$ the rat's retractor penis muscle contains a terminal nervous network, which does not degenerate either when the motor or the inhibitory post-ganglionic fibres to it are cut. His experiments are still in progress.

But, accepting this, we are still far from any real knowledge of the processes involved in inhibition. One a priori possibility may be disposed of. The decrease of rigidity in the inhibited muscular tissue shows that inhibition is not caused by the development of a contractile force, acting in a direction opposed to the normal one and overpowering it. We are then brought to the conclusion that certain nerve impulses - the inhibitory nerve impulses-are able to lessen or to stop the chemical change in the tissue which leads to contraction. As this chemical change is accompanied by a setting free of energy, it is spoken of as a dis-assimilatory or katabolic change, so that inhibitory nerve impulses may be said to lessen or to stop some katabolic change in the tissue. Beyond this we cannot at present go safely. Gaskell ${ }^{3}$ has propounded the theory that inhibitory nerves cause assimilation or anabolism, and that the anabolic process stops for the time the katabolic process. And it appears to be implied that the two processes are mutually antagonistic. Gaskell, however, does not enter into a discussion of the relation which he takes to exist between the two processes.

It does not seem probable that, in general, anabolism interferes with katabolism. The histological changes which take place in the majority of glands during secretion afford good evidence that the two processes go on actively at the same time. No inhibitory nerve fibres have, however, been shown to exist in the glandular tissues; and, so far, the question is left open, whether anabolism does not stop katabolism in the tissues in which inhibition does occur.

It is clear, if inhibition is caused by anabolism, that, as regards the unstriated muscle of the arteries, anabolism goes on up to a certain limit simultaneously with katabolism, for by a proper gradation of the stimulus applied to dilator nerves, all degrees of decrease of tone in the arteries can be obtained.

To account for this, we might suppose that, with increasing strength of stimuli, more and more molecules are involved. On this supposition it follows that a given molecule can only be concerned with one process at a time, and that the predominance of one process in the cell necessarily means a diminution of the other. And, consequently, the more fully contracted an artery, the less should be the assimilative processes going on in it. Here we are met by the serious difficulty that in some

${ }^{1}$ Langley, Journ. Physiol., Cambridge and London, 1885, vol. vi. p. Si.

2 "Proc. Phys. Soc.," 1898, p. xxxvii., Journ. Physiol., Cambridge and London, vol. xxii.

3 lbid., 1886, vol. vii. ए. 46. 
cases the small arteries can be kept for many hours, ${ }^{1}$ and probably for days and months, in a state of strong contraction; and it is not to be believed that the breaking down thereby involved could go on if there were not some corresponding fresh formation.

We seem then driven to suppose, on the lines of the theory, that though anabolism interferes with katabolism, katabolism does not interfere with anabolism. But in such cases it is hard to avoid the conclusion that different molecules are affected. And then we are at a loss for an explanation of how anabolism in one molecule can stop katabolism in another molecule.

What is required, in the first place, is some means of determining whether anabolism is increased, or not, during inhibition; and if it is, to what extent it is increased. It need hardly be said that increased strength of contraction after inhibition is not necessarily a sign of increased anabolism; for the continuance of a previously existing rate of anabolism, unaccompanied by katabolism, would be sufficient to produce this result.

Dastre and Morat ${ }^{2}$ regard the sympathetic ganglia as exercising a tonic action upon the blood vessels; they state that vaso-dilator action diminishes or disappears as nerve strands are stimulated more and more peripherally, and they conclude that the vaso-dilator fibres run to the sympathetic ganglia and inhibit their tonic action. Thus their view of inhibition is essentially that of Claude Bernard. The general question of tonic action we discuss later ( $p$. 676). Here we may consider the particular cases on which Dastre and Morat rest their view. These concern-(1) the vaso-dilator fibres for the ear in the rabbit, cat, dog; (2) the vaso-dilator fibres for the foot of the dog.

1. According to Dastre and Morat, stimulation of the eighth cervical and first thoracic nerve roots causes primary dilatation of the vessels of the ear. Since stimulation of the annulus of Vieussens and of the cervical sympathetic causes nearly always, if not always, contraction of the vessels of the ear, they consider that the vaso-dilator fibres coming from the spinal cord end chiefly in the ganglion stellatum; they consider that this ganglion gives off numerous vaso-constrictor fibres, which run to the ear by way of the cervical sympathetic. In my experiments, stimulation of the eighth cervical nerve had no effect upon the vessels of the ear. In the cat, the first thoracic nerve produced sometimes, but not usually, a slight vaso-constrictor effect; this was sometimes followed by an after-dilatation of the same kind as, though very much less than, that caused by stimulation of the cervical sympathetic. Further, a study of the effects produced by nicotin seems to me to show that the ganglion stellatum sends, at least as a rule, no vaso-constrictor fibres to the ear by way of the cervical sympathetic.

2. Dastre and Morat find that stimulation of the sympathetic, above the second and third lumbar ganglia, causes primary dilatation of the vessels of the digits of the hind-foot, whilst stimulation below these ganglia causes primary contraction. They consider that the second and third lumbar ganglia send vaso-constrictor fibres to the vessels of the foot, and that the tonic action of these ganglia is inhibited by vaso-dilator fibres running to them from the spinal cord. I have not been able to observe such a difference, except in so far that more care is required to avoid reflex dilatation when stimulating above than when stimulating below the ganglion. And the action of nicotin in the cat shows, I think, that the second and third lumbar ganglia send no nerve fibres of any kind to the foot.

${ }^{1}$ Cf. Eve, Journ. Physiol., Cambridge and London, 1896, vol. xx. p. 344.

2 "Recherches expérimentales sur le système nerveux," Paris, 1884. 


\section{Automatism of Peripheral Ganglia.}

It perhaps hardly needs to be pointed out that, if the peripheral ganglia are devoid of automatism, no essential difference is thereby established between their nerve cells and those of the central nervous system. For many nerve cells on the central nervous system have, so far as we know, no automatic action; and in other cases in which automatic action appears to exist, it is uncertain how far it is simply apparent, and due in reality to afferent impulses.

The experiments to which we may look to throw light on the question as regards those nerve cells which are normally in tonic activity, fall into three classes:-

1. We may cut the fibres running to a ganglion, and later cut those running from it, and observe if the second section increases the paralytic effects of the first section.

2. We may compare the extent of the paralysis produced, when in one animal the nerve fibres running to a ganglion are cut, and in another animal the nerves running from the ganglion.

3. We may compare the extent of the paralysis when the nerve fibres are cut, as in (2), but on the right and left side of an animal instead of in different animals.

Before dealing with the experiments which have been made on these lines, we may note that the peripheral ganglia of adult animals do not, so far as we know, depend for their nutrition on receiving impulses from the central nervous system. In some cases, in the central nervous system, removal of the normal impulses to the nerve cells causes them to atrophy. The nerve cells of the peripheral ganglia remain functionally active for a very long time after severance of their pre-ganglionic fibres. It was noticed by Budge ${ }^{1}$ that a year after section of the cervical sympathetic, stimulation of the superior cervical ganglion still caused dilatation of the pupil, although the nerve itself had not regenerated. Further, so far as the reagents in use show, the nerve cells retain their normal appearance.

1. It may be doubted whether the first form of experiment mentioned above is one which will give convincing results, unless there is a considerable interval of time between the two operations, for it cannot be said with certainty how long it takes for the stimulating effect of the first section to disappear completely. So far, at any rate, the method has not yielded anything decisive on the question.

Some interesting observations have been made on the effects of removal in the frog ${ }^{2}$ of the sympathetic ganglion which sends fibres to the pupil. They do not, however, allow any well-founded conclusion to be drawn as regards the automatism of the nerve cells.

Dastre and Morat ${ }^{3}$ state that, in the dog, section of the cervical sympathetic does not cause flushing of the bucco-facial region, whilst excision of the superior cervical ganglion does. I have not been able to find any such constant difference in the effect of the two operations.

Dastre and Morat found a greater dilatation of the vessels of the ear (and

${ }^{1}$ Budge, "Ueber die Bewegung d. Iris," Braunsehweig, 1855, S. 125. Budge, however, attributed the effect to pupillo-dilator fibres ruming to or through the ganglion from the hypoglossal.

2 Liégeois, Comprt. rend. Soc. de biol., Paris, 1862, p. 71 ; Vulpian, "Lecons sur l'appareil vaso-moteur," p. 312 ; ef. also Budge, Arch. $f$. physiol. Heilh., Stuttgart, 1852 , S. 773 ; Tuwim, Arch.f. d. ges. Physiol., Bonn, 1881, Bd. xxiv. S. 115.

3 "Système nerveux vaso-moteur," Paris, 1884; Arch. de pliysiol. norm. et path., Paris, 1892, p. 171 . 
of other parts of the head) on cutting centrally of the ganglion stellatum than on cutting peripherally of it, and on cutting centrally of the inferior cervical ganglion than on cutting peripherally of it. They found, also, a greater dilatation of the vessels of the foot of the dog on cutting the sympathetic chain centrally, than on cutting it peripherally, of the second and third lumbar ganglia. Hence they concluded that these ganglia exercise a tonic action on the parts mentioned. The considerations which lead me to consider the experiments inconclusive are given above (p. 675).

According to François-Franck, ${ }^{1}$ section in the dog of the ciliary nerves containing constrictor fibres for the sphincter iridis, causes greater dilatation of the pupil than section of the third nerves.

2. The second method has not given any certain results as regards the superior cervical ganglion, and it is on this that experiments have been chiefly made.

Bernard ${ }^{2}$ stated that excision of the superior cervical ganglion gives greater and more permanent paralytic effects than section of the cervical sympathetic. It is not, however, clear that Bernard excised a sufficiently long portion of the nerve to prevent regeneration, or, indeed, did more than state a general impression. Schiff, ${ }^{3}$ Callenfels, ${ }^{4}$ and PyeSmith ${ }^{5}$ found no difference between the result of the two operations.

3. Callenfels made three experiments upon rabbits by the third method, i.e. he cut the cervical sympathetic on one side and excised the superior cervical ganglion on the other. In two cases he found the temperature of the ear higher on the side on which the nerve only was cut; in one case the temperature was lower on this side. Tuwim, ${ }^{6}$ in similar experiments in rabbits, found no difference in the flushing of the ear on the two sides; nor in similar experiments in frogs did he find any difference in the flushing of the two sides of the tongue.

Jegorow, ${ }^{7}$ however, states that, in a series of experiments on the dog, the dilatation of the pupil was markedly greater after excision of the ciliary ganglion than after section of the third nerve a little centrally of the ciliary ganglion.

On the whole, the evidence at present leads us to conclude that autonomic ganglia, after separation from the central nervous system, do not exercise any considerable tonic action on unstriated muscle, but that it is possible they have a slight action.

Apart from tonic action, it is not improbable that peripheral nerve cells, severed from the central nervous system, may at times stimulate the tissues to which they send fibres. The nerve cells and fibres remain responsive to chemical and mechanical stimuli, and it is not unlikely that substances circulating in the blood and the movements of the body now and then serve as stimuli to the local nervous mechanism, and so set in action the peripheral apparatus.

So far, we have considered nerve cells which normally exercise a tonic influence on the tissues with which they are in connection. Of the others, there is little that need be said here. There is some evidence that the submaxillary ganglion passes into a state of slight tonic activity after section of the chorda tympani, for, so far as the experi-

1 Trav. du lab. de Marry, 1880, tome iv.

2 Op, cit., supra, p. 65\%. 3 Op. cit., supra, p. 657.

+ Op. cit., supra, 1. 65\%. ${ }^{5}$ Op. cit., supra, p. 65\%.

${ }^{8}$ Arch. f. d. ges. Physiol., Bonn, 1881, Bd. xxiv. S. 115; ef. also Budge, up. cit., 1885 , S. 125 .

${ }^{7}$ Arch. sluves de biol., Paris, 1887, tome iii. p. 322. 
ments have gone, dyspnœea increases and chloroform decreases the paralytic secretion. ${ }^{1}$ But the sympathetic ganglia supplying the hairs do not appear to acquire any tonic action after severance from the spinal cord.

Lastly, it remains to mention the belief once held, that the beat of the heart is caused by rhythmic impulses from the local ganglia. This view is now generally abandoned. The discussion of it belongs elsewhere. We may, however, notice the possibility that the local ganglia have even after section of the vagus, a tonic inhibitory action upon the heart.

\section{On Peripheral Ganglia as Centres for Definite Areas.}

It is conceivable that the peripheral nerve cells should be collected together according to their function, so that all the vaso-constrictor nerve cells which send their axons to a given area should be collected into one ganglion, the vaso-dilator nerve cells in another ganglion, the pilo-motor in a third, and so on. But in fact, within the limits of each division of the autonomic system, there is no instance in which this occurs. It occurs only when a tissue receives fibres from two divisions of the autonomic system; then the ganglia of the two systems are separated, and they may have different functions. What we find is that a ganglion which sends nerve fibres to a particular area sends to it all the varieties of nerve fibres, received by the area from the autonomic system to which the ganglion belongs. When an area receives axons from the sympathetic and from the cranial system, or from the sympathetic and from the sacral system, the axons from the two systems have each their own ganglion, and some variety of nerve cell may be, and indeed commonly is, present in the ganglion of the one, which is not present in the ganglion of the other.

Each ganglion, then, may be regarded as a group of nerve cells supplying fibres of various functions to a definite area. In some cases, and within the limits of each autonomic system, all nerve cells supplying a given area are collected together to make one ganglion, but this simple arrangement is commonly departed from in one, or in both, of two ways. For there is a tendency for the nerve cells supplying an area to be arranged in several small ganglia instead of in one larger one. Thus, instead of a single ciliary ganglion, there may be one main ganglion and several accessory ganglia. So the ganglia for the various abdominal and pelvic viscera usually consist of a main ganglion and of accessory ganglia. And even the vertebral ganglia may be in two parts, a small part occurring in the course of a grey ramus.

Further, as we have seen, there is some overlapping in the areas supplied by the several ganglia. The overlapping may be constant and considerable, as in the case of the ganglia sending axons to the hindlimbs; it may be constant and slight, as in the case of some of the thoracic ganglia sending fibres to the skin of the back; or it may be variable, both in occurrence and extent, as in the case of ganglia which may send fibres by a white ramus as well as by a grey one.

\section{Reflex Actions from Autonomic Ganglia.}

Experimental observations on reflex actions from autonomic ganglia were first made by Bernard with regard to the ganglion called by him

${ }^{1}$ Langley, Journ. Physiol., Cambridge and London, 1885, vol. vi. p. 71. 
the submaxillary ganglion. These and subsequent observations we have discussed in the article on "Secretion of Saliva." And we came to the conclusion that the supposed reflex secretion was partly due to stimulation of recurrent fibres and partly perhaps to stimulation of one branch of a dividing fibre.

Sokownin ${ }^{1}$ showed that when in the cat the nervous connections of the inferior mesenteric ganglion are severed, with the exception of the hypogastric nerves, and then one hypogastric nerve is cut and its central end stimulated, contraction of the bladder on the opposite side is produced. And it was found by Anderson and myself ${ }^{2}$ that the stimulation causes also other effects, namely, contraction of the internal
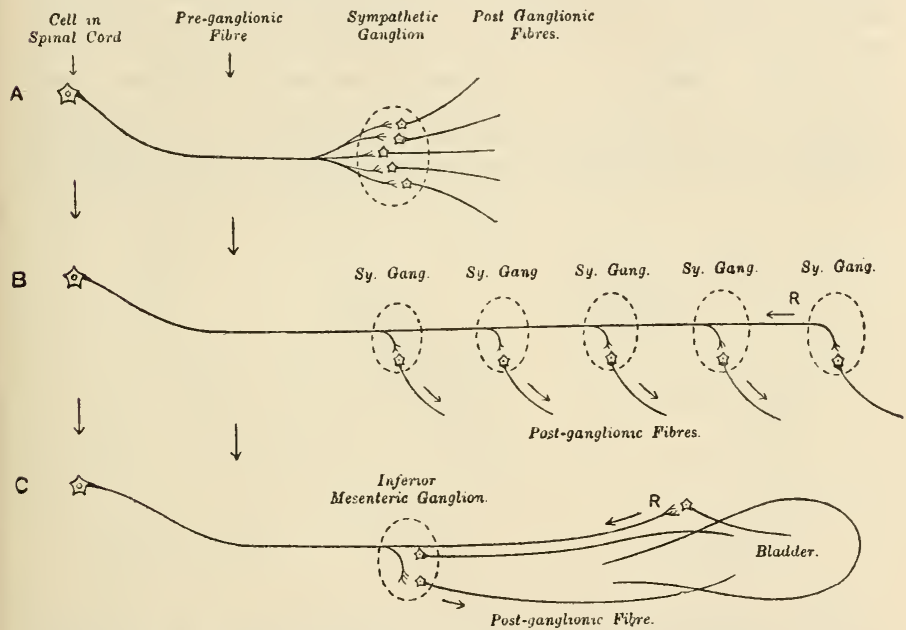

Frr. 315.-B and $\mathrm{C}$ show the probable mechanism of pseudo-reflexes; B, uncrossed, from the sympathetic chain ; C, crossed, from the hypogastric nerves. With the arrangement given in A (some compound ganglia), pseudo-reflexes are not obtained.

sphincter of the anus, pallor of the mucous membrane of the end of the rectum, sometimes slight pallor (very rarely contraction) of the uterus on the opposite side, and ocasionally slight pallor and contraction of the external generative organs.

We may for convenience speak provisionally of these actions as reflex actions, and proceed to consider the nervous mechanism by which they are produced. The injection of a small amount of nicotin puts a stop to the reflexes, although it does not stop the effects of stimulating the peripheral ends of the hypogastric nerves, so that we may conclude that the reflexes are normally produced by impulses which pass through

1 Areh. f. d. ges. Physiol., Bonn, 1874, Bd. viii. S. 600 ; Jahresb., ü. d. Fortsehr. d. Anat. u. Physiol., Leipzig, 1877 , Bd. vi. Abth. 3, S. 87. Cf. also H. Nussbamm, ibiel., 1879, Bd. viii. Abth. 2, S. 64 ; Nawrocki and Seabitschewsky, Arch. f. 1. grs. Physiol., Bown, 1891, Brl. xlix. S. 141.

2.Joum. Physiol., Cambridge and London, 1894, vol. xvi. p. 410. 
peripheral nerve cells, and chiefly through nerve cells in the inferior mesenteric ganglion. The same effect is produced by degenerative section of the inferior splanchnics, i.e. the nerve strands running from the spinal cord to the inferior mesenteric ganglion. Further, the observations tend to show that the same effect is also produced by degenerative section of the roots of the nerves which send efferent tibres to the various viscera in question. And we may conclude that the reflexes are produced by efferent fibres proceeding from the spinal cord, and having some connection with the nerve cells of the inferior mesenteric ganglion. In this case the method of connection can in principle hardly be other than that shown in C, Fig. 315. Some of the efferent fibres from the spinal cord (pre-ganglionic fibres), instead of sending all their terminal branches to the inferior mesenteric ganglion, send one or more to it, and one or more down the hypogastric of the same side to aberrant, more peripheral, ganglia; of the former, some are in connection with cells which send their axons down the opposite hypogastric. Thus, on stimulation of the central end of one hypogastric, impulses pass up the motor fibres, and spread out in their branches to some of the cells of the inferior mesenteric ganglion of the opposite side.

B

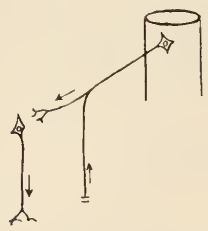

Pseudn- reflex
A

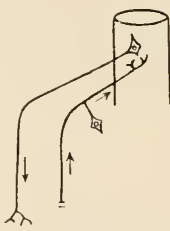

Reflex

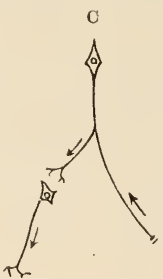

Cornmon Sclieme

Fic. 316.

The mechanism of these actions is not that which has been ordinarily understood as the mechanism of a reflex action. In the latter, special afferent fibres form an essential part of the machinery. So that it is clearly advisable to use some other term than "reflex" for those actions which are brought about by stimulating the central ends of efferent fibres. A good term is not very easy to find. I shall speak of them as pseudo-reflex actions. ${ }^{1}$

Morphologically, a pseudo-reflex action does not differ in principle from a reflex action which is produced by stimulating a nerve trunk. This will be seen on comparing the diagrams, A, B, and C of Fig. 316. A represents a simple reflex action, and $\mathrm{B}$ a pseudo-reflex action, and each of these can be reduced to the form $\mathrm{C}$.

In normal conditions, it does not seem probable that pseudo-reflex actions are often brought into play. So far as the experiments have gone, a nervous impulse set up in a post-ganglionic fibre cannot pass back through the nerve cell, so that the only way of setting up a pseudo-reflex action is by excitation of pre-ganglionic fibres, i.e. of nerve trunks and of ganglia, and not of peripheral endings in the autonomic tissues. Fig. 317 will serve to make this point clear.

${ }^{1}$ They might perhaps be called "deflected" or "deflex" actions. 
Other instances of the same kind of action occur in the body. We have seen that in the eat no pilo-motor fibres run ordinarily to the sympathetic chain bclow the third lumbar nerve, and that the pilo-motor fibres from the twelfth thoracic to the third lumbar nerves run downwards. But if any part of the lumbar sympathetic chain be stinulated, erection of hairs takes place in the areas of the skin innervated by two, three, or four of the ganglia above the point stimulated. ${ }^{1}$ The action can be produced after destruction of the spinal cord, or after section of the white rami, but is annulled by injection of nicotin ; and nicotin applied locally to any one ganglion annuls the effect in the area supplied by that ganglion. As with the hypo. gastric pseudo-reflexes, so here we may put on one side the posterior root fibres as being con. cerned with the action, since stimulation of these has no pilo-motor effect. It cannot be due to commissural fibres proceeding from lower ganglia to higher ones, since degenerative section of the sympathetic below the third lumbar white ramus abolishes the action below the point of section, but degenerative section between the ganglia does not abolish the effect of stimulating the upper eut end. It can tlien only be produced by descending motor fibres, the mechanism being diagrammatically as shown in B, Fig. 316.

Similar pseudo-reflexes occur in the upper thoracic region of the sympathetic chain. We have seen that there are no descending pilomotor fibres in the sympathetic in the region

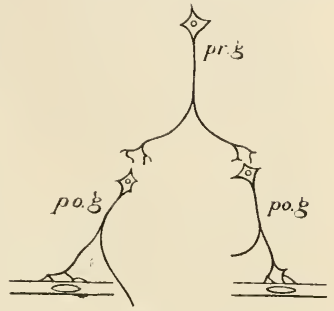

FIG. 317.-Impulse set up in the endings of a branch of the postganglionic fibre ( $p o . g$ ), though it may irradiate to the other endings of the same fibre, cannot pass back to the pre-ganglionic fibre $(p r \cdot g)$, and so cannot produce a pseudo-reflex action of the kind described in the text.

between the ganglion stellatum and (about) the sixth thoracic ganglion. But on stimulating any part of this region, after section of the white rami, erection of hairs is obtained in the areas supplied by the two to six ganglia below the point stimulated, and the action is stopped by injection of nicotin.

The pilo-motor pseudo-reflexes from the sympathetic chain, which we have just described, differ, it will be noticed, from those obtained from the hypogastric nerve, in being produced on the same side of the body as the stimulation, instead of on the opposite side (cf. B, Fig. 315). They are further interesting, as giving an indication of the number of ganglia to which a single nerve fibre sends branches. Generally speaking, there are fewer in the lumbar region than in the upper thoracic region of the sympathetic. Further experiments are required before any very definite statement can be made with regard to this; but, so far as the experiments have gone, it appears that the fibres ruming to the lumbar ganglia send branches to three ganglia as a rule; and the fibres to the thoracic ganglia generally send branches to four or five ganglia.

According to François-Franck, ${ }^{2}$ stimulation of the central end of one limb of the annulus of Vieussens, after severance of the connections of the ganglion stellatum with the spinal cord, causes dilatation of the pupil. He attributes this to a stimulation of sensory fibres, causing a reflex action in the ganglion stellatum. In the cat I have not found a dilatation of the pupil in these cireumstances; usually there was no effect, but occasionally the stimulation caused retraction of the nictitating membrane, and a slight separation of the eyelids. I take these effects to be produced by motor fibres, and to be pseudo-reflex and not reflex effects. They can hardly be due to an excitation of nerve cells in the ganglion stellatum, since the local application of nicotin to

' Langley and Anderson, Journ. Physiol., Cambridge and London, op. cil.

2 Arch. de physiol. norm. et path., Paris, 1894. 
this ganglion does not stop them. Injection of nicotin, or local application of nicotin to the superior cervical ganglion, does put a stop to them. We may conclude, then, that the nerve cells which are set in action are in the superior cervical ganglion, and that the reflexes are produced by fibres which divide in the trunk of the sympathetic, one division running to the anterior and the other to the posterior limb of the annulus of Vieussens. I have not found any effect on the eye, when the central end of the nerve which is given off by the ganglion stellatum to the heart and lungs is stimulated, although this contains numerous sensory fibres.

François-Franck also describes a reflex contraction of the pupil by way of the long ciliary nerves and the ciliary ganglion. Anderson and myself made one or two experiments on the cat and dog, but were unable to confirm this result.

It has been mentioned above, that after degenerative section of the inferior splanchnic nerves, stimulation of the central end of one hypogastric nerve has no effect; and we have deduced from this, that in all probability nervous impulses in post-ganglionic fibres cannot travel back through nerve cells and affect other nerve cells. It is clear, at any rate, that the impulses cannot excite all the nerve cells in the ganglion, for in that case stimulation of the central end of the hypogastric in the given circumstances would cause inhibition (or contraction) and pallor of the descending colon and rectum, since the nerves for those structures and the hypogastric nerve fibres arise in part from the same ganglia.

The experiment may also be taken as showing that the post-ganglionic fibres do not divide and send a branch to each hypogastric nerve; this makes it probable that post-ganglionic fibres only divide near their terminations, and thus we should not expect to find any wide spreading out of effects by an action on post-ganglionic fibres, analogous to that which may be obtained by an action on pre-ganglionic fibres.

Some other experiments, to observe whether stimulation of the central end of post-ganglionic fibres could cause a reflex effect, were made by Anderson and myself. We found no effect on stimulating the central end of one of the short ciliary nerves, nor on stimulating the central end of any one of the branches of the superior cervical ganglion.

\section{ON the Number of Nerve Cells on the course of a Pre-Ganglionic Fibre, and on Commissural Fibres.}

In the preceding account I have assumed that a nervous impulse, in its passage from the central nervous system to the periphery, passes through one nerve cell only of the sympathetic type, and that such nerve cells are not comnected by commissural nerve fibres. Both of these assumptions are opposed to general belief. Those who have considered that the nervous impulses pass through nerve cells at all, have taken for granted that in many cases they pass through more than one nerve cell; and most observers have taken for granted that the sympathetic ganglia are connected by commissural fibres. In this section I shall give some of the reasons in favour of my own view. In doing so; it must be remembered that I consider enteric nerve cells to be of a different type from the sympathetic nerve cell. ${ }^{1}$

We have good evidence, in the case of particular ganglia, that all the pre-ganglionic fibres which are able to affect the ganglion run to it direct and end in connection with its nerve cells. This is, for example, the case with the superior cervical ganglion, the ciliary ganglion, and the coccygeal ganglion.

$$
{ }^{1} \text { Cf. p. } 668 .
$$


It is hardly necessary to consider the evidence in detail, its general nature is indicated by what has already been said with regard to these ganglia.

We have good evidence, also, that the fibres given off by these ganglia run to the peripheral termination, without having nerve cells on their course.

Consequently, if a pre-ganglionic fibre has more than one nerve cell in its course, the second or other additional cells must be in the same ganglion as the first nerve cell, and the total number of cells with axons terminating in the ganglion must be great. But such arrangement is entirely unsupported by histological appearances. We may consequently adopt the simpler view, that a nervous impulse, in passing from the central nervous system to the periphery, passes through one nerve cell only.

The two questions, of the number of autonomic nerve cells on the course of a nervous impulse, and of the presence of commissural fibres, are closely connected. For if commissural fibres exist, and if they can be put in action by the pre-ganglionic fibres, it follows that a nervous impulse on its way to the periphery must in some cases, at any rate, traverse more than one nerve cell. The facts which bear on the question of commissural fibres are of several kinds.

1. We may consider, first, some cases in which it can be shown that commissural fibres do not exist. We have seen that the nerve cells of the ganglion stellatum in the cat send vasomotor and secretory fibres to the fore-foot, and vasomotor and pilo-motor fibres to the skin of the lower part of the neck and upper part of the thorax. If the superior cervical ganglion sends commissural fibres to any of these nerve cells, stimulation of the central end of the cervical sympathetic must canse observable changes in the foot and in the skin of the back. In fact, the stimulation has no effect. It is clear, then, that the superior cervical ganglion does not send commissural fibres to the great majority of cells of the ganglion stellatum. And, so far as the experiments have gone, it does not send commissural fibres to any of the cells of the thoracic ganglia.

The inferior cervical ganglion is small and comparatively unimportant, but as it is placed near the ganglion stellatum, it might be expected to send fibres to this ganglion. On stimulation of the central ends of the annulus of Vieussens-the white rami being cut-I have not found any effect on the blood vessels or glands of the foot, or on the hairs of the back; so that the inferior cervical ganglion sends no commissural fibres to those nerve cells of the ganglion stellatum which govern the abovementioned structures.

Stimulation of the sympathetic chain, just above the first coccygeal ganglion, has no effect on the hairs or blood vessels supplied by the lumbar or by any higher ganglia, so that the first coccygeal ganglion does not send fibres to the vasomotor or pilo-motor cells of these ganglia.

Stimulation of the upper thoracic region of the sympathetic does not affect the lumbar ganglia; nor does stimulation of the lumbar region affect the upper thoracic. These parts of the sympathetic cannot then be joined by commissural fibres.

So far, we have spoken of the ganglia of the sympathetic chain. There are similar facts with regard to the pre-vertebral and the vertebral 
ganglia. Stimulation of the central end of the splanchnic nerves-the spinal cord being destroyed-has no effect upon the hairs, i.e. the solar ganglia and the inferior mesenteric ganglia do not send commissural fibres to the pilo-motor nerve cells of the vertebral ganglia.

We find, then, that in a considerable number of cases the nerve cells of one ganglion do not send nerve fibres to the nerve cells of other ganglia. And, in consequence, there cammot be any general system of connection of ganglia with one another by commissural fibres.

Moreover, whenever an effect is obtained by stimulating the connecting strand between two ganglia, the effect may always be referred un good grounds to stimulation of fibres other than commissural fibres, namely, either of the peripheral ends of pre-ganglionic fibres, ${ }^{1}$ or of the central ends of the pre-ganglionic fibres, ${ }^{2}$ or of post-ganglionic fibres. Since, then, commissural fibres are in no case necessary in order to account for phenomena, and in particular cases it can be shown that they do not exist, we may reasonably adopt the view that commissural fibres are absent from the sympathetic system.

2. It can be shown that the pre-ganglionic fibres, if they excite nerve cells which pass on the stimulus to commissural fibres, can only do so to a very limited degree. From the twelfth thoracic to the lowermost effective spinal nerve (fourth or fifth lumbar), the preganglionic fibres of a given spinal nerve excite the nerve cells of the ganglia below, but do not excite the nerve cells of their own segmental ganglia. ${ }^{4}$ Thus, stimulation of the nerve roots of the third lumbar nerve causes erection of hairs in the region of the fourth lumbar ganglion, but none in the region of the third lumbar ganglion, i.e. excitation by pre-ganglionic fibres of the pilo-motor nerve cells of the fourth lumbar ganglion does not set in action commissural fibres passing to any more anterior ganglia above. And similarly with the other ganglia, up to the twelfth thoracic.

In some other cases the limitation of the stimulus to particular nerve cells is still more striking. In all compound ganglia it is obvious that stimulation of certain of the pre-ganglionic fibres running to the ganglia excites some only of the nerve cells, and no increase in the strength of the stimulus can cause irradiation of nervous impulses to the other cells of the ganglion. And the nerve cells which cannot then be brought into action may be nerve cells of the same class as the cells which are in a state of excitation. Of this we may give an example. In the cat, at times, when the arrangement of nerves is posterior, the fourth lumbar nerve causes erection of hairs on the tip of the tail; the nervous impulses pass through nerve cells in the coccygeal ganglion; other nerve cells in the coccygeal ganglia will, on stimulation, cause erection of hairs in the greater part of the rest of the tail; but no stimulation of the fourth lumbar nerve will affect this region. Hence, pilo-motor nerve cells, set in action by the fourth lumbar nerve, send no commissural fibres to the other pilo-motor nerve cells of the coccygeal ganglion.

Occasionally facts of the same kind can be shown in simple segmental ganglia. Thus in the cat the second and third sacral ganglia contain two sets of vasomotor cells; one set send their axons to the ano-genital region, and cause contraction of the arteries in this region;

\footnotetext{
${ }^{1}$ Cf. p. 634 et seq.
}

${ }^{3}$ Cf. P. 679 et seq.

${ }^{2}$ Cf. p. 629.

${ }^{4}$ Cf. p. 634 . 
the other set send their axons to the skin over the end of the sacrum, and cause contraction of the arteries there. When the arrangement of nerves is anterior, the stimulation of the fifth lumbar nerve causes contraction in the arteries of the ano-genital region, but not in the arteries of the skin over the end of the sacrum, i.e. the two sets of vasomotor cells in these ganglia are not connected by commissural fibres; and it seems safe to conclude that the nerve cells which are excited by preganglionic fibres send their axoms to peripheral structures, and in no case send them to other nerve cells.

It is to be noticed that if commissural fibres between ganglia are put in action at all, it is practically certain that they must be put in action by pre-ganglionic fibres, for they are not put in action by afferent spinal fibres, nor are they by the fibres which run from the ganglia to the periphery (cf. p. 682), and there is no other class of fibre present except the pre-ganglionic fibre.

3. After nicotin has been injected into the blood vessels, stimulation of the sympathetic between the vertebral ganglia has in most cases no effect. Such effect as is produced can be referred to post-ganglionic fibres. The action of nicotin, then, only allows us to conclude that commissural fibres, if present, are paralysed by nicotin. It does not directly affect the question of the presence of such fibres.

4. The results of experiments by the degeneration method are in their nature more conclusive, though not many have so far been made. When the lumbar sympathetic is cut below the last white ramus, and time is allowed for degeneration, all the sympathetic chain below the cut passes into a state like that which is produced by injection of nicotin. On stimulating between any two ganglia there is no effect, or only such as is produced by post-ganglionic peripherally running fibres. It might perhaps be supposed that commissural fibres degenerate after severance of the connections of the spinal cord with the sympathetic ganglia, but this hypothesis hardly requires serious consideration, for we have seen that the nerve cells giving off postganglionic fibres remain unaffected in function by separation from the spinal cord. We may then take the experiment as showing that there are no commissural fibres in the lower part of the sympathetic chain. And there can be little doubt that similar experiments upon the rest of the sympathetic would lead to a similar conclusion.

\section{The Size of Nerve Fibres as a Characteristic of the}

\section{Autonomic Systeir.}

Bidder and Volkmann divided the medullated nerve fibres of the body into two classes, according to their size and certain other characters. The smaller fibres they called sympathetic, the larger cerebrospinal fibres. They considered that most if not all the sympathetic fibres arose from the spinal and sympathetic ganglia. Bidder and Volkmann's use of the term sympathetic for all small medullated fibres was not generally adopted, and chiefly because it appeared clear that in mammals many small medullated fibres arose from the central nervous system.

But the statement that the nerve strands connected with the sympathetic and other peripheral ganglia contained many small medullated and very few large medullated fibres, was in the main accepted. 
Several subsequent observers pointed out that the medium and large fibres of the sympathetic, so far as they could be followed, ran past the ganglia and did not end in them. But no great attention was paid to the size of sympathetic nerve fibres until Gaskell's work in 1887 . He argued that all efferent "visceral" fibres are small fibres. And this view we may accept. The only point that can be urged against it is, that the actual termination of many of the medium and large fibres is not yet known.

The converse of this view, namely, that all small efferent medullated fibres are "visceral," i.e. autonomic, cannot at present be affirmed. Thus the anterior roots of the nerves to the limbs, which have not been shown to exercise any autonomic function, contain some small fibres, though but few.

A further matter, on which it is premature to make any dogmatic statement, is as to the size of the afferent fibres of the sympathetic and other parts of the autonomic system. It may, I think, be regarded as certain that the afferent fibres occurring in the sympathetic are not fibres of any one size. Small medullated fibres can be traced into the white rami of the sympathetic from the posterior roots of the spinal nerves; and the nervus accelerans, which consists exclusively or almost exclusively of non-medullated and small medullated fibres, gives, on stimulation, all the usual reflex effects. On the other hand, in the cat many of the larger nerve fibres of the sympathetic trunk can be traced into Pacinian bodies. And the depressor nerve, which is especially a nerve connected with autonomic reflexes, consists in great part of medium and of large nerve fibres. It is not unlikely, as regards the sympathetic system, that the medium and large fibres end in the serous membranes, and give rise to local pain, and that the small fibres end in connection with unstriated muscle and glandular tissue, and give rise to referred pain (cf. p. 689). But until the actual endings of the several afferent fibres have been ascertained, we cannot even say which are directly connected with autonomic reflexes, and which only indirectly by way of the somatic system.

It may be mentioned that the cutaneous nerves contain numerous small fibres which run to them direct from the spinal nerves.

\section{Afferent Autonomic Fibres. Referred Pain in Visceral Disease.}

Connection of afferent sympathetic fibres with spinal ganglia. -Some histological observations by Cajal and by Dogiel have been taken to imply that the afferent sympathetic fibres have a special connection with the spinal ganglia.

Cajal has described large nerve fibres as passing from the sympathetic chain, and giving off branches which penetrate between the cells of the spinal garglia; and he suggests that these fibres form nerve-endings around the cells. Dogiel ${ }^{1}$ traces small medullated and non-medullated nerve fibres from the primary ventral division of the spinal nerve into the spinal ganglion. He considers that these fibres arise from "sensory" cells of the sympathetic ganglia. He finds that the fibres divide and form pericellular endings, and as a rule, if not always, in connection with a new

1 Thternat. Monatschr. f. Anat. ". Physiol., Leipzig, 1897, Bd. xiv. In this paper, references to other observations bearing on the question will be found. 
type of posterior root cell, namely, one which has the general characters of Golgi's "sensory" type. The cell is small, its axon divides into a number of branches, and each branch ends in connection with typical unipolar cells. Thus Dogiel considers that a few afferent sympathetic fibres can stimulate a considerable number of posterior root cells. It will be noticed that a point of primary importance, namely, the origin of the branching fibres from the sympathetic, rests on wholly insufficient evidence. Dogiel's theory must be regarded as purely provisional, for there are certain facts which are inconsistent with it. Section of the inferior splanchnics, ${ }^{1}$ the lower lumbar sympathetic chain, ${ }^{2}$ or of a white ramus ${ }^{2}$ does not, as a rule, cause degeneration of any medullated fibres in the central ends of the nerves. Sometimes a few degenerated fibres may be followed for a short distance, but these appear to belong to small grey bundles and to pass off to peripheral tissues. This, of course, requires close investigation. The result, however, tends to show that the pre-vertebral ganglia do not send any medullated fibres to end free in the spinal ganglia, and that the vertebral ganglia do not send any by a white ramus. The result does not exclude the possibility of medullated fibres passing from a vertebral ganglion to a spinal ganglion by a grey ramus, but the absence of physiological evidence of the presence of sensory fibres in the grey rami is against it.

We may at any rate conclude that the majority of the afferent sympathetic fibres have their trophic centre in the cells of the spinal and not in the sympathetic ganglia.

It is well to bear in mind that the evidence, that the fibres between the spinal cord and the sympathetic chain are all medullated, is not entirely conclusive. The evidence simply establishes a high degree of probability. Beck (1846) describes a few "gelatinous" fibres in both anterior and posterior roots. Gaskell does not find any in the anterior roots, but does find some in the posterior roots. No doubt the probability is, that such non-medullated fibres as occur in the nerve roots run to the blood vessels of the spinal cord, but the proof of this is defective.

Relative number of afferent and efferent fibres.-The number of efferent and of afferent medullated fibres in a nerve strand can be determined by cutting the anterior roots of the nerves which supply it, and counting later the number of degenerated and of sound fibres. By this method,it appears that about one-tenth only of the medullated fibres in the hypogastric nerves are afferent, ${ }^{3}$ and the proportion of afferent fibres in the several splanchnic nerves is probably not very different from this. In the pelvic nerve, the proportion of afferent fibres is considerably greater, and in the cat approximately one-third of the whole number. ${ }^{4}$

The cervical sympathetic contains no afferent fibres proper to it, for stimulation of its central end does not cause pain, nor any reflex action. Sometimes a few afferent fibres from the vagus join the cervical sympathetic, but they run down the neck, and do not accompany the nerve in its peripheral course. The grey rami of the vertebral ganglia have also few, if any, afferent fibres accompanying the efferent postganglionic fibres to their peripheral endings.

1 Langley and Anderson, Journ. Physiol., Cambridge and London, 1894, vol. xvii. p. 184.

Langley, ibid., 1896, vol. xx. p. 55.

${ }^{3}$ Langley and Anderson, ibid., 1894, vol. xvii. p. 185.

4 Ibid., 1895, vol. xix. p. 377. 
General distribution of afferent fibres of autonomic nerves.-In the sympathetic every white ramus contains afferent fibres, for stimulation of the central end of even the smallest, readily causes a reflex body movement and change of blood pressure. The white rami form the only definitely proved channel for afferent impulses passing by the sympathetic. It is true that, as we have seen, there is histological ground for believing that a few afferent fibres pass to the spinal cord by way of the grey rami, but we may leave these out of account, since, in the experiments made so far, no reflex of any kind has been obtained by stimulating them.

The afferent fibres of the white rami do not run to the head, nor to any appreciable extent to the skin of the body. They run to the thoracic and abdominal viscera.

Such afferent autonomic fibres as run to the skin of the head and body must pass to it direct by the cerebro-spinal nerves. The deeper autonomic structures of the head probably receive afferent autonomic fibres from the seventh and the ninth nerves.

It is probable from the experimental evidence, so far as this goes, that the peripheral distribution in the viscera of the afferent fibres of each white ramus is essentially the same as that of the efferent fibres of the ramus, so that the sensory connection of the various viscera with the spinal nerves differs but little from the scheme given earlier for the motor connection.

At the same time, it must be borne in mind that the observations in man with regard to the cutaneous areas of tenderness and pain, which occur in disease of the several viscera (cf. p. 689), tend to show that the afferent fibres for any one organ arise from fewer white rami than do the efferent fibres. It is possible, however, that the referred tenderness ordinarily occurs only in the areas of those nerves which send a considerable number of afferent fibres to the affected organs, and that wider connections of afferent fibres with the several organs may be found, as clinical attention is more directed to the question. The results obtained so far are not concordant, but it is fairly well established that afferent fibres to the heart and lungs, the stomach, the liver, the intestine and kidney, form successive and overlapping series.

The peripheral distribution of the afferent and of the efferent sacral autonomic nerves appears also to correspond. Thus, on section of the pelvic nerve, degenerated fibres can be traced to the structures in which effects are produced by stimulation of the nerve, but degenerated fibres cannot be traced-according to the observations of Anderson and myself ${ }^{1}$ - to the uterus, which is unaffected by stimulation of the pelvic nerve.

Sensitiveness of the viscera. - It has been long known that the thoracic and abdominal viscera are very insensitive to certain forms of stimulation, such as handling and cutting, and this fact formed the basis of the theory put forward by Reil in 1811, that the sympathetic ganglia serve to suppress sensory impulses, and were only partial conductors of sensibility. Nevertheless, the splanchnic nerves, superior and inferior, and the pelvic nerve, when pinched or stimulated electrically, are apparently as sensitive as any nerve in the body, and we know that certain diseases of the viscera give rise to exquisite pain. There is 1 Jouru. Physiol., Cambridge and London, 1895, vol, xix. p. 272. 
comparatively little localisation of sensation in the viscera, and it is doubtful whether there is any proper sense of touch.

Referred pain.-At times diseases of the viscera give rise to pain in certain parts of the skin. This is spoken of as referred pain. Approximate explanation of its occurrence was put forward by Ross. ${ }^{1}$ On his view, the irritation of the visceral fibres of an organ is conducted to the grey matter of the cord, and there diffuses to the roots of the corresponding somatic nerves, and thus causes an associated pain in the area of distribution of these nerves. We may adopt the essential part of this view, namely, that when the afferent autonomic fibres of a nerve are affected, the primary referred pain will be in the area of the cutaneous fibres of the nerve.

It was shown by Mackenzie ${ }^{2}$ and by Head ${ }^{3}$ that visceral disease also causes areas of the skin to be more sensitive to slight stimuli, i.c. it may give rise to referred tenderness as well as to referred pain.

On this basis it is clear that referred pain will occur in the skin areas of those roots which have white rami, of those from which the pelvic nerve arises, and of those nerves, if any, with which the vagus afferent fibres are intimately connected. And there should not be primary referred pain in any others kin areas. Indeed, Ross-influenced by Gaskell's work on the visceral system-brought forward some facts to show that this was the case.

The question was followed up by Head, who has shown that, whilst primary referred pain and tenderness occur in large parts of the body, they do not occur in all. They do not occur in certain parts of the limbs, and we have seen that some of the nerves for the limbs send no fibres either to the cranial, sympathetic, or sacral autonomic system.

From what we have said earlier, with regard to the white rami and the pelvic nerve in man, we should expect the lumbo-sacral nerves, in the areas of which no primary referred pain occurs, to be ordinarily the third lumbar to the first sacral inclusive, sometimes also the second sacral, and possibly at other times the second lumbar. According to Head, the nerves are the second, third, and fourth lumbar. If this is so, either the afferent impulses pass by the grey rami, or the afferent sympathetic fibres of a nerve may cause primary referred pain outside the somatic area of the nerve to which they belong. Referred pain by way of the vagus occurs in part of the region of the fifth cranial nerve and of the upper cervical nerves $;^{4}$ hence, we must suppose that the roots of the vagus have intimate connection with the roots of the fifth and of the upper cervical nerves. Certain deep structures of the head, such as the iris, tooth pulp, tongue, cause referred pain in the skin region of the fifth nerve, and, taken together, in the whole region of the fifth.

Affections of certain of the thoracic abdominal organs frequently cause referred pain both in the body and in the head or neck, i.e. both above and below the brachial gap. This is naturally explained by the supposition that the organs receive fibres both from the sympathetic and from the vagus.

1 Bruin, London, 1888, vol. x. p. 351.

Med. Whron., Manchester, August 1892; cf. also Junil. Puth. und Bucleriol., Edin. and London, 1893, vol. i. p. 332.

${ }^{3}$ Brain, London, 1893 , vol. xvi. p. 1 ; 1894 , vol. xvii. p. 339 . Cf. further on this subject the article by Sherrington on "Cutaneous Sensibility" " in this volume.

Head, op cit., 1894.

VOL. II. -44 
Head finds that each area below the brachial gap, when associated with an area above the brachial gap, is associated with the same one; and, further, that the two associated areas may be the seat of referred pain from disease in different organs, for example, the stomach and the liver. Since each organ receives afferent fibres from more than one white ramus, we might expect that the fibres sometimes of one, sometimes of another, white ramus would be especially affected, and so cause referred pain in a different area of the skin; but it is difficult to see why there should be any correlated variation in the seat of referred pain above the gap, except on the unlikely supposition that the afferent fibres of each white ramus are connected peripherally with the fibres of a particular strand of the vagus. Head suggests that in some cases the correlation oceurs by means of the spinal cord. According to Head, the skin of the third and fourth cervical segments does not become the seat of referred pain from disease of any organ of the heal or neck; when it is affected from disease of the thoracic or abdominal viscera, it has correlated with it an area of pain in the head.

This correlation of tender areas above and below the brachial gap must, on the view we are taking of the matter, cease when organs are reached which do not receive fibres both from the vagus and the splanchnics. We do not know whether the lowest white ramus to send fibres to the intestines in man is the tenth, eleventh, or twelfth thoracic. All that we can say then, theoretically, is that the tenth, eleventh, or twelfth thoracic segment should be the last to be correlated with a tender region in the head or neck. According to Head, the tenth thoracic segment is the last.

We might also expect that there would similarly be double areas of referred pain, from disease of the descending colon and of such viscera as receive afferent fibres by way of the sympathetic and by way of the pelvic nerve. Of this, however, we can at present say very little.

We have taken, as the first step to an explanation, the view that irritation by disease of the afferent fibres of the white ramus of any nerve causes somehow irritation of the cutaneous sensory fibres of the nerve, and that thus tenderness or pain within the skin area of the nerve is produced.

Some difficulty seems to have been felt in accepting this view, since Head finds that the areas of referred pain and tenderness overlap little or not at all, and Sherrington finds that the sensory areas of the spinal nerves overlap considerably. But that the band of referred pain should be narrower than the band of cutaneous distribution of a nerve, seems natural enough. There is good reason to believe that the edges of the band of cutaneous distribution of a nerve receive fewer fibres than the central portion, and thus the central parts would naturally pass first into a state of over-sensitiveness. In visceral disease, though the fibres of a particular white ramus may be more affected than any other, it can never be affected alone, and it is not unreasonable to suppose that the irritation of other rami reaches the level at which a referred tenderness is produced before the irritation of the first is of such intensity that it causes referred tenderness in the parts of the skin which are bnt scantily supplied with somatic fibres from the corresponding spinal nerve.

There is nothing to show that if one ramus alone could be irritated, the region of skin tenderness would not coincide with the region of sensory supply of the somatic fibres of the nerve to which the ramus belongs. It must be mentioned, however, that Head, who has done most 
valuable work on this subject, considers that the skin areas of the body correspond to segments of the spinal cord, and not to the peripheral distribution of the posterior roots.

Ross, as we said, supposed that the visceral fibres affect the cutaneous fibres by some action on the grey matter of the spinal cord. This supposition does not at present rest on any satisfactory histological basis.

If the arrangement suggested by Dogiel (cf. p. 686) exists, it affords a fairly satisfactory basis for explaining the referred pain in visceral disease, so far as regards the trunk of the body. For referred pain in the head, however, an explanation based on some action in the central nervous system does not seem avoidable.

It must be borne in mind that a transference of impulses might take place in the spinal ganglia in other ways than that just mentioned, for example, by the collaterals which some of the nerve fibres are said to give off to the cells of the spinal ganglion. And it may be noticed that, so far as we know, diseases of the viscera do not cause referred pain in the muscles, and that diseases in the areas of the somatic nerves do not cause referred pain in the viscera.

\section{Growth of Pre-Ganglionic Cranial Fibres into Sympathetic GaNGLIA.}

We have seen (p. 652) that the pre-ganglionic fibres of the sympathetic, after section, readily re-form connections with sympathetic nerve cells. We have seen also that the fibres may in certain circumstances form connections with sympathetic nerve cells of a different class. The general similarity which exists between all pre-ganglionic
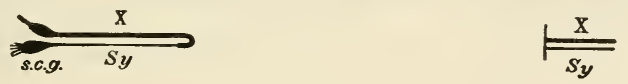

FIG. 318.

fibres suggested the possibility that cranial autonomic fibres might be able to form synapses with sympathetic cells. And experiment has shown that this is in fact the case. ${ }^{1}$ If the central end of the vagus be joined to the peripheral end of the cervical sympathetic (as in Fig. 318), it will in about a month make connection with the cells of the superior cervical ganglion of such nature that stimulation of the peripheral end of the nerve will cause dilatation of the pupil, contraction of the vessels of the ear, and the other effects which are normally produced by stimulation of the cervical sympathetic itself. In this formation of new connections some of the fibres of the vagus must change their function; since there are normally no pilo-motor fibres in the vagus, and some are found in it after union with the cervical sympathetic.

Probably a more striking instance of change of function is afforded by joining the central end of the lingual nerve ${ }^{2}$ with the peripheral end of the cervical sympathetic. The lingual nerve contains vaso-dilator and secretory fibres derived from the chorda tympani. After it has been allowed time to unite with the cervical sympathetic, it is found on stimulation to cause, amongst other effects, constriction of the arteries of the ear. It is difficult here to avoid the conclusion that some of the

${ }^{1}$ Langley, Proc. Roy. Soc. London, 1898, vol. 1xii. p. 331 ; Journ. I'hysiol., Cambridge and London, 1898, vol. xxiii. p. 240.

One experiment only of this nature has as yet been made. 
vaso-dilator fibres of the chorda tympani have become connected with vaso-constrictor nerve cells of the superior cervical ganglion, and have in consequence ceased to be vaso-dilator and become vaso-constrictor nerve fibres. The experiments point to the conclusion that any preganglionic fibre in the body is capable of forming functional connection with any nerve cell of the sympathetic type wherever found.

\section{General Comparison of the Cranial, Sacral, and Sympathetic Autonomic Systems.}

We have seen that the sympathetic system sends post-ganglionic fibres to the whole of the skin of the body. This distribution is in striking contrast to that of the cranial and the sacral system, which send no fibres to the skin, or only such as spread out into it from an adjoining mucous membrane. In this point, then, the cranial and sacral autonomic systems resemble one another and differ from the sympathetic system.

As I have said earlier, a comparison of the distribution of the postganglionic fibres shows, if we take the matter broadly, that while the sympathetic system supplies the whole of the body, the cranial autonomic system supplies (with the exception of the eye) certain structures at the anterior end of the alimentary canal, and the sacral system supplies certain structures at the posterior end of the alimentary canal.

We may put the facts, so far as we know them, in a tabular form. Some are still in dispute.

Autonomic structures having a double nervous supply, sympathetic and cranial or sympathetic and sacral.-The nuscular coats of the whole of the gut.

The glands opening into the gut, namely, salivary glands, gastric glands, pancreas, probably liver.

Parts at the mouth end of the gut; blood vessels of mucous membrane of mouth and pharynx; heart; unstriated muscle of lungs.

Parts at anal end of gut; blood vessels of rectum and anus; blood vessels and unstriated muscle of external generative organs; muscle coats of bladder.

Autonomic structures having a single nervous supply only, namely, from the sympathetic system.-The blood vessels, glands, and unstriated muscle of skin.

The blood vessels of the portion of the gut between the mouth and rectum, and of the glands opening into this portion; possibly the spleen.

The internal generative organs.

It has been asserted ${ }^{1}$ that in the gut, the internal generative organs, and the bladder, there is an essential difference between the character of the nerve fibres received from the sympathetic and of those received from the vagus or from the pelvic nerve, of such sort that the sympathetic fibres cause contractions of the circular muscular coat and inhibition of the longitudinal muscular coat; whilst the vagus and pelvic nerve cause inhibition of the circular muscular coat and contraction of the longitudinal muscular coat. A considerable number of observations which have been made on the several organs, show that this is not the case. And

\footnotetext{
${ }^{1}$ v. Basch and Hofmann, Hcd. Jahrb., Wien, 1877, S. 465 (on uterus); Fellner, ibid., 1883, S. 571 (on reetum); and Centralbl. $f$. d. med. Wissensch., Berlin, 1887, S. 258 (on uterus); Ehrmann, Med. Jahrb., Wien, 1885, S. 111 (on sniall intestine); v. Zeissl, Arch. f. d. ges. Physiol., Bonn, 1893, Bd. liii. S. 560 (on bladder).
} 
in the course of an investigation into the innervation of the pelvic viscera by Anderson and myself, results were obtained entirely in-

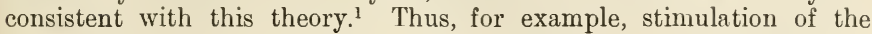
sympathetic causes the most striking longitudinal contraction of the vas deferens, and in the rabbit the most obvious inhibition of the longitudinal coat of the descending colon; and, on the other hand, stimulation of the pelvic nerve causes in the rabbit, in certain circumstances, complete and powerful contraction of the circular coat of the descending colon and rectum. In one case only is there some prima facie ground for the theory. The pelvic nerve causes great contraction of the rectococcygeal muscle; and the sympathetic, if it has any effect at all, which is not quite certain, has an inhibitory effect.

We give in the following table a summary of the chief actions of the sympathetic nerve on the one hand, and of the cranial and sacral autonomic nerves on the other, in the regions of double supply. There are certain points which are still doubtful, but the facts will serve to emphasise some conclusions with regard to the autonomic nerves.

\begin{tabular}{|c|c|c|}
\hline Tissue. & $\begin{array}{l}\text { Effect of Stimulating the } \\
\text { Cranial and Sacral Fibres. }\end{array}$ & $\begin{array}{l}\text { Effect of Stimulating the } \\
\text { Sympathetic Fibres. }\end{array}$ \\
\hline$\cdot \quad \cdot \quad \cdot \quad \cdot$ & Inhibition. & $\begin{array}{l}\text { Increase in rate and } \\
\text { strength. }\end{array}$ \\
\hline $\begin{array}{l}\text { Blood vessels of bucco-facial region of } \\
\text { dog }\end{array}$ & ? Dilatation. & $\begin{array}{l}\text { Dilatation, and in cer- } \\
\text { tain circumstances } \\
\text { contraction. }\end{array}$ \\
\hline $\begin{array}{l}\text { Blood vessels of salivary glands, } \\
\text { and most of buccal mucous ment- } \\
\text { brane }\end{array}$ & Dilatation. & Contraction. \\
\hline Salivary glands & Secretion. & Secretion. \\
\hline Muscular coats of alimentary canal . & $\begin{array}{l}\text { Chiefly contraction, } \\
\text { sometimes appar- } \\
\text { ent inhibition. }\end{array}$ & $\begin{array}{l}\text { Chiefly inhibition, } \\
\text { sometimes con- } \\
\text { traction. }\end{array}$ \\
\hline Bladder . & Strong contraction. & Feeble contraction. \\
\hline External generative organs & Inhibition. & Contraction. \\
\hline $\begin{array}{l}\text { Blood vessels of anal mucous mem- } \\
\text { brane and of external generative } \\
\text { organs }\end{array}$ & Dilatation. & Contraction. \\
\hline
\end{tabular}

A consideration of the facts given in the foregoing table shows that there is no constant contrast between the function of the sympathetic fibres on the one hand, and of the cranial and sacral on the other. In the double supply the fibres from each source may have the same ection, or they may have a different action. If they have a different action, either one or the other may be motor, and either one or the other may be inhibitory.

When, however, the fibres of one set arise from several nerves, we never find any difference in function in the fibres from the several nerves. Thus, all the sympathetic white rami which send fibres to a

${ }^{1}$ Journ. Physiol., Cambridge and London, 1895, vol. xviii. p. 104 (on descending colon); vol. xix. p. 74 (on bladder); vol, xix. p. 130 (on uterus and vas deferens). 
tissue have an action upon it, which may differ in degree, but does not differ in kind; similarly, a root of the pelvic nerve which has an action on a tissue, whether excitory or inhibitory, has the same kind of action as the other roots of the nerve.

This fact gives some probability to the view that the cranial and sacral autonomic centres in the central nervous system are not simply anterior and posterior parts of the sympathetic autonomic centres-parts separated from the sympathetic main region by the development of the nerves to the limbs-but are distinct centres developed at a different time and under different conditions.

We have, however, seen reason to believe that the sympathetic, the cranial, and the sacral autonomic systems resemble one another in a fundamental point of structure. Their efferent portions consist of a number of similar elements. Each element consists of two parts. One part consists of a nerve cell, which has its cell body in the central nervous system, and sends its axon, the pre-ganglionic fibre, outwards; the pre-ganglionic fibre divides, each division forming nerve-endings in connection with a peripheral nerve cell. The other part consists of the nerve cells in connection with which the pre-ganglionic fibre ends; the axons of these, the post-ganglionic fibres, form nerve-endings in the contractile and secretory tissues. Whilst accepting this as a general scheme, it is hardly necessary to say that the evidence for its universality is very inadequate. And it will be at once recalled, that nothing definite is yet known as to the nerve cell connection of the cranial nerve fibres to the gut.

The relation of the enteric nervous system to those just mentioned is at present only a matter of guess-work.

The experiments on regeneration, recounted above, throw a new, though doubtful, light on the question of the specific difference of nerve fibres having different functions. On the one hand, the experiments suggest that the nerve cells, which normally produce different efferent effects, remake their normal connections more quickly and permanently than abnormal ones, so that we should suppose that some difference exists between the different classes of nerve cells. On the other hand, it seems clear that the processes of both pre-ganglionic and ganglionic nerve cells have the power, within wide limits, of making connections other than the normal ones, and so acquiring non-normal functions. And this makes it possible that the functions of the several autonomic nerves are in part, it may be in very large part, dependent upon the particular structures with which they have an opportunity of coming in contact. Thus the laws of growth may have a larger rôle in the determination of function than physiological differences in nervous structures.

On this, as on many other physiological questions, we must hold suspended judgment. It is, however, hardly less important, and to many minds it is certainly more interesting, to formulate the problems to be solved than to dwell on the facts which are known. 


\section{ADDENDA.}

Nerve supply to the muscular coats of the gut (ef. p. 693, p. 642 ct seq., p. 663).-- Recent observations ${ }^{1}$ have strengthened the general conclusions arrived at in the text, that the cranial autonomic system sends motor fibres to both coats of the gut, and that the sympathetic system sends inhibitory fibres to both coats. With regard to this, it is to be borne in mind that the motor effect produced by stimulating a particular nerve-strand varies somewhat in different animals in different parts of the gut, and in different circumstances. The observations strengthen also the view that the cranial system sends some inhibitory fibres, and the sympathetic system some motor fibres, to both coats, but it is clear that these fibres vary in number in the nerves to the different parts of the gut and in different animals; and although it is perhaps probable that such fibres occur generally in mammals, further evidence is required to place this view on a sound basis.

Origin of splanchnic fibres supplying the stomach and intestine (p. 644).- In the rabbit I have found the splanchnic inhibitory fibres for the cardiac sphincter of the stomach arise from the fifth to the ninth thoracic nerves inclusive. Bunch ${ }^{2}$ in the dog finds that the vasomotor fibres for the small intestine leave the spinal cord by the second thoracic to the fourth lumbar inclusive. For reasons indicated in the text (pp. 464-465) I think the origin is probably less extensive. Bunch considers that the same spinal nerves, and especially the upper ones, send vaso-dilator fibres to the small intestine.

Nerve-fibres supplying the coronary vessels (p. 642, p. 663). -Maass ${ }^{3}$ states that in the cat vaso-dilator fibres pass to the coronary artery from the ganglion stellatum, and that vaso-constrictor and some vaso-dilator fibres pass to it from the vagus.

Nerve supply of the bladder (p. 646, p. 667).-Stewart 4 gives an account of a careful investigation of the innervation of the bladder of the cat. He confirms the statement that the sacral and sympathetic nerves supply both circular and longitudinal fibres of the bladder, and finds further evidence of inhibitory fibres for the bladder in the hypogastric nerves.

Origin from spinal cord of pelvic nerve (p. 666, p. 689).Harman ${ }^{5}$ finds that in man the pelvic nerve receives its fibres chiefly from the third sacral spinal nerve; usually it receives fibres also from the second and fourth sacral nerves, but sometimes from only one of them. The number of medullated fibres from the several sources is given.

The enteric nervous system (p. 668).-An important observation

${ }^{1}$ Langley, Journ. Physiol., Cambridge and London, 1898, vol. xxii. p. 905 (motor and inhibitory vagus fibres for the stomach of the rabbit, especially for the cardiac splineter). Cf. the earlier ohservations of Openchowski, Arch. f. Physiol., Leipzig, 1889, S. 549. Cf. also Curtado and Gnyon (observations on dog), Joum. de physiol., etc., Paris, 1899, tome i. p. 38; Bayliss and Starling, Journ. Physiol., Cambridge and London, 1899, vol. xxiv. p. 99 (motor and inhibitory vagus fibres for the small intestine of the dog; inhibitory fibres only were found in the splanchnic, bnt ef. Bunch (infra); Buneh, ibid., vol. xxv. p. 22 (motor and inbibitory fibres for the small intestine of the dog both in vagus and in splanchinic).

${ }^{2}$ Journ. Physiol., Cambridge and London, 1899, vol. xxiv. p. 72.

3 "Exper. Untersuch. ueber d. Innervation der Kranzgefisse," Rostock, 1899.

+ Am. Journ. Physiol., 1899, vol. ii. p. 182 ; vol. iii. p. 1.

5 Journ. Anat. and Physiol., London, 1899, vol. xxiii. p. 386. 
on the small intestine of the $\operatorname{dog}$ has been made by Bayliss and Starling. They find, after section of the vagi and removal of the solar ganglia, that mechanical stimulation of the intestine causes contraction above and inhibition below the point stimulated; the contraction passing down as a peristaltic wave, having an inhibited region in front of it. After local application of cocain or injection of nicotin, this effect is no longer obtained. They take the results to show that the enteric nervous system exercises reflex functions comparable to those of the brain and spinal cord, the nerve cells receiving afferent impulses, and sending inhibitory impulses downwards and motor impulses upwards. This hypothesis, however, can hardly be regarded as more than provisional. 


\title{
THE CEREBRAL COR'TEX.
}

\author{
By F. A. SCḦ̈FER.
}

Contents.-General Functions, p. 697-Results of Removal of whole Cortex, p. 698-of one Hemisphere, p. 704-Results of Artificial Excitation, p. 705Character of Muscular Contractions resulting from Excitation, p. $707-0$ ther Phenomena resulting from Excitation, p. 713-Effects on Respiration, p. 713On Circnlation, p. 714-On the Pupil, p. 715-On the Secretions and on the Muscular Tissue of the Viscera, p. 716-Heat Centres, p. 717-Epilepsy, p. 718-Special Localisations - The Motor Areas and Centres, p. 722Sensory (?) Functions of Motor Areas, p. 724-Results of Stimulation and Removal of Particular Areas, p. 732-Associated Movements, p. 735-The Principal Parts of the Motor Cortex of the Monkey, p. 736-The Head and Eyes Area, 738-Face Area, p. 740-Arm Area, p. 743-Leg Area, 744-Trunk Area, p. 745-The Motor Centres in the Orang, p. 745-The Motor Centres in Man, p. 747-The Sensory Areas of the Cortex, p. 749-The Visual Area, p. 752 - The Auditory Area, p. 761-The Olfactory and Gustatory Areas, p. 763-The Localisation of Tactile Sensibility, p. 766-Latent Regions of the Cortex, p. 769-Hypothesis of Association Centres of Flechsig, p. 771-Aphasia, p. 774 - Connections of Cortical Centres with one another and with Lower Centres, p. 776 .

\section{General Functions. ${ }^{1}$}

Thr cerebral cortex is the seat of the intellectual functions, of intelligent sensation or consciousness, of ideation, of volition, and of memory. That this is so is proved by the observation in man of cases of defective cerebral development and destructive disease. An individual with defective cerebral development is idiotic, in proportion to the amount of defect. It has not hitherto been shown that idiocy is associated especially with defect of any one particular region or of one hemisphere more than the other ; probably in all cases of marked idiocy the defect is bilateral. Similarly, an individual with extensive destruction of the cortex cerebri, the result of accident or disease, becomes mentally defective, the memory is lost, sensations are no longer rightly appreciated, ideas are confused, and the volitional power is disordered, diminished, or absent. These changes occur, not only as the result of affections of the cortex, but also from lesion of the fibres which connect the cortex with the "lower levels" of the central nervous system.

The term "level" in connection with the central nervous system was introduced by Hughlings Jackson to imply stages of complexity of the sensori-

${ }^{1}$ An admirable historical and critical account of the functions of the brain, including many references to the views and researehes of both older and more modern investigators, is given by Professor J. Soury in the article "Cerveau," in Richet's "Dict. de physiol.," Paris, 1897, fases. 5 and 6 . For a fuller discussion of many of the questions raised in the recent rapid progress of researeh in this branch of physiology, than ean well be introduced here, the student may be referrerl to that article; and the more so because the views which are taken by Professor Soury are, on some questions, different from those which are advocated here. See also "Le systeme nerveux," by the same author, Paris, 1899, which is an extension of the above article. 
motor functions of the organs designated. ${ }^{1}$ Thus those portions, such as the spinal cord and bulb and its immediate prolongations, upon which the performance of simple reflex actions, unattended necessarily by consciousness, depend, are regarded as forming the first or lowest level; above these come the centres, the action of which is more eomplex, and, in the first instance at least, volitional, and necessarily attended or preceded by conscious sensations; these centres form the second level; the third or highest level of Hughlings Jackson is that part of the central nervous system which is conceived to be the seat of memory and ideation. But however probable, it has not yet been proved that the domicile of the highest functions is anatomically separate from that of the functions with which the second level is concerned. Both are unquestionably dependent, in man at least, upon the integrity of the cortex, and it is very uncertain whether any special region of the cortex (e.g. the frontal lobe, as Hughlings Jackson supposes to be the ease) is especially associated with the functions ascribed to his "lhighest level." This subject will be further diseussed later.

\section{The Results of Removal of Both Cerebral Hemispheres.}

The results of experimental removal or destruction of the whole cerebrum in animals vary according to the kind of animal and its position in the scale of organisation. These results, as they have been described by various physiologists, and especially by Flourens and Goltz, have been so excellently summarised by Ferrier ${ }^{2}$ that no apology is needed for quoting part of his description at length :- "Deprived of its cerebral hemispheres" (the corpora striata and optic thalami being included in the removal), " the frog ${ }^{3}$ will maintain its normal attitude, and resists all attempts to displace its equilibrium. If laid on its back it will immediately tum on its face, and regain its station on its feet. If placed on a board, and the board be tilted in any direction, the animal will make the appropriate movements to throw its centre of gravity within the base of support. If its foot be pinched, it will hop away. If it is thrown into the water, it will swim until it reaches the side of the vessel, and then clamber up and sit perfectly quiet. ${ }^{4}$ If its back be stroked gently, it will utter loud croaks, and this with such uniformity on each application of the stimulus, that, as Goltz indicates, a chorus of

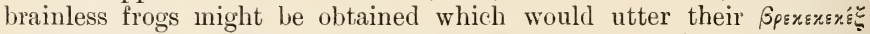

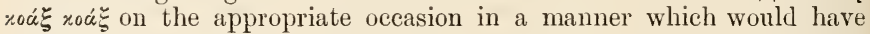
delighted the heart of Aristophanes. ${ }^{5}$ Indeed, in many respects, it would be difficult to say that the removal of the hemispheres had caused any alteration in the usual behaviour of the animal. If placed in a vessel of water, the temperature of which is gradually raised, it will not quietly submit to be boiled like a frog which has only its medulla and spinal cord, but will leap out as soon as the bath becomes uncomfortably hot. If placed at the bottom of a pail of water, it will

${ }^{1}$ Hughlings Jackson, "On the Relations of Different Divisions of the Nervous System to one another and to Parts of the Body," Brit. Med. Journ., London, 1898, vol. i. p. 65.

2 "Funetions of the Brain," second edition, 1856, 1) 109.

3 Goltz, "Beitr. z. Lehre v. d. Funetionen der Nerveneentren des Frosehes," Berlin, 1869.

${ }^{4}$ But if lowered gently into the water it will remain motionless (Onimms, Journ. de l'anat. et physiol. etc., Paris, 1870, p. 633 ; Eekhard, Hermann's "Handbuch," 1879, Bd. ii. Th. 2, S. 117 ; Goltz, loc. eit.).

5 The uniformity of this reflex is greater after removal of the thalamus than if the hemispheres and corpora striata only are removed (Goltz, loc. cit.; Paton, Edin. Med. and Surg. Journ., 1846, vol. 1xvi. p. 251). If another part of the skin be simultaneously and sufficiently stimulated, the reflex, as is the ease with many others, is inhibited. 
ascend to the surface to breathe. And not merely so, but if placed in a vessel inverted over a pneumatic trough, and containing a column of water sustained by barometric pressure, it will ascend to the surface as before; but not finding there the necessary atmospheric air, will work its way downwards, and succeed in making its escape out of the vessel to the free surface of the trough. There is a method in its movements. If an obstacle be placed between it and the light of a window, the frog will not spring blindly against it when its toe is pinched, but will clear it or spring to the side. It will alter the course of its leap, according to the position of the obstacle between it and the light. There is, so far, no difference between its behaviour and that of a frog in full possession of all its faculties. But yet a very remarkable difference is perceptible. The brainless frog, unless disturbed by any form of peripheral stimulus, will sit for ever quiet in the same place, and become converted into a mummy. All spontaneous action is annihilated. Its past experience has been blotted out, and it exhibits no fear in circumstances which otherwise would cause it to retire or flee from danger. It will sit quite still if the hand be put forth cautiously to seize it, but will retreat if a brusque movement is made close to its eyes. Surrounded by plenty, it will die of starvation; but, unlike Tantalus, it has no psychical suffering, no desire, and no will, to supply its physical wants."

There is a good deal of difference manifested if care be taken not to injure the optic thalami in removing the hemispheres. ${ }^{1}$ Under these circumstances, the frog, after the first effects of the operation have passed off, will show spontaneous movements without any manifest external stimulus, ${ }^{2}$ will feed itself by catching insects and the like, will bury itself on the approach of winter, in order to hibernate, and resume activity with the advent of spring, and in the breeding-season will give evidence of possessing normal sexual instincts. All these manifestations, as well as the power of balancing itself on a tilted board and the reaction on a turn-table, are either abolished or rendered imperfect on removing the optic thalami as well as the hemispheres. The latter are, in the frog, only to a very small degree developed, containing but a single layer of nerve cells; the thalami, on the other hand, are, in this animal, the chief centres for movements which are apparently voluntary, an importance which they probably owe to their connection with the visual organs on the one hand, and the tactile sense on the other, these being the senses by which the movements of these animals are mainly directed.

"The results which have been observed in fishes after similar ablation of the cerebral hemispheres are, cateris paribus, of the same nature as those seen in frogs. A fish so mutilated ${ }^{3}$ maintains its normal equilibrium in the water, and uses its tail and fins in swimming with as great precision and co-ordination as before. The brainless fish is, however, continually on the move, and there is also method in its movements. It will not run its head against any obstacle placed in its path, but turns to the right or left according to circumstances. Left to

${ }^{1}$ Schrader, Arch. f. d. ges. Physiol., Bonn, 1887. Bd. xli. S. 75 ; J. Steiner, "Die Functionen d. Centralnervensystem, u.s.w.," Erste Abth. "Der Frosel,," Bramnseluweig, 1885 .

2 This had already been shown by Desmoulins and Magendie, "Des systemes nerveux," 1825 , tome ii. p. 626 .

"Tulpian, "Leçons sur la physiol. du système nerveux," Paris, 1866, p. 683. 
itself in the water, it swims in a straight line, and, unlike other fishes which loiter by the way smelling at this and nibbling at that, it keeps on its course as if compelled by some irresistible impulse, and only stops when it reaches the side of the vessel, or when worn ont by pure nervous and muscular fatigue. Apparently there is some essential difference between the frog, which sits for ever quiet, and the fish, which goes on indefinitely, but the same explanation is applicable to both. In the one case there is no external source of irritation; in the other, the contact of the mobile water with the body surface acts as a continual external stimnlus to the natatory nechanism. As this is constant, so the fish contiuues to swim until arrested by some insurmountable obstacle or by pure nervous and muscular fatigue." 1

This description of the brainless fish is, however, only applicable to osseous fishes, and only to those osseous fishes from which the optic thalami have been removed as well as the corpora striata and hemispheres. For if only the hemispheres and corpora striata are cut away, the thalami and optic lobes being left intact, the behaviour of the animal is little, if at all, different from that of normal fishes. It moves to and fro in the water along with its fellows, nibbling at passing objects in the manner characteristic of these animals, distinguishing edible from non-edible material (worns from bits of thread resembling them), and even showing a preference for bait (wafers) of certain colours. $^{2}$ And still less is the description applicable to elasmobranch fishes, although the alteration here is in the opposite direction. For the shark, deprived of its hemispheres and corpora striata, remains perfectly immobile, even although the optic thalamus and optic lobes may be completely intact. The difference is due to the fact that the elasmobranch depends mainly upon its olfactory apparatus as a guide to its movements, and in removing the cerebral hemispheres the olfactory lobes are necessarily also removed. In fact, it has been shown by Steiner that renoval of the olfactory lobes alone, in the shark, has an effect similar to the removal of the hemispheres. The osseous fish, on the other hand, mainly depends upon influences reaching it through its visual organs, and the greater part of the central apparatus of vision remains intact. In reptiles also, the development of the cerebral hemispheres goes largely hand in hand with that of the olfactory apparatus. ${ }^{3}$

"The results of ablation of the cerebral hemispheres in pigeons have been described in great detail by Rolando, Flourens, Longet, Vulpian, and others. A pigeon so mutilated continues able to maintain its equilibrium and to regain it when disturbed. When placed on its back, it succeeds in regaining its feet. When pushed or pinched, it marches forward. Should it happen to step over the edge of the table, it will flap its wings until it regains a firm basis of support. When thrown in the air, it flies with all due precision and co-ordination. Left to itself, it seems as if plunged in profound sleep. From this state of repose it is easily awakened by a gentle push or pinch, and looks up and opens its eyes. Occasionally, apparently without any external stimulation, it

${ }^{1}$ Ferrier, loc. cit.

2 J. Steiner, "Die Functionen d. Centralnervensystem, u.s.w.," Zweite Abth. "Die Fische," Braunschweig, 1888.

${ }^{3}$ See Edinger, Anat. Anz, Jena, 1893, Bd, viii. S. 305. 
may look up, yawn, shake itself, dress its feathers with its beak, move a few steps, and then settle down quietly, standing sometimes on one foot and sometimes on both. Should a fly happen to settle on its head, it will shake it off. If ammonia be held near its nostrils, it will start back. Should the finger be brusquely approximated to its eyes, it will wink and retreat. A light flashed before its eyes will cause the pupil to contract; and if a circular motion be made with the flame, the animal may turn its head and eyes accordingly. It will start suddenly and open its eyes widely if a pistol be discharged close to its head."

"After each active manifestation called forth by any of these methods of stimulation, the animal again subsides into its state of repose. It makes no spontaneous movements. Memory and will seem annihilated. When irritated it may show fight both with wings and beak, but it exhibits no fear and makes no attempts at escape. It resists attempts to open its beak for the purpose of introducing nourishment, but, should its resistance be overcome, it swallows as usual. If fed artificially it may be kept alive for months, but left to itself it will die of starvation, like the frog or fish." 1

But in the bird also, ${ }^{2}$ if care be taken to remove only the cerebral hemispheres and basal ganglia without injury to the optic lobes or nerves, not only are spontaneous movements frequently manifested, but the animal (pigeon) gives indication that its movements are guided by visual impressions. Thus in the light or in day-time the animal walks continually about the room, ${ }^{3}$ avoiding all obstacles, ${ }^{4}$ even if transparent, such as glass objects, but in the dark remains quiescent and apparently asleep. It will even fly a little, so as to surmount small obstacles. There is sufficient understanding of things seen for selection of a sort to be exercised; Schrader found that his pigeons preferred to alight upon a perch to the floor; but they hardly ever flew spontaneously up from the floor on to a perch. ${ }^{5}$ Food, however, is not recognised, nor are enemies nor individuals of the opposite sex, although certain sexual phenomena, e.g. the cooing of the male bird, are retained. The animal manifests no fear. It responds mechanically to stimuli, like an automaton. If placed on a swinging cord, it balances itself perfectly with the to-and-fro movements, and this may be kept up for an hour or more; if then placed on the floor it still continues the balancing movements for a time. H. Munk ${ }^{6}$ found that pigeons deprived of their hemispheres were completely blind, but it would appear that whilst blind, in the sense of failing to comprehend the significance of objects, such animals will react to visual impressions through the medium of the optic lobes. In owls and falcons Schrader ${ }^{7}$ found much more marked disturbances of mobility, especially of the hind-limbs, after ablation of the hemispheres (or even of the anterior part only) than is the case with pigeons. It is noteworthy that these birds of prey employ their feet like hands to seize and hold their food, and their movements are therefore

${ }^{1}$ Ferrier, loc. cit.

2 Schrader, Aich. f. al. ges. Physiol., Bomn, 1888, Bd. xliv. S. 175.

3 Voit, Sitzungsb. d. math.-phys. C7. d. k.-bayer. Akad. d. Wissensch. zu Wimehen, 1868. S. 105 .

"Renzi, "Saggio di fisiol. sperin. sui centri nervosi," Ann. univ. di med. e chir., Milano, 1863, tomo clxxxvi. p. 179; ibid., 1894, tomo clxxxvii. p. 59; Schrader, loc. cit.

${ }^{5}$ Cf. M'Kendrick, "Recent Researches on the Nervous System," Edinburgh, 1874.

" "Functionen d. Grosshirurinde," Berlin, 1890, S. 279.

7 "Zur vergl. Physiol. d. Grosshirns," Strassburg, 1890. 
more truly of a voluntary character than the corresponding limbs of pigeons.

The results in mammals of complete removal of the cerebral hemispheres have been investigated in the guinea-pig and rabbit by many physiologists. The rabbit, after removal of the hemispheres, in a few minutes sits up and begins to move about in an apparently normal manner. ${ }^{1} \quad$ Its reflex excitability is increased. If the foot is pressed, it will kick and struggle violently, and may rush forward, as when the corpora striata are destroyed. ${ }^{2}$ But if the thalami be included in the lesion, the movements are much more limited, and, according to Christiani, ${ }^{3}$ progression is no longer possible. Recently the removal of the hemispheres has been effected in a very complete manner by Goltz in the dog. The following description is founded on Goltz's observations * upon an animal in which both cerebral hemispheres were completely removed in successive operations, the corpora striata and the optic thalami being also partly involved in the lesion. ${ }^{5}$ One optic nerve was also severed, and of course the olfactory lobes were included. The hemispheres were removed in three successive operations. The dog was kept alive for eighteen months after the final operation. After complete recovery from the last operation, the animal was able to walk in a perfectly normal fashion; towards the end of the period, however, its movements became gradually weaker, the hind-legs especially becoming emaciated and suffering loss of power. For the greater part of the day it walked restlessly up and down in its cage, but at night would go to sleep, rolled round in the manner usual with dogs. It then required a loud sound such as that of a horn, or rough shaking to awaken it. When awake it reacted to loud sounds by shaking its ears, to a glare of light by shutting its eyes, and by turning its head away. It reacted promptly and consequentially to tactile impressions. When its skin was pinched, it gave vent to its discomfort by snarling or barking just as a normal dog might do, and attempted to get away from the hand which was the source of discomfort, or failing to do this would turn round and bite at it, but in a clumsy manner and often without coming near it. If its feet were placed in cold water, they were quickly withdrawn. If one of its limbs was placed in an abnormal or uncomfortable attitude, it would never be allowed to remain so, although a dog with a lesion of the frontal cortex only does not replace a limb which has been put into such a position. If its fore-feet were placed upon the flap of a table, which was then gradually lowered, the legs would only be allowed to follow the flap for a short distance; the equilibrium was speedily recovered; a dog with the frontal centres only removed would fall under these circumstances. When one foot was accidentally hurt, the animal limped about for a time on three legs, in the manner usual with dogs.

Like the frog, fish, and bird in a like condition, the animal for months took no food spontaneously but had to be artificially fed by placing food at the back of its mouth, and even then often choked in the

1 Vulpian, "Lecons," etc., 1866.

2 See Nothnagel, Virchow's Archiv, Bd. lvii. s. 184, 206.

"Verhandl. d. physiol. Gesellsch. zu Berlin, Arch. f. Physiol., Lei]rig, 1884, S. 465.

4 "Der Hund ohne Grosshirn," Arch. f. d. ges. Physiol., Bom, 1892, Bd. li. S. 570 . Other experiments by Goltz of a like nature are deseribed in previous volumes of the same journal, but the removal was in no other ease so complete.

${ }^{5}$ Edinger, Neurol. Centralbl., Leipzig, 1893, Bd. xii. S. 327. 
process of swallowing. Eventually, it would itself take food if its head were placed over the dish containing it, and especially if the food came in contact with its snout. Meat which had been rendered bitter by quinine or colocynth was ejected with movements indicative of dislike. It also showed a feeling of satiety by refusing to take more than a certain quantity of food. For its size it required a very large amount of nourishment in order to keep up its weight; the extreme restlessness which it exhibited when awake tending apparently greatly to increase its katabolism. The higher psychical faculties were completely absent. The dog never showed any sign of recognition of persons or of other dogs, showed no fear of threatening movements; never wagged his tail, or showed any sign of pleasure on being stroked or spoken to; when wetted, shook himself vigorously but never attempted to lick himself dry, as a normal dog does; never used his fore-paws to hold a bone or to dig a hole for one (although there was even less paralysis apparent than in animals from which only the sigmoid gyrus is removed); never gave any sign of spontaneous mental activity, unless an increased restlessness and other signs of impatience when food was withheld for longer than usual, are to be in a measure so interpreted. The animal showed absolutely no sign of memory. Although its removal from the cage was the usual signal for feeding, it invariably resisted being taken hold of for this purpose, barking loudly and biting and struggling vigorously. During sleep it never showed any indications, as dogs often do, of dreaming. It exhibited no sexual instincts. Removal of the hemispheres had produced loss of all understanding and memory; the condition, in spite of the complexity of many of the phenomena which were still exhibited, was one of complete idiocy ; there was no sign of intelligence, and all the actions of the animal can be explained as reflex responses to immediate excitation. ${ }^{1}$

The result of experiments upon animals tends, therefore, generally to corroborate that of observations upon pathological cases in man; but it is clear that the higher the position of the animal in the scale of organisation, the more profound is the effect of removing the cerebrum. And it will presently appear that even partial bilateral lesions in monkeys and in man may, in some respects, and according to their extent and position, produce even more marked symptoms of loss of cerebral function, and especially of volitional movements, than in the animals above described.

There are, as Donaldson points out, ${ }^{2}$ anatomieal relations behind this differenee of effeet produced by lesions of the hemispheres. For the activity of the lower level eells is in all animals brought about by two sets of impulsesthe one set derived from the sensory nerves passing from their terminations in the grey matter of the lower level centres, either direetly to the motor eells or more probably through intermediary eells whieh play an important part in effeeting the eo-ordination required for purposeful movements. The other set of impulses, also in the first instance derived from the sensory nerves, pass to the eortex, and are thence sent down (perhaps along the fibres of the pyramidal traets) to the motor nerve cells, or rather probably also to the intermediary coordinating mechanism. In the lower anmals this second set plays an insignificant part in producing the ordinary co-ordinated movements of the animal;

1 H. Munk, Verhandl. d. physiol. Gesellsch. zu Berlin, Arch. f. I'hysiol., Leipzig, 1s94, S. 355 .

2 "American Text-Book of Physiology," p. 711. 
in the higher animals, an important part, so that in them the cutting of it off from the lower centre cells removes a great part of the impulses by which they are normally stimulated. ${ }^{1}$

\section{Effects of Removal of one Hemisphere.}

Goltz has published ${ }^{2}$ several accounts of dogs with very extensive lesions of the hemispheres, or from which one cerebral hemisphere has been wholly removed. ${ }^{3}$ Such animals, if kept a sufficient time after the removal, show in their ordinary movements an extraordinarily slight amount of motor paralysis, ${ }^{4}$ although apparently rendered incapable of performing such a purely volitional acquired action as the giving of the paw. Some of the differences of movement which are observable can be explained by the blindness which is produced on the homonymous half of the retina; but the contractions of muscles of the opposite side are rather less powerful than those of the same side. ${ }^{5}$ Sensation on the opposite side of the body is not lost, although somewhat blunted, and even the muscular sense of the paralysed side appear's to be still present. Whether the recovery of movement and of sensation which occurs in such an animal after a certain period, is due to a representation of both sides of the body in each cortex (as is unquestionably the case with the movements of some parts), and a vicarious taking up of the functions of the lost side by the other hemisphere, or whether the movements are merely association movements, which are called into play through commissural fibres in the lower centres, is still uncertain. It has, however, been ascertained in the dog and cat as well as in the monkey, that what is believed to be the volitional tract in the cord (crossed pyramidal tract), although mainly formed of fibres from the opposite hemisphere, also contains fibres which come from the homonymous hemisphere, ${ }^{6}$ and it may be through these fibres that such volitional movements as are preserved are carried on. Unilateral removal of the cerebral cortex, including the corpus striatum, is followed by degeneration and atrophy in specific parts of the lower portions of the central nervous system, which are either primary, resulting directly from removal of the nerve cells from which descending fibres originate, or from section of nerve fibres causing degeneration in their cells of

${ }^{1}$ Cf. Sherrington ("Proc. of Physiol. Congress," 1892, Centralbl. f. Physiol., Leipzig u. Wien, Bd. vi. S. 399), who found in the monkey that the reflex flexion of the hallux, which is caused by stimulation of the posterior roots of the sacral nerves, is abolished on cutting through the cervical cord.

2 In the Arch. f. d. ges. Physiol., Bonn, Bde. xiii., xiv., xx., xxvi., xxviii., and various other volumes up to the present time. An account of such an animal by Golt $\%$, and a report on the condition of the brain by J. N. Langley and A. S. Grimbaum, will be found in the Journ. Physiol., Cambridge and London, 1890, vol. xi. p. 606 . Cf. also Vitzou, A rch. de physiol, norm. et path., Paris, 1892, tome v.

${ }^{3}$ For observations upon the frog with one hemisphere and the corresponding thalanus removed, see Kato, "Thesis," Berlin, 1886 ; upon the pigeon see Lussana and Lemoigne, "Fisiologia d. centri nervosi encefalici," Padova, 1871, and M'Kendrick, loc. cit.; on the cat, R. Boyce, Phil. Trans., London, 1895, B, p. 321; and upon the rabbit (anatonical), Münzer and Wiener, Prag. med. Wchnschr., 1895; Honatschr. f. Psychiat. u. Neurol., Berlin, Bd. iii. S. 379. Frogs and pigeons show scarcely any noticeable defects, after recovery from the immediate effects of the operation, and rablits relatively little. There is nornally a tendency in moving to pass towards the side opposite to the lesion.

+ Immediately after the removal there is hemiplegia and hemianæsthesia, but this is gradually recovered from.

5 Boyee, Phil. Trans., London, 1895, B. This result was obtained with the contractions produced by absinthe, which stimulates the cortical centres.

${ }^{6}$ See article "Spinal Cord," by Sherrington, in this volume. 
origin; or, secondary, resulting from disuse of portions of the lower centres, in consequence of the lesion. The secondary changes are best observed when the lesion is performed in young animals, and may not show themselves at all in the adult.

The parts which are primarily degenerated or atrophied after removal of the whole cerebral cortex are the fibres of the pyramidal tract along their whole course ; fibres passing between the optic thalamus and the cortex; many of the cells of the thalamus and of the corpus Luysii; and many of the cells of the substantia nigra of the mesencephalon, all upon the same side of the brain. Degenerations also cross in the corpus callosum to the cortex of the opposite hemisphere, but how far the cells of that hemisphere are involved has not been ascertained. Secondarily, partial atrophy may be produced in the grey matter of the pons Varolii, in the quadrigeminal bodies, in the fillet, and in the red nucleus of the same side; and in the inferior and middle cerebellar peduncles, the hemisphere of the cerebellum, in the arciform fibres, and the nuclei of the posterior columns of the medulla oblongata of the opposite side. ${ }^{1}$

\section{The Results obtained from Artificial Excitation of tie Cortex Cerebri.}

Previously to 1870 , it was almost universally held that the cortex cerebri could not be excited artificially, that there was no localisation of particular functions in particular parts, that the cortex must act for each and all of its functions as a whole, and that lesions of the cortex produce, according to their extent, only a general depression of the functions of the cerebrum without special symptoms of local paresis. ${ }^{2}$ This doctrine was supposed to be firmly established by the experiments of Flourens, which led him to reject absolutely the localisation of function which had been formulated-on altogether insufficient evidence -by the phrenologists, and it remained for many years in almost undisputed possession of the field. A notable exception had, however, to be made, after it had been shown, by the masterly researches of Broca, ${ }^{3}$ that one important function of the cerebrum, that of producing articulate language, is intimately associated with the integrity of the posterior part of the third frontal convolution of the left hemisphere of the brain, and doubt had begun to be thrown upon the doctrine, at least in its rigid acceptation, by Hughlings Jackson, ${ }^{4}$ and by Bastian, ${ }^{5}$ the former of whom was led somewhat later, from the study of certain cases of epilepsy, following lesions of the brain in man, to the conclusion that the symptoms could best be explained by the assumption of functional localisations, the epileptiform contractions being regarded as the result of irritative lesions. ${ }^{6}$

${ }^{1}$ See on this subject, Langley, Journ. Physiol., Cambridge and London, 1890, vol, xi. p. 606 ; Langley and Sherrington, ibid., 1884, vol. v. p. 49; Monakow, Arch.f. Psychiat., Berlin, Bde. xii., xiv., xvi., xx., and xxvii.; other references in J. Soury, "Le systène nerveux," p. 642 ot seq.

2 Flourens, "Recherches expérimentales sur les propriétés et les fonctions du système nerveux," Paris, 1842, p. 99 ; Longet, "Anat. et physiol. du systime nerveux," Paris, 1842, tome i. J. 644; Matteucci, "Traité des phenomènes electrophysiologiques," Paris, 1843 , p. 242 ; Budge, "Untcrsuch. ueber d. Nervensystem," Frankfurt a/Main, 1842, Heft 2, S. 362 ; Scliff, "Lehrbuch d. Physiologie," 1858, Bd. i. S. 362.

3 :'Sur la siège de la faculté du langage articulé," Bull. Soc. anat. de Paris, 1861.

+ London Hosp. Rip., 1864, vol. i. p. 459.

" "On the Localisation of Function in the Cerebral Hemisplieres," Journ. Ment. Sc., London, Jan. 1869.

" "A Study of Convulsions," St. Andreu's Med. Grall. Assoc. Trans., London, 1870, vol. iii.

VOL. II. -45 
This was the condition of the subject when, in 1870 , were published the experiments of Fritsch and Hitzig, ${ }^{1}$ which clearly showed that the doctrine of the non-excitability of the cortex was erroneous. Fritsch and Hitzig found that electrical excitation of the dog's brain, at certain definite points upon the convolutions in the immediate neighbourhood of the crucial sulcus, is responded to by movement of parts on the opposite side of the body, that more prolonged stimulation produces local convulsive movements of the same parts, which may be followed by general epileptiform convulsions, and that removal of the localised brain area is followed by paralytic symptoms in the parts of the body or in the limbs which had previously responded to their excitation. From these experiments it became obvious-(1) That parts of the cerebral cortex can be directly excited to action by artificial stimulation; (2) that excitation of similar points in different animals, or upon opposite sides of the brain of the same animal, produces similar results; (3) that many parts of the cortex are apparently not thus directly excitable; (4) that the movements produced by artificial excitation are similar in their general character to those which are the result of voluntary movements of the animal; (5) that the voluntary movenients of the opposite side of the body are interfered with on removal of the excitable areas upon one side of the brain.

These facts, which were established by Fritsch and Hitzig by their experiments upon the dog's brain, were verified and amplified by those of Ferrier ${ }^{2}$ upon the brain both of the dog and monkey, and of many other mammals, and they have been abundantly tested and found to hold good for the human brain, in cases where by accident, disease, or the necessity of operation, the cortex has been laid bare.

Although it is difficult to obtain the movements in question by mechanical or chemical excitation, yet it is possible under some circumstances to do so $;^{3}$ and, as already stated, similar effects are obtained as the result of irritative lesions of the cerebral surface in man (Jacksonian epilepsy).

It was shown by Burdon Sanderson ${ }^{4}$ that if the cortex cerebri be removed at one of the excitable points, and the stimulating electrodes be placed upon the cut ends of the subjacent fibres of the corona radiata, a movement is evoked which is of a character precisely similar to that which had been previously called forth by excitation of the superjacent cortex itself. ${ }^{5}$ In non-narcotised animals the cortex is more excitable than the subjacent corona radiata, whereas in narcotised animals the reverse is the case. ${ }^{6}$ The brain of new-born dogs does not yield results on stimulation, but animals which are born in a more mature state, e.g. guinea-pigs, give cortical reactions. In the dog the effects of excitation begin to show themselves about the tenth

1 "Ueber die elektrische Erregbarkeit des Grosshirns," Arch. f. Anat., Physiol. $u$. wissensch. Med., 1870, S. 300.

2 West Riding Lun. Asyl. Rep., London, 1873; Proc. Roy. Soc. London, 1874, vol, xxii. p. 229 ; 1875, vol. xxiii. p. 409 ; "Functions of the Brain," 1876.

${ }^{3}$ François-Franck and Pitres, Progrès méd., Paris, Jan. 5, 1878 ; Landois, Wien. med. Presse, 1887, Nos. 7-9.

+ Proc. Roy. Soc. London, 1874, vol. xxii. p. 368.

${ }^{5}$ Cf. also Braun, in Beitr. z. Anat. u. Physiol. (Eckhard), Giessen, 1874, Bd. vii. S. 2 ; and Putnam, Boston Med. and S. Journ., 1874, vol. xei. p. 49.

"Richet, "Dict. de physiol.," tome iii. 1. 2, art. "Cervean." See on this and various other points connected with the subject of cortical excitation in the dog, François-Franck, "Leçous sur les fonctions motrices du cerveau," Paris, 1887. 
day. ${ }^{1}$ It is about this time that the fibres of the pyramidal tract begin to be
myelinated in the dog.2

On the Character of the Muscular Contractions which are
Evoked Fron the Cerebral Cortex.

If a tracing is taken by any form of myograph (Fig. 319) of the

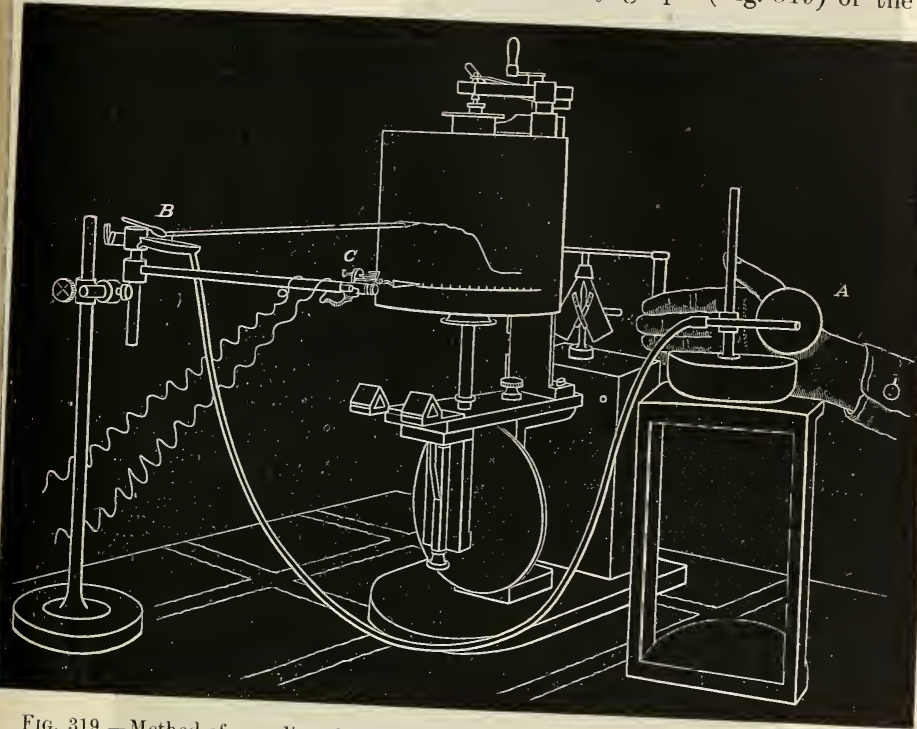

FIG. 319.-Method of recording the voluntary contraction-curve of opponens pollicis. $(A)$ tambour ; $(C)$ tine-signal.

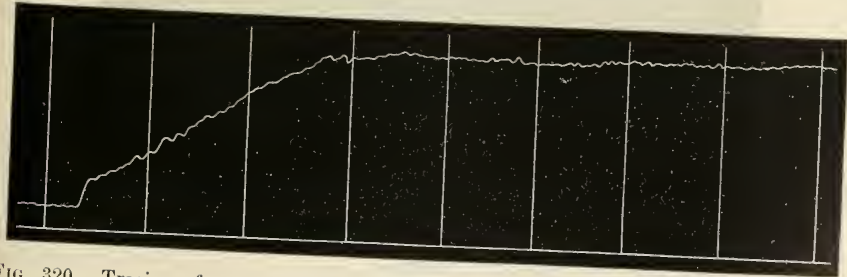

Fir. 320.-Tracing of a voluntary contraction of the thumb muscles, taken with a trans lines represent sceonds. To be read from surface. The intervals between the vertical voluntary contractic waves, irregular in ans of a muscle, the curve which is obtained exhibits in amplitude but fairly regular in rate, and exhibiting

${ }^{1}$ Soltmann, Jahrb. f. Kinderh., Leipzig, 1876, Bd. ix. S. 106 ; Steiner, Sitzungsb. d. k. ${ }_{2}$ Bechterewsen., Wien, 1895, S. 303. S. 513 
a rhythm of about ten or twelve contractions per second. ${ }^{1}$ Fig. 320 shows such a tracing taken on a slowly moving surface, and Fig. 321 another tracing with the excursion more amplified and recorded upon a more quickly moving paper. The rate of rhythm is very constant for the same individual, but varies in different individuals (from 8 to

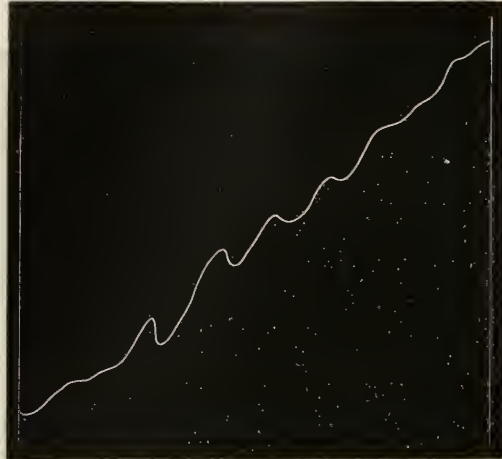

Frs. 321.- Part of a similar traeing, taken on a faster surface and with the movements more amplified.

13 per second), when the muscle is contracting against a small resistance. According to the observations of W. Griffiths ${ }^{2}$ it may become even more rapid (15 or 18 per second) with increase of the resistance up to a certain point, and then again diminish, and it undergoes similar changes of rate if the experiments be long-continued so as to produce fatigue. The vibration observable in the outstretched arm, when holding a weight, shows the same rate of rhythm as the muscle curve (Fig. $322)$.

Probably 10 per second may be taken as the average rate of rhythm, and 20 per second as the maximal rate. This would make the average interval between the consecutive nervous discharges from the cortical cells $0 \cdot 1$ second, and the ninimal interval 0.05 second. Richet found that in shivering the number of contractions per second in the muscles affected, was about 10 to 11

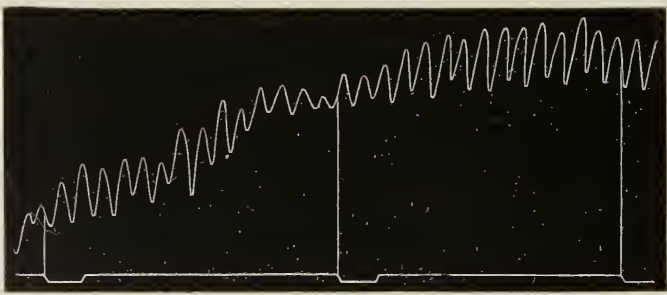

Fig. 322.-Vibrations of the outstretched arm holding a weight of about six kilos. The tracing was recorded by the apparatus shown in Fig. 319.-W. Griffiths.

per second, and did not exceed 13 per second, ${ }^{3}$ and that it is impossible to articulate at a rate of more than 11 syllables per seconc. That this rate originates in the cells of the brain appears probable from the fact, also pointed out by Richet, that it is impossible to think the act of articulation at a greater rate

${ }^{1}$ Schäfer, Journ. Physiol., Cambridge and London, 1885, vol. vii. p. 114 ; v. Kries, Arch. f. Physiol., Leipzig, 1886.

${ }^{2}$ Journ. Physiol., Cambridge and London, 1888, vol. ix. p. 39.

3 Trav. du lab. de Marey, 1895, tome iii. p. 17. 
than this ; and the same statement applies to the thinking the act of singing or of playing a series of musical notes. ${ }^{1}$ This would give, as the ordinary duration of the activity of the cortical cell, when involved in the performance of the simplest psychological action, about 0.09 second; but it is probable that with practice, and under other favourable conditions, it might be further reduced.

If the surface of the motor region of the cortex be excited by a faradic current, at a rate of rhythm either below or at about 10 to 12 per second, the muscles of the part with which the portion of the cortex stimulated is in connection respond by contractions, which succeed one

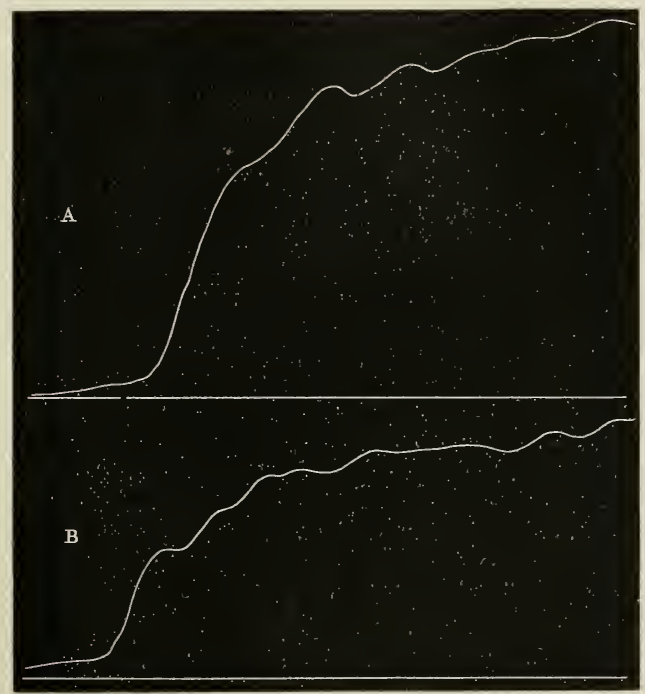

Fig. 323.-Myographic eurves from hamstring of monkey. (A) Natural contraction (voluntary); (B) contraetion caused by excitation of leg centre of cortex by rapid induced currents.

another at the same rate as the excitation. If, however, the excitation be more rapid than this, the response is no longer at the same rate -as in the case of excitation of the motor nerve fibre-but tends to reproduce the curve of voluntary contraction. ${ }^{2}$ This is illustrated in the tracings reproduced in Fig. 323. Of these A represents a voluntary contraction of one of the hamstring muscles of a monkey, the tendon of the muscle having been detached and fixed to the lever of a myograph; $B$ represents the contraction of the same muscle, as provoked by faradic excitation of the hamstring centre in the cerebral cortex, at a

${ }^{1}$ Richet, "Dict. de physiol.," Paris, 1898, tome ii. p. 10. Richet has also found, as the result of direct experiment upon the cerebral cortex of the dog, that in this animal the refractory period of the cortical cell is about $0.1 \mathrm{sec}$. (Ii'p. of Toronto Mceting Brit. Ass. Adv. Sc., London, 1897; Nature, London, 4th November 1897).

${ }^{2}$ Horsley and Schäfer, Journ. Physiol., Cambridge and Imindon, vol. vii. Figs. 323 and 324 are reproduced from the tracings given in this paper. 
rate of 60 per second; the two tracings show an identical rate of the waves which indicate successive muscular contractions.

The circumstance that a muscular response is obtained at the rate in question does not, of course, establish the fact that this rhythm is originated in the cortex itself. For excitation of the corona radiata, after removal of the superjacent motor cortex, or even of the cut spinal cord, produces a muscular response which has a precisely similar character and rhythm. Thus, in Fig. 324, A is the result of rapid stimulation of the cortex, $\mathrm{B}$ of the corona radiata, $\mathrm{C}$ of the cut spinal

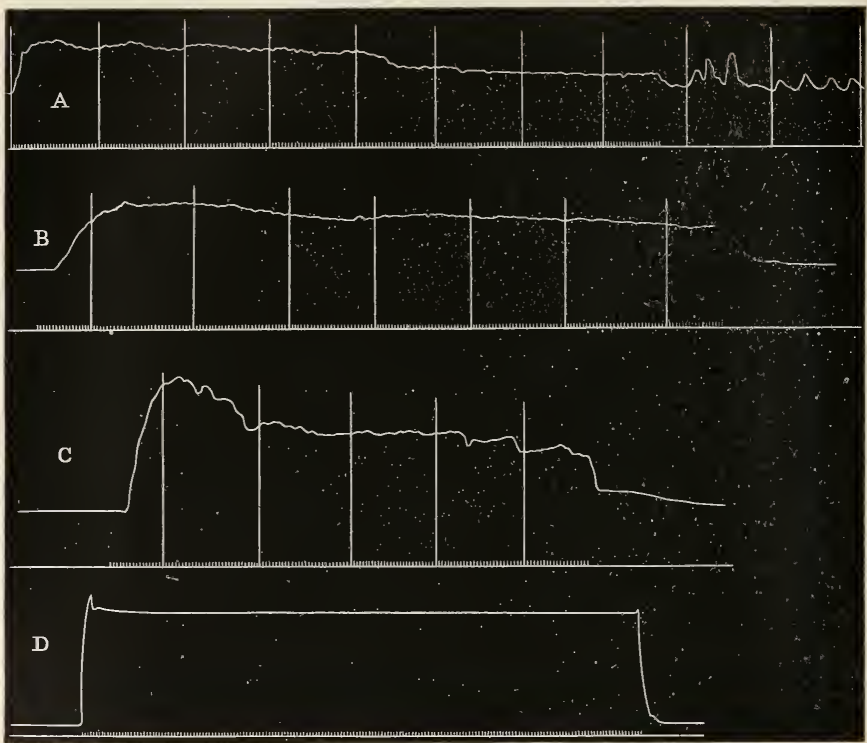

Fig. 324. - Tracings obtained from the same nuscle of a dog, on stimulation of different parts of the motor path. (A) Leg centre in cortex cerebri ; (B) corona radiata, after removal of cortex ; (C) cut spinal cord ; (D) eut motor nerve. The vertical lines (omitted in D) mark seconds. The teeth on the abscissa show the rate of excitation. In A the cessation of the excitation is followed by clonic epileptoid spasms of the muscle. All to be read from left to right.

cord, and D of the motor nerve, all in the same individual (dog), the same muscle being employed. The rate of stimulation in each case was at least 30 per second, but the response in $\mathrm{A}, \mathrm{B}$, and $\mathrm{C}$ was only at the rate of about 10 per second. In $B$ and $C$ this rhythm must have become established in the cells of the spinal cord; in A it may have origimated in the cells of the cortex. Or the cortical cells may have responded at the same rate as the excitation, and the change of rhythm may have occurred in the cells of the cord. It is, however, probable that the cells of the cortex respond to rapid or continuous excitation at about the same rate of rhythm as do the cells of the lower centres, since the rhythm of epileptoid contractions, which unquestionably originates 
in the cortex cerebri, ${ }^{1}$ although in itself slower than the normal rate of voluntary contractions, nevertheless shows indications of its curves being formed by the summations of excitation of a rate of about 10 per second. ${ }^{2}$

Although the character of the curves obtained by excitation of the cortex is similar to that obtained by excitation of the corona radiata, it must not be supposed that the effect is obtained by a spread of the stimulus to the subjacent fibres of the white matter. For there is a distinct delay in the lost time (period of latent excitation) of the muscular response, if we compare the commencements of the two curves; amounting, according to Bubnoff and Heidenhain, ${ }^{3}$ to about $0.0045^{4}$ in the unanæsthetised dog (Fig. 325), while with anæsthesia it is more prolonged, and eventually the response of the cortex ceases before that of the corona radiata. Further, as will presently appear, the excitation of the cortex is apt to be followed by epileptiform contractions of the muscles, which is never the case with stimulation of the coroula radiata.

Inhibition.-There is another function of the cerebral cortex which is no less important than that of originating movements, namely, that of inhibiting them. The restlessness of Goltz's decerebrised dogs, and perhaps that of the lunatic, may be due to the loss or diminution of this function. It has, moreover, been shown ${ }^{5}$ that in mammals there occurs, as an immediate result of removal of the hemispheres, a condition of tonic

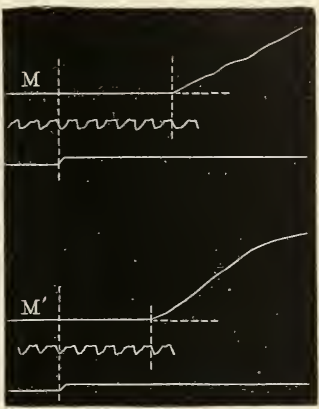

FIG. 325.-Period of latent ex. citation of a dog's musele on stimulation respeetively of the cortex cerebri, $\mathrm{M}$, and of the subjacent corona radiata, $\mathbf{M}^{\prime}$. Time traeing $\frac{1}{10} \sigma$ of a see. ond. - François-Franck and Pitres. ${ }^{6}$ contraction of certain groups of muscles of the trunk and limbs, the contraction being brought about by afferent impulses passing along the ordinary sensory channels; if one hemisphere only be removed, the tonus or rigidity only oceurs on the lomonyinous side of the body. An increase of the inhibitury function, on the other

${ }^{1}$ As is shown by the experiments of Goteh and Horsley (Phil. Trans., London, 1891, B, p. 267), who found that changes of electrical potential of the same rate as the elonic eontractions of the museles, passed along the spinal cord during the oceurrence of an epileptoid attack.

2 A similar observation has been made by Wolfenden and Dawson Williams regarding the rhythm of the eontractions in cases of disease aecompanied by muscular tremors, which frequently show a tendeney to fuse, so that the apparent rhythin is only about one-half the normal rate (Brit. Hed. Jou'n., London, 1888, vol. i. p. 1049). See also Herringham, Journ. Physiol., Cambridge and London, 1890, vol. xi. p. 481.

3 Arch.f. d. ges. Physiol., Bonn, 1881, Bd. xxvi. S. 187.

* It is interesting to note that this rednced lost time of the cerebral eortex is precisely the same as the redueed lost time of a simple reflex act, as determined by Wundt, and sinee in all probability the delay oeeurs, not in transmission through the nerve cell bodies, bint at the synapse or eonjunction of afferent fibres with them (sec p. 608), it may be eoncluded that when the eerebral surfaee is stimulated, the nervous impulses which are started pass to the projection cells from whieh the deseending fibres originate, through the intermediary of synapses formed between these cells or their dendrons and the more superfieial cells of the cortex, whieh are those aetually stimulated.

5 Sherrington, "Deeerebrate Rigidity, etc.," Jour'n. Physiol., Canbridge and Londun. 1898 , vol. xxii. p. 379 .

${ }^{6}$ François-Franck, "Leçons sur les fonctions motrices du cervean," Paris, 1887. 
hand, may be associated with a total temporary deprivation of volitional power, such as occurs in the condition known as hypnosis. ${ }^{1}$ That inhihition is an active function of the cortex, was shown by Bubnoff and Heidenhain, ${ }^{2}$ who occasionally got arrest of action on weak excitation. More recently it has been proved by Sherrington ${ }^{3}$ that electrical excitation of certain parts of the cortex may not only, as has long been known, produce contraction of definite muscles, but that simultaneously inhibition of tonically contracted antagonists may be also brought about. Thus if, after section of the third and fourth nerves on one side, say the left, so that only the external rectus is left in connection with the nerve centres, any of the parts of the left hemisphere which produce conjugate deviation of the eyes to the right be stimulated, not only does the right eye move to the right, but the left eye accompanies it, as far, at least, as the primary position; and this can, under the circumstances, only be effected by the inhibition of the left external rectus. ${ }^{4}$ The same will occur during voluntary movements, if the animal be kept alive a few days after the section of the nerves. Moreover, it is a familiar fact that we are constantly in the habit of exercising voluntary control over, and arresting reflex movements; and that this function is exercised by the cortex is shown by its absence when from any cause the cortex is in abeyance, e.g. during sleej. ${ }^{5}$

As to the manner in which inhibition is brought about, nothing is definitely known. It has been suggested by Lauder Brunton, that it may be regarded as a phenomenon analogous with that of interference in physics, in which case it would not be necessary to assume the existence of paths separate from those which conduct excitatory impulses. But it is well known that in the case of peripheral inhibition (heart, blood vessels), the paths are altogether different. And it has been shown by Sherrington, that inhibition of a muscle or group of muscles is not obtained by excitation of the same point of the cortex as provokes their contraction, hut rather in comnection with the point from which the contraction of the antagonist muscles is obtained, ${ }^{6}$ so that it appears certain that for this form of inhibition also there are separate nervous paths from those concerned in causing movement. It is probable that the action, whatever it may be, is exerted upon the lower nerve centres in the cord and bulb. Gad, ${ }^{7}$ with Orchansky, found that

${ }^{1}$ Inhibition through the cerebrum is not, however, the eause of the so-called hypmotism of aninals, of which the well-known experimentum mirabile of Kirseher may be taken as a type, for Verworn has shown that these effects are obtained equally well after removal of the cerebrum ("Die sogen. Hypnose der Thiere," Jena, 1898).

${ }_{2}$ Arch.f. d. ges. Physiol., Bomn, 1881, Bd. xxvi.

${ }^{3}$ Proc. Roy. Soc. London, 1893, vol. liii. p. 407; Journ. Physiol., Cambridge and London, 1894, vol. xvii. p. 27 ; H. E. Hering and Sherrington, Arch. f. d. ges. Physiol., Bonn, Bd. lxviii. S. 222 ; "Proc. Internat. Congress of Physiologists," Journ. Physiol., Cambridge and London, 1898, vol. xxiii. (Suppl.).

${ }^{4}$ This has been confirmed by aid of the graphic method by Topolanski, Arch. f. Ophth., Leipzig, 1898, Bil. xlvi. S. 452. It applies equally to the limb museles. The faet that when one set of museles contract the antagonists relax was noted by Charles Bell.

${ }^{5}$ According to the experiments of Famo upon the dog (Centralbl. $f$. Physiol., Leipzig u. Wien, 1895, Bd. ix. S. 466, and Arch. ital. de biol., Turin, 1895, tome xxiv. p. 438, removal of the whole frontal lobe, including the sigmoid gyrus, shortens the reaction time of reflexes, while stimulation of that lobe lengthens the time, i.e. tends to inhibit reflex movements. But destruction of the sigmoid gyrus alone produces no such effect.

${ }^{6}$ See also H. E. Hering and Sherrington, Arch. f. 1. ges. Physiol., Boun, Bd. lxviii. S. 222 .

7 “Verhandl. d. pliysiol. Gesellsch.," in Arch. f. Physiol., Leipzig, 1887, S. 363. 
the reaction time for contraction and for inhibition of skeletal muscle (masseter) was practically the same, namely, $0 \cdot 15$ second.

\section{Other Phenomena which result from Stimulation of the Cortex Cerebri.}

Besides the movements of voluntary muscles which are provoked by excitation of the motor area of the cortex, and which we shall study in detail in a subsequent section, various other phenomena are observed as the result of such excitation, prominent among these being alterations in the respiration, in the circulatory organs, in the pupil, and in some of the secretions. The probability of the production of sucl effects is clear from the influence which the emotions are well known to produce upon the various functions in question.

Influence of the cerebral cortex on respiration.-Danilewsky was the first to make observations upon respiration in this connection. ${ }^{1}$ On exciting the posterior part of the motor area of the dog, he obtained slowing and increase of amplitude of the movements; on applying the electrodes to the base of the brain, acceleration was producerl. Bochefontaine ${ }^{2}$ obtained usually acceleration of respiration.

Effects upon respiration were obtained by Franck and Pitres ${ }^{3}$ on excitation of the motor area only (in the dog), and were not very constant; sometimes the respirations were slowed, sometimes accelerated, sometimes increased in depth, sometimes diminished; sometimes with a tendency to an inspiratory, sometimes to an expiratory, tone; the variations, according to them, being due apparently more to the differences in strength of the stimulus than to the part of the motor area to which it was applied. They obtained no results on stimulation of the cortex outside the motor area. Similar results were obtained by v. Bechterew, ${ }^{4}$ whereas other observers ${ }^{5}$ have described special effects (expiratory or inspiratory) as being obtainable from different and fixed places in the cortex.

The differences described by these several observers appear to largely depend upon whether the animals were anisthetised or not, and upon the nature of the anresthetic. Richet, ${ }^{6}$ using chloralised dogs, found excitation of various points of the cortex, especially of the sigmoid gyrus, to suspend the respiration.

Connection of the frontal lobe with respiration.-H. Munk ${ }^{7}$ obtained in the dog, on placing the electrodes at a point on the anterior part of the frontal lobe, arrest of respiration in deep inspiration (often preceded by acceleration), whereas from a point on the under surface of that lobe there was also arrest, but in extreme expiration; sometimes, however, acceleration. In the monkey, also, stimulation of certain points on the frontal lobe produced either inspiratory or expiratory tetanus.

${ }^{1}$ Arch. f. d. ges. Physiol., Bonn, 1875, Bd. xi. S. 128.

${ }^{2}$ Arch. de physiol. norm. et path., Paris, 1876, p. 168.

${ }^{3}$ Loc. cit.

" "Physiol. d. motor. Hirnrindenregion," 1886; also (with Ostankoff) Neurol. Centralbl., Leipzig, 1894, S. 584.

${ }^{5}$ E.g. Unverricht, Neurol. Centrulbl., Leipzig, 1888, S. 274 ; Preobraschensky, Wien. klin. Wchnschr., 1890, S. 41-43; Schuchowski, Neurol. Centrulbl., Leipzig, 1898, S. 143.

6 "Circonvolutions cérébrales," Thèse de Paris, 1878.

7 Munk. Verhandl. d. physiol. Gesellsch. su Berlin, 1853; and "Funct. d, Grosshirnrinde," 1890 , S. 164. 
The effects on respiration produced by stimulation of this region have been worked out by W. G. Spencer. ${ }^{1}$ He found in the dog, cat, and rabbit, definite spots on the frontal lobe, excitation of which produce respectively (1) slowing and arrest of respiration, (2) acceleration, (3) inspiratory clonus, and (4) inspiratory tonus. The first of these is, in all animals tested, related with the part of the lower surface of the frontal lobe with which the olfactory lobe is connected, and a similar effect on respiration is obtained by stimulating, on the cut section of a hemisphere, the tibres of the anterior commissure. The arrest may occur in inspiration or expiration. When it occurs in inspiration, it is accompanied by a great rise of blood pressure.

Acceleration was obtained in the dog and cat by stimulation of the upper end of the supra-orbital sulcus, and in the rabbit by excitation of a point on the dorsal surface of the frontal lobe close to the longitudinal fissure. Inspiratory clonus was obtained from the junction of the olfactory bulb and tract, inspiratory tonus from many parts of the cortex, but most markedly from the middle of the motor area.

Most of these results are intimately related to the olfactory region of the cerebral cortex, and are probably to be referred to excitation of the tract which conveys olfactory sensations towards the lower centres (racinc moyenne of Broca). Spencer has himself shown that, except the inspiratory tonus effects (which follow the course of the pyramidal tract fibres), the other effects which he has described are traceable, by the excitation method, to the tegmental region. Probably, therefore, the points enumerated are not to be regarded as specific respiratory centres, but the movements obtained by their excitation are such as are ordinarily associated with stimulation of the olfactory nerve-endings, and are correlated with activity of the olfactory nervous apparatus.

Influence of cerebral excitation upon the circulation.-Schiff ${ }^{2}$ was the first to notice the effects on the circulation produced by excitation of the cortex, and they were subsequently (in 1875) shown by Vulpian to be of constant occurrence. Danilewsky ${ }^{3}$ obtained, on stimulation in dogs of the suprasylvian gyrus, vaso-constriction and cardio-inhibition. Bochefontaine ${ }^{4}$ showed that general vaso-constriction, usually accompanied by cardiac inhibition, but sometimes by cardiac acceleration, was produced by excitation of any part of the motor area ; observations which were confirmed by Richet, ${ }^{5}$ who, however, obtained these results chiefly from the anterior part of the sigmoid gyrus. On the other hand, Brown-Séquard, ${ }^{6}$ using heat as a source of excitation, obtained manifestations of vaso-dilatation. I myself, in some experiments (hitherto unpublished), performed several years since in conjunction with J. R. Bradford, constantly obtained vaso-dilatation as the result of stimulating certain regions of the cerebral cortex in monkeys anresthetised by chloroform or chloral, notably the mesial surface of the hemisphere anteriorly (gyius marginalis and gyrus

1 Phil. Trans., London, 1894, vol. elxxxv, B, 1) 609.

2 Quoterl by François-Franck, "Leçons sur les fonctions motrices du cervean," Paris, 1887, p. 163.

${ }^{3}$ Arch. f. d. yes. Physiol., Bonn, 1875, Bd. xi. S. 128.

4 Arch. de physiol. norm. et path. Paris, 1876, p. 142 ; 1883, p. 34.

5 Thèse de Paris, 1878.

6 Quoted from François-Frauck, "Leçons sur les fonctions motrices du cerveau," Paris, 1887 , p. 167 . 
fornicatus), while from some other parts of the cortex vaso-constriction was obtained. We did not, however, feel justified from our experiments in concluding that there was a definite localisation for the production of vaso-constriction and vaso-dilatation in the cortex, but supposed that we were exciting sensory regions, and that the difference of the results obtained was analogous to that obtained on stimulating different afferent nerves.

The circulatory effects of cerebral excitation have been very fully studied by François-Franck and Pitres. ${ }^{1}$ They obtained, as the results of simple excitations uncomplicated by epileptic after-effects, with quiet and very slightly anæsthetised animals (dogs), only constrictor effects upon the blood vessels, often followed, however, ly vaso-dilatation; the latter effect was never obtained primarily under these conditions: the effects were general, not loculised, and not confined to the opposite side: they were most marked when the electrodes were placed on the motor zone, but were also manifested when the adjacent regions were stimulated, but not if the stimulations were applied at some distance from that zone (unless epilepsy was proluced). The effects upon the heart-beat were very variable-sometimes it was accelerated, sometimes slowed-these differences appearing not to depend upon the seat of excitation, but partly upon the strength of current and partly upon the condition of excitability of the cortex.

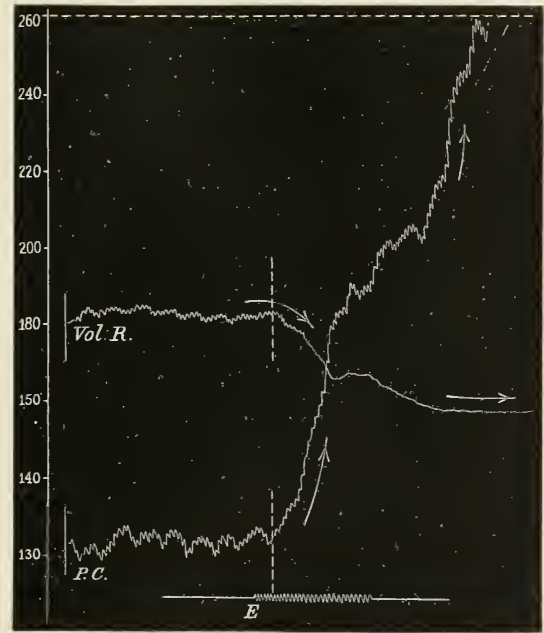

Fis. 326. - Efferts upon the volume of the kidney ( $V o l . R$. $)$ and arterial pressure $(P, C$.$) of exciting$ the motor area of the cerebral cortex of a dog. The very great rise of pressure in this experiment is due to the fact that the animal was atropinised, so that the cardio-inhibitory effects which are usually also producerl were alssent. - François-Franck and Pitres.

Effects upon the pupil.-Excitations which are sufficient to provoke epilepsy are always provocative of dilatation of the pupil, no matter to what part of the cortex they are applied (Franck and Pitres). On the other hand, weaker excitations do not affect the pupil, except when applied over the regions which are connected with movements of the eyes and head. Here it was found, both in the monkey and dog, by Ferrier, that if the electrodes were applied to the anterior part of the motor area, the movements of the head and eyes were usually accompanied by opening of the eyelids and marked dilatation of the pupils. The same is the case when the electrodes are applied over the posterior part of the hemisphere (visual region). On the other hand,

$$
1 \text { François-Franck, loc. cit. }
$$


excitation of the superior temporal convolution, which also produces movement of the head and eyes to the opposite side, was found by Ferrier to be accompanied by contraction of the pupils. The probability is, since dilatation of the pupil is ordinarily associated with convergence, and contraction with parallelism of the visual axes, that the pupillary effects of cerebral stimulation vary according as such stimulation tends to produce one or other of those conditions. But the matter is one to which little attention has hitherto been given.

It is noteworthy that, although in birds Ferrier was unable to obtain any movements of the limb or trunk muscles as the result of electrical stimulation of the hemisphere, nevertheless stimulation of a point at the vertex of the hemisphere produced "intense contraction of the opposite pupil, occasionally associated with turning of the head to the opposite side."

Effects upon the secretions and upon involuntary muscular tissue.-The effects of emotions upon the secretions, and especially the secretions of saliva and sweat, and upon the muscular tissue supplied through the sympathetic nervous system, especially that of the skin and hairs of the alimentary canal and of the urinary bladder, render it very probable that artificial excitation of the cortex should also produce similar effects. That excitation of the cortex in the dog produces a flow of saliva, was first shown by Bochefontaine, ${ }^{1}$ who obtained it from excitation of points scattered over the greater part of the motor area. The effect was usually most marked upon the gland (the submaxillary was employed) of the opposite side, but there was some secretion from the gland of the same side; the inpulses passed by the chorda tympani. ${ }^{2}$ There is also a marked flow as the result of artificially induced epilepsy. ${ }^{3}$ So far as the secretion of sweat is concerned, simple excitation of the cortex has yielded negative results, but Adamkiewicz has remarked the occurrence of abundant perspiration in partial cortical epilepsy in man, affecting the parts which were thrown into convulsions; and the same has been noted in the foot of the cat as the result of artificially produced epilepsy by Franck and Pitres. A certain number of observations have been made upon other secretions-urinary, gastric, biliaryby Bochefontaine ${ }^{4}$ and others, but these have been quite insufficient to determine whether any direct or localised relation subsists between special parts of the cortex and the secretions. The evidence is, on the whole, unfavourable to the conclusion that there are such localised relations, and the effects which are obtained seem to be produced by the cortex as a whole acting upon the bulbo-spinal centres.

Still fewer experiments have been made upon the influence of the cortex upon the "organic" muscles. The only ones that need here be referred to are those upon the bladder. Bochefontaine ${ }^{5}$ found that excitation in the dog of certain points in the neighbourhood of the crucial sulcus produced evacuation of the contents of the bladder in curarised animals. This observation has been

1 Arch. de physiol. norm. et path., Paris, 1876, p. 161.

${ }^{2}$ Cf. on this subject, Eckhard, Beiti: z. Anat. u. Physiol. (Erkhard), Giessen, Bd. vii. S. 199 ; and Neurol. Centrall,., Leipzig, 1889, S. 65 ; also v. Beehterew and Mislawski, ibid., 1888, S. 553. Further references will be found in the article "Salivary Secretion," by Langley, in the first volume of this work.

${ }^{3}$ Albertoni, quoted by François-Franck, loc. cit., P. 240 ; also Francois-Franek and Pitres, loc. cit. 
confirmed by François-Franck and Pitres, ${ }^{1}$ who find that the bladder is far more sensitive to the effects of cerebral excitation (in the motor region) than the vascular system, although the effects produced are somewhat irregular. v. Bechterew and Mislawsky also determined separately the effects of cortical stimulation upon the sphincter vesicie. They found that this was sometimes contracted, sometimes relaxed, as the result of such stimulation, effects comparable, as they point out, to the two kinds of influence, contraction and inhibition, which the will probably exerts upon that muscle.

Heat centres in the brain (?).-That injuries to the brain produced experimentally in animals cause a rise of body temperature, was shown by Tscheschischin. ${ }^{2}$ Eulenberg and Landois, ${ }^{3}$ and subsequently Hitzig, ${ }^{4}$ found that injuries of the cortex of the dog's brain in the neighbourhood of the crucial sulcus produce a rise of temperature, which is most marked on the opposite side of the body..$^{5}$ Similar observations were made by Wood, ${ }^{6}$ Pichet, ${ }^{7}$ Ott, ${ }^{8}$ and others. ${ }^{9}$ Aronsohn and Sachs ${ }^{10}$ found that punctures of the corpus striatum, in particular, cause a rise of temperature, and the same fact was affirmed independently by Ott, ${ }^{11}$ and has been confirmed by Girard, ${ }^{12}$ Baginsky and Lehmamn, ${ }^{13}$ and Hale White. $^{14}$ The observations of Hale White, which were made upon rabbits, tend to show that it is especially a lesion of the grey matter of the corpus striatum and not of the adjacent white matter of the hemisphere, nor of the optic thalamus, which is followed by the rise of temperature, and that this rise, which may amount to $3^{\circ}$ or $4^{\circ}$ or even $5^{\circ} \mathrm{F}$., lasts from a few hours to several days. Lesions of the crus cerebri and of the septum lucidum were also found by Hale White to be followed by a very considerable elevation of temperature, but injuries to the cortex cerebri to a less degree.

Injuries to various parts of the brain (cortex, corpus striatum, and crus cerebri) in man have also frequently been found to be associated with rise of body temperature. ${ }^{15}$ It is, however, very doubtful whether the facts observed warrant the assumption that the parts in question, which are apparently irritated by the lesion, are specific centres to determine the production of heat. For when the experiments

${ }^{1}$ Loc. cit.; see also v. Bechterew and Mislawsky (Neurol. Centralbl., Leipzig, 1888, S. 505), who obtained contraction of the detrusor vesice in the dog on stimulating a localised part of the posterior sigmoid gyrus. v. Bechterew obtained contraction of the sphincter vesice by stimulation of a point in the same gyrus a little further out (Neurol. Centralbl., Leipzig, 1893, Bd. xii. S. 81).

2 Aich. f. Amot., Physiol. u. wissensch. Med., 1866, S. 151; and Dentschis Aich. f. klim. Med., Leipzig, 1867, Bd. ii. S. 588.

"Virchow's Archiv, Bd. Ixvi. S. 489 ; Bd. lxviii. S. 245.

${ }^{4}$ Centralbl. f. d. med. Wissenseh., Berlin, 1876, s. 323.

5 This difference on the two sides was not fomm by Hale White and Washbourn in rabhits, Jow'm. Physiol., Cambridge and London, 1891, vol. xii. 1. 271.

6 "Fever," Smithson. Contrib. Knoul., Washington, 1880.

7 Soc. de biol., Paris, 29 Mars 1884.

${ }^{8}$ Jonin. Nerv, and Ment. Dis., N. Y., 1884.

${ }^{9}$ For references to earlier observations, see Riegel, Arch.f. d. grs. Physiol., Bonn, 1s72, Bd. v. S. 629 ; later papers are given by Hale White, Jomrn. Physiol., Cambridge and London, 1890, vol, xi. p. 1.

${ }_{10}$ Deutsche med. Wchnschr., Leipzig, 1884, and Arch.f. d. yes. Physiol., Boml, 1885, Bd. xxxvii. S. 232.

${ }_{11}$ Jourin. Nerv, and Ment. Dis., N. Y., 1884, 1887, 1888; and Brain, London, 1889.

12 Arch. de physiol. norm. et path., Paris, 1886, tome viii.

13 Tirchow's Archiv, 1886, Bd. evi. S. 258.

${ }^{14}$ Jourin. Physiol., Cambridge and Lonton, 1890, vol. xi. p. 1; ibil., 1891, vol. xii. p. 233.

${ }^{15}$ For a list of published cases, see Hale White, Brit. Med. Journ., London, 1894, vol. ii. p. 1093 ; ibill., 1897, vol. i. p. 1655. 
on this subject are examined, it is found that the results are closely dependent upon the establishment of an irritative lesion in parts which are either directly in, or in close proximity to, the path taken by motor impulses, and the resultant rise of temperature may therefore well be due to an increased activity, chemical or otherwise, of voluntary muscles, caused by irritation extending to the motor tract. ${ }^{1}$

\section{Epilepsy from Cortical Excitation.}

As already stated, Fritsch and Hitzig obtained, as the result of strong or prolonged stimulation of the excitable points which they had marked out in the dog's brain, after-actions of the muscles which had been called into activity; these after-actions took the form of clonic

A

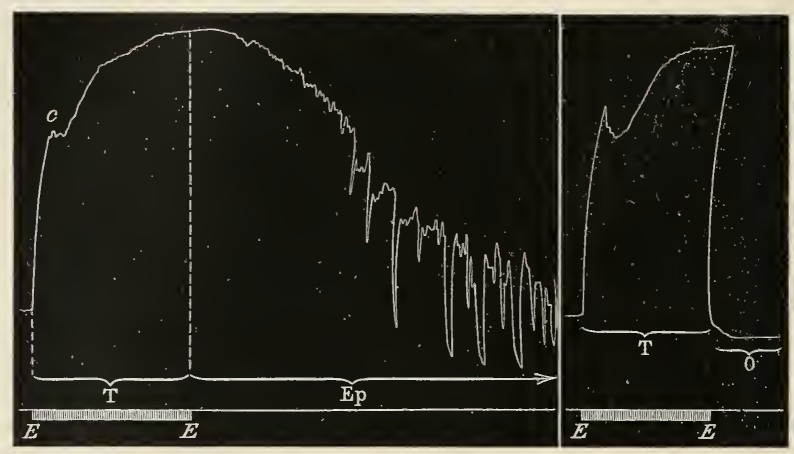

Fif. 327.-Curves obtained from a dog's muscle as the result of strong excitation (A) of the cortex cerebri, (B) of the subjacent corona radiata. E, E, period of excitation; T, contraction during excitation ; $\mathrm{E}_{\mathrm{p}}$ (in A), epileptoid contractions. In A the cessation of the excitation is followed by gradually developing epileptoid spasms, which appear to be formed by the fusion of smaller contractions. In $B$ the rajid fall of the muscle lever indicates the entire absence $(O)$ of an after-effect.-François-Franck and Pitres.

spasms, or even in some cases passed into well-characterised epileptic fits, spreading eventually to all the muscles of the body. The character of the after-contractions thus obtained is shown in the annexed tracing (Fig. 327, A), where it will be seen that each clonic spasm of a muscle is formed by the summation of a certain number of contractions, which succeed one another at the ordinary rhythm of discharge of the nerve centres. This summation occurs in the nerve cells of the cortex cerebri and not in the lower nerve centres, for, as already mentioned, it was found by Gotch and Horsley, by the aid of the capillary electrometer, ${ }^{2}$ that the passage of the nerve impulses down the spinal cord is accompanied by an electrical disturbance having the same rhythm as the muscular clonus.

${ }^{1}$ The formation of heat within the cortex itself has been studied by Mosso (Croonian Lecture, Phil. Trans., London, 1892, B). Cf. Hill and Nabarro, Journ. Physiol., Cambridge and London, 1895, vol. xviii. p. 218, and article "Animal Heat," this Text-Book, vol. i. p. 808 .

${ }^{2}$ Phil. Trans., London, 1891, B, p. 267. 
Other kinds of irritation of the cortex, and especially prolonged mechanical irritation (such as is liable to be produced by the irritation of a bony splinter from the inner table of the skull) will also produce epileptoid attacks, and the same result may follow experimental lesions of the cortex in animals. ${ }^{1}$ Irritation confined to the corona radiata is never followed by epilepsy; and if a portion of the excitable area of the cortex be removed, such, for example, as the centre excitation of which produces movement of one of the opposite limbs, and an epileptic fit be brought about by strong or prolonged excitation of another portion of the cortex, the limb in question remains quiescent." Epilepsy, therefore, is essentially a functional disturbance of the cortex cerebri. It does not occur if the Rolandic or motor region of the cortex be removed entirely, although it may be provoked by excitation of other portions of the cerebral surface ${ }^{3}$ than this, but not with the same ease and with a longer interval after the excitation.

Hughlings Jackson showed ${ }^{4}$ that in the pathological condition now named after him Jacksonian epilepsy, the convulsive movements in a fit follow a certain order or "march." Thus, if a fit begin by contraction of the thumb flexors, it first spreads to other muscles of the hand and forearm, next to the shoulder muscles, then to the muscles of the face, trunk, and leg in a definite order in each case, passing, if it begin in the hand, up the arm and doun the leg; if it begin in the toes, up the leg and down the arm. When the centres for movements which are bilaterally represented in the cortex are involved, the corresponding muscles of both sides are set in action, and in severe fits all the muscles on both sides of the body may be included. Ferrier's experiments ${ }^{5}$ upon monkeys and other animals entirely confirmed the observations of Hughlings Jackson upon man, showing that in each case the epileptoid attack began in the muscles corresponding to the motor centre which was stimulated, and spread thence with a definite "march." " The " march" is apparently dependent in each case upon the relative propinquity of the several centres in the motor region of the cortex, and, when they are equally close, upon their relative excitability; it is, however, not interfered with, according to Unverricht, ${ }^{7}$ by isolating the centres from one another, and the sequence must therefore be brought about-at least under these circumstances-by influences passing from one part to another through lower level centres. Section of the corpus callosum does not prevent the spreading of an artificially induced fit from the one side of the body to the other (Unverricht). In dogs, when the side of the brain opposite to that first set in action is involved, the fits always begin with the muscles of the hind-limb, wherever the excitation producing them might have been originally applied on the other side of the brain. ${ }^{8}$

${ }^{1}$ Hitzig, "Untersuch. neber d. Gehirn," Berlin, 1874, S. 271.

2 It was stated by Munk, and the statenent was confirmed by Bubnoff and Heidenlain (loc. cit.), that if during the progress of an epileptic attack, artificially produced, tlie cortical centre which was excited is rapidly extirpated, the attack inmediately ceases. But François-Franck and Pitres (loc. cit.) and Albertoni (loc. cit.) were unable to confirm this statement, and deny that the attack once commenced is modified by the ablation.

${ }^{3}$ François-Franck and Pitres (loc. cit.); Unverricht, Arch. f. Pyschiat., Berlin, 1883, Bd. xiv. S. 175 ; Rosenbach, Virchow's Archiv, 1884, Bd. xevii. S. 369. Albertoni (see Brain, London, 1878, vol. i. p. 139) came to a different conclusion regarding the sent of epilepsy, but on apparently insufficient grounds.

Lancet, London, 1873, vol. i. pp. 84, 162, 232.

5 West Riding Lun. Asyl. Rep., London, 1873.

${ }^{6}$ See also on this subject Albertoni, Ann. univ. di med. e chir., Milano, 1879 ; Luciani, Riv. sper. di freniat., Reggio-Emilia, 1876, p. 617; and Arch. per le mal. werv., 1881; Beevor and Horsley, Phil. Trans., London, 1887, vol. clxxviii. B.

7 Loc, cit.

${ }^{8}$ François-Franck and Pitres, loc. cit. ; Unverrieht, loc. cit. 
Landois ${ }^{1}$ found that convulsive movements can also be produced, in a regular march, by the application of chemical irritants to the surface of the brain.

The character of the muscular contractions in artificially provoked epilepsy has been studied in dogs by François-Franck and Pitres $;^{2}$ and in monkeys, rabbits, and dogs by Horsley and myself and others. ${ }^{3}$ FrançoisFranck and Pitres generally obtained in the animals they employed (which were usually allowed to partially recover from the influence of the anwsthetic for the purpose of the observation) an after-effect of a tetanic nature lasting for a few seconds; this was practically a continuation of the contraction which was evoked by the faradic current, but often of a more pronounced character. This "tetanic phase" was succeeded, usually quite suddenly but sometimes gradually (Figs. 327, 328), by the "clonic phase," characterised by the violent spasms of slow rhythm which have already been noticed, and which would last a variable time (from a few seconds to several minutes), slowly passing off but sometimes succeeded by a second or a third fit or even more. They note that the tetanic phase was occasionally absent, and this was the case also in many of the

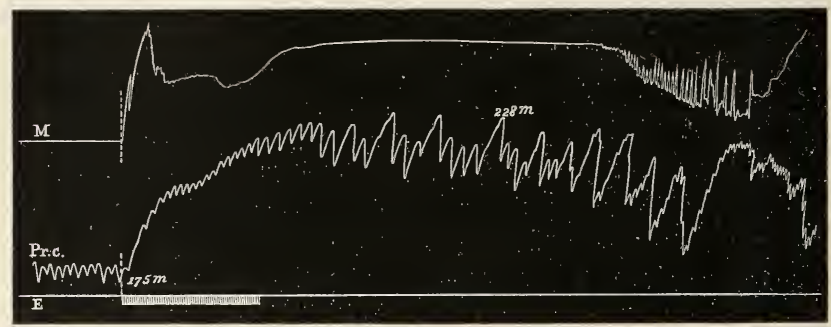

FIG. 328.-M, Curve of the contraction of a muscle during and after cortical excitation, showing the tetanic and epileptoid after-effect. Pr.c., carotid pressure. E marks the period of excitation.-Francois-Franck and Pitres.

experiments recorded by Horsley and myself, in which the animals were the whole time completely under the influence of the anæsthetic employed (chloroform or ether). As the attack subsides, the clonic spasms become slower and more irregular, and are often attended by short intervals of quiescence. In some cases the muscles affected pass towards the termination of the fit into a condition of contracture. Similar phenomena have been noted in attacks of cortical (Jacksonian) epilepsy in man. If the cerebral cortex be in a condition of abnormally increased excitability, the excitation of a sensory nerve may serve to provoke an attack of epilepsy. ${ }^{4}$ Likewise, if excitation of a sensory part of the brain (at least of some part other than the Rolandic region) fail to provoke epilepsy, an increase of excitability of the motor area, such as is brought about by exposure and previous stimulation, will render the excitation of the sensory area effective. These facts have some bearing upon the causation of idiopathic epilepsy in man, the fits in which, as is well known, are usually preceded by a sensation known as an aura,

1 Wien. med. Presise, 1887, Nos. 7 to 9.

2 Loc, cit.

3 Journ. Physiol., Cambridge and London, vol. vii.

4 François-Franck, "Leçons," 1. 119. 
which may be associated with any of the special senses, of which the tactile and visual are the most common, or may be of a vague dreamy character. In Jacksonian epilepsy the attack is frequently preceder by a sensation of numbness or tingling in the part in which the convulsions begin. There is, however, an important difference between the two kinds of epilepsy, in the fact that in the Jacksonian type the patient

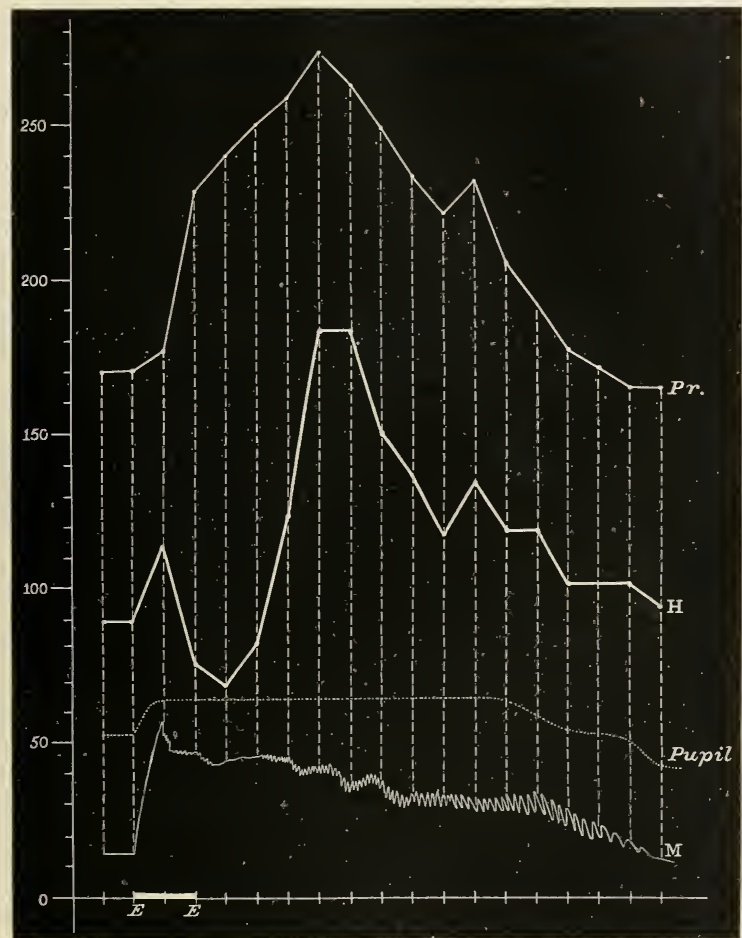

Fig. 329. - Curves showing the effects of cortical exeitation productive of epilepsy upon the blood pressure, the pulse rate, and the size of the pupil. $P r$. , carotid pressure, the height of the mercury column being marked in millimetres on the left of the figure; $\mathrm{H}$, the pulse rate; $\mathrm{M}$, curve of museular contraction; $E, E$, period of excitation, oceupying two seconds. - Francois.Franck and Pitres.

is conscious throughout the attack, whereas in idiopathic epilepsy, even in very slight attacks (petit $\mathrm{mal}$ ), unconsciousness is a prominent symptom.

Certain drugs, notably absinthe, produce, when injected into the vascular system, convulsive attacks which are scarcely distinguishable from the epileptic fits provoked by stimulation of the cortex cerebri. They occur, however, according to the observations of Magnan, ${ }^{1}$ even ${ }^{1}$ Arch. de physiol. norm. ot path., Paris, 1873, 1. 127.

VOL. $11 .-46$ 
after severance of the brain from the spinal cord: the action cannot, therefore, be only upon the cortex. ${ }^{1}$

François-Franck and Pitres (Figs. 328, 329) found that during an epileptiform attack in dogs, the blood pressure, which was raised during the excitation of the motor cortex which produced the attack, remained high, with irregular elevations and depressions as long as the fit lasted; while the pulse rate, which first rose a little and then fell considerably as the immediate result of the stimulation, rapidly mounted up during the commencement of the epileptoid attack until it was far above the normal rate, and again fell gradually during the clonic phase. The rise of blood pressure is independent of the pulse rate, and depends upon an active constriction of arterioles. There is usually a considerable postepileptic fall of blood pressure, and such a fall occasionally happens during the clonic phase of an attack. ${ }^{2}$

The effects upon respiration vary with the severity of the attack. In some cases the respiratory movements take on a clonic convulsive character, even during the tonic phase of the attack; in other cases they are altogether suspended during this phase, the thorax assuming an expiratory attitude; this condition being succeeded by one in which the respiratory movements are of a clonic and convulsive character.

The pupil usually dilates widely and remains dilated until near the end of the attack, the secretion of saliva is increased, the excitation operating through the chorda tympani, ${ }^{3}$ and occurring principally in the clonic phase, ${ }^{4}$ and a secretion of sweat has also been noticed during an attack. The effects upon other viscera are probably wide-spread, but have not as yet been sufficiently studied experimentally.

\section{SPECIAL LOCALISATIONS IN THE CORTEX CEREBRI.}

\section{The Motor Areas and Centres of the Cerebral Cortex.}

The terms "motor area" and "motor centre" are here used to imply those portions of the cerebral cortex which are directly connected by efferent projection fibres with the lower level centres (spinal cord, ${ }^{5}$ bulb and midbrain) from which impulses producing voluntary muscular action emanate. The use of the term "motor" for these regions does not necessarily exclude the possession in the same part of the cortex of some sort of conscious sensation, and especially of that which is known as the muscular sense, although, as we shall presently see, it is uncertain if this portion of the cortex actually does form part of the sensorium. But it is certain that the region in question is intimately connected with, and its integrity is essential for, the production of voluntary movements; nervous impulses for the production of these movements undoubtedly emanating from some of the cells in that region, which, as shown by Betz and Bevan Lewis, are characterised by

1 It was found by Horsley and myself (Journ. Physiol., Cambridge and London, vol. vii. p. 103) that an after-effect is very occasionally seen to succed prolonged or strong excitation of the eut spinal cord, which is similar in its character to the epileptoid spasms produced by excitation of the cortex cerebri, and whieh, like those spasms, may be preceded by a tonic phase (see tig. $6 \mathrm{~B}$, plate v., loc. cit.).

${ }^{2}$ François-Franck, loc. cit., p. 199.

3 Albertoni, "Papers from the Sienna Laboratory of Physiology," 1876.

${ }^{4}$ François-Franck and Pitres, loc. cit.

5 Termed also on this account the "cord-area" of the cortex by Sherrington, Journ. Physiol., Cambridge and London, 1885, vol. vi. p. 178 ; 1889, vol. x. p. 429. 
their size and grouping. ${ }^{1} \quad$ That such nervous impulses are normally set agoing by impulses derived from the periphery through sensory tracts, or from other regions of the brain through internuncial fibres, is certain, but this acknowledged fact does not justify one in terming the areas in question "sensory," for the typically motor cells of the anterior horn of the spinal cord are also called into activity by impressions reaching them from sensory tracts, and these are by common consent spoken of as motor cells. The application of the terms motor and sensory to cells in the central nervous system must be used rather for purposes of convenience than with a view to a rigid definition of function. If we define a motor or efferent cell as one which discharges towards a "lower level" or towards the periphery, a sensory or afferent cell as one which discharges towards the nerve centres or towards a higher level, it is still difficult, if not impossible, in the present state of our knowledge of the central nervous system, to say with regard to some cells whether they are to be regarded as discharging towards higher or lower levels. Nevertheless, when we find that excitation of the cells in a limited region of the cortex produces movements which are indistinguishable from voluntary movements of the muscles, and that extirpation of the same region produces permanent paralysis of those movements without permanent or it may be without appreciable loss of sensibility in the part affected, it appears not only convenient but imperative to think and speak of the region in question as the centre for the voluntary movements involved. ${ }^{2}$ And the more so because there are other regions of the cortex which, when artificially excited, produce special movements of a voluntary character, the power to execute which is, however, in these cases not lost on removal of the cortex of the regions in question, although a definite sensory defect (in the case of the occipital lobe, blindness) results. In spite, therefore, of the fact that movements have resulted from their stimulation, we are not justified in terming these portions of the cortex "motor," but may regard them as "sensory," and may look upon the movements as being set up by a "motor" discharging centre elsewhere, as the result of nervous impulses reaching it from the "sensory" region of the cortex.

In connection with this subject, the "isolation experiments" which have been performed by various observers ${ }^{3}$ deserve notice. These have consisted in cutting off the motor area from the rest of the cortex by an encircling incision passing through the grey matter and some little way into the white matter. In this manner it is believed that all fibres connecting this area with other portions of the cortex are severed, and only the fibres which pass in the corona radiata to or from the lower centres remain. If these fibres are both afferent and efferent,-sensory

${ }^{1}$ Betz, Centralbl. f. d. med. Wissensch., Berlin, 1874 and 1881 ; Bevan Lewis, Proc. Roy. Soc. London, 1878 ; and Brain, London, 1879, vol. i. p. 83.

2 "If we are to retain the terms sensory and motor cells, it wonld seem nore reasonable to adhere to the designation "motor" for those cclls which lie in the region of the eortex in which the first steps in the so-called voluntary notor proeesses begin."-C. K. Mills, "Nervous System and its Diseases," Philadelphia and London, 1898, p. 342.

${ }^{3}$ Exner and Vareth, Arch. $f$. d. ges. Physiol., Bonn, 1885, Bd. xxxvii. S. 523 ; ibid., 1887, Bd. xli. S. 349 ; ibid., 1889, Bd. xliv. ; Marique, "Rech. expér. sur le meeanisnie de fonctionnement des centres psyeho-moteurs du cerveau," Bruxelles, 1885 ; see also Brain, London, 1885, vol. viii. p. 536. In some of Marique's experiments severance of the motor eentres of the $\mathrm{dog}$, by a ent involving only the assoeiation fibres eonnected with the posterior parts of the brain, was found to produee almost as marker a motor paralysis as complete isolation. I have found, however, that this is not the ease in the monkey, nor did the cutting off in the same individual of the prefrontal area as well produce paralysis. 
and motor,--there seems no reason why the motor centres should not continue to act in response to tactile impressions. They do not, however, so act (although they are still capable of being excited by direct electrical excitation), but the isolation produces a paralysis as complete as if the centres in question were excised. The inference is that the motor centres are not themselves directly provided with sensory excitomotor fibres, but that they are set in action by impulses received by means of association fibres from sensory parts of the cortex.

Although this is the obvious inference, it must be pointed out that the results are open to another interpretation, namely, that the cutting off of the innumerable impulses which the motor centres are constantly receiving from the rest of the cortex, has rendered them less liable to respond to afferent impulses reaching them by the corona radiata. Such an explanation presupposes the maintenance of a kind of tonus in the motor centres of the cortex by impulses received through association fibres.

It must also be pointed out that these experiments are open to the criticism that the circumsection of an area very materially interferes with the blood supply of its cortex, and may therehy interfere also with its functional activity.

While there can be no objection, if it is preferred, to the employment of the term "psycho-motor" in place of the more simple term "motor," to denote the excitable centres in the Rolandic region of the cortex cerebri, the use of the term "sensori-motor" is to be deprecated, because it assumes, what has not yet been shown to be the case, that the region in question possesses, besides the faculty of receiving impressions from other portions of the cortex, and transmitting them as voluntary impulses to the lower level efferent stations in the bulb and cord, also that of perceiving sensory impressions directly received from lower level afferent stations; in other words, that it also functions as a region devoted to general (tactile) sensibility. This view has, it is true, been taken by Bastian, Dana, Flechsig, Mott, H. Munk, Schiff, Soury, and other distinguished neurologists, ${ }^{1}$ but as appears to me on insufficient evidence. It has found its strongest expression in H. Munk's division of the Rolandic area into so-called "sensory spheres" 2 (Fig. 330). Munk regards the movements which are evoked by excitation of the Rolandic region of the cortex as due entirely to the calling up of the sensations which ordinarily accompany those movements, and that the paralysis which results from extirpation of the parts in question is a paralysis of sensation; just as when all the sensory nerves (posterior roots) of a limb are cut, the power of voluntary movements, which are guided by sensations derived from the periphery, is greatly impaired, or in some cases appears to be entirely lost. In support of this view Munk asserts that removal of the part of the cortex, excitation of which produces

${ }^{1}$ Seliff was the first physiologist to regard the Rolandic zone as a purely sensory region, but he afterwards saw reason to give up this idea, and in an addendum to his collected papers (published in 1895) he definitely states that experiments liave shown that motor centres must exist in the cerebral cortex. The view originally taken by Hitzig was that the paralysis obtained is caused by loss of muscular sense or of loss of the consciousness called up by the imagining of movements ; later he admitted that loss of sensibility of tendons, ligaments, and articulations might also be a cause (Neurol. Centralbl., Leipzig, 1888).

${ }^{2}$ Munk has enunciated his views upon this question in a series of articles in the Arch. f. Physiol., Leipzig, 1877 to 1889 (Verhandl. d. physiol. Gesellsch. zu Berlin), republished with notes in book form in 1890; and more recently in the Sitzungsb. $d . k$. Akad. $d$. Wissensch. zu Berlin, 1892 and succeding years. 
movements of a limb or other part of the body, is followed by entire loss of sensibility in that limb. ${ }^{1}$

Even if this assertion of Munk's were true, it would by no means justify the exclusion of the expression " motor" from the designation of a part of the cerebral cortex which is directly functionally connected with the motor apparatus of the spinal cord and bulb. ${ }^{2}$ It is, however, not the case that sensibility is lost in the paralysed part, and it is difficult to comprehend that the assertion should have been made and that it should be reiterated. It is certain that in the monkey the careful and complete removal of the whole of the region of the cortex, excitation of which produces movements in the opposite hind-limb, may not be followed by any discoverable sensory paralysis of the limb in question $;^{3}$ although the limb is absolutely devoid of the power of executing any true voluntary movements, and although the entire removal of its cortex may be proved by the fact that it does not participate in an epileptic fit, induced by arti-

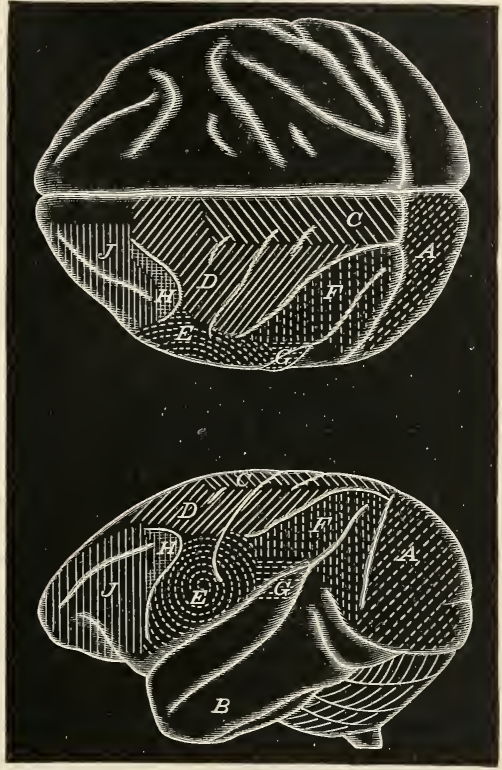

Fia. 330.-Brain of monkey, marked out into the so-called "scnsory areas". $A$, visual spliere; $B$, auditory sphere ; $C$ to $J$, tactile spheres. $C$ of leg; $D$ of arm ; $E$ of head ; $F$ of eyes ; $G$ of ear ; $H$ of neck; $J$ of trunk.-After H. Munk. ficial stimulation of other

1 " After total extirpation of the arm and leg area, tactile sensibility of the oprosite extrenities is permanently lost; a touch or light pressure is without any effect, neither tactile reflexes nor eye nor head movements are prodnced. The same is the case if the whole of the arm or leg region is removed in monkeys; tactile sensibility is permanently lost in the opposite arm or leg" (H. Munk, "Ueber die Fuhlsphare der Grosshirurinde, Funfte Mittheilung," Sitzungsb. d. k. Akad. d. Wissensch. zu Berlin, November 5, 1896). Further, "Schiff was right in affirming the existence of sensory disturbanees, local anrsthesias. The parietal lobe is therefore the tactile sphere, as the temporal is the auditory and the occipital the visual sphere. One can roughly say that, as the retina is projected on the visual so is the skin on the tactile sphere." "The affirmation that lesions of the cortex in the region of the parietal lobe produce purely motor disturbances, without accompanying sensory defects, has becn shown to be erroneons" (!).

2 Although as a matter of fact the fibres of the pyramidal tract do not terminate amongst the cells of the anterior horn of the spinal cord, as has hitherto been supposed, but amongst the cells of Clarke's column and of the base of the posterior horn (Sehäfer, "Proc. Physiol. Soc.," 18th March 1899, Journ. Physiol., Cambridge and London, vol. xxiv.), and their comnection with the motor cells mnst therefore be indirect, it is none the less the case that the impnlses they convey must be efferent, but whether motor or inhibitory, or both, is not known. Perhaps the fibres which go to the homonymous lateral column are inhibitory; while those to the heteronymous side are motor.

${ }^{3}$ Schäfer, Journ. Physiol., Cambridge and London, 1898, vol. xxiii. p. 310. 
parts of the cortex, which throws all the other muscles on the same side of the body into contraction. If the area, excitation of which produces facial movements, be cut away, there is paralysis of the cheek-pouch, but after recovery from the immediate effects of the operation the monkey still shows consciousness of the presence of food within the pouch, and occasionally empties it by pressure with the hand from outside or of the cheek against the shoulder. ${ }^{1}$

When there is paralysis of sensation produced by a lesion of the Rolandic region, it is not necessarily localised to the limb the muscles of which are deprived of voluntary motion, nor is it ever so complete as it would be if the paralysis of motion were due to sensory paralysis. $^{2}$ Charles Bell obtained paralysis of the facial muscles on cutting the trigeminal nerve; Exner and Pineles, ${ }^{3}$ paralysis of all the muscles of the larynx of the horse on cutting the superior laryngeal nerve; and Mott and Sherrington, ${ }^{4}$ paralysis of the upper limb of the monkey, on cutting all the posterior roots of the nerves supplying the limb. Mott and Sherrington found that if only one posterior root of the nerves proceeding to the upper limb were left, all the other sensory fibres being cut, the paralysis which they obtained from severance of all the posterior roots did not occur. Bastian ${ }^{5}$ has suggested that this effect is probably due to a diminution of excitability of the cells of the spinal cord, consequent on the absence of the advent of peripheral impressions; thus there would be a diminution of tonus of the muscles concerned. These cells and muscles are still capable of being excited to action by electrical excitation of the cortex cerebri, and are also brought into action in violent voluntary movements. ${ }^{6}$ Nor does sensory paralysis ever occur, as asserted by Munk, if the cortex connected with the movements of a limited part of the body, such as one limb, be alone removed. I have found 7 that if in successive operations even the whole of the motor cortex be removed in the monkey, there may be no appreciable effect upon the sensibility of the opposite side of the body, yet voluntary paralysis is complete. v. Bechterew ${ }^{8}$ makes a similar statement for the motor cortex of the dog and monkey. Mott ${ }^{9}$ came to the conclusion, from his experiments upon monkeys, that there was sometimes, after an extensive lesion in the Rolandic region, a certain amount of deficiency in the sensibility of the paralysed limb. But the method of testing this upon which he chiefly relied was one on which reliance cannot be placed. He fixed a "bull-dog" clip alternately to the skin of the sound and the paralysed limb, and found that it was usually at once removed from the sound limb but was frequently left on the paralysed side; he concluded that this showed a loss of tactile sensibility on the paralysed side. That this conclusion is erroneous is shown by the fact that

I.. Flood, Brit. Med. Joum., London, 1894, vol. ii. p. 189.

2 It is an ascertained fact that voluntary movements are directed by sensations and eannot be performed withont the guidance of some sort of sensation.

" Centrulbl. f. Physiol., Leipzig u. Wien, Bd. iv. S. 741. See also Exner, Arch. $f$. $d$. ges. Physiol., Bonn, 1891, Bd. xlviii. S. 592; and "Entwurf $7 n$ einer physiol. Erklärung der psych. Erscheinungen," Wien, 1894.

${ }^{4}$ Proc. Roy. Soc, London, 1895, vol. lvii. 1. 481.

6 Mott and Sherrington, loe. cit.

il lbid., 1895, vol. Ivii. ' Schäfer, loc. cit.

${ }^{8}$ Neurol. Centralbl., Leipzig, 1883, S. 409. This statement has, however, been somewhat modified by Bcchterew, see ibid., 1898, S. 139. See also "Die Leitungsbahnen im Gehirn u. Ruickemmark," Leipzig, 1894, S. 146 ; and Aufl. 2, 1899, S. 429 . Bechterew regards the parietal lobe as being especially associated with all forms of cutaneous and muscular sensibility.

${ }^{9}$ Journ. Physiol., Cambridge and London, 1894, vol. xv. 1). 464. 
animals display consciousness of and clearly localise a slight touch or gentle stroking of the paralysed limb, upon which a fairly strong clip will be left unnoticed. The reason why the pressure of the spring clip is felt upon the non-paralysed side is probably to be found in the fact that the muscles of that side are constantly in slight activity, and the variations in tension which this produces upon the skin converts the constant stimulus of the clip into a variable stimulus which is at once appreciated. On the paralysed side this of course does not occur. ${ }^{1}$

That the above is the correct explanation of the results with the "clip" test, is probable also from the fact that as soon as the " associated movements" of the paralysed limb return, the difference of behaviour of the two limbs to the clip is no longer apparent. ${ }^{2}$ Even where there appears a slight defect in the localisation of sensory impressions after removal of a localised portion of the Rolandic cortex, this by no means shows that the region in question is sensory, any more than defects of olfaction or vision after lesions of the trigeminal prove that olfactory and visual impressions are conveyed by this nerve. ${ }^{3}$ For after such lesions there invariably follows, as the result either of inactivity of the muscles or of vasomotor changes, a general swelling of the limb due to the accumulation of lymph; there is usually also dryness of the epidermis, and vaso-dilatation; any of which conditions would tend to affect its sensory functions. This will be further discussed when the clinical evidence has been alluded to.

The conclusion arrived at, therefore, so far as we can interpret experiments upon animals, is that removal of the part of the cortex with which the volitional movements of a limb are connected, either does not at all affect the tactile sensibility of the limb, or if a slight effect is produced it is in an indirect manner; the paralysis of motion cannot be the result of loss of tactile sensibility. ${ }^{4}$

It is difficult to determine in an animal questions which involve differences in general sensibility, and quite impossible to determine questions involving what has been called "muscular sensibility," or appreciation of muscular contraction through the position of the limbs. It is necessary, therefore, to turn to clinical experience to assist us in determining whether a lesion of the Rolandic region of the cortex, which in man as in monkey produces according to its extent paralysis of voluntary motion, is accompanied by loss of sensibility in the paralysed parts. The reply which clinical medicine has furnished to this question is not uncertain. Very many cases have been recorded in which sensibility has been most carefully tested, and in which not only has

\footnotetext{
1 The same explanation holds good for the results obtaincd by Mott from hemisection of the cord. He came to the conclusion that the paralysed limb in this case also was deficient in sensibility, because there was as a rule no tendency to remove a clip from $j$. I lave found, however, in monkeys with hemisection of the cord, that although these animals may not remove a chip from the paralysed side, they will react, by looking round or otherwise, to a touch or stroking of the paralysed fort, so that Mott's conclusion, which was based wholly upon the "clip" phenomenon, cannot be accepted without reservation in either case. Mott himself, in his hemisection experiments, got evidence of reaction to touch (Phit. Tians., London, 1892, vol. clxxxiii. B, p. 1).

2 Mott, ibid.

${ }^{3}$ Cf. on this subject, v. Bechterew, Neurol. Centrulbl., Leipzig, 1894, S. 252, 297.

${ }^{4}$ Schäfer, Journ. Physiol., Cambridge and London, 1898, vol. xxiii. 1) 310 . On the other hand, the tactile and general sensibility of a part may be completely abolislied by cocain, but the action of the muscles in no way affected. This is stated to be the case with the larynx by Beaunis (Bull. Soc. de psych. cxp)., Bordeaux, 1888, tome iii. 1. 14).
} 
there not been loss of sensibility in the paralysed limb, but in which no trace of deficiency in sensibility, as compared with the opposite side, could be detected. The fact that in numerous other cases defective sensibility has been found, in no way affects the argument. For $a$ single case of cortical lcsion, accompanied by muscular paralysis, careful examination fails to detect any loss or affection of tactile sensibility, is quite sufficient to invalidate the hypothesis that cortical muscular paralysis must have its origin in tactile paralysis. The position as based upon clinical observations is admirably summarised by Chareot and Pitres " : "Les paralysies d'origine corticale s'accompagnent parfois des troubles de la sensibilité cutanée ou musculaire; mais ces troubles sensitifs éventuellement associés aux paralysies motrices, n'ont aucun rapport constant et nécessaire avec les lésions de la zone motrice. Les centres moteurs corticaux de la région rolandique ne sont done pas des organes sensitivo-moteurs." C. K. Mills ${ }^{2}$ states that "innumerable cases have been reported of lesions of the motor cortex without the slightest impairment of sensibility. In several cases of excision of the human cortex in the Rolandic region by surgical operations, careful studies of the patients, by the writer and others, failed to show any impairnent of sensation." 3

This being so, how then are we to explain the occurrence of a certain number of cases (amounting, according to Charcot and Pitres, to about one-third of those which have been carefully recorded), in which the lesion of the Rolandic area is accompanied by a certain amount of sensory disturbance? This question is discussed by Charcot and Pitres as follows:-

"Not only are the sensory disturbances not exactly coterminous with the parts deprived of movement, but they also do not persist as do the motor paralyses. They are almost always fugitive and changeable. Would they be susceptible of alteration from one moment to another, of disappearing and reappearing under the influence of slight causes, if they actually depend on the destruction of corresponding perceptive centres? This is very improbable. Further, it is impossible, as some authors have attempted to do, to explain motor paralysis of cortical origin by primary or concomitant disturbances of the muscular sense, of tactile sensibility, or of the aggregation of sensations to which the term 'kinæsthetic " ${ }^{4}$ has been applied.'

1 "Les centres moteurs corticaux chez l'homme," Paris, 1895. Abundant statisties are here given as well as a succinct account of the history of the subject up to the date of publication. See also the cases quoted by Ferrier in "Cerebral Localisation," pp. 139-144.

2 “The Nervous System and its Diseases," Philadelphia and London, 1898.

"I have lately had the opportmnity of carefully testing a case in which, as the result of a gunshot wound of the mesial surface of the right hemisphere of the brain, was produced motor paralysis of the upper arm, trunk, and leg muscles (but not of the hand or face). Although the left foot was completely paralysed, the slightest touch could be felt upon it, and no difference in delicacy of discrimination of two points could be detected between it and the right foot. Localisation of the exact position of the point touched was imperfect upon the outer side of the leg and the dorsum of the foot only, but was perfect upon the inmer side of the leg and the plantar surface of the foot, although every part was equally and completely paralysed for voluntary motion. Such slight defect in localisation of tactile sensations as did exist was therefore independent of the muscular paralysis, and was perhaps due to the fact that a part of the cortex other than that involved in the production of the motor listurbance (or perhaps part of the thalamus opticus) was included in the area of disturbance.

4 The term "kinesthesis" or sense of movenent has been introduced by Dr. Charlton Bastian, to denote all those impressions which are evoked by muscnlar movements, inclnding "cutaneous impressions, impressions from muscles, fasciæ, tendons, and articular surfaces, and in addition a set of nufelt or bnt little felt impressions which guide the activity of the brain by the information (unconscious) which they afford ns as to the different degrees of 
"As a matter of fact, clinical medicine teaches in an unmistakable manner that anæsthesia of the limbs, whether superficial or deep, is not in itself a cause of motor failure. In anæsthesic hysteria the patients do not by reason of their anæsthesia lose the faculty of freely moving their limbs, even when their muscular sense is abolished and all forms of cutaneous and deep sensibility are lost. And it is equally true that the faculty of movement can be completely preserved in cases of hemianæsthesia, resulting from destructive lesions in the posterior part of the internal capsule. To us it appears that these facts are conclusive."

"There remains, however, to be explained why alterations of the motor zone are accompanied in almost a third of the number of cases where they occur by disturbance of sensation either cutaneous or muscular."

"Legroux and de Brun ${ }^{1}$ comnect these sensory symptoms with circulatory disturbances affecting points in the cerebral substance beyond those involved in the paralysing lesion. Ferrier regards them as being produced by co-existing lesions of sensory centres (elsewhere in the cortex) or of sensory tracts. But this combination of two lesions . . . is certainly exceptional."

"In our opinion the anæsthesic conditions which sometimes accompany motor paralyses of cortical origin are most frequently functional anæsthesias, analogous if not identical with hysterical anæsthesias; . . . they are superadded and accidental phenomena, not depending directly on the lesions of the Rolandic region, nor playing any pathogenic part in the production of the paralytic symptoms."

Besides this "functional" explanation, perhaps in correlation with it

contraction of all the muscles concerned in the production of any movement"; and he considers that the Rolandic region is a special seat for the nltimate reception of such impressions, and for their revival as an anteeedent to the excitation of the motor centres of the spinal cord and bulb.

Bastian's view lias been developed in a highly philosophical manner in a series of important articles, dating from some twenty-five years back up to the present time, and has been accpted, sometimes with and sometinies without acknowledgment, by many neurologists. As it has been often misapprehended, it nay be as well to point out that Bastian has not postulated that voluntary movements are produced by kinæsthetic inıpressions, but merely that the perfornance of such movements is guided by them ; and considering that in order properly to perform their functions the centres in the Rolandic area must receive such impressions, Bastian regards this area as a sensory area and its centres as sensory centres. By the same mode of reasoning, however, the centres in the anterior horns of the spinal cord from which the motor nerves originate, must also come under the same category. Bastian has not committed himself to the opinion that the Rolandic area is the sole portion of the cortex where kinesthetic impressions are perceived, but merely that part where these impressions or the memory of previous similar impressions are concentrated for the guidance of volitional impulses; which last are themselves originated by immediate or earlier sensory impressions, whether visual, auditory, tactile, gustatory, or olfactory. The difference, therefore, between Bastian's view of the Rolandic centres and that taken in the text, largely resolves itself into one of terminology. For full information regarding the kinæesthesis theory of voluntary action, the student will do well to consult Bastian's original works, and especially "The Brain as an Organ of Mind," p. 543; "Paralyses, Cerebral, Bulbar, and Spinal," p. 108 ; "Hysterical or Functional Paralysis," Appendices A and C; "The Muscular Sense," Brain, London, 1887 , vol. x. p. 1; "On Attention and Volition," ibid., 1892, vol. xv. p. 1. Cf. furtlier on the subject of the relation of motion to sensation, Petrina, Ztschr. f. Heilk:, 1881, Bd. ii. S. 375 ; Exner, "Localis. d. Functionen, etc.," 1881; Lisso, "Zur Lehre d. Local. des Gefuihls, ete.,"1882; Allen Starr, Am. Journ. Med. Sc., Phila., 1884, vol. lxxxviii. p. 114; Weir and Seguin, "Cerebral Surgery," Journ. Nerr. and Ment. Dis., N. Y., 1888; Dana, "Cortical Localisation of Cutaneous Sensations," N. Y., 1888; Med. Rec., N. Y., 1893, p. 578; Hitzig, Neurol. Centralbl., Leipzig, 1888, S. 249, 283 ; Darkschewitsch, Neurol. Centralbl., Leipzig, 1890, S. 714; Waller, Braiu, London, 1891, vol. xiv. p. 179, and 1892, vol. xv. p. 329; Madden, Journ. Nerv. and Ilent. Dis., N. Y., 1893 ; Starr and M 'Cosh, Am. Journ. Med. Sc., Phila., 1894, vol. cviii. p. 517 ; Wernicke, Arb. a. d. psych. Klin. in Breslau, Leipzig, 1895, Heft ii. S. 33 ; Bruns, Neurol. Centralbl., Leipzig, 1898, S. 856; Luciani and Sepilli, "Die Fnnetionslocalisation auf der Grosshirnrinde" (quoted from Ferrier, "Cerebral Localisation"); "Le système nerveux," by J. Soury, 1899.

1 “L'encephalon," 1884. 
there is more than one physico-anatomical explanation possible of the occurrence in some cases, especially where a lesion is large, of sensory disturbances affecting the paralysed side. For, first, the occurrence of a large lesion may undoubtedly, as suggested by Legroux and de Brun, affect the vascular supply of portions of the brain even remote from the part where the lesion itself occurs; or, in the case of a tumour, the pressure of the growth may make itself felt in remote parts. It is unquestionably the case that in extensive removals of cerebral cortex the effect of such removal may influence-temporarily if not permanently-even the whole of the same side of the brain; so that, for example, removal of the frontal lobe in the monkey has been found to produce both general hemianæsthesia and hemianopsia, ${ }^{1}$ and removal of the whole motor area at one operation the same combination of sensory disturbances. ${ }^{2}$ Secondly, defects of sensibility in a paralysed limb may be produced by the local effects of lymph accumulation, due to the loss of activity of the muscles of the part (see p. 727). And there is yet another way in which sensory defects indirectly following lesions of the motor region may be explained,-one which also offers an explanation of the fact that excitation of the Rolandic region of the brain is sometimes found to produce slight sensory symptoms, ${ }^{3}$ and also perhaps of the sensory "signal" which in some cases heralds the approach of an epileptiform fit originating in the same region. For since the volitional movements are guided and governed by afferent impressions received from all parts-from the skin, the muscles themselves and their tendons, and the other structures in the limbs which are affected by their action, the eye, the ear, and other special sense organs-it is certain that the parts of the cortex from which the volitional impulses emanate must by means of association fibres be in intimate connection with those parts of the cortex-some of which are known and others still unknown - which are involved in the perception of such sensory impressions. These association fibres must be assumed to be derived from cells in the sensory parts of the cortex, and since when a nerve fibre is stimulated conduction is in either direction, stimulation of the endings of these association fibres in the motor region may affect the sensory parts of the cortex through them. Further, it is a well-established fact that the severance of a nerve fibre, not only produces the Wallerian degeneration in its peripheral part, but also causes degenerative changes in the cell from which it takes origin; and especially is this the case with sensory cells, where these degenerative changes may proceed to complete atrophy. ${ }^{4}$ We should therefore expect that even

1 Ferrier and Turner, Phil. Trans., London, 1898, vol. exc. B, pp. 35, 36.

2 Mott, Journ. Physiol., Cambridge and London, 1892, vol. xv. p. 483, Exp. v.

${ }^{3}$ W. B. Rausom (Brain, London, 1892, vol. xv. p. 440) produced with a slight strength of emrent in a patient who had been trephined, and whose brain was stimulated by needle electrodes passed through the skin, a tingling sensation in the part, which would contract when the strength of the current was inereased. No effect was observed on tactile sensation. The effect may have been a vasomotor one. Cf. also C. L. Dana, Med. Rec., N. Y., 1893 , p. 578 , who in a similar experiment found that, simultaneously with the movement, a sensation of numbness and heaviness was produced in the limb nover (opposite arm), similar to the sensation experienced when a peripheral nerve is pressed. It is noteworthy that in neither case was the "idea of movement" called up, nor even such sensations as ordinarily accompany movement. The movements produced in Dana's case were very extensive, and with stronger stimulation were followed by general epileptic convulsions and loss of consciousness; prior to this the same numbness was felt in the opposite arm and shoulder, which were the parts first moved.

"Cf. article "Nerve Cell," this volume, p. 601. 
if a lesion be confined to a part of the cortex which is purely motor, there would be produced indirectly but at no long interval of time both functional and organic changes in parts which are purely sensory, and the extent to which these would be perceptible would probably depend largely upon the extent of the lesion in the motor region.

Redlich $^{1}$ has collected and analysed 132 cases of cerebral hemiplegia, with especial reference to the question of the condition of the muscular sense. In only twenty-eight out of the 132 was there any clear deficiency, and in all these there was defective general sensibility as well, although not always proportional to the defect of muscular sensibility. The large number of cases in which no defect of muscular sense can be determined, speaks strongly against the idea that cerebral hemiplegia is merely due to loss of that sense. ${ }^{2}$

Contracture.-There sometimes results after removal of the motor centres of the limbs in the monkey, and after lesions of the corresponding part of the brain in man, a condition of permanent contraction of some of the muscles, to which the term "contracture" has been applied. It is not found in other animals besides monkeys, and not always in these, being most frequently met with in those animals which are kept closely confined. According to H. Munk, ${ }^{3}$ contractures are of two kinds, namely-(1) those due to irritation of the cortical substance as the result of injury with incomplete removal-these are rare; and (2) those which occur after complete removal, and which are associated with the complete disuse of the limb. The condition appears to be due to the existence of a state of hypertonicity in the muscles affected, and has been compared by Soury ${ }^{4}$ to that which obtains in the congenital malady known as Thomsen's disease, in which the voluntary movements are characterised by prolonged tonic contractions of the muscles, the cause of the disease being in all probability due to some defect of the cells of the cortex. ${ }^{5}$ But, on the other hand, the hypertonicity may be due to a totally different cause, namely, the cutting off from the lower centres of the inhibitory impulses which they habitually receive from the cortex cerebri, while excitatory impulses which reach them from the cerebellum are still passing. Hence also it is found in man (and to a less degree in the monkey), that whereas in hemiplegia due to cerebral lesions, both contracture and exaggeration of spinal reflexes is liable to occur, in total transverse lesions of the spinal cord contracture never occurs, and the spinal reflexes are dininished or altogether abolished. ${ }^{6}$

1 Wien. klin. Wchnschr., 1893, Nos. 24-30.

${ }^{2}$ Legroux and de Brum, loc. cit., and Brain, London, 1885, vol. viii. 1. 554, classify the disorders of sensation met with in cerebral heniplegia into five groups-(1) Sensation unaffected, the paralysis purely motor; (2) anæsthesia affects the whole side; (3) anæsthesia is absolute in one extreme segment of a limb, less in the middle segment, and still less in the other extreme segment (c.q. absolute in foot or thigh, less in leg, still less in thigh or foot); (4) external (lateral) half of a limb, normal sensation, mesial half anresthetic ; (5) anæsthesia is in patches, which may shift from day to day. They find that the anresthesia, when it occurs, has no constant relation either of time or place to the motor paralysis, and are inclined to refer it to al terations in the cerebral circulation.

"Verhamll. d. physiol. Gesellsch. $\approx u$ Berlin in Arch. f. Physiol., Leipzig, 1895.

4Article "Cervean," in Richet's "Dictionnaire de pliysiologie," Paris, 1897, tome ii. 1. 891 .

5 Thomsen, Arch.f. Psychiat., Berlin, 1879 ; ilid., 1892, Bd, xxiv. S. $91 \mathrm{~s}$.

6 That this is the true explanation of many of these phenomcua was first shown by Bastian (Med.-Chir. Trans., London, 1890, p. 151). See further on this subject, Bruns, Arch. f. Psychiat., Berlin, 1893, Bd. xxv. S. 759 (literature); and in Neurol. Centralbl, Leipzig, 1897, S. 72. 
Detalled Riesults of Stimulating the Motor Cortex in Animals.

The results which were obtained by Fritsch and Hitzig (Fig. 331) in the dog have already been alluded to. Their experiments showed that there are certain definite points or centres, mostly situated on the convolution bounding the crucial sulcus-stimulation of which constantly produces $(a)$ movements of the head (contraction of neckmuscles); $(b)$ of the opposite forearm, extension and adduction; and, at an adjacent point, flexion and rotation (? supination); $(c)$ of the opposite hind-leg; $(d)$ of the facial muscles and eyes. They also obtained from intermediate but less circumscribed points contractions

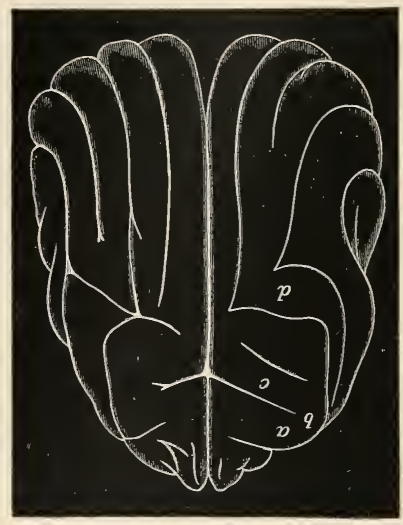

FIG. 331.--Diagram showing the chief results obtained by Fritsch and Hitzig (in 1870), on stimulation of the cerebral cortex of the dog. (a) Contraction of neck muscles; $(b)$ of arm muscles; $(c)$ of leg muscles; $(d)$ of facial muscles and eye movements. of the muscles of the back, tail and abdomen.

Many other physiologists ${ }^{1}$ have confirmed and extended the observations of Fritsch and Hitzig upon the dog. Ferrier was one of the first, and his results, which were obtained by an improved method, that of faradic excitation (whereas Fritsch and Hitzig employed for the most part the galvanic current), were on the whole confirmatory of those of the two German observer's, which they materially extended. They are embodied in the accompanying diagram, to the explanation of which the reader is referred. Ferrier obtained similar results in the jackal, and with slight modifications in the cat (Fig. 333). In rodents (rabbit, guinea-pig, rat), in which the hemispheres are smooth, stimulation of certain points of the external surface, in front of and above the fissure of Sylvius, gave the results which are shown in Fig. 334 , and very similar results have been arrived at for insectivora, ${ }^{2}$ for marsupials, ${ }^{3}$ and for monotremes. ${ }^{4}$ In birds only a single limited region at the vertex of the hemisphere was found by Ferrier to be responsive to excitation, and this caused contraction of the opposite pupil, occasionally associated with turning of the head to the opposite side. ${ }^{5}$ In the frog ${ }^{6}$ and fish,

1 Ferrier, Proc. Roy. Soc. London, 1874; "Functions of the Brain," London, 1876; Luciani and Tamburini, "Rich. s. centri psicomotori corticali," Reggio-Emilia, 1878-79, noticed ill Brain, London, 1879, vol. i. p. 529, and vol. ii. P. 234 ; H. Munk, Ges. Abhandl., Berlin, 1890; François-Franck and Pitres, various papers, the results of which are given by Franck in "Leçons sur les fonctions motrices dn cerveau," Paris, 1887 , p. 7 .

2 G. Mann, Journ. Anat. and Physiol., London, 1895, vol. xxx. p. 1.

${ }^{3}$ Ziehen, Centralbl. f. Physiol., Leipzig u. Wien, 1897, Bd. xi. S. 457.

${ }^{4}$ C. J. Martin, Joum. Physiol., Canbridge and L.ondon, 1898, vol. xxiii. p. 383.

5 Wesley Mills (Brit. Med. Journ., London, 1897, vol, i. p. 1485) and Boyce (communicatiou to Congress of Physiology at Cambridge, 1898) have succeeded in obtaining other movements of the head in birds.

${ }^{6}$ Ferrier, loc. cit. ; Langendorff, Centralbl. f. d. med. Wisscnsch., Berlin, 1876, S. 945.

7 Ferrier, loc. cit. 
excitation of the hemisphere produces contraction of muscles of the opposite side of the body, but the parts are too small to determine any exact localisation.

Of great practical value and interest were the observations which were made by Ferrier upon the brain of the monkey, in which the chief features of the conformation of the cerebral surface are so closely similar to those of the human brain, that it is possible to draw conclusions as to localisation of function in man, such as cannot well be done from experiments upon carnivora. The advantage of the

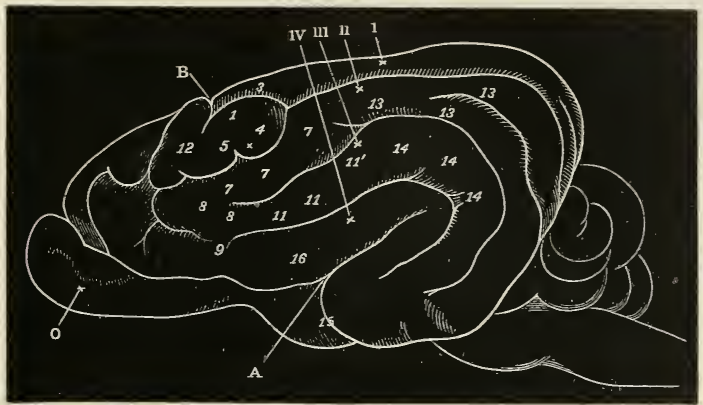

FIG. 332.-Diagram showing the results obtained by Ferrier on stimulation of the cerebral cortex of the dog. A, Sylvian fissure ; li, crucial sulcus, with sigmoid gyrus curving round it ; O, olfactory lobe ; I, II, III, IV, first, second, third, and fourth external gyri $;^{1}$ of these the first is continued anteriorly into the sigmoid (see Fig. 331); the second, third, and fourth blend in front to form the anterior composite gyrus. ${ }^{2}$ 1 , Opposite hind-limb advanced; 3 , tail moved laterally; 4 , retraction and adduction of opposite fore-limb; 5 , protruction of opposite forelimb with elevation of shoulder; $X$, between 4 and 5 , flexion of paw ; 7 , closure of oppositc cye and movement of eyeballs; 8 , retraction and elevation of opposite angle of mouth; 9 , opening of mouth and movements of tongue (sometimes accompanied by barking); 11, retraction of angle of month on both sides, and ear drawn forwards; $11^{\prime}$ (same as 7), closure of opposite eye; 12 , cyes open, pupils dilate, eyes and head move to opposite side; 13 , eyes (and head) move to opposite side; 14 , pricking, or sudden retraction, of opposite ear ; 15 , torsion of nostril on same side; 16, elevation of upper lip and dilatation of nostrils.

selection of monkeys as the subjects of experiment upon the brain, was immediately made evident by the precise results which were obtained as the result of stimulation of the cortex, and by the far-reaching effect which such experiments have had upon the diagnosis and surgical treatment of brain affections in man. The results obtained by Ferrier from stimulation of the Rolandic area of the monkey's brain are shown in Fig. 335. Ferrier's main results have been confirmed by many observers, ${ }^{3}$

1 This is Ferrier's nomenclature; by Leuret and Gratiolet the enumeration was taken from below up.

${ }^{2}$ Langley, Journ. Physiol., Cambridge and London, vol. iv. 1. 248. In this paper will be found a fill account of the fissures and convolutions of the log's brain, and of the nomenclature which has been adopted by various anthors.

${ }^{3}$ E.g. H. Munk, various papers in Verhamell. d. physiol. Gesellsch. ane Berlin, Arch. f. Physiol., Leipzig, collected in Ges. Abhandl., 1890; also in Ber. d. k. Alkul. d. Wissensch. zu Berlin; Horslcy and Schäfer, Proe. Roy. Soc. Lomdon, 1884; Thit. Trans., London, 1888, B ; and Festsehr. z. Carl Ludwiy, 1887 ; Beevor and Horsley, Phil. Trens., London, 1887 and 1888, B; A. Biedl, Wien. klin. Wchnschr., 1897, S. 635. 
and indeed are so easily obtained that it is almost impossible to fail in eliciting the response which he marked out as connected with certain

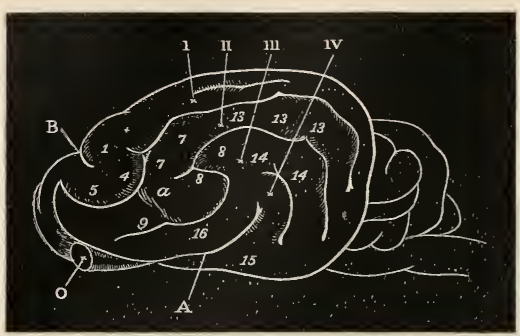

FIG. 333.- Diagram showing the results obtained by Ferrier from stimulation of the cerebral cortex of the eat. The numbers and letters have a similar meaning to those in the last figure, but all are not included, owing to the fact it was not found possible to differentiate so many distinet actions as in the dog. At the point marked $a$, there was produced a clutching action of the paw with protrusion of the elaws.

of the areas for the trunk and lower limb found that produces movements of a particular part or limb, is immediately followed, unless the movement is of a bilateral character, by paralysis of that part or limb: this had already been noted by Fritsch and Hitzig in the dog. But in the monkey the paralysis is, as a rule, much more marked and permanent, and in the case especially of the upper limb may proceed, as in monoplegia in man, to the development of a condition of contracture of the muscles and permanent immobility of the limb (see p. 731). In the leg, contracture does not as a rule occur, but after a time movements begin to reappear, until eventually it is difficult to determine that any motor paralysis exists; the same may also be the case with the arm. If, however, the whole portion of the Rolandic region which is connected with the particular limb has been removed or destroyed, unilateral movements of a truly voluntary nature do not reappear; the movements ${ }^{1}$ Horsley and Schäfer, Proc. Roy. Soc. London, 1884, vol. xxxvi. p. 437. areas of the Rolandic region. Apart from points of detail, the chief addition to our knowledge of the movements which he found to be provoked on stimulation of the cortex of this region, was the discovery that the excitable area extends to the mesial face of the hemisphere, to include the so-called marginal gyrus. Ferrier himself failed to obtain any definite response from this, but it was subsequently shown ${ }^{1}$ to contain extensions of the adjacent excitable areas of the external face of the hemisphere, including an important part cortex which on excitation

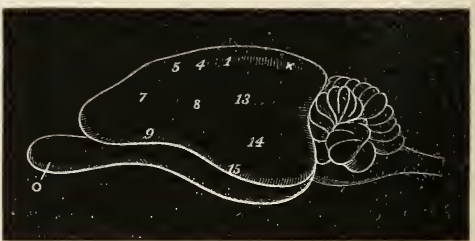

Fig. 334.-Diagram showing the results obtained by Ferrier on stimulation of the brain of the rabbit. 0 , Olfactory lobe ; $\times$, shallow suleus running parallel with median longitudinal fissure. 1, Advance of opposite hind-limb; 4, retraction and adduction of opposite fore-limb ; 5 , protraction of opposite fore-limb and elevation of shoulder ; 7 , retraction and elevation of angle of mouth (obtained over a large area); 8, closure of opposite eye; 9 , on orbital aspect-opening of mouth with movements of tongue; 13 , protrusion of opposite eyeball, and oceasionally turning of head to opposite side; 14 , sudden retraction and elevation or pricking of opposite ear; 15, torsion or closure of one or both nostrils (often associated with novement of ear). 
which are eventually manifested are those which are "associated" with the movements of the side of the body opposite to that which is paralysed, and appear to be brought about by the action of the other side of the brain acting through commissural fibres in the lower level centres. $^{1}$ The more delicate movements of the fingers and toes, which are never thus associated on the two sides, never reappear.

It is especially after removal of the leg area of the cortex that the associated movements become so completely restored, that, on superficial observation, it is almost impossible after a few weeks to detect any motor paralysis. But if the animal be held by the upper part of the body and swung gently forward towards the wire-work of a cage, or lowered rapidly towards the ground, the leg on the same side as the lesion will be put forward or downward to catch hold of the cage or touch the ground, but never the leg on the opposite side, however long the animal may have been kept after the lesion. This is, in fact, a true voluntary movement, and it is notrecovered, while all the associated movements which are used in ordinary progression have resumed to all appearance their normal characters.

In the same way it was shown by Hitzig that a dog from which the left sigmoid gyrus had been removed would, if suspended in the air by a cloth round its body, its legs being left free, immediately withdraw the left limbs, on a needle being brought near them as if for the purpose of pricking them ; but although, on

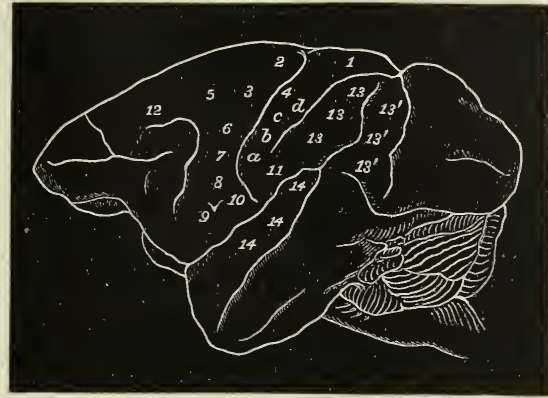

FIG. 335.--Diagram showing the results obtained by Ferrier from electrical excitation of the cerebral cortex of the monkey. 1, The opposite hind-limb is advanced as in walking. 2, Flexion with outward rotation of the thigh, rotation inwards of the leg, flexion of the toes. 3, Movements similar to 1 and 2 , and in some cases also the tail is moved. 4, The opposite arm is adducted, extended, or retracted, the hand pronated. 5, Extension forwards of opposite arm. $a, b, c, d$, Flexion of the fingers and clenching of the fist. 6 , Flexion and supination of forearm. $\bar{\tau}$, Retraction and elevation of the anglc of the mouth. 8, Elevation of the ala of the nose and upper lip. 9 and 10, Opening of the mouth with protrusion of tongue at 9 , and retraction at 10. 11, Retraction of the angle of the mouth. The action is that of the platysma inyoides. 12 , The eyes open widely, the pupils dilate, and head and eyes turn to the opposite side. 13, 13', The eyes move to the opposite side with an upward or downward deviation, according as the electrodes are on 13 or 13 ' respectively; normally also the pupils become contracted. 14, 14, Pricking of the opposite ear, head and eyes turn to the opposite side, pupils dilate widely. 15 (not shown in diagram), on the hippocampal gyrus (anterior and inner aspect), torsion of the lip and semiclosure of nostril of same side. approaching the right limbs with the needle, the animal showed signs of apprehension, these limbs were never moved away by themselves, nor except as a general movement and in association with those of the opposite side.

The areas, excitation of which on one side of the brain produces in the monkey movements involving muscles on both sides of the body, are-(1) The area for movements of the head and eyes; (2) the area concerned with movements of the tongue and cheeks, and movements of mastication and swallowing; (3) the larynx area; (4) the area

${ }^{1}$ Broadbent, Brit. and For. Med.-Chir. Rev., London, 1866, p. 477. 
concerned with closure of the eyelids. On the other hand, stimulation of the leg area, of the arm area, of the trunk area, and of most of the upper part of the face area, produces movements which are generally strictly confined to the muscles of the opposite side of the body. But in the dog and rabbit, according to Exner and Paneth, ${ }^{1}$ excitation of the whole of the cortical field of the facial produces

A.

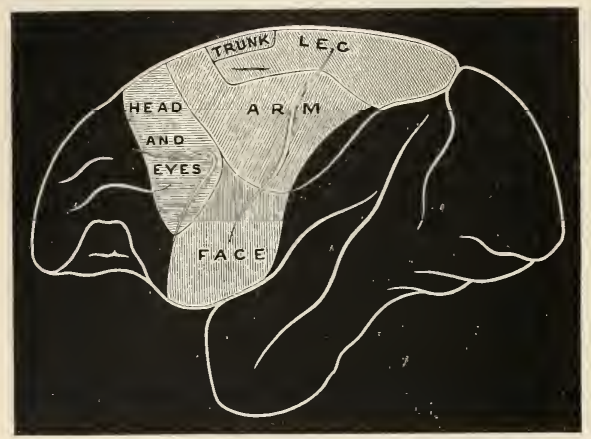

B.

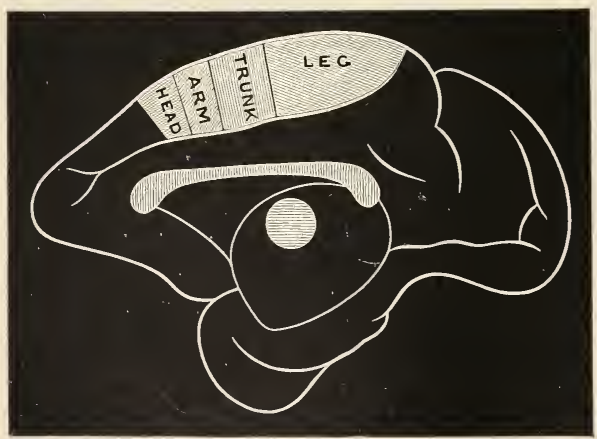

Fig. 336. Diagrans of the motor zone of the monkey's brain, showing the tive areas into which it is divisible. $A$, lateral or external surface; $B$, mesial surface. bilateral effects. And in the dog, according to v. Bechterew, ${ }^{2}$ excitation of the centre for the platysma myoides, and of the centre for lateral flexion of the trunk, produces contraction chiefly of the muscles of the same side of the body instead of those of the opposite side.

Details of the representation of muscular movements within the principal portions of the motor or Rolandic region of the monkey.-It is convenient for purposes of description to distinguish five areas of the motor cortex (Fig. 336), as follows -(1) The area connected with movements of the head and eyes; (2) the area connected with movements of the face, including those of the mouth, throat, and larynx; (3) the area connected with movements of the upper limb; (4) the area connected with movements of the trunk muscles, including those of
the tail ; and (5) the area connected with movements of the lower limb. These areas are not marked off from one another by fissures or other auatomical boundaries, nevertheless their limits, as determined by the excitation method, are sharp, and although, on exciting exactly at the boundary between any two areas, movements of both parts may result,

${ }^{1}$ Arch.f. d. ges. Physiol., Bonn, 1887, Bd. xli. S. 349.

2 Neurol. Centrulbl., Leipzig, 1888, S. 15. 
a very slight shifting of the electrodes to one or other side of the boundary will cause the movement to be limited to one part only, provided the excitation be of minimal intensity. If, however, the stimulus be strong or be too prolonged, a spread of effect is at onee produced.

Within the limits of the several areas above enumerated, varieties of movement are obtained which indicate a still further differentiation, or in other words an intra-areal localisation. ${ }^{1}$ It is obvious that these intra-areal localisations are likely to be most marked within the larger areas, and we should further expect the best differentiation in connection with those parts which are concerned with the more complex and precise movements directed by the will. These in the monkey are chiefly the movements of the upper limb, and especially the hand, and movements concerned with facial expression. Further, it is found that for many movements, if not for all, there is in each case a sort of focal point within the area, from which on the average of a large number of experiments the movement is more readily or more frequently obtained than elsewhere. This is illustrated in the accompanying diagram from Beevor and Horsley, in which each dot represents an instance in which a movement of the hallux was primarily obtained in a large number of experiments, and in which therefore the point about which the greatest number of dots are accumulated

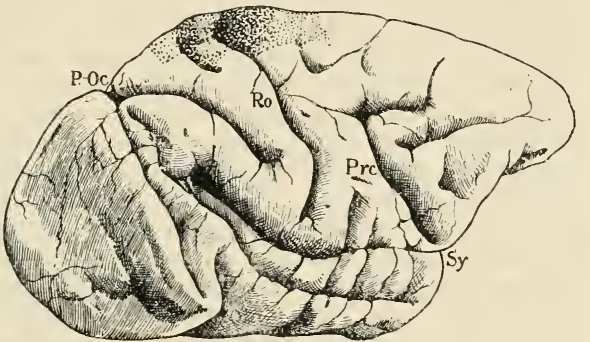

F1G. 337.-Diagram from Beevor and Horsley, slowing by relative shading the effect, in a number of individuals of Hacacus sinicus, of stimulation within the area where movements of the opposite hallux are elicited. has been termed the focal point for that movement. It is possible that its focal point does actually represent an absolutely localised centre of representation of each movement, but the fact that the movement is in many instances also got with extreme readiness in a circumjacent area of greater or less size, and that in many individuals it may be produced even more readily from other parts of such circumjacent area than the average focal point, seems to point to the conclusion, which is that usually accepted, that localisation of particular movements is rather connected with small areas of cortex than with mere points on the cerebral surface; in a few cases only do these areas seem to be so extremely limited in size as to merit being spoken of as points. It is further rare to find that the movement which is provoked is simple and uncomplicated by other movements, although occasionally this is so. Thus it is often possible by stimulation at the upper end of the Rolandic fissure to obtain a simple flexion or extension of the hallux, or by stimulation of the middle of the ascending parietal convolution to obtain simple flexion of the fingers, or at a little lower level simple opposition of

1 The intra-areal localisations have been largely worked out by Beevor and Horsley, Phil. Trans., London, 1887 and $1888, \mathrm{~B}$.

VOL. II. -4 ? 
the thumb. Much more commonly, however, the movements provoked are complicated by others. Thus in some individuals even minimal stimulation of what has been termed the hallux centre produces movements, not only of the hallux but also of other toes, and even of the foot and leg; and stimulation of the middle of the ascending parietal may cause not only flexion of the fingers but also extension of the wrist. Such movements may succeed one another, or they may be simultaneous. It has been usual to speak of the movement which is most often or most readily obtained on excitation of a particular localised area, as the "primary" movement, and the others as "secondary."

Undoubtedly the most striking character of many of the movements which are provoked by cerebral excitation is their co-ordinated and purposeful nature. In conformity with this, we find that they are rarely produced by contractions of a single muscle or group of muscles, but it is frequent for a succession of movements to occur, and these are very closely imitative of natural voluntary movements of the animal. Thus the successive movements involved in prehension are very easily called into play by excitation of certain portions of the arm area, and a series of complicated masticatory movements by excitation of the lower part of the face area. ${ }^{3}$ But if the excitation is only momentary, it is sometimes possible, as already mentioned, to obtain the contraction of only a single muscle, such as the opponens pollicis; nevertheless the movement produced in this case may be perhaps regarded as purposeful. On the other hand, it must be admitted that it is frequently difficult to recognise any purpose or any approach to the natural actions of the animal in some of the movements and combinations of movements which are provoked by artificial excitation of the cortex; but this is more especially the case when the cerebral surface is in an abnormally excitable condition, in which case the effect of the excitation appears to spread to areas which are not naturally involved.

These general remarks may serve as preface to the following details of the results obtained by stimulation and localised lesion respectively, within the limits of the motor sphere of the cortex, as previously defined. The results obtained by stimulation are shown in Figs. 337 and 338 .

1. The area connected with movements of the head and eyes and of the ear.- This area lies in front of a line which runs from below upwards, either coincident with or parallel to and just behind the lower limb of the precentral sulcus, at the top of which it turns sharply forward to reach the mesial surface of the hemisphere. It is bounded anteriorly by a line which runs nearly vertically through the anterior end of the precentral sulcus. It extends over on to the mesial aspect of the first frontal gyrus (marginal convolution). The part of the frontal lobe in front of this line (prefrontal region) is inexcitable, ${ }^{2}$ and the area itself is

${ }^{1}$ Fürstlier (Arch. f. Psychiat., Berlin, Bd. vi. S. 719) notes that in the rabbit chewing movements are especially easy to obtain over a relatively large surface, and Ferrier showed that in monkeys a protraction, followed by retraction of the arm and leg, as in elimbing, is very readily got over an extensive area. From this it would appear that the voluntary movements whieh are most habitually performed are usually those which are most easily obtained on artifieial excitation.

${ }^{2}$ Ferrier is of opinion that the inexcitable prefrontal region is nevertheless a prart of the same eentre as the excitable post-frontal area, for he finds that after a lesion of the prefrontal area alone there oeeurs descending degeneration in the mesial bundles of the 
most excitable in its middle and posterior part, and becomes less excitable as it is traced towards the prefrontal region. Its action is bilateral, excitation producing a movement of the head and both eyes towards the opposite side (conjugate lateral deviation), which may be simple or may be combined with an upward or downward inclination. Although the head and eye movements are usually obtained together, it sometimes happens that only the eyes are moved, and in other cases only the head as a whole; the latter appears to be especially the case if the part of the area which is close to the longitudinal fissure or the adjacent portion of the gyrus marginalis is stimulated.

The area in question has been the subject of special investigation by Mott, in conjunction with the writer. In our experiments ${ }^{1}$ we found that in the monkeys which have generally been used for stimulation experiments (Macacus rhcsus and Macacus sinicus), it was difficult to obtain any definite results other than the simple lateral conjugate deviation of the head and eyes to the opposite side, together with opening of the eyes and dilatation of the pupil, which had already been described by Ferrier and others. ${ }^{2}$ But by using a large species of Cercopithecus, in which this portion of the brain appears to be better displayed than in the macacque, we were able to show that excitation of the upper part of the area usually leads to downward as well as lateral deviation of eyes and head, of the lower part of the area to upward as well as lateral deviation; whereas excitation of a middle, narrow, wedge-shaped portion produces simple lateral deviation : sometimes the downward and upward results were almost unmixed with any lateral deviation (Fig. 337). The greatest effect for all the movements was obtained close to the angle of the precentral sulcus, which may therefore be regarded as the focus of the area. Stimulation of the posterior margin of the area, especially near the focal point, usually also elicits a sharp movement (either pricking or retraction) of the opposite ear or of both ears. The pricking or forward movement is especially caused by stimulation towards the upper part of this margin ; retraction of the ear, by stimulation towards the lower part.

Risien Russell ${ }^{3}$ endeavoured by a special method to differentiate the points on this area in the bonnet monkey, by stimulation after previous division of the lateral recti. He obtained in this way simple upward and downward movements of both eyes, from points situated close together and near the focus of the area; and by employing the same method in the dog and eat, in which animals stimulation of the head and eyes area only produces normally a simple lateral deviation, he was also able to elicit both an upward movement and a simple downward movement.

Inhibition effects. - Sherrington has shown ${ }^{4}$ that if in the monkey the third and fourth cranial nerves have been severed upon one side, say the left, so that the rectus externus remains the only unparalysed ocular muscle,

internal capsule and crusta, although not extending down to the pyramids of the medulla oblongata; and he interprets this to mean that the fibres from the prefrontal region pass to the nuclci of the motor nerves governing the eye movenents. But if this were the case the prefrontal region should not be inexcitable.

${ }^{1}$ Mott and Schäfer, Brain, London, 1890, vol. xiii. 1. 164 ; ef. Pick, Prag. med. Wchnschr., 1891.

${ }^{2}$ Ferrier, Proc. Roy. Soc. London, 1876 ; Horsley and Schäfer, Festschr. f. C. Ludwig,

Leipzig, 1887; and Phil. Trans., London, 1888, B ; Horsley and Beevor, ihid.

3 Journ. Physiol., Cambridge and London, 1894, vol. xvii. pp. 1 and 378.

4 Proc. Roy. Soc. London, 1893, vol. liii. pp. 411-420 ; Journ. Physiol., Cambridge and London, 1894, vol. xvii. [1. 27. 
excitation of the frontal (and of the occipital) cortex produces conjugate movements of both eyes towards the opposite side, i.e. from left towards right. Similarly by an appropriate experiment, the internal rectus can also be inhibited after section of the sixth nerve, so as to produce an outward movement of the globe. Inhibition of the tonus of the antagonistically acting eye muscles can thus be elicited from the cortex. The same may occur volitionally. The action does not take place necessarily through the opposite cortex, for it will occur if the area on the opposite side be removed, nor does it occur through the occipital area when the frontal is stimulated. And vice versî, on stimulation of the occipital area it does not occur through the frontal area, for these two areas can be severed from one another by a vertical incision through the brain, and the inhibition is still found to take place. The action is therefore directly on the lower centres. This is known also to be the case with the contraction effects, ${ }^{1}$ so that it would appear that the inhibitory phenomena follow the same paths as the excitatory.

Equal and simultaneous excitation of two similar points in the focal part of this frontal area in both hemispheres produces visual fixation, with the eyes either in the primary position or slightly convergent. If the electrodes are placed on the upper part of the area, the movement may be simply downwards; if on the lower part, simply upwards. ${ }^{2}$ A similar convergence is got, even if both right and left third and fourth nerves are previously divided. ${ }^{3}$ This effect must be due to inhibition of the tonus of both external rectus muscles.

In rare instances stimulation of the area on one side causes movements of the head and eyes to the same side, even if none of the nerves are severed. This may be due to the stimulus to inhibition being, under the immediate conditions of the experiment, more effectual than that to contraction.

Removal of the area on one side of the brain causes the head and eyes to be turned towards the side of the lesion, due doubtless to muscular paralysis. The effect passes off after a day or two, a statement which also applies to other bilateral movements. Nor is there any obvious permanent paralysis, even on removing the area on both sides. This is not to be wondered at, considering that movements of the head and eyes are elicited from other considerable areas of the cortex (occipital, temporal). ${ }^{4}$

2. The area connected with movements of the face, mouth, throat, and larynx.--This area embraces rather less than the lower half of the two central convolutions (Fig. 336). It is bounded above by a line joining the angle of the precentral sulcus with the lower end of the intraparietal sulcus, and extends below to the Sylvian fissure. ${ }^{5}$

1 Schäfer, Brain, London, 1888, vol. xi. ; Mott and Sehäfer, ibid., vol. xiii. p. 169.

- In Cercopithecus, Mott and Schäfer, loc. cit., p. 170.

3 Sherrington, loc. cit.

4 Extirpation in the dog of the centre for movements of the ocular muscles ( 7 in Fig. 332), also does not appear to have any permanent effect upon those movements. See Eckhard, Centralbl. f. Physiol., Leipzig u. Wien, 1898, No. 1; R. du Bois-Reymond and P. Silex, Arch. f. Physiol., Leipzig; 1899, S. 174. This last paper also contains an account of observations on the effects of cxcitation of the centre in the dog (see also on this subject, v. Bechterew, Arch. f. Physiol., Leiprig, 1899, S. 500). The extensive paralytic phenomena described by Grosglick as resulting from ablation of the frontal lobes (Arch. $f$. Physiol., Leipzig, 1895, s. 98), are probably due to an implication of other regions in the lesion.

${ }^{5}$ Beevor and Horsley deseribed (Phil. Trans., London, 1887, B, Plate vii., Figs. 7 and 8) primary movements of the thumb over the upper two-thirds of this area, but there must have been some umrecognised source of error in this observation. 
A considerable amount of differentiation is obtained within the area, as was observed already by Ferrier in his first experiments. Thus he got, on stimulation of the upper part of the area over the ascending frontal gyrus, retraction and elevation of the opposite angle of the mouth, due to the action of the zygomatic muscles, and from a part

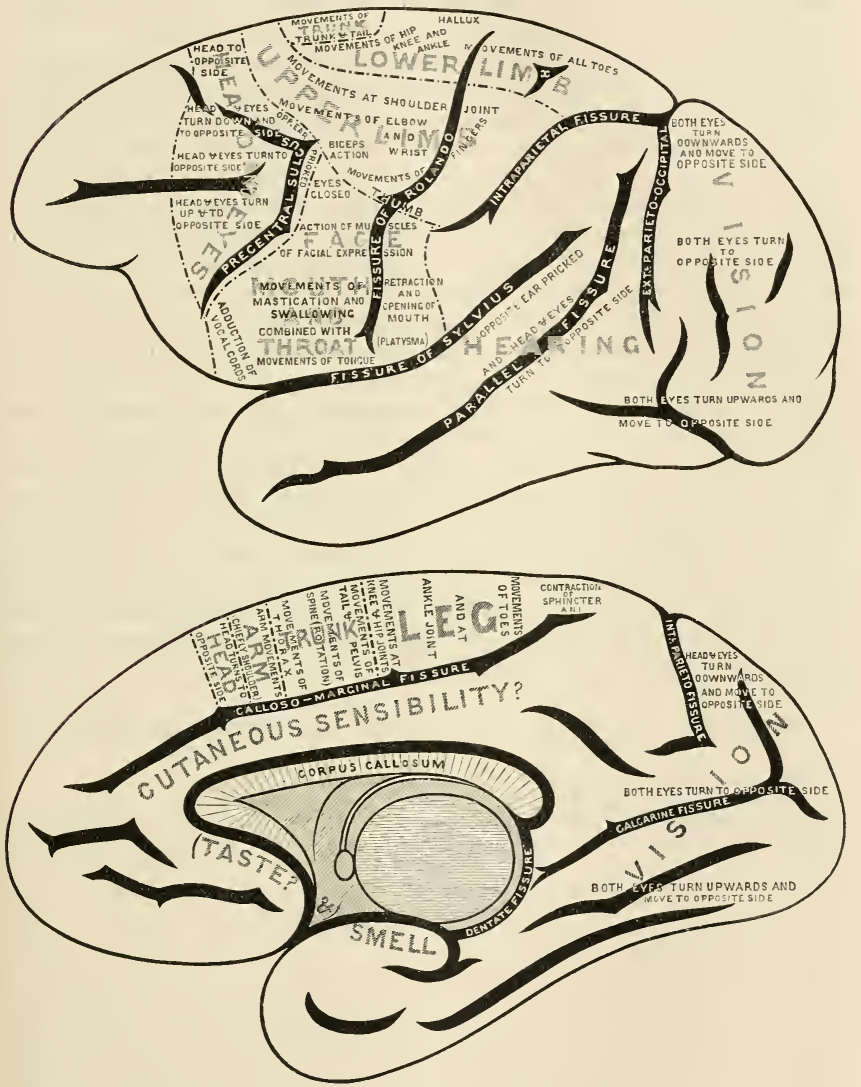

Fig. 338.-Diagrans of the lateral and mesial surfaces of the monkey's brain, showing the effects obtained from stimulation of the excitable areas.

just below this, elevation of the opposite ala of the nose and upper lip associated with depression of the lower lip. These movements when performed bilaterally produce a snarl, fully exposing the canine teeth; an expression very characteristic and frequent in monkeys when alarmed. Excitation of the part of the ascending parietal gyrus on a level with the two regions just described, produced "retraction of the angle of the mouth. The action is that of the platysma myoides, and 
when this is strong the head is drawn slightly to the side." 1 This muscle is also called into action in expressing sudden terror.

Stimulation below the centres above described produced, in Ferrier's experiments, opening of the mouth with protrusion and retraction of the tongue, the protrusion being obtained more anteriorly, the retraction more posteriorly. The movements were bilateral, and occasionally repeated even some time after the electrodes were withdrawn.

Subsequent observers have for the most part corroborated these statements of Ferrier, and have added the following facts:-(1) Stimulation of the upper part of the area, close to its upper boundary, produces, besides the movement of the opposite angle of the mouth, also closure of both eyes, ${ }^{2}$ but especially the opposite one. ${ }^{3}$ (2) Movement of the tongue nay be obtained even from the upper part of the area (thus showing a very extensive representation of this organ); here it takes the form of protrusion towards the opposite side, and may be combined with bilateral movements of the cheeks, ${ }^{4}$ whereas, over the lower part of the face area, retraction of the tongue is more common. In producing the lateral movements of the tongue, the same muscles on the two sides are not called into play; so that, although there is bilateral contraction it is not symmetrical. The cheek movements are more generally and easily obtained over the lower part of the area, and especially over the posterior part of the lower end of the ascending frontal, and over the lower end of the ascending parietal gyrus. They are there combined with rhythmic masticatory movements of the lower jaw and tongue, and with movements of the throat such as are made in deglutition. (3) Stimulation of the anterior part of the lower end of the ascending frontal convolution produces strong adduction of both vocal cords, ${ }^{5}$ but abduction of the cords is not obtainable from cortical stimulation in the monkey, although it can be obtained in the cat and dog. ${ }^{6}$

Destruction or removal of the facial area ${ }^{7}$ produces paralysis of

${ }^{1}$ Ferrier, "Functions of the Brain," 2nd edition, p. 242.

2 Schäfer with Horsley, Festschr.f. C. Ludvig, Leipzig, 1887, s. 280.

${ }^{3}$ Bcevor and Horsley, Phil. Trans., London, 1894, B. See on the subject of the cortical representation of the orbicularis oculi, Ziehen, Arch.f. Physiol., Leipzig, 1899, S. 158 .

*Horsley and Schäfer, loc. cit. Beevor and Horsley, loc. cit., also obtained a peculiar rolling movement of the tongue, with the dorsun turned to the opposite cheek.

s Semon and Horsley, Brit. Med. Journ., London, 1889, vol. ii. 1. 1383 ; and Phil. Trans., London, 1890 , 13, p. 187 .

${ }^{6}$ Risien Russell, Proc. Roy. Soc. Loudon, 1895, vol. lvii. p. $23 \overline{7}$; cf. also J. Brockiert, Flandie mét., tome xi. p. 769 ; and Klemperer, Aich.f. Lanyugol. u. Rhinol., Berlin, 1894, vol. ii. S. 329. Adduction of the vocal cords from cortical excitation was first obtained in the dog by $H$. Krause, on stimulation of the hase of the precrucial gyrus, where this is connected with the anterior composite gyrus (Arch. f. Physiol., Leipzig, 1884). Bilateral removal of the part in question was found by $\mathrm{H}$. Krause to produce marked alteration in or even abolition of the bark, growling or whining sounds only being produced. This confirms the prior observations of Soltmann (Jahrb. $f$. Kinderh., Leipzig, 1875, Bd. ix. S. 106). Masini ("Sui centri motori cort. d. larynge," Napoli, 1888) got unilateral effects from stimulation and ablation of Krause's centre, but his results have not been confirmed by others. Ferrier occasionally found that in the dog stimulation at the point marked 9 in Fig. 332 produced a loud bark, West Riding Lun. Asyl. Rrp., London, 1873. Duret, Compt. rend. Soc. de biol., Paris, 1877, and "Ettudes expér., ete.," Paris, 1878, found loss of the bark to result from bilateral extirpation of the region 9 of Ferrier (see Fig. 332), while mechanical excitation by pressure upon this region eaused a succession of barks. In connection with this, it is interesting to note that the bark of the dog has been acquired as the result of domestication, and nay be said to answer in a measure to "speech" in man.

${ }^{7}$ Everett Flood, Brit. Med. Journ., London, 1894, vol. ii. 1. 189. 
those muscles of the opposite side which act unilaterally on stimulation, but not of the bilaterally acting museles. The opposite cheek-pouch is paralysed and fills with food, but is occasionally emptied by pressure by the hand. Eventually the paralysis is recovered from, and it is emptied by its own muscular wall. If a second lesion is now established upon the intact side of the brain, this first cheek-pouch does not show renewed paralysis. ${ }^{1}$ With a bilateral lesion there is marked difficulty in swallowing and mastication, but the voice is not lost. The actions of the muscles are ultimately recovered. This may be due to parts of the grey matter in the deptl of the fissures within the area having been left, or it may be that the recovered movements are reflex, and not truly volitional.

3. The area connected with movements of the upper limb.This is an extensive area (Fig. 336) occupying the upper half of the central gyri (except their uppermost ends), and extending forwards in the first frontal gyrus, to the edge of the hemisphere, where it reaches the marginal part of that gyrus (Fig. 338). It is bounded in front by the area for the head and eyes, below by the facial area, behind by the intraparietal sulcus, and above by an imaginary line separating it from the leg area at about the level of a small horizontal suleus, which is very constant in the monkey's brain. Various complex movements of the arm and hand were described by Ferrier by stimulation within this area (Fig. 335). From the upper part, extending over both central gyri, the opposite hand was struck backwards, the arm being adducted, extended, and retracted, and the hand pronated. Further forwards (base of first frontal gyrus), extension forwards of the arm from the shoulder, as if trying to reach or touch something in front. Along the ascending parietal gyrus, clenching of the fist, beginning with the thumb and index finger, associated with action of the wrist. On the ascending frontal gyrus, in front of this, Hexion and supination of the forearm.

The retraction of the limb described by Ferrier is most readily got from the ascending parietal near the upper limit of the arm area. From the corresponding part of the ascending frontal it is inore usual to obtain, as a primary movement, a forward movement of the whole arm with raising of the shoulder, and this and other movements of the shoulder, combined secondarily with movements of the more peripheral parts of the arm, and especially with extension of the elbow, is found over the whole of the upper and anterior part of the area, merging in front into combined movements of the head and shoulder. This upper and anterior part of the arm area is therefore primarily concerned with shoulder movements, although, secondarily, other parts of the upper limb may be set in action by its stimulation. Below the shoulder region is a part of the area, occupying the expanded part of the ascending frontal gyrus just above the level of the angle of the precentral sulcus, which, as Ferrier showed (Fig. 335, 6), gives on stimulation a marked flexion of the arm at the elbow, combined with supination (biceps action). This is often combined as a secondary action with extension at the wrist and flexion of the fingers and thumb.

The primary effects obtained from stimulation of the ascending parietal in the lower two-thirds of its extent within the arm area are, as Ferrier stated, movements of the fingers and thumb. These, how- 
ever, are not confined to the ascending parietal, but extend to the corresponding margin of the ascending frontal, where indeed they may be alone represented. The thumb movements are most marked near the lowermost limit of the area, where, with a very weak excitation, a simple movement of the thumb, or one combined with that of the index finger (opposition), may readily be obtained. ${ }^{1}$

4. The area connected with movements of the lower limb.-This (Figs. 336, 338) occupies the upper end of the ascending frontal and ascending parietal gyri (including the parietal lobule) above the arm area, ${ }^{2}$ and the corresponding parts of the marginal convolution. ${ }^{3}$ Immediately in front of it is the area connected with movements of the trunk and tail, which in fact merges gradually into it. Stimulation over the posterior part of the area, namely, the upper end of the ascending parietal gyrus and the parietal lobule, and the adjacent part of the marginal gyrus, produces primarily movements of the opposite toes, which may or may not be associated with that of other parts of the limb. The movements of the hallux are especially represented close to the Rolandic sulcus at the upper end of the ascending frontal gyrus. ${ }^{4}$ It will be seen, therefore, that a large part of the leg area of the monkey is mainly connected with toe-novements, which indeed, as Ferrier found, are also readily, although not as a primary effect, elicited by stimulation even of the most anterior part of the area.

The movements of the ankle, knee, and hip are by no means sharply differentiated from one another in the leg area, being generally obtained in association, as indeed is the case in most voluntary movements of the limb. But it will be found that, on the whole, the representation of movements of toes, ankle, knee, and hip is from behind forwards; hip movements being most readily obtained from the front of the area, toemovements from the back, and knee- and ankle-movements from the middle part, the hip-movements being frequently combined with movements of the tail and of the pelvis. The analysis by stimulation of the leg centre by Beevor and Horsley ${ }^{5}$ gave uncertain results as to the focal points for the representation of these movements. They found that in by far the majority of cases the primary movement produced was that of flexion of all three joints, such, it may be added, as occurs when the limb is advanced in climbing. It is often followed, as in that action, by general extension. Extension of the hip, produced by contraction of the gluteal muscles, is more frequently got as a primary movement from excitation of the marginal gyrus near the anterior limit of the leg area, and flexion of the knee produced by contraction of the hamstrings a little behind this. ${ }^{6}$ Extirpation of the leg area is followed by, at first, complete paralysis of the limb, which hangs down limp in climbing, is dragged behind in walking, and in sitting is thrust passively forward. In the course of a week or two, however, the condition begins to show

${ }^{1}$ Horsley and Schäfer in Festschrift f. C. Luduig, Leipzig, 1887, S. 286 ; Beevor and Horsley, Phil. Trans., London, 1888, B, p. 232.

2 Ferrier, Proc. Roy. Soc. London, 1875, vol. xxiii.

${ }^{3}$ Horsley and Schäfer, ibid., 1884 , vol. xxxvi. p. 437.

${ }^{4}$ Beevor and Horsley, Phil. Trans., London, 1888, B. Both flexion and extension may be excited from the same spot. Sherrington found (in the monkey) that when flexion of the hallux was obtained, if the nerve passing to the flexors was eut, excitation at the same part of the cortex now produced extension.

5 Phil. Trans., London, $1888, \mathrm{~B}$.

${ }^{6}$ Horsley and Schäfer, Festschrift f. C. Ludwig, Leipzig, 1887, S. 278; and Phil. Trans., Lomlon, 1888, B, p. 10. 
improvement, and movements return, so that after a few weeks the animal climbs and runs about apparently as usual. Probably, however, the movements are now "associated" with those of the opposite side, and not truly voluntary; for if such an animal, apparently completely recovered, be held by the upper part of the body and lowered suddenly to the ground, or swung towards a cage, the leg which was paralysed is never put down to meet the ground or forwards to catch hold of the cage, a movement which is always made by an unparalysed leg. Sherrington ${ }^{1}$ has shown that in the monkey stimulation of a point in the posterior part of the lobus paracentralis, on the mesial surface, causes contraction of the sphincter ani.

5. The area for movements of the trunk and tail.-This is a comparatively small area, lying chiefly on the marginal convolution (Fig. $338)^{2}$ in front of the leg area, but coming to the external surface (Fig. 336) and extending over a small portion of the superior frontal gyrus ${ }^{3}$ as far as the limit of the arm area, which here bounds it laterally and anteriorly. ${ }^{4}$

Its excitation is followed by a bending of the tail to the opposite side, combined with a bending of the trunk or a rotation of the pelvis. The area is too small to give any well-marked differentiation on analysis by stimulation, but, on the whole, it is noticeable that the posterior part is especially connected with the movements of the lower part of the trunk and pelvis, associated with hip and leg movements: the anterior part with movements of the upper part of the vertebral column, associated with shoulder and arm movements. The trunk movements are occasionally bilateral, the tail or vertebral column being extended. Extirpation of the trunk area on one side produces, unless the lesion involve the adjacent areas (which it is somewhat difficult to avoid), very little obvious effect. If, however, it be removed on both sides simultaneously, there is for a time complete inability to sit erect, power over the trunk muscles being entirely lost. ${ }^{5}$

The motor centres in the anthropoid apes.-These have only been determined in a single case (orang-utan), ${ }^{6}$ and by the method of faradic excitation, but since the configuration of the brain in the anthropoids approaches still more closely to that of man than does that of the monkey, the evidence afforded is of great value and interest. It S. 399

l "Proc. Physiol. Cong.," Centralbl. f. Physiol., Leipzig u. Wien, 1892, Bd. vi.

${ }^{2}$ Horsley and Sehäfer, Proc. Roy. Soc. London, 1884, vol. xxxvi.

${ }^{3}$ Ferrier, ibid., 1875; Horsley and Schäfer, Phil. Tians., London, 1888, B, p. 9.

${ }^{4}$ On the centres for the trunk muscles in dogs, see Unverricht and Kusick, Ges. Abhandl. d. med. Klin. zu Dorpat, Wiesbaden, 1893; and H. Werner, Allg. Ztschr. f. Psychiat., etc., Berlin, 1895, Bd. lii. S. 134. These authors failed to confirm Munk's statement, that the centre for the trunk muscles is in the frontal lobes. They find that it lies between the centres for the muscles of the fore- and hind-limbs, and that its excitation provokes movements of the trunk muscles of the same side. Rothmann (Neurol. Centralbl., Leipzig, 1896, S. 1105) states, however, that the trunk movements described by those authors are really passive, and are due to contractions of hip and shoulder muscles of the opposite sidc. Rothmann, who examined the brains of four dogs from which Munk had removed the prefrontal lobe of one side, was unable to find any descending degeneration in the pyramidal tract either at or below the medulla oblongata. It is therefore highly inprobable that the centre for the trunk muscles is in this part of the dog's brain, as taught by Munk.

${ }^{5}$ Horsley and Schäfer, Phil. Trans., London, 1888, B, p. 13. This condition was not recovered from in the animals we operated on, but they were not kept under observation for more than three or four weeks.

${ }^{6}$ Beevor and Horsley, Phil. Trans., London, 1890, B, p. 129. 
was noticed by Beevor and Horsley-(1) That instead of the excitable sphere of the cortex being continuous, as in the monkey, it is, in the orang, interrupted here and there by spaces from which no effect could be obtained even by strong excitations; (2) that the amount of cortex which is excitable is relatively smaller in the orang than in the nonkey. Thus the first frontal gyrus was found to be inexcitable, and most of the upper part of the ascending parietal. Indeed, with the exception of an

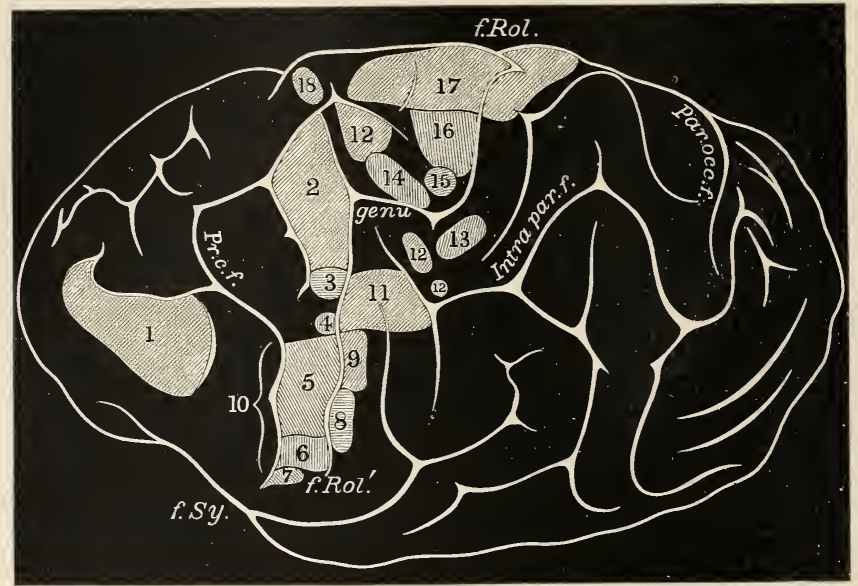

FIG. 339. -f. Rol., $f$. Riol.', fissure of Rolando, with well-markel genu ; Pr. $c . f$, preeentral fissure; $f$. $S y$., fissure of Sylvius; Intra par. $f$., intraparietal fissure ; $P a r$. occ. $f$, , external parieto-oeeipital fissure. 1, Both eyes move eonjugately to opposite side; 2, Eyes open, eyes and head to oplposite side ; 3, Closure of eyelids, those of opposite side most ; 4, Eversion of lower lip on opposite side ; 5 , Retraction of opposite angle of mouth ; 6, Rolling in of both lips, also opening of the mouth-this latter movenent is obtained a little further back than the former ; 7, Pursing of both lips; 8, Pouting of both lips ; 9, Elevation of upper lip on olposite side; 10, Movements of the tonguethese oeeurred over almost all the facial area; 11, Twitching of orbieularis oris of opposite side; 12 , Novements of the thumb, generally extension-it will be notieed that these were got at three several spots isolated from one another; 13, Extension of index finger; 14, Extension of all the tingers, with pronation or ulnar adduction of wrist: 15, Flexion of elbow ; 16, Adduetion of shoulder ; 17, Area for movements of lower limb. Movements of hallux, small toes, ankle, knee, and hip all occur at various points in this area (which extends over to the mesial surface), but movements of the hip and knee were not got quite as high as movements of the toes and ankle, and, on the other hand, their area of representation extended a little lower; 18, An ontlying spot where extension of toes was obtained.

area for eye-movements in front of the precentral sulcus, almost the only parts of the fronto-parietal lobe found to be excitable were the ascending frontal gyrus, the lower two-thirds of the ascending parietal gyrus, and the marginal gyrus opposite the upper end of the ascending frontal: (3) the tendency to provoke a sequence or march of movements from stimulation of one point only, which is so often met with in the monkey, is very much less marked in the orang. The 
accompanying diagram (Fig. 339) gives the main results of Beevor and Horsley's investigation.

The motor centres in man-Experimental evidence.-A few A.

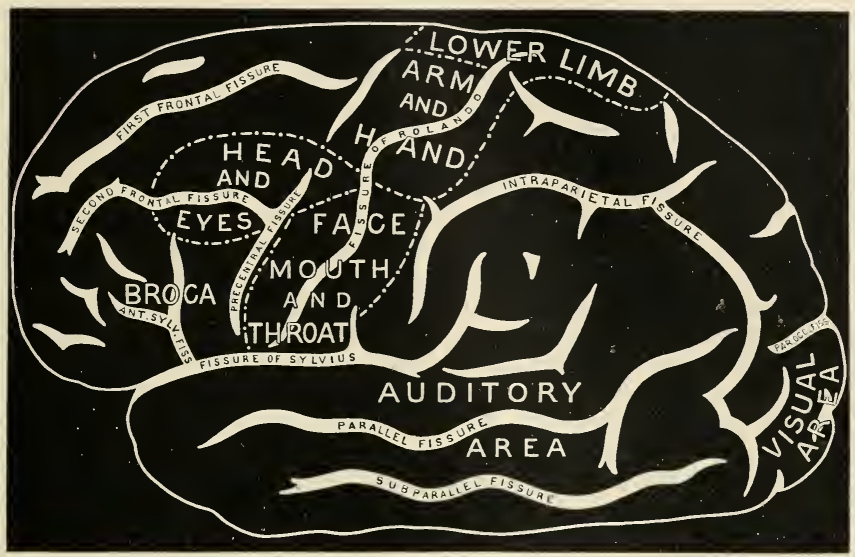

$B$.

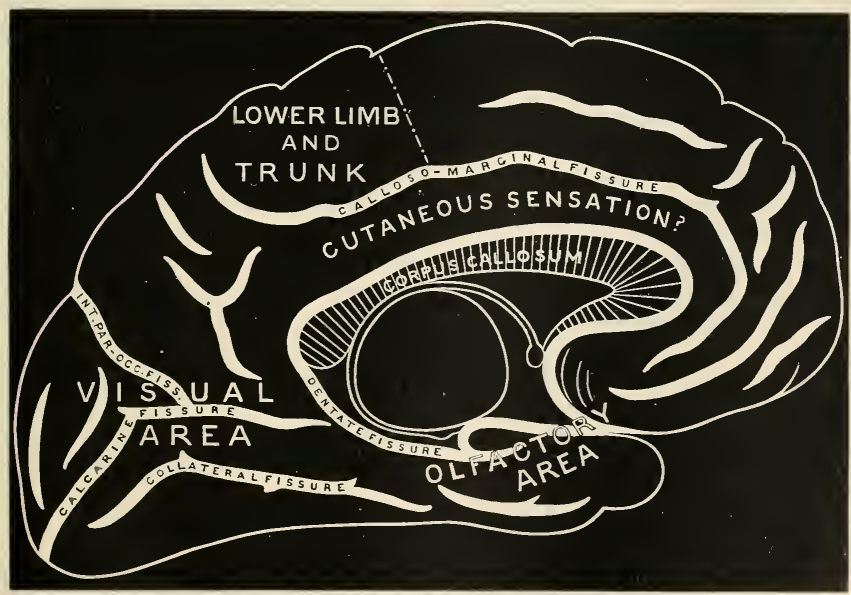

FIg. 340.-Diagrams indicating the probable localisation of the chief notor and sensory areas of the cerebral cortex of man. $A$, lateral surface; $B$, mesial surface.

experimental observations have been inade upon man, the cortex having been on several occasions stimulated over a limited area exposed for the 
purpose of operation. ${ }^{1}$ The results, as far as they go, conform with those made upon the monkey and orang. ${ }^{2}$ Both in the orang and in man a much greater strength of current than that employed for lower animals is needed to provoke movements, a result which appears to indicate that they are caused by excitation, not of the superficial but of more deeply lying parts of the cortex, which is much thicker than in the monkey. The convolutions are also more excitable near their convexity than near the bounding fissures. Both in the orang and in man it is difficult to provoke epilepsy by electrical excitation; and it may be added, it is easier to produce this condition in the dog than in the monkey, and in the rabbit than in the dog. Thus the lower the animal in the scale of intelligence the more easy is it to provoke epilepsy, which is characterised by a condition of abnormal excitability of and spread of excitation over the whole cortex.

Clinical evidence.-Abundant confirmation is fortheoning regarding the situation of the motor areas in man, as the result of clinical observation. On this subject a very large number of cases have been collected and published by Charcot and Pitres, ${ }^{3}$ to whose book the reader is referred for the details and diagrams by which the cases are illustrated. They show that the motor zone in man includes only the convolutions adjacent to the fissure of Rolando, namely, the ascending frontal, the ascending parietal, the paracentral lobule, and the Rolandic operculum. ${ }^{4}$ Lesions of any of these gyri, even if quite small, always cause permanent paralysis in one or several muscular groups of the opposite side of the body, and are followed by degeneration in the pyramidal tract. The areas are disposed in the following order from above down (Fig. 340, $A$ and $B$ ):-

That for the lower limb.-In the paracentral lobule and the upper fourth of the two central gyri. The area for the trunk muscles is closely associated with this one.

That for the upper limb.--In the middle part of the central gyri, extending from the leg area as far as the sharp downward bend of the fissure of Rolando.

That for conjugate movements of the eyes and head.-Occupying the ascending frontal gyrus just below the genu of the fissure of Rolando, and extending forwards into the base of the second and third frontal gyri.

That for the face, mouth, tongue, and throat.-In the lower third of the central gyri, and in the Rolandic operculum.

The motor area in man does not include Broca's convolution, i.e. the base of the third frontal gyrus, lesions of which, although productive of

\footnotetext{
1 See the cases quoted by Mills, Brain, London, 1889 ; Beevor and Horsley, Phil. Trans., London, 1890, B, pp. 152-156; and Ferrier, "Croonian Lectures," 1890, p. 34. Cf. also v. Bechterew, Arch. f. Physiol., Leipzig, 1899, S. 543.

${ }^{2}$ Gotch (Brit. Hed. Journ., London, 1893, vol. i. p. 1102) got movements of the head (to the same side) on stimulating a point on the ascending frontal gyrus a little below the genu of the bend of the Rolandic fissure. This is also got here in the orang, but not in the macaque.

3 "Les centres motenrs corticaux chez l'homme," Paris, 1895.

4 Whether the head and eye movements are represented here only or in a more advanced portion also, as in the anthropoid, does not appear clear as the result of clinical observations; these movements are rarely if ever wholly lost in cases of cortical paralysis.
} 
defects of speech, do not produce actual paralysis of the organs of speech (larynx, tongue, etc.). ${ }^{1}$

\section{The Sensory Areas of the Cerebral Cortex in the Monkey AND MAN.}

Besides the areas which have just been described, it is found that excitation of certain other regions of the surface of the hemisphere also produces definite movements. Thus, excitation of the superior temporal convolution of the monkey causes the animal to turn its head and eyes to the opposite side and to prick its ear, ${ }^{2}$ and a similar movement of the head and eyes is provoked by stimulation of the posterior part of the hemisphere. Ferrier also obtained "torsion of the lip and semiclosure of the nostril on the same side," when he excited a small area on the anterior and inner aspect of the uncinate gyrus; and he further states that sometimes, on exciting the lower extremity of the middle temporal convolution, " movements of the tongue, cheek-pouches, and jaws were induced, very like those which are characteristic of tasting."

Extirpation of the above areas is not, however, in any case followed by motor paresis, nor does there occur any long descending degeneration, like that down the pyramidal tract, as is always the case with a lesion of the excitable part of the parietal and frontal regions. On the other hand, there is indubitable evidence-at least in the case of the occipital region-that extirpation or lesion is productive of marked and permanent sensory disturbance (hemiopia). It has therefore been assumed by Ferrier and others, probably with reason, that in this case the movements which are produced are the result of and secondary to ideas of sensation which are called up by the stimulation, and it is inferred that the areas in question-at least those which have been most fully investigated-are portions of the cortex which are connected with the perception of, and response to, sensations derived from the organs of special sense, and differ in their mode of action from the "motor" areas by producing the movements in question in a more indirect manner. Other neurologists, on the other hand, have taken up the position that in all cases, whether the excitation is applied to the Rolandic or to the other areas, the movement produced is the result of subjective sensations, or ideas evoked by the excitation; by some it has been held that, in the case of the Rolandic area, the sensation thus excited is the sense of movement or muscular sense. If the result is brought about in a similar manner, the period of latent excitation of the muscles which are set in action should be about the same in both cases, whereas, if in the one case the transmission of impulses is more direct, there

${ }^{1}$ Amongst papers dealing with the subject of the localisation of motor centres in man, the following may be cited, in addition to those already referred to:-Séguin, Trans. Am. Neurol. Assoc., N. Y., 1877 ; Journ. Nerv. and Ment. Dis., N. Y., 1881 ; Gowers, Brain, London, 1878 , vol. i. p. 388 (congenital absence of hand, associated with atrophy of middle part of ascencling parietal) ; Bastian and Horsley, Brain, Londion, 1880, vol. iii. p. 113 (a similar ease to the above); Exner, "Local. 1. Funct. in d. Grosshirmr. d. Mensehen," Wien, 1881; and in Hermanu's "Handbuch," 1879, Bd. ii. ; Starr, "Localised Cerebral Disease," Am. Journ. Med. Sc., Plila., 1884; Keen, Am. Journ. Wed. Sc., Phila., November 1888; Phila. Med. Neu's, 1890, p. 381 ; Ferrier, "Cerebral Localisation," 1890 ; M'Bride, Journ. Nerv. and Ment. Dis., N. Y., 1890, vol. vii. 1. 512 ; C. K. Mills, Brain, London, 1899, vol. xii. pl. 233, 358.

2 Ferrier, Proc. Roy. Soc. London, 1s75, vol. xxiii. p. 426. 
should be a difference in the latency periods of the two. I have accordingly put this point to the test by the following method: ${ }^{1}$ - Two pairs of flat electrodes, carefully guarded with paraffin, are applied through trephine openings, the one to the frontal area, the other either to the occipital lobe or to the superior temporal gyrus. The wires from the electrodes (Fig. $341, F r$., Occ.) pass to a switch ( $S w$.), by turning

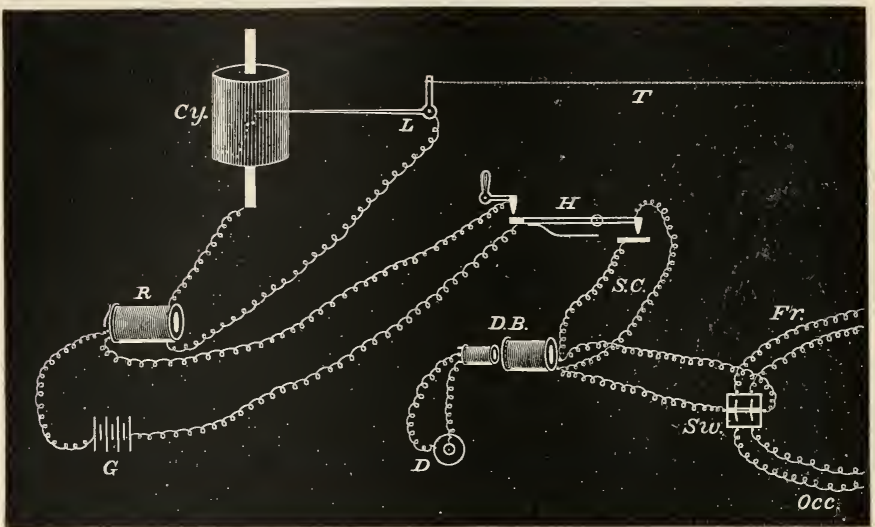

Fig. 341.-Diagram of the arrangement of apparatus for determining the comparative latency periods of two parts of the cerebral cortex. $G$, Grove battery working the Ruhmkorff coil ; D, Daniell cell working the du Bois coil, D.B.; S.C., short eireuit for du Bois coil. H, Helmholtz key arranged so that the Ruhmkorff eircuit is made at the same instant that the short eircuit of the du Bois coil is broken. The other parts of the diagram are described in the text.

which the areas under investigation can be alternately stimulated. The lateral movement of one of the eyes is recorded by attaching it by a thread $(T)$ to an aluminium lever $(L)$, through which sparks at the rate of 250 per second can be transmitted from a Ruhmkorff coil $(R)$ to the smoked paper on a rotating brass cylinder $(C y$.$) . The sparks are$

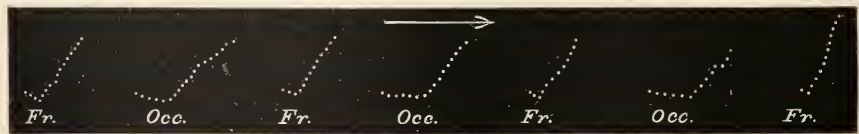

FIG.312.-Curves showing the comparative periods of lateney for the ocular museles, on stimulation of the frontal and occipital cortex respectively.

only allowed to commence from the moment of stimulation, and serve to mark the time as well as the curve described; for the lever is not allowed to touch the cylinder, differences in friction against the paper being thus eliminated-an important point when dealing with the latency of comparatively weak muscles.

The accompanying figure (Fig. 342) shows some of the tracings thus obtained from alternate stimulation of the frontal and occipital cortex;

' Internat. Monthly Journ. Anat. and Physiol., Leipzig, 1888, vol. v. p. 149. 
and the Tables I., II., and III. show the results of several experiments.

\section{TABLe I.}

\begin{tabular}{|c|c|c|c|c|c|c|c|c|c|c|}
\hline No. of Experiments & F. & o. & F. & 0. & F. & o. & F. & 0. & F. & $\theta$. \\
\hline I. & $\cdot 125$ & $\cdot 4$ & $\cdot 125$ & $\cdot 35$ & $\cdot 1$ & $\cdot 35$ & ; & ... & $\ldots$ & $\ldots$ \\
\hline II. & $\ldots$ & $\cdot 2$ & $\cdot 15$ & $\cdot 2$ & $\cdot 125$ & $\cdot 175$ & $\cdot 15$ & 2 & $\cdot 15$ & $\cdot 2$ \\
\hline III. & $\cdot 2$ & $\cdot 4$ & $\cdot 2$ & $\cdot 25$ & $\cdot 15$ & $\ldots$ & $\cdots$ & $\cdots$ & $\cdots$ & $\ldots$ \\
\hline IV. & $\ldots$ & $\cdot 35$ & $\cdot 1$ & $\cdot 2$ & $\cdot 1$ & $\ldots$ & $\ldots$ & $\ldots$ & $\ldots$ & ... \\
\hline V. & $\cdot 13$ & $\cdot 07$ & $\cdot 25$ & $\ldots$ & $\ldots$ & $\ldots$ & $\ldots$ & $\ldots$ & $\ldots$ & $\ldots$ \\
\hline
\end{tabular}

TABLE II.

\begin{tabular}{|c|c|c|c|c|c|c|c|c|c|c|}
\hline No. of Experiments. & $F$; & $\mathrm{T}$. & F. & T. & F. & T. & F. & T. & F. & T. \\
\hline I. & $\cdot 1$ & $\cdot 175$ & $\cdot 1$ & $\cdot 175$ & .075 & $\cdot 15$ & 075 & $\cdot 112$ & 075 & $\cdot 15$ \\
\hline II. & $\cdot 05$ & $\cdot 112$ & $\cdot 075$ & $\cdot 112$ & $\cdot 075$ & $\cdot 112$ & $\ldots$ & $\ldots$ & $\ldots$ & $\ldots$ \\
\hline III. & $\cdot 1$ & $\cdot 2$ & $\cdot 1$ & $\cdot 2$ & 075 & $\ldots$ & $\ldots$ & $\ldots$ & $\ldots$ & $\ldots$ \\
\hline IV. & $\cdot 2$ & $\cdot 25$ & $\cdot 15$ & $\cdot 3$ & $\cdot 2$ & $\cdot 15$ & $\cdot 1$ & $\ldots$ & $\ldots$ & $\ldots$ \\
\hline V. & $\ldots$ & $\cdot 25$ & $\cdot 12$ & $\cdot 25$ & $\cdot 12$ & $\cdot 15$ & $\cdot 05$ & $\ldots$ & $\ldots$ & $\ldots$ \\
\hline
\end{tabular}

TABLe III.

\begin{tabular}{|c|c|c|c|c|c|c|}
\hline No. of Experiments. & $\mathrm{T}$. & o. & T. & 0. & T. & 0. \\
\hline I. & $\cdot 2$ & $\cdot 2$ & $\cdot 2$ & $\cdot 15$ & $\cdot 15$ & $\cdot 15$ \\
\hline
\end{tabular}

In these Tables, $\mathrm{F}$ denotes excitation of frontal area; $\mathrm{O}$, of occipital lobe; T, of first temporal gyrus. The periods of latent excitation are given in seconds. The observations recorded along each horizontal line are immediately consecutive.

It will be observed that the periods of latency from the temporal and occipital regions are identical (Table III.), whereas, in the course of the same experiment, that from the frontal is markedly shorter than from either the temporal or occipital regions. ${ }^{1}$ In the latter case, therefore, there must be at least one other nerve cell (neurone)interpolated, if not more. One's first idea would be, that the action of the posterior (temporal and occipital) areas is through the frontal region. But both frontal regions may be completely removed, and the action will still be

${ }^{1}$ The variations in different experiments may be due to differences in the depth of anæsthesia. 
produced from the temporal and occipital regions. ${ }^{1}$ The extra cell or cells through which they operate must probably, therefore, be looked for in lower centres (thalamus ${ }^{2}$ or superior corpora quadrigemina?). It may therefore be concluded that the disposition of the tracts by which the nervous impulses which produce motion operate, is different in the case of the Rolandic areas and of the areas commected with visual and auditory sensations-a conclusion which has a far-reaching effect in connection with the question of the supposed sensory functions of the Rolandic cortex. It also militates against the hypothesis of Flechsig, ${ }^{3}$ that each sensory sphere is like the cortex of the Rolandic region; in having its own special mechanism for producing movements of the particular peripheral sense organ, and only of this one, and that each such sphere is therefore a motor area in the same sense as is the cortex of the Rolandic region.

We may next proceed to consider more in detail the several

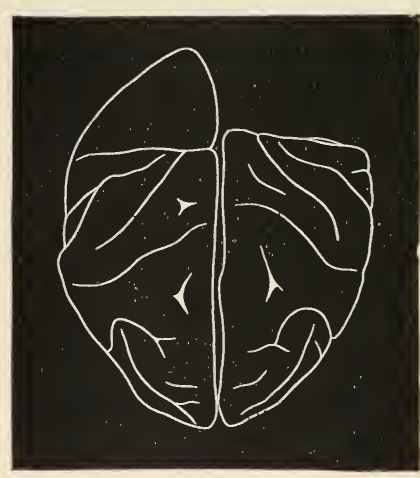

FIG. 343.-Brain of a monkey from which one occipital lobe had been entirely removed but the angular gyrus left intact. This animal was rendered permanently hemiopic. sensory areas which have been mentioned.

Visual area.-The parts comprised within this area are, in the monkey, the occipital lobe and the immediately adjacent portions of the temporal lobe, including the cuneus, lingual lobule, and posterior parts of the temporal convolutions upon the inner and under surfaces.

Ferrier was the first to describe in various animals (dog, jackal, cat, rabbit, monkey) the existence of an excitable zone in the posterior part of the hemisphere, stimulation of which causes movement of the eyes to the opposite side. In carnivora he placed this zone on the posterior part of the second convolution, in the monkey on the angular gyrus: and both alone and in conjunction with G. F. Yeo he subsequently found destruction of the angular gyrus to produce loss of vision in the opposite eye, and of both angular gyri complete blindness in both eyes. Hitzig described blindness of the opposite eye as the result of removal of the cortex of one occipital lobe in $\operatorname{dogs},{ }^{4}$ but later was led to conclude that a similar result might follow lesions of the anterior part of the brain also. ${ }^{5}$ On the other hand, Luciani and Tamburini ${ }^{6}$ asserted

1 Schäfer, Interwat. Monthly Jourm. Anat. and Physiol., Leipzig, 1888, vol. v. This has been confirmed by other investigators, e.g. Danillo, Rosenbach, Steiner and Obregia (Arch. f. Physiol., Leipzig, 1890, S. 260). The last observer also found that extirpation of the occipital lobes had no effect on the movements obtained from the excitation of the frontal lobes.

2 Various observers have described centrifugally conducting fibres as passing from the cortex of the occipital lobe, and terminating in the thalamus.

${ }^{3}$ Neurol. Centralbl., Leipzig, 1894, Bd. xiii. S. 674.

${ }^{4}$ Centralbl. f. d. med. Wissensch., Berlin, 1874, S. 548.

5 Arch. f. Psychiat., Berlin, 1883, Bd. xv. S. 270.

" "Sui centri psico-sensori corticali," Reggio-Emilia, 1879. See also on the effects of ablation of the occip,ital lobes in dogs, Vitzou, Arch. de physiol. norm. et path., Paris, 1893, p. 678. 
that destruction of the cortex of the angular gyrus and occipital lobe of one hemisphere produces not amblyopia but hemiopia, i.e. not blindness of the whole of the retina of the opposite eye, but blindness of the same side of the retina in both eyes. It was, however, shown by H. Munk ${ }^{1}$ that removal of the occipital lobe alone, in the monkey, produces homonymous hemiopia (blindness of the corresponding half of both retinæ), and that removal of both occipital lobes causes complete blindness, even although the angular gyri are intact; by Munk, therefore, the visual centre was located in this lobe alone. Subsequently the subject was investigated by me in conjunction with Sanger Brown* by the method of extirpation. The result of our experiments was to show that, as stated ly Munk, removal of one occipital lobe causes homonymous hemiopia, and of both lobes at first complete blindness; but the blindness was not permanent, unless the lesion extended somewhat in advance of what is generally taken to be the limit of

A.

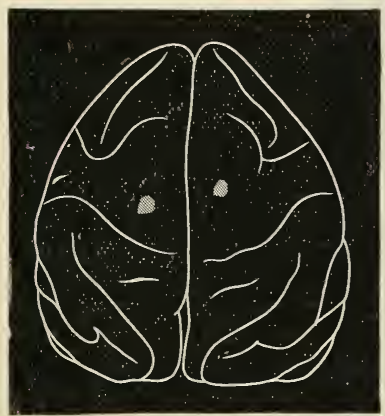

B.

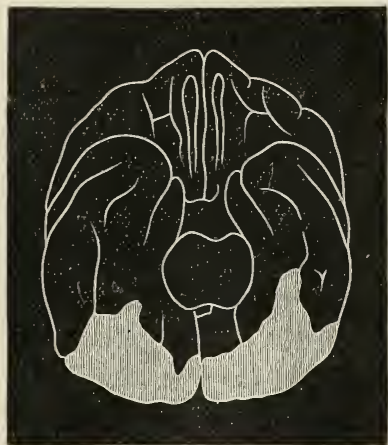

Fic. 341.-Views from above, $A$, and from below, $B$, of the brain of a monkey, from which both occipital lobes, and, on the under surface, part of the temporal lobes had been ent away. This animal was completely blind for several months, although both angular gyri were intact.

the lobe, on the inner and lower surface. The explanation of the difference between these results and those of Ferrier and Yeo is probably to be found in the fact that in their cases the removal is less complete-this is manifest from their figures. And the same remark applies to some experiments of Horsley and myself on this region. ${ }^{3}$ With regard to the angular gyrus, the experiments with Sanger Brown were entirely contradictory to those of Ferrier and Yeo. In animals in which the grey matter of this convolution was destroyed on one or both sides we could discover no defect of vision. If it were done on one side, there was neither blindness of the opposite eye nor hemiopia; if the eye of the same side were bandaged, the animal saw perfectly well with the opposite eye. In one case only, in which we scooped the whole of the convolution away, after opening up the fissures

1 "Die Functionen der Grosshirnrinde," 1890, S. 293. See also Sitzungsb. d. k. Akad. d. Wissensch., Berlin, 1890, S. 53.

${ }^{2}$ Sanger Brown and Schïfer, Phit. Trans., London, 1888, B, p. 303 ; Schäfer, Brain, London, 1887, vol. xi. p. 362 ; ibid., vol. xi. p. 145.

${ }^{3}$ Phil. Trans., London, 1888, B.

VOL. II. -48 
which bound it, we produced hemiopia, which lasted for a few days, gradually passing off. But in this case a considerable disturbance must have been mechanically produced in the occipital lobe by the operation, and this would be quite sufficient to explain the hemiopia. In no single instance in which I have performed a lesion in this, or any other part

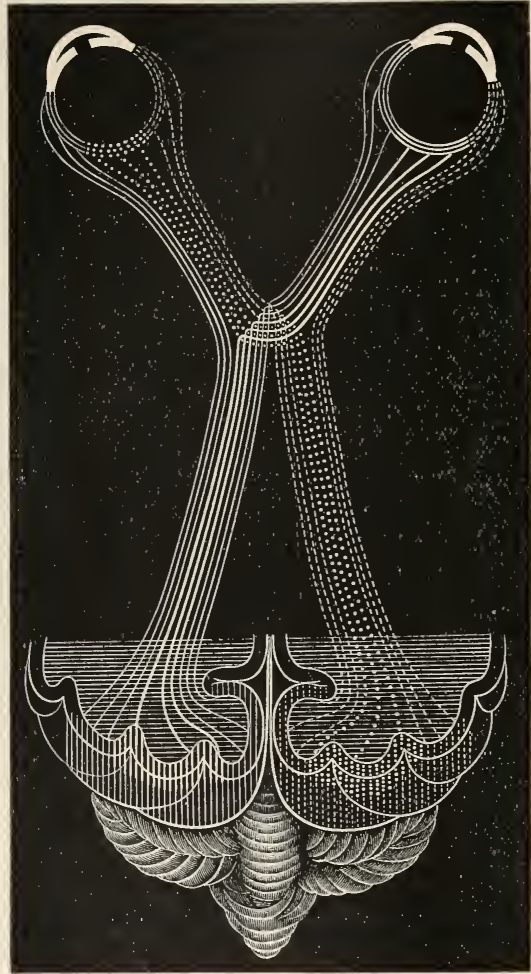

Fig. 345.-Diagram showing the connection between several parts of the retina and occipital lobes in the dog. - H. Munk. of the hemispliere, has the visual disturbance which resulted been confined to the opposite eye; it is invariably of a hemiopic character, producing partial blindness of both retinæ. ${ }^{1}$ Removal of the angular gyrus has no effect upon the sensibility of the opposite eyeball. ${ }^{2}$ After removal of the occipital lobe in a monkey, all optical reflexes, except the pupillary reflex, are abolished, so far as the blind side of the retina is concerned (Munk).

M'Kendrick ${ }^{3}$ found that destruction of the occipital lobe in the pigeon did not produce blindness of the opposite eye ; it was necessary to remove the whole hemisphere.

Localisation within the visual area. $-\mathrm{H}$. Munk attempted to determine this in the dog and monkey by effecting large partial extirpations of the occipital cortex. ${ }^{4}$ As the result of these experiments, he came to the conclusion that in the dog the upper zone of the retina corresponds with the anterior part of the visual

I As already stated, temporary hemiopia may be produced by extensive lesions of almost any part of the hemisphere, the cause being doubtless the vasenlar and meehanical disturbance which the removal occasions. It is, however, more apt to oceur from these eauses when the lesions are in the neighbourhood of the oceipital lobe.

2 Contrary to H. Munk, "Ueber die Functionen der Grosshirus, Gesammelte Mittheillungen," 4te Mittheilung, 1878. See further on the effects of removal of the occipital lobe in animals, Gilman Thompson and S. Brown, Rep. Loomis Lab., New York, 1890 ; also Vitzou, Arch. de physiol. norm. et path., Paris, 1893, p. 678; and "Proc. Physiol. Cong.," 1892, Centralbl. $f$. Physiol., Leipzig, u. Wien, Bd. vi. S. 400.

3 "Reent Researches on the Nervous System," Edinburgh, 1874.

4 "Gesammelte Abhandlungen," 1890 ; and Sitzungsb. d. k. Akad. d. Wissensch., Berliu, Jan. 1890, translated in Brain, London, 1890, vol. xiii. 
area, the lower zone with the posterior part, the mesial or internal zone with the mesial or internal part, and the lateral zone of the retina with the lateral part of the area, and that the part of the retina concerned with most distinct (central) vision is represented upon both visual spheres over an area which lies near the apex of the occipital lobe (in the monkey rather nearer the mesial surface); and he finds confirmation of these conclusions from the results of excitation performed in conjunction with Obregia. ${ }^{1}$ These results of Munk are embodied in the diagram on the previous page (Fig. 345). I myself, in conjunction with Sanger Brown, ${ }^{2}$ found in the monkey that bilateral injury of both mesial surfaces of the occipital lobes appeared to cause loss of central vision, peripheral vision being intact, and came to the conclusion, partly from this but mainly from the results of excitation of the occipital cortex, ${ }^{3}$ that the scheme given by Munk for the dog will not apply to the monkey's brain, in which it is more than probable that central vision is represented upon the mesial surface of the brain at the anterior part of the visual area, which would be in the neighbourhood of the anterior part of the calcarine fissure. And this appears certainly to be the part where visual perceptions are most obviously located in man (see p. 757).

Electrical excitation of the visual area.-As already remarked, it was stated by Ferrier that electrical excitation of the angular gyrus produces conjugate lateral movement of the eyes to the opposite side; with an upward deviation if the electrodes are placed on the anterior limb of the gyrus, with a downward deviation if placed on the posterior limb. ${ }^{4}$ These experiments were subsequently repeated by other observers, who for the most part failed to obtain the same result. ${ }^{5}$ I was myself never able to produce such moveinents on excitation of the anterior limb of the angular gyrus, but occasionally got the movement described by Ferrier when the electrodes were placed on the posterior limb and on the upper end of the middle temporal gyrus, which is continuous with the posterior limb. In both these cases there may have been a spread of current to the adjacent upper surface of the occipital lobe. If the electrodes are applied directly to the upper surface of the occipital lobe, conjugate deviation to the opposite side with downward direction of the visual axes is extremely well marked, and it is also produced by excitation of the upper part of the mesial surface of that lobe, and of the quadrate lobule immediately in front of the internal parieto-occipital fissure. If, on the other hand, the posterior extremity of the occipital lobe, its lower or tentorial surface, and the posterior and lowermost part of its mesial surface, be stimulated, the lateral deviation is combined with an upward movement. In both cases the downward or upward movement may be almost uncomplicated by lateral deviation; this depends upon the position of the electrodes. On the other hand, there is an intermediate zone, narrow on the mesial surface of the lobe, and broader upon the outer or convex surface, excitation of which is productive of

1 Vide infra.

2 Phil. Trans., London, 1888, B ; Brain, London, vol. x. p. 370.

${ }^{3}$ Schäfer, Brain, London, vol. xi. p. 1.

4 "Functions of the Brain," 2nd edition, p. 243.

${ }^{5}$ Luciani and Tamburini obtained in dogs conjugate deviation to the opposite side, both from the angular gyrus and from the convexity of the occipital lobe, but without any upward or downward deflection, "Centri psico-sensori corticali," Reggio-Emilia, 1879. 
simple lateral deviation. All the effects are strongest upon the mesial surface, especially towards the anterior limit of the lobe; it is here that the focal point for the movements is located. ${ }^{1}$

The movements are usually accompanied by dilatation of the pupils, but I have occasionally obtained, from stimulation on or near the quadrate lobule, marked contraction of the pupils, such as is produced when a strong light is thrown directly into the eye. Equal bilateral excitation produces, as with the frontal areas (p. 740), fixation of the

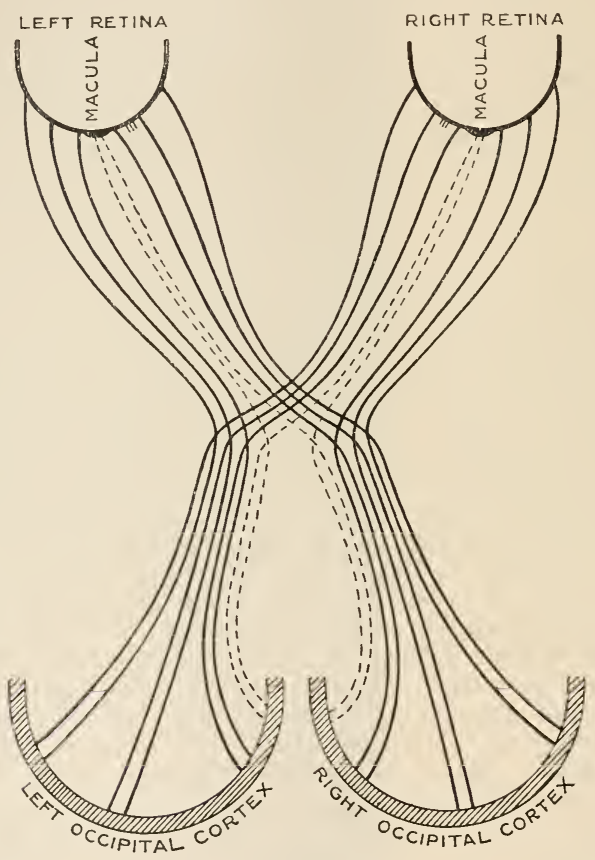

Fig. 346.-Diagram showing the probable relations between the parts of the retinie and the visual area of the cortex.

visual axes, with an upward or downward inclination according as the electrodes are placed on the lower or upper zones above described. ${ }^{2}$ If we assume that these various movements of the eyeballs are the result or concomitants of subjective visual sensations set up by the excitation,

${ }^{1}$ Schïfer, Bruin, London, 1888, vol. xi. p. 1. These observations have been confirmed in dogs ly Ohregia (with Munk), Arch. f. Physiol., Leipzig, 1890 (see also H. Munk, Sitzungsb. d. k. Akad. d. Wissensch., Berlin, 1890, S. 53, translated in Brain, London, 1890, vol. xiii. p. 45), and in the cat and rabbit by Steiner, Arch. f. d. ges. Physiol., Bomn, 1891, Bd. 1. S. 603 ; also in the monkey, by v. Bechterew, Neurol. Centralbl., Leipzig, 1897 , S. 720 , who affirms that similar movements of the eyes and pupils are obtainable by stimulation of the parietal lobe.

2 Mott and Schifer, Brain, London, 1890, vol. xiii. 
and that the movements take the direction towards which those sensations are projected, then the above facts, especially if taken in connection with the results of destructive lesions of the area, indicate a certain connection between the parts of the cerebral visual area and the retinæ. This connection may be stated as follows:-

1. The whole of the visual area of one hemisphere is connected with the corresponding lateral half of both retinæ.

2. The upper zone of the visual area of one hemispliere is connected with the upper zone of the corresponding lateral half of both retinz.

3 . The lower zone of the visual area of one hemisphere is comnected with the lower zone of the corresponding lateral half of both retine.

4. The intermediate zone of the visual area is connected with the middle zone of the corresponding lateral half of both retinx.

5 . The focal point of the visual area, which is placed on the anterior part of the mesial surface of the occipital lobe, is comnected with rather more than the corresponding half of the macula lutea of each retina.

These probable connections, in so far as they can be shown in a horizontal plane, are illustrated in the appended diagram (Fig. 346). They can be easily understood, if we imagine the visual areas of the two hemispheres to be joined together (with a little overlapping) at their mesial surface to form a single hemispherical cup of the size of the retina, and if we then imagine the two retine to be placed one inside the other in their natural position in contact with the cerebral cup thus formed. All corresponiting points, both of the two retinæ and of the cerebral visual area, will be in contact with one another (Fig. 347).

If we compare the scheme thus represented with that which is given by $\mathrm{H}$.

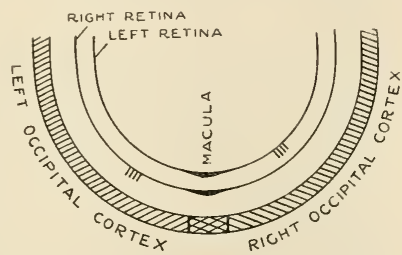

Fig. 347.-Diagram to indicate the corresponding points of the two retine and of the two halves of the visual cortex.

Munk for the dog (Fig. 345), we observe that the main point of difference is that Munk locates the focal point of each visual area at the apex of the occipital lobe. In accordance with this, he finds that destruction of this point on both sides causes loss of central vision. Whether this is the ease with dogs or not I camnot certainly say, but my experiments upon monkeys do not agree with this conclusion, nor does it find support from observations upon man. On the other hand, there is abundant clinical evidence to show-

1. That lesions of the occipital lobe produce disturbances of vision which are invariably of a hemiopic character.

2 . That lesions of the mesial surface of the lohe in the immediate neighbourhood of the calcarine fissure are those which for their size produce the most serious visual disturbances. On the other hand, lesions of parts near to but not involving these parts of the occipital lobe have not infrequently heen associated with defective appreciation of visual ohjects, and particularly with word-blindness.

3. Perimetric observations in man show that in cases of hemiopia, produced by lesions of the cortex (of one hemisphere), the line of demarcation between the blind and functional parts of the retina usually passes, not through the middle of the fovea but on the homonymous side; in other words, the forea is not involved in the hemiopic condition.

The visual area in man appears to be relatively less extensive than in the monkey. Séguin found that in by far the greater number of cases of hemiopia examined, the cortical lesion was in the neighbourhood 
of the cuneus. Monakow ${ }^{1}$ limits the visual area to the cuneus, the lingual lobule, and (probably) the two occipital gyri. The cuneus and lingual lobule are especially conneeted with the corpus geniculatum laterale, from which they receive fibres which run in the ventral part of the medullary centre of the occipital lobe; on the other hand, fibres pass from the cells of the visual area by way of the optic radiations to the superior corpora quadrigemina. All parts of the macula lutea are in man, according to Monakow, represented in both hemispheres, and over the whole visual cortex; hence, if the whole visual area of one hemisphere is destroyed, or even if the optic tract is completely degenerated, the macula lutea is not completely blind in any part.

According to Henschen, ${ }^{2}$ the cortical centre for vision in man (inclusive of colour vision) lies exclusively in the grey matter bounding the calcarine fissure, ${ }^{3}$ the upper lip of that fissure being connected with the upper quadrant of the retina, the lower lip with the lower quadrant, the macula lutea with the most anterior part of the area, and each macula being represented in both visual areas. Henschen describes the "visual" fibres of the optic tract as ending exclusively in the lateral geniculate body, the destruction of which produces hemiopia. From this fibres run in the ventral part of the optic radiations of Gratiolet to the cortex at the calcarine fissure. The remainder of the occipital cortex and the angular gyrus are considered by Henschen to be probably connected with special visual apperceptions, as is instanced by wordblindness accompanying lesions of these parts. ${ }^{4}$

Flechsig ${ }^{5}$ has shown that in man the fibres of the optic nerve become myelinated later than those of most of the other senses, viz. in the course of the first month of post-uterine life. Tracing them backwards, he finds the fibres of the optic tract passing directly to the lateral geniculate body, and thence to

${ }^{1}$ Arch. f. Psychiat., Berlin, 1891, Bd. xxiii. S. 609 ; 1892, Bd. xxiv. S. 229.

2 "Klin. u. anat. Beitr. z. Pathol. des Gehirns," Ujsala, 1890-94 ; and Brain, London, 1893, p. 170 ; "Sur les centres opt. cérébraux," Internat. Med. Cong., Rome, 1894.

3 The grey matter of the calcarine fissure, together with the immediately adjacent parts of the cuneus and lingual lobule, is characterised by the development of a stratum of white fibres in the middle of its thickness, which is known as the band of Gemmari, as well as by the thickness of the molecular laver, and the appearance of a very large number of stellate nerve cells, which form a layer of considerable thickness in conncetion with the band of Gennari. This layer corresponds with the laycr of granules in other parts of the cortex. This part has been tcrmed by Henschen the "cerebral retina"; he supposes that visual implessions are projected and reccived here, but are perceived elsewhere in the occipital lobe, and perhaps also in the parietal lobe. The structure of the visual cortex in man has been recently dealt with in a special treatise by Ramón y Cajal ("Die Schrinde," Leipzig, 1900), who draws especial attention to the presence of many stellate cells in the granule layer having descending instead of ascending axons. The fibres of the stripe of Gennari constitute, in all probability, according to Cajal, the eventual endings of the optic nerve fibres.

${ }^{4}$ See further, on visual representation in the cortex, Gowers, "Diseases of the Nervous Systcm," vol. ii. p. 142 ; Obersteiner, "Anatomy of Central Nervous Organs," p. 284 ; Exner, "Untersuch. u. d. Local. der Functionen in d. Grosshirnrinde des Menschen," Wien, 1881 ; Allen Starr, Am. Jonm. Med. Sc., Phila., 1884, vol. lxxxvii. p. 65 ; Ségnin, Arch. de neurol., Paris, 1886, p. 176; Nothnagcl and Naunyn, "Ucber die Localisation der Gehirnkrankheiten," Wicsbaden, 1887; Hun, Am. Journ. IIcd. Sc., Phila., 1887; Delépine, Trans. Path. Soc. London, 1890, p. 2 ; J. Soury, "Les fonctions du cerveau," 1892 ; and Rev. phil., Paris, 1895 and 1896 (see an account by Miss F. E. Welby in Brain, London, vol. xix. p. 432) : also article "Cerveau" in Richet's "Dict. de physiol.," 1897, pp. 898-952; Sachs, "Das Hinterhauptslappen," Leipzig, 1892 ; Donaldson, Am. Journ. Psychol., 1892, vol. iv. No. 4; Ewens, Brain, London, 1893, vol. xvi. 1. 475 ; Brissaud, Ann. d'ocul., Pruxelles, 1893, tomc cx. p. 321 : Vialet, "Les centres cérébraux de la vision, etc., "Paris, 1893; and Arch. d'ophth., Paris, tome xiii. p. 422; Holden, Arch.f. Ophth., Leipzig, Oct. 1896 ; Sharkey, Lance', London, 1897, vol. i. p. 1399; W. Harris, Brain, London, 1897 ; vol. xx.

5 " Localisation der geistigen Vorgänge," Leipzig, 1896. 
the superior corpora quadrigemina, but none directly to the thalamus. From the lateral geniculate body a stout bundle passes to the pulvinar thalami, but the fibres for the most part pass through this to join the optic radiations of Gratiolet and to pass in these to the margins of the calcarine fissure. Other fibres of the optic radiations, the origin of which is somewhat uncertain, spread out into the whole of the cuneus and lingual lobule, and extend over the posterior pole of the hemisphere to the basal surface (occipito-temporal region). No fibres of the optic radiations go to the gyrus angularis, which is therefore entirely excluded by Flechsig from the visual area of the cortex.

Steiner found that in the cat and rabbit the visual area does not begin to respond to electrical excitation until about the fourteenth or fifteenth day after birth, whereas response is obtained from the Rolandic area about the tenth day. In the $\log$ the response of the visual area is obtained still later (thirtieth to fortieth day). It is noteworthy, in connection with this observation, that puppies do not appear to recognise objects by sight until after about the twenty-third day after birth, and do not appear to recognise objects the images of which fall on the periphery of the retina until about the thirty-fourth day. ${ }^{1}$ It would appear from the observations of Raehlmann upon infants, that these do not recognise objects the images of which fall upon the centre of the retina until the fifth week after birth, and those which fall upon the periphery of the retina not until the fifth month.

The view has been taken by Ferrier, as the result of his experiments, that while the occipital lobe may be concerned with visual perceptions due to nervous impulses derived from the homonymous part of the same retina, the angular gyrus is especially connected with central vision of the opposite eye; and this view has been adopted by several other clinicians. His statement that blindness of the opposite eye, of a transient character, is caused by destruction of that gyrus, has, however, been confirmed only by one other observer, namely, Lannegrace, ${ }^{2}$ and is denied by all others who have experimented upon the subject; nor has it been shown in any animals with bilateral vision that lesion of one hemisphere only is productive of complete blindness of the opposite eye or of loss of central vision. It is true that cases of amblyopia, involving one eye only, accompanying cerebral lesions, are not unknown clinically, but they are not specially connected with lesions localised to the angular gyrus, and the symptom is always combined with anæsthesia of the globe. ${ }^{3}$ It appears certain that monocular defects of vision, when not due to injury of the optic nerve, are caused by a disturbance in the nutrition of the retinal elements (perhaps produced indirectly by an alteration in the blood supply), and are on a par with the defects in taste and smell which are brought about by lesions of the trigeminal nerve. ${ }^{4}$ The monocular defects which are observed in cases of functional or hysterical amblyopia may, according to Knies, ${ }^{5}$ be due to vasomotor disturbances (vaso-dilatation) affecting the conduction of the fibres of the optic nerve, where these pass through the foramen opticum. That the disturbance in these cases is not retinal, is shown by the fact that the ophthalmoscopic image is normal.

In progressive pathological lesions of the occipital lobe, loss or affection of

${ }^{1}$ Steiner, Sitzungsb. d. k. Akad. d. Wissensch., Wien, 1895, S. 303.

2 Arch. de méd. expér. et l'anat. path., Paris, 1889.

3 v. Bechterew, Neurol. Centralbl., Leipzig, 1894, S. 252 and 297.

${ }^{4}$ Defective vision in the opposite eye has been experimentally obtained by section of the ascending root of the fifth nerve in the medulla oblongata, and is aseribed by $\mathrm{v}$. Bechterew to vasomotor and trophic disturbance of the retinal elements (loc. cit.).

${ }^{5}$ Neurol. Centralbl., Leipzig, 1893, S. 570. 
colour vision upon the homonymous side of the two retinæ may precede the hemiopia; but it has not yet been determined that colour vision is dependent upon the integrity of a different part of the cortex than appreciation of form.

Occasionally hallucinations-in perfectly sane subjects-are present in hemiopia, the sensations originating on the blind side of the brain. In these cases either the lesion producing hemiopia is subcortical, or if cortical must be assumed to be incomplete. Such unilateral hallucinations, with or without hemiopia, are also sometimes seen in attacks of hemicrania. ${ }^{1}$

Cases of localised bilateral lesions of the internal surface of the occipital lobes, producing complete blindness, have been published by various observers. ${ }^{2}$ In some of these cases both lobes were affected simultaneously, in others first one lobe was affected, and a condition of hemiopia was produced, and then after an interval of time the other lobe, the condition being converted into double hemiopia or total blindness. In Forster's case central vision was still possible, although of a very imperfect character; it was found by Sachs, who made the examination of the brain, that a small portion of the grey matter of the cortex bounding the right calcarine fissure had not been involved in the destruction; but whether this was sufficient to account for the persistence of some central vision, cannot be definitely affirmed, especially since there have been several other cases of double hemianopsia in which some amount of central vision has persisted. $^{3}$ In this patient colour vision was absent, although the appreciation of form persisted, as shown by the fact that he could recognise common objects and even read and write. There was, however, a marked loss of memory of locality, and lack of power to appreciate the relative positions of objects, such as the position of countries and places on a map, the position and direction of the paths in a garden, or even the position of furniture and accustomed objects in his own room; although there was no such loss of memory of position of objects the appreciation of which was ordinarily determined by the sense of touch, such as parts of his own person, clothing, etc. In fact, although this patient still had some central vision, he was able to find his way about with far less facility than persons who are totally blind from destruction of the peripheral visual organs, and whose other senses have become sharpened by disuse of the visual sense. An animal (monkey, dog) rendered blind by removal of the occipital lobes is unable for an indefinite time to find his way freely about even the most familiar place without bumping against objects, and shows a great disinclination to move about; whereas it is a familiar fact that an animal which is blind in consequence of a peripheral lesion, speedily accommodates itself to the altered circumstances in which it is situated, and has no difficulty in finding its way about places with which it is familiar (H. Munk). Sachs ${ }^{4}$ attempts to explain some of the phenomena which were observed in Forster's case, by supposing that the appreciation of the quality of light coming from objects is effected in a manner different from that of the form and relative position of objects, that the perception of form and relative position involves motion of the eyes, and is associated with sensations produced by such motion, and that this perception is localised in other parts of the occipital lobes than those concerned in the appreciation of colour. Wilbrand finds it necessary, in consideration of various phenomena which have been observed clinically, to assume three centres of visualisation - one for the mere appreciation of luminosity, one for appreciation of colour, and one for appreciation of form and relative position of objects (space); he supposes that these may all be situated in the same region of the brain, but in different layers of the cortex. Holden ${ }^{5}$ noticed that partial loss of

1 J. Soury, article "Cervean," Richet's "Dict. de physiol.," p. 919.

2 Bouverct, Rev. gén. d'opht., Paris, 30th November 1897 ; Oulmont, Gaz. hebd. de méd. Paris, 1889, p. 607; Chauffard, Rev. de méd., Paris, 1888, p. 131; Forster, Arch. f. Ophth., Leipzig, 1890, Bd. i. S. 94 ; Sachs, Arb. «. d. psych. Klin. zu Breslau, Leipzig, 1895, S. 53 ; Dejerine and Vialet, Compt. rend. Soe. de biol., Paris, 1893, p. 983.

${ }^{3}$ See Soury, article "Cerveau," loc. cit., p. 949.

${ }^{5}$ Arch. f. Ophth., Leipzig, 1896. 
conductivity in the optic tract led to partial colour blindness, combined with a diminution of the power of appreciating the relative intensity of light, and interprets this observation as indicating the non-existence of a separate centre for colour appreciation. This interpretation cannot, however, be conceded, for the results may be explained by assuming that the nervous impulses for these qualities are conducted along different nerve fibres, which are differently affected by the conditions disturbing their conductivity - a phenomenon well known to occur in other mixed nerves. There is not sufficient evidence as yet to enable an authoritative opinion to be pronounced upon this matter. It has been suggested that the fact that in unilateral lesions of the cortex central vision is often unaffected, may be explained by supposing that the vascular conditions of the part of the cortex which is connected with the macula are more favourable, so that this particular portion of the centre is more resistant to pathological changes than the rest, without the necessity of assuming that the whole macula is represented upon both sides of the brain, but there is no evidence in favour of this suggestion. ${ }^{1}$

The auditory area of the cerebral cortex.-It was originally stated by Ferrier, ${ }^{2}$ and the statement has been confirmed by all subsequent observers, that stimulation in the monkey of the upper two-thirds of the inferior temporal convolution causes pricking of the opposite ear, turning of the head and eyes to the opposite side, and dilatation of the pupils. ${ }^{3}$ This was taken by Ferrier to indicate that this convolution is connected with the function of hearing (by the opposite ear), and in conformity with this view he found that if it was destroyed on one side, and the ear of the same side was stopped with wax, the animal was rendered deaf; and that if it was destroyed on both sides the animal became absolutely and permanently deaf. Ferrier therefore concluded that this gyrus, and this alone, is the cerebral seat of the auditory function.

I have elsewhere, however, shown that this deduction of Ferrier was founded upon an insufficient basis. ${ }^{4}$ On repeating his experiments, in conjunction with Sanger Brown, it was found that monkeys entirely deprived of the superior temporal gyri on both sides, and even of a very considerable additional amount of the temporal lobe, are by no means deaf, but still show signs of hearing quite distinctly, and of understanding the nature of the sounds heard." Even in the case upon which Ferrier chiefly relied in support of his opinion, the notes which he has published show that the monkey undoubtedly reacted to sounds. ${ }^{6}$

It is certain, therefore, that the function of sound-perception is not entirely localised to the superior temporal gyrus, although the focal point for it is probably there. Luciani and Tamburini ${ }^{7}$ extended it to the second temporal gyrus, because excitation of the upper end of this also gives the head-and eye-movement. H. Munk, chiefly from experiments on $\operatorname{logs}{ }^{8}$ ascribes the function to the whole of the temporal lobe. Luciani, ${ }^{9}$ in a later communication, says: "Our results tend to show

1 W. Harris, Brain, London, 1897, p. 307.

2 Proc. Roy. Soc. London, 1875.

${ }^{3}$ I have myself generally got retraction of the ear instead of pricking. Baginsky (with H. Munk) obtained raising of eyelids combined with movements of the opposite ear on stimulating the lowernost point of the temporal lobe in the log (Neurol. Centralbl., Leipzig, 1890 , Bd. ix. S. 458). Steiner (op. cit.) in the ralibit also obtainerl movement of the opposite ear, on excitation of the same part of the temporal lobe.

${ }^{4}$ Brain, London, 1888, vol. x. p. 362.

${ }^{5}$ Sanger Brown and Schäfer, Phil. Trans., London, 1888, B., p. 303.

${ }^{6}$ Brain, London, 1888, vol. xi. 1. 14 et seq.

7 "Sulle funzione del cervello ; seconda communicazione," 1879.

${ }^{8}$ Ges. Abhandl., Mitth. 4, S. 63.

9 "On Sensorial Localisations," etc., Brain, London, 1884, vol. vii. p. 195. 
that not only the whole cortical area of the temporal lobe, but probably also the cornu ammonis, forms an integral portion of the auditory sphere." With bilateral extirpation (in dogs) "at first the auditory troubles may amount to absolute deafness, but soon pass into a condition of obtuseness of hearing." In five monkeys the superior temporal gyrus was destroyed or removed on both sides by Sanger Brown and myself, and in one both temporal lobes were completely removed. In the last case there was produced for a time a condition of idiocy, dne probably to the extent of the lesion, but in none of the cases was hearing abolished; whether it was impaired it is quite impossible to say, since even normal monkeys will frequently take no notice of noises. ${ }^{1}$ At any rate these experiments, as well as those of Luciani, afford conclusive
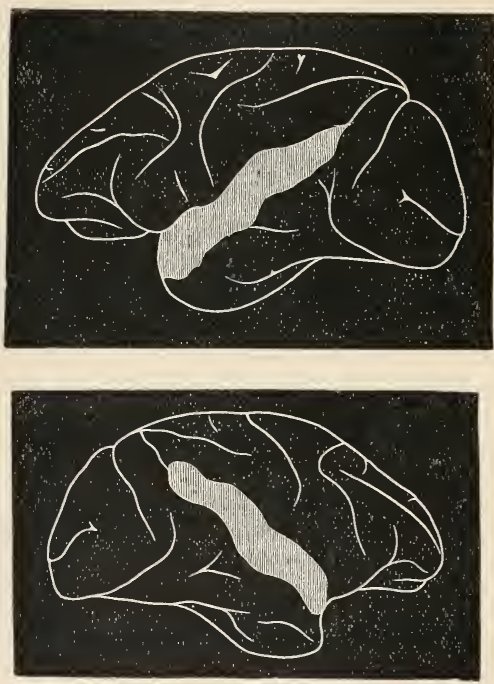

Fig. 348.-Diagrams of braiu of a monkey, showing complete removal of the superior temporal gyrus on both sides. Even when this was effected the animal retained a delicate sense of hearing. evidence that in animals the function of auditory perception is not entirely confined to the temporal lobe, although it has not been experimentally determined into what other portions of the cortex it extends. That its focal point is to be found in the superior temporal gyrus, and especially the upper part of that gyrus and the gyrus transversus anterior (which is concealed within the fissure of Sylvius, and is very early in making its appearance in man, Heschl), is probable, partly from the result of electrical excitation and partly from the observations of Flechsig (see below) on the time of myelination of the fibres; and also as the result of clinical and pathological evidence in man; in whom cerebral lesions productive of disturbances of auditory perception are generally found closely associated with the neighbourhood of the superior temporal gyrus. ${ }^{2}$

It has been shown by v. Monakow ${ }^{3}$ that the cortex of the temporal lobe, and especially of the first gyrus, is directly united with the mesial geniculate body, which again is connected directly with the inferior corpus quadrigeminum, and

${ }^{1}$ Since the publication of our experiments, Ferrier has given an account of a monkey on which he had performed the same operation, namely, removal of the superior temporal gyrus on both sides. Although he found that for a few days it appeared to take no notice of sounds, yet after a time (two or three weeks) it showed recovery of the sense of hearing, and even showed appreciation of the meaning of certain sounds, which it responded to by characteristic cries. It is therefore impossible to suppose that audition is localised to this gyrus in moukeys.

2 Ferrier, "Croonian Lectures," 1890, p. 80.

${ }^{3}$ Arch.f. Psychiat., Berlin, Bd. xxvii. S. 428 . 
this again by the lateral fillet and by some fibres of the reticular formation with the cochlear nerve. Flechsig 1 finds that in the ehild of two months, fibres are found undergoing myelination within the temporal lobe, connecting the mesial geniculate body almost exclusively with the transverse convolutions of the temporal lobe which are concealed within the Sylvian fissure. He regards these as the only corticipetal fibres concerned with audition, and is on this account disposed to locate the auditory area of the cortex in man exclusively in this situation, although apparently willing to admit that corticifugal (motor) fibres, set in activity by auditory impressions, nay emerge from a larger area. He describes a bundle of such fibres as passing from the temporal lobe by way of the lateral fourth of the crusta to the pons Varolii (temporo-pontine tract), and regards these as motor, carrying impulses for the movements of the ears, head, and trunk. The opinion that the fibres in question are respectively corticipetal or corticifugal rests, however, wholly upon the fact that those regarded as centripetal are developed at a relatively earlier period than the others. According to Held, although most of the fibres of the cochlear (auditory) nerve are interrupted in the dorsal and ventral auditory nuclei, the superior olivary nuclei of the same side, and the nuclei of the trapezium, of the fillet, and of the inferior quadrigeminal body of the opposite side, some fibres may pass directly to the cortex of the temporal lobe of the opposite side. ${ }^{2}$

Localisation within the auditory area.-Clinical evidence appears to point to the fact that the appreciation of words heard is intimately associated with the integrity of the first temporal gyrus of the left side, numerous cases having been recorded of "word-deafness" resulting from lesions of this convolution, especially of its posterior two-thirds. ${ }^{3}$ Luciani and Sepilli ${ }^{4}$ collected twenty cases of word-deafness, and of these fourteen were associated with lesions more or less extensive of the first and second temporal gyri on the left side of the brain. ${ }^{5}$

Munk states that in dogs in which the anterior part of the auditory area is destroyed there is deafness for tones of high pitch, while destruction of the posterior part of the area is attended by deafness to tones of low pitch. These statements are confirmed and amplified by v. Bechterew and Larionoff, ${ }^{6}$ who divide the auditory area into three zones, for high, medium, and low-pitched notes respectively. But the difficulties of such experiments are so great, that it would be wise to suspend one's judgment before accepting them as evidences of intra-areal localisation.

The olfactory and gustatory areas of the cerebral cortex.As Ferrier remarks: "The position of the olfactory centre may with great probability be inferred from anatomical considerations alone, apart from actual experiment." If with Broca we divide animals into those with a highly developed olfactory sense (osmatics, including the great majority of mammals) and those with feebly developed olfactory sense (anosmatics, including the monkey, man, and a few amphibious or marine mammals), we find that the parts of the brain which vary in development directly with this sense are-(1) The olfactory lobe (olfactory bulb and tract); (2) the posterior portion of the inferior surface of the frontal lobe; (3) the anterior portion of the hippocampal region, including the uncus, the

1 “Localisation der geistigen Vorgänge," ete., 1896.

2 H. Held, Arch.f. Anat. $u$. Entweklngsgeseh., Leipzig, 1893, S. 201.

${ }^{3}$ Naunyn, Verhandl. d. vi. Cong. f. immere Med., Wiesbaden, 1887, S. 132.

4 Quoted by Laquer (Neurol. Centralbl., Leipzig, J888, S. 337), who also gives the details of a well-observed ease in which the lesion was strictly limited to the first gyrus. For other cases bearing on this subject, sce Ferrier, "Cerebral Localisation," 1890.

${ }^{5}$ Manouvier found the riglit superior temporal gyrus to be small in a man who was from infancy deaf in the left ear (Bull. Soc. de psychol. physiol., Paris, 1890, tome v.). See also a case related by C. K. Mills in Brain, London, 1891, vol. xiv. p. 465.

${ }^{6}$ Areh. $f$. Physiol., Leipzig, 1899, S. 391. 
hippocampal gyrus, the hippocampus major, and the dentate convolution $^{1}$ (but not the amygdala); (4) a convolution termed the "bordering gyrus," ${ }^{2}$ which closely encircles the corpus callosum, and is connected at both ends with the olfactory lobe. Very little of this convolution is present in primates, the dorsal part only being represented by the "nerves of Lancisi," and the ventral part by the lamina of the septum lucidum, the peduncle of the corpus callosum, the fornix, and the fimbria; (5) the anterior part of the callosal convolution (gyrus fornicatus) $;^{3}$ (6) the anterior commissure.

The main connection of the olfactory nerve fibres is with the cortex of the same side of the brain. On physiological grounds, it appears to be doubtful whether there is any crossed connection between the two olfactory lobes, but anatomically such has been affirmed through the medium of some of the fibres of the anterior commissure (Meynert). This commissure is stated to be chiefly a means of connection between the hippocampal gyri, especially their anterior parts, and it also appears to connect the olfactory tracts with one another. ${ }^{4}$ According to the observations of Flechsig, ${ }^{5}$ the nerve fibres of the olfactory tract, which begin to be myelinated towards the end of the ninth month of foetal life, pass (1) partly to the whole of the hinder border of the base of the frontal lobe and to the front part of the gyrus fornicatus; $(2)$ partly to the uncus and to the adjacent inner pole of the temporal lobe, these two regions coming together below the island of Reil. From the frontal olfactory region, fibres pass by way of the stria tecta to the middle segment of the gyrus fornicatus, others into the septum lucidum, and then by way of the cingulum to the cornu ammonis. From the temporal olfactory region a strand of association fibres also goes into the cornu ammonis. From both regions, tracts (corticifugal) pass to the globus pallidus of the lenticular nucleus and to the thalamus.

Rámon y Cajal ${ }^{6}$ has traced fibres of the external auditory root, which arise as axons of the mitral cells of the olfactory bulb, into the uncus, where they terminate by arborising in the grey matter. Cajella ${ }^{7}$ describes the fibres of the mesial root as passing to end in the trigonum olfactorium by arborising amongst peculiar groups of pyramidal cells which are found in this region, and to which the name "olfactory islands" has been applied. According to the observations of Elliot Smith in monotremes, ${ }^{8}$ the axons of the mitral cells pass partly direct to the fimbria and fascia dentata, arborising amongst the cells of the fascia dentata (the axons of these cells ultimately go to the hippocampus); partly to the septum lucidum and peduncle of corpus callosum (gyrus subcallosus of Zuckerkandl; precommissural area of Elliot Smith), where they

${ }^{1}$ Hill, Phil. Trans., London, 1893, vol. elxxxiv. p. 389, states that in anosmatics the fascia dentata varies directly with the development of the olfactory organs.

2 Sec Quain's "Anatomy," vol. iii. pt. 1 ; also Zuckerkandl, "Ueber das Riechcentrum," Stuttgart, 1887.

3 There is, however, as Broca pointed out, an exception in the case of Cetacea, "in which, although the sense of smell is rudimentary or absent, the anterior portion of the callosal convolution is specially well developed" (Ferrier, "Functions of the Brain," p. 314). According to Ferrier, it is only the uncinate (anterior) portion of the hippocampal gyrus (hippocampal lobule) the development of which is correlated with that of the sense of smell. In man and in the dolphin (both anosmatics) the hippocampus is comparatively well developed. In reptiles, which have a strong olfactory sense, the cerebrum is almost entirely rejresented by the hippocanılal region (Edinger, Anat. Anz., Jena, 1893, Bd. viii. S. 305).

${ }_{4}$ Ganser, Arch. f. Psychiat., Berlin, 1878, Bd. ix. See also Dana, "On the central tracts of the olfactory nerves," New York Med. Journ., 1889.

5 "Die Localisation der geistigen Vorginge insbesondere der Sinnesempfindungen," Leipzig, 1896.

7 "Neue Darstellung vom histol. Bau des Centralnervensystems."

7 "La region ol fatoria," Madrid, 1893.

${ }^{8}$ Anet. Anz., Jena, 1895, Bd. x. S. 470. 
arborise around the dendrons of the pyramidal cells, the axons of which also then pass to the hippocampus to end in arborisations amongst the dendrons of its pyramidal cells (Fig. 349). If this be so, both the gyrus subcallosus and the fascia dentata are receptive areas of the second order for olfactory impressions.

Broca ${ }^{1}$ was of opinion that the whole of the limbic lobe (consisting of olfactory lobe, hippocampal lobe, and lobe of the corpus callosum) is related to the sense of snell, although he fully admitted the probability of its having also another unknown fumction, as shown by its development in some anosmatics. It has an intimate connection with the frontal lobe, and in Broca's opinion this last-named lobe owes its supposed importance as a special organ of intelligence to its connection with the olfactory region; since in most

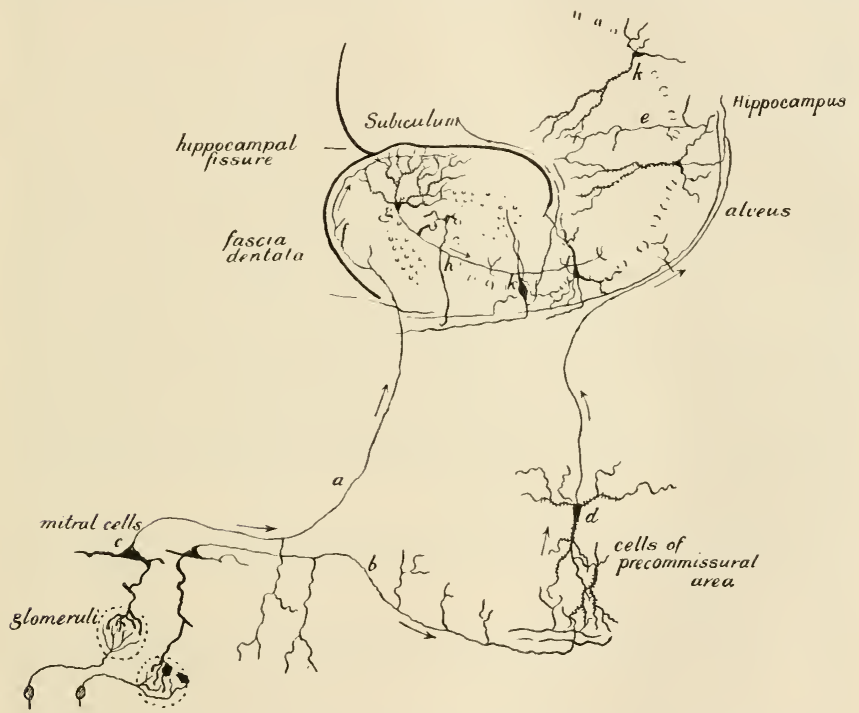

FIG. 349.-Diagram to show the connections of the olfactory organ with the hippocampal region.-Elliot Smith.

mammals the chief impressions derived from the external world are obtained through the medium of the olfactory sense. ${ }^{2}$

Ferrier found that " the effects of electrical irritation of the hippocampal lobule in the monkey and other animals are such as may be most reasonably interpreted as being indicative of subjective olfactory

1 "Mém. sur le cerveau," Paris, 1888. Broca's distinction of these regions of the brain (rhinencephalon, Turner) from all the rest of the cortex (pallium) has been followed by all subsequent writers (Schwalbe, "Neurologie" ; Zuckerkandl, "Ueber das Riechcentrum," 1887 ; Turner, Journ. Anat. and Physiol., London, vol. xxv. ; Retzius, "Das Menschenhirn," Stockholm, 1897. The rhinencephalon is phylogenically and ontogenically the oldest, and in many vertebrates remains throughout life the most important part of the cortex.

${ }^{2}$ P. Broca, "Local, cérébrales." "Rech. sur les centres olfactifs," "Mémoires," 1888, pp. $424,429$. 
sensation, namely, a peculiar torsion of the lip and nostril on the same side. Occasionally, however, the reaction is bilateral, and especially so in the rabbit. The reaction is that actually induced in these animals by the application of an irritant, such as a pungent odour, directly to the nostril. .. . But while we can be more or less certain, from the outward reactions, that subjective olfaction is aroused by stimulation, it is an exceedingly difficult problem to determine whether smell is lost by destruction of the same region." 1 In Ferrier's earlier experiments by this method, which were admittedly not very exact, he found, with destruction of the lower temporal regions on both sides, indications of impairment or abolition of the senses of smell and taste. In monkeys operated upon by myself, in conjunction with Sanger Brown, ${ }^{2}$ we were unable to detect such impairment, even when the whole of both temporal lobes was removed. We did not, it is true, remove the hippocampus major as well, and in some animals small pieces of the hippocampal convolution were left. Nevertheless, in spite of the very extensive lesion in this region, the animals unquestionably gave very distinct indications of still possessing both smell and taste. Ferrier has since repeated the operation upon another monkey, and obtained evidence of the loss of both senses for a period of about two months, after which time both senses appeared gradually to indergo recovery. ${ }^{3}$ There is, therefore, not complete localisation of those senses to these parts of the brain. Munk ${ }^{4}$ has recorded the case of a dog, rendered blind by destruction of the occipital cortex, which seemed also to have lost the sense of smell, and in which it was found post-mortem that the whole of the hippocampal gyrus upon each side was converted into a thin-walled cyst. Luciani ${ }^{5}$ got no evident deficiency of smell after extirpation of the temporal lobe in dogs, but found olfactory disorders to follow lesions of the gyrus hippocampi and of the hippocampus major combined.

A case of tumour in the right gyrus hippocampi has been recorded by Hughlings Jackson and Beevor, in which the patient suffered from subjective sensations of smell. ${ }^{6}$

It is probable, for various reasons, that the centre for the sense of taste is anatomically in close association with the olfactory centre, but neither experiments on animals nor clinico-pathological observations in man have thrown much light upon its localisation. Ferrier got occasionally movements of the lips, tongue, and jaws in the monkey and cat, on electrical excitation of the lower extremity of the temporal region, ${ }^{7}$ and interprets this as indicative of the calling up of subjective sensations of taste.

The localisation of tactile sensibility.-Ferrier was led by the results of his experiments upon the hippocampal region of the hemisphere, ${ }^{8}$ to the conclusion that tactile sensibility is in every case impaired or abolished, in proportion to the destruction of the hippocampal and inferior temporal region. It was, however, found by

1 "Croonian Lectures," 1890, p. $119 . \quad 2$ " Phil. Trans., London, 1888.

3 "Croonian Lectures," 1894. “ “Die Funetionen der Grosshirnrinde," S. 130.

5 Brain, London, 1885, vol. vii. p. 145. p. 126 .

., 1889, vol. xii. p. 340 . For other cases, see Ferrier, "Cerebral Localisation,"

${ }^{7}$ Flechsig has supposed, but without presenting any convincing evidence, that the sense of taste is connected with the anterior part of the gyrus fornicatus.

${ }^{8}$ Ferrier, Phil. Trans., London, 1875, pt. 2; Ferrier and Yeo, ibid., 1884, pt. 2. 
Horsley and myself that extensive lesions might be made in the hippocampus major without producing hemianæsthesia, although in other cases, when the lesion was more extensive and involved the greater part of the hippocampal gyrus, and a large part of the adjacent under surface of the temporal lobe, hemianæsthesia was very marked. The same result was obtained on destruction of the gyrus fornicatus, so that we were led to the conclusion that these convolutions, which form the greater part of the limbic lobe (especially the gyrus fornicatus) were the seat of perception of cutaneous sensations, and especially of tactile sensibility. Our experiments upon the gyrus fornicatus were confirmed by Munk, ${ }^{1}$ who was, however, of opinion that the anrsthesia is the effect of unavoidable injury to the neighbouring Rolandic (motor) region of the cortex, in which alone he localises tactile perceptions. Neither did we ourselves, however, nor Ferrier, ever obtain anæsthesia on directly injuring the motor cortex solely. Nevertheless, it must be borne in mind that in endeavouring to excise such deeply lying portions of the cortex as the gyrus fornicatus and hippocampal gyrus, it is necessary to raise up or draw aside the whole mass of the brain, and it is possible that the hemianesthesia we obtained in the experiments upon the hippocampus and gyrus fornicatus were due not to the actual injury to those parts, but to a general disturbance in the functions of the whole hemisphere (perhaps of the optic thalamus), which the manipulation of the brain might produce.

In order to test this point, I have recently ${ }^{2}$ performed experiments on the monkey in the following manner:- In a preliminary operation, the whole of the leg area of the cortex, together with the greater part of the remainder of the marginal convolution, was severed by a sharp knife passed vertically into the cerebral substance parallel with the longitudinal fissure. The only symptom produced was motor paralysis of the opposite leg; no anxsthesia could be detected; the animal responded, after the first day or two, to the slightest touch, both on the paralysed and on the non-paralysed side. After two or three weeks the brain was again exposed, and a cut was made along the same line as before, but deep enough to sever the fibres passing to and from the gyrus fornicatus. No additional symptoms whatever followed this second operation, although in two cases at least, from one-third to one-half of the gyrus fornicatus was severed from the rest of the brain. The experiment was, in one case, varied by removing the gyrus marginalis altogether in the first operation, and scooping away the middle part of the gyrus fornicatus in the second, but the result was the same.

These experiments are not conclusive, because the destruction of the gyrus fornicatus was not by any means complete, but, so far as they go, they are not in favour of this convolution being regarded as especially the seat of tactile sensibility; at least they tend to show that sensation is not localised to it. It is, however, possible that the whole of the limbic lobe, with the exception of the antero-inferior parts (which are probably devoted to smell and taste) may be the seat of cutaneous perception. It is natural to expect the senses of smell and taste to be in relation with that of cutaneous sensibility, since the organs of smell and taste are modifications of integumental structures. It has already been shown that the view of Munk, that tactile sensibility of the

${ }^{1}$ Sitzungsb. d. k. Akad. d. Wissensch. zu Berlin, 1892.

2 Journ. Physiol., Cambridge and London, 1898, vol. xxiii. p. 310. 
various parts is located in the motor areas of the Rolandic region, cannot be substantiated. This no doubt lands us in the unsatisfactory position that we are unable certainly to say in what part we are to localise cutaneous sensibility, or even if it is localised at all in the cortex. This difticulty may, however, arise from the great extent of cortex to which the tactile projection fibres are distributed. It is, I think, quite certain that the sensory projection of any part of the general surface of the body is not localised, as Munk teaches and as is believed by many neurologists, to the part of the Rolandic cortex stimulation of which

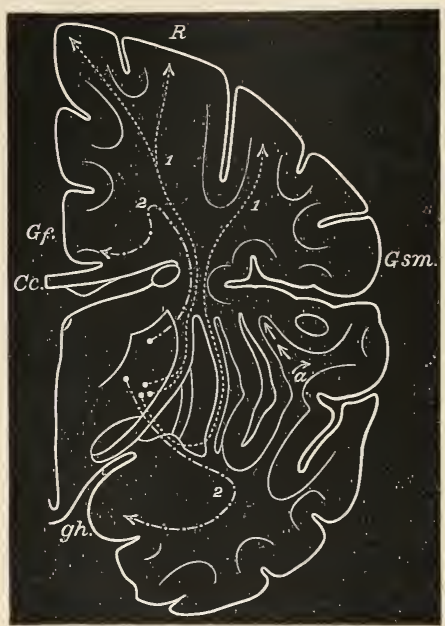

Fig. 350.-Diagram of a frontal section through the brain of a new-born ehild, to show the comrse and destination of fibres from the optic thalamus to the cortex. $C c$, Corpus callosum ; $G f$, gyrus fornicatus ; $g h$, gyrus hippoeampi ; Gsm, gyrus supraniarginalis; $R$, Rolandic region; 1 , 1 , fibres belonging to the first system; 2,2 , fibres belonging to the second system; $a$, fibres passing to the superior and transverse temporal gyri.-Flechsig. month of foetal life) are situated in the part of the internal capsule immediately behind the pyramidal fibres, and pass almost entirely to the two central or Rolandic gyri (but some go to the visual sphere); the second (Fig. 350, 2, 2), which appear about a month after the first, go partly to the Rolandic region, partly to the gyrus fornicatus and the cornu ammonis (the latter by way of the cingulum),

${ }^{1}$ Journ. Physiol., Cambridge and London, 1894, vol. xv. p. 469.

${ }^{2}$ Arch. f. Psychiat., Berlin, Bde. xiv., xvi., xxiii., xxv., xxvii.

3 Compt. rend. Soc. de biol., Paris, 1897 , p. 178.

4 "Die Localisation der geistigen Vorgänge insbesondere der Sinnesempfindungen des Menschen," Leipzig, 1896. 
and partly to the gyrus hippocampi; the third (which appear from one to several months after birth) go partly to the third frontal gyrus (by way of the fasciculus subcallosus), partly into the frontal lobe, almost to its apex; not ending here, however, but bending sharply backwards to the middle third of the gyrus fornicatus, to the anterior part of the first frontal gyrus, and to the foot of the second frontal gyrus. Almost all these fibres come from the lateral nucleus of the thalamus opticus, which constitutes, in Flechsig's opinion, a special station on the course of the afferent tracts towards the cortex, and which receives from below the chief part of the fillet layer, the superior peduncle of the cerebellum, the longitudinal bundles of the reticular formation, and part of the posterior longitudinal bundle. ${ }^{1}$ It appears obvious that these three systems, which are developed at considerable intervals from one another, cannot be regarded as having an identity of function, although it is not easy, in the present state of our knowledge, to indicate exactly what their several purposes may be. The fibres of the first system being distributed almost exclusively to the motor regions of the cortex, and becoming developed very early (a month before birth), may perhaps be regarded as excito-motor fibres for cerebral reflexes; they perhaps represent the fibres which convey impressions of muscular sense. Those of the second system, which are developed at about the time of birth, may well be those for the conveyance of cutaneous perceptions; their existence affords strong confirmation of the view that the limbic lobe is intimately connected with this sensory function. ${ }^{2}$ It is still more difficult to conjecture the special function of the third system of Flechsig, which is developed so much later than the others, and the myelination of which apparently extends over a considerable period of time, but it may perhaps be constituted merely by reinforcing fibres for the other two systems. ${ }^{3}$

\section{The Inexcitable Regions of the Cortex. Association Areas} of Flechsig.

It will be seen that if we mark off upon the surface of the cerebrum the areas from which a response to stimulation is obtainable, and to which we can with some probability ascribe a definitely localised function, whether motor or sensory, a large proportion-relatively much larger in man than in monkey, ${ }^{4}$ and becoming progressively smaller in descending the scale of intelligence in mammals-remains over. With regard to those portions of the surface which for the sake of brevity we may

1 According to Flechsig and Hösel, part of the main or upper fillet goes directly into the internal capsule and is connected with the central gyri, but this statement has not been confirmed by any other observer. Mott, after lesions of the fillet in the monkey, could trace no degencration beyond the thalamus (Brain, London, 1895, vol. xviii. p. 1).

2 There are also a certain number of published clinical cases which tend to favour the view that the limbic lobe, and espccially the gyrus fornicatus, is especially rclated to tactile sensibility (see Savill, Brain, London, 1891, vol. xiv. p. 270, and ibid., vol. xv. p. 448), but the importance of this evidence is diminished by the fact that extensive lesions of the gyrus fornicatus, which do not involve important neighbouring structurcs, are practically unknown.

${ }^{3}$ Flechisig appears to regard all these systems of fibres as connected with cutaneous sensibility, and considers that their distribution indicates exactly the extent of the area for cutaneous sensibility, which he accordingly locates in the several regions to which they pass, namely, the Rolandic region (including the two central gyri, the gyrus marginalis, and the bases of the first, sccond, and third frontal gyri), the gyrus fornicatus, cornu ammonis, and part of the gyrus hippocampi.

${ }^{4}$ Amounting, according to Flechsig, to two-thirds of the whole cortex in man.

VOL. II. -49 
speak of as the inexcitable or latent portions, since they are distinguished from the rest by the fact that their excitation produces no obvious response, and their excision no definite paralysis, their function is at present a matter of conjecture. ${ }^{1}$ What appears clear is, that any

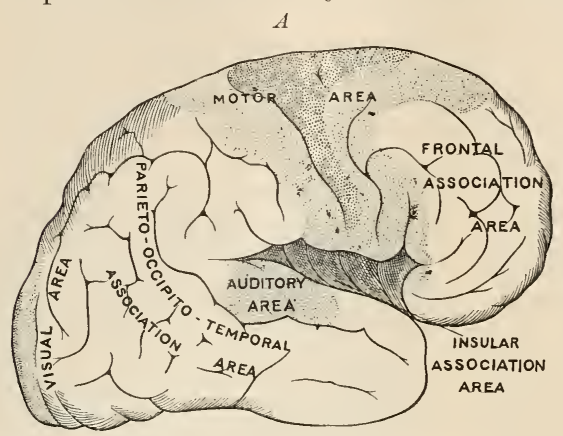

extensive lesion, especially bilateral, of these portions, leads to alterations in the intellectual condition of the patient, alterations which appear to vary directly with the amount destroyed. ${ }^{2}$ These inexcitable portions of brain surfaceare chietly massed in the prefrontal, in the post-parietal, in the temporal, and in man in the occipital, regions; to which

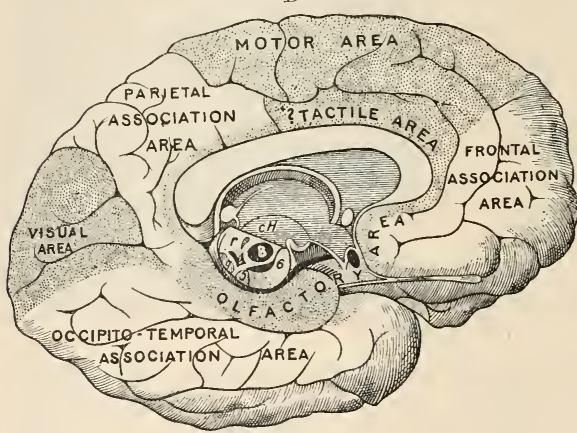
must be added the cortex of the island of Reil.

According to Flechsig, the fibres of the corona radiata of all these parts become myelinated far later than those of the excitable areas, and very few of the fibrespassdownwards as projection fibres, ${ }^{3}$ but nearly all belong to that class of internuncial fibres which were termed association fibres by Meynert, ${ }^{4}$ from the fact

Fic. 351.-Diagrams to sliow the supposed division of the cerebral cortex into projection and association areas, the former being shaded witli dots. A, lateral ; B, mesial surface.-Modified from Flechsig.

${ }^{1}$ An anatomical distinction hetween the excitable and latent areas consists in the fact that whereas the latter all belong to the five-layered cortical type of Meynert, the excitable regions, and to these must be arlded the gyrus fornicatus and hippocampal lobe, all possess, either in addition to or in substitution for the usual five layers of cells, ccrtain peculiarities of structure, c.g. the giant cells of the Rolandic area, the small stellate cells of the visual area, the special features of the lippocampal region, etc.

${ }^{2}$ It nust, however, be observed that this statement is probably true of the excitable regions also.

${ }^{3}$ Dejerine, however, finds that the prefroutal lobe is united to the internal nucleus of the thalamus by numerous fibres (Compt. rend. Soe. de biol., Paris, 1897, p. 178); and other authorities have come to a similar conclusion ("Versl. Deutsch. Naturf. u. Aerzte," Neurol. Centralbl., Leipzig, 1896, S. 995). Flechsig, ibid., 1897, S. 290, finds that the internal nucleus of the thalamus is developed proportionally with the development of the so-called association centres.

"Article "Brain" in Stricker's "Handbook"; also "Psychiatrie," Wien, 1884, and "Neue Studien neber die Associationsbundeln' des Hirnmantels," Sitzungsb. $d$. $k$. Akad. d. Wissensch., Wien, 1892, Bd. ci. 
that they connect either neighbouring or more distant parts of the cortex.

Most of the association fibres are comparatively short, but there are some well-known long tracts of this description, such as the supcrior longitudinal bundle passing between the Rolandic region and the parieto-occipital region; the perpendicular bundle passing between the parietal lobule and the temporo-occipital region; the anterior association bundle connecting the frontal and temporal lobes and passing across the bottom of the Sylvian fissure; and the inferior association bundle connecting the temporal and occipital lobes and the fibres of the fornix and cingulum (see Fig. 352).

According to Flechsig, the association fibres, which are both centrifugal and centripetal, serve largely to connect the inexcitable or association

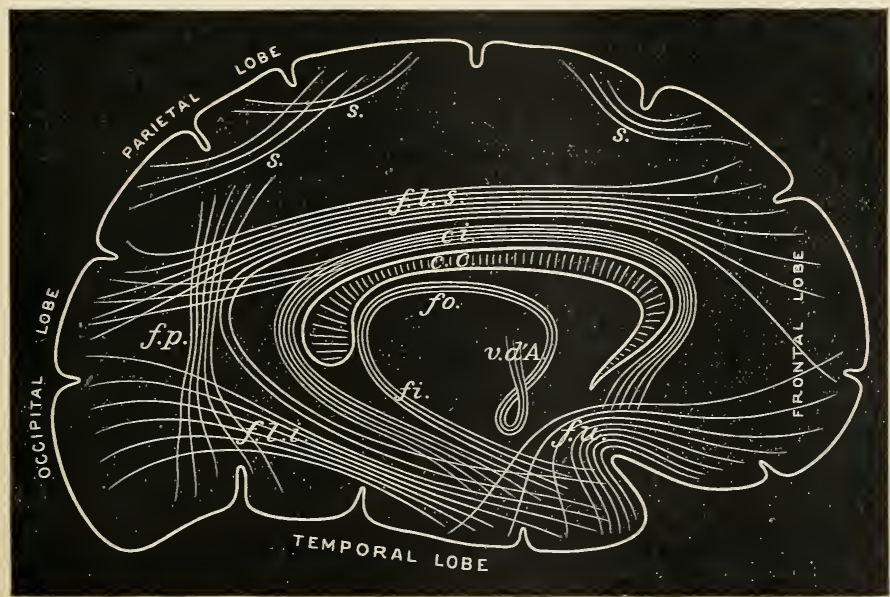

Fic. 352.-Diagram of the association fibres of the cerebral hemisphere. s., short fibres, connecting adjacent gyri ; f.l.s., fasciculus longitudinalis superior; f.l.i., fasciculus longitudinalis inferior; $f . u$., fascicnlus uncinatus ; ci., cingulum; f.p., fasciculus perpendicularis ; fo., fornix ; fi., fimbria ; v.d'A., bundle of Vieq d'Azyr.

areas with the excitable or projection areas, and through them also the various projection areas are indirectly interconnected and coagitated. Flechsig regards, therefore, these inexcitable regions of the cortex as constituting a great association centre, or rather three association centres, which he designates respectively the frontal, the paricto-occipitotemporal, and the insular centre, the chief function of these centres being to associate together impressions received from the adjacent sensory or projection areas. Dependent upon them is the due appreciation of the impressions recorded upon the several parts of the sensory cortex, and he also regards them as the seat of memory. ${ }^{1}$ Hence their lesion, although

${ }^{1}$ The portions of the association areas which lie immediately adjacent to the excitablc areas (which latter, according to Flechsig, are really sensory) are termed by Flechsig the "marginal zones." They receive from the excitable areas innumerable fibres of association, and according to Flechsig are concerned especially with the memory of sensory impressions. 
not productive of paralysis nor of any actual loss of sense, yet leads to inability to appreciate the impressions which are received through the sense organs, to loss of memory, and to a diminution of the intellectual faculties. $^{1}$ It has often been supposed that the frontal lobe is the special seat of the intellectual faculties $;^{2}$ chiefly on the ground that this lobe-at least the non-excitable anterior part (pre-frontal) -is much more developed in man than in the lower animals. But this is also true for the region which Flechsig terms the parieto-occipito-temporal association area $;^{3}$ and although cases in man are cited of lesions of the frontal lobes which have been followed by considerable mental and intellectual disturbance, this is equally a consequence of extensive lesions, especially bilateral lesions, of any part of the cortex. It is in fact more easy to produce a condition of semi-idiocy in monkeys from extensive bilateral lesions of the temporal lobes, than from complete severance of the prefrontal region; an operation which may indeed be effected without producing any very obvious symptoms. ${ }^{4}$

It is important, in effecting an extensive lesion of any part of the brain, to avoid if possible removing the cerebral substance, or interfering more than can be helped with its vascular connections. It is sufficient to cut off the part to be removed by a subcortical incision, so as completely to sever all its nervous connections with the rest of the brain, leaving the piece thus isolated in situ. In this manner much of the shock of the operation, and the mechanical and vascular disturbance of the whole hemisphere, which results from entire removal of a large piece of brain, and which may lead to erroneous conclusions being arrived at, ${ }^{5}$ are in some measure avoided. Operating in this way, I have in several cases completely removed in the monkey the whole of the inexcitable area of both frontal lobes without producing the slightest sign of the mental and intellectual dulness and alteration of character which has been regarded as pathognomonic of a lesion of this region. ${ }^{6}$ Many cases have also been described in man in which lesions of the frontal lobes have not been followed by any marked symptoms of this kind, and, on the other hand, they are apt to supervene with extensive bilateral lesions of other parts of the cortex. ${ }^{7}$

So much has been made of certain clinical cases in which an extensive lesion of the frontal lobes was followed by diminution of the intellectual

${ }^{1}$ For a criticism of Flechsig's views see Dejerine, Compt. rend. Soc. de biol., Paris, 1897, p. 178, who insists that numerous fibres pass from all parts of the cortex to the basal ganglia, or, in other words, that the association areas of Flechsig are in fact also projection areas. It must not be overlooked that the association of action of different parts of the cerebral cortex may equally well be carried out through fibres passing vertically in the corona radiata to and from the thalamus as by fibres passing tangentially from one portion of the cortex to another.

2 See below.

3 The parietal lobe, rather than the frontal, has been especially found to be developed in some men of genius. This has been the ease with great musicians, such as Bach and Beethoven, and also with men eminent in seience and philosophy, such as Döllinger, Gauss, Liebig, and Kant (ef. Ruidinger, "Ein Beitr. z. Anat. d. Affenspalte, u.s. w.," Bonn, 1882).

${ }^{4}$ Horsley aud Schäfer, Phil. Trans., London, 1888, B ; Schäfer, Trans. Internat. Cong. Psychol., London, 1893.

${ }^{5}$ Ferrier and Turner, for example, obtained on extirpation of the left frontal lobe in front of the precentral sulcus, deviation of both eyes to the left, paresis of the right arm and leg, left hemiopia and inpaiment of cntaneous sensibility over the whole of the right side, a dull, sleepy condition being also observed (Phil. Trans., London, 1898, B, 1) 365). It is impossible to suppose that all the functions which were impaired by the lesion had their seat in this lobe.

${ }^{6}$ Compare with these the results obtained by Bianchi, see Brain, London, 1895, vol. xviii. p. 498 ; also Grosglick, Arch. f. Physiol., Leipzig, 1895, S. 98. Such examples of a markedly contrary result are probably due to neglect of the above-mentioned considerations.

${ }^{7}$ Cf. Bruns, Nearol. Centralbl., Leipzig, 1898, S. 770, 848; Williamson, Brain, London, 1896 , vol. xix. p. 346 ; Bernhardt, "Symptomalogie u. Diagnostik der Hirugescliwiilste," Berlin, 1881 ; Oppenhein, "Die Geschwiilste des Gehims," Wien, 1896. 
faculties, and by a change for the worse in the general disposition of the individual, that it is important to ascertain what the clinical evidence on this point really amounts to. Welt ${ }^{1}$ has collected fifty-nine cases of lesions confined to the frontal region in man ; of these, forty-seven (or about 80 per cent.) showed no changes in intellectual capacity or in character ; and only twelve of the total number (or 20 per cent.) had such changes recorded against them. It is clear, therefore, that the doctrine of special localisation of the intellectual faculties in the latent or inexcitable portion of the frontal lobe rests on no sufficient basis.

We cannot, therefore, say that the intellectual faculties reside in this part more than in another; they are apparently bound up with the integrity of the whole organ. But what part of the changes of which they are the manifestation take place in the "sensory areas," what part in the "motor areas," and what part in what Flechsig has termed the " association areas," there is no evidence to show. That there is, however, some localisation in connection with the expression of intellectual processes in the association areas, is evidenced by the phenomena of aphasia to which attention will immediately be directed.

It must be borne in mind that although the inexcitable regions of the cortex yield no obvious result on stimulation, at least in the direction of the production of movement, they may nevertheless be concerned in the production of inhibitory phenomena, which are no less important in neural physiology than the phenomena of movement. For, after they are extirpated, and especially is this said to be the case with the prefrontal lobes, the time and intensity of spinal reflexes is stated to become shortened; while, on the other hand, stimulation of these regions, and especially of the prefrontal, is said, in the dog, to cause prolongation of latency and diminution in intensity of spinal reflex responses. ${ }^{2}$ It is further stated that these inhibitory influences travel both by the lateral and the anterior columns of the cord. Fano found that much more influence was exerted upon the reflex movements of the anterior than of the posterior limb, but that strong induced currents had in any case to be employed to obtain the inhibitory results. Very little effect was got by stimulating the occipital region, and none from the parieto-temporal. Neither ablation nor excitation of the motor region appeared to influence the spinal reflexes. This last statement appears improbable, and there can be little doubt that before drawing inferences of a general nature from these experiments they should be repeated, with every precaution to avoid fallacy, upon the monkey. ${ }^{3}$

It is noteworthy, confining our attention to the primates, although the statement appears to be one of general applicability to mammals, that as we ascend the scale of organisation we find the projection areas (both sensory and motor) of the cortex becoming relatively smaller and more concentrated, and the non-excitable ${ }^{\circ}$ or association areas occupying a larger proportion of the surface. This concentration is very obvious if we compare the Rolandic or motor areas of the monkey with those of the anthropoid ape and of man (Figs. 336,339 , and 340 ), and it applies not only to the whole motor area, but also to its individual components. It is also manifested in the auditory projection area,

1 Deutsches Arch. f. Klin. Med., Leipzig, 1888, Brl. xlii. S. 339.

${ }^{2}$ Fano, Atti d. r. Accal. d. Lincei, Roma, 1895, tomo ecxcii., Rendiconti, p. 115 ; Oddi, ibid., p. 118 ; Libertini, Arch. per le se. med., Torino, 1895, tomo xix.

${ }^{3}$ Goltz had long previously noted that dogs with the anterior half or fronto-parietal regions of the brain bilaterally removed, became much more excitable and restless, and shower a complete absence of self-control and an exaggeration of many reflex movements ; but there was of course much more than the prefrontal lobes lacking in these cases. 
which in man appears, as the result of clinical and anatomical observation, to be confined to the upper part of the first temporal gyrus and the transverse temporal gyrus, whereas in the monkey it certainly extends far beyond this limit (indeed, extirpation of the whole of both temporal lobes is not in the monkey sufficient to produce complete deafness). And the same principle is illustrated in the ease of the visual projection area, which in man appears, from clinical evidence, to be confined to the convolutions bordering on the calcarine fissure; while in the monkey it undoubtedly extends over the whole of the occipital lobe, which is relatively large in this animal. As regards the olfactory sense, the peripheral organ is so insignificant in all the primates that it is not to be expected that the cortical projection would be at all extensive in any. We have not sufficient knowledge of the exact localisation of the centres for taste and touch to draw a similar comparison.

\section{Aphasia.}

This term ${ }^{1}$ is used to express not only a loss or defect of articulate language, but also "a loss or defect in symbolising relations of things in any way." ${ }_{2}$ It occurs as the result of lesions upon the left side of the brain, and especially of the base of the third left frontal convolution. Three degrees may be distinguished, namely - (1) Defect of speech (partial aphasia); (2) loss of speech (eomplete aphasia); (3) loss of gesture language, i.e. of pantomime (amimia) as well as of speech (asemasia, ${ }^{3}$ Hamilton). In complete aphasia the patient does not speak intelligibly. He can only utter words without sense, or mere jargon.

Motor aphasia may show itself simply as a defect in or complete loss of utterance of spoken words (aphemia ${ }^{4}$ ), or simply as a defect in or complete loss of the ability to write intelligibly (agraphia), or as both these conditions combined.

The patient may understand what is said or read to him, and remember things told him. "This is important, for it proves that although speechless, the patient is not wordless." Apart from speech his articulatory and vocal organs may be unaffected. He may be able to sing. He smiles, laughs, frowns, and varies his voice so as to express emotions correctly. Although he has lost speech, he has not lost the images of things; he will point to any object which we name, and recognises drawings of objects. He may retain the propositional use of "yes" and "no," and either by these or by pantomime may intelligently assent or dissent to simple statements; he may have the interjectional or emotional use of certain words, such as oaths, which are uttered under appropriate circuinstances, but cannot be repeated at will; in rare cases a proposition is stated or a question is asked intelligibly, but always under the influence of emotion. Some of these instances "show that there is not only retention of some words but of some speech, by the right side of the brain." In point of fact, as Gowers insists, "the left hemisphere has by no means a monopoly of speech function. The right hemisphere contains structures of similar position and similar connections. These structures can supplement those in the left hemisphere. Loss of speech due to permanent destruction of the speech region in the left hemisphere, has been recovered from; and that this recovery was due to the

' á and фáaıs, speech.

${ }^{2}$ Hughlings Jackson, Brain, London, 1873, vol. i. 1. 311. The quotations and descriptions which follow are chiefly from the same source.

${ }^{3}$ From $\alpha$ and $\sigma \eta \mu a i \nu \omega$, an inability to indicate by signs or language.

${ }^{4} a$ and $\phi \eta \mu i$, I speak. 
supplemental action of the corresponding region of the right hemisphere, is proved by the fact that in some of these cases speech has been again lost when a fresh lesion occurred in this part of the right hemisphere. This supplemental action occur's in the sensory as well as in the notor functions. It occurs far more readily in children than in adults . . Hence it is probable that speech processes go on more equally in the two hemispheres in childhood than they do in adult life. It is also highly probable that there are individual differences in this respect among adults." 1 Two processes are involved in speech as in other forms of volitional expression, the one involving sensory, the other motor regions of the cortex. Thus the idea of words may be aroused through the senses of hearing, sight, or touch, and the parts of the brain where these perceptions occur are connected with the speech centre proper, which is an association centre, situated mainly if not entirely in the posterior part of the third left frontal convolution. ${ }^{2}$ Accordingly, we may have aphasia of certain kinds from inability to express words heard or words seen, produced by lesions in the sensory areas ("word - deafness," "word - blindness"). This is spoken of as "sensory aphasia" (Wernicke). Such forms of aphasia occur from lesions of the temporal region (especially the superior gyrus), and of the occipital region respectively, or both motor and sensory aphasia may be combined.

There may be inability to recollect words, to revive the word images (verbal amnesia). Patients with this affection can repeat words after you when bidden, which is not possible in cases of so-called motor aphasia.

Further, lesions producing aphasia or amnesia may occur in the fibres connecting the several centres ${ }^{3}$ - sensory and motor; this is illustrated by the

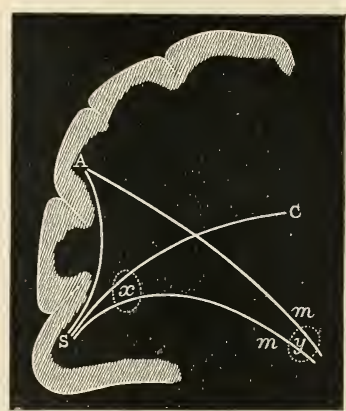

Fig. 353.-Diagram to show the manner in which lesions of internuncial fibres may produce aphasia.-Modified fronı Gowers. appended diagram (from Gowers), in which $S$ is the speech centre; $A$ the centre for movements of the hand; $S C$ fibres from the speech centre to the corpus callosun and opposite hemisphere; $S m, A m$, fibres to the internal capsule. A lesion at $x$ must cause permanent aphasia, since it involves both the fibres to the internal capsule and those to the corpus callosum, but would not cause agraphia (inability to write intelligibly), for impulses can still pass from $S$ to $A$; one at $y$ might cause only transient aphasia, the impulses from $S$ being able to pass through the corpus callosum to the opposite speech region.

There is abundant evidence that amnesia and even complete aphasia may be produced by lesions of the cortex of the left hemisphere elsewhere than in Broca's convolution. Thus, as has already been stated, ${ }^{4}$

\footnotetext{
${ }^{1}$ Gowers, "Lectures on the Diagnosis of Diseases of the Brain," London, 1885, p. 125. See a case reported by T. Barlow, Brit. Med. Journ., Loudon, 28th July 1877.

2 Broca, "Sur la siège de la faculté de langage articulé," Bull. Soc. anat. de Paris, 1861 and 1863 .

${ }^{3}$ De Watteville, Progrès méd., Paris, Mars 1875.

${ }^{4}$ Page 763. Cf. Hitzig, Arch. f. Psychiat., Berlin, 1884, Bd. xv. S. 274.
} 
it has been found that lesions of the temporal region, especially of the first and second temporal gyri and of the gyrus supramarginalis, are causative of the condition known as word deafness, ${ }^{1}$ i.e. words although heard are not understood, not " perceived," nor can words be recalled to memory. An individual with such a defect is unable to speak or to write intelligibly, although, if the visual centre is intact, he might be able to comprehend written language. And, as Bastian has pointed out, a lesion in the association fibres which connect the auditory word centre with the speech centre, would be equally productive of the same condition. ${ }^{2}$

Similarly, word-blindness (alexia) has been found to result from lesions in or near the left occipital region, written language becoming unintelligible, although spoken language may be comprehended. There is in these cases necessarily a condition of agraphia. Further, in the production of such acts as reading aloud or writing from dictation, both the visual and the auditory word centres as well as Broca's centre and the motor centres of the left hemisphere must all come into action, and a lesion of any one of these or of the association fibres connecting them one with another must be fatal to the proper performance of these actions. The cortex of the island of Reil of the left hemisphere has been supposed by Meynert to be a special association centre for language; it is better developed in the primates than in other animals, and has very few callosal fibres uniting it with the cortex of the opposite island.

Sensory aphasia may show itself as a defect in or loss of recollection of words, especially of names of people or things, although the idea of the thing is represented in consciousness (verbal amnesia), or it may show itself as word-blindness or word-deafness, or as a combination of two or more of these conditions. ${ }^{3}$

\section{The Connections of the Cortical Centres with one Another AND With THE LOWER Centres.}

\section{Connections of the frontal lobe and Rolandic area.-The Rolandic}

1 Wernicke, "Der aphasische Symptomencomplex," 1874. Bastian appears to liave been the first to point out that apliasia may result from disturbance in an auditory centre of the cortex ("On the Various Forms of Loss of Speech in Cerebral Disease," 1869), although the position of snch centre was at that time unknown, and its very existence jurely hypotlietical.

2 It is held by Herbert Spencer and by Bastian that words are revived in the cerebral hemispheres, as remembered sounds or visual impressions. This view appears more probable than that of Wundt and Bain, which considers that words are revived as faint impressions of the processes occurring in motor cells during their articulation.

3 The subject of apliasia is far too large and the literature mucl too voluminous to be dealt with here otherwise than in the briefest possible manner. The student is referred, for an account of all the varieties which occur and a full discussion of the conditions under which the different forms are produced, to the works of Bastian, "On the Various Forms of Loss of Speech, etc.," 1869; "Brain as an Organ of Mind," "Paralyses, Cerebral, Bulbar, and Spinal," London, 1886, and especially, "On Aphasia," ete., 1898 ; Wernicke, "Der aphasische Symptomencomplex," Breslau, 1874, and Fortschr. d. Med., Berlin, Bde. iii. and iv.; Ogle, St. George's Hosp. Rep., London, vol. ii. p. 83 ; Kussmaul, Ziemssen's "Cyclopxedia," London, 1877, and "Störungen der Sprache," 1885 ; Lichtheim, "On Aphasia," Brain, London, 1885, vol. vii.; Grashey, Areh.f. Psychiat., Berlin, 1885, Bd. xvi. S. 654. ; J. Ross, "On Apliasia," London, 1887 (which includes a brief and elear account of the various views that have been taken to explain the several forms of aphasia); Gowers, "Diseases of the Brain," London, 1893; Charcot, reported by Marie in Progrès méd., Paris, 1888, No. 5 ; Allen Starr, Brain, London, 1889, vol. xii. p. 82 ; Bateman, "On Aphasia," London, 1890 ; Dejerine, Compt. rend. Soc. de biol., Paris, 1891 ; Shaw, Brain, London, 1893, vol. xvi. p. 492 ; Byrom Bramwell, "Morison Lectures," Edinburgh, 1900. In several of these works the principal literature of the subject will be found referred to. 
(motor) areas of the cortex are connected with the mesencephalic, the pontine, the bulbar, and the spinal motor centres by means of the fibres of the pyramidal tract, which constitute the projection fibres of this portion of the cortex. These fibres take origin in some of the cells of the motor cortex-perhaps in the "giant cells," which were described there by Betz and by Bevan Lewis - and pass in the corona radiata directly to the middle two-thirds of the internal capsule. This middle two-thirds is the only part of the capsule which is directly excitable by artificial means; the anterior and posterior sixths being apparently inexcitable. The anterior sixth is largely composed of fibres which come from the prefrontal region of the hemisphere, while the posterior sixth appears to be chiefly composed of afferent (sensory) fibres, which probably have their origin for the most part in the thalamus. As the fibres of the pyramidal tract course downwards through the internal capsule they show a tendency to be arranged in such a way that the fibres which come from the region for the head and eyes and from that subserving movements of the tongue, are chiefly accumulated in the anterior half of the excitable region; those from the face, from the upper limb, from the trunk, and from the lower limb in the posterior half. ${ }^{1}$ There is, however, as the study of the detailed results obtained by Beevor and Horsley shows, ${ }^{2}$ a considerable overlapping of the representation of the several parts above enumerated, and this overlapping and intermingling tends to increase as the fibres are traced downwards, and becomes even more marked in the crusta. Here such arrangement as can be detected by localised stimulation shows that the order of fibres from within out is also face, upper limb, trunk, lower limb. ${ }^{3}$ The intermingling which obtains is strikingly exemplified by the results of degeneration after localised lesions of the cortex, since after lesions which are strictly limited to one of the excitable areas of the Rolandic cortex, degenerated fibres are found scattered over almost the whole of the excitable part of the capsule (although more numerous in some parts than in others), and over the whole of the excitable part of the crusta. ${ }^{4}$ Diagrams, therefore, representing the excitable (motor) part of the internal capsule, as divided up into segments, each of which contains fibres subserving only the movement of a particular part of the body, convey an erroneous impression of the actual distribution of the pyramidal fibres in the capsule. All that can be definitely stated is, that there is a greater accumulation of the fibres subserving the movement of this or that part in one section than in the others.

The pyramidal fibres, after arising from the cortical cells of the Rolandic area, first give off collaterals which pass into the adjacent grey matter, and perhaps others which pass into the corpus callosum, and are then continued into the internal capsule, the middle part of the crusta, and the pyramid bundles of the pons, to be collected in the medulla oblongata into the distinct masses which are known as the pyramids. Many fibres belonging to the pyramidal system end in the substantia nigra and in the grey matter of the pons and medulla oblongata. Arrived at the lower part of the medulla oblongata, the fibres of the pyramids enter the spinal cord in three tracts. By far the larger number pass directly at the decussation of the pyramids into the opposite lateral column of the spinal cord, forming the crossed lateral pyramidal fibres. A certain number pass into the lateral tract of the same side of the cord, ${ }^{5}$ and become intermingled with the fibres of the crossed

${ }^{1}$ François-Franck and Pitres, Compt. rend. Soc. de biol., Paris, 1877 ; Bcevor and Horsley, Phil. Trans., London, 1890.

2 See especially Figs. A to R, plates 5 to 9, Phil. Trans., London, 1890, B.

3 Beevor and Horsley, "Proc. Physiol. Cong.," Journ. Physiol., Cambridge and Londlon, 1898, vol. xxiii.

${ }^{4}$ Cf. Mellus, Proc. Roy. Soc. London, 1895, vol. Iviii. p. 206.

${ }^{5}$ François-Franck and Pitres, Progrès méd., Paris, 1880; Schäfer, Journ. Physiol., Cambridge and London, 1883, vol. iv.; Muratolf, Arch. f. Anat. u. Entucklngsyesch., 
pyramidal tract which have been derived from the opposite pyramid of the medulla oblongata (uncrossed lateral pyramidal filres). And lastly, a certain number remain in the anterior or ventral part of the cord, and are continued down for a variable distance in the anterior column of the sane side close to the anterior median fissure (uncrossed ventral pyramidal fibres). This direct or ventral pyramidal tract is not present as a distinct tract in the lower animals, not even in monkeys $;^{1}$ it is not known whether it occurs in the anthropoid apes. The fibres of the lateral pyramidal tract terminate amongst the cells at the base of the posterior horn, in the neighbourhood of the lateral horn, and amongst the cells of Clarke's column, ${ }^{2}$ not amongst the cells of the anterior horn as has generally been assumed.

Besides the connection with the lower centres, by means of the fibres which the pyraniids form, there appears to be another path of communication between the motor cortex and the nuclei of the motor nerves, by way of fibres which pass in the corona radiata and internal capsule to the nuclei of the pons, and perhaps to the substantia nigra, and thence by fibres which go either at once to the motor nuclei by way of the posterior longitudinal bundle and anterior descending tract of the cord, or indirectly with intercalation of the cerebellum. This second path of connection between the Rolandic area and the lower centres lias not, it is true, been absolutely proved, but its existence would be very probable if the statement of Starbinger ${ }^{3}$ is correct, that section of the pyramids (in the dog) produces no persistent loss of voluntary power over the muscles.

Cajal ${ }^{4}$ describes axons of many of the pyramidal cells of the frontal lobe as passing to the corpus striatum. After destruction of the frontal lobe in dogs and monkeys, Marinesco ${ }^{5}$ found numerous degenerated fibres in the corpus striatum and especially in the caudate nucleus. ${ }^{6}$ On the other hand, the corpus striatum receives numerous fibres from the thalamus and the subthalamic region, and probably sends out centrifugal fibres downwards, along the notor tract; but these have not hitherto been satisfactorily traced; some appear to go to the substantia nigra. The corpus striatum is generally believed to act as a centre for the ligher reflex movements, and to be in close association with the Rolandic area, but the experimental grounds for this belief are still lacking. ${ }^{7}$ Morphologically, the corpus striatum is regarded as a part of the cortex.

Corticipetal tracts pass, as shown by Flechsig, 8 to the Rolandic area from the lateral nucleus of the thalanus (see p. 768). Monakow further found that after extirpation of this part of the cortex in young animals, there was atrophy in the fillet and in the nuclei of the opposite posterior columns of the medulla oblongata. ${ }^{9} \quad$ Flechsig and Hösel ${ }^{10}$ state that part of the fibres of the

Berlin, 1893 ; Pitres, Aieh. de physiol. norm. et palh., Paris, 1894 ; Sherrington, Laneet, London, 1894, vol. i. ; Mellus, Proc. Roy. Soe. London, 1894, vol. lv. 1. 208; and 1895, vol. lviii. P. 206; Boyee, Phil. Trant., London, 1895, B, p. 321 ; Redlich, Neurol. Centralbl., Leipzig, 1897.

${ }^{1}$ Mellus (loc. eit.) oecasionally found a few degenerated fibres in the anterior column of the monkey, after lesions of the cortex.

2Sehäfer, "Proe. Phys. Soc.," Mareh 18, 1899, Journ. Physiol., Cambridge and London, vol. xxiv. Cf. Monakow, Arch.f. Psychiat., Berlin, 1895, Bd. xxvii.

${ }^{3}$ Jahrl. f. Psyehiat., Berlin, 1896, Bd. xv. My own experiments on the monkey have failed to eonfirm this statement.

${ }^{4}$ Cellule, Lierre et Louvain, 1892.

5 Compt. rend. Soe, de biol., Paris, 2 Fév. 1895.

${ }^{6}$ Cf. also on this subject H. Sachs, "Vorträge ü. d. Bau u. Thätigkeit d. Gehirns," Breslau, 1593 ; Seluukowski, Neurol. Centralbl., Leipzig, 1897, S. 524.

${ }^{7}$ Nothnagel, Virehow's Arehiv, 1873, Bde. lvii. and lviii.; and 1874, Bd. lx. ; Kïlliker, "Handbuch d. Gewebelehre," "Nervensystem," Leipzig, 1896, S. 622 ; Rezek, Jahrb.f. Psyehiat., Berlin, 1897, Bd. xvi. S. 40.

8 "Die Localisation, u.s.w."

${ }^{9}$ See also v. Bechterew, "Leitumgsbahnen," S. 158.

${ }_{10}$ Neurol. Centralbl., Leipzig: 1890, No. 14. 
fillet, and some perhaps also of the superior cerebellar peduncle, pass directly to the same area, chiefly to the ascending parietal gyrus, but this direct connection of fibres of the fillet with the cortex could not be found by Mott, and is also denied by Dejerine. ${ }^{l}$ The Rolandic areas are further commissurally counected with one another through the middle part of the corpus callosum, and by stimulation of these commissural fibres the grey matter of both sides can be simultaneously set in activity. ${ }^{2}$ If oue hemisphere be cut away and electrodes applied to the cut surface of the corpus callosum, the motor centres in the remaining hemisphere may be excited through the fibres of this commissure as readily as if the electrodes were applied directly to the grey matter, and the effects produced are similar, but not so easily isolated. That the movements which are produced by excitation of the callosal fibres are brought about through the cortex, and not by fibres which have been described as passing from the cortex of the one side across the corpus callosum directly to the internal capsule of the opposite side, ${ }^{3}$ is shown by the fact that they are not obtained if the Rolandic cortex is destroyed, although the connection between corpus callosum and internal capsule may not thereby be interfered with. Nor is degeneration set up in the opposite internal eapsule by removal of portions of the cortex of one hemisphere, although localised degenerations are thereby produced in the corpus callosum, and can be traced to the corresponding part of the Rolandic area of the opposite cortex. ${ }^{4}$ The fibres connecting the different motor centres of the two hemispheres through the corpus callosum, although massed together at certain places so as to give specific results on exciting these places, nevertheless show a certain amount of scattering, as can be ascertained by following the degenerations which result from localised cortical lesions (Sherrington). The corpus callosum also contains other fibres serving to connect the parts of the brain in front of and behind the Rolandic region. It is constituted, in fact, by a great mass of fibres, which must serve to effect an association of action between the two hemispheres. But the exact purpose of such association is by no means clear. Association of movement between the two sides can, and does, occur in the lower level centres, and although it is very probable that there is also association through the corpus callosum, it is nevertheless the case that this commissure can be completely divided in animals without any distinct symptoms being manifested. Many cases have been recorded of congenital absence of the corpus callosum in man. These are generally associated with a condition of greater or less idiocy, but this may be the result of a general lack of cerebral development, which usually accompanies absence of the commissure. In one or two cases of its complete absence, which have been published, no symptoms were noticed during life. ${ }^{5}$ The Rolandic area further receives numerous association fibres from other regions of the cortex.

The prefrontal (non-excitable) region of the cortex sends corticifugal fibres down in the anterior part of the internal capsule and in the mesial bundles of the crusta, to end in the grey matter of the nuclei pontis.

${ }^{1}$ Compt. rend. Soc. de biol., Paris, 1895, p. 285.

${ }^{2}$ Brown-Séquard, Compt. rend. Soc. de biol., Paris, 1879 ; Mott and Schäfer, Brain, London, 1890, vol. xiii. p. 174 .

${ }^{3}$ Hamilton, Proc. Roy. Soc. Loudon, 1884, p. 349. The fibres described by Hamilton appear to be really corticipetal fibres passing from the optic thalamus of one side across the corpus callosum, to the cortex of the oppositc side (Ferrier and Turner, Phil. T'rans., London, $1898, \mathrm{~B}, \mathrm{p} .31$ ).

+ Muratoff, A rch. f. Anat. u. Entwchlngsgesch., Leipzig, 1893, S. 97. Tschermak, however, found it to obtain in the cat, ibid., 1898, S. 291. But Mellus (Proc. Roy. Soe. Lomdon, 1895, vol. Iviii. p. 206) found in some cases, in the monkey, after lesions of the cortex of the one side, degenerated fibres in considerable numbers, running (presumably after having crossed by the corpus callosum) to the opposite internal eapsule.

${ }^{5}$ For abstracts of a large number of cases recorded by others, and an account of a case olserved by himself, see a paper by Alexander Bruce in Brain, London, 1889, vol. xii. p. 171 ; see also Dunn, Guy's Hosp. Rep., London, 1889, vol. xlvi. p. 117. 
According to Dejerine, ${ }^{1}$ lesions of this prefrontal region are also followed by degeneration in the stratum opticum of the thalamus, with subsequent atrophy of the mesial nucleus. Lesions of the parietal lobe and angular gyrus, on the other hand, cause, according to the same author, degenerations in the pulvinar and posterior part of the lateral nucleus. From the base of the third frontal convolution, and from the operculum, fibres pass into the mesial fasciculus of the crusta.

Connections of the visual area.-To the occipital lobe both corticifugal and corticipetal fibres can be traced. The corticifugal fibres pass chiefly to the pulvinar thalami and anterior quadrigeminal body of the same side, ${ }^{2}$ and are scattered as they pass from the cortex amongst the other fibres of the optic radiations. The occipital lobe also sends fibres to the crusta. ${ }^{3}$ The corticipetal fibres (optic radiations) arise from the lateral geniculate body, ${ }^{4}$ and also from the pulvinar thalami. They are traceable, according to Ferrier and Turner, not only to the occipital lobe, but also to the angular gyrus, whereas, according to Henschen and to Flechsig, they pass chiefly, if not entirely, to the cuneus and the lips of the calcarine fissure. M. and Mme. Dejerine have also described connections of the lingual and fusiform lobules with the posterior and inferior parts of the thalamus.

The occipital lobes are said to be commissurally connected with one another through the splenium of the corpus callosum $;^{5}$ according to Ferrier and Turner, the same statement applies to the angular gyri. The occipital lobe of one side is also connected with the temporal lobe of the opposite side by some of the fibres of the corpus callosum which form part of the tapetum (Sachs). There is, further, a well-marked tract of longitudinally coursing association fibres which passes forwards to connect the occipital with the frontal lobe, and shorter association fibres connecting it with nearer portions of the hemisphere.

The corticipetal tract for visual perceptions passes from the peripheral sense organs by way of the optic nerves and tracts to the corpora geniculata lateralia, thence as just stated by the optic radiations of Gratiolet to the visual area in the occipital lobes. According to Monakow, 80 per cent. of the fibres of the optic tract pass to the corpus geniculatum laterale and pulvinar, where they terninate, new fibres arising in these nuclei and continuing the optic path to the occipital lobe. Here these (corticipetal) fibres terminate mainly in the cuneus and the lips of the calcarine fissure. But, according to Flechsig, it is from the macula lutea that fibres pass-at least indirectly - to the calcarine fissure, not from the retina generally. ${ }^{6}$ v. Monakow has shown that extirpation of the occipital cortex in young animals leads to atrophy of the cells of the lateral geniculate body, whereas section of the optic tract does not affect the cells, but the grey matter in which they lie.

The manner in which the fibres pass from the lateral geniculate body to the visual cortex has been studied by Cajal, who finds that they are collected into a bundle situated at the upper and outer part of the cerebral peduncle, and continued into the internal capsule, whence they radiate into the occipital lobe ; here they are continued into the grey matter, where they appear to form the stripe of Gemnari. ${ }^{7}$ Other fibres of the optic tract go to the anterior corpora

1 Compt. rend. Soc. de biol., Paris, 2 Fév. 1897.

${ }^{2}$ v. Monakow, Arch. $f$. Psychiat., Berlin, Bde. xx., xxii.; Ferrier and Turner, Phil. Trans., London, 1898, B.

${ }^{3}$ Monakow, loc. cit. ; Dejerine, Compt. rend. Soc. de biol., Paris, 1893, p. 193.

" Flechsig, "Die Localisation der geistigen Vorgänge," Leipzig, 1896; Henschen, "Pathol. des Gehirns," Upsala, 1893.

${ }^{5}$ Dejerine, Compt. rend. Soc. de biol., Paris, 1892, p. 579; Vialet, "Les centres cérébraux de la vision," 1893.

6 "Gehirn und Scele," Leipzig, 1896.

7 Cajal, "Beitr. z. Studien des Med. Obl., u.s.w.," Leipzig, 1896 ; also "Die Sehrinde," Leipzig, 1900. 
quadrigemina; these probably chiefly serve to carry impulses for reflex movements of the eye.

Loss of the eyes in a new-born pup is followed by diminished development (atrophy) of the optic nerves and tracts, of the occipital lobes, of the superficial white matter of the anterior corpora quadrigemina, of the corpora geniculata lateralia, and of the pulvinares thalami, ${ }^{1}$ but not of the mesial corpora geniculata nor of the posterior corpora quadrigemina.

Connections of the auditory area.-Lesions of the first and second temporal gyrus are followed by descending degenerations in the part of the internal capsule which lies ventral to the lenticular nucleus and in the outermost part of the crusta. $^{2}$ These fibres terminate in the nuclei pontis. There are also commissural fibres passing by way of the corpus callosum to the temporal and to the occipital lobes of the opposite hemisphere (Sachs), and association fibres passing into the angular gyrus, and into the occipital lobe of the same hemisphere, and probably also into the Rolandic area and towards the speech centre. The course of these last has, however, not as yet been definitely traced. Ferrier and Turner found no degeneration in the posterior quadrigeminal bodies, nor in the fillet, after lesions of the first temporal gyrus ; v. Monakow, in experiments upon young animals, found atrophy of the mesial geniculate body to follow destruction of this convolution.

The corticipetal tract from the peripheral sense organ to the auditory area of the cortex, passes by the cochlear nerve to the auditory nucleus; other fibres arising thence probably terminate in the superior olive, the nucleus of the fillet, the posterior corpus quadrigeminum, and the mesial geniculate body of the opposite side; and others again, starting from these nuclei, carry the tract on to the cortex of the temporal lobe.

Connections of the olfactory region of the cortex.-The olfactory lobe (including the olfactory bulb and tract, and the part of the frontal lobe near the attachment of the olfactory tract) ${ }^{3}$ is connected with the two extremities of the limbic lobe. Fibres from the olfactory lobe pass by means of the outer root of the tract to the anterior part of the hippocampal gyrus, and other fibres have been traced from it into the fascia dentata. The inner root is connected with the anterior end of the gyrus fornicatus. Some of its fibres were described by Meynert as passing by way of the anterior commissure to the temporal lobe and hippocampal region of the opposite side, but the evidence of this "olfactory chiasnı" is insufficient. There is, however, as Ganser has shown, a commissural connection between the two olfactory regions of the cortex by means of the anterior commissure. These connections have been already noticed (see p. 764). There is, further, an important association bundle in connection with this region in the cingulum, which courses in the white matter of the gyrus fornicatus and gyrus hippocampi; its fibres probably connect those gyri with the cortex of the outer surface of the hemisphere (Beevor). ${ }^{4}$ Zuckerkandl ${ }^{5}$ has described a bundle of association fibres in the body of the fornix connecting the cornu ammonis with the temporal and olfactory lobes. ${ }^{6}$ The lippocampi of opposite sides are said to be connected commissurally by the "lyra." The fornix further carries fibres from the hippocampus to the corpus albicans. Most of these end here, but some are said to pass to the opposite side and to end in the grey matter of the third

${ }^{1}$ v. Gudden, Arch. f. Ophth., Leipzig, Bde. xx., xxi., xxv. ; Tagebl. $l .58$ Versamml. deutsch. Naturf. u. Aerzte in Strassburg, 1885 ; v. Monakow, Arch. f. Physiol., Leịzig, 1885, S. 329 ; Arch. f. Psychiat., Berlin, Bde. xiv., xvi., xx., xxiii., xxiv.

2 Zacher, Arch. f. Psychiat., Berlin, Bd. xxii. S. 654; Flechsig, op. cit.; Ferrier and Turner, op. cit.

3 See Quain's "Anatomy," vol. iii. pt. 1, p. 159.

4 Phil. Trans., London, 1891, B, p. 135.

5 " Das Riechbundel des Ammonshorns," A nat. Anz., Jena, 1888, Bd. iii. S. 15.

${ }^{6}$ See on the subject of the connections of the hippocampus with other parts, M. et Mine. Dejerine, Comipt. rend. Soc, de biol., Paris, 1897, 1. 587. 
ventricle. ${ }^{1}$ On the other hand, the axons from the cells of the corpus albicans (its mesial nucleus) bifurcate soon after leaving the cells, and while one set of branches pass by way of the bundle of Vicq d'Azyr to the anterior nucleus of the thalamus, the other set go to the tegmentum, and are ultimately traceable to the region of the trapezium in the pons Varolii; these form the pedunculomaxillary fasciculus of Rámon y Cajal, ${ }^{2}$ or the tegmental bundle of v.Gudden. From the lateral nucleus of the corpus albicans, other fibres, constituting the so-called peduncle of the corpus albicans, pass to the tegmentum, to end apparently in the central grey matter.

The hippocampus also sends fibres by way of the fornix and stria medullaris thalami to the nucleus habenulæ, whence other fibres arise and pass to the interpeduncular ganglion by way of Meynert's bundle (fasciculus retroflexus). ${ }^{3}$

${ }^{1}$ Kölliker, "Handbuch," 1896, Bd. ii.

2 "Beitr. z. Studien des Med. Obl., u.s.w.," 1896.

${ }^{3}$ D. S. Rámon, Aun. Soc. exper. de hist. natur., 1894. 


\title{
THE SPINAL CORD.
}

\author{
By C. S. Sherrington.
}

Contexts:-Reflex Action, p. 783-The Way In, p. 787-Recurrent Sensitivity, p. 788-The Way Out, p. 793-Relation of Afferent to Efferent Root Cell, p. 797-Reflex tonus, p. 799-Regeneration of Afferent Roots, 1. 804Functional Anatony of Mediate Spinal Cell-Systems, p. 801-The Spinal Grey Matter, p. 810-Reflex Capacity of Fragments of the Cord, p. 811The Spixal Aximal, p. 817-Rules of Spread in "Short" Reflexes, p. 820 -In Long Reflexes, p. 823-Influence of the Quality of the Stimulus, p. 825-.. Summation of Stinuli, p. 828-Intensity of the Stimulus, p. 828Character of the Muscular Response, p. 830-Influence of the Locus of the Stimulus, p. 832-"Local Sign" and Spinal Reflexes, p. 832-Fatality of Spinal Reflexes, p. 831-Influence of Condition of the Central Organ itself ; Vaseular Condition, p. 835-Drugs, p. 837-Influence of Associated Parts of the Central Organ ; Inhibition and "Bahnung," p. 837-Co-ordinate Character of the Movenents, p. 843-Phasic Tariation in Reflex Activity, p. 844Autotomy, p. 845-Spinal "Shock," p. 845-Difference between Skeletal and Visceral Musculature in regard to Shock, p. 817-Spinal Visceral Reflexes, p. 849-Urinary Bladder, p. 849-Defecation, p. 850-Sexual Spinal Reflexes, p. 851-Other Visceral Reflexes, p. 853-Reflexes affecting the Vascular Musculature, p. 854-Significance of spinal Reflexes, p. 856-SPINO-CEREBRAL AND Cerebro-Spinal Reactions, p. 860 - Conduction in the Cord, p. 861 -Spinal Traumatic Hyperæsthesia, p. 867-Toxic Functions of the CoRd, p. 868 Automatism, p. 869-"Knee-Jerk" and Allied Phenomena, p. 870-Tropнic Functions of the Cord, p. 875-Regeneration of the Spinal Cord, p. 878.

\section{Reflex Action.}

Spontaneously arising conversions of internal into external energy, such as characterise the life of certain cells, for instance those of cardiac muscle, are not apparent in the life of spinal cells. All purely spinal reactions seem reactions to environmental change. They are reflex in the sense that the environment is causally related to them, and that to the environment they return again. The initiation of some is less directly and demonstrably traceable from the enviromment than that of others. The former are by a convenient usage often termed "spontaneous." The latter or obviously reflex comprise for the most part the relatively simpler. The distinctions drawn between reflex and spontaneous reactions naturally alter from time to time. The tendency with increasing knowledge of physical and chemical agents has been for an increasing number of reactions to be included as reflex.

Among the properties of living matter (bioplasm), those which by their high development in the nervous system may be said to characterise the physiological qualities of that system, are excitability to certain kinds of stimuli, spatial transmission of the excited state, and ability to control the liberation of energy in tissues adjoint to them- 
selves. Hence the nervous system links together and co-ordinates the activities of the various organs. Its power of spatial transmission is technically known as its " conductivity."

In the physiological analysis of the reactions of the spinal system the unit reached is "the reflex." Upon it as basis our existing notion of the reactions of the nervous system is built. The whole neural system may be regarded as a superstructure resting upon two nerve cells, the two root cells, one afferent the other efferent. The afferent root cell is the alpha of every functional series of units in the system; the efferent root cell is the omega to which every series immediately or mediately leads. In every functional series of units there is a place or there are places where conduction involves change in quantity of the state of excitement transmitted, and alteration of its distribution both in space and time.

The places where such modification of the nerve impulses occur are spoken of as " nerve centres."

Stephen Hales first proved the spinal cord to be a seat of "reflection" as against other places where nerves seem to unite (for exaniple, the nerve plexuses). Grainger ${ }^{1}$ later indicated that in the spinal cord the grey matter is the seat of the reflection. It was then inferred that the essential element of the centre must be the nerve cell boly (perikaryon); later, an inextricable net of nerve cell branches was found surrounding and continuous with the bodies of the cells ( $\mathrm{J}$. Gerlach); to these together were attributed the reflecting and distributing properties of the nerve centre. More recently, from evidence that goes to show that the branches of the individual nerve cells do not anastomose into a continuum, but retain morphological individuality, although functionally linked, stress has been laid on the possible properties of the places of linkage or synapse. The places where nerve cells thus "hold hands" with each other are not necessarily near to the cell body, although usually so. It is as the, with few exceptions, exclusive seat of modification and redistribution of nerve impulses that brain and cord play such pre-eminent part in the functions of the nervous system. Comparable seats of modification occur also at places of linkage outside the central nervous system, e.g. the places of linkage of the spinal nerve cells with the sympathetic nerve cells in the sympathetic ganglia, and of the linkage of spinal nerve cells with muscle cells and epithelial cells in motor end-plates, glands, and sense organs. These seem the nearest approach outside the brain and cord to anything of real likeness to a nervous centre.

Afferent is the term applied to nerve cells, considered as conductors, along which the waves of change normally excited pass in a direction away from the extrinsic, i.e. non-nervous tissues. Efferent, conversely, is applied to nerve cells which conduct normally towards extrinsic, i.e. non-nervous tissues. The afferent root cells taken together form one great pathway into an intricate congeries of nerve centres which the cerebro-spinal organ encloses. The efferent root cells conversely together form one great pathway out. And within the cerebro-spinal organ the nerve centres are not only very numerous but are of very various rank. The cerebro-spinal organ is fundamentally a chain of segmental centres, each segmental centre primarily a seat of reflection and distribution connected with the local conducting paths between sense cells and musele cells of its own segment. The segmental centres have in the course of evolution of the nervous system come to be more and more put en rapport one with another by nerve cells overlying them, and dealing with them in groups. By means of these intermediate and overlying conductors, a nerve impulse may, besides embouching upon its own segmental muscle cells, travel over a concatenation of central nerve cells. The reactions which occur along nerve cell chains of a single link

1 "Observ. on the Struct. and Functions of the Spinal Cord," London, 1837. 
or of few links only are characterised by "monotony"; the reactions which employ long chains traversing many synapses are immensely variable. It is a cardinal feature in the architecture of the nervous system that the longest chains all include cerebral links. By rank in regard to nerve centres is understood such position as relieves from local or merely segmental work and involves general responsibility, i.e. for a series of segments or even for the whole body. A touchstone for rank of centre in this nervous hierarchy is the degree to which paths from separate loci and of differing sensual modality are confluent thither. A low centre lies at the focus of afferent paths from a limited locus and of one modality, e.g. from touch-spots in a small field of skin. $A$ high centre receives widely divergent multi-sensual paths, e.g. from organs for touch, heat, cold, pain, and muscular sense for the hand as a whole.

In addition to its power to modify the distribution of nerve impulses, both in space and time, the nerve centre exhibits a valve-like function, allowing conduction to occur through it in one direction only." This is called the "law of forward direction" in the central nervous system. That the direction of nerve impulses is not reversible along the neural chains, may be a function of the "synapse." This seems a bridge open to traffic from nerve cell A to nerve cell $\mathrm{B}$, but barred to passage in the opposite sense. That this valve action of the nerve centre is attributable to the synapse, is rendered likely by the similar valve-like connection between motor nerve fibre and muscle cell and between an efferent nerve and sense cell. How securely the circuits of the nervous system are valved against regurgitation, is shown by the Bell-Magendie law of the reactions of the spinal nerve roots.

In reflex action at its simplest, it is difficult to demarcate between functions of nervous and those of muscular or other contractile tissue. Muscular tissue, like nervous, transmits a state of excitement induced in it at one point to others,-and even across extracellular boundaries.

The reaction in which a local irritation of some point of the Stanniused heart induces co-ordinate contraction of the whole myocardium, is akin to reflex action. It is not yet clear whether the nervous or the contractile tissue is really responsible for the reflexes of the medusa bell or of the manubrium of Tiaropsis indicans, ${ }^{2}$ though cells resembling nervous have been demonstrated in the Medusce (O. and R. Hertwig, ${ }^{3}$ Schäfer $\left.{ }^{4}\right)$, as in the heart ventricle (Heymans). ${ }^{5}$ In the Ascidian, e.g. Ciona ${ }^{6}$ there is a characteristic "reflex" nexus between oral and aboral vents. When one aperture is touched, both shut. The musculature controlling each is of somewhat similar arrangement. The main ganglion of Ciona lies between the apertures, and is the nervous tie between the musculature of both. Yet, after ablation of the whole ganglion, the reflex, although the threshold stimulus required is heightened, is still elicitable. The nerve-path facilitates the reflex, but is not essential for it. The "reflex" activity of the striped muscle of the intestine of the tench 7 seems altogether independent of the spinal cord. In the dog recent observations by Goltz and Ewald ${ }^{8}$ show persistence of the "reflex" opening and closure of the rectal and vesical sphincter, after complete removal of the corresponding region of the spinal cord.

The simplest reflex arc imaginable would be one in which a single nerve conductor passed from some tissue recipient of environmental stimuli to some other tissue responsive to the nervous excitement in

1 W. James, "Boston Soc. of Nat. Hist., 1880," Scribner's Mag., 1888 ; "Text-Book of Psychology," London and New York, 1890, vol. ii. p. 580, ete.

2 Romanes, Phil. T'rans., London, 1877 ; "Roy. Inst. Discourse," May 1877.

3 "Das Nervensyst. u. die Sinnes organen d. Med.," 1878.

${ }^{4}$ Phil. Trans., London, $1878 . \quad{ }_{5}$ Arch. de pharmacod., Gand and Paris, 1896.

6 J. Loeb, "Untersuch. u. physiol. Morphol. d. Thiere," Würzburg, Bd. ii.

${ }^{7}$ Mahn, Arch.f.d. ges. Physiol., Bonn, 1892, Bd. lxxii. S. 273.

8 Ibid., 1896, Bd. Ixiii.

VOL. II. -5 O 
the conductor. Such an arc, formed by the intermediation of a single nerve cell between, for instance, a sensory tissue and a motile tissue, may exist in simple organisms, e.g. medusa, hydra, and perhaps in the viscera of vertebrates; but the reflex arc upon which the whole complex vertebrate nervous system is founded is less simple, and involves at least two nerve conductors and a linkage or synapse between them, as well as the recipient and the responsive tissues from which and to which respectively the two conductors carry. The peripheral part or path of the afferent conductor is one and the same, whether the arc employed be simple or complex. It forms the only channel of entrance into the whole nervous system. Similarly, the peripheral efferent conductor is the only channel out. Hence these two ways in and out must necessarily be treated introductorily to the reactions of the central nervous system.

It was in the seventeenth century that an idea truly allied to that which "reflex action" now denotes entered physiology. Some who then wrote on animal physiology express it, and among them, not earliest but most notoriously, Descartes. Previous to that century, certain "sympathies" between parts of the body had been noted, and had latterly been referred to communion established by nerves. In Cartesius' time (1650) the argument had become more precise. He pointed out that many of the nerves connected with the brain elicit, without affecting consciousness, involuntary movements. The involuntary closure of the eyelids in result of a threatened blow was one of various instances he gave. Such a process he designated a "reflected" one. Boyle brought to the question the Baconian touch-stone of experiment. $\mathrm{He}$ found the body of a viper for three days after decapitation "to be manifestly sensible of punctures, being put into a fresh and vivid motion, when it lay still before, upon being pricked." There is no evidence that Boyle thought such movements unaccompanied by sensation.

As to the seat of the reflexion, Descartes said the pineal gland, Willis the brain, nerve plexuses, and ganglia. Next century opinion came to be grouped into two camps, one maintaining the cerebro-spinal organ (Astruc, Hales, Perrault, K. Boerhaave, Haller, v. Swieten, Monro, and Whytt) to be the only seat of reflex action, the other the nerve plexuses and ganglia (Vieussens, $H$. Boerhaave, Vater, Meckel, and Gasser). Hales, rector of Teddington, contributed what is known as the fundamental experiment. He showed that the movements evoked by stimuli applied to the skin of the decapitated frog are lost irrevocably by destroying the spinal cord. Whytt's observations widened the notion "reflex," making it include salivation and contraction of the pupil. Muscular reactions he classified as-(1) produced by direct stimuli applied to muscles, (2) vital and involuntary, (3) voluntary. Each of these he considered accompanied by sensation. To Hales' fundamental observation he contributed a fundamental one on inhibition, noting that the reflex movements are inelicitable for the interval immediately succeeding the decapitation. Here again he invoked sensation, attributing the arrest to pain or fright. The part that Prochaska took in the development of the study appears overestimated; his experimental contributions are negligible; he held, however, like Descartes, that some reflex actions do not involve sensation.

The Bell-Magendie experiment was the next step, and its importance can hardly be overstated. The localisation of the ingoing chamnel in one root, and the outgoing in the other, was followed by the discoveries by Mayo (1823) of a small circumscript segment of the brain, which of itself, and alone, sufficed for the pupil reflex, and by Legallois (1826) of a small circumseript portion of the bulb, injury of which at once paralyses the respiratory movements, whereas other injuries do not. The "localisation" was carried further by Flourens and Longet. Prior to the work of these last, Marshall 
Hall's studies began, apparently in ignorance of much preceding knowledge. New points he ascertained are the tonic closure of the sphincters by reflex action, the cessation of strychnia convulsions on destruction of the spinal cord, and the easier excitation of reflexes by stimulating the end apparatus of nerves, than by stimulation of the nerve trunks themselves. In his own estimation, Hall's chief advance lay in the finding of evidence of separate nerve paths for reflexes unaccompanied by sensation, and apart from paths subserving sensation and volition. His evidence was not very satisfying, but it led to work by Grainger (1837), in which good proof was given that in the cord the sole seat of reflex centres is the grey matter; and, further, that each afferent root sends some fibres direct into the grey matter for reflex action, and some fibres upwards as a strand in the white matter, subserving the elaboration of sensation. Valentin suggested two years later that the nerve cells in the grey matter are structures for transferring impulses from the afferent to the efferent channels. Lastly, with the elder Waller's ${ }^{1}$ discovery of degeneration of nerve fibres after section, came demonstration of the functional and morphological continuity between the conducting channels and their respective groups of nerve cells. The question of the action of ganglia as reflex centres was, for the spinal ganglia, answered in the negative in Johann Müller's text-book (1837). For the ganglia of the sympathetic an answer in the affirmative was declared by Henle, ${ }^{2}$ Stilling, ${ }^{3}$ Claude Bernard, ${ }^{4}$ and others ${ }^{5}$; but except in a modified sense of the term "reflex centre," such as is afforded by the interpretation offered by Langley, ${ }^{6}$ its substantiation seems unlikely. The ganglia of invertebrata were at first supposed-chiefly owing to Newport's ${ }^{7}$ conclusions - to be like the spinal ganglia, not seats of reflexion; but Carpenter ${ }^{8}$ later proved them to have reflex powers, and pointed out their analogy with the spinal centres and brain, a view to which Newport subsequently gave adherence. ${ }^{9}$

\section{The Way IN.}

The peripheral afferent channel consists always of an elongated nerve cell, which by one of its ends extends to points accessible to the environment, including in the environment the "material me," and by the other end lies deeply embedded in linkage with other nerve cells of various rank, composing the machinery of the central nervous system. These nerve cells together form, therefore, the first part of the path travelled by waves of change constantly poured into the nervous system by the world of changing energy around it. These are the "primary afferent neurones" or the "afferent root cells." Their cell branches are as nerve fibres much commingled in most places with those of efferent and other nerve cells, but their cell bodies (perikarya) are collected apart into ganglia, which they thus form.

Assuming the whole organisation to consist of a fore and aft series of

${ }^{1}$ Phil. Trans., London, 1850, pt. 2, p. 423 ; Compt. rend. Acad. d. sc., Paris, 1851, tome xxxiii. p. 606 ; Arch.f.,Anat., Physiol. u. wissensch. Med., 1852.

2 "Patholog. Untersueh.," Berlin, 1840. 3 "Physiol. Untersueh.," Leipzig, 1840.

${ }^{4}$ Compt. rend. Acad. d. sc., Paris, 1862, tome ii. p. 341.

${ }^{5}$ Sokownin, Arch. f. d. ges. Physiol., Bonn, Bd. viii. S. 600 ; Nussbaum, Arb. d. Lab. d. Warschauer Med. Fac., 1879, Bd. v. S. 120 ; lately by Nawroeki and Skabitschewsky, Arch. f. d. ges. Physiol., Bonn, Bd. xii. S. 141; Langley and Anderson, Journ. Physiol., Cambridge and London, 1894, vol. xv. p. 11, and vol. xvi. p. 410 ; Griffiths, Journ. Anat. and Physiol., London, 1895, vol. xxix. p. 61; C. C. Stewart, Am. Journ. Physiol., vol. ii. p. 186.

"See this Text-Book, artiele "Sympathetie," p. 678.

7 Phil. Trans., London, 1832-43.

8 "The Physiology of the Nervous System of Invertebrated Animals," London, 1839.

9 "Nervous System of Myriapoda," Phil. Trans., London, 1843. 
segments, then the perikarya of the afferent root cells of each segment lie uncommingled with other nerve cells, and form the spinal ganglia (right and left) of that segment. And the branches thrust by the cells composing each spinal ganglion into the spinal cord, remaining unmixed with others, form a pure collection of afferent nerve fibres, known norphologically as the dorsal spinal root. This collection contains all the fibres from the sense organs in a segmental area of the body surface, which may be called a segmental skinfield (Fig. 354). It contains also all the fibres from the sense organs in the nusscular and visceral apparatus belonging to the same segment. Similarly, the afferent root cells of the fifth and eighth and intermedius cranial nerves intrude nerve fibres as unmixed afferent roots into the brain. In a few but important exceptions, e.g. the vago-glosso-pharyngeal roots, the afferent fibres are commingled with efferent. The primary afferent path

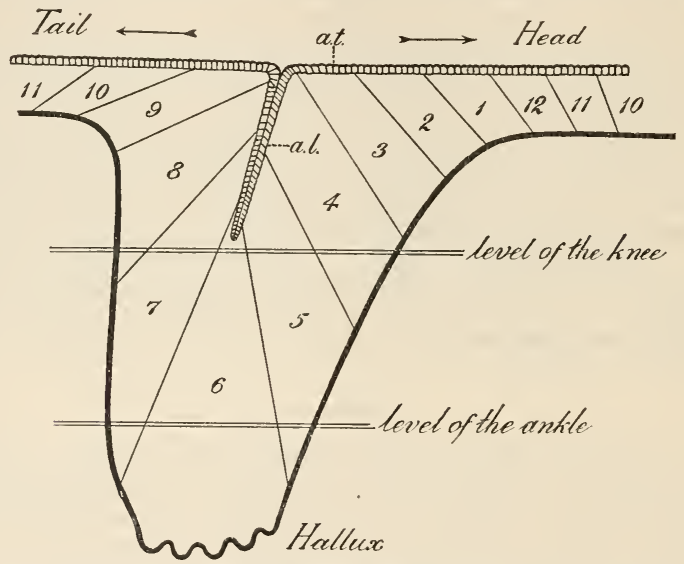

FIG. 354.-Diagram of the arrangement of segmental skin-fields in the hind limb of the monkey. The numbers indicate the fields of the spinal nerves, thoracic and post-thoracic. a.t, the axial line of the trunk, whether dorsal or ventral ; a.l, the axial line of the limb, whether dorsal or ventral. The "overlapping" of the fields is not represented.

for olfaction is peculiar, in that the bodies of its nerve cells are not aggregated, but, maintaining a very primitive disposition, ${ }^{1}$ lie scattered severally among cells of non-nervous type in the olfactory region of the body surface. $^{2}$ Their proximal branches are, however, before entering the central nervous system, collected into pure afferent roots, the olfactory nerves.

Recurrent sensitivity.-Magendie's later experiments led him to the view that the ventral root has some sensory fibres in it, derived from those comprising the dorsal root. Denied by many, ${ }^{3}$ this has been upheld by Bernard and Schiff, and the place of rebending of the sensory fibres has been referred to a region of the peripheral nerve, some little way beyond the place of union of the two roots in the nerve trunk. Severance of the dorsal root or of the neighbouring dorsal roots excludes the phenomenon. Schiff noted,

1 v. Lenhossék, "Sensibl. Fasern d. Regenwurms," Arch. f. mikr. Anat., Bomn, 1892, Bd. xxxix. S. 102

2 Grassi and Castronoro, ibid., 1889, Bd. xxxiv. S. 385.

3 J. Miiller, Tiedemann, Van Deen, Stannius, Retzius, and Backer, early repeating the Magendic experiment, do not mention it; Longet, Chauveau, and others mention it to deny it. 
after destruction of portions of the spinal cord (in birds), that the ventral roots were still found, when examined by the microscope, to contain a few undegenerated myelinate nerve fibres; these could be traced to the arachnoid and pia mater. Schäfer discovered that in the cat there are in the lumbar ventral roots a few nerve cells; these cells resemble the smaller of the cells of the spinal root ganglion; the number of these cells and the number of sound fibres remaining in the ventral spinal root, peripheral to its place of section, do not in many cases tally. A phenomenon somewhat similar to the slight sensitivity exhibited by the peripheral end of a severed ventral root, is met with on stimulating the peripheral ends of certain motor peripheral nerves after severance of the nerve nearer its origin. The branches of the facial of the dog, when examined in front of the parotid gland, exhibit it, unless the trigeminus is severed. The phenomenon is the more marked, the more peripheral the region of the nerve examined. Fibres can be traced by the degeneration method to loop back from the fifth nerve into these branches of the seventh.

Bell's ${ }^{1}$ and Magendie's ${ }^{2}$ observations, in so far as they dealt with the afferent channel, dealt with it only as a channel for exciting signs of sensation. Volkmann, ${ }^{3}$ later, showed that the dorsal root is also the channel for evoking reflex action after decapitation, and under circumstances, therefore, which there is reason to think exclude sensation. The discrimination between the functions of the roots was discovered, as above stated, in mammals. Wagner, ${ }^{4}$ Stannius, ${ }^{5}$ and Moreau ${ }^{6}$ found it to apply in fish; J. Muller ${ }^{7}$ and Panizza ${ }^{8}$ established it for the frog; and Panizza ${ }^{8}$ and Schiff ${ }^{9}$ for the bird.

The dorsal spinal root has been stated above to be a purely afferent tract of fibres. It does appear to be so in most animals; in some forms, however, it contains a few fibres which have origin in intraspinal cells, perhaps of efferent function. v. Wijhe ${ }^{10}$ holds that the ventral spinal roots of Amphioxus contain some afferent fibres. I 11 have brought forward some evidence in the same direction regarding the roots of the third, fourth, and sixth cranial nerves of mammals.

The histologists Ramón y Cajal ${ }^{12}$ and v. Lenhossék ${ }^{13}$ have, by use of the method of Golgi, discovered in the dorsal (afferent) spinal root, nerve fibres arising from cells in the grey matter of the spinal cord itself. This discovery made in the chick has been extended to the cord of certain teleosteans. ${ }^{14}$ The nerve cells which give origin to these fibres of the dorsal root lie for the most part in the dorsal portion of the ventral grey horn. They are multipolar, and the microscopists describe them as "motor." This suggests exception of the Bell-Magendie law of direction of transmission in the spinal nerve roots, and calls to mind the recent account ${ }^{15}$ of peripheral effects following excitation of the distal ends of the dorsal spinal nerve root of the frog. Steinach and Wiener detected visceral effects, Horton-Smith detected effects on skeletal

1 "An Idea of a New Anatomy of the Brain," 1811, republished in "An Exposition of the Natural System of Nerves," London, 1824.

2 Journ. de physiol. expér., Paris, 1822.

3 Arch. f. Anat., Physiol. u. vissenseh. Med., 1838, S. 15 ; ibid., 1841, S. 354.

4 "Handwörterb. d. Phys." 1846, Bd. iii. S. 366.

5 " Das periph. Nervensyst. d. Fisch.," Rostock, 1849.

${ }^{6}$ Compt. rend. Soc. de biol., Paris, 1858-1860.

7 "Handbuch d. Physiol.," 1834.

8 "Rieherche sperimentali sopra i nervi," Pavia, 1834.

9 "Lehrbuch. d. Physiol.," Lahr, 1858.

${ }^{10}$ Verhandel. d. k. Alad. v. Wetenseh., Amsterdam, January 1897.

11 Proc. Roy. Soc. London, $1897 . \quad 12$ Anat. Anz., Jena, 1890, S. 112.

13 Ibid., S. 360 . Later confirmations by Retzius, Biol. Lntersuch., Stockholm, 1892,

Bd. v. ; v. Gehuchten, Anat. Aw\%., Jena, 1893, S. 215 ; J. Martin, Cellule, Lierre and Louvain, 1895.

${ }^{14} \mathrm{v}$. Gehuchten.

15 Steinach, Lotos, 1893, Bd. xiv. ; Steinaeh and Wiener, Arch. f. cl. ges. Physiol., Bonn, Bd. lx. S. 593 ; Horton-Smith, Journ. Physiol., Cambridge and London, vol. xxi. p. 101. 
muscles only. The visceral effects have been confirmed by another observer, ${ }^{1}$ those on skeletal muscles have also been confirmed, ${ }^{2}$ though it is stated they must be regarded as the individual exception and not the rule. ${ }^{3}$ Stricker ${ }^{4}$ affirmed the demonstration of vaso-dilator fibres in the mammalian dorsal (posterior) roots. Veias ${ }^{5}$ and Joseph ${ }^{6}$ state, in contradiction to the original observations by Waller, ${ }^{7}$ that in the cat and rabbit injury to the dorsal root, proximal to its ganglion, causes peripheral degeneration of a number of nerve fibres, which can be followed into the distal nerve trunk. On the other hand, the original observations by Waller have been confirmed by many, ${ }^{8}$ both in the spinal region of outflow of fibres for the sympathetic system ${ }^{9}$ and in other regions. The position arrived at is therefore as follows : In Amphioxus, where all the nerve cells of both spinal roots are intraspinal, some of the fibres of the dorsal root may be efferent, though the majority of them are certainly afferent. ${ }^{10}$ In Petromyzon some ${ }^{11}$ of the fibres of the dorsal root spring not from cells of the spinal ganglion, but from intraspinal cells. These may be efferent. Also, in Pristiurus some of the fibres of the dorsal root arise from intraspinal cells, but in Myxine all arise from the extraspinal cells of the root ganglion. In the chick a few fibres of the dorsal root arise from multipolar intraspinal cells beyond all doubt, and in Rana peripheral effects result on exciting the peripheral ends of the dorsal root. In the mammal, efferent fibres appear to exist in certain dorsal nerve roots.

The total entrant path furnished by the whole collection of the afferent root cells is conveniently divisible by functional qualities into eight groups. These are-(1) from the retina; (2) from the olfactory surface; (3) from the cochlea; (4) from the vestibule and semicircular canals; (5) from the integument; (6) from the skeletal muscles (including their tendons) and from joints; (7) from the gustatory smrface; and (8) from viscera.

The conditions attending the initiation of nerve impulses in the fibres of this entrant path are examined in the articles on the several sense organs. These fibres appear amenable to the same general stimuli as are other nerve fibres. Though afferent, they are quite indistinguishable in their electro-motive reactions and microscopic structure from efferent fibres. Certain differences, probably quantitative rather than qualitative, are, it is true, found between the excitability of afferent and efferent nerve fibres. The main laws of "double conduction," rate of transmission, etc., treated of in the section on general physiology of nerve, apply, however, to them as to other peripheral nerve fibres.

The total number of separate channels composing the spinal entrant path is probably always greater than the number composing the total spinal efferent

I See Steinach, Arch.f. d. ges. Physiol., Bonn, Bd. Ixxi. S. 554.

2 Wana, ibid., Bd. lxxi. S. 555.

${ }^{3}$ H. E. Hering, ibid., Bd. 1xviii. S. 6.

${ }^{4}$ With his pupils Gartncr, Hasterlik, and Biedl. Morat and, more recently, Bayliss (Proc. Physiol. Soc., March 1900) have recorded results corroborating Stricker's. Bayliss obtained vaso-dilator effects in the hind-limb (dog) on stimulating electrically, niechanically, or chemically the peripheral cut ends of the fifth, sixth, and seventh lumbar and first sacral dorsal nerve roots.

5 " Beitr. z. Anat. u. Phys. d. spin. Gangl.," Munich, 1883.

${ }^{6}$ Arch.f. Fhysiol., Leipzig, 1887, S. 296. 7 "Nouvelle méthode, etc.," Bonn, 1852.

8 Bernard, "Système nerveux" (dog), 1858, tome i.; Kahler, Prag. med. Wchnschr., 18.84, S. 302 (dog); Singer and Miinzer, Denkschr. d. k. Akad. d. Wissensch., Wien, 1890, Bd. lvi. S. 570 (dog); Sherrington, Journ. Physiol., Cambridge and London, 1894, vol. xvii. p. 211 (cat and monkey); ibid., 1897, vol. xxi. p. 209.

${ }^{9}$ Sherrington, op. cit.

${ }^{10}$ J. F. Heymans and O. v. d. Stricht, "Sur le systeme nerveux de l'Amphioxus et sur la constitntion des racines sensibles," Bruxelles, 1898.

11 Freud, Nansen, Retzius, etc. 
path. In two frogs, Birge ${ }^{1}$ found 3781 and 5335 fibres in the afferent roots, and 3528 and 4283 respectively in the efferent roots. When the cranial roots are included, the excess of inferent channels over efferent channels becomes, of course, immensely greater still. It has been pointed out that, so far as examined, no spinal peripheral nerve trunk ${ }^{2}$ of macroscopic size is made entirely of efferent fibres, whereas nerve trunks entirely composed of afferent fibres are numerous enough.

Probably the nerve impulse travelling by the afferent root cell of the adult, with its T-shaped process, does not traverse the perikaryon but sweeps tangentially past it. That the perikaryon might be indifferent

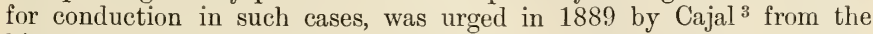
histology of cells of the mesencephalic roof of the bird. An experimental observation by Bethe ${ }^{4}$ (1896) seems to have placed this hypothesis beyond doubt for certain cells of Carcinus (second antenna). Observations by Steinach ${ }^{5}$ confirm the same view for the cells of the spinal ganglion. The conduction is, of course, ultimately dependent on the cell body, since the cell branches cannot maintain their vitality in its absence.

The application of nicotin ${ }^{6}$ to the spinal ganglion does not hinder the conduction of impulses through it, as in sympathetic ganglia; in the latter there is a synapse ${ }^{7}$ or linkage between two cells. There is a difference in the answer obtained by different observers as to the question of alteration of speed of travel of nerve impulses traversing a ganglion. Wundt ${ }^{8}$ found the reflex time markedly shorter from the spinal root than from the nerve trunk peripheral to the ganglion. Exner ${ }^{9}$ found the negative variation travel with unaltered speed through the spinal ganglion. Gad ${ }^{10}$ found a longer delay in the development of a respiratory reflex, when excited from the far side of the vagus ganglion, than could be accounted for by the greater length of nerve cord traversed. Moore and Reynolds ${ }^{11}$ have recently obtained on the spinal ganglion results confirmatory of Exner.

A nerve impulse traversing the afferent root cell passes when once within the spinal cord to various distances and in various directions, without leaving the root cell, and before impinging upon other nerve cells at the first intraspinal synapses. It is not known in many cases whether one afferent root cell may serve as entrant channel from sense organs of different kinds, e.g. whether one afferent nerve fibre from the skin is peripherally connected with tactual and with "cold" and "pain" organs. Probably each fibre is connected only with one species of sense organ. Similarly, it is not known whether one afferent root cell embouches intraspinally into only one or in to a number of functionally

1 Arch. f. Physiol., Leipzig, 1882, S. 476. See also Stilling, "Untersuch.," Kassel, 1857.

${ }^{2}$ Sherrington, Journ. Physiol., Cambridge and London, 1894, vol. xvii. The cervical sympathetic appears to consist wholly of efferent fibres. Schiff, Langley, etc.

${ }_{3}$ Rev. trimest. d. histol., tomes iii. and iv. pp. 67-78.

${ }^{4}$ Arch. f. mikr. A nat., Bonn, 1897-98, S. 50, 51.

${ }^{5}$ Arch. f. d. ges. Physiol., Bonn, Bd, Ixxviii.

${ }^{6}$ Langley and Anderson, Journ. Physiol., Cambridge and London, 1892, vol. xiii. p. 465.

7 For a description of this, see C. Hüber, Journ. Comp. Neurol., 1897, vol. vii. p. 72.

8 "Mechanik d. Nerven," 1876, Abth. 2, S. 567.

${ }^{9}$ Arch. f. Physiol., Leipzig, 1878, S. 567 ; Sitzungsb. d. k. Akad. d. Wissensch., Wien, 1877 , S. 729 .

${ }_{10}$ Arch. f. Physiol., Leipzig, 1889, S. 218.

${ }^{11}$ Fourth Internat. Cong. Physiol., reported in Journ. Ihysiol., Cambridge and London, 1898, vol, xxiii., Suppl. 
different intraspinal nerve paths. It seems likely that some afferent root cells do lead into spinal paths of various functional quality, e.g. into tactual, dolorific, and subconscious reflex.

The axon of the afferent root cell sometimes gives a "side-fibre" near its origin which recurves to apply itself to the perikaryon. ${ }^{1}$

In all the spinal nerves the ascending stems from the afferent fibres seem to be longer than the descending, but in the case of some of the cranial nerves, e.g. trigeminus, the descending are the longer. In each of the spinal nerves the ascending stems from certain of the root cells pass to the dorsal column nuclei of the bulb; thus, from the lowest nerves, these ascending stems extend through the whole length of the spinal cord. What proportion of the root cells of any particular afferent root possess, by means of long ascending stems, direct connection with the bulbar nuclei, is not known; the large afferent roots belonging to the limbs seem to furnish a larger number than the small afferent roots of other regions. The total region of embouchment of any one afferent root in the cord is therefore wide. It extends from probably a couple of spinal segments behind the surface attachment of the root anteriorly to the bulb itself.

It appears pretty certain that in front of (above) the segments composing the particular region abutting on the segment to which the ganglion of the root belongs, the ascending stem passes most of the segments without sending collaterals into them, and unconnected with them enters the dorsal nuclei of the bulb. Nine collaterals have been counted from one root fibre in the funiculus cuneatus. v. Lenhossék has not found any collaterals to fibres in the funiculus gracilis.

When, after transection of the bulb close to the calamus scriptorius, the extreme top of the dorso-median column is faradised, ${ }^{2}$ movements of the homonymous leg are evoked; in the monkey, movements of the foot. When the mesial part of the extreme top of the dorso-lateral column is similarly stimulated, movements of the homonymous hand result. In the same way, from the top of the dorso-mesial column, movement over the lowest intercostal spaces can be evoked. These novements appear to be reflexes, involving antidrome conduction down the ascending stems, and so, vii collaterals given off near the entrance of the root cell fibres, into the cord. The reactions indicate that the long ascending fibres are given off by thoracie as well as by limb roots, and that under the circumstances the collaterals by which reflexes are particularly facile are those given off by the stem fibres near the entrance of the root into the cord.

The date at which a nerve fibre completes its development, by acquiring a myelin sheath, ${ }^{3}$ indicates the time at which it becomes functionally active. The long ascending stems (fun. gracitis) of the afferent root cells do not become myelinate ${ }^{4}$ until much later than the medium and short fibres, except of Lissauer's marginal zone. The long ascending fibres would therefore pertain to a systcm characterising a more highly developed phase of existence than do the short intraspinal. The long ascending stems form, however, a very welldeveloped spinal column in systems even as low as that of the frog.

Besides the dorsal column nuclei of the bulb, the "column of Clarke" forms a nucleus of ending for many of the collaterals from ascending stems of afferent root cells in the lumbo-sacral region. And the lateral division of

1 C. Huber, Anat. An₹., Jena, Bd. xii.; J. Dogiel, ibid., 1896.

2 Sherrington, Proc. Roy. Soc. London, 1897.

${ }^{3}$ Flechsig, in C. Ludwig's laboratory, Leipzig, 1875; for latest extensions of method, see Ambronn \& Held, Arch.f. Physiol., Leipzig, 1896, S. 202.

${ }^{+}$Elechsig, op. cit., and especially Trepinski, Arch. f. Psychiat., Berlin, Bd. xxx. S. 54. 
the nucleus cuneatus seems to stand in similar relation to fibres from the roots at the top of the cord.

The cell-processes of the Gasserian ganglion, on entering the bulb in its pontine portion, bifurcate into long descending and short ascending stems. Some of the latter join the middle cerebellar peduncle ; most, however, end in the front (upper) part of the grey matter in the bulb called the sensory nucleus of the fifth. The former descend close outside this and give collaterals into it; the descending stems, grouped just lateral to the substantia Rolandi, course backwards, some of them even so far as the level of the second cervical segment. The petrous and jugular trunk ganglia of the glosso-pharyngeal vagus nerves are equivalent to spinal ganglia. The descending stems, resulting from the dichotomy of their fibres, run back as a circumscript bundle, fasciculus solitarius, into the hindmost fourth of the bulb. Both they and their shorter ascending stems give off and end in collaterals to the grey nucleus of ending of the glosso-pharyngeal vagus in the floor of the fourth ventricle.

The transverse extent of the region of intraspinal ending of the spinal afferent root includes in the uncrossed half of the cord the whole of the spongiosa, and some end in the substantia Rolandi of the dorsal horn. ${ }^{1}$ The root-collaterals traceable far across the spongiosa into the region of the efferent root cells in the homonymous ventral horn are often called the "reflex collaterals"; they perhaps constitute the ending of the afferent path for some of the simplest and most direct of spinal reflex movements. Root-collaterals form almost entirely the dorsal commissure especially in man (v. Lenhossék), and end in the crossed grey matter; but none contributc to the ventral commissure.

\section{The WaY OUT.}

The efferent root cells are intraspinal in position. They lie in the grey matter, chiefly in its ventral region. Each possesses numerous dendrons, and a single axon.

The grouping of the efferent root cells is segmental, in the sense that they lie in the cord at the same segmental level as the segmentally arranged nerve roots to which they contribute. But in some regions there is very considerable overlapping of the contiguous segmental fields to which they are peripherally distributed. In the limb and neck regions of the cord the root cells in the ventral horn are very distinctly collected into subgroups. These groups are probably assemblies of cells, that collectively innervate particular groups of muscles. Probably all the largest of the efferent root cells conduct to skeletal muscles. The axons of the vast majority of the motor root cells pass to muscles of the homonymous side. ${ }^{2}$ The medio-ventral group of the ventral horn is perhaps distributed to the muscles lying along the vertcbral axis. It seems, however, to include mediate cells, the "commissure group" " of v. Lenhossék, besides root cells. In the brachial and lumbo-sacral enlargements there is added to the grey matter of the ventral horn a huge lateral region, in which lie several subgroups of large efferent root cells. Of these there is one which, from its position in the frontal plane of the grey horn, is called the dorso-lateral subgroup. To it has been attributed the innervation of hand (and foot) muscles, especially of palmar (and plantar). The topography of this subgroup along the length of the cord agrees with the latter view, and with its atrophy after amputation of the end of the limb. After amputation even at shoulder or hip, or after the limb has been rendered apæsthetic by severance of the sensory roots, it is the group which suffers most severe regressive change. In the limb thus rendered apæsthetic one move-

1 v. Lenhossék, "Der fein. Bau d. Nervensystems," 2te Aufl., Berlin, 1895.

${ }^{2}$ A. S. F. Grünbaum, Journ. Physiol., Cambridge and London, 1894, vol. xvi. p. 365 ; Paladino, Arch. ital. de biol., Turin, tome xxii. p. 1. 
ment, the closure of the hand or foot, is, as a volitional movement, lost. The nutrition of this group seems therefore to be especially dependent on the maintenance of the local reflex arc (and perhaps reflex tonus). It is said ${ }^{1}$ that the reflex collaterals are more numerously traceable to this part of the ventral horn than to others. The efferent root cells innervating any one muscle do not seem to be collected into a definite group in the frontal planes of the cord. In the limb regions the root cells innervating each single muscle extend throughout the length of two, three, or more segments of the cord.

In the higher vertebrates each of the segmental spinal nerves which supplies the musculature of the limb leaves the spinal cord in the form of a row of rootlets, which together build up the motor root of the nerve. Excitation by electric currents of any one of these rootlets or filaments, that contribute to the formation of the motor root, produces a movement in the limb which

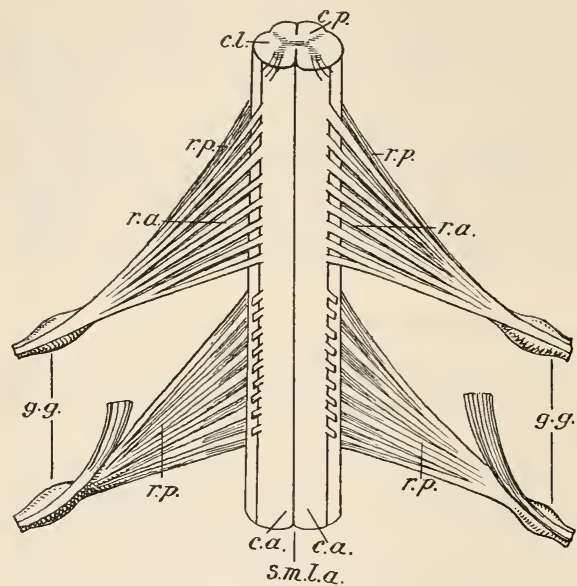

FIG. 355.-Diagram showing arrangement of the root filaments of two spinal nerves. c.a. The ventral white column of the cord; c.l. the lateral white column; c.p. the dorsal white column; s.m.l.a. the median ventral fissure of the cord; $r . a$. the ventral root; $r . p$. the dorsal root; g.g. the ganglion of the dorsal root. From van Gehuchten.

together show that nerve cells which innervate the diff The two observations to any one myotom in the limb lie commingled, so that any one section through the spinal cord at right angles to its length will meet nerve cells belonging to all the muscles represented in that segment. This is the same as saying that the nerve cells belonging to any one limb-muscle lie not gathered together at any one particular level of the spinal segment, but scattered throughout its length. And inasmuch as each muscle is innervated from several segments, the nerve cells for each muscle are scattered in a continuous series through the length of a series of spinal segments; and throughout this extent are commingled with the cells of a great number-in some cases as many as forty and more-of other muscles. It is therefore evident that no traumatic injury of the spinal cord can ever paralyse a single

${ }^{1}$ G. Mingazzini, Riv. sper. di freniat., Reggio-Emilia, 1892, tome xviii. Nos. 3-4; Lugaro, Riv. di patol. nerv., Firenze, 1896, tome i. p. 1. 
muscle alone and apart from its fellows. Even the severance of a whole single motor nerve root cannot paralyse a single limb-muscle; the effect of such an injury is to partially impair a large number of the inuscles.

The individual extrinsic eye muscles innervated by the oculo-motorius have been assigned to certain groups of cells in the nucleus of that nerve. The evidence is, however, conflicting. A demonstrably segmental grouping of the efferent root cells in the human spinal cord has been assumed by some anatomists $;^{1}$ the statement rests on some evidence of such a grouping in the eel ${ }^{2}$ and snake. ${ }^{3}$ Waldeyer assumes a similar arrangement in the cord of the gorilla. The careful examinations of the mammalian (including human) cord by Kaiser ${ }^{4}$ and v. Argutinski ${ }^{5}$ have shown that no segmental ${ }^{6}$ grouping of any of the cell columns is detectible; this conclusion stands in harmony with the results of the examination of the relation of the reflex movements to spinal segments. The results of an analysis of the spinal nerve supply of the muscles of the limbs show that the muscular tissue of the limb is arranged in a number of rays, a ray for each metamere contributing to the limb. It shows that of these rays the ones which lie tailmost in the fore-and-aft series are the longest, that is, extend to the extreme free apex of the limb, whereas the foremost, the most rostral, pass only, in the fore-limb, as far as the upper arm, the next only as far as the elbow, the next only as far as the wrist. The four last, or most aboral rays, all contribute to the musculature of the hand in the common macaque monkey (Macacus rhesus).

When we come to inquire how these units of the segmental architecture of the limbs, these muscular rays, are related to the physiological or functional units of the limb musculature, it is at once obvious that the extent and boundaries of the two do not coincide. The individual muscles of the limb are functional elements of its structure as a physiological machine. But each of these functional elements is compounded of portions of several rays or myotoms. Moreover, the boundaries between the myotoms do not correspond with the intervals of muscles, or even between muscle-groups.

The same want of segmental separation is evident when we examine the location of the nerve cells which innervate the individual muscles. In experiments on the plexuses, it is rare to find two consecutive individuals of the same species that possess a similar root distribution. The plexus in any species requires for description to be grouped into classes. For most purposes it suffices to distinguish a class in which the plexus is pre-fixed and a class in which it is post-fixed. Thus, in some individuals, the supinator brevis is innervated from the sixth and fifth cervical nerves, in others from the sixth and seventh. In the former case the plexus is of pre-fixed type. When one muscle or one part of the plexus is pre-fixed, all the rest are prefixed; and conversely, when one part is post-fixed, all are post-fixed. In the second instance given above, the supinator brevis is post-fixed, that is, its nerve cells are fixed in segments further aboral in the series than in the individuals with pre-fixed plexuses. Presumably, the muscle itself therefore is built of myotoms more aboral in the series than in the muscle in the pre-fixed individuals. Between the extreme cases of post-tixed type and the extreme examples of pre-fixed type extend a numerous series of intermediate individuals; in fact, any and every intermediate degree of type seems to exist. The extreme of post-fixed type passes over into the comparatively rare individual

'Schwalbe, "Lehrbuch d. Neurologie," in Hoffmann's "Lehrbuch der Anat. d. Menschen;" Waldeyer, "Das Gorilla-riickemmark," Berlin, 1889.

${ }^{2}$ Stieda, Ztschr. f. Wissensch. Zool., Leipzig, 1868, Bd. xviii.

${ }^{3}$ Luideritz, Arch.f. Physiol., Leipzig, 1881.

4 "Die Funktionen d. Ganglienzell. d. Halsmarkes," Haag, 1891.

${ }_{5}^{5}$ Arch.f. mikr. Anat., Bonn, 1896, Bd. xlviii.

${ }_{6}$ The cells of the columns, especially of that of the lateral horn, are collected into groups along the length of the cord, but the groups are much more numerous than are the segmental nerves. 
with an additional segment, e.g. the cat with fourteen ribs. This frequency of individual variations exhibits in an almost ludicrous aspect the view held, that each motor spinal root is a collection gathered together for a functional purpose, and representing a particularly highly co-ordinated movement of the limb. Contrary to any such hypothesis, it shows that for the functional mechanism of the limb it is immaterial whether this or that particular group of muscles is represented in a particular spinal root or not represented in it. The absolute segmental level is variable over the range of nearly a whole segment's length; the relative segmental position is inviolably preserved. Thus flexor carpi ulnaris may get fibres from as high as the sixth cervical segment, or it may not, but it never extends so well forward, so far rostrally, as flexor carpi radialis, and this latter never extends so far aborally as flexor carpi ulnaris, even when it is most post-fixed; that is, the region of outflow for the spinal nerve fibres to flexor carpi ulnaris lies rather more aborally than that of the outflow to flexor carpi ractialis, and this mutual segmental position of the muscles is maintained whether the plexus be post-fixed or pre-fixed.

In reflex movements, and in movements evoked from the cortex of the brain, the discharge of wotor impulses to a pluri-segmental muscle of the forearm or wrist or hand, is probably always pluri-segmental, and involves the whole length of the serial group of intraspinal nerve cells which innervates the muscle extending through its full number of segments of the cord. The afferent and cerebral channels of the cord treat the pluri-segmental motor stations or nuclei of these limb-muscles as entities of homogeneous structure, as, in fact, physiological units.

This serves to emplasise the physiological homogeneity of limb-muscle and nerve trunk, and the physiological heterogeneity, in spite of morphological unity, of the spinal nerve roots in the limb region of the body. The spinal nerve roots of the thoracic region are, from the physiological point of view, less heterogeneous. The peripheral nerve trunk is the physiological collection of nerve tibres, e.g. flexors collected together, vaso-dilators included with motors to muscles, etc. The nerve root is the morphological collection; it contains, commingled into one, such heterogeneities as adductors of the hallux with protrusor muscles of the pelvic floor.

Not all the efferent root cells are large. Many among the smaller intraspinal cells probably innervate the vascular and visceral musculature. A numerous set of such cells lies in a special lateral ridge of grey matter, extending in the cord (man and monkey) from second thoracic segment to second lumbar inclusive. This is the intermediate lateral cell-group lying in the lateral horn of spongiosa. Gaskell first pointed out the correspondence between the longitudinal spinal extent of this cell-group and the preponderance of minute myelinate fibres in the efferent roots, and the existence in those roots of pressor paths to the vascular musculature. The inference is, that the cell-group of the lateral horn is composed not of mediate (or column) cells but of the root cells innervating the musculature of the vessels (and viscera). The upper end of the group has close topographical correspondence with the upper limit of outflow of nerve fibres to the dilatator pupillæ. The direct demonstration that a lateral horncell gives an axon into the efferent spinal root has, however, not yet been supplied. But it has been proved ${ }^{1}$ that the minute nerve fibres passing by the efferent roots into the sympathetic do come from cells lying very strictly in the same segmental frontal level of the cord as the surface origin of the root filament which contains them. They in this point resemble the relation of the skeletal fibres to their cells of origin.

It has been laid down as a law, that the muscles of a given part of the limb and the sense organs in the overlying skin are supplied by the same spinal scgment, the affereut root cells being inchuded as belonging to a spinal

${ }^{1}$ Sherrington, Journ. Physiol., Cambridge and London, 1892, vol. xiii.; A. S. F. Grumbaum, ibid., 1894, vol. xvi. 
segment. But there are so many and such wide exceptions to this, that the rule as a law can hardly be said to exist. In respect to the proximal joints of the limbs, the muscles producing the chief movements in opposite directions are mainly represented at different segmental levels of the cord. Thus in the macaque the motor root cells for flexion at the elbow lie mainly in the fifth, sixth, and seventh cervical segments, those for extension at the elbow, chiefly in the seventh and eighth cervical and first thoracic segments. The motor root cells for opposite movements of the small joints at the free ends of the limb are, however, not thus segmentally separate, but lie commingled in the same segments of the cord. There are reasons for thinking, moreover, that the motor root cells for antagonistic movements, whether as in the latter joints segmentally commingled, or as in the former segmentally apart, are contemporaneously influenced by any reaction that, e.g. from brain or cord, initiates a co-ordinate movement in which they take part, the one set of cells being subjected to pressor, the other to inhibitory influence.

The fact that the ventral spinal roots and certain analogous cranial nerve roots form the only channels by which nervous impulses pass out of the cerebro-spinal organ to the extrinsic tissues, allows the efferent root cells, taken together, to be considered as one great efferent path for all nervous reactions. The telencephalon and diencephalon (primary cerebral vesicle) are the only portions of the nervous system whose root cells are solely efferent. The whole path is made up of the third, fourth, sixth, eighth, eleventh, and twelfth cranial nerves, of the unganglionated root of the fifth cranial and of all the ventral spinal roots. There are some minute nerve fibres in the sheath of the ventral root, which do not take origin from intraspinal cells; these fibres may be sympathetic. As already mentioned, in the ventral roots of the lumbar region of the cat, Schäfer discovered nerve cells, and similar have been seen in the same region in man. Whether these are afferent or efferent, and if the latter, whether sympathetic, is not certain.

Golgi discovered that from the axons of some spinal efferent root cells "side fibres" are given near its origin. The function of these is still unknown, though probably important. They may conduct centripetally into the axon. ${ }^{1}$

Restoration of movement after "nerve-crossing." - When two mixed nerve trunks (A and B), supplying antagonistic groups of muscles, are cut and "crossed," so as to secure union of the peripheral end of A with the central of $\mathrm{B}$, and vice versit, Flourens ${ }^{2}$ concluded that full control and co-ordination was regained by some central adjustment. R. H. Cunningham ${ }^{5}$ has, in the case of the fore-limb of the dog, re-examined this question carefully, and finds that on cortical excitation, and also in intentional co-ordinated novements, the co-ordination of the muscular contractions is not regained.

\section{Relation of Afferent to Efferent Roots.}

Besides the fact that excitation of the ventral spinal root excites contraction of the muscles directly through motor fibres, Bell and Magendie and their followers showed that its excitation evokes no signs of sensation and no reflex actions, and in this respect offers a second difference from the ganglionated root. This "law" of Bell and Magendie sheds light on the quality of the intraspinal are connecting the afferent nerves and roots with the efferent nerves and roots. It is evident that the nexus between afferent channel and efferent is of a kind allowing

\footnotetext{
'See v. Lenhossék, "Der feinere Bau d. Nervensystems," 2te Aufl. S. 129. He terms them "axo-dendrites."

2 "Recherches expér. sur propriét. et fonct. d. système nerveux," 1824, p. 272. Cf. Rawa, Arch. f. Physiol., Leipzig, 1885, S. 296.

${ }^{3}$ Am. Journ. Physiol., 1898, vol. i. p. 240.
} 
conduction in the direction from dorsal root to ventral root, but not conversely. This is the special case which gave the foundation of the law of conduction in the neural system termed the "law of forward direction," which has been already referred to (p. 785). ${ }^{1}$

The practically universal spinal irradiation obtained under strychnia bore out the view of a conducting network continuous in all directions throughout the grey matter. But when the property of "double-conduction" by nerve fibres had been ascertained, the "Bell-Magendie law" of the roots became more instructive. Since the dendritic processes of the efferent root cells join the general network, the Bell-Magendie law must indicate either that these dendrons are non-conductive, or that they, although conductive from the network into the motor cell, are not conductive back from the motor cell into the network. Gad (1884) ${ }^{2}$ especially insisted on the latter view; Golgi $(1882)^{3}$ on the former, considering the dendritic processes to be simply nutrient. Since the recent more general rejection of the theory of a continuous intraspinal network, Cajal (1889) and van Gehuchten (1891) ${ }^{4}$ have argued a "dynamical polarisation" of the nerve cell so that the sense of conduction is solely from dendron toward axon, never the reverse. They suggest the importance in this connection of the "articulation" between axon of one cell and dendron of next. The "valved" condition of the circuits may result from properties belonging to these places of "contact utile" or" "synapse." The "dynamic polarisation" of the cell seems to find expression in certain morphological characters of the cell, so that the two ends are in many cases recognisable by inspection. In accord with the constant failure to provoke reflex actions or signs of sensation by stimulation on the proximal side of the transection of an efferent spinal root, all attempts ${ }^{5}$ to similarly provoke from that root action currents in other nerve tracts have failed.

An immediate influence exerted by the efferent root cells on the motor root cells, as indicated by the skeletal muscles, is demonstrable in various ways. The posture of the hind-limb of the spinal frog, when the limbs are allowed to hang freely (Brondgeest's phenomenon), ${ }^{6}$ shows that the lengths of the flexor muscles of the limb are, under those conditions, slightly shorter than when the afferent roots of the sciatic plexus have been severed. Early experiments failed ${ }^{7}$ to show any lengthening of the muscles on section of their nerves, but the muscles were examined after arrest of the circulation and under extension by not inconsiderable weights. With lighter weight and intact circulation Wundt ${ }^{8}$ observed a lengthening after section of the muscular nerves, but, confronted by a somewhat similar lengthening, resulting as an aftereffect of simple contraction of the muscle, he hesitated to accept the lengthening consequent on nerve section as due to anything more than

I See W. James, loc. cit., Boston, 1880 ; see also J. Muiller's "Handbuch," 1834, Bd. i. Abth. 2.

${ }^{2}$ Tagebl. d. 66te Versamml. deutsch. Naturf. u. Aerzte; "Eulenberg's Real-encyc.," 1888, Bd. xvi. S. 673 .

3 "Studi istolog.", Milano, 1881; Gior. internaz. d. sc. med., Napoli, 1881, tomo iii.

4 Cellule, Lierre et Louvain, fasc. 1.

5 Hermann, "Lehrb. d. Physiol.," Aufl. xi. S. 6 ; Gotch and Horsley, Phil. Trans., London, 1891; Mislawski, Compt. rend. Soc. de biol., Paris, 1894, p. 488; Bernstein, Arch. f. d. ges. Physiol., Bonn, Bd. Ixxiii.

6 Hölland. Beitr. zu d. anat. u. physiol. Wissensch., Diisseldorf, 1860.

${ }^{7}$ R. Heidenhain, "Physiol. Studien," Berlin, 1856. This work contains full references to all the previous literature.

8 "Lehre v. d. Muskelbewegung," Braunschweig, 1858. 
the contraction caused by the stimulus when the nerve was cut. Cyon ${ }^{1}$ and Tschiriew ${ }^{2}$ obtained a lengthening after nerve section, the former also after section of the afferent spinal roots; and both, from their experiments, concluded in favour of a demonstrable reflex slight tonic contraction of muscle. v. Anrep's ${ }^{3}$ experiments discriminated between the two instances of lengthening observed by Wundt, and seem to conclusively establish a reflex tonus of the adductor magnus and semimembranosus muscles of the frog. Curari breaks the tonus down through the motor-end plate, morphia through the afferent spinal channel.

Cyon ${ }^{4}$ early insisted on the occurrence of a fall in the excitability of the motor root, as tested by faradisation and muscular contraction, ensuing upon severance of the afferent root. Harless ${ }^{5}$ had previously stated that the excitability of the sciatic nerve of the frog is lowered by section of the afferent spinal roots connected with it. Cyon's facts were disputed, ${ }^{6}$ but other observers have confirmed them, ${ }^{7}$ and they have been reaffirmed by himself. ${ }^{8}$ They have been extended to the mammalian roots, ${ }^{9}$ and the phenomenon seems well observable after cocainisation or section of the sacral roots of the dog.

Any interference with the nervous system which paralyses a skeletal muscle delays the onset of rigor mortis in it (Hermann ${ }^{10}$ ). Section of the motor spinal roots thus delays onset of the rigor; and so also does section of afferent roots, ${ }^{11}$ even if practised a short time only before destruction of the animal. It also causes some wasting of adult and some interference with growth of young muscles. ${ }^{12}$ When a limb has been rendered apæsthetic by severance of all its afferent roots, the muscles feel peculiarly limp and flaccid, ${ }^{13}$ and the passive mobility of the joints is greater than normal. The same condition is met with in many cases of tabes dorsalis ; ${ }^{14}$ the hip can, with the limb extended at the knee, be flexed to abnormal extent. This increased passive mobility is due no doubt to loss of tonus (hypotonia) in the limb muscles. At the same time it may be that not all skeletal muscles habitually possess such a tonus, and certain muscles appear at least to possess it in higher degree than others. A slight degree of tension of the musele is a necessary condition for the elicitation of the knee-jerk, and any damage which breaks the reflex arc

1 Ber. d. k. süchs. Gescllsch. d. Wissensch. Math.-phys. Cl., Leipzig, 1865.

2 Arch. f. Physiol., Leipzig, 1879.

3 Arch.f. d. ges. Physiol., Bonn, 1880, Bd. xxi. S. 226.

${ }^{4}$ Op. cit., S. 85.

5 Abhandl. d. bayr. Akad. Physik., 1858, Bd. xxxi.

${ }^{6}$ v. Bezold and Uspensky, Centralbl. f. d. mod. Wissensch., Berlin, 1867, S. 611 ; G. Heidenhain, Arb. a. d. physiol. Lab. zu WWürzburg, 1868, Bd. iii. S. 107.

7 Guttmann, Centralbl. f. d. med. Wissensch., Berlin, 1867, S. 689; Mareacei, Arch. per le sc. med., Torino, 1882, tome v. p. 283.

${ }^{8}$ Steinmann, Bull. Acad. imp. d. sc. de St. Pétersbourg, 1871, tome vii. p. 787 ; Cyon, Arch.f. d. ges. Physiol., Bonn, 1874, Bd. viii. S. 347.

9 E Belmondo e R. Oddi, "Intorno all" influenza cette radici spinali posteriori sull eccitabilità della anteriori," Lab. di Fisiol., Florence, 1890.

${ }^{10}$ v. Eiselsberg, Arch.f. d. ges. Physiol., Bonn, Bd. xxiv. S. 229 ; v. Gendre, ibid., Bd. xxxv. S. 45 ; Aust, ibid., Bd. xxxix. S. 241 ; Bierfreund, ibid., Bd. xliii. S. 195 ; J. R. Ewald, ibid., 1894, Bd. liv.; and Tammassia, Riv. sper. di freniut., Reggio-Enilia, 1882 ; and Atti. r. Ist. Veneto di sc., lctt. cel arti., tome iii. p. vi.

11 Sherrington, Proc. Roy. Soc. London, 1893, vol. liii. p. 408 ; Mott and Sherrington, ibid., 1895, vol. lvii. p. 481 .

${ }_{12}$ Mott and Sherrington, loc. cit.; H. E. Hering, Neurol. Centralbl., Leipzig, 1897, Bd. xvii.

${ }^{13}$ B. Stilling, Arch. f. physiol. Heilk., Stuttgart, 1842, S. 97 (frog); Mott and Sherrington, loc. cit.; H. E. Hering, Arch. f. expcr. Path. u. Pharmakol., Leipzig, 1898, Bd. xxxviii. S. 266.

${ }^{14}$ See, for instance, Jendrassik, Neurol. Centralbl., Leipzig, 1896, No. 17; and Fränkel, ibid., 1896, No. 8. 
maintaining the normal tonus of the vasti-crureus extinguishes the knee-jerk. The particular afferent channels on which the tonus and jerk depend in this case, have been traced to arise in the muscle itself. ${ }^{1}$ Cutaneous nerves seem not to be the constituents of the afferent spinal root which in respect to muscular tonus are of ehief importance. ${ }^{2}$ There is no asymmetry of facial expression, after section of the sensory root of the trigeminus, though in the rabbit the pinna drops ${ }^{3}$ on the side homonymous with the facial anæsthesia; but there is facial asymmetry after section of one facial nerve. The myogram obtained when the intact nerve of the quadriceps cruris is excited by a break shock shows a relaxation line much slower and more prolonged than when the cut nerve is similarly excited, and the relaxation line in the former case never, as in the latter, dips below the base line with an inertia fling. ${ }^{4}$ It is the inertia fling attaching to the movements, that is so characteristic of an ataxic limb. This has been admirably analysed by H. E. Hering ${ }^{5}$ in the case of his "Hebe-phenomen." in the frog. The contraction of the muscle when its afferent path is intact seems to set into activity some mechanism, lost after rupture of the are, that damps the inertia swing of the muscular movement. It may be that the tension developed in the muscle by its contraction excites reflexly in it a condition of tonus. Not every muscle seems, under all normal conditions, to conform to the statement that there is a constant neural tonus of reflex origin in it. When the elbow is well flexed, the biceps is so slack as to feel toneless. When the frog's leg is fully flexed at knee and extended at ankle, the exposed gastrocnemius can be seen to lie actually in slack folds; on being cut it does not gape. It may be that the neural tonus of skeletal muscles is only present in them so long as they are stretched, and only in such as are to a certain degree under stretch. The tonus will obviously be of chief advantage in such muscles as have to be in readiness to contract; the reflexly maintained spinal "sitting" posture of the frog entails, by passive stretch, tonus in the extensors, and holds them ready for the next movement-a spring. Not only is the posture of the pendent limbs of the spinal frog altered from normal by section of the afferent roots of the limb, but the sitting posture of the apæsthetic limb in the otherwise normal frog is abnormal, in the sense that the limb is less flexed at hip, and knee, and ankle. ${ }^{6}$ The apæsthetic fore-limb is, on the other hand, more flexed at elbow, and the digits are often bent under. The excitability of the cord and centres, under the influence of convulsants, is much depressed by section of the afferent roots $;^{7}$ it seems that in the spinal frog strychnin and picrotoxin ${ }^{8}$ fail to produce convulsions if all the afferent spinal roots be severed. Certainly, however, asphyxia can excite spinal convulsions when all the afferent roots of the cord or portion of cord under examination have been severed. ${ }^{9}$ The peculiar condition of extensor spasm of the limbs characterising decerebrate rigidity, after transection between diencephalon and mesencephalon, does not ensue in a limb the afferent spinal roots of which have been previously severed, and disappears at once in a limb on the severance of the afferent roots of the limb. ${ }^{10}$ The severance of other afferent roots does not destroy the rigidity of the limb. On the other hand, the section of an afferent root seems to make but slight change in the excitability of the motor cells of the corresponding efferent root; when tested by

${ }^{1}$ Sherrington, Proc. Roy. Soc. London, 1893, vol. lii.

${ }^{2}$ Mommsen. Virchow's Archiv, 1885, Bd. ci. s. 22.

${ }^{3}$ Filehne, Arch. f. Physiol., Leipzig, 1886, S. 440.

+ Tschiriew, ibid., 1879 , S. 82.

5 Arch. f. d. ges. Physiol., Bonn, 1897, Bd. Ixviii. S. I.

6 H. E. Hering, Arch. f. exper. Puth. u. Pharmakol., Leipzig, 1896, Bd. xxxviii. S. 276.

7 Luchsinger; H. E. Hering, Arch.f. d. ges. Physiol., Bonn, 1893, Bd. li. S. 614.

${ }^{8}$ H. E. Hering, ibid., op. cit.

${ }^{9}$ Luchsinger and others; also Sherrington, Phil. Trans., London, 1897.

${ }^{10}$ Sherrington, Journ. Physiol., Cambridge and London, 1898, vol. xxiii. p. 319. 
stimulation of the part of the cortex which produces movement through those cells, they seem slightly more excitable. ${ }^{1}$ It was noted above that excitation of the dorsal columns of the cord close to the bulb produces movements in the homonymous limbs. The section of the afferent roots of the limb renders this reaction much more difficult for that limb, ${ }^{2}$ though it is still as easy as before to evoke movements of the limb from the pyramidal tract. The significance of this result may be that the severance of the axon from the cell body and its cellulipetal processes, detaches the collaterals from their synapses with the motor cells. That the change induced is not located in the motor root cell itself, seems evident from the openness of the cortico-spinal synapse.

Panizza, ${ }^{3}$ who was the first after Magendie to fully confirm the Bell-Magendie law of the roots in warm-blooded animals, drew attention to the fact that though his experiments showed the dorsal (posterior) roots to be wholly afferent, yet the section of a number of the afferent roots brought marked impairment of the local motility. The movements of the apæsthetic limb are clumsy. The hind-limb in walking is lifted too high and set too far. Stilling ${ }^{4}$ reaffirmed these observatious after experimenting on the afferent roots of the frog. He wrote -

"The posterior roots have the following important functions:-(1) They maintain continuously the tonus of the muscles, or that action whereby even in time of rest a ceaseless readiness for movement, a ceaseless tension of the muscle fibres, is maintained. (2) The posterior roots transmit continuously the sensation of the condition of the muscles themselves. Each unsuitable, uncomfortable pose of the muscle is thereby brought to consciousness, and, as a result, corrected by the will or by reflex action." 5

Claude Bernard ${ }^{6}$ and Schiff $^{7}$ simultaneously later drew attention to the disturbance of movement produced by the section of the roots. Bernard pointed out that when the frog is held up by the body, the animal uses the apæsthetic hind-limb far less than the sound one to release itself, and that the creature springs with difficulty. He cut the afferent roots to one hindlimb in young puppies, and noted that the animal could no longer support its weight on that limb.

H. E. Hering ${ }^{8}$ has given a clear objectively written description of the postures and movements of the apæsthetic limb in the frog. When the animal is first approached - the day after operation - the apæsthetic limb is moved up closely to the body, the normal limb moving much less. In the execution of a small spring there is no obvious difference between the limbs; in execution of a longer spring the animal alights so as to have its head facing somewhat towards the side of the apæsthetic hind-limb. If both hind-limbs are apæsthetic, the spring is higher, but carries less far than normal; the turn with the one apæsthetic limb seems due to enfeebled extensor stroke. As the hindlimbs are drawn up into the sitting posture again, after the spring, the apæsthetic one is seen to be the later, and to be tilted up in so doing. This latter act constitutes Hering's "tilt-phenomenon," and he has shown by various

1 Sherrington, Phil. Trans., London, 1892, vol. lxxxviii. B; Mott and Sherrington, Proc. Roy. Soc. Loudon, 1895, vol. lvii. p. 481.

${ }^{2}$ Sherrington, ibid., 1897, vol. lxi. p. 245.

3 "Ricerche sperimentali sopra i nervi: Lettera del Professore Bartolomeo Panizza a Professore Maurizio Bufalini," Pavia, 1834. 'This appeared in German, annotated by G. Sehneemann, with a preface by Eisenmann, at Erlangen, 1836.

${ }^{4}$ Arch. $f$. physiol. Heilk., Stuttgart, 1842, S. 97.

${ }^{5}$ Stilling, loc. cit.

6 "Système nerveux," loc. cit., 1858.

7 "Lehrbuch d. Muskel- u. Nervenphysiologie," Lahr, 1858.

${ }^{8}$ Arch.f. exper. Path. u. Pharmakol., Leipzig, 1896, Bd. xxxviii. S. 266.

VOL. II. -5 I 
lesions of the muscles and nerves of the leg that it results when the tension of the antagonist muscles is abnormally low. He regards it as due to the loss of a reflex inhibition of muscular contraction, arising in afferent nerves of the "nuscular sense" order. It is therefore an ataxic effect. In swimming, although synchronous bilateral strokes are sometimes taken, the frog usually swims with the apæsthetic limb in permanent active extension. When both hind-limbs are apæsthetic, the frog does still occasionally swim with synchronous extensor strokes. Apæsthesia of the fore-limbs in the frog causes the frog to sit supported, not on the fore-feet, but on the fore-arm, often with the digits bent underneath. The spring is less high than usual, and for that reason less wile. When all four limbs have been rendered insensitive, the animal can still spring, but only a short distance, and often in attempting to spring upsets itself, rolling over sideways. Bickel ${ }^{1}$ has made observations on combination of apesthesia of one or more limbs with removal of one labyrinth. The forced postures regularly assumed after removal of a labyrinth were, as a rule, far less marked in the apæsthetic than in the normal limbs. Baldi ${ }^{2}$ in Luciani's laboratory observed dogs for long periods after severance of afferent roots of one or both limbs. When one hind-linb is apæsthetic, it is not at first used for walking; it seems unable to bear the weight of the body ; it is kept more or less flexed at hip and knee, and full extension is rarely executed by it. Later, it is used for walking, but is lifted too high, and sometimes set too far. With both hind-limbs apæsthetic, the fore-limbs at first simply drag the hind-limbs after them, these trailing with the dorsal aspect of the foot to ground. Later, as the hind-limbs are trailed, they make alternate movements of extension and flexion, and ultimately they may to some extent support the body, crawling as it were with very flexed knees, so that the rump droops too low. When the lowest three cervical and the first thoracic are the afferent roots cut, the animal limps, lifting the apæsthetic fore-limb from the ground. Later it comes at times to be used for progression, but the dorsum of the foot frequently doubles under, causing a fall or stumble. Baldi considers the ataxy the most marked symptom, and refers the chief gravamen of the lesion to rupture of the afferent channels from the peripheral organs of muscular sense. Combination of extirpation of one labyrintl with apæsthesia of the hind-limbs in the dog leads, when the latter operation precedes the former at a long interval, to a recrudescence of symptoms from which the apæsthetic limbs had partly recovered. After extirpation of both labyrinths, there appears far less power to compensate the disturbance of motility of apæsthetic limbs. ${ }^{3}$ An important observation by Bickel is that extirpation of one labyrinth causes the apresthetic tail to be twisted $90^{\circ}$ on its axis, and that this twist at once disappears when, weeks later, the other labyrinth is removed. Hering ${ }^{4}$ and Bickel have pointed out that the residual disturbance of motility, due to apæsthesia of both hindlimbs, is much increased by excluding retinal sensations, by bandaging the eyes or placing the animal in the dark.

Observations on the symptomatology of apresthesia of the limbs in the monkey ${ }^{5}$ show even more striking defects of motility. When the whole series of afferent roots of a limb are divided, the limb is useless for progression. The animals never use the apæsthetic limb in climbing either down or up a rope. The limb is chiefly moved in association with vigorous movements of the fellow normal limb. In climbing, while the normal movements are being executed by the normal arm, the apæsthetic, semiflexed at elbow, is often flung up behind the head.

\footnotetext{
1 Arch.f. d. ges. Physiol., Bonn, 1897, Bd. 1xvii. S. 299.

2 Spcrimentale, Firenze, Sept. 1885.

Ncurol., 1897, Bd. i. S. 4.

${ }^{5}$ Mott and Sherrington, Proc. lioy. Soc. London, 1895, vol. 1vii. p. 481.
} 
Hering ${ }^{1}$ made the following observations on such ataxic animals. When all the afferent brachial roots, except the last cervical, which I have shown to supply the skin of the whole hand, are severed, the monkey, in taking a piece of fruit offered him, exhibits zig-zag swaying of the out-thrust arm. If a maize-corn be placed on the ledge of the cage between the bars, the arm swaying, the more obviously the further it is extended, often fails to make its grasp between the right bars, and misses the object altogether. In picking small objects such as rice-corns out of the observer's hand, the normal monkey exhibits perfection of precision. He uses generally but finger and thumb, and rarely touches the hand on which the corn is lying. The monkey with its one afferent brachial root grasps clumsily with the whole hand, and often meets the observer's hand and not its content.

It has been pointed out that the movements of the proximal joints of the limb suffer less impairment of movement than those of the distal. It must be remembered that the number of nerve roots supplying the musculature of the proximal joints is greater than the number of roots supplying the distal. ${ }^{2}$ The posture of the aprsthetic hind-limb is an almost continuously maintained strong flexion at hip and knee; that of the fore-limb, which swings helplessly, flexion at elbow and wrist, with adduction at shoulder. Warrington has shown that severance of the afferent roots causes degeneration in certain of the motor root cells of the corresponding spinal region.

Charles Bell ${ }^{3}$ found that in the ass, after section of the supramaxillary divisions of both trigemini, the upper lip was no longer lifted in feeding, and the same phenomenon has been observed in the rabbit and cat. ${ }^{4}$ Bell questioned in consequence whether the nerve might not be motor. Experiments in Exner's laboratory ${ }^{5}$ led to the conclusion that in the horse a "paralysis" of the laryngeal museles of one side follows section of the corresponding sensory nerve of the larynx. This observation has, it is true, been disputed, but a most striking example of this kind is furnished by the ape. ${ }^{6}$ After section of all the afferent roots of the limb, the movement of grasp by hand or foot respectively is permanently lost. If even only one of the sensory roots of the three belonging to the hand and foot respectively is left to the limb, the loss of the grasp does not ensue, although there is marked ataxy. Inder the most inducive and favourable circumstances, the wholly apresthetic limb is never used for grasping. Yet, if the appropriate region of the cortex is excited, the grasp is elicited with apparently perfectly normal facility, and in the absinthe convulsions the closure of the hand and foot is as equally exhibited as by the fellow normal limb. ${ }^{6}$

Electrical change in the opposite afferent root has been in some cases found to accompany electrical excitation of the sciatic nerve of one side. ${ }^{7}$ In experiments on the frog, the electrical effect produced

${ }^{1}$ Neurol. Centralbl., Leipzig, loc. cit. Dr. J. L. Muskens tells me that after a time the ataxy subsides.

2 Sherrington, Journ. Physiol., Cambridge and London, 1892, vol. xiii. p. 1.

3 "Nervous System," 1820. Denied by Mayo, "Anat. and Physiol. Comment.," London, 1822.

${ }^{4}$ Schoeps, Arch. f. Anat. u. Physiol., Leipzig, 1827, S. 409.

5 Exner, Centralbl. f. Physiol., Leipzig u. Wien, 1889, Bd. iii. S. 115 ; Pineles, ibid., 1890, Bd. iv. S. 741 ; Móller, "Das Kehlkopfpfeifen d. Pferdes," Stuttgart, 1s88 ; Pineles, Arch.f. d. ges. Physiol., Bonn, 1891, Bd. xlviii. S. 17.

${ }^{6}$ Mott and Sherrington, loc. cit.; Hering and Sherrington, loc. cit.

7 Gotch and Horsley, Phil. Trans., London, 1891. 
in the opposite afferent root is very slight, inconstant, and whether significant of discharge of impulses appears doubtful. ${ }^{1}$

\section{Regeneration of the Afferent Root Fibres.}

Leaving aside the old question of the regeneration of peripheral ganglia as presenting no observations with methods adequate to settle it, ${ }^{2}$ there is the related one concerning regeneration of the afferent spinal roots. Eichhorst and Naunyn, ${ }^{3}$ after transection of the spinal cord, found nerve fibres traversing the cicatrix, which they considered traceable to an afferent root adjacent, being able to follow them to a spinal ganglion. After extradural section of the afferent root, a partial regeneration has been observed. ${ }^{4}$ Chipault ${ }^{5}$ has obtained in three puppies regeneration of the severed roots when they were sutured together immediately after section, the times elapsing between severance and suturing and the autopsy being from three to six months. The dorsal spinal columns, since they consist of afferent root fibres almost entirely, should show evidence of regeneration. There is, however, none such recorded. I ${ }^{6}$ looked for sigus of regeneration in a monkey's cord, in which the dorsal columns alone had been transected four months previously, but found no evidence of regeneration, although the scar was very limited in the longitudinal extent of the cord. Thirty-three days after section of the lumbar afferent roots proximal to their ganglia, I was surprised to meet, in the spinal stumps of the severed roots, numbers of minute myelinated fibres, less than $4 \mu$ in diameter, presenting the same appearance as the young nerve fibres in regenerating peripheral nerves. The ganglia had been excised, so that the new fibres could not be due to out-growth from them. The question of the regeneration of the afferent root is not without practical importance. Regeneration of the peripheral fibre-the cellulipetal conducting fibre-of the afferent root cell is a well-established fact, and is found to occur quite well in even advanced adult life. As tested by functional results, it takes place more quickly than the regeneration of the axon of the motor root cell. ${ }^{7}$

\section{Sketch of the Functional Anatomy of Mediate Spinal Systems.}

Although the two root cells, afferent and efferent, may suffice for certain reflex actions, it is probable that for most, even purely spinal, reflexes they are by themselves an insufficient apparatus. It is therefore convenient, before dealing with spinal reflexes in general, to consider other elements in the neural mechanism of the cord. Among the spinal cell systems, including in the word " cell" not only the cell body (perikaryon) but also all the nerve fibres which are its branches, there can be distinguished on the one hand the root systems and on the other the mediate systems. It is possible that the two root systems are themselves connected together, and that the simplest reflex are is formed by their direct linkage. But in many cases the reflex reaction seems to occur through one part of the afferent root system acting on a distant

1 E. M. Sowton, Proc. Roy. Soc. London, 1898, vol. 1xiv. p. 354.

2 The problem does not seem to have been touched in this half of the century. Valentin, "De functionibus nervorum cerebralium," 1839; Dupuys et Dupuytren, Bull. Acad. de méd., Paris, 1843-1844, p. 355 ; Schrader, "Exp. circa regenerat. in gangl. nerv. vuln. illatis in animal. instituta," Göttingen, 1850 ; Walther, "De regenerat. gangliorum," Bonn, 1853.

3 Arch. f. exper. Path. u. Pharmakol., Leipzig, 1874, Bd. ii. S. 37 .

Hahler, Prag. med. Wchnschr., 1884, S. 301.

5 "Études de chirurgie médullaire," Paris, 1893.

${ }^{6}$ Journ. Physiol.; Cambridge and London, 1893, vol. xiv. p. 271.

7 Ibid., 1894, vol. xvii. p. 218 . Cf. Kennedy, Phil. Trans., London, 1898, and Trans. lioy. Soc. Edin., 1899. 
part of the efferent root system through a "mediate" cell system. Such mediation may be vid one link-cell, or via a chain of link-cells. The spinal mediate cell systems may be classed as (1) intrinsic or purely spinal, and (2) extrinsic or spino-encephalic and encephalo-spinal.

Mediate cells of the spinal cord. - 1. Intrinsic mediate systems.These probably form a large proportion of the fibres of the cord, especially of the zone of white matter nearest to the grey. They contribute probably especially to the minute nerve fibres filling the area between the ventral horn and the base of the dorsal horn (lateral limiting layer). ${ }^{1}$ It seems that the fibres of the ventral part of the layer arise from cells of the ventral horn (Bruce). ${ }^{2}$ As a rule, the longer the course of a nerve fibre in the cord, the nearer its place, except at its immediate beginning or ending, to the periphery of the cord. ${ }^{3}$ (In the periphery of course is included the dorsal median septum.) Of the short fibres some pass tailward from their cells of origin, some pass headward. Certain that ascend far lie near the lip of the ventral median fissure.

A number of descending intrinsic spinal fibres are commingled with the pyramidal fibres in the pyramidal area of the lateral column. After complete degeneration of the pyramidal system, many sound fibres are left in the dog in that area, ${ }^{4}$ and in that area many fibres in the $\log$ are myelinated before the fibres of the pyramidal tract. ${ }^{5}$ Minzer, ${ }^{6}$ after semisection of the cord of a new-born rabbit at the nineteenth vertebra, performed total transection two vertebræ lower when the animal was grown, and found about as many fibres degenerate below the lesion on the semisected as on the intact side; the fibres omitting decussation must have been of intraspinal origin. I (with E. E. Laslett) have by semisection subsequent to total transection similarly found evidence, in the dog, of abundant spinal fibres descending in all regions of the ventro-lateral columus and extending through many segments. Münzer also in the new-born rabbit excised one hemisphere, and after spinal semisection in the grown animal found many degenerate fibres in the lateral column belind the lesion. $\mathrm{He}$ did not find injury of the anterior corpus quadrigeminum, nor of the cerebellum, produce spinal degeneration, and therefore many of the fibres degenerating are probably intrinsic spinal. ${ }^{7}$

Some intrinsic spinal nerve fibres, fasc. dorsalis proprius (Barker), probably both ascending and descending, lie among the root fibres of the dorsal column. (Flechsig's "oval centre" respected in tabes dorsalis; Schultze's "comma ${ }^{8}$

1 Sherrington, Journ. Physiol., Cambridge and London, 1893, vol. xiv. 1. 255 ; A. S. F. Grïbaum, ibid., 1894, vol. xvi. p. 368 .

2 Rev. neurol., Paris, tome iv. p. 698; Scot. Med. and Surg. Journ., Edinburgh, vol. i. p. 1.

3 Miescher, Arb. a. d. physiol. Anst. zu Leipzig, 1870, S. 183 ; Sherrington, Journ. Physiol., Cambridge and London, 1893, vol. xiv. p. 298; E. Flatau, Neurol. Centralbl.: Leipzig, 1897, Bd. xvi. S. 11.

${ }^{4}$ Sherrington, Journ. Physiol., Cambridge and London, 1885 , vol. vi. p. 177 ; 1893 , vol. xlv. p. 255 ; Löwenthal, "Inaug. Diss." Genève, 1885 ; Singer and Miinzer, Denkschr. d. k. Aliad. d. Wissensch., Wien, 1890 , Bd. lvii. S. 585.

s Sherrington, Journ. Physiol., Cambridge and London, 1885, vol. vi. p. 177.

${ }^{6}$ Prag. med. Wchnschr., November 1895. ${ }^{7}$ Minzer, loc. cit.; also Bd. xx. No. 4.

${ }^{8}$ Schultze's "comma tract" was first noted and figured by Charlton Bastian, Med. - Chir. Trans., London, 1867. Kahler and Pick, Arch. f. Psychiat., Berlin, 1880, also drew attention to it before Schultze, ibid., 1883. It is difficult to sunder it from the descending limbs of the dorsal root fibres. On the question of endogenous dorsal column fibres, see Daxemberger, Ztschr. f. Nervenheilk., 1897, S. 136 ; Gombault et Philippe, Arch. de méd. expér. et d'anat. path., Paris, 1894, tome vi. ; S. Blum, "Inaug. Diss.," Strassburg, 1895 ; Tootli, "Spinal Degenerations," London, 1889 ; Barbacci, Sperimentale, Firenze, 1891, tome iii.; Hoche, Neurol. Centralbl., Leipzig, 1896, Bd. xv., and Arch. f. Psychiat., Berlin, 1896, Bd. xxvii.; Alex. Bruce and Muir, Brain, London, 1896, vol. xix. p. 333 ; Miinzer and Wiener, Arch. f. exper. Path. u. Pharnakol., Leipzig, 1897, Bd. xxxv. S. 114 ; Dejerine and Thoari, Compt. rend. Soc. de biol., Paris, 1898 ; Zappert, Neurol. Centralbl., Leipzig, 1898, Bd. xvii. S. 105 ; Alex. Bruce, Brain, London, 1897, vol. xx.; also P. Marie, Redlich, Dejerine and Spiller, and Dufour, L. Mann, and others. 
tract," similarly intact in tabes, and a long descending tract noted by Hoche, the septo-marginal of Bruce and Muir; the tract found by Wiener and Muinzer ascending but of intraspinal origin.) Some of the descending fibres extend through seven and even twelve segments of the cord. Cajal has noted column cells contributing fibres to the dorsal columns. As a rule the long fibres are of larger diameter than the short.

Some of the mediate cells give off axons which cross from one lateral half of the cord to the other, while others do not. The former seem to be quite numerous, ${ }^{1}$ and their main passage of crossing to lie in the ventral white commissure. The median ventral cell group of the ventral horn of grey matter is probably a group of these mediate cells. ${ }^{2}$ Some mediate cells have been described as giving a bifurcating axon, one stem from which crosses the median sagittal plane, whereas the other does not. The number of mediate cells appears to be very great, affording a basis, therefore, for very various possibilities of direction of conduction in the cord. But it is impossible at present to say whether the majority of the mediate cells belong to the intrinsic (simply spinal) system, or to the spino-encephalic system, which is certainly very large.

2. Extrinsic mediate systems.-(a) Spino-encephalic.-(i.) Spinocerebellar.-Some intraspinal cells possess branches which extend headwards into the superior vermis of the cerebellum. One set of these forms an uncrossed spino-cerebellar system, the axons of which ascend in the dorsal part of the margin of the lateral column and in the restiform body. These cells of origin exist perhaps throughout the whole length of the cord, but are most numerous along the region between the first thoracic segment and the second lumbar (man). There the cells lie grouped together, forming the vesicular columu of Clarke. ${ }^{3}$ A small subgroup, probably belonging to the same system, lies in the upper cervical region, and as scattered cells in the lower brachial and other segments. It is more doubtful whether the group in the sacral region (Stilling's) is part of the system. Spinal lesions in the sacral region of man do not cause degeneration of the dorsal cerebellar tract. There is some reason to think that the lateral division of the nucleus cuneatus belongs to the same system. ${ }^{4}$ This system, called the dorso-lateral or restiformal spino-cerebellar, is remarkably developed in man, and in the monkey more than in other mammalian types examined, especially in its more posterior portion. Clarke's column forms a nucleus of ending for collaterals from the afferent roots of the lower limb, ${ }^{5}$ and for the pyramidal tract. ${ }^{6}$

Another spino-cerebellar system, mainly crossed (heteromeric), lies in the ventro-lateral edge of the lateral column. ${ }^{7}$ The cell-bodies whence these fibres

1 Cajal, van Gehuchten, v. Lenhossék, etc.

2 v. Lenhossék, "Der feinere-Bau des Nervensystems," Aufl. 2, Leipzig, 1895.

${ }^{3}$ Flechsig, "Leitungsbahnen im Gehirn u. Riickenmark," Lcipzig, 1876; Singer, Sitzungsb. d. k. Akad. d. Wissensch., Wien, 1881, 13d. Ixxxiii. S. 390 ; Löwenthal, Bull. Suc. vaudoise d. sc. nat., Lansanne, 1885, tome xxi. p. 92 ; Mott, Brain, London, 1890, vol. xiii. ; Tooth, "Spinal Degenerations," London, 1889 ; A. W. Campbell, Liverpool Med.-Chir. Journ., January 1894 ; Mott, Monatschr. f. Psychiat. u. Neurol., Berlin, 1897, Bd. i.; Laura, Areh. ital. de biol., Turin, 1882 ; Schäfer, "Proc. Physiol. Soc.," Joum. Physiol., Cambridge and London, 1899, vol. xxiv.; Löwenthal, Rec. zool. Suisse, 1886, tome iv., and others.

"Sherrington, Joum. Physiol., Cambridge and London, 1892, vol. xvi. ; Alex. Bruce, "Illust. of the Mid- and Hind-Brain," Edinburgh, 1892 ; A. Tschermak, Arch. f. Physiol., Leipzig, 1898.

${ }^{5}$ Leyden, "Klin. d. Rückenmarks krankh.," Bd. ii.; Lissauer, Fortsehr. d. Med., Berlin, 1884 ; Arch.f. Psychiat., Berlin, 1885, Bd. xii. S. 431 ; Krauss, Neurol. Ccntralbl., Leipzig, 1885, Bd. iv.; Hadden and Sherrington, Brain, London, 1888, vol, xi. ; J. Dejerine, Arch. dc physiol. norm. et path., Paris, 1888, tome i., and others.

${ }^{6}$ Schäfer, "Proc. Physiol. Soc.," Journ. Physiol., Cambridge and London, 1899, vol. xxiv. p. xxxii.

7 The existence of ascending fibres here was first noted by C. Bastian, Med-Chir. Trans., London, 1867 (with figurc). In 1879, Gowers called general attention to the degeneration 
rise are not with certainty identified; some may be contained in Clarke's column, ${ }^{1}$ but some lie at levels more caudal than the most caudal part of Clarke's column, ${ }^{2}$ and therefore more caudal than the main mass of the dorsolateral spino-cerebellar system. The fibres pass into the superior vermis ${ }^{3}$ of the cerebellum. The system is often called the ventro-lateral or conjunctival spino-cerebellar, because its fibres wind back into the cerebellum by the brachium conjunctivum.

(ii.) Other spino-encephalic systems.--Some spinal cells thrust branches up, it is said, into the mesencephalon, forming a spino-quadrigeminal ${ }^{4}$ system. The fibres of this system lie among the fibres of the ventro-lateral column in the region of the ventral root-zone. Many of them decussate to run forward through the bulb close to the ventro-lateral spino-cerebellar system; they reach the fillet. Instead of winding backwards and upwards to the cerebellum, they pass into the roof of the mesencephalon, and end more in the anterior than in the posterior corpus quadrigeminum. Others, it is said, proceed farther still, and enter the diencephalon, ending in the ventro-lateral nucleus of the optic thalamus, forming a spino-thalamic system. ${ }^{5}$ Throughout the dorsal longitudinal bundle of the cranial floor fibres exist, probably derived from the cells of the ventral grey horn of the contra-lateral side of the spinal cord, though probably only from the headmost region of the cord. Some ascending spinal fibres seem to end in the grey matter of the ventro-lateral region of the bulb. The ventro-lateral spinal column seems therefore to contain branches from intraspinal cells that reach each of the main divisions of the brain except the telencephalon, unless it be the $n$. lenticularis (Rossolymo).

(b) Encephalo-spinal.-Nerve cells of the formatio reticularis of the bulb and of the pons possess, there is reason to think, branches that pass back into the spinal cord. ${ }^{6}$ These are some of them direct and some crossed. The former run partly in the longitudinal bundle and partly in the reticular formation to the cord, forming a system which extends into the cord, the fibres in their spinal course lying in the ventral column to the median side of the motor root-zone. The crossed part of the system passes the median raphe near its origin, and descends in the lateral field of the reticular formation. Its spinal portion consists of fibres in the dorsal part of the lateral column where the fibres of the crossed pyramidal tract lie; this system seems to extend through the whole length of the cord.

A system of fibres is found in the ventro-lateral parts of the cord, which are stated to be derived from a group of bulbar cells (Deiter's nucleus) that forms

of the tract ("Diagnosis of Diseases of the Spinal Cord"), and it is often called the tract of Gowers. Singer examined it by experimental degenerations (Sitzungsb. $d . k . A k a d . d$. Wissensch., Wien, 1881, Bd. Ixxxiv. Abth. 3). He eonsidered it a spino-eerebellar tract, so also Schultze. Lowenthal (Bull. Soc. vaudoise d. sc. nat., Lausanne, 1885, tome xxi. p. 92, and "Diss.," Genève, 1885) was the first to prove its termination upward in the cerebellum, a result confirmed by Auerbaeh, Virchow's Archiv, 1890, Bd. exxi. ; Mott, Phil. Trans., London, 1891 ; Tooth, Brain, London, 1892, vol. xv.; Schäfer, “Quain's Anat.," 1893, vol. iii. p. 1, and others.

1 Sehäfer, "Proc. Physiol. Soc.," March 1899.

2 E.g., Gowers' case in "Diag. of Diseases of Spinal Cord." See also Tootl, op. cit., and St. Barth. Hosp. Rep., London, 1886, vol. xxi.

${ }^{3}$ Löwenthal, op. cit. ; Auerbaeh, op. cit. ; Mott, op. cit.

4 Mott, Brain, London, 1895, vol. x viii.; v. Sölder, Neurol. Centralbl., Leipzig, 1897 ; Thomas, Compt. rend. Soc. de biol., Paris, 1897, tome iii.; Rossolimo, Neurol. Centralbl., Leipzig, Bd. xvii.

${ }_{5}^{5}$ Mott., op. cit. ; H. Patrick, Arch. f. Psychiat., Berlin, 1893, Bd. xxv. S. 31 ; Journ. Nerv, and Ment. Dis., Feb. 1896 ; Fr. v. Sölder, op. cit.; Alex. Bruce, Brain, London, 1896, vol. xix. ; Edinger, "Vorlesungen u. d. Bau. d. nerv. Centralorg.," Leipzig, 1889 ; A. Tschermak, Arch. f. Physiol., Leipzig, 1898; Kohmstanm, Neurol. Centralbl., Leipzig, 1899 , Bd. xviii. S. 14.

${ }^{6}$ Held, Abhandl. d. k. Gesellsch. (l. Wissensch., Leipzig, 1892, Bd. xviii. S. 6; Fusari, Riv. sper, di freniat., Reggio-Enilia, 1896, tomo xxii. p. 417 ; А. Tschermak, Areh.f. Physiol., Leipzig, 1898, S. 291 ; Ramón y Cajal, "A puntos para el estudio, etc.," Madrid, 1895. 
an end-station for some of the fibres of the vestibular nerve branches. ${ }^{1}$ This bulbo-spinal system is, at least in the main, uncrossed. It extends into the lumbo-sacral region of the cord; its fibres in their spinal course lie among those of the periphery of the cord near the region of exit of the motor-root. Their collaterals enter the anterior horn. ${ }^{2}$ Some observers ${ }^{3}$ conclude that this system is derived from cerebellar cells. This can hardly be considered proven. There is some evidence also of a quadrigemino-spinal system $;{ }^{4}$ this is mainly crossed, the decussation occurring ventral to the central canal. The fibres pass backward near the raphe of the reticular formation. In their spinal course they lie among fibres of the ventral lip of the ventral column. The system extends to the lumbo-sacral region of the cord. The cells of the red nucleus ${ }^{5}$ also send, according to some authorities, fibres into the spinal cord as far as the lumbar region. This rubro-spinal system is a crossed one, its fibres decussating in the roof of the mesencephalon. The fibres in their spinal course lie in the central part of the lateral column somewhat ventral to the main portion of the pyramidal fibres.

Among the fibres passing tailward from the encephalon into the cord, those forming the cortico-spinal system or pyramidal tracts are perhaps the best known. Its fibres are branches from certain cells of the Rolandic cortex. They descend through the internal capsule, pes pedunculi, and pedal longitudinal fibres of the pons, and compose the ventral four-fifths and more of each pyramid of the bulb. The decussation of the pyramids is the crossing in bulk of the bundles of this tract at the caudal end of the bulb. The vast majority of its fibres cross at this place and shift into the dorsal part of the lateral column of the cord, lying separated (in man and most examined forms) ${ }^{6}$ from the surface of the cord only by the fibres of the dorsal cerebellar tract. Some of the crossed pyramidal fibres extend the whole length of the cord, reaching the most distal coceygeal region. ${ }^{7}$ In its course along the spinal cord it diminishes, owing to the ending of the constituent fibres at various levels. Its progressive decrease is far from regular, many fibres stopping short at some regions, comparatively few in others. The decussation of the crossed part of the system, especially in man, is not completed at the cephalic end of the cord, but continues back for a variable distance along the cord. The fibres which continue in the homonymous lip of the ventral spinal fissure before crossing form what is called the ventral pyramidal bundle, or Türck's bundle, and in man this has been seen extending even into the lowest sacral region. ${ }^{8} \mathrm{v}$.

1 Monakow, Arch.f. Psychiat., Berlin, 1883, Bd. xiv.; Ferrier and Turuer, Phil. Trans., Loudon, 1894, vol. clxxxv. B.; A. Thomas, Compt. rend. Soc. de biol., Paris, 1895, 1896 ; R. Russell, Phil. Trans., London, 1895, vol. clxxxvi. p. 633 ; Teljatnik, Neurol. Centralbl., Leipzig, 1897 ; R. E. Lloyd, Journ. Physiol., Cambridge and London, 1900, vol. xxv. p. 191.

Schäfer, "Proe. Physiol. Soc.,"Journ. Physiol., Cambridge and London, 1899, vol. xxiv.

${ }^{3}$ Marehi, Riv. sper. di freniat., Reggio-Emilia, 1886, tomo xii.; 1887, tomo xiii. ; Atti d. Accad., Firenze, 1891 ; Pelizzi, Riv. sper. di freniat., Reggio-Emilia, 1892 ; A. Thomas, "Le Cervelet," Paris, 1897 ; Biedl, Ncurol. Centralbl., Leipzig, 1895.

${ }^{4}$ H. Held, Neurol. Centralbl., Leipzig, 1890, S. 481; Arch. f. Physiol.,'Leipzig, 1893, S. 245 ; Boyee, Neurol. Centralbl., Leipzig, 1894, S. 466 ; Phil. Trans., London, 1895. But Mïnzer obtained no spinal degeneration after injury of anterior corpora quadrigemina in the rabbit or bird, Prag. med. Wchnschr., 1895.

${ }^{5}$ H. Held, Neurol. Centralbl., Leipzig, 1890 ; Arch. f. Physiol., Leipzig, 1891 ; Abhandl. d. k. Gesellsch. d. Wissensch., Leipzig, 1892, No. 6, Bd. xviii.; Ramón y Cajal, "Apuntos para el estudio, ete.," 1895; Kohnstamm, loc. cit.

6 Not in Macacus. E. P. France, Phil. Trans., Loudon, 1889 ; Sherrington, Journ. Physiol., Cambridge and London, 1889, vol. x.

${ }_{7}$ Sherrington, ibid., 1889, vol. x. ; Boyce, Phil. Trans., London, 1894, p. 186 ; Dejerine and Thomas, Arch, de physiol. norm. et path., Paris, 1896, tome ii. Previous observers found the tract end at the fourth lumbar level, ef. Tooth, "Spinal Degen.," London, 1890.

${ }^{8}$ Dejerine and Thomas, Arch. de physiol. norm. et path., Paris, 1896; it had been traced by Tooth, op. cit., 1889, to the second lumbar segment. In the monkey I have not with certainty found it farther back than the eighth cervical level; Redlich, to thirl cervical in the cat (Neurol. Centralbl., Leipzig, 1897). The actual level of the niain deeussation seems subject to much individual variation (Flechsig, "Leitungshahuen, etc.," Leipzig, 1876). 
Lenhossék, however, denies that the ventral pyramidal tract crosses; he considers it passes to the homonymous ventral horn.

Though the main bulk of the pyramidal system is crossed, it is in part uncrossed. Hence degeneration in both lateral columns follows unilateral lesions of cortex cerebri. ${ }^{1}$ The relative amount of the uncrossed and crossed degenerations varies much according as different regions of the motor cortex are the site of lesion. A minority of the pyramidal fibres, when arrived at the caudal end of the bulb, instead of sloping across the median sagittal plane, deviate ${ }^{2}$ to the homonymous lateral column, and lie there in a position symmetrical with the crossed area occupied by the main crossed division of the system. This uncrossed pyramidal system extends also the whole length of the cord.

The fibres of the pyramidal system are the latest in the cord to become myelinated ${ }^{3}$ and the slowest, after section in the cord, to show degenerative alteration. ${ }^{4}$ The pyramidal system, so far as is known, exists only in mammals. ${ }^{5}$ There is no other system which shows such increase in relative size as traced from lower to higher mammalian types. Hardly perceptible as a spinal tract in the rabbit, it in the monkey is much larger than in other laboratory forms, and in man is larger still. This relative increase is shown not merely by the tract occupying a larger proportion of the transverse area of the cord, but by its fibres being relatively more densely set within that area. Hence the "scar" left by degeneration of a pyramidal tract is in man not only relatively somewhat larger than in that of the dog, but far fewer sound nerve fibres remain in the sclerosed area.

In its spinal course the pyramidal system lies in the lateral column, nowhere actually abutting on the ventral grey cornu. The existence of a direct synapsis between the pyramidal axon and the ventral cornual root cell has been generally assumed, though rejected by some, and never definitely proven. ${ }^{6}$ After section of the tract, the degeneration of fine medullate fibres in the ventral cornu has been both asserted ${ }^{7}$ and denied. ${ }^{8}$ The collaterals

${ }^{1}$ Pitres, Bull. Soc. de physiol. de Bordeaux, 1880, tome i. p. 97 ; Gaz. hebd. de méd., Paris, 1881, p. 429 ; Progrès méd., Paris, 1882, p. 528 ; Arch. de physiol. norm. et path., Paris, 1884, tome iii. p. 142 ; Moeli, Arch. f. Psychiat., Berlin, 1883 ; Schäfer (Ferrier and Yeo's monkey), Journ. Physiol., Cambridge and London, 1883, vol. iv. ; Sherrington, ibid., 1885, vol. vi., 1889, vol. x. ; Löwenthal, "Diss.," Genève, 1885 ; Bianchi e Abundo, Psichiatria, Napoli, 1886 ; Marchi and Algeri, Riv. sper. di freniat., Reggio-Emilia, 1887, tomo xii. p. 3 ; Unverricht, Neurol. Centralbl., Leipzig, 1890, Bd. ix. S. 483 ; Sandmeyer, Ztschr. f. Biol., Miunchen, 1891, Bd. xxviii. S. 177 ; Singer and Miinzer, Denkschr. d. k. Akad. d. Wissensch., Wien, 1890.

2 W. Muratoff, Arch. f. Physiol., Leipzig, 1893 ; Sherrington, Lancet, London, Feb. 3, 1894 ; Neurol. Centralbl., Leipzig, 1894, Bd. xiii. S. 341 ; Mellus, Proc. Roy. Soc. London, 1894, vol. lv. p. 208; Boyce, Phil. Trans., London, 1895 ; Dejerine and Thomas, Arch. de physiol. norm. et path., Paris, April 1896; Rothmann, Neurol. Centralbl., Leipzig, June 1896 , and others.

${ }^{3}$ Flechsig and others.

* Schaffer, Neurol. Centralbl., Leipzig, 1895, Bd. ix. S. 386, found first sign of degeneration by the Marchi metliod on the fourteenth day in the cat ; see also Worotinsky, ibid.,Dec. 1897. On the ninth day in the cat I have found no sign of their degeneration by that method, but have on the twelfth day, and with carmine stain I got distinct evidence in the dog on the ninth day, Journ. Physiol., Cambridge and London, 1885, vol. vi. ; and Homen with acidfuchsin in the dog on the fifth day, Fortschr. d. Med., Berlin, 1885, S. 267. All observers are agreed that this onset is more tardy than that of degeneration in some other spinal fibres, especially those of Goll's column, where it is obvious in three to four days after spinal transection. Langley and Anderson suggest tliat small fibres are the earliest to show degeneration, "Proc. Physiol. Soc.," May 1894, Journ. Physiol., Cambridge and London, vol. xvi. p. 12.

${ }^{5}$ In the guinea-pig the pyramidal tract runs in the dorsal column of the cord.

6 v. Lénliossek, "Der feinere-Bau des Nervensystems," Aufl. 2, Leipzig, 1895; Monakow (Arch. f. Psychiat., Berlin, 1895, Bd. xxvii.) argue the intervention of a relay cell of the Golgi type, 2.

7 Sherrington, Journ. Physiol., Cambridge and London, 1890, vol. xi. p. 399 (Weigert's method); Mingazzini, Riv. sper. di freniat, Reggio-Emilia, 1892, tomo xviii.

${ }^{8}$ Starlinger, Jahrb. f. Psychiat., 1896, Bd. xv. ; Redlich, Neurol. Centralbl., Leipzig, 1897, Bd. xvi. S. 824 ; Schäfer, "Proc. Pliysiol. Soe.," Joum. Physiol., Cambridge and London, 1899, vol. xxiv. 
from this system pass in large numbers toward and into the base of the posterior horn and Clarke's column. ${ }^{1}$

\section{The Spinal Grey Matter.}

In the grey matter of the spinal cord lie the trophic centres, i.e. cellbodies or perikarya, for all the intrinsic spinal and spino-encephalic paths; also all the spinal synapses. ${ }^{2}$

Schiff ${ }^{3}$ supposed that "pain "-impulses ascend along the grey matter to the brain. The path for impulses connected with pain does (see "Cutaneous Pain," this vol., p. 965) plunge into the grey matter very soon after entering the cord; it then, probably, after a short course emerges into, in part at least, the opposite lateral column. The short in-andout traverse of the grey matter by this path is probably typical of all paths in the grey matter; they are probably all quite short. ${ }^{4}$ If all synapses lie in the grey matter, each path where it involves a passage from one link to another in a neural chain must enter the grey matter to establish its linkage; it probably soon emerges thence again. It is unlikely that there are spinal paths built up of any large succession of spinal links. Just as it seems that the spinal grey matter enters into the composition somewhere or another of every efferent path in the cord, including that of the simplest possible reflex, so it enters into the composition of every afferent path with the single exception of the remarkable long direct path of the dorsal column that first breaks into grey matter in the bulb; and therefore, speaking in conformity with the somewhat artificial scheme of delimitation generally employed, above the cord itself.

Very different in its appearance as regards detailed structure from the general spongiosa, is that portion termed gelatinosa which caps the dorsal horn. This gelatinosa probably forms an end-nucleus for a cell-system connected with some one particular set of sense organs. The gelatinous formation extends without break throughout the region of each segmental nerve, from the cranial fifth to the lowest coccygeal inclusive. It is extremely developed in the region of ending of the afferent root of the fifth cranial and in the spinal regions corresponding with the limbs. It is therefore probably less concerned with sense organs of viscera and muscles than with those of the skin; it may contain the first synapse for cutaneous pain. The paralysing effect of blood stasis in the lower part of the cord on cutaneous sensation agrees with this view. ${ }^{5}$ Certain chemical reagents, though acting but slightly on the peripheral nervous system with its adjunct motor endings and sense organs, extraordinarily affect the spinal cord. The difference probably rests on the grey matter of the latter with its abundant

${ }^{1}$ Schäfer, loc. cit.

${ }^{2}$ It is possible that some synapses occur outside the grey matter. Furtlier, Clarke's cell-column has a number of cells of aberrant position, especially in man, lying in the afferent root-zone.

3 "Plyssiologie," Lahr, 1858, Bd. i. S. 251 ; Arch. f. d. ges. Physiol., Bonn, 1882-1883, Bde. xxviii., xxix., xxx. ; also Wundt, "Grundzige d. physiol. Psychol.," Leipzig, 1893, Bd. i. S. 110-112; and Funke, Hermann's "Handbuch d. Physiol.," 18s0, Bd. iii. S. 2.

${ }^{4}$ Ciaglinski (Neurol. Centralbl., Leipzig, 1896, Bd. xvii.) finds degenerative evidence of a long ascending path in the central part of the spinal grey matter, but his observation stands unconfirmed.

5 Minzer and Wiener, Arch. f. exper. Path. u. Pharmakol, Leipzig, 1895, Bd. xxxv. S. 113 ; Langendorff, Arch. f. d. ges. Physiol., Bonn, 1898, Bd. lxxi. S. 401. 
perikarya and synapses. Among the agents are both depressants, as ether and chloroform, and stimulants, as strychnin.

Before it was established by Engelken ${ }^{1}$ and others that the intraspinal fibres are, like the peripheral nerves, excitable by various artificial stimuli as well as by natural, the spinal paths of conduction were spoken of as æsthesodic and kinesodic. These names (Schiff ${ }^{2}$ ) implied that the paths would transmit but would not initiate nerve impulses. ${ }^{3}$

The constant refuge sought at one period for an explanation of the difficulty connected with travel of impulses along the cord in spite of intervening incisions, was a liypothetical long patl up the continuum afforded by the grey matter. The grey matter is now no longer thought to offer such a continuum, and it is not at all probable that any conducting path continues long within it. ${ }^{4}$

Many of the fine fibrils contributing to the interlacement in the grey matter are "collaterals" from stem fibres. These appear to myelinate late and to degenerate early. The fine fibrils in the grey matter of the vesicular column of Clarke are usually much reduced in number, and may almost all disappear as a result of degeneration of the afferent spinal roots. ${ }^{5}$ After removal of the Rolandic area of the cortex in the monkey, ${ }^{6}$ I noted degeneration in some of the small fibrils of the grey matter of the ventral and lateral cornua of the cord. Mingazzini ${ }^{7}$ has studied the disposition of the decrease of the grey-matter fibrils in the human cord in lateral sclerosis. He concludes that the interlacement of all but the mesial part of the ventral horn is made almost entirely of pyramidal collaterals. To the postero-lateral group of the ventral horn pass, in addition to collaterals from the lateral tract, numbers of afferent root collaterals. Schäfer ${ }^{8}$ proves that the collaterals from the pyramidal tract pass toward Clarke's column and the base of the posterior horn; he denies that they go directly to the anterior horn. v. Lenhossék ${ }^{9}$ brings forward some evidence that even the "direct reflex collaterals" from the afferent roots do not reach actually to the efferent root cells, but act on them only through mediation of intervening cells or cell branches.

\section{Reflex Capacity of Fraghents of the Spinal Cord.}

Whytt ${ }^{10}$ was first to show that integrity of even a fraction of the length of the spinal cord suffices to enable reflex reactions to occur in response to skin stimuli, and therefore that reflex function does not depend on the integrity of the cord as a whole. Legallois ${ }^{11}$ extended similar observations to the mammalian cord. Striking examples of reflexes from a quite short isolated length of spinal cord are the sexual clasp by the fore-limbs of the male frog after section in front and

${ }^{1}$ Arch. f. d. ges. Physiol., Bonn, 1870, Bd. iii. S. 403 ; Biedermann, Sitzungsb. $l . k$. Akad. d. Wissenseh., Wien, 1883, Bd. lxxxvii. S. 3.

2 "Lehrbuch d. Muskel- u. Nerven-physiol.," Lahr, 1858.

${ }^{3}$ It must not be forgotten that we have still no proof of the exeitability of the grey matter of the cord in response to stimulation by artificial (eleetrieal and mechanieal) means.

${ }^{4}$ See to the contrary, Ciaglinski, note 4 , on previous jage.

5 Cf. Leyden, "Klin. d. Rüickenmark," Bd. ii.; Lissauer, Fortschr. d. Med., Berlin, 1884 ; Arch. f. Psychiat., Berlin, 1885, Bd. xvi. S. 431 ; Kranss, Neurol. Centralbl., Leipzig, 1885 ; Hadden and Sherrington, Brain, London, 1888, vol, xi. p. 325 ; Dejerine, Arch. de physiol. norm. et path., Paris, 1888, p. 331 ; Oppenheim, Arch. f. Psychiat., Berlin, 1889, Bd. xx. ; Tooth, "Spinal Degen.," 1889 ; Mott, Journ. Physiol., Cambridge and London, vol. xi. p. 12 ; Sherrington, ibid., vol. xiv. p. 255.

6 Journ. Physiol., Cambridge and London, 1890, vol. xi.

${ }^{7}$ Riv. sper. di freniat., Reggio-Emilia, 1892, tomo xviii. See also Fiirstner, quoted by v. Lenhossék, "Der feinere-Bau des Nervensystems," Aufl. 2, Leipzig, 1895.

8 "Proc. Physiol. Soc.," Mareh 1899, in Joum. Physiol., vol. xxiv. ${ }_{9}^{9}$ Op. cit., 405.

10 "On the vital and other involuntary motions of animals," Edinburgh, 1750.

11 " Euvres d. Car. Legallois, avee notes d. M. Pariset," Paris, 1830, tone i. 1. 135. 
behind the two nerve pairs of the brachial region, ${ }^{1}$ and the wagging of the monkey's tail after spinal transection in the anterior sacral region. $^{2}$ These are comparable with the sting reflex of the bee for which the hindmost abdominal ganglion pair is competent after severance of the longitudinal nerve-cords just in front of it. ${ }^{3}$ Mayo's ${ }^{4}$ celebrated experiment on what Grainger" calls "a segment of the cranial portion of the spinal cord," went far to draw attention to the fractional powers of the cerebro-spinal organ. Having isolated in the pigeon the "segment" composed of the optic tubercles and the crura cerebri (the optic and third nerves being attached) by section from the medulla oblongata and the brain, Mayo demonstrated that, on pricking the stump of the optic nerve adhering to the optic tubercle, the iris contracted.

Description of spinal function is best introduced by a glance at the organic reactions still possible in regions of the body whence the cord has been in toto removed. The destruction of the cord removes the corresponding region from all influence which the sense organs of its own or other parts can bring to bear on it; and also from the possibility of influencing, by means of its own sense organs, other parts. The rupture of these connections between it and the rest of the individual falls with very unequal severity on the various organs composing the part; it falls with especial severity on the skeletal musculature. It also much affects the circulation, at least for a time. If two frogs be placed under curari, so as to eliminate movements, and the brain and spinal cord of one be destroyed and of the other left intact, absorption of sodium chloride $(0.75$ per cent.) solution takes place much more copiously ${ }^{6}$ from the dorsal lymph sac of the frog with intact spinal cord than from that of the other. The destruction of the cord causes dilatation of the splanchnic vascular area, so that the major part of the blood remains almost stagnant in the portal blood vessels, and with the enfeebled circulation the absorption from the lymph sac is but slight. When in a mammal the lumbo-sacral legion of the cord has been exsected, ${ }^{7}$ the recovery of innervation by the tissues of the corresponding region appears, when tested by traumata, perfectly good. Ulcers occasioned by pressure, etc., heal readily when treated. The surface temperature is, after shock has passed by, maintained; so is the rectal. In the bitch the inguinal mammæ still yield milk in the puerperium. On application of cold to the skin, the blood vessels respond by constriction and later flushing. The hair is shed and renewed in due season. The rectum empties itself when distended; so also the urinary bladder, though not regularly. Pregnancy and labour occur, and in due course are brought to successful issue. The constrictor cunnce is not toneless, but fairly normally controls the vaginal orifice. The sphincter of the anus in time recovers tone, remains tight, and reacts vigorously to faradic stimulation. It also from time to time, especially immediately after defreation, exhibits the rhythmic relaxations and constrictions first 1866 .

${ }^{1}$ Spallanzani, "Opera," Modena, 1768 ; Goltz, Centralbl.f. d. med. Wissensch., Berlin,

${ }^{2}$ Sherrington, Phil. Trans, London, 1893, vol. clxxxiv. p. 641.

${ }^{3}$ Bethe, Arch. f. d. ges. Physiol., Bonn, 1897, Bd. lxviii. 136.

" Herbert Mayo, "Anat. and Phys. Commentaries," London, 1823, vol. ii. pp. 4, 18,

5 " Structure and Functions of the Spinal Cord," London, 1837.

${ }^{6}$ Goltz, Arch. f. d. ges. Physiol., Bonn, 1872, Bul. v. S. 53.

${ }^{7}$ Goltz and Ewald, ibid., 1896, Bd. Ixiii. 
noted by Gluge 1 in the spinal rabbit, later by Goltz ${ }^{2}$ in the dog. The skeletal musculature atrophies utterly, and comes to resemble connective tissue. The bones get brittle. Visible perspiration cannot-as far as noted in the dog-be induced.

These details serve to indicate the status obtaining in dogs preserved for even year-long periods after spinal exsection. The status is not, however, established forthwith. For a variable but not very prolonged period the ablation depresses the vascular function and tonus of the sphincters. These gradually recover. Congestion and œdema of the feet, diarrhœic stools, retentio urinc, patulous gaping of anal and vaginal ostia, are symptoms which in the course of some weeks subside. But the wasting of the striate musculature, save in these sphincters, is progressive and complete. If the spinal ablation involve the thoracic region, the tissue normally thick with muscle in the intercostal spaces becomes transparent and "as thin as paper." In the eel, when a length of the cord midway between head and tail has been destroyed, the musculature is permanently slack, although there is good co-ordination between the movements of the front and hind end of the animal in swimming. ${ }^{3}$ So in Lumbricus the ablation of a single abdominal ganglion leaves the local musculature permanently slack. ${ }^{4}$

These changes can be averted in any particular region by retention in that region of even a short fraction of the length of the spinal cord. And in addition, as discovered by Whytt, the solidarity of the whole cord is not necessary for the exhibition of local reflex reactions. When Amphioxus ${ }^{5}$ is separated by a couple of transections, each of the three pieces balances itself in water, and executes swimming movements that appear co-ordinate and result in an apparently-so far as the fragment represents the whole-normal locomotion, always with the head-end of the fragment foremost. Of the three pieces, that possessing "the head" exhibits movement spontaneously; but the two other pieces, although progressing co-ordinately under stimuli and maintaining normal posture, exhibit little spontaneity. The severed tail-fragment of Triton moves in response to contact. Reflex movements persist in the hind-limb of Rana when the piece between the sixth and eighth nerves is the only portion of cord undestroyed. Alternating side-to-side movements have been evoked from the tail of the monkey after spinal transection at the level of the penultimate sacral nerve root. These phenomena are comparable with the reflex attachment of the suckers provocable from even an amputated arm of Octopus on its contact with a solid object. ${ }^{*}$

In the frog, after spinal transection close below the brachial region, the reflex movements of the hind-limbs are distinctly co-ordinate. If a pedal digit be pressed, the limb is drawn up. The following is a usual sequence of events. First the dorsal flexors of the toes and tarsus act, then follows somewhat slow flexion at knee, then fairly synchronous contraction of the flexors of the hip and tarsus, so that the calf is deliberately placed against the thigh, the thigh in contact with the abdomen. Frequently the intact frog, in response to a similar stimulus, executes a movement to all appearance similar. Some groups

${ }^{1}$ Bull. Acad. roy. de méd. de Belg., Bruxelles, 1868.

${ }^{2}$ Arch. f. d. ges. Physiol., Bonn, 1873, Bd. vii. S. 582.

${ }^{3}$ Bickel, ibid., 1897, Bd. lxviii. S. 110.

${ }^{4}$ Maxwell, ibid., 1897, Bd. Ixvii.; Chicago Lab. Rep. of J. Loeb, 1898.

${ }^{5}$ Danilewski, Arch. f. d. ges. Physiol., Bonn, 1892, Bd. lii.

${ }^{6}$ W. B. Carpenter, "Priuciples of Physiology," 3rd edition, London, 1851. 
of muscles are more readily accessible to reflex stimuli than are others. The flexors of the knee and hip and ankle are more accessible than the extensors, and especially than the abductors of the digits. In the forelimb the adductors and the retractors of the upper-limb are the more accessible. The readiness with which reflexes can be elicited varies also with the region of the skin. As judged by pathic stimuli, e.g. by bits of paper soaked in acidulated water, the order of efficiency in descending scale runs in the frog somewhat as follows: anal region, first and second digits, plantar and dorsum pedis, outer side of knee, mesial half of dorsal aspect of lower half of leg, rest of leg except calf, outer side of thigh, upper half of calf, mesial side of ventral aspect of thigh, popliteal region.

The reflex of extension of the frog's hind-limbs is said to lie farther tailward than that of flexion. Gad ${ }^{1}$ found that after transection behind the eighth pair of spinal roots, i.e. behind the second of the three constituting the hindlimb plexus, all the conditions for the reflex of extension of the limb obtain. Sirotinin ${ }^{2}$ found the levels of maximum spinal excitability of the ilio-psoas semitendinosus and gastrocnemius to lie in the frog opposite respectively the fourth, fifth, and sixth vertebræ, both for mechanical and electrical stimuli. It is not clear, however, that the contractions he studied were always reflexly produced.

In the bird (pigeon) the post-thoracic spinal cord after isolation consummates various reflexes employing the musculature of the legs. The muscles are not toneless, but on the contrary tense. Contact with straw in the cage often suffices to excite wild "struggling" of the legs. ${ }^{3}$ If one of the dependent feet be, when the bird is lifted, pressed, it is drawn up, and the opposite leg is extended. If the extended leg is then gently passively flexed, extension of the contra-lateral, previously flexed, ensues. Not unfrequently, when the animal is lifted by the shoulders, alternate flexion and extension of the hind-limbs is started which may last more than a minute, flexion and extension alternating about twice a second. The tail feathers are immediately spread when the position of the bird, as held comfortably in the hand, is such that its head lies abnormally high, or the plane of the back is at greater inclination ${ }^{4}$ to the horizon than in its ordinary erect posture. ${ }^{5}$ Singer could not obtain inhibition of these reflexes by strong or pathic stimuli, as Goltz found possible in the dog.

In the mammal similar studies have been made. Evacuation of frees and urine ensue on rectal or vulval stimulation. Even with complete absence of the corresponding region of the spinal cord, these evacuations are more or less regularly maintained, the contents of the viscera exciting efficient contractions of the visceral museles. The contractions when the cord is present can be reflexly inhibited by strong cutaneous stimuli. Wagging movements of the tail can be elicited by stimuli to its skin. The musculature of the paralytic limbs is far from toneless. When the spinal transection lies in the lower thoracic region, the

${ }^{1}$ Verhandl. d. phys.-med. Gesellsch. in Würzburq, 1884, Bd. xviii. S. 8. Masius and Vanlair sech to have found section through the 8th root practically destroy the spinal reflexes for the parts of the limb not innervated from the 7 th. But their account is diffieult to follow; Mem. conron. Acad. roy. de med. de Belg., Bruxelles, 1869.

2 Arch. f. Physiol., Leipzig, 1887, S. 154.

${ }^{3}$ Sehitf, "Lehrbuch d. Pliysiol.," 1858, S. 196.

${ }^{4}$ Singer, Sitzungsb. d. k. Akad. d. Wissensch., Wien, 1884, Bd. Ixxxix.

${ }^{5}$ Goltz and Ewald, Arch. $f$. d. ges. Physiol., Bonn, 1896, Bd. lxiii. 
"knee-jerks," after the temporary period of "shock" is over, are more brisk than normal. Stimulation of the foot evokes drawing up of the leg and flexion at the hip and knee just as in the frog. The drawing up of the limb stimulated is accompanied usually by the pushing down of the opposite limb (active extension at hip, knee, and ankle), just as is often the case in the frog. To lift the animal by the fore-limbs frequently evokes rapid alternating flexion and extension of the hind-limbs like that observed in birds. The weight of the limbs probably produces tensions that are adequate and sufficient stimuli for apparatus of "muscular sense" within the limbs. The movements thus started can be brought to a standstill by strong "pathic" stimuli to skin, etc. When in the cat or monkey a "limb-region" of the cord has been isolated, reflex movements of the apex of the limb are easily obtained; the reflex movement of hallux and pollex of the monkey is commonly adduction-flexion, often combined with dorsal flexion of the basal-phalangeal joints. Extension of the wrist is not uncommon. In the cat and rabbit spreading of the digits in response to a touch on the foot is conmon; this is sometimes bilateral. Flexion at knee and adduction at hip are elicitable from almost the whole skin-field of the limb, but are feebler in the monkey than in the cat and $\log$. Irritation of the perineum elicits protrusion of the anus. Defæcation in the paraplegic monkey, as in the dog, soon becomes regular and efficient; it can sometimes be excited by irritation of the anus, but usually more effectually by pressure upon the bladder, and so probably upon the rectum, througl the ventral abdominal wall above the pelvis. The act is generally accompanied by elevation of the tail and flexion at hips and knees, and is followed by lateral movement of the tail. Micturition comes, after an interval immediately succeeding the spinal transection, to be fairly easily initiated and performed. On the crossed side to that excited, there occurs in the monkey quite frequently, as in the $\log$ and cat, extension at hip and knee synchronous with the homonymous flexion; adduction of the contra-lateral thigh occurs with perhaps peculiar frequency in the monkey. The cremasteric reflex is well seen in the monkey. In all types it is very usual for a touch on the side of the tail to evoke a lateral movenient of the tail, abducting it from the side stimulated, and for the tail to be depressed and drawn to the homonymous side on stimulation of the surface of the hind-limb.

If the isolated length of the spinal cord be thoracic, skin stimuli can in the dog evolse contraction of the lateral muscles, causing incurvation of the trunk with concavity toward the side of the excitation." Skin stimuli to the front of the thorax and back evoke "shaking" of the trunk, much like the movement made by a dog on coming out of water, twitching of the panniculus carnosus, and, by application of cold, a shivering confined to the musculature innervated by the isolated length of the cord. After high thoracic transection, rubbing the skin, especially on the side of the chest or scapula, evokes a "scratch" reflex, namely, rapid alternating flexions and extensions at ankle with protraction of the limb; this is a homonymous unilateral reflex, but reflexes obtained from the skin on the ventral surface of abdomen and thorax usually affect the limbs bilaterally.

Stimulation of a single afferent thoracic root evokes contraction in muscles, limited, roughly speaking, to the area of the corresponding

${ }^{1}$ Sherrington, Phil. T'rans., London, 1892. 
intercostal space; if the excitation be increased in intensity, muscles in the next space below and in the next space above likewise enter into contraction. The relation of skin (centripetal) imervation to muscle (centrifugal) innervation in this region is that the overlying skin is innervated from segmental nerves of a segmental level rather farther forward than the underlying muscle. ${ }^{1}$

If the isolated length of cord be brachial, the reflex movements readily obtainable by stimuli applied to the limb itself are, in the monkey, flexion-adduction of pollex, retraction at shoulder, extension at wrist, flexion at elbow, flexion of fingers, and protraction at shoulder. The hand, especially the palm, is a most efficient reflexigenous area.

From an isolated length of the cervical cord of the cat comprised between transections close above first cervical root and close below third cervical, retraction of the pinna can frequently be obtained with striking regularity and briskness, on excitation of its skin.

The most striking difference between the condition of a body-region with and without its spinal cord lies in the state of its skeletal musculature; in the former the muscles are not toneless ${ }^{2}$ nor altogether flaccid, nor do they atrophy beyond the effect of mere disuse. As regards the visceral and vascular musculature, there is far less difference between the two conditions. The visceral and vascular reflexes are, on the whole, most easily evoked by stimuli applied to reflexigenous regions that are themselves visceral or vascular, e.g. the greater reflex effect on blood pressure on exciting a thoracic root than on exciting merely its intercostal nerve. ${ }^{3} \quad$ Spinal reflexes playing upon skeletal musculature are on the other hand less easily excited from the viscera than from the skin and sense urgans of joints and muscles. The cutaneous stimuli which most potently evoke spinal reactions employing skeletal muscles, seem without doubt "pathic." Probably it is their "pathic" quality rather than their mere intensity as "pressure," etc., which renders them peculiarly efficient.

The functional powers exhibited by fragments of the length of the cord furnish the basis of a theory which regards the spinal organ as a linked chain of segmental centres. The morphological evidence of the segmentation of the body into metameres, each with its segmental nerve, collaterally supports this theory of its physiological segmentation. In lower forms the evidence of such is clearer than in higher; the theory is a good working hypothesis in light of which to study the functions of even such complex spinal organs as that of man. The spinal organ is certainly segmented in the sense that fractions of its length possess considerable functional solidarity. It is also in the same sense functionally divisible into lateral halves. The lumbo-sacral region of the cord can be split longitudinally with retention of the so-called "deep reflexes," e.g. "knee-jerks," unimpaired on either side. ${ }^{4}$ This recalls the relatively high degree of independence of function attaching to each of the pair of nerve cords in, for instance, the Arthropoda. ${ }^{5}$ It is the ill-understood phenomenon known as "shock" that especially tends to obscure the segmental analysis of the spinal functions in higher animals. The fact that the referred pains and areas of tenderness ${ }^{6}$ accompanying certain visceral affections are distributed over the skin surface in accordance with the scheme of distribution

I Sherrington, Phil. Trans., London, 1892.

? Marshall Hall, 1839.

${ }^{3}$ J. R. Bradford, Journ. Physiol., Cambridge and London, 1889, vol. $x$.

${ }^{4}$ Sherrington, Proc. Roy. Soc. London, 1892, vol. lii.

5 Bethe, Arch.f. d. ges. Physiol., Bonn, 1897, Bd. 1xviii.

${ }^{6}$ H. Head, Brain, London, 1893 , vol. xvi., and 1894, vol. xvii. 
of the cutaneous afferents of spinal roots rather than of the peripheral nerve trunks, furnishes evidence that there is still even in the most complex spinal organ some segmental partition in the framework of its function. The cutaneous and, where examined, muscular distribution of the afferent roots ${ }^{1}$ and the muscular distribution of the efferent roots is shown to be, even in the limb regions, as certainly and obviously segmental as is their fascicular connection with the spinal organ itself. The physiological architecture of even the mammalian limb exhibits therefore a segmental plan. On the other hand, the solidarity and homogeneity of the plurisegmental motor innervation of the individual limb muscles and of the plurisegmental centripetal bond between each practical unit of limb surface and spinal organ, warns against too lightly inferring that an ancestral segmentation is still upheld in the existing mechanisms of all the spinal regions of the vertebrate.

\section{The Spinal Animal.}

When,instead of being tested in parts, the spinal cord is experimentally examined as a whole, the reactions obtained are decidedly more complex. ${ }^{2}$

Amphioxus, after removal of its "head," on application of a stimulus, still swims forward and right side up, though not with normal precision. ${ }^{3}$ The decapitated eel swims well, turning, mounting, or diving, to normal appearance. Its equilibrium is, however, impaired, ${ }^{4}$ though when laid on its back it rights itself. ${ }^{5}$ The shark and ray still swim co-ordinately forward after decapitation or bulbar transection, but equilibrium is impaired. ${ }^{6}$

The "spinal" frog still retains tonus in the skeletal musculature, and can, it is said, spring." It is doubtful whether "spontaneous" movements, including respiratory, ever occur, even in animals kept for months under observation. When undisturbed, the creature sits with head abnormally low. The tonus of the muscles seems to have suffered some diminution, though in many cases it is practically impossible to obtain absolute assurance of this. During the period of shock immediately succeeding transection, it is obvious enough, but it is evanescent. The tonus of the extensors of the limbs seems to be more depressed than that of the flexors, at least at hip and knee. On an attenipt to passively draw the limb into extension, the whole animal may be pulled back without the limb being straightened. Similarly with the limbs of Astacus after transection behind the supra-œsophageal ganglion; the paralytic appendages are more flexed than normal at their "hip" joints," and the tail-fan is less spread. In the monkey, with thoracic transection I found that the hips and knees become strongly and permanently flexed after the lapse of six months. ${ }^{9}$ In a dog, six months after cervical transection, I found the ankles keep distinctly over-extended, but such rigidity is not the rule.

${ }^{1}$ Sherrington, Proc. Roy. Soc. London, 1892, vol. lii. : Phil. Trans., London, 1893.

2 Space unfortunately does not allow here of even the briefest sketch of the study of the reactions obtainable from the nerve-cord of Invertebrata. As contributory to a general conception of the "spinal" animal, these are of utmost importance. For recent work reference can be made to papers by Bethe, Loeb, and others, chiefly in Arch. f. d. ges. Physiol., Bonn, 1891, Bd. xlix. and onwards, and to Loeb's "Einleitung in die vergleichende Gehirnphysiologie," Leipzig, 1899.

${ }^{3}$ Danilewski, Arch. $f$. d. ges. Physiol., Bonn, 1892, Bd. lii.

${ }^{4}$ A. Bickel, ibid., Bd. lxviii. S. 111.

5 W. Pflïger, "Die sensor. Funcktionen d. Riickenm.," Berlin, 1853, S. 18.

${ }^{6}$ Loeb, Arch. f. d. ges. Physiol., Bonn, Bd. xlix.; Bethe, ibid., Bd. lxxvi., in part contra Steiner, op. cit.

${ }^{7}$ Pfliger, op. cit.; Schrader, Arch.f. d. ges. Physiol., Bonn, 1887, Bd. xli.

${ }^{8}$ Bethe, op. cit.

${ }^{9}$ Phil. Trans., London, 1897.

VOL. II. $-5^{2}$ 
In the spinal frog placed in water warm enough to form a skin stimulus, e.g. $36^{\circ}$ C., I have seen co-ordinate swimming, for a short time vigorous and executed with the normal bilateral stroke of the hind-limbs, and with movements of the fore-limbs. But the total co-ordination is distinctly less good than when metencephalon and myelencephalon remain. There is a tendency for the animal to dive deeper and deeper in the water; this seems due to the sunken position of the head: the loss of the semicircular canals cannot be without importance for this condition. Transection at the calamus almost immediately reduces the latent period for reflex movements of the foot as compared with the period when the section lies close in front of mesencephalon. If the reflex for a particular stimulus have been abolished by post-thalannic section, it is in a few minutes restored by post-calamic. But, on the other hand, the latency and liminal stimulus for the crossed reflex from one foot to the contralateral are less when transection is just above the calamus than when it is post-brachial, ${ }^{1}$ indicating some solidarity of the spinal organ as a whole in regard to limb movements. The spinal frog, when placed on its back, does not, as a rule, right itself, though it is said that the lizard and the salamander do; in some spinal frogs, inversion excites very active general movements much more than does any general stimulation of the dorsal skin when the animal is not inverted; but the inversion is not corrected. It is true that spontaneous movements are not observable with anything approaching the number and variety shown by the frog possessed of the lower part of the encephalon. At the same time one does see in the spinal frog from time to time changes of position that are not very clearly related to any detectible stimulus. Volkmann's ${ }^{2}$ experiment, namely, the placing of the hind-limb in a posture of extension during the period of spinal shock, with the result that, as the shock is recovered from, the limb is drawn up to the sitting posture, can hardly be cited, as he cited it, in instance of a movement without external stimulus. An external stimulus to the muscular sense organs of the limb is legitimately postulable. More "spontaneous" in character are occasional short fits of crawling, for which an external stimulus is less easy to assign. ${ }^{3}$ But the whole question is one of degree rather than of kind, if the definition above offered for spontaneity be accepted in the matter. It is to low forms, and especially to invertebrate, that resort has to be made for salient examples of spontaneity persistent after removal of the cephalic portion of the nervous system.

That the whole spinal cord presents no markedly greater competence in spontaneity than do mere regions of it, may be because its complete length includes no additional kind of sense organ above those distributed fairly equally throughout the body segments belonging to each of its component lengths.

In all the various examples in which co-ordinate progression has been observed as a function of spinal animals, the progression is always, in a sense, of one type only, namely, in the direction habitual to the species. The normal insect and Astacus $^{4}$ can go backwards as well as

${ }^{1}$ Rosenthal (in frog), Biol. Centralbl., Erlangen, 1881 ; Hallstèn, Arch. f. Physiol., Leipzig, 1886 ; Bickel (in Lacerta), Arch. f. d. ges. Physiol., Bonn, 1898, Bd. lxxi. S. 44.

2 Mïller, Arch.f. Anat., Physiol. u. wissensch. Med., 1838, S. 17.

3 Redi, "Opusc.,"Venezia, 1742, vol. iii. p. 290. The spinal tortoise crawls.

Foster, Huxley, Ward, Bethe. 
forwards; both when "spinal" go forwards only and not backwards. So also with Amphioxus and the eel, ${ }^{1}$ frog and tortoise.

The young puppy, after severance of the upper part of the medulla oblongata, has been found, ${ }^{2}$ on application of a moistened finger to the mouth, to suck the finger, wrapping the tongue round it, and at the same time pushing out the legs, as young animals do when sucking. The anencephalous fotus sucks when put to the breast.

The condition of the cat and dog after transection at the calamus scriptorius is not very dissimilar from that of the spinal frog, respiration being artificially maintained, and the animal's temperature kept from getting low. The arterial blood pressure is low at first, but that seems to have little immediate effect upon the reflex functions of the spinal cord. As the first "shock" passes off, a number of reflexes become patent, including combined novement of all four limbs, of such a character as to suggest that it represents a portion of the mechanism of progression.

\section{Special Reflexes.}

The cord may, in its relation to the sensifacient surface and the skeletal musculature, be considered divisible into right and left lateral halves, each subdivisible into regions of neck (cervical, including pinna), fore-limb (brachial), trunk (thoracic), hind-limb (crural), and tail (caudal). A reflex action in which the stimulus applied to a reflexigenous area in one of the above regions evokes a reaction in the musculature of another of the regions, is conveniently called a long spinal reflex. A reflex reaction in which the muscular reply occurs in the same region as the application of the stimulus, is conveniently called a short spinal reflex. Short spinal reflexes are, as a rule, more easily and regularly elicitable than are long spinal reflexes. It might further be convenient to allocate hard-and-fast boundaries to these regions, but such limits would of necessity be artificial and arbitrary. The scope of the delimitation is indicated and its purpose better served by comparing with one retina, say of the bird, the one lateral half of the skin ; and with the corresponding optic nerve, the corresponding lateral half of the spinal cord and bulb. Between these comparable surfaces a difference exists, in that the reflexigenous field of the skin, unlike the retinal, has instead of the one focal region of concentrated sensitivity, a couple at least of such foci, namely, the relatively highly sentient skin at the apex of each limb. But as the retina has muscles at call, so also the skin. The closeness of nexus between a retinal point and the visual musculature is graduate in degree, e.g. most close for the muscles of its own bulbus, next for those of the contralateral, then for the neck muscles, etc. Similarly there are degrees of nexal closeness between a point of skin and the related musculature; its connection is most close with muscles of its own limb, next with those of another limb or other region. The sole interest for physiology of the direction of nervous irradiation per se-apart from light it may incidentally cast upon the general physiology of nerve - lies in its elucidation of the machinery for working sentient surfaces. That the skin is a region which morphologically considered is composed of a segmental series, seems to have been allowed greater weight in the estimation of its reflexigenous functions than is altogether justified, at least in the higher vertebrata. That its segmental innervation demonstrably limits existing reflex spinal functions in the mammal, is not shown.

The direction of spread of impulses in spinal rellex actions.Regarding short spinal reflexes, and the directions taken by the ex-

${ }^{1}$ Bickel, Arch.f. d. ges. Physiol., Bonn, 1897, Bd. 1xviii.

2 Grainger, "Functions of the Spinal Cord," London, 1837. 
amples of intraspinal irradiation which they furnish, it is possible to make certain general statements. ${ }^{1}$

i. Broadly speaking, the degree of reflex spinal intimacy between afferent and efferent spinal roots varies direetly as their segmental proximity. Thus excitation of the central side of a severed thoracic root, e.g. seventh, evokes with especial ease contraction of muscles or parts of muscles innervated by the corresponding motor roots, and next easily muscles innervated by the next adjacent motor roots. The spread of short spinal reflexes in many instances seems to be rather easier tailward than headward. This may be related with the oblique correlation that so largely holds between the distribution of the afferent root in the skin, and the distribution of the efferent root in the underlying muscles. ${ }^{2}$

ii. Taken generally, for each afferent root there exists in immediate proximity to its own place of entrance in the cord (e.g. in its own segment) a reflex motor path of as low resistance as any open to it anywhere. Further, in response to excitation of even approximately minimal intensity, a single afferent root, or a single filament of a single root, evokes a spinal discharge of centrifugal impulses, which tends to occur not only through its own efferent root but through more than one efferent root, i.e. is plurisegmental. And this holds especially true in the limb regions. In the limb region the nerve root is therefore a morphological aggregate of nerve fibres, rather than a functionally determined assortment of impulse paths. The formation of functional collections of nerve paths (peripheral nerve trunks) out of morphological collections (nerve roots) seems to be the meaning of the limb plexuses.

iii. Motor mechanisms for the skeletal musculature lying in the same region of the cord, and in the self-same spinal segment, exhibit markedly unequal aceessibility to the local afferent channels as judged by pressor effects. ${ }^{3}$ For example, if the primary phase only of the reflex movement be considered, the flexors of the homonymous knee and the extensors of the contralateral are, in most animals, much more accessible than the extensors of the homonymous and the flexors of the contralateral. Inasmuch as at many joints the Hexors and extensors are both innervated by motor fibres contained in one and the same efferent root, it follows that the reflex movement obtained from excitation of an afferent root in many cases is quite dissimilar from the movement obtained by excitation of the corresponding efferent root, ${ }^{4}$ in spite of the rule of segmental proximity.

iv. Where a spinal reflex discharge is prolonged, it usually involves antagonistie sets of motor cclls alternately; it is in each of these several phases that statement iii. holds good, not in the whole discharge taken collectively as a single discharge. It then only applies when, as in very many instances, the reflex movement is not prolonged, and there is no secondary reversal.

v. The groups of motor nerve cells contemporancously discharged by spinal reflex action innervate synergic and not antergic museles. This

1 Sherrington, Phil. Trans, London, 1897.

2 Sherrington, ibid.; W. Page May, ibid., 1896.

3 It seems necessary to insert the qualification "pressor" before "effects" in the above statement, because there is some evidence ("reciprocal innervation") that inhibitory effects accompany the pressor effects, and it may be only in regard to pressor effect that in any particular ease the above statenent loolds.

t Sherrington, Phil. Trans., London, 1892 ; W. Page May, loc. cit., 1896. 
is the reverse of the view that since Winslow and Duchenne has been a common doctrine concerning muscular co-ordination. It controverts an argument adduced for the view that the limb movement evoked by excitation of an efferent root represents a highly co-ordinate functional synergism. ${ }^{1}$ The spinal reflex in its intraspinal irradiation develops a combined movement and synthesises a nuuscular harmony.

vi. It follows almost as a corollary from this, and from the rule of spatial proximity, that the spinal reflex movement elieitable in and from any one spinal region, will exhibit much uniformity despite considerable variety of the locus of incidence of the exeiting stimulus. Approximately the same movement, e.g. in the hind-limb flexion of the three great joints, will result, whatever piece of the limb surface be irritated. The seat of incidence of the stimulus will only influence the character of the general movement executed by the limb musculature, in so far that the flexion will tend to predominantly occur at that joint the flexor muscles of which are innervated by motor cells segmentally near to the entrance of the afferent fibres from the particular piece of skin the seat of application of the stimulus.

Regarding the course of irradiation in long intraspinal reflcxes, namely, those spinal reflexes that initiated from one of the abovementioned spinal regions spread over into others, Pflüger, in 1853, formulated four "laws." 2 He argued chiefly from a careful collation of the reports of a number of clinical cases. These "laws" have for many years been widely accepted. They are stated as follows:-

1. The law of homonymous conduction for unilateral reflexes. If a stimulus applied to a sensory nerve provokes muscular movements solely on one side of the body, that movement occurs under all circumstances and without exception on the same side of the body as the seat of application of the stimulus.

If, as is clear from the context in the original paper, by movement on the same side be meant contraction of muscles on the same side, this statement does not in reality very completely express the facts. It is in part an outcome of the rule of spatial proximity, but certain cases which conform to the latter yet offer striking exception to the former; for instance, when the tail is touched on one side, it very frequently is moved towards the opposite, and this in a great number of classes, from the fish to the mammal inclusive.

2. The law of bilateral symmetry of the reflex action. Then the change produced in the central organ by excitation of a sensory nerve has already evoked unilateral reflex, it, if it spreads farther, excites in the contralateral half of the cord only those motor mechanisms which are symmetrical with those already excited in the homonymous half of the cord. This statement, although true of a number of instances, fails to conform with fact in many, even perhaps the majority.

The important crossed reflex from the hind-limb of the bird and mammal does not conform to it; so similarly with the fore-limb. The asymmetry of the crossed reflexes of the limbs is important, because probably connected with the fundamental co-ordination of muscles for progression. Again, the wag reflex of the tail, and a reflex I have called the "torticollis reflex" (cervical region), afford important exceptions to the "law." And many other exceptions can be

${ }^{1}$ Ferrier and Yeo, Proc. Roy. Soc. London, 1881, vol. xxx.; P. Bert and Marcacci, Sperimentale, Firenze, Oetober 1881 ; J. R. Russell, Proc. Roy. Soc. London, 1892, vol. li. Ferrier has more recently (Brain, London, 1893) much modified his conclusions on this point.

2 "Die sensorische Funktionen d. Rückenmarkes," Berlin, 1853. 
found. In the spinal rabbit, on the other hand, the crossed reflex from one hindlimb to the other is often not an asymmetrical movement, but a symmetrical flexion; this seems to stand in obvious relation to the hopping mode of progression of the animal.

3. The law of unequal intensity of bilateral reflexes. When the excitation of a sensory nerve elicits reflex action involving both halves of the body, and the action is unequal on the two sides, the side of stronger contractions is always that homonymous with the seat of application of the stimulus.

This statement is in conformity with a number of instances. The following are examples. When bilateral retraction of the abdomen is excited from the skin of the chest, the contralateral retraction is much the less marked; in the bilateral protraction of the "whiskers" (cat, rabbit, dog) on excitation of the

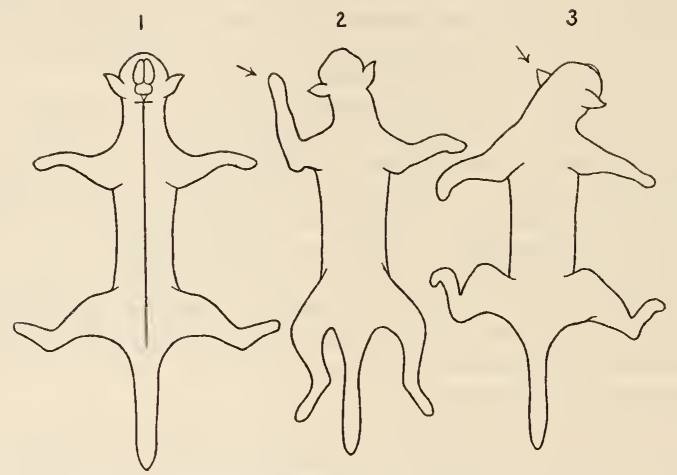

FIG. 356.-Diagram of the different movements of the head and fore-linib (spinal cat) caused by stimuli applied to the fore-paw and to the pinna respectively ; the former evokes a movement of the head toward the stimulus with protraction and lifting of the fore-foot, the latter a retraction of the fore-limb and a twisting of the head toward the opposite side ("torticollis reflex"). 1 , The pose of the spinal animal before the excitation ; 2, pose after stimulation of left fore-paw ; 3 , pose after stimulation of left pimin.

skin of the face, the crossed movement is the less ample. An interesting illustration, because it involves inhibitory as well as pressor influence, can be demonstrated in the spinal cat or dog thus ${ }^{1}$ :- The animal resting comfortably on its back, if one hind-paw be pressed, that leg will be flexed at hip, knee, and ankle, and if the stimulus be strong, or the reflex excitability good, the fellow hind-limb will be extended. If instead of one hind-paw both hindpaws be pressed, both hind-limbs are simultaneously flexed, and there is no trace of extension. The homonymous reflex is prepotent, therefore, and inhibits the crossed reflex.

But there are also a number of exceptions to this "law," among others, the abduction of the tail from the side stimulated, already referred to.

4. The fourth of Pfliiger's classical "laws" of spinal reflex action states that with associated spinal reflex centres the irradiation spreads more easily in the direction toward than in the direction away from the head.

${ }^{1}$ Sherrington, Phil. Trans., London, 1897. 
My own experience in the mammal is far from completely accordant with this statement; in, I think, the majority of instances, irradiation has spread more easily down than up the cord. It is easy to obtain reflex movements of the limbs and tail by excitation of the skin of the pinna, whereas the reverse is rare. To elicit by excitation of the hind-limb a movement of the fore-limb, is more difficult than by excitation of the fore-limb to elicit movement of the hind-limb. To elicit movement of the tail by excitation of the fore-limb, is easier than to move the fore-limb by excitation of the tail. The irradiation has in my experience been easier across the cord from hind-limb to hind-limb, than from lind-linb to fore-limb; but it is often easier down the cord from fore-limb to hind-limb, than across from fore-limb to fore-limb. In such reflexes also as the "shake" reflex (see p. 815), which implicate the trunk more than the limbs, the radiation is away from the head, for it is well obtained as a rump reflex when the skin of the slioulder is the part rubbed. ln the "scratching" reflex, too, the skin stimulus is applied far headward of the region of the muscular contraction evoked.

These so-called "laws" of reflex irradiation have been so generally accepted as to have obtained a doctrinal importance which they really hardly merit. It seems here less profitable to attempt adapting Pfliger's "laws" so as to make them better fit the observed facts, than to briefly describe the salient features of the long intraspinal reflexes as exhibited in an ordinary experiment on the spinal mammal.

When the animal is supported freely from above with its spinal axis horizontal, and the attitude of the limbs determined by gravitation, a point that early strikes the observer is that there are eight areas of skin whence spinal reflexes employing skeletal musculature can be provoked with pre-eminent facility. These areas are the soles, the palms, the pinnæ, and the tail and cloacal region. It is significant that seven of these areas are those which possess the greatest range of motility if the axis of the animal be considered fixed. From the pinna may be excited movements of all the limbs, the neck, the tail, and trunk. The irradiation from this reflexigenous area usually presents the following order-(1) Neck and homonymous fore-limb, (2) homonymous hind-limb, (3) tail and trunk on both sides, (4) contralateral hind-limb, (5) contralateral fore-limb. From the fore-foot can be excited, besides movements in the fore-limb itself, movements in the other limbs and tail. The facility of irradiation is usually in the following descending series-(1) homonymous hind-limb and the tail, (2) crossed hind-limb, (3) crossed fore-limb. The relative facility of spread of the reaction to the crossed fore-limb seems subject to much variation. In the frog the path between the two fore-limbs is, especially in the breeding season, very open and facile. In the cat and monkey, it seems to be much more open in the bulbo-spinal than in the spinal amimal. From the hind-foot the frequency and ease of irradiation into other spinal regions usually appears to exist in the following order - (1) crossed hind-limb and tail, (2) homonymous fore-limb, (3) crossed fore-limb. The facility of "spread" from one lateral half of the cord to the other is very dissimilar at different levels of the cord. It is particularly easy in certain parts of the tail region, and these motor mechanisms which are yoked together are for the most part, as with the flexion and extension of the hip and knee, of an asymmetrical kind. In the hind-limb region the crossed irradiation is also fairly free, and largely connects asymmetrical muscle-groups; but one of the most facile and persistent of all bilateral reflexes resulting from unilateral stimulation is the adduction of both thighs, a bilaterally symmetrical movement.

In the trunk the spread across the median plane is not so free in some animals as might have been expected: the yoking is of bilaterally symmetrical muscles. 
To excite movement of one fore-limb from the other is less easy than to excite one hind-limb from the other, at least in many animals. In the neck region irradiation across the median sagittal plane is fairly easy, and the yoking connects in large part asymmetrical muscles.

On the whole the long spinal reflexes are more variable and less certain and validly predictable than the short. They vary in a series of experiments, not only as to order of relative facility of direction of irradiation, but especially as to the sense of the movement elicited at the joint, whatever it may be, to which irradiation extends. Not unfrequently a region to which the reflex action usually demonstrably irradiates is altogether omitted, and omitted consistently throughout the whole of a lengthy experiment, although the spinal region in question has as far as known suffered no damage, nor indeed been

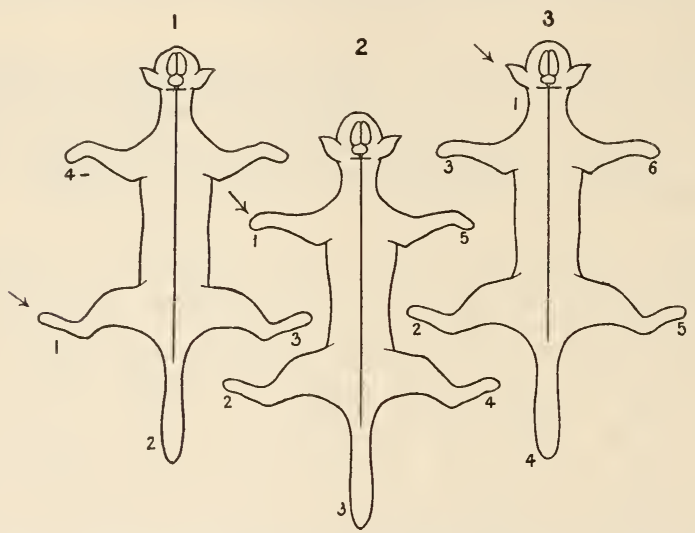

FIG. 357.-Diagram of irradiation of reflex movement in spinal cat in response to stimuli applied-1, to left hind-foot; 2 , to left fore-foot; 3 , to left pinna. The movements of the head are inchided in 1 and 2 . The numbers attached to the limbs, etc., indicate the order nsually taken by the parts in the "march" of the reaction.

directly implicated in any of the procedure. Thus excitation of the skin of the neck or pinna will sometimes spread back along the cord and produce movement in the tail or in the hind-limbs, and in doing so pass by the fore-limbs without evoking a twitch in either of them. The motor mechanisms of the fore-limb thus skipped over may show no sign, when examined by the local reflexes, of being less amenable than usual. Besides the limb movements, some other reflexes involve considerable co-ordination of trunk muscles, thus the "shaking" of the body on stimulating the skin of the back and shoulder. This seems analogous to the shaking of the head and ears on stimulating the ears, well seen in the bulbo-spinal dog. Related to it appears the "scratching" movement called from the hind-limb by rubbing the flank or shoulder.

There seems in the spinal mammal a disproportionate restriction of the range of spread of the reaction below the normal range. It is true that in response to a stimulus, e.g. of the skin of the normal animal, no 
obvious response may be forthcoming. Yet, in many cases, that does not make us for a moment disbelieve that there has been spread of nervous reaction to the excitation. We say "it was felt, but caused no movement." When movement occurs, it is interesting to note the diversity of range it habitually exhibits, the slightest movement of one part being associated with adjusted novements of others. The widest irradiation of a spinal reflex is poverty-stricken compared with such habitual normal reactions. Further, the incidence of the paralysis falls, as shown by the course of the spinal irradiation, unequally upon different regions of the musculature. 'The extensor nuuscles of the limbs, just as they suffer most depression of tonus, seem also, at least in regard to the primary phase of reaction, to be reflexly more paralytic than are the antagonistic flexors. But observations on this point are still scanty under circumstances where the initial spinal shock has passed off. There is some evidence that after a time the extensors of the hip recover much briskness of reaction even in the spinal mammal. ${ }^{1}$

In spinal reflexes, the course of irradiation, as compared with that of normal reactions elicited by a similar external stimulus, can be foretold with relative success. The limb on being touched will be Hexed at certain joints; this can be said of the spinal animal with far greater likelihood of its proving correct, than the normal animal; but even under the most rigorous conditions the fatality of the reaction in the spinal animal is far from absolute. The field of musculature provoked to action can be predicted with greater certainty than can the direction the movement will take. It cannot be definitely predicted that the normal cat when touched on the planta will move the limb touched; a movement of the eye or head may follow, and none of the limb. In the spinal cat, it can, however, be predicted with much certainty that such a stimulus will cause a movement of flexion of that limb the skin of which is stimulated.

Influence of the quality of the stimulus.-The external stimuli which are excitant of naked nerves, namely, those known as "general" nerve stimuli, e.g. mechanical, thermal, chemical, and electrical, are all efficient to evoke spinal reflexes. The adequate stimuli for the cutaneons end-organs are also efficient. Inasmuch as the end-organs have been adapted and specialised for certain external stimuli, the threshold value of the adequate stimuli is low as compared with that of the general stimuli, and this is especially evidenced in their reflexigenous power. This probably explains the fact first noted by Marshall Hall, ${ }^{2}$ that the stimulation of a nerve trunk is ceteris paribus less qualified to generate a reflex action than is the stimulation of the skin, although the stimulation of a nerve trunk represents the stimulation of a relatively large sensifacient surface.

At the same time it must be remembered that the "general " nerve stimuli stand in peculiar relation to pain, at least to cutaneous pain; and further, that pathic stimuli seem peculiarly efficient for producing spinal reflexes. The movement provoked may differ with the quality of stimulus: thus, the tail of the eel is made to approach by a tactile

1 The greater activity of flexors than extensors after spinal transection has been noted by Sanders-Ezn, Wundt. Lombard, and many others. But primary extension at hip is not uncommon when time has becn allowed for subsidence of shock. At the ankle-joints (dog) the paraplegic posture oftcn comes in the course of weeks to be rather over-extension than over-flexion.

2 "Memoirs on the Nervous System," London, 1837. 
stimulus, to withdraw by a pathic (Pflüger). It is curious that the sense organs of both skin and muscle seem to evoke reflexly the same movements one as other. Irritation of the skin of the foot evokes flexion at knee and hip; so also does stretching the muscular sense apparatus in the pedal muscles and ligaments. Certain reflexes are, however, peculiar to skin stimuli; such are the "scratch" reflex and the "shake" reflex in the spinal dog. These are elicitable by rubbing the skin of the trunk, especially of shoulder, back, and flank. They are rhy thmic alternating movements.

Mechanical stimuli applied to the skin were those earliest employed in the study of reflex actions. Not only the application of the mechanical stimulus but the cessation of its application is reflexigenous. ${ }^{1}$ Of chemical stimuli the application of acidulated water to the skin of the frog was methodised by Türck, ${ }^{2}$ and his plan has been used in many subsequent researches. The fluid is used by bringing it up to the foot or by bits of soaked paper of measured area allowed to rest on the skin a definite time, the skin being then washer with fresh water. The threshold value for chemical and for thermal stimuli applied to the skin of the spinal frog varies greatly in different experiments. The constant current both at opening and closure can excite reflexes under appropriate conditions. ${ }^{3}$ Pfliger's law regarding the production of the muscular twitch through the motor nerve by galvanism, is found to apply when examined in reflex preparations (frog). ${ }^{4}$ The reflex movement evoked by the break of the current is apt to be peculiarly prolonged. Single induction shocks can also excite reflexes, ${ }^{5}$ but to do so must be relatively very strong. Faradisation easily excites reflexes. Both with faradic and with mechanical stimuli, and both when the stimulation is of cutaneous and of muscular sense structures, it is very usually found that the discontinuance of a prolonged stimulation, especially when that stimulation is itself becoming or has become inefficient, provokes a fresh outburst ${ }^{6}$ of discharge from the spinal centres. After stretching a muscle by its tendon steadily and then suddenly relaxing its strain, a fresh reflex movement results; this is a phenomenon closely similar to that noted by Head ${ }^{7}$ in studying the effects of the vagus nerve on the activity of the respiratory centre for the diaphragm.

The spinal animal owes much of its interest as a field for study of 'reflex action to the combination it offers of a relatively homogeneous sensifacient surface comnected with a large and varied machinery of expression, its musculature. The spinal transection above cuts off all influences of light, sound, taste, smell, fron that machinery, and in large measure the very visceral organs. The projicient senses are gone. Tactile and thermal stimuli are well-nigh the only phenomena of the purely external world remaining to it, and of the "material me" the chief remaining phenomena are those adequate for the so-called muscular sense, and in some part the visceral. The quality of the stimulus, as determined by the particular avenue of sense through which it impinges on the central organ, would influence, one might think, the character of the reaction. The spinal frog, when its skin is

1 Sherrington, Phil. Trans., London, 1897.

2 Zlschr. d. k. -k. Gesellsch. d. Aerzte zu Wien, 1851, Bd. vii. S. 189.

3 Setschenow, "Ueber d. elektrische u. chemische Reizung d. scns. Ruickenm. d. Frosches," Graz, 1868; Fick, Arch. f. d. ges. Physiol., Bonn, 1870, Bd. iii. S. 326 ; Tarchanow, Bull. Acad. imp. d. sc. de st. Pétersbourg, 1871, tome xvi. p. 65.

${ }^{+}$Hallstèn, Arch. f. Physiol., Leiprig, 1885, S. 188.

${ }^{5}$ Setschenow, op. cit.; Fick, op. cit.

${ }^{6}$ Sherrington, Phil. Trans., London, 1897.

${ }^{7}$ Joum. Physiol., Cambridge and London, 1889, vol. x. 
removed, still shows reflexes in response to displacements of its limbs. The spinal bird exhibits "struggling" movements in its dependent limbs; so similarly the spinal mammal. Though stimulation of the skin is not thoroughly excluded in such observations, the exciting stimulus is probably in chief part of the nature of muscular tensions and positions. $^{1}$ When judged by effects on the rhythm of respiratory novement (the transection being in the bulb), and on arterial blood pressure, the faradisation of the central end of a purely muscular nerve in my experience, differs in its resultant reaction from that of a purely cutaneous, chiefly in exciting a less intense reaction, i.e. quantitatively rather than qualitatively. The mechanical stimulation of the muscles of the rabbit is however said to invariably cause a slight fall in arterial pressure, whereas the mechanical stimulation of the skin causes a rise. ${ }^{2}$ Bradford ${ }^{3}$ found that, as regards effect on blood pressure, the faradisation of the central end of an intercostal nerve was far less efficient than excitation of the parent trunk including the contribution from the sympathetic, that is, including visceral afferent fibres. The visceral afferent appears, therefore, to be peculiarly efficient for vasomotor reflexes.

When artificially applied "general" stimuli are employed, reflex movement is usually ${ }^{4}$ more easily evoked by excitation of the central end of the afferent spinal nerve root than by excitation on the central side of a severed peripheral nerve; this difference is often very marked indeed. But though it is easy to evoke spinal reflex movements through the afferent roots and their filaments, it must be remembered that the reflex so obtained is obtained under conditions unfitted to exemplify reflex spinal movements of normal occurrence. The mammalian spinal root is less a functional combination than a morphological one. To excite the whole of a single afferent root is to produce a condition probably never under normal circumstances existent. The root reflexes-of which a list is appended to this article-must, in order to be of use for the understanding of the working of the cord, be considered in relation with peripheral nerve reflexes, skin-spot reflexes, muscular sense reflexes, etc.

Among adequate stimuli, a class that are efficient for production of reflexes but of nature as yet insufficiently deternined by analysis, are those which institute and determine many reflexes of posture. The resumption of the right-side-up posture by the spinal eel, and by the headless Amphioxus or Mantis or Grillotalpa, and even by the amputated ray of Asterias, are reactions to contact (stereotropic) or to gravitation (geotropic); in the last-mentioned instance Loeb's ${ }^{5}$ experiments indicate stereotropism. Singer's reflex ${ }^{6}$ spreading of the tail feathers of the spinal bird is on a par with the above. In these and many other

${ }^{1}$ Reflex contraction of the gracilis can be regularly obtained by pinching the tibialis anticus tendon (cat). Fairly quick passive cxtcrision of the hind-limb of the spinal dog often causes activity (contraction) of the extensors of the same knee, sometimes of both knees. This occurs when the skin has been desensitized.

${ }^{2}$ E. A. G. Kleen, Skondin. Arch. f. Physiol., Leipzig, 1890, Bd. i. S. 247.

3 Journ. Physiol., Cambridge and London, 1889, vol. $\mathrm{x}$.

${ }^{4}$ Sherrington, Phit. Trans., London, 1897, in the mammal, but in the bird Vulpian has noted the converse ("Lecons sur le systeme nerveux," Paris, 1866.

${ }_{5}$ Arch. f. d. ges. Physiol., Bonn, 1891, Bd. xlix.; Preyer, Ifitth. a. d. zool. stat. zu Necopcl, Bd. vii. S. $96 . \quad$ Also Loeb, "Einleitung in d. vergleich. Gehimphysiol.," Leipzig, 1898.

${ }^{6}$ Vide supra, 1). 814. 
instances the assigning of the excitation to its particular group or groups of adequate stimuli has still to be done. Meanwhile it is well to keep in mind analogy with plant reactions, for instance, the reaction of vertical climbing shown by many insects (Coccinella) and by echinoderms (Cucumaria) with the "negative geotropism" of plasmodia of Myxomycetes. The extent to which, even in absence of connection with the highest parts of the nervous system, the lower reactions are recognisant of differences between different adequate stimuli, is illustrated by the rejection of bits of paper soaked in sea-water by the regenerated tentacles of Cerianthus, while bits of crab-flesh are accepted and carried toward the artificial month. ${ }^{1}$ The chelate limbs of Limulus and Astacus, ${ }^{2}$ after ablation of the supra-œsophageal ganglion, and in the former when the limb is isolated from the sub-œsophageal ganglion as well, react differently according as a piece of wood or of food is given to them; in the latter case, the reflex movement carries the morsel to the mouth, in the former the object is let fall. Again, the water-beetle (Dytiscus), after removal of the cephalic ganglia, remains motionless so long as it rests on a dry surface, but when placed in water executes the usual swimming motions with even excess of energy and rapidity. ${ }^{3}$

Summation of stimuli.-A single induction shock, the discharge of a Leyden jar, a single prick, etc., can excite a reflex action. Such brief " single" stimuli must, however, in order to produce reaction, be violent, indeed, so violent as to injure the sensifacient tissue too much for repetition. Even when thus strong, they are less efficient than less severe stimuli applied not singly but repeatedly. Stimuli far too weak to singly evoke reflex reaction, are easily able to do so if repeated. With separate electrical or mechanical stimuli, the reflex tends to occur after a given number of stimuli have been applied, although the time-intervals between the individual stimuli may be varied within wide limits,-e.g. ranging between 05 sec. and 4 sec. $^{4}$ There seems to result an addition of the effects of the separately inefficient stimuli up to a point at which they ultimately produce the effect,-e.g. the reflex discharge. This phenomenon is akin to the "latent addition" of subminimal stimuli by muscle and peripheral nerve. It has been suggested ${ }^{5}$ that perhaps no single stimulus whatever is in reality efficient to excite a refiex reaction, those instances in which a single stimulus appears to do so being explicable by the prolonged and repetitive stimuli set up by the accompanying injury done the sensifacient tissue. When applied to the spinal cord severed from the bulbar vasomotor centre, single induction shocks have no effect upon arterial pressure, or have only a minimal effect, even when the shocks are of extreme intensity. ${ }^{6}$ On the other hand, shocks of moderate intensity produce marked vasomotor effects when applied to the cord at a frequency of twenty per second. A repetition at the rate of twice or thrice a second makes them effective.

Intensity of the stimulus. - The threshold stimulus for exciting contraction of a muscle from the afferent spinal root is higher than

${ }^{1}$ Loeb, "Untersuch. z. physiol. Morphol. d. Thiere," Würzburg, 1891.

2 Bethe, Arch. f. d. ges. Physiol., Bonn, 1897, Bd. lxviii.

3 Carpenter, "Reflex Action in the Nervous System of Invertebrata," London, 1839.

4 J. Ward, Arch. f. Physiol., Leipzig, 1880.

5 W. Stirling, Arb. a. d. physiol. Anst. zu Leipzig, 1874, S. 223 ; Du Bois-Reymond, "Auslösung v. Reflexbeweg.," Berlin, 1880 ; S. Exner, "Entwurf einer physiol. Erklärung psychisch. Erscheinungen," Wien, 1894, Bd. ii. Abtl. 1, S. 49.

${ }^{6}$ Kronecker and Nicolaides, Arch. f. Physiol., Leipzig, 1883, s. 27-42. 
that for obtaining contraction by excitation from the corresponding efferent root; especially is the difference marked for very brief stimuli. The application of 2 grins. to an area of $1 \mathrm{sq}$. $\mathrm{mm}$. of the plantar skin of the spinal frog usually evokes a reflex response; this represents for the mechanical stimulus the threshold value efficient to evoke from that region a reflex reaction. This high threshold is probably determined by the "neuron-threshold" (Goldscheider ${ }^{1}$ ) at synapsis between afferent and efferent root cells; though it has been found actually lower for the reflex movement than for galvanometric effect led off from afferent nerve. ${ }^{2}$ But the efficiency of the stimulus, of course, much depends on its suddenness of application. A very gradually increased pressure will require values far above what is otherwise liminal. ${ }^{3}$ It is said that the spinal frog may be crushed without evincing a movement, if the crushing force be applied to it very gradually. Similarly, the application of warmth or cold to the frog's skin, if very gradual, ${ }^{4}$ excites no reflex reaction. If the temperature of water in which one limb of the spinal frog is immersed, be raised not more quickly than $\frac{1}{250}^{\circ} \mathrm{C}$. per second, no reflex ensues, even though the warming be carried up to the production of rigor caloris. Foster ${ }^{5}$ has, however, shown that a fallacy in this observation consists in neglecting the warming of the circulating blood which passes through the limb and so into the general circulation. The warmed blood much depresses the spinal reflex activity of the frog. When one toe only is immersed in the gradually warmed water, reflex movement ensues at a temperature of $35^{\circ} \mathrm{C}$.

The strength of stimulus exerts some influence on the extent and duration of the reflex movement excited; these tend to increase with increase of the stimulus. But there is no such regular relation between intensity of stimulus and intensity of reply as obtains in indirect excitation of skeletal muscle through its notor nerve. "The reflex arc, as judged by the contraction of its muscles, behaves somewhat like cardiac muscle in responding its approximate best or not at all." 6 In other words, internal conditions affect the amount of response much more than does mere intensity of external stimulus. This is no doubt the secret of the effectiveness of repetition of slight stimuli and of summation. If in the spinal mammal an external stimulus of an intensity that is usually efficient fails to excite a reflex, to increase its intensity is usually not of much avail.

Rosenthal ${ }^{7}$ has established the fact that there is a distinct relation between the intensity of the external stimulus and the latent time of spinal reaction, the latter becoming much shorter as the former is increased. Wundt ${ }^{8}$ has pointed out that strychnin still further limits the degree to which intensity of the external stimulus influences the intensity of the contraction in response; the evoked contraction tends to be always maximal, provided a certain period has elapsed since the preceding contraction. ${ }^{9}$ During this period the contrac-

I "Die Bedeutung der Reize," Leipzig, 1898.

2 E. Steinach, Arch. $f$. $l$. ges. Physiol., Bonn, 1895, Bd. lxiii.

${ }^{3}$ C. Fratscher, Jenaische Ztschr. $f$. Naturw., Jena, 1875, Bd. ix. S. 156.

4. Heinzmann, Arch. f. d. ges. Physiol., Bonn, 1872, Bd. vi. S. 222.

5 "Studies from the Physiol. Lab. of the Univ. of Cambridge," 1873, vol. i. p. 36. See also Tarchanow, Bull. Acad. imp. d. sc. de St. Pétersbourg, 1871, tome xvi. p. 226 ; Rudnow, Journ. dlya normal. i patol. gistologii, St. Petersburg, 1872, vol, v. p. 238.

${ }^{6}$ Sherrington, Phil. Trans., London, 1897.

7 Abhandl. d. k. Akad. d. Wissensch., Berlin, 1881.

8 "Untersuch. z. Mechanik. d. Nervencent.,"1876, Bd. i. Abth. 2, S. 70.

${ }^{9}$ G. L. Watson, Arch. f. Physiol., Leipzig, 1882, S. 49 ; and Journ. Physiol., Cambridge and London, 1881, vol. iii. p. 301. 
tion is dependent on the strength of the stimulus, being proportional to it at the beginning of the period and becoming gradually less so as the period advances. But the reflex responses referred to in these observations under strychnin are of the nature of gereral convulsions, and the liminal intensity of stimulus under strychnin is high.

The direction of the reflex movement is sometimes with intense stimuli the reverse of that evoked by weak stimuli. In the spinal eel a light touch on the side of the body causes that side to be approached toward the stimulus; an intense stimulus, e.g. hot iron, causes it to be withdrawn from the stimulus (Pfliger). The eel's touch reflexes exhibit "positive stereotropism" (Loeb), the pain reflexes "negative stereotropism."

The character of the muscular response.-The reflex contraction of a skeletal muscle may be almost as brief as the simple twitch due to momentary stimulation of the motor nerve, ${ }^{1}$ or it may be as prolonged as a long tetanic contraction. It has been suggested that every reflex contraction is tetanic in nature ${ }^{2}$; but this remains unproven, and is by some denied. ${ }^{3}$ Only in rare instances is a single muscle alone thrown into contraction if the contraction be vigorous. On the other hand, if the reflex be very weak, it often consists in a slight flickering in a single part of a single muscle; one can note this well in the gracilis muscle of the spinal mammal. The duration of the reflex contraction, unlike that of the contraction evoked by stimulation of the motor nerve, tends to continue after cessation of a brief stimulus and to fail before cessation of a prolonged stimulus. ${ }^{4}$ The reflex spinal movement, if prolonged, tends always, in my experience, to be of alternating character, offering in this respect a striking difference from a movement of similar duration evoked from the cortex. A movement from the initial posture of rest to another posture is obtained; this is the primary movement, and with it the reflex reaction, if of short duration, may come to an end. But with prolongation of the reflex action, the posture first attained is not maintained. A second movement, opposed in sense to the former, sets in. And this alternation of postures may be repeated a number of times. Examples are the wagging movement of the tail, and the repeated "kicks" of the spinal cat when one hind-paw is held. The rhythmic alternation is still more frequent in the "scratching" reflex, where a scrabbing movement of the ankle and foot occurs with alternations of 3 to 5 per second. Also in the "shaking" reflexes, where the animal shakes its ears or its body, as does a dog on coming out of water. In their phasic alternation of condition the motor cells pass through periods of discharge and quiescence; the contraction and the relaxation contemporaneously affect antagonistic muscles, and successively affect synergic muscles. Throughout the phases of this sequence, in which inhibition as well as excitation seems to play a part, it is only harmoniously acting regions of musculature that are at one and the same time in contraction. Thus in the rectus femoris muscle the part that flexes the hip contracts alternately with the part that extends the knee, and, on the other hand, synchronously with the other hip flexors

1 Wundt, op. cit. W.'s observations were made under weak strychnisation ; Stirling and Kronecker employed unpoisoned preparations.

2 Stirling, with Kronecker, loc. cit.

3 Wundt, "Untersuch. z. Mechanik. d. Nervencent," ]876, Bd. i. Abth. 2.

"Beaunis, "Rech. expér. sur les condit. de l'activité cérébrale," Paris, 1884, p. 106. 
and flexors of the knee. Each successive phase of the reflex movement is therefore co-ordinate in this sense.

The completion of the main movement executed in a spinal reflex is, in the mammal, very frequently succeeded by a brief "twitch" of contraction. This after contraction is sometimes of considerable amplitude, although "twitch"-like in brevity of duration; it is seen well at the ankle-joint of the spinal rabbit after reflexes induced by skin stimuli to the foot. This remaining after effect of a reflex tetanus excited from the cord is often quite prolonged, ${ }^{1}$ persisting even more than a minute.

It has been often noticed that a reflex excited in the spinal animal rapidly dwindles and ceases to be elicited if the stimuli applied at a locus be repeated rather frequently. This kind of after effect has been spoken of as fatigue, and in studying reflex actions by Türck's method it is necessary to allow pauses of many minutes between the reapplications of the stimulus. Owing to the rule of spatial uniformity, it is easy to bring the same motor apparatus into play from each of several afferent channels. When, after the stimulus applied to one of these channels has had its efficiency for producing the motor reaction greatly depressed or set aside altogether by frequent repetition, if the incidence of the stimulus be shifted to one of the other afferent channels playing on the same motor apparatus, the latter may still perfectly respond. ${ }^{2}$ This indicates that in the so-called "fatigue" of the reflex the block occurs in the afferent channel. It occurs probably at the intraspinal end of that channel, for the effect is seen when afferent nerve trunks are stimulated, as well as when sensory surfaces are used; and that nerve fibres themselves are practically unfatigable has been established (Bernstein, Bowditch, Waller, Wedenskii, and others).

But when more natural stimuli are applied, the reflex movement may persist much longer without becoming exhausted; for instance, the chelate appendage of an Astacus reduced to the spinal condition has been observed to prosecute continuously for many hours its reflex attempts to give the morsel it holds into the grasp of the maxillipedes. ${ }^{3}$

The contraction of the muscles in spinal reflexes is never, in my experience, very forcible. No very great opposition is required to restrain the movements, and the postures assumed by the limbs never attain the full extremes of flexion possible under vigorous willed action, or under excitation of the peripheral nerves. The average character of the contraction reflexly elicited from different muscles is obviously different for different muscles, e.g. that for the gastrocnemius and extensors of the hip is short and sharp as compared with that for the flexors of the ankle and hip, both in the frog and dog.

When a motor nerve is excited by induction shocks of not too high frequency (e.g. 42 per sec.), the contracting muscle vibrates at a similar rate of frequence. ${ }^{4}$ If the transection of the cord be excited instead of the motor nerve, the muscle vibrates, but with a frequency independent of that of the serial stimulus and far slower, e.g. about 10 per sec. ${ }^{5}$ A similar rate of vibration had been seen in spinal movements under strychnin. ${ }^{6}$

${ }^{1}$ de Boeck, Arch.f. Physiol., Leipzig, 1889.

2 Sherrington, Phil. Trans, , London, 1896.

${ }^{3}$ Bethe, Arch. f. d. ges. Physiol., Bonn, 1897, Bd. lxviii.

${ }^{4}$ Helmholtz, Monatsbl. Berl. Akad., 1864.

${ }^{5}$ Schäfer and Horsley, Journ. Physiol., Cambridge and London, 1886, vol. vii. p. 100 ; ef. v. Kries, Arch. $f$. Physiol., Leipzig, 1886; Kronecker and Stanley Hall, Arch. $f$.

Physiol., Leipzig, 1879, Suppl. S. 12, had previously obtained a much ligher result.
'Lovèn, Nord. med. Ark., Stockholn, 1879 ; Centralbl. $f$. $l$. med. II issenseh., Berlin, 1881. 
Influence of the location of the stimulus. - To the reactions elaborated by each sense probably in some measure there pertains, among other sensual qualities, a quality of sensual space. Of the four senses which pre-eminently furnish space-perception, one has its endorgans in the skin, another in the musculo-articular structures, and these are therefore in great measure spinal senses. It is not surprising, then, that per se the locus of the stimulus is for a spinal reflex an important determinant of the character of the resulting movement.

The rule of spatial proximity given above (under short spinal reflexes) partly expresses the influence of this factor in the reaction. Much that was mentioned regarding longer irradiation illustrates it further. But the importance of the locus, although high when broadly taken, does not appear obvious as attaching to small differences of location in a more or less homogeneous sensory field. Within a single large but functionally related sensifacient area, such as, for instance, the skin of any one limb, considerable areal variation of the incidence of the stimulus fails to at all markedly alter the resultant reflex movement. ${ }^{1}$ Thus, under appropriate conditions, a similar flexion of the hindlimb at hip, knee, and ankle is elicited from any small area taken in the skin surface of the limb. The flexions, though similar, are, however, demonstrably not identical. ${ }^{2}$ In the spinal monkey, excitation of the outer edge of the planta, while causing dorso-flexion at ankle, in doing so generally brings the perone into play more than is the case when the flexion is excited from the inner edge of the planta; then the tibialis anticus predominates, causing some inversion. In the frog, excitation of the skin of the knee on the dorsal and on the ventral aspect respectively, alike evoke flexion at hip, knee, and ankle, but in the former case the foot is somewhat everted, in the latter somewhat inverted. ${ }^{3}$

This influence of the location of the stimulus upon the character of the movement is well seen in the reflex reactions of the spinal frog. It furnishes a large part of the direct evidence of the so-called "purposive" character of spinal reflexes. The physiological study of reflexes must be objective, and from this point of view it is preferable perhaps to adopt the expression "local sign," although borrowing it from the psychologist, to denote a quality in virtue of which the character of the resulting movement is partly determined by the spatial position of the reflexigenous area whence initiated. In the spinal dog (cervical transection) the hind-foot is with fair accuracy brought to scratch the spot of the shoulder under irritation, although that spot lie behind the spinal lesion. The centripetal impulses from the spot-although they can yield no sensation-possess therefore in the above sense "local sign."

"Local sign" is in this sense a quality attaching to spinal and low-level reactions throughout an enormous range of different nervous systems. It is exemplified in the reflex actions obtaining between the manubrium and the nectocalyx of Sarsia, and even more strikingly in a naked-eye medusa, called on account of its localising reflexes Tiaropsis indicans. ${ }^{4}$ The manubrium deflects itself towards the stimulated part of the nectocalyx; its extremity

\footnotetext{
${ }^{1}$ This is expressed by that rule of spinal reflex action which states that there is uniformity of response despite spatial variety of provocation (vide supra, p. 821).

${ }^{2}$ If the rule of spatial proximity be considered, moreover, to interact with the previous rule, an explanation is offered for many of those minor differences obtaining in the broadly similar movements, there being a tendency for the muscles belonging to the immediate spinal vicinity of the skin stimulated to respond in preponderant degree.

${ }^{3}$ Schloesser, Arch.f. Physiol., Leipzig, 1880, S. 303.

${ }^{4}$ Romanes, Phil. Trans., London, 1879.
} 
is eventually brought with unerring precision to meet the concurrently contracted inbent portion of the nectocalyx. If one point of the nectocalyx be irritated, and while the manubrium is applied to that point, then another, the manubrium will leave the first point and move over to the second. In this way it may be made to indicate successively a number of points of irritation. "After a series of such irritations, the manubrium subsequently continues for some time to visit first one and then another of the points which have been irritated." A cut between the base of the manubrium and the point of irritation in the bell destroys the localisation, though movement occurs toward some part of the quadrant of the bell containing the site of stimulus; but the accuracy of the localisation is reduced. The reaction recalls the bending of the tentacles of Drosera ${ }^{1}$ in the direction needful to reach the seat of stimulation on the leaf. The headless bee stings in response to stimulation of the under-surface pretty accurately at the site of irritation." In the "spinal" crayfish, if one leg is caught it is flexed and drawn up, and soon all the others, if the leg is not released, are brought round it and push at the hand holding the limb. The yellow clover-fly has been observed, twelve hours after decapitation, to stand cleaning its wings with its hind-legs, and to clean its "three pairs of legs, rubbing them together in a determined manner, and raising its fore-legs vainly in air as if searching for its head to brush up." 3 But in Astacus the accuracy of localisation is much impaired on the crossed side by cutting the cross commissures combining the ganglia most closely concerned with the reaction. ${ }^{4}$ This recalls the effect of the tangential cut in the nectocalyx of Tiaropsis.

With the movement executed in a reflex reaction significant of "local sign" in the above sense, the afferent impulses involved seem naturally divisible into several groups according to their place of origin. There must be (1) a group originated at the seat of stimulus, (2) a group initiated in the motor and mobile organs reflexly set in action, and (3) in some cases a group originating at a distant spot to which the movement is directed. Regarding this last group, experiment illustrates its extinction, without extinction of the "local sign." Thus, in Astacus, ${ }^{5}$ after section of the nerve cords behind the mouth, when, therefore, the hind creature without mouth has lost all nervous connection with the front ereature possessing the mouth, food given to the claws of the hind creature is still at once and accurately carried by them to the mouth, and this often refuses to take the morsel brought. In the grasshopper, after extirpation of the supra- and subœsophageal ganglia (entire brain), the front leg is protracted, ${ }^{6}$ and in the normal way catches the antenna, and the usual movements of cleaning the antenna go on, though the antenna has entirely lost its innervation owing to the destruction of the brain. Regarding the second mentioned group of afferent impulses, H. E. Hering has made an interesting observation on the frog, and I have several times repeated it. The "cleansing" reflex of the spinal frog which brings the foot to a seat of irritation on the dorsal or perineal skin, is accurately executed after severance of the afferent spinal roots of the limb itself. In the same way, the bulbospinal frog brings the fore-limb to the snout when the snout is stimulated after section of the afferent roots of the fore-limb. Guiding sensations for directing the execution of these spinal cleansing reflexes are not essential; the sole important afferent factor as regards the local sign is restricted to the afferent nerves of the place of initiation of the whole reflex.

"Local sign," in the above sense, no doubt attaches to the motile

I Darwin, "Insectivorous Plants," London, 1875.

"Bethe, Arch.f. d. ges. Physiol., Bonn, 1897, Bd. Ixviii.

3 A. H. Swinton, "Insect Variety," London, 1880 ; see also Grainger, op. cit.

+ Bethe, Arch. f. d. ges. Physiol., Bonn, 1897, Bd. lxviii.

${ }^{5}$ J. Ward, Journ. Physiol., Cambridge and London, 1879, vol, ii., and others.

${ }^{6}$ Bethe, Arch. f. d. ges. Physiol., Bonn, 1897, Bd. lxviii.

VOL. II. -53 
apparatus, e.g. joints and muscles considered as sources of centripetal impulses, as well as to the skin. The evidence is, however, scanty. The assumption of posture by the spinal frog goes far, I think, toward establishing it. The spinal frog, if gently left under a bell-jar with one hind-limb extended, is found in the course of a variable time to have drawn the limb into flexion; and this it does when the skin of the limbs has been removed. Bethe's observations on insects show that reflex correction of the inverted position after decapitation is a contact reflex. The muscular unrest of a spinal frog (transection behind the eighth cranial nerve), when first inverted, is suggestively greater than when a piece of wood of weight equal to the frog rests upon the dorsal skin.

In connection with localising power and local sign and spinal reflexes, there is the widespread occurrence of stereotropic reactions in spinal animals, as already referred to; such reactions imply localisation. Thus the headless eel and Amphioxus swim "upright," the amputated ray of the starfish rapidly rights itself when inverted on a solid surface, ${ }^{1}$ and is restless when its ambulacral feet are not in contact with something firm. After removal of the cephalic ganglia, Grillotalpa and many insects, also Astacus, resist inversion and maintain the body's equilibrium. The balancing compensating movements of the tail of the bird ${ }^{2}$ after spinal transection, and the series of movements by which Cerianthus when inverted in a test-tube re-erects itself, are other instances. ${ }^{3}$

Fatality of spinal reflexes. - The movement resulting from each one of a series of slowly repeated stimuli applied under as far as practicable similar conditions to an afferent channel in a spinal animal, remains often approximately similar through a long series. It can be fairly predicted that the second response will be very like the first. The miformity of response is, as might be expected, best marked in the simplest reflexes, e.g. such as may be obtained from quite limited isolated fractions of the cord. In them the possible paths are most reduced, and the numbers of links (nerve cells) composing the nervous chains are snallest,-in other words, the synapses are fewest; in some, perhaps, there intervenes but one synapsis. These reflexes are the most "fatal," but their "fatality" is widely removed from absolute. It does not approach that with which similar movements follow repeated similar excitations of a motor nerve. In the frog the successive similar stimulation of a spot of skin, say on the second pedal digit, may regularly evoke flexion of the knee and ankle; but with that flexion may or may not be combined flexion of hip, and the degree of dorsal Hexion of the digits will vary.

The fatality of reflexes might be logically inferred from what Grainger ${ }^{4}$ described as their "resemblance to the motion which the function of the organ requires." In the intact complete individual the reaction of one part is as a rule subordinated to the advantage of the individual as a whole. The isolated local nervous system of spinal rank executes, so to say blindly, its special reaction uninfluenced by the condition of other parts of the nervous system. Michael Foster ${ }^{5}$ has pointed out that when the normal Astacus laid over on its back is trying to correct its posture, an object, even food, placed

${ }^{1}$ Romanes, op. cit. ; also Preyer, Demoor, Loeb, and others.

${ }^{2}$ Singer, Sitzungsb. d. k. Akad. d. Wissensch., Wien, 1884, Bd. lxxxix.

${ }^{3}$ Loeb, Arch. f. d. ges. Physiol., Bonn, 1895, Bd. lix.

4 "Funetions of the Spinal Cord," London, 1837, pp. 3, 101.

5 In Huxley's "Crayfish," London, 18s0, pp. 110, 111. 
between the chelæ, is either rejected forthwith, or is made use of for leverage in the attempt to return to position. The "spinal " crayfish similarly placed also "struggles," and will recover normal posture; but the placing of food in its chelæ renders idle the struggling movement of recovery, for there is thus evoked a "feeding" reflex which drafts off some or all of the limbs to carry the morsel to the mouth and tuck it between the jaws, leaving the remaining tilting movements of the limbs quite ineffective.

In the living severed halves of Hirudo, the reaction of the halves as regards the terminal sucker of each is different. Pressing the sucker of the front half against an appropriate surface usually does not cause it to attach itself, but simply eauses the head to be raised and moved vigorously with movements suggesting defence. Pressing the sucker of the "spinal" hind half causes the sucker to be reflexly attached to any appropriate surface (Loeb), ${ }^{1}$ in accordance with the spinal reflex nature of sucker reactions in general (Grainger). ${ }^{2}$ A touch on the abdomen or thorax of the decapitater bee causes it to sting itself at the spot touched, ${ }^{3}$ the creature dying from its own sting. The decapitated snake winds round a hot bar, ${ }^{4}$ just as it winds round a rabbit. ${ }^{5}$ The blind fatality of these reflexes justifies the expression that the animal reduced to the "spinal" condition is no longer an individual, but is a mere series of living segments.

Influence of condition of the central organ.-(1) Vascular condition.-A spinal frog in which the circulation has been arrested by ligation of the base of the heart, or in which the blood has been replaced by normal saline solution, will, by limb movements, continue to respond to skin stimuli for some thirty minutes if its temperature be low; for a shorter time only if at summer temperature. In the spinal rabbit the reflexes are abolished by arrest of the circulation in less than one minute. ${ }^{6}$ The reflex reactions of the mammal persist, however, much longer after arrest of the blood supply of the cord if the animal has previously been cooled. ${ }^{7}$ The vascular reflexes appear to persist longer than do those employing the skeletal musculature. After an interruption of the circulation in the rabbit by compression of the root of the aorta for forty seconds, the re-establishment of the circulation fails to restore the reflex function of the cord. After low thoracic ligation of the aorta when the hind-limbs are paralysed, stimulation of their skin can still evoke reflex movements from the fore-limbs. This may be due to the long upward nerve fibre path in Goll's column, which is not dependent on synapses in the spinal region.

When the abdominal aorta of the rabbit is clamped just behind the renal arteries for from thirty to sixty minutes, there ensues permanent sensory and motor paralysis of the hind-limbs, bladder, and rectum, which has its cause in the necrosis of the grey matter of the lumbar cord. ${ }^{8}$ When the animal is killed, five to six hours after the sixty minutes' compression, many of the

${ }^{1}$ Arch. f. d. ges. Physiol., Bonn, 1894, Bd. lvi. 1839.

2 "Functions of the Spinal Cord," London, 1837 ; also see Carpenter's Essay, London,

${ }^{3}$ A. Hethe, Arch. f. d. gcs. Physiol., Bonn, 1897, Bd. Ixviii.

${ }^{4}$ Osawa and Tiegel, ibid., 1877 , Bd. xvi. S. 90.

${ }^{5}$ Sigmund Exner, "Entwiirf ein. physiol. Erklär. psychisch. Erscheinungen," Wien, 1894.

6 Charles Richet, "Physiol. des muscles et des nerfs," Paris, 1882, p. 716.

7 Cl. Bernard, "Leçons sur le système nerveux," Paris, 1857.

${ }^{8}$ Ehrlich and Brieger, Ztschr.f. klin. Med., Berlin, 1884, Bd. vii. ; Singer, Sit $n$ ngsb. d. k. Akad. d. Wissenseh., Wien, 1887, Bd. xevi. ; Herter, Journ. Nerv. and Mcnt. Dis., N. Y., 1889 ; Spronk, Arch. de physiol. norm. ct path., Paris, 1888, Sér. 4, tome i. p. 1 ; Singer and Münzer, Denkschr. d. k. Akad. d. Wissensch., Wien, 1890, Bd. Ivii. ; Minzer and Wiener, Arch. f. exper. Path. u. Pharmaliol., Leipzig, 1895, Bd. xxxv. S. 114. 
spinal nerve cells, examined by Nissl's and other histological methods, are found scalloped in contour, shrunken, and with diffusely staining nucleus. Fixed twelve hours after the compression, all the cells are found altered; and fixed twenty-four hours after the compression, the outlines of all the cells are hardly discernible, and only their diffusely stained nuclei indicate their position. Not in every experiment is the whole grey matter of the region subjected to the stasis destroyed. The ventral horn of the grey matter suffers especially severely. The dorsal horn may remain comparatively unharmed. Clarke's column of cells seems, like the spinal ganglion cells, little harmed. When the cord is fixed seven to nine days after the compression, secondary degeneration is found to have occurred in a large region of distribution. Close round the ventral horns in the lumbar region is a dense ring of degenerated fibres, which are traceable headwards, and come to lie at the periphery of the whole ventrolateral column, except in its dorsal fifth and in the depth of the ventral fissure. Some of these ascending fibres have a contralateral origin, and pass through the ventral commissure. Fibres in the dorsal columns also degenerate. These are the "endogenous fibres" of the dorsal columns. ${ }^{1}$ As studied in the rabbit, they seem to be axons from cells of the dorsal grey horn, which enter the dorsal white column all along the limiting layer against the grey matter, and gradually pass into the dorsal mesial column, lying along the lip of the dorsal median fissure (septum). They can be traced forward to enter Goll's nucleus. The dorsal division of the cerebellar tract is little injured, but that is in part due to the fact that the cells (Clarke's column) of that tract for the most part lie headward of the region of stasis. The afferent roots are hardly at all degenerated.

Compression of the aorta of the dog close below the renal arteries does not usually produce spinal symptoms, ${ }^{2}$ although it does so in some cases. ${ }^{3}$ Fredericq ${ }^{4}$ and Colson ${ }^{5}$ produced paraplegia in the dog by blocking the thoracic aorta through one carotid. That motor paralysis should result from the stasis is only in accord with anticipation, seeing that degeneration of the motor root cells ensues; but the total anæsthesia that has been observed might not have been expected, in view of the intact condition of the afferent root cells. Only a very few fibres in the afferent roots ${ }^{6}$ degenerate after the compression, the spinal ganglion cells seeming more resistant than the intraspinal; and the dorsal columns seem, except for the endogenous fibres, intact. Cutaneous sensation seems therefore to employ a path which early in its intraspinal course involves the grey matter. Münzer and $\mathrm{Wiener}^{7}$ observed that in three of their twenty-one experiments one of the dorsal horns of grey matter was not necrotic. In each of these experiments the hind-limb of the same side as the sound dorsal grey horn was noted not to be anæsthetic; from its skin could still be provoked cutaneous pain.

Anæmia and asphyxia cause transient exaltation of the reflex reactions of the spinal cord. The spinal effect of excitation of the peripheral end of the vagus is explicable in this way. The same causes can produce spinal convulsions. When the spinal cord of the cat has been severed in the thoracic region, if the circulation be arrested in the isolated portion of the cord, contractions of the muscles of the hind-limbs and tail ensue. ${ }^{8}$ The actual sequence of the excitation of the musculature is not constant, but one posture is so frequently assumed as to be characteristic, namely, strong flexion of hip, combined with strong extension at knee and ankle; toward the end of the seizure, lifting of the tail and swaying of it laterally often occurs. These convulsions occur

${ }^{1}$ Singer and Muinzer, loc. cit.; Münzer and Wiener, loc. cit.; Sarbó, Neurol. Centralbl., Leipzig, 1895, Bd. xiv. S. 664 .

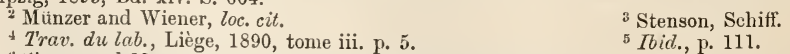

"Singer and Miinzer, loc. cit.; Müunzer and Wiener, loc. cit.

7 Arch. f. exper. Path. $u$. Pharmakol., Leipzig, 1895, Bd. xxxv. S. 114.

${ }^{8}$ Schiff, Arch. f. d. ges. Physiol., Bonn, 1883, Bd. xxx. S. 202. 
even although all the afferent roots of the isolated length of cord have been cut, and they are much less diminished by the root section than are the somewhat similar convulsions of strychnin poisoning. Contraction of the bladder and erection of the hairs are among the spinal effects of asphyxia in the mammal; in strychnin convulsions, on the contrary, erection of the hairs in my experience does not ensue; the spinal origination of the asphyxial erection is not, however, definitely proved.

During the condition of apnœa the reflex convulsions characteristic of strychnin and similar poisons do not, it is said, occur (Rosenthal and Leube, Uspensky).

(2) Drugs.- - Some drugs affect the activity of the spinal centres, certain of them greatly. Detailed account of their action lies beyond the scope of this article. Besides depressants of protoplasmic activity in general, e.g. chloroform, are certain alkaloids and salts, which selectively depress, e.g. morphia, or exalt, e.g. strychnia, the spinal centres. Material so unlike the normal constituents of nervous tissue can hardly be supposed to yield fresh nervous force. The influence must consist in facilitation of the discharge of force already latently present, and the rendering of the liberating forces more effective, tending to thwart inhibition. The action of strychnin on spinal nerve cells is comparable with that of atropin on cardiac muscle cells.

Influence of associated parts of the central organ-Inhibition and facilitation ("Bahnung") of spinal reflexes.-The response made by a reflex arc to an external stimulus is, as mentioned above, dependent as much on the internal condition of the arc as on the external conditions attaching to the stimulus. Reflex ares, both lower and higher, are inter-connected. The condition of any one is partly dependent on the condition of many others. The nervous system is a unity; in other words, each even local reflex action is in truth a reaction to all sensifacient stimuli incident on the individual at the moment in sum both as to space and as to past time. And those activities of the central nervous system which are customarily considered autochthonous, have also, on account of the inter-connection of the arcs, influence upon the reactions of other arcs. The influence arising either way may take expression in depression of the local reaction, e.g. the rendering a supraliminal stimulus liminal or subliminal, or, in promotion of the local reaction, rendering a previously subliminal stimulus liminal or supraliminal. The former kind of effect has, since the Weber experiment on the cardiac vagus, been known as "inhibition" (Hemmung, action moderateur), the latter has, by Sigmund Exner, who has particularly studied it, been termed "Bahnung," a felicitous expression not easily rendered into English. ${ }^{1}$

Inhibition of the heart's beat by excitation of the vagus nerve was discovered by the Weber's, and is described in Wagner's Dictionary of the following year (1846). E. H. Weber forthwith extended the notion to the case of the increased spinal activity witnessed after ablation of the brain. ${ }^{2}$ He suggested that removal of the brain removes a nervous mechanism which inhibits the reflex spinal machinery. This notion of the inhibition of the activity of one part of the central nervous system by the

1 Foster has rendered it by "augmentation," $\Lambda$. D. Waller by "canalisation," and the word "reinforcement" has been a good deal used for some of the examples covered by Exner's term. These three English terms are, however, not synonymous. "Facilitation" perhaps most nearly expresses what is intended.

${ }^{2}$ Wagner's "Handwörterb. d. Physiol.," Braunschweig, 1846, Bd. iii. S. 47. Cf. Volkmann, Arch. f. Anat., Physiol. u. vissensch. Mled., 1838, s. 87. 
activity of another part had, from its psychological aspect, long been expressed; for instance, in the "duobus doloribus simul abortis non in eodem loco vehementior obscurat alterum" of Hippocrates. ${ }^{1}$ As a working physiological thesis it only became accepted doctrine after Setschenow ${ }^{2}$ in 1863. He found that stimulation of the mid-brain and bulb, especially of the corpora quadrigemina, prolongs the reflex time. A frog, its cerebral hemispheres having been removed, withdraws its foot from a bath of acidulated water, if the above-mentioned parts are at the same time excited, much later than it does otherwise. The postulation of an inhibitory centre in the mesencephalon (and myelencephalon) was the first interpretation put upon Setschenow's observations. Then it was shown that similar inhibitions of spinal reactions could be obtained from foci in various other parts of the brain, ${ }^{3}$ and of the cord itself. ${ }^{4}$ The inhibitions are obtained either mediately from sensorial surfaces, or by immediate excitation of the foci themselves. Goltz ${ }^{5}$ introduced the view that a reflex centre, engaged in a particular reflex function, suffers impairment of activity for that particular reflex when it receives incitation from any other afferent channel. Schiff and Herzen teach that each spinal segment exerts an inhibitory influence on all segments lying further backward. Certain it is, a nerve cell thrown into activity may throw others not only into action but also out of action.

Where, as in the higher mammals, the cord possesses an immediate path from a region of the cerebral cortex, it is considerably influenced in its reflex functions by the activity of that cortex. Reflexes purely spinal are facilitated by the removal of this cortex or by the rupture of its descending path. The scratching reflex, which is easy to evoke from puppies and from the adult dog after high spinal transection, can, after ablation of the cortex cerebri, be abnormally easily and even uncontrollably elicited on the side of the body contralateral to the cerebral lesion. ${ }^{6}$ A monkey, in which a brachioplegia has been caused by ablation of the cortical arm area, when one month subsequent to the cerebral ablation the mesencephalon is transected, shows in the decerebrate condition an extraordinary degree of reflex responsiveness in the monoplegic limb to stimuli of the fellow fore-limb, and of the hind-limbs, ears, and other reflexigenous zones.

Various explanations have been offered for the facilitation of spinal reflexes which ensues on removal of the cortex. It has been argued (1) that the energy reflexly set free in the central nervous system is separable into that which is liberated in the brain and that liberated in the reflex spinal machinery, and that when the former liberation is no longer possible, the latter absorbs vicariously the excess. ${ }^{7}$ The view has nothing in its favour. It has been further argued (2) that the Wallerian degeneration of the pyramidal tract acts

1 "Aphorisns," Bk. ii. p. 46.

2 "Physiol. Studien ueber d. Hemmungsmechanismen f. d. Refl.," Berlin ; and with Paschutin, "Neue Versuche," Berlin, 1865; also Bull. Acad. imp. d. sc. de St. Pétersbourg, tome xx. p. 537 ; aud "Ueber d. elekt. u. chem. Reizung d. sensiblen Nerven n. Riickenmarks d. Frosches," Graz, 1868.

${ }^{3}$ L. N. Simonoff, Arch. f. Physiol., Leipzig, 1866, S. 545 ; J. Ott, Journ. Physiol., Cambridge and London, 1881, vol. iii. p. 163.

${ }^{4}$ A. Herzen, " Expériences sur les centres modérateurs de l'action réflexe," Turin, 1864 ; Lewisson, Arch. f. Physiol., Leipzig, 1869 ; Nothnagel, Virchow's Archiv, 1873, Bd. xlix.; Tarchanoff, Journ. dlya normal. $i$ patol. gistologii, St. Petersburg, 1872, vol. v. p. 285.

5 " Beiträge z. Lehre v. d. Fumctionen d. Nervencent. d. Frosches," Berlin, 1869.

${ }^{6}$ Gergens, Arch. f. d. ges. Physiol., Bonn, Bd. xiv. S. 340.

7 Brown-Séquard, "Sur la physiologie de la moelle epinière," 1855. 
irritatively upon the spinal centres "like a mild strychnisation" (Charcot). ${ }^{1}$ But the spinal exaltation has been shown by experiment to set in before Wallerian degeneration has time to occur, and this fact also excludes as a cause the local spinal hyperæmia ${ }^{2}$ which undoubtedly accompanies the process of Wallerian degeneration and the subsequent healing in the spinal tissue. The remaining hypothesis (3) is that the ablation removes a nervous centre which under normal conditions constantly restrains these spinal reflexes. But the reflexes become abnormally responsive not only after the loss of the cortex; early arrest of the development of the brain has the same effect, ${ }^{3}$ producing abnormal facility of the spinal reflexes, e.g. in agenesic porencephaly. And there is direct experimental evidence demonstrating that the excitation of the cortex can restrain spinal reflex action. ${ }^{4}$ The lowering of cerebral restraint is often well marked, even when the effect of the cerebral lesion on motility is slight or undemonstrable. It may be recalled that some (Marie, Mann (Breslau), Marinesco) regard the pyramidal tract as entirely an inhibito-motor path.

Even where the nervous system, as in lower vertebrata, possesses no immediate cortico-spinal tract, removal of the hemispheres nevertheless augments spinal reactions (Marshall Hall ${ }^{5}$ ). Other parts of the brain than the cerebral hemispheres also appear to exert restraint upon spinal reflexes: thus the mesencephalon and metencephalon, as shown by Setschenow's ${ }^{6}$ and Albertoni's ${ }^{7}$ experiments on the frog and toad. The stimulus whence springs the spinal inhibition by the optic lobes of the frog has been shown to act through the retina. ${ }^{8}$ In the $\operatorname{dog} I$ have seen a semisection of the posterior part of the bulb enormously increase the spinal reflexes on the same side as the section, e.g. the scratching reflex from the trunk and limbs.

Removal of the "cerebral" ganglia in invertebrata gives to the spinal arcs striking release from restraint. Of the halves of the transected Hirudo or Planarian the hinder crawls the more restlessly. " After "decerebration," the cephalopod Eledone enters into ceaseless motor activity, ${ }^{10}$ Dytiscus " executes swimming with great energy and rapidity, striking all its comrades to one side by its violence." 11 Limulus ${ }^{12}$ and Astacus ${ }^{13}$ constantly execute movements of preening and swinging with their limbs. The removal of restraint occurs on the homonymous side after unilateral lesion in these fornis; the effect is in so far like the extensor spasm supervening when one half of the mesencephalon is transected in the mammal. ${ }^{14}$

The condition of one part of the cord influences the reactions of the other parts. In this respect its homogeneity may be likened to that of the retina (simultaneous induction). Vulpian, on a priori grounds, argued, in his

${ }^{1}$ See also Vulpian, "Leçons sur les maladies du système nerveux," Paris, 1886 ; M. Lion, Ztschr. f. klin. Med., Berlin, Bd. ii. S. 310.

2 Erben, Wien. med. Wehnschr., 1890, S. 879.

${ }^{3}$ F. Schultze, "Beitrag z. Lehre v. d. angebor. Hirndefecten," Heidelberg, 1886 ; G. Anton, "Medic. Schriften," herausgegeb. a. d. Wien. klin. Wchnschr., No. xv., 1890 ; J. Arnold, Beitr. z. path. Anat. u. z. ally. Path., Jena, Bd. xi. S. 407.

${ }^{4}$ Simonoff, loc. cit.; Bubnoff u. Heidenhain, Arch. f. d. ges. Physiol., Bonn, 1881, Bd. xxvi. S. 137 ; S. Exner, ibid., 1882, Bd. xxviii. S. 487.

5 "Memoirs," London, 1838.

6 "Physiol. Studien ueber d. Hemmungsnechanismen f. d. Refl.," Berlin, 1863.

7 Arch. ital. de biol., Turin, 1887, tomo ix. p. 19.

s Langendorfi, Arch.f. Physiol., Leipzig, 1877, SS. 95 and 435.

${ }^{9}$ Loeb, Arch.f. d. ges. Physiol., Bonn, 1894, Bd. lvi.

${ }^{10}$ v. Uexkiull, Ztschr. $f$. Biol., 1895, Bd. xxxvi.

1 Carpenter, "General and Comparative Physiology," third edition, London, 1851.

12 Ida Hyde (Loeb's laboratory), Journ. Moryhol., Boston, 1894, vol. ix.

13 Ward, Journ. Physiol., Cambridge and London, 1879, vol. ii.

14 Sherrington, Phil. Trans., London, 1897. 
"Leçons sur la physiologie du système nerveux," that each part of the cord exerts a restraint upon the other parts.

In the spinal crayfish the movement of the leg is at once stopped by touching the tail. ${ }^{1}$ Goltz's reflex inhibition of the heart beat by striking the abdomen of the frog does not occur if at the same time the foot is pinched. The "spinal" snake, suspended by its head, makes rhythmical pendulous movements; these cease when the body is lightly touched. In the spinal dog a severe squeeze of the tail or hind-foot will interrupt micturition already in progress. Such a stimulus must be regarded as "pathic"; it often fails to inhibit, but it often succeeds. It is not observable in the bird, nor have I obtained it in the monkey. A more regularly obtainable example, which, moreover, does not require the use of strong stimuli, is the following. In the spinal cat (or frog) excitation of the skin of the foot, when the limbs hang down, causes drawing up of the homonymous and

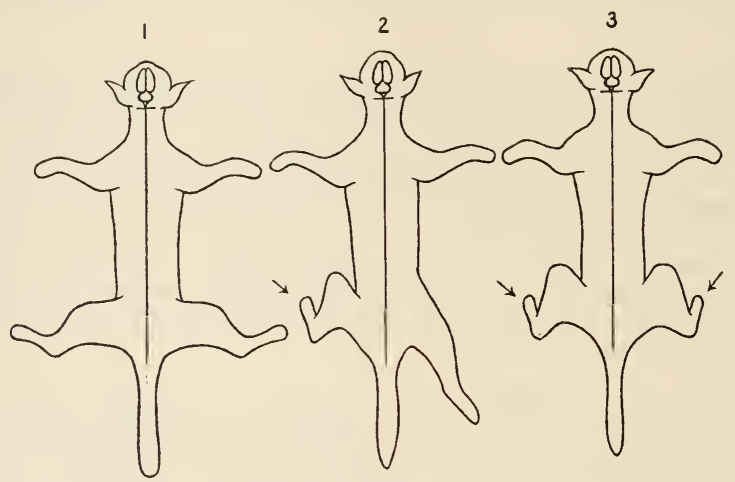

Fig. 358.-Diagram of the predominant uncrossed flexor reflex of the hind-limb inhibiting the erossed extensor reflex otherwise obtainable by stinulation of the opposite limb. 1, The initial pose of the spinal animal; 2, the pose assumed after stimulation of the left hind-foot, the flexors of the left hip, knee, and ankle, and the extensors of the right hip, knce, and ankle are in active contraction ; 3 , the pose assumed after simultaneous stimulation of both hind-feet. The extensor action of the hip, knee, and ankle that wonld appear from either side as a crossed reflex is bilaterally inhibited, and the antagonistic flexor reflexes bilaterally prevail.

extension of the contralateral limb. If both feet be stimulated simultaneously, the contralateral extension does not occur, the extensors are inhibited and the flexors of both sides are thrown into contraction. This experiment seems to be in some sort a converse to that in which one leg of a spinal frog is stimulated by a ligature or acid, while the other is lightly pinched. The latent time for the reaction of the pinched leg is then lengthened, or, with a particular strength of stimulus, the reaction does not occur.

After spinal transection, inhibition of skin reflexes by peripheral excitation of other surfaces is after a time unobtainable, so great becomes the reflex excitability. On stimulation of the pinna or fore-limb after semisection in the thoracic region, the movement of the hind-limb, which previous to semisection is less elicitable contralaterally than homonymously, I found became easier contralaterally.

It is probable that inhibition of one spinal "centre" by another plays a great part in the elementary co-ordination of actions executed under spinal

${ }^{1}$ J. Ward, Journ. Physiol., Cambridge and London, 1879, vol. ii. 
mechanisms. The augmentor innervation of one muscle group seems regularly to be accompanied by inhibition of the innervation of another muscle group; this mode of innervation I have termed "reciprocal." It seems a phenomenon common in spinal reactions (see above, p. 820).

In the spinal animal, inhibition due to excitation of a nerve path or centre is usually quite transient, and passes over into exaltation even during the continuance of the stimulation if that be fairly prolonged. This harmonises with the alternating and phasic character general in muscular reactions from the spinal animal.

Rosenthal ${ }^{1}$ insists on the longer latency of spinal reflexes evoked from the skin of one hind-limb in the muscles of the other when the transection is low than when high up. He found the reaction time for the above reflex in the spinal frog not altered by sagittal median longitudinal section of the crural region, but greatly lengthened by similar longitudinal section in the brachial. Bickel, ${ }^{2}$ using a crossed hind-limb reflex, finds the reflex time in the frog, salamander, and tortoise much greater when the cord has been transected (days previously) just behind the brachial plexus than when at the bulbo-spinal junction; after transection close anterior to the bulb, the reflex time is slightly shorter than when at the bulbo-spinal junction. de Boeck ${ }^{3}$ found in the rabbit that stronger stimuli were necessary to excite movement by application to the cord if the transection were below than if above the bulb.

Exner ${ }^{4}$ applied in the rabbit an electric stimulus to the cerebral cortex at a point whose excitation caused contraction of a certain muscle of the foot. The same muscle could also be caused to contract in response to electric stimulation of a given strength applied to the skin of the foot. When both cortex and skin were stimulated simultaneously, the extent of the contraction was greater than when either was stimulated alone. If the stimulus to the skin was so reduced that, alone, it was subliminal for the reaction of the muscle, the cortical stimulus rendered the skin stimulus efficient if applied at any interval less than about three seconds after the cortical stimulus. The efficient skin stimulus similarly rendered subliminal cortical stimuli efficient for a short period. When both stimuli were, taken singly, subliminal, each rendered the other subsequent one efficient throughout an interval of about an eighth of a second. It is true that there is nothing to prove in this experiment that the stimulus to the skin did not act via the cortex, in which case the interaction was between two cortical reactions, but the example even in that case serves by analogy to illustrate also spinal reinforcement.

Phenomena analogous to the "staircase" of contractions in cardiac and skeletal muscle are met in spinal reactions. When examining reflexes obtained from the afferent roots of the spinal animal, one often finds that, after beginning the experiment by excitation of a given spinal root in a limb region, the stimuli necessary being of a given strength, on returning to the same root after having excited reflexes by a neighbouring root, the strength of stimulus required has fallen to considerably lower than it had been originally, and this happens under circumstances which seem to preclude

${ }_{1}$ Abhandl. d. k. Akad. d. Wissensch. zu Berlin, 1873 ; ibid. 1875 ; Sitzungsb. d. phys.med. Soc. zu Erlungen, 1873; Verhandl. d. Cong. f. innere Med., Wiesbaden, 1884; Biol. Centralbl., Erlangen, 1885 ; Mendelsohn, Sitzungsb. d. k. Akad. d. Wissensch., Berlin, 1882 ; ibid., 1883 ; ibid., 1885 ; Rosenthal and Mendelsohn, Neurol. Centralbl., Leipzig, 1897.

2 Arch.f. d. ges. Physiol., Bonn, 1898, Bd. Ixx.

${ }^{3}$ Arch. $f$. Physiol., Leipzig, 1887, S. 166 ; cf. also Owsjannikow, Arb. a. d. physiol. Anst. zu Leipzig, 1874 .

${ }^{4}$ Arch.f. d. ges. Physiol., Bonn, 1882, Bd. xxvii. 
any other change in the conditions than the intercurrent stimulation of an adjacent root. Again, when a bilateral reflex is started by excitation of an area of skin or a nerve trunk, and, as often happens, the contralateral reflex is the lesser, the reflex at first obtained is unilateral and homonymous, but on then proceeding to excite the corresponding skin or nerve trunk of the opposite side, the reflex movement evoked is at once bilateral and stronger contralaterally. The stimulation of the first side has by its homonymous action "canalised" the contralateral part of the mechanism replying to the second side.

An instructive instance is yielded by the following. Excitation of the afferent root of either the eighth or seventh cervical nerve evokes in the spinal mammal sometimes flexion at elbow, - sometimes, on the contrary, extension. Excitation of the afferent root of the first thoracic nerve evokes almost invariably extension at elbow ; excitation of the afferent root of the sixth cervical evokes almost invariably flexion at elbow. If excitation of the afferent root of the eighth be preceded by excitation of the root of the first thoracic, the movement at elbow evoked by the eighth is almost always extension, and similarly though not so regularly by the seventh. If, on the contrary, the excitation of the seventh be preceded by excitation, not of the first thoracic, but of the sixth cervical, the movement it evokes at the elbow is almost sure to be flexion; and similarly though not so regularly with excitation of the eighth.

There are many sequences met in experimenting on the cord that the example just quoted typifies. Noteworthy is that the Hexors and extensors were never seen to be simultaneously thrown into contraction. I would express the general result by saying that sensory stimuli and reflex reactions which tend, when separate in time, to excite the same group of motor cells, canalise for and tend, when contemporary, to reinforce one another ; those which tend, when separate, to excite different but inter-related groups of motor cells, tend, when contemporary, to inhibit one another. ${ }^{1}$

The incitement to activity of functional group A of motor units seems colligate with inhibition of the functional group $B$, whose muscles are antagonistic to those innervated by $A$. By what mechanism this is ensured is not clear. Group A may have a path not only to the muscles but to the nerve cells of group B, by which it can depress the activity of the latter. Or each spinal path that embouches into A may also embouch mediately or immediately into $\mathrm{B}$, and while exciting A inhibit B. This latter does not necessarily imply two different kinds of disturbance in the paths opening upon $\mathrm{A}$ and $\mathrm{B}$ respectively; a difference between the condition obtaining at the moment in $A$ and $B$ motor units may determine a different reaction in them under an external influence which falls similarly on both. Whatever the detailed explanation, it is certain that co-ordination is a character common to all reflex actions from the unpoisoned spinal cord, and that reciprocal innervation largely expresses that co-ordination.

The movements that are in the spinal animal at various times elicited from one and the same area, and even same spot, of skin, vary considerably, and even at brief intervals. No phenomenon more often

${ }^{1}$ I am aware this differs from views expressed by Eekhard, Goltz, Sehiff, Herzen, Wundt, and others ; but see the suggestions of Deseartes, "De homine," Amsterdam, 1678, p. 34 ; of C. Bell, in a footnote, "Physiology of the Human Body," London, 1826, vol. iii.; also "Remarks on Respiratory Co-ordination," by S. J. Meltzer, Arch. f. Physiol, Leipzig, 1883 ; and New York Med. Journ., 1899; and ny notes on "Reeiprocal Innervation," in Proc. Roy. Soc. London, vol. lii. et seq., 1893-1898, and elsewhere. The suggestions of Descartes and Bell proposed, however, peripheral inhibition, not central. 
meets the observer than this, nor more often disturbs his observations on other points. It brings home to the imagination the constant manifold factors that must be playing upon the spinal cord, even under apparently the most uniform conditions. The play of the external environment upon organ is more or less under control of the experimenter. It is the varying condition of the central organ and of its bodily environments which creates his difficulties.

Under strychnin poisoning the whole skeletal musculature may be thrown into action from any one point of skin, and even when all the afferent roots have been severed, except that particular one conducting from the sole point excited. ${ }^{1}$ Each local afferent path must evidently lead to not merely one but many, and ultimately to the whole motor mechanism. The afferent path from a digit is thus, potentially at least, connected with the whole motor organ of the individual. Yet harmonious co-ordination of the contraction of the individual muscles in any functional group, and the harmonious mutual co-operation of functionally related muscle groups, characterises the natural movements of a normal animal. This mechanism has been symbolised by supposing in the cerebro-spinal organ between the various central connections open to the afferent path, resistances so graded and adjusted that only harmonious motor units and unit-groups become excited, and these each only in that degree due for execution of appropriate movement. It might have been supposed that with the cerebro-spinal organ maimed by subtraction from it of the entire brain, any afferent path entering the central nervous system, and there connected with not one but many-potentially all-the motor spinal units, would, the guidance of the brain and its great sense organs gone, act on the motor units both synergic and antergic so indiscriminately as to induce, if strongly stimulated, complete discharge of all of them, or, if weakly stimulated, feeble or incomplete discharge of all of them. Such discharges would of course produce unnatural novements, in which the action of one muscle or set of muscles would impair or defeat the action of another muscle or set of muscles. As a fact, the reactions of the skeletal musculature of the spinal animal display no inco-ordination of this kind. The afferent path evokes discharge only of harmoniously related motor groups. If it be stimulated strongly, the number of motor groups excited may be extensive and their situation involve the whole length of the spinal axis; but the groups excited contemporaneously are always harmoniously acting groups, e.g. flexors of hip with flexors of knee, and not groups mutually subversive of each other's action. Each individual muscle of each group, so far as analysed, seems in its action balanced and subordinated as in a "natural" movement. Nor does progressive weakening of the stimulation of the afferent path lead to the appearance of inco-ordination, as might have been expected by the excitation of the various groups becoming irregular and partial. As a fact, it leads only to the nonappearance of the reaction in a progressively increasing number of the harmoniously acting regions of the nusculature. No more striking contrast can be cited than that holding between this and the effect of excitation of a motor spinal root or of a filament of a motor root. The latter throws into contraction simultaneously muscles whose action is opposed; what movement results will be determined merely by which muscle of those in action pulls the hardest or has the better leverage.

\footnotetext{
${ }^{1}$ See Vul pian, "Leçons sur la physiologie du système nerveux," Paris, 1866.
} 
Even when the stimulus, instead of being restricted to a narrow sensory area or path, affects simultaneously large surfaces or wide channels, finally the whole skin, the motor discharge evoked is still confined wholly to harmoniously acting spinal units. Thus the spinal frog placed in water warm enough to stimulate the general skin, swims forward with balanced and symmetrical and synchronously bilateral strokes. The co-ordination of the action of the musculature of each limb, and that of the hind-limbs together, and even of fore-limbs with hind-limbs, is good; the aquatic locomotion is surprisingly elficient in view of such total loss of all the cranial senses.

These considerations clearly indicate that some set of spinal sense organs forms the chief basis of the mechanism which ensures such elemental muscular co-ordination as that under discussion. The fact that it is fairly preserved even in the skinned spinal preparation, indicates that the spinal sense in question does not depend on cutaneous sense organs. Its sense organs are probably those in the musculo-articular apparatus, since it seems hardly possible that the co-ordination is to so large an extent an innate quality of the motor unit groups themselves.

The integrity of the whole cord is not required to ensure co-ordination. Short isolated portions are enough. In Limulus four pairs of the abdominal ganglia isolated in a group can regulate the respiratory movements of the eight appendages co-ordinately, in total absence of the whole remainder of the nervous system. ${ }^{1}$ In spring the three upper spinal segments, isolated from the rest of the head and brain with their connected skin and muscles, execute the frog's sexual "clasp." In the cat and monkey, with spinal transection low in the sacral region, wagging of the tail from side to side by alternate co-ordinate contraction of its lateral musculature, is evoked from the local skin. In short, to employ the metaphor of resistances, in the reflex spinal organ the resistances are so disposed as to ensure synthesis not of a discord but always of a harmony of movement.

The influence which the condition of one part of the cord exerts upon others is seen in the effect which the initial posture of a limb can be demonstrated to exert upon the character of the reflex movement elicited from the limb. From the skin of the back of the proximal part of the thigh in the frog and $d \log$, the reflex response in the limb is flexion at the hip, if the resting posture of the limb at the time of application of the skin stimulus be one of extension, e.g. if the limb be hanging down. If, however, the resting posture of the limb be one of flexion at the time of application of the skin stimulus, the reflex movement of response is often an extension of the thigh at hip. The difference of the response may be due to the different condition of the musculo-articular sense organs in the two different initial postures, their difference reacting upon the excitability of the antagonistic motor mechanisms of the cord. The experiment is significant of the important part assignable to subconscious muscular sense reactions in regulating the activity of the skeletal musculature. It suggests an explanation for the alternating character of the movements executed by spinal reflexes.

Phasic variation in the reflex activity of the cord.-Although the relative invariability of the movement elicited by repetitions of a particular stimulus is a striking character of spinal reflexes in the mammal, and lends to them a machine-like quality of regularity, curious variety of result occurs, when they are examined in the same individual

'Ida Hyde (Loeb's laboratory), Journ. Morphol., Boston, 1894, vol. ix. 
from day to day. The very spot of skin that one day evokes nothing but flexion of all the toes, may the next evoke nothing but flexion of the hallux and extension of the other toes; and the next nothing, or again, only the movement obtained three days before. A stimulus usually eliciting dorso-flexion at ankle may on some days elicit in the same individual plantar flexion of the ankle. As a broad rule, spinal reflexes are more easily elicited when a well-nourished animal is hungry and expecting food, and less easily when it has just heavily fed. There is, so to say, a spinal hunger. The knee-jerk in the dog varies in the same way as the true reflexes in relation to time of feeding. But altogether apart from feeding time, on some days hardly a reflex can be elicited from the very animals that on other days yield a variety of reflexes with readiness. Conditions of individual age, and especially of body temperature and general nutrition, influence, as Freusberg points out for the lumbo-sacral reflexes of the dog, the facility of reflexes very greatly indeed.

Autotomy.-Among reflex reactions exciting contraction of muscles, an interesting group discovered by Fredericq, execute what he has called autotomy. ${ }^{1}$ In many animals, e.g. Asterias, Cometula, Ophiurus, Arachne, insects, and crustaceans, if a limb be pulled upon sufficiently forcibly or long, it is suddenly ruptured across and shed. In the same way some vertebrates (e.g. Anguis fragilis) shed the tail. The reaction is a reflex one, employing muscular contraction. It can be elicited not only by a blow or a erush, but by an induction shock or a chemical irritant. It requires in Asterias the mediation of the ganglion at the base of the ray; the circumbuccal nerve-commissures connecting ray with ray may all be severed without impairing the autotomy, although the reaction is then less easy to obtain. Fredericq's experiments show that the actual mechanism consists, at least in many cases, of a violent contraction of a muscle, so powerful as to break a proximal joint and rupture the tissue. In the crab the muscle employed is the great extensor of the forceps. This contraction does not seem at the disposal of the "will" of the animal. If the limb be gently though effectively fixed, the animal in all its endeavours to escape never performs autotomy. A sudden strong stimulus to the limb itself can provoke reflexly a more powerful contraction of the limb muscle than can be evoked by impulses emitted from the higher centres.

\section{SPINAL SHOCK.}

"Shock," like "collapse," a term more used by the clinician than the physiologist, is, like it, a term somewliat ill-defined in scope. In some forms of the clinical condition, circulatory disturbance and inspissation of the blood play part, but, as understood by the physiologist, "shock" is primarily a nervous condition. "If in a frog the spinal marrow be divided just behind the occiput, there are for a very short time no diastaltic actions in the extremities. The diastaltic actions speedily return. This phenomenon is 'shock.'” "2 Whytt had, a century previous to Hall, drawn attention to the same phenomenon, although assigning to it no descriptive term. The whole of that depression or suppression of nervous function which ensues forthwith upon a

\footnotetext{
${ }^{1}$ Léon Fredericq, Areh. de zool. expér., 1883 ; Arch. de biol., Gand, 1882, tome iii. p. 235 ; Rev. scient., Paris, 1886, p. 613; 1887, p. 1; Trav. du lab. de Marey, 1887-88, tonie ii. p. 201 ; ibid., 1894, tome iv.; Preyer, Mitth. a. d. zool. Stat. zu Neapel, 1887, Bd. vii. S. 203 ; Jean Demoor, Trav. d. l. stat. zool. d. Helder, 1891, p. 130 . For other references see papers by Fredericq.

2 Marshall Hall, "Synopsis of the Diastaltic Nervous System," London, 1850.
} 
mechanical injury of some part of the nervous system, and is of temporary nature, may be conveniently included under "shock." By Goltz it is considered as entirely a collection of inhibition phenomena. But it has its analogue, no doubt, in the "block" (Romanes, Gaskell) in a conducting tissue (medusa subumbrella, heart) ensuing on mechanical interference. Whether such phenomena are best included under the head of inhibitions is very questionable.

Among laboratory animals it is in the monkey that on the whole "spinal shock" appears at maximum. As studied in that animal, whether the site of spinal transection lies near the front or hind end of the thoracic region, appears to make slight difference to the general result, apart from determining the mere number of spinal segments displaying the phenomenon. The "shock" appears to take effect in the aboral direction only. ${ }^{1}$

Section below the brachial enlargement little if at all disturbs the reactions of the upper limb, although the number of headward rumning channels of conduction ruptured by such a section is enormous. Striking instances of the absence of headward spread of the depression due to "shock" are afforded by transections abutting on the lower edge of the fifth cervical seginent; these depress the respiratory activity of the phrenic motor cells hardly at all, even momentarily. On the aboral side of the transection, depression is profound. Analogously, the sudden cutting off of all that stream of centripetal impulses continually pouring for conscious and subconscious elaboration into the encephalon from the cutaneous, articular, and muscular sense organs of the tail, limbs, trunk, and neck, and from the viscera, does not seem at all to disturb the reactions of the head and brain. The animal immediately after the section will contentedly direct its gaze to sights seen through the window, or, if the section have been below the brachial region, may amuse itself by catching flies on the pane. Similarly, the head half of the worm Thysanozoon ${ }^{2}$ or Allolophobus ${ }^{3}$ is said to continue the movements of progression almost undisturbed by quick transection across its middle. The bee, if while it is sucking honey the abdomen is suddenly cut off, will rear itself for a moment and then quietly continue sucking. ${ }^{4}$ The exclusively aboral direction taken by shock seems universal in the nervous system. In the monkey, when the transection has been quite far forwards, e.g. at the first cervical level, there seems a tendency to drop off rapidly to sleep; but it is very doubtful whether that is due to any depression of the nervous organs in front of the section, other than that depression of function one might expect from the coincident fall of the arterial blood pressure and body temperature. In the cat a high transection, e.g. brachial or cervical, seems to frequently induce in front of the lesion not a depression but an exaltation of function, the region of the trigeminal distribution appearing hyperæsthetic. ${ }^{5}$ The reactions from the head organs in Astacus ${ }^{6}$ seem exalted by section of the nerve cord in front of the thoracic ganglia.

After high cervical transection, "shock" appears more severe in the fore-limbs than in the hind. For an hour or so it may be difficult to elicit any reflex movement from skin innervated behind the transection, whether by mechanical, thermal, or electrical stimuli. An apparently crossed reflex is, at least in my experience, among the earliest obtainable, namely, the crossed adductor reflex and the

1 Sherrington, Phil. Trans., London, 1897.

2 J. Loeb, Arch. f. d. ges. Physiol., Bonn, 1894, Bd. lvi.

3 W. W. Norman, ibid., 1897, Bd. lxvii.

5 Sherrington, Phit. Trans., London, 1897.

A. Bethe, ibid., Bd. Ixviii.

'Bethe, Arch. f. $d$. ges. Physiol., Bonn, 1897, Bd. lxviii. 
"crossed knee-jerk." An early reaction to emerge from the spinal shock is adduction-flexion of the hallux, in response to stimuli applied to the third, fourth, or fifth digits (plantar surface or sides), or to the skin of the sole, especially on its fibular side. The movement of the hallux is then slight and often tremulous. A similar reflex is obtainable in the hand. In the cat and $\log$ and rabbit an equivalent reaction seems to be the arching of the digits, and this is not unfrequently bilateral. Anal protrusion, in response to stimulation of the skin of the perineum, feeble abduction of the tail on pricking it, are also reactions which early emerge from the depression. The limbs at this time feel limp and the muscles more Haccid than they do later. Gradually these reflex movements become more vigorous, and others are added, c.g. the cremasteric, and the drawing up of the limb on stimulation of the foot. Extension of the limb is late to be obtained, and very rarely is obtained as a primary homonymous reaction. Crossed reflexes appear, and the march and duration of the individual reflexes gets more extensive. The disappearance of "shock" is very variously rapid in different individuals. In the same individual the progress is usually not steady; on some days reflexes are absent which had been gained, and reappear only later. The extensors of the hips and knees recover later than the flexors. In the spinal dog, after a brachial transection, it can perhaps be said that in five weeks the depression of shock has usually worn off. It is not renewed by a second transection practised behind the first one.

The cause of the "shock" cannot entirely be the fall of arterial pressure which a transection anywhere in front of the second thoracic level must entail, for the head does not participate in the shock, although participating in the lowered blood pressure; and when the transection is in the lower thoracic region, the shock distal to the lesion is about as severe as after cervical transection. Besides, section of both splanchnics does not cause shock. Neither is the "shock" explicable by fall of deep temperature, for it occurs where that is absent and has been almost avoided. It is noteworthy that, although in the monkey among laboratory animals it appears most severe, and is certainly particularly long lasting, it does not in that animal appear more severe as regards depression of function of urinary bladder, rectum, etc., than in other laboratory animals. It is especially well seen in the monkey that the motor root cell, though in spinal shock profoundly inaccessible to stimuli applied viâ skin or afferent nerve trunks, lies perfectly open to any applied via the pyramidal paths ; excitation of the pyramidal tract at the top of the cord readily evokes movements in the otherwise reactionless limbs. Also, excitation-mechanical, or by weak electric currents - of the central ends of the spinal afferent roots themselves readily evokes reflex movements, though far stronger stimuli fail absolutely when applied to the skin and afferent nerve trunks.

How long the phenomena of shock may persist, is a question on which very different views are held. Goltz is the founder of a school which works in the belief that the phenomena of shock may persist for months or even years. My own experience inclines me to think that the condition of a spinal cord, isolated by spinal transection, is often more normal a short time after the transection than it is when long periods of weeks and months have been allowed to elapse. The "shock" passes off, to be succeeded, it seems to me, by permanent alterations that in many ways resemble in their effects a recrudescence of shock. The decreasing depression merges in the onset of an increasing "isolation- 
dystrophy," due to the permanent withdrawal from the spinal cells of intluences they are normally accustomed to receive from other portions of the central nervous system. The most favourable time for the examination of the independent capabilities of the spinal cord is that when the sum of "shock" and "isolation-dystrophy" together is of smallest amount. That time, compounded as it is of two such variable factors, is itself extraordinary variable. In the monkey, "shock" lasts longer and the "isolation dystrophy" comes on earlier than in other animals commonly observed in the laboratory. It is the conjunction of the periods of these two phenomena which I imagine renders so difficult and so largely defeats attempts at observations of the proper spinal reactions of the monkey. If the overlap of the two is great, then no spinal reflexes or only the merest traces of them may be elicitable. In man it is natural to suppose that even more than in the monkey will spinal "shock" be severe and "isolation dystrophy" arrive speedily. Clinical observation supports and confirms this supposition. ${ }^{2}$ After total transverse lesions of the cord in man, the depression of function of the skeletal musculature is profound and practically permanent. On the other hand, the depression of visceral function seems hardly greater in man and the ape than in the rabbit and frog.

By his sense organs and their nerves the individual is put en rapport with the material universe. His sense organs constitute between his mind and that universe the only go-between. That universe for each individual is made up of two portions, one a part beyond his body, termed his environment, the other a part consisting of his own body, or more strictly of all that part of it that can affect his afferent end-organs. This latter part constitutes his "material me." It is divisible. Taking its sensibility as basis for its subdivision, it is a compound of skin, of motor apparatus, and of viscera. With the first of these are concerned perceptions obtained through the projecting as well as through the non-projecting senses. The senses concerned with the motor and visceral apparatus are solely non-projecting. The higher nervous centres are built, especially where most perfected, on the projecting senses far more than on the non-projecting. Of the non-projecting, the muscular much more closely than the visceral is linked with the powers of the great motor organ, the skeletal musculature, itself arisen chiefly as a machine for reacting to the projicient senses. Hence comes it that after destruction of the higher nervous centres, e.g.brain, the competence remaining to the spinal centres is found greatest for visceral reactions. From this point of view the visceral reflexes present the most perfect nervous reactions of the spinal animal. The bulbo-spinal animal, receiving as it does the afferent impulses of the vagus as well as of the thoracic and sacral nerves, may indeed be considered, as regards visceral function, a fairly perfect animal. The purely spinal animal does, however, exhibit some amount of damage to the nervous regulation of its viscera; this is chiefly of the nature of "shock," and temporary. The visceral shock appears to be little if at all greater in the ape and man than in the rabbit and frog, whereas in the two former the shock to the spinal mechanisms of the skeletal musculature is enormously greater than in the two latter types. The significance of the deeper depression of reaction into which the higher animal, as contrasted with the lower, sinks when made "spinal," appears this, that in the higher types more than in the lower the great projecting senses actuate the motor organ, and impel the motions of the individual. The deeper depression shows that as the individual ascends the scale of being, the more cognisant does it become of a circumambient universe that is "not me," and that that latter acquires a

1 Sherrington, Phil. Trans., London, 1897.

2 Bastian, Med.-Chir. Trans., London, 1891 ; Bruns, Neurol. Centralbl., Leipzig, 1893. 
more and more preponderant directive influence over those "reflexions," those expressions of the creature's neural states, its "doings." The relative slightness of the "visceral "part of spinal shock in higher animals indicates the extent to which the reactions of the visceral musculature and some of the reactions of the skeletal musculature accessory thereto are normally unconnected with higher conscient nervous organs.

\section{Spinal Visceral Reflexes.}

To the viscera belong three sets of peripheral afferent channels-(1) the vagus; (2) the spinal roots from first thoracic to second lumbar inclusive; (3) the second, third, and fourth sacral roots. The trophism of these channels depends on the vagal ganglia and the spinal ganglia mentioned. The efferent channels to the viscera lie in three corresponding sets.

The viscera are insentient under numerous traumatic and other injuries; in disease they nevertheless may cause pain. Much of this pain is "referred" to areas of the body surface, where the skin is then often found to be "tender," i.e. hyperalgesic. James Ross ${ }^{1}$ suggested that the pain is in such cases referred to parts supplied by sensory cutaneous nerve fibres ending in the same segments of the cord as do the afferent fibres of the viscus diseased. Head has examined the data for this hypothesis, and has established and elaborated it. ${ }^{2}$ The following conspectus of Head's results states the probable distribution of visceral afferent fibres of the spinal ganglia in man :-

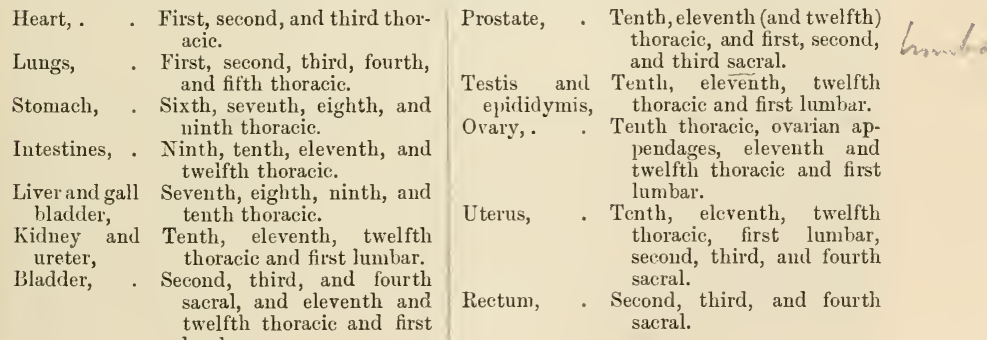

Urinary bladder.-The region of the cord from which efferent and to which afferent chamnels belonging to the urinary bladder pass is twofold, a sacral with which the second, third, and fourth sacral roots (man), and a lumbar with which the first, second, and third lumbar roots are connected. From this region, when isolated, reflex contractions and relaxations of the detrusor and sphincter can be elicited. The intravesical pressure which the sphincter withstands is lowered ${ }^{3}$ by spinal transection between the fifth and sixth lumbar segments of the cat (between sixth and seventh in rabbit).

These can therefore be said to form a spinal vesical centre. The upper limit of the region lies near the hindmost level of the origin of the second

1 " On the Segmental Distribution of Sensory Disorders," Brain, London, 1887, vol. x. p. 333 ; ef. further, Dana, New York Med. Journ., 1887.

${ }^{2}$ Brain, London, 1893, vol. xvi. ; 1894, vol. xvii. 1. 339.

${ }^{3}$ Masius, Bull. Acad. roy. d. sc. de Belg., Bruxelles, 1868; Kupressow, Arch. f. d. ges. Physiol., Bonn, 1872 , Bd. v. S. 292; Ott, Journ. Physiol., Cambridge and London, 1880 , vol. ii. p. 60 .

VOL. II. -54 
lumbar root (cat). ${ }^{1}$ The lower limit cannot be so distinctly determined. Transection below the sixth lumbar vertebra in the rabbit causes immediate escape of urine from the bladder. ${ }^{2}$ After spinal transection in the frog, rabbit, cat, dog, and monkey, as after tantamount injuries in man, micturition is interfered with. The exact share played in the suppression of function of the organ by the interference with the innervation of its intrinsic musculature and with that of other muscles, abdominal and perineal, accessory to the emptying of the organ, is hardly known. In the first days after the transection, the bladder can only be competently evacuated by artificial means. This is easily effected, especially in the female, by pressure on the distended viscus through the abdominal wall. The pressure should be light, and quickly applied, and not maintained for long; it appears to act by exciting a brief reflex micturition, no doubt of an imperfect character. The tail is raised, the anus somewhat protruded, the knees are in the bitch flexed, and a brief gush of urine is allowed to escape under the pressure of the hand on the abdominal wall. The pressure alone is quite inefficient to expel the urine, for it can be greater when first applied and ineffective, than later when effective; until the reflex ensues, the pressure cannot void the bladder of its contents; and although the pressure is maintained, the urine after the brief gush is not voided. It is then best to desist from the abdominal compression for a minute or so, and reapply it somewhat suddenly. Sometimes, instead of a brief gush, the whole contents are allowed to be discharged. It has been shown ${ }^{3}$ by comparing rate of escape with intravesical pressure, that when the bladder is reflexly voided, in response to stimulation of a sciatic nerve central to section, the reflex reaction involves two acts, contraction of the detrusor and relaxation of the sphincter (in addition to concomitant movements due to skeletal muscles). The impression given to the hand when the bladder is emptied by pressure over the hypogastrium is less that of contraction of the viscus than of a resistance low down on the pelvic floor suddenly yielding. I have noted that, in the monkey, variation of the level of the spinal transection anywhere between sixth cervical and fourth lumbar appears to make no noticeable difference in the degree of depression of the act of micturition, although in the latter case the hypogastric afferent and efferent paths are intact, in the former not. This depression seems as severe in the rabbit and cat as in the monkey. For many days the urine may have to be evacuated either by inducing the reflex in the above-mentioned way, or by the catheter. Later, the urine in the monkey as well as in other laboratory animals comes to be voided "spontaneously" from time to time. In some cases in the dog the urine even soon after transection is voided in small quantities, very frequently and apparently without obvious external stimulation having occurred, and without the bladder being even moderately filled. Defæcation frequently accompanies the micturition; the spinal micturition is followed by spinal wagging of the tail.

Goltz and Ewald, ${ }^{4}$ in their observations on dogs in which the lower region of the spinal cord had been removed in toto, found the bladder empty itself spontaneously from time to time months after removal of the cord; but the bladder had usually to be emptied by artificial means, and cystitis frequently occurred. Though some degree of retention of urine is a common symptom after total transverse lesion of the cord in man, and usually a permanent one, I have never seen cystitis ensue after spinal transection in laboratory animals, although the animals have been kept under observation many months.

Defæcation.- In the tortoise, after spinal transection behind the second of the three roots of the sciatic plexus, defæcation occurs in the sense that fæces

${ }^{1}$ Colin C. Stewart, Ani. Journ. Physiol., Boston, 1899, vol. ii. p. 182.

2 Masius, Bull. Acad. roy. d. sc. de Belg., Bruxelles, 1868.

3 Alfons Haue, Arch. f. d. ges. Physiol., Bonn, 1899, Bd. lxxiii. S. 453.

4 Ibid., 1896, Bd. lxiii. S. 362 . 
are expelled. ${ }^{1}$ Tampons of cotton-wool introduced into the rectum excite their own expulsion, if they be relatively large. The expulsion is accompanied by movements of both hind-limbs and tail. When the level of transection lies above the sciatic plexus, excitation with smaller tampons, or by electrodes applied just within the anus, evokes a complete act of defæcation. The posterior end of the body is raised on the extended hind-limbs, the tail is raised, and after expulsion of the material from the rectum follow movements of nettoyage by the hind-limbs. Defæcation, in the sense of regular expulsion of fæcal matter, occurs without difficulty in manmals after transection in the upper lumbar region, or higher. The external sphincter is patulous in some cases for a few hours or days after the spinal transection, but more usually recovers tone a few minutes after the operation, or never loses it. Goltz and Ewald found the tonus return even after exsection of the lumbo-sacral region of the cord. The rhythmic contractions of the sphincter noted ${ }^{2}$ to set in after high lumbar spinal transection in the dog, can be inhibited by strong sensory stimuli of adjacent parts. ${ }^{3}$ Gowers, ${ }^{4}$ in describing the condition of the rectal sphincter in disease of the spinal cord, says two states can be distinguished by introduction of the finger. If the lumbar centre is inactive, there is a momentary contraction, due to local stimulation of the sphincter, and then a permanent relaxation. If, however, the reflex centre and motor nerves from it are intact, the introduction of the finger is followed "first by relaxation and then by gentle, firm, tonic contraction. I have verified this by introducing an india-rubber cylinder instead of the finger, and registering the pressure on the cylinder by connecting it with a recording apparatus. The relaxation is then found to be preceded by a very slight brief contraction, and to be followed by unbroken tonic contraction." The relaxation may also be readily produced by stimuli to the mucous membrane above the sphincter. Incontinentia alvi is described as usual in man after total transverse lesion of the cord; it is not at all usual in the monkey.

Sexual reflexes.-The uterus.-As is well known, uterine contractions may be induced by applying heat or cold to the abdomen, by passing a catheter, by hot or cold rectal injections, by drawing back the perineum, or by putting the child to the breast. In animals, such contractions are strongly excited by faradisation of the central end of the first sacral nerve. ${ }^{5}$ As the irritation in these instances is not applied to the uterine tissue or to efferent nerve channels, the resultant uterine reactions must be induced reflexly. Röhrig ${ }^{6}$ states that asphyxia does not produce uterine contractions or abortion if the lumbar region of the spinal cord is destroyed, although it does produce those events if the cord is cut across in the thoracic region. Cases have been from time to time reported in which parturition has occurred and run its course in normal fashion in paraplegic women, the interruption of the cord being complete, and as low as the tenth thoracic level. Marshall Hall argued from such instances that uterine action is a reflex spinal one connected with a lumbar "centre." In these cases parturition is, of course, unattended with pain, although the labour "pains" are strong and efficacious. In one case, during the second stage, the "pains" recurred about every two minutes, each lasting about forty-five seconds. The patient perspired after each pain, as if she had been exerting herself. There was an indistinct tight feeling referred to the epigastrium at the height of each pain ;

${ }^{1}$ A. Bickel, Rev. méd. de la Suisse rom., Genève, 1897 ; J. Ott, Journ. Physiol., Cambridge and London, 1879 , vol. ii. p. 54 .

${ }^{2}$ Gluge, Bull. Acad. roy. d. sc. de Belg., Bruxelles, 1868 ; Goltz, Arch. f. d. gcs.

Physiol., Bonn, 1873, Bd. vii. S. 582.

${ }^{3}$ Goltz, loc. cit. ; J. Ott, loc. cit.

4 "Diseases of the Nervous System," London, vol. i.

${ }^{5}$ Cyon, Arch. f. d. ges. Physiol., Bonn, 1874, Bd. viii.

${ }^{6}$ Virchow's Archiv, 1879, Bd. lxxvi. S. 1. 
by the recurrence of this indistinct sensation of "tightness" she became able to say when a "pain" was present. ${ }^{1}$ Goltz ${ }^{2}$ made observations on a bitch ; - spinal transection was practised at the lower thoracic region; six months later "heat" came on, attended by the usual phenomena, psychical as well as physical. Impregnation was effected, the animal became gravid. The pregnancy was normal; the mammary glands enlarged as usual. Parturition followed in due course. Goltz and Ewald ${ }^{3}$ have more recently observed in a bitch, whose spinal cord from the lower thoracic region backward they had exsected eight months previously, pregnancy follow coitus and terminate with successful parturition. The animal suckled the puppies normally. The mammary glands thus share in the pregnant and lying-in states when there can be no spinal communication between the pelvic organs and the breast. In the case of the patient reported by Routh, ${ }^{4}$ it was so, although the destruction of the cord in the region of the seventh thoracic nerve was, as confirmed by autopsy, complete. The breasts enlarged during the pregnancy, and during the puerperium the mammary function was performed with normal punctuality and persistence. The quality of the milk was good. The relation of parturition to the spinal cord is probably more intimate in the second stage than the first. The communication is with the lumbar region through the three most anterior lumbar sympathetic ganglia. Connection of the lumbar region of the cord with the brain is evidently not essential for the co-ordination of uterine reactious, and even the lumbar "centre" can be altogether dispensed with. More than one observer has, however, noted that section of the lower part of the cord, made late in pregnancy or during labour, arrests labour.

Eckhard ${ }^{5}$ found the secretion of milk from the udder of the goat uninfluenced in quantity or quality by section of all the nerves of the organ. The influence of the spinal reactions on the mammary secretion remains unknown; but that the secretion can be influenced through the cord, is indicated by clinical observations which connect disturbance of the mammary secretion with strong emotions, perhaps mediately through vasomotor action. A prolonged contraction of the muscular and erectile tissue of the nipple and areola, elicitable by firm compression of the nipple and areola, has been described as "nipple reflex," ${ }^{6}$ but it has yet to be shown that the mechanical stimulus does not act by direct excitation of the contractile tissues involved. In the rhesus monkey I have seen menstruation come on and recur regularly after spinal transection at the twelfth thoracic level.

Erection and seminal ejaculation.-Among the very few reflexes which, after total transverse lesion of the cord, are in man preserved even from immediately after incidence of the injury onward, are the sexual reflexes of erection and seminal ejaculation. They occur especially in young adults. Erection is evoked, not only by touching the genital surfaces, but also by pressure on the bladder, or scrotum, or the perineal and anal skin, and from the front of the thigh. ${ }^{7}$ Ejaculation is much less usual than erection. Priapism is of usual occurrence, and probably explicable simply by the vasomotor paralysis.

In the spinal mammal erection can be reflexly elicited. In the dog, after spinal transection above the lumbar region, movements due to the skeletal musculature are easily excited from certain geuital regions. Touching the preputial skin evokes bilateral extension at the knees and ankles, and to a less extent at the hip-joints; accompanying this there is depression of the tail.

${ }^{1}$ See A. Kouth, Trans. Obst. Soc, London, 1897.

${ }_{2}$ Arch. f. d. ges. Physiol., Bonn, 1874, Bd. ix. S. 552.

3 Jbid., 1896, Bd. Ixiii. S. 362.

${ }^{4}$ Loc. cit.

${ }^{5}$ Bcitr. z. Anat, u. Physiol. (Eckhard), Giessen, 1855, Bd. i. S. $12 ; 1877$. Bd. viii. S. 117. Röhrig believed slight reflex effects could be observed. Virchow's Archiv, 1876, Bd. Ixvii.

6 J. B. Hellier, Brit. Mcd. Journ., London, November 7, 1896.

7 T. Kocher, Mitt. a. d. Gronzgeb. d. Med. u. Chir., Jena, 1896 Bd. i. S. 556. 
If through the skin more posteriorly the glans penis be pressed, the posterior end of the body is curved downwards, pushing the penal bone forward. These reflex movements suggest themselves as belonging to the act of copulation, and this suggestion is strengthened by failure to obtain similar movement on touching the analogous parts of the spinal bitch.

At breeding-time the male frog covering the female clasps her with his fore-limbs during four to ten days so vigorously, that much force is required to separate him from her. Decapitation of the male does not, as a rule, suffice to do so. The sexual posture is maintained as a spinal reflex. ${ }^{1}$ The cord may be divided both in front and behind the brachial region without interrupting the reflex. Experiment shows that from the spinal male frog at the breeding-season, and also at other times, the reflex is elicited by any object that stimulates the skin of the sternal and adjacent region. In the intact animal, on the contrary, other objects than the female are, when applied to that region, at once rejected, ${ }^{2}$ even although they be wrapped in the fresh skin of the female frog, and in other ways made to resemble the female. The development of the reflex is not prevented by removal of the testes, but removal of the seminal reservoirs is said to depress it, and their distension, even by indifferent fluids, to exalt it. If the skin of the sternal region and arms is removed, the reflex does not oceur. Severe mutilations of the limbs and internal organs does not inhibit the reflex, neither does stimulation of the sciatic nerve central to its section. The reflex is, however, depressed or extinguished by strong chemical and pathic stimuli to the sternal skin, at least in many cases. The tortoise exhibits a similar sexual reflex of great spinal potency, and Tarchanoff ${ }^{3}$ and Albertoni ${ }^{4}$ discovered simultaneously that even weak excitation of the optic lobes (mesencephalon) immediately inhibits the reflex in both frog and tortoise; without exciting contraction in other muscles, those exciting the sexual clasp are at once relaxed, and the male drops from the female. Stimuli applied to the optic thalami occasionally produce the same effect, but other parts of the brain and cord fail to give the reaction.

Other visceral reflexes. - As regards other viscera than the above, little concerning their relations with the spinal cord remains to be said, further than that already stated actually or by implication in the chapters on digestion, renal action, vasomotor distribution, and on the sympathetic nervous system. A frequent and troublesome sequel to high spinal transection, e.g. in the brachial region, in the $\operatorname{dog}$, is an alteration in the character of the fæces, these becoming fluid, frequent, copious, and laden with mucus. The explanation lies, perhaps, in vasomotor disturbance having caused congestion of the intestines. The same result follows exscetion of pieces of the thoracic region of the cord. Brachial transection (i.e. above brachial roots and below phrenic) in the rabbit immediately gives rise to obvious increase of peristaltic activity in the intestines. ${ }^{5}$ This does not follow a bulbar transection in front of the vagus root. Whether the cord gives secretory nerves to the intestines, has been discussed in another part of this work. ${ }^{6}$ In frogs the secretion of urine is said to continue normally after the whole spinal cord has been destroyed. ${ }^{7}$ In the dog, inasmuch as the flow of urine is abolished when the aortic pressure falls to $40 \mathrm{~mm}$. $\mathrm{Hg}$, and as it has been frequently noted to fall

${ }^{1}$ Spallanzani, "Opera sopra la riprodnzioni animali," Modena, 1768 ; "Exper. sop. la generaz. dei animali e veget.," 1785.

${ }^{2}$ Goltz, Centralbl. $f$. d. med. Wissensch., Berlin, 1865 , No. 19 ; 1866, No. 18 . This statement will not apply, however, to the male toad.

${ }^{3}$ Arch. f. d. ges. Physiol., Bonn, 1887, Bd. xl. S. 340.

+ "Man. di fisolog. d. homo," Milano, 1887; Arch. ital. de biol., Turin, 1887, pt. 1.

${ }^{5}$ C. Bernard, "Leçons sur le système nerveux," Paris, 1858 , tome i. p. 378.

6 J. S. Edkins, "Meehanism of Sceretion of Intestinal Juice," this Text-Book, vol. i. p. 555 .

${ }^{7}$ Bidder, Arch.f. Anat., Physiol. u. wissensch. Med., Berlin, 1844, S. 347. 
to below that after cervical transection, it might have been supposed that suppression of urine would, for some hours at least, succeed the transection. This, however, does not, in my experience, happen; the secretion of urine, if ever stopped, soon recommences, and the regular emptying of the bladder is one of the earliest and constantly recurring cares required from the attendant.

The vagus appears to be the afferent and efferent nerve to the pancreas, but the common bile duct, though opening together with the pancreatic, possesses afferent fibres from the spinal ganglia. If a small quantity of saline be injected into the common bile duct so as to distend the duct, a reflex alteration of arterial pressure results, of very marked and regular character; the afferent channels for this reflex pass through a large number of the thoracic spinal roots, especially perhaps those from the sixth to the twelfth, and I find the reflex not obviously lessened by section of both vagi. The liver and bile ducts have frequently, from Haller's experiments onwards, been found insensitive to artificial stimuli; this and the insensitivity of the liver under incision or puncture by the surgeon, both stand in contradiction to the pains caused by biliary calculi. The ease and certainty with which mechanical stimulation of the duct by a little fluid excites a strong vascular reflex, is of interest in this connection. The reflex still ensues when the fluid is prevented from entering the gall bladder. In the tench the musculature of the intestine is of the striped variety, but no spinal reflexes involving it can be found. ${ }^{1}$

Spinal reflexes affecting the musculature of the blood vessels. -On severance of cord from bulb, arterial pressure falls, owing to damage done not to the innervation of the heart, ${ }^{2}$ but to that of the blood vessels, ${ }^{3}$ especially of the smaller arteries. Goltz earliest drew attention to the partial character of the atonia of the blood vessels of the spinal frog, and its conversion into completeness by destruction of the spinal cord. In the dog he transected the spinal cord at the hind-end of the thoracic region, and waited until vascular tonus of the hind-limbs was restored; destruction of the lumbo-sacral region of the cord he found then renew the vascular dilatation in the hind-limb regions. Schlesinger ${ }^{4}$ obtained in the spinal rabbit, after doses of strychnin, slight reflex alterations in general arterial pressure; and similar have been sometimes obtained without the use of strychnin. In the rabbit the reflex has given heightening of pressure, in the dog depression. Nussbaum ${ }^{5}$ obtained in the curarised spinal frog reflex contraction of the blood vessels of the plantar web; Vulpian ${ }^{6}$ obtained a similar result in the crossed hind-limb, after transection of the spinal cord of the $\operatorname{dog}$ in the mid-thoracic region, on stimulating the sciatic nerve central to section of it; fall of temperature, as evidenced by a thermopile and galvanometer, was taken as index of the vaso-constriction. The same result has been noted in the rabbit. In order to obtain these reflexes, the lower part of the thoracic region of the cord must lie below the transection; the lumbo-sacral region of the cord does not of itself suffice. After spinal transection at the hindmost thoracic segment, Goltz ${ }^{7}$ found mechanical stimulation of the glans penis cause erection; this result was not obtained after subsequent destruction of the lumbo-sacral region of the cord. Similarly, excitation of the sciatic nerve, proximal to its section, causes flushing of the skin of the contralateral hind-foot. After spinal transection at the third cervical level, excitation of the brachial plexus cords, proximal to their

${ }^{1}$ Mahn, Arch.f. d. ges. Physiol., Bonn, 1899, Bd. lxxii.

2 v. Bezold, "Untersuch. i. d. Innerv. d. Herzens," Leipzig, 1863, Bd. ii.

${ }^{3}$ Ludwig u. Thiry, Sitzungsb. d. k. Akad. d. Wissensch., Wien, Bd. xlix. S. 421.

+ Med. Jahrb., Wien, 1874 , S. 20 ; ef. also Snirnow, Centralbl. f. d. med. Wissensch., Berlin, 1886; and Ustimowitch, Arch.f. Physiol., Leipzig, 1880.

5 Arch. f. d. ges. Physiol., Bonn, 1874 , Bd. x. S. 374.

6 "Leçons sur l'appar. vasomoteur," Paris, 1875 , p. 288.

Arch.f. d. ges. Physiol., Bonn, 1874, Bd. viii. S. 463. 
section, produces a fall of general arterial pressure. This depressor action is probably a reaction upon the abdominal viscera.

The fact that no obvious difference to the degree of fall of blood pressure is made by varying the position of a transection in the cervical region, led Stricker ${ }^{1}$ to exclude that region from the distribution of his spinal vaso-constrictor centres. The further fall of arterial pressure, caused by cutting the splanchnics after cervical transection, indicates that the upper thoracic region also contains vaso-constrictor centres. Bradford and Dean ${ }^{2}$ demonstrated outflow of vasoconstrictor nerves to the lungs in the upper thoracic roots, and Bayliss and Bradford ${ }^{3}$ demonstrated vaso-constrictor fibres to the brachial limb, leaving by the fourth to the ninth thoracic roots. This, together with the wellknown upper thoracic origin of the cervical sympathetic, ${ }^{4}$ proves, in conjunction with the fact that the spinal cells of the sympathetic lie at the same segmental level as the exit of their fibres from the cord, ${ }^{5}$ the existence of various spinal vasomotor mechanisms throughout the upper thoracic region of the cord. Vulpian's and Kabierski's ${ }^{6}$ above-mentioned results placed similar centres in the lower thoracic region, a location confirmed by Bradford's identification of the roots containing vaso-constrictors to the kidney, and Bayliss and Bradford's determination of those supplying the vessels of the hind-limb. The outflow of vaso-constrictors agrees, as pointed out by Gaskell, ${ }^{7}$ with the region of white rami communicantes and of preponderance of small myelinate fibres in the ventral root cells. The details concerning this vascular nerve supply do not belong to this portion of the book; excerpts are given here only as indicating the location of spinal centres that control the calibre of blood vessels and are capable of being influenced reflexly. The spinal vasomotor centres not only control smaller areas than does the bulbar centre, but are probably less accessible or excitable than it. The rise in arterial pressure which accompanies asphyxia fails altegether or occurs later after cervical transection than when the bulbar centre remains in connection with the cord. ${ }^{8}$ In the latter case the rise begins in 5 to 20 seconds, and reaches its maximum 15 to 40 seconds later. After spinal transection, the rise does not set in until 80 to 130 seconds after respiratory movement has ceased, and does not reach its full height until 100 to 120 seconds later. The reaction of the isolated spinal centres seems therefore an altogether slower process than the bulbar. Probably the ordinary reflex reactions, in response to excitations of different nerves, ete., play chiefly through the bulbar centre. The most remarkable afferent nerve, in regard to effect on arterial pressure, is the depressor, and this, a branch of the vagus, passes probably direct to the bulbar centre. The afferent path for spinal nerves must suffer interruption in the shape of synapses somewhere in the cord en route to the bulbar centre, for certainly the long dorsal-column direct path does not subserve vasomotor reflex action. Excitation of the whole sciatic trunk central to its section does not, with the dorsal columns of the cord alone intact, evoke any change in blood pressure. The path seems to ascend in the lateral part of the ventro-lateral column. ${ }^{9}$ The path descending from the bulbar centre to

1 Med. Jahrb., Wien, 1878, S. 21 ; ibid., 1886, S. 6.

2 Journ. Physiol., Cambridge and London, 1894, vol. xvi. p. 34 ; Proc. Roy. Soc. London, 1889 , vol. xlv. p. 369.

3 Journ. Physiol., Cambridge and London, 1894, vol. xvi. p. 10.

4 See especially, J. N. Langley's paper, Phil. Trans., London, 1892, with full literature to that date.

5 Sherrington, Journ. Physiol., Cambridge and London, vol. xiii., "Proc. Physiol. Soc.," Feb. 1892.

${ }^{6}$ Arch. f. d. ges. Physiol., Bonn, 1877, Bd. xvi. s. 527.

7 Journ. Physiol., Cambrirlge and London, 1885, vol. vii. p. 1.

${ }^{8}$ Kowalewsky and Adamik, Centralbl. f. d. med. Wissensch., Berlin, 1868, S. 582; Luchsinger, Arch. f. d. ges. Physiol., Bonn, 1878, Bd. xvi. S. 518 ; Konow and Stenbeck, Skandin. Arch. f. Physiol., Leipzig, 1889, Bd. i. S. 407.

${ }^{9}$ Dittmar, Arb. a. d. pleysiol. Anst. zh Leipzig, 1870, S. 18 ; 1873, S. 419. 
subsidiary spinal lies also in that region of the transverse area of the cord, and, though mainly uncrossed, is partly crossed. Lateral semisection of the cord in the cervical region has been found to depress the arterial pressure very little; the temperature of the skin of the head, forearm, hand, leg, and foot becomes for the time higher on the side of the semisection ${ }^{1}$ than on the opposite; contrarily, that of the trunk, shoulder, upper arm, and thigh is lower on the side of the lesion; this latter is probably due to the depression of tonus in the skeletal musculature. But the subject is still not clear. A wound of the dorsal column, for instance, produces a rise of temperature in the foot of the homonymous side, although there then can be no question of breaking of vaso-constrictor paths. After transverse lesions of the human spinal cord, the surface veins of the lower limbs are swollen, and the temperature of the feet is often higher than that of the hands. ${ }^{2}$ Priapism is also a common symptom, and probably indicates vasomotor paralysis rather than heightened reflex excitability.

The heart and blood vessels can be regarded not only in virtue of their musculature as organs of expression for reflexes, but also to some extent as constituting a region whence reflexes can be initiated. Heger ${ }^{3}$ injected into the crural artery, the crural vein being tied, a minute quantity of nitrate of silver solution, a salt to which it is believed the capillary wall is impermeable. The quantity injected was believed sufficient to extend into the capillaries. The injection caused an immediate alteration of the general blood pressure, even when the sciatic nerve was the only structure undivided at the root of the limb. The heart itself is a reflexigenous region of potency. Harvey's observations on Viscount Montgomery showed, as have all similar opportunities, that mechanical contact, even with some pressure applied from without, is an inadequate stimulus to the sense organs of the normal heart as regards the production of sensation. The afferent channels of the heart, however, are not, so far as yet known, any of them spinal; all, as far as investigated, run in the vagus nerve. From the endocardium and the pericardium, both visceral and parietal, under mechanical and chemical excitation, muscular, respiratory, and vascular reflexes have been provoked. ${ }^{4}$ From the cardiac branches of the vagus have been excited, under certain circumstances, not only their well-known effects on blood pressure (through bulbar vasomotor centre) and on rhythm of cardiac beat, but general reflexes affecting the skeletal musculature. ${ }^{5}$ The efferent channels connected with the cardiac afferent are therefore far from confined to vascular nerves. But further statement of vascular and heart reflexes belongs to the chapters on the circulation. ${ }^{6}$ Increased frequency of heart beat can, it seems, be evoked as a spinal reflex.

Significance of spinal reflexes.-The reactions of the skeletal musculature obtainable from the "spinal" animal in response to stimuli applied to its sense organs, exhibit often such obvious propriety as replies to those stimuli that they are termed "purposive." Some have regarded them as evidence of "conscious choice." 7

${ }^{1}$ v. Bezold, "Ueber d. gekreuzt. Wirk. d. Ruickenmarks," Berlin, 1859 ; Schiff, "Lehrbuch d. Nerven- u. Muskel- Physiol.," Lahr, 1859.

2 Th. Kocher., Mitt. a. d. Grenzgcb. d. Mled. u. Chir., Jena, 1896, Bd. i. S. 555.

${ }^{3}$ Bcitr. z. Physiol. C. Ludwig gew., Leipzig, 1887, S. 193.

${ }^{4}$ Budge, Arch. $f$. physiol. Heilk., Stuttgart, 1846, Bd. v. S. 588; Goltz, Virchow's Arehiv, 1863, Bd. xxvi. S. 5 ; Gurboki, Areh. f. d. ges. Physiol., Bonn, 1872, Bd. v. S. 289 ; François-Franck, Trav. du lab. dc MTarey, 1880, tome iv. p. 382.

${ }^{5}$ Bindge, Arch. $f$. physiol. Heilk., Stuttgart, 1846, Bd. v. S. 588; Goltz, Virchow's Archiv, 1863, Bd. xxvi. S. 5 ; Gurboki, Arch. f. d. ges. Physiol., Bonn, 1872, Bd. v. S. 289.

"The spinal "sweat" centres and their eonnections are also treated elsewhere in this work (see Waymouth Reid, vol. i. 1, 676).

7 E. Pflüger, "Untersuch, neber d. sensor. funct. d. Ruckenm.," Berlin, 1854. 
A drop of acidulated water applied to the skin over the inner femoral condyle of the frog, elicits the drawing up of the limb; at all joints except the digital, flexion occurs. The foot and digits thus sweep over the irritated point of skin as though to remove the source of irritation. The opposite leg is at the same time extended. If the foot of the irritated limb be amputated, the stimulus evokes a movement similar to the previous, but, on account of the absence of the foot, inefficient to remove the irritant. There then often ensues a drawing up of the opposite limb, extension of that limb being converted into flexion, and the foot of this second limb is swept over the irritated area of skin; to do this thoroughly requires some adduction. When the seat of irritation is on the skin of the back, a corresponding result is not infrequently obtained. The tail of a spinal animal is often abducted in response to "painful" stimuli applied to its lateral surface, but it is said the strychnin convulsion evoked by a similar stimulus always causes the tail to move toward the side stimulated; that is to say, if the stimulus be a painful one, it is in the former case taken out of harm's way; in the latter case plunged further into injury.

It is, of course, as impossible to disprove as to prove that psychical events accompany or that they do not accompany the nervous reactions of the spinal animal. To argue, as has been done, that conscious choice directs reactions solely spinal, is merely to raise a question perhaps interesting, but at present, and seemingly for ever, insoluble. The Darwinian theory of evolution and adaptation to the environment suggests how "purposive" neural mechanisms might arise.

A well-known controversy (Pfluiger, Lotze) as to the psychical powers of the cord, occurred prior to the advent of the Darwinian theory. Long previously, Prochaska had written: "The general condition which dominates the reflexion from sensorial impressions (i.e. those affecting his sensorium commune) to motor nerves is the instinct of the preservation of the individual." For "the individual" Grainger substituted "the organ." Lotze's position may be illustrated by the following quotation:- "The using of the limbs, in so far as the local direction of them goes, is no independent and self-completed labour of the will, but only the employment of an ahready present mechanism, the action of which the mind merely wills to go or not to go, but by no manner of means can regulate in all its details. We find arranged and interconnected in groups combined movements serving to protect the organism from harm, and in these the tendency to action in response to stimuli is so strong, that in many cases the mind cannot suppress the tendency even by the willing of antagonistic movement, e.g. coughing, sneezing. In fact, how ill were it for our bodily welfare, in many circumstances, did its defence depend on intellectual decision instead of on a mere machine."

As to the interpretation of the "purpose" of spinal reflex movements, it is more difficult than with higher reflexes, because the movements are often of more limited extent. The wider the field of a combined movement-if not convulsive but co-ordinate-the less dubiously does it indicate its object. But there is at best much room for fancy, and great caution is required. The spinal reflex "drawing up" of the limb that forms the usual response to a moderate stimulus to the foot, is generally and can be with plausibility construed as an effort to lift the irritated foot out of the way of harm. The stimulus is indeed usually of an intensity sufficient, were the cerebro-spinal system intact, to cause sensation of unpleasant quality. But it is possible to interpret this reflex as the local part of a larger reflex of progression. That alternative obtains support from the usually concomitant reflex extension of the fellow-limb of the opposite side. Further, the drawing up and protraction of the stimulated leg is usually succeeded by its extension, namely, by the phase of movement that 
would normally succeed flexion in the performance of progression. Again, in examining the instances adduced to illustrate the supposed purposive protective movement, resulting in removal of an irritant from the skin, it must be remembered that the flexion of hip, knee, and ankle in the frog is the regular primary response to stimulation almost anywhere in the skin of the limb or loin. And the same movement is seen. when the spinal preparation is altogether deprived of skin; also when the afferent roots to the limb are severed, the stimulus being applied to the skin of the loin. The alternative movement (flexion of the contralateral leg) ensuing when the primary movement of reaction has been performed-that it has been performed uselessly may be only of influence in so far as it means prolongation of the stimulusmay be simply the second phase of the spinal reflex, which always tends to be of alternating character, especially when a stimulus is prolonged. Besides, it must be granted that the ablation of the foot, or the pressure necessary to restrain it if not ablated, is in itself an additional stimulus. A suggestive context to the experiments of so-called "spinal choice" is furnished by the revival to action of a phrenic nucleus, after being thrown out of action by severance from its own lialf of the bulbar centre. Severance of the contralateral phrenic nerve then brings it into action again. ${ }^{1}$

Considered in the light of the Darwinian theory, the normal connections between any afferent path (e.g. from a piece of skin), and the motor apparatus conjoined with it, can only be such as to lead to a purposive movement. The whole ground of "purpose" is speculative. The physiologist can only state that the "spinal" animal exhibits visceral digestive reflexes, performs excretory and sexual functions, executes a number of movements preservative of the cutaneous sensorial surface, and in simpler animal types shows maintenance of habitual posture and movements preparatory for and partly executive of progression. The inclusion of the bulb with the spinal cord does not in this respect essentially alter the statement. More actions in which the skeletal musculature is co-ordinated immediately with and for the service of visceral organs are added, such as the taking of food, including oxygen, the out-putting of the pulmonary excretion, and the regulation of the activity of heart and blood vessels. But of any further reaction toward defence or self-preservation than is already included by implication in the above essentially visceral reactions, there is scanty evidence. Perhaps hunger and thirst, so far as certain subconscious nervous conditions pertain to them, may be present, but, as far as is observable, they initiate no movement; that is, in the spinal and in the bulbo-spinal animal the skeletal musculature as a motile mechanism lies still beyond their reach. Grainger's conclusion ${ }^{2}$ was that spinal cutaneous reflex movements "are either of a preservative character, or resembling the motions which the function of the organ requires." From the skin of the spinal creature, reflex movements resembling those executed by the normal individual in "preening" or cleansing itself, are of widespread occurrence. Together with the "preening" actions of the "spinal" fly, grasshopper, astacus, etc., there fall into this category the movements by which the "spinal" frog wipes irritants from its back and face; the "nettoyage" by the tortoise, the posturing of the hindlimbs and tail of the "spinal" dog concurrently with reflex evacuation of the freces, tending to keep the body from being soiled, and the "scratching" and "shaking" reflexes of the spinal dog. All these are

1 W. T. Porter, Journ. Physiol., Cambridge and London, 1895, vol. xvii. p. 455.

2 "Functions of the Suinal Cord," London, 1837. 
preservative of the part whence they are initiated. The conjunctival reflex closing the palpebral fissure, essentially a cutaneous and from the broad point of view a spinal reflex, is similarly preservative of the organ whence initiated.

In some instances, however, preservation of the individual, rather than of the organ, seems the key to the reflex spinal reaction. In many cases, such an interpretation has been given to signs, that when examined without prejudice, are of themselves quite equivocal. But in reflex autotomy, where the organ is sacrificed and the individual is freed from capture, there seems no other valid meaning. The preservative reflex here seems to offer one of the strongest guarantees that there is some solidarity in the spinal reflex creature,- that it is still an individual, that it has still some personality.

The spinal reflexes significant of locomotion seem never to give indication of any other locomotion than that of the most usual kind. Thus they yield always a going forwards, not a going backwards. They share markedly that character of purely spinal reactions of the skeletal musculature, namely, feebleness as compared with the prototype. It suggests itself that they may contribute chiefly toward preparatory posture in readiness for onset of action executed by the musculature under the driving of the higher centres. Thus the well-known reflex spinal posture of the frog is flexion of the hind-limbs, with the extensors of the joints taut and ready for spring. Again with the primary reflexes from the pedal ends of the limbs of the mammal. These apparently relate to progression-walking. But they do not take the form of the propelling stroke, the extensor push, that thrusts the body forwards. On the contrary, they flex the limband lift it from the ground. This is no doubt the phase of the limb's movement in progression which requires least of force; it resembles in so far the expiratory phase preceding the inspiratory of respiratory movement; it may almost be likened to the diastole of the heart; and like both these it is preparatory for a succeeding phase of greater muscular effort. That phase succeeds also in the spinal animal, but it is less easy to obtain, and recovers from shock less soon. The subject is speculative, but certainly in the assumption and maintenance of posture pure reflex spinal reaction upon the skeletal musculature plays an important part.

In opining whether spinal reactions are conscious or not, the following considerations arise. After total rupture across the spinal cord in man, the reactions of the isolated portion are never conscious. Where nervous reactions have to be studied entirely objectively, as in the lower animals and in young children, a criterion that may be well taken as test of their conscious character seems associative memory. ${ }^{1}$ Associative memory would seem to be a postulate for the very existence of perception. Consciousness can be regarded as a sequence of processes, namely, of ideas, and of states of passage between such. Where even simplest ideas, i.e. raw perceptions are not, there cannot be consciousness. Animal movements that are appropriate not only for an immediate but also for a remote end indicate associative memory. The approach of a $\log$ in answer to the calling of its name, the return of an animal when hungry to the place where it has been wont to receive food, such movements may be taken as indicative of consciousness, since they indicate the working of associative memory. Examined by

1 Cf. J. Loeb, Arch. f. d. ges. Physiol., Bonn, 1894, Bd. lvi. 
this criterion, all purely spinal reactions fail to evince features of consciousness. If two frogs, the one spinal, the other intact, be placed together in water which is then gradually warmed, the intact animal, as the water approaches $35^{\circ} \mathrm{C}$., exhibits movements significant of effort to escape. But the spinal frog will not move, though the temperature rise to even fatal height, so long as the ascent be gradual. A spinal frog placed direct from cold water into water at $35^{\circ} \mathrm{C}$. exhibits at once lively movements, but these, in view of their above-mentioned absence under the other condition, are no sign of associative memory. They may therefore be unconsciously reflex. Not so the reactions of the intact frog. ${ }^{1}$

Such a view of the relation of spinal reflexes to consciousness corrects a tendency to see in such reflexes the germs of volitional acts. The mainsprings in the evolution of volition are feeling and perception. From reactions unconscious, and therefore devoid both of feeling and of perception, volitions cannot have sprung. On the other hand, our individual experience shows how readily volitional acts by repetition and practice ultimately become actions involving neither attention nor even consciousness, - create, in fact, habitual reflexes. The spinal reflexes may be regarded as descended from volitional acts, inherited and therefore instinctive habits of simplest order, testifying to a so-tosay primitive process akin to memory in the spinal cord. ${ }^{2}$ It might be thought that, arisen thus, they would be most in evidence in the spinal organ of the most elaborated nervous systems. As a fact, they are there less obtainable and seem there less active than in simpler systems. This may be due to the greater solidarity of the whole nervous system in its higher than in its lower forms. The greater solidarity renders the more severe the injury wrought by mere isolation of any part from the whole. And this the more so that in higher animals the great projecting senses of the cranial region dominate the spinal cord and actuate the motor organ, the skeletal musculature of the whole body, more than in lower forms.

\section{Spino-Cerebral and Cerebro-Spinal Reactions.}

The spinal cell systems are not concerned only with reactions confined in their extension to the cord. They also transmit, on the one hand, impulses to nerve cells in the brain, and, on the other, receive impulses from nerve cells in the brain. The cell systems in the brain seem able to discharge all efferent root cells, especially those of the skeletal musculature. This is implied in the old remark that " to move things is all mankind can do." On account of its connections with the brain, the spinal cord has therefore to be studied in respect of (1) the elaboration of cutaneous, muscular, and visceral sensations; and (2) contractions of the skeletal, vascular, and visceral musculature in response to stimulation of the cranial sense organs; and (3) as the instrument of emotional and intended movements. Many of the reflex ares commencing in the spinal afferent root cells lead via encephalon back to the spinal cord again, involving cerebral links. These reflex ares, in their course, react on and are reacted on by ares based upon

1 F. Goltz, "Beitr. z. Lehre v. d. Nerv. d. Frosehes," Berlin, $186 \mathrm{~s}$.

${ }^{2}$ Hering, "Ueber d. Gedichtniss als allg. Funct, d. organisirt. Materie," Sitzungsb. $d$. k. Akad. i. Wissensch., Wien, 1870. 
cephalic sense organs. Cerebral confluence of long-conduction arcs seems of high importance in neural evolution. Complete transection of the cord ruptures, of course, all these long-conducting paths for all regions on the caudal side of the transection. After partial transections, the motor and sentient state of the distal region gives information as to the course that impulses must take.

Lateral semisection of the cord. -The results of semisection have been studied at various levels of the cord. As regards the paths interrupted, it is found that nerve fibres degenerate on the cephalic side of the lesion in both the dorsal and ventro-lateral columns. The degeneration in the former is completely homonymous, and extends up to the bulb, reaching the dorsal column nuclei,-Goll's only, if the semisection be not anterior to the thoracic levels; both Goll's and Burdach's, if the semisection be in the cervical region. ${ }^{1}$ The number of degenerating fibres gradually diminishes with increase of distance from the level of transection, and as it is traced headwards concentrates toward the median line. In the ventro-lateral column the degeneration implicates fibres ascending to the superior vermis of the cerebellum by the restiform and retrotlexed conjunctival cerebellar tracts, and to the mesencephalic roof and to the optic thalamus in lesser quantity. ${ }^{2}$ The degeneration in the ventro-lateral column is heavier on the homonymous side, but is not confined to it. From lesions low in the thoracic region, ascending fibres degenerate in the ventral lip of the column, which seem to end in the cervical region of the cord and lower part of the bulb. ${ }^{3}$

Gotch and Horsley have examined the action currents ${ }^{4}$ arising on stimulation of the sciatic nerve, after semisection at the lower thoracic region, and these are found to agree fairly with the data ascertained by degeneration. Seventy-seven per cent. of the angular measure of the galvanometric deflection appeared to belong to the dorsal column. Of the remaining 23 per cent. rather less than four-fifths was referable to the rest of the homonymous half of the cord. A discrepancy between the observations by the galvanometric and by the Wallerian method lies in the existence of action currents in the contralateral dorsal column, to the extent of one-fourth of that observed in the uncrossed, whereas no Wallerian degeneration occurs in the contralateral dorsal column. The degeneration and the action current in the homonymous dorsal column both conform with the course of the ascending stems of the dorsal root fibres. After spinal semisection at the lower thoracic region, stimuli applied to the skin of the foot are more effective for producing movement of the fore-limb when applied to the foot homonymous with the lesion, than when contralateral. There is no crossed path in the dorsai column at the level of semisection to account for this, and it still occurs when much of the grey matter and ventro-lateral column have been excluded. In the action-current observations it was noticed that semisection at the bottom of the thoracic region reduced the galvanometric deflection obtained from the cord a few segments higher when the homonymous sciatic was faradised. Three observations were made; the reduction varied from 56 to 85 per cent. of the angular deflection. When the crossed sciatic was faradised, the deflection in one of the observations was somewhat greater than before the semisection, in the other two it fell to 63 and 90 per cent. of the previous angle. Most of this was probably referable

1 Vide supra, 1. 792.

${ }^{2}$ Vide supra, p. 806.

"Sherrington, Journ. Physiol., Cambridge and London, 1893, vol. xvii.; Laslett and Warrington, Brain, London, 1899, vol. xxii. p. 586.

4 Gotch and Horsley, Thil. Trans., London, 1891. 
simply to the section of the roots in the dorsal column. After low thoracic semisection, the angle of galvanometric deflection obtained from the cord a few segments higher was, under sciatic faradisation, less when the nerve was homonymous than when contra-lateral. In the eight observations the defect of deflection on the uncrossed side varied between 26 and 78 per cent., and was presumably for the most part referable to the severance of the root-fibres in the dorsal column. A semisection some segments behind the faradised cut end of the cord in the thoracic region abolished the action current, however, in the homonymous sciatic nerve. What relation the degree of galvanometric deflection bears to the aggregate size or number of a spinal fibre system is not ascertained. One would think it more influenced by number of fibres than by size of fibres. For one and the same fibre system it presumably varies in a direct sense with the physiological intensity of the reaction, but in what ratio is difficult to estimate. One difficulty in interpreting in terms of normal spinal conduction the spread of action-current, is the fact of double conduction. Thus, when the cord is excited in the lower thoracic region, the chief channel of spread of action currents both up and down seems to be the dorsal column.

Observations, taking note of not only the relative amount but also the physiological quality of the reactions for which the cord is blocked or still patent after semisection, find special difficulty in regard to the quality of the long dorsal column path. Its severance has in various hands, ${ }^{1}$ and practised at various levels, made no striking alteration at all either in movement or in signs interpretable of sensation. There is clear evidence that the path belongs to an afferent chain of which the next neural link begins in the dorsal column nuclei of the bulb, and leads in the main by the crossed mesial fillet to the thalamus and, less directly, to cerebral cortex (especially its post-central and fornicate gyri). The clinical evidence collected about the physiological quality of the dorsal column is of negative character. Lesions in it, either unilateral or bilateral, have occurred without any obvious disturbance of sensation; at utmost a slight clumsiness in the movements of hand or foot. After arrest of the circulation in the lower region of the spinal cord, Mïnzer and Wiener ${ }^{2}$ found that "pathic" stimuli applied to the skin of the hind-limbs were not effective to provoke signs of sensation, and Langendorff, 3 that excitation of the afferent roots as well as light tactile stimuli to the skin, even under slight strychnisation, remained ineffective. The argument from these stasis experiments rests on the supposed earlier paralysis of grey than of white matter by the stasis, and infers that, therefore, tactual and painful sensorial impulses must embouch into grey matter near the entrance of the root conveying them into the cord; the further inference is that the long dorsal column path is not the path used in the reactions of touch and pain. But I have seen strong pathic stimuli evoke reactions when applied to the skin of the hind-limbs at a time when, by arrest of the circulation in the cord, those limbs themselves were flaccid and paralytic; here the reaction probably occurred via the long dorsalcolumn path. After transection of all the columns, except the dorsal, signs of sensation can still be evoked from the parts innervated by segments posterior to the section, as also from the cerebral face of a

${ }^{1}$ Miescher, Arb. a. d. physiol. Anst. zu Leipzig, 1870, S. 404 ; Nawrocki, ibid., 1871, S. 585 ; Dittmar, ibid., 1873, S. 479 ; Woroschiloff, ibid., 1874 , S. 99.

2 Arch.f. exper. Path. u. Pharmakol., Leipzig, 1895, Bd. xxxv. S. 114.

${ }^{3}$ Arch. f. d. ges. Physiol., Bonn, 1898, Bd. lxxi. S. 401. 
section of the column. ${ }^{1}$ Schiff concluded that the stinuli, even when such as calculated to evoke painful sensations, no longer evoke signs indicative that any painful quality attaches to the reactions manifested. Herzen ${ }^{2}$ and others confirm this. Chiefly for this reason the long dorsal-column path is often considered "tactual." Anatomists ${ }^{3}$ have written of it as "reserved for conscious sensation." Some authorities treat it as acknowledgedly connected with muscular sense. Its nerve fibres seem in the monkey to give reflex collaterals to the motor root cells of the hand and foot muscles especially, for electrical excitation of the path at the top of the cord excites those movements. The function of the long path must be considered still unproven; my own opinion inclines to relate it to muscular rather than to tactual sense. There is much in favour of H. E. Hering's view that it serves to conduct centripetal impulses which, arising in the apparatus of movement, regulate by unconscious reactions the movements of the skeletal musculature. ${ }^{4}$

Concerning the ventro-lateral columns, there is good evidence that in their lateral portion runs down the path concerned with the direct regulation of muscular contraction, while in the same region headward, runs the path from all sensory apparatus in its first relay after the first synapse. Woroschiloff's experiments ${ }^{5}$ showed that, at the level of the last thoracic vertebra, this lateral region is patent to downward impulses that move the hind-limb for progression, and upward that connect its skin with encephalic nervous centres, so as to allow reactions indicative of unimpaired sensitivity of the limb's surface. When at the lowest thoracic level the only part of the cord remaining unsevered is the lateral region of each ventro-lateral column, the rabbit, as judged by its movements and its reactions to sensible stimuli, is to all appearance still in a normal state. In the lateral region also, and apparently exclusively in it, ascend paths for vasomotor reflexes from the sciatic nerve. Direct faradisation of the ventro-lateral column ${ }^{6}$ in areas as circumscript as practicable produces, according to the locality of application to the face of the section, movement of the wrist, fingers, trunk, hip, knee, and digits, all on the homonymous side. To evoke movements is far easier from certain spots of the cross-section than from others. The easiest foci lie in the ventral root-zone, and the next easiest in the dorsal half of the lateral column. The path descendent from each half of the bulbar respiratory centre lies in the homonymous lateral column, ${ }^{7}$ and in the phrenic region either descendent path gives branch paths across to the contralateral motor cells. ${ }^{8}$ The descending path connecting the brain with the lumbo-sacral "centre" for the motor nerves of the urinary bladder lies similarly in the dorsal part of the

${ }^{1}$ Schiff, "Lehrbuch," 1858; also “ Recueil d. mém.," Lausanne, 1896, tome iii. ; Longet, "Systeme nerveux," Paris, 1860, tome i. p. 274 ; Eigenbrodt, "Ueber d. Leitungsgesetze i. Ruckenm.," Giessen, 1849.

${ }^{2}$ Cf. Note to French edition of Waller's "Human Physiology," Paris, 1898.

${ }^{3}$ v. Kölliker, "Handbuch d. Gewebel. d. Menschen," Leipzig, 1896, 6te Aufl., 13d. ii. S. 116 ; Waldeyer, Deutsche med. Wchnschr., Leipzig, 1891, S. 61. ; v. Lenhossék, "Der feinere Bau des Nervensystems," Berlin, 1895, S. 397.

${ }^{4}$ Prag. med. Wchnschr., 1896, Bd. xxi. S. 41; also Leyden, Virchow's Archiv, 1869, Bd. xxxvii. ; Frenkel, Neurol. Centralbl., Leipzig, 1897, Bd. xvi. S. 688 ; Miinzer' and Wiener, ibid., 1899, Bd. xviii. S. 21.

5 Arb, a. d. physiol. Anst. zu Leipzig, 1874.

${ }^{6} \mathrm{Gad}$ and Flatau, Ncurol. Centralbl., Leipzig, 1897.

7 Schiff, "Lehrbuch," 1858 ; Langendorff, Wertheimer, Gad and Marinesco, later.

${ }^{8}$ Porter, Journ. Physiol., Cambridge and London, 1895, vol. xvii. p. 455. 
lateral column, ${ }^{1}$ and on arriving at the spinal vesical centre gives branch paths across the median sagittal plane of the cord, forming therefore bilateral connection with the local centre, which is itself bilateral. The ascending path linking the sense organs of the hind-limbs with the bulbar vasomotor centre and the descending path from the latter to the thoracic efferent root cells of the vascular musculature, lie both of them in the lateral column, ${ }^{2}$ the descending in that of the side homonymous with their main ending, the ascending perhaps mainly in the side crossed from the peripheral sense organs possessing access to them (see below, p. 867).

Observations with the method of Wallerian degeneration yield clearly one negative point in regard to the ventro-lateral column, namely, that none of its fibres are afferent root cell fibres. They furnish also positive evidence that of its fibres many of the ascending pass to the cerebellum, entering it on the same side as the column in which they lie; and that probably a certain smaller number pass to the mesencephalon and diencephalon, as also to the upper part of the spinal cord itself. Of the ventral portion of the ventro-lateral column, especially its part between the ventral fissure and the exit of the ventral root bundles, little is known relative to function. Bilateral section of this region produces, so far as observed, no detectable impairment in the animals examined. ${ }^{3}$ Kürschner, ${ }^{4}$ Longet, ${ }^{5}$ Volkmann ${ }^{6}$ found mechanical and weak faradic excitation of this region, or above it, when it alone of the whole thickness of the cord remained, produce movements of the muscles far behind the level of section. Brown-Séquard concluded, after a similar transection, that some signs of sensation were still elicitable from regions behind the lesion. In agreement with these observations, the Wallerian degeneration method demonstrates fibres descending in this column from both roof and Hoor of mesencephalon, ${ }^{7}$ from metencephalon, ${ }^{8}$ and in mammals from the mantle of the hemisphere itself. The degeneration method also shows fibres ascending in this column in large numbers from spinal cells even many segments posterior $;^{9}$ these fibres are for the most part small, the descending for the most part large.

It was long ago ${ }^{10}$ known that an effect of lateral semisection of the cord is to impair the motor and sentient functions of the parts of the body innervated by the spinal nerves behind and on the same side as the section. It was more recently ${ }^{11}$ noted that the impaired motility becomes in course of time partly restored. Further study of the motor and sensory defects led to the belief that, while the path subserving intentional movement descends the same side of the cord as the muscles which contract, the sensitivity of each half the body involves a double

1 C. C. Stewart, Am. Journ. Physiol., Boston, vol. ii. p. 198; also I. Ott, Mcd. Bull., Phila., 1894, vol. xvi. p. 410 ; Mosso and Pellacani, Atti d. $r$. Accad. d. Lincci. Cl. di sc." fis., mat. c nat., Roma, 1881, tonı xii. p. 3 ; Budge, "Untersuch. ueber d. Nervensystem," 1859, Bd. i. S. 184 ; Ztschr. f. rat. Med., 1864 (3), Bd. xxi. S. 1.

2 Dittmar, Arb. a. d. physiol. Anst. zu Leipzig, 1873, S. 479.

${ }^{3}$ Miescher, op. cit.; Woroschiloff, op. cit.

"In the translation of Marshall Hall's "Memoirs," Marburg, 1840.

5 "Système nerveux," Paris, 1860, tome ii.

6 Wagner's "Handwörterbuch ; " so also Fick, Arch. f. d. ges. Physiol., Bonn, 1869, Bd. ii.

7 Held, Arch. f. Anat. $u$. Entwcklngsgcsch., Leipzig, 1893; Boyce, Phil. Trans., London, vol. clxxxvi. B, p. 321.

${ }^{8}$ Marehi, Ferrier and Turner, A. Thomas, etc., vide supra, p. 808.

9 Sherrington, Journ. Physiol., Cambridge and London, 1893, vol. xiv.

10 Hippocrates, $\pi \rho \circ \rho_{\eta} \iota \kappa \omega \nu . \beta$. xxvi. ; Galen, "De locis affectis" (young pigs and mon. keys), vol. iv. cap). 4 ; "De administ.," vol. viii. pp. 7, 8, 9.

11 Cooper, Med.-Chir. Trans., Lomion (dog), 1803, vol. i. p. 200. 
path employing the two halves of the cord, ${ }^{1}$ though the major division descends on the side homonymous with the peripheral sense organs. ${ }^{2}$ Bilaterality of the path was next accredited ${ }^{3}$ as well to the descending spinal conduction for intentional movement of the skeletal musculature of each side-half of the body; the homonymous path was stated as in sensation to be the chief one, that is, the side homonymous with the musculature innervated. It was noted, ${ }^{4}$ especially in the frog, that the impairment of the volitional motility and of the sentient function of the limb is, when the semisection lies though headward yet near to the nerves to the limb, greater than when placed further forward. After semisection in the cervical region, a certain power of volitional movement is retained for the hind-limb homonymous with the semisection. Attention was drawn, especially by the long series of experiments by Chauveau, ${ }^{5}$ to a great contrast existing in the homonymous side, between heightened reflex activity and defect in true sensifacient power. This increase of reflex excitability was noted to gradually increase rather than to subside with time. It was also observed that the temperature of the homonymous hand and foot (skin) rises after cervical semisection, but that the temperature of the axilla and groin falls (muscle). ${ }^{6}$

In a series of experiments by Weiss ${ }^{7}$ the difference between early and late symptoms was carefully recorded, the semisection lying at the hindmost thoracic segment in the dog. The exact extent of the lesions was verified by subsequent microscopical examination. (1) On the homonymous side the condition for the first day or so was: Motor pover ; "volitional" gone, reflex greatly impaired ; the hind-limb hangs extended at knee and ankle, adducted at hip: Sentient power; very defective. Later, in the course of some weeks, there ensued restoration of function: motor power, for standing, walking, running, became almost perfect, and the movements of the affected limb were well associated with the movements of the other limbs. As regards the sentient functions, mechanical and electric stimulation of the skin and of the nerve trunks of the limb showed deficiency; movements evoked reflexly in the limb by skin stimuli, though often vigorous, were never accompanied by whining. (2) In the contralateral limb the motility was at first somewhat impaired, but was soon restored to normal. The sentient functions, at first markedly depressed, as compared with the fore-limb, in course of time returned in part, though permanently subnormal. The sensory defect exists, according to Weiss ${ }^{8}$ and Botazzi, ${ }^{9}$ for all kinds of cutaneous sensation and for "muscular." A second semisection practised after recovery from the first, and on the opposite side one spinal segment further headward, abolished permanently motility under "volition" and all sentient power in both hind-limbs, and in as

${ }^{1}$ v. Deen, "Physiol. d. l. moelle épinière" (frog), Leide, 1841.

2 Budge, "Einfluss d. Centraltheil. d. Nervensystems" (cat and frog), 1842, S. 157.

3 Stilling, "Funct. d. Rückenmarkes": (cat), 1842.

"Eigenbrodt, "Die Leiturgsgesetze d. Ruickenmarks" (frog and dog), 1849 ; Kölliker, Czermak, and Corti (rabbit), "Mikr. Anat.," Leipzig, 1850, Bd. i. S. 438 ; v. Bezold, "Ueber d. gekreuzt. Wirkung. d. Ruickenmarkes" (frog, pigeon, rabbit, guinea-pig, cat, dog), Berlin, 1859.

5 Wonit. d. hôp., Paris, 1857, p. 1065.

${ }^{6}$ v. Bezold, op. cit. (cat and $\operatorname{dog}$ ), 1859 ; see also Schiff, "Lehrbuch d. Nervensystem," 1858-1859, S. 272; and Mott, Phil. Trans., London, 1892.

7 Sitzungsb. d. k. Akad. d. Wissensch., Wien, 1879, Bd. lxxx. Abth. 3. Homén's experiments (Helsingfors, 1885) yielded results on the dog identical with those of Weiss. The secondary degenerations were carefully studied as well as the functional condition.

${ }^{8}$ Loc. cit.

${ }^{9}$ Centralbl. $f$. Physiol., Leipzig u. Wien, 1894, S. 530.

VOL. II. -55 
far as could be judged all parts postjacent to the section. Further experiments of this kind ${ }^{1}$ on the dog show that when in the hindmost thoracic region an interval of some six or seven spinal segments is allowed between the semisections, recovery of motility of the hind-limbs in walking and progression may ultimately, although long delayed, be almost as complete as after single semisection. Restoration of sensitivity is longer delayed and less perfect. The incipient effect of the second semisection is to destroy locomotor and volitional motility and sentient power altogether in both hind-limbs. In one experiment three semisections were successfully accomplished,-- the foremost at the highest lumbar segment; the second, three spinal segments lower and on the opposite side; the third, the final, on the same side as the first and six spinal segments behind it. The power to stand was in time recovered. The strongest stimuli to the hind-limbs failed to evoke any sign of pain, as also did faradisation of the sciatic nerve. Faradisation of the spinal cord in the cervical region excited no movement in the hind-limbs. On more than one occasion irritation of the skin of the neck evoked scratching movements of the hind-paw. But I have myself seen this remarkable phenomenon after total transection at the eighth cervical level; the movement in front of the spinal severance is clearly mechanically conveyed to parts behind the lesion, and there in turn excites the spinal "scratch" reflex.

The conditions produced by spinal semisection have been studied recently in the ape (Mott, ${ }^{2} \mathrm{~W}$. A. Turner, ${ }^{3}$ Schäfer $\left.{ }^{4}\right)$. The seat of the lesion has been cervical, thoracic, or lumbar. Mott found impairment of "volitional" movement in the homonymous limb or limbs, but with time some restoration, just as noted in the $\operatorname{dog}{ }^{5}$ especially of associated movements. The defect of sentient power appeared at first considerable when tested by the application of a spring clip to the homonymous side; but there was much less impairment for tactual, pricking, and thermal stimuli. With the recovery of movement there gradually ensued considerable recovery of response to the pressure of a clip. ${ }^{6}$ Muscular sense was thought to be impaired on the homonymous side. Mott also noted allochiria ; ${ }^{7}$ a spring clip applied on the homonymous side occasionally directed attention not to the place stimulated, but to one symmetrically situated on the opposite side of the body. As to the effects of semisection, there obtained, for some years after publication of observations by Brown-Séquard, 8 a view fraught with practical importance from the support it seemed to obtain from clinical observations. It was held by Brown-Séquard that the path subserving cutaneous sensation ascends the spinal cord entirely contralateral to the initiating sense organs, the decussation of the afferent channels occurring soon after their entrance in the cord. Paralysis of intended movement without cutaneous anæsthesia on the one side, and on the opposite cutaneous anæsthesia without volitional paralysis, is

1 Osawa, “Inang. Diss.," Strassburg (Goltz's laboratory), 1882 ; Borgherini, "Beitr. z. Kennt. d. Leitungsb. im Rückenmark," Wien, 1886.

2 Phil. Trans., London, 1892.

3 Brain, London, 1892, vol. xv.

" "Proc. Physiol. Soc.," March 1899, Journ. Physiol., Cambridge and London, vol. xxiv. p. $\mathrm{xxii}$.

5 Eigenbrodt, op. cit., 1849 ; v. Bezold, op. cit., 1859 ; Weiss, op. cit., 1880 ; Homen, op. cit., 1885 .

${ }^{6}$ Cf. on this point, Schäfer, Journ. Physiol., Cambridge and London, 1898, vol. xxir.

7 See Obcrsteiner, Brain, London, 1882, vol. iv. p. 153.

${ }^{8}$ Compt. rend. Soc. de biol., Paris, 1850, tome i. p. 192. 
a clinical condition of acknowledged occurrence ensuing upon spinal injuries. That in man such symptoms have been satisfactorily demonstrated to be associated with uncomplicated and accurately limited local destruction of one lateral half of the cord, cannot, however, be affirmed. Experiments on animals have led some observers ${ }^{1}$ to uphold the view that Brown-Séquard first put forward and for some time held. The general consensus of opinion and the greater weight of evidence have been, however, both before and since Brown-Séquard's original report, against it. That important ascending paths do cross in the cord is beyond question. That they are ascending does not prove that they are "sensory;" the necessity of interpolating the remark emphasises the drawback attending the time-honoured misuse of the term "sensory" -as of "volitional" -in such connection. The increase of arterial pressure, caused by faradising the central end of one sciatic nerve, the cord having been at the hindmost thoracic level severed except for one lateral column, is greater ${ }^{2}$ if the nerve be contralateral to the undivided column than if homonymous. This does not, I think, prove that the path, even for impulses ascending to the bulbar vasomotor centre, is mainly contralateral; it is as well explained by supposing lumbar relay centres below the semisection more irritable on the side of the semisection than on the comparatively normal side. Similarly, in experiments ${ }^{3}$ in which movements of the fore-limb were used as index of ascendent conduction, the results in this connection merely indicate that a spinal path up from the hind-limb has become contralateral at the level of the lower thoracic region; the experiments do not necessarily show that the crossed path is the main one. There is the Van Deen ${ }^{4}$ experiment in the frog: a lateral semisection of the cord close in front of the crural plexus (say right), then a semisection of the opposite side close behind the brachial plexus. On stimulating the skin of the left fore-limb, movement of each of all the limbs is evoked; on stimulating the skin of the right hind-limb, movement of each of all the limbs is also evoked. Vulpian ${ }^{5}$ affirmed that this is true even when only one column of white matter connects the front and posterior halves of the animal. He extended similar observations to the dog. A semisection on the right side in the lumbar region produced what he considered hyperæsthesia in the right hind-limb, and slight depression of sensory function in the left. A second semisection on the left side at the fourth cervical level, practised a few hours after the former semisection, caused the hyperæsthesia of the right hind-limb to disappear, but produced marked hyperæsthesia of the left fore-limb.

These results naturally lead to consideration of the well-known zonal hyperæsthesia occurring as the result of various kinds of spinal lesion. It was noted, originally by Fodéra, ${ }^{6}$ that after partial transection of the cord, stimuli applied to a zone of skin just posterior to the lesion evoked signs of sensations more readily than normally. Fodéra concluded also that the muscles behind the section are more excitable by local reflex channels than they normally are. The hyperæsthesia of Fodéra is now a phenomenon universally acknowledged. It includes hyperalgesia. It seems to ensue when the spinal lesion involves the extreme dorsal part of the lateral column. ${ }^{7}$

1 Ferrier, "Funetions of the Brain," 2nd edition ; W. A. Turner, loc. cit.

2 Miescher, Arb. a. d. physiol. Anst. zu Leipzig, 1870. ${ }^{3}$ Worosehiloff, ibid., 1874.

4 "Traité sur la physiologie d. 1. moelle épin.," Leide, 1841.

5 "Leçons sur le système nerveux," Paris, 1866. ${ }^{6}$ Journ. de physiol. expér., Paris, 1822.

7 Martinotti, Arch. f. Physiol., Leipzig, Suppl., 1890. 
Türck first showed, in the frog, that it is demonstrable behind a total transection as well as after a partial lesion. I have seen it extremely marked in the dog after high spinal complete transection, e.g. in the brachial region. The lightest touch on the skin of the shoulders or arms appeared to cause acute sensation, though there was no redness or sign of vascular disturbance in the skin. It has been suggested that the hyperæsthesia is due to vasomotor disturbance; but Foster ${ }^{1}$ points out that irritation or paralysis of the cervical sympathetic is not shown to cause any disturbance of sensation in the skin of the ear, and a one-sided hyperæmia of the cord itself is difficult to postulate. It has been noted inmediately after the establishment of the lesion, which is against its being of inflammatory origin; it does certainly usually become more marked a few days after the establishment of the lesion. Its persistence for many weeks excludes its explanation by "injury currents" set up in the spinal nerve fibres. Woroschiloff's seems the best explanation, namely, the removal of tonic inhibitory influences; although to state this is, in our present knowledge, to say little definite. In the cat, immediately after high spinal transection, e.g. from the first cervical level as far back as the sixth, a peculiar condition is often observable in front of the section, e.g. in the sensorial field of the trigeminus. A single touch on the snout elicits vigorous licking of the spot touched; the mere approach of the warm can used to protect the animal from cold evokes screwing-up of the eyes and mouth; salivation is often profuse; a touch on the vibrissæ produces exaggerated facial movement; there often seems to be some photophobia. But in the dog and monkey, although there are indications of a somewhat similar condition, they are but slight. Perhaps related to the Fodéra hyperæsthesia is that heightened sensitivity, which a field of skin, supplied by a portion of an afferent spinal root, exhibits after severance of the rest of the root and of adjoining roots in front of and behind it. Similarly the kneejerk (cat) is extremely brisk when the two segments to which its reflex arc belongs are isolated from all the rest of the cord, by spinal transections just in front and behind. This reaction recalls the observation by Brown-Séquard, that after section of the dorsal columns it is the posterior face of the section that is the more sensitive, and that of the two next adjacent dorsal spinal roots it is the one on the posterior side of the lesion that is the more excitable. Here injury currents were perhaps playing a part, for the observations were all made soon after the establishment of the lesion. But it has been shown that a semisection carried out on the same side a few segments further back reduces the hyperæstletic symptoms, Vulpian ${ }^{2}$ says actually to inexcitability, excito-motility as well as sentience suffering extinction.

\section{Tonic Functions of the CoRd.}

Among the reflex functions of the spinal cord are some that, on account of the comparatively prolonged and steady character of their centrifugal effect upon the extrinsic tissues, are described under the term "neural tonus." It is in certain parts of the musculature that this tonus lies best open to examination. The sphincters of the anus, urinary bladder, and larynx, by a continued degree of steady unwilled contraction, guard their respective orifices. Destruction of the spinal cord abolishes their action for a time, but in the instances of the anal and vesical sphincters an efficient amount of tonic contraction is eventually regained. The sphincter muscles can, like other muscles, be excited reflexly through the cord to that status of decreased extension and increased tension which constitutes tetanic or voluntary contraction. But in addition to these intercurrent relatively brief contractions, they exhibit under

\footnotetext{
1 "Text-Book of Physiology," 1891, pt. 3. $\quad{ }^{2}$ Compt. rend. Soc. de biol., Paris, 1855.
} 
the so to say resting condition of their spinal centres a certain tension and contraction sufficient to offer resistance to the opening of the orifices they surround. Depression of function of the spinal cord, c.g. in spinal shock, diminishes greatly, at least for a time, the tonic contraction of the sphincter muscles. Destruction of the cord renders the orifices for a time patulous. ${ }^{1}$ Similarly, the tonic contraction of the vascular musculature is for a time ${ }^{2}$ decreased by section of spinal nerves or destruction of the spinal cord; lateral semisection of the cord in the neck is followed by more or less permanent narrowing of the homonymous pupil. Section of the vagus produces in the dog an increased frequency of heart-beat. These phenomena, like the rhythmic discharges of the respiratory centre, point to a continued activity of the bulbo-spinal centres, probably in result of some extrinsic, constant, or frequently repeated stimulus. The tonic action of some of the spinal efferent root cells that embouch into the sympathetic, although marked, is not apparently so dependent on the afferent root cells of their own locality as is that of the motor root cells innervating skeletal musculature. I have repeatedly found no perceptible difference in size between the pupils, after complete section of all the afferent roots of one side in the cilio-spinal region; nor when the cervical sympathetic has been cut weeks subsequent to complete spinal transection at the seventh cervical level. I have seen careful section of all the afferent roots of both sides, from the second thoracic to the thirteenth inclusive (cat), leave the arterial pressure practically unaltered. In accord with such observations is the greater difficulty of obtaining, from the second and third thoracic afferent roots in the spinal mammal, a pupillar dilatation than a contraction of the skeletal musculature.

As with other centrifugal nerve discharges, especially with rhythmically recurrent, there arises the question here whether the initiation is peripheral and reflex, or whether autocthonous and outcome of the independent individual metabolism of the nerve cells that themselves discharge. The rhythmic explosion in the muscle cells of the heart and their tonicity, expression of a more continuous discharge, seems shown autocthonous in origin. So also, among nerve cells, the explosive discharges of the bulbar respiratory seem to offer counterpart to that of cardiac cells among the muscular. In some instances the tonus of spinal nerve cells may be autocthonous in origin, evolved by "inner stimulation" of the "centre" itself. In other instances, spinal neural tonus is distinctly of peripheral initiation and demonstrably reflex.

The resting length of an innervated muscle varies. ${ }^{3}$ The bladder accommodates itself to various volumes of contents. ${ }^{4}$ If urination be deferred, when need is felt, the feeling of need will pass by, and Mosso found the intravesical tension then fall to that which previously obtained under a much smaller content of urine. The need for micturition felt at waking is perhaps chiefly due to increase of tension resulting from increase of neural tonus, as the activities of the cord return with the general awakening of the nervous system.

${ }^{1}$ Cf. Goltz and Ewald, Arch. f. d. ges. Physiol., Bonn, 1896, Bd. 1xiii. S. 362 ; Kronecker and Arnold, Rep. Brit. Ass. Adv. Sc., London, 1899.

${ }^{2}$ Goltz, Arch.f. d. ges. Physiol., Bonn, Bd. ix. S. 174, etc.

3 Tschiriew, Arch. f. Physiol., Leipzig, 1879 ; v. Anrep, Arch.f. d. ges. Physiol., Bonu, 1880, Bd. xxi. S. 226 . Cf. also Cyon, Ber. d. k. Sächs. Gesellsch. d. Wissensch., math.phys. Cl., Leipzig, 1865.

${ }^{4}$ A. Mosso and Pellacani, Arch. ital. de biol., Turin, 1881, tomo i. pp. 96, 291. 
The lengthening which some muscles have been proved to exhibit after separation from the motor root cells, may be taken as evidence that, in the state which is called "rest," their fibres are not perfectly at rest in the sense of devoid of contraction. Their state is then, moreover, due to indirect excitation through the nervous system. The sensible changes in the environment are so numerous and frequent, that an unceasing reflex activity of the nervous system is not surprising. It is, however, surprising, on the view generally taken, to find that in regard to the skeletal musculature the reflex effect takes the form of an equable, minute, continuous contraction. The fundamental observation on this subject is Brondgeest's ${ }^{1}$ experiment.

In addition to its neural tonus, the muscle has of course, in common with all living cell tissue, its vegetative tonus, on which Virchow has laid stress. The doctrine of a spinal tonus expressed by muscles is older than Brondgeest's experiment. J. Müller wrote in his text-book (1837), "The muscles, even in the condition of rest, are continually being acted on by their nerves." Marshall Hall, two years later, pointed to the difference between the tension of muscles, in the decapitated rabbit when the cord is destroyed, and when undestroyed. In the "spinal" animal the tonus of the muscles, especially of some muscles, is less than in the normal condition; but tonus does still exist. As a rule, it may be said that the "spinal" tonus of the flexors is greater than those of the extensors; in other words, a spinal transection depresses the tonus of the extensors more than that of the flexors, at least at first. This is seen not only in the spinal mammal, but in the crustacean and the insect. $^{2}$ It is typically illustrated by Brondgeest's fundamental experiment.

Chloroform and ether abolish this neural influence; so also does severance of the afferent roots. The neural influence is exerted chiefly on the muscles from which reflex contraction can in the spinal animal be evoked first and with most facility. Great depression of the tonus of the flexors is produced by section of afferent spinal roots alone, as well as by section of the efferent. $^{3}$ When the afferent spinal roots of a limb have been severed, the muscles of the limb feel flaccid, more flaccid than the muscles of the spinal animal. Some influence acts on the cord through the afferent roots causing portions of the limb musculature to be to a certain degree steadily braced and more tightly strung than when completely denervated. The influence does not, however, originate in the cutaneous sense organs, ${ }^{4}$ or at least there only in minor degree. Its maintenance appears to be one of the functions - a subconscious function - of the organs of "muscular sense." The severance of the afferent roots of a limb, besides causing ataxy of its "intended movements," renders certain of them impossible ${ }^{5}$ besides these obviously sensual impairments of the local skeletal musculature, are others bearing more on subconscious reflex influence on the muscles. The section of the afferent roots delays the onset of rigor mortis ${ }^{6}$ much as does section of their motor nerves. It also acts upon the extensor muscles of the limbs, for, after transection in front of the mesencephalon, decerebrate rigidity ${ }^{7}$ does not occur in the limb the afferent roots of which have been divided.

The knee-jerk and allied phenomena.-The reflex neural tonus of the skeletal musculature influences not only the resting length and tension of the inuscles, but also their direct excitability to a particular form of mechanical stimulation. When the leg is held supported in a

${ }^{1}$ See p. 798, supra; "Acad. Preisschrift," Utrecht, 1860.

2 Cf. J. Loeb and Bethe, op. cit., supra.

3 Narshall Hall maintained (1839) that tonus and reflex action are but modifications of one and the same function of the cord.

${ }^{4}$ Mommsen, Virchow's Archiv, Bd. ci. S. 22.

$\checkmark$ Exner and Pineles, Arch. f. d. ges. Physiol., Bonn, Bd. xlviii. S. 17 ; Mott and Sherrington, Proc. Roy. Soc. Lonidon, 1895, vol. lv.

${ }^{6}$ Sherrington, ibid., 1893, vol. lii.

7 Sherrington, Journ. Physiol., Cambridge and London, 1897, vol. xxii. 
posture of slight flexion at hip and knee, and all its muscles lie at rest, a tap on the patellar ligament evokes a short contraction of the quadriceps cruris, ${ }^{1}$ so that extension at the knee ensues. This is the phenomenon known as the "knee-jerk." Others similar exist in other' pieces of musculature, e.g. at the tendo Achillis for the triceps suræ, and at the extensor tendons of the wrist, and the masseter. The kneejerk may serve as their type. Muskens ${ }^{2}$ finds deficiency of jerk a usual, though not invariable, accompaniment of subnormal tension of the Achilles tendon; conversely he finds bypertonus of the triceps surce to co-exist with exaggerated jerk. He estimates degree of tonus by measurement of the pressure required for displacement of the tendon, the ankle resting in semi-flexion. The study of the time-phases of the muscular contraction has shown it to have all the characters of a simple twitch (Brissaud, ${ }^{3}$ Eulenberg, ${ }^{4}$ MacWilliam ${ }^{5}$ ). The interval elapsing between the application of the tap and the appearance of the contraction is too short to permit of the reaction being considered a reflex ${ }^{6}$ via the afferent root, the cord, and the motor root cells. The time is about the same as that of the contraction response on direct electrical excitation of the muscle, i.e. less than a quarter of that of the briefest reflex time. In accord with Sternberg's supposition that the mechanical excitability of the muscle varies directly as the jerk, Muskens ${ }^{7}$ observes the tap elicit in the hypotonic calf muscle a slow and merely local contraction instead of the normal quick response of the whole muscle.

A sine qua non for the elicitation of the "jerk" is slight tension of the muscle which is to respond; and the stimulus to the reaction is a sudden slight passive increase of the tension. Gowers has suggested, ${ }^{8}$

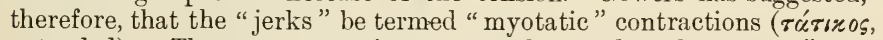
extended). They are sometimes termed "tendon-phenomena," but tendons are not essential. The terms "tendon reflexes" and "deep reflexes" have obvious objections as applied to the "jerk phenomena," for they are not proved to be reflex, although there do exist, in addition, undoubted reflexes from tendons and other deep structures.

On analysing ${ }^{9}$ the spatial distribution of the phenomenon in the muscles of the thigh, it is found to involve chiefly the vastus medialis and adjacent crureus, and when not strong to be entirely limited to them. When isolated in those muscles, it is at once abolished by severance of the afferent nerve fibres belonging to those particular muscles, whereas combined severance of all the other afferent nerve paths of the limb does not abolish it. The phenomenon, though not a reflex act, is therefore dependent on a reflex spinal arc, ${ }^{10}$ the afferent part of which is based on sense organs in the muscle itself and its appurtenances.

${ }^{1}$ C. Westphal, Arch. f. Psychiat., Berlin, 1875, Bd. v. S. 803 ; W. Erb, Bcrl. klin. Wehnschr., 1875, S. 26.

3 "Recherches physiol.," Paris, $1880 . \quad$ "Ztschr.f. klin. Med., Berlin, Bd. iv. S. 179.

${ }^{5}$ Centralbl. f. d. med. Wissensch., Berlin, 1887.

6 Waller, Lancet, London, 1881, vol. ii. p. 83 ; Brain, London, 1881, vol. iii. p. 179 ; Journ. Physiol., Cambridge and London, 1890, vol. xi. p. 384 ; de Watteville, Brit. Med. Journ., London, 1882 , vol. i. p. 736 ; Brain, London, 1886 , vol. viii. p. 516 ; Gowers, Med.Chir. Trans., London, vol. lxii. p. 269 ; Burckhardt, Festschr. z. Andenken an A. v. Haller dargebracht, Bern, 1877 ; Eulenberg, loc. cit. ; Jendrássik, Neurol. Centralbl., Leipzig, 1885, S. 412 ; Gotch, Journ. Physiol., Cambridge and London, 1897, vol. xix. ; also, Rosenheim, van T. Meulen, etc.

7 J. L. L. Muskens, loc. cit. $\quad 8$ "Diseases of the Nervous System," London, 1886, vol. i.

${ }^{9}$ Sherrington, Journ. Physiol., Cambridge and London, 1892, vol. xiii.; Proc. Roy. Soc. London, 1892, rol. li.

${ }^{10}$ Prévost, Rev. méd. de la Suissc rom., Genève, 1881; Westıłal, Bcrl. klin. Wchnschr., 
A great interest attaches to the knee-jerk and similar reactions as indices of the condition of the nervous system. Extinguished when the neural tonus of the muscle is extinguished (e.g. by completc rupture of the reflex arc) or is replaced by the active innervation of intentional movement, these "jerk" phenomena are indicators delicate enough to show many shades of spinal state.

Under apparently like circumstances, there are, in any large collection of healthy individuals, not in-

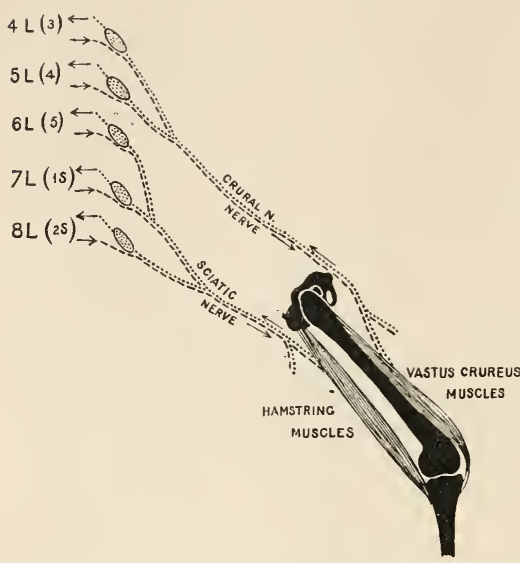

Ffc. 359. - Seheme of the arrangement of the nerves and muscles of ehief importanee in the knee-jerk, as determined in the Macaque monkey. The numbers $4 \mathrm{~L}-8 \mathrm{I}$, indieate the post-thoracie spinal roots of Macaeus, and the numbers in brackets the corresponding lumbar and sacral roots of man. The arrows pointing to the right indieate efferent paths, those pointing to the left afferent paths.

considerable differences of knee-jerk. In a very few persons, apparently healthy, the knee-jerk is at times inelicitable ${ }^{1}$ even under most favourable conditions. Of alterations in the jerk, some are referable to conditions directly affecting the local reflex arc itself. Thus, a second tap given soon after a first will generally evoke a more ample jerk. ${ }^{2}$ A series of taps too feeble to singly evolie the jerk, will, when repeated at intervals from a fifth to a tenth of a second, become efficient. Conversely, after the jerk has been elicited for a number of times, it tends to fail for a short period, despite the taps being continucd as before. Local fatigue of the extensor muscles diminishes the jerk $;^{3}$ general fatigue of the nervous system ${ }^{4}$ at first increases, but if severe, later diminishes the jerk. The rendering of the limb bloodless by the Esmarch bandage causes the knee-jerk in about fifteen minutes to disappear. ${ }^{5}$ "Pathic" stimuli ${ }^{6}$ applied to the skin increase the jerk, and friction of the skin of the leg, ${ }^{7}$ and the cold bath $^{8}$, are means of increasing the jerk in patients in whom it is defective.

1881 ; Schreiber, Arch. f. cxper. Path. u. Pharmakol., Leipzig, 1885, Bd. xviii. S. 270 ; Sherrington, Proc. Roy. Soc. London, 1892, rol. Ii. ; Sehultze and Fürbringer, Centralbl. f. d. med. Wissenseh., Berlin, 1875, S. 929.

1 Eulenberg without the now known reinforeements failed to elieit the jerk in sixteen out of 238 healthy ehildren; Pelizæus and Remak with use of reinforcements found the jerk present in eaeh of 2403 healthy ehildren examined in succession, Areh. $f$. Psychiat., Berlin, 1885, Bd. xiv. S. 402 and 167

${ }^{2}$ Heller, Berl. Klin. Wchnschr., 1886, S. 903 ; Meyer, ibid., 1888, S. 23 ; Sehreiber, loc. cit.

${ }^{3}$ Muhr, Centralbl. f. Nervenh.u. Psyehiat., Coblenz u. Leipzig, 1878 ; Jendrássik, Deutsehes Arch. f. klin. Med., Leipzig, Bd. xxxiii. S. 177 ; Eisenlohr, Festschr. f. Eröffn. d. ncu allgcm. Krankent. z. Hamb. Eppendorf., 1889 ; Sternberg, "Sehnenreflexen," Wien, 1893.

${ }^{4}$ Brissaud, op. cit. ; Marmian, Riv. clin. di Bologna. 1884, p. 415; Sehuster, "Diagnostik d. Rüekenmarkskrankheiten," Berlin, 1884 ; Erlenmeyer, "Ueber statische Reflexkrimpfe," Leipzig, 1885 ; Sternberg, Centralbl. f. Physiol., Lcipzig u. Wien, 1887, Bd. i. S. 81.

5 Sternberg, "Die Sebnenreflexen," Wien, 1893, S. 69.

${ }^{6}$ Weir Mitehell and Lewis, Med. News, Phila., 1886; Järiseh u. Sthiff, Med. Jahrb., Wien, 1882.

\footnotetext{
7 Schreiber, op. cit.

${ }^{8}$ Dunge, “Diss.," Boln, 1889.
} 
Westphal ${ }^{1}$ noted that in patients in whom, after epileptic seizure, the knee-jerk was inelicitable, it becarne elicitable after a reflex had been evoked from the leg by pricking the plantar skin. The duration of the reinforcement of the jerk by a local stimulus is variable, but may be long persistent if the stimulus have been strong.

Faradisation of the central end of the contralateral sciatic nerve reinforces the knee-jerk. ${ }^{2}$ Excitation of the central end of the nerves of the flexor muscles of the kuee ${ }^{3}$ (which in the cat are collected into a trunk free from cutaneous nerve fibres) strongly inhibits the jerk, causing at the same time a relaxation of the extensor muscles of the knee if they are then-e.g. under decerebrate rigidity - in tonic contraction. Mechanically stretching the kuee flexors-isolated in such a way as to prevent the pull upon them affecting the freedom of the knee-joint-similarly inhibits the jerk. On discontinuance of the stimulus, the jerk is much reinforced, and soon is so even during the stimulus, if that be prolonged beyond a certain time. Section of the nerves of the knee flexors is followed by a long lasting, slight augmentation of the jerk, ${ }^{4}$ so also is section of the whole sciatic nerve. Faradisation of the cutaneous nerves ${ }^{5}$ of the limb also generally depresses the jerk, but not so regularly or potently as the excitation of the nerves from the muscles antagonistic to the knee extensors. Bowditch and Warren ${ }^{6}$ found sensible stimuli to skin and mucosæ, e.g. sudden application of cooled air to conjunctiva, skin of neck, and uasal passages, reinforce the jerk if applied simultaneously with or not more than 0.2 second to 0.4 second before the tap on the patellar tendon. If applied earlier than that, they exerted an inhibitory or depressor influence, which reached its maximum about 1 second after application of the sensible stimulus. Stimulation of the skin of the knee, either of the side whence the jerk was elicited or of the contralateral side, caused little reinforcement, but when applied at appropriate intervals, a distinct inhibition was noticeable even when the stimulus preceded the tap for the jerk by fully three seconds. With other stimuli tried, no effect on the jerk was traceable, if the sensible stimulus preceded the knee tap by two seconds. A sudden sound reinforced the jerk, and never depressed it ; it reinforced most when it preceded the tap by 0.2 second to 0.3 second. A flash of light also reinforced; the best interval being 0.1 second to 0.3 second ; even at appropriate intervals it caused little inhibition.

Arrest of the circulation in the lumbar region of the cord was shown by Prevost ${ }^{7}$ to first exalt, and later depress and abolish the knee-jerk. Stimulation of the peripheral end of the cardiac vagus in the dog and monkey similarly exalts the jerk, ${ }^{8}$ no doubt by impeding the circulation. Chloroform and ether inhalation depress the jerk, and finally extinguish it, the speed of extinction being about the same in the spinal as in the intact animal, but in the latter, after cessation of the administration of the anæsthetic, recovering the more quickly. ${ }^{9}$

In the monkey, spinal transection usually depresses the jerk for a longer time than in the cat and $\log ^{10}$ Occasionally in the monkey, after transection at the lower thoracic region, the jerk is not elicitable for a week or so after transection. In the cat and dog it is elicitable in a quarter of an hour or less. After transection in the thoracic region, the jerk does not disappear immediately, but only in the course of a minute or so ; ${ }^{11}$ similarly, the knee-jerk has been elicited immediately after decapitation in man. ${ }^{12}$ In man, complete transverse destruction of the cord, even well above the lumbar region, seems usually to permanently abolish the kneejerks; buta certain number of well-authenticated cases show that it is not always so.

I Arch. f. Psychiat., Berlin, 1875, Bd. v. S. 803.

3 Sherrington, Proc. Roy. Soc. London, 1892.

${ }^{2}$ Sternberg, op. cit.

${ }^{4}$ Sherrington, ibid.

${ }^{5}$ Sternberg, op. cit. $\quad{ }^{6}$ Journ. Physiol., Cambridge and London, 1890, vol. xi. p. 25.

7 Op. cit., 1881; see also Hughlings Jackson and R. Russell, Brit. Med. Journ., London, 1893 , vol. ii.

${ }^{8}$ Sherrington, Journ. Physiol., Cambridge and London, 1892, vol. xiii.

9 Sherrington, ibid.

1 Sherrington, ibid.

10 Sherrington, ibid.

12 Barbé, Compt. rend. Soc. de biol., Paris, 1S85, p. 533. 
The spinal reflex mechanism connected with the knee-jerk of each side is unilateral, and lies in its own half of the cord. In the cat and dog it lies in the fifth and sixth iumbar segments; in the monkey (Macacus), in the fourth and fifth, chiefly the latter; and in man in the third and fourth, chiefly the latter. It has been experimentally shown that the lumbar cord can be split lengthwise along the median sagittal plane without impairment of the jerk of either side. ${ }^{1}$ Spinal transection above the lumbar region renders the jerk, after the short period of depression, more brisk than normal. After transection at the junction of diencephalon and mesencephalon, the briskness of the knee-jerks is enormously increased. A single tap on the tendo patellae will excite a series of jerks, and often the relaxation of the muscle between the jerks is only imperfect. ${ }^{2}$ Such a condition of jerk is sometimes called "tremospasm." Raising the intracranial pressure has been noted to have a like effect on the "jerk."3 Cortical excitation has also been found to exalt the jerk. After ablation of the Rolandic cortex, the contralateral knee-jerk becomes usually the more brisk. ${ }^{4}$

Jendrássik ${ }^{5}$ noted that a willed movement of the arm, if carried out by the subject at the time of the elicitation of the knee-jerk, augments it. It was later remarked ${ }^{6}$ that to get the full effect of the reinforcement, the patellar tap must be delivered at just the right moment after the reinforcing act. Bowditch and Warren, ${ }^{7}$ in their research, found the reinforcing influence to last only from 0.22 second to 0.6 second, and to be followed, in most persons, by a more persistent period of inhibitory influence, the normal degrec of jerk being recovered in 1.7 seconds to 2.5 seconds. The willed movement of another part, no doubt, removes the attention from the limb whence the jerk is to be obtained; in directing attention to any part of the body, there is a strong, in some persons an irrepressible, tendency to innervate the skeletal musculature of the part. How it is that even feeble excitation (viâ cerebral paths) of the motor root cells prevents the muscle from giving the twitch, in response to the patellar tap, is not clear. The fluctuation in the condition of the motor root cell, from minute to minute, as wakeful life proceeds, is well illustrated by the fluctuation of the knee-jerk, as for instance observed by Lombard ${ }^{8}$ under ordinary daily events. Swallowing, talking, the hearing of a child crying in a near room, the declamation of a poem, an itching of the ear,-events such as these seem to be mirrored as regards their influence on the motor root cells for the skeletal musculature by reinforcements of the knee-jerk. In sleep the knee-jerk is, when sleep is deep, usually unobtainable, ${ }^{9}$ but sensory stimuli, too feeble to wake the sleeper, make the jerk elicitable for a short interval. The perturbations of the condition of the extensor muscle of the knee and its local reflex arc brought about by sensory stimuli, customarily regarded as without consequence to, or almost indifferent as regards that region, show to what extent the whole nervous system is consolidated, and how continually its state of tension is braced or slackened by stimuli whose influence we do not recognise, or recognise only in one partial aspect. Fatigue, hunger, and enervating weather decrease the average knee-jerk, while rest, nourishment, and invigorating weather increase the a verage knee-jerk. In the winter frog the knee-jerk is difficult to elicit,

1 Sherrington, Journ. Physiol., Cambridge and London, 1892, vol. xiii.

2 Brown-Séquard, Journ. de la physiol. de l'homme, Paris, 1858, tome i. p. 472 ; Ordenstein, Thèse, Paris, 1867 ; Bouchard, Arch. gén. de méd., Paris, 1866, tome ii. p. 290; Dubois, Thèse, Paris, 1868; Charcot et Joffroy, Arch. de physiol. norm. et path., Paris, 1869, tome ii. ; Strauss, "Des Contractures," Thèse, Paris, 1875; Erb, Arch. f. Psychiat., Berlin, 1875 , Bd. v. S. 792 ; C. Westphal, ibil., Bd. v. S. 803 .

${ }^{3}$ Adamkiewicz, Sitzungsb. d. k. Akad. d. Wissensch., Wien, 1883 (3), Bd. xxxviii. S. 231.

${ }^{4}$ Ziehen, Arch. f. Psychiat., Berlin, 1890, Bd. xxi. S. 863.

5 Neurol. Centralbl., Leipzig, 1885, S. 412.

${ }^{6}$ Mitchell and Lewis, Med. News, Phila., 1886, Feb. 13 ; Lombard, Journ. Physiol., Cambridge and London, 1889, vol. x. p. 122.

${ }^{8}$ Journ. Physiol., Cambridge and London, loc. cit. ; Am. Journ. Psychol., 1888, vol. viii. ; Arch. f. Physiol., Leipzig, 1889, Suppl., S. 292.

${ }^{9}$ Bowditch and Warren, loc. cit. 
but during the breeding season it is easily evoked. ${ }^{1}$ There is a diurnal variation of the jerk; it is at its maximum, as a rule, soon after breakfast.

A crossed effect is of common occurrence. It is often an adduction at hip rather than an extension at knee. ${ }^{2}$ Its time has been found much longer ${ }^{3}$ than the ordinary knee-jerk time, and sufficient to allow of its being a true reflex movement; but there are also reasons against accepting it as such. ${ }^{4}$

\section{Trophic Functions of the Spinal Cord.}

Magendie ${ }^{5}$ showed that intracranial section of the trigeminus is followed in many instances by keratitis, ulceration of lip, palate, etc. Similarly, broncho-pneumonia ensues and proves fatal after bilateral vagotomy. ${ }^{6}$ Severance of the nerves of a limb produces wasting not only of its muscles but of its bones and ligaments. ${ }^{7}$ Section of the spermatic nerve produces atrophic changes in the testis. $^{8}$ Ablation of the superior cervical ganglion leads, in the turkeycock, to atrophy of the corresponding half of the comb. ${ }^{9}$ Section of the cervical sympathetic trunk has led in young rabbits to hypertrophy of the corresponding pinna, ${ }^{10}$ although in many no definite difference results. ${ }^{11}$ Schiff ${ }^{12}$ and S. Mayer ${ }^{13}$ found the hair on the side of the nerve-section grow the more quickly. Section of nerves of the salivary glands leads to the results described in Langley's article. ${ }^{14}$ The most striking of all the changes in the nutritive condition induced by separation from the nerve centres is, however, that which ensues in the skeletal muscles. In two or three weeks the muscle fibres begin to become smaller, and after some months or years they have dwindled out of recognition, leaving a tissue to all appearance like mere connective tissue.

The rate of this change is very different in different muscles, ${ }^{15}$ and in different fibres of the same muscle. In the "red" muscles and "red" fibres of muscles the change is slower than in the "pale." In none of the fibres of the extrinsic eye muscles of the monkey did I find, sixty days after section of their nerves, more than a slight increase of the interfibrillar granules that osmic acid blackens; the difference from normal was hardly discoverable. ${ }^{16}$ The intrafusal muscle fibres of the sensory organs (muscle spindles) of the skeletal muscles belong to the "red" variety of fibres, and are extreme examples of that variety. I am not convinced that there is any change in these muscle fibres, even where the nerve to the muscle has been severed three years previously, ${ }^{17}$

${ }^{1}$ Mommsen, Arch. f. Psychiat., Berlin, 1881, Bd. xv. ; and Virchow's Archiv, 1885, Bd. ci. S. 22 .

2 Hinsdale and Taylor, Internat. Med. Nag, 1895 .

${ }^{3}$ Burckhardt, Festschr. zum Andenken an Albrecht v. Haller, Bern, 1877; Goteh, Journ. Physiol., Cambridge and London, 1896, vol. xx. p. 322.

${ }^{4}$ Prévost and A. Waller, Rev. méd. de la Suisse rom., Genève, 1881.

5 Journ. de physiol. expér., Paris, 1824.

6 Legallois, "Expériences sur le principe de la Vie," Paris, 1812. The literature to 1877 is given in O. Frey's "Preisschrift," Jeipzig, 1877.

7 Sehiff, Compt. rend. Soc. de biol., Paris, 1854 , p. 1050.

8 Obolensky, Centralbl. f. d. med. Wissenseh., Berlin, 1867, S. 497.

${ }^{9}$ Legros, "Des nerfs vasomoteurs," Paris, 1873.

${ }^{10}$ Bidder, Centralbl. f. Chir., Leipzig, 1874; Stirling, Joum. Anat. and Physiol., London, 1876, vol. x. p. 511.

${ }^{11} \mathrm{C}$. Bernard, eited by Ollier, with Ollier's own experiments; Ollier, Journ. dc la physiol. de l'homme, Paris, 1863, tone vi. p. 107 ; Cohnheim, "Vorlesung. ueber allgem. Path.," Berlin, 1877, Bd. i. S. 599.

12 “Untersuch. z. Physiol. d. Nervensyst.," Frankfort a/Main, 1855, S. 166.

${ }^{13}$ Hermann's "Handbuch," 1879, Bd. ii. Abth. 1, S. 205.

14 This "'Text-Book," rol. i. p. 519.

${ }_{15}$ P. Knoll, Sitzungsb. d. l. Akad. d. Wisscnsch., Wien, 1893.

${ }^{16}$ Journ. Physiol., Cambridge and London, 1895, vol. xv.

17 Proc. Roy. Soe. London, 1896, vol. lx. 
and its regeneration prevented. Accompanying this histological change, and in a sense heralding it, are the well-known alterations in the electrical reaction of the muscle, which characterise the "reaction of degeneration." The muscle comes to respond to electrical stimuli in a manner apparently qualitatively different from normal. The excitability of the muscle to induced currents is abolished, that to the constant current is exaggerated. In regard to the response to the latter, the normal order of appearance of contraction, as the strength of the current is gradually increased from subliminal, begins with the kathode to the muscle and at the making of the circuit. To obtain a contraction when the anode is applied to the muscle and the current is made, requires a stronger current. In a well-marked reaction of degeneration, the anodal contraction, at make, appears with a weaker stimulating current than that required for the kathodal contraction at make. The contraction is greatly prolonged. Galvanotonus is easily produced.

Disease of sensory as well as of motor nerves is accompanied by change in the condition of nutrition of peripheral tissues. Alterations, both of atrophic and inflammatory kind, occur in the skin in relation to primary changes in nerve trunks. Of the latter alterations the course of herpes zoster is one example. The primary lesion seems an inflammatory condition of the spinal ganglion; ${ }^{1}$ on this ensues an eruption more or less occupying the field of skin for which the ganglion cells provide sensory chamnels. There is severe pain referred to the area of skin. Head's recent researches ${ }^{2}$ have placed beyond doubt the connection between this cutaneous eruption and focal inflammation of corresponding spinal ganglia. Occasionally a similar herpes ensues on irritation of the ganglia in spinal caries.

When the afferent spinal roots belonging to a limb are severedproximal to the ganglia-there is distinct wasting in much of the musculature of the limb; and, especially in the lower limb, ulceration is prone to occur on the outside of the ankle-joints. In man, where injury of the spinal cord upsets the condition of the skeletal musculature more than in animals, and where, therefore, the disturbance to the skin is probably also proportionately greater, there is especial tendency for ulceration (bed-sores) of the skin and tissues to occur; but these are met with as the result of similar lesions in laboratory animals.

When all these cases are examined, so far as by present methods they can be, they seem to yield evidence that every nerve and nerve centre that can influence the proper and specific activity of a tissue-and that is the sole function of nerves-possesses, in so far, a trophic influence on that tissue. If by trophic nerve be understood no more than this, all nerves are trophic nerves. That, on the other hand, any nerve directly modities the nutrition of a tissue independently of inducing vascular changes in it, or influencing those particular metamorphoses of material for which the tissue is the machine specifically adapted, there is no proof. The function and the nutrition of these physiological machines form an inseparable unity. Such a doctrine is a just corollary to the cell theory. Under the notion of normal nutrition is understood maintenance of a certain average form and certain average chemical composition. When the maintenance of the nutrition of one cell is directly and greatly dependent on the maintenance of another, the inference amounts to certainty that the latter governs the function of the former. This consideration shows clearly how, far more than any

1 v. Bärensprung, 1861.

${ }^{2}$ Allbutt's "System of Medicine," London, 1899, vol. viii. p. 635. 
other peripheral tissue, the skeletal muscles are governed by the efferent root cell system of nerve cells. No other tissue, except skeletal muscle, practically dies down, in consequence of injury of the root cell system.

The inflammation so prone to occur in exposed sensifacient surfaces, when their nerve chanuels are broken, does not stand in such direct relation to the nerve injury. The anæsthesia and analgesia of the surface lead to the unperceived accumulation of irritant effects. The most exposed part of the apæsthetic limb is the seat of ulceration, as the result of long-continued and undue-because unperceived-pressure. The keratitis often ensuing on section of the trigeminus can be avoided by careful protection of the eye from dirt. The ulceration about the lips after a similar unilateral lesion, has been noted to extend quite freely across the median line, so that there seems no difference between the essential resistance of the anæsthetic and of the normal tissue. The broncho-pneumonia, after vagotomy, seems entirely explicable by irritation of the respiratory tract from particles inhaled or entering from the digestive tract, the former tract being no longer securely guarded from this accidental leakage of material from the latter.

Not only are the nerve cells in the above cases trophic for peripheral tissues, but some nerve cells are trophic for other nerve cells, some nerve cell systems trophic for other nerve cell systems. It will have been remarked, from the foregoing paragraph, that the direction of the trophic influence is the same as that of the normal nerve conduction, that is, for instance, from motor root cell to muscle fibre, and not from muscle fibre to motor root cell. So in the cell systems of the cord and brain. Section of an optic nerve leads to trophic changes in the cells of the pulvinar, internal geniculate body, anterior corpus quadrigeminum, etc. If the section be made before the growth of the dependent cells is complete, their further growth is hindered. Thence results Gudden's or agenesic atrophy. A series of many links of a nerve cell chain may be affected by it, indeed the whole chain from end to end. But the damage must, in order to effect that result, be at the end which is the seat of excitation of its normal function. The sensory root cell is the alpha of each and every neural series, whatever the function and whatever the complexity, On the afferent root cell is therefore based the trophism of the whole nerve system, so far as there is a nervous system possessing solidarity as a whole, above and beyond the individual entity of the component cells. After severance of the afferent roots, the motor root cells of their spinal region soon present recognisable structural change. ${ }^{1}$ The nucleus of the motor root cell becomes markedly excentric in position, the Nissl bodies become very small or disappear, or lose their normal colour reaction; the cell becomes large, and the nucleus may even disappear. These changes do not occur with all the ventral horn cells, but only with certain of them ; the larger number of cells reveal no distinct alteration, and in the cells of the lateral horn no change is detected. The nutritive condition of the afferent root cells themselves is to some extent governed by the sensifacient organs that regularly play upon their distal extremities. Hence probably the atrophy of the

1 W. B. Warrington, Journ. Physiol., Cambridge and London, 1897, vol. xx. A full bibliography is given in Warrington's paper. For further aspects of the question of the relation between the visible structure of the nerve cell and its functional condition, reference must be made to the article "Nerve-Cell," in this volume. 
dorsal column, and of the grey mater consecutive to amputation of a limb. Of the spinal cell groups in the limb region, one belonging to the motor root cell system appears to suffer more than the rest. This is the dorsal-lateral group in the ventral horn,- which there is reason to think innervates in the crural region the muscles of the foot, in the brachial region the muscles of the hand. Whether the amputation of the limb has been at wrist or shoulder, at ankle or hip, this is the group that exhibits the chief atrophy. It is probably only after functional influence of one nerve cell on another, or on a peripheral tissue, has become established, that the nutrition of the latter is affected by the action of the former. In an amyelous fœetus, possessing of course no efferent spinal roots at all, the skeletal musculature may appear as well developed as in a normal fœtus of similar term. ${ }^{1}$

Trophic influence in a direction contrary in sense to the ordinary functional relationship and connection, is very obscure. The herpes zoster due to an inflamed spinal root ganglion, unless, as is possible, of reflex production, seems to be an example of this exercise of influence, so to say, "against stream." The cutting of the facial nerve is said to cause recognisable alteration of the structure of the root cells of that purely motor nerve. The section of a ventral spinal root seems also to affect its cells of origin. ${ }^{2}$ Transection of the spinal cord in young animals entails even in course of a few weeks atrophy of the cell-group of Clarke's column. These injuries are, it is true, applied actually to a portion of the cell itself which they are found to alter; but the phenomenon, which is one now under active investigation, admits at present of little that is satisfactory in way of explanation. The tearing out of the motor roots is said to be more productive of the central changes than is their simple section.

\section{Regeneration of the Spinal Cord.}

It has been asserted that the spinal cord of the frog ${ }^{3}$ and pigeon, ${ }^{4}$ after complete transection, may, in the course of a few weeks, show reunion, nerve cells and nerve fibres existing in the tissue bridging the lesion. Figures ${ }^{5}$ given of the cells believed to be nervous suggest connective tissue fibroblasts or neuroglia cells rather than nerve cells. The supposed regeneration is in accord with a number of observations of a not altogether convincing kind, none of them recent, in which restitution of "volitional movement" and of "conscious reaction" in relation with the regions behind the transection, appeared to occur. ${ }^{6}$ It must be remembered that some recovery of function is possible without regeneration of the tissue, ${ }^{7}$ and reflex movements can be only with difficulty discriminated from intentional.

The whole question bases itself on the discovery by Spallanzani, in $1768,{ }^{8}$

${ }^{1}$ Sherrington, Journ. Physiol., Cambridge and London, 1894, vol. xvii.; v. Leonava, Neurol. Centralbl., Leipzig, 1896, Bd. xv.

2 Warrington, loc. cit.; Marinesco, Neurol. Centralbl., Leipzig, 1898.

${ }^{3}$ Masius and Van Lair, Centralbl. f. d. med. Wissensch., Berlin, 1869, No. 39 ; Bull. Acad. roy. de méd. de Bclg., Bruxelles, 1870, tome xxi. (Mém. couronnée).

"Brown-Séquard, "Expér. sur les plaies d. l. moelle épinière," Gaz. méd. de Paris, 1849 , p. 232 ; "Régén. d. tiss. d. l. moelle épinière," ibid., 1850, p. 250 ; "Sur plusieurs cas de retour des fonctions, etc.," ibid., 1851, 1. $47 \overline{7}$.

${ }_{5}$ Masius and Van Lair, Bull. Acad. roy. de méd. de Belg., Bruxelles, 1870, tome xxi. pl. ii. fig. 5 .

'Prochaska, "Opera Minora," Vienna, 1800 ; Legallois, “ EEuvres,"Paris, 1824 ; Flourens, Ann.d.sc. nat., Paris, 1828, tome xiii. p. 113 (in the duck); Ollivier, "Traité d. maladies de l. moelle épinière," tome i. p. 249, 3rd edition (in the cat); Arnemann, Journ. compl. du dict. d. sc. méd., Paris, 1836, tome xxv. p. 214 (original, a rare work); "Versuche ueber d. Regeneration," Göttingen, 1797 ; Dentan, "Inaug. Diss.," Bern, 1873 ; Masius and Van Lair, loc. cit. ; Brown-Séquard, loc. cit. 7 'See section on spinal conduction, p. 865, supra. 8 " Prodromi sulle riproduzioni animali : Riproduzione della coda del girino," Modena, 1768. 
that the spinal cord is reproduced in the lizard in the regeneration of the tail. His description has been many times confirmed, and its fact thoroughly established. ${ }^{1}$ The regeneration takes place even when the spinal cord has been transected above the tail, in the thoracic region. ${ }^{2}$ But although the new formation of both ependyma cells and nerve cells in the caudal cord is clear and abundant, there is much less restitution of both these elements in the cicatrix in the thoracic region. ${ }^{3}$ Regeneration of the cord in the tailregion of Lacerta viridis is less complete ${ }^{4}$ than in that of Triton, and some are doubtful whether the nerve cells reappear in the former, ${ }^{5}$ though several observers regard this as unquestionable. ${ }^{6}$ In the tadpole the regeneration only includes the ependyma cells. ${ }^{7}$ In the adult frog, in spite of the observations of Masius and Van Lair, Sgobbo finds no karyomitosis or other signs of nervous regeneration at the transection of the cord. Similarly, the results of Brown-Séquard in the pigeon are contradicted by the careful observations by Piccolo and Santa Silena, ${ }^{8}$ and by Sgobbo. ${ }^{9}$ After semisections and total transections, these latter observers find in the pigeon no neoformation of nervous elements, but degeneration of nerve fibres extending for various distances, although the ependyma cells of the central canal remain almost unaltered, quite close up to the seat of trauma. It seems an established fact, that in the higher vertebrata, no karyomitosis goes on in the spinal nerve cells after that early stage of devolopment at which the dorsal white columns are sufficiently formed to cause a distinct dorsal median fissure to the cord. ${ }^{10}$ But as to spinal regeneration, numerous observations upon dogs have led to somewhat discordant conclusions. Regeneration of cells has been described; ${ }^{11}$ also the extension of nerve fibres across the scar without any regeneration of nerve cells ${ }^{12}$ whilst by others neither regeneration of nerve cells nor extension of nerve fibres into the scar could be found. ${ }^{13}$ Karyomitosis has been found in the ependyma cells of the central canal at the exit of lesion, but none in the nerve cells proper. The neuroglia cells of adult animals normally show some karyomitosis. ${ }^{14}$ The origin of the new nerve fibres that in some cases traverse the scar has not been ascertained.

1 Müller, "Ueber Regeneration, u.s.w.," Würzburg, 1864 ; Collueci, "Intorno all riproduzione degli arti e della coda nei tritoni," Mem. Accad. d. sc. d. Ist. di Bologna, (4) tomo iv. p. 301 ; Barfurth, Anat. Anz., Jena, 1888, S. 312 ; Caporoso, "Sulla rigenerazione d. midollo spinale," 1888 ; Magini, "Sulla rigenerazione d. nidollo spinale caudale nel triton," Bull. d. r. Accad. med. di Roma, 1890, tomo xvi. p. 88; Sgobbo, "Sulla rigenerazione del midollo, etc.," Psichiatria, Napoli, 1890, p. 295. Cf. S. Flexner, Journ. Morph., Boston, vol. xiv. p. 337 .

${ }^{2}$ Sgobbo, op. cit. ${ }^{3}$ Ibid. ${ }^{4}$ Magini, op. cit. ${ }^{5}$ Magini and Sgobbo.
${ }^{5}$ Colori, Mem. d. r. Accad. d. sc. d. Ist. di Bologna, 1858, tome ix. p. 345 ; Müller,

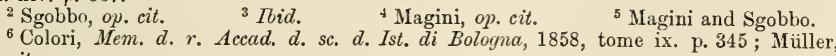

${ }^{2}$ Sgobbo, op. cit. ${ }^{3}$ Ibid. ${ }^{4}$ Colori, Magini, op. cit. ${ }^{5}$ Magini and Sgobbo.
${ }^{5}$. $r$. Accad. d. sc. d. Ist. di Bologna, 1858, tome ix. p. 345 ; Müller,

${ }^{2}$ Sgobbo, op. cit. ${ }^{3}$ Ibid. ${ }^{4}$ Colori, Magini, op. cit. ${ }^{5}$ Magini and Sgobbo.
${ }^{5}$. $r$. Accad. d. sc. d. Ist. di Bologna, 1858, tome ix. p. 345 ; Müller, op. cit.

7 Barfurth, Sgobbo.

8 "Sulla midolla spinale," Gior. de sc. nat. ed econ., Palermo, 1876, tome xi.

${ }^{9}$ Loc. cit.

${ }^{10}$ Ludwig Merk, Denkschr. d. k. Akad.d.Wissensch., Wien, 1887 ; Tirelli, Ann. difreniat., Turin, 1897, tomo v. p. 9; Monti and Fieschi, Arch. ital. de biol., Turin, tomo xxiv. p. 401.'

11 Deutan, "Inaug. Diss.," Bern, 1875; Masius, Arch. de biol., Gand, 1880, tome i. p. 696 ; Baer, Dawson and Marshall (Howell's laborat.), Journ. Exper. Med., Baltimore, 1898, vol. iii. p. 1.

${ }_{12}$ Eichlorst and Naunyn, Arch. f. exper. Path. u. Pharmakol., Leipzig, 1874, Bd. ii. S. 225; Eichlıorst, Ztschr.f. klin. Med., Berlin, 1879, Bd. i. S. 284.

${ }_{13}$ Westphal and Schiefferdecker, Virchow's Archiv, 1876, Bd. lxvii. S. 542 ; Reckling. hausen, loc. cit.; Sgobbo, loc. cit.; Kerestzzeghy and Hanss, Beitr. z. path. Anat. u. z. Ally. Path., Jena, 1892, Bd. ii. S. 33 ; Fiirstner and Knoblauch, Arch. f. Psychiat., Berlin, 1893, Bd. xxiii. S. 132 ; Friedmann, ibid., 1888, Bd. xix. S. 244.

${ }_{14}$ Nissl, Vcrsl. d. sudwest deutsch. psychiat. Vereins, Karlsruhe, 1893. See Neurol. Centralbl., Leipzig, 1894, Bd. xiii. S. 98. 
A P P E N D I X.

Table of Effects of Excitation of the Dorsal (Afferent) and Ventral (Efferent) Roots of some of the Spinal Nerves in Macacus rhesus. ${ }^{1}$

\begin{tabular}{|c|c|}
\hline DoRsal RoOTS. & Ventral RoOts. \\
\hline 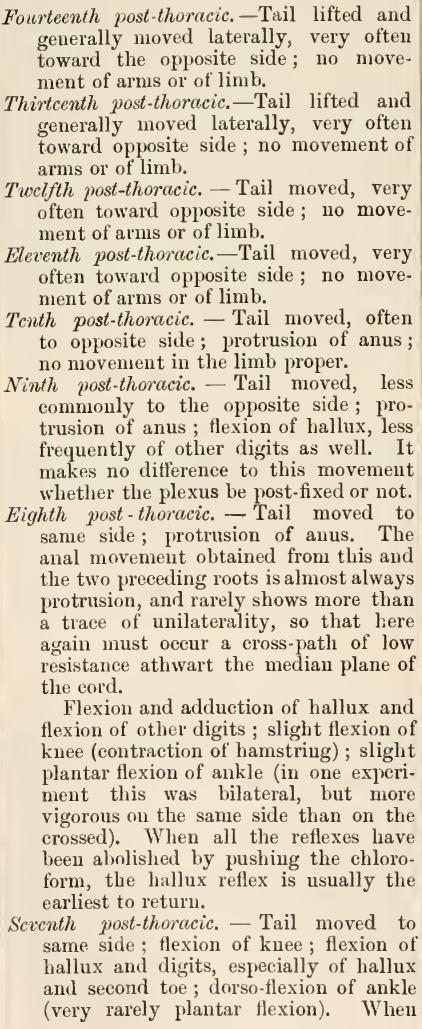 & $\begin{array}{l}\text { Tcnth post-thoracic. - Tail moved laterally } \\
\text { toward the side of excitation. } \\
\text { Ninth post-thoracic.-Tail moved laterally. } \\
\text { In some individuals slight external rota- } \\
\text { tion of thigh, and adduction-flexion of } \\
\text { hallux and toes ; contraction of bladder. }\end{array}$ \\
\hline
\end{tabular}

${ }^{1}$ Sherrington, Journ. Physiol., Cambridge and London, 1892, vol, xiii. ; Phil. Trans., London, 1896. 


Dorsau Roots.
the filaments of this large root are
separated into three sets, made up re-
spectively of the anterior, middle, and
posterior groups, no distinet difference
between the replies obtained from each
of the three is observable; crossed
plantar flexion of ankle.

Sixth post-thoracic.-Tail moved to same side; flexion of knee, flexion of hip, with some internal rotation; flexion of liallux and of other digits; less easily, dorsoflexion of ankle witl tilting up of the outer edge of the foot; adduction of thigh, easily obtainable as a crossed reflex.

Fifth post-thoracic.-Flexion at hip ; flexion at knce (inner hamstrings and gracilis seem particularly active); flexion of hallux, less of toes ; slight dorsoflexion at ankle at times; adduction at hip as a crossed effect; movement of tail, not constant. Knee-jerk.

Fourth post-thoracic.-Curving of body so that the lind-limbs point toward side stimulated; flexion at hip; flexion at knee; flexion of hallux occasionally, and still less frequently flexion of digits ; quite rarely slight dorsoflexion at ankle; adduction at hip, occasionally with erossed adduction. Knee-jerk.

Third post-thoracic.-Curving of body as with fourth root, but nore so; flexion at hip, rarely flexion at knee ; occasionally flexion of hallux, and oceasionally of other digits as well; drawing 11 of testis (cremaster).

Second post-thoracic.-Curving of body as above; flexion at hip; drawing up of testis (cremaster); very occasionally flexion of hallux.

First post-thoracic.-Muscles of flank contract; retraction of abdominal wall low down ; flexion at hip.

Twelfth thoracic.-Muscles of flank and abdomen contract; flexion at hip, but not regularly or in all individuals.

Eleventh thoracic.-Muscles of flank and abdomen and intercostal space; with moderate strength of stimulation never any movement in linib.

Seventh thoracic.-Muscles of back and side of ehest; sonc of the more superficial respond more readily than do the iutercostals. The intercostals that most readily respond are those of the seventh and eighth spaces, and next to them of the sixth space. It is difficult to evoke contraction in the crossed intercostals.

Fifth thoracic.-Muscles of back and side of chest; intercostal spaces involved are chicfly fifth and sixth; occasionally some retraction of shoulder.

Fourth thoracic.-Retraction of shoulder ; slight dilatation of homonymous pupil ; occasionally contraction of triceps.

Sixth post-thoracic. - Extension of hip; adduction of thigh; weak flexion at knee ; dorsoflexion at ankle ; extension of toes ; adduction of hallux.

Fifth post-thoracic.-Adduction at hip ; extension at knee; slight flexion at ankle ; slight extension of hallux.

Fourth post-thoracic.-Retraction of lower part of abdominal wall; flexion and adduction at hip; exteusion at knee.

Third post-thoracic.-Retraction of lower part of abdoninal wall; flexion at hip ; contraction of round ligament of uterus and of eremaster.

Second post-thoracic. - Retraction of ab dominal wall ; slight flexion at hip.

First post-thoracic. - Retraction of abdominal wall; no movement in the limb itself.

VOL. II. -56 


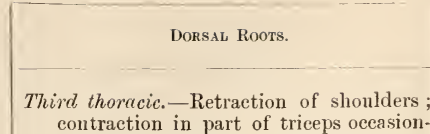
contraction in part of triceps occasion-
ally well marked; dilatation of homonymous pupil.

Sccond thoracic.-Movement at shoulder, slight flexion of thumb and digit; sometimes contraction in triceps ; sometimes dilatation of homonymous pupil.
\end{abstract}

First thoracic. - Movement at shoulder, certainly sometimes retraction; contraction in part of triceps ; adduction and flexion of thumb and other digits; extension of elbow, sometimes flexion at elbow; with stronger stimulation slight flexion and some pronation at wrist.

Eighth cervical.-Adduetion and flexion of thumb, flexion of other digits, flexion nore often extension at wrist ; drawing inward and downwards of shoulder, retraction of upper arm. At elbow rarely extension, sometimes flexion; during the latter movement the supinator longus acts, but the biceps acts very little. When the examination of the root is conducted by splitting it in three fore-and-aft divisions, extension at elbow and triceps contraction are less common from the foremost than from the hindmost division; and flexion and supinator longus action more common from the foremost than from hindmost division.

Serenth cervical. - Adduetion and flexion of thumb and flexion of other digits; flexion of elbow ; retraction of shoulder.

Sixth ccrvical.-Flexion of elbow ; adduction and flexion of thumb; flexion of other digits and retraction of shoulder less marked than with Root 7 .

Fifth cervical.-Flexion at elbow ; movement at shoulder, sometimes retraction, sometimes drawing up; adduction and flexion of thumb. Sometimes the movement at shoulder is simply adduetion.

\section{Vestral Roots.}

Second thoracic.-Retraction of shoulder ; slight flexion of wrist ; flexion of fingers and thumb with opposition of the latter; sometimes slight pronation at wrist; lateral eurving of the spinal column. Dilatation of pupil ; eye opens.

First thoracic.-Retraction of shoulder slight lateral flexion and retraction of neck; slight extension at elbow, flexion at wrist with pronation ; flexion of fingers and thumb with opposition of the latter: there is usually a slight abduetion of wrist toward nlnar side.

Eighth cervical. - The shoulder drawn downwards (latissimus dorsi). The adduetion is not so extreme as in case of Root 7 . Rotation inwards of arm ; flexion and pronation of wrist ; flexion of fingers, and of thumb with opposition.

Seventh cervical.-Retraction and strong adduetion at shoulder with some inward rotation of arm: the arm is earried across the body. Extension of elbow; slight flexion at wrist and some pronation; slight flexion of the fingers. The shoulder is drawn downwalds. Slight retraction and lateral flexion of neck.

Sixth cervical. - Moderate adduction at sloulder; strong flexion of elbow; slight extension of digits and hand (not in every individual). Some supination of wrist; slight extension of wrist in most individuals, but in a few flexion and not extension of wrist. In one individual flexion of fingers was obtained from this root, but that may have been due to mechanical effect of extension of wrist, which if extreme in Macacus partially flexes the fingers. Very slight lateral flexion of neck toward side stimulated and slight retraction of neck and head.

Fifth corvical.-Elevation, abduetion, and some outward rotation of the shoulder ; flexion of elbow, the wrist becoming slightly supine; slight radial abduction of wrist; very slight lateral flexion of neck toward side stimulated, 


\begin{tabular}{|c|c|}
\hline DoRsal Roots. & Ventral Roots. \\
\hline $\begin{array}{l}\text { Third cervical. - Elevation of shoulder ; } \\
\text { drawing down of head to same side } \\
\text { flexion of elbow feeble and occasional. } \\
\text { The flexion of elbow is in great neasure } \\
\text { supinator longus action : twisting of } \\
\text { head and neck to opposite side. } \\
\text { Second cervical. - Drawing up of shoulder. } \\
\text { down of head to sane side ; turning of } \\
\text { chin toward opposite shouldcr with } \\
\text { neck. Neither in this root nor in pre- } \\
\text { ceding did moderate stinuli evoke con- } \\
\text { traction in the muscles of the hyoid } \\
\text { region. } \\
\text { No dorsal root. }\end{array}$ & $\begin{array}{l}\text { with some (slight) retraction of neck } \\
\text { and head. } \\
\text { Fourth cervical. - Elevation of shoulder, } \\
\text { dragging it headward and toward the } \\
\text { spinal column. Slight lateral flexion } \\
\text { toward side stimulated with marked } \\
\text { retraction. When the shoulder is fixed, } \\
\text { slight turning of the head toward } \\
\text { the opposite side previously obtainable } \\
\text { becomes more pronounced. } \\
\text { Third cervical. - No movement of the limb } \\
\text { proper. Lateral flexion of the neck } \\
\text { toward the side stimulated, with } \\
\text { marked retraction and a little turning } \\
\text { of the neck, so that chin is thrust } \\
\text { upward toward opposite side. } \\
\text { Second cervical.-No movement of the } \\
\text { limb proper. Lateral flexion of the } \\
\text { neck toward the side stimulater, with } \\
\text { some retraction. Little or no rotation } \\
\text { of the head, but the chin may be } \\
\text { turned slightly toward opposite axilla. } \\
\text { First cervical. - No movement of limb } \\
\text { proper. Lateral flexion of the neck } \\
\text { toward the side stimulated without } \\
\text { rotation of the head. }\end{array}$ \\
\hline
\end{tabular}




\title{
THE PARTS OF THE BRAIN BELOW CEREBRAL CORTEX, VIZ. MEDULLA OBLONGATA, PONS, CEREBELLUM, CORPORA QUADRIGEMINA, AND REGION OF THALAMUS.
}

\author{
By C. S. Sherrington.
}

Contents. - The Mrelexcephalon or Region of the Medulla Oblongata, p. 884-The Root Cell Systems in, p. 881-The Bulbo-spinal Frog, p. 886-The Bulbo-spinal Mammal, p. 886- "Centres" in the Bulb, p. 887-Conduction Paths in the Bulb, p. 888-The Metencephalon or Region of the Pons VAROLII AND CEREBELLuM, p. 890-Reactions in the Frog, p. 890-And Rabbit, p. 890-The Poxs, p. 891-The Root Cell Systems in, p. 891- "Centres" in, p. 891-Conduction Paths in, p. 893-The Cerebellum, p. 893-Excitation of, p. 893-Lesions of, Unilateral Ablation, p. 895-Ablation of the whole Cerebellum, p. 897-Destruction of the Middle Lobe, p. 898-Longitudinal Bisection, p. 898-Ablation in the Bird, p. 898-Ablation in Lower Vertebrates, p. 899-Phenomena caused by Section of the Cerebellar Peduncles.Section of Anterior Peduncle, p. 900 Section of Middle Peduncle, p. $900-$ Section of the Posterior Peduncle, p. 901-Bilateral Section of the Peduncles, p. 901 - Phenomena of Cerebellar Origin in Human Disease, p. $901-$ Inferences drawn from Comparative Anatomy, p. 902 - Functions of the Cerebellum, p. 903 - The MIEsencephalon or Region of the Corpora QUadrigemina, p. 911-The Root Cell Systems in, p. 911-Excitation of the Corpora Quadrigemina, p. 912-Reactions of Animals after Ablation of all the Encephalic Regions in front of the Mesencephalon, p. 912-Lesions of the Corpora Quadrigemina, p. 912-Decerebrate Rigidity, p. 913-Conduction Paths through the Mesencephalon, p. 915-THE DiencepHalos or Region of тне Optic Thalajus, p. 916-Visual Connections of, p. 916-Excitation of, p. 917-Destructive Lesions of, p. 917.

\section{The Mrelencephalon or Region of the Medulla Oblongata.}

THE roof of this portion of the encephalon contains neither nerve cells nor nerve fibres. From the present point of view, therefore, it is functionless. The floor, on the other hand, forms the bulb, and exercises manifold neural functions.

Belonging to the afferent root cell system of this part are the ganglion cells of the nervus intermedius (geniculate ganglion), receiving impulses, perhaps gustatory, from the tongue region; the ganglion cells of the cochlear and vestibular nerves, conducting impulses from the organs for hearing, geotropism and space-relations of the head; the ganglion cells of glosso-pharyngeal and vagus nerves, conducting impulses from alimentary and respiratory mucous membranes and from heart; and occasionally ganglion cells belonging to the hypoglossal nerve, their distribution being unknown. The afferent root cell system of this region brings no impulses from skin, except from a tiny patch at the 
meatus of the ear. In addition, the region contains the end-nuclei which receive the fibres of the above afferent root cells (the descending stems of the

$A$.

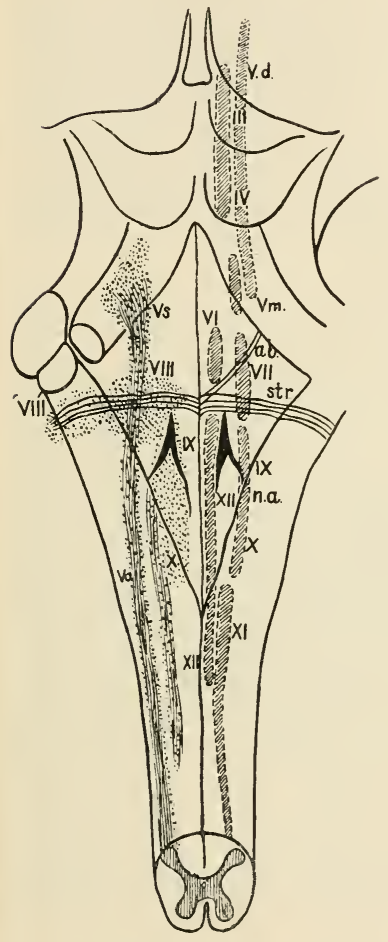

B.

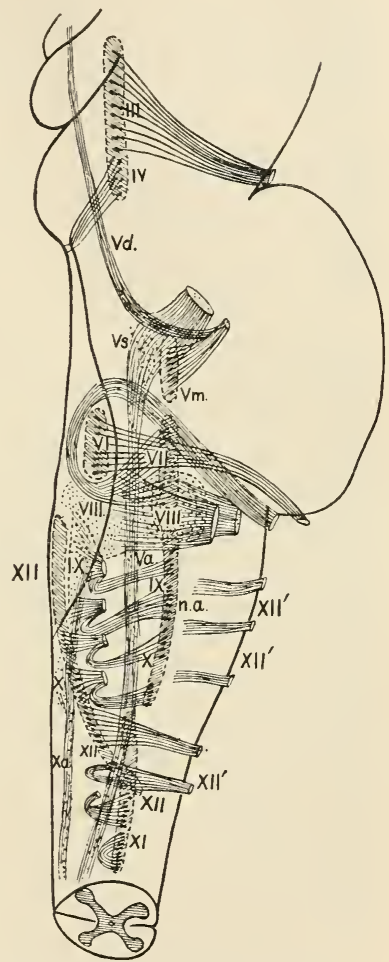

FIG. 360.-Diagrams to show the situation of the chief nerve nuclei in the medulla oblongata and pons near the floor of the fourth ventricle. $A$, from behind; $B$, profile view of the right half, the medulla and pons being supposed to be transparent. The efferent or motor nuclei are shaded with oblique lines, the afferent or sensory nuclei with dots. In $A$ the efferent or motor nuclei are represented on the right side only, the afferent or sensory on the left. III, IV, oculomotor and trochlear nucleus; Vd, descending root of the fifth nerve; Vs, so-called sensory nucleus of the fifth ; Va, ascending root of the fifth ; Vm, motor nucleus of fifth nerve; VI, nucleus of abducens; VII, nucleus of facial; n.VII, root of facial eurving round abducens nucleus ; VIII., inner or dorsal nucleus of auditory; VIII', outer or ventral nucleus of auditory; IX, X, vago-glosso-pharyngeal nucleus; n.a, nucleus ambiguus, accessory or efferent vago-glosso-pharyngeal nucleus; XI. nucleus of spinal accessory ; $\mathrm{XII}$, nucleus of hypoglossal ; $\mathrm{XII}^{\prime}$, issuing roots of hypoglossal.-Schäfer (twice the natural size).

afferent fibres of the vagus, tract. solitarius, partly pass to crossed nuclei, nuc. commissurales, and the end-nuelei also for three other afferent roots. First, for the afferent root cells (in trigeminus), leading from the frontal, ocular, nasal, facial, buccal, and lingual skin; second (Goll's nucleus), for afferent 
root cells, leading from the segmental nerves of the whole body below the brachial region; third (Burdach's nucleus), for afferent root cells, leading from all the brachial and cervical nerves.

The efferent root cell system discharges impulses to various regions of the alimentary secretory epithelium, from mouth to pancreas inclusive (chorda tympani, Jacobson's nerve, and branches of vagus), and the musculature of the alimentary canal from the soft palate along the pharynx and osophagus to and inclusive of the stomach, ${ }^{1}$ including the respiratory diverticulum (larynx, trachea, and lungs), and the tongue. The nucleus ambiguus is the cell group whence arise most, if not all, of the efferent fibres of the glosso-pharyngeal, vagus, and accessory nerves; the rootlets of the last named are entirely efferent. Cajal ${ }^{2}$ has found that some of the axis cylinders from the nucleus ambiguus pass to the rootlets of the crossed glosso-pharyngeal nerve, though the great majority enter the homonymous nerve: the same condition he found in lesser degree to hold in relation to the vagus rootlets.

In the frog, removal of the encephalon down to the level of the octavus nerve leaves the animal with regular and apparently normal respiratory movement and circulation, but with considerable impairment of its locomotor powers. It rests sprawling with head sunk. It crawls only under obvious external stimulation. ${ }^{3}$ Locomotion is easier to provoke than when the transection lies at the caudal end of the fourth ventricle. ${ }^{4}$ It will usually, when laid on its back, try to right itself, and usually succeeds. This evidences the existence of geotropic sense and of considerable muscular co-ordination. The co-ordinating action of the frog's myelencephalon on the skeletal musculature, may be likened to that of the ring commissure of Asterias. When placed in the tank upside down, a united action of the five rays soon rights the animal. But if without injury to the individual nerve systems of the several rays, the circumbuccal nerve ring be severed between the adjacent rays, ability to correct the inverted posture is destroyed. ${ }^{5}$ The individual action of the rays is vigorous, but they largely neutralise each other's mechanical effect. The inco-ordinate activity fails to arrive at the result attained when due correlation is ensured by the nerve-ring. The actions of the individual rays must therefore, when the nerve-ring is intact, influence each other. The convergence of afferent spinal paths from the slieletal musculature and of paths from the vestibular nerve towards the region which possesses this co-ordinating influence, is suggestive of their co-operation toward the result.

The mammal, after similar removal of all encephalon in front of the bulb, still breathes with regularity, and possesses good circulatory adjustments. There is still some degree of co-ordinate movement of progression, but such movement is difficult to provoke. When the transection lies below the junction of the middle and lowest thirds of the fourth ventricle, it becomes in some mammals ${ }^{6}$ extremely difficult to evoke by any sensory stimulus a movement in which all four limbs come into play. I would remark that, by this transection, the vestibular

1 Bichat, "Anat. gén.," Paris, 1812, tome iii. p. 360 ; Budge, Nov. acta phys.-med. Acad. nat. curios, 1860 , vol. xxvii.

2 "Origen de los nervios vago y glosso faringeo," Madrid, 1895.

3 Schrader, Arch. f. d. ges. Physiol., Bonn, 1887, Bd. xli.

${ }^{4}$ Noted in salamander also by Pfluger, "Die sensorische Functionen des Rückenmarkes," Berlin, 1852.

${ }_{5}^{5}$ G. Romanes, Phil. Trans., Loudon, 1881 ; J. Demoor and Marcellin Chapeaux, Trav. de l. stat. zool. d. Helder, 1891 .

G Owsjannikow, A.b. a. d. physiol. Anst. zu Leipziy, 1874, S. 314. 
apparatus is cut off from the cord. The fall of arterial blood pressure, consequent on the interference with the bulbar vasomotor centre, is not responsible for the difference in the reflexes.

Ablation of the rest of the bulb annuls the respiratory movements, depresses the arterial blood pressure, and destroys a number of vasomotor reflexes that were previously possible. The cardio-inhibitory centre connected with the vagus and its accessory nerve is also destroyed, and the reactions through it of sensory nerves upon the heart are of course lost with it.

In the bulb lie also, in major part, the nervous mechanisms guarding and working the apparatus of the great anterior opening of the body, by which material from the environment is seized, tested, and admitted as pabulum for digestion. The respiratory apparatus belongs to this same system, although with additional excretory functions, and in higher groups partly subservient to emotional and mental expression. Nothing perhaps shows more clearly the want of functional solidarity of the individual "segments," as morphologically defined, than the profound degree to which the nervous "centre" for the ingestion of food extends across the boundary between metencephalon and myelencephalon in the floor of the fourth ventricle. This "centre" pervades both, and in its spatial distribution, as in that of the bulbar mechanism of speech, little can be traced of any boundary between them, although its fractions can be located more or less independently. These are often referred to as "centres" of deglutition, phonation, vomiting, coughing, hiccough, etc. With all its complexity, the mechanism is essentially that of a great oral sphincter. Special details are given concerning them in various appropriate sections of this work. The visceral sensory surfaces and alimentary epithelium connected with the myelencephalon extend far backward. The reflex centres for movement of the cesophagus, and for secretion by the gastric ${ }^{1}$ and pancreatic glands, ${ }^{2}$ lie in the caudal part of the bulb, in connection with the vagus and its accessory nerve. After removal of the entire encephalon in the frog, the intestines enter into a condition of long-continued peristaltic activity. If, however, the bulb be left, a status of apparently abnormal quietude obtains in the intestine. $^{3} \quad$ A point of general interest in the physiology of the great "alimentary" nerve centre in the bulb is the high degree to which it employs inhibition. Each subdivision of it is depressible by " inhibitory" fibres from some afferent nerve trunk or another; thus, the respiratory portion by fibres in the superior laryngeal nerve, the deglutition portion of it by fibres in the superior laryngeal, and partly in the inferior laryngeal, the secretory portion by fibres in the afferent branches of the gastric and thoracic vagus, and so on. ${ }^{4}$

The term centre has been used with perhaps especial laxity in speaking of the bulb. A "convulsion" centre has been, among other centres, postulated for the bulb. Widespread convulsions of the skeletal inusculature can be produced by chemical stimulation there. ${ }^{5}$ A drop of 10 per cent. sodium-

${ }^{1}$ Pawlow, Arch. f. Physiol., Leipzig, 1895 (with Schowmow-Simanowski).

2 Pawlow, ibid., 1893, Suppl. (in Russian, 1888).

${ }^{3}$ Steinach, Arch. f. d. ges. Physiol., Bonn, 1895, Bd. 1x., S. 593 ; 1898, Bd. 1xxi. S. 523.

+ See especially Kronecker \& Meltzer, Arch. f. Physiol., Leipzig, 1881, S. 465 ; ilid., 1883, Suppl. S. 328 ; also Monatsbl. $d$. Berl. Ahad. d. Wissensch., 1881 ; also later papers from Kronecker, Bern Laboratory, and S. J. Meltzer, Trans. New York Acal. Med., Jan. 1899.

${ }^{5}$ Henbel, Arch. f. d. ges. Physiol., Bonn, 1874, Bd. ix. ; Lapinsky, ibid., 1899, Bd. lxxiv. S. 47 . 
chloride solution, or a crystal of kreatin placed on the floor of the fourth ventricle of the frog, produces, after a minute or so, a cry which ushers in a seizure, during which the fore-limbs are crossed under the sternum, the hindlimbs at first flexed at hip and extended at knee and ankle, until the animal rolls backward. Then extension at hip follows. The muscular contraction, at first clonic, later becomes tonic. The whole seizure lasts about one minute. The seizure is less intense, if the cerebral hemispheres have previously been removed. Convulsions camnot be similarly excited from the spinal cord itself.

It is in the bulb that nerve cells are met that more than any others in the neural axis seem to have automatic in contradistinction to reflex activity. The bulbar respiratory cells, like the muscle cells of the heart, seem to be able to rhythmically convert stores of their potential into kinetic energy apart from any spur by external stimuli, although profoundly responsive to these latter.

The bulb is indicated by various data as the part of the nervous system in which has been earliest developed, on a grand scale, that kind of communion between constituent neural units, which concentrates function and combines in co-ordination a number of various working factors. There seem to have been early centred in it regulating nervous mechanisms for certain reactions of fundamental import to the very existence of the individual. It thus gave, so to say, opportunity and guarantee for the response of the neural axis in front of it to the call for building up, in the course of ages, under the direction of the sense organs, especially those of sight and smell, the organs of perception, memory, and intellect, with culmination in the mammalian cerebrum and mind. On the other hand, behind it, somewhat similarly, proceeded a development of neural mechanisms for a great senso-motile organ, the skeletal musculature with its bony levers and its overlying skin, each lateral half of which may be considered as equivalent to the senso-motor mechanism of one eye or of one ear. As vertebrate evolution has gone on, the great motile organ has come more and more to lie at the disposal of the upper hierarchies of nerve powers composing the supreme senses, especially the visual with all its spatial quality. Hence the bulb stationed between these two developments is threaded by strands for their conjunction, pathways for travel up and down between them.

Concerning the bulb as a conductor, the following résumé must suffice. There descends into the cord, from the opposite half of the mesencephalon, ${ }^{1}$ a path that probably co-ordinates movements of the body with stimuli impinging on the retina. From the end stations of the vestibular nerve ${ }^{2}$ there descends into the cord an unerossed path, that probably relates the body movements with the sensations originated in the semicircular canals. Paths also, both uncrossed and crossed, descend into the cord from the red nucleus ${ }^{3}$ and the large diffuse nuclei of the reticular ${ }^{3}$ formation of both middle and hind-brain ; the functions of these are even more conjectural. In mammals a path descends from the cerebral hemisphere into and through the bulb to reach the motor root cell system. This makes the pyramids, and is largest in the highest forms of nervous system. It appears wanting in vertebrata below mammals (Singer, 1881). ${ }^{4}$

${ }^{1}$ Held, Neurol. Contralbl., Leipzig, 1890, Bd. ix. S. 481 ; Arch.f. Physiol., Leipzig, 1893, S. 254 ; Boyce, Phil. Trans., London, 1895, vol. clxxxvi. 1. 321 (communicated 1894).

2 See Ferrier and W. A. Turner, Phil. Trans., London, vol, clxxxv.

${ }^{3}$ Neurol. Centralbl., Leipzig, 1890, Bd. ix. S. 481.

${ }^{4}$ Sitzungsb. d. k. Akal. d. Wissenseh., Wien, 1881, S. 393 ; Pitres, Arch de physiol. norm. et path., Paris, 1886, Sér. 3, tome vii., and others. 
In the guinea-pig the pyramid path crosses into the dorsal column of the cord. The excitation of each pyramid evokes movements chiefly in the contralateral skeletal museles, especially of the limbs, but to some extent also in the homonymous. ${ }^{1}$ Complete severance of both the pyramids does not, however, produce even in so high a type as the dog any obvious permanent paralysis of movement," nor does it prevent in the dog excitation of the Rolandic cortex, producing movement both on the crossed or uncrossed limbs. ${ }^{3}$ The pyramidal paths decussate in major part at the caudal end of the bulb. The decussation, when examined by excitation, extends over a longer ${ }^{4}$ area than anatomical

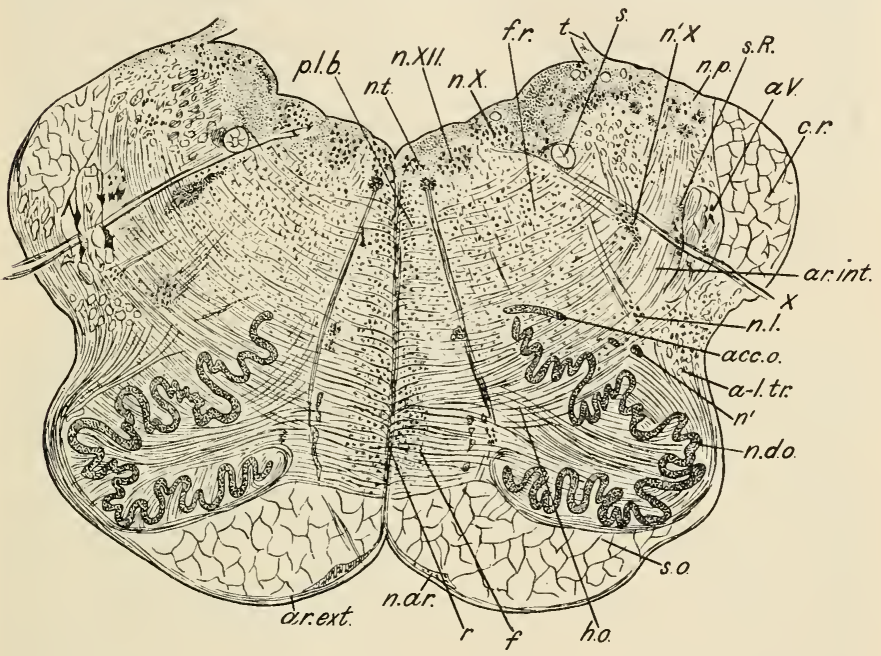

FIG. 361.-Transverse section of the medulla oblongata somewhat above the middle of the olivary body. p.l.b, posterior longitudinal bundle ; n.t, nucleus of funiculus teres; $n . X I I$, nucleus hypoglossi ; $n . X$, nucleus vagi; $n^{\prime} X$, nucleus ambiguus ; $s$, fasciculus solitarius; $n . p$, nuclens posterior (cuneatus); f.r, formatio reticularis; $t$, tænia; s.R, substantia Rolandi ; $a . V$, ascending root of fifth; $c . r$, corpus restiforme; ar.int, internal arcuate fibres; $X$, issuing root of vagus ; $n . l$, nucleus lateralis ; $x^{\prime}$, groups of large cells, perhaps betonging to nucleus lateralis; $a$-l.t $r$, antero-lateral ascending tract; n.d.o, nucleus dentatus olivæ; acc.o, accessory olivary nucleus ; s.o, siliqua olivæ; $h . o$, hilum olivæ; $p$, pyramid ; $f$, fillet ; $r$, raphe ; $n$.ar, nucleus of arcuate fibres; ar.fxt, external arcuate fibres.-Schäfer (from a photograph). Magnified 5 diameters.

examination would lead one to suspect. Pyramidal fibres have been traced ${ }^{5}$ into the hypoglossal nucleus, but not into the other motor uuclei of this region, and only into the contralateral hypoglossal. By some ${ }^{6}$ a descending spino-cerebellar

1 Wertheimer and Lepage, Arch. de physiol. norm. et path., Paris, 1897, tonie ix. p. 168.

2 Starlinger, Neurol. Centralbl., Leipzig, 1895, Bd. xiv. S. 390, and ef. various papers from Goltz's laboratory.

${ }^{3}$ Wertheimer and Lejage, Arch. de physiol. norm. et path., Paris, 1896, tome viii.

+ Balighian, Beitr. z. Anat. u. Physiol. (Eckhard), Giessen, 1879, Bd. viii. ; Staffany, ibid., 1888, Bd. xii. ; Wertheiner and Lepage, Arch. de physiol. norm. et path., Paris, 1897 , tome ix. p. 168.

5 Romanow, Neurol. Centralbl., Leipzig, 1898, Bd. xvii. S. 593.

"Biedl, ibid., 1895, Bd. xiv.; A. Thomas, "Il cerveletto," Florence, 1897. This is in accord with the original statement by Marchi, 1888 . 
path is also believed to exist. The mesial longitudinal bundle is in part a descending path from visual and auditory centres to spinal, especially cervical, motor mechanisms (Cajal, van Gehuchten).

Of the paths ascending through the bulb from the spinal cord, the ehief are-(1) The continuation from the dorsal columns of root fibres, that by a second link pass from the dorsal column nuclei to the fillet of the crossed side; (2) the two spino-cerebellar; one (the restiformal) chiefly uncrossed from the cord and entering the inferior peduncle of the cerebellum, the other (the conjunctival) entering with the superior peduncle of the cerebellum. With this latter pass probably spino-bulbar, spino-mesencephalic, and spinothalamic paths toward end-stations in and in front of the bulb itself. (3) The mesial longitudinal bundle contains fibres ascending (Held, Tschermak) from v. Lenhossék's commissural cell-group of the ventral horn of the cervical cord to reach motor cerebral nuclei, especially of the eye-muscles. The path from the dorsal column nuclei by the crossed fillet extends to the diencephalon (optic thalamus) and in part to the cerebral cortex, especially its parietal region. Of the function of the inferior olive little is known: its connection with paths from the vestibular nerve is suggestive.

\section{The Metencephalon or Region of the Pons Varolit and Cerebellum.}

If in the frog all the brain anterior to the metencephalon be removed, the animal can still walk, spring, and swim. The croak reflex is elicitable, but not readily; it is annulled when the metencephalon is destroyed. The animal reacts on the turn-table perfectly, but this reaction is destroyed when the ablation is extended to the root of the trigeminus. Goltz's reaction to the tilted board is perfect as regards head movement, imperfect as regards correction of the posture of the body by crawling up the board. The animal, if undisturbed, remains inert; if the ablation include the cerebellum and fore-part of the metencephalic floor, the creature becomes active, crawling about until it reaches some corner that blocks further progress. The condition resembles that of the annelid Nereis after removal of the supraasophageal ganglion. ${ }^{1}$ It jumps very rarely. That its locomotor coordination is good, is shown by its climbing over obstacles successfully; in swimming it rarely uses the bilateral stroke, but swims "like a dog." 2 In the condition of which continual crawling is characteristic, there is extraordinary activity of the swallowing reflex. Anything touching the muzzle is snapped at, taken into the mouth, and swallowed. The "embrace" reflex is in the male similarly active, more so than in the normal animal, even at the height of the breeding season. The respiratory movements appear normal. Even when the front part of the metencephalon has been removed, the frog at once rights itself when placed on its back.

In the rabbit similar ablation apparently destroys the power of progression. A condition likened to strychnisation ${ }^{3}$ sets in, and the skin of the head and trunk seem "hyperæstletic." When the muzzle is touched the homonymous fore-limb is lifted toward it, and when that is restrained the contralateral fore-limb is brought up as though as an alternative manœuvre.

\footnotetext{
1 S. S. Maxwell, Arch. f. d. ges. Physiol., Bonn, 1897, Bd. Ixvii. S. 263 (Loeb's Laboratory Archives, Bd. ii.).

2 Schrader, Arch. f. d. ges. Physiol., Bonn, 1887, Bd. xli.

${ }^{3}$ Christiani, Verhandl. d. physiol. Gesellsch. zu Berlin, in Arch. f. Physiol., Leipzig, 1884.
} 
For further description of the functions of the region, it is convenient to follow the morphological subdivision of it into a floor and roof, the former the pons of higher vertebrates, the latter the cerebellum.

\section{The Pons.}

All the root cell system of the metencephalon belongs to its floor. The afferent root cells include the collection of the trigeminal root conducting impulses from the cutaneous sense organs of the forehead and face and from the mucous membranes of the nose, eye, and mouth. It is not clear whether the afferent root cell system in the metencephalon includes any units from sense organs of muscle. If the eighth nerve be reckoned to this region, the system includes the sense organs for the spatial sense of the head, and in air-breathers the sense organ for hearing, which in mammals comes to rival in importance that of vision itself.

The efferent root cell system of the region discharges into muscles that execute prehension of food and mastication, facial expression (emotion), and that oral modification of throat sounds that contributes so largely to vocal expression and to speech. It is probable that the frontalis and orbicularis palpebrarum muscles get their motor innervation only through and not originally in this region. Lateral movement is also in part furnished to the retina from this region. Sone of the fibres in the facialis root come from cells of the crossed facialis nucleus. ${ }^{1}$

For the details of the special physiology of these nerves, reference can be made to the appropriate sections of this work. Out of those details features which rise as salient characteristics of the function of the region may be briefly stated thus. Into it extends the cephalic end of the great neural system for the reception of impulses generated in sense organs of the skin and its appendages, and since much of this region lies inaccessible to the visual field, their importance is in so far unique. The cephalic end of the skin connotes "most headward pain." Tactual space perception of the head directs prehension of food, though least in man. Among its sense organs are those contributory to the testing of objects in regard to their suitability for food, and alongside these lies a main part of the system for discharge into the muscular organs for the prehension and buccal examination and preparation of food. In this region enter the impulses from the organ that, comparably with the visual, forms the basis of an enormous superstructure of highest nervous reaction, and a medium for communication of feelings and ideas. Thirdly, into it pour the vestibular impulses informing as to position of the head, and so in great measure as to position of the body as a whole, and thus regulating the activity of great fields of skeletal musculature.

A number of "centres" are described in this metencephalic floor, many of which can be inferred directly from its efferent root cell groups. Among the reflexes localised within it may be mentioned the following:-Closure of the eyelids on touching the conjunctiva, in man

${ }^{1}$ Lugaro, Untersuch. z. Naturl. d. Menseh. u. d. Thiere, Bd. xv. ; Cajal, "Apuntes para el estudio del bulbo, etc.," Madrid, 1895 ; van Gehuchten, "Le systeme nervenx," 2nd edition, Gand, 1896 ; v. Pugliese and v. Milla, Riv. sper. di freniat., Regrio-Emilia, 1896, tomo xxii. p. 805 ; Dejerine and Theobari, Compt. rend. Soc. de biol., Paris, 4th Dee. 1897 ; G. Marineseo, Rev. neurol., Paris, 1898, tome iv. p. 30. 
of either eye (conjunctival reflex). Various stimuli, especially to the region of the first and second divisions of the fifth nerve, provoke this. Its period has been found to be particularly short, even as low as 035 sec. (Mayhew), ${ }^{1}$ more frequently, 04 sec. A flash of light produces the same movement less quickly 08 sec. (Garten). ${ }^{2}$ Excitation of the pinna or dura mater also elicits the closure. Excitation of the buccal mucous membrane near the canine tooth has been noted to very constantly evoke retraction of the lip (reflexus ultimus, Dastre). Twitching of the pinna oll stimulating its skin is also obtained in part from this region, and is a very constant reflex in the cat. ${ }^{3}$ All these are reflex actions not early

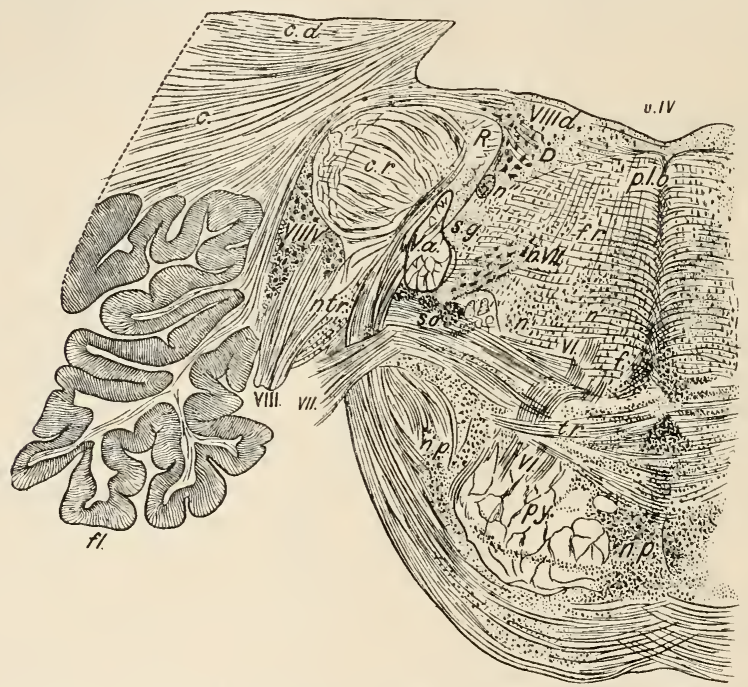

FIG. 362,-Transverse section of pons Varolii through the origin of the auditory nerve. $v .1 \mathrm{~V}$, fourth ventricle; $c$, white matter of cerebellar hemisphere; c. $d$, corpus dentatun cerebelli ; $f$, flocculus ; c.r, corpus restiforme ; $R$, Roller's ascending auditory bundle ; $D$, Deiter's uncleus ; VIII, issuing root of auditory nerve; VIII.d, dorsal nucleus; VIII.v, rentral (accessory) nucleus of auditory; $n . t r$, small-celled nuclcus traversed by fibres of the trapezium ; $t r$, trapezium ; $f$, fillet; $1 . l . b$, posterior (mesial) longitndinal bundle; $f . r$, formatio reticularis; $n \cdot n^{\prime} . n^{\prime \prime}$, nuclei in formatio reticularis; $V$. a, ascending root of fifth ; $s . g$, substantia gelatinosa ; s.o, npper olive ; $V I I$, issuing root of facial; $n . V I I$, nucleus of facial; VI, root bundles of abducens; $p y$, lyramid bundles; $n . p$, nuclei pontis. - Schäfer (from a photograph). Magnified abont 4 diameters.

annulled by narcotics. The conjunctival seems to involve the myelencephalon as well as the metencephalon. ${ }^{4}$ In the rabbit the tonus of the muscles of the pinna falls after severance of the sensory root of the trigeminus. ${ }^{5}$ Binswanger ${ }^{6}$ has called attention to the existence of

\footnotetext{
1 Journ. exper. Med., N. Y., 1897, vol. ii.

2 Arch. f. d. ges. Physiol., Bonn, 1898, Bd. lxxi. S. 488.

${ }^{3}$ Sherrington, Proc. Roy. Soc. London, March 1893.

+ Exner, Arch. f. d. ges. Physiol., Boun, 1874, Bd. viii. S. 520.

${ }^{5}$ Filehne, Arch.f. Physiol., Leipzig, 1886, S. 432.

'Arch. f. Psychiat., Berlin, 1888, Bd. xix.
} 
mechanisms in the tegmental region of the pous that can, on excitation, evoke convulsive movements of the trunk, neck, and limbs, and can be easily brought into action reflexly by stimulation of the trigeminal nerve.

As a conductor the floor of the metencephalon contains the mesial fillet, with its constituents from the dorsal column nuclei, etc., as elsewhere described. Near it the lateral fillet contains ascending paths from the end nuclei of the auditory nerves, chiefly contralateral. The superior olive is probably of auditory function, to judge from its close connection with this path. In the more lateral region, ascend paths from the ventrolateral column of the spinal cord, chiefly from mediate cells in the opposite half of the grey matter. Some of this path is probably connected with "pain." They are on their way to the roof of the metencephalon (cerebellum) and the mesencephalon. Transection of the dorsal portion of the metencephalic floor to one side only of the median sagittal plane produces (monkey) complete anæsthesia (including analgesia) of the contralateral half of the body, without any paralysis. $^{1}$ Of the paths connected with the cerebellum a most notable is, the ascending of the inferior perluncle coming from the lateral column of the cord and Clarke's column of cells (uncrossed mediate cells of the cord). There is also a path conducting upwards from this region into the crossed corpus striatum. ${ }^{2}$ There descend into the pons three main paths from the cerebral cortex, one from the frontal cortex which ends in the grey matter of the pons, a second from the temporal cortex which ends in the grey matter of the pons, and a third, the pyramidal, which only in small part terminates in the pons, in its motor nuclei. Of these the fronto-pontine is the most lateral, the temporopontine the most median. Among the fibres of the pyramidal tract which end in the pons, some have been traced to the crossed nuclei of the motor fifth and seventh, and some also to the homonymous nuclei. From the "reticular formation " of the pons a descending path passes into the ventro-lateral columns of the cord, partly decussating in its descent; the crossed path lies more dorsal in the cord than the uncrossed. Of their function nothing ean with certainty be said. There pass through the reticular formation of the pons paths descending from the mesencephalon (red nucleus and anterior corpus quadrigeminum) to the ventro-lateral column of the spinal cord. Both these paths are mainly crossed ones. The posterior longitudinal bundles form two more paths, chiefly descending. They in part mediate between the motor cell groups of the ocular and other muscles. This explains, perhaps, the elicitation of eyemovements, like those obtainable from the anterior corpus quadrigeminum, from the floor of the fourth ventricle behind the nucleus abducens. ${ }^{3}$

\section{The Cerebellum.}

Excitation.--Ferrier ${ }^{4}$ discovered (1873) that faradic excitation of the cortex of certain parts of the superior vermis, and postero-superior part of the lateral lobe, evokes in the dog and cat conjugate movement of the eyes toward the homonymous side; in the rabbit the movement obtained from the lateral lobe was sometimes not conjugate. Similar conjugate movements he obtained later ${ }^{5}$ in the monkey, also in that animal pure conjugate upward and downward movements of the eyes elicitable from the middle line of the superior vermis. From the flocculus was elicited

${ }^{1}$ Ferrier and Turner, Phil. Trans., London, 1898.

2 Wallenberg, Neurol. Centralbl., Leipzig, 1898, Bd. xvii. S. 293.

3 Knoll, Sitzungsb. d. k. Akad. d. Wisserusch., Wien, July 1886, S. 239.

4 West Riding Lun. Asyl. liep., London, 1873, vol. iii.

" "Functions of the Brain," London, 1876. 
conjugate rotation of the eyeballs around their anterior-posterior axes. With the movement of the eyeballs occurs constriction of the pupils, chiefly of the homonymous eye, and turning of the head in the same direction with the eyes. Movement of the limbs of the same side as the excitation was also elicited. In the pigeon he obtained drawing of the head backward and to the homonymous side, flapping of the hoinonymous wing, and flexion of the homonymous leg. In the carp he found curving of the tail to the homonymous side, spreading of the homonymous fins, and movement of the homonymous eye. He pointed out that the relation between the cerebellum and the musculature of limbs and trunk is therefore, unlike that between the latter and the cerebrum, an homonymous one; and similarly that cerebellar connection with the eye muscles is, as regards relations of laterality, the reverse of that possessed by the cerebral hemisphere. His experiments appear to have been the earliest to decide these important points. Hitzig, ${ }^{1}$ Nothnagel, ${ }^{2}$ and Dupuy $^{3}$ also have obtained definite muscular reactions by localised excitation of the cerebellum, and believe the excitations originate sensations of a painful character.

It has been found, independently by M. Löwenthal and myself, ${ }^{4}$ that excitation of the cerebellum, as of various other parts of the central nervous system, can not only excite contraction of the muscles, but can inhibit contraction. Certain forms of tonic spasm in various parts of the body relax on excitation of the cerebellar surface. When "decerebrate rigidity" has developed, it can be inhibited by faradisation of the anterior (cerebral) surface of the cerebellum at points (in the monkey) over a large area extending from near the middle line far out toward the lateral border of the cerebellar surface. ${ }^{5}$ Faradisation at these points causes immediate relaxation of the rigid neck and tail muscles, and of the rigidly fixed joints of the limbs, especially of the fore-limb, and especially of the same side as the site of excitation in the cerebellum.

Lesions of the cerebellum.-The earliest experiments in which successful recovery was obtained from ablation of the cerebellum (and cerebrum) in higher animals, seem to be those by du Verney in $1697 . .^{6}$ He noted that there remained still some "use of sense." Little progress was then accomplished until Rolando ${ }^{7}$ (Sassari, 1809); from that time onward a number of workers followed in this field. ${ }^{8}$ Flourens affirmed that removal of even considerable portions of the

1 "Untersuch. ü. d. Gehirn," Berlin, 1874.

2 Centralbl. f. d. med. Wissensch., Berlin, 1876.

${ }^{3}$ Compt. rend. Soc. de biol., Paris, 1885-1887.

${ }^{4}$ Sherrington, Proc. Roy. Soc. London, 1896, vol. 1x. p. 382 (in abstract); Löwenthal and v. Horsley, ibid., 1897, vol. lxi. p. 20 . My observations were made on the elbow, knee, neck, and tail of the monkey, cat, and rabbit; Löwenthal and Horsley's on the elbow of the dog.

${ }^{5}$ Sherrington, Journ. Physiol., Cambridge and London, vol. xxii. p. 319.

${ }^{2}$ Phil. Trans., London, vol. xix. p. 226.

7 "Saggio sopra l. vera struttura d. cervello, ete."

${ }^{8}$ Between du Verney and Rolando the chief experimenters studying destructive lesions of the cerebellum were :- Lorry, Hist. Acad. roy. d. sc., 1749, tome iii. p. 344 ; Haller and his pupils, especially Zimmermann and Zinn, and Saucerotte later in 1769. After Rolando the more important contributions in the same line have been the following, and chiefly on them the present section of this article is based :- Flourens, "Rech. exp., etc.," Mcm. Acad. d. sc. de l'Inst. de France, Paris, 1822 ; Magendie, Journ. de physiol. expér., Paris, 1825, tome iv. p. 399 ; Desmoulins, "Anat, des systèmes nerv.," Paris, 1825 ; Hertwig, "Exper. quædam," Inaug. diss., Berlin, 1826, S. 151 ; Serres, "Anat. compar. d. cerveau appliquée à la physiologie," Paris, 1826 ; Bouillaud, Arch. gén. de méd., Paris, 1827, tome xv. p. 64 ; 
cortex does not entail clear or obvious symptoms; but that pronounced disturbances ensue on injuring the deep structures. Experiments by Thomas ${ }^{1}$ show, however, that purely cortical ablations of cerebellum produce symptoms similar in type, though not so severe, as those entailed by injury of the deep nuclei and tracts. Very extensive removals of cortex induce symptoms of full and long-lasting severity.

Unilateral ablation.-The phenomena observable in a mammal after ablation of a lateral (e.g. right) half of its cerebellum may be briefly sketched thus.

No symptom is noticeable until the deep chemical narcosis employed during the operation has diminished. The animal then lies on the right side, with neck and trunk curved concavely to the right, and with some torsion of the unsupported head, so that the occiput tends to the right, but the muzzle rather to the left of the median position, and the left ear rather higher than the right. The limbs, especially the fore-limbs, are stiffly extended, the right more than the left. There is some abduction of the right limbs. There is often conjugate deviation of the eyeballs to the left. The animal cannot stand; it falls to the right side, that is, so that its right side is undermost. It may, if it try to move, roll round the long axis of its body, with a movement continued in the same direction as its fall. The movement of rotation may be accompanied by lateral nystagmus, the slower slipping over of the eyeballs being from right to left, the return snatches in the reverse direction. The majority of observers believe that muscular weakuess as well as spasm exists, especially in the right limbs. The next three or four days witness considerable recovery from some of these symptoms, and it is found that the tendon phenomena are brisk in the spastic limbs, therefore

Budge, "Untersuch. u. d. Nervensystem," 1841, Bd. i. S. 21 ; Longet, "Anat. et physiol. du système nerveux," Paris, 1842; Renzi, Gazz. mcd. ital. lomb., Milano, 1857, 1858 ; "Funzioni del cervelletto,"'Ann. univ. di med., Milano, 1864 ; Lissana, Gazz. med. ital. lomb., Milano, 1851, 1853 ; Journ. de la physiol. de l'homme, Paris, 1862 ; Med. contemp., Napoli, 1886, tome ii. ; Lussana and Lemoigne, Arch. de physiol. norm. ct path., Paris, 1877, Sér. 3, tome iii. ; Schiff, "Lehrbuch d. Muskel u. Nervell-pliysiol.," Lahr, 1858 ; Arch. f. d. ges. Physiol., Bonn, 1883, Bd. xxxii. ; Brown-Séquard, Journ. de la physiol. dc l'homme, Paris, 1859-1862 ; Wagner, "Krit. 11. exp. Untersuch. u. d. Funct. d. Gehirns," Güttingen, 1858-1860; Dalton, Am. Journ. Mcd. Sc., Phila., 1861 ; Schwann, Eckliard, Beitr. z. Anat. u. Physiol. (Eckhard), Giessen, Bd. viii. S. 149 ; Curschmann, "Beitr. z. Physiol. d. Kleinhirnschenkel," Giessen, 1863; Deutschcs Arch. f. Klin. Med., Leipzig, Bd. xii. S. 356 ; Dickinson, Brit. and For. Med.-Chir. Rev., London, 1865, vol. xxxvi. ; Vulpian, "Physiol. génér. et compar. d. syst. nerv.," Paris, 1866; Weir Mitchell, Am. Journ. Mcd. Sc., Phila., 1869, p. 320 ; Stefani, "Fisiologia del cervelletto," Ferrara, 1877 ; Ferrier, "Functions of the Brain," London, 1876 ; Blansehko, "Das Sehcentrum d. Frosches," Berlin, 1880 ; Baginsky, Biol. Centralbl., Erlangen, Bd. ii. S. 725 ; Arch. f. Physiol., Leipzig, 1881 ; Luciani, "Atti di Accad. med. fisica Fiorentina," sperimentalc, Firenze, 1882, 1883, etc. ; "Linea gen. del fisiol. del cervelletto," Florence, 1884; "Il Cervelletto," Florence, 1891 ; Bianchi, Riv. spcr. di freniat., Reggio-Emilia, 1882, tome viii. p. 436 ; Steiner, "Untersuch. 11. d. Pliysiol. d. Froschhirns," Brunswick, 1885 ; Goltz, ef. Luciani, op. cit., p. 180 ; Pugliatti, "Recherche sopra le fisiol. d. cervelletto," Messina, 1885 ; Fano, "Saggio sperinentale," Florence, 1884 ; "Sul nodo deambulatorio," Genoa, 1885 ; Borgherini, liv. sper. di freniat., Reggio-Emilia, 1888, tome xiv.; 1891, tome xvii. ; Fasola, "Scervellazioni parziali e totali," Reggio-Emilia, 1889 ; Laborde, Compt. rend. Soc. de biol., Paris, 1890 ; Lange, Arch. f. d. ges. Physiol., Bonn, 1891, Bd. 1. S. 615 ; Ferrier and Aldren Turner, Proc. Roy. Soc. London, 1893, vol. liv. ; Phit. Trans., London, vol. ev. ; R. Russell, Proc. Roy. Soc. London, 1894, vol. 1v. ; Phil. Trans., London, vol. cv. ; Biedl, Neurol. Ccntralbl., Leipzig, 1895, Bd. xvi. S. 434 ; A. Thomas, Compt. rend. Soc. de biol., Paris, 1895, 1896, 1897 ; "Le cervelet," Paris, 1897 ; Lnciani, "Rec. studi sulla fisiol. d. cervelletto," Reggio-Emilia, 1895 ; Luciani, Arch. ital. de biol., Turin, tome xxi. ; Atti d. 11 Cong. med. internaz., Roma, tomo ii.

1 Loc. cit. 
especially in the right. When suspended, the animal now exhibits less plemrosthotomus (concavity, to the right). The right forepaw often lies folded in the supine position, a sign significant of paresis. It can now be seen, if the animal tries to raise itself on its fore-limbs, that tremor, which may be present even when the animal is at rest, prevents the attempt, becoming very severe. The hind-limbs seem weaker than the fore-limbs, but the extensor rigidity is more marked in the fore-limbs (in the dog and cat, etc.; in the monkey (Luciani, Ferrier and Turner) the rigidity assumed is flexor, not extensor (at elbow and knee). Some days later the power to stand is regained, the fore-limbs becoming able to carry the

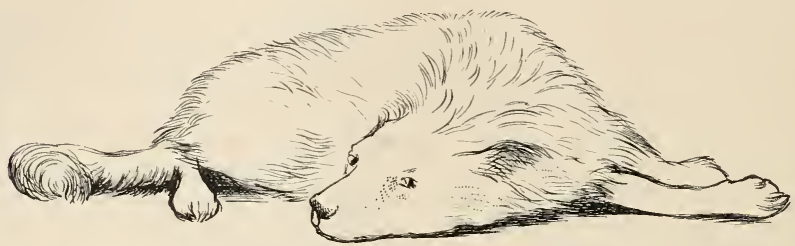

Fig. 363.-Posture assumed after removal of the right half of the cerebellum.Thomas (from an instantaneous photograph).

body earlier than the hind-limbs. When first attempting to walk, the hind-quarters drag or crouch, so that in the latter case the rump stands lower than the shoulder. Tremor and lateral oscillation, especially of neck and trunk, disturb every effort. If an obstacle be met in walking, the tumble occurs to the right, although the right limbs are widely abducted. Progression follows not in a straight line, but the main direction is broken by curves, is "festooned" to the right. The paces taken by the widely abducted legs are short. The whole gait is a reeling one. The right paws are lifted and set down again with an abnormal abruptness and with exaggerated muscular action, as compared with the less abnormal side. The knee-jerk is often found to be more

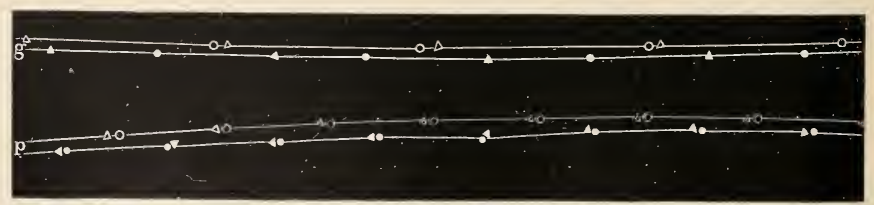

F1G. 364.- Foot-prints of a normal dog. $p$, walking ; $g$, galloping.-Luciani.

brisk on the homonymous side. Placed in water, the animal swims better than it can walk; in swimming, the direction of progression is curved toward the left; the left side of the body is plunged deeper than the right. In attempting to walk up steps, the left foot is used first. After the eyes are bandaged, any attempt to remove the bandage is made with the left foot.

The head and neck are at every effort so disturbed, by swaying and tremor, that feeding is impossible unless the head be held fixed for it. The muzzle is plunged deeply into the food, or sways about helplessly over it. The inco-ordination is not obviously increased by bandaging 
the eyes. The performance of every movement quickly produces great fatigue. Sight, hearing, and cutaneous sensation seem normal. Gradual improvement succeeds in effacing more or less completely the worse degrees of the above disturbances; they persist obviously in running longer than walking; an unwonted movement is apt to produce them again, after they have disappeared from the more ordinary movements; thus the $\mathrm{dog}$ in shaking itself after a swim is apt to shake itself off its balance, falling to the right. It is especially long before the dog recovers his habitual bark. Entire disappearance of the symptoms has not been obtained. Some glycosuria is usual in the early stage, and the animal is for a long time thin, voracious, and depressed; perhaps the longest of all symptoms to persist is tremor, when a strongly - willed movement is executed. Cerebellar tremor is one of the most exquisite examples of "intention tremor," i.e. of tremor marking movement that is "willed."

Ablation of the whole cerebellum. -The disturbance ensuing immediately upon ablation of the whole cerebellum appears at first sight less severe than that following removal of a lateral half of it. Opisthotonos, retraction of the neck, throwing up of the chin, tonic extension and abduction of the limbs, especially of the

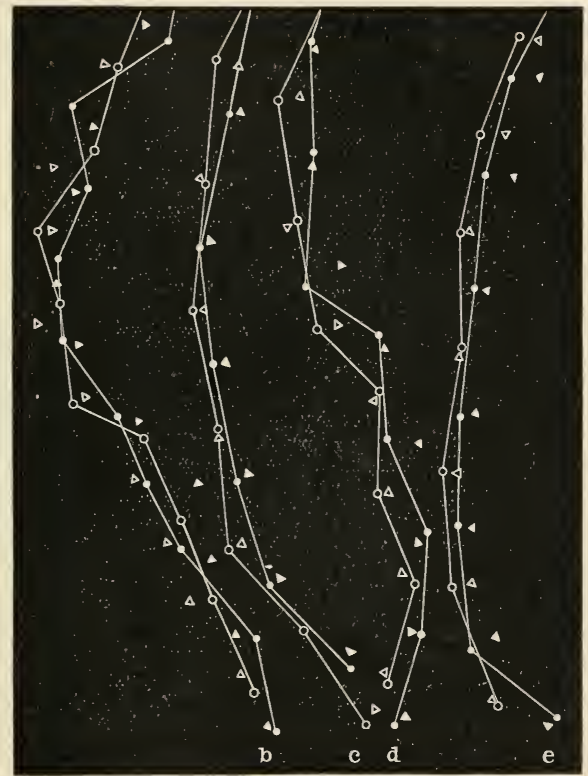

FIG. 365.-Footprints of a dog ten days after ablation of the middle lobe of the cerebellum. - Luciani. fore-limbs, a re simply the bilateral duplication of the former picture, the asymmetrical distortion of which is reduced to symmetry by doubling the insult. The progress toward recovery is, as might be expected, more slow than after unilateral ablation. Every attempt to move induces tremor and oscillation, and long remains abortive. Swimming is much easier to these animals than is walking (Luciani)." "I kept for eight months a water-dog from which I had removed the cerebellum; he made hardly any progressive movement during all that time except when I placed him in water" (Magendie). ${ }^{2}$ The muscular power of the body is weakened in all regions (Luciani), most notably in the hind-limbs and

$$
1 \text { Loc. cit. }
$$$$
2 \text { "Physiologie," Paris, } 1817 .
$$

VOL. II. -57 
in those which fix the vertebral column. Unsteadiness is most marked in the movements of the neck. A residuum of disturbance of motility is permanent; but in Luciani's dog, which lived for two years after ablation of the whole cerebellum (except for two small pieces adjoining the floceuli), improvement (compensation) progressed for about a year. The hind-quarters remained weak, tremor persisted in the muscles which fix the head, and the movement of the eyes and limbs, especially of the fore-limbs, were not perfectly harmonic. The senses and intellect remained perfect, and the animal could swim, run, jump, and feed itself. On the whole, perhaps the most significant symptom of cerebellar ablation is the tremor which disturbs willed movements- "intention tremor." I have myself seen it in the dog persisting two years after an ablation of almost the whole cerebellum. The animal when resting, e.g. lying in his cage, exhibited no tremulance; but on being called to activity, especially when released from his cage, and free and evidently pleasurably excited by his freedom, one saw almost his every motion shaken by tremor.

Destruction of the middle lobe.-The main phenomena following

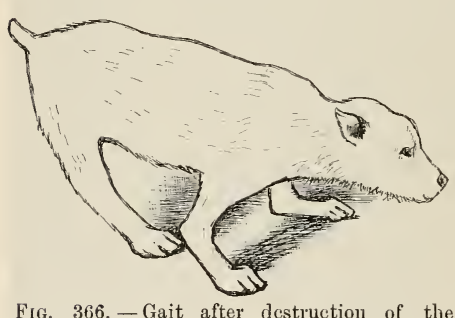

FIG. 366. - Gait after destruction of the vermis; the fore-limbs are abducted, the hind are abducted and protracted. Thomas (from an instantaneous photograpli). its two lateral halves by a clean incision is followed by disturbances closely like those following ablation of the vermis, but of less severe and less persistent character. "The animal seemed alternately impelled to right and to left, without preserving a fixed position; it first rolled a turn or two to one side, and then changed, as for relief, to turn as many times in the opposite direction." 2 As an early symptom it generally induces a tonic extensor rigidity, comparable with the extensor form of decerebrate rigidity. ${ }^{3}$

Ablation of the cerebellum in the bird.-In the pigeon, ${ }^{4}$ ablation of the cerebellum similarly produces malposture and a status in which each intentional movement is hampered or thwarted by uncontrollable deviation from the desired direction; abrupt oscillations seem grafted upon the movements executed. It is impossible for the animal to perch, the legs and talons being stiffly extended. In attempting to walk, the limbs become seized with extensor spasm; the animal falls; wild struggling of limbs, body, and head sets in, to terminate only in utter exhaustion. There is obvious difficulty in bending

1 A. Thomas, loc. cit.

2 Magendie, loc. cit.

" Sherrington, Journ. Physiol., Cambridge and London, vol. xxii. p. 319.

+ Flourens' classical observations (1822) were chiefly on the pigeon. 
the neck downward, and when the head is at last sufficiently lowered it is liable to be suddenly and uncontrollably drawn back (Schiff, Lange ${ }^{2}$ ); hence the animal cannot at first feed itself. The difference between this condition and that resulting from ablation of the cerebral hemispheres is very marked. "Take two pigeons," wrote Longet, "from one remove the cerebrum, from the other only half the cerebellum; next day the former will be firm on his feet, the latter will reel and struggle like a drunken man." ${ }_{3}$ But the severity of the disturbance soon diminishes. ${ }^{4}$ It is notable that tlight very rapidly recovers. Pigeons soon tly adroitly and turn apparently perfectly in the cramped space of a room. Walking is more slowly and less perfectly regained. In the decerebellate pigeon it can be seen that the front digits are over-extended when it walks, and the hind digit does not touch the floor at all. ${ }^{5}$ Perching remains a difficulty for a long time; at the moment of alighting, the legs and talons become suddenly rigid in extension, defeating as it were their own intention. The move-

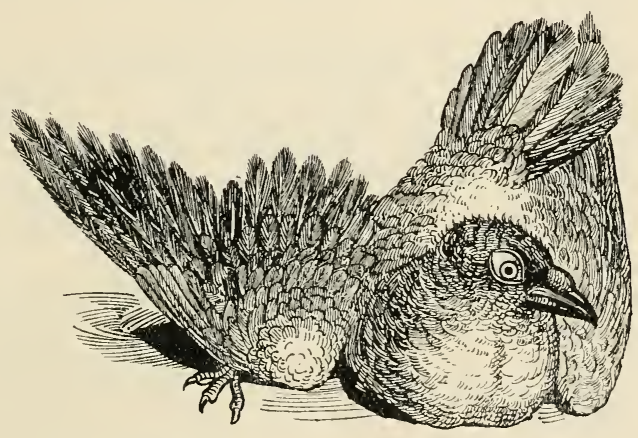

FIG. 367.-Pigeon after removal of the cerebellum (Dalton).

ments are most distraught when the creature is excited and seems at greatest pains to execute them.

Ablation of the cerebellum in lower vertebrates.-In the lizard and tortoise, little disturbance appears to result from ablation of the cerebellum. $^{6}$ Dickinson says that when in the snake the lateral connections of the cerebellum are severed on one side, the animal's posture becomes twisted to a spiral.

In the frog the cerebellum is small, a simple transverse band jutting over the fourth ventricle. Many observers state that its removal or destruction occasions no obvious departure from normality. But Budge ${ }^{7}$ noted that after ablation of one lateral half, e.g. right, the animal sits with the right side lower than the left, with the spine concave to the right, and with the digits of the left hind-foot extended. The animal can be made to lie on its right side, but never on its left. In crawling or springing forwards, there is deviation towards the right. Goltz ${ }^{8}$

\footnotetext{
1 Op. cit. $\quad 2$ Op. cit.

${ }^{3}$ Op. cit. ${ }^{4}$ Lueiani, op. cit.

5 Lange, loc. cit.

6 Desmoutin

7 Loc. cit.

${ }^{8}$ Loc. cit.
} 
further found that after removal of the cerebrum and optic lobes, ablation of the cerebellum rendered the movements of progression, which previously were quite possible, unsteady and well-nigh impossible.

In fish, all observers ${ }^{1}$ seem agreed no obvious disorder of locomotion ensues on destruction of the cerebellum, unless deep and perhaps extraneous connections are injured. Loeb and Steiner have recently confirmed this in Squalius and in the shark, in both of which the cerebellum is large.

Phenomena caused by section of the cerebellar peduncles.Section of the anterior (superior) peduncle.-Section of the anterior peduncle, after it has crossed the median line and is in the tegmentum, causes rolling round the long axis from the side of the lesion toward the sound side (Longet, Lafargue, Lussana, Lemoigne, ${ }^{2}$ etc.), as does injury to the posterior part of the optic thalamus (Flourens, Schiff, Lussana, etc.). ${ }^{3} \quad$ The homonymous limbs are abducted, the crossed adducted (Lussana). ${ }^{4}$ The direction of progression swerves toward the crossed side.

Its section before the decussation, near its exit from the cerebellum, causes disturbances similar to the foregoing, but in the opposite direction (Lussana, etc.). ${ }^{5}$ Ferrier and Turner ${ }^{6}$ noted also tilting of the chin to the crossed side, the occiput being drawn to the homonymous side, and (in monkeys) flexion and adduction of the homonymous limbs, with tremor in them, increased on exertion, and reluctance to use them. Intense perturbation and swaying of the body on the slightest attempt at movement was present at first, but soon passed off. The knee-jerk was brisker on the homonymous side.

Section of the middle peduncle.-Rolling round the long axis of the body ${ }^{7}$ and deviation of the eyeballs ${ }^{8}$ have long been known and studied in connection with this peduncle. Much controversy has taken place as to the direction of the rotation. Magendie described the rotation as occurring from the sound side toward the injured, the deviation as toward the sound side, the homonymous eye turning forward and downward, the crossed backward and upward. The same result was obtained by Schiff, Budge, Lussana, ${ }^{9}$ etc., when following Magendie's mode of operation, also by Ferrier and Turner, ${ }^{10}$ following a different and more exact method of section. Magendie's result is similar to that obtained by lateral ablation of the cerebellum itself. Longet's method, ${ }^{11}$ in other hands as well as in his own (Schiff, Lussana, Lemoigne), ${ }^{12}$ causes rotation from the side of the lesion. Rotation in a similar sense to Longet's is produced by Bernard's glycosuric piqûre, ${ }^{13}$ by evulsion of the facial nerve, ${ }^{14}$ scratching the pons in neurectomy of the trigeminus. It is also seen sometimes as a first and quite transitory effect of Magendie's section, ${ }^{15}$ and is then soon replaced by the reverse and comparatively permanent rotation.

\footnotetext{
${ }^{1}$ Desmoulins, Vulpian, Philipeau (with Vulpian), Ferrier, Bandelot, Steiner, loc. cit. But Dickinson reported "loss of balance."

2 Lafargue, "Inaug. Thèse," Paris, 1838 ; the others, loc. cit.

4 Loc. cit. ${ }_{5}^{5}$ Loc. cit.

7 Pourfour du Petit, "Lettres, etc.," Namur, 1710.

${ }^{8}$ Magendie, loc. cit.

${ }^{11}$ Longet, op. cit. ${ }^{12}$ Loc. cit.

13 "Leçons sur la physiol. du système nerveux," Taris, 1858.

14 Brown-Séquard.

${ }^{15}$ Schiff, loc. cit. ; Budge, "Physiol. des Menschen," Leipzig, 1862, S. 755.

${ }^{3}$ Loc. cit.
${ }^{6}$ Loc. cit.
${ }^{10}$ Loc. cit.
} 
The difference between the effects of Magendie's and of Longet's sections is not one of mere description. Magendie's section implicates especially the posterior part of the pons, and Longet's especially the anterior ; the explanations offered by Schiff ${ }^{1}$ and Longet ${ }^{2}$ do not appear to me satisfactory.

Suppose the right peduncle cut. The animal bends its neck toward the right shoulder, its chin toward the left (as though the left sternocleido-mastoid muscle had been paralysed), the right shoulder is turned toward the breast, the left toward the back, and thus the cephalic half of the animal has turned through an arc of about $90^{\circ}$ on the pelvic half. This is so long as the animal rests quiet; when it tries to move, the torsion of the body is converted into actual revolution about the long axis. The rate of rolling has been described to be as high as fifty turns a minute. It may persist for days, but gradually passes off; it leaves a permanent inability to turn the head to the left (Lussana). ${ }^{3}$

With the rolling and ocular deviation usually coexist incurvation of the trunk to the side of the lesion, some lateral nystagmus, adduction and ataxy and tremor of the homonymous limbs, abduction of the crossed limbs. In the dog and rabbit the limbs are extended, but in the monkey the homonymous limbs are flexed (Ferrier and Turner). ${ }^{4}$

Section of the posterior peduncle.-This experiment has been practised very frequently from the time of Rolando ${ }^{5}$ and Magendie ${ }^{6}$ onwards, and there exists little or no discrepancy in the observations. The phenomena ensuing are incurvation of neck and trunk to the side of the lesion, falling to that side, deviation of the eyeballs from that side; adduction of the homonymous limbs, with ataxy and tremor in them; abduction of the crossed limbs; tendency to roll toward the side of the lesion, and some twisting of the head, so that occiput points to lesion, chin in the direction of the eyes. Ferrier and Turner, ${ }^{7}$ the only observers who have employed monkeys, noted in that animal tonic flexion in the homonymous limbs, tonic extension in the crossed; but this characteristic position was not so persistent as incurvation of trunk, tendency to roll, and tremor on movement.

Bilateral section of the peduncles.-It has been shown by many observers, e.g. Magendie, Lussana, that the rolling movenent ensuing on section of one peduncle can be cut short by a symmetrical section of the opposite peduncle.

Phenomena of cerebellar origin in human disease.-On turning to the symptomatology of cerebellar disease for help toward the understanding of the functions of the organ, we find that the clinical material must be much sifted, to yield secure data for the purpose. No detailed attempt of that kind can have space here. But the following can be said:-A reeling gait, oscillations of the body which may impart a zigzag direction to the walk, difficulty in standing upright owing to unsteadiness of limb, are common phenomena in cerebellar disease. Occlusion of the eyes does not augment the unsteadiness or "reeling," hence there is no "Romberg's sign." The arms are less unsteady than the legs, but the handwriting is shaky. Sometimes there is swaying of the head, and very usually a peculiar "scanning" quality of speech, in which the syllables come haltingly evolved. Giddiness may be

1 "De vi motoria baseos eneephali," etc., Francofurtum a/Main, 1845.

${ }^{2}$ Loc. cit. ${ }^{3}$ Loc. cit. ${ }^{4}$ Loc. cit. ${ }^{5}$ Loc. cit.

${ }^{6}$ Loc. cit., and "Leçons sur la physiol. du système nerveux," Paris, 1841, tome i. p. 261.

7 Loc. cit. 
troublesome; also nystagmus (sometimes lateral, sometimes not). Nystagmus, unsteadiness, and giddiness may be associated, but are often apart, and there seems no necessary cerebellar connection between them. ${ }^{1}$ Tetanoid rigidity with retraction of the head has been noticed. ${ }^{2}$ Illusions of rotatory movement, actual imperative movement of head and eyes to one side, or actual imperative rotation of the body about its own long axis, ${ }^{3}$ have been well recorded symptoms, especially where the lesion has been found localised in a middle peduncle. Where the lateral lobe has been the site of lesion, there have been among the symptoms ${ }^{4}$ paresis of the homonymous arm and of the legs, and in those limbs instability and tremor in volitional effort; conjugate deviation of eyes, and of the head away from the side of the lesion; lateral nystagmus, the jerks being toward the side of the lesion: these signs, co-existing with an intact cutaneous sensibility, and without alteration of psychical condition, compose a series absolutely accordant with Luciani's ${ }^{5}$ descriptions of the symptoms in the dog and monkey. Just as, e.\%., in Luciani's dogs, the legs are set widely apart, as if to enlarge the basis of support, and the steps taken are abrupt and short.

Especially valuable from the present standpoint are observations upon cases of atrophy or agenesis of the cerebellum. Occasionally there come to light congenital defects, amounting to absence of one cerebellar hemisphere. ${ }^{6}$ In such instances the huge lefect has, in more cases than one, occasioned practically no symptoms whatsoever. Movement has been neither uncertain nor tremulous. In one case, the right being the hemisphere lacking, the person had "a habit" of keeping the head turned somewhat to the left; in childhood, it was stated there had been "restlessness" of the head. Of unsteadiness of attitude or of gait, of weakness of the limbs, nystagmus, peculiarity of speech, vertigo, or defective sensation or intelligence, it is certain there was no trace. The knee-jerks were normal and equal right and left. Further, in other instances there has been arrest of growth or atrophy of almost the whole, indeed of the whole, cerebellum. ${ }^{7}$ Not a hundredth part of the cerebellum has remained, and yet ability existed to stand, to walk, to handle and lift objects; in fact, a very fairly normal condition of volitional motility was preserved. Noteworthy in the records of such cases is absence of detection of any trace of impairment of cutaneous sensibility. The remarkably negative character of even extreme cases of atrophy or agenesis is paralleled by the frequency with which cerebellar lesions of even large size but non-irritative type await unsuspected a post-mortem revelation.

Inferences regarding function of the cerebellum drawn from comparative anatomy.--Unlike that of the cerebral hemispheres, the development of the cerebellum does not consistently and directly increase with progress upward in the grades of animal hierarchy. The cerebellum is found developed in extremely various degree in closely allied forms. Many forms higher, on general grounds, have simpler cerebella than some which are much lower. The crawling reptiles possess less cerebellum than the great swimmers, the Teleosteans and Selachians. In birds the cerebellum is relatively enormous, but even in them it consists entirely of the median vermis. In mammals the lateral lobes appear, small in the Marsupials, larger in the Carnivora, largest in the apes and man. The lateral lobes have

${ }^{1}$ Gowers, "Diseases of the Nervous System," London, 1886, vol, ii.

2 Hughlings Jackson, Medical Mirror, London, 1869 . ${ }^{3}$ Magendie, loc. cit.

4 Acland and Ballance, St. Thomas's Hosp. Rep., London, 1896, vol. xxiii.

5 Loe. cit.

${ }^{6}$ Lallement's ease, Bull. Soe, anat. de Paris, 1862 ; Hitzig's case, Arch. de neurol., Paris, 1884 ; Neubürger and Edinger's case, Berl. klin. Wchnschr., 1898, S. 69.

7 Combettes, Bull. Soc. anat. de Paris, 1831 ; Shuttleworth, Brit. Mcd. Journ, London, 1885 , etc. 
been supposed to be developed in connection with the part played by the limbs in locomotion. But their absence in birds and frogs and their remarkably large development in the Cetacea negatives such a view; the experiments above related have moreover shown that the vermis is connected with limb as well as with trunk muscles, and the lateral lobes with the trunk as well as with limb muscles. The deduction has been made, that size of cerebellum is related to degree of locomotivity. But the smallness of the frog's cerebellum is against that. Its size does, however, seem connected with range and variety of movement in some way ; the cerebellum of the albatross is very large among birds. On the whole the suggestions given by mere gross anatomy have been in the case of the cerebellum more than usually unfortunate.

In the development of the organ it is interesting that even the azygoid median lobe makes its appearance as a pair of lateral structures. Further, that the organ is by its ontogeny a portion of the sensory region of the neural tube. Histological completion of its tissue, to judge by date of myelinisation, occurs first in the vermis and in the flocculi, ${ }^{1}$ and is in the lateral lobes very much later; this agrees with the late appearance of the lateral lobes in the phylogenetic history of the organ.

Functions of the cerebellum.-It remains to attempt to form a conception of the functions of the cerebellum. For such an attempt two steps are to be taken : the tracking down the fundamental mischief at the root of the raw "symptoms" catalogued above; the drawing from this mischief inferences regarding the share taken by the cerebellum in the work of the nervous system.

The former problem may best be attempted by discussing an able analysis offered by Luciani. As first pointed out by Flourens, injury of the cerebellum causes inco-ordination of movement-ataxia. Much of the clumsiness of movement of the first (ten) days after cerebellar injury, Luciani ascribes to the tonic spasm existent in certain muscles, or, if not actually existent, liable to appear at the moment of active innervation. This tonic spasm and overaction renders certain groups of musculature unmanageable. This spastic rigidity affects especially the lateral and extensor muscles of the trunk and neck, and the extensor and abductor muscles of the fore-limbs (dog; in monkey the flexors and adductors). ${ }^{2}$ The more permanent inco-ordination evident after the period of rigidities has passed is considered by Luciani to be associated with, and probably due to, three defects of neuro-muscular action, namely, to parasthenia ${ }^{3}$ or defective force, paratonia or defective tone, and to astasia or unsteadiness of contraction. The three defects accompany each other, and affect, after total ablation of the cerebellum, the muscles of the body everywhere (Luciani), but predominantly the muscles of the loins and hind-limbs, and the muscles fixing the head.

Concerning the parasthenia, Luciani points to his direct dynamometrical observations; he found, e.g., the movements of the homonymous limbs weaker than those of the crossed side; his dogs in swimming circled toward the crossed side, although in walking they deviated toward the homonymous side.

Concerning the paratonia, Luciani points to the slackness of the

1 Alexander Bruee, Brain, London, 1895, vol. xviii.

2 Luciani, Ferrier, and Turner, loc. cit.

${ }^{3}$ Luciani has applied the full negative in his nomenclature, but as he does not use the terms in the sense of complete deprivation, I venture here to modify his "asthenia" and "atonia" to "parasthenia" and "paratonia." 
muscles, their flaccid feel, the semiflexed droop of the limbs, which, as the animal stands, sometimes appear to give way under it, especially if the animal's attention be suddenly diverted. An illustration of the paratonia is afforded by the slow slipping over of the eyeballs to the crossed side, after the return jerk of nystagmus has replaced them in the normal "forward" pose. The tonus of the lateral muscles pulling toward the lesion seems insufficient to balance the tonus in the muscles which swing the eyeballs in the opposite direction.

The astasia of muscles, a tremor heightened or only appearing when the musculature is set into action, Luciani attributes to imperfect fusion of the simple contractions, which on the "tetanus" theory compose willed contractions. It affects especially the muscles fixing the head, and occurs in monkeys more markedly than in dogs. This is his explanation of the "intention tremor." Some features of the peculiarity of movement in both the early and later periods are no doubt due to actions intended to correct the defects and disabilities. The animal more or less rapidly learns by the experience of many failures, e.g. falls, deviations in a particular direction, etc., to correct its mistakes as they arise. Among such compensatory acts may be probably the spreading of the limbs to right and left to give to the body a wider basis of support; also, perhaps, the incurvation of the trunk toward the side which is the weaker. The dog that as a result of removal of the right cerebellar hemisphere forges to the left in swimming, learns to swim straight by keeping the lumbo-sacral part of the vertebral column curved appropriately to the right. By such compensatory innervation as this the ataxia is gradually more or less successfully hidden. Luciani found that ablation of the Rolandic area of the cortex of the cerebral hemisphere largely hinders the development of this compensation for cerebellar defect. He urges on various grounds that the cerebellum has an important influence upon the activity of the Rolandic region of the cerebral cortex, the left half of the cerebellum acting on and with the right hemisphere of the cerebrum. The influence he describes to be of a tonic and trophic nature, modifying the excitability of the Rolandic cortex in a way comparable with that in which the afferent spinal root modifies the excitability of the motor nerve cell. Bianchi, as a test of the excitability, is reported to have faradised the sensori-motor cortex some days after ablation of the crossed half of the cerebellum, without obtaining results which to his mind establish clearly a departure from the normal excitability of the cortex. Similar experiments have been made by R. Russell and Luciani, the former concluding that the excitability may be raised, the latter concluding that the excitability is in some points of the Rolandic area exalted, and in others depressed. Luciani finds his "cerebellar influence on the cortex," trophic as it is and thereby affecting excitability, chiefly effective on the Rolandic region.

Regarding the field of distribution of the motor effects, both of spastic and of asthenic type, it is clear that each lateral half of the cerebellum mainly affects its own homonymous half of the body; it is in the eyeballs and hind-limbs that the more marked instances of any bilaterality of its action are seen. It may be, however, that almost the whole musculature is represented in each half of the cerebellum, and Luciani points out that after total ablation of the cerebellum the defect is everywhere greater than after lateral ablation. 
There is no evidence that purely cerebellar lesions impair instinct or intelligence.

Regarding sensory defects, Luciani, one of the earliest to hold that sensory disturbances are caused by destruction of the Rolandic cortex, finds none after ablation of the cerebellum, confirming in this respect the original observations of Rolando and Flourens. Clinical observations, in this respect especially valuable, are accordant with their views. There is, however, clearly one sensory disturbance, namely, vertigo; animals often indicate it by their actions, patients describe it. It may be asked whether the vertigo is not secondary to abnormal movements of the eyes, etc. It is not so, for it occurs in absence of the eyeballs, and is said clinically to oceur sometimes apart from any obvious inco-ordination. To interpret this vertigo, disturbances other than cerebellar occasioning vertigo may be recalled. These include, besides ocular squint, many spatial positions and movements unwonted to the body: the looking from a height, the gliding over ice, sea travel, to some even travel by train or the covering of one eye. Common to all these conditions is the synchronous rise of perceptions of spatial relations between the self and the environment which have not, or have rarely before, arisen in synchronous combination. The tactual organs of the soles and the muscular sense organs of limbs and trunk are originating perceptions that indicate that the self is standing on the solid earth, yet the eyes are at the same time originating perceptions that iudicate that solid earth is far away below the standing self. The combination is hard to harmonise at first; it is at least not given as innately harmonised. The conflicting senses occasion the feeling of giddiness. The cerebellum has paths to it from most, if not from all, afferent nerve roots. But to one of these it stands associated especially closely, so closely that, as its place in the neural axis implies, it may be regarded as having been evolved above that root, namely, the vestibular, the nerve of the semicircular canals, the sense organs furnishing data for the appreciation of positions and movements of the head. Traumatic like other abnormal irritations of this nerve or of the cerebellum originate sensations at variance with those derived from other sources contributing to the space perceptions of the moment. The movement of the body becomes thus imperfectly adjusted to the spatial requirements of the act it would perform. Giddiness also supervenes. Giddiness appears a symptom of only the irritative period of cerebellar lesions; and is less marked in cerebellar than in labyrinthine or eighth nerve lesions. Since with huge cerebellar defect all conscious sensation may remain good, the failure of the sensorial adjustment of the body's movements to outer space-relations in consequence of cerebellar lesion must be ascribed to loss of impulses which regulate body-movements without being on their way into or within the cerebellum elaborated to conscious sensations.

Though some degree of imperfection of the movements persists long or always, the above loss of direct unconscious co-ordination may in large part be compensated, it would seem, by conscious sensations (perceptions). The movements thus compensatorily regulated, although correct, strike the observer as under constant guidance by attention and wanting the natural ease of automatic consummation.

Clinical experience even more than experimental indicates that if 
the lesion occur early before the mechanisms of the nervous system have lost in some degree their pristine elasticity, and if sufficient length of time be then allowed them, injury to the cerebellum, even though large, can be to an extraordinary extent compensated, and its evil overcome. Man with one-half of his cerebellum congenitally gone can pass muster among his fellows as normal both in mind and body. Such adaptive compensation is as striking as the instances that occur in animals after removal of portions of the cerebral hemisphere ${ }^{1}$ or removal of the otic labyrinth. ${ }^{2}$ Luciani's searching investigations have indicated that it is in the crossed hemisphere of the cerebrum itself that this compensatory mechanism must be looked for, at least in major part.

The second step toward interpretation of the cerebellum is already partly prejudiced in the procedure toward the first. The main fact available from the study of the structure of the organ is its extraordinarily rich connection with afferent nerves. The main phenomena displayed by cerebellar excitation and destruction are, on the other hand, not sensory but motor in kind. The action of the cerebellum must lie, therefore, somewhere within this gap.

A notion tacitly admitted above, in dealing with the disturbances ensuing on cerebellar lesion, supposes that ablation is followed by "irritative" signs at first, and then by signs of pure defect. Luciani has dealt with the discrimination between the two. He finds the former accentuated when the lesion involves the peduncles. It must be remembered that a peduncular lesion is the exact kind of injury which breaks the greatest number of paths with the least mechanical disturbance. If I take his meaning rightly, its argument is as follows. The cerebellum is an organ acting in a tonic and trophic way upon the sensori-motor (Rolandic) sphere of the cortex cerebri and on other parts. An immediate accompaniment of a mechanical lesion of the cerebellum is excitement of the organ in the vicinity of the lesion. This affects organs influenced by cerebellar activity, the effect simulating increased cerebellar action. This "irritative" effect of the lesion lasts, maintained by chemical and mechanical changes at the seat of injury, for a considerable time, some days at least. To it succeeds a period in which the excitation due to irritation having subsided, the results of deficient action of the cerebellum lie ummasked. There appear to me objections to this view. (1) The symptoms ascribed to irritation are hardly sufficiently the converse of those of defect, $e . g$., the spastic extension and abduction of limb characteristic of the first stage might on this hypothesis be expected to give way to marked flexion and abduction in the second. (2) The immediate though almost momentarily transient effect of severing a peduncle is the production of an attitude truly the converse of that which very quickly later, on completion of the severance, ensues in permanence. ${ }^{3}$ It would be useful to know what is the movement elicited by electrical excitation of the peduncles. If the attitude be the converse of that produced by ablation, the explanation of the latter cannot well be irritative. Ferrier's excitations of cortex

\footnotetext{
${ }^{1}$ Goltz, "Die Vorrichtungen des Grosshirns," Arch. f. d. ges. Physiol., Bonn, 1876, Bd. xiii., onwards.

${ }^{2}$ Cyon, ibid., 1874, Bd. viii.; Ewald, ibid., 1887, Bd. xli.; 1889, Bd. xliv., and "Physiol. Untersuch. ueber d. Endorgan d. N. octavus," Wiesbaden, 1892.

${ }^{3}$ For instance, see Budge, "Lehrbuch d. speziel. Physiol. d. Menschen," 8te Aufl., Iseipzig, 1862 ; Lussana, loc. cit.
} 
cerebelli produced in the head and eyes a turning to the homonymous side, the reverse direction to that produced by ablation even in the so-called irritative stage. Through the variety of forced attitudes assumed under cerebellar lesions, there is repeated a group of features, suggesting that the cerebellum reacts upon some focus of innervation in which the motor elements of that group are knit together to an entity. The turning of the head, the lateral deviation of the eyeballs, the pleurosthotonus, the extension of elbow, can all be evoked from the extreme posterior end of the optic thalamus, as if from a focus for a co-ordinate movement in which these components existed. It is perhaps on this that a cerebellar influence is exerted. As to the direction of its influence, it seems probable that the clean removal by a skilled experimenter of a portion of cerebellum leads, though its effect at first be compounded of irritation and deficiency, almost at once to a result in which deficiency is dominant. The interpretation of the irritative symptoms would then be, that in the organ from which Luciani's tonic and trophic cerebellar influence was removed, there ensued for a time an overaction, comparable to the profuse paralytic secretion of a submaxillary gland after section of its nerves-comparable, in fact, to all escape from control; such overaction would of itself entail after a time exhaustion. This phenomenon would come under the class of "isolation dystrophies" already spoken of. Luciani, in describing the influence which the cerebellum exerts upon the cerebral cortex, compares it luminously to that exerted by the afferent spinal root upon the motor nerve cells. It would represent his view hardly fully, perhaps, to say that the cerebellum maintains the tone of certain "muscular" regions of the cerebral cortex. From the construction of the cerebellum it is clear that it may act as an adjuvant to the Rolandic cortex in one or all of several ways as follows:-

1. The afferent impulses gathered up from the cord, the trigeminus, and especially the vestibular nerve, may, acting through the cerebellar cortex, and then the corpus dentatum and crossed rubro-thalamic nuclei, finally react on the cerebral cortex, mainly of the side opposite to that whence they started. Such a path of action is implied in the view of cerebellar action sketched by Gowers. Impulses coming from muscles and ligaments, originated by contractions and tensions, impulses from the semicircular canals and from the muscles of the eyes, impressions not affecting consciousness, reach the cerebellar cortex, and thence react mainly on the Rolandic region of the cerebral cortex. Such a view does not deal with the middle peduncle nor with the afferent portion of the posterior. It brings forward clearly, however, a cerebellar factor in the "tonus" of the cerebral cortex.

2. The afferent impulses gathered up from the cord and cranial nerves, especially from the vestibular, may, acting through the vermis, react upon the cerebello-spinal and vestibulo-spinal efferent systems, and through these last contribute a necessary factor to the normal tonus and excitability of the spinal and cranial motor root cells. If this factor in the normal condition of the motor root cells were wanting, they might be expected to reply in various abnormal ways under excitation from the cortex.

3. Each half of the cerebellum receives a great incoming tract from the pontine nuclei across the median line. There is little doubt that these nuclei are themselves end stations for fibres efferent from centres 
further forward, among others from the cerebral cortex. By functional discharge of certain upper and cortical regions, the crossed cerebellar hemisphere may, one can believe, be excited. The cerebellar excitation so evoked can recoil by way of the anterior peduncle on the cerebral hemisphere whence it started. After severing both the anterior (superior) peduncles and the tracts from cerebrum to the pontine nuclei, $\mathbf{I}$ found 1 "decerebrate rigidity" set in, a condition-opisthotonos, extensor rigidity of limbs, retraction of neck, etc.-closely like that ensuing on ablation of the cerebellum. It would seem that interruption of these peduncles approaches in effect to removal of the cerebellum, a fact which lays stress on the importance of the above cerebro-cerebellar circuit as a factor in cerebellar action. On the other hand, the cerebellar activity excited by action of the crossed cerebral hemisphere can, to judge by the structural connections, react on the cranio-spinal motor root nerve cells of its own side, i.e. of the side to which the pyramidal crossing will mainly carry the impulses generated from the cortex cerebri. Thus, one and the same cerebral discharge may perhaps pour upon the motor root nerve cells a combination of cerebral and cerebellar impulses. Destructive lesion of the cerebellum, by rendering the latter impulses impossible, may upset the co-ordination of the movement to be executed.

No fact is clearer in the physiology of the central nervous system than that many movements of great taxic complexity, e.g. of progression, etc., can be carried out in a co-ordinated manner in the absence of the Rolandic cortex and of the cerebral cortex altogether. It is also clear that lesions of the cerebellum greatly disturb these movements; the impression that they disturb them preponderantly is perhaps illusory. There is some evidence that these movements are related to the thalamencephalon, much in the same way as higher movements, such as grasp of hand, are related to the cerebral cortex. With the thalamencephalon the cerebellum has the same broad structural linkage as with the cortex, only the linkage seems structurally closer and more abundant. It is to these ties, rather than to those connecting it with the cerebral cortex, that I would relate the disturbance of attitude and locomotion so prominent among the results of cerebellar ablation.

The so to say highly technical movements ordered by the cerebral cortex employ comparatively limited aggregates of musculature, e.g., speech employing the muscles of the oral and laryngeal regions, grasp employing the muscles of a single limb. The muscular mechanisms of progression and pose, the innervation of which may, as above suggested, be centred together, perhaps in the thalamencephalon, are of wider spatial distribution, and employ not one side of the trunk but both sides, not one limb but all four. Often they probably involve the musculature of the entire body. It is because of its intimacy of connection with such movements as these that the cerebellum is an organ which justifies Hesiod's or $\sigma w$

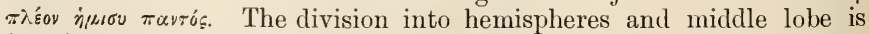
founded merely upon gross anatomy. The organ commonly, probably always, "functions" as a whole. Luciani is right to insist on this, and the reason that it "functions" as a whole seems clearly because it is so largely a piece of mechanism that deals with the innervation, not

${ }^{1}$ Sherrington, Proc. Roy. Soc. London, 1896, vol. 1x. 
of this or that piece of musculature, but of the nusculature of the body as a whole. The earlier observers, Flourens, Bouilland, etc., stated this when they spoke of the cerebellum as an organ of "equilibration."

In Rolando's "saggio" the cerebellum was likened to Volta's then newly invented electric pile, and was argued to augment the power of movements initiated by the cerebrum. Its removal was shown to leave the innervation of certain parts weakened. This view, apart from the crude simile, is to-day accepted in great part; witness Luciani's parasthenia. The rolling about the long axis of the body and other phenomena of similar kind are generally explained by weakened innervation on one side, leading to upset of balance in assumption of attitudes which demand bilaterally symmetrical innervation; the sound side pushes over the weak side. Some ${ }^{1}$ have, however, urged that the phenomenon is rather due to abnormal over-action on the part of the stronger side; there is plenty of analogy for this latter view, although specific proof is wanting. Such analogy is found in the fact that severance of afferent spinal roots and that semisection of the spinal cord heighten the spinal reflexes, long and short, in one-half of the cord. $^{2}$ As to the exact nature of the depression of action on the one side, and of the questionable increase of action on the other, there seem as yet insufficient data to discuss these profitably here.

Flourens analysed differently from Rolando. He had, by reason of Legallois's and his own researches on the bulbar nœud vital, been faced by the existence of real foci in the nervous system, capable of marshalling a complexus of muscles for orderly accomplishment of a single end. Indeed, Legallois and he had discovered what Bell, having guessed at, had actually groped for by dissection, but, neglecting more delicate and potent means, had failed to reach, namely, the focal centre guiding and co-ordinating the movements of respiration. Flourens translated the disturbances ensuing on destruction of the cerebellum to mean loss of a part possessing ability to co-ordinate the innervations which guide and execute complex movements. By his doing so, the idea of nervous co-ordination was, it seems to me, formally introduced into physiology.

Lussana and Carpenter were the earliest to literally express what Flourens' inco-ordination and the vast afferent connections of the organ conspire to suggest, that we have here in the cerebellum a part of the machinery for, in the widest acceptation of that term, the muscular sense. Impressions originating in the sensifacient organs of muscles, tendons, joints, and ligaments, and those arising in the apparatus of the labyrinth, appear to be transmitted directly or indirectly to the cerebellum. Therein is elaborated reflexly an adjustment of the bodily "me" to the existent and constantly varying spatial relations between that "me" and its environment. The connection of the cerebellum with the vestibular nerve, and so with the labyrinth, is as marked as is the connection between each of these structures and the tonus of the muscles of the homonymous half of the body. Destruction of each lowers the muscular tonus; and I would suggest that reflex muscular tonus bears to these structures the same relation as the reflex movement bringing a luminous image into more central vision bears to the eye and nervous visual centres.

${ }^{1}$ E.g., Brown-Séquard, loc. cit.; Vulpian, loc. cit.

${ }^{2}$ See above, p. 865 . 
"Muscular sense" is a complexus. By its processes, out of a number of contributory impressions collected from various channels, are synthesised specific perceptions. As in the physiological basis of other senses, so here a number of impressions have, apart from and devoid of psychical accompaniment, a reflex influence upon motor (muscular) innervation. It is with this reaction that the cerebellum is, it would seem, employed. That it is apparently devoid of psychical accompaniment need not imply that the impressions concerned in it are crude and inelaborate. The seeming want of reaction of so much of the cerebellar structure under artificial stimulation, and the complex relay system revealed in the histology of the cerebellum, suggest that the impressions are elaborate.

But the results of destruction of the cerebellum show plainly that much co-ordination of muscles is possible in absence of the organ. The ataxies of an apæsthetic limb and of the tabetic patient present instructive differences from cerebellar ataxy. In this latter, there is no ignorance of the malpositions; they are acutely recognised and even anticipated. The dog prepares to lean against the wall to obviate the fall that he expects will happen on that side. In the former ataxia the malposition often remains unnoticed until attention is directed to it by its falling within the central region of vision. The aprsthete and the tabetic are without sensations which the decerebellate possesses, and to the former deprivation of vision, a sense that contributes, not improbably as importantly as the labyrinthine, to the co-ordination of innervation requisite for equilibrium, means a severer loss. Hence Romberg's symptom-staggering on closure of the eyes-occurs with the former but not with the decerebellate condition. The amount of recovery of execution of movement possible to animals deprived of cerebellum is a measure of the potency of the supplementary means for guidance of muscular innervation; many of these may be conscious. In decerebellate animals a phenomenon regarding which observers are unanimous is the profound lassitude which forthwith succeeds execution of movements ordinarily quite unfatiguing. This is probably in large part a mental fatigue, due to strained endeavour to continually obviate, or correct, constantly recurring distortions of movements, keenly perceived at their genesis to be imperfect.

To sum up: the cerebellum is the organ rather of a particular class of reactions than of a particular sense. Its reactions have their sources in the sensifacient organs of various senses, but especially of those pregnant with spatial quality, and in those subserving the "muscular sense," in the widest meaning of that term. It supports the tonus of the majority, perhaps of all, the cranio-spinal motor root cells, of some more than others, e.g., those connected with eye muscles, neck muscles, muscles of the spine. It preponderantly helps to secure co-ordinate innervation of the skeletal musculature, both for maintenance of attitude and for execution of movements. So far as the geotropism and stereotropism of the animal can be "centred" at any one limited field of the central nervous system, that field is cerebellar. It supports habitual posture, and is at least as importantly associated with the movements dependent on the lower cerebral centres (e.g. walking, running), as with those elaborated in connection with the highest (e.g. technical movements). 


\section{Mesencephalon or Region of the Corpora Quadrigemina.}

An afferent root cell system has not been identified in this region, though there are reasons for thinking it exists in connection with sensory organs in the ocular muscles. ${ }^{1}$

The efferent root cell system comprises the motor cells innervating all the eye muscles, except the lateral rectus and the dilatator pupillæ. Some of the cells of the oculo-motorius are crossed, ${ }^{2}$ that is, contribute the nerve fibre process to the nerve trunk of the crossed side; but to which of the eye muscles the crossed fibres pass is not yet ascertained. The fibres of the trochlearis decussate in their peripheral course on the roof, the mesencephalon. Whether the decussation is total is not yet ascertained. The smaller root cells of the oculomotorius send their nerve fibres to the ciliary ganglion, whence, as from a sympathetic ganglion, fresh nerve cells emit fibres (but in this case, unlike the sympathetic elsewhere, myelinated) to the ciliary muscle and constrictor pupillæ. Among the units of the motor root cell system are perhaps to be reckoned those that supply the upper facial muscles, frontalis, corrugator, and orbicularis palpebrarum $;^{3}$ the nerve fibres from these root cells do not, however, emerge from the central nervous system in the mesencephalon, but pass down to join those which innervate other facial muscles, and leave the metencephalon in the facial nerve.

The distinction between roof and floor in the mesencephalic region, though clear enough, does not so lend itself to experimental opportunity as in the parts of the brain previously dealt with. The nervous masses of the roof form in mammals the corpora quadrigemina, the hinder pair of which are the enlarged equivalents of two comparatively small nuclei, occupying only a small hinder edge of the lobi optici of fish and Sauropsida. The relatively huge size of the front pair in reptiles and birds harmonises with the preponderant rôle played by vision in those animals.

Excitation of the mesencephalic roof succeeds under stimuli of all kinds, electrical, mechanical, thermal, and chemical; electrical stimuli are most efficient. In fish, ${ }^{4}$ the tail is drawn toward the side excited, and the dorsal and anal fin are spread and inclined towards that side; the pectoral fin is spread, and there is movement of the eyeball of the same side. In the frog the head is thrown to the opposite side and upward, and croaking may be elicited. The heart is usually inhibited, but not strongly. ${ }^{5}$ If telencephalon and diencephalon have been previously ablated, weak and prolonged excitation of the mesencephalic roof by sodium chloride increases (Setschenow) ${ }^{6}$ greatly the latency of move-

${ }^{1}$ Sherrington, “Proe. Phys. Soc.," June 1894, Joum. Physiol., Cambridge and London, 1894, vol. xvi.; Proc. Roy. Soc. London, 1897, vol. lxi. 1. ‘47; 1898, vol. Ixiii.

${ }^{2}$ Perlia, Arch. f. Ophth., Leipzig, Bd. xxxv. ; Spitzka, Trans. Phila. Neurol. Soc., 1885 ; Edinger, "Vorlesungen ueber d. nerv. Centralorg.," 1889 ; Kölliker, Sitzungsb., d. phys.-med. Gesellsch. zu W Würzburg, 1892 ; van Gehuchten, Cellule, Lierre et Louvain, 1892, tome viii.; Cramer, "Beitr. z. fein. Anat. d. Briicke," Jena, 1894; van Gehuchten, Rev. neurol., Paris, 1898, tome vi.

3 Mendel, Neurol. Centralbl., Leipzig, 1887, S. 537 ; H. Tooth and W. A. Turner, Brain, London, 1891, vol. xiv. p. 490; Oppenheim, Arch. f. Psychiat., Berlin, 1892, Bd. xxiv. S. 758 ; Muratoff, Neurol. Centralbl., Leipzig, 1892, Bd. x. S. 514 ; W. A.

Turner and Bulloch, Brain, London, 1894, vol. xvii.

4 Ferrier, "Functions of the Brain," London, 1876.

5 Wilson, Journ. Physiol., Cambridge and London, 1890, vol. xi.

6 "Physiologische Studien," Berlin, 1863. 
ments evoked by stimuli to the skin. The latency may be increased eight-fold, or the reaction may even be suppressed altogether. This "inhibition" holds good for "pathic" stimuli, but not for pure "tactile." 1 In connection with this inhibition of spinal reflexes and the close ties between the mesencephalic roof and the retinæ, it is interesting that in the blinded frog the croak reflex can be elicited with machine-like regularity. ${ }^{2}$

In birds, ${ }^{3}$ excitation of the optic lobes evokes contralateral pupillar dilatation, spreading, raising, and flapping of the contralateral wing, and movement of both legs. Faradic stimulation of an anterior corpus quadrigeminum in the rabbit and in carnivora ${ }^{4}$ produces dilatation of the contralateral, and later of the homonymous pupil, conjugate deviation of the eyes upward and to the opposite side, retraction of the ears and angles of the mouth. To these effects are added pricking of the crossed ear, and vocalisation if the electrodes are applied to the posterior instead of the anterior corpus. In the $\log ^{5}{ }^{5}$ but not in the rabbit, ${ }^{6}$ it has been possible to elicit different co-ordinated eye movements from different points of the anterior corpora; from the sulcus between them, return of the divergent bulbs to parallelism without any change in the pupils; from a posterior point, elevation of the eyes with dilatation of both pupils; further back still, convergence with contraction of both pupils. After sagittal section in the median plane, the excitation affects only the homonymous eyeball. In all the conjugate reactions, the contraction of one muscle is accompanied by relaxation of the tonus of its antagonist. But the eyeball movements elicited are not always conjugate; and sometimes one only is elicitable from the whole of both the anterior corpora. ${ }^{7}$ In the monkey, Ferrier ${ }^{8}$ discovered reactions similar to those of the dog, with the addition of wide opening of the palpebral fissures, elevation of the eyebrows, and turning of the whole head in the direction of the gaze, i.e. away from the side of excitation. Excitation of the posterior corpora in the monkey produces vocalisation, the sounds ranging among many varieties of cries.

Ablation. - The frog, after removal of all parts in front of the mesencephalon, but with the latter intact, continues to exhibit occasional movements that are "spontaneous." 9 It swims and springs co-ordinately, but it no longer reacts by climbing to the tilted board. The condition of the animal resembles torpor; let down gradually into water, it floats without stirring, although it still can swim. Probably vision and smell are gone. Croaking is easily elicited. On stroking the flanks, not only is croaking elicited, but the animal gradually rises on its four limbs with curved back and muscles in tonic contraction. It remains so for many minutes after the stimulus to the flank skin has been discontinued, even for half an hour..$^{10}$ Destruction of the mesencephalon renders the croak reflex difficult to elicit, and altogether abolishes the tonic reflexes, though the latter are still possible in the

1 Setschenow and Paschutin, "Neue Versuche," Berlin, 1865; and Setschenow, Mem. Acad. imp. d. sc. dc St. Pétersbourg, tome xx. p. 357 ; and "Ueber d. elekt. u. chemisch. Reizung. d. sensibl. Ruickenmarksnerven," Berlin, 1868.

${ }^{2}$ Langendorff, Arch.f. Physiol., Leipzig, 1877.

3 Ferrier, "Functions of the Brain," London, 1876.

${ }^{5}$ Adamik, Arch.f. Ophth., Leipzig, 1872, Bd. xviii. S. 153.

${ }^{6}$ Knoll, Sitzungsb. d. k. Akad. d. Wissensch., Wien, July 1886, S. 239.

7 Knoll, ibid.

$8 \mathrm{Op}$. cit.

9 Volkmann, art. "Gehirn," Wagner's "Handwörterbuch," Braunschweig, 1844, S. 582.

10 Verworn, Arch. f. d. gcs. Physiol., Bonn, 1897, Bd. lxv. S. 63. 
fore-limbs after destruction of the front part alone of the mesencephalon. After transection through the posterior part of the region, pressure on the foot often excites a crawling backward; with the lobes intact it always excites a spring forward. Circus movements and forced asymmetrical postures frequently attend partial lesions. After transection behind the mesencephalon, the cutaneous reflex time to Türck's stimulus (acid water) is still abnormally long, though shorter than when the lobes are stimulated, or the transection lies in front of mesencephalon. ${ }^{1}$

Removal of the mesencephalic roof in Testudo palustris induces extraordinary activity. ${ }^{2}$ The creature does not stumble against objects, and evidently retains some visual power. The retraction of the head on the shell being tapped, a normal reaction, drops out. When put into a tank, instead of diving at once and hiding, the animal swims continually at the surface. The ablation reduces the latent time for movement of leg in response to stimulus of head from 08 sec. to 05 sec., and the liminal stimulus is lowered.

In birds, destruction of one optic lobe impairs, ${ }^{3}$ but does not completely destroy, the visual power of the contralateral eye. ${ }^{4}$ Destruction of an anterior corpus quadrigeminum abolishes ${ }^{5}$ reflex contraction of the pupil to light, "Whytt's reflex." ${ }^{6}$ It is removal of the surface layer of the lobes that seems to impair visual sensation, while it is the deeper lesions which seem to most easily produce forced movements. Transection, close in front of mesencephalon, leaves vocalisation fairly intact. ${ }^{7}$ I found it still obtainable in the monkey, even after transection close behind the anterior corpora; transection behind the posterior bodies seems to abolish it. The cry is a prolonged one, and in all the reflexes obtained from the monkey (cat, dog, etc.), after transection at the front limit of mesencephalon, there is a peculiar feature in regard to their time character. ${ }^{8}$ The reflex movements produced are executed with a curious deliberate slowness, and the attitudes assumed are long maintained. It has been concluded by Ziehen ${ }^{9}$ that the tonic component of the epileptic discharge into muscles is not cortical but subcortical. The "cataleptoid reflexes," elicitable after transection at the mesencephalon, recall to mind his conclusions. The state of constrained immobility, so-called "hypnotic," into which many animals, e.g. frog, hen, can by skilled handing be brought, has been shown by Verworn ${ }^{10}$ to be accentuated in decerebrate individuals.

And it is after complete transection here that the condition called deeerebrate rigidity ${ }^{11}$ supervenes. In this state the respiratory

1 Setschenow, op. cit.

2 Fano, "Contributo speriment. all. psicho fisiol. d. lobi ottici nel testuda palnstre," Reggio Emilia, 1886.

${ }^{3}$ Stefani, First Internat. Cong. Physiol., Basle, 1889.

+ Münzer and Wiener, Monatschr. f. Psychiat. u. Neurol., Berlin, 1898, Bd. iii. S. 402 ; Flourens, Renzi, etc., considered the blindness total.

${ }^{5}$ H. Mayo, "Auat. and Physiological Commentaries," loc. cit., London, 1823 ; Flourens, "Expér. sur le système nerveux," Paris, 1825 ; Budge, "Ueber d. Beweg. d. Iris," Braunschweig, 1855 , S. 64.

"Robert Whytt, "Works," Edinburgh, 1768.

7 Onodi, Berl. klin. Wehnschr., 1894, S. 48.

8 Sherrington, Proc. Roy. Soc. London, 1896, vol. lx.

9 Arch. $f$. Psychiat., Berlin, 1887, Bd. xviii.

10 "Beitr. z. Phys. d. Centralnervensystem," Jena, 1898; and Heubel, Arch. f. d. ges. Physiol., Bonn, 1877, Bd. xiv.; Danilewsky, ibid., 1881, Bd. xxiv.

11 Sherrington, Proc. Roy. Soc. London, 1896, vol. lx. ; Löwenthal and Horsley, ibui., 1897, vol. lxi.; Sherrington, Journ. Physiol., Cambridge aud London, 1897, vol. xxii.; Phil. Trans., London, 1897.

VOL. II. $-5^{8}$ 
movements proceed regularly, and the pulse remains quiet and full, but certain groups of muscles become rigid. The elbows and knees are stiffly extended, and do not allow of the usually easily made passive flexion. The tail is stiff and straight instead of pliable and drooping; the neck and head retracted. The condition seems more accentuated in the fore-limb than in the hind. Wrist and ankle are much less affected, the digits not at all. Passive movement of the limb hastens the development of this prolonged spasm and re-develops it when it has subsided. The spasm seems dependent on an action-perhaps a tonic action - of the afferent spinal roots of the region implicated by the spasm. Section of the afferent roots of the arm, prior or subsequent to the transection of the mesencephalon, prevents or abolishes the rigidity in it. The influence thus based upon the afferent roots seems to descend from a region above the lowest third of the bulb, for unilateral transection at that level abolishes the rigidity or prevents it, and on the same side as the section. The inhibition influence from the optic lobes of the frog has been noted to be crossed. ${ }^{1}$

In this condition of decerebrate rigidity reflexes can be elicited which actuate the neck, trunk, and the four limbs as a whole, and treat them as components of a single motile apparatus. There must, therefore, in the mammal exist behind the diencephalon a nervous mechanism, which can co-ordinate the action of the great regions of skeletal musculature (including the tail), and the character of the combined movements resulting leaves little room for doubt that the combination is subservient of quadrupedal progression. The combined movement treats the pair of fore-limbs in such a manner that the one is flexed and protracted, the other is extended and retracted at the same time, and similarly when the one limb is flexed at hip and knee, its fellow is extended at those joints. Further, there is a diagonal relation between the limbs in the combined movement of such a kind that a fore-limb and the diagonal hind-limb are flexed together or extended together. It results, therefore, that there are two possible postures that the reflex can produce as regards the limbs-(1) Left fore-limb and right hind-limb flexed, with right fore-limb and left hind-limb extended; (2) left fore-limb, left hind-limb extended, with right fore-limb and left hindlimb flexed. Which of these is produced is determined by the seat of stimulus. The stimulus regularly provokes flexion in the limb stimulated as a starting-point, and affects the other three limbs in the way above stated. These reflexes are frequently "alternating" in character, so that successive flexions and extensions of the limbs follow in sequence, extension and retraction succeeding to flexion and protraction; and in this alternation the diagonal symmetry characteristic of the primary movement is maintained through the succession. The rigidity ensues after a semisection of the region, and is most marked on the side of section. It can then be inhibited in various parts of the musculature, by stimulation of appropriate parts of the Rolandic area of the crossed hemisphere.

A point of importance attaching to decerebrate rigidity is the field it offers for examination of the play of inhibition, and this is exemplified in the execution of the combined limb reflexes just mentioned. Thus, to give a single example, if the skin of the left hand be the locus of excitation, there ensues inhibition of the left triceps brachii, with con-

${ }^{1}$ Langendorff, Arch. f. Physiol., Leipzig, 1877. 
traction of the flexors of the elbow, and inhibition of the right extensor cruris, with contraction of the hamstring muscles; while, on the contrary, there ensues inhibition of the flexors of the right elbow with contraction of the triceps brachii, together with inhibition of the right hamstring muscles, together with contraction of the right extensor cruris. In this reflex there is also bending of the neck and turning of the head toward the left side, somewhat as if to meet the protracted and raised left arm.

Besides reflexes obviously associated with an "act," as the above are with quadrupedal progression, others can be elicited when the mesencephalon is the highest part of the encephalon remaining, some of them less easy of interpretation. The following may be mentioned as fairly significant. Irritation of the pinna in the dog and cat evokes with the progression reflex a turning of the head and neck away from (not as with fore-foot stimuli toward) the side stimulated. This movement frequently culminates in the shaking of the head from side to side as though to shake the ears. ${ }^{1}$ Mechanical stimuli to the nostrils provokes a fit of sneezing, but not, as far as I have seen, of coughing.

Tracts of conduction.-Paths that ascend in the mesencephalon and partly end in it, lie in the fillet, and these come partly from the ventro-lateral column of the spinal cord, partly from the end-nuclei of the auditory nerve. A large path from the cerebellar hemisphere crosses the midsagittal plane to enter and in part end in the red nucleus of the tegmental region. Severance of the tegmental region and fillet on one side of the midline produces complete anæsthesia and analgesia of the skin of the contralateral half of the body, the analgesia, at first total in degree, gradually subsides to partial. The posterior longitudinal bundles running up and down the region seem mainly commissural and associational between the ocular nuclei.

The main descending paths of the region are the mesencephalo-spinal, a crossed path traceable from the anterior and posterior corpora quadrigemina into the ventral part of the opposite ventro-lateral spinal column $;^{2}$ I would interpret this as a projection system belonging to vision. Then there are in the mammal, the cerebral descending paths, fronto-pontine, temporo-pontine, and pyramidal. These in their descent are seen to give fibres to the substantia nigra just dorsal to them, and from the pyramidal pass fibres, probably indirectly, to the motor root cell system, represented here by the oculo-motorius and trochlearis nuclei; some fibres pass to the crossed and some to the liomonymous nerves. Cajal ${ }^{3}$ showed that efferent fibres pass out of this region into the retina by way of the optic tracts and nerves, and these have been traced by the degeneration method from the optic lobe of the bird into the crossed optic nerve. ${ }^{4}$ Conversely from the ganglion cell layer of the retina of the bird passes a path into the "optic nuclei," dorsal and ventral, of the surface layer of the crossed optic lobe. ${ }^{5}$ In some birds (e.g. owl) the connection is probably partly uncrossed; in mammals it seems to be always partly uncrossed, ${ }^{6}$ and the ratio of the size of the uncrossed to the crossed portions is particularly large in

1 Christiani notes this reflex, "Zur Physiologie d. Gehirns," Berlin, 1885.

${ }^{2}$ H. Held, Neurol. Centralbl., Leipzig, 1890, Bd. ix.; Arch.f. Anat. u. Entwoklngsgesch., Leipzig, 1893 ; R. Boyce, Phil. Trans., London, 1895 (communicated 1894) ; Ferrier and W. A. Turner, ibid., 1894; Münzer and Wiener (in bird), Monatschr. f. Pyschiat. u. Neurol., Berlin, 1898, Bd. iii.; Boyce and Warrington, Proc. Roy. Soc. London, 1898, vol. lxiv.

3 Anat. Anz., Jena, 1889 ; Cellule, Lierre et Louvain, 1893, tome ix.

4 Jelgersma, Neurol. Centralbl., Leipzig, 1895, S. 290; Wallenberg, ibid., 1898 ; Boyce and Warrington, Proc. Roy. Soc. London, 1898, vol. lxiv.; and Fourth Internat. Cong. Physiol., Canıbridge, Sept. 1898.

${ }_{5}$ Münzer and Wiener, Monatschr. f. Pyschiat. u. Neurol., Berlin, 1898, Bd. iii. S. 379.

${ }^{6}$ Monakow, Arch.f. Psychiat., Berlin, 1892, Bd. xxiv. 
animals in which the visual axes are parallel, e.g. man. Paths of which the functions are less well indicated than the above run to the homonymous and contralateral corpora striata, to the homonymous half of the cerebellum, to the motor nuclei of the bulb, chiefly of the crossed side. ${ }^{1}$ The mesencephalon also receives a path from the corpus striatum, ${ }^{2}$ but in the bird none from the pallium (cortex cerebri). ${ }^{3}$

\section{The Diencephalon or Region of the Thalamus Opticus.}

This region includes as its main divisions, studied in regard to function, the optic thalamus, subthalamic body, and lateral geniculate body. The two latter bodies may be considered grey nuclei somewhat more definitely separable than, but otherwise comparable with, the remains of some twenty (Nissl, v. Monakow) grey nuclei composing the thalamus. Of the first and last named only, has more definite knowledge been acquired. The region relatively to its bulk sends but very few fibres in a caudal direction; on the other hand, it is an abundant source of fibres passing into the telencephalon (both corpus striatum and cortex). It is connected with the Rolandic region of the cortex by a system of fibres that, among cortical fibres, become myelinated somewhat early (ninth foetal month), and by later developing systems with-(1) The gyrus hippocampi, cingulum, and gyrus fornicatus (first post-natal month), and (2) with the frontal convolutions (fourth post-natal month). It is also connected with the cortex of the insular region, and sends an especially numerous fibre system to the occipital and temporal cortex, including pre-eminently that of the calcarine and angular gyri. The researches of Dejerine ${ }^{4}$ prove that there is practically no region of the cortex of the hemisphere which does not receive nerve fibres from the optic thalamus, and that, on the other hand, there are no fibres from the thalamus to the pes pedunculi cerebri.

Panizza ${ }^{5}$ and Jos. Swan ${ }^{6}$ almost simultaneously discovered that destruction of an eye is followed by shrinkage of the contralateral optic thalamus. In the lower vertebrata the corpora bigemina are the chief regions of reception of the fibres of the optic "nerve." In the higher mammalia diencephalic nuclei of ending of the optic nerves assume larger and larger proportions as compared with mesencephalic. ${ }^{7}$ In the rabbit the main nucleus is still the anterior corpus quadrigeminum; in man the lateral geniculate body preponderates. Only in primates is the pulvinar large; in other mammalia it is small. All goes to indicate the growing importance of the cortical mechanism concerned with vision in the evolution of the sense. Of the four masses of grey matter into which in mammals the thalamus is broadly and easily divisible, it is the hindmost (pulvinar) that is directly in receipt of fibres from the optic

${ }^{1}$ Miinzer and Wiener, Monatschr. f. Psychiat. $u$. Neurol., Berlin, 1898, Bd. iii.

2 Edinger, "Vorlesungen neber d. Bau d. nerv. Centralorg.," 1896, 5te Aufl. ; Münzer and Wiener, loc. cit.

${ }^{3}$ Edinger, Mümzer and Wiener, Boyce and Warrington.

${ }^{4}$ Compit. rend. Soc. de biol., Paris, 1893, p. 193; ibid., Feb. 1897 ; also 1898 with E. Long ; also Dejerine's "Anat. d. eentres nerveux," Paris, 1894.

${ }^{5}$ Mem. r. 1st. Lomb. di se. e lett., Milano, 1856, tomo v. p. 375 ; "Observations sul nervo ottico," April 19, 1855 (bird and man). Panizza also drew attention in this same memoir to the fact that an atrophy of the oceipital region of the cortex, and in less degree of part of the parietal, ensues in man on loss of an eye in ehildhood.

6 "On the Origin of the Optic Nerve," London, 1856. The observation is on the horse, pl. viii. fig. 5 .

${ }^{7}$ Gudden, Ganser, Monakow, Edinger, etc. 
"nerve"; and from pulvinar pass fibres to the occipital and to the angular regions of the cortex. ${ }^{1}$ The lateral geniculate body also sends fibres to the occipital cortex, including the borders of the calcarine fissure. ${ }^{2}$

In the lateral grey mass of the thalamus end many of the fibres of the fillet; they enter especially the ventral and posterior part of the lateral mass, and lie around the median centre of Luys. Their region there is known as the "field of the fillet." And it is from the lateral grey mass of the thalamus that corticipetal fibres pass to the parietal and falciform (mesial) regions of the cortex. From the front end of the thalamus pass fibres to the frontal region of the cortex. A large and, both phylogenetically and ontogenetically, early developed system of fibres connects the thalamus with the corpus striatum. This in mammals connects especially with the head of the nucleus caudatus; but the thalamus is also connected with the nucleus lenticularis (ansa lenticularis). It also receives fibres from the superior cerebellar peduncle.

Excitation of the thalamus.-Excitation of the thalamus by weak faradisation produces little obvious result. ${ }^{3}$ Ferrier ${ }^{4}$ says: "I have not observed any outward manifestation on irritation with a current sufficient to excite active contraction when applied to the corpus striatum "; except that, in a monkey, when the electrodes were applied to the mesial surface near the soft commissure, spasmodic extension of the legs resulted, and in a rabbit movements of the eyes ${ }^{5}$ and twitching of the opposite ear. These phenomena might, he adds, "be regarded as indications of irritation of sensory centres."

Destructive lesions and ablations of the thalamus.-Observers, from Rolando, ${ }^{6}$ Panizza, ${ }^{7}$ and Renzi ${ }^{8}$ onwards, ${ }^{9}$ have, after experimental lesion of owe thalamus, noted visual defect and dilatation of the contralateral pupil, the latter a merely temporary symptom. The observation by Hughlings Jackson ${ }^{10}$ in 1875 of a case in which, post-mortem, a softening of the right optic thalamus was discovered, confined to the posterior part of the thalamus, and uncomplicated by destruction elsewhere, was of fundamental assistance in indicating the functions of the thalamus. The symptoms observed were diminution of tactual sensibility on the left side of the body, impairment of common sensitivity in the left nostril, slight impairment of taste on the left side of the tongue, and of hearing in the left ear, and loss of the left half of the field of vision of each eye, i.e. blindness of the right half of each retina. Experimental work on the subject thoroughly substantiates the teaching of this case. ${ }^{11}$

The defect of vision for the contralateral retina seems, at first at

1 v. Monakow, "Gehirnpathologie," Wien, 1897 ; and Arch. f. Psychiat., Berlin, 1888-92, Bde. xx. xxii. xxiv.; Vialet, "Centres eérébraux de vision," Paris, 1893; Ferrier and Turner, Phil. Trans., London, 1897.

2 See especially Ferrier and Turner, ibid.; v. Monakow, op. cit.; Henschen, "Klin. Beiträge," Upsala, 1892.

3 See for older literature in Eckhard, Hermann's "Handbuch," 1880, Bd. ii. Abth. 1.

4 "Functions of the Brain," London, 1876, 1. 161.

5 Topolanski, Arch. f. Ophth., 1898.

6 "Saggio sopra il cervello," Sassari, 1809.

8 "Sagrio di fisiologia" Ann univ, di med e chir. Milano, 1860, ${ }^{7}$ Loc. cit.

${ }^{9}$ Lussana, "Monografia d. Vertegine," Milano, 1872.

${ }^{10}$ London Hosp. Rep., 1875, vol. viii.

11 Ferrier ("Functions of the Brail," 1876), Lemoine (Thèse de Paris, 1880). Ferrier and Ferrier and Turner (Phil. Trans., London, loc. cit.), in the monkey, Lo Ionaeo (Arch. ital. de biol., Turin, 1898, tomo xxx.), and Sellier and Vergier (Arch. de physiol. norm. et path., Paris, Sér. 5, tome x. p. 706) in the dog, arrive at conclusions that, although somewhat varying in detail, in the main harmonise witl eacl other and the above. (See also Fournie, "Recherches expérimentales," Paris, 1873). 
any rate, more than a hemianopia, ${ }^{1}$ i.e. a hemianopia plus amblyopia of the whole field. The hemianopia does not involve the central region of the visual field of either eye. Severance of corpus callosum leads to no permanent objective impairment ${ }^{2}$ Ferrier and Turner and Lo Monaco, making use of this fact, have proceeded to open the lateral ventricle subsequent to partial section of corpus callosum, and with the thalamus well exposed have destroyed the posterior part of it. There resulted total blindness of the contralateral eye, impairment of tactual and dolorous sensitivity in the crossed half of the body, and slight defect of force in movements executed by the contralateral limbs. In Hughlings Jackson's case, muscular weakness on the crossed side was noted. Symptoms both in the $\operatorname{dog}^{3}$ and monkey ${ }^{4}$ abate in the course of some weeks; the total crossed blindness becoming amblyopia. The hemianopia and defect of power of skin localisation appears permanent. The older observers ${ }^{5}$ on experimental thalamic lesion obtained very regularly great disturbance of motility, such as forced movements of various kind and degree. It was in experimenting on the thalamus that Magendie discovered the forced movement which he styled mouvement de manége (circus movement). Recent ${ }^{6}$ observers have not confirmed "forced movement" as a necessary part of the symptomatology of thalamic lesion. Neither is paralysis; the utmost impairment of the motor power amounting only to a slight hemiparesis. Many observers ${ }^{7}$ have noted signs of disturbance of the muscular sense.

The effects of removing the cerebral hemispheres, either along with or without simultaneous removal of the diencephalon, have already been described (see article, "Cerebral Cortex"). In both cases there is loss of intelligence, in higher mammals imbecility. There is a great difference between the animal without telencephalon but with diencephalon, and the animal deprived of both telencephalon and diencephalon, especially in regard to the facility and completeness of performing complex muscular movements. The most plausible explanation seems that which supposes, that with diencephalon remaining there is still possible a conjoint influence of visual impressions with impressions derived from other senses (tactual, muscular, and labyrinthine), which makes itself felt upon the machinery of the skeletal musculature: when the diencephalon is lost, the contribution from the retina largely disappears.

Experimental lesions of the thalamus cause distinct rise of body temperature, apparently without vasomotor disturbance. ${ }^{8}$

One territory of the thalamus (corp. genic. lat.) functions as a station between relay cells from the nerve cells (retinal) of vision and nerve cells conducting through the "optic radiations" to the visual field of the

\footnotetext{
${ }^{1}$ Ferrier and Turner, Lo Monaco.

${ }^{2}$ Koranyi, Arch. f. d. ges. Physiol., Bonn, 1890, Bd. xlvii. S. 35; Muratoff, Neurol. Centralbl., Leipzig, 1893, Bd. xii. S. 714 ; Lo Monaco (Luciani's Laborat.), Riv. di patol. nerv., Firenze, A pril 1897.

${ }^{3}$ Lo Monaco, Arch. ital. de biol., Turin, 1898, tomo xxx. p. 198.

4 Ferrier and Turner, Phil. Trans., London, 1897, vol. clxxxix.

5 Magendie, Longet, Schiff, Renzi, Lussana and Lemoigne.

${ }^{6}$ Nothnagel, Virchow's Archiv, Bd. Ixii. S. 201; Ferrier, "Funetions of the Brain," loc. cit.; Ferrier and W. A. Turner, Phil. Trans., London, loc. cit.; Lo Monaco, loc. cit.; Sellier and Verger, loc. cit.

7 Meynert especially insisted on this. See also Ferrier, "Functions of the Brain," 1876, p. 248.

${ }^{8}$ Halc White, Lanct, London, 1894, vol. ii. ; Tangl, Arch. f. d. ges. Physiol., Bonn, 1895, Bd. lxi. S. 559.
} 
cortex. Another of its territories (ventral nucleus) similarly mediates between relay cells from nerve cells (spinal) of muscular and cutaneous sense and nerve cells conducting to the somæsthetic field of the cortex. In both these cases the thalamic nucleus projects upon the cortex a sense field in which the body is largely bilaterally represented. The other territories and nuclei of the thalamus are believed similarly to project other sensorial fields upon the cortex. The thalamus is therefore a high intermediary centre between the sense organs and the cortex. There is evidence of a particular dependence of emotional and mimetic expression upon action of the thalamus. In some cases regions of the face are paralysed for emotional but not for willed movements; in other cases the converse occurs. This has been found related to presence or absence of concomitant thalamic lesions. ${ }^{1}$

${ }^{1}$ Nothnagel, later Rosenbach, Neurol. Centralbl., Leipzig, 1886, Bd. v. S. 241 ; Stromeyer, "Topische Diagnostik," 1897, S. 251 ; Barlow, Brit. Med. Journ., London, 1877, vol. ii. ; S. Kiritzew, Neurol. Centralbl., Leipzig, 1891, Bd. x.; Bechterew, Mislawski, and others. 


\section{CUTANEOUS SENSATIONS.}

\section{By C. S. Sherrington.}

Contents :-The Sense of Touch, p. 921-Touch-Spots, p. 921-The Adequate Stimulus, p. 924-Weber's Law in respect to Tactual Sensations, p. 928-The Spatial Quality of Touch, p. 934-Theory of "Localising" Power of Touch, p. 938-Projection of Touch, p. 941-Illusions in Tactual Judgments, p. 943Reaction Time for Touch, p. 945-The Sense. of Temperature, p. 945"Cold" and "Warmth" as Distinct Species of Sense, p. 945-Distribution of Apparatus for Thermal Sensations in the Skin, etc., p. 946-The Adequate Stimulus, p. 950-Other Stimuli, p. 954-Paradoxical Sensation of Cold, p. 954-Some Characters of "Cold" and "Warmth" Sensations, p. 955-Relation of Thermal Sensation to Pain, p. 955-Influence of Areal Extent of Stimulus upon Intensity, p. 956-The Pliysiological "Zero-temperature" and "Adaptation," p. 957-The Degree of Projection of Thermal Sensation, p. 958 - The Thermal Sense in relation to Thermotaxis, p. 959-The Liminal Difference of Intensity of Stimulus, p. 959--Reaction Time, p. 962 -Antagonism of Sensations of "Cold" and "Warmth" p. 965-Paths of Conduction, p. 965-Comson Sensation, p. 965-Cutaneous Pains considered in relation to Pain in general, p. 966-Relation of Pain to Intensity of Reaction, p. 966--Quality of Cutaneous Pain, p. 967-Scope of the term "Common Sensation," p. 969-The Viscera and Common Sensation, p. 970The Musculo-articular Apparatus and Common Sensation, p. 972-Nature of "Physical Pain," p. 973-Action of Painful Stimuli, p. 974-Character of the Motor Reactions provoked, p. 974-The Central Neural Mechanism for Cutaneous Pain, p. 976--Tickling, p. 976-Dolorous Reflexes compared with Tactual, p. 977-The Spinal Path of Pain, p. 977-Associated (Referred) Pains, p. 981-Interference of Pain with other Sensations, p. 984-The Peripheral Path of Pain, p. 984-" Pain-Spots" in the Skin, p. 984-Pain and Nerves of Muscular Sense, p. 989-Pain and Visceral Nerves, p. 990-Hunger as a type of Visceral Sensation, p. 991-Evolution of Visceral Pain, p. 993-Cutaneous Pain and Adequate Stimuli, p. 994-Specific End-organs or Undifferentiated Endings, p. 995-The Forms of "Excess" of Stimulus, p. 997-Summation in Production of Pain, p. 998- "Inertia" of the Neural Apparatus of Pain, p. 998 - The Brain and Physical Pain, p. 1000.

Sense spots. - The surface of the skin is found to be a mosaic of tiny sensorial areas. The elements of this mosaic are set not actually edge against edge as in the retina; between each element and its neighbours of like function extends a relatively wide interval, insentient when examined by stimuli of little above liminal intensity (Blix). ${ }^{1}$ The more locally limited and the nearer to minimal the stimuli, the smaller appears each individual sensifacient field; ultimately, hy carrying the tests to

${ }^{3} Z t$ schr. f. Biol., 1885, Bd. xxi. S. 152. Also Goldscheider, Monatsh. f. prakt. Dermat., Hamburg u. Leipzig, 1884, Nos. 7-10; Arch. f. Physiol., Leipzig, 1885, Suppl. ; Arch. f. Psychiat., Berlin, 1887, Bd. xviii. S. 659 ; and Eulenburg's "Real-Encyclop. d. ges. Heilkunde," 3te Aufl., art. "Empfindung." 
further refinement, the individual fields may be reduced to mere "spots." Each of these "spots" is found to subserve a specific sense,-touch, cold, warmth, or cutaneous pain. Each doubtless coincides with the site of some sensorial "end-organ," or with a tiny cluster of such. Rather, indeed, than to a mosaic may the skin be likened to a sheet of water wherein grow water-plants, some sunken and some floating. An object thrown upon the surface moves the foliage commensurately with the violence of its impact, its dimensions, and with their propinquity to its place of incidence. Where the foliage grows densely, not a pebble striking the surface but will meet some leaf; and beyond that or those directly struck, a number will be indirectly disturbed before equilibrium of the surface is reestablished.

Throughout almost all regions of the skin "touch spots," "cold spots," "warmth spots," and "pain spots" lie strewn in intercommingled fashion. In some districts one variety predominates, in other districts another. On the whole, "pain spots" seem to be the most numerous, and certainly "warmth spots" are the least so.

\section{Touch.}

Touch means, in physiology, a sensation evoked, under normal conditions, by mechanical stimulation from the skin; under abnormal conditions it may also be evoked by a variety of other stimuli.

Touch spots.-If in an area of the skin, e.g. of the calf, the hairlets be cut short, and the surface minutely tested from point to point by pressing on it perpendicularly a bristle, a certain number of spots are found, at which the slight pressure employed (about 33 grms. on the square millimetre, applied to an area equal to the cross-section of a fine
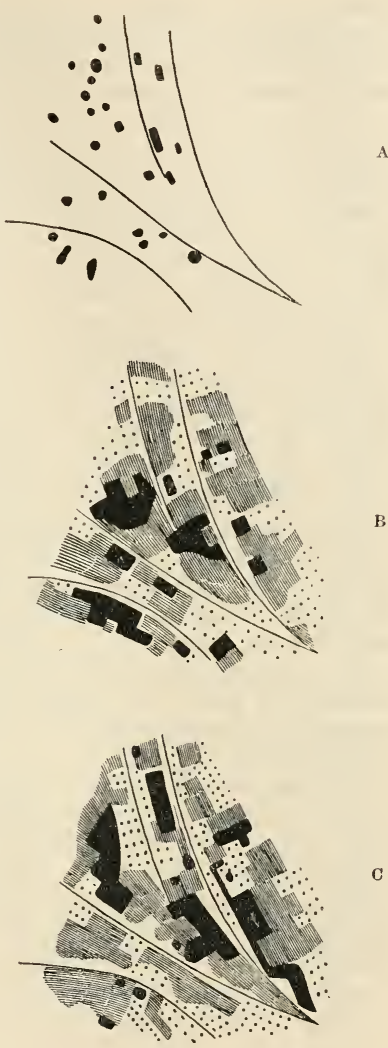

FIG. 368.-Map showing the relative distribution of the sensitivity to toueh, warmth, and cold in the palm of the left hand. In A the whole surface is, when tested by a small cork applied by a spring, found of approximately equal sensitivity except in the areas marked black; these are relatively insentient for touch. In B the areas of sensitivity to warmth stimuli are represented by degrees of shading, the most sensitive by the black shading, the next by the lined areas, the next by the dotted areas, and those of least sensitiveness by the blank spaees. In $\mathrm{C}$ the topography of the distribution of sensitivity to cold stimuli is indicated in the same way as that to warm stimuli in B.-From Goldscheider. 
bristle) $)^{1}$ is perceptible. These spots are called "touch spots." In a square centimetre of such skin the touch spots are about fifteen in number. If into one of these "spots" a fine needle be thrust, there is excited a strong sharply localised sensation of pressure, unaccompanied by smart or sting or any painful quality. Goldscheider ${ }^{2}$ describes the impression as having a "shotty" character, as of a little hard body embedded in the skin, and there pressed upon. The sensation is unaccompanied by "cold" or "warmth," even when a cold or warm needle is employed.

Almost invariably there are one or more "touch spots" close to the place of emergence of each hair; they lie usually on the side from which the hair slopes. It is usual for "touch spots" to be arranged in short lines, radiating from the mouth of a hair follicle. In an area of the forearm (back) containing fifteen hairs, Goldscheider figures seventy-eight "touch spots"; also, on the flexor aspect, 147 "touch spots" to twentytwo hairs; and sixty-six "touch spots" to thirty-eight hairs in a small area of the scalp. In hairless regions-which compose about one-fiftieth of the total body surface of man-the "touch spots" also lie in short chains radiating from certain points.

The "touch spots" are much more numerous in some regions than in others. An area of the dorsum of the ungual phalanx of a finger contains about seven times as many as an equal area from between the shoulders. Regions comparatively poor in "touch spots" are the flexor aspect of the upper arm, the upper third of the thigh, the leg above the inner malleolus, the neck, and in general the skin over subcutaneous surfaces of bone. In some of these regions, roughly oval intervals, measuring a centimetre along the major axis, exist between the individual " touch spots."

Neither are all regions of skin equally freely furnished with " touch spots," nor do some regions contain any purely tactual apparatus at all, if we agree to sunder from pure "touch" the sensations which are elicitable from "pain spots." It is obvious that as there are scattered upon the cutaneous surface sensory end-organs of four different functional groups, e.g. touch, cold, warmth, and pain, the organs of these four classes can be in different regions commingled in different proportions. Nowhere are touch organs the only end-organs present, nor are they in any region interspersed with only one other of the three remaining kinds of sense spots. Together with "cold spots" and "warmth spots," they contribute to a ternion combination for the surface of the cavity of the mouth; throughout that cavity pain spots are few, and from certain parts of the inside of the cheek are wanting altogether. ${ }^{3}$ Elsewhere the touch organs where they occur lie commingled with individual organs of all three of the other kinds. But they do not occur in quite all the mucous and cutaneous regions in which representatives of the other kinds are present. They are absent from the cornea ${ }^{4}$ and conjunctiva of the upper lid, ${ }^{5}$ and from the glans penis. ${ }^{6}$

${ }^{1}$ v. Frey, "Beitr. z. Sinnesphysiol, d. Haut," Ber. d. k. sitchs. Gesellsch. d. Wissensch. z. Leipzig, math.-phys. Classe, July 1894, Dec. 1894, March 1895, August 1897.

2 Arch. f. Physiol., Leipzig, 1885, Suppl., S. 11.

${ }^{3}$ Kiesow, Phil. Siud., Leipzig, 1893, Bd. ix. S. 510 ; Arch. ital. de biol., Turin, 1897, tomo xxvi.; ఛ. Frey, Ber. d. k. sächs. Gesellsch. d. Wissensch. zu Leipzig, math.-phys. Classe, loc. cit.

${ }^{4}$ Hoggan, Linnean Soc. Journ. Zool., London, vol. xvi. p. 82 ; v. Frey, loc. cit.

5 v. Frey, loc. cit. Nagel says they are very few, Arch. f. d. ges. Physiol., Bonn, 1895, Bd. lix. S. 570 .

${ }^{6}$ v. Frey, loc. cit. 
When the skin of the frog is stimulated by light blows with a tiny blunt-pointed lever, some spots are found, as judged by electrical response of the sensory nerves, to be easily excitable, and others to be inexcitable. Punctiform distribution of the tactual surface seems thus proved in the frog's as well as in the human skin. ${ }^{1}$

As to the tactual sensitivity of the cornea, there exists difference of opinion. Some observers ${ }^{2}$ maintain that pure tactual sensations can be elicited from it and from the conjunctiva as well, although all regard the palpebral conjunctiva under the upper eyelid as very poorly tactual. The difference is less a difference between actual observation than of interpretation of observation. The latter turns on the question of the quality of weak sensations evoked by contact stimuli. Some consider even the weakest of these to have unpleasant quality, when elicited from cornea and conjunctiva. If the differentiation between $(\alpha)$ pure tactual sensations and $(\beta)$ sensations of contact of ineradicably painful quality, be admitted at all, then there can be little hesitation in allowing that the cornea (and parts of the conjunctiva), although richly endowed with " pain spots," are destitute, or almost destitute, of " touch spots." 3 The passage of the upper eyelid over the eyeball in blinking is seen but is not felt as touch, except at the moment of meeting of the borders of the two lids. The eyelids being closed, the point of a blunt pencil pressed upon the cornea through the upper eyelid is not felt at the corneal surface, although clear enough on the skin of the eyelid, and although, as the pencil is pressed by one's self, the fingers by secondary touch easily feel the resistance of the hard cornea against the point of the pencil.

As to the identification of the particular end-organ, among those structurally recognised in the skin, to which "touch" may be specifically assigned, no doubt the short hairs are most sensitive tactual apparatus. To them are distributed nerve fibrils which end in the hair papilla, and others which encircle the hair follicle, in many instances composing a rich ring-like arrangement near the level of the openings of the sebaceous glands into the hair follicle. Further, ${ }^{4}$ Meissner's corpuscles have for long been regarded as tactual, and with much probability of justice. They seem to take the place of sensory hairs in hairless parts. Hairs are by some ${ }^{5}$ regarded as homologues of certain cutaneous sensory apparatus found in fish and amphibians. Such a relationship conforms well with a certain degree of physiological analogy that can be discovered between hairs and Meissner corpuscles.

The following data are useful for comparison with those above given regarding the distribution of touch spots :-

Average Number of Meissner's Corpuscles to $1 \mathrm{sq}$. mm. of Skin Surface. ${ }^{6}$

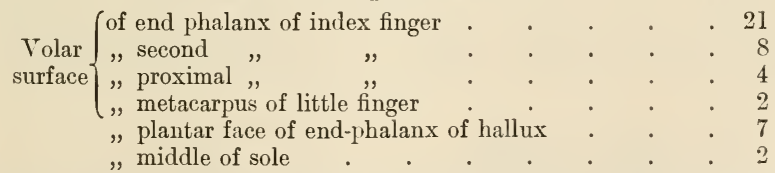

One corpuscle occurs on the average in every $35 \mathrm{sq}$. $\mathrm{mm}$. of skin over the front of the forearm; von Frey and Kiesow located 99 toucl-spots in 6 sq. cm. of the

1 Steinach, Arch.f. d. ges. Physiol., Bonn, Bd. Ixiii. S. 495, etc.

2 G. Fuchs, Mcd. Jahrb., Wien, Bd. iv. ; A. Molten, "Inaug. Diss.," Erlangen, 1878 ; H. H. Donaldson, Mind, London, 1885, vol. x. p. 399 ; Max Dessoir, Arch. f. Physiol., Leipzig, 1892, S. 175 ; Nagel, Arch. f. d. ges. Physiol., Bonn, 1895, Bd. lix. S. 570.

${ }^{3}$ Probably there is a considerable amount of individual variation, see Nagel, loc. cit.

4 "Beitr. z. Anat. u. Physiol. d. Haut," Leipzig, 1853.

5 Maurer, Morphol. Jahrb., Leipzig, Bde. xviii., xx.

6 Meissner, "Beitr. z. Anat. u. Physiol. d. Haut," Leipzig, 1853. 
front of distal end of the forearm. In the toes, on the average, one papilla out of every four contains a corpuscle (Meissner). It is not clear that the so-called "tactile cells" (Merkel) are concerned with touch, but in addition to the Meissner corpuscles there occur in the papillæ of the palmar and digital skin the "plume-organs" of Ruffini, so called on account of their shape. ${ }^{1}$ These possess a nerve-ending quite different from that of either the simple or the compound Meissner organs; the network of terminal nerve filaments is much more rich and complex in the "plume-organs," and is not enclosed in such a thick or definite capsule as that of the Meissner organs. Beneath the organs of the papillæ there lie at various depths the nerve-endings discovered by Ruffini, ${ }^{2}$ the "elastic tissue spindles." Numerous and of wide distribution, these are very probably tactual. Deeper still occur the Golgi-Màzzoni corpuscles, and in some regions fair numbers of Pacini corpuscles.

The adequate stimulus.-The "touch spots" are found to be the most sensitive points of the skin surface They are readily excited, not only by pushing the skin surface inward, but also by pulling it outward (v. Frey). When of small amplitude, a push at a touch spot cannot be distinguished from a pull of equal amplitude. Meissner ${ }^{3}$ years ago pointed out that the hand immersed in a fluid at body temperature fails to feel the contact of the fluid, although the fluid pressure may be far above that of liminal intensity for touch. It seems clear that the adequate stimulus for touch organs consists in deformation of the skin surface. Fluid exerting everywhere a pressure normal to the face of the submerged skin does not deform the surface, except at a boundary of submerged and unsubmerged parts. At that boundary it is, as Meissner observed, felt. Elsewhere it bears on the sides as on the summits of the skin ridges equally. On the contrary, a solid surface presses primarily on the ridge summits and not on the ridge sides. The solid, therefore, causes deformation, and its contact stimulates the tactual surface, and is perceived.

A bristle attached to the prong of a vibrating tuning-fork can be used for stimulating a "touch spot" intermittently. The resulting sensations retain a quality of intermittence, even at high frequency of repetition of the stimulus. The vibrations of strings are recognisable as such by the finger, even at a frequency of 1552 vibs. per second. ${ }^{4}$ Induced currents evoke distinctly intermitting sensation, at a repetition of 130 per second. ${ }^{5}$ A wheel with toothed edge gives sensation of smoothness at its edge when the teeth meet the skin in succession at the rate of 480 to 640 per second. ${ }^{6}$ As compared with the sensation obtained from pain spots, "touch" is quicker both of development and subsidence. ${ }^{7}$

\footnotetext{
1 Monitore zool. ital., 1895, tomo vi. p. 8 ; "Di nuove forme d. termin. nerv. n. strato pap. e. subpap. d. cute," Siena, 1898.

${ }_{2}$ Atti. d. r. Accad. d. Lincci. Cl. di sc. fis., mat. c nat., Roma, 1893, tomo vii.; aud "Ulteriori ricerche sugli organ. nerv. term. n. conn. sottocut. d. polpast. d. uomo.," Ricerche u. lab. di anat. norm. d. r. Univ. di Roma, 1896, tomo v. fase. 3 ; Sfameni, Anat. Anz., Jena, Bd. ix. S. 22.

${ }^{3}$ Ztschr. f. rat. Mcd., Dritle Reihe, Bd. vii. S. 92 . Meissner further relates that when a very accurately cast mould of the finger, made in paraffin, is carefully reapplied, it excites no "touch," except at its margin.

4 Landois, "Lchrbuch d. Physiol. d. Menschen," 1880 ; Preyer, "Empfindungsvermögen," Bonn, 1866.

${ }_{5}^{5}$ v. Frey, loc. cit. Cf. Bloch, Trav. du lab. dc Marcy, Paris, 1897.

${ }^{6}$ Valentin, Arch. $f$. physiol. Hcilk., Stuttgart, 1852, pts. 3 and 4, Bd. xi. ; Sergi, Ztschr. f. Psychol. u. Physiol. d. Stinncsorg., Hamburg 11. Leipzig, 1892, Bd. iii.

7 Goldscheider, loc. cit. ; Nagel, loc. cit.; v. Frey, loc. cit.
} 
The first instance in which a variation of the resting currents of a sensory nerve was obtained by application of an adequate stimulus to the end-orgalls was in the case of the eye. ${ }^{1}$ Working with cooled (hypersensitive) nerves, Steinach has obtained negative variation of the resting sciatic current by dealing a light blow to the skin of the frog's foot with a graduated lever. Within limits the extent of the negative variation varies directly with the force of the blow applied.2

Fontana ${ }^{3}$ showed in 1780 that by gradually increased compression a nerve can, without exciting it, be paralysed as a conductor. The first application of the pressure is felt, and then the sensation subsides, if the compression be steady and not too rapidly increased. Similar pressure applied to the skin evokes a sensation which does not so subside,- the sensation continues. The inner stimulus must therefore be tetanic in character. This difference between nerve and end-organ does not, however, hold if the stimulus to the latter is but little above liminal,then the touch is felt for a moment after contact, and speedily subsides. ${ }^{4}$ It may be again felt for a moment at the sudden discontinuance of this stimulus ${ }^{5}$ in this the reaction resembles many reflexes obtainable from the spinal cord, where discontinuance ${ }^{6}$ of a prolonged stimulation evokes a renewal or an increase of a reflex reply that had ceased or become feeble. " Touches" evoked by brief stimuli, even of very slight intensity, especially from the region of the nose, may persist for very long, or leave a very protracted after sensation. The prolongation of the sensation is of an itching, tickling quality, and may continue even for minutes. This secondary sensation may be due to a local vascular reflex initiated by the stimuli, for the itching spot can generally be seen to be flushed. ${ }^{8}$

The principle of "adaptation," laid stress upon by Hering and G. E. Müller, in the visual and muscular senses, plays a part also in tactual. After the skin has been tested by a number of light weights, a heavy weight appears disproportionately heavy; and light weights disproportionately light, after a series of much heavier. The large areas over which contact of the skin with clothing is habitual become, even under attention, almost insentient of the contact. A plate carrying artificial teeth is for the first few hours almost unbearable to the mouth, and provokes reflexly for a time excessive salivation, etc. In a comparatively short period the intensity of the reaction to the stimulus dwindles to an extraordinary extent. A similar adaptation is experienced when eye-glasses, spectacles, etc., are first worn.

The method of application of the adequate stimulus for touch has been, in the vast majority of instances, the application of pressure. This has been done with weights in a scale-pan; ${ }^{9}$ with a spring balance (the baræsthesiometer) ${ }^{10}$ with a pulsating elastic tube, distended by hydrostatic means; ${ }^{11}$ by a mercurial balance, enabling rapid variations of pressure to be made

1 W. Kühne and J. Steiner, Untersuch. a. d. physiol. Inst. d. Univ. Heidelberg, 1881, Bd. iv. The earlier work by Holmgren and $\mathrm{H}^{\prime}$ Kendrick dealt with the retinal currents.

${ }^{2}$ Arch.f. d. ges. Phusiol., Bonn, 1896, Bd. lxiii. S. 495, etc.

3 "De corpor. animal., ete.," Firenze.

${ }^{4} \mathrm{Kiesow}$, Arch. ital. de biol., Turin, 1896, tomo xxvi. p. 417.

5 Cf. also Steinach, op. cit., S. 514.

${ }^{6}$ Head, Journ. Physiol., Cambridge and London, 1889, vol. x. p. 279.

7 Sherrington, Phil. Trans., London, 1896.

"Dohrn, "De varia variarum cutis, etc.," Diss., Kiel, 1859 ; Ztschr. $f$. rat. Med., 1861 , Bd. x. S. 339.

${ }^{10}$ Eulenberg, ibid., S. 360 ; Berl. klin. Wchnschr., 1869, Bd. vi. S. 469.

11 Goltz, Centralbl. f. d. med. Wissensch., Berlin, 1863, S. 273. 
without shock ${ }^{1}$ by a combination of a delicate spring and beam balance ${ }^{2}$ by applying fine elastic filaments, glass, wool fibres, hairs, etc. ${ }^{3}$ An adjustable projection from a smooth subspherical surface has been introduced by Graham Brown. v. Frey ${ }^{4}$ has been, as far as I know, the first to employ measured degrees of traction as stimuli.

Liminal intensity of stimulus.-When a solid body of known weight, with one side smooth and of known area, is warmed to the skin temperature, and allowed, in virtue of its own weight, to rest smooth side downward on the supported hand, it causes a sensation of touch of a certain intensity, i.e. it "touches" with a certain degree of intensity. The intensity of the physical stimulus is easily written in such instances as values of "pressure." The resulting sensations are often, especially when the intensity is somewhat high, termed "pressure sensations." Such pressure sensations are, however, to be regarded merely as degrees of touch sensation.

Kammler ${ }^{5}$ measured the least weights with contact surface of $9 \mathrm{sq}$. $\mathrm{mm}$. capable of exciting touch sensation at different skin regions. He found 002 grm. liminal for the forehead, temple, and back of hand and forearm; 003 grm. for flexor aspect of forearm; $005 \mathrm{grm}$. for lips, chin, eyelids, and abdomen; 01 grm. for palmar surface of fingers; $1 \mathrm{grm}$. for ankle and finger-nail. It must be remembered, however, that for one and the same region it is found, in actual practice, that the liminal stimulus varies considerably, even when tested by contact areas $7 \mathrm{~mm}$. in diameter; thus, in the pulp of the finger, at some areas, the liminal intensity is four times greater than at others. ${ }^{6}$ Where thick and unyielding, the skin will, coeteris paribus, require greater pressure to deform its surface than where thin and supple. The pressure required to evoke sensation is therefore, if deformation of surface (v.s.) be the immediate stimulus, a mode of expression that has for each surface to be interpreted.

The expression liminal stimulus includes three important values of stimulus, namely, the least stimulus that never fails to evoke sensation, the least stimulus that in the whole series of observed cases does evoke sensation, and the least stimulus that in half the number of observed cases evokes sensation. These are respectively the upper, lower, and median limina.

The short hairs of the skin much enhance its tactual sensitivity. On 9 sq. mm. of skin from which the hairs had been shaved, the liminal stimulus was found to be 36 mgrms., whereas, on the same surface, before it was shaved, 2 mgrms. was the liminal stimulus. The liminal stimulus for the touch spots about a short hair is three to twelve times greater than for the hair itself, i.e. the hair is three to twelve times more sensitive than the "spots." The liminal stimulus for the hair progressively increases as it is progressively cut shorter and shorter. ${ }^{7}$ Each short hair is a lever, of which the long arm outside the skin acts at an advantage upon the touch organs at the root. The short hairs are probably the most sensitive tactual organs of the body.

1 Landois, loc. cit.

2 v. Frey, see Kiesow, loc. cit.

3 Hensen, "Vortrag gegen den sechsten Sinn," Kiel, 1893; v. Frey, op. cit., July 1894.

Op. cit., 1897.

s "Exper. d. variarum cutis regionum min. pondera sent. virtute," Diss., Vratislaviæ, 1858 ; also with Aubert in Untersuch. z. Naturl. d. Mensch. u. d. Thiere, 1859, Bd. v. Scripture finds the limina here obtained "quite too low for most people," "New Psychology," London, 1897, p. 284.

${ }_{6}^{6}$ Kiesow, loc. cit.

${ }^{7}$ v. Frey, loc. cit. 
More critical than the larger areal methods of touch testing are observations by a method introduced by Hensen (1893). ${ }^{1}$ By using the bending of a glass-wool fibre, one end of which rested on the skin (face), Hensen found a pressure of less than 001 grm. easily perceptible. A somewhat artificial but otherwise convenient way of expressing the intensity of stimulus thus used, is to give the weight per sq. mm., which will exert, on an area equal to the cross-section of the fibre or biistle used, the pressure actually exerted by the fibre or bristle employed in the observation. The following results obtained by $\mathrm{v}$. Frey, using Hensen's method, are expressed in that way. The contact surfaces ranged between $\frac{1}{500} \mathrm{sq} . \mathrm{mm}$. and $\frac{1}{16} \mathrm{sq} . \mathrm{mm}$.

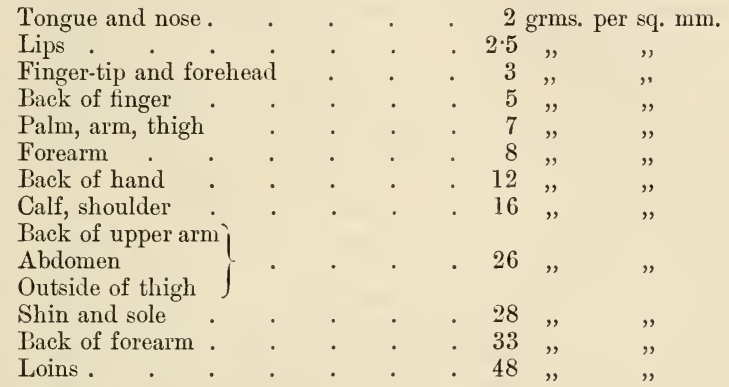

Applying weights to $1-10$ sq. mm. area of skin of the frog's foot, Steinach ${ }^{2}$ found 2 grm. the liminal weight.

To excite a naked nerve, the minimal mechanical stimulus is in experiment ' 2 grm. moving at $140 \mathrm{~mm}$. a second (Tigerstedt). For the touch spot, v. Frey finds $\cdot 2$ grm. at $\cdot 17 \mathrm{~mm}$. a second minimal. The limen is lowest with a contact area of about $.5 \mathrm{~mm} .{ }^{3}$

$I_{i}$ is probable that tactile stimuli of subliminal intensity can by repetition become summated to liminal intensity. If a point of skin be faradised with the secondary coil of the inductorium too distant from the primary for any single shock to evoke sensation, a sensation can still be evoked from the skin. ${ }^{4}$ That is, electric shocks individually too feeble as stimuli, repeated in series eventually evoke sensation. The stimuli are in this experiment not, however, of the specific quality for touch organs; but the phenomenon of "latent summation" is clearly shown.

An important determinant of the intensity of the limina stimulus, is the speed of its application. When quickly applied, the pressure required is less. ${ }^{6}$ The energy of the stimulus is the criterion.

When touch spots and pain spots are tested with mechanical stimuli, e.g. pressure with a bristle, the liminal stimulus for touch lies lower than for pain; the quotient $\frac{\text { touch-limen }}{\text { pain-limen }}$ varies from $\frac{1}{3}$ in the forearm to ${ }_{100}^{1}$ in the finger-tips. ${ }^{7}$ Examined by faradic currents, the quotient of

1 "Vortrag gegen den sechsten Sinn," Kiel, 1893.

${ }^{3}$ v. Frey and Kiesow, Ztschr. f. Psychol. u. Physiol. d. Sinnesorg., Hamburg u. Leipzig, 1899 , Bd. xx. S. 147.

${ }^{4}$ v. Frey, Ber. d. k. sächs. Gesellsch. d. Wissensch. z. Leipzig, Math. -Phys. Classe, loc. cit.

"Richet, "Reeherehes sur le sensibilité," Thèse inaugurale, Paris, 1877.

${ }^{6}$ Stratton, Phil. Stud., Leipzig, Bd. xii. S. 556; Kiesow, Arch. ital. de biol., Turin, 1897, tomo xxvi. p. 440 ; Blix, Ztschr. f. Biol., 1885, Bd. xxi.

$7_{\mathrm{v}}$. Frey, loc. cit. 
excitability is altered to more than unity, the "pain threshold" lying lower than the " touch threshold."

Many other conditions affecting the skin affect the liminal stimulus. Cold blunts the sensibility at "touch spots," but less than at "pain spots." To stretch the skin much lowers its sensitivity at "touch (and pain) spots," deformation of the skin surface is then, of course, less facile. By rubbing and by scratching, it is easy to raise the liminal stimulus for a local region threefold. Hence the rubbing or scratching to "remove irritation." By practice and attention the liminal stimulus for a patch of skin may be soon reduced to half its original value. ${ }^{1}$

As to drugs, cocain ${ }^{2}$ locally applied (5 per cent. solution injected subcutaneously), depresses all forms of cutaneous sensitivity, after causing evanescent hyperalgesia. Tactual sensitivity is more depressed than painful, and less than thermal. Applied to the conjunctiva, cocain abolishes pain more speedily than tactual and than cold sensation. Carbolic acid ( 5 per cent.) acts similarly to, but less strongly than, cocain. In subcutaneous injections of 2.5 per cent. solution, it can locally completely paralyse all cutaneous sensation. Chloroform, applied with a sponge, after a temporary burning sensation, leaves sensibility, especially to temperature, blunted for a longer period. Orthoform acts as does cocain, but its action lasts longer; unlike cocain, it is not toxic. ${ }^{4}$ Menthol, after producing a temporary hyperæsthesia, especially of the "cold spots," depresses sensibility, especially for cold. The feeling of local coolness, caused by applying menthol to the skin, is due less to evaporation than to hyperæsthesia of the end-organs for cold. ${ }^{5}$

The liminal difference of intensity.-That smallest increment or decrement of stimulus which occasions a noticeably different degree of sensation can serve as criterion of fineness of gradation of a sense. E. H. Weber, ${ }^{6}$ in measuring such "differences," found it best to apply the comparable stimuli successively to the same surface of skin. He used the two distal phalanges of two adjacent fingers, which, with the whole arm, lay fully supported on a rigid surface. The time interval between two successive stimuli must be brief, e.g. 5 secs., and regular, in comparing pairs successively. The duration of the stimuli should be equal. No thermal stimulus should accompany the tactile. By measuring the stimuli, giving "just noticeable differences," Weber found the limit of discrimination for tactile stimuli, evoking sensations from the volar skin of ungual phalanx, was reached when the weights used as stimuli were to each other as 29 to 30 . But the ratio was not the same for all skin regions, and it differed in different individuals. The ratio for a given region and individual remains approximately constant throughout a considerable range of absolute value of external stimulus. This is the basis of Weber's "law"; it applies best to stimuli of moderate intensity. With tactile stimuli, as the stimulus decreases to near liminal intensity, the ratio of increment or decrement required for perceptible difference has to be much greater than for stimuli of moderate intensity; thus in the palm 1.66 grms. and 1 grm. Similarly, in the case of very intense stimuli, the ratio is departed from. With moderate weights, a difference of pressure is perceptible when the ratio of alteration is smaller than

${ }^{1}$ v. Frey, loc. cit.

2 Goldscheider, Monatsh. f. prakt. Dermat., Hamburg u. Leipzig, 1886, No. 2, Bd. v.

${ }^{3}$ Goldscheider, loc. cit.

${ }^{5}$ Goldscheider, loc. cit.

${ }^{6}$ Wagner's "Handwörterbuch," Braunschweig, 1846, Bd. iii. Abth. 2, S. 511, etc. 
when either very small or very large weights are used; that is, sensitiveness to pressure difference is keenest under moderate stimulation. With slower rates of change the threshold difference is lower than with quicker. ${ }^{1}$ The average threshold difference, the initial pressure being 5 grm. on 20 sq. mm. of the second phalanx of the finger, was found with instantaneous addition to be $35 \mathrm{grm}$.

\section{Liminal Difference in Various Skin Regions. ${ }^{2}$}

Forehead

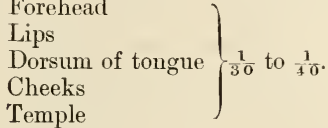

Finger nail

Back of forearm, of leg, and of thigh

Back of hand

Back of middle and proximal phalanges of fingers

Volar side of finger

$$
\text { , , , } \quad \text { forearm }
$$

Upper arm

Dorsum of foot

$$
\text { , toes }
$$

Plantar surface of toes

Sole of foot

Surface of leg and thigh

more than $\frac{1}{10}$

Methods.-The "liminal difference" for touch has been estimated in various ways-(1) As by Weber, referred to in the above paragraph. Of two stimuli, easily judged to be of different intensity, one is diminished or increased to the extinction of perceptible difference; or, of two indistinguishable stimuli, one is increased or diminished (in the above case by addition to or subtraction of weight from it), so that a diflerence between the two stimuli can just be noticed. When the test starts from equality, the difference remains longer unnoticed than when tests go towards equality. The direetion of change, i.e. whether decrease or increase, may be known to the subject or not known. The former is the "conscious" method, the latter the "halfconscious." The direction being unknown, the changes may be of regular order or utterly irregular, the latter giving the "unconscious" method. The values of liminal difference found are different for these different methods. These are quick methods, but less precise than others to be mentioned. The two modifications of the method can be used together, and the mean liminal differences ascertained by each compared together. This combination constitutes the method of smallest difference. ${ }^{3}$ The method as used by Weber is known as method of just noticeable difference. (2) Method of right and wrong answers. ${ }^{4}$ - Two equal stimuli are, under favourable conditions, judged as a rule to be equal, although in certain proportion of instances to be slightly different. Two unequal stimuli are judged to be unequal with greater

${ }^{1}$ Hall and Motora, Am. Journ. Psychol., 1887, vol. i. p. 72 ; Stratton, loc. cit.; Seashore, Stud. Yale Psychol. Lab., New Haven, 1896, vol. iv.; Heinzmann, Arch. f. d. ges. Physiol., Bonn, 1872, Bd. vi. S. 222 ; Fratschner, Jenaische Ztschr. f. Med. u. Naturw., Leipzig, Bd. ii. S. 130 .

2 A. Eulenberg, Ztschr. f. rat. Med., 1861, Bd. x.

s G. E. Miiller, “'Zur Grundlehre d. Psychophysik,” S. 11.

" Fechner, "Elem. d. Psychophysik," Bd. i. S. 71. Fechuer elaborated this method, but it appears to have originated in Vierordt's laboratory, see Hegelmayer, also Renz and Wolff in Arch. f. physiol. Heilk., Stuttgart, 1856, Bd. xiv.; and Aun. d. Phys. u. Chem., Leiprig, Bd. xeviii. S. 600.

VOL. II. -59 
certainty the more they are unequal. When the difference between them is above a certain degree, they are constantly judged to be unequal. As the inequality between the stimuli is reduced more and more, less and less correctly is judgment made as to their inequality. The distinction between the stimuli is in each case either right or wrong. Occasionally no answer can be given, the judgment remains indeterminate. Such instances are dealt with by being added half to right answers, half to the wrong. For each value of the difference between the stimuli the ratio between the number of right answers and the total number of answers is determined $\frac{r}{t}$. The delicacy of perception of difference is inversely proportional to the differences of stimulus which produce equal ratios $\frac{r}{t}$. Thus if in two equal sets of experiments, e.g. on two individuals, $\frac{1}{35}$ and $\frac{1}{70}$ of difference of external stimulus elicited the same ratio of right to total answers, e.g. 80 per cent., the liminal difference of stimulus is half as great, and the delicacy of judgment of difference is twice as great in the second as in the first. (3) Method of mean error. ${ }^{1}$ - It is sought to make the two stimuli equal, that is indistinguishable, by use of the single sense under examination, in this instance touch. At the end of each attempt the objective stimuli judged equal are physically measured, e.g. on the balance. The smaller the difference between the stimuli so measured, the greater the delicacy of judgment of difference, the lower the liminal difference; always supposing the number of observations made is sufficiently great. (4) Method of apparently equal intervals. ${ }^{2}$ - Three stimuli are arranged in series by the subject, so that the difference between the first and second appears to him equal to that between the second and third. (5) Method of apparently doubling the stimulus (Merkel). - The subject tries to make one stimulus seem just double another. The two stimuli chosen are then physically compared. In regard to the two last metlods, it has been objected that considerable intervals are compared one with another by a different law from that applying to barely noticeable intervals.

It was during his study of touch that E. H. Weber discovered and first investigated the law that bears his name. The visual is perhaps the sense best suited for its accurate observation. For that reason its full discussion must be left to "Vision," but a brief sketch of the law and its significance, and of the superstructure of theory built on it, seems demanded here. Our sense is provided with no exact measure of itself. Yet we can compare sensations of the same quality, and express opinions as to their differences. Thus, of two touches, one may feel "heavy," the other "light." The difference may be wide or not, e.g. the light touch may be little lighter than the heavy, or much lighter. It is usual to consider this kind of difference between easily comparable sensations to be a difference of quantity. The heavier toucl is considered a stronger sensation than the lighter. When the thus assumed quantity of sensation is on the same hypothesis supposed to be increased, and the assumed increase is obtained without increase of the area or duration of application of the stimulus to the sensifacient surface, the increase of sensation is considered an increase of intensity of sensation. The difference between the sensation of a light touch implicating an area of skin and of a heavy touch implicating the same area, is therefore considered to be a difference of intensity. How much more intense one sensation is than another cannot be known. Whether a rifle's report be five or twenty times louder than a pistol's we cannot that way know. We can only roughly judge the degrees of difference. All we know is that a different sensation results when the physical stimulus is intense from what results when the plyysical stimulus, otherwise similar, is less intense. That

${ }^{1}$ Fechner, "Elem. d. Psychophysik," Bd. i. S. 120. For calculation, see Bruns, Phil. Stud., Leipzig, 1893, Bd. ix. S. 1.

2 Plateau, Bull. Acad. roy. $d$. sc. de Belg., Bruxelles, 1873, tome xxxiii. p. 376. 
the sensation from the former is the more intense is an inference from knowledge of laws connecting the sensation with the outward cause of sensation. We are only at the beginning of such knowledge. We do know that a more intense light applied to the excised eye causes a greater change in the electrical condition of the optic nerve than does a similar but less intense light; and that a heavier tap on the web of the pithed frog causes a greater swing of the needle of a galvanometer connected with the sensory nerve than does a lighter tap. Once within the penetralia of the central nervous system, we have, however, still no knowledge of the matter. A first step towards the problem would be to obtain an exact measure for sensation itself. The stars invisible by day are brightly perceptible by night. Yet they constitute to the untired morning eye an external stimulus physically at least as potent as to the eye at night. One and the same stimulus, according to the circumstances under which it operates, will be perceptible either more or less or not at all. If to the stimulus of the starlight there be added the overwhelmingly greater stimulus of daylight, the sensations from the retinal points at which the stimulus is sunlight + starlight, cannot be distinguished from the sensation generated by the rest of the retina where the stimulus is daylight alone. As daylight fails, the brightest stars become visible, followed by those less and less brilliant as the twilight deepens. A stimulus to be felt may be so much the weaker, the weaker the coexistent stimulation of the sensory organ, and must be so much the stronger the stronger the coexistent stimulation. Evidently sensation does not alter in identically the same progression as does stimulus. If a stimulus of intensity (1) causes a sensation comnted as 1 , a stimulus of intensity (2) evidently does not evoke a sensation 2, or stimulus (3) sensation 3, etc. Were it so, a sensible stimulus added to a pre-existent strong stimulus would provoke as great a difference (increase) of sensation as if added to a weak stimulus. Intensity of sensation, therefore, increases less than directly proportionately with increase of the intensity of the external stimulus. By noting for each strength of stimulus the addition required to evoke a just perceptible alteration of sensation, a series of quantities is obtained expressing the law according to which sensation alters when stimulation is increased. This expression is the so-called "law" of Weber. It says that a given stimulus is perceived less when added to a large stimulus than to a small one, or that an addition to a large stimulus is perceived less than an addition to a small one, unless it, relatively to the stimulus, is as great. The "law" may be phrased variously in physiological theory. It is true that it may be interpreted as not physiological at all, but psychological. The disproportion between increment of stimulus and increment of sensation may take place in purely psychological events and processes. Wundt is of that opinion. $\mathrm{He}$ points to the wide occurrence of such a ratio in all psychical activity as outcome of the relativity inherent in every conscious process. Waller finds the response in a nerve trunk directly stimulated, as judged by action eurrent, increase much more nearly directly as the increase of external stimulus than does the response from muscle when nerve is stimulated, or from nerve when retina is adequately stimulated. ${ }^{1}$ Waller's evidence seems to point to the law being in part a function of nerve cell endings; probably, therefore, applicable to synapses as to motor plates. Delbøuf considers the law an expression of an ever-increasing proportion of loss of effect in the central nervous system, due to "fatigue." The notion of fatigue has been in the physiology of the senses expanded so as to include phenomena to which the term is perhaps little suitable, e.g. Helmholtz's theory of successive contrast. Delbœuf's seems similarly a strained use of the term. A process need not be of the nature of fatigue to make an ever larger part of the work of the stimulus

${ }^{1}$ Brain, London, 1895, vol. xviii.; see also Fick, "Untersuch. u. elekt. Reizung," Braunschweig, 1869; Hermann, "Handbuch d. Physiol.," Bd. i. Abth. 1, S. 107 ; J. J. Muller, Arb, a.d. Züriche Hochschule, 1869; Dewar and M'Kendrick, Nature, London, July 1873 ; F. C. Müller, Arch. f. Physiol., Leipzig, 1886. 
"go to overcoming resistances, and an ever smaller part to the production of the feeling-bringing state" (James). When the pans of a balance are already loaded, but in equilibrium, it takes a larger weight added to one of them to incline the beam. As James says, Weber's law may be a sort of law of friction in the neural machine. The molecules of the sensorial and neural cells are complexes of atoms and atom-groups, spinning, oscillating, and colliding. They can be thought of as embracing hundreds rather than tens of atoms, each possessing its motion. The more violent the oscillations of an atom-group, or the further the group's centre of oscillation from the systemic centre of the molecule, the greater risk of its detachment altogether from the system. Each atom or atom-group, with its kinetic and potential energy, when either of these energies, or both together, exceed a certain amount, must leave the region of molecular affinities, and the molecule be in so far decomposed. Constancy of composition exhibited by a substance, and the uniformity with which it reacts in our testings, is no sign of a dead sameness and calm within it, but merely means that the energy of its molecular life is distributed so as to present a constant average. Among the million molecules of living matter subjected to test en masse by even our most delicate physiological experiments, there will at any given time be a certain number in the act of decomposing, and a certain number more on the verge of doing so. There will also be a certain number in the act of creation. The majority of the molecules will be in that average phase of the molecular existence in which the atomic movements most perfectly harmonise, somewhere about the mean between the extreme phases of the molecular condition of the particular substance in question. In addition to the molecules in this mean phase exhibited by the majority, there will be numbers in condition approximate to that eritical for decomposition, and numbers the condition of which is more than usually stable. If a formula analogous to Maxwell's for the conditions of a gas is with modification, as urged by Ebbinghaus, applied to the conditions of the molecules of protoplasm, the numbers of molecules in degrees of more than average instability and of more than average stability, arrange themselves about the majority possessed of the particular stability which is average for the chemical substance in question, approximately symmetrically. When the adequate stimulus impinges on such a substance, one may suppose the energy of movement of all the particles accessible to it to be increased. But the external result and the result upon the chemical constitution of the substance must be different in the different molecules. Those molecules already at verge of decomposition are doubtless easily and at once broken up, and likely enough also a number less near the critical condition; a number of which the stability is mean for the substance may also be decomposed, and if not decomposed brought nearer toward easy decomposition. If the stimulus is weak, the number of molecules sufficiently liable to be decomposed by it is relatively small. When the stimulus is sufficient to induce decomposition of the molecules of mean stability, they being the most numerous, the condition as regards strength of stimulus will be peculiarly favourable for producing a high ratio of released force to releasing force. Since a great number of the molecules approximate to the mean molecular condition of the substance, relatively small gradations in intensity of moderate stimuli will cause relatively large differences of effect. As the stimulus is progressively increased in strength, the further number of molecules involved in decomposition becomes progressively less. Finally, even the last and most stable that can be decomposed by the stimulus are reached. The "maximal" stimulus is thus arrived at, beyond which further increase of stimulus fails to cause further increase of effect. The reactions at base of Weber's law can probably be pictured somewhat on the lines of such an hypothesis as the above, but the total reaction must be the sum of an as yet unknown number of such simpler ones.

It must never be forgotten that Weber's law deals with judgments. 
The comparison of one sensation with a second of similar quale, but of dissimilar quantum, involves more than the mere neural process concerned with a simple sensation. From the very outset it works with ideas based on perceptions, these latter themselves elaborated from sensory impressions. The "law" expresses a striking relation found to hold approximately between the quantity of physical action at one end of the complex chain and the psychical action, as examined by introspective comparison, at the other. Moreover, the process of "attention," variable as it is, has to be used to focus the perceptions, which are therefore apperceptions of variable intensity. On the other hand, the conditions appear simpler in so far that by the "law of relativity" every sensation is ab initio in some sort a judgment and has implicit the terms of a comparison.

If it be assumed that the just noticeable difference of quantity of stimulus always evokes in a given sense the same quantity of sensation, and if it be further assumed that the "quantity" of sensation evoked by the liminal intensity of the stimulus is the same quantity as that evoked by each liminal difference of intensity, it may be said that the intensity of sensation increases in arithmetic progression when the external stimulus increases in geometrical. The intensity of the sensation varies as the logarithm of the stimulus (Fechner). It has been urged that, as a logical consequence of such assumptions, when 100 grms. is laid on the palm, and to it another 100 grms. added, and when, secondly, instead of 100 grms. a weight of 200 grms. is placed there, and to it another of 200 grms. added, the two weights added must in the two cases appear of equal weight; ${ }^{1}$ but in fact they are felt to be different.

The so-called "psychophysical law" (Fechner) is an attempt to supply an absolute measure for sensation. It has yet to be shown, however, that it introduces anything more than an arbitrary scale of measurement. Stimulus $(x)$ and sensation $(y)$ are, somewhat remotely, connected as cause and effect. $x$ is a quantity capable of measurement, and of numerical expression. Of $y$ no measure in the ordinary sense can be found, and the statement that one value of $y$ was double of another, would have, apart from special conventions, no definite meaning. Sensation like temperature is no doubt variable in degree, but changes of sensation, unlike changes of temperature, do not, so far as known, produce measurable changes in any object. Anything comparable with a thermometric scale cannot, therefore, be constructed for sensations. So long as there is no independent measure of sensation, the "psycho-physical law" cannot be spoken of as either a right or wrong interpretation of fact, although it may offer a convenient mode of representing, numerically, intensity of sensation.

The strength of an adequate stimulus may-its duration remaining the same-alter in three ways. It can act with more or less intensity on a certain definite number of end-organs; it can act with an unvarying intensity upon a more or a less extensive number of end-organs; or, by combination of both the preceding, its intensiveness and extensiveness may simultaneously and independently vary. It is noteworthy that the physiological results are in the three cases less dissimilar than might have been thought. Paths of conduction in the nervous system communicate in virtue of the branching of nerve cells, and across nerve cell junctions. The endings of nerve channels frequently embouch into common mechanisms, - that is, their areas of end-distribution overlap. Further, the impulses excited in nervous reactions tend to "irradiate" in nerve centres. By "irradiation" or "overflow," variation in pure intensity of stimulus produces some of the same effects as variation of the stimulus in extensiveness. In virtue of "overlap," variation in the mere spatial extension of the stimulus

${ }^{1}$ Hering, Sitzungsb. d. k. Akad. d. Wissensch., Wien, 1875, Bd. lxxii. Abth. 3, S. 310. This communication contains also the experiments testing Weber's law, carried out under Hering's direction by Löwit and Biedermann. 
produces by summation some of the effects of variation of stimulus in intensity. In examining Weber's "law," and Fechner's corollary thereto, the variation of the value of the stimulus should be ascertained to refer either purely to its intensiveness or purely to its extensiveness. The examination of Weber's law began as regards tactual sense exclusively as an examination of intensity. ${ }^{1}$

The spatial quality of touch.-Simultaneous tangible stimuli, alike except as to site of incidence, have in many cases the property of evoking sensations distinct one from another. With this property is intimately bound up much of that perception of the spatial relations between enviromment and organism which the latter possesses. By some it has been argued that all the numerous sensations contributory to space perception possess, by associative experience or as a native attribute, a quality of locality, a "local sign." 2 The degree to which "locality" attaches to lifferent sensations is various. Touch and sight possess it in high degree. In contradistinction to the organs of muscular sense and the semicircular canals, the skin and eye may be considered the external organs for space perception. From this point of view the two offer similarities of arrangement-1. The nerveendings are spread out in a single plane for the reception of stimuli which likewise are of plane extension. 2. ( $\alpha$ ) Excitation of each point of the sensifacient surface is colligate with a sensation different from that ensuing on simultaneous excitation of any other point of the surface, $(\beta)$ provided the second point be more than a minimal distance from the former point. 3. An object impresses the skin over an area the extension of which in the plane of contact is identical with its own; in the retina, the area, although of smaller extension, owing to the ocular dioptric mechanisms, similarly conforms to that offered by the object. 4. The spatial extension of the object generates, therefore, a set of simultaneous sensations; these can be compounded and, thanks to $2 \beta$, as a continu 5. For each position assumed by these two sense organs, the perceptions attaching to each distinct point of their sensifacient surfaces come by experience to correspond with a definite place in the environment.

The localising delicacy of touch has been measured in two ways-(1) The skin of a blindfolded person is touched with a blunt needle,--the person touched has then to indicate the position of the place touched. (2) The blunted points of a pair of eompasses ${ }^{3}$ are laid lightly and simultaneously on the skin, and the smallest distance of the points apart at which they are perceptibly separate is measured (Weber). ${ }^{4}$ If the points are sufficiently elose together, a single sensation results. The liminal distance can be determined, either by the method of just noticeable difference, or by the method ${ }^{5}$ of right and wrong answers. ${ }^{6}$ For the latter about thirty pairs of blunt needles are set, each pair in a little holder at a certain fixed distance apart. The distances should range from about $3 \mathrm{~mm}$. to $80 \mathrm{~mm}$. The answers obtained are "single," "double," "uncertain." The last are treated as half "single,"

${ }^{1}$ For fuller discussion of the Weber-Fechner "law" in regard to touch, reference can be made to Wundt's "Grindzuge d. physiol. Psychol.," Leipzig, 1893, Bd. i.; " Vorlesungen ucber die Menschen- u. Thier-seele," Leipzig, 1892, translated by Creighton and Titehener, London, 1894. Also, ef. Helmholtz, "Handbuch d. physiol. Optik," Berlin, 1897.

2 Lotze, "Medizin. Psychol.," Leipzig, 1852.

${ }^{3}$ Compasses used for thus testing the skin are often termed asthesiometers.

${ }^{4}$ Arch. f. Anat., Physiol. $u$. wissensch. Med., Leipzig, 1835, S. 152 ; and in Wagner's "Handwörterbueh," loc. cit.

${ }_{5}$ Vierordt, Ztschr. f. Biol., 1870, Bd. vi. S. 58 ; G. E. Miuller, Arch. f. d. ges. Physiol., Bonn, 1879, Bd. xix. S. 191.

${ }^{6}$ Not necessarily as a step toward the examination of the Weber-Fechner psychophysical "law." 
half "double." But with certain observers ${ }^{1}$ and in certain series ${ }^{2}$ of observations they have not occurred. With increasing separation of the points of contact the proportion of right answers to total answers increases ; at a certain separation the answers are all right. Throughout the observations single contacts are intermingled with double contacts.

When "touch spots" are sought out for excitation with the compass points, liminal distances are found much smaller than the average liminal distance of the region.

Some "æsthesiometers" allow of the reading of the pressure applied to each of the points. ${ }^{3}$

In vividness and delicacy of local sign, touch resembles the muscular and visual senses. It, however, falls far short of the last, being at best more than three thousand times less delicate when skin distances are compared with retinal.

Table of Liminal Distances for the "Sense Spots," in Millimetres."

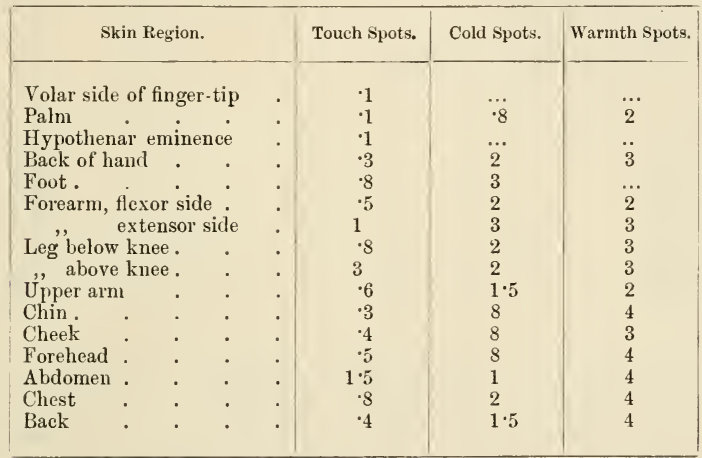

As said above, the "touch spots" tend to be arranged in tiny systems radiating from centres. The compass points are perceived to lie apart with especial distinctness, when applied to "touch spots" of two such separate systems. The above list shows that the liminal distance is least for "touch spots," and greater for "warmth spots" than for "cold spots." The direction of the axis joining any two stimulated "touch spots" is judged with greater nicety than is that of the line joining temperature spots of similar distance apart. ${ }^{5}$

The same has been noted with "average liminal distance." 6 Weber's method, as applied by himself, ${ }^{7} \mathrm{C}$. Vierordt, ${ }^{8}$ and others, ${ }^{9}$ is, however, of special value for giving an idea of the "average liminal distance" for a cutaneous region. It is this rather than the special details of liminal distance in relation to the distribution of "touch spots" that yields data valuable for dealing with the majority of space perceptions elaborated from tactual sensation. In estimating the

${ }^{1}$ Kottenkamp and Ulrich, Volkmann. 2 Hartmann.

${ }^{3}$ Griesbach, Arch. f. d. ges. Physiol., Bonn, 1897, Bd. lxviii. S. 67.

${ }^{4}$ Goldscheider, Arch. f. Physiol., Leipzig, 1885.

5 Goldscheider, ibid.

${ }^{6}$ Czermak, Untersuch. z. Naturl. d. Mensch. u. d. Thiere, Bd. i. S. 188.

7 Wagner's “Hanilwörterbuch," loc. cit. ${ }^{8}$ Tübingen Diss., 1871-1875.

${ }^{9}$ Kottenkamp and Ulrich, Ztschr. f. Biol., 1870, Bd. vi. S. 37 ; Paulus, ibid., Bd. vii. S. 237 ; Knőller, ibid. ; Riecker, ibid., 1873, Bd. ix. S. 95 ; 1874, Bd. x. S. 177 ; Hartmann, ibid., 1875 , Bd. xi. S. 79. 
"average liminal distance," certain precautions must for accuracy be taken. When the stimuli are successive instead of simultaneous, the distance is much reduced, e.g. from $20 \mathrm{~mm}$. to $5 \mathrm{~mm}$. on dorsum of hand. ${ }^{1}$ The æsthesiometer points must both be at skin temperature. The average liminal distance varies greatly in different skin regions. When children and adults are compared, it is found to be less in the former.

Table of Average Liminal Distances in Millimetres. ${ }^{2}$

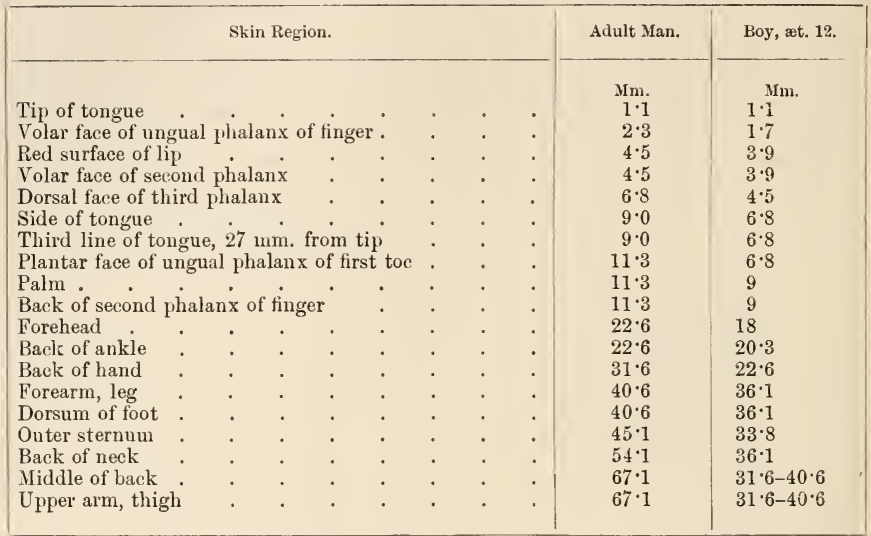

In the limbs, the "average liminal distance" for touch is greater parallel with the long axis of the limb than across it. The distance can be reduced by practice. The blind, who partially supply by other senses their deficiency of visual space-perception, recognise small shapes, e.g. letters, coins, with enhanced nicety by practised touch. ${ }^{3}$ Type-setters similarly discriminate between letters, correctly and rapidly by toucl. Under repeated examination by Weber's method, an area of skin will, in even a few hours, reduce its average liminal distance by a half. ${ }^{4}$ The "education" is at first slow, then quicker, and finally slower, dwindling to cessation when a certain decrement of the distance has been effected. The decrement obtained by "education" is completely lost by a few months of want of practice. In those inaccessible and relatively immobile regions of the body surface, where the liminal distance is very

${ }^{1}$ When two pressure stimuli are applied simultaneously near together, the areas of deformation of the skin surface are likely to meet and overlap. This will not occur when the same two stimnli are applied to the same two points successively. This, however, is an explanation insufficient to account entirely for the reduction of the average liminal distance obtained by applying the stimuli successively instead of simultaneously. Cf. Judd, Phil. Stud., Leipzig, Bd. xii. S. 409 ; v. Frey, Sitzungsb. d. phys.-med. Gesellsch. zu Würzburg, 1899, Bd. xii.

2 The observations on the adult are condensed from Weber's list in the "Handwörterbuch," with a few additions from the "Programmata," 1834, fase. 1. The observations on the boy are from Landois, "Lehrbuch," 1881, 2te Aufl. S. 929 . The distances given are the minimal from which double sensation is obtained, when stigmatic excitation of selected touch spots is not undertaken.

${ }^{3}$ Czermak, loc. cit. ; Stanley Hall on Laura Bridgeman, Mind, London, April, 1879.; The question has been recently examined by F. Galton, "Inquiries into Human Faculty," London, 1883.

* Volkmann. Ber. d. k. sächs. Gessellsch. d. Wrissensch., Leipzig, 1858. 
great, it cannot be reduced so speedily or largely as in the fingers. ${ }^{1}$ Education of an area of skin in one lateral half of the body reduces the average liminal distance, not only in and around the area itself, but in the symmetrically placed area of the other half of the body as well. Similarly, the learning of a movement by the right hand imparts a partial ability to perform it with the left. ${ }^{2}$

Delicacy of tactual space perception at any point of the surface of the body seems in part a function of its mobility (C. Vierordt). ${ }^{3}$ It depends both on the variety of direction and on the linear amplitude of the movements of the part. It increases with the distance of the part from the axis of rotation. In the arm the delicacy increases continuously from acromion to finger-tip, but at unequal rate between the different joints. If the localising power for touch at the acromion be valued at 100 , it increases to 2582 at the finger-tips. In the upper arm it increases to 151 , in the forearm to 272 , in the hand exclusive of digits to 659 , in the thumb to 2417 , and in the middle digit to 2582 . These values in each segment (e.g. forearm) of the limb can be considered the sum of two quantities. One of the quantities depends on the distance from the main axis of movement of the limb as a whole, e.g. from shoulder. The other is determined by the distance from the axis of movement of the segment itself, e.g. from elbow. Even in each separate finger the rate of increase of delicacy is different in the different segments and greatest in the terminal. The relation between delicacy of sense and degree of mobility is least easily examined in the head, because of the complexity of movement of that region. The difference is only as 100 to 160 between all mobile (but not motile) parts of the head's surface. Yet Vierordt's theory seems to apply very perfectly there also. ${ }^{4}$ Thus there is a continuous and regular decrease of average liminal distance along the cheek from ear to lip. In the head and in the arm the average liminal distance is not only inversely as the mobility of the part, when passively partaking in movements of the head upon the neck, but is further reduced by the active movements of the part itself, if mobile. Thus the average liminal distance decreases rapidly from base of tongue to tongue-tip. Again, its measure is less for the surface of the lower than the upper lip, for the upper than the lower eyelid; lower eyelid and upper lip are the less mobile.

Among conditions affecting the fineness of localisation by touch, are the following. It is decreased by anæmia of the skin and by venous hyperæmia $;^{5}$ also by too frequent testing, and by mental fatigue,-thus, three hours of school classes will double some threshold distances on the face ${ }^{6}$ also by cold and by excessive stretching of the skin. ${ }^{7}$ In relation to the last-named point, there is an experiment of some theoretical interest. If the skin of the neck be stretched by throwing back the head as far as possible, the distance of two skin-points can in certain regions of the neck be doubled. The average liminal distance in that region is, however, not doubled, but only increased 8 per cent. ${ }^{8}$ The passage of a

\footnotetext{
${ }^{1}$ Funke, Hermann's "Handbuch," 1880, Bd. iii. Abth. 2, S. 382. But see Volkmann, op. cit.

2 Weber, loc, cit. ${ }^{3}$ Arch. f. d. ges, Physiol., Bonn, 1869, Bd. ii. S. 297.

${ }^{4}$ Riecker, Ztschr. f. Biol., 1873, Bd. ix. S. 95 ; 1874, Bd. x. S. 177.

5 Alsberg, "Inaug. Diss.," Marburg, 1863.

${ }^{6}$ Griesbach, Arch. $f$. Hyg., München u. Leipzig, Bd. xxiv. S. 124 ; Varmor, "Thèse inaug., " Berne, 1896.

7 v. Frey, loc. cit.

${ }^{8}$ G. Hartmann, Ztschr. f. Biol., 1875, Bd. xi.
} 
subliminal electric current between points only little above liminal distance apart, causes simultaneous touch sensations from those two points to lose their separateness, and fuse. ${ }^{1}$ Narcotics markedly increase the average liminal distance. ${ }^{2}$

The sense of touch locality is disturbed and injured, disproportionately to damage of other tactile qualities, by lesions of the cortex of the cerebral hemisphere. In a hemiplegic patient the sense of touch in the affected limb is sometimes but little impaired, when tested by the liminal intensity or liminal difference of intensity; but when the patient is required to localise, great, often enormous, deficiency is found (Westphal), so that a touch on the hand may be perceived as though located on the upper arm.

Theory of "localising" power of "touch."-A something attaches to or is inherent in a "touch" which gives it place, establishing it within ideal space. There is reason to think (see "Muscular Sense" and "Spinal Cord") that something analogous may attach to even very simple reactions not involving consciousness, i.e. to what are sometimes called neuroses in distinction from psychoses.

This quality of "place" is by some regarded as native to and intrinsic in touch (Czermak, Vierordt), which thus possesses a simple spatial quale. Others (Lotze, Meissner, etc.) have regarded it as the outcome of experience of association, of sensations concomitant with each touch sensation; the associate groups centred on touch sensations generated at sufficiently separated spots of skin are, it is argued, sufficiently different to be perceptibly distinct. Each such little compound system of sensation serves as differentiating and recognisable sign, labelling the "touch" attached to it with "locality," or, in Lotze's language, giving it "local sign." Lotze's scheme of local signs was based mainly on muscular sense; Meissner attribnted it in tactual sense to physiological differences in the structure of the skin of different regions.

A difficulty in the way of accepting locality as a native quality of simple touch, is its relativity. At the root of locality lies discrimination of place, and to discriminate in place between even only two touches implies perception not alone of the excited points, but of the extensiveness of an unexcited interval. Thus localisation would commence in duality. Weber insisted from the outset on the perception being not only of two points, but always also of the interval between. How is it, then, that a single touch comes to be localised? Previous experience has taught the recipient subject that when either point was impressed by an object, the same object usually touched also certain immediately neighbouring points. Each point becomes thus associated witli an area of circumjacent points, the associated fading in strength as the distance from the point increases. The point about which the associated area thus centres has its own peculiarity or shade of feeling. Wundt writes: "If we touch first the cheek and then the palms, each time with the same pressure, the sensation is, notwithstanding the similar stimulation, markedly different in the two. So if we compare the feeling of the palm with that of the back of the hand, in short, of any two distant parts of the skin with each other. Moreover, we easily remark, by attentively observing, that spots even near together differ in respect of quality of feeling." Each has its "local sign." But the feeling of each point is, as above said, associated by experience with the feeling of the circumjacent. Its sensation revives consciousness of the whole region round about. The "local sign" of the central point, therefore, suggests the feeling of the entire district, and its locality is given by judgment of the relation between it and other points in the revived regional perception.

${ }^{1}$ Suslowa, Ztschr. f. rat. Yed., 1863 (3), Bd. xvii. S. 155.

${ }^{2}$ Lichtenfels, Sitzungsb. d. k. Akad. d. Missensch., Wien (2), Bd. xvi. S. 3. 
In the limbs and mobile parts, when a spot of less discrininative sensitivity is touched, instinct noves the member, so that it brings to the object the part where its own sensitivity is delicate, e.g. the finger-tips. It is apparent to most of us that if, with closed eyes, we attend to the consciousness of the resting arm, the hand, and especially the fingers, are the parts we are most aware of, best outlined, so to say, in consciousness. There arise lines of habitual association between all points of the member and its sensitive tip. James writes: "I think anyone must be aware when he touches a point of his hand or wrist that it is the relation to the finger-tips of which he is usually most conscious."

"Muscular" sensations may be regarded as varying in intensity according to extensity of movement. A more intense sensation accompanies a larger movement; a more prolonged sensation a longer lasting movement. Touch sensations may be regarded as varying in quality with the place of the skin whence they arise. Touch sensations are yoked with "museular" in four main modes. (1) The same tactual point is moved successively over various points of an object, i.e. the same touch in regard to quality is yoked with a series of "muscular" sensations differing in intensity ; "active touch." (2) An object is allowed to move over a series of tactual points, the tactual surface being held stationary, i.e. one muscular sensation is combined with a series of tactual ; "passive touch." (3) One tactual surface is moved over another, i.e. one set of "muscular" sensations is yoked with a double simultaneous series of tactual; "double touch." (4) The movements of the body, apart from contact with objects, are combined with corresponding series of tactual sensations due to stretching and folding of the skin. Disparate sensations, if connected frequently, become "associated," so that genesis of one involves reproduction of the other. In thinking of our arm's movements we always in some degree recall its field of touch. "Muscular" sense is more delicate for shoulder than for hand, yet in recalling a movement of the arm we habitually recall the hand more than the shoulder. Conversely, perception of a tactual point is fraught with "muscle" sensations linking to it other tactual points whose perceptions frequently precede or succeed or accompany it. These sensual links lend themselves well to measurement, because differing in many instances in mere intensity rather than in quality. The measure of their differences yields by associative experience a basis of estimation of the spatial distances of the tactual points. Tactual space includes the idea of the skin as a continuous surface, although its organs are punctiform. This results from infinitely graded muscle sensations accompanying infinitely graded rectilinear movements possible in various directions in a plane.

It is noteworthy how little vision seems to contribute to tactual localisation; some of the most delicately localising touch surfaces never come within the visual field at all. Tactual localisation differs from auditory and gustatory in that, whereas a single drop of fluil on the tongue may give a mixed sensation, and two sounds may be perceived to come from the same place, two compound touches of different quality, e.g. a blunt and a sharp, applied simultaneously in the same sensory circle, do not combine to any simultaneous sensation, but alternate, as in the struggle of different colours presented to identical spots of the two retinæ.

Weber ${ }^{1}$ described the whole surface of the skin as resoluble by analysis into "sensory circles." If for any part of the skin we measure exactly the distance between compass points the sensations aroused by which appear single, and make this measure in many directions about a single point, we obtain for this part of skin a figure of rounded form within which simultaneous touches appear single. Such an area is a Weher's

1 E. H. Weber, loc. cit. ; also "Tastsinn u. Gemeingefühl," Leipzig, 1852, S. 106. 
"sensory circle." It is in most regions oval, since discrimination is most delicate usually in some direction at right angles to the direction of least delicacy, e.g. across the limb. It might be supposed that in each "sensory circle" the two stimuli generate an indivisible sensation, because they both excite the terminal field of a single sensory nerve fibre,-that in fact each "sensory circle" is the area of ending of a single nerve fibre. But a sensory circle where large, e.g. in the back, with a diameter of more than six $6 \mathrm{cms}$., contains more than 200 touch spots; and histological examination shows that no cutaneous nerve fibre breaks up into anything like that number of end-fibres. Besides, if the sensory circles be represented by brackets $(a+b+c)(d+e+f)(g+h+i)$ containing cutaneous points $a, b$, and $c$, and if $a+b+c$ represent the ending of one sensory nerve fibre, and $d+e+f$ the ending of another, and if the explanation of the sensory circle be that two simultaneous touches cannot be felt as separate, if affecting only one and the same nerve fibre, the "average liminal distance" (that is, the size of the "sensory circle") will vary greatly in one and the same skin region, according as the simultaneous stimuli fall on points $c$ and $d$ or $f$ and $g$, or on $a$ and $c$, or $d$ and $f$. The sensory circles must be larger than the anatomical units of the sensory surface. Moreover, a few hours' use and practice can reduce the size of the sensory circles in the finger to one quarter their previous area. No morphological alteration could be effected in that time. Weber urged that for two simultaneous touches to be distinguishable, a certain number of unstimulated nerve-endings must intervene between the stimulated ones. The now recognised existence of "touch spots," each coinciding with a tiny cluster of or a single tactile endorgan, bears out Weber's view. When the compass points are felt as double, a striking part of the sensation is the feeling of an interval between the two points. The smaller "average liminal distance" for the fingers than for the back, is not explicable entirely by greater number of "touch spots" per unit surface in the former than in the latter. A sensory circle in the back may contain many more than 200 touch spots, while a digital sensory circle may contain many less than 200 . "Local sign" seems possessed in higher degree, therefore, by sensations generated through digital "touch spots" than through "touch spots" in the back.

The division of the skin into a mosaic of tiny sensifacient areas, each of diameter less than the radius of a " sensory circle," explains perception of contact along an unbroken line, even when the object in contact with the skin has spatial gaps, e.g. edge of comb or saw, so long as the gaps are less wide than are the "sensory circles."

That two stimuli, identical in quality and quantity, evoke when applied to two different sensory circles tactual sensations correspondingly different, is certainly referable to occurrences situated in the higher nervous "centres." A cutaneous nerve trunk stimulated in its course evokes tactual sensations referred to the skin area of the peripheral distribution of the nerve. The sensation evoked, although one towards which the end-organs have not collaborated, possesses in considerable degree its "local sign." It is noteworthy that at the distal ends of the limbs, where local sign is better developed than in the proximal parts of the limb, the nerve roots in their distribution to the skin overlap especially widely. ${ }^{1}$ This means that the area of embouchment into the central

1 Türck, Denkschr. a. k. Akad. a. Wissensch. Wien, 1855 ; Sherrington, Phil. Trans., London, 1892. 
nervous system of the sensory channels for a patch of the palm is greater than for an equal patch from the shoulder. It has been pointed out that in the limb the amount of sensory root overlap of distribution corresponds broadly with the degree of "local sign." But in other parts there are striking exceptions to this, e.g. the tongue; though there again the area of embouchment of the sensory channels into the grey matter is very large (descending root of fifth cranial). The paths of the spinal columns, including the tactual, embouch into and diffuse in the spinal grey matter. It is the wide spatial extent of the deep (central) connections of tactual paths, after their entrance into the cord and brain, that probably form an essential character of the physiological mechanism, correlated with the development of "local sign."

That the determination of the average liminal distance is by central as well as by peripheral factors, is shown not only by the rapid effect of
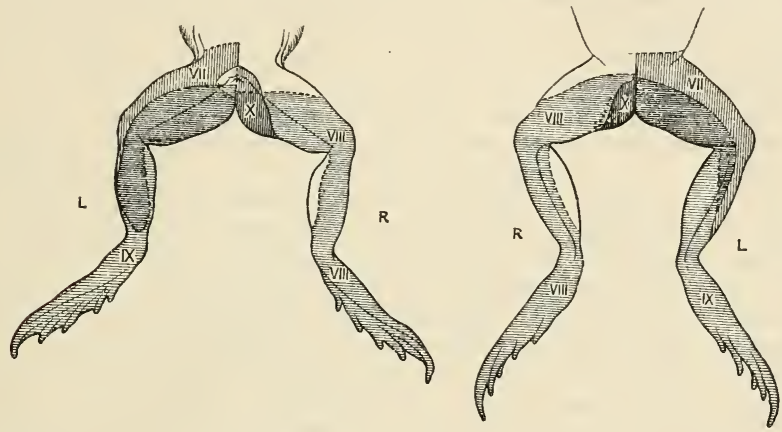

FIG. 369.-Map of the skin distributions of the seventh, eighth, ninth, and tenth afferent nerve roots of the frog. The test used in determining the distributions was occurrence or absence of response (movement) of the "spinal" animal to mechanical stimuli applied to the skin when all but one of the roots in question had been severed.-Method of remaining æsthesia.

local "education," and by the local reference of tactual nerve fibres stimulated in their course, but also by the small increase of the distance when the skin is stretched even to twice its unstretched length (see above, p. 937), and by its general increase under mental fatigue.

Of two lines seen under like conditions, a longer may be regarded as a greater stimulus than a shorter. A surface of $2 \mathrm{cms}$. in contact with the skin may be considered, cateris paribus, a greater stimulus than a surface of but $1 \mathrm{~cm}$. That Weber's law holds for comparison of pressures applied to skin, does not imply that it will hold for comparison of areas applied to the skin. The examination of the scope of the "law" in the latter direction has been less followed than in the former in its application to "touch." Fechner concluded that the law is inapplicable for areas of contact."

Projection of "touch."- -Sense perceptions inform at first hand of alterations in the condition of sensifacient organs. Since these alterations are, under normal circumstances, habitually occasioned by external objects, we habitually consider many of them as qualities of 
environmental objects, that is, we project them. The force with which the quality inheres is different in different senses. Visual perceptions seem almost unconquerably the properties of objects in space round about. Among the cutaneous senses, touch is that one most possessed of objectivity. When an external object is touched by or touches the finger, there arises, as an immediate rather than as really a mediate result, a presentation of a something not " $I$," endowed with quality, "hard," soft," "smooth," etc. Complete emancipation from the involuntary "projection" is not possible for us. In some cases the degree of projection of touch is very marked. Thus the perceptions of touch evoked by contact of objects with hairs are referred to the insentient free portions of the hairs themselves, even to their very tips. Similarly with the teeth. Again, the surgeon, as far as touch is concerned, actually lengthens his fingers by using a probe; a rod pressed by the fingers by one end upon a yielding or hard object gives two sensations, - one to the finger's where it meets them, and a second referred to the point of contact of the rod with it. In this we have a germ of the projicience so marked in visual sense of space. ${ }^{1}$

That identical muscular sensations may be accompanied by nonidentical cutaneous, is probably a main factor in giving the latter "objectivity." In all movements that involve the cutaneous surface, that surface being a sensifacient one, if those movements occasion adequate stimulation of the surface, cutaneous sensations will be generated in addition to the muscular sensation inherent in the movement. The two senses together will react to an object in greater range of quality than will either alone; the two will obtain more criteria for judging the object than will either one; the two are alike endowed preeminently with "local sign," and "projection" is an adjunct of the intuition or perception of space. When different objects or one object under different circumstances, evoke sensations, tactual and muscular, which vary (as to extension, intensity, and especially "local sign") not harmoniously, but in almost infinite combination, the compound perceptions resulting form a store of data especially favourable for the judging of space. The very variety of the variously congruent combinations of muscular sense and touch, the irregular flux of correspondence and of non-correspondence between the two, comes to imbue the perceptions of the latter with projection.

In the retina the extension of the sensifacient surface in space is such as to preclude in the same individual any one retinal point ever supplying the adequate stimulus to any other, i.e. no part of the two retince can be seen by any other part. With the skin it is different. One part of its surface may supply adequate stimuli to other parts, and often does. A very sinall fraction only of the human surface is inaccessible to touch by the individual's own hand. It results thence that we regard the cutaneous sensifacient surface-although it, like the retina, belongs to the sentient "self"- as, relatively to that, an external thing, and come to endow it, as we endow other objects of the environment, with the qualities of our sensations. Thus on this, the so-called "double touch" is built, so to say, a bridge from "outside of me" to "me" and "self." Hence tactual and the thermal cutaneous sensations, although "projected," are less so than are visual.

Illusions in tactual judgments of space. - Two " touches" a certain

${ }^{1}$ E. H. Weber, loc, cit. 
distance apart appear further apart if tested where the "local sign" is strong than where it is weak. The points of a pair of compasses, when moved with a sufficient speed over a length of skin, appear to get wider apart as they pass from skin less endowed with local sign to skin more endowed, e.g. from ear to lip. ${ }^{1}$ Moved in the opposite direction, they seem conversely to close together. Moreover, if moved with uniform speed, they appear to travel faster as they pass over the more perceptive region, e.g. towards lip. ${ }^{2}$

When two skin-points ( $a$ and $b$ ) are simultaneously touched with the compass-tips, the distance between them appears greater than if one compass point travels-not too slowly-from $a$ to $b$. The quicker the travel the shorter appears the distance; the slower the travel the longer the distance; so that it may, if quite slow, appear longer than if $a$ and $b$ be simultaneously touched. ${ }^{3}$ It is easier to read letters as they are traced on the skin than letters already fully shaped and pressed upon the skin. It must be remembered here that for the reason given above (p. 936, note 1), the deformation of the skin will be greater in the former case than in the latter.

If a body, e.g. a ball, be touched with a rod, the eyes being blindfolded, and its periphery followed by touching it with the rod, the dimensions appear smaller the longer the rod used. ${ }^{*}$

Owing to the inequality of the "average liminal distance" when measured along various axes in a single region of skin, it is easy to be deceived about the form of surface in contact with the skin. The crosssection of a circular tube pressed on the skin appears transversely oval; whereas contact with a true oval, and its longer axis lengthwise, along the limb, appears circular. Again, experiment shows that if a metallic tube, with triangular, circular, or rectangular cross-section, is pressed on the skin, it is not easy, unless the tube be much larger than the "sensory circles" of the skin in question, to distinguish its shape. A circle can be "felt as circular" by the tongue-tip, if the diameter be not less than $3.3 \mathrm{~mm}$.; but to be felt to be circular by the skin of the abdomen it must measure $55 \mathrm{~mm}$. across. ${ }^{5}$

An experiment, which as long ago as the days of Aristotle was of recognised psychological interest, is the following. If one crosses the first and middle fingers, and then places between them a small round body, e.g. a pea, the pea is "felt double," as though it were two peas. The illusion is strengthened by moving the surfaces of contact. The two sides of the fingers in use are in their ordinary position invariably turned away from each other. Experience has taught that they usually touch two different objects, and this association may be, in the unpractised person, sufficient to override the correcting perception furnished by the muscular seuse.

If a penholder be held between the lips, it can be felt to be straight. If one of the lips be displaced sidewise, the holder seems broken. This illusion vanishes in front of a mirror. So also with transplanted portions of skin : in the nose made from a flap of frontal skin, touches continue for months to be referred to the forehead.

Tactual judgments of form.-For judgment of the shape of objects and their dimensions by touch, we possess four methods of acquiring data,-

\footnotetext{
1 Weber, loc. cit.

2 Vierordt, loc. cit.

${ }^{3}$ Feehner, Vierordt, loc. cit.

+ Tourtual, eited by Vierordt, "Gründriss d. Physiol.," Tüibingen, 1877.

5 Vierordt, loc. cit.
} 
1. The object in contact excites a certain number of tactual sensations of definite intensities and imbued with "local signs." These component sensations are combined, either (1) simultaneously, or (2) successively as well as simultaneously. 2. The successive component sensations can be acquired by actively moving the tactual surface, to increase or renew the simultaneously-given groups of sensations, and in so doing the muscular sense is combined with the tactual in obtaining criteria for judging the shape and size of the object by the distances moved. 3. The tactual perceptions can be accompanied by visual. 4. The tactual perceptions can be accompanied by both muscular and visual.

The man blind from birth, on whom Cheselden performed his historic operation, on first obtaining his sight described the visual field as flat and its contents as " seeming to touch his eyes." Children cured of congenital cataract at the age of 6 and 7 , when, in spite of blindness, well acquainted through their other senses with the form and number of objects, and able to remember, recognise, and describe them, have still, on first obtaining vision, to touch objects in order to know what and how many they are. When shown a box, a coin, two fingers, etc., they do not recognise what they are, nor their squareness, roundness, or that the fingers are two (not one or three); after touching them over (tactual and muscular sensations), they recognise the species, form, and number at once. ${ }^{1}$ Judgment of direction and distance has similarly to be translated into visual signs from tactual and muscular. ${ }^{2}$ The child for many days after recovering sight, if in a strange room, feels its way between the furniture as though in a dark place. Faulty naming of objects seen, that are perfectly correctly named when touched, is said to be traceable in such children for some years.

The degree of importance of tactual sensation for guidance and execution of movement is very various. To enervate the foot induces no obvious defect in the stepping of the horse. A bird, in which the cutaneous nerves of the foot have been paralysed by section, will still grasp the perch, and sleep balanced on the enervated foot. ${ }^{3}$ On the other hand, Bell found the prehensile movements of the lips of the horse were abolished by section of the sensory nerves to the face. A monkey, in which the fifth cranial nerve of one side has been cut, and sensation lost there, keeps, in drinking from a bowl, moving round it toward the side away from the side of section. ${ }^{4}$ The guiding sensations for drinking with the lips are therefore almost exclusively supplied by touch.

Hering has devised a cylinder æsthesiometer for testing delicacy of judgment of surface form by touch. A set of reels (usually twelve), each about $8 \mathrm{~cm}$. long and $15 \mathrm{~mm}$. in diameter, are closely wound with silvered wire, each reel with a differently-sized wire, the coarsest wire being $1 \mathrm{~mm}$. thick. One reel has an absolutely smooth polished turned surface. The test is carried out by finding with which of the reels the sensation of roughness or smoothness begins or ends. To the finger-tips of many persons, whose hands are not much employed for rough labour, the coils of wire of $11 \mathrm{~mm}$. thickness give a distinctly "uneven feel."

${ }^{1}$ W. Uhthoff, Ztschr. f. Psychol. u. Physiol. d. Sinnesorg., Hamburg u. Leipzig, 1898, Bd. xiv. S. 200.

${ }^{2}$ Francke, Beitr. z. Augenh., Hamburg u. Leipzig, 1894, Bd. xvi. S. 1; Raehlmann, Ztschr. f. Psychol. u. Physiol. d. Sinnesorg., Hamburg u. Leipzig, 1895, Bd. viii. S. 401.

${ }^{3}$ A. Chauveau, Brain, London, 1891, vol. xiv.

${ }^{4}$ Sherringtou, Phil. Trans., London, 1892. 
Touches may be perceived as of separate locus, though their mutual direction be imperceptible; a line $9 \mathrm{~mm}$. long is perceived by touch as linear, but for the direction to be perceived must exceed $12 \mathrm{~mm}$. Alphabets for the blind have linear or punctate letters. The threshold of space for recognition of line direction is more than twice as high as for successive points. The dimensional distances being the same, the point alphabet is therefore the easier, especially if the points be taken in succession as in the New York horizontal point-letters; the Braille point-letters are vertical.

The central paths and centres concerned with the conduction and elaboration of the nervous reactions of "touch" are dealt with in the sections dealing with the "spinal cord" and the "cerebral cortex."

Reaction-Time.-The reaction-time for touch is longer on the average than that for audition, shorter than that for vision. The average time observed by Exner was $\cdot 1337$ seconds, by Hankel 1546 seconds, by Baldwin $\cdot 125$ seconds, by Hirsch $\cdot 182$ seconds, by Wundt $\cdot 213$ seconds. The reaction-time for electric skin stimuli is shorter than for tactile, thus Wundt 201 seconds $;^{1}$ for tactile shorter than for cold and warm. But the experiments have too many psychological factors to warrant discussion here.

\section{Cutaneous Senses of Cold and Warmth.}

Recent evidence has led to the cutaneous sensations of cold and warmth being regarded as specific and distinct from tactual sensations, and from each other.

"Cold" and "warmth" as distinct species of sense.-This view implies that even a "localised" cold (or warmth) sensation possesses no tactual element, and that a cold (or warm) stimulus, e.g. the point of a cooled (or heated) metal pencil applied to a "cold spot" (or to a "warmth spot" respectively), acts there not at all as a tactile stimulus. Much has been brought forward in support of such a view. It is in some ways more in accord with what we should expect from introspective examination, to regard the sensations we derive from the skin as all of them sensations of greater or less tactual intensity, some of them, however, inseparably accompanied, as it were coloured, by qualities of sensation which we call "cold" and "warmth," others not so accompanied or coloured. There is much to show that while we possess sensations of brightness quite uncoloured, the sensations of colour we possess are all of them inseparable from sensation of brightness. If each sensation of "cold" or "warmth" is a concomitant inseparable from a tactual sensation, it is simpler to treat it not as a separate species of sensation, but as a quality of tactual sensation. The weight of evidence seems at present, however, to be against this. It can be urged against it that thermal stimuli unaccompanied by any tactile element (e.g. radiant heat), or accompanied by only such as lie far below the threshold value of tactile stimuli, can yet excite strong "cold" and "warmth" sensations. On the other hand, to some ${ }^{2}$ even warm vapour moving over the skin gives a sensation that they describe as having some tactual character as well as the character of "warmth."

\footnotetext{
1 "Physiolog. Psychol.," Leipzig, 1893, Bd. ii. S. 223.

${ }^{2}$ See Eugenio Tanzi, "Sulle sensazioni del freddo e del caldo," Milano-Torino, 1886.

VOL. II. -60
} 
Observations by Blix $^{1}$ and Goldscheider ${ }^{2}$ first led to any general adoption of an analysis of the sense of temperature into distinct specific senses of "cold" and "warmth" respectively. These observers discovered that pre-eminently at certain points of the skin a cool object evokes sensation of "cold," and at certain others a warm object with special facility evokes sensation of "warmth." This discovery was reached by application to the skin of minutely localised stimuli, e.g. use of the points of cooled or warmed metal pencils, or minute drops of ether or oil. "Spots" of skin exist at which an object, very lightly applied, fails to evoke the sensation of touch, but, if cold, evokes the sensation of cold; and at these points any stimulus, if sufficiently intense, evokes the sensation of cold. ${ }^{3}$ Also other spots are similarly sensifacient for "warmth." These "cold spots" and "warmth spots" are therefore considered, in accordance with the theory of specific energies, to be the seat of specific end-organs of nerves for two senses, namely, of cold and warmth.

Weber wrote: ${ }^{4}$ "When the hand is benumbed, there oftentimes may arise from it a subjective sensation of warmth, but never, I believe, of cold." Herzen ${ }^{5}$ noted that his arm, when numbed by compression of its nerve trunks, could feel warmth, e.g. the warmth of his other hand, but not cold, e.g. the cold of a marble table. Faradisation of a nerve trunk evokes sensations of cold and of warmth referred to the area of distribution of the nerve.

Herzen ${ }^{6}$ has reported the following condition of a patient (in whom post mortem examination showed destruction of the dorsal columns of the spinal cord and the periphery of the dorsal part of the lateral columns in the cervical region); in the skin of the lower limbs there existed loss of tactual impressions, but not of painful; contact with objects at temperatures between $27^{\circ} \mathrm{C}$. and $60^{\circ} \mathrm{C}$. was felt, and the degree of warmth of them could be well estimated; objects at temperatures below $27^{\circ} \mathrm{C}$. evoked no sensation whatever.

Distribution of apparatus for thermal sensations in the skin, etc. 1. Distribution of "cold spots" and "warmth spots."-Over all the cutaneous surface of the body, when examined in areas not too minute, "cold spots" much outnumber "warmth spots." Observers are unanimous in finding the latter more difficult to mark and delimit. The "spots" are arranged in short, slightly curved, lines, which radiate from centres. These centres coincide with hairs, or, strictly speaking, with the points of the surface which lie immediately over the papillæ of hairs. Thus it comes about that each set of lines radiates from a point near the exit of a hair on the side opposite from that to which it slants. The "cold spots" and "warmth spots" are usually but slightly interspersed with each other along any individual ray, but "cold" rays and

1 Ztschr.f. Biol., 1884, Bd. xxi. S. 152.

2 Arch. f. Physiol., Leipzig, 1885, Suppl. S. 1. Goldscheider's discovery was made before, but published after, the appearance of Blix's paper. The analysis achieved by Blix and Goldscheider was elearly suspected by Czermak ("Physiol. Stud.," Wien), in 1855 , as the result of experiments of his own. This is the more noteworthy because at that date leading physiologists, including E. H. Weber, taught that even tactual and thermal sensations were evoked in common from one and the same set of nerve organs and fibres. E. H. Weber drew this interpretation from the fact that cold bodies feel heavier than warm bodies.

${ }^{3}$ Goldscheider, loc. cit., v. Frey, Ber. d. k. sächs. Gesellsch. d. Wissensch. zu Leipzig, math.-phys. Classe, loc. cit.; admitted in a qualified manner only by Kiesow, Phil. Stud., Leipzig, 1895, Bd. xi., but reaffirmed by S. Alritz, Skandin. Arch. f. Physiol., Leipzig, 1897, Bd. vii. S. 321 ; denied formerly by Dessoir, Arch. $f$. Physiol., Leipzig, 1892, and Nagel, Arch. f. d. gcs. Physiol., Bonn, 1895, Bd. lix. S. 570.

"Wagner's "Handwörterbuch," Braunschweig, Bd. iii.

${ }^{5}$ Arch.f.d. gcs. Physiol., Bonn, 1886, Bd. xxxvii.

${ }^{6}$ Ibid., loc. cit. 
"warmth" rays diverge from common centres. In some places, besides the centres coinciding with hairs, there are others apart from them.

The number of "spots" varies greatly, from minute field to field, even in the same local district of skin. Tested by surfaces of 3 to 4 $\mathrm{mm}$. diameter, the same small area will contain patches where sense of cold is more acute than sense of heat, side by side with patches where sense of warmth is more acute than of cold; and interspersed with these, lie other patches paræsthetic or anæsthetic to either or both. These last are possibly places where nerve trunks pass up into the skin, ${ }^{1}$ and may be likened to multiple blind spots in a retinal field.

Neither cold nor warmth sensations can be evoked from the cornea (except at its margin), ${ }^{2}$ nor ean they from the dentine or tooth pulp. ${ }^{3}$ In the conjunctiva, "cold spots" are everywhere much more numerous than "warmth spots." A reflex closure of the eyelid is much easier obtained by a cool object as stimulus to the conjunctiva than by a hot one, and the reflex in the former case is much more difficult to inhibit. ${ }^{4}$ Very cold air easily causes lachrymation ; very hot air, e.g. in a Turkish bath, has, on the contrary, little effect. The glans penis, although devoid of true "touch spots," is richly beset with "cold spots"; on the other hand, "warmth spots" are very sparse there. ${ }^{5}$

As to identification of the structural characters of the specific end-organs for "cold" and "warmth," it is suggestive that in those regions (conjunctiva, glans penis) whence "cold" sensations can be readily and almost exclusively evoked, the structures known as "end-bulbs" are numerous. These, as found in the conjunctiva, seem to be the simplest examples, as regards histological structure, of the type of end-organ, of which the Pacini corpuscle is the most complex. Though particularly numerous in the above-mentioned regions, ${ }^{6}$ they occur also in the skin elsewhere, ${ }^{7}$ and in the mucosa of the mouth. In the glans penis and clitoris they are larger than in the conjunctiva, and are often of compound construction. It must be added, however, that end-organs somewhat similar in general appearance are found near joint-surfaces ("articular end-bulbs"), whence, so far as we know, neither "cold" nor "warmth" sensations can be evoked. There is reason to think that the end-organs for cold in the glans penis lie particularly near the surface; on the other hand, that in the finger-tips they lie particularly deeply embedded in the skin. ${ }^{8}$ Ruffini's, ${ }^{9}$ end-organs in the finger-pulp, in which he finds nerve terminations resembling those of a Golgi tendon organ, may, it is suggested, ${ }^{10}$ be organs for "cold." Tomsa's 11 " nerve knots," sometimes called " genital corpuscles," 12 may without difficulty be related to the end-bulbs, of which they in some respects appear the most developed form. In that case it might be said that in all likelihood in "end-bulbs" we have the terminal organs for "cold sensations."

The long reaction time and the difficulty and want of sharpness of delimitation of "warmth spots" point to a deeper situation in the skin for the end-

1 Goldscheider, loc. cit.

${ }^{2}$ v. Frey, loc. cit. Nagel says that in some individuals there are "cold spots" over the corneal surface, Arch. f. d. ges. Physiol., Bonn, 1895, Bd. lix. S. 570. He never found corneal "warmth spots," or obtained from the cornea a warmtl sensation.

${ }^{3}$ v. Frey, loc. cit.

5 Max Dessoir's statement (Arch. f. Physiol., Leipzig, 1892), that the glans penis does not possess temperature sense, is certainly wrong.

${ }_{6}^{6}$ Especially along the niargin of the cornea, Dogiel, Arch. f. mikr. Anat., Bonn, 1891, Bd. xxxvii. S. 602 .

${ }^{7}$ Smirnow, Intcrnat. monatschr. f. Anat. u. Physiol., Leipzig, 1893, Bd. x. S. 241 ; also v. Frey, loc. cit.

${ }^{8}$ Kiesow and v. Frey, Ber. d. k. sächs. Gesellsch. d. Wissensch. zu Leipzig, March 1895.

9 Arch. ital. de biol., Turin, 1894, tome xxi. p. 249.

11 Sitzungsb. d. k. Akad. d. Wissensch., Wien, 1865 , Bd. li. S. 83.

12 This name, given by W. Krause to the organs discovered by Tomsa, is misleading, as a specific "genital" sense seems implied, a proposition which is quite untenable. 
organs of "warmth" than of "cold." Ruffini ${ }^{1}$ describes, in the deeper layers of the skin of the hand, end organs of larger size, and cylindrical form, placed more deeply than any other end-organs there. These organs of Ruffini have been found also in the skin of the eyelid and of the upper arm, and therefore appear of pretty general distribution. v. Frey suggests that they are the organs of "warmth spots."

Instruments employed for applying thermal stimuli to the skin are usually called thermo-asthesiometers. Many forms of them have been employed. Drops of warm or cold fluid, ${ }^{2}$ heated or cooled metal pencils, ${ }^{3}$ wires, ${ }^{4}$ tiny plates of known surface and thickness, and made of silver, ${ }^{5}$ or other metal of known thermal capacity and conductivity, or offering electrical resistance, have been employed. Kronecker introduced a modification of his perfusion cannula for this purpose, and Miescher-Ruysch further elaborated the same instrument. For many observations the Kronecker-Miescher thermo-æsthesiometer is the most handy and the best.

It consists of a conical tube (German silver) $45 \mathrm{~mm}$. long, divided longitudinally by a partition, which is incomplete below at the apex of the cone. The end of the cone is a circular platinum plate $1 \mathrm{~mm}$. in diameter. One compartment of the instrument is connected with an inlet, to which an indiarubber tube can be attached. The other compartment is connected in similar fashion with an outlet. Warmed or cooled water that is made to flow through the instrument, can only pass from the inlet compartment into the outlet compartment by the hole at the apex of the cone. The bulb of a fine thermometer is placed in the compartment that leads to the outlet; it is so mounted that it is not affected by the warmth of the hand holding the instrument. The whole instrument weighs 50 grms., and is held like a drawing pencil. The water supplied to it should be filtered clear, and not hard. Control experiments with the instrument have shown that the temperature of the apex is, when water more than $26^{\circ} \mathrm{C}$. above the temperature of the room flows through at 15 c.c. per minute, estimable within $\cdot 1^{\circ} \mathrm{C}$. if $\cdot 1^{\bullet}$ be subtracted from the reading of the thermometer at any moment. When used at temperatures less different from that of the room, the correctness is greater.

Some observers ${ }^{6}$ have used radiant heat for excitation.

2. The degree of thermal sensitivity of different regions of skin.-This is determined partly by the thickness of regional distribution of the "cold spots" and "warmth spots," as described in the preceding paragraphs, partly also by the sensifacient efficiency of the individual thermal "spots," also in some respects by the relative thermal conductivity of the skin, which is chiefly conditioned by the thickness of its epidermis and its moisture. Only by testing with surfaces of not much less than $100 \mathrm{~mm}$. sq. can the grade of general sensibility of a skin region be ascertained. ${ }^{7}$

Weber's estimations ${ }^{8}$ were carried out by testing the degree to which, at various regions of the body surface, distinction ${ }^{9}$ can be made

${ }^{1}$ Atti d. r. Accad. d. Lincei Cl. di sc. fis., mat. e nat., Roma, November 1893.

2 Goldscheider, loc. cit.

3 Goldscheider, loc. cit.

${ }^{4}$ v. Frey, loc. cit.; F. Kiesow, Phil. Stud., Leipzig, 1898, Bd. xiv. S. 583.

5 Sydney Alrutz, Skandin. Arch. $f$. Physiol., Leipzig, 1897, Bd. vii.

${ }^{6}$ Rauber, Centralbl. $f$. d. med. Wissensch., Berlin, 1869, S. 273; Tanzi, "Sulle sensazioni del freddo e del caldo," Torino-Milano, 1886.

${ }^{7}$ Goldscheider, loc. cit.

9 Nothnagel's estimations of regional thermal sensitivity were made in the same way; so also Eulenburg's, Monatsh. f. prakt. Dermat., Hamburg u. Leipzig, 1885, Bd. iv., and Ztschr. f. klin. Med., Berlin, Bd. ix. 
between glass phials filled with oil and warmed, or cooled in water, but of course applied dry. But the regional degree of development of thermal sensitivity in the skin may be more truly estimated in another way. If media with a contact surface of about 100 sq. mm., and at temperatures more than $15^{\circ} \mathrm{C}$. to $17^{\circ} \mathrm{C}$. below or above that of the area of skin to be tested, be employed for an appropriate time, they excite from the skin maximal sensations ${ }^{1}$ respectively of cold or warmth, which cannot be increased by further increase of the physical intensity of the stimulus. The maximal sensation thus evoked varies much in intensity in different regions.

Twelve grades can be readily distinguished in "cold" sensation, and eight grades in "warmth" sensation. ${ }^{2}$ The following scale was obtained by using brass cylinders of $1 \mathrm{~cm}$. diameter. ${ }^{3}$ The scale commences with the places yielding lowest maximal intensity of sensation :-

\section{Cold Sensations.}

1. Tips of fingers and toes, malleoli, ankle.

2. Other parts of digits, tip of nose, olecranon.

3. Glabella, chin, palm, gums.

4. Occiput, patella, wrist.

כ. Clavicle, neck, forehead, tongue.

6. Buttock, upper eyelid.

7. Lower eyelid, popliteal space, sole, cheek.

8. Inner aspect of thigh, arm above elbow.

9. The intercostal spaces along the region of the axillary line.

10. Mammary areola.

11. Nipple, flank.

12. Certain areas of the loins and abdomen.

The period of application of the brass surface for obtaining "warmth maxima" has to be more prolonged than that required for testing "cold maxima," 1.5 seconds instead of $\cdot 5$ second.

\section{Warmth Sensations.}

0 . Lower gum, mucosa of cheek at lower second molar, cornea.

1. Tips of fingers and toes, cavity of mouth, conjunctiva, patella.

2. Remaining surface of digits, middle of forehead, olecranon.

3. Glabella, chin, clavicle.

4. Palm, buttock, popliteal space.

5. Neck.

6. Back.

7. Lower eyelid, cheek.

8. Nipple, loin.

The observations, to a certain extent, bear out what might have been supposed, namely, that those portions of the body surface habitually protected by clothes would possess higher sensitivity to cold than parts habitually naked. The clothed parts of the body are, however, as will be seen later from other observations, less embued with power to discriminate between small degrees of difference of cold than are many other and unclothed parts.

When fluids quite coll, e.g. at $2^{\circ} \mathrm{C}$, or quite hot, e.g. at $50^{\circ} \mathrm{C}$, , are swallowed, sensations of cold or of warmth are felt in the mouth and at the

${ }^{1}$ Goldscheider, Arch. f. Psychiat, Berlin, 1887, Bd. xviii. S. 659.

2 Goldscheider, ibid.

3 The method is called by Goldscheider a method for testing the "absolute sensitivity." 
fauces and part of the pharynx, but not from the alimentary canal farther down. When hot fluids are drunk, a sensation is obtained which is referred to the pit of the stomach. E. H. Weber taught that this sensation has no quality of "warmth," but is obscurely painful; but Schlesinger gives distinct evidence to the contrary. ${ }^{1}$ A clyster introduced per anum evokes no sensation of cold until it is discharged, when, if at $15^{\circ} \mathrm{C}$., it elicits a strong sensation of cold from the anal skin. ${ }^{2}$ I once had opportunity to test the mucous membrane of the cæcum for sensibility to cold and warmth. I used small test-tubes containing water warmed (up to $50^{\circ} \mathrm{C}$. at highest), and cooled (at lowest, to temperature of melting ice). The patient experienced no sensation whatever from any of them. ${ }^{3}$ I also have had opportunity to examine the small intestine by its peritoneal surface, also the surface of the liver in the same way; in neither case did the patient experience any sensation whatsoever. When very cold air is inspired through the nose, the sensation of cold evoked is confined to the nostril, the floor of the nasal chamber, and the upper surface of the soft palate. ${ }^{4}$ In the hotter rooms of a Turkish bath, the feeling of heat which is so marked at the nostrils is very slight inside the nasal chamber; similarly, the very hot air does not cause lachrymation, although very cold air easily does so. From the granulating surfaces of wounds and cutaneous cicatrices thermal stimuli evoke no sensations of either cold or warmth, although, if strong, they may produce sensations of pain. ${ }^{5}$

The adequate stimulus. - In the cutaneous sensations of cold and of warmth is exemplified with especial distinctness the physiological law, that in the nervous system and its immediate agents, change of condition, rather than absolute condition, acts as stimulus. The warmth of the skin excites specific sensations chiefly when it undergoes alteration. When a point of skin becomes cooler, then does it provoke a thermal sensation. So long as its temperature remains unaltered, it does not, except under extreme conditions, generate any thermal sensations.

Under ordinary circumstances, a continuous stream of heat of fairly steady intensity passes from the interior of the body outward through the skin. So long as the conditions for this flow remain constant, the temperature of end-organs in the skin also remains constant, and different though the temperature of the different thermal end-organs in different skin regions may be, no difference between them is felt. The stream of heat flowing outward through the skin may be altered by alteration of conditions either within or without. To take the latter case first: the heat-stream may be checked by covering the free surface of the skin with clothes; the temperature of the thermal organs in the clothed skin will then rise, and the skin there will "feel warm," although the clothes are no warmer than the skin was. On the other hand, the loss of the heat streaming from the skin may be increased by simply cooling, without otherwise altering, the surrounding medium. The temperature of the thermal end-organs will then fall, and they will produce sensation of cold. The heat-stream flowing through the skin will be influenced not

1 Weber described the sensation evoked from the stomach by very cold fluid as possessing slightly the quality of "cold," Wagner's "Handworterbuch," Bd. iii., Schlesinger, "Die Syringomyelie," Wien, 1895, S. 23.

2 Weber found that if the clyster were quite cold and retained for a time, a sensation of coolness is felt in the anterior abdominal wall; he supposed this dne to actual cooling of the skin of the abdomen, ibid.

3 Weber touched the mucosa of the sigmoid flexure with a hot iron through an artificial anus; no sensation was perceived, ibid.

4 Weber, loc. cit.

${ }^{5}$ Cf. Weber, loc. cit., and others; see Goldscheider, "Ueber den Schmcrz," Berlin, 1894. 
only by the temperature of the medium, e.g. air, in contact with its surface, but also by other conditions of the medium, in so far as they affect its power of abstracting (or of supplementing) the skin's heat. This power will in any substance depend not only on (1) its temperature, but also on (2) its thermal conductivity, (3) its specific heat, and, in the case of any particular object, (4) on the nature of its contact with the skin, and (5) its interference with, or addition to, radiation. The degree to which an object acts as a "cold" stimulus increases coteris paribus with the degree to which it is cooler than the skin it acts on. Thus water at $20^{\circ}$ is a stronger "cold" stimulus than water at $25^{\circ}$, but it does not follow that it is a stronger "cold" stimulus than mercury at $25^{\circ}$, although certainly a stronger "cold" stimulus than oil at $25^{\circ}$. That is, the activity of the object as a "cold" stimulus depends on its thermal conductivity as well as on its temperature. Hence, of water and oil at the same temperature (below the skin temperature), the latter is cateris paribus the weaker stimulus, because it conducts less; of water and mercury at the same temperature (below the skin temperature), the former is cateris paribus the weaker stimulus, the latter conducts better, and more than sufficiently to counteract its lower specific heat. Capacity for heat is also a factor. ${ }^{1}$ The nature of the contact is also a determinant of the intensity of the stimulus; continuous surfaces of contact are more effectual than are broken. ${ }^{2}$ Also, of course, renewal of contact is very important. The very air around us in a room, the temperature of which, the skin having become thermally adapted to it, is giving us no sensation, does, if it move more quickly over a skin-surface, very readily produce sensation of cold, i.e. we "feel a cold draught." Sensation of cold can also occur without contact with cold substances, namely, by increased radiation. Thus we may feel bitterly cold in a room the temperature of which is high, if the walls are nevertheless very cold, e.g. in a mountain hut. In harmony with these facts are others regarding the "adequate temperature." Given an area of skin fully adapted "for the conditions of heat-supply and heat-loss under which it is living, it then, ex definitione, produces no sensation either of "cold" or "warmth." An object which, when in contact with that skin, evokes no sensation either of "cold" or "warmth," is of the "adequate temperature" (Hering). But the "adequate temperature" is not one definite temperature; it is a short range of temperatures above and below a mean. This range is far greater for oil than for mercury. The better heat-conductivity of the latter makes it difficult to so adjust it to the temperature of an area of skin, e.g. finger, that it shall not in unit time abstract from or add to the heat of skin sufficiently to cause a perceptible lowering or raising of the temperature of the end-organs. The experimental difficulty is increased by variation of the adequate temperature of different areas of skin, and also of one and the same area of skin. ${ }^{3}$ These facts all point to the intensity of a "cold" stimulus of given contact area being dependent on the amount of heat which it can withdraw from the skin in unit time; in other words, on the degree to which

${ }^{1}$ Hering, Hermann's "Handbuch," Leipzig, 1880, Bd. iii. Abth. 2.

2 Hering gives numerous examples of this, ibid.

3 Thus Hering fomd the adequate temperature of the little finger regularly somewhat higher, and that of the index finger regularly somewhat lower, than that of the middle finger, ibid. 
it can cool the cutaneous end-organs. It is assumed, in consequence of the results of the spatial analysis of the sensifacient surface, that lowering the skin temperature does not stimulate "warmth" organs, and that conversely the "cold" organs are not excited by a heightening (unless very excessive) ${ }^{1}$ of the skin temperature.

The complexus of attributes (temperature, thermal conductivity, specific heat, nature of surface, etc.) which conibine to constitute the value of an object as a "cold " stimulus when the object is of temperature below the "adequate," combine to constitute its value, conversely, as a "warmth" stimulus when the object is above the "adequate" temperature. The thermal senses are often termed "the sense of temperature," and rightly, in so far that it is "temperature " which, ranging on either side of the "adequate temperature" as zero, determines the species of the sensation; the other thermal qualities, such as conductivity, etc., only affect the quantity or intensity of the sensation. Alteration in skin temperature sufficient in speed and degree to evoke sensation, may be brought about by internal as well as by external changes. Thus, a flushed cheek "feels hot."

Weber urged that the actual process of falling or rising of the temperature of the cutaneous end-organ constitutes the adequate stimulus for the temperature sense. Hering has, on the contrary, put forward the view that the difference existing between the temperature of the end-organ and a physiological zero of temperature, to which it in its skin region is for the time being "adapted," constitutes the stimulus for temperature sense. Vierordt suggested that the transmission of a certain quantity of heat through the skin is the objective cause of all temperature sensations. The direction, according to him, as well as the intensity, of the transmission determines the sensation, the sensation, in his view, being altogether independent of the alterations of the objective temperature of the skin. The fact that a "warmth" sensation can be produced by a sudden hyperæmia of the skin, as well as by a warm object applied from without, controverts Vierordt's view that direction of transmission of heat determines the sensation.

If a metal disc, a little above $0^{\circ} \mathrm{C}$., be applied to the skin, e.g. of the forehead, for 30 seconds, the resulting "cold sensation" persists fully 20 seconds after removal of the disc. The "cold sensation," therefore, endures while the locally depressed temperature of the skin is rising; and, conversely, after strong stimulation by warmth. This offers a difficulty to Weber's view. Facile evocation of positive after sensations is characteristic of the temperature senses; in this respect both resemble the cutaneous pain sense. Further, the physical stimulus, the change in temperature, not only stimulates the nerve-endings, but actually alters their temperature; and if their temperature be much reduced or heightened, their excitability for a fresh stimulus is lessened. The nipples are exquisitely sensitive to warmth; if one be cooled, and then to both the same warm stinulus be consecutively applied, of the resultant warm sensations, that evoked from the cooled nipple is not the greater but the less. ${ }^{2}$ The finger held in water at $40^{\circ} \mathrm{C}$. for 10 seconds, and then, together with the dry finger of the other hand, placed in water at $15^{\circ} \mathrm{C}$., "feels the cold" less than does the unwarmed finger. Further, if the finger of one hand $(\mathrm{A})$ be held in water at $32^{\circ} \mathrm{C}$., and the corresponding finger of the other hand (B) be at

${ }^{1}$ v. Frey, loc. cit., states that from "cold-spots," by exeitation with hot objects, "cold sensations " can be evoked. So also S. Alrït, Skandin. Arch. f. Physiol., Leipzig, 1897, Bd. vii. S. 321 ; F. Kiesow, Phil. Stud., Leipzig, 1898, Bd. xiv. S. 585.

2 Goldscheider, loc. cit. 
the same time held in water at $15^{\circ} \mathrm{C}$., and if then both are placed in water at $40^{\circ} \mathrm{C}$., the finger of $\mathrm{A}$ feels the warmth better than that of $\mathrm{B}$. These and similar observations show that each temperature stimulus of above a certain intensity involves, besides its action as a stimulus, other consequences for the temperature end-organs. First, it excites end-organs (and their nervous apparatus) for which it is adequate, and therefore involves, especially in view of the long-drawn character of the after sensation, a temporary reduction of the excitability of the apparatus for a second stimulus : the sense organs of temperature are peculiarly easily tired. Secondly, it alters the temperature of the skin in a direction which tends to lessen the difference of temperature between the physical stimulus and the recipient organs, and therefore to reduce the intensity as a stimulus of any repetition of the original or a similar kind of stimulus. Thirdly, by altering the temperature of the skin, if the alteration be carried beyond a small range close above and below the normal skin temperature, the excitability of both the cold and warmtl organs is markedly lessened by the alteration.

An experiment laid stress upon as instancing "adaptation" and "contrast" is the following:-A mercury bath is warmed to such a temperature as to feel to the finger neither warm nor cold. From it the finger is transferred to mercury at the temperature of the room. After half a minute or so, the finger, on being transferred to the batl at skin temperature, then "feels it warm." Similarly, if the finger of one hand be held in water at $15^{\circ} \mathrm{C}$., and the corresponding finger of the other in water at $35^{\circ} \mathrm{C}$, and then both are transferred to water at $25^{\circ} \mathrm{C}$, the same water is felt warm by one finger, cold by the other. It must be remembered that Hering wrote prior to recognition of the separateness of the physiological apparatus for cold and for warmtl sensations. I do not see that the above presents difficulty for Weber's view if the mechanism be postulated to be double, as may now be done. Weber's view, equally with Hering's, accounts for bodies cooler than the skin giving rise to "warmth" sensation. But there are experiments of Weber's own which seem to me to argue strongly against his view. If, for instance, at the ordinary temperature of the room, one presses a cold piece of metal $\left(2^{\circ}\right.$ to $8^{\circ}$ C.) for 20 seconds against the palm, the temperature of the skin there falls $5^{\circ}$ to $8^{\circ} \mathrm{C}$. The sensation is painful. After removal of the metal, the cooled patch of skin gets warmer again, rapidly at first, later more slowly. Even in eight minutes' time it does not fully reach its previous temperature, although its temperature is rising all the time. Throughout the whole of that period, although the objective temperature of the skin is rising, a cold sensation is clearly felt. A converse experiment can be performed with warm metal. These results offer difficulty to Weber's view, but can be explained by Hering's. In these cases the periods seem altogether too long to be explicable by positive after sensations, even combined with concomitant lowering of excitability.

Again, as we sit before a fire, we can for hours continue to enjoy sensations of warmth from a surface of skin exposed to it. But we cannot suppose that under such circumstances a continual rising of the temperature of the skin and its thermal organs is lastingly going on. And, conversely, long-continued sensations of cold, produced under conditions that do not vary, are facts of experience which Weber's theory of the adequate stimulus for the thermal sense fails to meet successfully.

Hering's appears the most probable theory. It seems to me altered by the recent dual analysis of the thermal sense only in so far that we must suppose that the difference between the temperature of the end-organ and its physiological zero temperature (settled by adaptation) -which difference is, in his view, the cause of sensation-acts, when the existing temperature is higher, only on the one set of end-organs (apart from paradoxical sensation); when the existing tenıperature is lower, only 
on the other set of end-organs. It is important to note, however, that, as thus modified, the physiological sensory reaction and the psychological sensory reaction experienced when a skin-surface is changing from a condition of, as regards sensation, "warm" to "less warm," are both totally distinct from the physiological and psychological reactions concomitant with the transition from "cold " to "more cold," although the physical process initiating both reactions is the same, and although to both, in ordinary parlance, the term "cooling" can be equally correctly applied. And similarly with the comparison between the transitions from "cold" to "less cold," and from "warm" to " more warm."

Weber ${ }^{1}$ suggested, as the internal stimulus of temperature sensations, hypothetical conditions resulting from the fact that bodies undergoing changes in temperature undergo changes in volume. Most substances with rise of temperature expand, and, conversely, as their temperature falls, contract; and the change in volume is greater in the case of fluids than of solids. The sensorial end-organs of the skin are composed of fluid parts and of more solid parts. It may therefore be "that as tension acting on the parts of the skin in one definite direction generates sensations of touch from certain cutaneous organs, so also distension or collapse, with diminution or increase of tension coincidently in several directions, in certain skin organs generates sensations of cold and warmth." It is in such a manner as this that, Weber ${ }^{2}$ suggested, cold stimuli can summate with tactile stimuli for the production of pressure sensations.

The wall of the aorta was found by $\operatorname{Roy}^{3}$ to eontract in length when warned. The pressure exerted between a fibrous capsule and fluid contents within it, as the temperature of the whole rose and fell, might alter considerably; or heat may affect the end-organs mediately through pressures generated in the tissues round about them.

Other stimuli.-Other nervous excitants than thermal are able to excite the apparatus for "cold" and "warmth" sensations in the skin. It is a question how far they excite the end-organs or the nerves. The liminal value for electric stimuli (faradic) applied to the conjunctiva is higher for "cold" sensations than for "pain." 4 Pressure on the ulnar nerve trunk at the elbow evokes, obscurely, however, sensations of cold or tingling warmth, referred to the ulnar side of the hand more especially. Cold applied to the nerve trunk at the elbow, apart from the sensation of cold produced locally at the place of application, occasions, according to some observers, no sensation of cold ${ }^{5}$ in the hand; in the experience of many, however, although tactual tingling referred to the ulnar side of the hand is the main result, a certain amount of cold sensation referred to the same region is felt also. Such observations are among the most easily demonstrable in illustration of the importance of the end-organ or the efficiency of the adequate stimulus, and for its connection with "specific nerve energy."

Paradoxical sensation of cold.-A warm surface of a certain temperature applied to a "cold spot" produces a sensation of cold-paradoxical cold sensation. ${ }^{6}$ The temperature of the metal applied to elicit the cold sensation has to be about $45^{\circ}$ to $50^{\circ} \mathrm{C}$. For the skin at large, $45^{\circ} \mathrm{C}$. is usually

${ }^{1}$ Loc. cit. $\quad{ }^{2}$ Loc. cit., but see also Klug, Arb. a. a. physiol. Anst. zu Leipzig, 1876.

3 Journ. Physiol., Cambridge and London, 1882, vol. iii. p. 128.

${ }^{+}$v. Frey, Ber. d. k. sächs. Gesellsch. d. Wissensch. zu Leipzig, math.-phys. Classe, loc. cit.

5 Ludwig, "Lehrbueh der Physiol. d. Mensehen," Leipzig, 1859, 2te Aufl., Bd. i.

'Alf. Lehmann, "Die Hauptgesetze d. Gefühlsleben," Leipzig, 1892, S. 35; v. Frey, loc. cit. 
efficient; for the nipple, $40^{\circ}$ C. suffices. Alriitz ${ }^{1}$ found $48^{\circ}$ C. for the elbow, and $52^{\circ} \mathrm{C}$. for the forehead, sufficient. According to Alriitz, the paradoxical cold sensation can also be evoked in the following ways:-(1) By applying to the inner side of the elbow, or to the front of the thigh, a silver plate (Thunberg's) ${ }^{2}$ of 4 c.c. surface and $25 \mu$ thick, heated to $100^{\circ} \mathrm{C}$. A short cold sensation is immediately felt, succeeded by a "hot" sensation, and sometimes by a painful one. With thicker plates the final sensation of pain is of regular occurrence. (2) By fatiguing the warmth spots. A piece of metal at $52^{\circ}$ to $54^{\circ} \mathrm{C}$. is laid on the front of the forearm. It evokes a hot sensation, then this declines, and a cold sensation is felt pricking through. (3) After a bath of water at $37^{\circ}$ to $39^{\circ}$ of some duration, the foot is passed quickly through very hot water. The first feeling that then ensues is of "cold so distinct and so pure as to be indistinguishable from cold water" ${ }^{3}$ this subsides, and after it is felt a smarting painful sensation. Cold stimuli, even severe, are not found by Alritz to give "warmth" or "heat" sensations.

Some characters of "cold" and "warmth" sensations.-Contact under feeble pressure with an object of the "adequate temperature" elicits from neither a "cold spot" nor a "warmth spot" any tactual sensation. But if the contact, although feeble, be with an object sufficiently cool or hot, it will elicit a corresponding thermal sensation (Goldscheider), which may be combined with sensations which are in part at least tactual. At many, though not at all, thermal "spots," it can also be shown that analgesia exists, at least so far as to tests by pricking with a needle. When tested by a highly-heated metal point, able to elicit almost unbearable smarting, a thermal spot evinces little, or much less, pain, although if the "spot" be a "warmth spot," a hot feeling arises. Goldscheider considers the sensation ordinarily evoked by contact with an object hot enough to be "painful," to be compound of (1) warm sensations of high intensity, due to strong adequate stimulation of "warmth spots"; (2) tactual sensations evoked by contact in accordance with conditions laid down in the previous chapter; (3) pain sensations evoked by the physical heat exciting pain-nerves and their endings, in virtue of its property as a general nerve stimulus." The "burning," painful sensation of a hot body, on this view, owes its painful quality, not to over-excitation of "warmth spots," but to concomitant excitation of pain-nerves by the heat.

When the temperature of the cuticle rises to $48^{\circ}-49^{\circ} \mathrm{C}$., or falls to $12^{\circ}-11^{\circ}$ C., pain, in addition to thermal sensation, results. ${ }^{5}$ The above are average figures. The extreme temperatures at which stimuli, when applied to the skin, still evoke purely, i.e. not painful, thermal sensations (if one may for brevity use thermal in that meaning), varies under various conditions-(1) It is different in different skin regions; ${ }^{6}$ (2) it depends greatly on the duration of application of the stimulus. The hand dipped into water at $50^{\circ} \mathrm{C}$. feels for the first moment a pure "warmth" sensation, without any painful character. The sensation then decreases somewhat, and then increases, rapidly becoming painful. A silver plate of about 4 c.c. surface can be laid on the skin at a temperature of above $110^{\circ} \mathrm{C}$., and allowed to cool there, witlout causing pain, if it be only $01 \mathrm{~mm}$. thick : if it be $5 \mathrm{~mm}$. thick, it will evoke pain and

\footnotetext{
${ }^{1}$ Skandin. Arch.f. Physiol., Leipzig, 1897, Bd. vii. ; also líiesow, op. cit.

${ }^{2}$ Skandin. Arch. f. Physiol., Leipzig, 1897, Bd. vii.

3 Mind, London and Edinb., 1898, vol. vii. p. 141.

+ Goldscheider, loc. cit.

5 Weber, loc. cit.

${ }^{6}$ For details, see Donath, 'UUeber d. Grenze, etc.," Arch.f. Physiol., Leipzig, 1884, S. 694.
} 
" burn." For a similar reason, a hot glass or a live coal can be touched with impunity to pain if the contact last but a very short time. (3) It depends to a remarkable degree on the size of the area of application of the stimulus. This last point entails separate description.

As stated above (under Touch), a stimulus applied to a sensifacient surface, like retina or skin, has an influence on sense not only in virtue of its intensity, but also in virtue of its extension in space as well as in time. So that, coeteris paribus, a stimulus affecting a larger area evokes more sensory reaction than one affecting a smaller area. In accordance with the overlapping arrangement of the mechanisms of the nervous system, a stimulus simply in virtue of greater extent of application to the sensifacient surface evokes, at least in some cases, greater intensity of sensation. This is markedly exemplified in the cutaneous senses of "cold" and "warmth."

If one finger of one hand and the whole other hand are dipped simultaneously into the same cold or warm fluid, the sensation of cold or warmth is not equally intense in the two places; it is stronger in the larger surface. Weber wrote: 1 "This greater strength of impression, which results from the same impression acting upon a greater number of sensory points, is easily confused with that strength of impression which, under other circumstances, results from the fluid having a higher or lower temperature. Cold water, therefore, feels colder to the whole hand, warm water warmer, than to a single finger, in spite of the subject nevertheless knowing that the temperature of the water is the same. If one dips a finger of one hand and the whole other hand into water the temperature of which one is ignorant about, one is deceived. Water at $29^{\circ} .5 \mathrm{R}$. is, if the whole hand is dipped in it, thought to be warmer than water at $32^{\circ} \mathrm{R}^{2}$ into which one dips a single finger. Similarly, one is deceived in case of water at $17^{\circ} \mathrm{R}$. and $19^{\circ} \mathrm{R}$.

It is an important observation of Weber's, that the reinforcing influence which one thermal stimulus, e.g. of cold, has upon the intensity of another (of the same species) simultaneously applied, is greater when the places of application of the two stimuli lie near than when they are far apart. For instance, the difference between the temperatures of two equal surfaces can be distinguished with greater nicety when the surfaces are large than when small, but when the two smaller surfaces are applied not far apart from one another, the discrimination is more exact than between the larger surfaces if the latter be applied a considerable distance from eacl other.

So is it with the pain concomitant of cold and warmth sensations occasioned by thermal stimuli of excessive temperature. The bath comfortably hot to the finger of the nurse, may be very uncomfortably hot to the immersed child or patient. The finger can be kept for any length of time in water at $48^{\circ} \mathrm{C}$., but the whole hand held in, soon feels prolonged immersion painful. On the view taken by Goldscheider, therefore, in pain sense, as in thermal sense, mere areal increase of stimulation induces higher intensity of sensory reaction.

Besides the above factors determining the temperatures at which stimuli cause pain, as well as act on thermal sense, are of course others such as specific heat and the thermal conductivity of the object.

The objective temperature of the sensifacient thermal organs of an area of skin, in which we are conscious of neither cold nor warmth, is called the physiological zero temperature. It is not a constant objective temperature; it differs in different areas of skin,

${ }^{1}$ Loc. cit.

${ }^{2} \mathrm{Gad}$ says water at $27^{\circ} \mathrm{C}$. to the whole hand feels warmer than water at $40^{\circ} \mathrm{C}$. to the finger. This overstates the effect in my own experience, but my sensation gives figures nearer to Gad than to Weber. 
and in the same area of skin, at different times. Its value is determined by "adaptation." When an area of normal skin is resting at such a thermal equilibrium, i.e. is generating in its own sense organs of cold and warmth no trace of sensation detectable by us, that skin has its thermal sense organs in a state of "complete adaptation" to the thermal conditions of the moment. In a comfortable room we feel at no part of the body either hot or cold, although different parts of the surface are at different temperatures, with almost $10^{\circ}$ C. between them, e.g. lobe of ear $28^{\circ} \cdot 8 \mathrm{C}$., tongue $37^{\circ} \cdot 6 \mathrm{C}$. When we pass from such a room to another which is colder, we experience at first a feeling of cold. But this feeling after a time, if the room be not too cold, passes off. Hering says that under such conditions the temperature of the skin, physically measured, is somewhat lower in the second room. But the thermal sense organs within a certain range adjust themselves to the altered conditions, and a fresh physical temperature is taken for the physiological zero. On then returning to the former room, we experience a sensation of warmth; and this persists in decreasing degree until the physiological zero temperature has been re-established at the original skin temperature-as measured by the thermometer-once more. This adaptation there is no doubt is much greater in range and speed in some individuals than in others.

The range of physical temperature through which the subjective zero temperature may oscillate in virtue of "adaptation," can also be illustrated by experiments such as those given above on p. 953 , in which a portion of body surface is brought into contact with objects of various temperature and thermal quality. But it plays a more important part in the formation of certain perceptions than of others. The sensations of cold and warmth evoked from the cutaneous organs are in respect to projection like those of touch, intermediate in kind between visual and muscular. In respect to projection, it is easy to distinguish two groups of thermal sensations, the one group much more projected than the other. Many of our thermal sensations are habitually concomitant with tactual, e.g., we touch a warm object to feel if it is hot, etc.

It has been shown that a cold spot does not evoke a sensation of "touch," if contact of a test-object with it be sufficiently feeble. It does not, however, appear to have yet been shown that by contact more intense no sensation akin to touch is producible at a thermal spot. The glans penis and the cornea, endowed practically only with pain spots and cold spots, yet yield sensations in response to contact which possess some, though perhaps remote, likeness to touch; and these sensations, evoked from these parts by thermal stimuli acting in contact with them, are different from those evoked by thermal stimuli applied without contact, and the difference appears to me one of quality. These sensations in which the tactual and the thermal senses combine are to some extent projected; and to such an extent, even where the pure tactual component must be slight, e.g. in examining fluids (see above, p. 924), as to suggest that the thermal end-organs themselves display some reaction to tactile objects. The warmth perception resulting from contact with a hot body is referred to the hotness of the hot body; the expressions " burning hot" "scalding hot," "comfortably warm," etc., are applied not to any part of "me," but partly to the skin and partly to the heated object. It is noteworthy that in the great majority of instances the thermal sensations accompanied by sensible contact are obtained actively, that is to say, by muscular movements which bring the sensifacient surface into contact with the 
objects that furnish the stimuli. Thus it is, probably, that Funke ${ }^{1}$ comes to place thermal sensations among thuse that do not belong to the passive experience of the organism, but to the active and the most precise. He was thinking of those thermal preceptions in which the quality of the sensation appears to consciousness incorrigibly and forthwith as a property of the object.

But another, and perhaps purer, group of thermal sensations emphasises the attributes that Volkmar, ${ }^{2}$ unlike Funke, ascribes to thermal sense, namely, its origin in passive experience, and its lack of projection into the environment. This group takes origin in reactions to thermal stimuli supplied for the most part through the air. Most such intangible thermal stimuli experienced are applied to large surfaces, and are diffuse in the sense of not sharply limited in area. On a cold morning we complain "we feel cold," in a hot theatre that "we are too warm"; we refer the excessive cold or warmth to ourselves, and not to the surrounding air. It is in relation to this group of thermal sensations that projection is obscure. Inasinuch as a man's body is in part objective to his own "self," a noumenon, and sensible, coming partly into the range of his various specific sensual fields, thermal sensations can be referred by the "I" to the material "me." Most of the intangible thermal stimuli evoke sensation thus referred. Such sensations are strikingly prone to be "pleasurable" or the reverse. And even here the admixture of tactile quality with thermal stimulus has the effect of imbuing the resultant sensations with a projected quality. The "double touch" which contributes largely to spatial perception of the material "me," is still more potent when thermal sensations are compounded with it. The ear feels cool to the hand, the hand at the same time feels warm to the ear. In this case the sensations, although perceived to be due to the temperatures of the body itself, acquire a peculiar quality of the character of a projection.

It is in the forming of judgments upon this group of unprojected, or only slightly projected, "cold" and "warmth" sensations that "adaptation" often becomes especially deceiving. The oscillations of the physiological zero of temperature rob us of all steady criterion for judgments. Hence, in common parlance, we refer to the environment conditions which are internal, as well as fail to refer to it conditions which belong to it. A person coming in from a windy walk often feels a room uncomfortably warm which is not so to others; exercise has increased the circulation in his skin and tended to warm it, but the moving outer air has rapidly conducted off the extra heat; on entering the house, the stationary air there does this less quickly, and his feeling of warmth is referred to "the oppressive heat of the room." Hence opening windows and sitting in a draught with its accompanying risks; though keeping quiet for five minutes, until the circulation has returned to normal, would attain the same end without danger. ${ }^{3}$ In the cold stage of the ague-fit, although the blood itself is warmer than usual, the skin, precluded from it by vaso-spasm, and for that reason pallid, shrunken, and cool, develops an intense feeling of chill. The teeth chatter and knees shake. The cold sensation there is not due to unusual coldness of the environment, but to a starving of the skin from its heat by cutting off the internal supply. The patient, however, in spite of himself, refers the causation to the environment; but not the "cold"; he complains "how cold I am." A room often feels at the same time cold to one person, warm to another; just as the same water feels cold to the finger of one hand, warm to a finger of the other hand, the previous thermal conditions of the two hands having been dissimilar. Though neither ear nor hand may at a given

${ }^{1}$ Hermann's "Handbuch," Bd. iii. Abth. 2, S. 249, etc.

2 "Lehrbuch. d. Psychol.," 1886, Bd. i. S. 303.

3 This nature of the perception is shown perhaps better in other languages than ours, " avoir chaud," "avere freddo," etc. 
moment be producing any thermal sensation, finger and ear being each fully adapted to their physiological zero temperatures, on bringing the finger in contact with the ear, the finger may feel the ear to be cool, the ear feel the finger to be warm.

Chiefly among the great group of intactual unprojected thermal sense reactions lie those which reflexly help to regulate the general temperature of the homoiothermous animal. These reactions can scarcely be considered to come within the scope of this chapter so fitly as under that of animal warnth. Although reactions of which the initiation is due to the operation of the afferent mechanism of the specific senses of "cold" and "warmth," it seems certain that some of these reflexes do not directly influence the stream of consciousness. They are unconsciously executed, except when unusually intense. When sufficiently intense to affect consciousness, they are especially liable to assume pleasurable or painful characters of "feeling."

The liminal difference of intensity of stimulus.--Starting from the temperature sensation evoked by an adequate stimulus of threshold intensity, a very considerable range of intensities of sensation can be distinguished by incremerits of stimuli, and that without otherwise producing any change in the quality of sensation. Between certain limits of temperature the values of the threshold differences are very small. Above, and especially below, those limits the values of the threshold differences become much greater. Fechner, by applying successively to the same area of skin two objects different in temperature but otherwise similar, estimated the smallest difference of temperature (of water) perceptible by the thermal organs of the skin at the area in question. He found the minimal perceptible difference smallest with stimuli between the temperatures of $12^{\circ} .5 \mathrm{C}$. and $25^{\circ} \mathrm{C}$. Using water and the skin of the finger, he discovered that when the finger had been allowed sufficient time in the first specimen of water for, as would now be said, some "adaptation" of its end-organs to the temperature of that water, it could detect differences of temperature to a degree which did not affect his thermometer, although graduated in twentieths of Réaumur units. The range of temperature $\left(12^{\circ} .5\right.$ C. to $25^{\circ} \mathrm{C}$.) between which Fechner found the liminal difference smallest must have applied entirely to "cold" sensation, since the skin of the hand possesses normally a temperature ranging between $27^{\circ}$ C. and $29^{\circ}$ C. ${ }^{1}$ Alsberg, ${ }^{2}$ however, found the threshold difference smallest with stimuli (water) between the temperatures of $35^{\circ} \mathrm{C}$. and $39^{\circ} \mathrm{C}$, a range which, as the highest skin temperature is about $32^{\circ}$ C., ${ }^{3}$ must have applied to "warmth sensation." It might have been expected that the range of temperatures at which the threshold difference was smallest would have lain close above and below the "adequate temperature." Some observers ${ }^{4}$ have indeed obtained this result, notably Nothnagel, who found the range lie between $27^{\circ} \mathrm{C}$. and $33^{\circ} \mathrm{C}$.

As with tactual intensity, the threshold difference between two thermal stimuli applied successively to the same area of skin is much less than between stimuli simultaneously applied to similar but not identical skin areas, even if they lie near together. ${ }^{5}$

Hensen, ${ }^{6}$ to whom is owing the introduction of the hair-æsthesiometer for

${ }^{1}$ Kunkel, Sitzungsb. d. phys.-med. Gesellsch. zu Würzburg, 1886, S. 79.

2 E.g. Lindemann, "Diss.," Marburg, 1863.

4 Deutsches Arch. f. klin. Med., Leipzig, Bd. ii. S. 284.

5 Weber, loc. cit. $\quad{ }^{5}$ Arch. $f$. Ohrenh., 1893, Bd. xxxv. S. 161. 
examination of touch, employed the following method for observing the differential limen or the absolute limen of cold sensation. By opening a valve, a steady blast of air at the temperature of the room was allowed to pour from a pressure-bag at a velocity of $14 \mathrm{cms}$. per second along a lengthy tube of 3.5 mm. diameter. This draught, at $120 \mathrm{cms}$. away from the end of the tube, was distinetly detectable by the moistened skin of the face to be cool. It was sufficient to just bend a glass-wool fibre held at $90 \mathrm{cms}$. from the opening of the tube. By measuring the elasticity of the fibre by pressure on a chemical balance, the force of the draught was found, at $90 \mathrm{cms}$. from the opening, to be much less than 01 mgrm. A thermometer wrapped in moist linen at $90 \mathrm{cms}$. began to fall after 11 seconds, and soon had fallen $\cdot 06^{\circ} \mathrm{C}$. The sensation of cold was quite clearly perceptible after 7.5 seconds of the draught. The stimulus was probably quite intangible, for it scarcely caused flickering in a candle-flane $110 \mathrm{cms}$. distant from the mouth of the tube.

Both by the methods of successive stimuli and of simultaneous stimuli, the differential limen of stimulus value is very different in different regions of the skin. This is clear from Weber's and from Nothnagel's observations. Weber ${ }^{1}$ determined the degree to which at various regions of the body surface distinction can be made between glass phials filled with oil and then warmed or cooled in water, but applied dry. He sought in this way to estimate the degree of thermal sensitivity of the skin regions. The method is not the best adopted for that purpose; but for the questions under consideration his results are of interest. They were as follows:-

Thermal discrimination is at its maximum in the face, especially in the eyelids and cheeks. The eyelids are more sensitive at the canthi than between. The lips, less sensitive than eyelids and cheeks, are, like the former, more sensitive toward their angles of junction than in the middle. This is the reverse of the arrangement of tactual spatial sense. The thermal sensitivity of the nose is least at its tip, and greatest along the lower part of the outer edge of the nostril. It is very poor inside the nostril: in the external auditory meatus it is, on the eontrary, well developed. The skin just in front of the tragus has greater thermal sensitivity than the lip,-again the exact reverse of the distribution of the space-sense of touch. Along the median line of the body, thermal sensitivity is everywhere poor, alike in face, chest, abdomen, and back. Weber found the proximal part of the proximal phalanx of the palmar face of the index finger more sensitive than the corresponding surface of the other fingers; the ball of the thumb more sensitive than the hypothenar region. The skin over the olecranon he found more sensitive than that of the rest of the arm; the skin over the trochanter major more sensitive than that over the crista ilii.

In considering these results, it must be remembered that Weber does not seem to have paid attention to what Hering ${ }^{2}$ terms "the adequate temperature." There is much evidence that the excitability of a sense organ is at any time partly determined by antecedent stimulation. As the visual organ adjusts itself with a different pitch of excitability, becomes differently attuned, under influence of light and of dark respectively, so the thermal sense organs at different temperatures of the environment. When a skin surface under its living conditions of continual accession of heat and loss of heat generates sensation neither of "cold" or "warmth," it is described as "fully adapted"

1 Loc. cit., S. 554.

${ }^{2}$ Hermann's "Handbuch," 1880 , Bd. iii. S. 430. 
to the environment of the moment. For another medium, on contact with it, to elicit from it no sensation either of cold or of warmth, that medium must be of what Hering calls its "adequate temperature." The temperature of the skin being different in different regions, and of different thermal conductivity in different regions, these physical differences, apart from others of physiological nature, necessitate a different "adequate temperature" for media applied at different skin-regions. The distance of the thermal stinuli employed by Weber from the adequate temperature of the part examined, he did not ascertain. It must have varied considerably. If, therefore, the Weber law hold good in any degree for intensity of thermal stimuli, as for intensity of other adequate stimuli, the estimations of thermal sensibility, carried out by his method of difference between thermal stimuli, cannot be properly exact when the "adequate temperatures " are disregarded.

Nothnagel ${ }^{1}$ also examined the degree of thermal sensitivity of the skin by a method similar to Weber's; he also disregarded the "adequate temperatures." He found the eyelids, cheeks, and temples the most sensitive parts of the face; but he found their sensitivity equalled in the skin of the sides of the abdomen. The least sensitive part of the face he found to be the bridge of the nose. The ventral surface of the chest he found more sensitive below than above, the back less sensitive than the ventral aspect of the trunk. The middle line of the body he found everywhere relatively insensitive. $\mathrm{He}$ found the upper arm more sensitive than the forearm, the forearm more sensitive than the hand ; the same diminution of sensitivity in the peripheral direction obtains in the lower limb, but the whole lower limb is less sensitive than the upper. The dorsun of hand and foot he found more sensitive than palm and sole. ${ }^{2}$

The smallest difference perceptible between the temperatures of the metal (copper) dises used by Nothnagel were for different regions of skin examined in different individuals, as follows : ${ }^{3}-$

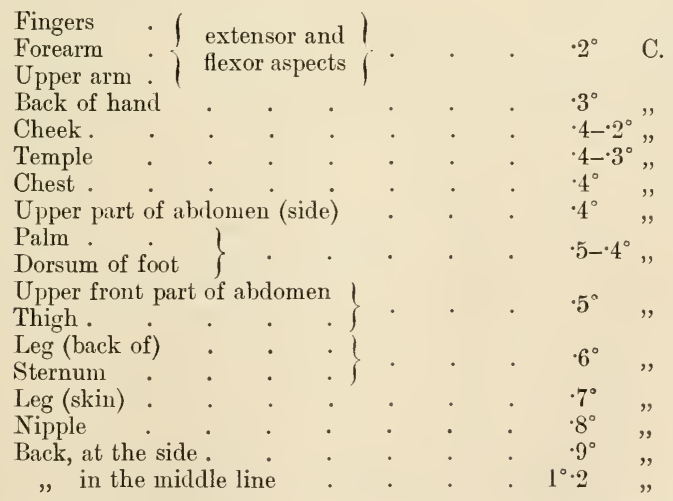

Subsequent to the discovery of "cold spots" and "warm spots," similar investigation of the thermal sensitivity of the various regions

1 Deutsches Arch. f. Klin. Med., Leipzig, Bd. ii. S. 284.

${ }^{2}$ But compare E. H. Weber's observation, loc. cit., given below, p. 962.

${ }^{3}$ Loc. cit., with some observations by Goldscheider, loc. cit., added.

VOL. II. -6 I 
of body surface was renewed by the "difference" method. The results were confirmatory. The differences varied between $2^{\circ} \mathrm{C}$. and $1^{\circ} 1 \mathrm{C}$. for different regions of skin.

It would hardly, perhaps, have been expected that regions endowed with very high absolute sensitivity should be poorly endowed with ability to distinguish difference. Yet a comparison of the performances of the ungual phalanx and nipple shows it to be so. Evidently in testing ability to distinguish, a very complex process is being examined. Absolute sensitivity must, of course, enter into it, but "experience" is probably just as important. The following is an example:-One and the same "cold" stimulus, applied successively to the nipple and the finger, evokes a more intense cold sensation from the former than from the latter, because the "absolute sensitivity" of the nipple is the greater. But with the finger a difference of temperature between two similar objects can be noted, the temperatures of which stand only $\cdot 2^{\circ} \mathrm{C}$. apart, while for detection by the nipple their difference must be $8^{\circ} \mathrm{C}$. Speaking generally, the threshold difference is low in value in regions where "touch" possesses strong local sign. As instanced above, it is a fact, elicitel by nore than one method, that sensitivity to cold and warmth increases as the skin of the limbs is examined in ascending direction from the apex of the limb toward the trunk.

It might have been supposed that that surface of the body habitually protected by clothes would have possessed higher sensitivity to cold than would parts habitually naked. On the contrary, it is found that the face is more sensitive to cold than are many clothed parts of the trunk and limbs. The clothed parts of the body surface are less practised in changes of temperature than the unclothed; the appreciation of degree of change of temperature by the clothed parts has been found correspondingly slight.

Anæmia of the forefinger by holding it above the head diminishes the minimal interval perceptible between two temperatures. ${ }^{1}$ Passive hyperæmia seems to affect it little. ${ }^{2}$ Long application of heat or cold greatly damages the discrimination. ${ }^{3}$

Reaction-time.-On application of a cold object to the skin, the resulting sensation of cold occurs after only a short period of latent excitation. ${ }^{4}$ In its short latency it resembles a "touch." On the contrary, the generation of a warmth sensation is a longer process; the sensation, like that of pain, develops slowly. ${ }^{5}$ When the hand is dipped in cold or hot water, the back of the hand perceives the temperature sooner than does the palm. Weber concludes this is because the palmar epidermis is the thicker. ${ }^{6}$ If both hands are plunged into a deep vessel containing water at $3^{\circ}$ to $4^{\circ} \mathrm{C}$., and the palms lie toward each other without touching, the cold is felt first by the backs of the hands, but after about eight seconds the feeling of cold is greater in the palms than in the backs; hence from the palms, although a higher maximal cold sensation can be evoked, a longer period of latent excitation is gone through: the difference in all likelihood lies in difference of rate of conduction to the end-organs.

If an object at temperature $T$ be applied to the surface of the epidermis, and $\theta$ be the physical temperature of the thermal end-organs, which are at the time at their physiological zero-temperature, $\theta_{1}$ can represent the temperature which the end-organs will take after a certain duration of the application.

I Alsberg, loc. cit.

${ }^{3}$ See Nothnagel, loc, cit.; also Goldscheider, loc. cit.

${ }^{2}$ Alsberg, loc. cit.

${ }^{5}$ Herzen, Arch. f. d. ges. Physiol., Bonn, 1886, Bd. xxxvii.

${ }^{6}$ Loc. cit.; see also for the thermo-conductivity of the skin, Klug, Ztschr. f. Biol., 1874 , Bd. x. S. 73. 
Let it be supposed that the change in temperature is sufficient to cause sensation, e.g. of "warmth." The difference between $\theta$ and $\theta_{1}$ expresses the intensity of the excitation. But $\theta_{1}$ will not be reached instantaneously. Before it is reached, the end-organs will have possessed a number of different intermediate temperatures- $t, t_{1}, t_{2}$, etc. If $t_{3}$ be the thermal state of liminal difference, that is, just sufficient to evoke sensation, it may perhaps be regarded as that which is just sufficient to be a stimulus at the end-organ. On that supposition, all the time passed between the production of the thermal state $t$ and the thermal state $t_{3}$ is lost, not in nervous sensifacient action, but in mere application of the stimulus. The reaction-time, in the ordinary acceptation of the term, commences at the production of $t_{3}$. This period preliminary to the initiation of the sensorial reaction can be reduced by the use of strong thermal stimuli.

The reaction-time differs according as the source of the thermal stimulus comes into actual contact with the skin or not. With strong "intangible" stimuli, the following periods have been found: ${ }^{1}$ -

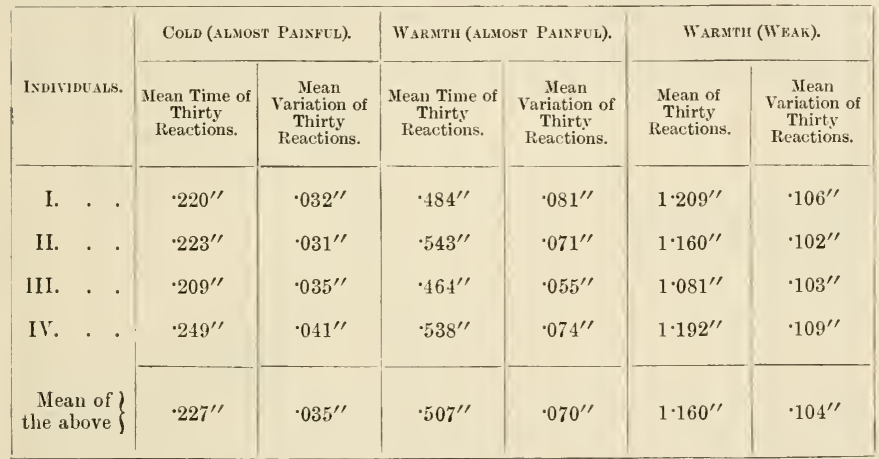

The stimulus used for cold was volatilisation of methyl chloride; that for warmth, the heat from a candle. The reaction-times for the tangible thermal stimuli were found to be shorter-very much shorter. The stimuli used were metal discs, cooled or heated.

\begin{tabular}{|c|c|c|c|c|c|c|}
\hline \multirow[b]{2}{*}{ IXDIVIDEALS. } & \multicolumn{2}{|c|}{ Coud. } & \multicolumn{2}{|c|}{ WARMTII. } & \multicolumn{2}{|c|}{ TOUCH ALONE. } \\
\hline & $\begin{array}{l}\text { Mean of } \\
\text { Fifty } \\
\text { Reactions. }\end{array}$ & $\begin{array}{c}\text { Mean } \\
\text { Variation of } \\
\text { Fifty } \\
\text { Reactions. }\end{array}$ & $\begin{array}{c}\text { Mean of } \\
\text { Fifty } \\
\text { Reactions. }\end{array}$ & $\begin{array}{c}\text { Mean } \\
\text { Variation of } \\
\text { Fifty } \\
\text { Reactions. }\end{array}$ & $\begin{array}{c}\text { Mean of } \\
\text { Fifty } \\
\text { Reactions. }\end{array}$ & $\begin{array}{c}\text { Mean } \\
\text { Variation of } \\
\text { Fifty } \\
\text { Reactions. }\end{array}$ \\
\hline I. & $\cdot 149^{\prime \prime}$ & $\cdot 013^{\prime \prime}$ & $\cdot 180^{\prime \prime}$ & $\cdot 019^{\prime \prime}$ & $\cdot 127^{\prime \prime}$ & $.009^{\prime \prime}$ \\
\hline II. . . & $\cdot 144^{\prime \prime}$ & $\cdot 013^{\prime \prime}$ & $\cdot 127^{\prime \prime}$ & $\cdot 013^{\prime \prime}$ & $\cdot 161^{\prime \prime}$ & $\cdot 010^{\prime \prime}$ \\
\hline III. - . & $\cdot 132^{\prime \prime}$ & $\cdot 009^{\prime \prime}$ & $\cdot 175^{\prime \prime}$ & $\cdot 021^{\prime \prime}$ & $\cdot 130^{\prime \prime}$ & $\cdot 017^{\prime \prime}$ \\
\hline IV. . & $\cdot 130^{\prime \prime}$ & $\cdot 014^{\prime \prime}$ & $\cdot 165^{\prime \prime}$ & $\cdot 015^{\prime \prime}$ & $\cdot 121^{\prime \prime}$ & $\cdot 012^{\prime \prime}$ \\
\hline $\left.\begin{array}{r}\text { Mean of } \\
\text { the above }\end{array}\right\}$ & $\cdot 137^{\prime \prime}$ & $\cdot 018^{\prime \prime}$ & $\cdot 162^{\prime \prime}$ & $017 "$ & $\cdot 129^{\prime \prime}$ & $\cdot 012^{\prime \prime}$ \\
\hline
\end{tabular}

^ Tanzi, "Sulle sensazioni del freddo e del caldo," Milano-Torino, 1886. 
These figures confirm the longer reaction period for warmth sensation, that appears to have first been drawn attention to by Herzen.

The quality of "locality" in thermal sensations.-When heat is applied to the skin at separate areas, there is found to be considerable power of "localising" the places of application. The thermal senses of the skin possess, therefore, like the tactile sense, a spatial quality. Rauber ${ }^{1}$ investigated the question by placing warmed metal balls in perforated wooden plates, which latter were applied to the skin. He found sensory circles for the "warmth" sense corresponding with Weber's circles for touch. He did not test cold sensations. This has been done by Goldscheider, ${ }^{2}$ who has, moreover, worked not with the average liminal distance, but with "cold spots" and "warmth spots."

With excitation of a cold or a warmth spot, as with that of a touchspot, there develops, in consciousness, not only a presentation of quality and quantity regarding the stimulus, but also of locality. By applying punctiform stimuli to pairs of cold spots or pairs of warmth spots, a high degree of "local sign" is found to attach to the resultant sensations. Goldscheider found this quality higher for "cold" than for "warmth" spots.

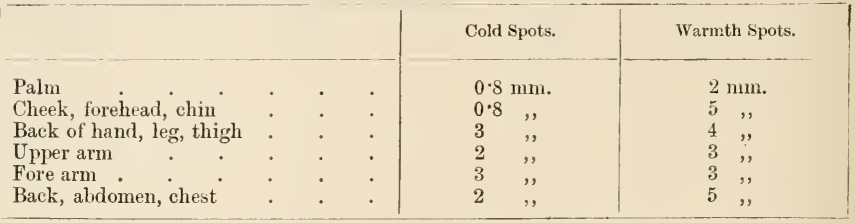

The origin and use of such refinement of localising power for cold and warm stimuli is difficult to understand. It may be that it is necessary in order to obtain perceptions of continuous areas of small dimension. It is to be noted that when a skin surface is excited by heat, the sensation is referred not to a number of individual spots of warmth in the skin, but to a continuous surface,-although the area stimulated is made up of a punctiformly arranged series of sentient places. This is expressed by the overlap of the sensory circles. The more intense the thermal stimulus, the finer the power of localisation (Klug) both for cold and warmth sensations. Two thermoæsthesiometers at the same temperature, when placed simultaneously on the skin, produce a sensation perceptibly double when they lie nearer together, if their temperature be $5^{\circ} \mathrm{C}$. than if it be $20^{\circ} \mathrm{C}$. The distance varies little with stimuli between $20^{\circ} \mathrm{C}$. and $40^{\circ} \mathrm{C}$. The average liminal distance is less with stimuli at $5^{\circ} \mathrm{C}$. than with stimuli at $50^{\circ} \mathrm{C}$. Beyond that temperature the stimuli become painful. To examine, therefore, whether mere distance of the temperature of the stimulus from that of the skin was the cause of the greater efficiency of the "cold" than of the "warmth" stimulus, Klug applied the two stimuli to the arm in a bath in which its skin-tcmperature had been lowered. The average liminal distance was then found smaller for stimuli at $50^{\circ} \mathrm{C}$. than at $5^{\circ} \mathrm{C}$. But the differences obtained in the distance were only small; and the universally richer distribution of "cold" spots than "warmth" spots, together with Goldscheider's positive results, make it clear that localisation is somewhat finer for the cutaneous "cold" sense than for the cutaneous "warmtl " sense.

An important point, somewhat allied to that of localisation, has been mentioned above, namely, that simultaneous thermal stimuli, if of the same species, e.g. cold, produce reinforcement of the physiological intensity one of

${ }^{1}$ Centralbl. f. d. med. Wissensch., Berlin, 1869, S. 273.

2 Loc. cit. 
another; and Weber showed that such stimuli, when applied to surfaces near together, mutually reinforce one another more than when farther apart.

Antagonism of sensations of "cold" and "warmth."-If by the thermo-resthesiometer a sensory circle for cold or warmth has been carefully delimited, and if then two fairly equal thermal stimuli, one cold, one hot, be simultaneously applied within the limits of the circle, a curious sensation results. Czermak, who performed the experiment with use of small test-tubes filled with oil and warmed or cooled, says, "one then distinguishes the sensation due to the cold test-tube quite clearly from that due to the warmed; but, owing to there being no hint of the relative neighbourhood of the two, one is thrown into a peculiar and indescribable confusion," which only disappears when the distance between the two stimuli is sufficiently increased. Klug, using Kronecker's fine-pointed thermo-æsthesiometers, found the average liminal distance not diminished by making one cold, the other warm. When the two points lay at less than that distance, he felt a duplex sensation, but of temporal, not of spatial duplicity. He describes the sensation as alternating cold and warmth, -in fact, resembling the sensation obtained by presenting different colours to identical areas of the two retinæ simultaneously.

Paths of conduction.-The facts mentioned above make it more than probable that the afferent nerve fibres for "cold" and for "warmth" respectively are distinct. The same conclusion is supported by pathological evidence. Herzen ${ }^{1}$ found, in experiments on the cat, that after transection of the dorsal spinal white column, the foot on the side of the lesion was not withdrawn when a piece of ice was applied to it, although the animal at once withdrew its opposite foot from contact with a similar stimulus. ${ }^{2}$

The sundering of the species of cutaneous sensations one from another in spinal disease most frequently takes the form of partial, often severe, impartment of pain and thermal sense, while touch remains intact. It used to be said that in disturbance of cutaneous sense the thermal sensations were the last and least to suffer. Goldscheider ${ }^{3}$ declares that rather the reverse is true. In tabes, syringomyelia, neuritis, etc., it is not very uncommon to find distinction drawn by the disease between sensations of cold and warmth, the one failing, the other not. Isolated impairment of "warmth" is more common than of "cold." 4 Certainly the effect of drugs upon skin sensitivity has marked effects on the thermal elements.

\section{The Skin and Common Sensation.}

The skin contributes, as might be expected, to so-called "common sensation." Its contributions are especially characteristic in certain pains, sexual feelings, the "feelings" of tickling, shivering, shuddering, and the like. From no part of the body can pain more urgent be provoked than from the skin. A question, arising almost necessarily at the outset in dealing with the physiology of cutaneous pain, regards the relation between it and other sensations of cutaneous origin. Is cutaneous pain the product of a specific sense of pain ? Data for complete satisfaction of this problem seem still insufficient. It is necessary, in order

${ }^{1}$ Arch. $f$. $r$. ges. Physiol., Bonn, 1866, Bd. xxxvii.

2 The conduction paths for thermal sensations are referred to in the article "Spinal Cord," p. 866.

${ }^{3}$ Loc. cit. $\quad$ + Schlesinger, "Die Syringomyelia," Wien, 1895. 
to state the problem adequately, to include with it some reference to other pains than the cutaneous. The general trend of the evidence will serve to indicate the direction in which the answer to the special case under consideration will in all likelihood ultimately be found.

Some authorities attach to the skin sensory impressions of "pure contact" 1 and of "movement," 2 which they regard as different from tactual or thermal. Such sensations, however, in so far as they differ from simple tactual impressions, seem to do so rather because of the nature of complex elaborations from tactual impressions. Hence they will not be dealt with here as specific and distinct from touch. That common sensation arising in the skin, of which the chief characteristic is pain, cannot be so dismissed.

Regarding the physiological nature and mechanism of pain there may be taken one of the three following views-(1) Pain may be a feeling inherent in and appearing under certain circumstances in any, each, and all of the separate senses. ${ }^{3}$ (2) Or it may be a quality conditionally arising in and limited to certain only of the species of sense. ${ }^{4}$ (3) Or it may itself be a particular species or mode of sensation, the product, and the only product, at least in certain varieties, of a specific sense. $^{5}$ On the last-named view, it should be generated by the reaction of a special neural mechanism, possessing specifically distinct peripheral nerve-ends, and specifically distinct sensorial mechanisms in the central nervous system.

Common experience teaches that a spot of skin, under moderate pressure, gives merely touch, while under extreme pressure it feels pain. The popular and the at first sight most natural, indeed almost intuitive, view of the causation of cutaneous pain, is that which regards it as resulting from excessive stimulation or reaction of the sensifacient apparatus of the tactual and other species of cutaneous sense. In such a supposition the belief is implicit, that a certain extreme degree of excitement in a sensory centre produces pain. Pain on this view is but a character or function common to various species or orders of sense, and not a sensation of species apart and distinct from the reactions of all other senses. On this view the sensations of each sense can among themselves be different, not only in virtue of difference of intensity, of quality (timbre, colour, etc.), of "local sign," etc., but also by difference of "affective tone" in relation to agreeablenesss and disagreeableness. Such an opinion is consonant with the general experience, that sensations of every species, although in moderation pleasant, become unpleasant when their intensity exceeds a certain degree.

It can be said that lights, sounds, odours, tastes, cold, heat, etc., must, considered as sensations, be "moderate" to be enjoyable. Of conditions that

${ }^{1}$ Meissner, "Beitr. z. Anat. u. Physiol. d. Haut," Leipzig, 1853.

2 Vierordt, Ztschr. f. Biol., 1876, Bd. xii. S. 226.

3 Hagen, "Psychol. Untersuch.," Braunsehweig, 1842, S. 59 ; Lotze, "Med. Psychol.," S. 233 ; Külpe, $V$ rtljschr. f. wisscnsch., Bd. xi. S. 424 ; Alf. Lehmann, "Die Hauptgesetze d. Gefühlslebens," Leipzig, 1892, S. 143 ; Wundt, "Grunaziige d. physiol. Psychol.," Leipzig, 1893.

${ }^{4}$ See, for instance, C. A. Strong, Psychol. Rev., N.Y. and London, 1894, vol. ii. p. 329 ; 1896, vol. iii. ए. 64 .

5 See Munsterberg, "Beitr. z. exper. Psychol.," No. 4 ; v. Frey, "Beitr. z. Physiol. d. Schmerzsinnes," Ber. d. k. sächs. Gescllsch. d. Wissensch. zu Leipzig, malh.-phys. Classe, July 1894, Dec. 1894, March 1895 ; Kiesow, Phil. Stud., Leipzig, 1894, Bd. ix. ; and, with reservations, Goldscheider, "Ueher den Schmerz," Berlin; and previous papers, Arch. $f$. Physiol., Leipzig, 1885 ; Arch. $f$. Psychiat., Berlin, 1887; and "Ueber die Behandlung des Schmerzes," Berl. klin. Wchnschr., 1896, No. 3. 
determine the pleasurable ("positive") or displeasurable ("negative ") tone of sensation, among the most powerful are "intensity and duration." I An intense light or a piercing note evoke sensory disturbances of distinctly "negative tone." With the minimal sensation the agreeableness is nil, but that character reaches its maximum before the total sensation is itself by any means maximal.

Reference is not intended above to the pain in the eye which, apart from visual sensation, can be caused by very strong illumination. That such pain can be caused by light quite apart from any effect upon visual sensation, is proved by its elicitation from totally blind eyes. ${ }^{2}$ It is probably a phenomenon allied to photophobia. Photophobia may occur in the totally blind, and is independent of, although influenced by, the retina and optic nerve. The mechanism of "light-pain" and photophobia is not clear : they are phenomena which led E. H. Weber ${ }^{3}$ to question whether, recurring to an older view, there are not nerve fibres of conmon sensation in the optic nerve. Magendie ${ }^{4}$ detected no reactions whatever on scratching the retina in mammals, amphibia, or fish, but in birds, body-movements and contraction of the pupil followed regularly. It is noteworthy that retinitis may run its course completely without pain, whereas inflammatory processes of the conjunctiva produce intense photophobia.

There are, however, certainly other conditions besides mere intensity and duration which can make sensory impressions agreeable and disagreeable. Some sensations of taste, e.g. bitter, sour, certain odours, possess an unpleasant character-a "negative tone"-almost at the limen of the sensible intensity of the stimulus. Here the disagreeableness seems to be allied, not to the intensity, but to the "timbre" or "colour" or quality of the sensation. Among all simple sensations it is in those of the highest and most projicient senses, e.g. sight, that implication of disagreeable and agreeable seems least an obligatory function of the timbre. Hence one may demur when asked to acceptaffective tone as an immediate quale inherent in all presentation. ${ }^{5}$ When of moderate intensity the majority of simple visual sensations do not, in virtue of mere colour, involve apart from complex associative experience-either delight or pain. Such visual sensations are from the present standpoint "neutral." With the sense of hearing it is different. The concord $c, e, g$, produces a sensation of positive tone, and is as distinctly elicitive of pleasure as are the notes $c$ and $d$, simultaneously struck, provocative of urgent disagreeableness, of-as the very name insists-discord. Between its extremes, imbued with "positive" and "negative" tone, the timbre scale (apart from the intensity scale) of sensation extends a neutral zone untinted, so to say, by either pain or pleasure. On this view a pleasant warmth differs from a painful burn, as the affective tone one of colour, e.g. orange, from the affective tone of another colour, e.g. violet, or as that of one complex tone differs from that of another.

But in accepting the above view, that pain is but a quality of sensation which, under appropriate circumstances of intensity or quality of stimulus, can arise in the domain of any and each sense, certain difficulties are encountered. It must be admitted that pain arises more frequently and more easily and is much more intense in connection with certain senses than with others. If "sense pain," like "local sign," be considered to be a quality of sensation, the admission has to be granted that, although qualities discoverable in a large group of

1 Wundt's "Grundziige," Leipzig, 1893.

2 Ph. v. Walther, Journ. d. Chir. u. Augenh, Berlin, Bu. xxx. S. 360.

3 Wagner's "Handworterbuch," Bd. iii. Abth. 2, S. 564.

${ }^{4}$ Journ. de pluysiol. expér., Paris, 1825, tome iv. pl. 180 and 310.

5 Marshall, Mind, London and Edinb., 1594, N. S., vol. iii. p. 533 ; and "Pain, Pleasure, and Esthetics," London, 1894. 
the senses, they are in certain hardly existent at all." "Painful" may therefore be a quality potential to a certain group of senses, but not general and common to each and all the senses. Nor does it seem to stand in such antithesis to "the pleasurable," as to be potential constantly in those species or orders of sensations, in which the latter is existent or potential. That is to say, a pleasure-pain series seems for some senses an unproven attribute. "Some species of sensations, e.g. certain tastes and odours, seem "unpleasant," even at the threshold of the sensible intensity of their stimuli; and, similarly, other's seem in some individuals to possess from very limen up to maximum a pleasurable, certainly no painful, character, e.g. the taste of "sweet." If stress be laid on this aspect of the question, it can be urged that some species of sensation are characterised in common by the attribute agreeable, and others in common by the attribute disagreable; and that neither the one character nor the other are qualities common to all species of sensation.

The disagreeableness of a vivid colour contrast or of discordant notes is akin to pain but is really a degree of "physical pain?" Is it strictly expressible as a fraction of the agonising torture of a scalded limb? "The nerves which subserve smell, taste, hearing, and sight cannot produce pain; violent irritation of the optic nerve produces intense visual sensations but not pure "pain." 3 "The pain which we feel when the finger is cut is a wholly different thing from the pain given to the most delicately musical ear by even the most horrible discord." 4

With regard to smell, the comparatively poor development of olfactory sense in man makes it very hazardous to assert that in that case "disagreeableness" cannot reach an intensity which, on the first-mentioned theory, is a "pain" equal in violence to any cutaneous or other. The fact that, e.g. in the case of the skunk, emissions of an odour can protect the emitter from carnivorous foes in its "struggle for existence," is evidence of the violence of the unpleasantness that an olfactory sensation can be plausibly argued to attain.

Pain may be only an extreme case of disagreeableness, but some forms of disagreeableness are much more complex mental states than others. The full scope of the terms "agreeable" and "disagreeable" is too wide for the discussion here. They attach to manifold processes ${ }^{5}$ besides simple sensations and perceptions, processes built on "affective tone," whether "negative" or "positive," and attributed to whatever psychical process, as on a foundation. What psychical process, it can be asked, is not? The sight of blood is disagreeable by reason of associated experience of disagreeable sensations. If the intellectually disagreeable is of later development than the physically disagreeable, ${ }^{6}$ it can be argued that all "disagreeable" has arisen out of the physically painful. "Doubtless in lower animals pain is almost the only stimulus." 7

Here it is necessary to guard against expanding the set problem by unwary use of the word "pain." This word will be here limited to phenomena of which everyday parlance speaks as bodily or "physical"

\footnotetext{
${ }^{1}$ On points in this connection, Helmholtz, "Physiol. Optik," Berlin, 1896, sec. 26.

${ }^{2}$ See, for instance, C. A. Strong, Psychol. Rev., N.Y. and London, 1894, vol. ii. p. 329.

3 Vierordt, "Grundriss," Tiubingen, $187 \%$.

${ }^{4}$ Foster, "Text-Book of Physiology," 1900, part iv.

5 Lehmann, "Hauptgesetze des nienschlichen Gefithlslebens," Leipzig, 1892.

6 W. James, "Text-Book of Psychology," London, 1892, p. 69.

7 Ibid.
} 
pain. "Painful" is the affective colour of many psychical states both more complex and less complex.

There are intellectual processes with negative affective tone, and simple sensations with negative affective tone. Of the latter, those belonging to the projicient senses are less strongly coloured than those of the non-projicient. It is the "sense pains" of the nonprojicient senses that constitute bodily or "physical" pain. Physical pain is so eminently obtainable from the skin, that about the question of cutaneous pain discussion as to existence of a specific sense of pain is chiefly turning.

Admitted that the disagreeableness of an intense light or of a discord is not fully comparable with the "pain" of scalded skin, that admission is not tantamount to allowing that cutaneous pain is the product of a specific "pain sense." It can be said that all parts of the body that can produce pain are endowed with nerve fibres normally ministering to a group of senses that can be classed together as yielding common sensation, and that these nerve fibres only under stimulation excessive either in intensity or duration or spatial extent, produce pain. By common sensation is understood that sum of sensations referred, not to external agents but to the processes of the animal body. Its "object" is the body itself-the material "me." Sensations derived from the bodily tissues and organs possess strong affective tone; while the sensations of special sense are relatively free from affective tone.

The scope of the term "common sensation" no doubt has varied somewhat with different authorities." Adopted in contradistinction to "special sensation," the ordinary meaning of the word does not immediately explain its technical use. All afferent nerve fibres were considered to supply impressions to the common sensation of consciousness; the nerves of the special senses were considered to supply, over and above the common sensation which they each supplied in common, impressions of special sensation, each peculiarly its own, optic nerve, visual, etc. Magendie's experiments (1825) of scratching the retina with a needle, exciting the eighth cranial nerve, etc., did something toward removing from the special sense nerves the ascription of functions of common sensation. Johann Miiller, ${ }^{2}$ no doubt under the influence of Kant's philosophy, brought forward the law of the specific energies of sense. Earlier writings by Purkinje, Elliot, Charles Bell, and Autenrieth had paved the way to this; and the limit of the law has been affected since by the Darwinian principles of evolution and adaptation. J. Miuller went so far as not to concede the existence of common sensation (Gemeingefühl) as distinct from touch. He reckoned to the category of tactual the sense impressions derived

${ }^{1}$ For instauce, compare E. H. Weber, Wagner's "Handwörterbueh," Bd. iii. S. 2, with J. Henle, "Allg. Anat.," Leipzig, 1841, S. 728. By some (e.g. K. Vierordt, "Grundriss," Tubingen, 1877 , S. 488), the term is made to include all those sense impressions in which we can discover or observe alteration of our own state of impressibility. By this definition are included in common sensation the so-called "subjective" sensations, originated in the special (so-ealled "objective") senses by internal stimulation, i.e. by conditious arising in the body itself. In this conncetion it may be remembered that the excitation of a skin nerve in its course (e.g. faradisation of ulnar trunk at elbow), it was at one time tanght evoked pain only, not sensations of touch. To refute the teaching, the experiment has only to be repeated. The sensation obtained is usually not painful, although always "strangc." Its strangeness is no doubt explicable by the combination in simultaneous exeitement of nerve fibres in groupings never previously experienced, dependent on their mere position in the nerve trunk. Some are included and some left out in unwonted fashion. Hence the punctiform character of the reference. The above wider scope of the term comnon sensation (Gemeingefühl in weiterem Sinn) seems inconvenient and is not adopted here.

2 "Zur vergleichenden Physiologie des Gesichtsinnes," 1826 ; "Ueber dic phantastischen Gesichterscheinungen"; and "Handbuch der Physiol.," 1832 to 1840. 
from viscera and muscles. Later, E. H. Weber ${ }^{1}$ brought forward reasons for sundering sensations of touch from those of common sensation. Weber was among the earliest (1846) to successfully analyse common sensation into component orders. ${ }^{2}$ He seems to have regarded common sensation as simply the sum of visceral and nuscular components severally distinguishable. Wundt insisted on the summation not being simple, but involving the building of a more or less complex superstructure by psychical synthesis, the synthesis in so far resembling a chemical synthesis, in that from it results an entity as individual as the component entities.

Total common sensation is the result of many component sensations. It has at basis multitudinous simple impressions, its raw material, whence in the psychical factory the concesthesis is produced. It is the sense of the afficirte Lciblichkeit, and is in some ways the antithesis of the Kantian innere Sinnc. The sensations contributing to it might be supposed to take origin in the most various sensifacient parts and organs of the body. As a matter of fact, it is found that stimuli exciting the apparatus of the special senses, visual and auditory, under ordinary circumstances give but little toward "common sensation." The sensations that arise in internal organs and viscera, on the contrary, contribute a great deal. Hence it may be said that it is built of what have been called ${ }^{3}$ entoperipheral feelings in contradistinction to the epiperipheral. What indeed the viscera contribute to "sense" goes entirely toward "common sensation"; under ordinary circumstances, however, their total contribution to consciousness is but small. The prevailing aspect of healthy perceptive consciousness looks not toward the material "me" but beyond it, or it may be said has a direction of outlook not of inlook. "Common sensation" plays, therefore, a subordinate rôle in healthy conscionsmess. The feelings of appetite for food, of need for evacuation of fxees or of urine, etc., all occupy but little of the conscious day. But when visceral sensations are strong, then common sensation plays no subordinate part in consciousness. The forms of special sensation may then be pushed into the background, or attached as mere accessories to the pre-eminent "feeling." This potential intensity-related to emotion-which is latent in visceral sensation will be adverted to again.

Numerous observations, experimental and clinical, from those by Haller ${ }^{4}$ and his pupils, through those by Bichat ${ }^{5}$ and Budge ${ }^{6}$ down to the present day, ${ }^{7}$ show clearly that the viscera, the serous membranes, the mucous membranes, except those lining the inouth, nose, throat, rectum, vagina, and entrance to the urethra, and the abdominal contents, except the kidneys in slight degree, and the generative glands, cannot by irritation with directly and variously applied mechanical, thermal, and chemical stimuli, be demonstrably proven to call forth pain or signs indicative of pain. The kidneys, ovary, and testis, under mechanical compression, do reply, but the first-named does so only very slightly. From their experimental observations on the visceral structures and from

${ }^{1}$ See, among other works, Wagner's "Handwörterbueh," 1846, Bd. iii. S. 1. S. 728 .

$2 \mathrm{~J}$. Hen'e in 1841 wrote of it as an "ungesonderte Chaos," "Allg. Anat.," Leipzig,

${ }^{3}$ H. Spencer, "Principles of Psychology," London, 1881, vol. i. pp. 166 and 250, etc. 1752 .

" "De partibus c. h. sensibilibus et irritabilibus," "Comment, soc. reg.," Göttingen,

5 "Anatomie générale," 1812, tomes i. and iii.

${ }^{6}$ Physiol. des Menschen," Leipzig, 1862.

7 See discussion, Lancet, London, 1892, vol. ii., Gee, Tait, and Sherrington ; also H. Head, Brain, London, 1893, vol. xvi. p. 126. 
similar on muscles, tendons, and joints, carried out with like negative result, Haller, and also Bichat in part, ${ }^{1}$ conclnded that these organs are insentient. Clinical experience shows that the viscera may be freely incised in the unanæsthetised patient, ligated, or pinched, without in the most evanescent or slightest degree affecting the attentive consciousness. The movement of the stomach and diaphragm, one against another, with large excursion and considerable speed, cannot under ordinary circumstances be perceived.

$\Lambda$ ttention cannot be induced to that direction, far less be focussed there. Yet to imagine all these structures insentient were certainly mistaken,-one is misled by the impotence of inadequate stimuli. The heart in its beating produces normally no "feeling," but it nevertheless possesses the in some ways most powerful afferent nerve in the whole body, and under certain conditions evokes all engrossing pain. The voluntary muscles contain numbers of afferent nerve fibres and sense organs, ${ }^{2}$ and can produce urgent sensation. All parts of the alimentary canal, under the vicissitudes of disease, give evidence of capability to affect sense even with pain. Excitations of the central end of the splanchnic nerve, and of many other visceral nerves, uniformly evoke evidence of pain. ${ }^{3}$ In the abdominal and thoracic sympathetic cord are numbers of myelinated fibres, which are the axons of nerve cells in the spinal ganglia, contributing to the afferent spinal roots. The white rami communicantes of the sympathetic are afferent as well as efferent, for by them fibres pass from the visceral nerve-chain into the spinal ganglia and roots. I find a lower thoracic white ramus (tenth thoracic) in a small cat to contain about 150 myelinated sensory fibres; such fibres are therefore fairly numerous in the abdominal sympathetic. ${ }^{4}$ They vary much in size, some being amongst the largest fibres in the sympathetic, but more are among the very small (12 $\mu$ to $1.5 \mu$ or less in the small cat). Some of the larger probably belong to the Pacini corpuseles, numerous in the neighbourhood of the pancreas, uterns, and elsewhere. The existence of abundant afferent channels from the viscera into the central nervous system is therefore as definitely proved by physiological researcli as is the existence of visceral pain demonstrated to bedside observation. Yet the impulses generated in the afferent nerves of the viscera, although they embonch into the central nervous system (vin the spinal and the vagal ganglia), do not appear under ordinary circumstances to evoke conscious reactions. Some authorities teach that they generate constantly "obscure sensations." "If the whole of our abdominal viscera were removed, we should be aware of the loss." 5 The supposition is one not of course to be tested by experience. Rather than that the viscera constantly generate obscure sensations, it would seem to me that some of them at least initiate intermittently with pliasic regularity sensations that are far from obscure, as well as viscero-motor, secreto-motor, and other reflexes outside the pale of consciousness. Sensations would seem to arise from the viscera under ordinary circumstances chiefly, as might be expected, in relation to exercise of their normal functions. The functional "activity" of most viscera is phasic ; sensations accompanying the "activity" phase seem pleasurable, not the reverse. In the prolonged pliase of pause for preparation, preceding the pliase of "action,"

1 Bichat did not deny the sensitivity of muscles. Sehiff, however, did so absolntely, "Muskel- und Nervenphysiologie," Lahr, 1858, and so have others.

"Sherrington, Journ. Physiol., Cambridge and London, 1893, vol. xvii. ; Brit. Med. Junrn., London, 1893, vol. ii.

${ }^{3}$ Bichat, "Anatomie générale," Paris, 1802 ; Budge and others.

4 There appear to be none in the cervical sympathetic. Bichat remarked on the insensitiveness of the superior cervical ganglion and the cervical sympathetic, "Anatomie générale," Paris, 1802, tome i. p. 24. Cf. Budge and Waller; also Langley, Phil. Trans., London, 1892, vol. clxxxiii. 1). 117. I found all (except seven) the nerve fibres in the cervical sympathetic of the monkey degenerate after section of the moto spinal roots of the lowest cervical and highest six thoracic nerves.

5 Foster, "Text-Book of Physiology," 1891, pt. 4, p. 1421. 
the call for repetition of the "activity" of the organ gradually springs up and cumulatively increases. The endings of the afferent visceral nerve fibres are in this phase excited with increasing urgency, by adequate stimuli, of the quality of which we are ignorant, but these stimuli may be in many cases of mechanical kind. Summation perhaps plays a greater part in rendering them effective than in the case of the adequate stimuli of the organs of the special senses. The sensations they evoke seem almost all of them imbued with displeasurable "tone," and they can culminate in physical pain.

An essential constituent of common sensation derives from the locomotor apparatus of the body, e.g. muscles, joints, etc. But the contribution by this factor is not so copious as might have been supposed. There is a large diversion of its stream of ingoing impulses to channels feeding the production of presentations of environmental agents. In fact, the sensations from muscles which influence, by their contractions, the various sense organs, may almost be said to be lost in contributing a further quality to the sensations of the sense organs which the muscles in question influence; for these they standardise their spatial quale. They, many of them, acquire thus in a secondary manner some projection. But their justifiable inclusion in common sensation is shown by nothing more clearly than by their relation to pain. Under circumstances for the most part exceptional, they contribute "fatigue," "cramp," "rheumatic" pains, etc., to common sensation.

Cutaneous sensation occupies in some ways an intermediate place between the forms of special sensation and of common sensation. ${ }^{1}$ Cutaneous sensation has, according to its species, a variable degree of projection, but never that degree attaching to special sensation. The ether vibrations which, impinging on the retina, evoke perception of a light shining at a distance, impinging on the cheek, evoke perception of a warm spot of skin. Even most " touches" are perceived not so much as pure signs of the environment, as of our conscious "self" reacting to an agent. But the position of cutaneous sensations in regard to common sensation will depend on to what extent the cutaneous sensations be regarded as separable into specific kinds. If the view taken in the articles on "touch" and "cold" and "warmth" sensations be accepted, then the specific "warmth" and "cold" senses are, especially the former, further from the special and projicient senses than is the pure "touch sense"; the converse is their order of propinquity to common sensation. On the view that all these three senses can each produce physical pain, all three are clearly contributory to common sensation.

On this view the, as they have been called, "2 "regular" stimuli provoke sensations less related to common sensation than do irregular excessive stimuli applied to the same cutaneous apparatus. The important point for the present argument is, that on this view the limits of physical pain include certainly several senses, a group of senses. Its most familiar ministers are perhaps cutaneous nerves, but in respect to it other nerves, those of deep structures, have to be placed beside them. Hence the classification of pains into "superficial" and "deep" ${ }^{3}$ and, on the above view, the existence of the latter class is of itself a guarantee further that non-painful sensations arise from the deeplying structures of the body.

1 "I can truthfully say, I feel the paper at the end of this pen; but with pain it is less that I feel pain than that, in Condillac's phrase, 'I am pain' ; language recognises this in such diction as 'I ache", (see Fechner's observation, Wagner's "Handwörterbuch," 1846, Bd. iii. S. 2).

"See A. Fick, "Physiol. des Menschen," Wien, 1882, S. 133.

${ }^{3}$ Xavier Bichat, "Ścusation externes et internes," "Anatomie générale," Paris, 1802, tome i. p. 165 . 
Under many circumstances on these deep sensations are grafted "pains." It is customary to consider such circumstances as lead to pain "abnormal," and therefore to treat the "pains" occasioned as reactions to abnormal occurrences. If to do so be but a propitiatory compliment to a well-intentioned scheme that helps to minimise harm, well and good; but with an environment infested by conflict, disease, and mischance, to call such "pains" abnormal reactions is merely a question of personal taste in the adjustment of the relativity of terms. With an organ so open to injury as the skin, the application of a certain percentage of stimuli noxious, from excess of intensity or duration, may be regarded as strictly normal. Of the more sheltered deep structures of the body, to arbitrarily say the same is, at least, as defensible as to say the reverse. The consideration possesses meaning for any attempt to trace the evolutionary history of "pain sensations."

It is obvious that the sensory structures, cutaneous and deep, which possess the power of calling up pain (in that term's restricted sense adhered to here) have as common attribute the non-projection of sensations. The kinds of sensation which the mind most fully projects, the "objective" sensations, as sometimes termed, seem always reactions to objects (noumena) that can affect as identical stimuli simultaneously, more than a single individual mind. Although a sensation obtained from the skin does, in many cases, include perception of an external agent causally connected with the sensation, it seems invariably also to include perception of the "material me" (afficirte Leiblichkeit). In such cases the agent acting on the skin is a noumenon that can affect as an identical stimulus successively, but not simultaneously, more than a single mind. The senses which deal with agents not similarly appellant to several minds at once are deficient in projection. I feel the radiant energy from a lamp with the back of my hand, I see it with my eye ; the latter's sensation does nothing to remind me of my own existence, the former's calls my attention to my hand. And there are a large number of instances of excitation of cutaneous, muscular, and visceral sense nerves in which no external agent whatever is perceived, and the perception relates exclusively to the "material me." In these cases the stimuli are furnished by objects, agents, which neither successively nor simultaneously can affect, as identical stimuli, more than a single mind. That one is the " $\mathrm{I}$ " resident with the particular "me," of whose material the stimulant agents are themselves part (actions). Concerning this category of internally initiated sensations, it will be obvious from the foregoing, that for their description the external world offers no adequate symbols whatsoever. There is no true basis for a convention of terms by which they can be made describable. Some of them, however, after a partial fashion, offer approach to conventional description, in virtue of their frequence among numerous individuals. An empirical standard for comparison of them becomes practicable, in proportion to their frequence and extension among a community. Hence skin-pains are more describable than others, for applications of painful stinuli to the skin are so common as to be-as above urged"normal." Indeed, the skin may be said to have evolved for itself a special sense of its own injuries. But where, as in consequence of disease, from time to time and from individual to individual, the deep organs vex the "normal" flow of consciousness, sensations are experienced which are, so to say, singularly individual and doomed to remain indescribable. The sensible universe has no suitable symbols for their expression. In this difficulty it is true that with intellectual health the sentient mind reflecting on the body often can, as in the case of projected sensations, rightly interpret that which it does feel. But this it does not with the same assurance, there being no such accessible touchstone of rightness or of wrongness for criterion. Yet one attribute of these sensations, otherwise indescribable though they be, the mind is never at a loss about. There is always distinct in them a pleasurable or a displeasurable "tone." 
Our sensations have as their end knowledge of the external world, but knowledge of that world in relation to ourselves. Mind rarely, probably never, perceives any object with absolute indifference, that is, without "feeling." In other words, affective tone is the constant accompaniment of sensation. Every form of common sensation is based on perceptions of an altered condition of the body itself. In connection with this comes the fact that all forms of common sensation possess significantly pre-eminent attributes of physical pleasure or physical pain. These reactions of the sensifacient organs, yielding perception of altered conditions of the material "me," the mind seems to view with less of philosophical indifference than it can usually behold others, e.g. those of the so-called "objective" senses. Few forms of common sensation leave the mind " unmoved." All are linked closely to emotion, and, as that term implies, all operate powerfully on motor mechanisms. Certain muscles-the exponents of expression-are almost uncontrollably excited. The reaction plays also upon the local musculature in a manner curiously imperative.

The facility of path of these motor reflexes colligated to pain, hints at their antiquity, or at their having been formed by some neural method particularly able to, as it were, make a good road. Each reaction that employs a neural path seems to smoothe it by sheer act of travel. This is true even of slight impulses-light traffic-and more true of heavy. Pain reactions are to be regarded as very heavy traffic. Their impressions summate with peculiar ease, take correspondingly long periods to subside, and, to judge by their inertia, move generally masses of neural material relatively great. Such impressions might wear a road with quite especial speed. Many spinal reflexes imply, so to say, well-worn habits based on ancient pain reactions. One is almost emboldened to figuratively imagine them as connate memories of the spinal cord. The majority of them seem to be protective reactions that in organisms of high neural type are accompanied by "pain."

Pain seems the psychical adjunct of protective reflexes. A pain reaction is in so far like a nauseous taste attaching to a poison. The temperature at which the skin begins to evoke pain $\left(48^{\circ} \mathrm{C}\right.$. $)$ is that at which nerve substance begins to suffer injury. ${ }^{1}$ The sensation caused by the pressure of a sharp point (e.g. needle) on the skin is felt to be painful just below the pressure sufficient for the instrument to break into the skin, i.e. the stimulus becomes painful just before it does injury. True, that the material "me" usually affects little the flow of healthy consciousness. Nevertheless, on the existence of that material "me" depends the very existence of that consciousness. It is the material "me" of which Lotze's trodden worm thinks when it contrasts its own suffering self against the whole remaining universe. The directness of nexus between excitation of pain on the afferent side and certain especially localised movements on the efferent, is argued to be the "instinctive" outcome of this, and of "protective" significance. The plan of defence often lies patent in the character of the movement. The tail is reflexly twitched away from the side to which the painful stimulus is applied. ${ }^{2}$. The pain reflex causes the pinched foot to be withdrawn. The epigastric pain of hunger can be relieved by putting food into the stomach; the faucial pain of thirst by the swallowing of fluid. Both " needs," so long as the cerebral hemispheres are in function,

${ }^{1}$ E. H. Weber, loc. cit.

${ }^{2}$ E. Pfluger, "Die sensorischen Functionen des Rückenmarkes," Berlin, 1853. 
excite the active search for food. The drawing in of the muscle of the abdominal wall, which is the motor reply elicitable by the testicle, may be interpreted as protective in reference to its original intraabdominal location.

How far the pain sensation is a part or an accompaniment of the reflex reaction, which by calling upon motility protects the individual, is questionable. Ordinarily the two are concomitant and colligated. Many ${ }^{1}$ hold the motor reaction to be secondary to the mental. There are, however, those who hold that in the case of the "feelings" the psychical effect is secondary to the efferent vascular ${ }^{2}$ and visceral. ${ }^{3}$ Of the coarser emotions it has been argued by James that the feeling does not cause, but is caused by the bodily expression. The bodily changes, according to him, follow directly the perception of the exciting fact, and our feeling of the same changes as they occur is the emotion. Certain experiments furnish evidence-not highly satisfactory - that all pleasurable states of consciousness are accompanied by bodily movements of extension, and all painful by movements of flexion. These movements may be very slight. Münsterberg ${ }^{4}$ concludes that the feeling of agreeableness is the mental accompaniment and outcome of reflexly produced movements of extension, and disagreeableness of the movements of flexion. Of the pain-feeling and the painreflexes which are colligated results of certain stimuli, the latter, the reflexes, seem to persist under conditions of mutilation of the central nervous system, that are by nost accepted to preclude the possibility of conscious reactions, and in disease where feeling of pain is lost. ${ }^{5}$ Granting the weight of evidence preponderates against the possession of capacity for pain by animal forms of low neural organisation, ${ }^{6}$ vestiges of the motor reflexes accompanying pain in higher forms are nevertheless possessed by them. The motor part of the total "pain reaction," both somatic and visceral, may have the more ancient evolutional history. Sense pain of high intensity may therefore be a means comparatively recently evolved, and now in evolution, for the better protection of the higher organisms. It is probable, however, that in the earlier grades of evolution of sense all species of sensation were more equally endowed with affective tone. The skin, unlike the sheltered viscera, is bare to the vicissitudes of the environment. Its more abundant opportunity has led in it to the more complete evolution and education of a sense of pain. Feeling of pain works beneficially for the preservation of the organism ${ }^{7}$ possessing it, and since it is in action before, and is maintained until the end of sexual naturity, favours directly the preservation of the species. In the struggle for existence it is doubtless one of the most potent weapons nature has devised. But it seems to predicate a certain advanced grade of neural organisation. There is little evidence that it is present in lowly organisms. To those possessing it-the higher animal forms-it dispenses an enormous competitive advantage. It is perhaps the primary determinant of "will." 8 It is noteworthy that in the human race there is some evidence that it is more developed in civilised than in uncivilised populations. The theory of utility is merely a conscious

${ }^{1}$ C. Bell, "Anatomy and Philosophy of Expression," London, 1806; H. Spencer, "Principles of Psychology," London, 1855, vol. ii. ; C. Darwil, "Expression of the Emotions," London, 1873; Duchenue, "Mechan. d. physionomie humaine," Paris, 1862 ; Gratiolet, "Physionomie," Paris, 1866, etc.

2 Lange, "Ueber Genuthsbewegungen," Leipzig, 1887 ; Sergi, "Dolore e piacere," Milano, 1894 ; Riv, di sociol, 1896 , tome iii. p. 23.

3 James, Mind, London and Edinb., 1884, vol. ix. ; "Psychology," vol. ii. p. 457 ; also Psychol. Rev., N. Y. and London, 1894, vol. ii.

4 "Lust and Unlust," "Beitr'. z. exper. Psychol.," No. 1.

${ }^{5}$ See H. Weber, op. cit., p. 520 (footnote).

${ }^{6}$ See Loeb, Arch. f. d. ges. Physiol., Bonn, 1894, Bd. Ivi. ; W. W. Norman, ibid., 1897, Bd. lxvii.

7 H. Spencer, op cit., vol. i.

${ }^{8}$ See Brentano, "Psychol.," 1874, ch. viii. 
reckoning upon it." "Economics as a science bases itself upon the conscious choice of pleasure." 2

Nothing said above disallows of the term " common sensation" being taken to embrace the whole office of the cutaneous senses, touch, cold, warmth, as well as the senses of the musculo-articular apparatus, and of the viscera, on the assumption that all these share in common the incapacity to "project" their sensations, and to provoke, under conditions of excessive stimulation, pain. On such a view the nerves of tactual and thermal sense, under appropriate circumstances, produce cutaneous pain; and the nerves which furnish the sensory impressions at basis of the perceptions of the "muscular sense" likewise, under appropriate conditions, produce the "ache" of muscular fatigue, the "gnawing" pain of hunger, the "cutting" pains of visceral cramp, etc. So also, on the same view, the feeling of thirst and the pain of cardialgia may both be the manifestations of the same gastric nerve fibres. But on this view the old difficulty encountered above, regarding the specificity of pain, has to be met with again, although in modified form.

Pain as a skin sensation is suigeneris, incapable of confusion with the sensations evoked by other, usually lesser, stimuli. It is not as if some painful component inherent in cutaneous sensation from the limen upwards increased, and increased disproportionately as the reaction grew more violent. There is no trace of the painful in a moderate " warmth sensation." Though musical notes of different pitch can be confused by a mind not musically trained, there is no chance of confusion in any mind between sensations of, for instance, pure "cold" and "pain." A pain sensation evoked by pressure from the skin does not quà sensation appear to introspection as an intensified touch sensation, though the stimulus for the former may be merely the intenser application of a contact. The degree of warmth sensation that a burn gives need not be more intense as a sensation of "warmth" than is a painless warmth sensation; in fact it is often less so, for the new sensation of pain suppresses sensations that might be simultaneously evoked.

The statement arrived at, therefore, comes to be: If the forms of common sensation as a group be characterised by want of projection and potential pain, among cutaneous sensations are certain which fall into the category of common sensation. The skin, therefore, contributes to common sensation; that is, nerves of common sensation are distributed to the skin. But it does not conversely follow that all cutaneous nerves are nerves of "common sensation." Are the nerves of common seusation identical with those of touch and warmth and cold, or with one or other among them, or with none of them?

The central neural mechanism for cutaneous "common sensation," e.g. pain, is certainly largely distinct from that of touch and temperature. This seems clearly indicated on examining both pain and the "feeling" of tickling. Tickling, it is sometimes taught, results from stimulation of the purely tactual nerves. The mechanism of the matter probably is that weak tactual stimuli call into reflex activity unstriated muscles of the skin. The contraction of these excites the feeling of shivering which combines with that of the light touches. In that case it is conceived to be a peculiar psychical elaboration from tactual and muscular impressions. It may be so, and it may stand in somewhat the same relation to the touch spots of the skin as does pain to the pain

1 See Patten, "Theory of Soeial Forces," Philadelphia.

${ }^{2}$ Witmer, "Twentieth Century Practice of Medicine," 1897, vol. xi. p. 919. 
spots. It is certainly a form of common sensation, and as such less comparable with a touch perception than a pain. In it a series of light, painless, mechanical stimuli, applied over a surface of skin, evokes hardly controllable action of muscles ordinarily secure to the will's mastery. This recalls the uncontrollable reaction of so-called volitional museles under pain. Summation of stimuli, which forms a characteristic part of the process of production of pain, plays also a prominent rôle in the production of the tickling "feeling." In both occurs, in consequence of summated sensory stimuli, uncontrollable nervous discharge into a muscular field with elements of triple kind, namely, sympathetic (cardiac and goose-skin reflex), ${ }^{1}$ respiratory, and volitional, the last including characteristically the muscles of emotional expression,--indeed, as all three kinds concurrently react along with the emotion, all may in a sense be termed emotional. The involuntary motor nerves played on are sympathetic, e.g. the pilomotor, sudorific, cardiac, vasomotor, etc. ${ }^{2}$ The pulse is altered; the volume of the arm decreases under pain, that of the brain increases. The semi-voluntary are those of respiration; the voluntary, those of expression, and those that move the part which is the seat of application of the stimulus. An ordinary "touch" does not in this way, by a touch-perception, affect the visceral, respiratory, and emotional muscles. The two reactions may be briefly contrasted. A touch evokes uncontrollably a perception of some part of the environment, may or may not evoke a movement, and does not affect the visceral and mimetic musculature. A cutaneous pain evokes uncontrollably a movement (of defence), and uncontrollably an emotion with its obligate visceral and mimetic movement of expression ; it may or it may not evoke a perception of the environment, although it evoke a reference to the body itself.

The central lines of propagation of the disturbance, provoked by ordinary touch on the one hand, and by painful touch or tickling touch on the other, seem therefore different. The latter irradiates into motor paths, other than those to which the former travels; and even where the directions of the irradiation in the two cases partially lead toward the same centres, these are reached, in the case of tickling and pain, by lines of approach seemingly less open to inhibition from above than those employed by the reactions of pure tactual or thermal order.

Here may be adduced the well-known observation by Schiff, ${ }^{3}$ confirmed by Herzen. ${ }^{4}$ If in the lower cervical region the cord be transected, with exclusion of its dorsal columns, each touch or light handling of the skin, behind the segment of transection, calls forth signs that the animal "feels." The rabbit raises its head, moves its "whiskers," opens its eyes, or gives other signs which, taken together, amount to unequivocal evidence. On the other hand, if the touch stimulus be intensified and converted into a painful stimulus, even of the most extreme kind, there is no further response than that evoked at outset by the touch. Similarly, Schiff states, if a nerve trunk be bared and then lightly taken between forceps, there is an initial start, and after that any further pinching or compression of the nerve remains ineffectual. It is argued from Schiff"s observation that "painful" impressions are conducted, not viô the long ascending afferent root fibres of the dorsal columns, but vic $\hat{\imath}$ the grey matter. The experiment certainly shows that if Schiff's interpretation of the signs, as indicating touch and pain, be accepted, conduction for the former occurs along the dorsal white columns, and that the evidence of pain remaining

${ }^{1}$ J. Mackenzie, Brain, London, 1893, vol. xvi.

2 A. Mosso, "Circolazione nel cervello humano," Milano, 1881 ; "La paura," Milano, 1886 ; Alf. Lehmann, "Die Hauptgesetze d. Gefuihlslebens," Leipzig, 1892 ; Feré, Rev. Phil., Paris, tome xx. ; E. A. Wright, Brain, London, 1895, vol. xviii. p. 217.

3 "Physiologie," Lahr, 1858 ; "Recueil des mém.," Lausanne, 1896, tome iii.

${ }^{4}$ Arch. f. d. ges. Physiol., Bonn, 1886, Bd. xxxviii.; also, "Eléments d. physiol. humaine," par A. Waller, trad. par A. Herzen, Paris, 1898 (note on 1. 601).

vOL. II.-62 
negative, in spite of employment of strong "painful" stimuli, conduction of pain impressions does not travel along the dorsal white columns. It appears probable that the path for pain involves a spinal relay cell, or several such, instead of the long direct root-ganglion path.

Budge concluded that a powerful stimulus produces-(1) a local reflex, by short fibres entering the grey matter, in the immediate vicinity of the entrance of the afferent root in the cord; and (2) a "feeling" of pain from centres in the bulb, reached by conduction through and up along the grey matter of the spinal cord. That the path of upward conduction for pain lay in the spinal grey matter is a conclusion in which, on the basis of his own experiments, he follows Stilling, Schiff, and Brown-Séquard. ${ }^{1}$

Schiff ${ }^{2}$ and also Budge ${ }^{3}$ concluder from vivisection experiments-in which, as the latter carefully points out, the character of movements is the only guide that the observer has regarding the provocation of pain-that only those fibres of the afferent spinal roots provoke pain, which end soon after entering the cord in the grey matter of the cord. Budge, especially, exhaustively examined by irritation the various portions of the central nervous system, to determine those which, when so examined, seem, as judged by the reactions, to provoke pain. He obtained no evidence of painful reaction from the cerebral hemisphere, including corpus striatum. The optic thalamus gave dubious indications, which Budge was inclined to attribute to experimental implication of the neighbouring cerebral peluncle. The deeper and median parts of the corpus quadrigeminum anterius, he concluded, could occasion severe pain, so also the corpus quadrigeminum posterius. From the corpora quadrigemina to the lower end of the fourth ventricle was a region, irritation of which always seemed to evoke pain, unless the irritation were confined to grey matter. Removal of the corpora quadrigemina on one side (after ablation of the hemisphere on that side, which was unproductive of effect as regards pain) in the rabbit produced at first marked diminution of sensitivity to pain on the same side of the body (rabbit), followed by hyperalgesia on that side.

Hence Schiff ${ }^{4}$ wrote of the grey matter as "rsthesodic." Budge 5 describes, as an experiment for demonstrating the insensitivity of grey matter, the pricking with a needle of the grey matter of the calamus scriptorius; the animal remains perfectly unmoved thereby, a touch on the adjoining white matter evokes a violent reaction.

Other experiments which bear on the central path of conduction in the cord, are those in which various degrees of transverse and longitudinal section of the corl have been performed. Stilling, Schiff, and Budge (see above) believed that the impressions eliciting pain crossed to the grey matter, opposite the side of their entrance, and ascended in it to the bulb. They were under influence of Gerlach's supposed network of conducting fibres in the grey matter. For them the grey matter was a continuum, as it is not now believed to be. There is now no evidence of any but short fibres in the grey matter, and any prolonged conduction up it, if possible, would involve transition across many synapses; and every synapse probably introduces a resistance, so that the resistance of such a path, if the path were possible, would probably be enomously high. Experiments show the grey matter incapable of conducting a long distance. ${ }^{6}$ Brown-Séquard, ${ }^{7}$ Ferrier, ${ }^{8}$ and Aldren

${ }^{3}$ Gaz. méd. de Paris, 1854, $1855 . \quad$ 2 "Physiologie," Lahr, 1858, Bd. i. S. 242, etc. 3 "Untersuch. ueber d. Nervensystem," 1841, Bl. i. S. 10 ; and, finally, "Physiol. des Menschen," Leipzig, 1862.

"Op. cit. 5 "Physiol. des Menschen," Leipzig, 1862.

S. 340 . Sitzungsb. d. k. Akad. d. Wissensch. math.-naturv. Cl., Wien, 1879, Bd. 1xxx.

7 "Lectures on the Physiology and Pathology of the Nervous System," Philadelphia, 1860 , 1. 30 ; Journ. de l'anat. et physiol., etc., Paris, 1869, tome vi.; Lancet, London, 1868, vol. ii.

8 “Functions of the Brain," London, 1886, p. 51 ; also Lancet, London, 1890, vol. i. p. 1416. 
Turner, ${ }^{1}$ believe that pain impulses cross almost immediately after entrance into the cord, and pass up the opposite half of the spinal cord. Miescher ${ }^{2}$ and Woroschiloff ${ }^{3}$ arrived at the result that, in the rabbit, the impulses ascend the lateral columns, and $\mathrm{I}^{4}$ obtained the same result in the dog, using blood pressure reflexes as sign. Weiss, ${ }^{5}$ Homén, ${ }^{6}$ Osawa, ${ }^{7}$ Mott, ${ }^{8}$ Bottazzi, ${ }^{9}$ and Holtzinger ${ }^{10}$ conchude the conduction involves both lateral columus, the tautomeric about as much as the crossed. In all this experimental work on animals the observer has to work through signs of subjective states incomparably inferior in most instances to the verbal communication establishable with an intelligent human subject. Clinical pathology, therefore, can render great service here.

Vieusseux ${ }^{11}$ - a Swiss physician (1818), suffering from spinal disease-noted with surprise that the fingers of his right hand, though analgesic to such degree that they bore without pain any degree of pressure or wounding, could feel and judge a patient's pulse quite well. Since then, especially since E. H. Weber's researches, ${ }^{12}$ many cases of "dissociation" paralysis of the skin, have been observed as the result of spinal diseases, including pressure lesions, traumatic injuries, syringomyelia, tabes dorsalis, leprosy, etc. In certain stages of ether narcosis a similar phase of dissociative paralysis occurs. ${ }^{13}$

This sundering of the different species of skin sensations one from another in spinal disease, most frequently takes the form of partial, often severe, impairment of the pain and thermal sensation, while tactual remains normal or nearly so. Mechanical stimuli are felt by the skin with normal delicacy as "touches," while stimuli that normally are painful are felt merely as touches. This, I find, occurs at the edge of an isolated spinal root skin field. It also vecurs in diseases of the spinal grey matter (e.g. syringomyelia, gliosis).

In these latter it forms, with certain features of distribution, a pathognomonic condition. Contact with the finger-tip, a brush, the head of a pin, is normally perceived and properly localised; but with this may coexist complete analgesia, to mechanical as well as thermal irritants. The patient no longer feels a pin-prick or a strong faradic current as pain. Ability to distinguish differences of temperature may be totally gone. The sense of pain of the deeper parts is also impaired, so that fractures of the bones are no longer felt to be painful, and extensive surgical operations can be performed on the patient without pain. The mucous membranes often participate in the impairment of temperature sense, so that patients may eat too hot food without noting its harm. Careful observation of the exact size and position of the lesion in the cord producing this condition gives the following indications as to the position of the painpath. Certain eases ${ }^{14}$ seem to prove pretty clearly that destruction limited to one dorsal (posterior) grey cornu in a segment of spinal cord entails loss of sensation for pain (and temperature) in the corresponding segmental slin field (not a peripheral nerve field) on the same side of the body. Instances show that where both dorsal grey horns have been injured, but one more than the other,

1 Brain, London, 1891, vol. xiv. p. 496.

2 Arb. a. d. physiol. Arst. zu Leipzig, 1870, S. 172,

3 lbid., 1874, S. 99.

${ }^{4}$ Sherrington, Brain, London, 1886, vol. ix. p. 342.

5 Sitzungsb. d. k. Akad. d. Wissensch., Wien, 1879, Bd. lxxx. Abth. 3.

${ }^{6}$ Compt. rend. Soc. de biol., Paris, 1883, p. 23.

7 Thesis, Strassburg, 1882.

8 "Proc. Physiol. Soc.," 1891, Journ. Physiol., Cambrilge and London ; and Phil. Trans, , London, 1892 , pp. 1-58.

${ }^{y}$ Centralbl. $f$. Physiol., Leipzig u. Wien, 1894 Bd., S. 530.

10 Neurol. Centralbl. (original), Leipzig, 1894 , S. 642.

11 Mehlis, "Commentatio de morbis," ete., Gottingen, 1818.

12 Wagner's "Handwörterbuch," 1846, Bd. iii. S. 2, especially case reported on S. 520.

${ }^{13}$ Longet, Arch. gén. de méd., Paris, 1847, tome lxxiii. p. 374.

${ }^{14}$ Dejerine and Sottar, Compt. rend. Soc. de biol., Paris, 1892, tome i. 1. 716 ; Rossolymo, Arch. f. Psychiat., Berlin, 1890, Bd. xxi. S. 997 ; Fürstner and Zacher, ibid., 1883, Bd. xiv. S. 422 ; Oppenheim, ibid., 1893, Bd. xxv. S. 315 ; E. Asinus, "Ueber Syringomyelia," Biblioth. med., Cassel, 1893, Bd. c. S. 1 ; M. Lachr, Arch. f. Psychiat., Berlin, 1897, Bd. xxviii. S. 773 ; H. Schlesinger, "Die Syringomyelia," Leipzig-Wien, 1895. 
the severer analgesia is on the same side as the severer lesion, but touch has remained good on both sides. This agrees so far with the path traced by Schiff and Budge to short fibres of the spinal root that plunge into the grey horn. The continuation of the path cannot, lowever, remain long in the grey matter, because the grey matter may be quite destroyed, and hollowed out at a level, and yet no complete analgesia result in the parts further below the site of the lesion. ${ }^{1}$ The path therefore leaves the grey matter soon after entering it. In the grey matter a column cell must be introduced into the chain of conduction, because section of posterior roots leads to no degeneration that goes through the grey matter and on into the white beyond. ${ }^{2}$ Laehr $^{3}$ points out, on the basis of the Brown-Séquard paralysis, that in certain such cases the loss of sensation on the side opposite to the loss of motility is a loss of pain and temperature sensation. The conduction upwards of impulses producing vasomotor reflexes was found by Miescher ${ }^{4}$ and myself 5 to occur in the ventrolateral parts of each lateral column. It has been suggested that the ventral division of the cerebellar tract (Gower's' tract) is a pain-path. ${ }^{6}$ It may be so, although the connection with the cerebellum does not support this suggestion; yet, because part of the tract ends in the cerebellum, it does not follow that all of it does so. But we do know that the ventral cerebellar tract is partly crossed. There is the bundle of crossed fibres, described by Grunbaum ${ }^{7}$ (by degeneration after median longitudinal section), lying at the periphery between the two cerebellar tracts. Its upward destination is unknown. Even if the pain-path employ these crossed fibres, the pain fibres of the roots do not transgress the median line of the grey matter, but end on their own side. It is quite possible that the path is partly crossed, partly not. The tautomeric segmental effect of the lesions confined to grey matter, and the crossed hemianæsthetic effect of the lesions which include white matter, indicate clearly that outside the grey matter is a collected strand of pain fibres, reinforced from segment to segment by contributions from column cells in the grey matter. The main path so formed may likely enough be a crossed path, but there seems to be an uncrossed as well. Gowers has recorded a case in which a unilateral hæmorrhage in the lateral colunn at the upper cervical region produced complete analgesia and thermanæsthesia of the opposite side, without disturbance of touch. The path of conduction for warmth impressions, it is suggested, lies near to but is not entirely commingled with that for pain. ${ }^{8}$

The above statements suggest strongly that there is in the spinal cord a more or less distinct "pain-path," into which the impulses generated at certain "skin spots" enter with comparative ease. Among the features of the path are these: It is less open than others to reactions of moderate and brief kind, that is, it requires stimuli intense or long-continued; the efficiency of stimuli that are long-continued is due to the high degree of summating power possessed by the path; the path is largely a crossed path, and soon enters, crosses, and leaves the grey matter; in doing so a column cell is intercalated in the conduction chain. What is the relation, if any, of this path to impulses subserving the pain initiated by deep organs? Is it a skin pain-path or a path for sense pain in general? There is a good deal of evidence in favour of the conduction of the centripetal impulses from muscles by the

1 M. Laehr, up. cit.; also Schlesinger, op. cit.

${ }^{2}$ Singer, Sitzungsb. d. k. Akad. d. Wisscnsch., Wien, 1882, Bd. lxxxiv. Abth. 3; Tooth, Goulstonian Lectures, Lancet, London, 1889, vol. i. ; Mott, Brain, London, 1895, vol. xvii.; Mott and Sherrington, Proc. Roy. Soc. London, 1895, vol. lvii.

${ }^{3}$ Op. cit. "Op. cit.

6 Gowers, "Diseases of the Nervous System," London, vol. i.

7 Journ. Physiol., Cambridge and London, 1894, vol. xvi.; see also Mott, Brain, London, 1895, vol. xvii.

${ }^{8}$ H. Schlesinger, op. cit. 
long root fibres of the dorsal spinal column, without decussation right up to the dorsal column nuclei. Muscular sensation is not affected by the diseases that implicate the above-described spinal path,- - at least,muscular co-ordination is not obviously disturbed by them. The severest muscular cramps and fatigue do not induce associated skin sensations.

Some individuals never have a presentation of a certain sense without the occurrence of a presentation of another sense, hence "coloured audition," photosms," etc. These are called "secondary sensations," the word sensation being employed in its broad sense to cover presentational perceptions. ${ }^{1}$ A somewhat similar phenomenon, also familiar to many, is the "referred" or "associate" sensation, Mitempfindung. In it excitement of one sensifacient organ or part produces, besides its own proper sensation, a sensation referred to another part. As not the "reference" but the "association" is the peculiarity, the reference being only the ordinary process, which in this case by its operation calls attention to the association, the term "referred" is not altogether happily applied to the phenomenon. "Sympathetic," the term used at the beginning of this century, ${ }^{2}$ seems better, and is especially appropriate because of the proneness of associate sensations to be of the nature of pain. An often cited associate sensation is the tickling in the nose which is felt on looking at a brilliant light $;^{3}$ here probably the association is less between the optic nerve and the nasal branches of the trigeminus than between the branches of the trigeminus supplying the eyeball and those supplying the nose. Pain in the shoulder associated with liver disease was similarly regarded, and a fanciful speculation offered in explanation for it by the anthropotomist. What Americans call "the ice-cream nerve"-pain on the forehead on eating a cold substance-is a better example. By applying a "mustard leaf" over the front of the upper part of the sternum, I can produce, in myself, the sensation of a patch of hot, umpleasantly tingling character, referred to the inner side of each upper arm, just above the inner condyle; each patch seems oval, the long axis directed up the arm, and extending for about 3 in. During the phenomenon I cannot really rid myself of the belief that the skin there must be looking red, but no flush is found. In this case the associated sensations and the stimulus applied lie, as shown by delimitations of the cutaneous segmental-nerve areas, ${ }^{4}$ both of them within the same segmental area.

In connection with viscera, the phenomenon takes forms that might almost be spoken of as "illusions of pain." The associate pains"sympathies" literally_of visceral disease have been recorded, and dwelt on by M. Traube, ${ }^{5}$ Quincke, Hilton, ${ }^{6}$ Brown-Séquard, ${ }^{7}$ Dana, ${ }^{8}$ Head, J. Mackenzie, ${ }^{9}$ and others, but it is especially since the work of Head ${ }^{10}$ that the previously isolated facts have been systematically extended and ably correlated, so as to assume scientific importance. Pains are initiated by the afferent nerves of viscera in certain skin areas. The importance of this for the present argument lies in the fact that the central pain-path seems shown by this evidence to be to some extent a

1 "Secondary sensation" has been used by Gad and Goldscheider in another and preferable sense. See Arch. $f$. Physiol., Leipzig, 1892, and this article, p. 998.

2 Johann Miiller, Purkinje, ete.

3 Miiller's "Elements of Physiology," 1832-1837, vol, i.

* Sherrington, Phil. Trans., London, 1892.

${ }^{5}$ See Bruns, Ztschr. f. Psychol. u. Physiol. d. Sinnesorg., Hamburg and Leipzig, 1896, Bd. ix. S. 134 .

" "Rest and Pain," London, 1855.

7 "Researches on Epilepsy," Boston, 1857.

${ }^{8}$ New York Med. Journ., July 1887.

9 Brain, London, 1894, vol. xvi.

${ }^{10}$ Brain, London, 1893, vol. xvi. pp. 1-133; 1894, vol. xvii. pp. 339-480; 1896, vol. xix. pp. $153-276$. 
channel leading in common from visceral and from cutaneous afferents. To Head we owe the knowledge that the spatial arrangement of the radiation is, largely at least, of segmental character. Each viscus stands in relation with a definite patch of skin. The patches thus marked out do not correspond with any of the areas of distribution of the peripheral nerves, as far as known to gross human anatomy, but resemble and in fact strikingly agree in disposition with the segmental skin areas, found to belong to the spinal nerve roots, i.e. spinal ganglia. The segmental character of the "reference," and the likelihood that the connection or communication occurs where the column cell is intercalated, that is, at the synapse in the conduction chain, both suggest that the seat of "reference" is in the spinal grey matter itself.

The spinal ganglia contain, of course, the afferent visceral nerve fibres of the white rami and also the afferent nerve fibres from the skin, etc. Regarding the manner and place of "radiation," Müller, as early as 1834, wrote, "either of two hypotheses may be adopted." The radiation may occur in the spinal ganglion, by escape there of excitement from one nerve cell to another, the conductors being there no longer isolated by protective sheaths. Or, "the sympathetic sensations are the result of the radiation of the irritation from the fibres prinarily affected upon the roots of other fibres in the brain or spinal cord, just as in the production of reflex movements impulses conveyed by sensitive nerves into the cord are communicated to motor nerves; the only difference being that, when sympathetic sensations are produced, the radiation of the impulses does not reach motor but only sensory fibres, or, at any rate, acts on sensory at the same time as on motor nerves." "This is another proof that the same sensation is produced at whatever point of its length a nerve fibre is irritated." The mere radiation of an impulse will therefore suffice to produce sympathetic sensations. "The extension of irritation in the spinal corl, from the roots of the sensory to the roots of the motor nerves, and the fact that sensory nerves without ganglia, e.g. optic-in the instance where a strong light causes a sensation of tickling in the nose-exhibit radiation of sensations, shows that the radiation must take place not in the ganglia but in the central nervous system." ${ }^{1}$ The seat of radiation is considered by Head also to be intraspinal, by Kyri, ${ }^{2}$ however, to be intraganglionic. As above stated, I myself should regard it as at the site of synapsis between the visceral spinal ganglion cells and column cells of the grey matter that continue the thermalgesic chain of conduction. The referred sensation is not one of touch, but of pain, -is, in fact, a "sympathy." The segmental patch of skin to which the reference is made may be hyperalgesic ; generally, not the whole of it, but a part, is also hyperæsthetic to warmth; this is called by Head "the maximal point." In fact, the visceral part of the spinal segment communicates its own excitement to a certain portion of the somatic part of the segment. Thus Head finds that disease of the kidney and ureter cause reference of pain and hyperalgesia in skin, the sensory innervation of which is from the tenth, eleventh, and twelfth thoracic spinal ganglia. Similarly with disease of the cardiac end of the stomach and the skin fields of the sixth and seventh thoracic spinal ganglia. But not all spinal segments innervate viscera. Several neither give fibres to nor receive fibres from the viscera, - that is, possess no white ramus communicans. It is especially in the limb regions that the visceral component of the spinal segment is wanting. Head finds no referred pains in the fifth, sixth, seventh, and eighth cervical, nor in the second, third, or fourth lumbar segments. The afferent nerves of the viscera compose to a certain extent a twofold system, - a cranial and a spinal, the former being the vagus, the latter

1 "Elements of Physiology," translated by W. Baly, London, 1837, vol. i.

${ }^{2}$ Centralbl.f. Gynäk., Leipzig, 1894; and Versamml. deutsch. Naturf., etc., Wien, 1894. 
the white rami communicantes. Head shows that affections of the thoracic and abdominal viscera, but not of the pelvic, produce pain and tenderness not only in the borly, but also over certain areas of the scalp. These headaches, he finds, stand in direct and constant relation to the position of the area of referred pain and tenderness on the surface of the body. The headache corresponds with this or that dorsal segmental area. The skin areas of referred pain in some cases overlap distinctly but not to the same extent as do the areas of segmental nerves, when delimited by the method of "remaining æsthesia." I Hear ${ }^{2}$ includes in his considerations the trophic nerves of the skin. "I do not, therefore, think we shall be far wrong in assuming that trophic nerves to the viscera bear a similar relation to the sensory sympathetic fibres as the trophic nerves to the skin bear to the fibres for pain. If this be so, it will help to explain the effect produced by counter-irritation of the skin on the activity of the viscera. Conversely, it will also explain the occasional improvement in pains of a purely nervous origin by some visceral irritation, e.g. the removal of lightning pains over the first, second, third, and fourth sacral areas (rectum) in a tabetic, by the administration of a purge."

Another set of referred pains studied by Head, ${ }^{3}$ are those in which each organ in the head (nose, eye, ear, teeth, tongue, nostrils, larynx, brain) refers a pain to one or more areas on the surface, where the skin may in consequence become actually tender. Thus lesions, not of the conjunctiva and anterior layer of the cornea, for they cause simply local pain, but of the substantia propria of the cornea, and of all parts of the globus behind it, back to the retina, produce pain, frequently tenderness, of areas of skin over the upper half of the face and the anterior half of the cranium from Head's maxillary to his vertical areas inclusive.

Again, in the instance of the ear, the meatus auditorius does not cause referred pain, but local pain purely. Lesions of the membrana tympani and mildle ear cause referred pain and tenderness over the liyoid area. Tension in the middle ear, sucl as occurs in the first stage of otitis media before the membrane is perforated, causes referred pain and superficial tenderness over the vertical and occasionally over the parietal area of the scalp, in addition to that over the liyoid area.

Head's discovery of the segmental distribution of the skin areas of hyperalgesia was early confirmed by J. Mackenzie, ${ }^{4}$ and by J. Kyri. ${ }^{5}$ The former has pointed out that the pilomotor nerves over a limited area may be rendered abnornally sensitive by visceral disease,-a "goose-skin" reflex becomes more readily elicitable than in health. The pilomotor efferents belong to the sympathetic,-issuing by the white rami. ${ }^{6}$ The general radiation of a sensation is well exemplified, as $\mathrm{J}$. Miiller pointed out, by the associate sensations of the sexual orgasm. For details of the anatomical distribution of Head's areas, the original papers should be consulted. ${ }^{7}$

The central paths of the pain impulses from the skin and viscera are therefore closely connected with one another. The spinal connections of the nerves for warnth and cold, and for trophic disturbances in the skin, must also be in somewhat close association, especially

1 Sherrington, Phil. Trans., London, 1892.

2 Brain, London, 1893, vol. xvi. p. 129.

+ Ibid., vol. xvi. pp. 321 and 515 .

3 Ibid., 1893, vol. xvi. p. 339 , ete.

5 Loc. cit.

${ }^{6}$ Langley and Sherrington, Journ. Physiol., Cambridge and London, 1891, vol. xii. 1. 278 ; also Langley, ibid., 1893, vol. xv. p. 176.

7 Brain, loc. cit. 
those for warmth, with pain. On the other hand, the nerves for pure touch, and perhaps those of the muscular sense, must be fairly separate centrally from those of pain.

It has been pointed out ${ }^{1}$ that the sensations of the intellectual senses have a power of cohesion, of integrating; whereas "hunger, thirst, nausea, and visceral feelings in general cohere little with one another and with other feelings, and thus integrate but feebly." They interfere and are incompatible with and even displace other sensations. Cutaneous "pain" soon and easily overpowers, cancels, and obscures other specific qualities of sensations arising simultaneously. We know from experience that from certain stimuli certain specific sensations must under ordinary circumstances arise, yet when pain is added to these they are inhibited, not felt. This argues for the suppression of one reaction by intervention of a second of a different kind, rather than for any alteration of mere intensity in the earlier. It is as if with a certain violence of stimulus a new sensation ousted a co-existent from all rôle in consciousness.

The central neural mechanism for cutaneous pain is certainly therefore largely distinct from that of touch, and to a certain extent from cold and warmth sensations. There is evidence also that its peripheral mechanism is to a certain extent distinct. The existence of separate afferent nerve fibres with specific dolorific end-organs would completely accord with the third hypothesis mentioned above at the outset. The chief arguments in its support can briefly be cited as follows.

There are some regions of the skin that are analgesic, though they possess good tactual sense (e.g. the buccal mucosa opposite the second lower molar (Kiesow)). ${ }^{2}$ Other regions exist which are by a number of observers judged to be anesthetic as regards pure "touch," although acutely sensitive for pain (cornea) (see p. 987). Analysis of the functions of the skin as a sensifacient surface distinguishes within it elemental points, not only for "touch," "cold," and "warmth" sensations, but also others, there is evidence, for " pain." Goldscheider early declared that the "cold spots" and "warmth spots" can by no amount of excitation be proved to initiate pain. On touching one of the latter with a heated cone, a "feeling with high degree of heat quality but without painful quality" 3 is produced; although at other places than "temperature spots," contact with the cone-point can be extremely painful. The peculiar "burning" character of the pain produced by contact of the skin with a hot object, is explained as due to the admixture of sensations arising from the stimulated "warmth spots," with pain arising from the stimulated "pain spots." It is stated ${ }^{4}$ that "touch spots" are similarly analgesic, although it is not yet altogether clear that touch of a painful quality cannot be excited from them by intense stimulation. It is to be remembered that the "spots" from which, according to both observers, pure pain can be so easily excited are extremely numerous, and it is difficult so to apply with minute areal limitation an intense mechanical stimulus that it shall not affect "pain spots" in the vicinity of the touch spots.

When a small region, e.g. 1 sq. cm. of skin, in which the hairlets

\footnotetext{
${ }^{1} \mathrm{H}$. Spencer, "Principles of Psychology," vol. i. p. 187.

${ }^{2}$ Kiesow, Phil. Stud., Leipzig, Bd. ix. S'. 540 ; ibid., Bd. xiv. S. 567 ; v. Frey, Ber. d. $k$. süchs. Gesellsch., Leipzig, July 1894.

${ }^{3}$ Op. cit.

${ }^{4}$ Goldscheider, v. Frey, op. cit.
} 
have been cut close, is examined with bristle æsthesiometers (see "Touch Spots"), a certain number, e.g. fifteen touch spots are found, all easily detected by using pressure intensities of less than 40 (3-3.3) grms. per sq. mm. No further touch spots are then revealed by increasing the intensity of stimulation even three- or fourfold. But when the intensity is increased to something over 200 grms. per mm. (occasionally as low as 150), there appear a large number of other "spots," at each of which smart reaction occurs. These spots are scattered pretty evenly, and, unlike touch spots, bear no obvious relation to the position of the hair follicles. ${ }^{1}$ The sensation produced at each is pricking and painful, and in many of them the pain caused is, in the opinion of some observers, ${ }^{2}$ quite unattended by any sensation of pressure. From each of these spots, when pricked, very lively, in some cases " hardly bearable," pain is evoked. These spots are called "pain spots" (Goldscheider, v. Frey).

Among the advantages of the hair-æsthesiometer (Hensen, ${ }^{3} v$. Frey ${ }^{4}$ ) are(1) Exactitude of local application, i.e. "stigmatic stimulation"; (2) impossibility of exceeding with it a certain easily measured intensity, which is its maximum capacity. In practice it is perfectly easy, when the hair is mounted properly, to ensure employing its maximal power at each application. Hensen and v. Frey use a chemical balance to calibrate the hair instruments. v. Frey takes as index of the stimulus the pressure exerted by the hair into its cross-section, expressed in fractions of a sq. mm. Nagel objects ${ }^{5}$ that a truer index would be the force that lias to be exerted to bend the hair. $\mathrm{He}$ says that stimuli of unequal pressure but equal force are not perceivedin using the hair-æsthesiometer-to be different.

There are many forms of algesimeter besides the "hair" pattern. A simple needle is the most generally used, and offers many advantages for the clinician. If the needle has actually to enter the skin to evoke pain, that is of itself a sign of hypalgesia in any cutaneous region. The pinching up of a fold of skin will often successfully evoke pain when needle-pricks, however deep, do not. Quantitative results are the object of algesimeters. Bjornstrom's ${ }^{6}$ instrument is a form of pincher, the pressure of which can be read from a scale. Hess's ${ }^{7}$ and Motschutkowsky's ${ }^{8}$ instruments are standard needles, the degree of depth of insertion of which into the skin can be read off on a scale. Bernhardt's method is by faradisation of the skin, the measure of the stimulus being the distance of the secondary coil from the primary.

Differences between the reactions obtainable from pain spots and touch spots are found as follows:-

1. The liminal sensibility of mechanical stimuli (pressure) is higher for pain spots than for touch spots. ${ }^{9}$ This the above-given example illustrated. The ratio $\frac{\text { touch limen }{ }^{10}}{\text { pain limen }}$ varies in different regions of the skin; thus it is $\frac{1}{3}$ for the arm, $\frac{1}{100}$ for the finger-tips. Hence, when both are equally tightly squeezed, a fold of palmar skin gives less pain than a fold from the fore-arm.

v. Frev, loc. cit.

2 . Frey, loc. cit.

3 "Gegen den sechsten Sinn," Kiel, 1893 ; and Arch. f. Ohrenh., 1894, Bd. xxxv. S. 161.

+ op. cit.

5 Arch. f. d. ges. Physiol., Bonn, 1895, Bd. lix. S. 595.

6 "Algesimetrie," Upsala, 1877.

7 Verhandl. d.X. internat. med. Cong., Berlin, 1890 ; Deutsche med. Wchnschr., Leipzig, 1892 , Bd. $x$.

${ }^{8}$ Ncurol. Centralbl., Leipzig, 1895, Bd. xiv. S. 146.

${ }^{9}$ Goldscheider, op. cit.

${ }^{10} v$. Frey, op. cit. 
The lowest limina for pain are found in the temple ${ }^{1}$ and the dorsal skin of the finger-joints. ${ }^{2}$ Next, perhaps, come the tongue-tip and the forehead. The hand on the ulnar side is more sensitive for pain than on the radial. Glans penis, buttock, ankle, are among the least. On the whole, the pelvic region is as poorly sentient to pain as any part of the body surface. Flexor sides are, on the whole, less sensitive than extensor in regard to pain. ${ }^{3}$ The topography of "pain" in the skin, as regards its degrces of delicacy, certainly differs much from the topography of "touch," but the accounts yielded by various observers with algesimeters of different kinds, are very discrepant. ${ }^{4}$ The following are Bernhardt's figures, obtained by his faradic method; the figures represent the distance of the sccondary coil from the primary :-

\begin{tabular}{llll|llll} 
Tongue-tip & . & . & 141 & Back of hand & . & . & 99 \\
Cheek & . & . & 125 & Fore-arm . & . & . & 93 \\
Back & . & . & 116 & Instep & . & . & 92 \\
Sternum & . & . & 114 & Pulp of finger & . & . & 84 \\
Buttock & . & . & 111 & Palm . & . & . & 75 \\
Thigh & . & . & 102 & Toe-tip . & . & . & 65
\end{tabular}

Goldscheider has examined the regional distribution of heat-pain.

2. The liminal intensity for electrical stimuli (break-induction shocks applied by unipolar method) is higher for touch spots than for painspots. $^{5}$

It is impossible to say whether these electrical stimuli to the skin excite the end-organs or the nerve fibres. The anomalies of the results of electric excitation in various parts of the skin show clearly that the skin is not equipped with organs adapted for such stimuli. In the palm, for the current to suffice to excite touch, it must be applied at the mouths of the sweat glands. ${ }^{6}$

Liminal intensity of pain stimuli is highest where the skin is thick and lies on thick muscle far from bone $;^{7}$ this is with mechanical stimuli, and where the local deformation is not well estimated. Palm, thigh, and ankle, after temple, are parts where the limen is highest."

3. Cooling the skin paralyses the pain spots sooner than the touch spots, although causing preliminary hyperæsthesia of both. ${ }^{9}$

Cocain locally applied to the tongue suppresses touch, taste, cold, warmth, and pain sensations, the order of paralysis in time being earliest temperature sensation, then taste, then touch, and finally pain. ${ }^{10}$ According to Shore, pain is suppressed before touch. ${ }^{11}$ The statement ${ }^{12}$ that cocain paralyses in the eyeball only the pain spots, is not confirmed. ${ }^{13}$ The behaviour of cocain in rendering the cornea analgesic, and yet allowing sensations of touch, though impaired, is important for deciding the true position of pain from pain spots. Carbolic acid, ${ }^{14}$ in 5 per cent. solution on skin or tongue, acts in the samc way as cocain, but not so strongly. By subcutaneous injection in 2.5

${ }^{1}$ Harold Giffing, Psychol. Rev., N. Y. and London, Feb. 1895.

2 O. Motschutkowsky, Neurol. Centralbl., Leipzig, 1895, Bd. xiv. S. 140.

${ }^{3}$ Hess, ibid., 1895, Bd. xiv. S. 548.

+ The degree of yieldinguess of the skin, by reason of whether it lies elosc on bone or Hoats on muscle, etc, , is of great influence (see H. Giffing, op. cit.; also, for other causes of difference, Hess, op. cit.).

${ }^{5}$ v. Frey, op. cit. $\quad{ }^{6}$ v. Frey, op. cit.

${ }^{8}$ Gitting, op. cit.
${ }^{11}$ Joum. Physiol., Cambridge and London, 1892, vol. xii. p. 207 . The discrepancy

7 Giffing, op. cit. between Goldscheider and Shore may be reconciled by Kiesow's observation, that deep pain disappears, but not surface pain (Phil. Stud., Leipzig, 1894, Bd. ix. S. 510), in the mincosa of the lip.

12 H. Donaldson, Mind, London and Edinb., 1885, vol, x.

${ }^{13}$ v. Frey, op. cit.; Nagel, op, cit.

${ }^{14}$ Goldscheirler, op. cit. 
per cent. solution, it can locally completely paralyse all endings, including "painful." Chloroform, ${ }^{1}$ locally applied with a sponge, after a temporary burning sensation, leaves all forms of sensibility locally lowered. It acts on different sensations in the same order of degree as cocain, but especially suppresses heat-pain. Menthol, ${ }^{2}$ after causing a temporary hyperæsthesia of cold spots, depresses sensibility of all forms. Orthoform, ${ }^{3}$ in subcutaneous injection, after preliminary local pain, causes local analgesia. Guaiacol acts less quickly, but otherwise similarly and more lastingly, than cocain. ${ }^{4}$

4. The faradisation of pain spots, at any frequency above twenty shocks per second, evokes a continuous sellsation; faradisation of touch spots, at rates of 130 shocks per second, evokes a discontinuous vibratory sensation. A similar difference between the length of duration of the reaction is also traceable by single shocks or mechanical stimuli; the pain sensation fades more gradually away. ${ }^{5}$

5. Not only ninute, but also regional, distribution is different in the case of the two sets of "spots." The cornea and ocular conjunctiva are rich in pain spots, but have no touch spots; so also the glans penis. ${ }^{6}$ The eyelid sweeps over the cornea without being felt. On the other hand, a patch of mucous membrane inside the cheek is without pain spots, ${ }^{7}$ although provided with touch spots; indeed, the whole of the inside of the mouth is poorly provided with pain spots, although much of it is extremely rich in touch spots. There is a patch of the mucosa of the cheek at which faradic currents, sufficient to violently tetanise the facial muscles and radiate over all the region of the upper jaw, cause no trace of unpleasant sensation at the point of application.

In examining the buceal mucosa for pain by electric stimuli, it must be remembered that there moisture renders stigmatic application difficult, and with increase of area the density of the current falls. Comparison of dolorific effect obtained by stigmatic stimuli elsewhere with this buccal stimulation is therefore complex; the buccal stimuli would be effectually less intense in application.

6. The sensation from a touch spot is referred to the surface of the skin; that from a pain spot seems to radiate into the depth as well as over the skin surface, and is less clearly qualified with "local sign." s

7. The "latent period" of skin pain is greatly longer than that of touch.

From clinical pathology there is testimony corroborative of the pliysiological. A difficulty, however, arises with the pathological evidence. The pain stimulus being of intenser nature, allows the inference that the selective action of disease on the fibres of a peripheral nerve may consist in limiting the range of their response to the stimulus, without causing it to lose its functions altogether. The plausibility of this suggests itself most in the cases where there is analgesia without total anæsthesia of a part. Pressure on the ulnar nerve trunk will cause partial or total anæsthesia for pain and tcmperature,

1 Goldscheider, op. cit.

2 Goldscheider, op. cit.; Sherrington, "Interméd. d. biol.," Paris, 1897, tome i. p. 84.

${ }^{3}$ H. de Varigny, ibid., 1897 , tome i. p. 125 . Orthoform is an ether from amidooxybenzoic acid.

${ }^{4}$ Loiselle, ibid., 1897, tome i. p. 157.

${ }^{5}$ Nagel, op. cit.; v. Frey, op. cit. Butcf. Bloch, Trav. du lab. de Marey, Liège, 1897, tome iii.

${ }^{6}$ There is a difference of opinion as to whether any touch can be felt on the cornea without pain. Nagel has pointed out that touches can be felt on the conjunctiva and cornea without any painful or disagreeable quality in them. It is the ease with which the corneal endings provoke pain which is denoted by the term "pain spots" as used in the above paragraph. See also Motter, "Diss.," Erlangen, 1878; M. Dessoir, Arch.f. Physiol., Leipzig, 1892, S. 175.

${ }^{7}$ Kiesow, Phil. Stud., Leipzig, 1894, Bd. ix. S. 510 ; v. Frey, loc. cit.

${ }^{8}$ E. H. Weber, op. cit. 
with little or no diminution in sensibility to contact, with intact "motor" sensations and normal localisation. ${ }^{1}$ But, as a fact, other combinations of selective loss not unfrequently occur, for which mere gradation of injury is not so plausible an explanation; thus, complete loss of temperature sensations in forearm and hand, with pain and contact preserved, due to injury of the shoulder. ${ }^{2}$ On reunion of a cutaneous nerve trunk after trauma, the different specific sensations return at different rates, usually cold before pain and pain before warmth. ${ }^{3}$ An exhaustive and most interesting analysis of the changes in the sense spots of the arm, due to pressure of a cervical rib on the brachial plexus, has been described from the case of himself by L. F. Barker. ${ }^{4}$ He found groups of sense spots of one kind persisting in places where none of other sense species remained. Most of the sense spots in these selectively anæsthetic areas reacted to normal threshold stimuli, as could be particularly well proved for the pain spots. In places "cold pain" and "warmth pain" were elicitable, but not "cold" or "warmth" sensation; that is, ice gave pain but no cold, a heat of $47^{\circ} \mathrm{C}$. also pain but no "warmth " sensation..$^{5}$ The skin had thus in so far been transmuted to the sensory condition of the mucosa of the stomach, where the ice that is painfully cold to the lips causes pain but no sensation whatsoever of cold.

In toxic neuritis, ${ }^{6}$ and in tabes dorsalis, as well as in the above pressure diseases, the injury to the peripheral nerves may act selectively in regard to the pain spots.

It would seem that we may with some probability allocate the pain sense to certain kinds of the multifold structures described as nerve-endings in the skin-(1) The pain-endings lie more superficially than the other endings. This is strongly indicated by the lowness of their threshold electrical stimulus, which cannot be due to a greater electrical excitability of the pain nerve fibres, since when the ulnar trunk is galvanised the touch fibres are the most easily excited. (2) The central part of the cornea appears to be endowed with pain sensation only. It is found to possess histologically only one form of nerve-ending, namely, free nerve filaments ramifying amidst the epithelial cells. It is therefore probable that the free nerve filaments ${ }^{7}$ in the epidermis form the apparatus.

For the present issue the two most distinctive differences between the sensory reactions obtainable at touch spots and pain spots respectively seem (1) the (not universally accepted) absence of painful quality from the maximal reactions of touch spots; $(2)$ the relatively poor tactual quality of the reactions excited at pain spots, although even the minimal reaction is there said to possess painful quality. I cannot unhesitatingly find in my own perceptions that excitation of a pain spot, e.g. by a bristle xesthesiometer, ever evokes pain pure without any tactual sensation. The fact that there coexist in the skin two kinds of sense spots, excitable by the same sort of mechanical stimulus, and that the one kind requires for excitation a very distinctly

${ }^{1}$ A. Herzen, Arch.f. d. ges. Physiol., Bonn, 1886, Bd. xxxvii.; E. Biernaeki, ref. in Neurol. Centralbl., Leipzig, 1893, Bd. xii. S. 369 .

${ }^{2}$ Piek, Prag. med. Wchnschr., 1888, Bd. xiii. S. 81.

3 Chareot, Compt. rend. Soc. de biol., Paris, 1892, p. 941.

${ }^{4}$ Journ. Exper. Med., N. Y., 1896, vol. i.

${ }^{5}$ For a number of eases of lepra nervosa exhibiting selective sensory paralyses, see Laehr, Arch. f. Psychiat., Berlin, 1897, Bd. xxviii. S. 772 ; Pitres, Gaz. d. h6p., Paris, 1892, tome lxv. p. 1287 ; Rosenbaeh, Neurol. Centralbl., Leipzig, 1884, Bd. iii. S. 361 ; H. Schlesinger, Deutsche Ztschr.f. Nervenh., Leipzig, 1892, Bd. ii. S. 230 ; Marestant, Rev. de méd., Paris, 1891, tome xi. p. 781 ; Thibièrge Bruhl, Gaz. hcbd. de méd., Paris, 1891.

${ }^{6}$ See Beau, Arch. gén. de méd., Paris, 1848, tome lxxvi.

${ }_{7}$ Merkel, Arch. f. mikr. Anat., Bonn, 1875, Bd. xi. S. 636 : Ranvier, Compt. rend. Soc. de biol., Paris, 1880, tome xli. 1. 1087; Dogiel, Arch. f. mikr. Anat., Bown, 1891, Bd. xxxvii. S. $602 ; 1893$, Bd. xli. S. 585 . 
higher threshold of intensity of stimulus than does the other, does not supply a final criterion for judging the question under consideration. There may coexist in the skin two sets of true tactual organs, the one more sensitive to touch than the other.

Some observers who admit distinction between "pressure spots" (called here "touch spots") and "pain spots," affirm the perception of a quality of "contact" in skin sensation in addition to qualities of "pressure" and of "pain." 1 They find "contact" quality in the sensations both from "pain spots" and "pressure spots." 2 Some affirm it also-and I agree with them in this -in the sensations evoked at temperature spots, ${ }^{3}$ although less prominent in the perception than is the quality of cold or warmth respectively. Tactile and temperature stimuli are, it may be recalled, not invariably discriminable when applied to some regions of the skin of the back. It is true, however, that if one only out of the four sets of end-organs in the skin can evoke the pain psychosis, then that one can quite appropriately be considered a specific "pain organ." Nor in the skin especially is there any teleological difficulty in the acquirement by the body of a specific set of nerve fibres and nerve organs for sensations of pain. Thus it is found that certain forms of common sensation (e.g. sexual voluptas, tickling), peculiarly potential of pleasurable emotion, have been evolved in special connection with certain fields of skin which are not pre-eminently sentient for ordinary "touch."

Let it be granted that the above evidence still leaves it open to regard the skin as possessing, apart from tactual and thermal sense, a specific dolorific sense as well. What have the other sensifacient regions of the body, which can as unequivocally as the skin initiate physical pain, to offer in comment upon such a view? There are "deep" pains as certainly as there are cutaneous or "superficial." Deep structures occasioning pain are the organs of bodily movement and the viscera. Are these structures also endowed with specific nerve fibres for a painsense. The afferent nerves of the organs of bodily movement are the ministers of the so-called muscular sense. (For detailed account of them and their function, reference must be made to the article on "Muscular Sense"). They are, as regards their distribution to tissue, and probably also the details of their function, divisible into several subgroups. The pain, which under unusual circumstances is evoked from the parts supplied by this sense, may either express the excessive reaction of specific "muscular sense" nerves, or the reaction of separate pain fibres, admixed with the fibres proper to "muscular sense," just as it was granted, for the argument above, "pain fibres" exist admixed among the other afferent cutaneous nerve fibres.

The stimuli which provoke pain from the deep structures, such as muscles and joints, appear to be mechanical in quality; in fact, the same in kind as those which normally must furnish the impressions at basis of the muscular sense. Haller's ${ }^{4}$ original experiments vouch for the inadequacy of an exhaustive number of chemical and thermal stimuli. More than that, his experiments, together with other observations, show that certain mechanical insults to muscle and tendon are quite impotent to excite pain.

In reading those earlier experiments, one is struck by two circumstances

${ }^{1}$ Kiesow, Ztschr.f.Psychol. u.Physiol.d.Sinnesorg., Hamburg u.Leipzig, 1896, Bd. x.S.139.

2 Dessoir, 1892, Nagel, 1895, Kiesow, 1896, and, if I understand him correctly, Goldscheider ("Ueber deu Schmerz," Berlin, 1894) throughout his analysis of skin senses, $1885-1895$.

${ }^{3}$ Kiesow, loc. cit.

4 “De Partibus," Göttingen, 1752. 
regarding the mechanical irritations employed, which might detract from their efficiency as excitants, namely-(1) The thoroughly abnormal character of the production of the strains in many cases, e.g. twistings, foldings, etc., which the body itself cannot produce; (2) the absence of the opportunity for prolonged summation. Against such negative evidence is the fact that muscles stretched passively for a prolonged time become painful, ${ }^{1}$ and that the passive stretching of muscle is a most efficient excitant for normal spinal reflexes through its afferent nerves. ${ }^{2}$ It would appear that only mechanical conditions, akin to such as obtain in museles during their functional activity, can initiate from them sensation and pain. The structure of muscle spindles certainly points to the adequate stimulus being of a mechanical kind, ${ }^{3}$ and that of tendon organs points in the same direction.

Extreme and abnormal actions of muscles furnish the best instances of muscular pain. Weber ${ }^{4}$ felt the muscles of his horizontally held arm become painful in 300 seconds, unbearably so in 900 seconds. At the same time contractions as vigorous as those of cramp may be caused without the painful sensation of cramp. ${ }^{5}$ To attribute the sensation of cramp merely to compression of sensory nerves passing through the muscles, and not to sensory nerves of their own, is certainly not permissible. The pain of muscular cramp is distinctly perceived to be in the contracting muscles, that is, is referred to the contracting muscles. The adequate stimulus seems under certain circumstancesand among these excessive duration appears eminently important-to elicit pain from the muscular sense. A specific set of nerve fibres and end organs, devoted solely to production of pain, although to the above extent supported by analogy from the skin, does not appear a warrantable postulate for muscles.

The feeling of muscular fatigue is a form of common sensation that has undoubtedly considerable "negative tone." It presents, in accordance with its amount, practically infinite shades from the scarcely unpleasant up to the extremity of distressful. It is a state certainly largely due to the regularly employed specific nerves of muscular sense. In its relation to them it is well comparable with the feeling of liunger in relation to the visceral nerves.

The viscera offer still another field for analogy. There we find regions of the body, usually only in very subordinate degree sensifacient, that under certain circumstances evoke extremity of pain. The impulses embouching via the vagus and afferent spinal roots from the visceral nerves upon the central nervous system appear hardly at all to elicit ordinarily conscious reactions. When abnormally they do so, they seem almost invariably to produce pain as their result. It is as though particular afferent nerves, which usually are not in the strict meaning of the term sensory nerves at all, can on occasion become "sensory," even to the extent of convulsing the whole mind. Some authorities, as above mentioned, teach that the visceral nerves are generating constantly "obscure sensations." ${ }^{6}$ It would rather seem that these nerves intermittently with phasic regularity launch sensory impressions (as well as viscero-motor, secreto-motor, and other reflexes) that do not trouble the stream of consciousness at all. These

1 Cf. E. H. Weber, op. cit. ; also, Schiff, "Muskel-u. Nervenphysiologie," Lahr, 1858.

${ }^{2}$ Sherrington, Proc. lloy. Soc. London, 1893, vol. lii.

3 Sherrington, Journ. Physiol., Cambridge and London, 1894, vol. xvii. ; and C. Huber, Jonurn. Comp. Neurol., 1898, vol. viii. p. 169.

${ }^{4}$ Op. cit. $\quad{ }^{5}$ Vulpian, "Leçons sur la physiol. du système nerveux," Paris, 1866.

"See Vulpian, op. cit.; Foster, "Text-Book of Physiology," London, 1891, part iv. 
sensations are all contributory to common sensation, and therefore potentially possess the attribute with which all forms of common sensation are intensely imbued, namely, "affective tone," - and physical pain is stronger than physical pleasure. The relation of the stomach to the "feeling of hunger" is a helpful instance.

The prominent features of the "feeling" hunger are-(1) peculiar sensations, referred to the epigastrium (stomach); (2) general "sinking" or "feeling of weakness"; (3) desire for food. Regarding the first the sensations are of an ill-localised gnawing character, principally referred to the epigastrium but not confined to that region, for the pharynx shares in them. The nature of the stimulus provoking these local sensations is not clear. It is often said to be "emptiness of the stomach." Introduction of food into the stomach rapidly stops the feeling, even before much absorption of the taken food can have occurred. Hunger is temporarily relieved by introducing non-uutritious material into the stomach. It has been urged, on the other hand, that a rabbit after twenty-four hours' abstinence is very hungry, although its stomach will be then found to still contain much food. It is said to take three days' abstinence to empty the stomach of the domestic rabbit. But here the stomach after abstinence, although distended, is not so distended as before, and the absolute value of the limen of "relaxation stimulus" may legitimately be variously placed. Also, it has been urged that many persons do not feel hungry in the morning after the longest abstinence of the twenty-four hours ; - most English do, but few French or German, a difference related to difference in national habit regarding meal-time. In duodenal fistula hunger has been observed as a marked symptom, even when the stomach is full. On the other hand, in gastric catarrh hunger may be absent although the stomach remain empty for hours. The nature of the stimulus causing the hunger sensations, referred to the epigastrium, is therefore not clear. Tension of the stomach wall (or relaxation) is, however, indicated by various of the above evidences as playing some part in it. Normal tensions, and abnormal, are well known to be efficient as stimuli for the afferent nerves of hollow viscera $(e, g$. urinary bladder, heart, etc.). The tension caused by the engorgement of the mucosa with the proper digestive granules developed in the cells, may give rise to this local sensation in the case of the stomach.

As to the sensory nerve channel for the local sensation of hunger, likely enough it is the vagus, but very possibly the stomach receives sensory nerve fibres also from the thoracic spinal ganglia, vît the rami communicantes. After section of both vagus nerves, rabbits continue to feed well, and therefore must have appetite. ${ }^{1}$ So also, after section of the cervical cord or of both the splanchnic nerves, and after extirpation of the ganglia coliaca and meseraica. ${ }^{2}$ Neither the vagus nor the splanchnic afferent channels are therefore necessarily the single channels for the feeling of hunger; but that after their section the local feeling of hunger exists, is only presumed. Section of merely a few muscular nerves would hardly relieve the body from the heavy feeling of fatigue consequent on a long march; would section of the vagi remove the oppression of impending asphyxia? Section of the sensory nerves of the pharynx and œsophagus does not prevent the occurrence of thirst, but moistening the fauces does temporarily allay thirst.

The dog exhibits hunger after complete removal of the stomach, ${ }^{3}$ so

${ }^{1}$ Leuret and Lassaigne, "Rech. physiol. et chim. p. servir a l'histoire de la digestion," Paris, 1825, p. 211 ; Sédillot, "Du nerf pneumog. et ses fonctions," Paris, 1829 ; Longet, "Physiol. d. système nerveux," Paris, 1860, tome ii. p. 535 ; Budge, "Lehrbuch d. speciel. Physiol. d. Menschen," Leipzig, 1862, S. 691. These refute Brachet's conclusions, "Recherches s. 1. fonct. d. systeme nerveux," Paris, 1837, p. 219.

Budge, Nova acta phys.-med. Acad. nat. curios, 1860, tomo xxvii.

3 Ludwig and Thanhofer, quoted from Beaunis, "Les sensations internes," Paris, 1889, p. 34 . 
here is, together with the local excitation, a general condition produced. As to this latter, again, its nature is not clear. A man is usually less hungry after his ten hours' fast, which has included the night's rest, than after a four hours' abstinence in the working day. The stomach is certainly as emptied in the former case as in the latter. But the greater metabolism that has intervened in the latter brings about a general change which causes hunger. To say this change is want of food-material in the body, is merely to restate the problem. It has been argued that it is want of blood or lymph. Where in duodenal tistula, despite replenishment of the stomach with food, hunger develops and persists, it is relieved speedily by introducing the products of gastric digestion into the upper end of the small bowel. ${ }^{1}$ Similarly thirst is, without drinking, rapidly assuaged by the injection of fluid into a vein. ${ }^{2}$ These effects resemble the production of, and relief from, general muscular fatigue, given by transfusion of blood in Mosso's well-known experiment. ${ }^{3}$ The blood is somewhat inspissated in moderate hunger. ${ }^{4}$ The relief occurs so rapidly, that it is argued that it may be due to alteration of the blood rather than of the lymph. How quickly large quantities of fluid may be transferred from the blood to the lymph, is shown by experimental observations. ${ }^{5}$ The feeling of hunger is relieved, is even converted into a pleasurable satiety, before the blood is saturated with absorbed material. The chief use of the local peripheral reference of sensation in hunger seems as an index for regulating the amount of intake, and thus preventing excess. It has been remarked that animals in which the vagi have been cut, so far from not evincing hunger and taking insufficient food, take an excessive quantity. But I have seen in the dog distinct diminution of appetite ensue on double vagotomy, after cervical spinal transection.

The peripheral regions to which the feeling is chiefly referred, stomach and throat, are innervated mainly by the ninth and tenth cranial pairs. "Hunger" feeling (and "thirst" feeling) are held, therefore, to originate in impressions elaborated by the bulbar centres receiving the roots of those nerves. These centres are perhaps specially sensitive to those qualities of the circulating blood which depend on intake of food, much as the bulbar centre receiving the lung branches of the vagus is specially sensitive to the respiratory quality of the blood. It can hardly be supposed that in the nerve cells of these centres impulses are actually initiated by conditions of the blood supplied to them. Meynert, it is true, argued that functional hyperæmia of the brain is felt as pleasurable and anæmic conditions of the brain as the reverse, the central elements perceiving their own nutritive condition. ${ }^{6}$ But the condition of their blood affects the excitability of nerve centres; it may be supposed to increase the excitability of those into which embouch the afferent nerves of the gullet and the stomach. Some nerve centres are remarkably increased in excitability by moderate hunger, just as conversely the knee-jerks are found to be diminished shortly after a heavy meal. The thirst which is caused by some drugs subsides after a time without the taking of liquid into the body ; it has been argued here that the effect is due to temporary increased central activity, due to temporary alteration of the blood. It has been several times remarked that the stomach and intestines of fasting animals exhibit remarkably active peristaltic action. A condition of the general blood or lymph can be easily conceived to not only affect the nerve centres centrally, by affecting the nutrition of their ganglion cells, but also by peripheral changes exciting

\footnotetext{
${ }^{1}$ Busch, Virchow's Archiv, Bd. xiv. S. 140.

2 Dupuytren, "Leçons orales," Paris, 1832.

3 A. Mosso, Atti d. r. Accad. d. Lincei Cl. di sc. fis., mat. e nat., Roma, May 1887.

Lloyd-Jones, Journ. Physiol., Cambridge and London, 1891, vol. xii. p. 299.

${ }_{5}^{5}$ Sherrington and Copeman, ibid., 1890, vol. xi., "Proe. Physiol. Soc.," p. viii., ibid., 1893, vol. xiv.

"Klinisehe Vorlesungen über Psychiatrie," Wien, 1890, S. 6.
} 
sensory nerve-endings. It has been urged that a change, e.g. a diminution in the volume of the circulating blood, may be perceptible by means of the heart nerves. ${ }^{1}$ Deficiency of lymph might certainly induce a relaxation of many membranes to which afferent nerve fibres are distributed, e.g. capsules of Pacini corpuscles, etc. Such changes may be thought too weak to produce effect; yet a character of common sensation is a pre-eminent tendency of the impressions building it to fuse, to summate, to cumulate. This is as unmistakable in pain, as in tickling, the sexual "feeling," etc. This explains why mere excessive duration of a stimulus which at the outset lies far below the limen for pain, may bring it up beyond that limen and make it effective, even insufferable, e.g. a tight boot or hat. Hence it is impossible to deny that visceral stimuli, that for a short time are of negligible intensity, may, if continued, develop potency for sensation.

From the above it is evident that the nature of the condition of the body which is the source of the feeling of hunger, is not definitely known, nor is the manner in which the condition can react on sense. Nevertheless, the kind of condition which excites the "feeling" is clear enough. It is a condition in which requisite supply of material for maintaining normal life is withheld, in which injury is threatened, and to which the dativus incommodi of the grammarians is applicable. A need arises like the besoin de respirer, and becomes irrepressible, i.e. excites to action. Hunger eventually becomes painful, even more than, conversely, satiety is pleasant. The dogs from which the cerebral hemispheres had been removed by Goltz became restless as feeding-time drew near. This restlessness subsided when the usual quantity of food had been taken, and the dogs then invariably "refused" further quantities of food. In other words, the restlessness was part of the hunger reflex, and so far, like the movements of "swallowing" brought out by food placed in contact with the dog's muzzle, could only be excited in the hungry state. So also the male pigeon, after removal of the hemispheres, will in spring coo all day long, and show distinct signs of sexual excitement. But his activity is entirely indifferent toward the hen-bird placed near; he leaves her unnoticed. The muscular restlessness (the motor adjunct of the "hunt" for food) stands evidently in the same relation to this visceral sensation of painful "tone" as does the flexion of the hip and knee (withdrawal of the foot) in relation to the cutaneous sensation of painful "tone" initiated by a pinch of the foot.

The use of the discussing of the feeling of hunger to the argument here is, that it broadly indicates how the production of painful sensations by the same visceral afferent nerves as those ordinarily employed in unpainful and in even unconscious neural reflex reactions, comes about. That in some instances the visceral pain is due to tension, forms, to my thinking, not a difficulty, but merely indicates that the normal adequate stimulus is of a mechanical kind. ${ }^{2}$

A further difficulty for supposing that the viscera are provided with specific nervous apparatus for a "pain-sense," is the following:-

"It may happen to a man to suffer pain in a particular region or tissue of the body once only in the course of his lifetime, or possibly not even once; nay, we may suppose that in this or that region or tissue pain is felt once only in one individual among a large number of persons." 3 How, if this pain requires, to carry it out, a special mechanism, including special.afferent nerve fibres, can, in the case in

'Budge, Nora acta phys.-med. Acad. nat. curios, 1860, tomo xxvii. ; "Physiologie," Leipzig, 1862, S. 815 .

2 See C. A. Strong, Psychol. Rev., N. Y. and London, 1896, vol. iii. p. 64, contra H. R. Marshall, ibid., 1895, vol. ii. p. 594. Cf. also E. H. Weber, Wagner's "Handworterbuch," Bd. iii. (2) S. 580.

${ }^{3}$ M. Foster, "Text-Book of Physiology," London, 1900, part iv.

VOL. II. -63 
question, such a mechanism of pain have originated? Or, when originated, be preserved intact (although unused whole generations through), in order occasionally, perhaps once in a lifetime, to come into use?

This difficulty is in the main, though not entirely, avoided by the view that visceral pain results from excessive stimulation of afferent structures normally employed for reflex actions, accompanied only in some instances by sensations at all, and not, under usual circumstances, by feelings of physical pain.

Further, it must be remembered that some of the most salient pains of visceral disease are of the nature of "sympathies," "associate" or " referred" sensations (Mitempfindungen). ${ }^{1}$ These are clearly merely adjuncts of excessive neural reaction, - not always the effect of excessive stimulation,--and yield no support to a hypothesis of specific visceral pain-nerves. The visceral nerves seem connected with the central neural mechanism for pain only through a path offering high resistance. The central paths of pain are probably in all cases high-resistance paths, but the resistance seems different in degree in different pain-paths. It seems lowest in a certain set of the cutaneous paths. The resistance, it seems, can be overcome in various ways, by intensifying the stimulus, and also by repeating it (summation). When nerve impulses are enabled, e.g. by summation, to travel high-resistance paths, they show a tendency to escape to neighbouring channels. Borrowing language from electrical termino$\operatorname{logy}$, the tension of the charges seems to be high, and the ordinarily efficient insulation then allows some leakage. In physiological parlance, by virtue of the neural laws of irradiation and intermingling (overlap), the visceral afferent nerves, thrown into excitement by excitation excessive in intensity or duration (conditions in which pains arise) impart excitement to juxtaposed nervous apparatus, and especially to the dolorific nerves of the skin. There is nothing in the socalled referred pains of the viscera, either in regard to their mechanism or their occurrence, that argues for the existence of specific pain-nerves in the viscera.

As far, therefore, as analogy from the pains of the viscera and the organs of muscular sense can guide, we are not given warranty for an expectation that cutaneous pain shall be the outcome of the stimulation of specific "pain-nerves." And if that supposition be adopted, logicai justice leads to the following dilemma. The adequate stimuli for touch and thermal sense can evoke pain from the skin. A persistent touch, e.g. the light pressure of a hat, not painful at first, may in time become so. According to Weber, the merely areal increase of application of heat to the skin can convert a non-painful heat stimulus into a painful. Those who infer that painful cutaneous sensations are called up only by specific pain-nerves will be consequent, if they suppose that the skin possesses at least two species ${ }^{2}$ of pain fibres, equipped with specific end-organs, for which tangible and thermal stimuli respectively are adequate. Analgesia for tactile stimuli sometimes co-exists in the same skin with hyperalgesia for thermal stimuli. $^{3} \quad$ Painful mechanical stimuli have been in similar experiments noted to elicit wholly different reflexes from those evoked by painful thermal stimuli. ${ }^{4}$

Weber felt this difficulty when he adopted the long-current opinion that nerves of "touch" and thermal sense yield pain when excited not mediately

${ }^{1}$ See above, p. 981.

2 From the analogy of the existence of separate nerve-endings in the skin for sensation of "cold" and "warmth" it would be probable that more than two, namely, three species of pain-endings in skin would be demanded for pains from mechanical and thermal stimuli. Two is, however, taken in the text as the smaller hypothesis.

3 Strong, Psychol. Rev., N. Y. and London, 1896, vol. iii.

4 E. Bruecke, "Vorlesungen ueber Physiologie," Wien, 1874, Bd. ii. S. 240. 
through their end-organs but immediately along their course. He argued that the pain felt when the ulnar nerve is cooled by holding the elbow long enough in ice-cold water, is caused by excitation of the nerves of temperature sense. But in that experiment, sensations tactual and thermal as well as dolorous, arise, referred to the peripheral distribution of the ulnar trunk. The experiment admits just as well of explanation by the hypothesis of special pain nerves irritable in their course.

It may be argued, however, that even on the view of the existence of specific pain end-organs in the skin, it is not obligatory to suppose that, though they respond equally both to tangible and thermal stimuli, they are in reality of more than a single kind. These stimuli are of the nature of general nerve excitants. They only excite the pain sensations when they are applied in relatively high intensity, and excite it cateris paribus in proportion to the height of grade of their intensity. Such excitants of sufficient intensity, when applied to any naked nerve fibre, motor as well as sensory, and in its course, as well as at its ramified endings, will excite it. What is necessary, therefore, in the skin is that the endings of the hypothetical specific pain-nerves should be amenable both to tangible and thermal stimuli, and this might possibly be the case in one of two ways. They might possess end-organs so highly differentiated in regard to specific nerve energy, that any stimulus affecting them at all irritated pain-reaction. Or they might, while their central connections obeyed to the letter the well-worn law of specific nerve energies, be provided with peripheral end-organs so little differentiated as to allow with facility a considerable range of quality of general nerve excitants to play upon them with tolerable effect. The main difficulty is that the effect is so great. Choice between the two views will depend largely upon the relative value attached to two different functions seeming to belong to every sensifacient end-organ. The sensorial end-organ is an apparatus by which an afferent nerve fibre is rendered distinctively amenable to some particular physical agent, and at the same time rendered less amenable to, i.e. is shielded from, other excitants. It lowers the value of the limen of one particular kind of stimulus, it heightens the value of the limen of stimuli of other kinds.

Especially in regard to the latter result of adaptation, has the original Müllerian law of specific nerve energies required reconsideration. The BlixGoldscheider cold spots of the skin evoking cold sensations reply ${ }^{1}$ to stimuli of pressure and heat as well as of cold; that is, non-adequate as well as adequate stimuli fairly easily evoke from them their specific sensation. On the other hand, there are in the tongue individual papillæ from which only a certain suitable set of tasteable substances can evoke a sensation, - always the specific sensation,- - and all other stimuli fail to evoke any sensation whatsoever. ${ }^{2}$ The warmth spots of the skin are in the condition of these tongue papillæ; but it may be that the pain spots are more in the condition of the cutaneous cold spots in this respect, and even overpass them. Somewhat against this is the histological evidence of the skin regions which are preeminently dolorific, pre-eminently possessing so-called "naked" nerve fibril sensorial endings. If simplicity of histological structure in a sensorial endorgan be any guide to absence from it of functional differentiation, histology indicates the explanation of the presumed amenity of pain spots to various

\footnotetext{
1 Denied by M. Dessoir and Nagel, affirmed by Goldseheider, v. Frey, and Kiesow. My own experienee on this point supports the latter group of observers, and would include faradisation.

2 Oehrwall, Skandin. Arch. f. Physiol., Leipzig, 1891, Bd. ii. S. 1; cf. Kiesow, Phil. Stud., Leipzig, 1898, Bd. xiv. S. 591.
} 
physical stimuli, by want of differentiation rather than height of differentiation of end-organ as the more probable.

On the other hand, instead of supposing that the hypothetical specific pain nerves are each and all normally and delicately amenable to a range of variety of stimuli, for whose appreciation elsewhere a whole series of sense organs is required, it may be answered that there is no objection to supposing that the skin has two or three different species of specific pain nerves. For this belief there appears at present no decisive evidence, but there is the analogy with muscular and visceral nerves.

On the hypothesis of the existence of a specific pain-sense it would be expected that excitation of the cerebral cortex would give rise to some evidence of production of pain. The cerebral cortex, which contains, as is known, fields for the termination of so to say each avenue of sense, should on that view excite pain sensations. This does not appear in fact to be the case. The well-known experiments of Haller, Bichat, Budge, etc., besides bedside evidence, and the opportunities of electrical excitation, do not bear out the expectation. Epilepsy, so far as I can learn, hardly ever includes in its prodromata a "pain " aura, although other orders of sensation-visual, auditory, tactual, thermal, gustatory, and odorous-seem each laid under contribution.

On the other hand, the dogs from which Goltz had removed the cerebral hemispheres reacted to "painful" stimuli in a way which was interpreted as evidence of the persistence of physical pain. Such evidence is, however, after all, equivocal for the point, as is the reflex vocalisation noted in the rat-and I have heard it in the monkey-elicitable even in the first hour after cerebral ablation, anterior to the corpora quadrigemina. We reflect that in this condition the contrast of the sensible world as "me" and "the rest" seems from other evidence gone, and the animal has become "impersonal." In all these cases the working of the machinery of expression is but a dubitable sign for the actual existence of the feeling which is under ordinary conditions "expressed."

On the whole, it would appear as if the deep sensory nerves, the visceral and the musculo-articular, and of the superficial sensory nerves, the cutaneous, possess the power of evoking physical pain; and it appears significant for this fact that these are the sensory channels which inform the sentient self of the condition of that half of its sensible universe, its bodily "me belonging to self." It is impossible to think that the normal functions of three sets of afferent nerves of such different distribution and physiology as the visceral, musculo-articular, and cutaneous, are all the same, although the dolorific actions from them appear so similar. Bodily pain would therefore seem less a single specific order of sensation than, like local sign, a quality attaching in common to a certain non-universal group of specific sensations. Pain has no one specific stimulus with which it can be correlated. This view of course relinquishes the conception of any specific pain-sense, also, strictly speaking, of "pain" nerve fibres. But I recognise that it may be convenient to distinguish, when certain apparatus especially readily ministers to pain, some nerves as "dolorific" or " painnerves"; as in the skin where certain nerves develop sensations with pain quality dominant, almost to the exclusion of all other (as muscle nerves evoke dominant local sign). This usage will at least serve as a

\footnotetext{
${ }^{1}$ See, as a rare instance, the case given by Pierce Clark, Am. Journ. Insan., Utica, N. Y., July 1897.
} 
protest against the teaching that pain stimuli and pain sensations are agents and reactions lying beyond the sacred pale of "normal" life.

Having discarded the supposition of separate afferent nerve fibres with their specific end-organs, entrusted specifically with carrying "painful" impressions to a "pain centre," it is finally worth considering what meaning can be still attached to the phrase "pain centre." There might exist such a specific pain centre, to be reached by currents of overflow from other sense centres, when the violence of their inner excitenient had surpassed a certain pitch. Or, again, a certain extreme degree of inner excitement might superimpose the feeling of pain upon the sensation elaborated by each of the sense centres itself, ${ }^{1}$ although unequally in degree in the centres of the different senses.

The rate at which agreeableness and disagreeableness vary with intensity of a sensation, may roughly be represented by a curve about a horizontal line marking the threshold of sensibility. If below the line be disagreeable, the curve at starting from the horizontal rises, at first more slowly than sensational intensity, then faster, and reaches its maximum distance above, that is, on the agreeable side of neutrality, before sensation is near its acme. After its maximum the agreeableness soon drops and sinks below the horizontal zero level into the realm of the disagreeable or painful. Into this it plunges, becoming, after a certain depth, finally asymptotic to the horizontal level. For some sensations-as bitter, sour, and certain smells-- the curve does not ascend at all, but enters the realm of disagreeable from its outset. Among these sensations must be included those from the pain spots of the skin. The sensation of pain spots seems to resemble most nearly in this respect such olfactory sensations as in highly osmatic animals certain odours, e.g. that of the skunk, evoke. How intimately vexation of the stream of consciousness by pain stands in relation to intensity of the stimulus, is no way shown better than by simply increasing the number of nerve fibres, under application of a stimulus non-painful when at work upon a limited number. Minuteness of local application of stimuli to the skin does not appear to have yet reached a degree at which it becomes probable that the sensation is caused by excitation of a single nerve fibre alone. The nerve fibres to the skin are in their distribution very intermingled. I found that in most regions of skin, every, even small area, obtains nerve fibres from two successive spinal roots, and in not a few regions of skin the number of spinal roots furnishing the areas is three. ${ }^{2}$ Hence in a skin field where only one root remains, punctiform stimulation at the field's edge is less bound up with pain than that nearer the field's centre, the latter implicating a greater number of nerve fibres. In regard to the pain produced by cold and warm stimuli size of the skin-surface, i.e. the number of the sensifacient nerve-endings influences in the directest manner the product of the sensation. A finger-joint can be kept comfortably in water at $48^{\circ} .7 \mathrm{C}$., but the whole hand plunged into the same water soon becomes painful. Similarly with water at $6^{\circ} \cdot 2 \mathrm{C}$. The "touch spots" in a skin area do not work all from the same sensibility limen; increase of pressure on the same field will, within limits, excite fewer touch-organs if light than if heavier.

That the seat of the neurosis underlying the painful in a sensation is not identical with that of the production of the regular form of the sensation, seems shown by the evidence given of the difference of the central paths of conduction for pain and for touch. The grounds were given above for the belief that the pain-path involves especially the spinal grey matter and the white matter immediately abutting upon that. In connection with this is the prominent part which summation plays in the production of pain.
${ }^{1}$ Lotze, "Med. Psychol.," S. 245 ff.
${ }^{2}$ Phil. Trans., London, 1892. 
Pain sensations summate at less frequency than touch sensations, and even more easily than muscle twitches.

Richet ${ }^{1}$ has pointed out that, in faradising the skin, a distance of the secondary coil from the primary can be found at which neither single makeshocks nor break-shocks are perceptible, but a rapid series of these individually imperceptible shocks evokes a strongly unpleasant sensation. In certain clinical cases, ${ }^{2}$ rhythmic repetition of a liminal stimulus, e.g. touches with a brush or needle point, after a certain number of seconds, evokes a pain which swells wavelike to a crisis and then subsides, often recrudescing to a second or even third crisis. When the point of a pin is lightly pressed on the skin, there succeeds, after the immediate pricking feeling, an interval without sensation, followed by a second pricking feeling. This second sensation, which has been termed the "secondary," differs from the first in having no touch-quality mixed with it; it seems "to come from within." No "secondary sensation" follows stimulation with a single induction shock; it does, however, follow when a short series, or even two induction shocks, ${ }^{3}$ are applied for not less than $\frac{1}{12}$ of a second. It is therefore due to summation in the latter case, and probably in the former also.

The inertia of the organs for pain seems peculiarly great. Faradic excitation, with shocks at higher rate of frequency than 20 per second, causes continuous sensation of pain, and the period of latent excitation for pain is relatively long. I found that by focussing the heat-rays from a lamp upon a skin area of about $25 \mathrm{sq}$. $\mathrm{mm}$. (on the back of the hand), and allowing a perforated screen to intermittently intercept the radiation, the heat-pain begins to be perceptibly intermittent when, the times of play and of interception being equal, the intermittence falls below the rate of thirteen per minute. An intensity of this intermittent radiation stimuli, at first not painful and yielding sensations strikingly discrete, soon becomes dolorific, and then the sensations remain discrete no longer but are fused more or less together.

Weber ${ }^{4}$ counted the seconds elapsing before the finger- or tongue-tip had to be withdrawn on account of pain from water at various temperatures. The warmth gradually became painful in many instances when not so at first,-e.g. with water at $44^{\circ}$ Reaumur, whence the finger was withdrawn in from twentyone to twenty-eight seconds. He found the fingers of the left hand slightly more sensitive than those of the right. The rounded end of an iron rod, four Paris lines $\left(\frac{1}{3}\right.$ in.) thick, cooled to $3^{\circ} \cdot 2$ Reaumur, and applied to the forehead eleven lines from the median plane evoked painful sensation in ten seconds; that is, the sensation caused began to be painful in ten seconds, and similarly, from the upper eyelid, in eight seconds became insufferable. In these cases pain almost is summation.

"When all the spinal paths are in normal condition, impulses of moderate strength will take the simple white paths, and only impulses of excessive strength will overflow into the branching side paths through the grey matter." 5 The essential part of this idea is (1) a summating path offering high resistance to conduction, and (2) a low resistance path of more mobile neural protoplasm, possessing less inertia. To identify the one with grey matter, the other with white, seems unnecessary and unfortunate. Every conduction path in the nervous system is composed both of grey and white matter, inasmuch as every nerve fibre is but the process of a nerve cell, and every nervous impulse is but a wave of change which travels a concatenation of nerve cells, and every (or almost every) synapse between nerve cells occurs in the grey matter. It is more useful to interpret the suggestion rather in the sense that when nervous

1 "Recherches expér. et cliniques sur la sensibilité," Paris, 1877.

2 Naunyn, Irch. f. exper. Path. u. Pharmakol., Leipzig, 1889, Bd. xv.; and Rosenbach.

${ }^{3}$ Goldscheider and Gad, Arch. f. Physiol., Leipzig, 1892.

"Wagner's "Handwörterbuch," 1846, Bd. iii. Abth. 2, S. 481.

${ }^{5}$ Wundt, "Physiol. Psychologie," 3te Aufl., Bd. i. S. 112 ; Goldseheider, "Ueber den Schmerz," Berlin, 1894. 
impulses are strong, they overflow from their ordinary channels of direct and facile conduction toward mechanisms of conscious reaction into channels less direct with consciousness, the latter being composed of a greater number of conducting neurones than the former. It is well known that the general rule of arrangement in the spinal cord is, that long nerve fibres lie outside, short nerve fibres inside next to the grey matter. Some of these latter are extremely short, and all belong to "relay cells." It is this sheet of short relay fibres, immediately abutting on the grey matter, that pain impulses-to judge from experimental and other data-especially in one part of their course employ. These short cell-paths, abutting on the spinal grey matter, effect their mutual linkage there. We know summation in connection with the nervous system only as a property attaching to cell-chains, often, it is true, to simple two-link chains. Hence summation may be a function of the transmission of impulses from one cell to another, - in short, a function depending on the synapse. It is in accord with this to find that a conducting chain of a particularly high number of links, i.e. built up of more nerve cells than usual, exhibits summation particularly markedly. That is, the inertia of the pain apparatus may be a peculiarity of the reaction of the "pain" nerve cell, or, on the other hand, may be simply the outcome of its consisting of many nerve cells. That pains are badly localised (E. H. Weber), thus painful touches not so well as less painful, harmonises also with the sanie general conception.

The physiological apparatus for cutaneous pain takes, as compared with that of other skin senses, a long time to get into movement, and when once moved requires, in comparison with other apparatus, a long time to return to equilibrium.

Connected with summation must be the phenomenon of delayed pain sensation ${ }^{1}$ commonly met with in tabetic cases. The pain produced by a prick with a needle is perceived only after a lapse of an abnormally long time; even five seconds may intervene between prick and pain. The sensations of touch and pain are thus isolated, a clear interval separating the two. The patient replies to the single prick with a double response "now," followed by "ah!" corresponding with the "touch" and the "pain." Schiff ${ }^{2}$ described a similar phenomenon as occurring in experiments where partial transection of the spinal cord has trespassed on the grey matter and narrowed it. According to him, the delay increased proportionally with the encroachment on the grey matter, just as the wave of conduction was delayed in the bell of medusa where the tissue was elcroached upon, and the path narrowed, in Romanes' experiments. ${ }^{3}$ Compression of a nerve trunk has also the effect of delaying the sensory reaction for which it is afferent. I myself met very marked instances of delay in experiments, ${ }^{4}$ when dealing with the analysis of sensory nerves into their separate root components. For instance, the reflex from a digital nerve which received afferent fibres from the seventh and eighth cervical roots, and from the first thoracic, was much delayed in occurrence after section of the first thoracic and eighth cervical dorsal (afferent) roots ; the latency was sometimes more than three seconds. Clinical observation ${ }^{5}$ has also detected the delay in injury or disease of peripheral nerves. Schiff's reference of it to narrowing the grey matter is a suggestion applicable, therefore, to only part of the problem. In my own experiments there was similarly a narrowing of the conduction patl, but the path was entirely a peripheral one. The explanation is probably connected with the increase of intensity of reaction obtained in so many cases

1 Osthoff, "Die Verlangsamung d. Schmerz-empf. bei Tabes dorsalis," Erlangen, 1874.

2 "Musket-u. Nervenphysiologie," Lahr, 1858.

3 Phil. Trans., London, 1880; also, "Jelly Fish, Star Fish, and Sea Urehins," London, 1885.

4 Phil. Trans., London, 1892.

5 Leyden and Goldscheider, "Erkrank. d. Riiekenmarks," Wien, 1895, Heft 1 ; Nothnagel's "Specielle Pathol.," Bd. x. 
by increase of the extent of the stimulus, i.e. by simple increase of the number of nerve fibres excited (cf. "Cutaneous Senses of Cold and Warmth," p. 956).

The stimuli which evoke pain may be characterised as "excessive." It might almost be asserted that "excess" is that quality of a stimulus in virtue of which it becomes "adequate" for the sense of pain. "Excessive " in this application connotes "harmful," or " to be avoided," e.g. by muscular action for resistance or escape. The "excess" of the stimulus may lie in its intensity, or in its extensity (spatial or temporal).

The conclusions thus reached may be briefly stated thus:-Affective tone is an attribute of all sensation, and among the attribute tones of skin sensation is skin-pain. Affective tone inheres more intensely in senses which refer to the body than in those which refer to the environment, that is, it is strongest in the non-projicient senses. It is therefore strong in the cutaneous senses, and in them is inversely as their projicience, therefore least in touch spots, more in thermal spots, most in the so-called "pain spots." The sensations from pain spots have weak tactual quality and strong affective quality, especially of negative tone. The pain produced by heat is probably evoked from "pain spots," and not from thermal spots; the pain produced from "pain spots" is produced not only by mechanical stimuli, but probably also by thermal, chemical, and electrical stimuli. Stimuli evoking skin-pain are broadly such as injure or threaten injury to the skin; the skin may be said to have gone far toward developing a special sense of its own injuries. The central conducting path concerned with these skin feelings seems a side-path into which the impressions from the various skin spots embouch with various ease, those from the "pain spots" especially easily. The physiological reactions connected with this sidepath are characterised by tendency to "summation," tendency to "collateral irradiation," slow culmination and slow subsidence. They often involve with their own activity that of adjacent sensory channels (associate pains, referred pains). and almost invariably of motor centres of visceral, facial. and other muscles of expression (emotional discharge).

Can bodily pains be of central nervous origin? The dog from which the cerebral hemispheres have been removed gives reactions, interpreted ${ }^{1}$ as evidencing pain. Budge and others ${ }^{2}$ have examined by experiment the effect of stimuli to various parts of the brain, with a view to discovering which parts can give rise to painful sensation, and which not. The result of their observations, chiefly made on the rabbit, has been negative as regards evidence of pain from all parts above the optic thalami. From the optic thalami the evidence was equivocal; what there was Budge was inclined to attribute to the adjoining crus cerebri. From the corpora quadragemina down to the lower end of the rhomb-encephalon inclusive, signs of pain were easily evoked. Pathology seems to prove the production of pain by irritative lesions of the thalamus. Support is lent by the close connection found clinically $^{3}$ and experimentally ${ }^{4}$ between the thalamus and bodily expression of emotion.

\footnotetext{
'Goltz, Arch. f. d. grs. Physiol., Bonn, 1892, Bd. li.; and IIunk's eritique, Arch.f. Physiol., Leipzig, 1892.

2 "Untersuch. neber d. Nervensystem," 1841, Bl. i. S. 10; "Physiol. d. Mensehen," Leipzig, 1862 ; Schiff, "Physiologie," Lahr, 1858, Bd. i. S. 242 ; Lussana, and Lussana and Lemoigne, Arch. de physiol. norm. ot path., Paris, 1860 ; Longet, Bechterew, ete.

${ }^{3}$ Nothnagel, Meynert, Rosenbach, Kiritzow, Mingazzini.

Bechterew, Outanskow, Mislawski.
} 
In one case ${ }^{1}$ a boring, tearing pain in one half of the body, accompanied by motor weakness the day following its onset, led to suicide two years after the attack. A lesion was found post-mortem in the thalamus (nucleus externus), adjoining the posterior third of the posterior segment of the internal eapsule, in the hemisphere opposite to the henialgesia. In another instance 2 there was " severe pain for a number of years in the right side of the body, and for five years weakness of the right side, with involuntary movements of the right hand and sometimes leg. On movement of the parts, pain could be producer in arm and leg throughout, but while at rest pain was chiefly in elbow and knee. The tactual sense was intact, although there was slight hyperæsthesia. Localisation power and the temperature and musele senses were intact, except slight numbness to cold. There was a high degree of hyperalgesia to pressure, pereussion, and all passive movements. A vertical section through the pulvinar of the left thalamus displayed on the edge of the middle and posterior third a round pea-sized eyst, filled, and a web of connective tissue, lying in the immediate neighbourhood of the internal capsule." In another case, ${ }^{3}$ a solitary tubercle in the right half of the pons was the only lesion found to account for severe pains existing in the left limbs, with complete anæsthesia of their skin. "anæsthesia dolorosa."

Cognition and feeling seem almost concomitant. To abstract either from other can only be done as a conventional simplification for logical purposes. There is therefore no a priori reason for expecting cognition and feeling to be respectively adjunct to spatially separate cerebral organs. As regards pain and the cortex, some argue that the feeling of bodily pain is elaborated in the Rolandic region, and that thence are initiated emotional movements, respiratory, vascular, and facial, etc. Pain aura in epilepsy seems to be very rare. ${ }^{4}$ It has been suggested ${ }^{5}$ that the severe pains of hysterical and hypochondriacal patients are, in some instances, of cortical origin. Witmer ${ }^{6}$ had a "painless" man for some time under observation. He had worked at a show as "the human pincushion." "He could be cut with a knife, or stuck with pins or needles, without showing the slightest sign of pain. I have known him to hold a red-hot coin in his hand without wincing, until it had burnt itself deep into the flesh. It is impossible to say positively whether this subject inhibited the expression of pain, or whether he inhibited the pain itself. He said he felt pain on ordinary occasions, when he had not made up his mind to be insensible to pain, but he reported that when once he had decided not to feel pain of the stimulus, the pain was no longer felt. There were areas of the skin which he could not render insensible to pain. I am inclined to believe he inhibited the sensation of pain, and not its exterual manifestations." Kant describes that he learnt to inhibit the pains of his gouty attacks. ${ }^{7}$ Cases of hysteria present phenomena analogous to the above. Pain disorders are much more readily produced by hypnotic suggestion than are disorders of any of the other senses, 8 except perhaps the muscular.

${ }^{1}$ Edinger, Deutsche Ztschr.f. Nerrenh., Leipzig, 1891, Bd. v. S 262.

2 Biernacki, Dcutsche med. Wh huschr., Leipzig, 1893, No. 52. Also Henschen's case, "Klin. Beitr. z. Path. d. Gehirns," 1890, Bd. i. S. 103.

3 Marot, Bull. Soc. anat. de Paris, 1875 ; also Nothnagel's case, "Diagn. d. Gehirnk.," Wien, 1879 , S. 586 .

4 For a case, see Pierce Clarke, Am. Jouru. Insan., Utica, N. Y., 1897.

${ }^{5}$ Edinger, op. cit.

6 "Twentictl Century Practice of Medicine," 1897, vol. xi. p. 939.

7 Letter to Hufeland, "Ueber d. Macht d. Gemuths."

${ }^{8}$ Witmer, op. cit., and Journ. Nerv. and Mcnt. Dis., N. Y., April 1894, p. 1. 


\title{
THE MUSCULAR SENSE.
}

\author{
By C. S. Sherrington.
}

Contents.-Definition of "Muscular Sense," p. 1002-Views regarding the nature of Muscular Sense, p. 1002-Charles Bell's "Sixth Sense," p. 1006-The Peripheral Apparatus of the Muscular Sense; Histological Data, p. 1006-Nerves of Muscles and Tendons, p. 1007-End-Bulbs and Pacinian Corpuscles, p. 1007 - Hypotheses as to the Mode of Excitation of the Peripheral End-Organs of the Muscular Sense, p. 1009-Muscle-Reflexes, p. 1011-The Performances of Muscular Sense, p. 1013-Perception of Posture, p. 1013-Perception of Passive Movement, p. 1014 - Perception of Active Movement, p. 1016-Objective Effects of Loss of Muscular Sense upon "Willed Movement," p. 1018 Subconscions Character of Reactions of Muscular Sense, p. 1019-Probable Importance of Tonus for Co-ordination, p. 1020-Apæsthesia produces A taxy of "Willed Movement," p. 1020-Senso-paralysis, p. 1021-Perception of Resistance, p. 1021-The Liminal Stimulus, p. 1021-The Liminal Difference, p. 1022-"Adaptation," p. 1024-Interpretation of Results, p. 1024-Fusion of Muscular with other Sensations, p. 1024.

IT is somewhat difficult to present a concise definition of the term "muscular sense." Authorities have not been perfectly concordant in their use of the term. It may perhaps best be taken to include all reactions on sensc arising in motor organs and their accessories.

\section{Views Regarding the Nature of Muscular Sense.}

At the outset of any inquiry into muscular sense arises a question omissible for other senses. In the very expression "a sense" physiology elsewhere postulates existence of an organ or organs placed peripherally, that is, outside the central nervous system, whence afferent channels convey to intrinsic "centres" impulses which there initiate the physiological and psychological reactions of the sense. In the case of the muscular sense, the existence of peripheral sense organs has been by many denied.

The view which dispenses with peripheral organs and afferent nerves for the muscular sense has had powerful adherents (Bain, Helmholtz, Lewes, Mach). It supposes that during movement, e.g. a willed movement, the outgoing current of impulses from brain to muscle is accompanied by a "sensation of innervation." Where a movement is to be precise, we do become, in fact, acutely aware immediately in advance of the amount and dircetion it is to assume. The very discharge into the motor nerves seems thus itself to possess a sensual quality.

An objection to this hypothesis is that it sunders sharply the sensations of passive from those of active movement, whereas there is strong ground for believing the two intimately allied; see below, p. 1016. 
Different exponents of "sensation of innervation" suggest different views of its mechanism. The discharge of motor nerve cells, as per se of sensual quality, is one suggestion (J. Müller, ${ }^{1}$ Helmholtz, ${ }^{2}$ Wernicke,${ }^{3}$ and formerly Wundt). ${ }^{4}$ Another regards the motor nerve cell as possessing cellulifugal branches besides its axon, and supposes the discharge of the cell in that way to run centripetally in the nervous system as well as outwardly; among these, Meynert, ${ }^{5}$ H. Munk, ${ }^{6}$ Exner, ${ }^{7}$ and formerly, if I mistake not, Bastian. ${ }^{8}$ For others, again, the motor nerve fibre itself is the site of refluent impulses from as well as to muscle (Arnold, ${ }^{9}$ Lewes ${ }^{10}$ ), at once the afferent and efferent channel for the "sensori-motor circuit" of Charles Bell." The "sensation of innervation" theory is connected with, but should be sharply distinguished from, that cortical revival of sensible and remembered effects of past movements, which seems to form the initiatory phase of the process of willed muscular action. As a component of the muscular sense (kinæsthetic) factor of a willed movement, Bastian ${ }^{12}$ recognises a central process, revival of a kinæsthetic image, and a peripheral process consisting of guiding kinæsthetic impressions, which flow in from muscle, etc., and from eye, and influence the motor discharge, although in a subconscious way. The former component is psychologically a memory image of the sensations that accompanied the movement when executed formerly, and especially when in process of being learnt. To this position Münsterberg ${ }^{13}$ stands near. For Wundt, ${ }^{14}$ centromotor elements lie in connection with centro-sensory, and these latter are excited not only by influent impulses generated by peripheral muscular sense organs, but also by the motor discharge in the centro-motor elements along their central connections. This superficially approaches the immediate consciousness of one's own movement, championed by Trendelenberg ${ }^{15}$ and George, ${ }^{16}$ and combatted by Lotze ${ }^{17}$ and Harless. ${ }^{18}$ A difference between Bastian and Wundt lies in the former supposing that for the guiding kinæsthesis there collaborate peripherally arising impressions with remembered peripherally arisen; while the latter supposes peripherally arising sensations to combine with centrally arising, not distinctly stating those to be kinæsthetic revivals. For Wundt the notor cerebral cell presumably reacts by cellulifugal processes upon sensory cerebral cells; Bastian years ago made a similar suggestion for the motor cornual cells of the spinal cord, and Exner carries the latter notion to extended use in the text and schemata of his recent "Entwurf." 7

Since Hume, it has been admitted that the perceived results of our volition are the outward ends obtained, and not the inward action of the neuromuscular machinery. But discrimination between weights is found possible

1 "Handbuch d. Physiol.," 1840, Bu. ii. S. 500.

2 "Physiol. Optik," 2te Aufl., 1896, S. 742, 947 ; and also in 1st edition.

3 "Ges. Aufsatze," 1893, S. 6 ; "Grundzuige d. physiol. Psychol.," Leipzig, 4te Aufl., 1894, Bd. i. S. 423 ; also in 3te Aufl., 1887.

t "Beitr. z. Theorie d. Sinneswahrnehmung," Leipzig, 1861, S. 409.

5 "Das Zusammenwirken d. Gehirntheile," Verhandl. d. X. internat. med. Cong., Berlin, 1890.

6 "Ueber Funktionen d. Grosshirnrinde (Mitt. iii. 1878), 1881, S. 43, 52.

7 "Entwurf z. einer physiol. Erklirung d. psych. Erschcinmngen," Wien, 1894, S. 170.

${ }^{8}$ Brit. Med. Journ., London, 1869, vol. i. ; "Brain as an Organ of Mind," 1880.

9 "Ueber die Verrichtung. d. Wurz. d. Ruickenm.," 1844, S. 112.

10 "Physiology of Common Life," Edinburgh, 1859, vol. ii. ; and in "Essays."

"1 Phil. Trans., London, 1830 ; and "The Hand," 3rd edition, London, 1834, p. 216.

12 Proc. Roy. Soc. London, 1895, vol. lviii. p. 89.

13 "Beitr. z. exper. Psychol.," 1889, Heft 1; "Die Willenshandlung," Freiburg, 1888, Bd. i. S. 145 .

14 "Grundziige d. physiol. Psyehol.," 4 te Aufl., 1893, Bd. i. S. 431 ; "Vorlesung. ueber

d. Mensch. und Thierseele," 2 te Aufl., 1892, s. 145 .

15 "'Logische Untersuch.," 2te Aufl., 1846, Bd. ii. S. 196.

16 "Lehrbuch d. Psychol.," Berlin, 1854, S. 231.

17 "Med. Psychol.," 1852, S. 305; "Kleine Schriften," Bü. iii. S. 389.

${ }^{18}$ Fichte, Ztschr. f. Philos., N. S., Bd. xxxviii. S. 66. 
when the movements are brought about by direct faradisation of the nerve trunks and muscles. ${ }^{1}$ The liminal difference is, however, then much greater than under volition. It is less under faradisation of the nerve than under galvanisation of the nerve, and less under galvanisation of the nerve than of the muscle; but even with nerve faradisation it is 2.5 times greater than with volition. Waller ${ }^{1}$ considers this amount of discrimination no greater than ean be accounted for by touch alone.

Wundt, when upholding the "sensation of innervation," appealed to the "heaviness" of a paretic limb. But it can be replied that a paretic limb feels heavy because its movements are hampered as when weighted. Vulpian and also Ferrier ${ }^{2}$ have pointed out that when a hemiplegic patient tries to close his paralysed hand, he in his endeavour, without willing to do so, performs the action with the sound one. The muscles of the glottis and the respiratory muscles invariably become active under effort; as the effort increases, its motor discharge becomes more and more diffuse.

A phenomenon cited by Helmholtz is the following:- "When the right external rectus is paralysed, the right eye can no longer rotate to the right. So long as it turns only to the nasal side it makes regular movements, and the correct position of objects is perceived. When it should be rotated outwardly, however, it stays still in the primary position, and the objects appear flying to the right, although the position of eye and retinal image are unaltered." The left sound eye is covered. "In such a case," Helmholtz ${ }^{3}$ goes on to say, "the exertion of the will is followed neither by actual movement of the eye, nor by contraction of the muscle in question, nor even by increased tension in it. The act of will produced absolutely no effect beyond the nervous system, and yet we judge of the direction of the line of vision, as if the will had exercised its normal effects. We believe it to have moved to the right, and, since the retinal image is unchanged, we attribute to the object the same movement we have erroneously ascribed to the eye. . . These phenomena leave no room for doubt that we only judge the direction of the line of sight by the effort of will with which we strive to change the position of our eyes. . . . We feel, then, what impulse of the will, and how strong a one, we apply to turn the eye into a given position." But the interpretation neglects (G. E. Müller, W. James) the movement of the covered eyeball as a source of peripheral sensation.

Not only is evidence of sensation of innervation unconvincing, but facts it fails to explain are explicable by the counter view of peripheral sense organs. Of a patient in which the entire arms and trunk down to the navel were insensible, superficially and deeply, but the arms not paralysed, it was noted as follows:- "We ${ }^{4}$ take three bottles, - two empty weighing 250 grms. each, the third full of $\mathrm{Hg}$ and weighing 1850 grms. The patient declares he finds them all weigh alike. An experiment was performed, in which he could be informed neither by sight nor by hearing. He even declared, holding in his hand the bottle full of mercury, that he found it have no weight. We place successively in his hand (his eyes bandaged), a piece of modelling wax, a stick of wood, a thick rubber tube, and newspaper folded lengthwise. These he squeezes. He feels no difference of resistance; he does not even perceive that anything is in his hand."

\footnotetext{
1 Beruhardt, Areh. f. Psychiat., Berlin, 1872, Bd. iii. S. 618 ; Ferrier (and Brunton), "The Functions of the Brain," 1876, and 2nd edition, 1886; Waller, Brain, London, 1891, vol. xiv.; and "Proc. Phys. Soc.," Journ. Physiol., Cambridge and London, 1892, vol. xiii.

2 "Functions of the Brain," 1876, p. 227.

3 I quote from W. James' translation in "Principles of Psychology," vol. ii. p. 507, which is from the 1st edition of the "Physiol. Optik." The passage remains unaltered in the 2 nd erlition, 1). $744(1896)$, which is a part of the volume lying beyond that revised by Helumholtz before his last illness.

${ }^{4}$ Gley et Maxilier, trans. by W. James, Rev. phil., Paris, tome xxiii. p. 442.
} 
But there are differences in the effect of anæsthesia on motor power. In some cases the anæsthetic patients cannot move their limbs at all; in some they cannot move them when their eyes are shut. ${ }^{1}$ In some, accurate movement is only possible by help of visual perceptions; with closed eyes ataxy becomes extreme. But there is a class of cases in which, although a limb has lost all sensitivity even for passive movement, voluntary movements can be accurately executed even when the eyes are closed. The patients can, for example, write accurately at will although their eyes are closed, and though they have no feeling of the writing taking place, and do not know when it begins or stops." This class is formed of hysterical and hypnotic cases. At first sight these examples furnish the strongest evidence in favour of the "feeling of innervation" view. No other guiding impressions resident or remote appear to exist. Appeal to the speaking intelligence of the patient does not give evidence of any. But those who have especially studied these cases urge that the condition is not one of true or physiological anæsthesia. They regard these patients as victims of divided consciousness; a part of their consciousness containing, for instance, the kinæsthesia of a limb or lateral part of the borly, becomes split off from the remaining consciousness. In the split-off part the kinæsthetic impressions and sensations may nevertheless remain to produce their usual effects, including co-ordination of movement.

The thumb and the three outer fingers are kept fully extended by pressing their tips down on a table edge, and the index is flexed fully except at its end joint, at which, under the circumstances, flexion is mechanically impossible. If, then, the effort to flex the end be made without looking at the finger, most persons believe the movement intended is carried out. The peripheral sensations of the existing position are overcome by those of the flexed position revived for the initiation of willed movement. ${ }^{3}$ Similarly, Exner's illusion,biting on an unyielding plate giving sense of penetration, although no movement is really effected. Loeb's ${ }^{4}$ interesting observations on willed movements seem to me to illustrate rather that two simultaneous movements are not, when asymmetrically placed, appreciable very delicately in regard to extent and direction, and that judgment of them is easily biassed by the precurrent kinzsthetic idea revived in the willing of the movement. They seem to me to show a somewhat surprising deficiency in muscular sense working under certain conditions, but not to prove that that sense is based on "feeling of innervation," still less to disprove that it is based on centripetal impressions excited in the acting peripheral organs during the movement.

Our conclusion is therefore that "sensation of innervation"-provided that memory revivals of muscular sensations of peripheral origin be not included under that term-remains unproven. Many psychologists argue for it; many, however, do not accept it. ${ }^{5}$

A second view held regarding "muscular sense" considers it to result from sensations derived from the sense organs of the other "five senses," and to have, strictly speaking, no apparatus of its own. Changes in tension of the skin (Schiff) at flexures, etc., are reported by the cutaneous afferent nerves, and yield perceptions of the movements they accompany, of postures, etc. The delicacy of the "five senses" is such that in unsuspected ways they make us aware of the effects of even minute movements (Hensen). ${ }^{6}$ A grave objection to this view

${ }^{1}$ Bastian, Brain, London, 1887, vol. x. p. 1 ; Binet, Rev. phil., Paris, tome xxv. p. 478.

2 Chareot, "(Envres complétes," Paris, tome iii. p. 467.

${ }^{3}$ It is right to state that this is not the interpretation of the experiment submitted by Dr. Sternberg, who devised it ; he regards it as evidenee of "sensation of innervation."

${ }^{4}$ Arch. f. d. ges. Physiol., Bonn, 1890, Bd. xli.; also 1892, Bd. xlvi.

' Bastian, Brit. Med. Journ., London, 1869, vol. i. p. 461 ; James, op. cit.; Münsterberg, op. cit.

6"Gegeu den sechsten Sinn," Berlin, 1893. 
is the very slight impairment of muscular sense which follows cutaneous anæsthesia, and the very great impairment following injury to the deep sensory nerves of a limb, in spite of visual assistance.

There remains, then, a third view, namely, that the muscular sense is based on a specific set of sensations obtained by specific sense organ: in muscles, tendons, joints, and all the accessory organs of movement. To this view adherence is given in the following account.

Charles Bell ${ }^{1}$ was the first to definitely postulate the existence of a muscular sense on a physiological parity with the other senses. After his writings it began to be referred to as the sixth sense. He was not, of course, the first to suppose that consciousness of the positions and movements of the body and of its parts is based on sensations. Neither did he, in speaking of " muscular sense," suggest that the muscles are the exclusive site of origin of its sensations ; but he was the first to postulate the existence of special afferent peripheral nerves for impulses evoked by the movements and postures of the body.

It is obvious that any extensive action of the skeletal musculature must, when it occurs, affect various organs of sense. Such effects may be separated into "resident" and "remote." 2 The hand when moved at the wrist-joint provokes mechanically, by tensions and relaxations of muscles and of their tendons, ligaments, and the like, a number of sensory end-organs belonging to those structures. It also affects, by stretching and flexing the skin, sense organs within it. If the movement bring the part against an object, or against another part of the subject, touch, and in the latter case the so-called "double touch," results. Its movement may bring the part moved into the field of vision. These last-named tactual and visual effects constitute the "remote" results: on the contrary, the sensations of muscle and joint -in fact, of the motor organs in play-are the "resident" effects.

We may treat of the muscular sense as of other senses. The parts of its apparatus may be distinguished as peripheral, internuncial, and central ; among its phenomena may be distinguished centripetal impulses, sense impressions, sense perceptions, judgments, and ideas.

\section{The Peripheral Apparatus of the Muscular Sense.}

Under this heading may be included the afferent nervous apparatus of the locomotor organs, muscles, joints, and bones. Evidence that structures are afferent may be indirect, e.g. inferred from histology, or direct, obtainable by physiological experiment. To begin with the histological evidence.

Nerves of bone.-Bones are indubitably well supplied with nerves, - the long, the short, and the flat bones alike. The nerve fibres are derived, according to Kölliker's ${ }^{3}$ summary, from the spinal roots and from the sympathetic. One-third of the fibres measure above $10 \mu$ diameter. They "branch and end in a manner that quite recalls the behaviour of sensory spinal fibres" (Kölliker). ${ }^{4}$ In my degeneration experiments on the nerves of the limb, I found many sound myelinated fibres in the nerve perforating the tibia, though all motor root fibres and all sympathetic myelinated fibres had been destroyed by degeneration. ${ }^{5}$

Periosteum.-The periosteum is supplied with nerves, though sparsely.

1 Phil. Trans., London, 1830.

"W. James, "Principles of Psychology," London, 1891, vol. ii. p. 494.

i "Handbueh d. Gewebelehre," 1889, Bd. i.

${ }^{\circ}$ Sherrington, Journ. Physiol., Cambridge and London, 1894, vol. xvii. 
he fibres measure $9 \mu-4 \mu$, and undergo terminal subdivision (e.g. in fossa ifraspinata scapulce hominis. ${ }^{1}$ The periosteum near the ends of bones, where abuts on the joint and ligaments, conveys nerves destined for the latter.

Nerves of the muscles and tendons.-The nerves of muscles derive irge numbers of their fibres from the spinal ganglia. In the limb muscles of he monkey and cat I found from a half to a quarter of all the nerve fibres to re sensory. ${ }^{2}$ The size of the sensory nerve fibres varies between $18 \mu$ and $2 \mu$

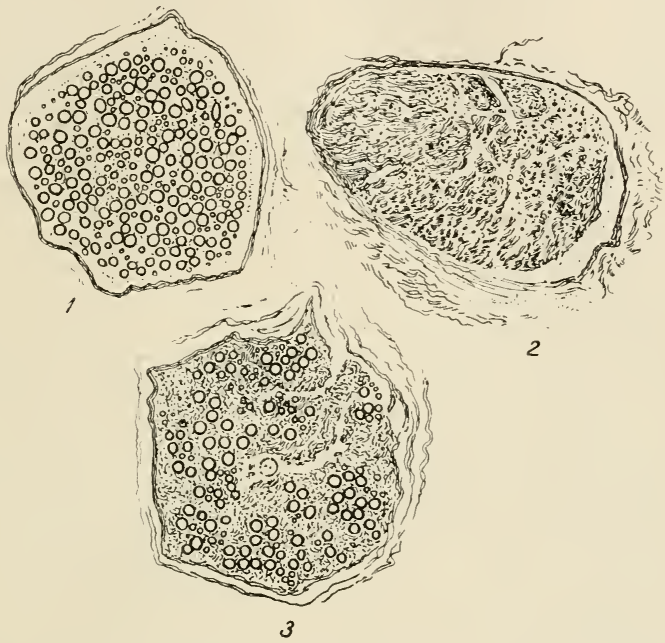

FIG. 370.-Transverse sections of a nerve-twig supplying a portion of the vastus medialis muscle (knee-jerk muscle) of the cat. From micro-plotographs of osmic acid preparations. 1. Normal condition of the nerve. 2. Condition three months after severance of all the ventral spinal roots, and of the corresponding spinal ganglia contributing to the nerve. No myelinate nerve fibres remain. 3. Condition three months after severance of all the ventral spinal roots, the spinal ganglia remaining intact. A number of healthy nerve fibres both large and small remain. These belong to the spinal ganglia, and are afferent.

or less. These sensory fibres end in end organs-muscle spindles, ${ }^{3}$ Golgiorgans, ${ }^{4}$ modified Pacini-organs, ${ }^{5}$ Pacini-organs. ${ }^{6}$

Interosseous membrane.-Between the limb bones the nervi interossei of the leg and forearms give numerous twigs to those membranes. The nerve fibres end in free endings (Kölliker), in end-bulbs and in Pacini bodies.

Muscle spindles.-Hassal (1851) seems to have earliest noted these structures. They were first distinctly described by Kölliker (1862) and by Kuhne (1863).

${ }^{1}$ See Kölliker, loc. eit.

2 Journ. Physiol., Cambridge and London, 1894, vol. xvii. p. 211 ; and Brit. Med. Journ., London, 1893, vol. ii. p. 685 .

${ }^{3}$ Hassall, cited by Trinchese, Mem. r. Acead. d. se. d. Ist. di Bologna, 1830.

${ }^{4}$ Rollett, Sitzungsb. d. k. Akad. d. Wisscnsch., Wien, 1876 ; Golgi, Mcm. d. r. Accad. se., Torino, 1880 , tome xxxii. p. 359.

${ }^{5}$ Ciaccio, Mem. r. Acead. d. sc. d. Ist. di Bologna, 1889, tomo x. ; Mazzoni, ibid., 1891, tomo i.; Ruffini, Atti d. r. Accad. d. Jincei, Roma, 1892.

${ }^{6}$ Rauber, Festschr. f. Bischoff, 1883 ; Sherrington, Proc. Roy. Soc. London, 1897, vol. 1x. ; Ruffini, loc. cit., and Sperimentale (Accad. med.-fis. fiorentina), Firenze, April 1897; Brain, London, 1897, vol. xx. 
The last-named gave them the name here adopted. They were not known to $b$ sensorial until Ruffini (1892) succeeded in histologically analysing their nerve endings, and I (1893) in tracing the majority of the nerve fibres to the spina ganglia. The muscle spindle is a fusiform organ, in which for clearness 0 . description I distinguish an equatorial and two polar regions. Along its axis lies a bundle of striated muscle fibres, two to ten in number. In the monkey and man it is rare to find a single intrafusal muscle fibre only. I have seen in man as many as twelve muscle fibres in one spindle. In the lizard and snake, ${ }^{1}$ however, the muscle spindle contains usually but a single fibre. The intrafusal muscle fibres differ from ordinary muscle fibres in being smaller and not undergoing any marked degenerative change after destruction of the nerve trunk innervating the muscle. ${ }^{2}$ They seem in some respects extreme instances of the red variety of muscle fibre, but the intrafusal fibres are even in the very reddest muscles of the rabbit smaller than are the ordinary fibres of those muscles. They contain many central nuclei, and at the equatorial regions of the spindle a richly nucleated core of nonstriated material encroaches on the striated structure of the fibre, leaving only a peripheral striped sheet, which recalls that of a developing fibre; and in places even that peripheral zone may be wanting. The intrafusal muscle bundle emerges from the capsules sometimes at both ends, often only at the distal pole, it liaving in the latter case ended in tendon while still within the capsule. The capsule consists of fibrous membranes, which externally are connected with the well-developed sheath of Henle of the entering nerve fibres. At the poles the capsule merges into perimysium internum. It is separated internally from the intrafusal muscle bundle by a space, the periaxial space, widest in the equatorial region. The intrafusal muscle bundle is supplied by numerous nerve fibres, some of which are probably motor. The most obvious nerve-ending is one in which a large myelinated fibre having crossed the periaxial space, lost its myelin, and divided dichotomously more than once, terminates in a ribbon-shaped axis cylinder, which winds many times round an intrafusal muscle fibre forming close coils. This is the "annulo-spiral ribbon " ending of Ruffini. Another form of ending, also sensorial, is Ruffini's "flower-spray" ending, which coexists with the annulo-spiral. ${ }^{3}$ Both these kinds of endings are situated in the equatorial region, but outside that region are found nerve-endings, somewhat like ordinary motor end-plates; these are probably motor. The motor nerve fibres of skeletal muscle are as a rule larger than sensory nerve fibres; in the spindles the sensory nerve fibres are larger fibres than the motor. Cipollone ${ }^{4}$ has recently shown that an intrafusal muscle fibre in the lizard commonly has not one but two motor end-plates, one at each end of it. Just as, unlike other fibres of skeletal muscle, the intrafusal muscle fibres do not undergo degeneration after destruction of the nerve trunk, ${ }^{5}$ so also, unlike other muscle fibres, they do not participate in the hypertrophy produced by functional exercise. ${ }^{6}$ Muscle spindles have now been studied in various animal types (e.g. frog, lizard, pigeon, rat, guinea-pig, rabbit, cat, dog, monkey, man, etc.). Those of the frog and lizard are smallest and structurally simplest, and often contain but one muscle fibre apiece. ${ }^{7}$ Those of the monkey and man are largest and most complex, and rarely contain but a single muscle fibre. In the eat and monkey I found it not uncommon for the spindles to be conjoined in pairs and even triplets. Their tendon-bundles with special frequency enter into Golgi-organs, ${ }^{8}$ with these again possess accessory Pacini 1897 .

${ }^{1}$ Cipollone, "Ricerche sull' anat. d. terminazioni nervose nei muscoli striati," Rona,

Sherrington, Journ. Physiol., Cambridge and London, 1894, vol. xvii. p. 211.

${ }^{3}$ See also an excellent account by C. Sihler, Arch. f. mikr. Anat., Bonn, 1895, Bd. xlvi. S. 709.

4 Loc cit. ${ }^{5}$ Sherrington, loc. cit.

6 Horpurgo, Virchow's Archiv, 1897, Bd. cl. S. 554.

${ }^{7}$ Cipollone, loc. cit. $\quad 8$ A. Cattaneo, Arch. ital. de biol., Turin, 1888, vol. x. 
bodies of simple type. ${ }^{1}$ They are not equally distributed through the musculature. Huber ${ }^{2}$ agrees with me in thinking that they are disproportionately frequent in the small distal muscles of the limbs. Kölliker noted fifteen spindles in each belly of the omohyoideus. I failed to find ${ }^{3}$ any at all in the following muscles:-Recti and obliqui oculi, levator palpebre superioris, the intrinsic muscles of the tongue and larynx. Cipollone ${ }^{4}$ notes their absence from the muscles of facial expression, although present in the masticatory muscles (pterygoids, masseter).

Kiihne ${ }^{5}$ has insisted on certain resemblances between the muscle spindle and the electric organ, which is stated to be derived from muscular tissue. Kerschner ${ }^{6}$ argues the muscle spindle to be an organ in which the "action current" of muscle forms the stimulus to the sensory nerve fibres.

The Golgi-organ of tendons. - These sensorial end-organs were earliest described by Rollett, ${ }^{7}$ but for the first time fully by Golgi, who studied them in mammals, birds, and reptiles. The organ consists of terminal arborisations of one or of two myelinated nerve fibres, the arborisations permeating as a richly branching tree, the thickness of a tendon bundle, which is somewhat sharply marked off from the surrounding tendon bundles. The terminal arborisation is of great size and complexity, and extends in some cases for $500 \mu$ along the tendon bundle. The organ has often a distinct capsule, and is well supplied with blood vessels. Like the muscle spindles, they seem particularly large and complex in the higher mammals. The tendon bundle in which the Golgi-organ lies ean often be traced to receive the insertions of muscle fibres that belong to muscle spindles. The Golgi-organ affects particularly that region of the general tendon which adjoins the flesh of the muscle. These organs are found in the tendons of the eye muscles, which, as I have pointed out, are muscles devoid of spindles; but the great number of sensory nerve-endings in the eye muscles are smaller and simpler than the ordinary tendon organs.

Large end-bulbs, or, as they are called by the Italian authorities, "modified Pacinian bodies," are also met in muscle tendons, especially in the sheaths of tendons, and of the muscles. For description of them reference can be made to Ciaccio, ${ }^{8}$ Mazzoni, ${ }^{9}$ or Ruffini. ${ }^{10}$

Pacinian corpuscles occur in connection with tendons, also in the septa, and in the perimysium internum of muscles, and even embedded in the fleshy tissue. ${ }^{11}$

The individual contributions toward muscular sense of these different sets of end-organs can only be conjectured. In the skeletal muscles there are, as we have seen, three sets of sensory organs, e.g. muscle spindles, tendon organs, Pacini corpuscles modified and typical. The fact that of these, one set, the spindles, are contractile, suggests that they function in signalling active movements rather than passive. Throughout the body it is remarkable how great a part mechanical actions play as stimuli to protoplasm. It has been argued that the action current is the immediate physical stimulus for the sensorial endings in muscle. That is plausible, although some of the arguments brought forward, such as the initiation of the kinæsthetic idea in the latent period of the muscular response, are weak. The possibility that the immediate stimulus consists of chemical products of the muscle has been discussed by G. E. Müller and Schumann, ${ }^{12}$ but remains little more than a suggestion. If suggestion is profitable, it seems to me more likely that the immediate stimulus of the muscle spindle is of a mechanical kind, produced by the change in form of the

1 Cattaneo, loc. cit. 2 Journ. Comp. Neurol., 1898.

${ }^{3}$ Journ. Physiol., Cambridge and London, 1894, vol. xvii.

${ }^{4}$ Loc. cit., p. 211.

${ }^{5}$ Virchow's Archiv, Bd. xxx. S. 206.

${ }^{6}$ Ber. d. naturw.-med. Ver. in Innsbruck, 1897, Bd. xxiii.

${ }^{7}$ Loc. cit., 1876, in the frog. ${ }^{8}$ Loc. cit. ${ }_{9}^{9}$ Loc. cit. ${ }^{10}$ Loc. eit.

${ }^{1}$ Rauber," "Festschr. f. Bischoff," 1883 ; Ruffini, loc. cit. ; Sherrington, loc. cit.

12 Arch. f. d. ges. Physiol., Boun, 1889, Bd. xlv. S. 37.

voL. II. -64 
muscle fibre when it contracts. It is true that change of form of the spindle must occur to sone extent, not only when the muscle shortens, owing to contraction, but also when its points of attachment are approximated by other causes (e.g. passive movement). But the change is, for the same degree of approximation of its attachments, not quite the same when passively obtained, either as to extent or character. The rectilinear course of the fibres is not so maintained. When the ankle of the frog is fully plantar-flexed, the sural muscle lies actually in folds. But the muscle spindle is peculiar among sensory end-organs, in that the part to which the actual nerve-ending is applied is not only contractile but extensile. Each lengthening and shortening of the muscle, within degrees which do not overstep the limits of extensility and elasticity of the muscle fibres, ehanges the figure of the intrafusal muscle fibres. Ruffini's anmulo-spiral ending is a form of nerve-ending special to the spindle. There is no ground for thinking the nerve fibril as extensile and elastic as a muscle fibre. The tendril-like rings of the nerve fibril must, if so, be subject to strain when the muscle fibre they clasp becomes thicker, and when they are pulled apart from each other as the muscle fibre elongates. It is noteworthy, however, that in some cases the muscle fibre, to judge from its less distinct striation there, is less contractile at the place of the nerve-ending than elsewhere. The striated and fibrillated substance of the fibre is there largely replaced by the voluminous nuclei I have described; these lie in a central core of finely granular material, surrounded only by a striated zone, recalling the appearance of a Purkinje cardiac fibre. In some cases the striation of the muscle fibres seems to fail at the nerve-ending, though in many it is perfectly continuous throughout.

Beside changes in form, changes in tension must also occur in the spindles; in the tendon organs the changes in tension must be much greater than the change in form, the tendon bundle being but little extensible or elastic. Its tension will wax and wane with the tension throughout the muscle. The stimulus for the tendon organ may well be mechanical; the nerve-endings that penetrate the little tendon bundle (Golgi's tendinetta) of the organ may be compressed as the muscle contracts or is passively stretched. It is easy to understand how the organs might react under both passive and active movements of the limb. The Pacini corpusele is often interpreted as being adapted for stimulation by compression. The modified form is more usual in muscles than is the typical form. It oceurs in some places, e.g. sheaths of museles, where there is likelihood of its being laterally compressed. The ordinary Pacini, embedded in muscle, is admirably placed for being compressed, especially when, as sometimes, seated in the retiring angle between a septum or aponeurosis and obliquely inserted muscle bundles.

From the above we might expect tension to largely determine museular sense perceptions. It must be admitted, however, that observations showing that the liminal excursion for passive movements is practically unaffected by the initial position of the joint, is against tension being of such great importance. The same position or movement of a joint may involve very different degrees of tension, according as it is favoured or hindered by outward forces (traction of weight, ete.), including muscular; yet the limen of judgment remains the same; the weighting of one of the compared hands makes, according to Loeb, very little difference to the illusions he studied as to excursion and direction of "willed" movements. This difficulty in the way of regarding the perceptions of muscular sense as based on elemental sensations of tension, may be partly met by supposing that, while some of the sense organs have tension for adequate stimulus, some have change of form with or apart from tension. Impressions from tension-organs (isometric), checked against simultaneous impressions from form-organs (isotonic) or organs of joints, might supply a judgment based on their ratio, which might give the required ideas. One and the same sensation of muscle-form or of joint-novement, summed with 
one and the same sensation of tension of muscle (including tendon), would, if memorable, make a kinæsthetic complex for judging and standardising comparable complexes. ${ }^{1} \quad$ Sense organs of joints certainly contribute importantly to the various kinds of museular sense perceptions.

The skin does not much assist muscular sense. Cutaneous anresthesia inflicts little injury on the sense of movement or posture. That it has some influence, however, is undeniable. Of two equal weights, one hollow and therefore the larger, lifted successively, the larger seems the lighter, ${ }^{2}$ even if the test be made when the eyes are shut. Tested with the eyes open, ${ }^{3}$ the larger weight seems more distiuctly the lighter than when touch is appealed to apart from vision. A pound of lead is, as regards sensation, heavier than is a pound of feathers.

Sensitivity of muscles, joints, and bones, as evidenced by reflexes. - The muscular structures, including tendon, appear insensitive under many mechanical, thermal, electrical, and chemical stimuli, known to be efficient in other cases.

Haller and his pupils concluded that neither tendon nor muscle is sentient. Bichat considered the former slightly sensitive. Schiff, ${ }^{4}$ Bernstein, ${ }^{5}$ and Bloch ${ }^{6}$ have all, more recently, maintained the insensibility of muscle. Rollett, ${ }^{7}$ after finding nerve-endings in tendon, tried to elicit reflexes from tendons, but failed to obtain any. Reflexes can, however, be initiated from these structures. One very constantly obtainable is initiated by eompressing the tibialis anticus tendon (cat) just above the annular ligament; the response is given by the adductor muscle of the thigh.

The tendon reflexes are misleadingly so called. Thus, in their prototype the "knee-jerk," the tendon is not necessary to the reaction, and the reaction has so short a latency that it cannot be reflex. There are, however, nearly thirty named "tendon reflexes" studied in the human body, and some of these may be true reflexes. It is not possible to decile the point until the latent period has been measured in each instance.

From the muscles themselves, in spite of former negative results, there is now evidence of the obtaining of reflexes. Vasomotor reflexes can be evoked by kneading exposed muscles in the curarised rabbit. ${ }^{8}$ The effect is usually a fall of general blood pressure. Electrical stimulation of the muscles and nerves less constantly produces a fall, often a rise. The knee-jerk can be inhibited by stretching or compressing any of the hamstring muscles, ${ }^{9}$ even when the muscle is completely freed from all connection with the body except through its nerve trunk and its blood vessels. I noted movements of the opposite eye and pricking of the ears (cat), produced by gently stretching the inferior oblique muscle, freed from all connections but its nerve and blood vessels, and suspended between two pairs of ivory-tipped forceps. ${ }^{10} \mathrm{~A}$ twitch can be evoked from the tibialis anticus, isolated but for nerve and blood vessels, by jarring it, especially lengthwise. The sudden relaxation of a passively stretched muscle I have often seen evoke a reflex, c.g. the

1 See also in regard to this, Delabarre, "Inaug. Diss.," Freiburg, 1891.

2 Charpentier, Arch. de physiol. norm. et path., Paris, 1891, Sér. 5, tome iii. p. 122.

${ }^{3}$ Gilbert, Stud. Yale Psychol. Lab., New Haven, 1894, vol. ii. 1. 43; Seashore, ibid., 1895, vol. iii. p. 1.

4 "Muskel- u. Nervenphysiol.," Lahr, 1858, S. 157.

5 "Untersuch. ueber d. Nerven- u. Muskelsystem," 1871, S. 239.

"Rev. scient., Paris, 1890, tome xlv. p. 294.

7 Eulenburg's "Real-Eneyc.," 1888, Bd. xiii. S. 581.

8 Kleen, Skandin. Arch. f. Physiol., Leipzig, 1890, Bd. i. S. 247.

${ }^{9}$ Sherrington, Proc. Roy. Soc. London, 1893, vol. lii. 
letting go of the stretched sural triceps often causes reflex contraction of the pretibial inuscles.

Contrary to older observations, ${ }^{1}$ it now seems proven that feeble reflexes can be elicited by irritation of joint surfaces, mechanical or thermal. Goldscheider ${ }^{2}$ found the effect increase the deeper he penetrated towards the bone. Striking the articular end of a bone elicits reflexes, but these are not truly joint reflexes, for they persist after all the nerves to the end of the bone have been destroyed. ${ }^{3}$ So also with periosteal reflexes, they are not truly elicited from the nerveendings in periosteum. Both these latter sources of reflexes-reflexogenous regions, as they are sometimes termed-are really dependent on distant bone, to which the mechanical shock is conveyed, and there excites the nerves distributed. Sternberg has obtained reflex contraction of the pretibial nuscles (in rabbit) by tapping the bared or lowercut surface of the tibia, after the lower part of that bone has been severed from the upper by exsection of a piece from the shaft.

In other experiments, direct excitation of the proximal ends of the nerves of muscles, etc., has been employed. Vasomotor reflexes have been observed to result. In the cat, as I have shown, ${ }^{4}$ the excitation of the proximal end of the cut nerve trunk of the group of hamstring muscles-a nerve trunk of exclusively muscular distribution-reflexly affects the innervation of the antagonistic vastus and crureus to the extent of temporarily abolishing the knee-jerk dependent on the spinal tonus of those muscles. Similarly, excitation of the same nerve trunk causes reflex inhibition of the rigid spasm of the extensor cruris, occurring under "decerebrate rigidity." Stimulation of the central end of a twig of this nerve trunk, the rest of the trunk being uneut, produces reflex contraction of the remainder of the hamstring group, and reflex relaxation of the already contracted antagonistic group, the extensors of the knee.

This is a result comparable with that seen in the self-regulative innervation of the respiratory museles, ${ }^{6}$ and with that given by the afferent nerve of the heart muscle, which inhibits reflexly the constrictors of the arterioles, i.e. the antagonistic muscle. The depressor nerve stands in some respects as prototype of the sensory nerves of all muscle. The vasomotor reflex, following excitation of a muscle by kneading, is said to be invariably lowering of general arterial blood pressure. ${ }^{7}$ Certainly that result does often ensue, but not invariably on exciting directly the nerve of a muscle electrically; I have then seen the blood pressure rise as frequently as fall. Alteration of pulse frequence is noted among the effects, ${ }^{8}$ the frequency is at first lessened (through the vagus centre), and later increased (through the nervus accelerans). ${ }^{9}$ It is easy to affect respiratory rhythm by excitation of the central ends of muscular nerves.

That the nerves of muscle are competent to produce pain, is proved by the pain of muscular eramp and other facts. Attempts to assign the pain to the dragging upon or the compression of nerve trunks coursing through or on or between muscles, or to their stimulation by "action currents" of muscles, fail for obvious reasons. It is difficult to satisfy one's self that such reflex effects as these, vaso-

${ }^{1}$ Haller and his pupils, Bichat, etc.

3 Sternberg, "Sehnenreflexe," Wien, 1893.

${ }^{4}$ Proc. Roy. Soc. London, 1893, vol. lii.

${ }^{2}$ Arch.f. Physiol., Leipzig, 1890, S. 380. May 1899.

${ }^{7}$ Kleen, Skandin. Areh.f. Physiol., Leipzig, 1890, Bd. i. S. 247.

8 Asp, Ber. d. k. sïchs. Grsellsch. d. Wissensch., Leipzig, 1867.

${ }^{9} \mathrm{Jacob}$, Arch. f. Physiol., Leipzig, 1893. 
motor and respiratory, are elicited by intensities of stimulus not beyond the bounds of normal, or exaggerated, so as to be dolorous. To discriminate between the two possibilities, I have stimulated the peripheral ends of the cut motor roots of the nerves of the hamstring muscles (cat), the afferent roots remaining intact. It is then found that even when the anæsthesia is shallow the muscles can be thrown into a very vigorous and complete tetanic contraction, maintained even for 30 seconds, or more, without producing noticeable effect on the general arterial blood pressure or on respiratory rhythm. When the stimulation is increased sufficiently, and the contraction is therefore very severe, slight effects on blood pressure and respiration are obtainable, but the attendant risk of some escape of the stimulating current to the sensory roots is not entirely excluded.

From the point of view of muscular sense proper, more interesting than the reflex effects upon vasomotor and respiratory mechanisms, are those upon the local musculature. These latter exemplify certain degrees of co-ordination, including "reciprocal innervation." From skin and the cutaneous nerves similar reflexes can be excited, although it would seem less readily.

\section{The Performances of Muscular Sense.}

The perceptions of muscular sense may be grouped into (i.) those of posture, (ii.) of passive movement, (iii.) of active movement, and (iv.) those of resistance to movement. Changes in consciousness accompany all movements of the body which occur at speeds above a certain liminal, and over distances of beyond a certain liminal extent. Most movements of the body alter the position of one part of it relatively to another, as well as alter the body's position relatively to its environment, without altering the mutual space-relationship between different parts of the body. The latter class may, from the physiological point of view, be termed "solely extra-relative." All these kinds of movements are perceptible by the mind, in virtue of the space perceptions with which it is furnished, by the combined labours of the senses of sight, touch, and the organs of the labyrinthine and muscular senses. But with solely extra-relative movements the muscular sense is usually little concerned, and consideration of them can be omitted from any brief exposition of "muscular sense."

Perception of posture.-This is not the place to discuss how the position of the body, considered as a whole, in reference to the earth's centre, and contradistinguished from all else which is "environment," is perceived. For that, much else besides the muscular sense is instrumental. To that, the muscular sense contributes limited perceptions of the relative positions of the corporeal parts, e.g. of the limbs, their joints, etc. The muscular sense is no doubt a large contributor toward the perception of the mechanical equilibrium of the body, and in that way toward the regulation and maintenance of its stability. The necessary corrective movements that, during locomotion, prevent gravity pulling us prone, or as we sit, stand, or maintain postures natural to us (our usustatus, ususitus, usumotus), involve perceptions of the relative position of our corporeal parts are guided by-one might almost say supplied by - the muscular sense. These perceptions, though not for the most part "objects" of attention, determine actions, and can be attended to at once, should need arise. That in the dark one hand, passively placed, can be touched on any desired finger-tip by a finger of the other hand, illustrates our possession of a sense of posture largely 
provided by "muscular sense." Posture, passively maintained, is found to be nearly as accurately perceived as when actively maintained. Sense of posture is very defective in limbs the sensory nerve roots of which are diseased. The appreciation of difference between postures of the limb is not so delicate as to account for the minute alterationsless than an angular degree-at a joint perceived in Goldscheider's experiments. There the perception is one of movement rather than of successive assumption of different postures,-an important distinction, paralleled in the visual and tactual senses.

Besides purely muscular, articular sensations also probably contribute to perception of posture. This has been said ${ }^{1}$ to be shown by the position of the fingers being as sensible when the muscles are paralysed as when they are normal,-_but in reality such observations fail to prove or negative the assertion.

Perception of passive movement.-The minimal adequate stimulus for sense of passive movement may be expressed in angular measurement of a movement about a joint. Leyden formd that movements of $55^{\circ}$ to $1^{\circ}$ at the hip-joint are perceptible $;^{2}$ but we owe most of the measurements on this subject to the careful work of Goldscheider. ${ }^{3}$ The liminal excursion for the shoulder-joint is $\cdot 2^{\circ}$ to $\cdot 4^{\circ}$, the speed of movement not being less than $3^{\circ}$ per second. Degree of sensitivity, as judged by the size of the liminal excursion, places the joints in the following order, ${ }^{4}$ beginning with the least sensitive:-

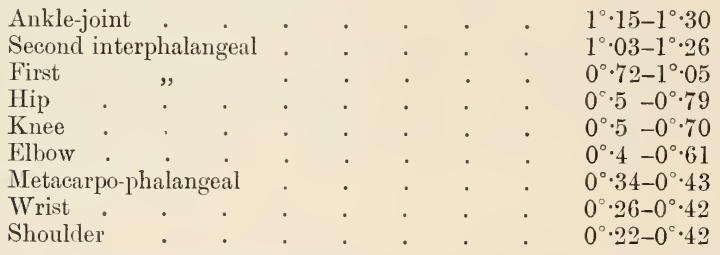

Arranging the joints in order of speed of movement required for the limen of perceptible excursion, the list is as follows, ${ }^{4}$ beginning with the least sensitive :-

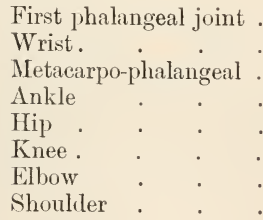

$12^{\circ} \cdot 5 \quad$ per second.
$3^{\circ}-9^{\circ}$
$4^{\circ}$
$2^{\circ}-3^{\circ} \cdot 5 \quad$,
$1^{\circ} 6-3^{\circ} \cdot 2 \quad$,
$1^{\circ}-2^{\circ} \cdot 5 \quad$,
$0^{\circ} \cdot 7-1^{\circ} \cdot 4 \quad$,
$0^{\circ} \cdot 3-1^{\circ}$,

A much smaller $\left(\frac{1}{4}\right)$, and especially a much slower $\left(\frac{1}{15}\right)$, movement is just noticeable at the great proximal joints of the limb than at the sinall peripheral. The delicacy of sense of movement progressively

1 Duchenne, "Conseience musculaire," Paris, 1853.

2 Virchow's Archiv, 1869, Bd. xlvii. S. 32.

${ }^{3}$ Goldscheiler excluded eutaneous sensations of pressure, as far as possible, by casing the segment of the limb in a gutta-percha sheath filled with water. The limb was moved by hydranlic means, and the movement was recorded graphically on a magnified scale upon a rotating cylinder, so that its time and its amplitude of excursion were known.

${ }^{4}$ Goldscheider, Arch.f. Physiol., Leipzig, 1889. 
diminishes from the attached base to the free apex of the arm. The natural movements at a joint are usually slow, in proportion as the lever working at that joint is long. The natural movements at the shoulder and knee are slower than those at the digital joints. Movements of such speed as those of the fingers in writing hardly occur at hip or at shoulder. Speed and excursion mutually support one another as provocative of sensation. A movement too slow to be felt, if of small excursion, becomes sensible if its excursion be prolonged. With equal movements nearly liminal in amplitude, an increase of speed of execution converts an imperceptible into a perceptible. By multiplying together the liminal excursions and the least speeds necessary to obtain them, numerical measures of the liminal performance of the nuuscular sense are obtained, and these, as far as concerns passive movements of the upper limb, run as follows:-

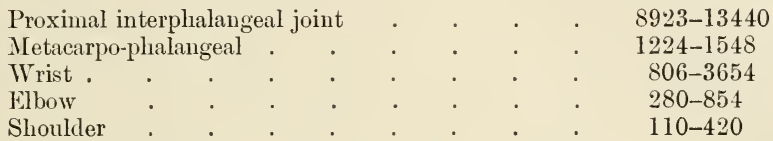

The performances by the shoulder are, when thus reckoned, more than forty times as delicate as those by the fingers.

Goldscheider, mainly on account of the disturbance and defect of the sense of passive movement occasioned by faradisation of the joints, concluded that the deep sensitiveness of joints is a chief sensory element in the production of perception of passive movement. Touch and vision are not essential to the perception of the movements. Goldscheider denied importance to the afferent nerves of muscle, because the liminal angle for a joint remains the same for various initial positions; but this is not a very cogent argument.

The liminal speeds for given joints starting from various initial positions are exemplified as follows:-

$\begin{aligned}, \quad \text { with arm } 45^{\circ} \text { below horizontal } & \text { above horizontal (not a pure } \\ & \text { movement at shoulder but } \\ & \text { involving scapula and } \\ & \text { vertebral column as well) }\end{aligned}$

Hip-joint, thigh at $180^{\circ}$ with trunk, the leg

$\begin{array}{ll}0^{\circ} \cdot 35 & \text { per seco } \\ 0^{\circ} \cdot 30 & , \\ & \\ 0^{\circ} \cdot 33 & , \\ 0^{\circ} \cdot 45-0^{\circ} \cdot 36 & , \\ 0^{\circ} \cdot 3-0^{\circ} \cdot 25 & , \\ 0^{\circ} \cdot 5 & , \\ 0^{\circ} \cdot 33 & , \\ 0^{\circ} \cdot 6 & , \\ 0^{\circ} \cdot 38 & , \\ 0^{\circ} \cdot 42 & , \\ 0^{\circ} \cdot 6 & , \\ 0^{\circ} \cdot 6 & , \\ 0^{\circ} \cdot 8 & , \\ 0^{\circ} \cdot 5 & , \\ 0^{\circ} \cdot 9 & , \\ 0^{\circ} \cdot 9 & , \\ 1^{\circ} \cdot 4 & ,\end{array}$
extended . . . .

, thigh at $90^{\circ}$ with trunk . . . .

" thigh at $180^{\circ}$ with trunk, and at the same time abducted $40^{\circ}$. . .

thigh at $90^{\circ}$ with trunk, and at the same time abducted $40^{\circ}$. thigh at $50^{\circ}$ with trunk thigh nearly fully extended, thigh adducted to $40^{\circ}$. . . thigh at $180^{\circ}$ with trunk-adduction . " $"$-abduction . , ", , -abduction.

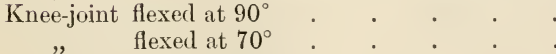
Ankle-joint flexed at $90^{\circ}$ 
That Goldscheider is justified in giving an important rôle to joints in the sense of passive movement, is borne out by the inferiority of accuracy of perception of passive movement, when the limb, e.g. the leg is stretched sufficiently to just separate the joint surfaces ${ }^{1}$ one from another, as compared with the accuracy when the joint surfaces play normally over one another. That active movements are slightly better perceived than are passive, may in part be due to the greater compression at joints which accompanies the former, evoking presumably more joint sensation. But the sensations derived from muscles and tendons are certainly also largely contributory to perceptions of passive movement.

Just as sensation of movement seems an elementary product of vision and of touch, so also in the muscular sense. An image in the periphery of the visual field may remain unseen, so long as it does not move, but should it move it will occasion a sensation forthwith, - a perception referred to "something moving." Two points of contact with the skin, if both within the liminal distance of sensible separation, are not distinguished as differing in locality, but if one of the points move over skin, though the movement be even toward the other, its alteration of locality is felt at once, and a sensation of movement results, though entirely within the limit of a single "Weber-circle." The feeling of movement of a finger is obtainable from a finger in which the sense of posture has been (temporarily) quite obliterated by strong faradisation. A movement is easily felt to have occurred, even when the direction in which the part has been moved is not perceived. The importance of the speed of movement for perception of movements of approximately liminal perceptibility is hardly intelligible if the perception depends purely on inference from sensations of locality, of comparison between two sensations, one of the locus a $q u o$ and a second of the locus ad quem. In the case of the fingers the duration of liminal movements, perceptible as movements, is so short (-02 second) that it is almost impossible for two sensations, one of end and one of beginning, to be differentiated and dealt with. These arguments, brought forward by Goldscheider, seem to me to justify the supposition that simple sense-fusions of "movement" can be yielded by the muscular just as by visual and tactual sense. But this does not, of course, alter the probability, amounting in some cases to certainty, that in many of our usual passive movements the excursion and duration of which are far greater than the almost liminal movements studied by Goldscheider, our perceptions of them include perceptions of the initial posture, of the ultimate posture, and of intermediate positions also, and supply the basis for judgments.

Perception of active movement.-The accuracy of judgment of actively performed movements, that is, of movements occasioned by active contraction of the muscles, is almost always examined in the form of "willed" movement, and is based only in part on the performances of the muscular sense. In experiments on the sensible limen of willed movement, as regards excursion and speed, Goldscheider found the liminal value slightly finer than for passive, although the difference was almost negligible. Thus the average for the end finger-joint with active movement is $1^{\circ} \cdot 02-1^{\circ} 09$, for the passive $1^{\circ} 0-1^{\circ} \cdot 8$. That active movements are slightly better perceived than passive, may in part be due to the compression at joints that accompanies active movements more than passive, and therefore generates presumably more jointsensation.

It is possible to execute subliminal willed movements, that is, willed movements so small in excursion as to be insensible to muscular sense. These can be evoked by thinking vividly of the movement.

\footnotetext{
${ }^{1}$ Lewinsky, Virchow's Archiv, 1879, Bd. Ixxvii. S. 145.
} 
Amount of active movement, its extent and direction, is under many circumstances erroneously judged. A subject desired to compress a dynamometer half a dozen times in succession, with all his strength, is surprised to find he has not exerted the same force at last as at first. ${ }^{1}$ In Loeb's ${ }^{2}$ experiments, one arm was required to perform a movement of similar extent and direction to a movement simultaneously performed by the other, but from an initial position asymmetrical with the latter's. The discrepancy between the movement executed and that judged to have been made, illustrates the shortcomings of the muscular sense under certain conditions. Loeb found the discrepancy in extent and direction between the willed movement and the executed movement, as shown by the difference between the copying movement and the copied movement, vary proportionately with the degree of shortening of the muscle at the commencement of the copying act. The shorter the active muscle already was at outset of the movement, the greater the deficit in its extent of performance, as compared with the intended movement, as measured by that of the movement copied,-in other words, the more its actual performance was over-valued; and conversely. He found that analogous conditions similarly distort the perception of direction of movement.

In view of observations by E. H. Hering and myself, ${ }^{3}$ it would be preferable were these results to be stated, not in terms of degrees of shortening of a muscle, but in distance from limit of movement in a particular direction. Thus the sensible effects of lengthening (relaxation) of muscles would be included as well as those of shortening (contraction). From Loeb's research the initial position of the joint appears of importance from the point of view of active (willed) movement, though Goldscheider showed it affects little the sensible limen of passive movement. An illusion studied by Loeb is this: from symmetrical points the two hands are moved simultaneously, horizontally in the same direction, e.g. both toward the right, with the intention that both should traverse the same rectilinear distance; the hand moving inward toward the median plane of the body is then found to traverse a greater distance than the other. The high delicacy of perception of movement, both passive and active, revealed by Goldscheider's work, contrasts with the large errors of estimation of amount and direction of movement instanced by such illusions as these. This argues for existence of a selisation of movement per se as an elemental product of the muscular sense, and not as a judgment based on comparison of discrete sensations of position. The extent of the illusions seems partly due to simultaneity being a relatively disadvantageous condition for comparison between perceptions. When the willed movement is a successive, not a simultaneous, imitation, the error is not so great. The error varies considerably with the individual. Also in Loeb's illusions there is the bias given to the judgment by the intention to make the movements equal. But the lack of definiteness of the muscular sensations received is strikingly illustrated. ${ }^{4}$

$A$ person stands, eyes shut, facing a vertical screen. $A$ and $B$ are two points on the screen that are placed symmetrically with regard to the median vertical plane of the body, and each about $25 \mathrm{cms}$. from that plane. The vertical heights of the two points are the same. The person has to outline, eyes shut, two similar simple figures, simultaneously with both hands. The hands are to be moved simultaneously in the same direction, e.g. both to right, and the figures are to be made as nearly as possible identical. The left hand starts from point $A$, the right from $B$. Ascending or descending parts of the

1 Delbouf, Rev. phil., Paris, 1881, tome xii. p. 526.

2 Arch.f. d. ges. Physiol., Bonn, 1890, Bd. xli.; 1892, Bd. xlvi.

3 Ibid., 1897, Bd. lxviii. $\quad$ Ibid., 1892, Bd. xlvi. 
outlines are drawn fairly equal. If, then, the attempt be repeated but with the starting-point $\mathrm{B}$ considerably above $A$, the ascending part of the outline of the right-hand figure is smaller than the corresponding of the left-hand figure; the descending part, on the contrary, is longer.

Symmetrical muscular movements executed with the intent to be, and after performance judged to be, equal, show habitual preponderance of one side, right or left, in different persons (Stanley Hall), and this without dependence on right-handedness or left-handedness, ${ }^{1}$ but rather upon muscular development due to influence of occupation. Bloch, however, states that, in measuring the number of pages of a book between the finger and thumb, the distance is under-estimated by the left hand with right-handed people, but by the right hand with lefthanded people.

It seems that speed of movement is not very correctly judged. In the above-mentioned illusions the total duration of the movement is equal, and the speed of movement is not detected to be different. Loeb found that the weighting of a hand-thereby introducing a resistance-did not much affect the result. He concluded from this that mere tension of muscle was not a chief basis for the sensation. Exner's illusion ${ }^{2}$ points in the same direction. If a plate of vulcanite be placed between the teeth and bitten upon, the sensation given is as though the jaws moved perceptibly toward one another, although the plate be really quite unyielding; the illusion is obtained of the teeth sinking slightly into the substance bitten. Sternberg's illusion ${ }^{3}$ mentioned above is comparable with these.

\section{Objective Effects of Loss of Muscular Sense upon "Willed" Movements.}

The office of the muscular sense in regard to willed movement can also be studied to a certain and fortunate degree objectively. It is found that the destruction of sensitivity in particular regions brings about objectively observable disturbances of movement. A region totally deprived of sensation by section of its afferent nerves, is termed apcesthetic, and the effects of apresthesia upon the musculature of the part are three-(1) paralysis, (2) ataxia, (3) atonia. Certain elementary adjustments of muscular action occur reflexly without awakening attention or in the slightest degree seeming to trouble at all the stream of consciousness. Yet these have, inferiorly and remotely, relationship to the highest achievements of the muscular sense, such as the manipulative finesse of the human thumb and finger. The muscular sense embraces the part played by sources of afference in the taxis of muscular activity, even where the reactions lie not amenable to introspection. The physiologist derives a certain advantage from this, in that it offers an objective mode of studying the activities of the afferent apparatus of muscular sense by watching the objective effects of it on the characters of muscular movements.

The unconscious reflex actions of the adult may be taken to represent those reactions accruing to him phylogenetically or ontogenetically, which, though of ancient origin, have with little metamorphosis remained on the average useful to him as an adult member of his species. As to

1 II. Cremer, "Inang. Diss.," Würkurg, 1887.

2 See Sternberg, Arch.f. d. ges, Physiol., Bonn, 1895, Bd. lix.

3 Ibid. 
how consciousness comes not to be involved in them, there are several probabilities to offer. Unhesitating fatality and speed are characters which consciousness might only impede, though, for certain nervous reactions, these very characters would be beneficial to the race. Another suggestion would be, that division of attention appears, from the constitution of mind, to undermine all exquisiteness of performance.

Excitation of the afferent nerve fibres in the nerve trunk of a skeletal muscle produces, apart from and more readily than those general reflexes, vasomotor, etc., above-mentioned, special reflexes upon the local inusculature. The above-mentioned general reflexes are only positively indicative of that degree of common sensation in which sense-feeling is dominant and painful. The local reflex effects on the musculature obtained from afferent muscle nerves by weaker stimuli, are more special in character, and more significant for the study of muscular sense, especially in its relation to co-ordination. If, when the cranio-spinal axis has been transected just in front of the pons, and "decerebrate rigidity" has set in, the purely muscular nerve trunk of one of the flexor muscles of the knee is cut, and its central end excited by ligation or faradisation, the existing strong tonic contraction of the extensor muscles of the knee is at once relaxed, ${ }^{1}$ and the fellowmuscles of the flexor group, previously inactive and flaccid, are at the same time thrown into contraction. The same result may be brought about also by stretching or compressing the muscles thenselves, so long as their afferent nerves are intact. The excitation of the sensory nerve fibres from the flexor muscles, besides provoking contraction of the fellowflexors, inhibits the contraction (and even the tonus) of the antagonist group. In this experiment the sensory impulses arising in one muscle influence the activity of the motor nerve cells governing its fellowmuscles, and of those governing its antagonists, augmenting the former, diminishing and even annulling the latter. Acting thus, the centripetal impulses from the muscle produce an important item of spatial co-ordination, which I have termed "reciprocal innervation." 2 This effect of the centripetal impulses from the muscle is not accompanied, it would seem, by psychosis. Kinæsthesis, in the psychological sense, seems precluded where the whole cerebrum had been removed. Muscular "sensations" can hardly have been present; indeed, similar observations can be made even when the whole brain has been cut off by transection at the top of the spinal cord itself. The co-ordination of the movements of the spinal frog is suggestive of regulation by afferent nerves,--probably muscular. I found the initial posture of the limb in the spinal animal (e.g. frog) distinctly affect the character of the reflex movement elicitable from the limb even in the absence of cutaneous organs. The coordination was therefore evoked by impulses from the peripheral organs and nerves of muscular sense, in the absence of all the nervous machinery from which one is accustomed to consider consciousness indissoluble.

How little ordinary consciousness is involved by the lower performances of muscular sense, is instanced by the untying of the hands during sleep, even although the knots used for confining them be of a quite complex kind. The two distinct cortical regions related to the movements of the eyeballs accords with the evidence mentioned above in favour of the regulation of those movements by intrinsic muscular sensations (frontal cortex), as well as by visual (retinal) sensations (occipital cortex).

${ }^{1}$ Sherrington, Proc. Roy. Soc. London, 1893, vol. lii.

${ }^{2}$ Ibid., 1896, vol. lix. 
Another influence exerted by "afference" ("Centripetalität," H. E. Hering) upon the motor innervation of skeletal muscle has to be remembered. The blocking all afferent channels lowers the muscular tonus, as seen in the excessive passive mobility of the limb, and in the delay of rigor mortis in its muscles. ${ }^{1}$

The mechanical tension of a muscle which results from its reflex tonus seems important ${ }^{2}$ for the proper execution of its rôle in a combined movement. The action of a muscle $A$, if unprevented by external resistances, inevitably produces centripetal impulses, which react on the neural tonus, and therefore on the mechanical tension of its antagonist muscles B. More than that, the action of the muscle A, if unprevented by external resistance, inevitably not only stretches its antagonist $\mathrm{B}$ passively, but in so doing causes a discharge of centripetal impulses in $\mathrm{B}$, by mechanically exciting B's end-organs, and that discharge modifies, probably usually augments, the neural tonus of $B$. This regulates the course of the movement, and is preparatory to a next step in the secuence of movement, usually of the reverse direction to the initiatory.

Probably, however, there are other factors still in the ataxy of an apæsthetic limb. Its ataxy is different to that of cerebellar disturbance, and that of disseminated sclerosis. Analysis of it discovers disturbance in the extent, the direction, and the speed of the movement as compared with a normal movement of similar intention. The apæsthetic limb of the frog is flung upward, much as the leg of the tabetic patient is in walking advanced too high and too far. ${ }^{3}$ I have seen the aprsthetic fore-limb of a cat, when raised to assist the mouth in the management of a large morsel, oftentimes miss the cat's mouth, and even its head altogether. ${ }^{4}$ Similarly in the working of the hand, although it itself retain a large part of its sensorial innervation, if the rest of the limb be aprthestic, it often misses the object at which it grasps, and may pass an inch or so to one side of its intended goal. ${ }^{5}$ The limb can be seen to swerve from side to side in the progress of its movement. The speed of normal willed movements has been shown to be uniform from soon after start to close upon finish. ${ }^{6}$ The jerky movements of afferent ataxy are obviously not carried out with even approximately uniform velocity.

The centripetal impulses, loss of which produces loss of skeletal tonus, arise not in the skin but in the deep tissues. Those afferent channels, rupture of which produces this ataxia, are not principally cutaneous. A frog in which the hind-limbs have been rendered apresthetic, exhibits marked ataxia in those limbs; but a frog from which the skin of the hind-limbs has been removed, ${ }^{7}$ moves with no obvious want of precision. The bird in which the skin of the foot has been insensitised by nerve section, will sleep perfectly balanced on the insensitive foot. ${ }^{8}$ The horse which goes lame from tenderness of the hoof, trots normaliy after neurotomy has rendered the whole foot insen-

I Sherrington, Proc. Roy. Soc. London, 1893, vol. lii.

2 B. Stilling, Arch. f. physiol. Heilk., Stuttgart, 1842, Bd. i. S. 97 , and many later observers, especially H. E. Hering, C. Bell, C. Bastian, Cyon, S. Exner, Mott and Sherrington.

${ }^{3}$ H. E. Hering, Arch. f. exper. Path. u. Pharmakol., Leipzig, 1896, Bd. xxxviii. S. 276 ; and Arch.f. d. ges Physiol., Bonn, 1898, Bd. Ixx. S. 559.

${ }^{4}$ Sherrington, Journ. Physiol., Cambridge aud London, vol. xxii. 1898, p. 319.

${ }^{5}$ H. E. Hering, Neurol. Centralbl., Leipzig, 1897, Bd. xvi.

${ }^{6}$ Loeb and Korányi, Areh. $f$. $d$. ges. Physiol., Bonn, 1890, Bd. xlvi. S. 101.

${ }^{7}$ A. Bernard, "Leçons sur la physiol. dı système nerveux," Paris, 1858, p. 254.

${ }^{8}$ Chauveau, Brain, London, 1891, vol. xiv. 
sitive. Cocainisation of the vocal cords does not hamper the management of the voice. ${ }^{I}$ The cocainised eyeballs can be well trained in any desired direction in a perfectly dark room. ${ }^{2}$ In tabes dorsalis, in which the chief lesion concerns the afferent roots, locomotor ataxy is a characteristic, and in this disease the early loss of the knee-jerk indicates the early breakdown of the afferent nerve fibres of the muscles of the lower limb. Besides the consequent loss of tension, the loss of what Bastian calls "guiding" sensations is probably a factor in the inco-ordination.

To the organs of muscular sense is largely traceable local "feeling of fatigue." In tabes dorsalis the patient is often abnormally resistant to the induction of this feeling in certain groups of muscles, even on prolonged exercise of them (compare above, pp. 972 and 990).

Finally, there is the phenomenon of senso-paralysis. Charles Bell noted that after section of the supramaxillary (purely afferent) divisions of both trigemini, power to use the upper lip for prehension (ass) is lost. In the monkey the "grasp" of hand or foot in lost in an apæsthetic limb. ${ }^{3}$ These instances suffice to indicate that in their relation to afference some movements stand very differently to others.

Exner finds it impossible, without the help from a retinal image, to at all accurately converge the eyes for an object, which, though unseen, e.g. in a dark room, has its direction and distance well perceived, since it is held before one in one's hand.

Perception of resistance.-Muscles are physiological machines for antagonising various forces by elastic tension. In so doing, certain changes in consciousness are brought about, and among these is a "sense of resistance." To hold between the fingers a thread that carries a weight, gives a sensation as of the existence outside the fingers of a something only describable as offering resistance. It is, however, difficult to think of "resistance" as a quite elementary sensation. It can perhaps be simplest thought of as sensation of tension; but discrimination between resistances is based on ratios between degrees of tension sensations and other sensations, such as posture, excursion, and speed of movement, and on touch. More accurate judgment of the weight of an object can be found by lifting it than by allowing it simply to rest on the supported hand. Touch is contributory, but the basis of the judgment seems to be those sensations accompanying the muscular action which are called kinæsthetic. When the finger presses down the beam of a balance, counterweighted by weights ranging between 200 and 2000 grms., a constant fractional increase of about $\frac{1}{13}$ is found to be the liminal sensible difference, if active movement of the finger be not employed, but that fraction falls to $\frac{1}{1^{9}}$ when the finger is pressed down by willed movement. ${ }^{*}$

Liminal stimulus. - The load affording the limen of sensible resistance for the elevators of the shoulder has been found ${ }^{5}$ to be in a given case $7 \cdot 5$ grms., hung at the tip of the forefinger, the arm being horizontal and joint movement limited to the shoulder. With movement at the elbow only, the load had to be increased to 9 grms. ; and with movement in the finger only, to

${ }^{1}$ H. Beaunis, Compt. rend. Soc. d. psychol. physiol., Paris, 1887.

2 Sherrington, Proc. Roy. Soc. London, 1897, vol. 1x.

${ }^{3}$ Mott and Sherrington, ibid., 1895, vol. Ivii.; cf. also Exner, Arch. f. d. gcs. Physiol., Bonn, 1892, Bd. l.

4 Merkel, Phil. Stud., Leipzig, Bd. v. S. 287.

${ }^{5}$ Goldscheider and Blecher, Arch. f. Physiol., Leipzig, 1893. 
24 grms. The static leverage being taken into account, the observations indicate that the delicacy of perception of resistance as measured by the minimum perceptible is greater for peripheral than for proximal segments of the limb.

Liminal difference.-H. E. Weber, measuring the just noticeable difference between weights successively lifted by the same arm, found that difference one-fortieth part of the lesser of the two weights compared. The law which lie had previously induced from observations on tactile stimuli, he therefore recognised as applicable to the muscular appreciation of resistances. That law states that the increase of stimulus required to be perceptible as an increase is not any fixed quantity, but one that bears a constant ratio to the total stimulus.

Weber's observations on the application of his law to kinæsthetic sensatious rest on experiments that deal with only a small number of compared weights. He attempted to exclude participation of touch in the discrimination by hanging the weight in a cloth, the gathered ends of which were compressed as tightly as possible between the fingers. In that way he sought to keep the tactile stimulus maximal and invariable throughout. Since then fuller series and stricter methods have been pursued. Fechner in his historical research ${ }^{1}$ recorded 24,576 separate judgments on weights of vessels, which could be filled in various degree and lifted each by a wooden handle. Hitzig's ${ }^{2}$ kinæsthesiometer has been frequently employed; it is a set of balls outwardly alike, but individually loaded so as to be of different weights. Merkel ${ }^{3}$ used pressure on a balance-beam; Jacobi ${ }^{4}$ preferred a form of steel-yard, one end of which was lifted by the hand. Goldscheider ${ }^{5}$ employed a weight attached by a cord running over a pulley. Langlois and Richet ${ }^{6}$ observed as index to the fimeness of the muscular sense in the respiratory mechanism, the smallest rise in the mercury column of a manometer which caused perceptible impediment to the expiration of the observer.

The observations obtained by these methods can be dealt with by-(1) The methods of just discernible differences; (2) the method of average error; (3) the method of right and wrong answers; (4) the method of intervals apparently equal; or (5) the method of doubling the stimulus. These methods have been already alluded to.

In Jacobi's observations, ${ }^{7}$ the liminal difference remained pretty constantly $\frac{1}{20}$ throughout a long range of weights. Experiment finds, in fact, that in the muscular, as in the other senses, the liminal difference is not expressible throughout the range of even work-a-day stimuli as a constant fraction of the total stimulus. Experiments in Hering's laboratory ${ }^{8}$ on the liftings of eleven weights, ranging from 250 to 2750 grms., showed the liminal sensible increment to vary from $\frac{1}{21}$ at 250 grms. to $\frac{1}{114}$ at 2500 grms.; at 2750 grms. to rise to $\frac{1}{98}$ again. Merkel found that for weights below 200 grms. and above 2000 grms., and moved by the finger, the discriminative power was less than for the range between those figures. On the contrary, neither Fechner, ${ }^{9}$ nor Miiller and Schumann ${ }^{10}$ found the discriminative delicacy decline with weights even far heavier than 2 kilos.

${ }^{1}$ Ber. d. k. sächs. Gesellsch. d. Wissensch., Leipzig, 1860, S. 76 . The vessels used are still in the University, Gottingen.

2 Neurol. Centralbl., Leipzig, 1888, Bd. vii.

3 Op. cit.

4 Arch. f. exper. Path. u. Pharmakol., Leipzig, 1893, Bd. xxxii. S. 49.

5 Arch. $f$. Physiol., Leipzig, 1891.

6 Rev. phil., Paris, 1890 , p. 557.

T Arch.f. exper. Path. u. Pharmakol., Leipzig, 1893, Bd. xxxii. S. 49.

8 Sitzungsh. d. math.-phys. Cl. d. k.-bayer. Akad. d. Wissensch. zu München, 1875, Bd. lxxii. Abth. 3, S. 342, ete. (Hering's own experiments and those of his pupils, Biedermann and Loewit, agree.)

${ }^{9}$ Op. cit.

${ }^{10}$ Arch. f. d. ges. Physiol., Bown, 1889, Bd. xlv. 
The following fractions express the results of actual observations :-

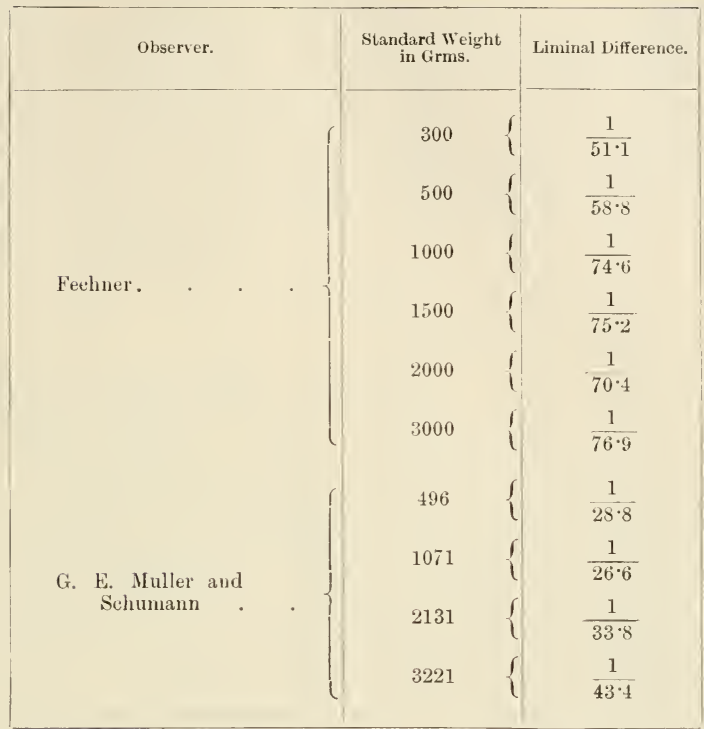

In comparing a weight-the so-called "standard weight"-with others, it is not a matter of indifference whether the lifting of the standard precede or follow that of the weight to be judged against it. Hence the well-known " time error." There is a tendency to undervalue the succeeding weight of the pair. This tendency is stronger with shorter intervals between the successive lifts than with longer, and with heavier weights than with lighter. It appears when the comparison is between right and left hand lifts, though not so markedly as with lifts confined to right or left alone. Fatigue affects the time error in the direction of reducing it. An explanation offered for the time error is that, especially when the successive lifts follow each other rapidly, the motor organs are still in some degree of excitement, or at any rate the path is smoothed and favourably prepared for repetition, when the second impulse to lift occurs; hence more innervation is employed than is intended, and the weight is judged to move more easily. That it occurs with alternatehanded experiments is one more instance of the smoothing a motor path on one side by use of the symmetrical motor path on the opposite, e.g. of the collaboration of right and left hemispheres. On this view of the causation of the time error the effect of fatigue is obvious. There is also a spatial error. The weights to be lifted cannot be so placed as to lie to hand in quite the same manner. The difference of the position from which the weight is to be picked up exerts a complex influence. ${ }^{1}$

Discrimination is usually sharper when weights are compared in ascending order, i.e. from lighter to heavier than vice versâ. G. E. Müller and Schumann have pointed out the great influence of precurrent effect upon the judgment of a succeeding one. A weight, 826 grms., when compared ten times, with all precautions for rendering judgment unbiassed, against a "standard" weight 626 grms., was once pronounced the lighter, twice pronounced equal,

${ }^{1}$ Sunkel, “Inang. Diss.," Marburg, 1890. 
and on seven occasions the heavier. A weight, 876 grms., compared with that of 626 grms., was on all of ten occasions judged the heavier. The "standard" weight, 626 grms., and a weight of 2476 grms., were then lifted alternately, each of them thirty times, the "standard" weight preceding the heavier weight. The "standard" was then thrice lifted alternately with weights 926 grms., 876 grms., 826 grms. Then the standard was lifted ten times alternating with the weight 2476 grms.; and finally, thrice in alternation with weights 926,876 , and 826 . The ten alternatives with the 2476 weight, and the three with the 926,876 , and 826 , were then once more gone through. In the latter part of the experiment the weight $876 \mathrm{grms}$. was thrice judged to be lighter than 626 grms., and seven times to be equal with it; and weight 926 grms. was on nine occasions judged equal to weight 626 grms., and once judged to be lighter than it. This phenomenon is an example of what is termed "adaptation" or "adjustment."

The motor innervation having become "adapted" for a heavier weight, the perceptions obtained from the peripherally arising impressions resemble in judgment such as would arise under usual circumstances, were a lighter weight than that actually raised to be the object of a smaller effort. Under the more intense motor innervation the weight is actually lifted as quickly as a lighter weight would be were the motor innervation less intense.

Hering 1 has pointed out how manifold must be the sensations on which estimation of lifted weights is built. Comparisons of perceptions of the speed with which the lifted weights are moved, of the fields of muscular action invoked to maintain the balance of the body as the weight is moved, as well as of skin sensations of pressure and pull, all play a part. A light weight taken up in expectation of its being a heavy one seems to "fly up." An onlooker can often, by watching the speed of lift, tell whether the weight under trial is lighter or heavier than the "standard." Indeed, discrimination between the weights, by lifting them, may be less a matter of discrimination between kinæsthetic sensations of tension than between kinæsthetic sensations of movementspeeds. $^{2}$ But Loeb's experiments (above-mentioned) show that discrimination of speed of willed movement is poor. Novement as such is perceived by the muscular sense. A further datum has been insisted on by Jacobi, namely, the latent period of the movement; that is, the length of interval between the signal for willing of the movement and the commencement of the movement which is willed. For a given weight a definite latency corresponds with a definite innervation force, and with equal efforts the latency is proportional to the amount of the weight. Again, the momentum of the mass moved, and the facility with which the initiated movement can be arrested, may also be a factor in forming the judgment, and will vary with the load lifted. A term "sense of tension" may have to replace "sense of resistance." Of observations on sensible muscle-tensions few exist. Sense of resistance to muscular action may be based on compared sensations of joint movements and of changes of muscle-tension, with or without comparison with sensations signifying changes of muscle form. In these ways may be obtained the distinct perceptions we have of the different ways-with ease, or against difficulty-in which the same posture is maintained, or movements identical as regarls excursion and velocity are executed.

\section{Fusion of Muscular Sensations with other Sensations.}

The perceptions furnished by the muscular sense deal largely with the mutual relations between motile parts. By collaboration of muscular sense with certain other senses (especially sight and touch), those

1 In a letter to Fechner.

2 G. E. Miller and Schumann, Arch.f. d. ges. Physiol., Bonn, 1889, Bd. xlv.; Sunkel, "Inaug. Diss., Marburg," 1890. 
senses gain spatial quality measurable in terms of the intensities of the muscular sensations.

Concomitant muscular sensations have been regarded as endowing sensations of other modality with spatial quality. Lotze developed his scheme of "local signs" " from the supposition that visual space-perception rests on measurement of retinal differences in terms of intensities of muscular sensations afforded by muscular movements required, when the various retinal points are replaced by the fovea for purposes of attentional vision. Münsterberg ${ }^{2}$ goes so far as to write, "Every content of consciousness requires a muscular impression"; and it has been said "only when we move a limb do we become conscious of its existence." 3

A longer line can be visually just distinguished to be longer than a shorter when the difference amounts to $\frac{1}{50}$ of the length of the latter. If we look through a horizontal slit toward a white sereen, and without being able to see any other objects perceive a vertical thread, each eye will of itself take up such a position that the image of the thread falls on the yellow spot. The visual axes intersect at the thread. On bringing the thread nearer or removing it farther from the eyes, the angle of intersection of the visual axes is changed as the eyes follow the thread. If the thread is moved very little, the rotation of the eyes is so slight that the accompanying movement sensation is not noticeable. It is found that the increment of movement, which just suffices to cause noticeable sensation, amounts to approximately $\frac{1}{50}$ of the total excursion counted from the parallel position as zero. That perception of spatial magnitude is thus directly proportional to the liminal difference of sensation of movement possessed by the eye, suggests that the sensation of movement serves as criterion for the space-perception.

Again, we possess the finest sensitivity of our eyeball movements when the visual axes are practically parallel,-a rotation of about $1^{\prime}$ is then noticeable. If we suspend two black threads near together in front of a white screen, and fixate them with one eye, and then retire gradually from them, though keeping them constantly fixated, the apparent distance between the threads constantly lessens as the retinal image grows smaller. At a certain distance the two threads appear one. There is a certain magnitude of the retinal image of two points (i.e. a certain magnitude of the visual angle between them) below which they cannot be perceived as separate. The two images fuse to one at the moment when the distance between their retinal images is so small that the eye has to turn only about $1^{\prime}$ in order to bring first one thread and then the other on the same retinal point. The limit to which the eye can attain in cognition of spatial distance is identical with the limit of its apprehension of the sensation of its own movement.

These are quoted as instances of the grounds upon which the fusion of muscular sensations of various intensity with perceptions of certain other senses is regarded as endowing the latter with the quality of "space."

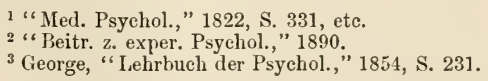




\section{VISION.}

By W. H. R. Rivers.

Contents :-The Dioptric SysteM, p. 1026-Accommodation, p. 1033-The Pupil, p. 1042-The Retina and Visual Sensations.--Phiysiological Retinal Processes, p. 1045-Visual Sensations, p. 1052-Adaptation and Induction, p. 1056 -The Duration of Stimulus and Sensation, p. 1067-The Brightness of Colour Sensations, p. 1076-Mixture, p. 1081-Vision of Different Regions of the Retina, p. 1083 -Colour-Blindness, etc., p. 1088-Binocular Phenomena, p. 1098-Functions of the Retinal Structures, p. 1099-Theories of Colour-Vision, p. 1105-Brnocular Vision, Movements of the Eyes, and Spatial PerCEPTION, p. 1122 - Novements of the Eyes, p. 1129-Spatial Perception, p. 1132.

\section{THE DIOPTRIC SYSTEM.}

Static and dynamic refraction.--In the first part of this article the refractive apparatus of the eye will be considered,-first in a condition of rest, and then as adjusted to vision at different distances and with different luminosities.

In order to study the formation of the retinal image, and the conditions by which it is modified, it is necessary to simplify the complex optical system of the eye, and reduce it mathematically to a pair of surfaces,-Listing's schematic eye,--or, still further, to a single surface,Listing's reduced eye. For this reduction certain data are necessary, namely, the radii of eurvature of the refractive surfaces, the distances of these surfaces from one another, and the refractive indices of the transparent media. The cornea and the aqueous humour are so nearly of the same refractive index, that the refraction at the posterior corneal surface may be neglected, and this leaves three surfaces needing investigation, namely, the anterior surface of the cornea, and the anterior and posterior surfaces of the lens.

The ophthalmometer.-The radii of curvature and the distances of the surfaces from one another might be determined on the dead eye, but, owing to the alteration caused by the diminution of the intra-ocular pressure at death, this is not satisfactory. One difficulty in their neasurement during life arises from the unavoidable movements of the living eye, but this difficulty has been overcome by the ophthalmometer first devised by Helmholtz, in which the image reflected from any of the refractive surfaces of the eye may be measured, and the curvature caleulated from its size.

The principle upon which Helmholtz's instrument depends, is the aplarent deviation of an object seen obliquely through a glass plate. 
In Fig. 371 an observer at $l$ will see $O$ through the plate $a a$ as if it were situated at $O^{\prime}$, and through the plate $b b$, at $O^{\prime \prime}$. An object seen through the two plates will appear doubled, the double images deviating in opposite directions. The amount of deviation of each depends on the thickness of the plate $h$ on the angle of incidence, and on the refractive index.

$$
O O^{\prime}=h \frac{\sin (\alpha-\beta)}{\cos \beta}
$$

If the angle the plates make with one another be so adjusted that the twoimages just touch at their inner edges, the distance $O^{\prime} O^{\prime \prime}$ will be equal to the diameter of the object, each image having been moved outwards through half its diametel.

Radius of curvature of the cornea. -In order to find the curvature of the cornea, the eye to be examined is placed about 2 metres from the ophthalmometer, in front of which is a screen bearing two luminous objects, $a$ and $b$, in Fig. 372, the distance between $a$ and $b$ being about half a metre. Four points of light will be seen reflected from the cornea, and the plates are adjusted till the two middle points coincide, so that only three points are seen. The size of the image $\beta$ may be calculated by the formula given (or more commonly in practice is found from a table prepared for the instrument), and the radius of curvature of the cornea is calculated from the formula $r=\frac{2 x \beta}{y}$ where $x=$ the dis-

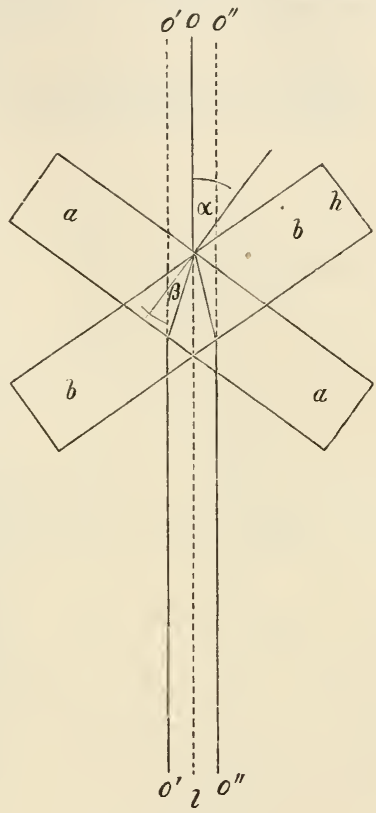

Fig. 371 .

tance between the cornea and the object $a b$, and $y=$ the size of the object, namely, the distance between $a$ and $b$. By this method Helmholtz found the radius of the central part to be 7.829 $\mathrm{mm}$. Other investigators have given rather smaller figures.

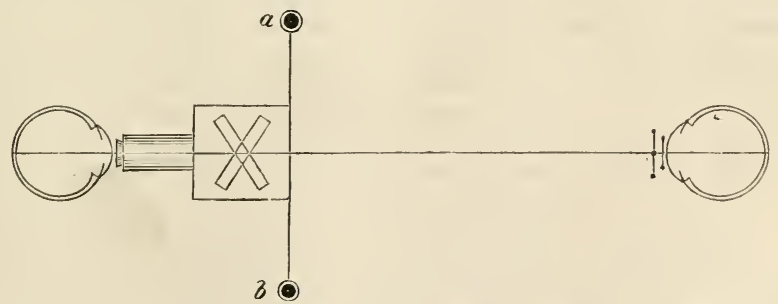

Fig. 372 .

In another form of ophthalmometer, devised by Coccius, the plates are replaced by a double image prism, and the images are brought into apposition 
by altering the size of the object; $\beta$ is then a constant for the instrument; and $y$ being found, the radius is calculated from the same formula. In Landolt's ophthalmometer the doubling of the object is brought about by means of two prisms, while in the instrument of Nandelstamm and Schöler the reflected image is directly measured by means of microscope and micrometer.

Position and curvature of the anterior lens surface.-The distance between the anterior surface of the cornea and the anterior surface of the lens was measured by Donders with the corneal microscope. In

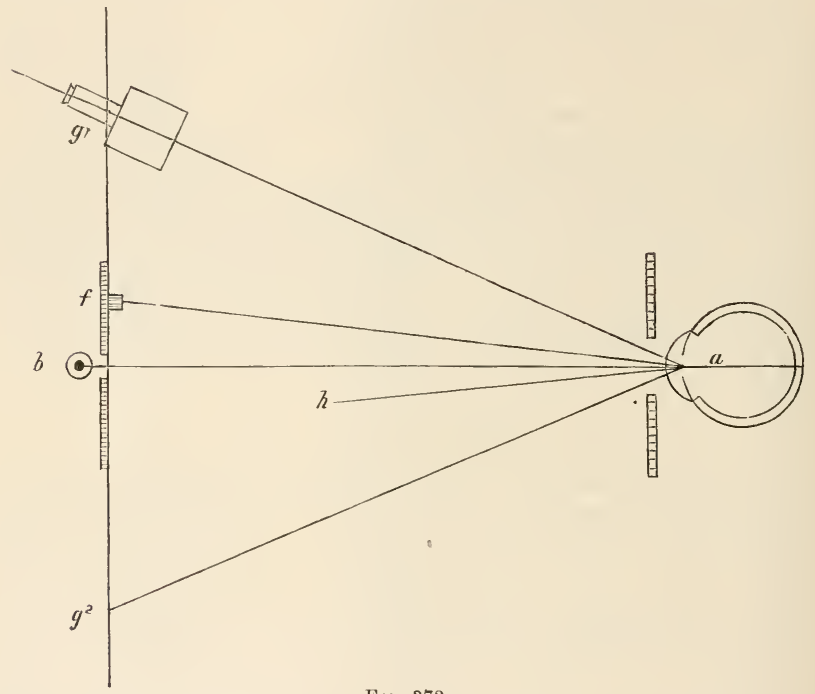

FIG. 373.

the method used by Helmholtz advantage was taken of the facts that the anterior lens surface corresponds with the plane of the pupil, and that the reflected image of an object in the corneal axis appears to lie behind the cornea at a distance equal to half its radius of curvature. With the ophthalmometer at $g^{\prime}$ in Fig. 373 , the corneal reflection of a luminous object at $b$ is doubled. The pupil will be seen double at the same time,

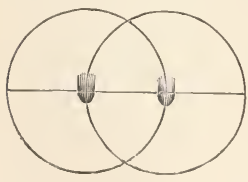

FIG. 374 . and a fixation point, $f$, is moved till a position of the observed eyeball is found at which the doubled corneal images correspond with the edges of the doubled pupil (Fig. 374). This is repeated with the ophthalmometer at $g^{2}$, and the point where the lines $g^{\prime} \alpha$ and $g^{2} a$ intersect will show the position of the pupil. In calculating the position, account has to be taken of the difference between the real and apparent positions of the pupil and of the angle fah, between the corneal axis and the line of vision (see p. 1031). The distance is found to he about $3.6 \mathrm{~mm}$., the figures given by different investigators varying from 2.9 to $4.0 \mathrm{~mm}$. 
The image of the anterior lens surface is too faint and ill-defined to give satisfactory results by doubling, and Helmholtz determined the curvature of this surface by making the lens and corneal images of the same size. This was done by having luminous objects of different sizes for cornea and lens. The size of a reflected image being proportional to the focal distance of the reflecting surface, the radius of curvature of the lens can be calculated from the sizes of the objects, and the known radius of the cornea. When the eye is accommodated for distant objects, it has been found to be about $10 \mathrm{~mm}$., the figures of different observers varying from 7.9 to $11.9 \mathrm{~mm}$.

Position and curvature of the posterior lens surface. - The position of the posterior lens surface is found by producing a light reflection from the surface, and then exchanging the position of the luminous object and the observing eye. In each case it is arranged that the lens image shall be covered by a corneal reflected image, and the position of the lens image will then be at the point of section of the two lines of vision of the observer passing through the two corneal images. The position of the latter being found, the position of the lens image may be calculated, and is about $7.5 \mathrm{~mm}$. behind the cornea.

The curvature of the posterior surface may be found in the same way as that of the anterior, or the apparent size of the reflected image froin the posterior surface may be measured, and the real size calculated from the properties of the refractive media lying in front of it. ${ }^{1}$ The radius is about $6 \mathrm{~mm}$., when the eye is accommodated for distant vision.

The cardinal points. - In addition to the above data, it is necessary to know the refractive indices of the media. The index of the aqueous is approximately equal to that of the vitreous, measurements on fresh eyes by Hirschberg, using Abbe's refractometer, giving that of the aqueous as 1:337, of the vitreous 1:336. The index of the lens varies in its different layers, the refractive power being greatest at the nucleus, and diminishing outwards. The mean index adopted by Listing, derived from the results of Helmholtz and Krause, was 1.4545 ; but other investigators have given smaller values. In calculating the schematic eye, Listing used rather larger values than those given above for the distances between the refractive surfaces and for the radius of the cornea, and calculated the following positions of the cardinal points :-The anterior principal focus $12.8326 \mathrm{~mm}$. in front of the anterior surface of the cornea; the posterior principal focus $14.6470 \mathrm{~mm}$. behind the posterior surface of the lens; the first principal point $2.1746 \mathrm{~mm}$., the second $2.5724 \mathrm{~mm}$., behind the anterior corneal surface; and the first nodal point $7580 \mathrm{~mm}$., the second $3602 \mathrm{~mm}$. in front of the posterior surface of the lens. In the reduced eye, the foci are the same as those of the schematic eye, while the single principal point is $2 \cdot 3448 \mathrm{~mm}$. behind the anterior surface of the cornea, and the single nodal point $4764 \mathrm{~mm}$. in front of the posterior surface of the lens.

Centre of rotation of eyeball.-This may also be found with the aid of the ophthalmometer. The instrument is placed in the middle of a graduated horizontal are, along which a fixation point can be moved. A fine hair is suspended vertically in front of the observed

\footnotetext{
${ }^{1}$ For more detailed deseription of these and the previous methods, refer to Arch. $f$. Ophth., 1855, Bd. i. Abth. 2, S. 1; "Handbueh f. d. ges. Augenh.," 1874, Bd. iii. S. 204, ete. ; or "Traite complet d'opht.," by de Weeker and Landolt, 1880, tome i. p. 738.
} 
eye, which is rotated till the hair covers first one and then the other border of the cornea. The angle through which the eyeball has moved, namely, the angle yxy' in Fig. 375, may be read off on the scale, and is about $56^{\circ}$. The diameter of the cornea $y y^{\prime}$ may be found with the ophthalmometer by doubling the cornea, by altering the plates till the doubled images from the centre of the cornea correspond with the edges of the

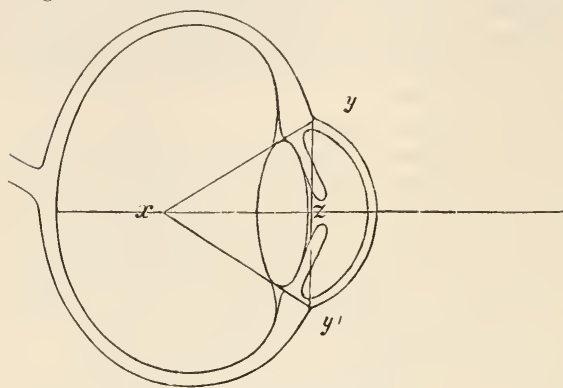

Fig. 375.-Donders. doubled cornea (as in Fig. 374 , with the difference that the circles represent the doubled cornea, and not the pupil). The angle yxz and the side $y z$ being known, $x z$, the distance from the centre of rotation to the basis of the cornea, may be found, and the height of the cornea, i.e. $2 \cdot 6 \mathrm{~mm}$., being added, will give the distance of the centre of rotation behind the summit of the cornea. By this method Donders ${ }^{1}$ found the distance in emmetropic eyes to average $13.54 \mathrm{~mm}$. In myopic eyes the centre was found to be $14.52 \mathrm{~mm}$., and in hypermetropic $13.22 \mathrm{~mm}$. behind the anterior corneal surface.

According to J. J. Miiller ${ }^{2}$ and Berlin, ${ }^{3}$ the centre of rotation is not a fixed point, and recedes from the cornea when the lines of fixation are directed upwards. The former determined the path of a definite point of the cornea during movement of the eyeball, while the latter arranged a number of fine objects, so that they appeared in line with one another in different positions of the eyeball, the point of section of the different lines corresponding with the centre of rotation.

Optical irregularities.-Before considering some further points

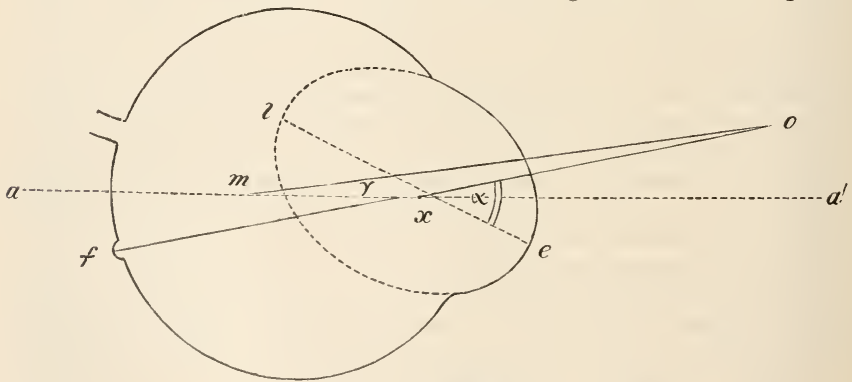

Fic. 376.--Landolt.

about the refractive system, certain definitions will be necessary. The optical axis of the eyeball ( $\alpha \alpha^{\prime}$ in Fig. 376) is the line which passes

1 "Accommodation and Refraction of the Eye," 1864, p. 181.

2 Arch. f. (phth., 1868, Bd. xiv. Abth. 3, S. 183.

${ }^{3}$ Ibid., 1871, Bd. xvii. Abth. 2, S. 150. 
through the nodal point and the centre of rotation of the eyeball. If the different refractive surfaces were exactly centred, as we shall see is not the case, it would pass through the summits of the three chief curved surfaces. The line of vision of is the line passing from the fovea centralis through the nodal point of the reduced eye, and continued to the point of fixation external to the eyeball. The line of fixation om is the line passing from the external fixation point to the centre of rotation of the eyeball. A fourth line, called by Helmholtz ${ }^{1}$ the Visirlinie, passes from the point of fixation to the centre of the pupil; owing to the frequent eccentricity of the pupil, this may differ considerably from the lines of vision and fixation.

The cornea is ellipsoidal, ${ }^{2}$ and the major axis of the corneal ellipse, $e l$, may not correspond to the summit of the cornea. The angle between the line of vision and the major axis of the corneal ellipse is known as the angle $\alpha$ (oxe in Fig. 376); the angle between the optic axis and the line of fixation, as the angle $\gamma$.

The deviation of the line of vision from the optical axis, and the imperfect centring of the eye, are shown by the following experiment of Helmholtz. $^{3}$ In Fig. $377, a b$ is a graduated scale with apertures at $a$ and $b$; the observer at $a$ sees the three images of a light at $b$, reflected from cornea and lens of an eye at $d$. If the line of vision corresponds with the optical axis, the observer should see the anterior lens image midway between the others, when the observed eye is directed to the centre of the scale, $c$. It is found that the point of fixation has to be shifted to one side, i.e. the nasal side of $c d$, and slightly above the plane $a b d$. If the eye were properly centred, and the observer and light at $a$ and $b$ exchanged places, the anterior lens image should still be midway between the others. In none of the eyes examined by Helmholtz was this the case, and this observation

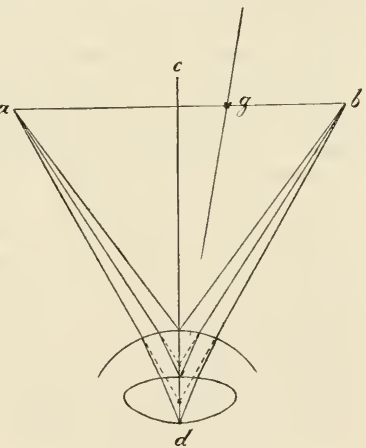

Fig. 377 . has been confirmed by Tscherning. ${ }^{4}$

As shown by the experiment described, the optic axis cuts the posterior pole of the eyeball on the inner side of the yellow spot and slightly above it. The angles $\alpha$ and $\gamma$ may be determined with the ophthalmometer. A light to serve as object for the reflected image is placed over the instrument, which is furnished with a graduated arc and movable fixation point. The position of the eyeball is found which gives an image exactly in the centre of the cornea, i.f. the cornea

1 "Handbuch d. physiol. Optik," 2te Aufl., Hamburg u. Lcipzig, 1896, S. 115.

2 The gencrally accepted view, that the cornea is ellipsoidal, has been denied by Sulzer (Arch. d'opht., Paris, 1891, tome xi. p. 419) and others. The form of the surfuce has bcen determined by calculation from observations of the curvature at a certain number of points. From examination of a large number of points of the corneal surface with the ophthalmometer of Javal and Sclioitz, Sulzer came to the conclusion that the central prart is approximatcly splierical; that at a certain distance from the centre the cornea becomes flatter, the nasal side more than the temporal, and the upper more than the lower half.

3 "Handbuch d. physiol. Optik," 2te Aufl., s. 108.

${ }^{4}$ Ztschr. f. Psychol. u. Physiol. d. Sinnesory., Hamburg u. Leipzig, 1892, Bd. iii. S. 475. 
is doubled by altering the plates till the reflected images of the flame fall on opposite edges of the cornea, as shown in Fig. 374. The position of the fixation point on the graduated are will give the angle $\gamma$, and the angle a may be calculated, since in Fig. 378, vc, the distance from the centre of rotation to the graduated are, is known, and also $k c$, the distance from the nodal point to the centre of rotation. The method is not strictly accurate, for the centre of the cornea does not, as already men-

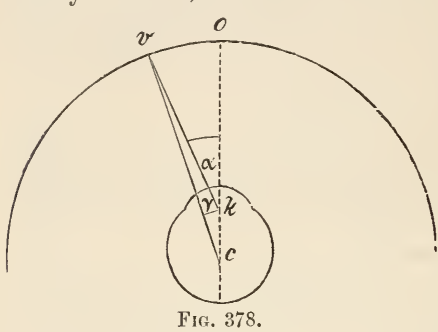
tioned, necessarily correspond with the corneal axis, and modifications of the method have been devised to overcome this difficulty. ${ }^{1}$

According to Donders, ${ }^{2}$ in emmetropic eyes the angle $\alpha$ is about $\tilde{5}^{\circ}$, so that, when the lines of vision are parallel, the optic axes would diverge $10^{\circ}$. The deviation was found to be greater in hypermetropic, and less in myopic eyes, in which the deviation may be nil, and the optic axes may even converge. If the optic axis and the axis of the corneal ellipse coincide, the angles $\alpha$ and $\gamma$ vary together, and the difference between them diminishes, the further the point of fixation is from the eye.

In addition to the three reflected images used in the ophthalmometric methods, a fourth may be observed reflected from the posterior surface of the cornea. It may be seen by placing the flame well on one side, and magnifying the corneal image with a lens.

An entoptic image may also be seen, owing to the fact that a certain amount of the light reflected from the posterior surface of the lens is reflected again from the posterior surface of the cornea. The light from the anterior surface of the lens comes to a focus near the posterior surface of the lens, ${ }^{3}$ and, owing to its distance from the retina, is not observed entoptically. The light from the posterior surface of the lens, when again reflected, comes to a focus near the retina, and gives rise to an entoptic image. When a flame is held on one side of the line of vision in a dark room, a faint inverted image may be seen on the opposite side of the line of vision, which moves in the opposite direction to that of the flame. This image may in some cases be the basis of the occurrence of monocular diplopia.

Myopia and hypermetropia.-Theoretically, myopia might be due to the curvature of any of the refractive surfaces being greater than normal, to increase of the refractive index of one of the media, or to elongation of the eyeball. Similarly, hypermetropia might be due to diminished curvature, diminished refractive index, or to shortening of the eyeball. In nearly all cases, change in the shape of the eyeball is believed to be the most important factor, although altered curvature may account for many slight examples of these defects. It has been suggested that cases of transitory myopia in certain inflammatory conditions of the eyeball may be due to increased refractive index of the aqueous. The refraction may differ in the two eyes, and this condition is known as anisometropia.

Astigmatism.-This term is employed for two kinds of defect,

${ }^{1}$ Arch. f. Ophth., 1865, Bd. xi. Abth. 2, S. 257.

2 "Anontalies of $A$ ceommodation and Refraction," 1864, p. 183.

${ }^{3}$ Ztschr. f. Psychol. u. I'hysiol. d. Sinnesorg., Hamburg u. Lei lzig, 1892, Bd. iii. s. 429. 
namely, for irregularities of cornea or lens,-irregular astigmatism; and for differences in the refraction of different nieridians of the corneal and lens surfaces,-regular astigmatism. One kind of irregular astigmatisn is due to the structure of the lens, and shows itself in the "starlike" appearance of any minute luminous point. In most eyes, again, a fine luminous line appears, when carefully examined, to be doubled; or multiplied still more. This may be observed well in the case of the horns of the new moon. This appearance is supposed to be the basis of some of the recorded cases of monocular diplopia or polyopia.

Regular astigmatism may depend on a defect either of the lens or of the cornea, the latter being the more common. The existence and amount of corneal astigmatism may be determined by a modification of the ophthalmometer devised by Javal and Schiötz. ${ }^{1}$ Thomas Young ${ }^{2}$ discovered that the defect in his own case was due to the condition of the lens, by finding that it persisted when the refractive power of the cornea was abolished by covering it with a layer of water. The meridians of greatest and least refraction are always at right angles to one another, but may be at any angle to a vertical section through the eyeball. The defect prevents the occurrence of a true punctiform focus, and produces two linear foci, and between them an interfocal region, in one part of which the focus is approximately circular. This circular focus lies nearer the anterior than the posterior linear focus. In the average emmetropic eye the vertical meridian is more convex than the horizontal, the difference not amounting to more than $5 \mathrm{D}$, and in most cases of marked astigmatism the vertical is also the more convex meridian.

Chromatic aberration.-The difference of focus for the ends of the spectrum was calculated by Helmholtz. ${ }^{3}$ Using Listing's reduced eye, and assuming that its refraction is equal to that of water, he found a difference of $\cdot 434 \mathrm{~mm}$., so that an eye accommodated for red at infinite distance would be accommodated for violet at a distance of $71.3 \mathrm{~cm}$. Matthiessen ${ }^{4}$ found the distance between the two foci still greater ( 58 to $.62 \mathrm{~mm}$.); and Einthoven, ${ }^{5}$ who has recently calculated the cardinal points of the eye for different parts of the spectrum, also finds the values with the reduced eye too small. Einthoven found that there was no appreciable difference in the position of the posterior nodal point for different colours.

The effect of this difference of focus may be most readily observed on looking, with inexact accommodation, through violet glass transmitting red rays, especially with a half-covered pupil. It is also seen with fine black or white lines. It is much increased when the pupil is dilated. It is said that chromatic aberration is more easily observed by emmetropic and hypermetropic than by myopic eyes.

\section{ACCOMmodation.}

Changes in lens.-The changes which have been observed during accommodation will first be described. The anterior surface of the lens increases in curvature. while the eurvature of the posterior surface

1 "Beitr. z. Psychol. u. Physiol. d. Sinnesorg." (Helmholtz Festgruss), Hamburg and Leipzig, 1891, S. 39.

2 Phil. Trans., London, 1801, p. 57.

3 "Handbuch d. physiol. Optik," 2te Aufl., S. 157.

4 Compt. rend. Acad. d. sc., Paris, 1847, tome xxiv. p. 875.

5 Arch. f. d. ges. Physiol., Bonn, 1896, Bd. lxii. S. 166. 
is very slightly altered. This may be observed by means of the reflected images seen with the phakoscope, and the amount of change may be accurately measured with the ophthalmometer. The central point of the anterior surface is found to move forwards, the amount of movement averaging $5 \mathrm{~mm}$. The radius of curvature of the anterior surface diminishes from an average of $10 \mathrm{~mm}$. to one of $6 \mathrm{~mm}$., while in cases of hypermetropia the increase of curvature may be even greater. Knapp ${ }^{1}$ found that in four individuals the amount of accommodation, calculated from the change of curvature of the lens, agreed fairly closely with the actually observed range of accommodation.

According to Tscherning, ${ }^{2}$ however, the increase of curvature does not take place uniformly over the whole anterior surface, but is greater for its central part. He came to this conclusion after repeating an experiment of Thomas Young, in which he used a plano-convex lens with squares marked out on the plane surface,- the aberroscope. On holding this $10 \mathrm{~cm}$. from the eye, and looking at a light, most individuals see the outer lines curved with the convexity inwards, while, on accommolating, the curved lines become straight or even curved outwards. Tscherning regards this as showing that in its resting state the refraction is greater at the periphery of the lens than at the centre, while during accommodation the increase of refraction is relatively less at the periphery, so that the whole lens may become equally refractive, or the central part even more refractive than the periphery. With more exact measurements, by means of his ophthalmophakometer, Tscherning found that while the radius of curvature of the central parts altered from $10 \mathrm{~mm}$. to 5 or $6 \mathrm{~mm}$. during accommodation, a point on the periphery, $1.4 \mathrm{~mm}$. from the centre, only showed a radius of $8 \mathrm{~mm}$. during accommodation, while in the more peripheral parts there was actual and not merely relative flattening. ${ }^{3}$ Crzellitzer ${ }^{4}$ has since repeated and confirmed these olsservations.

The curvature of the posterior surface is found to be very slightly increased, the observations of numerous workers showing a change from about $6 \mathrm{~mm}$. to $5 \mathrm{~mm}$. in radius.

Most investigators have found no alteration in the position of the lens, i.e. the position of the posterior surface. Reich ${ }^{5}$ and Tscherning ${ }^{6}$ have, however, each described slight recession of the posterior surface, about $1 \mathrm{~mm}$, and Hensen and Voelckers ${ }^{7}$ observed recession of the lens in animals, shown by the movements of a style placed against its posterior surface. Tscherning also described a slight movement downward of the lens during accommodation. The question has been very thoroughly investigated by Hess, ${ }^{8}$ who finds that, when the eye is accommodated, there is considerable mobility of the lens. In cases of absent iris (after iridectomy) under the influence of eserin, Hess observed a marked shaking of the lens with every movement of the head, the phenomenon disappearing after instillation of atropin. The movement was most marked with the head inclined backwards. For observations on the normal eye, Hess took advantage of the fact that eserin acts on the

1 Arch. f. Ophth., 1860, Bd. vi. Abth. 2, S. 1.

${ }^{2}$ Arch. de physiol. norm. et path., Paris, 1894, tome vi. p. 40.

${ }^{3}$ Ibid., 1895, tome vii. p. 158.

4 Arrh. f. Ophth., 1896 , Bd. xlii. Abth. 4, S. 36.

5 Ibid., 1874, Bd. xx. Abth. 1, S. 207.

7 Centralbl. f. d. med. Wissenseh., Berlin, 1866, S. 721

${ }^{8}$ Arch. f. Ophth., 1896, Bd. xlii. Alith. 1, S. 288. 
ciliary inuscle before it affects the iris, and applied eserin after using homatropin. The normal eye showed similar marked shaking, the observation being especially striking when opacities of the lens existed. The shaking was also observed subjectively. On moving the head from side to side, and then stopping the movement, an observed needle appeared to make three or four oscillations after cessation of movement of the head. The same phenomenon was observed in entoptic observation of the lens. The mobility of the lens during marked accommodation, produced voluntarily or by means of eserin, was ascribed by Hess to slackening of the suspensory ligament. Hess ${ }^{1}$ also confirmed Tscherning's observation of a downward movement of the lens during accommodation, but found further that the direction of the movement depended on the position of the head, and that the movement did not occur when the head was inclined so that the iris was horizontal, showing that it was due to gravity. The movement of the lens was determined by the apparent movement of a point of light which moved with the head, and had a constant position relative to the eye; during strong accommodation there was an apparent rise of the point of light, showing a downward movement of the retinal point stimulated. The amount of movement of the lens, with strong voluntary accommodation, was found to be about $3 \mathrm{~mm}$., while in the eserinised eye, on moving the head from shoulder to shoulder through an angle of $180^{\circ}$, the displacement of the lens amounted to $1 \mathrm{~mm}$.

Hess also found a certain amount of mobility in the antero-posterior direction, and investigated its amount by determining the near point with the head inclined forwards and backwards. With voluntary accommodation he found in several individuals that in the two positions of the head, the near point varied to an extent corresponding to a difference of refraction of $34 \mathrm{D}$ to $\cdot 48 \mathrm{D}$, and calculated that this corresponded to a displacement of the lens antero-posteriorly of 15 to $\cdot 21 \mathrm{~mm}$. With the eye well under the influence of eserin, the refraction, as shown by the near point, changed to the extent of $\cdot 61$ to $\cdot 81 \mathrm{D}$, showing a displacement of the lens of $\cdot 26$ to $\cdot 36 \mathrm{~mm}$. With relaxed accommodation $n o$ change in the near point with altered position of the head could be observed. Hess suggests that the previous observations of recession of the posterior lens surface may have beell due to the position of the head.

Crzellitzer ${ }^{2}$ has confirmed the observations as to the shaking of the lens, but supposes that this is due to a muscular tremor and not to slackness of the zonula. This explanation cannot, however, apply to the other observations of Hess.

Several observers have noted irregularities of the border of the lens in albinos and in cases of absent iris, such as might be due to greater pressure on certain parts than on others. Hess ${ }^{3}$ observed that this appearance, which he describes in most cases as a wavy border, becomes quite uniform after the instillation of eserin. When the lens is observed from in front in similar cases, its outer zone is seen as a circular band, bright by reflected, dark by transmitted light. This appearance is due to total reflection, and has been used as an indication of change of shape of the lens. The breadth of the border depends on the angle at which the two lens surfaces meet, increased breadth showing increased

${ }^{1}$ Arch. f. Ophth., 1897, Bd. xliii. S. 477.

${ }^{3}$ Arch. f. Ophth., 1896, Bd. xlii. Abth. 1, S. 288.

2 Loc. cit., S. 90. 
thickness of the lens. The border becomes broader during accommodation, and it is said that the lens can also be observed to diminish in size concentrically. ${ }^{1}$

Changes in suspensory apparatus.-The free mobility of the lens, and the changes observed in the regularity of its edge, are evidence that the suspensory ligament is slack during accommodation. In cases of absent iris, a fine radially striated appearance may be observed in the position of the ligament, and Hess found that this striation became indistinct after the use of eserin. In animals, Hensen and Voelckers ${ }^{2}$ found that a style placed behind the ligament showed that it became slack during accommodation. Schön ${ }^{3}$ has objected that this is only true of the fold of the ligament which runs to the posterior surface of the lens, which he supposes to become bent on itself, while the anterior stronger process remains tense.

The behaviour of the ciliary processes during accommodation has been much discussed. Then observed from in front in albinos and in cases of absent iris, Becker ${ }^{4}$ noted recession in near vision, but most other observers have seen the processes move forwards, and at the same time approach the optic axis. They are also said to swell slightly. When observed in animals through a window in the sclerotic, the ciliary region is seen to sink inwards during accommodation (Hensen and Voelckers). ${ }^{2}$ The space seen between the ciliary processes and the edge of the lens appears to remain approximately constant during accommodation (Hjort, ${ }^{5}$ Hess).

Hensen and Voelckers ${ }^{6}$ found that during accommodation the choroid moved forwards. They obtained this result in an extirpated human eye as well as in animals. They found that a needle showed a movement of the choroid, amounting to $\frac{1}{2} \mathrm{~mm}$. at the equator of the eyeball, diminishing from this point, so that no movement was observed in the ciliary and macular regions. The retina moved with the choroid. The circular or oval phosphene, seen on sudden relaxation of an accommodative effort in the dark, has been ascribed to the movement of the choroid. It is probably due to traction near the posterior pole of the eyeball, for Berlin $^{7}$ found that, when produced simultaneously with an ordinary pressure phosphene, the latter appeared outside the ring of the accommodation phosphene.

In addition to the associated contraction of the pupil during accommodation, the iris is pushed forwards by the bulging lens. If the eyeball is observed laterally, the pupillary edge may be seen to move forwards while the peripheral zone recedes. Helmholtz $^{8}$ found the amount of movement forwards about $\cdot 4 \mathrm{~mm}$.

Tscherning ${ }^{9}$ observed a greater recession of the middle than of the outermost zone of the iris, but other observers have failed to confirm this.

Thomas Young ${ }^{10}$ found that his range of accommodation was as great when his cornea was covered with water as under normal conditions, and

\footnotetext{
1 Graefe u. Saemisch, "Handbuch d. ges. Augenh.," Bd. vi. S. 470.

2 "Experimentaluntersuch. iiber d. Mechanismus d. Acc.," Kiel, 1868.

3 Arch.f. d. ges. Physiol., Bonn, 1895, Bd. lix. S. 448.

4 Med. Jahrb., Wien, 1863 (2), Bd. xix. S. 159.

Klin. Monatsbl. f. Augenh, 1876, Bd. xiv. S. 205.

6 Arch. f. Ophth., 1873, Bd. xix. Abth. 1, S. 156.

7 Ibril., 1874, Bd. xx. Abth. 1, S. 89.

8 "Handbuch d. physiol. Optik," 2te Aufl., S. 141.

9 Arch. de physiol. norm. et path., Paris, 1894, tome vi. p. 40.

${ }^{10}$ Phil. Trans., London, 1801, p. 57.
} 
also observed no changes during accommodation in the images reflected from the cornea. Later measurements with the ophthalmometer have failed to show any change in the radius of curvature of the cornea.

Intra-ocular pressure.-The condition of intra-ocular pressure during accommodation has been the subject of much difference of opinion. According to one view, the pressure in the vitreous rises while that of the aqueous falls during accommodation. This view is based chiefly on cases described by Förster. ${ }^{1}$ In cases in which the anterior chamber had been opened, the cornea was noticed to flatten during accommodation, pointing to lessened pressure within; while, in a case of perforation of the cornea, the aqueous filling the opening receded during accommodation. In these cases the general intra-ocular tension was abnormally lowered, and it is doubtful whether the same phenomena would occur in the normal eye, and this doubt is increased by the fact that Förster notes that they occurred after the use of atropin. Manometrical observations, however, in both cavities of the eyeball during accommodation have failed to show any noticeable difference, ${ }^{2}$ while it has been found that injection of fluid into one cavity raises the pressure of both equally. The free mobility of the lens during strong accommodation, described by Hess, would be impossible if there were any difference of pressure in front of and behind the suspensory ligament.

Hensen and Voelckers ${ }^{3}$ found that in animals an artificial protrusion of the vitreous through a puncture increased during accommodation.

Nervous mechanism.-The chief point of interest is the supposed function of the sympathetic as the nerve of accommodation for far vision. Jessop ${ }^{4}$ observed recession of the anterior lens surface on stimulating the long ciliary nerves, after the ciliary muscle had been moderately contracted by pilocarpin. Morat and Doyon ${ }^{5}$ assert that when the sympathetic is stimulated in a dog or cat, under the influence of morphia or pilocarpin, the anterior lens image enlarges, and that a needle placed against the anterior lens surface indicates flattening of the lens. They ascribed this effect to the inhibitory influence of the sympathetic on the ciliary muscle. On the other hand, Langley and Anderson ${ }^{6}$ failed to observe either movement of the choroid, backward movement of the anterior lens surface, enlargement of lens images, or increase of distance between them. Heese ${ }^{7}$ also failed to obtain any separation of the lens images on stimulation of the sympathetic. In the cat, with which animal Morat and Doyon obtained their must marked results, Heese noticed a diffused halo round the lens image, which he ascribed to dispersion rendered more obvious by the dilatation of the pupil, and supposed that this may have misled the French observers.

Unequal and meridional accommodation.-By the former term is meant accommodation of a different amount in the two eyes; by the latter, of a different amount in the two meridians of the same (astigmatic) eye. Observations of Hering and Donders are generally held to have disproved the existence of the former, but A. E. Fick ${ }^{8}$ has recently attempted to show that

${ }^{1}$ Klin. Monatsbl. f. Augenh., 1864, Bd. ii. S. 368.

2 For recent confirmation of this, see a paper by Hess, Arch. f. Ophth., 1898, Bd. xlvi. S. 243 .

${ }^{3}$ Centralbl. f. d. med. Wissensch., Berlin, 1866, S. 721.

${ }^{4}$ Ophth. Rev., London, 1887, vol. vi. p. 163.

${ }^{5}$ Arch. de physiol. norm. et path., Paris, 1891, Sér. 5, tome iii. p. 507.

${ }^{6}$ Journ. Physiol., Cambridge and London, 1892, vol. xiii. p. 583.

7 Arch. f. d. ges. Physiol., Bonn, 1892, Bd. lii. S. 563.

8 Arch. Ophth., N.Y., 1889, vol. xviii. p. 292. 
it occurs in cases of mnequal refraction. He combined printed type in the stereoscope, arranging that the gaps in the type presented to one eye should be supplied by that presented to the other eye, and found that the print could be read with a difference of 2 to 3 D. Hess ${ }^{1}$ has, however, by means of careful stereoscopic experiments, shown that the older view is probably correct. He used fine threads at slightly different distances, so that they would be seen lying side by side in binocular vision, and found a difference of distance amounting to $25 \mathrm{D}$ was sufficient to render one of the two threads indistinct or even invisible. Hc found that cases of slight anisometropia showed no more evidence of unequal accommodation than did normal vision; when fully corrected, a difference of $5 \mathrm{D}$ was not compensated for by accommodation. Hess ascribes Fick's results partly to the use of too coarse tests, and partly to his neglect of the influence of the pupil. It is possible in myopia for a distant object to appear more distinct with the eye accommodated for near vision than with the unaccommodated eye, owing to the contracted pupil in the former case more than compensating for the increased diffusion circle.

Hess also calls attention to the influence of rivalry in such experiments (see p. 1122).

Greeff $^{2}$ investigated the same question by a different and very simple method. If an opaque body is lield before the eyes, part of the visual field will be seen by one eye, part by the other eye, and part by both eyes. With lifferent refraction in the two eyes, one part of the field will be indistinet if the difference is not compensated for by accommodation ; and Greeff found by this method that both in natural and artificial anisometropia there was not the slightest evidence of unequal accommodation.

The evidence seems also to be against the existence of meridional accommodation. Its occurrence has been advocated (1) on the ground of experiments in which astigmatism is imitated by means of glasses, (2) on account of the marked and often sudden changes of vision which occur in cases of natural astigmatism, and (3) because astigmatism is sometimes found to have increased after instillation of atropin. Hess ${ }^{3}$ has recently failed to find any evidence of unequal contraction of the ciliary muscle sufficient to overcome astigmatism of a fraction of a dioptre. Astigmatics often assist vision by closing the eyelids, so as to convert the palpebral aperture into a slit, and this fact may explain some of the cases which have been brought forward in favour of meridional accommodation. Hess has called attention to another possibility. It has generally been supposed that astigmaties always accommodate for one or other of their linear foci. Hess finds that this is not the case, but that accommodation normally occurs for that point in the interfocal region where the focus is approximately circular. Using a photographic apparatus rendered astigmatic by a cylindrical lens, Hess ${ }^{4}$ found that the clearest image oceurred at the circular focus; and eareful observations of the eyes of astigmatics showed that, when they saw most distinctly, their refraction was adjusted approximately to the circular focus. The variations in astigmatic vision, which have been ascribed to meridional accommodation, may very well have been due to variations in the adjustment of the eye to different parts of the interfocal region. If meridional accommodation occur in cases of astigmatism, it might be expected to lead to anatomical changes in the form of localised hypertrophies of the ciliary muscle. No such appearances have been recorded.

Theories of accommodation.-In the widely accepted theory of Helmholtz, it is supposed that during relaxation of accommodation the suspensory ligament is in a state of tension, thereby compress-

Arch.f. Ophth., 1889, Bd. xxxv. Abth. 1, S. 157.

2 Arch. f. Augenh., Wiesbaden, 1891, Bd. xxiii. S. 371.

Arch.f. Ophth., 1896, Bd. xlii. Abth. 2, s. 80.

t Loc cit. Refer to IIess's paper for the photographs illustrating this. 
ing the anterior surface of the lens. The longitudinal fibres of the ciliary muscle are supposed to have their more fixed attachment in front, and on contraction to pull the choroid forward, relaxing the ligament, and releasing the pressure on the lens. The circular fibres are supposed to prevent distortion of the ciliary processes by making them follow the retreating lens.

The observations of Hensen and Voelckers on animals are all in favour of this theory, and especially the occurrence of movement of the choroid forwards. The recent observations of Hess seem to afford convincing evidence in favour of the main feature of the theory, namely, slackening of the suspensory ligament during accommodation. Recently the theory has been attacked in several quarters; one objection made by Schön is based on the supposed difference of pressure in the aqueous and vitreous humours in the eye adjusted for near vision. Schön points out that the ability of the contracting ciliary muscle to pull the choroid forwards depends on the intra-ocular pressure, and that it is impossible that both rise of pressure in the vitreous and slackening of the suspensory ligament should take place from the same cause. As we have seen, however, there is no satisfactory evidence that the pressure in the vitreous becomes higher than that in the aqueous humour.

The greatest difficulty of the Helmholtz theory lies in the rôle assigned to the circular fibres. It has been found by Iwanoff ${ }^{1}$ that the circular fibres are hypertrophied in cases of hypermetropia, in which accommodation has been over-used, and are atrophied in myopia, in which less than the normal effort is required. This change is said not to be constant, but such changes are only to be expected in those cases in which the refractive anomaly has been left uncorrected. If this observation is accepted, the circular fibres must be of more importance than is assigned to them by the theory. On the other hand, the circular fibres are absent or almost absent in nearly all mammals in whom the mechanism of accommodation is probably of essentially the same nature as in man. Schön has objected that numerous pathological conditions associated with hypermetropia and over-action of accommodation are not intelligible if the process of accommodation is one of relaxation of tension.

When accommodation was found to depend on a change in the form of the lens, Cramer supposed that the increased curvature of the central part of the lens was brought about by means of the pressure of the iris on the peripheral parts. This was soon disproved by the observation that accommodation took place quite normally when the iris was absent, but this flattening of the periphery of the lens by pressure and bulging of the centre is the essential feature of the two recent theories of Tscherning and Schön.

Tscherning ${ }^{2}$ supposes that the posterior attachment of the meridional fibres of the ciliary muscle is more fixed than the anterior, and that when these fibres contract they pull on the suspensory ligament, rendering it more tense, thereby flattening the peripheral parts of the lens, while the centre becomes more convex. He states that when traction is made on the suspensory ligament in the extirpated eye, the change in shape of the lens corresponds to that taking place during accommodation. Crzellitzer, however, who has devised a special ap-

${ }^{1}$ Arch. f. Ophth., 1869, Bd. xv. Ah,th. 3, S. 284.

${ }_{2}$ Arch. de physiol. norm. et path., l'aris, 1894, tome vi. p. 40 . 
paratus for exerting uniformly circular traction on the zonula, failed to make satisfactory observations on the peripheral parts of the lens.

Tscherning neglects in his theory the eircular fibres of the ciliary muscle. In S'chön's theory, ${ }^{1}$ on the other hand, they play an important part. Schön supposes that the zonula becomes more tense during accommodation, being compressed by the ring fibres, assisted by contraction of the meridional fibres. He regards the lens capsule, zonula, and choroid, as together forming an elastic and continuous covering of the globular eyeball, and the result of eireular pressure round one pole of this globe would cause protrusion of the part within the ring of pressure, namely, the anterior lens surface. The posterior surface of the lens would be prevented from altering in form by the increased pressure in the vitreous, which Schön supposes to be present. While Schön's theory is satisfactory in supplying the circular fibres with a function, it goes too far, for it leaves the other parts of the muscle-the only parts existing in most animals-without any direct function in accommodation. ${ }^{2}$ The theories of both Schön and Tscherning involve increased tension of the suspensory ligament during accommodation. Schön supposes that the observation of Hensen and Voelckers, showing relaxation of the zonula, arises from the folding upon itself of the posterior fold of the ligament, and does not concern the more important fold going to the anterior surface of the capsule. Both theories must fall, if Hess has given a correct explanation of his observations.

Before the changes in the lens were described by Helmholtz, one of the favourite theories was, that accommodation for near objects depended on increase in length of the antero-posterior diameter of the eyeball, brought about by the external pressure of the contraction of the extra-ocular muscles associated with convergence, together with internal traction by the ciliary muscle. On the discovery of the lens changes, the theory was discarded, but it has recently been supposed to be a subsidiary factor, and to be the explanation of the apparent accommodation which has been observed in cases of aphakia, and also when the ciliary apparatus is supposed to be completely paralysed by atropin. Knapp's observations show that in normal conditions the changes in the lens are sufficient wholly to account for the range of accommodation. Any change in the shape of the eyeball would be accompanied by change in the curvature of the cornea; all agree that the latter does not occur in normal eyes, and Silex ${ }^{3}$ has found no change in a boy who seemed to have some power of accommodation after removal of the lens.

Accommodation in birds, reptiles, amphibia, fishes, and cephalopods.- The comparative physiology of accommodation has been very thoroughly investigated by Beer. In birds ${ }^{4}$ he has found that the cornea consists of two distinct lamellæ, and that Crampton's muscle produces retraction of the inner or posterior lamella. In some birds (owls, hawks, buzzards) he found with the ophthalmometer that the peripheral parts of the cornea were flattened, while the curvature of the central part increased. The lens showed increased curvature on stimulation of the ciliary muscle. On dividing the ligamentum pectinatum, the lens was found to have increased its curvature, and stimulation of the muscle then had no influence on the curvature. There was no

${ }^{1}$ Arch. f. d. ges. Physiol., Boun, 1895, Bd. lix. S. 427.

${ }^{3}$ Schou supposes that the meridional fibres assist in maintaining and regulating intraocular pressure.

Arch. Ophth., N. Y., 1889, vol. xviii. p. 274.

Arch, f. d. ges. Physiol., Bonu, 1893, Bd. liii. S. 175. 
appreciable alteration in the curvature of the posterior surface of the lens. Beer concluded that in far vision the anterior surface of the lens is compressed by traction exerted by the ciliary processes (which in the bird are directly attached to the lens capsule). This traction is kept up by the tension of the ligamentum pectinatum; when the ciliary muscle contracts, it draws back the inner lamella of the cornea, and the tension of the ligamentum pectinatum being relaxed, the anterior lens surface is allowed to become more convex. This mechanism has the same principle as that formulated by Helmholtz for the human eye, but differs greatly in its details. In some birds the same mechanism supplements the increased refraction of the lens by increasing the curvature of the central part of the cornea.

In reptiles (except snakes), Beer ${ }^{1}$ found that the mechanism of accommodation closely resembles that of birds. In most snakes, on the other hand, no change in the form of the lens was observed, but a forward movement of the whole lens was found to take place. This movement was brought about by means of a striped nuscle composed of circular fibres, which exerts pressure on the vitreous during contraction, and forces forward the most movable part of the wall of the vitreous chamber, namely, the lens. When the vitreous chamber has been opened, contraction of the muscle causes retraction of the lens. Among the snakes examined, only one was found (Tropidonotus tessellatus) in which the anterior surface of the lens became more convex, but this change was also probably accompanied by a bodily movement of the lens forwards. Most of the amphibia examined ${ }^{2}$ were found to accommodate in the same way as snakes, by means of a forward movement of the lens. Some were found to be approximately emmetropic in air, others in water; but in no case was the amount of accommodation present sufficient to enable an animal to see distinctly in both media. In some snakes and amphibia, the power of accommodation seemed to be absent.

In fishes, Beer found the mechanism to be of an absolutely different nature." It had previously been found that the fish is myopic in water, and Beer confirmed this, using the methods of skiascopy and direct ophthalmoscopy, the myopia varying from about 3 to $12 \mathrm{D}$ in water in most fishes, and from 40 to 90 $\mathrm{D}$ in air. With the ophthalmoscope Beer found a decrease of the myopia on electrical stimulation of the eye. With the phakoscope and ophthalmometer he found no change in the curvature of the lens, but distinct movement of the lens backwards and slightly to the temporal side during stimulation. He found that this movement was due to the campanula, which could be seen to contract; while retraction of the lens did not occur after cutting the campanula or its tendon. The lateral movement of the lens was due to the position of the campanula on the temporal side, and through the space between the pupil and the lens Beer was able to observe a corresponding lateral movement of the retinal image. This mechanism seemed to be limited to the Teleostean fishes; no movement of the lens could be detected in the Elasmobranchs examined.

In Teleostean fishes, the eye when at rest is shown to be adjusted for near vision, and accommodation for far vision is brought about by movement of the lens backwards. Beer ${ }^{4}$ has found that this is also true of the cephalopod eye, though the mechanism of retraction is different. The determination of the refraction was very difficult, but Beer satisfied himself by skiascopy that myopia existed at rest, varying from 2 to $10 \mathrm{D}$, being greatest in the octopods. On electrical stimulation the myopia decreased; no alteration in the curvature of the lens could be detected; and, by means of a needle in the lens, graphic tracings, showing retraction of the lens during stimulation, were obtained. The retraction was found to be due to the ring muscle first

'Arch. f. d. ges. Physiol., Bonn, 1898, Bd. lxix. S. 507.

2 Ibid., 1898, Bd. Ixxiii. S. 501.

+ 1bid., 1897, Bd. Ixvii. S. 541.

3 lbid., 1894, Bd. Iviii. S. 523.

VOL. II. -66 
described by Langer, in which the fibres run meridionally, and on contraction pull the lens backwards. Beer found that the increased pressure on the chamber behind the lens, due to the contraction of this muscle, produced bulging of the lax posterior pole of the eyeball, but that the recession of the retina so caused was too small to neutralise the recession of the lens. Both in fishes and cephalopods the iris was found to have no influence on the process of accommodation. There is no evidence of anything like a mechanism of accommodation in the compound form of eye.

\section{The PuPIL.}

Pupillometry.-In measuring the size of the pupil, it must be remembered that it is magnified by the refractive action of the cornea and aqueous humour. The methods ordinarily used have the disadvantage that they involve the near approach of an object to the eye, and consequent shading. This difficulty may be overcome for exact measurements by the use of a telescope with a scale (Schadow ${ }^{1}$ ), or by observing the image of the pupil reflected from a mirror. Schirmer ${ }^{2}$ used the image reflected from one side of a prism seen against a scale, which was reflected internally from another side of the prism which had been covered with mercury. Hess ${ }^{3}$ employs a method in which the virtual image of a scale appears in the plane of the pupil. The most exact method is with the ophthalmometer, on the same principle as described on p. 1030 (Fig. 374). Two other special methods are useful under certain circumstances. One first employed by Bcllarminoff ${ }^{4}$ is to photograph the pupil under different conditions, and for this the magnesium flashlight has been used by du Bois-Reymond ${ }^{5}$ and Haycraft. The other method first employed by A. Fick ${ }^{6}$ is to observe the entoptic inage of the pupil. A fine hole in a screen at a short distance from the eye will be seen as a diffusion circle, which varies in size with the size of the pupil. If two such holes are used, at such a distance from one another that the two diffusion circles just touch, the diameter of the pupil is approximately equal to the distance between the two holes. The exact relation is influenced by the distance of the holes from the eye, and to a considerable extent by the condition of refraction of the eye. The method is especially useful as a means of detecting slight changes in the size of the pupil; when the circles just touch, enlargement of the pupil will make them overlap, and contraction will make them separate from one another.

Changes in the pupil.-Change in the size of the pupil takes place under four chief conditions, namely (1) with change in the illumination; (2) with change in convergence and accommodation; (3) in response to sensory stimuli in different parts of the body; and (4) in connection with psychical changes, and especially emotional conditions. The pupil is also affected by various drugs and in various abnormal conditions of the nervous system.

The amount of contraction depends on the intensity of the light falling on the eye, and Haycraft ${ }^{7}$ has recently shown, using the photographic flash light method, that the relation is of the same kind as that

\footnotetext{
1 Arch. f. Ophth., 1882, Bd. xxviii. Abtl., 3, S. 183.

2 Ibiil., 1894, Bd. xl. Abth. 5, S. 8.

${ }^{3}$ Ber. ü. d. Versamml. d. ophth. Gesellsch., 1893, s. 235.

${ }^{4}$ Arch. f. d. ges. Physiol., Bonn, 1885, Bd. xxxvii. S. 107.

5 Arch. f. Physiol., Leipzig, 1888, S. 394.

${ }^{6}$ Arch. f. Ophth., 1856, Bd. ii. Abth. 2, S. 70.

${ }^{7}$ In a communication to the Physiological Society not yet published.
} 
existing between stimulus and sensation, namely, that the amount of contraction of the pupil is proportional to the logarithm of the intensity. Schirmer ${ }^{1}$ found the condition of adaptation of the eye to be of great importance, and that, in different conditions of adaptation of the two eyes, the same illumination may contract the pupil of one, and dilate that of the other eye. With maximal adaptation to the dark, Schirmer found that the pupil maintained the same diameter, usually between 3 and $4 \mathrm{~mm}$., within wide ranges of illumination (i.c. between 100 and 1100 mc.). ${ }^{2}$ Schirmer has proposed to call this the physiological pupilwidth, and Silberkuhl ${ }^{3}$ has employed it for measurements of the pupil under different conditions. The pupil is said to contract more when light falls on the central retina than on the periphery. This is probably true under ordinary conditions, but has not been investigated in dark adaptation, when a different result would probably be obtained (see p. 1083). 'The size of the retinal area stimulated has been found to be of influence, but there are no exact observations on this point also. The pupil has been said to become smaller and to contract less with advancing age. It is also said to be larger in myopic than in emmetropic and hypermetropic eyes, but Schadow ${ }^{4}$ and Silberkuhl ${ }^{5}$ have failed to find any appreciable difference. Silberkuhl found no difference in cases of unequal refraction.

Each pupil contracts not only when light falls directly upon it, but also when light falls on the pupil of the other eye. These reactions are known as the direct and consensual reactions respectively. ${ }^{6}$ It is said that the consensual reaction takes place somewhat later than the direct, but this point seems to be doubtful.

The contraction of the pupil with accommodation and convergence is an associated movement. Following E. H. Weber, it is often supposed that the contraction is especially associated with convergence, but this is not the case. The pupil contracts with accommodation without any change in convergence, as well as with convergence without accommodation. The latter may be shown by experiments with prismatic glasses, while the former is shown by an experiment due to Hering ${ }^{7}$ in which the pupil is observed by the entoptic method. Each eye looks through a fine hole in a screen, so that a binocularly combined image is seen; if a second hole is made in each screen, in one above and in the other below the original hole, three pupillary diffusion circles may be observed in a line, and are seen to change in size when the accommodation is changed without alteration of convergence. According to a recent paper, however, by Verwoort, ${ }^{8}$ this result is due to the imperfect exclusion of convergence; and this author believes that Weber was right in associating the reaction of the pupil with convergence (see p. 1131, for the relation between accommodation and convergence).

Mechanism of contraction.-The contraction of the pupil, due to the action of the sphincter muscle, depends, in birds and mammals, upon a reflex starting from the retina, and having its centre in the floor of the aqueduct of Sylvius. In fishes and amphibia, the pupil con-

${ }^{1}$ Arch. f. Ophth., 1894, Bd. xl. Abth. 5, S. 8.

$21 \mathrm{mc}$, is the illumination given by one candle at a distance of 1 metre.

${ }^{3}$ Arch. f. Ophth., 1896, Bd. xlii. Abth. 3, S. 179.

${ }^{4}$ Ibid., 1882, Bd. xxviii. Abth. 3, S. 183.

5 Loc. cit.

${ }^{6}$ Steinach (Arch. f. d. ges. Physiol., Bonn, 1890, Bd. xlvii. S. 289) has found that the consensual reaction does not occur in animals with total dccussation of the optic nerves in the chiasma.

7 "Lehre v. binoc. Sehen," 1868, S. 134.

${ }^{8}$ Arch. f. Ophth., 1899, Bd. xlix. S. 348. 
tracts to light in an extirpated eyeball. It has been disputed whether this is due to direct action on the iris or to an intra-ocular reflex. Steinach ${ }^{1}$ has shown that the reaction is greatly influenced by the state of the eye, and is very marked when the animal has previously been kept for some time in the dark. The iris of the extirpated eye will then contract to light, when the whole of the posterior pole of the eye, including the retina, has been removed by cauterisation. With local stimulation of the pupil by light, Steinach found that the contraction began at the part stinulated and slowly spread to other parts. The reaction, however, did not occur on stimulation of the peripheral part of the iris, and was limited to the part near the pupillary border. In the eel, salamander, and frog, Steinach found that the muscle fibres of the sphincter were pigmented, fine yellowish brown pigment granules appearing to lie between the muscle fibrillæ, and he ascribes the influence of light to its direct action on the pigment. Steinach investigated the influence of the different parts of the spectrum, and found that this corresponded with the absorption of the pigment, the maximum effect being in the green about $F$, while the red end of the spectrum as far as $C$ produced no contraction.

Mechanism of dilatation.-There has been till recently much uncertainty as to the cause of the dilatation of the pupil. It has been ascribed to the contraction of radial muscular fibres, to inhibition of the sphincter, and to vaso-constriction, acting either by emptying the iris of blood, or by producing longitudinal contraction of its radial arteries. Langley and Anderson ${ }^{2}$ have shown that there is a substance in the iris which contracts radially, but have left it an open question whether this substance is in the form of unstriped muscle fibres. They found that local stimulation of the sclerotic produced dislocation of the pupil, which could only be explained by radial contraction, and that local dilatation of the pupil might be observed to occur simultaneously with local contraction of the sphincter. They found that a strip of iris, isolated except at its ciliary attachment, shortened on stimulation of the cervical sympathetic to as much as half its length; and they showed that this shortening was not accompanied by any appreciable change in the blood vessels. Heese, ${ }^{3}$ about the same time, took tracings of the contraction of radial strips of the iris with the sphincter removed, and on stimulation of the cervical sympathetic obtained curves, corresponding, as regards form and latent period, with those of unstriped muscle fibres. The question still remains, whether this radial contraction is accompanied by simultaneous inhibition of the sphincter. Langley and Anderson failed to obtain any evidence of relaxation of the sphincter on stimulation of the sympathetic. Waymouth Reid ${ }^{4}$ has, however, found definite differences in the electrical variation of a demarcation current, when the electrodes are placed concentrically near the pupil and radially near the periphery of the iris. When the third nerve was stimulated, there was a negative variation in the concentric position and a positive variation in the radial position; when the sympathetic was stimulated, the concentric position gave a positive, the radial gave a negative, variation. Reid concludes that contraction of the sphincter is accompanied by inhibition of the radial contractile substance, and that contraction of the latter is accompanied by inhibition of the sphincter.

1 Arch. f. d. ges. Physiol., Bonn, 1892, Bd. lii. S. 495.

${ }^{2}$ Journ. Physiol., Cambridge and London, 1892, vol. xiii. p. 554. Refer to this paper for a full account of the literature of the subject.

3 Arch. f. d. ges. Physiol., Bonn, 1892, Bd. lii. S. 535.

4 Journ. Physiol., Cambridge and London, 1894-5, vol. xvii. p. 433. 


\section{THE RETINA AND VISUAL SENSATIONS.}

\section{Physiological Retinal Processes.}

Visual purple.-The most obvious change which occurs when light acts upon the retina is the bleaching of the visual purple or rhodopsin contained in the outer limbs of the rods. The existence of this substance was first described by Boll in 1876, and its properties have been most fully investigated by Kïhne. ${ }^{1}$ In the process of bleaching an intermediate substance, visual yellow is formed. Visual purple varies in colour to some extent in different animals, inclining more to violet in some, including man. The red colour in some cases is ascribed by Kühne to mixture with visual yellow. Green rods occur amongst the others in amphibia. The bleaching is limited to the part of the retina on which light falls, and produces an optogram, or image of an external object, both in the living and the recently killed animal. The eye should be kept from the light for ten minutes, then exposed for about three minutes in a room lighted by one window, so that an image of the window falls on the retina; the eyeball should then be extirpated by the light of a sodium flame, and the retina laid in a 4 per cent. solution of alum. The eye of a recently killed animal will then show a welldefined colourless optogram, while in an eye exposed during life it will be still pink and less sharply defined.

The bleaching of visual purple during life cannot be brought about in any other way than by the direct action of light. Exposure of one eye only to light does not lead to any change in the purple colour of the other eye, nor is any change produced by exposure of the body to light.

Regeneration of visual purple takes place in both the living and extirpated eye, when kept in the dark; it also occurs in red light. Boll ${ }^{2}$ supposed that red light acts as a stimulus to regeneration, but from experiments with optograms Kühne found that, with moderate intensities, regeneration takes place at the same rate in red light as in the dark, and with brighter red light more slowly. Regeneration seems to depend upon some substance derived from the pigment epithelium, to which Kühne has given the name rhodophyllin. If a piece of the retina be separated from the underlying pigment layer, bleached by light, and then replaced on the pigment, regeneration takes place, and even without replacement regeneration inay occur, probably owing to the particles of pigment retained between the rods. In some cases this takes place when no pigment seems to be present, especially in the lower half of a retina placed vertically, and Kühne supposes that in this auto-regeneration, which takes place very slowly, rhodophyllin is present in the rods. Regeneration is uninfluenced by light applied to the other eye, or to another part of the same eye. Both bleaching and regeneration are thus independent of the nervous system, and visual purple has been found to be present a year after division of the optic nerves. Regeneration is hastened by pilocarpin and muscarin, probable owing to increase of the secretory activity of the pigment epithelium.

Rhodophyllin is apparently, like visual purple itself, soluble in bile salts, for a solution of visual purple in bile salts is capable of regeneration after

\footnotetext{
1 Thersuch. a. d. physiol. Inst. d. Univ. Heidelberg, 1878-82.

2 Arch. f. Physiol., Leipzig, 1877, S. 4.
} 
bleaching, and the process may be repeated two or three times. Boll supposed that the regenerating substance is derived from the coloured globules of the epithelial cells, and described the globules after regeneration as more faintly coloured. But no definite relation has been found by other observers between the action of light and the condition of either the coloured globules or the myeloid granules of the pigment epithelium. Regeneration takes place when there is no pigment, as in the albino rabbit, and in those mammals (man, ox, pig) in which there are no fat globules in the epithelium.

The times necessary for bleaching and regeneration of visual purple have been determined. In the living frog ten minutes' exposure to strong sunlight is necessary, while regeneration requires one to two hours; in a warm-blooded animal bleaching is far more rapid,-- according to Kühne, sixty times more rapid than in the frog. Solution of visual purple from the frog and mammal, however, bleach in about the same time. The difference in the two animals may depend partly on the temperature, partly on the much greater length of the rods in the frog, and consequent greater thickness of the purple layer. The size of the pupil is also of influence. Kühne found by experiments on the rabbit with one pupil dilated by atropin, that the amount of bleaching differed greatly in the two eyes. The effect of temperature on the time of bleaching differed to some extent in solutions obtained from the frog and rabbit, and Kiihne supposes that there are different visual purples as there are different hæmoglobins.

In order to investigate the absorption spectrum of visual purple, the retina must be treated with a solution of bile salts. Owing to the fact that bile has

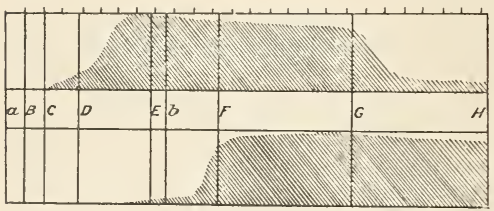

Fig. 379.-Kïhne. a powerful solvent action on red blood corpuscles, the solution of visual purple so obtained is mixed with hæmoglobin, especially in man and other mammals. ${ }^{1}$ Kühne $^{2}$ has recently described two methods for separating the hæmoglobin. In one, bile salts and visual purple are precipitated by an excess of magnesium sulphate, leaving the hæmoglobin in solution; in the other, a retina which has been hardened in alum is left for some hours in a 10 per cent. solution of sodium chloride, after which treatment with bile salts extracts visual purple only. No observations have yet been recorded on the spectrum of human visual purple dissolved with these precautions. The spectra of visual purple and visual yellow of the frog are shown in Fig. 379.

Köttgen and Abelsdorff ${ }^{3}$ have determined the absorption of visual purple in a large number of animals. They found that in mammals, birds (owl), and amphibia, the maximum absorption took place at $500 \lambda$, while in fishes the maximum was at $540 \lambda$. The curves representing the absorption in the two classes are shown in Fig. 380. Kühne's new method was not used, but the differences seem to be too great to be explained by the presence of hæmoglobin in one and not in the other. Köttgen and Abelsdorff failed to obtain any evidence of the existence of visual yellow in their investigations.

The influence of coloured light on visual purple is of great importance in relation to its possible function in colour vision. The red of the spectrum has least action, and, as already mentioned, in moderate intensity does

${ }^{1}$ In the frog a pure solution may be obtained, owing to the vessels in this animal being limited to the hyaloid (Kühne).

${ }^{2}$ Ztsihr. f. Biol., 1895, Bd. xxxii. S. 21.

${ }^{3}$ Zischr. f. Psychol. u. Physiol. d. Sinnesorg., Hamburg u. Leipzig, 1896, Bd. xii. S. 161. 
not hinder regeneration. When sufficiently intense, it acts, however, on visual purple, and will in time completely bleach the retina. That yellow light is also of very slight influence, is shown by the use of the sodium flame in experimental investigations on visual purple; its action, however, is greater than that of red light. Visual purple is most rapidly bleached by the part of the spectrum between $D$ and $E$, and from the maximum of action in this part of the spectrum there is a gradual diminution with diminishing wave-length. Kiihne investigated whether the slighter activity of the violet end was due to greater spreading out

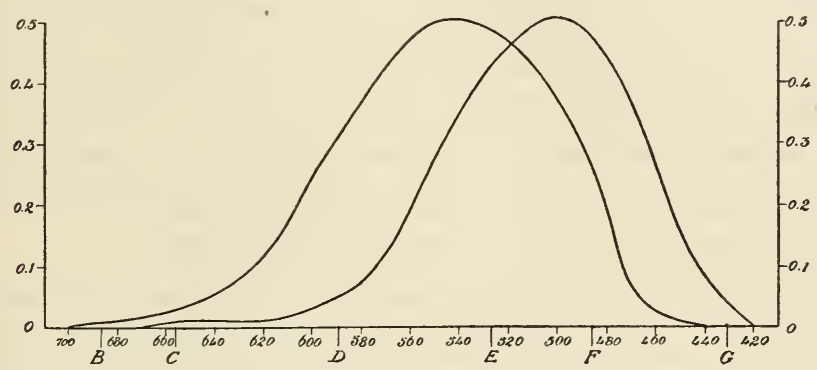

FIG. 380 .

of that end in the prismatic spectrum and consequent smaller intensity, but experiments with a grating spectrum also showed the greatest activity of the yellow-green rays. The degree of activity corresponds with the degree of absorption of monochromatic light by visual purple (Fig. 379). Visual yellow is acted on more strongly by the violet end in correspondence with its absorption spectrum, and consequently visual yellow is often absent as an intermediate product when visual purple is bleached by light of short wave-length. Fuchs and Kreidl ${ }^{1}$ have found that the Pöntgen rays have no influence on the visual purple of the frog, and Pergens $^{2}$ found that this was also the case after removal of the lens in Leuciscus rutilus. Gatti ${ }^{3}$ has found that the rays have no influence on the regeneration of visual purple. Fuchs and Kreidl found, on the other hand, that the yellow-green fluorescent light from the tube bleached visual purple very slowly.

The whitish-green fluorescence of the retina in ultra-violet light depends on the rods and on the bleached visual purple. The unbleached rod layer fluoresces very slightly and with a bluish tint; when completely colourless, the fluorescence is much stronger and greenish in appearance. Visual yellow, on the other hand, appears to be completely devoid of fluorescence. The cones and the rods about the ora serrata, which have no purple, do not fluoresce, and under ultra-violet rays the fovea appears as a dark spot. It is difficult to determine whether the solution of visual purple is fluorescent, since the bile in which it is dissolved fluoresces strongly (Ewald and Kiihne). ${ }^{4}$

The function of visual purple will be considered later in connection with the theories of light and colour vision. It will be sufficient to

\footnotetext{
1 Centralbl. f. Physiol., Leipzig u. Wien, 1896, Bd. x. S. 249.

${ }^{2}$ Klin. Monatsbl. $f$. Augenh., Stuttgart, 1897, Bd. xxxv. S. 354.

${ }^{3}$ Centralbl. $f$. Physiol., Leipzig u. Wien, 1897 , Bd. xi. S. 461.

4 Untersuch. a. d. physiol. Inst. d. Univ. Ileidelberg, Bd. i. S. 139.
} 
mention here the experiment of Kühne ${ }^{1}$ on the vision of frogs whose visual purple had been completely bleached. He found that they could evidently see, were able to catch flies in bright sunlight, and behaved very differently from blind frogs. He found that the great majority of normal frogs put themselves under green in preference to blue glass, when differences of temperature and intensity were excluded. Bleached frogs also preferred green glass, while blind frogs were found indiscriminately under each.

Other changes in rods. - In addition to the changes in colour, the outer limbs of the rods have been observed to change in size under the action of light. In frogs kept in the dark, there are distinct spaces between the rods; the distances, according to v. Hornbostel, ${ }^{2}$ varying from 5 to $8 \mu$; in frogs exposed to the light, the rods are separated only by narrow lines. The swelling, however, is only perceptible after nearly one hour's exposure. No increase in diameter occurs in red light.

Angelueci ${ }^{3}$ and Gradenigo ${ }^{4}$ describe contraction of the inner limbs of the rods under the action of light, so that they become shorter and thicker. In the dove, under the other hand, Stort ${ }^{5}$ described the inner limb as longer and thinner in the retina exposed to light.

The pigment epithelium.-The changes produced in the pigment epithelium by the action of light were first studied by Kühne, Boll, and Angelucei. In the eye of a frog which has been kept in the dark, the pigment layer separates easily from the rod and cone layer, and the brown pigment is found heaped up in the body of the epithelial cell and round the outer extremities of the rods, not reaching beyond the outer third of the length of the rod. In an eye which has been illuminated, the epithelium and rods are separated with difficulty; the body of the epithelial cell contains much less pigment, so that the nucleus is elearly seen; and the pigment is much more abundant between the outer limbs of the rods, and extends also between the inner limbs as far as the membrana limitans externa. In birds and mammals the pigment layer naturally separates more easily than in the frog, but in them the pigment shows the same change of distribution under the influence of light and dark. In the frog, about ten minutes of illumination are needed to bring the pigment to the membrana limitans externa, while one and a half to two hours are necessary for return to the dark position; these times correspond very closely with those necessary for bleaching and regeneration of visual purple respectively in the same animal, and point to the close connection of the two processes. The pigment, however, differs from visual purple in its behaviour to coloured light, the movement forwards taking place actively in red light. According to Angelucei, ${ }^{6}$ the violet end of the spectrum is that which acts with the greatest intensity, and there is, according to him, a gradual increase in the movement forwards with decreasing wave-length of the light used. According to A. E. Fick, ${ }^{7}$ the forward movement of the pigment, once set up by a short exposure to light, goes on in the dark. He found that frogs from the dark exposed to the light for one minute, and then kept in the dark for twenty minutes, showed forward position

\footnotetext{
1 Untersuch. a. d. physiol. Iust. d. Univ. Heidelberg, Bd. i. S. 119.
2 Ibid., Bd. i. S. 409.

${ }^{3}$ Untersuch. z. Notuil. d. Menseh. u. d. Thiere, 1892, Bd. xiv. S. 237.

4 Quoted from Angelueci.

5. Areh. f. Ophth., 1888, Bd. xxxiii. Abth. 3, S. 275.

${ }^{6}$ I'ntersuch. z. Naturl. d. Menseh. u. d. Thiere, 1892, Bd. xiv. S. 252.

${ }^{7}$ Arch. f. Ophth., 1891, Bd. xxxvii. Abth. 2, S. 1.
} 
of pigment, while in those killed immediately after the minute's exposure there was little change from the dark position. The forward position was found in frogs when respiration had been interfered with, either by keeping them in closed vessels or under water, or by mixing $\mathrm{CO}^{2}$ with the air breathed. In nearly all cases the lower half of the retina showed a more forward position of the pigment than the upper half, and Fick believes that this is due not only to the fact that the lower part of the retina is usually more exposed to light, but also to a greater tendency to forward movement.

The influence of the nervous system on the movement of the pigment will be considered in connection with the movements of the cones.

The cones.-The movement of the cones under the action of light was observed later by Engelmann and van Genderen Stort. ${ }^{1}$ While investigating the recession of the pigment in frogs in the dark, it was noticed that the cones were situated between the outer limbs of the rods, and not close to the external limiting membrane, as had been previously described. They were found to occupy the latter position in frogs exposed to the light. The change was found to be constant and to depend on contraction and relaxation of the inner part of the inner limb of the cone, which Engelmann named the cone-myoid. In the dark position, the ellipsoidal body of the cone is situated between the outer limbs as far back as the retracted pigment, and connected with the nucleus of the cone in the external nuclear layer by a narrow process, - the elongated cone-myoid. In the light position, the body of the internal limb was directly continuous with the nuclear portion. The degree of lengthening observed was greater the more speedy the process of hardening for examination, the cone-myoid having a tendency to contract on the action of the hardening reagent. The greatest difference in length was observed in frogs and fishes; it was less marked, though distinctly present, in birds and mammals. The contraction with light took place rapidly, the maximal contraction occurring in a few minutes; extension in the dark was more gradual. In the twin cones observed in the frog's retina, only one of the two showed differences of length. The degree of action of the different parts of the spectrum was not very fully investigated, but the violet end appears to be the most active. The influence of the coloured globules found in the outer ends of the inner limbs of the cones of birds and some reptiles was investigated. The degree of contraction did not seem to depend on the globules, for in the tortoise, with intensely coloured globules, very little, if any, change in length was observed. It was found that cones with red globules were affected most by green light, but, on the other hand, in the red area of the dove's retina, the cones with small red globules scattered through the whole length of the internal limb, shortened less actively than others in green light. The cones with orange, yellow, or green globules showed in general no distinctive action.

The Röntgen rays have no influence on the movements of cones or of pigment, both being found in the dark position when the retina has been exposed in a dark box to their influence (Pergens, Gatti).

Influence of nervous system on movements.-It has already been stated that the bleaching of visual purple is confined to the eye that is acted on by light. This is not the case with the movement

${ }^{1}$ Areh. f. d. ges. Physiol., Bonn, 1885, Bd. xxxv. S. 498; and Arch. f. Ophth., 1888, Bd. xxxiii. Abth. 3, S. 229. 
of the pigment and cones. Engelmann ${ }^{1}$ found that if only one eye of a frog was exposed to light, both eyes showed the forward position both of pigment and cones. After removal of the brain, the changes were limited to the eye acted on. Engelmann concluded that there were centrifugal "retino-motor" fibres in the optic nerve. The same position as that due to light was also brought about by means of strychnin, and by the application of tetanising currents to the eye. Engelmann also found that the same forward movement occurred on stimulation of the skin. A frog with the anterior half of the body protected from light, and the posterior half exposed, showed the light position, especially of the pigment. A. E. Fick ${ }^{2}$ objected that variations in the position of pigment occurred in dark frogs; that results similar to those of Engelmann occurred after section of the optic nerve; and that the experiment showing the influence of skin stimulation was vitiated by the interference with respiration caused by the covering of the anterior half of the animal. Fick only observed the position of the pigment. Nahmmacher ${ }^{3}$ observed the position of the cones in a large number of frogs. He used chemical stimulation of various parts with and without previous section of the optic nerve. After stimulation of the optic chiasma, he found forward position in 82.3 per cent. of the cases in which the optic nerve had not been divided, and only 18.4 per cent. in those with divided nerve. Further evidence in favour of centrifugal influence was derived by Engelmann from the electrical behaviour of the two eyes, and the weight of evidence seems to be distinctly in favour of the existence of "retino-motor" fibres in the optic nerve.

There seems now to be definite anatomical evidence of the existence of such centrifugal fibres, which pass from the nerve fibre layer directly into the inner reticular layer, and then either terminate in free endings near the border of the inner nuclear layer (Ramón y Cayal), or join the cells in that layer (Dogiel).

Microchemistry.-Certain other differences between a retina which has, and one which has not, been exposed to light have been noted. The reaction of the former is said to be acid, that of the latter alkaline. Birnbacher ${ }^{4}$ found the illuminated retina stained yellow with eosin, while the dark retina was coloured an intense pink. With Biondi-stain the cones of the former were coloured green, those of the latter yellow. Dor ${ }^{5}$ has tried the effect of coloured light; with red light he found a difference in the staining of rods and cones; while with blue light there was no such difference. The rod and cone nuclei have been described as differing greatly in the illuminated and non-illuminated retina, being round and deficient in chromatin in the former, elongated and with abundant chromatin in the latter case (Mann, ${ }^{6}$ Pergens ${ }^{7}$ ).

Retinal currents.-The electrical condition of the retina, and its modifications on the action of light, have been much investigated, but their exact nature is still undetermined. When the optic nerve is connected directly with the retina, a current of rest is indicated; if connection is made with the outer side of the retina, i.e. with the rods, the nerve is positive, the retina negative; if, on the other hand, the

I Arch.f. d. ges. Physiol., Bonn, 1885, Bd. xxxv. S. 498.

2 Vrtljschr. d. naturf. Gesellsch. in Zürich, 1890, S. 83; and Arch. f. Ophth., Bd. xxxvii. Abth. 2 , S. 1.

3 Arch.f. d. ges. Physiol., Bonn, 1893, Bd. liii. S. 375.

4 Arch. f. Ophth., 1894, Bd. xl. Abth. 5, S. 1.

5 Ann. d'ocul., 1896, tome exv. p. 443.

${ }^{6}$ Journ. Anat. and Physiol., London, 1895, vol. xxix. p. 105.

7 Ann. Soc. roy. d. sc. mél. et nat. de Bruxelles, 1896, tome v. p. 389. 
inner nerve fibre side of the retina is connected, the retina is positive, the nerve negative. If the inner and outer side of the retina are connected, the former is positive, the latter negative.

If the optic nerve is connected with the eyeball, the direction of the current varies with the part of the eyeball; if with the cornea, the nerve is negative; if with the sclerotic, the nerve is positive. This is probably due to the fact that when connection is made with the sclerotic, the effect is the same as if it were connected with the rods; while in the case of the cornea, conduction takes place through the ocular media to the inner surface of the retina.

When light falls on the retina, this current undergoes a more or less complicated variation. When the isolated retina is employed, the changes are as shown in Fig. 381 ; on the action of light, a positive succeeded by a negative variation; on cutting off light, a positive variation. The relative amount of the variations depends on the condition of the retina; in some cases the negative variation is considerable, while the preceding positive phase is very slightly marked; in other cases the negative phase is not sufficient to bring the current back to the condi-
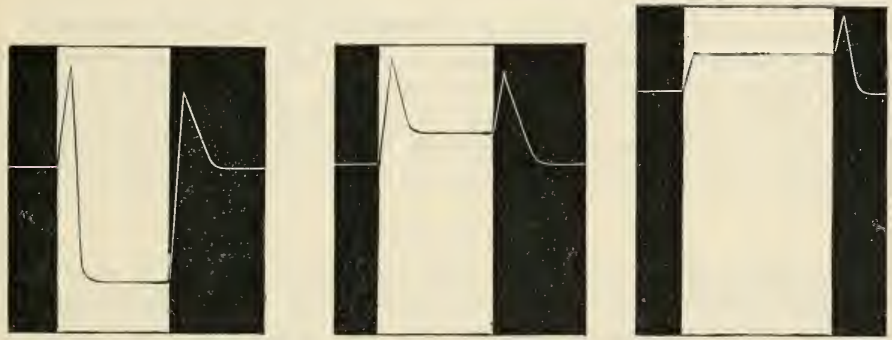

Fıg. 381.-Steinerand Kühne. Fig. 382.-Steinerand Kühne. Fıg. 383.-Steinerand Kuhne.

tion at the time of the stimulus (Fig. 382). According to Kiihne and Steiner, ${ }^{1}$ this occurs when the retina is especially fresh, and they regarded it as probably the nearest approach to the normal condition; and this seems more probable from the fact that when the whole eyeball is used the negative phase is entirely absent (Fig. 383); and this phase is probably due to injury in removal of the retina. The retina is extremely sensitive to light, and shows a change with light of an intensity only just sufficient to produce sensation in the living eye. The duration of the light stimulus is of influence on the variation; the three changes described also occur with instantaneous illumination, and according to Fuchs ${ }^{2}$ the positive phase is much shorter with instantaneous than with longer illumination.

Holmgren ${ }^{3}$ found that the retinal current did not depend on visual purple, and that the changes occurred in bleached eyes; but, according to Kuihne and Steiner, ${ }^{4}$ the reaction is more intense when visual purple is present, while the bleached eye showed a marked negative phase, and sometimes even absence of the positive phase. These changes may, however, have been due to fatigue changes other than those of visual

1 Untersuch. a. d. physiol. Inst. d. Univ. Heidelberg, Bd. iv. S. 72.

2 Arch. f. d. ges. Physiol., Boun, 1894, Bd. lvi. S. 408.

3 Untersuch. a. d. physiol. Inst. d. Univ. Heidelberg, Bd. ii. S. 81.

${ }^{4}$ Ibid., Bd. iii. S. 354 . 
purple. The times taken by the various phases have been studied by Fuchs; he found a latent period of .0004 to .0061 sec.; a duration of the positive phase varying from 007 to $0181 \mathrm{sec}$; the time to the end of the negative phase varied from $\cdot 0089$ to $0352 \mathrm{sec}$.

Engelmann and v. Grijns ${ }^{1}$ found that light applied to one eye of a frog caused, after a short latent period, a marked variation of the current in the other eye, the change being an increase in the negativity of the equator in relation to the cornea. The same result is obtained with chemical stimulation of one eye. Conduction by the glandular skin of the head was excluded. The influence of other movements was also excluded by the use of curari, which was found not to prevent the movement of cones and pigment.

The effect of coloured light on the electrical variation is a point of very great importance which has not yet been satisfactorily investigated. Dewar and $\mathbf{M}$ 'Kendrick ${ }^{2}$ found that yellow light produced the largest variation, green next, and then red and blue. These and similar results obtained by other observers are probably dependent on the different intensities of the coloured lights. All colours appear to give a variation in the same direction.

Dewar and $\mathrm{M}^{\prime}$ Kendrick ${ }^{3}$ found that the relation between luminosity of the stimulus and electrical variation was approximately the same as that existing between stimulus and sensation. When the luminosity increased from one to a hundred units, the variation was multiplied from three to six times. Waller ${ }^{4}$ has investigated the relation more thoroughly, and has found a logarithmic relation between stimulus and variation.

\section{Visual Sensations.}

Introductory.-The facts of light and colour vision will now be considered, avoiding, as far as possible, reference to the physiological structures and processes concerned, and only those general and theoretical questions will be discussed which do not involve any special theory of vision. After the facts have been described, an account will be given of the known relations between visual sensations and retiual elements, and of the chief physiological theories of vision.

Visual sensations may be divided into three groups, namely, sensations of light, of colour, and of form. The perception of form is closely associated with other kinds of spatial perception, in which binocular vision and movements of the eyes are the chief physiological factors, and will be more fully dealt with in the last part of this article. In this part it will only be considered in so far as it seems to be a function of the retina without movement, as in the discrimination of two points. The sensibility for light or luminosity is very closely associated with that for colour, but the two will be considered separately as far as possible.

The character of a sensation which follows stimulation of the retina always depends on two factors, - the nature of the stimulus, and the condition of the part of the retina stimulated. Each of these factors is complex, and consequently the character of the sensation may be influenced by many circumstances, including the nature of previous stimulation of the retina; the size and position of the area stimulated; the duration of the stimulus, and the rate at which individual stimuli

1 “Beitr. z. Psych. u. Phys. Sinn.," 1891, S. 197.

2 Trans. Roy. Soe. Edin., 1876, vol. xxvii. p. 141.

3 lbid., p. 156.

${ }^{+}$Brain, London, 1895, vol. x viii. p. 200 ; also more recently in Proc. Roy. Soc. London, 1900. 
succeed one another; the nature of the stimulation of other parts of the same retina, of the other retina, and even of other sense organs. The influence of abnormal conditions of the retina ${ }^{1}$ forms an important division of the subject. In addition to visual sensations arising in the usual way from stimulation by light, those due to mechanical or electrical stimulation of the eyeball have also to be considered.

Sensations of light are capable of being arranged in a continuous series, passing from the deepest black or dark to the brightest white or light; the sensations are said to differ from one another in brightness or luminosity.

Sensations of colour, on the other hand, differ from each other in three ways, - in colour-tone or hue, in brightness or shade, and in saturation or tint.

By degree of saturation is meant the extent to which a sensation of colour is fused with one belonging to the black-white series. Spectral colours have a high degree of purity or saturation, but it is possible to experience sensations of more than spectral saturation (see p. 1059).

The three characteristics of colour sensations correspond to three differences in the physical nature of the light stimulating the eye, namely, wave-length, intensity, and complexity. The correspondence, however, is not exact, and it is important to use the three physical terms only when speaking of the stimulus, and to reserve the terms, colour-tone, brightness, and saturation or purity for the sensations. It is often useful to follow the example of painters, and to speak of the colours depending on long wave-length as warm, and of those of short wave-length as cold colours.

Colour-tone, brightness, and saturation do not vary independently of one another. Brücke ${ }^{2}$ found that addition of white light to a coloured light alters its colour-tone, changing it towards red, and ascribed this to a preponderance of long-wave rays in white light, which is not noticed under ordinary conditions, but becomes noticeable when the light is added to another colour. Rood, ${ }^{3}$ on the other hand, found that the only colour which remained unchanged was a greenish yellow; those on the long-wave side become more red on diminution of saturation, while those on the short-wave side deviated towards violet. He notes that all the changes were such as would be produced by the addition of violet. Alteration of brightness affects colour-tone and saturation. This, however, is closely connected with adaptation of the eye, and must be considered later.

Quantitative standards.-In order to compare the sensibility of one part of the retina with another, or of the same part under different conditions, certain methods of exact estimation have been employed. One of these is to determine the mimimal stimulus capable of being perceived, the threshold of sensibility. In the use of colourless light there is only one such threshold; coloured light at low intensitics appears colourless (see p. 1079), and consequently one has here to distinguish between the point at which the coloured light is seen as light, the absolute threshold, and the point at which its colour can be recognised, the specific or chromatic threshold. The interval between

${ }^{1}$ For the sake of brevity, the word "retina" is here and elsewhere frequently used instead of "cerebro-retinal apparatus." Since we are almost wholly ignorant in what part of the sensory system the changes determining the speeific nature of a sensation take plaee, the latter term would be more aeeurate.

${ }^{2}$ Sitzungsb. d. k. Akad. d. Wissensch., Wien, 1865, Bd. li. Abth. 2, S. 461.

${ }^{3}$ Am. Journ. Sc., New Haven, 1880, vol. xx. p. 81 . 
the two thresholds is sometimes called the photo-chromatic interval, and differs in extent for different colours, being absent, or almost absent, in the case of red, and greatest for green, light. Another method of comparing sensibility is to find the amount by which a stimulus must be increased or decreased, in order to give a sensation which is perceived as different. The just perceptible difference or threshold of discrimination is a unit which is very commonly used in the measurement of sensibility. In the case of colourless light, this threshold is again only of one kind; for coloured light the threshold of discrimination may be determined for differences of colour-tone, brightness, and saturation.

It is also necessary to have some standard by means of which to compare different colours in respect of brightness. This is a matter of considerable difficulty, and the methods which have been devised depend on certain peculiarities of vision ; the subject of hetero-chromatic photometry must be postponed till these have been considered.

A method of comparing different colours in respect of saturation, which is due to Hering, may be mentioned now, as it is capable of being employed without reference to the special theory in connection with which it is used by Hering. Under certain conditions a coloured light may appear colourless, and the white or grey with which a coloured light is matched under these conditions is known as its white "value" (Valenz). For the present the white value of a given light may be defined as the light with which it would be matched when seen with the dark-adapted eye at a low intensity (see p. 1078).

The number of colour-tones which can be discriminated in the spectrum is about 160 , and others have to be added for the purple series. It has been found that the eye is most sensitive to change of colour-tone at two places, about the lines $D$ and $F$, and is less sensitive between and on either side of these points. According to König, ${ }^{1}$ the just perceptible difference at $640 \lambda$ is $1 \cdot 28 \lambda$; at $590 \lambda, \cdot 26 \lambda$; at $540 \lambda$, $.68 \lambda$; at $490 \lambda, \cdot 16 \lambda$; and at $430 \lambda$ it is $1.06 \lambda$. Uhthoff ${ }^{2}$ obtained similar results, while others have found the degree of sensitiveness at $D$ as great as or greater than at $F$.

The number of degrees of brightness and saturation of a given colourtone, which can be discriminated, has not been satisfactorily determined. Taking more or less rough estimates, v. Kries ${ }^{3}$ has calculated that the total number of different light and colour sensations which we are capable of experiencing would amount to about 500,000.

Limits of the spectrum.-It has been much disputed whether the limitation of the visible spectrum is due to insensitiveness of the retina to infra-red and ultra-violet rays, or whether the invisibility is due to absorption before reaching the retina. As regards the red end, this question seems to have been decided by Aschkinass, ${ }^{4}$ who has investigated the absorption of the ocular media by means of a spectrobolometer, and found that the absorption at the visible limit only amounts to 5 per cent., and that complete absorption does not occur till $1400 \lambda$. At the other end the limitation seems to be to a considerable extent due to absorption; the limits of different individuals vary here much more than at the red end, and are probably due to differences of pigmentation of the yellow spot and of the lens. The spectrum

1 Arch.f. Ophth., 1884, Bd. xxx. Abth. 2, S. 178.

2 Arch. f. Physiol., Leipzig, 1889, S. 171.

3 Ibid., 1882, Suppl., S. 58.

${ }^{4}$ Ztschr. f. Psychol. u. Physiol. d. Sinnesorg., Hamburg u. Leipzig, 1896, Bd. xi. S. 44. 
shortens at this end with increasing age, apparently owing to increased pigmentation.

According to Helmholtz, ${ }^{1}$ the ultra-violet spectrum as far as the line $R$, is visible under normal conditions, being indigo blue with slight and bluish grey with greater intensity. Its visibility is said to be increased by removal of the lens, ${ }^{2}$ and it is said that this part of the spectrum then appears nearly or quite colourless. In cases in which the lens has been removed, it is also said that Röntgen rays give rise to a visual sensation, but the evidence for this is unsatisfactory. The invisibility of the infra-red rays is probably due to insensitiveness of the retina, while the ultra-violet rays fail to be seen, partly, at any rate, owing to absorption by the ocular media.

The exact limits of the visible spectrum show a certain amount of variation in individuals with normal vision, and greater variations in abnormal vision.

Visual acuity.-This is usually defined by the smallest visual angle which the line between two points must subtend, in order that the points may be distinguished as two. It has also been defined by the smallest angle at which the eye can recognise the form of an object. In practice it is most commonly tested by the power of distinguishing letters, the visual acuity being expressed by the formula $\mathrm{V}=\frac{d}{D}$, where $D$ is the distance at which the letters employed subtend an angle of five minutes, and $d$ is the greatest distance at which these letters can be recognised, thus $V=\frac{5}{5}$ means that letters which subtend an angle of five minutes at 5 metres can be read at 5 metres. Such a method, though generally in use, is not satisfactory physiologically, for the perception of the form of relatively complicated objects like letters involves other than retinal factors, and Guillery ${ }^{3}$ has proposed as the test of visual acuity the smallest extent of a black point on a white ground which can be perceived at a given distance, and finds that this method gives satisfactory results. Such a point has been called the "physiological point" (Aubert). The subject of visual acuity is one of great practical importance, for it has become customary to use it much more largely as a test of the condition of the retina than either the light or colour sense.

The relation between visual acuity and intensity of illumination has been much investigated. The acuity increases with increased brightness, and this fact has been made the basis of a method of colour photometry (p. 1077). According to Posch, ${ }^{4}$ visual acuity increases rather more quickly than the logarithm of the intensity of illumination, and König ${ }^{5}$ has recently found a similar logarithmic relation. König also finds that at very low luminosities the relation between acuity and intensity differs from that at ordinary luminosities.

Extension and sensibility.-There is a definite relation between the extent of the retina stimulated and the brightness of the resulting sensation. Förster ${ }^{6}$ was the first to point out that brightness and visual angle supplement each other; that the smaller the one the greater must the other be in order that perception shall take place. According to Charpentier, this relation only holds for areas below a

\footnotetext{
1 "Handbuch d. physiol. Optik," 2te Aufl., S. 279.

2 Compt. rend. Acad. d. sc., Paris, 1883, tome xevi. p. 509.

${ }^{3}$ Arch.f. Augenh., Wiesbaden, 1891, Bd. xxiii. S. $323 .{ }^{4}$ Ibid., 1876, Bd. v. S. 14.

${ }^{5}$ Sitzungsb. d. k. Akad. d. Wissensch., Berlin, 1897, S. 559.

6 "Ueber Hemeralopie u. die Anwendung eines Photometers," Breslau, 1857.

7 "La lumière et les couleurs," Paris, 1888, p. 138.
} 
certain limit, corresponding approximately to the dimensions of the fovea. Not only is the threshold of stimulation thus influenced by the size of the retinal area, but also the threshold of discrimination for light and colour. Donders ${ }^{1}$ proposed to use the visual angle as the readiest means of measuring the colour sense, and Guillery has recently applied this method in the investigation of the sensitiveness of different parts of the retina (p. 1086). Within certain limits it seems as if a definite amount of light stimulating the retina produces the same sensory effect, whether it is spread over many or concentrated on a few retinal elements. ${ }^{2}$

It has been found that when coloured light stimulates a very small area of the retina, it may appear colourless, i.e. there is a a photochromatic interval for stimulation of small extent. This was investigated especially by Aubert, ${ }^{3}$ who found the appearance of colour depended greatly on the nature of the background,-yellow appearing very early on a dark, very late on a bright, background, while green appeared equally soon on both. The disappearance of the colour of small areas depends partly on the intensity of the colour, and has been made the basis of a method of colour photometry. The question is, however, complicated by the difference between vision with the fovea and with the surrounding part of the retina.

If a small field of coloured light is reduced in size till it appears colourless, it has been found that its colour may be restored, if other equally small areas are simultaneously illuminated with the same light. ${ }^{4}$ It seems as if a subliminal excitation in one retinal area were capable of being reinforced by similar excitations in other parts of the retina (see p. 1066).

\section{ADAPTATION AND INDUCTION.}

Adaptation.-The two chief factors on which the excitability of a given area of the retina depends, are the nature of the preceding stimulation and the condition of the surrounding retina.

The condition of excitability of the retina, depending upon the nature of the stimulation, is called adaptation. The visual apparatus has the power of adjusting itself to differences of illumination, just as it has to differences of distance. The adjustment to differences of illumination depends on two factors, - the size of the pupil, and the condition of excitability of the retina. The relation between the condition of the pupil and retinal adaptation is not yet quite clear. Schirmer ${ }^{5}$ has shown that the two are connected, the size of the pupil depending on the illumination to which the eye has previously been exposed, as well as on the existing illumination (see p. 1043).

Retinal adaptation was first studied by Aubert, ${ }^{6}$ who investigated the extent to which the sensitiveness to minimal stimulation was affected by complete exclusion of light from the eye. He found that the increase was rapid at first, the sensitiveness being multiplied fifteen to twenty times in the first two minutes. The increase took place more slowly later, and the sensitiveness had almost reached its maximum in abont

1 Arch. f. Ophth., 1877, Bd. xxiii. Abth. 4, S. 282.

2 Abney has recently (Proc. Roy. Soc. London, 1897, vol. lxi. p. 330) found a definite relation between the threshold and the diameter of the retinal area stimulated, the smallest diameter, and not the area, being the determining factor.

3 "Physiol. d. Netzhaut," Breslau, 1865, S. 108.

${ }^{4}$ See A. E. Fick, Arch.f. d. ges. Physiol., Bonn, 1888, Bd. xliii. S. 445.

5 Arch. f. Ophth., 1894, Bd. xl. Abtl. 5, S. 8.

6 "Physiol. d. Netzhaut," Breslau, 1865, S. 25. 
twenty minutes, but continued to increase slightly for two hours, when it was thirty-five times greater than at first. Charpentier ${ }^{1}$ found the rapidity of the increase corresponded to that described by Aubert, but described the amount of increase as much greater, the eye completely adapted to darkness being able to see light one thousand (in one case two thousand five hundred) times less intense than after exposure to bright light. In adapting the eye to various illuminations, he found that the sensitiveness increased much more rapidly as complete darkness was approached. According to Charpentier, the increase of sensitiveness is wholly due to the retinal condition, and is unaffected by differences in the size of the pupil. Adaptation to bright illumination after being in the dark, takes place much more rapidly than adaptation to darkness.

One effect of dark adaptation is to increase the vividness of the intrinsic light of the retina, and this increase may introduce difficulties in ascertaining the threshold of sensibility for light. The two effects do not necessarily increase pari passu, and Aubert thought it probable that they were independent of one another, the intrinsic light being of central origin, while the increase of sensitiveness is due to the condition of the retina (p. 1096).

The retina is not only capable of adaptation to difference of luminosity, but also to differences of colour. The light which enters the eye is always more or less coloured. If coloured glasses are worn, the retina becomes adapted to the colour, and objects after a time again appear colourless. When the glasses are removed, objects appear coloured in the colour complementary to that of the glasses, and it is not till the eye has become again adapted to white light that objects appear colourless. Brïcke ${ }^{2}$ has shown that in the normal condition our eyes are always more or less adapted to red, owing to the light which passes through the iris and sclerotic, the effect being especially great with lateral illumination. It is then much more noticeable, because different in the two eyes. A mixture of red and green which is yellow in daylight, will appear reddish to the eye adapted to the dark. This source of error may be minimised by wearing a black, funnelshaped shade to keep off lateral light when experiments in colour vision are carried out in a lighted room.

After-images. - When the adaptation is local, the appearances which occur are known as after-images, and may be seen in the absence of any objective light stimulus. When a limited surface is accurately fixed, it may appear to become brighter or darker according to circumstances; if coloured, it may appear to change in colour-tone, brightness, or saturation. If after a certain period of fixation the eyes are closed or directed to a grey surface, larger than the fixed surface, a part of the visual field will be seen, corresponding in form to the latter. This after-image may be of the same brightness as the original, or the reverse, and is known as positive or negative, in the same sense as in photography. If coloured, it may be of the same colour as the original, or it may be complementary. The term negative is often applied to the latter, but it is better to limit that term to denote brightness, and to speak of coloured images as homochromatic or complementary. The after-image of a colourless surface may be coloured, usually in some variety of green, and that of a coloured surface may not be exactly comple-

1 "La lumière et les couleurs," Paris, 1888, p. 150.

2 Ann. d. Phys. u. Chem., Leipzig, 1851, Bd. Ixxxiv. S. 418.

VOL. II. -67 
mentary to the original. This anomalous coloration is usually due to the abnormal nature of the white light used, or to the influence of the light passing through the sclerotic and iris. After-images are very delicate means of observing slight differences of colour, partly owing to their relatively low degree of brightness, which permits slight differences of colour to be discriminated, partly to the absence of differences of marking and texture, which in many surfaces mask slight differences of colour. It is usually possible to get rid of anomalous colour by excluding lateral light. In some cases, however, it is possible that unusual coloration may be due to an abnormal condition of the retina (Hilbert). ${ }^{1}$

If a fixed surface is gradually replaced by a different surface, the change which has been taking place during fixation may gradually pass into the after-image. If the change is sudden, there is in the ordinary method of observation an interval between the disappearance of the surface and the appearance of the negative or complementary image. This interval is partly filled up by phenomena to be described later, requiring special means of observation. If the eyes are kept steadily fixed, the afterimage may be observed to undergo periodical variations. The negative (or complementary) image disappears and reappears, the intervals being sometimes observed to be occupied by a positive (or homochromatic)image. Plateau, ${ }^{2}$ who first described the appearance, observed nine variations, and a still larger number may be seen. The earlier variations occur at intervals of about three to four seconds, the later variations more rapidly, and the latter resemble in many respects the variations of minimal sensations which have been usually aseribed to variations of attention. These periodical variations of after-images were referred by Helmholtz ${ }^{3}$ to movements of the eyes. They do not, however, accompany the ordinary blinking movements of the eyelids, and the regularity of their occurrence is against this supposition. After-images are, however, influenced by movements of the eyes. Any sudden movement, or sudden change of accommodation, will make an after-image disappear; but during gradual changes after-images may be kept steadily in view.

The shortest time of exposure necessary to produce an after-image effect is very small. A coloured patch on a rotating dise will give rise, at certain rates, and with bright illumination, to a ring in the complementary colour of the patch. The after-image effect is strong enough to more than neutralise the effect of the original excitation, and shows that the complementary image occurs with exposure of a small fraction of a second.

According to Exner, ${ }^{4}$ there are three parts of the spectrum which are much less altered in appearance by fatigue than other parts, namely, red from the end of the spectrum to between the lines $C$ and $D$, green between $E$ and $b$, and blue about $G$. On the other hand, v. Kries ${ }^{5}$ found yellow, green, and blue least changed in colour-tone, red and yellow-green changing their hue in the direction of yellow, blue-green and violet in the direction of blue. Hess ${ }^{6}$ found that the appearance of a spectral light after fatigue corresponded in general to that of a mixture of this light with the complementary of the

${ }^{1}$ Ztschr. f. Psychol. u. Physiol. d. Sinnesorg., Hamburg u. Leipzig, 1893, Bd. iv. S. 74.

2 Ann. d. Phys. u. Chem., Leipzig, 1834, Bd. xxxii. S. 543.

3 "Handbuch d. physiol. Optik," 1867 , S. 364.

4 Arch.f. d. ges. Physiol., Bonn, 1868, Bd. i. S. 375.

5 Arch.f. Physiol., Leipzig, 1882, Suppl., S. 113.

${ }^{6}$ Arch. f. Ophth., 1890, Bd. xxxvi. Abth. 1, S. 1. 
fatiguing light. If the eye is exposed to a coloured light, and then exposed to its complementary light, the latter appears more saturated.

Burch 1 has recently found that when light of great intensity is used, characteristic fatigue effects are produced with four parts of the spectrum, namely, red from $A$ to $B$; green in the neighbourhood of $E$; blue about halfway between $F$ and $G$; and violet at and beyond $H$. In each case the eye is found to be blind to the fatiguing colour; thus after fatigue to green, the red of the spectrum is seen to pass directly into the blue. Fatigue to yellow caused disappearance of both red and green, so that only blue and violet were left. After fatigue by three of the four parts of the spectrum only one colour was seen. The red so obtained extended from $A$ to a little beyond $b$; the green from $C$ nearly as far as $G$.

Persistent positive image.-Under certain conditions, a positive after-image is seen, which persists for a considerable time. It differs from the ordinary after-image in not undergoing the definite periodical variations of the latter, although similar irregular variations may be observed as it becomes faint. Such a positive image is seen with the dark-adapted eye, and may be very well seen soon after waking. It occurs with very short stimulation.

The most marked positive image is that which occurs after very intense stimulation. It is bright and well-defined when the eyes are closed, but appears as a dark cloud when seen against a bright surface. Its duration depends on the intensity of the stimulus, and it may persist for minutes or hours. In its later stages it may become coloured, and may also undergo irregular variations. In cases of very intense stimulation, a similar phenomenon has been observed to persist for very long periods, and even for life; and there does not appear to be any clear distinction between this definitely pathological phenomenon and the ordinary persistent image. The latter possibly only occurs when the stimulation has been sufficiently intense to disturb the normal functions of the retina.

Another phenomenon which has been observed with strong stimulation of the retina, is coloured waning of the after-image (farbiges Abllingen). The image of white light may be seen to appear in a succession of colours, beginning with bright greenish blue, passing on to blue, violet, pink, and then changing to a dirty orange and yellow-green. The exact nature of the coloration varies with the intensity and duration of the stimulus. The colours have been referred to differences of persistence (p. 1068), and may also in some cases be connected with the colour adaptation of the retina.

Corona of after-image.-The after-image change in a retinal area influences and is influenced by the changes going on in surrounding parts of the retina. The most marked instance of this is the corona of an after-image. If a white patch on a black ground is fixed, the black after-image will be seen to be surrounded by a light halo. If watched, this halo may be seen to vary; at one moment it is a narrow border, at the next it broadens out, and undergoes periodical changes analogous to those of the after-image itself. If one fixes two white patches separated from one another by a narrow black strip, the part of the after-image corresponding to the strip appears very bright, and may be regarded as due to the superposition of two halos. In he

1 Journ. Physiol., Cambridge and London, 1898, vol. xxii. p. xii. and Phil. Trans., London, 1899, vol. exei. p. 1. 
after-image of a black patch on a white ground, the black corona of the light after-image is much less marked. Similar appearances occur in coloured images.

On the other hand, the after-image change in a limited part of the visual field is influenced by the brightness of the surrounding parts. This was described by Hering under the name of induction. When black and white surfaces adjoin one another, the black may be observed to become brighter and the white darker on fixation. A grey patch on a ground brighter than itself will appear to become lighter when fixed; on a ground darker than itself it will appear to become darker. Martius has recently called attention to this phenomenon, and proposed it as a method of colour photometry (p. 1078).

Contrast. - The facts just given are closely connected with the subject of contrast. These appearances may be regarded as examples of reciprocal action of retinal areas on one another, and this reciprocal action is the basis of contrast. Contrast may be simultaneous or successive. Two adjoining or neighbouring parts of the visual field are influenced in their brightness and colour by this reciprocal action. This action is so rapid as to be almost instantaneous, and is then known as simultaneous contrast. During longer observation, the difference between the two parts of the field may become considerably greater, and this successive contrast is due, partly to the change mentioned at the end of the last section (Hering's successive induction), partly to movement of the eye, and consequent development of after-image effects.

Pure simultaneous contrast is most marked at first, and if steady fixation is kept up, rapidly diminishes. It is also most marked when the contrasting surfaces join one another. This maximum effect of

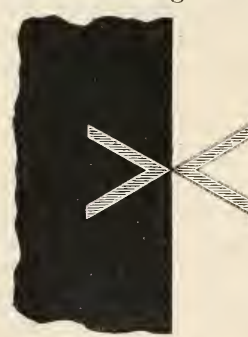

FIG. 384.-Hering. contrast at the line of junction, however, must not be confused with marked borders, which occur during fixation, and are due to slight involuntary movements of the eyes, and consequent stinulation by one surface of the border of the retinal area previously exposed to the other surface.

Simultaneous contrast occurs both for light and colour. Simultaneous light contrast is shown in a striking manner by an arrangement, such as in Fig. 384, in which a grey cross is placed on a surface, half of white paper and half of black velvet. The two parts of the cross will be seen

to differ greatly in apparent brightness. A grey, observed against a variable background, changes in apparent brightness with every change of the background; and it may be shown, by looking through a small aperture, that these changes in brightness are not due to variations in the size of the pupil. It is an interesting example of light contrast, that a change in the illumination of a room which is not directly observed, may show itself as a change in the apparent brightness of a shadow, although there may have been no change in the actual illumination of the shadow. The delicacy of Rumford's method of photometry depends on contrast, the shadows showing slight changes of brightness much more readily than the surrounding illuminated surface.

Simultaneous colour contrast shows itself most readily on a grey 
surface adjoining a coloured surface, especially when the grey surface is of medium intensity. The contrast effect is much aided by the absence of other differences between the contrasting surfaces. Differences of texture or of glaze, inequalities of surface, and similar details, obscure colour contrast very greatly. Nearly all grey papers are more or less coloured, but the colour may not be obvious on simple inspection, and may only become apparent when a disc made of the paper is rotated. A rapidly-rotating disc presents a uniform surface, which is better adapted for the discrimination of slight coloration. Hering ascribes to the same factor the visibility at a distance of a grease spot on cloth, which is imperceptible when looked at closely. It is for the same reason that colour contrast appears so well on a rotating dise, and that contrast is so much favoured by covering the induced and inducing surfaces with transparent tissue paper. The same factor also accounts for the distinctness of contrast effects in the after-image. If the afterimage of Fig. 384 is observed, the two parts of the cross will be found to differ in brightness more than in the original.

It was formerly believed that contrast occurred most readily with moderate degrees of saturation, and it was supposed that the favourable effect of covering the contrasting surfaces with tissue paper was due to lessened saturation. Rollett ${ }^{1}$ and Hering, ${ }^{2}$ on the other hand, found that contrast increased with saturation. The subject has been quantitatively studied by Kirschmann, ${ }^{3}$ and by Pretori and Sachs. ${ }^{4}$ Two chief methods have been used. A surface coloured by contrast may be compared with an objectively coloured field; or advantage may be taken of the fact that the effect of a saturated surface on a less saturated surface of the same colour is to abolish the colour of the less saturated surface, and the contrast effect may be measured by finding the amount of colour in the less saturated surface which can just be neutralised. The latter method has been found to be the more satisfactory, and the above observers agree in finding that the contrast effect increases with increase of saturation of the inducing surface. According to Kirschmann, the former seemed approximately to increase in arithmetical, when the saturation increased in geometrical, progression. Pretori and Sachs found a definite relation between the white value of the reacting surface and the amount of colour that could be neutralised, the two increasing proportionally.

The influence of the extent of the contrast inducing surface was also investigated quantitatively by Kirschmann, who used two dises at a certain distance from one another. In the case of brightness contrast, it was found that the contrast effect increased with increase of extent of the inducing surface, the relation between the two being approximately logarithmic. Colour contrast was also found to increase with extent of the inducing surface, and apparently in the same ratio as brightness contrast.

If two surfaces of different colour adjoin a third suitable surface, the latter may be seen to be coloured by contrast, in the colour which is complementary to that which would be produced by mixture of the two inducing colours.

${ }^{1}$ Sitzungsb. d. k. Akad. d. Wissensch., Wien, 1867, Bd. Iv. Abth. 2, S. 344.

${ }^{2}$ Arch. $f$. d. gcs. Physiol., Bonn, 1887, Bd. xli. S. 1.

3 Phil. Stud., Leipzig, 1891, Bd. vi. S. 417.

${ }^{4}$ Arch. f. d. ges. Physiol., Bonn, 1895, Bi. 1x. S. 71. 
Irradiation.-When a black square on a white ground is compared with a white square of the same size on a black ground, the white square seems the larger. This depends on irradiation, and is seen under various conditions. It is the cause of the apparent notch seen in the edge of a dark object against a flame. Owing to its relatively extensive contour, print is a favourable object for observing the influence of irradiation. The phenomenon has been ascribed to various causes, such as propagation of the physiological process to surrounding elements, spherical aberration, and diffraction at the pupil. There is little doubt that it is a physical phenomenon due to diffusion, for it is much more marked with a large pupil. Printed type appears very considerably reduced in size with a pupil dilated by atropin, and is restored to its normal appearance by means of a small artificial pupil. ${ }^{1}$ Irradiation has also been referred to chromatic aberration, but it is said to occur with monochromatic light.

Homochromatic induction. ${ }^{2}$ - Under certain circumstances, an induced colour may be of the same hue as the inducing colour. This occurs especially with small black or white surfaces on a strongly coloured ground. This homochromatic induction probably depends simply on dispersed light $\left(\right.$ Blix $\left.^{3}\right)$. Aars ${ }^{4}$ has recently described a form of colour mixture which depends on the same phenomenon. He found that a narrow band of colour seen from a distance is altered in colourtone when it separates two larger coloured surfaces, the induction being homochromatic. The phenomenon occurred most readily with inducing colours of short wave-length, a fact in favour of dependence on dispersion; and the mixture described by Aars is essentially of the same nature as that which occurs on mixing pignents together.

Temporal and spatial induction.-Both after-image and simultaneous contrast may be regarded as manifestations of a process of physiological induction. Stimulation of any retinal area induces an altered condition of excitability in that area (temporal induction), and an altered condition in surrounding areas (spatial induction). The change in the appearance of a surface during fixation, the after-image, and successive contrast, are examples of temporal induction. Simultaneous contrast, and some other appearances which depend on reciprocal action of different retinal areas, are examples of spatial or areal induction. The corona of an after-image depends on both temporal and spatial induction.

Hering ${ }^{5}$ has used induction in a different and more limited sense. By simultaneous induction he means the change during fixation in adjoining black and white surfaces; by successive induction, the persistence of the change after removal of the stimulating surfaces.

Theory of after-image.-There have been two chief rival theories of after-image. In the theory first advanced by Scherffer, of which Fechner and Helmholtz have been the chief advocates, it is supposed that after-images are due to fatigue; that the retina is relatively passive; that in the case of light the after-image is due to general diminished activity of the fatigued area; while in the case of colour it is due to

1 Mind, London and Edinb., 1896, vol. , p. 71.

2 This plienomenon has been named by Blix, "isochromatic induction." Isochromatic is not only a term used in physics in a different sense, but is also incorrect in this connection, meaning rather "of equal colour value."

3 Skandin. Arch.f. Physiol., Leipzig, 1895, Bd. v. S. 13.

4 Ber. internat. Cong. Psychol., Munchen, 1896, S. 188.

5 "Zur Lehre vom Lichtsinu," S. 36. 
visibility of those rays to which the area has not been fatigued. An after-image seen in complete darkness is ascribed to visibility of part of the intrinsic light of the retina.

The other view, advocated by Plateau and Hering, ascribes a more active rôle to the retina, and supposes that a condition is set up antagonistic in nature to the original excitation. Plateau supposed that the variations of an after-image were due to an oscillatory process in the retina on its way to a state of rest.

The great difficulty of the former view lies in explaining the brightness of the after-images which may be seen in complete darkness. The brilliancy of these, and especially of coloured images, may be very considerable. On the fatigue hypothesis, the latter are explained as due to part of the intrinsic light, that part corresponding to the exciting rays being absent. The brightness of such an image is, however, much greater than that of the whole intrinsic light, even after prolonged stay in the dark. That the brightness of an image should be greater than that light of which it is a part, would seem an insuperable difficulty to the fatigue hypothesis, but Helmholtz brought in the influence of contrast to explain it. We have nothing with which to compare the intrinsic light of the retina, while the after-image is seen against the darker background corresponding to the rest of the retina, and its apparent brightness is heightened by contrast, i.c. the phenomenon is explained on psychological grounds.

Another objection to the fatigue hypothesis is, that after-images are observed most readily in the early morning, when the liability to fatigue should be slight. They also occur especially well in the young and vigorous. The periodical variations of after-images cannot be explained by fatigue, especially the appearance of a positive image between two negative phases.

Hering has devised a number of experiments directed against the fatigue explanation. ${ }^{1}$ If a small saturated red disc on a somewhat darker grey ground is fixed, and after an interval both disc and ground are shaded, the disc will appear bluish green and much brighter than the ground. ${ }^{2}$ In this experiment the retinal area in question is still being objectively stimulated by red light. The blue-green after-image would on the fatigue hypothesis be due to the visibility of part of the small amount of white light reflected by the red disc.

Hering has compared an after-image with objective light of the same colour. On the white half of a black and white surface he placed a small red disc $5 \mathrm{~mm}$. from the line of junction of the black and white halves; on the black ground, $10 \mathrm{~cm}$. from this line, a blue-green disc. After fixation of the line of junction close to the red disc for one minute, he fixed a point on the black surface $5 \mathrm{~mm}$. from the blue-green disc, so that the subjective blue-green image could be compared with the objective blue-green disc. With suitable general illumination the after-image appeared brighter and more saturated than the disc. Here an area of the retina "fatigued" to red produces a brighter and more saturated blue-green sensation than one previously allowed to rest by exposure for one minute to a black surface, and then stimulated by blue-green light. According to Hering, this can only be due to some inherent capacity of the visual organ. The nature of this capacity must be left till his special theory is considered.

${ }^{1}$ For these and numerous other after-image and contrast experiments, see Arch. $f . d$. ges. Physiol., Bonn, Bd. xliii. S. 264 and 329.

2 This experiment was also carried out by Plateau, to whom it was suggested by Wheatstone (Bull. Acad. roy. de méd. de Belg., Bruxelles, 1875, tome xxxix. p. 100). 
The seat of after-images is another question of interest. The influence of movement of the eyeball upon after-images, and the fact that pressure on the eyeball hinders the appearance of an after-image (p. 1098), have been supposed to be in favour of their peripheral origin. Rimpler ${ }^{1}$ found that the sensations arising from stimulation of the stump of the optic nerve, after extirpation of the eyeball, were not followed by after-images. On the other hand, Parinaud ${ }^{2}$ has argued in favour of a central seat, from the fact that after excitation of one eye, a negative after-image may be seen in the other eye. This, however, is simply due to the difficulty of knowing with which eye we are receiving an impression (see p. 1136). Parinaud also states that after-images do not appear to move when the eyeball is mechanically displaced, but this observation is very doubtful.

Theory of contrast.-The old view of Johannes Miiller, that contrast depended on reciprocal action of retinal areas, was at one time almost completely neglected: Contrast was referred by Helmholtz to purely psychological factors, and especially to supposed processes of unconscious inference.

In the case of colour contrast, Helmholtz's mode of explanation may be illustrated by what is usually known as Meyer's experiment, although in its essentials it was described by J. Miiller. ${ }^{3}$ If a small patch of grey paper is placed on a coloured (green) surface, it becomes tinged with the complementary colour (pink) by contrast. This pink coloration is greatly increased by covering with transparent tissue paper. Helmholtz ${ }^{4}$ supposed that, in looking at such a surface, we seem to be looking at a background through a coloured (green) medium, the grey patch appears part of the background, and is regarded as an object seen through the green medium. It is a matter of experience that an object which appears white through a green medium must be pink, and the patch is therefore seen as pink. If a grey patch, corresponding in intensity to that under the tissue paper, is held in front of the cover, it is very little, or not at all, coloured by contrast; and, according to Helmholtz, this is due to the fact that it is regarded as an independent object, and is therefore not affected in the same way as if it were part of the background.

The mirror contrast experiment of Ragona Scina, and the colour contrast produced by rotating such a disc as Fig. 385, were explained on the same lines. In the case of the latter, the explanation was somewhat forced, it being supposed that the colours of the dise were regarded as variations of a uniform colour, such as might be produced by shadows, coloured reflections, staining by coloured liquids, or soiling by coloured powders. "In order to produce an objectively grey spot on a greenish surface, a reddish pigment would be required." Coloured shadows were regarded by Helmholtz as giving the most convincing evidence in favour of the psychological nature of contrast. On a surface illuminated both by daylight and lamplight, a shadow thrown by an object from the yellow lamplight will appear blue by contrast. Helmholtz observerl through a tube the border of the coloured shadow, and, when the blue colour liad developed, moved the tube, so that the eye was wholly exposed to the shadow. He found that the blue colour persisted, although there was now no contrast inducing light, and disappeared on removing the tube from the eye. It was supposed that the blue colour, produced by the illusion of judgment, persisted till removal of the tube allowed comparison with other colours, and rendered a new judgment possible.

\footnotetext{
1 Centralbl. f. d. med. Wissensch., Berlin, 1882, S. 4.

${ }^{2}$ Gaz. d. hop., Paris, 1882, p. 459.

3 "Handbuch d. Physiol.," 1837, Bd. ii. S. 369.

4 "Handbuch d. physiol. Optik," 1867, S. 407.
} 
This mode of psychological explanation has been criticised by Hering, and its application to these phenomena shown conclusively, by means of variations of the experiments, to be unwarranted. ${ }^{1}$ In the case of all, the contrast colour may, by suitable means, be made so intense, that observers, unaware of the nature of the experiment, may not know which is the objective and which the subjective colour. In the case of Meyer's experiment, the fact that the grey patch in front of the cover is so little affected by contrast, is due to the effect of differences of texture, etc., obscuring the colour difference. Hering found that if the grey patch was carefully covered with tissue paper, so that it exactly resembled the other patch, both showed the contrast colour equally well. Another argument used by Helmholtz was, that a black line marking off the patch from the surrounding area, much diminished the contrast colour;

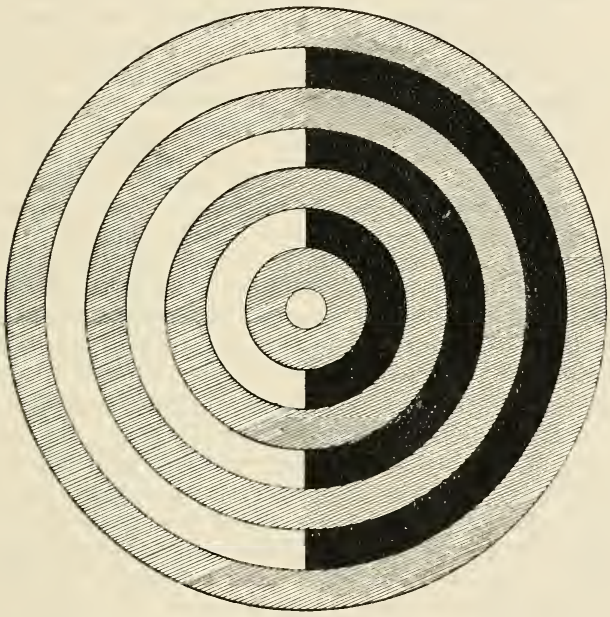

Fig. 385. -The shaded rings represent the coloured portions of the disc. The other rings give on rotation a grey of medium brightness which is coloured by contrast.

but this is to be expected if, as generally acknowledged, contrast colour is most marked immediately adjoining the inducing surface. In the case of mirror contrast, Hering has devised a form of the experiment in which observers may see the most pronounced contrast effects, while wholly unaware that they are looking through a coloured medium.

Hering has shown that Helmholtz's results with the coloured shadow are due to the lack of careful fixation, and to the effect of successive contrast. After steady fixation through a tube of the line of junction of the illuminated surface and blue shadow, the after-image is scen on removing into the shadow, or on extinguishing the yellow light, the bluc colour showing in the lalf previously illuminated, while the half previously rendered blue by contrast becomes colourless, or may even be tinged yellow. On removal of the tube, or better, on opening the tube so as not to disturb fixation, the after-image is still seen on the larger background.

Hering ${ }^{2}$ has devised a binocular experiment which conclusively disproves

1 Arch. f. d. gcs. Physiol., Bonn, 1887, Bd. xl. S. 172 ; and Bd. xli. S. 1 and 358.

2 Ztschr.f. Psychol. u. Physiol. d. Sinnesorg., Hamburg u. Leipzig, 1890, Bd. i. S. 18. 
the explanation of Helmholtz. One eye looks through red and the other through blue glass at a white surface, which appears violet, owing to binocular colour mixture (1. 1124); a black patch on the white surface is doubled by converging the eyes for a nearer point, and the double images seen against the violet ground are coloured differently by contrast, - that seen by one eye being blue-green, and that seen by the other, yellow. This experiment would show that two separate illusions of judgment may occur simultaneously, one for each eye. The occurrence of mixture of contrast colours is also in favour of their physiological origin.

A simple but striking experiment, illustrating both temporal and spatial induction, is given by Hering. ${ }^{1}$ Place two broad white strips of paper on black velvet, so that a strip of the black, about $5 \mathrm{~mm}$. wide, is left between them. If a point is fixed on the black strip, this will be seen to become lighter, and, on removing the white strips to one side, after fixation for thirty seconds, the black strip will become much brighter, and, with suitable adjustment of the general illumination, may appear brighter than the objective white strips. The surfaces previously covered by the white strip are black by successive contrast, but the blackness does not seem great enough to account for the brightness of the middle strip. The same appearance may be observed with closed eyes. Hering regards the experiment as showing that the visual organ, by its own inherent capacity, can produce a greater brightness in one part of the field than is produced in another part by white light.

This experiment is still more striking if coloured (red) strips on a black ground are used. On removing the red strips, a red after-image is seen corresponding to the black strip between them, and this occurs when the green colour of the surrounding surface produced by successive contrast is hardly noticeable. This appearance is obviously closely related to the corona of the after-image.

According to Hering, the reciprocal relation of retinal areas is a fact of the most fundamental importance in vision. Its function is to counteract the effect of their regularly diffused light which surrounds every retinal image, and the reciprocity is therefore most marked at the junction of the reacting surfaces. That objects are seen with sharp outlines is due to this reciprocal action, and to the production of a retinal condition opposed in nature to the original excitation. Hering regards it as incredible that a phenomenon of such fundamental importance as contiast should not depend on some physiological mode of relation between retinal (cerebro-retinal) areas. The nature of this physiological relation will be discussed later.

Plateau, ${ }^{2}$ who advocated a physiological explanation of both afterimage and contrast, referred them to oscillations in time and space respectively. The periodical variations of an after-image were examples of the former kind of oscillation, while he regarded irradiation and contrast as due to a similar oscillatory condition, passing outwards in the retina from the place of stimulation.

The phenomenon described on p. 1056, in which subliminal excitation in a small retinal area is reinforced by stimulation of other areas of the retina by the same kind of light, seems to be an instance of induction opposed in nature to the ordinary form. It ceases, however, to be contradictory, if one assumes that the opposed state induced in the retina by stimulation of one part, reacts on the inducing surface. When a number of small retinal areas are stimulated, the induced change will be more intense than in the case of only one area, and the effect of the reaction may be sufficient to raise the colour of the small areas above the threshold.

${ }^{1}$ Arch.f. d. gcs. Physiol., Bonn, 1888, Bd. xliii. S. 266.

${ }^{2}$ Bull. Acad. roy. de méd. de Belg., Bruxelles, 1876 , tome xlii. p. 684. 
While there is now no doubt that the general phenomenon of contrast nay be referred to a process of physiological induction, there are certain features of contrast which are at present wholly unintelligible physiologically, and these can only be expressed as facts in psychological terms. Thus, the obscuration of slight differences of colour or brightness in two objects by other differences, such as inequalities of surface and differences of texture, can only at present be given as a psychological fact. Waller ${ }^{1}$ has described an experiment, in which, when a coloured surface is placed on either side of the middle third of a grey strip, the contrast colour in the strip is barely perceptible. If, now, the middle third is marked off by placing a pin across the strip at each end, the contrast colour appears distinctly. One can only give this as an example of the psychological fact that colour contrast is favoured when the induced surface is sharply marked off from surrounding parts. Sherrington ${ }^{2}$ has pointed out that individuals may see a difference of colour between two surfaces, due to contrast, until they are convinced that the two surfaces are objectively alike, and may then fail any longer to see the difference in colour. In this, as in other cases, the psychological factors tend to mask the appearances which depend on the physiological reciprocity of adjoining retinal areas.

\section{The Duration of Stimulus and Sensation.}

Duration and sensibility.-In the section on after-image the factor "duration" has been to some extent considered. In this case, however, attention was limited to the altered condition of sensibility which remains for considerable periods after retinal stimulation. In this and the following sections facts will be considered which illustrate the relation of the duration of the stimulus to the duration of the sensation. However short the duration of the stimulus, the sensation which arises lasts for an appreciable time, and this phenomenon may be termed persistence of vision. It has also been termed positive after-image, but it would probably be better to limit the term "after-image" to those more or less prolonged manifestations dealt with in the previous sections.

It has been found that the threshold of sensibility depends upon the duration of the stimulus within certain limits, i.e. for durations between $\cdot 002$ and $\cdot 125 \mathrm{sec}$. (Charpentier). ${ }^{3} \quad$ A feeble light of short duration, which is below the threshold of stimulation, may become visible by increasing its duration; the duration necessary within the limits mentioned being inversely proportional to the intensity, so that doubling duration has the same effect on sensibility as doubling intensity. For these short intervals the effects of light on the retina appear to accumulate as they do on a photographic plate. The same inverse relation also holds between intensity and extent of retina stimulated (p. 1055), so that summation of feeble stimuli seems to occur both in time and space, and, according to Charpentier, for a given sensation a certain amount of light is necessary, and within the limits it matters little whether this amount is extended over a larger or smaller area, or reaches the eye in a longer or shorter time. Adaptation of the retina to the dark shortens the duration necessary for a sensation in the same way that it lowers the threshold. According to Charpentier, with very short duration coloured light is seen as colourless, i.e. for coloured light there is an interval between the absolute and chromatic thresholds of duration, but this has

1 Journ. Physiol., Cambridge and London, 1891, vol. xii. p. xliv.

2 lbid., 1897 , vol. xxi. p. 41.

3 Arch. d'opht., Paris, 1890, tome x. p. 108. 
been denied. According to Kunkel, there is a photo-chromatic interval as regards duration in the case of green and blue, but not in the case of red.

Persistence of vision.-When the retina is affected by a momentary light-stimulus of ordinary intensity, the sensory process, owing to inertia, takes a certain time in reaching its maximum, and then fades away, rapidly at first, more gradually later. These three periods may be known as the periods of rise or waxing (Anklingen), maximum, and fall or waning (Abllingen) of the sensory curve. The period of rise has been most fully investigated by Exner, ${ }^{1}$ who used an instrument devised by Helmholtz. Two surfaces were exposed in rapid succession,- - the first, a white square on a black surface; the second, a uniform white surface. It was found that if the first excitation had reached its maximum, while the second was still in the period of rise, the appearance would be that of a light square on a dark ground; if, on the other hand, both had passed the maximum, the first excitation would have fallen to a lower level than the second, and the appearance would be that of a dark square on a light ground. Between these two appearances there would be a moment at which the surface would appear uniform, and this appearance would correspond to a condition when one excitation had not quite reached the maximum, while the other had just passed it. By this method Exner found that the time taken in reaching the maximum varied with the intensity. The more intense the stimulus, the more rapid the rise of the sensory curve, and the relation between the two appeared to be such that if the intensity increased in geometrical progression, the time in which the curve reached its maximum decreased in arithmetical progression. The period of waxing in the case of different colours was determined by Kunkel " using Exner's method. He determined the times for red, green, and blue; when these colours were made of approxinately equal brightness, he found that the period of rise was shortest for red and longest for green, the times taken in reaching the maximum for red, blue, or green being 0573 sec., 0916 sec., and 133 sec. respectively.

The periods of maximum and of fall are not capable of such exact investigation. By varying the intensity of his two excitations, Exner endeavoured to determine the course of the curve and the total duration of the sensory process, and found the maximum to be of very short duration followed by a gradual fall, the total duration varying with the intensity, the size of the retinal image, and the part of the retina stimulated. It is probable that the period of waning is different for different colours, but there are no exact data on this point.

Talbot-Plateau law.-One of the chief methods which has been employed to investigate the nature of the sensory curve is that of intermittent retinal stimulation. When momentary stimuli follow one another with sufficient rapidity, the effect is a continuous sensation of uniform brightness. The brightness of this sensation is that which would have arisen if the amount of light intermittently reaching the retina had been uniformly distributed over the whole period of stimulation. This proposition, which is known as the law of Talbot ${ }^{3}$ and Plateau, ${ }^{4}$ has been experimentally investigated. Helmholtz found

${ }^{1}$ Sitzungsb. d. k. Akad. d. Wissensch., Wien, 1868, Bd. lviii. Abth. 2, S. 601.

2 Arch.f. d. ges. Physiol., Bonn, 1874, Bd. ix. S. 197.

${ }^{3}$ London, Edinburgh, and Dublin, Phit. Mag., London, 1834, Ser. 3, vol. v. p. 327.

${ }^{+}$Ann. d. Phys. u. Chem., Leipzig, 1835, Bd. xxxv. S. 457. 
that a disc, such as in Fig. 386, on rotation gave a uniform grey, and that the brightness of the grey was the same as that of a surface obtained by mixing the double images of black and white strips, doubled by means of a piece of Iceland spar. ${ }^{1}$ The law has been found to be approximately true for moderate intensities, though Fick 2 found that there were slight deviations. For low intensities continuous illumination gave a brighter sensation than intermittent; with stronger illumination, intermittent illumination had the greater effect, while at the upper limit of intensity employed by him the law held good. Investigators of this question have usually employed dises seen by reflected light. O. Griinbaum, ${ }^{3}$ using direct light, has found that the law does not hold above a certain intensity, the brightness of a light diminished in

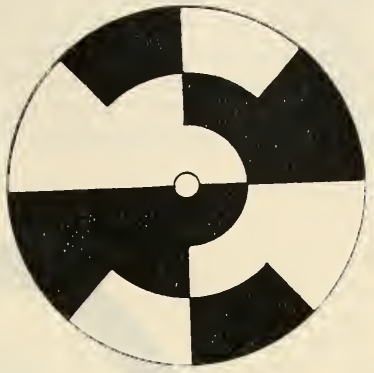

Fig. 386. intensity by rotating before it alternate open and black sectors being greater than that of the same light diminished to an equal degree in physical intensity ly means of Nicol's prisms.

Flicker sensation. - When the intermittent stimulation is not sufficiently rapid to cause complete fusion, a characteristic flickering appearance is seen, and the point at which this flicker passes into a continuous sensation has been used as a means of determining the conditions which influence visual persistence. The flickering appearance alters in character with the rate of stimulation. With slow rates of rotation of a disc divided into sectors, these can at first be distinguished; increase of the rate produces a coarse and unpleasant Hicker, accompanied at certain rates by a peculiar glittering appearance, and this gradually passes into a fine tremulous appearance before complete fusion occurs. Some observers have used the point of cessation of the coarse, others of the fine flicker, and it has not yet been clearly established that the two points of transition follow the same laws. It is difficult to make a clear distinction between coarse and fine flicker, and the point of cessation of the latter is that with which the most satisfactory results may be obtained.

The point of fusion depends in the first place on intensity; being raised by increase of intensity, i.e. more rapid stimulation is required. It has been supposed that as the intensity is increased in geometrical progression, the rapidity of stimulation necessary for fusion increases in arithmetical progression, so that with high luminosities the point of fusion is not appreciably affected by further increase. Grübaun has found that this is only true up to a certain limit, and that as intensity is increased, a point is reached at which the point of fusion begins to fall. This only occurs when the stimulus has reached a considerable intensity, corresponding with that at which the same observer finds the Talbot-Plateau law no longer holds good. It seems probable that both changes are connected with the prolonged positive or blinding image

1 "Handbuch d. phys. Optik," 2te Aufl., S. 483.

2 Arch. f. Anat., Physiol. u. wissensch. Med., 1863, S. 754.

3 Journ. Physiol., Cambridge and London, 1898, vol. xxii. p. 433. 
which follows intense stimulation of the retina (p. 1059). It is here supposed that periods of stimulation alternate with periods of complete absence of stimulation. Flicker also occurs when periods of greater and less excitation alteruate, and the point of fusion is then lowered by diminution of the difference of intensity in the two periods.

There are different opinions as to the influence of the relative duration of the periods of excitation and non-excitation. Charpentier ${ }^{1}$ found that increase of duration of the stimulation raised the point of fusion, while Marbe ${ }^{2}$ found the point of fusion lowered when the more intense stimulus was prolonged in relation to the less intense.

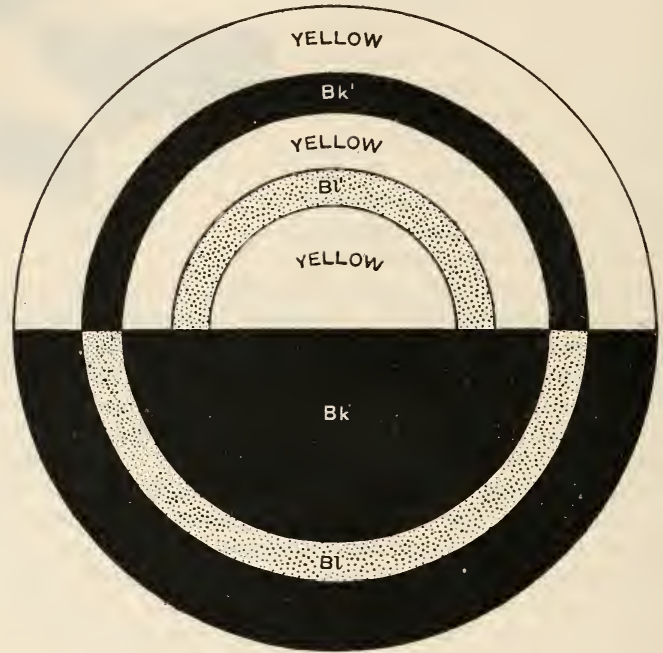

FIG. 387.--Sherrington.

Sherrington ${ }^{3}$ has recently shown that the factors described in the previous sections as spatial and temporal induction, have an enormous influence on the point of fusion. Using a yellow, blue, and black disc (shown in Fig. 387), in which $B l$ and $B k$ were of the same objective brightness as $B l^{\prime}$ and $B k^{\prime}$, he found that the rate at which flicker ceased in the two rings differed very greatly; $B l B k^{\prime}$ requiring a much higher rate than $B l^{\prime} B k$, the brightness of $B l$ and the darkness of $B k^{\prime}$ being increased by simultaneous contrast, or spatial induction. By means of this and similar experiments, he shows conclusively that the point of cessation of flicker depends on what one may call the "physiological" intensity, and not on the physical intensity, and also furnishes a conclusive proof that simultaneous contrast rests upon a physiological basis. By means of the disc shown in Fig. 388, Sherrington further shows that temporal induction influences the point of fusion. If this disc be rotated in the same direction as a clock hand, flicker persists longer

1 Arch. d'opht., Paris, 1890, tome x. p. 340.

2 Phil. Stud., Leipzig, 1896, Bd. xii. S. 279.

3 Journ. Physiol., Cambridge and London, 1897, vol. xxi. p. 33. 
in the ring corresponding with the white space between the two black teeth, and with the black tooth between the two white notches than in the two rings enclosing it; on reversing the direction of rotation, flicker ceases sooner in this middle ring. In this experiment, the whiteness and blackness of the teeth and notches are intensified by contrast, and the effect of changing the direction of rotation shows that the fusion takes place less readily when ordinary white precedes intensified black, and ordinary black precedes intensified white, than in the opposite order. In the one case the intensified black and white have been preceded by ordinary black and white respectively, which will, by lowering sensi-

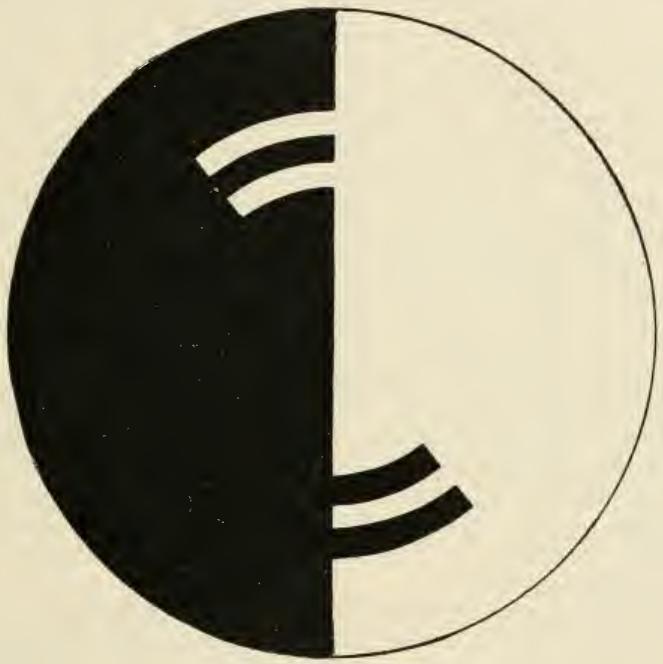

Fig. 388.--Sherrington.

bility, have lessened their intensification; while, in the other case, the intensified black and white will have been preceded by white and black respectively, and this, by increasing sensibility, will further augment the intensification due to spatial induction, and bring about an increased difference in the physiological intensities of the successive stimuli.

The influence of contrast or induction thus demonstrated by Sherrington gives a clue to a difficulty in regard to flicker which has been much discussed. Filehne ${ }^{1}$ found that if discs of black and white sectors were rotated, the number of sectors on the disc was of influence. If a disc with four sectors be rotated $n$ times per second in order to produce fusion, a disc with sixty-four sectors might be expected to require $\frac{n}{16}$ rotations to eliminate flicker. A higher number is, however, found necessary. His results have been confirmed by other observers. The size of the aperture through which the rotatory disc is observed is found to be of influence. The phenomenon has been explained by the fact that in the slower rotation necessary with the larger number of sectors, the movement of the contours is of influence (Marbe), ${ }^{2}$ or that

${ }^{1}$ Arch. f. Ophth., 1885, Bd. xxxi. Abth. 2, S. 20.

2 Loc. cit., p. 288 , 
it depends on movement of the eyes, and therefore becomes much less marked if the dise is observed through a slit (Schenck). ${ }^{1}$ Griinbaum ${ }^{2}$ has recently worked out a definite relation between the breadth of the sector and the diameter of the aperture through which the disc is observed, and there is little doubt that the phenomenon is due to the influence of contrast. The larger the breadth of the aperture in relation to that of the individual sector, the larger will be the fraction of the total revolution of the dise during which black and white will be exposed simultaneously, and will, by reciprocal intensification of each other, increase the rapidity of stimulation necessary to abolish flicker.

Two other factors found to be of importance are the size and position of the retinal area stimulated. Exner ${ }^{3}$ found that the duration of the sensory process varied with the size of the retinal image; the duration decreasing in arithmetical, when the size increased in geometrical, progression ; and Charpentier ${ }^{4}$ similarly found that increase in the size of the retinal image raised the point of fusion. These observers have also found that flicker disappears less readily on the peripheral retina, especially in the dark-adapted eye, while Exner found that fusion took place more readily for the fovea than for an area $1.33 \mathrm{~mm}$. from it. Bellarminoff ${ }^{5}$ has further found a higher frequency necessary for fusion on the nasal than on the temporal half of the retina for certain kinds of light. Exner ${ }^{6}$ found the point of fusion to be lowered by pressure on the eyeball (p. 1098).

Increase in the size of the retinal image and removal of the image to the peripheral retina act in the same way as increase of intensity; and the influence of these factors, and of adaptation, induction, and duration of the stimulus, all seem to be instances of a general law, that the point of fusion of intermittent stimuli, so as to produce a continuous sensation, depends, not on the physical intensities of the stimuli, but on their physiological intensities, as determined by the condition and nature of the stimulated retinal area.

The previous considerations have applied to colourless sensations. The point of fusion in the case of colour sensations seems to depend wholly upon the brightness of the colour, and to be independent of its colour tone. If coloured light stimulates the retina intermittently, it is found that the brighter the colour, the greater is the rate of stimulation necessary to cause fusion. This has led to the adoption of flicker as the basis of a method of heterochromatic photometry; and the subject will be more fully considered later, when we come to the means of determining the brightness of a colour.

Recurrent images. - It has so far been assumed that the course of the sensory curve is a simple rise and fall. Facts must now be considered which show that the curve is more complex, and that in nearly all conditions of stimulation there is a secondary process, which takes place later than that already considered, and gives rise to what may best be known as a recurrent image, to distinguish it from the after-images described on p. 1057. The recurrent image was first noticed by Purkinje, as an elliptical strip following a moving light. It has usually been

${ }^{1}$ Arch. f. d. ges. Physiol., Bonn, 1896, Bd. lxiv. S. 165.

2 Journ. Physiol., Cambridge and London, 1897, vol. xxi. p. 396.

${ }^{3}$ Sitzungsb. d. k. Akad. d. Wissensch., Wien, 1868, Bd. lviii. Abth. 2, S. 601.

4 Arch. d'opht., Paris, 1890, tome x. p. 340.

5 Arch. f. Ophth., 1889, Bd. xxxv. Abth. ], S. 25.

6 Arch.f. d. ges. Physiol., Bonn, 1878, Bd. xvi. S. 407.

7 "Beobachtungen u. Versuche z. Phys. d. Sinn.," S. 111. 
observed by moving the source of stimulation, when the recurrent appears to follow at a certain distance the primary image ; this may be done most readily by means of a revolving mirror. The same phenomenon may also be observed with a momentary stationary stimulus. By the former method some observers have described the recurrent as immediately following the primary image, while others have seen the two separated by a dark interval. This difference has been shown by Charpentier ${ }^{1}$ and v. Kries ${ }^{2}$ to depend on the intensity of the stimulus, and on the adaptation of the eye.

Most observers have described the interval as about $\frac{1}{5}$ second: according to Charpentier it may vary from $\frac{1}{4}$ to $\frac{1}{30}$ second with intensity. By altering intensity alone v. Kries did not produce so great a difference, and he regards the length of the interval as chiefly dependent on adaptation. He found that in complete dark-adaptation the recurrent image followed the original immediately, and was brighter than to the light-adapted eye. v. Kries believes, however, that the two appearances do not depend simply on shortening of the interval, and he states that, with moderate dark-adaptation, two images may be seen, one following immediately, the other at an interval. The duration of the stimulus is also of influence; increased duration shortens the interval, and lengthens the recurrent image (Bosscha). ${ }^{3}$ The size of the recurrent image varies with intensity (Charpentier), attaining with strong light five or six times the size of the original.

The coloration of the image has been differently described. Some have seen it as colourless, whatever the nature of the stimulus; others as faintly complementary, others as violet or reddish. Hess ${ }^{4}{ }^{4}$ on the other hand, describes it as having the same colour as the original, but so faint that it can only be detected by comparing double images. According to Charpentier, it is violet with low, colourless with high, intensity; while v. Kries describes the ordinary image as faintly complementary, the image of the dark-adapted eye as colourless.

The interval between the original and recurrent image has been described as of abnormal darkness (Bidwell), or as faintly tinged in the complementary colour (Hess).

When coloured light is used for stimulation, the recurrent image varies with the part of the spectrum. Bidwell ${ }^{5}$ and v. Kries have failed to see any recurrent image following spectral red, and according to others it is very faint with this part of the spectrum. The brightest image is obtained by using green light, and, according to Hess, the curve representing the brightness of the image for different parts of the spectrum corresponds closely with the curve of brightness of the spectrum for the dark-adapted eye at low intensity.

v. Kries has found that the image is not seen in direct vision; that, as the moving image crosses the region of central vision, it disappears momentarily; and, using the stationary method, he has found that the region of the visual field in which it is absent has a diameter of 35 to $38 \mathrm{~mm}$. at 1 metre distance. ${ }^{6}$ v. Kries has also found that there

${ }^{1}$ Compt. rend. Acad. d. sc., Paris, 1896, tome cxxii. p. 87.

${ }^{2}$ Ztschr.f. Pyschol. u. Physiol. d. Sinnesorg., Hamburg u. Leipzig, 1896, Bd. xii. S. 81.

3 Arch.f. Ophth., 1894, Bd. xl. Abth. 1, S. 22.

4 Arch. f. d. ges. Physiol., Bonn, 1891, Bd. xlix. S. 190.

5 Proc. Roy. Soc. London, 1894, vol. lvi. p. 132.

${ }^{6}$ Hess (Arch. f. Ophth., 1897, Bd. xliv. S. 445) denies this, while others (Samojloff and Hamaker) have confirmed v. Kries' observation. Hess also denies that the influence of adaptation is as described by v. Kries.

VOL. II. -68 
was no recurrent image in a case of night blindness, and in one of total colour-blindness.

Hamaker ${ }^{1}$ has recently brought forward evidence which shows that the phenomenon is more complex than has been supposed. In addition to the recurrent image, several observers have seen others, and Hamaker clearly distinguishes four stages: the primary original image, followed immediately by a short "tail"; a secondary image, separated from the former by a dark interval; a tertiary image, lasting for two or three seconds; and a quaternary image, which is probably identical with the ordinary negative after-image. Some of the discrepancies between different observers may have been due to confusion between the secondary and tertiary images. The influence of adaptation and of other conditions is not the same for the two images.

In this and previous sections, four distinct phenomena have been distinguished which are ordinarily classed together as after-images, namely, the negative image due to local adaptation; the quasi-pathological positive image; the persistence due to inertia ; and the recurrent image of Purkinje. These are four quite distinct conditions, and it has been a great source of confusion in the past, that they have all had the same name. The term after-image should be confined to the phenomenon due to local adaptation, while the other three phenomena should be termed the persistent positive image, retinal persistence, and the recurrent image. A fifth phenomenon, which is sometimes confused with after-image, is the memory-image, which in many individuals (of the visual type) not only remains after the impression, but may be recalled.

Charpentier's band.-Another phenomenon pointing to the complexity of the sensory curve, is that known as Charpentier's dark band. $^{2}$ If a black disc which has a white sector is rotated slowly in strong illumination, the side of the white sector which first penetrates the black ground shows a black band separated from the black ground by a similar white band. The band occurs about $\frac{1}{72}$ of a second after the beginning of the passage of the white, and lasts about the same time. It may often be seen to be followed by a series of similar less distinct black bands at uniform distance. Charpentier ascribes these bands to retinal oscillations, which he supposes to have a constant rapidity, each total oscillation lasting about $\frac{1}{36}$ of a second. The oscillations are supposed to be of two kinds, one more rapid being polarised in the direction of the yellow spot, while the other radiates in all directions.

Other appearances with intermittent stimulation.--Some other phenomena of interest occur with intermittent retinal stimulation. The flicker which occurs before complete fusion is, at a certain stage, of a glittering character, and gives the appearance of a brightness considerably greater than that of the continuous sensation; the Talbot-Plateau law being only true after complete fusion. Brücke ${ }^{3}$ described the maximum brightness with black and white sectors as occurring when there were $17 \cdot 6$ stimuli per second, and ascribed the appearance to the reinforcement of each white sector by the positive (recurrent) image of the preceding sector.

Exner ${ }^{4}$ described certain subjective appearances with intermittent stimulation as occurring especially with red, green, and blue light, while other colours appeared to be decomposed by this means, violet light

${ }^{1}$ Ztschr. f. Psychol. u. Physiol. d. Sinnesorg., Hamburg u. Leipzig, 1899, Bd. xxi. S. 1.

2 Arch. de physiol. norm. et path., Paris, 1892, Sér. 5, tome iv. p. 541; and 1896, tome viii. 1. 677 .

${ }^{3}$ Sitzungsb. d. k. Akad. d. Wissensch., Wien, 1864, Bd. xlix. Abth. 2, S. 128.

${ }^{4}$ Arch. f. d. ges. Physiol., Bonn, 1868, Bd. i. S. 375. 
giving a red and blue figure. These appearances required intense light, and Burch ${ }^{1}$ has recently described the spectrum as showing the three colours, red, green, and blue, most strongly with intermittent light of great intensity.

Another phenomenon which has lately attracted much attention is the appearance of colours, when a disc of black and white sectors is rotated. These colours were first observed by Fechner ${ }^{2}$ in 1838, and are often called by his name. He used a disc, shown in Fig. $389,{ }^{3}$ and found that different individuals varied greatly in the distinctness with which they saw the colours. The most usual appearance which accompanies flicker at a certain stage is one of a more or less definite pattern in yellow and blue. The colours distinctly increase in vividness with prolonged observation and with the brightness of the illumination. In recent modifications of this experiment the appearance of colour has been found to be more definite, if narrow black bands are placed on the white sectors, or, better still, if such bands are observed through a gap in the disc. All gradations of colour may be observed depending on the relative position of the black sectors, the white sectors, and the bands. The most definite colour which is seen is red, and Bidwell ${ }^{4}$ has shown that this is the same phenomenon as one described by him, in which a bright object on a dark background appears, when suddenly exposed, to be surrounded by a narrow red border lasting about $\frac{1}{10}$ of a second. With brighter illumination a distinct blue-green appears,

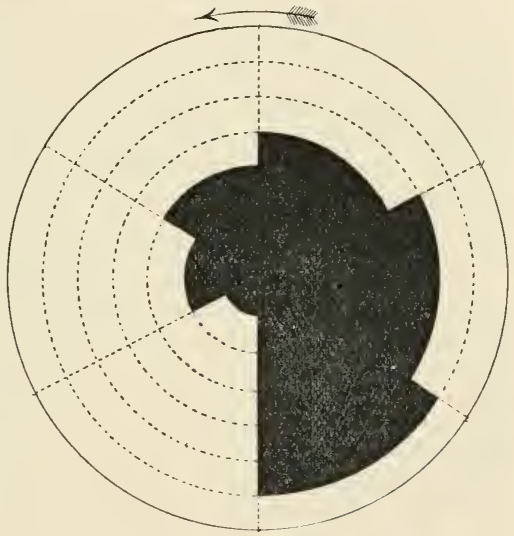

FIG. 389. and Bidwell ${ }^{5}$ has referred this to the after-image of the red effect. This appears to be an instance of the phenomenon already mentioned, in which the after-image effect may neutralise or overpower the colour of the original excitation to which it owes its origin. Some of these subjective colours are said to be visible with monochromatic illumination.

Fechner explained the phenomenon by supposing that the different colours made their appearance at different rates, in the same way that the coloured waning of after-images showed that they disappeared at certain rates. This explanation was adopted by Helmholtz, ${ }^{6}$ who supposed that the process in the case of red and violet was more rapid than for green, a supposition confirmed later by Kunkel (see p. 1068).

${ }^{1}$ Journ. Physiol., Cambridge and London, 1897, vol. xxi. p. 431.

${ }^{2}$ Ann. d. Phys. u. Chem., Leipzig, 1838, Bd. xlv. S. 227.

${ }^{3}$ Fechner calls attention to the fact that, when this figure is revolved slowly in the direction of the arrow, the black seems to extend beyond its limits; when in the opposite direction, the black seems to shrink, the latter effect being the more marked.

+ Proc. Roy. Soc. London, 1896, vol. 1x. p. 368.

s Ibid., 1897, vol. Ixi. p. $268 . \quad$ "Handbuch d. pliys. Optik," 1867, S. 380. 
It is doubtful whether the coloured bands can be explained in this way, although the distinctness of the red in Bidwell's experiments is to be expected if this colour reaches its maximum with the rapidity ascribed to it by Kunkel.

Theory of fusion with intermittent stimulation.-At first sight the most obvious explanation of fusion of discontinuous stimuli, so as to produce a continuous sensation, is derived from the analogy of muscular tetanus. The rise and fall of the sensory curve takes an appreciable time, and if a new stimulus comes in before the curve due to the previous stimulus has had time to fall, the result would be a continuous line; the occurrence of flicker would then be due to the fact that a slower rate allowed the individual curve to fall slightly before the second stimulus arrived. If this were the case, however, the intensity of the resulting sensation, with complete fusion, should be the same as that of each individual sensation at or near to its maximum. The fact known as the law of Talbot and Plateau seems therefore to negative this simple view, and render's necessary some other explanation. In order to reconcile the fusion of intermittent stimuli with the Talbot-Plateau law, it seems necessary to adopt the assumption that a less intense stimulus, occurring at a certain interval after a more intense stimulus, acts immediately in preventing the excitation set up by the former from reaching its maximum, and leads to a state of oscillation about a mean excitation, which may produce the appearance of flicker, or if slight enough may be unnoticed (remain below the threshold), and lead to the appearance of continuity in the sensory process.

As in the case of after-image, persistence has been ascribed to both peripheral and central processes. Exner ${ }^{1}$ found that with intermittent electrical stimulation of the eyeball, light sensations were still discontinuous with a rapidity of sixty per second, while, with stimulation by light, fusion took place with about twenty-four stimuli per second. Assuming that the electrical stimuli acted on the optic nerve fibres, Exner regarded the above fact as proof of the retinal origin of persistence. Filehne ${ }^{2}$ objected that, under certain conditions, sixty or more light stimuli per second give a discontinuous sensation; and Grübaum ${ }^{3}$ has shown that the figure is still higher. Exner's proof, then, loses its validity; and there is at present no definite evidence as to the seat of visual persistence. Sherrington has shown the dependence of persistence on spatial induction or contrast, and, consequently, if the latter is central, the former must be so also. The seat of induction generally, however, is also still an open question.

\section{The Brightnfess of Colour Sensations.}

Colour photometry.-Numerous methods have now been devised to enable different colours to be compared in respect of brightness. The difficulty in doing this is enhanced by the fact that the relative brightness of different colours varies with the absolute intensity, a fact first noted by Purkinje, and usually known by his name.

The most widely employed method of colour photometry has been the simple direct comparison of coloured with colourless light. This method was employed by Fraunhofer in 1817, and the extensive researches of $\mathrm{Abney}^{4}$ and König ${ }^{5}$ on

1 Arch. f. d. ges. Physiol., Bonn, 1875, Bd. xi. S. 414.

2 Arch. f. Ophth., 1885, Bd. xxxi. Abth. 2, S. 20.

3 Journ. Physiol., Cambridge and London, 1897, vol. xxi. p. 398.

4 Phil. Trans., London, 1886, vol. clxxvii. p. 423; and 1888, vol. clxxix. p. 547.

5 "Beitr. z. Psych. u. Phys. d. Sinn.," 1891, S. 311. 
the brightness curves of normal and abnormal vision are based on this direct comparison. At first sight it seems very difficult to say with any degree of accuracy that a coloured field has the same brightness as a colourless field; and some, including Helmholtz, have bcen unable to make satisfactory observations of this kind, but most of those who have used the method agree that it is practicable, and gives more consistent results than might be expected from its purely subjective nature. Rood ${ }^{1}$ and Abney tested their results by mixing two complementaries, of which they had determined the white equivalents, and found that the grey so produced was equal to the sum of the white equivalents of the components.

As might be expected, this direct method of comparison appears to be especially easy in the more simple colour system of the colour-blind.

The direct method must, however, always be more or less arbitrary; G. E. Müller ${ }^{2}$ has lately objected that certain colours have a "penetrative" quality, which may be confused with briglitness; and it is certainly very dangerous to draw conclusions in respect to slight individual differences of colour vision from such a method. Numerous indirect methods have now been described to determine the relative brightness in a more objective way.

One of these methods, devised by Vierordt, ${ }^{3}$ consists in finding the amount of white light which must be added to a coloured light, to render the colour of the latter imperceptible. It is, in fact, a method by which the brightness of a part of the spectrum is determined by finding its threshold of saturation. Vierordt's scale of intensities for the spectrum agrees roughly with Fraunhofer's. A similar method was employed by Draper ${ }^{4}$ with very different results. This observer found that with the prismatic spectrum red was the brightest colour, while with the diffraction spectrum all parts were equally bright, all colours disappearing with the same amount of added white light.

Another method is based on the principle that the degree of visual acuity depends upon intensity. This is interesting as the earliest method employed, having been used by Herschel in $1800,^{5}$ who determined the distinctness with which he could with the microscope observe fine details of opaque objects illuminated by the different parts of the spectrum, and placed the maximum intensity in the yellow and green. This method has been most thoroughly investigated by Macé de Lepinay and Nicati, ${ }^{6}$ who found for the longer wave half of the spectrum that it gave results agreeing fairly with those of Fraunhofer and Vierordt. They considered two lights as equally bright when the visual acuity in them was of the same value.

One of the most satisfactory methods is known as flicker photometry. The point at which intermittent stimuli give rise to a continuous sensation seems to depend on brightness and to be independent of colour-tone. The measurement of the intensity of sensation by means of time was suggested by Talbot ${ }^{7}$ in 1834 , and was first put in practice by Schafhäutl ${ }^{8}$ and Rood. ${ }^{9}$ Probably the most simple application of the method is to rotate open sectors before a ficld halffilled with the colour whose brightness is to be determined, while the other half shows colourless light which can be varied in intensity. The two halves are judged to be equally bright, when both show a just perceptible flicker. Results obtained by this method agree closely with those obtained by other methods, and fairly closely with those derived from direct comparison.

1 Am. Journ. Sc., New Haven, 1878, vol. xv. p. 81.

2 Ztschr. f. Psychol. u. Physiol. d. Sinnesorg., Hamburg u. Leipzig, 1896, Bd. x. S. 28.

3 "Die Anwendung des Spektralapparats zur Messung d. Stärke des farbigen Lichts," Tübingen, 1871.

${ }^{4}$ London, Edinb. and Dublin Phil. Mag., London, 1879, Ser. 5, vol. viii. p. 75.

5 Phil. Trans., London, 1800 , vol, xe. p. 255.

${ }^{6}$ Ann. de him., Paris, 1881, tome xxiv. p. 289 ; and 1883 , tome xxx. p. 145.

7 London, Edinb. and Dublin Phil. Mfag., London, 1834, Ser. 3, vol. v. 1. 327.

8 Abhandl. d. bayr. Akad., 1855, Bd. vii. S. 467.

${ }^{9} \mathrm{Am}$. Journ. Sc., New Haven, 1893, vol. xlvi. 1. 173. 
A very simple allied method ${ }^{1}$ depends on a phenomenon described by Jastrow. If a rod be moved in front of a rotating disc on which a colour and a grey are being mixed, coloured bands will be seen following the rod, except with a certain grey in the case of each colour. The grey found to give no bands with a given colour agrees very closely with that which ceases to flicker at the same time as that colour.

Another method differing in principle is to determine the distance at which small areas of different colours become invisible. This has lately been used by Haycraft ${ }^{2}$ with results agreeing very closely with those of the flicker method.

The dependence of the amount of contraction of the pupil on intensity is the principle of another method, first advocated as a general photometric method by Gorham. ${ }^{3}$ The size of the pupil is observed entoptically, and this method has, in the case of coloured papers, been found to give results closely agreeing with the flicker method. ${ }^{4} \mathrm{~A}$ different application of the method has been made by Haycraft, ${ }^{5}$ who measured the size of the pupil, when exposed to different parts of the spectrum, on photograms obtained by the flash-light, and obtained a curve of luminosity of the spectrum agreeing with that of flicker.

Another method proposed by Martius ${ }^{6}$ depends on the fact that a coloured patch becomes darker on fixation when on a background darker than itself, lighter when on a lighter ground. Accordingly, the intensity of a grey on which a coloured patch appears to become neither lighter or darker may be taken as a measure of the brightness of the colour. Martius found it more convenient to use a grey patch on a coloured ground owing to absence of disturbance by the complementary colour of the after-image, and the brightness of a series of coloured papers was found by this method to agree fairly with the results of Fraunhofer and Vierordt. The results, however, differ considerably from those of other methods.

The various methods which have been described give results which, on the whole, agree with one another. Hayeraft found a close agreement between the flicker and distance methods, and the flicker, band, and pupil methods also give concordant results, and agree approximately with the results of direct comparison. The assumption that the attribute which is being measured by these methods is brightness, rests partly on the correspondence with direct comparison, but more on the fact that a method, such as flicker, seems to be reliable as a means of determining the brightness of different physical lights of the same colour, in which case its results can be checked by the ordinary physical methods of photometry. As it measures brightness in that case, we may conclude that it also measures brightness in the case of lights of different colours, although it is difficult to exclude the possibility that it may also be in some degree affected by the wave-length. In the section on retinal persistence and flicker, it was stated that probably flicker depends wholly on intensity and not on wave-length, and this statement rests to a great extent on the reliability of flicker as a photometric test.

Purkinje's phenomenon. - One difficulty of colour photometry is at the same time a phenomenon of great interest. The relative brightness of different colours varies with the absolute intensity. Purkinje observed that a red and a blue, which appeared equally bright with ordinary illumination, differed in brightness at lower intensities, the

\footnotetext{
1 Journ. Physiol., Cambridge and London, 1897, vol. xxii. p. 137.

2 Ibid., vol. xxi. p. 126.

3 Proc. Roy. Soc. London, 1884, vol. xxxvii. p. 425.

4 Journ. Physiol., Cambridge and London, 1897, vol. xxii. p. 137.

${ }_{5}^{5}$ Communication to Physiological Society, not yet published.

6 " Beitr. z. Psych. u. Phil.," Leipzig, 1896, Bd. i. S. 95.
} 
blue becoming the brighter. This is a special case of a more general phenomenon, which has since been named after Purkinje, namely, that with decrease of the intensity of the spectrum, the short wave end becomes relatively brighter. The question how far this change is a function of the altered intensity as such, and how far of the altered condition of adaptation of the eye, will be considered later.

It has already been stated that, with the exception of red, all colours appear colourless at a low intensity, and Purkinje's phenomenon attains its maximum effect when the intensity has been so far reduced as to produce a colourless spectrum. The brightest part of the spectrum is then found to be in the green at $E$, or slightly on the violet side of $E$, instead of about $D$, as at ordinary luminosities. The fact that the interval between the absolute and chromatic thresholds is greatest for green, stands in relation to this phenomenon. Further, the red end of the spectrum is shortened, and is relatively much darker than in the

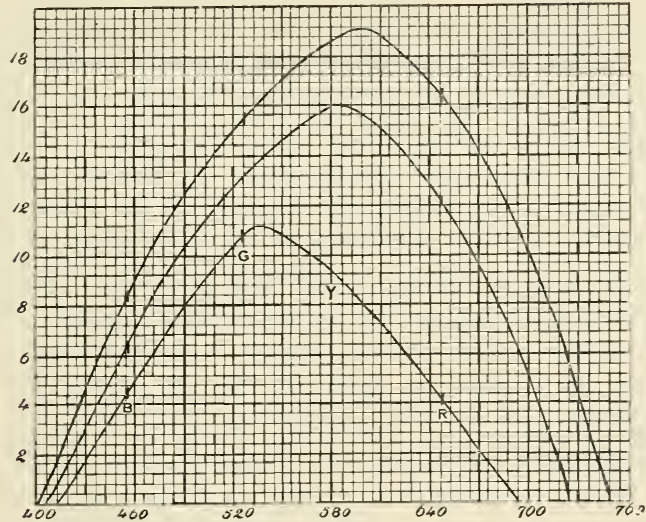

Fig. 390.-In this figure the red end of the spectrum is on the righthand side, the blue on the left. The ordinates show the number of revolutions of a semi-dise per second which were found necessary to extinguish flicker. The upper curve represents a bright spectrum, the lower a dark spectrum, and the middle curve one of intermediate intensity.-Hayeraft.

ordinary spectrum, while the violet end is relatively brighter. The curve of luminosity, as determined by flicker, is given in Fig. 390 together with the curves of luminosity at ordinary intensities, and in an intermediate condition.

The order in which the colours disappear on lowering the intensity of the spectrum is said to be that yellow and blue go first, violet then becoming invisible, while green persists longest. If the intensity of a spectrum is increased above the ordinary limits, it also becomes colourless, and under these circumstances yellow and blue persist, after red, green, and violet have disappeared.

The loss of colour at high or low intensities does not take place suddenly, but is preceded by a gradual decrease of saturation. Hering has pointed out that if a red and blue be compared while the intensity 
is being gradually lowered, the blue will appear to become whiter and the red blacker, and the difference in saturation is in Hering's opinion the basis of the changes in apparent intensity.

Most of the work on Purkinje's phenomenon has been done by direct comparison of the brightness of different colours. Macé and Nicati used the acuity method of colour photometry, and Haycraft has recently investigated Purkinje's phenomenon for coloured papers by the flicker and distance methods. The distance method gave curves for the different colours which agreed very closely with those obtained by the flicker method. One point about which there is difference of opinion concerns the upper limit of Purkinje's phenomenon, and it is also doubtful whether the relative brightness of different parts of the spectrum varies to any appreciable extent at ordinary luminosities. This and other questions require investigation with spectral colours, and with one of the objective methods of colour photometry; probably flicker photometry would give the most satisfactory results.

We may now return to consider whether Purkinje's phenomenon is a function of intensity or of adaptation. It is generally agreed that adaptation of the eye to the dark is a necessary condition for the favourable observation of the phenomenon, but, according to Hering, ${ }^{1}$ it is the essential factor. He varied the two conditions independently of one another, using two adjoining rooms separated by a partition, with round holes filled with coloured glass. By altering the illumination of a screen in one room, the intensity of the coloured patches could be varied, while by alteration of the illumination of the other room the eyes of the observer in this room could be brought to any degree of adaptation. It was found that if the eye remained in a condition of light-adaptation, red and blue when lowered in intensity became gradually blacker without change in relative brightness. Of the two, the red retained its colour slightly the longer, and appeared slightly brighter. On the other hand, adaptation of the eye to the dark brings about the phenomenon, without any change in the actual illumination of the patches themselves, the blue becoming whiter and brighter than the red. Hering points out that, in addition to the ordinary adaptation on prolonged stay in the dark, a momentary adaptation occurs on lowering the illumination, and this also produces the Purkinje phenomenon, though in a less marked degree than with prolonged adaptation.

Weber's law.-The relation of the difference of stimulus, which produces a just perceptible difference of sensation, to the total stimulus, has been frequently investigated for vision. The method most widely employed has been to observe the point at which a shadow illuminated by one light just disappears (or becomes visible) against a background illuminated by two lights. In 1760, Bouguer found by this method that the difference was independent of the absolute intensity. Another method is to use Masson's disc, a white disc on which there is a narrow black band, which gives a grey ring on rotation. The breadth of the band is found, which gives a just perceptible ring with different illuminations. The results of star photometry have also been used, on the supposition that the differences between the different magnitudes represent equal differences of sensation, but this method does not appear to be satisfactory. The difference of stimulus necessary for a just perceptible difference of sensation presents great variations, which

\footnotetext{
1 Arch. f. d. ges. Physiol., Bonn, 1895, Bd. 1x. S. 519.
} 
probably depend chiefly on the method of the investigator. It is generally put at $\frac{1}{100}$ of the total stimulus, but the delicacy of discrimination appears to be greater, the figure given by Helmholtz being $\frac{1}{167}$; by Aubert, $\frac{1}{186}$; by Schirmer, $\frac{1}{217}$; and by Ole Bull, $\frac{1}{240}$. In some individuals, the delicacy of discrimination is even higher than this.

Aubert, ${ }^{1}$ using the shadow method, found that as the illumination diminished the threshold of discrimination increased; and Helmholtz, ${ }^{2}$ using Masson's dises, found a similar departure from Weber's law at low luminosities. Kräpelin ${ }^{3}$ and Schirmer, ${ }^{4}$ on the other hand, found that the threshold of discrimination is constant when due attention is paid to adaptation, and that, with maximal adaptation to the illumination at which the observations are made, Weber's law is true within wide limits. Kräpelin found that the results were uninfluenced by the size of the pupil.

\section{Mixture.}

Colour mixture.-The mixture which occurs when an area of the retina is simultaneously stimulated by different kinds of light, may be effected in several ways. In an old method, due to Lambert, a coloured patch is placed on each side of a plate of glass held vertically, so that one patch is seen by reflected, the other by transmitted, light. The relative intensities of the two components may be varied by varying the angle at which the line of vision cuts the glass.

Mixture due to double refraction may be seen if two patches are observed through a crystal of Iceland spar, so that two of the four patches produced coincide.

In pigment colours the most usual method is by means of the colour wheel, - a method depending upon visual persistence. Spectral colours may be allowed directly to combine on a retinal area, or may be converged by means of a lens on a screen.

The colour triangle.-The representation of the facts of colour mixture by means of a geometrical diagram is due to Newton. He adopted a circle with its circumference divided into parts corresponding to the colours of the spectrum. At the centre of gravity of each of the segments corresponding to these parts, he placed a circle, with its area proportional to the amount of light of each colour entering a given mixture. The point in the diagram representing the centre of gravity of all these circles gives the nature of the mixture. A radius drawn through it gives the hue, and the distance of the point from the centre the degree of saturation.

This method of calculating the results of colour mixture was verified

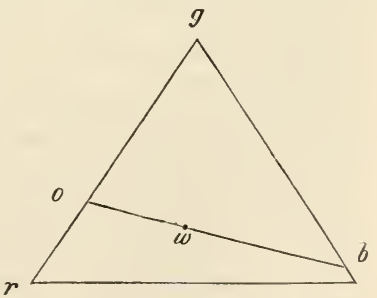

Fig. 391. experimentally by Maxwell. In a colour diagram each point represents a colour. The resultant of a mixture of any two colours is found by dividing the line joining the two points of the diagram inversely as the quantity of each; thus the resultant of mixture of two parts of red with

\footnotetext{
1 " Physiol. d. Netzhaut," S. 52.

2 "Handbuch d. physiol. Optik," 1867, S. 315.

${ }^{3}$ Phil. Stud., Leipzig, 1885, Bd. ii. S. 306.

4 Arch. f. Ophth., 1890, Bd. xxxvi. Abth. 3, S. 121.
} 
one of green would be represented in Fig. 391 by $o$ when $o g=2 o r$; if $R$ = quantity of red and $G=$ quantity of green, $R \times r o=G \times o g$.

If this resultant colour is mixed with a third $(B)$, the resultant of the mixture of the three will be on the line $o b$, at a point determined by the quantities of each. Maxwell found that by taking these components in different proportions, one may determine the colour corresponding to every point of the triangle of which the three colours form the angles. If three suitable spectral colours are chosen, it will be possible to represent all other colours by means of such a triangle. Complementary colours in such a diagram will be placed at a distance from one another, and the resultant of their mixture to form white will be represented by a definite position within the triangle; the lines joining any two complementaries must pass through this definite point; the lines joining the point for white with any point of one side of the triangle will represent all degrees of saturation of the colour of that point from spectral purity to white. In Fig. 391, if $O$ and $B$ are complementaries and mixed together in approximately equal proportions to match white, $w$ will represent the point for white, and points between $o$ and $w$ will represent orange in its various grades of saturation. The fact that it is possible to find three colours, and by means of their geometrical representation to deduce the results of their mixture in any proportions, is the basis of one of the chief theories of colour vision, and will be returned to in that connection and also in connection with colour blindness.

Laws of colour mixture.-The validity of this procedure depends on certain assumptions which were formulated by Grassmann, ${ }^{1}$ as follows :-

1. Every impression of light may be imitated by mixing a homogeneous colour of a certain intensity with colourless light of a certain intensity.

2. If one of the two mingling lights be continuously altered (whilst the other remains unchanged), the impression of the mixed light also continuously changes.

3. Two colours, each of which has a constant tint and a constant intensity of the intermixed white, also give constant mixed colours, no matter of what homogeneous colours they may be composed.

A fourth assumption which Grassmann regarded as less well established was-

4. The total intensity of a mixture is the sum of the intensities of the lights mixed.

Grassmann's propositions have been investigated experimentally. The third and fourth are those about which there is most doubt. The third proposition implies that a mixture good at one intensity should hold good for all intensities, i.e. multiplication of both sides of an equation by the same factor should not affect the validity of the equation. According to several observers, this proposition is not true, and the alteration of an equation with altered intensity is usually described as a departure from Newton's law or rule.

The most marked examples of this deviation occur in the colourblind, and will be considered later (p. 1090). According to König and his followers, it also occurs in normal vision. Thus Tonn ${ }^{2}$ found that the complementary to a red of $670.8 \lambda$ varied with the intensity, shifting from $511.8 \lambda$ to $547 \cdot 3 \lambda$ when the intensity was reduced to a fortieth. The complementary to $586.5 \lambda$, on the other hand, showed no change

${ }^{1}$ London, Edinb. and Dublin Phit. Mag., London, 1854, Ser. 4, vol. vii. p. 254.

Z Ztschr.f. Psychol. u. Physiol. d. Sinnesorg., Hamburg u. Leipzig, 1894, Bd. vii. S. 279. 
with intensity. The alteration of an equation with intensity is, according to Ebbinghaus ${ }^{1}$ and Franklin, ${ }^{2}$ shown by matching a grey, formed by mixture from red and blue-green with one formed from yellow and blue. If these greys are made of equal intensity with ordinary illunination, it is found that, on lowering the illumination, the grey from the red and blue-green becomes decidedly the brighter, and the grey derived from blue and yellow requires a considerable addition of white light to enable it again to match the other. According to Hering, ${ }^{3}$ these changes with intensity do not occur if the fields used are of small extent, and are ascribed by him to the influence of the macular pigment; to differences in its absorption at different intensities. ${ }^{4}$

The experimental investigation of Grassmann's fourth proposition has been rendered difficult by our want of a suitable means of determining the intensities of the components in colour mixture. Rood and Abney, using single direct comparison of coloured with colourless light as a test of brightness, have confirmed Grassmann's fourth proposition, but the subject requires reinvestigation, with the aid of more satisfactory methods of colour photometry.

When two spectral colours, which lie nearer to one another in the spectrum than complementary colours, are mixed, the resultant has the tone of an intermediate part of the spectrum, but is generally less saturated, and usually the less saturated, the farther apart the two colours. The smallest loss of saturation occurs when warm colours are mixed, while the greatest loss of saturation occurs when green is one of the components of the mixture.

Another proposition, which may be added to those of Grassmann, has been enunciated by v. Kries ${ }^{5}$ and Hering. ${ }^{6}$ Colour mixtures good for one condition of retinal excitability are good for all conditions of excitability. A match between red and blue-green on the one side, and yellow and blue on the other, holds good after adaptation of the eye to red, as well as in the natural condition. With marked dark-adaptation, however, this rule may possibly be no longer true.

\section{Vision of Different Regions of the Retina.}

Light sense of peripheral retina.-One of the factors which determines the character of the sensation due to a certain stimulus is the region of the retina stimulated. The peripheral retina will first be compared with the central region taken as a whole, and then the special differences will be considered which occur in the central region, characterising the vision of the fovea and macula. Both sets of differences may be considered under the three heads of light, colour, and form.

The condition of adaptation is of the greatest importance in relation to the sensibility to light. Those observers who have not paid any special attention to adaptation have found a gradual decrease of sensitiveness from centre to periphery, while in dark-adaptation the threshold of

${ }^{1}$ Ztschr.f. Psychol. u. Physiol. d. Sinnesorg., Hamburg u. Leipzig, 1893, Bd. v. S. 173.

2 Proc. internat. Cong. exp. Psych., London, 1892, p. 104.

${ }^{3}$ Arch.f. d. ges. Physiol., Bonn, 1893, Bd. xlvii. S. 277.

${ }^{4}$ In a recent paper (ibid., 1898, Bd. Ixx. S. 297) Tschermak, working under Hering, has found changes in colourless matches with alteration of adaptation, and ascribes the deviations to this factor, and not to the direct influence of altered intensity.

${ }^{5}$ Arch. f. Physiol., Leipzig, 1878, S. 517.

${ }^{6}$ Arch. f. d. ges. Physiol., Bonn, 1888, Bd. xlii. S. 488. 
sensibility is the same in the central (except fovea) and peripheral retina, and also in different parts of the latter.

Some observers have employed the threshold as the test, and others the just observable difference, while Guillery has used the extent of the retinal image which enables discrimination of difference. No observer has compared the threshold and difference methods, both with and without dark-adaptation.

According to Schön, ${ }^{1}$ there is a marked difference between the light sensibility of the nasal and temporal halves of the retina. He found that a point $45^{\circ}$ from the fovea on the temporal side required, for the production of a sensation of a certain degree of brightness, three times as much light as the corresponding point on the nasal side. Guillery has also found the temporal half less sensitive, but the difference he describes is very much less than that of Schön. Neither observer mentions the conditions of adaptation.

Exner and Bellarminow have found that flicker is more marked in the periphery than in the centre of the visual field, and have inferred from this that the sensation produced by a definite stimulus is there more intense. Bellarminow, further, has found that flicker occurs more readily with stimulation of the nasal than of the temporal half, thus supporting the view of Schön. Schön has advanced a theory of binocular vision founded on this difference between the temporal and nasal halves of the retina (p. 1138).

Colour-sense of periphery.-The colour vision of the peripheral retina has been investigated by many observers with very divergent results. The divergency depends to a very great extent on neglect of such factors as adaptation, the brightness and degree of saturation of the coloured light employed, the size of the area stimulated, and the nature of the background. The sensibility is generally tested by passing over the retina from periphery to centre, or vice versâ, patches of coloured light, and noting, first, the limits where the different colours begin or cease to be perceived; and, secondly, the changes in colour-tone, saturation, or brightness which the patches appear to undergo. In general, it may be said that the brighter and more saturated the colour, and the larger the area stimulated, the greater is the distance from the centre at which a colour can be recognised. It is generally agreed that at ordinary intensities no colours are perceived at the extreme periphery, all being perceived as some shade of grey, depending on the kind of light and on the background; but, according to many observers, including Landolt ${ }^{2}$ and Charpentier, ${ }^{3}$ all colours can be recognised as far peripherally as white, if the light is made sufficiently intense.

According to Hering, the grey with which a given colour is matched at the extreme periphery of the retina is the same as the grey with which it is matched at a low intensity, and he has used this match as one means of determining the white value of a colour. v. Kries ${ }^{4}$ has lately determined the white value of both pigment and spectral lights for the periphery of the retina and for the dark, and found a great difference between the two, the periphery values corresponding fairly well with the normal brightness values by heterochromatic photometry.

1 Arch. f. Ophth., 1876, Bd. xxii. Abth. 4, S. 49; 1878, Bd. xxiv. Abth. 1, S. 27.

2 Klin. Monatsbl. f. Augenh., 1873 , Bd. xi. S. 376.

3 "La lumière et les couleurs," Paris, 1888, p. 183.

+Centralbl. f. Physiol., Leipzig u. Wien, 1897, Bd. x. S. 745. 
There is considerable difference of opinion on the question of the exact limits of the fields for different colours, but there is now fairly general agreement in placing the limits for blue and yellow distinctly outside those for red and green. Nearly all agree that the limits are not fixed, and are greatly influenced by intensity and the other factors mentioned.

Another important feature of peripheral colour vision is the change in tone which a coloured light appears to undergo as it passes over the retina from centre to periphery. Here again there is fairly general agreement that blue and yellow become colourless without undergoing any change in tone, while reds and greens pass through a stage in which they are seen as yellow or blue before becoming colourless. Violet is seen as blue, orange as yellow. Some observers, however, have seen yellow change to green peripherally.

On passing a large number of pigment colours over the retina from periphery to centre, Hess ${ }^{1}$ found that nearly all showed change of colourtone. The colours fell into two groups: one group appeared yellow and the other blue in the periphery. Each of these groups could be again divided into those which appeared red and those which appeared green as they approached the centre. In addition to these four groups, Hess found four colours which showed no change in colour-tone: these four colours formed two complementary pairs, yellow and blue, red and green. The red and green which did not change in tone were found with more difficulty than the yellow and blue, nearly all the pigments used showing a yellow or blue tone at some time in their course over the retina. The red which showed no change was bluer than the red of the spectrum. With spectral colours the same general changes were observed, but only three kinds of light could be found which showed no change in tone, in the yellow at $574: 5 \lambda$, in the green at $495 \lambda$, and in the blue at $471 \lambda$. In order to produce a constant red, a certain amount of blue had to be mixed with the extreme red of the spectrum, the latter always being seen as yellow on the peripheral retina. As in the case of pigment colours, the constant yellow and blue, and red and green of the spectrum were found to be complementary pairs.

Hess also investigated the peripheral limits for the colours which did not change in tone, and found that both the white value and the "colour value" were of influence. A green and red pigment were said to have the same colour value when they had to be mixed together in equal proportions in order to make grey. When the white and colour values were made equal, Hess found that the peripheral limit of the unchangeable red corresponded with that of the green, and of the yellow with the blue. According to Hering and Hess, the outermost zone of the retina is relatively insensitive to all colours, the middle zone is insensitive to red and green, and only the central zone has normal colour vision. These zones are often spoken of as zones of total colour-blinduess, of redgreen blindness, and of normal colour vision respectively, but by this it is not meant that the zones are absolutely insensitive. It would, as Hess suggests, be more correct to speak of them as zones with weak colour-sense, since, with sufficiently strong intensity, all colours can be perceived to the extreme periphery. Hegg ${ }^{2}$ has more recently confirmed Hess's conclusion, that the borders for red and green coincide when attention is paid to equal chromatic and white values. He found the

1 Arch. f. Ophth., 1889, Bd. xxxv. Abth. 4, S. 1.

${ }^{2}$ Ibid., 1892, Bd. xxxviii. Abth. 3, S. 145. 
limit for yellow about $1^{\circ}$ smaller than that for blue, and refers this to the difference of dispersion of the two kinds of light.

Guillery ${ }^{1}$ has recently investigated the colour-sense of the peripheral retina, by using as his measure the extent of the retinal image which enables the colour to be recognised. He found for each colour gradual increase necessary from centre to periphery. The figures for yellow and blue showed a close agreement, but differed considerably from those for red and green, which corresponded well with each other, thus confirming the result of Hess by an entirely different method. It may be mentioned here that the photochromatic interval is found to be greater for the peripheral than for the central retina, and that Hess found that a colour equation good for the central (extramacular) region also held good for the periphery.

Visual acuity of peripheral retina.-The difference between central and peripheral retina in respect of visual acuity, is far greater than for other kinds of retinal sensibility. It has been tested by finding the distance at which two points can be discriminated, and also by means of letters. Grœnouw ${ }^{2}$ and Guillery ${ }^{3}$ have also used lately the size of the "physiological point" as a test. Acuity is most intense in direct vision, and drops very rapidly at first, and then more slowly towards the periphery. The low degree of acuity in the periphery can be greatly improved by practice (Dobrowolsky and Gaine ${ }^{4}$ ), differing in this respect from the sensibility for light and colour. The diminished acuity has been referred to four factors,-anatomical differences, want of practice, greater spherical aberration, and diminished intensity in indirect vision. It is probable that the first three of these causes all take part in the production of the difference. Koester ${ }^{5}$ has found that, with dark-adaptation, visual acuity is equally good in all parts of the retina, except in the fovea where it is lowered; while in light-adaptation there is an enormous increase for central vision.

Macular vision.-So far, in comparing the peripheral with the central retina, it has been assumed that the central area has uniform sensibility. This is very far from being the case. The macula, and still more the fovea, differ in their behaviour both to light and colour stimuli from that part of the central region around them which for distinction may be called the "paracentral " region.

The influence of the macula is chiefly due to its yellow pigmentation, and shows itself chiefly in colour mixture, the relative intensity of different parts of the spectrum being altered owing to the absorptive action being greater for some colours than for others. The relative degree in which the different parts of the spectrum are absorbed by the pigment of the extirpated retina was studied by Sachs. ${ }^{6}$ Hering had previously found no absorption of red, and Sachs found the absorption still very slight at $D$, with a slight increase between $D$ and $E$, which became more rapid between $E$ and $F$, reaching a maximum near $F$, then falling off slightly to the end of the violet. The influence of the macula on colour matches has been studied by Hering. ${ }^{7}$ He found that

1 Ztschr.f. Psychol. u. Physiol. d. Sinnesorg., Hamburg u. Leipzig, 1896, Bd. xii. S. 267.

2 "Ueber die Sehschürfe d. Netzhautperipherie," Wiesbaden, 1892.

${ }^{3}$ Ztschr.f. Psychol. u. Physiol. d. Sinnesorg., Hamburg u. Leipzig, 1896, Bd. xii. S. 243.

${ }^{4}$ Arch.f. d. ges. Physiol., Bonn, 1876, Bd. xii. S. 411.

5 Centralbl. $f$. Physiol., Leipzig u. Wien, 1896, Bd. x. S. 433.

${ }^{6}$ Arch. f. d. ges. Physiol., Bonn, 1891, Bd. 1. S. 574.

7 Ibid., 1893, Bd. liv. S. 277. 
a large colourless field, produced by mixture of spectral red and bluegreen, became red if reduced in size, and that a similar small colourless field became green if increased in size. Placing a screen with two apertures before such a colourless field, he found that one aperture fixed directly appeared redder and darker than the other seen indirectly. Hering explains the changes which take place in the relative intensity of colour matches on alteration of the absolute intensity by the influence of macular pigmentation (p. 1083). Breuer ${ }^{1}$ has recently compared colour equations for the central and paracentral regions, and found that his results were in agreement with the measurements of Sachs.

Foveal vision.-If the area of the central region used in direct vision is still further reduced in size, several striking differences are found to exist between its behaviour and that of the paracentral region. The observation of the nature of vision with this region is impeded by the great difficulty of guaranteeing accurate fixation. There seems to be an instinctive tendency to look at a luminous point in such a way as to see it most distinctly, and in experiments on foveal vision this tendency has to be overcome. It is an old observation of astronomers (Herschel, Arago) that faint stars are seen best when observed indirectly, and it is now generally agreed that the threshold of stimulation for light is higher for the fovea than for surrounding parts. This diminished sensitiveness to light seems to extend to the whole of the macula, but is much more marked in its central part. Breuer found that the amount of light absorbed by the macular pigment, which probably extends throughout the foveal region, was not great enough to account for the loss of sensitiveness shown by the fovea. The difference of sensitiveness between the fovea and surrounding part is greatly increased in dark-adaptation of the eye.

The colour vision of the fovea is approximately normal. Several observers have described it as less sensitive for colour than the rest of the retina, especially for blue, and König ${ }^{2}$ has lately described the fovea as blue-blind. It is a fact that a small blue field of low intensity may become invisible on direct fixation, and this is probably due to the influence of the macular pigment, which probably extends to the fovea, supplemented by the low sensibility to light of that region. At ordinary intensities, there is no doubt that blue is well perceived in foveal vision.

A feature in which the fovea has, however, a distinctive character is the absence or diminution of the photochromatic interval which occurs for the rest of the retina. According to A. E. Fick, ${ }^{3}$ Parinaud, ${ }^{4}$ and König, ${ }^{5}$ there is no interval between the absolute and specific thresholds for coloured light in the fovea, while others (Charpentier ${ }^{6}$ and Koster ${ }^{7}$ ) find that it exists, though less marked than in indirect vision.

A disputed point is whether Purkinje's phenomenon occurs in the fovea. In addition to the ordinary difficulties, its investigation is rendered unsatisfactory by the difficulty of comparison of different colours as regards brightness, especially for very small areas; but, according to König ${ }^{8}$ and v. Kries, ${ }^{9}$ Purkinje's phenomenon is not true of the fovea, and v. Kries notes that a small

${ }^{1}$ Ztschr.f. Psychol. u. Physiol. d. Sinnesorg., Hamburg u. Leipzig, 1897, Bd. xiii. S. 464.

${ }^{2}$ Sitzungsb. d. k. Akad. d. Wissensch., Berlin, 1894, S. 577.

${ }^{3}$ Arch. $f$. d. ges. Physiol., Bonn, 1888, Bd. xliii. S. 481.

4 Ann. d'ocul., 1894, tome cxii. 1. 228.

5 Ibid.

- Arch. d'opht., Paris, 1896, tome xvi. p. 337.

7 Arch.f. Ophth., 1895, Bd. xli. Abth. 4, S. 1.

${ }^{9}$ Ibicl., Bd. xlii. Abth. 3, S. 95.

8 Loc. cit. 
blue field loses greatly in intensity on approaching the fovea, while a similar red field does not. Hering ${ }^{1}$ has found that small fields of red and green, which appear equally bright when fixed centrally, become of different brightness when observed indirectly, and points out that Purkinje's phenomenon may be produced by a change from centre to periphery of the retina.

The alteration of a colour match, with diminution of the intensity, does not, according to v. Kries, take place in foveal vision (see pp. 1090 and 1104). Hering ${ }^{2}$ has stated that the deviations from Newton's law become less marked the smaller the area of the retina stimulated, and are absent when the field is made sufficiently small. Another feature of foveal vision, according to v. Kries, ${ }^{3}$ is the absence of the recurrent image of Purkinje (p. 1073).

Wilbrand ${ }^{4}$ and Franklin ${ }^{5}$ have described in the normal eye the existence of a small blind area in the neighbourhood of the fixation point, its exact position and shape varying in different observers.

\section{Colour-Blindness, etc.}

Colour-blindness.-This is by far the most important abnormality of vision, from the theoretical point of view. It is customary to distinguish between congenital and acquired colour-blindness. The former has been more fully studied, and its varieties seem to be better defined, than those due to disease, although for theoretical purposes the latter have advantages that the others lack. Congenital colour-blindness occurs in three chief forms: a rare form, in which all colours are confused, usually known as total colour-blindness, or monochromatic vision; the common form, in which there is confusion between red and green; and a very rare form, in which yellow is confused with blue. The latter forms are often classed together as examples of dichromatic vision.

Total colour-blindness.-There are now on record a considerable number of cases of this defect, which are undoubtedly congenital. Any colour may be confused with any other,-red with green, and yellow with blue. The spectrum is seen as a band of light differing in its different parts in brightness only; and by suitable adjustment of the relative intensities a match may be made between any two parts. The red end is in all, or nearly all, cases considerably shortened, while the violet end has the same limit as to the normal eye. A most striking feature is the nature of the curve of luminosity shown in Fig. 392. The brightest part of the spectrum in nearly all cases ${ }^{6}$ is the green about the line $E$, the actual maximum varying with the source of illumination, while the red end is relatively darker, the violet end relatively brighter than in the normal eye. As Hering ${ }^{7}$ was the first to point out, the curve of luminosity corresponds with the curve of luminosity of the normal eye at low intensities and with dark-adaptation. Matches made by Hering with dark-adapted eye were found to be good for his case of total colourblindness, with the exception of slight differences in the blue and violet, probably referable to slight differences of macular pigmentation. This correspondence has been abundantly confirmed by other observers.

${ }^{1}$ Arch. f. d. ges. Physiol., Bonn, 1895, Bd. 1x. S. 533.

2 Ibid., 1893, Bd. liv. S. 277.

${ }^{3}$ Ztschr. f. Psychol. u. Physiol. d. Sinnesorg., Hamburg u. Leipzig, 1896, Bd. xii. S. 81.

4 "Die Erholungsausdehnung des Gesichtsfeldes," 1896, S. 42.

${ }^{5}$ Sitzungsb. d. k. Akad. d. Wissensch., Berlin, 1894, S. 589.

${ }^{6}$ The most definite exception is a case described by Magnus (Centralbl. $f$. prakt. Augenh., Leipzig, 1880, Bd. iv. S. 373), in which the maximum brightness was at $D$.

${ }_{7}$ Arch. f. d. ges. Physiol., Bonn, 1892, Bd. xlix. S. 563. 
v. Kries ${ }^{1}$ has found that the curve of luminosity of total colourblindness is not affected by change of intensity, and that colour equations are also not affected by change of intensity. Uhthoff ${ }^{2}$ has found that the equation of Ebbinghaus and Franklin (p. 1083), which is correct for the normal eye, is not so for the achromatic eye, the mixture of red and blue-green being much the brighter; in fact, the appearance of this match to the achromatic eye is the same as that found for the normal eye in the condition of dark adaptation.

Nearly all the recorded cases have presented certain features which are not found in other forms of colour-blindness, namely, diminished visual acuity, photophobia, and nystagmus. Their presence has led to total

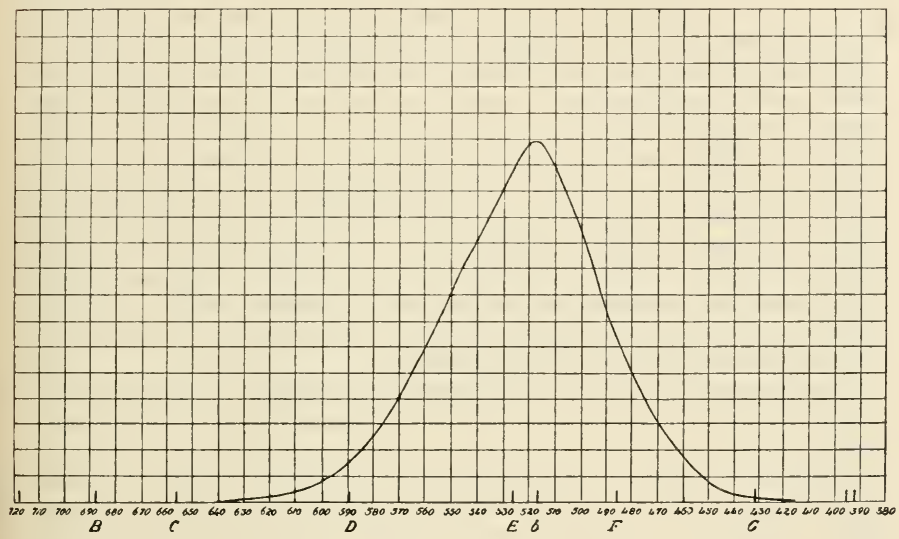

FIG. 392.-König.

colour-blindness being regarded as a pathological condition, originating probably in early life, and differing altogether in nature from the common form of colour-blindness. As will be seen later (p. 1102), however, there is a possible explanation of these features, which makes them consistent with a developmental rather than a strictly pathological anomaly. The diminution of visual acuity seems to exist only for ordinary illumination; with feeble light, vision may be as acute or even more acute than normal, especially at first, and the visual acuity in different parts of the retina shows a very close agreement with that of the normal eye when adapted to the dark. The photophobia seems to be nearly always present in some degree; nystagmus has been recorded in most cases. Nettleship ${ }^{3}$ found a central scotoma of considerable extent in several cases of this kind, and a small absolute central scotoma has been found by many recent observers. One feature in which this differs from the common form of colour-blindness is its frequent occurrence in females. It may occur in several nembers of one family.

Red-green blindness. - In considering the common form of colourblindness, it is especially difficult to avoid terms which imply theory, but

${ }^{1}$ Ztschr. f. Psychol. u. Physiol. d. Sinnesorg., Hamburg u. Leipzig, 1897, Bd. xiii. S. 295.

2 Ibid., 1899, Bd. xx. S. 326.

${ }^{3}$ St. Thomas's Hosp. Rep., London, 1880, vol. x. p. 37.

VOL. $11 .-69$ 
since there is no doubt that the most obvious characteristic is confusion between red and green, the condition may, without prejudice, be spoken of as red-green blindness. It is now generally agreed that to individuals with this defect the spectrum is divided into two parts, separated by an interval which can be matched with white, known as the neutral band. In most cases the neutral band is very narrow, and its existence can only be demonstrated with a spectrum in which each part is free from mixture with other rays, or it may be demonstrated more roughly by finding where the colour of one end passes into that of the other. This neutral band is situated in the green or blue-green, somewhere between the lines $b$ and $F$, the wave-lengths $505 \lambda$ and $492 \lambda$. In other cases the neutral band is broader, and more easily demonstrated. On either side of the neutral band the spectrum appears to be of one colour, differing in its different parts in brightness and saturation only. By suitable adjustment of intensities any part of one half can be matched with any other part of that half, and a match may also be made between two parts of one half by adding white light to the one that appears more saturated. The colour of each half appears to increase in saturation from the middle outwards. Further, in all cases of this defect, it is possible to find a red and a green, each of which may be matched with white; this may be done with spectral, and less easily with pigment colours; the red which is so matched is one in which a certain amount of blue has to be added to the extreme red of the spectrum. In any given case the red and green so found are complementary, and mixed together make white to the normal eye.

Another feature common to all cases of red-green blindness is, that a colour equation which is good for normal vision is good for dichromatic vision.

Cases of red-green blindness seem to show the changes in colour matches with alteration of intensity more distinctly than in normal vision. The first observation of the kind was made by Preyer, ${ }^{1}$ who found that the match between the neutral band and white was no longer good on change of intensity, the neutral band shifting its position towards violet when the intensity was increased. This was confirmed by König and Brodhun, ${ }^{2}$ and denied by Hering, who pointed out, however, the great difficulty of determining the position of the neutral band, owing to its varying with the character of the source of light and the colour adaptation of the eye; while, if the eye is adapted to the dark in order to get rid of the latter factor, there is great diminution of the sensitiveness for slight differences of colour upon which the determination of the neutral point depends.

Brodhun ${ }^{3}$ investigated the influence of intensity on a number of equations in which a mixture of light of $615 \lambda$ and $460 \lambda$ was matched with intermediate parts of the spectrum. He found that a match with light of $480 \lambda$ was not affected by change of intensity, but, as the wavelength of the homogeneous light increased, marked changes occurred; thus, with an intensity of one unit, light of $560 \lambda$ was matched with a mixture of 49 parts of $615 \lambda$, and 428 parts of $460 \lambda$; while, with an intensity of 32 units, the proportions were 76 and 11.4 respectively.

'Arch.f.d.ges. Physiol., Bonn, 1881, Bd. xxv. S. 41.

2 Sitzungsb. d. k. Akad. d. Wisscnsch., Berlin, 1887, S. 311.

${ }^{3}$ Ztschr. f. Psychol. u. Physiol. d. Sinnesorg., Hamburg u. Leipzig, 1893, Bd. v. S. 323. 
Hering ${ }^{1}$ has objected to the results of König and Brodhun, that they were made with too large a field, and he found that with so small a field that the area stimulated was limited to the macula, matches at one intensity were good for any other. v. Kries and Nagel, ${ }^{2}$ using a small field without absolutely accurate fixation, have compared equations with the eye adapted to bright light and to darkness, and have found a very marked difference in the two conditions. With a still smaller field, and with accurate central fixation, it was found that there was a central area of the visual field in which there was no deviation from Newton's law, and a match was good at all intensities. Two dichromats (Nagel and Stark) were able to measure this central region, and found that at 1 metre from the eye its vertical diameter was between 30 and $40 \mathrm{~mm}$., these limits corresponding closely with those of foveal vision.

The two kinds of red-green blindness.-The features described are common to all cases in which red and green are confused. In respect of certain other features, these cases fall into two groups, which, in accordance with a special theory, have commonly been known as red-blind and green-blind. In the present state of the subject it is better to use terms which do not involve theory, and v. Kries has lately suggested that the names protanopic and deuteranopic shall be substituted for red-blind and green-blind. These names have the disadvantage that they give no clue to the nature of the difference of vision in the two groups. The most obvious difference is in the relative brightness of the red end of the spectrum. To one group it is very dark, or even black (i.e. the spectrum is shortened), while to the other its brightness is approximately as great as to the normal eye, and this difference may be expressed by the terms scoterythrous and photerythrous. ${ }^{3}$ In the scoterythrous group the spectrum is more or less shortened at the red end, the amount of shortening being, however, influenced by intensity and by adaptation, especially adaptation to red. Consequently, a colour-blind case must always be compared with a case of normal vision under exactly similar conditions. In this group the maximum brightness is shifted towards $E$, as compared with normal vision. According to Abney, it is about $559 \lambda$. In the photerythrous group the maximum brightness is about $D$, and the spectrum has approximately the same relative brightness and the same red limit as in normal vision.

The neutral band has been said to be nearer the violet end in the scoterythrous group, but this does not seem to be the case. In all cases it lies between 505 and $492 \lambda$, but König found that its position varied within these limits, independently of the other differences. According to v. Kries, the differences in the two groups only amount to 4 to $6 \lambda$, and this may easily be obscured by the factors mentioned in the last section.

It is a point of great theoretical importance, whether these two groups are sharply marked off from one another, or whether they are connected by transitional cases. As regards shortening, maximum brightness, and neutral band, there is no definite evidence in favour of a distinct separation without transitional cases; but in the case of certain other features the evidence appears to be conclusive.

${ }^{1}$ Lotos, Prag, 1885, Bd. vi. S. 142; Arch.f.d. ges. Physiol., Bonn, 1893, Bd. xlvii. S. 277.

2 Ztschr. f. Psychol. u. Physiol. d. Simesorg., Hamburg u. Leipzig, 1896, Bd. xii. S. 1.

3 I am indebted to Dr. Verrall of Trinity College, Cambridge, for suggesting these designations. They do not imply that either group see red, but only that the light which is red to the normal eye is relatively dark to one and relatively light to the other group. 
In 1879 , v. Kries and Küster ${ }^{1}$ determined the amounts of spectral red and blue necessary to match a certain blue-green, and found that the cases examined fell into two sharply-defined groups. Recently, v. Kries ${ }^{2}$ has determined in twenty cases the amount of lithium-red necessary to match sodium-yellow, and has found two distinct groups, the members of each differing very little from one another but very greatly from those of the other group, i.e. to ten parts of the yellow the members of one group required $36: 3$ to 40 parts of red light, while those of the other varied from 196 to 225 .

Donders ${ }^{3}$ compared the relative intensities of lithium-red, sodiumyellow, and thallium-green in twenty cases of colour-blindness, and found that they fell into two widely separated groups, the relative intensities of the three lights showing a close correspondence in photerythrous and normal vision.

In two scoterythrous and two photerythrous cases, v. Kries ${ }^{4}$ obtained matches of a mixture of blue and red light with a number of intermediate homogeneous lights. Representing the amounts of red and blue light respectively by curves, v. Kries found that the red curves of the two scoterythrous cases corresponded closely with each other, and similarly those of the two photerythrous cases; but the red eurves of the two groups differed greatly from one another, the former having its maximum at $571 \lambda$, while that of the latter was at $603 \lambda$. The blue curves of all four, on the other hand, showed a much closer correspondence with each other.

According to Hering and Hess, there is an almost exact correspondence between the vision of total colour-blindness and that of the extreme periphery of the normal retina, and also between red-green blindness and the red-green blind zone of the normal eye. Hess found that colour matches good for his red-green blind zone were good for two red-green blind cases of the photerythrous type.

v. Kries ${ }^{5}$ has lately brought forward evidence of a very different relation between the two. He finds that the curve of brightness of the spectrum at the extreme periphery of the normal eye corresponds closely with that for the paracentral region, the maximum brightness being between $608 \lambda$ and $589 \lambda$, while the curve differs enormously from that of congenital total colour-blindness. He finds that the periphery values of the normal eye differ very greatly from those of the scoterythrous group of red-green blindness, the curve of the latter having its maximum at $558 \lambda$. The values of a photerythrous case agree much more closely with the normal, but show a greater deviation than is found between different cases of normal vision. Polimanti ${ }^{6}$ has also found that the values obtained by the flicker method for lights between $664 \lambda$ and $526 \lambda$ differ in the normal and colour-blind eyes. This was especially marked in a scoterythrous case, in whom the maximal value (i.e. brightness) was at $565 \lambda$ by the flicker method, while the maximum for a normal eye was at $589 \lambda$. A photerythrous case agreed inuch more closely with the normal, the maximum being in this case, however, displaced somewhat towards the red end of the spectrum.

Weak colour-vision and individual variations.-In addition to

1 Arch.f. Physiol., Leipzig, 1879, S. 513.

2 Ztschr.f. Psychol. u. Physiol. d. Sinnesorg., Hamburg u. Leipzig, 1897, Bd. xiii. S. 259.

${ }^{3}$ Arch.f. Physiol., Leipzig, 1884, S. $528 . \quad{ }^{4}$ Loc. cit., S. 274.

${ }^{5}$ Ztschr. f. Psychol. u. Physiol. d. Sinnesorg., Hamburg u. Leipzig, 1897, Bd. xv. S. 247.

${ }^{6}$ Ibid., 1899, Bd. xix. S. 263. 
these well-defined cases, others are met with which seem to show weakness rather than absence of certain colour sensations. They behave in the same way as in the complete form, but only confuse colours when they possess a low degree of intensity or saturation. Such cases, as might be expected, differ from the more complete cases in the limits of the spectrum and in the form of their luminosity curves.

Again, besides these intermediate cases, others occur in which there does not seem to be any confusion of colours, but which are in certain ways anomalous. These cases are often described as individual variations. They were first observed by Rayleigh, ${ }^{1}$ who found that if a number of individuals matched a mixture of spectral red and green with spectral yellow, some required a much larger proportion of green than the others, while some, on the other hand, required a much larger proportion of red. The yellows matched by such individuals appear very different to the normal eye. In addition to these marked variations, slight differences occur, and these may often be found between the two eyes of the same individual.

The subject has been fully investigated by Donders ${ }^{2}$ and Hering. ${ }^{3}$ Donders examined the proportions in which light corresponding to the lithium and thallium lines was mixed to match sodium light. In sixty cases, fifty-six gave results agreeing fairly, the variations being within moderate limits. In the other four cases the proportions were very different, the proportion of green to red being very much larger, 48.50 as compared with $23 \cdot 77$. Donders, however, unlike Rayleigh, found that these cases showed a weakened colour-sense of the same kind as in the common form of colour-blindness. Donders then examined several cases of weak colour-sense, and found that, as regards the match in question, five belonged to the second category, requiring a larger proportion of green; three other cases of weak colour-sense, on the other hand, belonged to the first category; while one case, belonging to the second category as regards Rayleigh's match, appeared to have normal colourvision. In the complete form of red-green blindness, it was found that Rayleigh's match was no longer possible.

Donders supposed that the results depended on a difference in the relative intensities of lithium and thallium light, but found that this was much less than could account for the condition.

Maxwell ${ }^{4}$ found that considerable variations occurred in the colour equations made by different individuals, and referred them to corresponding differences in macular pigmentation. This explanation has been applied and developed by Hering, not only for individual variations, but also to explain the two forms of red-green blindness. His investigations will be considered later in connection with his theory.

Blue-yellow blindness.-Cases in which there is confusion between yellow and blue are very rare. The two chief cases have been recorded by v. Vintschgau ${ }^{5}$ and Hering, ${ }^{6}$ and by Donders. ${ }^{7}$ The first case was very completely investigated. It was found that both yellow and blue could be matched with grey and with each other, while a match could not be made between red and green. The spectrum was shortened at each end. There was a broad neutral grey band in the yellow, extending from $598 \lambda$ to $568 \lambda$, and the short wave-end also pre-

\footnotetext{
1 Nature, London, 1882, vol. xxv. p. 64.

2 Arch. f. Physiol., Leipzig, 1884, S. 518.

3 Lotos, Prag, 1885, Bd. vi. S. 142.

+ Phil. Trans., London, 1860, vol, cl. 1) 76.

5 Arch.f. d. ges. Physiol., Bonn, 1891, Bd. xlviii. S. 431; and 1894, Bd. lvii. S. 191.

${ }^{6}$ Ibid., 1894, Bd. Ivii. S. $308 .{ }_{7}^{7}$ Brit. Med. Journ., London, 1880, vol. ii. p. 768.
} 
sented a neutral region beyond the point where colour could be perceived. The most saturated green was seen at $532 \lambda$, and the place of maximum brightness was more towards the red end, between $558 \lambda$ and $565 \lambda$. The case recorded by Donders was very similar, the spectrum being shortened at each end, and the neutral band in the yellow was even broader than in the other case, extending to one-third of the length of the spectrum.

Unilateral cases.-The subjective nature of the sensations experienced by the congenitally colour-blind can, in ordinary cases, only be a matter of speculation. Individuals, who see the spectrum from the red end to the neutral band in only one colour, may nevertheless, under many circumstances, distinguish red from yellow and yellow from green, but accurate observers recognise that they are enabled to do this owing to differences of saturation. Red is especially distinguished as a dark colour with a very high degree of saturation. Most observers, such as Dr. Pole, ${ }^{1}$ who has given a classical account of his own condition, believe that the two colours they experience correspond to the yellow and blue of the normal eye. A few cases have been recorded in which the congenital defect was unilateral, and the sensations of the defective could be compared with those of the normal eye. One such case investigated by v. Hippel ${ }^{2}$ and Holmgren, ${ }^{3}$ was of the ordinary type, in which red and green are confused. There is some doubt as to the subgroup to which this subject belonged; according to Holmgren, he was a typical example of the group in which the spectrum is shortened at the red end; while, according to v. Hippel, ${ }^{4}$ he could distinctly see as far as the rubidium line $y$, which he called faint yellow. Both agree that the two colours of this case corresponded with the yellow and blue of the normal eye, the yellow corresponding to the colour of $D$, and the blue to the indium or cæsium line of the normal eye.

In another unilateral case recorded by Holmgren, blue and yellow were confused; the neutral band was just on the green side of $D$; the two colours compared with those of the normal eye were red and green, the red corresponding to carmine and the green inclining to blue. There was no sensation corresponding to the yellow of the normal eye.

In a third unilateral case recorded by Becker, ${ }^{5}$ the condition was one of total or almost total colour-blindness. The defect was said to have existed from an early age. All colours were confused, with the exception of brown, which seems to have been correctly recognised. The case differed in several respects from typical total colour-blindness. There was no shortening of the red end, and slight shortening at the violet end. The point of maximum brightness was almost exactly at the sodium line. Visual acuity was normal, and no mention is made of photophobia or nystagmus.

In other unilateral cases which have been described, the defect was probably acquired.

Acquired colour-blindness.-As regards the subjective nature of the sensations experienced, it might be expected that acquired cases would be more useful than congenital, since the individual would have the memory of his normal vision as a standard. These cases, however, have been much less fully studied. Acquired colour-blindness

1 Phil. Trans., London, 1859, vol. cxlix. p. 323.

2 Arch. f. Ophth., 1880, Bd. xxvi. Abth. 2, S. 176 ; and 1881, Bd. xxvii. Abth. 3 , S. 47 .

${ }^{3}$ Centralbl. $f$. d. med. Wissensch., Berlin, 1880, S. 898 and 913.

${ }^{4}$ Arch.f. Ophth., 1879, Bd. xxv. Abth. 2, S. 205.

${ }^{5}$ Centralbl. $f$. prakt. Augenh., Leipzig, 1888, S. 353. 
occurs in two chief forms; and it may begin by peripheral constriction of the colour fields, the defect spreading inwards, or as a central scotoma spreading outwards. In both cases red and green seem to be almost always the first colours to go, blue and yellow remaining. In acquired total colour-blindness the evidence seems to be in favour of the maximum brightness being at $D$, and not at $E$, as in the congenital form.

One distinction of importance in localised defects of retinal sensibility may be mentioned here. These defects usually occur either as cases of hemianopia or as scotomata, usually central. In some cases there is complete absence of vision in the defective part of the retina; the condition resembles that of the blind spot; and often the patient may only be made aware of his defect by special means, although in the case of hemianopia the defect is usually marked enough to attract attention spontaneously. In other cases the defective part of the field may be occupied by a cloud (Schwarzsehen). This appears to be common in central scotoma, but does not seem to have been recorded in hemianopia.

Santonin vision.-Abnormalities of colour-vision are found under certain other conditions. A normal individual, who has worn coloured spectacles, or been in coloured illumination for a long time, will confuse colours in a way that may resemble colour-blindness. More important is the condition produced by certain drugs, and especially by santouin. This produces yellow vision in bright light, and violet vision in the dark, while in bright light, black objects often appear to have violet borders. The violet has been regarded as an after-image effect, but it seems to occur when the eyes have been altogether kept in the dark after taking the drug. The violet end of the spectrum is shortened. There does not appear to be any marked change in other parts of the spectrum, nor anything corresponding to the neutral band of yellowblue blindness; in fact, some observers have described the yellow of the spectrum as more intense than normal. Haidinger's brushes, and especially the yellow part, become more distinct than normally; and a definite yellow spot may be seen at the fixation point, apparently corresponding to the macula (König). ${ }^{1}$ Schultze ${ }^{2}$ supposed that the santonin effect depends upon coloration of the macula, and in favour of this is the increase in the distinctness of Haidinger's brushes, which probably depend upon the yellow spot; while, according to Preyer, ${ }^{3}$ the shortening of the violet end of the spectrum only occurs with direct vision, the violet of the spectrum again becoming visible when observed indirectly. Rose, ${ }^{4}$ on the other hand, advocated the view that it depends on an affection of the nervous system, and failed to find coloration of any part of the eye sufficient to account for the phenomena. It seems probable that the condition is of a different nature to yellow-blue blindness.

Picric acid is said also to produce yellow vision, and yellow vision has been described in jaundice.

Night-blindness and the vision of albinos.-Night-blindness is a condition of theoretical interest. It occurs in several abnormal conditions of the retina and choroid, but is especially well marked in retinitis pigmentosa, in which the retinal pigment layer appears to be chiefly and primarily affected. It also occurs after exposure to too strong light,

\footnotetext{
${ }^{1}$ Centralbl. f. praht. Augenh., Leipzig, 1888, S. 353.

2 "Ueber den gelben Fleek der Retina," Bonn, 1866.

${ }^{3}$ Arch.f. d. ges. Fhysiol., Bonn, 1868, Bd. i. S. 299.

+ Virchow's Archiv., 1860, Bd. xviii. S. 29.
} 
especially in lowered conditions of nutrition, and has also been observed in connection with diseases of the liver (Parinand). ${ }^{1} \quad$ There seems to be little doubt that the essential feature of the condition is abnormal slowness of dark-adaptation. It has been found that a night-blind individual may attain the same degree of sensitiveness at low intensities as normal, but requires for it a much longer period of adaptation.

Schirmer ${ }^{2}$ objects to the view that night-blindness depends simply on adaptation of abnormal slowness, on the ground that, immediately after going into feeble illumination, the night-blind see much worse than normal individuals. This may, however, be connected with the momentary adaptation described by Hering (p. 1080). Treitel ${ }^{3}$ found that the bright-adapted eye, when placed suddenly in feeble illumination, behaved in many ways like the night-blind eye.

Photophobia, such as that which occurs in albinos, may be regarded as an abnormal condition of the upper limit of adaptation. It does not disappear with a small artificial pupil. Schirmer ${ }^{4}$ has found that in this condition the threshold is normal, and dark-adaptation takes place normally in both rate and extent.

Subjective colour sensations.-The most frequent of these is red vision, or erythropsia. It has frequently been described as occurring after removal of the lens for cataract and after strong stimulation, such as that of light reflected from snow. It has been observed in conjunction with night-blindness. It has been produced experimentally by Fuchs ${ }^{5}$ by exposure to snow-light with an artificially dilated pupil. The white squares of a black and white chessboard pattern appeared red, while the black squares were greenish. The condition has been referred to entoptic vision of visual purple, but no satisfactory explanation is yet forthcoming.

Cases of other kinds of subjective colour-vision have been described, such as green vision (Somya), ${ }^{6}$ either affecting the whole visual field or in the form of coloured globes. Blue vision often occurs after removal of the lens for cataract. It is connected with the yellow coloration of the lens.

Intrinsic light (of the retina?).-This increases in intensity with dark-adaptation of the eye, and the variations in its appearance have been described by Aubert.7 Aubert regarded it as probably of central origin, and the same view has been advocated by others. Filehne ${ }^{8}$ found that the intrinsic light was continuous over the central scotoma of the visual field which existed in his own eye, and since his defect was probably due to affection of the nervous path, rather than of the retina, he held this to point to the central origin of the intrinsic light. It has been suggested (Dufour) ${ }^{9}$ that absence of vision and eloudy vision (p. 1095) may be characteristic of cortical and subcortical hemianopia respectively; and if found to be so, the fact would point to the central origin of the intrinsic light, but the clinical evidence on this point is at present scanty and conflicting.

1 Arch. gén. de méd., Paris, 1881, Sér. 7, tome vii. p. 403.

2 Verhandl. d. X. internat. med. Cong., Berlin, 1890, Bd. iv. Abth. 10, S. 58.

3 Arch. f. Ophth., 1887, Bd. xxxiii. Abth. 4, S. 73.

4 Verhandl. d. X. internat. med. Cong., Berlin, 1890, Bd. iv. Abth. 10, S. 58.

5 Arch. f. Ophth., 1896, Bd. xlii. Abth. 4, S. 207.

${ }^{6}$ Ztschr. f. Psychol. u. Physiol. d. Sinnesorg., Hamburg u. Leipzig, 1894, Bd. vii. S. 305.

7 "Physiol. d. Netzliaut," S. 333.

8 Arch.f. Oplith., 1885, Bd. xxxi. Abth. 2, S. 1.

${ }^{2}$ Rev. méd. de la Suisse Rom., Genève, 1889, tome ix. p. 445. 
The relation of after-image to the intrinsic light of the retina has been already mentioned. According to Helmholtz, after-images are modifications of intrinsic light; according to others, they are independent phenomena, the former being of peripheral, the latter of central origin (G. E. Müller). ${ }^{1}$

Electrical stimulation of eye.-Not only is the eye stimulated by variations in the strength of a constant current applied to it, but, during the passage of the current, sensations arise whose quality depends upon the direction of the current. With an ascending current $^{2}$ the visual field becomes brighter, with a descending current the field becomes darker, even when its brightness is only due to the intrinsic light of the retina. The field is also coloured, the exact nature of the coloration having been described in different ways. With an ascending current, violet has usually been seen; with a descending current, a yellow-reddish according to Helmholtz $;{ }^{3}$ greenish according to recent careful experiments by G. E. Müller, ${ }^{4}$ and a number of good observers. The position of the optic dise is dark in the former case, bright and bluish in the latter, effects due apparently to spatial induction. When the stimulation ceases, the bright violet field becomes dark and yellowish; the dark yellow field becomes bright and bluish (temporal induction). The sensation which arises on sudden closing of the current has a higher intensity, but has the same character as that which continues after closure (Purkinje, G. E. Müller); thus, with closure of ascending and opening of descending current, there is a bright violet flash; with opening of ascending and closure of descending current, there is a dark greenish-yellow sensation.

An interesting point, determined by G. E. Müller, is that the condition of retinal adaptation does not appreciably influence the effect of electrical stimulation.

Mechanical stimulation and pressure blindness.-The phosphene produced by localised pressure on the accessible parts of the eyeball is blue and yellow, this being in agreement with the greater peripheral extension of the fields for these colours.

The results of mechanical stimulation of the optic nerve are very important in relation to the doctrine of specific nervous energy. It is generally supposed that when the optic nerve is cut during extirpation of the eyeball, in a conscious individual, a flash of light is experienced. This belief rests only on tradition. In 1882, SchmidtRimpler ${ }^{5}$ found that no flash occurred in several cases in which the eyeball was removed without an anesthetic, and there is no satisfactory evidence that mechanical stimulation of the nerve produces a light sensation. On the other hand, Schmidt-Rimpler found that electrical stimulation of the stump of the optic nerve after extirpation, produced in some cases a light sensation, which was described by one individual as of the same nature as that which he had seen when treated by electricity before removal of the eyeball.

If mechanical pressure is exerted on the eyeball for some time, a condition almost amounting to blindness is induced, in which objects can

1 Ztschr.f. Psychol. u. Physiol. d. Sinnesorg., Hamburg u. Leipzig, 1897, Bd. xiv. S. 40.

2 I.e., with the anode over the eye

3 "Handbuch d. physiol. Optik," 2te Aufl., S. 243.

${ }^{4}$ Ztschr. f. Psychol. u. Physiol. d. Sinnesorg., Hamburg u. Leipzig, 1897, Bd. xiv. S. 329. Refer to this paper for a full account of this subject.

${ }^{5}$ Centralbl. $f$. d. med. Wissensch., Berlin, 1882, Bd. xx. S. 1. 
no longer be distinguished (Donders). Exner ${ }^{1}$ found that if a field half white and half black be observed while the eyeball is being pressed, the white half becomes quite dark. If a white square is then placed on the black half, it will be seen. Exner explained this experiment by supposing that, while looking at the white half, the store of material in the retina had been used up, and, owing to interference with blood supply, could not be regenerated, while on the black half the retinal material was not used up, and permitted vision of the white square. Kühne ${ }^{2}$ has pointed out that the vision of the white square may be due to its greater distinctness, owing to contrast on the black ground, and found that pressure might produce blindness without the presence of light. Exner also found that pressure on the eyeball influenced the point of fusion of intermittent retinal stimuli; a black and white disc, which still flickers to a normal eye, appearing grey to a pressed eye. $\mathrm{He}$ found that it prevented the appearance of after-images and shortened the existence of those already present.

\section{Binocular Phenomena.}

Adaptation.-Certain binocular relations are of importance in connection with the theories of light and colour sensation. The condition of adaptation of one eye is independent of that of the other eye. It has been clearly established that dark-adaptation of one eye does not increase the sensibility of the other eye. Charpentier ${ }^{3}$ at first thought that it did so, but found that the apparent increase of intensity was due to the consensual increase in the size of the pupil admitting more light to the eye, and was absent with a small artificial pupil.

Binocular contrast.-The influence of stimulation of one recina upon the condition of the other is best shown by producing double images of a white strip on a black ground. If, now, a coloured glass is held before one eye, and a grey glass before the other, the strip seen by the latter will appear in the colour complementary to that of the glass. Another method is to hold before one eye a card with a small hole, which will also be coloured complementarily to the colour of a glass held before the other eye. When the colour stimulus applied to the one eye is removed, the colour of the strip or of the hole will change to the colour of the glass, and this contrast colour is more marked than that which is seen at first, i.e. binocular contrast is more marked during the afterimage effect than during the original excitation.

Another example of binocular colour contrast is known as Fechner's side-window experiment. If one stands so that light falls laterally on one eye, and a white strip of paper be doubled against a dark ground, it will be seen that the strip belonging to the more illuminated eye is darker than that belonging to the other eye, while the former is tinged green and the latter red. These features become more marked the longer the difference between the illumination of the two eyes is allowed to last. The darkness of the strip seen by one eye is explained by the "fatigue" of this eye being greater than that of the other. The different coloration of the two strips is explained partly by the fact that the light which passes through the sclerotic of the more illuminated eye, acquires a red colour (see p. 1057), and partly by binocular contrast. As

1 Arch.f. d. ges. Physiol., Bonn, 1878, Bd. xvi. S. 407 ; and 1879, Bd. xx. S. 614.

2 Untersuch. a. d. physiol. Inst. d. Univ., Heidelberg, 1882, Bd. ii. S. 46.

3 "La lumière et les conleurs," Paris, 1888, p. 177. 
Hering points out, binocular contrast gives a very delicate means of determining the condition of excitability, i.e. of adaptation of the two eyes.

Ebbinghaus $^{1}$ has described an after-image seen by one eye after stimulation of the other eye, the after-image being of the same colour as the original; and the same phenomenon, as already mentioned, may be seen in connection with binocular contrast. This kind of after-image must be distinguished from the negative after-image which is ascribed to one eye after stimulation of the other, this phenomenon depending simply on the difficulty of telling with which eye an observation is being made(seep.1136).

The subject of binocular colour mixture will be discussed later.

In order to explain the phenomena of binocular contrast, it is necessary either to assume a sympathetic relation between the two retinæ or to ascribe them to cerebral processes. The movements, described by Engelmann, of the cones and pigment in one eye, when light is applied to the other eye, give a possible physiological basis for the retinal origin of the phenomena; but these movements probably take place too slowly, even in warm-blooded animals, to explain the almost instantaneous occurrence of binocular contrast, On the other hand, Engelmann and v. Grijns found that the electrical variation of the opposite eye occurred after a short latent period (p. 1052), and a retinal process underlying this may be the basis of the phenomenon. The alternative is that binocular contrast depends on a cerebral process, and since there seems little reason to differentiate the binocular from the ordinary form of colour contrast, this alternative would render probable the central origin of contrast generally.

\section{Functions of the Retinal Structures.}

The sensitive layer.-The facts of light and colour-vision having been reviewed, their relation to retinal structures and processes may now be considered. The chief theories of vision are based on the hypothetical existence of certain visual substances, and do not attempt to identify these substances with any known retinal structures. Quite apart from these theories, however, there are some grounds for assigning certain visual functions to certain retinal structures. The reasons which establish the rod and cone layer as the primarily sensitive part of the retina may be dealt with first.

The changes which have been described in the rods, cones, and pigment epithelium may be taken as evidence that these structures are the sensitive elements of the retina, and that the chief processes by which light vibrations are transformed into nervous impulses take place in them. Further evidence is derived from the entoptic appearances known as Purkinje's figures. These are due to shadows thrown by the retinal vessels on the sensitive elements behind, and are under ordinary circumstances invisible, owing to local adaptation. The distance of the

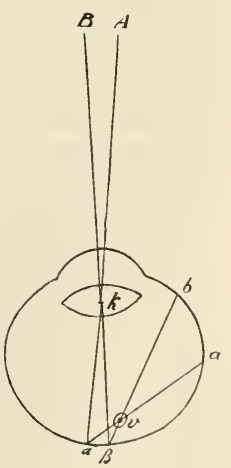

FIG, 393 . elements behind the vessels may be calculated from the apparent movement of the figures on moving the source of illumination. I'urkinje described three methods of seeing the figures: by concentrating light with 
a lens on the sclerotic, the point of light being as fine as possible and at some distance from the cornea (Fig. 393 ); by illuminating the eye laterally, so that the light enters the pupil and produces an image on the nasal side of the retina, while the light from this lateral image in its turn throws shadows of the retinal vessels (Fig. 394); and, thirdly, by holding a card against a brightly illuminated background, with a small hole in the anterior focus of the eye. In each case the source of illumination should be moved; by the first and third methods the apparent vertical movement of the figures will be in the same direction as the movement of the source of illumination; by the second method it will be in the opposite direction. In calculating the position of the sensitive elements, the first method is the most satisfactory, and was used by Heinrich

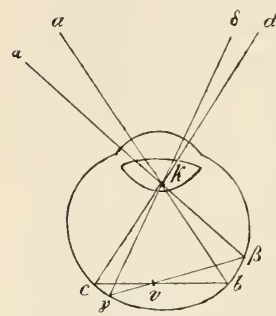

Fig. 394 .

Miiller, to whom we owe the experiment. In Fig. 393, $\alpha \beta$ may be calculated directly from $A B$, the apparent movement on a screen at a known distance; if $b \beta$ and $a \alpha$ be joined, their point of intersection, $v$, will give the position of the vessel. Muiller measured the distance, $b v$ and $a v$, on frozen eyeballs, and took the mean of the variations met with; the distances, $\alpha v$ and $\beta v$, could then be calculated. In his own eye the distance thus ascertained varied from 17 to 32 mm., for the eye of three other observers from $\cdot 19$ to 33 . The distance between the vessels and the rod and cone layer in man was found to be between $\cdot 2$ and $\cdot 3 \mathrm{~mm}$.

König and Zumft ${ }^{1}$ have made use of a modification of Purkinje's third method to determine the depth of the sensitive elements for white and monochromatic lights. They held a screen with two holes closely before the eye, and obtained two shadow figures of the same vessel. These were projected on a scale, and the depth of the sensitive layer calculated from the distance of the projected figures on the scale and that between the two holes. They give the following figures:-For white light the distance from the vessel to the sensitive layer was $4120 \mathrm{~mm}$.; for light of $670 \lambda, \cdot 4402 \mathrm{~mm}$.; of $535 \lambda, \cdot 4141 \mathrm{~mm}$.; of $434 \lambda, \cdot 3643 \mathrm{~mm}$. Satisfactory results were only obtained with one observer. They seemed to show that the greater the wave-length, the more deeply seated is the sensitive layer. The difference between their extreme measurements, namely, $.0759 \mathrm{~mm}$., is rather more than the thickness of the rod and cone layer, and König supposes that red light may act on the retinal pigment.

Leber and Koster ${ }^{2}$ were unable to obtain two images with two holes, as in König's method, and also failed to confirm his results by another method. From König's results it should follow that Purkinje's images, projected on a field half red and half blue, should show a difference of parallax; their relative rapidity should be as $22: 19$. They failed to observe any difference, although they found by separate experiments that a difference of $22: 19$ in rapidity was capable of being perceived.

Evidence as to the seat of the sensitive elements of the retina has also been derived from the degree of delicacy of local discrimination in the retina. The visual angle necessary to enable two points to be distinguished has been tested by observations on stars and on fine parallel lines. The figures obtained by different observers vary from $50^{\prime \prime}$

${ }^{1}$ Sitzungsb. d. k. Akad. d. Wissensch., Berlin, 1894, S. 439.

2 Arch.f. Ophth., 1895, Bd xli. Abth. 1, S. 1. 
to $147 \cdot 5^{\prime \prime}$. With fine black wires against the sky, separated from each other by a distance corresponding to the diameter of the wires, Helmholtz found for his own eye an angle of $63^{\prime \prime} \cdot 75$ necessary for discrimination. This corresponds in Listing's eye to a retinal distance of '00463 mm. The thickness of the cones in the macula has been determined by several observers, the figures given varying from .0015 (H. Müller) to 0054 (Kölliker). In making his observations, Helmholtz noticed at a certain distance the appearances shown in Fig. 395, $A$. He explained the appearance by the arrangement of the cones, as is shown in Fig. $395, B$.

From the figures above obtained Helmholtz calculated the number of cones which should be found on a square millimetre,

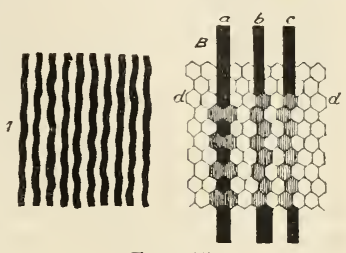

Fig. 395 . namely, 13,466; and this agrees very closely with the number of cones in a square millimetre of the fovea as counted by Sulzer, ${ }^{1}$ about 13,500 . Observations obtained by du Bois-Reymond ${ }^{2}$ also agree very closely. This observer determined the distance at which a number of (460) holes in a sheet of metal were discriminated when looked at against the sky, and found that in different observers the points were perceived when they ranged from 7400 to 14,900 per square millimetre of the retinal surface. These observations, together with the nature of the vision of the peripheral retina, make it most probable that visual acuity is dependent on the cones, i.e. at ordinary luminosities.

Function of visual purple.-Till comparatively recently, no systematic attempt had been made to bring visual sensations into relation with any known retinal substances. When visual purple was discovered, it was hoped that it would become the basis of the physiological theory of vision, but its absence from the place of most distinct vision was so great a difficulty that it was soon neglected as an important factor in vision.

In 1867, however, before visual purple was discovered, the occurrence of rods alone in nocturnal animals had led Max Schultze ${ }^{3}$ to the conclusion that rods were in some way concerned with vision at low intensities. The chemical behaviour and great instability of visual purple suggested the same idea to Kiihne. ${ }^{4}$ Parinaud ${ }^{5}$ was led by the consideration of pathological night-blindness to regard visual purple as the basis of vision at low intensities, and found this confirmed by the nature of foveal vision. Charpentier ${ }^{6}$ held at one time similar views. More recently the same idea has been much more fully developed, especially by v. Kries, ${ }^{7}$ who appears to have independently reached conclusions identical with those of Parinaud.

According to this view, the great increase of sensitiveness to light of the dark-adapted eye is due to accumulation of visual purple. The fact that adaptation to brightness takes place with much greater rapidity than to darkness, agrees with the fact that visual purple is bleached so much more rapidly than it is regenerated; and there is, further, a rough correspondence between the lates of the two processes,

${ }^{1}$ Sitzungsb. d. k. Akad. d. Wissensch., Wien, 1880, Bd. Ixxxi. S. 7 .

${ }^{2}$ Arch.f. Ophth., 1886, Bd. xxxii. Abth. 3, S. 1.

- Arch. f. mikr. Anat., Bonn, 1866, Bd. ii. S. 175.

${ }^{4}$ Journ. Physiol., Cambridge and London, 1878, vol. i. p. 189.

5 Compt. rend. Acal. d. sc., Paris, 1881, tome xciii. p. 286 ; and 1885, tome ci. p. 821.

6 Ibid., i878, tone Ixxxvi. p. 1341.

7 Ztschr. f. Psychol. u. Physiol. d. Sinnesorg., Hamburg u. Leipzig, 1895, IBd. ix. S. 81. 
although much importance cannot be attached to this, owing to the fact that our knowledge of the rapidity of the changes in visual purple is derived chiefly from observations on cold-blooded animals.

The difference between the absolute and the chromatic thresholds for coloured light would be due to the sensitiveness of visual purple at low intensities being greater than that of the chromatic apparatus; and it is in accordance with this view that there should be no photochromatic interval for red light, which does not act appreciably on visual purple. Purkinje's phenomenon (p. 1078) would be due to the greater action of light of short wave-length on visual purple.

If vision with the dark-adapted eye at low intensities depends wholly on visual purple, the curve of luminosity of the spectrum should correspond with the curve of absorption of visual purple. In the absence of observations on the spectrum of human visual purple in a pure condition, this point is at present undecided.

The similar spectrum of total colour-blindness leads to the assumption that in this condition visual purple is the only substance present, and that the totally colour-blind individual is dependent for his vision wholly on this substance. The features of total colour-blindness, which have led to its being regarded as a pathological condition, namely, photophobia and diminished visual acuity at ordinary illumination, become quite natural if vision depends only on visual purple. Further, there should be an absolute central scotoma corresponding to the fovea, and this has been in several cases recorded, and may exist in all, probably varying in size in different cases. The nystagmus which is a frequent symptom may be consequently explained by absence of a place of most distinct vision, and therefore of steady fixation. It is in relation to this anomaly that this theory of the action of visual purple may be capable of objective verification; for, if true, the retina of a totally colour-blind eye might show absent or ill-developed cones. There is at present no record of the anatomical condition in this defect.

The departure from the law of colour mixture with adaptation to low intensities would also be ascribed to the influence of visual purple, a new factor coming into play as the intensity is reduced. In order that these deviations may be explained on this hypothesis of the function of visual purple, it is necessary that that side of a colour equation which becomes the brighter at low intensities should have the greater action on visual purple (the greater rod-value); and, according to v. Kries, ${ }^{1}$ this is the case. To give one instance: in a match of a mixture of spectral red and green with homogeneous yellow, the mixed field becomes relatively brighter on diminution of intensity, owing to the especially high rod-value of the green, both red and yellow having relatively very low rod-values.

Another phenomenon, which is referred by v. Kries to visual purple, is the recurrent image. He ascribes it to the greater inertia of visual purple, so that this substance reacts to light appreciably later than the other photochromatic substance or substances, the latent period becoming, however, shorter as the intensity is increased. The fact observed by both Bidwell and v. Kries, that there is no recurrent image with red light, is very much in favour of this view, and so is the influence of adaptation on the phenomenon. In a case of night-blindness, v. Kries found that no recurrent image was perceived, although other equally

${ }^{1}$ Ztschr. f. Psychol. u. Physiol. d. Sinnesorg., Hamburg u. Leipzig, 1896, Bd. ix. S. 81 ; and Bd. xii. s. 1 . 
unpractised individuals perceived it easily. According to v. Kries, there is no recurrent image in total colour-blindness (again only one case examined); but this is denied by Hering and Hess. Hamaker's observations (p. 1074) seem to show that the phenomenon is complicated by other processes going on at the same time.

The slow rate of dark-adaptation upon which night-blindness depends would be due to slow formation of visual purple. The two chief causes of this condition, namely, disease of the retinal pigment layer, and action of too strong light, would naturally affect the formation of the substance, while its occurrence in jaundice and in diseases of the liver would be explained by the solvent action of bile salts on visual purple. Pilocarpine is said to have a beneficial effect on night-blindness, ${ }^{1}$ and Kühne found that this drug hastens regeneration of visual purple (p. 1045). Schirmer ${ }^{2}$ has observed slowness of adaptation in cases of detached retina, and compares this condition with the slowness of the auto-regeneration described by Kühne.

Certain other facts are consistent with the theory. Exposure of one eye to light has no effect on the dark-adaptation of the other eye, while Engelmann found that exposure of one eye led to no change in the visual purple of the other eye. G. E. Muiller has found that the sensations arising from electrical stimulation of the eyeballs are unaffected by the condition of adaptation, and Kühne found that visual purple was not influenced by electrical stimuli. The fact that Dor (p. 1050) found a difference in the staining of rods and cones, after the action of red light, none after blue light, is explicable if the former acts only on the cones, the latter on both rods and cones. The observations of Koester and König on visual acuity (pp. 1055 and 1086) are explicable, if the acuity of darkadaptation depends on the rods, that of bright adaptation on the cones.

Foveal vision and absent visual purple.--The absence of rods and visual purple from the fovea gives a means of verifying the truth of the hypothesis. The difficulties of studying foveal vision have been already mentioned. Another difficulty is the uncertainty as to the anatomical limits of the area of the retina which contains no visual purple. Most observers give the foveal depression a diameter of 2 to $4 \mathrm{~mm}$., while others assign it larger values. For the present purpose, however, the importance of the fovea lies in the exclusive presence of cones. Comparatively few measurements have been made of the area free from rods. Koster ${ }^{3}$ has recently examined four cases, and found the area in which rods were entirely absent varied from 44 to $901 \mathrm{~mm}$, while rods were comparatively scanty in a still larger area. Koster has suggested $5 \mathrm{~mm}$. as the probable average diameter of the rod-free area, and $8 \mathrm{~mm}$. as that of the area in which cones predominate. There is still greater uncertainty as to the area from which visual purple is absent, for Kiihne's observations seemed to show considerable individual differences, while, if his observation is correct, that the rods in the macula are devoid of purple, still larger values than those of Koster must be assigned to the area which has no visual purple.

One feature of foveal vision about which there is no doubt, is the high threshold for light. This low degree of sensitiveness to light, which is especially marked in the dark-adapted eye, would be a natural

$1^{\circ}$ Kuschbert, Deutsche med. Wchnschr., Leipzig, 1884, S. 324.

${ }^{2}$ Verhandl. d. X. internat. med. Congress, Berlin, 1890, Bd. iv. Abth. 10, s. 63.

${ }^{3}$ Arch. f. Ophth., 1895, Bd. xli. Abth. 4, S. 1. 
deduction from the theory. A second feature is the absence of the photochromatic interval for coloured light of low intensity. According to some observers, the interval is only diminished, and v. Kries supposes it possible that the colour of a light may not be perceived at very feeble luminosities when it acts on the chromatic apparatus proper, although the interval in this case is very much less than that which exists where visual purple is present. A third feature in accordance with the theory, is the absence of Purkinje's phenomenon. This rests not only on the observations of $v$. Kries and other advocates of the theory, but also on the independent observation of Hering, that the phenomenon may be produced by going from the centre to a more peripheral part of the visual field. According to Tschermak, both Purkinje's phenomenon and the deviations of matches occur in central vision, but to a less marked degree than elsewhere. ${ }^{1}$ Nagel and Stark (colour-blind) have found that the deviations from Newton's law do not occur for the fovea, and they have been able to measure the area of the visual field within which colour equations hold good at all intensities, and find that it corresponds closely with the measurements of the rod-free area made by Koster. The existence of an absolute scotoma in total colour-blindness has already been mentioned. Lastly, according to v. Kries, the recurrent image cannot be seen in an area of the visual field corresponding to the fovea.

This theory of the function of visual purple has been criticised by Hering and Hess, ${ }^{2}$ who have objected to the theory mainly in regard to its proposed explanation of total colour-blindness. In two cases of total colour-blindness examined by themselves, and in one examined by Pflüger, no central scotoma was found. In view of the considerable individual differences which exist in the distribution of rods and cones within the macular area, of the difficulty of detecting a small central scotoma, and of the possibility that in such abnormal individuals the absent or defective cones may be replaced by rods, the failure to detect a central scotoma in some cases does not tell seriously against the theory. Hering and Hess also found in one case of total colour-blindness that, when dark-adapted, the sensitiveness increased from the centre to the periphery of the fovea, as in the normal eye. This fact is, however, not inconsistent with the theory. Hering and Hess also found that one of their cases, a good observer, saw a recurrent iniage. Uhthoff $^{3}$ has since given a full description of a case in which there was a definite central scotoma, and other features which were all in harmony with the hypothesis that vision depended on visual purple.

Some of the peculiarities have been referred to the influence of the pigment of the macula, which probably extends throughout the fovea. Its absorptive power might explain the diminished sensitiveness to light, though, according to Breuer, the diminution is too great to be accounted for in this way.

The accumulation of evidence in favour of this theory of the function of visual purple is further supplemented by the comparative evidence which first led Schultze in this direction. Krause ${ }^{4}$ has found that Schultze was wrong in supposing that rods were exclusively present in nocturnal animals, cones being

${ }^{1}$ Arch.f. d. ges. Physiol., Bonn, 1898, Bd. lxx. S. 297.

2 Ibid., 1898, Bd. lxxi. S. 105.

${ }^{3}$ Ztschr. f. Psychol. u. Physiol. d. Sinnesorg., Hamburg u. Leipzig, 1899, Bd. xx. S. 326.

+ Internat. Monatschr. f. Anat. u. Physiol., Leipzig, 1886-1895. 
also present in all the instances examined. Krause, however, finds that nocturnal animals are characterised by the length of the outer limbs of the rods. Krause did not pay especial attention to the presence or absence of visual purple; but there is no doubt that it is present in fishes, in nocturnal animals, and in those who live mainly underground. It is very abundant in the owl, and absent or scanty in birds whose habits are wholly diurnal. The greater length of the outer limbs of the rods found by Krause in nocturnal animals would give greater thickness in the layer of visual purple. The only known instance of a nocturnal animal whose retina is devoid of visual purple is the bat. Its minute rods show, according to Kiihne, only a trace of a purple colour, but the experiments of Spallanzani and others have shown that the bat is dependent to a very slight extent upon vision, and the imperfect condition of the rods in this animal may well be the result of disuse.

If dark-adaptation depends primarily on visual purple, the question remains, whether the changes in the piginent and cones have a similar function. Their rate of occurrence is much the same as that of the bleaching and regeneration of visual purple, but the fact that the changes of cones and pigment are not limited to the eye acted on, is against their connection with adaptation being so close as is that of visual purple. There is no doubt that one function of the pigment is to supply material for regeneration of visual purple, and it can probably do this quite as efficiently when situated round the outer part of the outer limb of the rods, as in the dark position. In the light position also, it probably acts by absorbing light and preventing too violent action on the sensitive structures, and its absence will explain the photophobia of albino vision, and also the photophobia which often accompanies night-blindness in abnormal conditions of the retina.

Cerebral centres for light and colour-vision.-This is a subject on which our knowledge is very scanty. It has been supposed ${ }^{1}$ that recorded cases in which there has been hemianopia for colours with retained light sense, are in favour of a separate cerebral centre for colour, and similar cases in which red-green blindness occurs as a hemianopic defect may similarly be held to point to separate cerebral representation of different colours. The facts known are, however, far too few to justify any definite conclusion.

\section{Theories of Colour-Vision.}

Introductory.-If the theory of the function of visual purple considered in the preceding section is correct, it still leaves the question of colour-vision almost untouched. But certain anomalous features of colour-vision are explained by it; and if accepted, it will necessitate some modification of, at least, one of the prevailing theories of colour-vision.

There are two important assumptions common to these theories. It is assumed that the manifold colour sensations that we experience are derived from a limited number of components, these components being known as primary, fundamental, or elementary colour sensations. Any given colour sensation is to be regarded as arising by a process of fusion of two or more primary sensations. The idea appears to have had its origin in the facts of colour mixture, and it derives its strongest support from colour-blindness, which seems to be most naturally explained by the absence of one or more of the components. Although the idea is earlier than that of specific nervous energy, the two are closely connected, and the former is a necessary consequence of the latter.

The second assumption is, that the visual process is primarily photo-

${ }^{1}$ Schneller, Arch. f. Ophth., 1882, Bd. xxviii. Abth. 3, S. 73.

VOL. II. -70 
chemical; that light acts primarily on certain visual substances; and that, further, these substances are limited in number corresponding to the components of sensation. The behaviour of visual purple and the analogy of photography have probably largely contributed to the general acceptance of this view, and certain of the facts of colour-vision, and especially those of persistence and induction, naturally suggest a process of this kind.

The two chief theories of colour-vision are component theories, ${ }^{1}$ and assume the existence of a limited number of visual substances. There is no direct evidence of the existence of such substances. They are purely hypothetical factors, which enable the facts of light and colour-vision to be expressed simply and consistently.

The chief theory at present advocated which rejects the idea of components is that of Wundt, who holds that the series of colour sensations which pass into one another by insensible gradations have their basis in a series of changes in one. complex substance, which similarly pass gradually into one another.

Young-Helmholtz theory.-The theory of Thomas Young, adopted by Helmholtz and Maxwell, is a component theory, and is closely

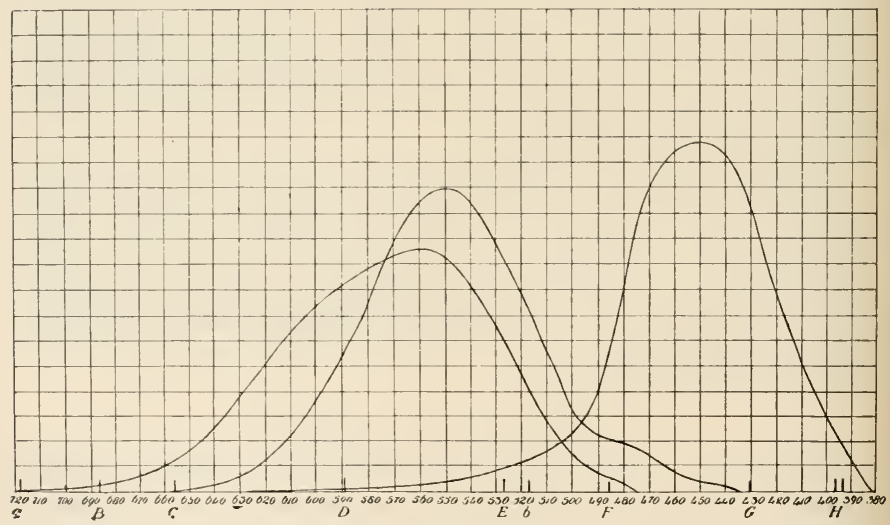

FIG. 396.-König and Dieterici.

connected with the doctrine of specific nervous energy. In its earlier form it was supposed that there were three kinds of nerve fibre in the retina corresponding to three primary colours. In its modern form these have been replaced by three photochemical substances, with three corresponding fundamental sensations. Each of the three substances is acted on by light of all wave-lengths, but on one the action of the long-wave end of the spectrum greatly predominates, on another the middle, and on the third the short-wave end. The action of the spectrum on each of the three substances is usually represented by curves such as those in Fig. 396.

${ }^{1} \mathrm{G}$. E. Müller has recently used the term "component theory" in another sense. A component theory is, according to him, one in which the sensation white is regarded as a mixture of component chromatic sensations. Used in this sense, the Young-Helmholtz is a component theory, while Hering's theory is not. 
With predominant decomposition of olle substance the sensation red is experienced, of the others green and violet or blue. The colours intermediate between these, and the purple series connecting the two ends of the spectrum, depend on the relative amounts of decomposition of the three substances. According to this theory, the white-black series of sensations has no separate physiological basis, but arises when all three chromatic substances are excited in an equal degree. Black is the sensation which accompanies absent or subliminal decomposition of the substances.

The primary colours.-There has been much difference of opinion as to the choice of the three primary colours. Those most commonly adopted have been red, green, and violet. In addition to evidence derived from colour mixture, their adoption has been supported by the fact that, when the intensity of the spectrum is lowered, these three colours remain visible after yellow and blue have disappeared. Donders and König have attached importance to the existence at each end

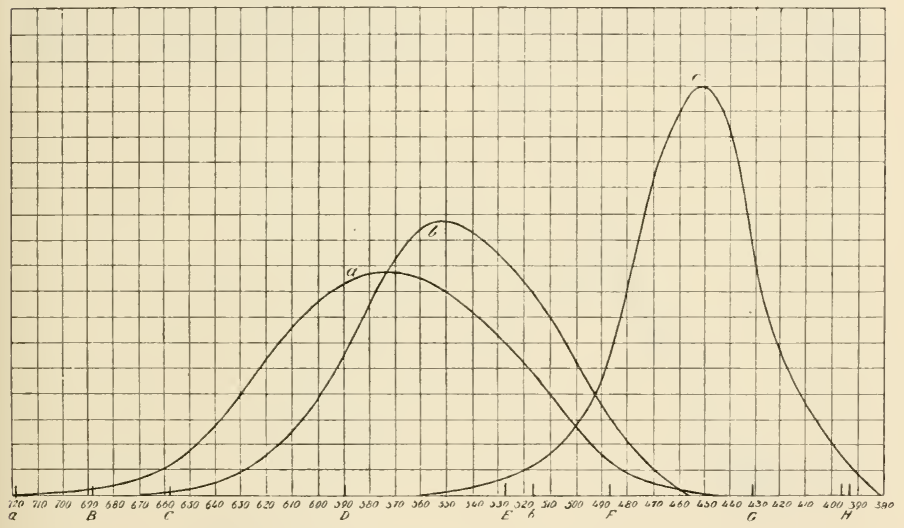

Fic. 397.-The cnrve $c$ is the elementary curve of the sensation of the short-wave end of the spectrum, and was approximately the same in both groups of red-green blindress. The curve $b$ is that of the sensation from the long-wave end in the scoterythrous group, while the curve $a$ is that of the photerythrous group.-König and Dieterici.

of the spectrum of a region showing only change in saturation and no change of colour-tone, and they suppose that at each end only one fundamental sensation is present, namely, red and violet. Maxwell adopted blue as the third primary, and Exner ${ }^{1}$ came to the sane conclusion, on the ground that red, green, and blue were the colours least changed in tone by fatigue. ${ }^{2}$ More recently Helmholtz and König have arrived at more exact conclusions as to the nature of the fundamental sensations.

König and Dieterici ${ }^{3}$ distinguish between elementary and fundamental sensations. By the former König means the smallest number of elements to which it is possible to reduce the manifold colour sensations we experience,

1 Arch. f. d. ges. Physiol., Bonn, 1868, Bd. i, S. 373.

2 On the ground of his fatigue experiments, described on p. 1059, Burch regards red, green, blue, and violet as primary colour sensations.

${ }^{3}$ Ztschr. f. Psychol. u. Physiol. d. Sinnesory., Hamburg u. Leipzig, 1893, Bd. iv. S. 241. 
and he regards this reduction as an experimental procedure which does not necessarily carry with it any physiological significance. By fundamental sensations, on the otler hand, he means the colours which correspond to simple physiological peripheral processes. In order to arrive at the elementary sensations, he constructs elementary sensory curves in the following way. He assumes the division of the spectrum into five regions: two end regions (red end to $655 \lambda$ and $430 \lambda$ to violet end), in each of which only one elementary sensation is present; two intermediate regions ( $655 \lambda$ to $630 \lambda$ and $475 \lambda$ to $430 \lambda$ ), in each of which two of the elcments are present; and a middle region $(630 \lambda$ to $475 \lambda)$, in which all three elementary sensations are present together. The intermediate regions are characterised by the fact that every colour-tone in each of them can be matched by mixing the light at the point where it joins the middle region with light from the corresponding end region. In dichromatic vision there will be only three regions, namely, the two end regions, and a middle region in which the two elementary sensations of the dichromatic eye occur together.

Starting from these assumptions, König has calculated from a series of equations the curves of excitability of the three elementary sensations of the normal eye (Fig. 396), and the two of the dichromatic eye (Fig. 397). The short

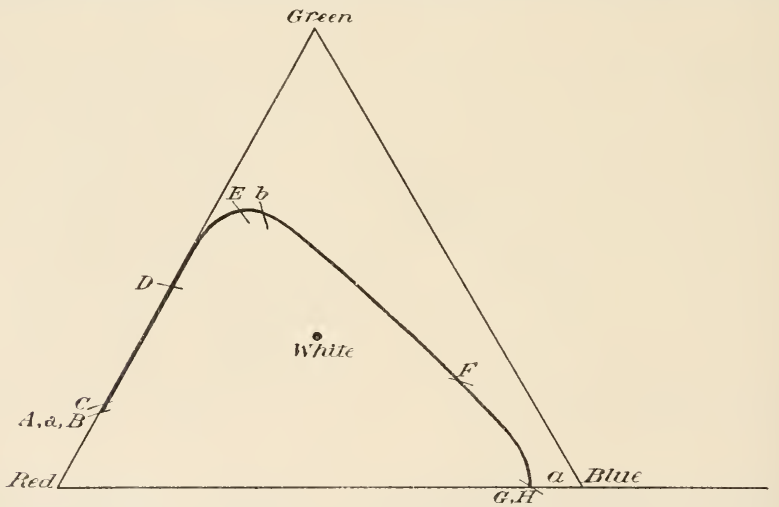

FIG. 398. - The curve represents the colours of the spectrum, while points at the three angles represent the fundamental colours.

wave or cold curve of the latter was the same in all cases, and corresponded with the blue or violet curve of the normal eye. The warm curve of the colour-blind differed in the two groups, and the two curves corresponded fairly closely with the red and green curves of the normal eye. On the basis of these results, König deduced the three fundamental colours by means of the colour triangle, and concluded that they were a red lying outside the spectrum, being rather more purple than the red of the end region, a green at $505 \lambda$, and a blue at $470 \lambda$. The green is much more saturated than spectral green, as is seen by the figure of König's colour triangle (Fig. 398).

Helmholtz ${ }^{1}$ calculated the fundamentals mathematically by another method, from the degree of sensibility for differences of colour-tone in each part of the spectrum. He has taken as his data the observations of König and Brodhun, ${ }^{2}$ on the degree of sensitiveness of the eye for changes of colourtone, and has arrived at the conclusion that the three elementary colours are a

1 " Handbuch d. physiol. Optik," 2te Aufl., S. 449.

${ }^{2}$ Ztschr. f: Psychol. u. Physiol. d. Sinnesorg., Hamburg u. Leipzig, 1892, Bd. iii. S. 97. 
highly saturated carmine-red, bluer than spectral red; a green, lying between 540 and 560 in the yellow-green; and a blue, corresponding to ultramarine; all three fundamental colours being much more saturated than the colours of the spectrum. Their relation is shown in Fig. 399, in which the curved lines represent the spectrum, $x, y$, and $z$ the three fundamentals, the distance of the spectral curve from the sides of the triangle representing the difference of saturation. This difference in saturation is held to show that light of any wave-length acts on all three photochemical substances; the different colours depending simply on the quantitative relations of the three processes.

It will be noticed that the red and blue fundamentals of Helmholtz correspond with those of König deduced by a different method, and also, as will be seen later, with the fundamental red and blue of Hering arrived at in a

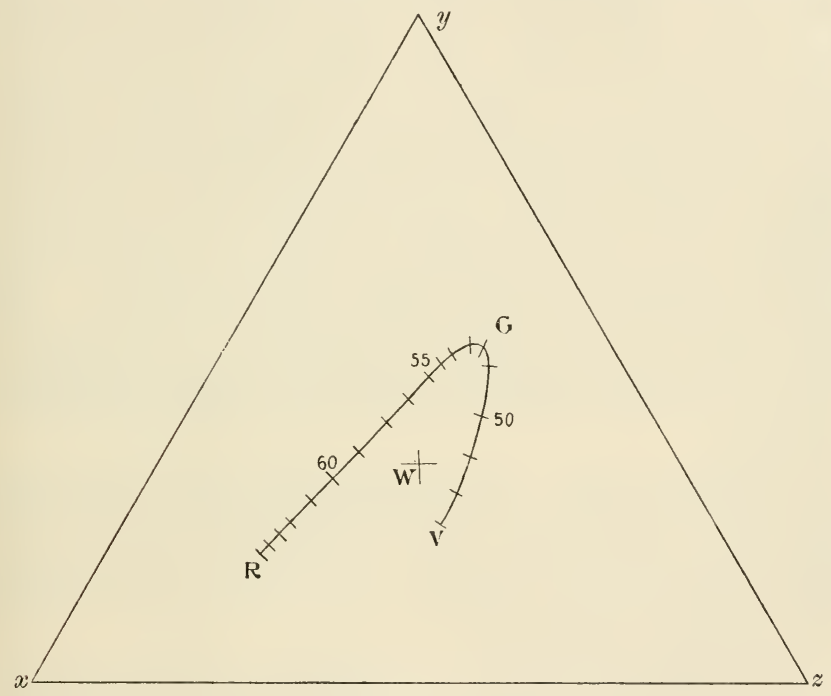

FIG, 399.-Helmholtz.

wholly different way. The greens of Helmholtz and König, on the other hand, differ considerably, $505 \lambda(K)$ and 540 to $560 \lambda(H)$; both, however, lie between the fundamental yellow and green of Hering (see p. 1114). ${ }^{1}$

Adaptation and induction.-Of all the facts of light and colourvision, the most difficult to explain by the Young-Helmholtz theory are adaptation and induction. Adaptation was almost completely neglected by Helmholtz, and he apparently did not regard it as anything requiring special explanation. He explained after-image phenomena by fatigue, supplemented when necessary by psychological explanations. In addition to the arguments already brought against the fatigue explanation in general, there are other special objections to it when combined with the hypothesis that the sensation white is a complex of chromatic sensations. It is a necessary consequence of this hypothesis

${ }^{1}$ For criticism of König's methods by Hering, sce Arch. f. d. ges. Physiol., Bonn, 1893, Bd. xlvii. S. 310 ; and 1895, vol. 1x. S. 540. 
that diminution in the sensibility for white light should involve diminution in sensibility for coloured light, and vice versa. ${ }^{1}$ It is found, however, that the two kinds of excitability vary independently of one another. If a large coloured (red) surface be observed after prolonged fixation of a black dise on a white ground, the part of the coloured field previously exposed to black appears much less saturated than the rest of the coloured surface. According to this theory, fatigue for white involves fatigue for red, but this experiment would mean that a part of the retina fatigued for red gives a better red sensation than one which has been exposer to black, i.e. has not been fatigued at all.

The influence which fatigue of the retina to one colour has on the colour-tone of different parts of the spectrum, has been much discussed in relation to the Helmholtz theory. Hering regards these facts as irreconcilable with the theory. The yellow of the spectrum does not change in colour-tone with fatigue; it only undergoes change in saturation, and the same is the case with blue (v. Kries, Hess, etc.). But since by this theory yellow is due to predominant excitation of the red and green substances, and blue to that of the green and violet substances, blue should change in tone, after fatigue to yellow, and appear violet, i.e. of the two processes upon which blue depends only the green has been affected (or affected to any extent) by the fatigue to yellow. Again, to a retima fatigned for spectral blue, violet appears red (Exner, Hess). Violet light by this theory has a slight action on the red substance, and yet here the action of the red substance predominates over and masks the action of the violet substance which has only been partially fatigued by blue light, and is being stimulated by its own special stimulus, namely, violet light.

Helmholtz $^{2}$ held that these and other similar objections only apply if the fundamental colours are to be found in the spectrum, and that they are met by the assumption already mentioned, that light of any wavelength acts on all three substances; the difference between the action of different lights being only quantitative. Hess, ${ }^{3}$ on the other hand, holds that the changes of colour-tone after fatigue are incompatible with any form of the three-colour theory.

Helmholtz never attempted to explain contrast physiologically, and was content with the psychological explanations already considered. v. Kries has also regarded contrast as chiefly a psychological matter, and as unimportant for a physiological theory of vision.

Colour-blindness.-The facts of colour-blindness, together with the condition of the peripheral retina, may next be considered. At one time the facts of colour blindness seemed to be strongly in favour of the theory, and, according to Maxwell, colour equations, in the common form of the defect, were consistent with the absence of one of the three normal components. In the scoterythrous group the red component was supposed to be absent, while in the photerythrous group the green component was wanting, and the two groups have since been commonly known as red-blind and green-blind respectively. The rare cases of confusion between yellow and blue were held to depend on absence of the violet component.

At an early stage, however, doubts were raised. The correspondence

1 Arch.f. d. gcs. Physiol., Bonn, 1888, Bd. xliii. S. 329.

2 "Handbuch d. physiol. Optik," 2te Aufl., S. 517.

3 Arch. f. Ophth., 1893, Bd. xxxix. Alth. 2, S. 45. 
between colour-blindness and the peripheral retina was made by Fick ${ }^{1}$ the basis of a suggestion that, instead of assuming absence of a component, it would be more in agreement with the correspondence in question, if it were assumed that in these conditions there was an altered condition of excitability of the physiological bases of the components; that in the redgreen blind condition the red and green parts of the spectrum might excite two of the processes in an equal degree, so that their curve of excitability might nearly or altogether coincide with one another; while in the extreme periphery of the retina, and in total colour-blindness, light of any wave-length would act equally well on all three substances.

The chief advocates of the three-colour theory (Holmgren, v. Kries, König) have now accepted the position, that the subjective sensations of the red-green blind most probably correspond with the yellow and blue of the normal eye; while nearly all agree that the white of the colourblind agrees with the white of the normal eye; and the old idea, that colour-blindness depends simply on absence of one or more components, has been almost entirely given up by advocates of the theory.

In the second edition of the "Physiologisches Optik," S. 373, Helmholtz assumed that in the red-blind zone the substance sensitive to red has become similar to that sensitive to green, while at the extreme periphery all three substances have become like one another. As an alternative hypothesis, Helmholtz suggested a psychological explanation,- - that, when one of the fundamentals is absent, we interpret the colours which are still perceptible, as the colours of a line which, in the colour triangle, would pass through white parallel to the line joining the two fundamentals still present. According to Helmholtz, these would pass from yellow through white to blue.

König ${ }^{2}$ has advanced the view, that the curves of the three elementary sensations vary with the absolute intensity. These variations are held to be the basis of Purkinje's phenomenon, and of the characteristic spectrum of low intensity. He further supposes that the visual substances of the various forms of colour-blindness behave at ordinary intensities in the same way as those of the normal eye at lower intensities. He gives a series of luminosity curves of the spectrum at different intensities, and shows their correspondence with a number of curves in different individuals, some of whom had dichromatic, others normal trichromatic, others anomalous trichromatic systems.

The correspondence of the spectrum of low intensity with that of total colour-blindness was explained by supposing that in the latter condition the three substances behaved at all intensities in the same way as in the normal eye at low intensities, i.e. all three substances were acted on equally by light of any wave-length.

v. Kries has supposed that, in colour-blindness, the three components of the retinal process are present, and that the defect depends upon abnormal action of the nerve paths, or nerve centres.

Retinal basis for the theory.-More recently, König ${ }^{3}$ has endeavoured to bring the Young-Helmholtz theory into relation with definite retinal substances. König explains Purkinje's phenomenon, and

${ }^{1}$ Verhandl. d. phys.-med. Gesellsch. in Würzburg, 1873 ; see also Arch. f. d. ges. Physiol., Bonn, 1896, Bd. lxiv. S. 313. Helmholtz had, however, previously made a similar suggestion himself, "Handbuch d. physiol. Optik," 1867, S. 848 .

"Beitr. z. Psyeh. u. Phys. d. Sinn.," 1891, S. 311.

${ }^{3}$ Sitzungsb. d. k. Akad. d. Wissensch., Berlin, 1894, s. 57 . 
the departures from Newton's law, by supposing that visual purple, with feeble decomposition, gives rise to a colourless sensation, but that with stronger decomposition the visual yellow formed is the basis of the blue component of the Young-Helmholtz theory. The former supposition has already been considered, and the latter was based on a correspondence between the curve of absorption of visual yellow with the curve of the elementary blue sensation calculated by König and Dieterici, and on the supposed blue-blindness of the fovea.

König places the red and green substances in the pigment epithelium, and regards the cones as dioptric mechanisms. It would follow from König's hypothesis that in red-green-blindness the fovea should have only monochromatic vision.

In 1884 an attempt was made by Holmgren ${ }^{1}$ to prove the truth of the three-colour theory by stimulation of minute retinal points. He found that a minute yellow point of light appeared red at some points of the retina, green at others, and concluded that this depended on the stimulation of elements for red and green respectively. Hering, ${ }^{2}$ however, showed that Holmgren's results were due to his use of impure light, and did not occur with true homogeneous yellow light. Isaachsen ${ }^{3}$ also, working under König, failed to confirm Holmgren's results. Holmgren, ${ }^{4}$ later, repeated his experiment with homogeneous spectral light, and with a number of observers, some of whom saw the phenomenon, others with difficulty, or not at all. He obtained very indefinite results with blue light.

If the recent theory of the function of visual purple is correct, the Young-Helmholtz theory will not be weakened; in fact, the acceptance of the former will to some extent assist the latter theory, for it relieves the latter from the difficult necessity of explaining total colour-blindness and Purkinje's phenomenon. If these depend upon the visual purple in the rods, which function independently of the chromatic apparatus, the cones may be the basis of the colour vision. But though visual purple may relieve the Young-Helmholtz theory of some of its difficulties, those connected with the explanation of after-images and contrast, and the subjective sensations of the colour-blind, still remain.

Hering's theory.-The starting-point of Hering's theory was the failure of Helmholtz to give a physiological explanation of contrast. Hering also objected to some of the fundamental principles of Young's theory. Any theory of sensation must have a physical, a physiological, and a psychological aspect; and Hering objected to the psychological aspect of the three-colour theory. In 1865 , Mach ${ }^{5}$ had pointed out that the black-white series of sensations differed wholly in quality from the chromatic series, and that, to be consistent with psycho-physical parallelism, the two series should have a separate physiological basis. Consciousness is totally unable to analyse the sensation white into any coloured components. He further pointed out that in the series of colour sensations there are four which appear simple and undecomposable, namely, red, yellow, green, and blue,-and that each of these colours should have a separate physiological basis. Hering adopted the same attitude, and pointed out that two of the original three primary colour sensations of Helmholtz were manifestly complex; that

\footnotetext{
${ }^{1}$ Cong. périod-intemat. d. sc. méd. C. r., Copenhagen, 1884, tome i. p. 93.

2 Arch.f. d. ges. Physiol., Bonn, 1887, Bd. xl, s. 1.

3 Ibid., 1888, Bd. xliii. S. 289.

4 Skandin. Arch. f. Physiol.. Leipzig, 1889, Bd. i. S. 152 ; and Bd. iii. S. 253.

${ }^{5}$ Sitzungsb. d. k. Akad. d. Wissensch., Wien, 1865, Bd. lii. Abth. 2, S. 320.
} 
violet is obviously a mixture of red and blue, while spectral red, if isolated so as to exclude contrast from the strong yellow of the spectrum, manifestly contains a yellow component. ${ }^{1}$

Hering advanced novel views about the sensation of blackness. He objected to the generally accepted view, that this sensation could be defined as that accompanying absence of external stimulation. He pointed out that with total exclusion of light from the eye, i.e. in the dark-adapted eye, the sensation was one of a considerable degree of brightness, and was named by him the sensation of mean grey. Black proper only occurs, according to Hering, under the influence of external stimulation, i.e. under the influence of simultaneous or successive contrast. The after-image of a white patch, or a black patch seen on a white ground, give us a special quality of sensation, which we recognise as present in the mean grey, but in a less degree; and it is this special quality of sensation which Hering regards as black proper. Since black is a special quality of sensation, as well-rlefined and undecomposable as white or red, it is in accordance with psychophysical parallelism that it should have a separate physiological basis.

Hering, then, regards the whole series of our visual sensations as made up of six primary sensations; but the most novel and fundamental feature of his theory is the arrangement of these six sensations in three pairs, the two members of each pair being antagonistic in nature to each other, and depending on physiological processes of an opposite kind. Hering usually speaks of his theory as the theory of antagonistic colours.

Each of the three pairs of sensations depends upon the action of a special substance, the three substances being called the black-white, the red-green, and the blue-yellow respectively. The processes of an opposed nature taking place in these substances are the two kinds of metabolism. In most kinds of stimulation of the retina, all three substances are set in action, and the character of the sensation is determined by the relative amount of action of each. This relative amount determines the "weight" of each primary sensation in the complex sensation. Both the general character of the sensation and the "weight" of each element depend on the condition of excitability of the substances, as well as on the value of the stimulating light. The ter'm "value" (Valenz), which has been used in the earlier part of this article to express a fact, has, when used by Hering, a special meaning. The white value of a light expresses its action on the black-white substance, and its colour value on one or both of the chromatic substances. All coloured lights, except those corresponding to the four primary colours, have three values, corresponding to their action on the three substances.

Hering ${ }^{2}$ always distinguishes carefully between the optical value of a light and its physiological value (Moment), which depends on the optical value and the condition of excitability of the visual organ.

The exact colours which were originally chosen by Hering were those which appeared simple to psychological analysis, namely, a red in which no trace of either blue or yellow could be perceived, a yellow in which no trace of red or green could be perceived, and similarly pure green and

${ }^{1}$ Wundt has objected that the ehoice of red, yellow, green, and blue is purely arbitrary ; that all sensations are equally simple; and that one has as much right to regard red as a nixture of purple and orange, or blue as a mixture of violet and blue-green, as to regard orange or violet as a mixture of red with yellow or bluc. The new fundanicntals of Helmholtz and könig are not open to Hering's objections.

2 Arch.f. d. ges. Physiol., Bonn, 1887, Bd. xli. S. 29. 
blue. The colours so adopted on purely psychological grounds were found later to correspond with the colours which were found not to change in colour-tone in different regions of the retina, namely, a red more purple than the extreme red of the spectrum (and obtained by mixing blue light with the latter), a yellow at $574.5 \lambda$, a green at $495 \lambda$, and a blue at $471 \lambda$.

Hering's theory of metabolism.-In order to consider the application of Hering's theory to the conditions of excitability of the visual substances, it will be necessary to enter more fully into his general theory of metabolism. ${ }^{1}$ When a substance is protected from exterual stimulation, it undergoes spontaneous or autonomous katabolism and anabolism, which are equal to each other; the substance is in a condition of autonomous equilibrium. If the substance is affected by a stimulus to katabolism, it undergoes a descending change. Hering now calls the katabolism allonomous; the substance becomes less capable of performing work, and is regarded as at a low potential. With this there is a spontaneous tendency to return to a condition of autonomous equilibrium, or of mean potential, i.e. its autonomous anabolism becomes stronger. Allonomous katabolism is therefore accompanied by autonomous anabolism, and the latter becomes the greater, the longer the katabolic stimulation lasts. Sooner or later, with constant stimulation, the autonomous anabolism becomes equal to the allonomous katabolism, and a new condition of equilibrium is set up, which Hering speaks of as allonomous equilibrium at a low potential.

If, on the other hand, the substance is affected by a stimulus to anabolism, it undergoes an ascending change. The substance becomes more capable of work, and is at a high potential. There is a spontaneous tendency to return to a condition of mean potential, and the allonomons anabolism is therefore accompanied by antonomous katabolism, which sooner or later, with constant stimulation, produces allonomous equilibrium at a high potential.

It is the direction of the change, i.e. whether ascending or descending, which determines the nature of the sensation; the ascending change in the three substances being the basis of black, blue, and green respectively; the descending, of white, yellow, and red. These general conceptions may now be applied in detail to the hypothetical visual substances of Hering. In the case of the white-black substance, the sensation, corresponding to equilibrium, between katabolism and anabolism, is the neutral grey, i.c. a grey of the brightness of that seen after prolonged stay in the dark. A descending change from a condition of higher to one of lower potential determines a sensation brighter than this neutral grey; the degree of brightness depends on the rapidity of the change, or, in other words, on the degree in which katabolism preponderates over anabolism. An ascending change from lower to higher potential determines a sensation darker than the neutral grey, the depth of darkness again depending on the rapidity of the ascending change.

Adaptation and induction.-The conditions of allonomous equilibrium are the basis of adaptation. When the black-white substance is completely adapted to the dark, it is in the condition of mean potential, corresponding to autonomous equilibrium. During the day the eye is always adapted to the surrounding illumination. The exact condition of adaptation must vary to some extent with the continual changes of external illumination to which one is normally exposed, but for practical purposes the condition of adajtation may be regarded as

${ }^{1}$ Cf. vol. i. pp. 868-870. 
changing very slowly, and in any definite interval of time as corresponding to the mean illumination during that period. The white-black substance is then in a condition of allonomous equilibrium at a low potential. The brighter the illumination to which the eye is adapted, the lower is the potential at which equilibrium occurs. During the daytime the white-black substance is always to be regarded as in a condition of relatively low potential. The condition of allonomous equilibrium at a high potential is exceptional in the case of the black-white substance, owing to the fact that there is no external stimulus to anabolism. Black proper, according to Hering, only occurs under the influence of simultaneous or successive contrast; and the best example of allonomous equilibrium at relatively high potential, in the case of the black-white substance, occurs in Hering's "simultaneous induction." If a black square on a white ground is fixed, the whole surface after a time is seen as a uniform grey. The black-white substance corresponding to the black square will have undergone an ascending change, that corresponding to the white a descending change, and the condition of uniform grey of the whole surface corresponds to equilibrium, which in the former case is at a high potential, and in the latter at a low potential. The potential of the former will only, however, be higher than that of the eye completely adapted to the dark, if the experiment is carried out with the eye adapted to the dark. In the case of the white-black substance, it would seem as if allonomous equilibrium might in ordinary life take place at very different levels of potential, less than that of the autonomous condition; and that allonomous equilibrium, at a potential higher than that of the dark-adapted eye, only occurs under very exceptional conditions. This might be urged as an objection to the terminology adopted by Hering. It is due, however, to the absence of proper external stimuli to anabolism, and this difficulty does not occur in Hering's treatment of the chromatic substances.

When the red-green and yellow-blue substances undergo the descending change, the corresponding sensations are red and yellow respectively. When they undergo the ascending change, they are green and blue respectively. The rapidity of the change (or the predominance of one process over the other) partly determines the "weight" of the particular element in question in the sensational complex, or, in other words, the purity or saturation of the colour; the other factor determining the purity being the degree of simultaneous stimulation of the other substances.

The conditions of adaptation to coloured light may be readily referred to different conditions of allonomous equilibrium. When the chromatic substances are in a condition of equilibrium, they do not contribute to the quality of the sensational complex. They are equally in a condition of autonomous equilibrium, whether the eye has been wholly unstimulated or whether the stimulation las been exclusively by mixed colourless light. When the eye, after exposure to red light, no longer sees objects red, the red-green substance has become adapted to the light, and is in a condition of allonomous equilibrium, but at a low potential; on the other hand, in adaptation to green light, this substance is in allonomous equilibrium at high potential. With removal of the light to which the eye has become adapted, the complementary after-image colour is due to the autonomors change back to a condition of mean potential. After-images occupying limited portions of the visual field are due to local adaptation, and are explained on the same lines as general adaptation. The after-images seen with complete exclusion of light from 
the eye, which formed the great difficulty of the fatigue theory, are here referred to autonomous changes. Hering has formulated the nature of the active opposed change, which Plateau had previously suggested as the basis of after-image in distinction from the passive condition advocated by Fechner and Helmholtz.

The absence of colour in all kinds of light at low intensity is, according to Hering, a function of the dark-adaptation of the eye rather than of the low intensity of the stinulus, and is due to the fact that in the dark the white-black substance rises greatly in potential, while the chromatic substances remain in the condition of autonomous equilibrium. Consequently, the weight of the chromatic is very small compared with that of the colourless elements, and the former remain under the threshold. The altered distribution of brightness of the colourless spectrum will be considered later.

While the ordinary negative or complementary after-image may easily be explained by the adoption of Hering's views of metabolism, the same cannot be said of the prolonged positive image which occurs after looking at the sun or other strong illumination. Hering proposes the following explanation. ${ }^{1}$ When looking at the sun there is not only very violent katabolism, but also a considerable increase of anabolism, so that not only is the visual substance used up, but also the material for anabolism. Consequently, in the darkened eye, the sensation depends on very feeble katabolisn due to intrinsic stimuli and still feebler anabolism. The substance is at a very low potential, but the sensation is one of brightness. If now the retina is illuminated, the increase of katabolism in the blinded area will be very slight, but its anabolism will be very much increased by the katabolism going on in the rest of the retina (see Explanation of Contrast). Anabolism will be in excess of katabolism, and the image will appear dark. Given the assumption that the material for anabolism is used up, this explanation follows perfectly from the theory; but it is not quite easy to understand how this consumption can take place during the very short exposure necessary for a blinding image. The phenomenon clearly borders on the pathological, and is of essentially the same nature as the persistent scotoma which has in many cases followed direct vision of the sun. Its explanation belongs to the pathology rather than the physiology of metabolism.

The relation of Hering's theory to the question of fatigue is a problem belonging to general physiology, but it may be briefly considered here. Some of the objections which have been most strongly urged against the theory have involved this question. Donders pointed out that during the day the eye is exposed for hours to light which gives a more intense sensation than that of the mean grey, and that the eye should consequently become fatigued. The answer to this is the occurrence of allonomous equilibrium, and from one point of view this may be regarded as fatigue, since the substance is then less capable of work. This condition, however, must be rigorously distinguished from such a condition as that resulting from exhaustion of the material for anabolism and also from accumulation of metabolic products. During the continuous daylight of a northern summer, the visual substance oscillates about a condition of equilibrium at a low potential, but the supply of new and the removal of waste material go on with a regularity suited to the needs of the substance at its comparatively low level of potential.

1 “Zur Lehre vom Lichtsinn," S. 10\%. 
Simultaneous contrast, and the other phenomena which I have included under spatial induction, are explained physiologically by Hering on the same lines as the phenomena of temporal induction. When a retinal area undergoes katabolism under stimulation, the process of katabolism may be regarded as acting as a stimulus to anabolism in the same area; it is due to this tendency of allonomous katabolism to promote autonomous anabolism that allonomous equilibrium occurs. In the same way, allonomous anabolism in a retinal area may be regarded as acting as a stimulus to autonomous katabolism in that area. Contrast is simply explained by supposing that this tendency to promote the opposite kind of change is not limited to the area stimulated, but extends to adjoining areas, and that this action is most marked at the line of junction of the reacting areas, and diminishes from here outwards. Hering formulates the nature of the physiological reciprocity of retinal areas, advocated by Johannes Müller, by making an assumption which is perfectly consistent with his general theory.

The strong point of Hering's explanation is, that it brings temporal and spatial induction into the closest relations with one another; each of the two processes helps to make the occurrence of the other more intelligible. In both kinds of induction we may see the working of a general law, that the occurrence of destructive metabolism at once determines an increase in constructive metabolism, which hinders the living substance from undergoing too great or too rapid decomposition; and that, on the other hand, constructive metabolism determines an increase in the tendency to spontaneous decomposition, which sets a limit to assimilation, and prevents it from reaching a degree beyond the needs of the organism.

Specific brightness. - Hering's treatment of the subject of brightness may be considered here. It is interesting, as the only feature in which the theory has suffered any important modification since first formulated. In the "Lehre zum Lichtsinn" Hering thought it probable that the brightness of a visual sensation was wholly due to its white component, and that if we were able to see the four primary colours in a state of purity they would appear of equal brightness. For this assumption has now been substituted the idea of specific brightness of the colours. ${ }^{1}$ The two colours, red and yellow, which are due to katabolism, are supposed to contribute positively to the total brightness, while the contribution of green and blue is negative and diminishes the total brightness of the sensation. This assumption is especially applied to explain the changes in the spectrum with lowered intensity. In the eye adapted to the dark, the black-white substance will have been raised to a condition of high potential, while the chromatic substances will not be appreciably affected; consequently, the "weight" of the colourless component of the sensation will be relatively much increased, and with slight stimulation the coloured components will remain under the threshold. The chromatic substances will also cease to contribute to the brightness of the sensations, and the curve of luminosity of the spectrum will be that of the black-white substance. According to Hering's theory, then, the spectrum of low intensity is the spectrum of the black-white substance. With increase of intensity the specific brightness of the chromatic processes begins to affect the curve of luminosity; red and yellow contribute positively, green and blue negatively, and the consequence is a shifting of the maximum brightness of the spectrum towards the former, until yellow again becomes the brightest part. From this

${ }^{1}$ Hillebrand, Sitzungsb. d. k. Akad.d. Wissensch., Wien, 1889, Bd. xeviii. Abth. 3, S. 70. 
it follows that in the long-wave half of the spectrum, the white value of a colour should be less than the brightness as determined by the methods of heterochromatic photometry, while in the short-wave half the reverse should be the case. This was shown to be true by Sachs, ${ }^{1}$ who used the method of pupil photometry; and with the flicker method the same relation between brightness and white value is found.

The colourless sensation which occurs when two complementary coloured lights are mixed, depends on the combined action of the coloured lights on the black-white substance; one of the two complementary lights acts as a katabolic, the other as an anabolic, stimulus; and with a suitable intensity of each, these stimuli neutralise each other, and the chromatic substance or substances remain in a condition of equilibrium. Hering has repeatedly verified by experiment the proposition that the white value of the grey produced by mixing two colours is equal to the sum of the white values of those colours, the brightness of the grey depending wholly on the action of the black-white substance. Abney and Rood have also found that the brightness of a grey produced by mixing two colours is equal to the sum of the brightness of those colours as determined by heterochromatic photometry. If both propositions are correct, it would mean that the positive contribution made by the specific brightness of the warm colour is exactly neutralised by the negative contribution of the cold colour.

Peripheral retina and colour-blindness.-According to Hering, the peripheral retina of the normal eye shows the same conditions as are found in colour-blindness. In total colour-blindness, and in the outermost zone of the retina, only the black-white substance is present; the middle zone of the retina is regarded as red-green blind, and the smaller individual differences are of the same nature as those which distinguish macular from extramacular vision. Since the curve of luminosity of the spectrum at a low intensity depends, according to the theory, on the black-white substance, it should correspond with that of total colour-blindness, and Hering found this to be the case. He was able in 1892, on the basis of his theory, to foretell the matches that would be made by the case of total colour-blindness he was about to examine. Hering has also supposed that the white values of coloured light were the same for the extreme periphery of the retina as for total colourblindness, but according to v. Kries (p. 1084) this is not the case.

Red-green blindness and the vision of the middle zone of the peripheral retina are ascribed by Hering to absence of the red-green substance. $^{2}$ The investigations of Hess on the vision of the peripheral retina and the nature of vision in acquired colour-blindness, and in Hippel's unilateral case, are strong evidence in favour of Hering's view. The chief difficulty in the way of the theory is the existence of two distinct classes of this form of colour-vision. Hering's explanation is, that these differences are of exactly the same order as the individual variations first described by Rayleigh, and depend like them upon differences of pigmentation of the macula and of the lens. A large number of very careful observations were made by $\mathrm{Hering}^{3}$ on Prof. Biedermann and Dr. Singer, who were well-marked examples of individual variation. He regarded the former as relatively yellow-sighted,

1 Arch. f. d. ges. Physiol., Boun, 1892, Bd. lii. S. 79.

2 More exactly, the condition of the peripheral retina is regarded as one of feeble redgreen sense.

${ }^{3}$ Lotos, Prag, 1885, Br. vi. S. 142. 
and the latter as relatively blue-sighted, these conditions depending on relatively greater and less pigmentation respectively. Hering regards the photerythrous group as relatively yellow-sighted, and corresponding to this he found that Biedermann's matches were good for photerythrous cases. The scoterythrous group, on the other hand, would be relatively blue-sighted, and Singer's matches were found to be good for them, but required alteration for Biedermann. Hering prepared a number of dried human retinæ, and found when Singer looked through the macular region of one of these that Biedermann's matches appeared correct, and by the same means Hering was able to convert a scoterythrous into a photerythrous case. Hering also found that the extramacular region of his own retina gave results which stood in the same relation to those of his central region as the results for the central region of scoterythrous stood to those of central photerythrous vision. Biedermann and Singer carried out a series of observations on their peripheral vision, and Hering expected to find that the differences between them would disappear. They remained present, however, though considerably diminished in amount, and Hering ascribed this to differences in the pigmentation of the lens, which he found to be of a distinct yellowgreen colour, not only in adults but in the newly-born child. ${ }^{1}$

If this explanation be correct, it might be expected that there would be a gradual transition of one class into the other, corresponding with a graded series of degrees of pigmentation, but the evidence seems to be against this. Hering himself was inclined to think that the cases he examined centred round Biedermann and Singer, rather than round a point between them. It is quite possible, however, that the variations of pigmentation may be discontinuous; and, in the absence of direct investigation of the question, the existence of two distinct groups by no means destroys the validity of the proposed explanation.

From his examination of individual variations, it seemed probable to Hering that there is a relation between macular (and lens) pigmentation and development of the colour sense. Biedermann had a very highly developed colour sense (high degree of discrimination and wide limits of visibility of spectrum), while Singer had a weak red-green sense, and much more limited spectrum; and in cases of red-green blindness it seemed as if the group with more pigmentation (yellow-sighted or photerythrous) had a more highly developed blue-yellow sense, and Hering supposes that the shortening of the spectrum in the scoterythrous group may be due to weakness of the yellow sensation.

v. Kries has recently written strongly against Hering's view. He points out that the most marked distinction between the two groups of red-green blindness is at the long-wave end of the spectrum, while the macular pigment, as Sachs has shown, only begins to show any appreciable absorption in the yellow-green. In order to determine the influence of macular pigmentation in a number of cases of dichromatic vision, he matched blue-green light of $490 \lambda$ with a mixture of blue $(460 \lambda)$ and green $(510-517 \lambda)$; the quotient $\frac{\text { blue }}{\text { green }}$ showed no regular difference between the two groups of red-green blindness; in fact, the extremes were both in the scoterythrous class.

According to Hering's theory, there should be in yellow-blue blindness two neutral bands, without shortening of either end of the spectrum. Hering

1 The greenish yellow colour of the lens in childhood is seen best if obscrved in water placed in a white porcelain vessel. The greenish yellow colour changes to yellow in adult life. 
explains the shortening in the case recorded by Vintschgau and himself, partly by a co-existing weakness of the red-green sense, and partly by a quantitative anomaly. He found evidence that the colourless region at the violet end had a red value, for when violet light was added to a red which was below the chromatic threshold, the mixture became red, while addition of violet light to a green above the chromatic threshold produced a mixture which appeared colourless.

Hering's theory and the retina.-It might be supposed that, according to Hering's theory, the katabolic colours should produce an electrical change in the retina in an opposite direction to the anabolic colours. Red and yellow should cause a variation of one kind, and green and blue of the opposite kind. This is not the case. This supposition, however, neglects the black-white substance which is acted on by all kinds of light, and the variation due to this would mask any difference in the behaviour of the chromatic substances. In order to support the theory, it would only be necessary to show that a green of a definite white value gives a smaller variation than a red of equal white value. Another factor to be taken into account is the influence of spatial induction, which, by causing the opposite kind of change in the nonstimulated area, would still further obscure the direct results of stimulation.

The recent views considered on pp. 1101-1105 must, if correct, modify the theory to some extent, at any rate in the form in which it is usually understood. According to these views, the spectrum of low intensity of total colour-blindness is the spectrum of visual purple, while the effect of dark-adaptation depends on accumulation of this substance. This suggests that Hering's black-white substance and visual purple may be one and the same thing; against this is the undoubted fact that the production of colourless sensation by colour mixture holds for the fovea; the colourless sensation cannot here be due to the combined white values of the coloured lights. It must be remembered, however, in answer to this and other difficulties, that Hering has never regarded his theory as an expression of retinal processes. In one place ${ }^{1}$ he expressly states that his theory has nothing to do with the first immediate changes in the peripheral organ. If Hering's substances are regarded as central, these difficulties to a great extent disappear. Under ordinary conditions, the central black-white action could be excited by two kinds of peripheral activity, i.e. changes in visual purple, or changes in the chromatic apparatus. As already mentioned, the existence of binocular colour contrast suggests the central origin of contrast in general, and, if spatial induction is central, temporal induction has probably the same seat.

Hering has not only left the actual seat of the psycho-physical processes undecided, but, further, has only adopted the conception of three special substances, in order to assist the exposition of his views. He would prefer ${ }^{2}$ to speak of only one psycho-physical substance, capable of manifold qualitatively different kinds of anabolism and katabolism, each kind being capable of analysis into three components, corresponding to three chief varieties of anabolism and katabolism.

G. E. Müller ${ }^{3}$ has lately suggested certain modifications of Hering's theory, by means of which he thinks certain difficulties may be removed. He supposes that visual purple acts as a sensitizer to the visual substances proper, and that, in

${ }^{1}$ Arch.f. d. ges. Physiol., Bonn, 1887, Bd. xli. S. 39.

${ }^{2}$ Sitzungsb. d. k. Akad. d. Wissensch., Wien, 1889, Bd. xeviii. Abth. 3, S. 73.

3 Ztschr. f. Psychol. u. Physiol. d. Sinnesorg., Hamburg u. Leipzig, 1896, Bd. x. S. 1 and 321 ; and 1897, Bd. xiv. S. 1 and 161 . 
addition to the three kinds of visual substance with their seat in the periphery, there is a central black-white excitation which is the basis of the intrinsic light, and is capable of being set in action by change in the chromatic substances in the periphery as well as by change in the peripheral black-white substance.

Ebbinghaus ${ }^{1}$ attempted to bring the theory into relation with retinal structures. He supposed that visual purple was Hering's blue-yellow substance, that it existed alone in the rods, and together with some other substance which neutralised its colour in the cones. These views were supported by the correspondence of the absorption curves of visual purple and visual yellow with the elementary curves for yellow and blue in the red-green blind.

Other theories of colour-vision.-The theory of Donders ${ }^{2}$ occupies a position intermediate between those of Helmholtz and Hering. Donders recognised the claims of red, yellow, green, and blue to be regarded as primary, or, as he called them, simple colours. On the other hand, he held the facts of colour mixture to show a threefold physiological process. The colours corresponding to these three physiological energies he called fundamental colours, and he adopted as such red, green, and violet. He supposed that these energies had their seat in the periphery, while the processes in the visual centres had a fourfold nature, corresponding to the four simple colours. He further supposed that a single peripheral process might affect more than one central process, so that a fundamental colour, as violet, might correspond to two simple colours, as red and blue. For his central processes Donders adopts a hypothesis in terms of molecules. The sensation white corresponds to complete, colours to partial, dissociation of the molecules; this partial dissociation gives rise to molecules whose secondary dissociation is the basis of the colours complementary to those arising through the primary dissociation. Black corresponds to a condition where dissociation and repair are equal, but both are reduced to a minimum. Donders did not attempt to apply his theory to the whole range of colour phenomena, but used it chiefly in reference to colour-blindness. A somewhat similar molecular hypothesis has been advanced by Ladd Franklin. ${ }^{3}$

In 1881, Preyer $^{4}$ advanced a theory which, in many respects, resembled that of Hering. In this theory analogies were drawn with the temperature sense of the skin, and assumptions were made as to the seat of the different kinds of sensation, the rods being regarded as photogenous, and the cones as chromatogenous elements.

The theory of Wundt ${ }^{5}$ has been already mentioned. He assumes the existence of separate substances for light and colour sensations, but supposes that the latter depend on different kinds of change in one complex substance. In order to support his theory, attempts have been made to show that colour-blindness may occur in a far greater variety of forms than is commonly supposed (Kirschmann) ${ }^{6}$ Contrast is explained psychologically, while after-images are referred to fatigue assisted by contrast.

A theory of a different nature has been advanced by Charpentier. ${ }^{7}$ He distinguishes between two kinds of retinal elements,-photo-æsthetic elements of a rudimentary nature, which have to do with sensations

${ }_{1}$ Ztschr. f. Psychol. u. Physiol. d. Sinnesorg., Hamburg u. Leipzig, 1893, Bd. v. S. 145.

2 Arch. f. Ophth., 1881, Bd. xxvii. Abth. 1, S. 55 ; and 1884, Bd. xxx. Abth. 1, S. 15.

${ }^{3}$ Mind, London and Edinburgh, 1893, vol. ii. p. 473.

${ }^{4}$ Arch. f. d. ges. Physiol., Bonn, 1881, Bd. xxv. S. 31.

5 "Grundziige d. phys. Psych.," 1893, Bd. i. S. 535.

6 Phit. Stud.. Leipzig, 1893, Bd. viii. S. 173 and 407.

7 "La lumiere et les couleurs," Paris, 1888, p. 265.

VOL. II. -7 I 
of simple luminosity, having little to do with localisation; and more specialised visual elements, the basis of distinct perception. $\mathrm{He}$ supposes that colour is due to the simultaneous activity of the two kinds of retinal element; to interference between the two sets of vibrations started by their stimulation. He formerly believed visual purple to be the basis of the photo-rsthetic activity, but since this substance is absent from the fovea, and since the presence of only one kind of element would involve the colour-blindness of this part of the retina, he has lately ${ }^{1}$ given up the idea that visual purple is the source, or the only source, of the photo-æsthetic activity. The other kind of vibration he supposes to be set up by thermo-electric action between the cones and the retinal pigment. Charpentier thinks it probable that the retinal oscillations described by him (p. 1074) depend on transverse vibration of the free ends of the rods and cones.

\section{BINOCULAR VISION, MOVEMENTS OF THE EYES, AND SPATIAL PERCEPTION.}

Introductory.-The point of especial interest in this part is the physiological basis of visual perception of space. Its consideration involves psychological factors to a much greater extent than the subject of the second part of this article. The physiological factors are of two kinds, namely, movements of the extrinsic and intrinsic ocular muscles with the central motor processes connected with their innervation, and the binocular mechanism by means of which the two eyes are brought into physiological association with each other, and are made to act in many ways as if they were one organ. Certain facts about these two sets of factors must first be considered.

Binocular combination.-In studying binocular vision, it is necessary to have some means of presenting different objects to the two eyes. This may be done withont apparatus, by placing the object on the lines of direction of the eyes, either beyond or nearer than the fixation point, when the combined image is seen accompanied by the lateral images. The observation of the combined image may be assisted by screens to hide the lateral images. Some individuals, especially myopes, obtain binocular combination most readily with the lines of vision crossed beyond the objects, and with normal vision this method is usually rendered more easy by wearing weak convex glasses. Probably most individuals, however, obtain more satisfactory results by fixing a point nearer than the objects to be combined. Of the different forms of stereoscopic or haploscopic arrangement, which have been used for the same purpose, the most suitable for physiological experiments is Wheatstone's, especially in the modified form of Hering's haploscope.

Retinal rivalry.-When the fields presented to the two eyes are different, a phenomenon known as "retinal rivalry" occurs. If two glasses differing in brightness are held, one before each eye, the combined field may be observed to become periodically lighter and darker; with glasses of different colour, the field may change periodically in colour. The greater the difference in brightness between the two fields, the more marked is the rivalry. When there is in one field an

${ }^{1}$ Arch. d'opht., Paris, 1896, tome xvi. p. 337. 
object with well-marked contours, this will tend to prevail in the struggle between the two fields, and will not disappear periodically, although the background may continue to do so. If each field contain an object with contours, and these coincide, there will be marked rivalry, especially if the objects differ greatly in form. At times only one of the objects will be seen, while at other times parts of the two objects will be seen together. Rivalry may occur when one eye is closed; if a sheet of white paper is observed close to the eyes while one eye is closed, a periodical darkening of the paper occurs, which is due to the influence of the intrinsic light of the closed eye (Purkinje, Hering). In 1864, Hering ${ }^{1}$ pointed out that the behaviour of black in retinal rivalry was evidence in favour of black being a positive sensation.

The explanation given of retinal rivalry has been chiefly psychological. Helmholtz referred the phenomenon to the influence of attention, this being applied at one moment to the field of one eye, and at the next to the field of the other. The variations may take place with considerable regularity, and the periods of variation show some correspondence with those of the variations of after-images. We are at present wholly in the dark as to the physiology of this phenomenon.

Brightness of binocular field.-The apparent brightness of a given illumination is not the same for one and for two eyes; according to old observations, a light seen with two eyes appears about one-tenth brighter than when seen with one eye alone. When the illumination of the two eyes is different, the apparent brightness of the combined field varies with the intensity, and with the difference of illumination of the two eyes. Fechner ${ }^{2}$ found that if a dark glass were held before one eye, the apparent brightness of the field increased when this eye was closed or screened, and diminished again on reopening the eye. In this experiment the brightness of the sensation increases when the amount of light entering the eyes is diminished, and decreases when it is augmented, and it is consequently known as "Fechner's paradoxical experiment." After a time the brightened field may be observed to darken again owing to rivalry. If the glass used lets through much light, this effect does not occur, and the field brightens and darkens with increase and decrease of illumination. For each individual, Fechner found an indifferent point, corresponding to a glass with which the brightness of the combined field remained the same whether the eye was closed or open. Such a glass was found on the average to be one which transmitted two-thirds of the light.

The greatest amount of darkening oecurred with a glass which let through about $\frac{1}{25}$ of the light, and on either side of this point glasses could be found with which the combined field was equally bright; thus a glass transmitting $\frac{1}{8}$ of the light gave a combined field of the same brightness as one transmitting $\frac{1}{50}$, and such glasses were said by Fechner to be of conjugate intensity. It has been supposed that the paradoxical experiment is due to the pupil; closing the eye behind the dark glass would lead to a consensual widening of the pupil of the other eye, and might in this way brighten the field. Fechner found, however, that the experiment succeeded with a small artificial pupil, and the phenomenon also occurs as well with atropinised pupils as in the normal condition. No satisfactory physiological explanation has been given. It has been supposed that the brightness of the combined field is the

1 “ Beitr. z. Physiologie," Leipzig, 1864, Heft 5, 5. 309.

${ }^{2}$ Abhandl. d. k. sächs. Gesellsch. d. Wissenseh., 1861, Bd. v. S. 416. 
mean of that of the two eyes, and the phenomenon has also been referred to the influence of attention, which is supposed to be concentrated on the open eye when the darkened eye is closed. Fechner supposed that it depended on antagonism between the two retinæ.

Binocular colour mixture.-When a different colour stimulus is applied to each eye, the mixture-colour may be observed during the transitions from one colour to the other which depend on rivalry. With suitable adjustment of the two stimuli in respect of intensity, the rivalry may be very much lessened or abolished, and the mixture-colour persists. The existence of true binocular colour mixture, however, has been denied by Helmholtz ${ }^{1}$ and others, but there can be no doubt of its existence, the mixture-colour being in all respects comparable with the colour produced by mixture on the colour wheel or by the double-image prism. It may be seen with simple binocular combination; with the stereoscope; and by a method devised by Hering, ${ }^{2}$ in which a patch of light is seen in a dark box containing two coloured glasses, arranged in such a way that each eye looks through a different coloured glass. The patch of light is most conveniently produced by covering an aperture in the floor of the box with ground glass, and looking through the box towards a brightly illuminated surface. Binocular colour mixture may also be observed in the combined after-image of the two eyes. ${ }^{3}$

In experiments showing the rivalry of contours, the parts where the images of the two eyes join have a peculiar lustrous appearance. This is also seen in the binocular combination of black and white fields, the combined image looking like graphite. If a red and blue pattern be observed with a red glass before one eye, and a blue glass before the other, the same lustre is very marked. The natural lustre of certain objects has been referred to the same cause. It is supposed that objects with a certain degree of roughness of surface reflect rays in irregular directions, so that corresponding points of the two eyes are stimulated by light of different degrees of intensity.

Corresponding points.-The physiological basis of single vision with the two eyes is the mechanism of corresponding or identical points. These points may be defined as those parts of the two retinæ which receive the two images of the same distant point of light (star). If one retina be placed in front of the other, so that the fover are in contact, and the temporal half of one retina covers the nasal half of the other, any point in one retina will cover its corresponding point in the other, and such points have therefore also been called covering points. Since the nasal half of each retina has a more extended field than the temporal half, only the central part of the visual field is binocular.

J. Müller ${ }^{4}$ demonstrated the parts of the two retinæ which corresponded to one another by means of pressure phosphenes. He found that a phosphene produced by pressure on the outer side of one eye was projected, so that it was localised in the same position in space as one due to pressure on the inner side of the other eye. Hering ${ }^{5}$ showed that an after-image of one eye has exactly the same localisation in the visual field of the other eye as the original of the after-image to the

1 "Handbuch d. pliys. Optik," 1867, S. 776.

2 Hermann's "Handbuch," 1879, Bd. iii. S. 593.

${ }^{3}$ Proc. Camb. Phil. Soc., 1895, vol. viii. p. 273.

4 " "Zur vergleichenden Physiologie des Gesichtsinnes," Leipzig, 1826, S. 73.

5 “Beitr. z. Physiologie," Leipzig, 1863, Heft 3, S. 182. 
first eye. The method most widely used is that of substitution, which depends on identical localisation with the stereoscope or haploscope.

In considering the mechanism of corresponding points, it is necessary to have some definite terminology for different parts of the retina. It is now usual to regard each retina as made up of a number of points arranged in vertical and horizontal sections. One point corresponds with another, when it lies in the same vertical and horizontal sections in the two eyes. Points which do not correspond are termed "disparate" points. If a point lies in the same horizontal section in the two eyes, but in different vertical sections, the points are termed horizontally disparate, or as having horizontal disparation. If they lie in the same vertical but in different horizontal sections, they have vertical disparation.

It has been found that the strictly vertical meridians of the two eyes do not correspond with one another. If the double images of two lines are observed, it is found that these appear to be vertical and parallel to one another, when the lines diverge from one another upwards, so as to form an angle of $2^{\circ}$ with one another. This "physiological incongruence" of the two retinæ varies in amount in different individuals; the deviation of apparently vertical lines may reach $2^{\circ} \cdot 30^{\prime}$, but is much less and may be absent in myopia.

According to Volkmann, ${ }^{1}$ there is a similar incongruence between the horizontal meridians, amounting, however, only to half a degree, the meridian of each eye inclining slightly downwards and outwards.

Another kind of incongruence has been described by Kundt, ${ }^{2}$ who found that when he halved a horizontal line seen with one eye, the outer half was made constantly too large, and Hering ${ }^{3}$ supposes that this is due to difference in the distance between corresponding points on the two sides of the retina.

Single and double vision.-When retinal disparation is marked, two images of an external point are seen. The nature of the disparation and diplopia depends on the position of the point in relation to the fixation point. When nearer, the double images are crossed or homonymous, i.e. the image of the right eye appears to the left, and that of the left eye to the right; when the point is beyond the fixation point, the diplopia is uncrossed or heter- $a$ onymous. The retinal disparation is similarly termed crossed and uncrossed respectively.

When the disparation is slight, fusion of the two images oceurs, and single vision results. This was first shown by Wheatstone, ${ }^{4}$ and the amount of disparation with which single vision can occur has been investigated by Volkmann. ${ }^{5} \quad \mathrm{He}$ combined vertical lines (Fig.

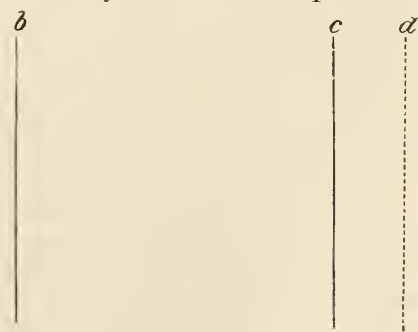

FIG. 400.

400 ), in which $a, b$, and $c$ were fixed, and $d$ movable; $a$ and $b$ being presented in the stereoscope to the left, $c$ and $d$ to the right eye. He found

1 "Physiol. Untersuch. im Gebiete d. Optik," Leipzig, 1864, S. 206.

${ }^{2}$ Ann. d. Phys, u. Chem., Leipzig, 1863, Bd. exx. S. 118.

3 Hermann's "Handbuch," Bd. iii. S. 362. "Phil. Trans., London, 1838, p. 371.

${ }^{5}$ Arch. f. Ophth., 1859 , Bd. v. Auth. 2, S. 1. 
that the amount of difference between $a b$ and $c d$, which still allowed single vision, varied with the distance between $a$ and $b$, and was also considerably diminished by practice; when $a b$ equals $5.3 \mathrm{~mm}$., observed at a distance of $16 \mathrm{~cm}$., he found the limit when $c d$ was less than $a b$ to be $1.84 \mathrm{~mm}$. when $c d$ was greater than $a b$, it was $2 \cdot 27 \mathrm{~mm}$. With horizontal lines the amount of disparation consistent with single vision was much less, being under similar conditions, only $42 \mathrm{~mm}$. and $\cdot 75 \mathrm{~mm}$. Fusion into single vision takes place much more readily with horizontal than with vertical disparation, and consistently with this result he found that a circle fused more easily with a smaller ellipse when the shorter axis of the ellipse was horizontal. From experiments on fusion in different meridians, he found that the tendency for disparate lines to fuse diminished with their deviation from the vertical.

Wheatstone supposed that it followed, from the occurrence of single vision with disparate points, that double vision could occur with corresponding points, and in favour of this described the following experiment. In Fig. 401 the image of the thin line would, in binocular combination, fall in the left eye on points corresponding to those of the thick line in the right eye, but Wheatstone found that the two thick lines fused, giving an

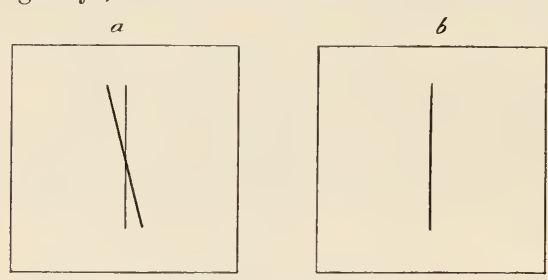

FIG. 401.- Wheatstone.

inclined line, seen in relief crossed by the thin vertical line. This experiment has been used by him and by others as evidence against any necessary physiological connection between corresponding points. The experiment has been very fully criticised by Hering, ${ }^{1}$ who has found that if the

vertical line presented to the right eye be distinguished by a mark, and care be taken that the two vertical lines are seen with corresponding points, and with accurate fixation, the two vertical lines either fuse or can be observed independently of the oblique line. Care has also to be taken to ensure that both eyes are being used. Hering points out that the stereoscopic appearance of the cross exists when it is observed with only one eye, and is especially marked when drawn on glass, there being a natural inclination for two lines crossing each at an acute angle to be seen stereoscopically. Other experiments in favour of double vision with corresponding points, brought forward by Nagel and Wundt, have been similarly shown by Hering to he inconclusive.

It has been supposed that corresponding points are identical in function, and also that the nerve fibres from corresponding points are connected with the same nerve cell. Single vision with disparate points, and double vision with corresponding points, have both been brought forward as facts inconsistent with identity. If the existence of double vision with colresponding points were proved, the doctrine of identity would have to be given up. Single vision with disparate points, on the other hand, only requires a modification of the doctrine, to the effect that, while single vision is necessary with stimulation of corresponding points, it is possible with that of disparate points. In the latter case the single image is not only in many cases capable of analysis into double

1 "Beitr. z. Physiologie," Leipzig, 1862, Heft 2, S. 81. 
images, but there is a further distinction that the single image receives a different localisation, and is seen either nearer to or further from the eye than the single image of corresponding points. This difference of localisation was first observed by Wheatstone, ${ }^{1}$ and, as will be seen later, there is a close connection between disparation and spatial localisation.

Panum ${ }^{2}$ proposed a modification of the doctrine of corresponding points, in which it was supposed that to each point in one eye there corresponded a definite sensory region (Empfindungskreis) in the other.

The horopter.-The horopter is the name applied to the sum of those points of space which, in a given position of the eyes, fall on

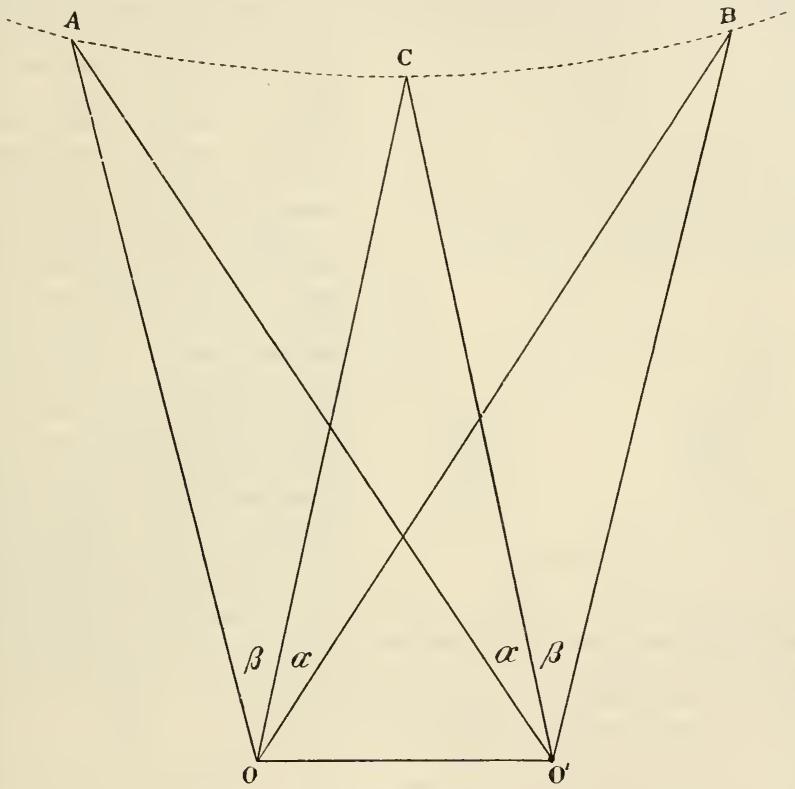

Fig. 402.-Hillebrand.

corresponding points in the two retinæ. The form of the horopter has chiefly been determined mathematically, but is also to a certain extent capable of experimental investigation. According to J. Miiller, the points forming the horopter lie in a circle passing through the fixation point and the nodal points of the two eyes, and also in a line at right angles to the plane of the circle, passing through the fixation point. In this theoretical construction it is assumed that corresponding points are identical ; that no wheel rotation occurs in movements of the eyes; that the retina is a spherical surface ; and that the centre of rotation of the eye coincides with the nodal point. None of these assumptions are correct, with the doubtful exception of the first, and consequently the theoretical horopter changes

${ }^{1}$ Loc. cit., p. 374.

2 "Physiol. Untersuch. über das Sehen mit zwei Augen," Kiel, 1858, S. 62. 
with every change of convergence, and does not exist in asymmetrical positions of the eyes. In the experimental investigation of the horopter, it is not sufficient to determine the points in space which in any given position of the eyes are seen singly, since single vision occurs not only with corresponding but also with slightly disparate points. Hering ${ }^{1}$ determines the form of the horopter by finding the points which are seen singly and in the same vertical plane as the point fixed. He distinguishes between the vertical and the horizontal horopter; the vertical horopter being made up of lines which fall on corresponding vertical sections of the retina, and the horizontal on corresponding horizontal sections. The total horopter is the part common to both vertical and horizontal horopters. In the determination of the vertical horopter, Hering uses vertically

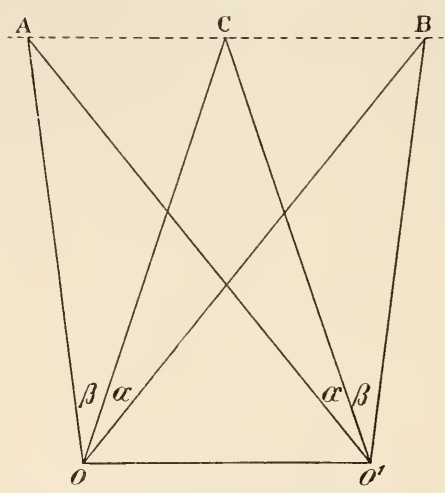

Fig. 403.-Hillebrand. suspended threads, the ends of which are not exposed, seen against a uniform background. He finds that if these threads are viewed with both eyes, and arranged so that they appear to be in a plane parallel to the frontal plane, at a certain distance, less than 2 metres from the eyes, they form a plane; nearer than this they form a curved surface concave to the face, the concavity increasing with approach to the eyes; beyond this distance they form a surface convex to the face. Helmholtz $z^{2}$ also found that threads apparently in a plane really formed a concave cylindrical surface near the eyes, and became a plane surface only at a certain distance, which he found to vary in different individuals; and he explained the facts partly by erroneous estimation of degrees of convergence. He also found that if small objects were fixed on the threads, these were arranged in a plane and not a curved surface; but Hillebrand ${ }^{3}$ has shown that this is not the case if the objects are at irregular distances from one another ; Hillebrand also found that the arrangement as described by Hering occurs with any degree of convergence. According to Hering, the curved surfaces are the real form of the horopter at different distances from the eyes, and depend on the special kind of retinal incongruence described by Kundt and himself (see p. 1125).

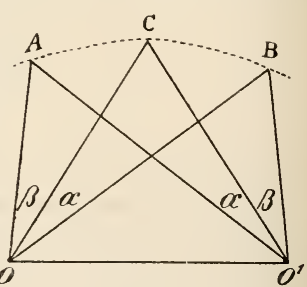

Fig, 404.-Hillebrand.

Hillebrand illustrates the form of the horopter by Figs. 402, 403, and 404 , in which $O$ and $O^{\prime}$ represent the nodal points of the two eyes. In all three figures the angle $\alpha$ is less than the angle $\beta$, corresponding to the incongruence in which a distance on the nasal half of the retina is greater

1 "Beitr. z. Physiologie," Leipzig, 1864, Heft 5, S. 296.

2 "Handbuch d. physiol. Optik," $186 \pi$, S, 654.

${ }^{3}$ Ztschr. f. Psychol. u. Physiol. d. Sinnesorg., Hamburg u. Leipzig, 1893, Bd. v. S. 1. 
than its corresponding distance on the temporal half. There will be a certain distance, at which the point of intersection of the indirect lines of vision will lie in the same vertical plane as the fixation point (Fig. 403); nearer than this they will lie nearer (Fig. 404), beyond this they will be farther (Fig. 402) than the fixation point. The horopter, as experimentally determined, is more extended than the theoretical or mathematical horopter, which Hering speaks of as the skeleton of the real horopter.

\section{Movements of THE EYEs.}

Methods of measurement.-The centre round which the eyeball rotates has already been dealt with (p. 1029). The plane passing through the two lines of fixation is known as the plane of fixation. In investigating the movements of the eyes, it is important to exclude movements of the head, and this is done most satisfactorily by means of a fixed board with a cast into which the teeth of the observer fit accurately. The head may then be fixed in any position, and the amount of ocular movement measured by means of movable fixation points on graduated scales.

The movement of the eyeballs which presents the greatest difficulty is the wheel or swivel rotation round the optic axis. Numerous methods have been devised for observing it and for measuring its amount. It may be observed directly by watching a marking of the iris, a conjunctival vessel, or a mark placed on the conjunctiva (J. Müller, 1826). It has been doubted whether the conjunctiva follows the movements of the eyeball with sufficient exactness for this purpose, but this seems to be the case. Another method is to make use either of regular or irregular astigmatism. In the latter the movement of the star-like figure may be observed by comparing it with a stationary thread. In regular astigmatism the amount of rotation may be measured by finding the cylindrical glass which corrects the defect in different positions; for satisfactory results, probably a degree of astigmatism of over $1 \mathrm{D}$ is necessary. Another method which has been employed satisfactorily for exact measurements is the degree of rotation of the blind spot. The method, due to Ruete, which has been most widely used, is the observation of the apparent rotation of an afterimage. A linear after-image will show the changed direction of any retinal meridian on which a linear image has been formed, and this method is well adapted for quantitative results.

Another method by means of which any difference in the swivel rotation of the two eyes may be detected, is the observation of halfimages of a doubled vertical line. This method is very delicate, and gives more exact results than the after-image method, but the error due to retinal incongruence (p. 1125) must be taken into account.

Listing's law.-W' Wen the head is fixed, there is, according to Listing, only one position of the lines of fixation from which the eyeball is able to move upwards and downwards and from side to side without any swivel rotation. This position is known as the primary position of the line of fixation. Listing's law states that when the eyeball moves from the primary position to any secondary position, the axis round which the movement of the eyeball takes place is one at right angles to a plane passing through the line of fixation in both its primary and its secondary positions. In other words, the axis round which oblique movements take place lies in the same plane as the axes round which simple horizontal and vertical movements take place. 
The law was first formulated by Listing ${ }^{1}$ on theoretical grounds, and was shown experimentally by Helmholtz to be true for distant vision.

Helmholtz used the after-image method, in which the observer should be seated opposite a screen at sufficient distance to render the convergence inappreciable. The position of the head must be found

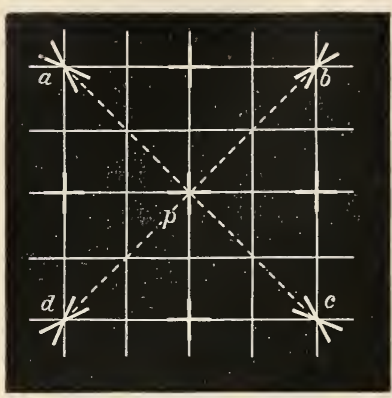

Fig. 405.-Hering. from which the after-images of a vertical and a horizontal strip do not deviate from a vertical and a horizontal line respectively on the screen with rertical and lateral movements of the eyes. When this position is found, the line of fixation of each eye is in the primary position, and the correspondence of the after-images with the lines shows that Listing's law is true for simple vertical and horizontal movements of the eyeball. In order to show that the same is true for oblique directions, the screen should be capable of rotation in a vertical plane round its centre, and the afterimages of oblique strips will be found not to deviate from lines on the screen of corresponding obliquity. If, on the other hand, a fixed screen is used, such as is shown in Fig. 405, the after-images of horizontal and vertical strips will appear distorted, as shown in that figure. This distortion is apparent, and depends on the fact that the lines of the screen, which really cross at right angles, give rise to retinal images in which the lines cross obliquely. Nevertheless the objective lines are seen at right angles, and the obliquity is ascribed to the after-image, as is represented in Fig. 406.

Donders found that it made no difference whether the visual axis was moved from the primary to a secondary position in a straight line or in any other way, and this fact was referred to by Helmholtz as Donders' law. The apparent distortion of the after-image is exactly the same for each eye.

Listing's law only applies to parallel or approximately parallel positions of the visual axes. With convergence there is evidence of a swivel rotation of the eyeballs outwards, i.e. an increase of the normal divergence upwards of the median vertical sections of the two retine.

According to Donders, ${ }^{2}$ the swivel rotation of convergence may be shown by a simple experiment, in which a horizontal line, doubled by con-

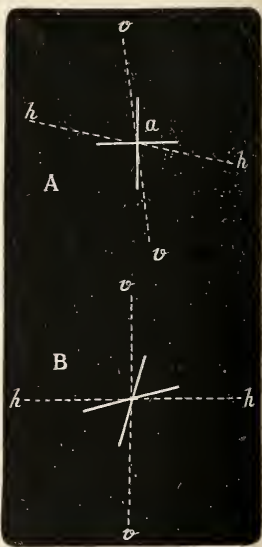

Fig. 406.-Hering. vergence for a nearer point, gives two images which form an angle open downwards. The amount varies considerably in different individuals. In seventeen cases Donders found it to vary from $1^{\circ} \cdot 2$ to $5^{\circ} \cdot 5$.

1 See Ruete's “Lehrbuch d. Ophth.," 1857.

2 Arch.f. d. ges. Physiol., Bonn, 1876, Bd. xiii. S. 418. 
Nagel ${ }^{1}$ and Hering ${ }^{2}$ have found that certain small deviations from Listing's law occur, when it is necessary, for the sake of single vision, to keep the images of an external object on corresponding points.

Compensatory eye movements. -The movement of this kind about which there has been most doubt, is the swivel rotation which takes place when the head is inclined to one side. Such a movement in the opposite direction to the inclination of the head was first described by John Hunter. Its existence has been denied, but the results of many investigations show that there is a partial compensation only, the angle of rotation of the eyeball being given by different observers from $\frac{1}{6}$ to $\frac{1}{12}$ of the angle of inclination of the head. The subject has recently been investigated by W. A. Nagel, ${ }^{3}$ using the blindspot method. He found that the amount of compensation varied from $\frac{1}{5 \cdot 2}$ to $\frac{1}{11 \cdot 8}$, the rotation of the eyeball decreasing relatively as the inclination of the head increased.

The compensatory movements of the eyeballs are influenced by the condition of the labyrinth, and especially of the semicircular canals, and have been much used as a means of investigating the functions of these organs, both in man and other animals. The movements are abolished by destruction of both labyrinths, and either appear not to be affected or are exaggerated with destruction of one side.

Nage ${ }^{4}$ has investigated the compensatory swivel rotation in a number of animals, and with more extensive rotation of the head than has been studied in man, and has found considerable differences in different animals. The rabbit showed as much as $90^{\circ}$ of rotation of the eyeball round its axis; while in birds, with great mobility of the neck, compensatory eye movements may be completely absent.

Associated movements.--Some movements of the eyeballs are more closely associated than others. The parallel movements of the two eyes together are very closely associated, and not only continue when one or both eyes have become blind, but were observed by Donders ${ }^{5}$ in a case of complete congenital blindness. The exact association between the movements of accommodation in the two eyes has already been considered (p. 1038). There is a very close association between convergence, accommodation, and contraction of the pupil on the one hand, and divergence, relaxation of accommodation, and dilatation of the pupil on the other; but these movements are capable of dissociation to a considerable extent. Distinct vision with convex or concave glasses depends on a certain degree of this dissociation, accommodation altering while the convergence remains constant, while, by means of a prism, convergence or divergence may be made to occur without accompanying accommodation. The same dissociation occurs when well-defined double or combined images of objects are seen by diverging or converging the eyes. The ease of dissociation may be greatly increased by practice. The amount of accommodation which can take place with a given convergence of the visual lines is known as the relative range of accommodation. Donder's ${ }^{6}$ found that with increasing convergence the relative range at first increased and then decreased, till with maximum convergence it became nil.

1 "Das Sehen mit zwei Augen," Leipzig, 1861. 2 Hermann's "Handbuch," Bd. iii. S. 504.

"Ztschr. f. Psychol. u. Physiol. d. Sinnesorg., Hamburg n. Leipzig, 1896, Bi. xii. S. 331. See this paper for a full account of the subject.

+ Loc cit. $\mathrm{S}$. 346

${ }^{5}$ Arch.f. d. ges. Physiol., Bonn, 1876, Bd. xiii. S. 283.

6 "Anomalies of Accommodation and Refraction," p. 113. 
Using the method of binocular combination, Dixon ${ }^{1}$ found that the variation in the angle of convergence which he could make without altering his accommodation, decreased with the distance of the objects. At $100 \mathrm{~cm}$. he found that he could vary his convergence to an amount corresponding to $6 \mathrm{D}$, without alteration of accommodation.

How far the association between convergence and accommodation persists in monocular vision, has not been satisfactorily determined.

The axes of the eyeballs during sleep are usually described, following J. Müller, as directed upwards and inwards. Raehlmann and Witkowski, ${ }^{2}$ however, found that any position might occur, and that inco-ordinated movements of a special character were common during sleep. The movements were very slow, and might lead to any kind of divergence, horizontal or vertical, of the lines of vision. They also found that movement of one eye only, occurred frequently. Henke ${ }^{3}$ and others have found that while going to sleep crossed and slanting double images may be observed, indicating divergence and swivel rotation of such a kind that the vertical meridians converge upwards.

As already mentioned, co-ordinated movements persist in the blind, even in congenital blindness, although in some cases minor degrees of inco-ordination have been observed after many years.

In newly-born children, most observers have failed to observe co-ordinated eye movements till some time after birth. Raehlmann and Witkowski ${ }^{4}$ found the movements often inco-ordinated and much like those in sleep, but more rapid. They failed to observe movements of fixation in the first ten days. Two such excellent observers as Donders ${ }^{5}$ and Engelmann, however, observed definite binocular fixation with alteration of convergence in a child a few minutes after birth, but Hering has pointed out that this result can only be expected exceptionally, since most children pass the early days of life almost in a condition of sleep.

These various cases have all been discussed in connection with the question whether the association of eye movements is innate or acquired, a question important in connection with theories of the genesis of spatial perception. The observations of Donders are sufficient evidence that the association is not acquired in the life of the individual, but it is also certain that the association is not so fixed as to be incapable of being dissolved under many conditions.

\section{Spatial Perception.}

Visual localisation.-This has been referred to factors of three kinds,- to sensations derived from movements of the eyeballs and of accommodation; to sensations, or modifications of sensations, arising from the simultaneous stimulation of the two retinæ; and, thirdly, to a number of factors of a more purely psychological nature. The sensations of movement have been referred to peripheral changes set up by movements of the eyeballs, and also to central changes connected with innervation. The psychological factors are of great importance, and in vision with one eye and at great distances are the chief or only means of localisation. They also, however, influence the process of localisation under all ordinary conditions, and in order to investigate the physiological factors they must be excluded. This is done by using very fine

${ }^{1}$ Mind, London and Edinburgh, 1895, vol. iv. p. 205.

2 Arch.f. Physiol., Leipzig, 1877, S. 454. ${ }^{3}$ Arch.f. Ophth., 1864, Bd. x. Abth. 2, S. 181.

4 Loc. cit., p. 459. ${ }^{5}$ Arch. f. Ophth., 1871, Bd. xvii. Abth. 2, S. 34. 
lines or points or falling objects, in which the factor of size is reduced to a minimum, or is unknown. Fine suspended threads are better than lines on paper, since in the latter case other markings or inequalities of the paper surface may be of influence.

Two kinds of localisation must be rigorously distinguished from one another, namely, localisation relative to the fixation point, and localisation of the fixation point itself ; or, more strictly, of the fixation point relative to the individual. The apparent position of a fixed point does not necessarily correspond with its actual position, and it would be more accurate to follow Hering, and to speak of localisation relative to the nucleus or central point (Kernpunkt) of visual space and localisation of the nucleus itself. The surface containing the nucleus which appears parallel to the plane of the face is called by Hering the nuclear plane (Kernfläche), and corresponds to the horopter of actual space. Since, however, fixation point and nucleus coincide with one another under ordinary conditions, the former designation may be employed.

Localisation relative to the fixation point.-A point may be localised on either side of, above or below, and nearer or farther than the fixation point. The three dimensions may be termed breadth,

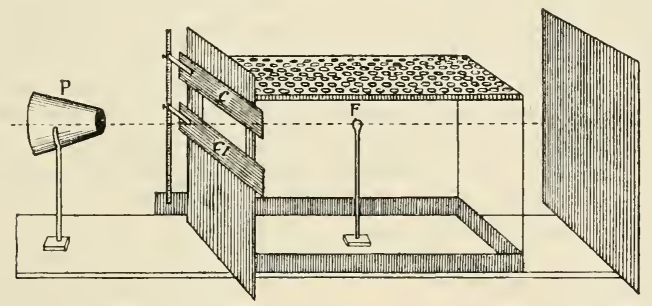

FIG. 407.-Greeff.

height, and depth respectively. It is the third which has been most fully investigated, and about which there is most difference of opinion. It is a familiar experiment that the relative distances of two objects (needle and thread) is estimated much better with two eyes than with one, and the great importance of binocular vision is shown most conclusively by means of Hering's fall experiment. ${ }^{1}$ An observer looks through a broad tube at a bead on a vertical thread which acts as the fixation point. The psychological factors are excluded by using falling balls of different colours and unknown size. It is found that, with only one eye open, the observer is unable to distinguish whether the falling ball is nearer or farther than the bead, while with two eyes the accuracy of perception is very considerable, the just perceptible difference of distance being about $\frac{1}{50}$ of the distance of the fixation point. It has been objected, that perception might depend on some other factors, such as the apparent rate of movement of the ball. In order to overcome this and other objections, Greef ${ }^{2}$ has modified the apparatus, as shown in Fig. 407, so that at all distances the ball is seen to fall through the same distance at the same rate. The ball is always visible for less than 02 sec., which excludes the influence of movement of the eyes. With

1 Arch.f. Anat., Plysiol. u. wisscnsch. Med., Leipzig, 1865, S. 152.

2 Ztschr. f. Psychol. u. Physiol. d. Sinnesorg., Hamburg u. Leipzig, 1892, Bd. iii. S. 21. 
this apparatus Greeff found with monocular vision that the right and wrong answers were approximately equal, while with both eyes the wrong answers formed a very small percentage of the total, varying from 2 to 6 per cent., according to the distance of the fixation point, $\mathrm{F}$, the width of the slit, $c c^{\prime}$, and the distance between the falling ball and the fixation point. He found the experiment succeeded at any distance of the fixation point up to 20 metres, at which distance the falling ball harl to be not less than $35 \mathrm{~cm}$. from the fixation point. Taking the just perceptible difference at $\frac{1}{50}$, Greeff found that this corresponded to a retinal distance of 002 to $.003 \mathrm{~mm}$. Diminished visual acuity diminished the influence of binocular vision. At one time Greeff believed that no cases of squint gave good results with the experiment, but later ${ }^{1}$ has found that cases do occur in which it seems that the capacity for performing the fall experiment can be acquired after tenotomy. It seems also that such cases may be able to give correct answers with the fall experiment, though unable to combine stereoscopically (Simon). ${ }^{2}$

The fall experiment shows conclusively that there is a binocular mechanism of some kind for the perception of relative distance. It also shows that this mechanism is independent of movements of the eyes, the time during which the falling ball is exposed being too short for a movement either of the eyeballs or of accommodation to be completed. That perception of depth is independent of movement, is also shown in other ways. Dove ${ }^{3}$ found that the appearance of distance in stereoscopic combination occurred with instantaneous illumination. This was doubted by Donders, ${ }^{4}$ but on careful investigation he found that, with a suitable position of the eyes, the stereoscopic effect might occur with a single electric spark. Usually more than one was necessary; the first spark probably only enabled the observer to fix suitably.

As a proof that binocular relief is independent of movement of the eyes, Wheatstone ${ }^{5}$ showed that it may be seen in the after-image. $\mathrm{He}$ exposed broad coloured lines on a complementary ground, and found that the binocular after-image showed itself in bold relief in the intervals of the alternation due to rivalry. A similar observation was made by Rogers, ${ }^{6}$ who found that the appearance of relief in the after-image also occurred when the lines had been presented to the eyes successively.

Localisation of fixation point.-It is generally supposed that we localise the fixation point in its real position, namely, at the point of intersection of the lines of fixation, and that this localisation depends on our knowledge of the position of the eyeballs, derived either from muscle sensations of peripheral origin, or from "sensations of innervation." There is no doubt, however, that in the absence of retinal factors our knowledge of the position of the eyeballs is very defective. Very large errors may be made in localising a point of light in complete darkness (Exner). ${ }^{7}$ In cases of blindness due to optic atrophy, after the eyeballs have been directed to one side, they may slowly return to the median position without the knowledge of the owner, who still believes he is looking to one side

${ }^{1}$ Klin. Monatsbl. f. Augenh., 1895, Bd. xxxiii. S. 352.

${ }^{2}$ Centralbl. $f$. prakt. Augenh., Leipzig, 1896, Bd. xx. S. 174.

${ }^{3}$ Sitzungsb. d. k. Akad. d. wissenseh. zu Berlin, 1841, S. 251.

+ Areh. f. Ophth., 1867, Bd. xiii. Abth. J, S. 29.

5 Phil. Trans., London, 1838, p. 392.

${ }^{6}$ Am. Journ. Sc., New Haven, 1860, vol. xxx. p. 387.

7 Ztschr. f. Psychol. u. Physiol. d. Sinnesorg., Hamburg u. Leipzig, 1896, Bd. xii. S. 321. Sherrington, on the other hand, found that the eyes ean be directed to a given point in complete darkness with considerable accuracy (Proe. Roy. Soc. London, 1898, vol. lxiv. p. 120. 
(Raehlmann and Witkowski). ${ }^{1}$ Under certain conditions, as in stereoscopic combination, we localise quite independently of the degree of convergence.

Accommodation and localisation in depth.-Several investigations have been carried out to determine whether sensations of movement may act as subsidiary factors in localisation. It is impossible to test the influence of convergence experimentally, since the binocular retinal mechanism cannot be excluded when both eyes are used. In experiments with one eye the psychological factors must be absolutely excluded. In order to do this most completely, Hillebrand, ${ }^{2}$ following a suggestion of Hering, has used the dividing lime between two surfaces. The observer looks through an aperture at a fixed white screen, one-half of which is hidden by a black screen with sharply cut edge which is capable of being moved towards or away from the eye. It is found that the observer is totally unable to tell the distance of the black screen, or whether it is moving towards or away from him, or even whether it is moving at all. Wundt ${ }^{3}$ found that he was able to detect the approach of a vertically suspended thread when there was no appreciable change in its apparent size, and supposed that this was due to the influence of accommodation. He was only able to detect recession of the thread from the eye when it had undergone a visible change in size. Arrer ${ }^{4}$ has also obtained results with the the thread experiment, which he thinks can only be explained by the influence of movement factors. Hillebrand found when his screen was exposed at intervals at different distances, that some individuals were able to detect whether the screen had been moved towards or away from the eye during the interval, and this has been confirmed by Dixon ${ }^{5}$ and Arrer. It is not easy to feel quite certain that psychological factors have been absolutely excluded, and the evidence shows that if sensations arising from accommodation are of any influence at all in localisation, this influence is very slight and limited to certain individuals.

Hillebrand argued that, owing to the close association between convergence and accommodation, his experiment disproving the influence of accommodation also disproved the influence of convergence in depth perception, but the association is not close enough to justify this argument, and the possibility still remains that convergence may have some influence, although there is no positive evidence in favour of it.

The cyclopean eye.-. Some of the chief investigators of binocular vision (Helmholtz, Hering, and less definitely Donders) have adopted the idea that we localise objects in space in reference to the middle point between the two eyes, and as if seen by an eye in that position. If two parallel lines are drawn at a distance apart, corresponding to the interocular distance, and are observed so that one line lies in the line of vision of each eye, the combined image of the two lines will be seen in the median plane between the two eyes, as if it were coming from a middle eye. The same appearance is produced with converging lines, provided that the lines of vision converge to a corresponding degree. ${ }^{6}$ This tendency is so strong, that, in monocular vision,

1 Arch.f. Physiol., Leipzig, 1877, S. 463.

2 Ztschr.f. Psychol. u. Physiol. d. Sinnesorg., Hamburg u. Leipzig, 1894, Bd. vii. S. 97.

3 "Beitr. z. Theorie d. Sinneswahrnahrung," 1862, S. 105.

${ }^{4}$ Phil. Stud., Leipzig, 1896-1897, Bd. xiii. S. 116 and 222.

5 Mfind, London and Edinburgh, 1895, vol. iv. p. 195.

${ }^{6}$ For other experiments illustrating the cyclopean eye, see Towne, Guy's Hosp. Rep., London, 1865, vol. xi. p. 144 ; and Le Conte, "Sight," 1883, p. 213. 
the nearer of two objects may be localised in the line passing from the farther object to the root of the nose, and not in the line passing to the open eye. This reference to the cyclopean eye is not constant; and by practice in using one eye, as in shooting, it is probable that localisation in space may take place in reference to the visual direction of that eye.

In connection with the idea of the cyclopean eye, it is interesting that we are unable to tell with which eye we are seeing an object. If a piece of black pasteboard with a pinhole in the middle is noved about till the hole is in front of one eye, it may appear to be midway between the two eyes, and mistakes are frequently made in naming the eye which is exposed to the light (Rogers). ${ }^{1} \quad$ An after-inage obtained by exposure of one eye may appear to be seen with the other eye. The familiar experiment in which a tube on the right side of an opaque object seems, when the right eye is applied to the tube, to penetrate the object, is another example. On the other hand, Helmholtz ${ }^{2}$ noticed that if one of two stereoscopic photographs had on it a spot, it gave rise to an appearance of dimness in the combined picture, and that he found himself involuntarily rubbing the eye of the same side as the spot. Helmholtz supposed that this indicated that he was sensible of the eye stimulated.

Psychological factors in localisation.-These are often known as the empirical factors, but since this name seems to assume that the other factors are not based on experience, it is perhaps better to avoid this nomenclature.

The psychological factors play a very important part in localisation of the fixation point, but are also of great importance in localisation relative to the fixation point. They are the only factors in question for localisation beyond a certain distance from the eyes, namely, that distance at which the binocular retinal mechanism ceases to be of value, probably about 20 metres. The appearance of distance produced in paintings is due exclusively to the application of devices based on these psychological factors, and it is on this account that the appearance of distance in pictures, as was pointed out by Francis Bacon, is more narked when only one eye is used. Our ideas of distance and size are very closely related; it is probable that in general the idea of distance is primary, and that our idea of the size of an object depends on the estimated distance of the object (p. 1140), but, when the size is known, it may greatly influence depth perception. This is shown in a striking way in Hering's fall experiment. Observers tend to localise the larger balls nearer than the fixation point, the smaller farther than the fixation point, and they may be unconscious of the factor upon which their erroneous localisation has depended. Linear and aerial perspective have great influence on the apparent distance of an object, while the distribution of light and shade is especially important in producing the appearance of relief and solidity. The parallactic movements of objects at different distances on moving the head may also be important factors in depth perception.

Theories of localisation.-Most of the older theories ignored the distinction between relative and absolute localisation. At one time it was generally accepted that the estimation of distance depended on movement; one object was supposed to appear nearer than another,

${ }^{1}$ Am. Journ. Sc., New Haven, 1860, vol. xxx. p. 404.

2 "Handbuch d. physiol. Optik," 1867 , S. 744. 
because movements of convergence and accommodation were necessary in order to see it distinctly. This view is altogether negatived by experiments, and there is at present no evidence to show that movement factors take any part whatever in relative localisation, while they seem to be comparatively unimportant in the process of absolute localisation in adult life." $\quad$ The importance of binocular vision in spatial perception was first shown by Wheatstone, who, however, adopted a psychological view of the binocular mechanism, supposing that the estimation of distance depended on a mental conıparison of the two dissimilar images of the two eyes, and this mode of describing the binocular mechanism in psychological terms is still commonly adopted.

Local signs.- This term, which is due to Lotze, is often used in connection with this subject. Lotze supposed that in the skin differences of quality of the sensation arising from stimulation of different parts, were the basis of localisation, the differences of quality being produced by differences of texture and thickness of the skin and of underlying parts, and these differences of quality were spoken of as "local signs." In the case of the retina Lotze supposed that the local signs were movements or rather impulses to movement. $\mathrm{He}$ applied the idea, however, only to estimation of height and breadth, and not of depth, referring the latter to more psychological factors. When a definite point of the retina is stimulated, a reflex movement occurs which brings the image of the stimulating object on the place of most distinct vision. Each retinal point will then become associated with a definite movement, and Lotze supposed that the starting of this definite movement was the local sign in the case of the retina, and acted in the same way as the difference of quality in touch as the basis of localisation.

Hering's theory of the binocular mechanism.-The most satisfactory physiological theory of the binocular mechanism is, that relative depth-perception depends on double images, or rather on retinal disparation. This view was first advanced by Panum, ${ }^{2}$ and has been most fully developed by Hering. ${ }^{3}$ According to this view, it is held that retinal disparation is the physiological basis of the idea of nearer or farther than the fixation point, the difference depending on whether the disparation is crossed or uncrossed. An object is seen nearer than the fixation point when it stimulates points with crossed disparation. The appearance of nearness occurs both when double images are seen and when the disparation is so slight that the double images are fused into one. Further, the greater the disparation, the greater is the apparent distance of the object from the fixation point. In the same way, uncrossed retinal disparation acts as the basis of the idea of farther than the fixation point. It must be clearly understood that our ideas of distance are not held to depend on double images, which, as a matter of fact, few people ever see, or probably ever have seen; but retinal disparation is held to be the physiological basis of our ideas of relative distance in much the same way that certain chemical changes in the retina are held to be the physiological basis of our ideas of colour; in both cases there is, of course,

1 To what extent they take part in the genesis of spatial perception is another matter which cannot be considered here.

${ }_{2}$ Arch. f. Anat., Physiol. u. wissensch. Mrd., 1861, S. 63 and 178; and "Physiol. Untersuch. über das Sehen mit zwei Augen.," 1858.

3 "Beitr. z. Physiol.," 1864, Heft 5 ; and Arch. f. Anat., Physiol. u. wissensch. Med., 1865 , S. 152 .

VOL. II. -72 
no direct consciousness of the nature of the retinal mechanism. Localisation above or below, to right or to left of the fixation point, is similarly held to depend directly on the nature of the retinal stimulation. In the exposition of his theory Hering uses the expression "value" ("Werth"), and ascribes to each retinal element a certain height, breadth, and depth value. The localisation of a point relative to the fixation point (Kernpunkt) is determined by these three values. Two corresponding points have identical height and breadth values and opposed depth values. The opposed values cancel one another, so that the external point appears in the horopter. Points situated on the opposite sides of the median section, but at equal distances from it, have the same depth value and opposed breadth values, so that the external point is seen in the line passing from the cyclopean eye through the fixation point, and at a distance from the latter, depending on the nature and amount of the disparation. Hering's theory has met with much opposition from psychologists, because it has been held to imply innate ideas of distance. As Hering points out, the anatomical mechanism of retinal disparation is certainly innate, and therefore the function of that mechanism must also to some extent be innate.

Hering further supposes that retinal disparation acts as a stimulus to movement, the movement being of a kind adapted to abolish the disparation. Crossed retinal disparation will then act as a stimulus to reflex convergence and accommodation, while uncrossed retinal disparation will act as a stimulus to divergence and relaxation of accommodation. Similarly, breadth and height values will act as stimuli to horizontal and vertical movements respectively. If Hering's values are regarded as "local signs," it will be seen that he would differ from Lotze in regarding the sensory side of the reflex action as the local sign, while Lotze supposed that the motor side was the local sign.

Hering regards retinal rivalry, and the fact that contours prevail in the rivalry, as most important factors in the mechanism of depth perception. In order that contours shall prevail, it is necessary that they should be sharp and well defined, and it is on this account that he believes contrast to be of such fundamental importance in vision (p. 1066).

Hering denies that muscle sensations have anything whatever to do with localisation of the fixation point itself. The idea of nearness which may accompany convergence is regarded by him as rather the cause than the consequence of the movement. The distance of the fixation point is referred by him to the influence of retinal values, acting together with the psychological or experience factors. So far as the position of the head and eyes is of importance, localisation depends, not on their actual position, but on the idea which we have of their position.

Schön's theory of the binocular mechanism.-Another view as to the nature of the binocular mechanism has been advanced by Schön. ${ }^{1}$ This view only applies to indirect vision, and is based on the difference in sensibility of the nasal and temporal halves of the retina. According to Schön, the nasal half is much more sensitive, especially to light, than the temporal half of the same or of the other eye. Consequently, corresponding or slightly disparate points of the two retinæ will produce sensations of different intensity, when stimu${ }^{1}$ Arch. f. Ophth., 1876, Bd. xxii. Abth. 4, S. 31 ; and 1878, Bd. xxiv. Abth. 1,
S. 27 . 
lated by the same point; and Schön supposes that this difference of intensity, assisted by differences of quality due to differences of sensibility to colour, acts as the basis of the ideas of distance. Schön, in fact, suggests that differences of quality act as local signs in the retina, in much the same way that Lotze supposes them to act in the skin. The objections to this theory are, first, that it is doubtful whether the differences between the nasal and temporal halves described by Schön exist. Other observers have found slight differences, but very much less than those described by Schön; secondly, the sensibility of the retina is continually varying with the condition of adaptation, and varying adaptation should produce variations in our estimation of distance, which is not found to be the case; and lastly, this explanation, as Schön himself acknowledges, only applies to indirect vision, for images of objects in the same line as the fixation point will fall either on the nasal halves or on the temporal halves, which have, according to Schön, equal sensitiveness.

To explain this latter kind of localisation, Schön suggests another explanation, another kind of local sign, which he calls the "rivalry sign." Since images of an object beyond the fixation point fall on the more sensitive nasal halves, and those of objects nearer the fixation point on the less sensitive temporal halves, the former will more easily prevail in the rivalry; and Schön supposes that this difference acts as the basis of estimation in the case of direct vision.

Perception of form.-The physiological basis of localisation in space has now been considered at some length, and especially of localisation in depth. This localisation in space is the chief factor in various kinds of more complex perception, and these, namely, the perception of form and size, can now be more briefly considered.

Certain very simple cases of perception of form were dealt with in the second part of this article, and in such cases the process seems to be comparatively simple. In the case of very small objects, such as letters, it is doubtful how far the form of the retinal image is the sole physiological factor, and how far perception depends on minute movements of the eyes following the outline of the object. In the case of large objects, movements must be of greater influence. The phenomenon of metamorphopsia is evidence of the view that the retinal factor is very important. This occurs in retinitis, especially in the early stages; the forms of simple objects are found to be distorted, a straight line appearing curved or irregular. Its occurrence in the early stages of retinitis points to its dependence on inflammatory exudation, disturbing the relative position of retinal elements, so that a row of such elements which have previously been in a straight line becomes distorted. A linear retinal image will therefore fall on retinal elements which have normally occupied a curved or irregular position, and have been previously stimulated by curved or irregular objects, and a straight line will consequently appear curved or irregular.

The perception of form in three dimensions, i.e. of solidity, depends primarily on the physiological binocular mechanism for relative distance, but is very greatly influenced by psychological factors, and especially by the distribution of light and shade, as is shown by Wheatstone's pseudoscope and by other experiments in which reversal of the apparent direction of incidence of light produces illusions of relief.

Perception of size.-The two chief factors on which depends the 
perception of the size of an object are the size of the retinal inage and the estimated distance of the object. The importance of the latter factor is shown by an after-image experiment. If the after-image of an object is projected on a moving screen, it will be seen to change in size, becoming smaller as the screen approaches the eyes, larger as it recedes. The retinal image upon which the after-image depends remains constant in size, and the changes in apparent magnitude depend on the projection. Emmert ${ }^{1}$ measured the after-image at different distances, and found the linear size of the image equal to the linear size of the object, multiplied by the distance at which the image was seen. $\mathrm{He}$ found that the changes were the same when accommodation was paralysed, and occurred equally well in monocular and binocular vision. The estimated distance of the after-image is therefore probably dependent on psychological factors. It is known that judgments of size are greatly influenced by these factors; the large size of objects seen indistinctly in a fog is a familiar example of the influence of aerial perspective.

Another instance which has excited much discussion is the apparently greater size of the sun and moon at the horizon than at the zenith. This is usually brought into relation with the apparent flattening of the sky; the horizon appearing farther away than the zenith, the moon appears larger at the former. A favourite explanation of the larger size at the horizon is that in that position it can be compared with intervening objects; while, according to another, the appearance is an instance of the illusion that a space filled with objects appears longer than an unfilled space (see p. 1141). Another explanation for which there is much to be said refers the larger size at the horizon to greater indistinctness, consequently greater estimated distance and greater apparent size. Helmholtz ${ }^{2}$ found that a reflected image of the moon at the zenith, seen so that it appeared near the horizon, was not enlarged. Scharwin and Novizki ${ }^{3}$ have observed corresponding changes in the size of an after-image, when projected at horizon and zenith. It is probable that the apparently greater size at the horizon is due to more than one factor, greater indistinctness being the most important.

Micropsia and macropsia.-Illusions of size appear in certain conditions which throw some light on the process of perception. The metamorphopsia of retinitis is often accompanied, especially in the early stages, by micropsia. Objects may appear reduced to as much as one-fifth their natural size. This micropsia is referred to the influences of inflammatory exudation separating the retinal elements, so that a group of elements which are normally covered by the retinal image of a small object require a very much larger object in order to be covered, and the latter consequently appears diminished in size. Occasionally macropsia is observed, and this is referred to crowding of retinal elements together; it is, however, much rarer than micropsia, and the increase in size is comparatively slight.

Similar illusions of size may occur under numerous other conditions. An appearance of diminished size of small objects (printed type) may be due to indistinct vision, the outer part of the type being hazy, while the central part is seen distinctly. A more important effect is due to irradiation, ${ }^{4}$ and is

${ }^{1}$ Klin. Monatsbl. f. Augenh., 1881, Bd. xix. S. 443.

2 "Handbuch d. physiol. Optik," 1867, S. 631.

${ }^{3}$ Ztschr.f. Psychol. u. Physiol. d. Sinnesorg., Hamburg u. Leipzig, 1596, Bd. xi. S. 408.

${ }^{4}$ Mind, London and Edinb., 1896, N.S., vol. v. p. 71. 
well marked in black type. Such type appears very much reduced in size with a dilated pupil, and a difference of apparent size to the two eyes may be due to inequality of the pupils. This kind of micropsia disappears with an artificial small pupil, and does not occur for white objects on a dark ground.

One kind of micropsia which has attracted much attention is that which occurs after instillation of atropin. This may be due to the dilated pupil and irradiation, but also occurs when this factor is excluded. It is then probably an instance of a physiological micropsia, which occurs when an object is observed lying beyond the fixation point, and is associated with an appearance of greater distance. Objects nearer than the fixation point, on the other hand, appear enlarged, and with this may be connected the macropsia which occurs after instillation of eserin. Donders ${ }^{1}$ and others have supposed that the micropsia of atropin is due to greater effort of accommodation and consequent idea of greater nearness, and that with this idea of greater nearness is associated one of smaller size. This explanation is open to the two objections that the micropsia is usually accompanied by an appearance of greater distance, and that it occurs with complete paralysis of accommodation, and Donders himself gave up this explanation in $1886 .^{2}$ The micropsia which occurs with commencing presbyopia seemed to be in favour of this explanation, but this probably depends on indistinct vision.

Geometrical illusions.-A number of illusions, usually classed together under the above heading, are of considerable interest for

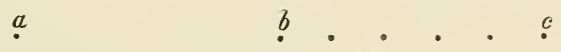

FIG. 408.

the theory of the physiological basis of spatial localisation. A few of the more important are shown. In Fig. 408 the unfilled distance $a b$ appears shorter than $b c$. In Fig. 409 the line $a b$ is really continuous with $c d$, but appears as if continued to $e$. In Fig. 410 the parallel lines appear to converge at each end.

Figs. 409 and 410 and many similar illusions, including the well-known Zöllner's pattern, may all be referred to the erroneous estimation of angles, namely, to the overestimation of acute angles and the underestimation of obtuse angles.

The influence of various conditions on these illusions has been determined. The illusions are present with instantaneous illumination, although, according to Helmholtz, the effect is less marked than when movement of the eye is allowed. They are present in the after-image (Hering). Zollner found that the maximum apparent deviation of the parallel lines in his pattern occurred when

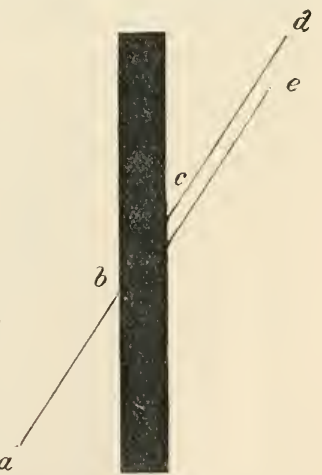

FIG. 409. the cross lines were at an angle of $30^{\circ}$. The illusion is marked when his figure is observed with the eye at right angles to the paper; it becomes more marked when looked at obliquely from in front, and disappears when observed obliquely from either end. The illusion is

1 Nederl. Lancet, 1851, S. 607.

2 Ber. Ophth. Gevellsch., Heideỉberg, 1886, s. 82. 
very slight close to the eye, becomes more marked as it is removed, and then decreases, till at a certain distance it is no longer visible. Kundt ${ }^{1}$ determined the distance at which the illusion of Zölner's pattern disappeared for different observers, and varied the size of the figure and number and angle of the cross lines. This illusion disappeared at a smaller distance from the eye when the number of cross lines was increased, and at a smaller distance with an angle of $20^{\circ}$ than with

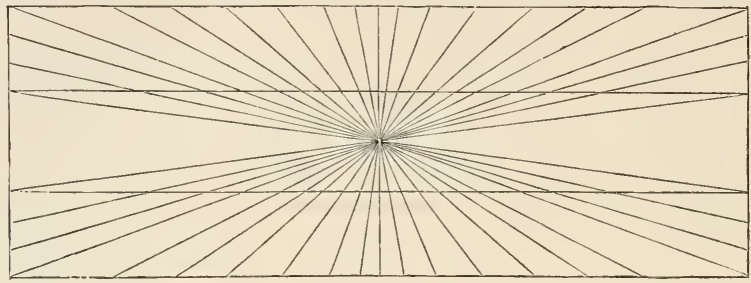

FIG. 410.

one of $32^{\circ}$ or $45^{\circ}$. There was no illusion with an angle of $70^{\circ}$. The results of different observers differed to some extent, but the variations under the different couditions were the same for all. In Fig. 409, Kundt found the illusion increased by distance.

The illusions described have been referred to retinal factors, to the influence of movement, and to purely psychological factors. The

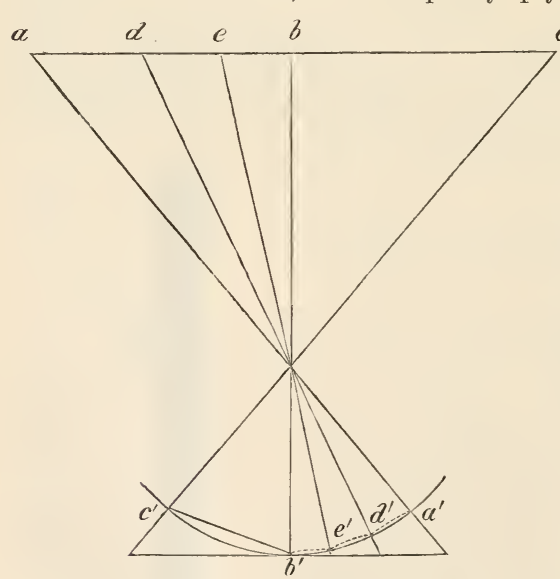

Fig. 411.-Kundt. physiological factor to which they have been referred by Hering ${ }^{2}$ and Kundt is the curvature of the retina. Their mode of explanation may be illustrated by the simple illusion in which an empty space appears shorter than an equal space partially filled with intervening points. It is supposed that our estimation of length, when two points of the retina are stimulated, is a function of the chord of the segment of the retina included between the two points. If this is the case, our judgment of the distance $(a d c b)$ in Fig. 411 will depend on the sum of the component retinal chords $\left(b^{\prime} e^{\prime}, e^{\prime} d^{\prime}\right.$, and $\left.d^{\prime} \alpha^{\prime}\right)$, while that of the uninterrupted distance $(b c)$ will depend on $b^{\prime} e^{\prime}$, and the interrupted distance will consequently be over-estimated.

The wrong estimation of angles is explained by Hering ${ }^{3}$ on the

\footnotetext{
1 Ann. d. Phys. u. Chem., Leipzig, 1863, Bd. exx. S. 118.

2 "Beitr. z. Physiol.," 1861, Heft 1, S. 65. 3 Ibid., S. 71.
} 
same lines, and his explanation is illustrated by Fig. 412, in which $n$ and $n^{\prime}$ represent vertical and oblique sections of the same eye, looking at the point $s$ in the triangle. Owing to the curvature factor, $a b$

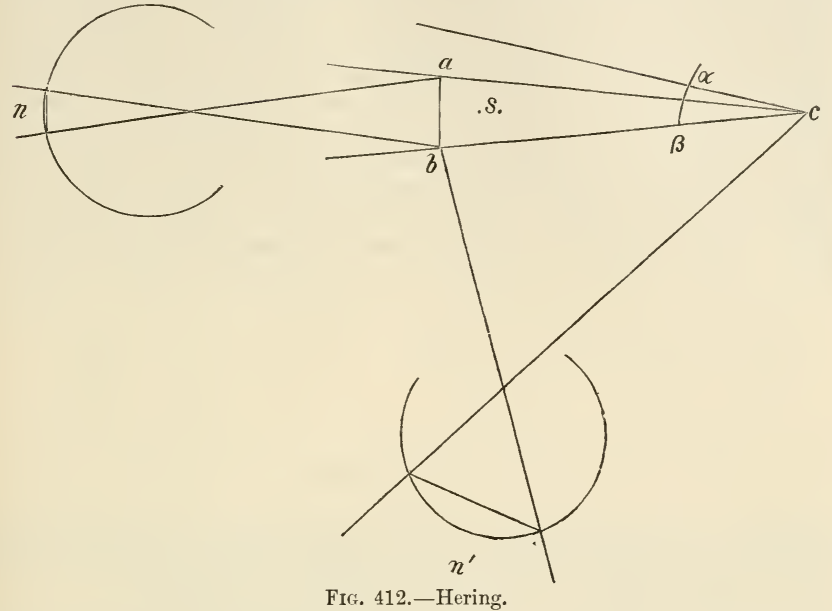

will be relatively less under-estimated than $c b$, and the angle $b c a$ will be seen too large as $\beta c \alpha$. If this explanation is correct, all triangles will appear in different relations except one in which all three angles are equal, namely, $60^{\circ}$, and consequently this explanation involves the under-estimation of angles under $60^{\circ}$, and the over-estimation of angles over $60^{\circ}$, and, as already mentioned, it is about this angle that the illusion of Zöllner's pattern ceases to be seen.

The illusion of Fig. 408 has also been referred to the influence of movement. It is supposed that the eye in passing from $b$ to $c$ is interrupted, and the movement taking place either with more difficulty or more slowly than in passing from $a$ to $b$ gives rise to an appearance of greater distance. Similarly, the fact that vertical lines are over-estimated relatively to horizontal lines has been referred to greater difficulty of vertical movements. Unsatisfactory attempts have also been made to refer the wrong estimation of angles and the various related illusions to movement factor's.

On the other hand, these illusions have been referred exclusively to psychological factors. Lipps ${ }^{1}$ has shown that the illusion that a square is longer vertically than horizontally is greatly assisted by the

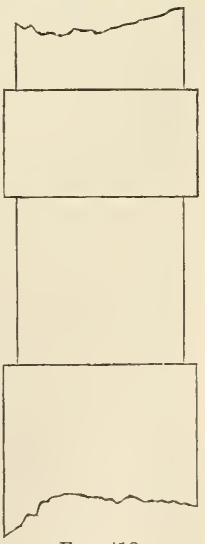

FIG. 413. addition shown in Fig. 413, and he endeavours to explain this and all the other illusions by supposing that they suggest certain mechanical activities.

Another illusion which has recently excited much discussion is that 1 "Beitr. z. Psychol. u. Physiol. d. Simesorg.," Hamburg u. Leipzig, 1891, S. 219. 
shown in Fig. 414. It has been referred to the erroneous estimation of angles, and to psychological factors. The most satisfactory explanation is that our judgment of the length of the line $a b$ is intluenced by the idea of other imaginary lines, $a^{\prime} b^{\prime}, a^{\prime \prime} b^{\prime \prime}$. The illusion is diminished by making the base line $a b$ more obvious, and increased by making the end lines more distinct. This illusion has been investigated quantitatively by Heymans, who found the illusion increased with the acuity of the angle. It increased, but not continuously with increase in length of the end lines, but attained its maximum when the end lines were between 20 and $40 \mathrm{~mm}$. (with a base line of $75 \mathrm{~mm}$.). Heymans ${ }^{1}$ supposed that this result contradicted the above explanation, but it is to be expected that the distances near the base line would influence

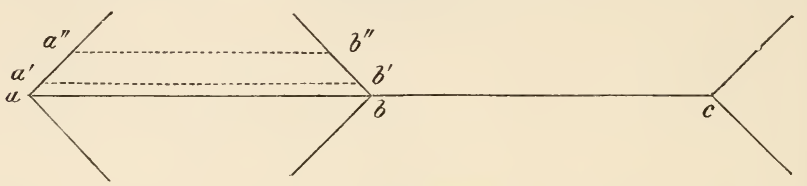

Fig. 414.-Auerbach.

perception to a much greater extent than those more remote, and that when the two lines at each end became longer than the base line, a new kind of figure would be produced and new factors introduced. ${ }^{2}$

Colour stereoscopy.-In a red and blue pattern on a black background one of the two colours may be observed to stand out in relief. The phenomenon occurs for other colours, but is more marked the farther the two colours lie from one another in the spectrum. The first observers of this appearance saw red (or yellow) stand out in relief, but it has been found that to some individuals blue appears nearer, and that there is little difference in the numbers of those who see red or blue in relief respectively, while to others the phenomenon is very indistinct, or is not observable under ordinary conditions. In marked cases (Donders) the colour may appear to stand out as much as $20 \mathrm{~cm}$. from the background at a distance of 4 metres. The appearance only occurs to a marked extent with binocular vision, but under certain conditions may be observed with one eye, though much less distinctly. It has been found by Einthoven ${ }^{3}$ to be to a considerable extent dependent on the eccentricity of the pupil; individuals with the pupil situated on the temporal side of the optic axis see red in front of blue, while those with nasal pupils see blue in relief. This comes out much more clearly if the pupils are dilated with atropin, and a small artificial pupil placed before each eye. When the artificial pupils are separated from one another, red is in relief, while, as they are approached to one another, blue starts forward. This may also be observed by separating and approaching to one another lenses held before the eyes. Hess made use of this fact to test the movements of the lens described by him (see p. 1035). With convex lenses, situated temporally, blue is seen in front; when situated nasally, red appears in front. Hess put eserin in one eye, and found, on inclining the head to the same side, thus making

${ }^{1}$ Ztschr. f. Psychol. u. Physiol. d. Sinnesorg., Hamburg u. Leipzig, 1896, Bd. ix. S. 221.

2 For reference to the chief papers on this illusion, see ibid., 1896, Bd. ix. S. 16.

3 Arch.f. Ophth., 1885, Bd. xxxi. Abth. 3, S. 211. 
the lenses farther apart than normal, that blue stood out, while with the head inclined to the opposite side red was in relief.

The explanation originally given by Donders was based on the supposition that all individuals see red in front of blue. A greater effort of accommodation is necessary to focus red distinctly, and Donders supposed that this greater effort gives rise to the idea of greater nearness. The correct explanation was given by Einthoven, who referred the appearance primarily to chromatic aberration, acting together with the deviation between the optic axis and the line of vision. In an eye accommodated for blue, the red rays will form a diffusion circle round the focus for blue, and owing to the deviation of the optic and visual axes, as in Fig. 415, the focus for blue will not be in the centre, $x$, of the diffusion circles, but towards its nasal side, i.e. with a median pupil. If the pupil is temporal, this eccentricity will be increased; if

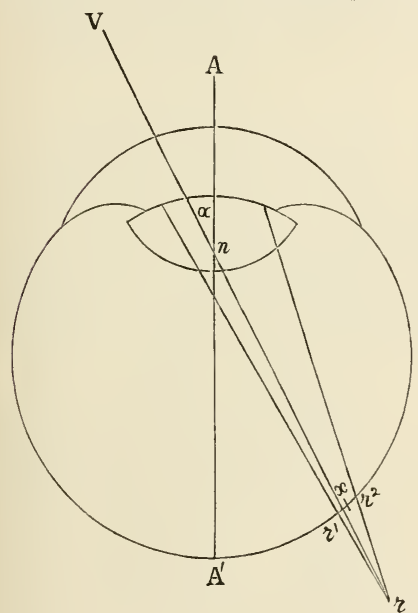

FIG. 415.

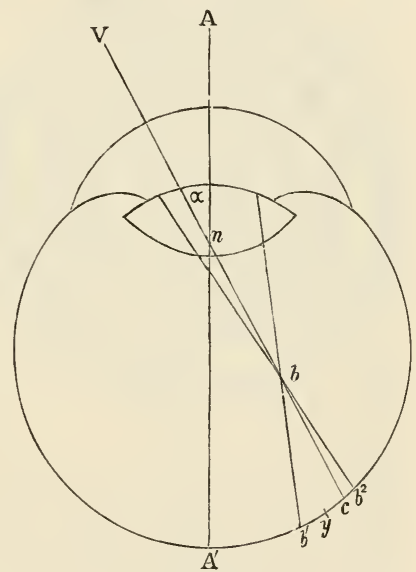

Fig. 416.

slightly nasal, it will be compensated for; while, with marked nasal pupil, the focus for blue will be on the temporal side of the diffusion circle. In an eye accommodated for red, as shown in Fig. 416, the focus for blue will have the same relation to that for red as in the eye accommodated for blue. Assuming that red is localised in the centre of its diffusion circle, the foci for red and blue in one eye will be on different points, while in binocular vision the red foci will be on disparate points. With median pupils the red focus will be on the temporal side of blue in each eye, and the disparation will be crossed; consequently, red will appear the nearer. With temporal pupils the disparation will be greater, and red will appear in stronger relief. With marked nasal pupils, on the other hand, the red focus will be on the nasal side of the blue focus in each eye; consequently, the disparation will be uncrossed, and red will appear further away than blue, i.e. blue will appear in relief.

The less marked appearance of relief which occurs in monocular 
vision has been explained by Einthoven ${ }^{1}$ differently. If a series of alternate red and blue strips are looked at with one eye and an eccentric pupil, one of the colours will appear in relief. The raised colour depends on the nature of the eccentricity and on the direction from which the strips are observed. Einthoven points out that the red and blue will be displaced laterally on the retina giving rise to dark and light borders which appear to separate the strips, and have the same effect as shadows. The colour relief, depending on the distribution of the light and shade, will consequently vary with the direction from which the colours are regarded.

Another appearance which may be referred to the same factors as colour stereoscopy, is the apparent movement of different parts of a coloured (especially red and blue) pattern. Such a pattern, when moved, especially in peripheral vision, gives a flickering appearance, which in some people produces dizziness and even vomiting. The apparent difference of movement is naturally explained by the difference of focus for different colours, and greater excursion of the more peripheral focus on movement (Schapringer). ${ }^{2}$

Visual perception of movement.-A moving object must have a certain velocity to be seen as moving. Aubert ${ }^{3}$ has found this velocity in direct vision to be between $1^{\prime}$ and $2^{\prime}$ in the second. At about $1^{\prime}$, movement is only seen after some seconds; while with slower movement, namely, about $40^{\prime \prime}$ per second, the object appears to be at rest. The retinal distance corresponding to $1^{\prime}$ being about $4.36 \mu$, the threshold of movement for the fovea corresponds fairly closely with the limit of visual acuity. It was found difficult to investigate the just perceptible difference of velocity, but for small velocities it was found to be about the same as the threshold, namely, 1'. For peripheral vision, Aubert found that the angular velocity had to be increased for movement to be seen, but the decrease of sensibility for movement from centre to periphery was proportionally very much less than the decrease in visual acuity. On the other hand, there is evidence that the peripheral retina is especially sensitive to movement. It is a familiar experience in perimetric observations that at the extreme periphery of the retina an object is not seen at all till it moves, and that this movement may be seen by an individual who is unable to describe in any way the object which is moving. According to Exner, ${ }^{4}$ there is a tendency to see any kind of change (change of intensity) in the periphery as movement. It is generally supposed that seeing a movement is due to a more or less complicated mental process; that a body is seen at $a$ at the moment $t$, at $a^{\prime}$ at the moment $t^{\prime}$, and at $a^{\prime \prime}$ at the moment $t^{\prime \prime}$, and that it is inferred that the body has moved from $a$ to $a^{\prime \prime}$. Exner ${ }^{5}$ supposes that there is in addition direct sensibility to movement, immediate sensation of movement. Animals often fail to see an object till it moves, even when it is quite close; and it is to be expected that seeing movement in peripheral vision should be one of the most primitive properties of the visual apparatus.

Certain after-image phenomena, which are consistent with direct

${ }^{1}$ Brain, London, 1893, vol. xvi. p. 191.

${ }^{2}$ Ztschr. f. Psychol. u. Physiol. d. Sinnesorg., Hamburg u. Leipzig, 1893, Bd. v. S. 385.

${ }^{3}$ Arch.f. d. ges. Physiol., Bonn, 1886, Bd. xxxix. S. 347 ; and 1887, Bd. xl. S. 459.

${ }^{4}$ Arch. f. Ophth., 1886, Bd. xxxii. Abth. 1, S. 233.

${ }^{5}$ Sitzungsb. d. k. Akad. d. Wissensch., Wien, 1875, Bd. lxxii. Abth. 3, S. 156. 
sensibility of the retina to movement, usually receive a more complicated psychological explanation. Helmholtz ${ }^{1}$ explained the well-known railway carriage and waterfall illusions, by supposing that an observer becomes accustomed to regard the voluntary impulses set up when looking at a moving object, as those adapted for the fixation of a resting object. When the eyes are turned to a resting object, the motor impulses continue, and bring about movements of the eyes; and since the observer supposes that his eyes are stationary, objects appear to him to move in the opposite direction to the previously observed objective movement. Helmholtz believed that this illusion did not occur with accurate fixation.

This explanation cannot apply to the modification of the illusions observed with a spiral disc (Fig. 417). When rotated this gives the appearance of circular bands moving inwards and outwards, according to the direction of rotation. When the rotation is stopped, the rings appear to move in the opposite direction, and if any object is observed it will appear to swell or diminish in size. If a grey screen is observed, or if the eyes are closed, the visual field shows a peculiar streaming appearance inwards or outwards, according to the direction of rotation.

As a modification of the experiment, Dvořák ${ }^{2}$ laid on a large disc with a spiral a smaller concentric disc with the spiral in the opposite direction, and on

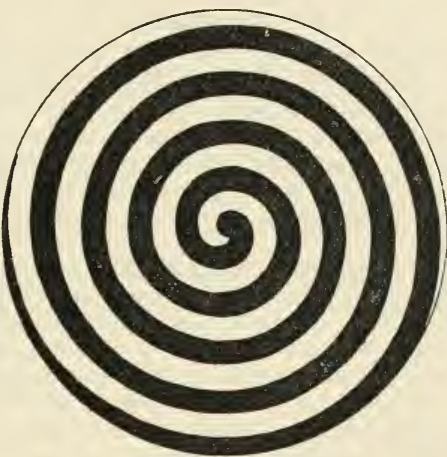

FIg. 417.

this a smaller dise with the spiral in the same direction as the first. The after-image showed, with apparent movement, the opposite directions in the different parts. The appearances occurred with accurate fixation, guaranteed by obtaining a well-defined after-image of a white patch in the centre. According to Helmholtz's explanation, this would involve different ideas of movements simultaneously present for different parts of the retina.

There seems no doubt that these illusions depend on an after-image change of some kind taking place in the retinal (cerebro-retinal) apparatus, and are independent of movements of the eyes. Dvorák has investigated other conditions to find if change of stimulation can produce an afterimage, and has found that if the illumination of a room is alternately raised and lowered, that the alternation appears to continue for a time after the illumination is made constant; during the objective alternation he noticed contraction and dilatation of the pupil, which did not continue during the subjective alternation. These subjective alternations may, however, have been of the same nature as the periodical variations of the after-image.

When a figure made up of narrow concentric black and white rings

1 "Handbuch d. physiol. Optik," 2te Aufl., S. 746.

${ }^{2}$ Sitzungsh. d. k. Akad. d. Wissensch., Wien, 1870, Bd. lxi. Abth. 2, S. 257. 
is moved in the same way, as in rinsing out a basin, the figure appears to rotate round its centre. One of the factors upon which this depends appears to be the unequal distinctness of vision in different meridians, due to astigmatism. If a number of such figures be placed round a ring with teeth projecting inwards, and the whole moved in the same way, the inner toothed ring will appear to rotate in the opposite direction to that of the figures, and more slowly.

A peculiar illusion of movement was first described by Charpentier, ${ }^{1}$ and later by Aubert ${ }^{2}$ and Exner; ${ }^{3}$ by the latter it is referred to "autokinetic" sensations. If a small feebly illuminated object be observed in a room, otherwise completely dark, the object will appear to move with a certain rapidity in a definite direction. Charpentier found its angular velocity about $2^{\circ}$ to $3^{\circ}$ per second, and that the total movement might be through as much as $30^{\circ}$. He found that the appearance was not due to unconscious movement of the eyes, and occurred, though in a less marked degree, when other control points of light were present. Its direction was influenced by the idea of a certain direction; a loud sound caused a movement towards the supposed direction of the sound, and direction of the attention to the floor caused movement downwards, to the roof upwards. If due to movement of the eyes, the apparent movement should have been in the opposite direction. Aubert found also that a real movement of the object might not be seen.

Localisation in animals.-The question of the mechanism of the estimation of space relations in animals below man is one of great difficulty. One can hardly believe that the more psychological motives of localisation, which play so important a part in man, can be of any importance here; on the other hand, the most definite physiological factor, the binocular mechanism, is out of the question in some animals, as birds, and must be of a special nature in other animals. Only man and some primates have the two features, optic axes capable of convergence and a single central fovea. Other animals have structural peculiarities which have been regarded as of a foveal nature, but we know nothing of their function. It is quite possible that binocular mechanisms may exist wholly different from that found in man. Loeb ${ }^{4}$ has suggested that the marked astigmatism found in many animals may play a part in space perception. The astigmatism is so considerable that the form of the retinal image must be appreciably affected by movements of an object nearer to or away from the eye, and it is quite conceivable that these changes in form might act as the basis of localisation.

${ }^{1}$ Compt. rend. Acad. d. sc., Paris, 1886, tome cii. pp. 1155 and 1462.

2 Arch.f. d. ges. Physiol., Bonn, 1887, Bd. xl. S. 477 and 623.

3 Ztschr. f. Psychol. u. Physiol. d. Sinnesorg., Hamburg u. Leipzig, 1896, Bd. xii. s. 313.

4 Arch.f. d. ges. Physiol., Bonn, 1887, Bd. xli. S. 371. 


\section{THE EAR.}

\section{By John Gray M'Kendrick and Albert A. Gray.}

Contents :-T'he External Ear, p.1149-The Auricle,p.1149-The External Auditory Meatus, p. 1150-Movements of the Auricle, p. 1152-The Middle Ear, p. 1152-The Tension of the Membrana Tympani, and its Regulation, p. 1152Movements of the Membrana Tympani in response to Sound Pressures, p. 1153The Movements of the Malleus, p. 1155-The Movements of the Incus, p. 1155 - The Movements of the Stapes, p. 1156-The Tensor Tympani, p. 1156-The Stapedius, p. 1157-The Movements of the Ossicles as a whole under the Influence of Sound-waves, p. 1158-The Magnitude of the Movements of the Ossicles, p. 1159-Transmission of Sound to the Labyrinth otherwise than by the Ossicles, p. 1160-Regulation of Atmospheric Pressure in the Tympanum, p. 1161Auditory Reflexes, p. 1162-Binaural Audition, p. 1163-The INternal EAR, p. 1164-General Mechanism of Internal Ear, p. 1164-The Utricle and Saccule, p. 1166-The Cochlea, p. 1168-Pitch, p. 1169 -Beats, p. 1169 -Beat Tones, p. 1170 - Audibility as affected by Intensity, p. 1171-The Analytic Properties of the Ear, p. 1171-Resonance, p. 1177-Probable Action of the Cochlea, p. 1179Upper Partial Tones and the Theory of Dissonance, p. 1186-Combination Tones, p. 1188-Objections to the Theory of Helmholtz, p. 1190-Other Theories, p. 1190-The Semicircular Canals, p. 1194.

\section{The External Ear.}

The auricle.-The most natural supposition is that the auricle reflects sound-waves into the external meatus. But cases of absence of the auricle have been recorded without any appreciable diminution in the power of hearing, ${ }^{1}$ and persons from whom the external ear has been removed have been found to possess normal hearing power. Experiments upon the auricle, in which the depressions on its surface were filled up, have given contradictory results. ${ }^{2} \mathrm{M} \cdot$ Kendrick $^{3}$ found that covering the external ear with plaster of Paris, leaving the entrance to the canal free, weakened the intensity, and altered the quality of tones. It must be observed that in such experiments the mass of the auricle itself would not be able to vibrate to the same extent as in the normal condition, and hence an apparent diminution of hearing may have been due to a loss of vibrations conducted by the solid structures, and not to the loss of the reflecting action of the auricle.

Long ago, Boerhaave attempted to show mathematically that the concha acts as a parabolic reflector, collecting and throwing vibrations into the meatus. Savart, ${ }^{4}$ however, demonstrated experimentally that this is not the case. Still there is evidence that the concha does in a slight degree affect acuteness of hearing by reflection. Thus ${ }^{5}$ if a small tube, curved like a

${ }^{1}$ Allen Thomson, London and Edinburgh Month. Journ. Med. Sc., April 1847; Toynbee, "Diseases of the Ear," London, 1868, p. 14.

2 Schneider, "Inaug. Diss.," Marburg, 1855 ; Harless, Wagner's "Hanclwörterbuch d. Physiol.," 1853 , Bd. iv. S. 422.

3 “"Text-Book of Physiology," vol. ii. p. 672.

${ }^{4}$ Journ. de physiol. expér., Paris, 1824, tome iv. p. 183.

5 Politzer, "Diseases of the Ear," 1894 , p. 710. 
hunting-horn, is inserted into the meatus in such a way that its wide opening is directed backwards to the concha, hearing is assisted, the sound-waves which strike the concha being reflected upon the instrument, which then directs them inwards.

A more delicate mode of testing this question is to sound a compound tone, and observe whether it changes in quality, according to its position in relation to the ear. Thus, if the auricle reflects the fundamental tone more strongly than the partials, or the converse, then a change in the quality of the tone should be perceptible when alterations are made in the position of the source of sound. Experiment shows that such a difference in quality is found to occur. Thus S. P. Thompson ${ }^{1}$ has pointed out that the ticking of a watch placed in the middle line behind the head appears to have a slightly different quality from that which it possesses when held in the corresponding position in front of the head, the higher tones being relatively more pronounced in the former case. Gray ${ }^{2}$ has found that by putting the hand in front of the ear, with the hollow of the palm directed backwards, while the watch is behind the head, the relative increase in the intensity of the higher tones is annulled, and the sound has the same quality as when held in front of the head.

Mach ${ }^{3}$ has suggested that the pinnæ may act as resonators for the higher partials of compound tones. S. P. Thompson ${ }^{4}$ is of opinion that the cartilaginous rim at the outer margin of the pinna may catch sound-waves that have been reflected from the ground in an upward direction. Politzer ${ }^{5}$ asserts that not only the concha but also the tragus exercises a certain influence upon soundwaves. By placing a small dise behind the tragus, and thus directing its apex outwards, he found that acuteness of hearing was appreciably increased. This increase he attributes to the larger surface which the tragus offers to waves which have been reflected forward from the concha, and which are finally reflected into the meatus. It must be stated, however, that some authorities ${ }^{6}$ hold that the auricle does not act as a reflector of sound at all.

Another function of the auricle, probably quite as important as its acoustic function, is its protective influence. The mouth of the meatus is somewhat twisted, and since the tragus projects backwards over it, the difficulty of foreign bodies obtaining entrance is much increased. The stiff hairs which are present in the lower animals, and frequently also in the human subject, at the entrance to the meatus, no doubt aid in this protective function, by preventing the entrance of dust, insects, etc., into the outer canal.

The external auditory meatus.-The functions of the external auditory meatus are (1) to conduct waves of sound to the membrana tympani, and (2) to afford protection to this membrane and the delicate structures in the middle ear.

As regards its first function, the question of its action upon the reflection of sound again comes into consideration, and authors are not wanting who find in its various curves a means of reflecting the waves of sound to the drum-head. Such curves exist at two places, one at the depression on the posterior wall of the cartilaginous meatus, immediately after its commencement, and the other at the concavity which occupies the anterior and inner wall of the meatus, and which is subtended by the tympanic membrane. Politzer ${ }^{7}$ states that the latter concavity is in the form of a parabolic curve, and will thus reflect sound-waves very powerfully on to the membrane. The

${ }^{1}$ London, Edinburgh, and Dublin Phil. Mag., London, 1882, vol. xiii. p. 413.

2 When no reference is given to statements by $\mathrm{M}^{\circ}$ Kendrick and Gray, it will be understood that these are the result of experiments and observations made for the purpose of this article.

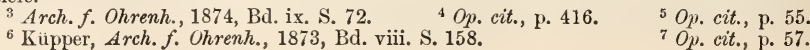

${ }^{6}$ Küpper, Arch. f. Ohrenh., 1873, Bd. viii. S. 158.
} 
value of these cavities, however, for thus concentrating and directing sound-waves, is problematical, as obliteration of the external one by displacement of the auricle backwards has no detrimental effect upon hearing (Gray).

The width of the meatus varies much in different individuals; in rare cases the greater part of the drum-head may be seen by looking directly into the meatus, without any displacement of the cartilaginous structures. These anatomical variations have not been found to produce any appreciable effect upon hearing. Further, the lumen of the tube may be considerably narrowed by putting in small pellets of wax without any noticeable diminution in the intensity with which sounds are heard. ${ }^{1}$

The protective function of the meatus is more important than any function it may perform of reflecting waves of sound upon the membrane. The tube protects the membrane in two ways-(1) By its varying curvature and by its length, and (2) by the secretion of cerumen. As regards the first, it is obvious that any foreign body entering the meatus will come first into contact with one wall and then with another, and may thus be arrested. Further, as the meatus is of considerable length (averaging $24 \mathrm{~mm}$.) in proportion to its transverse area, the air in its cavity maintains a fairly uniform temperature. This temperature is only slightly affected even when the external air is very cold, or when strong winds or draughts blow upon the ear.

By the secretion of cerumen, the entrance of foreign bodies, especially insects, is prevented. Dust and small particles of foreign matter readily stick to the cerumen, and are thus hindered from passing inwards.

The chemical analysis of cerumen shows water, fats, and a yellow pigment, the nature of which has notyet been determined. Cerumen has a bitter taste and an aromatic odour. Probably those qualities deter insects from entering the meatus. They are rarely found in the cavity.

Cerumen is formed, usually in small quantities, by the tubular glands found in the skin lining the walls of the cartilaginous portion. At the outer part of the meatus the glands are scattered throughout the whole circumference of the tube, but on passing inwards we find that those on the inferior and anterior wall rapidly diminish in number, and cease to exist before the osseous meatus is reached. On the posterior and superior wall they are found further along the tube, and a few even exist in the osseous meatus.

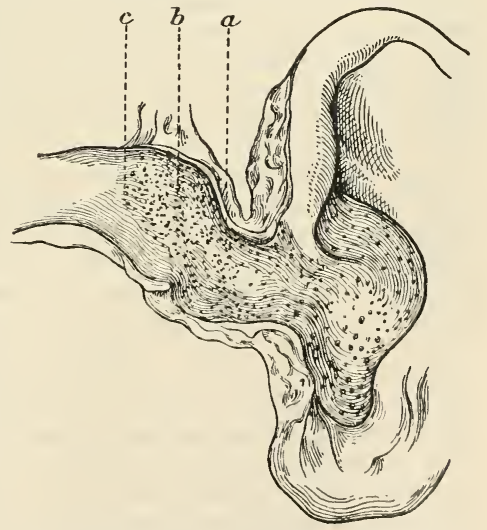

FIG. 418.-Posterior wall of the cartilaginous and osseous meatus. $a$, Orifices of glands on the cartilaginous portion; $b$, boundary between osseous and cartilaginous meatus; c, termination of the triangular space occupied by the orifices of the glands.-Politzer.

Now, since the cartilaginous portion of the tube slopes downwards, from within outwards, it is obvious that the force of gravity will tend to draw the cerumen

${ }^{1}$ Politzer, op. cit., p. 57 ; and Arch. f. Ohrenh., Bd. ix. S. 72. 
downwards and outwards. Since the osseous portion of the meatus runs from above downwards and inwards, it is evident that, if glands existed in this portion, the cerumen would collect around the tympanic membrane, with a result disastrous to the transmission of sound.

The outward flow of cerumen is also aided by the continually repeated movements of the cartilaginous meatus which occurs during mastication.

Movements of the auricle. - The movements of the auricle in the human being are for the most part insignificant. They have no effect on hearing. The muscles of the tragus and antitragus tend to contract the orifice of the meatus, while those of the helix produce the opposite effect. Many animals have the power of moving the opening of the auricle in the direction from which the sound comes. Thus the horse pricks its ears when it hears a sound, and no doubt its appreciation of the direction of sounds is thereby assisted.

Though none of the muscles of the pinna are, as a rule, under the control of the will, yet some individuals have the power of moving that structure as a whole. No doubt, by attention and practice, a man may acquire the power of moving the auricle slightly; but at best these movements are small as compared with those of the lower animals. Politzer ${ }^{1}$ states that an unconscious movement of the ear occurs in many persons when they listen to a very feeble noise. In some animals a reflex contracture of the muscles of the pinna occurs when the ear is suddenly stimulated by sound.

\section{The Middle Ear.}

The function of the middle ear is to receive sound-waves occurring in the air, and to transmit the effect of these to the labyrinth. The reception of the waves naturally comes first under consideration, and, since the tympanic membrane is the only structure concerned in this part of the process, an accurate knowledge of its anatomical structure, position, and tension in the living being are necessary for a full appreciation of its important function.

The tension of the membrana tympani, and its regulation.Although, as a matter of fact, the membrana tympani is a stretched niembrane, yet, from the acoustic point of view, it does not act like the membrane of a drum. If it did so act, waves of sound would cause it not only to vibrate, but to continue vibrating after the tone had ceased, and thus confusion and indistinctness of the sound would result. The membrane is also perfectly elastic in all its parts, although the amount of movement of various parts is not the same, owing chiefly to the fact that the relative number of radiating and circular fibres varies in different parts. Thus the central portions are composed almost entirely of radial, while the peripheral parts are formed chiefly of circular fibres. If the air in the external meatus be rarefied and condensed alternately by means of a pneumatic speculum, it will be observed that certain parts of the membrane undergo much greater alterations in position than others. Mach and Kessel ${ }^{2}$ first demonstrated that this change in position of the membrane was greatest in the posterior segment.

Some writers have asserted that the membrane has a tone of its own. Thus Politzer ${ }^{3}$ names the tone as $e^{4}\left(m i^{4}\right)$, having a vibration frequency

${ }^{1}$ Op. cit., p. 56.

2 Sitzungsb. d. k. Akad. d. Wissensch., Wien, 1874, Bd. lxix. S. 221.

${ }^{3}$ Op. cit., p. 58. 
per second of 2560, and König places the pitch of the proper tone two octaves higher, namely at $m i^{6}$, the frequency of which is 10,240 . But it is very doubtful if the membrane can be said to have any proper tone in the living body; if it has such, the pitch is in all probability nearer that assigned to it by König than by Politzer.

The tension of the drum-head is maintained in the living body by the tensor tympani: like other muscles, it possesses a constant tonicity. As the tendon of the muscle is inserted into the handle of the hammer a short distance below the horizontal axis of rotation of the bone, it is obvious that it must exert a constant force tending to draw the membrane inwards.

The cone which is formed by the inwardly drawn membrane has its walls convex outwardly. This is due to the arrangement and relative length and strength of the circular fibres. This mechanism may be illustrated by an experiment upon a spider's web, which is also composed of circular and radiating fibres. If the centre of the web be drawn out at right angles to its plane, it will be found that the sides of the cone thus formed are convex in a direction opposite to that towards which the apex of the cone points (Gray).

Secchi ${ }^{1}$ maintains, as the result of experiment, that there exists in the living subject a pressure in the tympanic cavity of $4 \mathrm{~mm}$. above that of the external air. This statement has neither been confirmed nor denied by other investigators, but it is difficult to see how it can be brought into line with wellascertained facts regarding the tympanum.

Movements of the membrana tympani in response to sound pressures. - If the drum-head had been a uniformly flat stretched membrane, the amplitude of its movements in response to the varying pressures of sound-waves would be greatest in the centre, while it would diminish as the periphery of the membrane was approached. A variety of conditions combine to render the movement much more complex. These are the peculiar curvature of the membrane, the fact that it is weighted by the hammer, and the pull which the tensor tympani exerts upon it through the hammer. Helmholtz ${ }^{2}$ was the first to investigate this problem in a satisfactory manner.

As the outward curvature of the radial fibres is slight, each fibre may be regarded as the long arm of a lever, while the handle of the

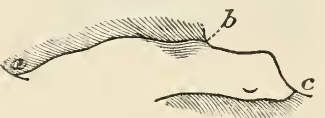

FIG. 419.- $a$, Outer margin of meatus; $b$, $c$, tympanie ring with the membrane between, showing its eurvatures. - Helmholtz.

hammer is the short arm. This mechanism secures that a slight pressure of the air, corresponding to a sound-wave, exerts a considerable force upon the malleus. To aid in understanding the mechanism, it will be easier to consider, first, the effect of pressure upon a single radial fibre. The fibre may be regarded as inextensible and slightly curved outwards; hence variations in pressure on the convexity of the curve will cause the degree of curvature to change, while the length of the arc will remain the same.

1 Trans. Internat. Med. Cong., Berlin, 1891 ; Rọme, 1894.

2 "Ueber die Mechanik der Gehörknöehclehen," "Wissensehaftliehe Abhandlungen," Bd. ii. S. 503 and 515; "Die Mechanik der Gehörknöchelehen und des Tronmelfells," Arch. f. d. ges. Physiol., Bonn, 1868, Bd. i. S. 1 ; see also the translation of the latter work by A. H. Buek and N. Smith, New York, 1873. The description is taken from Helmholtz's work.

VOL. II. -73 
In other words, the radius of the arc and the chord of the arc will change, while the length of the are remains constant. But the length of the arc may be regarded as the length of a radial fibre ; hence-

$$
l=2 r \sin ^{-1}\left(\frac{\lambda}{2 r}\right)
$$

where $l=$ length of fibre, $r=$ radius of the circle of curvature, and $\lambda=$ chord of the arc $l$, because $\frac{\lambda}{2 r}$ is the sine of half the angle at the centre belonging to

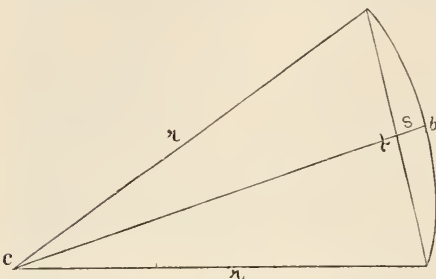

FIG. 420.

the are $l$. This equation may also be written -

$$
\lambda=2 r \sin \left(\frac{l}{2 r}\right) .
$$

Now, if we subtract the one equation from the other, we have-

$$
l-\lambda=2 r\left\{\frac{l}{2 r}-\sin \left(\frac{l}{2 r}\right)\right\}
$$

which gives the difference between the chord of the are and the curve.

But as the curve is very slight, $r$ is large in comparison with $l$, and the divisions becoming rapidly very small as the sine in the formula is developed by the involution of its are. Hence-

$$
\sin \frac{l}{2 r}=\frac{l}{2 r}-\frac{1}{6}\left(\frac{l}{2 r}\right)^{3}
$$

and from this the preceding equation becomes-

$$
l-\lambda=\frac{1}{24} \frac{l^{3}}{r^{2}} \cdot \text {. . . . . }
$$

Again, let $s$ be the distance of the centre of the are from the centre of the chord, then the degree of curvature is found by the equation-

So that

$$
\frac{r-s}{r}=\cos \frac{l}{2 r} \text {. }
$$

$$
\begin{aligned}
s & =r-r \cos \frac{l}{2 r} \\
& =r\left(1-\cos \left(\frac{l}{2 r}\right) .\right.
\end{aligned}
$$

By evolution of the cosine-

$$
s=\frac{1}{8} \frac{l^{2}}{r} \cdot \text {. . . . . . . . }
$$

Now eliminate $r$ from equations 1 and 2 , and we obtain

$$
l-\lambda=\frac{8}{3} \frac{s^{2}}{l}
$$

This equation gives the amount of shortening of the chord which occurs when the curve of the are is increased; that is to say, it gives the extent to which the two ends of the fibre are drawn together. Now, if $s$, the displacement of the middle of the fibre, be very small in comparison with $l$, then $l-\lambda$ obviously becomes very small in comparison with $s$. Conversely, the very small increase in the magnitude $l-\lambda$ must cause a relatively great increase of 
$s$; that is to say, it must cause a relatively great displacement of the centre of the fibre.

Again, if $t=$ the tension of the fibre, $p=$ the pressure on each unit of its length, and $r=$ the radius of curvature, then-

$$
t=p r
$$

and the forces which act upon both ends of the fibre must be equal to the pressure which acts upon the diameter of the semicircle through a width equal to that of the fibre. ${ }^{1}$ Therefore

$$
2 t=2 p r .
$$

Hence the greater the radius of curvature, the greater will be the alterations in tension of the fibre, caused by changes in the pressure of the air. Further, as the radial fibres are those which are attached to the malleus, it is evident that the variations in the tension of the fibres cause movements of the bones when sound-waves strike the drumhead. Thus a very small change of pressure in the air causes a considerable change in the tension of the fibres; and further, in accordance with the laws regulating the action of the lever, as the force which the fibres exert upon the handle of the malleus increases, the amplitude of movement of that bone diminishes. In this way the special form of the drum-head secures a maximum of efficiency for tones of the feeblest intensity.

The movements of the malleus. - The movements of the malleus are of two, possibly of three kinds. An accurate knowledge of the anatomy of the ligaments and articulations is necessary for their comprehension.

In the first place, as the malleus is firmly attached to the membrana tympani along the manubrium, it is obvious that the movements of the membrane would tend to carry the bone inwards as a whole. This movement, however, is to a great extent prevented by the ligaments attached to the head and neck of the malleus, and in the living subject it is exceedingly minute. A second and also comparatively unimportant movement of the malleus is that of rotation on the axis passing through the length of the bone from above downwards. This movement is produced by the tensor tympani, the tendon of which is not inserted into the bone straight, but somewhat at an acute angle or on one side. Hence, when the muscle contracts, it not only pulls the handle of the malleus inwards, but also rotates it round an axis passing from the head of the bone downwards to the tip of the manubrium. This movement, however, is very insignificant.

By far the most important movement of the malleus is one of rotation round a horizontal axis, which passes through the anterior ligament and head of the malleus, the body of the incus, and the short process of the incus (see Fig. 421 p. 1158). When the manubrium is pushed inwards, the part of the malleus above this axis must move outwards. The ligaments, especially the external and superior, prevent extensive movements of the malleus.

The movements of the incus.-This bone is attached to neighbouring structures at three places. Two of these attachments are true joints, namely, the malleo-incudal and the stapedial; the third is where the short process of the incus is connected with the posterior wall of the tympanic cavity. The malleo-incudal joint is peculiar both as to

\footnotetext{
${ }^{1}$ Helmholtz, op. cit.
} 
structure and mode of action. The posterior surface of the rounded head of the hammer fits into the somewhat saddle-shaped concavity of the anterior surface of the anvil. At the lower margin of the joint the prominent edge of the malleus fits on the lower edge of the articular surface of the incus in such a way, that with every movement of the edge of the malleus inwards the incus is carried inwards at the same time; whereas, when the malleus moves outwards, the incus behind is not compelled to follow the malleus to the full extent of its movements.

The short process of the incus fits into a slight depression on the posterior wall of the tympanic cavity, close to the entrance to the mastoid cells, where it is fixed by a ligament. The tip of the short process is covered with cartilage, and a certain degree of movement is possible at the point of contact with the temporal bone.

The remaining connection of the incus is that of its long process with the stapes. This presents the usual features of a joint. Its capsule contains a considerable amount of elastic fibre, and it is somewhat firmer and stronger on its inferior aspect. The movements of the incus, under the influence of sound-waves, are similar to those of the malleus, but less in extent. The bone rotates round an axis, which passes from the tip of the short process through its body, and which, if continued, would pass through the neck of the malleus, and just above the cog-like edge of the articular surface. Thus, as has already been pointed out, when the handle of the malleus moves inwards, the greater part of the body of the incus and of the head of the malleus move outwards together, and the long process of the incus moves inwards. The result of this lever-like mechanism is that, when the membrana tympani and the handle of the malleus move inwards, the base of the stapes is pushed into the oval window.

The movements of the stapes.-This bone is fixed in the fenestra ovalis by the annular ligament. It is placed almost at a right angle to the long process of the incus, and one would at first sight imagine that it must slightly follow the to-and-fro movements of that process. It does not, however, move exactly in this way, because the fibres of the annular ligament are more tense below than above the foot-plate of the bone, and hence movement is more free along the upper edge of the footplate than along the lower edge.

Before we discuss the movements of the ossicles, as constituting a chain or lever passing from the membrana tympani to the fenestra ovalis, it is necessary to describe the action of the intrinsic muscles of the ear.

The tensor tympani. - When this muscle contracts, the membrana tympani becomes more tense. A careful consideration of the exact position of its insertion into the bone shows that the tendon is attached to the anterior surface of the inner edge of the manubrium, and not to its posterior surface; contraction of the muscle must tend to rotate the malleus on its vertical axis. Further, the point of insertion is much nearer the axis of rotation than the attachment of the radial fibres of the tympanic membrane. Thus the mechanical arrangement is that of a lever of the third order, in which the power (contracting force of the muscle) is placed at a short distance from the fulcrum, while the weight (resistance or tension of the fibres of the membrane) is at a considerable distance. Hence the force with which the muscle renders 
the membrane tense will be much less than its actual power, while, on the other hand, the amplitude of movement at the point of resistance will be considerable.

Again, when the muscle is in a state of contraction, it renders all the ligaments of the ossicles tense, with the exception of the superior ligament of the malleus. This effect may be seen in the human subject, if the tympanum be opened during rigor mortis.

The normal stimulus which causes the tensor tympani to contract is the pressure of sound-waves on the membrana tympani. Although the innervation of the membrana has not been conclusively established, there is little doubt it is supplied with sensory nerves by the fifth, and also by the tympanic plexus, formed by fibres derived from the otic ganglion, from the petrosal ganglion of the glosso-pharyngeal, and from the carotid plexus. ${ }^{1}$ When pressure is made on the membrana, there is irritation of these sensory nerves, followed by a reflex contraction of the tensor tympani, supplied by a motor filament from the motor division of the fifth nerve. ${ }^{2}$ This response to sound was first discovered by Hensen and Bockendahl. ${ }^{3}$ They attached a light signal to the tendon of the muscle in the dog, and observed a movement when a note was sounded. Pollak ${ }^{4}$ found that when one ear was stimulated by sound-waves, the tensor tympani on the opposite side was also thrown into contraction. He also found that the reflex contraction was most marked when a tone of high pitch was sounded, deep tones causing only very slight contractions. The tensor tympani also contracts during yawning. In a few rare cases, the muscle appears to be under control of the will. ${ }^{5}$

The stapedius.-Henle ${ }^{6}$ was of opinion that the stapedius, when it contracted, held the plate of the stapes firmly in its place, and prevented it being forced into the fenestra ovalis, when strong pressures were communicated to it fron the membrana tympani. On the other hand, Politzer ${ }^{7}$ endeavoured to demonstrate that contraction of the muscle draws the plate of the stapes out of the fenestra ovalis, and, by releasing the tension of the chain of bones, produces relaxation of the membrana tympani. According to this view, the stapedius is the antagonist of the tensor tympani. The stapedius is supplied by a branch of the facial nerve. We have no direct evidence of any reflex excitation of the stapedius, but one would expect it to respond in a manner analogous to the response of the tensor tympani. Lucae $^{8}$ suggests that it may respond to tones of high pitch, and he also mentions that contraction of certain muscles of the face, especially of the orbicularis palpebrarum, causes a simultaneous contraction of the stapedius.

The movements of the ossicles as a whole under the influence of sound-waves. - When a sound-wave strikes the membrana tympani, that structure will be driven inwards during the positive phase; that is to say, during the time when the pressure is greater than

1 Bischoff, "Mikroskopische Analyse der Kopfnerven," Munich, 1865.

2 Politzer, Jahresb. ii. d. Leistung. . . in d. Anat. u. Physiol., Berlin, 1860, S. 583 ; and Voltolini, Virchow's Archiv, Bd. lxv. S. 467.

3 Arch.f. Ohrenh., Bd. xvi. S. 253.

+ Med. Jahrb., Wien, 1886.

${ }^{5}$ Sehwartze, Arch. f. Ohrenh., Bd. ii. ; Lucae, ibid., Bd. iii. ; and others.

6 " Handbuch d. Anat. des Menschen," Bd. ii. "Eingeweidelehre," S. 749.

7 Wien. med. Wchnschr., Bd. xvii. S. 1657 ; Bd. xviii. S. 113.

8 Politzer, "Diseases of the Ear," 1894, p. 69. 
atmospheric pressure. As the handle of the malleus is attached to the membrana tympani, it must also move inwards; but as this bone rotates round its axis, the head of the malleus is carried outwards, and it carries the upper part of the body of the incus along with it. The incus also rotates round an axis passing through the lower part of the body, so that the long process passes inwards, driving the stapes before it. During the negative phase of the sound-wave-that is to say, when the pressure is less than atmospheric pressure-the reverse action takes place, with this difference, that the malleus can unlock itself from the incus and swing some distance outwards without carrying the incus along with it.

In the transmission of the effect of a sound-wave from the membrana tympani to the labyrinth, there is a change in the amount of force, and a corresponding change in the amplitude of the movement. We may regard the mechanism of transmission as serving the purpose of con-

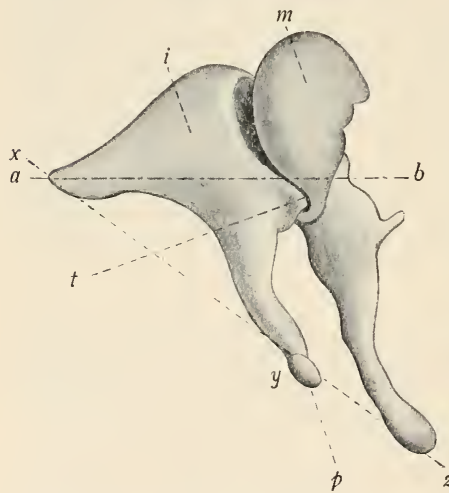

FIG. 421.-To show the relations of the malleus and incus to one another.-Helmholtz. $m$, Malleus ; $i$, incus ; $p$, its long process; $t$, tooth of incus fitting against ridge of malleus; $a-b$, axis of rotation of the bones; $x, y, z$, line joining short process of incus with end of handle of malleus. veying periodical variations of pressure, occurring in the air, into a minute cavity or space full of fluid, in which the terminal organ of hearing exists. It is extremely difficult to transmit sound-waves from air into a fluid, especially if the cavity containing the latter be of very small dimensions. This result is attained with a minimum loss of energy by the chain of bones. The chain may be regarded as a lever, in which the manubrium of the malleus forms the long arm, the axis of rotation passing through the malleus and incus forms the fulcrum, and the long process of the incus, carrying the stapes, forms the short arm. The pressure of the sound-wave, applied by the membrana tympani to the manubrium of the malleus, may be regarded as the force, while the resistance is at the base of the stapes, by which the pressures are communicated to the labyrinth. The mechanism is therefore a lever of the second order. Careful measurements have shown that the length of the long arm is to the length of the short arm as $1 \cdot 5: 1$. The resulting force at the stapes is therefore correspondingly increased, while the amplitude of the movement at the stapes is correspondingly less than at the tip of the manubrium. Thus the pressures are conveyed with great efficiency from the membrana tympani to the labyrinth, and the amplitude of the oscillation is diminished so as to be adapted to the small capacity of the labyrinth. Further, it is to be observed that as the drum-head is nearly twenty times larger than the fenestra ovalis, the energy of the movements of the membrana tympani is concentrated on an area twenty times smaller; hence the pressure of a sound-wave, acting on the membrana, 
is increased thirty-fold $(1.5 \times 20)$, when it acts on the plate of the stapes.

Before leaving the subject of the transmission of pressures corresponding to sound-waves, from the air to the labyrinth, we may consider the effect which the dimensions of the conducting apparatus have on the transmission. The first who drew attention to this subject was Weber, ${ }^{1}$ and it has been further investigated by von Helmholtz. ${ }^{2}$ By dividing the velocity of sound in air by the number of vibrations per second, we obtain the wavelength. It is thus ascertained that the wave-length of sounds of the lowest pitch recognised by the human ear is about $40 \mathrm{ft}$., that the lowest tone of the organ has a wave-length of about $37 \mathrm{ft}$., that the wave-length of the middle $c$ of the piano is above $4 \mathrm{ft}$, that the highest tone of the piccolo stop of the organ has a wave-length of $3.5 \mathrm{in}$., while the wave-length of the highest tone audible to the ear is at least $\cdot 5 \mathrm{in}$. Thus it is evident that the total length of the solid and fluid masses of the ear is only a small fraction of the wave-length of ordinary perceptible sounds. Hence we must regard the ossicles and the intra-labyrinthine fluid, not as forming an independent vibrating mass, but as one body which has practically no proper vibration period. If, for example, it were possible to throw the ossicles into periodic vibrations, the tone resulting thereby would be so high in pitch as to be beyond the limits of hearing. The movement of the chain of ossicles, there. fore, forms only a small part of a sound-wave.

The magnitude of the movements of the ossicles.-The movements of the bones of the ear may be studied experimentally in the dead animal by various methods. Politzer ${ }^{3}$ opened the cavity of the tympanum, and fixed bits of fine glass thread to the various ossicles; the free ends of the threads were then adjusted to a surface of a recording drum. When a sound was allowed to fall on the membrana tympani, the glass threads oscillated and traced curves on the smoked paper covering the drum. The movement of greatest amplitude was found to be at the tip of the manubrium, $0.76 \mathrm{~mm}$; the movement at the tip of the long process of the incus was $0.21 \mathrm{~mm}$.; while the greatest amplitude at the stapes was $\cdot 0714 \mathrm{~mm}$. A similar series of measurements were made by Mach and Kessel ${ }^{4}$ and by Buck ${ }^{5}$ in 1870 . In the following year the matter was re-investigated by Burnett. ${ }^{6}$ He opened the tympanum, illuminated the interior by a strong beam of light, sprinkled the ossicles with powdered starch, so as to secure brightly luminous points, fixed a tube into the external auditory meatus so as to convey vibrations from an organ-pipe to the outer surface of the membrana tympani, and then observed the excursions of the shining particles when the organ-pipe was sounded. The chain of bones vibrated in excursions bearing a fixed relationship to each other. Particles on the fenestra rotunda were seen to vibrate synchronously with oscillating particles on the stapes. Further, the excursions at the fenestra rotunda were equal to those of the stapes, and sometimes they were equal to those at the point of the manubrium. The amplitude of movement at the base of the stapes and round window varied from 0.001 to $0.032 \mathrm{~mm}$., considerably less than the measurement made by Politzer. Burnett also made the interesting observation that variations in the labyrinthine pressure modified the amplitude of movement at the stapes, an increase of

1 Weber, Verhandl. d. süchs. Gesellsch. d. Wissensch., 1851.

2 "Mechanism of the Ossieles of the Ear," p. 9. ${ }^{3}$ Arch. f. Ohrenh., 1864, Bil. i.

${ }^{4}$ Loc. cit.

6 "Treatise on the Ear," p. 95. 
pressure diminishing, while a diminution of pressure increased the amplitude. It is more than probable that in all of these experiments the amplitude has been over-estimated, as the restraining influence of the tensor tympani and stapedius does not come into play after death. Very feeble sounds no doubt cause a movement of the stapes so small as to be invisible to the microscopic powers that can be employed in such observations. The greatest movements occur with tones of medium pitch.

An interesting experiment may also be performed, in which the cat's tympanum is used as a manometric capsule, after the well-known method of König. Two small glass tubes are fixed with sealing-wax into holes trephined in the tympanic bulla, and a tube car'rying a conical mouthpiece is also inserted into the external meatus. Gas is led into the cavity by one of the small tubes, and it issues from the cavity by the other tube to a small gas jet. When sound-waves, such as those of organ-pipes, tuning-forks, or of the human voice, are conveyed by the mouthpiece to the membrana tympani, flame pictures, similar to the well-known flame pictures of König, may be seen on rotating a rectangular mirror in front of the flame. ${ }^{1}$

Transmission of sound to the labyrinth otherwise than by the ossicles.-The earlier physiologists, and in particular Scarpa, were of opinion that sound passed from the tympanic membrane into the air in the tympanum, and was transmitted to the labyrinth by the fenestra rotunda, which was termed a secondary tympanum. Experiments made by Weber-Liel, ${ }^{2}$ by a method very similar to that of Burnett, already described, supported this view, but on the whole it was not accepted by the majority of physiologists. In 1894, Secchi ${ }^{3}$ advocated the same view, on the ground that, as the tympanum is a closed cavity, changes in the position of the membrana tympani must be accompanied by changes in the pressure of the air within the cavity. These variations of pressure in the cavity are distributed over its walls, and as these are rigid everywhere except at the fenestra rotunda, the variations of pressure could be transmitted to the fluid in the labyrinth by the fenestra rotunda. It must be remembered, however, that, from the mechanical arrangements already described, almost all the energy of the membrana tympani is conveyed to the fenestra ovalis by the chain of bones. And if we suppose that pressures are communicated simultaneously to the fenestra ovalis and to the fenestra rotunda, to the first by a chain of bones, and to the second by the air in the tympanum, any inward pressure on the fenestra rotunda will be more than annulled by the inward pressure on the fenestra ovalis, and that therefore there must be an outward movement at the fenestra rotunda, when there is an inward movement at the fenestra ovalis, a result which is confirmed by experimental observation (M'Kendrick). Finally, interference with the movements of the chain of bones has a marked effect upon the perception of sound, which could not be the case if they did not play the more important part in the conduction of sound. At the same time, it may be admitted that in exceptional cases, in which the chain may be absent, hearing may still exist, in consequence of aerial pressures being communicated to the membrane covering the fenestra rotunda.

1 M'Kendriek, “Text-Book of Physiology," vol. ii. p. 687.

${ }^{2}$ Centralbl. f. d. med. Wissensch., Berlin, 1876, Bd. xiv. S. 17.

${ }^{3}$ Loc. cit. 
Secchi's views have, up to a certain point, received confirmation from the recent careful observations of Bezold. ${ }^{1}$ The latter has found that, in disease causing rigidity or loss of the ossicles, the hearing is seriously affected as regards the lower and middle notes, while the upper notes are heard perfectly well. Thus the sufferers may not hear loud thunder, but the whistling of a canary may be clearly heard at a considerable distance. Hence Bezold holds that the ossicles are indispensable for the transmission of the lower notes, but take no part in the conduction of the upper ones.

There is still another route by which sound pressures may be transmitted from the external air to the labyrinth, namely, by the bones of the head. This is the case not only when the sounding body is applied to the cranium, but also when the sounds are transmitted through the air. Politzer ${ }^{2}$ found that when the external auditory meatus on both sides was filled with glycerine, and the nose and mouth firmly closed, conversation could be understood at a distance of 0.5 metre. It is also known that tones are perceptible even in cases where the stapes is ankylosed to the edges of the fenestra ovalis, if the tones are of sufficient intensity. As sound-waves are conducted through the solid and fluid parts of the body, they must directly reach the labyrinth without passing through the external or middle ears. Therefore, if the structures in the labyrinth are normal, it is impossible to exclude completely sound-waves from being perceived when transmitted by the bones of the head.

Regulation of atmospheric pressure in the tympanum.-As the membralla tympani is very thin and fibrous, the pressure of the air on each side of it must affect its capability of vibrating when subjected to periodic sound pressures. On one side it is freely exposed to the air, while on the other it is in contact with a confined mass of air in the tympanic cavity. As the air in the latter might be to some extent absorbed, and consequently have its pressure reduced, and as, on the other hand, the pressure of the air on the outer surface is liable to considerable variations, there must be some arrangement for establishing from time to time an equilibrium of pressure on the two sides of the membrane. This is accomplished by the Eustachian tube, passing from the tympanic cavity to the posterior part of the pharynx. This tube occasionally opens so as to allow the air of the pharynx, which communicates by the oral and nasal openings, to pass into the tympanum, and to exert its pressure on the inner surface of the membrana tympani. It is usually held that the Eustachian tube is closed during ordinary breathing, and that it is opened only at the moment of deglutition. Cleland ${ }^{3}$ and others, ${ }^{4}$ however, maintain that the tube is open during rest, and closed during deglutition.

An experiment performed by Politzer ${ }^{5}$ throws some light upon the matter now under consideration. He constructed a small glass manometer, the short limb of which passed through an indiarubber cork. A few drops of a coloured fluid were introduced into the manometer. The cork was pressed

1 “Ueber d. functionelle Pruf. d. menschl. Gehör.," Wiesbaden, 1897, S. 118-122.

${ }^{2}$ Arch. f. Ohrenh., 1864, Bd. i. S. 350.

3 Journ. Anat. and Physiol., London, vol. iii. p. 97.

${ }^{4}$ Luschka holds that the tensor opens the tube and the levator closes it. "Der Schlundkopf d. Mensehen," Tiibingen, 1868, S. 45 and 47. Sce also Hammerschlag, Wien. med. Wchnschr., Nos. 39 and 40, 1896; and Lueac, Arch.f. Ohrenh., Bd. xlii. S. 178.

5 "Diseases of the Ear," p. 64. 
tightly into the external auditory meatus. It was then found that, after closing the nostrils, the first stage of deglutition caused the fluid to rise slightly in the manometer. The rise was quickly followed by a considerable fall during the second portion of the act, and the fluid remained at the level to which it had fallen until deglutition with the nostrils opened was again performed. This would appear to indicate that, when the nostrils were closed, the air in the pharyngeal cavity communicated with that in the tympanic cavity at the beginning of swallowing, thus causing a rise of pressure. Further, that in the later portion of the time of swallowing, the tube still seemed to be open, so as to allow of some of the air being sucked out of the tympanic cavity, causing a fall of pressure. The tube then was closed and remained closed until the next process of deglutition.

With the same instrument, Politzer found that in many individuals slight

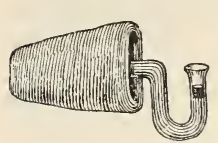
fluctuations occurred, synchronous with expiration and inspiration, even when these acts were quietly performed, showing that the tube, in some persons, may be slightly open, even when the muscles of deglutition are not in action. He also states that air passes more readily from the tympanum to the pharyngeal eavity than in the reverse Fig. 422.- Ear mano- the tympanum to the pharyngeal cavity than in the reverse
meter.-Politzer. larger cavity.

It has also been observed that in a case of paralysis of all the muscles attached to the eartilaginous portion of the tube, a falling inwards of the membrana tympani occurred, from absorption of air in the tympanic cavity and consequent diminished pressure, thus showing that the muscles are necessary for the passage of air into the tympanum (Gray).

We may conclude that, during rest, the condition of the Eustachian tube varies from a position of slight opening to gentle closure. When swallowing oceurs there is a firm closure of the tube, caused by the upward pressure of the levator palati. This is quickly followed by the tube becoming widely opened, chiefly by the action of the tensor palati.

\section{Auditory Reflexes.}

As has already been noticed (p. 1157), Pollak ${ }^{1}$ has observed the peculiar fact, that when a sound is led to one ear, a reflex contraction of the tensor tympani of the opposite ear occurs; high notes produce this reflex better than low ones.

Lucae $^{2}$ maintains that a reflex contraction of the stapedius occurs simultaneously with voluntary contraction of the facial muscles, particularly the orbicularis palpebrarum. This produces a deep humming noise in the ear, and a relaxation of the tympanic membrane. According to Politzer, ${ }^{3}$ during this contraction the perception of the middle and the deeper notes of the tuning-fork is destroyed. The present writers, however, cannot corroborate this latter statement.

Politzer ${ }^{4}$ and Helmholtz ${ }^{5}$ have observed that during yawning a reflex contraction of the tensor tympani occurs, causing slight temporary deafness.

A peculiar aural reflex has been described by Gray. ${ }^{6}$ If a watch or

\footnotetext{
1 Med. Jahrb., Wien, 1886.

3 "Diseases of the Ear," 1894, p. 69.

4 Arch. f. Ohrenh., Bd. iv. S. 23.

5 Arch.f. d. ges. Physiol., Bonn, 1868, Bd. i. S. 1.

6 Proc. Roy. Soc. Edin., 1897, vol. xxi. p. 443. 
tuning-fork of fairly high pitch be held a few inches from one ear, while the chain of ossicles of the other ear is pushed gently inwards by a probe or by pressing the finger into the meatus, thus condensing the air within, the tuning-fork or watch will be better heard. This is probably due to increased labyrinthine pressure in one ear, causing a reflex contraction of the intra-tympanic muscles of the opposite ear.

Urbantschitsch ${ }^{1}$ has stated that the stimulation of one ear by sound causes increased sound perception in the other ear. This may be true under some circumstances, but certainly not under all, for, as Politzer ${ }^{2}$ says, a loud sounding tuning-fork held opposite one ear destroys the perception for a weak sounding fork held opposite the other ear.

Various writers have recorded cases of subjective colour sensations accompanying audition, and these cannot be considered pathological, for they have occurred in perfectly healthy individuals. ${ }^{3}$

\section{Binaural Audition.}

Binaural audition, in the ordinary sense of the term, does not affect the quality of sounds, but there is little doubt that it does affect the intensity with which sounds are perceived. With the exception described above (p. 1163), we hear a sound louder with two ears than with one. Urbantschitsch ${ }^{4}$ attributes this to the passing of subjective irritation from the auditory centre of one side to that of the other. Politzer, ${ }^{5}$ however, maintains that this explanation is superfluous, and considers that the superiority of binaural over monaural audition is due to the well-known physiological law of "summation of sense."

But the greatest difference between binaural and monaural audition is, that by the former we are enabled to judge of the direction of sound with considerable accuracy, whereas in hearing with one ear we are almost completely deprived of this faculty. Our judgments as to the direction of sounds are formed, for the most part, from the different degrees of intensity with which they are heard by the two ears.

In 1874 , Mach $^{6}$ put forward the theory that the pinna of the ears act as resonators for the higher notes, and that their resonating capacity varies according to their position relative to the waves of sound.

Lord Rayleigh ${ }^{7}$ holds that diffraction of the sound-waves will occur as they pass round the head to the ear remote from the source of sound; thus the partial tones will reach the two ears with different degrees of intensity, and consequently there will be a difference in the quality of the sound as heard by the two ears. From this difference in quality we draw our inference as to the direction of the sound.

S. P. Thompson ${ }^{8}$ has advanced a theory somewhat similar to that of Lord Rayleigh. His view of the matter is, that we estimate the direction of a sound by the difference of intensity with which those partials are heard which have the greatest effect in producing the difference of quality with which the sound is heard by the two ears. This hypothesis is in keeping with the fact that the direction of a compound sound can be much more accurately estimated than the direction of a simple note, particularly if it be of low pitch.

\footnotetext{
1 “"Lehrbuch d. Ohrenh.," 1890, S. 416.

2 "Diseases of the Ear," 1894, p. 608.

3 Baratoux, "L'audition coloriée," Paris, 1888 ; Binet, Rev. d. deux mondes, 1892.

4 Loc. cit.

${ }^{6}$ Arch. f. Ohrenh., 1874, Bd. ix. S. 72. Mag., London, 1882, vol, xiii. p. 406.
${ }^{8}$ London, Edinburgh, and Dublin Phil. Masic.

5 op. cit., p. 608 .
} 
It is possible, as suggested by Gray, ${ }^{1}$ that when a sound-wave falls on the membrana while the intrinsic muscles are in a state of tension, a slight change may be produced in this tension. This change will be often different in the two ears, and thus we may judge of the direction of a sound by the muscular sense. If this be so, then our powers for estimating the direction of sound are ultimately referable to the muscular sense.

\section{The Internal Ear.}

\section{By JoHn G. M'Kendrick.}

The internal ear consists of a complicated series of sacs and tubes filled with fluid. In certain situations the walls of the sacs contain highly differentiated epithelial structures, which are intimately related to the terminal filaments of the auditory nerve. The problem is to explain how the pressures transmitted by the foot of the stapes affect these terminal structures in such a way as to excite sensations corresponding to the pitch, intensity and quality of tones. It is evident that the subject may be approached from the physical or from the physiological side. The physical phenomena of sound fall within the scope of the principles of mechanies; but when we attempt to apply those principles to an explanation of the functions of the internal ear, we find the task surrounded with difficulties.

We shall in this article discuss the question purely as regards its physiological aspects, presuming an acquaintance with the anatomical structure of the internal ear, ${ }^{2}$ and also with the general principles of acoustics. $^{3}$

General mechanism of internal ear.-As the base of the stapes is opposite the vestibule in which lie the utricle and saccule, it is evident that these latter structures are the first to receive the mechanical impulses communicated by the chain of bones. The utricle (communicating with the semicircular canals) and saccule (communicating by the canalis reuniens with the ductus cochlearis) are surrounded by the perilymph. It must also be noted that the cavity of the vestibule containing perilymph opens on the one hand into the scala vestibuli, while on the other it is in free communication with the perilymphatic space around the ampullæ and the tubes of the semicircular canals. These perilymphatic spaces in the vestibule, around the canals, and in the scalæ of the cochlea, are contained in corresponding cavities in the bone, the walls of which are, except at the fenestra rotunda, perfectly rigid. The fenestra rotunda, however, is covered by a membrane. Suppose no fenestra rotunda had existed, any pressure of the base of the stapes inwards would have produced no effect on the membranous structures, as the fluid would be practically incompressible; in other words, the base of the stapes, in such conditions, would have been immovable. As a portion of the wall of the bony cavity is yielding, namely, the fenestra rotunda, it is clear that an inward movement of the base of the stapes becomes possible, and this inward movement will always be accompanied by an outward movement of the membrane covering the round window. In this manner, pressures or impulses can be communicated

1 Proc. Roy. Soc. Edin., 1897, vol. xxi. p. 443.

2 Quain's "Anatomy," 9th ed., vol. iii. part 3, p. 71.

${ }^{3}$ Rayleigh, "The Theory of Sound," 2 vols. London, 1896. 
to the membranous sacs which contain the endolymph, and in which we find the nerve-endings.

It is also of importance to observe that, as already pointed out (p. 1159), the dimensions of the internal ear are so small as to form only a small part of the wave-lengths even of tones of high pitch. The whole of the membranous labyrinth may be regarded as part of any wave acting on the ear, and the waves are not arrested by the labyrinth as waves of light are arrested by the retina, but they sweep onwards through the bones of the head. The fact of the labyrinth being so small relatively to the size of the wave, makes no difference to the result, so that the labyrinth is acted on in the same way whether a wave of 30 feet in length, such as is produced by the longest pipe in a modern organ, or a wave of two-thirds of an inch, produced by the highest note of a piccolo flute, falls upon the ear. The nerve-endings are still smaller, but they also act as minute portions of any wave, and any reasoning as to the effect of such waves is quite irrespective of the small dimensions of the receiving organs in the internal ear.

If, now, we consider a wave of sound as a series of states of condensation and states of rarefaction, travelling on continually in one direction ; and, further, if we remember that the motion of each individual particle forming the wave is very small, and is alternately backwards and forwards, in the same line as that in which the wave travels, - we see that the movements, inwards and outwards, of the base of the stapes correspond to these oscillations, or, in other words, to increase and diminution of pressure with each wave. Further, the length of time occupied by each excursion inwards and outwards of the base of the stapes will correspond to the frequency of the waves, the amplitude of the movement of the stapes to the amplitude of the wave, while the way in which the base of the stapes moves out and in will correspond to the form or character of the wave communicated to it. We also know, from physical experiments, that the frequency of the movements determines pitch, the amplitude intensity or loudness, and the form quality of tone. No doubt it is difficult to appreciate what is termed form of wave when applied to waves of sound, because our ideas of form of wave are derived from our knowledge of waves in which particles move in a direction more or less at right angles to the direction in which the wave travels, as in waves on the surface of water; and it is difficult to conceive of vibrations of particles in the same direction as that in which the wave progresses, as in a wave of sound. ${ }^{1}$ Consequently all writers assist the mind in forming ideas of waves by the use of curves representing waves in which the particles vibrate transversely; but it must never be forgotten that the movements so represented are totally unlike those occurring in waves of sound.

Suppose the base of the stapes to be at rest, and that any movement inward is called a positive movement, and any movement outward a negative movement, the positive movement would correspond to a positive pressure or condensation, and the negative movement to a negative pressure or rarefaction. Suppose the bone is now moved inwards and outwards through a certain distance, at the same rate, from the two extreme points of its excursion, positive and negative. This is what we may term a simple pendular movement or vibration. But it is easy to conceive that, in moving inwards from its position of rest, it might not move to the extreme point of its positive excursion, but only a short distance, say half the distance, when it might move, for an instant, in the opposite direction; then again reverse its movement, and proceed to the limit of its positive excursion; then swing back to the position of rest; then move in the negative direction to the extreme point of excursion; and then, finally, return to the position of rest. This would

${ }^{1}$ Sir G. B. Airy, "On Sound," 2nd edition, London, 1871, p. 22 ; also plate i. figs. 6,7 , and 8 . 
not be a pendular, but a compound vibration; and one can readily understand that, while each such compound movement always was executed in a given time and through a given amplitude, it might be varied in character; or, in other words, while the time and the amplitude of the movement were constant, the form of the movement might be varied to almost an infinite degree, corresponding to the number and intensity of the components that produce the compound movement.

\section{The Utricle and Saccule.}

The movements of the base of the stapes, as we have seen, may vary in number, in amplitude, and in character or form, and these correspond to the pitch, intensity, and quality of any tone. These movements are communicated to the perilymph, and by it to the sacs of the membranous labyrinth. As already mentioned, the utricle and saccule are the first to receive the impacts, and from them the impulse is transferred to the ampullæ on the one hand, and to the ductus cochlearis on the other. The base of the stapes is chiefly opposite to the utricle, although it partly abuts against the saccule. On the wall of the utricle, immediately in front, there are no nerve-endings or modified epithelium, but on the back wall we find the crista acustica with its characteristic bristle cells, the points of which are directed towards the base of the stapes. In front of these bristle cells lie a wellknown mass of otoliths. The pressures must be communicated, in the first instance, to the otoliths, and by them to the bristle cells. As the bristle cells are fixed, while the otoliths are capable of moving backwards and forwards in a fluid, it is clear that an impulse from the base of the stapes may cause the otolith-mass to oscillate, and, by thus making a series of impacts on the points of the bristle cells, the excitation of the latter may be intensified by summation. ${ }^{1}$ Further, the thin membrane bearing the bristle cells will readily move to the impacts of the stapes, and the heavy otolithic mass, by virtue of its inertia, will move more slowly at first, but it will continue to oscillate, and thus keep up stimulation. The long and extremely light hairs or bristles also appear to be well adapted for sympathetic resonance. ${ }^{2}$ Helmholtz points out that the bristles in the crista, being of such minute mass, cannot long continue in independent motion. ${ }^{3}$ The ampullæ, being relatively wide cavities with narrow exits, are suitable for producing a central current, which partly passes into eddies, and these would deflect the bristles, causing them to oscillate. A movement of the whole mass of the bristles, floating in the fluid, would not serve the same purpose. Discontinuous streams of different strengths and in different directions would do so effectively. ${ }^{4}$ Others have suggested that the otoliths act as dampers $;^{b}$ but when we consider that a damping mechanism is only specially necessary for the perception of tones in music, and that otoliths are found in the ears of animals so low in the scale as to make it improbable that they appreciate such tones, this function appears to be unlikely.

Considerable doubt still exists as to the specific functions of the

1 Müller, “Elements of Physiology,” trans. by Baly, London, 1842, vol. ii. p. 1289.

${ }^{2}$ Helmholtz, "Sensations of Tone," translation by Ellis, London, 1875, p. 206.

${ }^{3}$ Helmholtz, ibid., p. $21 \%$.

"Helmholtz, Monatsb. d. Berl. Acad. d. Wissensch., April 23, 1868.

5 Paul Meyer, "Études histologiques sur le labyrinth membraneux des reptiles et des oiseaux," 1864, p. 129. 
utricle and saccule. The most primitive form of internal ear is undoubtedly a sac containing fluid in which an otolithic mass is immersed, and having on the wall hair-like processes related to the terminations of a nerve (Aurelia aurita, Unio, Phialidium, Pterotrachea). Such an arrangement is adapted to receive impulses from the surrounding water. ${ }^{1}$ In the lower vertebrates we find a single sac, into which open the semicircular canals; still higher we find the beginning of the ductus cochlearis; and, lastly, a division of the single sac into two, with a great development of the ductus cochlearis. These facts point to the sacs being organs for the reception of waves giving rise to sensations of sound as sound, and without reference to pitch or quality. Animals furnished with internal ears in which the cochlea is absent or only developed to a slight extent, no doubt have sensations that correspond to tones and noises, but these would differ only as regards intensity. Such sensations would be similar to visual sensations of luminosity, without reference to colour. To analyse auditory sensations, a more complicated mechanism is necessary, and hence we find the gradual evolution of a cochlea.

In man and the higher animals there can be no appreciation of sound irrespective of pitch and quality, because the movements of the stapes will not only call into action the nerve-endings in the utricle and saccule, but also those in the cochlea. One cannot conceive the vestibular portion of the internal ear acting alone. As, however, the two sacs are each supplied by a special nerve, it is not improbable that movements of the base of the stapes acting on both may give rise to two distinct effects, but there is no experimental evidence bearing upon this point.

When we take into account the minute size of the parts, and their situation in the temporal bone, the results of direct experiments on the ear of a living animal must be received with caution. Still the evidence, meagre as it is, is in favour of the view above set forth. Thus, Flourens destroyed all the nervous filaments to the labyrinth except those supplying the saccule and utricle, without observing deafness. Others have observed disease of the cochlea causing its destruction, but without complete loss of hearing. ${ }^{2}$ Destruction of the semicircular canals does not cause deafness. Gellé states that after he destroyed the cochlea in guinea-pigs, deafness came on only after inflammatory changes had invaded the structures in the vestibule. ${ }^{3}$

Recently Lee ${ }^{4}$ has argued that as fishes have no papilla acustica basilaris, and as, with few exceptions, they are dumb, it is highly probable they have no sense of hearing. Actual experiment appears to support this contention. The papilla acustica basilaris appears first in amphibia in an offshoot from the lagena, and, in the higher animals, it becomes part of the organ of Corti. If this view be correct, then the cristæ and maculæ acusticæ have nothing to do with hearing, but serve for the perception of movements, such as those of rotation and translation through space, which are infinitely coarser than those which are the physical basis of sound. Lee has also endeavoured to show that the organs in the lateral line of fishes and the maculæ and cristæ acusticæ

${ }^{1}$ For an excellent account of the comparative anatomy of the internal ear, see article "Audition," by Gellé, in Richet's "Dict. de physiol.," tome i. pp. 914-919. Figures showing the internal ears of these animals are given in this article.

2 Valsalva, Moos and Steinbrugge, Ztschr. f. Ohrenh., Wiesbarlen, tome xi. p. 281.

3 "Fonctions du limacon," Etudes d'otologie, 1880, tome i.

4 Am. Joum. Physiol., Boston, 1898, vol. i. 1. 128. 
have a common function, namely, the appreciation of mass-movement. Beard ${ }^{1}$ had already argued in favour of the parts of the internal ear and the organs of the lateral line being phylogenetically related. According to these somewhat speculative views, when animals became air-breathers, a part of the ear, the papilla acustica basilaris, was gradually evolved for the perception of the delicate vibrations of sound, while even in the higher vertebrates the nerveendings in the saccule and utricle may still have to do with only the perception of mass movements. While it may be that fishes are deaf, it is difficult to believe that hearing is associated in the higher animals solely with the cochlea. In certain fishes there are connections in the form of ossicles between the air-bladder and the acoustic labyrinth, ${ }^{2}$ and in these it is possible the air-bladder may act as a resonator, as Johannes Muiller thought. ${ }^{3}$ In the higher forms of vertebrates the intricate relations between the membrana tympani and the saccule and utricle by the chain of ossicles indicates that these sacs, while they may retain their primitive function of appreciating mass movements, are also the recipients of those more delicate movements caused by sound-waves impinging on the membrane.

\section{The Cochlea.}

It is remarkable that some of the older anatomists conjectured, long before the discovery of the organ of Corti, that this part of the internal ear was in some way analogous to a musical instrument, having wires of varying lengths tuned to tones of different pitch. ${ }^{4}$ Helmholtz, however, was the first to put forth a theory as to its mode of action, and whether this theory be accepted or not, all discussions of the subject must take the work of Helmholtz as a starting point. ${ }^{5}$ Let us inquire, in the first place, what the ear is capable of doing. We can hear musical tones and noises, we have a peculiar auditory sensation to which we give the name of beats, and we have the power of analysing a musical tone into its components. These are sensations capable of being tested by direct experiment.

For this purpose, the inquirer should be furnished with-(1) A set of forks ranging in pitch from 128 to 1024 vibs. per second, say $u t_{2}, u t_{3}, s o l_{3}$, $u t_{4}, m i_{4}, s o l_{4}, t i_{4}, u t_{5} ;(2)$ forks corresponding to $r e_{3}, m i_{3}, m i_{3}{ }^{2}, f a_{3}, l a_{3}$, $l a_{3} b ;(3)$ a set of resonators, as devised by Helmholtz, corresponding to those forks ; (4) the manometric analyser, for the series of forks enumerated in (1); (5) a double syren; (6) a set of steel cylindrical bars, for tones of very high pitch ; and (7) a few rectangular brass clips, capable of sliding freely on any of the forks, with a tightening screw. ${ }^{6}$

Pitch.-As determined by the syren, a sensation of musical tone begins with about 30 vibs. per second. It is, however, difficult to appreciate the pitch of musical intervals in the lower ranges, and it is only with about 40 vibs. per second that pitches are clearly

1 Zool. Anz., Leipzig, 1884, Bd. vii. S. 140.

2 Owen, "Comparative Anatomy," vol. i. p. 342.

3 " Physiology," vol. ii. p. 1245.

4 "Anatomy," by John and Charles Bell, 1816, vol. iii.

${ }^{5}$ Rayleigh, op. cit., vol. ii. p. 432.

6 The best acoustical apparatus is supplied by Dr. Rudolf König, Quai d'Anjou, Paris. An excellent set of apparatus, consisting of bellows, overtone apparatus, difference tone apparatus, apparatus for beats, and fine rich reed tones, is made by Appunn of Hannau, near Frankfort. Appunn's apparatus consists almost entirely of reeds, with appropriate resonators. It is described in "Sprache und Ohr," by Oskar Wolf, Braunschweig, 1871, S. 15. Sec also M'Kendrick, Proc. Roy. Soc. Edin., 1873-74. 
distinguishable. When a tone of lower pitch than 30 is sounded, the ear instinctively picks up the octave, probably because the octave is faintly produced by the instrument. ${ }^{1}$ From these tones of low pitch, as we listen to sounds rising in pitch to the upper limits, there is no break in our sensations, but a gradual passage through tones of ever-increasing pitch. This experience is similar to that of running the eye along a pure spectrum. When we get above 4000 vibs. per second, there is again much difficulty in detecting musical intervals, but we observe the gradual rise of pitch, till we reach, with cylindrical steel bars, tones corresponding to 20,000 to 30,000 vibs. per second. Sensitive flames give out even higher perceptible tones. ${ }^{2}$

In these experiments, the sounds are heard in succession, but an interesting question arises as to the smallest difference in pitch that can be appreciated by the ear when beats are produced by two tones sounding at the same moment.

Beats.--If we simultaneously sound two forks that are in unison, say two forks $u t_{4}$, the waves coincide and one sound is heard. Let us then place on one of the forks, near the end of one of its prongs, a brass clip (see above), and sound the fork by bowing. It will be found to be considerably flattened as regards pitch. If we then sound the two forks simultaneously, a rapid, rattling, beating sound is heard, peculiar in its character, unlike a tone, but conveying no idea of two sounds. Let us then slip the elip farther down the prong. On again sounding the two forks, a slow soft throb is heard, and the number of throbs can be readily counted. If we know the pitch of the higher note, the pitch of the lower is at once determined by subtracting the number of beats per second from the number representing the pitch of the higher note. Thus, if one fork makes 100 vibs. while the other makes 101 vibs., then 100 waves from one source are superposed on 101 equal waves from another source. When the two waves are in the same phase, one wave increases the amplitude of the other. But after 50 waves from the first fork have passed, $50 \frac{1}{2}$ waves will have passed from the second fork. The two waves will then be in opposite phases; that is to say, the onward movement of the particles of the first wave will be neutralised by the backward movement of those of the second wave, and the particles will be at rest. This rest may last through several waves, and then the waves again change gradually, until they again coincide after 100 waves have passed from the first fork. The same phenomenon will occur with every 100 waves. There will thus be a waxing and waning of the auditory sensation. ${ }^{3}$ The number of beats per second is equal to the difference of the frequency of the tones. As an interval depends on the ratio of frequencies, it is evident that the number of beats given by two tones nearly in unison will be doubled if the two tones are sounded an octave higher. It is thus possible, by means of beats, to ascertain the sensitiveness of the ear, even to fractions of a vibration per second, and it appears that in no part of the scale can 0.2 vib. per second be distinguished with certainty. The sensitiveness varies with pitch. Thus, at 120 vibs. per second, $0 \cdot 4$ vib. per second; at 500, about 0.3 vib. per second; and at $1000,0.5$ vib. per sec. can be distin-

${ }^{1}$ Preyer, "Physiol. Abhandl.," Jena, 1876; van Schalk, quoted by Raylcigh (op. cit.), fixes the lower limit at 26 vibs., Arch. néerl. d. sc. exactes, tome xxix. p. 87.

² Rayleigh, op. cit., vol. ii. p. 433.

${ }^{3}$ Airy, op. cit., p. 215 ; Rayleigh, op. cit., vol. i. p. 22.

VOL. 1I. -74 
guished. ${ }^{1}$ These results are a remarkable testimony to the sensitiveness of the ear.

König has investigated the phenomena of beats by large tuning-forks specially devised to produce the purest tones by simple pendular movements, and he has found that with such it is possible to obtain primary and secondary sets or orders of beats, and even beats of high orders. When two simple tones interfere, the primary beats belong to either an inferior or superior set, corresponding in number to the two remainders, positive and negative, found by dividing the frequency of the higher tone by that of the lower. ${ }^{2}$ Thus, suppose two tones having frequencies of 40 and 74 . Then $74 \div 40=1+$ a positive remainder of 34 ; or $74 \div 40=2-6$, a negative remainder of 6 . König finds that if two such forks are sounded there are two sets of beats, one at 34 per second and the other at 6 per second. The inferior beat is heard distinctly when its number is less than half the frequency of the lower primary, but if its number is greater, the superior beat comes out the stronger.

Beat tones.-Much discussion has taken place as to what occurs when very rapid beats fall on the ear. Lagrange and Thomas Young held that they blend into a tone, but Helmholtz and others maintain that this is not the case. It is possible to experience a double sensation at the same time, both of a beat tone and of individual beats; just, as in listening to a syren, we may, as it were, feel the puff's and hear the beginning of a musical tone. König has undoubtedly given strong evidence in favour of the existence of true beat tones. He uses pure tones, instead of the mixed tones of the syren and of reeds. These beat tones are best heard when large forks of high pitch are struck. Thus, take $u t_{6}=2048$ and $r e_{6}=2304$. Then $2304-2048=256=u t_{3}$. Strike $u t_{6}$, then $r e_{6}$, and $u t_{3}$ sounds. This is an example of the grave or inferior beat. Again, take $u t_{6}=2048$ and $s i_{6}=3840$. Then $(2048)_{2}-3840$ $=256$, a negative remainder $=u t_{3}$, as before. Sound both forks and $u t_{3}$ will be heard. Again, take $u t_{6}=2048$ and $s o l_{6}=3072$. Then $3072-2048=1024=u t_{5}$. Sound $u t_{5}$ separately, so that the sound may catch the ear. Then sound $u t_{6}$ and $s o l_{6}$, and we hear $u t_{5}$ ringing out.

Strong as this evidence appears to be, it does not satisfy some observers. Lord Rayleigh, ${ }^{3}$ in particular, declares that the question "must still be regarded as an open one," and he states that no evidence has been adduced that the intensity of the beat tone is proportional to that of the generators.

Audibility as affected by intensity.-In considering the action of sound on the minute mechanisms in the cochlea, it is important to take into account the degree of sensitiveness of the ear, both as to amplitude and as to energy of vibration. When an open organ-pipe is sounded, there is a condensation and rarefaction of the air, and there is a position of maximum condensation, data regarding which can be determined experimentally. From these data, Töpler and Boltzmann ${ }^{4}$ concluded that "plane waves, of pitch 181, of which the maximum condensation is $6.5 \times 10^{-8}$, are just audible." These observers also stated the matter thus: The ear is affected by vibrations of molecules of the air

\footnotetext{
1 Preyer, op. cit. ; Ellis, Proc. Mus. Assoc., 1877, p. 1.

2 König, "Quelques expériences d'acoustique," Paris, 1882, 1. 87. An excellent account of König's researches is given by Silvanus P. Thompson, Notices Proc. Roy. Inst. Gr. Britain, London, June 13, 1890.

${ }^{3}$ Rayleigh, op. cit., vol. ii. p. 469.

${ }^{4}$ Ann. d. Phys. u. Chem., Leipzig, 1870, Bd. cxli. S. 321.
} 
not more in amplitude than $0004 \mathrm{~mm}$. at the ear, or $0 \cdot 1$ of the wave length of green light, and the energy of such a vibration on the drumhead is not more than $\frac{1}{543}$ billionth kilo., or $\frac{1}{17}$ of that produced upon the same surface of the retina by a single candle at the same distance.

Lord Rayleigh has investigated the question by two methods. ${ }^{1}$ By the first, he estimated that the amplitude of the movement of the aerial particles, with a sound just audible, "was less than the ten-millionth of a centimetre," and that under favourable conditions "an amplitude of $10^{-8} \mathrm{~cm}$. would still have been audible." 2 By the second method, he not only determined the amplitude, but he estimated the energy emitted when the sound was just becoming inaudible, at $42 \cdot 1$ ergs per second. Further, in considering the amplitude or condensation in the progressive aerial waves, at a distance of $27 \cdot 4$ metres from the fork, in the second experiment, he states that $s$, the maximum condensation, $=6.0 \times 10^{-9}$, a result showing "that the ear is able to recognise the addition and subtraction of densities far less than those to be found in our highest vacua." Lastly, Lord Rayleigh contrasts the energy-emission of a source of light with that of the fork in the second experiment, and arrives at a conclusion similar to that of Töpler and Boltzmann, namely, "that the streams of energy required to influence the eye and ear are of the same order of magnitude." ${ }^{3}$

The analytic properties of the ear.-When we listen to musical sounds, even the untrained ear perceives that they may differ much in character although they may be of the same pitch. Thus the note $c$ as produced on a violin, a harp, a tuning-fork, a clarionet, an oboe, or by the human voice, gives rise to a peculiar sensation, characteristic of the instrument. No doubt this may be partly due to certain noises often produced by the particular instrument; but these noises quickly fade away and disappear as we move away from the source of sound, while the character of the sound still remains. It is well known that physically the waves of such sounds are not simple pendular vibrations, but are more or less complex vibrations, produced by certain tones of shorter wave-length being combined with the first or fundamental wave. When we listen to certain sounds having a character or quality, even the inexperienced ear can detect the fact that the sensation is not that of listening to one sound but to a mixture of sounds, higher in pitch than the tone that seems to give pitch to the sound. For example, if we bow $u t_{2}$ somewhat roughly, we hear, in addition to the tone of the fork, certain high ringing tones which rapidly die away. These tones are peculiar, and do not belong to the harmonic scale of the fork. The forks examined by Helmholtz showed a frequency of the first partial from 5.8 to 6.6 times that of the proper tone. ${ }^{4}$ When we listen to the syren, we are also often conscious of tones much higher in pitch than those accounted for by the stop we happen to be using and the speed of the syren disc. If we sound strongly a reed having a frequency of 64 vibs. per second, as in Appunn's apparatus, and place a large zinc cone over the reed, we hear a vast number of tones, all of a pitch above 64 vibs. per second, and if we sing a note or series of notes while the reed is sounding, we have the curious sensation of always being in harmony with

1 Proc. Roy. Soc. London, 1877, vol. xxvi. 1. 248 ; London, Edinburgh, and Dublin Phil. Mag., London, 1894, vol. xxxviii. p. 366.

2 Rayleigh, op. cit., vol. ii. p. 435.

3 Ibid.; footnote, p. 438.

${ }^{4}$ Ibid., vol. i. p. 59. 
the tones of the reed. These experiments show-(1) That many sounds which an unobservant person might consider simple are in reality complex; and (2) that the ear (and by this term we mean, at present, ear, nerve, and brain) has a power of analysis, or of resolving a compound tone into its constituents.

Physically, such a compound tone is built up of a fundamental or prime tone, and of other tones known as over-tones, over-partial tones or harmonics. Taking the tuning-fork $\left(u t_{2}\right)$ in the series as the prime tone, the partials are the following:-

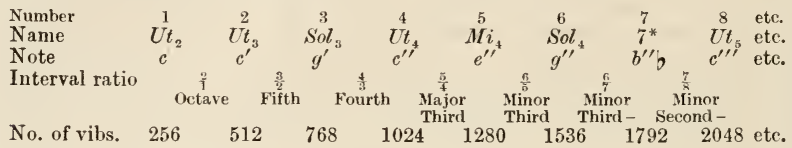

The upper partials become more and more faint, and pass on to tones naking $9,10,11$, etc., times the number of vibrations of the prime tone.

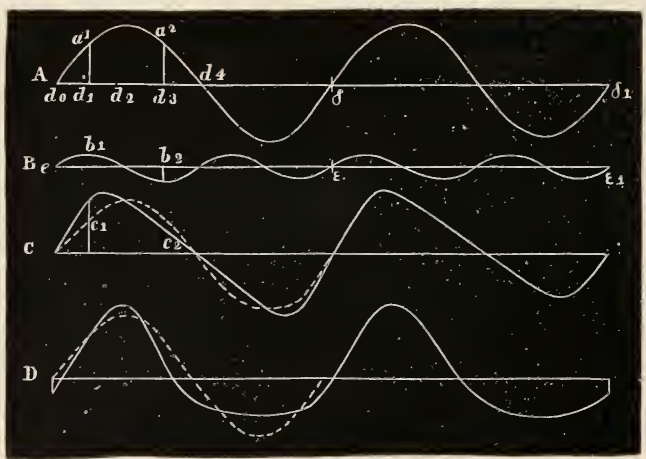

FIG. 423. - Various forms of wave-curves.

If we sound all these forks together, the motion is periodic but is compounded of the motions represented by the waves of each of the partials; as, when two or more sources of sound move the air simultaneously, the resulting disturbance must be the sum of what would be caused by each source acting separately. The scnsation is that of a compound musical tone having a period (and therefore pitch) of the lowest or prime tone. The sensation, however, is not simple, but it is capable of analysis, when, by an effort at attention, we sift out its constituents. This process of resolution is easier for certain persons than for others, but even those who are not musical soon develop the power. As well put by Helmholtz, the power of analysis largely depends on attention. In ordinary life it is not necessary thus to analyse compound tones, and indeed it would be a distraction to do so, as our pleasure from musical sounds does not spring from this analysis but from a general recognition of their character, pitch, sequence, and harmony.

Thus we find two classes of tones-(1) Those incapable of further 
analysis, and therefore termed pure or simple tones; and (2) those that may be resolved into partials or compound tones. As first shown by Ohm, the only form of vibration incapable of being resolved into simpler constituents is that of a pendulum or tuning-fork, and the curve so obtained is the curve of sines. ${ }^{1}$ Such a curve is seen in Fig. $423, \mathrm{~A}$. It represents the pendular motion of the air in the external meatus excited by a tuning-fork in motion. The horizontal line represents time, while the ordinates $\left(d_{1}, d_{2}\right)$ show the amount of displacement of the air. Suppose now another tone, represented by curve $B$, an octave above the first, is sounded, there will then be two vibrations of $\mathrm{B}$ for one of $\mathrm{A}$. It will be seen that by superposing $\mathrm{B}$ on $\mathrm{A}$, so that $d_{1} a_{1}$ in $\mathrm{A}$ is added to $b_{1}$ in $\mathrm{B}$, the ordinate $c_{1}$ in $\mathrm{C}$ is obtained, and by subtracting $b_{2}$ in $\mathrm{B}$ froni $d_{3} a_{2}$ in $\mathrm{A}, c_{2}$ in $\mathrm{C}$ is obtained; and so on with reference to other points, until the curve (C) represents the combination of $\mathrm{A}$ and $\mathrm{B}$. It will be seen that $(C)$ is periodic, and that its periods are those of $A$; or, in other words, the motion of the air produced by two tones (A and B) is also periodic, because one of the simple tones makes twice as many vibrations as the other in the same time. Now suppose that $e$ in $B$ were moved to the right so that $e$ was below $d_{1}$ in $A$, then the compound curve would be that shown in $D$. Thus, by combining $A$ and $B$ in various ways, many curve forms might be obtained, but these could always be resolved into $\mathrm{A}$ and $\mathrm{B}$, in which the frequency of $\mathrm{B}$ is always double that of $\mathrm{A} .{ }^{2}$ These curves represent the motions of the air when two tuning-forks, say $u t_{3}$ and $u t_{4}$ are sounded, or when a flute is gently blown so as to give a note of that pitch. But the ear can readily hear the tones of the

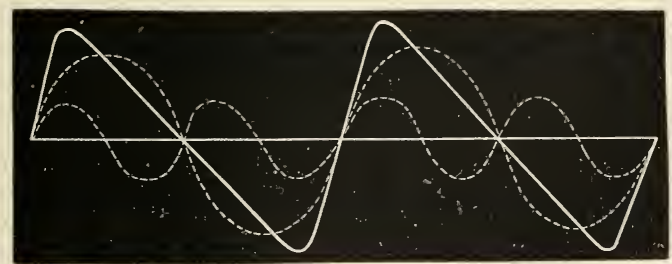

FIG. 424. - Form of wave produced by combining two simple waves.

two forks. It is more difficult to analyse the sound as produced by the flute, but if we assist the ear by using two resonators, one tuned to $u t_{3}$ and the other to $u t_{4}$, all difficulty disappears, and we can analyse the somnd. The arjoining figure (Fig. 424) shows (the thick line) the resultant wave -produced by superposing a tone and its octave. Now, just as the ear can pick out different musical tones when several instruments are sounded, so in favourable conditions it can resolve a musical tone into a series of partials. This power is succinctly expressed in Ohm's law as follows: Every motion of the air which corresponds to a composite mass of musical tones is capable of being analysed into a sum of simple pendular vibrations, and to each such single vibration corresponds a simple tone, sensible to the ear, and having a pitch determined by the periodic time of the corresponding motion of the air. ${ }^{3}$ But we have seen

\footnotetext{
${ }^{1}$ Helmholtz, op. cit., p. 35 ; Rayleigh, op. cit., vol. i. p. 17.

2 Helmholtz, op. cit., p. 46 . 3 lbid., p. 51.
} 
that, by combining tones that differ in phase and in amplitude, we can produce a great variety of wave-forms. These wave-forms, however, can always, according to Fourier's mathematical law, be resolved into the constituents. This law is expressed as follows: Any given regular periodic form of vibration can always be produced by the addition of simple vibrations having vibrational numbers, which are once, twice, thrice, four times, etc., as great as the vibrational number of the given motion. Applying this to the motion of the air close to the ear, we find that any such motion, corresponding to a musical tone, may be always, but for each case only in a single way, shown to be the sum of simple motions, corresponding to the partial tones of this musical tone. ${ }^{1}$

The interesting question now arises whether the ear, having to deal with waves varying almost infinitely in form, is differently affected by such waves, according as their form represents various modes of pressure (pushes and pulls) on the drum-head and conducting mechanism. Suppose we sound the harmonic series of forks from $u_{2}$ to $u t_{5}$, we hear a rich harmonious sound, and we can analyse the sensation, and pick out the tone of any particular partial, more especially if it is slightly strengthened by a touch of the bow. By varying the order and the intensity of the partials, we can produce a very large number of waveforms, but the general character of the harmonious sound, so far as we can judge, remains the same. This would appear to indicate, with an experiment comparatively so rough, that the ear takes no cognisance of phase. Helmholtz investigated the question in a similar way, by means of his vowel tone apparatus. ${ }^{2}$ This apparatus consists of a harmonic series of forks, electrically driven, and so arranged that they can be sounded in any order and with various intensities, according to changes produced in the size of the orifices of their appropriate resonators. ${ }^{3}$ The resonators are thus put slightly out of tune, their resonance is weakened, and thus the phase is altered. Helmholtz stated the result as follows:-Differences in musical quality of tone depend solely on the presence and strength of partial tones, and in no respect on the differences in phase under which these partial tones enter into composition.

It must be admitted that this mode of investigation is not satisfactory, inasmuch as when, in successive experiments produced by pressing on the keys of the instrument, we apparently have the same tonal result, we cannot be sure that there is no difference in sensation, as we have no criterion by which to judge. We apparently hear the same compound tone, but it may be slightly different from the one we just heard an instant before. Accordingly, it is better to investigate this question by experiments with intervals slightly inharmonious. This has been done by Lord Kelvin. ${ }^{4}$ Suppose we take the two forks, $u t_{2}=256$ and $u t_{3}=512$, and sound these together, we have a sound composed of a tone and its octave. If, then, we slightly Hatten $u t_{3}$, so that its vibration number is 510 , we hear, not a roughness corresponding to 254 beats, but a slow beat of only 2 per second. The sensation is

${ }^{1}$ As to Fourier's theorem, see Donkin's "Acoustics," pp. 60-66; O'Kinealy, London, Edinburgh, and Dublin Phil. Mag., London, vol. xlviii. p. 95 ; Ohm's "System der Mathematik," Bd. ix. S. 286 ; Airy, op. cit., p. 160 ; Rayleigh, op. cit., vol. i. pp. 118, 202.

2 Helmholtz, op. cit., p. 606. Apparatus shown in M'Kendrick's "Physiology," vol. ii. p. 691 .

${ }^{3}$ Helmholtz, op. cit., p. 174.

${ }^{4}$ Proc. Roy. Soc. Edin., 1878, vol. ix. p. 602 ; also "Popular Lectures and Addresses," vol. ii. p. 395 . 
that of an interval with a certain relation of phases, but after a short period the phase relationship will change and will pass through every possible value. The cycle is audible; there are beats on the imperfect harmony. Now it might be said that these beats were produced between 510 and the first partial of $u t_{2}$, namely, $u_{3}=512$, but as the beats are always most distinct when $u t_{2}$ is sounded very feebly, this appears to be out of the question. In like manner, beats may be heard on the approximate harmonies, $2: 3,3: 4,4: 5,5: 6,6: 7,7: 8,1: 3,3: 5$, and they all "fulfil the condition of having the whole period of the imperfection, and not any submultiple of it, for their period." It is also easy to produce beats on a major chord in which one of the forks is slightly flattened. For example, sound $u t_{3}, m i_{3}$, and $s o l_{3}=4: 5: 6$; then slightly flatten $\mathrm{sol}_{3}$ or $m i_{3}$, and a peculiar beat will be heard as if a wheel were being turned against a surface, one small part of which was rougher than the rest; the effect is always loudest when the tone of the forks is allowed to almost die away, and one of the forks is then gently touched with the bow.

From the point of view of these investigations, the question under discussion assumes an aspect different from that present to the mind of Helmholtz. A harmony (by which we mean an octave, fifth, major chord, etc.) is a sound which, without being a simple tone, has the variations of air pressure strictly periodic, and, according to Fourier's harmonic analysis (analysis of periodic variations), any periodically varying quantity may be looked on as the sum of quantities, varying separately according to the simple harmonic law, in periods respectively equal to the main period, half the main period, a third of the main period, a fourth of the main period, and so on. Thus, the variation of the air pressure of a harmony is the sum of the variations of simple tones, one having a period equal to the period of the harmony, a second one-half, a third one-third, and so on. A harmony is therefore composed of simple tones, if produced by such instruments as well-bowed tuningforks. On listening to such a complex tone or sound, a tone may predominate in the sensory impression, and the pitch of the sound is referred to the main period of this tone, the other tones merely giving a special character to the sound. In musical harmony, however, one tone does not thus predominate, and a sound of two, three, or four, or more tones, having commensurable periods, is heard, and the pcriod of the harmony is the least multiple of the pcriod of its constituent tones. The harmonic number of a tone in a harmony is denoted by the number of times that the period of the harmony contains this particular tone. Again, the quality or character of a harmony depends on the amplitudes of the tones forming it, the frequency period of these tones, and the relation of their phases. It is most important to observe the effect of phase. Thus the instants of maximum pressure or of minimum pressure may coincide, or there may be a coincidence of the maximum pressure of one tone with the minimum pressure of the other. If, now, the tone of a perfect binary harmony be very slightly sharpened or flattened, and if the two tones are sustained so as to secure perfect uniformity as to pitch and intensity, the effect is an imperfect harmony, with a slow changc of phasc relation through a cycle, and there is a variation of the quality of the tone recurring periodically on the imperfect harmony. This is the beat in the imperfect harmony, and it indicates that the ear does distinguish between an increase of pressure on the drum-head and 
diminution of pressure, or between a push and a pull ; or, in other words, it is affected by phase. ${ }^{1}$ The fact, however, that it is affected by phase, does not greatly interfere with the analytic power already discussed, because when one listens to such an imperfect harmony with appropriate resonators, one can still resolve the harmony into its constituents.

S. P. Thompson ${ }^{2}$ has discussed experimentally certain points regarding phase. By leading the sound of a tuning-fork through a tube to each ear separately, so that the phases could not mix in the air, and in such a way that the sound waves struck each tympanic membrane in exactly opposite phase simultaneously, the sound seemed to be localised at the middle of the back of the head. If, on the other hand, the sound-waves struck each tympanic membrane in the same phase simultaneously, the sound appeared to be localised in the ears.

Again, if the sounds of two tuning-forks, which, when sounded together, gave beats, were led to the two ears separately, in such a way that they could not mix in the air, beats of great intensity were still perceived and appeared to be localised in the middle of the back of the head. In this experiment the peculiar fact was noticed, that the silences were not true ones, but rather that the sound appeared to be going on all the time. Interference must therefore have occurred within the ear, or in the nerve-paths, and thus it is obvious that the ears can appreciate difference of phase.

During recent years some experimenters ${ }^{3}$ have attributed these "subjective beats" to interference occurring, not in the ear itself or nerve-paths, but in the bones of the head. If the latter view is correct, then the experiments described above cannot be considered as proving that the ear or ears are able to appreciate difference of phase.

The question of the influence of phase has also been investigated by König, ${ }^{4}$ by means of an instrument termed a wave-syren, an invention of his own; and there can be no doubt that his observations are not only of great interest, but that they present the greatest difficulties in the way of the theory of Helmholtz. He first drew to scale the curves obtained by compounding partials up to the tenth member of the series; and he modified the curves so that they were compounded first with zero difference of phase, then with all the upper members moved one quarter, then with a difference of half a wave, and lastly with a difference of three-quarters. The sounds of all these curves, according to Helmholtz, should be exactly alike, although they differed in form and position. These curves were cut out on the circumference of metallic cylindrical hoops, which were then mounted on an axis and rotated rapidly. Against these toothed edges (or edges showing sinusoidal curves) air was blown under pressure through narrow slits, as the curves passed in front of the slits; and thus sounds varying in phase from a quarter to three-fourths of the wave-length were obtained. It was found that they varied in quality; with a phase of a $\frac{1}{4}$, the sound was loud of a peculiar shrill quality ; those of $\frac{3}{4}$ difference were gentle and soft; while the curves of 0 and $\frac{1}{2}$ had a quality intermediate in character. These were no doubt minor differences, and, according to Lord Rayleigh, "the results are in harmony with the view that would ascribe the departure from Ohm's law involved in any recognition of phase relations to secondary causes." 5 It is clear, however, that the influence of phase is not so absolutely negative as Helmholtz supposed.

The phonograph, better than any other instrument, illustrates many of these phenomena. The marks on the wave-cylinder correspond to the pitch,

${ }^{1}$ Kelvin, op. cit.; see also, at the end of the paper, eurves showing the variations of pressure with imperfect harmonies.

${ }^{2}$ London, Edinburgh, and Dublin Phil. Mag., London, 1877, vol. iv. p. 274 ; 1878, vol. vi. p. 383 ; 1881 , vol. xii. p. 351 .

${ }^{3}$ K. L. Schäfer, Arch.f. d. ges. Physiol., Bonn, 1895, Bd. lxi. S. 544.

+ Konig, "Quelques expériences d'acoustique," Paris, 1882, p. 236.

- Rayleigh, op. cit., vol. ii. p. 469. 
intensity, and character of the sounds given to the phonograph, and which are reproduced with remarkable fidelity. The number of marks in a given time determines pitch; the depth of the marks, amplitude or loudness; and the character or form of the mark, to the character or quality of tone. These marks can now be carefully traced and analysed. ${ }^{1}$

Resonance.-The principle of resonance is of great importance, both as explaining the mode of working of resonators employed in the analysis of compound tones, and as suggesting the probable mode of action of the cochlea. The material systems with which we have to do in this question may be subjected to various modes of vibration, all or any of which may coexist at any particular moment. Thus a system may show free or forced vibrations. The period of a free vibration depends on the constitution of the system itself; the vibration is that made by the system when disturbed from the position of equilibrium and left to itself. A forcer vibration, on the other hand, has a period determined solely by the external force acting on the system. So long as the external force acts, the forced vibration continues, but a free vibration quickly dies away. Further, a vibrating system of one degree of freedom may have the amplitude of its movements reduced by damping. Damping will soon extinguish a free vibration, and its influence is felt on a forced vibration, when there is an approach to isochronism. Now, when a forced vibration is excited in any one part of a system, all the other parts are also influenced, and a vibration of the same period is excited, whose amplitude depends on the constitution of the system as a whole. $^{2}$ If a part of the system is specially affected within a certain limit of amplitude, it is in the position of a system having one degree of freedom acted on by a given force and independent of the natural period. Resonance usually occurs when there is an approximate equality of periods between the vibrating body and the resonator. In some cases, the amplitude within which resonance is possible may be considerable; in others, very small; and much depends, as regards delicacy of resonance, on the degree of damping that may be called into play. The best example of resonance may be given by taking two forks in perfect unison, say two forks, $u t_{4}$; then, when one fork is bowed, the other, placed in any part of the room, will respond; while a slight change of pitch (produced by fixing a slider on the limb of one of the forks) will prevent response, even although the deviation from unison may cause only one beat per second. Tuning-forks are susceptible of sympathetic vibration to a remarkable degree, notwithstanding the difficulty of setting their mass in motion, because they admit of a long accumulation of minute impulses; but for this reason there must be precise agreement between the pitches of the two forks. It is also observed that, if a fork is thus set agoing, it continues sounding for a considerable time. On the other hand, stretched membranes or strings give forth tones that rapidly diminish in intensity, and they are readily set into sympathetic vibration, which, however, is not limited to a particular pitch. Consequently, such bodies may be influenced by several tones. If a body has a vibration that dies away quickly, say after 8 or 10 vibs., it is not necessary that the next impulses acting upon it should be of exactly the same period as those producing the first movement.

${ }^{1}$ See "Voice Production" in the following article of this volume, alsn the references therein given.

${ }^{2}$ Rayleigh, op. cit., vol. i. p. 70. 
Helmholtz has shown in a table the difference in pitch in terms of an equally tempered tone ( $\frac{1}{6}$ of an octave) necessary to reduce the intensity of sympathetic vibration to $\frac{1}{10}$ of that produced by perfect unison $(A)$, and the number of vibrations after which the intensity of tone, in a body whose sound is allowed to die out, diminishes to $\frac{1}{10}$ of its original amount $(B) .^{1}$ The table is as follows:-

A.

$\begin{array}{lrr}\text { 1. } & \frac{1}{8} \\ 2 . & \frac{1}{4} \\ \text { 3. } & \frac{1}{2} \\ \text { 4. } & \frac{3}{4} \\ \text { 5. } & 1 \\ \text { 6. } & 1 \frac{1}{4} \\ \text { 7. } & 1 \frac{1}{2} \\ \text { 8. } & 1 \frac{3}{4} \\ 9 . & 2\end{array}$

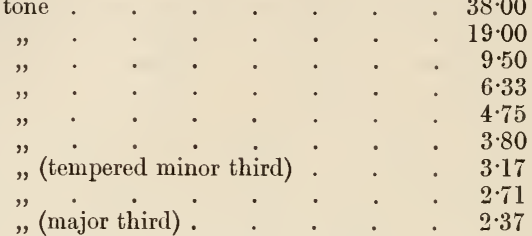

$B$.

$38 \cdot 00$

$9 \cdot 50$

$6 \cdot 33$

$\cdot 75$

$3 \cdot 80$

$2 \cdot 71$

$2 \cdot 37$

That is to say, suppose a body were started into vibration by an exact unison, and that the resonance was reduced to $\frac{1}{10}$ of its original amount, 38 vibs. would be executed before the intensity of the free vibration was reduced to $\frac{1}{10}$ in the case of $\frac{1}{8}$ tone, and so on. Now, if the ear required 38 vibs. before the sympathetic resonance was reduced $\frac{1}{10}$, as the time occupied for these vibrations for $A$ would be $\frac{1}{3}$ second, for $a \frac{1}{6}$ second, for $a^{\prime} \frac{1}{12}$ second, it is evident that such a state of things would disturb musical effect, because the first note would not have died away before the end of the second note. This would certainly occur if we executed a shake on a piano of eight or ten notes a second, so that each note would be sounded four or five times. But it is well known that below $A$ the sensation excited by such shakes is rough and unpleasant, indicating, in the opinion of Helmholtz, "that the vibrating parts of the ear are not damped with sufficient force and rapidity to allow of successfully effecting such a rapid alternation of tone." Now, if a body is thrown into vibrations by sympathetic resonance, it has the vibration frequency of the exciting tone, but when the exciting tone ceases, it goes on moving at the rate of its own proper tone. If, then, the ear vibrated as a single system, and continued its movements for a sensible time, it would move at its own rate, which, as has been shown, is independent of the rate of the exciting tone. Thus two tones of a shake, either in high or low tones, would not mix, but they might blend with that of a third tone, that of the ear itself, said by Helmholtz to be between $e^{\prime \prime \prime \prime}$ and $g^{\prime \prime \prime \prime}$, or, say, $f^{\prime \prime \prime \prime}$.

To set this aspect of the question in a clear light, we cannot do better than quote from Helmholtz. ${ }^{2}$ "Now, if a shake of 10 notes in a second be made on A, of which the vibrational number is 110 , this tone would be struck every $\frac{1}{5}$ of a second. We may justly assume that the shake would not be clear, if the intensity of the expiring sound were not reduced to $\frac{1}{10}$ of its original amount in this $\frac{1}{5}$ of a second. In this case, after at least 22 vibs., the parts of the ear which vibrate sympathetically with A must descend to at least $\frac{1}{10}$ of their intensity of vibration as their tone expires, so that their power of sympathetic vibration cannot be of the first degree in the table, but may belong to the second, third, or some other higher degree. That the degree cannot be very much higher, is shown, in the first place, by the fact that shakes and runs begin to be difficult, even in tones which do not lie very low. . . . We may, on the whole, assume that the parts of the ear which vibrate sympathetically have an amount of damping power corresponding to the third degree of our table, where the intensity of sympathetic vibration with a semitone difference of pitch is only $\frac{1}{10}$ of what it is for a complete unison."

1 Helmholtz, op. cit., p. 213 ; also Rayleigh, op .cit., vol. i. p. 52, where the mathematical theory of damping is fully explained.

2 Helmholtz, op. cit., p. 215. 
Probable action of the cochlea.-We have already seen that the impact of sound-waves must produce a variable pressure in the fluids in the internal ear. Each movement inwards of the base of the stapes, acting like a piston, must cause a pressure in the perilymph, which must pass up the scala vestibuli. This pressure must act on the ductus cochlearis, pressing it from the membranc of Reissner down on the membrana basilaris to the fluid in the scala tympani; and, finally, it must cause a movement outwards of the membrane covering the fenestra rotunda. It is not necessary to consider that a wave will pass through the helicotrema from one scala to the other, as it is clear that any pressure in the scala vestibuli must act in the manner indicated. In all probability, the helicotrema serves the office of equalising pressures between the two scalæ, just as the aqueductus vestibuli does for the fluids in the saccule and utricle, and the canalis reuniens for the fluids in the saccule and the ductus cochlearis. These variations of pressure will be made with each movement of the stapes corresponding to a vibration, and it is evident that the pressures may vary in number, in intensity, and in character in a way exactly corresponding to the pitch, intensity, and quality or character of tones. Such being the case, there are only three ways in which the ductus cochlearis, which contains the nerve-endings, may be affected. Either (1) small vibratile bodies may exist between the pressures sent into the organ and the filaments of the auditory nerve, each vibratile body having a frequency period of its own; or (2) individual nerve fibres may be directly excited by waves of a definite period; that is to say, there may be differences in the nerve fibres, so that they have a selective action ; or (3) the organ may be affected as a whole, all the nerve fibres being affected by any variations of pressure, and thus the power of analysis, which is admitted, is relegated from the peripheral to the cerebral organs.

The first hypothesis seems a priori to be probable, for the following reasons :-(1) The existence of such bodies would give a natural explanation of many if not all of the phenomena; $(2)$ the evidence of comparative physiology points to a gradually increasing complexity in the structure of all the terminal organs of special sense, as if there arose a necessity for differentiation and discrimination in the effects of various kinds of stimuli; and (3) investigations into the action of all the sense organs, such as those of touch and temperature in the skin, of light and colour in the retina, of taste in the tongue, and of smell in the olfactory region-all indicate specialisation of function in the peripheral apparatus. It is not necessary to enlarge on the general evidence indicated under (2) and (3).

The theory that vibrators exist in the cochlea is associated with the name of Helmholtz, because, although it was a conception that flitted before the minds of Thomas Young, Johannes Miiller, and others, it was first clearly enunciated and discussed by him. ${ }^{1}$ It may be shortly stated as follows :-(1) In the cochlea there are vibrators, tuncd to frequencies within the limits of hearing, say from 30 to 40,000 or 50,000 vibs. per second. (2) Each vibrator is capable of exciting its appropriate nerve filament or filaments, so that a nervous impulsc corresponding to the frequency of the vibrator is transmitted to the brain, not corresponding, necessarily, as regards the number of nervous impulses, but in such a way that when the impulses along a particular nerve fibre reach the brain, a

${ }^{1}$ Helmholtz, op. cit., pp. 217-226. 
state of consciousness is aroused which does correspond with the number of the physical stimuli and with the period of the auditory vibrator. (3) The mass of each vibrator is such that it will be easily set in motion, and after the stimulus has ceased it will readily come to rest. (4) Damping arrangements exist in the ear, so as to quickly extinguish movements of the vibrators. (5) If a simple tone falls on the ear, there is a pendular movement of the base of the stapes, which will affect all the parts, causing them to move; but any part whose natural period is nearly the same as that of the sound will respond on the principle of sympathetic resonance, a particular nerve filament or nerve filaments will be affected, and a sensation of a tone of a definite pitch will be experienced, thus accounting for the discrimination of pitch. (6) Intensity or loudness will of course depend on the amplitude of movement of the vibrating body, and consequently on the intensity of nerve stimulation. (7) If a compound wave of pressure be communicated by the base of the stapes, it will be resolved into its constituents by the vibrators corresponding to tones existing in it, each picking out its appropriate portion of the wave, and thus irritating corresponding nerve filaments, so that nervous impulses are transmitted to the brain, where they are fused in such a way as to give rise to a sensation of a particular quality or character, but still so imperfectly fused that each constituent, by a strong effort of attention, may be specially recognised. The last statement gives an explanation of the analytic powers of the ear.

The structure of the ductus cochlearis meets the demands of this theory. It is highly differentiated, and its parts appear suitable for executing independent vibrations. The minute size of the structures does not present any difficulty; because, however minute the vibrators might be, if they had different periods, they must act in obedience to the same principles of resonance as larger bodies do outside the ear. In 1863 , Helmholtz was of opinion that the different degrees of tension in the arches of Corti indicated capacity for vibrating at different periods. Soon afterwards it was shown by Hasse $^{1}$ that these rods do not exist in birds, animals presumably capable of appreciating tones; and Hensen ${ }^{2}$ pointed out that the membrana basilaris consists of transverse fibres, which vary in length from $0.04125 \mathrm{~mm}$. at the base of the cochlea to 0.495 min. at the hamulus. This led Helmholtz to state that "it is probably the breadth of the membrana basilaris in the cochlea which determines the tuning." 3 He pointed out that the membrane was in a state of considerable tension transversely, while it had only little tension in the longitudinal direction, and that such a membrane had very different properties from that of a membrane which had the same tension in all directions. The membrana, from its structure, behaves like a system of stretched strings, bound together by a semifluid substance. Each string or fibre would act independently of the others, and would be set into vibration by an impulse to the fluid in the scala vestibuli, corresponding to its period. Consequently, if a part of the membrana were called into action, one of its radial fibres, corresponding to the exciting tone, would vibrate, and the vibrations would extend with diminishing strength on the adjacent portions of the membrane. Possibly some of the structures on the surface of the membrane might act as dampers. In this way the parts of the membrane near the

1 " De cochlea avium," Kiel, 1866.

${ }^{2}$ Ztschr. f. wissensch. Zool., Leipzig, Bd. xiii. S. 492.

${ }^{3}$ Op. cit., p. 218. 
fenestra rotunda would be adapted for the higher, while those near the vertex of the cochlea would be suitable for deep tones. Corti's arches are therefore of secondary importance, serving either as supporting structures, or for transmitting vibrations of parts of the basilar membrane to the rows of hair cells placed on their backs. When we take into account the fact that the filaments of the cochlear nerve do not terminate in the rods, nor in the fibres of the basilar membrane, nor even in the hair cells, but, as shown by Retzius, ${ }^{1}$ in fine filaments lying against the hair cells, we must consider how vibrations of the fibres of the basilar membrane could affect these filaments.

The short hairs of the hair cells project through apertures in the membrana reticularis, as if it were necessary to cause any oscillation communicated to the hair cells to be always in the same direction. Resting upon the upper surface of the membrana reticularis is the membrana tectoria; in sections the latter appears often to be separated from the former by a considerable interval; but this is not always the case, and in some sections the two may be seen to be almost in apposition. The question whether the two are in actual contact at any time has not been settled by histologists. Suppose that, in accordance with the view of Helmholtz, a segment of the basilar membrane were thrown into sympathetic vibration, it would move in a direction at right angles to the direction of its fibres. These movements would be communicated to the structures lying on its upper surface; and if we suppose the arches of Corti to be elastic, such movements would be transmitted to the hair cells. These would move in the line of their long axis, -in other words, their hairs would move up and down in the meshes of the membrana reticularis, and strike against the under surface of the membrana tectoria. A reaction would take place from the latter, and thus the delicate nerve-endings between the hair cells would receive pressures corresponding in frequency to the oscillations of the membrana basilaris. In the cochlea of birds and amphibia, the mechanism is practically the same, but in consequence of the membrana basilaris not being highly differentiated, there cannot be the nice appreciation of pitch of tone which exists in the higher animals. There is every reason to suppose that a bird has a power of discriminating pitch only through a narrow range. The number of notes birds use in song is very limited, and even those which can be taught to whistle tunes, like piping bullfinches, can only use a very few tones. Parrots, mocking-birds, and minas no doubt can imitate many tones, and it would be interesting to examine the structure of the cochlea in these birds, an investigation not yet carried out.

The theory of the action of the cochlea above described is supported by the results of certain experiments and by clinical observations; but the parts involved are so minute as to make it difficult to be accurate as to the portion actually destroyed or modified. All such results, therefore, must be received with great caution. The experiments of Baginsky ${ }^{2}$ on dogs show that, when the upper parts of the cochlea are destroyed, deafness for low tones results, while destruction of the basal portions causes deafness for high tones. Stepanoff, ${ }^{3}$ however, could not

${ }^{1}$ Biol. Untersuch., Stockholm, Bd. v. S. 35, Tafel xvii. fig. 4.

2 Sitzungsb. d. k. Akad. d. Wisscnsch., Wien, 1863, S. 685 ; Virchow's Archir, Bd. xciv. S. 65 ; Centralbl. f. Physiol., Leipzig u. Wien, 1888, S. 299.

${ }^{3}$ Monatschr. f. Ohrenh., Berlin, 1888, s. 85 . 
find any loss of perception for low tones, when in guinea-pigs the upper parts of the cochlea were destroyed. He tested hearing by the doubtful expedient of observing a reflex movement of the auricle when a sound was heard, and he found that the animals reacted even after the perilymph and endolymph were flowing out of the broken cochlear apex. This was too rough an experiment, and does not appear to have much value.

Hensen $^{1}$ has demonstrated that in the supposed auditory organ of arthropods certain hairs vibrate in sympathy with certain tones, and not with others. In certain crustacea these hairs number about 468 ; the largest is about $\cdot 72 \mathrm{~mm}$. long, and the shortest $\cdot 14 \mathrm{~mm}$. The hairs vibrated within a range of three octaves. In Mysis, one of the hairs answered strongly to $d=$ and $d \neq$, more weakly to $g$, and very weakly to $G$. Its pitch was between $d^{\prime \prime}$ and $d^{\prime \prime}=$, or the second partial of $d^{\prime}$ to $d^{\prime \prime} \neq$, the third of $g$ to $g \#$, the fourth of $d$ to $d \neq$, and the sixth of $\mathrm{G}$ to $\mathrm{G}=$. Another hair answered strongly to $a \neq$, more weakly to $d \neq$ and $A=$. Its tone, therefore, was about $\alpha \varpi^{2}$

There is a certain amount of clinical evidence on this question. One case is on record where an individual showed during life a total want of perception of high tones, while tones low in pitch were audible. The necropsy showed that there was atrophy of the fibres of the nerve furnishing the lowest whorl of the cochlea. ${ }^{3}$ In boilermaker's deafness the hearing power for high tones is particularly affected. In one such case, a necropsy showed that the lowest whorl was diseased. ${ }^{4}$ It is the general opinion of aurists that in cases of partial deafness from diseases of the inner ear, the loss of audibility is for the higher tones, and, as a rule, postmortem examination shows changes in the basal portion of the cochlea. A case is recorded of a man, æet. 52 , suffering from locomotor ataxia, who was deaf to the ticks of a watch and to ordinary speech. The fork $(c)$ was heard by bone conduction, while $c^{\prime \prime}$ was inaudible. After death the left ear showed the cochlear division of the nerve to be degenerated, and the corresponding organ of Corti also much altered. On the right, however, there still existed a large bundle of nerve fibres at the apex of the cochlea, and the corresponding part of Corti's organ was intact. The rest of the nerve and cochlea were degenerated, as on the left side. ${ }^{5}$

On the other hand, cases have been cited in which the whole cochlea of one side has been exfoliated, and yet the individual was able to distinguish tones with that ear. These are extremely doubtful, and the observation is open to the objection that the patient probably heard, by bone conduction, with the sound ear. There can be no doubt that exfoliation of both cochlex causes complete deafness for tones, although there may still be something like a sensation of sound. ${ }^{6}$

I have constructed a model of the cochlea, consisting of a rectangular box, with glass sides and top, which is completely filled with water. It is divided horizontally by a glass partition, to represent the basilar membrane (it is not necessary to imitate the membrane of Reissner); the upper

1 Hermanu's "Handbuch," 1880, Bd. iii. Th. 2, S. 107; Hensen, "Stud. ueber das Gehörorgan der Decapoden," Leipzig, 1863 ; also Ztschr. f. wissensch. Zool., Leipzig, Bd. xiii. S. 398 .

2 Helmholtz, op. cit., p. 225.

${ }^{3}$ Moos and Steinbrügge, Ztschr. f. Ohrenh., Wiesbarlen, Bd. x. S. 1.

" Habermann, Arch. f. Ohrenh., Wiesbaden, Bd. xiii. S. 1.

${ }^{5}$ Ibid., Bd. xxxiii., S. 2 ; also Centralbl. f. Physiol., Leipzig u. Wien, 1892, S. 776.

${ }^{6}$ Gruber, Allg. Wien. med.Ztg., 1864, Bd. ix. Nos. 41, 43, 45, ; also Bezold, Ztschr. f. Psychol. u. Physiol. d. Sinnesorg., Hamburg u. Leipzig, 1896, Bd. xiii. 
compartment will then represent scala vestibuli and the lower scala tympani. A hole is then cut in the end opposite each scala, and over this hole a thin elastic membrane is stretched. The upper hole is the fenestra ovalis, and the lower the fenestra rotunda. A hole is also cut in the horizontal glass plate, and covered by membrane. This will represent a segment of the basilar membrane. On this membrane a small weight rests, connected with a fine watch-spring, which has its own period of vibration. Then, by means of a horizontal piston, representing the base of the stapes, moved by a wheel, a series of pressures are given to the membrane covering the oval window. With each inward pressure there is a movement downwards of the membrane in the horizontal glass plate, and a movement outwards of the membrane covering the round window. When the piston moves outwards there is a reversal of the movements of the membranes. If now the wheel moving the piston be rotated rapidly, to imitate simple pendular vibrations the steel spring, resting on the membrane, representing a segment of the membrana basilaris, at once begins to oscillate at its own period when the number of movements of the piston reach that period. If they go faster, then the spring soon ceases to oscillate. Suppose the period of the spring to be 12 per second, and another hole to be cut in the horizontal plate, and covered with membrane, on which rests a weight, with a spring tuned to the octave of the first, that is 24 per second. By means of a wheel having an excentric motion, the piston may be caused to execute a compound movement, produced by two movements in the ratio of $2: 1$ to imitate the movements of the base of the stapes when a sound and its octave act upon it. If, now, the wheel be rotated, when it moves at a rate equal to that of the first spring (12 per second), it is seen that both springs are oscillating; that is to say, the compound harmonic motion of $2: 1$ is analysed by the springs, each taking its own period. It would only be a matter of trouble and expense to construct a more complete analyser to imitate the cochlea. ${ }^{1}$

Beauregard and Dupuy ${ }^{2}$ find that when the cochlea is stimulated by sound, a current of action occurs in the auditory nerve. Non-polarisable electrodes are placed on the membrana tympani and the cut surface of the peripheral portion of the auditory nerve. In guinea-pigs the current was much less marked when low tones were used than with high ones. The authors maintain that their experiments prove that sound is analysed in the ear. ${ }^{3}$

An obvious question in discussing the Helmholtz theory of the cochlea is as to whether the histological evidence as to the number of possible vibratile structures is such as will satisfy the demands of theory. Helmholtz attempts to answer this question on the basis of E. H. Weber's statement, that practised musicians can "perceive even a difference of pitch for which the vibrational numbers are as 1000 to 1001 ," or the $\frac{1}{64}$ of a semitone, a smaller interval than that between two of Corti's arches, on the assumption that there are about $33 \frac{1}{3}$ for each semitone in each cochlea; and he accounts for the apparent deficiency by the explanation that if a tone came between the pitch of two of the arches "it would set them both in sympathetic vibration, and that arch would vibrate the more strongly which was nearest in pitch to the proper tone." This would also explain how it is that when we listen

${ }^{1}$ No description of the apparatus has been hitherto published; in its construction I have been much indebted to suggestions made by Professor Crum Brown.

${ }_{2}^{2}$ Arch. internat. de laryngol. etc., Paris, July and August 1896.

3 These observations require repetition. I liave myself often searched for such an action current, but without success. 
to the syren, as its dise revolves faster and faster, our sensations go on not by leaps and bounds but continuously. Since the time when Helmholtz wrote the "Tonempfindungen," histological evidence has accumulated, and now we can give more ample data on which to form an opinion on this question. ${ }^{1}$

Suppose we take the lower limit of perception of tone to be that corresponding to nearly 16 , and the upper limit nearly 50,000 vibs. per second, sensible discrimination of pitch diminishes towards each end of the range, more especially towards the upper limits. By noticing the smallest changes in different determinations, and by the method of computing right and wrong cases, in observations on the comparison of differences, experiments have shown that between 64 and 1024 vibs. per second the least noticeable difference is so small as 0.2 vibs. Kuilpe gives 0.4 at 32 and 0.3 at 2048 vibs. per second. It is a striking testimony to the delicacy of the ear that between 32 and 2048 vibs. per second we can observe a difference of less than 1 vib. per second. Even the most skilled ear cannot observe large differences between 12,288 and 16,384, and it may be safely stated that only about 23 tones can be distinguished beyond a vibration frequency of $4096 .^{2}$ From these data we can compute the number of tones the human ear can distinguish. Thus, within the range of human audition, we can hear about 97 tomes between 16 and 64 vibs. per sec., 4800 between 65 and 1024, 6144 between 1025 and 4096 , and 23 on the upper side of 4097 . This gives a total of 11,064 tones heard by the human ear. It is interesting to notice in this connection that the pitch of the highest tone of the piccolo stop of the organ is 4096 vibs. per sec., while the deepest tone of the contra-bassoon is 27 . These are the highest and the lowest tones used in music.

The dimensions of the ductus cochlearis, the number of the rods of Corti, and the number of hair cells have been determined by competent observers. At my request, Dr. William Snodgrass made transverse sections of the cochlear division of the auditory nerve of an adult who was not deaf and who did not die of a disease affecting the ear, and he carefully counted the number of nerve filaments. ${ }^{3}$ These were found to be about 14,000 . It is obvious that all such estimations must be approximate.

The following shows the general result of computations relating to this question :-

1. Audible Sounds, the Pitch of which can be Discriminated • 11,064

Less those below 64 and above 4096 .

Leaving . $\quad$, . $\quad . \quad 10,954$

Say. $: \quad \cdot \quad \cdot 11,000$

2. Distribution of Sounds in Six Octaves used ix Music$\frac{11,000}{6}=1833$ for each octave $\frac{1833}{12}=153$ for each semitone.

3. Musician's Ear can detect Difference of $\frac{1}{64}$ Semitone$\frac{153}{64}=2 \cdot 4$ for each $\frac{3}{64}$ semitone.

1 M'Kendrick, Trans. Roy. Soc. Kidinburgh, 1896, vol. xxxviii. p. 780.

2 Külpe, "Outlines of Psychology," translated by Titchener, 1895, p. 106.

${ }^{3}$ Result not hitherto publislıed. 
4. Nerve Fibres in Cochlear Divisiox of Auditory Nelive, 14,000.

Range of audition, 11 octaves.

$\frac{14,000}{11}=1273$ nerve fibres for each octave.

Assuming that the number of auditory filaments is the same for each of the eleven octaves -

$1273 \times 6=7638$ for 6 octaves used in music.

$\frac{1273}{12}=106$ nerve fibres for each semitone.

$\frac{106}{64}=$ less than 2 nerve fibres for $\frac{1}{6 \pm}$ of a semitone.

5. Fibres in Membiana Basilahis (Retzius)-

$\frac{24,000}{11}=2182$ basilar fibres for each octave.

$2182 \times 6=13,092$ for 6 octaves used in music.

$\frac{2182}{12}=180$ basilar fibres for each semitone.

$\frac{180}{64}=$ less than 3 basilar fibres for each $\frac{1}{6 \pm}$ semitone.

6. Hair Cells-Inner, 3487 ; Outer, $11,700=15,237$ -

$\frac{15,237}{11}=1385$ cells for each octave.

$1385 \times 6=8310$ hair cells for 6 octaves used in music.

$\frac{1385}{12}=115$ cells for each semitone.

$\frac{115}{64}=7$ css than 2 for cach $\frac{1}{64}$ semitone.

7. Corti's Rods. InNer, 5590 ; OUter, $3848=9438$.

$\frac{9438}{11}=858$ rods for cach octave.

$858 \times 6=5148$ rods for 6 octaves.

$\frac{858}{12}=71$ for each semitone.

$\frac{71}{61}=$ at least one rod for each $\frac{1}{1+}$ semitone. As ench rod forms half of a

61 Corti's arch, there will be nearly one arch for each $\frac{1}{6 !}$.

There are thus in the cochlea a sufficient number of possible vibratile masses to satisfy theory.

Careful measurements of parts of the cochlea have also shown remarkable differentiation, indicating that we have an organ which camnot be supposed to act only as a whole. These measurements show, for example, that the breadth of the basilar membrane increases towards the apex; that the osseous lamina spiralis diminishes in brealth as the basilar membrane increases; that from base to apex, both the

VOL. II. -75 
pillars of Corti lengthen; that from base to apex both inner and outer hair cells increase in size; that from base to apex the hairs increase in length, while the increase is greater in the hairs of the outer cells; and that the membrana tectoria increases in breadth from base to apex. $^{1}$ Further, if we take man, the cat, and the rabbit, all of which presumably hear tones, but probably have very different powers of discrimination of tones, we find remarkable differences in detail, as given in the following table by Retzius : ${ }^{2}-$

\begin{tabular}{|c|c|c|c|c|}
\hline & & Man. & Cat. & Rabbit. \\
\hline Ear teeth . & . & 2,490 & 2,430 & 1,550 \\
\hline Holes in habenula for nerves & . & 3,985 & 2,780 & 1,650 \\
\hline Inner rods . & . & 5,590 & 4,700 & 2,800 \\
\hline Outer rods . & . & 3,848 & 3,300 & 1,940 \\
\hline Inner hair cells (one row) . & . & 3,487 & 2,600 & 1,600 \\
\hline Outer hair cells (several rows) & . & 11,750 & 9,900 & 6,100 \\
\hline Fibres in basilar membrane & . & 23,750 & 15,700 & 10,500 \\
\hline
\end{tabular}

Upper partial tones and the theory of dissonance.-We have seen that a compound tone is built up of a number of partial tones, the frequency numbers of which are multiples of that of the first or fundamental tone. It has also been shown that when two tones sufficiently near in pitch are simultaneously sounded, beats are produced. If such beats are few in number, so as to be readily counted, the sensation of waxing and waning is not disagreeable; but if they are sufficiently numerous it may be impossible to count them, and the sensation is disagreeable. Such a sensation is dissonance. The sensation is most disagreeable when the ear is affected by about 33 beats per second ; if they are more numerous, the sensation is rough and unpleasant. Further, even when the frequency of beats is much greater than the number of vibrations required to produce the sensation of a tone, the sensation is never uniform, but is of a rough intermittent character. In other words, the sensation of a tone cannot be produced by the intermittent impulses on the drum-head, due to beats. If now we sound an interval on an instrument giving forth compound tones, such, for example, as an octave, each tone will have its corresponding partials; and as these come closer and closer together the higher they are in the series, it is clear that they may come within beating distance, and thus give a certain harshness to the sound. The beating distance may, for tones of medium pitch, be roughly fixed at a minor third; this interval, of course, will expand for intervals on low tones, and contract for intervals on high ones. Thus the same interval in the lower part of the scale may give slow beats that are not disagreeable, while in the higher part it may cause harsh and unpleasant dissonance. As a rule, the partials up to the seventh are beyond beating distance, but above this they soon come close together. In the neighbourhood of the tenth, the interval may be about a tone, of the sixteenth a semitone, and still higher they come so close together as to cause dissonance. This fact explains the harsh but brilliant quality of some of the tones of a trumpet. Suppose that one of the tones of the interval is slightly out of tune, then all the partials will be correspondingly affected, and the

1 M'Kendrick, op. cit., p. 782.

2 Retzius, "Das Gehörorgan der Wirbelthiere," Bd. ii. S. 356. 
dissonance will be most disagreeable, even with compound tones having few partials, such as those of the flute, still more with those having numerous partials, like the tones of reeds, as in a harmonium. ${ }^{1}$ Helmholtz has applied this view to an explanation of the satisfying character of certain musical intervals, as contrasted with the discordant character of others. Thus, unison $\frac{1}{1}$, minor third $\frac{6}{5}$, major third $\frac{5}{4}$, fourth $\frac{4}{3}$, fifth $\frac{3}{2}$, minor sixth $\frac{8}{5}$, major sixth $\frac{5}{3}$, and octave $\frac{2}{1}$, are all concords; while a second $\frac{9}{8}$, minor seventh $\frac{16}{9}$, and major seventh $\frac{15}{8}$, are discords. The smoothest interval is the octave, next the fifth, then the fourth, major third, and so on.

There can be no doubt that this explanation satisfies the case, with intervals sounded on instruments giving forth compound tones, but it does not appear to do so when an interval is composed of sounds produced by instruments like large stopped organ pipes, in which there are no over-tones. By careful bowing, few partials may be produced by well-constructed tuning-forks, and these soon die away, leaving the pure proper tone of the fork. In such cases the interval of a minor seventh should be as concordant as that of a fifth, but it is not so. On the theory of the cochlea already described, there is no difficulty in explaining these facts, because, if it possesses analytic powers by sympathetic resonance, it must respond to the prime tones and partials of both of the notes forming the interval. The only thing unexplained is, why the sensation should be disagreeable when two portions of the membrana basilaris sufficiently near are thrown into vibration. The sensation of a beat is not due to the stimulation of one part of the membrana, but the peculiar wave of pressure of two tones, causing a beat, is analysed. While a beat is sounding, listen with an appropriate resonator to one of the constituent tones of the beat, and it will be at once heard. For some unexplained reason, however, if two nerves sufficiently near are simultaneously stimulated, or if they are stimulated in the intermittent manner peculiar to beats, the sensation is disagreeable. Helmholtz compares it with that caused by a flickering light on the eye. Something similar I have found to be produced by simultaneously stimulating the skin, or margin of the lip, by bristles attached to tuning-forks giving both beats. If the frequency of the forks is great, the sensation is that of a most disagreeable tickling. It may be that the instiuctive effort at analysis of tones close in pitch causes the disagreeable sensation.

Combination tones. - The law that the oscillations of elastic bodies, and of the air, produced by several sources of sound, are the sum of the individual motions from each source, only holds good when the bodies are of infinitely small dimensions, and when the vibrations themselves are also infinitely small. If vibratile bodies are not infinitely small, then other phenomena may be observed. Helmholtz ${ }^{2}$ considered these phenomena with reference to the theory of dissonance and the action of the ear. If two tuning-forks, representing a fifth, are properly bowed, sounding the fork of lower pitch first and that of higher pitch afterwards, we may hear a weak lower tone, the pitch of which is an octave below that of the first fork. This is known as a combination tone. Such tones are divided into two classesdifferential tones, in which the frequency is the difference of the frequencies of the generating tones; and summational tones, having a frequency which is the sum of those of the tones producing them.

1 For numerous examples, see Sedley Taylor, "Sound and Music," London, 1873, p. 166, et ssq.; also, Helmholtz, oj. cit., p. 272.

${ }^{2}$ Op. cit., p. 229. 
The following table shows the pitch of several differential tones of the usual intervals : ${ }^{1}$ -

\begin{tabular}{|c|c|c|c|c|c|c|}
\hline \multicolumn{3}{|c|}{ Interval. } & & $\begin{array}{l}\text { Ratio of } \\
\text { Frequency. }\end{array}$ & Difference. & $\begin{array}{l}\text { Combinational Tone is deeper than the } \\
\text { deeper generating Tone by }\end{array}$ \\
\hline $\begin{array}{l}\text { Octave } \\
\text { Fifth } \\
\text { Fourth } \\
\text { Major third } \\
\text { Minor third } \\
\text { Major sixth } \\
\text { Minor sixth }\end{array}$ & $\begin{array}{l}\dot{ } \\
\dot{5} \\
\dot{5} \\
\dot{ }\end{array}$ & $\begin{array}{l}\dot{5} \\
\dot{5} \\
\dot{ }\end{array}$ & $\begin{array}{l}\cdot \\
. \\
.\end{array}$ & $\begin{array}{l}1: 2 \\
2:: 3 \\
3: 4 \\
4: 5 \\
5: 6 \\
3: 5 \\
5:: 8\end{array}$ & $\begin{array}{l}1 \\
1 \\
1 \\
1 \\
1 \\
2 \\
3\end{array}$ & $\begin{array}{l}\text { A unison. } \\
\text { An octave. } \\
\text { A twelftl. } \\
\text { Two octaves. } \\
\text { Two octaves and a major third. } \\
\text { A fifth. } \\
\text { A major sixtl. }\end{array}$ \\
\hline
\end{tabular}

Differential tones, first noticed by Sorge about 1740, are usually associated with the name of Tartini. ${ }^{2}$ Summational tones were discovered by Helmholtz. ${ }^{3}$ It is clear that there must be differential tones of several orders, according as they are produced between the generating tones themselves, between the differential tone and each of the generators, and so on. ${ }^{4}$ It is not difficult to detect differential tones, but this is not the ease with summational tones. Helmholtz heard them first with the syren and harmonium, and afterwards with organ pipes and tuning-forks. On the other hand, Hermann and many others assert that they have not been able to hear these tones. ${ }^{5}$ With Appumn's apparatns, which includes a large series of reeds, which can be sounded with great force, I ean hear them with the use of the appropriate resonator. Without the resonator, they are almost inaudible, sometimes quite inaudible, because, probably, they are lost in the loud-sounding generators. It is clear that a resonator can have no effect on a tone sensation that is purely subjective. This proves that they have an objective existence, and that they are not produced in the ear itself. Some have contender that, as regards differential tones, these may be produced by a blending of beats. This is open to the objections-(1) That it is doubtful if any number of beats can produce a sensation of tone-although it is held by Hermann and other critics that "the ear is capable of recognising as a tone any periodicity within certain limits of frequency "; ${ }^{6}(2)$ that the explanation does not include the existence of summational tones; and (3) that, by using appropriate sympathetic membranes, Helmholtz was able to demonstrate the existence outside of, and quite independently of, the ear, of both kinds of combination tones. Further, Helmholtz states that "whenever the vibrations of the air or of other elastic bodies, which are set in motion at the same time by two generating simple tones, are so powerful that they can no longer be considered infinitely small, mathematical theory shows that vibrations of the air must arise which have the same vibrational numbers as the combination tones." 7

By other experiments, however, it may be shown that, in certain circumstances, combination tones may be produced by the mechanism of the ear itself. If two tones are sounded from sources very close together, the combination tone is strong; but if the sources are wide apart, it is weak or inaudible. Helmholtz held that the unsymmetrical form of the membrana

1 Helmholtz, op. cit., p. 231.

2 Sorge, "Vorgemach musikalischer Composition," 1740 ; Helmholtz, op. cit., p. 229.

${ }^{3}$ A win. d. Phys. u. Chem., Leipzig, Bd. xcix. S. 497 ; Monatsb. Berl. Acad., May 22, 1856. As to the mathematical theory of such tones, see Helmholtz, op. cit., app. xii. p. 621 .

${ }^{4}$ Hallström, Ann. d. Phys. n. Chem., Leipzig, 1832, Bd. xxiv. S. 438.

5 Arch. f. d. ges. Physiol., Bonn, 1891, Bd. xlix. S. 499.

${ }^{6}$ Rayleigh, op. cit., vol. ii. 1). 461; also Hermann, op. cit., S. 514.

${ }^{7}$ Helmholtz, op. cit., p. 235. Forsyth and Sowter have recently furnished evidence of the objective reality of combination tones, Proc. Roy. Soc. London, vol. lxiii. p. 396 . See also Riicker and Edser, London, Edinburgh, and Dublin Phil. May., London, 1895, vol. xxxix. p. 341 . 
tympani favours the production of minute vibrations superposed on those of the membrane proper, and thus gives rise to weak combination tones. The loose formation of the malleo-inculal joint might have a similar effect; that is to say, if acted on by two very powerful tones, minute motions might be produced (correspouding to the pitch of the combinational tones) which would be added to the combined motion of the two generators. This explanation can only be admitted when the generators are loud; but, as Hermann urges, differential tones may be heard when the generators are very weak. Lord Rayleigh supports Helmholtz in the statement that "loud generators are necessary." This is cousistent with the results of experiments made with Appunn's apparatus. It is a reasonable objection, however, to experiments with reeds, that the tone supposed to be a combination tone may really be a loud partial of one or other of the reeds. A combinational tone is well brought out, according to Helmholtz, "when two clear and powerful soprano voices execute passages in thirds."

The importance of these combinational tones in the theory of hearing is obvious. If the ear can only analyse compound waves into simple pendular vibrations, of a certain order, how can it detect combinational tones, which no doubt can be heard, and yet do not belong to that order? Or, the question may be put, Can combinational tones explain consonance and dissonance, produced by tones that are simple, and are destitute of partials? Take the octave, and sharpen the upper note, so that the octave is slightly untrue. Suppose the lower tone has a frequency of 100, and the upper 201 vibs. per second, a combinational tone of 101 is produced, and this, with the lower generator, will produce one beat per second. The beats cannot be avoided, unless the octave is pure. Thus the octave, produced by simple tones, is a concord bounded by discords. Again, investigate a fifth slightly out of tune. Here we have, say, the generators produced by 200 and 301. The combinational tone of the first order is 101 ; it will produce a combinational tone of the second order by the differential tone of the first order, 101, beating with 200 ; the lowest generator thus giving a differential tone of the second order, having a frequency of 99 , and there will also be two beats between the differential tone of the second order, 99, and the differential tone of the first order, 101. Thus a fifth is a consonance less sharply cut off than the octave, owing to the feebleness of the combinational tone of the second order. ${ }^{1}$ Thus we see that combinational tones are produced when the notes of intervals are sounded strongly on instruments free from partials; and that these combinational tones may produce beats with either of the generators, or among themselves; and these beats, feeble as they may be, produce that feeling of less and less consonance, until we come to intervals that are truly dissonant. One might suppose in the case of tones that abound in partials, that combinational tones might be produced by the partials, and that thus a new source of beats might lead to confusion and discord; but theory shows that dissonance due to combinational tones produced between partials never occurs except when it has alrearly taken place by the action of the partials among themselves.

According to this view, combinational tones are not produced by beats, but are true sounds superadded to that of the generators. The ear, therefore, in dealing with them, vibrates in some part of its mechanism with each generator, accorling to Ohm's law, while it is also

${ }^{1}$ For other examples, see Sedley Taylor, op. cit., 1. 181. 
affected by the combinational tone itself, according to its frequency. This view will satisfy the theory both as regards differential and summational tones.

Objections to the theory of Helmholtz.-In addition to those already considered, the following may be mentioned. Rutherford ${ }^{1}$ points out that a compound tone is heard as one sound; and he argues that if Helmholtz were correct, the note is first analysed by the cochlea into its constituents, and is then reconstructed in the brain, a process apparently unnecessary. The obvious reply is that, as a matter of fact, any compound tone may be analysed by the aid of resonators, and if of sufficient intensity, it does not appear to give rise to a simple sensation; but by a strong effort of attention the apparently simple sensation can always be more or less fully resolved into sensations corresponding to the partials. Waller ${ }^{2}$ adduces the result of an experiment of Hermann ${ }^{3}$ as a strong objection. Hermann observed that a beat tone might be heard which did not excite a resonator or fork tuned to its pitch. If such be the case, Waller maintains that it is improbable it could act on the fibres of the basilar membrane, if these were supposed to respond as resonators. No doubt if a differential tone really exists in the air, and is not generated in the ear, and yet is unable to set an appropriate resonator in vibration, the theory of hearing founded on sympathetic resonance would receive a severe blow. It does not follow, however, that in Hermann's observation the resonator was unaffected. It did not respond to such an extent as to enable a tone to be heard, although the ear could hear the true differential tone, if sufficiently near the sources of the sounds. This only proves that, with the means at our disposal, the ear is more sensitive than the resonator. Further, as has already been pointed out, it is possible to tune resonators in such a way as to enable them to respond to differential tones, irrespective of the ear. It has also been shown that combinational tones may be generated in the middle ear, in which case it is obvious they would not act on a resonator. Thus, either on the supposition that such tones are generated in the air, or, on the converse, that they are produced in the middle ear, the experiment does not invalidate the sympathetic theory of hearing. ${ }^{4}$

Other theories.-As opposed to the theory of Helmholtz is the one known as the telephone theory, advocated by Rutherford. Rinne $^{5}$ had already, in 1865 , offered cogent objections to the analysis theory, and had suggested that the cochlea acted as a whole. This view was supported twenty years later by Voltolini, ${ }^{6}$ and, in 1886 , Rutherford ${ }^{7}$ stated it with greater fulness, illustrating the function of the cochlea by the analogy of a telephone, and, in particular, attempting to show experimentally that nervous impulses of different rates of frequency can be transmitted along the auditory nerve.

According to Rutherford, all the hairs of the auditory cells vibrate

1 Rep. Brit. Ass. Adv. Sc., London, 1886.

${ }^{3}$ Arch. f. d. ges. Physiol., Bonn, Bd. xlix. S. 499.

${ }^{4}$ Gray suggests that the intratympanic muscles may act in such a way as to produce differential tones.

${ }^{5}$ Ztschr.f. rat. Med., Dritte Reihe, Bd. xxiv. S. 12-64. An excellent analysis is given in "Die Lehren von den Funktionen der einzelnen Theile des Ohrlabyrinths," by Stanislaus von Stein, trans. from Russian by C. von Krzywicki, Jena, 1894, S. 141.

'Virchow's Archiv, Bd. c. S. 27-41; see also von Stein, op. cit., S. 153.

7 Rep. Brit. Ass. Adv. Sc., London, 1886 ; also Journ. Anat. und Physiol., London, vol. xxi. p. 166 ; see also address on "Tone Sensation," Brit. Med. Journ., London, 1898, vol, ii. p. 353. 
equally to every note, and the hair cells transform sound vibrations into nerve vibrations, or impulses, similar in frequency, amplitude, and character to the sound vibrations. There is thus no analysis of sound in the peripheral organ. Now, there can be no doubt that a wire connecting two telephones, or connecting a microphone transmitter with a telephone receiver, will carry a series of electric impulses, or waves, corresponding in number, form, and character to the waves of musical tones falling on the microphone or first telephone. Thus the tones corresponding to the vibrations of the disc of a phonograph may be transmitted to a telephone, and be there reproduced with remarkable fidelity. The analogy, therefore, between the fibres of the auditory nerve and such a wire is admissible. But the analogy breaks down when we consider the fact of analysis. The distal telephone does not analyse the waves reaching it, and if its movements could be graphically recorded, a matter merely of experimental difficulty, they would reproduce exactly the waves falling on the proximal telephone or the microphone transmitter. When we listen to the distal telephone, we hear all the sounds corresponding to these waves, and we can at once analyse these, sifting the one from the other; that is to say, in the experiment, the ear is, after all, the ultimate analyser. Further, one can conceive a device by which the waves of sound given forth by the second telephone might be analysed, but such a device could only be constructed on the principles of sympathetic resonance.

Waller ${ }^{1}$ proposes to explain the mechanism of the cochlea as follows: The basilar membrane, as a whole, vibrates to every tone, thus repeating the vibrations of the membrana tympani; and since the hair cells move with the basilar membrane, they produce what may be called pressure patterns against the tectorial membrane, and filaments of the auditory nerve are stimulated by these pressures. With the exception of the use of the phrase "pressure patterns," this is much the same as the view of the mechanism of the cochlea already described, except that I hold that, in conformity with Ohm's law, there is an analysis by the cochlea, whereas, according to Rutherford, the tones are not analysed by the cochlea, but by the brain; while Waller's view admits of a certain degree of peripheral analysis (pressure patterns), but also relegates ultimate analysis to the brain.

Judging from the effect of rapid stimuli sent along a nerve to a muscle, it might be supposed that a number of nervous impulses, corresponding to a tone of high pitch, could not call forth a response from the terminal apparatus in the nerve centre at the same rate. For when more than about thirty stimuli are sent to a muscle, it passes into a state of complete tetanus, and no matter how many more stimuli per second are sent along the nerve, the muscle remains in the same state. As Rutherford, ${ }^{2}$ however, has pointed out, although the effect upon the muscle, so far as contraction goes, is the same no matter how rapid the stimuli sent to it may be, provided there are more than 30 per second, yet on listening over the muscle while the nerve is being stimulated, a sound is heard, the pitch of which varies according to the number of stimuli per second sent into the nerve. This had been already pointed out by Lovén, who regarded the notes produced as being due to electrotonic variations in the nerve. Thus, when forty stimuli per second are used, a tone

${ }^{1}$ Proc. Physiol. Soc., Jan. 20, 1891.

${ }^{2}$ Loc. cit. 
is heard having a pitch equivalent to 40 vibs. per second. Rutherford, indeed, found it possible to employ as many as 352 stimuli per second; and, on listening over the muscle, to hear a tone of a pitch of that number of vibrations per second. More than 352 stimuli per second did not produce a tone but only a noise. This, however, does not, according to Rutherford, invalidate his theory, for, as he points out, a muscular fibre is a very different thing from a nerve fibre or a nerve cell, and he is of opinion that stimuli of far greater frequency than that recorded might be transmitted along a nerve. Rutherford further instances the case of the insect's wing, such as that of the honey-bee, in which 460 impulses per second pass along the motor nerves to the muscles of the wing.

The most obvious objection to any theory ${ }^{1}$ which dispenses with peripheral analysis, is that it leaves the exceedingly elaborate structure of the organ of Corti, and indeed of the cochlea as a whole, out of account; or, to put the matter in another light, it assigns to that organ a comparatively simple function (like that of a vibrating membrane), and one which could be performed by a more simple structure. Further, the holder of such a theory, while recognising the analytic powers we undoubtedly possess, must refer these powers to the cortex cerebri, and practically admit that the problem cannot be solved. Ohm's law may be subject to limitations, but at present there is no substitute for it. Moreover, the cochlea becomes more elaborate in all its parts as we ascend the animal scale, until in man, who doubtless possesses greater powers of analysis than any other animal, the number of hair cells, fibres of the basilar membrane, and arches of Corti, are all much increased. Finally, it has been shown that on purely physical grounds the organ of Corti may be considered to be an analytic apparatus, a view consistent with physical theory, and with all we know of the mode of action of the other peripheral sense organs.

A further question, however, arises, namely, as to whether it is possible for the cochlea to analyse a compound tone on any other principle than that of sympathetic resonance. Various attempts have been made to explain its mode of action in another way. Hurst ${ }^{2}$ has suggested that with each movement inwards and ontwards of the stapes, a peculiar wave is generated which travels up the scala vestibuli, through the helicotrema into the scala tympani, and down the basilar membrane to the fenestra rotunda. This wave is not a mere undulation of the basilar membrane, but it causes movements of fluid to and fro in each scala, and these produce a peculiar wave of pressure. As the one wave ascends while the other descends, a movement (or pressure) of the basilar membrane occurs at the point where they meet, and the movement is chiefly in the direction of the tectorial membrane, so that this membrane strikes suddenly on the hair cells and thus irritates the nerves. The point at which the waves meet will depend upon the pitch of the note, or, in other words, upon the time interval between the two waves. In this way, and without sympathetic resonance, the cochlea would, within limits, respond to tones of different pitch. The intensity of the movement of the tectorial membrane against the hair cells would, of course, correspond to intensity of tone. The mode of action of the cochlea as an analyser is not, I think, sufficiently explained by this theory. Thus, if $e^{\prime}$ and $g^{\prime}$ be sounded, we have a fifth, with a vibration ratio of $\frac{3}{2}$. A compound wave-movement is transmitted, and this

${ }^{1}$ A full statement of all the speculations of physiologists as to the cochlea will be found in von Stein's book, op. cit., S. 138-155.

2 "A New Theory of Hearing," Trans. Liverpool Biol. Soc., 1895, vol. ix. pl. 321-353. 
compound movement must affect the membrana basilaris. Even supposing Hurst's theory is correct, this movement, or its effects, must pass up the membrane of Reissner and down the basilar membrane, but how does this explain the fact of analysis? Further, as Hurst himself observes, accorling to his theory, the "region of the cochlea where the stimulation of high tones occurs is near the apex ; low ones producing a stimulation, near the base." This is contrary to the generally accepted view, founded mainly on pathological evidence, that the seat of stimulation by high tones is in the basal region, while that of low tones is in the apical, and Hurst's explanation appears to me insufficient.

More recently an ingenious view has been set forth by Max $\mathrm{Mayer}^{1}{ }^{1}$ in which the principle of sympathetic vibration is also discarded. Nayer supposes a wave to travel up the scala vestibuli, and to press the basilar membrane downwards. As it meets with resistance in passing upwards, its amplitude diminishes, and thus the distance up the seala through which the wave progresses will be determined by its amplitude. Further, the wave during its progress will irritate a certain number of nerve terminations ; consequently, a tone of feeble intensity (amplitude) will irritate only the fibres lying near the fenestra ovalis a certain number of times per second, while, if the same note be sounded loudly, the wave will travel further up the scala and irritate a large number of nerve fibres the same number of times per second. Thus the pitch will be the same, but the intensity or loudness will be greater. Pitch, according to this view, will depend on the number of stimuli per second, while loudness will depend on the number of nerve fibres irritated. How can such a

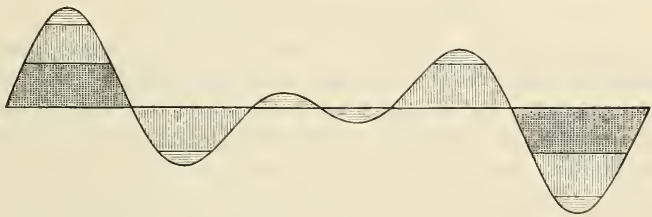

FIG. 425.-Compound wave form according to Max Mayer.

mechanism act as an analyser? Suppose a compound wave (such as shown in Fig. 425) to travel up the scala vestibuli, the two tones producing such a curve have a vibration frequency of the ratio $2: 3$, and it will be observed that there are three maxima and three minima. Now, as the wave passes up the scala, the parts shaded horizontally will die away first, and there will be left two maxima and minima. Let the wave travel still further up, and the part shaded vertically will disappear, leaving only one maximum and minimum. Finally, as the wave travels further, this also will disappear. Thus the nerve fibres in these different portions of the basilar membrane will be affected, and each will be affected a different number of times per second. The compound tone will therefore be resolved into three tones having vibration frequencies in the ratio of $3: 2: 1$. Accorling to Mayer, when two tones of the relationship $3: 1$ are sounded, we actually hear these tones of the relationship $3: 2: 1$,-the tone indicated by 1 being the so-called differential or beat tone. Similarly, if two tones of the ratio $9: 4$ be sounded, we resolve into tones of the ratio $9: 4: 1$ (the 1 being obtained by $4 \times 2=8$, and $9-8=1$ ); and if we construct a curve in the same way as shown in Fig. 425, and cut off successive maxima and minima, we liave the same ratio of maxima and minima, namely, $9: 4: 1$. Finally, Mayer, as the result of experimental inquiry, denies the existence of combination tones, the vibration frequencies of which are the sum or the difference

${ }^{1}$ Ztschr. f. Psychol. u. Physiol.. d. Sinnesory., Leinzig, Bd. xvi. and xvii. ; also Vcrhandl. d. physiol. Gesellsch, zu Berlin, February 18, 1898, s. 49 (in Aich. f. Physiol.). 
of the two generating tones. Such tones are, according to him, entirely subjective. On the other hand, when two notes are sounded, a new series of tones appear, as was previously pointed out by König. Thus, suppose two tones of $5: 8$ are sounded, then there are heard $(\alpha) 8-5=3$ (tone corresponding to 3 ); also $(\beta) 5 \times 2-8=2$ (tone corresponding to 2 ); and also $(\gamma) 5 \times 3-8 \times 2=1$ (tone corresponding to 1 ), and so on. Such occurs even whell the generators have no overtones, and their intensities depend on the varying strengths of the two primary tones.

Thus Max Mayer agrees with Hurst in imagining a series of waves being transmitted along the scalæ, instead of the scalæ forming part of one wave. The two differ in respect that Max Mayer supposes, on physical grounds, that the amplitude must diminish from base to apex of the cochlea, while Hurst argues, also from the physical point of view, that the amplitude must increase. Mayer's theory wholly rests on the supposition of diminished amplitude. I would urge two objections to both theories: (1) it is impossible to conceive of minute waves following each other in rapid succession in the minute tubes forming the scalæ; and (2) neither theory takes into account the remarkable differentiation of structure found in the epithelium of the organ of Corti. Further, each push out and in of the base of the stapes must cause a movement of the fluid in the scalæ as a whole. It appears to me, therefore, that the theory of sympathetic resonance gives a more satisfactory explanation of the facts.

\section{The Semicircular Canals.}

These organs, regarding the functions of which there are still differences of opinion, are anatomically in close connection with the internal ear, and indeed form an integral part of it. The membranous tubes, with their ampullæ, communicate freely with the cavity of the utricle, just as the saccule opens into the ductus cochlearis. In the ampullæ the adherent portion of the membranous structure projects into the cavity of the ampulla as a transverse septum or sharp ridge, which partially divides the ampulla into two. On the summit of this ridge we find a highly specialised epithelium, forming the crista acustica ampullæ, and which presents characters similar to those shown by the maculæ in the utricle and saccule. These general anatomical facts support the supposition that the canals and ampullæ are connected with the sense of hearing.

On the other hand, there is a suggestion that they and the structures lying in the vestibule have functions different from that of the cochlea, in the fact that the cochlear and vestibular nerves pursue a somewhat different course when they reach the brain, and apparently have different centres. ${ }^{1}$ Too much weight, however, must not be given to this statement, as the nerve to the posterior semicircular canal passes along the cochlear branch, while those to the two remaining canals accompany the vestibular branch. Further, while one can understand that the structures in the utricle, on receiving impulses from the base of the stapes, may act as peripheral organs for the perception of sounds, without reference to pitch or character, it has as yet been found impossible to suggest any rational view as to the action of the canals and ampulle with regard to auditory impressions. Helmholtz ${ }^{2}$ thought that in the ampullæ eddies of fluid pressure might follow the pushes inwards and outwards of the base of the stapes, thus causing currents or pressures to pass transversely across the bristles on the hair cells in the

1 Flatau, "Atlas of the Human Brain," 1894, p. 17, plate i. fig. 13.

${ }^{2} O_{1}$. cit., p. 217 . 
crista in the utricle; but while we admit this ingenious suggestion, it does not appear to account for the presence of such bristles in the cristæ of the ampullæ themselves, nor does it account in any way for the remarkable form of the canals.

Let us consider the base of the stapes moving inwards and outwards with the varying pressures caused by waves of sound. We have already seen how impulses must thus be communicated to the cochlea and the saccule. They must also be communicated to the utricle, into which we find the five openings of the ampullæ of the canals. It is evident that the pressures must act equally on all the ampullæ, and we can conceive the pressure tending to compress or push back the fluid at the mouth of each ampulla. If the pressure were less at the mouth of one ampulla than at the mouth of the other, there would be a flow of fluid in the canal. But as the pressure at the mouth of each canal must be the same, and as the fluid is incompressible, no movement of the fluid can take place, but there will be an increase of pressure on the walls of the ampullæ, and the effect of this will be to cause the ampulla and the other end of one canal to separate slightly, and to press the wall bearing the nerve-endings against the osseous wall. If the tube were slightly elastic, such a movement would undoubtedly take place. Thus, with a positive pressure the ampulla and the other end of the canal would tend to move away from each other, thus, $\leftarrow \longrightarrow$, and, with a negative pressure towards each other, thus, $\rightarrow \leftarrow$. In this way one can conceive of sound-waves acting on the ampullæ. ${ }^{1}$

Again, as the three canals lie in the three directions of space, it has sometimes been held that they may have to do with the appreciation of the direction of sound. An obvious objection to this view is, that as a matter of fact our perception of the direction of sound, or, in other words, of the position in space of the sounding body, is by no means accurate, and that we look with the eyes for the source of the sound, and instinctively direct the ears or the head, or both, in the direction from which the sound appears to proceed. We usually judge of the direction of a sound by making two or more simultaneous or successive observations. If the sound be heard more loudly in the right ear than in the left, we turn the head to the right, and by repeated observations judge of the direction; if the sound be produced at any point equidistant from both ears, we cannot tell its position unless we see what produces it. It is conceivable that we might have been furnished with an organ by which the source of sound might have been at once located, without moving the head, but we do not possess this faculty. ${ }^{2}$

The relation of the canals in the two ears is remarkable, and must have some physiological significance. This has been carefully investigated in the human subject by Crum Brown, ${ }^{3}$ who devised a strict method by which the angles formed by the planes of correspouding canals were measured. The results were as follows:--(1) The horizontal (external) canal has its plane sensibly at right angles to the mesial plane, and therefore the two (right and left) horizontal canals are sensibly in the same plane. (2) The planes of the superior (vertical) and posterior (vertical) canals of the same side make nearly the same angle with the

${ }^{1}$ Suggestion by Dr. II'Kendrick. Not previously published.

${ }^{2}$ See also Preyer, Areh.f. d. ges. Physiol., Bonn, 1887, Bi. xl. S. 586.

3 Nature, London, 1878 , vol. xviii. p. 663 ; also NI'Kendrick's "Physiology," vol. ii. Pp. $694-702$. 
mesial plane, but the right superior and the left posterior canals are not quite parallel-the posterior making with the mesial plane a somewhat greater angle than the superior-as much as $8^{\circ}$ more in one instance. Thus the $\frac{\text { right }}{\text { left }}$ superior canal is nearly parallel to the $\frac{\text { left }}{\text { right }}$ posterior, and in each pair of approximately parallel canals the ampulla of one canal is at the one end, and that of the other at the other end. To sum up-(1) The six canals are sensibly parallel, two and two; and (2) the two horizontal canals are on the same plane, while the superior canal on one side is nearly parallel with the posterior canal of the other. These facts point to the two sets of canals and ampullie acting as one organ, in a manner analogous to the action of two retine for single vision.

For many years the view has been developing that the semicircular canals communicate nervous impulses, which give a consciousness of the position of the head in space, and thus bring into play the muscular mechanisms necessary for equilibrium. Suppose that the canals and ampullie were free to move in the perilymph surrounding them, a very slight movement of the head in space might cause a small pull or pressure on the nerve-endings in the ampulle, and thus a nervous impulse would be aroused in the nerve fibres distributed to these structures. Such a mechanism would be equivalent to a variation of pressure; but, as we have seen that sound-waves also correspond to variations of pressure, it is evident that such variations might be produced by movements of the stapes or by movements of the head as a whole. Thus the ultimate mechanism of hearing, and that assumed to be the function of the semicircular canals, is essentially the same in kind, only, according to this view, the pressures communicated by the base of the stapes give rise to sensations of hearing, while those brought about by the varying tensions of the ampullie originate those vague sensations which preside over and regulate the muscular movements required to maintain equilibrium.

These views may be traced to two important researches, the one by Purkinje $^{1}$ in 1820 , and the other by Flourens ${ }^{2}$ in 1828. Purkinje directed attention to the well-known vertigo that follows rapid rotation of the body in the erect position in a vertical axis. The vertigo is felt, often with nausea, on suddenly stopping the rotatory motion. During rotation, if the eyes are open, objects appear to be moving in an opposite direction to that of the real movement; and when the movement of the body is arrested, a sensation of movement in the same direction continues for a few instants, and external objects still appear to be moving in the opposite direction. This may be termed visual vertigo. It has its counterpart in tactile sensations, when there is a tactile vertigo, on touching bodies during the rotation. In these experiments we may suppose external objects to be rotating round an imaginary axis, and, as we have seen, the rotation may be in the same direction as, or in the opposite direction to, that round the vertical axis of the body. Purkinje made the important observation, that the position of the imaginary axis of rotation for external objects depends on the axis of rotation executed at the head ; and that if we change the position of the head, after having arrested the movement of the body, we find that the axis of imaginary rotation

${ }^{1}$ Med. Jahrb., Wien, 1820, Bd. vi. ; also Bull. der sehlesischen Gesellsch., 1825-1826.

${ }^{2}$ Mém. Acad. d. sc. de l'Inst. de Franec, Paris, 1828, tome ix. p. 5 ; also "Recherehes expérimentales sur les propriétés du système nerveux," Paris, 1842, p. 438. 
remains unaltered. It was also noted that the sensation of movement was experienced even when the eyes were closed, and when no tactile sensations were possible; nor could Purkinje avoid observing the disturbance of equilibrium all have experienced, and the involuntary tendency to steady one's-self by seizing hold of external objects. Purkinje explained the phenomena by supposing that when rotation takes place, the brain, of soft consistence, and surrounded by a thin layer of fluid, has a tendency to drag behind the movements of the walls of the cranium, and that the dragging might, without tearing the brain substance, exercise tension on the cerebellum and cerebral peduncles. He did not associate the phenomena with the semicircular canals; but he supposed that, as during rotation the images of external objects flash across the retinæ, the objects appear to be in movement, and the ocular muscles contract spasmodically or intermittently to secure fixation. A struggle takes place between the sensations excited by retinal stimulation and the nervous impulses connected with the muscles. This struggle, in the language of Purkinje, along with the tension on the brain itself, and especially on its peduncles, gives rise to vertigo and the peculiar sensations of movement.

As bearing on this subject, the following interesting experiments, first studied by Purkinje, may be readily performed. Stand erect and rotate round a vertical axis for some minutes, and then stop. Surrounding objects appear still to move round, and there may be vertigo. The direction of the apparent motion depends on the direction of the preceling real motion, and is always opposite to it, and the axis about which the apparent motion takes place is always that line in the head which was the axis of the preceding real rotation. Bend the head forwards, rotate, and then stop. In this case the apparent motion is round a vertical axis. Again, bend the head forwards, rotate, stop, and then raise the head so as to look forwards, and the apparent motion will take place round a horizontal axis, the horizontal axis having been vertical when the real rotation took place, and the floor will seem to rise on one side and to fall on the other. Again, certain relations of these movements to visual phenomena are of interest. During real rotation round a vertical axis, at first the eyes move by a series of jerks; that is to say, they remain fixed for an instant, then follow the head, again they remain fixed, again move with a jerk, and so on. After a little time, however, if the rotation is continued, the eye does not continue fixed for even an instant, but moves more slowly than the head, then quickly makes up to it in speed, then falls behind. At last both head and eye move together. If, then, the rotation stops, the movements of the eyeballs are renewed, and gradually become less and less until they cease. The oscillatory movements of the eyeballs are the effect of the visual vertigo. When the real rotation ceases, the person has still a sensation that his head is turning round, but he feels also that his body is at rest relatively to his head; he sees that objects are at rest relatively to his head, and he conchudes that as his head is turning round, his body, and external objects, must be rotating also, and his eyes oscillate exactly as they would do if these movements were real. That these oscillations of the eyeball may be related to irritations of the semicircular canals, is rendered probable by the ohservation of Cyon, that irritation of the canals excites oscillatory spasms of the ocular muscles at the rate of from 20 to 150 per minute. The eycuballs oscillate about an axis perpendicular to the plane of the irritated canal. Oscillatory movements of the eyeballs of a similar kind may be observed in rapid railway travelling when the person looks at near objects, and probably they form no inconsiderable part of the cause of the fatigue in such circumstances. ${ }^{1}$

1 M'Kendrick's "Physiology," vol. ii. p. 701. 
Flourens, in 1828, announced that injury to the semicircular canals causes motor inco-ordination and disturbance of equilibrium, and that section of the canals produces a rotatory movement of a kind corresponding to the canal that had been divided; in other words, that division of a membranous canal causes rotatory movements round an axis at right angles to the plane of the divided canal; that is to say, the body of the animal always moves in the direction of the cut canal. Further, he was led to suggest that, as section of the canals produces disturbances of the same kind as those following injury of the cerebellum, they also have to do with co-ordination of movement.

These observations of Flourens have been corroborated by many other observers. Vulpian ${ }^{1}$ thought that the phenomena are due to an auditive vertigo, which acts on all the organs. Brown-Séquard stated that the same phenomena are caused by irritation of the auditory nerve, but this was denied by Schiff. ${ }^{2}$ In 1869, Löwenberg, ${ }^{3}$ after numerous experiments, more especially on the horizontal and vertical canals, in which they were not only divided but subjected to mechanical and chemical excitation, conchuded that the difficulties of co-ordination of movement are due, not to paralysis, but to excitation, that these movements are reflex and unconscious, and that the centres for the reflex excitations are in the optic lobes (bird).

An important contribution was made to the subject by Goltz, ${ }^{4}$ who, although he devised no new experiments of importance, and relied mainly on the observations of Flourens, was the first to clearly formulate the conditions necessary for co-ordination, namely-(1) A central co-ordinating organ, (2) centripetal fibres with their peripheral terminations, and (3) centrifugal fibres with their terminal organs. A lesion of any one of these portions of the mechanism produces inco-ordination. Goltz admitted the contention of Flourens, that the canals are necessary for the equilibration of the body, but he went farther, and contended that they have mainly to do with the equilibrium of the head. The mechanism was stated to be as follows:-The endolymph exercises a stronger pressure on the walls of the ampullæ when the movements of the head bring these to a low level; this pressure irritates the nerves of the ampullæ and excites centripetal impulses, which reflexly cause movements resulting in the equilibration of the head. If, then, the canals are divided or injured, disordered movements ensue from the head losing equilibrium. This theory is often termed the hydrostatic theory of Goltz, and it assumes that the movements of the body are regulated entirely by the more or less conscious appreciation we have of the position of the head in space.

The subject was next investigated by Cyon, ${ }^{5}$ who found the general loss of co-ordination of movement after section of a canal to be due to the disordered movements of the head. Thus, when the head of a pigeon was fixed so that the beak was directed upwards and the

1 "Leçons sur la physiologie du système nerveux," Paris, 1866, p. 600.

2 "Lehrbuch der Physiologie," 1858.

3 Arch. f. Augen- $u$. Ohrenh., Bd. iii.

4 Arch. f. d. yes. Physiol., Bown, Bd. iii. S. 172.

5 Ibid., 1873, Bd. viii.; "Cours de physiologie," St. Petersbourg, 1873-74, tome ii.; "Methodik der physiologischen Experimente," St. Petersburg, 1876, S. 540-547 ; "Rapports physiologiques entre le nerf acoustique," etc., Compt. rend. Acad. d. sc., Paris, 1s76, tome Ixxxii. p. 856 ; “Les organes périphériques du sens de l'espace," ibid., Paris, 1877, tome lxxxv. p. 1284; also "Recherches expérimentales sur les fonctions des canaux semicirculaires," Thèse, 1878. 
occiput downwards, the bird showed irregularity of movement and a loss of equilibrium, as if one of its canals had been divided. If the apparent position of surrounding objects is determined by the position of the head, any sudden change in position might cause for a moment disturbance of motion, and the same result might be expected to follow a strabismus, at all events for the first few minutes. Cyon adjusted glass prisms before the eyes of birds, so as to cause a squint, and the result was loss, for a short time, of co-ordinating power. He also found that after the disturbance of motion following section of the horizontal canal on one side, consisting of oscillations in a horizontal plane and round the vertical axis of the head, and of loss of equilibrium, supporting the head, by placing the finger below the beak, at once caused the movements to disappear, and the bird became calm. Flight after such an injury was almost impossible. At the end of eight or ten days, all the symptoms described disappeared, except that there was still some awkwardness in flying, but eventually this also disappeared, and the movements of the bird were normal. The phenomena following section of the vertical canals were of the same nature, the only difference being as regards direction of movements. The movements of the head were now from below upwards or from above downwards in a vertical plane and around a horizontal axis, and the movements of the body, instead of being mouvements de manége, or round a vertical axis, as after division of a horizontal canal, were now backwards and forwards, as if around a horizontal axis passing through the body. As Cyon held that the perception of the position of bodies in space depends on the position of the head, his general conclusions resi largely on this view. Thus (1) to maintain equilibrium, we must have an accurate notion of the position of the head in space; (2) the function of the semicircular canals is to communicate impressions that give an accurate representation of this position - each canal having a relation to one of the dimensions of space; (3) disturbance of equilibrium follows section; (4) involuntary movements following section are due to abnormal excitations ; (5) abnormal movements occurring a few days after the operation, are caused by irritation of the cerebellum.

It might be argued, as indeed was done by Böttcher, ${ }^{1}$ that the phenomena of Flourens were secondary, and that they were caused by irritation of neighbouring parts of the brain, more especially of the cerebellum. This view, however, is disproved by the fact that the phenomena of Flourens appear immediately after section, whereas similar phenomena, caused by irritation of the cerebellum, do not appear until several days after the irritation. It is contradicted also by the observation that if we divide, not two symmetrical canals, but on the one side a horizontal and on the other a vertical canal, or if we cut two canals on the same side, the phenomena of Flourens do not appear. It is clear, therefore, that secondary lesions of the brain have nothing to do with the phenomena.

Hitherto we have considered the results obtained by experimental physiologists. The functions of the canals have, however, been investigated in another way, by physical experiment and by the application of purely theoretical considerations. This has been done more especially

${ }^{1}$ Arch. f. Ohrenh., Bd. ix.; see also Baginsky, Biol. Centralbl., Bd. i. S. 438 ; Jacobson, Arch. f. Ohrenh., 1881-2, Bd. i.; 1894, Bd. xxii.; Högyes, Arch. f. d. ges. Physiol., Bonll, 1881, Bd. xxvi. S. 558. 
by Mach, ${ }^{1}$ Crum Brown, ${ }^{2}$ and Breuer, ${ }^{3}$ and they have been led to emit theories similar to each other, and all based on the notion of the canals being organs for sensations of acceleration of movement or for the sense of rotation. Mach was the first to point out that the phenomena of Purkinje, already described, were in all probability related to the semicircular canals. He showed that when the body is moved in space, in a straight line, we are not conscious of the velocity of motion, but of variations in this velocity. Similarly, if the body is rotated round a vertical axis, we perceive only angular acceleration, and not angular velocity. The sensations produced by angular acceleration last longer than the acceleration itself, and the position of the head during the movements enables us to determine direction. By a special apparatus, consisting of a chamber rotating round a vertical axis and surronnded by a scale, Mach investigated the nature of vertigo. He examined critically all the organs that might be supposed to be the seat of the sensations of vertigo, or rather its cause, such as the nerves, skin, connective tissues, bones and muscles, and the displacement of the mass of blood caused by rotation, and found it impossible thus to account for the phenomena. It will be remembered that Purkinje attached great importance to the movements of the eyeballs, and he supposed that vertigo consists in a displacement of the visual field, produced by the movements of the eyeballs, and which we refer to external objects that appear to be moving, just as we judge of the changes in position of external objects by the sensations excited by contractions of the ocular muscles, as we follow objects with the eye. This explanation did not satisfy Mach, and he inclined to the explanation offered by Breuer, namely, that the movements of the eyeballs are of a reflex character, and that the reflexes are excited by irritation of the nerves distributed to the ampullie. The movements are, in a sense, compensatory, and they are in the opposite direction to the rotation of the head. When the movement of the head stops, then the eyeballs return to their normal position; but, during this movement backwards, external objects will appear to move in the visual field in a direction opposite to the direction of the rotation of the eye. A sensation is then excited of objects moving in a direction opposite to that of the body, and vertigo is the result. Mach found, however, that this did not fully explain the phenomena, as in certain experiments there was, after rotation, an apparent movement of external objects, even when the retina was stationary, and he finally put forward the following theory:Suppose in a body, B, there is a cavity on the walls of which there are nerve-endings, and that this cavity contains a solid or liquid, A. By its weight, $A$ will exercise a greater pressure on one part of the walls of the cavity than on the others, and it will thus determine the position of the body, B, relative to the vertical. With each acceleration of $B, A$ will press in the opposite direction, and this contrapressure will be added to the acceleration due to weight, so that the direction of the pressure as well as its intensity will change in the cavity. In like manner, with each angular acceleration communicated to $\mathrm{B}$, A will oppose a rotation in the opposite direction. Thus, B will obtain knowledge both of its

${ }^{1}$ Sitzungsb. d. k. Akad. d. Wissensch., Wien, 1874; also "Grundlinien der Lehre von den Bewegungsempfindungen," Leipzig, 1875.

2. Journ. Physiol., Cambridge and London, vol. viii. p. 32\%.

3 Med. Jahrb., Wien, 1874, 1875, S. 72, 87. 
position and of its progressive acceleration in a straight line, and, in the case of rotation, angular acceleration will also be indicated. The vestibule and semicircular canals in Mach's scheme constitute B; the vestibule possibly having to do with the sense of acceleration of movement in a straight line, while the semicircular canals serve for angular acceleration. During each angular acceleration around an axis passing through the plane of the canal, there will be a traction or pull in the opposite direction on the nerves of the ampullæ, and this will give rise to the sense of angular acceleration. Each excitation produced in this manner, or, as regards the nerves of the ampullæ, in accordance with the law of the "specific energies" of nerves, in any other way, will give rise to a sense of rotation. Thus Mach, like Goltz, attaches importance to the fluid in the canals, and by varying pressures of this fluid, produced by angular rotation, and always in a direction opposite to the rotation of the body, sensations of movement are excited, which in turn cause the vertigo of Purkinje and the phenomena of Flourens.

The hydrodynamical theories advanced by Breuer and Crum Brown differ only in detail from that of Mach. All three observers uphold a dynamical theory, as opposed to the statical theory of Goltz. Suppose the three canals to be full of fluid, a rotation of the head in one direction will cause currents of fluid in the canals in the opposite direction, and the amount of flow in each canal will depend on the plane in which the head is turned and on the rate of rotation. As the auditory hairs exist in the crista of the ampullæ, we can conceive that they will be affected by the currents, and produce a sensation of rotation of the head in the plane of the canal in which the flow takes place, and in a direction opposite to it. If the rotation of the head continue, the flow of the endolymph backwards will be arrested by friction, and if the head then suddenly stop, the endolymph will continue to flow in the direction in which the head was previously rotated, and a sensation will be felt of a rotation of the head and body in a direction opposite to that of the first rotation. Crum Brown ${ }^{1}$ sums up the matter thus-

"We have two ways in which a relative motion can occur between the endolymph and the walls of the cavity containing it-(1) When the head begins to move, here the walls leave the fluid behind; (2) when the head stops, here the fluid flows on. In both cases the sensation of rotation is felt. In the first this sensation corresponds to a real rotation, in the second it does not; but in both it corresponds to a real acceleration (positive or negative) of rotation, using the word acceleration in its technical kinematical sense."

Mach attributes the effects to variations of pressure in the canals rather than to an actual flow of fluids, while Crum Brown differs from both Mach and Breuer on the following points:-(1) In attributing movement or variations of pressure, not merely to the endolymph, but also to the walls of the membranous canals and to the surrounding perilymph; and (2) in regarding the two labyrinths as one organ, all the six canals being required to form a true conception of the rotatory motion of the head. As already pointed out, the six canals are sensibly parallel, two and two. The two horizontal canals are on the same plane, while the superior canal on one side is nearly parallel with

'Nature, London, 1878, vol. xviii. p. 633 ; review of Cyon's work, loc. cit.

VOL. II. -76 
the posterior canal of the other. Crum Brown states the matter thus:-

"Further, in each of these three pairs (right and left horizontal, right superior and left posterior, right posterior and left superior), the two canals are so placed that when rotation takes place about the axes to which they are perpendicular, one of the two canals moves, with its ampulla preceding the canal, so that the flow or tendency to flow [or pressure] is from ampulla to canal, while in the other the ampulla follows the canal, and the flow or tendency to flow [or pressure] is from canal to ampulla. If, then, we suppose that flow from ampulla to canal-or, adopting Mach's view, increase of pressure in the ampulla-alone stimulates the hair cells, while no effect is produced by flow in the opposite direction-or by diminution of pressure in the ampullawe have in the six canals a mechanical system capable of giving us an accurate notion of the axis about which rotation of the head takes place, and of the sense of rotation."

Cyon, ${ }^{1}$ in later researches, has performed certain experiments which, it must be confessed, throw doubt on both the statical and dynamical theories already described. He has punctured the canals and drawn off the endolymph, injected the canals with gelatin, and plugged them with slender filaments of laminaria, which soon became swollen. Yet by these severe proceedings, by which great variations of pressure must be produced, none of the phenomena of Flourens were observed. The obvious objection to these experiments is, that they were so rough as to entirely destroy the mechanism they were intended to exanine. Again, Cyon has repeatedly observed, after making a minute puncture in a canal, that there was a minute pulsation, caused either by a small artery supplying the canal, or by the pulsations of the brain itself through the fluid in the arachnoid with which the membranous canals communicate; and he suggests that, if Mach's view be correct, we ought in this way to have rhythmic excitations of the nerve-endings in the ampulla. As it is not likely, however, that such pulsations would have any effect, unless the canal were punctured, the observation is of no great importance; at all events, it is not a valid objection to Mach's theory.

It is well known that a rabbit may show the phenomena of Purkinje after having been rotated on a turn-table. Cyon took advantage of this fact and performed the experiment on animals in which the auditory nerves had been divided, and he states that the results were the same as in normal animals. This observation would at first sight appear to seriously compromise the statical and dynamical theories, but what it really does is to show that some of the phenomena of Purkinje may, as he himself supposed, be due to the action of rotation on the brain itself. If so, one would expect to see these phenomena after the division of the auditory nerves, and the fact does not invalidate the reasoning on theoretical and experimental data regarding the function of the canals already described.

Cyon contends that the semicircular canals only indirectly assist in giving a knowledge of spatial relations. He holds that knowledge of the position of bodies in space depends on nervous impulses coming from the contracting ocular muscles; that the oculomotor centres are in intimate physiological relationship with the centres receiving impulses from the nerves of the semicircular canals; and that the oculomotor centres, thus excited, produce the movements of the eyeballs,

1 Thèse, op. cit., pp. 41, 42, 43. 
which then determine our notions of spatial relations. The canals are thus the peripheric organs of the sense of space, and the sensations caused by excitation of the nervous terminations in the ampullæ assist in forming our notions of the three dimensions, the sensations excited by each canal corresponding to one dimension. Cyon, however, does not show how the ampullie are excited in the first instance. Further, the mechanism assumed to exist is much more complicated than that required for the static or dynamic theories. The latter explain in a satisfactory manner the cause of the sensation of rotation, without touching the much larger question of the origin of our notions of space; and whether one adopts, with regard to this problem, the intuitional or empiric theories, there remains the fact that a comparatively simple function has been suggested for the canals. The interpretation of spatial relations does not, in all probability, depend on one organ alone, but on the combined effect of nervous impulses coming from many organs-eye, ear, skin, tendons, and muscles; and to say that the canals are the only organs having to do with our knowledge of spatial relations is to present a fragment of the truth.

The view of Cyon as to the influence of the canals on the movements of the eyes, is supported by some recent experiments carried out by Lee. ${ }^{1}$ By passive rotation of a dog-fish round different axes, compensating movements of the eyes and fins (especially the pectoral fins) were produced. It was observed that if the fish were rotated in the plane of one of the canals, exactly the same movements of the eyes and fins occurred as were produced by experimental operation and stimulation of the ampulla of that canal. Lee is of opinion that each ampulla has a principal and a subordinate function, which produce opposite effects on the eye movements. Thus, if the chief function of one ampulla is to move the eyeball upwards, the subordinate effect is to rotate it downwards. By pressing with a blunt needle upon the ampulla, either of those functions may be called forth. For example, gentle pressure upon the anterior ampulla causes the eyeball of the same side to roll slightly downwards, and that of the opposite side slightly upwards. This is the subordinate function. A stronger pressure upon the same ampulla causes the eyeball of the same side to roll strongly upwards, and that of the opposite side to roll strongly downwards-this is the principal function. It would appear that the compensatory movements of the eyes and fins, when the fish is rotated, are produced by the principal function of a pair of canals, while the reactions following the compensatory movements are produced by the subordinate functions of a pair of canals. On electrically stimulating different parts of the cut surface of the auditory nerves, results were obtained the same as when the stimulus was applied to the corresponding ampulla, and the reverse of the results obtained by mere section of the same nerve. Further, when rotation occurs in a plane different from that of any of the canals, two pairs of canals are stimulated to varying degrees by the pressure of the endolymph, and the result corresponds to the algebraic sum of the stimuli to the various ampullæ. These striking results strongly support the dynamic theory.

The researches of Ewald ${ }^{2}$ merit attention, as he has been led to extend the influence of the canals. He divides the labyrinth function-

1 Journ. Physiol., Cambridge and London, vol. xvii. p. 192.

2 "Untersuch. ü. das Endorgan des Ncrvus octavus," Wiesbaden, 1892. 
ally into two portions, the "hearing labyrinth" and the "muscular-tone labyrinth," the former consisting of the cochlea, and the latter of the semicircular canals, and the structures in the vestibule. After many experiments, he concluded that all the muscles of the body are kept in a state of tone by means of the labyrinth, and that injury to it most affects those muscles whose movements are most delicate, such as those of the eye and larynx. After plugging the canals, abnormal flaccidity of all the muscles ensued. He further states that if the labyrinth of an animal be injured, and the animal be then killed, the usual rigor mortis does not, in most cases, occur. In some cases, however, it appeared to come on earlier. While Ewald thus relates the labyrinth to muscular tonicity, he does not deny that sound-waves stimulate the nerve-endings, and he suggests that in this way rhythmic movements of the muscles, as in dancing to music, may be facilitated.

In 1883 , Sewall ${ }^{1}$ carried out instructive experiments on cartilaginous fishes. The animals selected were young sharks and skates. He found that merely cutting through all the canals on one side produces no disturbance of equilibrium. The same negative result follows section of the vertical canals on both sides. On injuring the utricle and removing the otoliths, there was occasionally no disturbance of equilibrium; but in the majority of instances it was observed that the fishes dived and swam downwards in circles towards the injured side. The surest way, however, of producing disturbance of equilibrium was to injure the saccule, when the fish kept swimming round in a circle in the vertical plane. Operations on the ampullæ gave somewhat ambiguous, though generally positive, results. Thus, injury to the anterior vertical ampulla tended to produce rotation in the long axis of the body, while similar injury to the longitudinal ampulla tended to produce somersault movements. When the nerve supplying a horizontal ampulla was cut, there was no disturbance of equilibrium.

In the larger sharks serious injuries to the labyrinth often produced little or no disturbance of equilibrium; but the most common results were nystagmus and vomiting. Sewall cautiously concludes that the results of experiments are not sufficient to justify the opinion that the labyrinth is the peripheral organ of equilibrium.

It is remarkable that Steiner ${ }^{2}$ came to the conclusion that in the dog-fish the canals had nothing to do with equilibrium, while we have seen that Lee obtained remarkable positive results (p. 1203). In another research, Lee ${ }^{3}$ found that, after cutting the auditory nerve in dog-fish on one side, rolling movements round the longitudinal axis towards the injured side occurred. Somersault movements were absent. On cutting the auditory nerves on both sides, the sense of statical equilibrium was lost, and the animal Jay on its back, belly, or side indifferently, and swam in these positions. On stimulating the ampulla of the anterior canal, an upward rotation of the eye on the same side, and a downward rotation on the opposite side, were noticed. On dividing the nerve to this ampulla, the opposite effect was produced on the eyes, and if both nerves were cut the fish dived downwards. On cutting the nerves to the posterior ampulla on both sides, the fish swam upwards near the surface, sometimes even putting its head out of the water.

1 Journ. Physiol., Cambridge and London, vol. iv. p. 339.

${ }^{2}$ Deutsche merl. Wchnschr., Leipzig, 1889, No. 47.

3 Journ. Physiol., Cambridge and London, 1894, vol. xv. 
These experiments undoubtedly prove that the ampullæ contain sense organs connected with the sense of equilibrium. Lee is of opinion that the otoliths and maculæ form the organ for statical equilibrium.

The light thrown upon the subject by pathological evidence is scanty. There is no doubt that any disease or injury of these structures, occurring rapidly, produces giddiness, staggering, nystagmus, vomiting, noises in the ears, and more or less deafness, though sometimes some of the symptoms may be absent. But all these, with the exception of the deafness, may merely be reflex symptoms, and even the deafness is probably due to coincident affection of the cochlea.

On the other hand, a slowly progressive affection of the labyrinth may ultimately leave the canals and vestibule transformed into a bony mass, in which no trace of the original soft structures remains. In such cases there may be no perceptible disturbance of equilibrium, no nystagmus or giddiness, nor any other symptom of that nature. ${ }^{1}$ If, therefore, we look upon the canals and vestibule as organs whose function is the maintenance of equilibrium, then we must admit that they are not indispensable for that purpose, but that some other organ is able to carry on the function.

From the foregoing, it will be seen that the subject is involved in obscurity. Certain symptoms follow injury to the canals, such as loss of equilibrium, giddiness, nystagmus, and often vomiting; and we may assume that these symptoms are not due to coincident injury of the brain. Injury to the cochlea produces no such symptoms. Are we to conclude, therefore, that the function of the former structures is to make us conscious of the position of the head in space, or the maintenance of equilibrium, or the maintenance of muscular tone, etc.? One difficulty lies in the fact that all the symptoms may be merely reflexes caused by injury, and may not be due to loss of function. The vestibular nerve is in much closer connection than the cochlear portion with those regions of the central nervous system (such as the cerebellum) which, when injured, cause such symptoms.

Whether the canals are concerned in the act of hearing is not known. Injury to these structures in animals does not appear to cause deafness, but we cannot definitely say that hearing is unaffected. In the human subject, on the other hand, it is extremely doubtful whether the canals and vestibule are ever affected without causing deafness; but it must be remembered that these structures are rarely affected without the cochlea suffering coincidently, so that the deafness may be caused by disease of the latter structure. ${ }^{2}$

${ }^{1}$ Politzer, "Diseases of the Ear," 1894, p. 606.

${ }^{2}$ A précis of the enormous literature on the functions of the semicircular canals is given by von Stein, op. cit., S. 172-522. He has collected the results of many investigations, and although the material is presented in considerable eonfusion, the book is a mine of literary wealth. 


\title{
ON VOCAL SOUNDS.
}

\author{
By John Gray M'Kendrick and Albert A. Gray.
}

Contents :-Voice Production, p. 1206-The Singing Voice, p. 1206-Tone Intensity, p. 1207-Tone Pitch, p. 1207-The Range of the Voice, p. 1207-The Muscular Mechanisms which affect the Vocal Cords, p. 1209-Voice Registers, p. 1210The Quality of the Voice and Vowel Tones, p. 1215-Theories of Vowel Tones, p. 1216-Wave-forms of Vowel Tones, p. 1220-The Fourierian Analysis of Wave-forms, p. 1221-Phonograms, p. 1224-General Conclusion, p. 1233The Consonants, p. 1233-Effect of the Cavities in connection with the Larynx and Pharynx upon the Voice, p. 1235-Whispering, p. 1236.

\section{Voice Production.}

VOICE is produced by the vibrations of the vocal cords, and developed by the resonance of the upper cavities. It is to be distinguished from speech, which is the production of sounds intended to express ideas. Many of the lower animals have voice, but none have the power of speech in the sense in which man possesses that faculty, or, at all events, the range of animal speech is very limited. There may be speech without voice, as in whispering, or voice without speech, as in singing notes without words.

The singing voice.-One of the functions of the larynx is the production of the singing voice, and hence it is to be looked upon as a musical instrument; but when an attempt is made to compare it with the various instruments used by musicians, we find that it is quite different from any of these. It is most nearly allied to reed instruments, the essential factor in both being that the air is made to pass through a narrow chink, and in doing so it is cut up into a series of successive puffs. The similarity, however, ends here; for in reed instruments changes in pitch are obtained by alterations in the dimensions of the resonator which is attached to them; while in the case of the larynx the pitch is regulated primarily by changes in the length, tension, and thickness of the membranous tongues themselves. The action of the vocal cords, however, may be compared with that of the performer's lips in playing a brass instrument, and his lips may further be likened to membranous tongues. It is to the membranous yielding nature of the vocal cords that the peculiar softness of the human voice is to be attributed; we find that in reed instruments in which the tongues are made of brass or metal, the tone is apt to be cutting, on account of the presence of large numbers of high upper partials. ${ }^{1}$

The larynx, therefore, may be compared roughly to a reed instrument, in which changes in pitch are produced by alterations in the tension, length, thickness, and breadth of the cords themselves, and possibly,

${ }^{1}$ Helmholtz, "Sensations of Tone," translated by Ellis, 3rd ed., p. 151. 
also, by variations in the strength of the current of air, and not at all, or only in the slightest degree, by changes in the size or shape of the adjacent cavities.

Tone intensity.-The intensity or loudness of tone will be determined by the amplitude of the vibrations of the margins of the membranes (vocal cords), and its pitch will be influenced by arrangements which cause an increase or decrease of the tension of the margins of the membranes. The pitch may also be raised by increasing the force of the blast of the air, since this will also increase the mean tension of the margins of the elastic membranes.

With tones of medium pitch, the pressure in the trachea has been found to be about $160 \mathrm{~mm}$. of water; with tones of high pitch, $920 \mathrm{~mm}$; and with tones of very high pitch, $945 \mathrm{~mm}$; while in whispering it may fall to a pressure of only $30 \mathrm{~mm}$.

Tone pitch.-Pitch, so far as the vocal cords are concerned, depends on the number of vibrations per second, or, to put it in another way, on the length of time occupied by each vibration. This again will be determined by the length, thickness, elasticity, and degree of tension of the cords, and on the strength of the blast of air. Other conditions remaining constant, the greater the length of the cords the lower will be the pitch.

The range of the voice.-The range of the human voice is about three octaves, from $f \alpha_{1}$ (87 vibs. per second) to sol $_{4}(768$ vibs.). In men the vocal cords are more elongated than in women, in the ratio of $3: 2$, so that the male voice is of lower pitch, and is usually stronger. At the age of puberty, the larynx grows rapidly, and the voice of the boy "breaks" in consequence of the lengthening of the cords, generally falling an octave. A similar change, but less in amount, occurs at the same period in the female. At puberty in the female there is an increase of about one-third in the length of the glottis, but it is nearly doubled in the male.

Some of these changes have been carefully studied by Paulsen. ${ }^{1}$ In girls, in the sixth year, the pitch ranges from $a$ to $f^{\prime \prime}=$. From this period there is a slow increase of range in the lower voice tones, while a more rapid extension occurs in the range of the upper tones. The lowest limit of pitcl occurs about the age of 12 in the girl's voice, and the highest is reached in the eleventh year. In the boy's voice the range about the age of 6 years is more limited, lying between $b$ and $f^{\prime \prime}$. At first the upper range is extended rapidly, reaching the highest point about the twelfth year, and the lower range increases more rapidly than in girls, reaching the lowest pitch in the thirteenth year. The shrieking voice of a child may, according to Stevens, ${ }^{2}$ rise as high as $g^{\prime \prime \prime}(3072$ v. s. per second), and sometimes screams may cven reach from 5000 to $6000 \mathrm{v}$. s. per second (v. s. = single vibrations).

In advanced life the upper tones gradually weaken, and ultimately disappear, while the character of the voice also changes, owing to loss of elasticity caused by calcification (or ossification), which first begins about middle life in the thyroid cartilage, then appears in the cricoid, and much later in the arytenoids. Eunuchs, as a rule, retain the voice of childhood; and by careful training in normal persons it is sumetimes possible to so modify the development of the larynx that an adult male can still sing the soprano parts used in cathedral choirs.

The ranges of the different varieties of the human voice are shown in the

${ }^{1}$ Arch. f. d. ges. Physiol., Bonn, Bd. lxi. s. 407.

2 Phys. Rev., N.Y., 1895, vol. iii. p. 230. 
following diagram, where the dotted lines give the ran ge of certain remarkable voices, and the figures represent the number of vibrations per second. ${ }^{1}$

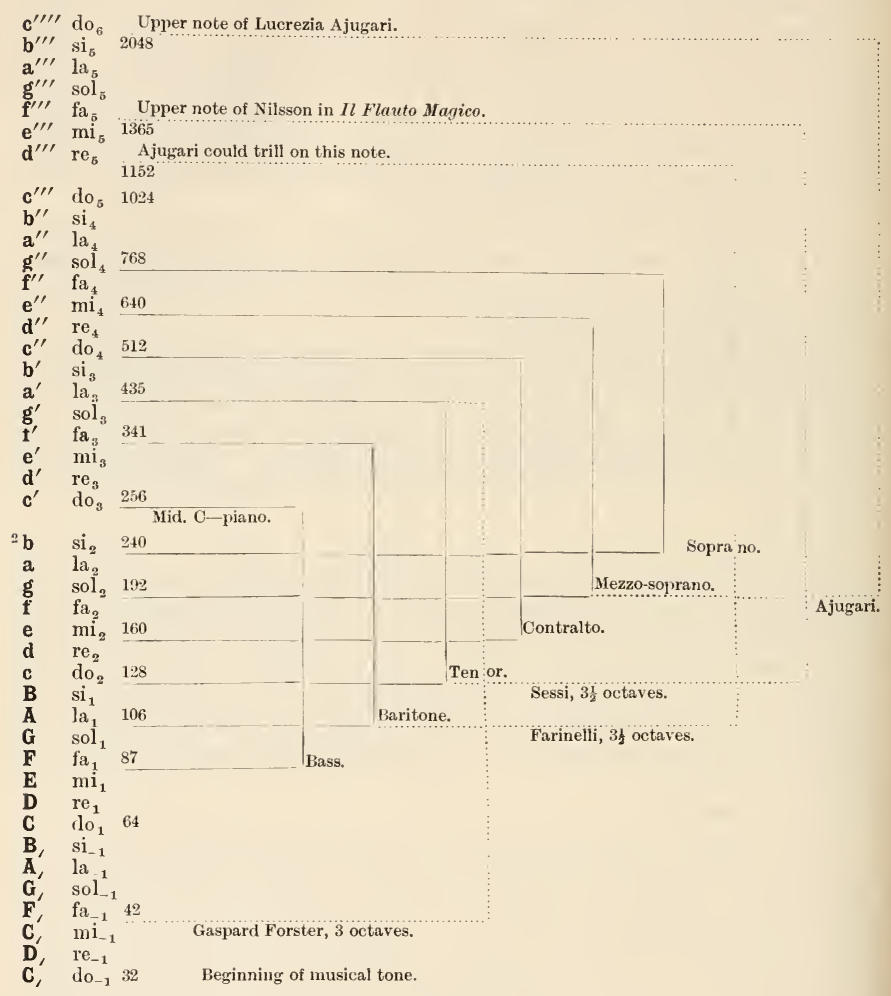

There is thus a range for ordinary voices of nearly two octaves, and certain rare voices may have a range of three and a half octaves. The deepest tone on record is that of Forster, $f a_{-1}$ ( 42 vibs. per second), and the highest, that of Ajugari, on the authority of Mozart, $d o_{6}$ (2048 vibs. per second). The table shows that the lowest note of Forster is not much above the pitch at which the perception of tone begins, and that from this note to the highest of Ajugari is a range of nearly six octaves. From the lowest note of an ordinary bass voice to the highest of an ordinary soprano the range is only about three octaves. In this connection, it is interesting to observe that the range of the human ear is from $d o_{-1}$ (32 vibs. per second) to $d o_{10}(33,768$ vibs. per second), or eleven octaves.

${ }^{1}$ From article "Voice" in "Encyc. Brit.," 9th edition, vol. xxiv. p. 275, by John G. M'Kendrick. The letters used to designate the tones have been added.

2 German writers use $h$ instead of $b, b$ instead of $b b$, ais for $a \not$, and gis for $y \not$, etc. 


\section{The Muscular Mechanisms which affect the Vocal Cords.}

There is no doubt that the muscle which produces the greatest effect in tightening the vocal cords, and thus raising the pitch of the voice, is the thyro-cricoid. This muscle, acting from the thyroid cartilage as a fixed point, rotates the cricoid in such a manner that its anterior part is elevated towards the thyroid, and the posterior part, which bears the arytenoid cartilages, is depressed and further removed from the angle of the thyroid cartilage, thus stretching the thyro-arytenoid ligaments, the free edges of which, covered by mucous membrane, form the vocal cords. There is, further, no doubt that the lateral crico-arytenoid muscles must tend to rotate inwards the vocal processes of the arytenoid cartilages, and thus to approximate or adduct the cords, and the posterior crico-arytenoids must antagonise the lateral, rotate the vocal processes outwards, and abduct the cords. The arytenoideus muscle, on the other hand, brings the arytenoid cartilages bodily together, and at the same time may somewhat tend to depress their vocal processes. The action of the thyro-arytenoideus muscle is more complex, and will be immediately considered.

All the muscles except the thyro-cricoid, which receives its nerve from the superior laryngeal, are innervated through the inferior laryngeal branch of the vagus, the fibres being derived from the accessory roots.

The inferior laryngeal contains, therefore, both the adductor and abductor nerves. When profoundly anæsthetised, stimulation causes abduction of the cord, but if the anæsthesia is slight, then the same stimulation will cause adduction. ${ }^{1}$

The tonic contraction of the abductors, produced by activity of a centre in the medulla oblongata, is normally stronger than that of the adductors, so that during rest the glottis is slightly open; on section of the inferior laryngeal nerve, the cords come together and the glottis tends to close. The bulbar centre is under the dominance of a centre in the Rolandic region of the cerebral cortex. ${ }^{2}$

With regard to the action of the thyro-arytenoid muscle, until recently it has usually been held by English writers that the muscle, when contracting as a whole, lowers pitch; whereas German physiologists consider that the pitch of the voice is raised by it.

Wyllie $^{3}$ found that, in the exsected larynx, when the cords were approximated and the arytenoid cartilages pushed forward, the pitch of the artificial voice, produced by blowing up through the trachea into the larynx, was considerably raised. But it is not definitely stated by Wyllie what is meant by pushing the arytenoid cartilages forward. If these cartilages, as a whole, are pushed forward, and thus approximated to the thyroid, the vocal cords cannot be tightened. Wyllie, however, appears to mean that if the upper parts of the arytenoid cartilages be pushed forward, a movement of rotation about a horizontal axis takes place and the vocal processes are depressed, thus giving rise to increased tension of the cords. It is difficult to see how the vocal processes could be depressed during phonation, for the articular surfaces of the cricoid cartilage are so placed that one would expect the vocal processes of the arytenoids to rise during phonation. Neumann, ${ }^{4}$ however, found by experiment that the cord sank during plionation.

${ }^{1}$ Semon, Brit. Med. Journ., London, 1886, vol. ii. pp. 405 and 415.

2 See article "Cerebral Cortex." 3 "Disorders of Speech," 1894, p. 482.

${ }^{4}$ Monatschr. f. Ohrenh., Berlin, October 1894. 
We must, however, remember that pressure of the arytenoid cartilages from behind cannot be compared accurately with the action of the thyro-arytenoid muscle acting in front. The external part of the thyroarytenoid might tend to cause depression of the vocal process; but it is very difficult to see how the internal portion of that muscle can produce such an effect; and the small thyro-arytenoid of Sömmering, when present, will have an opposite effect.

Many writers, while admitting that parts of the thyro-arytenoids relax the cords, consider that other portions will cause increased tension. ${ }^{1}$ Jacobson, ${ }^{2}$ who has made a careful examination of the function of this muscle, points out that those fibres which run from the outer edge of the vocal process backwards and outwards will aid in causing abduction of the cord. But we cannot admit the monualified statement that the thyro-arytenoid causes increased tension of the cords. No doubt such fibres, running outwards from their insertion in the vocal cords, could, by pulling on the edge and making it curved instead of straight, tighten the cord. Indeed, the elliptical shape which the rima glottidis assumes, after passing from the chest into the middle register, may indicate some such action. If the part of the muscle which is in contact with the vocal cord contracts as a whole, it will tend to make the cord more rigid; and if the arytenoids were at the same time fixed, the pitch would rise.

\section{Voice Registers.}

The voice has been divided into three registers, namely, the lower or
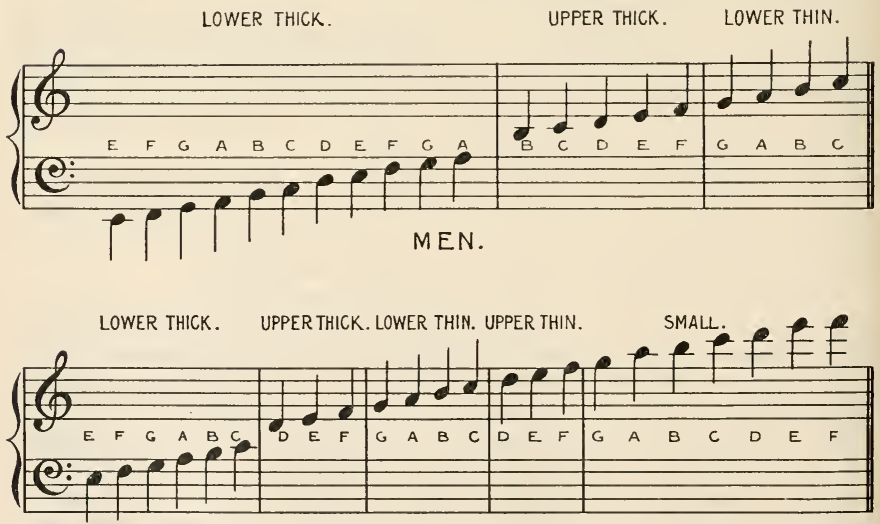

WOMEN AND CHILDREN.

FIG. 426.-Diagram showing range of registers of human voice.

chest, the middle, and the small or head register. The average range of pitch within each register is shown in the accompanying diagram (Fig. 426).

Chest register. - The chest register belongs more to the male than

1 Foster, "Text-Book of Physiology," 1891, part 4, p. 1460.

2 Arch.f. mikr. Anat., Bonn, 1887, Bd. xxix. 
the female singing voice; but in the contralto of the latter it is also used extensively, and it is the voice used in ordinary conversation. If the larynx be examined while the lowest note of this register is being uttered, it will be seen that the epiglottis is kept far back, and, to a certain extent, hides the larynx. In suitable cases, however, it may be seen that between the vocal cords there is an elliptical opening, which extends from the posterior laryngeal wall right up to the apex of the thyroid cartilage in front. During the sounding of such a note, the larynx is fixed by the sterno-thyroid and the thyro-hyoid muscles, and the arytenoid cartilages are brought into apposition by the arytenoideus. We may assume, then, that the note is produced by the vibration of the vocal cords in their whole length, breadth, and thickness (Fig. 427).

If the singer be directed to proceed singing up the scale, it will be observed that the vocal processes of the arytenoid cartilages become rotated inwards. This is brought about by means of the lateral crico-

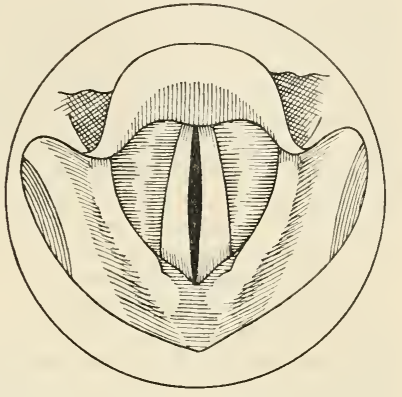

FIG. 427.-Aperture of glottis during production of low note of lower register. -Bosworth. arytenoid muscles, and it continues until these two prominences are in contact, when an elliptical opening exists, extending from the point where they touch one another to the angle of the thyroid cartilage in front. If the singer continues to ascend the scale, this elliptical opening becomes more and more contracted, until the chink of the glottis is represented by a linear slit. This condition is brought about

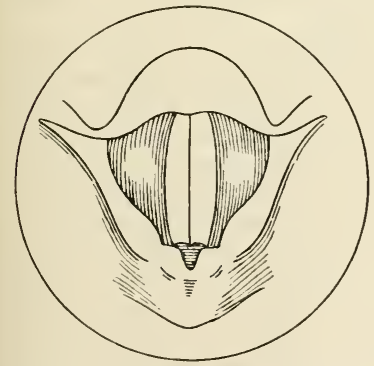

FIG. 428.-A - Aperture of glottis during production of high note of lower register.-Bosworth. by contraction of the thyro-cricoid muscle, and the notes uttered during this stage are said to belong to the upper thick register; the highest note obtainable by this register has now been reached. It should be added that, as the note rises in pitch, the epiglottis is carried further forward, so that the cords are more easily seen (Fig. 428).

During the utterance of the thick register, the false cords are more widely apart than at any other time.

Having reached the highest tension which it is possible to produce, by the mechanism just described, the singer must now employ a different one in order to pass higher up the scale, and this is done by making use of the mechanism of the midlle register.

Middle register.-On passing from the highest note of the lower register to the lowest of the middle register, a very sudden change occurs, which not only gives a sense of relief to the singer, but is usually detected by the ear. This change consists in the relaxation of the anterior fibres of the thyro-cricoid muscle, which may be felt by the finger 
when it is placed over the thyro-cricoid membrane. This relaxation of the thyro-cricoid muscle is accompanied by contraction of the thyro-arytenoid, with the result that the cord is rendered narrower, and now only those parts lying near the edge vibrate. The tension of the cords is still maintained by the posterior fibres of the thyro-cricoid and by the thyro-hyoid. ${ }^{1}$

According to Bosworth, the sense of relief in passing from the lower to the middle register is due for the most part to the fact that less muscular effort is required to throw the narrow portion of the cord into vibration, than was necessary in the lower register to set the cord into vibration in its whole breadth. The natural result of this diminution of the vibrating mass is that the voice loses considerably in volume; at the same time, however, it gains in softness. In this register the larynx appears to become narrower than in the lower, and simultaneously with this narrowing the ventricular bands approach one another. It is quite possible that the diminution in the breadth of the vocal cords themselves may be in large part merely apparent, their outer portion being hidden by the ventricular bands, so that they may be really as broad as in the lower register.

The rise in pitch which occurs during the transition from the lower to the middle register is, however, undoubtedly due in part to a thinning of the vocal cords. This is best seen by using Voltolini's method of illuminating the larynx, that is, by placing a strong light directly in front of the thyroid cartilage, instead of reflecting it through the mouth and pharynx. When the mirror is put into the throat, the cords now appear pink or red in colour, and changes in their thickness can easily be observed. In the middle register they are beautifully transparent, but much less so in the lower.

On ascending in the middle register, the cords gradually become more and more tense as the anterior fibres of the thyro-cricoid musele again come into play, and the crico-thyroid space becomes obliterated. The pitch can be raised no further by this mechanism. It must be added that, in the whole of the middle register, the vocal processes of the arytenoid cartilages are kept in contact by the action of the lateral crico-arytenoid and the inter-arytenoid muscles.

Head register.-Having reached the highest note which can be

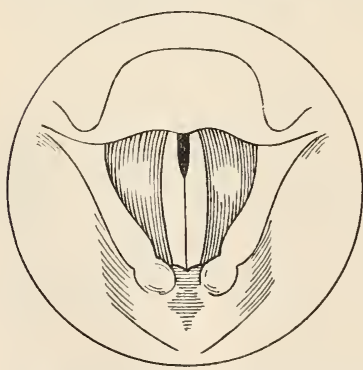

FIG. 429. - Aperture of glottis during production of a note in the head register.-Bosworth. produced in the middle register, the mechanism must now be changed again, if a series of higher notes is required. This new mechanism is called the head register. By far the most striking change that takes place is, that the vocal cords are now brought closely in contact along their posterior halves, while there remains an oval orifice at the front. Thus the only vibrating portions of the cord are those which bound this opening (Fig. 429).

The tension of the vocal cords in this register is still maintained by the thyro-cricoid and thyro-arytenoid muscles. As the pitch rises the small orifice in front becomes shorter and narrower, until at the highest notes only the anterior third of the cords may be vibrating.

In the preceding description of the registers, that classification has ${ }^{1}$ Bosworth, "Diseases of the Throat and Nose," vol. ii. p. 469. 
been followed which is, on the whole, the most generally accepted. As regards the action of the larynx, however, during the different registers, though there is some agreement as to the principal changes which oceur, many parts are as obscure as the amount of literature on the subject is large.

Lehfeldt, ${ }^{1}$ when experimenting upon larynges removed from the dead body, found, by accident, that when a weak blast of air was driven through the glottis, the pitch of the note produced was higher than when the blast was strong. From this discovery he inferred that the falsetto (thin) register was produced by a weak current of air, while a strong current was necessary for the production of the chest register. He further maintained that the reason why the note produced by the strong blast was deeper than that produced by the weak one, was because in the first case the cords were made to vibrate in their whole extent, while the weak current was unable to effect this.

Johannes Muiller ${ }^{2}$ adopted this view, and its truth is still maintained by some authorities. Lehfeldt further supposed that the outer fibres of the thyro-arytenoid musele might hinder the outer portions of the cords vibrating, while the inner portions were free to vibrate. There is nothing inconsistent in this from an acoustical point of view. Oertel's ${ }^{3}$ view, promulgated in recent times, is somewhat akin to this; he holds that when the falsetto voice is produced, nodal lines are formed in the vocal cords, parallel to their edges.

Garcia, ${ }^{4}$ from observations with the laryngoscope, which he introduced, held that, in the lower part of the chest register, the whole of the cord, along with the vocal process of the arytenoid cartilage, is thrown into vibration; while, as the voice ascends in pitch, only the ligamentous portion of the cord vibrates, the vocal processes being in contact. Behnke, ${ }^{5}$ on the other hand, maintains that the vocal processes of the arytenoid cartilages do not vibrate at all during phonation. In recent years, however, it has been definitely shown that these cartilaginous structures do partake in the vibration of the cord. Hodgkinson ${ }^{6}$ insufflated finely powdered indigo into the larynx, and, by observing the positions which it assumed on the cord during phonation, he was able to demonstrate the fact that, in the deeper note of the lower register, the vibrating margin extended from the thyroid cartilage in front to a point behind the junction of the ligamentous and cartilaginous portions of the cord. Hodgkinson's experiment further disproved the assertions of some authorities, who maintain that contact of the vocal processes is essential for phonation. In this respect Hodgkinson's view is in agreement with that of Wesley Mills. ${ }^{7}$

As regards the mechanism of the middle register, observers are fairly unanimous upon one point, namely, that the vocal processes of the arytenoid cartilages are in contact throughout. As regards minor points, opinions vary considerably; this may be due to the fact that the mechanism itself differs in individuals.

\footnotetext{
1 "Inaug. Diss.," 1835.

2 "Physiology," translated by Baly, London, 1837-42.

3 "Ueber d. Mechan. des Brust- u. Falsett-Registers," Stuttgart, 1882.

4 "Beobacht. ueber d. menschl. Stimme," Monatschr. f. Ohrenh., Berlin, 1878, Nos. 1 to 6.

5 "Mechanism of the Voice," 1880, p. 88.

${ }^{6}$ Brit. Med. Journ., London, 1895, vol. ii. p. 482.

7 Proc.Am. Ass. Adv. Sc., 1882.
} 
Martels ${ }^{1}$ affirms that, in the chest register, the whole cord vibrates, while in the upper register only the mucous membrane is in motion. Lermoyez ${ }^{2}$ agrees to a certain extent in this view of Martels, and states that the pitch is altered solely by variations in the tension of the cords.

The falsetto register, according to Martels, is a flute sound; the chest register, on the other hand, is comparable to the sound produced by a reed instrument.

Although almost all observers agree that the posterior portion of the glottis is closed during the production of the upper registers, the condition of the anterior part is not so satisfactorily settled. Browne and Behnke ${ }^{3}$ state that even in the highest register the anterior portion of the glottis is open, while the larger posterior portion is tightly closed. This register, according to these writers, is only used by women and boys. Wesley Mills, ${ }^{4}$ however, states that the anterior as well as the posterior portion of the glottis is closed in the head voice ; that is to say, that it is the same mechanism as that which is used by men for the production of their high falsetto voice.

French ${ }^{5}$ has obtained photographs of the larynx, as it appears during the different registers, which should tend to modify very considerably some of the views hitherto prevalent concerning the production of the different registers. He found that, in proceeding from the lowest to the highest notes of the lower register, the vocal cords became lengthened by $\frac{1}{8} \mathrm{in}$.; this was in a contralto singer. When this singer passed to the middle register, the cords became shortened by $\frac{1}{16}$ in., this shortening being followed by another increase in length, as the upper part of the middle register was approached.

From the photographs of the larynx during the production of the lower notes of the head register, French infers, contrary to most anthorities, that the edges of the ligamentous cords are not in contact at any portion; so that air passes through the whole length of the ligamentous glottis. During the upper notes of this register, however, it is probable that the cords are closed in their posterior portions.

According to French, it is a general rule, to which, however, there are many exceptions, that in the lower notes of each register the vocal cords are short and wide, and the glottis is open both in its ligamentous and cartilaginous portions. On ascending the scale, the cords increase in length and decrease in width. ${ }^{6}$

1 "Physiol. de la phonation," Rev. bibliog. univ. d. sc. méd., Paris, 1885, Nos. 13 and 15 .

2 "Etude expér. sur la phonation," Thèse de Paris, 1886, p. 199.

3 "Voice, Song and Speech," 1893, p. 135.

${ }^{4}$ Loc. cit.

5 Verhandl. d. internat. med. Cong., Berlin, 1890.

${ }^{6}$ For further information on the subject of the vocal registers, the reader is referred to Semon's exposition, published in the Proceedings of the Royal Institution, 1891. The following are some of the works on this subject:-Behnke, "The Mechanism of the Human Voice," 1880; Bennett, "The Falsetto Voice," Med. Rec., N. Y., Nov. 1, 1893; Browne and Behnke, "Voice, Song and Speech," 1893; Castex, "Hygiene de la voix parlée ct chantée," Paris, 1894 ; Fournié, Rev. méd. frane. et étrang., Paris, 1881 ; French, "The Action of the Glottis in Singing," New York Med. Journ., Jan. 1891; Garnault, "Cours théorique et pratique de physiol.," ete., 1896 ; Gavarret, "L'audition et la phonation" ; Gouguenhein and Lermoyez, "Physiol. de la voix et du chant," 1885 ; Garcia, "Bcobachtungen ueber die menschliche Stimme," Monatschr. f. Ohrenh., Berlin, 1878; Gruitzner, "Physiologie der Stimme u. Sprache"; Hermann's "Handbuch," 1879 ; Hodgkinson, Brit. Med. Journ., London, 1895, vol. ii. ; Gordon Holmes, "The Physiology of the Vocal Registers in Male and Female," IFed. Times and Gaz., London, 1885, p. 476; Franklin H. Hooper, Ann. d. mal. de l'oreille, du larynx, etc., Paris, 1890 ; Hooper, "'Tension 


\section{The Quality of the Voice and Vowel Tones.}

The quality of the human voice depends on the same laws as those determining the quality, klangfarbe, or timbre of the tones produced by any musical instrument. Tones of a mixed character-that is to say, composed of a fundamental and partials-are produced by the vibrations of the true vocal cords, and certain of these partials are strengthened by the resonance of the air in the air passages and in the pharyngeal and oral cavities. So strongly may certain of these partials be reinforced, as to obscure or hide the fundamental tone, and give a peculiar character to the sound. But there are still many difficulties in the way of a true interpretation of voice tones. Thus we observe that we may sing a scale, using one sound for each note, such as $l a, l a, l a$, etc. Or, by pulling the mouth in a certain position, we can pronounce the vowel sounds, $a h, \bar{c}, \bar{e}, o, o u$ (pronounced as the $u$ in prune). As we do so, we notice that each sound appears to the ear to have a pitch of its own, different from that of the others. Helmholtz ${ }^{1}$ gave the characteristic pitch of the several vowel tones as follows:-

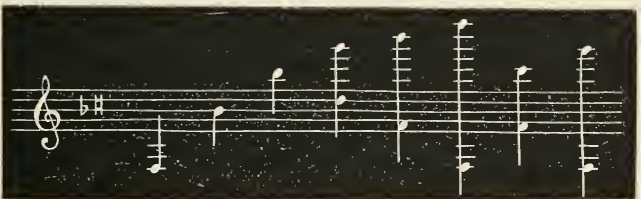

\begin{tabular}{|c|c|c|c|c|c|c|c|c|c|c|c|}
\hline Vowels & • & • & . & $\mathrm{OU}$ & $\mathrm{O}$ & $A$ & AI & $\mathrm{E}$ & I & $\mathrm{EU}$ & $\mathrm{U}$ \\
\hline Tone & . & . & . & $f a_{2}$ & $s i \ddot{b}_{3}$ & $\mathrm{sib}_{4}$ & $\underset{\text { or }}{\operatorname{sol}_{\overline{5}}}$ & $\begin{array}{l}s i \phi_{\bar{b}} \\
\text { or }\end{array}$ & $\begin{array}{l}r e_{6} \\
\text { or }\end{array}$ & $\begin{array}{l}d o_{5} \\
\text { or }\end{array}$ & $\begin{array}{c}\operatorname{sol}_{5} \\
\text { or }\end{array}$ \\
\hline No. of vibs. & - & . & . & 170 & 470 & 940 & $\begin{array}{c}\mathrm{re}_{4} \\
1536 \\
\text { or } \\
576\end{array}$ & $\begin{array}{c}f a_{3} \\
1920 \\
\text { or } \\
341\end{array}$ & $\begin{array}{c}f a_{2} \\
2304 \\
\text { or } \\
170\end{array}$ & $\begin{array}{c}f a_{3} \\
1024 \\
\text { or } \\
341\end{array}$ & $\begin{array}{c}J u_{2} \\
1536 \\
\text { or } \\
170\end{array}$ \\
\hline
\end{tabular}

FIG. 430.-Pitch of vowels according to Helmholtz.

of the Vocal Bands," Arch. Laryngol., N.Y., 1883; Illingworth, "Mechanism of the Voice," 1882 ; Jelentfy, Arch. f. d. ges. Physiol., Bonn, 1880, Bd. xxii. : Kosehlakoff, "Ueber die Schwingungstypen der Stimmbinder," Arch. f. Physiol., Leipzig, 1886"; Krzywicki, Verhandl. d. Berl. laryngol. Gesellsch., 1891-1892; Lermoyez, "Etude expér. sur la phonation," Paris, 1886; Lunn, "Philosophy of Voice," 1886 ; Morell Mackenzie, "Hygiene of the Vocal Organs," 1890 ; Mandl, "Hygiène de la voix parlíe ou chantée," 1879 ; Martel, Rev. bibliog. univ. d. sc. méd., Paris, 1885 ; Merkel, "Anat. u. Physiol. des menschlichen Stimm- u. Sprachorgans," Leipzig, 1863 ; Meyer, Arch. $f$. Physiol., Leipzig, 1889 ; Michael, "Die Bildung des Gesangs-register," 1887 ; Mills, Proc. Am. Ass. Adv. Sc., 1882 ; J. Muller, "Physiology," translated by Baly, London, 1837-42; Neumann, Wien. med. Presse, 1893, No. 24 ; Oertel, "Ueber dic Mechanismus des Brustund Falsett-registers," 1882, "Laryngo-stroboscopische Examination," Nünchen. med. Wchnschr., 1895, No. 11 ; Rethi, "Tension of the Cords," Monatschr. f. Ohrenh., Berlin, 1894 ; Carl Seiler, "Physiology of Voice and Speech," Philadelphia, 1888; Seiler, "The Voice in Singing," 1881; Semon, Notices Proc. Roy. Inst. Grat Britain, 1891; Stockhausen, "Gesangsmethode," Leipzig, 1884; Störk, "Sprechen und Singen," Wien, 1881.

1 "Sensations of Tone," translated by Ellis, 1875, p. 165 . Sec also same work, p. 163, footnote 1, for results obtained by Merkel, Reyher, Hellwag, Flurake, and Donders. 
König ${ }^{1}$ has given a different evaluation of the pitch of vowel sounds thus :-

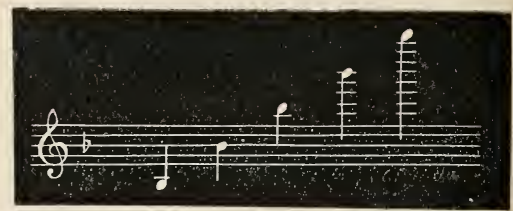

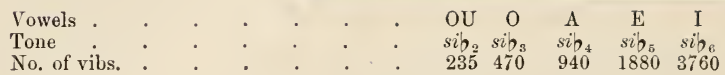

FIG. 431.-Pitch of vowels according to König.

Donders, ${ }^{2}$ who was the first to observe that the cavity of the mouth for different vowels is tuned to different pitches, gave $U$ as $f_{1}, O$ as $d_{1}$, $A$ as $b^{\prime} b, O$ (like $u$ in tugh) as $g^{2}, \dot{U}$ (like $u$ in lune, Fr.), as $a^{\prime \prime}, E^{\prime}$, as $c^{\prime \prime \prime}$ and $I$ as $f^{\prime \prime \prime}$.

Again, we may sing a vowel on a scale and still recognise the vowel in each note. Thus, if we sing $\alpha$, or 0 , or $i$, on a scale beginning with $c$, the ear catches the sound of $a$, or $o$, or $i$, in each note. Such tones as we have described are termed vowels, or we might call them vowel tones.

Other sounds associated with peculiar conditions of the buccal cavity, and which may be strengthened by the laryngeal sound, are those known as consonants. These may be labials, as $F, V$, or $P$, linguals, as $S, T$, $D$, and gutturals, as $J, K$, or $G$, Ger. The physical nature of consonantal sounds has not yet been sufficiently investigated. We shall first proceed to discuss the nature of vowel tones, more especially with the view of determining the cause of quality of vowel tone, and the reason why a vowel tone always appears to have the same quality, whatever may be the pitch of the note on which it is sung.

Theories of vowel tones.-The investigation of vowel tones may be considered to date from the experiments of Willis ${ }^{3}$ about 1829 , who imitated the larynx by means of a reed, above which he placed a resonating cavity, and his conclusions are very similar to those put forward by Hermann at the present day. About 1837, Wheatstone ${ }^{4}$ made certain further observations, and advanced a theory, and in later times the subject has been studied by Donders, Helmholtz, König, Hermann, and many others.

The invention of the tinfoil phonograph by Edison in 1877, and the improvement of the instrument by the labours of Edison, Graham Bell, and others, in more recent years (until we now possess, in the wax-cylinder phonograph, a far better mechanism), have led to the re-investigation of the whole question of vowel tone by Fleeming Jenkin, Ewing, Hermann, Pipping, Boeke, Lloyd, M'Kendrick, and others. The difficulties in the way of accounting for some of the phenomena of vowel tones will be appreciated when we state that these and other competent observers

${ }^{1}$ Compt. rend. Acad. d. sc., Paris, 1870, tome lxx. p. 931 ; also "Quelques expériences d'acoustique," 1882 , p. 47.

2 "De physiologie der spraakklanken," 1870, S. 9.

${ }^{3}$ Cambridge Phil. Trans., 1829, vol. iii. p. 231 ; also Ann. d. Phys. u. Chem., Leipzig, Bd. xxiv. S. 397 .

${ }_{4}$ Westminster Review, Oct. 1837. 
are ranged in two camps-those who uphold the theory of relative as opposed to those who contend for the theory of fixed pitch. Assuming that a vowel is always a compound tone, composed of a fundamental and partials, those who uphold the relative pitch theory state that, if the pitch of the fundamental tone is changed, the pitch of the partials must undergo a relative change; while their opponents contend that whatever may be the pitch of the tone produced by the larynx, the pitch of the partial that gives quality or character to a vowel is always the same, or, in other words, vowel-tones have a fixed pitch.

There are many methods of investigating this problem, but these may be grouped in two divisions-first, experimental methods, by which the pitch of the oral cavity, in the position suitable for the production of any given vowel, may be determined; and, second, mathematical methods, by which the curve or wave-form, representing a certain quality of vowel-tone, may be analysed into its components, in accordance with Fourier's theorem.

One of the early experiments of Willis ${ }^{1}$ favoured the fixed-pitch theory. A piece of watch-spring was held by forceps against a revolving toothed wheel. A compound tone was produced, which of course retained the same pitch so long as the wheel revolved uniformly. Now, by keeping the wheel revolving at a uniform rate, and at the same time changing the length of the portion of the spring which was allowed to vibrate, Willis found that the qualities of various vowels were obtained with considerable distinctness.

Willis also used reed pipes attached to cylindrical chambers of variable length, and altered the quality of tone by increasing or diminishing the length of the resonant chamber. The shortest tubes gave $I$, then $E, A, O$, to $U$. In this way he determined the pitch of the vowel as it sounds in words. ${ }^{2}$

As already stated, Donders ${ }^{3}$ was the first to show that the cavity of the mouth, as arranged for the giving forth of a vowel, was tuned as a resonator for a tone of a certain pitch, and that different pitches corresponded to the forms of the cavity for the different vowels. This he discovered, not by the use of tuning-forks, but by the peculiar noise produced in the mouth when the different vowels are whispered. The cavity of the mouth is then blown like an organ-pipe, and by its resonance reinforces the corresponding partials in the rushing windlike noise. The question was then taken up by Helmholtz, ${ }^{4}$ and treated in his usual masterly fashion. To determine the pitch of the cavity of the mouth, considered as a resonance cavity, he struck tuningforks of different pitches, and held them before the opening of the mouth, say widely opened. Then, the louder the proper tone of the fork was heard, the nearer "it corresponded with one of the proper tones of the included mass of air." As the shape of the moutl could be altered at pleasure, according to the vowel to be emitted, it was easy to

1 op. cit., p. 231.

2 Ellis. See footnote in "Sensations of Tone," by Helmholtz, p. 170; also article "Sound" in Encyc. Metropol., par. 375, by Sir John Herschell.

${ }^{3}$ Op. cit.; also Arch. f. d. holländ. Beitr. z. Nat- u. Heilk., Utreeht, 1857, Bd. i. S. 157 ; see also references to older observers in Helmholtz's "Sensations of Tone," p. 162 (footnote).

4 "Ueber die Vokale," Arch.f. d. holländ. Beitr. z. Nat-u. Heilk., Utrecht, 1857, Bd. i. S. 354-355; also "Ueber die Klangfarbe der Vocale," Gel. Anz. d. k. bayr. Akad. d. Wissensch., 1879, S. 537-541; Ann. d. Phys. u. Chem., Leipzig, Bd. cviii. S. 280 ; also in Wissensch. Abhandlungen, Bd. i. S. 395-397 : also "Sensations of Toue," eliap. v. p. 153.

VOL. II. -77 
discover the pitch of the included mass of air for each vowel. He came to the conclusion that " the pitch of the strongest resonance of the oral cavity depends solely upon the vowel for pronouncing which the mouth has been arranged." He also found the same resonances for men as for women and children. He then carefully examined the form of the oral cavity for each vowel, and showed how very slight changes would account for the quality being slightly altered for different dialects.

Helmholtz also showed that the tones of the human voice have a peculiar relation to the human ear. Thus the upper partials of $I$ are in the neighbourhood of $e^{\prime \prime \prime \prime}$ up to $g^{\prime \prime \prime \prime}$, and the human ear itself is tumed to one of these pitches; that is to say, by its resonance it favours the perception of these tones. Powerful male voices produce these partials strongly. Thus, a bass voice singing $e^{\prime}$ produced the seventh partial $d^{\prime \prime \prime \prime}$, eighth $e^{\prime \prime \prime \prime}$, ninth $f^{\prime \prime \prime \prime}=$, and the tenth $y^{\prime \prime \prime \prime}=$. Some of these partials, such as $e^{\prime \prime \prime \prime}$ and $f^{\prime \prime \prime \prime}=$ may clash, and by producing dissonance, give harshness to the voice. This illustration will show how Helmholtz, following out the theory applicable to all musical instruments, endeavoured to explain the quality of different voices. His theory as to vowel-tone is summed up in the following sentence- "Vowel qualities of tone consequently are essentially distinguished from the tones of most other musical instruments, by the fact that the loudness of their partial tones does not depend upon the numerieal order, but upon the absolute piteh of those partials; thus, when I sing the vowel $A$ to the note $E_{0}$, the reinforced tone $b^{\prime \prime}$; is the twelfth partial tone of the compound; and when I sing the same vowel $A$ to the note $b^{\prime} b$, the reinforced tone is still $b^{\prime \prime} b$, but is now the second partial of the compound tone sung." 1

Further, Helmholtz endeavoured to demonstrate the correctness of his view by synthetically combining the tones of certain tuning-forks in his well-known vowel tone apparatus. ${ }^{2} \mathrm{He}$, in early experiments, used eight forks, the first being the fundamental tone $s i_{1} b$, and the others the first seven partials. Thus-

$$
s i_{1} b, \quad s i_{2} b, \quad f a_{3}, \quad s i_{3} b, \quad r e_{4}, \quad f a_{4}, l a_{4} b, \quad s i_{4} b,
$$

The vowel $O$ is well sounded with this apparatus, when we sound $s i_{3} b$ (characteristic of $O$ ) strongly; more feebly $s i_{2} b, f a_{3}, r e_{3}$, and the fundamental softly. $O U$ is good with $s i_{1} b$ strong, and the partials feeble. Using in another apparatus

$$
s i_{2} b, \quad s i_{3} b, \quad f a_{4}, s i_{4} b, \quad r e_{5}, f a_{5}, l a_{5} b, \quad s i_{5} b,
$$

$O U$ was given with $s i_{2} b$ (the fundamental) alone. $O$ was sounded by fundamental $s i_{2} b$ moderate, $s i_{3} b$ strong, and $f a_{4}$ weak. If we sound $s i_{2} b$ (fundamental) along with $s i_{3} b$ and $f a$ moderate, and $s i_{4} b$ and $r e_{5}$ strongly, we obtain $A$. This vowel is characterised by $s i_{4} b$, along with the partials $s i_{3} b$ and $s i_{2} b$. To obtain $E$, give $s i_{2} b$ and $s i_{3} \rho$ moderate, and $f a_{3}, l a_{3} b$, and $s i_{3} b$ as strong as possible. The characteristic partial of this vowel is $s i_{3} b$. We have performed many experiments with this apparatus, and find the results obtained by Helmholtz to be consistent with our experience. Much depends, in the appreciation of this experiment, on careful attention, practice, and a good ear.

König investigated the subject with the aid of his manometric

${ }^{1}$ Helmholtz, "Sensations of Tone," p. 172.

2 For figure and deseription of this famous apparatus, see M'Kendrick's "Physiology," vol. ii. p. 691 . 
flame apparatus. This method is useful, because it shows the different forms of the sound-wave, without change of period, corresponding to a

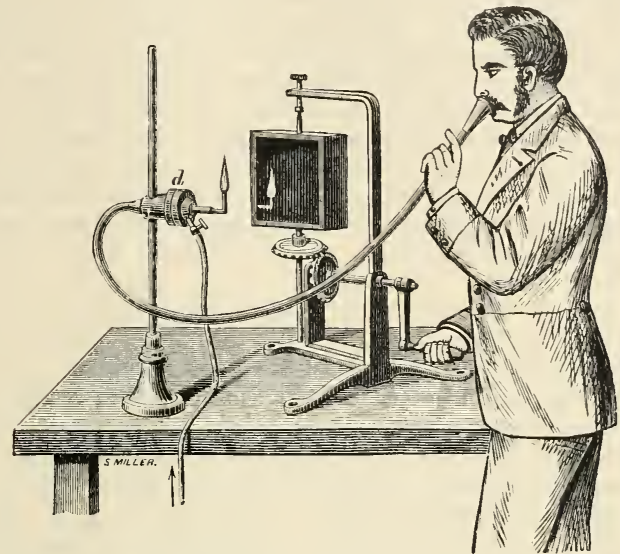

Fig. 432. - König's apparatus for illustrating the quality of vowel tones by a manometric flame.

tone of determinate pitch, when the vowels are sung upon that tone. The apparatus is shown in Fig. 432.

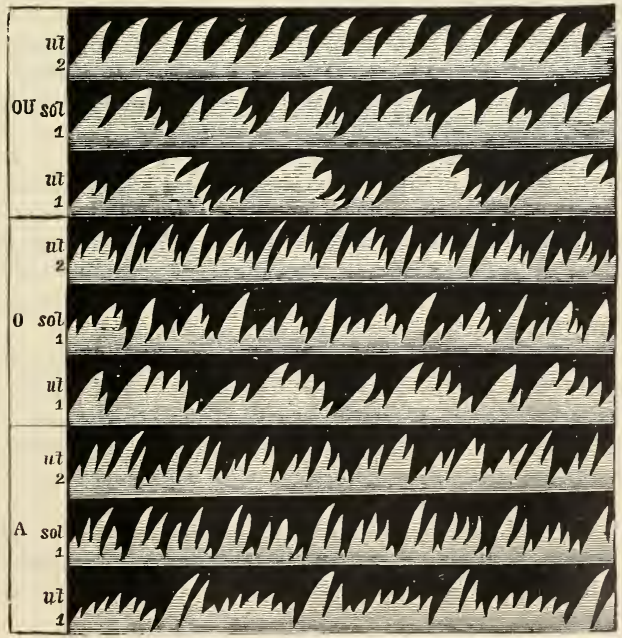

FIg. 433. - Flame pictures of the vowels $O U$, $O$, and $A$. - König.

The next figure shows the forms taken by the flame when the three notes $u t_{1}, s_{1} l_{1}$, and $u t_{2}$ are sung with the vowels $A, O$, and $O U$. 
Each of these pictures is composed of two groups of teeth, reproduced again and again, and thus showing a regular periodic vibratory movement. The three pictures of $u t_{1}$ contain eight groups of teeth, while those of sol $_{1}$ contain twelve, and those of $u t_{2}$ sixteen. As the length of all these pictures corresponds to the same duration of a vibratory state, it is evident that the period of the vibratory movement, or the length of the wave characteristic of a tone of determinate pitch, is independent of the vowel upon which the sound is emitted. But the form of the wave characteristic of the tone of given pitch varies much with the vowel upon which it is sung. This alteration of the form of the wave, while the period is constant, must be due to the superposition of a tone developed in the mouth, characteristic of the vowel, upon the tone emitted by the $\operatorname{larynx} .^{1}$

According to König, $O U$ is always easily emitted with $s i_{2} b$, and the neighbouring tones $l a_{2}, s o l_{2}$ with the lower partials, si $b, m i_{1} b$. $O$ is emitted with $s i_{3} b$, the partials $l a_{3}, s o l_{3}$, and the lower partials $s i_{2} b, m i_{2} b, s i_{1} b$. A comes with $s i_{4} \emptyset$, with $l \alpha_{4}$, with $s o l_{4}$, along with the lower partials, $s i_{3} b, m i_{3} b, s i_{2} b$. $\quad E$ and $I$ are not easily sounded on low tones, as their characteristic partials are very high. On tones lower than $u t_{3}$, the female voice turns involuntarily to $O$ or $O U$, which have $s i_{3} b$ and $s i_{2} b$ as their characteristic partials. Above $f a_{4}$ it is $A$, characterised by $s i_{4} b$, which is most readily given. Above $s i_{4}$ the voice passes into $E$ and $I$. These olservations of Konig are harmonious with the conclusions of Helmholtz, and favour the fixed pitch theory.

Hallock ${ }^{2}$ has recently employed a device founded on that of König. Eight resonators in harmonic series were each connected with a manometric capsule, and photographs were taken of the eight bands of flame pictures, reflected in a mirror, when the vowels were sung before the resonators. ${ }^{3}$ From these photographs, the partials present in any vowel tone within the range of the resonators could be determined. Of course the higher partials, on which, as pointed out by Helmholtz, much depends, were not detected.

Wave-forms of vowel tones.--We must now turn to the evidence adduced by an analysis of the wave-forms of vowel tones. To appreciate this evidence, the following statements must be kept in view :-

1. Pitch depends on the length of time in which each single vibration is executed, or, in other words, on the vibrational number of the tone.

2. Musical tones are higher, the greater their vibrational number; that is to say, the shorter the vibrational period.

3. The sensation excited by a periodic vibration is a musical tone. This tone is usually compound, the constituents being partial tones.

4. Only one form of vibration, like that of a pendulum or the limbs of a tuning-fork, can give rise to a simple tone, destitute of partials. This is a simple pendular vibration, and the sensation is a simple tone.

5. A compound tone is the sensation produced by the simultaneous action of several simple tones, with definite ratio of pitch. Such a compound tone corresponds physically to a wave of more or less complex form.

\footnotetext{
1 Gavarret, "Phénomènes physiques de la phonation et de l'audition," Paris, 1877, p. 394

${ }_{2}$ Am. Ann. Photo., 1896, p. 21.

${ }^{3}$ For a figure of such an analyser, see M'Kendrick's " Physiology," fig. 418, vol. ii. p. 686.
} 
6. Such a compound wave is capable of being analysed into a number of simple pendular vibrations, and each pendular vibration corresponds to a simple tone, having a pitch determined by the periodic time of the corresponding motion of the air (Ohm's law).

7. It is evident that such combinations of simple waves may give rise to an infinite variety of wave-forms, but, according to Fourier's theorem, " "any given regular periodic form of vibration can always be produced by the addition of simple vibrations, having vibrational numbers, which are once, twice, thrice, four times, etc., as great as the vibrational number of the given motion."

8. If we know the amplitudes of the simple vibrations and the difference of phase, then any regularly periodic motion can be shown to be the sum of a certain number of pendular vibrations; in other words, the compound wave may be analysed into its constituents.

The Fourierian analysis of wave-forms.-The method of applying the Fourierian analysis may be shortly stated. ${ }^{2}$ Suppose a single wave $(A A$ or $B B)$ is taken and referred to rectangular axes $(O X, O Y)$, aloscisse of equal length are measured off along $O X$, ordinates are drawn to meet the curves, and these are very accurately measured. For a simple curve, such as $A A$, few ordinates suffice; but if the curve is complex in form, the number of measurements must be increased until no considerable curvature exists between the summits of any two successive co-ordinates. When a sufficient number of ordinates has been taken, an analysis carried to one-half that number of partials, or even somewhat less, suffices to define the curve. The mathematical process for evaluating the amplitude and phase of a certain number of partials, say the first twelve, is given by Fleeming Jenkin and Ewing in their well-known paper. ${ }^{3}$ A

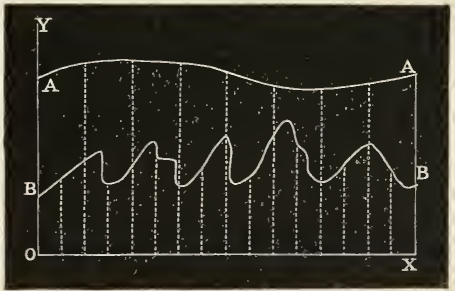

Fic. 434.-Diagram showing the first step in analysing wave-forms. curve may be rebuilt graphically from its analysis, but the process is laborious when the partials are numerous. If we know the pitch of the fundamental tone, then the pitch of any partial is ascertaince by multiplying its vibrational number by the figure representing the ratio of its amplitude to that of the fundamental tone. For example, take the analysis of a Swedish $i$ (resembling the French), sung by Pipping at a pitch of 293 vibrations per second.

An inspection of this figure shows that two reinforcements are very pronounced, the one on the seventh and eighth, and the other on the fourth partial. Each of these partials has to do with the resonance cavity to which it corresponds, and in which it has been developer to a greater or less extent. In the case of the vowel $i$, one of the reson-

1 Donkin, "Acousties," Fourier's theorem proved, pp. 65-71; illustrated, pp. 56-65. See also Everett, "Vibratory Motion and Sound."

${ }_{2}$ The authors are much indebted to Dr. R. J. Lloyd for aid in the discussion of this diffieult subject.

${ }^{3}$ Trans. Roy. Soc, Edin., vol. xxviii. p. 750. 
ances is obtained in the narrow tube formed between the tongue and the hard palate. This resonance has a very narrow range, for the tube will not respond to any partial having a pitch much different from its own. A rounded cavity, on the other hand, such as is formed when we articulate

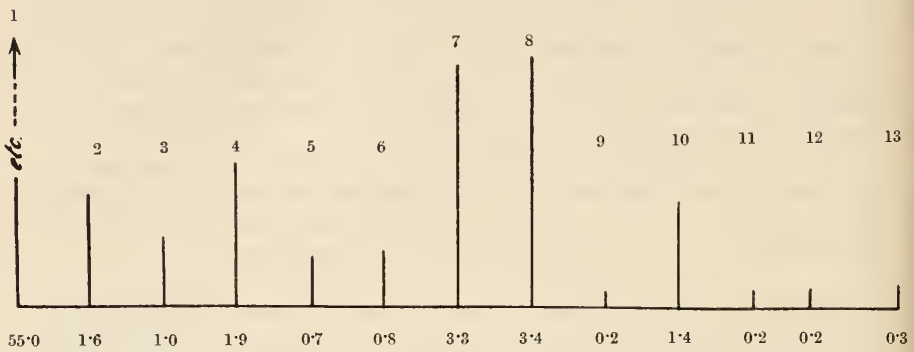

FIG. 435.--Diagram showing the amplitude, as indicated by the height of the ordinates, of the partials of the Swedish $i$, sung at pitch of 293 vibs.

$a a$, will have a much greater range of resonance. ${ }^{1}$ The valuation of the pitch of any resonance which only reinforces a single partial, is very simple, and is expressed by the equation-

$$
n=N p,
$$

where $N$ is the vibrational number of the fundamental tone, $p$ the number of the partial, and $n$ is the vibrational number of the resonance, or, in other words, its pitch. In the above figure, for example, the isolated reinforcement of the tenth partial in the vowel sung at 29:3 v. d. shows the existence of a resonance of $293 \times 10=2930$ vibs. Where two partials are equally reinforced by one cavity, the case is not so simple. Approximately, it is expressed by the equation-

$$
n=N \frac{p+p}{2}
$$

Thus the resonance which reinforces the seventh and eighth partials is about $293 \times \frac{15}{2}=2198$ v. d. Even here there are minor considerations involved, which can only be seen clearly after examining one or two more general and more complex cases.

Let us take first an instance of German long $a$, sung and analysed by Hermann. ${ }^{2}$ The pitch is 98 vibs., and the analysis is only carrier to ten partials, so that it does not display the higher resonances of this vowel, except slightly on the tenth partial.

\footnotetext{
${ }^{1}$ Lloyd, "On the Fourierian Analysis of Phonographic Tracings of Vowels," Proc. Roy. Soc. Edin., 1898.

2 "Phonophotographisch. Untersuch.," Bd. i.-v. ; Arch.f. d. ges. Physiol., Bonn, Bd. xlv. S. 582 ; Bd. xlvii. S. 44 ; Bd. xlvii. S. 347 ; Bd. liii. S. 1 ; Bd. lviii. S. 255, contain many analyses and much controversy abont the Fonrierian analysis. No. 2 contains an account of a method of lightening the labour of these analyses ; also, "Bemerkungen zur Vocale-frace" (ibid., Bd. xlviii. S. 181); also, "Vocale im Telephon und Microphon" (ibid., Bd. xlviii. S. 543) ; also, "Weitere Untersucl. ueber das Wesen der Vocale" (ibid., Bd. lx. S. 169) deals chiefly with short (German) vowels.
} 
This analysis displays only one considerable reinforcement, but it extends in varying strength over the sixth, seventh, eighth, and ninth partials. The resonance chamber in this case is the inter-velar or pharyngeal cavity. Call this the $\alpha$-resonance. How can we value the proper pitch of that resonance? Hermann has employed what he terms a centre-of-gravity calculation; that is to say, he proceeds as if he were finding the centre of gravity of four

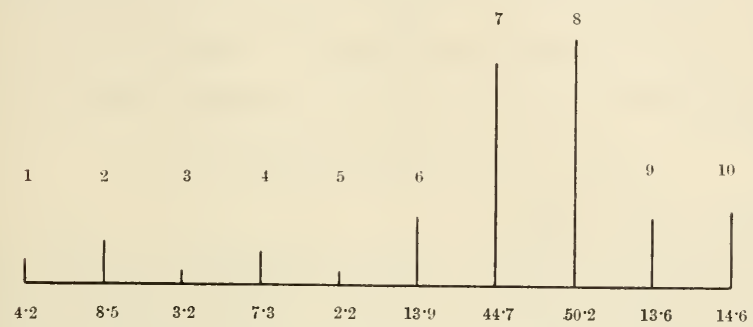

F1G. 436. -Diagram showing the amplitude in the partials of the German long $a$, sung and analysed by Hermann.

heavy points, situated, as above, on the axis of $x$, and each weighing as many units as there are units in the respective amplitudes. Having thus discovered the "mean partial," he multiplies the funlamental by it to get the value of the resonance itself. Both operations are combined in the equation-

$$
n=N \frac{p^{\prime} a^{\prime}+p^{\prime \prime} a^{\prime \prime}+p^{\prime \prime \prime} a^{\prime \prime \prime}+\text { etc. }}{a^{\prime}+a^{\prime \prime}+a^{\prime \prime \prime}+\text { etc. }}
$$

where $N$ and $n$ are again the pitch-numbers of the note and of the desired resonance, respectively; $p^{\prime}, p^{\prime \prime}, p^{\prime \prime \prime}$ are successive integers, the numbers of the reinforced partials; and $a^{\prime}, a^{\prime \prime}, a^{\prime \prime \prime}$ are the amplitudes found for these partials respectively. For the resonance displayed in the above Fig. 436, the process is as follows:-

$$
n=98 \times \frac{(6 \times 13 \cdot 9)+(7 \times 44 \cdot 7)+(8 \times 50 \cdot 2)+(9 \times 13 \cdot 6)}{(13 \cdot 9+44 \cdot 7+50 \cdot 2+13 \cdot 6)}=737 \text { vibs. }
$$

Pipping ${ }^{1}$ objects to the assumption that the partials, as in the above figure, are equidistant from one another. It is true that they are equidistant, when considered as an arithmetical progression. Thus, first partial, 98 vibs.; second partial, 196 vibs. ; third partial, 294 vibs., ete. But musical distances are not measured in aritlimetical, but in geometrical progression, and the equidistant partials are not the first, second, third, fourth, etc., but the first, second, fourth, eighth, etc. The abscisse in the figure ought not, therefore, to be measured off in proportion to the numbers $1,2,3,4$, ete. ; but to $\log _{2} 1$, $\log _{2} 2, \log _{2} 3, \log _{2} 4$, etc., or simply $\log 1, \log 2, \log 3, \log 4$, etc., seeing the

1 "Ueber die Tonfarbc der Vocale," Ztschr. f. Biol., Bd. xxvii. S. 1 (being detailed analysis of twenty-four vowel phonograms); also, "Zur Lehre von den Vocalklangen," ibid., Bd. xxxi. S. 524-583, containing controversy witl Hermann as to real meaning of Fourierian analysis; also, "Ueber die Theorie der Vocale," Acte soc. scicnt. Fennice, Bd. $\mathrm{xx}$. part 11, replies to Lloyd, and gives more analyses. 
base does not matter if the right mutual proportion is maintained. The result is shown as follows :-

1st Octave.

2nd Octave.

3rd Octave.

4th.

1st Partial.

2nd.

3 rd.

4th.

5 th.

6th. 7th. Sth. 9th. 10th.

Frg. 437.-Diagram showing the application of Pipping's method.

If we adopt this view, we get the following equation to find $p$, the mean partial :-

and then $n=\Lambda_{p}^{r}$, as before.

$$
\log p=\frac{a^{\prime} \log p^{\prime}+a^{\prime \prime} \log p^{\prime \prime}+a^{\prime \prime \prime} \log p^{\prime \prime \prime}, \text { etc. }}{a^{\prime}+a^{\prime \prime}+a^{\prime \prime \prime}, \text { ete. }}
$$

This method of Pipping's has been further developed by Lloyd, ${ }^{1}$ because he was of opinion that Pipping's method left frequency out of account. Look again at the above figures. The amplitudes are $4 \cdot 2,8 \cdot 5,3 \cdot 2$, etc.; but the first-named amplitude is traversed only once in each period, while the second is traversed twice, the third three times, and so on. The general expression for the whole amplitude traversed in each period is $a p$, i.e., amplitude multiplied by frequency. It seems a right prineiple to estimate the strength of the reinforcements of each partial in terms of the whole amplitude traversed, and if so, the multiplied amplitude $\left(a^{\prime} p^{\prime}, a^{\prime \prime} p^{\prime \prime}\right.$, etc.) will have to be substituted for the single amplitudes $\left(a^{\prime}, a^{\prime \prime}\right.$, etc.) in the last equation. Thus-

to find the mean partial.

$$
\log p=\frac{a^{\prime} p^{\prime} \log p^{\prime}+a^{\prime \prime} p^{\prime \prime} \log p^{\prime \prime}+a^{\prime \prime \prime} p^{\prime \prime \prime} \log p^{\prime \prime \prime}+\text { ete. }}{a^{\prime} p^{\prime}+a^{\prime \prime} p^{\prime \prime}+a^{\prime \prime \prime} p^{\prime \prime \prime}+\text { ete. }}
$$

Comparing the three methods of Hermann, Pipping, and Lloyd, it is evident that Pipping's will always give a result somewhat lower, and Lloyd's will give a result somewhat higher, than Hermann's. In the present case, shown in the above figure, the difference between the three calculations is not great. The first (Hermann) gives 737 vibs., the second (Pipping) 732 vibs., while the third (Lloyd) is 741 vibs. With high partials the differences become less and less, but with lower partials the differences are so great as to be, in Lloyd's opinion, "of vital importance," the object of these calculations being to find ont what is the true pitch of the resonating cavity that may reinforce several or many partials.

Phonograms.-We are now in a position to examine the methods that have been adopted to obtain graphic tracings of the wave-forms (phonograms) corresponding to vowel tones, so as to submit these to the Fourierian analysis.

Donders, ${ }^{2}$ in 1870 , was the first to apply the phonautograph of Leon Scott $^{3}$ (invented in 1856) to the investigation of the curves produced by the sounds of vowels. In 1878, Fleeming Jenkin and Ewing ${ }^{4}$ succeeded in obtaining tracings of the records of vowel sounds on the tinfoil

${ }^{1}$ Reply to Pipping's review of the author's articles on "Speech Sounds," in Phonetischc Stud., 1890-1892 (Ztschr. f. d. französische Sprache, Bd. xri. S. 211); also, "The Genesis of Vowels," Journ. Auat. and Physiol., London, vol. xxxi. p. 233; also, "Interpretation of Phonograns of Vowels," ibid., p. 240.

2 "De physiologie der sprachklanken in het bijzonder van die der nederlandische taal," Utrecht, 1870.

${ }^{3}$ E. Leon Scott, Compt. rend. Acad. d. sc., Paris, tome liii. p. 108.

"Fleeming Jenkin and Ewing, "On the Harmonic Analysis of certain Vowel Sounds," Trans. Roy. Soc. Edin., vol, xxviii. p. 745 . 
phonograph, and the curves were submitted to harmonic analysis. This was the beginning of the present discussion. These two observer's obtained good curves, even with the imperfect instrument, and the curves were submitted to analysis, so as to determine the amplitude of their constituents up to the sixth partial, and the process of measurement and calculation was applied to more than one hundred curves. The sixth partial was not a high limit to reach, but the phonograph, at that date, did not record the higher partials. For example, it could not reproduce the sound of $I$ in machine, nor the French or German $U$. Twelve values for $y$ (the lengths of the co-ordinates for one period chosen) gave the data for calculating the amplitude and phases of the first six partials. Professor Tait supplied the authors with the solutions of the simultaneous equations for twelve values of $y$, and the results are given in a series of tables, in which the predominance of certain partials is unmistakable. In this way each vowel was examined. The conclusions arrived at were, on the whole, favourable to the constant-pitch theory, and were thus summed up: "It is clear that the quality of a vowel sound does not depend either on the absolute pitch of reinforcement of the constituent tones alone, or on the simple grouping of relative partials independently of pitch. Before the constituents of a vowel can be assigned, the pitch of the prime must be given; and, on the other hand, the pitch of the most strongly reinforced partial is not alone sufficient to allow us to name the vowel. To do this, we must also know the relation of the constituent partials to one another." 1 Again, "The ear is guided by two factors, one depending on the harmony or group of relative partials, and the other on the absolute pitch of the reinforced constituents. The ear recognises, as it were, when it hears a vowel sung, at any pitch, the kind of oral cavity causing the reinforcement. As to the question, "Is the resonance cavity for a vowel sound constant at all pitches ?" the authors are cautious; and their opinion appears to be that whilst the resonance cavity for a vowel sound has an absolute pitch, it may have a certain effect in reinforcing other subordinate tones or partials. While experimentally it may be shown that a constant cavity may produce a vowel-like tone, say $O$, over a wide range of pitch, it is probable that the resonant cavities of the human being are slightly adjustable, so as to bc, as it were, tuned to the pitch on which the vowel is sung. In other words, Fleeming Jenkin and Ewing hold that both the relative and the absolute factors enter into the composition of a vowel, a conclusion not far from the truth.

The subject was taken up by Hermann ${ }^{2}$ about 1890 , and he used the much improved wax-cylinder phonograph. He succeeded in obtaining photographs of the curves on the wax cylinder, a beam of light reflected from a small mirror attached to the vibrating disc of the phonograph being allowed to fall on a sensitive plate while the phonograph was slowly travelling. The curves thus obtained, representing the waveforms of the vowel tones (Fig. 438), ${ }^{3}$ were submitted to analysis, with the view of estimating the pitch of the mean partial or the formant, as it is called by Hermann, according to the method already described. Hermann also pointed out that the quality of a vowel tone varies considerably, according to the rate at which the cylinder was

${ }^{1}$ Jenkin and Ewing, op. cit., p. 770.

2 op. cit.

3 The curves in Figs. 438 and 441 were kindly sent to Dr. M'Kendrick by Professor Hermann. They are here introduced simply as examples. 
rotated. This should obviously not be the case, were the relative pitch theory correct. It is curious that even with competent observers there should be such difficulty in deciding this apparently simple question of fact, some asserting that there is no difference in quality, and others as positively stating that there is a difference when the cylinder is caused to move slowly. M'Kendrick, after many experiments, has come to the conclusion that there is a difference, but not so great as to disguise the quality of the vowel. To produce any appreciable change in quality, the cylinder must be driven at an enormous speed. The ear can always distinguish $O$ or $A$ or $E$ at different rates, but the quality is undoubtedly altered. The sound of a vowel never passes into the sound of another vowel.

Hermann presents the fixed-pitch theory in a modified form, and states that there is for each vowel a characteristic tone, a formant. Not only does Hermann support the fixed-pitch theory, but he maintains

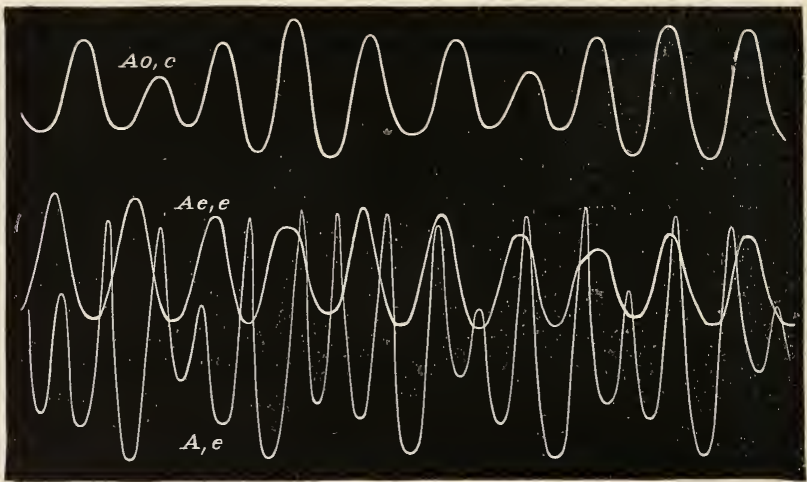

Fig. 438. - Examples of vowel curves obtained by Hermann.

that the formant need not necessarily be a partial of the fundamental. Sometimes it is such a note, but more often not. ${ }^{1}$ The pitch of the formant, however, may vary considerably; indeed, with the same prime, it may vary as much in certain cases as several semitones. Fig. 439 shows, in musical notation, the pitch of the vowel, according to Hermann. ${ }^{2}$

Sauberschwartz ${ }^{3}$ has investigated the subject by an ingenious application of the laws of the interference of sound. Certain vowels were sung into the mouthpiece of a long tube, to which other short tubes of definite length were attached. By closing the outer ends of certain of these tubes, various partials could be extinguished by interference, and the listener at the other end of the tube observed an alteration in the quality of the vowel. Thus, by extinguishing the formant of $A$, the sound approximated to that of $O$, and became somewhat nasal. With $E$ there are two characteristic formants, and

' Hermann, Arch.f. d. ges. Physiol., Boun, 1895, Bd. lxi. S. 178.

2 Ibid., Bd. xxxv. S. 42.

${ }^{3}$ Hermann, lbid., 1895, Bd. lxi. 
Sauberschwartz found that if one of these was extinguished the quality of the vowel was not much altered, but that when both were shut out the vowel was much changed in quality and approximated to $U$ or $O$. Sauberschwartz in general supports Hermann.

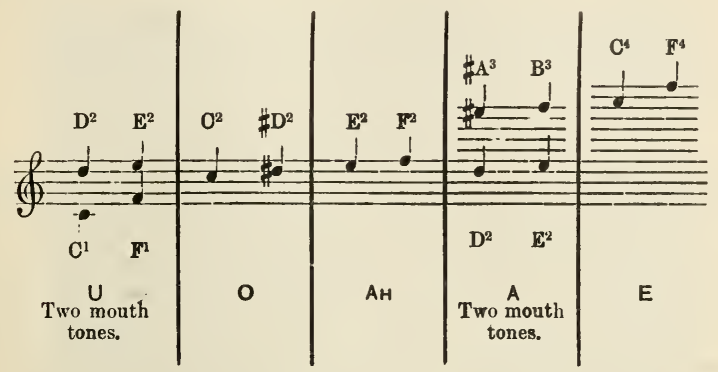

Fir. 439.-Pitch of the vowels according to Hermann.

Boeke $^{1}$ of Alkmaar has not only devised an ingenious and most accurate method of obtaining curves from the wax cylinder of the phonograph, but he has applied the Fourierian analysis with striking results in general support of the fixed-pitch theory." ${ }^{2}$ The method consists in measuring microscopically the transverse diameter of the impressions on the surface of the phonograph cylinder, on different (generally equidistant) parts of the period, and in inferring from these measurements the depth of the impressions on the same spot, or, in other words, deriving from them the curve of the tone which produced the impression.

Thus the depth $(d)$ of the impressions on a certain point may be easily deduced from its breadth (b) on the same point. Suppose $E G D F$ to be the transverse section of the phonograph marker, and $H K$ the longitudinal section of the surface, $A C B D$ the transverse section of

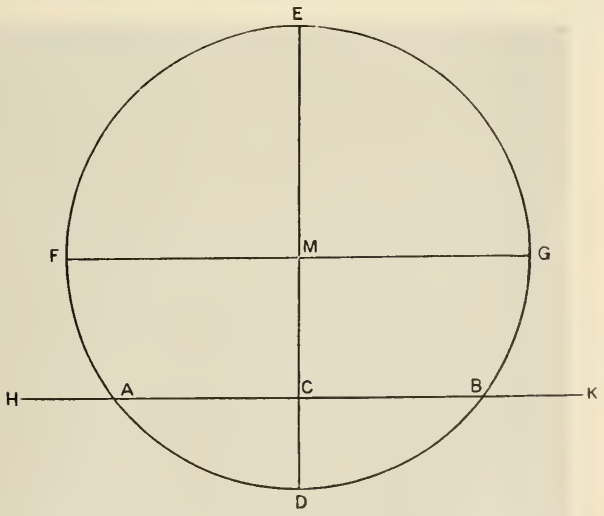

Fig. 440.-Diagram showing Boeke's theory of measurement of depth of eurve.

the groove which the marker ploughs on the wax surface, $A B=b$ its breadth, $C D=d$ its depth; and $F G=E D=2 r$, being the diameter of the cylinder; if the axis of the recording marker were tangential to the

1 "Microskopisehe Phonogramstudien," Arch. f. d. ges. Physiol., Bonn, Bd. 1. S. 297 ; also Proc. Roy. Soc. Edin., 1898.

2 The writers have to thank Dr. Boeke for unpublished notes on the subject, and also for many interesting analyses. 
surface of the cylinder, the prolongation of its edge would cut the axis of the cylinder, and we would have the equation-

$$
\begin{gathered}
\left(\frac{1}{2} b\right)^{2}=d(2 r-d) . \\
\text { Or } \quad d^{2}-2 d r+\frac{1}{4} b^{2}=0 \ldots \ldots
\end{gathered}
$$

From which we deduce-

$$
d=r \pm \sqrt{\left(r+\frac{1}{2} b\right)\left(r-\frac{1}{2} b\right)}
$$

As the equation (1) is that of an ellipse, the axes of which are $2 r$ and $r$, we see that the breadth and depth of the impression have the same ratio to each other as an abscis to its ordinate in the above-mentioned ellipse. It is true that the supposition as to the axis of the marker does not hold good as regards the phonograph, as it makes an angle (a) with the tangent, but the only difference which this makes is that they should be multiplied by the constant term, $\cos \alpha$, to obtain their real value. As the marker connected with the glass plate of the phonograph is constantly moving up and down, the value of the angle (a) changes at each moment ; but seeing the greatest depth of the impressions cut out on the wax surface does not exceed $0.02 \mathrm{~mm}$., these slight variations are of no consequence. By an elaborate and most careful system, the curves, corresponding to the ordinates obtained as above, are plotted out on millimetre paper, and then a magnification of about one hundred is worked out.

Discussion of examples of phonograms.-Examples of curves obtained by Hermann are shown in Figs. 438 and 441. These show

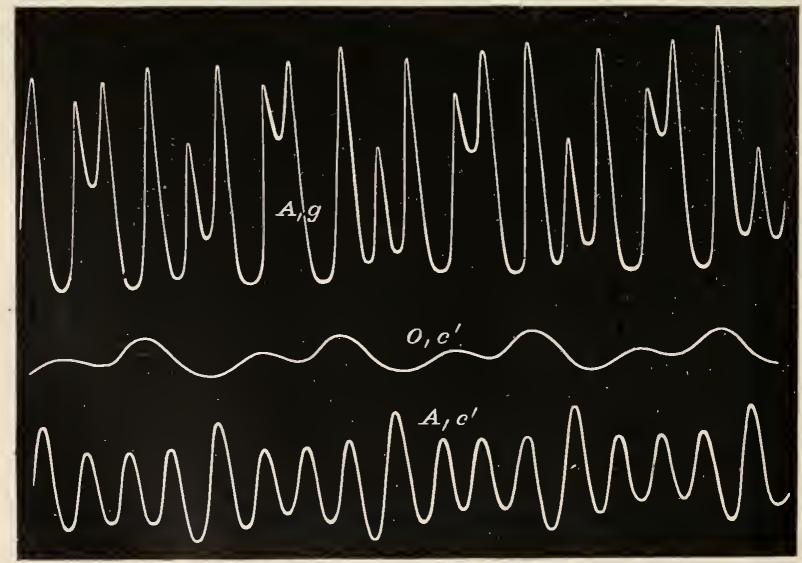

FIG. 441. - Examples of vowel eurves obtained by Hermann.

curves of the vowel $A$, sung on the notes $e, g, c^{\prime}$; of the vowel 0 , sung on the note $c^{\prime}$; and of the vowels $A e$ and $A o$, sung on the notes $c$ and $e$.

Curves of vowel tones may also be obtained on a large scale by 
M'Kendrick's ' phonograph recorder. In Fig. 442 we see curves obtained from the tracings on the wave-cylinder representing the tones of various instruments.

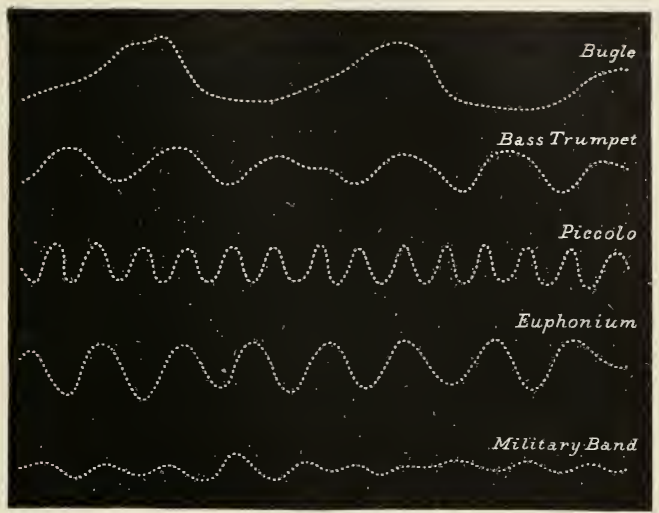

FIG. 442.-Exanıles of tracings obtained by M'Kendrick's phonograph recorder. The dots represent drops of ink from a small syphon. One inch shows the vibrations occurring in the $\cdot 1480 \mathrm{sec}$.

The following analyses supplied by Boeke are very instructive. The first is the analysis of the tones of a cornet. Observe how the intensity of the partials gradually diminishes:-

$\begin{array}{lcccccccccccc}\text { Note } & 1 & 2 & 3 & 4 & 5 & 6 & 7 & 8 & 9 & 10 & \text { Partials. } \\ f=170 \text { vibs. } & 1 & 1.05 & 1.22 & 1.15 & 1.01 & 0.80 & 0.53 & 0.28 & 0.13 & 0.10 & , \\ c^{\prime}=256 & , & 1 & 0.92 & 0.81 & 0.53 & 0.39 & 0.20 & 0.07 & 0.04 & 0.06 & 0.04 & , \\ g^{\prime}=384, & , & 1 & 0.76 & 0.46 & 0.14 & 0.09 & 0.06 & 0.07 & 0.02 & 0.01 & 0.01 & , \\ c^{\prime \prime}=512 \quad,, & 1 & 0.92 ? & 0.30 & 0.14 & 0.15 & 0.09 & 0.07 & 0.06 & 0.03 & 0.02 & ,,\end{array}$

Contrast this analysis with that of the vowel $\bar{a} \bar{a}$ sung by Boeke, æt. 50 , on the notes $f$ and $c^{\prime}$, and the same vowel sung on the notes $g^{\prime}$ and $e^{\prime \prime}$, sung by his son, æt. 12 , both like the vowel in the word "heart," it will be seen from the analytical numbers that the formant of the son's vowel $\bar{a} \bar{a}$ was almost the same as that of the father, although the pitch of his voice was exactly an octave higher.

\section{Man, cet. 50, singing $\bar{a} \bar{a}$.}

$\begin{array}{lcccccccccccc}\text { Pitch } & 1 & 2 & 3 & 4 & 5 & 6 & 7 & 8 & 9 & 10 & \text { Partials. } \\ f 170.6 \text { vibs. } & 1 & 0.86 & 0.46 & 1.74 & 1.90 & 1.55 & 0.51 & 0.54 & 0.43 & 0.44 & , \\ c^{\prime} 256 \quad, & 1 & 0.49 & 1.96 & 1.25 & 0.60 & 0.56 & 0.23 & 0.05 & 0.06 & 0.10 & \text { ", }\end{array}$

1 "Observations on the Phonograph," Trans. Roy. Soc. Edin., vol. xxxviii. part. 4 ; also Proc. Roy. Soc. Edin., 1896-7, opening address; also, "Sound and Speech Waves us revealed by the Phonograph," London, 1897. 
Boy, cet 12, singing $\bar{u} \overline{\text {. }}$

$\begin{array}{lccccccc}\text { Pitch } & 1 & 2 & 3 & 4 & 5 & 6 & \text { Partials. } \\ g^{\prime} 384 \text { vibs. } & 1 & 1.92 & 2 \cdot 67 & 0.45 & 0.17 & 0.06 & , \\ e^{\prime \prime} 640,, & 1 & 8.09 & 1 \cdot 45 & 0.53 & & & ,\end{array}$

Dr. Lloyd has examined these figures and supplied the writer with the following table:-

\begin{tabular}{|c|c|c|c|c|c|}
\hline \multirow{2}{*}{ MAN's $\bar{c} \bar{u}$. } & \multirow{2}{*}{$\begin{array}{c}\text { PARTials } \\
\text { REINFoRCED. }\end{array}$} & \multicolumn{2}{|c|}{$\begin{array}{l}\text { EVALUATED GY Hermasi's } \\
\text { METHOD. }\end{array}$} & \multicolumn{2}{|c|}{$\begin{array}{l}\text { Evaluated by Pipping's Niethod } \\
\text { (= LLOYd's Formula). }\end{array}$} \\
\hline & & $\begin{array}{c}\text { Mean } \\
\text { Partial. }\end{array}$ & V. 1.1 & $\begin{array}{c}\text { Mean } \\
\text { Partial. }\end{array}$ & v. D. \\
\hline 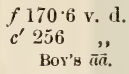 & $\begin{array}{l}4-6 \\
3-4\end{array}$ & $\begin{array}{l}4 \cdot 96 \\
3 \cdot 39\end{array}$ & $\begin{array}{l}846 \\
868\end{array}$ & $\begin{array}{l}4 \cdot 90 \\
3 \cdot 35\end{array}$ & $\begin{array}{l}836 \\
859\end{array}$ \\
\hline $\begin{array}{l}g^{\prime} 384 \text { v. d. } \\
c^{\prime \prime} 640, y\end{array}$ & $\begin{array}{l}2-4 \\
1-3\end{array}$ & $\begin{array}{l}2 \cdot 82 \\
2 \cdot 04\end{array}$ & $\begin{array}{l}1084 \\
1307\end{array}$ & $\begin{array}{l}2 \cdot 75 \\
1 \cdot 98\end{array}$ & $\begin{array}{l}1057 \\
1267\end{array}$ \\
\hline
\end{tabular}

A study of these figures will show (1) that the man's resonance rises slightly (half semitone) in ascending 7 semitones in the middle of his register; (2) that the boy's resonance rises 3 semitones in ascending 9 semitones in the upper half of his register; and in the mid-register the boy's resonance is to the man's as $5: 4$. This indicates that, as we ascend a scale in singing a vowel, the pitch of the oral cavity slightly changes.

Lloyd holds a view differing from those already described. Vowels, according to him, owe their character, not to the resonance of a partial or partials of a certain fixed pitch, but to the relative pitch of two or more partials. This view accounts for a fact which is not explained by the other theories, namely, that the articulation of a vowel seems to be the same for a child as for an adult. Thus, in the vowel $A$ as in "fat," sung on a note having a pitch of 134 v. d., Lloyd finds two partials, the lower of which he terms the pharyngeal or $\alpha$-resonance, and the other the oral or 3 -resonance. Of these the lower, for $A$ in "fat" has a vibrational number of 736 , while the upper has 1121 . The ratio of these two partials is therefore $\frac{1121}{736}=1.47$. This is termed by Lloyd the radical ratio, and it determines the nature of the vowel. Again, the radical ratio of the Swedish long $A$ is 1.35 .

The following curves were sent to the writers by Dr. Boeke. They are the curves, taken by Boeke's method, of the vowel $A$ (as in "fat"), sung by M'Kendrick, Pipping, Boeke, and Hermann on a pitch of $u t_{2}=128$ v. d., and $u t_{3}=256 \mathrm{v}$. $d$., and are instructive as showing the same vowel sung on the same pitch by men of different nationalities. The results of harmonic analysis giving, in plotted lines, the amplitude of the partials, are shown graphically in Fig. 444. The numerals at the beginning of each line in Figs. 443 and 444 correspond. In Fig. 444 the dots represent partials, and the height of the dot above the mean represents the amplitude of the corresponding partial. There is a likeness between the $A c$ of $\mathrm{M}^{\prime}$ Kendrick and Pipping, and also between M'Kendrick's $A c^{\prime}$ and Boeke's $A c^{\prime}$, both in form and analysis.

Lloyd, in support of his view of a cleavage in the reinforcements, which is

${ }^{1}$ V. D. = a double vibration, or, as expressed in England, a complete vibration. 


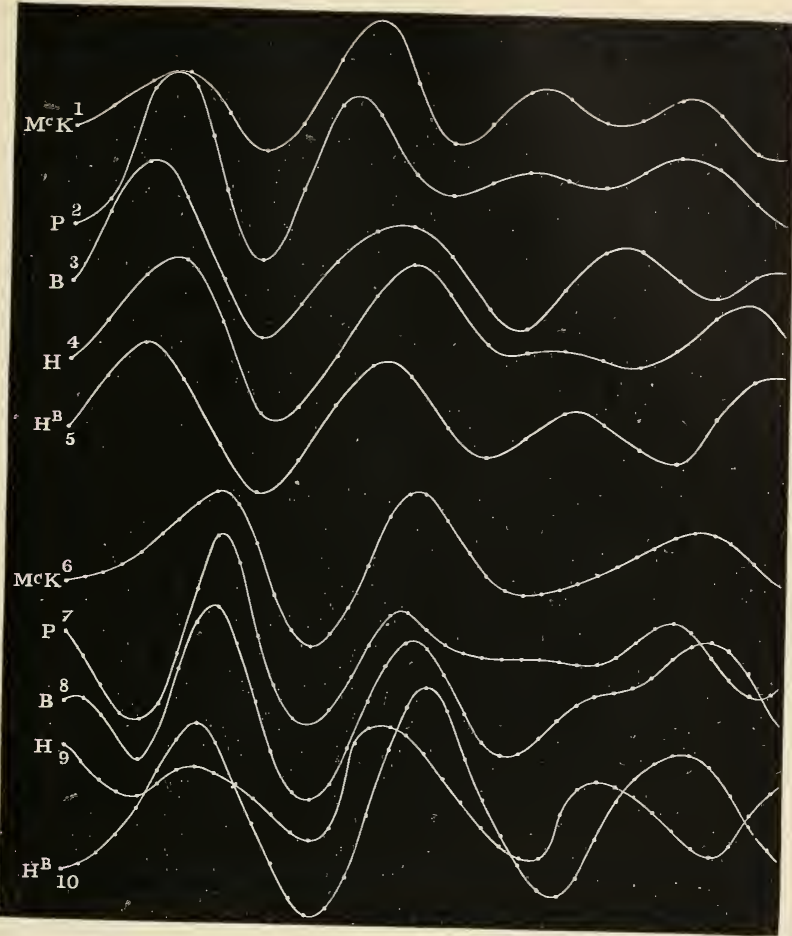

Fig. 443.-Curves by Boeke. For description, see text.

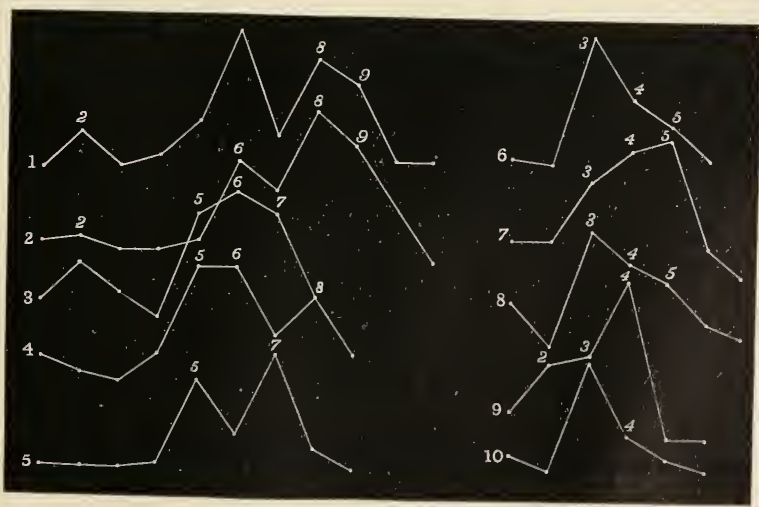

Fig. 444.-Diagran showing harmonic analyses of the curves in Fig. 443. 
the sign of two separate resonances, has reported upon two examples of $O$ sung by Hermann, both at 132 v. d. The amplitudes were as follows :-

\begin{tabular}{llllrrrrrrr} 
V. D. & 1 & 2 & 3 & \multicolumn{1}{c}{4} & 5 & 6 & 7 & 8 & 9 & 10 \\
132 & $6 \cdot 7$ & $10 \cdot 7$ & $18 \cdot 4$ & $14 \cdot 6$ & $18 \cdot 1$ & $4 \cdot 7$ & $2 \cdot 5$ & $1 \cdot 3$ & $1 \cdot 0$ & $0 \cdot 5$ \\
132 & $7 \cdot 8$ & $23 \cdot 4$ & $11 \cdot 2$ & $7 \cdot 0$ & $17 \cdot 0$ & $3 \cdot 6$ & $2 \cdot 1$ & $1 \cdot 2$ & $0 \cdot 6$ & $1 \cdot 0$
\end{tabular}

To these he adds two other examples of the same vowel, one sung at 148 v. d. by M'Kendrick, and the other at 128 v. d. by Boeke.

$\begin{array}{ccccccccc}\text { V. D. } & 1 & 2 & 3 & 4 & 5 & 6 & 7 & 8 \\ 148 & 1 & 1 \cdot 12 & 1 \cdot 73 & 0.19 & 1.90 & 0.97 & 0.35 & \ldots \\ 128 & 1 & 2.32 & 5.81 & 2 \cdot 10 & 5.48 & 0.32 & 0.55 & 0.76\end{array}$

The fourth partial in each of these four analyses shows a palpable falling off in strength, as compared with its neighbours on either side, and this falling off marks in each ease the gap between an $\alpha$-resonance of 300 to $400 \mathrm{v}$. d. and a $\beta$-resonance of 600 to $800 \mathrm{v}$. d. The vowel $O$, however low it may be sung, can hardly have more than two partials, intermediate to the two culminations. In three out of the four cases above given it has only one intermediate partial. This common partial must be subject to a strong influence from both resonances at one time. All the other partials are situated so much nearer to the one resonance than to the other, that they may be regarded as being under the sole influence of the former.

The common partial, on the other hand, receives a stimulus from each; and it must be remembered that these stimuli are mutually independent, as the one operates in the oral and the other in the pharyngeal cavity, and that it is a matter of chance whether, in any phonogram analysed, these two stimulations operate to exaggerate or to conceal each other in the tabulated numerical strength of the partial. Thus it comes to be a question of phase.

Lloyd has evaluated the $\alpha$ - and $\beta$-resonances of the vowel $(O)$ as above given, and stated the result in the following table:-

\begin{tabular}{|c|c|c|c|c|c|c|c|c|c|}
\hline \multirow{2}{*}{\multicolumn{2}{|c|}{ Sung }} & & & \multirow{2}{*}{ V. D. } & \multicolumn{2}{|c|}{ Partials Rkinforced. } & \multicolumn{2}{|c|}{ Mean Resonance. } & \multirow{2}{*}{$\begin{array}{c}\text { Radical } \\
\text { Ratio. }\end{array}$} \\
\hline & & & & & $\alpha$ & $\beta$ & $\alpha$ & $\beta$ & \\
\hline Hermann & & . & . & 132 & $2-4$ & $4-7$ & 421 & 653 & 1.553 \\
\hline Hermann & . & . & . & 132 & $1-3$ & $4-7$ & 290 & 672 & $2 \cdot 318$ \\
\hline M'Kendrick & . & . & . & 148 & $1-4$ & $4-7$ & 365 & 812 & $2 \cdot 221$ \\
\hline Boeke . & . & . & 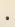 & 128 & $2-4$ & $4-6$ & 391 & 615 & $1 \cdot 573$ \\
\hline
\end{tabular}

These four available instances give an average of $368 \mathrm{v}$. d. for $\alpha$-resonance, of $688 \mathrm{v}$. d. for $\beta$-resonance, and of 1.916 for the radical ratio. A sounder method would be to take from one voice a sufficient number of $O$ analyses to constitute an average, thus eliminating the chances which cause the common partial to vary to such a noticeable degree.

In some vowel tones the intensity of the fundamental or prime tone is weaker than one of the upper partials. Helmholtz lays special emphasis on this observation, and he put the statement conversely, namely, that vowel tones differ from those of ordinary musical instruments in that one of the upper partials is more marked than the prime. Hermann also supports this view, and, in a communication to the writers, Boeke expressly states that his analyses bring out the same fact. On the other hand, Auerbach ${ }^{1}$ maintains that the prime tone is always the strongest. Lord Rayleigh ${ }^{2}$ is also of this opinion,

1 Ann. d. Phys. u. Chem., Leipzig, 1876, Erganzungsband, Bd. viii. S. 177.

2 "Theory of Sound," 1896, vol. ii. p. 477. 
while he admits that in the vowel $A$, the fundamental is not heard so loudly as in other vowels. Hermann suggests that when the prime tone is heard of very weak intensity it may exist only in the ear, but Lord Rayleigh shows that even then it does exist in the external air.

General conclusion.-It would appear, on the whole, that the truth does not rest entirely on the side of one theory, but that both are partially true. The view of Donders, that each vowel has a mouth cavity of unchangeable and fixed pitch is too exclusive, and, on the other hand, it cannot be denied (as is virtually done by those who uphold the relative-pitch theory) that each vowel has a predominant partial or predominant partials which give it a definite character, and which must be produced by the mouth cavity as a whole, or by the double resonance of portions of the cavity, as contended by Lloyd. When a vowel is spoken, either separately or as it exists in a word, the complex tone is produced by the vocal cords. The resonance chambers above assume a certain form, and one or more partials peculiar to the vowel are so strengthened as to give such character to the vowel-tone as to enable the ear at once to identify it. As the form of the mouth cavity varies in individuals and in different races, the partials may not be identical in each case, but they will be so near a common pitch as to enable the ear at once to identify the vowel. If, however, men of different nationalities attempt to fix the absolute pitch of the partial, they will not agree, as has been found to be the case. Again, in singing a vowel in a scale, very slight alterations in the form of the resonance cavities take place. In rising from the lower to the higher notes, the larynx rises, and more or less of a muscular strain acts on the walls of the resonance cavities. Thus there must be a slight change in the volume of these cavities, leading to the development of partials different from those formed by the cavity at rest or in speech, but still so sufficiently near as not materially to alter the quality of the tone. Consequently the ear still recognises the tone of the vowel, even when sung at a high pitch, and it may even recognise the special quality of a particular voice. Sometimes, though rarely, the quality may become richer as the voice rises in pitch; usually for each voice there is a register in which the voice has its maximum of good quality. It is not surprising, therefore, that a singer almost instinctively chooses such vowels as best suit the resonating arrangements of his or her voice, and avoids tones, vowels, and words containing vowels that would force the production of notes of inferior quality. On the whole, we are of opinion that Lloyd's theory of double resonance accounts best for the true nature of vowel-tones. ${ }^{1}$

\section{The Consonants.}

The classification of the consonants suggested by $\mathrm{Wyllie}^{2}$ will be adopted here.

It will be observed that they are arranged from above down, according to the part of the oral cavity in which they are produced. Again, the rows are themselves divided into three vertical columns, the

${ }^{1}$ Another method of examining the marks on the wax cylinder of the phonograph, as regards speech, but without reference to analysis, is given with numcrous figures in "La parole d'après le tracé du phonographe," par H. Marichelle, Paris, 1897.

2 "Disorders of Speech," 1894, p. 8.

VOL. $11 .-78$ 
voiceless oral consonants, the voiced oral consonants, the voiced nasal resonants.

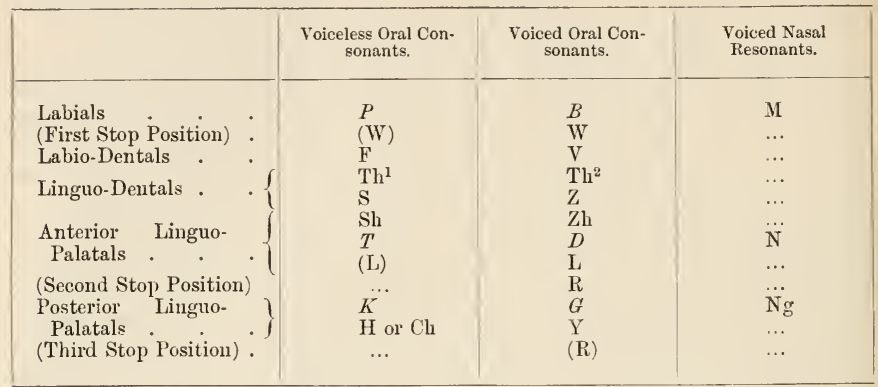

The voiceless $W$ and the voiceless $L$ have been given above within brackets, the former being now almost confined to Scotland, and the latter being peculiar to Wales. The burring or uvular $R$ is also given within brackets.

For example, the voiceless labial $p$ corresponds with the voiced labial $b$ and the voiced nasal resonant $m$. When the consonant is voiceless, it communicates an explosive character to the succeeding syllable, as in "pitch"; but if it comes at the end of a syllable it gives a sharp sudden termination to that syllable, as "stop." If the labial consonant be the voiced $b$, the explosive beginning or sharp sudden termination of the syllable is not so marked as in Bob. The difference between these two letters depends in part upon the firmness with which the orbicularis oris closes the mouth. $M$ is produced in the same way as $b$, with the difference that in the former the air is allowed to escape through the nose. Voiceless $w$ is produced in almost exactly the same manner as $p$, and the voiced $w$ in the same way as $b$; the only difference is that, in the two w's the mouth is not completely closed, and a fricative sound accompanies the consonant. The voiceless $w$ occurs in "which"; the voiced $w$ in such words as "water." $F$ and $v$, the former voiceless, the latter voiced, are produced by the passage of the breath between the lower lip and the upper incisor teeth. Similarly, the voiceless and voiced th, as in "think" and "thou" respectively, are formed by the breath rushing through between the tip of the tongue and the incisor teeth. $S$ and $z$ are produced in almost the same way as $t h$ (voiceless) and th (voiced) respectively; in pronouncing the $s$ and $z$, however, the tip of the tongue touches the tooth nearer its insertion, and indeed often touches the gum rather than the tooth itself. Sh and $z h$ (as azure) are respectively the voiceless and voiced oral consonants produced by the tongue and anterior part of the palate; while $t$ and $d$ are produced by the same structures, but with the stoppage between tongue and palate more complete than when $s h$ and $z h$ are uttered. $N$ is produced in the same way as $d$, but with nasal resonance superadded. $L$ and $r$ are also produced at this position; in the former case the tip of the tongue is applied to the palate while the breath escapes on each side; $r$ is produced by the vibratory movement of the tongue against the palate. The remaining consonants are produced by the posterior portion of the tongue and the palate. $K$ and $g$ are voiceless and voiced consonants, respectively, while $n g$ is $g$ with nasal resonance added. It is 
interesting to observe that in Greek, when two such consonants as $k$ and $g$ occur together, nasal resonance is added to one of them, e.g. $\sigma 0 \gamma \gamma \alpha \lambda \omega$, pronounced sunkalo. $H, c h, y$, and the burring $r$ are also produced at this position.

It is only by a proper appreciation of the method of production of the consonants that Grimm's law ${ }^{1}$ of the permutations of these letters can be thoroughly understood. Thus frater in the Latin is represented by brother in the Low-German, and pruoder in the Old High-German. It is not difficult to see why this should be, since $f, b$, and $p$ are produced by very similar muscular movements. So also the Greek $\left(\chi \eta^{\prime} v\right)$ is represented by the LowGerman "goose," and the Old High-German "Kans," all three initial consonants being produced by very similar muscular mechanisms.

The acoustic analysis of the consonant sounds has not yet been undertaken, and it is clear that considerable difficulties must be encountered in such an investigation. Tracings representing the consonant sounds have been obtained by Blake, ${ }^{2}$ but for the purposes of analysis these are not satisfactory.

\section{Effect of the Cavities in CONNECTion with the LaRynx and Pharynx upon the Voice.}

It needs but little consideration to understand that the various cavities, which are in more or less direct connection with the mouth and larynx, must have at least some effect upon the quality of the voice. It is, however, difficult to say exactly what these effects are.

Moura ${ }^{3}$ has attempted to show that the trachea has very considerable influence upon the pitch of the voice, but it is clear, from physical considerations, that he attributes too much importance to the phonetic rôle of the trachea. In the first place, its proper tone is far removed from the lower notes of the human voice; and, secondly, although it is a more or less rigid structure, its elasticity must be small when compared with the rigid character of substances used as resonators, especially since its inner surface is covered with mucous secretion. Nevertheless, it is probable that the trachea may resonate to some of the notes of the voice, and thus produce an effect upon their quality; the pitch, on the other hand, is determined by the vocal cords themselves.

It is doubtful if the laryngeal ventricles have any effect upon the voice in the human subject, on account of their small size. In some animals, such as the howling monkey, the orang, and the horse, it is possible that they may act as cavities reinforcing the voice. The loud deep roar of the lion, on the other hand, comes from a larynx in which the ventricles are absent. ${ }^{4}$ In the human subject the laryngeal ventricles probably perform a function unconnected with the voice. Wyllie ${ }^{5}$ has shown, on the dead subject, that if the false cords be partially approximated, and a blast of air be driven up against them from below, the effect upon these structures is to drive them further inwards, and hence close the glottis more completely in a valve-like fashion. The action, indeed, is somewhat similar to that which occurs when the mitral valve

\footnotetext{
${ }^{1}$ Morris's “Historical English Grammar," 1882, p. 46.

2 Blake, Am. Journ. Otol., N. Y., July 1879.

3 Paris Soc. d. Laryngol., Rhinol., etc., March 1893.

4 Wolff, "Diss. Anat.," p. $9 . \quad{ }^{5}$ Edin. Med. Journ., 1866, vol. xii. p. 214.
} 
of the heart is closed during systole, the valves being distended from below. According to Wyllie, therefore, the principal function of the false cords is to close the glottis tightly against an expiratory blast of air, and thus enable the animal to fix the ribs for effort. This view is borne out by the researches of Brunton and Cash, ${ }^{1}$ who not only confirmed Wyllie's statements by new experiments, but carried the investigation into the realms of comparative physiology. They point out that in those animals whose habits required fixation of the thorax, for striking or climbing, the ventricular bands (false cords) are well developed; and, conversely, animals which do not require strong fixation of the thorax, have ventricular bands of insignificant proportions. They further observed, that in animals in which vomiting is easily performed, the ventricular bands are well developed, and thus enable the animal to fix the thorax for the effort in a much more effectual way than in those animals in which the ventricular bands are small or absent.

The oral, pharyngeal, and nasal cavities have considerable influence upon the voice. This has already been discussed. In general, however, it may be said that these cavities, the pharyngeal and nasal in particular, confer a clear ringing character upon the voice; when they are affected by catarrh the voice has a muffled quality.

\section{WHISPERING.}

Arnott, ${ }^{2}$ in 1826 , defined whispering as articulation without voice, and for a long time it was supposed that during this act the cords were wide apart, allowing the breath to pass freely. Brücke was the first to dispute this statement, maintaining that the vowels are always produced in the larynx even in whispering. This view, at first sight, appears to be supported by an observation made by Czermak. He examined the larynx with the mirror during whispering, and found that an open triangular space is present at the posterior portions of the glottis, while the anterior portions of the vocal cords take up positions very similar to those which they assume during ordinary vocalisation. The air rushing through this triangular area, according to him, produces the fricative noise characteristic of whispering. ${ }^{3}$

The matter is not, however, by any means settled by this observation, since the open condition of the mouth necessary for laryngoscopic investigation would prevent true whispering, which is invariably performed with an almost closed buccal cavity. Moreover, it has been noticed that, after excision of the larynx, whispering is still possible. ${ }^{4}$ Although it may be questioned whether this is altogether the same as ordinary whispering, nevertheless, to judge by one's own sensations in performing the act, the part played by the vocal cords appears to be insignificant, if not altogether negligible.

1 Journ. Anat. and Physiol., London, vol. xvii. p. 363.

2 "Physics," 5th edition, p. 640.

${ }^{3}$ Czermak, Sitzungsb, d. k. Akad. d. Wissensch., Math. Nat. Cl., Wien, 1866, Bd. lii.

4 Macdonald and Symonds, Brit. Med. Journ., London, 1889, vol. i. p. 996. 


\title{
THE SENSE OF TASTE.
}

\author{
By J. B. Haycraft.
}

Contents:--The Anatomical Mechanisms concerned in the Production of Taste, and the Regions of Mucous Membrane which are involved, p. 1237-Taste Sensations, p. 1240-The Action of Drugs on Taste, p. 1241-Modality and Quality of Taste, p. 1242-The Relationship between Taste Sensations and the Chenico-Physical Properties of the Stimulus, p. 1244-After-Tastes and Successive and Simultaneous Contrast, p. 1244.

Anatomical.-The anatomical mechanism concerned in the production of the sensation of taste consists, in the first place, of certain sensory end-organs within the cavity of the mouth and throat, and, in the second place, of sensory nerves proceeding from these, and entering the base of the brain, where they form connection with fibres from other parts of that organ. The sensory surfaces in which the sensory nerves of taste arise are as follows - the edges, upper surface, and root of the tongue, the palate, the pillars of the fauces, the posterior surface of the epiglottis, and the arytenoid cartilages of the larynx. ${ }^{1}$ These sensory surfaces are also connected with nerves that are concerned with the production of the sensations of touch, temperature, and pain.

The gustatory or taste bulbs found in the tongue and posterior surface of the epiglottis are in all probability sensory end-organs; they are distributed as follows:- On the front of the tongue, where they are rare, and lodged in the tops or sides of some of the fungiform papillæ, and at the root of the tongue, where they are more numerous, and are lodged in special gustatory papillæ.

In man there are some eight or ten of these gustatory or circumvallate papillæ; while in the rabbit the bulbs are lodged within foliated portions of the mucous membrane, called the papillæ foliatæ, placed one on each side of the root of the tongue. They are found in a similar situation in the human tongue, but not on special foliate papillæ. Gustatory bulbs are also found upon the posterior surface of the epiglottis, and it was this discovery that induced Nicholson to test the gustatory properties of the epiglottis.

Our belief that the bulbs are end-organs of taste, and not of common sensibility, rests upon the following evidence. They are found solely on the sensory surfaces of taste. Their highly specialised structure, presenting many homologies with the end-organs of the special senses, suggests their performance of some special function. We should on this account expect them to be organs of taste, rather than organs of a widely distributed sensory mechanism, like that of general sensibility, which is elsewhere devoid of these structures. More direct evidence is given by the effect of division of the glosso-pharyngeal nerve, which

${ }^{1}$ P. Nicholson, Virchow's Archiv, Bd. cxxiii. S. 398-401. 
undoubtedly carries nerve fibres of taste from the back of the tongue, whereas branches of the fifth are concerned in the transmission of ordinary sensory impulses from this situation. M. v. Vintschgau and Hönigschmied ${ }^{1}$ found that within a few months subsequent to the division of the glosso-pharyngeal nerve in the rabbit, the bulbs entirely disappeared on the side on which the division was made.

While it is pretty evident that the bulbs are end-organs of taste, and that they are the terminations of the taste fibres which pass up the branches of the glosso-pharyngeal nerve, it is possible, of course, that these fibres have other terminations as well. As regards the front of the tongue where the bulbs are few in number, and where the taste-

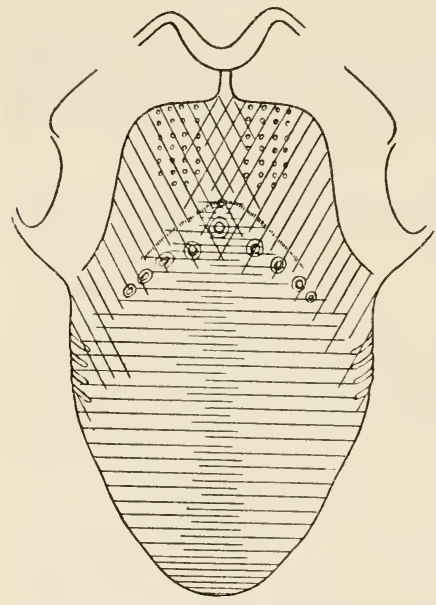

FIG. 445.-Horizontal lines indicate distribution of lingual including chorda tympani ; oblique lines indicate distribution of glossopharyngeal ; the larger circles indicate circumvallate papille; the smaller circles are placed over the area supplied by the superior laryngeal. nerve above the point of junction of the chorda has no effect upon taste. On the other hand, division of the chorda tympani, and disease of the middle ear iuvolving this nerve, lead to loss of taste on the affected side. Hence there is little doubt that the taste fibres pass from the lingual nerve into the chorda tympani on their way to the brain. As regards the further course of the fibres, there is much conflicting evidence. According to some observers, the fibres pass again into the course of the fifth nerve to reach the brain, while others

1 Arch. f. d. ges. Physiol., Bonn, 1877, Bd. xiv. S. 443 ; Hermann's "Handbuch," 1879, Bd. iii. Th. 2, S. 145 ; also see F. Hermann, Centralbl. f. Physiol., Leipzig u. Wien, 1877 , Bd. ii. S. $503-4$.

${ }^{2}$ Mader, Centralbl. f. d. med. Wissensch., Berlin, 1879, S. 395.

3 Neurol. Contralbl., Leipzig, 1882, S. 104 ; also see F. Saltzer, Arch. f. klin. Chir., Berlin, Bd. xxxvii. Heft 3. 
regard the sensory portion (pars intermedia) of the seventh nerve as the path followed by the taste fibres.

The idea that the taste fibres reach the brain by the fifth nerve roots was held so long ago as 1876 by Erb, ${ }^{1}$ and has been strongly maintained by Schiff, ${ }^{2}$ and advocated in this country by Gowers and Turner. ${ }^{3}$ According to these observers, the taste fibres leaving the lingual by the chorda tympani pass into the seventh nerve and so to the geniculate ganglion. Thence they are continued into the great superficial petrosal and Vidian nerves to the spleno-palatine ganglion, through which they pass into the second division of the fifth nerve, and so to the brain. The evidence brought forward is as follows: Intracranial lesion or section of the facial nerve has no effect upon taste. Section of the fifth nerve roots, on the other hand, leads to loss of taste on the anterior part of the tongue (Schiff). ${ }^{4}$ Disease of the root of the fifth nerve also abolishes taste in the front of the tongue, and H. Salomonsohn ${ }^{5}$ found loss of taste subsequent to pressure of a tumour upon the second division of the fifth nerve. Ferguson ${ }^{6}$ has described loss of taste in the front of the tongue, following the pressure of a tumour upon the Vidian nerve.

In favour of the idea that the taste fibres, which leave the lingual nerve by the chorda tympani, reach the brain by the facial nerve (or pars intermedia), we have the following observations :-Extirpation of the entire Gasserian ganglion in man, and intracranial section of the second and third divisions of the fifth nerve are often unaccompanied by any loss of taste (MacTiffany, Krause, and Lynn Thomas). ${ }^{7}$ In lower vertebrates the seventh nerve is undoubtedly a nerve of taste (Strong). ${ }^{8}$ Developmentally the sensory fibres of the chorda tympani and of the great superficial petrosal nerves are a portion of the facial nerve in man as in lower vertebrates. ${ }^{9}$

In discussing the contradictory results, as regards taste, which may follow disease or section of the fifth nerve roots, Dixon ${ }^{10}$ points out the close proximity of the geniculate ganglion to the Gasserian, and of the facial to the fifth nerve-roots; he views the loss of taste which in some cases follows the extirpation of the Gasserian ganglion or lesion of the fifth nerve roots, as due to an inflammatory or other involvement of the geniculate ganglion or of the root of the facial nerve. He draws attention to the strong developmental and anatomical reasons for looking upon the facial nerve in man as the nerve of taste for the anterior part of the tongue, and contends that by assuming a lesion of the seventh

1 "Handbuch d. Krankh. d. periph. Nerven."

${ }^{2}$ Schiff, Rev. méd. de la Suisse Rom., Genève, tome vii. p. 54.

${ }^{3}$ Gowcrs, "Diseases of the Nervous System," vol. ii. p. 224, and Turner in Allbutt's "System of Medicine," 1899, vol. vi. p. 752, "Diseases of the Cranial Nerves."

${ }_{4}^{4}$ Neurol. Centralbl., Leipzig, 1882, S. 73.

5 "Diss.," Berlin, 1888.

$6 \mathrm{Med}$. News, Phila., Oct. 1890.

7 MacTiffany, "Intracranial Neurectomy and Removal of the Gasscrian Ganglion," Ann. Surg., St. Louis, 1894; Krause, "Die Neuralgie des Trigeminus," 1896 ; Lynn Thomas, Brit. Med. Journ., London, 1899, vol. ii., Report of Sumner Mceting of British Medical Association, 1899.

8 "The Cranial Nerves of Amphibia," Journ. Morphol., Boston, 1895.

${ }^{9} \mathrm{His}$, "Die ersten Nervenbahnen beim menschlichen Embryo," and "Die morpholo. gische Betrachtung der Kopfuerven," Arch. f. Anat. u. Entwchlngsgesch., Leipzis, 1887 ; Dixon, "The Development of the Branches of the Fifth Cranial Nerve in Man," Trans. Roy. Irish Acad., Dublin, 1896.

io Edin. Med. Joum., 1897, vol. i. pp. 395, 628 ; and "The Sensory Distribution of the Facial Nerve in Man," Jouri. Anat. and Physiol., London, 1899, vol. xxxiii. p. 471. 
nerve roots, rather than one of the fifth nerve, many of the pathological cases recorded receive an equally satisfactory explanation.

As the case is one that cannot be determined by experiments on lower animals, the positive evidence of the retention of taste in man, after section of the fifth nerve roots, is of great interest.

It is generally admitted that the fibres of taste from the back of the tongue pass up the branches of distribution of the glosso-pharyngeal nerve. It is not certain whether they ascend to the brain by the same nerve, and many believe that they pass by a rather circuitous path to the fifth nerve. It is frequently stated that disease involving the trunk of the glosso-pharyngeal nerve never abolishes taste, though cases have been recorded in which it is maintained that taste was lost under these conditions. ${ }^{1}$ It is supposed that the taste fibres ascend in the tympanic branch (nerve of Jacobson), and reach the fifth through the small superficial petrosal, and through branches of the tympanic plexus. In support of this view, it is urged that disease of the fifth nerve is often followed by loss of taste on the back of the tongue, and that disease of the middle ear, which is especially liable to affect Jacobson's nerve, may be followed by a similar result. On the other hand, in direct contradiction to this view, we have the observations of MacTiffany, Krause, and Lynn Thomas above mentioned, as well as the embryological evidence, that the nerve of Jacobson is developed as a branch of the glosso-pharyngeal, and so would not be expected to carry impulses from that nerve into the course of the fifth. ${ }^{2}$

The tongue may be paralysed as to its nerves of general sensibility, whilst the sense of taste may remain normal, and in other cases it may be paralysed as to taste while its general sensibility remains normal. In the case of smell, on the other hand, paralysis of the nerves of general sensibility, due to injury or diseases of the fifth, produces dryness and certain trophic changes which cause anosmia, although the fibres of the olfactory nerve are not directly affected.

While the lingual and glosso-pharyngeal nerves carry both nerves of taste and of general sensibility, the very root of the tongue is supplied, in part at any rate, by a nervous twig from the superior laryngeal branch of the vagus, about whose function at present but little is known.

Taste sensations.-Many of our so-called tastes are sensations arising from stimulation of the nasal mucous membrane, but a certain residue are quite odourless, and appeal alone to the mucous membrane of the mouth. Salt, sweet, bitter, acid, are true tastes, and so probably are metallic and astringent, though the nerves of tactile sensibility have much to do with the feeling of astringency.

Our power of tasting is much greater on some parts of the gustatory mucous membrane than on others, being generally greater at the root than at the tip of the tongue, and very slight over the middle line of the tongue anteriorly. Our gustatory sensations exhibit, however, great individual differences, more so perhaps than is the case with any other sense organ, and although the above statement is the rule, there are many exceptions; for example, in the case of some persons, the front of the tongue is extremely sensitive, while in others again it is hardly so at all.

${ }^{1}$ F. Pope, Lancet, London, 1889 , vol. ii. p. 458.

${ }^{2}$ Dixon, Trans. Roy. Irish Acad., Dublin, 1896. 
It is a matter of common experience that bitter substances are tasted best at the back of the tongue, and on that account no one would think of sipping a glass of beer. Sweet substances are tasted best by the tip and sides of the tongue, and so perhaps are salines and acids. All parts of the tongue are, however, sensitive to all four tastes, and bitter if it is strong enough-as quinine powder, for instance-can be tasted on the front of the tongue. The difference appears, therefore, to be one of degree, and suggests that for each taste there are special end-organs present, and that they are unequally distributed over the tongue. We may suppose, for instance, that there are many end-organs for bitter sensations towards the back of the tongue, and very few at the front. In support of this view much evidence is forthcoming. Let us take first the reaction time for the various sensations. M. v. Vintschgau and Hönigschmied ${ }^{1}$ give the following reaction times in seconds for the base as compared with the tip of the tongue:-

\begin{tabular}{|c|c|c|c|c|}
\hline & & Salt. & Sugar. & Quinine. \\
\hline Tip & . & 597 & $\cdot 752$ & $\cdot 993$ \\
\hline Base & . & $\cdot 543$ & $\cdot 552$ & $\cdot 502$ \\
\hline
\end{tabular}

The most outstanding fact in the above table is the difference between the reaction time of quinine at the base and tip of the tongue. This corresponds with the observable difference of sensitiveness of these parts, the less sensitive one requiring a longer application of the stimulus to produce an appreciable result in sensation. This result is verified by Öhrwall, ${ }^{2}$ who finds that the reaction time of bitter substances is long at the front of the tongue, while at the base it is the same as for other substances.

Öhrwall, in the same paper, has described the effects of stimuli applied to individual papillæ. He used porous points soaked in sapid substances, and with these he was able to apply stimuli to a single papilla, to the exclusion of the rest. Öhrwall found that the filiform papillæ are insensitive to taste, not so the fungiform papillæ. Of 125 papillæ tested by him, twenty-seven reacted neither to acid, bitter, nor sweet; sixty reacted to all three; and of the remaining thirty-eight, some reacted to acid and sweet but not to bitter, others to bitter and acid and not to sweet, and others to sweet, but not to bitter and acid. On placing an electrode in contact with a papilla, and passing through it a constant electrical current, an acid papilla discharged an acid, and a sweet papilla discharged a sweet taste. These results are again consistent with the separate existence of bitter, sweet, etc., end-organs. Some papillæ are devoid of any end-organs, other's possess them all, and others again possess one or two varieties.

Action of drugs. -By the action of drugs we can abolish certain tastes more than others, so as to leave the mouth practically insensible to certain stimuli. This again suggests the presence of different kinds of end-organs, some of which are more affected than others. Cocaine

Bd. ${ }^{1}$ xx.

1 Arch. f. d. ges. Physiol., Bonn, 1875, Bd. x. S. 1 ; and v. Vintschgau, ibid., 1879,

${ }^{2}$ Skandin. Arch.f. Physiol., Leipzig, 1890, Bd. ii. న. 1. 
when applied to the tongue destroys both sensibility to touch and to taste, ${ }^{1}$ and it is also said to destroy the sense of temperature. ${ }^{2}$ It appears that this drug destroys the power of tasting bitter substances most readily, ${ }^{3}$ and does not affect the power of tasting sweet, salt, and sour substances to the same degree. It also abolishes tastes electrically produced. A decoction of the leaves of Gymnema sylvestre, according to most observers, paralyses the sense of bitter and of sweet, ${ }^{4}$ while others maintain that all tastes are affected, though bitter and sweet tastes more especially. Gymnema abolishes the bitter tastes that can be electrically produced.

Modality and quality.-Some observers maintain with Öhrwall that the difference between the sensations of bitter, sweet, etc., are differences not of quality but of modality. Referring to the definition of the term, as given by Helmholtz, its introducer, ${ }^{5}$ we find that modality is that difference which exists between the sensations of different senseorgans, such as, say, sweetness, warmth, and colour. We can neither compare with each other sensations which differ in modality, nor do they pass into each other by intermediate stages, as do sensations which are associated with the same sense-organ and which differ from each other in quality. We cannot say that a sweet taste is more like blue than red; here is an instance of modality. Orange is, on the other hand, more like yellow than blue, and can pass into yellow by intermediate sensations; here is then an instance of quality. Accepting this definition of Helmholtz, it is difficult to understand how the difference between, say, bitter and salt, can be described as one of modality. A mixture of salt solution and syrup tastes sweetish salt, and one can by graduated mixtures pass from salt to sweet. In the same way we can pass from bitter to salt, or from bitter to sweet. A large number of sapid substances give rise to these intermediate sensations; thus sulphate of magnesia is a saline bitter, a grape is acid sweet, etc. Just as we can say, that the colour orange is more like yellow than blue, so we can institute comparisons between the taste sensations and say that bitter-sweet is more like sweet than it is like acid. It is probable that we have in the tongue special end-organs for bitter, sweet, acid, salt, and perhaps some others. Many sapid substances stimulate but one of these end-organs, while others stimulate more than one, giving rise in sensation to a fairly large number of qualitative differences.

The law of the specific energy of the senses, as taught by Johannes Müller, ${ }^{6}$ is a law which deals with modality and not with quality. As he put it, each sense-organ, however stimulated, gives rise to its own specific sensations; the eye to the sensation of light, but never of hearing and smell; the ear to sensations called sounds, never to sensations of light or taste. This law is also true of taste; and stimuli, whether they are sapid substances, electrical currents, or mechanical

1 Brunton, "Text-Book of Pharmacology," p. 878.

2 Gollscheider, Monatsh. f. prakt. Dermat., Hamburg, u. Leipzig, 1886, No. 2.

3 Mosso and Adduco, Gior. d. r. Accad. di med. di Torino; 1886; Shore, Jonm. Physiol., Cambridge and London, 1892, vol. xiii. p. 191.

${ }^{4}$ Hooper, Nature, London, April 14, 1887 ; Shore, Journ. Physiol., Cambridge and London, 1892, vol. xiii. p. 191; see also Kiesow, op. cit.; L. Hermamn, Arch. $f$. d. ges. Physiol., Bonn, 1891, Bd. xlix. S. 579.

5 "Handbuch d. physiol. Optik," 1894, S. 584.

6 Johannes Miuller was perhaps the first to distinguish between modality and quality. See Müller's "Physiology," English translation, vol. ii. p. 1059. 
irritation, if capable of producing sensations, produce those of taste, not those of smell or hearing. ${ }^{1}$

Since Müller's time the law of specific energy of the senses has come to mean far more than he intended, and although it is still called Müller's law it is quite contrary to his teaching. ${ }^{2}$ It has become a law regarding quality instead of modality; of nerve fibre rather than of nerve. It is maintained by many recent writers that each nerve fibre with its end-organ has a specific energy, which means that it can, when stimulated, give rise to a certain definite sensation, and no other. If, then, we have more than one kind of sensation, of light, taste, smell, etc., we must have more than one kind of nerve fibre of light, taste, smell, etc., and as many nerve fibres as we have sensations. This law is at the basis of the Young-Helmholtz theory of vision, and ClerkMaxwell puts it as follows: ${ }^{3}$

"All the evidence we have on the nature of nervous action goes to prove that, whatever be the nature of the agent which excites the nerve, the sensations will differ only in being more or less acute. Now we may perceive with our eyes a faint red light, which may be made stronger and stronger until our eyes are dazzled. We may then perform the same experiment with a green light or a blue light. We shall then see that our sensations of colour may differ in other ways, besides in being stronger and fainter. The sensations of colour cannot be due to one nerve only."

The facts which we have just reviewed in relation to taste lend support to this neo-Miillerian law of specific energy of the senses, for there appear to be, at any rate in certain cases, special end-organs and nerves associated with the production of each specific taste. Whether this law is really a general law of sensation can only be determined when it is shown to be true in the case of other senses; as to this the evidence is as yet very meagre.

As we have indicated, the end-organs of taste can be stimulated by agencies other than sapid bodies. Mechanical blows and friction act as stimuli; a pencil or glass rod rubbed against the side of the root of the tongue produces a distinctly bitter taste, and acid and sweet tastes are alleged to have been produced by tapping the front of the tongue. Electricity is a very powerful stimulant, for a current too weak to produce an effect upon the skin or the eye will produce a distinct sensation of taste upon the tongue.

It was once thought that the action of the electric current was electrolytic, and that the ions were tasted, but this is negatived by the fact that distinct tastes are produced by slight alternating induction shocks, a condition under which there can be little electrolytic change. The electric currents act probably upon the end-organs, and do not directly stimulate the nerves, for cocaine and gymnema abolish the electric taste when applied to the surface of the tongue.

In experimenting with electrical stimulation of the tongue, one electrode, preferably a non-polarisable one, is placed on the dorsum of

I Such stimuli generally, of course, stimulate the nerves of tactile sensibility as well, and produce the specific sensations associated with their stimulation.

2 Many quotations might be given from Miiller's "Physiology," in support of this statement; the following has bcen selected, as bearing directly upon taste, p. 1066 :- "The nerves of taste and smell are capable of being cxcited to an infinite variety of scnsatious by external causes; but each taste is due to a determinate condition of the nerve excited by the external cause."

3 "Collected Papers," vol. i. pp. 446-447. 
the tongue, and the other electrode, generally flattened, is placed below. The anode upon the dorsum is the stronger stimulus, and its effects are specifically different from the cathode. The action of the anode appears to produce a warm acid feeling at the tip of the tongue, while the cathode is said to produce an alkaline or metallic taste. ${ }^{1}$ The results appear to vary, however, with the position of the electrode placed upon the dorsum of the tongue. At the back, bitter and metallic bitter tastes are produced, while in front the effect varies with the nature of the papillæ stimulated. Öhrwall finds the acid papillæ react readily with the anode, while the cathode produces a specific reaction with the bitter and sweet papillæ. The natural stimuli of the organ of taste are certain sapid substances dissolved in the juices of the mouth. Insoluble substances can only affect the tongue by mechanical pressure, for whell suspended in water they have no effect.

Chemico-physical characters of sapid substances.-It is frequently stated that there is no relationship between the tastes produced by sapid substances and their chemico-physical characters. This is, however, very questionable, as the following facts indicate. ${ }^{2}$ If we take one of Mendelejeff's groups, containing elements having similar chemicophysical properties, and if we combine these elements each with one other common element to form a series of sapid salts, we shall find the tastes of these salts are the same, or very similar. If, for instance, we select the elements in Group I (L.Na.K.Cu.Rb.Ag.Cs.Au.) and combine them, to form $(a)$ chlorides and $(b)$ sulphates, we shall find that the soluble chlorides are all salt, and the sulphates are saltish bitter. The chlorides of Group II (Mg.Ca.Zn.Sr.Cd.Ba.Hg.) are saltish bitter, with a warm pungent taste. Sodium and potassium compounds of Groul, VII (F.Cl.Mn.Br.I.) are salt, passing in the higher members of the group to saltish bitter. Amongst the organic substances which produce an acid taste, such as acetic, oxalic, or tartaric acid, we find the presence of a compound radicle, $\mathrm{CO} . \mathrm{OH}$; ; and it is probable that just as these substances owe certain of their chemico-physical properties to the presence of this radicle, they also owe to it their acid taste. The alcoholic bodies, like glycerin, glucose, saccharin, contain a radicle, $\mathrm{CH}_{2} . \mathrm{OH}$., and this radicle, the presence of which characterises the group from a chemical point of view, has much to do in all probability with the production of taste, for these bodies when they are sapid are sweet.

It is true that we not infrequently find that similar tastes are produced by sapid bodies of quite dissimilar chemico-physical characters; thus, sugar and acetate of lead are sweet. We cannot therefore affirm that similar tastes prove the existence of similar chemico-physical properties in the stimuli, though in many cases, at any rate, we can say that substances with similar chemico-physical properties have similar tastes. In the case of sight we have an analogous condition. Similar stimuli give rise to similar sensations, but the same sensation may be produced by dissimilar stimuli. A grey or a yellow sensation can be produced in a variety of ways, by very dissimilar stimuli; thus, grey can be produced by red together with blue-green spectral rays, or by orange together with blue spectral rays, and so on.

After-taste and successive and simultaneous contrast.--When a sapid substance acts upon the tongue, the taste may be followed by after-

${ }^{1}$ M. v. Vintschgau, Arch.f. d. ges. Physiol., Bonn, Bd. xx.; L. Hermann, loc. cit.

2 J. B. Hayeraft, Proc. Roy. Soc. Edin., vol. xiii.; and Brain, London, vol. x. p. 145. 
effects which have been compared with the after-images in the organ of vision. M. v. Vintschgau ${ }^{1}$ describes the metallic bitter of the taste which sometimes remains subsequent to the breaking of a current through the tongue, and which, during its passage, produced a metallic acid sensation.

Of greater interest, however, is the after-effect produced by a sapid substance itself. It has long been known that after tasting common salt the tongue is more sensitive to the taste of sugar; so much so, that a very weak solution of sugar, too weak to be distinguished as a sweet liquid, produces a taste, if it is taken after washing out the mouth with salt and water. In the same way, acid stimuli render the tongue more susceptible to sweet substances, and after tasting weak sulphuric acid, even distilled water appears somewhat sweet. ${ }^{2}$ Not only does common salt produce after-susceptibility to sweet, but many other substances act in the same way. Thus, after gargling with chlorate of potash, tapwater tastes sweet with a trace of acid (M. v. Vintschgau). A still more curious case is mentioned by J. Frentzel, ${ }^{3}$ and one that can be readily verified. If the mouth be rinsed out with a weak solution of sulphate of copper, and if the experimenter then smokes a pipe or cigarette, a well-marked sweet taste is developed.

Simultaneous contrasts also appear to be capable of demonstration in the case of taste, as in the case of vision. If a sapid substance be applied at one region of the tongue, other parts of the organ are rendered more susceptible to other stimuli. Thus, if one border of the tongue be rubbed with salt, the other border will give a reaction with sugar out of all proportion to the strength of the stimulus employed. Salt and acid give likewise simultaneous contrast, but it is questionable whether any facts can be brought forward to show that bitter sensations are affected by other stimuli simultaneously applied.

Taste sensations take some time before they are generated, and the reaction time for taste is comparatively slow. This can readily be understood when we remember that the sapid particles have to diffuse into the somewhat viscous fluids bathing the mouth, before they can gain access to the end-organs of taste. We taste more quickly when we rub the tongue against the roof of the mouth, or when we rub the sapid solution upon the mucous membrane by means of the finger. For the same reason, it is difficult at once to free the mouth from a sapid material which has had free access to it; and a clinging taste is frequently got rid of only after repeated rinsings of the mouth with water or such mechanical expedients as the chewing of bread-crumb.

Our power of localising the spots at which stimuli are applied is fairly developed in the case of taste, for the sensory surface is also furnished with the nerves of common sensibility, so that sapid stimuli are never applied without at the same time stimulating our nerves of touch. It is on this account that we can learn by everyday experience that bitter substances are best tasted at the root, and sweet substances are best tasted at the tip, of the tongue; for when the bitter sensation develops, we know by our sense of touch that the sapid material has reached the back of the tongue. Sweet sensations, on the other hand, are developed almost as soon as we become conscious of the presence of the sapid substance on the tip of the tongue.

1 Arch. f. d. ges, Physiol., Bonn, 1879, Bd. xx. S. 81.

${ }^{2}$ F. Kiesow, Phil. Stud., Leipzig, Bd. x. S. 3 and 4.

${ }^{3}$ Centralbl. $f$. Physiol., Leipzig u. Wien, Bd. x. S. 3. 


\title{
THE SENSE OF SMELL.
}

\author{
By J. B. Haycraft.
}

Contents :- The Anatomical Data, p. 1246-The Passage of Air through the Nose, p. 1248 - Olfactometry, p. 1249 - Hyperosmia and Anosmia, p. 1251-The Classification of Odours, p. 1252-The Law of Specific Energy of the Senses, p. 1253-The Relationship between Smell and the Chemico-Physical Properties of Odorous Bodies, p. 1254-The Physical Condition of the Stimulant, p. 1256 -The Simultaneous Action of Two Odours, p. 1257-The Psychology of Smell, p. 1258.

Anatomical data.-That sensory surface of the body which is modified in such a manner as to render it impressionable to odorous particles, is found in the upper or olfactory region of the nose. On opening the nasal cavities, the upper region is seen to be lined by a mucous membrane which has a yellow or brown-yellow colour, due to

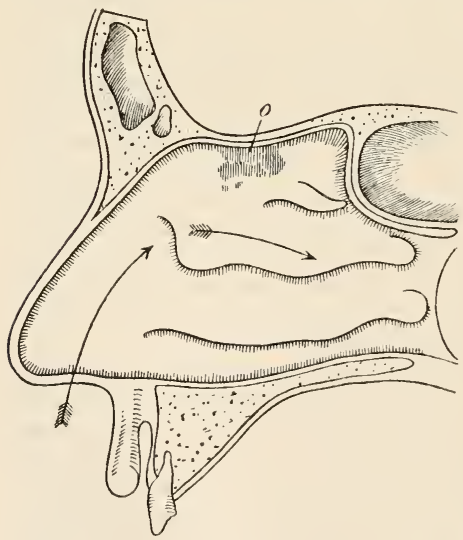

FIg, 446 . the presence of pigment within it. This yellow membrane is generally believed to be olfactory, and is so termed. It covers the superior and part of the middle turbinated bones, and that portion of the septum which lies at the same level. Max Schultze ${ }^{1}$ was, however, of opinion that the true olfactory mucous membrane, namely, that membrane which contains the olfactory end-organs, has in man a much more limited distribution, and never reaches even to the lower margin of the superior turbinated bone. This has recently been confirmed by the very careful investigations of A. von Brunn, ${ }^{2}$ who finds true olfactory epithelium only on an area little more than $1 \mathrm{~cm}$. across, situated in the upper part of the superior turbinated, together with another area of much the same size upon the septum opposite (see Figs. 446, 447, o).

1 "Bau der Nasenschleimhaut," Halle, 1862, S. 75.

2 Arch.f. mikr. Anat., Bonn, 1892, Bd. xxxix. S. 632. 
There are two kinds of epithelial cells to be found in the olfactory mucous membrane, as is also the case in many other sensory epithelia, such as the retina, the auditory, and the gustatory. They are, in the first place, the supporting cells, and between them lie the true olfactory cells. Each of the latter is provided with some seven or eight ciliumlike processes, ${ }^{1}$ which project from the free surface of the mucous membrane into the serous fluid which moistens it. The olfactory cells are prolonged internally into processes which become the fibres of the olfactory nerve. These fibres, which are nonmedullated, arborise within the olfactory bulb, within glomeruli formed by these arborisations and the dendrons of the bulb cells. Central processes of the latter carry the olfactory impulses to their centres within the brain.

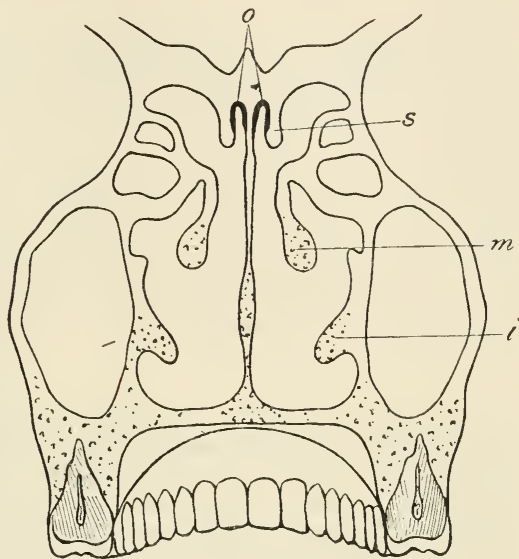

FIG. 447.

The fifth is the nerve of common sensibility to the nose, and in the case of disease or section of this nerve, irritants such as pepper, chlorine, and ammonia produce no irritating effect. When loss of smell likewise occurs, this is

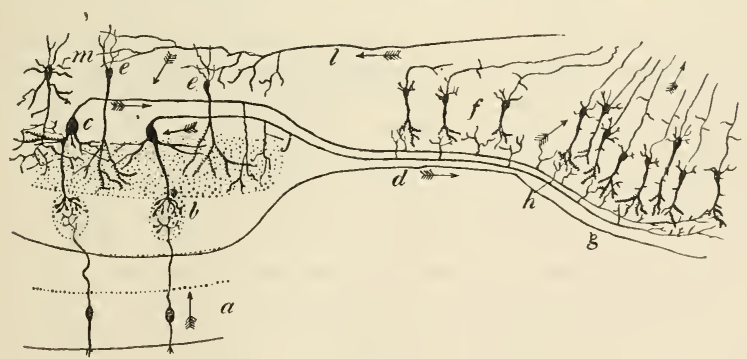

FIG. 448. - Diagram of olfactory path (Cajal). $\quad a$, the olfactory cells; $b$, olfactory glomeruli with synapses between their central nerve-processes and the dendrons of the mitral cells $(c)$ in the olfactory lobe; $d$, fibres passing from mitral cells to arborise with dendrons of cells of cerebral cortex, $f ; j$, collaterals from these fibres; $e, m$, other cells in the olfactory lobe; $l$, centrifugal fibres derived from cortical cells and terminating in the olfactory lobe.

probably due to dryness of the mucous membrane, and is thus only indirectly. dependent upon division or disease of the nerve.

Where the olfactory nerves are themselves divided, diseased, or pressed

${ }^{1}$ von Brunn, loc. cit., S. 638. 
upon by a tumour, anosmia may follow. The olfactory nerves are occasionally absent, and in these cases we find congenital anosmia, though this is said not to be invariably the case. ${ }^{1}$ In the very exceptional cases in which sensation is present, it has been suggested that fibres of the fifth nerve officiate as nerves of smell.

In the ease of man, the monkeys, and some cetacea, the olfactory senseorgan is evidently one which has undergone involution. This is evident, not only from a study of the nose itself, but also from a study of the central nervous system. The olfactory bulbs and tracts are but rudimentary organs, when compared with the olfactory lobes of macrosmatic mammals. In the nose of man, the three turbinals represent the chief remnants of the much more complex organ which must have existed in ancestral forms. ${ }^{2}$ The sensory surface must have greatly diminished in extent, and now occupies, as has already been described, but a small patch of the upper region of the nasal cavity. It is probable, therefore, that the sense of smell was more acute in the ancestral forms, and that it played a relatively great part in the psychology of man's ancestry, a condition which we find to-day among the macrosmatic mammalia.

The passage of air through the nose.-The organ of smell is stimulated by odorous particles which reach the nose, and enter it either by the anterior or by the posterior nares. If a bottle containing a volatile oil be unstoppered in a room, the oil volatilises, and the particles gradually diffuse into the room. The dispersion of these particles by diffusion may be aided by agitating the air, as with a fan, or by a draught, and by displacements of air due to differences of temperature, etc. ${ }^{3}$

The outside air in the neighbourhood of the face is drawn through the nose, either in the course of ordinary inspiration, or by sniffing. If this air contains a sufficiency of odorous particles, we are sensible of the odour.

The air stream was supposed by the older observers to pass chiefly along the lower region of the nose on the upper surface of the palate, and to take, in fact, an almost straight course from the anterior to the posterior nares. More recent observers have shown that the main air-stream on entering the nostrils at first ascends almost vertically, due probably to the horizontal position of the inlet; it then bends round, and sweeps backwards and downwards at the level of the middle turbinals, and leaves the nose by the posterior nares.

The earliest experiments were made by E. Paulsen. ${ }^{4} \mathrm{He}$ divided the head of a cadaver in the mesial plane, and placed tiny squares of red litmus paper upon the mucous membrane of the nasal cavity. The two halves were then replaced in juxtaposition, and air saturated with ammonia vapour was drawn through the nose by means of a pump attached to the trachea. On separating the halves of the head once more, and looking into the exposed nasal cavities, he could see the direction which the main air-current had taken, for this was indicated by the blueness of the litmus paper. H. Zwaardemaker ${ }^{5}$ performed a

1 See Gowers' "Diseases of the Nervous System," vol. ii. p. 140.

2 Schwalbe, Schrift. d. phys-ökonom. Gesellsch. zu Königsberg, 1882, Bd. xxiii. ; Zuckerkandl, "Das periphere Geruchsorgan der Saugethiere," Stuttgart, 1887 ; Seydel, Morphol. Jahrb., Leipzig, Bd. xvii.

${ }^{3}$ See an excellent article on the "Diffusion of Volatile Substances," by Justus Gaule, "Physiol. des Nase und ihrer Nebenhöhlen," Wien, 1896.

${ }^{4}$ Sitzungsb. d. k. Akad. d. Wissensch., Wien, 1882 (3), Bd. lxxxv. S. 348.

5 "Die Physiologie des Geruchs," Leipzig, 1895, S. 49. 
somewhat similar experiment with the head of a horse, and G. Franke with the human cadaver. ${ }^{1}$ They divided the head through the mesial plane, and applied a glass plate, so that it closed one nasal cavity, replacing the septum. On drawing smoke through the nose, they could observe through the glass the direction that it took.

While these observers are not quite agreed as to the exact height that the main stream of air reaches, they are at one in placing it below that portion of the nose in which von Brunn has localised the position of the olfactory epithelium.

This olfactory epithelium is, in fact, placed in a narrow chink above the main stream of air, and the question arises, how do the odorous particles reach it? Diffusion no doubt will account for the entrance of many particles, for this is a rapid process, and will occur during the period of the shortest sniff. The air, however, within the true olfactory region must suffer, at any rate, partial displacement towards the posterior nares, and this air will in its turn be displaced by fresh air entering the nose and laden with odorous particles. The pressure of air during the commencement of inspiration is less at the posterior nares than the pressure of the outside air, and therefore less than that of the air within the whole cavity of the nose. There will consequently be a displacement of the outside air, and of that of the whole cavity of the nose towards the posterior nares. Braune and Clasen $^{2}$ have indeed experimentally shown what is a priori a certainty, namely, that the air even within the antrum of Highmore suffers partial displacement during each act of inspiration. We may conclude, therefore, that although the main stream does not reach the level of the true olfactory mucous membrane, yet over this surface there is nevertheless a definite motion of the air. This motion may be difficult to recognise by the methods of Paulsen and his followers, but it may be quite sufficient to appeal to the exquisitely delicate sense-organs of the olfactory nerve.

Olfactometry.-It is a matter of great theoretical as well as of practical interest to determine how small a quantity of a substance can excite the sense of smell. It will be remembered that Kirchoff and Bunsen were able to distinguish by means of the spectroscope as little

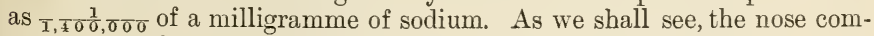
pares most favourably with the eye. A grain of musk will scent a room for a very long time, and the actual quantity drawn into the nose during any given inspiration must therefore be inconceivably small. E. Fischer and Penzoldt ${ }^{3}$ placed a weighed quantity of an odorous substance, such as mercaptan, in a room. The air was mixed with a fan, and the experimenter entered the room from time to time until he was able to smell the substance. In these investigations it was found that mercaptan, with a dilution of 1 to $50,000,000,000$, could be detected. By calculation it was found that the actual weight of mercaptan, in the 50 c.c. of air, inspired by the experimenter, was $\frac{1}{160,000,000}$ of a milligramme.

By methods such as the above, which give the smallest amount of a substance which can produce sensation, we can arrange odorous bodies in the order of their potency. We can also test the sensibility of the

\footnotetext{
I Arch. f. Laryngol. u. Rhinol., Berlin, 1893, Bd. i. S. 236.

${ }^{2} Z$ tschr. f. Anat. u. Entwcklngsgesch., Leipzig, 1876, Bd. ii. S. 1.

${ }^{3}$ Sitzungsb. d. phys.-rned. Soc. zu Erlangen, 1885-86, Bd. xviii. S. 7. VOL. II. -79
} 
nose under various physiological conditions, and test the acuteness of the sensations of those supposed to suffer from partial or complete loss of sensation. For the latter purpose we have to determine the quantity of a substance which can just be detected by a normal individual, and then determine in the case of another person with blunted sensations how much the stimulus must be increased.

In this line of research Valentin was a pioneer, ${ }^{1}$ and the methods adopted since his time are but modifications and improvements of his methods. Valentin took a weighed quantity of an odorous substance and diffused this into a known quantity of air. Of this air he took a portion, and again and again diluted it until it could just and only just be detected by the person experimented upon. In other experiments water was used to effect this dilution instead of air.

(1)

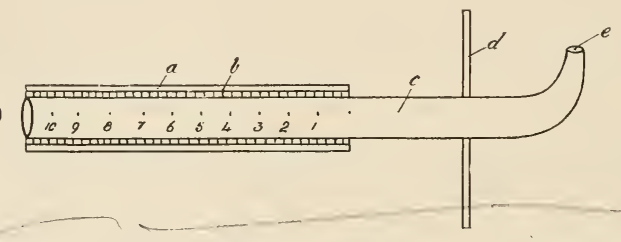

(2)

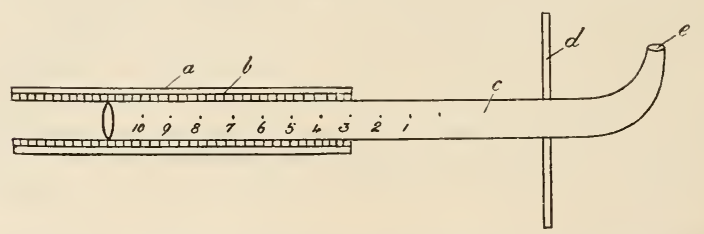

Fig. 449.-Diagram of Zwaardemaker's olfactometer- $a$, external protecting tube of glass; $b$, tube of indiarubber, giving off odorous particles from its inner surface when this is exposed; $c$, inner graduated glass tube through which air is drawn; $d$, profile view of wooden shade ; $e$, end of tube placed in the nostril. In 1 , the inner tube is pushed home, none of the indiarubber is exposed and nothing is smelt. In 2 , the tube is pulled out for three divisions, each being $77 \mathrm{~cm}$. long, so that $2 \cdot 1 \mathrm{cms}$. or three olfacties of tube are giving off particles of odorous indiarubber into the inner tube, through which the air is drawn. ${ }^{2}$

Fröhlich ${ }^{3}$ placed odorous bodies in a stoppered bottle, and tested the acuteness of smell by determining the distance at which the odour could be detected. The odorous particles diffusing from the bottle would become more and more scattered the greater the distance from the bottle, and would require, therefore, a more acute organ of smell to detect their presence when the bottle was held at some distance from the nose. While this is a good practical method, by means of which we may compare the organ of smell in the case of one individual with that of another individual, it is useless when we wish to compare the

1 "Grundriss d. Physiologie," s. 515.

${ }^{2}$ Other methods have been used by Charles Henry, Compt. rend. Acad. d. Sc., Paris, 1891, tome exii. p. 344 ; N. Savelieff, Neurol. Centralbl., Lcipzig, 1893, S. 340; and Reuter, Ztschr. f. klin. Med., Berlin, 1893.

${ }^{3}$ Sitzungsb. d. k. Akad. d. Wissensch. math.-naturw. Cl., Wien, 1851, Bk. vi. 
potency of one odour with that of another, the reason being that no two odours agree in their rate of diffusion. For the latter purpose there is probably no better method than the one already alluded to, namely, that of Fischer and Penzoldt, who caused a weighed quantity of an odorous body to diffuse into the air of a room (which was agitated by means of a fan) until it produced a sensation. The quantity of the body necessary to produce the sensation was a measure of its potency.

Perhaps the most important recent advance has been made by H. Zwaardemaker, who has invented a very useful olfactometer, which can be used for physiological and clinical purposes, and by means of which the sharpness of smell may readily be expressed in smell units or olfacties.

Zwaardemaker's olfactometer may be described as follows, but for a more detailed description the reader should consult his work : ${ }^{1}$

Indiarubber tubing has a distinct odour, and in Zwaardemaker's instrument the air inhaled by the nostrils is drawn over the surface of indiarubber, and the amount of this surface is so adjusted that its odour can only just be perceived. The extent of this surface is measured, and is a quantitative test of the acuteness of the sense-organ in the individual tested. The tubing, two or three inches in length, is fitted inside a wider tube of glass, which prevents any odorous particles from leaving its outer surface. Another longer glass tube, just wide enough to fit within the indiarubber tube, is pushed within it. When this is pushed from one end to the other of the indiarubber tube, both sides of the latter are covered (Fig. 449,1), and no particles of the indiarubber diffuse into air aspirated by the nostrils. On drawing the inner tube a little way out of the indiarubber tube, a certain portion of the latter is exposed (Fig. 449, 2), and odorous particles are inspired, and produce a sensation if they are in sufficient quantity. With his olfactometer Zwaardemaker found that a normal individual could perceive on the average the odour of the indiarubber when $7 \mathrm{~cm}$. long was exposed. The inner glass rod was therefore graduated, each graduation being $\cdot 7 \mathrm{~cm}$. An individual who ean only detect an odour when the tube is drawn out to the second division, exposing $1.4 \mathrm{cms}$. of the tube, has half the normal sensitiveness; if he can perceive the odour only when it is, as in the figure, drawn out to the third division, he has but one-third the normal sensitiveness. The physical quantity or stimulus, namely, the number of odorous particles given off into the inspired air by $\cdot 7 \mathrm{~cm}$. of indiarubber, Zwaardemaker takes, therefore, as a unit of measurement, and this is termed an "olfactie." Ordinary indiarubber has too faint an odour to permit of its use, except in those cases where the deviation from the normal is considerable. In testing cases of partial anosmia, a tube is used made of a substance which is twenty-four times as powerful a stimulus as indiarubber, itself. This is "Kunsthorn " or "Guttapercha ammoniacum," called in English " hoof cement," and used by the veterinary surgeon for strengthening the hoof. By means of two instruments, one containing a tube of indiarubber, the other a tube of hoof cement, we can stimulate the nose from one up to two or three hundred olfacties. Z waardemaker further found that in many cases of anosmia certain odours might be perceived almost to the normal extent, while others hardly acted as stimuli at all. To investigate these cases, he constructed tubes of odorous substances, some smelling of bees-wax, others of asafoetida, etc. ${ }^{2}$

Hyperosmia and anosmia.-It appears from olfactometric observations that hyperosmia is not of very rare occurrence. Increased sensitiveness is common in hysteria and other nervous affections, and certain

1 “Die Physiol. des Geruchs," Leipzig, 1895.

${ }^{2}$ Arch. f. Laryngol. u. Rhinol., Berlin, Bd. iv. 
drugs are capable of inducing it. One of the most important of these is strychnine, which may, in a diluted form and locally applied, be given for the treatment of anosmia. Cocaine is said to produce hyperosmia before it causes partial or complete loss of the sensation.

Anosmia is not of infrequent occurrence. It may be complete and congenital, as in cases where the olfactory nerves are absent; and partial anosmia may occur from a variety of causes, such as stenosis of the nasal passages, catarrh and disease of the mucous membrane, and various nervous affections. Habitual smoking produces partial anosmia, mor phia and cocaine locally applied rapidly induce it. It is questionable whether the strongest odour, pure and simple, can produce complete anosmia. When this is induced by the vapour of ammonia, we have to deal with an odorous body which is at the same time a powerful irritant; and the complete anosmia which may result is, in all probability, due to inflammation of the olfactory mucous membrane itself.

There are some persons who, while acutely sensitive to most odour's, are incapable of recognising others. There are many individuals who are unable to smell methyl-alcohol, hydrocyanic acid, and mignonette. Cloquet ${ }^{1}$ mentions the case of an individual who was unable to smell vanilla, although sensitive to the perfume of flowers; while Gründe, quoted by Cloquet, mentions a man insensible to everything but decaying and fæcal matter.

The classification of odours.-In judging of the acuteness of smell, we have almost entirely confined our attention to our power of being stimulated by different quantities of an odorous material. But almost no two odorous bodies smell exactly alike, and we are able to experience throughout life an almost infinite number of odours differing from each other in quality. Thus we can distinguish the odour of almost every kind of flower, fruit, beast, etc. Various attempts have been made to group these sensations together, but without very satisfactory results. Albrecht v. Haller ${ }^{2}$ divided odours into (1) sweet smelling, (2) foul smelling, (3) neither the one nor the other. This, which is a classification on purely physiological lines, gives us very little information, and is rather a classification of the feelings that accompany sensation than of the sensations themselves. Fourcroy and Lorry have each classified odours, but their basis is not physiological, it is purely chemical. Linné, whose work is best known, classifies odours into the following seven classes :-

1. Aromatic odours, such as turpentine, peppermint, and lavender.

2. Fragrant odours, such as those of the lily, jasmine, vanilla.

3. Ambrosial odours, such as musk and ambergris.

4. Alliaceous odours, such as chlorine, sulphuretted hydrogen, asafœtida.

5. Goaty odours (Odores hircini), such as those of cheese, cat's urine, rancid oil.

6. Repulsive odours (Odores tebri), such as hyoscyamus, and many of the family of the deadly nightshade.

7. Nauseating odours, such as those of fæces, putrid flesh, etc.

The above classification, which is drawn up upon partly a chemical and partly a physiological basis, would hardly find general acceptance, and indicates most forcibly the fact that any rigid classification is at present quite impossible. In the case of the sense of taste, it is probable that there are only four sensations-those called sweet, sour, bitter, and salt; and these four sensations are universally recognised and have definite and well understood names. Were it 
possible in the same way to group bodies that produce similar or allied smells, mankind would long ago have introduced a well-understood smell nomenclature. This has not been done, and odours are called by the names of bodies which give them out, and of these there are an infinite number.

The law of specific energies of the senses.-As far as we know, the sense of smell obeys the law of the specific energies of the senses, as taught by Johannes Miiller; that is to say, however stimulated the nose may be, an odour is invariably produced. While the usual stimulation is naturally brought about by the action of odorous particles, we can also stimulate the nose electrically and produce the sense of odour. If the cavities of the nose are filled with normal saline, and one electrode from an electric battery be dipped into the solution, while the otler is placed upon the forehead, an odour something like that of phosphorus is experienced whenever the intensity of the current is changed. ${ }^{1}$ The action of a mechanical stimulation has not yet been investigated. How far the sense of smell obeys the neo-Muillerian law of the specific energy of the senses, is as yet an undecided question. By this law, every sensation which differs from every other sensation, except as regards its quantity or intensity, must have its own nerve fibre and end-organ. Putting it another way, a nerve fibre only transmits impulses producing one kind of effect. It has been assumed that this neo-Miillerian law is true of all the senses, although the evidence is at present certainly inconclusive in the case of almost all of them. It is therefore still upon its trial, and we have to examine the evidence in the case of smell. Were we able, as in the case of the sense of taste, to classify our odours into certain groups, and were we able to find evidence that portions of the olfactory mucous membrane are sensitive, each particular portion to the odours of a particular group, then we should be in a fair way to establish this law in the case of smell. This, as we have seen, we are unable to do. How far cases of partial anosmia have a bearing upon this question, it is dificult to say. We liave seen that some persons are unable to smell mignonette, hydrocyanic acid, violets, etc. It may be that in these cases one of the special sets of nerves necessary for the transmission of the impulse set up by the action of this odour is absent. It may be equally possible that the protoplasm of the end-organ of the nerve-on the supposition that there is but one kind of end-organ-may exhibit an individual variation of such a nature as to prevent its reaction to that particular stimulus. This evidence is therefore inconclusive in our present state of ignorance, as to what actually occurs when the endorgan is stimulated.

Aronsohn ${ }^{2}$ found, on fatiguing the nose by the vapour of ammonium sulphide, that sulphuretted hydrogen, and the vapours of chlorine and bromine, produced very diminished effects, while ethereal odours could be perceived as before. Again, in another experiment, when the nose was fatigued by the odour of camphor, he found that ether and eau de Cologne could not be smelt. These experiments are of great interest, and show that various odorous bodies are capable of producing similar fatigue effects upon the olfactory organ. It does not follow, however, that a group of bodies so acting fatigues one kind of end-organ to the exclusion of the others. The fatioue that they produce may be that of all the end-organs to allied kinds of

${ }^{1}$ E. Aronsohn, Centralbl.f. d. med. Wissensch., Berlin, 1888.

${ }^{2}$ Arch. $f$. Physiol., Leipzig, 1884. 
stimuli. The very uniform appearance of the olfactory end-organs indicates, indeed, that they are all concerned in the reception of all the stimuli which produce olfactory sensations; and we may at any rate conclude that the neo-Müllerian law requires, in the case of smell, additional evidence before its acceptance can be granted.

Relationship between smell and chemico-physical properties of odorous bodies.-From general analogy we should expect that there will be found to exist some sort of resemblance between the sensations produced by stimuli of similar physico-chemical properties. This connection is at first sight by no means apparent, and was only clearly understood after Mendelejeff had classified the elements into natural groups under what is known as the Periodic Law. ${ }^{1}$ Taking one of these groups, we find that they consist of elements having similar general chemico-physical properties; thus in the sixth group we have oxygen, sulphur, chromium, selenium, molybdenum, and tellurium. In ascending one of these groups, beginning in the sixth group with oxygen, we find that the molecular weight increases, and that there is a progressive change in the chemico-physical properties of each member of the group. We also find that the odd and even members respectively of the group resemble each other more particularly. Thus in group six we find that, in their chemico-physical properties, sulphur, selenium, and tellurium closely resemble each other. Now what we can say as to the chemico-physical properties of these elements, we can also affirm as to their physiological properties, as regards the sense of smell, and also of taste. Thus in group six, sulphur, selenium, and tellurium, when compounded with hydrogen, with methyl, or with ethyl, etc., have strong and disagreeable odours. They are not the same, although there are points of resemblance, especially as regards their offensiveness. The odours of the selenium compounds are, however, recognisably intermediate between those of sulphur and those of tellurium, just as its molecular weight occupies a position between these two. There are other groups having elements which give rise to smell; thus in group seven we have chlorine, bromine, and iodine. These odours are not unlike each other, the odour of bromine linking the lower chlorine to the higher iodine. Among these compounds may be instanced chloroform, with its fragrant odour; bromoform, with a fragrant odour, mixed with another odour which we recognise as that of the higher iodoform.

Leaving Mendelejeff's groups, and studying groups of organic bodies like the monatomic alcohols or the fatty acids, having similar chemicophysical properties, but showing a progressive change in their characters as we pass from the lower to the higher members of the group, we shall find that there exists in like manner a change in respect of the sensations they are capable of producing.

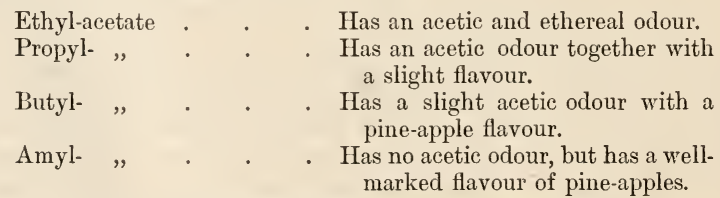

${ }^{1}$ J. B. Haycraft, "The Sense of Smell," Proc. Roy. Soc. Edin., 1887, vol. xiv. p. 207 ; and Brain, London, 1889, vol. xi. p. 166. 
This last example will best illustrate how difficult it was to correlate smell with the chemico-physical properties of odorous substances. No one would group the sensation of ethyl-acetate with that of amyl-acetate; they are very unlike, yet they are linked together by the smells of the intermediate members of the group. It is only by studying a whole group of related elements, or compounds, that it is possible to make out any comnection at all. We may say then that smell follows the periodic law, and that the power of existing smell, when present, is found to vary in the same way as the chemico-physical properties of substances.

Such a statement is not vitiated by the fact that certain substances, having dissimilar chemico-physical properties, produce the same smell. This only shows that the nose has not a sufficient power of analysis to distinguish in this case the two stimuli from each other. In the case of vision, two very dissimilar stimuli may affect the eye, and produce identically the same sensation. Thus a mixture of red and blue-green spectral rays will produce a sensation called grey, identical with that produced by a mixture of orange and blue. In the case of taste, where there are but a limited number of sensations, we find many instances of a like nature. Our previous conclusions, although they place the power of producing smells on all-fours with the chemicophysical properties of chemical substances, do not commit us to the statement that odorous substances act chemically upon the olfactory end-organs. This is quite another matter, and must be discussed on its own merits. While it is quite possible that the differences in quality of smell may be due to the different chemical decomposition produced upon the protoplasm of the endorgan, it does not necessarily follow that this is the case. Odorous bodies have dynamical properties which might fully account for their action as stimuli. Odorous bodies have no doubt definite vibratory periods, and they absorb in great amount the heat rays. Spectroscopic investigation indicates, although the evidence is as yet somewhat fragmentary, that the elements-or their compounds-in one of Mendelejeff's groups are similar in the character of their molecular vibrations; and Carnelley ${ }^{1}$ has shown that a progressive change of vibrational pitch occurs on passing from a lower to a higher member of a group. Much the same is true of the alcohols, and of the fatty acids; the alcohols, for instance, have characteristic absorption-bands which shift towards the red end of the spectrum on ascending the group. William Ramsay ${ }^{2}$ showed that a substance must have at least fifteen times the molecular weight of hydrogen in order that it may be smelt at all. The light gases of the atmosphere are odourless; so is marsh gas (8), ethane (15), olefiant gas (14), hydrocyanic acid (15), methyl-alcohol (16), the latter two of which are smelt by some persons and not by others. Bodies of similar constitution, but with heavier molecules, are frequently odorous; and as a rule the strength of the odour increases with the molecular weight. Thus, while methane and ethane are odourless, propane (22) can be smelt. While hydrocyanic acid is odourless, the higher members or nitriles have characteristic smells. While methylalcohol is odourless, the higher members have stronger and stronger odours. Recently J. Passy ${ }^{3}$ has shown by olfactometric measurements the relative potencies of some of the alcohols, and his results are given in the following table :-

$\begin{array}{llllr}\text { Methyl-alcohol } & . & . & . & 1 \\ \text { Ethyl- ", } & . & . & . & 4 \\ \text { Propyl- ", } & . & . & . & 100 \\ \text { Butyl- } " & . & . & . & 1000 \\ \text { Amyl- }, & . & . & . & 10,000\end{array}$

${ }^{1}$ London, Edinburgh, and Dublin Phil. May., 1884, ser. 5, vol. xviii.

${ }^{2}$ Nature, London, 1882, vol. xxvi. p. 187. 'SCompt. rend. Soc. de biol., Paris, 1893. 
In both sight and hearing we have this interesting fact, namely, that vibrations, which are either enormously rapid or comparatively slow, are in both cases incapable of acting as stimuli. We have, according to Ramsay, an analogy in the case of smell, for bodies of low molecular weight, and having therefore a high vibrational period are odourless. In the case of smell there are no known bodies of slow vibrational periods incapable of producing a sensation; but in the case of taste there are indications that there exist both a lower as well as an upper margin to our sensation.

The physical condition of the stimulant.-E. H. Weber ${ }^{1}$ maintained that if you allow a 10 per cent. watery solution of eau de Cologne to flow into the nose, it fails to produce an effect upon the sense of smell; and he concluded that if we are to smell them, odorous particles must be carried by the air into the cavities of the nose. Recently Aronsohn ${ }^{2}$ found, on repeating these experiments, that such solution is highly irritating to the mucous membrane of the nose, and produces partial anosmia. It is supposed that the olfactory cells swell up, become waterlogged, and are no longer sensitive to stimuli, and on account of this action Aronsohn explains Weber's inability to smell the watery solution of the eau de Cologne. In other experiments Aronsohn replaced the water by normal saline, and states that under these conditions the scent produces its normal effect. In all these experiments we must bear in mind that the true olfactory region of the nose is now known to be situated in quite the upper part of what is really nothing more than a narrow chink. It is therefore a matter of very considerable difficulty to displace the air from this chink and replace it with the scent in a fluid form. But with this successfully accomplished, I myself, and three other persons upon whom I have experimented, have been unable to perceive any odour at all in the case of 5 or 10 per cent. solutions of eau de Cologne. The experiment may best be performed as follows:-The person to be experimented upon lies upon his back on a bench with his head hanging downwards over the end, so that the olfactory region of the nose is lowest. Two glass funnels are connected by tubing with a T-junction, and from the third limb of the $\mathrm{T}$, another tube passes into one nostril. Warm normal saline is allowed to flow from one funnel until both nasal cavities are filled, and the air-free solution falls upon the ground. This is then displaced by warm scented normal saline.

The following experiment indicates that although the nose is filled with odorous particles, yet they are incapable of affecting the olfactory nerves, and only do so when the air containing them is set in motion. The subject of this experiment performs several acts of respiration in rapid succession, so as to render himself apnœic. Under these conditions he is then able to remain perfectly immovable, without breathing, for from one to three minutes. A bottle containing ammonia is unstoppered under his nose; the vapour diffuses into the nose, is irritative, but cannot be smelt. If, after waiting say two minutes, he then pinches his nose so as to imprison the ammonia vapour already within it, walks into the fresh air, removes his fingers from the nose, and inspires through it, the ammonia is at once smelt. These experiments may be repeated with eau de Cologne, camphor, bergamot, etc., and indicate that odorous particles do not stimulate the end-

1 Arch. f. Anat., Physiol. u. wissensch. Med., 1847, s. 342.

2 "Exper. Untersuch. z. Physiol. des Geruches," Inaug. Diss. 
organs simply by their presence, even when in the gaseous condition, they must be moved over the olfactory surface.

The simultaneous action of two odours.-When two odours diffuse into a room, we can generally recognise them both, or the odour partakes of the nature of each. If one of them $(A)$ is small in amount, and the other $(B)$ greatly preponderates, we may only just recognise $A$. On increasing the quantity of $A$ the sensation changes, and we may recognise the two odours; and on increasing $A$ still further, we may lose the sensation of $B$ in the preponderance of that of $A$. But this is not always the case, for certain odours appear to antagonise each other; and if two such antagonistic odours are mixed, either one of them alone is smelt or nothing at all. Thus iodoform may be antagonised by balsam of Peru, the odour of musk by that of the smell of almond. This antagonism may of course be merely a chemical or physical action outside the body of one odour upon the other. Zwaardemaker, however, maintains that there is in some cases a purely physiological antagonism. He has constructed a double olfactometer, consisting of two olfactometers of the form already described. Two odours in any desired quantity can thus be led (without mixing) one into each nostril, so that each odour will stimulate its own portion of olfactory mucous membrane. The best results were obtained when the vapour of a 2 per cent. solution of acetic acid was led into one nostril, and the vapour of a 1 per cent. solution of ammonia into the other. If ammonia was alone smelt, he increased the strength of the acetic acid vapour, until a point was reached at which there was no odour perceptible.

The following is a list of physiologically antagonistic odours :-

\author{
Musk and oil of bitter almonds. \\ Caoutchouc and paraffin. \\ Volatile oils and iodoform. \\ Ammonia and acetic acid.
}

If these statements are true, and we can hardly doubt the results obtained by so experienced an investigator as Zwaardemaker, we have here what appears to be a unique fact in the physiology of the senses. We have to suppose that two stimuli, each one capable by itself of producing an effect on consciousness, when they act together mutually antagonise one another's action. There are other cases, in other senseorgans, in which one stimulus may modify the effect of another ; but in no other case that I am aware of do two separate stimuli destroy each other's effect on consciousness. This discovery modifies, too, one definition of modality as used by Helmholtz. Modality is the difference which exists between the sensations of the different sense-organs, and these are distinguished by their inability to pass into each other by intermediate stages. By quality, however, is meant the difference which exists between sensations of the same sense-organ, and these are distinguished by their power of passing into each other by intermediate links. As this is not the case with the smell of ammonia and acetic acid-for, according to Zwaardemaker, you either smell the one or the other, or nothing at all-the differences betwcen them must be one of modality rather than of quality, although they belong to one and the same sense-organ.

The psychology of smell.-We detect the presence of most of 
the substances taken into the mouth by the nerves of common sensibility; and our experience leads us therefore to associate taste with the direct application of certain bodies to the surface of the tongue. Most of these bodies are scented, and the odorous particles they give off affect the olfactory mucous membrane at much the same time as the substances reach the mouth. We do not, of course, know that the odorous particles are within the nose, and we associate therefore the sensation, which is really a combined olfactory and gustatory sensation, with the presence of the substance within the mouth, and we conclude that our sensation is one of taste. Thus we say we taste fruit, meat, etc., although the olfactory component of the sensation is really the greater one of the two; we project our olfactory sensation into our mouth. In the case of odorous bodies which are not eaten, like the rose, our experience leads us to associate our sensations with the object itself, generally felt and seen just in front of us. The odorous particles which proceed from the rose do not excite the nerves of common sensibility; and the fact which localises their actions as stimuli to the sensory surface of the nose is the experience that the scent is best felt when air is voluntarily sniffed through it. In most cases, therefore, we project our sensations outside the body, and this is expressed by the saying that "the rose smells." Persons who pay any close attention to their sensations, and are accustomed to detect odours by sniffing with their eyes closed, are conscious of the sensation, as something connected with the interior of the nose; and in their cases they can hardly be said to project their sensations. ${ }^{1}$

${ }^{1}$ As works of further reference, the reader is referred to Zwaardemaker's book, "Die Physiol. des Geruchs," Leipzig, 1895 ; and "Physiol. des Nase und ihrer Nebenhöhlen," by Justus Gaule, Wien, 1896. The older references will be found in v. Vintschgau's artiele in Hermann's "Handbueh," Bd. iii. Th. 2, S. 225. 
INDICES. 



\section{INDEX OF SUBJECTS.}

Abdominal muscles influence of, on circulation, . $\quad 44,52,91,92,93,94$, $127,129,141,147$

Aberroscope, . . . . . 1034 Absinthe, effect of, on nervous system, 139, 721,722

Acceleration of heart, 55. See also Heart. ,, respiration. See Respiration, frequency of.

Accelerator nerves. See Nerves, Accelerator.

tone, 55,56

Accomnodation, visual. Sce Eye.

Acetate of lead, cffect of, on nerve cells, 604 Acid, lactic, effect of, on heart muscle, . 195 , in exhaustion of muscle, . 389 ", , rigor mortis, . . . 393

Aconitine, effect of, on electrical response

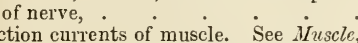

Action currents of muscle. See Muscle,
electrical response of. ", nerve. See Nerve, electrical response of. ,, spinal cord,

Adaptation, visual. See Retina.

Adductor preparation, double, . . 353

Adequate temperature, - . 951, 960

Esthesiometer, 925, 935, 944, 948, 959, 985

Esthesodic paths, . . . 811, 978

Affective tone, $\quad 966,974,997,1000$ curve of, . . . 997 of different sensatious, 966 , $967,968,969,973,975,990$ influence of duration of
stimulus 1000 on, .967 , , , intensity of stimulus on, . 967,972 , quality of stimulus on, . 967,968 from locomotor apparatus, proctor

of psychical processes, 968,969 relation of movements of

flexion and extension to, 975

,

, from viscera, $969,970,971,972$

Afferent reflex channels,

, root fibres. Sce Nerve root fibres.
Afferent root cells. See Root cells.

PAGE

After-images. See Sensation, visual.

Afterruthenband, . . . . . 349

Agraphia, . . . . . 774, 776

Air bladder, . . . . . . . 1168

, compressed, respiration of . . 128

, entry of, into veins, . . . . 120

,. rarefied, respiration of, . . . 128

Albatross, cerebellum of, . . . 903

Allino, vision in, . . . . 1095

Alcohol, effect of, on arterial pressure, . 80 ,, ," ,, heart, . . . 52 ", $\quad, \quad$, nerve, : 491, 492, 535

,, , , nerve cell, . . 604

:, ,, ,, reaction time, . 611

Alexia, . . . . . 776

Algesimeter, . . . . . 985

Alimentary tract, muscular mechanisms

of . 313

Alkalies, cffect of, on heart muscle, $19 \dot{9}, 225$

Alkaloids, effect of, on electrical response

of nerve . . . . . . 536

Allochiria, . . . . . . 866

Allolophobus, spinal shock in, . . . 846

Amacrin cells, . . . . . 593

Amblyopia from cortical lesions, . 752, 753,

759,760

." , lesions of thalamus, 918

Ammonia, cffects of, on nerve, : $\quad$ : 491

Ammesia, verbal, . . . . 775,776

Amphiarthrosis, . . . 233

Amphibia, action of ear of, . . 1167, 1181

,, ,, pupil retlex in, . 1043

, ", sympathetic nerve

, $\quad$ : $\quad$ cells in, . . 655

,, ," vagus nerve in, . 215

Amphioxus, after decapitation, $817,819,827$,

, movements of, after section

of cord, . . . 813

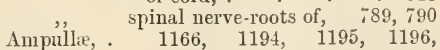
$1198,1201,1202,1203,1204$,

1205

Amyl nitrite, influence of, on arteries, . 138 ,, ," dicrotic

wave, . 110

Anabolic nerves, . . . . 220, 221

Anabolism, . : $189,221,220,674,675$

Anacrotic wave, . . . . 108, 111

Anæmia, effect of, on ncrve cells, . . 604 
1004

1005 ,, muscular

sense, 1006,1011

from lesions of cortex, 766,767

", ," , mesenceph-

alon, 915

,, , , , nerves, 987, 988

," , , pons, $\quad$, spiual cord, 893

, in leprosy, ". . . 988

Anæsthetics, effects of, on electrotonic

$\begin{array}{rr}\text { currents, } & 552 \\ \text { nerve } & 491\end{array}$

Analgesia from lesions of mesencephalon, . . 915

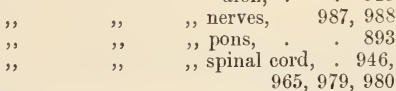

Anelectrotonus, 495, 496, 497, 506, 507, 509, 512,546

Angle, visual, - $1055,1056,1100$

Anguis fragilis, autotomy in, . . 845 Angular gyrus. See Gyrus.

Animal mechanism,

Anisometropia,

Anodon, nerves of

1032,1038

Ano-genital region, sympathetic nerves

of, . . $635,646,667,684,693$

Anosmatic animals,

Anosmia, . - 1248, 1251, 1252, 1253

Ansa lenticularis,

Anschlagszuckungen, . . . . 367

Anthropoid apes, motor area of, . 745,773

Antiarine, effect of, on heart, . 196, 223

Antipyrin, effect of, on nerve cells, . 604

Antrum, pyloric, . . . 321, 322

Anus, nerves of, : : $\quad 336,337,692,693$ , splineter of, 336,337 . See also Rectum, Defacation.

Aorta, blood-flow in, . . . 4 42, 84 , compression of, effect on afferent

nerve-

roots, 836

, , $\quad \begin{gathered}\text { roots, } \\ \text { pulmon- }\end{gathered}$

aryeir-

e ula-

tion, , 151

, , , , spinal

cord, 835 ,

", , , $\quad \begin{gathered}\text { spinal } \\ \text { ganglia, } 836\end{gathered}$ 836,862

,, effect of heat on, . . . 954

, ligature of, effect on respira-

tion, . .

", pressure in, relation of, to pul-

Apæsthesia,

monary pressure, $\quad 149,150$

" effect of, on muscle, 1018,1020

influence of retinal sensa-

tions in, . . 802

in limbs, 793, 799, 800, $80 \dot{1}, 802$,

803
in tail, . $\quad . \quad 802$

with excision of labyrinth, 802

Apes, cerebellum in, . . . . 902

, lateral semisection of cord in, 866

" motor area in, . . 745,773
PAGE

774

complete, $\quad 774$

," motor, . $\quad . \quad$. $\quad .774$

,, partial, . . . . 774

" sensory, . . . 775,776

Aphemia, . . . 774

Apnoa, . . . . . $\quad$. 289,291

,, ehemical, . . . . 301

", effect of, on spinal reflexes, . 837

,, expiratory, . . . . 299

," inspiratory, . . . $\quad .300$

,. mechanical, . . . 298, 301

," spuria, . . . . . 305

,, vagi, . . . . 301

,, vera, . $\quad . \quad$. $\quad 301$

Apomorplin, emetic action of, . . 326

Aqueductus vestibnli, . . . $\quad .1179$

Aqueous humour. See Eye.

Arachne, autotomy in, . . . . 845

Are, reflex, . $\quad 785,786,804,837,860$

Arciform fibres, degeneration in, . 705

Area, association. See Association areas, Cerebral cortex.

,, auditory. Sec Cerebral cortex.

," motor. See Cerebral cortex.

," olfactory. See Cerebral cortex.

,, precommisural,

, Rolandic. See Cerebral cortex, Motor area.

sensory. See Cerebral cortex.

,, tactile, . . .

", visual. See Cerebral cortex.

Areas, projection, . . 771, 772, 773

Arm, centre for movenents of. See

Cerebral cortex.

Arsenic, effect of, on nerve-cells, . . 604

Arterial pressure, 77 . Sce also Pulse.

aortic, relation of, to

pulmonary, . . 149

conditions affecting, 80,82 ,

curve of. See Curvc.

ill aorta, . . 43, 78

, carotid artery, . 94

,, cerebral circulation, 145 ,

146,147

,, cold-bloodedanimals, 80

,, different animals, . 80 arteries, 81,94

effect' of alcohol on, . 80 artificial respiration on,

127,128

asphyxia on, 77 ,

$307,308,855$

bleeding on, 75,77

ehloroform

on, . 80

distension of

bile ducts

on, . 854

epilepsy on,. 722

exercise on, . 80

massage on, . 155

tranfusion on, 74 ,

section of cer-

75,76

vical

cord

on, . 856 
Arterial pressure, effect of section of PAGE

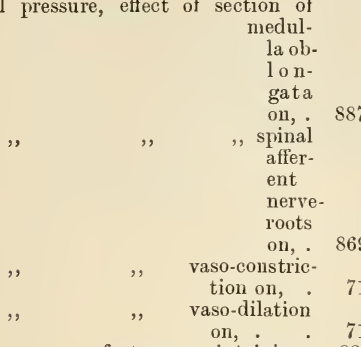

factors maintaining, 82 ,

in femoral artery, . 81

influence of accelerator nerves on, 53

,, , afferent

nerves on, 166

, amount of

blood on,

depressor

nerve on, 59,60

, diastolic

filling of

heart on, . 51

force of heart

on, . 41,50

gravity on, 81 , $90,92,94$

muscle rc-

flexes on, 1011,

1012

posture on, 80 ,

$81,90,92,94$

, reflex

856

," , respiration

on, $93,122,127$, 128,129

,, ,, spinal cord

on, . 854,855

temperature

on, . $\quad 80$

vagus on, 53,55 ,

56,57

vascular

tone on, . 136

on dicrotic

wave, 111 pulse

curve, 111, 112

,, velocity

of pulse-

wave, . 102

, volume

of limbs, 158

mean, constancy of, 81,82 measurement of, . 77 normal, . . 80 post-mortem fall of, . 71 pulmonary, 148, 149, 151,

, relation to aortic, . 149 record of, 78. See also

Pulse curve.
Arterial pressure, relation of arterial vol-

PAGE

$$
\begin{aligned}
& \text { ume to, . } \\
& \text {,, to capillary } \\
& \text { pressure, 116, } \\
& 117,118 \\
& \text {, , , heart fre- } \\
& \text { quency, 53, } \\
& \text {, intra. } \\
& \text { cranial, } 146 \\
& \text {,, systolic } \\
& \text { output, } 48 \text {, } \\
& 50,53,73 \\
& \text {, volume of } \\
& \text { arteries, } 73 \text {, } \\
& \text { 74, } 119 \\
& \text {,, wave-mo- } \\
& \text { tion, . }
\end{aligned}
$$

Artery or arteries, 72. See also Pulse.

blood flow in. See

Blood flow.

branching of, . . 62

breaking strain of, . 74

carotid, blood flow in, $84,85,86,145$

, ligation of, 148

, pressure in, 81

continuous contrac-

tion of,

coronary, 164. See

also Coronaryar.

teries.

elasticity of, $72,86,102$, 103,111

extensibility of, 73,74

femoral, blood flow in, 86

,, pressure in, 81

of brain,. . . 148

, heart, . . . 164

,, muscles, distensi-

bility of, . . 158

, organs. See Circulation.

,, skin, distensibility of, .

pulmonary, disten-

sion of, 154

ligation of, 149

150,154

pressure

in. See

Arter-

ial pres

sure.

radial. See Pulse.

resilience of, . . 74

rhythmic contraction

of, - mesenteric,

ligature of, .

systemic, relation of,

to pulmonary sys-

tem and systemic

veins, .

vasomotor mechanism

of, 136 . Sce also

Vasomotor.

velocity head in, 64,66 volume of, . 73,74 
PAGE

Artery or arteries, wave-motion in, . 96 See also Pulse.

Arthropods, auditory organs of, . . 1182

Articular end-bulbs, . . . . 947

Articulation. See Joint, also Voice. maximum rate of,

Artifieial respiration, effect on circulation,

Ascidia, reflexes in, . . . . $\begin{array}{r}126,129 \\ 785\end{array}$

Asemasia, . . . . 774

Asphyxia, . . . . . 307

causes of, . . . $\quad 307$

dysjnceic stage of, . . $\quad$. 307 effects of, on arterial pressure,

$139,307,855$

,, ,, capillary pres-

sure, . . 117

," heart, $41,50,307$
, intestinal move-

ments, . . 331

,, , , spinal reflexes, . 836

, , uterus, . . 851

exhaustion stage of, . . . 307

expiratory spasnis of, . $\quad 279$

hyperpnœic stage of, . . 307

in plethoric animals, . . 77

reducing substances in, . 290

,' reducing substances in, 2.290

Aspiratory action of thorax, 44, 91, 120, 122

Association areas of cortex, $769,771,773$,

775,776

\begin{tabular}{|c|c|c|c|}
\hline \multirow{2}{*}{, } & & \multirow{2}{*}{\multicolumn{2}{|c|}{$\begin{array}{l}\text { frontal, } \\
\text { influence of, on spinal }\end{array}$}} \\
\hline & , & & \\
\hline & & reflexes, . & 773 \\
\hline ", & ", & insular, . & 771 \\
\hline ," &, & nıarginal zones of, . & $\pi 71$ \\
\hline$"$ & , & parieto-oceipito-tent- & \\
\hline$"$ & & strueture of, & 770 \\
\hline , & tibres, & $\cdot$ & 771 \\
\hline ", & , & anterior, . & 77 \\
\hline " & , & of auditory area, & \\
\hline ", & ,. & inferior, & \\
\hline ", & $"$ & of motor area, & \\
\hline , & ,", & ", olfactory area, & \\
\hline " & , & perpendieular, & \\
\hline & & superior longitu & \\
\hline
\end{tabular}

Astacus, reflexes in, $817,818,828,831,833$, $834,839,840$

spinal shock in, 846 . See also Crayfish.

Astasia, . . . . . 903, 904

Asterias, autotomy in, . . . . 845

,, movements of, . . . 886

". reflexes in, . . 827,834

Asthma, . . . . . 311, 312

Astigmatism, . . 1032, 1038, 1148

," irregular, . . . 1033

, $\quad$ regular, . $\quad: \quad .1033$

Ataxia in apæsthetic limb, . . 1020

,. from lesions of cerebellum . 903

" " loss of muscular sense, 1018, 1020

Atlanto-occipital joint,

$\Delta$ tropin, action of, on cardiac nerves, 222,224

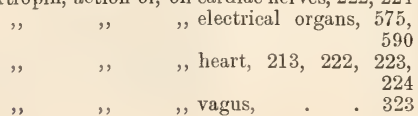

PAGE

Atropin, action of, on visual perception, 1141 Auditory area. See Cercbral cortex.

, nuclei,. . . 763, 781, 885

, sensation. See Sensation.

Auerbach, plexus of, . $327,329,668,672$

Augmentation, . . . . . 837

Angmentor nerves, 216. See also Nerves, Accelerator.

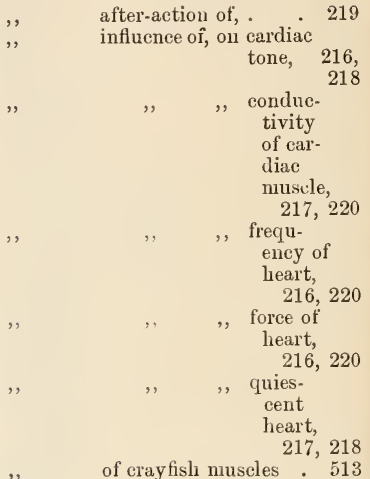

Aura, epileptic, . . . $\quad 702,996,1001$ Aurelia, ear of, . $\quad$. $\quad$. $\quad .1167$ Auricle. See Ear.

,, of heart, . . . 2, 3,22 ,", curve of contraction of, 172 ," effect of heat on, - 172 , , fibrillar contraction of, 192 , influence of vagus on, 204 , $205,207,213,215$

,, pressure in. See Pressure in heart.

Auriculo-ventricular valves. See Valves.

Autonomic ganglia, 660. See also Sympathetic ganglia.

as centres for de-

finite areas, . 678 automatism of, 676 ciliary, $650,660,664$, $677,678,682,911$ ncrve cells of, 664 , 668, 677. See also Sympathetic. of enteric system, 668,672 ,, pelvic nerve, 667 , other than cranial, 668,673 sacral, and sympathetic, 671 otic, $661,664,1157$ reflex actions from, 138,678 spheno-palatine, $651,661,662,664$, 1239

subdivision of, . 678 sublingual, . 661 submaxillary, 661,664 , $672,673,677,679$ 
Autonomic ganglia of tenth and eleventh cranial nerves, 197,200 , 201, 601, 663, 664, $668,793,884,1157$ nerve fibres, to abdominal

viscera, 663, 688,

See also 689,692

Sympathetic. afferent,

, connec-

tion of

with

spinal

ganglia, 656

., distri-

bution

of, . 688

relative

number

of, in

nerves, 687

, trophic

centres

for, . 203

to ciliary muscle, 660

commissural, . 682

degeneration of, 665

See also Sympa-

thetic.

direct, . . 668

, of brachial plexus, 669 ,

,, ,, lumbo-

sacral

plexus, 669 ,

,, ,, third

cervical

nerve, - 669

of fifth nerve, - 662 growth of cranial,

into sympathetic

ganglia,

691

to heart, $197,198,200$ $202,642,663$

medullated, 664, 665 $667,685,687$

to mucous mem-

branes, 660,661 , $662,664,667,692$

See also Sympathetic.

non-nedullated, 665 , $667,686,687$

number of nerve cells on course of, . . 682

of pelvic nerve, $\quad 666$ connection of,

with cord, 666, 667

stimulation of, $\quad 667$ to orbital gland,

660,661

, pelvic viscera, 667 ,

See also Sym688,692

pathetic.
Autonomic nerve fibres to pharynx, 601, 624,

regeneration of, 655

See also Sympa-

thetic.

sacral, . $666,6 \mathrm{~s} 8$

to salivary glands,

132, 141, 660, 661, 664,692 . See

also Sympathetic.

of seventh and

ninth nerves, 660 , 664,688

to sphineter pu-

pillæ, . 660,662

size of, $664,665,667$,

685

summary of

stimulation

effects of, . 693

of tenth and

eleventh nerves, 197 , $200,663,664,665$,

, third nerve, 660 ,

to thoracic viscera,

$663,688,689,692$

See also Sympa-

thetic.

, tongue, 624, 628 , $660,661,664$

of twelfth nerve, 664

vasomotor. See

Vasomotors, Vaso-

constrictors, Vaso-

dilators.

,, nervous system, $660,692,694$

,, eranial, 660, 691,

$692,694,695$

, enteric, 660,668 ,

694,695

inhibitory

action of, 672 ,

696

sacral, 660,666 ,

692,694

structures

with double

supply from,

, , , single

supply

from, 692

,, summary

of stimula

tion effects

of,. $\quad 693$

, sympathetic.

Sce Sympa-

thetic.

Autotomy, . . . . $\quad 845,859$

Axis cylinder, $4 \dot{5} 2,4 \dot{5} 6,479,52 \dot{1}, 567,592$,

, of rotation of joints, 235, 236, 237, 238,

,, optical. See Eye.

$239,240,241$

Axoll, .

BAG of membranes, foetal, . . . 351 
Bahnung,

Ball-and-socket joint

Band of Gennari, .

transverse, of pylorus,

Baræsthesiometer,

Bat, retina of,

Bed-sores,

Bee sting, reflex of, shock in,

Bidder's ganglion,

Bile ducts, effect of distension of, on arterial pressure, . . 854

reflexes from, . . 854

Biliary secretion, influence of cortex on, 716

Bineural cells,

Binocular vision. See Vision.

Bioplasm,

Bipolar cells,

Birds, ablation of cerebellum in, : 898

,. ,, ,, cerebral hemispheres

, " cerebral hemispheres 700,701

,, , optic lobes in, 700,701

action of inhibitory nerves of, . 215

ccrebellum of

902,903

cochlea of .

Golgi-organs of .

1180,1181

$\cdot 1009$

mesencephalon of, . . . . 912

motor area of,

732

optic lobes of,

911,912

pignient of eye of

.1048

pupil reflex in,

. 1043

retinal cones in,

. 1049 visual purple in,

. 1105

Bladder,

afferent root fibres from, $\quad \cdot \quad 849$ centre for contraction of, 345,849 contraction of, $341,344,345,346$ detrusor musele of, $339,345,346$ effect of section of eord on, $\quad 340$ ,, stimulation of hypogastric nerve on, .

, lumbar nerve-root. on, 343 , pelvic nerve on, . 344 , sacral nerve roots

on, . . 344 ,, sigmoid gyrus on, 346 $\begin{array}{lll}\text {, tension on, } & \text {. } & 341 \\ \text { anglion cells of, . } & \text {. } & 342\end{array}$ inhibition of sphineter of, 344,345 ,

inhibitory nerves of, . . 695 mechanism of closure of, $\quad . \quad 340$ motor nerves of, $343,345,346,667$, $679,692,693,695$ movements of, . . 339, 341 muscular tissue of, . . $\quad . \quad 339$ pressure in, $340,341,342,345,346$ relaxation of, . $\quad 341,342,344$ sacral nerve fibres to, . . 342 sphineters of, $339,340,342,344$, 345,346 sympathetic fibres to, $342,646,659$ $692,693,695$
tonic contraction of, $\quad$. 340,341

Blood, amount of,

,, in limbs, .

, lungs,
Blood, amount of, in organs,

PAGE

, , , influence of, on arterial

pressure, 74

,, systolic

output, 74

circulation of. See Circulation, Blood flow.

complemental, . . . . 128

during hunger . . . . 992

dyspnœic, effect of, on vasomotor

tone, . 138

influence of, on vasomotor tone, 136 posture on distribution of, . . 95

intracranial, . . . . 144

murmurs, . . . . . 33

pressure of. See Blood pressure.

residual, in heart, . $7,10,53,308$ , lungs, . 128

$\begin{array}{lll}\text { supplemental, . } \quad . \quad & \text {. } & 128\end{array}$

tidal, 128

velocity of. See Blood flow.

viseosity of, . . . 65, 76,97

Blood capacity of organs . . . 155

Blood flow, . . . . 62, 82

$$
\text { in aorta, . . . } 42,84
$$

, arteries,. . 67, 82, 84, 113

,, brain . . 146

,, capillaries, * . 113,114

, carotid artery $84,86,145$

,, coronary arteries, . 13,20

165,166

curve of velocity of, . $\quad 85,87$

dynamies of, . . . 62

effect of section of vagi on, . $\quad 86$ vasomotor changes

on, . . . 85

in femoral artery, . $\quad . \quad 86$

influence of transfusion on, 76

local variations in, . 83,87

in lungs, 125, 126, 12 $7.128,129$ , muscles, . . . 155

measurement of, . . . 83

velocity of, in aorta, . . 42

,, arteries, 82, 84

, capillaries, . 114

estimation of, . 66

factors affecting

82,87

in pulmonary

artery, . . 43

,, renal artery, $\quad 160$

,, veins, . 82,121

," record of, . 86,87

in veins, $\quad 82,113,118,121$

,, cause of, . 121

, effect of move.

ment on, 120,121

, splenic con-

traction on, 121

,, posture on, 120

,, respiration

on, . . 121

,, peristalsis

on, . 121

", velocity of, $\begin{gathered}\text { rate of, . } \\ \text {, }\end{gathered}$

,, in venous sinuses, . . 145

Blood pressure in arteries. See Arterial pressure. 
Blood pressure in capillaries. See Capil-

PAGE

$$
\begin{aligned}
& \text {, heart. See Pressure } \\
& \text { in heart. } \\
& \text {, veins. See Vicnous } \\
& \text { pressure. }
\end{aligned}
$$
laries.

Bones, articular surfaces of. See Joints.

,, atrophy of, after nerve section, . 875

, nerves of, afferent, . . . 1006

,, reflexes from, . . . . 1012

, sensitivity of, . . . . . 1011

Brachial plexus, variations in, . . 633

, , vasomotor fibres of,

Brain, 697. See also Cerebellum, Cerebral

$$
156,669,670
$$

circulation, Cerebral cortex, Dieneephalon, Medul-

la, Meseneephaton, Meteneephalon, Pons, Thalamus.

,, amount of blool in, . . . 144

,, arterial pressure in, . . . 145

,., supply of, . . 148

,, blood flow in, . . . . 146

, capillaries of, . . . . 112

,. eapillary pressure in, . . 116, 118

,. circulation in, 141. See also Cere-

bral cireulation.

diastole of, .

142,143

, effect of respiratory movements on, 142 ,

143

,. movements of, . $141,142,143,145$

,. systole of, . . . . . 143

, variations in diameter of, . . 145

,, vasomotor nerves of, $146,168,618,624$

venous pressure in, . $143,145,146$

Bristle cells,

. 1166,1181

Broca's convolution, . . 748, 775, 780

Bromide of potassium, effect of, on lieart

minscle,

Bromehial breathius

, muscles, action of, $\quad . \quad 309$

,, , , innervation of, . 309,310

, tolle, . . . . . 311

,, tubes, effects of constriction of, 311

Bufo, eardiac tone in,

Bulb. See Medulla oblongata.

", olfactory,

, taste. See Tongue.

Bulbo-spinal animal,

1247,1248

Bulbus arteriosis, rhy thmic contractions

of, . . $\quad 177,178,180,182,184$

Bundle, association. See Association fibres, Faseieulus.

, longitudinal, . . $890,893,915$

,, of Meynert, . . . . 782

" tegmental, . . . 782

", of Türek, $\quad . \quad$. $\quad . \quad 808$

," of Vicq d'Azyr, : . $\quad$. 782

Burdacl, nucleus of, . $\quad . \quad$. $\quad . \quad 886$

CAFFEIN, dilletic action of, .

Calcarine fissure, . $\quad 755,757,758,759,780$

Calcium chloride, action of, on heart, 225, 226 ,$\quad$ salts, influence of, on electrical response of nerve,

Calorific nerves,
Canalis auricularis, $178,179,180,182,183$,

," reuniens, . . . . . . 1179

Canalisation, . . . . 837, 842

Canals, semicircular. See Scmicireular eanals.

Caoutchouc, contraction of, . . 396, 397

Capacity of vascular system, . . . 70

Capillaries, . . . . . 112

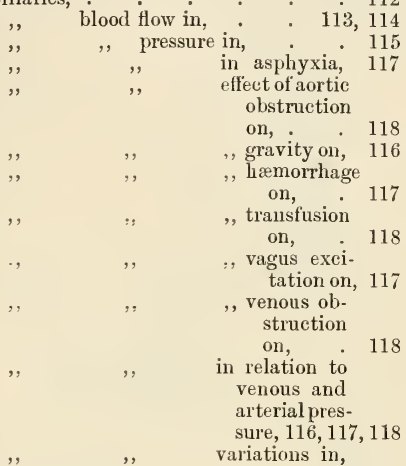

", 116,117

,, of brain, pressure in, . 116, 117

, circulation in, . . . 112

,. of intestines, pressure in, . 117

,, , liver, pressure in, . . 117

,, ,, lungs, flow in, . 125, 126

, pulse in, . . . . 114

,, rate of flow in, . . 114

," reflexes initiated from, . 856

,, sectional area of, . . 115

, size of, . . $\quad 112,114$

Capillary contact, . . . . . 460

,, electrometer, : : 417,418

Capsule, internal, 777. See also Internal

eapsule.

Carbolic acid, effect of, on skin sensations,

928,986

Carbonic acid, effect of, on nerve, 491, 492 , $535,536,537$, 540,553

, , , , , respiration,

,. oxide, ,. ,, nerve, 289, 290

Carcinus, nerve cells of, ', nerve, • 492

Cardinal point, . $\quad . \quad$. $\quad . \quad 1029$

Cardiogram, . . . . . . 36

Cardiograph, . . . . . . $\quad . \quad 35$

Cardiometer, . . . 49

Carnivora, cerebellum in, : . . 902 corpora quadrigemina in, . 912

Carotid artery. See Arteries.

Carp, cerebellum of, . . . , 894

Carpal joint, . . . . . 241

Cartilages, articular. Sec Joints. of larynx. See Larynx.

Cat, ablation of hemispheres in, . . 704

,, centre for movements of vocal corls in, 742

, cerebellim of, . . . 893

,ychlea of, $\quad$. $\quad . \quad$. $\quad . \quad 1186$

," knee-jerk in,. . . . . 874 
Cat, motor area in,

,, muscle spindles in,

", reflexes in,

reflex pinna-twitch of, .

spinal,

", , shock in,

, visual area in,

Cataleptoid reflexes,

Catelectrotonus, 507, 508, 509, 546, 548, 550

Catgut, contraction of, . . . 396, 397

Cells, "amacrin,"

,, interstitial,

," niediate, of cord,

66.593

$805,806,810$

722,723

", nerve. See Nerie cells.

," olfactory,

", root. See Root cells.

, sensory,

, spiral, ," tactile,

Central vision

Centre or centres, uervous,

$\begin{array}{lll}\text {, } & \text {, } & \begin{array}{c}\text { accelerator. See Centrc } \\ \text { auditory. See Cerebral } \\ \text { cortex, auditory. }\end{array} \\ \text { association. See As. } \\ \text { sociation areas. } \\ \text { cardiac, }\end{array}$

Centre for colour vision, $758,760,761,1105$

PAGE
1105

,, convulsion, . . . 887,893

,$\quad$ for contraction of pupil, . 716, 1043
,$\quad$ cortical. Sce Cerebral cortex.

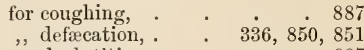

,, deglutition, . . . 887

,erection of penis, : . $\quad .160$

,, gastric secretion,. . . 887

", generative functions, . . 348

genito-spinal, . . . . 336

of gravity of body, $25 \dot{7}, 25 \dot{9}, 260$, $261,262,264,270$

,, , head, . . 258, 261

", $\quad$ ", limbs, $\quad$. $\quad 258,261$

", ", trunk, . . 258

gustatory. Sce Cerebral cortex.

for hallux movements, . 737, 738

heat, . . . . 717

for hiccough, . . . $\quad . \quad 887$

for kinæsthetic impressions, . 729

,, light-vision, 1105. See also

Cicroral cortex, Visual area.

local, other than cranial, sacral,

and sympathetic, . . . 671

in medulla oblongata, . . 887

motor, 722, 723. See also Cere-

bral cortex.

for micturition, - . $\quad 345,346$

,, movements of bladder, 345,849

,,, ear, . . 739

," , intestine, : 734

," , $\quad$ cesophagus, . 887

olfactory. See Cerebral cortex.

oval, . . . 805

for pain, . . . . . 997

,, pancreatic secretion, . . 887

,, parturition, . 351, 851, 852

, phonation,. . . . 887

," platysma, : $\quad$. $\quad 736,741$

iil pons, . . . . 891

psychomotor, . . . . 724

reflex, sympathetic ganglia as, 138,678 reflex. See Peflex centres, Medulla

oblongata, Pons, Corpus striatum.

respiratory, 284, 285, 286, 287, 288, $289,291,292,309,321,863,887$

of rotation of joints, . . 235, 237 segmental, . . . 784 sensori-motor, . . . . 724 sensory. See Cerebral cortex.

for sliace perception, . . 760 for speech, . . . 705,775 sweat, . . . 856 for taste. See Cerebral cortex.

trophic, for visceral afferent nerves, . 203 vaso-constrictor, : $136,854,855$ vaso-dilator, . . 61 vasomotor, : . $\quad 136,887$ bulbar, $136,139,855,887$ ,, conduction paths to, from cord, 855,864 exhaustion of, . 95 influence of asphyxia

on, . . 307 , ccrebral cortex on, , on ecrebral circulation,. 147, 148 
Centre, vasomotor, influence of circula-

PAGE tion on, . ,, compensating for gravity, 94,95 ,, depressor nerve on, 60 ,, respiration on, . 129 ,, urea on, . , 160 spinal, $137,160,854,855$ sympathetic, . . 138, 157,160 vesical, . . . 849,863 visual. See Cerebral cortex.

for voluntary movements. See Cerebral cortex. , vomiting,

Centripetalitä

Centrosome,

Cerebellum,

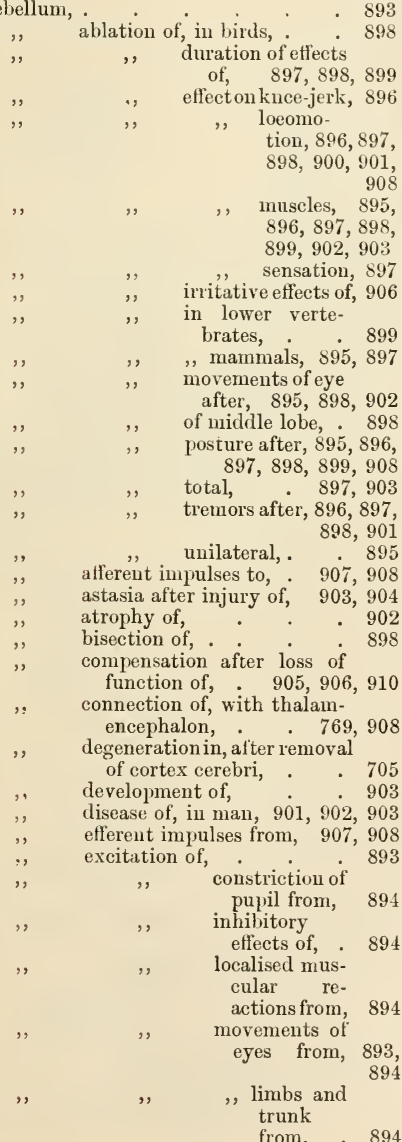

Cerebellum, excitation of, sensory effects

$$
\text { of, . } 894
$$

functions of, . . 903,910 impulses to, from eerebral cortex, . . . 908 in different animals, . . 902 influcuce of, on activity of motor area, 904, 906, 907,908 on optic thala-

nius, . 907

lesions of, . . . 894, 901 effect of, on speech, 901 inco-ordination of movement after, 903 908, 909, 910 parasthenia after, 903 paratonia after, . 903 sensoly disturb-

ances from, 903, 904 spastic rigidity from, . 903 vertigo from, 901, 902,

mediate spinal fibres to, 806,807 myelination of, . . . 903 peduncles of, anterior, 769,900 , 908 ,, connection of, with Gasserian ganglion, 793 ,, motor area, $\quad 779$

$$
\begin{gathered}
\text {," , thala- } \\
\text { mus, } 769 \\
\text { middle, }
\end{gathered}
$$$$
\text { ", middle, . } \quad \text { posterior, . } \quad \text {. } 900
$$$$
\text { ", section of, } 900,901,908
$$$$
\text { ,, ,, effect onloco- }
$$
motion, 900, 901 effeet on kneejerk, eye moveinents after, 900 , 901 irritative effects, . 906 posture after, 900,901 tremorsafter, 900,901

,, relation of, to sensation, 897,905
,,$\quad$ museular sense to, 909 ,

Cerebral circulation, 141,168 . See also Brain.

compensatory mechanism of, 146 conditionsaffecting, 147 ,

influence of arterial 168 pressure on, 145,146 ,

, respiration on, 143,147 splanehnic vascular area in, 147 
Cerebral circulation, influence of venous

PAGE

pressure

on, 146,147

methods of investi-

gation, 142, 144, 145

principles of, . 145

in strychnine

spasms, . . 146

vasomotor mechan-

ism of, $146,147,168$, 618,624

Cerebral cortex, . 697

ablation of, bilateral, 698,703 711

in birds, 700,701 ,

degenerations

after, 704, 738,

effects of, on

motor

func-

tions, 698

, on psychi

calfunc-

tions, 699

$701,703,704$

, , sen-

sory

func-

tions, 704

, , spinal

aetivity, 837,838

in fishes, 699,700

, frog, 698,699

704

, mammals, 702

703,704

$\begin{array}{rr} & \text { unilateral, } \\ & 704,\end{array}$

association areas of,

769

See also $A s$ -

sociatiou

areas, Associ-

ation fibres.

,, ,, in different

animals, . 77 :

,, , effects of

lesions of, 770

771,773

excitation of, 770

", , function of, 771 ,

772,773

,, ,, influence of,

on reflexes, 773

,, ,, marginal

zones of, . 771

,, ,, myelination

of, . 770

,, filres of, 771,779 ,

780,781

auditory area of, . $\quad 761$

ablation of, 761 ,

association

fibres of,

762

commissural

fibres of, . 781
Cerebral cortex, auditory area of, con-

nection of,

with genic-

ulate body

762,763,
781

,, ,, with inter-

nal ear, 763 ,

781

,, ,, with nuclei

pontis, . 781

corticifugal

fibres of, . 763

, corticipetal

fibres of, . 781

, in different

animals, . 773 ,

774

, focal point of, 761 ,

,, lesions of, . 763

, localisation

within, . 763

myelination

iil, . 762,763

stimulation

of, . 761

cells of, . . 722, 723

minimal dura-

tion of, ae-

tivity of, . 709

rate of discharge

fronl, . 708,710

refractory period of,

connection between dif-

ferent centres

of, $\quad 771,776$

with bulbar

centres, 777,778

,, cerebellum, 907,908

commissural, 764 $779,780,781$

with eorpora quadrige-

mina, . 780

,, corpus stri-

atum, 778

diencephalon, . 916

genieulate body, 780 ,

internal ear, . $\quad 781$

lower centres, . 776

mesencephalic

centres, . 777

optic thalamus, 768 , $778,779,780,916$,

917

pontine centres, 777 ,

$778,779,781$

retina, . 780

spinal centres, 777 ,

778. See also

Spinal cord.

substantia nigra, 778

cord-area of, . . 722

effect of absinthe on, . 721

excitability of, $705,706,748$, 
PAGE

Cerebral cortex, excitation of, $705,706,709$ $712,713,716$ $718,732,749$

Cerebral cortex, localisation of function in,

PAGE 770

705,722

749,752

761,763

eonjugate

eye-move-

ment from,

$712,716,739$, $740,748,749$, $752,755,756$

effect of, 761

on heart, 55 , 714

,, ,, knee-

jerk, 874

,,,, organie

mus-

cles, 716

,, , pupil, 715

,, , secre-

epilepsy

tions, 716

from, 706 , 718,748

See also

Epilepsy.

vasomotor,

714,715

functions of, general, 697 , 770,772

inhibitory, 711 , 739,773

motor, . 722

", sensory, : 749 gustatory area of, . 763,766 heat-centres of, . . 717 heat-production in, . 718 inexeitable region of, . 769 influence of, on body temperature, 717 cireulation,

,, involuntary muscle, 716 , reflextime, $\quad 712$ ,, respira tion, 713 ,

,, secretions, 716 ,, spinal re-

flexes, 837 , 838,839 , 841,843 inhilitory action of, 711,712 , 739,773 insensitivity of, 978,996 , 1000 isolation experiments on, 723 lesions of, aphasia after, 774 , effect on intelleet, 697,699 , $703,770,772$,

, tactile, 773 sense, 938

lost time of, motor area of

,,
,,
,,
,,

,, birds, 732

, cat, . 732

cells of, 722 , 723,777

commissural

fibres of, 779

comnection

of, with

bulbar

centres, 777,778

,, ,, corpus

stria-

tum, 778

,, , dien-

cepha-

lon, 916

,, ,, mesence-

phalic

centres,

,, , poutine

centres,

$77 \%, 7 \% 8$

,, , spinal

centres,

777,778

,, , substan-

tia nigra,

, , thala-

$$
778
$$

mus, 768,778

degenera-

tious follow.

ing ablation

of, $\quad 811$

excitation of, 732,738

,, bilateral

effects

of, 735 ,

736,739 , 741,742 , 
Cerebral cortex, motor area of, excitation of, charac-

ter of

move-

ments

from, 738

, , in apes,

,, ,, man,

, primary

move-

ments

from,

738, 743 ,

,, second-

ary

move-

nents

from, 738

743,744

,, sensory

syin p-

toms

from,

730

for face,

mouth,

throat, and

larynx, 736, 740,748

,, projection

fibres of

focal proints 777

in, $\quad 737$

in dog, 732,736

, fish, - 732

,, frog, . 732

, gtimea-

pig, . 732

for head,

ear, and

cyes, 736 ,

738,748

", projection fibres of,

iufluence of 77 cerebellum on, 904, 906, 907,908

in insee-

tivora, 732

,, jackal, 732

lesions of, 705 ,

706,717 ,

726,727 ,

728, 730 ,

731,738 ,

748

,, aphasia

after, 774 ,

, contrac-

ture

after, 731 ,
Cerebral cortex, motor area of, lesions of

sensory

symp-

toms

after, 725 ,

728,729 , 730,731

for lower

limbs, 736,

744,748

, projec-

tion

fibres

of, . 777

, upper, 736 ,

743,748

in man, 747,

, marsu-

pials, 732

,, monkey,

733,734 ,

735,736 ,

738,773

,, nono-

tremes,

732

, rabbit, 732 ,

736, 738

, rat, . 732

projection

fibres of, 777 ,

relation of, 778

to muls-

eular

sense, 749

, to pain,

1001

,, , tac-

tile

sense,

767,768 ,

769

tonus in, 724

for trunk

and tail, 736 ,

745

muscular contraction

evoked from, . 707, 709

olfactory area of $\quad .763$

,$\quad$ associa-
tion
fibres
of, 781
,, commis-
sural
fibres of,
764,781

prefrontal region of, 738,770 ,

$772,773,777, \quad 779$

reduced lost-time of, $\quad \begin{aligned} & 780 \\ & 711\end{aligned}$

refractory period for, . 615

relation of pain to, . 1001

sensory areas of, . 723,749

spheres of, 724,725

tactile area of, . . 766

, centripetal

fibres of, 769 
PAGE

Cerebral cortex, visual area of, 752, 774, 1105 , commissural

fibres of, 780

, connection

of, with cor-

pora quad.

rigemina,

759,780

,, ,, with

genicu-

late

body, 758,780 , ,, ,, retina,

, $\quad$, , ,, retina, 780

,, ,, ,, thala-

mus

, develop-

ment of

excitabi-

lity in,

,, for differ-

ent kinds

of visuali-

sation, . 760

, electrical

stimula-

tion of, . 755

, focal point

of,

756

, localisa-

tion in, 754

, in $\operatorname{man},{ }_{758}, 757$,

, relation of,

to mac-

ula, 757,758 ,

761

$$
\text { , } \quad \text {, , , retina, 754, }
$$

ganglia of invertebrates, inlibi-

758

tion by,

hemispheres, 697 . See also $^{\circ}$

Cerehral cortex.

ablation of, bilate-
ral,

ral, 698

unila-

teral, 704

association fibres

of, 770,771 . See

also Association

fibres.

commissural

fibres of. See

Corpus callosum,

Commissure.

corona radiata of, 706

$710,711,719$

degenerations after

ablation of,

704

gyri of. See Gyri.

lobes of. See Lobes.

pressure gauge,

retina, .

Cerebro-spinal fluid,

Cerianthus, reflexes in,

Cerumen,
Ceruminous glands,

PAGE

Cetacea, cerebellum of,

1151

. $\quad 903$

, scnse of smell in, .1248

Charpentier, band of, . . . . . 1074

Chest register. See $V$ oice.

Cheyne-Stokes respiration, . . 139, 309

Chiasma, olfactory, . . . . 781 optic, . . $754,756,75 \bar{i}, 1043$

Chloroform, effect of, on arterial pressure, $\quad 80$ , , , , cutaneous sense, $\begin{array}{r}928, \\ 987\end{array}$

, $\quad$, , , depressor nerve, . 60

,, , , , electrical organs, 575

," $\quad, \quad$, , heart, . $41,52,58$

,, , , ,, knee-jerk, . . 873

," , , , nerve, 491, 492, 534, 870

, ,, , splenic contrac-

tion, . . 163

,, , , , vascular tone, 95,135 ,

Choroid coat. See Eye.

138

Chorde tendiner, . . . . . 9, 10

Chromatin . . . . 594, 599,600

Chromatolysis, : $599,600,601,603,604$

Chromatophil granules, . . . 599

Chronograph, . . . . 264, 265

Chronophotograph, . . $265,267,268$

Ciliary ganglion. See Ganglion.

,, muscle. See Muscle.

, nerve. See Nerve.

," processes. See Eye.

Cingulum, . . . $764,768,771,781$

Ciona, reflexes in, . . . . 785

Circle of Willis, . . . . 145, 148

Cireulation, 1. Sce also Blood, Blood flow, Blood pressure.

in arteries, 72 . See also Arteries, Arterial pressure.

of artificial fluids in heart, 224

eapacity of, . . 70

capillary, 112. See also

Capillaries.

cerebral, 141. See also Cerebral circulation, Brain.

coronary; 164. See also Coronary arteries.

dynamies of, . . 62,67 effect of cerebral excitation

on, . . . 714

, destruction of cord on,

812,813

,, cpilepsy on,. . . 722

,, gravity on, . . 90, 92, 93

, liamorrhage on, $17,75,117$

,, obstruction in systemic system, . $\quad 72,107$

,, pulmonary obstruction

on, . . 72, 107

,, section of medulla on,

,, of posture on, . $\quad 90,92$

cnergy of, . . . 42

general principles of, . . 68

in generative organs, . . 160

", head,. . . 141

", heart, 164. See also Heart.

hepatic, 
Circulation, hepatic, influence of respiration on, 162

spleen on, 162 hyd̈rokinetic mean pressure of, . 70,71 hydrostatic mean pressure of, . . . 70 influence of gravity on, 90,92 , $93,94,95$ ,, gyrus fornicatus on, . 714 ,, ,, marginalis on, 714 ,, intestinal movements on, . . 330, 331 nervous system on. Sce Centres, cardiac Centres, vasomotor, Vasomotor nerves.

,, respiration on, 91, 93, 122, $125,127,128,129$

. sigmoid gyrus on, . 714 in intestines, . . . 160 ,, kiriney . . . 159 , limbs, . . . 155 ,, lungs. Sec Circulation, pulmonary.

mechanism of, . . . mesenteric, . . . 160 model of, . . . 68,90 panercatic, . . . 164 peripheral resistance in, . 72 pulnınary, . . . 148 , in artificial respiration, 126, 149 conditions affecting, . 126,150 congestion of, 72 , influeuce 150,15 arterial pressure on, 150,151 , respiration on, $122,126,127,12 !$ innervation of, 15 relation of, to systenic, 71,154 vasomotor meehanism of, 151

152,154

renal,

, effect of drugs on, . 160 in salivary glands, . 141

,, skin, . . . . 158

,, special parts, . . 141

„, spinal animal, . . 819

,, spleen, . . . 162

time. See Circulation-time. 88

in tongue, .

vasomotor, mechanism of, $54,59,130$. See also Centre, eardiae, Centre, vasomotor, Vusomotors.

in veins, 118. Sec also Veins.

Circulation-time,

$$
\begin{aligned}
& \text { influcnce of vasonotor } \\
& \text { mechanism on, } \\
& \text { in kidney, . } \\
& \text {, portal system, } \\
& \text { of whole blood,. }
\end{aligned}
$$

Clarke's column,

PAGE

$806,807,810,811,836,878$

Clitoris, . . . . . . 347 ,, sensory end-organs in, . . 947

Cocaine, effect of, on cutaneous sense, 928, 986 ,, ,, , gustatory sense, 1241,

$\begin{array}{rr} & 1243 \\ & 329\end{array}$

," ., :, intestine, . . 329

," ", ", olfactory sense, : 1252

Coccinella, geotropism of, . . . 828 Coccygeal ganglion, $634,635,639,682,683$

Cochlea, 1168. Sec also Sensation, auditory. action of,

684 afferent fibres to cortex from, . 781 of amphibia, . . . . 1181 analytical function of, 1168,1171 , $1180,1189,1191,1192,1193$ of birds, . 1180,1181 damping mechanisn of, 1178,1180 duct of, $1166,1167,1179,1180$, 1184 effect of lesions of, 1167, 1181, 1182 hair-cells of, $1181,1184,1185$, $1186,1190,1191,1192$

helicotrema of, . . . 1179 lamina spiralis of, . . 1185 membrana basilaris of, 1179,1180 , $1181,1185,1186$, $1190,1192,1193$ reticularis of, . 1181 ," tectoria of, 1181,1186 , 1192 membrane of Reissner of, . 1179 model of, . . 1182, 1183 lesonance of, $\quad 1178,1187,1190$, $1192,1194,1218$ rods of Corti of, $1180,1181,1182$, $1184,1185,1186,1192$ scala tympani of, . 1179 vestibuli of, 1164,1179 sensitiveness of, . . 1169,1170 to different modes of wavc-pressure, 1174 , 1175,1176

vibrators of, . $1179,1180,1181$, 1183,1190

Coetticient of elasticity of arteries, 72,73 ,, friction,. . . . 234 642,645 plexus, : $\quad \cdot \quad \cdot \quad \cdot \quad \cdot \quad \cdot 642$ Coffee, influence of, on reaction time . 611 Coitus, . . . . 347 , aflerent impulses in, . . . . 348

, centre for movements of, . . 348

, contraction of vesiculre in, 347,349

", effect of section of cord oll, . 348

," movenents of uterus in, $374,348,349$

," movenients of uterus in,, 37 vas deferens in, 347,349

,' nervous mechanism of, . . 348

,' reflex movements of, in spinal

dog, . . $852,853,854$

Cold, sense of. See Sensation.

Cold-blooded animals, arterial pressure

in, . . . . . 80 Cold-spots, $\quad 921,935^{\circ}, 945^{\circ}, 946,955^{\circ}, 964$, $984,989,995$ 
Collateral fibres of afferent spinal roots,

PAGE $792,793,794,806,811$ , , , , dorsal columns, . 863 , , , , grey matter of cord, 811 , , , ,, pyramidal tract, 777,806 , 810,811 , , reflex, . . . 793 "ganglia of sympathetie, . . 617 Colon, •. . . . . 335,336 ,, nerves of, . . . $\quad 336,337,646$ ,, vasomotors of, . . . . 161 Colour, elementary, . $\quad 1107,1108,1111$ , fundamental, . 1107, 1108, 1121 ,, primary, $1105,1106,1107,1112,1113$,

1121. See also S'ensation, visual. Colour-blindness, . $\quad 1088,1092,1118$

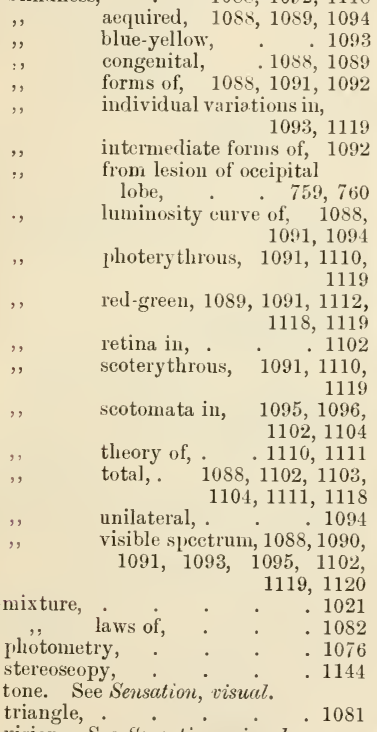

Colour-mix ture, blue-y congenital, $\quad .1088,1089$ forms of, 1088, 1091, 1092 individual variations in, intermediate forms of, $109^{\circ}$ rom lesion of oceipital lobe, . . 759, 760 1091, 1094 photery throus, 1091, 1110 , 1119 red-green, 1089, 1091, 1112, retina in, . . . 1102 scoterythrous, 1091,1110 ,

1119 1102,1104 theory of, . $.1110,1111$ $1104,1111,1118$ nilateral, . 109 ible spectrum, 1088, 1090 1119,1120 ,, laws of, . 1082 , pliotometry, . . . . 1076 ", tone. See Sensation, visual. ", vision. See Seisation, visuul.

Columns of Clarke, $136,601,725,778,792$, $806,807,810,811,836,878$ ," of eleetrieal organs, . . . 563 ,, ,, Goll, degenerations in, 809, 861 ,, ,, spinal eord. See Spinal Cord.

Columnæ carneæ,

Cometula, autotony in, . . . 845

Comma tract, . . . 805

Commissural cell group of ventral horn of cord, . . 890

C,$\quad$ fibres of synpathetie, 682,683 Commissure, anterior, . . 714, 764, 781 $\begin{array}{ll}, \quad \text { dorsal of cord, } & \text { v } \quad . \quad 793,806\end{array}$ Conprensator,

Complemental blood, ·. • • • • $\quad \cdot 128$

Compressed air, respiration of, . 128

Conduction in brain. See Medulla, Pons, Mesencephalon, Crusta, Internal capsule.
Conduction in nerve, 784,798 . See also

PAGE Nerve.

$$
\begin{array}{r}
, \quad \text { cells, } 483,605,606,607, \\
613,680,682,791, \\
798 \\
, \quad \text { spinal cord. See Spinal } \\
\text { cord. }
\end{array}
$$

Cone-myoid,

Cones, retinal. See Retina.

Conjugate deviation of eyes, $739,740,748$, $749,755,761,893,894,895,900,901$,

Conjunctiva, sense spots of, . . 947, 987 , sensory end-organs of, 922, 923, 947 $\begin{array}{lll}\text { vasomotors of, } & \text {. } & \text {. } \\ \text { Conjunctival reflex, } & \text {. } & 892,919\end{array}$

\begin{tabular}{c} 
spino-cerebellar mediate \\
system, \\
\hline
\end{tabular}

Conscionsness, . $\quad . \quad . \quad . \quad 697$ ,, in epile sisy, to co-ordination of movenent, . . 1019 relation of spinal reflexes

Contraetile stress. See Mrusele,

Contraetion of Musele. See II susele.

Contractionsuclle, 859,860

Contraeture,

. 435

Coutrast effeets, sustatory,

,, visual. See Sensution, visual.

Convergenee. See Eye.

Convolutions. See Gryri.

Convulsion centre, · . . 887, 893

Convulsions, spinal, . . . . . 836

C from strychnine, . . 837

,, spinal. See Spinial coril.

, voeal. See Vockl corls.

Corethre larra, contraction waves in, . 384

Cornea, 1026

,, accommodation in, . . . 1037

, axis of, . . . . 1031, 1032

,, ill birds, . . . . . 1040

, curvature of, 1026, 1027, 1037, 1040,

1041

, form of, . . . . . .1031, 1032

,, irregularities in, . . . 1033

,, pain-spots of, . . . . 923

, refraetion-index of, . . .1026, 1033

, sense-spots of, . 984, 986, 987, 988

", sensory end-organs of, . 922,923

,. thermal sensations in, : 947,957

Curona radiata, . . 706, 710, 711, 719

Coronary arteries, . . . . 164 ,, blood How in, 13, 20, 165 ,

, eireulation in, . $\quad 164$

$\begin{array}{lll}\text {,, } & \text { during eardiac eycle, } 13,\end{array}$

", 20,85

, $\quad$ effeet of ligature of, 165 ,

, vasomotors of, $\quad 162,1963$,

Corpora bigemina, . . . . . 916

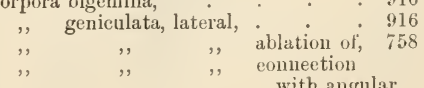
with angular gyrus, • 780 
Corpora geniculata, lateral, connection with occipital

lobe, , optic tract,

effect of excision of eye on, . relation to visual area, 759 mesial, effect of ablation of first temporal gyrus on,

, relation to auditory area,

quadrigemina, 893, 911. See 762,763

also Mesencephalon. anterior, ablation of, . 913

,, , effeet of, on vision, 913

, effect of excision of eyes on,

,, stimulation of,

f,

connection of, with

912
auditory area, 763 ,, occi-

pital lobe, 780

,, , , optic

tract, 780, 781

, , , , spinal cord, 915

, , , , visual area, 758,759

degeneration in, after ablation of cortex 705 , removal of eye, 781

influence of, on spinal reflexes, . 838,839 posterior, . . 781 ,, excitation 12 region of . 911 sensitivity of, 978,1000

Corpus albicans, , callosum,

,, , congenital absence of, 779 ", degcneration in, after cortical lesions, 705, 779 fibres of, from motor cortex, . 777,779 , from occipital cortex, . 780,781
Corpus callosum, fibres of, from temporal cortex, . . 780, 781

function of, . . 779 peduncle of, . . 764 section of, . . 779 splenium of, . . 780 stimulation of, . . 779 '. 907

Luysii, . $\quad . \quad 705,917$

striatun, . . . . 778

, connection of, with frontal lobe, 778 , with thalamus, 778 degeneration in, after frontal lesions, . 778

fibres of, to motor tract 778 influence of, on body temperature, . 717 insensitivity of, . 978 reflex function of, . 778 Corpuseles, genital, . . . . . 947

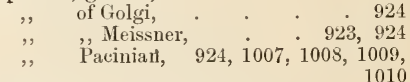

Cortex cerebri. See Cerebral cortex.

Corti, organ of, 1180, 1181, 1182, 1184,

Cosine lever, $1185,1186,1192$

Coughing, . $\quad . \quad+\quad . \quad+\quad . \quad 308$ centre for, . . . . 887

Cranial autonomic system. See Autonomic system.

,$\quad$ nerves. See Nerres.

, nerve roots. See Nerve roots.

Crayfish, muscles of, . . . 377, 513 , nerves of, $480,512,513$ See also Astacus,

Cremasteric reflex, after section of cord, 815

Crista acustica, . . . 1194,1195

Croak-reflex, . . . . 890, 912

Crocodile, accelerator nerves of, . . 199 heart of, . . . . 214

Crossed innervation, . . $\quad 334,337,345$

Crura cerebri, . . . . 717, 739

Crusta, connectiou of, with Broca's convolution, . . . 780 degeneration in, after lesions of motor cortex, . . . 777 fibres of, from auditiory area, . 781 ,, , oceipital lobe, . 780 , $\quad$, prefrontal region, 779

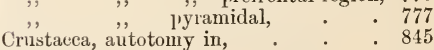
Crying, . . . . 308 Cucumaria, movements of, . $\quad . \quad 828$ Cuneus, 752, 758, 759, 780 Curari, cffect of, on electrical organs, 574, 590 ,, , , muscle tonus, . . 799 ," ,, nerves, 168, 222, 224, 324,536 ,, ,, ,, splenic contraction, 163 Currents, electrical, in electrical organs.

Sce Elcctrical Organs.

," $\quad$,, $\quad$,, muscle. See Muscle

", $\quad$, , retina, 1050, 1051,

Curve of action of spectrum on visual 1052,1120 substances, . 1106 
PAGE

Curve, chronographic, .

265

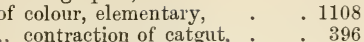

", contraction of catgut, - diaphragm, 283, 296

," intestincs, 327, 328,

," , , , $\quad$, nusele, $\quad 358,800$

ing, . 367

anodic, 433

arrested, 367,

368

cardiac, 39 ,

172,440

catholic, 433

cortical, 707 ,

$709,710,711$

of crayfish,

513,514

epileptiform,

$711,718,720$

in fatigue, 380

inertia, 361,

370

jury, 417

isometric,

$362,366,368$, 440,442

isotonic, 358

$366,368,376$,

$388,440,442$,

800

-jerk, . 370

in tetanus, 379

$385,386,510$, voluntary

707,709

,, ,, ,, œesophagus, 317, 318

,, energy of muscular contraction, 362 ,

,, extension of muscle, . 241, 404

,, impulse, eardiac, . . 39

", luminosity, 1079, 1102, 1117, 1118

myocardiographic, $\quad 39$

negative variation in electrical

organs, 577,578

, musele, $\begin{array}{r}579 \\ 417 \text {, }\end{array}$

$418,419,420$,

$421,422,424$, 446,448

, nerve, 495,532

$533,548,555$

, retina, 1051, 1052

of pressure, arterial, "60, $69,78,92,123$,

$127,139,147,151$

See also Pulse.

, cardiac, 18, 19, 20, 22, 23 ,

cerebral, . 142, 147, 148

capillary, . $\quad 69$

venous, $94,124,140,148$ respiratory, . $282,296,283,310$ of rigor mortis . . . . 392 ,, sounds of heart, . $\quad . \quad 30,32$ splyygmographic. Sce Pulse.

Traube-Hering, . . 138, 307 of sensation, visual, $1068,1072,1073$,
Curve of tone, affective,

PAGE

, vowel.

997

". " $\quad 1219,1220$

, , velocity of blood flow . 35,87

, , volume of kidney, . . . 159

,, , , , limbs, . . 156, 157

," , , spleen, . . . 163

Cutaneous sensation. See Sensation.

Cyclopean eye, . . . . . 1135

Cylindrical tubes, fluid-flow in, . $\quad$. 63

Cystitis after lesions of cord, . . 850

Dartos tunic, . . . . 348,635

Deafness from cortical lesions, $761,762,763$

,, , ear lesions, . . . 1167

Decerebrate rigidity, . * . $\quad . \quad 913$

from ce rebella

lesions, . 898,908

effect of, on re-

flexes, . 914, 915

,, section of

afferent roots

ou, $800,870,914$

,, semisection

of bulb oll, $\quad 914$

inhibition of, 894,914

limb-postures in, 914 movements of pro-

gression in, . 914 muscles in, . 711, 914

Decussation of olfactory fibres, . . 781

,, ,, optic nerve, $75 \dot{4}, 756,757$,

1043

, ,, pyramids, . $777,808,889$

Defæcation, 335. See also Rectum.

, action of perineal muscles

in, . . . . 336

centre for, . $\quad 336,850,851$

effect of lesions of cord

on, . $812,814,815,850,851$

respiratory movements in, 336

Deflex action, . . . . 680

Degeneration of nerve fibres, $47 \dot{9}, 52 \dot{2}, 547$, 601,603
,, $\quad$, $\quad$ primary, $\quad 704$
$\begin{array}{lll}\text {,, } & \text { primary, } & 704 \\ \text {,, } & \text { secondary, } & 705\end{array}$

", reaction of, in muscle, 439,876

Deglutition, . . . . 314 action of cardiac sphincter in, 318 , constrictors of pharynx in, . 316 cosophagus in, . 316 , $317,319,320$

afferent nerves for, . . 320 centre for, . . . 320, 887 closure of air passages in, 315,316 isthmus faucium in, 315 naso-pharynx in, . 315 \begin{tabular}{ll} 
luration of, & 319 \\
\hline
\end{tabular} effect of extirpation of epiglottis on, section of cesopliageal nerves on, . 320

efferent nerves for, . . 320 inhibition of esophagus in, 318 of liquids, . - . 319 movements of laryux in, 315,316 . 319,320 tongue in, 314,315 ,

," , $\quad$ tongue in, 314, 315, nervous mechanism of, . 319 
Deglutition, reflex stimuli for, . $\quad \begin{array}{r}\text { PACE } \\ 319\end{array}$

relation of, to respiration, . 321

of solids, . . . 319

sounds of, . . . . $\quad$. 319

stages of, . . . . 314

time-relations of movements

of, . . 319

voluntary stinuli for, $\quad$. 319

Deiter's nucleus, . . . . 807

Deliriun cordis, . . . . . 165

Delphine, action of, on heart, . . 224

Denarcation currents. See Nerve, Nusele, currents of rest of

Dendrite, . . . . 592, 798

Dendron, $592,593,596,599,605,607,655$

Depressor nerve, . . $59,154,855,1012$ , in animals . . . 59

, effect of curari on, 168

," , strychnine on, 60 suprarenal extract on, . 60

, excitation of, $59,60,61,134$,

167 extent of influence of, . 61 , fatigue of, . . 60 , fibres of, : . 665,686 ., influence of, on arterial

pressure, 59,60

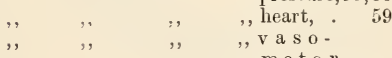

motor

$\begin{array}{lll} & \text { centre, . } & 60 \\ , . \quad \text { in man, . . } & 60\end{array}$

," mode of action of, . 60

, section of, . . . 60

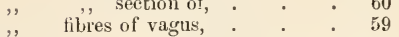

Diaphragm, . $314,319,320,325,326$ See also Respiration.

Diastole of brain,

142,143

,, ,, heart, $2,3,44$

See also Diastolic filling.

,, auricles, . . . 2, 3

$\begin{array}{lll}, " & \text { time-relations of }, & 39 \\ , & , \quad & \text { ventricles, } 2,3,4,7,38,39\end{array}$ ,", spleen,

Diastolic filling of heart, . . . 44

$$
\begin{aligned}
& , \text {, forces effecting, } 44 \\
& \text {," } \quad, \quad, \quad \text { influence of } \mathrm{ab} \text { - } \\
& \text { dominalpres- } \\
& \text { sure on, } 44,127 \\
& \text {, on arterial } \\
& \text { pressure, . } \\
& \text { eardiac sys- } \\
& \text { tole on, } \\
& \text { intrathoracic } \\
& \text { pressurc on, } 44,45 \text {, } \\
& \text { of ligation of } \\
& \text { veins on, } \\
& \text { negative car- } \\
& \text { diac pressure } \\
& \text { on, . } 44 \\
& \text { respiration on, 44, } \\
& 94,125,127,129 \\
& \text { on systolic } \\
& \text { output, }
\end{aligned}
$$

Dierotic noteh. See Pulse wave.

Diencephalon, 916. See also Thalamus, Corpora genicu-

$$
\text { latu. }
$$

Diencephalon, connection of, with optic

PAGE nerve, . . 916, 917 fibres of, to cerebral cortex, 916 grey niatter of, . 916,917 in lower vertebrates, . 916 ,, niammals, . 916,917

Differential rheotome, . . . 414, 415 time, . . . . 614

Digestive tract, neuro-muscular mechan.

ism of, . . 313

Digitalin, effect of, on heart, 196, 223, 224

. , ," ,, nerve, . . 536

Dioncea, contractile struetures in, . . 450

Dioptric system, . . . . . 1026 Diplopia, . . 1032, 1033, 1125, 1137 ,, heteronymons, . 1125, 1137 ,, homonymous, . 1125,1137

Dise, electric, . . . . 563, 590

Disparation, retinal, . $\quad 1125,1137,1138$

Dissociation of cye-1novements, . . 1131 paralysis, . 979

Distance, perception of. See Pereeption.

Diving birds, vena cava of, . . . 72

Dog, bark of, . . . . . 742

,, cerebellar peduncles of, . . 901

,, cerebellum of, . $893,895,897,904$

,, cerebral cortex of, $702,704,706,709$, $732,733,736,740,742,745$, $752,754,755,757,759,761,763$ corpora quadrigemina of, . . 912 knee-jerk in, . . . . 874

", mesencephalon of: $\quad . \quad$. $\quad .912$

,, muscle spindles in, . . . 1008

," lyramids of, . . . . 778

,. suinal, . . $819,832,840$

", ," cord of, $812,813,836,852,865$,

$866,86 \%, 879$

,, thalamus of, . . . . 918

,, vaso-dilators of bucco-facial region of, 618

Dolphin, olfactory area of, . . . 764

Donders, law of, . . . . . . 1130

Double vision. See Diplopia.

Dove, retinal rods in, . . . . 1048

Dromograpl, . . . . . 84

Drosera, movements of, . . . 853

Ductus cochlearis, $1166,1167,1179,1180$,

Duodenum, vasomotors of, . . . 161

Dynamies of blood, . . . . $\quad$. 62

Dynanometer, . $\quad . \quad . \quad 243$

Dyspncea, · . . 287, 290, 307, 312

Dytiscus, cephalic ganglia of, $\quad 828,839$

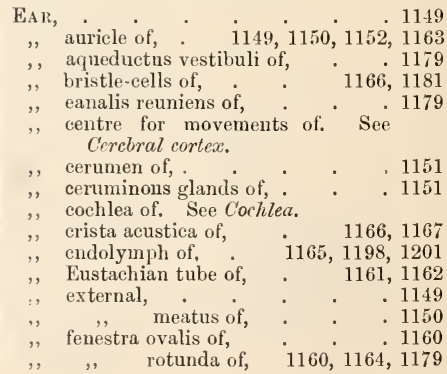


PAGF

. 1155

Ear, incus of, articulations of, $1155^{\circ}, 1156,1189$ movements of, . 1155,1156 internal, 1164. See also Cochlea,

Scmicircular canals.

afferent fibres to cortex from, 781 ,' in lower animals, 1167, 1168 labyrinth of, 1164. See also Coch-

$$
\begin{aligned}
& \text { lea, Semicircular } \\
& \text { canals. }
\end{aligned}
$$

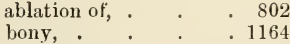

membranous, . . 1164

relation of size of sound-

waves to, . . 1165

macula acustica of, $1167,1168,1205$

malleus of, . . . . . 1155 articulations of, 1155,1156 ,

ligaments of 1189

,, movements of, $\quad 1155,1156$ manoneter, . 1162 middle, ossicles of . 1155,1156,1157

, in fishes, . . 1168

,, influence of labyrinth

pressure on movements of, . . 1159 movements of, $\quad 1157,1165$ magnitude of, $\quad 1159$ relation to sound waves, . 1159 transmission of sound otoliths of, by, $\quad 1166,1167,1205$ perilymph of, . . 1164, 1179 perilymphatic spaces of, 1164, 1166, 1201 resonance of, $1178,1187,1190,1192$, 1194,1218 sacenle of, . . . 1164,1166 semicircular canals of. See Semicircular canals.

stapedius musele of, . $\quad 1157,1162$ stajes of,

,, annular ligament of,

- 1156

, novents of, 1156, 1165, 1166 ,, compound,

pendular,

1165,1180

tensor tympani muscle of, 1153,1155 ,

action of, on ossi-

1156

cles, .

1156,1157

nervous mcchanism

for, . . . 1157

normal stimulus for, 1157,1162 tragus of, 1150,1152 transmission of sound to auditory

$$
\text { nerve in, } 1179 \text {, }
$$
1181,1191

labyrinth of, 1150 ,

1152,1158

1159,1160 ,

1161,1164

1165
Ear, tympanic membrane of,

PAGF action of sound on, 1153, 1154, $1155,1157,1189$

,, , , , eurvature of, 1153,

, = , , , elasticity of, 1152

,, , , , innervation of, 1157

,, , , , movements of, 1153

,, , , , production of

combination

tones by, . 1189

, , , , $\quad$ structure of $\begin{array}{r}1152, \\ 1153\end{array}$

, , , , , tension of, 1152 1153,1155 1156,1162

," tympanum of, ," tone of, . 1152

,, tympanum of, . $\quad . \quad$ pressure in, $\quad . \quad 1152$

, , , , regulation

tricte 1164, 1166, 120 $620,624,656,658,669$ 675,676

, vestibule of, $1164,1201,1204,1205$
214

Eel, heart of,

,, sphineter iridis of, $\quad . \quad . \quad 1044$

,, spinal, . . $817,818,830,834$

,' , cord of, . . . 795, 813

Eflerent root cells. See Root cells.

,, root fibres. See Nerve-root fibres.

Effort, sense of

Elasmobranchs, cerebrum in, $\cdot{ }^{\circ} \cdot 605$

," inhibitory nerves in, . 216 Elastic equilibrium of musele. See Nuscle.

,, tissue spindles,

924

,$\quad$ tubes, fluid flow in, . $\quad . \quad 67$

, , , wave-motion in, 96, 98, 99

Elasticity of arteries, $\quad 72,86,102,103,111$

,, ,, lungs, . 122, 124, 275, 312

, veins,

Elbow-joint,

nerve cells, $565,566,566,588$ organs, 561

," after-effects of activ-

ity of, . 572,577 , 583,586

effects of activity of, . 571 nature of activity of,. 590 central nervous con-

nections of, $\quad 565,566$, $567,58 \mathrm{~s}$

currents of, 568, 571, 579, 583. See also Electrical. oryuns, response of.

electric dise of, 563,564 , $565,566,567,568,570$ $584,590,591$

, plate of, 563,564 , $565,566,56 \%$, $568,570,584$ 590,591

excitation of, 569,570 $571,572,573$

,, direct, $572,577,590$

", ," indirect, . 572, 577

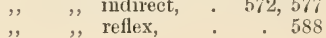


Electrical organs, excitation of, self, $\quad \begin{gathered}\text { PAGE } \\ 579,\end{gathered}$ $582,586,589$ fatigue of, $574,586,589,591$ of Gymnotus, . 561, 562, $565,569,570,588$ influence of drugs on, 574 , 575,590 ,, arrest of circulation on . . 574 latent period of, . 576 of Malapterurus, 561, 562, $567,5 \dot{69}, 570,572,574$, $577,579,582,583,585$, $588,589,590$ meandering layer of, 564 , 565,566 of Mormyrus, $\quad 561,565$, 569,588 museular tissne of, 564 , natural discharge of, $\quad 569$, 570,588 nerves of, $456,481,482$, $483,563,564$, $565,566,567$, 588,591

polarisation of . . 584 polarising eurrents for, 584 of Raia, 561, 562, 563, 564, $569,575,590$ reflex time of, . . 589 refractory period of, . 582 response of, $571,579,586$ eonditions affecting, 572 curve of, 577 , 578,579 ., E.M.F. of, 583 , 584 influcnee of direction of eurrent on, 582 , , inteusity of stimulus on, $\quad 573$ , repetition of stimulus on, 582 , temperature on, 577,581 multiple, 579 reflex, 588, 589 residual effeet of, . 583 time-relatious of, 575,589 structure of, $562,563,568$, of Torpedo, 561, 562, 565 , $566,569,570$, $571,574,575$, $577,579,582$, $583,585,586$,

Electro-eardiograni, 588

Electrodes, non-polarisable, • • 448 Electrolysis in noist eonductors, : $\quad 540$ , nerve, . $540,544,557$
Electrometer, capillary, PAGE

. 417, 418 Electrotonus, 494, 540, 546. See also Nerve. Eledone, nerves of, . . 482, 530, 532 ," reflexes in, . . . . 839 Ellipsoidal joints, . . . . . $\quad$. 240 Emetics, $: 326$ Emys, heart of, . . . . . 196 End-bulbs, - : $\quad 947,1007,1009$

Endolymph. See Ear.

End-organs, sensory. See Skin, Sensation, Petinu, Cochlea, Tongue, Nose, Muscular Sense.

Enteric nervous system, . 668, 694, 695 Enterograpli, . . . . . 327

Epididymis, $\quad . \quad 5 \quad . \quad . \quad 549$

Epigastric ganglion, . . . 617

Epiglottis, . . 316, 1211, 1237

Epileysy, . . . . 718 ,, aura of, $\quad{ }^{\circ} \quad 720,996,1001$ , in brain lesions, $705,706,718,748$ , from excitation of eord, $\quad 722$ ,, , , eortex, 718, 748 ," effect of, on cirenlation, - 722 ,, ,, , eonsciousness, . 721 , $\quad$, , pupil, . . 722 , , , ,

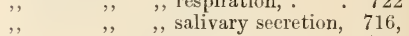
", ', ", salivary secretion, 716,

, , , , sweat secretion, 716,

,, Jaeksonian, . 706, 719, 720, 721 ," "mareh" of spasms in, 719,720 ," rliythm of musenlar contraction

in, . . 710,718 Equilibrium-length of musele. See Mrusile.

Equation, personal, . . . . 608 Ereet position of body, $\quad$. $^{*} \quad$. 231,260 Ereetion, . 347 ,, action of ischio - cavernosus muscle in, . . . . 347 of elitoris $\quad: \quad: 347$ ," $\quad$, feathers, $\quad . \quad \cdot \quad \cdot \quad \cdot \quad \cdot 618$

,, ", hair. See Sympathetic neve fibres, pilo-motor.

influence of vaso-dilatation in, 347 museles concerned in, . . 348 of penis, . . . . 347 , , quills, . . . 618,625

Ergograph, . . . . . . 387 Erythropsia, $\quad . \quad+\quad . \quad . \quad . \quad 1096$

Eserin, effect of, on vision, . . . . 1141 Ether, dissociation paralysis from inhalation of, . . . . . 978 ,, effect of, on electrieal organs, . 575 ," ,, , knee-jerk, . . 873

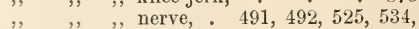
,, ,. neural tonus of musele, $\quad 870$ Eustachian tube, . . . 1161, 1162 Exeitation, law of, $\quad \cdot \quad 435,469,515$ Exeito-motor voluntary impulses, 724, 728,

Exhaustion. See Fatigue.

Expiration, 279. See also Respiration.

$$
\begin{gathered}
\text { in bronchial constriction, } \quad 312 \\
, \quad \text { diaphragm in, 276,279, } 296 \\
\text { effeet of excitation of inferior } \\
\text { laryngeal }
\end{gathered}
$$$$
\text { nerve on, } 305
$$ 
Expiration, effect of excitation of sensory nerves on, 305

$$
\begin{gathered}
\text {, } \quad \text { superior } \\
\text { laryngeal } \\
\text { nerve on, 293, } \\
\\
\\
\text {, inflation of lungs on, 294, }
\end{gathered}
$$

forced, 279 influence of corpora quadrigemina on, . . . 306 mirmur of, . . . 281 muscles of, $\quad . \quad 278,279$ ribs in, . . . 278,279 total force of, . . 281,282

Extensibility of arteries, . . . 73, 74

$\begin{array}{cccc}\text {, } & \text {, muscle. See Muscle. } \\ \quad, & \text {, veins, . } & \cdot \quad \cdot \quad 118\end{array}$ Eye," "veins, • • 118 ,, accommolation of, . . . 1033 ,, , aqueous humour iil, 1037, 1039 in amphibia, 1040 , 1041 ssociation of pupil-contractioll with, 1043 1131, 1132 in cephalopods, 1040 , changes in suspensory apparatus, 1036, 1038, 1039,1040 ,, choroid coat during, 1036, 1039 , , ciliary process during, 1036

in fishes, 1040, 1041 changes in formi of lens during, 1040 ,, iris during, 1036 inflnence of, in space rerception, 1135, 1137, 1138

lens in, 1033, 1034, $1035,1039,1040$, meridional, $\quad 1037$, nervous mechanism of, . 1037 range of, . 1131 in reptiles, 1040, 1041 theories of, 1038 unequal, 1037, 1038 vitreous humour during, 1037, 1039, 1040,1041 1031, 1032 1031,1032 angle $\alpha$ of,

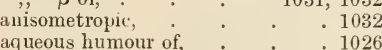
aqueous humour of, refraction - in dex of, 1026, 1029, 1032 astigmatic, . $1032,1033,1148$ accommodation in, 1037, 1038
Eye, astigmatic, foci of,

PAGE

$$
\begin{array}{lrrr}
\text { axis of, optical, } & . & . & . \\
\text { centre of rotation of, } & . & . & 1031 \\
\text { centring of, } & . & 1029 \\
\text { chromatic aberration in, } & . & 1031 \\
\text { ciliary muscle of, . } & 1039, & 1040,1041 \\
\text { compound, . } & . & . & 1042
\end{array}
$$
cones of. See Retina.

convergence of, association of pupil-

contraction with,

1131,1132 cornea of. See Comea.

Cyclopean, . . . . . 1135 dioptric system of, . . 1026 electrical stimulation of, 1097, 1103 excision of, contraction of pupil after, . . 1044 degenerations following, . . . 781 effect of, on occipital lobc, . 916 ,, optic thal.

amus, . 916

pupil-reflex after, . 1043

$$
\begin{aligned}
& \text { foci of, . } \quad \text {. } \quad 1029,1033,1039 \\
& \text { anterior, . . . } 1029 \\
& \text {, circular, . . 1033, } 1038 \\
& , \text { for different colours, } 1033 \\
& \text {, linear, . . 1033, } 1038
\end{aligned}
$$
form of, irregularities in, . . 1032

hypermetropic, . . 1032 ,, acconmodation

$$
\text { in, . . . } 1034
$$
centre of rotation

$$
\text { of, . . . } 1030
$$

ciliary nuscle in, . 1039 optical axis of, . 1032

innervation of muscles of, . . 911

intermittent electrical stimulation

of, . . . . 1076

iris of, nerves to, $617,619,620,621$, $656,658,659,660,662$ See also Pupil.

lens of,

1026 curvature of, . 1028,1029 , $1033,1034,1039,1040,1041$ distance from cornea, 1028,1029 . $1034,1035,1041$ irregularities of form, 1033, 1035,1036 structure, . 103: mobility' of, 1034, 1036, 1041 pigmentation of, 1054, 1118, 1119 refraction-index of, 1026,1029 , removal of, effect on colour vision, . 1055, 1096

line of fixation of, . . . . 1031 ," vision of, . . . . 1031 , , relation to corneal axis, . 1031 , , , , optical axis, 1031 mechanical stimulation of, . . 1097 movenients, . . 1129 of accommodation. Sce Eye, accommodation. associated, 1043, 1131, 1132 centres for. See Cerebral cortex. compensatory, 1131 VOL. II. -8 I 
Eye, movements, conjugate, 712,716 $739,740,748,749,752$, $755,756,761,893,894$, $895,900,901,912$ of convergence, 1130,1131 , $1132,1135,1137,1138$

Donder's law of, . . 1130 horizontal. . . 1129 from lesions of cerebel-

lum, . 893, 894, 895, $898,900,901$, 902

, semicircular canals, 1131, $1197,1203,1204,1205$ Listing's law of, 1129, 1131 measurement of, . 1129 oblique, . . . 1129 relation of, to visual

illusions . . 1143 rotatory, round optic axis, . .1129, 1131 during slecp, . . 1132 from stimulation of medulla oblongata, . 893 ,, mesencephalon, 911, 912

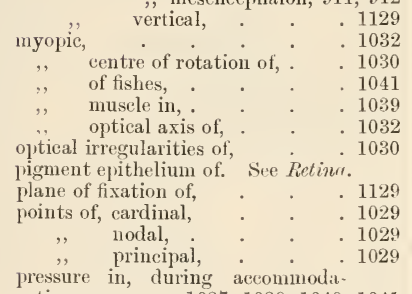
vertical 1129

FACE, motor centres for. See Cerebral Cortex.

vasomotors of, 141 . Sce also Sympathetic nerre fibres.

Facilitation of spinal reflexes,

837,838 841,842

Fieces, effect of lesions of cord on, 851,853 Falcon, brain of, . . . . 701 Fallopian tubes,
Fasciculus dorsalis proprius, peduneulo-naxillary, . $\quad . \quad 782$ retroflexus, . . 782 solitarius, . . . 285,793 subcallosus, 769. See also Bundle.

Fatigue, effect of, on localising power of skin, . . 9 937, 941 of muscle, 385. Sce also IIuscle.

,, olfactory organ, .

,, retina. See Retina.

sense of, . 1253

", thermal sensory organs, $: 953$

Feathers, ereetion of . . . . 618

Fechner, law of, . . . . . . 933

Femur, rotation of, . . . . 238

Fenestra ovalis, . $\quad . \quad$. $\quad . \quad 1160$ rotunda, $\quad 1160,1164,1179$

Filiform papillæ, . $\quad . \quad$. $\quad .1241$

Fillet . . 705, 763, 769, 778, 779, $781,862,890,893,915$,

Fimbria, . . . . . 764

Fishes, air bladder of, . . . . . . 1168 cerebellum of, . . : 900 cerebrum of, . $\quad 699,700,732$ ear of, . 1167, 1168, 1203, 1204 eye of, . . 1043, 1049, 1105 optic lobes of, . . . . 911 organs of lateral line of, 1167,1168 thalamus of, . . . 670,699

Fissure, calcarine, . $\quad 755,757,758$ jalpebral, . . . 699,681

Flame manometer, . . . . . 1218

Flexure, sigmoid,. . . . 335, 336

Flicker plıotometry, . . . . . 1076 sensation. See Sensation, visual.

Flocculus, . . . . . 894, 903 Fluid, flow of, . . . . 62

, ,, in branching tubes, . 67

," , , , cylindrical tubes, 63, 64

,, ,, ,, clastic tubes, . . 67

, , , , tubes of varying diammeter,

66 viscosity of, . . . $63,65,98$

Focal points of anditory area $\quad$ 761, 762 , , , motor area, . . . 737 ,. ., , visual area, . . . . 756 Foci, visual. See Eye.

Form, perception of. See Pererption.

Formatio reticularis, . . $286,807,888$ Fornix, . . 764, 771, 781, 782

Fourier, law of, . . . . . 1174

Fourierian analysis, $\quad . \quad 5 \quad . \quad \cdot \quad \cdot 1221$

Fovea, See Retina and Scnsation, visual.

Friction coefficient, . . . . 234 of joint surfaces, . . . . 234

Frigorific nerves, . . . . . 619

Frog, alimentary canal of, . . . 335

, bulbo-spinal, . . . 886

," cerebellum of, : $\quad \cdot \quad 899,903$

,, crebrum of, : $629,698,704,732$

$\therefore$ eye of, . $1044,1048,1049$ heart-tonus of, : $\quad . \quad 197$ knee-jerk of, . . . . 874 medulla of, : $\quad 886,888,890$ mesencephalon of, . . . 911 muscle-spindles in, : . 1008 reflexes in,. . $813,814,844,853$ 
PAGE

Frog, spinal, $\quad 817,818,819,826,832,834$, $835,840,844$ ,, $\quad$,, cord of, $811,814,867,878,879$ , ," nerve-roots of, . . . 790 , tactual sensation in, . . 9 923, 927 , thalamus of, . . . 698,699 Frontal association centre, . . . . 771 , lobe. See Lobe.

Fronto-pontine fibres, . . . . 893, 915

Fundus of stomach, . . . . 322

Fungiform papillæe, . . . . 1237, 1241

Fusspunkt, . . . . . $\quad . \quad 376$

GALL-BLADDER, afferent nerves from, . 849 Ganglion or ganglia, autonomic. See Autonomic
ganglia, Sym-
pathetic ganglia.

of bladder, 342

cerebral, of inver-

tebrates, 813,839 cervical. See

Sympathetic

ganglia.

ciliary, 200, 650, 660, $664,677,682,911$ of eranial nerves.

Sce Autonomic ganglia.

Gasserian, 793, 891, 141,1239

geniculate, 884,1239 of heart, 169, 180, $182,183,186$, $192,197,198$, $200,202,678$

,, Bidder's, 169, 170, $171,175,178$, 179,183

, development of, - 177, 203

,, Ludwig's, 169, 175

, relation of, to cardiac nerves, . 197 ,, Remak's, 169, 170, $171,173,175$ ,, of tortoise,

of hypoglossal 173,174 nerve . 664,884 inferior mesenteric. See Sympathetic ganglia.

influence of, on

transmission of impulses, - 791 interpeduncular, 782 of intestines, 327,329 , 668,672 jugular, - 663,793 otic, $661,664,1157$ petrous, . 793 of pelvic plexus 667 , prevertebral.

See Sympathetic ganglia.
Ganglion or ganglia, semilunar. See Sympathetic ganglion, Solar ganglion.

solar. See Sympathetic ganglion.

spheno-palatine, 651 , $661,662,664,1239$

spinal, • 787,788

, action of nico-

tin on, . 791

,, conduction by cells of, 791

, connection of a ff e $r$ e nt sympathetic fibres with, 686

,, effect of compression of aorta on, 836

, influence of, on reflex time, 791

$\therefore$, relation of, to referred pain, 982

,, trophic effects of lesions of 876,878

,, visceralafferent fibres of, 203, 849 stellate. See

Sympathetic ganglia.

of stomach,

, , tenth and ele-

venth nerves, 200, 201, 601, 663, 664, $668,793,849,884$,

1157

sublingual, . 66 submaxillary, 66,664 , $672,673,677,679$ sympathetic. See Sympathetic ganglia.

of ureters,

vertebral. See Sympathetic.

Ganglion-cells of cochlear nerve, . . 884 ,", vestibular nerve, : 884 Geninule, ." vestibular nerve, · $\quad$. $\quad 593$ Generative organs, $347^{\circ}$ Sce also Coitus, Erection.

, $\quad$ effect of excitation

," $\quad$ effect of excitation

", of pelvic nerve on, 348

, , , , , pudic

nerveon, 348

, , , hypo-

gastric nerves on, . 349

external, . 160,347 internal, . 160,347 muscular mechanism of, . . . 347 sacral autonomic nerves to, $160,348,667$, 692,693 
PAGE

Generative organs, sensory nerves of, . 349 sympathetic nerves of, $160,348,349,635$, $646,659,679,692$

Geniculate body. See Corpora geniculata. ganglion,

884,1239

Genital corpuscles,

, sense,

Genito-spinal centre,

Gennari, band of, .

Geotropism, .

Glands, ceruminous, gastric, nerves of,
lachrymal, nerves of, mammary, effect of section of nerves of mucous, nerves of, . . . 618 orbital, nerves of, . . 660,661 salivary, circulation in, . . 141 , secretory nerves of, 618,620 , $660,661,692,993$ vasomotor nerves of, 132 , $141,617,619,620,624$, $626,660,661,672,674$

692,693

sweat,

618,625

Glans penis, sense-spots of, $922,947,986,987$

Globus pallidus,

Glomeruli, olfactory,

Glottis. See Larynx.

Glycosuric puncture,

764
1247

Golgi organs, 924, 1007, 1008, 1009, 1010

Goll, column of, degenerations in, . 809

, nucleus of, . . . . . 885

Goose-skin, . . . . 625

Gorilla, efferent root-cells of, . . 795

Gowers, tract of, 807

Gradient, pressure, . . . $\quad 64,66$

Granules, chromatophil, _ . . 599 Nissl, . . . . 599,600

,, tigroid, $\quad . \quad . \quad . \quad .599$

Grey rami, . . . . . 628,629 distribution to allo-genital region . 636 , limbs, 629, 632, 637,638 trunk, 632,637 ,

" " $\quad$ fibres of, $649,651,687,688,689$
$, \quad \quad, \quad 638$ ", ," origin of, from ganglia, . 629 Grillotalpa, reflexes in,

Fuaiacol, effect of, on cutaneous sensa-

Gininea-pig, cerebrum of,

27,834 muscle spindles in ," pyramidal tract of, . 809,889 Gustatory area. See Cerebral cortex. sense. See Sensation.

Gynincma, effect of, on taste, .1242, 1243 Gymnotus, elcctrical organs of, 481, 561, 562, $565,569,570,588$ Gyrus or gyri, angular, $752,753,754,755$, $758,759,780$ central, · $\quad \dot{6} 768,769$ fornicatus, $714,764,766,767$, $768,769,770,781$ hippocampal, $764,766,767$, $769,770,781$ marginal, . $714,764,769$
Gyrus or gyri, sigmoid, 713, 714, 717, 764, 765 suprasylvian, . . 714 temiporal, $749,755,761,762$,

763,781 uncinate, . $749,763,764$

HeMAUTOGRAPH, . . . 18, 100

Hæmic murmurs, . $\quad$. $\quad$. $\quad$. 33

Hæmodromograph, . . . . . 84

Hæmodynamies, . . . . . $\quad .62$

Hæmodynamometer, . . . . 78

Hæmorrhage, effect of, on artcrial pressure, $\quad 75,77$ , , eapillary pressure, . 117

, , , , respiration, 309

Hair, erection of.' See Symprthetic nerve-fibies, pilomotor.

Hair - cells, 1181, 1184, 1185, 1186, 1190, 1191,1192

,, effect of nerve section on, . $\quad 875$

," tactual functions of, . 923, 926, 942

Hairs, auditory, . . . 1201,1202

Hallucinations in hemiopia, . . 760

Hallux, centre for movements of, . 737,744

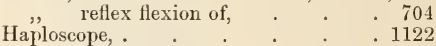

Head, circulation in, . . . . 141

,, equilibration of, 1196, 1198, 1199,

1201, 1202

,2 motor area for. Sce Cerebral cortex.

register. See Voiee.

,, vasomotors of, 141 . See also Sympathetic nerve-fibres, Autonomie nerve-fibres.

Hearing. See Sensation, auditory, Ear. centre for. See Cerebral eortex.

Heart, .

acceleration of. Sce Aurgmentor. nerves, Centre eardiac accelerator, Nerves aecelerator, Heart, frequency. action of,

afferent root-cells for,

auricles of. See Auricles.

auriculo-ventricular muscular ring of, 178,179 , 180,182 , 183,186

valves of. See

Valves.

automatic action of, . $169,173,176$

beat, . . 169

,, cause of, $169,170,180,186,224$

,' influence of, on cerebral circulation, circulating fluid on, . 224 on coronary circulation, . 165 drugs oll, 195, 196,

intracardiac pressure on, 170

, relation to peculiarities of cardiac muscle, . . 188 in warm-blooded animals : 186 blood-supply of, - $164,165,166$ 
PAGE

Heart, centre for. See Centre, cardiac.

circulation in,

$$
\text { , of artificial fluids }
$$$$
\text { in, . . } 224
$$

contraction of, : $\quad 2,3,4,5,6,7$

See also Systole,

Systolic output.

muscle of. See

diastole of. See Diastole.

Muscle, cardia:

diastolic filling of

dilated, tension in, . 40,41

effect of alcohol on, . $\quad 52$

,, asphyxia on, $41,50,52,307$,

308

, ehloroform on, $41,52,58$

, excitation of mesence-

phalon on, . . 911

, gravity on, . $\quad 3,4,31$

,, intrathoracic pressure on,

$44,45,125$

,, transfusion on, . 75,77 energy of, .

entry of air into, . . . 120

exhaustion of, . . . 150

force of, . . $40,42,50,51,441$

,. conditions affecting, 41,165

, influence of augmentor nerves on, $\quad 216,220$

,, ,, temperature on, . 172

, ., vagus on, 203, 204, 205, $207,208,209,210,212$,

form of, 1,20

frequency, influence of accelerator centre on, . 55 afferent impulses on, $55,57,58$, $59,8.56$

arterial pres-

sure on, $50,53,56$ augmentor

nerves on, 216 220

cortical ex-

eitation on $55,714,715$

coronary cir-

culation on, 165

exercise on, 58

heat on, . 172

intra-cardia:

pressure on, 58

respiration on,

58,129

vagus on, 54,57 ,

$58,203,204,205,208,220$

See also Centres, cardinc.

ganglia of. See Ganglia.

heat-production by,

heat-rigor of,

$4,5,10$

of Helix,

impulse of, 33. See alsolmipulse.

influence of afferent nerves on, 55,57 ,

58,59

muscle reflexes on

1012,1013

vagus on, 54, 57, 58, 203,

204, 205, 207, 208, 209,

$211,213,220$
Heart, inhibition of,

PAGE

ligature of. See Stanius heart.

", measuremients of, in systole and

diastole,

movements of, : $\quad \cdot \quad 2,3,4$

muscle of. See Cardiac muscle.

musculature of, . . . $4,6,7$

nerves of, accelerator, $53,55,56,58$, $198,199,200$, $642,649,663$,

665

, $\quad$ inhibitory, 54, 198, 203,663 ,

sympathetie, $617,618,623$, $624,642,659,675$

nutrition of, . . . . 224

pressure in. See Pressure in

heart.

reflexes of,

$54,57,59,856$

regurgitation in, . . 11,12

sensitivity of, . . . 856,971

sinus, contractility of, $172,183,185$,

,, extension of, . . . 173

sounds of, . . . . 28

suspension of, . . . . . $\quad$. 6

systole of. Sec Systole.

systolic output of, . . . 48

of tortoise, . . . . 173

"Treppe" phenomenon in, 192, 214,

215,222

valves of. See Valves.

veins of, rhythnic contractility

of, . $\quad 178,179,190$

ventricles of. See lentricles.

volume of, changes in, . . 49

work of, . 40,43

Heat centres, . . . . . 717

,, production in cerebral cortex, . 718

, , , , heart, . . . 44

., , , musele. See iruscle, thermal response.

Heat production, in Vol. I.

," ,. , nerve, . . 453, 454

,, rigor, . . . 4 4, 5, 10,390

,, sensation of. See Sensation.

systole, . . . 4, 4,10

Hebe-phenomen, . . . . . 800

Helicotrema, . . . . . . $\quad .1179$

Helix, muscle tonus of, . . . 377,378

Hemianresthesia in cortical lesions, $\quad 767$

Hemiopia from brain lesions, $749,753,754$, $757,758,768,917,918$

Hemiplegia, disorders of sensation in, . 731 spinal reflexes in, . 731

Hemispheres. See Cerebral hemispheres.

Hemmung, . . $83 \pi$

IIepatic circulation, . . . . 161

Herpes zoster, .

Hertz waves, stimulation of nerve by, . 462

Heterodromous eurrents, . . . 585

Hiccough, . . . . . 887

Hinge-joint, : $:{ }^{*} \quad{ }^{*} 234,238$

Hip-joint, . . 231, 232, 235, 238

Hippocampus major, $762,764,765,766,767$,

Hirudo, reflexes in, $\quad 769,781,782$,
835,839

Homodromous currents, . . . 585

Hooke's law, . . . . 355 
Horopter, · .

Humerus, rotation of, . . . . 238

Hunger, · · . . . 991, 992

Hyperæsthesia in lesions of cord, . . 867

Hyperalgesia in lesions of cord, . . 867

Hypermetropia. See Eye.

Hyperosmia,. . . . . . 1251

Hyperpnœa, . . . . . 307

Hypnosis, . . . . . 712,913

Hypogastric plexus, $337,338,342,344,348$,

349

Hypoglossal nuclets, 885,889

IDEATION, . $\quad .697,698$

Idiocy, brain in, . . . . 697, 703

Idiomuscular contraction. See Muscle.

Ileum, vasomotors of, . . . . 161

Impulse of heart, . . . . 33

$, \quad \quad, \quad$ influence of, on heart
sounds, $28,30,31$

,$\quad$, $\quad$ influence of, on heart
sounds, $28,30,36$

,$\quad$, $\quad$ influence of, on heart
sounds, $28,30,36$

," $\quad$," $\quad$ origin of, , posture on, 34

", $\quad$, position of, $\quad . \quad$. 33

Impulses, nervous, conduction of. See

\section{Conduction.}

influence of ganglia on . . 791

inhibitory, : 674

irradiation of, 819,820 law of conduction

of, $\quad . \quad 606,785,798$

rate of discharge of,

Incisor teeth, from cortex, 708, 710

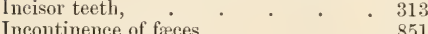

Ineus. See Ear.

urine, 342

India-rubber, contraction of, $\quad . \quad 396,397$

Indifferent point, . . . . 494, 495

Induction, visual. See Sensation, visual.

Inlibition, · • • . 672, 711, 914 of ciliary musele, . $\quad . \quad 618$ cortical, . $711,712,739,773$ of decerebrate rigidity, .

, lieart. See Heart.

, intestinal movements, 329,331 , $332,333,334,335,663,693$, 695,696

from medulla oblongata, . 887 of muscle tonus, . 1012,1019

,, muscles of erayfish, . . 513

,, esophageal movements, - 318

,, pain, . . . 1001

,, reflexes, $\quad 712,77 \dot{3}, 81 \dot{4}, 815$,

$842,911,912$

, respiration, - $303,304,321$

relation of, to shock, . . 846 of stomach movements, $31 \dot{8}, 322$,

sphincter cardix, $\quad 323,324$

iridis, $\quad 618,1044$

pylori, . 322,323

vesieæ, $344,345,695$

Inlibitory centre, 711. Sce also Centre,

$$
\text { cardiac. }
$$

nerves, action of, $203,672,673$

Innervation, crossed,

$$
334,337,345
$$

Innervation, reciprocal $842,1013,1019$ ,, sensation of, $1002,1003,1004$, 1005,1134

Inscriptiones tendineæ, . . . 429

Insectivora, motor area in, . . . 732

Inspiration, . . . . . . 275

in bronchial constriction, . 312

," diaphragm in, $275,276,277,296$

effect of collapse of lungs on, 295

, inflation of lungs on, 295,

298,302

,. vagus excitation on, 293,

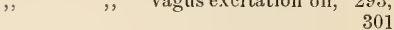

, , , , , section on, 296

,, forced, . . . 279

, influence of corpora quadri-

gemina on, . 306

,, thalamus on, : 306

murmur of, . . 281, 311

muscles of, . . . 277,279

" centre for, . . 284

ribs' in, . . $275,276,277$

total force of, . . 281, 282

Insular association centre, . . . 771 Intellect, effect of ablation of hemi-

spheres on, 699,703

lesions of association

areas on, 770

$771,772,773$

, excitable

areas on,
relation of frontal lobe to, $765,770,772$,

773

,, $\quad, \quad$ parietal lobe to . 772

", seat of, . - $697,770,772,773$

Intention tremor, . . . 897, 903

Intermediary nerve-cells, . . . 592

Internal capsule, . . . . . 777

fibres of, from genienlate

body, . 780

, motor

cortex, 777 , 778,779

occipital

lobe, . 780

prefrontal

region, 739 , 777,779

, temporal

lobe, . 781

$,, \quad,, \quad, \quad$, thalamns, 777

Interosseous membranes, afferent nerves

of, . . . . . . . 1007

Interpednucular ganglion, . . . $\quad$. 782

Interstitial cells, . . . . 660, 672

Intestines, . . . . . . . 326

" action of movements of, on
food, . food, . - 2.330 $\begin{array}{ll}\text { afferent fibres of, } & \text {.' } 688,849\end{array}$ capillary blood pressure in, 117, 118 centre for movements of, . 334 circulation in, . . 160 condition of, in fasting, - 328 curve of contraction of, 327,328 ,

effect of asphyxia on, . . 331

,, local anæmia on, . 330

, nicotin on, . . 329

function of muscular coats of, 327 
PAGE

Intestines, influence of brain on, . . 334 , inhibition of, $329,330,331,332$, $333,335,696$ inhibitory nerves of, $333,334,335$ motor nerves of, - $331,333,334$, $663,667,693,695,696$ movement of, . $326,327,328$ myogenic contraction of, . 329 nerve-plexuses of, . . 327,330 nerves of, in frog, . . 335 peristalsis of, $120,329,330,853$ reflex influences on, . . 331 response of, to loeal stimuli, . 329

, rhythmic movements of, $\begin{array}{r}328, \\ 330, \\ 332\end{array}$

,, structure of, . . . . 327

, sympathetic nerves of, . 333,643 , $644,645,646,659,692,695$ ,, tone of, . . 328, 331, 335 ,. vasomotors of, . . . 160 Intrinsic light, - 1063, 1696, 1121, 1123 Iris, dilator contractile tissue of, . . 1041 ,, during accommorlation,

,, movements of. See Pupit.

,, referred pain from,

,, vasomotors of,

Island of Reil,

. 1036

Isolation dystrophy,

689

621,617

770,776

Isthmus fancium during deglutition, . 315

JAC'KAL, motor area in,

Jacksonian epilepsy, . 706, 719, 720, 721

Jaundice, effect of, on vision, . 1095, 1103

Jaw, movements of, in mastication, . 313

Jejunum, vasomotors of, . . . 161

Jerk curves, . . . . . $\quad$. 370

Joints, . . . . . . 233

,, atlanto-occipital, . . . 231

," axes of, $235,236,238,239,240,241$

,, ,, resultant, . . 236, 237

," ball-and-socket,. $\quad . \quad$. 234,235

,, earpal, . . . . . 241

, centre of rotation of, . . . . $\quad$. 235

,, elassification of, . . . . 233

,, contact of surfaces in, . . 234

,, elbow, . . . . . 23?

," ellipsoidal, $\quad . \quad$. $\quad . \quad 240$

,, friction in, . . . . . 234

", hinge, . . . . 234, 238

,, hip, . . . 231, 232, 235, 238

, influence of atmospheric pres-

sure on, . 235

," knee, . . . . . . 237, 240

," ligaments of, : . $\quad$. $234,235,238$

,, mechanism of, . . . . 233

," metacarpo-phalangeal, : $\quad 235$

,, radio-nlnar, . . . 239, 240

," reflexes fronı, : . $\quad . \quad . \quad 1012$

,, saddle, . . . . . 240

," screw, . . . . 239

,, sensitivity of, $1011,1014,1015,1016$

," sensory end-organs of, . . 947

,, shoulder, . . . . 237, 238

", temporo-maxillary, : . . 313

", trapezo-metacarpal, : . : 241

Jugular ganglion. See Ganglia of tenth nerve.

Katabolic Nerves,

220,221

liatabolism, .
Kataerotic wave,

PAGE

Kernflüche,

108,112

Kernpunkt, .

. 1133

1133

Kidney, afferent autonomic fibres of, 688,849 circulation in, . . . . 159

time of, . . 89 sympathetic fibres of, $643,645,659$ Kinæsthesiometer, . . . . 1022 Kinæsthesis, . . . $728,1003,1005$ Kinesthetic impressions, centres for, . 729 relation to volitional impulses, $\quad 729$

Kinesodic paths, . . . . 811

Kinee-jerk, . : : . $\quad . \quad 870,871$ augmentation of, . $\quad 873,874$ conditions affecting, $872,873,874$, 875 erossed, . . . . 847 during sleep, . . . 874 effect of cerebellar lesions on, 896 , 900 lesions of cord on, 868,873 section of afferent roots on, . individnal variations in, $8 \dot{45}, 872$ influence of cortex on, . 872,874 muscle tension on, 799 , $800,871,872$ inhibition of, $873,874,1012$ in spinal animal, . $\quad .815,816$ time of, . . . $8 \pi 1,1011$ Knee-joint, . . . . . . 237, 240

Kymographion, 78

LABIALS, 1234

Labio-dentals,

Labyrinth. See Cochlea, Semicircular canals.

Lacerta, spinal cord of, . . . 879

Lachrymal gland. . . . 618

Lactic acid in exhausted mnsele, . . 389

Lamina spiralis, . . . . . 1185

Lancisi, nerves of, . . . . . 764

Larynx, . . . . . . 1206

cartilages of, arytenoid, 1209,1210 ,

1211,1237

cricoid, $120 \%, 1209$

thyroid, 1207,1209 changes in, with age, . 1207 in deglutition, $315,316,319,320$ epiglottis of, . . . 1211 false cords of, . $\quad 1211,1212$ fixation of, in eliest register, . 1211 glottis of, in defreation, . . 336 ,, ,, hearl register, 1212, 121 ,, , middle register, 1212 , ,, ,, parturition, . 351 , , , vomiting, . . 321 muscles of, - . . 1209 nerve-entre for movements of, 735 , $736,740,1209$ nerves of, . . 480,624, 1209 regulation of piteh by, 1206,1213 in respiration, . . . 279, 304 ventricles of, : . $\quad 1235,1236$ in voice [rorluction, is 1206, 1235 voeal cords of. See Focal cords. 
Larynx, vocal processes of, 1209, 1210, 1211 1212,1213 ,, whispering, . 1236

Latent period of cortical excitation of muscle, . 749, 750, 751 of excitation of electrical

$$
\text { organs, . } 576
$$

musele, 381 , 430,445

nerve, . 510

nerve-

endings, 483,

skin, 987,998 ,

vasomotor

nerves, 135

for reflexes in spinal ani-

mal, 818

vasomotor, 167

Lateral" ganglia of sympathetic, . . 617

,, line, organs of, . 1167, 1168

," sclerosis,. . . 811

Laughing, · . $\quad$. $\quad$. $\quad$. $\quad 308$

Law of colour-mixture, . . . 1082

conduction in nerve-cells, . . 605

contraction, Pfliger's, 503, 508, 513 ,

515,557

degeneration, Wallerian, . . 479

Donders, . . . 1130

excitation, . . $435,469,515$

Fechner, . . - 933

forward dircetion, . $\quad 606,785,798$

Fourier, . . 1174

Hooke, 354

Listing, · . . 1129, 1131

Poiseuille, 64

Ritter-Valli, . . . . 477

specific encrgies, $\quad 1242,1243,1253$

spinal reflexes, . . . 821,823

Talbot-Platean, $\quad 1068,1069,1076$

Torricelli, . . $\quad 62$

Weber, $928,930,93 \dot{1}, 932,94 \dot{1}, 961$,

1022,1080

Lead acetate, effect of, on nerve cells, . 604

Lens. See Eye.

Leprosy, anæsthesia in, . . . . 988

Levels of central nervous system, . $\quad 697,698$

Lever, cosine, . . . 356

Light, physical characters of, 1053, 1054 ,' sensation of. See Sensation,

$$
\text { visual. }
$$

Ligaments, . . . . 234, 235, 238

Limbs, amount of blood in, . . . 155 , amputation of, effect on dorsal

columns, 878

$$
\text { , efferent }
$$

root cells, 793

apresthetic, $793,799,800,801,802$

circulation in, 803,1020

crossed reflexes of, . $\quad . \quad+821$

eurve of volume of, . $\quad . \quad 158$

extensors of, in spinal animal, 817,825

flexors of, in spinal animal, 817,825

motor centres for. See Cerebral

cortex.

nicvements of, angular, 229, 230, 233

$$
\text { penlular, . } 269
$$$$
\text { rotatory, . . } 233
$$

Limbs, movements of in running, PAGE from stimulation of dorsalcolumn of bulb, . 792 in walking, 267,268 posture of, in decerebrate rigidity, 914 significance of nerve-plexuses of, 82 , spinal nerve supply to muscles of, 795 , 797,803 sympathetic nerves to. See Sympathetic.

tonus of flexors and extensors of, . . . 870 trophiceffects of nerve-section on, 875 vasomotors of, $155,156,158,625$, $657,670,675$

\section{, volume of,} 657,070
.$\quad 158$ Limulus, reflexes in, · . $8 \dot{28}, 8 \dot{39}, 844$ Line of fixation, . $\quad . \quad$. $\quad$. $\quad .1031$ , vision. See Eye.

Linguo-dentals, . . . . . 1234

," -palatals, . . . . . 1234

Lissauer, marginal zone of, . . . $\quad$. 792

Listing, law of, . . . $\quad 1129,1131$

Liver, capillaries of, . . . 112,118

circulation in, . . . 161

,,, influence of spleen on, . . 162 sympathetic nerves of, $64 \dot{2}, 64 \dot{4}, 645$, 692 vascularity of, . . . . 162 vasomotors of, $\quad 161,643,644,692$

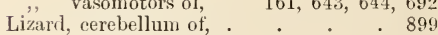
muscle spindles of, . . . 1008 spinal cord of, . $\quad . \quad .879$

Lobe, electrical, . . . . 566, 588 frontal, $763,764,765,769,770,771,775$ ,, ablation of, $751,752,772,778$ ," centripetal fibres of, . 769 connection of, with corpus

$$
\begin{aligned}
& \text { stri- } \\
& \text { atum, } 778 \\
& \text { lower } \\
& \text { centres, } 776 \\
& \text { occipital } \\
& \text { lohe, } 780
\end{aligned}
$$

influence of, on reflexes, . 712

,", influence of, , respiration, 713 ,

inhibitory action of, . $\quad 740$ prefrontal region of. See

Prefrontal.

relation of, to intellect, 765,772 ,

peecl-centre of $\quad 705,773$

limbic, speech-centre of, $765,767,769,781$

lingual, : . $\quad 752,758,759,780$

occipital, : * $\quad 752,758,759$ ablation of, $749,752,753,754$, $755,757,760,780$ association fibres of, 771,780 connection with corpora

quadri-

gemina, 780

crusta, 780

, frontal

lobe, 780

,, genicu-

late

body,. 780 
Lobe, occipital, connection with opposite

$$
\begin{gathered}
\text { lobe, } 780 \\
\text { retina, } 780 \\
\text { temporal } \\
\text { lobe, } 780, \\
\text { thala- } 781 \\
\text { mus, } 780, \\
916,917
\end{gathered}
$$

corticifugal fibres of, $\quad 780$ corticipetal fibres of, . 780 effect of excision of eye o11, 781 excitation of, . 749,755 inexcitable region of, . 770 ," lesions of, . $759,760,776$ olfactory,

commissural fibres of, 764,781 commection with gyrus den-

tatus, 781 , fornicatus, 78] , hippo-

$$
\text { limbie }
$$$$
\text { campi, } 781
$$$$
\text { lobe, }
$$$$
\text { opposite }
$$$$
\text { temporal }
$$$$
\text { lobe, . } 781
$$

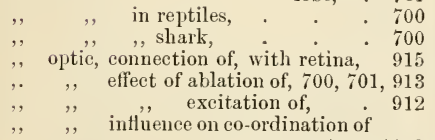
movements, . 1198

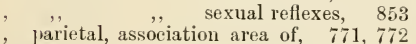
,, ,, degenerations in, after thalamic lesions, 780 eye movements from excitation of, $-756$ relation to intellectual functions, $7 \tau 2$ quadrate, sensation,, 726 temporal, $752,759,761,76 \dot{2}, 770,771$. 775,776 ablation of, . $766,767,781$ connection of, with nuclei pontis, 781 crossed connection with oc-
cipi-
tal lobe, 780 , 781 $,, \quad,, \quad,, \quad,, \begin{gathered}\text { olfac- } \\ \text { tory }\end{gathered}$ tory 781 , , stimulation of, . . 766 Localisation, 832, 833, 834, 987, 999, 1025 Localisation, cerebral. Sec Cerebral cortex.

Longitudinal bundle, $769,771,778,890,893$,

Lost time: Sce Reaction time.

Luciani's groups, .

226

Ludwig, ganglia o!, $\quad . \quad$. $\quad$. 169,175

Lumbo-sacral plexus, variations in, 633,635 , ,, vasomotor fibres of, 669
,

Luminosity curve, 1088, 1091, 1094, 1117

1118

Lungs, . . . . . . 274

afferent nerves of, . . 688,849

,, amount of blood in, . . . 128

, autonomic nerve supply of, 642,663 ,

664, 692

, blood capacity of, . . 155

,, , flow in, 125, 126, 127, 128, 129

, in child, . . . . . 275

, circulation in. See Pulmonary cireulation.

, effect of bronchial constriction on, 309

," elasticity of, . $122,124,275,312$

,. in expiration, . . 274, 276, 279

, inflation of, negative after-effect of, .

, in inspiration, : $274,2 \dot{7} 5,280,281$

, motor nerves of, . . 663,664

,, movements of, . . . . 280

,, pressure in, . . . . 281

,, vasomotor's of, . $151,152,154,642$

,, volume of, . . . . 154

Lyra . . . . . . . 781

Macacus Rhesus, spinal nerve-roots of, 880

Macropsia, . . . 140

Macrosmatic animals . . . $\quad$. 1248

Macula acustica. See Ear.

lutea. See Retinu.

Malaptemurus, electrical organ of. See Eleetrical Oryan.

Malleus. See Ear.

Malonic nitrite, effect of, on nerve-cells, 604

Manmmals, ablation of cerebellum in, 895, 897

,, cerebellum of, . . . 902

, action of vagus in, . . . 215

,., cerebrum in, . $\quad 702$

,, diencephalon of, . 916, 117

,, electromotive phenomena of leart of, . . 447,448 Golgi-organs of, . . 1009

,, pigment-epithelium of, ${ }^{\circ} \quad .1048$

,, pupil-reflex in, . . . 1043

,, $\quad$ retinal cones of, : $\quad . \quad$. 1049

section of medulla of, . . 886

,, sympathetic nerve-cells in, . 655

," visnal purple of, . . . $1040^{\circ}$

Mammary secretion. Sec Milk seeretion.

Manometer, air, . . . . . 15

,, differential, . . . 18

ear, $: 1162$

," flame, $\quad$. $\quad$. $\quad$. $\quad .1218$

, fluid,. $\quad: \quad . \quad 16$

,, maximum and minimum, . 16

," mereurial, . . 15, 78

,,$\quad$ spring, : : $15,16,17,18$

Mantis, reflexes in, . . . . 827

Marginal convolution, . $\quad \cdot \quad 714,764,769$

, zone of Lissaner, : . . $\quad$. 792

,$\quad$ zones of association areas, . 771

Marsupials, cerebellum of, . . . 902

,, motor area of, . . . 732

Massage, effect of, on blood-flow in
muscles,

Mastication, . $\quad . \quad$. $\quad . \quad 313$

," afferent nerves for, pisy of 313 tongue on, . . . 313 
Mastication, effect of palsy of buecina-

$$
\text { tor on, palsy of }
$$$$
\text { tongue on, . } 313
$$

efferent nerves for, . . 314

functions of teeth in, . 313

movements of jaw in, : 313

$$
, \text { tongue in, . } 313
$$

muscles of, . . 313

Meatus, external, of ear, . $\quad 1150,1151$

Mechanism, animal, . . . 228

of circulation, $\quad \cdot \begin{array}{r}1 \\ \quad \cdot \quad \cdot \quad\end{array}$

, closure of bladder,
concerned in taste,
$\quad 1237$

of digestive tract, . . 313

, internal ear, . . . 1164

, joints, . . . 233

,, micturition, . . . 344

, respiration, . . 274

,, urinary tract, . . 338

, visual accommodation.

See Eye.

, voice production, . 1209

Mediate systems. See Spinal cord.

Medulla oblongata,

automatism of nerve

cells of, . . . 888

centres in, . $54,136,887$

chemical stimulation of, 887

conduction paths in, ascending, $\quad 890$

from dorsal

colunins

of cord, $\quad 890$

to motor

area, 890

spino-

bulbar, 890

spino-

cere-

bellar,

$806,807,890$

spino-

niesen-

cephalic, 890

spino-thalamic, 807,890

,. lescending, 888

,, from audi-

tory area, 890

,, , cerebral

hemi-

splieres,

777,808

888,890

, comnected

with re-

tinal im-

pulses, 888

,, from nuclei

of reticu.

lar forma-

tion, 888

,, , nuelei of

vestibn-

lar nerve,

,, , red nu-

cleus, 808 ,

888
Medulla oblongata, conduction paths in

PAGE

\section{descending}

spino-cere-

bellar, 889

, from visual

arca, $\quad 890$

connection of, with

spinal a fferent root

cells, - $\quad \overline{7} 92,793,861$

degenerations in, after

ablation of cortex, 705

electric lobes of, 566,588 eye movements from

stimulation of, . 893 general functions of, 888 inlibitory action of, 199,887

nuclei in, $861,862,885$. 886,887

connection

with mo-

tor area, 778

reflexes of, . 892

root-fibres of, afferent, 884

, from aliment.

ary tract, 884

brachial and

cervical

nerves, 886

., ,, lieart, . 884

, , internal

ear, . 884

respiratory

tract, 884

segmental

nerves

below

brachial, 886

skin, 884,885

, , , skim, 884,885

, efferent, 886

,, ,, to alimien-

tary

canal, 886

heart, 886

," ,, , , lieart,

tory tract, 886

semisection of $\quad 914$ sensitivity of, 978,1000 transection of, 886,887 ,, effect on alimen-

tary tract, $\quad 887$

,, ,, circulation, 886 ,

, ,, muscular co-

ordination, 886

, ,, respiration, 285 , $305,309,886$,

, in frog, . . 886

,, ,, mammal, : 886

Medullary sheath, Medullated nerve fibres, post-ganglionic, 650

of sympathetic, 648 , $650,651,652,685$

Medusa, reflexes in,

Meissner, corpuseles of, ${ }^{\circ} \quad$. $\quad .923$ plexus of, $\quad: \quad \cdot 668,672$ 
Uembrana basilaris, $1179,1180,1181,1185$ $1186,1190,1192,1193$ ,, reticularis, . . . 1181 , tectoria, . $1181,1186,1192$ Membrane, nictitating, $617,619,620,622$, tympanic. See Ear.

656,681

Membranes, fotal, $699,701,703$
$697,698,771$ seat of, $697,698,771$

Menstruation, after section of cord, 85.

Menthol, effect of, on cutaneous sensa-

tion, . . 928, 987

Mesencephalon, 911. Sce also Corpora quadrigemina, Crusta.

ablation of, . . . 912 conduction in, ascending, 915

$$
\begin{aligned}
& , \text {, , from audi- } \\
& \text { tory nuc- } \\
& \text { lei, } \\
& \text {, , , , cerebel- }{ }^{915} \\
& \text {, corpus } \\
& \text { stria- } \\
& \text { tum, } 916 \\
& \text {,, posterior } \\
& \text { longitu- } \\
& \text { dinal } \\
& \text { bundles, }
\end{aligned}
$$
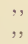$$
\text { , }
$$

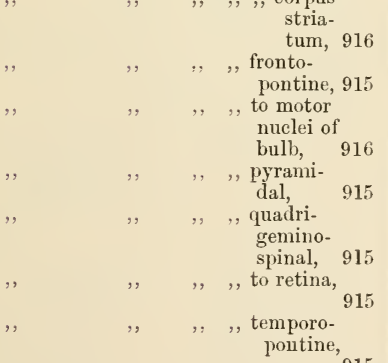

excitation of, in birds, . 912

$$
\begin{array}{ll}
\text {,, dog, } & 912 \\
\text { effect on } & \\
\text { leart, } & 911 \\
\text {,, pupil, } & 912 \\
\text {,, spinal } & \\
\multicolumn{2}{c}{\text { reflexes, }}
\end{array}
$$$$
911,912
$$$$
\text { cye move- }
$$$$
\text { ments from, }
$$$$
\text { in frog, } 911,912
$$$$
\text { ," } \quad \text { ", in frog, } \quad 911
$$

Mesencephalon, excitation of, motor

PAGE

effects, 911, 912

in rabbit, . 912 roof of, 911

vocalisation

effects, . 912

lesions of, decerebrate

rigidity after,

913

,, effect on locomotion,

913,914

reflexes, . 912,

$913,914,915$
$, \quad \quad, \quad$ vision, $\quad 913$

", ", vocalisation, 913

", partial, . . 913 nuclei of, . . 911,915 root-cells of afferent, . 911 ,, , efferent, . 911 root-fibres of, cfferent, . 911 ,, to ciliary ganglion, 911 orbital muscles,

transection of, . . 913

Mesenteric artery, ligation of, . . . 331

, circulation, . . 160

", ganglion, inferior, . 160, 342, $349,617,636,645,646$, $647,651,679,684$

,, $\quad$, superior, $342,642,645$ ", plexus, . 331, 336, 338, 642

Mesentery, vasoniotors of, . . . 659 Metabolism, Hering's theory of, 1114. See also Metabolism in $\mathrm{Vol}$. I.

Metencephalon, 890. See also Pons, Cerebellum. transection of,

effect on nuuscles, 890 ,, reflexes, 890 , respiration, 890 in frog, 890

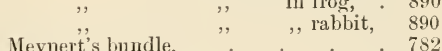
Micropsia, . $\quad . \quad$. 1140 Micturition, 339. See also Bladder, Urethra.

$$
\begin{aligned}
& \begin{array}{l}
, \quad \text { action of abdominal muscles } \\
\text { in,. . . . } 345,346
\end{array}
\end{aligned}
$$

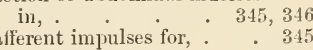

$$
\begin{aligned}
& \text { after section of cord, . . } 340 \text {, } \\
& 345,850 \\
& , \text {, nerves of } \\
& \text { bladder, - } 342 \\
& \text { centre for, . } \quad 345,346,849 \\
& \text { in child, . } 339 \\
& \text { effect of destruction of cord } \\
& \text { on, } \quad \text { S12, 814, } 850 \\
& \text { stimulation of cord } \\
& \text { on, } 346 \\
& \text { crura cerebri } \\
& \text { on, } \quad 346 \\
& \text { medulla ob- } \\
& \text { longata, } 346 \\
& \text { factors in, . . } 34 \% \\
& \text { ", from stimulation of nerves, . } 344 \\
& \begin{array}{r}
, \quad \text { influence of cortex on, } 346,716,717 \\
349,
\end{array}
\end{aligned}
$$


Micturition, mechanism of, . . . 345 reflex, $\quad 339,345,346,869$

Milk" secretion, in spinal animal, 852 . See also Milk secretion in Vol. I.

Mitempfindung,

• • • $\quad 68,90$ ,,$\quad$, nerve,
$,, \quad, \quad$, polarisation in, $541,542,543$
541,542, $543,544,547,549,550$

Moments, . . . . 249, 252, 254 Monkey, cerebellim of, . . 893, 901 cerebral cortex of, . $703,706,733$, $734,735,736,738,744,749$, $752,754,755,757,761,774$ corpora quadrigemina of, . 912 knee-jerk in, . . . . 874 mesencephalon of, . . . 912 muscle spindles of, . . 1008 pyramidal tract of, . 778,809 reflex flexion of hallux in, . 704 spinal cord of, . 812,813 ,, shock in, . $846,847,848$

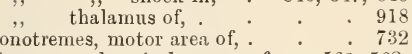

Monotremes, motor area of, $565,569,588$

Morphia, effect of, on muscle tone, . 799

$$
, \quad, \quad, \quad \text {, negative variation }
$$
in nerve, . 536

$,, \quad, \quad$, reaction time, . 611

Motor aphasia, , sense of smell, . 1252

, area. See Cerebral cortex.

," tract. Sec Pyramidal tract.

Mouth, autonomic nerves of,

, motor centres for movements of, . $\quad 736,738,740,748$

, negative jressire in, . . 314

, resonance of, . $1217,1222,1223$, $1225,1230,1233$

, secretoly nerves of, . $618,660,661$

, sense spots of, . . . . 98i

, sensory end-organs of, 922 ,

947. See also Tongue.

,. vasomotors of,

Movements of body, $\quad . \quad+\quad 228,24 \pi, 250$

$$
\begin{array}{r}
, \quad \text { in ruming and } \\
\text { walking, . } 267,268,269
\end{array}
$$

co-ordinaterl, from excitation of cortex, . 738 relation of conscious. ness to, . 1019 , muscular sense to, 1019 in spinal animal, 1019 influence of thalamus on, 618

in a parsthetic limbs, . 1020 of joints. See Joints.

, limbs, . 229, 230, 233 scuse of. See Perception. voluntary, centre for. See Cerebral cortex, motur area. influence of muscular sense on, 1018

Murmurs, hæmic,
Murmurs, vesicular, · . . 281, 311

Muscarin, effect of, on heart, 196, 222, 223,

, , , , , respiration, . 309

Muscle or Muscles, $\quad$. $\quad$. $\quad 352$ abdominal, influence of, on circulation, 44, 52 , $91,92,93,94,127$, $129,141,147$

, respication, . 279

abduetor of crayfish-claw, 480, 513 ability of, to generate tension, 368 , produce shorten-

ing, . . 368 accelerator urinæ, $\quad$. 340,345 action of, on joints, $234,238,241$, 255,256 as machine, . . 241 methods of studying, . 254, 255 principle of lever applied to, 249,251 voltaic currents on, 426 , 435,436 . See also Nuscle, polarisation of.

adductor, of crayfish-claw, 480, 513 ,. , larynx, . . 480 alæ nasi, . . . 280, 284 in apæsthetic limbs, . . 799 ary-epiglottidean, . . 316 arytenoid, $316,1209,1211,1212$ atrophy of, after nerve section, 875 , 876,877

blood-capacity of, . . 155 bronchial, . . . 309, 310 buccinator, . . . 313 bulbo-cavernosis, . 347,348 cardiac, . . . 439 automatic action of, 173 , $176,177,178,179,187$, 188,194 compared with skele-

tal, . . 449 conductivity of, 191, 193, $194,207,209,210,213$, $215,216,217,220$ contractile force of, . 204, 208, 209, 210, 212, 215, 220,223 contraction of, $16 \bar{\tau}, 439$ ,, auriculo-ventrieular panse in, 182 block of, 180,181 , $182,183,187,190$, $191,192,194,207$, $209,217,220,227$ ,. compensatory pause in, . 191 curve of, 39, 440, 442 , effect of asphyxia on, 307,308 , circulating fluid on, 224 heat on, 172 , reflex stimulation of vagus on, 185 , 
Muscle, cardiac, contraction of,

PAGE

cffect of water on,

185,186

fibrillar, . 192

influence of in-

tracardiac pressure

on, $\quad 170,440$

, resistance

on, 241, 244,

246,441

isometric, . 440

isotonic, . 440

rccord of, 39,172

relation to stim-

ulus, $189,449,450$

sequence of, . 171 ,

$180,181,183,184$,

$186,187,188,190$

,, from series of

induction cur-

rents, . . 191

, in Stannius

heart, . 169, 170,

$175,176,178,184$,

187

, from strong te-

tanic currents, 192

wave of, 171,181 ,

$182,183,184,185$, $186,187,190,191$,

$\begin{array}{ll}\text { development of, } & 194 \\ & 177\end{array}$ duration of excitatory

state of, - 444, 445

effect of acids on . 195 alkalies on, 195 ,

antiarine on, 196

223

atropine on, 222 ,

223, 224

delphine on, 224

digitalin on, 196 , 223,224

ligation of

coronary

arteries on, 192

193

muscarine

on, $196,222,223$,

224

nicotine on, 199 ,

201, 202, 221

potassic bro-

mide on, . 193

proteids on, 225,

226

saline solu-

tions on, 225,226

suprarenal

extract on, 193

veratrine on, 196 ,

electrical response of, 443 ,

curve of, 446 ,

dura-

448

tion of, 445
Muscle, cardiac, electrical response of, from

excita-

tion of

cardiac

nerves,

212,218

in in-

jured

ventricle,

latent

period

of, .445

in mammals, 447

mono-

phasic, 449

propa-

gation

of, 474

excitability of $172,191,194$,

204, 210, 213, 215,449

influence of augmentor

nerves on, 197 ,

$198,199,200$,

$216,218,219$

220

vagus on, 197 ,

$198,199,200$,

201, 203, 204,

205, 207, 208,

209, 210, 211,

$212,213,214$,

$215,218,219$,

220

mechanical response of, 439

, , propa-

gation

of, 443 ,

444

metabolic processes of, 189

221

reaction of, to stimuli, 171 ,

$176,188,191,192,193$, 194,449

refractory phase of, 189 , 190,449

relaxation of, $\quad 210,218$, 219,221

rhythm of develop-

ment of, . . 175

rhythmicity of, 169,170 $171,175,176,177,178$,

$179,180,184,187,188$, $190,194,196,208,210$, $215,216,217,220,226$,

stimuli of, normal, 172 ,

173

tension of, $\quad 440,441$

tonicity of, $194,210,213$,

$215,216,218,221,222$

ciliary, $618,660,911,1039$

compressor naris, . . 280 constrictor of pliarynx, 315, 316, 319 ,, pupillæ, . . 911 ,, urethre . . 34s contractile stress of, $363,368,374$, $375,376,379,397$ 
Muscle, contraction of, arrested, 367. See also Musele twiteh. characteristics of, 188 , continuous, fron 189,449 action of chemi cals, 437 , of central origin, $437,711,731$

,, from compression, 438 , , desiccation, 437,438

,, discrimination of varictics of, 437 ,, localised, 384, 391, $392,431,432$

,, from passage of constant cur-

rent, - 425, 437, $510,511,513,516$

, in strychuine poisoning, 425, 437

, tetanic,. - 353

See also Muscle, tetanised.

., from veratrine, 425,428

,, voluntary, 438 $707,708,709$ cortical, 707, 709, 731 738 curve of, 707 $709,710,711$

effect of exhaustion on. See Muscle, cxhaustion. epileptiform, 710, 718, 720,722

force of, $242,243,252$ heat-production

,, relation to in, 394,397 work done, $398,399,400$

idio-nuscular, 384,391 $392,431,432$ influence of doubly refracting particles in,

$$
\begin{array}{r}
\text { tempera- } \\
\text { ture on, } 369, \\
371,373, \\
374,375, \\
376 \\
\text { tension } \\
\text { on, 369, } 370, \\
371,372,374 \\
\text { thalamus } \\
\text { on, } 918
\end{array}
$$

inhibition of, 711,712 ,

$713,739,1012,1019$

irregular, . 393

instantancous. See

Muscle twiteh.

isometric, 353,358 , 362,370 isotonic, $354,358,371$, $372,373,374,376$
Muscle, contraction of, in lesions of cere-

bellum, . 904 maximal, $358,373,375$ myotatic, - . 871 paradoxical, . 548 period of, . 363,376 , of latency of, $381,711,749$, 750,751 relaxation of, 363,376

peristaltic. See Peristalsis.

Pflüger's law of, 503, $508,513,515,557$

prolonged. See supra, Contraetion, eontinuous.

rapidity of,

reaction time of.

See Reaction-time.

reflex, - 830, 831

relation of work to load, . 399, 400 rhythm of, in shivering, . 708 secondary excitation in, 363,364 , 372

stair-case phenomenon in, . 381 theory of, 354,363 , $368,371,372,376$, $379,394,395,397$, 402

in Thomsen's disease, 731

transformation of chemical energy in, unstriped mus.

cle, $\quad 439$ voluntary, 707,708 ,

wave of, $383,415,435$ , propaga-

tion rate of, 383,384 $385,388,435$ slow, 384 contracture of, after lesions, of cortex, . 711, 731, 734 corrugator supercilii, . . 911 of Crampton, . . . 1040 ,, crayfish-claw, . . 480, 513 crico-arytenoid, 280, 316, 1209, 1211 .

$$
\text { currents, . . . . } 407
$$

,, of action, 407,414 . Sce also Iuscle, electrieal response.

, injury,

,, rest,

in parallel-fibred muscle, 408, 410 oblique-fibred nuscle, 408, 410 secondary, . . 426 cylinder doctrine of, . $\quad . \quad$. 411 death of, . $\quad . \quad 390$ 
PAGE

Muscle, in decerebrate rigidity, . . 914 detrusor urinæ, $\quad 339,717,849,850$ digastric, . .313 dilatator naris, . . . 280 distensibility of arteries of, $\quad 158$ effects of apæsthesia on, 1018, 1020 , destruction of cord on, 813 , 816 lesions of cerebellum on, $895,896,897,898,899$ section of afferent nerve roots oll, 798,799 $801,802,803,870$ $876^{\circ}$ motor nerves on, $798,799,800$ elastic equilibrium of, . . 360 elasticity of, $355,360,362,363,376$, 390 electrical properties of, - 407, 413 ,, ,, in excited state, 413 See also Muscle, Electrical response. influence of arrangement of fibres on, 408, 410 relation to functional capability, 412,413

, duration of,

415,416 , 417,421

, , to inclirect continuous excitation,

, in injured muscle, 416, 420, 422, 423, 424

,, to instantaneousstimuli, 413, 414 ,, monophasic,

, rate of pro416,420 pagation of, 414,415 , 420,435

Muscle, electrical response of,

PAGE

,
,
,
,
,

equilibiun ,", wave of, . 415 mechanical response to, 379 ,

, , in strych nine spasms, 425, , summation of, $\quad 423$

, in tetanus, 422 ,

,, , curve of,

, time relations of, 379 ,

$$
414,420
$$

, in veratrinised muscle, 425 equilibrium leugth of, excitatory, 360 , $374,377,378$ , influence of exlraustion on, 385,386 , 388 377,378

relation to tension, 360, 361,362 , 385

erector clitoridis, penis, excitation of, - 348 347,348 $352,363,375$ cliemical effects of, 394 , $400,403,406$ by currents of different directions, 434 direct, 353, 383, 389, electrical effects. See Ifuscle, clectrical response.

indirect, $353,377,383$, 389,390

by induction eurrents, $353,438,439$ isometric, 353. Sce also Muscle twitch. isotonic, 354. Sec also ifuscle twitch. inhibitory, . . $3 \pi 1$ 
PAGE

Muscle, excitation of, maximal, 358, 373, 375 mechanical effects.

See Muscle, me-

chanical response.

period of latency of, $381,711,749,750$,

polar. See Muscle, polar excitation. secondary, 363,364 , 372 , muscular, $436,437,438$ neuro-mus cular, 436 , 437,438

by series of instan-

taneous stimuli, 353

single instan-

taneous stimulus, 358

staircase pheno-

menon in, . 381

submaximal, . 375

, influence on length $\lambda, 375$

, summation of, iuflu-

summated, iuflu-
ence on length $\lambda, 375$

in tetanus, 377,378 , 379

thermal effects of.

See Muscle, ther-

mal response.

by voltaic currents, 431 , $432,434,435,436$,

wave of, $383,415,435$, 436,438

excitatory process of, $352,360,363$, $364,372,375$ $376,378,379$ $402,403,412$,

, contractile

component of, 363, 364,

,, influence of mechanical conditions on, . 363 neural tonus

on, . 870

tempera-

ture on, 369 , $371,373,374$, 375,376 , tension on, $363,364,369$, $370,371,372$, 374

under iso-

metric con-

ditions, 363

under iso-

tonic con-

ditions, 363

oxidation

in, . 406
Muscle, excitatory process of, relaxation

$P A G E$

component

of, 363,364 ,

376

,, toxic pro-

ducts of, 389

,, transforma-

tions of

chemical

energy in, 393

exhaustion of, . . . 385,389

causes of, . 389

effect of, on re-

sponse to

single ex-

citation, 388

, , on rigor

mortis, 392

393

,, , thermo-

genetic

processes, 400

,, , volun-

tary con-

traction, 708

,, , working power, . 400

daring polarisation, . 430

in different kinds of effort, 406

excitatory equili-

brium length

in, $\quad 385,386,388$

extensibility in, 385

indirect excita-

bility in, . 389

in man, . . 387

rate of, . 386,387

relation of acid

reaction to, . 389

tension to length

in, . 385

,', to work done, 387
extensibility of, $, \quad 241,368$

in after - loading contraction, . 368

,, arrested con-

traction, . 368

,, exhaustion, . 385 ,

389,390

', ,' curve of, 357,404

$356,357,385,404,405$

facial,

. 726

frontalis, $\quad$. $\quad$. $\quad$. 891,911

geniohyoid, . . . . 313

genioglossus, . . . 313, 314

of heart. Sec Muscle, cardiac.

heat-rigor of, . . . . 390 hyoglossus, . . . . 313

influence of thalamus on, $\quad 918$

intereostal, . $\quad 277,278,279$

ischio-cavernosus, . . 347,348

latent excitation of, $381,711,749$, 750,751

length of. See Muscle, equilibrium length of.

in isotonic contraction,

$358,373,374$ 
PAGE

Muscle, length of normal, . $\quad 357,377,869$ ,$\quad$ relation to excitatory
state, . $372,375,376$ length $\Lambda$ of, . . $377,378,385$ , $\quad \lambda$ of, . $\quad 373,374,375,378$ levator ani, : . $\quad 336,337,345$ ,, palati, . . 315,1162 levatores costarum, . . 277 of limbs, spinal nerve-supply to, masseter, . 795, 797, 803 mechanical response of. Sé Muscle, contraction.

mylohyoid, $155,313,315,317,319$, myotatic contraction of, . $\quad 871$ nerves of, $\quad 639,1007,1019$ neural tonus of, . $\quad 870,1020$ orbicularis palpebrarum, . 891, 911 oxidation processes in, $379,394,403$ conditions affectiug, 406 relation to work done, 399,400

palato-glossus, . $313,314,315$ papillary, -pharyngeus . $7,8,10,23$ paralysis of, in cord lesions, . 794 pectoralis, . . . 279 pharyngeal, : . $\quad 315,316,319$ of pinna, . 1152 platysma, . . . 736,741 polar excitation of, . 428, 431 ,, anodicopening, 436 , ,, clos. ", ing, 434 ,, cathodic, . 431 ", localisation of effects of, 431 polarisation of, . . 426,427 anodic a $\mathrm{fter}$ effect, . 428,431 cathodic aftereffect, . 428, 431 currents of, . 426 ,, ,, opposed, 426 , 427 ,, positive, 427 , 428 dipliasic effects of, 428 eflect of, on conductivity . 431 ,, tendinous inseriptions on, 429 excit a tor $y$ efficets of, 428,431 , $432,434,435$

fatigue effects of, . . influence of, on excitability, 429,430
Muscle, polarisation of, influence of

PAGE strength and dura$t$ i o $n$ of current on, 426 , 428 polar effects of, 428,
$429,431,433,436$ $429,431,433,436$
.$\quad 428$ preparation, double adductor, : 353 isometric, . 353,358 ", isotonic, - 353, 358

pterygoid, . . . 313 reaction of degeneration of, 439,876 to resistance, . 361,362 recto-coccygeus, . . 667,693 reflexes, . . . 1011, 1012 ,, influence of, on arterial pressure, 1011 1012 heart, 1012

, $10 \mathrm{ca} 1$ musculature, . 1019 ,, respiration, 1012, 1013

refractory period of, . 449 relation of, to efferent root-cells, 793 ,

,, between thermogenetic and inngenetic processes of, . 398 relaxation of, . . $363,369,376$ excitatory, - 377,378 ,, ,, influence of tem-

$$
\begin{array}{r}
\text { perature on, } 369, \\
371,376 \\
\text {, , , tension on, } 369,
\end{array}
$$
$370,371,372$

of respiration. See Respiration. retractor penis, $348,349,635,636$,

rhombus,

rigor mortis of, . . $\quad 390$

$$
\begin{aligned}
& \text {, ,, cause of shorten- } \\
& \text { ing in, . 390, } 391 \\
& \text {, cessation of, . } 392 \\
& \text {, curve of, . . } 392 \\
& \text {, in } \mathrm{d} i \mathrm{ff} \text { e } \mathrm{r} \text { e } \mathrm{nt} \\
& \text { muscles, . } \\
& \text {, influence of ex. } \\
& \text { haustion on, } 392 \text {, } \\
& 393 \\
& , \quad, \quad, \quad, \text { nervous } \\
& \text { system on, } 391 \\
& 799,870,1020 \\
& \text {,, ,, ,, putrefaction } \\
& \text { on, . . } 392 \\
& \text {, ,, ,, semicircular } \\
& \text { canals on, . } 1204 \\
& \text {, nature of, . } 393 \\
& \text {, relation to exci- } \\
& \text { tation, 390, } 391 \\
& 393 \\
& \text {,, ,, ,, idio - nuscu- } \\
& 1 \text { a r co ll- } \\
& \text { traction, 391, } 392 \\
& \text { sacro-lumbalis, . . . . } 279
\end{aligned}
$$

scalenus, 
Muscle, sensation from. See Muscular sense.

sensitivity of, . $971,989,996,1011$ sensory end-organs of, 1007, 1008,

, nerves of, . . . 1007

serratus magius,

, posticus, . . 277,279

shatter of, ․ . . 372

shortening of, during contraction, 244 ,

skeletal, efferent root-cells for, . 793 sympathetic nerves of, 639

," . 1191

sphincter. See Sphincter, Bladder,

Rectum.

in spinal animal, . . 817,825 spindle, $990,1007,1008,1009,1010$ stapedius, . . 1157,1162 sterno-hyoid, . . . . 279 , -mastoid, . . . 279 , -thyroid. . . . 279 stimuli of. See Muscle, excitation.

styloglossus, . . . 313,315 in tabes dorsalis, $\quad$. $\quad$. 799 temporal, . . 313

tension of, . . . . $\quad . \quad 363$ curve of, . . 367,370 graphic record of changes in, . . 364 influence of, on contraction, 369 , $370,371,372$, on relaxa. tion, 369,370 371,372 relation to resistance, 362 , 363 thermal response, 400 , 401,406

secondary excitation by . . 363, 364, 372 in tetanic contraction, 355 , twitch, 360, 361, 362, 363 tensor palati, . . . . 1162 tympani. See Ear. tetanus of,

353,378 curve of, $\quad 379,385,386$ decremental effect in, . 423 in epileptilorm spasms, 718 ,

equilibrium length in. See Muscle, equilibrium length.

extension curve of, . 404 fatigue in, . . 385,386 genesis of, . . . 378 hicat production in, 401, 402 isonetric, . . . 353 isotonic, . $\quad 354$ Pitter's, . . 437, 510 stummation of excitation effects in, 378,379 ', of stimuli in, 377,378
work in, $\quad 354,355,357$ with diminishing load, $355,356,403$
Muscle, tetanus of, work in, with increas-

ing load, 404

, , after previous ex-

tension, 357,358

work-diagram of, 357,405

thermal response of, . 399, 401

effect of ex-

haustion on, 400

estimation of,

in isometric 398,399

conditions, 400 , 401,406

in isotonic

conditions, 401

relation of,

to exter-

nal condi-

tions, 403,

$404,405,406$

, frequen-

cy of stimulation, 402, 403

., , inogene-

tic processes, 398

,, ,, strength of sti-

mulus, 400,401

,, , tension, $400,401,406$

,, ,, work done, 400 $401,404,406$ in tetanus, 401, 402

thermal section of, . . . 411 thyro-arytenoid, 316, 1209, 1210,

1212,1213

,, -cricoid, . 1209, 1211, 1212 ,, -hyoid, . . . 280, 316 tollus of, . . . . . 377 afferent nerve impulses

for, . . . 1020 in apresthetic limb, $\quad .1020$ ,, ccrebellar lesions, . 903 ,, crayfish muscle, . $37 \%$ effect of anresthetics on, 870 excitatory diminution

of, . $\quad 377,378$ in heart of Helix, $\quad 377$ influence of afferent impulses on, 726 central nervous system oll, . . 377 on co-ordination of movement, . 1020 muscular sense on, . 870 semicircular canals on, . 1204

neural, - $800,869,870$ reflex, $799,800,909,910$ , inhibition of, . 1019 ", formation of chemical into

mechanical energy in, . . 393 
PAGE

Muscle, tremors of, . . 711, 897, 903 trophic effects of nerve section

in, . . . . . 876

twitch, . . . . $\quad 358,384$ curve of, after-loading, $36 \tau$,

,, arrested con-

traction of, 368, 369,

373,374

,. isometric, 362,365 ,

368,370

sotonic, 358,359 ,

$365,368,373,374$,

$375,376,388,800$

duration of, $383,384,388$

effect of after-loading

on, $\quad$ - 366, 379

arrest of con-

traction on, 367 ,

$369,370,371$,

372,373

exhaustion on, 388

gradual in -

crease of

tension ou, 370

371

jerk on, 370,371 .

sudden in-

crease of

tension on, 367 ,

$369,370,371,372$

unloading

during,

varying me-

clianical

conditions, 363,

366

energy-curve of, . 362,367 equilibrium length of.

See Muscle, equili-

brium length of.

excitatory process in, 360 , $363,372,376$

inertia curve of, . 361,370 influence of tempera-

tiure on, $373,374,375,376$

, increasing load on, 359 , $367,368,369,371$

isometric, $358,362,370,401$

isotonic, $358,371,373,374$

under isometric and iso-

tonic conditions successively, .

366

,, isotonic and iso-

metric conditions sue-

cessively, $363,369,371$,

jerk-curve of, . . 371

period of contraction in, 363

relaxation in, 363

relation of load to lift

in, . . . 359

, of tension to length

in, . . . 361, 362

release curve of, . . 374

with supported load, . 379

in unloaded muscle, 373,360

work of, . $\quad 359$

,, -diagram of, $\quad 359$
Muscle, unstriped, contraction of, $\quad \begin{array}{r}\text { PAGE } \\ 439\end{array}$

efferent root-cells for, 796

influence of cortex on, 716

vasoniotor reflexes from, 1011, 1012

vasomotors of, . - $155,160,640$

of Wilson,

work of, 245 . See also iruscle

tetanus, Muscle twitch.

zygomatic, centre for, . . 741

Muscular sense, . $\quad . \quad$. 1002

," ,, adaptation in, . . 1024

,, afferent nerves for, 1006 ,

1007,1019

a taxia from loss of, 1018,1020

conduction of, in cord,

effect of cutaneous anæs-

thesia oll, 1006, 1011

,, lesions of thal. amus on,

, loss of, on willed movements, . 1018

estimation of, 1014, 1022 ,

1023

in hemiplegia, . . 731

influence of, on muscle

tonus, . . . 870

liminal difference for, 1004 ,

1022

nature of, $1002,1005,1006$

paralysis from loss of, . 1021

perceptions, 1013

of a ctive

movement, 1013,1014

erroneous judgments from, 1017 . $1018,1023,102$

fusions of, with other

sensations, 1024

liminal stim.

nli for, 1014 , $1015,1016,1021$

of passive movement, 1013,1014

,, posture, 1013

,, resistance, 1013,1021

summation of stimuli

for, . 1017

peripheral end-organs for, 1007,1008 , $1009,1010,1011$

,, neural apparatus

of, $\quad 1002,1006$

reflexes of, $826,827,832$ relation of cerebellum to, 910

cere br $\mathrm{m}$ m

to, $\quad 749,769$

to co-ordina-

tion of move-

ment, . 1019

, local sign, 1025

semicircular

canals to, 1204

to space percep-

tion, 1134, 1138 
Muscular sense, relation of, to voluntary

PAGF movement, . . 722, 724, 729, 731

Musculo-articular sense organs, influence

of, on reflexes,

Mustard, • • 844

Mrelencephalon, $: 884$

Myelination in cerebellum, . . . 903 of centripetal fibres of cortex, . . 768,769 ,, collateral fibres of spinal grey matter, . . . 811 cortical fibres, . . 916 in flocculus, . . . 903 of funiculus gracilis, . $\quad$. 792 in inexcitable regions of cortex, . 770 of nerve-fibres, . . . 792 , olfactory tract, . . 764

,, optic nerve, . . . 758

, pyramidal tract, . 809

," stems of afferent rootcells,

in temporal

Myocardiograph,

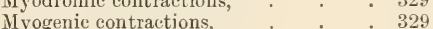
Myograph, . . . . . 364, 432 Myopia. See Eye.

Myotatic contraction, . . . . 871 Myotonograph, . . $\quad 365$ Myxine, nerve roots of, . . . 790 Myxomycetes, geotropism of, . $\quad 828$

NASO-PHARynX, closure of, in deglutition, 315

Necrosis of grey matter of cord, - $\quad 835,836$

Negative variation. See Nerve, electrical response, Muscle, electrical response.

Nereis, cephalic ganglion of,

Nerve or Nerves, , accelerator, : . $53,55,56,58$,

$\cdot \quad 890$
$\cdot \quad \dot{4}$
531
$198,199,200,642,649$, $663,665,686,693$ of adductor muscle of Anodon, . 221 , of adductor muscle of A nodon,

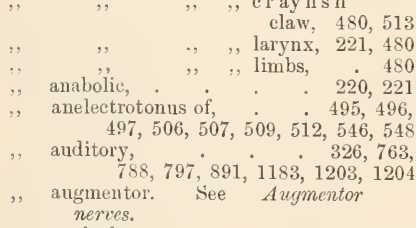

,, auricnlar, . . 141, 624, 656, 669

, aurieulo-temporal, . . . 141

,, autonomic. See Autonomic.

axis cylinder of, . $\quad 452,456,479$, $521,567,592,601$

of brenchial muscles, . $\quad 309$ ealorific, . . . . $\quad 619$ ratelectrotonus of, $\quad 49 \dot{5}, 49 \dot{7}, 506$,

cells. See Nerve cells. $546,548,550$

cervical, third, vasomotors of, . 669 eliorda tympani, . 132, 134, 141 , $326,626,664,672,673,674$, $677,886,1238,1239$
PAGE

Nerve, ciliary, . $623,660,677,682,1037$ , $\mathrm{CO}_{2}$ production in, . . 454,540 ," cochlear, $\quad 763,781,884,1167,1184$,

1185,1205

colonic, . . . . 646 conductivity of, $\quad 451,452,459,486$ in afferent nerves,

conditions affecting, . 458,477 in different nerves, 480,482 $456,605,785,798$ effect of anrsthetics on, . 491 ,, arrest of circulation on, 477 ,

,, hases on, . 466 ,, $\mathrm{CO}_{2}$ on, 491, 492

., desiccation on, 464,488

,, electrical currents on, 493, 499 504

, Hertz - waves oll, . . 462 , polarisation on, 493, 499, 504

See also Nerve polarisation. ,, salts on, 466, 487, ,, severance from nerve cells on, 478,479

influence of intensity of stimu-

lus on,. 458 ,, temperature

on, 455,484

in medullated nerves, 482, 486 ,, nerve cells, - 483 ,, non - medul-

lated nerves, 482 rate of, $455,457,482$, $484,486,487$, 501,530

in terminal ar-

borisations, . 482

coronary, 174, 181, 203, 205, 211,

of crayfish claw, $221,480,512,513$ crossing, . . . . 797 crural, . . $\quad 154,640,656$ currents of action, 524. See also Nerve, electrical response.

demarcation. See Nerve, currents of rest.

electrotonic. See Nerve, electrotonic currents.

of rest, in afferent and efferent nerves, 521 in differentnerves 520 experimental demonstration of, 519 
PAGE

Nerve currents of rest, influence of arrest of circulation on,

, connection

with nerve

cell on,

, electrotonus

on, . 546

, on excitability,

,, on polar excitation, .

,, previous ex-

citation on,

, site of section

on,

,, temperature

on, . .

methods of pro-

ducing,

in non-medul-

lated nerves,

degeneration of, $479,522,547,601$,

depressor, . . . . 59

dorsal, of penis, . . . $\quad .636$

effects of constant current on, . 493

See also Nerve polarisation.

efferent visceral system of, . . 197

of electrical organ, . 481, 482, 564, $565,566,567$

electrical resistance of, . . 520 response of, . 459,524

, , in afferent nerves, 525,925

,, after-effects of, 531, 537

, , conditions affecting,

527,533

., curve of,

$529,530,532$,

534,552

, diphasic effects of, . 531,

532,533

,, discontinuous

character of, 527,528

duration of

527,528

,, electrolytic character of, 546

E. M.F. of, . . 546

, influence of alkaloids

on, . . . 536

,, anæsthetics on, . 534

", , $\mathrm{CO}_{2}$ on, . 535, 537,

539,540

,, ,, demarcation difference on, . 527

, ,, electrotonus on, - 550

,, , intensity of stimulus on, . 533

,2, previous activity

on, . $536,538,540$

, salts on, . . 536

, , temperature on, . 534

, in medullated nerve, 528

, nature of,

, in non-medullated

nerve, $526,527,532,554$ , prolonged, 537, 556, 557

, propagation-time of, 530 ,

,, from reflex stimula.

534,791

tion,
Nerve, electrical response of, relation of, to excitatory state, 525,526

relation of, to strength

of stimulus, . $\quad 469,931$

,, to repeated stimula-

tion, . . 528

, to single stimulus, . 529

, in stale nerves, 538,540

, from stimulation of

sensory end-organs,

525,925

, with two surface

contacts, 531

electrolysis in, $458,540,544,557$

electromotive changes in, 518

See also

Nerve,

currents of rest.

,, excitatory,

453, 524

See also

Nerve,

electrical

response.

,, secondary, 556

elcetrotonic currents of,

influence of anæs-

thetics on, 552 ,

553

, character of nerve on, 554

condition of

nerve on, 547

excitation

on, 547,550

intensity of

current

on, . 547

, polarisability

on, $\quad 547$

, proximity

of poles, 547

on resting

currents, 546 ,

547

, separation of

poles, . 547

,, temperature, 555

in non-medullated

nerves,

polar development

of, : $\quad 547$

propagation of, $\quad 547$

time relations of, . 547 electrotonus of,

electromotive

changes of, . 546

influence of, on

currents of

rest, . 546,547

,, on electrical

response, . 550

physiological

conditions on, 550

endings, excitatory time of, 483,496

,, scnsory. Sce Sensation.

excitability of, $451,452,458,459,486$

conditions affeet-

ing, . 466,477 
Nerve, excitability of, in crayfish, . 480,481 , different animals, 481 , nerves, 480, 481 effect of anæsthetics on . 491 , arrest of eireulation on, 477,478 , bases on, . 466 .. $\mathrm{CO}_{2}$ on, 491, 492 , desiccation on, 464,488 , eleetrical currents on, $494,498,504$ nerve injury on, 499,652 , 665 ,, salts on, 466,488 , 489,490

severance

from nerve cell on. 478,479 temperature on, . 484 in flexor and ex-

tensor nerves, 480 local, $\quad 488,489,492$ measurement of, 459 polarisation

changes in, 494, 498,

Ritter-Valli law of, . . . 477 of vaco-sympathe-

tic of frog, . 481

excitation,

y acicts

bases, : : 466

chemical agents, 459 $464,465,466$, $473,482,485$ condenser dis-

charges, . 485, 506 , desicuation,. 464 effect of, on polarisa-

tioll, . 550,551 efficieney of, conditions affecting, 468 , $469,470,471,47 \%$ $473,503,505,557$ estimation of 468 electrical, $459,462,46 \%$ l,y galvanic currents, 462 , $169,470,472,503,506$ 510

, induced eurrents, 459 , $460,463,471,485$ 506,507

influence of, on electro-

tonus, 550

latent, $\quad 483,496,510$

law of, 469,470 mechanical, $459,467,472$, 473,482 , 485,927

by neutral salts, 464,466 ,, organie substances, 466
Nerve excitation, polar. See Nerve,

PAGE

polar cxeitation.

relation of electrical

response to, 469 , to polarisation, 557

thermal, . - 459,473

by undulatory eur-

rents, . 461,485

unipolar, . . . 463

excitatory state of, $451,452,458,487$ , chemical

changes of, 453

, effect of temperature on, 455 , 470

, electromotive changes of, 453

, nature of, 557,55

1) benomena of, $453,525,526$ , propagation of, $452,455,457$, $458,482,484$ $486,487,490$, $499,501,504$

relation to electrical response, 525, 526 thermal changes during, 453,454

facial, $660,688,789,878,884,885,886$, $891,900,911,1157,1239$

fibrils, $596,597,598$

aflerent, excitability of', . 790 ,$\quad$ autonomic, 664,667 . 685,686

elfect of section of, ou nerve

cells 600 efferent excitability of, : 790 retino-motor, . 1050 size of, . . 1006, 1007 specific energy of, 694,1106 . 1243,1253 sympathetic, $648,650,651,685$ visceral. . 686,688 fifth, $54,141,320,326,662,726,759$ $788,793,797,810,875,877$, $885,891,892,1021,1157$. $1238,1239,1240,1247$

fourth, . . $789,797,911,915$ frigorific, . . . . 619 function of, . . . . 451 senito-anal, . . . . $636^{\circ}$ glośso-pharyngeal, $1+1,305,320,321$, $326,624,660,664$ $688,793,884,886$ 1240

of leart, 54, 59, 166, 197, 203, 642, 663 See Heart, nerves of.

hypoglossal, 134, 141, 320,624, 636, $646,64 \overline{7}, 649,664,679$, $687,797,884$ impulses. See Impulses.

indefatigability of, $454,473,536,598$ inhibitory, $672,673,674$

,, of eardiac splincter, . 695

,, ,, crayfish claw, . 513

,, ,, heart, 54, 203, 663, 664, 665,693 
PAGE

Nerve, inhibitory, of intestines, 663, 694, 695,

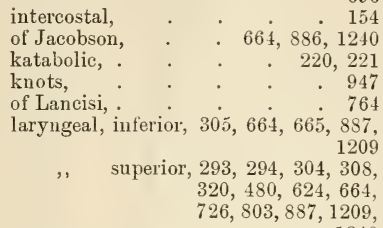

, eathodic, 504, 506

,, eause of, . 507
Nerve, polar excitation of, closing, $\quad$ PAGE

$$
\begin{aligned}
& , \text {, , ,, in crayfish, . } 513 \\
& , \text { by descendiug } \\
& \text { current, } 472,503 \text {, } \\
& 507 \\
& \text {, induced cur- } \\
& \text { reuts, . 506, } 507 \\
& \text {, influence of cur- } \\
& \text { rent inten- } \\
& \text { sity on, . } 503 \\
& \text { duration of } \\
& \text { current oll, } 505 \text {, } \\
& 506 \\
& \text {, , nerve length } \\
& \text { on, . 505, } 506 \\
& \text { number of } \\
& \text { poles, . } 506 \\
& \text {,, , polar scpar- } \\
& \text { ation, . } 505 \\
& \text {,, ,, polarisation } \\
& \text { intensity, } 505 \\
& \text {.. in man, . } 515 \\
& \text { opening } 503,504 \\
& \text {, intluence of } \\
& \text { demarca- } \\
& \text { tion cur- } \\
& \text { rent on, } 508 \\
& \text {,2 ,, ,, polarisa- } \\
& \text { tion cur- } \\
& \text { rent on, } \quad 509
\end{aligned}
$$

polarisation, 493. See also Nerve, electrotonus of, electrotonic currents of. after-effects of, 537,543 , 545,556

currents of, $543,545,556$ conductivity ehanges

in, . . 493, 499 by demarcation eur-

rent, . . . 499 effects of, $540,543,545$

during cur-

rent flow, 545

electrolytic, 540,

545

excitation

on, 550,551

idio-polar, 544 polar, 494, 499, $501,502,503$, $540,546,556$

excitability changes

during, - 494, 498 external, : 493,541 Hering's model of, 541,543 Hermann's model of, 541,542

influence of duration of current, 49.5, $497,498,500$, $502,505,506$ intensity of eurrent, 495, 500, 502, 505, 
Nerve, polarisation, influence of nerve

PAGE length, - 495, 505, internal, 493, 494, 540, $541,542,543$,

in non-medullated nerve, . 499, 543 , positive, . 537,556 relation of, to excita-

tion. . 557 time relations of electrotonic effects, 496 , 497,549 ,, extrapolar effects, 496,497 by transverse currents,

post-cellular, ,, -ganglionic. See Sympathetic nerve fillres.

pre-cellular,

pre-ganglionic. See Sympathetic nerve fibres.

pudic,

$348,349,636$ pupillo-constrictor, . . 660,662 ,, -dilator. See Sympathetic nerve fibres.

reaction of, chemical, . . 454 refractory period of, . . . 476 regeneration of, $167,479,602,603$, 652,804

of retractor penis, . . . 221 root-fibres, 787,793 . See also Nerve roots.

afferent, bulbar, 884,885 ,

886

cranial, 788, 789, $790,791,792$, 793,810

of fifth

nerve, . 793

glosso

pharyngeal, 793

,, vagus, . 793

spinal, 787,788 ,

$789,790,791$

, ascend.

ing

stems of, 792

, , collaterals

of, 792,794

806,811

,, degenera

tion of, 646 ,

647,648

, effects of

anæmia

on. 836

,, ,, excita-

tion

of, . 880

, , section

of, 726 ,

799,801 ,

802,869 ,

870,871 ,

876,877

914
Nerve root-fibres, afferent spinal, effects of excita-

bility

of, $\quad 790$

, number

of, 790,791

path of, to

bulbar

vaso-

motor

centre, 855

, regenera-

tion of, 804

, relation

to effer-

ent, . 797

,, vaso-

motor

direct, 670

, visceral, 849

efferent,. . 793,794

in dorsal roots, 789,790

effects of excitation of . 880

section

of afferent

roots on, 799

excitability of, 790

number of

fibres of, 790,791

relation to

efferent, 797,820

vasomotor

direct, . 670

visceral, . 202

See also Autonomic nerves.

roots, cranial . . . . 788

dorsal spinal, . . . 788

, of chick, . 789,790

,, degeneration

from section of, 790

, effect of section on cord,

,, on effer-

ent cells, 800,803

,, on moti-

lity, 801,

802,803

,, , rigor

mortis, 799

,2 stimula-

tion of

sciatic

nerve

on, . 803

motor fibres of,

789,790

of Myxine, - 790

,Petromyzon, 790

,, , Pristiurus, . 790

, effect of regen-

eration of, 804

vaso-dilator

fibres of, 790

visceral fibres

of, . $\quad 840$ 
PAGE

Nerve roots, spinal,

88,794 ,, of Amphioxus, . 789, 790 ,, ,, birds, , discovery of functions of, . 786, 789 , of fish, . $788,789,793$ ,, ,, frog, . 789, 790, 791 ,, number of fibres in, 791 , relation of afferent to efferent, . 797,820 , rootlets of, . . 794 ,, ventral, . . 797

, , effect of section of afferent roots on, . 799 on arterial pressure, 869 ," kneejerk, 871 , pupil, 869 , rigor mortis, 799,870

,, , nerve cells of, . 789 , 797 shaker, - 467 sciatic, $58,134,136,137,156,158,161$, $167,480,626,652,656,665$, $670,799,803$

scrotal, 636 secretory. See Sympathetic nerve fibres, Autonomic nerve fibres. sensory, effect of section of, on movements, , , nerve cells,

negative variation in, from excitation of sensory organs, sixth, . . . 789, 797 spinal, connection of, witl sympathetic. See Sympathetic.

roots of. See Nerve roots. rootlets of, . . . table of excitation effects of,
variations in root origins of,

of, . . . 633 vasomotor fibres in, . 668 to limb muscles, 795, 797, 803 accessory, $320,663,797,886$ splanchnic, 50, 58, 160, 161, 164, 324, $331,333,335,338,642$, $644,647,648,649,687$, $688,695,971$

inferior, $645,646,648,649$, $680,687,688$ of spleen, . . . . . 163,663 , stoniach. See Stomach.

stimuli. See Nerve excitation. sympathetic. See sympathetic.

thermal response of, . . 454 third, $660,664,669,789,797,911$, of toad, Nerve, trophic, . . . . $\quad \begin{array}{r}\text { PAGE } \\ 876\end{array}$ union of, after section, $457,458,480$ ,, of ureter, . . . . 338 ,. vagus, action of, on heart, $53,54,57,58$, 203, 204, 205, 206, 207, 208, $209,210,211,212,213,214$, $215,219,220,221,481,856$ afferent root-tibres of, 793,849 , 854,856 after-action of, . 219,220 autonomic fibres of. See Autonomic nerves.

bronchial fibres of, $\quad 309,310$ centre of, . $\quad 54,885,886$ constituent fibres of, 200,649 , 788 degenerative section of, 663,665 depressor fibres of, . . 59 effect of atropine on, . 222 nicotine on, 199, 202 efferent root-cells of, . 886 experimental union with sympathetic . 691 fibres of, to stomach, 323,324 , ganglia of. See Ganglia. $326,335,887$ influence of, on arterial pressire, $53,56,57,152$ , intestines, 161 , $331,334,335$ , pancreas, 164, , respiration, 288 , 292, 296, 301, 302, $303,304,320$

intracranial stimulation of, . . 204, 205 referred pain through, . 689 regeneration of, . . 665 trophic effects of section

$$
\text { of, . . . 875, } 877
$$

vasomotor. See Vasomotor nerves.

vestibular, $884,888,907,909,1205$ vidian,

Nerve cell or cells, . 1239 . . . 592, 784 afferent, . . . 572, 784 automatism of, . . 888 axon of, . . . 592 bineural, . . . . 593 bipolar, . . . $\quad 593$ centrosome of, . . . 594 clianges in, on excitation, 599 , 600,605 eliromatin of, $\quad 594,599,600$ chromatolysis in, $599,600,601$, of Clarke's column, atrophy 603,604

$$
\text { of, }
$$

classification of, . . 592 conduction by, $483,605,607,613$, $680,682,791,798$ of cortex, duration of activity

$$
\text { rate of discharge }
$$$$
\text { from, } \quad 708,710
$$
refractory period

$$
\text { of, . . . } 709
$$

on course of pre-ganglionic

fibres, 
Nerve cell or cclls, distributing 592 dynanical polarisation of, . 798 effect of anrmia on, - 604,836 drings on, . 604,607 section of sensory fibre on, 601, 603, 604 motor fibre on, 600,603 ,, toxines on, . . 604 efferent. . 592, 784, 793 electrical, . $\quad 565,566,567,588$ of enteric system, . . 668 fibrils of, $592,593,596,597,598$ , degeneration of, 601 , 602,603

\section{, effect of excitation} on, . . . 605 regeneration of, . 602 ,

of ganglia of cranial nerves, 664 influence of nervous impulses on, . $6 \pi \hat{r}$ ,' temperature on, 604 local ganglionic, $\quad \cdot \quad \cdot \quad 572$ mediate of cord, $805,806,810$ of motor cortex, $722,723,777$ neuron of, . . . 592, 593 neurone theory of, - 593,594 Nissl bodies of, . . 599,600 nucleolus of, . $\quad$. 594 nucleus of, . 594, 600, 603 number of, involved in reflexes, $609,610,611,612$ perikaryon of, . . 594, 604 relation to

nerve im-

pulses, 604, 791 , nutrition, 604 ," $\quad$, reflex impulses, 604 of pelvic gauglia, . . 668 , peripheral autonomic

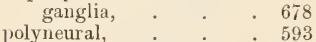
processes of, 592, 593, 594, 596, $599,605,607,655,687,798$ protoplasm of, . . 594, 599 refractory period of, . . 614 regeneration of, $600,603,692$, $693,878,879$ sensory type of, . . . 687 spinal, 655

,, effcet of compression of aorta on, 836 strychnine

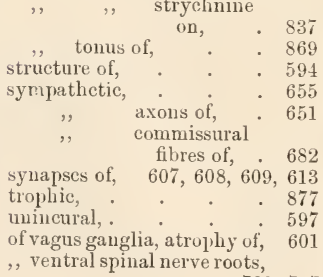

PAGE

Nervi erigentes, $160,626,637,658,666,673$. See also Nerve, pelvic.

Nervous system, autonomic. See Autonomic.

central, . $697,783,884$ levels of, 697,698

," central, levels of, $697,698,88$
,,$\quad$ sympathetic. See Sym-
pathetic.
visceral.

Neural tonus of muscle, $868,869,870,872$

Neuraxon, . . . . . . 592

Neurite, . . . . . . 592

Neuritis, anæstlesia in, . . . 988

Neuron, . . . . . 592,593

Neurone theory, . $\quad . \quad 593,594,597,598$

Nicotin, effect of, on cardiac nerves, 221,223 ,

,. :, ", efferent ganglia, , $\begin{aligned} & 224 \\ & 201\end{aligned}$

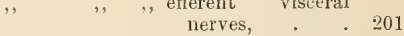

,, hepatic circulation, 161

,, intestine, . . 329

, nerve cells, . 202, 607

", semilunar ganglia,. 160

sense of smell, . 1252

,, spinal ganglia, 791

,, sympathetic,, 621, 622

$\begin{array}{llll}, & \text {," } & \text {, sympathetic }, & 621,622 \\ , & \text {, vagus nerve, . } & 199,202\end{array}$

Nictitating membrane, $617,619,620,622$,

656,681

Night blindness, . . $\quad 1095,1102,1105$

Nissl bodies, . . . . 599, 600

Nitrate of sodium, diuretic action of, . 160

Nitrous oxide, effect of, on nerve, . 492

Nodal point, visual, . . . . 1029

Noud vital, . . . . . . . . 285

Non-polarisable electrodes, . . . 409

Normal-Stellung, . . . . 258, 260

Nose, 1246. See also Sensation, olfactory.

,, olfactory cells of, . . .1247, 1249

, mucous membrane of, . 1246

", $\quad$, nerves of, 1247, 1248, 1252

," ", region of, . . . 1246

," , sensitiveness of, 1249,1250 ,

1251,1252

passage of air through, . 1248 resonance of, . $1234,1235,1236$ turbinal bones of, . $\quad 1248$ vasomotors of, . $\quad 618,623,662$

Nostril, contraction of, . . . 618

Notch, dicrotic, . . . 20, 37

Nucleus or Nuclei, ambiguus, . $\quad . \quad 886^{\circ}$ auditory, $763,781,885$ of Burdach, . . 886 caudatus, $\quad 78,917$ commissurales, . 885 cuneatus, . 793,806 of Deiters, . 807

,, facial uerve, 885,886

, fourth ,, . . 911

", Goll, . . . 885

habenulæ, : 782

of hypoglossal nerve,

885,889

lenticularis, . $\quad 917$

Luysii, : . 917

of medulla oblongata, 778 , $861,862,885,886,887$ , mesencephalon,

911,915 
PAGE of pons, $778,779,781$,

$891,893,907$
,

,

,

, red, $705,808,888,893$, sensory, of fifth
nerve,. of thalamns, $\quad 769,770$, 778,780 third nerve, . 911 ,, trapezium,. . 763 , vagus, 54, 292, 885, 886

Nyctalopia, $1095,1102,1105$

Nystagmus, 895, 898, 902, 1089, 1102, 1205

Occipital Lobe. See Lobe.

Octopus, nerves of, Odours, 1252 . $1254,125 \pi$. See also $482,530,532$

Sensation, olfactory.

Esophagns, action of, in deglutition, 316, 317 centre for movements of, . 887 contraction of parts of, . 318 muscle fibres of, . 317,318 nerves of, $320,335,663,664,665$ pressure in, . . . 317 reflex inhibition of, . . 318

Olfactie, , stimmlation of, . 320,321

Olfactometry, : $1249,1250,1251,1257^{\circ}$

Olfactory area. See Cercbral cortcx.

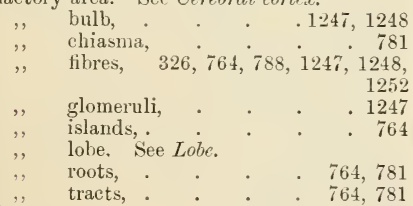

Olive,. . . $763,781,890,893$

Oncograph, . . . . 162

Oncometer, . . . . . . . 162

Ophiurus, autotomy in, . . . 845

Ophthalmometer, . . $1026,1027,1028$

Opisthotonos from cerebellar lesions, 897,898

Optic lobes. See Lobe.

, nerve. See Nerve.

,, thalamus. See Thalamus.

,, tract, connection with corpora geniculata, . 758,780

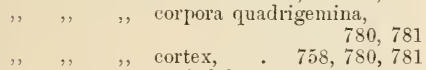

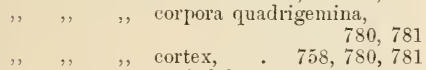

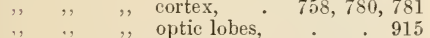
, , degeneration of, after excision of eye, section of, . . . $\quad .780$

Op'tical axis. See Eye.

Optogram, . . . . . . 1045

Orang, motor area of, . . . . 745

Orbital gland, 660,661

Organs of lateral line, : $\quad . \quad . \quad 1167,1168$ Orthoform, effect of, on seusation, 928,987 Os uteri during parturition, . . 350,351 Osmatic animals, . . . . 763 Ossicles, auditory. See Ear. Otic ganglion, Otoliths,

$1166,1167,1205$
Otter, vena cava of,

Owl, ablation of cortex in, . . . 701

, connection between retina and optic lobe in,

,, eye of, .

1040

\section{Pacinian Corpuscles,}

$686,924,1007$, $1008,1009,1010$

Pain centre, 997

,, conduction-path of. See Sensation of pain.

, nerves, $988,989,990,994,996$

, referred,

," ", skin areas of, . $689,690,691$

," in visceral disease, . 686,688 , 689,690 sensation of. See Sensation. spots, 921, 923, 984, 985, 987, $988,989,995,1000$

Palate, gustatory function of, . . 1237 Pallium,

Palpebral fissure, .

$619,656,681$

Panereas, autonomic nerve-supply of, . 692 ,, centre for secretion of, . . 887 secretory nerves of, . . 663 See also Vol. 1. vagus fibres to, vasomotors of,

$164,644,645$ 1167,1168

Papilla acustica,

Papillæ, gustatory. See Tonfue.

Papillary muscles, . . . $\quad 7,10,23$

Paradoxical contraction, . . . 548

Parasthenia, $\quad 903$

Paratonia, . . . .903

Parelectronomy, . . . . . . 423

Parietal lobe. See Lobe.

Parieto - occipito - temporal association area,

Parturition, 349. See also Uterus.

,, action of abdominal museles

$$
\begin{aligned}
& \text { in, . . . . . } 351 \\
& \text { cause of onset of, . } \quad 350 \\
& \text {," centre, . . 351, 851, } 852 \\
& \text {," effect of lesions of cord on, } 351 \text {, } \\
& 812,851,852 \\
& \text { influence of foetus on, } 350,351 \\
& \begin{array}{lll}
, & \text { influence of foetus on, } & 350, \\
, & \text { nervous mechanism of, } \quad . \quad 351
\end{array} \\
& \text {,, stages of, . . . } 351 \\
& \text { ", uterine contractions in, } 350,351
\end{aligned}
$$

Peduncles of cerebellum. See Cerebel-

$$
\text { lum. }
$$

corpus albicans, .

782

$$
\text { , callosum, }
$$

Peduncnlo-maxillary fasciculus,

Pelvic nerve. See Nerve.

$$
\text { plexus, } 636,646,647,651,667,668
$$

Pendulum-movements, $\quad 269,328,329,330$

Penis, erection of, 347 . See also Eicction.

ligature of veins of,

347

". nerves of, $160,348,636,659,667$ retractor muscle of, $635,636,667,674$

P'ereeption of breadth, . 1133, 1137, 1138 distance, absolute, 1133, 1134. $1136,113 i$ intluence of accommodation on, 1135 , 1137,1138 
Perception of distance, influence of bin-

\author{
PAGE
}

ocular vision

on, 1133 ,

1134,1137

conver-

$\mathrm{g}$ e n e e

on, 1135 ,

1137,1138

dispara-

tion oll, 1137,

1138

eye-move-
ments
on, 1133,
1134,1135,
1136,1137
,,$\quad$ head-move-

", ments

on, $\quad 1136$

. $\quad$, museular ${ }^{1138}$

$\mathrm{s}$ e n s e

on, 1134

, $\quad$ retinal 1138

rivalry

on, . 1138

sensation

of inner-

vation

oll, 1134

sensitive-

ness of

ret in a

on, 1138 ,

1139

psychological fac-

tors in, 1136, 1137 relation of, to ideas

of size, . . 1136

relative, 1133,1136

1137,1138

retinal factors in, 1134

theory of, . . 1136

of form, . 1139

, height, 1133, 1137, 1138

, movement, 1146, 1167, 1168 acceleration

of 1200,1201 active, 1013, 1016 passive, 1013 ,

position of

head, 1196, 1198,

$1199,1201,1202$

posture, 1013,

rotatory, $\quad \begin{array}{r}1014 \\ 1200 \text {, }\end{array}$ 1201,1202

, resistance, . 1013, 1021

size,

space, . 934, 936, 938, 942,

$1013,1025,1122$,

$1132,1163,1164,1195$

in animals, 1148

centre for, . . 760

illusions of, 942 ,

$1140,1141,1147$

influence of mus-

cular sense on, . 1164
Perception of space, physiological fac-

PAGE tors in, . 1122, 1132

point of reference

for, . $\quad .1133,1135$

psychological factors in, . . 1136

relation to seni-

circular canals, 1202 ,

1203

theories of, . . 1136

ikaryon, 594, 784, 787, 788, 791, 792, 810 See also Nerve-cell.

Perilymph, . . . . . 1164, 1179

Periosteum, sensory nerves of, . . 1006

Peristalsis, . . $171,180,183,329$, 330,853

Perpendicular bundle, . . . . 771

Persistence of visual sensation, 1067, 1068, $1069,1074,1076$,

Petromyzon, spinal nerves of, . . 790

Petrous ganglion, . . . . 793

Pflïger's law of contraction, . 503, 508, 513,

515,557
821,823

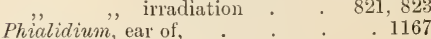

Phonation. See Voùce.

Plionantograph, . . . . . 1224

Phonogram, . 1224, 1228

Phonograph, . $\quad$. $\quad 1216,1225,1229$

Phosphenes, . . . . . 1097

Photo-resthetic elements of retina, 1121

Plioto-chromatic interval. See Sensaition, isisual.

Photo-hæmatochometer, . . . 145

Photometry, colour, . . . $\quad .1076$

flicker, . . . 1076

Photophobia, 967, 1089, 1096, 1102, 1105

Phrenograph, . . . . . 283

Physiological point, visual, $\quad .1055,1086$

Picric acid, yellow vision from, . . 1095

Piezometer,. . . . . 63

Pigeon, cerebellumi of, . . . 894,898

cerebral cortex of, $700,701,704,754$

,. muscle spindles of, . . . 1008

optic lobes of, . . . 700,701

, semicircular canals of, . 1199

spinal cord of, $814,827,834,878,879$

Pigment, retinal. See Retina.

Pillars of fauces, in deglutition, . . 315 gustatory functions of, 1237

Pilocarpine, effect of, on retina, . . 1103

Pilomotor nerves. See Sympathetic nerves.

Pinna, . . 1149, 1163

," reflex twitch of, $\quad . \quad$. 892

retraction of, . . 618

Pitot's tubes, $\quad . \quad . \quad . \quad . \quad 56$

Placenta, . . . 351

Plane of fixation, visual, $\quad . \quad . \quad 1129$

Planes of body, . . 228, 229, 230, 231

Plate, electric, . . . . . 563

Plateau of eardiogram, . . . . $\quad 37$

, ventricular pressure-curve, 18, 19 ,

Plethora, effects of, on circulation, . $\quad 77$

Plethysmograph, . . . 156, 162

Pleurosthotonos, . . . $\quad 895,896$

Plexus of Auerbach, . $\quad 327,329,668,672$

,, brachial, . . $156,633,669,670$

celiac, $156,638,669$

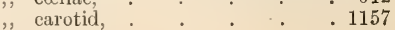


Plexus, hypogastric

2,344 ,

348,349

$\begin{array}{ll}, & \text { lumbo-sacral, . } \\ ,, & \text { mesenteric }\end{array} \quad 331,336,338,642$

, nesophageal,

", pelvic, $636,646,647,651,667,668$

,, post-fixed, . . . . 795

,, pre-fixed, . . . . . 795

,, pulmonary, . . . 320

,, renal, . . . 642

,, solar, . . . 331, 334, 342

,, spermatic, . . . . 338

". . . 1157,1240

Plexuses of limbs, significance of, 820

Plume-organs, . . . . 924, 1008

Pneumatograph, . . . . . . 282

Pneumograph, . . . . . 282

Points, cardinal, of eye, $\quad 1209$

, corresponding, of retina, 757,1124 ,

,, nodal, of eye, . . . . 1029

, principal, of eye, . . 1029

Poiseuille, law of,

1029
64

Polar excitation of sensory organs, 516,517

Polarisation of electrical organ, . . 584

, muscle. See Muscle.

", nerve. See Nerve.

, nerve-cells, dynamical, . 798

Polarising currents, heterodromous, . 585

," homodromous, : 585

Polyneural cells, . . . . . . 593

Pons, . . . . 891

,, afferent root fibres of, . . . . 891

,, , , , to auditory organ,

," , , , mucous mem-

, muscle sense

organs, . 891

, skin of head, 891

,, spatial sense

organs, $\quad 891$

centres in, ,, . . . 891, 893 conduction paths in, ascending, 893

$$
\begin{aligned}
& \text {,, , , , from auditory } \\
& \text { end-nuclei, } 893 \\
& \text {, to corpus } \\
& \text { striatum, } 893 \\
& \text {, from dorsal } \\
& \text { column } \\
& \text { nuclei, } 893 \\
& \text { spino-cerebel- } \\
& \text { lar, . } 893 \\
& \text {, spino-mesen- } \\
& \text { cephalic, } 893 \\
& \text {, descending, } 893 \\
& \text {,, , , from corpora } \\
& \text { quadrige- } \\
& \text { mina, . } 893 \\
& \text {, , from fronto- } \\
& \text { pontine, } 779 \text {, } \\
& 893 \\
& ,, \text {, in posterior } \\
& \text { longitudinal } \\
& \text { bundles, } 893 \\
& \text {, , , pyramidal, } 777 \\
& \text {,, ,, from red } \\
& 778,893 \\
& \text { nucleus, } 893
\end{aligned}
$$

reticular

formation, 893

, temporo-pon-

tine,

893

degeneration in, after ablation of

cortex, . . . . 705

, efferent root-fibres of, . . 891

, , , , , for eye move-

ments, . 891

,, facial expres-

sion,

,, movements

of moutl, $\quad 891$

,, nuuscles of

mastication, 891

, functions of, . . . . . 891

," nuclei of, . . . 891, 893, 907

,, reflexes of, . . . . 891,892

transection of, . 893

Porencephaly, . . . . . 839

Portal vein, ligation of, . $\quad 45$ vasomotors of, . $140,161,643$

Position of body,

$$
\text { , }{ }^{\prime} \text { erect body, · } \quad \cdot \quad 231,260
$$

Post-cellular fibresormal, . 231, 258, 260

,, -fixed plexuses, $\quad . \quad \cdot \quad \cdot \quad \cdot \quad \cdot \quad 795$

,, ganglionic fibres. See Sympathetic

nerve fibres.

Posture, perception of,

1013,1014

,, reflexes,

Potassium salts, effect of, on muscle, 193, 225 ,

$$
\text { , , , , nerve, 465, 489, }
$$

490,536

Pre-cellular fibres, . . . . 623

Pre-commissural area, . . . . . 764

Pre-dicrotic wave, . . . . 111

Pre-fixed plexuses, . . . . 795

Pre-frontal region of cortex, $738,770,772,773$,

$777,779,780$

Pre-ganglionic fibres. See Sympathetic

nerve fibres.

Pregnancy,

Pressure, abdominal, influence of, on circulation, . 44, 52, 91, 92, $93,127,129,141,147$ arterial. See Arterial pressure.

capillary. See Capillaries.

of circulation, mean hydrokinetic, 70,71 ,, liydrostatic, 70,71

intracardiac, . . 14, 40

$\begin{array}{cc}, & \text { auricular, } 22,51,150 \\ , & , \quad \text { curve of, } 22\end{array}$

," $\quad$,, influence

of valves

on, 9

maximal, 24 negative,

23,119

diastolic, $41,50,51$

52,72 ,

75,77

influence of, on

heart-beat, 56 58,170 
Pressure, intracardiac, influence of, isometric contraction of heart, 440 methods of recording, ventricular, is,

, curve of, 18, 20 , during

diastole, 25 , , , rising tension, 19, 36,38 ,, , systolic output,

20,22 ,. , maximal, 22 , , , negative, 25 intracranial, $143,145,146,309$ intrapulmonary, $125,126,127$, 128,281 intrathoracic, $44,47,94,120$
$122,124,127$ amount of, . 124 in child, . 124 during respira-

tion, 275,281 .

influence on 307,312 arterial pressure, 122 , $124,125,127$,

, heart, 44, 45,

, intrathor-

acic vessels, 125

,, pulse curve, of respination on, 124,127

,, ,, systole on, 47 ,

intraocular. measurement of, 124

intravesical, $340,341,342,345$, 346

lateral, in tubes, negative auricular, $63,66,67,98$ in heart 23,119 ,, ,, mouth,

$$
\text { ," ,, thorax, }
$$
ventricular,

- $23,25,44$

. 314

$44,275,281$

$25,27,28$ asoj'hageal, .

in veins. See lenous pressure.

Pressure curve. See Curve.

$$
\begin{array}{ll}
, & \text { gange, cerebral, } \\
,, & \text { gradient, } \\
,, & \text { head of, }
\end{array}
$$

Prevertubral ganglia. See Sympathetic ganglia.

\section{Priapism,}

Principal points, visual,

Pristiurus, nerve-roots of,

Processes of nerve-cell. cells.

Projection areas,
$771,772,773$
$941,942,957,958$ $972,973,976,1258$

Pronation, . . . 238, 240

Prostate, nerves of, . . . . $\quad$. 849

Proteids, action of, on heart, . 225, 226

Pseudo-reflexes, . . . 680,681

Psychical powers of spinal cord, . . 857

Psycho-notor centres, . . . . 724

Pterotrachea, ear of, . 1167

Pulmonary artery. See Artery.

, circulation. See Circulation.

plexus,

pressure. See Pressure, intrapulmonary.

Pulse, . compressibility of, 99 contraction of, . . . 99 103

,. analysis of, . . . 108

," from different arteries, . 112

, effect of obstructed circulation on, . . . 107 factors determining form of,

99,112 features of, . . 103,112 influence of arterial pressure

on, $103,104,112$ thoracicpressure

oll, . . 106 vence comites

on, $104,107,112$

, radial, . . 104 , respiratory undulations of, 107 dicrotism of. See Pulse wave.

effect of epilepsy on, . . . 722 frequency of, . . . 99, 100

in animals, . . 101 average, . . 101 eonditions affecting, 100 , relation of, to diastole, . . 39 variations of, 100,101

fulness of as index of vasomotor tone, . 95 radial, $10:$ ,, influence of venæ comites on, . . . 103, 104 regularity of, $\quad$. $\quad$. $\quad . \quad 99$ size of, . . 99 in smaller arteries, . . . 97 speed of, . . . . 99 strength of, $\quad . \quad$. $\quad .99$ venous, . . 114,122 wave,. $. \quad .96,104,108$ ,. anacrotic. . . . 108, 111 ," conditions affecting, . 110, 111 ,, dierotic, $86,96,108,110,111$ ,. katacrotic, . . 108, 112 ,, length of, . . . . 103

, negative, : $\quad .98$ , predicrotic, . . . 111

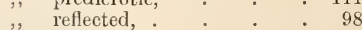
," velocity of : : 102, 103 Pulvinar," . . . $780,916,917$ Pupil, 1042. See also Iris.

,, during accommodation, 1042, 1043 eontraction of, centre for, . . 1043 
PAGE

Pupil, contraction of, consensual, . 1043

\begin{tabular}{|c|c|c|}
\hline , & ," & direct, . . 1043 \\
\hline , & , & in excised eye, \\
\hline , & , & $\begin{array}{l}\text { influence of adap- } \\
\text { tation on, } 1043,1044\end{array}$ \\
\hline ," & , & , age on, : 1043 \\
\hline ", & , & $\begin{array}{l}\text { ", stimulus inten- } \\
\text { sity on,. }\end{array}$ \\
\hline , & , & mechanisn of 1043 \\
\hline " & , & $\begin{array}{l}\text { novements associ- } \\
\text { ated with, } \quad .1043, \\
1131\end{array}$ \\
\hline ," & ," & $\begin{array}{r}\text { nerves for, } \quad 660,662, \\
1044\end{array}$ \\
\hline ,. & , & reflex, $812,913, \begin{array}{r}1043, \\
1044\end{array}$ \\
\hline , & ", & $\begin{array}{l}\text { from retinal sti- } \\
\text { mulation, } 1042,1043\end{array}$ \\
\hline ;, & ", & $\begin{array}{c}\text {, stimulation } \\
\text { floeculus, }\end{array}$ \\
\hline
\end{tabular}

, dilatation of, $617,619, € 21,623,656$, $658,659,662,681,912$, $917,1044,1131$ nerves for, 617,619 , $620,621,652,656,658$, $659,662,1044$

eccentricity of, - 1114

", * * 1114

, , lesions of thalamus on, $\quad 917$

,. $\quad, \quad$ section of spinal afferent

roots on, . . 869

semisection of cord on,. 869 stimulation of cerebral

cortex on,

15,756 optic lobes oll, $\quad 912$ sclerotic

oll, . 1044

mcasurement of, . $\quad .1042$

size of . . 1042, 1043

,, physiological, . . . 1043

,, relation to adaptation, 1056 ,

1057

Pupillometry,

1042

Purkinje, phenomenon of, 1078, 1080, 1087 , $1099,1100,1102,1104,1111$

Purple, visual. See Retina.

Pylorus. See Stomaeh.

Pyramidal tract, 776, 777,808 . See also Pyramids.

in animals, $809,888,889$ collaterals of, $777,806,810$,

811

connection with Clarke's

column, . 806,810 degeneration of, 705,805 ,

direct, 809

fibres of, from corpus striatum, . 778 lateral, : 777,778 in guinea-pig, . . 889 inhibitory functions of, 839 in medulla, . 888, 889 „, mesencephalon, . 915 myelination of, 707,809 in pons, . . 893 ,, spinal cord, $725,808,809$ ventral, . 778,808
Pyramids, 777, 888, 889. See also

PAGF: Pyramidal tract. decussation of,

, excitation of,

$777,808,889$

847,889

Pyrosis, section of

778,889

QUADRATE LOBULE, quadrigemina.

Quadrigemino-spinal mediate system, . 808 Quills, erection of, . . . 618,625

RABBIT, cerebellum of, .

, cerebral cortex of, $\quad \begin{array}{r}702,732,736, \\ 738,756,759,\end{array}$

, cerebral cortex of, $\quad \begin{array}{r}702,732,736, \\ 738,756,759,\end{array}$ 761

cochlea of, . . . . 1186 corpora quadrigemina of,. $\quad 912,916$ metencephalon of, . . 890 muscle spindles of, . . . 1008 papillæ foliatæ of, . $\quad 1237,1238$ pyramidal tract of, . . 809 spinal cord of, . . 835,836 sympathetic nerves to ear in, . 624 thalamus of, . . . 702

Rabies toxine, effect of, on nerve cells, . 604 Radial artery. See Artery. pulse. See Pulse.

Radio-ulnar joints

239,240

Radius, pronation of, . . . 238,240

Rami, grey. See Grey rami.

,, white. See White rami.

Rarefied air, respiration of, . . . 128

Rat, motor area of, . . . . 732

,, muscle spindles of, . . . 1008

Ray, electrical organs of, $561,562,563,564$,

$569,575,590$

, movements of, after decapitation, . 817

Reaction of degeneration, . . 439,876 time, . . 608, 610,611

,, conditions affecting, . 611

,, for gustatory sense, 1241,1245

,, ,, muscular contraction,

inhibition, 711,713

,

711,713

, reduced, . $609,612,711$

, reflex, . $\quad 609,711,712$

,, for tactual sense, . . 945

, , thermal sense, . . 962

,, total, . . . 611

Reciprocal innervation, . 842, 1013, 1019

Recording apparatus of Hurthle, . . 78

Rectum, 336. See also Defacation.

, autonomic nerve-supply of, 646,667 , 679,692

effect of excitation of cord on, . 336 inhibitory nerves of, . . 337 motor nerves of, - . 336, 337 spinal afferent root tibres of, - 849 sphineters of, 336, 337 . See also Sphincter ani.

sympathetic nerves of,

Recurrent sensitivity,

Red uucleus. See Nucleus.

Reduced eye, . . . . 1026, 1029 Refcrred pain, $686,688,689,690,691,849$, Reflex action, 783. See also Reflexes. 
Reflex action, afferent chaunels of, 787,788 789, 790. See also Nerve routs, afferent.

efferent ehannels of, 793,797

,. historical account of, . 786

,, relation of afferent to efferent channels, . . 797 arc, . $\quad 785,786,804,837,860$ centres, . . 787 ,, bulbar, . . . . 887 , pontine, . . 891, 892 , spinal, $137,811,817,819,849$, $850,851,852,853,854,855$ contraction of muscle, . 830,831 , influence of the muscic on, 814 , location of stimulus on,

, skin - region on, $\quad 816$ ,, stimulns on,

$816,825,828$

Reflexes, 783 . See also Reflex action.

auditory,

. 1162

from autonomic ganglia, 678,679

, bone,

1012

," blood capillaries : . 856

bulbar,

$54,57,58,856$

cardiac,

cataleptoid, . . . . 913

cerebral, excito-motor fibres of, 769

in collaterals, . . . 793

conjunctival, . . . . 947

of corpus striatum, . . . 778

cortical inhibition of, . 712,713

erenasteric, . 815

in decerebrate rigidity, . 914, 915

effect of section of mesencephalon

on,

. 890

of electrical organs, . . 588

in enteric nervous system, 696

of erection, . . . 852,854

"hallux,

", hind-limb of frog, . . 814

influence of mesencephalon on,

$912,913,914,915$

from joint surfaces,

latent period for, in spinal animal, 818 muscle,

1011,1012 affecting local musculature, effect on arterial pres-

sure, . 1011, 1012 heart, . 1012, 1013 respiration, 1012 ,

1013

optical,

754,781

from pain sensation, $974,975,977,993$ pontine,. . 891,892 pupil, rectal,

$7 \dot{85}, 8 \dot{10} 0,851$

reduced,

reduced,

609

root,

1012,1013

root, . . . 827

of secretion, . . . . $\quad .679$

from semicircular canals, 1198 sexual, . . 851, 852 spinal, $783,804,811,819,844,849,856$ , affecting blood vessels, . 854
Reflcxes, spinal, after-contraction in, $\quad 831$ alternating character of, 820 , $830,841,844$ bilateral, . $822,823,842$ crossed, . 821, 822, 823 co-ordination in, 813,817 , $842,843,844$

facilitation of, $837,838,841$.

fatality of, . . . 834

fatigue in . $\quad .831$

from fragments of cord, 811 , $813,814,815,816,844$ general conditions affecting,

845

in hemiplegia, . . 731

influence of anæmia on, 836 ,

apucea on, $\quad 837$

,, arrest of circula-

tion on, 835,862

,, asphyxia on, . 836

, association centres

on, . 773

associated parts

of cerebro-spinal

centres on, . 837

, bulb on, 838,839 cerebral cortex on, 838

, condition of cen-

tral organs on, 835

corpora quadri-

gemina on, 838,839

, drugs on, . 837

, excitation of cortex on, . 841 mesenceph-

" $\quad$ mesenceph-
alon on, 911,
912

, $\quad$ alon on, 911,
912

,, mid-brain on, 838 ,

,, muscular sense on, other parts of cord

844

on, $\quad 840$

shock on, $846,847,848$ starvation on, 845 strychuine on, 829 , 830,843

,, summation of stimuli on, 828 inhibition of, $814,815,837$, 842

irradiation of, $819,820,821$ local sign in, $832,833,834$ long, $819,821,822,823,824$ phasic variation in, 820,830 ,$$
\begin{aligned}
& 841,844 \\
& \text { postural, } \quad . \quad 827,844
\end{aligned}
$$

purposive, $832,856,857,858$ relation of, to conscious-

ness, . $\quad 859,860$ rules of, : $820,821,832$ after section of cord, 731 , $811,813,814$

See also Spinal animal. sense-organs for, . . 844 short, . . 819,820 significance of, . $\quad 856$ 
,, , staircase phenomenon in, 841 from stimulation of

limbs, 823,840 , pinna, 823,824 ,. synergic effects of, 820,812 , uniformity of 813 ,. variations in, $\quad . \quad 821$ ," vasomotor, $13 \dot{7}, 85 \dot{4}, 855$, $856,80^{\circ} 3,861$ ,, visceral, . . 848,849 of 'sting of bee, . $\quad . \quad 812$ tendon, . $\quad . \quad$. 871,1011 time of, influence of cord level

on, . 841 ganglia ou, . 791 mesencephalon on, 911,912 prefrontal cortex on, . 773 stimulus

torticollis, on, . 829 on, $609,612,711$

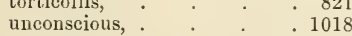
uterine, : $\quad . \quad: \quad .851$ vasomotor, bullar, . . 855 spinal, $816,82 \dot{7}, 854$, $855,856,863,864$ from muscle, 1011,1012 vesical, . . . $785,849,850$ visceral,. $\quad . \quad 816,827,849$

Reflexus ultimus,.

. 892

Refraction, visual. See Eye.

Refractory period of electrical organ, . 614 ,, ,, muscle, . . . 449 ," $\quad, \quad$ nerve, $\quad$, -cell, $614,615,709$ Regeneration of nerve-cells,. . 600,603 , 878,879 , fibres, 479, 602, 603, $652,654,665,804$ spinal cord, .

Registers, vocal. See Voice.

Regurgitation in heart,

Reil, island of,

Reissuer, membrane of,

Reizwelle,

Remak, ganglia of,

Renal circulation,

,, ganglia,

Reptiles, cerebellum of,

$$
\begin{aligned}
& \text { - } 11,12,32 \\
& 770,776 \\
& \text {. } 1179 \\
& 415,435 \\
& 169,170,171,173 \\
& \text { - } 159 \\
& 642,645 \\
& \text { - } 642 \\
& \text {. } 902
\end{aligned}
$$$$
\text { corpora quadrigemina of, . } 911
$$$$
\text { Golgi organs of, . . . } 1009
$$$$
\text { inhibition in, . . . } 215
$$$$
\text { olfactory area of, : : } \quad 764
$$$$
\text { lobes of, . . } 700
$$

Residual blood in heart, . . $7,10,53$

Resilience of arteries, $\quad$. . $\quad \begin{array}{r}128,308 \\ 74\end{array}$

Resistance, perieption of, . . 1013, 1021

Respiration, 274. See also Inspiration,

$$
\text { Expiration. }
$$

Respiration, acessory movements of

279 action of intercostals in, . 277 ,

278,279

288,292 , $295,302,304$ apnœic, . $289,298,299,300$, 301,305 artificial, effect of, on circu-

lation, . . 126,129 asphyxial. See Asphyxia.

automatic regulation of, 295, 306 automatism of centre for, . 289 centre for, $284,285,286,287$, $288,291,292,309,321,887$ Cheyne-Stokes,. . 139, 309 of compressed air, . . 128 curve of, . . . 282, 296 diaphragm in, . $275,276,277$, $279,282,283,296$ diaphragmatic, influence on circulation, . $127,129,149$ dyspnoic, . 289, 290, 307, 312 frequency of, 281, 290, 291, 293, $294,298,303,304,305,307$, influence of afferent im713,714 pulses on, 288, 289, 291, 292, 303,304 amount of $\mathrm{CO}_{2}$ on, 289,290 , O on, 289, 290, 291,292

on arterial pressure, $93,122,123$. 127,129

, blood content of lungs, 128,129 blood-state on, 289 , 291, 292 striction on, 309, 311

cerebral anæmia on, . 309

on cerebral circulation, 143,147 , circulation, 44, $58,91,93,122$, $125,126,127,129$ collapse of lungs on, 295, 298, 303 on curve of limb volume, ,, diastolic fill. ing of lieart, 44 , $125,127,129$

frequency of heart, 58, 123, 129 epilepsy on, . 722 excitation of allterior commissure

$$
\text { on, . }
$$
cortex on, 713,714 glossopharyngeal nerve, 305 ,

VOL. II. $-8_{3}$ 
Respiration, influence of excitation of

PAGE

laryngeal

nerves, 293

294,304

305,308

,, sensory

nerves, 305

inflation of lungs on, $295,298,299$

intracranial pres-

sure on,

on intrapulmon-

ary pressure, 125

, intrathoracic

pressure,

portal circu-

lation,

$$
124
$$

systolic out-

$\begin{array}{ll}\text { put, } & 129 \\ \text { musearin on, } & 309\end{array}$

nuusele reflexes

on, .1012, 1013

negative venti-

lation of lungs

on, . 300

reducing sub-

stances on, . 290

section of cord

on, . 284,286

nedulla ob-

longata on, 285 , $287,305,309$, $886,88 \pi$

, metenceph-

alon on, . 890

vagi on, 288,293 .

$294,295,296,297$

sex on,

temperature of

medulla ob-

longata on, . 291 on vagus centre, 129 inhibitory action of vagu

on, venous flow, 121 laboured, . $\quad 276,279,280$ lungs in, See Lungs.

modified movements of, . 308 movements of ale nasi in, 280 $\begin{array}{ll},, & \text { larynx in, } 279,304 \\ ,, & \text { vocal cords }\end{array}$ in, . . 280

nervous mechanism of, . 283 yseudo-respiratory move-

ments of, of rarefied air, . 128 record of movements of, . 282 relation of, to deglutition, 321 rhythm of, 281, 288, 291, $292,294,307,309$ rima glottidis in, 280, 308, 309 in sleep, . 309 sounds of, . . . 281 of swallowing, . $\quad 314,319,321$ thorax in. See Thorax. types of. $\quad 280$

Respiratory bundle, : $\quad . \quad$ : $\quad$. 285 reflexes, . 1012,1013 tract, root cells for, aftierent, 881
Respiratory tract, root cells for, eflerent, 886 undulations of arterial pressure curve, 92, 107, 123, 128

Restiformal spino-cercbellar mediate sys-

tem, . . . 806,890

Retching, . . . . . . 324

Reticular formation, . . . . 883, 893

Retina, 1045. See also Sensation, visual.

$$
\text { action of stains on, - 1050, } 1103
$$
adaptation of, 1056, 1057, 1067, 1083 , $1096,1097,1101,1103$, 1105 binocular, . 1098 for brightness, . 1057 ,, colour, . 1057 influence of, on acuity, 1086

,, foveal vision, 1087

,, light sensitivity, 1083 , recurrent local, 1057,1099
in Purkinje's phenomenoll, . 1080 relation of size of

pupil to, 1056,1057 theory of, 1109,1114 , 1120

blind area of, . . . 517, 1088 cerebral, : . $\quad 758$ changesin, duringaccommodation, 1036 chemical reaction of, $\quad .1050$ in colour blindness, . . 1088, 1102

See also Colour-blindness.

cones of, 1049, 1099, 1101, 1103, 1105, 1122 action of light on, . 1049 cone-myoid of, . 1049 function of, 1101, 1112, 1121 nuclei of, . . 1050 staining power of, 1050, 1103

,' staining powcr of, 1050,1103 connection of, with cortex, 754,755 ,
$757,758,780$
optic lobes, 915 corresponding points of, 757,1124 , $1126,1127,1138$

covering points of, . . . 1124 currents of..$\quad 1050$

, effect of light on, $\quad 1051$,
1052 variation in, 1051,1052 , 1120

eurvature of, as factor in illusions, 1142 ,

1143

disparation of, . $\quad 1125,1137,1138$ excitability of, . $1056,1057,1062$ fatigue of, 1058, 1059, 1062, 1063, 1070, $1098,1107,1110,1116$

fluorescence of, . . . 1047 fovea of, 1086, 1087, 1101, 1103, 1112 functions of, . . . . 1099 influence of colour on, . . 1046 light on, 1045,1048 , 1049,1051 ,, sensibility of. See Retina, adaptation. 
PAGE

1045 pilocarpine on

. 1045

," intrinsic light of, $1063,1096,1121$,

, 112 :

macula lutea of, $1031,1054,1086$, $1095,1101,1104$ , relation to visual area, $757,758,761$ 780

movements of cones of, 1049, 1050 pigment - epithel-

ium of, 1048,1050

nerves of common sensation of, 967 in nocturnal animals, 1101, 1104, 1105 paracentral region of, 1086, 1087, 1092 photo-xsthetic elements of, . 1121 physiological incongruence of, . 1125 jrocesses of, . 1045 pigment of, $1086,1087,1093,1095$, $1104,1105,1118$
epithelium of, 1045,1046,
1099,1100,
epithelium of, 1045,1046,
1099,1100,

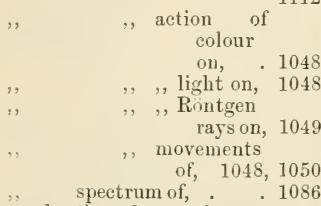

reciprocal action of parts of, 1060,1062 1064,1066 relation of, to invisible rays of spectrum,

- 1054 rhodophyllin of, $\quad . \quad+\quad .1045$ rhodopsin of, . . . . 1045 rods of $1099,1100,1101,1103,1104$,

$1105,1121,1122$

,effect of light on, 1045, 1047.

,, nuclei of, . . . 1050 sensitive layer of, . $\quad . \quad .1099$ , depth of, for colours, . 1100 sensitivity of, $1053,1054,1055,1056$ $1081,1138,1146$ specific nerve-fibres of, stimulation of, 1052, 1053,1055 , 1056. See also Sensation, visual, Eyje.

after-image effects, 1057. Sec also Sensation, visual. intermittent, 1068, 1069, 1072,1074 1076 minimal duration of, 1067 by mixed colours, . 1081 multiple subliminal, 1056 ,

1066 polar, . . 517, 518 punctate, . . 1112 summated, . 1055, 1067 vasomotor sympathetic fibres to, 618 visual differences of different parts of, 1033. See also Sensation, visual.
Retina, visual purple of,

bleaching of, 1045 $1046,1047,1101$ function of, 1101, influence of 11120 colour on, . 1046 , Röntgen rays on, 1047 regeneration of 1045 , $1046,1047,1101$ relation of retinal

currents to, 1051 , to fovea, . 1103 spectrum of, 1046 , 1047

,, , substances of, $1106,1111,1113$ $1117,1118,1120$

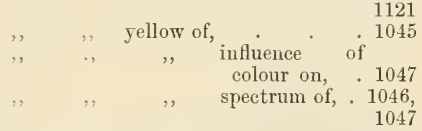

zone of normal colour-vision

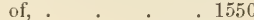
red-green blindness of, 1088 , 1092,1118

total colour-blindness of, . . . 1085, 1118

Retinal rivalry, . . . . . 1138 Retinitis, . . . 1095, 1139, 1140 Rheonome, . . . . . . 471 Rheoscope, physiological, . . . 436 Rheotome, . . 414, 415,426, 427, 460 Rhinencephalon, . . . . . 765 Rhodophyllin, . . . . . . 1045 Rhodopsin, . . . . . . 1045 Ribs, movenients of, $\quad . \quad 275,276,277,279$ Rigidity, decerebrate. See Decrebrate rigidity.

Rigor mortis. See Muscle.

Ritter's tetanus, . . . . 437, 510

Ritter-Valli law, . . . . . 477

Rods, retinal. See lietina.

Rolandic area. See Cerebral cortex, motor area.

Romberg's symptom, . . . . . 910 Root cells, . . 592,784 ,, afferent, $592,784,787,788,790$, for alimentary tract, 884 bulbar, . . 884 for brachial and cervical nerves, . . 886 , heart, . . . 884 conduction by, $\quad 791$ effect of limb amputation on,

for internal car, . 884 segmental nerves below brachial, . $\$ 86$ , skin, . $884,885,886$ efferent, " $592,784,793,796,797$ arrangement of, in cold, 793, 794, 795, 797 for alimentary tract, 866 of bulb, . . . 886 dorso-lateral sub-group of, . 
Root cells, efferent, effect of limb amputation on, . 793 scction of afferent roots on, section of facial $800,803,877$ nerve on, . 878 for eye muscles, . 795 , heart, . 202 relation of, to muscles, 794 for skeletal muscles, 793 , 794,795

Root fibres. "See Nerve root fibres.

unstriped muscles, 796 , reflexes, . . . 827 ,, systems, spinal, . . . . 804 Roots of cranial nerves. See Nerie roots.

, olfactory, . . . . 764, 781 , of spinal nerves. See Nerve roots.

Rotations, axes of, 235, 236, 237, 238, 239,

\begin{tabular}{|c|c|c|}
\hline ", & centre of, & $\theta^{\prime}$ \\
\hline , & of femur, . & \\
\hline & , humerus, & . \\
\hline
\end{tabular}

Rubro-spinal mediate system, : 808 Running,

$264,268,270$

Saccule, . . . 1164, 1166, 116 ' Sacral autonomic system. See Autonomic.

Saddle joint,

Salamander, iris of, . . . . 1044 Salivary glands, autonomic ncrve-supply of, . . . 692 effect of stimulation of cortex on, . 716,722 secretory nerves of, 618,620 , $660,661,693$. See also Salivary secretion in Vol. I.

vasomotor nerves of, . 132 , $141,617,619,620,624$, $626,660,661,674,693$

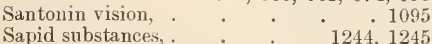

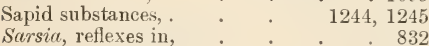

Sauropsida, optic lobes of, : $\quad \cdot \quad \cdot \quad 911$

Scala tympani, . . . . . . 1179

, vestibuli, . . . $\quad 1164,1179$

Schematic eye, . . . $\quad$. 1026,1029

Schleuderzuckungen, . . . . 370

Scotomata, . 1095, 1096, 1102, 1104

Scratching reflex, . . $838,839,866^{\circ}$

Screw joints, . . . . $\quad$. 239

Scrotum, dartos tunic of, . . $\quad 348,635$

Seal, vena cava of, $\quad . \quad$. $\quad .72$

Secretions. See also Vol. I.

Secretion, gastric, centre for, . . . 887 influence of cerebral cortex

on, . . . 716, 722 pancreatic, centre for, . . 887 reflex, . . . . 679 salivary, influence of cortex on, · 716,722 Salivary glands.

of sweat, influence of cortex on, . $\quad 716,722$ nerves of. See

Sympathrtic neres. $788,982,983$

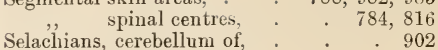

Selbst-tetanisirung, . . . . 579

Semen, ejaculation of, . . $347,349,852$

transmission of, to ovum, . 348

Semicircular canals, . . . . 1194 ampullæ of, 1166,1194 , $1195,1196,1198$, $1201,1202,1203$, 1204,1205

auditory hairs of, 1201 ,

connection of, with auditory sense, 1194, $1195,1204,1205$ with vertigo, $1196,1197,1200$, 1205

crista acustica of, 1194, 1195

effects of section of, 1198, 1199

excitation effects of, 1198 eye movements from

irritation of, 1197, 1203,

1204,1205

function of, 1196,1199 , $1200,1201,1205$

head movcments after

section of, 1198, 1199

influence of, on muscle

tone, 1204 , rigor mortis, 1204

lesions of, 1167, 1197,

1198, 1199, 1202, 1204,

1205

nerves of, 1194,1205

planes of, 1195, 1196, 1201 relation of, to equili-

bration

of head,

1196,1198 , 1199,1201 ,

1202

inco-or-

dination

of move-

ment, 1198 , 1199,1205

, main-

tenance

of equili-

brium, 1196,

1198,1199 ,

1204,1205

, muscu-

lar sense,

1204

, sense of

accelera-

tion of

move-

ment, 1200 ,

1201

, sense of

rota-

tion, 1200 ,

1201, 1202 
Semicircular canals, relation of, to spatial perception, 1202 , 1203

See also Perception.

, in the

$t w o$

ears, 1195

1196,1201

section

of, 1198,1199

Semilunar ganglion. See Sympathetic ganglia.

valves. See Valves.

Sensation or Sensations,

affective tone of. See Affect

ive tone.

auditory, 1168. See also Ear.

affective tone of, 967,968

associated visual

sensations, . 1163

of beats, 1168,1169 , 1175,1185 1187,1189

binaural, . 1163 centre for. See

Cerebral cortex.

course of impulses

to cortex, . . 781

dissonance in, 1186, 1189

evolution of organs

of, . 1167, 1168

function of auricle in 1149 , 1150,1152

, cochlea in, 1167 , 1168. See also Cochlea.

,, external nieatus in, $\quad 1150,1151$

,, labyrinth in, 1164 See also Cochlea. Semicircular canals.

,, middle ear in, 1152 ,

,, utricle and saccule in, 1167 , 1168,1204

influence of lesions of eochlea on, 1181 , 1182

cochlear

nerve

on, .1167

ossicles

on, . 1161

thalamus

on, . 917 tone-phase

in, .1175

1176

localising power of, 1163

1164,1195

of londness, 1164, 1165, $1167,1180,1192$,
Sensation, auditory, of piteh, 1164, 1165

1166,1167

range of, . . 1208

reaction time of, . 611

reflex, . . 1162

relation of semi-

circular eanals

to, . 1194,1205

sympathetic reson-

ance in, . 1178 , 1180,1187

of tone quality, 1164 ,

1165,1167

, tones, 1168, 1180 ,

1192. See

also Tones.

, co m bin a-

tional, 1188, 1189 , 1193,1194

,, compound, 1172 , 1180,1190 , 1192,1193

, cont in uous eharacter of, 1184

differential, 1188

, effect of coch.

lear lesions

on, . 1182

,, high, . . 1193

, liminal differ-

ences for, 614 , 1169,1184 stimnli

for, 1168,1169

$1170,1171,1184$

,, low, . 1193

,, partial, 1173 ,

, relation of num.

ber of vibra-

tions to, 118?,

1185

, summational, 1188

,, theories of, 1179, $1190,1192,1193$

during yawning, . 1162

of cold, 921, 945, 955. See

also Skin, Sensation, thermal.

, in alimentary tract, 950

, conduction patlis for, 965

", in different regions, . 948, $949,950,955,960$, 961,962

effect of lesions of cord

on, . . 946 end-organs for, $\quad 946$, $947,953,957$ estimation of, $\quad 948,960$ liminal distances for, 935 maximal, . 949,960 paradoxical, . . 954 reaction time for, . 962

common, . 965, 969,970,976 affective tone of, . 9636 , $967,974,975,991$, 1000 . See also Affective tone. auditory, 
Sensation, common cutaneous, . . 965 , $972,976,991$ central ne u ral mechanism of, . 967 , 977,984 ,. motor reactions of, 974 , 975,976 , 977,993 1000

nerves of, . . 976 visceral, . 970,976 , 990,991 visual, . . 970 , of contact, . . . . 966, 989 cutaneous, 920 . See also Skin. areas of, . . 920 of cold. See Sensation of cold. common. See Sensation, common. conduction of, in cord, $862,863,866$ of contact. - 926, 966 , dissociation of, $\quad \begin{array}{r}989 \\ 979\end{array}$ influence of congestion of cord on, . 810,836 drugs on, 928 of movement, . 966 , pain. See Sen. sation of pain. paralysis of, from lesions of notor cortex, 724, 726, 727, $7 \cdot 28$ projection of, 972,973 , regular and irregular stimuli of, 972 of tickling, . . 976

, touch. See Sensation, tactual.

, warmth. See Sensation of warmth.

dissociation paralysis of, . 979 of effort, of fatigue, gustatory, . aflective tone of, 967 , afferent nerves for, $1237,1238,1239$ 1240 after-effects of, . 1244 , 1245

contrast effects in, 1245 electrical stimuli of, $516,1243,1244$ influence of drugs

on, 1241,1242 lesions of fifth nerve on, 1239,1240 , glossopharyngeal on,
PAGE

Sensation, gustatory, influence of lesion of Vidian nerve on, . 1239 , section of chorda tympani on, 1238 , , , of lingual nerve on, 1238 mechanical stimuli

of, . . . 1243 modality of, . 1242 peripheral organs of, 1237, 1241, 1242, 1243. See also Tongue.

polar stimulation of, . . . 516 quality of, . . 124: reaction time for, 1241 , 1245 relation to olfac-

factory sense, . 1258 sapid stimuli for, 1244 , sensory surfaces for, 1245 1237 varieties of, 1240, 1241,

of hearing. See Sensation, auditory.

,, hunger,. . 9 991, 992 , innervation, 1002, 1003, 1004, 1005,1134

integrating power of, . . 984 of movement, 966 . See also Perecpition.

muscular. See Muscular scnse. olfactory, 1246. See also Nose, olfactory. acuteness of, $\quad .1249$ 1250 affective tone of, . 968 afferent channels for; $764,124 \overline{7}, 1248,1252$ in animals, . 1248 centre for. See Ceretral cortcx. in disease, 1251, 1252 fatigue effects in, 1253 individual variations in, 1252, 1253 influence of drugs

$$
\begin{gathered}
\text { on, lesions of } \\
\text { tifth nerve } \\
\text { on, . } 1247 \\
\text {, olfactory } \\
\text { nerves on, }
\end{gathered}
$$
1247,1248
of organs
1248

involution of organs of, specific encrgies in, . . 1253 from mixed odours, 1257 peripheral endorgans for, 1247, 1249, 1253 pliysiological antagonism in, 
Sensation, olfactory, projection of PAGE psychological factors

in, $\quad \cdot 1257$
quality of,
relation of, to gusta-

tory sense, . 125

sensory surfaces for, 1246,1248

stimuli for, 1252 , 1253 , 1254,1256

,, chemico-physical relations of, . 125

,, classification of, . . 1252

,, electrical, . 1253

, liminal, .1249, $1250,1255,1256$

,, physical relations of, . 1256

of pain, 966, 969, 996. Sce also Skin.

adequate stimulus for, . . 1000

associated, . . 981

central neural mech-

anism of, $976,977,984$

character of stimuli of, . . 1000 conduction of, in cord, . 810, 893, 977, $978,979,980,981,982$, $983,994,997,998$ cutaneous, $965,966,972$ $975,976,1000$ deep, . 972, 973,989 delayed, . 999 in different regions, 985 , 986,987

estimation of, . 985 inertia of neural meehanisn of, 998, 999 influence of drugs on, 986 ,, stimulus on, 987 , 997,998

interference of other sensations by, . 98

latent period of, 987,998 , 999

liminal, . 985, 986, 988 local sign in, . 987, 999 from locomotor organs, $972,976,980,989,990$ in lower animals, . 975 motor reflexes from, 974 . $975,977,993$

from muscles, 989, 996 ,

nature of nerve-endings for, 979 $988,994,995$ peripheral neural mechanism for, 984, 994 , 995,996

ratio to tactual sensa-

tion, . .985 referrerl, 9s1, 982, 983, 994,1000 relation to brain, 1000 ,
Sensation of pain, relation to non-

P'AGE

rrojicient

senses, . 973, 976,1000

protective

reflexes, 974 975,977

stimulus, 994 $997,998,999$

sensations contributory to, $972,973,976,989$, 990

significance of, 973,974 , 975

from special sense

organs, . 967, 968

in spinal disease, 946, 965 , 979,980

superficial, - 972,989 sympathetic,. 981, 994 from viscera, . 970,971 , $972,980,981,982$ $983,989,990,993$, 994,996 voluntary inhibition

of, . 1001 seat of, $697,698,722,724,726$,

\section{sceondary,}

of sight. See Sensation, visual.

,, smell. See Sensation, olfac-

tory.

sympathetic,

tactual, 921. See also Shin.

981,994

,, adequate stimulus for, 924 933, 994

,, after-effect of, . . 925

,, centre for. See Cerebral cortex.

, $\quad$ in different regions, 922 , $923,926,927,928,929$, $935,936,937,940,941$ double, . . 1006 end-organs for, 921,922 ,

See also SZin. estimation of, . 925, 927 in frog, . 923,927 functions of hairs in, 923 ,

illusions of 926

influence of drugs on, 928 , lesions of cord on, 946 , 965,979

,, ,, cortex, oll, . 938 , repeated stimuli on, 921 , 927,987

,, stimulus on, $925,927,930$, 931,933

judgment of direction by, 944,945 form by, 944

, , $\quad$, liminal differences for, 614 , $928,935,936$, $937,940,941$. 

985,986

, local sign in, 934, 935, 938, $940,941,942,943$ projection of, . 941,942 ratio to pain sensa-

tions, . . . 985 reaction time of, 927,928 ,

relation to muscular sense, . 939, 942, 944 skin-conditions affect-

ing, . . 926, 928 spatial discrimination by, $934,936,937,938$, $941,942,1025$

„tetanic character of, . 925 of taste. See Sensations, gusta-

tory.

,, temperature, 945, 952. See

also Sensations, thermal.

thermal, 921, 945. See also Sensation of cold, Sensation of warmth. adequate stimulus for, . . 950, 994 after-effects of, . 952 antagonism in, . 965 conditions affecting, 950 , $951,952,953,954,960$, 962

, in lesions of cord, 946,965 ,

, liminal differences 979 for, . $\quad 959,964$

, local sign in, . . 964

", projection of, . 957,958

,, relation to pain sensa-

tions, - 955,956

, stimuli for, $945,946,948$, $949,954,955,958,963$

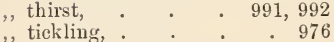

,, touch. See Sensations, tactual.

visceral, $\quad 970,971,976,990,991$ , neural mechanism of, 993 , 994, 996 refcrred, $981,982,983$ 994,1000

visual, 1052. See also Eye, Vision.

abnormalities of, 1088, 1095, 1096. See also Colour - blindness. acuity of, . .1055 in colour-

blindness, 1089 estimation of, . 1055 in peripheral retina, 1086 , relation to 1103 cones, 1101, 1103 , illumina. tion, 1055 alfective tone of, 967,968
Sensation, visual, after-images of, 1057,1062 , 1074, 1075, 1098, 1099, 1141,1147

, complementary, 1057,1058

,. corona of, $\quad 1059$,

, effect of eye movements on, 1058, 1064

,, , tension on 1064,1098

, homochromatic, 1057,1058

, negative, 1057 , $1058,1074,1099$

,, positive, 1057, 1074,1116

,, seat of, 1064,1097 ,

1099

,, theory of, 1062, 1063, 1097, 1109, $1115,1116,1121$

, variations in, 1058 , $1059,1063,1066$

in albinos, . 1095 associated auditory sensations, . . 1163 binocular combination in, 1122. See also $V$ ision.

of black, 1107, 1113, 1114, 1121,1123

, blue, 1108, 1109, 1110,

$1112,1113,1114,1115$, 1117,1121

brightness of, 1053, 1054 , $1055,1057,1058$, $1059,1076,1084$

,, in binocular vision, 1123,1124

,, ,, colour-blindness, 1088, 1091, 1092, 1094, 1095

, effect of contrast on, 1060 ,

1061

, in flicker, . 1074

,, ,, intermittent stimulation, 1068

,e relation to acuity, 1055 , 1068

,, ,, visual angle,

1055 ,, theory of, 1117,

centre for. Sce Cere-

bral cortex.

Charpentier's band in, 1074 of colour, $1652,1053,1056$, 1072,1074

,, cerebral centre for, . 1105

, from elcetrical stimulation, 1097 
PAGE

Sensation, visual, of colour, elementary, 1107 ,

1108,111 ,, curve of, 1108
1087

, fundamental, 1107 1108,1121

, in low intensity

spectrum, 1107 $1111,1117,1118$,

, from mechanical stimulation, 1097 at niasula, . 1086

,, in peripheral

retiua, 1084, 1085, $1092,1095,1118$

,, primary, 1105, 1107, 1112,1121

,, simple, . 1121

, stereoscopic, . 1144

,, subjective, . 1096

", theories of, 1105, $1106,1112,1121$

of colour brightness, 1076

, curve of, 1079 ,

, estimation of, 1077,1078

, relative, 1078,

1079

,, specific, 1117

colour mixture in, 1081 , 1086,1120

binocular, 1124

, law of, 1082, 1102

,, theory of, 1118,

1121

, tone of, 1053, 1054, 1057,1058 , 1059,1067 1083

, , in different

parts of re-

tina, 1085, 1114

, effect of con-

trast on, 1060 1061,1064

, ,, fatigue

$$
\text { on, } .1110
$$

, relation to

visual angle, 1055

conditions affecting, 1052

contrast in, . . 1060

,, binocular, 1098,

1099

,, conditions

affecting, 1061

estimation

of, . 1061

psychological

factors in, 1067

simultaneous, 1060,1062 , 1070,1117

successive, 1060 ,

1062

theory of, 1064 ,

$1110,1120,112$

eurve of, 1068, 1072, 1073 ,
Sensation, visual, in different parts of

retina, . . 1083, 1146

diplopia in, 1032, 1033,

1125,1137

duration of, . . 1067

effect of pressure on cye on, . 1097

, repetition of stimuli on, 1068, 1069,1072

, retinal fatigue

on, 1058,1059

Fechner's colours in, 1075 ,

1123

flickering, 1069, 1076, 1077

, in different re-

gions of retina,

1084

,, influence of area of stimulation on, 1072

duration of stimulus, 1070

, induction on, .1070

," intensity of stimulus,

1069

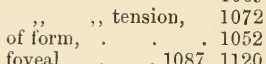

foveal, liminal stimu-

lus for, 1087, 1103

photochromatic interval for, 1087

1104

relation to

visual purple, 1103

fusion of disparate points in, 1125 , 1126,1127

,, point of, 1069, 1070, 1072,1098

, in, theory of, 1076 of green, 1107, 1108, 1109

$1112,1113,1114,1115$ 1117,1121

,grey, 1113, 1114, 1118

induction in, 1060,1062 ,

1076

homochroma-

tic, . 1062

spatial, 1062, 1066 , $1070,1117,1120$

temporal, 1062 , 1066, 1070, 1117,

1120

," theory of, 1109 ,

1114,1115

irradiation in, 1062,1140

of light, . . 1052, 1053

cerebral ecntre

for, . 1105

from elcetrical

stimulation, 517 ,

1097

at foven, . 1087

, maeula, 1086 , 
Sensation, visual, of light in peripheral retina, 1083 from stimulation of optic nelve, lininal differences for, $614,1054,1055$, 1056,1080 , 1100 ., stimulus for, 1053 , 1067,114 ,. ,absolute, 614 , $1053,1056,1057$, $1067,1079,1087$ 110 ,, ,, chromatic, 1053 , $1056,1067,1079$ $1087,1102,1103$, 1110 local sign in, 1132,1137 , $1138,1139,1148$ macular, . 1086, 1087 memory image of, . 1074 metabolic processes in, 1114 of movement, . . 1146 persistence of, 1067, 1068, $1069,1074,1076$ photochromatic interval of, 1054, 1067, 1068, $1079,1086,1087,1102$,

physiological point of, 1104 1055,1086 polar excitation of, . 517 primary, . . 1113 psycho-physical sub-

stances of

Purkinje's phenomenon

in, $1078,1080,1087$, $1102,1104,1111$

reaction time for, 611 recurrent images of, 1072 $1088,1103,1104$

, , from coloured

light, 107 , conditions affecting,

, relation to visual ficld,

1073 , , purple, 1102

of red, $1107,1108,1109$, $1112,1113,1114$ $1115,1117,1121$ relation to ultra-violet

spectrum, . 1054, 1055 retinal inage in, 1045 , 1139,1140 , rivalry in, 1122 , 1124,1138 saturation of, 1053,1054 , 1059,1079 1083,1084 1094,1115 effects of, on contrast, 1061 liminal, 1077
Sensation, visnal, space perception in.

PAGE See Perception. summation of stimuli in, 1055,1067 Talbot-Plateau law of, $1068,1069,1076$ time relations of, . 1068 of violet, $1107,1110,1121$ visnal angle of, 1055,1056 , 1100 Weber's law of, $\quad 1080$ of white, 1107, 1109, 1114, 1121 ,, yellow, 1110, 1013, 1114, $1115,1117,1121$

of warmth, 921, 945. See also

Sensation, the'mal, skin.

in alimentary tract, 950 conduction jath for, 965 ,

980

in different regions, 948 , $949,950,955,960,961$, 962 estimation of, 948,949 , 960,961

influence of lesions

of cord on, . $\quad 946$

liminal distances for, 935 maximal, . 949,960 reaction time for, . 962

Scnse, genital," 947

,, spots, 920. See also Skin.

Senses, specific energies of, . . . 1253

Septo-marginal tract, . . . . 806

Septum lucidum, . . . . 717,764

Serous membranes, nerves of, . . 686

Sexual reflexes, . . . . 851, 890

Shaking reflex, . . . . . . 830

Shark, cerebellum of, . . . . . 900

,, cerebrum of, . . . 700,817

Shivering, 708

Shock. Sec Spinal shock.

Shoulder-joint, . . . . 237, 238

Sight. See Sensation, visual.

Sigmoid flexure, . . . $\quad 335,336$ gyrus, : $\quad: \quad 713,714,717$ 764,765

Sinus ganglia. See Ganglia of Remak.

extension, . 179

of heart, . . $172,183,185,190$

Sinuses of Yalsalva, . . . 12, 14

," venous, of eranium : : 143,145 Size, sense of. See Percention.

Skeletal muscles, sympathetic nerves to, 639

Skin, 920. See also Sensation, cntaneous.

,, adaptation of, . . 925, 957,960

, circulation in, . 158

," cold spots of, $921,93 \dot{5}, 94 \dot{5}, 94 \dot{6}, 955$. $964,984,989,995$

, distribution of cold and warmth spots in, . . . . . 946 elastic tissue spindles of, . . 924 , end-organs for cold sensation, 946, 947, 953,957 ., warmth sensation, 946 , $947,948,953,957$

,. Golgi corpuscles of, . . . 924 ," localising power of, : 934, 938, 964 ,, , ,, central factors in, 
Skin, localising power of, conditions afrect-

\section{PAGE} ing, $936,937,938$,

in different regions, 935, 936, 937, 940, 941

, , , , theory of, 938, 942 „, Meissner's corpniscles of, . 923, 924 ", nerve-endings in, for pain sensation,

, Pacinian corpuscles of, . . 924 pain nerves of, . . 988, 994, 996 ,, spots of, 984, 985, 987, 988, 989, 995,1000 physiological zero-temperature of, 956 , plume-organs of plume-organs of, . . 924, 1008 referred pain in, . $689,690,691,982$,

regular and irregular stimuli of, 972 relation of, to common sensation, 965 segmental areas of, . . . 788 scnse spots of, - . . 920,935 sensory areas of, . . . . 920 ,, circles of, $939,940,943,964$ ," end-organs of, 921,922, 923, $924,946,947,948,953,957$ sympathetic nerves of. See Sympethetic.

tactile cells of,

tactual sensitivity of different regions, 922, 923, 926, 927, 928, 929, $935,936,937,940,941$

thermal sensitivity of different regions, - $948,955,960,961,962$ touch spots of, $921,922,923,924,935$, $940,984,985,987,988,989$ trophic changes in, from nerve lesions, . . . 876, 877 warmth spots of, $921,935,945,946$, $955,964,984,989,995$

Sleep, cause of, . . . . 608 ,, eye movements during, : . 1132 , respiration during, . . . $\quad . \quad 309$ Snell. See Sensation, olfactory, Odours.

Snake, cerebellum of, . . . . 899

, efferent root-cells of, . $\quad . \quad 795$ muscle spindles of, : $\quad . \quad .1008$

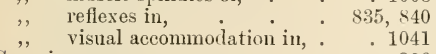
Sneezing, . . . . 308 Sodium ehloride, action of, on heart, 225,226 ,, nitrate, action of, on kidncy sceretion . . . 160 salts, action of, on nerve, 46. 465 , $489,490,536$

Solar ganglion. See Sympathetic ganglia.

,, plexus, . . . 331, 334, 342 Sound, cardiac,

,, sensation of. See Sensation, auditory, Touss.

Sounds in deglutition, . of heart, . $\quad 28,38$ ,, curve of, : : $\quad 30,32$ ," first, : : $\quad 29,38$ ," relation to impulse curve, 36 in niusele, second, . . 31, 36, 38 respiratory, : . $\quad$. 281, 311
Specific energies of nerve fibres, . 694, 1243, 1253

,' $\quad$,' senses, 1242, 1243, 1253 Speetrum, luminosity curves of, 1088, 1091, $1094,1117,1118$ visible, limitation of, 1054,1055 of visual purple, . 1046, 1047 ,, ,, yellow, : 1046,1047

speech, . . . . . . 901 . 901

,, defects of. See Aphisia.

Spermatic plexus, . . . . 338

Spheno-palatine ganglion, 651, 661, 662, 664 Sphincter ani, $336,337,646,667,679,745$,

antri pylorici, . $785,851,868$ cardiac, $318,321,325,326,695$ pupillie, 660, 1044. See also Pupil contraction. pyloric, $\cdot \quad \cdot 322,323$ vaginæ, . . . 348 vesicæ, $339,340,342,344,345$, $346,717,785,849$, 850,868

Sphincters, effect of lesions of cord on, $812,813,851,869$

Sphygmogran," spinal shock on, . 869

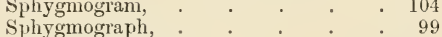
Sphygnomanometer; . . . . $\quad$. 79,80 Sphygmoscope, . . . . 14, 17 Spinal animal, $817,818,819,824,825,826$, $832,835,841,842,843,844,848$, $856,858,870,1019$ convulsions, . . . .836 cord, . $\quad . \quad . \quad . \quad 783$ centres of. See Reflexcentirs. Clarke's column of, . 136,806 , $807,810,811,836$ commissmre of, dorsal, . 793 ventral, . 806 conduction in, . . . 861 ,, esthesodic, : 811,978 ,, from afferent roots, 791, 792,793 ,, association areas, 773 anterior descending tract, . . 778 to bull, : : 861,862 ,, cerebellum, 861, 864 from cortex, $777,778,863$, ilorsal columis, . $\quad 864,865$ 862,863 grey matter of, . 793,810 intraspinal, . 861,864 kinesodic, . . 811 , to mesenepphalon, 861,864 ", of pain, 810 . Sce also Sensution of pain. , from respiratory centre, 863 ", scusory, 810, 862, 863, 864, $865,866,977,978,979,980$, $981,982,983,997,998$ to thalamus, . S61 ,, of vasomotor impulses, 137 , $861,863,864$

, ventro-lateral columins of, . $\quad 773,861,863$, 864 
Spinal cord, couduction to vesical

PAGE

centre, 863,864

,, of voluntary impulses, 777 ,

$863,864,865$

See also Spinal cord,

mediate system.

connection of, with sympa-

thetic system. See Sym-

pathetic.

degeneration in, after lesions

of cortex, 705

, necrosis

of grey

matter, 836

,, semi.

section, 861

, destruction of, effect on cir-

culation, 137 $138,812,813$

, defecation,

$812,814,815$

, micturition,

, muscles,

$$
812,814
$$

813,816

,, parturition,

, reflexes, $\quad 786$

,, secretions, 812,813

, sphincters,

$812,813,869$

,, tissues,

812,813

efferent root-cells of, 793,794 ,

795,797

electrical cells of, . $\quad 565,567$ epileptoid spasms from

exeitation of, .

722

functions of, conducting.

861. See also Spinal

cord, conduction.

,, psychical, 857, 859, 860

,, reflex. See Reflexes.

,, tonic, . . 868, 869

trophic, 875

grey matter of, . . $\quad 793,810$

collaterals of, 811

conduction

by, 810,811 .

977,978 ,

979,980

effect of

chemicals on, 800,811

excitability of, 800,811

in lateral

sclerosis,

necrosis of

835,836

influence of amputation of

limb on, . 878

blood stasis on, 810,862 section of afferent

roots on, . 800

lesions of, anresthesia after, 866 ,, in dorsal columns, 862,868

from, liyperiesthesia
Spinal cord, lesions of, influence of, on cut-

PAGE

aneous sense, 946 , $965,979,980,999$

,, onknee-jerk, 873,874

,, ,, vascular

tone, 854,869

notor paralysis

after, . . 794

mediate systems of, . . 804

bulbo-

spinal, 807 ,

$808,863,864$ cortico-

spinal, 808

ence-

plialo-

spinal, 806 ,

$807,863,864$

extrinsic, 806,861

intrinsic,

805,810 ,

$836,861,864$

nerve

cells of,

$805,806,810$

pyrami.

dal, 808 ,

809. See

also $P y$ -

ramidal

tract.

quadri-

gemino-

spinal, 808 ,

864

rubro-

spinal, 808

spino-

cerebellar,

806,861 , 864,890

spino-

con-

junc-

tival, 807 861,890

spino-

dorso-

lateral, 806

spino-

ence-

phalic,

$805,806,807$,

$810,861,862$

863

spino-

quad-

rige-

minal,

$807,861,890$

spino-

restifor-

mal, 806, 861,890

spino-

thala-

mic,

807,861

862,890 
Spinal cord, mediate systems of, spino-

ventro-

lateral, 807

reflexes of. See Reflexes, spinal.

regeneration of,

$\begin{array}{ccc}\text { ", dorsal } & \text { columns, } 804 \\ \text { regions of, } & 819\end{array}$

section of, effect on circula-

tion, 117 , 137

knee-jerks, 815

reflcxes, 811 ,

813,814 ,

815

respiration,

in lumbo-sacral

region, 137,849 ,

850,851 ,

852

shock after, 845 .

See also Spinal shock.

through upper

limit. See

Spinal animal.

segmental character of, . 816

semisection of,

action currents

after, . 861

degenerations

atter, . 861

influence on

circulation, $\quad 856$

, motor and

sensory

functions, 864,866

, pupil, . 869

substantia gelatinosa of, . 810

, , , spongiosa of, . 793 ganglia. See Ganglia.

nerve roots. See Nerve roots.

reflexes. See Reflexes.

root systems, 804. See also

Nerve root fibres, Root cells.

shock,

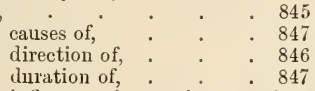

influence of, on reflexes, 846 ,

, $\quad$, skeletal muscles,

sphincters, . 869

viscera, . 848

isolation dystrophy tiom, 848

in man, . 848 ,, monkey, . $846,847,848$ synapses, . $\quad .810$

Spindles, elastic tissue, · · $\quad$. 924

,, -cerebellar $, \quad 806,890$

"encephalic system," . $805,806,807$

,, -quadrigeminal ,, . . 807,890

-thalamic $\quad 807,890$

Spiral cells,
Splanchnic nerves. See Nerve.

PAGE

,, vascular area, . 50,62, 91, 93,

$94,136,138$,

$147,158,161$

Spleen, changes in volume of, $162,163,164$

,. circulation in, . . . 162

, influence of, on venous circula-

tion, . . . . 120, 162 nerves of, $163,164,643,645,663$ systole and diastole of, . . 162

splenium, . . . . . . 780

Squatius, cerebellum of, $\quad . \quad \therefore \quad .900$

Staircase phenomenon, $192,214,215,222$,

$380,381,841$

Stannius heart, . $169,170,175,178,184$,

Stapes. See Ear.

Stereoscopy, . . . . . . 1122, 1144

Stereotropism, . $\quad$. $830,834,910$

Stethometer, . . . . . 282

Sting-reflex, . . . . . 812

Stomach, 321. See also Vomiting.

ablation of, . . . . 991

antrum of, . . . 321, 322

capillaries of, . . . 112

cause of movements of, . . 323

centre for secretion of, . . 887

condition of, in fasting, . 321

contractions of, . . 321, 323

dilatation of cardia of, . 321,325

direct stimulation of, . . 323

efferent root cells for, . . 886

fundis of, . . . 322

influence of distension of, on

heart, . 58

emotions on, 323,324 ,

excitation of vagus

716

on, . $\quad 323,324$

intestinal repletion

on, . . .

severance of nerves

of, . $\quad . \quad 323$

local ganglia of, . . . 323

movements of, : $\quad \cdot 321$

.. food in, . . 322 $323,324,335,663$, $664,692,693,695$ secretory, - 663,664 sensory, . 324, 688, 849,991

passage of fluids through, . 323 peristalsis of, - $322,323,325$

pyloric portion of, . 321,322

sphincter of, : 322,323

reflex inhibition of, $\quad . \quad 324$

, stimulation of, . . 323

relaxation of, $\quad . \quad .321$

structure of, . . $\quad 321$

tonic contraction of, . 321,323

transverse band of, . 321,322 ,

323,325

Stria tecta, . . . . 764

Stromuhr, . . . . . 83

Strontium salts, effect of, on nerve, 536

Strychnine, effect of, on intracranial

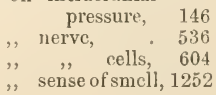


Strychine, effect of, ou spinal cord, 89,829 $830,837,843$

Sublingual ganglion, . . . . 661 Submaxillary ganglion, $\quad 661,664,672,677$ gland, nerves of. See Sympathetic, Autonomic nerve fibres; also Vol. I.

Substantia gelatinosa, . . . . 810 810 nigra, . $\quad 705,777,778,915$

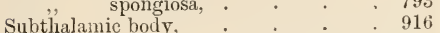

Sucking, . . . . 314 , action of intraoral pressure on, 314 , movements of tongle in, . 314 Supination, . . . . 238, 240 Supplemental blood, • . . . 128 Suprarenal extract. See Vol. I.

effect of, on depressor nerve, 60 ,. on heart muscle, . 193 ,, spleen, . 163 vaseular tone, 136,138

Sympathetic, cervical, . . . . 617 ,, early observations on, 618 , , , experimental union of, with lingnal, 691 , , , , vagus, 691 fibres of, efferent, 791 non-medullated, . 649 size of, . 648 , 649, 791, 971

, ganglia of. See Sympathetie ganglia.

, origin of, from cord, 619

, regeneration of, 652,653

," section of, 130, 131, 132, $603,619,656,658$, 676,677

stimulation of, . 617,619 $622,640,675,687$, 1037,1044

, tonic action of, . 619,656 , 675,677 ganglia, . . . . 617 automatism of, . 676 as centres for definite areas, . 678 cervical, inferior, 197 , 617,622 , 642,683 superior, 130 $131,138,141$, $617,621,623$ $624,649,651$ $652,653,654$, $656,658,664$ $669,674,676$, $677,682,683$ $691,875,971$

coceygeal, . 630,634 635,639 682,683

684

entiac, . 642, 645 commissural fibres

of, . $\quad 682,683$
Sympathetic ganglia, connection of, with

spinal nerves, . 617 , $620,621,630,634$, $635,636,638,642$, $644,645,646,647$

developmental connection with spinal, effect of nieotin

on, . $160,621,622$ epigastric, . . 617 growth of cranial fibres into, . 691

lumbar, 157, 627, 628, $629,630,634,636$ $675,681,684$

mesenteric, inferior, $160,342,349$ $617,636,645$ $646,647,651$, 679,681 superior, 342 , 642,645

origin of grey rami from, 629

post-ganglionic

fibres of. See

Sympathetic

nerve fibres.

prevertebral, 617,646 , $647,649,683$

reflexes from, 138,678 relation of, to sen-

sory impilses, . 688 renal,. . 642,645 sacral, $157,160,625$. $629,634,636$, $639,673,684$

selective arrangement of fibres in, 636 653

solar, . 160, 164, 617 , $642,643,644$ $648,649,684$

stellate, $157,197,200$, $620,624,630,634$, $635,638,642,647$, $649,656,669,675$. $677,681,683,695$ subdivision of, 678 thoracie, 156, 157, 622, $624,627,628$, $629,630,634$, $635,681,971$

vertebral, - 617,625 , $631,647,648$ $650,678,683$

\section{comnections}

of white

rami with, 638

lumbar, . $58,156,635,640$, $641,675,681,683$ fibres of, $\quad$. 648,971
nerve fibres, nerve fibres, afferent, of bone, 1006 ,, course of, 688 ,, distribution of, 688 
Sympathetic nerve fibres, afferent,

PAGE relative number of, . 687

,, trophic cells of, 686

of bladrler, . 646 , $659,692,693$ internal carotid

on, - 622, 624, 651,652

to ciliary muscle, . 618,

commissural, $\begin{array}{r}1037 \\ 682\end{array}$

683

connection of,

with cranial

nerves, 623,624

degeneration

of, $622,652,655$

efferent,

cervical region, 791

,, in grey rami,. 637

", in white rami, . 629, $637,644,688$

of Fallopian

tube, . 646 in grey rami, 627 , $628,632,636,637$, $638,639,688,689$ of head, 141, 617, $623,659,692,693$ " heart, 166, 197, 198,642

inhibitory, . 675 , of internal genitals, . 617 , 646,688 of intestines, 160 , 643, 644, 645, 646,659 " iris, 141, 617, $619,620,621$, $652,656,658$, 1044 ,, kiduey, 159, 643, 645,659 „, liver, 161, 643 ", lungs, 152, 642 medullated, . 648 ", loss of medulla by, . 648 , $649,650,665$ of $\mathrm{mucous}$ membranes, 141, $617,618,619$ $620,623,692$, 693 skeletal

museles, 155, 639 nerve cell con-

nections of, 621, $623,629,630$,
Sympathetic nerve fibrcs, non - medul-

PAGE lated, 648,649 , $650,651,652$ of orbital nuuscles, . 620 origin of, from cord, 619, 620, $630,634,636,638$ $642,643,644,645$ 646

permanence of scetion-effects of, . . 655 pilomotor, 618,619 , $620,623,625,628$ $630,659,678,681$, 684

post-ganglionic, 623 , 692,694

, to $\mathrm{ab}$ dominal viscera, 642

, of inferior mesenteric ganglion, 646 , medullated, 649 , 650

, regeneration of, 65 , relation of, to pseudo-reflexes, 680 , 682

,, to skin, 624, 627,628 , 636,638 , 692

,, of superior cer-

vical

ganglion, 623 , 624

, to thoracic viscera, 642 pre-ganglionic, 623 ,

, for abdominal viscera, 642 , 645,680

, to aber-

rant

cclls,

,, of eer-

vical

symin-

thetic, 619 , 620,623

, loss of medulla by, $648,+449$. 650,665 
Sympathetic nerve fibres, pre-ganglionic,

nerve

cells on

course

of, 201,682

, regener-

ation of, 652

relation

to spinal

roots, 658

, relation

to white

rami, 637

, selective

arrange-

ment

of, 636,639 ,

653

,, for

skin, 620 ,

623, 630,

634,636 ,

637

, , , thoracic

vis-

cera, 642

, varia-

tions in

origin

of, 632,633 ,

636

of rectum, 646,679

regeneration of, 652 ,

of retina, - 618

,s s livary

glands, 132, 141,

$617,618,620$.

$624,692,693$

, serous mem.

branes, . 606

size of, $648,685,979$

of skin of ano-

genital

region, 635 ,

$646,659,684$

., ,, head, 617 ,

$618,619,624$ 658,659 , 675,676

trunk and

limbs, 625 , 627,629

of sphincter

634

ani, . 646, 679

, spleen, 164, 643,

summary of

stimulation

effects of, . 693

of sweat glands, 618

$625,629,630$, $63+, 658$

tongue, 141,618 ,

, utcrus, 617, 688

, vasomotor.

See Vuso-

motor.
Sympathetic nerve fibres of viscera, 617,688

PAGE

,, abdon1-

inal, 642

645,692 ,

693,695

,, pelvic, 645 ,

646,659

679,692

693

, thoracic, 642 ,

649,692 ,

693

nervous system,

616,617

comparison

with other

autonomic

systems, 692

functions of, 617 .

$618,625,626$,

$635,642,643$,

$644,646,688$,

ganglia of.

693

See Sympa-

thetic gan-

glia.

nerve cells

of, 623,655 , 682,694

nerve fibres

of. See

Sympa-

thetic nerve

fibres.

systems re-

lated to, 659 , 692

tonic action

of, 619,655 , $672,675,676$. 677 pain,
Synapse, $\quad 607,608,613,784,785,798,810$

Synchondrosis, . . . . 233

Syren, . . . $\quad 1168,1176,1188$

Syringomyelia, . . . . . . 979

Systole of brain, . . . . . . 143

heart, 2, 3, 4, 7, 38. See also Systolic output.

duration of, . . 38

form of heart in, $\quad \dot{2}, 3,20$

heat, . . $4,5,10$

inefficient, . . 22

influence of, on filling of

auricle, $\quad 47$

heart-fre-

quency on, 39

position of semilunar

valves in, . . 13

pressure during. See

Pressure. spleen,
Systolic output of heart, $\quad$ - $20,22,38,48$ ," amount of, 43,49 ", estimation of, 42,48 , 49,84

, influence ofalcohol on, - 52 , a mount of

blood on, 74 
Systolic output of heart, influence of

PAGE eardiac

nerves on, $5: 3$

diastolic

filling on, 51

, on dicrotic

wave, 111

, , respiration

on, 125,129

,, transfu-

sion on, 75

, ,, from left ven-

tricle, . 43,51

,, $\quad$,, obstructed, 72, 441,

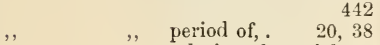

,., relation of arterial

pressure to, 48,50 ,

53,73

,, ,, from right ven-

tricle,

,

,, tension of muscle during,

Swallowing. See Deglutition.

Sweat centres, . . . . 856

$, \quad, \quad$ influence of cortex on, . 716 ,

722

,, nerves, $618,625,629,630,634,658$

Tabes Dorsalis, 799, 805, 806, 979, 988, 999,

Table of amounts of muscular shortening, 245 arterial pressures . . . 81 auricular pressures, . . 24 autonomic nerve supply, . 692 circulation time, . . 88 connections of vertebral ganglia, 634 consonants, . . . 1234 currents of electrical organs, . 569 differential tones, . . . 1188 distribution of Meissner's corpuscles,

duration of systole, $\quad \cdot \quad 38,39$ effects of excitation of nerve

roots, $880,881,882,883$ respiration on circu.

lation,

,, stimulation of autonomic fibres,

129

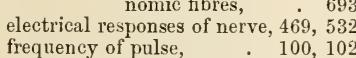
functions of autononic nerves, 693 liminal differences for muscular sense, $\quad 1023$ ,,,$\quad$ in sense organs, for thermal sense, $\quad 961$ ," distances for sense spots, 929 $935,936,964$ stimuli for muscular sense, 1014 thurements of cochlear structures, periods of latent excitation of muscle,
Table of Pfliuger's law of contraction, PAGE polar excitation of nerve, . 516 polarisation after-effects, $\quad 545,586$ position of joints, . . . 231 pulmonary arterial pressures, . 149 reaction times for thermal sense, 963 response to nerve excitation, . 503 spinal origins of sympathetic, 620 systolic output, time relations of deglutition, . $\quad 319$ variations in pulse rate, . 100, 102 ,, ,, vena cava pressurc, 72 velocities of blood flow, 84, 86, 102 ,, ,, pulse wave, . 102, 109 venous pressures, . $\quad 120$ visceral fibres of spinal ganglia, 849

Tachogram, . 87

Tachypncea,. . . . . . 291

Tactile area. See Cerebral cortex.

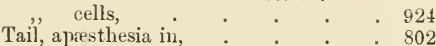

, antotomy of, . . . . 845

,, reflex movements of, $812,814,815,821$, 830,844

Talbot-Plateau law, . 1068, 1069, 1076

Tapetum, . . . . . . 780

Tartar emetic, emetic action of, . . 326

Taste, centre for. See Cerebral cortex.

sensations. Sce Sensations, gustatory.

Teeth, incisor,

, molar, . . . . . 313

", referred pain from, : $\quad . \quad$. 689

,, sense of temperature in, . . 947

Tegmental bundle, . . . . 782

Teleostei, cerebellin of, . . . . 902

vagus nerve of, . . . 215

Telephone currents, stimulation of nerve

by, . . . . . 462,475

Temperature, effect of lesions of thalamus on, . . . 918 sense of. See Sensations.

Temporal convolutions. See Gyri. lobe. Sce Lobe.

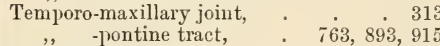

Tench, intestines of, . . . 785, 854

Tendinous inscriptions, . . . 429

Tendon, Golgi organs of, . 1009, 1010 reflexes, 871, 1011. See also

Knee-jerk.

,, sensitivity of, . . . 1011

,, sensitivity of, . . . 1011

Tension sensory nerves of, . . . 1007

Testis, afferent root fibres for, . . 849

,, atrophy of, from section of spermatic nerve, . . . . 875

Tetanomotor, . 467

Tetanus. Sce Muscle.

toxine effect of, on nerve cells, 604

Thalamus optieus, . . . . 916 , ablation of, $699,700,702,918$ , comnection with corpus albicans, 782 striatum, 778 ,

cortex, . 768,769 , 916,917

, , , motor area, . 778

VOL. II. -84 
Thalamus opticus, connection with

PAGE occipital lobe, 780,917 olfactory area, . 764 optic tract,

916,917

,, prefroutal region, 780 sensory tracts, . 769 superior cerebellar peduncle, . 917 degeneration in, after excision of eye, ,, lesions of gyrus, 780 parietal lobe, . 780 , removal of cortex, . 705 effect of ablation of cortex on, $\quad 700,702,705$

, excision of eye on, . 781 excitation of, . . . 917 fillet-fibres of, . . . 917 functions of, . 768, 918, 919 grey matter of, . . 917 influence of cerebellum on, 907 insensitivity of, . 978, 1000 lesions of, effect on audition, . 917 ,, , eutaneous sense, 917,918

,, ,, emotional expression, 919 ,, ,, muscles, 918

,,,, $\mathrm{mus}$. c nl a r sense, 918 ,, , pupil, 917 ,, , taste, 917 , , , t e m. perature, 918 , in vision,

917,918

pain from, 1000,1001 mediate spinal fibres to, . 807 muclci of, lateral, . 769,778 , mesial, . . 780 region of, . . . 916 relation to sensation, 768,917 Thermo-æsthesiometer, 768, 917 Thirst, 991,992

Thomsen's disease,

Thorax, 274. See also Ribs. aspiratory action of, .

$44,91,120$, 122,129

in child,

275

, cxpiration, . . . 274, 279 , inspiration,. 274, 275, 276, 278 pressure in. See Pressure, intrathoracic.

in rickets,

Thorus of dendron,

T'hysanozoon, spinal shock in,

Tiaropsis, reflexes in,

Tickling sensation,

Tidal blool,
593

$785,832,833$ 976 128
Tigroid granules,

Time, differential

"reaction. See Reaction time.

reflex. See Reflexes.

volitional,

Toad, nerves of,

Tone or Tones, 1168. See also Voice, tones of. affective. See Affective tone.

amplitude of, . . 1175, 1221

beat, . 1170, 1174, 1175, 1188, 1190

", combinational, . 1187, 1188, 1190

, compound, . 1172, 1173, 1220, 1121

". differential, . . 1187, 1188, 1190

,, Fourierian analysis of, . . 1221

", fundamental, . . . 1172

liarmonic, . . . . . 1172

," number of, . . . 1175

", harmony of, . . . . . 1175

over,. . . . . 1172

,, partial, 1171, 1172, 1173, 1174, $1215,1217,1218,1220$. See also Tones, Fouricrian analysis of, Phonograms.

phase of, . . $1175,1176,1221$ pitch of, 1168, 1178, 1215, 1216,

$1217,1218,1220,1221$. See also Tones, Fourierian analysis of.

$$
\text { prime, }
$$

" quality of, . $\quad \cdot \quad$. $\quad 1174,1176$,

sensations of. See Sensations, $1215,1216,1218$ auditory.

simple, $\quad 1173,1175,1220,1221$ summational, . . . . 1187 vocal production of. See Voice.

waves of, . 1174, 1176, 1220, 1221 curves of, 1172, 1173, 1193. See also Phonograms.

Tongue, 1237. See also Sensation, gustatory.

afferent root cells for, . . 884 autonomic nerves of, $618,624,660$, $661,662,664$ centre for movements of, . 735 742,748 circulation in, . . . . 141 circumvallate papille of, . $\quad 1237$ in deglutition, . . 314, 315 effect of diugs on, . $\quad 1241,1242$ filiform papillæ of, . . . 1241 fungiform papilla of, . . 1241 gustatory nerves of, 1237, 1238, 1240 sensitiveness of different parts of, . 1240 , 1241,1244

in mastication, - 313 muscles of, papillie foliatæe of . . . 1237 paralysis of, . . . 313,1240 referred pain fron, . . . 689 in sucking, 314 taste bulbs of, vasomotors of,

1237,1238 - 141 Tonometer, 15,210 Tonus, accelerator, $\quad \cdot \quad \cdot \quad . \quad+55$ ,, bronchial, . . . . . 311 , gastric,

, intestiual,

. $\quad 328,330,335$ 
Tonus of muscles, . . . . $\quad \begin{array}{r}\text { PAGE } \\ 377\end{array}$ afferent nerve impulses for, . . 1020 in apesthetic limbs, 1020 of crayfish, . . 377 of heart, - 194, 210, 216, $218,221,222,377,378$ influence of afferent impulses on, . 726 , anesthetics on, 870 ,, cerebellar lesions on, . . 904 on co-ordination, 1020 muscle-sense on, 870 section of afferent roots on, . 870 neural, . $868,869,870$ ,, influence on $\mathrm{ex}$ citability, 870 reflex, $\quad$, knee-jerk, 892
ing $800,909,910$ ,, inhibition of, . 1019 in 'spinal animal, $81 i, 870$ skelctal, . . . 377 of sphincter ani, . . $\quad 336$ ,, spinal cord, . $\quad . \quad 868,869$ , synmathetic system, - 619,655 , vagal, $672,675,676,677$ $55,129,294,311$ vasomotor, . $131,132,133,135$, $136,137,138,619$, $656,672,675,677$
influence of cord on, 854,869 ," vesical, ,$\quad$ influence of cord on, 854, 869
. Torpedo, electrical organ of. See

\section{Electrical organ.}

Torricelli, law of, .

Tortoise, . . . 821

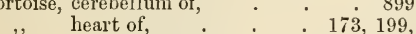
mesencephalon of, 205, 214 reflexes in, . . . . 853 retina of, $\quad . \quad 1049$ spinal cord of, . . $\quad .850$ "'sen. See Sensations, tactial.

Touch-spots,

$921,922,923,935$, $940,984,985,988,989$

Toxines, effect of, on nerve cells, . . 604 Trachea, resonance of, . . . . 1235 Tract, comma, . . . . . 805 ,, of Gowers, $\quad . \quad . \quad . \quad .807$ olfactory, . $\quad . \quad+\quad . \quad 764$ optic, . . . 758,915 pyramidal. See Pyramidal tract. septo-marginal,

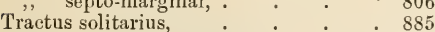
Tragus, . . . $\quad .1150,1152$ Transfusion, effect of, on arterial pressure, 74 ,

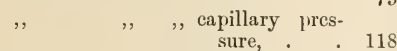

$$
\begin{aligned}
& \text { ", , heart, : } 75,77 \\
& \text {,, , , , velocity of blood } \\
& \text { flow, . } 76 \\
& \text {, venous pressure, } 76
\end{aligned}
$$

Tremors,

PAGE

Tremospasn,

Treppe phenomenon, . $192,214,215,222$

Triton, reflexes in, . . . . 813

spinal cord of, . . . . $\quad$. 879

Trophic changes from lesions of afferent

roots, 876

nerves, 875

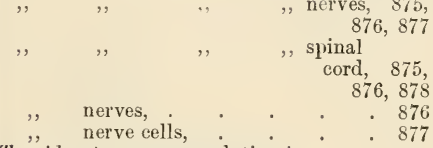

Tropidonotus, accommodation in, . 1041

Trunk, motor centres for, See Cerebral cortex.

, movements of, from stimulation

of dorso-lateral columns of bulb, 792 sympathetic nerves to, $157,625,627$,

629,634

Tubes, branching, fluid-flow in, . . 67

,, cylindrical, , . 63,64

,, elastic, , . . 67

,$\quad$," wave motion in, . 96, 98,99

, lateral pressure in, - 63, 66, 67, 98

, of varying width, fluid-flow in, . 66

Tunica dartos, . . . . 348, 635

Turbinal bones, . . . . . . . 1248

Tirck, bundle of, . . . . . 808

Twitch. See Muscle.

Tympanum. See Ear.

Uncinate GrRest, $\quad$ · $\quad 749,763,764$ Uncus, . $\quad . \quad$. $\quad$. $749,763,764$

Unio, auditory organ of, . . . 1167

Urea, effect of, on nerve, $\quad$. $\quad$. 466

," ,, , renal circulation, . 160

$,, \quad, \quad$, vasomotor centre, . 160

Ureter, . . . . . . 338

, afferent root fibres for, . . 849

, effect of excitation of splanchnics

on, . . . 339

,, , ligation of, . . . 339

," local ganglia of, . . . 338

,, movements of, . . . . 338

, nerves of, . . . . . 338

,

" sensory nerves of, : : $\quad .339$

Urethra, accelerator muscle of', - 340,345

,, constrictor of, . . 340,346

," contractions of, in coitus, . 347

, influence of, in retaining urine, 340

,, in micturition, . . 345,346

", muscles of, . . 339,348

,, Wilson's muscle of, : . 340

Urinary bladder. See Bladder.

,, sceretion, effect of cord lesions on, 853

$, \quad, \quad$ influence of cortex on, 716

," tract, mechanism of, . . 338

Urine, incontinence of, . . . 342

,, retention of, . . . . 850

Uterus, afferent nerves of, . . $\quad 349,849$

," cervix of, during labour, : 350

," in coitus,. . $347,348,349$

, contractions of, . $350,351,851$

," efferent nerves of, . . 349,667

, in parturition, 350, 351. Sce also Parturition. 
Vasomotor nerves, antagonism of, . PAGE , round ligament of, . sympathetic nerves of, . . 646 Utricle, $1164,1166,116 \bar{\tau}, 1204$

TAGINA,

349,350

Vagus nerve. See Nerve.

Valsalva, sinuses of,

Valves of heart,

$\begin{array}{ccc}, & \text { auriculo-ventricular, } 8,29 \\ , & , & \text { closure of, } \\ 8,9,10,24,38 \\ ,, & \text { opening of, } 24 \\ \text { sacculation } & \text { of, 9, 10, } 34\end{array}$

during rising tension, . 19 influence on lieart sounds,

- 12$$
\text { , }
$$

,, closure of, 12,20 , 37,38 influence on heart sounds, 31 opening of, 21, 23, position during systole, 13

Vas deferens, of veins, - . 91, 120 Vaseular system, eapacity of, $\quad \cdot 70,71,75$ effect of plethora on, . 77 ", tone. See Vasomotor tone.

Vascularity of organs, .

133,135

Vaso-constriction,

effect on general circu

lation,

microseopical study of 11

Vaso-constrictor nerves, 132, 134, 135, 136. See also Vasomotor nerves. of muscles, . 640 $\begin{array}{ll}\text {, } & \text {, penis, } \quad 348\end{array}$ Vaso-dilatation, . . " . : 132 eflect on general circulation, . 71 microscopical study of, 114

Vaso-dilator nerves, $132,134,135,136,626$, 672,675 action of of bucco-facial region, $618,626,659,676,693$ degeneration of, . 134 of dorsal nerve roots, 790 excitability of, . 134 fatigue of, . 134 of lind-limb, . 790 , muscles, . . 641 latent period for, . 135 of mylo-hyoid muscle, 641 ,, penis, . . 348 , salivary glands, 626 , $660,661,674$ , skin, . . . 626 Vasomotor centres. 'See Centres. mechanism, nerves, 131, 132. See V Tasoconstrictor, Vasodilator nerves.

," action of, . of braclialplexus, 669,670 , brain, . 146, 148, 168, 618,624

classification of, . 167 of conjunctiva, . 617,619 coronary, . $166,663,665$ degeneration of, . 134 distribution of, . 132 of ear, 130,131, 132, 138, $617,619,620,624,656$ $658,669,675,676$

effect of different cur-

rent strengtlis on, 167

, temperature on, 134 ,

electrical stimulation 135,167 of, $\quad 134,135,167$ ,, external genitals, . 667 , 679,693 of eye, . . . 141 ,f face, . . . 141 fatigue of, . 134 of head, - 141, 617, 619 , $620,623,658$

, heart, . . . 166 , intestines, $160,644,645$, iris, 667,695

", kidney, . . 159

, larynx, . . 624

,, latent period for, . 135

, limbs, 155, 156, 625, 630 , $634,654,656,670,675$,

, liver, 161, 643, 644, 645 ,, lumbo-sacral plexus, 669 ," lungs, $151,152,154$,

,, mesentery, . . 659 , moutl, $141,618,623$, $660,661,662,693$ ,museles, 155, 156, 640 ,, nasal fossæ, 618,623 ,

in nerve roots, . 670 of orbital gland, . 661 ,, pancreas, $163,644,645$ path of fibres of, in cord, . . 137 of penis, . . 160,348 ,, pharynx, . 624,641 ,portal vein, 161, 640,

643 pulmonary, 151, 152, 154, regeneration of, . 167 of salivary glands, . 132, $141,617,624,626,661$, $668,672,674,693$ simultaneous excitation

of, . . 134 in spinal nerves, 133,134 , 167,668 of spleen, - . 164 , tongue, 141, 624, 660, $661,662,664$ ,, third cervical nerve, 669 , trunk, 157,625,634 ", veins, . 140,625 
PAGE

Vasomotor reflexes, $157,166,167,816,827$ $854,855,856,863,864,1011$,

1012

tone, 131, 135, 136, 137, 138, 619, $656,672,675,677,854,869$

Vein or Veins,

,, , cause of, . $\quad$. 121

," influence of abdominal

muscles

on, 91,92

141

, , , , , , movement

on, 120,121

, peristalsis

on, . 120

, posture on,

, respiration

on, . 121

splenic con-

tractions

on, . 120

," blood' pressure in. See Venous pressure.

breaking strain of, capacity of cercbral,

143,145 circulation in, . . . . 118 effect of intra-thoracic pressure on, 125 , transfusion on, . . 75 entry of air into, . . . 125 extensibility of, . . . . 118 of heart, contractility of, . . 190 innervation of, . . 140,625 in obstructed circulation, . 72,160 of penis, ligature of, . . . 347 portal, effect of transfusion on, . 76 , ligature of, . . 45 ,, vasomotors of, $140,161,643$ structure of,

systemic, relation of, to systemic arteries,

variations in voliume of $\quad 90,120$

Velocity head,

,, in arteries, ligation of pressure, after transfusion, . 76 ,, during respiration, 122

, 2 during respiration,
$,, \quad, \quad$ vaso-constric-

tion and vaso-dilatation 71

liead of, . 71 intluence on limb. volume, . . 158 intra-thoracic, . 119 relation to intracranial pressure, $\quad 146$ variations in, . 72

sectional area of, . : $\quad 103,104$ Venæ comites, ladial, · · · 103, 104
Venous pressure, Venous pressure, cerebral, : $143,145,146^{\circ}$ in different veins, . 120 influence of posture on, 90,
Vcnous pressure, influence of respiration

PAGE

$$
\begin{gathered}
\text { on, } \quad \begin{array}{c}
\text { transfusion } \\
\text { on, }
\end{array} \\
\text { post-mortem fall in, : } 76 \\
\text { relation to capillary pres- } \\
\text { sure, 116, } 117, \\
\text { intracranial } \\
\text { pressure, } 118 \\
\text { in vena cava. See Vena } \\
\text { caca. }
\end{gathered}
$$

," pulse, . . . 114, 119, 12\%

," sinuses of head, . . 143,145

, system, capacity of, . . $\quad 71,75$

Ventricles of heart, cavities of, . . . 5

, , compensatory pause 191

curve of contraction

of, . $\quad 39,172$

diastole of, . 2, 3, 4, 44 See also Diastolic filling of heart.

fibrillar contractions

of, . 192,193

force of, . . . 441

influence of direct stimulation of, . . 214 heat on con-

traction of, 172 vagus on, 213

214,215

period of contraction of, 38

diastole of, 38

rising tension

in, $19,36,38$ systolic output of, 20,22 ,

pressure in. See

Pressure, intracardiac. rlyythm of develop-

ment of, 175 excitation of, 175

rhythmical power of, 176 , $187,188,194$

synchronism of, . 7 systole of, $2,3,4,22,38$,

systolic output from.

See Systolic output.

Treppe-phenomenon in,

laryux

192

1235,1236

Veratiin, effect of, on lieart, . 196, 223

nerve, $236^{\circ}$

Verbal amnesia, . . . . . 775,776

Verkurangshroft, . . . . 376

Vermis, superior, . . . . 893

Vertebral ganglia. See Sympathetic gungliut.

Vertigo, auditive, $1196,119 \overline{7}, 1198,1200$, $1201,1202,1205$

in cereliellar lesions, 901, 902, 905 tactile,

visual,

190,1197

Vesical centre. See Biludder, Micturition.

Vesiculne scminales, . . . 347,349

Vesicular nurmur, . . . 281,311 
Vestibule, 1164, 1201, 1204, 1205 Viscera, nerves of, $197,200,201,203,617$, $642,659,686,688$ referred pain from, $689,690,981$, $982,983,994$ reflexes from, $816,827,848,849$ $688,849,970,971$, $993,994,996$

Viscometer, shock from,

848

Viscosity of blood,

Vision, 1026. See also Sensation, visual.

, binocular,
$, \quad, \quad$ double,
,$\quad$ single, 1125,1126
,$\quad$ theory of,
", central, . $755,757,759,76$
See also Sensation, visual,
foreal.
centre for. See Cerebral cortex.
colour sense in. See Sensation,

,, colour sen
visual.

, double, . . 1032, 1033, 1125

,, influence of mesencephalon on, . 913

," , thalamus on, 917, 918

," organs of. See Eye, Retina.

,. scuse of light in. See Sensation, visual.

,, space perception in. See Pereeption.

Visirlinie,

1122

1125

37,1138

97

Voice, baritone, . . . . . 1208

bass, .

, breaking of, . . . . 1207

, contralto, . . . 1208,1210

, in eunuchs, . . . . 1207

", falsetto, . . 1213,1214

," influence of age on, . . $\quad .1207$

," , air cavities on, . $\quad .1235$

", ", sex on, . 1207, 1210

,. loudness of, . . . 1207

", mechanism of production of, 1206,1209

," mezzo-soprano, . . . . 1208

,, organs of. See Laryux, Vocal cords.

pitch of, $1207,1209,1210,1212,1213$, 1214,1220 quality of, . . . $\quad 1215,1218$ range of, . . . 1207, 1208 registers, . . . . 1210

,, chest, . $1210,1213,1214$

", liead, . . . 1212, 1214

," middle, : 1211,1213

," sinall, . . . 1210

;, thick, . . . . 1211

$\because \quad$ thin, : $\quad: 1213$

relation to intra-tracheal pressure, 1207 singing, . . 1206 soprano, . . . . . . 1208 tenor, . . . 1208 tones of, 1215. See also Tones.

, compound, $1215,1217,1218$, 1031

Visual angle,

, area. See Cerebral cortex.

" purple. See Retina.

," sensations. See Sensations.

", substances. See Retina.

," yellow. See Retina.

Vitreous humour. See Eye.

Tocal cords, .

. 1206

$,, \quad, \quad$ abduction of, : $\quad 1209,1210$

, , , action of laryngeal muscles

on, $1209,1210,1211,1212$,

1213

adduction of, . . . 1209

alterations in length of, in different register's, 1214

, thickness in different registers, . . 1214 centre for movements of, . 742 during respiration, . . 280 influence of inferior laryngeal nerve on,

. 1209 relaxation of, . . 1210

tightening of, $1209,1210,1212$ vibrations of, $1206,1207,1208$ , in elicst register, 1211 , 1213,1214 ,", head register, 1212 , ,, , middle register, 1212 , influence of strength of blast on, . 1213

in voice production, 1206, 1207 ,, whispering, . 1236 leyisters. See Voice.

Voice,

sounds. See Voicc. consonantal, 1220

influence of air passages

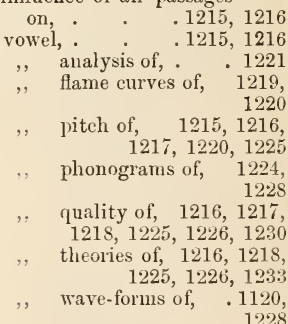

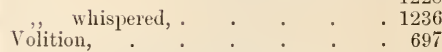

," effect of cortical lesions ou, . 697 , $698,699,701,703,704,722,723$ See also Volitional movements.

Volitional contraction of muscle, . 707 , curve of, $\begin{array}{r}707, \\ 709\end{array}$ ,, effect of fatigue on, .708

, , resistance on, . 708

, rhythm of, 708 movements, centre for. Sce

Cerebral cortex.

effect of lesions of cerebellum

on, $897,898,902$ section of

pyramids on,. 778 
Volitional movements, excito-motor im-

PAGE pulses for, 723,724

728,729 , 730

relation of kinæs-

thetic impres-

sions to, 723,724 ,

725,726 ,

$72 \pi, 728$,

729,730

, muscular sense

to, 722,724

729,731

1018

.. time, . . . . 612

tract. See Pyramidal tract.

Vomiting,

afferent impulses for, : $\quad$ : 326

of central origin, . . . 324

centres for, . . 326,887

direct, . . . . 324

in disease of semicircular canals,

efferent impulses for, . . $\quad 326$

essential factors in, . . 325

glottis during, . . . 324

movements of stomach in, . 325

muscles concerner in, . 324,325 reflex, . . . 324, 326

Vowel tones. See Voice.

Vulva, tone apparatus,

WALKING,

Wallcrian degeneration,

Warmth. See Scinsations. spots. See Skin.

Water brash, . • . • . 326

Wattles, erection of, : $\quad: \quad \div 618$

Waves, . . . . . 96,97,98 , anacrotic, . . . 108,111

", in arteries, $\quad . \quad .96,97,99$
Waves of contraction of heart muscle,

PAGE

$171,180,181,182$

$183,184,185,186$,

$18 \pi, 190,191,194$

, intestine, 328, 329

", muscle. See

Muscle contraction.

, resophagus, . 318

dicrotic." See Pulsc ware

of expansion, . • . $96,97,99$

, input, . . . . 98

katacrotic, : : 108, 112

of light, . . . . . . 1053

negative, . $\quad$. $\quad .96,98$

plateau, . . . . $18,19,37$

positive, · . . . $\quad 96,98$

predicrotic, . . . . 111

of pulse. See Pulse wavc.

reflected, . . . 98,99

of sound. See Voice, Tones.

velocity of, . . . 97,98

Wave syren, . . . . . . 1176

Weber, law of, $928,930,931,932,941,961$, 1022,1080

Whispering, . . . . 1236

White rami, $200,629,637,638,644,649,687$, $688,689,690,693,971$

Whytt's reflex, . . . . 913

Word blindness, . . $757,758,775,776$

"deafness, . . . $763,775,756$

Work of heart, . . . . 40,43

, , contracting muscle, 245. Sce also Mrusclc tetanus, Muscle twitch.

, diagram, . $246,357,359,401$

YAWNING, . . . . 309

Yellow, spot. See Retina.

, visual. See Rictina, macula.

ZeITstarie, $\quad . \quad \div 390$

Zygomatic muscles, centre for, . . . 741 


\section{INDEX OF A UTHORS.}

PAGE

AARs on induction,

1062

Abelous on fatigue of muscle, . $\quad 389,390$

Abelsdorff on rhodopsin, . . . 1046

Abercrombie on intracranial blood, . 144

Abney on vision, . 1056, 1076, 1077, 1083,

1091,1118

Abundo on pyramidal tract, . . . 809

Acland on cerebellum, . . . . 902

Adami on arterial pressure, . . 80

, , heart, . $6,10,11,18,39,41,49$, $53,166,188,219$

Adamkiewiczal centre, . . . 59

Adamkiewicz on intra-thoracic pressure, 125

$$
\begin{array}{lll}
\Rightarrow \quad \text {, knee-jerk, } \quad . \quad & \text {. } 874 \\
, & \text {, sweat secretion, . } & 716
\end{array}
$$

Adamiik on corpora quadrigemina, $\quad 912$

,, ,, muscular reflexes, . . 855

, , vasomotor centres, . . 137

Aducco on taste, . . . . 1242

, , vagal centre, . . 58

Aeby on muscular contraction, . . 384

Airy on sound, . . 1165, 1169, 1174

Albanese on excitability of nerve, . 480

$$
\text { , , lieart beat, }
$$

Albertoni on epilepsy,

$$
, \text {, salivary secretion, }
$$

,, ,, spinal reflexes,

Albrecht on nerve stimuli,

Alexander on vasomotor nerves,

Algeri on pyramidal tracts, .

Alruitz on cold spots, : $946,948,952,955$

Alsberg on thermal sense, . . 959,962

$$
,, \quad, \text { touch, . . . } 937
$$

Ambronn on myelination, . . . . 792

Anderson on accommodation, : 1037

, autonomic fibres of third

$$
\text { nerve, . . } 660
$$

,, effect of nicotin on spinal ganglia

hyjogastric nerve, . . 687

iris. . 1044

, lumbo-sacral plexus, . 633

nerve degencration, . 479,652

nerves of retractor penis, . 221 ,

349

viscera, 201, 203,336 ,

$337,343,348,349$,

$646,647,659$

pelvic nerve, $666,66 \overline{7}, 688,693$

, Iseudo-reflexes, $679,681,682$

, l'yramidal tract, . . 809
Anderson on sympathetic ganglia, $\quad$ PAGE

nerves, 618,621 ,

$636,646,649,651$

, vasomotor nerves, $138,160,626$

Angelucci on retina, . . . . 1048

v. Anrep on motor nerves, . . . 799

, , muscle, . . . 869

, ", vasomotor nerves, . 134, 135

Ansehitz on chronograph, . . . 265

Anton on spinal reflexes, . . . 839

A páthy on nerve cells, . . . 596, 597

Arago on vision, . . . . . 1087

v. Argutinski on spinal root cells, . 795

Arloing on nerve degeneration, . . 479

,, vagus nerve, . . 665

Arnemann on section of cord, . $\quad .878$

A rnold on muscular sense, . . . 1003

, ,, neural tonus, . . . 869

, spinal reflexes, . . . 839

Arnott on whispering, . $\quad . \quad$. $\quad 1236$

Arnstein on nerve cells, . . . 655

A ronsohn on heat centres, . . $\quad 717$

, , , sense of smell, . . 1253, 1256

Arrer on visual localisation, . . . 1135

d'Arsonval on electrodes, . . . 493

, nerve excitation, . 462

. 1054

496,549

- 979

. 1012

1012
.$\quad 786$

. 1096

.1146 ,

1148

, ", touch, . visual sensation, . $\quad 1055, \begin{array}{r}926 \\ 1056,\end{array}$

1057,1081

Auerbach on reaction time, . . 611, 614

. 807

. 1232

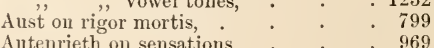

Autenrieth on sensations, . . . $\quad .969$

Babuchis on nerve, $\quad 457$

Backer on spinal nerve roots, . . 788

Bacon on vision, 1136

Badaud on vasomotors of lungs, . . 151

Baer on regeneration of cord 489

Baginsky on auditory area, . . . . 761

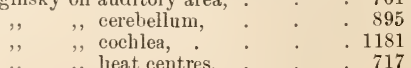


PAGE

Baginsky on semieircular eanals,

Bain on museular sense,

,', verbal reeollection, .

Baldi on apesthesia,

Baldwin on reaetion times,

Balfour on sympathetic ganglia,

Balighian on pyramids,

Ballanee on eerebellum,

Ballet on chromatolysis,

Ballowitz on electrieal organs,

Baneroft on vasomotor nerves,

Bandelot on cerebellum,

Baranowski on polarisation, .

Baratonx on vision,

Barbacei on mediate spinal fibres,

Barbé on knee-jerk,

v. Bärensprung on herpes,

Barfurth on regeneration of cord, .

Barker on faseiculus dorsalis,

,, ,, nerve eell,

,, ,, sense spots,

Barlow on aphasia,

$$
\text { , , thalamus, }
$$

Barnard on arterial pressure,

$$
\text { ,, depressor nerve, . }
$$$$
\text { ,, heart, }
$$$$
\text { ,, pulse eurve, }
$$

,, vena cava pressure,

$$
\text { ", vaseular tone, }
$$

1199

1002

776

802

945

202

889

902

604

566,567

625

900

549

1163

805

873

876

879

805

604

988

775

919

92

62

120

,' , vaseular tone, .

v. Baseh on arterial pressure,

,, erossed inmervation, . . 345

,, intestinal movements, 331, 333

,, manometer, . . 17, 18

,, nerves of uterus, . . 692

, sphygmomanometer, . $\quad 79$

Bastian on aphasia,

,, ,, eomma traet, : . $\quad .805$

,, ,, eontractures, . . . . 731

,, ,, functions of brain, . . 705

,, , motor centres, . . 724, 729

,, , museular palsy, . . . 726

," , ,

, , ,. tonus, . . . 1020

, , sense of effort, . . . 605

, , shock, . . . 848

," , tract of Gowers, . . . . 806

Bateman on aphasia, . . . . 776

Baumgarten on aurieulo-ventricular valves,

Bayliss on augmentor nerves, $\quad 2 \dot{1} \overline{7}, 21 \dot{9}$ , , blood pressure, $18,19,70,72,117$

,, , depressor nerve, . $60,61,62,134$

, heart, . . 187, 447, 448

, intracranial pressure, . 145

, nerves of intestine, $334,695,696$

,, semilunar valves, . . . 21

,., transfusion, . . . 76

, vasomotor nerves, $140,156,158$, $161,635,643$,

790,855

Baxter on cardiae systole, 39

Beard on organs of lateral line, . . 1168

Beau on pain,

988

Beaumont on stomaeh,

321

Beaunis on liunger,
Beaunis on sensory paralysis, . . 727

Beauregard on cochlea, . . . 1183

v. Bechterew on amblyopia, . . . 759

,, bladder, . . 346, 717

,, eerebral eortex, $713,726,727$,

$736,740,748$

$756,763,778$

, nerves of intestine, 334, 643

,, pain, . . . . 1000

, pyramidal traet, . . 707

,, salivary seeretion, . 716

,, thalanus, . . . 919

Beck on electrical response of nerve, . 525

,, , nerve eentres, . . . . 605

," ,, ,, roots, . . . . 687

Beeker on vision, : . . . . .1036, 1094

Bedart on ankle-joint, . . . . 253

Beer on aceommodation, $1040,1041,1042$

,, ,, bronehial museles, . . 309, 310

Beevor on epilepsy, . . . . . 719

, , internal eapsule, : $\quad$. 777

,, ,, intra-areal localisations, . . 737

,, , motor area, . . $733,739,740$,

$742,744,745$,

$746,747,748$

Behnke ", olfaetory area, . . 766, 781

Bell on eochlea, . . . . . 1168

,, , nuseular inhibition, . . 712

," ", sense, . . 1003, 1006

," ,, ,, tonus, \& . . 1020

,, ,, nerve roots, . 658, 786, 789, 797

,, ,, phonograph, . . 1216

,, , reflexes, . . . . 786,842

,. , sensations, . . . 969, 975

", ,, senso-paralysis, . $726,803,1021$

, , , sphineter vesicæ, . . . 339

Bellarminoff on vision, $1042,1072,1084$

Belmondo on spinal nerve roots, . . 799

Bennett on voeal registers, . . . 1214

Bergrün on intestinal movements, . 334

Berlin on aeeommodation, . . . 1036

," ,, eye movements, . . . 1030

Bernard on inhibitory nerves, . . 672

,, intestinal movements, . 853

", museular sense, . . 1020

", nerve roots, - $788,790,801$

", pseudo-reflexes, . . 678

", reflexes, . 166,835

", sympathetie nerves, $619,621,657$,

$677,787,875$

, , vasomotor , $\begin{array}{r}131,132,136, \\ 141,156,670\end{array}$

Bernhard' on eerebral cortex, $\quad$. $\quad$. 772

1004

$955,986^{\circ}$

, ", museular sense,

,, pain,

955,986
.$\quad 290$

, lieart, 58,170

Bernstein on dyspncea, .

$416,417,423,1011$

nerve, $453,455,473,475,476$. $482,495,528,530,531$, $547,548,550,55 \&, 831$ roots. . 798

resuiration,

798
124

rheotome,

414

bert on bronchial muscles, . $\quad 309$

,, , nicturition, . . . . 345

,, , nerve,

$45 \%$ 
PAGE

Bert on nerve roots,

821

Bethe on nerve cells, . . 594, 596, 597, $598,604,791$

,, ., ., cord of invertebrata, 817, 818,

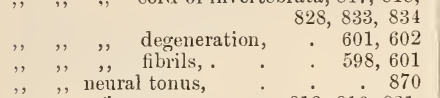

", ", reflexes, . $\quad 812,816,831$,

$833,834,835$

, , shock, . . . . . 846

," ," spinal cord, . . . . 817

Bethellieim on eoronary arteries, . . 165

Betz on intestinal movements, . . 331

,, , nutor nerve cells, . $72 \cdot 2,723,777$

Beutner on arterial pressure, $22,148,149$

v. Bezold on accelerator nerves, . . 199

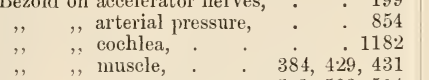

, , muscle, . . 384, 4.29, 431

", nerve, a , a

, ,, ,, roots, . . . 799

", ", spinal cord, . . . 865,866

, ,, tymjanmm, . . . . 1161

, ,, vasomotor centre,

,, , , $\quad$ reflexes,

Bianchi on cerebellum,

- 136

856

895,904

772

$\begin{array}{ccr}\text { ", pyramidal tract, . } & \text {. } & 809 \\ ,, \quad, \text { nerves of stomach, } & . & 1012 \\ & . & 886\end{array}$

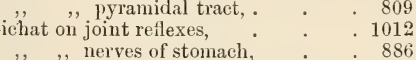

,, , nerves of stomach, $\quad 9 \dot{7} 0,971,986$

", , sensitivity of muscle, . . 1011

Bickel on apresthesia, . . . . 802

,, ,, defrecation, . . . 851

,, ,, spinal cord, $813,817,818,819,841$

Birluer on ciliary ganglion, . . . 664

,, ,, heart ganglia, : . 169,183

,, ", nerves, . . . . 685

, ," secretion of irinc, $\quad . \quad 853$

,', , sympathetic nerves, $648,650,875$

Bidwell on vision, 1073, 1075, 1102

Biedermamn on colour vision,

. 1119

,, electrical organs, . 568

", muscle, 185, 186, 385, 428,

$429,430,431,433,434$,

$436,437,438,439$ nerve, $221,297,453,470,488$, $489,506,509,511,512,513$,

$515,520,521,526,528$,

$552,553,554,555,811$

sense of resistance, . 1022

,Weber's law, . 933

r. Biedl on bulbo-spinal fibres, . . 808

, cerebellum, . . . 895

, luotor area, . . 733

nerve roots, . . . 790

spino-cerebellar fibres, 889

Biertreund on rigor mortis, . 391, 392, 799

Biernacki on prain, . . . . \$\$8, 1001

Bifli on sympathetic, : . $\quad$. $\quad$. 619

Bilharz on eleetrical organs, . . . $\quad .567$

Binet on muscular sense, : . . 1005

$$
\text { , vision, }
$$

Binswanger on fions,

birge on nerve roots,

Birubacher on retina,

Bischoff on blood,

1163

892

791

1050

tymoin

lijornströn on algesimeter, .

1157
Blake on circulation time, PAGE

1235

895

1021

100

1062

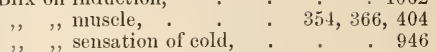

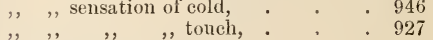

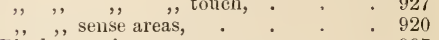

Bloch on pain, . . . . . . 987

,, ," perception of movement, . 1018

," , sensitivity of muscle, . . 1011

", ", touch, . . . . . 924

Blum on mediate spinal fibres, . . 805

Bochefontaine on cerebral cortex, $713,714,716$

$\begin{array}{lll}, \quad \text {, coronary arteries, 165, } & 193 \\ , \quad \text {, intestinal movements, } & 334 \\ \text {, } \quad \text {,, sympathetic nerves, . } & 618\end{array}$

Bockendahl on tympanic membrane, . 1157

de Boeck on spinal cord, . 831, 833, 841

Boeke on phonation, 1216, 1227, 1229, 1230

Boerhave on concha, . . . . 1149

,$\quad$, reflexes, . $\quad . \quad$. $\quad 786$

Bohr" on coronary circulation, . . 165

flu Bois-Reymond on centre for ocular muscles, . 740

,, compensator, . 409

,, cones, . . 1101

,, electrical organs, 569, $570,571,583,585$,

586,587

., intercostal muscles, 279

,, joints, . 241

,, muscle, 353, 411, 413, $422,423,426,427$,

, nerve, $435,453,463$,

$467,469,481,494$,

$505,518,520,521$,

$524,525,546,548$,

556,560

\begin{tabular}{lll}
,, & , pupil, & \\
,, &, reflex stimuli, & 812 \\
\hline & & 828
\end{tabular}

Bokai on pulmonary vasomotor's, · · 151

Boll on electrical organs, $\quad$,, retina, . $\quad 1045,1046,1048$
.

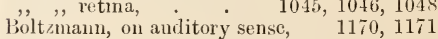

Bonuzzi on nerve roots, . . . 670

Booker on respiratory centre, . . 306

Borellus on centre of gravity of body, . 257

Borgherini on cerebellum, . . . 895

,, $\quad$, spinal cord, . . . . 866

Boruttan on muscular contraction, . 383

, , nerve, $453,496,526,530,531,532$, $546,547,549,550,554$,

Bosscha on recurrent images, $\quad 555,55$

Bosworth on voice registers, . $\quad . \quad 1212$

Botazzi on heart muscle, . . . 197

," , pain,. . . . . 979

", spinal cord,. $. \quad . \quad 865$

Bötteher on semicircular canals, . . 1199

Bouchard on knee-jerk, . . . 874

Bonguer on vision, . . . . . 1080

Bouillaud on cerebellum . . . 894,909

Bouveret on amblyopia, . . 760

Bowditeh on heart muscle, 170, 141, 192, 214,

$215,221,381$

, knce-jerk, . . 873,874 
PAGE

Bowditch on nerve, . . . 473, 831 ,, vasomotor nerves, $134,135,626$ Boyce on cerebral cortex, . 704, 732, 916 ,, ,, meseneephalo-spinal fibres, 864,888 ,

,, ,, pyramidal fibres, . $778,808,809$ ," ," quadrigemino-spinal fibres, . 808 Boycott on nerve, . . . . 475 Boyle on reflexes, . . . . . . . . 786 v. Braam Houckgeest on gastric nerves, 323 , intestinal movements, 328, 331

Brachet ,', ,, nerves, 331, 333 Bradford on arterial pressure, $71^{\circ}, 148,149$,

$$
\begin{aligned}
& \text {,, , cervical sympathetic, . } 622 \\
& \text {, , influcnce of cortex on cir- } \\
& \text { culation, } \\
& \text {, }, \text { vasomotor nerves, * } 714 \\
& \text {," ," , }
\end{aligned}
$$
Bramwell on aphasia, . . . . 776 ,, , cardiogram, : . $\quad 37$

Braun on corona radiata, . . . 706

Braune on centre of gravity of boly, 231, 257,

, , , chronograph, . . $\begin{aligned} & 258,260 \\ & 265,266\end{aligned}$ ", ", joints,. . 234, 237, 240,261 ,, , nasal fossæ, . . . 1249

, , pressure in femoral vein, . 120 ,, ,, walking, . . . . 267

Brentano on pain, . . . . $\quad .975$

Breuer on foveal vision, : $\quad 1087^{\circ}, 1104$ ,, , macular vision,

, ," semicireular canals,

,, vagus nerve, .

. 1087

1200,1201

294,303

Brieger on neerosis of grey matter of cord, 835

Brissaud on knee-jerk, , , visual area,

871,872

. . 758

Broalbent on heart sounds, . . . $\quad 29$

, , ", notor paralysis, . . 735

Broca on limbic lobe, . . . . 765 ,, , olfactory centre, . . $\quad 763,764$ ,, ,, speech centre,

Brockiert on centres for movements of vocal cords,

705,775

Brodhun on colour vision, . $\quad 1090,1108$ Brodic on heat-rigor, . . . 390

Brondgrest on spinal cord, . . 798,870

Brown on bronchial muscles, . $\quad 309$ ,, , capillary pressure, _. . 115 , , cerebral cortex, $758,754,75 \dot{5}, 761$,

762,766

, , senicircular eanals, 1195, 1200,

1201,1202 , on touch,

926

Browne on voice registers,

1214

Brown-Séquard on associated pain, . 981 , , auditory vertigo, . 1198 , cerebellum, . 895, 909 , cercbral cortex, . 714 , cervical sympathetic, 619
Brown-Séquard on corpus callosum, PAGE

$$
\begin{aligned}
& \text {,, } \quad, \text { facial nerve, . } \quad 900 \\
& \text {, , knee-jerk, . . 874 } \\
& \text {, } \quad \text {, respiratory centre, . } 286 \\
& \text {, , rigor mortis, . 391, } 393 \\
& \text {, } \quad \text {, spinal cord, } 838,864,866 \text {, } \\
& \text {,, vagus nerve, . . } 663 \\
& \text {, vasomotor nerves, 131,151, }
\end{aligned}
$$

Bruce on cerebellnm, . . . . . 902

,, ,, corpus callosum, : . . . 779

, ,, lateral limiting layer, : . 805

', ', nediate spinal fibres, $805,806,807$

Bricke on colour tone, . . . . 1053

,, ,, flicker sensation, . . . 1074

, , heart, . . . . . 3

,, , pain, . . . . . 994

,, ,, retinal adaptation, . . 1057

,, ,, rigor mortis, . . . 390

,, ,, semilunar valves, . . 13

", whispering,. . . . 1236

Bruhl ou sensory paralysis, . . . 988

de Brun on cortical paralysis, $729,730,731$

v. Brunn on olfactory cpithelium, 1246, 1247

Bruuner on arterial pressure, . . 71

Bruns on cerebral cortex, . . . 772

, , , contractures, . . . . 731

,, referred pain, . . 981, 982

,, , relation of motion to sensation, $\quad 729$

," shock, . . . 848

," ,, touch, . . . . . . 930

Brunton on blood in muscles, . . 155

,, ,, cortical inlibition, . . 712

,., muscular sense, . . 1004

,, ", taste, . . . . 1242

" $\quad$ " voice, $\quad . \quad$. $\quad .1236$

Bubnofl on epilepsy, . . . 719

,, ,, lost time of cerebral cortex, 610,

,, ,, spinal reflexes, . . . 839

Buchner on nerve, - . . 467

Buck on anditory ossicles, $705,895,899,900,906$

Budge on brain, . $705,895,899,900,906$
, cervical sympathetie, . 622,656 ,

", , cervical sympathetie, $657,622,656,677$

,. ., defecation, . . . 326

,, , micturition, : . . 346

," nerves of intestines, . . 331

", ," ,, stomach, . . 886

", ", pain, ". 970,97i, 978, 1000

, , pupil reflex, . . . 913

", ", pupillo-dilator fibres, . 621, 623,

652, 659

refractory period for muscle, 190

, respiratory centre, . . 306

, Ritter-Valli law, . . . 477

," spinal cord, . . . . 865

", vagns herve, : . . . 991

," vasomotor rellexes, . . $856^{\circ}$

, vesical eentrc, . . . . 86.1

, volume of blook, : : : $\quad .993$

Bulgett on rasomotor nerves, . . 135

Buisson on sphygmograph, . . . 100

liulgik on visceral nerves, . $\quad 163,64: 3,645$

Bull on visual sense, . . . . . 1081

Bulloch on facial muscles, . . . 911

Bunch on nerves of intestinr, $161,332,334$,

$644,645,646,695$

Bureh on capillary electroneter, . . 418 
Buich on colour vision, . $\quad 1075,110^{7}$ ,, , electrical organs, $571,572,574,575$, $576,578,579,584$,

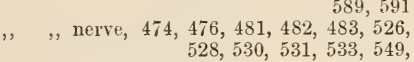
$528,530,531,533,549$,
552,582 ,, , undulatory currents, Burckhardt on knee-jerk,

Burdach on semilunar valves,

Burnett on auditory ossicles,

Busch on hunger, . $8 \dot{71}, 875$

CAJAL on dynamical polarisation of cells, 798 ,, encephalo-spinal fibres,. . 807

,, mesencephalon, · . 915

,, mesial longitudinal bundle, . 890

,, nerve cells, 593, 595, 605, 608, 655, $778,791,806$ , , fibres, . . . . 201 ", nucleus ambiguus, $\quad$ • $\quad$. 886 ,olfactory tract, . . . 764 ,, pedunculo-maxillary fasciculus, 782 , retina,. . 1050 , rubro-spinal fibres, . . 808 ,, sympathetic fibres, . . 686 $\begin{array}{cc} & 758,780 \\ \text { Callenfels on sympathetic nerves, } & . \\ 653,657\end{array}$

Campbell on Clarke's column, $\quad 677$

Cannon on deglutition, . . . 319 ,$\quad$, , refractory period for nerve cells,

615

, , , stomach, . . 322, 324, 325

Caporoso on regeneration of cord, . 879

Cappie on circulation, . . . . 118

Carnelley on Mendelejeff's groups, . 1255

Carpenter on cerebellum, . . 909

,, ganglia of invertebrates, 787,828

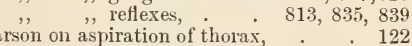

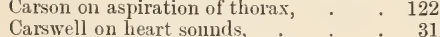

Cartesius on reflexes, . . . 786

Cash on intestinal movements, . . $\quad 329$

,, , muscular contraction, . . 384

,", voice, . . . . 1236

Castex on vocal registers, . . . 1214

Castronovo on olfactory cells, : . $\quad .738$

Caton on excitation of nerve centres, . 605

," nerve, . . . . 525

Cattaneo on muscle spindles, $\quad .1008,1009$

Cattell on reaction time, . . . 612

Cavazzani on circle of Willis, . . 145 ", vasomotor nerres, . 140, 160,

Cavendish on electrical organs,

Ceradini on semilunar valves,

Chantemesse on cliromatolysis,

Chajeaux on Asterias, .

Chareot on aphasia,

cortical paralyses,

knee-jerk,

motor area,

muscular sense,

pain,

spinal reflexes,

('harpentier on musenlar sense, , nerve excitation,
Charpentier on visual purple,

PAGE sense, $1055,1057,1067$, $1070,1072,1073,1074$, $1084,108 \pi, 1098,1121$,

Chauflard on amblyopia . . . 760

Chauveau on blood flow, . . $\quad 84,85$

, " " cardiograph muscles, . 155

, , cardiograph, : $\quad$ coronary arteries, $\quad$. 13,26

", , heart, $7,9,10,14,15,18,20$,

$$
22,23,24,28,30
$$

$\begin{array}{llll}, & \text {, muscular sense, } \quad . & .1020 \\ \text {, nerve, } & . & 482\end{array}$

" " ,, roots, $: . \quad .788$

," , ", pulmonary arterial pressure,

, , semilunar valves, . . $\begin{array}{r}149 \\ 21\end{array}$

", $\quad$, spinal cord, . . . 865

, , touch, . . . 944

", ", vesicular murmur, . . 311

Clieyne on "Townsend case, . . . 46

Chipault on nerve roots, . . . . 804

Chirac on coronary arteries, . $\quad . \quad$. 165

Christ on pulse, . . . . . 101

Christiani on ccrobral hemispheres, 702,890

$$
\begin{array}{lll}
\text {," } \quad \text { mesencephalon, } & \text {. } & 915 \\
\text {, respiratory centre, . } & . & 306
\end{array}
$$

Ciaccio on Pacini organs, . . 1007, 1009

Ciaglinski on spinal grey matter, . 810,811

Cipollone on muscle spindles, . 1008, 1009

Clarke on pain aura, . . . 996, 1001

Clasen on nasal fossæ, . $\quad$. $\quad$. 1249

Cleland on Eustachian tube, $\quad . \quad 1161$

Cloquet on sense of smell, . . . 1252

Coats on vagus nerve, . . . 204, 294

Coccius on ophthalmoneter, . . . 1027

Cohnheim on arterial pressure, . . 145

,, , , capillary circulation, 113,114

, , , cervical sympathetic, . 875

, , , coronary arteries, . 165, 193

", , oncometer, . . 159

" , transfusion, $\quad$. $\quad . \quad 75,77$

, ., venous pressure, . . 121

Cohnstein on arterial pressure, . . 80

", , blood flow in reins, . . 121

Colberg on bladder, . . . . 340

Colin on arterial pressure, . . . 22

Collucci on regeneration of cord, . . $\quad 879$

Colori $\quad, \quad$. . 879

Colson on spinal cord, ." . . . . 836

Combettes on cerebellum, . . $\quad$. 902

Consiglio on vasomotor nerves, $\quad . \quad 627,657$

Contejean on cardiac pressure, . . 18

Cooke on heart beat, . . . 225, 226

Cooper on spinal cord, . . . . . 864

Copeman on lymph, : $\quad$. $\quad$. $\quad$. 992

Corti on spinal cord, . . . . . 865

Cossy on nerve roots, $\quad . \quad . \quad . \quad 670$

Coste on pulse, . . . . . . 101

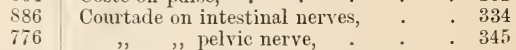

728 Couvreur on vasomotor nerves of liungs, 151

874 Cox on nerve cells, . . 596, 601

748 Cramer on accommodation, . . . 1039

1005 ,, , perception of movement, . 1018

$988 \quad, \quad$ ", third nerve, . . 911

839 Crzellitzé on accommodation, 1034, 1035,

$1011 \quad 1039$

Cumuingham on nerve crossing, . . 797 
PAGE

• . 895 Curtado on nerves of alimentary canal, 695 Cybulski on arterial pressure,

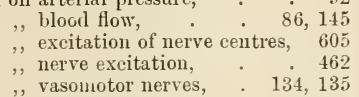

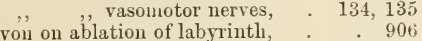

, , accelerator nerves, . . . 199

,", blood flow in veins, . . 121

", , depressor nerve, . . . $\quad . \quad 59$

", muscle length, . . . 869

", ," , tonus, : . . 1020

, nerve roots, . . 670,799

", ,, senicircular canals, $1197,1198,1199$,

1202,1203

$\begin{array}{lll},, \quad \text { uterine contractions, } \quad . \quad & \text {. } 851 \\ ,, \quad \text { vasomotor nerves, . } & \text {. } 156\end{array}$

$\begin{array}{cccc},, & \text {, vasomotor nerves, . } & \text {. } & \text {. } 156 \\ \text {, , , } & \text {, } & 167\end{array}$

Czermak on julse, $\quad . \quad$. $\quad .100$

,, , spinal cord, $\quad . \quad \cdot \quad . \quad 865$

" $\quad$ ", thermal sense, . . . . 965

, , , tonch, . . . 935, 936, 938

", , whispering, . . . . 1236

DALTON on cerebellum,

Dana on associated pain,

,, ,, motor area,

895

849,981

724,730

, olfactory tracts,

. 764

," ," relation of motion to sensation, 729

Danilewsky on cerebral cortex, 713, 714, 752 , excitation of nerve
centres, . . 605 ,, hypnotic state, . . 913 :, nerve, . 501, 505, 506, 525

", spinal cord, sew motion to

Darkschewits
sensation, .

\begin{tabular}{l} 
Darwin on Drosera, \\
\hline
\end{tabular}

,, , sensations, . . . . . 975

Dastre on arterial pressure, . $\quad . \quad 133$

,, ,, cervical sympathetic, $618,621,623$,

,, , erural nerve, . . . 657

," ," inhibitory nerves, $\quad . \quad$. 672

," $\quad$," reflexus ultimus, . $\quad . \quad$. $\quad$. 892

", ,, transfusion, . . . . 76

,, ,, vasomotor nerves, 141, 158, 626,

, , , , tone, . . . 138

Davies on aspiration of thorax, . . 122

Dawson on regeneration of cord, . . $\quad 879$

Daxemberger on mediate spinal fibres, . 805

Deahna on vasomotor reflexes, . . 166

Dean on arterial pressure, . $71,148,149$ ,, ,, intracranial pressure, . . 145 ,, ,, vasomotor nerves, $151,152,153,154$,

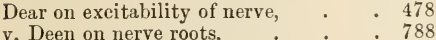

,. , spinal cord, . . 865,867

Dehio on "chromatolysis, . . . 604

Deiters on nerve cells, . . . 592

Dejerine on amblyopia, . . $\quad 760$

,, aphasia, . . . 776

association areas, . $\quad 772$

"Clarke's column, . 806, 811

", dissociation paralysis, $\quad .979$

", facial nerve, . . 891

", fillet,. $\quad: \quad$ : 779
PAGF

781

805

780

pyramidal tracts, $\quad 708,809$
thalamus, - $\quad 768,770,916$
visual area,

pyramidal tracts, $\quad 708,809$
thalamus, - $\quad 768,770,916$
visual area,

$\begin{array}{lll}\text { ", visual area, } & \text {. } & 780 \\ \text { e on muscular sense, } & \text {. } & 1011\end{array}$

$\begin{array}{lr}\text { Delabarre on muscular sense, } & \cdot \\ \text { De la Mure on cerebral pulsation, } & 1011 \\ \text { Delber } & 142\end{array}$

Delbceuf on perception of movement, . 1017 Weber's law, . . . 931

Delépine on visual area, . $\quad . \quad 758$

Demoor on Asterias, . . . . . 886

,, ,, autotomy, . . . . 845

,, nerve cells, . . . 608

,, , reflex movements, . . 834

, ". stereotropism, . . 834

Dentan on spinal cord, . . 878,879

Deseartes on reflexes, . . . 786, 842

Desmoulins on cerebellum, . . 894,899 , cerebral hemispheres, . 699

Dessoir on cold spots, . . 946, 947, 995

,, , cornea, . . . . 923

", ", pain, . . . . . 987, 989

Dewar on electrical response in nerve, . 931 ,, retinal currents, . . . 1052

Deyber on nerve cells, . . . . 608

Dickinson on cerebellum, - $895,899,900$ ,, cervical sympathetic, 618,621 , visceral nerves, $160,201,202$,

646

Dieterici on colour vision, . . . 1107

Dittmar on dorsal column of cord, . 862 ,, , vasomotor centre, . 136, 864

," ," ," reflexes, . . 855

, , , $\quad$ tracts, . . 137

Dixon on eye movements, . . . 1132

", taste, . . . . 1239,1240

,' , visual localisation, . . 1135

Dobrowolsky on visual acuity, : . 1086

Dogiel on blood flow, . . . $\quad 83,84$

,, , heart sounds, . . . 29

", ", nerve cells, . . $\quad 596,601,655$

", ,, fibres, . . . 686,687

", ", paín nerves,. . . . . . . 988

", . . . . . . 1050

," , root cells, . . . . 792

, "., sense spots, . . . . 947

, ,, ureter, . . . . 338

Dolimen on dyspncea, . . . . 290

Dohrn on touch, . $\quad$. $\quad . \quad$. 925

Donaggio on nerve cell, . . $\quad 597$

Donaldson on cerebral hemispheres, . 703

,. ,, cornea, . . . . 923

," ," pain, . . . . 986

", ", ventricular diastole, . 25

,

Donders on accommodation, . $\quad . \quad 1037$

$\begin{array}{cccc}, \quad, \text { cardiac diastole, } & \text {. } & 26 \\ , & , & \text {. } & 38\end{array}$

," centre of rotation of eye, : 1030

", colour vision, 1056, 1092, 1093, $1094,1107,1116$, $1121,1144,1145$

cyclopean eye, . . . 1135

, differential time, . . 614

", on electrical response of

muscle, • 444

eye movements, $1130,1131,1132$

", fatigue of muscle, . 388 
Denders on intra-thoracic pressure, 122,124

124
281

Marey tambonr,

micropsia, .

optic axes,

phonograms,

position of lens,

pressure on eye,

reaction time,

sucking,

visual localisation

"vowel tones, $1215,1216,1217$,

Donkin on Fourier's theorem,

Dor on retina,

1174,1221

$.1050,1103$

Dove on visual localisation, .

Doyon on accommodation,

," , cervical sympathetic, . . 618

, ," vasomotor nerves of eye, . 141

Draper on colour sense, . . . . . 1077

Dreser on metabolism in nerve, . . 454

Drogoul on coronary arteries, . . 165

Dubois on knee-jerk, . . . . 874

Duchenne on muscular co-ordination, . 821 „, perception of posture, . 1014 sensations, . 975

Dudgeon on sphyginograph, . . . 100

Dufour on intrinsic light, . . 1096

,. , mediate spinal fibres, . . 805

Duncan on viscosity of blood, . $\quad 65$

Dunge on knee-jerk, . . . . 872

Dunglison on heart sounds, . . . 28

Dunn on corpus callosum, . . . $\quad 779$

Dupuy on cerebellum, . 894

,, ,, cervical sympathetic, . . 618

", cochlea, . . . . 1183

Dupuys on peripheral ganglia, . . 804

Dupuytren on peripheral ganglia,. . 804

,, , thirst, . . . . 992

Duret on motor centre for larynx, . 742

Dutil on chromatolysis, . . . 604

Duval on slecp, . . . . . 608

Dvoř́k on visual illusions, . . . . 1147

Dziedziul on vasomotor nerves, . 134, 626

EbBingraus on binocular vision, . . 1099

$\begin{array}{cccc}\text { ". } \quad \text {, colour vision, } & .1082, & 1121 \\ \text { Eckhard on centre for ocular muscles, } & . & 932 \\ & .740\end{array}$

$\begin{array}{cccc}\text { ". } \quad \text { ", colour vision, } & .1082, & 1121 \\ \text { Eckhard on centro for ocular muscles, } & . & 932 \\ & 740\end{array}$ cerebcllum, : 895 cerebral hemispheres, . 698 ercetion, . . . 347 fifth nerve, . . . 662 heart-bcat, $170,171,183,209$ inhibitory centre, . . 54 milk secretion, . nerve excitation, 464, 466, 489 nervi erigentes,. . 160, 348 reflexes,

60,348
.$\quad 842$ retractor penis, . . . 349 salivary secretion, . . 716 thalamus, . . . 917 vagus nerve, . . . 204

Edigren oll cardiogram.

Edinger on bronchial muscles, $35,36,38$

102,108 cerebellum, ccrebral hemispheres, corpus striatum, olfactory arca,
Edinger on optic thalamus, .

PACE

.916

, , pain, . . . 1001

,, , spino-thalamic fibres, . . 807

," , third nerve, . . . 911

Edison on phonograph, . . . 1216

Edkins on nerves of intestine, . . 853

Edser on combinational tones, . . 1188

Edwards on influence of temperature on body,

Ehrlich on metabolism,

,I, necrosis of spinal grey matter, 835

Ehrmann on nerves of intestine, . 334, 692

Eichhorst on regeneration of cord, . 879

,, ,, , nerve, 602, 804

Eigenbrodt' on spinal' cord, . $\quad \delta 63,865,866$ Einbrodt on arterial pressure, . 123, 128

Einthoven on bronchial muscles, . 309, 310 ,

,, chromatic aberration, . 1033

,, on electrical phenomena

of heart, . . . 448

,, heart sounds, . . . 30

, stereoscopy, 1144, 1145, 1146

v. Eiselsberg on rigror mortis, . . 779

Eisenlohr on knee-jerk, . . . 872

Eisler on lumbo-sacral plexus, . . 633

Elliott on sensations, . . . . . 969

Emmert on perception of size, . . 1140

Engelken on nerve fibres, . . . 811

Engehmann on axis-cylinder, . . 598

, , bulbus arteriosus, . . 178

, cones, . 1049, 1050, 1099

„. contractility of veins, . 179

, electrical crgans, . . 563

,, eye movements, . . 1132

", heart muscle, $182,185,190$, $194,443,444,446,447$

, intestinal movements, 329,331

,. nerves, . . 331

", ", muscle, . $380,395,39 \dot{6}, 397$,

$432,433,439$

," , nerve, . . 475, 510, 523

,, , retinal currents, . . 1052

,, , ureter, . . . . 338

", ", visual purple, . . 1103

Engstrom on vagal centre, . . . 55

Erb on compression of nerve, . . 480

,, ,, knee-jerk, . . . . 871

," taste, . . . . . 1238,1239

Erben on degeneration of ncrve, . . 839

, , reflexes, . . . 839

Erichsen on coronary arteries, : . $\quad$. 165

Erlenmeyer on knee-jerk, . . . 872

d'Espine on cardiac pressure, : . 19

Eulenburg on heat centres, . . . 717

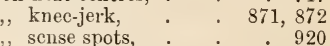

$\begin{array}{lll}\text { ", thermal sense, } \quad . \quad & \text {. } & 920 \\ \text {, } & 948\end{array}$

, thermal sensc,

925,929

Eve on arterial contraction, . . . 675

, , nerve cells, . . . 600

Everett on Fourier's theorem, . . 1221

Ewald on ankle-joint, . . . . 251

,, ,, electrical organs, . . . . 566

,", labyrinth, . : . : 906

", ", micturition, : $\quad \cdot \quad \cdot \quad$. $\quad$. 850

,, ,, neural tonus, . . . $\quad 869$

," , parturition, . . . . 852

", ", rectal sphincter, : . 785,851 
PAGE

Ewald on rhodopsin, . . . . 1047

,, ,, rigor mortis,

799

,, semicircular canals,

spinal cord,

,, vasomotor centres,

", viscosity of blood,

Ewart on electrical organs,

Ewens on visual area,

Ewing on Fourier's theorem,

,, ,, phonograms,

, ,, vowel tones, .

1203,1204

812,811

- $13 \pi$

65

563

. 758

1221

$.1224,1225$

. 1216

Exner on Buhnung,

, conductivity of nerve, . 483, 791

,, conjunctival reflex,

,, colour vision,

- 892

, differential time,

,, flicker sensation,

,, isolation experiments,

,, laryngeal muscles, .

", ', nerves, .

, muscular sense, 1003, 1005, 1018

,, tonus, . . . 1020

,, nerve cells, . . . . 604

,, perception of movenients, 1146 ,

,, persistence of vision,

1148

1068 ,

1072,1076

, pressure effects in eye, . . 1098

,, reaction time,

, reflexes,

$609,612,828$

$835,839,841$

relation of motion to sensation, 729

,, senso-paralysis, . . . 1021

, spinal tonus,. . 870

,, visible spectrun, . . . . 1058

visual area, . . . $\quad 75 \mathrm{~s}$

,, , localisation,. . . 1134

Faivre on arterial pressure,

', ", heart, .

80,149

9,30

317

Falkenheim on intracranial pressure, . 145

Fano on cerebellum, . . . 895

,, ,, heart beat, . . 227

,", ,, muscle, . . 182, 1960, 197

,, mesencephalon, . .913

", reflexes, : $: 773$

Fasola on cerebellum, . . . . . 895

Fechner on after-images, . . 1062

,, binocular vision, 1098, 1123, 1124

, coluur vision,, 1075

,, pain,

972

"sensation, . 933,941, 943

, sense of resistance, . . 1022

", thermal sense, . $\quad 959$

,, touch,

929,930

337,692

Fellner on nerves of rectum,

Fenoglio on coronary arteries,

- 165

Fenwick on papillary muscles,

10

Feré on sensations,

977

1239

Ferrier on ablation of frontal lobe, 730,772

hemispheres, 698, 700, 701

", angular gyrus, . 780

, auditory area, . $761,762,781$

", cerebellum, . $893,895,896,900$.

901,903

conduction in bulb,

corpus callosum, .

888
Ferrier on cortical paralyses,

PAGL

, Deiters' nucleus,

, , , epilepsy, . . . 719

,. ,, functions of brain, . . 706

geniculate bodies. $\quad . \quad 917$

gustatory area, . . . 766

influence of cortex on pupil, 715 ,

mesencephalon, . 911,912,915

metencephalon, . . . 893

motor area, . 732, 733, 734, 738,

$739,741,742,743$,

$744,745,748,749$

muscular sense, . . . 1004

olfactory area, $763,764,765,76 f$

paill, . . . . 978

prefrontal region,. . . 738

pupillo-dilator fibres, . . 621

,, sensory centres, . . . 749

,, spinal cord, . . . . . 867

.,, nerve roots, . . 821

,, tactile area,. . . $\quad 766,767$

", thalamus, . . . 917,918

, visual area, . $752,755,759,780$

,, word-deafness, . . . 763

Fick on accommodation, . . . . 1037

,, , , cardiac pressure, . . . 14,18

,", colour blindness, : . . . . 1111

,., ,. sense, . . . . 1056

,, ,, eutaneous sensation, . . 972

,. ,, dyspucea, . . . . 291

,$\quad$, foveal vision, . . . . $\quad .1087$

,, joints, . . . . 233, 257

,, manometer, . . . . 15

muscle, 244, 245, 246, 247, 256, 354, $356,357,358,359,360,361,362$, $364,365,394,395,397,398,401$,

$403,404,438$

nerve, . 458,500,506,507,931

pendulum movement of limbs, $\quad 269$

pupil, . . . . . 1042

reflexes, . . . 826

retina, . $\quad 1048,1049,1050$

,", Talbot-Platean law, . . 1069

Fieschi on spinal cord, . . . . . 879

Filehne on ear muscles, . . . 892

,, , flicker sensation, $\quad 1071,1076$

," ," intrinsic light, . . . 1096

,, , muscle tonus, . . . 800

Finger on micturition, . . . . 345

Fischer on centre of gravity of body, . 231, $257,258,260$

, chronograpl, . . 265, 266 joints, 234, 237, 240, 252, 261 sense of smell, . 1249,1251 walking, . . 267

Flatau on chroniatolysis, . . . 604 nerve cells, . $\quad 598$

spinal cord, . . . 805,863

vestibular nerve, . .1194

Flechsig on association centres, $769,770,771$

, , auditory area, . . . 781

centripetal fibres of cortex. 768 ,

Clarke's columul,

fillet, $769,7 \% 8$

gustatory area, . . . 766

motor area, . . . 724

myelination, . . . 792

olfactory tract, . . . $\quad$. 
Flechsig on optic nerve, PAGE

805

$\mathrm{s} 08, \mathrm{~s} 09$ ,, oval centre,

,, pyramidal tracts,

, sensory spheres,

temporal gyri,

, visual area,

Fleisch on negative variation in nerve 525

Fleischl on nerve,

Flemning on nerve cells,

Fletcher on retractor penis, .

$$
, \text { vasomotor nerves, }
$$

$$
\text { ,, water-rigor, }
$$

Flexner on regeneration of cord,

Flint on heart valves,

Flood on motor area,

Flörake on vowel tones,

Flourens on cerebelluni,

$$
\text { . } 1215
$$$$
726,742,743
$$$$
894,898,900 \text {, }
$$

903,909

,, cerebral hemispheres, 698, 700 ,

, , internal ear, . . . . 1167

optic lobes,

reflexes, . . . 786

,2 respiratory centre, . 284, 285

, senicircular canals, . 1196, 1198

", spinal cord, . . . 878

Fodéra on spinal cord,. . . . . . 867

Fontana on nerve, . . . . . 925

Förster on accommodation, . . . 1036

,, ,, amblyopia, . . . . 760

, visual acuity, . . . 1055

Forsyth on combinational tones, . . 1188

Foster on asplyyxia, . . . . 307

,, , Astacus, . . . 818, 835

", augmentation, . . . 837

", ", heart beat, . : : . 170

, pain, . . . . . 968

", skeletal tonc, . . . $\quad .377$

," spinal cord, . . $829,834,868$

," , synapse, . . . 607,623

, , visceral sensations, 971, 990, 993

,", vocal cords, . . . . 1210

Fourcroy on odours, . . . . . 1252

Fournié on thalamus, . . . . 917 vocal registers, : . 1214

France on pyramidal tracts, . . . 808

Francke on touch, . . . . . 944

François-Franck on arterial pressure, 53, 148 , bronchial muscles, . 310 on cerebral cortex, . 706 ,

$713,715,716$,

717,732

ciliary nerves, 677,682 epilepsy, $719,720,722$ ganglion stellatum, 681 heart,

", ,

internal capsule, : 777

, lust time of cerebral cortex,

,, pupillo-dilator nerves,

, pyramidal fibres, . 777

, vagal centre, $54,55,57$

, vasomotor nerves, . 154

$158,160,161,164,623$,

644,645
Franeois-Franck on vasomotor reflexes, PAGE

167,856

,, venous pressure, . 119

Franke on nasal cavities, . . . 1249

Fränkel on tabes dorsalis, . . . 799

Franklin on vision, . 1082, 1088, 1121

Frascatorius on heart, . . . . 2

Fratscher on reflexes, . . . . 829

Fratschner on touch, . . . . $\quad .929$

Fraunhofer on colour sense, . $\quad . \quad 1076$

Fredericq on autotomy, . . . 845

, blood stasis in cord, . . 836

, cardiac pressure, 9, 14, 18, 19 ,

$22,23,27$

$$
\text { , cardiogram, . . } 36,37
$$

, nerve currents, . . 521, 526

,, respiratory centre, . 291, 305

,, semilunar valves, . $\quad 21$

," sphygmoscope,. . . I7

, , vagus centre, . . . 129

,venous pressure, . . 119

French on vocal registers, . . . . 1214

Frenkel on dorsal columns of cord, $\quad .863$

Frentzel on taste, . . . . . 1245

Freud on spinal nerve roots, . . . 790

Freusberg on reflexes, . . . . 845

v. Frey on cardiac pressure, 14, 15, 18, 23, 24

,, , cardiogram, . . . 36,39

, , contraction of muscle, . 197, 379 ,

380

,, ,, diastole, . . . . . 26

," pain, . . 966, 985, 986, 987

," ," thermal sensation, 947,948, 952,

", thermal sensation, $947,948,952$,

, touch, . 922, 924, 925, 926, 927, $928,935,937,984,985,986$

,, vagus nerve, . . . 875

,, vasomotor nerves, . . 134

," wave motion, . $\quad . \quad 99$

Friedmann on arterial pressure, : • $\quad$ • 92

, , , regeneration of cord, . 879

Fried'rich on 'Ritter's tetanus, . . 437

Fritsch on electrical organs, . $565,566,567$,

, motor area, . 706, 718, 732, 733

Fröhlich' on sense of smell, . . . 1250

Frommann on nerve cells, . . . . 595

Fuchs on colour sense, . $\quad . \quad$. 1096

, cornea, . . . . . 923

,, nerve currents, $521,525,530,531$

," ,, retinal currents, . . 1051, 1052

, rhodopsin, . . . 1047

Funke on pain, . . . . . . 810

,, ,, pulmonary circulation, . . 126

,, , reaction of nerve, . . . 454

," thermal sense, : . . $\quad$. 958

", ", touch, . . . . . 937

Fïrbringer on knee-jerk, : . . $\quad$. 872

Fürstner on centre for mastication, . 738

$$
\text { ,, ,, dissociation paralysis, . } 979
$$

$$
\text { ", spinal cord, . 811, } 879
$$

Fusari on encephalo-spinal fibres, . . 807

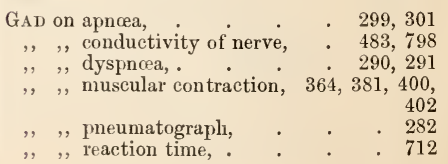


PAGE

Gad on respiratory centre,

,, ,, secolldary sensations,

,, ,, spinal cord,

, , thermal sense, .

,, ,, vagus nerve,

Gaine on visual acuity, .

Galen on pulsation of brain, . ,, spinal cord,

Galton on touch, .

Gamgee on viscosity of blood,

Ganser on anterior commissure,

,, , olfactory tracts,

$$
\text { ", thalamus, }
$$

Garcia on vocal registers,

Garnault on vocal registers, .

Garten on conjunctival reflex, ,, electrical organs, .

Gärtuer on blood sinuses,

$$
\text { , nerve ruots, }
$$

Gaskell on augmentor nerves,

, cardiac muscle, . 169, 170, 172, $173,176,179,180,182,184$, $185,187,193,195,196,205$, $210,222,223,846$

, , , inhibition, . . . 219,674

," , nelve roots, . . . 687

,, vagus nerve, $199,200,201,203$, 204, 212, 213, 219, 220, 663,

665

,, vasomotor nerves, $152,155,160$, $167,348,640,641,659,796$,

855

,, ,, visceral nerves, . . 637,686

Gasser on reflexes,

Gatti on cones,

,", rhodopsin,

. 786

. 1049

1047

Ganle on cardiac pressure, $22,23,24,25,26$

,, ,, heart beat,

26,225

, ,, manometer,

,, ,, sense of smell,

16

Gavarret on vocal registers,

1248,1258

. 1214

Gee on pain,. $\quad .9220$

v. Gehuchten on facial nerve, . . 891 , mesial longitudinal

bundle, . . . 890 , nerve cell, $59 \dot{5}, 600,601$, $602,603,605,655$,

798,806

, $\quad, \quad$, roots, . . 789

," , third nerve, . . 911

Geigel" on cerebral circulation, . . 146

Gellé on internal ear, . . . . 1167

Geluk on heart sounds, . . . 30

v. Gendre on rigor mortis, . . . . 799

Genouville on bladder, . . . . 341

George on muscular sense, . . 1003, 1025

Gergens on reflexes, . . . . 838

Gerlach on bronchial muscles, . . 309

$$
\text { , " nerve cells, . . } \quad 607,784
$$

,, rigor mortis,

Gibson on cardiac movements,

Gierke on respiratory centre, .

Giffing on pain,

Gilbert on muscular sense,

Girard on heat centres, .

Glause on cardiac muscle,

Gley on fibrillar contractious, , , muscular sense,

VOL. II. -85

38

285

986

1011

717

222,223

- 193

. 1004
Gluge on rectal sphincter, . . 813,851

Goldscheider on chromatolysis, . . 604

,, nerve cell, . . . 598

, pain, 966, 985, 986, 987, 989,

999

,, perception of movement, 1014,

$1015,1016,1017$

reflexes, . . 829, 1012

secondary sensations, 981,998

, sense of resistance, 1021, 1022

,, sjots, $920,922,926,928$,

935

, thermal sense, $946,947,948$,

$949,952,955,961,962,964$. $965,984,995,1242$

," touch, . . . . 924

Goldstein on 'dyspmœa, . $\quad . \quad$. $\quad$. 291

Golgi on nerve cells, 593, 594, 597, 605, 607,

797,798

, ,, sense organs,

1007,1009

sympathetic fibies, . . . 651

Goltz on arterial pressure, . . . 854

,, , cardiac nerves, . . . 57

,, ,, pressure, 22, 23, 24, 25, 26 , cerebellum, . . . 895, 899

:, cerebral hemispheres, 698, 702, 704,

773,906

, defecation, . . . . 336

, erection, $\quad . \quad$. 160,854

", gastric nerves, . . . 323

,hunger, . . . . 993

, inhibition, . $58,838,840,842$

, manometer, . 16

, micturition, . . . 345,850

, neural tonus, . . . 869

,, pain, . . . 996, 1000

parturition, . $\quad 351,852$

, semicircular canals, . . 1198

i, shock, . . $846,847,848$

,, sphincter ani, $\quad 785,813,851$

, spinal cord, $812,813,814,842,853$,

, , touch, . . . . . 925

, vasomotol centres, . . 137 nerves, $134,626,657,671$,

Gombault on mediate spinal fibres $\quad 856$

Gorham on colour sense, . . . 1078

Gotcl on action currents in cord, . . 861

, , , conduction of nerve impulses, 613 ,

,, ,, cortical contractions, . . . 711

,, ,, electrical organs, 561, 563, 571, 572 , $574,575,576,577,578,579,584$. $585,587,588,589,591$

,, , knee-jerk, . . . 871,875

", motor cortex, . $\quad$. $\quad .748$

", ,, muscle, . : . . 386, 422

", ,, nerve, 451, 45ं $461,472,474,476$, $481,482,483,485,487,501$, $502,504,521,522,523,525$, $526,530,531,533,549,552$

roots, . . 798, 803
", reflex time, . . . 609

Gottstein on vasomotors of muscles, . 640

Gottwalt on auricular pressure, . . 9

Gouguenleim on vocal registers, . . 1214

Gowers on anosnia, . . . 1248

,, , aphasia, : . $774,775,770$

", "ercbellum, . . . . 902,907 
Gowers on knee-jerk,

PAGE

,, ,, motor area,

", , pain,

, ,, rectal sphincter,

", ,, spinal cord, .

, ,, taste,

,, visual area,

Gradenigo on retinal rods,

Graefe on accommodation,

Grainger on reflexes, $784,787,833,834,835$ , spinal cord, $812,819,857,858$

Grandis on lost time of cortex, . . 610

Grashey on aphasia, . . . . $\quad 776$

,, ,, cerebral circulation, . . 146

, dicrotic wave, . . 109

Grassi on olfactory cells, . . . 788

Grassmann on colour mixture, . . 1082

Gratiolet on motor area, . . . 733

, , sensations, . . . 975

Graves on pulse, . . . . . 101

Gray on auditory sensation, . . .1164, 1190

,, ,, ear, . 1149, 1150, 1151, 1153, 1162

rocal sounds, 1206

Greeff on accommodation,

1038

visual localisation, . 1133, 1134

Gréhan' on breaking strain of vesscls, 74, 119 , systolic output, . . . 48

Griesbach" on touch, . . . 935, 937

Griffiths on bladder, $339,340,341,344,345$

,, ,, cortical contractions, . . 708

," , sympathetic ganglia, . . 787 $\begin{array}{ccc}\text { v. Grijns on cones, } \quad . \quad & . & . \\ ,, \quad, \text { retinal currents, } & \text {. } & .1052\end{array}$

Grinım on vomiting centre, . . . . 326

Grœnouw on visual acuity, . . . . 1086

Grosglick on frontal lobe, . . $\quad 740,772$ ,, transfusion,

Grouiroes on heart,

Gruber on cochlea,

Grimbaum on cerebral hemispheres, . 704 , efferent root fibres, . 793,796 flicker sensation, . 1072, 1076 ,, lateral limiting layer, . 805 spinal corrl, . . . 980 , Talbot-Plateau law, . 1069

Griinde on sense of smell, . . . 1252

Griinhagen on fifth nerve, . . . 662 , nerve, 488, 491, 494, 496, 510,

$546,547,549$

Grunmach on pulse,

103

Gritzner on intestinal movements, . 330

, , nerve, $464,465,466,478,489$, $491,508,525,557$

$\begin{array}{llr}\text { ", } \quad \text { " vasomotor ncrves, } & \text {. } & 167,640 \\ & \text {, vocal registers, . } & 1214\end{array}$

$\begin{array}{rrr}\text {," } \quad \text {, vasomotor ncrves, } & \text {. } & 167,640 \\ & . & 1214\end{array}$

v. Gudden on exeision of eye, . . 781

, tegmental bundle, . . 782

thalamus, . . 916

Guillery on visual acuity, $1055,1056,1084$,

1086

Gulland on vasomotors of brain, . 146, 168

Furboki on vaseular reflexes, . . 856

Guttmanu on heart,

(tuy on pulse,

$$
\text { nerve roots, } \quad \cdot \quad \cdot \quad \cdot \quad 799
$$

(iuyon on nerves of alimentary tract, 334, 695 , pelvic nerve,

IIABERMAN on coclilea,

Hadion on Clarke's columin,
Hagen on pain,

PAGE

. 669

$\begin{array}{cccc}\text { Hales on arterial pressure, } & \text {. } & \text {. } & 77,122 \\ ,, \text { arterioles, } & \text {. } & \text {. } & 130\end{array}$

," , breaking strain of arteries, : 74

, ,, capillary circulation, . . 114

,, ,, circulation, . . . . 67

,, heart, . . . . 42,52

, , spinal reflexes, . . 784,786

,, ., venous pressure, . . . . 119

Halford on lieart,. $\quad . \quad$. $\quad . \quad$. 4,29

Hall on muscular contraction, . . 831

,, ,, neural tonus, . . . . 870

", ", perception of movement, . . 1018

,2, reaction time, . . . 611

, reflexes, . $\quad . \quad 787,825,839$

,., shock, . . . 845

,, , touch, : . : $927,929,936$

,, ,, uterine contraction, . . . 851

Haller on cerebellum, . . . . 894

,, cerebral pulsation, . . 142

," heart,. . 34, 169, 171

,, odours, . . . . . 1252

, pain, . . 970,971,989

,, reflexes, $\quad$. 786,1012

,, sensitivity of organs, . 854, 1011

Halliburton on oncometer, . . . 159

Hallion on vasomotor nerves, $158,160,161$,

$164,644,645$

Hallock on vowel tones,

1220

Hallstèn on reflexes, . . . . 818,826

Hallström on differential time, . . 1188

Hamaker on vision, . 1073, 1074, 1103

Hamberger on intercostal muscles, 277, 278

Hamcl on cedema. . . . 68

Hamilton on corpus callosum, . . 779

Hammerschlag on Eustachian tube, . 1161

Hankel on reaction time, . . . 945

Hanss on regeneration of cord, . . 879

Harless on centre of gravity of body, . 259

,, ., ear, . . . . 1149

", ", muscular sense, . $\quad$. $\quad .1003$

,, :, nerve, . . 464, 488, 799

Harmann on pelvic nerve, . . . 695

, " white rami, . . . 638

Harnack on vomiting centre, . . 326

Harris on visual area, . . . 758,761

Hartmann on touch, . . . 935, 937

Hartwcll on intercostal muscles, . 278

Harvey on heart, $1,2,7,28,34,57,856$

Hasse on cochlea,. $. \quad . \quad . \quad .1180$

$$
\text { ,, muscle spindles, . . . } 1007
$$$$
\text { nerve roots, . . } \quad .790
$$

Haue on micturition, . . . . 850

Haughton on muscle force, . . . $\quad$. 243

Havet on nerve cells, . . . 608

Hayeraft on animal mechanies, . $\quad 228,259$

$$
\text { ,, colour sense, . . . } 1078
$$$$
\text { ", heart. . 4, 5, 10, 11, } 29
$$$$
\text { , pupil, . . . } 1042
$$$$
\text { ," sense of smell, : . } \quad .1246,1254
$$$$
\text { ", taste, . . . 1237, } 1244
$$

Head on gastric nerves, . . . 324

," ,, herpes, . . . 524,576

," , nerve currents, . 524, 531, 538

", ,, refcrred pain, $689,690,816,849,981$,

$982,983,970$

," , respiration, 283, 296, 297, 298, 299,

$301,302,303$

,, , spinal reflexes,

925

," $\quad$," vagus nerve, . $\quad . \quad$. $\quad$. 826 
PAGE

Heese on accommodation, . . . . 1037

, iris,

Heflter" on heart bcat, .

1044

Heger on blood flow in lungs, 128

$$
\text { ,, nerve cell, }
$$

, vascular reflexes,

Hegs oll eolour vision,

340

, epilepsy, heat-production in muscle, 398 ,

400

,, nerve, . . 454, 467, 481

" , roots, . . 798, 799

,, nerves of Anodon,. . 221

,, reduced lost time, . 610,711

, spinal reflexes, . 839

,, vagus nerve, . . 199, 204

, vasomotor nerves, 158,640 ,

, , , , reflexes, . 167

Heinzmann on reflexes, · · 829

Held" on cochlear nerve, $\quad . \quad$. $\quad .763$

, , conduction in bulb, . . 888

", ,, encephalo-spinal fibres, . . 807

", , mesencephalon, . . . 915

", ", mesial longitudinal bundle, . 890

,, ,, nerve cells, . . . 595, 607

,, ,, quadrigemino-spinal fibres, . 808

,. rubro-spinal fibres, . . 808

, ventro-lateral column of cord, . 864

Heller on knee-jerk,

Hellier on nipple-reflex,

Helmholtz on accommodation, 1036, , after-images, 1058, 1062, 1063

, analytical power of ear, 1171, $1173,1174,1176$

, auditory reflexes, . . 1162

", beat tones, . . . 1170

, bristle cells, . . . 1166

", ehromatic aberration, . 1033

cochlea, 1168, 1179, 1180, 1181,

1183

, colour vision, $1010,1075,1077$,

$1106,1107,1108,1109,1111$,

1124

,, combinational tones, 1187,1188 ,

1189

contrast,. . . 1064, 1065

, cyclopean eye, . . 1135, 1136

", electrical stimulation of

eye, . . . . 1097

eye movements, . . 1130

lioropter, . . . 1128

, internal ear, . . . 1159

lens, 1029

, membrana tympani, 1153, 1155

modality, . . 1242

muscle, 247, 362, 380, 381, 388, $398,414,421,831$

muscular sense, 1002, 1003, 1004

nerve, $454,455,458,486,549$

ophthalmometer, . 1026, 1027

pain,

perception of size, : 1140

, polar excitation of visual organs,

, retina,
Helmholtz on semicircular canals, PACE

$$
\text { , sound, . } 1178,1187,1206
$$

,, specific energy of nerve

fibres,

1243

Talbot-Plateau law, 1069

, visible spectrum, . . 1055

"Visirlinic, 1031

,, visual illusions, . 1141, 1147

, vowel tones, 1215, 1216, 1217,

1218,1232

Weber's law, . . 934, 1081

Henke on anklc-joint, . . . . 253

,, , erect posture, . . . 261

", , eye movements, . . . . 1132

nuscular force, . . . 243

Henle on arteries, . . . . . 131

,2, common sensation, . 969,970

" "sphincter vesice, . . 340, 345

". ., sympathetic ganglia, . . 787

Henriques on coronary cireulation, . 165

, pulnionary vasomotors, . 151

Henry on sense of smell, . . . 1250

Henschen on geniculate bodies, . . 917

$$
\text { ", pain, . . . } 1001
$$

". visual area, $\quad 758,780$

Hensen on accommodation, 1034, 1036, 1037,

1039

,, auditory hairs, . . . . 1182

membrana basilaris, . . 1180

", muscular sense, . . . 1005

thermal sense, . . 959

touch,. $.926,927,985$

, tympanic membrane, . . 1157

Hering on acceleration of heart, . . 58

, accommodation, . . 1037

, after-images, $\quad 1063,1124$

,, apæesthesia, 799, 800, 801, 802, 803,

1020

,, arterial pressure, . . . 139

,, binocular vision, 1099, 1124, 1137,

1138

., circulation time, . . . 88

", colour blindness, 1088, 1090, 1091 ,

$1092,1093,1103$

vision, 1054, 1080, 1082, 1084, 1085, 1109, 1110, 1112, $1113,1114,1115,1116,1117$, $1118,1119,1120,1123$

,, contrast, . . .1061, 1065

", convulsions, . . 800

", cortical inhibition, . . . 712

,, cyclopean eyc, . . . 1135

dorsal columns, . . 863

"eye movements, . . . . . .

foveal vision, . . 1088, 1104

"Hebe-phenomen, . . . 800

"horopter, . . 1128, 1129

", induction, : $1060,1062,1066$

intrinsic light, . . . 1123

macular vision, . . 105t, 1087

nyograph, . . . 432

"nerve, $429,453,463,470,508,525$, $528,541,543,582,591$

$$
, \text { roots,. } 790,799
$$

perception of movement, . 1017

pupil, . 1043

sensc of resistance, . . 1021

retinal pigment, . . . $1086^{\circ}$

spinal centres, . . . 289

, reflexes, : . 833,860 
Hering on tetanus, PAGE

,, ,, thermal sense, 950, 952, 953, 957,

960

touch,

933,944

vagus nerve,

294,303

, visual illusions, . . . 1141, 1142

,. ,, incongruence, . 1125, 1126

,, , , localisation, . . 1133

", ", purple, . . . 1104

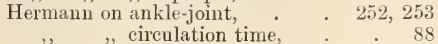

cochlea, . . . 1190

,, differential time, . . 614

tones, 1188,1189

, Fourier's theoren, 1221, 1223,

1224

musele, 384, 410,416,417, 423,

434

nerve, $453,494,496,497,526$,

$531,541,542,545,547$,

$548,549,550,551,552$,

$556,557,559,591,931$

. roots, . . . 798

phonograms, . . 1225,1228

rigor mortis,

391,799

taste,

$1238,1242,1244$

vowel tones, $1216,1226,1232$,

Herringham on cortieal contractions, 1233

Herschel on vision, . . . 1077, 1087

Hersehell on vowel tones, . . 1217

Herter on necrosis of spinal grey matter, 835

Hertwig on cerebellum, . . 894 Meduse, . . . 785

Herzen on dorsal columins of cord, . 863

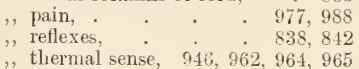

" ," thermal sense, $946,962,964,965$

Heschl on temporal gyri,

i034, 1035,1036 ,

$1037,1038,1039$

colour blinuness, $1092,1103,1118$ vision, 1085, 1086, 1110, 1118

lens, . . . . 1144

pain, . . . 985,986

pupil, $\quad . \quad . \quad . \quad . \quad 1042$

recurrent images, . . . . 1073

visible spectrum, . . . 1058

visual purple, . $\quad . \quad$. 1104

Hesse on heart,

Heubel on convulsion centre, $\quad \begin{array}{r}4,5,10 \\ \quad \quad 887\end{array}$

,, hypnotic state, . $\quad$. 913

Heyman on heat production in muscle, 400

Heymans on heart, . . . 785

nerve roots, $\quad: \quad . \quad 790$
visual illusions, $\quad . \quad 1144$

Hilbert on "' after-images, • 1144

, eardiogram, .. . $\quad 37$

Hill on asphyxia,

blood in muscles, pressure,

cardiometer,

cereliral cireulation,

$\therefore \quad 50$

circulation,

CO. in cerebral activity? . 1

\begin{tabular}{lr} 
depressor nerve, : $\quad \cdot \quad 454$ \\
\hline
\end{tabular}

diastolic filling of beart, : $\quad 45,46$

effert of chloroform on circulation,

entry of air into veins,

Hill on fascia dentata, . . . 764

,. ,, gemnulæ, . . . . 593

,, heat production in brain, . . 718

,, movements of brain, . . . 142

,, , pulse, . . . 104, 111

,, ,, Traube-Hering curves, . . 139

,, ,, vagus inhibition, . . . 56

,, ,, vascular tone, . . . 136, 141

.. , vasomotor centre, . . . 140

Hillebrand on colour vision, . . . 1117 , horopter, . . . 1128

, visual localisation, . $\quad .1135$

Hiltón on associated pain, . . . 981

Hinsdale on knee-jerk, . . . . . 875

v. Hippel on colour-blindness, . . 1094

Hippocrates on inhibition, . . . 838 spinal cord, . . . 864

Hirsch on reaction time, . . . . 945

Hirschberg on nerve, . . . 485, 491

,, vitreous humour, . . 1029

Hirschbruch on touch, . . . 926,928

His on fifth nerve, $\quad$. $\quad$. $\quad$. 1239 heart beat, . . . 179

Hitrig on aphasia, . • • · 202

,, , cerebellum, . . . $\quad$. 894,902 ,, cerebellum, c $732,734,735,752$

., heat centres, . . 717

", relation of motion to sensation, 729

", sense of resistance. . . 1022

Hjort on accommodation, . . . . 1036

Hoche on mediate spinal fibres, . $805,806^{\circ}$

Hodge on nerve cells, . . . 599, 600

Hodgkinson on voice register's, . .1213, 1214

Hoffa on vagus nerve, . . . . 293

Hofmann on uterine nerves, . . 692

Hofmeister on stomach, . . 321,322

Hoggan on touch spots, . . . 922

Högyes on semicircular canals, . . . 1199

Hoisholt on latent period for muscle, . 383

Holden on visual area,. . . 758,760

Holmes on vocal registers, . . . . 1214

Holmgren on colour blindness, . . 1094

," , , vision, . . .1111, 1112

," , nerve cells, . . . 597

Holowinski on heart, . . . 1051

Holtzinger on pain, $\quad . \quad$. $\quad . \quad$. 979

Homén on pain, . $\quad . \quad$. $\quad . \quad$. 979

,, pyramidal tract, . . . 809

spinal cord,. $\quad 865,866$

Hönigschmied on taste, . . . 1238, 1241

Hooper on taste, . . . . . 1242

,, vocal registers, . . . . 1214

Hoorweg on nerve, . . . 4 462, 472

v. Hormbostel on retinal rods, $\quad . \quad 102,110$
$\quad .1048$

Horsley on action currents in cord, . 861

,, cerebellun, . . . 894

, conduction of nerve impulses,

$613,615,718,831$ cortical contractions, - 709, 711 decerebrate rigidity, . . 913 epilcpsy, . • $719,720,722$ internal capsule, . . . 777

, intra-areal localisations, . 737 motor area, $733,734,739,740$, $742,744,745,746$, $747,748,749$ 
PAGE

Horsley on nerve, 457, 480, 521, 522, 523, 525

,,$\quad$, , roots,
,$\quad$, prefrontal region,
,$\quad$, tactile area,
, visual area,

Horton-Smith on nerve roots,

Hösel on fillet,

Howell on heart,

,, vasomotor nerves,

Howells on excitability of nerve,

Hitber on muscle spindles,

,, ,, nerve cells,

,, , sympathetic ganglia,

Hultkrantr on elbow-joint,

Hume on volition,

Hun on visual area,

Hunt on sciatic nerve,

Hunter on arteries,

,, , , eye movements,

,, ,, nerve cells,

Hurst on cochlea,

Huirthle on arterial pressure,

$$
\begin{aligned}
& \text {,, cardiac pressure, } \\
& \text {," cardiogram, } \\
& \text {," dicrotic wave, } \\
& \text {," manometer, } \\
& \text {,, nerve excitation, } \\
& \text {," semilunar valves, } \\
& \text {,, systolic output, }
\end{aligned}
$$

Hutchinson on intercostal muscles,

Huxley on Astacus, , respiration,

Hyde on reflexes,

526,530

798,803

- 772

. 767

- 753

- 789

769,778

$56,225,226$

- 135

990,1009

655,792

- 791

- 239

. 1003

758

167

74,130

. 1131

607

1192, 1193, 1194

$81,133,145$

$14,15,18,20$

30

38

108

16,18

462

21

22

277

280

818

839,844

1215

1112

Isaachsen on colour vision,

Iwanoff on accommodation,

JAcksox on aphasia,

$$
\begin{aligned}
& \text {,, brain, } \\
& \text {, cerebellum, } \\
& \text {, epilepsy, } \\
& \text {, knee-jerk, . } \\
& \text {, olfactory area, : } \\
& \text {, thalamus, . }
\end{aligned}
$$

Jacob on muscle reflexes,

Jacobi on intestinal ncrves,

, sense of resistance, .

Jacolsson on fluid flow in tubes,

1039

,, , intra-thoracic pressure,

774

$697,698,705$

- 902

- 719

- 873

766

917,918

. 1012

332,333

1022,1024

,, semicircular canals,

,, venous pressure,

, vocal cords,

125

. 1199

120

1210

de Jager on intracardiac pressure, $22,25,27$ , pulmonary circulation, 126,149

James on affective tone,

968

, , law of forward conduction, 785,798

,, ,, muscular sense,

$1004,1005,1006$

,, , nervous impulses, .

,, ,, pain,

, , touch,

,", Weber's law,

Jannsen on chronograph,

Järisch on knee-jerk,

Jastrow on colour sense,

Javal on ophthalmometer

Jegorow on ciliary ganglion,

,, ,, pilomotor nerves,
Jegorow on sweat nerves,

PAGE

Jelenffy on vocal registers, . . . 1215

Jelgersma on optic lobes, . 915

Jendrássik on knee-jerk, . . $871,872,874$

,, ,, tabes dorsalis, . . 799

Jenkin on Fourier's theorem, . . . 1221

," ,, phonograms, . .1224, 1225

,, ,, vowel tones, . . . . 1216

Jenner on coronary arteries, . . . 165

Jessop on acconmodation, . . . . 1037

,, cervical sympathetic, . . 618

Joffroy on knee-jerk, . . . . 874

Johanssen on acceleration of heart, . 56

Johannsson on transfusion, . . . . 77

Jolyet on conductivity of nerve, . . 482

,, , superior maxillary nerve, . 662

Jones oll vasomotor nerves, . . . 625

Joseph on nerve roots, . . . . 790

, vagus nerve, . 663

Ju'd on" touch, . . . . . 936

Jurgensen on entry of air into veins, . 120

KABIERski on vascular reflexes, . . 855

Kahler on comma tract, . . . 805

,. , nerve roots, . $\quad 790,804$

", regeneration of nerve, . 602,804

Kaiser on contraction of heart, . 178

,, ,, muscular tension, . 364, 372, 374, $375,376,380$

,, ,, root cells, . . . . 795

Kammler on touch, . . . . . $\quad$. 926

Kant on pain, : . $\quad 1001$

Kato on cerebral hemispheres, . . 704

Kauders on systolic output, . $\quad .51,53$

Kaufmann on blood in nuscles, . . 155

Keen on motor area, . . . . 749

Kelvin on audition, . . . 1174, 1176

Kennedy on coronary circulation,. 165

,, degeneration of ncrve, . 602

, regeneration of nerre, . 804

Kent on heart, . . . . . 186

Kerestzzeghy on regeneration of cord, . 879

Kerschner on muscle spindles, . . 1009

Kessel on membrana tympani, . . 1152

, , ossicles of ear, . . . . 1159

Keyt on pulse, . . . . . 102

Kiesow on cold spots, $946,947,945,952,955$,

,, ,, pain, . . 966, 986,98i, 989

" , taste, . . . . . 1242,1215

, $\quad$, touch, . $922,925,926,927,984$

kiritzow on pain, . . . . . 1000 ,, thalamus, . . . . 919

Kirscher on liypnotisn, . . . . 712

Kirschmann on vision, : $\quad 1061,1121$

Kleen on blood in nuscles, . . . 155 reflexes, . . $82 \pi, 1011,1012$

Kïmucusiewicz on arterie-, . . . 139 , hæmotrhage, . . 75

Klemperer on vocal cords, . . . . 712

Klug on coronary arterics, . . . 13

Ku thermal scise, $954,962,964,965$

finap' on accommodation, . . 1034, 1040

linies on amblyopia, . . . . 759

Knoblauch on regencration of cort, . 879

Kinoll on apnora, . . . . . 301

., arterial pressure, . . . . 149

". ., atropliy of musele, . : . . $\quad 875$

," , , corpora quadrigemina, . . 912

", ", fourth ventricle, . . . 893 
PAGE

Knoll on intracranial pressure, . . 145

, vagus nerve, . . . . 215

Knoller on tonch, . . . 935

Knortz on museular force, . . . . 243

Kobert on eardiae muscle, . . . 223

Kocher on reflexes,

852,856

Koester on visual acuity, : . 1086, 1103
Kohrausch on sphincter vesice, .

Kohnstamm on muscular contraction, . 364

, , spino-thalamic fibres, . 807

v. Kolliker on cones, . . . . 1101

corpus striatum, . . 778

", dorsal columns of eord, . 863

, electrical response of musele, . . 443 fornix, . 782

muscle spindles, . 1009 nerve cells, . 608,655 ,excitation, . . 464 nerves of bone, .1006, 1007 , pre- and post-cellular fibres,

spinal cord, : $\quad . \quad 865$

sympathetic fibres, . 651

third nerve,. . . 911

Künig on colour blindness, 1090, 1091, 1095 vision, $1054,1076,1082$, $1107,1108,1111,1112$

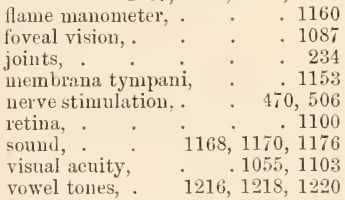

vorwel tolles, $1216,1218,1220$

Konow on depressor nerve,

$$
\text { ,, ,, vasomotor centres, }
$$

$$
\text { reflexes, }
$$

Korinyí on corpus callosum,

855

orpus callosum,
colitional movements,

918

1020

Kornitzer on cardiac impulse,

Koschlakoft on voeal registers,

Koster on foveal vision,

$$
\text { retina, . }
$$

1087,1103

Kottenkamp on touch,

1100

Kottgen on rhodopsin, 935

Kowalewsky on pulmonary cirenlation, 127

$$
\text { vasomotor centres, }
$$
reflexes,

Kräpelin on vision,

137

855

lirause on nerve knots,

1081

respiratory bundle,

retinal rods,

taste,

Krauss on Clarke's column,

Krawsoff on eortical lost time,

Krehl on eardiac pressure, heart,

8, 14, 18, 23

$6,10,12,186,188$

Kreidl on eardiar nerves,

$$
\text { rhodopsin, }
$$

1. hries on blood flow,

$$
\begin{aligned}
& \text { capillary pressure, } \\
& \text { colour blindness, }
\end{aligned}
$$

742

806,811

610

. 1047

87,89

115,116

1089, 1091, vocal cords,

v, Kries on dicrotic wave, . $108,109,110$

, differential time, . . 614

fluid flow in tubes, . . 63

foveal vision, $1087,1088,1104$

, manometer, . . . 78

, muscular contraction, 363, 364, $366,36 \pi, 368,369,831$

, nerve stimulation, . 471, 472

,, predicrotic wave, . . 111

," reaction time, . . 611

,, recurrent images, . . 1073

, visual purple, 1101, 1102

, volitional impulses, . . 615

, wave motion, . . 97, 98, 99

Krimer on vasomotor mechanism, . . 130

Kronecker on bulbar centres, . . 887 eapillary contact, . . 461

, deglutition, . $305,316,317$

318,319

fatigue of muscle, . . 386

heart beat, $189,191,225,226$

muscular contraction, 192,384 ,

nerve stimulation, . $\quad 476$

., neural tonus, . . 869

, phrenograph, . $\quad 283$

, reflexes, . . 828,830

, sense spots, 948

rzywicki on voeal registers, . . . 1215

Kuhlwetter on nerve roots, . . . 670

Külne on motor nerve-endings, . 496, 591

., muscle, . . 437, 438

", ", ,

," nerve, . . 456, 458, 466, 521,$$
\text { pressure on eye, 525, 582, } 925
$$

, ,, retina, . . 1048, 1051, 1105

,, , rhodopsin, 1045, 1046, 1047, 1048,

1101, 1103

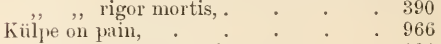

,", tone perception, . . . 1184

kundt on horopter, . . . . . 1128

visual incongruence, . . 1125

", ," illusions, . . . 1142

Kunkel on action of salts on muscle, . 489

, thermal sense, . . . 959

vision 1068,1075

Knorz on ankle-joint, : $\quad \cdot \quad \cdot 251,253$

Kupfier on intestinal nerves, . 331, 333

Kiipuer on pinna, . . . . 1150

Kupressow on intravesical pressure, . 849

Kiirscbner on spinal eord, . . . 864

Kusick on motor centres, . . . 745

Knssmaul on aphasia, . $\quad . \quad+\quad 776$

,, convulsions, . . . 307

micturition, . . 345

liuster on colour blindness, . . . 1092

Kiyri on referred pain, . . . 982,983

LABORDE on cerebellum, . . . 895

$\begin{array}{lll}, \quad \text {, entry of air into veins, } & . & 120 \\ , & \text {, vagal centre, . . } & 54\end{array}$

1092,1103

vision, $1054,1058,1083$,

$1084,1110,1111,1118,1119$ eortical contractions, . 708
Laehr on sensory paralyses, · $\quad 979,980,988$

Laennec on heart sounds, . . . 28

Lafargue on cerebellim, . . . . 900

Lagrange on beat tones, . . . 1170

v. Lair on regeneration of cord, . 878,879

Lallement on cerebellum, . . . 902
Laffont on superior naxillary nerve, : 662 
PAGE ,, ,, nerve cells, 600 , , , stimulation, . . 474 Landergren on 'renal cireulation, . . 160 Landgraf on pulmonary arteries, . 150, 154 Landois on cerebral eortex, . . . 706 ," ,, epilepsy, . . . . $\quad$. 719 , heat centres, . . . 717 , pulse,. . . 102, 108 ,, systole, . . . . 38 ", toueh,

Landolt on eolour vision, . . . 1084 ,, , lens, . . . . . 1029 ,, ,, ophthalmometer, Langdon on nerve stimulation, Lange on eerebellum,

. 1028

. 475 895,899

Langendorff on Clieyne-Stokes respiration, 309 , eortieal lost time, . 610 ,, heart, . . 176, 226 ", nerve stimulation, 467,470 , optic lobes, . . . 914 ,, respiratoly centre, . 286 ,, rigor mortis, . . 391 ,, spinal eord, . $810,839,862$, $863,864,912$

,, vagus nerve, . . . 301 Langer on aecommodation, : . $\quad .1042$ Langley on aceommodation, . . . . 1037 , autonomic system, $660,669,671$ ,, on eerebral hemispheres, 704, 705 , cervical sympathetie, . 618, 621 , $622,624,640,651,791,855$ , commissural sympathetic fibres,

682

, conduetivity of nerve, . . 458

,, degeneration of nerve, 479,652 , effect of nieotin on ganglia, 202, $608,621,791$ , , poisons on heart, . 223 ,, grey rami, . $628,629,637$ , growth of cranial fibres into

sympathetic ganglia,

, hypoglossal nerve, . . 687

, iris, . . . . 1044

,, lumbo-sacral plexus, . . 633

,, motor eentres, . . . 733

, pelvie nerve, $342,666,667,688$,

693

,, pilomotor nerves, $618,621,623$, $627,659,983$ ,, jre-ganglionie and post-gan-

glionic fibres, . . . 623 , psendo-reflexes, . $670,680,681$,

pupillo-dilator fibres, $\quad 682$

,pyranidal traet,. . . . 809

,, regeneration of nerve, 603,653 ,

654,655

, retractor penis, . . 221, 349
, salivary glands, . $\quad 875$

,, salivary glands,. . . 875

$\begin{array}{ll}\text {," splanehnie nerves, . } & 687 \\ \text { submaxillary ganglion, } & .678\end{array}$

$\begin{array}{lll}\text {,, submaxillary ganglion, } & . & 678 \\ \text { sweat nerves, } & . & 627\end{array}$

", sympathetic of ano-genital region, . 635,646 nerves, . 616,649 ,

659

,, , , vagus nerve,

664,665
Iaugley on vasomotor merves, motor nerves, $138,141,157$ $160,626,627,640,643,674$,

675

, visceral nerves, . $202,203,324$, $336,337,343,344,348,349$, $643,646,647,648,695,971$

Langlois on fatigue of musele, ·.

,, ,, sense of resistanee, : $\quad \begin{array}{r}389 \\ -\end{array}$

Lannegraee on visual area, . $\quad 759$

Lapinsky on eonvulsion centre, . . 887

Laquer on word-deafness, . . . . 763

Larionoff on auditory area, . $\quad . \quad 563$

Laserstein on taste organs, . . . . 517

Laslett on degeneration in cord, . . $\quad .861$ mediate spinal fibres, . . 805

Lassaigne on vagus nerve, . . . . 991

Lathan on white rami, . . . 638

Latsehenberger on pulmonary cireulation, . . 126 Laulanié on heart, vasomotor reflexes, * 166

Laura on Clarke's eolumn, . . . . 806

Leber on retina, . . . . . . 1100

Le Conte on eyclopean eye, . . . . 1135

Lee on interual ear, . . . . . 1167

,, ,, organs of lateral line, : . $\quad .1167$

,, ,, semicireular eanals, 1203, 1204, 1205

Legallois on regeneration of cord, . 878

$$
\begin{array}{lllll}
, \quad & \text {, respiration, } & . & 284, & 786 \\
, \quad & \text {, spinal reflexes, } & . & & 811 \\
\text {, } & \text {, vagus nerve, } & . & . & 875
\end{array}
$$

Legros on superior eervieal ganglion, . 875

Legroux on eortical paralyses, $729,730,731$

Lehfeldt on vocal sounds, . . . 1213

Lehmann on affective tone, . . . 968

,, , lieat centres, : : . $\quad 717$

,, , pain, . . . . . 966

,, pulse, . . . . 100

,, ,, sensations, . . 954,977

Leicher on musele, . . . 435

, nerve stimulation, . . $\quad 472$

Leitersdorffer on ereet posture, . . . 263

Lemoigne on cerebellum, . . $\quad 895,900$

$$
\begin{aligned}
& \text {,, , eerebral hemispheres, . } 704 \\
& \text {, , pain, . . . . } 1000 \\
& \text { "Thalamus, } 918
\end{aligned}
$$

v. Lénhossék on dorsal columns of cord, 792,

863

, nerve eells, $594,595,655$,

$793,797,806$

,, , roots, 789, 793, 811

," olfactory cells, . . 788

", pyramidal tract,. . 809

Leonard on vasomotor nerves, . . 135

v. Leonava on amyelous fotus, . . 878

Lepage on pyramils, . . . . 8s9

Lepinay on colour sense, . . . 1077

Lépine on sleep, . . . . . 608

134,662

Lermoyez on roeal registers, . 1214, 1215

Leube on a junca, . . . . . 837

," convulsions, . . . 837

Leuret on notor area, . . . . 733

,., , vagus nerve, . . . . 991

Levi on herve eells, . . . . 594, 596

Lewandowsky on vagus nerve, . 301

Lewasehew on vasomotor nerves, 156,627 ,

Lewes on muscular sense, . . 1002, 1003 
Lewinsky on perception of movement, Lexis on knee-jerk, ,, , motor nerve cells, . $\quad 722,723,777$ Lewisson on reflexes, . . . 838 Lewy on capillaries, . . . . . 112 , intracranial pressure, 146 ,, , viscosity of blood, .

Leyden on Clarke's column, .

$$
\begin{aligned}
& ,, \quad, \text { dorsal columns of cord, } \quad .864 \\
& \text { ", ," pain, . . . . } 999 \\
& \text {,, , perception of movement, . } 1014 \\
& \text {, , pulsation of brain, . . } 142
\end{aligned}
$$

Leydig on nerve cells, . . . . 598

Libertini on reflexes, . . . . . 773

Lichtenfels on touch, . . . . 938

Lichtheim on aphasia,. . . . . 776

,, , pulmonary arteries, 149,150,

Liégeois on sympathetic ganglia, . . 676

Liel on auditory sensations, . . . . 1160

Lindemann on thermal sense, . . 959

Limné on odours, . . . . . 1252

Lion on reflexes, . . . . . . 839

Lipps on visual illusions, . . . . 1143

Lissauer on Clarke's column, . 806,811

Lisso on relation of motion to sensation, 729

Listing on eye movements, . . . 1129, 1130

, , lens, . . . 1029

Lloyd on "encephalo-spinal fibres, . $\quad 808$

,, , Fouricr's theorem, . .1222, 1224

", phonograms, . . . 1230, 1232

, vowel tones, . 1216, 1230, 1233

Lloyd-Jones on hunger, $1216,1230,1233$

Locke on cathodic after-effect, . . . 429 , heart beat, . . . . 226

Lodge on nerve stimulation, . . . 476

Loeb on cerebellum, . . . . 900

,, ,Ciona, . . . . . $\quad .785$

,, ,, consciousness, . . . . 859

", muscular sense, : . 1005,1006

,, ,, nerve-cord of invertebrates, . 817

,, ,, stimulation, . . . 462

,, ,, neural tonus, . . . . 870

,, , pain, . . . . . 975

," , perception of movement, 1017, 1018

,, ,, reflexes, $827,828,830,834,835,839$

,, , spinal cord of shark, . . 817

,, ,, stereotropism, . . 834, 835

," , Thysanozoon, . . . . 846

," , vision, . . . . . . 1148

, , volitional movements, . 1020, 1024

Loiselle on pain,

Lombard on knee-jerk, : . . . 874

$$
\text { ,, reflexes, : : : } \quad: 825
$$

Lo M'onaco on thalamus, · . 9 917, 918

Londe on chronograph, . . . 265

Long on thalamus, . . . . . $\quad$. 916

Longet on bronchial muscles, - : 309 , cerebellum, . $895,899,900,901$ cerebral hemispheres, . 700,705 columns of cord, . . 863,864 deglutition, .

dissociation paralyses, . $\quad 979$ gastric ncrves, . . . 323 nerve roots, . . . 788 pain, . . . 1000 reflexes, . . . $\quad 786$ thalamus, vagus nerve,
PAGE

Lorenzini on electrical organs, . . 561

Lorry on odours, . . . . . . 1252

Lortet on blood flow, . . . 84,85

Lotze on local sign, $934,938,1025,1137,1138$

,, ,, muscular sense, . . . 1003

," ,, pain, . . . 966,997

," , spinal cord, . . . . . . 857

Lovén on erection, . . $\quad 347$

,, ,, great auricular nerve, . 624,699

,, , intra-thoracic pressure, . . 47

", muscle sounds, . . . 1191

", reflex contraction, . 831

,", tetanus, . . . . 426

, vasomotor reflexes, . . 167

Löwenberg on semicircular canals, . 1198

Löwenthal on cerebellum, . . . 894

,, Clarke's column, . . 806

", decerebrate rigidity, . 913

", mediate spinal fibres, . 805

, pyramidal tract, . . 809

,, tract of Gowers, . . 807

Lower on heart valves, .

Löwit on bulbus artcriosus, . . 178

,. , sense of resistance, . . 1022

,, , vagus nerve, . . . 217, 221

,, Weber's law, . . . . . 933

Löwy on cerebellum, . . . . . 894

,, cerebral pulsation, . . . 142

, respiratory centre, . . $\quad 306$

Loye on transfusion, . . . . 76

Lucae on auditory reflexes, . . . . 1162

,, Eustachian tube, . . . 1161

," stapedius, . . . . 1157

,, tensor tympani, . . . . 1157

Luclisinger on acceleration of heart, . 56 , action of poisons on heart, 222 ,

, convulsions, . . . 800

," heart beat, . . . 171

,, nerve, . . . . 491

,, sweat nerves, . . . 623

, vascular reflexcs, . . 855

Lnciani on 'cerebellum, 895, 896, 897, 899, $902,903,904,905,906,907$

cerebral cortex, $732,752,755$,

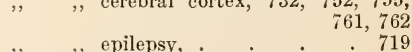

," $\quad$, heart, $\quad \cdot \quad \cdot \quad \dot{25}, 224,226$

," ,, olfactory area, . . . 766

$, \quad, \quad$ relation of motion to sensation,

, word-deafness, : $\quad \therefore \quad 763$

Lideritz on root cells, . . . . 795

Ludwig on acceleration of heart, . . $\quad 56$

, arterial pressure, $78,122,123,854$

, blood flow, . . . 83

depressor ncrve, . . . $\quad 59$

, ganglia of heart, . . . 169

, heart, . $\quad 3,4,5,9,29,34,171$

hunger, . . . . 991

, intestinal movements, . 328

," , , , intestinal movements, nerves, . 331, 334

", ", thermal sense, . . . 954

,", vagus nerve, : . $\quad$. 199, 293

,. vasomotor centres, . . 136

Lugaro on facial nerve, . . . 891

,, nerve cells, . 596, 600, 601, 604

,, reflex collaterals, . . 794

", sleep, . . . . 608

Lunn on vocal registers, . . . . 1215 
PAGE

Lussana on cerebellum, $895,900,901,906,909$ ,, , cerebral hemispheres,

,, pain,

,, thalanius,

, vasomotor reflexes, 167

.1000

917,918

Luschka on Eustachian tube,

. 1161

MAAS on coronary arteries, . . . 695

Macalister on elbow-joint, . . . 239

M'Bride on motor area, _ . . . 749

II 'Cosh on relation of motion to sensa-

tion,

Macdonald on nerve, $, 461,472,485,504$ ,. , whispering, . . . 1236

Macé on colour sense, . 1080

MacGillavry on bronchial muscles, . 309

Mach on colour vision, . . . . 1112

, concha,

1112

,, membrana tympani,

,, muscular sense,

1152

1002

1159

, ossicles of ear,

, pinna,

1163

", semicircular canals, .1200, 1201

M'Kendrick on Appunn's apparatus, . 1168

\begin{tabular}{|c|c|c|}
\hline , & ,, cerebral hemisphe & leres, 701,704 \\
\hline , & , cochlea, & . $\quad 1182$ \\
\hline ," & , ear, . . & 1149,1164 \\
\hline ,' & ,, fenestra rotunda, & . 1160 \\
\hline & ", menbrana tectori & . 1186 \\
\hline & ," nerve currents, & 931,1183 \\
\hline & ,, phonograph, & . 1229 \\
\hline & ,, retinal currents, & . 1052 \\
\hline & ,, semicircular canal & . 1195 \\
\hline & ,, visual area, . & 754 \\
\hline & ,, vocal sounds, & $.1206,1208$ \\
\hline & ,, vowel tones, : & 1216,1226 \\
\hline & on referred pain, . & $689,981,983$ \\
\hline & ," skin reflexes, . & - $\quad 977$ \\
\hline & ay on ta & 1239,1240 \\
\hline & rary artertes, & . 165 \\
\hline
\end{tabular}

,. ., heart, $173,185,18 \overline{7}, 18 \dot{8}, 192$

214, 215

," ," vagus nerve, . : 214,215

Madden on relation of motion to sensa-

tion,

729

. 1238

Magendie on arterial pressure, . . 122 cerebellum, 894, 897, 898, 900

901,902

, cerebral hemispheres, 699

,, deglutition,

,, fifth nerve, . . . 875

314,315

,, heart sounds, . . . 30

, nerve roots, $658,786,788,789$,

797

,, reflexes, . . . 786

,, regeneration of cord, . 879

retina, . 967,969

,, thalamus, . . . 918

, transfusion, 75

,, venous blood-flow, . . 121

vomiting

, , vomiting,

Maggiore on muscular contraction,

Magini on cardiac pressure, .

Magnan on effects of absinthe,

Magnus on colour blindness,

Magrath on coronary circulation, .
Mahn on intestine, $\quad 785,854$

PAGE

Maier on ureters, . . . 337

Mall on arterial tension, $\quad 70$

,, , heart, . . . . . 50

,, , intestine, . $327,329,330,331$

,, , portal vein, . . . 140,643

Malpighi on capillary circulation, 112

Manca on vasomotor nerves, . . 140, 161

Mandelstamm on ophthalmometer, . 1028

Mandl on vocal registers, . . . 1215

Maun on mediate spinal fibres, . . 805

,, motor centres, - . 732

, nerve cells, . . $596,599,600$

,, pyramidal tract, . . . 839

," retina, . . . 1050

Manouélian on nerve cells, . . $\quad .608$

Manouvier, on auditory area, . . 763

Maragliano on vasomotor reflexes, . . 167

Marbe on visual scnse, . . . 1070, 1071

Marcacei on nerve roots, . . 799,821

Marehand on heart beat, . . 171, 183

Marchi on mediate spinal fibres, $808,809,889$

v. Marckwald on respiration, 283, 288, 305

Marcuse on nerve stimulation, . . 505

Marěs on nerve, . . . . 461, 507

Marestant on sensory paralyses, . . 988

Marey on arterial pressure, . . 56, 128

,, , blood flow, . . . $\quad 85,86$

,, , cardiograph, . . . $\quad 35,36$

, extensibility of arteries, . 73

", , fluid flow in tubes, . . 67

,. , heart, $7,14,15,18,19,20,22,23$,

24,28

,, ,, myograph, . . . . . 364

,, , refractory period, . . . 190

,, ,, pneumograph, . . . 282

,, , semilunar valves, . . . . 21

,, , sphygmograph, . . 99,100

,, , vasomotor reflexes, . . 166

, , walking, 264, 265, 266, 268, 269,

Margulies on apæsthesia, . . . . 802

Mariannini on cardiac impulse, . $\quad . \quad 33$

Marichelle on phonograms, . . . 1233

Marie on mediatc spinal fibres, . $\quad 805,839$

Marinesco on chromatolysis, . 599, 604

,, , facial nerve, . . . 891

", $\quad$, frontal lobe, $\quad$ nerve cells, $\quad$. $\quad 595,600$

", ", pyramidal tract, . $\quad . \quad 539$

,", respiratory centre, . $\quad 286$

", ", ventro-lateral colmmis, . 863

Marique on cerebral cortex, . . . 723

Marlow on electrical response of nerve, . 525

Marmian on knce-jerk, . . . 872

Marot on pain, . . . . . 1001

Iarshall on affective tone, . $\quad .967$

\begin{tabular}{lll}
, , regeneration of cord, . & 879 \\
\hline & 993
\end{tabular}

Iartell on vocal registers, . . 1214, 1215

Martin on coronary arteries, . . 13

, , , intercostal museles, . 277, 278

", ". heart, . . 25, 53,56

, , motor area, . . . . 732

", nerve roots. . . . 789

", respiratory centre, : $\quad 306$

", ". vasomotors of heart, . . 166

Martinotti on nerve cell, . . $\quad 597,607$

,, ,, spinal cord . . . . 867

Martius on after-images, . . . 1060 
Martius on cardiogram,

PAGE

37,38

1078

, colour sense,

v. Marxow on nerve centres,

,, :, polarisation of nerve,

Maschek on nerve stimulation,

Masini on vocal cords, .

Masius on defæcation,

$$
\begin{aligned}
& \text {,, intravesical pressure, } \\
& \text {,, regeneration of cord, } \\
& \text {,, spinal reflexes, }
\end{aligned}
$$

Masoin on intercostal muscles,

Matteucci on brain,

$$
\text { ,, musele, }
$$

Matthias on muscle,

. 1033

Maurer on hairs, .

Maxilier on muscular sense, .

Maxwell on colour blindness,

vision, $1081,1052,1106$,

$$
\begin{aligned}
& \text {, , ganglia of Lumbricus, . } 813 \\
& \text {,, ", Nereis, . . . . } 890
\end{aligned}
$$

, specific energy of nerve fibres, 1243

May on nerve roots, . . . $\quad 820$

Mayer on cochlea,

1193,1194

$$
\begin{aligned}
& \text {,, ,, fiftl nerve, } \\
& \text {,, , heart } \\
& \text {, intestinal movements, } \\
& ,,,, \text { nerves, . . } \\
& \text {,', micturition, . } \\
& \text {, muscle, } \\
& \text {, nerve section, } \\
& \text {, stomach, }
\end{aligned}
$$$$
\begin{array}{r}
662 \\
51,58
\end{array}
$$$$
331,333
$$

- 323

Mayhew on conjunctival reflex, . . 892

Mayo on arteries,

$$
\begin{aligned}
& \text {, pupil reflex, . . } 786,812,913 \\
& \text {, supramaxillary nerve, } 803
\end{aligned}
$$

Mazzoni on Pacini organs, . . $100 \dot{7}, 1009$

Meckel on reflexes, . . . 786

Mehlis on pain, . . $\quad$. $\quad . \quad 979$

Meissner on touch, . 923, 924, 938, 966

Mellus on corpus callosum, . . . 779

,, ,, internal capsule,

,, ,, pyramidal fibres,

Meltzer on bulbar centres,

$$
\begin{aligned}
& \text {, , deglutition,. } \\
& \text {," heart beat, . } \\
& \text {,, , respiration,. }
\end{aligned}
$$

778,809

S57

$316,318,319$

189

$304,305,1012$

842

911

Mendelssolnin on nerve currents, . . . 521

$$
\text { , reflexes, . . . } 841
$$

v. Mering on stomach, . $\quad .323$

Merk on spinal cord, . . . . $\quad$. 879

Merkel on pain nerves, . . . . . 988

$$
\begin{aligned}
& \text {," , sense of resistance, . 1021, } 1022 \\
& \text {,, , touch, } \\
& \text {, , vocal registers, } \\
& \text {, vowel tones, } \\
& 1215
\end{aligned}
$$

Merunowicz on heart beat

170,224

v. Meulen on knee-jerk,

871

Heyer on centre of gravity of body, . 257

$$
\begin{aligned}
& \text { contrast, } \\
& \text { erect posture, } \\
& \text { heart, } \\
& \text { joints, } \\
& \text { knee-jerk, } \\
& \text { nerve stimulation, } \\
& \text { cell, }
\end{aligned}
$$

$\cdot 1166$

", ,, vocal registcrs, . . . . 1215

Meynert on anæmia of brain, _ . 992

,, anterior commissure, . . 764

, association fibres, . . 770

, island of Reil, . . . 776

muscular sense, . . . 1003

, olfactory roots, . . . 781

pain,. . . 1000

, thalamus, . . . 918

1215

301,305

979

", sense spots, : - $\quad .948$

,, spinal cord, $805,862,864,867$

,, vasomotor reflexes, . . 980

v. Nilla on facial nerve, 891

Mills on cerehral cortex, $72 \dot{8}, 732,74 \dot{8}, 749$,

763

,, ,, sensory and motor cells, . . 723

,, , vagus nerve, . . . . 214

", , voice registers, 1213, 1214, 1215

Milne-Edwards on pulse, . . 101, 102 ,, superior cervical ganglion,

130

Mingazzini on grey matter of cord, . 811

$$
\text { ,, pain, . . } 1000
$$

, pyramidal tracts, . $\quad 809$

,, reflex collaterals, . . 794

Mink" on diastole,

26

346,717

, intestinal nerves, $\quad$. $\quad 334,643$
,, nerve roots, .

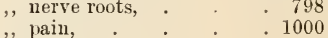

,, respiratory centre, . . 286

, salivary sccretion, $\quad .716$

,, thalamus, . . . 919

Mitchell on cerebellum, _ . 895

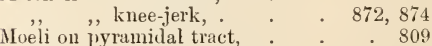

Moens on cardiac pressure, . . . 26

,, ,, pulse, . . . . . . 103

,, , semilunar valves, . . . 12

", wave motion, $\quad$. $\quad 97,98$

Mogk on venous pressure, . . . 119

Moller on laryngeal nerves, . . . 803

Molten on cornea, . . . . . 923

Mommsen on excitability of nerve, . 511

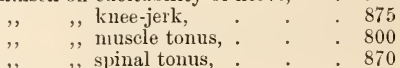

v. Monakow ou cerebral cortex, $705,758,762$,

$778,780,781$

„, Deiter's nucleus, . . 808

,, excision of eye, . . 781

geniculate bodies, . . 917

", optic lobes, . . . 915

", tract, . . . 780

,, pyramidal tract, . 778,809

,, thalamus, . $768,916,917$

Mönckeberg on nerve cells, . 594, 598, 601

Monckwald on deglutition, . $319,320,321$

Monro on intracranial blood, . . 144

, , reflexes, . . 786

Monti on nerve cells, . . . . $\quad$. 608

,2, spinal cord, . . . $\quad 879$

Noore on conductivity of nerve, . 483,791

,, nerve cells, . . . 604

", ", plethysmograph, : : . 162

,, , spleen, . 163, 164, 643, 645 
PAGE

Moore on vagus nerve, .

663

Moos on cochlea,

1167,1182

Morat on accommodation,

1037

,, arterial pressure, . . . 133

,, cervical sympathetic, 618, 621, 623,

crural nerve, $\quad 624$

, gastric nerves, . $\quad . \quad 323,324$

,, inhibitory nerves, . . . 672

," nerve roots, . . . . 790

,, nerves of genital organs, . 348

,, sympathetic ganglia, . 675,676

, vasomotor nerves, $138,141,158,626$, $659,669,671$

Moreau on electrical organs, .

574

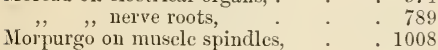
Morris on Grimm's law, . . . . 1235 Moser on deglutition, . . . . . 319

Mosso on arterial pressure, . . . 80 ,, ,, bladder, . . . 864,869 ," ,, eardiac diastole, . . . . 25

,, ,, cerebral activity, . . . . 25

,, ,, effect of gravity on circulation, $\quad 95$

," ,, fatigue, . 387, 359, 598, 605, 992

,, , heat production in brain, . 718

,, , intra-thoracie pressure, . . 47

,. , micturition, . . . . 346

," cesophagus, . . . . $\quad .320$

,, , pulsation of hrain, . . 142

,, ", respiration, . . . 280, 309

,. , sensations, . . . . 977

," , taste, . . . . . . 1242

Motora on tonch, . $\quad . \quad$. $\quad . \quad .929$

Motschutkowsky on pain, . . 985, 986 Mott on apresthesia, . . 799, 802, 803 , , , cerebral cortex, 724, 730, 739,740,

, Clarke's column,

$756,76 \mathrm{~s}$

, eorpus callosum,

806,811

, fillet,

,, motor paralysis,

,, musele tonus, .

,, nerve roots,

,, oncometer,

,, pain,

,, rigor mortis,

,, root cells,

, senso-paralysis,

,, spinal cord, $865,866,870$

,, spino-quadrigeminal tibres, . 807

,, ,, thalamic fibres, . . 807

,, ", traet of Gowers, . . . 807

Motter on pain,

- 987

. 1235

- 872

805,506

1103

1097

1168

- $1166^{\circ}$

. 1179

", bristle cells, .

, cochlea,

," eolour visiou,

$1077,1106,1120$

,, cones, .

1101
1064

,, dyspnca, . . . $\quad . \quad 289$

,, electrical response of muscle, 443 ,

931 ", eye movements, $1030,1129,1132$
Mïller on horopter, PAGE

1127

,, law of forward conduction, . 798

,, muscular sense, 1003, 1004, 1009

,, nerve roots, . . . 788, 789

,, neural tonus, . . . 870

", phosphenes,. . . . 1124

,, pilomotor nerves, . . . 618

,, regeneration of cord, . . 879

,, retina, . . . . 1100

, sense organs, . . . 487

, , , , of resistance, $1022,1023,1024$

," ", specific encrgy of senses, 969,1242 ,

1253

, , spinal ganglia, . . . 787,982

,, ,, sympathetic pain, . 981, 983

,, , touch, . . . . 929,934

", vocal sounds, ".

Munk on bulbus arteriosus, . . 178

,, ,, cerebral cortex, 701, 703, 713, 724, $725,726,732,733,745$, $753,754,755,757,760$, $761,763,766,767,768$

,, ,, contractures,. . . . 731

,, ,, epilepsy, . . . . . 719

," , museular sense, : . $\quad .1003$

Miinsterberg on muscular sense, 1003, 1005,

1025

Miinzel on arterial pressure, • • 966, 975

Miinzer on cerebral hemispheres, . $\quad$. 704

,, ,, mediate spinal fibres, $805,806,808$,

„, mesencephalon, . . 915, 916

,. necrosis of spiual grey matter, 835 ,

, ., Mecrosis of spinal grey inatter, 885,

,, , nerve roots, . . . . 790

,, „, optic lobes, . . . . 913

," , spinal cord,. . $\quad 810,862,863$

Muratoff' on cor'pus callosum, . 779,918

$$
\text { , , , facial nuscles, . . } 911
$$

,, , , pyramidal fibres, . $\quad 779,809$

Murou on entry of air into veins, . . 120

Murray on cardiogram, . . . 37

Muskens on apæsthesia, . . . 803

, . . . . . . 871

Muybridge on chronograph, . . . 265

Nabarro on blood in muscles, . . 155 , carbonic acid in cerebral activity, . . . 454 , heat production in brain, . 718

Nigele on pulse, . . . . . 102

Nagel on cold spots, . . $\quad 946,947,995$

, . colour blindness, . . . 1091

,", double vision, : . . . 1126

,. ., eye movements, . . . . . 1131

,, , foveal vision,. . . . . 1104

, pain, . 985, 987

,, ", rigor mortis, . . . 391, 392

, touch spots, . . . 922,923

Nahmmiacher on cones, . . . . . 1050

Namias on earliac impulse, . . . 33

Nansen on nerve roots, . . . . . 790

Nassc on defiecation, . . . . 33ti

,., intestinal movenents, . . 331

Natanson on capillary pressure, . . 116

Namyn on intracranial pressure, . . 145

, pain, . . . 998

," regeneration of cord, . . 879

,, nerve, 60:2, 804 
PAGE

,$\quad \cdot \quad+768$

Nawrocki on dorsal columns of cord, . 862

, ,, nerves of bladder, . . 342

", , pseudo-reflexes, $\quad . \quad$. 679

, , ", pupillo-dilator fibres, . 621

", , sympathetic ganglia, : 787

, thermal response of museles, 401

Nélis on nerve cells, . . . 601, 602

Nettleship on colour blindness, . . 1089

Neubuirger on cerebellum, . . . . 902

Neumann on polar excitation of nerve, . 506 , vocal cords, . . . 1209 ", , registers, . . . 1215

New on enterograph, . . . . 328

Newport on ganglia of invertebrates, . 787

Newton on colour triangle, . . . 1081

Nicati on colour sense, . . . $\quad .1077,1080$

Nicholson on taste, . . . . 1237

Nicolaides on reflexes, . . . . $\quad .828$

Fich ", vasomotor tracts, . . 137

Nicolls on bloot flow, . . . . 86

$$
\text { ,, ,, elasticity of arteries, : . } 74
$$

," lıæmic murmurs, . . . 33

,, , hæmodynamics, . $63,65,66,68$

, , wave motion, . . 96, 97

, work of heart, . . . 42

Nissl on "ehromatolysis . $\quad . \quad$. $\quad . \quad 604$

$$
\text { ," , nerve cell, . . 597, 598, 599, }
$$

$$
\text { ,, ,, neuroglia, . . . . } 879
$$

" , thalamus, $\quad . \quad \therefore \quad .916$

Norman on Allolophobus, . . . 846

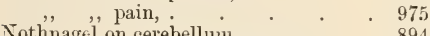

Nothnagel on cerebellum, . . . 894

, ,, cerebral hemispheres, . 702

,, corpus striatum, . . 778

,, intestinal movements, . 330

," paill, . .999, 1000, 1001

,, spinal cord, . . . 838

," thalamus, . . 918,919

", thermal sense, . 948, 959, 961,

, visual area, . . . 758

Novi' on cortical lost time, . $\quad . \quad . \quad 610$

Novizki on perception of size, . . 1140

Nuël on inhibition, . . . . 204

Nussbaum on pseudo-reflexes, . . $\quad .679$

," , sympathetic ganglia, . 787

Nysten on rigor mortis, . . 390

Obersteiner on allochiria, . . . 866 ,, visual area, . $\quad .758$

Obolensky on spermatic nerve, : $\quad 875$

Obregia on cerebral cortex, . $752,755,756$

Oddi on spinal reflexes,. $\quad . \quad$. 773

," , , , roots, . . . . 799

Oelirwall on taste, $995,1240,1242,1244$

Oertel on vocal sounds, . . 1213,1215

Ogle on aphasia, . . . . 776

,, ,, sympathetic nerves,..$\quad 657$

Ognefl' on electrical organs, . $\quad$. $\quad 566$

Ohin on sound.

Ohrn on heart beat,

$.1173,1174$

O'Kinealy on Fourier's theorcm, · 226

Oldag on nerve stimulation, . . . 470

", , vagus nerve, . . . . . 301

Oliver on depressor nerve, : . $\quad$. $\quad$. $\quad 60$

,, , pulse,

,, , suprarenal extract,

79,95

138,193
Ollier on cervical sympathetic, PAGT

Ollivier on section of cord $\cdot \quad 875$

Omodei on vomiting, . . . . . 325

Onimus on cerebral hemispheres, . . 698

Onodi on mesencephalon, . . . 913

," ., sympathetic ganglia, . . 202

Openchowski on nerves of alimentary

tract, . . . 696

," pulmonary vasomotors, 151

,, stomach, . 323,325

Oppenheim on 'Clarke's column, . . $\quad$. 811

$$
\begin{array}{llll}
\text { " , cortical lesions, } & \text {. } & 772 \\
\text {, dissociation paralysis, } & . & 979 \\
\text {, } \quad \text {, facial muscles, } & . & 911
\end{array}
$$

Orchansky on reaction time, . . $\quad .712$

Ordenstein on knee-jerk, . . . 874

Osawa on pain, . $\quad . \quad 5 \quad . \quad 5979$ , spinal cord, : $\quad . \quad$. $\quad 835,866$

Ostankoff on cerebral cortex, $\quad . \quad 713$

Osthoff on pain, . $\quad . \quad+\quad .999$

Ostroumoff on vasomotor nerves, . 156,626 ,

Ott on defrecation, . . . . 851

,, ,, heat centres, . . . . . 717

,, , intravesical pressure, . . . 849

,, , spinal reflexes, . . . . 838

,", vesical centre, . $\quad . \quad+\quad . \quad$. 864

Oulmont on amblyopia, . . . 760

Outanskow on pain, . . . . 1000

Overend on papillary muscles, . . 10

Owen on air bladder, . . . 1168

Owsjanuikow on spinal cord, . 841,886 ,, , vasomotor centres, . 136

PAcini on electrical organs, . . 565, 568

Page on electrical response of muscle, . 444 ,

Pagliaui on cardiac diastole, 445, 446

Pal on intestinal movements, $\quad . \quad$. $\quad 334$

Paladino on root cells, . . . . 793

Pandi on chromatolysis, . . . . 604

Paneth on motor area, . . . . 736

,, , sympathetic nerves, . . 657

Panizza on nerve roots, . . 789,801

916,917

Panum on vision, . $\quad . \quad$. $\quad .1127,1137$

Parinaud on after-images, . . 1064

. 1087

. 1096

. 1101

- 912

- 838

. 1255

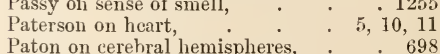

Patrick on spino-thalamic fibres, · : $\quad$. 807

Patten on pain, . . . . . 976

Paulsen on phonation, . . . $\quad .1207,1248$

Paulus on touch, . . . . . 935

Pawlow on arterial pressure, . . . 82

,, , gastric secretion,. . . $\quad .887$

221

54

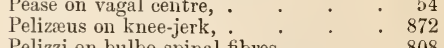

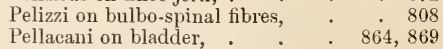

Peltier on polarisation currents, . . 427

Penzoldt on sense of smell, . . .1249, 1251

Pergens on cones, . . . . . 1049, 1050

,, rhodopsin, : $\quad . \quad .1047$ 
PAGE

Perlia on third nerve, . . . . 911

Perrault on reflexes, $\quad . \quad . \quad . \quad$. 786

Petit on cerebellum, . . . . $\quad$. 900

,, ,, cervical synipathetic, . . 618

Petrina on relation of motion to sensa. tion,

Pfeiffer on pulmonary circulation, - 126

Pflüger on colour blindness, . . . 1104

, , dyspnoea, . . . . 290

". intestinal nerves, . . . 333

, ," nerve, . 453, 478, 494, 503, 504,

507,548
5

,, nerves of salamander, . . 886

, pain, . . . . 974

,, polarisation of muscle, . . 436

", reducing substances of blood, 290

,, respiratory centre, . . 291

," spinal corl, . $817,821,826,830$,

$$
\begin{array}{ll} 
& 856,857 \\
& 132,659
\end{array}
$$

, , , vasomotor nerves, - 132, 659

Philipeau on cerebellum, . . . 900

Philippe on mediate spinal fibres, . . 805

Piccolo on regeneration of cord, . . . 879

Pick on comma tract, . . . . 805

,, „, motor area, . . . . . 739

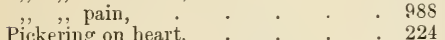

Pineles on laryngeal nerves, . $\quad \cdot \quad 726,803$

,. , spinal tonus, . . . 870

Piotrowsky on circulation in penis, . 160

$\begin{array}{llll}, & \text {," }, \text { tongue, } & . & 141 \\ , & \text {, muscular tone, } & . & 377 \\ ,, & \text { nerve, . } & . & 491\end{array}$

Pipping on sound, . $1216,1223,1224$

Pitres on cerebral cortex, $706,713,715,716$,

$$
\text { , } \quad \text {, cortical paralyses, . } \quad 717,732,748
$$$$
\text { "epilepsy, } \quad 719,720,722
$$$$
\text { ," internal capsule, . . . } 777
$$

", lost time of cerebral cortex, . 610

", pyramidal fibres, . $777,809,888$

,, sensory paralysis, . . 998

Plateau on after-inages, . 1058,1063 ,

, , muscles of insects, . . $\begin{array}{r}1066 \\ 243\end{array}$

", touch, . . . . 930

", ". visual sense, . . . . . 1068

Ploschko on nerve cells, . . . 655

Poiseuille on arterial pressure, $78,81,122$ ,, ,, blood flow, . . . 89

", circulation timie, : $\quad . \quad 88$

", fluid flow in tubes, . . $\quad 64$

", pulmonary circulation, . 125

Pole on colour blindness, . . . . 1094

Polimanti on colour blindness, . . 1092

Politzer on auditory reflexes, .1162, 1163

,, binaural audition, . . 1163

", concha, . . 1149, 1150

,, ear movements, . . . 1152

"Eustachian tube, .1161, 1162

", external meatus, . $\quad .1150,1151$

,, membrana tympani, . 1152, 1157

,, ossicles of ear, . . . 1159

", semicircular canals, : . 1205

", sound transmission, . . 1161

, stapedius, . 1157

Pollak on auditory reflexes, . . . . 1162

, , tensor tympani, : . 1157

Pope on taste, . . . . . 1240

Popielski on conductivity of nerve, : 484
Porter on cardiac pressure, PAGE

$9,19,23$,

24,27

. 165

. 16

858,863

287

21

663

166

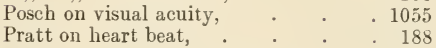

Preobraschensky on cerebral cortex, . 713 ,, vasomotors of kidney, . . 159

Pretori on contrast, . $\quad . \quad$. 1061

Prévost on knee-jerk, . . $\quad 871,873,875$ ,, spheno-palatine ganglion, 651,662

Preyer on audition, . . . . 1169,1170

," ., autotomy, . . . . 845

, $\quad$, colour blindness, . . . $\quad .1090$

,",$\quad$ vision, . . . 1121

, , reflexes, . . . 827,834

," ", Santoniu vision, . . . $\quad .1095$

, , semicircular canals, . . . 1195

,, , stereotropism, . . . 834

, ", touch,. . . . . 924

Pribram on cardiac inhibition, _. 58

Prochaska on spinal cord, . $786,857,878$

Protopopow on ureters, . . . 338

Przybylski on pupillo-dilator fibres, . 621

Pugliatti on cerebellum, . . . 895

v. Pugliese on facial nerve, . . . $\quad$. 891

Pugnat on nerve cells, . . . . $\quad$. 600

Pupin on $\quad$. . . . 608

Purkinjc on colour vision, 1076, 1078, 1099

,, , electrical stimulation of eye, 1097

", , intrinsic light, . . . 1123

". ", recurrent images, . . 1072

" "

", ", sympathetic pain, : . 981

,, , vertigo, . . . 1196, 1197

Putnam on corona radiata, . . . 706

Pyc-Smith on sympathetic nerves, 657,677

QUINCKE on associated pain, . . 981

Quinquaud on breaking strain of blood 126 vessels, . . 74,119

,, systolic output, . . 48

RABL-RUCKHARD on slecp, . . . 608

Rach oll respiratory centre, . . . 288

Raehlmani on eye movenients, : . : 1132

,, , touch, . . . . 944

," $\quad$,, visual area, . . . $\quad$. 759

", ", , localisation, . 1135

Rámon on funiculus retroflexus, . . 782

Ramsay on sense of smell, . . .1255, 1256

Ranke on blood in muscles, . . . 155

fatigue of muscle, . . . 389

nerve, . . . . 489

Ransoni on action of poisons on heart, . 224

, , , motor area, . . . 730

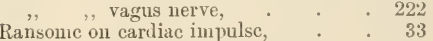

Ranvier on degeneration of ncrve, . 602

, heart beat,. . . 189

,, pain nerves, . . . 988

,, regeneration of nerve, . 479 
Ranvier on vasomotor reflexes,

Rauber on Pacini organs, , thernial sense,

Rawa on nerve crossing,

Rayleigh on auditory sense, . ,, colour blindness,

167
1099

$100 \%, 1009$

964

797

1163,1171

$.1093,1118$

, $\quad$, sound, 1164, 1169, 1170, 1173, $1174,1176,1177,1188,1189$,

1232,1233

Rebatel on coronary arteries,

13,26

Rechberg on

Recklingliansen reseneration of eorl, 879

Redi on spinal frog,

Redlich on hemiplegia, .

818

731 mediate spinal fibres, . 778,805 ,

Reich on accommodation, 808, 809

Reid on heart beat,

,, ,, iris, .

,, sympathetic nerves, .

Reil ou sympathetic ganglia,

Renak on ganglia of heart, .

$$
\text { ,, knee-jerk, }
$$

Rénant on nerve cells, .

Renz ou touch,

Renzi on cerebellum,

,, ,, cerebral hemispheres,

, , optic lobes,

, , thalamus,

Rethi on vocal registers,

Retzius on coclilea,

$$
\begin{aligned}
& \text {, , electrical organs, . } \\
& \text {,, limbic lobe, } \\
& \text {, nerve cells, } \\
& , \text {. , roots. }
\end{aligned}
$$

Reuter on sense of smell,

Reyfisch on bladder,

Reyher on vowel tones,

Reyuolds on nerve,

,, ,, cells,

Rezek on corpus striatum,

Richet on cerebral cortex, 706, 708, 709, 713

$$
\begin{aligned}
& , \text {, , heat centres, } \\
& \text {,, , pain, . } \\
& \text {,, , reaction time, } \\
& \text {,, reflexes, }
\end{aligned}
$$

688

169

872

650

608

929

895

701

913

917,918

1215

- 1186

563

765

596, 655

$788,789,790$

- 1250

340,346

. 1215

483,791

604

778

714

614

,, sense of resistance, . . 1022

, summation of stimuli, . . 380

, , touch,

Riecker on touch, .

Riegel our auricular pressure,

$$
\text { ,, ,, bronchial nuscles, }
$$

, ,, eontraetion of arteries,

, ,, lieat centres, .

, venous pressure,

Rimpler on after-images, ,, optic nelve,

ger on action of poisons on heart,

$$
\text { , heart beat, }
$$

Ritter on excitability of nerve,

livers on vision,

Robertson on nerve cells,

Rogers on cyclopean eye,

$$
, \text { visual localisation, }
$$

Rolimann on metabolism in nerve,

Röhrig on mill secretion,
Rolando on cerebellum, . $\quad 894,901,909$ ,, eerebral hemisphere, . . 700

$$
\text { ", "thalamus, . . . . } 917
$$

Rolleston on cardiac pressure, . 14, 18, 25

$$
\text { ,, ,, nerve fibres, . . . } 598
$$

Rollett oll contrast, . . . . 1061

,, ,, Golgi organs, $\quad . \quad .1007,1009$

," , muscular contraction, . 384, 385

,, , nerve,. . . . 480, 487

", sensitivity of tendon, . . 1011

- Romanes on Asterias, . . . . $886^{\circ}$

, heart beat, . $180,222,846$

,Hedusa, . . . . 999

,, stereotropism, . . . 834

Tiaropsis, : 785,832

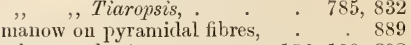

Romberg on heat, . . 186, 188, 202

Rood on colour vision, 1053, 1077, 1083, 1118

Rose on santonin vision, . . . 1095

Rosenbach on cortical stimulation, . 752

$$
\text { , epilepsy, . } \quad 719
$$$$
\text { „, pain, . . 998, } 1000
$$$$
\text { ,, sensory paralysis, . . } 988
$$$$
\text { ," thalamus, . . . } 919
$$

Rosenberger" on heart ganglia, . . . 169

Rosenheim on knee-jerk, . . . 871

Rosenthal on heart beat, . . 169,170

$$
\begin{aligned}
& \text {,, inhibition, . . . } 219 \\
& \text {,, muscular foree, : . } 243
\end{aligned}
$$$$
\text { , nerve, . } \quad .460,504
$$$$
\text { , reflexes, - 818, 829, 837, } 841
$$$$
\text { ,, respiration, 282, 283, 288, 289, }
$$
$290,293,294,300,301,303$,

, sphincter vesice, . $\quad 340$ $\begin{array}{ccc}\text { Ross on aphasia, . } & \text {. } & 516 \\ \text {. } & \text {. } & 776\end{array}$ $\begin{array}{lll}\text {, referred pain, . } \quad . \quad 689,691,849 \\ \quad . \quad & 226\end{array}$

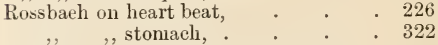

Rossolymo on dissociation paralysis, $\quad 979$ ,, , spino-quadrigeminal fibres, 807

Roth on nerve stimulation, . . 475,476

Rotlimann on wotor centres, . . 745

," , pyramidal tract, . . 809

Rouanet on heart sounds, * · $\quad \begin{array}{r}31 \\ \text { Roussy on coronary circulation, } \quad 165,193\end{array}$

$\begin{array}{lll}\text { Routh on uterine contractions, } & \text {. } & 852\end{array}$

Roy on aorta, . . . . . 954

,, ,, arterial pressure, . . . 80

", "bladiler, : . 342

,", bronchial museles, . . . 309

, , eapacity of veins, . . . 118

,, ,, eapillary pressure, . . . . 115

, , eardiometer, . . . 49, 43

,, ,, extensibility of arteries, . . 73

, heart, $6,10,11,18,25,41,188,216$,

219

, ,, myocardiograph, . . . . 39

,, , oncometer, . . . 159, 162

, , size of brain, : . $\quad . \quad$. 145

", ", size of brain, $\quad \cdot \quad \cdot \quad \cdot \quad \cdot 162$

," "Traube-Hering curves, . . 139

$\begin{array}{llr}\text { ", Traube-Hering curves, . . } & \text {. } & 139 \\ \text {, vagal centre, . } & \text {. } & 59\end{array}$

", "vasomotors of heart, . . 166

Rücker on combinational tones, : $\quad .1188$

Rudinger on intellectual faculties, . 772

Rudnow on reflexes, . . . . 829

Ruete on eye movements, . . . 1129

Ruffini on Pacini organs, $1007,1008,1009$ 
Ruffini on plume organs,

Ruine on cochlea,

Rusch on apncea, .

Russell on cerebellum,

,, encephalo-spinal fibres,

,, knee-jerk,

, motor centres,

, nerve roots,

Rutherford on cochlea, .

1190, 1191, 1192

, compound tones,

, , muscle sounds,

1190

, polarisation of nerve, 501,502

,, vagus nerve, . . . 663

SACHs on amblyopia, .

760

,, ,, colour vision,

$.1118,1119$

, contrast,

1061

, electrical organs, . . . $\quad .565$

, , frontal lobe, . . . . $\quad$. 778

, heat centres, .

717

,, occipital lobe,

,, retina, .

780,781

. 1086

758

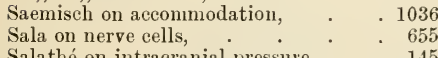

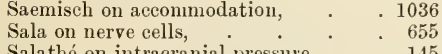

Salathé on intracranial pressure, : $\quad$ : 145

Salkowski on pupillo-dilator nerves, . 621

Salom ," vasomotor nerves, . . 621

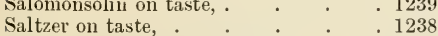

Samojloff on recurrent images, . . . 1073

Samways on auricular systole, . . 22

10,41 $\begin{array}{lr}\text {, cardiac pressure, } & 10,41 \\ \text { polarisation of nerve, } \quad . \quad 549\end{array}$

Sandborg on heart

. 6,28

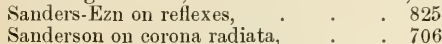
, , , diphasic curve of museular contraction, .

, , electrical organs,

", , , $\quad$ response of muscle,

", $\quad$, $\quad$ response of muscle,

419

563

, , galvanometric examination of muscle,

446 ,, latent period of excitation, 382 ,, muscle,

stethometer, * 282

Sandmann on bronchial muscles, . . 310

Sandmeyer on pyramidal tract, . . 809

Santa Silena on regeneration of cord, . 879

Sappey on joints,. . . . . 240

Sarbó on chromatolysis, . . . . 604

, , spinal cord, . . . . . 836

Sauberschwartz on vowel tones, 1226,1227

Sancerottc on cerebcllum, . . . 894

Savart on concha, . . . . . 1149

Savelicff on sense of smell, . . . . 1250

Saville on limbic lobe, . $\quad . \quad$. $\quad .769$

Scabitschewsky on bladder, . . . 342

$$
\text { ," ', pseudo-reflexes, . } 679
$$

Scarpa ou auditory sense, . . . 1160

Schadow on pupil,

1042,1043

Schäfer on auditory area, . . 761, 762 ,, ,, bulbo-spinal tibres, . . 808 ," , cerebral cortex, 697, 714, 723, 772

," , Clarke's column, . 806, 807, 811

, , , conduction of nervous impulses,

613,615
Schäfer on corpus callosum -

779

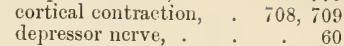

epilepsy, . . . 720,722

, , latent period of cortical excitation,

750,752

Medusw, . . . . 785

, motor centres, . 726, 727, 728,

$733,734,739,740,742,744,745$

muscular contraction, . . 831

," nerve cells, . . 592, 601, 789

,, olfactory area, . . . 766

", plethysmograph,. . 140, 162

", prefrontal region, . . $7 \tau^{2}$

," pyramidal tibres, . $\quad 725,778$,

reflex time, .

. 610

$\cdot 286$

163, 164, 643,645

,, suprarenal extract, . . 193

, tactile area,. . . . 767

,, tract of Gowers, . . . $\quad .807$

,, vagus nerve, . . . 663

, visual area,. . $\quad 753,754,755$,

756,757

volitional impulses, . 615

Schafer, K. L., on subjective beats, . 1176

Schaffer on chromatolysis, . . . 604

Schafhäutl on visual acuity, . . . . 1077

v. Schalk on audition, . . . . 1169

Schapringer on stereoscopy, . . . . 1146

Scharwin on perseption of size, . . 1140

Schelske on vagus nerve, . . 198, 217

Schenck on flicker sensation, . . 1072

, , , museular contraction, 364, 365, $369,370,371$,

372,376

,, , nerve stimulation, . . 475

Scherffer on after-images, . . . 1062

Schiefferdecker on regeneration of cord, $\quad 879$

Schiff on bronchial nuuscles, . . . 309 cerebellum, . $\quad 895,899,900,901$

,, cerebral cortex, : . 705,714

,, ,, contraction of arteries, . . 138

,", deglutition, . . . . 315

, , gastric nerves, . . 324

,, great auricular nerve, . 624,669

, heart beat, . . 171, 183

, ,, inhibition, . . . . 198, 204

,, intestinal movements, . . 331

,", nerves, . . . 334

, knee-jerk, . . . . 872

, ,, motor area, . . . . . 724

museular contraction, . 385, 431

," ,, sense, . . . 1005

necrosis of spinal grey matter, 836

nerve, . . $454,521,653,875$

, roots, . . $788,789,801$

pain, . 810,977,978,990, 999

vilomotor nerves, . . . 618

rigor mortis, . $\quad 391,392,393$

sensitivity of muscles, . 971,1011

spinal cord, . $811,814,836,838$,

$842,856,863,865$

splenic contractions, . . 164

symipathetic, . $657,677,791$

taste, . . . 1239

thaliums, 918

vagus nerve, . . . 663,665 
PAGE

Schiff on vasomotor nerves, . $131,132,156$, $624,625,627,658,670$

,, ,, vertigo, .

1198

, , vomiting, . . $\quad 325$

Schiötz on ophthalmometer, . . . 1033

Schipiloff on rigor mortis, . . . 393

Schirmer on adaptation, . . . 1103

, ,, night blindness, . . 1096

.,, pupil, . 1042, 1043, 1056

," visual sense, . . . 1081

Schklavewsky on capillary circulation, 113

Schlesinger on arterial pressure, . . 854

$$
\text { ,, ,, sensory paralysis, 979, 980, }
$$

, thermal sense, . . 965

Schloesser on reflexes, . . . . 832

Schmey ou fibrillar contractions, . . 192

Schmidt on reducing substances in blood, 290

Sehmiedeberg on vagus nerve, . . 199

Schneider on ear, . . . . . 1149

Schoeps on supramaxillary nerve, . . 803

Schöler on ophtlialmometer. . . 1028

Schön on accommodation, 1036, 1039, 1040

, , binocular vision, . 1138, 1139 visual aenity,

1138, 1139

Schönlein on electrical organs, $571,574,575$, $576,577,579,582$, $583,585,587,588$,

590

, inuscular tension, . . 365

nerve, . . 481

Schott on nerve stimulation, . . 472

Schowmow-Simanowski on gastric secre-

tion,. . . . 887

Schrader on ablation of brain, $699,701,890$

, bulbo-spinal animal, . . 886

, regeneration of ganglia, . 804

,, spinal animal, . . 817

Schreiber on knee-jerk, . . . 872

Schukowski on cerebral cortex, . $\quad 713,778$

v. Schulten on vasomotors of eye, 141

v. Schulthess-Rechberg on coronary ar-

teries,

Schultre on cerebral lesions,

, , comma tract, . . . 805

,, ,, electrical organs, . 565, 566

knee-jerk, . . 872

nerve cells, . . . 594

olfactory surface, . . 1246

reflexes, . . . 839

retinal rods, . 1101, 1104

santonin vision, . . . 1095

tract of gowers, . $\quad . \quad 807$

Schumann on muscular sense, . . 1009

, sense of resistance, 1022,1023 ,

1024

Schuster on knee-jerk, .

- 872

Schuitz on stomach,

321,322

765

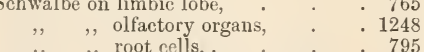

Schwann on cerebellum, . . . 895 nuscular contraction, . 243 ,

361,362

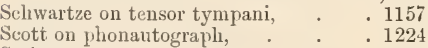

Seripture on touch, . . . . . $\quad$. 926

Seashore on muscular seuse, . . . . 1011 , touch,

929
PAGE

Sedgwick on coronary arteries, . . 13

Sedillot on vagus nerve, . . . . 991

Sée on coronary arteries, . . . 193

Séguin oll hemiopia, . . . 757,758

motor centres, . . . 749

, relation of motion to sensa-

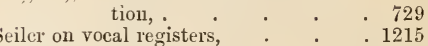

Sellier on thalamus, . . . . 917, 918

Semon on excitability of nerve, . . 480

, , vocal cords, . . . 742, 1209

", , , registers, . 1214,1215

Senac on cardiac impulsc, . . . 34

Sepili on relation of motion to sensa-

tion, . . . 729

word-deafness, $\quad$. $\quad$. 763

Sequeira on pulse, . . . . 104, 111

Sergi ou pain, . . . . . . 975

,, ,, toucli, . . . . . . 924

Serres on cerebellum, . . . . 894

Setsclienow ou mesencephalon, 911, 912, 913

$\begin{array}{lll}, & \text {, polar excitation of nerve, } 505 \\ , & , \text { reflexes, } 826,838,839\end{array}$

Sewall on nerve stimulation. $\quad 475$

,, ,, semicireular canals, . . 1204

Seydel on olfactory organs, . . . 1248

Sfameni on touch spots, . . . 924

Sgobbo on regeneration of cord, . . 879

Sharkey on visual area, . . . 758

Shaw on aphasia, . . . . . 776

Sheen on centre of gravity of body, . $\quad 259$

Slierrington on ablation of hemispheres, 705

,, amyelous fotus, . $\quad 878$

,, apæsthesia, 799, 802, 803, 1020

, centre for splincter aui, 745

cerebellum, . . 894, 898

cervical sympathetic, . 622

, Clarke's column, - 806, 811

, contraction of bladder, . 342

, contrast, . . . 1067

, convilsions, . . $\quad 809$

, cord area, . . . 722

, corpus callosum, . . 779

, cortical inhibition, 712,739 ,

740

,, cutaneous sensations, 689, $\begin{array}{r}690, \\ 920\end{array}$

, decerebrate rigidity, 711,800 , $839,908,913$

dissociation paralysis, . 979

dorso-median columins of

$\begin{array}{cc}\text { cord, } & 792 \\ \text { grey matter of cord, } & 811\end{array}$

hallux refles, . . 704

hunger, . . 992

innervation of chest wall, 816

knee-jerk, $800,816,871,872$,

$873,874,1011$

lateral limiting layer, . 805

lumbo-sacral plexus, . 633

lymph, . . 992

mediate spinal fibres, . 805

medulla, . 884

mesencephalon, . 911, 913

inenstruation, . . 852

mictmition, . . 850

motor area, . . . 744

muscle atrophy, . . 875

990, 1011,

1012,1013

Secchi on tympanum, : $1153,1160,1161$ 
PAGE

Sherrington on muscle spindles, 990,1008 , 1009

, muscular sense, 1002, 1021

,, nerves of bladder, . . 343

", , bone, . . 1006

", ,, generative or-

gans, . 348

tendons, $100 \bar{i}, 1008$

nerve fibres,

,, roots, $789,790,796,799$,

$801,803,804,880$

nucleus cuneatus, . . 806

pain, $970,979,980,987,998$,

paralysis of limb-muscles, 726

, perception of movement, 1017

, pilomotor nerves, 618,619 ,

$620,621,983$

pinna reflex, . . 892

preganglionic fibres, . 658

pupil, . . 621, 869

, pyramidal fibres, $778,808,809$

, , reciprocal innervation, 841 , $842,1012,1019$

rectal sphincter, . . 851

, regeneration of nerve, . 602

., rigor mortis,. . 799,870

reflex contractions, 830,831

segmentation in limbs, . 817

sensitivity of muscle, . 971

, senso-paralysis, . . 1021

shock,. . 846, 847

,, skin areas, . . 940,997

,, spinal cord, 783, 804, 812, 815, $817,818,820,822,823$, $826,827,829,839,840$, $841,842,854,861,862$, $864,866,868,870,925$ nerves, $628,629,880$

", ", nerves, 628, 629, 880

", ,, pain, 981, 982,

,, thermal sense, . . 950

, touch, . . . . 944

,, variations in size of brain, 145

, vasomotor reflexes, . 980

vasomotors of penis, . 160

visceral afferent fibres, . 971

visual induction, 1070,1071 ,

1076

Shore" on pain" ," localisation, . 1134

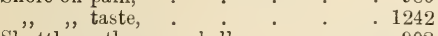

Shuttleworth on cerebellum, . $\quad 902$

Sibsoll on lieart, . . 2 $\quad 2,3,10,29$

Sihler on muscle spindles, . . . 1008

Silberkuhl on pupil, . . . . . 1043

Silex on acconmodation, . . 1040

, , centres for eye muscles, . . 740

Simon on visual localisation, . . 1134

Simonoff on spinal cord, . . . 838, 839

Singer on Clarke's column, . . . 806

,, colour vision, . . . $\quad .1119$

, nlediate spinal fibres, . $\quad 805$

,, necrosis of spinal grey matter, 835 ,

nerve roots, . 790

pain, . . 980

, posture reflexes, . . . 827

". pyramidal tracts, . . 809,888
Singer on spinal reflexes, . PAGE

814

831

$\begin{array}{ll}\text { Sirotinin on spinal reflexes, . } & 814 \\ \text { Skabitschewsky on sympathetie ranglia, } & 787\end{array}$

Skoda on cardiac impulse, . . . 34

Smirnow on arterial pressure, . . 854

,, nerve cells, . . . 655

,, sense spots, . . . 947

Smith on alimentary canal of frog, . 335

,, , check ligaments of horse, . 262

,, ,, heart beat, . . . . 170

,, nerve roots, . . . . 659

,, , olfactory tract, . . . . 764

,, ,, respiration, . . . . 280

Snodgrass on cochlea, . . . . . 1184

Sokownin on pseudo-reflexes, . . 679

," , sympathetic ganglia, . 787

v. Sölder on spino-quadrigeminal fibres, 807

, , , , thalamic fibres, . 807

Solokow on action of poisons on heart, . 222

Soltmann on motor area, . . 707, 742

,, , vagal tone, . . . 55

Sommerbrodt on heart, . . . $\quad .58$

Somya on green vision, . . . . 1096

Sorge on differential tones, : $\quad . \quad .1188$

Sottar on dissociation paralysis, . . 979

Soukanoff oll nerve cells, . . . 608

Soury on cerebral cortex, $697,705,724,758$

,, ,, contractures, . . . . 731

,, hemiopia, . . . . 760

,, ,, relation of motion to sensation, 729

Sowter on combinational tones, . . 1188

Sowton on nerve roots, . . . . 804

Spallanzani on regeneration of cord, . 878

,$\quad$,, spinal reflexes, . 812, 853

,, vision, . . . . 1105

Spallitta on vasomotor nerves, - $62 \overline{7}, 657$

Spehl on blood capacity of organs, . 155

,, , , flow in lungs, . . . 128

Spencer on frontal lobe, . . . . 714

, , respiratory centre, . . 306

," ,, seusations, . . 970,975, 984

". $\quad$, verbal recollection, . . 776

Spiegelberg on parturition, . . . 350

Spiller on mediate spinal fibres, . . 805

Spitzka on third nerve, . . . . 911

Spring on cardiac diastole, . . $\quad 26$

Spronk on necrosis of spinal grey matter, 835

Staffany on pyramids, . . . 889

Stannius on heart beat, . . 169, 175 , nerve roots, . . 788,789

Stark on colour blindness, . . . . . 1091

, , foveal vision, . . . . $110 \%$

", ", electrical organ, . . . . 563

Starling on arterial pressure,
, augmentor nerves, $\quad 217,219$

, augmentor nerves, $\quad$ capillary pressure, $\quad$. $\quad$. 117

", heart, 18, 19, 187, 447, 448

", intestinal nerves, $334,695,696$

," mechanism of digestive tract, 321

,, , , generative or-

gans, - 347

", , , , respiration, . 274

semilumar valves, . . 21

transfusion, . . 76

", vasonotor nerves, $140,161,643$

", vena rava pressure, . . 72

VOL. II. -86 
Starlinger on pyramids,

Starr on aphasia, .

, , motor area,

, ", motor area,

$$
, \text {, visual area, }
$$

Stefani on hlood vessels of muscles, . 158

$$
\begin{aligned}
& \text {, , , eardiac pressure, } \\
& \text {, cerebellum, } \\
& \text {, optic lobes, . } \\
& \text {, vasomotor tone, } \\
& \text {,, bulbar centres, } \\
& \text { nerve, } \\
& \text {, , loots, } \\
& \text {, pupil, } \\
& \text {, reflexes, } \\
& \text {, touch, }
\end{aligned}
$$

$$
\text { ,, semicircular canals, . . } 1205
$$

Steinach on alimentary canal of frog, . 335

Steinbrìgge on cochlea,

Steiner on cerebellum,

cerebral cortex, $699,700,707,752$,

", $\quad 756,759,761$

$485,525,526$

$659,789,790$

1043,1044

829

$923,925,927$

1167,1182

895,900

, , œsopliagus, . . . 320

.. , respiration of swallowing, . 304

", semicircular canals, . . 1204

,. , sensory nerves, . . . . 925

,, spinal cord, . . . . 817

Steimman on blood flow in veins, . . 121

, nerve roots, . . . 799

Stenbeck on depressor nerve, . . 60

,, vasomotor centres, . . 137

," , , , reflexes, . $\quad . \quad 855$

Stenson on necrosis of spinal grey matter,

Stepanoff on cochlea,

1181

,, , knee-jerk,

, muscular sense,

Stevens on vocal sounds, . . . 1207

1012

$871,872,873$

1005,1018

Stewart on chromatolysis,

604

, , circulation time,

, conduction in nerve cells, : 791

, nerves of bladiler, polarisation of nerve,

,, sympathetic ganglia,

, systolic output,

,, vagus inhibition,

, vesical centre,

Stieda on efferent root cells,

Stilling on apresthesia, .

$$
\begin{aligned}
& \text {, muscular tonus, . } \\
& \text {, nerve roots, . } \\
& \text {, pain, . } \\
& \text {, spinal cord, } \\
& \text {,, sympatlietic ganglia, } \\
& \text {, vasomotor nerves, }
\end{aligned}
$$

Stirling on cervical sympathetic,

$$
\begin{aligned}
& \text { inuscular contraction, } \\
& \text { reflex contractions, }
\end{aligned}
$$

$$
\text { stimuli, }
$$

Stockhausen on vocal registers,

Stolnikow on systolic output,

Störk on vocal registers,

Stort on retina,

Stratton on touch,

Strauss on knee-jerk,

v. d. Stricht on nerve roots, .
Stricker on accelerator centre,

$$
\text { , nerve roots, }
$$

, nerve roots, - $65 \dot{8} 8,670,790$

,, ,, vasomotor centres, . . 855

Stroebe on regeneration of nerve, . . 479

Stromeyer on thalamus, . 919

Strong on pain, . . . 966, 968, 994

$$
\text { ,, taste, . . . . } 1239
$$$$
\text { visceral sensations, . } 993
$$

Stuart on deglutition, . . . . 315, 316

$$
\text { , kymograph,. . . . } 78
$$

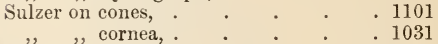

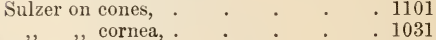

Sunkel on sense of resistance, 1023, 1024

Suslowa on touch, . . . . 938

Sustschinsky on vagal centre, . $\quad 55,56$

Swan on thalamus, . . . . . 916

v. Swieten on reflexes, . . . . . 786

Swinton on reflexes, . . . . . 833

Symonds on whispering, . . . 1236

TAIT on pain, . . . . . . 970

1225

Talbot on visual sense, . $\quad . \quad 1068,1077$
Talma on heart sounds. $\quad . \quad 32$

,. ,, pulmonary circulation, . . 127

Tamburini on auditory area, . . 761

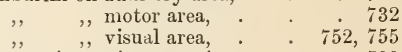

Tammassia on rigor mortis, . . . 799

Tangl on thalamus, . . . . 918

Tanzi on reaction time, . . . . 963

,, , sense spots, . . . 945, 948

Tarchanoff on spinal reflexes, $826,829,838$,

,, ,, vagal centre, . . . 54

Tartini on differential tones, . . . 1188

Taylor on knee-jerk, . . . . 875

, sound, . . 1187, 1189

Teljatnik on encephalo-spinal fibres, . 808

Teuner on convulsions, . . . 307

Thane on lumbo-sacral plexus, : . 633

Thanofer on hunger, . . . . . 991

Thebesius on semilunar valves, : . 13

Theobari on facial nerve, . . . . 891

Thiry on arterial pressure, . $\quad . \quad$. 854

", , dyspnœa, . . . . 289

vasomotor centres, . . . 136

Thoari on mediate spinal fibres, . . 805

Thoma on branching of arteries, . $\quad 62$

56 Thomas on cerebellum, $894,895,898$
864 , mediate spinal fibres, 807,808 ,

$\begin{array}{rr}849,864 & \quad, \quad \text { mediate spinal fibres, } \quad 807,808, \\ 795 & 809,889\end{array}$

$799,801 \quad$, , taste, . . . 1239, 1240

Thompson on audition, $\quad 1150,1163,1170$,

$791,801 \quad 1176$

$978 \quad, \quad$, , vasomotor nerves, . . 140

$865 \quad$, , visual area, . . . 753

787 Thomsen ou contractures, . . . 731

131 Thomson on circulation, . . . 72

$875 \quad, \quad$, ear, . . . . . 1149

384 , , vasomotor mechanism, . 130

830 Thunherg on thermal sense,. . . 955

828 Thurston on cardiac systole, . . 38

1215 Tiedemann on nerve roots, . . . . 788

48 Tiegel on muscular contraction, . . 381

$1215 \quad$,, ,, polarisation of nerve, . . 501

$1048,1049 \quad$, reflexes, . 835

927, 929 Tigerstedt on circulation, $1,83,84,89,129$ $874 \quad,, \quad,, \quad, \quad$ in kidneys, . 160

$790 \quad$ ", ", fifth" nerve, . . . 662 
PAGE

Tigerstcdt on heart, . 19, 48, 49, 50, 187 , , nerve, 478, 482, 495, 498, 501, $509,557,927$

, , period of latent excitation, 381

", "pulse, . . . 100, 102

, , tetanomotor, . . . 467

,, , transfusion, . . . 77

Timofeew on accelerator tone, . . 56

Tirelli on spinal cord, . . . . 879

Tomsa on nerve knots, . . . . . 947

Tonn on colour nixture,

Tooth on Clarke's column,

. 1082

806,811

, facial muscles,

, mediate spinal fibres, . . $\quad . \quad 805$

,, pain,

pyramidal traet

", tract of Gowers, : $: 807$

Tópler on auditory sensation, 1170,1171

Topolanski on cortical inhibition, . 712

,, , thalamus, . . . 917

'Torricelli on fluid flow in tubes, . . 62

Tourtual on touch, . . . . 943

Towne on cyclopean eye, . . . . 1135

Toynbee on ear, . . . . . . . 1149

Traube on arterial pressure, . . . . 139

, , associated pain, . . . 9 981

," ," dyspncea, . . . . 289

Treitel on night blindness, : . . . 1096

Trendelenberg on muscular sense, . 1003

Trepinski on funiculus gracilis, . . 792

Tschermak on colour mixture, . . 1082

, , , corpus callosun,, . . 779

,, encephalo-spinal fibres, . 807

", foveal vision,.. . . 1104

", mesial longitudinal bundle, . 890

,, nucleus cuneatus, . . 806

herning on accommodation, 1034, 1036 ,

$\begin{array}{lr}1039, & 1040 \\ \text { Tscheschischin on heat centres, } \quad .717\end{array}$

Tschirjew on accelerator centre, . $\quad 55,56$ , ", muscle, . . . 800,869 ", $\quad,$, nerve, on section of motor nerves, 799

Tuckett on 'degeneration of nerve, 652,665 ,, , regeneration of nerve, . 654,665 Tunniclifte on blood in muscles, . . 155

,, , cardiac diastole, . . 25

Tunstall on refractory period in nerve cells,

Türck on skin areas,

615

940 ", $\quad$, spinal cord, . . . . . $\quad$. 826,868

Turier" on cerebellum, $895,896,900,901,903$ ,, cerebral cortex, 730, 772, 780, 781

, chromatolysis, . . 604

, conduction in bulb, . $\quad .888$

,, corpus callosum,. . . $\quad .779$

, facial muscles, . . . . 911

", geniculate bodies, $\quad$. $\quad$. 917

," heart sounds, . . . 28

", mesencephalon, : : . 915

,, metenceplalon, . . . 893

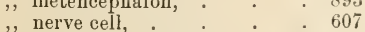

nucleus of Deiters, . 808

", nain, . . . $\quad$. 978

", rhinencephalou, : : : 765

, spinal cord . . . 866,867

, taste, . . . . . 1239
Turner on thalamus, . . . 917,918

Tuwim on sympathetic nerves, . 676,677

v. UExкüLL on nerve, . $467,468,482,483$ ,, reflexes, . . . 839

Uhthoff on colour vision, '1054, 1089, 1104 ," , touch, . . . . 944

Ulrich on touch, . $\quad . \quad$. $\quad . \quad 935$

Unverricht on cerebral cortex, . 713,745 ,, epilepsy, . . . 719 ,, pyramidal tract, . . 809

Urbantschitsch on anal reflexes, . . 1163

Uspensky on apnœa, . . . . 837

,, convulsions, . . . 837

,, ., nerve roots, . . . 799

VALENTIN on reflexes, . . . . 787

$\begin{array}{ll}, \text { regeneration of ganglia, } & 804 \\ ,, \text { scnse of smell, } & 1250\end{array}$

,, touch, . . . . 924

Valsalva on cochlea, . $\quad . \quad . \quad .1167$

Vanlair on reflexes, . . . . $\quad . \quad 814$

,, ,, vagus nerve, . . . . 665

Vareth on cerebral cortex, . . . . 723

de Varigny on pain, . . . . 987

Varmod on touch, . . . . 937

Vas on nerve cell, . . . . 599, 600

Vater ou reflexes, . $\quad . \quad . \quad . \quad .786$

Veias on nerve roots, . . . . 790

Vergier on thalamus, . . . . 917,918

du Verney on cerebellum, . . . 894

Veruon on musele proteids, . . . 390

Verwoort on pupil, . . . : . 1043

Verworn on hypnotism, . . . 712,913 , mesencephalon, . . 912

Vialet on "eerebral cortex, . $758,760,780$

,, , thalamus, . . . . 917

Vierordt on blood flow,. . . . 48

,, ,, capillary circulation, . 114, 115

,, ., chronograph, . . . 265

", circulation time, . $\quad .88$

,, $\quad$, colour sense, . $\quad .1077$

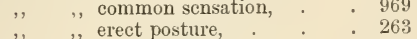

," pain,. . . . . 968

,$\quad$, pulse, . . . $\quad . \quad 99$

,, ", sense of movement, . . 966

,, thermal sense, . . . 952

,, ," touch, 934, 935, 937, 938, 943

Vieussens on pain, . . . . 979 , reflexes, . . . 786

v. Vintsch"gau on colour blindness, 1093

$$
\begin{aligned}
& \text {, , , conductivity of nerve, } 458 \\
& \because \quad \quad \text {, reaction time, } \quad . \quad \text { sense of smell, . } \quad .1258 \\
& \text {," taste, .517, 1238, 1241, }
\end{aligned}
$$

1244,1245

Virchow on muscle tone, . . . $\$ 70$

Vitzou on cerebral hemispheres, - $\begin{array}{r}704, \\ 752,754\end{array}$

Voelckers on accommodation, 1034, 1036 ,

1037,1039

Voit on cerebral henispheres, . . 701

Volkmanı on arterial prossure, . . so

,, blood flow, . . 4s, 83

," breaking struin of arterics, 74

ciliary ganglion, . . 664

$\because$ fatigue of muscle, : : 386

," intercostal nuscles, . 277

,", medullated nerves, . . $\quad 655$ 
Volkmann on mesencephalon nerve roots, spinal cord, . $818,837,864$ sympathetic fibres, . 648,650 touch, . . 935, 936, 937 venous pressure, . . 119 visual incongruence. . 1125

Volkmar on "thermal sense, . . . 958

Volta on muscular contraction, . . 353

Voltolini on cochlea, . . 1190

, tympanic membrane, . 1157

Vulpian on action of poisons on heart, . 224

, arterial pressure, . 854,855

cerebellum, . $895,900,909$

,, cerebral cortex, 699, 700, 702, 714

,", cranial nerres, 660, 661, 662, 663

,, inhibitory nerves, . . 672

", muscular sense,. $\quad$. 1004

, nerve roots, . . 671

,, semicircular canals, . . 1198

, spinal cord, $827,839,843,867$,

, sympathetic nerves, - 618,676

vasomotor centres, . . 137

, visceral sensations, . . 990

WAGNER on accelerator centre, . . 55

, cerebellum, . $\quad . \quad 895$

,, electrical organs, . . 566

,, lateral sinuses, . . 145

, nerve roots, . . . 789

, reflexes, . . 837

Waldenburg on respiration, . $\quad . \quad 128$

Waldeyer on dorsal column of cord, $\quad 863$

,, , nerve cells, . . . 795

, neurone, . . 593, 594

Wallemberg on corpus striatum, . $\quad . \quad 893$

," ,, optic lobes, . . . . 915

Waller on canalisation, . . . 837

, cardiac pressure, . . . 19

, cochlea, . . . 1190,1191

contrast, . . . . 1067

, degeneration of nerves, 601, 652,

, fatigue of muscle, . $\quad 388,389$

," nerve, . . . 831

heart, . 187,448

knee-jerk, : . 871,875

, metabolism in nerve, . 454, 537,

muscular sense, . . 1004

nerve, $462,468,472,474,485$,

$486,498,516,524,533$,

$534,536,537,538,540$,

$553,554,555,575,931$

pulmonary circulation, . 150, 151

regeneration of nerves, . 652

, relation of motion to sensation,

retinal currents, 729

sense of effort, $\quad \cdot \begin{array}{r}1052 \\ -\end{array}$

spinal nerve roots, . . 790

sympathetic nerves, $619,622,623$,

651,652

vagus nerve,

vasomoto: mechanism, 131, 132

visceral nerves, . . 971

Walsh on electrical organs, : . $\quad . \quad 561$

v. Waltler on lain, . . $\quad .967$

, regencration of ganglia, . 804
IVana on nerve roots, . $\quad 790$

Ward on reflexes, $818,828,833,839,840$

Warfield on heart, . . . . 56

Warren on knee-jerk, . . . . 873,874 ,, vasomotor nerves, 134, 135, 626

Warrington on ehromatolysis, . . 604 , , degeneration in cord, . 861

,, nerve roots, . . . 803

,, optic lobes, : . $\quad 915,916$

, root cells, . . 877,878

Washbourn on heat centres,. . . 717

Waters on alimentary canal of frog, . 335

Watson on reflexes, . . $\quad 829$

de Watteville on aphasia, . . . 775

,, knee-jerk, . . 871

,, polar excitation of nerve,

516

Weber on auditory sensatious, $\quad 1160,1183$

, centre of gravity of body, . 257

," circulation, . . 68,70

", common sensation, 969, 970,974, 975

,, compression of thorax, . - 45

," crect posture, . . . 260

", inhibition, . 54, 198, 203, 837

", internal ear, . . . . 1159

", joints, $\quad 235,240,251$
2, movements of body, $\quad . \quad 228$

", muscular contraction, 242, 244, $245,360,385$

, pain, 979, 987, 990, 993, 994, $\frac{998,}{999}$

, photophobia, . . 967

pulse, . . . $\quad . \quad 102$

", pupil, : $\quad . \quad$. $\quad . \quad 1043$

", semilunar valves, . . . 12

,, sense of resistance, . . 1022

,", smell, . . . 1256

,' thermal sense, $946,948,950,952$, $953,954,955,956,959,960,961$,

962,965

touch, 928, 929, 930, 934, 935, 936 ,

$937,938,939,940,942,943,946$

vasomotor mechanisn, . . 130

," walking and running, 264, 268, 269

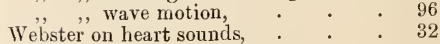

de Wecker on lens, $\quad . \quad$. $\quad . \quad 1029$

Wedemeyer on intra-thoracic pressure, . 47

Wedenski on nerve, - $473,476,528,831$

Wegner on vasomotor nerves, . . 623

Weidenfeld on intercostal museles, . 279

Weir on relation of motion to scnsation, 729

Weiss on nerve, . . . 481, 497, 550

,, , pain, . . . . . 979

,, spinal cord, . $\quad . \quad$. 865,866

Welby on brain, . . . . 758

Welt on frontal lobe, . . . . 773

Werigo on nerve stimulation, : . 475

Werner on motor centres, . . . 745

Wernicke on aphasia, . $\quad . \quad 7 \div 5,776$

,, nuscular sense, . . 1003

," relation of motion to sensa-

tion, . . . 729

Wertheim on elasticity of arteries, . 73 , , extensibility of muscle, . 241

Werthcimer on pyramids, . . . 889

", respiratory centre, . cord, . 
$\mathrm{PA}(\mathrm{EE}$

Werziloff on nerve roots, . . 677

Westphal on knee-jerk, . $\quad 871,873,874$ ,,$\quad$, regeneration of eord, $\quad 879$ " . . . . . 938

Weyl on electrical organs, . . . . 454

Weyrich on aurieular pressnre, . . 119 ,, intra-thoracic pressure, . 47

Wheatstone on after-images, . 1063

, , disparation, 1125, 1126, 1127

," , spatial perception, 1134, 1137

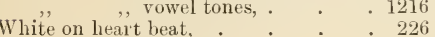

, , heat centres, . $\quad . \quad$. $\quad$. 717

". thalamus, . . 918

Whytt on reflexes, - $786,811,813,913$ ., on shock, . . . 845

Wiedershelm on nerve cells, . . . 608

Wiener on alimentary eaual of frog, . 335 ,, , cerebral hemispheres, . . 704

", ", mediate spinal fibres, . 805,806 ,

863
915,916

, , , optic lobes, . $\quad . \quad$. $\quad .913$

", spinal cord, . $810,8 \dot{3} 5,836,862$

$\begin{array}{lll}\text { v. 'Wijhe on spinal nerve roots, } & \text {. } & 659,789 \\ \text { V } & 789\end{array}$

Wilbrand on blind spot, . . . 1088

,., visual centre, : . $\quad .760$

Williams on heart sounds, : . $\quad 29,31$

,, , muscular tremors, . . 711

Williamson on cerebral cortex, . $\quad$. 772

Willis on reflexes, . . . . 786

, , , vowel tones, . . . . $\quad .1216,1217$

Wilson on mesencephalon, . . . 911

,, , optic lobes, . . . . 610

Winslow on museular co-ordination, . 821

Wintrich on heart sounds, . . . 29

Wintringham on veins, . . . 119

Witkowski on eye movements, . . 1132

Witmer ", visual loealisation, .

. 1135

-976, 1001

Wolfenden on museular treinors, : $\quad$ • 711

Wolff on touch, . . . . . 929

, , voice, . . . . . . 1235

Wood on heat centres, . . . . 717

Woods on cardiac pressure, . . . . 41

Wooldridge on heart beat, . . . 187

,, ,, nerves of heart, . 57, 59

Worm-Miiller on cardiac systole, . $\quad 6$

Worosehiloff on pain, . . . . . $\quad .979$ ,, $\quad$, spinal cord, - $862,863,867$, idal tract, . 809

Wright on sensation, . . . . 977

Wundt on colonr vision, 1106, 1113, 1121

,, ,, common sensation, . . 970

,, , double vision, . . . $1126^{\circ}$

," muscle, . . 385, 388, 467

,, , muscular sense, . . 1003,1004

, , , nerve, . . . 4 483, 549

,, ,, , cells, . . . . 604

, , pain, . . $810,966,967,998$

,, , reflexes, . $825,829,830,842$

, ,, reflex time, $609,611,612,614$,

791, 945

$, \quad,$, section of motor nerves, . 798

,, , touch, . . . . . 938

,, ,, vagus nerve, . . . . 198

,, ,, verbal recollection, . . $\quad .776$

, , visual localisation, . . 1135

,, Weher's law, . . 931, 934

Wyllie on vocal cords, . $\quad$. 1209

," , voice, . . . 1233, 1235, 1236

YEO on spinal nerve roots, . . . 821

, , , tactile area, . . . 766

", ", visual area, . . . . . 752

Young on aceommodation, . . . 1036

,, ,, astigmatism,. . . . 1033

," beat tones, . . . $\quad .1170$

,, , cochlea, . . . . . 1179

,. $\quad$ eolour vision, : . $\quad .1106$

, , specific energy of nerve fibres, 1243

ZACHER on auditory area, . . . 781

," , dissociation paralysis, . . 979

Zappert on mediate spinal fibres, . . 805

v. Zeissl on micturition, . . . 342

$\begin{array}{lll}\text { Ziehen on epilepsy, . . . . . } & 913\end{array}$

, , knee-jerk, . . . . 874

, „motor centres, . . 732, 742

Zinmermann on cerebellum, . . 894

Zinn on cerebellum, . . . . 894

Köllner on visual illusions, . . . . 1141

Zuckerkandl on limbic lobe,. . 764, 765

,, ,, olfactory area, . . 781

,, , organs, . . 1248

Zumft on retina, . . . . . . 1100

Zuntz on arterial pressure, . . $\quad 80,128$

,, , blood flow in veins, . . 121

", ", systolic output, . . . 48, 49

.., vasomotor reflexes, . . 166

Zwaardemaker on extensibility of arteries, 73 ,, olfactometer, 1251, 1257, 



\section{YOUNG J. PENTLAND'S RECENT PUBLICATIONS.}

CONTRIBUTIONS to CLINICAL MEDICINE. By M'CALL ANDERson, M.D., F.F.P.S. (Glas.), Professor of Medicine in the University of Glasgow; Physician, Glasgow Western Infirmary. 8vo, cloth. pp. xvi., 416, with 28 Illustrations. Price Ios. 6d. nett.

TEXT-BOOK of GENERAL BOTANY. By DR. W. J. BEHRENS. Translation from the Second German Edition. Revised by Patrick Geddes, F.R.S.E., Professor of Botany in the University of Dundee. Second Edition, 8vo, cloth, pp. viii., 374, with 408 Illustrations, finely engraved on wood, and 4 analytical tables. Price 5s.

DISEASES of the EYE: A Practical Treatise for Students of Ophthalmology, By George A. Berry, M.B., F.R.C.S.Ed., Ophthalmic Surgeon, Edinburgh Royal Infirmary ; Lecturer on Ophthalmology, Royal College of Surgeons, Edinburgh. Second Edition, thoroughly Revised and Enlarged. 8vo, pp. xvi., 728. Illustrated with Wood Engravings and Coloured Plates from Original Drawings. Price 25s. [Pentland's Medieal Serics, Volume Seeond.

THE ELEMENTS of OPHTHALMOSCOPIC DIAGNOSIS. FOR the use of Students attending Ophthalmic Practice. By George A. Berri, M.B., F.R.C.S.Ed., Ophthalmic Surgeon, Edinburgh Royal Infirmary; Lecturer on Ophthalmology, Royal College of Surgeons, Edinburgh. Crown 8vo, cloth, pp. xii., 83. Price 3s. 6d.

THE NATIONAL MEDICAL DICTIONARY, INCLUDING ENGLish, French, Gerian, Italian, and Latin Technical Terms used in Medicine and the Collateral Scimnees, and a Skries of Tables of useful data. By John S. Billings, A.M., M.D., LL.D., Harv. and Edin., D.C.L. Oxon., Member of the National Academy of Sciences; Surgeon, U.S.A., etc., with the collaboration of W. O. ATwATER, M.D. ; Frank Baker, M.D. ; C. S. Minot, M.D.; Jalies M. Flint, M.D.; R. Lorini, M.D.; Washington Matthews, M.D.; S. M. Burnet, M.D. ; J. H. Kidder, M.D. ; H. C. Yarrow, M.D. ; William Lee, M.D. ; W. T. Councilman, M.D. In two Imperial 8 vo Volumes, containing about 1600 pages. Price 50s. nett, carriage free.

DISEASES of the HEART and THORACIC AORTA. By BrRoN Bramwell, M.D., F.R.C.P.Ed., Lecturer on the Principles and Practice of Medicine, and on Practical Medicine and Medical Diagnosis, in the Extra-Academical School of Medicine, Edinburgh; Physician to the Edinburgh Royal Infirmary. Large 8vo, cloth, pp. xvi., 783. Illustrated with 226 Wood Engravings, and 68 pages of Lithograph Plates, exhibiting 91 Figures-317 Illustrations in all. Price 25s.

INTRACRANIAL TUMOURS. By BrROM BRAMWELL, M.I), F.R.C.P.Ed., Lecturer on the Prineiples and Practice of Medicine in the ExtraAcademical School of Medicine, Edinburgh; Physician to the Edinburgh Royal Infirmary. 8vo, eloth, pp. xvi., 270, with 116 Illustrations. Price 14s.

ILLUSTRATIONS of the NERVE TRACTS in the MID and HIND BRAIN AND THE CRANIAL NERVES ARISING THEREFROM. By Alex. Bruce, M.D., F.R.C.P.Ed., Iecturer on Pathology in the School of Medicine; Assistant Physician, Royal Infirmary, Edinburgh, Oblong 4to, cloth, Illustrated with 28 Coloured Plates and Engravings in the text. Price 50s. nett. 
THE MURMURS of MITRAL DISEASE. By EDWARD MANSFIELD Brockbank, M.D., M.R.C.P, Late Resident Medical Officer, Manchester Royal Infirmary, and Birmingham General Hospital. 8vo, cloth, pp. viii., 48, with 5 Illustrations. Price 3 s. 6d.

THE PRINCIPLES of TREATMENT, AND ThEIR APPLICATIONS in Practical Medicine. By J. Mitchell Bruce, M.D., F.R.C.P., Physician to and Lecturer on the Principles and Practice of Medicine, Charing Cross Hospital ; Consulting Physician to the Hospital for Consumption, Brompton ; Examiner in Medicine, University of Cambridge. 8vo, cloth, gilt top, pp. xviii., 616. Price I6s.

[Pentland's Medieal Scries, Volume Eighth.

DISEASE in CHILDREN : A Manual For STudents and Practitioners. By James Carmichael, M.D., F.R.C.P.Ed., Physician, Royal Hospital for Sick Children; University Lecturer on Disease in Children, Edinburgh. Crown 8vo, cloth, pp. xvi., 520. Price Ios. 6d. [Pentland's Students' Manuals.

TUBERCULOUS DISEASE of the BONES and JOINTS. By W. Watson Cheine, M.B., F.R.S., F.R.C.S., Professor of Surgery, King's College, London. 8vo, cloth, pp. xvi., 374. Illustrated with numerous Wood Engravings throughout the text. P'rice I4s. nett.

THE TREATMENT of WOUNDS, ABSCESSES, and ULCERS. By W. Watson Cheyne, M.B., F.R.S., F.R.C.S., Professor of Surgery, King's College, London. Second Edition. Crown 8vo, cloth, pp. xvi., 197. Price 3s. 6d.

ATLAS of the DISEASES of the SKIN. By H. RADCLIFFE Crocker, M.D., F.R.C.P., Physician to the Department for Diseases of the Skin, University College Hospital, London. In 16 Fasciculi, containing 96 plates, exhibiting 238 Figures in colours from Original Drawings specially prepared for the work, with Descriptive Letterpress. Price 2Is. each nett. Or in 2 large handsome Folio Volumes, Half Morocco, gilt tops. Price fir, i8s. nett.

* * Subscribers' N'anes are now being reccived.

MANUAL of PRACTICAL ANATOMY. By D. J. CUNNINGHAM, M.D., Professor of Anatomy and Chirurgery, University of Dublin. Second Edition. In 2 Vols., Crown 8vo, eloth. Volume First-Upper and Lower Limb; Abdomen. Volume Second-Thorax; Head and Neck. Illustrated with 372 Engravings and 2 full-page Plates. Price 12s. 6d. each.

[Pentland's Students' Manuals.

GEOGRAPHICAL PATHOLOGY : AN INQUIRY INTO THE GEOGRAphical Distribution of Infective and Chimatic Diseases. By Andrew Davidson, M.D., F.R.C.P.Ed., late Visiting and Superintending Surgeon, Civil Hospital ; Professor of Chemistry, Royal College, Mauritius. In 2 Vols, large 8vo, pp. xvi., 1008, Illustrated with Maps and Charts. Price 3is. 6d.

HYGIENE and DISEASES of WARIM CLIMATES, IN A SERIES of Articles bi Eminent Authorities. Edited by Andrew Davidson, M.D., F.R.C.P.Ed., late Visiting and Superintending Surgeon, Civil Hospital ; Professor of Chemistry, Royal College, Nauritius; Author of Geographieal Pathology. Royal 8vo, cloth, pp. xvi., 1016. Illustrated with Engravings throughout the text and fullpage Plates. Price 3is. 6d.

A TREATISE on OBSTETRICS for STUDENTS and PRACTITIONERS. By EDward P. DAvis, A.M., M.D., Professor of Obstetrics and Diseases of Infancy in the Philadelphia Polyclinic, ete. Large 8vo, eloth, pp. 553. Illustrated with 217 Engravings, and 30 Plates in colours and monochrome. Price I6s. nett.

TEXT-BOOK of NERVOUS DISEASES. BY AMERICAN AUTHORS. Edited by Francis X. Dercum, M.D., PH. D., Clinical Professor of Nervous Diseases in the Jefferson Medical College of Philadelphia. Royal 8vo, pp. xvi., 1056, with 341 lingravings in the text, and 7 Coloured Plates. Price 25s. nett.

TOXIC AMBLYOPIAS: Their Classification, Histori, Symptoms, Pathology, and Treatment. By G. E. De schiveinitz, A.M., M.D., Professor of Ophthalmology in the Philadelphia Polyclinic; Clinical Professor of Ophthalmology in the Jefferson Medical College, Philadelphia. Royal 8vo, cloth, pp. '238, with $46^{\prime}$ Figures in the Text and 9 full-page Plates. Price I6s. nett. 
EDINBURGH MEDICAL JOURNAL. Edited by G. A. Gibson, M.D.. D.Sc., F.R.C.P.Ed. Issued monthly, with Illustrations. Subscription $£$ r, Is. • per annum (in advance), post free. For the Colonies and Abroad, 24s. per annum (in advance), post free. Single numbers, 2s. eacl. By post, 3 d extra.

THE FUNDUS OCULI, with an OPHTHALMOSCOPIC ATLAS, illustrating its Physiological anin Pathological Conditions. By W. Adams Frost, F.R.C.S., Ophthalmic Surgeon, St. George's Hospital ; Surgeon to the Royal Westminster Ophthalmic Hospital, London. In one handsome 4to Volume, extra cloth, gilt top, with 47 Plates, exhibiting 107 beautifully Coloured Figures and numerous Engravings in the text. Price $£ 3,3$ s. nett,

DISORDERS of the MALE SEXUAL ORGANS. By EUGENE Fuller, M.D., Instructor in Genito-Urinary and Venereal Diseases, New York PostGraduate Medical School. Large 8vo, pp. viii., 241, with full-page Plates and Illustrations in the text. Price $9 \mathrm{~s}$.

DISEASES of the HEART and CIRCULATION. By G. A. GIBSON, M.D., D.Sc., F.R.C.P.Ed., Physician to the Royal Infirmary; Consulting Physician to the Deaconess Hospital; Lecturer on Medicine at Minto House, and on Clinical Medicine at the Royal Infirmary, Edinburgh. 8vo, cloth, gilt top, pp. xxii., 932, with 210 Illustrations. Price 24s.

[Pentland's Medical Series, I'olume Sixth.

TEXT-BOOK of MEDICINE. By British Teachers. Edited by $(\mathrm{x}$. A. Grbson, M.D., D.Sc., F.R.C.P.Ed., Physician to the Royal Infirmary ; Lecturer on Medicine, School of Medicine, Edinburgh. In Press, Royal 8vo, pp. xxiv., 1250, with Illustrations.

*** The following, among others, will contribute:-Professors Thomas OLIver; Sir William Gowers, Drs. J. Mitchell Bruce, F. W. Mott, Hector Mackenzik, Hale White, Lauder Brunton, Sidney Martin, Rose Bradford, W. Pasteur, Patrick Manson, J. W. Moore, W. Allan Jamieson, J. O. Afrleck, Wiluilu Russeld, Alex. Bruce, R. W. Philip, the Edtor, \&c. \&c.

PHYSICAL DIAGNOSIS : A Guide to Methods of Clinical InvestiGation. By G. A. Gibson, M.D., D.Sc., F.R.C.P.Ed., Physician to the Royal Infirmary; Lecturer on the Principles and Practice of Medicine in the Edinburgh Medical School; and William Russeli, M.D.. F.R.C.P.Ed., Assistant Physician to the Royal Infirmary of Edinburgh ; Lecturer on Pathology and Morbid Anatomy in the Edinburgh Medical School. Third Edition in preparation.

[Pentland's Students' Manuals.

HYDATID DISEASE in its CLINICAL ASPECTS. By JAMES Graham, M.A., M.D., late Demonstrator of Anatomy, Sydney University ; Medical Superintendent, Prince Alfred Hospital, Sydney. 8vo, pp. xvi., 204, with 34 full-page Coloured Plates. Price I6s.

A SYSTEM of PRACTICAL THERAPEUTICS. By VARIOUS Authors. Edited by Hobart Ayory Hare, M.D., Clinical Professor of Diseases of Children, and Demonstrator of Therapeutics in the University of Pennsylvania ; Physician to St. Agnes Hospital, Philadelphia. Assisted by Walter Chrystie, M.D., late Physician to St. Clement's Hospital, and Instructor in Physical Diagnosis in the University of Pennsylvania. Six Volumes, Royal 8vo, of about 500 pages each. Uniform with the System of Gyncecology and Obstetrics. Price per Volume I2s. 6d., carriage free.

* * Detailed Prospectus sent free by post on application.

Two Supplementary Iolumes, bringing the work up to date. Price 12s. 6d. each, earriage paid.

HANDBOOK of OBSTETRIC NURSING. By F. W. N. HAULTAIN, M.D., F.R.C.P.Ed., Lecturer on Midwifery and Diseases of Women, School of Medicine; Physician for Diseases of Women, Deaconess Huspital; Obstetric and Gynecological Physician, Royal Dispensary; Examiner, Royal College of Physicians, Edinburgh; and J. HAIG FERguson, M.D., F.R.C.P.Wd., Lecturer on Midwifery and Diseases of Women, School of Medicine and Jubilee Institute for Nurses ; Wxaminer in Midwifery, University of Edinburgh and Royal College of Physicians : late President, Royal Medical Society, Edinburgh. Third Edition. Crown 8vo, eloth, pp. xvi., 264. with Coloured Plate and 37 Wood lingravings. Price 5 s. 
PHYSICAL and NATURAL THERAPEUTICS : The Remedial Uses of Atmospheric Pressure, Climate, Heat and Cold, Hydrotherapeutic Measures, Mineral Waters, and Electricity. By Georges Hayem, M.D., Professor of Clinical Medicine in the Faculty of Medicine, Paris. Edited by Hobart Amory Hare, M.D., Professor of Therapeutics and Materia Medica, Jefferson Medical College, Philadelphia. Large 8vo, cloth, pp. 426, with 113 Illustrations in the text. Price I4s.

HUMAN MONSTROSITIES. By Barton Cooke Hirst, M.D., Professor of Obstetrics in the University of Pennsylvania ; and Georan A. Pikrsol, M.D., Professor of Embryology, and Histology in the University of Pennsylvania. In handsome Folio, containing about 150 pages of text, Illustrated with Engravings and 39 full-page Photographic Plates from Nature. In 4 Fasciculi. Price 25s. each, carriage free.

DISEASES of the SKIN: A MANUal FOR Students and PractiTioners. By W. Allan JAmieson, M.D., F.R.C.P.Ed., Extra Physician for Diseases of the Skin, Edinburgh Royal Infirmary; Consulting Physician, Edinburgh City Hospital; Lecturer on Diseases of the Skin, School of Medicine, Edinburgh. Fourth Edition, Revised and Enlarged, 8vo, cloth, gilt top, pp. xvi., 678, with Woodeut and 9 doublepage Coloured Illustrations. Price 2Is. [Pentland's Mcdical Series, Volume First.

Botany: A Concise Manual for Students of Medicine and Scinnce. By Alexander Johnstone, F.G.S., Lecturer on Botany, School of Medicine, Edinburgh. Crown 8vo, cloth, pp. xvi., 260, with 164 Illustrations and a Series of Floral Diagrams. Price $6 \mathrm{~s}$.

[Pentland's Students' Manuals.

THE JOURNAL Of PATHOLOGY and BACTERIOLOGY. EDITED, with the collaboration of distinguished British and Foreign Pathologists, by German Sims Woodhead, M.D., Professor of Pathology, Cambridge. Assisted in special departments by Allan Macfadien, M.D. Edin.; Sinney Martin, M.D. Lond. (Pathological Chemistry) ; S. G. SHAтrock, F.R.C.S. (Morbid Anatomy and Histology); G. E. Cartwright Wood, M.D. Edin. (Bacteriology). Issued at Quarterly Intervals. Subscription 2Is. per annum (in advance), post free. Vols. I.-VI. Royal 8vo., pp. xvi., 500, with numerous Illustrations, many coloured, extra cloth, gilt top. Price 25s. each nett.

NEW PRONOUNCING DICTIONARY of MEDICAL and SCIENTIFIC TERMS. By John M. Keating, M.D., LL.D., Fellow of the Royal College of Physicians, Philadelphia; Editor of Cyclopcedia of the Diseases of Children. Large 8vo, pp. 818. Second Impression. Price 7s. 6d. nett.

CLINICAL GYN屟COLOGY, MEDICAL and SURGICAL. By American Authors. Edited by John M. Keating, M.D., LL.D., and Henry C. Coe, M.D., M.R.C.S., Professor of Gynæcology, New York Polyclinic. Two Volumes, Royal 8vo, cloth, pp. xviii., 994, Illustrated with full-page Plates and Engravings in the text. Price 25s. nett.

TEXT-BOOK of ABDOMINAL SURGERY: A CLINICAL MANUAL for Practitioners and Students. By Skene Keith, F.R.C.S.Ed., assisted by George E. KeIth, M.B. 8vo, cloth, pp. xvi., 508. With 34 Illustrations. Price I6s.

[Pentland's Medical Series, Volume Fourth.

RENAL GRoWths: Their Pathology, Diagnosis, and Treatment. By T. N. Kelynack, M.D. (Vict.), M.R.C.P. (Lond.), Pathologist, Manchester Royal Infirmary ; Demonstrator and Assistant Lecturer in Pathology, Owens College, Manchester. 8vo, cloth, pp. xvi., 269, with 96 Illustrations. Price I2s. 6d. nett.

THE PARASItes of MAN, and the Diseases which Proceed rrom them. A Text-Book for Students and Practitioners. By Rudolf Leuckart, Professor of Zoology and Comparative Anatomy in the University of Leipsic. Translated from the German with the Co-operation of the Author, by Willian E. Hoyle, M.A. (Oxon.), M.R.C.S., F.R.S.E., Curator of the Museums, Owens College, Manchester. Natural History of Parasites in General. Systematic Account of the Parasites Infesting Man: Protozoa-Cestoda. Large 8vo, cloth, pp. xxviii., 772, Illustrated with 404 Engravings. Price 3Is. 6d.

THE RADICAL CURE of HERNIA, HYDROCELE, and VARICOCELF. By Charles Barrett Lockwoon, F.R.C.S. Crown 8vo, eloth, pp. xii., 279, with Illustrations. Price 4s. 6d. 
TRAUMATIC INFECTION. HUNTERIAN LECTURES DELIVERED AT the Roral College of Surgeons of England. By Charles Barrett Lockwood, F.R.C.S., Hunterian Professor, Royal College of Surgeons of England; Assistant Surgeun to St. Bartholomew's Hospital ; Surgeon to the Great Northern Central Hospital. Crown 8 vo, eloth, pp. xii., 138, Illustrated with 27 Wood Engravings in the text. Price 3 s.

ASEPTIC SURGERY. By Charles BarRetT LOCKwood, F.R.C.S. Second Edition. Crown 8vo, eloth, pp. xvi., 263. Price 4S.

DISEASES of the THROAT, NOSE, and EAR. By P. MCBRIDE, M.D., F.R.C.P.Ed., Lecturer on the Diseases of the Ear and Throat, Edinburgh School of Medicine; Aural Surgeon and Laryngologist, Royal Infirmary, Edinburgh ; Surgeon, Edinburgh Ear and Throat Dispensary. Third Edition, thoroughly Revised and largely Re-written. 8vo, pp. xvi., 744, with Coloured Illustrations from Original Drawings. Price 25s.

[Pentland's Medical Series, Volume Third.

\section{ATLAS of VENEREAL DISEASES: A SERIES OF Illustrations} From Original Paintings, with Descriptions of the Varied Lesions, their Differential Diagnosis and Treatyent. By P. H. MacLaren, M.D., F.R.C.S.Ed., Surgeon, Edinburgh Royal Infirmary ; formerly Surgeon in charge of the Lock Wards, Edinburgh Royal Infirmary; Examiner in the Royal College of Surgeons, Edinburgh. In one handsome Royal 4to Volume, extra cloth. Price 63s. nett.

SYSTEM of GYNECOLOGY and OBSTETRICS, BY AMERICAN Authors. Edited by Matthew D. Mann, A.M., M.D., Professor of Obstetries and Gynecology in the Medical Department of the University of Buffalo, N.Y.; and BArToN Cooke Hirst, M.D., Associate Professor of Obstetrics in the University of Pennsylvania; Obstetrician to the Philadelphia Maternity Hospital; Gynecologist to the Orthopædic Hospital. In 8 very handsome Volumes, Royal 8vo, cloth, of about 400 pages each, fully Illustrated with Engravings and Coloured Plates. Price I2s. 6d. each nett. $\quad *^{*}$ A Detailed Prospectus will be sent to any address on application.

FUNCTIONAL and ORGANIC DISEASES of the STOMACH. By Sinnky Martin, M.D., F.R.S., F.R.C.P., Assistant Physician and Assistant Professor of Clinical Medicine at University College Hospital; Assistant Physician to the Hospital for Consumption and Diseases of the Chest, Brompton. 8vo, cloth, pp. xx., 506, Illustrated with numerous Engravings throughout the text. Price I6s.

[Pentland's Medical Series, Volume Fifth.

THE NERVOUS SYSTEM and ITS DISEASES: A PRACTICAL Treatise on Neurology for the Use of Physicians and Students. By C. K. Mrlls, M.D., Professor of Mental Diseases and of Medical Jurisprudence in the University of Pennsylvania ; Clinical Professor of Neurology in the Women's Medical College, Pennsylvania. 8vo, cloth, pp. xxx., 1056, with 459 Illustrations. Price 25s.

ON the RELATION of the NERVOUS SYSTEM to DISEASE AND DISORDER IN THE VISCERA. BeING THE Morison Lectures Delivered before the Royal College of Phisicians in Edinburgh in 1897 and 1898. By Alexander Morison, M.D. (Edin.), F.R.C.P. (Edin.), Physician in Charge of Out Patients to the Great Northern Central Hospital and the Children's Hospital, Paddington Green; Physician to the St. Marylebone General Dispensary. Svo, cloth, pp. xvi., 132, with 39 Illustrations. Price 7s. 6 d.

A SYSTEM of GENITO-URINARY DISEASES, SYPHILOLOGY, AND DERMAToLOGY. Edited by Prixce A. Morrow, M.D. Six Vols., Large 8vo, of abont 550 pages each, fully Illustrated. Price per vol. I4S., carriage free.

MANUAL of BACTERIOLOGY. By RoBerT MUIR, M.I), F.R.C.P.Ed., Professor of Pathology, University of Glasgow ; and JAMEs Ritchik. M.D., B.Sc., Lecturer in Pathology, University of Oxford. Second Edition, throughly Revised and largely Re-written. Crown 8vo, cloth, pp. xx., 564, with 126 Illustrations in the text. Price I2s. $6 \mathrm{~d}$.

MANUAL of MIDWIFERY. By R. MlLne Muriay, M.B., F.R.C.P.Ed., Assistant Physician Royal Maternity and Simpson Memorial Hospital ; Lecturer on Midwifery and Diseases of Women, School of Medicine, Edinburgh. Crown 8vo, with numerous Illustrations, pp. 500 or thereby. Preparing. 
PRESCRIBING and TREATMENT IN THE DiseASES OF INFANTS ANd Children. By P. E. MusketT, L.R.C.P. \& S. Fd., late Surgeon to the Sydney Hospital ; formerly Senior Resident Medical Officer, Sydney Hospital. Third Edition, Revised and Enlarged. $18 \mathrm{mo}$, limp roan, for Pocket, pp. xvi., 336. Price 6s. 6d.

PRACTICAL TREATISE on MEDICAL DIAGNOSIS. By JOHN H. Musser, M.D., Assistant Professor of Clinical Medicine in the University of Pennsylvania; Physician to the Philadelphia and the Presbyterian Hospitals. Royal 8vo, cloth, pp. viii., 881, Illustrated with 162 Woodcuts and 2 Coloured Plates. Price 24s.

MALIGNANT DISEASE of the THROAT and NOSE. By DAVID Newman, M.D., Laryngologist to the Glasgow Royal Infirmary; Assistant Surgeon to the Western Infirmary; Examiner in Pathology in the University of Glasgow. 8vo, pp. xvi., 212, with 3 Illustrations. Price 8s. 6d.

TEXT-BOOK of OPHTHALMOLOGY. By W. F. Norris, A.M., M.D, and C. A. Oliver, A.M., M.D. Royal 8 vo, pp. viii., 622, Illustrated with 5 Coloured Plates and 357 Woodcuts. Price 25 s.

LEAD POISONING, in ITS ACUte AND Chronic Forms. The Goulstonian Lectures nelivered in the Royal College of Physicians. By Thomas Oliver, M.D., F.R.C.P., Physician, Royal Infirmary, Newcastle-on-Tyne; Professor of Physiology, University of Durham; Honorary Physician, Newcastle-onTyne Dispensary and Industrial Schools. 8vo, pp. xii., 122, with 32 Illustrations, mostly in colours. Price ros. 6d.

THE PRINCIPLES and PRACTICE of MEDICINE. By WILLIAM OsLer, M.D., F.R.C.P., L.L.D. (Edin.) F.R.S., Professor of Medicine in the Johns Hopkins University, and Physician-in-Chief to the Johns Hopkins Hospital, Baltimore. Third Edition, thoroughly Revised and largely Re-written, Large 8vo, cloth, pp. xviii., 1182, with Charts and Illustrations. Price 24s.

LECTURES on ANGINA PECTORIS and ALLIED STATES. By Williay Osler, M.D., F.R.C.P., Professor of Medicine, Johns Hopkins University, Baltimore. 8 vo, cloth, pp. viii., 160. Price 6s. nett.

A TREATISE on SURGERY, bY AMERICAN Authors, for StTudents and Practitioners of Surgery and Medicine. Edited by Roswell Park, A.M., M.D., Professor of the Principles and Practice of Surgery in the Medical Department of the University of Buffalo, New York, etc. Two Vols., Large 8vo, eloth, pp. 1603, with 807 Engravings and 38 full-page Plates in colours and monochrome. Price 34s. nett.

THE SCIENCE and ART of OBSTETRICS. By Theophilus PARVIN, M.D., LL.D., Professor of Obstetries and Diseases of Women and Children in Jefferson Medical College, Philadelphia, and one of the Obstetricians to the Philadelphia Hospital. Third Edition, thoroughly Revised. Large 8vo, cloth, pp. 701, with 269 Wood Engravings, and 2 Coloured Plates. Price $18 \mathrm{~s}$.

BERI BERI: Researches concerning its Nature and Cause, and the Means of its Arrest. By C. A. Pekelharing, Professor in the Faculty of Medicine, University of Utrecht; and C. WINKLER, Lecturer in the University of Utrecht. Translated by James CanTlie, M.A., M.B., F.R.C.S. 8vo, cloth, pp. xvi., 160, Illustrated with full-page Coloured Plates from Original Drawings. Price ros. 6d. nett.

Pediatrics: The Hygienic and Medical Treatment of Diseases in Childien: By Thomas Morgan Rotch, M.D., Professor of Diseases of Children, Harvard University. Two Vols., Large 8vo, pp. 1124, with 450 Illustrations in the text, and 8 full-page Coloured Plates. Price 25s. nett.

TEXT-BOOK of PHYSIOLOGY. By British Physiologists. Edited by K. A. Sснӥнгr, F.R.S., Jodrell Professor of Physiology, University College, London. Two Vols., Royal 8vo, pp. xxiv., 960 or thereby, with numerous Illustrations in the text. Volume First. Price 3is. 6d.

* * The following, among others, have contributed the Articles:- Professor BuRDon Sanderson (Oxford), Professor Gamgee, Dr. Gaskell (Cambridge), Professor Gotch 
(Oxford), Professor Sherrington (Liverpool), Professor M'Kendrick (Glasgow), Professor Halliburton (King's College), Professor Haycrafte (Cardiff), Dr. Penbrey (Charing Cross), Dr. Starling (Guy's), Dr. Rivers (Cambridge), J. N. Langley (Cambridge), the Editor, \&c. \&c.

DISEASES of the MOUTH, THROAT, and NOSE, INCLUDING RiIINOscopy and Methons of Local Treatment. By Philip Schech, M.D., Lecturer in the University of Munich. Translated by R. H. BLAIKIE, M.D., F.R.S.E., formerly Surgeon, Edinburgh Ear and Throat Dispensary; Late Clinical Assistant, Ear and Throat Department, Royal Infirmary, Edinburgh. 8vo, cloth, pp. xii., 302, with 5 Wood Engravings. Price 9 s.

ELEMENTS of PHARMACOLOGY. By Dr. Oswald SCHMIEDEBERG, Professor of Pharmacology, University of Strasburg. Translated by Thomas Dixson, M.B., Lecturer on Materia Medica in the University of Sydney, N.S.W. 8vo, cloth, pp. xii. 223, with 7 Illustrations. Price 9s.

SURGical ANATOMY: A Manual for Students. By A. Marmaduke Sheild, M.B. (Cantab.), F.R.C.S., Senior Assistant Surgeon, Aural Surgeon and Teacher of Operative Surgery, Charing Cross Hospital. Crown 8vo, cloth, pp. xii., 226. Price 6s. [Pentland's Students' Manuals.

MEDICAL GYNECOLOGY : A Treatise oN THE Diseases OF WOMEN hrom the Standpoint of the Phisician. By Alexanier J. C. Skene, M.D., Professor of Gynecology in the Long Island College Hospital, Brooklyn, New York. 8 vo, cloth, pp. vi., 530, with Illustrations in the text. Price 2IS.

ILLUSTRATIONS of ZOOLOGY, INVERTEBRATES AND VERTEbrates. By William Ramsay Smith, B.Sc., formerly Demonstrator of Anatomy, Edinburgh School of Medicine, Minto House ; Late Senior Assistant to the Professor of Natural History, University of Edinburgh ; and J. Strwart Norwell, B.Sc. Second Edition, Crown 4 to, extra cloth, gilt top, with 70 Plates, exhibiting over 400 Figures. Price 7s. 6 d.

PRACTICAL GUIDE to the EXAMINATION of the EYE. By Simeon Snell, F.R.C.S.Ed., Ophthalmic Surgeon, Sheffield General Infirmary; Professor of Ophthalmology, School of Medicine, Sheffield. Crown 8vo, cloth, pp. 172, with 88 Illustrations. Price $5 \mathrm{~s}$.

DISEASES of the DIGESTIVE ORGANS in INFANTS and CHILDREN. With Chapters ON THE Investigation OF Disease AND ON THE General Management of Children. By Louis Stark, M.D., Late Clinical Professor of Diseases of Children in the Hospital of the University of Pennsylvania; Physician to the Children's Hospital, Philadelphia. Second Edition, Post 8vo, cloth, pp. 396, with 12 Illustrations. Price Ios.

EPIDEMIC OPHTHaLmia : Its Symptoms, Diagisosis, and Managiement. By Sydnet Stephenson, F.R.C.S.Ed., Surgeon, Ophthalmic School, Hanwell. 8 vo, cloth, pp. xvi., 278, Illustrated throughout the text. Price 9s. nett.

LECTURES on GIDDINESS and HYSTERIA in the MALE. By Sir Thojas Grainger Stewart, M.D., LL.D., Late Physician in Ordinary to Her Majesty the Queen for Scotland, and Professor of the Practice of Physic and of Clinical Medicine in the University of Edinburgh. Crown 8vo, eloth, pp. x., 89, with 13 Illustrations. Price 2s. 6d. nett.

APPENDICITIS and PERITYPHLITIS. By (HARLES TALAMON, Physician to the Tenon Hospital, Paris. Translated from the French by RIchari d. A. Berre, M.B., C.M., late President of the Royal Medical Society, Edinburgh. Crown 8vo, cloth, pp. viii., 239. Price 6s.

THE PATHOLOGY and TREATMENT Of VENEREAL DISEASES. By Robrit W. Taylor, M.D., Clinical Professor of Venereal Diseases at the College of Physicians and Surgeons (Columbia College), New York; Surgeon to Bellevue Hospital ; and Consulting Surgeon to the City (Charity) Hospital, New York. Large 8vo, cloth, pp. 1002, with 230 Illustrations and 7 Coloured P'lates. Price 22s. nett.

OUTLINeS of ZOOLOGY. By .. Anthun Thomson, M.A., Regius Professor of Natural History in the University of Aberdeen. Third lidition, Revised and Enlarged. Crown 8vo, cloth, pp. xx., 820, Illustrated with 332 ligures in the text. Price I5s.

[Pentland's Students' Manuals. 
PRACTICAL GUIDE to MEAT INSPECTION. By ThOMAS WALlEY, M.R.C.V.S., formerly Principal of the Edinburgh Royal (Dick) Veterinary College; Professor of Veterinary Medicine and Surgery, \&c. Fourth Edition, thoroughly Revised. In Press.

DISEASES of the LIVER, GALL BLADDER, and BILIARY SYSTEM ; their Pathology, Diagnosis, and Surgical Treatment. By H. J. WARING, M.S., B.Sc., F.R.C.S., Demonstrator of Operative Surgery and Senior Demonstrator of Anatomy, St. Bartholomew's Hospital ; Senior Assistant Surgeon, Metropolitan Hospital ; Surgeon to the Belgrave Hospital for Children, London. 8vo, cloth, pp. xvi., 386, with 58 Illustrations. Price I2s. 6d. nett.

MANUAL of OPERATIVE SURGERY. By H. J. WARING, M.S., B.Sc., F.R.C.S., Demonstrator of Operative Surgery and Senior Demonstrator of Anatomy, St. Bartholomew's Hospital; Surgeon, Metropolitan Hospital; Surgeon to the Belgrave Hospital for Children, London. Crown 8vo, pp. xxiv.,'661, with 420 Illustrations in the text. Price 12s. 6d.

RESEARCHES in FEMALE PELVIC ANATOMY. By J. ClaRENCE Webster, M.D., F.R.C.P.Ed., Assistant to the Professor of Midwifery and Diseases of Women and Children, Edinburgh University. 4to, cloth, Illustrated with 26 fullpage Coloured Plates from Original Drawings. Price 30 s.

TUBO-PERITONEAL ECTOPIC GESTATION. By J. Clarence Webster, M.D., F.R.C.P.Ed., Assistant to the Professor of Midwifery and Diseases of Women and Children, Edinburgh University. 4to, cloth, uniform with above, Illustrated with 11 Plates, mostly in colours, from Original Drawings. Price I6s.

ECTOPIC PREGNANCY. By J. Clarence Webster, M.I., F.R.C.P.Ed., formerly Assistant to the Professor of Midwifery and Diseases of Women and Children in the University of Edinburgh. 8vo, cloth, pp. xvi., 374, with 22 pages of Plates and Figures throughout the text. Price I2s. 6d. nett.

PRACTICAL and OPERATIVE GYNECOLOGY. By J. Clarence Webster, M.D., F.R.C.P.Ed., formerly Assistant to the Professor of Midwifery and Diseases of Women, University of Edinburgh. Crown 8vo, cloth, pp. xvi., 296, with 54 Illustrations. Price 7s. 6d.

[Pentland's S'tudents' Manuals.

DISEASES of WOMEN: A TexT-BOOK FOR STUDENTS AND PRACtitioners. By J. Crarence Webster, B.A., M.D., F.R.C.P.Ed., Demonstrator of Gynæcology, M'Gill University; Assistant Gynæcologist, Royal Victoria Hospital, Montreal. Crown 8vo, pp. xxiv., 688, with 241 Illustrations. Price I4S.

DIABETES MELLITUS and its TREATMENT. By RICHARD T. Williamson, M.D., M.R.C.P., Medical Registrar, Manchester Royal Infirmary; Assistant to Professor of Medicine, Owens College, Manchester. 8vo, cloth, pp. xvi., 417, with 18 Illustrations (two Coloured). Price I2s. 6d. nett.

TEXT-BOOK of OBSTETRICS, INCLUDING THE PATHOLOGY AND Therapeutics of the Puerperal State. Designed for Practitioners and Students OF Medicine. By Dr. F. Winckex, Professor of Gynæcology and Director of the Royal Hospital for Women; Member of the Supreme Medical Council and of the Faculty of Medicine in the University of Munich. Translated from the German under the supervision of J Clifton EDgar, A.M., M.D., Adjunct Professor of Obstetries in the Medical Department of the University of the eity of New York. Royal 8vo, cloth, pp. 927, Illustrated with 190 Engravings, mostly original. Price 28s.

PRACTICAL PATHOLOGY : A MANUAL FOR STUDENTS AND Practitioners. By G. Sims Woodhead, M.D., M.R.C.P.Ed., Director of the Laboratories of the Royal Colleges of Physicians (London) and Surgeons (England). Third Edition, Revised and Enlarged, 8vo, cloth, pp. xxiv., 652, with 195 Coloured Illustrations, mostly from Original Drawings. Price 25s.

\section{YOUNG J. PENTLAND,}

EDINBURGH : II TEVIOT PLACE.
LONDON :

38 WEST SMITHFIELD, E.C. 




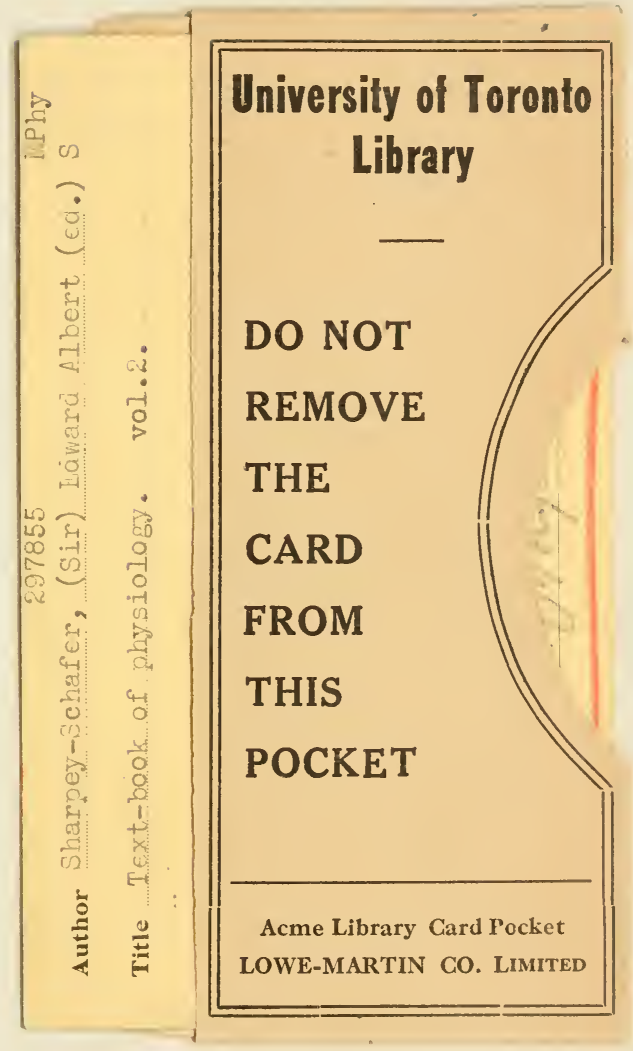


